SANDIA REPORT

SAND98-0180/2

Unlimited Release

Printed June 1998
REQEIVED

1 JUL 071998

\title{
Guidebook for Performance Assessment Parameters Used in the Waste Isolation Pilot Plant Compliance Certification Application \\ Volume II: Appendices
}

Susan M. Howarth, Mary-Alena Martell, Ruth Weiner, Charlene Lattier

Prepared by

Sandia National/Laboratories

Albuquerque, New Mexico 87185 and Livermore, California 94550

Sandia is a multiprogram laboratory operated by Sandia Corporation, a Lockheed Martin Company, for the United States Department of Energy ứnder Contract DE-AC04-94AL85000.

Approved for public release; further dissemination unlimited.

(1) Sandia National Laboratories

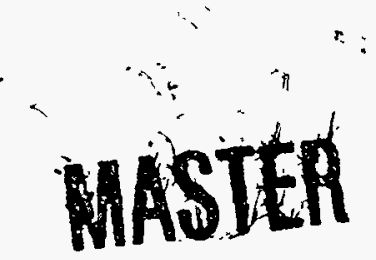

DCCUMEST IS UNHAMITED 
Issued by Sandia National Laboratories, operated for the United States Department of Energy by Sandia Corporation.

NOTICE: This report was prepared as an account of work sponsored by an agency of the United States Government. Neither the United States Government nor any agency thereof, nor any of their employees, nor any of their contractors, subcontractors, or their employees, makes any warranty, express or implied, or assumes any legal liability or responsibility for the accuracy, completeness, or usefulness of any information, apparatus, product, or process disclosed, or represents that its use would not infringe privately owned rights. Reference herein to any specific commercial product, process, or service by trade name, trademark, manufacturer, or otherwise, does not necessarily constitute or imply its endorsement, recommendation, or favoring by the United States Government, any agency thereof, or any of their contractors or subcontractors. The views and opinions expressed herein do not necessarily state or reflect those of the United States Government, any agency thereof, or any of their contractors.

Printed in the United States of America. This report has been reproduced directly from the best available copy.

Available to DOE and DOE contractors from

Office of Scientific and Technical Information

P.O. Box 62

Oak Ridge, TN 37831

Prices available from (615) 576-8401, FTS 626-8401

Available to the public from

National Technical Information Service

U.S. Department of Commerce

5285 Port Royal Rd

Springfield, VA 22161

NTIS price codes

Printed copy: A99

Microfiche copy: A01

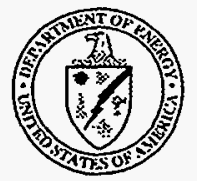




\section{DISCLAIMER}

Portions of this document may be illegible in electronic image products. Images are produced from the best available original document. 
SAND98-0180/2

Unlimited Release

Printed June 1998

\title{
Guidebook for Performance Assessment Parameters Used in the Waste Isolation Pilot Plant Compliance Certification Application
}

\author{
Volume II: Appendices \\ Susan M. Howarth, Mary-Alena Martell \\ Regulatory Compliance Department \\ Ruth Weiner \\ Transportation Systems Department \\ Sandia National Laboratories \\ PO Box 5800 \\ Albuquerque, NM 87185-0773 \\ Charlene Lattier \\ GRAM, Inc. \\ Albuquerque, NM 87112
}

WPO\# 47127

\begin{abstract}
The Waste Isolation Pilot Plant (WIPP) Compliance Certification Application (CCA) Performance Assessment (PA) Parameter Database and its ties to supporting information evolved over the course of two years. When the CCA was submitted to the Environmental Protection Agency (EPA) in October 1996, information such as identification of parameter value or distribution source was documented using processes established by Sandia National Laboratories WIPP Quality Assurance Procedures. Reviewers later requested additional supporting documentation, links to supporting information, and/or clarification for many parameters. This guidebook is designed to document a pathway through the complex parameter process and help delineate flow paths to supporting information for all WIPP CCA parameters. In addition, this report is an aid for understanding how model parameters used in the WIPP CCA were developed and qualified. To trace the source information for a particular parameter, a dual-route system was established. The first route uses information from the Parameter Records Package as it existed when the CCA calculations were run. The second route leads from the EPA Parameter Database to additional supporting information.
\end{abstract}




\section{ACKNOWLEDGMENTS}

The authors thank the other members of the CCA Parameter Team: Lori Dotson and Deborah Coffey, Karen Schmeige (SAIC), and J.T. Schneider and Gretchen Newman (GRAM, Inc.) for their help during the parameter development process and subsequent CCA parameter reviews and audits. Their feedback provided the framework for this guidebook.

The authors also thank Lori Dotson, Diane Hurtado, and Martin Tierney for their helpful review comments and Carol Crawford for her thorough reference and literature review. In addition, the authors thank Tim Peterson (Tech Reps, Inc.) for his help in shaping this document into final form. 


\section{Errata and Reference Tracking Information for Volume II}

\begin{tabular}{|c|c|}
\hline Page & Change / Reference Information \\
\hline & Appendices A and B \\
\hline A3-37 & The material PAN_SEAL is listed in the records database as PAN_S_l. \\
\hline A3-37 & The WPO Number for ID 2604 is incorrect. The correct ID is WPO\#32612. \\
\hline B1-3 & The material PAN_SEAL is listed in the records database as PAN_S_1. \\
\hline \multirow[t]{2}{*}{ B2-10 } & The material PAN_SEAL is listed in the records database as PAN_S_1. \\
\hline & Appendix D \\
\hline $\mathrm{D}-4$ & Item 20. "H-19" is hyphenated in the records database entry for WPO\#44763. \\
\hline $\mathrm{D}-6$ & Item 1. Correct the spelling of the word "Berkeley." \\
\hline D-6 & Item 2. Insert a hyphen between "Nevada" and "Reno." \\
\hline \multirow[t]{2}{*}{ D-6 } & $\begin{array}{l}\text { Item 5. Insert a hyphen between "Nevada" and "Las Vegas." Insert a space after } \\
\text { "EA" in "EA 95-04". }\end{array}$ \\
\hline & Appendices F and G \\
\hline F-13 & $\begin{array}{l}\text { In reference } 4 \text {, Frank Hansen's surname is misspelled and the memo date is } \\
\text { incorrect. The correct memo date is } 8 / 2 / 96 \text {. }\end{array}$ \\
\hline F-17 & $\begin{array}{l}\text { Third footnote. This document is on file in the SWCF as WPO\#44202 and it is } \\
\text { dated February } 27,1997 .\end{array}$ \\
\hline G-4 \& 5 & $\begin{array}{l}\text { Page G-4/G-5. Blom, 1989, p. 216. This page is on file in the SWCF as } \\
\text { WPO\#41116. }\end{array}$ \\
\hline $\mathrm{G}-4,5,8 \& 10$ & Tierney, 1990. SAND90-2510 is on file in the SWCF as WPO\#23860. \\
\hline G-8 & Sandia WIPP Project. SAND $92-0700 / 3$ is on file in the SWCF as WPO $\# 23529$. \\
\hline G-9 \& 12 & $\begin{array}{l}\text { Iman and Shortencarier, 1984. This report is on file in the SWCF as } \\
\text { WPO\#41121. }\end{array}$ \\
\hline G-14 & $\begin{array}{l}\text { Blom, 1989, p. } 232 \text { is not cited in the memo reference list. The citation for this } \\
\text { callout is: Blom, G. } 1989 \text {. Probability \& Statistics: Theory and Applications. } \\
\text { New York, NY: Springer-Verlag. }\end{array}$ \\
\hline G-21 & Glimm, 1991. This journal article is on file in the SWCF as WPO\#44601. \\
\hline \multirow[t]{2}{*}{ G-21 } & Sandia WIPP Project. SAND92-0700/3 is on file in the SWCF as WPO\#23529. \\
\hline & Appendix K \\
\hline $\mathrm{K}-3$ & Change the callout for "Chan, 1992" to read "Chan et al., 1992." \\
\hline $\mathrm{K}-3$ & $\begin{array}{l}\text { Change the callout for "Hansen and Ahrens, 1996" to read "Ahrens and Hansen, } \\
\text { 1995." }\end{array}$ \\
\hline$\overline{\mathrm{K}-3}$ & $\begin{array}{l}\text { Initial capitalize all of the title words in "Initial Pore Fluid Pressure, Pore } \\
\text { Pressure" to match other entries on the page. }\end{array}$ \\
\hline $\mathrm{K}-19-\mathrm{K}-18$ & $\begin{array}{l}\text { Replace the "Compacted Crushed Salt References" list with the reference list } \\
\text { provided on the next two pages. The revised list incorporates all necessary } \\
\text { corrections and formatting changes. }\end{array}$ \\
\hline
\end{tabular}




\section{Compacted Crushed Salt References}

Ahrens, E.H., and F.D. Hansen. 1995. Large-Scale Dynamic Compaction Demonstration Using WIPP Salt: Fielding and Preliminary Results. SAND95-1941. Albuquerque, NM: Sandia National Laboratories.

Brodsky, N.S. 1994. Hydrostatic and Shear Consolidation Tests with Permeability Measurements on Waste Isolation Pilot Plant Crushed Salt. SAND93-7058. Prepared by RE/SPEC Inc., Rapid City, SD. Albuquerque, NM: Sandia National Laboratories.

Brooks, R.H., and A.T. Corey. 1964. "Hydraulic Properties of Porous Media." Hydrology Paper No. 3. Fort Collins, CO: Colorado State University. (WPO\#41117)

Callahan, G.D., M.C. Loken, L.L. Van Sambeek, R. Chen, T.W. Pfeifle, J.D. Nieland, and F.D. Hansen. 1995. Evaluation of Potential Crushed-Salt Constitutive Models. SAND952143. Prepared by RE/SPEC Inc., Rapid City, SD. Albuquerque, NM: Sandia National Laboratories.

Chan, K.S., S.R. Bodner, A.F. Fossum, and D.E. Munson. 1992. "A Constitutive Model for Inelastic Flow and Damage Evolution in Solids Under Triaxial Compression," Mechanics of Materials. Vol. 14, no. 1, 1-14.

Davies, P.B. 1991. Evaluation of the Role of Threshold Pressure in Controlling Flow of WasteGenerated Gas into Bedded Salt at the Waste Isolation Pilot Plant. SAND90-3246. Albuquerque, NM: Sandia National Laboratories.

Hansen, F.D. 1995. Untitled Memorandum to T. Jones, INTERA Inc. November 30, 1995. Albuquerque, NM: Sandia National Laboratories. (WPO\#29905)

Haverkamp, R., and J.Y. Parlange. 1986. "Predicting the Water-Retention Curve from ParticleSize Distribution. 1. Sandy Soils Without Organic Matter," Soil Science. Vol. 142, no. 6, 325-339.

Holcomb, D.J., and D.W. Hannum. 1982. Consolidation of Crushed Salt Backfill Under Conditions Appropriate to the WIPP Facility. SAND82-0630. Albuquerque, NM: Sandia National Laboratories. (WPO\#26463)

Holcomb, D.J., and M. Shields. 1987. Hydrostatic Creep of Crushed Salt With Added Water. SAND87-1990. Albuquerque, NM: Sandia National Laboratories. (WPO\#26469)

Hopmans, J.W., and B. Overmars. 1986. "Presentation and Application of an Analytical Model to Describe Soil Hydraulic Properties," Journal of Hydrology. Vol. 87, no. 1-2, 135-143.

Lappala, E.G., R.W. Healy, and E.P. Weeks. 1987. Documentation of Computer Program VS2D to Solve the Equations of Fluid Flow in Variably Saturated Porous Media. WaterResources Investigations Report 83-4099. Denver, CO: U.S. Geological Survey. (WPO\#45627)

Mayer, G., F. Jacobs, and F.H. Wittmann. 1992. "Experimental Determination and Numerical Simulation of the Permeability of Cementitious Materials," Nuclear Engineering and Design. Vol. 138, no. 2, 171-177.

Mualem, Y. 1976. "A New Model for Predicting the Hydraulic Conductivity of Unsaturated Porous Media," Water Resources Research. Vol. 12, no. 3, 513-522. 
Parker, J.C., R.J. Lenhard, and T. Kuppusamy. 1987. “A Parametric Model for Constitutive Properties Governing Multiphase Flow in Porous Media," Water Resources Research. Vol. 23, no. 4, 618-624.

Pfeifle, T.W., and P.E. Senseny. 1985. "Permeability and Consolidation of Crushed Salt From the WIPP Site." RSI-0278. Prepared for Sandia National Laboratories, Albuquerque, NM. Rapid City, SD: RE/SPEC Inc. (WPO\#36824)

Pihlajavaara, S.E. 1991. "Long-Term Gas Permeability Properties of Concrete in Wet Repository Conditions," Nuclear Engineering and Design. Vol. 129, no. 1, 41-48.

Rawls, W.J., D.L. Brakensiek, and K.E. Saxton. 1982. "Estimation of Soil Water Properties," Transactions of the ASAE. St. Joseph, MI: American Society of Agricultural Engineers. 1316-1320. (WPO\#42417)

RE/SPEC. 1994. "Determination of Intact Density of WIPP Salt Specimens." Calculation File 325/3.4/01. Prepared for Sandia National Laboratories, Albuquerque, NM. Rapid City, SD: RE/SPEC Inc. (WPO\#42723)

RE/SPEC. 1995. "WIPP Crushed-Salt Database for Constitutive Model Evaluations."

Calculation File 325/4/03. Prepared for Sandia National Laboratories, Albuquerque, NM. Rapid City, SD: RE/SPEC Inc. (In Records Package WPO\#40871)

RE/SPEC. 1996a. "Shear Consolidation Testing Performed to Supplement the WIPP CrushedSalt Database." Calculation File 325/12.1/01. Prepared for Sandia National Laboratories, Albuquerque, NM. Rapid City, SD: RE/SPEC Inc. (In Records Package WPO\#41392)

RE/SPEC. 1996b. "Mechanical and Hydrologic Properties Testing of Dynamically-Compacted Crushed Salt." Calculation File 325/12.1/02. Prepared for Sandia National Laboratories, Albuquerque, NM. Rapid City, SD: RE/SPEC Inc. (In Records Package WPO\#41392)

Sjaardema, G.D., and R.D. Krieg. 1987. A Constitutive Model for the Consolidation of WIPP Crushed Salt and Its Use in Analyses of Backfilled Shaft and Drift Configurations. SAND87-1977. Albuquerque, NM: Sandia National Laboratories.

Stephens, D.B., M. Unruh, J. Havlena, R.G. Knowlton, Jr., E. Mattson, and W. Cox. 1988.

"Vadose Zone Characterization of Low-Permeability Sediments Using Field

Permeameters," Ground Water Monitoring Review. Vol. 8, no. 2, 59-66.

van Genuchten, M.Th. 1980. "A Closed-Form Equation for Predicting the Hydraulic"

Conductivity of Unsaturated Soils," Soil Science Society of America Journal. Vol. 44, no. 5, 892-898.

van Genuchten, M.Th., and D.R. Nielsen. 1985. "On Describing and Predicting the Hydraulic Properties of Unsaturated Soils," Annales Geophysicae. Vol. 3, no. 5, 615-628.

Wosten, J.H.M., and M.Th. van Genuchten. 1988. "Using Texture and Other Soil Properties to Predict the Unsaturated Soil Hydraulic Functions," Soil Science Society of America Journal. Vol. 52, no. 6, 1762-1770.

Zeuch, D.H., D.J. Zimmerer, and M.E. Shields. 1991. Interim Report on the Effects of BrineSaturation and Shear Stress on Consolidation of Crushed, Natural Rock Salt from the Waste Isolation Pilot Plant (WIPP). SAND91-0105. Albuquerque, NM: Sandia National Laboratories. (WPO\#26465) 


\begin{tabular}{|c|c|}
\hline Page & Change / Reference Information \\
\hline & Appendix M1 \\
\hline M1-21 & Memo: Vaughn/Tierney-10. This memo is on file in the SWCF as WPO\#43088. \\
\hline $\begin{array}{l}\text { M1-34-36, \& } \\
168\end{array}$ & $\begin{array}{l}\text { The material PAN_SEAL (listed as material id for ids } 252-266 \text { and ids } 2731 \text { - } \\
2734 \text { ) is listed in the records database as PAN_S_1. }\end{array}$ \\
\hline M1-87 & Hydraulics/ASCE-3/70. This journal is on file in the SWCF as WPO\#41125. \\
\hline M1-150 & ID 2604. The correct WPO\# for this ID is WPO\#32612. \\
\hline M1-158 & D 2663. The correct WPO\# for this ID is WPO\#32117. \\
\hline M1-189, 191 & $\begin{array}{l}\text { Nuclides and Isotopes. The } 1989 \text { edition of this document is on file in the SWCF } \\
\text { as WPO\#41566. }\end{array}$ \\
\hline M1-241 & $\begin{array}{l}\text { D 3106. SAND91-0893/3-MEMOBRUSH. Memo is located on pp. A-25-A- } \\
36 \text { of SAND91-0893/3. }\end{array}$ \\
\hline M1-257 & Fox-1. See the correction for page M4-3 below. \\
\hline $\begin{array}{l}\text { M1-267, 269, } \\
271\end{array}$ & $\begin{array}{l}40 \text { CFR 191. 50 FR } 38066 \text { is on file in the SWCF as WPO\#39132. Use either } \\
\text { "50 FR 38066" or "WPO\#39132" instead of 40CFR191. }\end{array}$ \\
\hline M1-287 & $\begin{array}{l}\text { ID } 3470 \text { is on file in the SWCF as WPO\#38209 (listed as WPO\#38214 in } \\
\text { original list). }\end{array}$ \\
\hline M1-293 & $\begin{array}{l}\text { According to the Source Cross-Reference listing (Appendix M4), } \\
\text { FEDERAL_REGISTER is a callout to } 61 \text { FR 5224, which is on file in the SWCF } \\
\text { as WPO\#41579. Use either "61 FR 5224" or "WPO\#41579" instead of } \\
\text { "FEDERAL_REGISTER." }\end{array}$ \\
\hline M1-293 & Memo:Leonard/Marietta-2. This memo is on file in the SWCF as WPO $\# 40850$. \\
\hline M1-293 & Memo:Leonard/Marietta-3. This memo is on file in the SWCF as WPO\#40849. \\
\hline \multirow[t]{2}{*}{ M1-295 } & WID. For citation information, see the correction for page M4-7 below. \\
\hline & Appendix M2 \\
\hline M2-2 & $\begin{array}{l}\text { ID } 3310,3312,3319,3307,3311 \text {. The word "Parameters" needs to be initial } \\
\text { capitalized in the description. The date on WPO\#36771 is March } 29,1996 \text {. In } \\
\text { the column where WPO\#35852 and WPO\#35856 are listed, a closing quotation } \\
\text { mark needs to be inserted at the end of the title. }\end{array}$ \\
\hline M2-2 & $\begin{array}{l}\text { ID } 2283,2284,2285 \text {. The punctuation before "Brown" needs to be changed to a } \\
\text { semicolon. }\end{array}$ \\
\hline M2-2 & $\begin{array}{l}\text { D } 3134,3186,3187,3159 \text {. Per the records database, WPO\#35134 is a one-page } \\
\text { email (not a handwritten 4-page note). The title for the handwritten note and the } \\
\text { email is the same. }\end{array}$ \\
\hline M2-3 & $\begin{array}{l}\text { D 3173. WPO\#38367 is an April } 1996 \text { draft copy of SAND94-0472. The title, } \\
\text { "Mixed Brooks and Corey Two-Phase Characteristic Curves," is listed in the } \\
\text { records database as WPO\#38391. This memo is also reprinted in SAND94-0471 } \\
\text { (pp. F-3 \& F-4). The closing quotation mark should follow the word "Curves." }\end{array}$ \\
\hline M2-3 & $\begin{array}{l}\text { ID 3173. Per the records database, WPO\#35134 is a one-page email (not a } \\
\text { handwritten 4-page note). The title for the handwritten note and the email is the } \\
\text { same. }\end{array}$ \\
\hline
\end{tabular}




\begin{tabular}{|c|c|}
\hline Page & Change / Reference Information \\
\hline M2-3 & $\begin{array}{l}\text { ID 3134, 3186, 3187. Entry for WPO\#40514. Insert "p." between the WPO\# } \\
\text { and " } 3-52 \text { "; insert a space after the word "Task"; insert the word "Analysis" after } \\
\text { "PA" and the word "the" after "Supporting"; transpose the closing quotation } \\
\text { mark and the period. }\end{array}$ \\
\hline $\mathrm{M} 2-4$ & $\begin{array}{l}\text { ID } 3171,3135 \text {. Per the records database, WPO\#35134 is a one-page email (not a } \\
\text { handwritten 4-page note). The title for the handwritten note and the email is the } \\
\text { same. }\end{array}$ \\
\hline $\mathrm{M} 2-4$ & ID 3184, 3190, 3191. Entry for WPO\#39626. Initial capitalize the word "Filled" \\
\hline$\overline{\mathrm{M} 2-4}$ & $\begin{array}{l}\text { ID 3456, 3473. Entry for WPO\#38217. The hyphen after BRAGFLO should be } \\
\text { an underline (BRAGFLO_DBR). }\end{array}$ \\
\hline M2-4 & ID 3473. Correct the spelling of the word "blowout." \\
\hline M2-4 & $\begin{array}{l}\text { ID 3245. Entries for WPO\#40521, 35695. Insert a closing quotation mark at the } \\
\text { end of the document title. }\end{array}$ \\
\hline $\mathrm{M} 2-4$ & ID 3470, 3472. WPO\#38214. Consistently capitalize title words in these entries. \\
\hline M2-5 & $\begin{array}{l}\text { ID } 3184,3190,3191 \text {. The author name "Harar" should be corrected to "Harr" in } \\
\text { the citation. }\end{array}$ \\
\hline M2-5 & $\begin{array}{l}\text { ID 3473. Initial capitalize the word "Using"; insert the word "Silica" before the } \\
\text { word "Sands". The NMERI report number needs to be changed to: } \\
\text { 1996/7/32250; insert a space between "Mar" and "96." }\end{array}$ \\
\hline M2-5 & $\begin{array}{l}\text { ID } 3245 \text {. In the entry for WPO\#36723, change the code name to: CUTTINGS_S } \\
\text { (insert an "S" before the underline). }\end{array}$ \\
\hline M2-6 & $\begin{array}{l}\text { D 3256, 3247, 2254. In the entry for WPO\#36766, change the code name to: } \\
\text { CUTTINGS_S (insert an "S" before the underline). }\end{array}$ \\
\hline M2-6 & $\begin{array}{l}\text { ID } 3246 . \text { In the entry for WPO\#40521, insert a closing quotation mark after the } \\
\text { word "Sources". }\end{array}$ \\
\hline M2-6 & $\begin{array}{l}\text { ID } 3471 \text {. WPO\#38214. Consistently capitalize title words in this entry to match } \\
\text { IDs } 3470 \text { and } 3472 \text { on page M2-4. }\end{array}$ \\
\hline M2-7 & $\begin{array}{l}\text { ID 3256, 3247. Initial capitalize the word "Using"; insert the word "Silica" } \\
\text { before the word "Sands"; change the NMERI report number to: 1996/7/32250; } \\
\text { insert a space between "Mar" and "96." }\end{array}$ \\
\hline M2-7 & $\begin{array}{l}\text { ID } 3256 \text {. In the entry for WPO\#36766, change the code name to: CUTTINGS_S } \\
\text { (insert an "S" before the underline). }\end{array}$ \\
\hline M2-7 & $\begin{array}{l}\text { ID 27. In the discussion of Domega, correct the company name to Baroid } \\
\text { Drilling Fluids Inc; capitalize "SAND" in "SAND89-2408". }\end{array}$ \\
\hline M2-7 & $\begin{array}{l}\text { ID 2254. Insert "WPO\#" before " } 35695 \text { "; correct the spelling of Partheniades' } \\
\text { surname. The entry should read: Partheniades, E., and R.E. Paaswell. } 1970 . \\
\text { "Erodability of Channels w/Cohesive Boundary," J. of Hydraulics Div., Proc. of } \\
\text { the ASCE. Vol. 96, no. HY3, 755-771. }\end{array}$ \\
\hline M2-8 & $\begin{array}{l}\text { ID 61. In the entry for WPO\#41247, change Freeze and Cherry citation to: } \\
\text { Freeze and Cherry, } 1979 \text { (Groundwater). }\end{array}$ \\
\hline M2-8 & $\begin{array}{l}\text { ID 3194. In the entry for WPO\#41662, insert a space between " } 41662 \text { " and } \\
\text { "Memo" and initial capitalize the word "definition." }\end{array}$ \\
\hline
\end{tabular}




\begin{tabular}{|c|c|}
\hline Page & Change / Reference Information \\
\hline M2-8 & $\begin{array}{l}\text { ID 3194. Entry for WPO\#44401. Popielak et al., 1983 (TME-3153) is on file in } \\
\text { the SWCF as WPO\#42085. Insert a space after the ampersand. }\end{array}$ \\
\hline M2-9 & ID 2612. Remove the comma after the word "Parameters." \\
\hline M2-9 & $\begin{array}{l}\text { ID 3194. Entry for WPO\#30994. Delete extra spaces between the words } \\
\text { "showing" and "proposed." }\end{array}$ \\
\hline M2-17 & $\begin{array}{l}\text { ID } 3144,3156,3168 . \text { Per the records database, WPO\#35134 is a one-page email } \\
\text { (not a handwritten 4-page note). The title for the handwritten note and the email } \\
\text { is the same. }\end{array}$ \\
\hline M2-18 & $\begin{array}{l}\text { ID } 67,68,69 . \text { In the entry for WPO\#31070, initial capitalize the word } \\
\text { "Reservoir". }\end{array}$ \\
\hline M2-18 & ID 2918. In the entry for WPO\#31082, insert a hyphen between "SWCF" and "A." \\
\hline M2-18 & $\begin{array}{l}\text { ID 2918. In the entry for WPO\#37149, correct the spelling of the word } \\
\text { "contiguous." }\end{array}$ \\
\hline M2-18 & $\begin{array}{l}\text { ID 2334. In the entry for WPO\#30640, make "Seals" plural in the phrase "Shaft } \\
\text { Seals System". }\end{array}$ \\
\hline M2-18 & D 2334. Entry for WPO\#41809. Insert a space after "p." \\
\hline M2-19 & $\begin{array}{l}\text { ID } 3180 \text {. Per the records database, WPO\#35134 is a one-page email (not a } \\
\text { handwritten 4-page note). The title for the handwritten note and the email is the } \\
\text { same. }\end{array}$ \\
\hline M2-19 & ID 64. Insert "WPO\#” before "40434". \\
\hline M2-19 & ID 2334. Initial capitalize "Knowles"; also see note for page M2-27. \\
\hline M2-21 & $\begin{array}{l}\text { ID } 2335,2336,3009,3010,3011,2351,2352,2353,2368 . \text { Initial capitalize } \\
\text { "Knowles"; also see note for page M2-27. }\end{array}$ \\
\hline $\mathrm{M} 2-22$ & $\begin{array}{l}\text { ID } 2370,3078,3079,3080,2385,2386,2387,2402,2403 . \text { Insert a space after } \\
\text { "p"; the word "Seals" is plural in the phrase "Shaft Seals System". }\end{array}$ \\
\hline M2-23 & $\begin{array}{l}\text { ID } 2370,3078,3079,3080,2385,2386,2387,2402,2403 . \text { Initial capitalize } \\
\text { "Knowles"; also see note for page M2-27. }\end{array}$ \\
\hline M2-24 & $\begin{array}{l}\text { ID } 2404,2419,2420,2421,2436,2437,2438,2317,2318 . \text { Insert a space after } \\
\text { "p."; the word "Seals" is plural in the phrase "Shaft Seals System". }\end{array}$ \\
\hline M2-25 & $\begin{array}{l}\text { ID 2404, 2419, 2420, 2421, 2436, 2437, 2438, 2317, 2318. Initial capitalize } \\
\text { "Knowles"; also see note for page M2-27. }\end{array}$ \\
\hline M2-26 & $\begin{array}{l}\text { ID } 3147,3185,3192,3193 \text {. Per the records database, WPO\#35134 is a one-page } \\
\text { email (not a handwritten 4-page note). The title for the handwritten note and the } \\
\text { email is the same. }\end{array}$ \\
\hline M2-26 & $\begin{array}{l}\text { ID } 3185,3192,3193 . \text { In the entry for WPO\#39626, initial capitalize the word } \\
\text { "Filled." }\end{array}$ \\
\hline M2-26 & $\begin{array}{l}\text { ID } 2319,2464,3148,2467,2470,2471,2472 . \text { In the entries for WPO\#30640, } \\
\text { the word "Seals" is plural. In ID } 2319 \text {, insert a space after "p." }\end{array}$ \\
\hline M2-26 & ID 2467. Correct the spelling of Wakeley's surname. \\
\hline M2-26 & $\begin{array}{l}\text { ID 120. Change the WPO\# to WPO\#41118, which is where p. } 55 \text { from this book } \\
\text { is filed; insert a comma after Freeze's surname and a period after Cherry; insert a } \\
\text { hyphen in "Prentice-Hall"; insert a period and space between the "p" and " } 55 . "\end{array}$ \\
\hline
\end{tabular}




\begin{tabular}{|c|c|}
\hline Page & Change / Reference Information \\
\hline M2-27 & $\begin{array}{l}\text { ID 2319. Initial capitalize "Knowles"; also note that fuller citations for these } \\
\text { callouts are not included in the Source Cross-Referencing listing. }\end{array}$ \\
\hline M2-27 & $\begin{array}{l}\text { D } 3148 . \text { In the entry for WPO\#35134, remove the extra quotation mark (after } \\
\text { date). }\end{array}$ \\
\hline M2-28 & $\begin{array}{l}\text { ID 861. In the entry for WPO\#40516, initial capitalize the word "Flow"; in the } \\
\text { description of the } 5 / 2 / 96 \text { memo from Wallace to Tierney, remove the text in } \\
\text { parentheses. This memo is on file in the SWCF as WPO\#39365. }\end{array}$ \\
\hline $\mathrm{M} 2-28$ & $\begin{array}{l}\text { ID 142. WPO\#32290 is a memo from Lori Dotson to Martin Tierney (the author } \\
\text { and recipient names are reversed). Insert the word "Units" after "NonSalado". }\end{array}$ \\
\hline $\mathrm{M} 2-28$ & ID 3487, 3484, 3483. Insert "WPO\#” after "PRP." \\
\hline M2-30 & ID 3485. Remove the comma after "WPO\#37225." \\
\hline $\mathrm{M} 2-30$ & ID 3419. Initial capitalize the word "Parameter." \\
\hline $\mathrm{M} 2-30$ & ID $171,172,173$. The word "Spallings" is plural. \\
\hline $\mathrm{M} 2-30$ & $\begin{array}{l}\text { ID } 161,162,163 \text {. The memo title for WPO\#43088 is: Dewylake logs of intrinsic } \\
\text { permeability. Pace, } 1990 \text { is reprinted in SAND89-2408. Pages A-159 through } \\
\text { A-164 contain letter 1a, a } 9 / 11 / 90 \text { letter to J.W. Berglund, "Bar graphs } \\
\text { representing range of values for drilling operations near WIPP Site." Pages } \\
\text { A-167 through A-170 contain letter 1b, a } 9 / 18 / 90 \text { letter to Berglund, "Changes to } \\
\text { bar graphs." }\end{array}$ \\
\hline M2-31 & ID 162. Add “WRP" between "USGS" and "83-4016": USGS-WRI-83-4016. \\
\hline M2-31 & ID 172,173 . The word after "A-169" should be "of," not "or." \\
\hline M2-34 & ID 225. Insert a closing quotation mark at the end of the memo title. \\
\hline M2-34 & ID 3501,3500 . The date of WPO\#42852 should be $11 / 14 / 96$. \\
\hline M2-34 & $\begin{array}{l}\text { ID 3497. This document is printed in: Federal Register. Vol. } 61, \text { no. } 28,5224- \\
5245.61 \text { FR } 5224 \text { is on file in the SWCF as WPO\#41579. }\end{array}$ \\
\hline M2-35 & $\begin{array}{l}\text { D 253. Remove the spaces after "worksheet" and "date" and insert a space } \\
\text { after each of these commas. }\end{array}$ \\
\hline M2-36 & $\begin{array}{l}\text { ID } 3429,3433,3459,3457,3458,3460,3461,3315,3316,3240 \text {. WPO\#42261 } \\
\text { is a two-page memo dated } 11 / 13 / 96 \text {, "Additional Source Information for Mobile } \\
\text { Colloidal Actinides". WPO\#37522 is a } 4 \text { page memo with the authoirs/title/date } \\
\text { cited here. }\end{array}$ \\
\hline M2-37 & ID 256. The word "Seal" should be initial capitalized. \\
\hline M2-38 & ID 3317. Initial capitalize the word "Parameters." \\
\hline M2-38 & $\begin{array}{l}\text { ID } 297,298 \text {. Insert a space after Larry Sanchez's initials and initial capitalize the } \\
\text { word "Use." }\end{array}$ \\
\hline M2-38 & $\begin{array}{l}\text { ID 2832, 2831, 2826. Remove the comma before "et" and insert a hyphen } \\
\text { between "Low" and Boiling." }\end{array}$ \\
\hline M2-38 & $\begin{array}{l}\text { ID 2883. Change the citation of Nuclides and Isotopes to: Nuclides and Isotopes: } \\
\text { Chart of the Nuclides. } 14^{\text {th }} \text { ed. p. } 52 \text {. In the last box for ID } 2883 \text { insert: San } \\
\text { Jose, CA: General Electric. }\end{array}$ \\
\hline M2-38 & ID 2865. WPO\#44691 is page E-7 from the $65^{\text {th }}$ edition of this Handbook. \\
\hline
\end{tabular}




\begin{tabular}{|c|c|}
\hline Page & Change / Reference Information \\
\hline M2-40 & $\begin{array}{l}\text { D } 2825,2830 \text {. Remove the comma before "et" and insert a hyphen between } \\
\text { "Low" and "Boiling". }\end{array}$ \\
\hline M2-40 & ID 3503. WPO\#40133 is dated 6/26/96. \\
\hline M2-40 & ID 520, 559, 579. Change "Anh2" to "Anhydrite Two-Phase." \\
\hline M2-40 & $\begin{array}{l}\text { I 2158. Move the closing quotation mark to follow the word "Values" (not the } \\
\text { memo date). }\end{array}$ \\
\hline M2-42 & ID 522, 561, 582. Change "Anh2" to "Anhydrite Two-Phase." \\
\hline M2-42 & ID 544. Replace the text after "Pkg 8" with: "Salado Halite Porosity." \\
\hline $\mathrm{M} 2-42$ & ID 547, 548, 549. Rick Beauheim is also an author of this memo. \\
\hline $\mathrm{M} 2-42$ & ID 580, 560, 521. Insert "\#”' between "WPO\#” and " 31186 ". \\
\hline M2-43 & $\begin{array}{l}\text { ID } 2177 \text {. Move the closing quotation mark to follow the word "Values" and fix } \\
\text { the memo date of WPO } \# 44704: 3 / 31 / 97 \text {. }\end{array}$ \\
\hline M2-44 & $\begin{array}{l}\text { ID } 2178,2159,2170 . \text { Move the closing quotation mark to follow the word } \\
\text { "Values" and fix the memo date of WPO\#44704:3/31/97. }\end{array}$ \\
\hline M2-44 & ID 2789, 2784, 2774, 2790, 2785. Change "Anh2" to "Anhydrite Two-Phase". \\
\hline $\mathrm{M} 2-45$ & $\begin{array}{l}\text { ID 2169. Move the closing quotation mark to follow the word "Values" and fix } \\
\text { the memo date of WPO\#44704:3/31/97. }\end{array}$ \\
\hline M2-46 & ID 2775. Change "Anh2" to "Anhydrite Two-Phase". \\
\hline M2-46 & D 2180. Correct the spelling of "Shephard." \\
\hline M2-46 & $\begin{array}{l}\text { D 524, 563, 586, 526, 565. Move the closing quotation mark to follow the word } \\
\text { "Values" and fix the memo date of WPO\#44704:3/31/97. }\end{array}$ \\
\hline M2-56 & ID 587, 566. Insert "\#” between "WPO\#" and "42351". \\
\hline M2-57 & $\begin{array}{l}\text { D } 3143,3155,3167,3179 \text {. Per the records database, WPO\#35134 is a one-page } \\
\text { email (not a handwritten 4-page note). The title for the handwritten note and the } \\
\text { email is the same. }\end{array}$ \\
\hline M2-57 & ID 587, 566. Change "Anh2" to "Anhydrite Two-Phase." \\
\hline M2-58 & ID 527. Insert "\#" between "WPO\#" and "42351." \\
\hline M2-59 & ID 527. Change "Anh2" to "Anhydrite Two-Phase." \\
\hline M2-59 & $\begin{array}{l}\text { ID 588, 567, 528. After pkg 11, insert the following replacement text: "Salado } \\
\text { anhydrite effective porosity." }\end{array}$ \\
\hline M2-59 & $\begin{array}{l}\text { ID 591, 592, 593, 570, } 571 \text {. Correct the spelling of "Howarth" and insert a } \\
\text { closing quotation mark after the word "Permeability." }\end{array}$ \\
\hline M2-60 & ID 572, 531, 532, 533. Insert a closing parenthesis in the entry for WPO\#41677. \\
\hline M2-60 & $\begin{array}{l}\text { D 596, 575, 536, 597, 576, 537, 598, 577, 538, 599. Change "Anh2" to } \\
\text { "Anhydrite Two-Phase." }\end{array}$ \\
\hline M2-61 & $\begin{array}{l}\text { ID 572, 531, 532, 533. Insert a closing quotation mark after the word } \\
\text { "Permeability." }\end{array}$ \\
\hline M2-62 & ID 2939. Correct the spelling of the name "Vaughn." \\
\hline M2-62 & $\begin{array}{l}\text { ID 2516, 2533, 2550,2567, 2809. Mike Lord is the author of WPO\#39058. } \\
\text { Also the word "calculations" is plural in these entries. }\end{array}$ \\
\hline
\end{tabular}




\begin{tabular}{|c|c|}
\hline Page & Change / Reference Information \\
\hline M2-64 & $\begin{array}{l}\text { ID } 3076,2381,2398,2415,2432,2449,2313,2466,2483,3057,2279 . \text { Close up } \\
\text { the space between the initials in "L.D. Hurtado." }\end{array}$ \\
\hline M2-66 & All entries. The word "Seals" is plural in these entries. \\
\hline M2-68 & All entries. The word "Seals" is plural in these entries. \\
\hline M2-68 & $\begin{array}{l}\text { ID } 2529,2546,2563,2580,2597,2993,2512,3015,2343,2360 \text {. Mayer et al., } \\
1992 \text { is on file in the SWCF as WPO\#41122. The citation for this entry is: } \\
\text { Mayer, G., F. Jacobs, and F.H. Wittmann. 1992. "Experimental Determination } \\
\text { and Numerical Simulation of the Permeability of Cementitious Materials," } \\
\text { Nuclear Engineering and Design. Vol. 138, no. 2, 171-177. }\end{array}$ \\
\hline M2-70 & $\begin{array}{l}\text { ID } 2377,3083,2394,2411,2428,2445,2462,2326,2479,2495,3064 \text {. Mayer et } \\
\text { al., } 1992 \text { is on file in the SWCF as WPO\#41122. See previous entry for citation. }\end{array}$ \\
\hline $\mathrm{M} 2-70$ & ID 344, 345. Insert a space after "p."; close up the space after "SAND." \\
\hline M2-72 & $\begin{array}{l}\text { ID 346. Insert a space after "p."; replace the "s" s" after "permeability" with a } \\
\text { period; close up the space after "SAND." }\end{array}$ \\
\hline M2-72 & $\begin{array}{l}\text { ID } 3262,3263,3265,3264,3266,3389,3390,3391,3392,3393 \text {. Entries for } \\
\text { WPO\#37791. Correct the spelling of "Bynum"; insert the words "to be" between } \\
\text { the words "Solubility" and "Used". The word "Calculations" is plural. }\end{array}$ \\
\hline M2-72 & ID 3265. Per the records database, the parameter id is SOLSIM. \\
\hline M2-74 & ID 2907,2898 . Make the "p." lowercase in these citations. \\
\hline M2-74 & D 2909. Insert a space after Wang's initial. \\
\hline M2-74 & ID 651. The word "Spallings" is plural in this citation. \\
\hline M2-74 & $\begin{array}{l}\text { ID 2041, 2274, 1992, 1993, 2040, 2044, 1994, 2043, 2275. Change "CAD" to } \\
\text { "CAO" in these entries. The correct report number is: DOE/CAO-95-1121. }\end{array}$ \\
\hline $\mathrm{M} 2-75$ & ID 2907. Internal punctuation is missing in this entry. \\
\hline $\mathrm{M} 2-75$ & $\begin{array}{l}\text { ID } 651 \text {. The text for ID } 651 \text { on p. K2-74 ends with a period. The text on M2-75 } \\
\text { starts mid-sentence. Remove the second "NY" in the Streeter and Wylie entry. } \\
\text { Correct the spelling of the word "Book." Streeter and Wylie, p. } 297 \text { is on file in } \\
\text { the SWCF as WPO\#45492. }\end{array}$ \\
\hline M2-76 & $\begin{array}{l}\text { ID } 1995,2228,2042,2046 \text {. Change "CAD" to "CAO" in these entries. The } \\
\text { correct report number is: DOE/CAO-95-1121. }\end{array}$ \\
\hline M2-76 & $\begin{array}{l}\text { ID } 663,664,665 \text {. In the entry for WPO\#30921, the word after "Documentation" } \\
\text { should be changed to "of"; in the entry for WPO\#30925, insert the word "the" } \\
\text { after "Documentation." }\end{array}$ \\
\hline \multirow[t]{2}{*}{ M2-78 } & D 670, 2741. Brooks and Corey, 1964 is on file in the SWCF as WPO\#41117. \\
\hline & Appendix M4 \\
\hline M4-2, M4-3 & $\begin{array}{l}\text { 40CFR191. The title for the } 1985 \text { version is different from the current title. The } \\
\text { title should be changed to: Environmental Standards for the Management and } \\
\text { Disposal of Spent Nuclear Fuel, High-Level and Transuranic Radioactive } \\
\text { Wastes. The text to put in the Journal column is: Federal Register. Vol. 50, no. } \\
\text { 182, 38066-38089. Add the WPO\# to the Ref ID field: WPO\#39132. }\end{array}$ \\
\hline M4-2, M4-3 & $\begin{array}{l}\text { CRC-14. Add "The Chemical Rubber Co." to the publisher box and "Cleveland, } \\
\text { OH" to the location box. }\end{array}$ \\
\hline
\end{tabular}




\begin{tabular}{|c|c|}
\hline Page & $\begin{array}{c}\text { Change / Reference Information } \\
\end{array}$ \\
\hline M4-2, M4-3 & $\begin{array}{l}\text { Federal_Register. Supply the following title in the title box: } 40 \text { CFR 194: } \\
\text { Criteria for the Certification and Re-Certification of the Waste Isolation Pilot } \\
\text { Plant's Compliance with the } 40 \text { CFR } 191 \text { Disposal Regulations. The text to put } \\
\text { in the Journal column is: Federal Register. Vol. } 61 \text {, no. 28, 5224-5245. Add the } \\
\text { following WPO\# to the Ref D field: WPO\#41579. The author is U.S. EPA. }\end{array}$ \\
\hline M4-2, M4-3 & $\begin{array}{l}\text { Finley-1. Add the report number and page citation to this entry: SAND92- } \\
0700 / 5 \text {, p. A-5 through A-8. }\end{array}$ \\
\hline M4-2, M4-3 & $\begin{array}{l}\text { Fox-1. The } 1934 \text { publication date is incorrect. The first edition was published in } \\
\text { 1973, 2nd edition in 1978, 3rd edition in 1984, and 4th edition in } 1992 \text { Pages } \\
\text { 404-409 from the first edition are on file in the SWCF as WPO\#41622. Add the } \\
\text { following author information: R.W. Fox and A.T McDonald. }\end{array}$ \\
\hline M4-2, M4-3 & $\begin{array}{l}\text { Hydraulics/ASCE-3/70. Add the following to the journal box: Vol. } 96, \text { no. } \\
\text { HY3, 755-771. Add the following WPO\# to the Ref ID field: WPO\#41125. }\end{array}$ \\
\hline M4-2, M4-3 & $\begin{array}{l}\text { Memo: Leonard/Marietta-2. This memo is on file in the SWCF as WPO\#40850. } \\
\text { Add the WPO\# to the Ref D field. }\end{array}$ \\
\hline M4-2, M4-3 & $\begin{array}{l}\text { Memo: Leonard/Marietta-3. This memo is on file in the SWCF as WPO\#40849. } \\
\text { Add the WPO\# to the Ref ID field. }\end{array}$ \\
\hline M4-2, M4-3 & $\begin{array}{l}\text { Memo: Vaughn/Tierney-10. This memo is on file in the SWCF as WPO\#43088. } \\
\text { Add the WPO\# to the Ref } \mathrm{D} \text { field. }\end{array}$ \\
\hline M4-2, M4-3 & $\begin{array}{l}\text { Novakmemo-1. In the journal box a space needs to be inserted between "Waste" } \\
\text { and "Isolation"; the spelling of the words "Model" and "Parameters" need to be } \\
\text { corrected; the report number needs to be added: SAND92-0700/3. In the author } \\
\text { box, correct the spelling of "Papenguth." }\end{array}$ \\
\hline M4-4, M4-5 & $\begin{array}{l}\text { Nuclides/Isotopes. Replace the title information with the following: Nuclides } \\
\text { and Isotopes: Chart of the Nuclides. } 14^{\text {th }} \text { ed. This document is on file in the } \\
\text { SWCF as WPO\#41566. Add the WPO\# to the Ref ID field. }\end{array}$ \\
\hline M4-4, M4-5 & Obert-1. Page 541 from this book is on file in the SWCF as WPO\#43092. \\
\hline M4-4, M4-5 & $\begin{array}{l}\text { SAND86-7167. Replace the word "Flossing" with the phrase "Flow in"; add a } \\
\text { space after the parenthesis in the title. SAND86-7167 is on file in the SWCF as } \\
\text { WPO\#28486. }\end{array}$ \\
\hline M4-4, M4-5 & $\begin{array}{l}\text { SAND89-0462. Insert a comma after the word "Transport." SAND89-0462 is } \\
\text { on file in the SWCF as WPO\#24125. }\end{array}$ \\
\hline M4-4, M4-5 & SAND89-2408. This report is on file in the SWCF as WPO\#27724. \\
\hline M4-4, M4-5 & SAND89-7068/1. On file in the SWCF as WPO\#24085. \\
\hline M4-4, M4-5 & $\begin{array}{l}\text { SAND91-0893/3. On file in the SWCF as WPO\#26419. Insert the text } \\
\text { "December 1991" after the word "Plant". Add the report number to the Journal } \\
\text { box. Add "WIPP PA Division" to the Author box. }\end{array}$ \\
\hline M4-4, M4-5 & $\begin{array}{l}\text { SAND91-0893/3-MemoBeauheim. This memo is located in Appendix A of } \\
\text { SAND91-0893/3, which is on file in the SWCF as WPO\#26419. Correct the } \\
\text { spelling of "Data" and "WIPP." }\end{array}$ \\
\hline
\end{tabular}




\begin{tabular}{|c|c|}
\hline Page & Change / Reference Information \\
\hline M4-4, M4-5 & $\begin{array}{l}\text { SAND91-0893/3-MemoBeauheim2. This memo is located in Appendix A of } \\
\text { SAND91-0893/3, which is on file in the SWCF as WPO\#26419. In the title box, } \\
\text { replace "1991" with "1992". In the journal box, insert a space after "December" } \\
\text { and "Performance." }\end{array}$ \\
\hline M4-4, M4-5 & $\begin{array}{l}\text { SAND91-0893/3-MemoBrush. This memo is located in Appendix A of } \\
\text { SAND91-0893/3, which is on file in the SWCF as WPO\#26419. In the journal } \\
\text { box, insert a period after "Data." }\end{array}$ \\
\hline M4-4, M4-5 & $\begin{array}{l}\text { SAND92-0479. On file in the SWCF as WPO\#23526. Delete the word "the" } \\
\text { before "Waste" in the title box and the period after "Trauth" in the author box. }\end{array}$ \\
\hline M4-4, M4-5 & $\begin{array}{l}\text { SAND92-0700/3. On file in the SWCF as WPO\#23529. Spell out the word } \\
\text { "Volume" in the title box. }\end{array}$ \\
\hline M4-4, M4-5 & $\begin{array}{l}\text { SAND92-0700/3-MemoPeterson. This memo is reprinted in Appendix A of } \\
\text { SAND92-0700/3, which is on file in the SWCF as WPO\#23529. Correct the } \\
\text { spelling of the word "Parameters" in the journal box. Insert a space after the } \\
\text { word "through." }\end{array}$ \\
\hline M4-4, M4-5 & $\begin{array}{l}\text { Streeter-1. Page } 297 \text { is on file in the SWCF as WPO\#45492. The } 6^{\text {th }} \text { edition is } \\
\text { available at UNM CSEL. }\end{array}$ \\
\hline M4-4, M4-5 & Streeter-2. The $2^{\text {nd }}$ edition is available at UNM CSEL. \\
\hline M4-6, M4-7 & $\begin{array}{l}\text { Vapor-Liquid_Equilibria. The information listed in the Journal box is an edition } \\
\text { statement. That information should be moved to the title box: "Vapor-Liquid } \\
\text { Equilibria for Mixtures of Low-Boiling Substances. Chemistry Data Series v. 6." } \\
\text { Correct the spelling of Knapp's surname. The publisher is: DECHEMA; } \\
\text { Scholium International. The place of publication is: Frankfurt, Germany; Great } \\
\text { Neck, NY. }\end{array}$ \\
\hline M4-6, M4-7 & Vaughn:Worksheet-1. Add "Sandia National Laboratories" to the publisher box. \\
\hline M4-6, M4-7 & $\begin{array}{l}\text { Vaughn:Worksheet-4. Add "Sandia National Laboratories" to the publisher box } \\
\text { and "Albuquerque, NM" to the location box. }\end{array}$ \\
\hline M4-6, M4-7 & $\begin{array}{l}\text { Vaughn:Worksheet-4A. Add "Sandia National Laboratories" to the publisher } \\
\text { box. }\end{array}$ \\
\hline M4-6, M4-7 & $\begin{array}{l}\text { WPO\#22443, WPO\#26051, WPO\#30598, WPO\#30599, WPO\#30600, } \\
\text { WPO\#30601, WPO\#30603, WPO\#30605, WPO\#30606, WPO\#30607, } \\
\text { WPO\#30608, WPO\#30611, WPO\#30641, WPO\#30642, WPO\#30643. Add } \\
\text { "Sandia National Laboratories" to the publisher box and "Albuquerque, NM" to } \\
\text { the location box. }\end{array}$ \\
\hline M4-6, M4-7 & $\begin{array}{l}\text { WPO\#30703. Add "J.D. Schreiber" to the author box and "31-Jan-96" to the } \\
\text { date box. Add "Sandia National Laboratories" to the publisher box and } \\
\text { "Albuquerque, NM" to the location box. }\end{array}$ \\
\hline M4-6, M4-7 & $\begin{array}{l}\text { WPO\#30713, WPO\#30721. Add "Sandia National Laboratories" to the } \\
\text { publisher box and "Albuquerque, NM" to the location box. }\end{array}$ \\
\hline M4-6, M4-7 & $\begin{array}{l}\text { WPO\#30819. The date in the records database for this item is February 7, } 1996 . \\
\text { Add "Sandia National Laboratories" to the publisher box. }\end{array}$ \\
\hline M4-6, M4-7 & WPO\#30921. Add "Sandia National Laboratories" to the publisher box. \\
\hline M4-8, M4-9 & WPO\#30925. Add "Sandia National Laboratories" to the publisher box. \\
\hline
\end{tabular}




\begin{tabular}{|c|c|}
\hline Page & Change / Reference Information \\
\hline M4-8, M4-9 & $\begin{array}{l}\text { WPO\#31041. Replace the word "in" that precedes "BRAGFLO" with "on"; add } \\
\text { "Sandia National Laboratories" to the publisher box and "Albuquerque, NM" to } \\
\text { the location box. }\end{array}$ \\
\hline M4-8, M4-9 & $\begin{array}{l}\text { WPO\#31070, WPO\#31072, WPO\#31082, WPO\#31083. Add "Sandia National } \\
\text { Laboratories" to the publisher box and "Albuquerque, NM" to the location box. }\end{array}$ \\
\hline M4-8, M4-9 & WPO\#31084. Add "Sandia National Laboratories" to the publisher box. \\
\hline M4-8, M4-9 & $\begin{array}{l}\text { WPO\#31168, WPO\#31171, WPO\#31174, WPO\#31175, WPO\#31185, } \\
\text { WPO\#31186, WPO\#31217, WPO\#31218, WPO\#31220, WPO\#31221, } \\
\text { WPO\#31235, WPO\#31368. Add "Sandia National Laboratories" to the } \\
\text { publisher box and "Albuquerque, NM" to the location box. }\end{array}$ \\
\hline M4-8, M4-9 & $\begin{array}{l}\text { WPO\#31379. Add middle initial "E." to "P. Drez" the author box; add "CTAC" } \\
\text { to the publisher box. }\end{array}$ \\
\hline M4-8, M4-9 & $\begin{array}{l}\text { WPO\#31904, WPO\#32038. Add "Sandia National Laboratories" to the } \\
\text { publisher box and "Albuquerque, NM" to the location box. }\end{array}$ \\
\hline M4-8, M4-9 & $\begin{array}{l}\text { WPO\#32281, WPO\#32287, WPO\#32288. Add "Sandia National Laboratories" } \\
\text { to the publisher box. }\end{array}$ \\
\hline M4-8, M4-9 & $\begin{array}{l}\text { WPO\#32290. Add the word "Units" to the end of this memo title. Add "Sandia } \\
\text { National Laboratories" to the publisher box and "Albuquerque, NM" to the } \\
\text { location box. }\end{array}$ \\
\hline M4-10, M4-11 & $\begin{array}{l}\text { WPO\#32291. Correct the spelling of the word "Addendum" in the title box. } \\
\text { Replace "Lori" with "L.J." in the author box. Add "Albuquerque, NM" to the } \\
\text { location box. }\end{array}$ \\
\hline M4-10, M4-11 & $\begin{array}{l}\text { WPO\#32328. Correct the report number in the journal box: DOE/CAO-95- } \\
1121, \text { Rev. } 2 \text {. Add "DOE/CAO" to the publisher box and "Carlsbad, NM" to the } \\
\text { location box. }\end{array}$ \\
\hline M4-10, M4-11 & $\begin{array}{l}\text { WPO\#34618. In the title box, insert a space between "McFadden" and "(DOE)"; } \\
\text { insert first initial "D." before "R. Anderson" and correct the spelling of the word } \\
\text { "Waste". In the author box, add McFadden's middle initial "H." Add } \\
\text { "DOE/CAO" to the publisher box and "Carlsbad, NM" to the location box. }\end{array}$ \\
\hline M4-10, M4-11 & $\begin{array}{l}\text { WPO\#35134. Add the word "Concerning" as the first word of the title. Add } \\
\text { "Sandia National Laboratories" to the publisher box. }\end{array}$ \\
\hline M4-10, M4-11 & WPO\#35148. Add "Sandia National Laboratories" to the publisher box. \\
\hline M4-10, M4-11 & $\begin{array}{l}\text { WPO\#35183, WPO\#35193, WPO\#35194, WPO\#35266, WPO\#35268. Add } \\
\text { "Sandia National Laboratories" to the publisher box and "Albuquerque, NM" to } \\
\text { the location box. }\end{array}$ \\
\hline M4-10, M4-11 & WPO\#35269. Add "Sandia National Laboratories" to the publisher box.. \\
\hline M4-10, M4-11 & $\begin{array}{l}\text { WPO\#35270. In the author box, replace "Jim" with "J." Add "Sandia National } \\
\text { Laboratories" to the publisher box and "Albuquerque, NM" to the location box. }\end{array}$ \\
\hline M4-10, M4-11 & $\begin{array}{l}\text { WPO\#35271. Change the spelling of "Modeling" in the title box. Insert the date } \\
\text { "19-Feb-96" in the date box. Add "Sandia National Laboratories" to the } \\
\text { publisher box and "Albuquerque, NM" to the location box. }\end{array}$ \\
\hline M4-10, M4-11 & WPO\#35330. Add "Sandia National Laboratories" to the publisher box. \\
\hline
\end{tabular}




\begin{tabular}{|c|c|}
\hline Page & Change / Reference Information \\
\hline M4-10, M4-11 & $\begin{array}{l}\text { WPO\#35695 = WPO\#47189. This memo is in the records database as } \\
\text { WPO\#47189. It is not listed in the table of contents for WPO\#35695. Delete the } \\
\text { text in parentheses in the title box. Change the text in the author box to read: } \\
\text { J.W. Berglund. Add "Sandia National Laboratories" to the publisher box and } \\
\text { "Albuquerque, NM" to the location box. }\end{array}$ \\
\hline M4-10, M4-11 & $\begin{array}{l}\text { WPO\#35835. In the author box, close up the space between Bynum's initials. } \\
\text { Add "Sandia National Laboratories" to the publisher box. }\end{array}$ \\
\hline M4-10, M4-11 & $\begin{array}{l}\text { WPO\#35850, WPO\#35852. Replace Papenguth's first name with the initials } \\
\text { "H.W." Add "Sandia National Laboratories" to the publisher box and } \\
\text { "Albuquerque, NM" to the location box. }\end{array}$ \\
\hline M4-10, M4-11 & $\begin{array}{l}\text { WPO\#35854. Replace the incorrect first name in the author box with the initials } \\
\text { "M.K." Add "Sandia National Laboratories" to the publisher box and } \\
\text { "Albuquerque, NM" to the location box. }\end{array}$ \\
\hline M4-10, M4-11 & $\begin{array}{l}\text { WPO\#35855, WPO\#35856. Replace Papenguth's first name with his initials, } \\
\text { "H.W." Add "Sandia National Laboratories" to the publisher box and } \\
\text { "Albuquerque, NM" to the location box. }\end{array}$ \\
\hline M4-10, M4-11 & WPO\#36041. Add "Sandia National Laboratories" to the publisher box. \\
\hline M4-12, M4-13 & $\begin{array}{l}\text { WPO\#36051. Add "Sandia National Laboratories", to the publisher box and } \\
\text { "Albuquerque, NM" to the location box. }\end{array}$ \\
\hline M4-12, M4-13 & $\begin{array}{l}\text { WPO\#36053. Replace "SNL" with "Sandia National Laboratories" in the } \\
\text { publisher box. }\end{array}$ \\
\hline M4-12, M4-13 & WPO\#36058. Add "Sandia National Laboratories" to the publisher box. \\
\hline M4-12, M4-13 & WPO\#36203. Correct the spelling of the word "Castile" in the title box. \\
\hline M4-12, M4-13 & WPO\#36205. Add "Sandia National Laboratories" to the publisher box. \\
\hline M4-12, M4-13 & $\begin{array}{l}\text { WPO\#36301. Add the text "D. Stoelzel" to the author box and the date "3-Apr- } \\
96 \text { " to the date box. Add "Sandia National Laboratories" to the publisher box and } \\
\text { "Albuquerque, NM" to the location box. }\end{array}$ \\
\hline M4-12, M4-13 & $\begin{array}{l}\text { WPO\#36320, WPO\#36380. Add "Sandia National Laboratories" to the } \\
\text { publisher box. }\end{array}$ \\
\hline M4-12, M4-13 & $\begin{array}{l}\text { WPO\#36455. Add middle initial "L.".to "R. Beauheim" in the author box. Add } \\
\text { "Sandia National Laboratories" to the publisher box and "Albuquerque, NM" to } \\
\text { the location box. }\end{array}$ \\
\hline M4-12, M4-13 & $\begin{array}{l}\text { WPO\#36456. Add middle initial "L." to "R. Beauheim" in the author box. Add } \\
\text { "Sandia National Laboratories" to the publisher box. }\end{array}$ \\
\hline M4-12, M4-13 & $\begin{array}{l}\text { WPO\#36489. Transpose the author's initials and surname to read "M.G. } \\
\text { Wallace." Add "Sandia National Laboratories" to the publisher box and } \\
\text { "Albuquerque, NM" to the location box. }\end{array}$ \\
\hline M4-12, M4-13 & $\begin{array}{l}\text { WPO\#36491. Correct the spelling of the word "Resistance" in the title box. } \\
\text { Add middle initial "G." to "M. Wallace." Add "Sandia National Laboratories" to } \\
\text { the publisher box and "Albuquerque, NM" to the location box. }\end{array}$ \\
\hline M4-12, M4-13 & WPO\#36723. Add "Sandia National Laboratories" to the publisher box. \\
\hline
\end{tabular}




\begin{tabular}{|c|c|}
\hline Page & $\begin{array}{l}\text { Change / Reference Information } \\
\end{array}$ \\
\hline M4-12, M4-13 & $\begin{array}{l}\text { WPO\#36766. Initial capitalize the word "Use" in the title box. Replace "Hans } \\
\text { w." with "H.W." in the author box. Add "Sandia National Laboratories" to the } \\
\text { publisher box and "Albuquerque, NM" to the location box. }\end{array}$ \\
\hline M4-12, M4-13 & $\begin{array}{l}\text { WPO\#36771. Replace "Hans W." with "H.W." in the author box. Add "Sandia } \\
\text { National Laboratories" to the publisher box and "Albuquerque, NM" to the } \\
\text { location box. }\end{array}$ \\
\hline M4-12, M4-13 & $\begin{array}{l}\text { WPO\#36772. Insert the word "Halites" after the word "Salado" in the title box. } \\
\text { Add "idpram: PRMX_LOG" to the end of the title. Add "Sandia National } \\
\text { Laboratories" to the publisher box. }\end{array}$ \\
\hline M4-12, M4-13 & $\begin{array}{l}\text { WPO\#37061. Close up the space between the author's initials. Add "Sandia } \\
\text { National Laboratories" to the publisher box. }\end{array}$ \\
\hline M4-12, M4-13 & $\begin{array}{l}\text { WPO\#37147. Replace "Jon" with "J." in the author box. Add "Sandia National } \\
\text { Laboratories" to the publisher box. }\end{array}$ \\
\hline M4-12, M4-13 & WPO\#37148. Add "Sandia National Laboratories" to the publisher box. \\
\hline M4-12, M4-13 & $\begin{array}{l}\text { WPO\#37223, WPO\#37225. Add "Sandia National Laboratories" to the } \\
\text { publisher box and "Albuquerque, NM" to the location box. }\end{array}$ \\
\hline M4-14, M4-15 & $\begin{array}{l}\text { WPO\#37227, WPO\#37228. Add "Sandia National Laboratories" to the } \\
\text { publisher box and "Albuquerque, NM" to the location box. }\end{array}$ \\
\hline M4-14, M4-15 & $\begin{array}{l}\text { WPO\#37230, WPO\#37231. Add McCord's middle initial "T." in the author } \\
\text { box. Add "Sandia National Laboratories" to the publisher box and } \\
\text { "Albuquerque, NM" to the location box. }\end{array}$ \\
\hline M4-14, M4-15 & WPO\#37314. Add Siegel's middle initial " $D$. " in the author box. \\
\hline M4-14, M4-15 & $\begin{array}{l}\text { WPO\#37400. Add the following to the end of this title: Support the WIPP CCA. } \\
\text { Close up the space between the author's initials in the author box. Add "Sandia } \\
\text { National Laboratories" to the publisher box. Correct spelling of title word } \\
\text { "Solution." }\end{array}$ \\
\hline M4-14, M4-15 & $\begin{array}{l}\text { WPO\#37401, WPO\#37402. Add "Sandia National Laboratories" to the } \\
\text { publisher box. }\end{array}$ \\
\hline M4-14, M4-15 & $\begin{array}{l}\text { WPO\#37404. Replace "Larry" with "L." in the author box. Add "Sandia } \\
\text { National Laboratories" to the publisher box and "Albuquerque, NM" to the } \\
\text { location box. }\end{array}$ \\
\hline M4-14, M4-15 & $\begin{array}{l}\text { WPO\#37465. In the title box, insert a space between "T." and "Corbet". In the } \\
\text { author box, insert Corbet's middle initial "F." }\end{array}$ \\
\hline M4-14, M4-15 & WPO\#37522. Insert Papenguth's middle initial "W." in the author box. \\
\hline M4-14, M4-15 & $\begin{array}{l}\text { WPO\#37533. In the title box, make the } \mathrm{m} \text { in "Cm" lowercase and add the } \\
\text { following to the end of the title: to Support the WIPP CCA. Add "Sandia } \\
\text { National Laboratories" to the publisher box. }\end{array}$ \\
\hline M4-14, M4-15 & $\begin{array}{l}\text { WPO\#37542, WPO\#37713. Add "Sandia National Laboratories" to the } \\
\text { publisher box. }\end{array}$ \\
\hline M4-14, M4-15 & $\begin{array}{l}\text { WPO\#37799. Close up the space between Bynum's initials in the author box. } \\
\text { Add "Sandia National Laboratories" to the publisher box. }\end{array}$ \\
\hline M4-14, M4-15 & WPO\#37804. Add "Sandia National Laboratories" to the publisher box. \\
\hline
\end{tabular}




\begin{tabular}{|c|c|}
\hline Page & Change / Reference Information \\
\hline M4-14, M4-15 & $\begin{array}{l}\text { WPO\#37822. Insert Siegel's middle initial "D." in the author box. Add "Sandia } \\
\text { National Laboratories". to the publisher box. }\end{array}$ \\
\hline M4-14, M4-15 & $\begin{array}{l}\text { WPO\#37970, WPO\#38214. Add "Sandia National Laboratories" to the } \\
\text { publisher box. }\end{array}$ \\
\hline M4-16, M4-17 & WPO\#38217. Add "Sandia National Laboratories" to the publisher box. \\
\hline M4-16, M4-17 & $\begin{array}{l}\text { WPO\#38231. Change "Dissolve" to "Dissolved" in the title box. Transpose the } \\
\text { author's initials and surname to read "L.H. Brush." Add "Sandia National } \\
\text { Laboratories" to the publisher box. }\end{array}$ \\
\hline M4-16, M4-17 & $\begin{array}{l}\text { WPO\#38274, WPO\#38333, WPO\#38337, WPO\#38343, WPO\#38344, } \\
\text { WPO\#38449, WPO\#38450, WPO\#38454. Add "Sandia National Laboratories" } \\
\text { to the publisher box. }\end{array}$ \\
\hline M4-16, M4-17 & $\begin{array}{l}\text { WPO\#38455. Insert Tierney's middle initial "S." in the author box. Add } \\
\text { "Sandia National Laboratories" to the publisher box. }\end{array}$ \\
\hline M4-16, M4-17 & $\begin{array}{l}\text { WPO\#38456, WPO\#38568, WPO\#38569. Add "Sandia National Laboratories" } \\
\text { to the publisher box. }\end{array}$ \\
\hline M4-16, M4-17 & $\begin{array}{l}\text { WPO\#38769. Move "Solutions Engineering" from the author box to the } \\
\text { publisher box. Add "Colorado" to the location box. }\end{array}$ \\
\hline M4-16, M4-17 & WPO\#38801. Add "Sandia National Laboratories" to the publisher box. \\
\hline M4-16, M4-17 & $\begin{array}{l}\text { WPO\#38819. Insert "USDOE/CAO" in the publisher box and "Carlsbad, NM" } \\
\text { in the location box. }\end{array}$ \\
\hline M4-16, M4-17 & $\begin{array}{l}\text { WPO\#38928. Correct the date of this memo to } 6 / 7 / 96 \text {. This memo is a one-page } \\
\text { record. }\end{array}$ \\
\hline M4-16, M4-17 & WPO\#38928 $\left(2^{\text {nd }}\right.$ entry $)=$ WPO\#38939. \\
\hline M4-16, M4-17 & WPO\#38941. Add "Sandia National Laboratories" to the publisher box. \\
\hline M4-18, M4-19 & $\begin{array}{l}\text { WPO\#39058, WPO\#39065. Add "Sandia National Laboratories" to the } \\
\text { publisher box. }\end{array}$ \\
\hline M4-18, M4-19 & WPO\#39169. Insert "INTERA Inc." in the publisher box. \\
\hline M4-18, M4-19 & $\begin{array}{l}\text { WPO\#39178, WPO\#39179. Add "Sandia National Laboratories" to the } \\
\text { publisher box. }\end{array}$ \\
\hline M4-18, M4-19 & $\begin{array}{l}\text { WPO\#39260. Insert "1996" in the date box. Add "Sandia National - } \\
\text { Laboratories" to the publisher box and "Albuquerque, NM" to the location box. }\end{array}$ \\
\hline M4-18, M4-19 & $\begin{array}{l}\text { WPO\#39353, WPO\#39355. Add "Sandia National Laboratories" to the } \\
\text { publisher box. }\end{array}$ \\
\hline M4-18, M4-19 & $\begin{array}{l}\text { WPO\#39365. Insert the author's initials as "M.G." and delete the lowercase } \\
\text { "m"; add "Sandia National Laboratories" to the publisher box and "Albuquerque, } \\
\text { NM" to the location box. }\end{array}$ \\
\hline M4-18, M4-19 & $\begin{array}{l}\text { WPO\#39371. Replace the code name before the word "Skin" with } \\
\text { "SECOTP2D". Add "Sandia National Laboratories" to the publisher box. }\end{array}$ \\
\hline M4-18, M4-19 & $\begin{array}{l}\text { WPO\#40133. Insert the author's middle initial "M." in the author box. Add } \\
\text { "Sandia National Laboratories" to the publisher box. }\end{array}$ \\
\hline M4-18, M4-19 & $\begin{array}{l}\text { WPO\#40199. Insert the author's middle initial "S." in the author box. Add } \\
\text { "Sandia National Laboratories" to the publisher box and "Albuquerque, NM" to } \\
\text { the location box. }\end{array}$ \\
\hline
\end{tabular}




\begin{tabular}{|c|c|}
\hline Page & Change / Reference Information \\
\hline M4-18, M4-19 & $\begin{array}{l}\text { WPO\#40432, WPO\#40434. Add "Sandia National Laboratories" to the } \\
\text { publisher box. }\end{array}$ \\
\hline M4-18, M4-19 & $\begin{array}{l}\text { WPO\#40486. Replace Palmer Vaughn's first name with the initial "P." and } \\
\text { Mike Lord's first name with the initial "M." Add "Sandia National } \\
\text { Laboratories" to the publisher box and "Albuquerque, NM" to the location box. }\end{array}$ \\
\hline M4-18, M4-19 & WPO\#40511. Add "Sandia National Laboratories" to the publisher box. \\
\hline M4-18, M4-19 & $\begin{array}{l}\text { WPO\#40512. Transpose Bynum's initials and surname to read "R.V. Bynum." } \\
\text { Add "Sandia National Laboratories" to the publisher box and "Albuquerque, } \\
\text { NM" to the location box. }\end{array}$ \\
\hline M4-18, M4-19 & $\begin{array}{l}\text { WPO\#40514. Transpose Vaughn's initials and surname to read "P.M. Vaughn." } \\
\text { Add "Albuquerque, NM" to the location box. }\end{array}$ \\
\hline M4-18, M4-19 & $\begin{array}{l}\text { WPO\#40515. Transpose Shinta"s initials and surname to read "A.A. Shinta." } \\
\text { Add "Albuquerque, NM" to the location box. }\end{array}$ \\
\hline M4-18, M4-19 & $\begin{array}{l}\text { WPO\#40516. Transpose Ramsey's initials and surname to read "J.L. Ramsey." } \\
\text { Add "Albuquerque, NM" to the location box. }\end{array}$ \\
\hline M4-18, M4-19 & $\begin{array}{l}\text { WPO\#40520. Transpose Stoelzel's initials and surname to read "D.M. Stoelzel." } \\
\text { Add "Albuquerque, NM" to the location box. }\end{array}$ \\
\hline M4-20, M4-21 & $\begin{array}{l}\text { WPO\#40521. Insert a space between "Task" and "5" in the title box. Transpose } \\
\text { Berglund's initials and surname to read "J.W. Berglund." Add "Albuquerque, } \\
\text { NM" to the location box. }\end{array}$ \\
\hline M4-20, M4-21 & $\begin{array}{l}\text { WPO\#40524. Transpose Helton's initials and surname to read "J.C. Helton." } \\
\text { Add "Albuquerque, NM" to the location box. }\end{array}$ \\
\hline M4-20, M4-21 & WPO\#41131. Replace the word "Draft" with "Report" in the title box. \\
\hline M4-20, M4-21 & $\begin{array}{l}\text { WPO\#41556, WPO\#41557, WPO\#41558. Replace "Mary-Alena" with "M.A." } \\
\text { in the author box. Add "Sandia National Laboratories" to the publisher box and } \\
\text { "Albuquerque, NM" to the location box. }\end{array}$ \\
\hline M4-20, M4-21 & $\begin{array}{l}\text { WPO\#41559. Replace "Christine" with "C.T." and "Larry" with "L." in the } \\
\text { author box. Reformat the date as " } 28-O c t-96 \text { ". Add "Sandia National } \\
\text { Laboratories" to the publisher box and "Albuquerque, NM" to the location box. } \\
\text { In the title box, remove the word "Request." }\end{array}$ \\
\hline M4-20, M4-21 & $\begin{array}{l}\text { WPO\#41560. Insert a space between "for" and "Use" in the title box. Reformat } \\
\text { the date as "28-Oct-96". Add "Sandia National Laboratories" to the publisher } \\
\text { box and "Albuquerque, NM" to the location box. }\end{array}$ \\
\hline M4-20, M4-21 & $\begin{array}{l}\text { WPO\#41662. Replace "Jon" with "J." in the author box. Add " } 30-O c t-96 \text { " to } \\
\text { the date box. Add "Sandia National Laboratories" to the publisher box and } \\
\text { "Albuquerque, NM" to the location box. }\end{array}$ \\
\hline M4-20, M4-21 & $\begin{array}{l}\text { WPO\#42046. Replace "Larry" with "L." in the author box. Add "Sandia } \\
\text { National Laboratories" to the publisher box and "Albuquerque, NM" to the } \\
\text { location box. }\end{array}$ \\
\hline M4-20, M4-21 & $\begin{array}{l}\text { WPO\#42257. Add "Sandia National Laboratories" to the publisher box and } \\
\text { "Albuquerque, NM" to the location box. }\end{array}$ \\
\hline M4-20, M4-21 & $\begin{array}{l}\text { WPO\#42263. Add "1996" to the date box. Add "Sandia National Laboratories" } \\
\text { to the publisher box and "Albuquerque, NM" to the location box. }\end{array}$ \\
\hline
\end{tabular}




\begin{tabular}{|l|l|}
\hline \multicolumn{1}{|c|}{ Page } & \multicolumn{1}{|c|}{ Change / Reference Information } \\
\hline M4-20, M4-21 & WPO\#42681. Add "Sandia National Laboratories" to the publisher box. \\
\hline M4-20, M4-21 & $\begin{array}{l}\text { WPO\#42701. Insert the following in the title box: "Effectiveness of Passive } \\
\text { Institutional Controls in Reducing Inadvertent Human Intrusion into the Waste } \\
\text { Isolation Pilot Plant for Use in Performance Assessments." Add the following to } \\
\text { the journal box: "WIPP-CAO-96-3168." Add "WIPP/CAO" to the publisher } \\
\text { and "Carlsbad, NM" to the location box. }\end{array}$ \\
\hline M4-20, M4-21 & $\begin{array}{l}\text { WPO\#42714. Replace "Mary-Alena" with "M." and "Charlene" with "C." in the } \\
\text { author box. Add "Sandia National Laboratories" to the publisher box and } \\
\text { "Albuquerque, NM" to the location box. }\end{array}$ \\
\hline M4-20, M4-21 & $\begin{array}{l}\text { WPO\#42750. Insert a space between "of" and "Use" in the title box. Replace } \\
\text { "Mary-Alena" with "M." and "Charlene" with "C." in the author box. Add } \\
\text { "Sandia National Laboratories" to the publisher box and "Albuquerque, NM" to } \\
\text { the location box. }\end{array}$ \\
\hline M4-20, M4-21 & $\begin{array}{l}\text { WPO\#42751. Remove the word "the" that precedes the word "Use" in the title } \\
\text { box. Replace "Mary-Alena" with "M." Add "Sandia National Laboratories" to } \\
\text { the publisher box and "Albuquerque, NM" to the location box. }\end{array}$ \\
\hline M4-20, M4-21 & $\begin{array}{l}\text { WPO\#42752. Replace "Mary-Alena" with "M." and "Charlene" with "C." in the } \\
\text { author box. Add "Sandia National Laboratories" to the publisher box and } \\
\text { "Albuquerque, NM" to the location box. }\end{array}$ \\
\hline M4-20, M4-21 & $\begin{array}{l}\text { WPO\#43751. Add "Sandia National Laboratories" to the publisher box and } \\
\text { "Albuquerque, NM" to the location box. }\end{array}$ \\
\hline Appendix N \\
\hline N-3 & The correct WPO\# for CL_M_T2 is WPO\#32117. \\
\hline
\end{tabular}


Page intentionally blank 


\section{Appendix A1}

\section{Materials Dictionary}

A1-1 
Materials

\begin{tabular}{|c|c|}
\hline Material ID & Material Description \\
\hline AC225 & Actinium 225 \\
\hline AC227 & Actinium 227 \\
\hline$A C 228$ & Actinium 228 \\
\hline$A M$ & Americium \\
\hline$A M+3$ & Americium III \\
\hline$\overline{A M 241}$ & Americium 241 \\
\hline AM243 & Americium 243 \\
\hline ASPHALT & Asphalt column \\
\hline AT217 & Astatine 217 \\
\hline BA137 & Barium 137 \\
\hline BA137M & Barium 137 Metastable \\
\hline BH_CREEP & Creep Borehole Fill \\
\hline BH_OPEN & Borehole Unrestricted \\
\hline BH_SAND & Borehole filled with silty sand \\
\hline $\mathrm{Bl} 211$ & Bismuth 211 \\
\hline $\mathrm{B} \mid 212$ & Bismuth 212 \\
\hline BI213 & Bismuth 213 \\
\hline BI214 & Bismuth 214 \\
\hline BLOWOUT & BRAGFLOW Direct Brine Releases \\
\hline BOREHOLE & Borehole and Fill \\
\hline BRINESAL & Salado Brine \\
\hline CASTILER & Castile Brine Reservoir \\
\hline CAVITY_1 & Cavity for Waste Areas \\
\hline CAVITY_2 & Cavity for Non-waste Areas \\
\hline CAVITY_3 & Cavity for Shaft \\
\hline CAVITY_4 & Cavity for Borehole \\
\hline CELLULS & Cellulose \\
\hline CF & Califorinum \\
\hline CF252 & Californium 252 \\
\hline CLAY_BOT & Shaft Bottom Clay \\
\hline CLAY_RUS & Rustler Compacted Clay Column \\
\hline$C_{L} L_{2} T 1$ & Lower Salado clay: 0 to 10 years \\
\hline CL_L_T2 & Lower Salado clay: 10 to 25 years \\
\hline CL_L_T3 & Lower Salado clay: 25 to 50 years \\
\hline CL_L_T4 & Lower Salado clay: 50 to $10 \mathrm{~K}$ years \\
\hline CL_M_T1 & Upper Salado clay: 0 to 10 years \\
\hline CL_M_T2 & Upper Salado clay: 10 to 25 years \\
\hline CL_M_T3 & Upper Salado clay: 25 to 50 years \\
\hline CL_M_T4 & Upper Salado clay: 50 to 100 years \\
\hline CL_M_T5 & Upper Salado clay: 100 to $10 \mathrm{~K}$ years \\
\hline CM243 & Curium 243 \\
\hline CM244 & Curium 244 \\
\hline CM245 & Curium 245 \\
\hline CM248 & Curium 248 \\
\hline CONC_MON & Concrete Monolith \\
\hline CONC_PLG & Concrete Plug, surface and Rustler \\
\hline CONC_T1 & Concrete column: 0 to 400 years \\
\hline CONC_T2 & Concrete column: 400 to $10 \mathrm{~K}$ years \\
\hline $\mathrm{CS}$ & Cesium \\
\hline CS137 & Cesium 137 \\
\hline CULEBRA & Culebra member of the Rustler formation \\
\hline DEWYLAKE & Dewey Lake Red Beds \\
\hline
\end{tabular}


Materials

\begin{tabular}{|c|c|}
\hline Material ID & Material Description \\
\hline DRILLMUD & Drilling Mud \\
\hline DRZ_0 & Disturbed rock zone; time period -5 to 0 years \\
\hline DRZ_1 & Disturbed rock zone; time period 0 to 1000 years \\
\hline EARTH & Earthen Fill \\
\hline EXP_AREA & Experimental Area \\
\hline FORTYNIN & Forty Niner Member \\
\hline FR221 & Francium 221 \\
\hline GLOBAL & Information that applies globally \\
\hline $\mathrm{H} 2$ & Hydrogen Gas \\
\hline IMPERM_Z & Impermeable Zones \\
\hline MAGENTA & Magenta Member \\
\hline ND143 & Neodymium 143 \\
\hline NITRATE & Nitrate \\
\hline $\mathrm{NP}$ & Neptunium \\
\hline$N P+4$ & Neptunium IV \\
\hline $\mathrm{NP}+5$ & Neptunium V \\
\hline NP237 & Neptunium 237 \\
\hline NP239 & Neptunium 239 \\
\hline OPS_AREA & Operations Region \\
\hline PA231 & Protactinium 231 \\
\hline PA233 & Protactinium 233 \\
\hline PA234M & Protactinium 234 Metastable \\
\hline PAN_SEAL & Panel Seal \\
\hline PB & Lead \\
\hline PB209 & Lead 209 \\
\hline PB210 & Lead 210 \\
\hline PB211 & Lead 211 \\
\hline PB212 & Lead 212 \\
\hline PB214 & Lead 214 \\
\hline PHUMOX3 & Proportionality Constant, +3 State, Humic Colloids \\
\hline PHUMOX4 & Proportionality constant with humic colloids for actinide in oxidation state IV \\
\hline PHUMOX5 & Proportionality constant with humic colloids for actinide in oxidation state $V$ \\
\hline PHUMOX6 & Proportionality constant with humic colloids for actinide in oxidation state VI \\
\hline PM147 & Promethium 147 \\
\hline $\mathrm{PO} 212$ & Polonium 212 \\
\hline $\mathrm{PO} 213$ & Polonium 213 \\
\hline PO214 & Polonium 214 \\
\hline $\mathrm{PO} 215$ & Polonium 215 \\
\hline $\mathrm{PO} 216$ & Polonium 216 \\
\hline $\mathrm{PO} 218$ & Polonium 218 \\
\hline PU & Plutonium \\
\hline $\mathrm{PU}+3$ & Plutonium III \\
\hline$P U+4$ & Plutonium IV \\
\hline PU238 & Plutonium 238 \\
\hline PU239 & Plutonium 239 \\
\hline PU240 & Plutonium 240 \\
\hline PU241 & Plutonium 241 \\
\hline PU242 & Plutonium 242 \\
\hline PU244 & Plutonium 244 \\
\hline $\mathrm{AA}$ & Radium \\
\hline RA223 & Radium 223 \\
\hline RA224 & Radium 224 \\
\hline
\end{tabular}


Materials

\begin{tabular}{|c|c|}
\hline Material ID & Material Description \\
\hline RA225 & Radium 225 \\
\hline RA226 & Radium 226 \\
\hline RA228 & Radium 228 \\
\hline REFCON & Reference Constant \\
\hline REPOSIT & Repository regions outside of Panel region \\
\hline RN219 & Radon 219 \\
\hline RN220 & Radon 220 \\
\hline RN222 & Radon 222 \\
\hline SALT_T1 & Shaft salt column compacted: time 0 to 10 years \\
\hline SALT_T2 & Shaft salt column compacted: time 10 to 25 years \\
\hline SALT_T3 & Shaft salt column compacted: time 25 to 50 years \\
\hline SALT_T4 & Shaft salt column compacted: time 50 to 100 years \\
\hline SALT_T5 & Shaft salt column compacted: time 100 to 200 years \\
\hline SALT_T6 & Shaft salt column compacted: time 200 to $10 \mathrm{~K}$ years \\
\hline SANTAROS & Santa Rosa Formation \\
\hline SHFT_DRZ & Disturbed Rock Around Shaft \\
\hline SM147 & Samarium 147 \\
\hline SOLAM3 & Solubility Multiplier for $\mathrm{Am}+3$ \\
\hline SOLMOD3 & Oxidation state III model \\
\hline SOLMOD4 & Oxidation state IV model \\
\hline SOLMOD5 & Oxidation state $V$ model \\
\hline SOLMOD6 & Oxidation state VI model \\
\hline SOLPU3 & Solubility Multiplier for $\mathrm{Pu}+3$ \\
\hline SOLPU4 & Solubility Multiplier for Pu+4 \\
\hline SOLTH4 & Solubility Multiplier for Th+4 \\
\hline SOLU4 & Solubility Multiplier for U+4 \\
\hline SOLU6 & Solubility Multiplier for U+6 \\
\hline SR & Strontium \\
\hline SR90 & Strontium 90 \\
\hline STEEL' & Generic steel in waste \\
\hline SULFATE & Sulfate \\
\hline S_ANH_AB & Salado anhydrite beds $A$ and $B$, intact and fractured \\
\hline S_HALITE & Salado halite, intact \\
\hline S_MB138 & Salado marker bed 138 , intact and fractured \\
\hline S_MB139 & Salado marker bed 139 , intact and fractured \\
\hline TAMARISK & Tamarisk Member \\
\hline TH & Thorium \\
\hline $\mathrm{TH}+4$ & Thorium IV \\
\hline TH227 & Thorium 227 \\
\hline TH228 & Thorium 228 \\
\hline TH229 & Thorium 229 \\
\hline TH230 & Thorium 230 \\
\hline TH231 & Thorium 231 \\
\hline TH232 & Thorium 232 \\
\hline TH234 & Thorium 234 \\
\hline TL207 & Thallium 207 \\
\hline $\mathrm{U}$ & Uranium \\
\hline $\mathrm{U}+4$ & Uranium IV \\
\hline$U+6$ & Uranium VI \\
\hline U233 & Uranium 233 \\
\hline U234 & Uranium 234 \\
\hline U235 & Uranium 235 \\
\hline
\end{tabular}




\section{Materials}

\begin{tabular}{|l|l|}
\hline \multicolumn{1}{|c|}{ Material ID } & \multicolumn{1}{c|}{ Material Description } \\
\hline U236 & Uranium 236 \\
\hline U238 & Uranium 238 \\
\hline UNNAMED & Unnamed Lower Member of Rustler Formation \\
\hline WAS_AREA & Waste emplacement area and waste \\
\hline Y90 & Yttrium 90 \\
\hline ZR90 & Zirconium 90 \\
\hline
\end{tabular}

\section{A1-5}




\section{Page intentionally blank}




\section{Appendix A2 \\ Parameter Property Dictionary}


Parameters

\begin{tabular}{|c|c|c|}
\hline Parameter ID & Parameter Description & Units \\
\hline ABERM & Area of Berm Placed Over Waste Panel & $m^{\wedge} 2$ \\
\hline ABSROUGH & Absolute roughness of material & $\mathrm{m}$ \\
\hline ACF_CH4 & Acentric Factors - $\mathrm{CH} 4$ & NONE \\
\hline ACF_CO2 & Acentric Factors - $\mathrm{CO} 2$ & NONE \\
\hline ACF_H2 & Acentric Factors $-\mathrm{H} 2$ & NONE \\
\hline ACF_H2S & Acentric Factors - H2S & NONE \\
\hline ACF_N2 & Acentric Factors - N2 & NONE \\
\hline ACF_O2 & Acentric Factors - $\mathrm{O} 2$ & NONE \\
\hline AL2 & $\log 2$ & NONE \\
\hline APORO & Waste permeability in CUTTINGS model & $m^{\wedge} 2$ \\
\hline APOROS & Culebra Advective Porosity & NONE \\
\hline AREA_CH & Area For CH Waste Disposal in CCDFGF Model & $\mathrm{m}^{\wedge} 2$ \\
\hline AREA_RH & Area for RH waste disposal in CCDFGF model & $\mathrm{m}^{\wedge} 2$ \\
\hline AREA_ZRO & Area in Waste Panels Not Used For Disposal (CCDFGF Model) & $m^{\wedge} 2$ \\
\hline ASDRUM & Surface area of corrodable metal per drum & $m^{\wedge} 2$ \\
\hline ATMPA & Conversion from std. atmosphere to $\mathrm{Pa}$ & $\mathrm{Pa}$ atm \\
\hline ATWEIGHT & Atomic Weight in $\mathrm{kg} / \mathrm{mole}$ & $\mathrm{kg} / \mathrm{mole}$ \\
\hline AVOGADRO & Avogadro's number , & $1 / \mathrm{mol}$ \\
\hline BBLG & Conversion from barrel to gallon & $g a l / b b l$ \\
\hline BKLINK & Klinkenberg B Correction Parameters for $\mathrm{H} 2$ gas & $\mathrm{Pa}$ \\
\hline CAPHUM & Maximum Concentration of Actinide with Mobile Humic Colloids & moles/liter \\
\hline CAPMIC & Maximum Concentration of Actinide on Microbe Colloids & moles/liter \\
\hline CAP_MOD & Model number, capillary pressure model & NONE \\
\hline CEMENT & Waste Cementation Strength & $\mathrm{Pa}$ \\
\hline CITOBQ & Curie to Becquerel Conversion & $\mathrm{Bq} / \mathrm{Ci}$ \\
\hline CLIMTIDX & Climate Index & NONE \\
\hline CLOSMOD & Closure Surface Model & NONE \\
\hline COLDIA & Drill collar diameter in CUTTINGS model & m \\
\hline COMPRES & Brine Compressibility & $P a \Lambda 1$ \\
\hline COMP_RCK & Bulk Compressibility & $\mathrm{Pa}^{\wedge} 1$ \\
\hline CONCINT & Actinide Concentration with Mobile Actinide Intrinsic Colloids & moles/liter \\
\hline CONCMIN & Actinide Goncentration with Mobile Mineral Fragment Colloids & moles/liter \\
\hline CORRMCO2 & Inundated corrosion rate for steel without $\mathrm{CO} 2$ present & $\mathrm{m} / \mathrm{s}$ \\
\hline CORRWCO2 & Inundated corrosion rate for steel with $\mathrm{CO} 2$ present & $\mathrm{m} / \mathrm{s}$ \\
\hline CUMPROB & Cumulative Probability & NONE \\
\hline DARM2 & Conversion from darcy to $\mathrm{m}^{\wedge} 2$ & m^2/darcy \\
\hline DAYSEC & Conversion from days to seconds & s/day \\
\hline DCELLCHW & Average density of cellulosics in $\mathrm{CH}$ waste & $\mathrm{kg} / \mathrm{m}^{\wedge} 3$ \\
\hline DCELLRHW & Average density of cellulosics in $\mathrm{RH}$ waste & $\mathrm{kg} / \mathrm{m}^{\wedge} 3$ \\
\hline DIAMMOD & Modem or current diameter & Im \\
\hline DIRNCCHW & Bulk density of iron containers, $\mathrm{CH}$ waste & $\mathrm{kg} / \mathrm{m}^{\wedge} 3$ \\
\hline DIRNCRHW & Bulk density of iron containers, $\mathrm{RH}$ waste & $\mathrm{kg} / \mathrm{m}^{\wedge} 3$ \\
\hline DIRONCHW & Average density of iron-based material in $\mathrm{CH}$ waste & $\mathrm{kg} / \mathrm{m}^{\wedge} 3$ \\
\hline DIRONRHW & Average density of iron-based material in RH waste & $\mathrm{kg} / \mathrm{m}^{\wedge} 3$ \\
\hline DISPT_L & Transverse dispersivity & m \\
\hline DISP_L & Longitudinal dispersivity & m \\
\hline DNSFLUID & Brine Density & $\mathrm{kg} / \mathrm{m}^{\wedge} 3$ \\
\hline DNSGRAIN & Material Grain Density & $\mathrm{kg} / \mathrm{m}^{\wedge} 3$ \\
\hline DOMEGA & Drill string angular velocity $(0)$ & $\mathrm{rad} / \mathrm{s}$ \\
\hline DPHIMAX & Incremental increase in porosity relative to intact conditions & NONE \\
\hline DPLASCHW & Average density of plastics in $\mathrm{CH}$ waste & $\mathrm{kg} / \mathrm{m}^{\wedge} 3$ \\
\hline DPLASRHW & Average density of plastics in $\mathrm{CH}$ waste & $\mathrm{kg} / \mathrm{m}^{\wedge} 3$ \\
\hline
\end{tabular}


Parameters

\begin{tabular}{|c|c|c|}
\hline Parameter ID & Parameter Description & Units \\
\hline DPLSCCHW & Bulk density of plastic liners, $\mathrm{CH}$ waste & $\mathrm{kg} / \mathrm{m}^{\wedge} 3$ \\
\hline DPLSCRHW & Bulk density of plastic liners, RH waste & $\mathrm{kg} / \mathrm{m}^{\wedge} 3$ \\
\hline DPOROS & Diffusive Porosity for Culebra Dolomite & NONE \\
\hline DAROOM & Number of drums, per room, in ideal packing & NONE \\
\hline DRUBBCHW & Average density of rubber in $\mathrm{CH}$ waste & $\mathrm{kg} / \mathrm{m}^{\wedge} 3$ \\
\hline DRUBBRHW & Average density of rubber in RH waste & $\mathrm{kg} / \mathrm{m}^{\wedge} 3$ \\
\hline DTORT & Diffusive Tortuosity & NONE \\
\hline EPAREL & EPA Release Limit & Ci/wuf \\
\hline ETHICK & Effective Thickness & $m$ \\
\hline EXPKLINK & Klinkenberg b correction parameters for $\mathrm{H} 2$ gas & NONE \\
\hline F3M3 & Conversion from $\mathrm{ft}^{\wedge} 3$ to $\mathrm{m}^{\wedge} 3$ & $m^{\wedge} 3 / t^{\wedge} 3$ \\
\hline FBETA & Factor beta for microbial reaction rates & NONE \\
\hline FCE & Cementation Scaling Factor & NONE \\
\hline FGE & Gravity effectiveness factor in CUTTINGS model & NONE \\
\hline FPICD & PIC multiplicative factor for human intrusion by driling & NONE \\
\hline FPICM & PIC multiplicative factor for human intrusion by mining & NONE \\
\hline FSE & Stress effectivenss factor in CUTTINGS model & NONE \\
\hline FTM & Conversion from feet to meter & $\mathrm{m} / \mathrm{ft}$ \\
\hline FTORT & Fracture Tortuosity & NONE \\
\hline FVW & Fraction of Repository Volume Occupied By Waste In CCDFGF Model & NONE \\
\hline GAS_MIN & Gas rate cut-off & mscf/day \\
\hline GRATMICH & Gas Production Rate, Microbial, Humid Conditions Relative to Inundated Rate & $\mathrm{mol} / \mathrm{kg}^{*} \mathrm{~s}$ \\
\hline GRATMICl & Gas production rate, microbial, inundated conditions - & mol/kg"s \\
\hline GRAVACC & Standard gravitational acceleration & $\mathrm{m} / \mathrm{s}^{\wedge} 2$ \\
\hline GRIDFLO & Index for Selecting a Brine Pocket & NONE \\
\hline GT13 & Conversion from gallon to in^3 & in^3/gal \\
\hline HALFLIFE & Halflife & s \\
\hline HMBLKLT & Culebra Half Matrix-Block Length & $m$ \\
\hline HREPO & Height of repository at burial time in CUTTINGS model & $m$ \\
\hline HRH & Emplaced Height of Remote Handled Waste in CCDFGF Model & $\mathrm{m}$ \\
\hline HUMCORR & Humid corrosion rate for steel & $\mathrm{m} / \mathrm{s}$ \\
\hline IFRX & Index for fracture perm. enhancement in X-direction & NONE \\
\hline IFRY & Index for fracture perm. enhancement in Y-direction & NONE \\
\hline IFRZ & Index for fracture perm. enhancement in Z-direction & NONE \\
\hline INPORO & Default value for initial repository porosity in CUTTINGS model & NONE \\
\hline INVCHD & Inventory of Contact Handled Design & $\mathrm{Ci}$ \\
\hline INVRHD & Inventory of Remote Handled Design & $\mathrm{Ci}^{\circ}$ \\
\hline INV_AR & The area of the repository in the CUTTINGS model & $m^{\wedge} 2$ \\
\hline KGAS & Ratio of specific heats for Hydrogen in CUTTINGS model & NONE \\
\hline KGLB & Conversion from $\mathrm{kg}$ to $\mathrm{lb}$ & $1 \mathrm{~b} / \mathrm{kg}$ \\
\hline KMAXLOG & Log of Maximum Permeability in Altered Anhydrite Flow Model Anhydrites & $\log \left(m^{*}=2\right)$ \\
\hline KPT & Flag for Permeability Determined Threshold & NONE \\
\hline L1 & Drill collar length in CUTTINGS model & m \\
\hline L2 & Drill pipe length when repository penetrated, CUTTINGS model & $\mathrm{m}$ \\
\hline LAMBDAD & Drilling Rate Per Unit Area & $\left(\mathrm{km}^{\wedge}-2\right) \mathrm{yr}^{\wedge-1}$ \\
\hline LBKG & Conversion from $\mathrm{lb}$ to $\mathrm{kg}$ & $\mathrm{kg} / \mathrm{hb}$ \\
\hline LOGSOLM & Log of the Radionuclide Solubility & Log(moles $/)$ \\
\hline MAXFLOW & Maximum blowout flow & s \\
\hline MDO & Molecular diffusion in pure fluid & $\mathrm{m}^{\wedge} 2 / \mathrm{s}$ \\
\hline MEA_STOR & Measured Storativity & NONE \\
\hline MINERT & Mining rate from 40 CFR 194 & $y \mathrm{y}^{\wedge-1}$ \\
\hline MINFLOW & Minimum blowout flow & 5 \\
\hline
\end{tabular}


Parameters

\begin{tabular}{|c|c|c|}
\hline Parameter ID & Parameter Description & Units \\
\hline MINP_FAC & Mining Transmissivity Multiplier & NONE \\
\hline MKD_AM & Matrix Partition Coefficient for Americium & $\mathrm{m}^{\wedge} 3 / \mathrm{kg}$ \\
\hline MKD_NP & Matrix Partition Coefficient for Neptunium & $\mathrm{m}^{\wedge} 3 / \mathrm{kg}$ \\
\hline MKD_PU & Matrix Partition Coefficient for Plutonium & $\mathrm{m}^{\wedge} 3 / \mathrm{kg}$ \\
\hline MKD_TH & Matrix Partition Coefficient for Thorium & $\mathrm{m}^{\wedge} 3 / \mathrm{kg}$ \\
\hline MKD_U & Matrix Partition Coefficient for Uranium & $\mathrm{m}^{\wedge} 3 / \mathrm{kg}$ \\
\hline MW_CH2O & Molecular Weight - $\mathrm{CH} 2 \mathrm{O}$ & $\mathrm{kg} / \mathrm{mol}$ \\
\hline MW_FE & Molecular Weight - FE & $\mathrm{kg} / \mathrm{mol}$ \\
\hline $\mathrm{MW} \mathrm{H}_{2}$ & Molecular Weight - $\mathrm{H} 2$ & $\mathrm{~kg} / \mathrm{mol}$ \\
\hline MW_H2O & Molecular Weight - $\mathrm{H} 2 \mathrm{O}$ & $\mathrm{kg} / \mathrm{mol}$ \\
\hline OMEGAA & Constants for RKS EOS & NONE \\
\hline OMEGAB & Constants for ADS EOS & NONE \\
\hline OXSTAT & Index for the Oxidation State & NONE \\
\hline PARTDIA & Waste Particle Diameter in CUTTINGS Model & $m$ \\
\hline PASCP & Conversion from $\mathrm{Pa}^{*} \mathrm{~s}$ to $\mathrm{cP}$ & cP/Pa*s \\
\hline PBRINE & Prob. that Drilling Intrusion In Excavated Area Encounteres Pressurized Brine & NONE \\
\hline PCT_A & Threshold Pressure Linear Parameter & $\mathrm{Pa}$ \\
\hline PCT_EXP & Threshold pressure exponential parameter & NONE \\
\hline PC_CH4 & Critical Pressure of $\mathrm{CH} 4$ & $\mathrm{~Pa}$ \\
\hline PC_CO2 & Critical Pressure of $\mathrm{CO} 2$ & $\mathrm{~Pa}$ \\
\hline PC_H2 & Critical Pressure of $\mathrm{H} 2$ & $\mathrm{~Pa}$ \\
\hline PC_H2S & Critical Pressure of H2S & $\mathrm{Pa}$ \\
\hline PC_MAX & Maximum allowable capillary pressure & $\mathrm{Pa}$ \\
\hline PC_N2 & Critical Pressure of N2 & $\mathrm{Pa}$ \\
\hline PC_O2 & Critical Pressure of $\mathrm{O} 2$ & $\mathrm{~Pa}$ \\
\hline PF_DELTA & Incremental pressure for full fracture development & $\mathrm{Pa}$ \\
\hline PHUMCIM & Proportionality Const.,Humic Colloids, Castile Brine, MgO controlls pH & moles/liter \\
\hline PHUMSIM & Proportionality Const. of Actinides in Salado Brine w/Humic Colloids, Inorganic & moles/liter \\
\hline $\mathrm{PI}$ & Mathematical constant: $\mathrm{PI}$ & NONE \\
\hline PIPED & Drill pipe diameter in CUTTINGS model & $m$ \\
\hline PI_DELTA & Fracture initiation pressure increment & $\mathrm{Pa}$ \\
\hline PLGPAT & index for Plugging Pattem After Drilling Intrusion & NONE \\
\hline PMLT_HI & Log triangular distribution high value for permeability & $\log \left(m^{\wedge} 2\right)$ \\
\hline PMLT_LO & Log triangular distribution low value for permeability & $\log \left(m^{\wedge} 2\right)$ \\
\hline PMLT_MD & Log triangular distribution mode for permeability & $\log \left(m^{\wedge} 2\right)$ \\
\hline PORE_DIS & Brooks-Corey pore distribution parameter & NONE \\
\hline POROSITY & Effective porosity & NONE \\
\hline PO_MIN & Minimum brine pressure for capillary model $\mathrm{KPC}=3$ & $\mathrm{~Pa}$ \\
\hline PRESSURE & Brine far-field pore pressure & $\mathrm{Pa}$ \\
\hline PRMX_LOG & Log of intrinsic permeability, $X$-direction & $\log \left(m^{\wedge} 2\right)$ \\
\hline PAMY_LOG & Log of intrinsic permeability, Y-direction & $\log \left(m^{\wedge} 2\right)$ \\
\hline PAMZ_LOG & Log of intrinsic permeability, Z-direction & $\log \left(m^{\wedge} 2\right)$ \\
\hline PROBDEG & Prob. of plastics\&rubber biodegradation in event of microbial gas generation & NONE \\
\hline PROPMIC & Moles of Actinide Mobilized on Microbe Colloids per Moles Dissolved & moles/liter \\
\hline PSIPA & Conversion from psi to pascal & $\mathrm{Pa} a^{*} \mathrm{n}^{\wedge} 2 / \mathrm{b}$ \\
\hline PSUF & Surface atmospheric pressure at elevation $1039 \mathrm{~m}$ in CUTTINS model & $\mathrm{Pa}$ \\
\hline$\overline{\text { QINIT }}$ & Initial quantity of material in waste & moles \\
\hline $\mathrm{R}$ & Gas constant $R$ & $\mathrm{~J} / \mathrm{mol}{ }^{*} \mathrm{~K}$ \\
\hline RADN_DRZ & DRZ outer radius at each shaft & $\mathrm{m} / \mathrm{m}$ \\
\hline REF_PRES & Reference pressure for porosity & $\mathrm{Pa}$ \\
\hline REF_TEMP & Reference Temperature & $\mathrm{K}$ \\
\hline RELP_MOD & Model number, relative permeability model & NONE \\
\hline
\end{tabular}


Parameters

\begin{tabular}{|c|c|c|}
\hline Parameter ID & $\begin{array}{ll}\text { Parameter Description } \\
\end{array}$ & Units \\
\hline RE_CAST & External drainage radius for the Castile formation & $\mathrm{m}$ \\
\hline RGAS & Gas Constant for Hydrogen & $\mathrm{N}$ *m/kg/degK \\
\hline RHOS & Waste Particle Density in CUTTINGS_S Model & $\mathrm{kg} / \mathrm{m}^{\wedge} 3$ \\
\hline RHW_AR & The total area of the remote-handled waste in the CUTTINGS model & $m^{\wedge} 2$ \\
\hline ROOM & Equivalent radius of one room in CUTTINGS model & $\mathrm{m}$ \\
\hline ROUGHP & Friction factor for very rough pipe in CUTTINGS model & NONE \\
\hline RPANEL & Equivalent radius of one panel in CUTTINGS model & m \\
\hline RSH_AIR & Air-supply shaft radius $(3.09 \mathrm{~m})$ & $\mathrm{m}$ \\
\hline RSH_EXH & Air-exhaust shaft radius $(2.3 \mathrm{~m})$ & $\mathrm{m}$ \\
\hline RSH_SAL & Salt-handling shaft radius $(1.8 \mathrm{~m})$ & $\mathrm{m}$ \\
\hline RSH_WAS & Waste-handling shaft radius $(3.5 \mathrm{~m})$ & $\mathrm{m}$ \\
\hline ATK & Conversion from Rankine to $\mathrm{K}$ & Krankine \\
\hline SAL_USAT & Average saturation, unsaturated zones & NONE \\
\hline SAT_IBAN & Initial Brine Saturation & NONE \\
\hline SAT_RBRN & Residual Brine Saturation & NONE \\
\hline SAT_RGAS & Residual Gas Saturation & NONE \\
\hline SAT_WICK & Index for computing wicking & NONE \\
\hline SECYR & Seconds to years Conversion & $\mathrm{yr} / \mathrm{s}$ \\
\hline SKIN_RES & Skin Resistance & NONE \\
\hline SOLCIM & Solubility Mult. in Castile Brine, Inorganic Chem Controlled by $\mathrm{Mg}(\mathrm{OH}) 2-\mathrm{MgCO} 3$ & moles/liter \\
\hline SOLSIM & Solubility Mult. in Salado Brine, Inorganic Chem Controlled by $\mathrm{Mg}(\mathrm{OH}) 2-\mathrm{MgCO} 3$ & moles/liter \\
\hline STOIFX & Stoichiometric factor $-\mathrm{X}$ & NONE \\
\hline SUFTEN & Surface tension of brine in CUTTINGS model & $\mathrm{N} / \mathrm{m}$ \\
\hline TA & Time Active Institutional Controls at WIPP Site Are Effective & yr \\
\hline TAUFAIL & Effective shear strength for erosion (rfail) & $\mathrm{Pa}$ \\
\hline TC_CH4 & Critical temperature: Methane (CH4) & $K$ \\
\hline TC_CO2 & Critical temperature: Carbon Dioxide (CO2) & K \\
\hline TC_H2 & Critical temperature: Hydrogen $(\mathrm{H} 2)$ & $\mathrm{K}$ \\
\hline TC_H2S & Critical temperature: Hydrogen Sulfide (H2S) & $\mathrm{K}$ \\
\hline TC_N2 & Critical temperature: Nitrogen (N2) & $\mathrm{K}$ \\
\hline TC_O2 & Critical temperature: Oxygen (O2) & $K$ \\
\hline THCK_CAS & Thickness of the Castile formation & $\mathrm{m}$ \\
\hline THICK & Thickness of feature or layer & $\mathrm{m}$ \\
\hline TPICD & Time over which passive institutional controls reduce rate of drilling & yr \\
\hline TPICM & Time over which passive institutional controls reduce rate of mining & yr \\
\hline TRANSIDX & Index for selecting realizations of the Transmissivity Field & NONE \\
\hline TAEPO & Temperature of repository in CUTTINGS model & $\mathrm{K}^{\circ-}$ \\
\hline VISC & Hydrogen Viscosity in CUTTINGS Model & $\mathrm{Pa} a^{*}$ \\
\hline VISCO & Viscosity & Pa*s \\
\hline VOLCHW & BIR total volume of $\mathrm{CH}$ waste & $m^{\wedge} 3$ \\
\hline VOLRHW & BIR total volume of $\mathrm{RH}$ waste & $m^{\wedge} 3$ \\
\hline VOLUME & Total Reservoir Volume & $m^{\wedge} 3$ \\
\hline VOLWP & Uncompacted Volume of Waste Panels in CCDFGF Model & $m^{\wedge} 3$ \\
\hline VPANLEX & Excavated volume of one panel & $m^{\wedge} 3$ \\
\hline VAEPOS & Excavated storage volume of repository & $m^{\wedge} 3$ \\
\hline VROOM & Volume of one room in repository & $m^{\wedge} 3$ \\
\hline WTF & Mass fraction of salt in brine & NONE \\
\hline WUF & Unit of Waste & Curies \\
\hline YLDSTRSS & Yield Stress Point & $\mathrm{Pa}$ \\
\hline YRSEC & Conversion from mean solar or tropical year to seconds & s/yr \\
\hline ZCINK & Zero Celcius in Kelvin & $K$ \\
\hline
\end{tabular}


Page intentionally blank 
Appendix A3

\section{Parameter Tables}


Tables Keyed to Material Region Areas of Grid

Table

Title

1 Borehole, Blowout, Drillmud

1A Borehole Concrete Plug

1B Borehole Open

1C Borehole Silty Sand

1D Borehole Creep

2 Shaft DRZ

3 Earthen Fill

4 Rustler Compacted Clay

5 Asphalt

6 Shaft Concrete

7 Shaft Crushed Salt (time periods 1 - 6)

8 Upper Shaft Clay (time periods $1-5$ )

9 Lower Shaft Clay (time periods 1 - 4)

10 Bottom Clay Component

11 Concrete Monolith

12 Santa Rosa Formation

13 Dewey Lake Red Beds

14 Forty Niner Member

15 Magenta Dolomite

16 Tamarisk Member

17 Culebra Dolomite

18 Unnamed Lower Member

19A Salado Halite

19B Salado Brine

$20 \quad$ Marker Bed 138

21 Anhydrite Beds A\&B

22 Disturbed Rock Zone

23A Waste Panel

23B Waste Chemistry

24A Rest of Repository

24B Predisposal Cavities

25 Panel Closure

26 Operations Region

27 Experimental Area

$28 \quad$ Marker Bed 139

29 Castile Formation

30A Castile Reservoir

30B Castile Brine

31 Global

32 Reference Constants 
1. Borehole, Blowout, Drillmud

\begin{tabular}{|c|c|c|c|c|c|c|c|c|c|c|c|c|c|}
\hline ID & orial & ter & Units & Distribution & Maan & ledian & Deviation & Low & High & Entry Date & Impt & WPO & Codes \\
\hline 3251 & BLOWOUT & PSUF & $\mathrm{Pa}$ & CONSTANT & $.95 E+04$ & $8.95 \mathrm{E}+04$ & $0.00 E+\infty 0$ & $8.95 \mathrm{E}+04$ & $.95 E+04$ & $4 / 25 / 96$ & $4 A$ & 37093 & CS \\
\hline 3252 & .OWOUT & VISC & $\mathrm{Pa}^{*} \mathrm{~s}$ & CONSTANT & $9.20 E-06$ & 9.20E-06 & $0.00 E+\infty$ & $9.20 E-06$ & 9.20E-06 & 4/25/96 & 3 & 37094 & CS \\
\hline 3258 & LOWOUT & TAEPO & $\mathrm{K}$ & CONSTANT & $3.00 E+02$ & $3.00 E+02$ & $0.00 E+\infty$ & $3.00 \mathrm{E}+02$ & $3.00 E+02$ & $4 / 25 / 96$ & $4 A$ & 37101 & cs \\
\hline 3473 & LOWOUT & THCK_CAS & $m$ & CONSTANT & 1.23E+01 & $1.23 E+01$ & $0.00 E+00$ & 1.23E+01 & 1.23E+01 & $6 / 6 / 96$ & 4B & 38213 & DBR \\
\hline 3257 & LOWOUT & SUFTEN & $\mathrm{N} / \mathrm{m}$ & & $8.00 \mathrm{E}-02$ & $8.00 \mathrm{E}-02$ & $0.00 E+00$ & $8.00 \mathrm{E}-02$ & 8.00E-02 & $4 / 25 / 96$ & 3 & 37100 & CS \\
\hline 249 & OWOUT & RPANEL & $m$ & CONSTANT & $6.09 E+01$ & $.09 E+01$ & $0.00 E+00$ & $6.09 E+01$ & $.09 E+01$ & $4 / 25 / 96$ & 4B & 37091 & CS \\
\hline 3248 & OWOUT & ROOM & $m$ & CONSTANT & $1.71 E+01$ & $1.71 E+01$ & $0.00 E+00$ & 1.71E+01 & $1.71 E+01$ & $4 / 25 / 96$ & $4 \mathrm{~B}$ & 37090 & CS \\
\hline 3247 & BLOWOUT & RHOS & $\mathrm{kg} / \mathrm{mm} 3$ & CONSTANT & $2.65 E+03$ & $2.65 \mathrm{E}+03$ & $0.00 E+\infty 0$ & $2.65 E+03$ & $2.65 E+03$ & $4 / 25 / 96$ & 4B & 37089 & CS \\
\hline 3259 & & APORO & $m^{\prime} 2$ & & & $1.70 E-13$ & $0.00 E+00$ & $1.70 E-13$ & $1.70 E-13$ & $25 / 96$ & 1 & 37102 & CS \\
\hline 3456 & BLOWOUT & RE_CAST & $\mathbf{m}$ & CONSTANT & $1.14 E+02$ & $1.14 E+02$ & $0.00 E+00$ & $1.14 E+02$ & $1.14 E+02$ & $6 / 6 / 96$ & 4B & 38208 & DBR \\
\hline 3246 & BLOWOUT & PARTDIA & $m$ & LOGUNIFORM & 2.35E-02 & $2.80 E-03$ & 4.24E-02 & 4.00E-05 & $2.00 E-01$ & $5 / 3 / 96$ & 1 & 37088 & CS \\
\hline 3472 & BLOWOUT & MINFLOW & $\mathbf{s}$ & CONSTANT & $2.59 E+05$ & $2.59 E+05$ & $0.00 \Sigma+\infty$ & 2.59E+05 & $2.59 E+05$ & $6 / 6 / 96$ & 4B & 39211 & DBR \\
\hline 3471 & BLOWOUT & MAXFLC & s & CONSTANT & $9.50 E+05$ & $9.50 E+05$ & $0.00 E+\infty$ & $9.50 E+0.5$ & $9.50 E+05$ & $6 / 6 / 96$ & $4 B$ & 38210 & $\mathrm{DBR}$ \\
\hline 3254 & BLOWOUT & KGAS & NONE & & $1.41 E+\infty$ & $1.41 E+\infty$ & $0.00 E+00$ & $1.41 E+00$ & $1.41 E+\infty$ & $4 / 25 / 96$ & 3 & 37096 & cs \\
\hline 3260 & BLOWOUT & INPORO & NONE & CONSTANT & 8.49E-01 & $8.49 E-01$ & $0.00 E+00$ & $8.49 E-01$ & 8.49E-01 & $4 / 25 / 96$ & $4 B$ & 37103 & cs \\
\hline 3250 & BLOWOUT & HREPO & $m$ & CONSTANT & $3.96 E+\infty 0$ & $3.96 E+00$ & $0.00 E+00$ & $3.96 E+00$ & $3.96 E+00$ & 4/25/96 & 4B & 37092 & CS \\
\hline 3470 & BLOWOUT & GAS_MIN & mscf/day & CONSTANT & $1.00 E+02$ & $1.00 E+02$ & $0.00 E+\infty$ & $1.00 E+02$ & $1.00 E+02$ & $6 / 6 / 96$ & $4 B$ & 38209 & DBR \\
\hline 3255 & BLOWOUT & FSE & NONE & CONSTANT & $0.00 E+\infty$ & $0.00 E+00$ & $0.00 E+00$ & $0.00 E+00$ & $0.00 E+\infty$ & $4 / 25 / 96$ & 4B & 37097 & CS \\
\hline 3256 & BLOWOUT & FGE & NONE & CONSTANT & $1.81 E+01$ & $1.81 E+01$ & $0.00 E+\infty$ & 1.81E+01 & $1.81 E+01$ & $4 / 25 / 96$ & $4 B$ & 37098 & cs \\
\hline 3420 & BLOWOUT & FCE & NONE & & $1.00 E+\infty$ & $1.00 E+\infty 0$ & $0.00 E+00$ & $1.00 E+00$ & $1.00 E+\infty$ & $12 / 96$ & $4 B$ & 37668 & CS \\
\hline 3245 & BLOWOUT & CEMENT & $\mathrm{Pa}$ & CONSTANT & $6.90 \mathrm{E}+03$ & $6.90 E+03$ & $0.00 E+00$ & $6.90 E+03$ & $6.90 E+03$ & $4 / 25 / 96$ & 4B & 37087 & CS \\
\hline 3253 & BLOWOUT & RGAS & 'm/kg/ degk & CONSTANT & $4.12 E+03$ & 4.12E+03 & $0.00 E+\infty 0$ & 4.12E $\div 03$ & $4.12 E+03$ & $4 / 25 / 96$ & 3 & 37095 & cs \\
\hline 3239 & JREHOLE & INV_AR & $m^{\wedge} 2$ & CONSTANT & 1.12E+05 & 1.12E+05 & $0.00 \mathrm{E}+00$ & 1.12E+05 & $1.12 E+05$ & $4 / 25 / 96$ & $4 B$ & 37081 & $\mathrm{CS}$ \\
\hline 23 & TOLE & CAP_A & NONE & CONST & $2.00 E+\infty$ & $2.00 E+\infty$ & $0.00 E+00$ & $2.00 E+\infty 0$ & $2.00 E+\infty 0$ & $2 / 20 / 96$ & 4B & $31486 A$ & $B F$ \\
\hline 3242 & OOLE & COLDIA & $m$ & CONS & 2.03E-01 & 2.03E-01 & $0.00 E+\infty$ & 2.03E-01 & 2.03E-01 & 4/25/96 & $4 B$ & 37084 & CS \\
\hline 25 & & COMP_B & & & & & & 2.64E-09 & & $2 / 20 / 96$ & $4 A$ & 31510 & $B F$ \\
\hline 33 & & PRESSURE & $\mathrm{Pa}$ & & $0.00 E+\infty$ & & $0.00 E+00$ & $0.00 E+\infty 0$ & $0.00 E+\infty$ & $11 / 5 / 96$ & 5 & $31524^{\circ}$ & $B F$ \\
\hline 3414 & & & uries & CONSTA & $3.44 E+\infty$ & $3.44 E+\infty 0$ & $0.00 E+00$ & $3.44 \mathrm{E}+\infty$ & $3.44 \mathrm{E}+\infty$ & $12 / 4 / 96$ & 2 & $37137 \mathrm{~A}$ & CS \\
\hline 2254 & OREHOLE & TAUFAIL & & & & & & & & $4 / 25 / 96$ & 1 & 31536 & CS \\
\hline 42 & AREHOLE & SAT_RGAS & ONE & TANT & $E-01$ & $=-01$ & $0.00 E+\infty 0$ & $E-01$ & $E-01$ & $0 / 96$ & $4 A$ & 31535 & BF \\
\hline 41 & OREHOLE & SAT_RBRN & NONE & & 2.00E-01 & 2.00E-01 & $0.00 E+00$ & $2.00 E-01$ & & $10 / 31 / 95$ & $4 A$ & 31533 & $B F$ \\
\hline 3240 & OOLE & ROUGHP & ONE & CONS & $8.00 \mathrm{E}-02$ & $8.00 \mathrm{E}-02$ & $0 E+\infty$ & $E-02$ & & $5 / 96$ & 3 & 37082 & CS \\
\hline 3261 & OREHOLE & RHW_AR & $r 2$ & CONSTANT & 1.58E+04 & 1.58E+04 & $0.00 E+00$ & $1.58 E+04$ & $1.58 E+04$ & $4 / 25 / 96$ & 4B & 37104 & CS \\
\hline 40 & OREHOLE & AELP_MOD & ONE & CONSTANT & $4.00 E+\infty$ & $4.00 E+\infty$ & $0.00 E+\infty$ & $4.00 E+\infty$ & $4.00 E+00$ & $10 / 31 / 95$ & 4B & 31532 & $\mathrm{BF}$ \\
\hline 38 & OREHOLE & PTINDEX & ONE & UNIFORM & $5.00 E-01$ & $5.00 E-0 r$ & $2.89 E-01$ & $0.00 E+\infty$ & $1.00 E+\infty$ & $15 / 96$ & 5 & $31530^{\circ}$ & $B F$ \\
\hline 36 & DREHOLE & PAMZ_LOG & $g\left(m^{2} 2\right)$ & NURIMAL. & $-1.22 E+01$ & $-1.25 E+01$ & $-5.67 E+\infty$ & $-1.40 E+01$ & $-1.10 E+01$ & $10 / 31 / 95$ & 48 & 31528 & $B F$ \\
\hline 26 & 1OLE & DIAMMOD & $n$ & CONS & $=01$ & $=-01$ & $0.00 E+\infty 0$ & 3.11E-01 & E-01 & $2 / 96$ & $4 B$ & 31511 & $B F$ \\
\hline 34 & HOLE & RMX_LOG & $\left(m^{\wedge} 2\right)$ & NORMAL & $-1.22 E+01$ & $-1.25 E+01$ & $-5.67 E+00$ & $-1.40 E+01$ & $-1.10 E+01$ & $10 / 31 / 95$ & $A B$ & 31525 & $B F$ \\
\hline 27 & JREHOLE & DOMEGA & $d / s$ & CUMULATIVE & $8.63 E+00$ & $7.80 E+\infty 0$ & $3.16 E+00$ & $4.20 \varepsilon+\infty$ & $2.30 E+01$ & 9/95 & $4 B$ & 31512 & cs \\
\hline 31 & OREHOLE & POROSITY & NONE & CONSTANT & $5.00 E-02$ & $5.00 \mathrm{E}-02$ & $0.00 E+\infty$ & $5.00 E-02$ & 5.00E-02 & $2 / 21 / 96$ & $4 A$ & 31522 & $B F$ \\
\hline 30 & OREHOLE & PORE_DIS & MUINE & CONSTANT & $9.40 E-01$ & $9.40 E-01$ & $0.00 E+\infty$ & $9.40 \mathrm{E}-01$ & $9.40 \mathrm{E}-01$ & $2 / 20 / 96$ & $4 A$ & 31521 & $B F$ \\
\hline 32 & DREHOLE & PO_MIN & & Lemsola & $1.01 E+05$ & $1.01 E+05$ & $0.00 E+\infty$ & $1.01 E+05$ & $1.01 E+05$ & $2 / 21 / 96$ & $4 B$ & 31523 & $B F$ \\
\hline 3241 & OREHOLE & PIPED & & CONSTANT & $1.14 E-01$ & 1.14E-01 & $0.00 E+\infty$ & 1.14E-01 & $1.14 E-01$ & $4 / 25 / 96$ & 4B & 37083 & cs \\
\hline 3121 & BOREHOLE & PCT_EXP & NONE & CONSTANT & $-3.46 E-01$ & $-3.46 E-01$ & $0.00 E+\infty$ & $-3.46 E-01$ & -3.46E-01 & $2 / 21 / 96$ & $4 B$ & 36363 & $\mathrm{BF}$ \\
\hline 3120 & BOREHOLE & PCT_A & & CONSTANT & $5.60 E-01$ & 5.60E-01 & $0.00 E+\infty$ & $5.60 E-01$ & $5.60 \mathrm{E}-01$ & 2/21/96 & $4 B$ & $36362 A$ & $B F$ \\
\hline 29 & OREHOLE & PC_MAX & & CONSTANT & $1.00 E+08$ & $1.00 E+08$ & $0.00 E+\infty$ & $1.00 E+08$ & $1.00 E+08$ & $2 / 20 / 96$ & 4B & 31514 & $\mathrm{BF}$ \\
\hline 3243 & OREHOLE & L2 & $m$ & CONSTANT & $4.72 E+02$ & $4.72 E+02$ & $0.00 E+\infty 0$ & $4.72 E+02$ & $4.72 E+02$ & $4 / 25 / 96$ & $4 B$ & 37085 & CS \\
\hline 3244 & OREHOLE & L1 & & CONSTANT & $1.83 E+02$ & $1.83 E+02$ & $0.00 E+00$ & $1.83 E+02$ & $1.83 E+02$ & $4 / 25 / 96$ & $4 B$ & 37086 & CS \\
\hline 3122 & BOREHOLE & KPT & NONE & CONSTANT & $0.00 E+00$ & $0.00 E+\infty$ & $0.00 E+\infty$ & $0.00 E+\infty$ & $0.00 E+\infty 0$ & $21 / 96$ & 48 & 36361 & $\mathrm{BF}$ \\
\hline 35 & BOREHOLE & PRMY_LOG & $\log \left(m^{\wedge} 2\right)$ & NORMAL & $-1.22 E+01$ & $-1.25 E+01$ & $-5.67 E+\infty 0$ & $-1.40 E+01$ & $-1.10 E+01$ & $10 / 31 / 95$ & $4 B$ & 31527 & $\mathrm{BF}$ \\
\hline 173 & DRILLMUD & YLDSTRSS & & CUMULATIVE & $5.98 E+00$ & $4.40 E+\infty$ & $3.70 E+\infty 0$ & $2.40 E+\infty$ & $1.92 E+01$ & $1 / 9 / 97$ & $4 B$ & 32751 & CS \\
\hline
\end{tabular}


1. Borehole, Blowout, Drillmud

\begin{tabular}{|c|c|c|c|c|c|c|c|c|c|c|c|c|c|}
\hline ID & Material & Parameter & Units & Distribution & Mean & Median & Deviation & Low & High & Entry Dato & Impt & WPO & Codes \\
\hline 171 & DRILLMUD & DNSFLUID & $\mathrm{kg} / \mathrm{m} / 3$ & CUMULATIVE & $1.21 E+03$ & $1.21 E+03$ & $5.00 E+01$ & $1.14 E+03$ & $1.38 E+03$ & $1 / 9 / 97$ & 4B & 32748 & CS \\
\hline 172 & RILLMUD & VISco & Pa's & CUMULATTVE & 1.10E-02 & 9.17E-03 & 5.32E-03 & $5.00 E-03$ & $3.00 E-02$ & 1/9/97 & $4 B$ & 32750 & CS \\
\hline
\end{tabular}

\section{A. Borehole Concrete Plug}

\begin{tabular}{|c|c|c|c|c|c|c|c|c|c|c|c|c|c|}
\hline ID & Material & Paramater & Units & Distribution & Mean & Modian & Deviation & Low & High & Entry Date & Impt & WPO & Codes \\
\hline 3153 & CONC_PLG & SAT_RGAS & NONE & CONSTANT & $.00 E+\infty$ & $0.00 E+\infty$ & $0.00 E+\infty 0$ & $0.00 E+\infty 0$ & $0.00 E+00$ & $4 / 96$ & 4B & 36597 & BF \\
\hline 3152 & CONC_PLG & SAT_RBRN & ONE & CONSTANT & $00 \mathrm{E}+\infty$ & $0.00 E+\infty$ & $0.00 E+00$ & $0.00 E+\infty$ & $0.00 E+00$ & $3 / 14 / 96$ & 48 & 36596 & BF \\
\hline 3149 & PLG & RELP. & NONE & CONSTANT & $4.00 E+\infty$ & $4.00 E+\infty 0$ & $0.00 \mathrm{E}+00$ & $4.00 \mathrm{E}+\infty$ & $4.00 E+\infty 0$ & $3 / 14 / 96$ & $4 B$ & 36592 & BF \\
\hline 3193 & CONC_PLG & PRMZ_LOG & $\log \left(m^{\wedge} 2\right)$ & CONSTA & $-1.63 E+01$ & $-1.63 E+01$ & $0.00 E+00$ & $-1.63 E+01$ & $-1.63 E+01$ & $3 / 14 / 96$ & 1 & 36657 & $B F$ \\
\hline 3192 & ONC_PLG & PRMY_LOG & $\log \left(m^{\wedge} 2\right)$ & CONSTANT & $-1.63 E+01$ & $-1.63 E+01$ & $0.00 E+00$ & $-1.63 E+01$ & $-1.63 E+01$ & $3 / 14 / 96$ & 1 & 36656 & $B F$ \\
\hline 3185 & ONC_PLG & PRMX_LOG & $\log \left(m^{\wedge} 2\right)$ & CONSTANT & $-1.63 E+01$ & $-1.63 E+01$ & $0.00 \mathrm{E}+00$ & $-1.63 E+01$ & $-1.63 E+01$ & $3 / 14 / 96$ & 1 & 36642 & BF \\
\hline 3147 & CONC_PLG & POROSITY & NONE & CONSTANT & 3.20E-01 & $3.20 E-01$ & $0.00 \mathrm{E}+00$ & 3.20E-01 & $3.20 \mathrm{E}-01$ & $3 / 14 / 96$ & $4 B$ & 36589 & $\mathrm{BF}$ \\
\hline 3154 & CONC_PLG & PORE_DIS & NONE & CONSTANT & $9.40 \mathrm{E}-01$ & 9.40E-01 & $0.00 E+00$ & $9.40 \mathrm{E}-01$ & 9.40E-01 & $3 / 14 / 96$ & $4 B$ & 36598 & $\mathrm{BF}$ \\
\hline 3155 & CONC_PLG & PO_MIN & $\mathrm{Pa}$ & CONSTANT & $1.01 E+05$ & $1.01 E+05$ & $0.00 E+00$ & $1.01 E+05$ & $1.01 E+05$ & $3 / 14 / 96$ & $4 B$ & 36599 & $B F$ \\
\hline 3158 & CONC_PLG & PCT_EXP & NONE & CONSTANT & $0.00 E+00$ & $0.00 E+00$ & $0.00 E+00$ & $0.00 E+\infty 0$ & $0.00 E+00$ & $3 / 14 / 96$ & $4 B$ & 36603 & BF \\
\hline 3157 & CONC_PLG & PCT_A & $\mathrm{Pa}$ & CONSTANT & $0.00 E+00$ & $0.00 E+\infty 0$ & $0.00 \mathrm{E}+00$ & $0.00 E+00$ & $0.00 E+00$ & $3 / 14 / 95$ & $4 B$ & 36601 & $B F$ \\
\hline 3151 & CONC_PLG & PC_MAX & $\mathrm{Pa}$ & CONSTANT & $1.00 \mathrm{E}+08$ & 1.00E+08 & $0.00 E+00$ & $1.00 E+08$ & $1.00 E+08$ & $3 / 14 / 96$ & $4 B$ & 36595 & $\mathrm{BF}$ \\
\hline 3156 & CONC_PLG & KPT & NONE & CONSTANT & $0.00 E+00$ & $0.00 E+\infty 0$ & $0.00 \mathrm{E}+\infty$ & $0.00 E+00$ & $0.00 \mathrm{E}+00$ & $3 / 14 / 96$ & $4 B$ & 36600 & $\mathrm{BF}$ \\
\hline 3148 & CONC_PLG & COMP_RCK & $P a^{n-1}$ & CONSTANT & 1.20E-09 & 1.20E-09 & $0.00 E+\infty 0$ & 1.20E-09 & 1.20E-09 & $5 / 2 / 96$ & 4B & 36591 & $\mathrm{BF}$ \\
\hline 3150 & CONC_PLG & CAP_MOD & NONE & CONSTANT & $1.00 E+\infty$ & $1.00 E+00$ & $0.00 E+\infty$ & $1.00 E+00$ & 1.00E+00 & $3 / 14 / 95$ & $4 B$ & 36593 & BF \\
\hline
\end{tabular}

\section{B. Borehole Open}

\begin{tabular}{|c|c|c|c|c|c|c|c|c|c|c|c|c|c|}
\hline 10 & & & & & & & & ow & ligh & & Impt & VPO & Codes \\
\hline 141 & I_OPEN & AS & DNE & VT & $30 E+\infty$ & $\infty E E+\infty$ & $0.00 E+\infty$ & $0.00 E+00$ & $.00 E+\infty$ & & $4 B$ & 36571 & BF \\
\hline 140 & H_OPEN & BRN & NONE & vi & & $.00 E+\infty$ & $0.00 E+00$ & $0.00 E+\infty 0$ & $0.00 E+00$ & $3 / 14 / 50$ & $4 B$ & 36570 & $B F$ \\
\hline 137 & H_OPEN & ELP_MOD & & ST & $5.00 \mathrm{E}+00$ & $5.00 E+\infty$ & $0.00 E+\infty$ & $5.00 E+\infty 0$ & $5.00 E+\infty 0$ & $4 / 96$ & $4 \mathrm{~B}$ & 36567 & $\mathrm{BF}$ \\
\hline 187 & PEN & PRMZ & & $T$ & $9.00 E+00$ & $-9.00 E+00$ & $0.00 E+00$ & $-9.00 E+\infty 0$ & $-9.00 E+00$ & $4 / 96$ & $4 B$ & 36650 & $B F$ \\
\hline 186 & EN & $\overline{O G}$ & & CON & $-9.00 E+\infty 0$ & $-9.00 E+\infty 0$ & $0.00 E+00$ & $-9.00 E+00$ & $-9.00 E+00$ & 4/96 & $4 B$ & 36649 & $B F$ \\
\hline 3134 & H_OPEN & PRMX_LOG & $g\left(m^{\prime} 2\right)$ & $\mathrm{CON}$ & $-9.00 E+00$ & $-9.00 E+\infty$ & $0.00 E+\infty$ & $-9.00 E+\infty 0$ & $-9.00 E+00$ & $3 / 14 / 96$ & $4 B$ & 36564 & $B F$ \\
\hline 3135 & H_OPEN & POROS & NONE & CONSTA & 3.20E-01 & 3.20E-01 & $0.00 E+\infty$ & 3.20E-01 & 3.20E-01 & $3 / 14 / 96$ & $A B$ & 36565 & $\mathrm{BF}$ \\
\hline 3142 & H_OPEN & PORE_DIS & NONE & CONSTANT & $7.00 E-01$ & 7.00E-01 & $0.00 E+\infty$ & 7.00E-01 & 7.00E-01 & $3 / 14 / 96$ & $4 B$ & 36572 & $B F$ \\
\hline 3143 & H_OPEN & & & CONSTANT & $1.01 E+05$ & $1.01 E+05$ & $0.00 E+00$ & $1.01 E+05$ & $1.01 E+05$ & $14 / 96$ & $4 B$ & 36584 & BF \\
\hline 3146 & & PCT_EXP & & CONSTANT & & $0.00 E+00$ & $0.00 E+\infty$ & $0.00 E+00$ & $0.00 E+00$ & & $4 B$ & 36588 & $B F$ \\
\hline 3145 & BH_OPEN & & & CONSTANT & $0.00 E+00$ & $0.00 E+\infty$ & $0.00 E+00$ & $0.00 E+00$ & $0.00 E+00$ & & $4 B$ & 36586 & $B F$ \\
\hline 3139 & BH_OPEN & PC_M & & CONSTANT & $1.00 E+08$ & $1.00 E+08$ & $0.00 E+\infty$ & $1.00 E+08$ & $1.00 E+08$ & $3 / 14 / 96$ & 48 & 36569 & $\mathrm{BF}$ \\
\hline 3144 & BH_OPEN & KPT & VUINE & CONSTANT & $0.00 E+\infty$ & $0.00 E+00$ & $0.00 E+\infty 0$ & $0.00 E+\infty 0$ & $0.00 E+00$ & $3 / 14 / 96$ & $4 B$ & 36585 & $B F$ \\
\hline 3136 & BH_OPEN & COMP_RCK & Par-1 & CONSTANT & $0.00 E+00$ & $0.00 E+00$ & $0.00 E+\infty 0$ & $0.00 E+\infty 0$ & $0.00 E+\infty 0$ & $3 / 14 / 96$ & $4 B$ & 36566 & $\mathrm{BF}$ \\
\hline 138 & H_OPEN & CAP_MOD & NONE & CONSTANT & $1.00 E+\infty$ & $1.00 E+\infty$ & $0.00 E+00$ & $1.00 E+\infty 0$ & $1.00 E+00$ & $3 / 14 / 96$ & $4 \mathrm{~B}$ & 36568 & $B F$ \\
\hline
\end{tabular}


1C. Borehole Silty Sand

\begin{tabular}{|c|c|c|c|c|c|c|c|c|c|c|c|c|c|}
\hline ID & Material & Parameter & Units & Distribution & Mean & Median & Deviation & Low & High & Entry Dato & Impt | & WPO & Codes \\
\hline 3165 & BH_SAND & SAT_RGAS & NONE & CONSTANT & $0.00 E+00$ & $0.00 E+\infty 0$ & $0.00 E+\infty 0$ & $0.00 E+\infty 0$ & $0.00 E+\infty$ & $3 / 14 / 96$ & $4 B$ & 36615 & $B F$ \\
\hline 3164 & BH_SAND & SAT_RBRN & NONE & CONSTANT & $0.00 \mathrm{E}+00$ & $0.00 \mathrm{E}+00$ & $0.00 E+\infty 0$ & $0.00 E+\infty 0$ & $0.00 E+\infty$ & $3 / 14 / 96$ & 4B & 36614 & BF \\
\hline 3161 & BH_SAND & RELP_MOD & NONE & CONSTANT & $4.00 \mathrm{E}+00$ & $4.00 \mathrm{E}+00$ & $0.00 E+00$ & $4.00 E+\infty 0$ & $4.00 E+\infty$ & $3 / 14 / 96$ & $4 \mathrm{~B}$ & 36611 & BF \\
\hline 3191 & BH_SAND & PRMZ_LOG & $\log (m \wedge 2)$ & UNIFORM & $-1.25 E+01$ & $-1.25 E+01$ & 8.66E-01 & $-1.40 E+01$ & $-1.10 E+01$ & $3 / 14 / 96$ & 1 & 36655 & $B F$ \\
\hline 3190 & BH_SAND & PRMY_LOG & $\log \left(m^{\wedge} 2\right)$ & UNIFORM & $-1.25 E+01$ & $-1.25 E+01$ & 8.66E-01 & $-1.40 \mathrm{E}+01$ & $-1.10 E+01$ & $3 / 14 / 96$ & 1 & 36654 & $\mathrm{BF}$ \\
\hline 3184 & BH_SAND & PRMX_LOG & $\log \left(m^{1} 2\right)$ & UNIFORM & $-1.25 E+01$ & $-1.25 E+01$ & $8.66 \mathrm{E}-01$ & $-1.40 E+01$ & $-1.10 E+01$ & $3 / 14 / 96$ & 1 & 36641 & $B F$ \\
\hline 3159 & BH_SAND & POROSITY & NONE & CONSTANT & 3.20E-01 & 3.20E-01 & $0.00 E+\infty 0$ & 3.20E-01 & 3.20E-01 & $3 / 14 / 96$ & $4 B$ & 36605 & $\mathrm{BF}$ \\
\hline 3166 & BH_SAND & PORE_DIS & NONE & CONSTANT & $9.40 E-01$ & $9.40 \mathrm{E}-01$ & $0.00 E+00$ & 9.40E-01 & $9.40 E-0 t$ & $3 / 14 / 96$ & $4 B$ & 36616 & $\mathrm{BF}$ \\
\hline 3167 & BH_SAND & PO_MIN & $\mathrm{Pa}$ & CONSTANT & $1.01 E+05$ & $1.01 E+05$ & $0.00 E+00$ & $1.01 E+05$ & $1.01 E+05$ & $3 / 14 / 96$ & $4 B$ & 36617 & $B F$ \\
\hline 3170 & BH_SAND & PCT_EXP & NONE & CONSTANT & $0.00 \mathrm{E}+00$ & $0.00 E+\infty 0$ & $0.00 E+\infty$ & $0.00 E+00$ & $0.00 E+00$ & $3 / 14 / 96$ & $4 B$ & 36621 & $\mathrm{BF}$ \\
\hline 3169 & BH_SAND & PCT_A & $\mathrm{Pa}$ & CONSTANT & $0.00 E+\infty$ & $0.00 E+\infty$ & $0.00 \mathrm{E}+\infty$ & $0.00 E+00$ & $0.00 E+\infty 0$ & $3 / 14 / 96$ & $4 \mathrm{~B}$ & 36620 & $B F$ \\
\hline 3163 & BH_SAND & PC_MAX & $\mathrm{Pa}$ & CONSTANT & $1.00 E+08$ & $1.00 E+08$ & $0.00 E+\infty 0$ & $1.00 E+08$ & $1.00 E+08$ & $3 / 14 / 96$ & $4 B$ & 36613 & BF \\
\hline 3168 & BH_SAND & KPT & NONE & CONSTANT & $0.00 E+\infty$ & $0.00 E+00$ & $0.00 E+\infty 0$ & $0.00 E+00$ & $0.00 E+\infty 0$ & 3/14/96 & $4 B$ & 36619 & $\mathrm{BF}$ \\
\hline 3160 & BH_SAND & COMP_RCK & $P a \wedge 1$ & CONSTANT & $0.00 E+00$ & $0.00 \mathrm{E}+\infty 0$ & $0.00 E+00$ & $0.00 E+\infty 0$ & $0.00 E+00$ & $3 / 14 / 96$ & $4 B$ & 36610 & $B F$ \\
\hline 3162 & BH_SAND & CAP_MOD & NONE & CONSTANT & $1.00 E+\infty$ & $1.00 E+\infty$ & $0.00 E+\infty 0$ & $1.00 E+\infty 0$ & $1.00 E+00$ & 3/14/96 & $4 B$ & 36612 & BF \\
\hline
\end{tabular}

\section{D. Borehole Creep}

\begin{tabular}{|c|c|c|c|c|c|c|c|c|c|c|c|c|c|}
\hline ID & Material & Parameter & Units & Distribution & Mean & Median & Deviation & Low & High & Entry Date & Impt & WPO & Codes \\
\hline 3177 & BH_CREEP & SAT_RGAS & NONE & CONSTANT & $0.00 E+00$ & $0.00 E+00$ & $0.00 \mathrm{E}+\infty 0$ & $0.00 E+00$ & $0.00 E+00$ & $3 / 14 / 96$ & 4B & 36631 & $B F$ \\
\hline 3176 & BH_CREEP & SAT_RBRN & NONE & CONSTANT & $0.00 E+00$ & $0.00 E+00$ & $0.00 E+00$ & $0.00 E+00$ & $0.00 E+00$ & $3 / 14 / 96$ & 4B & 36630 & $B F$ \\
\hline 3173 & BH_CREEP & RELP_MOD & NONE & CONSTANT & $4.00 E+\infty 0$ & $4.00 \mathrm{E}+00$ & $0.00 E+\infty$ & $4.00 E+00$ & $4.00 E+00$ & $3 / 14 / 96$ & 48 & 36626 & $B F$ \\
\hline 3189 & BH_CREEP & PRMZ_LOG & $\log \left(m^{\prime} 2\right)$ & UNIFORM & $-1.35 E+01$ & $-1.35 E+01$ & $8.66 E-01$ & $-1.50 E+01$ & $-1.20 \mathrm{E}+01$ & $3 / 14 / 96$ & 1 & 36653 & $B F$ \\
\hline 3188 & BH_CREEP & PRMY_LOG & $\log \left(m^{2} 2\right)$ & UNIFORM & $-1.35 E+01$ & $-1.35 E+01$ & 8.66E-01 & $-1.50 E+01$ & $-1.20 E+01$ & $3 / 14 / 96$ & 1 & 36652 & $8 F$ \\
\hline 3183 & BH_CREEP & PRMX_LOG & $\log \left(m^{\wedge} 2\right)$ & UNIFORM & $-1.35 E+01$ & $-1.35 E+01$ & $8.66 E-01$ & $-1.50 \mathrm{E}+01$ & $-1.20 E+01$ & $3 / 14 / 96$ & 1 & 36640 & $\mathrm{BF}$ \\
\hline 3171 & BH_CAEEP & POROSITY & NONE & CONSTANT & 3.20E-01 & 3.20E-01 & $0.00 E+\infty$ & 3.20E-01 & 3.20E-01 & $3 / 14 / 96$ & $4 B$ & 36624 & $B F$ \\
\hline 3178 & BH_CREEP & PORE_DIS & NONE & CONSTANT & $9.40 E-01$ & $9.40 E-01$ & $0.00 E+00$ & 9.40E-01 & $9.40 E-01$ & $3 / 14 / 96$ & $4 B$ & 36633 & $B F$ \\
\hline 3179 & BH_CREEP & PO_MIN & $\mathrm{Pa}$ & CONSTANT & $1.01 E+05$ & $1.01 E+05$ & $0.00 E+\infty 0$ & $1.01 E+05$ & $1.01 E+05$ & $3 / 14 / 96$ & $4 B$ & 36634 & $\mathrm{BF}$ \\
\hline 3182 & BH_CREEP & PCT_EXP & NONE & CONSTANT & $0.00 E+\infty 0$ & $0.00 E+00$ & $0.00 E+\infty 0$ & $0.00 \mathrm{E}+00$ & $0.00 E+\infty$ & $3 / 14 / 96$ & $4 B$ & 36639 & $B F$ \\
\hline 3181 & BH_CREEP & PCT_A & $\mathrm{Pa}$ & CONSTANT & $0.00 E \div 00$ & $0.00 E+\infty$ & $0.00 E+\infty 0$ & $0.00 E+\infty$ & $0.00 E+\infty$ & $3 / 14 / 96$ & $4 B$ & 36637 & $\mathrm{BF}$ \\
\hline 3175 & BH_CREEP & PC_MAX & $\mathrm{Pa}$ & CONSTANT & $1.00 E+08$ & 1.00E+08 & $0.00 E+00$ & $1.00 E+08$ & $1.00 E+08$ & $3 / 14 / 96$ & 4B & 36628 & $B F$ \\
\hline 3180 & BH_CREEP & KPT & NONE & CONSTANT & $0.00 E+\infty 0$ & $0.00 E+\infty 0$ & $0.00 E+00$ & $0.00 E+\infty$ & $0.00 E+00$ & $3 / 14 / 96$ & 4B & 36636 & $B F$ \\
\hline 3172 & BH_CREEP & COMP_RCK & $\mathrm{Pa}^{\wedge}-1$ & CONSTANT & $0.00 E+00$ & $0.00 E+00$ & $0.00 E+\infty$ & $0.00 E+00$ & $0.00 E+\infty$ & $3 / 14 / 96$ & 4B & 36625 & $\mathrm{BF}$ \\
\hline 3174 & BH_CREEP & CAP_MOD & NONE & CONSTANT & $1.00 E+\infty$ & $1.00 E+00$ & $0.00 E+00$ & $1.00 E+\infty$ & $1.00 E+00$ & $3 / 14 / 96$ & 48 & 36627 & $\mathrm{BF}$ \\
\hline
\end{tabular}

$=$

\section{Shaft DRZ}

\begin{tabular}{|c|c|c|c|c|c|c|c|c|c|c|c|c|c|}
\hline ID & Material & Parameter & Units & Distribution & Mean & Modian & Deviation & Low & High & Entry Date & Impt & WPO & Codes \\
\hline 3133 & SHFT_DRZ & PRMX_LOG & $\log (m \wedge 2)$ & |TRIANGULAR & $-1.53 E+01$ & $-1.50 E+01$ & $6.24 E-01$ & $-1.70 \mathrm{E}+01$ & $-1.40 E+01$ & $3 / 1 / 96$ & 1 & 36563 & $B F$ \\
\hline
\end{tabular}




\section{Earthen Fill}

\begin{tabular}{|c|c|c|c|c|c|c|c|c|c|c|c|c|c|}
\hline ID & Material & Parameter & Units & Distribution & Mean & Median & Deviation & Low & High & Entry Date & Impt & WPO & Codes \\
\hline 2504 & EARTH & PRMY_LOG & $\log \left(m^{\wedge} 2\right)$ & TRIANGULAR & $-1.43 E+01$ & $-1.40 E+01$ & $1.03 E+\infty$ & $-1.70 E+01$ & $-1.20 E+01$ & 2/14/96 & 1 & 32928 & BF \\
\hline 2497 & EARTH & COMP_RCK & $\mathrm{Pa}^{\wedge}-1$ & CONSTANT & 3.10E-08 & 3.10E-08 & $0.00 E+\infty$ & 3.10E-08 & 3.10E-08 & $2 / 14 / 96$ & 1 & 32918 & $\mathrm{BF}$ \\
\hline 2706 & EARTH & KPT & NONE & CONSTANT & $0.00 \mathrm{E}+00$ & $0.00 E+\infty$ & $0.00 E+\infty$ & $0.00 E+00$ & $0.00 \mathrm{E}+00$ & 2/20/96 & $4 B$ & 32919 & BF \\
\hline 2498 & EARTH & PC_MAX & $\mathrm{Pa}$ & CONSTANT & $1.00 E+08$ & $1.00 E+08$ & $0.00 E+00$ & $1.00 E+08$ & $1.00 E+08$ & $11 / 3 / 95$ & $4 B$ & $32922 A$ & BF \\
\hline 2707 & EARTH & PCT_A & $\mathrm{Pa}$ & CONSTANT & 5.60E-01 & $5.60 \mathrm{E}-01$ & $0.00 E+00$ & $5.60 \mathrm{E}-01$ & 5.60E-01 & $2 / 14 / 96$ & 1 & 32920 & BF \\
\hline 2708 & EARTH & PCT_EXP & NONE & CONSTANT & $-3.46 E-01$ & $-3.46 E-01$ & $0.00 E+00$ & -3.46E-01 & $-3.46 E-01$ & $2 / 22 / 96$ & 1 & 32921 & $\mathrm{BF}$ \\
\hline 2501 & EARTH & PO_MIN & $\mathrm{Pa}$ & CONSTANT & $1.01 E+05$ & $1.01 E+05$ & $0.00 \mathrm{E}+00$ & $1.01 E+05$ & $1.01 E+05$ & $2 / 21 / 96$ & $4 B$ & 32925 & $\mathrm{BF}$ \\
\hline 2499 & EARTH & PORE_DIS & NONE & CUMULATIVE & $2.52 E+\infty$ & $9.40 E-01$ & $2.48 E+00$ & $1.10 \mathrm{E}-01$ & $8.10 E+\infty$ & $2 / 20 / 96$ & 1 & 32923 & $B F$ \\
\hline 2500 & EARTH & POROSITY & $m^{n} 3 / m^{n} 3$ & CONSTANT & 3.20E-01 & 3.20E-01 & $0.00 E+\infty 0$ & 3.20E-01 & 3.20E-01 & $2 / 14 / 96$ & 1 & 32924 & $B F$ \\
\hline 2496 & EARTH & CAP_MOD & NONE & CONSTANT & $2.00 E+00$ & $2.00 E+00$ & $0.00 E+00$ & $2.00 E+\infty$ & $2.00 E+\infty$ & 2/14/96 & $4 B$ & $32916 A$ & $B F$ \\
\hline 2503 & EARTH & PRMX_LOG & $\log \left(m^{n} 2\right)$ & TRIANGULAR & $-1.43 E+01$ & $-1.40 E+01$ & $1.03 E+00$ & $-1.70 E+01$ & $-1.20 E+01$ & $2 / 14 / 96$ & 1 & 32927 & $\mathrm{BF}$ \\
\hline 2512 & EARTH & SAT_RGAS & NONE & UNIFORM & $2.00 E-01$ & $2.00 E-01$ & $1.16 E-01$ & $0.00 E+\infty 0$ & $4.00 E-01$ & $2 / 14 / 96$ & 1 & 32964 & $\mathrm{BF}$ \\
\hline 2505 & EARTH & PRMZ_LOG & $\log \left(m^{\wedge} 2\right)$ & TRIANGULAR & $-1.43 E+01$ & $-1.40 E+01$ & $1.03 E+\infty$ & $-1.70 E+01$ & $-1.20 E+01$ & $2 / 14 / 96$ & 1 & 32944 & $B F$ \\
\hline 2509 & EARTH & RELP_MOD & NONE & CONSTANT & $4.00 E+\infty 0$ & $4.00 E+00$ & $0.00 E+00$ & $4.00 E+00$ & $4.00 E+00$ & $2 / 14 / 96$ & $4 B$ & $32952 A$ & BF \\
\hline 3032 & EARTH & RSH_AIR & $m$ & CONSTANT & $3.09 E+00$ & $3.09 E+00$ & $0.00 \mathrm{E}+00$ & $3.09 E+00$ & 3.09E+00 & $2 / 15 / 96$ & 48 & $32954 \mathrm{~A}$ & BF \\
\hline 3035 & EARTH & RSH_EXH & m & CONSTANT & $2.30 E+00$ & $2.30 E+00$ & $0.00 E+00$ & $2.30 E+\infty 0$ & $2.30 E+00$ & $2 / 15 / 96$ & 4B & $32956 \mathrm{~A}$ & $B F$ \\
\hline 3033 & EARTH & RSH_SAL & $m$ & CONSTANT & $1.80 E+\infty$ & $1.80 E+\infty 0$ & $0.00 E+\infty$ & $1.80 E+00$ & $1.80 E+\infty$ & $2 / 15 / 96$ & $4 B$ & 32957A & BF \\
\hline 3034 & EARTH & RSH_WAS & $m$ & CONSTANT & $3.50 \mathrm{E}+00$ & $3.50 E+\infty$ & $0.00 E+\infty 0$ & $3.50 E+\infty 0$ & $3.50 E+\infty 0$ & $2 / 15 / 96$ & 4B & 32959A & BF \\
\hline 2510 & EARTH & SAT_IBRN & NONE & CONSTANT & $8.00 E-01$ & $8.00 E-01$ & $0.00 E+\infty$ & 8.00E-01 & $8.00 \mathrm{E}-01$ & $11 / 3 / 95$ & 1 & 32961 & $\mathrm{BF}$ \\
\hline 2511 & EARTH & SAT_RBRN & NONE & CUMULATIVE & 2.50E-01 & $2.00 E-01$ & $1.76 E-01$ & $0.00 E+00$ & $6.00 E-01$ & $2 / 14 / 96$ & 1 & 32963 & BF \\
\hline 2502 & EARTH & PRESSURE & $\mathrm{Pa}$ & CONSTANT & $1.01 E+05$ & $1.01 E+05$ & $0.00 E+\infty$ & $1.01 E+05$ & 1.01E+05 & 2/20/96 & 1 & 32926 & BF \\
\hline
\end{tabular}




\section{Rustler Compacted Clay}

\begin{tabular}{|c|c|c|c|c|c|c|c|c|c|c|c|c|c|}
\hline ID & Materíal & Paramoter & Units & Distribution & Mean & Median & Deviation & Low & High & Entry Date & Impt & WPO & Codes \\
\hline 3010 & CLAY_RUS & PRMY_LOG & $\log \left(m^{\wedge} 2\right)$ & TRIANGULAR & $-1.89 E+01$ & $-1.83 E+01$ & 7.81E-01 & $-2.10 E+01$ & $-1.73 E+01$ & $2 / 13 / 96$ & 1 & 31887 & $\mathrm{BF}$ \\
\hline 3001 & CLAY_RUS & COMP_RCK & $P a \wedge-1$ & CONSTANT & 1.96E-09 & 1.96E-09 & $0.00 E+\infty 0$ & 1.96E-09 & 1.96E-09 & $2 / 13 / 96$ & 1 & 31878 & BF \\
\hline 3002 & CLAY_RUS & KPT & NONE & CONSTANT & $0.00 E+\infty$ & $0.00 E+\infty 0$ & $0.00 E+00$ & $0.00 E+\infty$ & $0.00 E+\infty$ & $2 / 20 / 96$ & $4 B$ & 31879 & BF \\
\hline 3003 & CLAY_RUS & PC_MAX & $\mathrm{Pa}$ & CONSTANT & $1.00 E+08$ & $1.00 E+08$ & $0.00 E+\infty 0$ & $1.00 E+08$ & $1.00 \mathrm{E}+08$ & $2 / 13 / 96$ & $4 B$ & $31882 A$ & BF \\
\hline 3004 & CLAY_RUS & PCT_A & $\mathrm{Pa}$ & CONSTANT & $5.60 E-01$ & $5.60 E-01$ & $0.00 E+\infty$ & 5.60E-01 & $5.60 \mathrm{E}-01$ & $2 / 13 / 96$ & 1 & 31880 & BF \\
\hline 3005 & CLAY_RUS & PCT_EXP & NONE & CONSTANT & $-3.46 E-01$ & $-3.46 E-01$ & $0.00 E+00$ & $-3.46 E-01$ & $-3.46 E-01$ & $2 / 13 / 96$ & 1 & 31881 & BF \\
\hline 3131 & CLAY_RUS & PO_MIN & $\mathrm{Pa}$ & CONSTANT & $1.01 E+05$ & 1.01E+05 & $0.00 E+00$ & $1.01 E+05$ & $1.01 E+05$ & $2 / 26 / 96$ & $4 B$ & 36561 & BF \\
\hline 3006 & CLAY_RUS & PORE_DIS & NONE & CUMULATIVE & $2.52 E+\infty$ & $9.40 E-01$ & $2.48 E+\infty 0$ & $1.10 E-01$ & $8.10 E+00$ & $2 / 20 / 96$ & 1 & 31883 & BF \\
\hline 3007 & CLAY_RUS & POROSITY & $m^{\wedge} 3 / m^{\wedge} 3$ & CONSTANT & 2.40E-01 & 2.40E-01 & $0.00 E+00$ & $2.40 E-01$ & 2.40E-01 & $2 / 13 / 96$ & 1 & 31884 & BF \\
\hline 3000 & CLAY_RUS & CAP_MOD & NONE & CONSTANT & $2.00 E+\infty 0$ & $2.00 E+\infty$ & $0.00 E+\infty 0$ & $2.00 E+00$ & $2.00 E+\infty$ & $2 / 13 / 96$ & 4B & $31877 \mathrm{~A}$ & BF \\
\hline 3009 & CLAY_RUS & PRMX_LOG & $\log \left(m^{\wedge} 2\right)$ & TRIANGULAR & $-1.89 E+01$ & $-1.83 E+01$ & 7.81E-01 & $-2.10 E+01$ & $-1.73 E+01$ & $2 / 13 / 96$ & 1 & 31886 & $B F$ \\
\hline 3015 & CLAY_RUS & SAT_RGAS & NONE & UNIFOAM & 2.00E-01 & 2.00E-01 & 1.16E-01 & $0.00 E+\infty 0$ & $4.00 E-01$ & 2/13/96 & 1 & 31896 & BF \\
\hline 3011 & CLAY_RUS & PRMZ_LOG & $\log \left(m^{\wedge} 2\right)$ & TRIANGULAR & $-1.89 E+01$ & $-1.83 E+01$ & 7.81E-01 & $-2.10 E+01$ & $-1.73 E+01$ & $2 / 13 / 96$ & 1 & 31888 & $B F$ \\
\hline 3012 & CLAY_RUS & RELP_MOD & NONE & CONSTANT & $4.00 E+\infty$ & $4.00 E+00$ & $0.00 E+00$ & $4.00 E+\infty 0$ & $4.00 E+00$ & $2 / 13 / 96$ & $4 B$ & $31889 \mathrm{~A}$ & BF \\
\hline 2996 & CLAY_RUS & RSH_AlR & $m$ & CONSTANT & $3.09 E+00$ & $3.09 E+00$ & $0.00 E+00$ & $3.09 E+\infty 0$ & $3.09 E+00$ & $2 / 13 / 96$ & $4 \mathrm{~B}$ & $31890 \mathrm{~A}$ & BF \\
\hline 2999 & CLAY_RUS & RSH_EXH & $m$ & CONSTANT & $2.30 E+00$ & $2.30 E+00$ & $0.00 E+\infty$ & $2.30 E+\infty 0$ & $2.30 E+\infty 0$ & $2 / 13 / 96$ & $4 \mathrm{~B}$ & $31891 \mathrm{~A}$ & $B F$ \\
\hline 2997 & CLAY_RUS & RSH_SAL & $m$ & CONSTANT & $1.80 E+00$ & $1.80 E+\infty 0$ & $0.00 E+00$ & $1.80 E+\infty 0$ & $1.80 E+00$ & 2/13/96 & $4 B$ & $31892 A$ & BF \\
\hline 2998 & CLAY_RUS & RSH_WAS & $m$ & CONSTANT & 3.50E+00 & $3.50 E+\infty 0$ & $0.00 E+00$ & $3.50 E+\infty 0$ & $3.50 E+\infty$ & $2 / 13 / 96$ & $4 \mathrm{~B}$ & $31893 \mathrm{~A}$ & $\mathrm{BF}$ \\
\hline 3013 & CLAY_RUS & SAT_IBRN & NONE & CONSTANT & 7.90 E-01 & 7.90E-01 & $0.00 E+00$ & 7.90E-01 & 7.90E-01 & $2 / 13 / 96$ & 1 & 31894 & BF \\
\hline 3014 & CLAY_RUS & SAT_RBRN & NONE & CUMULATIVE & $2.50 E-01$ & $2.00 E-01$ & $1.76 E-01$ & $0.00 E+\infty$ & $6.00 E-01$ & $2 / 13 / 96$ & 1 & 31895 & BF \\
\hline 3008 & CLAY_RUS & PRESSURE & $\mathrm{Pa}$ & CONSTANT & $1.01 E+05$ & $1.01 E+05$ & $0.00 E+00$ & $1.01 E+05$ & 1.01E+05 & $2 / 20 / 96$ & 1 & 31885 & $\mathrm{BF}$ \\
\hline
\end{tabular}




\section{Asphalt}

\begin{tabular}{|c|c|c|c|c|c|c|c|c|c|c|c|c|c|}
\hline ID & Material & Parameter & Units & Distribution & Mean & Median & Deviation & Low & High & Entry Date & Impt & WPO & Codes \\
\hline 2284 & ASPHALT & PRMY_LOG & $\log \left(m^{\wedge} 2\right)$ & TRIANGULAR & $-1.97 E+01$ & $-2.00 E+01$ & 6.24E-01 & $-2.10 E+01$ & $-1.80 E+01$ & & 1 & 31391 & $B F$ \\
\hline 2277 & ASPHALT & COMP_RCK & $P a \wedge-1$ & CONSTANT & 2.97E-08 & 2.97E-08 & & 2.97E-08 & 97E-08 & 96 & 1 & 31380 & BF \\
\hline 3238 & ASPHALT & DNSGRAIN & $\mathrm{kg} / \mathrm{m}^{\wedge} 3$ & CONSTANT & $2.02 E+03$ & $2.02 E+03$ & $0.00 E+\infty 0$ & $2.02 E+03$ & $2.02 E+03$ & & 1 & 36760 & BF/NU \\
\hline 2599 & ASPHALT & KPT & NONE & CONSTANT & $0.00 E+\infty$ & $0.00 E+00$ & $0.00 E+\infty 0$ & $0.00 E+00$ & $0.00 E+\infty 0$ & $2 / 20 / 96$ & $4 B$ & 31381 & BF \\
\hline 2278 & ASPHALT & PC_MAX & $\mathrm{Pa}$ & CONSTANT & $1.00 E+08$ & $1.00 E+08$ & $0.00 E+\infty$ & $1.00 E+08$ & $1.00 E+08$ & $11 / 3 / 95$ & 4B & $31385 A$ & BF \\
\hline 2600 & ASPHALT & PCT_A & $\mathrm{Pa}$ & CONSTANT & $0.00 \mathrm{E}+00$ & $0.00 E+00$ & $0.00 E+\infty 0$ & $0.00 E+00$ & $0.00 E+\infty 0$ & $16 / 96$ & 1 & 31382 & $B F$ \\
\hline 2601 & ASPHALT & PCT_EXP & NONE & CONSTANT & $0.00 E+00$ & $0.00 E+00$ & $0.00 \mathrm{E}+00$ & $0.00 E+\infty$ & $0.00 E+\infty$ & $2 / 13 / 96$ & 1 & 31384 & $B F$ \\
\hline 2281 & ASPHALT & PO_MIN & $\mathrm{Pa}$ & CONSTANT & $1.01 E+05$ & $1.01 E+05$ & $0.00 E+00$ & $1.01 E+05$ & $1.01 E+05$ & $2 / 21 / 96$ & $4 B$ & 31388 & $B F$ \\
\hline 2279 & ASPHALT & PORE_DIS & NONE & CUM & $2.52 E+\infty$ & $9.40 \mathrm{E}-01$ & $2.48 E+00$ & 1.10E-01 & $8.10 E+00$ & $2 / 21 / 96$ & 1 & 31386 & $\mathrm{BF}$ \\
\hline 2280 & ASPHALT & POROSITY & $m^{\wedge} 3 / m^{\wedge} 3$ & CONSTANT & $1.00 \mathrm{E}-02$ & $1.00 \mathrm{E}-02$ & $0.00 E+\infty 0$ & 1.00E-02 & $1.00 \mathrm{E}-02$ & $2 / 13 / 96$ & 1 & 31387 & BF \\
\hline 2276 & ASPHALT & CAP_MOD & NONE & CONS & $1.00 \mathrm{E}+\infty 0$ & $1.00 E+\infty 0$ & $0.00 E+\infty$ & $1.00 E+00$ & $1.00 E+\infty$ & 1/3/95 & $4 B$ & 31375A & BF \\
\hline 2283 & ASPHALT & PRMX_LOG & & TRIAR & $-1.97 E+01$ & $-2.00 E+01$ & 6.24E-01 & $-2.10 E+01$ & $-1.80 E+01$ & $2 / 13 / 96$ & 1 & 31390 & $B F$ \\
\hline 2292 & ALT & SAT_RGAS & NONE & UNIFC & 2.00E-01 & $2.00 E-01$ & 1.16E-01 & $0.00 \mathrm{E}+00$ & $4.00 E-01$ & $2 / 13 / 96$ & 1 & 31407 & $B F$ \\
\hline 2285 & ASPHALT & PRMZ_LOG & $\log \left(m^{\prime} 2\right)$ & TRIANGULAR & $-1.97 E+01$ & $-2.00 E+01$ & $6.24 E-01$ & $-2.10 E+01$ & $-1.80 E+01$ & $2 / 13 / 96$ & 1 & 31392 & BF \\
\hline 2933 & ASPHALT & RADN_DRZ & & CONSTANT & $1.63 E+\infty$ & $1.63 E+\infty 0$ & $0.00 E+00$ & $1.63 E+\infty$ & $1.63 E+\infty 0$ & 2/13/96 & 1 & 31395 & BF \\
\hline 2289 & ASPHALT & RELP_MOD & NONE & CONSTANT & $4.00 E+00$ & $4.00 E+\infty 0$ & $0.00 E+\infty$ & $4.00 \mathrm{E}+\infty 0$ & $4.00 E+00$ & $11 / 3 / 95$ & 4B & $31397 A$ & $B F$ \\
\hline 2929 & ASPHALT & RSH_AIR & m & CONSTANT & $3.09 E+00$ & $3.09 E+00$ & $0.00 E+00$ & $3.09 \mathrm{E}+00$ & $3.09 \mathrm{E}+\infty$ & $2 / 13 / 96$ & $4 B$ & $31399 \mathrm{~A}$ & BF \\
\hline 2932 & ASPHALT & RSH_EXH & $m$ & CONSTANT & $2.30 E+\infty$ & $2.30 E+\infty$ & $0.00 E+\infty$ & $2.30 E+00$ & $2.30 E+00$ & $2 / 13 / 96$ & $4 B$ & $31400 \mathrm{~A}$ & $B F$ \\
\hline 2930 & ASPHALT & RSH_SAL & $m$ & CONSTANT & $1.80 E+\infty$ & $1.80 E+\infty$ & $0.00 E+\infty$ & $1.80 \mathrm{E}+\infty 0$ & $1.80 E+00$ & $2 / 13 / 96$ & 4B & $31401 A$ & $B F$ \\
\hline 2931 & ASPHALT & RSH_WAS & m & CONSTANT & $3.50 E+\infty$ & $3.50 \mathrm{E}+\infty 0$ & $0.00 E+\infty$ & $3.50 E+\infty 0$ & $3.50 E+00$ & $2 / 13 / 96$ & $4 B$ & $31402 A$ & $B F$ \\
\hline 2290 & ASPHALT & SAT_IBRN & NONE & CONSTANT & $0.00 E+00$ & $0.00 E+00$ & $0.00 E+\infty$ & $0.00 E+00$ & $0.00 E+00$ & $2 / 13 / 96$ & 1 & 31403 & $\mathrm{BF}$ \\
\hline 2291 & ASPHALT & SAT_RBRN & NONE & CUMULATIVE & 2.50E-01 & $2.00 E-01$ & 1.76E-01 & $0.00 E+00$ & 6.00 E-01 & $2 / 13 / 96$ & 1 & 31405 & $\mathrm{BF}$ \\
\hline $2 \sigma_{0}$ & ASPHALT & PRESSURE & $\mathrm{Pa}$ & CONSTANT & $1.01 E+05$ & $1.01 E+05$ & $0.00 E+\infty 0$ & $1.01 E+05$ & $1.01 E+05$ & 2/20/96 & 1 & 31389 & $B F$ \\
\hline
\end{tabular}




\section{Shaft Concrete}

\begin{tabular}{|c|c|c|c|c|c|c|c|c|c|c|c|c|c|}
\hline ID & Material & Parameter & Units & Distribution & Mean & Median & Doviation & Low & High & Entry Date & Impt & WPO & Codes \\
\hline 2470 & CONC_T1 & PRMX_LOG & $\log \left(m^{n} 2\right)$ & TRIANGULAR & $-1.88 E+01$ & $-1.87 E+01$ & & $-2.07 E+01$ & $-1.70 E+01$ & 2/14/96 & 1 & 32583 & $\mathrm{BF}$ \\
\hline 2463 & CONC_T1 & CAP_MOD & NONE & CONSTANT & $2.00 E+\infty$ & $2.00 E+\infty$ & $0.00 E+00$ & $2.00 E+\infty$ & $2.00 E+\infty 0$ & $2 / 14 / 96$ & $4 B$ & $32549 \mathrm{~A}$ & $B F$ \\
\hline $24 \pi 7$ & CONC_T1 & SAT_IBRN & NONE & CONSTANT & $1.00 \mathrm{E}+\infty$ & $1.00 \mathrm{E}+\infty$ & $0.00 E+\infty 0$ & $1.00 E+\infty$ & $1.00 E+\infty$ & $2 / 28 / 96$ & 1 & $32621 \mathrm{~A}$ & $B F$ \\
\hline 3038 & CONC_T1 & RSH_WAS & $\mathbf{m}$ & CONSTANT & $3.50 \mathrm{E}+00$ & $3.50 E+00$ & $0.00 E+\infty$ & $3.50 E+\infty 0$ & $3.50 E+\infty$ & $2 / 15 / 96$ & $4 \mathrm{~B}$ & $32617 \mathrm{~A}$ & BF \\
\hline 3037 & CONC_T1 & RSH_SAL & $m$ & CONSTANT & $1.80 E+\infty 0$ & $1.80 E+\infty$ & $0.00 E+00$ & $1.80 \mathrm{E}+00$ & $1.80 E+\infty$ & $2 / 15 / 96$ & $4 B$ & $32609 \mathrm{~A}$ & BF \\
\hline 3039 & CONC_T1 & RSH_EXH & $\mathrm{m}$ & CONSTANT & $2.30 \mathrm{E}+\infty 0$ & $2.30 E+\infty$ & $0.00 E+\infty$ & $2.30 \mathrm{E}+00$ & $2.30 E+00$ & $2 / 15 / 96$ & 48 & $32608 \mathrm{~A}$ & BF \\
\hline 3036 & CONC_T1 & RSH_AIR & $\mathrm{m}$ & CONSTANT & $3.09 E+\infty$ & $3.09 E+00$ & $0.00 E+\infty$ & $3.09 E+\infty$ & $3.09 \mathrm{E}+\infty 0$ & 2/15/96 & $4 B$ & $32607 \mathrm{~A}$ & $\mathrm{BF}$ \\
\hline 2476 & CONC_T1 & RELP_MOD & NONE & CONSTANT & $4.00 E+\infty$ & $4.00 \mathrm{E}+00$ & $0.00 E+00$ & $4.00 E+00$ & $4.00 E+00$ & $2 / 14 / 96$ & $4 B$ & $32605 \mathrm{~A}$ & BF \\
\hline 3040 & CONC_T1 & RADN_DRZ & $\mathrm{m} / \mathrm{m}$ & CONSTANT & $1.00 E+\infty$ & $1.00 E+\infty$ & $0.00 \mathrm{E}+\infty$ & $1.00 E+00$ & $1.00 E+\infty$ & $2 / 15 / 96$ & 1 & 32600 & $B F$ \\
\hline 2478 & CONC_T1 & SAT_RBAN & NONE & CUMULATIVE & $2.50 \mathrm{E}-01$ & 2.00E-01 & 1.76E-01 & $0.00 E+00$ & $6.00 E-01$ & $2 / 14 / 96$ & 1 & 32623 & $B F$ \\
\hline 2471 & CONC_T1 & PRMY_LOG & $\log \left(m^{\wedge} 2\right)$ & TRIANGULAR & $-1.88 E+01$ & $-1.87 E+01$ & 7.55E-01 & $-2.07 E+01$ & $-1.70 \mathrm{E}+01$ & $2 / 14 / 96$ & 1 & 32585 & $\mathrm{BF}$ \\
\hline 2479 & CONC_TH & SAT_RGAS & NONE & UNIFORM & 2.00E-01 & $2.00 E-01$ & 1.16E-01 & $0.00 E+\infty$ & $4.00 E-01$ & $2 / 14 / 96$ & 1 & 32625 & $\mathrm{BF}$ \\
\hline 2469 & CONC_T1 & PRESSURE & $\mathrm{Pa}$ & CONSTANT & 1.01E+05 & $1.01 E+05$ & $0.00 E+\infty$ & $1.01 E+05$ & $1.01 E+05$ & $2 / 20 / 96$ & 1 . & 32580 & BF \\
\hline 2467 & CONC_T1 & POROSITY & $\mathrm{m}^{1} 3 / \mathrm{m}^{13}$ & CONSTANT & $5.00 \mathrm{E}-02$ & $5.00 E-02$ & $0.00 E+\infty$ & 5.00E-02 & $5.00 \mathrm{E}-02$ & $2 / 14 / 96$ & 1 & 32572 & $B F$ \\
\hline 2466 & CONC_TI & PORE_DIS & NONE & CUMULATIVE & $2.52 E+\infty$ & $9.40 E-01$ & $2.48 \mathrm{E}+\infty$ & $1.10 E-01$ & $8.10 E+00$ & 2/20/96 & 1 & 32570 & $\mathrm{BF}$ \\
\hline 2468 & CONC_T1 & PO_MIN & $\mathrm{Pa}$ & CONSTANT & $1.01 E+05$ & $1.01 E+05$ & $0.00 \mathrm{E}+00$ & $1.01 E+05$ & 1.01E+05 & $2 / 21 / 96$ & $4 B$ & 32576 & $\mathrm{BF}$ \\
\hline 2683 & CONC_T1 & PCT_EXP & NONE & CONSTANT & $-3.46 E-01$ & $-3.46 E-01$ & $0.00 \mathrm{E}+\infty 0$ & $-3.46 \mathrm{E}-01$ & $-3.46 E-01$ & $2 / 14 / 96$ & 1 & 32563 & $\mathrm{BF}$ \\
\hline 2682 & CONC_T1 & PCT_A & $\mathrm{Pa}$ & CONSTANT & $5.60 E-01$ & 5.60E-01 & $0.00 E+\infty$ & $5.60 \mathrm{E}-01$ & $5.60 \mathrm{E}-01$ & 2/14/96 & 1 & 32561 & $\mathrm{BF}$ \\
\hline 2465 & CONC_Tr & PC_MAX & $\mathrm{Pa}$ & CONSTANT & $1.00 E+08$ & $1.00 E+08$ & $0.00 E+\infty$ & $1.00 E+08$ & $1.00 E+08$ & $11 / 3 / 95$ & 4B & $32567 \mathrm{~A}$ & $\mathrm{BF}$ \\
\hline 2681 & CONC_T1 & KPT & NONE & CONSTANT & $0.00 E+00$ & $0.00 \mathrm{E}+00$ & $0.00 E+00$ & $0.00 E+\infty 0$ & $0.00 \mathrm{E}+00$ & $2 / 20 / 96$ & 4B & 32559 & $B F$ \\
\hline 2464 & CONC_T1 & COMP_RCK & $P a \sim_{1}$ & CONSTANT & 1.20E-09 & 1.20E-09 & $0.00 E+00$ & $1.20 \mathrm{E}-09$ & $1.20 \mathrm{E}-09$ & $5 / 2 / 96$ & $4 B$ & 32556 & $B F$ \\
\hline 2472 & CONC_T1 & PRMZ_LOG & $\log (m \wedge 2)$ & TRIANGULAR & $-1.88 E+01$ & $-1.87 E+01$ & 7.55E-01 & $-2.07 E+01$ & $-1.70 E+01$ & $2 / 14 / 96$ & 1 & 32587 & $\mathrm{BF}$ \\
\hline 2486 & CONC_T2 & PRMX_LOG & $\log \left(m^{\wedge} 2\right)$ & CONSTANT & $-1.40 E+01$ & $-1.40 E+01$ & $0.00 \mathrm{E}+\infty$ & $-1.40 E+01$ & $-1.40 E+01$ & 11/3/95 & 1 & 32662 & $B F$ \\
\hline 2494 & CONC_T2 & SAT_RBRN & NONE & CUMULATIVE & $2.50 E-01$ & 2.00E-01 & $1.76 E-01$ & $0.00 E+00$ & $6.00 E-01$ & $2 / 14 / 96$ & 1 & 32676 & $\mathrm{BF}$ \\
\hline 2493 & CONC_T2 & SAT_IBRN & NONE & CONSTANT & $1.00 E+\infty$ & $1.00 E+\infty$ & $0.00 E+\infty 0$ & $1.00 E+00$ & $1.00 E+\infty$ & $2 / 21 / 96$ & $4 A$ & 32675 & $\mathrm{BF}$ \\
\hline 3043 & CONC_T2 & RSH_WAS & $m$ & CONSTANT & $3.50 \mathrm{E}+\infty$ & $3.50 E+00$ & $0.00 E+00$ & $3.50 \mathrm{E}+00$ & $3.50 E+00$ & $2 / 15 / 96$ & $4 B$ & $32673 A$ & $B F$ \\
\hline 3042 & CONC_T2 & RSH_SAL & $m$ & CONSTANT & $1.80 E+\infty$ & $1.80 E+\infty$ & $0.00 E+\infty$ & $1.80 \mathrm{E}+\infty$ & $1.80 \mathrm{E}+00$ & $115 / 96$ & $4 B$ & $32672 A$ & $\mathrm{BF}$ \\
\hline 3044 & CONC_T2 & RSH_EXH & $\mathbf{m}$ & CONSTANT & $2.30 E+\infty$ & $2.30 E+\infty$ & $0.00 E+\infty$ & $2.30 \mathrm{E}+00$ & $2.30 \mathrm{E}+\infty$ & $115 / 96$ & 48 & $32671 \mathrm{~A}$ & $\mathrm{BF}$ \\
\hline 3041 & CONC_T2 & RSH_AIR & $m$ & CONSTANT & $3.09 E+\infty$ & $3.09 E+\infty 0$ & $0.00 E+\infty$ & $3.09 E+\infty$ & $3.09 \mathrm{E}+\infty$ & $2 / 15 / 96$ & $4 B$ & $32670 \mathrm{~A}$ & $\mathrm{BF}$ \\
\hline 2492 & CONC_T2 & AELP_MOD & NÓNE & CONSTANT & $4.00 E+\infty$ & $4.00 E+00$ & $0.00 E+\infty$ & $4.00 E+\infty$ & $4.00 \mathrm{E}+\infty$ & $2 / 14 / 96$ & $4 \mathrm{~B}$ & $32669 \mathrm{~A}$ & $B F$ \\
\hline 3045 & CONC_T2 & RADN_DRZ & $\mathrm{m} / \mathrm{m}$ & CONSTANT & $1.00 E+\infty$ & $1.00 E+00$ & $0.00 E+00$ & $1.00 E+\infty$ & $1.00 E+00$ & 2/15/96 & 1 & 32667 & $\mathrm{BF}$ \\
\hline 2480 & CONC_T2 & CAP_MOD & & & & & $0.00 E+00$ & & $2.00 E+00$ & 2/14/96 & $4 \mathrm{~B}$ & $32631 \mathrm{~A}$ & $B F$ \\
\hline 2487 & CONC_T2 & PAMY_LOG & $\log (m \wedge 2)$ & CONSTANT & $-1.40 E+01$ & $-1.40 E+01$ & $0.00 \mathrm{E}+00$ & $-1.40 E+01$ & $-1.40 E+01$ & $11 / 3 / 95$ & 1 & 32663 & $B F$ \\
\hline 2495 & CONC_T2 & SAT_RGAS & NONE & UNIFORM & $2.00 E-01$ & $2.00 E-01$ & $1.16 E-01$ & $0.00 E+\infty$ & 4.00E-01 & $114 / 96$ & 1 & 32678 & BF \\
\hline 2485 & CONC_T2 & PRESSURE & $\mathrm{Pa}$ & CONSTANT & $1.01 E+05$ & $1.01 E+05$ & $0.00 E+\infty$ & $1.01 E+05$ & $1.01 E+05$ & $20 / 96$ & $4 \mathrm{~A}$ & 32661 & BF \\
\hline 2484 & CONC_T2 & POROSITY & $3 / m^{\wedge} 3$ & CONSTANT & $5.00 E-02$ & $5.00 E-02$ & $0.00 E+\infty$ & $5.00 E-02$ & $5.00 E-02$ & $144 / 96$ & $=1$ & 32659 & $B F$ \\
\hline 2483 & CONC_T2 & PORE_DIS & ONE & CUMULATIVE & $2.52 E+\infty 0$ & $9.40 \mathrm{E}-01$ & $2.48 E+\infty$ & $1.10 E-01$ & $8.10 E+\infty$ & 2/20/96 & 1 & 32658 & BF \\
\hline 2808 & CONC_T2 & PO_MIN & & CONSTANT & $1.01 E+05$ & 1.01E+05 & $0.00 E+\infty$ & $1.01 E+05$ & $1.01 E+05$ & $121 / 96$ & $4 B$ & 32660 & BF \\
\hline 2688 & CONC_T2 & PCT_EXP & NONE & CONSTANT & $-3.46 E-01$ & $-3.46 E-01$ & $0.00 E+\infty$ & $-3.46 \mathrm{E}-01$ & $-3.46 \mathrm{E}-01$ & $1 / 4 / 96$ & 1 & 32656 & BF \\
\hline 2687 & CONC_T2 & $P C T=A$ & $\mathrm{~Pa}$ & CONSTANT & 5.60E-01 & $5.60 E-01$ & $0.00 E+\infty$ & $5.60 \mathrm{E}-01$ & 5.60E-01 & 2/14/96 & 1 & 32652 & $\mathrm{BF}$ \\
\hline 2482 & CONC_T2 & PC_MAX & a & CONSTANT & $1.00 E+08$ & $1.00 E+08$ & $0.00 E+\infty$ & $1.00 E+08$ & $1.00 E+08$ & $2 / 14 / 96$ & 4B & $32657 \mathrm{~A}$ & BF \\
\hline 2686 & CONC_T2 & KPT & NONE & CONSTANT & $0.00 E+\infty$ & $0.00 E+\infty$ & $0.00 E+00$ & $0.00 E+\infty$ & $0.00 E+\infty$ & $2 / 20 / 96$ & $4 B$ & 32640 & $B F$ \\
\hline 2481 & CONC_T2 & COMP_RCK & $\mathrm{Pa}^{\wedge-1}$ & CONSTANT & 1.20E-09 & 1.20E-09 & $0.00 E+00$ & $1.20 \mathrm{E}-09$ & 1.20E-09 & $5 / 2 / 96$ & 48 & 32638 & BF \\
\hline 2488 & CONC_T2 & PRMZ_LOG & $\log \left(m^{\wedge} 2\right)$ & CONSTANT & $-1.40 E+01$ & $-1.40 E+01$ & $0.00 E+00$ & $-1.40 E+01$ & $-1.40 E+01$ & $11 / 3 / 95$ & 1 & 32664 & BF \\
\hline
\end{tabular}


7. Grushed Salt

\begin{tabular}{|c|c|c|c|c|c|c|c|c|c|c|c|c|c|}
\hline ID & & & Jnits & & Mean & Median & Deviation & Low & High & try Date & Impt & WPO & \\
\hline 2526 & $L T_{-} T 1$ & REEP & NONE & CONSTANT & $.00 E+\infty$ & $4.00 E+00$ & $0.00 E+00$ & $4.00 E+00$ & $4.00 E+\infty 0$ & 10/27/95 & 4B & 33405 & $B F$ \\
\hline 2513 & & & NONE & CONS & $10 E+\infty$ & $2.00 E+00$ & $0.00 E+\infty 0$ & $2.00 E+\infty$ & $2.00 E+\infty 0$ & 10/27/95 & AB & $33356 \mathrm{~A}$ & BF \\
\hline 2529 & $L T+T 1$ & SAT_RGAS & NONE & UNIFORM & $2.00 E-01$ & $2.00 E-01$ & $1.16 \mathrm{E}-01$ & $0.00 E+00$ & $4.00 E-01$ & $2 / 13 / 96$ & 1 & 33420 & BF \\
\hline 2528 & & SAT_R & NONE & CUMULATIVE & $2.50 E-01$ & 2.00E-01 & |1.76E-01 & $10.00 E+00$ & $6.00 E-01$ & 2/13/96 & 1 & 33418 & BF \\
\hline 2527 & $L T T_{-} T 1$ & SAT_ & NONE & CONSTANT & $3.20 E-01$ & $3.20 E-01$ & $0.00 E+\infty 0$ & $3.20 \mathrm{E}-01$ & $3.20 E-01$ & $2 / 13 / 96$ & 1 & 33416 & $B F$ \\
\hline 2936 & SALT_TI & RSH_WAS & $\mathbf{m}$ & CONSTANT & $3.50 \mathrm{E}+00$ & $3.50 E+\infty 0$ & $0.00 \mathrm{E}+00$ & $3.50 \mathrm{E}+\infty 0$ & $3.50 \mathrm{E}+\infty 0$ & $2 / 13 / 96$ & $4 \mathrm{~B}$ & $33413 A$ & $B F$ \\
\hline 2935 & SALT_T1 & RSH_SAL & $\mathbf{m}$ & CONSTANT & $1.80 E+00$ & $1.80 E+\infty 0$ & $0.00 E+00$ & $1.80 \mathrm{E}+00$ & $1.80 E+\infty$ & $2 / 13 / 96$ & \begin{tabular}{l|l}
$4 B$ \\
\end{tabular} & $33411 \mathrm{~A}$ & $\mathrm{BF}$ \\
\hline 2934 & LT_T1 & RSH_AIR & $\mathbf{m}$ & CONSTANT & $3.09 E+00$ & $3.09 \mathrm{E}+\infty 0$ & $0.00 E+00$ & $3.09 \mathrm{E}+00$ & $3.09 E+00$ & $2 / 13 / 96$ & $4 \mathrm{~B}$ & $33407 A$ & $B F$ \\
\hline 2938 & $T T_{-} T 1$ & RADN_DRZ & $\mathrm{m} / \mathrm{m}$ & CONSTANT & $1.81 E+\infty$ & $1.81 \mathrm{E}+\infty$ & $0.00 E+00$ & $1.81 E+\infty$ & $1.81 E+\infty$ & 213/96 & 1 & 33401 & BF \\
\hline 2519 & & PRESSURE & $\mathrm{Pa}$ & CONS? & $1.01 E+05$ & $1.01 E+05$ & $0.00 E+\infty 0$ & $1.01 E+05$ & $1.01 E+05$ & $20 / 96$ & 1 & 33386 & BF \\
\hline 2517 & SALT_T1 & PORO & $m^{\wedge} 3 / m^{\wedge} 3$ & CONSTANT & $5.00 \mathrm{E}-02$ & $5.00 \mathrm{E}-02$ & $0.00 E+\infty 0$ & $5.00 E-02$ & $5.00 \mathrm{E}-02$ & $2 / 13 / 96$ & 1 & 33381 & BF \\
\hline 2744 & SALT_T1 & KPT & NONE & CONSTANT & $0.00 \mathrm{E}+00$ & $0.00 E+\infty$ & $0.00 E+\infty$ & $0.00 E+00$ & $0.00 \mathrm{E}+00$ & $2 / 20 / 96$ & $4 B$ & 33364 & BF \\
\hline 2514 & SALT_T1 & COMP & $P a^{n-1}$ & CONSTANT & $1.60 E-09$ & $1.60 E-09$ & $0.00 E+00$ & $1.60 \mathrm{E}-09$ & $1.60 \mathrm{E}-09$ & $2 / 13 / 96$ & 1 & 33359 & $B F$ \\
\hline 2937 & SALT_TI & RSH_EXH & $m$ & CONSTANT & $2.30 \mathrm{E}+00$ & $2.30 E+\infty$ & $0.00 E+00$ & $2.30 E+\infty$ & $2.30 E+\infty$ & $2 / 13 / 96$ & $4 B$ & 33410 & $B F$ \\
\hline 2939 & SAL & CUME & ONE & UNIFORM & $5.00 E-01$ & $5.00 E-01$ & $2.88 E-01$ & $0.00 E+00$ & $1.00 E+00$ & $2 / 13 / 96$ & $4 B$ & $33361 \mathrm{~A}$ & $\mathrm{BF}$ \\
\hline 2516 & & PORE & NONE & CUMULATIVE & $2.52 E+00$ & $9.40 E-01$ & $2.48 E+\infty$ & 1.10E-01 & $8.10 E+\infty$ & $2 / 22 / 96$ & 1 & 33380 & $B F$ \\
\hline 2515 & SALT_T1 & PC_MAX & $\mathrm{Pa}$ & CONSTANT & $1.00 E+08$ & $1.00 \mathrm{E}+08$ & $0.00 E+00$ & $1.00 E+08$ & $1.00 E+08$ & $10 / 27 / 95$ & 48 & $33373 \mathrm{~A}$ & BF \\
\hline 2745 & SALT_T1 & PCT_A & $\mathrm{Pa}$ & CONSTANT & $5.60 E-01$ & $5.60 E-01$ & $0.00 E+00$ & $5.60 E-01$ & $5.60 E-01$ & $2 / 13 / 96$ & 1 & 33369 & BF \\
\hline 2746 & SALT_TI & PCT_E & NONE & CONSTANT & $-3.46 E-01$ & $-3.46 E-01$ & $0.00 E+00$ & $-3.46 E-01$ & $-3.46 E-01$ & $2 / 13 / 96$ & 1 & 33370 & BF \\
\hline 2942 & SALT_T1 & PMLT_HI & $\left(m^{\wedge} 2\right)$ & CONST & $-1.23 E+01$ & $-1.23 E+01$ & $0.00 E+00$ & $-1.23 E+01$ & $-1.23 E+01$ & $2 / 13 / 96$ & 1 & 33375 & BF \\
\hline 2941 & LT_T1 & PMLT_LO & $\log \left(m^{\wedge} 2\right)$ & CONS & $-1.73 E+01$ & $-1.73 E+01$ & $0.00 \mathrm{E}+00$ & $\mid-1.73 E+01$ & $-1.73 E+01$ & $2 / 13 / 96$ & 1 & 33377 & BF \\
\hline 2940 & SALT_TI & PMLT_MD & $\log \left(m^{\wedge} 2\right)$ & CONSTANT & $-1.48 E+01$ & $\mid-1.48 E+01$ & $0.00 E+\infty$ & $-1.48 E+01$ & $-1.48 E+01$ & $2 / 13 / 96$ & 1 & $3337 \varepsilon$ & BF \\
\hline 2518 & SALT_TI & PO_MIN & $\mathbf{P a}$ & CONSTANT & $1.01 \mathrm{E}+05$ & $1.01 E+05$ & $0.00 E+\infty$ & $1.01 E+05$ & $1.01 E+05$ & $2 / 21 / 96$ & $4 B$ & 33382 & $B F$ \\
\hline 2534 & SALT_T2 & POROSITY & $3 / \mathrm{m}^{13}$ & CONSTANT & $5.00 \mathrm{E}-02$ & $5.00 E-02$ & $0.00 E+00$ & $5.00 E-02$ & $5.00 \mathrm{E}-02$ & $2 / 14 / 96$ & 1 & 33357 & $B F$ \\
\hline 2950 & SALT_T2 & PMLT_HI & $\log \left(m^{\wedge} 2\right)$ & CONSTANT & $-1.23 E+01$ & $-1.23 E+01$ & $0.00 \mathrm{E}+\infty 0$ & $\mid-1.23 E+01$ & $-1.23 E+01$ & $2 / 13 / 96$ & & 33440 & $B F$ \\
\hline 2545 & SALT_T2 & SAT_RBRN & NONE & CUMULATIVE & $2.50 E-01$ & $2.00 E-01$ & $1.76 \mathrm{E}-01$ & $0.00 E+\infty$ & $6.00 E-01$ & $2 / 14 / 96$ & & 33391 & $B F$ \\
\hline 2945 & $L T_{-} T 2$ & $\mathrm{RSH}_{\text {_ }}$ & m & CONSTANT & $3.50 E+\infty 0$ & $3.50 \mathrm{E}+\infty$ & $0.00 E+\infty 0$ & $3.50 E+00$ & $3.50 \mathrm{E}+00$ & $2 / 13 / 96$ & $4 B$ & $33387 \mathrm{~A}$ & $B F$ \\
\hline 2944 & ILT_T2 & RSH_SAL & $\mathrm{m}$ & CONSTANT & $1.80 E+00$ & $1.80 E+\infty 0$ & $0.00 E+\infty 0$ & $1.80 E+\infty 0$ & $1.80 E+00$ & $2 / 13 / 96$ & 4B & $33385 \mathrm{~A}$ & $B F$ \\
\hline 2946 & SALT_T2 & RSH_EXH & $\mathrm{m}$ & CONSTANT & $2.30 E+\infty 0$ & $2.30 E+\infty 0$ & $0.00 E+\infty 0$ & $2.30 E+00$ & $2.30 E+\infty 0$ & $2 / 13 / 96$ & 4B & $33384 \mathrm{~A}$ & $B F$ \\
\hline 2943 & SALT_T2 & RSH_A & $m$ & CONST & $3.09 E+00$ & $3.09 E+\infty$ & $0.00 E+00$ & $3.09 E+00$ & $3.09 \mathrm{E}+00$ & $2 / 13 / 96$ & $4 B$ & $33383 A$ & $B F$ \\
\hline 2543 & & RELP. & NONE & CONS & $4.00 E+\infty 0$ & $4.00 \mathrm{E}+00$ & $0.00 E+00$ & $4.00 E+\infty 0$ & $4.00 E+00$ & 2/14/96 & 4B & $33379 f$ & $B F$ \\
\hline 2947 & LT_T2 & RADN_DRZ & $\mathrm{m} / \mathrm{m}$ & CONSTANT & $1.11 E+\infty$ & $1.11 E+\infty$ & $0.00 \mathrm{E}+\infty 0$ & $1.11 E+\infty$ & $1.11 E+\infty$ & $2 / 13 / 96$ & 1 & 33374 & $B F$ \\
\hline 2546 & HLT_T2 & SAT_RGAS & NONE & UNIFORM & $2.00 E-01$ & $2.00 E-01$ & $1.16 E-01$ & $0.00 E+00$ & $4.00 E-01$ & $2 / 14 / 96$ & 1 & 33393 & $B F$ \\
\hline 2531 & SALT_T2 & COMP_RCK & $\mathrm{Pa}^{n-1}$ & CONSTANT & $1.60 E-09$ & $1.60 E-09$ & $0.00 E+00$ & 1.60E-09 & 1.60E-09 & $2 / 14 / 96$ & 1 & 33429 & $B F$ \\
\hline 2948 & SALT_- & PMLT_I & $\log \left(m^{\wedge} 2\right)$ & CONSTANT & $-1.48 E+01$ & $-1.48 E+01$ & $0.00 E+00$ & $-1.48 E+01$ & $-1.48 E+01$ & $2 / 13 / 96$ & 1 & 33445 & $B F$ \\
\hline 2533 & LT_T2 & PORE_ & NONE & CUMULATIVE & $2.52 \mathrm{E}+00$ & $9.40 E-01$ & $2: 48 E+00$ & $1.10 E-01$ & $8.10 E+\infty 0$ & $2 / 22 / 96$ & -1 & 33355 & $B F$ \\
\hline 2530 & SALT_T2 & CAP_MOD & NONE & CONSTANT & $2.00 \mathrm{E}+00$ & $2.00 E+00$ & $0.00 E+\infty 0$ & $2.00 E+\infty 0$ & $2.00 E+00$ & $10 / 27 / 95$ & 4B & $33424 A$ & $B F$ \\
\hline 2749 & SALT_T2 & KPT & NONE & CONSTANT & $0.00 \mathrm{E}+0.0$ & $0.00 \mathrm{E}+\infty$ & $0.00 E+\infty$ & $0.00 E+00$ & $0.00 E+\infty 0$ & $2 / 20 / 96$ & 48 & 33431 & $B F$ \\
\hline 2532 & SALT_T2 & $\overline{P C \_N}$ & & DNSTANT & $1.00 \mathrm{E}+08$ & $1.00 E+08$ & $0.00 E+00$ & $1.00 \mathrm{E}+08$ & $1.00 E+08$ & 10/27/95 & $4 B$ & 33438 & BF \\
\hline 2750 & LT_T2 & PCT_A & $\mathrm{Pa}$ & CONSTANT & $5.60 \mathrm{E}-01$ & $5.60 E-01$ & $0.00 E+00$ & $5.60 E-01$ & $5.60 E-01$ & $2 / 14 / 96$ & & 33433 & $B F$ \\
\hline 2751 & SALT_T2 & PCT_EXP & NONE & CONSTANT & $-3.46 E-01$ & $-3.46 E-01$ & $0.00 E+00$ & $-3.46 E-01$ & $-3.46 E-01$ & $2 / 14 / 96$ & 1 & 33435 & $\mathrm{BF}$ \\
\hline 2949 & LT_T2 & PMLT_LO & $\log \left(m^{\wedge} 2\right)$ & CONSTANT & $\mid-1.73 E+01$ & $-1.73 E+01$ & $0.00 E+00$ & $-1.73 E+01$ & $-1.73 E+01$ & $2 / 13 / 96$ & 1 & 33442 & BF \\
\hline 2535 & SALT_T2 & PO_MIN & $\mathrm{Pa}$ & CONSTANT & $1.01 E+05$ & $1.01 E+05$ & $0.00 E+00$ & $1.01 E+05$ & $1.01 E+05$ & $2 / 21 / 96$ & $4 B$ & 33362 & $B F$ \\
\hline 2549 & SALT_T3 & PC_MAX & $\mathrm{Pa}$ & CONSTANT & $1.00 E+08$ & $1.00 E+08$ & $0.00 E+00$ & $1.00 E+08$ & $1.00 E+08$ & 10/27/95 & $4 B$ & $33408 \mathrm{~A}$ & $B F$ \\
\hline 2952 & SALT_T3 & RSH_SAL & $m$ & CONSTANT & $1.80 E+\infty$ & $1.80 E+\infty$ & $0.00 E+00$ & $1.80 E+\infty$ & $1.80 E+00$ & $3 / 96$ & $4 B$ & $33441 A$ & $B F$ \\
\hline 2552 & LT_T3 & PO_MIN & $\mathrm{Pa}$ & CONSTANT & $1.01 E+05$ & $1.01 E+05$ & $0.00 E+00$ & $1.01 E+05$ & $1.01 E+05$ & $1 / 96$ & 4B & 33419 & $B F$ \\
\hline 951 & LT_T3 & RSH_AIR & $\mathrm{m}$ & CONSTANT & $3.09 E+\infty$ & $3.09 \mathrm{E}+00$ & $0.00 E+00$ & $3.09 E+\infty$ & $3.09 E+\infty 0$ & $2 / 13 / 96$ & AB & $33437 A$ & $B F$ \\
\hline 560 & SALT_T3 & RELP_MOD & NONE & CONSTANT & $4.00 E+\infty$ & $4.00 E+00$ & $0.00 E+00$ & $4.00 E+\infty$ & $4.00 E+\infty 0$ & 10/27/95 & $4 B$ & $33436 \mathrm{~A}$ & $B F$ \\
\hline 955 & SALT_T3 & RADN_DRZ & $m / m$ & CONSTANT & $1.00 E+00$ & $1.00 E+00$ & $0.00 E+00$ & $1.00 E+\infty$ & $1.00 E+\infty$ & $2 / 13 / 96$ & 1 & 33432 & $B F$ \\
\hline 551 & $-T_{-} T_{3}$ & POROSITY & $m^{\prime} 3 / m^{\prime} 3$ & CONS? & $5.00 \mathrm{E}$ & $5.00 E-02$ & $0.00 E+00$ & 5.00 & $E-02$ & 4/96 & 1 & 33417 & $B F$ \\
\hline 550 & LT_T3 & PORE_DIS & DNE & JMULATIVE & $2 E+\infty 0$ & & & & $8.10 E+00$ & $1 / 22 / 96$ & 1 & 33415 & $B F$ \\
\hline
\end{tabular}




\section{Crushed Salt}

\begin{tabular}{|c|c|c|c|c|c|c|c|c|c|c|c|c|c|}
\hline 10 & Materia! & Parameter & Units & Distribution & Mean & Median & Deviation & Low & High & Entry Date & Impt & WPO & Codes \\
\hline 2954 & SALT_T3 & RSH_EXH & $m$ & CONSTANT & $2.30 \mathrm{E}+\infty$ & $2.30 E+\infty$ & $0.00 E+\infty$ & $2.30 E+\infty$ & $2.30 \mathrm{E}+00$ & $2 / 13 / 96$ & $4 B$ & $33439 \mathrm{~A}$ & BF \\
\hline 2956 & SALT_T3 & PMLT_MD & $\log \left(m^{\wedge} 2\right)$ & CONSTANT & $-1.48 E+01$ & $-1.48 E+01$ & $0.00 \mathrm{E}+\infty$ & $-1.48 E+01$ & $-1.48 E+01$ & $2 / 13 / 96$ & 1 & 33414 & $B F$ \\
\hline 2957 & SALT_T3 & PMLT_LO & $\log \left(m^{\wedge} 2\right)$ & CONSTANT & $-1.73 E+01$ & $-1.73 E+01$ & $0.00 E+\infty$ & $-1.73 E+01$ & $-1.73 E+01$ & $2 / 13 / 96$ & 1 & 33412 & $B F$ \\
\hline 2958 & SALT_T3 & PMLT_HI & $\log \left(m^{\wedge} 2\right)$ & CONSTANT & $-1.23 E+01$ & $-1.23 E+01$ & $0.00 E+00$ & $-1.23 E+01$ & $-1.23 E+01$ & $2 / 13 / 96$ & 1 & 33409 & $\mathrm{BF}$ \\
\hline 2755 & SALT_T3 & PCT_A & $\mathrm{Pa}$ & CONSTANT & $5.60 \mathrm{E}-01$ & $5.60 \mathrm{E}-01$ & $0.00 \mathrm{E}+\infty$ & 5.60E-01 & $5.60 \mathrm{E}-01$ & $2 / 14 / 96$ & 1 & 33404 & $B F$ \\
\hline 2754 & SALT_T3 & KPT & NONE & CONSTANT & $0.00 E+00$ & $0.00 E+00$ & $0.00 E+\infty$ & $0.00 E+\infty$ & $0.00 E+00$ & $2 / 20 / 96$ & 4B & 33402 & $\mathrm{BF}$ \\
\hline 2548 & SALT_T3 & COMP_RCK & $P a \Lambda 1$ & CONSTANT & 1.60E-09 & $1.60 E-09$ & $0.00 E+\infty 0$ & $1.60 E-09$ & $1.60 E-09$ & $2 / 14 / 96$ & 1 & 33400 & $B F$ \\
\hline 2547 & SALT_T3 & CAP_MOD & NONE & CONSTANT & $2.00 E+00$ & $2.00 E+\infty$ & $0.00 E+00$ & $2.00 E+\infty$ & $2.00 E+\infty$ & $10 / 27 / 95$ & $4 \mathrm{~B}$ & $33397 \mathrm{~A}$ & $B F$ \\
\hline 2563 & SALT_T3 & SAT_RGAS & NONE & UNIFOAM & $2.00 E-01$ & 2.00E-01 & 1.16E-0i & $0.00 E+\infty 0$ & 4.00E-01 & $2 / 14 / 96$ & 1 & 33447 & $B F$ \\
\hline 2756 & SALT_T3 & PCT_EXP & NONE & CONSTANT & $-3.46 E-01$ & $-3.46 E-01$ & $0.00 E+00$ & $-3.46 E-01$ & $-3.46 E-01$ & $2 / 14 / 96$ & 1 & 33406 & $B F$ \\
\hline 2562 & SALT_T3 & SAT_RBRN & NONE & CUMULATIVE & 2.50E-01 & $2.00 E-01$ & 1.76E-01 & $0.00 E+\infty 0$ & $6.00 E-01$ & $2 / 14 / 96$ & 1 & 33446 & $B F$ \\
\hline 2953 & SALT_T3 & RSH_WAS & $m$ & CONSTANT & $3.50 \mathrm{E}+00$ & $3.50 E+\infty 0$ & $0.00 E+\infty$ & $3.50 \mathrm{E}+\infty$ & $3.50 \mathrm{E}+\infty$ & $2 / 13 / 96$ & $4 B$ & $33443 \mathrm{~A}$ & BF \\
\hline 2577 & SALT_T4 & RELP_MOD & NONE & CONSTANT & $4.00 E+00$ & $4.00 \mathrm{E}+\infty 0$ & $0.00 E+\infty$ & $4.00 E+\infty 0$ & $4.00 E+00$ & $10 / 27 / 95$ & $4 B$ & $33553 \mathrm{~A}$ & $\mathrm{BF}$ \\
\hline 2580 & SALT_T4 & SAT_RGAS & NONE & UNIFORM & 2.00E-01 & 2.00E-01 & 1.16E-01 & $0.00 E+\infty 0$ & $4.00 E-01$ & $2 / 14 / 96$ & 1 & 33565 & $8 F$ \\
\hline 2579 & SALT_T4 & SAT_RBRN & NONE & CUMULATIVE & 2.50E-01 & $2.00 E-01$ & 1.76E-01 & $0.00 E+\infty 0$ & $6.00 E-01$ & $2 / 14 / 96$ & 1 & 33564 & $\mathrm{BF}$ \\
\hline 2961 & SALT_T4 & RSH_WAS & $m$ & CONSTANT & $3.50 \mathrm{E}+\infty$ & $3.50 \mathrm{E}+00$ & $0.00 E+\infty$ & $3.50 \mathrm{E}+00$ & $3.50 E+\infty$ & $2 / 13 / 96$ & $4 B$ & $33561 \mathrm{~A}$ & $B F$ \\
\hline 2960 & SALT_T4 & RSH_SAL & $m$ & CONSTANT & $1.80 \mathrm{E}+00$ & $1.80 E+00$ & $0.00 \mathrm{E}+\infty$ & $1.80 E+\infty$ & $1.80 \mathrm{E}+00$ & $2 / 13 / 96$ & $4 B$ & $33560 \mathrm{~A}$ & $\mathrm{BF}$ \\
\hline 2959 & SALT_T4 & RSH_AIR & $m$ & CONSTANT & $3.09 E+\infty 0$ & $3.09 E+\infty 0$ & $0.00 \mathrm{E}+00$ & $3.09 E+\infty$ & $3.09 E+00$ & $2 / 13 / 96$ & $4 B$ & $33556 \mathrm{~A}$ & $B F$ \\
\hline 2963 & & RADN_DRZ & $\mathrm{m} / \mathrm{m}$ & CONSTANT & $1.00 \mathrm{E}+00$ & $1.00 \mathrm{E}+00$ & $0.00 E+00$ & $1.00 E+00$ & $1.00 \mathrm{E}+00$ & $2 / 13 / 96$ & 1 & 33540 & $\mathrm{BF}$ \\
\hline 2568 & & POROSITY & $m^{n} 3 / m^{n} 3$ & CONSTANT & 5.00E-02 & $5.00 \mathrm{E}-02$ & $0.00 \mathrm{E}+00$ & $5.00 \mathrm{E}-02$ & $5.00 \mathrm{E}-02$ & 2/14/96 & 1 & 33467 & $\mathrm{BF}$ \\
\hline 2567 & SALT_T4 & PORE_DIS & NONE & CUMULATIVE & $2.52 E+\infty 0$ & 9.40E-01 & $2.48 E+\infty$ & $1.10 E-01$ & $8.10 E+00$ & $2 / 22 / 96$ & 1 & 33465 & $\overline{B F}$ \\
\hline 2565 & SALT_T4 & COMP_RCK & $P_{2}{ }^{\prime-1}$ & CONSTANT & 1.60E-09 & 1.60 E-09 & $0.00 E+00$ & $1.60 \mathrm{E}-09$ & $1.60 \mathrm{E}-09$ & $2 / 14 / 96$ & 1 & 33453 & $\mathrm{BF}$ \\
\hline 2962 & SALT_T4 & RSH_EXH & $\mathrm{m}$ & CONSTANT & $2.30 E+00$ & $2.30 E+\infty$ & $0.00 E+\infty$ & $2.30 \mathrm{E}+00$ & $2.30 \mathrm{E}+00$ & $2 / 13 / 96$ & 48 & $33558 \mathrm{~A}$ & $\mathrm{BF}$ \\
\hline 2564 & SALT_T4 & CAP_MOD & NONE & CONSTANT & $2.00 E+00$ & $2.00 E+00$ & $0.00 E+00$ & $2.00 E+00$ & $2.00 E+00$ & $10 / 27 / 95$ & $4 B$ & $33449 \mathrm{~A}$ & $\mathrm{BF}$ \\
\hline 2569 & ALT_T4 & PO_MIN & $\mathrm{Pa}$ & CONSTANT & $1.01 E+05$ & $1.01 E+05$ & $0.00 \mathrm{E}+\infty$ & $1.01 E+05$ & $1.01 E+05$ & $2 / 21 / 96$ & $4 B$ & 33469 & BF \\
\hline 2759 & ALT_T4 & KPT & NONE & CONSTANT & $0.00 E+\infty$ & $0.00 E+\infty$ & $0.00 E+00$ & $0.00 E+\infty$ & $0.00 \mathrm{E}+00$ & $2 / 20 / 96$ & $4 B$ & 33454 & $\mathrm{BF}$ \\
\hline 2566 & ALT_T4 & PC_MAX & $\mathrm{Pa}$ & CONSTANT & $1.00 \mathrm{E}+08$ & $1.00 E+08$ & $0.00 E+\infty$ & $1.00 E+08$ & $1.00 E+08$ & $10 / 27 / 95$ & 48 & $33459 \mathrm{~A}$ & $\mathrm{BF}$ \\
\hline 2760 & SALT_T4 & PCT_A & $\mathrm{Pa}$ & CONSTANT & $5.60 E-01$ & $5.60 \mathrm{E}-01$ & $0.00 E+00$ & $5.60 E-01$ & $5.60 E-01$ & $2 / 14 / 96$ & 1 & 33456 & $\overline{B F}$ \\
\hline 2761 & SALT_T4 & PCT_EXP & NONE & CONSTANT & $-3.46 E-01$ & $-3,46 \mathrm{E}-01$ & $0.00 E+\infty$ & $-3.46 E-01$ & $-3.46 E-01$ & $2 / 14 / 96$ & 1 & 33457 & $\mathrm{BF}$ \\
\hline 2966 & SALT_T4 & PMLT_HI & $\log \left(m^{\wedge} 2\right)$ & CONSTANT & $-1.40 E+01$ & $-1.40 E+01$ & $0.00 \mathrm{E}+\infty 0$ & $-1.40 E+01$ & $-1.40 E+01$ & $2 / 13 / 96$ & 1 & 33460 & $\mathrm{BF}$ \\
\hline 2965 & SALT_T4 & PMLT_LO & $\log \left(m^{\wedge} 2\right)$ & CONSTANT & $-2.29 E+01$ & $-2.29 E+01$ & $0.00 E+00$ & $-2.29 E+01$ & $-2.29 E+01$ & $2 / 13 / 96$ & 1 & 33461 & $B F$ \\
\hline 2964 & SALT_T4 & PMLT_MD & $\log \left(m^{1} 2\right)$ & CONSTANT & $-1.72 E+01$ & $-1.72 E+01$ & $0.00 E+\infty 0$ & $-1.72 E+01$ & $-1.72 E+01$ & $2 / 13 / 96$ & 1 & 33463 & $\mathrm{BF}$ \\
\hline 2972 & SALT_T5 & PMLT_MD & $\log \left(m^{\wedge} 2\right)$ & CONSTANT & $-1.93 E+01$ & $-1.93 E+01$ & $0.00 E+00$ & $-1.93 E+01$ & $-1.93 E+01$ & $2 / 13 / 96$ & 1 & 33585 & BF \\
\hline 969 & SALT_T5 & RSH_WAS & $m$ & CONSTANT & $3.50 E+\infty 0$ & $3.50 E+\infty 0$ & $0.00 E+00$ & $3.50 E+\infty$ & $3.50 \mathrm{E}+00$ & $2 / 13 / 96$ & 48 & $33622 \mathrm{~A}$ & BF \\
\hline 2968 & SALT_T5 & RSH_SAL & $m$ & CONSTANT & $1.80 \mathrm{E}+\infty 0$ & $1.80 E+\infty 0$ & $0.00 E+00$ & $1.80 E+00$ & $1.80 E+00$ & $2 / 13 / 96$ & $4 B$ & $33620 \mathrm{~A}$ & $B F$ \\
\hline 2970 & SALT_T5 & RSH_EXH & $m$ & CONSTANT & $2.30 E+\infty 0$ & $2.30 E+\infty$ & $0.00 E+00$ & $2.30 E+00$ & $2.30 E+00$ & $2 / 13 / 96$ & $--4 B$ & $33618 A$ & BF \\
\hline 2967 & SALT_T5 & RSH_AIR & $m$ & CONSTANT & $3.09 E+\infty 0$ & $3.09 \mathrm{E}+00$ & $0.00 E+00$ & $3.09 E+00$ & $3.09 \mathrm{E}+00$ & $2 / 13 / 96$ & $4 B$ & $33616 \mathrm{~A}$ & $\mathrm{BF}$ \\
\hline 2594 & SALT_TS & RELP_MOD & NONE & CONSTANT & $4.00 E+\infty 0$ & $4.00 E+\infty 0$ & $0.00 E+00$ & $4.00 E+00$ & $4.00 E+00$ & $10 / 27 / 95$ & $4 B$ & $33614 A$ & $\mathrm{BF}$ \\
\hline 2971 & SALT_T5 & RADN_DRZ & $\mathrm{m} / \mathrm{m}$ & CONSTANT & $1.00 E+\infty$ & $1.00 E+\infty$ & $0.00 \mathrm{E}+\infty$ & $1.00 E+00$ & $1.00 E+00$ & $2 / 13 / 96$ & 1 & 33609 & $B F$ \\
\hline 2585 & SALT_T5 & POROSITY & $\mathrm{m}^{\prime} 3 / \mathrm{m}^{\prime} 3$ & CONSTANT & $5.00 E-02$ & $5.00 E-02$ & $0.00 E+00$ & 5.00E-02 & $5.00 E-02$ & $2 / 14 / 96$ & 1 & 33590 & BF \\
\hline 2597 & SALT_T5 & SAT_RGAS & NONE & UNIFORM & $2.00 E-01$ & $2.00 \mathrm{E}-01$ & 1.16E-01 & $0.00 E+00$ & $4.00 \mathrm{E}-01$ & $2 / 14 / 96$ & 1 & 33628 & $\mathrm{BF}$ \\
\hline 2596 & SALT_T5 & SAT_RBRN & NONE & CUMULATIVE & $2.50 E-01$ & 2.00E-01 & 1.76E-01 & $0.00 E+\infty 0$ & $6.00 E-01$ & $2 / 14 / 96$ & 1 & 33626 & $\mathrm{BF}$ \\
\hline 2973 & SALT_T5 & PMLT_LO & $\log \left(m^{\wedge} 2\right)$ & CONSTANT & $-2.29 E+01$ & $-2.29 E+01$ & $0.00 E+00$ & $-2.29 E+01$ & $-2.29 E+01$ & $2 / 13 / 96$ & 1 & 33583 & $\mathrm{BF}$ \\
\hline 2974 & SALT_T5 & PMLT_HI & $\log \left(m^{n} 2\right)$ & CONSTANT & $-1.54 E+01$ & $-1.54 E+01$ & $0.00 \mathrm{E}+\infty 0$ & $-1.54 E+01$ & $-1.54 E+01$ & $2 / 13 / 96$ & 1 & 33581 & $\mathrm{BF}$ \\
\hline 2766 & SALT_T5 & PCT_EXP & NONE & CONSTANT & $-3.46 E-01$ & $-3.46 E-01$ & $0.00 \mathrm{E}+00$ & $-3.46 E-01$ & $-3.46 E-01$ & $2 / 14 / 96$ & 1 & 33577 & BF \\
\hline 2765 & SALT_T5 & PCT_A & $\mathrm{Pa}$ & CONSTANT & $5.60 \mathrm{E}-01$ & $5.60 \mathrm{E}-01$ & $0.00 E+\infty$ & 5.60E-01 & $5.60 \mathrm{E}-01$ & $2 / 14 / 96$ & 1 & 33575 & BF \\
\hline 2583 & SALT_T5 & PC_MAX & $\mathrm{Pa}$ & CONSTANT & $1.00 E+08$ & $1.00 E+08$ & $0.00 E+00$ & $1.00 E+08$ & $1.00 E+08$ & 10/27/95 & $4 B$ & $33579 \mathrm{~A}$ & $B F$ \\
\hline 2764 & SALT_T5 & KPT & NONE & CONSTANT & $0.00 E+\infty$ & $0.00 \mathrm{E}+00$ & $0.00 \mathrm{E}+00$ & $0.00 E+\infty 0$ & $0.00 E+00$ & $2 / 20 / 96$ & $4 B$ & 33573 & $B F$ \\
\hline 2582 & SALT_T5 & COMP_RCK & $\mathrm{Pa}^{\wedge}-1$ & CONSTANT & 1.60E-09 & 1.60E-09 & $0.00 \mathrm{E}+\infty 0$ & 1.60E-09 & $1.60 \mathrm{E}-09$ & $2 / 14 / 96$ & 1 & 33572 & $\mathrm{BF}$ \\
\hline 2581 & SALT_T5 & CAP_MOD & NONE & CONSTANT & $2.00 E+\infty$ & $2.00 \mathrm{E}+\infty$ & $0.00 E+\infty$ & $2.00 E+\infty$ & $2.00 E+00$ & $10 / 27 / 95$ & $4 B$ & $33568 \mathrm{~A}$ & $B F$ \\
\hline 2809 & SALT_T5 & PORE_DIS & NONE & CUMULATIVE & $2.52 E+00$ & $9.40 E-01$ & $2.48 E+\infty$ & $1.10 E-01$ & $8.10 E+\infty 0$ & $2 / 26 / 96$ & 1 & 33588 & $B F$ \\
\hline
\end{tabular}




\section{Crushed Salt}

\begin{tabular}{|c|c|c|c|c|c|c|c|c|c|c|c|c|c|}
\hline ID & Material & Parameter & Units & Distribution & Mean & Modian & Deviation & Low & High & Entry Date & Impt & WPO & Codes \\
\hline 3125 & SALT_T5 & PO_MIN & $\mathrm{Pa}$ & CONSTANT & $1.01 E+05$ & $1.01 E+05$ & $0.00 E+\infty$ & $1.01 E+05$ & $1.01 E+05$ & 2/21/96 & $4 B$ & 36368 & $\mathrm{BF}$ \\
\hline 2992 & SALT_T6 & SAT_RBRN & NONE & CUMULATIVE & 2.50E-01 & 2.00E-01 & 1.76E-01 & $0.00 E+00$ & 6.00E-01 & $2 / 13 / 96$ & 1 & 33483 & $\mathrm{BF}$ \\
\hline 2990 & SALT_T6 & POROSITY & $\mathrm{m}^{\wedge} 3 / \mathrm{m}^{\wedge} 3$ & CONSTANT & $5.00 E-02$ & $5.00 E-02$ & $0.00 E+00$ & 5.00E-02 & 5.00E-02 & $2 / 13 / 96$ & 1 & 33657 & BF \\
\hline 2979 & SALT_T6 & RADN_DRZ & $\mathrm{m} / \mathrm{m}$ & CONSTANT & $1.00 E+\infty$ & $1.00 \mathrm{E}+\infty 0$ & $0.00 E+00$ & $1.00 \mathrm{E}+\infty 0$ & $1.00 E+00$ & 2/13/96 & 1 & 33476 & $\mathrm{BF}$ \\
\hline 2991 & SALT_T6 & RELP_MOD & NONE & CONSTANT & $4.00 E+00$ & $4.00 E+\infty 0$ & $0.00 E+\infty$ & $4.00 E+\infty 0$ & $4.00 \mathrm{E}+00$ & 2/13/96 & $4 B$ & $33478 \mathrm{~A}$ & BF \\
\hline 2975 & SALT_T6 & RSH_AlR & $m$ & CONSTANT & $3.09 \varepsilon+00$ & $3.09 E+\infty$ & $0.00 E+00$ & $3.09 E+00$ & $3.09 E+00$ & $2 / 13 / 96$ & $4 B$ & $33479 A$ & $B F$ \\
\hline 2978 & SALT_T6 & RSH_EXH & $m$ & CONSTANT & 2.30E+00 & $2.30 E+\infty$ & $0.00 E+\infty$ & $2.30 E+\infty$ & $2.30 E+\infty 0$ & $2 / 13 / 96$ & $4 B$ & $33480 \mathrm{~A}$ & BF \\
\hline 2989 & SALT_T6 & PORE_DIS & NONE & CUMULATIVE & $2.52 E+\infty$ & 9.40E-01 & $2.48 E+\infty 0$ & $1.10 \mathrm{E}-01$ & $8.10 E+\infty 0$ & $2 / 26 / 96$ & 1 & 33655 & $\mathrm{BF}$ \\
\hline 2977 & SALT_T6 & RSH_WAS & $m$ & CONSTANT & $3.50 \mathrm{E}+\infty 0$ & $3.50 \mathrm{E}+\infty$ & $0.00 E+\infty 0$ & $3.50 \mathrm{E}+\infty$ & $3.50 E+\infty 0$ & $2 / 13 / 96$ & $4 \mathrm{~B}$ & $33482 A$ & $\mathrm{BF}$ \\
\hline 2987 & SALT_T6 & PCT_A & $\mathrm{Pa}$ & CONSTANT & $5.60 E-01$ & 5.60E-01 & $0.00 E+00$ & 5.60E-01 & $5.60 E-01$ & $2 / 13 / 96$ & 1 & 33642 & $\mathrm{BF}$ \\
\hline 2976 & SALT_T6 & RSH_SAL & $m$ & CONSTANT & $1.80 E+00$ & $1.80 \mathrm{E}+\infty 0$ & $0.00 \mathrm{E}+00$ & $1.80 \mathrm{E}+00$ & $1.80 E+\infty 0$ & $2 / 13 / 96$ & $4 B$ & $33481 \mathrm{~A}$ & BF \\
\hline 3126 & SALT_T6 & PO_MIN & $\mathrm{Pa}$ & CONSTANT & $1.01 E+05$ & $1.01 E+05$ & $0.00 E+\infty 0$ & $1.01 E+05$ & $1.01 E+05$ & $2 / 21 / 96$ & $4 B$ & 36369 & $B F$ \\
\hline 2980 & SALT_T6 & PMLT_MD & $\log \left(m^{\wedge} 2\right)$ & CONSTANT & $-2.03 E+01$ & $-2.03 E+01$ & $0.00 E+\infty 0$ & $-2.03 E+01$ & $-2.03 E+01$ & $2 / 13 / 96$ & 1 & 33651 & $B F$ \\
\hline 2981 & SALT_T6 & PMLT_LO & $\log \left(m^{\wedge} 2\right)$ & CONSTANT & $-2.29 E+01$ & $-2.29 E+01$ & $0.00 E+\infty$ & $-2.29 E+01$ & $-2.29 E+01$ & $2 / 13 / 96$ & 1 & 33650 & $\overline{B F}$ \\
\hline 2988 & SALT_T6 & PCT_EXP & NONE & CONSTANT & $-3.46 E-01$ & $-3.46 E-01$ & $0.00 E+00$ & $-3.46 \mathrm{E}-01$ & $-3.46 E-01$ & $2 / 13 / 96$ & 1 & 33645 & $B F$ \\
\hline 2986 & SALT_T6 & PC_MAX & $\mathrm{Pa}$ & CONSTANT & $1.00 E+08$ & $1.00 E+08$ & $0.00 E+00$ & $1.00 E+08$ & $1.00 E+08$ & $2 / 13 / 96$ & $4 B$ & $33647 A$ & BF \\
\hline 2985 & SALT_T6 & KPT & NONE & CONSTANT & $0.00 E+\infty$ & $0.00 \mathrm{E}+00$ & $0.00 E+\infty$ & $0.00 \mathrm{E}+\infty$ & $0.00 E+00$ & $2 / 20 / 96$ & $4 B$ & 33640 & $B F$ \\
\hline 2984 & SALT_T6 & COMP_RCK & $P a \wedge 1$ & CONSTANT & 1.60E-09 & 1.60E-09 & $0.00 \mathrm{E}+00$ & $1.60 E-09$ & 1.60E-09 & $2 / 13 / 96$ & 1 & 33635 & $B F$ \\
\hline 2983 & SALT_T6 & CAP_MOD & NONE & CONSTANT & $2.00 \mathrm{E}+00$ & $2.00 E+\infty$ & $0.00 E+00$ & $2.00 E+\infty$ & $2.00 E+00$ & 2/13/96 & 48 & $33633 \mathrm{~A}$ & $\mathrm{BF}$ \\
\hline 2993 & SALT_T6 & SAT_RGAS & NONE & UNIFORM & 2.00E-01 & $2.00 E-01$ & 1.16E-01 & $0.00 \mathrm{E}+\infty$ & 4.00E-01 & $2 / 13 / 96$ & 1 & 33484 & $\mathrm{BF}$ \\
\hline 2982 & SALT_T6 & PMLT_HI & $\log \left(m^{\wedge} 2\right)$ & CONSTANT & $-1.77 E+01$ & $-1.77 E+01$ & $0.00 E+\infty 0$ & $-1.77 E+01$ & $-1.77 E+01$ & $2 / 13 / 96$ & 1 & 33648 & $\mathrm{BF}$ \\
\hline
\end{tabular}




\section{Upper Shaft Clay}

\begin{tabular}{|c|c|c|c|c|c|c|c|c|c|c|c|c|c|}
\hline ID & Material & Parameter & Units & Distribution & Mean & Median & Deviation & Low & High & Entry Date & Impt & WPO & Codes \\
\hline 3084 & CL_M_T1 & RSH_AIR & $m$ & CONSTANT & $3.09 \mathrm{E}+\infty$ & $3.09 \mathrm{E}+\infty$ & $0.00 E+\infty$ & $3.09 E+00$ & $3.09 E+\infty$ & $2 / 15 / 96$ & $4 B$ & $32056 \mathrm{~A}$ & BF \\
\hline 2378 & CL_M_T1 & CAP_MOD & NONE & CONSTANT & $2.00 E+\infty 0$ & $2.00 E+\infty 0$ & $0.00 E+00$ & $2.00 E+\infty$ & $2.00 E+\infty 0$ & $2 / 15 / 96$ & $4 B$ & $32028 \mathrm{~A}$ & $\mathrm{BF}$ \\
\hline 2394 & CL_M_T1 & SAT_RGAS & NONE & UNIFORM & $2.00 E-01$ & 2.00E-01 & $1.16 E-01$ & $0.00 E+\infty 0$ & 4.00E-01 & $2 / 15 / 96$ & 1 & 32062 & $\mathrm{BF}$ \\
\hline 2393 & $C L \_M_{-}{ }_{1} 1$ & SAT_RBRN & NONE & CUMULATIVE & $2.50 E-01$ & 2.00E-01 & $1.76 \mathrm{E}-01$ & $0.00 E+00$ & $6.00 E-01$ & $2 / 15 / 96$ & 1 & 32061 & BF \\
\hline 2392 & $C L \_M_{-} T 1$ & SAT_IBRN & NONE & CONSTANT & $7.90 E-01$ & 7.90E-01 & $0.00 E+00$ & $7.90 \mathrm{E}-01$ & 7.90E-01 & $2 / 15 / 96$ & 1 & 32060 & $\mathrm{BF}$ \\
\hline 3086 & CL_M_T1 & RSH_WAS & $m$ & CONSTANT & $3.50 E+\infty 0$ & $3.50 \mathrm{E}+\infty 0$ & $0.00 E+00$ & $3.50 E+\infty$ & $3.50 E+\infty 0$ & $2 / 15 / 96$ & 4B & $32059 \mathrm{~A}$ & $\mathrm{BF}$ \\
\hline 3087 & CL_M_T1 & RSH_EXH & $m$ & CONSTANT & $2.30 E+\infty 0$ & $2.30 E+\infty$ & $0.00 E+00$ & $2.30 E+00$ & $2.30 E+\infty$ & $2 / 15 / 96$ & $4 B$ & 32057A & $\mathrm{BF}$ \\
\hline 2391 & CL_M_T1 & RELP_MOD & NONE & CONSTANT & $4.00 \mathrm{E}+00$ & $4.00 E+\infty$ & $0.00 E+00$ & $4.00 E+\infty 0$ & $4.00 E+\infty$ & $2 / 15 / 96$ & 48 & 32055A & $\mathrm{BF}$ \\
\hline 3088 & CL_M_TI & RADN_DRZ & $\mathrm{m} / \mathrm{m}$ & CONSTANT & $1.71 E+\infty$ & $1.71 E+\infty$ & $0.00 E+00$ & $1.71 E+\infty$ & $1.71 E+\infty$ & $2 / 15 / 96$ & 1 & 32053 & $\mathrm{BF}$ \\
\hline 2387 & CL_M_T1 & PAMZ_LOG & $\log \left(m^{\wedge} 2\right)$ & TRIANGULAR & $-1.89 E+01$ & $-1.83 E+01$ & 7.81E-01 & $-2.10 E+01$ & $-1.73 E+01$ & $2 / 15 / 96$ & 1 & 32050 & $B F$ \\
\hline 2386 & CL_M_T1 & PRMY_LOG & $\log \left(m^{\wedge} 2\right)$ & TRIANGULAR & $-1.89 E+01$ & $-1.83 E+01$ & 7.81E-01 & $-2.10 E+01$ & $|-1.73 E+01|$ & $2 / 15 / 96$ & 1 & 32049 & $\mathrm{BF}$ \\
\hline 2380 & CL_M_T1 & PC_MAX & $\mathrm{Pa}$ & CONSTANT & $1.00 E+08$ & $1.00 E+08$ & $0.00 E+00$ & $1.00 \mathrm{E}+08$ & $1.00 E+08$ & $11 / 3 / 95$ & $4 B$ & $32043 A$ & $\mathrm{BF}$ \\
\hline 3085 & CL_M_T1 & RSH_SAL & $m$ & CONSTANT & $1.80 E+00$ & $1.80 E+\infty 0$ & $0.00 E+\infty 0$ & $1.80 E+\infty 0$ & $1.80 E+\infty 0$ & $2 / 15 / 96$ & $4 B$ & 32058A & $\mathrm{BF}$ \\
\hline 2656 & CL_M_T1 & KPT & NONE & CONSTANT & $0.00 \mathrm{E}+00$ & $0.00 E+\infty$ & $0.00 E+\infty$ & $0.00 E+\infty$ & $0.00 E+\infty$ & $2 / 20 / 96$ & $4 B$ & 32040 & $B F$ \\
\hline 2385 & CL_M_T1 & PRMX_LOG & $\log \left(m^{\wedge} 2\right)$ & TRIANGULAR & $-1.89 E+01$ & $-1.83 E+01$ & 7.81E-01 & $-2.10 E+01$ & $-1.73 E+01$ & $2 / 15 / 96$ & 1 & 32048 & $\mathrm{BF}$ \\
\hline 2657 & CL_M_T1 & PCT_A & $\mathrm{Pa}$ & CONSTANT & 5.60E-01 & 5.60E-01 & $0.00 E+\infty 0$ & $5.60 \mathrm{E}-01$ & 5.60E-01 & $2 / 15 / 96$ & 1 & 32041 & $\mathrm{BF}$ \\
\hline 2658 & CL_M_T1 & PCT_EXP & NONE & CONSTANT & $-3.46 E-01$ & $-3.46 E-01$ & $0.00 E+00$ & $-3.46 E-01$ & $-3.46 E-01$ & $2 / 15 / 96$ & 1 & 32042 & $\mathrm{BF}$ \\
\hline 2383 & CL_M_T1 & PO_MIN & $\mathrm{Pa}$ & CONSTANT & $1.01 E+05$ & $1.01 E+05$ & $0.00 E+\infty 0$ & $1.01 E+05$ & $1.01 E+05$ & 2/21/96 & $4 B$ & 32046 & $B F$ \\
\hline 2381 & CL_M_T1 & PORE_DIS & NONE & CUMULATIVE & $2.52 E+\infty 0$ & $9.40 E-01$ & $2.48 E+00$ & 1.10E-01 & $8.10 E+\infty$ & $2 / 20 / 95$ & 1 & 32044 & BF \\
\hline 2382 & CL_M_T1 & POROSITY & $m^{\wedge} 3 / m^{\wedge} 3$ & CONSTANT & 2.40E-01 & 2.40E-01 & $0.00 E+00$ & $2.40 E-01$ & 2.40E-01 & $2 / 15 / 96$ & 1 & 32045 & BF \\
\hline 2384 & CL_M_T1 & PRESSURE & $\mathrm{Pa}$ & CONSTANT & 1.01E+05 & $1.01 E+05$ & $0.00 E+00$ & 1.01E+05 & $1.01 E+05$ & $2 / 20 / 96$ & 1 & 32047 & $\mathrm{BF}$ \\
\hline 2379 & CL_M_T1 & COMP_RCK & $P a^{n-1}$ & CONSTANT & 1.81E-09 & 1.81E-09 & $0.00 \mathrm{E}+00$ & 1.81E-09 & 1.81E-09 & $2 / 15 / 96$ & 1 & 32039 & $B F$ \\
\hline 2404 & CL_M_T2 & PRMZ_LOG & $\log (m 2)$ & TRIANGULAR & $-1.89 E+01$ & $-1.83 E+01$ & 7.81E-01 & $-2.10 E+01$ & $-1.73 E+01$ & $2 / 15 / 96$ & 1 & 32125 & $\mathrm{BF}$ \\
\hline 2661 & $\mathrm{CL}_{-} \mathrm{M}$ & KPT & NONE & CONSTANT & $0.00 E+00$ & $0.00 E+00$ & $0.00 E+\infty$ & $0.00 E+\infty 0$ & $0.00 E+\infty$ & $2 / 20 / 96$ & $4 B$ & 32067 & $\mathrm{BF}$ \\
\hline 2411 & CL_M & SAT & NONE & UNIFORM & $2.00 \mathrm{E}-01$ & $2.00 E-01$ & 1.16E-01 & $0.00 E+\infty 0$ & $4.00 E-01$ & $2 / 15 / 96$ & 1 & 32137 & $\mathrm{BF}$ \\
\hline 2410 & CL_M_T2 & SAT_RBRN & NONE & CUMULATIVE & 2.50E-01 & 2.00E-01 & 1.76E-01 & $0.00 E+\infty$ & $6.00 E-01$ & $2 / 15 / 96$ & 1 & 32136 & $\mathrm{BF}$ \\
\hline 3091 & CL_M_T2 & RSH_WAS & $m$ & CONSTANT & $3.50 E+00$ & $3.50 E+\infty$ & $0.00 E+00$ & $3.50 E+\infty 0$ & $3.50 E+00$ & $2 / 15 / 96$ & 4B & $32134 A$ & $B F$ \\
\hline 3090 & CL_M_T2 & RSH_SAL & $m$ & CONSTANT & $1.80 E+\infty$ & $1.80 E+\infty 0$ & $0.00 E+00$ & $1.80 E+00$ & $1.80 E+00$ & $2 / 15 / 96$ & $4 B$ & $32133 A$ & $B F$ \\
\hline 3092 & CL_M_T2 & RSH_EXH & m & CONSTANT & $2.30 E+\infty$ & $2.30 E+\infty$ & $0.00 E+00$ & $2.30 E+00$ & $2.30 E+00$ & $2 / 15 / 96$ & $4 B$ & $32132 A$ & BF \\
\hline 3089 & CL_M_T2 & RSH_AIR & $m$ & CONSTANT & $3.09 E+\infty 0$ & $3.09 E+00$ & $0.00 E+\infty$ & $3.09 E+\infty 0$ & $3.09 E+00$ & $2 / 5 / 96$ & $4 B$ & $32131 \mathrm{~A}$ & $\mathrm{BF}$ \\
\hline 2408 & CL_M_T2 & RELP_MOD & NONE & CONSTANT & $4.00 E+00$ & $4.00 E+00$ & $0.00 E+\infty$ & $4.00 E+00$ & $4.00 E+00$ & $2 / 15 / 96$ & $4 \mathrm{~B}$ & $32130 \mathrm{~A}$ & $B F$ \\
\hline 3093 & CL_M_T2 & RADN_DRZ & $\mathrm{m} / \mathrm{m}$ & CONSTANT & $1.47 E+\infty$ & $1.47 E+\infty$ & $0.00 E+\infty$ & $1.47 E+\infty$ & $1.47 E+\infty$ & $2 / 15 / 96$ & 1 & 32128 & $\mathrm{BF}$ \\
\hline 2396 & CL_M_T2 & COMP_RCK & $P a \wedge 1$ & CONSTANT & 1.81E-09 & 1.81E-09 & $0.00 E+\infty 0$ & 1.81E-09 & 1.81E-09 & $2 / 15 / 96$ & 1 & 32066 & $B F$ \\
\hline 2402 & CL_M_T2 & PRMX_LOG & $\log \left(m^{\wedge} 2\right)$ & TRIANGULAR & $-1.89 E+01$ & $-1.83 E+01$ & 7.81E-01 & $-2.10 \mathrm{E}+01$ & $-1.73 E+01$ & $2 / 15 / 96$ & 1 & 32123 & BF \\
\hline 2399 & CL_M_T2 & POROSITY & $m^{1} 3 / m^{13}$ & CONSTANT & $2.40 \mathrm{E}-01$ & 2.40E-01 & $0.00 E+\infty$ & 2.40E-01 & 2.40E-01 & 2/15/96 & 1 & 32120 & $\mathrm{BF}$ \\
\hline 2398 & $\mathrm{CL}_{-} \mathrm{M}_{-} \mathrm{T2}$ & PORE_DIS & NONE & CUMULATIVE & $2.52 E+\infty$ & $9.40 E-01$ & $2.48 \mathrm{E}+\infty 0$ & 1.10E-01 & $8.10 E+00$ & 2/20/96 & $=1$ & 32119 & $\mathrm{BF}$ \\
\hline 2400 & CL_M_T2 & PO_MIN & $\mathrm{Pa}$ & CONSTANT & $1.01 E+05$ & $1.01 E+05$ & $0.00 E+00$ & $1.01 E+05$ & $1.01 E+05$ & $2 / 21 / 96$ & $4 B$ & 32121 & $\mathrm{BF}$ \\
\hline 2663 & CL_M_T2 & PCT_EXP & NONE & CONSTANT & $-3.46 E-01$ & $-3.46 E-01$ & $0.00 \mathrm{E}+00$ & $-3.46 E-01$ & $-3.46 E-01$ & $2 / 15 / 96$ & 1 & 32117 & $\mathrm{BF}$ \\
\hline 2395 & CL_M_T2 & CAP_MOD & NONE & CONSTANT & $2.00 E+\infty 0$ & $2.00 E+00$ & $0.00 E+\infty$ & $2.00 E+00$ & $2.00 E+00$ & $2 / 15 / 96$ & $4 \mathrm{~B}$ & $32064 A$ & $\mathrm{BF}$ \\
\hline 2397 & CL_M_T2 & PC_MAX & $\mathrm{Pa}$ & CONSTANT & $1.00 E+08$ & $1.00 E+08$ & $0.00 E+\infty$ & $1.00 E+08$ & $1.00 E+08$ & $10 / 26 / 95$ & $4 \mathrm{~B}$ & $32118 \mathrm{~A}$ & $\mathrm{BF}$ \\
\hline 2403 & CL_M_T2 & PRMY_LOG & $\log \left(m^{1} 2\right)$ & TRIANGULAR & $-1.89 E+01$ & $-1.83 E+01$ & 7.81E-01 & $-2.10 E+01$ & $-1.73 E+01$ & $2 / 15 / 96$ & 1 & 32124 & $B F$ \\
\hline 2662 & CL_M_T2 & PCT_A & $\mathrm{Pa}$ & CONSTANT & 5.60E-01 & 5.60E-01 & $0.00 E+00$ & 5.60E-01 & 5.60E-01 & $2 / 15 / 96$ & 1 & 32116 & $\mathrm{BF}$ \\
\hline 2417 & CL_M_T3 & PO_MIN & $\mathrm{Pa}$ & CONSTANT & $1.01 E+05$ & $1.01 E+05$ & $0.00 E+00$ & $1.01 E+05$ & $1.01 E+05$ & $2 / 21 / 96$ & $4 B$ & 32150 & $B F$ \\
\hline 2668 & CL_M_T3 & PCT_EXP & NONE & CONSTANT & $-3.46 E-01$ & $-3.46 E-01$ & $0.00 E+\infty$ & $-3.46 E-01$ & $-3.46 E-01$ & $2 / 15 / 96$ & 1 & 32144 & $B F$ \\
\hline 2667 & CL_M_T3 & PCT_A & $\mathbf{P a}$ & CONSTANT & 5.60E-01 & 5.60E-01 & $0.00 E+00$ & $5.60 \mathrm{E}-01$ & 5.60E-01 & $2 / 15 / 96$ & 1 & 32143 & $\mathrm{BF}$ \\
\hline 2414 & $C_{-} M_{-} T 3$ & PC_MAX & $\mathrm{Pa}$ & CONSTANT & $1.00 E+08$ & $1.00 E+08$ & $0.00 E+00$ & $1.00 E+08$ & $1.00 E+08$ & $10 / 26 / 95$ & $4 B$ & $32145 \mathrm{~A}$ & $\mathrm{BF}$ \\
\hline 2666 & CL_M_T3 & KPT & NONE & CONSTANT & $0.00 E+\infty 0$ & $0.00 E+00$ & $0.00 E+00$ & $0.00 E+\infty$ & $0.00 E+\infty$ & $2 / 20 / 96$ & $4 B$ & 32142 & $\mathrm{BF}$ \\
\hline 2413 & CL_M_T3 & COMP_RCK & $\mathrm{Pa}{ }^{\prime} 1$ & CONSTANT & 1.81E-09 & 1.81E-09 & $0.00 E+00$ & 1.81E-09 & 1.81E-09 & $2 / 15 / 96$ & 1 & 32141 & $\mathrm{BF}$ \\
\hline 2419 & CL_M_T3 & PRMX_LOG & $\log \left(m^{\wedge} 2\right)$ & TRIANGULAR & $-1.89 E+01$ & $-1.83 E+01$ & 7.81E-01 & $-2.10 E+01$ & $-1.73 E+01$ & $2 / 15 / 96$ & 1 & 32155 & $B F$ \\
\hline 2416 & CL_M_T3 & POROSITY & $\mathrm{m}^{\wedge} 3 / \mathrm{m}^{\wedge} 3$ & CONSTANT & 2.40E-01 & 2.40E-01 & $0.00 \mathrm{E}+00$ & 2.40E-01 & 2.40E-01 & $2 / 15 / 96$ & 1 & 32148 & $B F$ \\
\hline 2412 & CL_M_T3 & CAP_MOD & NONE & CONSTANT & $2.00 E+\infty$ & $2.00 E+00$ & $0.00 E+\infty$ & $200 E+\infty$ & $2.00 E+\infty 0$ & $2 / 15 / 96$ & $4 B$ & $32139 A$ & $\overline{B F}$ \\
\hline
\end{tabular}




\section{Upper Shaft Clay}

\begin{tabular}{|c|c|c|c|c|c|c|c|c|c|c|c|c|c|}
\hline ID & Material & Parameter & Units & Distribution & Mean & Median & Deviation & Low & High & Entry Date & Impt & WPO & Codes \\
\hline 2427 & CL_M_T3 & SAT_RBRN & NONE & CUMULATIVE & $2.50 E-01$ & 2.00E-01 & $1.76 E-01$ & $0.00 E+\infty$ & $6.00 E-01$ & $2 / 15 / 96$ & 1 & 32168 & $B F$ \\
\hline 2415 & L_M_T3 & PORE_DIS & NONE & CUMULATIVE & $2.52 E+\infty$ & $9.40 E-01$ & $2.48 E+\infty 0$ & 1.10E-01 & $8.10 E+\infty 0$ & $2 / 20 / 96$ & 1 & 32146 & $B F$ \\
\hline 2428 & CL_M_T3 & SAT_RGAS & NONE & UNIFORM & 2.00E-01 & 2.00E-01 & 1.16E-01 & $0.00 E+\infty 0$ & $4.00 E-01$ & 2/15/96 & 1 & 32169 & BF \\
\hline 3096 & CL_M_T3 & RSH_WAS & $m$ & CONSTANT & $3.50 E+00$ & $3.50 E+\infty$ & $0.00 \mathrm{E}+00$ & $3.50 \mathrm{E}+00$ & $3.50 E+00$ & $2 / 15 / 96$ & 4B & 32166A & $\mathrm{BF}$ \\
\hline 3095 & CL_M_T3 & RSH_SAL & $m$ & CONSTANT & $1.80 \mathrm{E}+00$ & $1.80 E+00$ & $0.00 E+00$ & $1.80 E+\infty 0$ & $1.80 E+\infty 0$ & $2 / 15 / 96$ & $4 \mathrm{~B}$ & 32165A & $B F$ \\
\hline 3097 & CL_M_T3 & RSH_EXH & $m$ & CONSTANT & $2.30 E+\infty$ & $2.30 \mathrm{E}+\infty 0$ & $0.00 E+\infty$ & $2.30 E+\infty 0$ & $2.30 \mathrm{E}+00$ & $2 / 15 / 96$ & 4B & $32164 \mathrm{~A}$ & $\mathrm{BF}$ \\
\hline 3094 & CL_M_T3 & RSH_AIR & $\mathrm{m}$ & CONSTANT & $3.09 E+\infty$ & 3.09E+00 & $0.00 E+00$ & $3.09 E+\infty 0$ & $3.09 E+\infty 0$ & $2 / 15 / 96$ & 4B & $32163 \mathrm{~A}$ & $B F$ \\
\hline 2425 & CL_M_T3 & AELP_MOD & NONE & CONSTANT & $4.00 E+\infty$ & $4.00 E+\infty$ & $0.00 E+\infty$ & $4.00 E+\infty$ & $4.00 E+\infty 0$ & $2 / 15 / 96$ & 48 & $32162 A$ & $\mathrm{BF}$ \\
\hline 3098 & CL_M_T3 & RADN_DAZ & $\mathrm{m} / \mathrm{m}$ & CONSTANT & $1.28 \mathrm{E}+00$ & $1.28 \mathrm{E}+\infty$ & $0.00 E+\infty$ & $1.28 E+\infty$ & $1.28 E+\infty$ & $2 / 15 / 96$ & 1 & 32160 & BF \\
\hline 2421 & CL_M_T3 & PRMZ_LOG & $\log \left(m^{\wedge} 2\right)$ & TRIANGULAR & $-1.89 E+01$ & $-1.83 E+01$ & 7.81E-01 & $-2.10 E+01$ & $-1.73 E+01$ & $2 / 15 / 96$ & 1 & 32157 & $B F$ \\
\hline 2420 & CL_M_T3 & PRMY_LOG & $\log \left(m^{\wedge} 2\right)$ & TRIANGULAR & $-1.89 \mathrm{E}+01$ & $-1.23 E+01$ & 7.81E-01 & $-2.10 E+01$ & $-1.73 E+01$ & $2 / 15 / 96$ & 1 & 32156 & $\mathrm{BF}$ \\
\hline 2442 & CL_M_T4 & RELP_MOD & NONE & CONSTANT & $4.00 E+\infty 0$ & $4.00 \mathrm{E}+\infty$ & $0.00 E+\infty$ & $4.00 \mathrm{E}+\infty 0$ & $4.00 E+00$ & $10 / 26 / 95$ & $4 \mathrm{~B}$ & $32209 \mathrm{~A}$ & $B F$ \\
\hline 2437 & CL_M_T4 & PRMY_LOG & $\log \left(m^{\wedge} 2\right)$ & TRIANGULAR & $-1.89 E+01$ & $-1.83 E+01$ & 7.81E-01 & $-2.10 E+01$ & $-1.73 E+01$ & 2/13/95 & 1 & 32203 & $\mathrm{BF}$ \\
\hline 2444 & CL_M_T4 & SAT_RBRN & NONE & CUMULATIVE & 2.50E-01 & $2.00 E-01$ & $1.76 E-01$ & $0.00 E+00$ & $6.00 E-01$ & $2 / 19 / 96$ & 1 & 32225 & $\mathrm{BF}$ \\
\hline 2921 & CL_M_T4 & RSH_WAS & $m$ & CONSTANT & $3.50 E+00$ & $3.50 E+\infty 0$ & $0.00 E+\infty$ & $3.50 E+00$ & $3.50 \mathrm{E}+\infty 0$ & $2 / 13 / 96$ & $4 B$ & $32215 A$ & $B F$ \\
\hline 2920 & CL_M_T4 & RSH_SAL & $\mathbf{m}$ & CONSTANT & $1.80 E+\infty$ & $1.80 E+\infty$ & $0.00 \mathrm{E}+00$ & $1.80 \mathrm{E}+\infty$ & $1.80 \mathrm{E}+00$ & $2 / 13 / 96$ & $4 B$ & $32214 A$ & $B F$ \\
\hline 2919 & CL_M_T4 & RSH_AIR & $\mathbf{m}$ & CONSTANT & $3.09 E+00$ & $3.09 \mathrm{E}+\infty 0$ & $0.00 \mathrm{E}+\infty 0$ & $3.09 E+00$ & $3.09 E+\infty 0$ & 2/13/96 & $4 B$ & $32212 A$ & $\mathrm{BF}$ \\
\hline 2923 & CL_M_T4 & RADN_DRZ & $\mathrm{m} / \mathrm{m}$ & CONSTANT & $1.11 E+\infty$ & $1.11 E+\infty$ & $0.00 E+00$ & $1.11 \mathrm{E}+00$ & $1.11 E+00$ & $2 / 13 / 96$ & 1 & 32207 & $\mathrm{BF}$ \\
\hline 2438 & CL_M_T4 & PRMZ_LOG & $\log \left(m^{\wedge} 2\right)$ & TRIANGULAR & $-1.89 E+01$ & $-1.83 E+01$ & 7.81E-01 & $-2.10 E+01$ & $-1.73 E+01$ & $2 / 13 / 96$ & 1 & 32204 & $B F$ \\
\hline 2445 & CL_M_T4 & SAT_RGAS & NONE & UNIFORM & 2.00E-01 & $2.00 E-01$ & 1.16E-01 & $0.00 \mathrm{E}+\infty 0$ & 4.00E-01 & $2 / 13 / 96$ & 1 & 32226 & $B F$ \\
\hline 2430 & CL_M_T4 & COMP_RCK & $P a^{n-1}$ & & 1.81E-09 & 1.81E-09 & $0.00 E+\infty$ & 1.81E-09 & 1.81E-09 & $2 / 13 / 96$ & 1 & 32193 & $\mathrm{BF}$ \\
\hline 2922 & CL_M_T4 & RSH_EXH & $\mathrm{m}$ & & $2.30 E+00$ & $2.30 E+\infty$ & $0.00 \mathrm{E}+\infty$ & $2.30 \mathrm{E}+\infty 0$ & $2.30 \mathrm{E}+\infty$ & $2 / 13 / 96$ & $4 B$ & $32213 \mathrm{~A}$ & $\mathrm{BF}$ \\
\hline 2436 & CL_M_T4 & LOG & $g\left(m^{\wedge} 2\right)$ & TRIANGULAR & $-1.89 E+01$ & $-1.83 E+01$ & 7.81E-01 & $-2.10 E+01$ & $-1.73 E+01$ & $2 / 19 / 96^{\circ}$ & 1 & 32202 & $\mathrm{BF}$ \\
\hline 2429 & CL_M_T4 & CAP_MOD & NONE & CONSTANT & $2.00 E+\infty$ & $2.00 E+\infty$ & $0.00 E+\infty$ & $2.00 \mathrm{E}+00$ & $2.00 E+00$ & $11 / 2 / 95$ & $4 B$ & $32191 \mathrm{~A}$ & $\mathrm{BF}$ \\
\hline 2671 & CL_M_T4 & KPT & NONE & CONSTANT & $0.00 E+00$ & $0.00 \mathrm{E}+00$ & $0.00 E+\infty$ & $0.00 E+\infty$ & $0.00 E+\infty 0$ & $2 / 20 / 96$ & $4 B$ & 32194 & $B F$ \\
\hline 2431 & CL_M_T4 & PC_MAX & $\mathrm{Pa}$ & CONSTANT & $1.00 E+08$ & $1.00 E+08$ & $0.00 E+\infty$ & $1.00 E+08$ & $1.00 \mathrm{E}+08$ & $10 / 26 / 95$ & $4 B$ & 32197A & $\mathrm{BF}$ \\
\hline 2672 & CL_M_T4 & PCT_A & $\mathrm{Pa}$ & CONSTANT & $5.60 E-01$ & $5.60 E-01$ & $0.00 E+\infty 0$ & $5.60 \mathrm{E}-01$ & 5.60E-01 & 2/13/96 & 1 & 32195 & $B F$ \\
\hline 2673 & CL_M_T4 & PCT_EXP & NONE & CONSTANT & $-3.46 E-01$ & $-3.46 E-01$ & $0.00 E+\infty 0$ & $-3.46 E-01$ & $-3.46 E-01$ & $2 / 13 / 96$ & 1 & 32196 & $\mathrm{BF}$ \\
\hline 2434 & CL_M_T4 & PO_MIN & $\mathrm{Pa}$ & CONSTANT & $1.01 E+05$ & $1.01 E+05$ & $0.00 \mathrm{E}+\infty$ & $1.01 E+05$ & $1.01 E+05$ & $2 / 21 / 96$ & $4 B$ & 32200 & $B F$ \\
\hline 2432 & CL_M_T4 & PORE_DIS & NONE & CUMULATIVE & $2.52 E+\infty$ & & $2.48 \mathrm{E}+00$ & $1.10 \mathrm{E}-01$ & $8.10 E+00$ & $2 / 20 / 96$ & 1 & 32198 & $B F$ \\
\hline 2433 & CL_M_T4 & POROSITY & $m^{\wedge} 3 / m^{\prime} 3$ & CONSTANT & 2.40E-01 & & $0.00 E+\infty$ & 2.40E-01 & 2.40E-01 & $2 / 13 / 96$ & 1 & 32199 & $B F$ \\
\hline 2461 & CL_M_T5 & SAT_RBAN & NONE & CUMULATIVE & $2.50 E-01$ & & 1.76E-01 & $0.00 E+00$ & $6.00 \mathrm{E}-01$ & $2 / 13 / 96$ & 1 & 32254 & $B F$ \\
\hline 2448 & CL_M_T5 & PC_MAX & $\mathrm{Pa}$ & CONSTANT & $1.00 E+08$ & $1.00 E+08$ & $0.00 E+00$ & $1.00 E+08$ & $1.00 E+08$ & $2 / 13 / 96$ & $4 B$ & 32234A & $B F$ \\
\hline 2454 & CL_M_T5 & PAMY_LOG & $\log \left(m^{\wedge} 2\right)$ & TRIANGULAR & $-1.89 E+01$ & $-1.83 E+01$ & 7.81E-01 & $-2.10 E+01$ & $-1.73 E+01$ & $2 / 13 / 96$ & 1 & 32240 & $B F$ \\
\hline 2925 & CL_M_TS & RSH_SAL & $m$ & CONSTANT & $1.80 E+\infty$ & $1.80 E+\infty$ & $0.00 \mathrm{E}+\infty 0$ & $1.80 E+00$ & $1.80 E+00$ & $2 / 13 / 96$ & $4 B$ & $32251 \mathrm{~A}$ & BF \\
\hline 2927 & CL_M_T5 & RSH_EXH & $m$ & CONSTANT & $2.30 \mathrm{E}+00$ & $2.30 \mathrm{E}+\infty 0$ & $0.00 \mathrm{E}+00$ & $2.30 E+\infty 0$ & $2.30 E+00$ & $2 / 13 / 96$ & $-4 B$ & $32250 \mathrm{~A}$ & $B F$ \\
\hline 2924 & CL_M_T5 & RSH_AIR & $m$ & CONSTANT & $3.09 E+00$ & $3.09 E+00$ & $0.00 \mathrm{E}+\infty 0$ & $3.09 E+00$ & $3.09 E+00$ & $2 / 13 / 96$ & $4 B$ & $32249 \mathrm{~A}$ & $B F$ \\
\hline 2459 & CL_M_T5 & RELP_MOD & NONE & CONSTANT & $4.00 \mathrm{E}+\infty 0$ & $4.00 E+\infty$ & $0.00 E+\infty$ & $4.00 E+\infty$ & $4.00 E+00$ & $10 / 26 / 95$ & $4 B$ & $32248 \mathrm{~A}$ & $\mathrm{BF}$ \\
\hline 2928 & CL_M_T5 & RADN_DRZ & $\mathrm{m} / \mathrm{m}$ & CONSTANT & $1.00 \mathrm{E}+\infty 0$ & $1.00 \mathrm{E}+00$ & $0.00 E+00$ & $1.00 E+00$ & $1.00 E+\infty$ & $2 / 13 / 96$ & 1 & 32245 & $\mathrm{BF}$ \\
\hline 2455 & CL_M_T5 & PRMZ_LOG & $\log \left(m^{\wedge} 2\right)$ & TRIANGULAR & $-1.89 E+01$ & $-1.83 E+01$ & 7.81E-01 & $-2.10 E+01$ & $-1.73 E+01$ & $2 / 13 / 96$ & 1 & 32241 & $\mathrm{BF}$ \\
\hline 2926 & CL_M_T5 & RSH_WAS & $m$ & CONSTANT & $3.50 E+\infty 0$ & $3.50 E+00$ & $0.00 E+\infty 0$ & $3.50 \mathrm{E}+\infty 0$ & $3.50 E+\infty$ & $2 / 13 / 96$ & $4 B$ & $32252 A$ & $\mathrm{BF}$ \\
\hline 2453 & CL_M_T5 & PRMX_LOG & $\log \left(\pi^{n} 2\right)$ & TRIANGULAR & $-1.89 E+01$ & $-1.83 E+01$ & 7.81E-01 & $-2.10 E+01$ & $-1.73 E+01$ & $2 / 13 / 96$ & 1 & 32239 & $\mathrm{BF}$ \\
\hline 2450 & CL_M_T5 & POROSITY & $\mathrm{m}^{13 / \mathrm{m}^{13}}$ & CONSTANT & 2.40E-01 & 2.40E-01 & $0.00 E+\infty$ & 2.40E-01 & 2.40E-01 & $2 / 13 / 96$ & 1 & 32236 & $\mathrm{BF}$ \\
\hline 2449 & CL_M_T5 & PORE_DIS & NONE & CUMULATIVE & $2.52 E+\infty 0$ & $9.40 E-01$ & $2.48 \mathrm{E}+\infty 0$ & 1.10E-01 & $8.10 E+\infty$ & $2 / 20 / 96$ & 1 & 32235 & $\mathrm{BF}$ \\
\hline 2451 & CL_M_TS & PO_MIN & $\mathrm{Pa}$ & CONSTANT & $1.01 E+05$ & $1.01 E+05$ & $0.00 E+\infty$ & $1.01 E+05$ & 1.01E+05 & $2 / 21 / 96$ & $4 B$ & 32237 & BF \\
\hline 2677 & CL_M_T5 & PCT_A & $\mathrm{Pa}$ & CONSTANT & 5.60E-01 & 5.60E-01 & $0.00 \mathrm{E}+\infty 0$ & $5.60 \mathrm{E}-01$ & 5.60E-01 & $2 / 13 / 96$ & 1 & 32232 & $\mathrm{BF}$ \\
\hline 2676 & CL_M_T5 & KPT & NONE & CONSTANT & $0.00 E+00$ & $0.00 E+\infty$ & $0.00 E+00$ & $0.00 E+00$ & $0.00 E+00$ & $2 / 20 / 96$ & $4 B$ & 32231 & $\mathbf{B F}$ \\
\hline 2447 & CL_M_T5 & COMP_RCK & $P a \wedge-1$ & CONSTANT & $1.81 E-09$ & 1.81E-09 & $0.00 E+00$ & 1.81E-09 & 1.81E-09 & $2 / 13 / 96$ & 1 & 32230 & $B F$ \\
\hline 2446 & CL_M_T5 & CAP_MOD & NONE & CONSTANT & $2.00 E+\infty 0$ & $2.00 E+00$ & $0.00 E+\infty$ & $2.00 E+\infty 0$ & $2.00 E+\infty$ & $11 / 2 / 95$ & 4B & $32228 \mathrm{~A}$ & BF \\
\hline 2462 & CL_M_T5 & SAT_RGAS & NONE & UNIFORM & $2.00 \mathrm{E}-01$ & 2.00E-01 & 1.16E-01 & $0.00 E+\infty 0$ & $4.00 E-01$ & $2 / 13 / 96$ & 1 & 32255 & BF \\
\hline 2678 & CL_M_T5 & PCT_EXP & NONE & CONSTANT & $-3.46 E-01$ & -3.46E-01 & $0.00 E+\infty$ & $-3.46 E-01$ & $-3.46 E-01$ & $2 / 13 / 96$ & 1 & 32233 & BF \\
\hline
\end{tabular}




\section{Lower Shaft Clay}

\begin{tabular}{|c|c|c|c|c|c|c|c|c|c|c|c|c|c|}
\hline 10 & aterial & & nits & & Mean & & & Low & High & & Impt & WPO & Codes \\
\hline 2341 & & & NONE & CONSTANT & $7.90 E-01$ & 7.90E-01 & $0.00 E+\infty 0$ & 7.90E-01 & $7.90 E-01$ & /13/96 & 1 & 31920 & BF \\
\hline 2327 & CL_L & $O D$ & NONE & CONSTANT & $.00 E+00$ & $2.00 E+00$ & $0.00 E+\infty$ & $2.00 E+\infty$ & $2.00 E+\infty 0$ & $11 / 2 / 95$ & $4 \mathrm{~B}$ & $31897 \mathrm{~A}$ & BF \\
\hline 2342 & CL_L_T1 & SAT_ & NONE & TTVE & $.50 E-01$ & $2.00 E-01$ & $1.67 E-02$ & $1.00 E+\infty 0$ & $6.00 E-01$ & $113 / 96$ & & 31921 & $B F$ \\
\hline 3019 & CL_L_TI & RSH_WAS & $\mathbf{m}$ & CONSTANT & $3.50 \mathrm{E}+00$ & $3.50 E+00$ & $|0.00 E+\infty 0|$ & $3.50 E+\infty 0$ & $3.50 \mathrm{E}+00$ & 2/20/96 & 4B & $31919 \mathrm{~A}$ & BF \\
\hline 3018 & CL_L_T1 & RSH_SAL & $\mathrm{m}$ & CONSTANT & $1.80 E+\infty 0$ & $1.80 E+\infty$ & $0.00 E+00$ & $1.80 E+\infty$ & $1.80 E+\infty 0$ & 2/20/96 & 4B & $31918 \mathrm{~A}$ & $B F$ \\
\hline 3020 & CL_L_T1 & ASH_EXH & $m$ & CONSTANT & $2.30 E+00$ & $2.30 E+\infty$ & $0.00 \varepsilon+\infty$ & $2.30 E+\infty$ & $2.30 \mathrm{E}+\infty 0$ & $2 / 15 / 96$ & $4 B$ & $31917 \mathrm{~A}$ & $B F$ \\
\hline 3017 & CL_L_TI & RSH_AIR & $\mathrm{m}$ & CONSTANT & $3.09 E+\infty$ & $3.09 E+\infty$ & $0.00 E+\infty$ & $3.09 E+\infty$ & $3.09 E+\infty$ & $2 / 15 / 96$ & $4 B$ & $31916 \mathrm{~A}$ & BF \\
\hline 2340 & CL_L_T1 & RELP_MOD & NONE & CONSTANT & $4.00 E+\infty$ & $4.00 E+\infty 00$ & $0.00 E+\infty$ & $4.00 \mathrm{E}+\infty$ & $4.00 E+\infty$ & $10 / 26 / 95$ & $4 B$ & 31915A & BF \\
\hline 3021 & $C L_{-} L_{-} T 1$ & RADN & $\mathrm{m} / \mathrm{m}$ & CONSTANT & $1.86 \mathrm{E}+\infty$ & $1.86 \mathrm{E}+00$ & $0.00 E+00$ & $1.86 \mathrm{E}+\infty$ & $1.86 E+\infty$ & $2 / 15 / 96$ & & 31913 & $B F$ \\
\hline 2336 & $C L_{-} L_{-} T 1$ & PRMZ & $\log \left(m^{n} 2\right)$ & TRIANGULAR & $-1.89 E+01$ & $-1.83 E+01$ & $7.81 E-01$ & $-2.10 E+01$ & $-1.73 E+01$ & $2 / 13 / 96$ & & 31910 & $B F$ \\
\hline 2335 & CL_L_T1 & PRMY & $\log \left(m^{\prime} 2\right)$ & TRIAN & $-1.89 E+01$ & $-1.83 E+01$ & 7.81E-01 & $-2.10 E+01$ & $-1.73 E+01$ & 2/13/96 & & 31909 & $B F$ \\
\hline 2641 & CL_L_T1 & KPT & NONE & CONSTANT & $0.00 E+00$ & $0.00 E+00$ & $0.00 E+00$ & $0.00 E+\infty$ & $0.00 E+00$ & $2 / 20 / 96$ & $4 B$ & 31900 & $B F$ \\
\hline 2333 & CL_L_TI & PRES: & $\mathrm{Pa}$ & CONSTANT & $1.01 E+05$ & $1.01 E+05$ & $0.00 \mathrm{E}+\infty 0$ & $1.01 E+05$ & $1.01 E+05$ & $2 / 20 / 96$ & 1 & 31907 & BF \\
\hline 331 & CL_L_T1 & POROSITY & $\mathrm{m}^{\wedge} 3 / \mathrm{m}^{13} 3$ & CONSTANT & $2.40 E-01$ & $2.40 E-01$ & $0.00 E+00$ & $2.40 E-01$ & $2.40 E-01$ & $2 / 13 / 96$ & & 31905 & $B F$ \\
\hline 2330 & CL_L_T1 & PORE & NONE & CUMUL & $2.52 E+\infty$ & $9.40 E-01$ & $2.48 E+\infty$ & $1.10 E-01$ & $8.10 \mathrm{E}+\infty$ & $2 / 20 / 96$ & 1 & 31904 & $B F$ \\
\hline 2332 & CL_L_T1 & PO_MIN & $\mathrm{Pa}$ & CONSTANT & $1.01 E+05$ & $1.01 E+05$ & $0.00 E+00$ & $1.01 E+05$ & $1.01 E+05$ & $2 / 21 / 96$ & 4B & 31906 & BF \\
\hline 2643 & CL_LLT1 & PCT_EXF & NONE & CONSTANT & $-3.46 E-01$ & $-3.46 \mathrm{E}-01$ & $0.00 E+\infty 0$ & $-3.46 E-01$ & $-3.46 E-01$ & $2 / 13 / 96$ & & 31902 & BF \\
\hline 2642 & CL_L_T1 & PCT_A & $\mathrm{Pa}$ & CONSTANT & $5.60 E-01$ & $5.60 \mathrm{E}-01$ & $0.00 E+00$ & $5.60 E-01$ & $5.60 E-01$ & $2 / 13 / 96$ & & 31901 & $\mathrm{BF}$ \\
\hline 2329 & CL_L_T1 & PC_MAX & $\mathrm{Pa}$ & CONSTANT & $1.00 E+08$ & $1.00 E+08$ & $10.00 \mathrm{E}+00$ & $1.00 \mathrm{E}+08$ & $1.00 E+08$ & $2 / 13 / 96$ & $4 B$ & $31903 \mathrm{~A}$ & $B F$ \\
\hline 2328 & CL_L_TI & COMP & $\mathrm{Pa}^{2}-1$ & CONS & $1.59 E-09$ & $1.59 E-09$ & $0.00 E+00$ & $1.59 E-09$ & $1.59 E-09$ & $13 / 96$ & & 31899 & $B F$ \\
\hline 2343 & CL_L_T1 & SAT_RGAS & NONE & UNIFORM & $2.00 E-01$ & $2.00 E-01$ & $1.16 \mathrm{E}-01$ & $0.00 \mathrm{E}+\infty$ & $4.00 E-01$ & $2 / 13 / 96$ & & 31922 & $B F$ \\
\hline 2334 & CL_L_T1 & PRMX_LOG & $\log \left(m^{\prime} 2\right)$ & TRIANGULAR & $-1.89 E+01$ & $-1.83 E+01$ & 7.81E-01 & $-2.10 E+01$ & $-1.73 E+01$ & $13 / 96$ & 1 & 31908 & BF \\
\hline 2352 & CLLL_T2 & PRMY_LOG & $\log \left(m^{\prime} 2\right)$ & TRIANGULAR & $-1.89 E+01$ & $-1.83 E+01$ & 7.81E-01 & $-2.10 E+01$ & $-1.73 E+01$ & $2 / 14 / 96$ & 1 & 31936 & $\mathrm{BF}$ \\
\hline 2353 & CL_L_T2 & PRMZ & $\log \left(m^{2} 2\right)$ & TRIANGULAR & $-1.89 E+01$ & $-1.83 E+01$ & $7.81 E-01$ & $-2.10 E+01$ & $-1.73 E+01$ & $2 / 14 / 96$ & 1 & 31937 & $B F$ \\
\hline 3026 & CL_L_T2 & RADN_DRZ & $\mathrm{m} / \mathrm{m}$ & CONSTANT & $1.16 E+\infty$ & $1.16 \mathrm{E}+\infty$ & $0.00 E+00$ & $1.16 E+\infty$ & $1.16 E+\infty 0$ & $2 / 15 / 96$ & 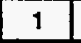 & 31959 & $\mathrm{BF}$ \\
\hline 2357 & CL_L_T2 & RELP_MOD & NONE & CONSTANT & $4.00 E+00$ & $4.00 E+00$ & $0.00 E+00$ & $4.00 E+00$ & $4.00 E+00$ & 114/96 & $4 B$ & 31962A & $\mathrm{BF}$ \\
\hline 3022 & CL_L_T2 & RSH_AIR & n & CONSTANT & $3.09 E+\infty$ & $3.09 \mathrm{E}+00$ & $0.00 E+00$ & $3.09 E+\infty$ & $3.09 E+00$ & $2 / 15 / 96$ & 4B & 31964A & $B F$ \\
\hline 2351 & CL_L_T2 & PRMX & $g\left(m^{\wedge} 2\right)$ & TRIANGULAR & $-1.89 E+01$ & $-1.83 E+01$ & 7.81E-01 & $-2.10 E+01$ & $-1.73 E+01$ & $2 / 14 / 96$ & 1 & 31935 & $B F$ \\
\hline 3023 & $C L_{-} L_{-} T 2$ & RSH_ & $m$ & CONSTANT & $1.80 E+00$ & $1.80 \mathrm{E}+\infty$ & $0.00 E+00$ & $1.80 E+\infty$ & $1.80 E+\infty$ & $2 / 15 / 96$ & $4 B$ & $31966 \mathrm{~A}$ & $B F$ \\
\hline 2346 & CL_L_T2 & $P C_{-} M$ & $\mathrm{~Pa}$ & CONS & $1.00 E+08$ & $1.00 E+08$ & $0.00 \mathrm{E}+00$ & $1.00 \mathrm{E}+08$ & $1.00 E+08$ & $2 / 14 / 96$ & $4 B$ & $31930 \mathrm{~A}$ & BF \\
\hline 3025 & CL_L_T2 & RSH_EXH & $m$ & CONSTANT & $2.30 E+00$ & $2.30 \mathrm{E}+00$ & $0.00 E+00$ & $2.30 E+\infty 0$ & $2.30 E+00$ & $2 / 15 / 96$ & $4 B$ & 31965A & $B F$ \\
\hline 348 & CL_L_T2 & POROSITY & $3 / \mathrm{m}^{\wedge} 3$ & CONSTANT & $2.40 E-01$ & $2.40 E-01$ & $0.00 E+\infty$ & $2.40 E-01$ & $2.40 E-01$ & 114/96 & & 31932 & $B F$ \\
\hline 2347 & $C L_{-} L_{-} T 2$ & PORE_DIS & NONE & CUMULATIVE & $2.52 E+\infty 0$ & $9.40 E-01$ & $2.48 E+00$ & 1.10E-01 & $8.10 E+00$ & $220 / 96$ & 1 & 31931 & BF \\
\hline 2349 & L_L_T2 & PO_MI & $\mathrm{Pa}$ & CONSTANT & $1.01 E+05$ & $1.01 E+05$ & $0.00 E+00$ & $1.01 E+05$ & $1.01 E+05$ & $2 / 21 / 96$ & 4B & 31933 & $B F$ \\
\hline 2647 & CL_L_T2 & PCT_A & $\mathrm{Pa}$ & CONSTANT & $5.60 \mathrm{E}-01$ & $5.60 E-01$ & $0.00 E+\infty$ & $5.60 E-01$ & $5.60 E-01$ & $2 / 14 / 96$ & 1 & 31928 & BF \\
\hline 2646 & CL_L_T2 & KPT & NONE & CONSTANT & $0.00 \mathrm{E}+\infty$ & $0.00 E+00$ & $0.00 \mathrm{E}+\infty$ & $0.00 \mathrm{E}+00$ & $0.00 \mathrm{E}+00$ & $20 / 96$ & $4 B$ & 31927 & $\mathrm{BF}$ \\
\hline 2345 & CL_L_T2 & COMP_RCK & & CONSTANT & $1.59 E-09$ & $1.59 E-09$ & $0.00 E+\infty 0$ & $1.59 E-09$ & $1.59 E-09$ & 2/14/96 & 1 & 31926 & $B F$ \\
\hline 2344 & CL_L_T2 & CAP_MOD & NONE & CONSTANT & $2.00 E+00$ & $2.00 \mathrm{E}+00$ & $0.00 E+\infty 0$ & $2.00 E+00$ & $2.00 E+00$ & $2 / 14 / 96$ & $4 \mathrm{~B}$ & $31924 \mathrm{~A}$ & $B F$ \\
\hline 2359 & CL_L_T2 & SAT_RBRN & NONE & CUMULATIVE & $2.50 E-01$ & $2.00 E-01$ & $1.76 E-01$ & $0.00 E+00$ & $6.00 E-01$ & 2/14/96 & & 31969 & $B F$ \\
\hline 360 & CL_L_T2 & SAT_F & ONE & UNIFORM & $2.00 E-01$ & $2.00 E-01$ & $1.16 E-01$ & $0.00 E+\infty$ & $4.00 E-01$ & $14 / 96$ & 1 & 31971 & $\mathrm{BF}$ \\
\hline 2648 & CL_L_T2 & PCT_E & NONE & CONSTANT & $-3.46 E-01$ & $-3.46 E-01$ & $0.00 E+00$ & $-3.46 E-01$ & $-3.46 E-01$ & $114 / 96$ & 1 & 31929 & $B F$ \\
\hline 3024 & $C L_{-} L_{-} T 2$ & RSH_WAS & $\mathbf{m}$ & CONSTANT & $3.50 E+\infty$ & $3.50 E+\infty 0$ & $0.00 E+00$ & $3.50 E+\infty$ & $3.50 \mathrm{E}+\infty$ & $2 / 15 / 96$ & 4B & $31967 \mathrm{~A}$ & BF \\
\hline 2376 & CL_L_T3 & SAT_RBRN & NONE & CUMULATIVE & $2.50 E-01$ & $2.00 E-01$ & $1.76 E-01$ & $0.00 E+\infty$ & $6.00 \mathrm{E}-01$ & 2/14/96 & 1 & 32004 & $B F$ \\
\hline 377 & CL_L_T3 & SAT_F & NONE & UNIFORM & $2.00 E-01$ & $2.00 E-01$ & $1.16 E-01$ & $0.00 E+\infty$ & 4.00E-01 & $2 / 14 / 96$ & 1 & 32005 & BF \\
\hline 3029 & CL_L_T3 & RSH_WAS & $\mathbf{m}$ & CONSTANT & $3.50 E+00$ & $3.50 \mathrm{E}+00$ & $0.00 E+00$ & $3.50 E+\infty 0$ & $3.50 \mathrm{E}+00$ & $2 / 15 / 96$ & 48 & $32002 A$ & $B F$ \\
\hline 3028 & $C_{\text {L_L_T3 }}$ & RSH_SAL & $m$ & CONSTANT & $1.80 E+00$ & $1.80 \mathrm{E}+00$ & $0.00 \mathrm{E}+00$ & $1.80 E+\infty$ & $1.80 E+00$ & $2 / 15 / 96$ & $4 B$ & $32001 \mathrm{~A}$ & $B F$ \\
\hline 3030 & CL_L_T3 & RSH_EXH & $\mathbf{m}$ & CONSTANT & $2.30 E+00$ & $2.30 E+00$ & $0.00 E+00$ & $2.30 E+\infty$ & $2.30 E+00$ & $2 / 15 / 96$ & 4B & $32000 \mathrm{~A}$ & $B F$ \\
\hline 027 & CL_L_T3 & RSH_AIR & & CONSTANT & $3.09 \mathrm{E}+\infty$ & $3.09 \mathrm{E}+00$ & $0.00 \mathrm{E}+\infty 0$ & $3.09 E+\infty$ & $3.09 E+00$ & $5 / 96$ & $4 B$ & $31999 \mathrm{~A}$ & $B F$ \\
\hline 2374 & CL_L_T3 & RELP_MOD & NONE & CONSTANT & $4.00 E+00$ & $4.00 E+00$ & $0.00 E+00$ & $4.00 E+00$ & $4.00 E+\infty 0$ & $2 / 14 / 96$ & $4 B$ & 31998A & $\mathrm{BF}$ \\
\hline 3031 & CL_L_T3 & RADN_DRZ & $\mathrm{m} / \mathrm{m}$ & CONSTANT & $1.00 E+00$ & $1.00 E+\infty$ & $0.00 E+00$ & $1.00 \mathrm{E}+00$ & $1.00 E+\infty$ & $2 / 15 / 96$ & 1 & 31996 & $B F$ \\
\hline 370 & CL_L_T3 & PRMZ_LOG & $\log \left(m^{\wedge} 2\right)$ & TRIANGULAR & $-1.89 E+01$ & $-1.83 E+01$ & $7.81 E-01$ & $-2.10 E+01$ & $-1.73 E+01$ & $2 / 14 / 96$ & 1 & 31993 & $B F$ \\
\hline
\end{tabular}




\section{Lower Shaft Clay}

\begin{tabular}{|c|c|c|c|c|c|c|c|c|c|c|c|c|c|}
\hline ID & Material & Parameter & Units & Distribution & Mean & Median & Deviation & Low & High & Entry Date & Impt & WPO & Codes \\
\hline 2369 & CL_L_T3 & PRMY_LOG & $\log \left(m^{n} 2\right)$ & TRIANGULAR & $-1.89 E+01$ & $-1.83 E+01$ & 7.81E-01 & $-2.10 E+01$ & $-1.73 E+01$ & 2/14/96 & 1 & 31992 & BF \\
\hline 2362 & CL_L_T3 & COMP_RCK & $\mathrm{Pa}^{\prime}-1$ & CONSTANT & 1.59E-09 & 1.59E-09 & $0.00 E+00$ & 1.59E-09 & 1.59E-09 & $2 / 14 / 96$ & 1 & 31976 & $\mathrm{BF}$ \\
\hline 2368 & CL_L_T3 & PRMX_LOG & $\log \left(m^{n} 2\right)$ & TRIANGULAR & $-1.89 E+01$ & $-1.83 E+01$ & 7.81E-01 & $-2.10 E+01$ & $-1.73 E+01$ & $2 / 14 / 96$ & 1 & 31990 & $\mathrm{BF}$ \\
\hline 2361 & CL_L_T3 & CAP_MOD & NONE & CONSTANT & $2.00 E+00$ & $2.00 E+\infty 0$ & $0.00 E+00$ & $2.00 E+\infty 0$ & $2.00 E+00$ & $2 / 14 / 96$ & $4 B$ & $31974 A$ & BF \\
\hline 2651 & CL_L_T3 & KPT & NONE & CONSTANT & $0.00 \mathrm{E}+00$ & $0.00 E+\infty$ & $0.00 E+00$ & $0.00 E+\infty 0$ & $0.00 E+00$ & $2 / 20 / 96$ & $4 B$ & 31978 & BF \\
\hline 2363 & CL_L_T3 & PC_MAX & $\mathrm{Pa}$ & CONSTANT & $1.00 E+08$ & $1.00 E+08$ & $0.00 E+00$ & $1.00 E+08$ & $1.00 E+08$ & 2/14/96 & $4 B$ & 31981A & $\mathrm{BF}$ \\
\hline 2652 & CL_L_T3 & PCT_A & $\mathrm{Pa}$ & CONSTANT & $5.60 \mathrm{E}-01$ & $5.60 \mathrm{E}-01$ & $0.00 E+\infty$ & $5.60 \mathrm{E}-01$ & 5.60E-01 & $2 / 14 / 96$ & 1 & 31979 & BF \\
\hline 2653 & CL_L_T3 & PCT_EXP & NONE & CONSTANT & $-3.46 E-01$ & $-3.46 E-01$ & $0.00 E+\infty$ & $-3.46 E-01$ & $-3.46 E-01$ & 2/14/96 & 1 & 31980 & $\mathrm{BF}$ \\
\hline 2366 & CL_L_T3 & PO_MIN & $\mathrm{Pa}$ & CONSTANT & $1.01 E+05$ & $1.01 E+05$ & $0.00 E+00$ & $1.01 E+05$ & 1.01E+05 & $2 / 21 / 96$ & $4 B$ & 31985 & $B F$ \\
\hline 2364 & CL_L_T3 & PORE_DIS & NONE & CUMULATIVE & $2.52 \mathrm{E}+\infty 0$ & 9.40E-01 & $2.48 E+\infty$ & 1.10E-01 & $8.10 E+\infty 0$ & $2 / 20 / 96$ & 1 & 31982 & BF \\
\hline 2365 & CL_L_T3 & POROSITY & $m^{n} 3 / m^{n} 3$ & CONSTANT & 2.40E-01 & 2.40E-01 & $0.00 \mathrm{E}+00$ & 2.40E-01 & $2.40 E-01$ & $2 / 14 / 96$ & 1 & 31984 & BF \\
\hline 3082 & CL_L_T4 & SAT_RBRN & NONE & CUMULATIVE & $2.50 E-01$ & $2.00 E-01$ & 1.76E-01 & $0.00 E+00$ & $6.00 \mathrm{E}-01$ & $2 / 15 / 96$ & 1 & 32025 & $\mathrm{BF}$ \\
\hline 3074 & CL_L_T4 & PCT_A & $\mathrm{Pa}$ & CONSTANT & $5.60 \mathrm{E}-01$ & $5.60 E-01$ & $0.00 E+00$ & 5.60E-01 & 5.60E-01 & $2 / 15 / 96$ & 1 & 32010 & $B F$ \\
\hline 3079 & CL_L_T4 & PRMY_LOG & $\log \left(m^{\wedge} 2\right)$ & TRIANGULAR & $-1.89 E+01$ & $-1.83 E+01$ & 7.81E-01 & $-2.10 E+01$ & $-1.73 E+01$ & $2 / 15 / 96$ & 1 & 32017 & $\mathrm{BF}$ \\
\hline 3066 & CL_L_T4 & RSH_SAL & $m$ & CONSTANT & $1.80 E+00$ & $1.80 E+\infty$ & $0.00 E+00$ & $1.80 E+00$ & $1.80 \mathrm{E}+00$ & $2 / 15 / 96$ & $4 B$ & $32023 \mathrm{~A}$ & $\mathrm{BF}$ \\
\hline 3068 & CL_L_T4 & RSH_EXH & $m$ & CONSTANT & $2.30 E+00$ & $2.30 \mathrm{E}+\infty$ & $0.00 E+00$ & $2.30 E+00$ & $2.30 E+\infty$ & $2 / 15 / 96$ & 4B & $32022 \mathrm{~A}$ & $B F$ \\
\hline 3065 & CL_L_T4 & RSH_AIR & $m$ & CONSTANT & $3.09 \mathrm{E}+00$ & $3.09 E+\infty 0$ & $0.00 E+00$ & $3.09 E+00$ & $3.09 E+00$ & $2 / 15 / 96$ & $4 B$ & 32021A & $\mathrm{BF}$ \\
\hline 3081 & CL_L_T4 & RELP_MOD & NONE & CONSTANT & $4.00 E+\infty$ & $4.00 E+\infty$ & $0.00 E+\infty$ & $4.00 E+\infty 0$ & $4.00 \mathrm{E}+00$ & $2 / 15 / 96$ & $4 B$ & $32020 \mathrm{~A}$ & $B F$ \\
\hline 3069 & CL_L_T4 & RADN_DRZ & $\mathrm{m} / \mathrm{m}$ & CONSTANT & $1.00 E+00$ & $1.00 E+\infty$ & $0.00 E+00$ & $1.00 E+\infty 0$ & $1.00 E+\infty 0$ & 2/15/96 & 1 & 32019 & $\mathrm{BF}$ \\
\hline 3080 & CL_L_T4 & PRMZ_LOG & $\log \left(m^{\wedge} 2\right)$ & TRIANGULAR & $-1.89 E+01$ & $-1.83 E+01$ & 7.81E-01 & $-2.10 E+01$ & $-1.73 E+01$ & $2 / 15 / 96$ & 1 & 32018 & $\mathrm{BF}$ \\
\hline 3067 & CL_L_T4 & RSH_WAS & $m$ & CONSTANT & 3.50E+00 & $3.50 E+\infty$ & $0.00 E+00$ & $3.50 \mathrm{E}+\infty 0$ & $3.50 E+00$ & $2 / 15 / 96$ & 4B & $32024 A$ & BF \\
\hline 3078 & CL_L_T4 & PRMX_LOG & $\log \left(m^{\wedge} 2\right)$ & TRIANGULAR & $-1.89 E+01$ & $-1.83 E+01$ & 7.81E-01 & $-2.10 E+01$ & $-1.73 E+01$ & $2 / 15 / 96$ & 1 & 32016 & $B F$ \\
\hline 3077 & CL_L_T4 & POROSITY & $m^{\wedge} 3 / m^{\wedge} 3$ & CONSTANT & 2.40E-01 & 2.40E-01 & $0.00 E+00$ & 2.40E-01 & 2.40E-01 & $2 / 15 / 96$ & 1 & 32014 & BF \\
\hline 3076 & CL_L_T4 & PORE_DIS & NONE & CUMULATIVE & $2.52 E+\infty$ & $9.40 \mathrm{E}-01$ & $2.48 E+\infty$ & $1.10 E-01$ & $8.10 E+\infty$ & $2 / 20 / 96$ & 1 & 32013 & BF \\
\hline 3070 & CL_L_T4 & CAP_MOD & NONE & CONSTANT & $2.00 \mathrm{E}+00$ & $2.00 E+00$ & $0.00 E+00$ & $2.00 E+\infty 0$ & $2.00 E+00$ & $2 / 15 / 96$ & 4B & $32007 \mathrm{~A}$ & BF \\
\hline 3075 & CL_L_T4 & PCT_EXP & NONE & CONSTANT & $-3.46 E-01$ & $-3.46 E-01$ & $0.00 \mathrm{E}+00$ & $-3.46 E-01$ & $-3.46 E-01$ & 2/15/96 & 1 & 32011 & $B F$ \\
\hline 3073 & CL_L_T4 & PC_MAX & $\mathrm{Pa}$ & CONSTANT & $1.00 E+08$ & $1.00 E+08$ & $0.00 E+00$ & $1.00 E+08$ & $1.00 E+08$ & $2 / 15 / 96$ & $4 B$ & $32012 A$ & BF \\
\hline 3072 & CL_L_T4 & KPT & NONE & CONSTANT & $0.00 E+00$ & $0.00 E+00$ & $0.00 \mathrm{E}+00$ & $0.00 E+\infty$ & $0.00 E+00$ & $2 / 20 / 96$ & $4 B$ & 32009 & $B F$ \\
\hline 3071 & CL_L_T4 & COMP_RCK & $P a \wedge 1$ & CONSTANT & 1.59E-09 & 1.59E-09 & $0.00 \mathrm{E}+00$ & 1.59E-09 & 1.59E-09 & $2 / 15 / 96$ & 1 & 32008 & $\mathrm{BF}$ \\
\hline 3083 & CL_L_T4 & SAT_RGAS & NONE & UNIFORM & 2.00E-01 & 2.00E-01 & 1.16E-01 & $0.00 E+00$ & $4.00 E-01$ & $2 / 15 / 96$ & 1 & 32027 & $\mathrm{BF}$ \\
\hline 3123 & CL_L_T4 & PO_MIN & $\mathrm{Pa}$ & CONSTANT & 1.01E+05 & $1.01 E+05$ & $0.00 E+00$ & $1.01 E+05$ & $1.01 E+05$ & $2 / 21 / 96$ & 48 & 36366 & $B F$ \\
\hline
\end{tabular}




\section{Bottom Clay Component}

\begin{tabular}{|c|c|c|c|c|c|c|c|c|c|c|c|c|c|}
\hline ID & aterial & Paran & Units & $\mathrm{on}$ & Mean & Median & Deviation & Low & High & Entry Date & Impt & WPO & Codes \\
\hline 316 & AY_BOT & PRESSURE & $\mathrm{Pa}$ & NT & $11 \mathrm{E}+05$ & $1.01 E+05$ & $0.00 E+00$ & $1.01 E+05$ & $1.01 E+05$ & 2/21/96 & $4 B$ & 31865 & BF \\
\hline 311 & AY_BOT & COMP_RCK & $\mathrm{Pa}^{\mathrm{A}}-1$ & & $E-09$ & 1.59E-09 & $.00 E+00$ & $59 \mathrm{E}-09$ & 1.59E-09 & $2 / 21 / 96$ & 4B & 31857 & BF \\
\hline 2636 & AY_BOT & KPT & NONE & & $10 E+00$ & $0.00 E+00$ & $0.00 E+00$ & $00 E+00$ & $0.00 E+00$ & 2/21/96 & $4 B$ & 31858 & BF \\
\hline 312 & AY_BOT & PC_MAX & $\mathrm{Pa}$ & ANT & $1.00 E+08$ & $1.00 E+08$ & $0.00 E+00$ & $1.00 E+08$ & $1.00 E+08$ & 11/3/95 & 4B & $31861 \mathrm{~A}$ & BF \\
\hline 637 & AY_BOT & PCT_A & $\mathrm{Pa}$ & & $5.60 E-01$ & 5.60E-01 & $0.00 E+00$ & 5.60E-01 & 5.60E-01 & 2/21/96 & 4B & 31859 & BF \\
\hline 638 & BOT & PCT_EXP & NONE & CONS & $-3.46 E-01$ & $\mid-3.46 E-01$ & $0.00 E+00$ & $-3.46 E-01$ & $-3.46 E-01$ & 2/21/96 & 4B & 31860 & $B F$ \\
\hline 315 & BOT & PO_MIN & & & & $1.01 E+05$ & $0.00 E+00$ & $1.01 E+05$ & $1.01 E+05$ & $2 / 21 / 96$ & 48 & 31864 & BF \\
\hline 310 & CLAY_BOT & CAP_MOD & IONE & CONS & $2.00 E+00$ & $2.00 E+00$ & $0.00 E+00$ & $2.00 E+00$ & $2.00 E+00$ & $11 / 3 / 95$ & 4B & 31855 & BF \\
\hline 314 & CLAY. & POROSITY & $3 / m^{\wedge} 3$ & CON: & 2.40E-01 & 2.40E-01 & $0.00 E+00$ & $2.40 E-01$ & $2.40 E-01$ & $2 / 21 / 96$ & $4 A$ & 31863 & BF \\
\hline 326 & CLAY_BOT & SAT_RC & NONE & UNIFC & $2.00 E-01$ & 2.00E-01 & 1.16E-01 & $0.00 E+00$ & 4.00 E-01 & $2 / 21 / 96$ & 1 & 31875 & BF \\
\hline 317 & CLAY_BOT & PRMX_LOG & $\log \left(m^{\wedge} 2\right)$ & TRIANGULAR & $-1.89 E+01$ & $-1.83 E+01$ & 7.81E-01 & $-2.10 E+01$ & $-1.73 E+01$ & $2 / 21 / 96$ & 1 & 31866 & BF \\
\hline 318 & CLAY_BOT & PRMY_LOG & $\log \left(m^{\wedge} 2\right)$ & TRIANGULAR & $-1.89 E+01$ & $-1.83 E+01$ & 7.81E-01 & $-2.10 E+01$ & $-1.73 E+01$ & $2 / 21 / 96$ & 1 & 31867 & BF \\
\hline 319 & CLAY_BOT & PRMZ_LOG & $\log \left(m^{\wedge} 2\right)$ & TRIANGULAR & $-1.89 E+01$ & $-1.83 E+01$ & 7.81E-01 & $-2.10 E+01$ & $-1.73 E+01$ & $2 / 21 / 96$ & 1 & 31868 & $B F$ \\
\hline 2323 & CLAY_BOT & RELP_MOD & NONE & CONSTANT & $4.00 E+00$ & $4.00 E+00$ & $0.00 E+00$ & $4.00 E+00$ & $4.00 E+00$ & $11 / 3 / 95$ & $4 B$ & 31872 & $\mathrm{BF}$ \\
\hline 24 & CLAY_BOT & SAT_IB & NONE & CONS & 7.90E-01 & $7.90 E-01$ & $0.00 E+00$ & 7.90E-01 & 7.90E-01 & $2 / 21 / 96$ & $4 A$ & 31873 & BF \\
\hline 25 & CLAY_BOT & SAT_RBRN & NONE & CUMULATIVE & 2.50E-01 & 2.00E-01 & 1.76E-01 & $0.00 E+00$ & $6.00 E-01$ & $4 / 12 / 96$ & $4 A$ & $31874 A$ & BF \\
\hline 13 & AY_BOT & ORE_DIS & NONE & CUMULATTVE & $2.52 \mathrm{E}+00$ & 9.40E-01 & $2.48 E+00$ & $1.10 \mathrm{E}-01$ & $8.10 E+00$ & $2 / 26 / 96$ & $4 \mathrm{~A}$ & 31862 & $B F$ \\
\hline
\end{tabular}

\section{Concrete Monolith}

\begin{tabular}{|c|c|c|c|c|c|c|c|c|c|c|c|c|c|}
\hline ID & Material & Parameter- & Units & Distribution & Mean & Median & Deviation & Low & High & Entry Date & Impt & WPO & Codes \\
\hline 3060 & CONC_MON & PRMY_LOG & $\log \left(m^{\wedge} 2\right)$ & CONSTANT & $-1.40 E+01$ & $-1.40 E+01$ & $0.00 E+00$ & $-1.40 E+01$ & $-1.40 E+01$ & $2 / 15 / 96$ & 1 & 32522 & BF \\
\hline 3052 & CONC_MON & COMP_RCK & $\mathrm{Pa}^{\wedge}-1$ & CONSTANT & 1.20E-09 & 1.20E-09 & $0.00 E+\infty 0$ & $1.20 E-09$ & 1.20E-09 & $5 / 2 / 96$ & $4 B$ & 32504 & $B F$ \\
\hline 3053 & CONC_MON & KPT & NONE & CONSTANT & $0.00 E+00$ & $0.00 E+00$ & $0.00 \mathrm{E}+00$ & $0.00 E+00$ & $0.00 E+00$ & $2 / 20 / 96$ & $4 B$ & 32507 & BF \\
\hline 3054 & CONC_MON & PC_MAX & $\mathbf{P a}$ & CONSTANT & $1.00 E+08$ & $1.00 \mathrm{E}+08$ & $0.00 \mathrm{E}+00$ & $1.00 E+08$ & $1.00 E+08$ & 2/15/96 & $4 B$ & $32512 A$ & BF \\
\hline 3055 & CONC_MON & PCT_A & $\mathrm{Pa}$ & CONSTANT & 5.60E-01 & 5.60E-01 & $0.00 E+00$ & $5.60 \mathrm{E}-01$ & $5.60 E-01$ & $2 / 15 / 96$ & 1 & 32508 & BF \\
\hline 3056 & CONC_MON & PCT_EXP & NONE & CONSTANT & $-3.46 E-01$ & $-3.46 E-01$ & $0.00 E+00$ & $-3.46 E-01$ & $-3.46 E-01$ & $2 / 15 / 96$ & 1 & 32510 & $\mathrm{BF}$ \\
\hline 3124 & CONC_MON & PO_MIN & $\mathrm{Pa}$ & CONSTANT & $1.01 E+05$ & $1.01 E+05$ & $0.00 E+00$ & $1.01 E+05$ & $1.01 E+05$ & $2 / 21 / 96$ & $4 B$ & 36367 & $\mathrm{BF}$ \\
\hline 3057 & CONC_MON & PORE_DIS & NONE & CUMULATIVE & $2.52 E+00$ & $9.40 \mathrm{E}-01$ & $2.48 E+00$ & 1.10E-01 & $8.10 \mathrm{E}+00$ & $2 / 20 / 96$ & 1 & 32514 & BF \\
\hline 3058 & CONC_MON & POROSITY & $m^{\wedge} 3 / m^{\wedge} 3$ & CONSTANT & $5.00 \mathrm{E}-02$ & $5.00 \mathrm{E}-02$ & $0.00 E+00$ & $5.00 E-02$ & $5.00 \mathrm{E}-02$ & $2 / 15 / 96$ & 1 & 32516 & $B F$ \\
\hline 3051 & CONC_MON & CAP_MOD & NONE & CONSTANT & $2.00 E+00$ & $2.00 E+00$ & $0.00 E+00$ & $2.00 E+00$ & $2.00 E+00$ & $2 / 15 / 96$ & $4 B$ & $32503 A$ & $B F$ \\
\hline 3059 & CONC_MON & PRMX_LOG & $\log \left(m^{\wedge} 2\right)$ & CONSTANT & $-1.40 E+01$ & $-1.40 E+01$ & $0.00 E+00$ & $-1.40 E+01$ & $-1.40 E+01$ & $2 / 15 / 96$ & 1 & 32520 & $B F$ \\
\hline 3064 & CONC_MON & SAT_RGAS & NONE & UNIFORM & 2.00E-01 & $2.00 E-01$ & 1.16E-01 & $0.00 E+00$ & 4.00E-01 & $2 / 15 / 96$ & 1 & 32546 & $\mathrm{BF}$ \\
\hline 3061 & CONC_MON & PRMZ_LOG & $\log \left(m^{\wedge} 2\right)$ & CONSTANT & $-1.40 \mathrm{E}+01$ & $-1.40 E+01$ & $0.00 E+00$ & $-1.40 E+01$ & $-1.40 E+01$ & $2 / 15 / 96$ & -1 & 32527 & $\mathrm{BF}$ \\
\hline 3050 & CONC_MON & RADN_DRZ & $\mathrm{m} / \mathrm{m}$ & CONSTANT & $1.00 E+00$ & $1.00 E+00$ & $0.00 E+00$ & $1.00 E+00$ & $1.00 E+00$ & $2 / 15 / 96$ & 1 & 32528 & $\mathrm{BF}$ \\
\hline 3062 & CONC_MON & RELP_MOD & NONE & CONSTANT & $4.00 \mathrm{E}+00$ & $4.00 E+00$ & $0.00 E+00$ & $4.00 E+00$ & $4.00 E+00$ & $2 / 15 / 96$ & $4 \mathrm{~B}$ & $32530 \mathrm{~A}$ & $\mathrm{BF}$ \\
\hline 3046 & CONC_MON & RSH_AIR & $m$ & CONSTANT & $3.09 E+00$ & $3.09 E+00$ & $0.00 E+00$ & $3.09 E+00$ & $3.09 E+00$ & $2 / 15 / 96$ & $4 B$ & 32532A & $\mathrm{BF}$ \\
\hline 3049 & CONC_MON & RSH_EXH & $m$ & CONSTANT & $2.30 E+00$ & $2.30 E+00$ & $0.00 E+00$ & 2.30E+00 & $2.30 E+00$ & $2 / 15 / 96$ & $4 B$ & $32534 \mathrm{~A}$ & BF \\
\hline 3047 & CONC_MON & RSH_SAL & $m$ & CONSTANT & $1.80 \mathrm{E}+00$ & $1.80 E+00$ & $0.00 E+00$ & $1.80 E+00$ & $1.80 E+00$ & $2 / 15 / 96$ & $4 B$ & 32537A & $B F$ \\
\hline 3048 & CONC_MON & RSH_WAS & $m$ & CONSTANT & $3.50 E+00$ & $3.50 E+00$ & $0.00 E+00$ & $3.50 E+00$ & $3.50 E+00$ & $2 / 15 / 96$ & $4 B$ & $32538 \mathrm{~A}$ & BF \\
\hline 3115 & CONC_MON & SAT_IBRN & NONE & CONSTANT & $1.00 E+00$ & $1.00 E+00$ & $0.00 E+00$ & $1.00 E+00$ & $1.00 \mathrm{E}+00$ & 2/28/96 & 1 & $32540 A$ & $B F$ \\
\hline 3063 & CONC_MON & SAT_RBRN & NONE & CUMULATIVE & $2.50 E-01$ & 2.00E-01 & $1.76 E-01$ & $0.00 E+00$ & $6.00 \mathrm{E}-01$ & $2 / 15 / 96$ & 1 & 32542 & $B F$ \\
\hline 3114 & CONC_MON & PRESSURE & $\mathrm{Pa}$ & CONSTANT & $1.01 E+05$ & $1.01 E+05$ & $0.00 E+00$ & $1.01 E+05$ & $1.01 E+05$ & $2 / 21 / 96$ & $4 A$ & 32518 & $\mathrm{BF}$ \\
\hline
\end{tabular}




\section{Santa Rosa Formation}

\begin{tabular}{|c|c|c|c|c|c|c|c|c|c|c|c|c|c|}
\hline ID & Material & meter & Units & Distribution & Mean & Median & Deviation & Low & High & Entry Date & Impt & WPO & Codes \\
\hline 343 & AANTAROS & PRESSURE & $\mathrm{Pa}$ & CONSTANT & $.01 E+05$ & $1.01 E+05$ & $0.00 E+\infty 0$ & $1.01 E+05$ & $1.01 E+05$ & $2 / 22 / 96$ & 1 & 33544 & $B F$ \\
\hline 337 & SANTAROS & COMP_RCK & $\mathrm{Pa}^{\mathrm{n}-1}$ & CONSTANT & 1.00E-08 & $1.00 E-08$ & $0.00 \mathrm{E}+00$ & $1.00 \mathrm{E}-08$ & $1.00 \mathrm{E}-08$ & $11 / 1 / 95$ & AB & 33487 & $B F$ \\
\hline 2768 & SANTAROS & KPT & NONE & |CONSTANT & $0.00 E+00$ & $0.00 E+\infty 0$ & $0.00 E+00$ & $0.00 E+00$ & $0.00 E+00$ & $2 / 20 / 96$ & $4 B$ & 33510 & BF \\
\hline 339 & SANTAROS & C_MAX & $\mathrm{Pa}$ & CONSTANT & $1.00 E+08$ & 1.00E+08 & $0.00 E+00$ & $1.00 E+08$ & $1.00 E+08$ & $2 / 20 / 96$ & 4B & 33530 & $B F$ \\
\hline 2769 & SANTAROS & PCT_A & $\mathrm{Pa}$ & CONSTANT & $0.00 E+00$ & $0.00 E+00$ & $0.00 E+00$ & $0.00 E+00$ & $0.00 E+00$ & $2 / 22 / 96$ & 48 & 33528 & BF \\
\hline 2770 & SANTAROS & PCT_EXP & NONE & CONSTANT & $0.00 E+00$ & $0.00 E+00$ & $0.00 E+00$ & $0.00 E+00$ & $0.00 E+00$ & $2 / 22 / 96$ & $4 B$ & 33529 & $B F$ \\
\hline 342 & SANTAROS & O_MIN & $\mathrm{Pa}$ & CONSTANT & $1.01 E+05$ & $1.01 E+05$ & $0.00 E+00$ & $1.01 E+05$ & $1.01 E+05$ & $2 / 21 / 96$ & $4 B$ & 33543 & $\mathrm{BF}$ \\
\hline 336 & SANTAROS & AP_MOD & NONE & CONS & $1.00 E+00$ & $1.00 E+00$ & $0.00 E+00$ & $1.00 \mathrm{E}+00$ & $1.00 E+00$ & $2 / 22 / 96$ & AB & 33485 & BF \\
\hline 341 & SANTAROS & OROSITY & NONE & CONSTANT & 1.75E-01 & 1.75E-01 & $0.00 E+00$ & 1.75E-01 & 1.75E-01 & $11 / 1 / 95$ & 4B & 33542 & $B F$ \\
\hline 352 & SANTAROS & SAT_RGAS & NONE & CONSTANT & 7.71E-02 & 7.71E-02 & $0.00 E+00$ & 7.71E-02 & 7.71E-02 & $2 / 20 / 96$ & $4 B$ & 33557 & BF \\
\hline 344 & SANTAROS & PRMX_LOG & $\log \left(m^{\wedge} 2\right)$ & CONSTANT & $-1.00 E+01$ & $-1.00 E+01$ & $0.00 E+00$ & $-1.00 E+01$ & $-1.00 E+01$ & $11 / 1 / 95$ & 4B & 33545 & $\mathrm{BF}$ \\
\hline 345 & SANTAROS & PRMY_LOG & $\log \left(m^{n} 2\right)$ & CONSTANT & $-1.00 E+01$ & $-1.00 E+01$ & $0.00 E+00$ & $-1.00 E+01$ & $-1.00 E+01$ & $11 / 1 / 95$ & $4 B$ & 33546 & $B F$ \\
\hline 346 & SANTAROS & PRMZ_LOG & $\log \left(m^{\wedge} 2\right)$ & CONSTANT & $-1.00 E+01$ & $-1.00 E+01$ & $0.00 E+00$ & $-1.00 E+01$ & $-1.00 E+01$ & $11 / 1 / 95$ & $4 B$ & 33547 & $B F$ \\
\hline 349 & SANTAROS & RELP_MOD & NONE & CONSTANT & $4.00 E+00$ & $4.00 E+00$ & $0.00 E+00$ & $4.00 E+00$ & $4.00 E+00$ & $11 / 1 / 95$ & 4B & 33550 & BF \\
\hline 350 & SANTAROS & SAT_IBRN & NONE & CONSTANT & 8.36E-02 & 8.36E-02 & $0.00 E+00$ & 8.36E-02 & 8.36E-02 & 2/22/96 & 4B & 33552 & $\mathrm{BF}$ \\
\hline 351 & SANTAROS & SAT_RBRN & NONE & CONSTANT & 8.36E-02 & 8.36E-02 & $0.00 E+00$ & 8.36E-02 & 8.36E-02 & $2 / 22 / 96$ & 4B & 33554 & $\mathrm{BF}$ \\
\hline 340 & SANTAROS & PORE_DIS & NONE & CONSTANT & $6.44 E-01$ & $6.44 \mathrm{E}-01$ & $0.00 E+00$ & $6.44 \mathrm{E}-01$ & 6.44E-01 & $2 / 20 / 96$ & $4 B$ & 33538 & $\overline{B F}$ \\
\hline
\end{tabular}

\section{Dewey Lake Formation}

\begin{tabular}{|c|c|c|c|c|c|c|c|c|c|c|c|c|c|}
\hline ID & Material & Parameter & Units & Distribution & Mean & Median & Deviation & Low & High & Entry Date & Impt & WPO & Codes \\
\hline 160 & DEWYLAKE & PRESSURE & $\mathbf{P a}$ & CONSTANT & $1.01 E+05$ & $1.01 E+05$ & $0.00 E+00$ & $1.01 E+05$ & $1.01 E+05$ & $2 / 8 / 96$ & 1 & 32733 & BF \\
\hline 154 & DEWYLAKE & COMP_RCK & $P a^{\wedge}-1$ & CONSTANT & $1.00 \mathrm{E}-08$ & $1.00 E-08$ & $0.00 E+00$ & $1.00 \mathrm{E}-08$ & $1.00 \mathrm{E}-08$ & 11/1/95 & 4B & 32802 & BF \\
\hline 2696 & DEWYLAKE & KPT & NONE & CONSTANT & $0.00 E+00$ & $0.00 E+00$ & $0.00 E+00$ & $0.00 E+00$ & $0.00 E+00$ & $2 / 20 / 96$ & 4B & 32703 & BF \\
\hline 156 & DEWYLAKE & PC_MAX & $\mathrm{Pa}$ & CONSTANT & $1.00 E+08$ & $1.00 E+08$ & $0.00 E+00$ & $1.00 \mathrm{E}+08$ & $1.00 E+08$ & 11/1/95 & $4 B$ & 32728 & BF \\
\hline 2697 & DEWYLAKE & PCT_A & $\mathrm{Pa}$ & CONSTANT & 2.60E-01 & 2.60E-01 & $0.00 E+00$ & 2.60E-01 & 2.60E-01 & $2 / 20 / 96$ & AB & 32725 & BF \\
\hline 2698 & DEWYLAKE & PCT_EXP & NONE & CONSTANT & $-3.48 E-01$ & $-3.48 E-01$ & $0.00 E+00$ & $-3.48 E-01$ & $-3.48 E-01$ & $2 / 20 / 96$ & 4B & 32727 & BF \\
\hline 159 & DEWYLAKE & PO_MIN & $\mathrm{Pa}$ & CONSTANT & 1.01E+05 & $1.01 E+05$ & $0.00 E+00$ & $1.01 E+05$ & $1.01 E+05$ & $2 / 21 / 96$ & 4B & 32732 & BF \\
\hline 153 & DEWYLAKE & CAP_MOD & NONE & CONSTANT & $2.00 E+00$ & $2.00 \mathrm{E}+00$ & $0.00 E+00$ & $2.00 E+00$ & $2.00 E+00$ & $2 / 20 / 96$ & 4B & 32797 & $B F$ \\
\hline 158 & DEWYLAKE & POROSITY & NONE & STUDENT & 1.43E-01 & 1.43E-01 & 7.66E-02 & $3.50 E-02$ & 2.48E-01 & 2/20/96 & 1 & 32731 & $B F$ \\
\hline 170 & DEWYLAKE & SAT_RGAS & NONE & CONSTANT & 7.71E-02 & 7.71E-02 & $0.00 E+00$ & 7.71E-02 & 7.71E-02 & $2 / 20 / 96$ & 4B & 32743 & BF \\
\hline 161 & DEWYLAKE & PRMX_LOG & $\log \left(m^{\wedge} 2\right)$ & CONSTANT & $-1.63 E+01$ & $-1.63 E+01$ & $0.00 E+00$ & $-1.63 E+01$ & $-1.63 E+01$ & 2/22/96 & 1 & 32734 & $B F$ \\
\hline 162 & DEWYLAKE & PRMY_LOG & $\log \left(m^{\wedge} 2\right)$ & CONSTANT & $-1.63 E+01$ & $-1.63 E+01$ & $0.00 E+00$ & $|-1.63 E+01|$ & $-1.63 E+01$ & $2 / 22 / 96$ & 1 & 32735 & BF \\
\hline 163 & DEWYLAKE & PRMZ_LOG & $\log \left(m^{\wedge} 2\right)$ & CONSTANT & $-1.63 E+01$ & $-1.63 E+01$ & $0.00 E+00$ & $-1.63 E+01$ & $-1.63 E+01$ & 2/22/96 & --1 & 32736 & BF \\
\hline 166 & DEWYLAKE & RELP_MOD & NONE & CONSTANT & $4.00 E+00$ & $4.00 E+00$ & $0.00 E+00$ & $4.00 E+00$ & $4.00 E+00$ & $11 / 1 / 95$ & 4B & 32739 & BF \\
\hline 167 & DEWYLAKE & SAL_USAT & NONE & CONSTANT & $8.36 E-02$ & $8.36 E-02$ & $0.00 E+00$ & 8.36E-02 & 8.36E-02 & $1 / 30 / 97$ & 5 & 32740 & BF \\
\hline 168 & DEWYLAKE & SAT_IBRN & NONE & CONSTANT & $1.00 \mathrm{E}+00$ & $1.00 E+00$ & $0.00 E+00$ & $1.00 \mathrm{E}+00$ & $1.00 E+00$ & $11 / 1 / 95$ & 4B & 32741 & BF \\
\hline 169 & DEWYLAKE & SAT_RBRN & NONE & CONSTANT & 8.36E-02 & 8.36E-02 & $0.00 E+00$ & 8.36E-02 & 8.36E-02 & $2 / 22 / 96$ & $4 B$ & 32742 & $B F$ \\
\hline 157 & DEWYLAKE & PORE_DIS & NONE & CONSTANT & 6.44E-01 & 6.44E-01 & $0.00 E+00$ & 6.44E-01 & 6.44E-01 & $2 / 20 / 96$ & 4B & 32730 & $\mathrm{BF}$ \\
\hline
\end{tabular}




\section{Forty Niner Member}

\begin{tabular}{|c|c|c|c|c|c|c|c|c|c|c|c|c|c|}
\hline ID & ial & leter & Units & Distribution & Mean & Median & Deviation & Low & High & Entry Date & Impt & WPO & Codes \\
\hline 2094 & FORTYNIN & SAT_R & NONE & & 2.00E-01 & $2.00 E-01$ & $0.00 E+00$ & 2.00E-01 & 2.00E-01 & $11 / 1 / 95$ & $4 \mathrm{~B}$ & 33024 & BF \\
\hline 2240 & ORTYNIN & AT_RBRN & NONE & & 2.00E-01 & 2.00E-01 & $0.00 E+00$ & $2.00 E-01$ & $2.00 E-01$ & $11 / 1 / 95$ & 48 & 33022 & $\mathrm{BF}$ \\
\hline 2093 & ORTYNIN & RELP_MOD & NONE & CONSTANT & $4.00 E+00$ & $4.00 E+00$ & $0.00 E+00$ & $4.00 E+00$ & $4.00 E+00$ & $11 / 1 / 95$ & $4 B$ & 33019 & BF \\
\hline 2901: & FORTYNIN & PRMZ_LOG & $\log \left(m^{\wedge} 2\right)$ & CONSTANT & $-3.50 E+01$ & $-3.50 E+01$ & $0.00 E+00$ & $-3.50 E+01$ & $-3.50 E+01$ & $2 / 11 / 96$ & $4 B$ & 33010 & $B F$ \\
\hline 2900 & FORTYNIN & PRMY_LOG & $\log \left(m^{\wedge} 2\right)$ & ONSTANT & $-3.50 E+01$ & $-3.50 E+01$ & $0.00 E+00$ & $-3.50 E+01$ & $-3.50 E+01$ & $2 / 11 / 96$ & $4 B$ & 33008 & BF \\
\hline 2899 & FORTYNIN & PRMX_LOG & $\log \left(m^{\wedge} 2\right)$ & TANT & $-3.50 E+01$ & $-3.50 E+01$ & $0.00 E+00$ & $-3.50 E+01$ & $-3.50 E+01$ & $1 / 96$ & 4B & 33002 & $B F$ \\
\hline 2088 & FORTYNIN & PORC & NONE & STUDENT & 8.20E-02 & $8.20 E-02$ & 1.03E-01 & & & & 1 & 32995 & $B F$ \\
\hline 087 & FORTYNIN & PORE_DIS & NONE & CONSTANT & 7.00E-01 & 7.00E-01 & $0.00 E+00$ & 7.00E-01 & $7.00 E-01$ & 11/1/95 & 4B & 32991 & $B F$ \\
\hline 18 & FORTYNIN & PO_MIN & $\mathrm{Pa}$ & CONSTANT & 1.01E+05 & $1.01 E+05$ & $0.00 \mathrm{E}+00$ & $1.01 E+05$ & $1.01 E+05$ & $2 / 21 / 96$ & 4B & 32997 & $B F$ \\
\hline 2717 & FORTYNIN & PCT_EXP & NONE & & $0.00 E+00$ & $0.00 E+00$ & $0.00 E+00$ & $0.00 E+00$ & $0.00 E+00$ & $11 / 14 / 95$ & $4 B$ & 32986 & BF \\
\hline 2716 & FORTYNIN & PCT_A & $\mathrm{Pa}$ & CONSTANT & $0.00 E+00$ & $0.00 E+00$ & $0.00 E+00$ & $0.00 E+00$ & $0.00 E+00$ & $11 / 14 / 95$ & 4B & 32984 & BF \\
\hline 2239 & FORTYNIN & PC_MAX & $\mathrm{Pa}$ & CONSTANT & $1.00 E+08$ & $1.00 E+08$ & $0.00 E+00$ & $1.00 E+08$ & $1.00 E+08$ & $11 / 1 / 95$ & $4 B$ & 32989 & $\mathrm{BF}$ \\
\hline 2715 & FORTYNIN & KPT & NONE & CONSTANT & $0.00 E+00$ & $0.00 E+00$ & $0.00 E+00$ & $0.00 E+00$ & $0.00 E+00$ & $2 / 20 / 96$ & 4B & 32982 & BF \\
\hline 2238 & FORTYNIN & COMP_RCK & $P a^{n}-1$ & CONSTANT & $0.00 E+00$ & $0.00 E+00$ & $0.00 E+00$ & $0.00 E+00$ & $0.00 E+00$ & $11 / 1 / 95$ & $4 B$ & 32979 & BF \\
\hline 2085 & FORTYNIN & CAP_MOD & NONE & CONSTANT & $1.00 E+00$ & $1.00 \mathrm{E}+00$ & $0.00 E+00$ & $1.00 \mathrm{E}+00$ & $1.00 E+00$ & $11 / 1 / 95$ & $4 B$ & 32975 & $\overline{B F}$ \\
\hline
\end{tabular}

\section{Magenta Dolomite}

\begin{tabular}{|c|c|c|c|c|c|c|c|c|c|c|c|c|c|}
\hline ID & Material & & & & Mean & Median & Deviation & Low & High & Entry Date & Impt & WPO & Codes \\
\hline 101 & MAGENTA & JRE & $\mathrm{Pa}$ & & & & & $17 E+05$ & 9.17E+05 & & 1 & 32539 & BF \\
\hline 3016 & MAGENTA & OMP_RCK & $a^{n}-1$ & & & & $1.42 E-10$ & 1.16E-10 & & & 1 & 33249 & $\mathrm{BF}$ \\
\hline 2725 & MAGENTA & KPT & NONE & TANT & $0 E+00$ & $.00 E+00$ & $0.00 \mathrm{E}+00$ & $.00 E+00$ & $0.00 E+00$ & $2 / 20 / 96$ & 48 & 33254 & BF \\
\hline 2098 & MAGENTA & & $\mathrm{Pa}$ & CONSTANT & $1.00 E+08$ & $1.00 \mathrm{E}+08$ & $0.00 E+00$ & $1.00 E+08$ & $1.00 \mathrm{E}+08$ & $11 / 1 / 95$ & 48 & 33263 & BF \\
\hline 2726 & IAGENTA & $C T_{-} A$ & $\mathrm{~Pa}$ & CONSTANT & 2.60E-01 & $2.60 E-01$ & $0.00 \mathrm{E}+00$ & 2.60E-01 & $2.60 \mathrm{E}-01$ & $2 / 20 / 96$ & 48 & 33257 & $B F$ \\
\hline 2727 & MAGENTA & & NONE & CONSTANT & $-3.48 E-01$ & $-3.48 E-01$ & $0.00 E+00$ & $-3.48 E-01$ & $-3.48 E-01$ & $2 / 20 / 96$ & $4 B$ & 33261 & $B F$ \\
\hline 2728 & AAGENTA & IN & $\mathrm{Pa}$ & CONSTANT & $1.01 E+05$ & $1.01 E+05$ & $0.00 \mathrm{E}+00$ & $1.01 E+05$ & $1.01 E+05$ & $2 / 21 / 96$ & $4 B$ & 32536 & $B F$ \\
\hline 2097 & AAGENTA & & NONE & CONSTANT & $2.00 E+00$ & $2.00 E+00$ & $0.00 E+00$ & $2.00 \mathrm{E}+00$ & $2.00 E+00$ & $11 / 1 / 95$ & 4B & 33239 & $8 F$ \\
\hline 2100 & IAGENTA & OROSITY & NONE & STUDENT & 1.38E-01 & 1.38E-01 & 8.23E-02 & $2.70 E-02$ & & & 1 & 32531 & BF \\
\hline 2109 & MAGENTA & HICK & $m$ & CONSTANT & $8.50 E+00$ & $8.50 E+00$ & $0.00 E+00$ & $8.50 E+00$ & $8.50 E+00$ & $1 / 30 / 97$ & 5 & $32568^{*}$ & $\mathrm{BF}$ \\
\hline 2102 & MAGENTA & PRMX_LOG & $\log \left(m^{\wedge} 2\right)$ & CONSTANT & $-1.52 E+01$ & $-1.52 E+01$ & $0.00 E+00$ & $-1.52 E+01$ & $-1.52 E+01$ & $2 / 13 / 96$ & 1 & 32545 & $\mathrm{BF}$ \\
\hline 2103 & MAGENTA & PRMY_LOG & $\log \left(m^{\wedge} 2\right)$ & CONSTANT & $-1.52 E+01$ & $-1.52 E+01$ & $0.00 E+00$ & $-1.52 E+01$ & $-1.52 E+01$ & $2 / 13 / 96$ & 1 & 32547 & $\mathrm{BF}$ \\
\hline 2104 & MAGENTA & PRMZ_LOG & $\log \left(m^{\wedge} 2\right)$ & CONSTANT & $-1.52 E+01$ & $-1.52 E+01$ & $0.00 E+00$ & $-1.52 E+01$ & $-1.52 E+01$ & $2 / 13 / 96$ & 9 & 32550 & $B F$ \\
\hline 2106 & MAGENTA & 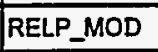 & NONE & CONSTANT & $4.00 E+00$ & $4.00 \mathrm{E}+00$ & $0.00 E+00$ & $4.00 E+00$ & $4.00 E+00$ & $11 / 1 / 95$ & $4 A$ & 32557 & $\mathrm{BF}$ \\
\hline 2241 & MAGENTA & SAT_RBRN & NONE & CONSTANT & 8.36E-02 & 8.36E-02 & $0.00 E+00$ & 8.36E-02 & 8.36E-02 & $2 / 22 / 96$ & $4 B$ & 32560 & $B F$ \\
\hline 2107 & MAGENTA & SAT_RGAS & & CONSTANT & & 7.71E-02 & $0.00 E+00$ & 7.71E-02 & & & $4 B$ & 32562 & $\mathrm{BF}$ \\
\hline 2099 & MAGENTA & PORE_DIS & NONE & CONSTANT & 6.44E-01 & $6.44 E-01$ & $0.00 \mathrm{E}+00$ & 6.44E-01 & $6.44 \mathrm{E}-01$ & $2 / 22 / 96$ & $4 \mathrm{~B}$ & 32493 & $B F$ \\
\hline
\end{tabular}




\section{Tamarisk Member}

\begin{tabular}{|c|c|c|c|c|c|c|c|c|c|c|c|c|c|}
\hline ID & Material & Parameter & Units & Distribution & Mean & Median & Deviation & Low & High & Entry Date & Impt & WPO & Codes \\
\hline 2192 & TAMARISK & SAT_RGAS & NONE & CONSTANT & 2.00E-01 & 2.00E-01 & $0.00 E+00$ & $2.00 E-01$ & 2.00E-01 & $11 / 1 / 95$ & $4 B$ & 34591 & BF \\
\hline 2245 & TAMARISK & SAT_RBRN & NONE & CONSTANT & 2.00E-01 & $2.00 \mathrm{E}-01$ & $0.00 \mathrm{E}+00$ & 2.00E-01 & $2.00 \mathrm{E}-01$ & 11/1/95 & 4B & 34589 & $B F$ \\
\hline 2191 & TAMARISK & RELP_MOD & NONE & CONSTANT & $4.00 \mathrm{E}+00$ & $4.00 \mathrm{E}+00$ & $0.00 E+00$ & $4.00 E+00$ & $4.00 E+00$ & $11 / 1 / 95$ & $4 B$ & 34588 & $B F$ \\
\hline 2916 & TAMARISK & PRMZ_LOG & $\log \left(m^{\wedge} 2\right)$ & CONSTANT & $-3.50 E+01$ & $-3.50 E+01$ & $0.00 E+00$ & $-3.50 E+01$ & $-3.50 E+01$ & $2 / 16 / 96$ & 4B & 34586 & BF \\
\hline 2915 & TAMARISK & PRMY_LOG & $\log \left(m^{\wedge} 2\right)$ & CONSTANT & $-3.50 E+01$ & $-3.50 E+01$ & $0.00 E+00$ & $-3.50 E+01$ & $-3.50 E+01$ & $2 / 13 / 96$ & $4 B$ & 34583 & BF \\
\hline 2914 & TAMARISK & PRMX_LOG & $\log \left(m^{\wedge} 2\right)$ & CONSTANT & $-3.50 E+01$ & $-3.50 E+01$ & $0.00 E+00$ & $-3.50 E+01$ & $-3.50 E+01$ & $2 / 13 / 96$ & $4 B$ & 34580 & $B F$ \\
\hline 2186 & TAMARISK & POROSITY & NONE & STUDENT & 6.40E-02 & $6.40 E-02$ & $9.56 \mathrm{E}-02$ & $0.00 E+00$ & $2.17 \mathrm{E}-01$ & $2 / 20 / 96$ & 1 & 34568 & $B F$ \\
\hline 2185 & TAMARISK & PORE_DIS & NONE & CONSTANT & 7.00E-01 & 7.00E-01 & $0.00 E+00$ & 7.00E-01 & 7.00E-01 & $11 / 1 / 95$ & $4 B$ & 34565 & BF \\
\hline 2796 & TAMARISK & PO_MIN & $\mathrm{Pa}$ & CONSTANT & $1.01 E+05$ & $1.01 E+05$ & $0.00 E+00$ & $1.01 E+05$ & $1.01 E+05$ & $2 / 21 / 96$ & 4B & 34571 & $B F$ \\
\hline 2795 & TAMARISK & PCT_EXP & NONE & CONSTANT & $0.00 E+00$ & $0.00 E+00$ & $0.00 E+00$ & $0.00 E+00$ & $0.00 E+00$ & $11 / 14 / 95$ & $4 B$ & 34562 & BF \\
\hline 2794 & TAMARISK & PCT_A & $\mathrm{Pa}$ & CONSTANT & $0.00 E+00$ & $0.00 E+00$ & $0.00 E+00$ & $0.00 E+00$ & $0.00 E+00$ & $11 / 14 / 95$ & $4 B$ & 34560 & BF \\
\hline 2244 & TAMARISK & PC_MAX & $\mathrm{Pa}$ & CONSTANT & $1.00 E+08$ & $1.00 E+08$ & $0.00 \mathrm{E}+00$ & $1.00 E+08$ & $1.00 E+08$ & $11 / 1 / 95$ & $4 B$ & 34563 & $8 F$ \\
\hline 2793 & TAMARISK & KPT & NONE & CONSTANT & $0.00 \mathrm{E}+00$ & $0.00 E+00$ & $0.00 E+00$ & $0.00 E+00$ & $0.00 E+00$ & $2 / 20 / 96$ & 4B & 34558 & $B F$ \\
\hline 2243 & TAMARISK & COMP_RCK & $P a^{\wedge}-1$ & CONSTANT & $0.00 \mathrm{E}+00$ & $0.00 E+00$ & $0.00 E+00$ & $0.00 E+00$ & $0.00 E+00$ & $11 / 1 / 95$ & $4 B$ & 34529 & $B F$ \\
\hline 183 & TAMARISK & CAP_MOD & NONE & CONSTANT & $1.00 E+00$ & $1.00 \mathrm{E}+00$ & $0.00 E+00$ & $1.00 E+00$ & $1.00 E+00$ & $11 / 1 / 95$ & $4 B$ & 34524 & $\mathrm{BF}$ \\
\hline
\end{tabular}

\section{Culebra Dolomite}

\begin{tabular}{|c|c|c|c|c|c|c|c|c|c|c|c|c|c|}
\hline ID & Material & Parameter & Units & Distribution & Mean & Median & Deviation & Low & High & Entry Date & Impt & WPO & Codes \\
\hline 2692 & CULEBRA & PCT_A & $\mathrm{Pa}$ & CONSTANT & $2.60 E-01$ & 2.60E-01 & $0.00 E+00$ & 2.60E-01 & $2.60 \mathrm{E}-01$ & $2 / 20 / 96$ & 48 & 32752 & $B F$ \\
\hline 119 & CULEBRA & CAP_MOD & NONE & CONSTANT & $2.00 E+00$ & $2.00 E+00$ & $0.00 E+00$ & $2.00 E+00$ & $2.00 \mathrm{E}+00$ & 11/1/95 & 4B & 32686 & $B F$ \\
\hline 120 & CULEBRA & COMP_RCK & $\mathrm{Pa}^{\wedge}-1$ & CONSTANT & $1.00 \mathrm{E}-10$ & $1.00 \mathrm{E}-10$ & $0.00 E+00$ & $1.00 \mathrm{E}-10$ & 1.00E-10 & $11 / 1 / 95$ & $4 \mathrm{~B}$ & 32688 & $B F$ \\
\hline 3483 & CULEBRA & DISP_L & $\mathbf{m}$ & CONSTANT & $0.00 E+00$ & $0.00 E+00$ & $0.00 E+00$ & $0.00 E+00$ & $0.00 E+00$ & $6 / 12 / 96$ & 1 & 38354 & TP \\
\hline 3484 & CULEBRA & DISPT_L & $m$ & CONSTANT & $0.00 E+00$ & $0.00 E+00$ & $0.00 E+00$ & $0.00 E+00$ & $0.00 E+00$ & $6 / 12 / 96$ & 1 & 38355 & TP \\
\hline 843 & CULEBRA & DNSGRAIN & $\mathrm{kg} / \mathrm{m}^{\wedge} 3$ & CONSTANT & $2.82 E+03$ & $2.82 E+03$ & $0.00 E+00$ & $2.82 E+03$ & $2.82 E+03$ & $6 / 10 / 96$ & 1 & 32689 & NU/TP \\
\hline 3486 & CULEBRA & DPOROS & NONE & CUMULATIVE & $1.60 \mathrm{E}-01$ & 1.60E-01 & 3.50 E-02 & $1.00 \mathrm{E}-01$ & 2.50E-01 & $6 / 13 / 96$ & 1 & 38357 & TP \\
\hline 3474 & CULEBRA & DTORT & NONE & CONSTANT & $1.10 E-01$ & 1.10E-01 & $0.00 E+00$ & 1.10E-01 & 1.10E-01 & $6 / 10 / 96$ & 1 & 38345 & TP \\
\hline 3462 & CULEBRA & ETHICK & $m$ & CONSTANT & $4.00 E+00$ & $4.00 E+00$ & $0.00 E+00$ & $4.00 E+00$ & $4.00 \mathrm{E}+00$ & $5 \pi / 96$ & 1 & 37727 & FLTP \\
\hline 123 & CULEBRA & FRCTRIDX & NONE & UNIFORM & $5.00 E-01$ & 5.00 E-01 & 2.89E-01 & $0.00 E+00$ & $1.00 E+00$ & $1 / 23 / 97$ & 5 & $32529^{\circ}$ & $B F$ \\
\hline 861 & CULEBRA & FTORT & NONE & CONSTANT & $1.00 E+00$ & $1.00 E+00$ & $0.00 E+00$ & $1.00 E+00$ & $1.00 E+00$ & $5 / 9 / 96$ & 4B & 32541 & TP \\
\hline 3485 & CULEBRA & HMBLKLT & $m$ & UNIFORM & 2.75E-01 & 2.75E-01 & 1.30E-01 & $5.00 E-02$ & 5.00E-01 & $6 / 13 / 96$ & 1 & 38356 & $T P$ \\
\hline 2691 & CULEBRA & KPT & NONE & CONSTANT & $0.00 E+00$ & $0.00 E+00$ & $0.00 E+00$ & $0.00 E+00$ & $0.00 E+00$ & $2 / 20 / 96$ & $4 B$ & 32555 & $\mathrm{BF}$ \\
\hline 3418 & CULEBRA & MEA_STOR & NONE & CONSTANT & 1.00E-05 & $1.00 E-05$ & $0.00 E+00$ & 1.00E-05 & 1.00E-05 & $5 / 1 / 96$ & 1 & 37664 & GI \\
\hline 3487 & CULEBRA & APOROS & NONE & LOGUNIFORN & 2.10E-03 & $1.00 \mathrm{E}-03$ & $2.50 E-03$ & 1.00E-04 & 1.00E-02 & $6 / 14 / 96$ & 1 & 38358 & $T P$ \\
\hline 143 & CULEBRA & PRMX_LOG & $\log \left(m^{\wedge} 2\right)$ & CONSTANT & $-1.37 E+01$ & $-1.37 E+01$ & $0.00 E+00$ & $-1.37 E+01$ & $-1.37 E+01$ & $2 / 13 / 96$ & -1 & 32775 & $\mathrm{BF}$ \\
\hline 3469 & CULEBRA & SKIN_RES & NONE & CONSTANT & $0.00 E+00$ & $0.00 E+00$ & $0.00 E+00$ & $0.00 E+00$ & $0.00 E+00$ & $5 / 9 / 96$ & 48 & 37735 & TP \\
\hline 151 & CULEBRA & SAT_RGAS & NONE & CONSTANT & 7.71E-02 & 7.71E-02 & $0.00 E+00$ & 7.71E-02 & 7.71E-02 & $2 / 20 / 96$ & $4 B$ & 32783 & BF \\
\hline 150 & CULEBRA & SAT_RBRN & NONE & CONSTANT & $8.36 \mathrm{E}-02$ & 8.36E-02 & $0.00 E+00$ & 8.36E-02 & 8.36E-02 & $2 / 22 / 96$ & $4 B$ & 32782 & BF \\
\hline 149 & CULEBRA & SAT_IBRN & NONE & CONSTANT & $1.00 E+00$ & $1.00 E+00$ & $0.00 E+00$ & $1.00 E+00$ & $1.00 E+00$ & $11 / 1 / 95$ & 48 & 32781 & BF \\
\hline 148 & CULEBRA & RELP_MOD & NONE & CONSTANT & $4.00 E+00$ & $4.00 E+00$ & $0.00 E+00$ & $4.00 E+00$ & $4.00 E+00$ & $11 / 1 / 95$ & 48 & 32780 & $8 F$ \\
\hline 3419 & CULEBRA & MINP_FAC & NONE & UNIFORM & $5.01 E+02$ & $5.01 E+02$ & $2.88 E+02$ & $1.00 E+00$ & $1.00 E+03$ & $5 / 1 / 96$ & 1 & 37666 & $\mathbf{F L}$ \\
\hline 144 & CULEBRA & PRMY_LOG & $\log \left(m^{\wedge} 2\right)$ & CONSTANT & $-1.37 E+01$ & $-1.37 E+01$ & $0.00 E+00$ & $-1.37 E+01$ & $-1.37 E+01$ & $2 / 13 / 96$ & 1 & 32776 & BF \\
\hline 137 & CULEBRA & PC_MAX & $\mathrm{Pa}$ & CONSTANT & $1.00 E+08$ & $1.00 E+08$ & $0.00 E+00$ & $1.00 E+08$ & $1.00 E+08$ & $11 / 1 / 95$ & $4 B$ & 32755 & $B F$ \\
\hline 142 & CULEBRA & PRESSURE & $\mathrm{Pa}$ & CONSTANT & $8.22 E+05$ & $8.22 E+05$ & $0.00 E+00$ & $8.22 E+05$ & $8.22 E+05$ & $2 / 8 / 96$ & 1 & 32774 & $B F$ \\
\hline 140 & CULEBRA & POROSITY & NONE & CONSTANT & $1.51 \mathrm{E}-01$ & 1.51E-01 & $0.00 E+00$ & 1.51E-01 & 1.51E-01 & 2/1/96 & 1 & 32769 & $B F$ \\
\hline 139 & CULEBRA & PORE_DIS & NONE & CONSTANT & $6.44 \mathrm{E}-01$ & 6.44E-01 & $0.00 E+00$ & 6.44E-01 & 6.44E-01 & $2 / 20 / 96$ & $4 B$ & 32764 & $B F$ \\
\hline 141 & CULEBRA & PO_MIN & $\mathrm{Pa}$ & CONSTANT & $1.01 E+05$ & $1.01 E+05$ & $0.00 E+00$ & $1.01 E+05$ & $1.01 E+05$ & 2/21/96 & $4 B$ & 32772 & $B F_{1}$ \\
\hline 2693 & CULEBRA & PCT_EXP & NONE & CONSTANT & $-3.48 E-01$ & $-3.48 E-01$ & $0.00 E+00$ & $-3.48 E-01$ & $-3.48 E-01$ & $2 / 20 / 96$ & AB & 32753 & $B F$ \\
\hline 2071 & CULEBRA & THICK & $m$ & CONSTANT & $7.75 E+00$ & $7.75 E+00$ & $0.00 E+00$ & $7.75 E+00$ & 7.75E+00 & $5 / 1 / 96$ & 1 & 32790 & BF/FL \\
\hline 145 & CULEBRA & PRMZ_LOG & $\log \left(m^{\wedge} 2\right)$ & CONSTANT & $-1.37 E+01$ & $-1.37 E+01$ & $0.00 E+00$ & $-1.37 E+01$ & $-1.37 E+01$ & $2 / 13 / 96$ & 1 & 32777 & BF \\
\hline
\end{tabular}




\section{Unnamed Lower Member}

\begin{tabular}{|c|c|c|c|c|c|c|c|c|c|c|c|c|c|}
\hline ID & Material & Parameter & Units & Distribution & Mean & Median & Deviation & Low & High & Entry Date & Impt & WPO & Codes \\
\hline 2226 & UNNAMED & SAT_RGAS & NONE & CONSTANT & 2.00E-01 & 2.00E-01 & $0.00 E+00$ & 2.00E-01 & 2.00E-01 & $11 / 1 / 95$ & $4 B$ & 34703 & $B F$ \\
\hline 2248 & UNNAMED & SAT_RBRN & NONE & CONSTANT & 2.00E-01 & 2.00E-01 & $0.00 E+00$ & 2.00E-01 & 2.00E-01 & $11 / 1 / 95$ & $4 B$ & 34702 & BF \\
\hline 2225 & UNNAMED & RELP_MOD & NONE & CONSTANT & $4.00 \mathrm{E}+00$ & $4.00 \mathrm{E}+00$ & $0.00 \mathrm{E}+00$ & $4.00 E+00$ & $4.00 E+00$ & $11 / 1 / 95$ & $4 B$ & 34701 & BF \\
\hline 2913 & UNNAMED & PRMZ_LOG & $\log \left(m^{\wedge} 2\right)$ & CONSTANT & $-3.50 E+01$ & $-3.50 \mathrm{E}+01$ & $0.00 \mathrm{E}+00$ & $-3.50 E+01$ & $-3.50 E+01$ & $2 / 13 / 96$ & $4 B$ & 34699 & BF \\
\hline 2912 & UNNAMED & PRMY_LOG & $\log \left(m^{\wedge} 2\right)$ & CONSTANT & $-3.50 E+01$ & $-3.50 E+01$ & $0.00 E+00$ & $-3.50 E+01$ & $-3.50 E+01$ & $2 / 13 / 96$ & $4 B$ & 34697 & $\mathrm{BF}$ \\
\hline 2911 & UNNAMED & PRMX_LOG & $\log \left(m^{\wedge} 2\right)$ & CONSTANT & $-3.50 E+01$ & $-3.50 E+01$ & $0.00 E+00$ & $-3.50 E+01$ & $-3.50 E+01$ & $2 / 13 / 96$ & $4 B$ & 34695 & $B F$ \\
\hline 2220 & UNNAMED & POROSITY & NONE & STUDENT & 1.81E-01 & 1.81E-01 & 1.27E-01 & 2.00E-03 & 2.73E-01 & $2 / 20 / 96$ & 1 & 34692 & BF \\
\hline 2219 & UNNAMED & PORE_DIS & NONE & CONSTANT & $7.00 \mathrm{E}-01$ & 7.00E-01 & $0.00 E+00$ & 7.00E-01 & 7.00E-01 & $11 / 1 / 95$ & $4 B$ & 34691 & $\mathrm{BF}$ \\
\hline 2802 & UNNAMED & PO_MIN & $\mathrm{Pa}$ & CONSTANT & $1.01 E+05$ & $1.01 E+05$ & $0.00 E+00$ & 1.01E+05 & $1.01 E+05$ & $2 / 21 / 96$ & $4 B$ & 34693 & $B F$ \\
\hline 2801 & UNNAMED & PCT_EXP & NONE & CONSTANT & $0.00 E+00$ & $0.00 \mathrm{E}+00$ & $0.00 E+00$ & $0.00 E+00$ & $0.00 E+00$ & $11 / 14 / 95$ & $4 B$ & 34689 & $B F$ \\
\hline 2800 & UNNAMED & PCT_A & $\mathrm{Pa}$ & CONSTANT & $0.00 E+00$ & $0.00 E+00$ & $0.00 E+00$ & $0.00 E+00$ & $0.00 E+00$ & $11 / 14 / 95$ & $4 B$ & 34688 & $\overline{B F}$ \\
\hline 2247 & UNNAMED & PC_MAX & $\mathrm{Pa}$ & CONSTANT & $1.00 E+08$ & $1.00 E+08$ & $0.00 \mathrm{E}+00$ & $1.00 E+08$ & $1.00 \mathrm{E}+08$ & $11 / 1 / 95$ & $4 B$ & 34690 & $\mathrm{BF}$ \\
\hline 2799 & UNNAMED & KPT & NONE & CONSTANT & $0.00 E+00$ & $0.00 \mathrm{E}+00$ & $0.00 E+00$ & $0.00 \mathrm{E}+00$ & $0.00 E+00$ & $2 / 20 / 96$ & $4 B$ & 34687 & $\mathrm{BF}$ \\
\hline 2218 & UNNAMED & COMP_RCK & $\mathrm{Pa}^{\wedge}-1$ & CONSTANT & $0.00 E+00$ & $0.00 E+00$ & $0.00 E+00$ & $0.00 E+00$ & $0.00 E+00$ & $11 / 1 / 95$ & $4 B$ & 34686 & $\mathrm{BF}$ \\
\hline 2217 & UNNAMED & CAP_MOD & NONE & CONSTANT & $1.00 E+00$ & $1.00 E+00$ & $0.00 E+00$ & $1.00 E+00$ & $1.00 \mathrm{E}+00$ & $11 / 1 / 95$ & $4 B$ & 34684 & $\mathrm{BF}$ \\
\hline
\end{tabular}

\section{A. Salado Halite}

\begin{tabular}{|c|c|c|c|c|c|c|c|c|c|c|c|c|c|}
\hline ID & & ter & Units & Distrit & Mean & Median & Deviation & Low & High & Entry Date & Impt & WPO & Codes \\
\hline 544 & _HALITE & OROSITY & NONE & CUMULATIVE & $1.28 E-02$ & $1.00 \mathrm{E}-02$ & 8.52E-03 & $1.00 \mathrm{E}-03$ & $3.00 \mathrm{E}-02$ & 2/1/96 & 1 & 34387 & BF \\
\hline 41 & & DCK & $\mathrm{Pa}^{A}-1$ & UNIFORM & $9.75 E-11$ & $9.75 E-11$ & $5.46 E-11$ & $2.94 \mathrm{E}-12$ & $1.92 \mathrm{E}-10$ & 2/1/96 & 1 & 34210 & BF \\
\hline 1703 & & & $\mathrm{~kg} / \mathrm{m}^{\wedge} 3$ & ONSTANT & $0.00 E+00$ & $0.00 E+00$ & $0.00 E+00$ & $0.00 E+00$ & $0.00 E+00$ & $1 / 23 / 97$ & 5 & $34211^{\circ}$ & BF \\
\hline 778 & & PT & NONE & CONSTANT & $0.00 E+00$ & $0.00 E+00$ & $0.00 E+00$ & $0.00 E+00$ & $0.00 E+00$ & $2 / 20 / 96$ & $4 B$ & 34233 & BF \\
\hline 1724 & HALITE & DISP_L & m & CONSTANT & $1.00 E+02$ & $1.00 E+02$ & $0.00 E \div 00$ & $1.00 E+02$ & $1.00 E+02$ & $2 / 12 / 96$ & $4 B$ & $34234^{\circ}$ & BF/NU \\
\hline 725 & HALITE & DISP_T. & m & CONSTANT & $5.00 E+00$ & $5.00 E+00$ & $0.00 E+00$ & $5.00 E+00$ & $5.00 E+00$ & 2/12/96 & 4B & $34235^{\circ}$ & NU/BF \\
\hline 42 & S_HALITE & & $\mathrm{Pa}$ & CONSTANT & $1.00 E+08$ & $1.00 E+08$ & $0.00 E+00$ & $1.00 E+08$ & $1.00 E+08$ & $11 / 1 / 95$ & $4 B$ & 34379 & $\mathrm{BF}$ \\
\hline 779 & S_HALITE & PCT_A & $\mathrm{Pa}$ & CONSTANT & 5.60E-01 & $5.60 E-01$ & $0.00 E+00$ & $5.60 \mathrm{E}-01$ & $5.60 \mathrm{E}-01$ & $2 / 22 / 96$ & $4 B$ & 34375 & BF \\
\hline 780 & S_HALITE & PCT_EXP & NONE & CONS & $-3.46 \mathrm{E}-01$ & $-3.46 \mathrm{E}-01$ & $0.00 E \div 00$ & $-3.46 E-01$ & $-3.46 E-01$ & $2 / 22 / 96$ & 4B & 34377 & $B F$ \\
\hline 540 & S_HALITE & CAP_MOD & NONE & CONSTANT & $2.00 E+00$ & $2.00 E+00$ & $0.00 E+00$ & $2.00 E+00$ & $2.00 E+00$ & $11 / 1 / 95$ & $4 B$ & 34208 & BF \\
\hline 543 & S_HA & is & NONE & CUMULA & $2.90 E+00$ & & $3.10 E+\infty 0$ & $2.00 E-01$ & $1.00 E+01$ & $11 / 1 / 95$ & AB & 34385 & BF \\
\hline 1742 & S_HALITE & JRTUSTY & NONE & CONSTANT & $1.00 \mathrm{E}+01$ & $1.00 E+01$ & $0.00 E+00$ & $1.00 E+01$ & $1.00 E+01$ & $2 / 12 / 96$ & 4B & $34428^{\circ}$ & NU \\
\hline 546 & S_HALITE & PRESSURE & $\mathbf{P a}$ & UNIFORM & $1.25 E+07$ & $1.25 E+07$ & $8.23 E+05$ & 1.10E+07 & 1.39E+07 & $2 / 8 / 96$ & 1 & 34394 & BF \\
\hline 547 & S_HALITE & PRMX_LOG & $\log \left(m^{\wedge} 2\right)$ & UNIFORM & $-2.25 E+01$ & $-2.25 E+01$ & $8.66 E-01$ & $-2.40 E+01$ & $-2.10 E+01$ & $3 / 6 / 96$ & $1 B$ & $34397 \mathrm{~A}$ & $B F$ \\
\hline 548 & S_HALITE & PRMY_LOG & $\log \left(m^{\wedge} 2\right)$ & UNIFORM & $-2.25 E+01$ & $-2.25 E+01$ & 8.66E-01 & $-2.40 \mathrm{E}+01$ & $-2.10 E+01$ & $3 / 6 / 96$ & $1 B$ & $34399 \mathrm{~A}$ & $\mathrm{BF}$ \\
\hline 549 & S_HALITE & PRMZ_LOG & $\log \left(m^{\wedge} 2\right)$ & UNIFORM & $-2.25 E+01$ & $-2.25 E+01$ & 8.66-6E-01 & $-2.40 E+01$ & $-2.10 E+01$ & $3 / 6 / 96$ & 18 & $34401 \mathrm{~A}$ & $B F$ \\
\hline 553 & S_HALITE & RELP_MOD & NONE & DELTA & $4.00 E+00$ & $4.00 E+00$ & $0.00 E+00$ & $1.00 E+00$ & $4.00 \mathrm{E}+00$ & 11/1/95 & $4 B$ & 34412 & $B F$ \\
\hline 554 & S_HALITE & SAT_IBRN & NONE & CONSTANT & $1.00 E+00$ & $1.00 E+00$ & $0.00 E+00$ & $1.00 E+00$ & $1.00 E+00$ & 11/1/95 & 4B & 34416 & $B F$ \\
\hline 555 & S_HALITE & SAT_RBRN & NONE & UNIFORM & 3.00E-01 & 3.00E-01 & 1.73E-01 & $0.00 E+00$ & $6.00 E-01$ & 11/1/95 & AB & 34418 & BF \\
\hline 556 & S_HALITE & SAT_RGAS & NONE & UNIFORM & $2.00 E-01$ & 2.00E-01 & 1.15E-01 & $0.00 E+00$ & $4.00 E-01$ & 11/1/95 & 4B & 34420 & $B F$ \\
\hline 545 & S_HALITE & PO_MIN & $\mathrm{Pa}$ & CONSTANT & $1.01 E+05$ & $1.01 E+05$ & $0.00 E+00$ & $1.01 E+05$ & $1.01 E+05$ & 2/21/96 & 4B & 34391 & BF \\
\hline
\end{tabular}

\section{B. Salado Brine}

\begin{tabular}{|c|c|c|c|c|c|c|c|c|c|c|c|c|c|}
\hline ID & Material & Parameter & Units & Distribution & Mean & Median & Deviation & Low & High & Entry Date & impt & WPO & Codes \\
\hline 57 & BRINESAL & WTF & NONE & STUDENT & 3.24E-01 & 3.24E-01 & 9.83E-03 & 3.07E-01 & 3.32E-01 & 2/20/96 & 1 & 31552 & BF \\
\hline 55 & BRINESAL & VIsco & $\mathrm{Pa}$ as & CONSTANT & $2.10 E-03$ & 2.10E-03 & $0.00 E+00$ & 2.10E-03 & $2.10 \mathrm{E}-03$ & 2/9/96 & 1 & 31548 & BF \\
\hline 52 & BRINESAL & RSLOPE & NONE & CONSTANT & $0.00 \mathrm{E}+00$ & $0.00 \mathrm{E}+00$ & $0.00 E+00$ & $0.00 E+\infty 0$ & $0.00 E+00$ & $1 / 23 / 97$ & 5 & $31544^{*}$ & BF \\
\hline 51 & BRINESAL & REF_TEMP & $\mathrm{k}$ & CONSTANT & $3.00 E+02$ & $3.00 E+02$ & $0.00 E+00$ & $3.00 E+02$ & $3.00 \mathrm{E}+02$ & $11 / 2 / 95$ & 4B & 31543 & $B F$ \\
\hline 50 & BRINESAL & REF_PRES & $\mathrm{Pa}$ & CONSTANT & $1.01 E+05$ & 1.01E+05 & $0.00 E+00$ & $1.01 E+05$ & $1.01 E+05$ & $11 / 2 / 95$ & 4B & 31542 & BF \\
\hline 49 & BRINESAL & DNSFLUID & $\mathrm{kg} / \mathrm{m}^{\wedge} 3$ & CONSTANT & $1.22 E+03$ & $1.22 E+03$ & $0.00 E+00$ & $1.22 E+03$ & $1.22 E+03$ & 2/1/96 & 1 & 31541 & BF \\
\hline 48 & BRINESAL & COMPRES & $P a^{\wedge}-1$ & CONSTANT & 3.10E-10 & $3.10 E-10$ & $0.00 E+00$ & $3.10 \mathrm{E}-10$ & $3.10 \mathrm{E}-10$ & $2 / 1 / 96$ & 1 & 31540 & $B F$ \\
\hline
\end{tabular}


20. Marker Bed 138

\begin{tabular}{|c|c|c|c|c|c|c|c|c|c|c|c|c|c|}
\hline ID & Aaterial & Parameter & Units & Distribution & Mean & Median & Deviation & Low & High & Entry Date & Impt & WPO & Codes \\
\hline 563 & S_MB138 & PF_DELTA & $\mathbf{P a}$ & |CONSTANT & $.80 E+06$ & $3.80 E+06$ & $0.00 \mathrm{E}+00$ & $3.80 E+06$ & $3.80 \mathrm{E}+06$ & $2 / 22 / 96$ & $4 B$ & 34516 & BF \\
\hline 559 & MB138 & CAP_MOD & ONE & NT & $.00 E+00$ & $00 E+00$ & $00 E+00$ & $2.00 E+00$ & $2.00 E+00$ & 2/11/96 & 4A & $4430 \mathrm{~A}$ & $B F$ \\
\hline 560 & S_MB138 & |COMP_RCK| & $a^{\wedge}-1$ & $\mathbf{I T}$ & 26E-11 & $26 E-11$ & $11 E-10$ & $1.09 E-11$ & 2.75E-10 & & $4 \mathrm{~A}$ & 34439 & $B F$ \\
\hline 1743 & MB138 & DNSGRAIN & $\mathrm{kg} / \mathrm{m}^{\wedge} 3$ & CONSTANT & $2.75 E+03$ & 2.75E+03 & $.00 E+00$ & $2.75 E+03$ & $2.75 E+03$ & $2 / 1 / 96$ & 1 & 34441 & BF/NU \\
\hline 2169 & S_MB138 & DPHIMAX & NONE & CONSTANT & 3.90E-02 & $3.90 E-02$ & $.00 \mathrm{E}+00$ & $3.90 E-02$ & $3.90 E-02$ & $2 / 22 / 96$ & 48 & 34442 & BF \\
\hline 2902 & S_MB138 & EXPKLINK & NONE & CONSTANT & $-3.41 E-01$ & $-3.41 E-01$ & $.00 E+00$ & $-3.41 E-01$ & $-3.41 E-01$ & 123/96 & $4 \mathrm{~A}$ & $34443 A$ & BF \\
\hline 2810 & S_MB138 & IFRX & NONE & CON & $1.00 E+00$ & $1.00 E+00$ & $0.00 E+00$ & $1.00 E+00$ & $1.00 \mathrm{E}+00$ & $1 / 30 / 96$ & 4B & 34465 & $\mathrm{BF}$ \\
\hline 2813 & S_MB138 & IFRY & NONE & NTT & $1.00 E+00$ & $1.00 E+00$ & $0.00 E+00$ & $1.00 E+00$ & $1.00 E+00$ & 1/30/96 & $4 B$ & 34466 & BF \\
\hline 2816 & S_MB138 & IFRZ & NONE & ANT & $0.00 E+00$ & $0.00 E+00$ & $0.00 E+00$ & $0.00 E+00$ & $0.00 E+00$ & 1/31/96 & $4 B$ & 34471 & BF \\
\hline 2170 & S_MB138 & KMAX & $\log \left(m^{\wedge} 2\right)$ & CONSTANT & $-9.00 E+00$ & $-9.00 E+00$ & $0.00 E+00$ & $-9.00 E+00$ & $-9.00 E+00$ & $2 / 22 / 96$ & 4B & 34476 & BF \\
\hline 2783 & S_MB138 & KPT & NONE & TANT & $.00 E+00$ & $0.00 E+00$ & $.00 E+00$ & $0.00 \mathrm{E}+00$ & $0.00 E+00$ & $2 / 20 / 96$ & 4B & 34479 & BF \\
\hline 561 & S_MB138 & PC_MAX & $\mathrm{Pa}$ & ANT & $1.00 E+08$ & & $0.00 E+00$ & $1.00 \mathrm{E}+08$ & $1.00 E+08$ & 2/1/96 & $4 A$ & $34512 A$ & BF \\
\hline 2904 & S_MB138 & BKLINK & $\mathrm{Pa}$ & VT & 71E-01 & E-01 & $0.00 E+00$ & 2.71E-01 & 2.71E-01 & 4/23/96 & $4 A$ & $34429 \mathrm{~A}$ & BF \\
\hline 2785 & S_MB138 & $P C T$ EXP & NONE & CONS & $-3.48 E-01$ & $8 E-01$ & $0 E+00$ & $-3.48 E-01$ & $-3.48 E-01$ & $2 / 8 / 96$ & $4 A$ & 34507 & BF \\
\hline 578 & S_MB138 & SAT_RGAS & NONE & STUDENT & 7.71E-02 & 7.71E-02 & 6.41E-02 & $1.40 \mathrm{E}-02$ & 1.97E-01 & $2 / 22 / 96$ & $4 A$ & 34546 & BF \\
\hline 565 & S_MB138 & PI_DELTA & & & & $2.00 \mathrm{E}+05$ & $0.00 E+00$ & 2.00E+05 & $2.00 E+05$ & 2/22/96 & $4 B$ & 34523 & BF \\
\hline 668 & S_MB138 & PO_MIN & & ANT & $1.01 E+05$ & $1.01 E+05$ & $0.00 E+00$ & $1.01 E+05$ & $1.01 E+05$ & 2/21/96 & 4B & 34531 & BF \\
\hline 566 & S_MB138 & PORE_DIS & NONE & ENT & 6.44E-01 & & $1.09 \mathrm{E}-01$ & 4.91E-01 & & 2/22/96 & $4 A$ & 34527 & BF \\
\hline 567 & S_MB138 & POROSITY & NONE & STUD & & & & & & & $4 A$ & 34530 & BF \\
\hline 569 & S_MB138 & PRESSURE & $\mathrm{Pa}$ & STUDENT & 1.16E+07 & $E+07$ & 1.27E+06 & 9.38E+06 & $1.29 E+07$ & 2/19/96 & $4 A$ & 34532 & BF \\
\hline 570 & S_MB138 & PRMX_LOG & $\log \left(m^{\wedge} 2\right)$ & 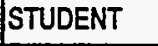 & $-1.89 E+01$ & $-1.89 E+01$ & 1.20E+00 & $-2.10 E+01$ & $-1.71 E+01$ & & $4 A$ & 34536 & BF \\
\hline 571 & S_MB138 & PRMY_LOG & $\log \left(m^{\wedge} 2\right)$ & STUDENT & -1.89E+01 & $-1.89 E+01$ & $1.20 \mathrm{E}+00$ & $-2.10 E+01$ & $-1.71 E+01$ & 2/11/96 & $4 \mathrm{~A}$ & 34537 & BF \\
\hline 572 & S_MB138 & PRMZ_LOG & $\log \left(m^{\wedge} 2\right)$ & STUDENT & $-1.89 E+01$ & $-1.89 E+01$ & $1.20 E+00$ & $-2.10 E+01$ & $-1.71 E+01$ & 2/11/96 & $4 A$ & 34538 & $\mathrm{BF}$ \\
\hline 575 & S_MB138 & RELP_MOD & NONE & DELTA & $4.00 E+00$ & $4.00 E+00$ & $0.00 E+00$ & $1.00 E+00$ & $4.00 \mathrm{E}+00$ & $2 / 8 / 96$ & $4 A$ & $34542 A$ & BF \\
\hline 576 & S_MB138 & SAT_IBRN & NONE & CONSTANT & $1.00 E+00$ & $1.00 E+00$ & $0.00 E+00$ & $1.00 E+00$ & $1.00 E+00$ & $2 / 11 / 96$ & 1 & 34544 & BF \\
\hline 577 & S_MB138 & SAT_RBRN & NONE & STUDENT & 8.36E-02 & 8.36E-02 & 5.01E-02 & 7.78E-03 & 1.74E-01 & $11 / 21 / 96$ & $4 \mathrm{~A}$ & 34545 & $B F$ \\
\hline 2784 & S_MB138 & PCT_A & & CONSTANT & 2.60E-01 & 2.60E-01 & $0.00 \mathrm{E}+00$ & 2.60E-01 & $2.60 E-01$ & $2 / 8 / 96$ & $4 \mathrm{~A}$ & 34505 & $B F$ \\
\hline
\end{tabular}




\section{Anhydrite Beds $A$ and $B$}

\begin{tabular}{|c|c|c|c|c|c|c|c|c|c|c|c|c|c|}
\hline ID & Material & Parameter & Units & Distribution & Mean & Median & Deviation & Low & High & Entry Date & Impt & WPO & Codes \\
\hline 2775 & S_ANH_AB & PCT_EXP & NONE & CONSTANT & $-3.48 E-01$ & $-3.48 E-01$ & $0.00 E+00$ & $-3.48 E-01$ & $-3.48 E-01$ & $2 / 8 / 96$ & $4 A$ & 34184 & $\mathrm{BF}$ \\
\hline 520 & S_ANH_AB & CAP_MOD & NONE & CONSTANT & $2.00 E+00$ & $2.00 E+00$ & $0.00 E+00$ & $2.00 E+00$ & $2.00 E+00$ & $2 / 8 / 96$ & $4 A$ & 34130 & BF \\
\hline 521 & $S_{-}$ANH_AB & COMP_RCK & $P a^{n}-1$ & STUDENT & $8.26 \mathrm{E}-11$ & $8.26 \mathrm{E}-11$ & 1.11E-10 & 1.09E-11 & 2.75E-10 & 2/14/96 & $4 \mathrm{~A}$ & 34135 & BF \\
\hline 1661 & S_ANH_AB & DNSGRAIN & $\mathrm{kg} / \mathrm{m}^{\wedge} 3$ & CONSTANT & $2.75 E+03$ & $2.75 E+03$ & $0.00 E+00$ & 2.75E+03 & $2.75 E+03$ & 2/12/96 & $4 A$ & 34137 & NU/BF \\
\hline 215B & S_ANH_AB & DPHIMAX & NONE & CONSTANT & 2.39E-01 & 2.39E-01 & $0.00 E+00$ & 2.39E-01 & 2.39E-01 & $2 / 22 / 96$ & $4 B$ & 34138 & $B F$ \\
\hline 2820 & S_ANH_AB & EXPKLINK & NONE & CONSTANT & $-3.41 E-01$ & $-3.41 E-01$ & $0.00 E+00$ & $-3.41 E-01$ & $-3.41 E-01$ & $4 / 23 / 96$ & $4 \mathrm{~A}$ & $34139 A$ & BF \\
\hline 2812 & S_ANH_AB & IFRX & NONE & CONSTANT & $1.00 E+00$ & $1.00 E+00$ & $0.00 \mathrm{E}+00$ & $1.00 \mathrm{E}+00$ & $1.00 E+00$ & $1 / 30 / 96$ & $4 B$ & 34158 & BF \\
\hline 2815 & S_ANH_AB & IFRY & NONE & CONSTANT & $1.00 E+00$ & $1.00 \mathrm{E}+00$ & $0.00 \mathrm{E}+00$ & $1.00 E+00$ & $1.00 \mathrm{E}+00$ & $1 / 30 / 96$ & $4 B$ & 34159 & $\mathrm{BF}$ \\
\hline 2818 & S_ANH_AB & IFRZ & NONE & CONSTANT & $0.00 E+00$ & $0.00 E+00$ & $0.00 E+00$ & $0.00 E+00$ & $0.00 E+00$ & $1 / 31 / 96$ & AB & 34160 & $\mathrm{BF}$ \\
\hline 2159 & S_ANH_AB & KMAXLOG & $\log \left(m^{\wedge} 2\right)$ & CONSTANT & $-9.00 E+00$ & $-9.00 E+00$ & $0.00 E+00$ & $-9.00 E+00$ & $-9.00 E+00$ & $2 / 22 / 96$ & $4 B$ & 34163 & $B F$ \\
\hline 2773 & S_ANH_AB & KPT & NONE & CONSTANT & $0.00 E+00$ & $0.00 E+00$ & $0.00 E+00$ & $0.00 E+00$ & $0.00 E+00$ & $2 / 20 / 96$ & $4 \mathrm{~B}$ & 34165 & $\mathrm{BF}$ \\
\hline 1684 & S_ANH_AB & MDISP_L & $m$ & CONSTANT & $1.00 E+02$ & $1.00 E+02$ & $0.00 E+00$ & $1.00 E+02$ & $1.00 E+02$ & $2 / 12 / 96$ & $4 B$ & $34166^{\circ}$ & BF/NU \\
\hline 1685 & S_ANH_AB & MDISP_T & m & CONSTANT & $5.00 E+00$ & $5.00 E+00$ & $0.00 E+00$ & $5.00 E+00$ & $5.00 E+00$ & 2/12/96 & $4 B$ & $34167^{\circ}$ & NU/BF \\
\hline 2819 & S_ANH_AB & BKLINK & $\mathrm{Pa}$ & CONSTANT & 2.71E-01 & 2.71E-01 & $0.00 E+00$ & 2.71E-01 & 2.71E-01 & $4 / 23 / 96$ & $4 A$ & $34129 \mathrm{~A}$ & $\mathrm{BF}$ \\
\hline 2774 & S_ANH_AB & PCT_A & $\mathrm{Pa}$ & CONSTANT & 2.60E-01 & 2.60E-01 & $0.00 \mathrm{E}+00$ & 2.60E-01 & 2.60E-01 & $2 / 8 / 96$ & $4 A$ & 34183 & $B F$ \\
\hline 1702 & S_ANH_AB & TORTUSTY & NONE & CONSTANT & $1.00 E+01$ & $1.00 \mathrm{E}+01$ & $0.00 E+00$ & $1.00 E+01$ & $1.00 E+01$ & $2 / 12 / 96$ & 48 & $34207^{\circ}$ & $\mathrm{NU}$ \\
\hline 524 & S_ANH_AB & PF_DELTA & $\mathrm{Pa}$ & CONSTANT & $3.80 \mathrm{E}+06$ & $3.80 E+06$ & $0.00 E+00$ & $3.80 E+06$ & $3.80 E+06$ & $2 / 22 / 96$ & $4 B$ & 34187 & BF \\
\hline 526 & S_ANH_AB & PI_DELTA & $\mathbf{P a}$ & CONSTANT & $2.00 E+05$ & $2.00 E+05$ & $0.00 \mathrm{E}+00$ & $2.00 E+05$ & $2.00 \mathrm{E}+05$ & $2 / 22 / 96$ & $4 B$ & 34190 & BF \\
\hline 529 & S_ANH_AB & PO_MIN & $\mathbf{P a}$ & CONSTANT & $1.01 E+05$ & $1.01 E+05$ & $0.00 E+00$ & $1.01 E+05$ & $1.01 E+05$ & $2 / 21 / 96$ & 4B & 34194 & BF \\
\hline 527 & S_ANH_AB & PORE_DIS & NONE & STUDENT & 6.44E-01 & 6.44E-01 & 1.09E-01 & 4.91E-01 & 8.42E-01 & $2 / 22 / 96$ & $4 A$ & 34192 & $B F$ \\
\hline 528 & S_ANH_AB & POROSITY & NONE & STUDENT & 1.10E-02 & 1.10E-02 & 3.04E-03 & 6.00E-03 & 1.70E-02 & $2 / 21 / 96$ & $4 \mathrm{~A}$ & 34193 & $8 F$ \\
\hline 531 & S_ANH_AB & PRMX_LOG & $\log \left(m^{\wedge} 2\right)$ & STUDENT & $-1.89 E+01$ & $-1.89 E+01$ & $1.20 \mathrm{E}+00$ & $-2.10 E+01$ & $-1.71 E+01$ & 2/11/96 & $4 A$ & 34196 & BF \\
\hline 532 & S_ANH_AB & PRMY_LOG & $\log \left(m^{\wedge} 2\right)$ & STUDENT & $-1.89 E+01$ & $-1.89 E+01$ & $1.20 E+00$ & $-2.10 E+01$ & $-1.71 E+01$ & $2 / 11 / 96$ & $4 \mathrm{~A}$ & 34197 & $\mathrm{BF}$ \\
\hline 533 & S_ANH_AB & PRMZ_LOG & $\log \left(m^{\wedge} 2\right)$ & STUDENT & $-1.89 E+01$ & $-1.89 E+01$ & $1.20 \mathrm{E}+00$ & $-2.10 \mathrm{E}+01$ & $-1.71 E+01$ & 2/11/96 & $4 A$ & 34198 & $\mathrm{BF}$ \\
\hline 536 & S_ANH_AB & RELP_MOD & NONE & DELTA & $4.00 E+00$ & $4.00 \mathrm{E}+00$ & $0.00 \mathrm{E}+00$ & $1.00 \mathrm{E}+00$ & $4.00 \mathrm{E}+00$ & $2 / 8 / 96$ & $4 A$ & 34201 & BF \\
\hline 537 & S_ANH_AB & SAT_IBRN & NONE & CONSTANT & $1.00 \mathrm{E}+00$ & $1.00 E+00$ & $0.00 E+00$ & $1.00 \mathrm{E}+00$ & $1.00 E+00$ & 2/8/96 & $4 A$ & 34202 & $\mathrm{BF}$ \\
\hline 538 & S_ANH_AB & SAT_RBRN & NONE & STUDENT & 8.36E-02 & $8.36 E-02$ & $5.01 E-02$ & 7.78E-03 & 1.74E-01 & $11 / 21 / 96$ & $4 A$ & 34203 & $\mathrm{BF}$ \\
\hline 539 & S_ANH_AB & SAT_RGAS & NONE & STUDENT & 7.71E-02 & 7.71E-02 & $6.41 E-02$ & 1.40E-02 & 1.97E-01 & $2 / 19 / 96$ & $4 A$ & 34204 & $B F$ \\
\hline 522 & S_ANH_AB & PC_MAX & $\mathrm{Pa}$ & CONSTANT & $1.00 E+08$ & $1.00 E+08$ & $0.00 E+00$ & $1.00 E+08$ & $1.00 E+08$ & 2/11/96 & $4 A$ & 34185 & BF \\
\hline
\end{tabular}




\section{Disturbed Rock Zone}

\begin{tabular}{|c|c|c|c|c|c|c|c|c|c|c|c|c|c|}
\hline ID & Materia! & Parameter & Units & Distribution & Mean & Median & Deviation & Low & High & Entry Date & Impt & WPO & Codes \\
\hline 2703 & DRZ_O & PCT_EXP & NONE & CONSTANT & $0.00 E+00$ & $0.00 E+00$ & $0.00 E+00$ & $0.00 E+00$ & $0.00 E+00$ & $11 / 14 / 95$ & $4 B$ & 32833 & $\mathrm{BF}$ \\
\hline 174 & DRZ_o & CAP_MOD & NONE & CONSTANT & $1.00 \mathrm{E}+00$ & $1.00 E+00$ & $0.00 \mathrm{E}+00$ & $1.00 E+00$ & $1.00 E+00$ & 2/12/96 & $4 B$ & 32754 & $\mathrm{BF}$ \\
\hline 186 & DRZ_o & RELP_MOD & NONE & DELTA & $4.00 E+00$ & $4.00 E+00$ & $0.00 E+00$ & $1.00 E+00$ & $4.00 E+00$ & $11 / 1 / 95$ & 4B & 32856 & $\mathrm{BF}$ \\
\hline 183 & DRZ_O & PRMZ_LOG & $\log \left(m^{\wedge} 2\right)$ & CONSTANT & $-1.70 E+01$ & $-1.70 E+01$ & $0.00 E+00$ & $-1.70 E+01$ & $-1.70 E+01$ & 11/1/95 & $4 \mathrm{~B}$ & $32850 \mathrm{~A}$ & $\mathrm{BF}$ \\
\hline 182 & DRZ_O & PRMY_LOG & $\log \left(m^{\wedge} 2\right)$ & CONSTANT & $-1.70 E+01$ & $-1.70 E+01$ & $0.00 E+00$ & $-1.70 E+01$ & $-1.70 E+01$ & $11 / 1 / 95$ & 4B & $32849 A$ & $\mathrm{BF}$ \\
\hline 181 & $D R Z_{-} 0$ & PRMX_LOG & $\log \left(m^{\wedge} 2\right)$ & CONSTANT & $-1.70 E+01$ & $-1.70 E+01$ & $0.00 E+00$ & $-1.70 E+01$ & $-1.70 E+01$ & $11 / 1 / 95$ & $4 B$ & $32847 A$ & $B F$ \\
\hline 178 & $D R Z=0$ & POROSITY & NONE & CUMULATIVE & 1.57E-02 & $1.29 E-02$ & 8.52E-03 & $3.90 E-03$ & 3.29E-02 & $2 / 12 / 96$ & 4B & 32839 & BF \\
\hline 188 & DRZ_o & SAT_RBRN & NONE & CONSTANT & $0.00 E+00$ & $0.00 \mathrm{E}+00$ & $0.00 E+00$ & $0.00 E+00$ & $0.00 E+00$ & $2 / 12 / 96$ & $4 B$ & 32860 & $B F$ \\
\hline 179 & DRZ_O & PO_MIN & $\mathrm{Pa}$ & CONSTANT & $1.01 E+05$ & $1.01 E+05$ & $0.00 E+00$ & $1.01 E+05$ & $1.01 E+05$ & 2/21/96 & $4 \mathrm{~B}$ & 32844 & BF \\
\hline 189 & DRZ_o & SAT_RGAS & NONE & CONSTANT & $0.00 E+00$ & $0.00 E+00$ & $0.00 \mathrm{E}+00$ & $0.00 E+00$ & $0.00 E+00$ & $2 / 12 / 96$ & $4 B$ & 32862 & $B F$ \\
\hline 2702 & $D R Z$ _o & PCT_A & $\mathrm{Pa}$ & CONSTANT & $0.00 E+00$ & $0.00 E+00$ & $0.00 E+00$ & $0.00 E+00$ & $0.00 \mathrm{E}+00$ & $11 / 14 / 95$ & AB & 32832 & $B F$ \\
\hline 176 & DRZ_o & PC_MAX & $\mathrm{Pa}$ & CONSTANT & $1.00 E+08$ & $1.00 \mathrm{E}+08$ & $0.00 E+00$ & $1.00 E+08$ & $1.00 E+08$ & $11 / 1 / 95$ & 4B & 32834 & BF \\
\hline 945 & DRZ_o & MDISP_T & $m$ & CONSTANT & $5.00 E+00$ & $5.00 \mathrm{E}+00$ & $0.00 \mathrm{E}+00$ & $5.00 \mathrm{E}+00$ & $5.00 E+00$ & $2 / 12 / 96$ & 48 & $32805^{\prime \prime}$ & BFNU \\
\hline 944 & DRZ_o & MDISP_L & $m$ & CONSTANT & $1.00 E+02$ & $1.00 \mathrm{E}+02$ & $0.00 E+00$ & $1.00 E+02$ & $1.00 \mathrm{E}+02$ & $2 / 12 / 96$ & $4 B$ & $32804^{*}$ & NU/BF \\
\hline 2701 & DRZ_o & KPT & NONE & CONSTANT & $0.00 E+00$ & $0.00 E+00$ & $0.00 E+00$ & $0.00 E+00$ & $0.00 E+00$ & $2 / 20 / 96$ & $4 B$ & 32801 & $B F$ \\
\hline 175 & DRZ_o & COMP_RCK & $P a^{\wedge}-1$ & CONSTANT & $7.41 \mathrm{E}-10$ & 7.41E-10 & $0.00 \mathrm{E}+00$ & 7.41E-10 & 7.41E-10 & $2 / 12 / 96$ & $4 B$ & 32758 & BF \\
\hline 177 & DRZ_o & PORE_DIS & NONE & CONSTANT & 7.00E-01 & $7.00 E-01$ & $0.00 E+00$ & $7.00 E-01$ & 7.00E-01 & $2 / 12 / 96$ & $4 B$ & 32837 & $B F$ \\
\hline 962 & DRZ_o & TORTUSTY & NONE & CONSTANT & $1.00 E+01$ & $1.00 E+01$ & $0.00 \mathrm{E}+00$ & $1.00 E+01$ & $1.00 E+01$ & $2 / 12 / 96$ & 48 & $32868^{*}$ & NU/BF \\
\hline 187 & DRZ_o & SAT_IBRN & NONE & CONSTANT & $1.00 E+00$ & $1.00 E+00$ & $0.00 E+00$ & $1.00 E+00$ & $1.00 \mathrm{E}+00$ & 11/1/95 & 4B & 32857 & $\mathrm{BF}$ \\
\hline 3129 & DRZ_1 & PCT_EXP & NONE & CONSTANT & $0.00 E+00$ & $0.00 E+00$ & $0.00 E+00$ & $0.00 E+00$ & $0.00 E+00$ & $2 / 21 / 96$ & 4B & 36560 & $\mathrm{BF}$ \\
\hline 205 & DRZ_1 & SAT_RBRN & NONE & CONSTANT & $0.00 E+00$ & $0.00 E+00$ & $0.00 \mathrm{E}+00$ & $0.00 E+00$ & $0.00 E+00$ & $11 / 1 / 95$ & 4B & 32912 & $B F$ \\
\hline 203 & DRZ_1 & RELP_MOD & NONE & DELTA & $4.00 \mathrm{E}+00$ & $4.00 \mathrm{E}+00$ & $0.00 \mathrm{E}+00$ & $1.00 \mathrm{E}+00$ & $4.00 E+00$ & $2 / 12 / 96$ & $4 B$ & 32910 & BF \\
\hline 200 & DRZ_1 & PRMZ_LOG & $\log \left(m^{\wedge} 2\right)$ & CONSTANT & $-1.50 E+01$ & $-1.50 E+01$ & $0.00 E+00$ & $-1.50 E+01$ & $-1.50 E+01$ & $11 / 1 / 95$ & 4B & $32907 A$ & BF \\
\hline 199 & $D R Z$ _ & PRMY_LOG & $\log \left(m^{\wedge} 2\right)$ & CONSTANT & $-1.50 E+01$ & $-1.50 E+01$ & $0.00 E+00$ & $-1.50 E+01$ & $-1.50 E+01$ & $11 / 1 / 95$ & 4B & $32906 \mathrm{~A}$ & $\mathrm{BF}$ \\
\hline 198 & DRZ_1 & PRMX_LOG & $\log \left(m^{\wedge} 2\right)$ & CONSTANT & $-1.50 E+01$ & $-1.50 E+01$ & $0.00 E+00$ & $-1.50 E+01$ & $-1.50 E+01$ & $11 / 1 / 95$ & 4B & $32905 \mathrm{~A}$ & $B F$ \\
\hline 195 & DRZ_1 & POROSITY & NONE & CUMULATIVE & 1.57E-02 & 1.29E.02 & 8.52E-03 & 3.90E-03 & 3.29E-02 & $2 / 12 / 96$ & $4 B$ & 32902 & $B F$ \\
\hline 196 & DRZ_1 & PO_MIN & $\mathrm{Pa}$ & CONSTANT & $1.01 \mathrm{E}+05$ & $1.01 E+05$ & $0.00 E+00$ & 1.01E+05 & $1.01 E+05$ & 2/21/96 & $4 \mathrm{~B}$ & 32903 & $B F$ \\
\hline 205 & DRZ_1 & SAT_RGAS & NONE & CONSTANT & $0.00 \mathrm{E}+00$ & $0.00 \mathrm{E}+00$ & $0.00 \mathrm{E}+00$ & $0.00 E+00$ & $0.00 \mathrm{E}+00$ & 11/1/95 & $4 B$ & 32913 & BF \\
\hline 3128 & $D R Z$ _ 1 & PCT_A & $\mathrm{Pa}$ & CONSTANT & $0.00 E+00$ & $0.00 E+00$ & $0.00 E+00$ & $0.00 E+00$ & $0.00 \mathrm{E}+00$ & $2 / 21 / 96$ & 48 & $36559 A$ & $B F$ \\
\hline 193 & DRZ_1 & PC_MAX & $\mathrm{Pa}$ & CONSTANT & $1.00 E+08$ & $1.00 E+08$ & $0.00 E+00$ & $1.00 E+08$ & $1.00 E+08$ & $11 / 1 / 95$ & $4 B$ & 32899 & $\mathrm{BF}$ \\
\hline 3116 & DRZ_1 & KPT & NONE & CONSTANT & $0.00 \mathrm{E}+00$ & $0.00 E+00$ & $0.00 \mathrm{E}+00$ & $0.00 E+00$ & $0.00 E+00$ & $2 / 20 / 96$ & 48 & 32870 & $\mathrm{BF}$ \\
\hline 191 & DRZ_1 & COMP_RCK & $P a^{n}-1$ & CONSTANT & 7.41E-10 & 7.41E-10 & $0.00 E+00$ & 7.41E-10 & 7.41E-10 & $2 / 12 / 96$ & $4 B$ & 32871 & $\mathrm{BF}$ \\
\hline 190 & DRZ_1 & CAP_MOD & NONE & CONSTANT & $1.00 E+00$ & $1.00 E+00$ & $0.00 E+00$ & $1.00 E+00$ & $1.00 E+00$ & $2 / 12 / 96$ & 48 & 32869 & $\mathrm{BF}$ \\
\hline 194 & DRZ_1 & PORE_DIS & NONE & CONSTANT & 7.00E-01 & 7.00E-01 & $0.00 \mathrm{E}+00$ & 7.00E-01 & 7.00E-01 & $2 / 12 / 96$ & $4 B$ & 32901 & BF \\
\hline
\end{tabular}


23A. Waste Panel

\begin{tabular}{|c|c|c|c|c|c|c|c|c|c|c|c|c|c|}
\hline ID & Materia! & Parameter & Units & Distribution & Mean & Median & Deviation & Low & High & Entry Date & Impt & WPO & Codes \\
\hline 2040 & WAS_AREA & DIRONCHW & $\mathrm{kg} / \mathrm{m}^{\wedge} 3$ & CONSTANT & $1.70 E+02$ & $1.70 E+02$ & $0.00 E+00$ & $1.70 E+02$ & $1.70 E+02$ & $2 / 13 / 96$ & 2 & 32468 & $B F$ \\
\hline 651 & WAS_AREA & ABSROUGH & $m$ & UNIFORM & $2.50 E-02$ & $2.50 E-02$ & $8.66 E-03$ & 1.00E-02 & $4.00 \mathrm{E}-02$ & $11 / 14 / 95$ & $4 B$ & 34980 & $\mathrm{CS}$ \\
\hline 2046 & WAS_AREA & DRUBBRHV & $\mathrm{kg} / \mathrm{m}^{\wedge} 3$ & CONSTANT & $3.30 \mathrm{E}+00$ & $3.30 E+00$ & $0.00 E+00$ & $3.30 E+00$ & 3.30E+00 & $2 / 13 / 96$ & 2 & 32483 & $\mathrm{BF}$ \\
\hline 2042 & WAS_AREA & DRUBBCHW & $\mathrm{kg} / \mathrm{m}^{\wedge} 3$ & CONSTANT & $1.00 E+01$ & $1.00 E+01$ & $0.00 E+00$ & $1.00 \mathrm{E}+01$ & $1.00 E+01$ & $2 / 13 / 96$ & 2 & 32481 & $B F$ \\
\hline 2228 & WAS_AREA & DPLSCRHW & $\mathrm{kg} / \mathrm{m}^{\wedge} 3$ & CONSTANT & $3.10 E+00$ & 3.10E+00 & $0.00 E+00$ & $3.10 E+00$ & 3.10E+OD & $2 / 13 / 96$ & 2 & 32480 & $B F$ \\
\hline 1995 & WAS_AREA & DPLSCCHW & $\mathrm{kg} / \mathrm{m}^{\wedge} 3$ & CONSTANT & $2.60 E+01$ & $2.60 E+01$ & $0.00 E+00$ & $2.60 E+01$ & $2.60 \mathrm{E}+01$ & $2 / 13 / 96$ & 2 & 32478 & BF \\
\hline 2275 & WAS_AREA & DPLASRHW & $\mathrm{kg} / \mathrm{m}^{\wedge} 3$ & CONSTANT & $1.50 E+01$ & $1.50 E+01$ & $0.00 E+00$ & $1.50 E+01$ & $1.50 \mathrm{E}+01$ & $2 / 13 / 96$ & 2 & 32476 & $\mathrm{BF}$ \\
\hline 2043 & WAS_AREA & DPLASCHW & $\mathrm{kg} / \mathrm{m}^{\wedge} 3$ & CONSTANT & $3.40 E+01$ & $3.40 E+01$ & $0.00 E+00$ & $3.40 \mathrm{E}+01$ & $3.40 E+01$ & $2 / 13 / 96$ & 2 & 32474 & $B F$ \\
\hline 657 & WAS_AREA & GRATMICI & mol/kg"s & UNIFORM & 4.91E-09 & 4.91E-09 & 2.65E-09 & 3.17E-10 & 9.51E-09 & 2/8/96 & $1 B$ & 34928 & $B F$ \\
\hline 2044 & WAS_AREA & DIRONRHW & $\mathrm{kg} / \mathrm{m}^{\wedge} 3$ & CONSTANT & $1.00 E+02$ & $1.00 E+02$ & $0.00 E+00$ & $1.00 E+02$ & $1.00 E+02$ & $2 / 13 / 96$ & 2 & 32469 & $B F$ \\
\hline 2804 & WAS_AREA & KPT & NONE & CONSTANT & $0.00 E+00$ & $0.00 E+00$ & $0.00 E+00$ & $0.00 E+00$ & $0.00 E+00$ & $2 / 20 / 96$ & 4B & 34942 & $B F$ \\
\hline 1993 & WAS_AREA & DIRNCRHW & $\mathrm{kg} / \mathrm{m}^{\wedge} 3$ & CONSTANT & $2.59 E+03$ & $2.59 E+03$ & $0.00 E+00$ & 2.59E+03 & $2.59 E+03$ & $2 / 13 / 96$ & 2 & 32467 & $\mathrm{BF}$ \\
\hline 1992 & WAS_AREA & DIRNCCHW & $\mathrm{kg} / \mathrm{m}^{\wedge} 3$ & CONSTANT & 1.39E+02 & 1.39E+02 & $0.00 E+00$ & 1.39E+02 & $1.39 \mathrm{E}+02$ & $2 / 13 / 96$ & 2 & 32466 & $\mathrm{BF}$ \\
\hline 2274 & WAS_AREA & DCELLRHW & $\mathrm{kg} / \mathrm{m}^{\wedge} 3$ & CONSTANT & 1.70E+01 & $1.70 E+01$ & $0.00 \mathrm{E}+00$ & $1.70 E+01$ & 1.70E+01 & $2 / 13 / 96$ & 2 & 32465 & $\mathrm{BF}$ \\
\hline 2041 & WAS_AREA & DCELLCHW & $\mathrm{kg} / \mathrm{m}^{\wedge} 3$ & CONSTANT & $5.40 E+01$ & $5.40 E+01$ & $0.00 E+00$ & $5.40 E+01$ & $5.40 \mathrm{E}+01$ & $2 / 13 / 96$ & 2 & 32464 & $\mathrm{BF}$ \\
\hline 653 & WAS_AREA & COMP_RCK & $P a^{n}-1$ & CONSTANT & $0.00 E+00$ & $0.00 E+00$ & $0.00 E+00$ & $0.00 E+00$ & $0.00 E+00$ & $2 / 8 / 96$ & 4B & 34987 & BF \\
\hline 3454 & WAS_AREA & CLOSMOD & NONE & CONSTANT & $4.00 E+00$ & $4.00 E+00$ & $0.00 E+00$ & $4.00 \mathrm{E}+00$ & $4.00 \mathrm{E}+00$ & $6 / 4 / 96$ & 4B & 38200 & BF \\
\hline 652 & WAS_AREA & CAP_MOD & NONE & CONSTANT & $1.00 E+00$ & $1.00 E+00$ & $0.00 E+00$ & $1.00 E+00$ & $1.00 E+00$ & $2 / 8 / 96$ & 4B & 34982 & $\mathrm{BF}$ \\
\hline 1994 & WAS_AREA & DNSGRAIN & $\mathrm{kg} / \mathrm{m}^{\wedge} 3$ & CONSTANT & $0.00 E+00$ & $0.00 E+00$ & $0.00 E+00$ & $0.00 E+00$ & $0.00 E+00$ & $1 / 8 / 97$ & 5 & $36967^{\circ}$ & $\mathrm{BF}$ \\
\hline 663 & WAS_AREA & PRMX_LOG & $\log \left(m^{\wedge} 2\right)$ & CONSTANT & $-1.28 E+01$ & $-1.28 E+01$ & $0.00 E+00$ & $-1.28 E+01$ & $-1.28 E+01$ & $2 / 8 / 96$ & 1 & 34877 & BF \\
\hline 2232 & WAS_AREA & VOLCHW & $m^{\wedge} 3$ & CONSTANT & $1.69 E+05$ & 1.69E+05 & $0.00 E+00$ & 1.69E+05 & $1.69 E+05$ & $2 / 13 / 96$ & 2 & 32484 & $\mathrm{BF}$ \\
\hline 2231 & WAS_AREA & SAT_WICK & NONE & UNIFORM & 5.00E-01 & 5.00E-01 & 2.89E-01 & $0.00 E+00$ & $1.00 E+00$ & $11 / 2 / 95$ & 4B & 34908 & $B F$ \\
\hline 671 & WAS_AREA & SAT_RGAS & NONE & UNIFORM & 7.50E-02 & 7.50E-02 & 4.33E-02 & $0.00 E+00$ & 1.50E-01 & $2 / 22 / 96$ & $4 B$ & 34905 & $B F$ \\
\hline 670 & WAS_AREA & SAT_RBRN & NONE & UNIFORM & 2.76E-01 & 2.76E-01 & 1.59E-01 & $0.00 E+00$ & 5.52E-01 & $2 / 21 / 96$ & 4B & 34902 & BF \\
\hline 669 & WAS_AREA & SAT_IBRN & NONE & CONSTANT & $1.50 E-02$ & 1.50E-02 & $0.00 E+00$ & 1.50E-02 & $1.50 E-02$ & 2/20/96 & 4B & 34894 & $\mathrm{BF}$ \\
\hline 668 & WAS_AREA & RELP_MOD & NONE & CONSTANT & $4.00 E+00$ & $4.00 E+00$ & $0.00 E+00$ & $4.00 E+00$ & $4.00 E+00$ & $2 / 8 / 96$ & 4B & 34890 & $B F$ \\
\hline 2823 & WAS_AREA & PROBDEG & NONE & DELTA & $2.00 E+00$ & $2.00 E+00$ & $0.00 E+00$ & $0.00 E+00$ & $2.00 E+00$ & $2 / 23 / 96$ & 4B & 34881 & $\mathrm{BF}$ \\
\hline 656 & WAS_AREA & GRATMICH & $\mathrm{mol} / \mathrm{kg} " \mathrm{~s}$ & UNIFORM & 6.34E-10 & 6.34E-10 & $3.66 E-10$ & $0.00 E+00$ & 1.27E-09 & $2 / 8 / 96$ & $1 B$ & 34923 & $B F$ \\
\hline 664 & WAS_AREA & PRMY_LOG & $\log \left(m^{\wedge} 2\right)$ & CONSTANT & $-1.28 E+01$ & $-1.28 E+01$ & $0.00 \mathrm{E}+00$ & $-1.28 E+01$ & $-1.28 E+01$ & 2/8/96 & 1 & 34878 & $\mathrm{BF}$ \\
\hline 2233 & WAS_AREA & VOLRHW & $m^{\wedge} 3$ & CONSTANT & $7.08 E+03$ & $7.08 E+03$ & $0.00 E+00$ & $7.08 E+03$ & $7.08 E+03$ & $2 / 13 / 96$ & 2 & 32485 & BF \\
\hline 662 & WAS_AREA & PRESSURE & $\mathrm{Pa}$ & CONSTANT & $1.01 E+05$ & $1.01 \mathrm{E}+05$ & $0.00 E+00$ & 1.01E+05 & $1.01 E+05$ & $11 / 2 / 95$ & 4B & 34876 & BF \\
\hline 660 & WAS_AREA & POROSITY & NONE & CONSTANT & $8.48 E-01$ & $8.48 E-01$ & $0.00 E+00$ & 8.48E-01 & 8.48E-01 & 2/8/96 & 48 & 34874 & $\mathrm{BF}$ \\
\hline 659 & WAS_AREA & PORE_DIS & NONE & CUMULATIVE & 3.25E+00 & $2.89 E+00$ & $1.27 E+00$ & $1.44 \mathrm{E}+00$ & $5.78 \mathrm{E}+00$ & $2 / 22 / 96$ & 4B & 34989 & $\mathrm{BF}$ \\
\hline 661 & WAS_AREA & PO_MIN & $\mathrm{Pa}$ & CONSTANT & 1.01E+05 & $1.01 E+05$ & $0.00 E+00$ & $1.01 E+05$ & $1.01 E+05$ & $2 / 21 / 96$ & AB & 34875 & BF \\
\hline 2806 & WAS_AREA & PCT_EXP & NONE & CONSTANT & $0.00 E+00$ & $0.00 E+00$ & $0.00 E+00$ & $0.00 E+00$ & $0.00 E+00$ & $2 / 8 / 96$ & $4 B$ & 34984 & BF \\
\hline 2805 & WAS_AREA & PCT_A & $\mathrm{Pa}$ & CONSTANT & $0.00 E+00$ & $0.00 E+00$ & $0.00 E+00$ & $0.00 E+00$ & $0.00 E+00$ & $2 / 8 / 96$ & $4 B$ & 34983 & BF \\
\hline 658 & WAS_AREA & PC_MAX & $\mathrm{Pa}$ & CONSTANT & $1.00 E+08$ & $1.00 \mathrm{E}+08$ & $0.00 \mathrm{E}+00$ & $1.00 E+08$ & $1.00 \mathrm{E}+08$ & $11 / 2 / 95$ & 48 & 34986 & $\mathrm{BF}$ \\
\hline 665 & WAS_AREA & PRMZ_LOG & $\log \left(m^{\wedge} 2\right)$ & CONSTANT & $-1.28 E+01$ & $-1.28 E+01$ & $0.00 E+00$ & $-1.28 E+01$ & $-1.28 E+01$ & $2 / 8 / 96$ & 1 & 34879 & $B F$ \\
\hline
\end{tabular}


23B. Waste Chemistry

\begin{tabular}{|c|c|c|c|c|c|c|c|c|c|c|c|c|c|}
\hline ID & Material & Parameter & Units & Distribution & Mean & Median & Deviation & Low & High & Entry Date & Impt & WPO & Codes \\
\hline 3220 & $A C 225$ & ATWEIGHT & $\mathrm{kg} / \mathrm{mole}$ & CONSTANT & $25 \mathrm{E}-01$ & 2.25E-01 & $0.00 E+00$ & 2.25E-01 & 2.25E-01 & $4 / 10 / 96$ & 3 & 36738 & CSINU \\
\hline 3321 & AC225 & EPAREL & Cirwuf & CONSTANT & $0.00 E+00$ & $0.00 E+00$ & $0.00 E+00$ & $0.00 E+00$ & $0.00 E+00$ & $4 / 16 / 96$ & 3 & 36868 & CS \\
\hline 3267 & AC225 & HALFLIFE & s & CONSTANT & $8.64 E+05$ & $8.64 E+05$ & $0.00 E+00$ & $8.64 E+05$ & $8.64 E+05$ & $4 / 16 / 96$ & 3 & 36795 & CSINU \\
\hline 3221 & AC227 & ATWEIGHT & $\mathrm{kg} / \mathrm{mole}$ & CONSTANT & $2.27 E-01$ & $2.27 E-01$ & $0.00 E+00$ & 2.27E-01 & $2.27 E-01$ & $4 / 10 / 96$ & 3 & 36739 & CS/NU \\
\hline 3364 & AC227 & EPAREL & Cirwuf & CONSTANT & $1.00 E+02$ & $1.00 E+02$ & $0.00 E+00$ & $1.00 \mathrm{E}+02$ & $1.00 E+02$ & $4 / 16 / 96$ & 3 & 36926 & CS \\
\hline 3268 & AC227 & HALFLIFE & $\mathbf{s}$ & CONSTANT & $6.87 E+08$ & $6.87 E+08$ & $0.00 E+00$ & $6.87 E+08$ & $6.87 E+08$ & $4 / 16 / 96$ & 3 & 36796 & CSINU \\
\hline 3222 & AC228 & ATWEIGHT & $\mathrm{kg} / \mathrm{mole}$ & CONSTANT & 2.28E-01 & 2.28E-01 & $0.00 E+00$ & $2.28 E-01$ & 2.28E-01 & $4 / 10 / 96$ & 3 & 36740 & CSINU \\
\hline 3322 & AC228 & EPAREL & Citwut & CONSTANT & $0.00 E+00$ & $0.00 E+00$ & $0.00 E+00$ & $0.00 E+00$ & $0.00 E+00$ & $4 / 16 / 96$ & 3 & 36869 & CS \\
\hline 3269 & AC228 & HALFLIFE & s & CONSTANT & $2.21 E+04$ & $2.21 E+04$ & $0.00 \mathrm{E}+00$ & $2.21 E+04$ & $2.21 E+04$ & $4 / 16 / 96$ & 3 & 36797 & CSINU \\
\hline 3457 & AM & CAPHUM & moles/liter & CONSTANT & 1.10E-05 & 1.10E-05 & $0.00 E+00$ & 1.10E-05 & 1.10E-05 & $5 / 8 / 96$ & 1 & 37721 & NU \\
\hline 3447 & AM & CAPMIC & moles/iter & CONSTANT & $1.00 \mathrm{E}+00$ & $1.00 \mathrm{E}+00^{\circ}$ & $0.00 E+00$ & 1.00E+00 & $1.00 E+00$ & $5 / 8 / 96$ & 1 & 37712 & NU \\
\hline 3310 & AM & CONCINT & moles/iter & CONSTANT & $0.00 E+00$ & $0.00 E+00$ & $0.00 E+00$ & $0.00 E+00$ & $0.00 E+00$ & $4 / 16 / 96$ & 1 & 36857 & NU \\
\hline 3441 & AM & CONCMIN & moles/iter & CONSTANT & $2.60 \mathrm{E}-08$ & 2.60E-08 & $0.00 E+00$ & 2.60E-08 & $2.60 E-08$ & $5 / 8 / 96$ & 1 & 37704 & NU \\
\hline 3311 & AM & PROPMIC & NONE & CONSTANT & $3.60 E+00$ & $3.60 E+00$ & $0.00 \mathrm{E}+00$ & $3.60 E+\infty 0$ & $3.60 E+00$ & $4 / 16 / 96$ & 1 & 36858 & NU \\
\hline 3444 & $A M+3$ & MDO & $\mathrm{m}^{\wedge} 2 / \mathrm{s}$ & CONSTANT & $3.00 \mathrm{E}-10$ & $3.00 E-10$ & $0.00 E+00$ & $3.00 E-10$ & $3.00 E-10$ & $5 / 17 / 96$ & 1 & 37708 & TP/NU \\
\hline 3482 & $A M+3$ & MKD_AM & $\mathrm{m}^{\wedge} 3 / \mathrm{kg}$ & UNIFORM & 2.60E-0i & 2.60E-01 & 1.40E-01 & 2.00E-02 & $5.00 E-01$ & $112 / 96$ & 1 & 8353 & NU/TP \\
\hline & AM241 & ATWEIGHT & $\mathrm{kg} / \mathrm{mole}$ & |CONSTANT & 2.41E-01 & 2.41E-01 & $0.00 E+00$ & 2.41E-01 & 2.41E-01 & $11 / 2 / 95$ & 3 & 31357 & $\begin{array}{l}\text { CS/FU } \\
\text { TP/NU }\end{array}$ \\
\hline 3363 & AM241 & EPAREL & Cirwuf & CONSTANT & $1.00 E+02$ & $1.00 \mathrm{E}+02$ & $0.00 E+00$ & $1.00 E+02$ & $1.00 E+02$ & 4/16/96 & 3 & 36925 & cs \\
\hline 3 & AM241 & HALFLIFE & $s$ & CONSTANT & $1.36 \mathrm{E}+10$ & $1.36 E+10$ & $0.00 E+00$ & 1.36E+10 & $1.36 E+10$ & 4/16/96 & 3 & 31358 & NU/CS \\
\hline 4 & AM241 & INVCHD & $\mathrm{Ci}$ & CONSTANT & $4.78 E+05$ & 4.78E+05 & $0.00 E+00$ & 4.78E+05 & $4.78 E+05$ & 10/29/96 & 2 & $31359 A$ & $\begin{array}{c}\text { CS/PN/ } \\
\text { NU }\end{array}$ \\
\hline 5 & AM241 & |INVRHD & $\mathrm{Ci}$ & CONSTANT & $9.44 E+03$ & $9.44 E+03$ & $0.00 \mathrm{E}+00$ & $9.44 E+03$ & $9.44 E+03$ & 10/29/96 & 2 & $31360 A$ & $\begin{array}{c}\text { NU/CSI } \\
\text { PN }\end{array}$ \\
\hline 3223 & AM243 & ATWEIGHT & $\mathrm{kg} / \mathrm{mole}$ & CON & & 2.43E-01 & $0.00 E+00$ & 2.43E-01 & 2.43E-01 & $10 / 96$ & 3 & 6741 & NU/CS \\
\hline 3365 & AM243 & EPAREL & Cihwof & CONST & & $1.00 E+02$ & $0.00 E+00$ & $1.00 E+02$ & $1.00 E+02$ & $16 / 96$ & 3 & 36927 & CS \\
\hline 6 & AM243 & HALFLIFE & s & & & $2.33 E+11$ & $0.00 E+00$ & $2.33 E+11$ & $2.33 E+11$ & $16 / 96$ & 3 & 31374 & ESINU \\
\hline 3415 & AM243 & INVCHD & $\mathrm{Ci}$ & CONSTANT & $3.25 E+01$ & $3.25 E+01$ & $0.00 E+00$ & $3.25 E+01$ & $3.25 E+01$ & $10 / 29 / 96$ & 2 & $37138 \mathrm{~A}$ & $\begin{array}{c}\text { NU/PNI } \\
\text { CS }\end{array}$ \\
\hline 3416 & AM243 & INVRHD & $\mathbf{C i}$ & CONSTANT & 2.27E-04 & 2.27E-04 & $0.00 E+00$ & 2.27E-04 & 2.27E-04 & $10 / 29 / 96$ & 2 & $37139 A$ & $\begin{array}{c}\text { NU/PNI } \\
\text { CS }\end{array}$ \\
\hline 3224 & AT217 & ATWEIGHT & $\mathrm{kg} / \mathrm{mole}$ & CONSTANT & 2.17E-01 & 2.17E-01 & $0.00 E+00$ & 2.17E-01 & 2.17E-01 & $4 / 10 / 96$ & 3 & 36742 & CSINU \\
\hline 3323 & AT217 & EPAREL & Cirwut & CONSTANT & $0.00 E+00$ & $0.00 E+00$ & $0.00 E+00$ & $0.00 E+00$ & $0.00 E+00$ & $4 / 16 / 96$ & 3 & 36870 & CS \\
\hline 3270 & AT217 & HALFLIFE & $s$ & CONSTANT & 3.23E-02 & 3.23E-02 & $0.00 E+00$ & 3.23E-02 & 3.23E-02 & $4 / 16 / 96$ & 3 & 36799 & CS/NU \\
\hline 3225 & BA137 & ATWEIGHT & $\mathrm{kg} / \mathrm{mole}$ & & & 1.37E-01 & $0.00 E+00$ & 1.37E-01 & 1.37E-01 & $4 / 10 / 96$ & 3 & 36743 & NU/CS \\
\hline 3324 & BA137 & EPAREL & Cihwuf & CONSTANT & $0.00 E+00$ & $0.00 E+00$ & $0.00 E+00$ & $0.00 E+00$ & $0.00 E+00$ & |116/96 & 3 & 36871 & CS \\
\hline 3271 & BA137 & HALFLIFE & s & CONSTANT & $1.00 E+38$ & $1.00 E+38$ & $0.00 \mathrm{E}+00$ & $1.00 \mathrm{E}+38$ & $1.00 E+38$ & $4 / 16 / 96$ & 3 & 36800 & CSINU \\
\hline 3226 & BA137M & ATWEIGHT & $\mathrm{kg} / \mathrm{mole}$ & CONSTANT & 1.37E-01 & 1.37E-01 & $0.00 E+00$ & 1.37E-01 & 1.37E-01 & $4 / 10 / 96$ & -3 & 36744 & CS/NU \\
\hline 3325 & BA137M & EPAREL & Citwuf & CONSTANT & $0.00 E+00$ & $0.00 E+00$ & $0.00 E+00$ & $0.00 E+00$ & $0.00 E+00$ & 4/16/96 & 3 & 36872 & cs \\
\hline 3272 & BA137M & HALFLIFE & $s$ & CONSTANT & $1.53 E+02$ & $1.53 E+02$ & $0.00 E+00$ & $1.53 E+02$ & $1.53 E+02$ & 4/16/96 & 3 & 36801 & CSINU \\
\hline 3227 & $B 1211$ & ATWEIGHT & $\mathrm{kg} / \mathrm{mole}$ & CONSTANT & 2.11E-01 & 2.11E-01 & $0.00 E+00$ & 2.11E-01 & 2.11E-01 & 4/10/96 & 3 & 36746 & NU/CS \\
\hline 3326 & $B \mid 211$ & EPAREL & Ci/wut & CONSTANT & $0.00 E+00$ & $0.00 \mathrm{E}+00$ & $0.00 E+00$ & $0.00 E+00$ & $0.00 E+00$ & $4 / 16 / 96$ & 3 & 36873 & CS \\
\hline 3273 & $B 1211$ & HALFLIFE & s & CONSTANT & $1.28 E+02$ & 1.28E+02 & $0.00 E+00$ & $1.28 E+02$ & $1.28 E+02$ & $4 / 16 / 96$ & 3 & 36803 & NU/CS \\
\hline 3228 & $\mathrm{BI} 212$ & ATWEIGHT & $\mathrm{kg} / \mathrm{mole}$ & CONSTANT & 2.12E-01 & 2.12E-01 & $0.00 E+00$ & 2.12E-01 & 2.12E-01 & $4 / 10 / 96$ & 3 & 36748 & NU/CS \\
\hline 3327 & BI212 & EPAREL & Cirwuf & CONSTANT & $0.00 E+00$ & $0.00 E+00$ & $0.00 E+00$ & $0.00 E+00$ & $0.00 E+00$ & $4 / 16 / 96$ & 3 & 36874 & CS \\
\hline 3274 & $\mathrm{Bl} 212$ & HALFLIFE & $\mathbf{s}$ & CONSTANT & 3.63E+03 & $3.63 E+03$ & $0.00 E+00$ & $3.63 E+03$ & $3.63 E+03$ & $4 / 16 / 96$ & 3 & 36804 & NU/CS \\
\hline 3229 & $\mathrm{Bl} 213$ & ATWEIGHT & $\mathrm{kg} / \mathrm{mole}$ & CONSTANT & 2.13E-01 & 2.13E-01 & $0.00 E+00$ & 2.13E-01 & 2.13E-01 & 4/10/96 & 3 & 36749 & NU/CS \\
\hline 3328 & $\mathrm{Bl} 213$ & EPAREL & Cirwuf & CONSTANT & $0.00 E+00$ & $0.00 E+00$ & $0.00 E+00$ & $0.00 E+00$ & $0.00 E+00$ & 4/16/96 & 3 & 36875 & Cs \\
\hline 3275 & $\mathrm{~B} 1213$ & HALFLIFE & s & CONSTANT & $2.74 E+03$ & $2.74 E+03$ & $0.00 E+00$ & $2.74 E+03$ & $2.74 E+03$ & $4 / 16 / 96$ & 3 & 36806 & CSINU \\
\hline 3230 & Bl214 & ATWEIGHT & $\mathrm{kg} / \mathrm{mole}$ & CONSTANT & 2.14E-01 & 2.14E-01 & $0.00 E+00$ & 2.14E-01 & 2.14E-01 & $4 / 10 / 96$ & 3 & 36750 & CSINU \\
\hline 3329 & $B 1214$ & EPAREL & Cirwuf & CONSTANT & $0.00 E+00$ & $0.00 E+00$ & $0.00 E+00$ & $0.00 E+00$ & $0.00 E+00$ & $4 / 16 / 96$ & 3 & 36876 & CS \\
\hline 3276 & BI214 & HALFLIFE & $s$ & CONSTANT & 1.19E+03 & $1.19 E+03$ & $0.00 E+00$ & $1.19 E+03$ & 1.19E+03 & $4 / 16 / 96$ & 3 & 36807 & CSINU \\
\hline 2994 & CELLULS & FBETA & NONE & UNIFORM & 5.00E-01 & 5.00E-01 & 2.89E-01 & $0.00 E+00$ & $1.00 E+00$ & $2 / 13 / 96$ & $4 A$ & 31826 & $B F$ \\
\hline
\end{tabular}


23B. Waste Chemistry

\begin{tabular}{|c|c|c|c|c|c|c|c|c|c|c|c|c|c|}
\hline ID & Material & Parameter & Units & Distribution & Mean & Median & Deviation & Low & High & Entry Date & Impt & WPO & Codes \\
\hline 838 & CF & LOGSOLM & $\log ($ moles $/)$ & CONSTANT & $0.00 E+00$ & $0.00 E+00$ & $0.00 E+00$ & $0.00 E+00$ & $0.00 E+00$ & $5 / 1 / 96$ & 4B & 31827 & NU \\
\hline 106 & CF252 & ATWEIGHT & $\mathrm{kg} / \mathrm{mole}$ & CONSTANT & $2.52 E-01$ & 2.52E-01 & $0.00 E+00$ & 2.52E-01 & $2.52 E-01$ & $11 / 2 / 95$ & 3 & 31828 & $\begin{array}{l}\text { TPICSI } \\
\text { FUNU }\end{array}$ \\
\hline 3330 & CF252 & EPAREL & Citwuf & CONSTANT & $0.00 E+00$ & $0.00 E+00$ & $0.00 E+00$ & $0.00 E+00$ & $0.00 \mathrm{E}+00$ & $4 / 16 / 96$ & 3 & 36877 & CS \\
\hline 107 & CF252 & HALFLIFE & s & CONSTANT & $8.33 E+07$ & 8.33E+07 & $0.00 E+00$ & 8.33E+07 & $8.33 E+07$ & $4 / 16 / 96$ & 3 & 31829 & NU/CS \\
\hline 108 & CF252 & INVCHD & $\mathrm{Ci}$ & CONSTANT & 1.12E-04 & 1.12E-04 & $0.00 E+00$ & 1.12E-04 & 1.12E-04 & $10 / 29 / 96$ & 2 & $31830 \mathrm{~A}$ & $\begin{array}{c}\text { NU/CS/ } \\
\text { PN }\end{array}$ \\
\hline 109 & CF252 & INVRHD & $\mathrm{Ci}$ & CONSTANT & 5.96E-05 & 5.96E-05 & $0.00 E+00$ & $5.96 E-05$ & 5.96E-05 & 10/29/96 & 2 & $31831 \mathrm{~A}$ & $\begin{array}{c}\text { PN/CSI } \\
\text { NU }\end{array}$ \\
\hline 3231 & CM243 & ATWEIGHT & $\mathrm{kg} / \mathrm{mole}$ & CONSTANT & 2.43E-01 & 2.43E-01 & $0.00 E+00$ & 2.43E-01 & $2.43 E-01$ & $4 / 10 / 96$ & 3 & 36751 & NU/CS \\
\hline 3366 & CM243 & EPAREL & Citwuf & CONSTANT & $1.00 E+02$ & $1.00 E+02$ & $0.00 E+00$ & $1.00 \mathrm{E}+02$ & $1.00 \mathrm{E}+02$ & $4 / 16 / 96$ & 3 & 36928 & CS \\
\hline 3277 & CM243 & HALFLIFE & $\mathbf{s}$ & CONSTANT & 8.99E+08 & $8.99 E+08$ & $0.00 E+00$ & 8.99E+08 & 8.99E+08 & $4 / 16 / 96$ & 3 & 36809 & NU/CS \\
\hline 3410 & CM243 & INVCHD & $\mathrm{Ci}$ & CONSTANT & $1.08 \mathrm{E}+00$ & $1.08 E+00$ & $0.00 E+00$ & $1.08 E+00$ & $1.08 E+00$ & $10 / 29 / 96$ & 2 & $37133 \mathrm{~A}$ & $\begin{array}{c}\text { NU/CSI } \\
\text { PN }\end{array}$ \\
\hline 3411 & CM243 & INVRHD & $\mathrm{Ci}$ & CONSTANT & $1.97 E+01$ & $1.97 E+01$ & $0.00 E+00$ & 1.97E+01 & $1.97 E+01$ & $10 / 29 / 96$ & 2 & $37134 \mathrm{~A}$ & $\begin{array}{c}\text { PN/CSI } \\
\text { NU }\end{array}$ \\
\hline 110 & CM244 & ATWEIGHT & $\mathrm{kg} / \mathrm{mole}$ & CONSTANT & 2.44E-01 & 2.44E-01 & $0.00 E+00$ & 2.44E-01 & 2.44E-01 & $11 / 2 / 95$ & 3 & 32331 & $\begin{array}{l}\text { CS/TPI } \\
\text { NU/FL }\end{array}$ \\
\hline 3331 & CM244 & EPAREL & Cirwuf & CONSTANT & $0.00 E+00$ & $0.00 E+00$ & $0.00 E+00$ & $0.00 E+00$ & $0.00 E+00$ & $4 / 16 / 96$ & 3 & 36878 & CS \\
\hline 111 & CM244 & HALFLIFE & s & CONSTANT & $5.72 E+08$ & $5.72 E+08$ & $0.00 E+00$ & $5.72 E+08$ & $5.72 E+08$ & $4 / 16 / 96$ & 3 & 32495 & NU/CS \\
\hline 112 & CM244 & INVCHD & $\mathrm{Ci}$ & CONSTANT & 7.36E+03 & $7.36 E+03$ & $0.00 E+00$ & |7.36E+03 & 7.36E+03 & 10/29/96 & 2 & $32496 \mathrm{~A}$ & $\begin{array}{c}\mathrm{PN} / \mathrm{NU} / \\
\mathrm{CS}\end{array}$ \\
\hline 113 & CM244 & INVRHD & $\mathrm{Ci}$ & CONSTANT & 7.36E+01 & 7.36E+01 & $0.00 E+00$ & 7.36E+01 & 7.36E+01 & $10 / 29 / 96$ & 2 & $32497 \mathrm{~A}$ & $\begin{array}{c}\begin{array}{c}\text { CSIPNI } \\
\text { NU }\end{array} \\
\end{array}$ \\
\hline 3232 & CM245 & ATWEIGHT & $\mathrm{kg} / \mathrm{mole}$ & CONSTANT & 2.45E-01 & 2.45E-01 & $0.00 E+00$ & 2.45E-01 & 2.45E-01 & $4 / 10 / 96$ & 3 & 36752 & NU/CS \\
\hline 3367 & CM245 & EPAREL & Cilwuf & CONSTANT & 1.00E+02 & $1.00 E+02$ & $0.00 E+00$ & $1.00 E+02$ & $1.00 E+02$ & $4 / 16 / 96$ & 3 & 36929 & CS \\
\hline 3278 & CM245 & HALFLIFE & $\mathbf{s}$ & CONSTANT & $2.68 E+11$ & $2.68 \mathrm{E}+11$ & $0.00 E+00$ & $2.68 E+11$ & $2.68 E+11$ & $4 / 16 / 96$ & 3 & 36811 & NU/CS \\
\hline 3412 & СM245 & INVCHD & $\mathbf{C i}$ & CONSTANT & 1.15E+02 & $1.15 E+02$ & $0.00 E+00$ & $1.15 E+02$ & 1.15E+02 & $10 / 29 / 96$ & 2 & $37135 \mathrm{~A}$ & $\begin{array}{c}\text { PN/NUI } \\
\text { CS }\end{array}$ \\
\hline 3413 & СM245 & INVRHD & $\mathbf{C i}$ & CONSTANT & 1.48E-05 & 1.48E-05 & $0.00 E+00$ & 1.48E-05 & 1.48E-05 & 10/29/96 & 2 & $37136 \mathrm{~A}$ & $\begin{array}{c}\text { CS/PNI } \\
\text { NU }\end{array}$ \\
\hline 3233 & CM248 & ATWEIGHT & $\mathrm{kg} / \mathrm{mole}$ & CONSTANT & $2.48 \mathrm{E}-01$ & 2.48E-01 & $0.00 E+00$ & $2.48 E-01$ & 2.48E-01 & $4 / 10 / 96$ & 3 & 36754 & $\mathrm{CS} / \mathrm{NU}$ \\
\hline 3368 & CM248 & EPAREL & Cirwuf & CONSTANT & $1.00 E+02$ & $1.00 \mathrm{E}+02$ & $0.00 E+00$ & $1.00 \mathrm{E}+02$ & $1.00 E+02$ & $4 / 16 / 96$ & 3 & 36930 & cs \\
\hline 115 & CM248 & HALFLIFE & s & CONSTANT & $1.07 E+13$ & 1.07E+13 & $0.00 E+00$ & 1.07E+13 & $1.07 E+13$ & $4 / 16 / 96$ & 3 & 32499 & CS/NU \\
\hline 2265 & CM248 & INVCHD & $\mathrm{Ci}$ & CONSTANT & 3.69E-02 & 3.69E-02 & $0.00 E+00$ & 3.69E-02 & 3.69E-02 & $10 / 29 / 96$ & 2 & $32500 \mathrm{~A}$ & $\begin{array}{c}\text { PN/NUI } \\
\text { CS }\end{array}$ \\
\hline 2266 & CM248 & INVRHD & $\mathrm{Ci}$ & CONSTANT & 2.15E-04 & 2.15E-04 & $0.00 E+00$ & 2.15E-04 & 2.15E-04 & $10 / 29 / 96$ & 2 & $32501 \mathrm{~A}$ & $\begin{array}{c}\begin{array}{c}\text { PN/CSI } \\
\text { NU }\end{array} \\
\end{array}$ \\
\hline 841 & cs & LOGSOLM & $\log ($ moles $/)$ & CONSTANT & $0.00 E+00$ & $0.00 E+00$ & $0.00 E+00$ & $0.00 E+00$ & $0.00 E+00$ & $5 / 1 / 96$ & $4 B$ & 32680 & NU \\
\hline 116 & CS137 & ATWEIGHT & $\mathrm{kg} /$ mole & CONSTANT & 1.37E-01 & 1.37E-01 & $0.00 E+00$ & 1.37E-01 & 1.37E-01 & 11/2/95 & 3 & 32332 & $\begin{array}{l}\text { CS/TPI } \\
\text { FLINU }\end{array}$ \\
\hline 3369 & CS137 & EPAREL & Cilwuf & CONSTANT & $1.00 E+03$ & $1.00 E+03$ & $0.00 E+00$ & $1.00 E+03$ & $1.00 E+03$ & $4 / 16 / 96$ & 3 & 36931 & CS \\
\hline 117 & CS137 & HALFLIFE & $s$ & CONSTANT & $9.47 E+08$ & $9.47 E+08$ & $0.00 E+00$ & $9.47 E+08$ & $9.47 E+08$ & $4 / 16 / 96$ & 3 & 32682 & CS/NU \\
\hline 2037 & CS137 & INVCHD & $\mathrm{Ci}$ & CONSTANT & $3.35 E+03$ & 3.35E+03 & $0.00 E+00$ & $3.35 E+03$ & $3.35 E+03$ & 10/29/96 & 2 & $32683 \mathrm{~A}$ & $\begin{array}{c}\mathrm{PN} / \mathrm{NU} / \\
\mathrm{CS} \\
\end{array}$ \\
\hline 118 & $\operatorname{cs} 137$ & INVRHD & $\mathrm{Ci}$ & CONSTANT & $8.98 E+0.4$ & 8.98E+04 & $0.00 \mathrm{E}+00$ & $8.98 E+04$ & $8.98 E+04$ & $10 / 29 / 96$ & 2 & $32684 \mathrm{~A}$ & $\begin{array}{c}\text { PN/NUI } \\
\text { CS }\end{array}$ \\
\hline 3234 & FR221 & ATWEIGHT & $\mathrm{kg} / \mathrm{mole}$ & CONSTANT & 2.21E-01 & 2.21E-01 & $0.00 E+00$ & 2.21E-01 & 2.21E-01 & $4 / 10 / 96$ & 3 & 36755 & CSINU \\
\hline 3332 & FR221 & EPAREL & Cilwuf & CONSTANT & $0.00 E+00$ & $0.00 E+00$ & $0.00 E+00$ & $0.00 E+00$ & $0.00 E+00$ & $4 / 16 / 96$ & 3 & 36879 & CS \\
\hline 3279 & FR221 & HALFLIFE & $\mathbf{s}$ & CONSTANT & 2.88E+02 & $2.88 E+02$ & $0.00 E+00$ & $2.88 E+02$ & $2.88 E+02$ & $4 / 16 / 96$ & 3 & 36812 & NU/CS \\
\hline 3235 & ND143 & ATWEIGHT & $\mathrm{kg} / \mathrm{mole}$ & CONSTANT & 1.43E-01 & 1.43E-01 & $0.00 E+00$ & 1.43E-01 & 1.43E-01 & $4 / 10 / 96$ & 3 & 36757 & CS/NU \\
\hline 3333 & ND143 & EPAREL & Cilwuf & CONSTANT & $0.00 E+00$ & $0.00 E+00$ & $0.00 E+00$ & $0.00 E+00$ & $0.00 E+00$ & $4 / 16 / 96$ & 3 & 36880 & Cs \\
\hline 3280 & ND143 & HALFLIFE & $s$ & CONSTANT & $1.00 E+38$ & $1.00 E+38$ & $0.00 E+00$ & $1.00 E+38$ & $1.00 E+38$ & $4 / 16 / 96$ & 3 & 36813 & CSINU \\
\hline 2906 & NITRATE & QINIT & moles & CONSTANT & 2.61E+07 & $2.61 E+07$ & $0.00 E+00$ & $2.61 E+07$ & 2.61E+07 & $2 / 22 / 96$ & 2 & 32335 & $\mathrm{BF}$ \\
\hline 3458 & $\mathrm{NP}$ & CAPHUM & moles/iter & CONSTANT & $1.10 \mathrm{E}-05$ & 1.10E-05 & $0.00 E+00$ & 1.10E-05 & 1.10E-05 & $5 / 8 / 95$ & 1 & 37723 & NU \\
\hline 3313 & NP & CAPMIC & moles/iter & CONSTANT & 2.70E-03 & 2.70E-03 & $0.00 E+00$ & $2.70 E-03$ & 2.70E-03 & $4 / 16 / 96$ & 1 & 36860 & NU \\
\hline
\end{tabular}


23B. Waste Chemistry

\begin{tabular}{|c|c|c|c|c|c|c|c|c|c|c|c|c|c|}
\hline ID & Material & Parameter & Units & Distribution & Mean & Median & Deviation & Low & High & Entry Date & Impt & WPO & Codes \\
\hline 3312 & NP & CONCINT & moles/liter & CONSTANT & $0.00 E+00$ & $0.00 E+00$ & $0.00 E+00$ & $0.00 E+00$ & $0.00 E+00$ & $4 / 16 / 96$ & 1 & 36859 & NU \\
\hline 3439 & NP & CONCMIN & moles/iter & CONSTANT & 2.60E-08 & $2.60 E-08$ & $0.00 E+00$ & 2.60E-08 & 2.60E-08 & $5 / 8 / 96$ & 1 & 37700 & $N U$ \\
\hline 3314 & NP & PROPMIC & NONE & CONSTANT & $1.20 E+01$ & 1.20E+01 & $0.00 E+00$ & $1.20 E+01$ & $1.20 E+01$ & $4 / 16 / 96$ & 1 & 36861 & NU \\
\hline 3477 & $N P+4$ & MKD_NP & $m^{\wedge} 3 / \mathrm{kg}$ & UNIFORM & 1.00E+01 & $1.00 \mathrm{E}+01$ & $5.50 E+00$ & $9.00 E-01$ & $2.00 E+01$ & $6 / 12 / 96$ & 1 & 38348 & NU/TP \\
\hline 3476 & $N P+5$ & MKD_NP & $m^{\wedge} 3 / \mathrm{kg}$ & UNIFORM & $1.00 E-01$ & 1.00E-01 & $5.70 E-02$ & 1.00E-03 & 2.00E-01 & $6 / 12 / 96$ & 1 & 38347 & NUTTP \\
\hline 246 & NP237 & |ATWEIGHT & $\mathrm{kg} / \mathrm{mole}$ & CONSTANT & 2.37E-01 & 2.37E-01 & $0.00 E+00$ & 2.37E-01 & 2.37E-01 & $11 / 2 / 95$ & 3 & 32336 & $\begin{array}{l}\text { FLCSI } \\
\text { TP/NU }\end{array}$ \\
\hline 3370 & NP237 & EPAREL & Cilwurf & CONSTANT & $1.00 E+02$ & $1.00 E+02$ & $0.00 E+00$ & $1.00 E+02$ & 1.00E+02 & $4 / 16 / 96$ & 3 & 36932 & cs \\
\hline 247 & NP237 & HALFLIFE & s & CONSTANT & $6.75 E+13$ & $6.75 E+13$ & $0.00 E+00$ & $6.75 E+13$ & $6.75 E+13$ & $4 / 16 / 96$ & 3 & 32579 & NUICS \\
\hline 248 & NP237 & INVCHD & $\mathrm{Ci}$ & CONSTANT & $6.19 E+01$ & $6.19 E+01$ & $0.00 E+00$ & $6.19 E+01$ & $6.19 E+01$ & $10 / 29 / 96$ & 2 & $32584 \mathrm{~A}$ & $\begin{array}{c}\text { NU/PN } \\
\text { CS }\end{array}$ \\
\hline 249 & NP237 & INVRHD & $\mathbf{C i}$ & CONSTANT & $2.95 E+00$ & $2.95 E+00$ & $0.00 E+00$ & $2.95 E+00$ & 2.95E+00 & 10/29/96 & 2 & $32593 \mathrm{~A}$ & $\begin{array}{c}\mathrm{NU} / \mathrm{CS} / \\
\mathrm{PN}\end{array}$ \\
\hline 3236 & NP239 & ATWEIGHT & $\mathrm{kg} / \mathrm{mole}$ & CONSTANT & 2.39E-01 & 2.39E-01 & $0.00 E+00$ & 2.39E-01 & 2.39E-01 & $4 / 10 / 96$ & 3 & 36758 & CSINU \\
\hline 3334 & NP239 & EPAREL & Cikwuf & CONSTANT & $0.00 E+00$ & $0.00 E+00$ & $0.00 E+00$ & $0.00 E+00$ & $0.00 E+00$ & $4 / 16 / 96$ & 3 & 36881 & CS \\
\hline 3281 & NP239 & HALFLIFE & $\mathbf{s}$ & CONSTANT & $2.04 E+05$ & $2.04 E+05$ & $0.00 E+00$ & $2.04 E+05$ & $2.04 E+05$ & $4 / 16 / 96$ & 3 & 36815 & CSINU \\
\hline 250 & PA231 & ATWEIGHT & $\mathrm{kg} / \mathrm{mole}$ & CONSTANT & 2.31E-01 & 2.31E-01 & $0.00 E+00$ & 2.31E-01 & 2.31E-01 & $11 / 2 / 95$ & 3 & 32337 & $\begin{array}{l}\text { FLNUI } \\
\text { CSITP }\end{array}$ \\
\hline 3371 & PA231 & EPAREL & Cirwuf & CONSTANT & $1.00 \mathrm{E}+02$ & $1.00 E+02$ & $0.00 E+00$ & $1.00 E+02$ & $1.00 E+02$ & $4 / 16 / 96$ & 3 & 36933 & CS \\
\hline 251 & PA231 & HALFLIFE & s & CONSTANT & 1.03E+12 & $1.03 E+12$ & $0.00 E+00$ & $1.03 E+12$ & $1.03 E+12$ & $4 / 16 / 96$ & 3 & 32929 & NU/CS \\
\hline 2267 & PA231 & INVCHD & $\mathrm{Ci}$ & CONSTANT & 4.62E-01 & 4.62E-01 & $0.00 E+00$ & $4.62 \mathrm{E}-01$ & 4.62E-01 & 10/29/96 & 2 & $32930 \mathrm{~A}$ & $\begin{array}{c}\text { NU/PN/ } \\
\text { CS }\end{array}$ \\
\hline 2268 & PA231 & INVRHD & $\mathrm{Ci}$ & CONSTANT & 5.63E-03 & $5.63 E-03$ & $0.00 E+00$ & 5.63E-03 & $5.63 E-03$ & $10 / 29 / 96$ & 2 & $32931 \mathrm{~A}$ & $\begin{array}{c}\mathrm{PN} / \mathrm{CS} / \\
\mathrm{NU}\end{array}$ \\
\hline 3237 & PA233 & ATWEIGHT & $\mathrm{kg} / \mathrm{mole}$ & CONSTANT & 2.33E-01 & 2.33E-01 & $0.00 E+00$ & 2.33E-01 & 2.33E-01 & 4/10/96 & 3 & 36759 & CSINU \\
\hline 3335 & PA233 & EPAREL & Citwuf & CONSTANT & $0.00 E+00$ & $0.00 E+00$ & $0.00 E+00$ & $0.00 E+00$ & $0.00 E+00$ & $4 / 16 / 96$ & 3 & 36882 & CS \\
\hline 3282 & PA233 & HALFLIFE & $s$ & CONSTANT & $2.33 E+06$ & 2.33E+06 & $0.00 E+00$ & $2.33 E+06$ & $2.33 E+06$ & $4 / 16 / 96$ & 3 & 36828 & NU/CS \\
\hline 3197 & PA234M & ATWEIGHT & $\mathrm{kg} / \mathrm{mole}$ & CONSTANT & 2.34E-01 & 2.34E-01 & $0.00 E+00$ & 2.34E-01 & 2.34E-01 & $4 / 10 / 96$ & 3 & 36660 & CSINU \\
\hline 3336 & PA234M & EPAREL & Cihwuf & CONSTANT & $0.00 E+00$ & $0.00 E+00$ & $0.00 E+00$ & $0.00 E+00$ & $0.00 E+00$ & $4 / 16 / 96$ & 3 & 36883 & CS \\
\hline 3283 & PA234M & HALFLIFE & $s$ & CONSTANT & $7.02 E+01$ & $7.02 E+01$ & $0.00 E+00$ & $7.02 E+01$ & $7.02 E+01$ & $4 / 16 / 96$ & 3 & 36829 & NU/CS \\
\hline 282 & PB & LOGSOLM & $\log ($ moles $/)$ & CONSTANT & $0.00 E+00$ & $0.00 E+00$ & $0.00 E+00$ & $0.00 E+00$ & $0.00 E+00$ & $5 / 13 / 96$ & 4B & 33213 & PN \\
\hline 3421 & PB209 & ATWEIGHT & $\mathrm{kg} / \mathrm{mole}$ & CONSTANT & 2.09E-01 & 2.09E-01 & $0.00 E+00$ & 2.09E-01 & 2.09E-01 & $5 / 2 / 96$ & 3 & 37670 & NU/CS \\
\hline 3337 & PB209 & EPAREL & Cirwuf & CONSTANT & $0.00 E+00$ & $0.00 E+00$ & $0.00 E+00$ & $0.00 \mathrm{E}+00$ & $0.00 E+00$ & $4 / 46 / 96$ & 3 & 36884 & cs \\
\hline 3284 & PB209 & HALFLIFE & $s$ & CONSTANT & 1.19E+04 & $1.19 E+04$ & $0.00 E+00$ & 1.19E+04 & 1.19E+04 & $4 / 16 / 96$ & 3 & 36830 & CSINU \\
\hline 283 & PB210 & ATWEIGHT & $\mathrm{kg} / \mathrm{mole}$ & CONSTANT & 2.10E-01 & 2.10E-01 & $0.00 E+00$ & 2.10E-01 & $2.10 E-01$ & $11 / 2 / 95$ & 3 & 32338 & $\begin{array}{l}\text { CSINU } \\
\text { FLTP }\end{array}$ \\
\hline 3372 & PB210 & EPAREL & Cirwuf & CONSTANT & $1.00 E+02$ & $1.00 \mathrm{E}+02$ & $0.00 E+00$ & $1.00 \mathrm{E}+02$ & $1.00 E+02$ & $4 / 16 / 96$ & 3 & 36934 & CS \\
\hline 284 & PB210 & HALFLIFE & $s$ & CONSTANT & $7.04 E+08$ & $7.04 E+08$ & $0.00 E+00$ & $7.04 E+08$ & $7.04 E+08$ & $4 / 16 / 96$ & -.3 & 33218 & NUICS \\
\hline 285 & PB210 & INVCHD & $\mathrm{Ci}$ & CONSTANT & $8.75 E+00$ & $8.75 E+00$ & $0.00 E+00$ & $8.75 E+00$ & $8.75 E+00$ & $10 / 29 / 96$ & 2 & $33221 \mathrm{~A}$ & \begin{tabular}{|c|}
$\mathrm{NU} / \mathrm{PN}$ \\
$\mathrm{CS}$ \\
\end{tabular} \\
\hline 286 & PB210 & INVRHD & $\mathrm{Ci}$ & CONSTANT & 1.14E-04 & $1.14 E-04$ & $0.00 E+00$ & 1.14E-04 & $1.14 E-04$ & $10 / 29 / 96$ & 2 & $33223 \mathrm{~A}$ & \begin{tabular}{|c} 
PN/NU/ \\
CS
\end{tabular} \\
\hline 3200 & PB211 & ATWEIGHT & $\mathrm{kg} /$ mole & CONSTANT & 2.11E-01 & 2.11E-01 & $0.00 E+00$ & 2.11E-01 & 2.11E-01 & 4/10/96 & 3 & 36705 & CSINU \\
\hline 3338 & PB211 & EPAREL & Cilwuf & CONSTANT & $0.00 \mathrm{E}+00$ & $0.00 \mathrm{E}+00$ & $0.00 E+00$ & $0.00 E+00$ & $0.00 E+00$ & $4 / 16 / 96$ & 3 & 36885 & CS \\
\hline 3285 & PB211 & HALFLIFE & $\mathbf{s}$ & CONSTANT & $2.17 E+03$ & $2.17 \mathrm{E}+03$ & $0.00 E+00$ & $2.17 E+03$ & 2.17E+03 & 4/16/96 & 3 & 36831 & CSINU \\
\hline 3201 & PB212 & ATWEIGHT & $\mathrm{kg} / \mathrm{mole}$ & CONSTANT & 2.12E-01 & 2.12E-01 & $0.00 E+00$ & 2.12E-01 & 2.12E-01 & $4 / 10 / 96$ & 3 & 36706 & NU/CS \\
\hline 3339 & PB212 & EPAREL & Cihwuf & CONSTANT & $0.00 E+00$ & $0.00 \mathrm{E}+00$ & $0.00 E+00$ & $0.00 E+00$ & $0.00 E+00$ & $4 / 16 / 96$ & 3 & 36886 & cs \\
\hline 3286 & $\mathrm{PB212}$ & HALFLIFE & s & CONSTANT & $3.83 E+04$ & $3.83 E+04$ & $0.00 E+00$ & $3.83 E+04$ & $3.83 E+04$ & $4 / 16 / 96$ & 3 & 36832 & CS/NU \\
\hline 3202 & PB214 & ATWEIGHT & $\mathrm{kg} / \mathrm{mole}$ & CONSTANT & 2.14E-01 & 2.14E-01 & $0.00 E+00$ & 2.14E-01 & 2.14E-01 & $4 / 10 / 96$ & 3 & 36707 & CSINU \\
\hline 3340 & PB214 & EPAREL & Citwruf & CONSTANT & $0.00 E+00$ & $0.00 E+00$ & $0.00 E+00$ & $0.00 E+00$ & $0.00 E+00$ & $4 / 16 / 96$ & 3 & 36888 & CS \\
\hline 3287 & PB214 & HALFLIFE & $\mathbf{s}$ & CONSTANT & $1.61 E+03$ & $1.61 E+03$ & $0.00 E+00$ & $1.61 E+03$ & $1.61 E+03$ & $4 / 16 / 96$ & 3 & 36833 & CSINU \\
\hline 3429 & PHUMOX3 & PHUMCIM & NONE & CUMULATIVE & $1.10 E+00$ & $1.37 E+00$ & 4.69E-01 & $6.50 \mathrm{E}-02$ & $1.60 E+00$ & $5 / 8 / 96$ & 1 & 37683 & NU \\
\hline 3433 & PHUMOX3 & PHUMSIM & NONE & CONSTANT & 1.90E-01 & $1.90 E-01$ & $0.00 E+00$ & $1.90 E-01$ & 1.90E-01 & $5 / 8 / 96$ & 1 & 37690 & NU \\
\hline
\end{tabular}


23B. Waste Chemistry

\begin{tabular}{|c|c|c|c|c|c|c|c|c|c|c|c|c|c|}
\hline ID & Material & Parameter & Units & Distribution & Mean & Median & Deviation & Low & High & Entry Date & Impt & WPO & codes \\
\hline 3430 & PHUMOX4 & PHUMCIM & NONE & CONSTANT & $6.30 E+00$ & $6.30 E+00$ & $0.00 E+00$ & $6.30 E+00$ & $6.30 E+00$ & $5 / 8 / 96$ & 1 & 37685 & NU \\
\hline 3434 & PHUMOX 4 & PHUMSIM & NONE & CONSTANT & $6.30 \mathrm{E}+00$ & $6.30 E+00$ & $0.00 E+00$ & $6.30 E+00$ & $6.30 E+\infty$ & $5 / 8 / 96$ & 1 & 37691 & NU \\
\hline 3431 & PHUMOX5 & PHUMCIM & NONE & CONSTANT & $7.40 E-03$ & $7.40 E-03$ & $0.00 E+00$ & $7.40 E-03$ & $7.40 E-03$ & $5 / 8 / 96$ & 1 & 37687 & NU \\
\hline 3435 & PHUMOX5 & PHUMSIM & NONE & CONSTANT & $9.10 E-04$ & $9.10 E-04$ & $0.00 E+00$ & $9.10 E-04$ & 9.10E-04 & 5/8/96 & 1 & 37694 & NU \\
\hline 3432 & PHUMOXE & PHUMCIM & NONE & CONSTANT & $5.10 E-01$ & $5.10 E-01$ & $0.00 E+00$ & $5.10 E-01$ & $5.10 E-01$ & $5 / 8 / 96$ & 1 & 37688 & NU \\
\hline 3436 & PHUMOX6 & PHUMSIM & NONE & CONSTANT & $1.20 E-01$ & $1.20 \mathrm{E}-01$ & $0.00 E+00$ & $1.20 E-01$ & $1.20 E-01$ & $5 / 8 / 96$ & 1 & 37695 & NU \\
\hline 1167 & PM & LOGSOLM & $\log ($ moles $/)$ & CONSTANT & $4.66 \mathrm{E}+00$ & $4.66 \mathrm{E}+00$ & $10.00 E+00$ & $4.66 E+00$ & $4.66 E+00$ & $11 / 2 / 95$ & 1 & $33227^{*}$ & BF \\
\hline 287 & PM147 & ATWEIGHT & $\mathrm{kg} / \mathrm{mole}$ & CONSTANT & $1.47 \mathrm{E}-01$ & 1.47E-01 & $0.00 E+00$ & |.47E-01 & 1.47E-01 & $11 / 2 / 95$ & 3 & 32339 & $\begin{array}{l}\text { TPICSI } \\
\text { NU/FL }\end{array}$ \\
\hline 3341 & PM147 & EPAREL & Citwuf & CONSTANT & $0.00 E+00$ & $0.00 E+00$ & $0.00 E+00$ & $0.00 E+00$ & $0.00 \mathrm{E}+00$ & 4/16/96 & 3 & 36889 & CS \\
\hline 288 & PM147 & HALFLLFE & $\mathbf{s}$ & CONSTANT & $8.28 E+07$ & $8.28 E+07$ & $0.00 E+00$ & $8.28 E+07$ & $8.28 E+07$ & $4 / 16 / 96$ & 3 & 33231 & NU/CS \\
\hline 2038 & PM147 & INVCHD & $\mathrm{Ci}$ & CONSTANT & $3.43 E-04$ & 3.43E-04 & $0.00 E+00$ & $3.43 E-0.4$ & $3.43 E-04$ & $10 / 29 / 96$ & 2 & $33233 \mathrm{~A}$ & $\begin{array}{c}\mathrm{CS} / \mathrm{PN} / \\
\mathrm{NU}\end{array}$ \\
\hline 289 & PM147 & |INVRHD & $\mathrm{Ci}$ & CONSTANT & $4.67 E-04$ & 4.67E-04 & $0.00 E+00$ & 4.67E-04 & $4.67 E-04$ & 10/29/96 & 2 & $33236 \mathrm{~A}$ & $\begin{array}{c}\text { CSIPN/ } \\
\mathrm{NU}\end{array}$ \\
\hline 3203 & PO212 & ATWEIGHT & $\mathrm{kg} / \mathrm{mole}$ & CONSTANT & 2.12E-01 & $2.12 E-01$ & $0.00 E+00$ & $2.12 E-01$ & 2.12E-01 & $4 / 10 / 96$ & 3 & 36708 & NU/CS \\
\hline 3342 & PO212 & EPAREL & Cirwuf & CONSTANT & $0.00 E+00$ & $0.00 E+00$ & $0.00 E+00$ & $0.00 E+\infty 0$ & $0.00 E+00$ & $4 / 16 / 96$ & 3 & 36891 & CS \\
\hline 3288 & PO212 & HALFLIFE & $s$ & CONSTANT & $3.00 E-07$ & $3.00 E-07$ & $0.00 E+00$ & $3.00 E-07$ & $3.00 E-07$ & $4 / 16 / 96$ & 3 & 36834 & NU/CS \\
\hline 3204 & PO213 & ATWEIGHT & $\mathrm{kg} / \mathrm{mole}$ & CONSTANT & 2.13E-01 & 2.13E-01 & $0.00 E+00$ & 2.13E-01 & $2.13 E-01$ & $4 / 10 / 96$ & 3 & 36709 & CSINU \\
\hline 3343 & PO213 & EPAREL & Cilwuf & CONSTANT & $0.00 E+00$ & $0.00 E+00$ & $0.00 \mathrm{E}+00$ & $0.00 \mathrm{E}+00$ & $0.00 E+00$ & $4 / 16 / 96$ & 3 & 36892 & CS \\
\hline 3289 & PO213 & HALFLLFE & $s$ & CONSTANT & $4.20 E-06$ & $4.20 E-06$ & $0.00 E+00$ & $4.20 E-06$ & $4.20 E-06$ & $4 / 16 / 96$ & 3 & 36835 & CS/NU \\
\hline 3205 & PO214 & ATWEIGHT & $\mathrm{kg} / \mathrm{mole}$ & CONSTANT & 2.14E-01 & 2.14E-01 & $0.00 E+00$ & $2.14 E-01$ & 2.14E-01 & $4 / 10 / 96$ & 3 & 36710 & NU/CS \\
\hline 3344 & PO214 & EPAREL & Citwuf & CONSTANT & $0.00 \mathrm{E}+00$ & $0.00 E+00$ & $0.00 E+00$ & $10.00 E+00$ & $0.00 E+00$ & 4/16/96 & 3 & 36893 & cs \\
\hline 3290 & PO214 & HALFLIFE & s & CONSTANT & $1.64 \mathrm{E}-04$ & $1.64 E-04$ & $0.00 \mathrm{E}+00$ & $1.64 E-04$ & 1.64E-04 & $4 / 16 / 96$ & 3 & 36836 & NU/CS \\
\hline 3206 & PO215 & ATWEIGHT & $\mathrm{kg} / \mathrm{mole}$ & CONSTANT & $2.15 E-01$ & 2.15E-01 & $0.00 \mathrm{E}+00$ & $2.15 E-01$ & $2.15 E-01$ & $4 / 10 / 96$ & 3 & 36711 & CSINU \\
\hline 3345 & PO215 & EPAREL & Cithurf & CONSTANT & $0.00 E+00$ & $10.00 E+00$ & $0.00 E+00$ & $0.00 \mathrm{E}+00$ & $0.00 E+00$ & $4 / 16 / 96$ & 3 & 36894 & CS \\
\hline 3291 & PO215 & HALFLIFE & $s$ & CONSTANT & $1.78 \mathrm{E}-03$ & $1.78 E-03$ & $0.00 E+00$ & $1.78 E-03$ & $1.78 E-03$ & $4 / 16 / 96$ & 3 & 36837 & $\operatorname{CS} / N U$ \\
\hline 3207 & PO216 & ATWEIGHT & $\mathrm{kg} / \mathrm{mole}$ & CONSTANT & 2.16E-01 & $2.16 E-01$ & $0.00 E+00$ & 2.16E-01 & $2.16 E-01$ & $4 / 10 / 96$ & 3 & 36712 & NU/CS \\
\hline 3346 & PO216 & EPAREL & Citwuf & CONSTANT & $0.00 \mathrm{E}+00$ & $0.00 \mathrm{E}+00$ & $0.00 \mathrm{E}+00$ & $0.00 \mathrm{E}+00$ & $0.00 \mathrm{E}+00$ & $4 / 16 / 96$ & 3 & 36895 & $\operatorname{cs}$ \\
\hline 3292 & PO216 & HALFLIFE & $s$ & CONSTANT & $1.50 E-01$ & $1.50 E-01$ & $0.00 \mathrm{E}+00$ & $1.50 \mathrm{E}-01$ & $1.50 E-01$ & $4 / 16 / 96$ & 3 & | 36838 & NU/CS \\
\hline 3208 & PO218 & ATWEIGHT & $\mathrm{kg} / \mathrm{mole}$ & CONSTANT & $2.18 \mathrm{E}-01$ & $2.18 E-01$ & $0.00 E+00$ & 2.18E-01 & 2.18E-01 & $4 / 10 / 96$ & 3 & 36713 & CS/NU \\
\hline 3347 & PO218 & EPAREL & Clisurf & CONSTANT & $0.00 E+00$ & $0.00 E+00$ & $0.00 E+00$ & $0.00 \mathrm{E}+00$ & $0.00 E+00$ & $4 / 16 / 96$ & 3 & 36896 & CS \\
\hline 3293 & PO218 & HALFLIFE & $s$ & CONSTANT & $1.83 E+02$ & $1.83 E+02$ & $0.00 E+00$ & $1.83 E+02$ & $1.83 E+02$ & $4 / 16 / 96$ & 3 & 36839 & CSINU \\
\hline 3459 & PU & CAPHUM & molesniter & CONSTANT & 1.10E-05 & $1.10 E-05$ & $0.00 E+00$ & $1.10 E-05$ & 1.10E-05 & $5 / 8 / 96$ & 1 & 37724 & NU \\
\hline 3315 & PU & CAPMIC & molesniter & CONSTANT & $6.80 \mathrm{E}-05$ & $6.80 E-05$ & $0.00 E+00$ & $6.80 E-05$ & $6.80 E-05$ & $4 / 16 / 96$ & 1 & 36862 & NU \\
\hline 3316 & PU & CONCINT & molesniter & CONSTANT & $1.00 E-09$ & $1.00 E-09$ & $0.00 E+00$ & $1.00 \mathrm{E}-09$ & $1.00 E-09$ & $4 / 16 / 96$ & 1 & 36863 & NU \\
\hline 3440 & PU & CONCMIN & molesniter & CONSTANT & $2.60 E-08$ & $2.60 \mathrm{E}-08$ & $0.00 E+00$ & $2.60 \mathrm{E}-08$ & $2.60 E-08$ & $5 / 8 / 96$ & 1 & 37703 & NU \\
\hline 3317 & PU & PROPMIC & NONE & CONSTANT & $3.00 E-01$ & $3.00 E-01$ & $0.00 E+00$ & $3.00 E-01$ & $3.00 E-01$ & $4 / 16 / 96$ & 1 & 36864 & NU \\
\hline 3442 & $\mathrm{PU}+3$ & MDO & $m^{\wedge} 2 / s$ & CONSTANT & $3.00 E-10$ & $3.00 E-10$ & $0.00 E+00$ & $3.00 E-10$ & $3.00 E-10$ & $5 / 17 / 96$ & 1 & 37705 & TPINU \\
\hline 3480 & $\mathrm{PU}+3$ & MKD_PU & $\mathrm{m}^{\wedge} 3 / \mathrm{kg}$ & UNIFORM & $2.60 E-01$ & $2.60 E-01$ & $1.40 E-01$ & $2.00 E-02$ & $5.00 E-01$ & $6 / 12 / 96$ & 1 & 38351 & NU/TP \\
\hline 3443 & $\mathrm{PU}+4$ & MDO & $m^{\wedge} 2 / s$ & CONSTANT & $1.53 E-10$ & $1.53 \mathrm{E}-10$ & $0.00 E+00$ & $1.53 E-10$ & $1.53 E-10$ & $5 / 17 / 96$ & 1 & 37708 & TPINU \\
\hline 3481 & $\mathrm{PU}+4$ & MKD_PU & $m^{\wedge} 3 / k g$ & UNIFORM & $1.00 E+01$ & $1.00 E+01$ & $5.50 E+00$ & $9.00 E-01$ & $2.00 E+01$ & $6 / 12 / 96$ & 1 & 38352 & TPINU \\
\hline 291 & PU238 & ATWEIGHT & $\mathrm{kg} / \mathrm{mole}$ & CONSTANT & $2.38 E-01$ & $2.38 \mathrm{E}-01$ & $0.00 E+00$ & 2.38E-01 & $2.38 E-01$ & 11/2/95 & 3 & 32340 & \begin{tabular}{|l} 
FLCS/ \\
TP/NU
\end{tabular} \\
\hline 3373 & PU238 & EPAREL & Cirwuf & CONSTANT & $1.00 \mathrm{E}+02$ & $1.00 E+02$ & $0.00 E+00$ & $1.00 E+02$ & $1.00 E+02$ & $4 / 16 / 96$ & 3 & 36935 & CS \\
\hline 292 & PU238 & HALFLIFE & s & CONSTANT & $2.77 E+09$ & $2.77 E+09$ & $0.00 E+00$ & $2.77 E+09$ & $2.77 \mathrm{E}+09$ & $4 / 16 / 96$ & 3 & 33245 & NU/CS \\
\hline 293 & PU238 & INVCHD & $\mathrm{Ci}$ & CONSTANT & $1.93 E+06$ & $1.93 E+06$ & $0.00 E+00$ & $1.93 E+06$ & $1.93 E+06$ & 10/29/96 & 2 & 33247A & \begin{tabular}{|c} 
NU/CSI \\
PN
\end{tabular} \\
\hline 294 & PU238 & INVRHD & $\mathrm{Ci}$ & CONSTANT & $1.08 E+03$ & $1.08 E+03$ & $0.00 E+00$ & $1.08 E+03$ & $1.08 E+03$ & 10/29/96 & 2 & $33251 \mathrm{~A}$ & $\begin{array}{c}\text { CS/PN/ } \\
\mathrm{NU}\end{array}$ \\
\hline
\end{tabular}


23B. Waste Chemistry

\begin{tabular}{|c|c|c|c|c|c|c|c|c|c|c|c|c|c|}
\hline ID & Material & Parameter & Units & Distribution & Mean & Median & Deviation & Low & High & Entry Date & Impt & WPO & Codes \\
\hline 295 & PU239 & ATWEIGHT & $\mathrm{kg} / \mathrm{mole}$ & CONSTANT & 2.39E-01 & 2.39E-01 & $0.00 E+00$ & 2.39E-01 & 2.39E-01 & $11 / 2 / 95$ & 3 & 32341 & $\begin{array}{l}\text { TP/CS/ } \\
\text { NU/FL }\end{array}$ \\
\hline 3374 & PU239 & EPAREL & Cinwuf & CONSTANT & $1.00 \mathrm{E}+02$ & $1.00 E+02$ & $0.00 E+00$ & $1.00 E+02$ & $1.00 E+02$ & $4 / 46 / 96$ & 3 & 36936 & CS \\
\hline 296 & PU239 & HALFLIFE & $\mathbf{s}$ & CONSTANT & $7.59 \mathrm{E}+11$ & $7.59 E+11$ & $0.00 E+00$ & $7.59 E+11$ & $7.59 E+11$ & $4 / 16 / 96$ & 3 & 33256 & CSINU \\
\hline 297 & PU239 & INVCHD & $\mathrm{Ci}$ & CONSTANT & $7.85 E+05$ & $7.85 E+05$ & $0.00 E+00$ & $7.85 E+05$ & $7.85 E+05$ & $10 / 29 / 96$ & 2 & $33260 \mathrm{~A}$ & $\begin{array}{c}\mathrm{CS} / \mathrm{NU} / \\
\mathrm{PN}\end{array}$ \\
\hline 298 & PU239 & INVRHD & $\mathrm{Ci}$ & CONSTANT & $1.03 E+04$ & $1.03 E+04$ & $0.00 E+00$ & $1.03 E+04$ & $1.03 E+04$ & 10/29/96 & 2 & $33262 \mathrm{~A}$ & $\begin{array}{c}\text { CS/NUI } \\
\text { PN }\end{array}$ \\
\hline 299 & PU240 & ATWEIGHT & $\mathrm{kg} / \mathrm{mole}$ & CONSTANT & 2.40E-01 & 2.40E-01 & $0.00 E+00$ & 2.40E-01 & $2.40 E-01$ & $11 / 2 / 95$ & 3 & 32342 & $\begin{array}{l}\text { FU/TPI } \\
\text { NU/CS }\end{array}$ \\
\hline 3375 & PU240 & EPAREL & Ci/wuf & CONSTANT & $1.00 E+02$ & $1.00 E+02$ & $0.00 E+00$ & $1.00 E+02$ & $1.00 E+02$ & 4/16/95 & 3 & 36937 & cs \\
\hline 300 & PU240 & HALFLIFE & s & CONSTANT & $2.06 E+11$ & $2.06 E+11$ & $0.00 E+00$ & $2.06 E+11$ & $2.06 E+11$ & $4 / 16 / 96$ & 3 & 33265 & NU/CS \\
\hline 301 & PU240 & INVCHD & $\mathrm{Ci}$ & CONSTANT & $2.09 E+05$ & $2.09 E+05$ & $0.00 E+00$ & 2.09E+05 & $2.09 E+05$ & $10 / 29 / 96$ & 2 & $33267 \mathrm{~A}$ & $\begin{array}{c}\text { PN/CSI } \\
\text { NU }\end{array}$ \\
\hline 302 & PU240 & INVRHD & $\mathrm{Ci}$ & CONSTANT & $5.05 E+03$ & $5.05 E+03$ & $0.00 E+00$ & $5.05 E+03$ & $5.05 E+03$ & 10/29/96 & 2 & $33268 \mathrm{~A}$ & $\begin{array}{c}\text { PN/NUI } \\
\text { CS }\end{array}$ \\
\hline 303 & PU241 & ATWEIGHT & $\mathrm{kg} / \mathrm{mole}$ & CONSTANT & 2.41E-01 & 2.41E-01 & $0.00 E+00$ & 2.41E-01 & $2.41 E-01$ & $11 / 2 / 95$ & 3 & 32343 & $\begin{array}{l}\text { TP/NU/ } \\
\text { FL/CS }\end{array}$ \\
\hline 3348 & PU241 & EPAREL & Ciknuf & CONSTANT & $0.00 E+00$ & $0.00 E+00$ & $0.00 \mathrm{E}+00$ & $0.00 E+00$ & $0.00 E+00$ & $1 / 16 / 96$ & 3 & 36898 & cs \\
\hline 304 & PU241 & HALFLIFE & $\mathbf{s}$ & CONSTANT & $4.54 \mathrm{E}+08$ & $4.54 E+08$ & $0.00 E+00$ & $4.54 E+08$ & $4.54 E+08$ & $4 / 16 / 96$ & 3 & 33292 & NU/CS \\
\hline 305 & PU241 & INVCHD & $\mathrm{Ci}$ & CONSTANT & $3.71 E+05$ & 3.71E+05 & $0.00 E+00$ & 3.71E+05 & $3.71 E+05$ & 10/29/96 & 2 & $33270 \mathrm{~A}$ & $\begin{array}{c}\text { PN/NUI } \\
\text { CS }\end{array}$ \\
\hline 306 & PU241 & INVRHD & $\mathrm{Ci}$ & CONSTANT & $2.28 E+04$ & $2.28 E+04$ & $0.00 E+00$ & $2.28 E+04$ & 2.28E+04 & $10 / 29 / 96$ & 2 & $33271 \mathrm{~A}$ & $\begin{array}{c}\mathrm{CS} / \mathrm{NU} / \\
\mathrm{PN}\end{array}$ \\
\hline 307 & PU242 & ATWEIGHT & $\mathrm{kg} / \mathrm{mole}$ & CONSTANT & 2.42E-01 & 2.42E-01 & $0.00 E+00$ & 2.42E-01 & 2.42E-01 & $11 / 2 / 95$ & 3 & 32344 & $\begin{array}{l}\text { TPIFU } \\
\text { NU/CS }\end{array}$ \\
\hline 3376 & PU242 & EPAREL & Cirwuf & CONSTANT & $1.00 E+02$ & 1.00E+02 & $0.00 E+00$ & $1.00 E+02$ & $1.00 E+02$ & $4 / 16 / 96$ & 3 & 36938 & CS \\
\hline 308 & PU242 & HALFLIFE & s & CONSTANT & $1.22 E+13$ & 1.22E+13 & $0.00 E+00$ & 1.22E+13 & $1.22 \mathrm{E}+13$ & $4 / 16 / 96$ & 3 & 33295 & NU/CS \\
\hline 309 & PU242 & INVCHD & $\mathbf{C i}$ & CONSTANT & $1.17 E+03$ & $1.17 E+03$ & $0.00 E+00$ & $1.17 E+03$ & 1.17E+03 & $10 / 29 / 96$ & 2 & $33274 \mathrm{~A}$ & $\begin{array}{c}\mathrm{NU} / \mathrm{PN} \\
\mathrm{CS}\end{array}$ \\
\hline 310 & PU242 & INVRHD & $\mathbf{C i}$ & CONSTANT & $1.50 E-01$ & $1.50 \mathrm{E}-01$ & $0.00 E+00$ & 1.50E-01 & $1.50 \mathrm{E}-01$ & 10/29/96 & 2 & $33272 \mathrm{~A}$ & $\begin{array}{c}\text { PN/CSI } \\
\text { NU }\end{array}$ \\
\hline 311 & PU244 & ATWEIGHT & $\mathrm{kg} / \mathrm{mole}$ & CONSTANT & 2.44E-01 & 2.44E-01 & $0.00 E+00$ & 2.44E-01 & 2.44E-01 & $11 / 2 / 95$ & 3 & 32345 & $\begin{array}{l}\text { CS/FL } \\
\text { NU/TP }\end{array}$ \\
\hline 3377 & PU244 & EPAREL & Cinwuf & CONSTANT & $1.00 E+02$ & $1.00 E+02$ & $0.00 E+00$ & $1.00 E+02$ & $1.00 E+02$ & 4/16/96 & 3 & 36939 & CS \\
\hline 312 & PU244 & HALFLIFE & s & CONSTANT & $2.61 E+15$ & 2.61E+15 & $0.00 E+00$ & $2.61 E+15$ & $2.61 E+15$ & $4 / 16 / 96$ & 3 & 33297 & NU/CS \\
\hline 2269 & PU244 & INVCHD & $\mathrm{Ci}$ & CONSTANT & 1.51E-06 & 1.51E-06 & $0.00 E+00$ & 1.51E-06 & 1.51E-06 & $10 / 29 / 96$ & 2 & $33450 \mathrm{~A}$ & \begin{tabular}{|c|} 
NU/CSI \\
PN \\
\end{tabular} \\
\hline 2270 & PU244 & INVRHD & $\mathrm{Ci}$ & CONSTANT & $8.50 \mathrm{E}-11$ & $8.50 E-11$ & $0.00 E+00$ & $8.50 E-11$ & $8.50 E-11$ & 10/29/96 & 2 & $33452 \mathrm{~A}$ & \begin{tabular}{|c|} 
PN/NUI \\
CS \\
\end{tabular} \\
\hline 313 & RA & LOGSOLM & $\log ($ moles $/)$ & CONSTANT & $0.00 E+00$ & $0.00 E+00$ & $0.00 E+00$ & $0.00 E+00$ & $0.00 E+00$ & $5 / 1 / 96$ & 4B & 33455 & NU \\
\hline 3209 & RA223 & ATWEIGHT & $\mathrm{kg} / \mathrm{mole}$ & CONSTANT & 2.23E-01 & 2.23E-01 & $0.00 E+00$ & 2.23E-01 & 2.23E-01 & $4 / 10 / 96$ & -3 & 36714 & NU/CS \\
\hline 3349 & RA223 & EPAREL & Cirwuf & CONSTANT & $0.00 E+00$ & $0.00 E+00$ & $0.00 E+00$ & $0.00 E+00$ & $0.00 \mathrm{E}+00$ & $4 / 16 / 96$ & 3 & 36899 & CS \\
\hline 3294 & RA223 & HALFLIFE & $\mathbf{s}$ & CONSTANT & $9.88 E+05$ & $9.88 E+05$ & $0.00 E+00$ & $9.88 E+05$ & $9.88 E+05$ & $4 / 16 / 96$ & 3 & 36840 & CSINU \\
\hline 3210 & RA224 & ATWEIGHT & $\mathrm{kg} / \mathrm{mole}$ & CONSTANT & $2.24 E-01$ & 2.24E-01 & $0.00 E+00$ & 2.24E-01 & 2.24E-01 & $4 / 10 / 96$ & 3 & 36715 & NU/CS \\
\hline 3350 & RA224 & EPAREL & Cikwuf & CONSTANT & $0.00 E+00$ & $0.00 E+00$ & $0.00 E+00$ & $0.00 E+00$ & $0.00 E+00$ & $4 / 16 / 96$ & 3 & 36901 & CS \\
\hline 3295 & RA224 & HALFLIFE & s & CONSTANT & $3.16 E+05$ & $3.16 E+05$ & $0.00 E+00$ & 3.16E+05 & $3.16 E+05$ & $4 / 16 / 96$ & 3 & 36841 & CSINU \\
\hline 3211 & RA225 & ATWEIGHT & $\mathrm{kg} / \mathrm{mole}$ & CONSTANT & $2.25 E-01$ & 2.25E-01 & $0.00 E+00$ & 2.25E-01 & 2.25E-01 & $4 / 10 / 96$ & 3 & 36716 & NU/CS \\
\hline 3351 & RA225 & EPAREL & Cirwuf & CONSTANT & $0.00 E+00$ & $0.00 E+00$ & $0.00 E+00$ & $0.00 E+00$ & $0.00 E+00$ & $4 / 16 / 96$ & 3 & 36902 & cs \\
\hline 3296 & RA225 & HALFLIFE & 5 & CONSTANT & $1.28 \mathrm{E}+06$ & $1.28 E+06$ & $0.00 E+00$ & $1.28 E+06$ & $1.28 E+06$ & $4 / 16 / 96$ & 3 & 36842 & NU/CS \\
\hline 314 & RA226 & ATWEIGHT & $\mathrm{kg} / \mathrm{mole}$ & CONSTANT & 2.26E-01 & $2.26 E-01$ & $0.00 E+00$ & 2.26E-01 & 2.26E-01 & $11 / 2 / 95$ & 3 & 32347 & $\begin{array}{l}\text { NU/CS } \\
\text { TP/FL }\end{array}$ \\
\hline 3378 & RA226 & EPAREL & Citwuf & CONSTANT & $1.00 E+02$ & $1.00 E+02$ & $0.00 E+00$ & 1.00E+02 & $1.00 E+02$ & $4 / 16 / 96$ & 3 & 36940 & CS \\
\hline 315 & RA226 & HALFLIFE & s & CONSTANT & $5.05 \mathrm{E}+10$ & $5.05 \mathrm{E}+10$ & $0.00 E+00$ & $5.05 E+10$ & $5.05 \mathrm{E}+10$ & $4 / 16 / 96$ & 3 & 33458 & CSINU \\
\hline 316 & RA226 & INVCHD & $\mathrm{Ci}$ & CONSTANT & $1.14 E+01$ & $1.14 E+01$ & $0.00 E+00$ & $1.14 E+01$ & 1.14E+01 & $10 / 29 / 96$ & 2 & $33462 \mathrm{~A}$ & \begin{tabular}{|c|} 
PN/NUI \\
CS
\end{tabular} \\
\hline 317 & RA226 & INVRHD & $\mathrm{Ci}$ & CONSTANT & 2.79E-04 & 2.79E-04 & $0.00 E+00$ & 2.79E-04 & 2.79E-04 & 10/29/96 & 2 & $33464 A$ & $\mid \begin{array}{c}\text { PN/CSI } \\
\text { NU }\end{array}$ \\
\hline
\end{tabular}


23B. Waste Chemistry

\begin{tabular}{|c|c|c|c|c|c|c|c|c|c|c|c|c|c|}
\hline ID & Material & Parameter & Units & Distribution & Mean & Median & Deviation & LOW & High & Entry Date & Impt & WPO & Codes \\
\hline 318 & RA228 & ATWEIGHT & $\mathrm{kg} / \mathrm{mole}$ & CONSTANT & 2.28E-01 & 2.28E-01 & $0.00 E \div 00$ & 2.28E-01 & 2.28E-01 & $2 / 12 / 96$ & 3 & 32348 & $\begin{array}{l}\text { FUTTI } \\
\text { NUICS }\end{array}$ \\
\hline 3352 & RA228 & EPAREL & Cirwut & CONSTANT & $0.00 E+00$ & $0.00 E+00$ & $0.00 E+00$ & $0.00 E+00$ & $0.00 E+00$ & $4 / 16 / 96$ & 3 & 36903 & $\mathrm{CS}$ \\
\hline 319 & RA228 & HALFLIFE & $s$ & CONSTANT & 2.11E+08 & 2.11E+08 & $0.00 E+00$ & 2.11E+08 & $2.11 E+08$ & $4 / 16 / 96$ & 3 & 33468 & NU/CS \\
\hline 2271 & RA228 & INVCHD & $\mathrm{Ci}$ & CONSTANT & $9.10 E-01$ & $9.10 \mathrm{E}-01$ & $0.00 E+00$ & 9.10E-01 & $9.10 E-01$ & $10 / 29 / 96$ & 2 & $33470 \mathrm{~A}$ & $\begin{array}{c}\text { PN/NUI } \\
\text { CS }\end{array}$ \\
\hline 2272 & RA228 & INVRHD & $\mathbf{C i}$ & CONSTANT & $9.23 E-02$ & 9.23E-02 & $0.00 E+00$ & 9.23E-02 & 9.23E-02 & $10 / 29 / 96$ & 2 & $36968 \mathrm{~A}$ & $\begin{array}{c}\text { PN/CSI } \\
\text { NU }\end{array}$ \\
\hline 3212 & RN219 & ATWEIGHT & $\mathrm{kg} / \mathrm{mole}$ & CONSTANT & 2.19E-01 & 2.19E-01 & $0.00 E+00$ & 2.19E-01 & 2.19E-01 & $4 / 10 / 96$ & 3 & 36719 & CSNU \\
\hline 3353 & RN219 & EPAREL & Cinurf & CONSTANT & $0.00 E+00$ & $0.00 E+00$ & $0.00 E+00$ & $0.00 E+00$ & $0.00 E+00$ & $4 / 16 / 96$ & 3 & 36904 & cs \\
\hline 3297 & RN219 & HALFLIFE & $\mathbf{s}$ & CONSTANT & $3.96 E+00$ & $3.96 E+00$ & $0.00 E+00$ & $3.96 \mathrm{E}+00$ & $3.96 E+00$ & $4 / 16 / 96$ & 3 & 36843 & CSINU \\
\hline 3213 & RN220 & ATWEIGHT & $\mathrm{kg} / \mathrm{mole}$ & CONSTANT & $2.20 \mathrm{E}-01$ & 2.20E-01 & $0.00 \mathrm{E}+00$ & 2.20E-01 & $2.20 E-01$ & $4 / 10 / 96$ & 3 & 36720 & NU/CS \\
\hline 3354 & RN220 & EPAREL & Cirwuf & CONSTANT & $0.00 E+00$ & $0.00 E+00$ & $0.00 E+00$ & $0.00 E+00$ & $0.00 E+00$ & $4 / 16 / 96$ & 3 & 36906 & CS \\
\hline 3298 & RN220 & HALFLIFE & $s$ & CONSTANT & $5.56 \mathrm{E}+01$ & $5.56 E+01$ & $0.00 E+00$ & $5.56 \mathrm{E}+01$ & $5.56 \mathrm{E}+01$ & $4 / 16 / 96$ & 3 & 36844 & CSINU \\
\hline 3214 & RN222 & ATWEIGHT & $\mathrm{kg} / \mathrm{mole}$ & CONSTANT & 2.22E-01 & 2.22E-01 & $0.00 E+00$ & 2.22E-01 & 2.22E-01 & $4 / 10 / 96$ & 3 & 36732 & NU/CS \\
\hline 3355 & RN222 & EPAREL & Cilwuf & CONSTANT & $0.00 \mathrm{E}+00$ & $0.00 E+00$ & $0.00 E+00$ & $0.00 E+00$ & $0.00 \mathrm{E}+00$ & $4 / 16 / 96$ & 3 & 36907 & $\mathrm{cs}$ \\
\hline 3299 & RN222 & HALFLIFE & s & CONSTANT & $3.30 E+05$ & $3.30 E+05$ & $0.00 \mathrm{E}+00$ & $3.30 E+05$ & $3.30 E+05$ & $4 / 16 / 96$ & 3 & 36845 & $\operatorname{cs} / N U$ \\
\hline 514 & SM147 & ATWEIGHT & $\mathrm{kg} / \mathrm{mole}$ & CONSTANT & 1.47E-01 & 1.47E-01 & $0.00 E+00$ & $1.47 E-01$ & 1.47E-01 & $11 / 2 / 95$ & 3 & 32455 & $\begin{array}{l}\text { NU/FU } \\
\text { CS/TP }\end{array}$ \\
\hline 3379 & SM147 & EPAREL & Cirwuf & CONSTANT & $1.00 E+02$ & $1.00 E+02$ & $0.00 E+00$ & $1.00 E+02$ & $1.00 \mathrm{E}+02$ & $4 / 16 / 96$ & 3 & 36941 & CS \\
\hline 515 & SM147 & HALFLIFE & s & CONSTANT & $3.38 E+18$ & $3.38 E+18$ & $0.00 E+00$ & $3.38 E+18$ & 3.38E+18 & $4 / 16 / 96$ & 3 & 34350 & $\begin{array}{l}\text { CSIFU } \\
\text { NUITP } \\
\end{array}$ \\
\hline 3263 & SOLAM3 & SOLCIM & NONE & CUMULATIVE & $1.80 \mathrm{E}-01$ & $-9.00 E-02$ & 3.68E-01 & $-2.00 E+00$ & $1.40 E+00$ & $5 / 24 / 96$ & 1 & $37106 \mathrm{~A}$ & PN/NU \\
\hline 3262 & SOLAM3 & SOLSIM & NONE & CUMULATIVE & $1.80 \mathrm{E}-01$ & $-9.00 E-02$ & 3.68E-01 & $-2.00 E+00$ & $1.40 E+00$ & $5 / 24 / 96$ & 1 & $37105 \mathrm{~A}$ & NU/PN \\
\hline 3402 & SOLMOD3 & SOLCIM & moles/iter & CUMULATIVE & 1.97E-07 & $6.52 E-08$ & 2.01E-07 & $6.52 E-09$ & 6.52E-07 & $24 / 96$ & 1 & $37125 \mathrm{~A}$ & PN/NU \\
\hline 3406 & SOLMOD3 & SOLSIM & moles/iter & CUMULATIVE & 1.76E-06 & 5.82E-07 & $1.80 E-06$ & 5.82E-08 & $5.82 E-06$ & $5 / 24 / 96$ & 1 & 37129A & PN/NU \\
\hline $34 Q 3$ & SOLMOD4 & SOLCIM & moles/iter & CUMULATIVE & 1.80E-08 & 6.00 E-09 & 1.90E-08 & 6.00E-10 & 6.00 E-08 & $4 / 24 / 96$ & 1 & 37126 & NU/PN \\
\hline 3407 & SOLMOD4 & SOLSIM & moles/liter & CUMULATIVE & $1.30 E-05$ & 4.40E-06 & 1.40E-05 & 4.40E-07 & 4.40E-05 & $4 / 24 / 96$ & 1 & 37130 & PNINU \\
\hline 3404 & SOLMOD5 & SOLCIM & moles/iter & CUMULATIVE & $6.70 \mathrm{E}-06$ & 2.20E-06 & 6.80E-06 & 2.20E-07 & 2.20E-05 & $4 / 24 / 96$ & 1 & 37127 & NUIPN \\
\hline 3408 & SOLMOD5 & SOLSIM & moles/liter & CUMULATIVE & 7.00E-06 & 2.30E-06 & 7.10E-06 & 2.30E-07 & 2.30E-05 & $4 / 24 / 96$ & 1 & 37131 & NU/PN \\
\hline 3405 & SOLMOD6 & SOLCIM & moles/iter & CUMULATIVE & 2.70E-05 & $8.80 E-06$ & 2.70E-05 & 8.80E-07 & $8.80 E-05$ & $4 / 24 / 96$ & 1 & 37128 & NU/PN \\
\hline 3409 & SOLMOD6 & SOLSIM & moles/iter & CUMULATIVE & 2.60E-05 & $8.70 E-06$ & $2.70 E-05$ & $8.70 E-07$ & 8.70E-05 & $4 / 24 / 96$ & 1 & 37132 & NU/PN \\
\hline 3264 & SOLPU3 & SOLCIM & NONE & CUMULATTVE & 1.80E-01 & $-9.00 E-02$ & 3.68E-01 & $-2.00 E+00$ & $1.40 E+00$ & $5 / 24 / 96$ & 1 & $37108 \mathrm{~A}$ & PN/NU \\
\hline 3265 & SOLPU3 & SOLSIM & NONE & CUMU & $.80 E-01$ & $-9.00 E-02$ & 3.68E-01 & $-2.00 E+00$ & $1.40 E+00$ & $5 / 24 / 96$ & 1 & $37109 A$ & PN/NU \\
\hline 3389 & SOLPU4 & SOLCIM & NONE & CUMULATIVE & $1.80 E-01$ & $-9.00 E-02$ & 3.68E-01 & $-2.00 E+00$ & $1.40 E+00$ & $5 / 24 / 96$ & 1 & $37111 A$ & NU/PN \\
\hline 3266 & SOLPU4 & SOLSIM & NONE & |CUMULATIVE & $1.80 \mathrm{E}-01$ & $-9.00 E-02$ & 3.68E-01 & $-2.00 E+00$ & $1.40 E+00$ & $5 / 24 / 96$ & 1 & $37110 \mathrm{~A}$ & PN/NU \\
\hline 3393 & SOLTH4 & SOLSIM & NONE & CUMULATIVE & $1.80 \mathrm{E}-01$ & $-9.00 E-02$ & 3.68E-01 & $-2.00 E+00$ & $1.40 E+00$ & $5 / 24 / 96$ & 1 & $37115 \mathrm{~A}$ & PN/NU \\
\hline 3390 & SOLU4 & SOLSIM & NONE & CUMULATTVE & 1.80E-01 & $-9.00 E-02$ & 3.68E-01 & $-2.00 E+00$ & $1.40 \mathrm{E}+00$ & $5 / 24 / 96$ & 1 & $37112 A$ & NUIPN \\
\hline 3392 & SOLU6 & SOLCIM & NONE & CUMULATIVE & 1.80E-01 & $-9.00 E-02$ & $3.68 E-01$ & $-2.00 E+00$ & $1.40 \mathrm{E}+00$ & $5 / 24 / 96$ & 1 & $37114 A$ & PNNU \\
\hline 3391 & SOLUG & SOLSIM & NONE & CUMULATTVE & $1.80 \mathrm{E}-01$ & $-9.00 E-02$ & 3.68E-01 & $-2.00 E+00$ & $1.40 \mathrm{E}+00$ & $5 / 24 / 96$ & 1 & $37113 A$ & PN/NU \\
\hline 1659 & SR & LOGSOLM & $\log ($ moles $/)$ & CONSTANT & $0.00 E+00$ & $0.00 E+00$ & $0.00 E+00$ & $0.00 E+00$ & $0.00 E+00$ & $5 / 1 / 96$ & 4B & 34352 & NU \\
\hline 3215 & SR90 & ATWEIGHT & $\mathrm{kg} / \mathrm{mole}$ & CONSTANT & 8.99E-02 & 8.99E-02 & $0.00 E+00$ & 8.99E-02 & $8.99 E-02$ & $4 / 10 / 96$ & 3 & 36733 & CS/NU \\
\hline 3380 & SR80 & EPAREL & Cimurf & CONSTANT & $1.00 E+03$ & $1.00 E+03$ & $0.00 E+00$ & $1.00 E+03$ & $1.00 \mathrm{E}+03$ & $4 / 16 / 96$ & 3 & 36942 & cs \\
\hline 517 & SR90 & HALFLIFE & s & CONSTANT & $9.19 E+08$ & $9.19 E+08$ & $0.00 E+00$ & $9.19 E+08$ & $9.19 E+08$ & $4 / 16 / 96$ & 3 & 34353 & CS \\
\hline 2039 & SR8O & INVCHD & Ci & CONSTANT & $2.77 E+03$ & 2.77E+03 & $0.00 E+00$ & $2.77 E+03$ & 2.77E+03 & $10 / 29 / 96$ & 2 & $34354 \mathrm{~A}$ & $\begin{array}{c}\text { PN/CS } \\
\text { NU }\end{array}$ \\
\hline
\end{tabular}


23B. Waste Chemistry

\begin{tabular}{|c|c|c|c|c|c|c|c|c|c|c|c|c|c|}
\hline ID & Material & Parameter & Units & Distribution & Mean & Median & Deviation & Low & High & Entry Date & Impt & WPO & Codes \\
\hline 518 & SR9O & INVRHD & $\mathrm{Ci}$ & CONSTANT & $8.45 E+04$ & $8.45 E+04$ & $0.00 E+00$ & $8.45 E+04$ & 8.45E+0.4 & $10 / 29 / 96$ & 2 & $34355 \mathrm{~A}$ & $\begin{array}{c}\text { NU/PN/ } \\
\text { CS }\end{array}$ \\
\hline 2907 & STEEL & CORRMCO2 & $\mathrm{m} / \mathrm{s}$ & UNIFORM & $7.94 \mathrm{E}-15$ & 7.94E-15 & 4.58E-15 & $0.00 E+00$ & $1.59 E-14$ & $2 / 8 / 96$ & 1 & 34357 & BF \\
\hline 2908 & STEEL & CORRWCO2 & $\mathrm{m} / \mathrm{s}$ & UNIFORM & 1.03E-13 & $1.03 E-13$ & $5.96 \mathrm{E}-14$ & $0.00 E+00$ & $2.06 E-13$ & $2 / 8 / 96$ & 1 & 34358 & BF \\
\hline 2910 & STEEL & HUMCORR & $\mathrm{m} / \mathrm{s}$ & CONSTANT & $0.00 E+00$ & $0.00 E+00$ & $0.00 E+00$ & $0.00 E+00$ & $0.00 E+00$ & $2 / 8 / 96$ & 1 & 34127 & $B F$ \\
\hline 2898 & STEEL & STOIFX & NONE & CONSTANT & $1.00 E+00$ & $1.00 E+00$ & $0.00 E+00$ & $1.00 \mathrm{E}+00$ & $1.00 \mathrm{E}+00$ & $2 / 6 / 96$ & $4 A$ & 34128 & $B F$ \\
\hline 2909 & SULFATE & QINIT & moles & CONSTANT & $6.59 E+06$ & $6.59 E+06$ & $0.00 E+00$ & $6.59 \mathrm{E}+06$ & $6.59 E+06$ & $2 / 22 / 96$ & 2 & 32457 & $\mathrm{BF}$ \\
\hline 3461 & $T H$ & CAPHUM & molesniter & CONSTANT & 1.10E-05 & 1.10E-05 & $0.00 E+00$ & 1.10E-05 & 1.10E-05 & $5 / 8 / 96$ & 1 & 37726 & NU \\
\hline 3318 & $T H$ & CAPMIC & moles/iter & CONSTANT & 1.90E-03 & 1.90E-03 & $0.00 E+00$ & 1.90E-03 & 1.90E-03 & $4 / 16 / 96$ & 1 & 36865 & NU \\
\hline 3319 & TH & CONCINT & moles/iter & CONSTANT & $0.00 E+00$ & $0.00 E+00$ & $0.00 E+00$ & $0.00 E+00$ & $0.00 E+00$ & 4/16/96 & 1 & 36866 & NU \\
\hline 3437 & $T H$ & CONCMIN & molesniter & CONSTANT & 2.60E-08 & 2.60E-08 & $0.00 \mathrm{E}+00$ & 2.60E-08 & 2.60E-08 & $5 / 8 / 96$ & 1 & 37697 & NU \\
\hline 3320 & $T H$ & PROPMIC & NONE & CONSTANT & $3.10 E+00$ & $3.10 \mathrm{E}+00$ & $0.00 \mathrm{E}+00$ & $3.10 E+00$ & $3.10 E+00$ & $4 / 16 / 96$ & 1 & 36867 & NU \\
\hline 3449 & $T H+4$ & MDO & $m^{\wedge} 2 / s$ & CONSTANT & 1.53E-10 & 1.53E-10 & $0.00 E+00$ & $1.53 \mathrm{E}-10$ & 1.53E-10 & $5 / 17 / 96$ & 1 & 37715 & NUTTP \\
\hline 3478 & $\mathrm{TH}+4$ & MKD_TH & $\mathrm{m}^{\wedge} 3 / \mathrm{kg}$ & UNIFORM & $1.00 E+01$ & $1.00 E+01$ & $5.50 E+00$ & $9.00 E-01$ & $2.00 E+01$ & $6 / 12 / 96$ & 1 & 38349 & NUTTP \\
\hline 3216 & TH227 & ATWEIGHT & $\mathrm{kg} / \mathrm{mole}$ & CONSTANT & 2.27E-01 & 2.27E-01 & $0.00 E+00$ & 2.27E-01 & 2.27E-01 & 4/10/96 & 3 & 36734 & NU/CS \\
\hline 3356 & TH227 & EPAREL & Cirwuf & CONSTANT & $0.00 E+00$ & $0.00 \mathrm{E}+00$ & $0.00 E+00$ & $0.00 E+00$ & $0.00 E+00$ & $4 / 16 / 96$ & 3 & 36909 & cs \\
\hline 3300 & $\mathrm{TH} 227$ & HALFLIFE & $s$ & CONSTANT & $1.62 \mathrm{E}+06$ & $1.62 E+06$ & $0.00 E+00$ & $1.62 E+06$ & $1.62 E+05$ & $4 / 16 / 96$ & 3 & 36846 & NU/CS \\
\hline 3217 & TH228 & ATWEIGHT & $\mathrm{kg} / \mathrm{mole}$ & CONSTANT & 2.28E-01 & 2.28E-01 & $0.00 E+00$ & 2.28E-01 & 2.28E-01 & $4 / 10 / 96$ & 3 & 36735 & $\operatorname{cs} / N U$ \\
\hline 3357 & TH228 & EPAREL & Cihwof & CONSTANT & $0.00 E+00$ & $0.00 E+00$ & $0.00 E+00$ & $0.00 E+00$ & $0.00 E+00$ & $4 / 16 / 96$ & 3 & 36910 & CS \\
\hline 3301 & TH228 & HALFLJFE & s & CONSTANT & $6.04 E+07$ & $6.04 E+07$ & $0.00 E+00$ & $6.04 E+07$ & $6.04 E+07$ & $4 / 16 / 96$ & 3 & 36847 & NU/CS \\
\hline 603 & TH229 & ATWEIGHT & $\mathrm{kg} / \mathrm{mole}$ & CONSTANT & $2.29 E-01$ & 2.29E-01 & $0.00 E+00$ & 2.29E-01 & 2.29E-01 & 11/2/95 & 3 & 34594 & $\begin{array}{l}\text { FUTTPI } \\
\text { NU/CS } \\
\end{array}$ \\
\hline 3381 & TH229 & EPAREL & Cihwuf & CONSTANT & $1.00 E+02$ & $1.00 E+02$ & $0.00 E+00$ & $1.00 E+02$ & $1.00 E+02$ & 4/16/96 & 3 & 36943 & $\mathrm{cs}$ \\
\hline 604 & TH229 & HALFLIFE & $\mathbf{s}$ & CONSTANT & $2.32 E+11$ & $2.32 E+11$ & $0.00 E+00$ & $2.32 E+11$ & $2.32 E+11$ & $4 / 18 / 96$ & 3 & 34595 & NU/CS \\
\hline 605 & TH229 & INVCHD & $\mathrm{Ci}$ & CONSTANT & $9.29 E+00$ & $9.29 E+00$ & $0.00 E+00$ & $9.29 E+00$ & $9.29 E+00$ & $10 / 29 / 96$ & 2 & $34596 \mathrm{~A}$ & $\begin{array}{c}\text { NU/PN/ } \\
\text { CS }\end{array}$ \\
\hline 606 & TH229 & INVRHD & $\mathrm{Ci}$ & CONSTANT & 6.83E-01 & 6.83E-01 & $0.00 E+00$ & 6.83E-01 & 6.83E-01 & $10 / 29 / 96$ & 2 & $34597 \mathrm{~A}$ & $\begin{array}{c}\text { CS/NUI } \\
\text { PN } \\
\end{array}$ \\
\hline 607 & TH230 & ATWEIGHT & $\mathrm{kg} / \mathrm{mole}$ & CONSTANT & 2.30E-01 & 2.30E-01 & $0.00 E+00^{\circ}$ & 2.30E-01 & 2.30E-01 & 11/2/95 & 3 & 34600 & $\begin{array}{l}\text { NU/CS/ } \\
\text { TP/FL }\end{array}$ \\
\hline 3382 & TH230 & EPAREL & Cihwof & CONSTANT & $1.00 E+01$ & $1.00 E+01$ & $0.00 E+00$ & $1.00 E+01$ & $1.00 \mathrm{E}+01$ & 4/16/96 & 3 & 36944 & CS \\
\hline 608 & TH230 & HALFLIFE & s & CONSTANT & $2.43 E+12$ & $2.43 E+12$ & $0.00 E+00$ & $2.43 E+12$ & $2.43 E+12$ & $4 / 16 / 96$ & 3 & 34601 & CS/NU \\
\hline 609 & TH230 & INVCHD & $\mathrm{Ci}$ & CONSTANT & 2.83E-01 & 2.83E-01 & $0.00 E+00$ & 2.83E-01 & 2.83E-01 & 10/29/96 & 2 & $34602 A$ & \begin{tabular}{|c|}
$\mathrm{CS} / \mathrm{PN} /$ \\
$\mathrm{NU}$
\end{tabular} \\
\hline 610 & TH230 & INVRHD & $\mathrm{Ci}$ & CONSTANT & 2.22E-02 & 2.22E-02 & $0.00 E+00$ & 2.22E-02 & 2.22E-02 & 10/29/96 & 2 & $34603 \mathrm{~A}$ & $\begin{array}{c}\text { CS/NUI } \\
\text { PN }\end{array}$ \\
\hline 3218 & TH231 & ATWEIGHT & $\mathrm{kg} / \mathrm{mole}$ & CONSTANT & 2.31E-01 & 2.31E-01 & $0.00 E+00$ & 2.31E-01 & 2.31E-01 & $4 / 10 / 96$ & 3 & 36736 & CSNU \\
\hline 3358 & TH231 & EPAREL & Cinurf & CONSTANT & $0.00 E+00$ & $0.00 E+00$ & $0.00 E+00$ & $0.00 E+00$ & $0.00 \mathrm{E}+00$ & $4 / 16 / 96$ & 3 & 36911 & cs \\
\hline 3302 & TH231 & HALFLIFE & s & CONSTANT & $9.19 E+04$ & $9.19 E+0.4$ & $0.00 E+00$ & $9.19 E+04$ & $9.19 E+04$ & $4 / 16 / 96$ & 3 & 36848 & NU/CS \\
\hline 611 & TH232 & ATWEIGHT & $\mathrm{kg} / \mathrm{mole}$ & CONSTANT & 2.32E-01 & 2.32E-01 & $0.00 E+00$ & 2.32E-01 & 2.32E-01 & $11 / 2 / 95$ & 3 & 32458 & $\begin{array}{l}\text { CS/NUI } \\
\text { FUTP }\end{array}$ \\
\hline 3383 & TH232 & EPAREL & Cikwuf & CONSTANT & $1.00 E+01$ & $1.00 E+01$ & $0.00 E+00$ & $1.00 E+01$ & $1.00 E+01$ & 4/16/96 & 3 & 36945 & CS \\
\hline 612 & TH232 & HALFLIFE & $\mathbf{s}$ & CONSTANT & $4.43 E+17$ & $4.43 E+17$ & $0.00 E+00$ & $4.43 E+17$ & $4.43 E+17$ & $4 / 16 / 96$ & 3 & 34605 & NU/CS \\
\hline 613 & TH232 & INVCHD & $\mathrm{Ci}$ & CONSTANT & 9.14E-01 & 9.14E-01 & $0.00 E+00$ & 9.14E-01 & 9.14E-01 & 10/29/96 & 2 & $34806 \mathrm{~A}$ & $\begin{array}{c}\text { PN/NUI } \\
\text { CS }\end{array}$ \\
\hline 614 & TH232 & INVRHD & $\mathrm{Ci}$ & CONSTANT & 9.26E-02 & $9.26 E-02$ & $0.00 E+00$ & 9.26E-02 & 9.26E-02 & 10/29/96 & 2 & $34607 \mathrm{~A}$ & $\begin{array}{c}\text { NU/CSI } \\
\text { PN }\end{array}$ \\
\hline 3219 & TH234 & ATWEIGHT & $\mathrm{kg} / \mathrm{mole}$ & CONSTANT & 2.34E-01 & 2.34E-01 & $0.00 E+00$ & 2.34E-01 & 2.34E-01 & $4 / 10 / 96$ & 3 & 36737 & CS/NU \\
\hline 3359 & TH234 & EPAREL & Cihwuf & CONSTANT & $0.00 \mathrm{E}+00$ & $0.00 E+00$ & $0.00 E+00$ & $0.00 E+00$ & $0.00 E+00$ & $4 / 16 / 96$ & 3 & 36921 & cs \\
\hline 3303 & TH234 & HALFLIFE & $\mathbf{s}$ & CONSTANT & $2.08 E+06$ & $2.08 E+06$ & $0.00 E+00$ & $2.08 \mathrm{E}+06$ & $2.08 \mathrm{E}+06$ & $4 / 16 / 96$ & 3 & 36849 & NUICS \\
\hline 3196 & TL207 & ATWEIGHT & $\mathrm{kg} / \mathrm{mole}$ & CONSTANT & 2.07E-01 & 2.07E-01 & $0.00 \mathrm{E}+00$ & 2.07E-01 & 2.07E-01 & 4/10/96 & 3 & 36659 & CS/NU \\
\hline 3360 & TL207 & EPAREL & Cihurf & CONSTANT & $0.00 E+00$ & $0.00 E+00$ & $0.00 E+00$ & $0.00 E+00$ & $0.00 E+00$ & $4 / 16 / 96$ & 3 & 36922 & CS \\
\hline 3304 & TL207 & HALFLIFE & s & CONSTANT & $2.86 E+02$ & $2.86 E+02$ & $0.00 \mathrm{E}+00$ & $2.86 E+02$ & $2.86 \mathrm{E}+02$ & 4/16/96 & 3 & 38850 & CSNA \\
\hline 3460 & U & CAPHUM & moles/iter & CONSTANT & 1.10E-05 & 1.10E-05 & $0.00 E+00$ & 1.10E-05 & 1.10E-05 & $5 / 8 / 96$ & 1 & 37725 & NU \\
\hline 3308 & $u$ & CAPMIC & moles/iter & CONSTANT & 2.10E-03 & 2.10E-03 & $0.00 E+00$ & $2.10 E-03$ & $2.10 E-03$ & $4 / 16 / 96$ & 1 & 38855 & NU \\
\hline
\end{tabular}


23B. Waste Chemistry

\begin{tabular}{|c|c|c|c|c|c|c|c|c|c|c|c|c|c|}
\hline ID & Material & Parameter & Units & Distribution & Mean & Median & Deviation & Low & High & Entry Date & Impt & WPO & Codes \\
\hline 3307 & $\mathrm{u}$ & CONCINT & moles/iter & CONSTANT & $0.00 E+00$ & $0.00 E+00$ & $0.00 E+00$ & $0.00 E+00$ & $0.00 E+00$ & $4 / 16 / 95$ & 1 & 36854 & NU \\
\hline 3438 & U & CONCMIN & moles/iter & CONSTANT & 2.60E-08 & 2.60E-08 & $0.00 E+00$ & $2.60 E-08$ & 2.60E-08 & $5 / 8 / 96$ & 1 & 37698 & NU \\
\hline 3309 & $\mathrm{u}$ & PROPMIC & NONE & CONSTANT & 2.10E-03 & 2.10E-03 & $0.00 E+00$ & $2.10 E-03$ & $2.10 \mathrm{E}-03$ & $5 / 24 / 96$ & 1 & 36856 & NU \\
\hline 3446 & $U+4$ & MDO & $m^{\wedge} 2 / s$ & CONSTANT & $1.53 E-10$ & $1.53 \mathrm{E}-10$ & $0.00 E+00$ & 1.53E-10 & $1.53 \mathrm{E}-10$ & $5 / 17 / 96$ & 1 & 37711 & NUTRP \\
\hline 3479 & $\mathrm{U}+4$ & MKD_U & $\mathrm{m}^{\wedge} 3 / \mathrm{kg}$ & UNIFORM & $1.00 \mathrm{E}+01$ & 1.00E+01 & $5.50 E+00$ & 9.00E-01 & $2.00 E+01$ & $6 / 12 / 96$ & 1 & 38350 & TPNU \\
\hline 3448 & $\mathrm{U}+6$ & MDO & $m^{\wedge} 2 / s$ & CONSTANT & 4.26E-10 & $4.26 \mathrm{E}-10$ & $0.00 E+00$ & 4.26E-10 & $4.26 \mathrm{E}-10$ & $5 / 17 / 96$ & 1 & 37714 & TP/NU \\
\hline 3475 & $\mathrm{U}+6$ & MKD_U & $\mathrm{m}^{\wedge} 3 / \mathrm{kg}$ & UNIFORM & 1.50E-02 & $1.50 E-02$ & $8.70 E-03$ & 3.00E-05 & $3.00 \mathrm{E}-02$ & $6 / 12 / 96$ & 1 & 38346 & NU/TP \\
\hline 632 & U233 & ATWEIGHT & $\mathrm{kg} / \mathrm{mole}$ & CONSTANT & 2.33E-01 & 2.33E-01 & $0.00 E+00$ & 2.33E-01 & 2.33E-01 & 11/2/95 & 3 & 32459 & $\begin{array}{l}\text { FUCS/ } \\
\text { NU/TP }\end{array}$ \\
\hline 3384 & U233 & EPAREL & Cirwut & CONSTANT & $1.00 E+02$ & $1.00 E+02$ & $0.00 E+00$ & $1.00 E+02$ & $1.00 E+02$ & 4/16/96 & 3 & 36946 & CS \\
\hline 633 & U233 & HALFLIFE & s & CONSTANT & $5.00 \mathrm{E}+12$ & $5.00 \mathrm{E}+12$ & $0.00 E+00$ & $5.00 \mathrm{E}+12$ & $5.00 \mathrm{E}+12$ & $4 / 16 / 96$ & 3 & 34662 & NU/CS \\
\hline 634 & U233 & INVCHD & $\mathrm{Ci}$ & CONSTANT & $1.79 E+03$ & $1.79 E+03$ & $0.00 E+00$ & $1.79 E+03$ & 1.79E+03 & $10 / 29 / 96$ & 2 & $34663 \mathrm{~A}$ & $\begin{array}{c}\text { PN/CSI } \\
\text { NU }\end{array}$ \\
\hline 635 & U233 & INVRHD & $\mathrm{Ci}$ & CONSTANT & $1.58 E+02$ & $1.58 E+02$ & $0.00 E+00$ & $1.58 E+02$ & $1.58 E+02$ & $10 / 29 / 96$ & 2 & $34664 \mathrm{~A}$ & $\begin{array}{c}\text { NU/PN/ } \\
\text { CS }\end{array}$ \\
\hline 636 & U234 & ATWEIGHT & $\mathrm{kg} / \mathrm{mole}$ & CONSTANT & 2.34E-01 & 2.34E-01 & $0.00 E \div 00$ & 2.34E-01 & 2.34E-01 & 11/2/95 & 3 & 32460 & $\begin{array}{l}\text { FLCSI } \\
\text { TPINU } \\
\end{array}$ \\
\hline 3385 & U234 & EPAREL & Cinwuf & CONSTANT & $1.00 E+02$ & $1.00 E+02$ & $0.00 E+00$ & $1.00 E+02$ & $1.00 \mathrm{E}+02$ & 4/16/96 & 3 & 36947 & CS \\
\hline 637 & U234 & HALFLIFE & s & CONSTANT & $7.72 \mathrm{E}+12$ & $7.72 E+12$ & $0.00 E+00$ & $7.72 E+12$ & $7.72 E+12$ & 4/16/96 & 3 & 34667 & $\mathrm{CS} / \mathrm{NU}$ \\
\hline 638 & U234 & INVCHD & $\mathrm{Ci}$ & CONSTANT & $7.08 E+02$ & $7.08 E+02$ & $0.00 E+00$ & $7.08 E+02$ & $7.08 \mathrm{E}+02$ & $10 / 29 / 96$ & 2 & $34668 \mathrm{~A}$ & $\begin{array}{c}\text { NU/PNI } \\
\text { CS }\end{array}$ \\
\hline 639 & U234 & INVRHD & $\mathrm{Ci}$ & CONSTANT & $4.29 E+01$ & 4.29E+01 & $0.00 E+00$ & $4.29 E+01$ & $4.29 E+01$ & $10 / 29 / 96$ & 2 & $34669 \mathrm{~A}$ & $\begin{array}{c}\text { NU/PN/ } \\
\text { CS }\end{array}$ \\
\hline 640 & U235 & ATWEIGHT & $\mathrm{kg} / \mathrm{mole}$ & CONSTANT & 2.35E-01 & 2.35E-01 & $0.00 E+00$ & 2.35E-01 & 2.35E-01 & 11/2/95 & 3 & 32461 & $\begin{array}{l}\text { FUTPI } \\
\text { NU/CS } \\
\end{array}$ \\
\hline 3386 & U235 & EPAREL & Citwuf & CONSTANT & $1.00 \mathrm{E}+02$ & $1.00 \mathrm{E}+02$ & $0.00 E+00$ & $1.00 E+02$ & $1.00 E+02$ & 4/16/96 & 3 & 36948 & cs \\
\hline 641 & U235 & HALFLIFE & s & CONSTANT & $2.22 E+16$ & $2.22 E+16$ & $0.00 E+00$ & $2.22 E+16$ & $2.22 \mathrm{E}+16$ & $4 / 16 / 96$ & 3 & 34671 & $\mathrm{CS} / \mathrm{NU}$ \\
\hline 642 & U235 & INVCHD & $\mathrm{Ci}$ & CONSTANT & $1.28 E+01$ & $1.28 E+01$ & $0.00 E+00$ & $1.28 E+01$ & $1.28 E+01$ & $10 / 29 / 96$ & 2 & 34672A & $\begin{array}{c}\text { CSIPN/ } \\
\text { NU }\end{array}$ \\
\hline 643 & U235 & INVRHD & $\mathrm{Ci}$ & CONSTANT & $4.63 E+00$ & $4.63 E+00$ & $0.00 E+00$ & $4.63 E+00$ & $4.63 E+00$ & 10/29/96 & 2 & $34674 \mathrm{~A}$ & $\begin{array}{c}\text { NU/PNI } \\
\text { CS }\end{array}$ \\
\hline 644 & U236 & ATWEIGHT & $\mathrm{kg} / \mathrm{mole}$ & CONSTANT & 2.36E-01 & 2.36E-01 & $0.00 E+00$ & 2.36E-01 & 2.36E-01 & $11 / 2 / 95$ & 3 & 32462 & $\begin{array}{l}\text { CSIFU } \\
\text { TP/NU } \\
\end{array}$ \\
\hline 3387 & u236 & EPAREL & Cirwuf & CONSTANT & $1.00 \mathrm{E}+02$ & $1.00 E+02$ & $0.00 E+00$ & $1.00 E+02$ & $1.00 E+02$ & $4 / 16 / 96$ & 3 & 36949 & CS \\
\hline 645 & U236 & HALFLIFE & s & CONSTANT & $7.39 E+14$ & $7.39 E+14$ & $0.00 E+00$ & $7.39 E+14$ & & $4 / 16 / 96$ & 3 & 34676 & NU/CS \\
\hline 2216 & U236 & INVCHD & $\mathrm{Ci}$ & CONSTANT & $5.69 E-01$ & 5.69E-01 & $0.00 E+00$ & 5.69E-01 & $5.69 E-01$ & $10 / 29 / 96$ & 2 & $34677 \mathrm{~A}$ & \begin{tabular}{|c|} 
NU/CSI \\
PN
\end{tabular} \\
\hline 646 & U236 & INVRHD & $\mathrm{Ci}$ & CONSTANT & $1.03 \mathrm{E}-01$ & 1.03E-01 & $0.00 E+00$ & 1.03E-01 & 1.03E-01 & $10 / 29 / 96$ & 2 & $34678 \mathrm{~A}$ & \begin{tabular}{|c|}
$\mathrm{NU} / \mathrm{PN} /$ \\
$\mathrm{CS}$ \\
\end{tabular} \\
\hline 647 & U238 & ATWEIGHT & $\mathrm{kg} / \mathrm{mole}$ & CONSTANT & 2.38E-01 & 2.38E-01 & $0.00 E+00$ & 2.38E-01 & 2.38E-01 & $11 / 2 / 95$ & 3 & 32463 & $\begin{array}{l}\text { FUCSI } \\
\text { TP/NU } \\
\end{array}$ \\
\hline 3388 & $\mathrm{U} 238$ & EPAREL & Citwuf & CONSTANT & $1.00 E+02$ & $1.00 \mathrm{E}+02$ & $0.00 E+00$ & $1.00 \mathrm{E}+02$ & $1.00 \mathrm{E}+02$ & $4 / 16 / 96$ & 3 & 36950 & CS \\
\hline 648 & U238 & HALFLIFE & s & CONSTANT & $1.41 \mathrm{E}+17$ & $1.41 \mathrm{E}+17$ & $0.00 E+00$ & $1.41 E+17$ & $1.41 \mathrm{E}+17$ & $4 / 96 / 96$ & $=3$ & 34680 & CSINU \\
\hline 849 & U238 & INVCHD & $\mathrm{Ci}$ & CONSTANT & 3.96E+01 & $3.96 E+01$ & $0.00 E+00$ & 3.96E+01 & $3.96 E+01$ & 10/29/96 & 2 & $34681 \mathrm{~A}$ & \begin{tabular}{|c}
$\mathrm{NU} / \mathrm{PN} /$ \\
$\mathrm{CS}$ \\
\end{tabular} \\
\hline 650 & U23B & INVRHD & $\mathrm{Ci}$ & CONSTANT & 1.05E+01 & $1.05 E+01$ & $0.00 E+00$ & 1.05E+01 & $1.05 E+01$ & 10/29/96 & 2 & $34682 \mathrm{~A}$ & \begin{tabular}{|c|}
$\mathrm{PN} / \mathrm{NU} /$ \\
$\mathrm{CS}$ \\
\end{tabular} \\
\hline 3198 & Y90 & ATWEIGHT & $\mathrm{kg} / \mathrm{mole}$ & CONSTANT & 8.99E-02 & 8.99E-02 & $0.00 E+00$ & 8.99E-02 & 8.99E-02 & $4 / 10 / 96$ & 3 & 36703 & CS/NU \\
\hline 3361 & $Y 90$ & EPAREL & Citwuf & CONSTANT & $0.00 E+00$ & $0.00 E+00$ & $0.00 E+00$ & $0.00 E+00$ & $0.00 \mathrm{E}+00$ & $4 / 16 / 96$ & 3 & 36923 & CS \\
\hline 3305 & Y9O & HALFLIFE & s & CONSTANT & $2.30 E+05$ & $2.30 \mathrm{E}+05$ & $0.00 \mathrm{E}+00$ & $2.30 \mathrm{E}+05$ & $2.30 E+05$ & 4/16/96 & 3 & 36851 & CS/NU \\
\hline 3199 & ZR90 & ATWEIGHT & $\mathrm{kg} / \mathrm{mole}$ & CONSTANT & 8.99E-02 & 8.99E-02 & $0.00 E+00$ & 8.99E-02 & 8.99E-02 & $4 / 10 / 96$ & 3 & 36704 & NU/CS \\
\hline 3362 & ZR90 & EPAREL & Cikwuf & CONSTANT & $0.00 E+00$ & $0.00 E+00$ & $0.00 E+00$ & $0.00 E+00$ & $0.00 E+00$ & $4 / 16 / 96$ & 3 & 36924 & CS \\
\hline 3306 & ZR90 & HALFLIFE & $s$ & CONSTANT & $1.00 \mathrm{E}+38$ & $1.00 E+38$ & $0.00 E+00$ & $1.00 E+38$ & $1.00 E+38$ & $4 / 16 / 96$ & 3 & 36852 & CS/NU \\
\hline 228 & $\mathrm{H} 2$ & VISco & Pa's & CONSTANT & 8.93E-06 & 8.93E-06 & $0.00 E+00$ & 8.93E-06 & 8.93E-06 & $3 / 14 / 96$ & 3 & $32334 \mathrm{~A}$ & BF \\
\hline
\end{tabular}


24A. Rest of Repository

\begin{tabular}{|c|c|c|c|c|c|c|c|c|c|c|c|c|c|}
\hline ID & aterial & Parameter & Units & Distribution & Mean & Median & Deviation & Low & High & Entry Date & Impt & WPO & Codes \\
\hline 2117 & REPOSIT & DIRONCHW & $\mathrm{kg} / \mathrm{m}^{\wedge} 3$ & CONSTANT & $1.70 \mathrm{E}+02$ & $1.70 E+02$ & $0.00 E+00$ & $1.70 E+02$ & $1.70 \mathrm{E}+02$ & $2 / 13 / 96$ & 2 & 32442 & BF \\
\hline 2110 & REPOSIT & CAP_MOD & NONE & CONSTANT & $1.00 E+00$ & $1.00 E+00$ & $0.00 E+00$ & $1.00 \mathrm{E}+00$ & $1.00 E+00$ & $2 / 8 / 96$ & $4 B$ & 33285 & $B F$ \\
\hline 2123 & REPOSIT & DRUBBRHW & $\mathrm{kg} / \mathrm{m}^{\wedge} 3$ & CONSTANT & $3.30 \mathrm{E}+00$ & $3.30 \mathrm{E}+00$ & $0.00 E+00$ & $3.30 E+00$ & $3.30 E+00$ & $2 / 13 / 96$ & 2 & 32451 & $B F$ \\
\hline 2122 & REPOSIT & DRUBBCHW & $\mathrm{kg} / \mathrm{m}^{\wedge} 3$ & CONSTANT & $1.00 \mathrm{E}+01$ & $1.00 E+01$ & $0.00 E+00$ & $1.00 E+01$ & $1.00 E+01$ & $2 / 13 / 96$ & 2 & 32450 & $B F$ \\
\hline 2995 & REPOSIT & DPLSCRHW & $\mathrm{kg} / \mathrm{m}^{\wedge} 3$ & CONSTANT & $3.10 E+00$ & 3.10E+00 & $0.00 E+00$ & $3.10 E+00$ & $3.10 E+00$ & $2 / 13 / 96$ & 2 & 32449 & BF \\
\hline 2121 & REPOSIT & DPLSCCHW & $\mathrm{kg} / \mathrm{m}^{\wedge} 3$ & CONSTANT & $2.60 \mathrm{E}+01$ & $2.60 \mathrm{E}+01$ & $0.00 \mathrm{E}+00$ & $2.60 \mathrm{E}+01$ & $2.60 E+01$ & $2 / 13 / 96$ & 2 & 32447 & $\mathrm{BF}$ \\
\hline 2120 & REPOSIT & DPLASRHW & $\mathrm{kg} / \mathrm{m}^{\wedge} 3$ & CONSTANT & $1.50 \mathrm{E}+01$ & $1.50 E+01$ & $0.00 E+00$ & $1.50 \mathrm{E}+01$ & $1.50 \mathrm{E}+01$ & 2/13/96 & 2 & 32446 & BF \\
\hline 2128 & REPOSIT & GRATMICI & mol/kg"s & UNIFORM & 4.91E-09 & 4.91E-09 & 2.65E-09 & 3.17E-10 & 9.51E-09 & $2 / 8 / 96$ & 18 & 33235 & BF \\
\hline 2118 & REPOSIT & DIRONRHW & $\mathrm{kg} / \mathrm{m}^{\wedge} 3$ & CONSTANT & $1.00 \mathrm{E}+02$ & $.00 \mathrm{E}+02$ & $0.00 E+00$ & $1.00 E+02$ & $1.00 \mathrm{E}+02$ & $2 / 13 / 96$ & 2 & 32443 & $B F$ \\
\hline 2736 & REPOSIT & KPT & NONE & CONSTANT & $0.00 E+00$ & $0.00 E+00$ & $0.00 E+00$ & $0.00 E+00$ & $0.00 E+00$ & $2 / 20 / 96$ & 4B & 33238 & $B F$ \\
\hline 2116 & REPOSIT & DIRNCRHW & $\mathrm{kg} / \mathrm{m}^{\wedge} 3$ & CONSTANT & $2.59 \mathrm{E}+03$ & $2.59 E+03$ & $0.00 E+00$ & $2.59 E+03$ & $2.59 E+03$ & $2 / 13 / 96$ & 2 & 32440 & $\mathrm{BF}$ \\
\hline 2115 & REPOSIT & DIRNCCHW & $\mathrm{kg} / \mathrm{m}^{\wedge} 3$ & CONSTANT & $1.39 \mathrm{E}+02$ & $1.39 E+02$ & $0.00 \mathrm{E}+00$ & 1.39E+02 & 1.39E+02 & $2 / 13 / 96$ & 2 & 32439 & $\mathrm{BF}$ \\
\hline 2114 & REPOSIT & DCELLRHW & $\mathrm{kg} / \mathrm{m}^{\wedge} 3$ & CONSTANT & $1.70 E+01$ & $1.70 E+01$ & $0.00 E+00$ & $1.70 E+01$ & $1.70 E+01$ & $2 / 13 / 96$ & 2 & 32436 & BF \\
\hline 2113 & REPOSIT & DCELLCHW & $\mathrm{kg} / \mathrm{m}^{\wedge} 3$ & CONSTANT & $5.40 E+01$ & $5.40 E+01$ & $0.00 E+00$ & $5.40 E+01$ & $5.40 E+01$ & $2 / 13 / 96$ & 2 & 33298 & BF \\
\hline 2112 & REPOSIT & COMP_RCK & $P a^{\wedge}-1$ & CONSTANT & $0.00 E+00$ & $0.00 \mathrm{E}+00$ & $0.00 E+00$ & $0.00 E+00$ & $0.00 E+00$ & $2 / 8 / 96$ & $4 B$ & 33290 & $B F$ \\
\hline 3455 & REPOSIT & CLOSMOD & NONE & CONSTANT & $4.00 E+00$ & $4.00 E+00$ & $0.00 E+00$ & $4.00 E+00$ & $4.00 E+D 0$ & $6 / 4 / 96$ & $4 B$ & 38207 & $\mathrm{BF}$ \\
\hline 2119 & REPOSIT & DPLASCHW & $\mathrm{kg} / \mathrm{m}^{\wedge} 3$ & CONSTANT & $3.40 E+01$ & $3.40 E+01$ & $0.00 E+00$ & $3.40 \mathrm{E}+01$ & $3.40 \mathrm{E}+01$ & $2 / 13 / 96$ & 2 & 32444 & $\mathrm{BF}$ \\
\hline 2131 & REPOSIT & PRMX_LOG & $\log \left(m^{\wedge} 2\right)$ & CONSTANT & $-1.28 E+01$ & $-1.28 E+01$ & $0.00 E+00$ & $-1.28 E+01$ & $-1.28 E+01$ & $2 / 8 / 96$ & $4 A$ & 33255 & $\mathrm{BF}$ \\
\hline 2141 & REPOSIT & VOLCHW & $m^{\wedge} 3$ & CONSTANT & 1.69E+05 & $1.69 E+05$ & $0.00 E+00$ & 1.69E+05 & 1.69E+05 & $2 / 13 / 96$ & 2 & 32452 & BF \\
\hline 2137 & REPOSIT & SAT_RGAS & NONE & UNIFORM & 7.50E-02 & 7.50E-02 & 4.33E-02 & $0.00 E+00$ & 1.50E-01 & $2 / 22 / 96$ & $4 B$ & 33286 & BF \\
\hline 2741 & REPOSIT & SAT_RBRN & NONE & UNIFORM & 2.76E-01 & $2.76 \mathrm{E}-01$ & 1.59E-01 & $0.00 E+00$ & 5.52E-01 & $2 / 20 / 96$ & $4 B$ & 33283 & $B F$ \\
\hline 2740 & REPOSIT & SAT_IBRN & NONE & CONSTANT & 1.50E-02 & $1.50 E-02$ & $0.00 E+00$ & 1.50E-02 & 1.50E-02 & $2 / 11 / 96$ & 4B & 33277 & $B F$ \\
\hline 2135 & REPOSTT & RELP_MOD & NONE & CONSTANT & $4.00 E+00$ & $4.00 E+00$ & $0.00 E+00$ & $4.00 \mathrm{E}+00$ & $4.00 E+00$ & $2 / 8 / 96$ & 4B & 33275 & $\mathrm{BF}$ \\
\hline 2824 & REPOSIT & PROBDEG & NONE & DELTA & & $2.00 E+00$ & $0.00 E+00$ & $0.00 E+00$ & $2.00 E+00$ & $2 / 23 / 96$ & $4 B$ & 33264 & $\mathrm{BF}$ \\
\hline 2127 & REPOSIT & GRATMICH & mol/kg"s & UNIFORM & 6.34E-10 & $6.34 \mathrm{E}-10$ & $3.66 \mathrm{E}-10$ & $0.00 E+00$ & 1.27E-09 & $2 / 8 / 96$ & 1B & 33234 & $\mathrm{BF}$ \\
\hline 2132 & REPOSIT & PRMY_LOG & $\log \left(m^{\wedge} 2\right)$ & CONSTANT & $-1.28 E+01$ & $-1.28 E+01$ & $0.00 E+00$ & $-1.28 \mathrm{E}+01$ & $-1.28 E+01$ & $2 / 8 / 96$ & $4 A$ & 33258 & BF \\
\hline 2142 & REPOSIT & VOLRHW & $\mathrm{m}^{\wedge} \mathbf{3}$ & CONSTANT & $7.08 E+03$ & $7.08 E+03$ & $0.00 E+00$ & $7.08 E+03$ & $7.08 E+03$ & $2 / 13 / 96$ & 2 & 32453 & $\mathrm{BF}$ \\
\hline 2130 & REPOSIT & POROSITY & NONE & CONSTANT & 8.48E-01 & 8.48E-01 & $0.00 E+00$ & 8.48E-01 & 8.48E-01 & $2 / 8 / 96$ & $4 B$ & 33250 & $\mathrm{BF}$ \\
\hline 2129 & REPOSIT & PORE_DIS & NONE & CUMULATTVE & $3.25 E+00$ & 2.89E+00 & $1.27 E+00$ & $1.44 E+00$ & $5.78 \mathrm{E}+00$ & $2 / 21 / 96$ & $4 B$ & 33248 & $B F$ \\
\hline 2739 & REPOSIT & PO_MIN & $\mathrm{Pa}$ & CONSTANT & 1.01E+05 & 1.01E+05 & $0.00 E+00$ & 1.01E+05 & 1.01E+05 & $2 / 21 / 96$ & $4 B$ & 33252 & $B F$ \\
\hline 2738 & REPOSIT & PCT_EXP & NONE & CONSTANT & $0.00 E+00$ & $0.00 E+00$ & $0.00 E+00$ & $0.00 E+00$ & $0.00 E+00$ & $2 / 8 / 96$ & $4 B$ & 33242 & $B F$ \\
\hline 2737 & REPOSIT & PCT_A & $\mathrm{Pa}$ & CONSTANT & $0.00 \mathrm{E}+00$ & $0.00 E+00$ & $0.00 E+00$ & $0.00 E+00$ & $0.00 E+00$ & $2 / 8 / 96$ & 48 & 33240 & BF \\
\hline 2242 & REPOSIT & PC_MAX & $\mathrm{Pa}$ & CONSTANT & $1.00 E+08$ & $1.00 E+08$ & $0.00 E+00$ & $1.00 E+08$ & 1.00E+08 & $11 / 1 / 95$ & $4 B$ & 33246 & $B F$ \\
\hline 2133 & REPOSIT & PRMZ_LOG & $\log \left(m^{\wedge} 2\right)$ & CONSTANT & $-1.28 E+01$ & $-1.28 E+01$ & $0.00 E+00$ & $-1.28 E+01$ & $-1.28 E+01$ & $2 / 8 / 96$ & $4 A$ & 33259 & $B F$ \\
\hline
\end{tabular}


24B. Predisposal Cavities

\begin{tabular}{|c|c|c|c|c|c|c|c|c|c|c|c|c|c|}
\hline ID & Material & & nits & tion & Mean & edian & ion & Low & High & Entry Date & Impt & WPO & Codes \\
\hline 84 & CAVITY_1 & RMY_LOG & $g\left(m^{\wedge} 2\right)$ & CONSTANT & $1.00 E+01$ & $-1.00 E+01$ & $0.00 E+00$ & $-1.00 E+01$ & $-1.00 E+01$ & $2 / 20 / 96$ & $4 B$ & 31681 & $B F$ \\
\hline 76 & AVITY_1 & $O D$ & IONE & CONSTANT & $1.00 E+00$ & $1.00 E+00$ & $0.00 E+00$ & $1.00 E+00$ & $.00 E+00$ & $11 / 2 / 95$ & AB & 31626 & $B F$ \\
\hline 89 & IVITY_1 & $\overline{B R N}$ & $\overline{O N E}$ & ONSTANT & $.00 E+00$ & $0.00 E+00$ & $0.00 E+00$ & $0.00 E+00$ & $0.00 E+00$ & $2 / 17 / 96$ & 4B & 31687 & BF \\
\hline 3099 & AVITY_1 & BRN & IONE & CONSTANT & $.00 E+00$ & $0.00 E+00$ & $0.00 E+00$ & $0.00 E+00$ & $0.00 E+00$ & $2 / 17 / 96$ & 4B & 31686 & BF \\
\hline 85 & CAVITY_I & LOG & $g\left(m^{\wedge} 2\right)$ & CONSTANT & $-1.00 E+01$ & $-1.00 E+01$ & $0.00 E+00$ & $-1.00 E+01$ & $-1.00 E+01$ & $2 / 20 / 96$ & $4 B$ & 31682 & $B F$ \\
\hline 90 & CAVITY_1 & 3AS & ONE & CONSTANT & $.00 E+00$ & $0.00 E+00$ & $0.00 E+00$ & $0.00 E+00$ & $0.00 E+00$ & $2 / 17 / 96$ & $4 B$ & 31688 & BF \\
\hline 83 & CAVITY_1 & $\angle O G$ & $g\left(m^{\wedge} 2\right)$ & CONSTANT & $-1.00 E+01$ & $|-1.00 E+01|$ & $0.00 E+00$ & $-1.00 E+01$ & $-1.00 E+01$ & $2 / 20 / 96$ & 48 & 31680 & $\mathrm{BF}$ \\
\hline 32 & CAVITY_1 & RESSURE & $\mathrm{Pa}$ & CONSTA & $1.01 E+05$ & $1.01 E+05$ & $0.00 E+00$ & 1.01E+05 & $1.01 E+05$ & $11 / 2 / 95$ & $4 \mathrm{~B}$ & 31679 & BF \\
\hline 2612 & CAVITY_1 & KPT & NONE & CONSTANT & $0.00 E+00$ & $0.00 E+00$ & $0.00 E+00$ & $0.00 E+00$ & $0.00 E+00$ & $2 / 17 / 96$ & 4B & 31650 & BF \\
\hline 38 & CAVITY_1 & 100 & ONE & CONSTANT & $4.00 E+00$ & $4.00 E+00$ & $0.00 E+00$ & $4.00 \mathrm{E}+00$ & $4.00 E+00$ & $11 / 2 / 95$ & 4B & 1685 & BF \\
\hline 7 & CAVIT & CK & & $\mathrm{CON}$ & $0.00 E+00$ & $0.00 E+00$ & $0.00 E+00$ & $0.00 E+00$ & $0.00 E+00$ & $11 / 2 / 95$ & 4B & 31628 & $B F$ \\
\hline 30 & CAVITY_1 & $\Gamma$ & ONE & CONSTANT & $1.00 E+00$ & $1.00 E+00$ & $0.00 E+00$ & $1.00 E+00$ & $1.00 E+00$ & $11 / 2 / 95$ & 48 & 31677 & BF \\
\hline 8 & $Y \_1$ & $x$ & & CON & $0 E+08$ & $1.00 E+08$ & $0.00 E+00$ & $1.00 E+08$ & $1.00 E+08$ & $11 / 2 / 95$ & $4 B$ & 31673 & $B F$ \\
\hline 2613 & AVITY_1 & CT_A & $\mathrm{Pa}$ & CON & $.00 E+00$ & $0.00 E+00$ & $0.00 E+00$ & $0.00 E+00$ & $0.00 E+00$ & $2 / 17 / 96$ & 4B & 31670 & $B F$ \\
\hline 2614 & CAVITY_1 & CT_EXP & NONE & CONSTANT & $0.00 E+00$ & $0.00 E+00$ & $0.00 E+00$ & $0.00 E+00$ & $0.00 E+00$ & $2 / 17 / 96$ & 4B & 31672 & BF \\
\hline 81 & CAVITY_1 & $O_{-}$ & $\mathrm{Pa}$ & CONS & $1.01 E+05$ & $1.01 E+05$ & $0.00 E+00$ & $1.01 E+05$ & $1.01 E+05$ & $2 / 21 / 96$ & 4B & 31678 & BF \\
\hline 79 & CAVITY_1 & POR & NONE & $\mathrm{CON}$ & 7.00E-01 & 7.00E-01 & $0.00 E+00$ & $7.00 E-01$ & $7.00 E-01$ & $11 / 2 / 95$ & 4B & 31675 & $B F$ \\
\hline 95 & CAVITY_2 & POR & ONE & CONS & $1.00 E+00$ & $1.00 E+00$ & $0.00 E+00$ & $1.00 E+00$ & $1.00 E+00$ & $11 / 2 / 95$ & 4B & 31747 & $\mathrm{BF}$ \\
\hline 103 & CAVITY_2 & RELP. & NONE & CONSTANT & $4.00 E+00$ & $4.00 \mathrm{E}+00$ & $0.00 E+00$ & $4.00 \mathrm{E}+00$ & $4.00 E+00$ & $11 / 2 / 95$ & $4 B$ & 31759 & $\mathrm{BF}$ \\
\hline 100 & CAVI & LOG & $g\left(m^{\wedge} 2\right)$ & CONSTANT & $-1.00 E+01$ & $-1.00 E+01$ & $0.00 E+00$ & $-1.00 E+01$ & $-1.00 E+01$ & $2 / 17 / 96$ & $4 B$ & 31754 & BF \\
\hline 99 & CAVITY_2 & |PRM & $\log \left(m^{\wedge} 2\right)$ & CONSTANT & $-1.00 E+01$ & $-1.00 E+01$ & $0.00 \mathrm{E}+00$ & $-1.00 E+01$ & $-1.00 E+01$ & $2 / 17 / 96$ & $4 B$ & 31752 & $B F$ \\
\hline 98 & 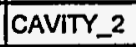 & IG & & CONS & $-1.00 E+01$ & $-1.00 E+01$ & $0.00 E+00$ & $-1.00 \mathrm{E}+01$ & $-1.00 E+01$ & $2 / 17 / 96$ & $4 B$ & 31751 & BF \\
\hline 97 & CAVITY_2 & PRE & $\mathrm{Pa}$ & CONSTANT & $1.01 E+05$ & $1.01 E+05$ & $0.00 E+C 0$ & $1.01 E+05$ & 1.01E+05 & $11 / 2 / 95$ & $4 B$ & 31750 & $\mathrm{BF}$ \\
\hline 3100 & AVITY_2 & $\mathrm{RN}$ & IONE & ICONSTANT & $0.00 E+00$ & $0.00 E+00$ & $0.00 E+00$ & $0.00 E+00$ & $0.00 E+00$ & $2 / 17 / 96$ & $4 B$ & 31760 & $\mathrm{BF}$ \\
\hline 34 & AVITY_2 & is & & ANT & & 7.00E-01 & $0.00 E+00$ & 7.00 E-01 & 7.00E-01 & $12 / 95$ & AB & 31746 & $B F$ \\
\hline 96 & CAVITY_2 & - non & & CON & $.01 E+05$ & $1.01 E+05$ & $0.00 E+00$ & 1.01E+05 & $1.01 E+05$ & $11 / 2 / 95$ & $4 B$ & 31749 & $\mathrm{BF}$ \\
\hline 618 & -2 & CT EXP & ONE & CONSTANT & $0.00 \mathrm{E}+00$ & $0.00 E+00$ & $0.00 \mathrm{E}+00$ & $0.00 E+00$ & $0.00 \mathrm{E}+00$ & $11 / 14 / 95$ & $4 B$ & 31741 & BF \\
\hline 2617 & CAVITY_2 & CT & $a$ & CONSTANT & $0.00 E+00$ & $0.00 \mathrm{E}+00$ & $0.00 E+00$ & $0.00 E+00$ & $0.00 \mathrm{E}+00$ & $114 / 95$ & $4 B$ & 1740 & $\mathrm{BF}$ \\
\hline 93 & AVITY_2 & $P$ & & CONSTANT & & $1.00 E+08$ & $0.00 E+00$ & $1.00 \mathrm{E}+08$ & $1.00 E+08$ & $11 / 2 / 95$ & $4 B$ & 31743 & $B F$ \\
\hline 616 & AVITY_2 & PT & DNE & CONSTANT & $00 E+00$ & $0.00 E+00$ & & & $0.00 E+00$ & Son & 4B & & $B F$ \\
\hline 104 & CAVITY_2 & BRN & ONE & CONS & $.00 E+00$ & $0.00 E+00$ & $0.00 E+00$ & $0.00 E+00$ & $0.00 E+00$ & & $4 B$ & 1761 & $\mathrm{BF}$ \\
\hline 11 & CAVITY_2 & AOD & DNE & CONS & $1.00 E+00$ & $1.00 E+00$ & $0.00 E+00$ & $1.00 E+00$ & $1.00 E+00$ & 17000 & $4 B$ & 1692 & $B F$ \\
\hline 05 & CAVITY_2 & GAS & ONE & CONSTANT & $0.00 E+00$ & $0.00 \mathrm{E}+00$ & $0.00 E+00$ & $0.00 E+00$ & $0.00 E+00$ & $1 / 196$ & 48 & 31763 & BF \\
\hline 10 & CAVITY_2 & COMI & $a^{n}-1$ & CONSTANT & $0.00 E+00$ & $0.00 E+00$ & $0.00 E+00$ & $0.00 E+00$ & $0.00 E+00$ & $11 / 2 / 95$ & 4B & 31694 & BF \\
\hline 2055 & AVITY_3 & LOG & $g\left(m^{\wedge} 2\right)$ & CONSTANT & $-1.00 E+01$ & $-1.00 E+01$ & $0.00 E+00$ & $-1.00 E+01$ & I & 2/20/96 & $4 B$ & 31182 & $B F$ \\
\hline 2049 & CAVITY_3 & $O D$ & DNE & CONSTANT & $00 E+00$ & $1.00 E+00$ & $.00 E+C 0$ & $1.00 E+00$ & $1.00 \mathrm{E}+00$ & & $4 B$ & 31767 & $\mathrm{BF}$ \\
\hline 2235 & AVITY_3 & BRN & & CONSTANT & & $0.00 \mathrm{E}+00$ & $0.00 \mathrm{E}+00$ & $0.00 E+00$ & $0.00 E+00$ & $2 / 17 / 96$ & $4 B$ & 31788 & $\mathrm{BF}$ \\
\hline 3102 & AVITY_3 & & & CONSTANT & $0.00 E+00$ & $0.00 E+00$ & $0.00 \mathrm{E}+00$ & $0.00 E+00$ & $0.00 \mathrm{E}+00$ & $2 / 17 / 96$ & 4B & 31786 & $\mathrm{BF}$ \\
\hline 2056 & AVITY_3 & nimi__Lo & & CONSTANT & $-1.00 E+01$ & $-1.00 E+01$ & $0.00 E+00$ & $-1.00 E+01$ & $-1.00 E+01$ & 2/20/96 & $4 B$ & 31783 & BF \\
\hline 2059 & CAVITY_3 & GAS & ONE & CONSTANT & & & $0.00 E+00$ & $0.00 E+00$ & $0.00 E+00$ & (1) & $4 B$ & 34700 & $B F$ \\
\hline 2054 & CAVITY_3 & LOG & $\log \left(m^{\wedge} 2\right)$ & CONSTANT & $-1.00 E+01$ & $-1.00 E+01$ & $0.00 E+00$ & $-1.00 E+01$ & $-1.00 E+01$ & 200190 & $4 B$ & & BF \\
\hline 3101 & CAVITY_3 & SSURE & & CON & 1.01E+05 & 1.01E+05 & $0.00 E+00$ & $1.01 E+05$ & $1.01 E+05$ & 2/17/96 & 4B & 31780 & BF \\
\hline 2620 & CAVITY_3 & KPT & ONE & CONSTANT & $0.00 E+00$ & $0.00 E+00$ & $0.00 \mathrm{E}+00$ & $0.00 E+00$ & $0.00 E+00$ & $2 / 17 / 96$ & 4B & 31770 & BF \\
\hline 2052 & CAVITY_3 & PORE_DIS & NONE & CONSTANT & 7.00E-01 & 7.00E-01 & $0.00 E+00$ & $7.00 E-01$ & 7.00E-01 & $2 / 17 / 96$ & $4 B$ & 31777 & $B F$ \\
\hline 2623 & CAVITY_3 & PO_MIN & & CONSTANT & 1.01E+05 & $1.01 E+05$ & $0.00 E+00$ & $1.01 E+05$ & $1.01 E+05$ & 2/17/96 & 4B & 31770 & $B F$ \\
\hline 2622 & CAVITY_3 & PCT_EXP & NONE & CONSTANT & $0.00 E+00$ & $0.00 E+00$ & $0.00 E+00$ & $0.00 E+00$ & $0.00 E+00$ & 2/17/96 & 4B & 01074 & $B F$ \\
\hline 2621 & CAVITY_3 & PCT_A & $\mathrm{ra}$ & CONSTANT & $0.00 E+00$ & $0.00 E+00$ & $0.00 E+00$ & $0.00 E+00$ & $0.00 E+00$ & $2 / 17 / 96$ & 4B & 31773 & $B F$ \\
\hline 2234 & CAVITY_3 & PC_MAX & $\mathrm{ra}$ & CONSTANT & $1.00 E+08$ & $1.00 E+08$ & $0.00 E+00$ & $1.00 E+08$ & $1.00 E+08$ & $2 / 17 / 96$ & $4 B$ & 31775 & $\mathrm{BF}$ \\
\hline 2053 & CAVITY_3 & POROSITY & NONE & CONSTANT & $1.00 E+00$ & $1.00 E+00$ & $0.00 E+00$ & $1.00 E+00$ & $1.00 E+00$ & $2 / 17 / 96$ & 4B & 31778 & $\mathrm{BF}$ \\
\hline 2058 & CAVITY_3 & RELP_MOD & NONE & CONSTANT & $4.00 E+00$ & $4.00 \mathrm{E}+00$ & $0.00 E+00$ & $4.00 E+00$ & $4.00 E+00$ & $2 / 17 / 96$ & 4B & 31785 & $\mathrm{BF}$ \\
\hline 2051 & CAVITY_3 & COMP_RCK & $\mathrm{Pa}^{\wedge}-1$ & CONSTANT & $0.00 E+00$ & $0.00 E+00$ & $0.00 E+00$ & $0.00 E+00$ & $0.00 E+00$ & $2 / 17 / 96$ & $4 B$ & 31769 & BF \\
\hline
\end{tabular}


24B. Predisposal Cavities

\begin{tabular}{|c|c|c|c|c|c|c|c|c|c|c|c|c|c|}
\hline ID & Material & Parameter & Units & Distribution & Mean & Median & Deviation & Low & High & Entry Date & Impt & WPO & Codes \\
\hline 3103 & CAVITY_4 & PRESSURE & $\mathrm{Pa}$ & CONSTANT & $1.01 E+05$ & $1.01 E+05$ & $0.00 E+00$ & $1.01 E+05$ & $1.01 E+05$ & 2/17/96 & $4 B$ & 31815 & $B F$ \\
\hline 2065 & CAVITY_4 & PRMX_LOG & $\log \left(m^{\wedge} 2\right)$ & CONSTANT & $-1.00 E+01$ & $-1.00 E+01$ & $0.00 E+00$ & $-1.00 E+01$ & $-1.00 E+01$ & $2 / 20196$ & $4 B$ & 31817 & $B F$ \\
\hline 2066 & CAVITY_4 & PRMY_LOG & $\log \left(m^{\wedge} 2\right)$ & CONSTANT & $-1.00 E+01$ & $-1.00 E+01$ & $0.00 E+00$ & $-1.00 E+01$ & $-1.00 E+01$ & $2 / 20 / 96$ & 4B & 31818 & $B F$ \\
\hline 2067 & CAVITY_4 & PRMZ_LOG & $\log \left(m^{\wedge} 2\right)$ & CONSTANT & $-1.00 E+01$ & $-1.00 E+01$ & $0.00 E+00$ & $-1.00 E+01$ & $-1.00 E+01$ & $2 / 20 / 96$ & 48 & 31819 & $B F$ \\
\hline 2069 & CAVITY_ 4 & RELP_MOD & NONE & CONSTANT & $4.00 E+00$ & $4.00 E+00$ & $0.00 E+00$ & $4.00 E+00$ & $4.00 \mathrm{E}+00$ & $2 / 17 / 96$ & 4B & 31821 & $B F$ \\
\hline 2237 & CAVITY_4 & SAT_RBRN & NONE & CONSTANT & $0.00 E+00$ & $0.00 E+00$ & $0.00 E+00$ & $0.00 E+00$ & $0.00 E+00$ & 2/17/96 & 4B & 31823 & $B F$ \\
\hline 2064 & CAVITY_4 & POROSITY & NONE & CONSTANT & $1.00 E+00$ & $1.00 E+00$ & $0.00 E+00$ & $1.00 E+00$ & $1.00 E+00$ & $2 / 17 / 96$ & 4B & 31802 & $\mathrm{BF}$ \\
\hline 3104 & CAVITY_4 & SAT_IBRN & NONE & CONSTANT & $0.00 E+00$ & $0.00 E+00$ & $0.00 E+00$ & $0.00 E+00$ & $0.00 E+00$ & $2 / 17 / 96$ & 4B & 31822 & $\mathrm{BF}$ \\
\hline 2063 & CAVITY_4 & PORE_DIS & NONE & CONSTANT & 7.00 E-01 & 7.00 E-01 & $0.00 E+00$ & 7.00E-01 & 7.00E-01 & $2 / 17 / 96$ & $4 B$ & 31801 & $\mathrm{BF}$ \\
\hline 2628 & CAVITY_4 & PO_MIN & $\mathrm{Pa}$ & CONSTANT & $1.01 E+05$ & $1.01 E+05$ & $0.00 \mathrm{E}+00$ & $1.01 \mathrm{E}+05$ & $1.01 E+05$ & 2/17/96 & 4B & 31803 & $\mathrm{BF}$ \\
\hline 2627 & CAVITY_4 & PCT_EXP & NONE & CONSTANT & $0.00 E+00$ & $0.00 E+00$ & $0.00 E+00$ & $0.00 E+00$ & $0.00 E+00$ & 2/17/96 & 48 & 31798 & $B F$ \\
\hline 2626 & CAVITY_4 & PCT_A & $\mathbf{P a}$ & CONSTANT & $0.00 E+00$ & $0.00 E+00$ & $0.00 E+00$ & $0.00 E+00$ & $0.00 E+00$ & $2 / 17 / 96$ & $4 B$ & 31797 & $B F$ \\
\hline 2236 & CAVITY_4 & PC_MAX & $\mathrm{Pa}$ & CONSTANT & $1.00 E+08$ & $1.00 E+08$ & $0.00 E+00$ & $1.00 E+08$ & $1.00 E+08$ & $2 / 17 / 96$ & 48 & 31800 & $\mathrm{BF}$ \\
\hline 2625 & CAVITY_4 & KPT & NONE & CONSTANT & $0.00 \mathrm{E}+00$ & $0.00 E+00$ & $0.00 E+00$ & $0.00 E+00$ & $0.00 E+00$ & $2 / 17 / 96$ & $4 B$ & 31796 & $\overline{B F}$ \\
\hline 2060 & CAVITY_4 & CAP_MOD & NONE & CONSTANT & $1.00 E+00$ & $1.00 E+00$ & $0.00 E+00$ & $1.00 E+00$ & $1.00 E+00$ & $2 / 17 / 96$ & 48 & 31791 & $\mathrm{BF}$ \\
\hline 2070 & CAVITY_4 & SAT_RGAS & NONE & CONSTANT & $0.00 E+00$ & $0.00 E+00$ & $0.00 E+00$ & $0.00 E+00$ & $0.00 E+00$ & $2 / 17 / 96$ & 4B & 31824 & BF \\
\hline 2062 & CAVITY_4 & COMP_RCK & $\mathrm{Pa}^{\wedge}-1$ & CONSTANT & $0.00 E+00$ & $0.00 E+00$ & $0.00 E+00$ & $0.00 E+00$ & $0.00 E+00$ & $2 / 17 / 96$ & $4 B$ & 31794. & $B F$ \\
\hline
\end{tabular}




\section{Panel Closure}

\begin{tabular}{|c|c|c|c|c|c|c|c|c|c|c|c|c|c|}
\hline ID & Material & Parameter & Units & istribution & Mean & Median & Deviation & Low & High & Entry Date & Impt & WPO & Codes \\
\hline 258 & PAN_SEAL & PRESSURE & $\mathrm{Pa}$ & CONSTANT & $1.01 E+05$ & $1.01 E+05$ & $0.00 E+00$ & $1.01 E+05$ & $1.01 E+05$ & $2 / 6 / 96$ & $4 B$ & 33118 & $B F$ \\
\hline 253 & PAN_SEAL & COMP_RCK & $P a^{n}-1$ & CONSTANT & 2.64E-09 & 2.64E-09 & $0.00 E+00$ & $2.64 E-09$ & 2.64E-09 & $2 / 20 / 96$ & $4 B$ & 32935 & $B F$ \\
\hline 2731 & PAN_SEAL & KPT & NONE & CONSTANT & $0.00 E+00$ & $0.00 \mathrm{E}+00$ & $0.00 E+00$ & $0.00 E+00$ & $0.00 E+00$ & 2/20/96 & $4 B$ & 33089 & $\mathrm{BF}$ \\
\hline 254 & PAN_SEAL & PC_MAX & $\mathrm{Pa}$ & CONSTANT & $1.00 E+08$ & $1.00 E+08$ & $0.00 E+00$ & $1.00 E+08$ & $1.00 E+08$ & 11/2/95 & $4 B$ & 33109 & $B F$ \\
\hline 2732 & PAN_SEAL & PCT_A & $\overline{\mathrm{Pa}}$ & CONSTANT & 5.60E-01 & $5.60 E-01$ & $0.00 E+00$ & $5.60 E-01$ & $5.60 \mathrm{E}-01$ & $2 / 20 / 96$ & $4 B$ & $33107 \mathrm{~A}$ & $B F$ \\
\hline 2733 & PAN_SEAL & PCT_EXP & NONE & CONSTANT & $-3.46 E-01$ & $-3.46 \mathrm{E}-01$ & $0.00 E+00$ & $-3.46 E-01$ & $-3.46 E-01$ & $2 / 20 / 96$ & 4B & 33108 & $B F$ \\
\hline 257 & PAN_SEAL & PO_MIN & $\mathrm{Pa}$ & CONSTANT & 1.01E+05 & $1.01 E+05$ & $0.00 E+00$ & $1.01 E+05$ & $1.01 E+05$ & $2 / 21 / 96$ & 4B & 33115 & $B F$ \\
\hline 252 & PAN_SEAL & CAP_MOD & NONE & CONSTANT & $2.00 E+00$ & $2.00 E+00$ & $0.00 E+00$ & $2.00 E+00$ & $2.00 E+00$ & $2 / 20 / 96$ & 4B & 32933 & $\mathrm{BF}$ \\
\hline 256 & PAN_SEAL & POROSITY & NONE & CONSTANT & $7.50 E-02$ & $7.50 E-02$ & $0.00 E+00$ & 7 & $7.50 \mathrm{E}-02$ & $11 / 2 / 95$ & $4 B$ & 33113 & BF \\
\hline 266 & PAN_SEAL & SAT_RGAS & NONE & CONSTANT & $2.00 E-01$ & 2.00E-01 & $0.00 \mathrm{E}+00$ & $2.00 E-01$ & $2.00 \mathrm{E}-01$ & $11 / 2 / 95$ & $4 B$ & 33141 & $B F$ \\
\hline 259 & PAN_SEAL & PRMX_LOG & $\log \left(m^{\wedge} 2\right)$ & CONSTANT & $-1.50 E+01$ & $-1.50 E+01$ & $0.00 E+00$ & $-1.50 E+01$ & $-1.50 E+01$ & $2 / 20 / 96$ & $4 B$ & 33120 & $B F$ \\
\hline 260 & PAN_SEAL & PRMY_LOG & $\log \left(m^{\wedge} 2\right)$ & CONSTANT & $-1.50 E+01$ & $-1.50 E+01$ & $0.00 E+00$ & $-1.50 E+01$ & $-1.50 E+01$ & $2 / 20 / 96$ & 4B & 33122 & BF \\
\hline 261 & PAN_SEAL & PRMZ_LOG & $\log \left(m^{\wedge} 2\right)$ & CONSTANT & $-1.50 E+01$ & $-1.50 E+01$ & $0.00 E+00$ & $-1.50 E+01$ & $-1.50 E+01$ & $2 / 20 / 96$ & $4 B$ & 33123 & $B F$ \\
\hline 264 & PAN_SEAL & RELP_MOD & NONE & CONSTANT & $4.00 \mathrm{E}+00$ & $4.00 E+00$ & $0.00 E+00$ & $4.00 E+00$ & $4.00 E+00$ & $11 / 2 / 95$ & $4 B$ & 33131 & $\mathrm{BF}$ \\
\hline 2734 & PAN_SEAL & SAT_IBRN & NONE & CONSTANT & $1.00 E+00$ & $1.00 E+00$ & $0.00 E+00$ & $1.00 \mathrm{E}+00$ & $1.00 \mathrm{E}+00$ & $2 / 28 / 96$ & $4 B$ & $33134 \mathrm{~A}$ & $B F$ \\
\hline 265 & PAN_SEAL & SAT_RBRN & NONE & CONSTANT & $2.00 E-01$ & 2.00E-01 & $0.00 E+00$ & $2.00 E-01$ & $2.00 \mathrm{E}-01$ & $11 / 2 / 95$ & $4 B$ & 33139 & $B F$ \\
\hline 255 & PAN_SEAL & PORE_DIS & NONE & CONSTANT & $9.40 E-01$ & $9.40 E-01$ & $0.00 E+00$ & $9.40 \mathrm{E}-01$ & $9.40 E-01$ & $2 / 20 / 96$ & 4B & 33112 & $B F$ \\
\hline
\end{tabular}

\section{Operations Region}

\begin{tabular}{|c|c|c|c|c|c|c|c|c|c|c|c|c|c|}
\hline ID & Material & Parameter & Units & Distribution & Mean & Median & Deviation & Low & High & Entry Date & Impt & WPO & Codes \\
\hline 13 & OPS_AREA & PRESSURE & $\mathrm{Pa}$ & CONSTANT & $1.01 E+05$ & $1.01 E+05$ & $0.00 E+00$ & $1.01 E+05$ & $1.01 E+05$ & $2 / 16 / 96$ & $4 B$ & 32629 & BF \\
\hline 8 & OPS_AREA & COMP_RCK & $\mathrm{Pa}^{\wedge}-1$ & CONSTANT & $0.00 E+00$ & $0.00 E+00$ & $0.00 E+00$ & $0.00 E+00$ & $0.00 E+00$ & $2 / 16 / 96$ & $4 B$ & 32606 & BF \\
\hline 2604 & OPS_AREA & KPT & NONE & |CONSTANT & $0.00 E+00$ & $0.00 E+00$ & $0.00 E+00$ & $0.00 E+00$ & $0.00 E+00$ & $2 / 16 / 96$ & $4 B$ & 32614 & $\mathrm{BF}$ \\
\hline 9 & OPS_AREA & PC_MAX & $\mathrm{Pa}$ & CONSTANT & $1.00 \mathrm{E}+08$ & $1.00 E+08$ & $0.00 E+00$ & $1.00 \mathrm{E}+08$ & $1.00 \mathrm{E}+08$ & $2 / 16 / 96$ & $4 B$ & 32619 & BF \\
\hline 2605 & OPS_AREA & PCT_A & $\mathrm{Pa}$ & CONSTANT & $0.00 E+00$ & $0.00 E+00$ & $0.00 E+00$ & $0.00 E+00$ & $0.00 E+00$ & $2 / 16 / 96$ & $4 B$ & $32616 \mathrm{~A}$ & BF \\
\hline 2606 & OPS_AREA & PCT_EXP & NONE & CONSTANT & $0.00 E+00$ & $0.00 E+00$ & $0.00 E+00$ & $0.00 E+00$ & $0.00 E+00$ & $2 / 16 / 96$ & $4 B$ & 32618 & $B F$ \\
\hline 12 & OPS_AREA & PO_MIN & $\mathrm{Pa}$ & CONSTANT & $1.01 E+05$ & $1.01 E+05$ & $0.00 E+00$ & $1.01 E+05$ & $1.01 E+05$ & $2 / 16 / 96$ & 4B & 31474 & $B F$ \\
\hline 7 & OPS_AREA & CAP_MOD & NONE & CONSTANT & $1.00 E+00$ & $1.00 E+00$ & $0.00 E+00$ & $1.00 E+00$ & $1.00 E+00$ & $2 / 16 / 96$ & $4 B$ & 32604 & $\mathrm{BF}$ \\
\hline 11 & OPS_AREA & POROSITY & NONE & CONSTANT & $1.80 E-01$ & $1.80 E-01$ & $0.00 E+00$ & 1.80E-01 & $1.80 E-01$ & $2 / 16 / 96$ & $4 B$ & 32626 & $B F$ \\
\hline 22 & OPS_AREA & SAT_RGAS & NONE & CONSTANT & $0.00 E+00$ & $0.00 E+00$ & $0.00 E+00$ & $0.00 E+0.0$ & $0.00 E+00$ & $2 / 16 / 96$ & 4B & 32641 & BF \\
\hline 14 & OPS_AREA & PRMX_LOG & $\log \left(m^{\wedge} 2\right)$ & CONSTANT & $-1.10 E+01$ & $-1.10 E+01$ & $0.00 E+00$ & $-1.10 E+01$ & $-1.10 E+01$ & 2/16/96 & $4 B$ & 32630 & BF \\
\hline 15 & OPS_AREA & PRMY_LOG & $\log \left(m^{\wedge} 2\right)$ & CONSTANT & $-1.10 E+01$ & $-1.10 E+01$ & $0.00 E+00$ & $-1.10 E+01$ & $-1.10 E+01$ & $2 / 16 / 96$ & 48 & 32632 & $B F$ \\
\hline 16 & OPS_AREA & PRMZ_LOG & $\log \left(m^{\wedge} 2\right)$ & CONSTANT & $-1.10 E+01$ & $-1.10 E+01$ & $0.00 E+00$ & $-1.10 E+01$ & $-1.10 E+01$ & $2 / 16 / 96$ & 48 & 32633 & $\mathrm{BF}$ \\
\hline 19 & OPS_AREA & RELP_MOD & NONE & CONSTANT & $4.00 E+00$ & $4.00 E+00$ & $0.00 E+00$ & $4.00 E+00$ & $4.00 \mathrm{E}+00$ & $2 / 16 / 96$ & 48 & 32634 & $B F$ \\
\hline 20 & OPS_AREA & SAT_IBRN & NONE & CONSTANT & $0.00 E+00$ & $0.00 E+00$ & $0.00 E+00$ & $0.00 E+00$ & $0.00 E+00$ & $2 / 16 / 96$ & AB & 32637 & BF \\
\hline 21 & OPS_AREA & SAT_RBRN & NONE & CONSTANT & $0.00 E+00$ & $0.00 E+00$ & $0.00 E+00$ & $0.00 E+00$ & $0.00 E+00$ & $2 / 16 / 96$ & 48 & 32639 & $B F$ \\
\hline 10 & OPS_AREA & PORE_DIS & NONE & CONSTANT & 7.00E-01 & $7.00 \mathrm{E}-01$ & $0.00 E+00$ & $7.00 E-01$ & $7.00 E-01$ & 2/16/96 & 4B & 32624 & BF \\
\hline
\end{tabular}




\section{Experimental Area}

\begin{tabular}{|c|c|c|c|c|c|c|c|c|c|c|c|c|c|}
\hline ID & Material & Parameter & Units & Distribution & Mean & Median & Deviation & Low & High & Entry Date & Impt & WPO & Codes \\
\hline 213 & EXP_AREA & PRESSURE & $\mathrm{Pa}$ & CONSTANT & $1.01 E+05$ & $1.01 E+05$ & $0.00 E+00$ & $1.01 E+05$ & $1.01 E+05$ & $11 / 2 / 95$ & $4 B$ & 32948 & BF \\
\hline 208 & EXP_AREA & COMP_RCK & $P a^{\wedge}-1$ & CONSTANT & $0.00 E+00$ & $0.00 E+00$ & $0.00 E+00$ & $0.00 E+00$ & $0.00 \mathrm{E}+00$ & $11 / 2 / 95$ & $4 B$ & 32972 & BF \\
\hline 2711 & EXP_AREA & KPT & NONE & CONSTANT & $0.00 E+00$ & $0.00 E+00$ & $0.00 \mathrm{E}+00$ & $0.00 E+00$ & $0.00 E+00$ & 2/16/96 & $4 B$ & 33009 & BF \\
\hline 209 & EXP_AREA & PC_M & $\mathrm{Pa}$ & CONSTANT & $1.00 \mathrm{E}+08$ & $1.00 E+08$ & $0.00 E+00$ & $1.00 E+08$ & $1.00 E+08$ & $11 / 2 / 95$ & $4 B$ & 33040 & $B F$ \\
\hline 2712 & EXP_AREA & PCT_A & $\mathrm{Pa}$ & CONSTANT & $0.00 E+00$ & $0.00 E+00$ & $0.00 E+00$ & $0.00 E+00$ & $0.00 E+00$ & $11 / 14 / 95$ & $4 B$ & 33037 & $B F$ \\
\hline 2713 & EXP_AREA & PCT_EXP & NONE & CONSTANT & $0.00 E+00$ & $0.00 E+00$ & $0.00 E+00$ & $0.00 \mathrm{E}+00$ & $0.00 E+00$ & $11 / 14 / 95$ & 48 & 33039 & BF \\
\hline 212 & EXP_AREA & PO_MIN & $\mathrm{Pa}$ & CONSTANT & $1.01 E+05$ & $1.01 E+05$ & $0.00 E+00$ & $1.01 E+05$ & $1.01 E+05$ & $2 / 21 / 96$ & $4 B$ & 32946 & $B F$ \\
\hline 207 & EXP_AREA & CAP_MOD & NONE & CONSTANT & $1.00 E+00$ & $1.00 E+00$ & $0.00 E+00$ & 1.00E+OD & $1.00 \mathrm{E}+00$ & $2 / 17 / 96$ & 48 & 32969 & $B F$ \\
\hline 211 & EXP_AREA & POROSITY & NONE & CONSTANT & 1.80E-01 & 1.80E-01 & $0.00 E+00$ & $1.80 \mathrm{E}-01$ & 1.80E-01 & $2 / 17 / 96$ & $4 \mathrm{~B}$ & 32945 & $B F$ \\
\hline 222 & EXP_AREA & SAT_RGAS & NONE & |CONSTANT & $0.00 E+00$ & $0.00 E+00$ & $0.00 E+00$ & $0.00 E+00$ & $0.00 E+00$ & $2 / 17 / 96$ & 48 & 32968 & $\mathrm{BF}$ \\
\hline 214 & EXP_AREA & PRMX_LOG & $\log \left(m^{\wedge} 2\right)$ & CONSTANT & $-1.10 E+01$ & $-1.10 E+01$ & $0.00 E+\infty 0$ & $-1.10 \mathrm{E}+01$ & $-1.10 E+01$ & $2 / 17 / 96$ & $4 B$ & 32951 & $\mathrm{BF}$ \\
\hline 215 & EXP_AREA & PRMY_LOG & $\log \left(m^{\wedge} 2\right)$ & CONSTANT & $-1.10 E+01$ & $-1.10 E+01$ & $0.00 E+00$ & $-1.10 E+01$ & $-1.10 E+01$ & $2 / 17 / 96$ & 48 & 32953 & $B F$ \\
\hline 216 & EXP_AREA & PRMZ_LOG & $\log \left(m^{\wedge} 2\right)$ & CONSTANT & $-1.10 E+01$ & $-1.10 E+01$ & $0.00 E+00$ & $-1.10 E+01$ & $-1.10 E+01$ & $2 / 17 / 96$ & $4 B$ & 32955 & $\overline{B F}$ \\
\hline 219 & EXP_AREA & RELP_MOD & NONE & CONSTANT & $4.00 E+00$ & $4.00 E+00$ & $0.00 E+00$ & $4.00 E+00$ & $4.00 E+00$ & $11 / 2 / 95$ & $4 B$ & 32962 & $\mathrm{BF}$ \\
\hline 220 & EXP_AREA & SAT_IBRN & NONE & CONSTANT & $0.00 E+00$ & $0.00 E+00$ & $0.00 E+00$ & $0.00 E+00$ & $0.00 E+00$ & $2 / 17 / 96$ & 48 & 32965 & $\mathrm{BF}$ \\
\hline 221 & EXP_AREA & SAT_RBRN & NONE & CONSTANT & $0.00 E+00$ & $0.00 E+00$ & $0.00 E+00$ & $0.00 E+00$ & $0.00 E+00$ & $2 / 17 / 96$ & $4 B$ & 32967 & BF \\
\hline 10 & EXP_AREA & PORE_DIS & NONE & CONSTANT & $7.00 E-01$ & 7.00E-01 & $0.00 E+00$ & 7.00E-01 & $7.00 E-01$ & $11 / 2 / 95$ & $4 B$ & 33045 & $B F$ \\
\hline
\end{tabular}

\section{Marker Bed 139}

\begin{tabular}{|c|c|c|c|c|c|c|c|c|c|c|c|c|c|}
\hline ID & Material & Parameter & Units & Distribution & Mean & Median & Deviation & Low & High & Entry Date & Impt & WPO & Codes \\
\hline 2180 & S_MB139 & PF_DELTA & $\mathrm{Pa}$ & CONSTANT & $3.80 E+06$ & $3.80 E+06$ & $0.00 E+00$ & $3.80 E+06$ & $3.80 \mathrm{E}+06$ & $2 / 22 / 96$ & $4 B$ & 34850 & BF \\
\hline 579 & S_MB139 & CAP_MOD & NONE & CONSTANT & $2.00 E+00$ & $2.00 E+00$ & $0.00 E+00$ & $2.00 E+00$ & $2.00 E+00$ & $2 / 11 / 96$ & 1 & 34559 & $\mathrm{BF}$ \\
\hline 580 & MB139 & COMP_RCK & $P a^{A}-1$ & STUDENT & $8.26 E-11$ & $8.26 E-11$ & $1.11 \mathrm{E}-10$ & $1.09 \mathrm{E}-11$ & 2.75E-10 & $2 / 14 / 96$ & 1 & 34574 & $\mathrm{BF}$ \\
\hline 1784 & MB139 & DNSGRAIN & $\mathrm{kg} / \mathrm{m}^{\wedge} 3$ & CONSTANT & $2.75 E+03$ & $2.75 E+03$ & $0.00 E+00$ & $2.75 E+03$ & 2.75E+03 & $2 / 1 / 96$ & 1 & 34579 & BFINU \\
\hline 2177 & S_MB139 & DPHIMAX & NONE & CONSTANT & $3.90 E-02$ & 3.90E-02 & $0.00 E+00$ & $3.90 \mathrm{E}-02$ & 3.90E-02 & $2 / 22 / 96$ & 4B & 34582 & BF \\
\hline 2903 & S_MB139 & EXPKLINK & NONE & CONSTANT & $-3.41 E-01$ & $-3.41 E-01$ & $0.00 E+00$ & $-3.41 E-01$ & $-3.41 E-01$ & $4 / 23 / 96$ & 1 & $34799 A$ & BF \\
\hline 2811 & S_MB139 & IFRX & NONE & CONSTANT & $1.00 E+00$ & $1.00 E+00$ & $0.00 E+00$ & $1.00 E+00$ & $1.00 E+00$ & $1 / 30 / 96$ & 4B & 34818 & $\mathrm{BF}$ \\
\hline 2814 & S_MB139 & IFRY & NONE & CONSTANT & $1.00 E+00$ & $1.00 E+00$ & $0.00 E+00$ & $1.00 E+00$ & $1.00 E+00$ & $1 / 30 / 96$ & 48 & 34819 & $B F$ \\
\hline 2817 & S_MB139 & IFRZ & NONE & CONSTANT & $0.00 \mathrm{E}+00$ & $0.00 E+00$ & $0.00 E+00$ & $0.00 \mathrm{E}+00$ & $0.00 E+00$ & $1 / 31 / 96$ & 4B & 34820 & $\mathrm{BF}$ \\
\hline 2178 & S_MB139 & KMAXLOG & $\log \left(m^{\wedge} 2\right)$ & CONSTANT & $-9.00 E+00$ & $-9.00 \mathrm{E}+00$ & $0.00 \mathrm{E}+00$ & $-9.00 E+00$ & $-9.00 E+00$ & $2 / 22 / 96$ & $4 B$ & 34823 & BF \\
\hline 2788 & S_MB139 & KPT & NONE & CONSTANT & $0.00 E+00$ & $0.00 E+00$ & $0.00 \mathrm{E}+00$ & $0.00 \mathrm{E}+00$ & $0.00 E+00$ & $2 / 20 / 96$ & $4 B$ & 34825 & BF \\
\hline 582 & S_MB139 & PC_MAX & $\mathrm{Pa}$ & CONSTANT & $1.00 E+08$ & $1.00 E+08$ & $0.00 E+00$ & $1.00 E+08$ & $1.00 \mathrm{E}+08$ & $2 / 1 / 96$ & 1 & 34848 & $B F$ \\
\hline 2905 & S_MB139 & BKLINK & $\mathrm{Pa}$ & CONSTANT & 2.71E-01 & 2.71E-01 & $0.00 E+00$ & 2.71E-01 & 2.71E-01 & $4 / 23 / 96$ & --1 & $34557 \mathrm{~A}$ & $\mathrm{BF}$ \\
\hline 2790 & S_MB139 & PCT_EXP & NONE & CONSTANT & $-3.48 E-01$ & $-3.48 E-01$ & $0.00 \mathrm{E}+00$ & $-3.48 E-01$ & $-3.48 E-01$ & $2 / 8 / 96$ & 1 & 34844 & $\mathrm{BF}$ \\
\hline 599 & S_MB139 & SAT_RGAS & NONE & STUDENT & 7.71E-02 & 7.71E-02 & 6.41E-02 & $1.40 \mathrm{E}-02$ & 1.97E-01 & $2 / 22 / 96$ & 1 & 34508 & $\mathrm{BF}$ \\
\hline 586 & S_MB139 & PI_DELTA & $\mathrm{Pa}$ & CONSTANT & $2.00 E+05$ & $2.00 E+05$ & $0.00 E+00$ & $2.00 E+05$ & $2.00 E+05$ & $2 / 22 / 96$ & $4 B$ & 34856 & $B F$ \\
\hline 589 & S_MB139 & PO_MIN & $\mathrm{Pa}$ & CONSTANT & 1.01E+05 & $1.01 E+05$ & $0.00 E+00$ & $1.01 E+05$ & $1.01 E+05$ & $2 / 21 / 96$ & $4 B$ & 34862 & $B F$ \\
\hline 587 & S_MB139 & PORE_DIS & NONE & STUDENT & 6.44E-01 & 6.44E-01 & 1.09E-01 & 4.91E-01 & 8.42E-01 & $2 / 22 / 96$ & 1 & 34859 & $B F$ \\
\hline 588 & S_MB139 & POROSITY & NONE & STUDENT & 1.10E-02 & 1.10E-02 & $3.04 \mathrm{E}-03$ & $9.00 \mathrm{E}-03$ & $1.70 \mathrm{E}-02$ & $2 / 27 / 96$ & 1 & 34860 & $B F$ \\
\hline 590 & S_MB139 & PRESSURE & $\mathrm{Pa}_{-}$ & STUDENT & $1.16 E+07$ & 1.16E+07 & $1.27 E+06$ & $9.38 E+06$ & T.29E +07 & 9/17/96 & 1 & 34863 & $B F$ \\
\hline 591 & S_MB139 & PRMX_LOG & $\log \left(m^{\wedge} 2\right)$ & STUDENT & $-1.89 E+01$ & $-1.89 E+01$ & $1.20 \mathrm{E}+00$ & $-2.10 E+01$ & $-1.71 E+01$ & $2 / 1 / 96$ & 1 & 34865 & $\mathrm{BF}$ \\
\hline 592 & S_MB139 & PRMY_LOG & $\log \left(m^{\wedge} 2\right)$ & STUDENT & $-1.89 E+01$ & $-1.89 E+01$ & $1.20 E+00$ & $-2.10 E+01$ & $-1.71 E+01$ & $2 / 1 / 96$ & 1 & 34866 & $\mathrm{BF}$ \\
\hline 593 & S_MB139 & PRMZ_LOG & $\log \left(m^{\wedge} 2\right)$ & STUDENT & $-1.89 E+01$ & $-1.89 E+01$ & $1.20 E+00$ & $-2.10 E+01$ & $-1.71 E+01$ & $2 / 1 / 96$ & 1 & 34868 & $B F$ \\
\hline 596 & S_MB139 & RELP_MOD & NONE & DELTA & $4.00 E+00$ & $4.00 E+00$ & $0.00 E+00$ & $1.00 \mathrm{E}+00$ & $4.00 E+00$ & $2 / 8 / 96$ & 1 & 34500 & $\mathrm{BF}$ \\
\hline 597 & S_MB139 & SAT_IBRN & NONE & CONSTANT & $1.00 \mathrm{E}+00$ & $1.00 E+00$ & $0.00 E+00$ & $1.00 E+00$ & $1.00 E+00$ & $2 / 11 / 96$ & 1 & 34503 & $\mathrm{BF}$ \\
\hline 598 & S_MB139 & SAT_RBRN & NONE & STUDENT & $8.36 \mathrm{E}-02$ & $8.36 \mathrm{E}-02$ & 5.01E-02 & 7.78E-03 & 1.74E-01 & $11 / 21 / 96$ & 1 & 34506 & BF \\
\hline 2789 & S_MB139 & PCT_A & $\overline{\mathrm{Pa}}$ & CONSTANT & 2.60E-01 & 2.60E-01 & $0.00 E+00$ & 2.60E-01 & 2.60E-01 & $2 / 22 / 96$ & 1 & 34843 & $B F$ \\
\hline
\end{tabular}




\section{Castile Formation}

\begin{tabular}{|c|c|c|c|c|c|c|c|c|c|c|c|c|c|}
\hline ID & Material & Parameter & Units & & Mean & & & Low & High & Entry Date & Impt & WPO & Codes \\
\hline 244 & IMPERM_Z & SAT_RGAS & NONE & CONSTANT & $0.00 E+00$ & $0.00 E+00$ & $0.00 E+00$ & $0.00 E+00$ & $0.00 E+00$ & $11 / 2 / 95$ & 4B & 33230 & $B F$ \\
\hline 243 & IMPERM_Z & BRN & NONE & |CONSTANT & & & $0.00 E+00$ & $0.00 E+00$ & $0.00 E+00$ & $11 / 2 / 95$ & $4 B$ & 33226 & BF \\
\hline 241 & IMPERM_Z & ELP_MOD & NONE & CONSTANT & $4.00 E+00$ & $4.00 E+00$ & $0.00 E+00$ & 4.00E+00 & $4.00 E+00$ & $11 / 2 / 95$ & 48 & 33222 & $B F$ \\
\hline 238 & IMPERM_Z & PRMZ_LOG & $\log \left(m^{\wedge} 2\right)$ & CONSTANT & $-3.50 E+01$ & $-3.50 E+01$ & $0.00 \mathrm{E}+00$ & $-3.50 E+01$ & $-3.50 E+01$ & 2/22/96 & 4B & 33214 & $B F$ \\
\hline 237 & IMPERM_Z & PRMY_LOG & $\log \left(m^{\wedge} 2\right)$ & CONSTANT & $-3.50 E+01$ & $-3.50 E+01$ & $0.00 E+00$ & $-3.50 E+01$ & $-3.50 E+01$ & $2 / 22 / 96$ & $4 B$ & 33209 & BF \\
\hline 236 & IMPERM_Z & PRMX_LOG & $\log \left(m^{\wedge} 2\right)$ & CONSTANT & $-3.50 E+01$ & $-3.50 E+01$ & $0.00 E+00$ & $-3.50 E+01$ & $-3.50 \mathrm{E}+01$ & $2 / 22 / 96$ & $4 B$ & 33205 & $B F$ \\
\hline 233 & IMPERM_Z & POROSITY & NONE & CONSTANT & $5.00 \mathrm{E}-03$ & $5.00 E-03$ & $0.00 E+00$ & $5.00 E-03$ & 5.00E-03 & $11 / 2 / 95$ & AB & 33188 & $B F$ \\
\hline 232 & IMPERM_Z & PORE_DIS & NONE & CONSTANT & 7.00E-01 & $7.00 \mathrm{E}-01$ & $0.00 E+00$ & 7.00E-01 & 7.00E-01 & $2 / 27 / 96$ & 4B & 33185 & BF \\
\hline 234 & IMPERM_Z & PO_MIN & $\mathrm{Pa}$ & CONSTANT & $1.01 E+05$ & $1.01 E+05$ & $0.00 E+00$ & $1.01 E+05$ & $1.01 E+05$ & $2 / 21 / 96$ & 48 & 33191 & BF \\
\hline 2722 & IMPERM_Z & PCT_EXP & NONE & CONSTANT & $0.00 E+00$ & $0.00 E+00$ & $0.00 E+00$ & $0.00 E+00$ & $0.00 E+00$ & 11/14/95 & 4B & 33174 & BF \\
\hline 2721 & IMPERM_Z & PCT_A & $\mathrm{Pa}$ & CONSTANT & $0.00 E+00$ & $0.00 E+00$ & $0.00 E+00$ & $0.00 E+00$ & $0.00 E+00$ & $11 / 14 / 95$ & 4B & 33172 & BF \\
\hline 231 & IMPERM_Z & PC_MAX & a & CONSTANT & $1.00 E+08$ & $1.00 E+08$ & $0.00 \mathrm{E}+00$ & $1.00 E+08$ & $1.00 E+08$ & $11 / 2 / 95$ & 4B & 33177 & BF \\
\hline 2720 & IMPERM_Z & KPT & NONE & CONSTANT & $0.00 E+00$ & $0.00 E+00$ & $0.00 E+00$ & $0.00 E+00$ & $0.00 E+00$ & $2 / 20 / 96$ & $4 B$ & 33119 & BF \\
\hline 230 & IMPERM_Z & COMP_RCK & $\mathrm{Pa}^{\mathrm{A}}-1$ & CONSTANT & $0.00 E+00$ & $0.00 E+00$ & $0.00 E+00$ & $0.00 \mathrm{E}+00$ & $0.00 \mathrm{E}+00$ & $11 / 2 / 95$ & $4 B$ & 33064 & $B F$ \\
\hline 29 & IMPERM_Z & CAP_MOD & NONE & CONSTANT & $1.00 \mathrm{E}+00$ & $1.00 E+00$ & $0.00 E+00$ & $1.00 \mathrm{E}+00$ & $1.00 E+00$ & $11 / 2 / 95$ & $4 B$ & 33059 & BF \\
\hline
\end{tabular}

\section{A. Castile Reservoir}

\begin{tabular}{|c|c|c|c|c|c|c|c|c|c|c|c|c|c|}
\hline ID & aterial & & & & lean & ledian & & Low & High & Entry Date & Impt & WPO & odes \\
\hline 4 & ER & & ONE & & 8.70E-03 & 8.70E-03 & 5.70E-03 & 2.00E-03 & $1.60 \mathrm{E}-02$ & 1/31/96 & 1 & 31610 & BF \\
\hline & & & & $\mathrm{CON}$ & $0 E+00$ & 2.00E+00 & $.00 E+00$ & $10 E+00$ & $2.00 E+00$ & & 4B & & $\mathrm{BF}$ \\
\hline 61 & & & $g\left(P a^{\wedge}-1\right)$ & TRIA & $-9.80 E+00$ & $\mid-1.00 E+01$ & $6.80 E-01$ & $-1.13 E+01$ & $-8.00 E+00$ & & 1 & & BF \\
\hline 194 & ER & $\overline{0}$ & DNE & DELTA & $1.60 E+01$ & $1.60 E+01$ & $.00 E+00$ & $1.00 E+00$ & & $3 / 21 / 96$ & 4B & $36658 \mathrm{~A}$ & GF/BF \\
\hline 2608 & ASTILER & $\overline{\mathrm{PT}}$ & ONE & CON & $00 E+00$ & $0.00 E+00$ & $0.00 E+00$ & $0.00 E+00$ & $.00 E+00$ & $2 / 20 / 96$ & 4B & 31583 & $\mathrm{BF}$ \\
\hline 62 & CASTILER & & $\mathrm{Pa}$ & $\mathrm{CON}$ & $1.00 E+08$ & $1.00 E+08$ & $0.00 E+00$ & $1.00 E+08$ & $1.00 \mathrm{E}+08$ & $11 / 2 / 95$ & $4 B$ & 1607 & BF \\
\hline 2609 & & & $\overline{\mathrm{Pa}}$ & $\mathrm{CON}$ & $5.60 E-01$ & 5.60E-01 & $0.00 E+00$ & $5.60 \mathrm{E}-01$ & 5.60E-01 & $11 / 14 / 95$ & 4B & 31605 & $B F$ \\
\hline 2610 & & & ONE & CONSTANT & $-3.46 E-01$ & $-3.46 E-01$ & $0.00 E+00$ & $-3.46 \mathrm{E}-01$ & $-3.46 E-01$ & $11 / 14 / 95$ & $4 B$ & 31606 & $B F$ \\
\hline & ILER & & & CUMULATIVE & $4.00 E-01$ & 4.01E-01 & $6.30 E-02$ & $2.45 E-01$ & 5.68E-01 & $1 / 9 / 97$ & $4 B$ & $31554^{*}$ & BF \\
\hline & ER & & & & & $7.00 E-01$ & $0.00 E+00$ & $7.00 E-01$ & 7.00E-01 & $11 / 2 / 95$ & $4 B$ & 31609 & BF \\
\hline 2918 & ASTILER & ME & 3 & CONSTANT & $4.00 E+06$ & & & & $4.00 E+06$ & $3 / 20 / 96$ & 1 & $31625 \mathrm{~A}$ & $B F$ \\
\hline & CASTILER & & & ULAR & $1.36 \mathrm{E}+07$ & 1.27E+07 & $1.25 \mathrm{E}+06$ & $1.11 E+07$ & 1.70E+07 & $3 / 21 / 96$ & 1 & $31612 \mathrm{~A}$ & BF \\
\hline & $\overline{\text { CAS }}$ & & $\log \left(m^{n} 2\right)$ & TRIANGULAR & $-1.21 E+01$ & $-1.18 E+01$ & $1.01 E+00$ & $-1.47 \mathrm{E}+01$ & $-9.80 E+00$ & $2 / 12 / 96$ & 1 & 31613 & $B F$ \\
\hline 20 & CASTILER & & & TRIANGULAR & $-1.21 E+01$ & $-1.18 E+01$ & $1.01 \mathrm{E}+00$ & $-1.47 E+01$ & $-9.80 E+00$ & $2 / 12 / 96$ & 1 & 1614 & $\overline{B F}$ \\
\hline 60 & CASTILER & PRMZ_LOG & $\log \left(m^{\wedge} 2\right)$ & TRIANGULAR & $-1.21 E+01$ & $-1.18 E+01$ & $1.01 E+\infty 0$ & $-1.47 E+01$ & $-9.80 E+00$ & 2/12/96 & $=1$ & (2) & BF \\
\hline 72 & CASTILER & RELP_MUD & NONE & CONSTANT & $4.00 E+00$ & $4.00 E+00$ & $0.00 E+00$ & $4.00 E+00$ & $4.00 E+00$ & $11 / 2 / 95$ & 48 & 31619 & BF \\
\hline 73 & ILER & & ONE & CONSTANT & $1.00 E+00$ & $1.00 E+00$ & $0.00 E+00$ & $1.00 E+00$ & $1.00 E+00$ & $11 / 2 / 95$ & $4 B$ & 31620 & BF \\
\hline 74 & CASTILER & & NONE & CONSTANT & 2.00E-01 & $2.00 E-01$ & $0.00 E+00$ & $2.00 E-01$ & $2.00 \mathrm{E}-01$ & $11 / 2 / 95$ & $4 B$ & 31621 & $\mathrm{BF}$ \\
\hline 75 & CASTILER & & NONE & CONSTANT & 2.00E-01 & 2.00E-01 & $0.00 E+00$ & $2.00 E-01$ & 2.00E-01 & $11 / 2 / 95$ & $4 B$ & 31622 & $\mathrm{BF}$ \\
\hline 65 & CASTILER & PO_MIN & $\mathrm{Pa}$ & CONSTANT & $1.01 E+05$ & $1.01 E+05$ & $0.00 E+00$ & $1.01 E+05$ & $1.01 E+05$ & $2 / 21 / 96$ & $4 B$ & 31611 & $\mathrm{BF}$ \\
\hline
\end{tabular}

\section{B. Castile Brine}

\begin{tabular}{|c|c|c|c|c|c|c|c|c|c|c|c|c|c|}
\hline ID & Material & Parameter & Units & Distribution & Mean & Median & Deviation & Low & High & Entry Date & Impt & WPO & Codes \\
\hline 44 & BRINECST & DNSFLUID & $\mathrm{kg} / \mathrm{m}^{\wedge} 3$ & CONSTANT & $1.22 E+03$ & $1.22 E+03$ & $0.00 E+00$ & $1.22 E+03$ & $1.22 E+03$ & $1 / 30 / 97$ & 5 & $31538^{\circ}$ & $B F$ \\
\hline 43 & BRINECST & COMPRES & $P a^{\wedge}-1$ & CONSTANT & $9.00 \mathrm{E}-10$ & $9.00 E-10$ & $0.00 E+00$ & 9.00E-10 & $9.00 \mathrm{E}-10$ & $1 / 30 / 97$ & 5 & $31537^{\circ}$ & $B F$ \\
\hline
\end{tabular}


31. Global

\begin{tabular}{|c|c|c|c|c|c|c|c|c|c|c|c|c|c|}
\hline ID & Material & Parameter & Units & Distribution & Mean & Median & Deviation & Low & High & Entry Date & Impt & WPO & Codes \\
\hline 225 & GLOBAL & TRANSIDX & NONE & UNIFORM & $5.00 E-01$ & $5.00 \mathrm{E}-01$ & 2.89E-01 & $0.00 E+00$ & $1.00 \mathrm{E}+00$ & $11 / 2 / 95$ & 4B & 33055 & BF \\
\hline 3498 & GLOBAL & TPICM & yr & CONSTANT & $6.00 E+02$ & $6.00 E+02$ & $0.00 E+00$ & $6.00 E+02$ & $6.00 E+02$ & $6 / 27 / 96$ & 4B & 38737 & GF \\
\hline 3499 & GLOBAL & TPICD & $y r$ & CONSTANT & $6.00 E+02$ & $6.00 \mathrm{E}+02$ & $0.00 E+00$ & $6.00 E+02$ & $6.00 E+02$ & $6 / 27 / 96$ & 1 & 38738 & GF \\
\hline 3491 & GLOBAL & TA & $y r$ & CONSTANT & $1.00 \mathrm{E}+02$ & $1.00 E+02$ & $0.00 E+00$ & $1.00 E+02$ & $1.00 \mathrm{E}+02$ & $6 / 26 / 96$ & $4 B$ & 38730 & GF \\
\hline 3495 & GLOBAL & PLGPAT & NONE & DELTA & $0.00 \mathrm{E}+00$ & $0.00 \mathrm{E}+00$ & $0.00 E+00$ & $1.00 \mathrm{E}+00$ & $3.00 E+00$ & $6 / 26 / 96$ & 1 & 38734 & GF \\
\hline 3493 & GLOBAL & PBRINE & NONE & CONSTANT & $8.00 E-02$ & $8.00 \mathrm{E}-02$ & $0.00 E+00$ & $8.00 \mathrm{E}-02$ & $8.00 E-02$ & $6 / 26 / 96$ & 1 & 38732 & GF \\
\hline 3417 & GLOBAL & OXSTAT & NONE & UNIFORM & $5.00 \mathrm{E}-01$ & $5.00 E-01$ & 2.89E-01 & $0.00 E+00$ & $1.00 E+00$ & $5 / 1 / 96$ & 1 & 37663 & $N U$ \\
\hline 3497 & GLOBAL & MINERT & $y r^{n}-1$ & CONSTANT & $1.00 \mathrm{E}-04$ & 1.00E-04 & $0.00 E+00$ & 1.00E-04 & 1.00E-04 & $6 / 27 / 96$ & 1 & 38736 & GF \\
\hline 3494 & GLOBAL & LAMBDAD & $\left(k m^{\wedge}-2\right) y r^{\wedge}-1$ & CONSTANT & 4.68E-03 & $4.68 \mathrm{E}-03$ & $0.00 E+00$ & 4.68E-03 & 4.68E-03 & $6 / 26 / 96$ & 1 & 38733 & GF \\
\hline 3500 & GLOBAL & FPICM & NONE & CONSTANT & 1.00E-02 & 1.00E-02 & $0.00 E+00$ & 1.00E-02 & 1.00E-02 & $6 / 27 / 96$ & $4 B$ & 38742 & GF \\
\hline 3501 & GLOBAL & FPICD & NONE & CONSTANT & 1.00E-02 & $1.00 \mathrm{E}-02$ & $0.00 E+00$ & 1.00E-02 & 1.00E-02 & $6 / 27 / 96$ & 4B & 38743 & GF \\
\hline 223 & GLOBAL & CLIMTIDX & NONE & CUMULATIVE & $1.31 E+00$ & 1.17E+00 & 3.48E-01 & $1.00 E+00$ & $2.25 E+00$ & $5 / 1 / 96$ & 1 & 33031 & FL. \\
\hline
\end{tabular}


32. Reference Constants

\begin{tabular}{|c|c|c|c|c|c|c|c|c|c|c|c|c|c|}
\hline ID & Material & Parameter & Units & Distribution & Mean & Median & Deviation & Low & High & Entry Date & Impt & WPO & Codes \\
\hline 2877 & REFCON & MW_CACO3 & $\mathrm{kg} / \mathrm{mole}$ & CONSTANT & $1.00 E-01$ & $1.00 E-01$ & $0.00 E+00$ & $1.00 \mathrm{E}-01$ & $1.00 E-01$ & $12 / 4 / 96$ & 5 & $32390^{\circ}$ & $\mid \begin{array}{c}\text { TP/BFI } \\
\text { FUNUI CS }\end{array}$ \\
\hline 2883 & REFCON & F3M3 & $m^{\wedge} 3 / f^{\wedge} 3$ & CONSTANT & 2.83E-02 & 2.83E-02 & $0.00 E+00$ & 2.83E-02 & 2.83E-02 & $2 / 6 / 96$ & 3 & 32384 & $\begin{array}{c}\text { TPICSI } \\
\text { FL/NU/BF }\end{array}$ \\
\hline 2881 & REFCON & FTM & $\mathrm{m} / \mathrm{ft}$ & CONSTANT & 3.05E-01 & $3.05 E-01$ & $0.00 E+00$ & 3.05E-01 & 3.05E-01 & $2 / 6 / 96$ & 3 & 32385 & $\begin{array}{c}\text { TPIFU } \\
\text { NU/CS/BF }\end{array}$ \\
\hline 3492 & REFCON & FWW & NONE & CONSTANT & $3.86 \mathrm{E}-01$ & 3.86E-01 & $0.00 \mathrm{E}+00$ & $3.86 E-01$ & 3.86E-01 & $6 / 28 / 96$ & AB & 38731 & GF \\
\hline 2889 & REFCON & GRAVACC & $\mathrm{m} / \mathrm{s}^{\wedge} 2$ & CONSTANT & $9.81 E+00$ & $9.81 E+00$ & $0.00 E+00$ & $9.81 E+00$ & $9.81 E+00$ & $2 / 6 / 96$ & 3 & 32386 & $\begin{array}{c}\text { NU/BFI } \\
\text { TP/FU CS }\end{array}$ \\
\hline 2884 & REFCON & GT13 & in^3/gal & CONSTANT & 2.31E+02 & $2.31 E+02$ & $0.00 E+00$ & $2.31 E+02$ & $2.31 E+02$ & $2 / 6 / 96$ & 3 & 32387 & $\begin{array}{c}\text { FUTPI } \\
\text { BF/CS/NU }\end{array}$ \\
\hline 3502 & REFCON & HRH & $m$ & CONSTANT & $5.09 E-01$ & 5.09E-01 & $0.00 E+00$ & 5.09E-01 & 5.09E-01 & $6 / 27 / 96$ & 4B & 38744 & GF \\
\hline 3503 & REFCON & ABERM & $m^{\wedge} 2$ & CONSTANT & $6.29 E+05$ & $6.29 E+05$ & $0.00 E+00$ & 6.29E+05 & $6.29 E+05$ & $6 / 27 / 96$ & 4B & 38745 & GF \\
\hline 2885 & REFCON & LBKG & $\mathrm{kg} / \mathrm{b}$ & CONSTANT & 4.54E-01 & 4.54E-01 & $0.00 E+00$ & 4.54E-01 & 4.54E-01 & $2 / 6 / 96$ & 3 & 32389 & $\begin{array}{c}\text { FUTPI } \\
\text { NU/CS/ BF }\end{array}$ \\
\hline 2882 & REFCON & DARM2 & $m^{\wedge} 2 / d a r c y$ & CONSTANT & $9.87 E-13$ & 9.87E-13 & $0.00 E+00$ & $9.87 E-13$ & $9.87 E-13$ & $2 / 6 / 96$ & 3 & 32379 & $\mid \begin{array}{c}\text { BFIFU } \\
\text { TPICSINU }\end{array}$ \\
\hline 2875 & REFCON & MW_CAO & $\mathrm{kg} / \mathrm{mole}$ & CONSTANT & 5.61E-02 & $5.61 E-02$ & $0.00 E+00$ & 5.61E-02 & $5.61 E-02$ & $12 / 4 / 96$ & 5 & $32391^{*}$ & $\begin{array}{c}\text { TP/CS } \\
\text { NUUBF/ FL }\end{array}$ \\
\hline 2876 & REFCON & MW_CAOH2 & $\mathrm{kg} / \mathrm{mole}$ & CONSTANT & 7.41E-02 & $7.41 E-02$ & $0.00 E+00$ & 7.41E-02 & 7.41E-02 & $12 / 4 / 96$ & 5 & $32392^{*}$ & $\begin{array}{c}\text { FL/TPI } \\
\text { BF/NU/CS }\end{array}$ \\
\hline 2866 & REFCON & $\mathrm{MW} \_\mathrm{CH} 2 \mathrm{O}$ & $\mathrm{kg} / \mathrm{mole}$ & CONSTANT & $3.00 E-02$ & $3.00 E-02$ & $0.00 E+00$ & $3.00 E-02$ & $3.00 E-02$ & $2 / 13 / 96$ & 3 & 32393 & $\begin{array}{c}\text { NU/CSI } \\
\text { TP/BF/FL }\end{array}$ \\
\hline 2860 & REFCON & MW_CH4 & $\mathrm{kg} / \mathrm{mole}$ & CONSTANT & $1.60 E-02$ & 1.60E-02 & $0.00 E+00$ & $1.60 \mathrm{E}-02$ & $1.60 E-02$ & $12 / 4 / 96$ & 5 & $32394^{\circ}$ & $\begin{array}{c}\mathrm{CS} / \mathrm{FU} \\
\mathrm{BF} / \mathrm{NU} / \mathrm{TP}\end{array}$ \\
\hline 2859 & REFCON & $M W \_C O 2$ & $\mathrm{~kg} / \mathrm{mole}$ & CONSTANT & 4.41E-02 & 4.41E-02 & $0.00 E+00$ & 4.41E-02 & 4.41E-02 & $12 / 4 / 96$ & 5 & $32395^{\circ}$ & $\begin{array}{c}\text { FLIBFI } \\
\text { TP/NU CS }\end{array}$ \\
\hline 2865 & REFCON & MW_FE & $\mathrm{kg} / \mathrm{mole}$ & CONSTANT & 5.58E-02 & 5.58E-02 & $0.00 E+00$ & 5.58E-02 & 5.58E-02 & $2 / 13 / 96$ & 3 & 32396 & $\begin{array}{c}\text { CS/TPI } \\
\text { NU/FU BF }\end{array}$ \\
\hline 2873 & REFCON & MW_FE3O4 & $\mathrm{kg} / \mathrm{mole}$ & CONSTANT & 2.32E-01 & 2.32E-01 & $0.00 E+00$ & 2.32E-01 & 2.32E-01 & $12 / 4 / 96$ & 5 & $32397^{*}$ & $\begin{array}{c}\mathrm{CS} / \mathrm{FU} \\
\mathrm{TP} / \mathrm{NU} / \mathrm{BF}\end{array}$ \\
\hline 2886 & REFCON & KGLB & $\mathrm{lb} / \mathrm{kg}$ & CONSTANT & 2.20E+00 & $2.20 E+00$ & $0.00 E+00$ & $2.20 E+00$ & $2.20 E+00$ & $2 / 6 / 96$ & 3 & 32388 & $\begin{array}{c}\text { TPICSI } \\
\text { BF/NU/FL }\end{array}$ \\
\hline 3496 & REFCON & AREA_RH & $m^{\wedge} 2$ & CONSTANT & $1.58 E+04$ & $1.58 E+04$ & $0.00 E+00$ & $1.58 E+04$ & $1.58 \mathrm{E}+04$ & $6 / 27 / 96$ & 4B & 38735 & GF \\
\hline 2833 & REFCON & ACF_CH4 & NONE & CONSTANT & 1.00E-02 & 1.00E-02 & $0.00 E+00$ & 1.00E-02 & 1.00E-02 & $2 / 12 / 96$ & 3 & 32349 & $\begin{array}{c}\text { CS/TPI } \\
\text { BFIFU NU }\end{array}$ \\
\hline 2832 & REFCON & ACF_CO2 & NONE & CONSTANT & 2.31E-01 & 2.31E-01 & $0.00 E+00$ & 2.31E-01 & 2.31E-01 & $2 / 12 / 96$ & 3 & 32350 & $\begin{array}{c}\text { FL/BFI } \\
\text { NU/TP/ CS }\end{array}$ \\
\hline 2831 & REFCON & ACF_H2 & NONE & CONSTANT & $0.00 E+00$ & $0.00 E+00$ & $0.00 E+00$ & $0.00 E+00$ & $0.00 E+00$ & $2 / 12 / 96$ & 3 & 32351 & $\begin{array}{c}\text { TPICSI } \\
\text { FUBFINU }\end{array}$ \\
\hline 2835 & REFCON & ACF_H2S & NONE & CONSTANT & $1.00 E-01$ & $1.00 \mathrm{E}-01$ & $0.00 E+00$ & $1.00 E-01$ & $1.00 E-01$ & $2 / 12 / 96$ & 3 & 32352 & $\begin{array}{c}\text { BF } \pi P I \\
\text { FUCSINU }\end{array}$ \\
\hline 2834 & REFCON & ACF_N2 & NONE & CONSTANT & $4.50 \mathrm{E}-02$ & 4.50E-02 & $0.00 E+00$ & 4.50E-02 & 4.50E-02 & $2 / 12 / 96$ & 3 & 32354 & $\begin{array}{c}\text { NU/BFI } \\
\text { FLTP/ CS }\end{array}$ \\
\hline 2836 & REFCON & ACF_02 & NONE & CONSTANT & 1.90E-02 & 1.90E-02 & $0.00 E+00$ & $1.90 E-02$ & 1.90E-02 & $2 / 12 / 96$ & 3 & 32355 & $\begin{array}{c}\text { CS/FU } \\
\text { NU/TPI BF }\end{array}$ \\
\hline 3113 & REFCON & ACTCONST & $\mathrm{kg} / \mathrm{Ci}$ & CONSTANT & $1.13 E+13$ & $1.13 E+13$ & $0.00 E+00$ & $1.13 E+13$ & 1.13E+13 & $12 / 4 / 96$ & 5 & $32356^{*}$ & CS/BF \\
\hline 3132 & REFCON & DRROOM & NONE & CONSTANT & $6.80 E+03$ & $6.80 E+03$ & $0.00 E+00$ & $6.80 E+03$ & $6.80 E+03$ & $2 / 26 / 96$ & 3 & 32372 & BF \\
\hline
\end{tabular}


32. Reference Constants

\begin{tabular}{|c|c|c|c|c|c|c|c|c|c|c|c|c|c|}
\hline ID & Material & Parameter & Units & Distribution & Mean & Median & Deviation & Low & High & Entry Date & Impt & WPO & Codes \\
\hline 3489 & REFCON & AREA_CH & $m^{\wedge} 2$ & CONSTANT & 1.12E+05 & $1.12 E+05$ & $0.00 E+00$ & $1.12 E+05$ & $1.12 E+05$ & $6 / 26 / 96$ & $4 B$ & 38728 & GF \\
\hline 2887 & REFCON & DAYSEC & s/day & CONSTANT & 8.64E+0.4 & $8.64 E+04$ & $0.00 E+00$ & $8.64 E+04$ & $8.64 E+04$ & $2 / 6 / 96$ & 3 & 32383 & $\begin{array}{c}\text { FLTPI } \\
\text { BF/CS/NU }\end{array}$ \\
\hline 3488 & REFCON & AREA_ZRO & $m^{\wedge} 2$ & CONSTANT & $4.13 E+03$ & $4.13 E+03$ & $0.00 E+00$ & 4.13E+03 & $4.13 E+03$ & $6 / 26 / 96$ & $4 B$ & 38727 & GF \\
\hline 3106 & REFCON & ASDRUM & $m^{\wedge} 2$ & CONSTANT & $6.00 \mathrm{E}+00$ & $6.00 E+00$ & $0.00 E+00$ & $6.00 E+00$ & $6.00 \mathrm{E}+00$ & $2 / 17 / 96$ & 4B & 36370 & BF \\
\hline 2890 & REFCON & ATMPA & Palatm & CONSTANT & $1.01 E+05$ & $1.01 E+05$ & $0.00 E+00$ & $1.01 E+05$ & $1.01 E+05$ & $2 / 6 / 96$ & 3 & 32358 & $\begin{array}{c}\text { TP/BFI } \\
\text { FLNU/CS }\end{array}$ \\
\hline 3109 & REFCON & AVOGADRO & $1 / \mathrm{mol}$ & CONSTANT & $6.02 E+23$ & $6.02 E+23$ & $0.00 E+00$ & $6.02 E+23$ & $6.02 E+23$ & $2 / 17 / 96$ & 3 & 32359 & BFINU \\
\hline 2879 & REFCON & BBLG & $\mathrm{ga} / \mathrm{bbl}$ & CONSTANT & $4.20 E+01$ & $4.20 E+01$ & $0.00 E+00$ & $4.20 \mathrm{E}+01$ & 4.20E+01 & $2 / 5 / 96$ & 3 & 32360 & $\mathrm{BF}$ \\
\hline 3111 & REFCON & СITOBQ & $\mathrm{Bq} / \mathrm{Ci}$ & CONSTANT & $3.70 E+10$ & 3.70E+10 & $0.00 E+00$ & $3.70 \mathrm{E}+10$ & $3.70 E+10$ & $2 / 17 / 96$ & 3 & 32377 & NU/BF \\
\hline 2872 & REFCON & MW_FEOOH & $\mathrm{kg} / \mathrm{mole}$ & CONSTANT & 8.89E-02 & 8.89E-02 & $0.00 E+00$ & 8.89E-02 & 8.89E-02 & $12 / 4 / 96$ & 5 & $32400^{*}$ & $\begin{array}{c}\text { FUTP/ } \\
\text { NU/BF/CS }\end{array}$ \\
\hline 2897 & REFCON & $\mathrm{AL2}$ & NONE & CONSTANT & 6.93E-01 & 6.93E-01 & $0.00 E+00$ & $6.93 E-01$ & $6.93 E-01$ & $2 / 6 / 96$ & 3 & 32357 & $\mathrm{BF}$ \\
\hline 2829 & REFCON & TC_H2S & $K$ & CONSTANT & $3.74 E+02$ & $3.74 E+02$ & $0.00 E+00$ & $3.74 E+02$ & $3.74 E+02$ & $2 / 5 / 96$ & 3 & 32430 & $B F$ \\
\hline 2870 & REFCON & MW_FECO3 & $\mathrm{kg} /$ mole & CONSTANT & 1.16E-01 & 1.16E-01 & $0.00 E+00$ & 1.16E-01 & 1.16E-01 & $12 / 4 / 96$ & 5 & $32398^{\circ}$ & $\begin{array}{c}\text { TPNUI } \\
\text { CSIFU BF }\end{array}$ \\
\hline 2896 & REFCON & $\mathrm{PI}$ & NONE & CONSTANT & $3.14 E+00$ & $3.14 E+00$ & $0.00 E+00$ & $3.14 E+00$ & $3.14 \mathrm{E}+00$ & $2 / 6 / 95$ & 3 & 32422 & $B F$ \\
\hline 2892 & REFCON & PSIPA & $P a^{\star} \operatorname{in}^{\wedge} 2 / b$ & CONSTANT & $6.89 E+03$ & $6.89 E+03$ & $0.00 E+00$ & $6.89 E+03$ & $6.89 E+03$ & $2 / 6 / 96$ & 3 & 32423 & $\begin{array}{c}\text { FL/BFI } \\
\text { TP/CS/NU }\end{array}$ \\
\hline 2893 & REFCON & $R$ & $\mathrm{~J} / \mathrm{mol}^{*} \mathrm{~K}$ & CONSTANT & $8.31 E+00$ & $8.31 E+00$ & $0.00 E+00$ & 8.31E+00 & $8.31 E+00$ & $2 / 6 / 96$ & 3 & 32424 & $\begin{array}{c}\text { NU/CSI } \\
\text { BF/FL TP }\end{array}$ \\
\hline 2891 & REFCON & RTK & Krankine & CONSTANT & 5.56E-01 & 5.56E-01 & $0.00 E+00$ & 5.56E-01 & 5.56E-01 & $2 / 6 / 96$ & 3 & 32425 & $\begin{array}{c}\text { TP/NUI } \\
\text { FL/BF/CS }\end{array}$ \\
\hline 3112 & REFCON & SECYR & yris & CONSTANT & 3.17E-08 & 3.17E-08 & $0.00 E+00$ & 3.17E-08 & $3.17 E-08$ & 2/17/96 & 3 & 32426 & $\mathrm{BF}$ \\
\hline 2827 & REFCON & TC_CH4 & $\mathrm{K}$ & CONSTANT & $1.91 E+02$ & $1.91 E+02$ & $0.00 E+00$ & $1.91 E+02$ & $1.91 E+02$ & $2 / 5 / 96$ & 3 & 32427 & $\mathrm{BF}$ \\
\hline 2840 & REFCON & PC_N2 & $\mathrm{Pa}$ & CONSTANT & $3.39 E+06$ & $3.39 E+06$ & $0.00 E+00$ & $3.39 E+06$ & $3.39 E+06$ & $2 / 5 / 96$ & 3 & 32419 & $\mathrm{BF}$ \\
\hline 2825 & REFCON & TC_H2 & $K$ & CONSTANT & $4.36 E+01$ & $4.36 E+01$ & $0.00 E+00$ & $4.36 E+01$ & $4.36 E+01$ & $2 / 5 / 96$ & 3 & 32429 & $B F$ \\
\hline 2841 & REFCON & PC_H2S & $\mathrm{Pa}$ & CONSTANT & $9.01 E+06$ & $9.01 E+06$ & $0.00 E+00$ & $9.01 E+06$ & $9.01 E+06$ & $2 / 5 / 96$ & 3 & 32418 & $\mathrm{BF}$ \\
\hline 2828 & REFCON & TC_N2 & $\mathrm{K}$ & CONSTANT & 1.26E+02 & $1.26 E+02$ & $0.00 E+00$ & $1.26 \mathrm{E}+02$ & $1.26 E+02$ & $2 / 5 / 96$ & 3 & 32431 & $\mathrm{BF}$ \\
\hline 2830 & REFCON & TC_O2 & $K$ & CONSTANT & $1.55 E+02$ & 1.55E+02 & $0.00 E+00$ & $1.55 E+02$ & $1.55 E+02$ & $2 / 5 / 96$ & 3 & 32432 & $\mathrm{BF}$ \\
\hline 3490 & REFCON & VOLWP & $m^{\wedge} 3$ & CONSTANT & 4.36E+05 & 4.36E+05 & $0.00 E+00$ & 4.36E+05 & 4.36E+05 & $6 / 26 / 96$ & $4 B$ & 38729 & GF \\
\hline 3107 & REFCON & VPANLEX & $m^{\wedge} 3$ & CONSTANT & $4.61 E+04$ & $4.61 E+04$ & $0.00 E+00$ & $4.61 E+04$ & $4.61 E+04$ & $2 / 17 / 96$ & $4 B$ & 33273 & BF \\
\hline 3108 & REFCON & VREPOS & $m^{\wedge} 3$ & CONSTANT & $4.36 E+05$ & $4.36 E+05$ & $0.00 E+00$ & $4.36 \mathrm{E}+05$ & $4.36 E+05$ & $2 / 17 / 96$ & 48 & 33276 & BF \\
\hline 3105 & REFCON & VROOM & $m^{\wedge} 3$ & CONSTANT & $3.64 E+03$ & $3.64 E+03$ & $0.00 E+00$ & $3.64 E+03$ & $3.64 E+03$ & $2 / 17 / 96$ & 4B- & 33280 & $B F$ \\
\hline 2888 & REFCON & YRSEC & s/yr & CONSTANT & $3.16 E+07$ & $3.16 E+07$ & $0.00 E+00$ & $3.16 E+07$ & $3.16 E+07$ & $2 / 6 / 96$ & 3 & 32434 & $\begin{array}{c}\text { BF/TPI } \\
\text { FLCS/NU }\end{array}$ \\
\hline 2826 & REFCON & TC_CO2 & $K$ & CONSTANT & $3.04 E+02$ & $3.04 E+02$ & $0.00 E+00$ & $3.04 E+02$ & $3.04 E+02$ & $2 / 5 / 96$ & 3 & 32428 & $B F$ \\
\hline 2878 & REFCON & MW_NACL & $\mathrm{kg} / \mathrm{mole}$ & CONSTANT & $5.84 E-02$ & $5.84 \mathrm{E}-02$ & $0.00 E+00$ & 5.84E-02 & 5.84E-02 & $12 / 4 / 96$ & 5 & $32410^{\circ}$ & $\begin{array}{c}\text { FUCSI } \\
\text { BF/TP/NU }\end{array}$ \\
\hline 3110 & REFCON & ZCINK & $K$ & CONSTANT & $2.73 E+02$ & $2.73 E+02$ & $0.00 E+00$ & $2.73 E+02$ & $2.73 E+02$ & $2 / 17 / 96$ & 3 & 32435 & NU/BF \\
\hline 2874 & REFCON & MW_FES & $\mathrm{kg} / \mathrm{mole}$ & CONSTANT & 8.79E-02 & 8.79E-02 & $0.00 E+00$ & $8.79 E-02$ & $8.79 E-02$ & $12 / 4 / 96$ & 5 & $32401^{\circ}$ & $\begin{array}{c}\text { NU/FL } \\
\text { CS/BF/TP }\end{array}$ \\
\hline 2869 & REFCON & MW_FES2 & $\mathrm{kg} / \mathrm{mole}$ & CONSTANT & 1.20E-01 & 1.20E-01 & $0.00 E+00$ & $1.20 E-01$ & 1.20E-01 & $12 / 4 / 96$ & 5 & $32402^{\circ}$ & $\begin{array}{c}\mathrm{NU} / \mathrm{CS} / \\
\mathrm{BF} / \mathrm{TP} / \mathrm{FL}\end{array}$ \\
\hline 2858 & REFCON & MW_H2 & $\mathrm{kg} / \mathrm{mole}$ & CONSTANT & 2.02E-03 & 2.02E-03 & $0.00 E+00$ & 2.02E-03 & 2.02E-03 & $2 / 13 / 96$ & 3 & 32403 & $\begin{array}{c}\text { BF/CSI } \\
\text { FUTP/NU }\end{array}$ \\
\hline 2864 & REFCON & MW_H2O & $\mathrm{kg} / \mathrm{mole}$ & CONSTANT & $1.80 E-02$ & $1.80 E-02$ & $0.00 E+00$ & 1.80E-02 & $1.80 E-02$ & $2 / 13 / 96$ & 3 & 32405 & $\begin{array}{c}\text { NU/BFI } \\
\text { CS/TPI FL }\end{array}$ \\
\hline
\end{tabular}


32. Reference Constants

\begin{tabular}{|c|c|c|c|c|c|c|c|c|c|c|c|c|c|}
\hline ID & Material & Parameter & Units & Distribution & Mean & Median & Deviation & Low & High & Entry Date & Impt & WPO & Codes \\
\hline 2862 & REFCON & MW_H2S & $\mathrm{kg} / \mathrm{mole}$ & CONSTANT & $3.41 E-02$ & 3.41E-02 & $0.00 E+00$ & 3.41E-02 & 3.41E-02 & $12 / 4 / 96$ & 5 & $32406^{*}$ & $\begin{array}{c}\text { CSIFU } \\
\text { TP/BFINU }\end{array}$ \\
\hline 2867 & REFCON & MW_H2SO4 & $\mathrm{kg} / \mathrm{mole}$ & CONSTANT & $9.81 E-02$ & $9,81 E-02$ & $0.00 \mathrm{E}+00$ & $9.81 E-02$ & 9.81E-02 & $12 / 4 / 96$ & 5 & $32407^{*}$ & $\begin{array}{c}\text { TPIBFI } \\
\text { CSIFUNU }\end{array}$ \\
\hline 2842 & REFCON & PC_O2 & $\mathrm{Pa}$ & CONSTANT & $5.08 E+06$ & $5.08 E+06$ & $0.00 E+00$ & $5.08 E+06$ & $5.08 E+06$ & $2 / 5 / 96$ & 3 & 32420 & $B F$ \\
\hline 2861 & REFCON & MW_N2 & $\mathrm{kg} / \mathrm{mole}$ & CONSTANT & $2.80 \mathrm{E}-02$ & $2.80 E-02$ & $0.00 E+00$ & $2.80 E-02$ & $2.80 \mathrm{E}-02$ & $12 / 4 / 96$ & 5 & $32409^{*}$ & $\begin{array}{c}\text { FUNUI } \\
\text { BF/CS/ TP }\end{array}$ \\
\hline 2871 & REFCON & MW_FEOH2 & $\mathrm{kg} / \mathrm{mole}$ & CONSTANT & 8.99E-02 & 8.99E-02 & $0.00 E+00$ & 8.99E-02 & 8.99E-02 & $12 / 4 / 96$ & 5 & 32399* & $\begin{array}{c}\text { NU/BFI } \\
\text { FUCS/ TP }\end{array}$ \\
\hline 2863 & REFCON & MW_O2 & $\mathrm{kg} / \mathrm{mole}$ & CONSTANT & 3.20E-02 & 3.20E-02 & $0.00 E+00$ & 3.20E-0.2 & $3.20 \mathrm{E}-02$ & $12 / 4 / 96$ & 5 & $32411^{*}$ & $\begin{array}{c}\text { FLNNUI } \\
\text { CS } / T P / B F\end{array}$ \\
\hline 2894 & REFCON & OMEGAA & NONE & CONSTANT & 4.27E-01 & 4.27E-01 & $0.00 E+00$ & 4.27E-01 & 4.27E-01 & $2 / 6 / 96$ & 3 & 32412 & BF \\
\hline 2895 & REFCON & OMEGAB & NONE & CONSTANT & 8.66E-02 & |8.66E-02 & $10.00 E+00$ & 8.66E-02 & |8.66E-02 & $2 / 6 / 96$ & 3 & 32413 & BF \\
\hline 2880 & REFCON & PASCP & CP/Pa*s & CONSTANT & $1.00 E+03$ & $1.00 E+03$ & $0.00 E+00$ & $1.00 \mathrm{E}+03$ & $1.00 E+03$ & $2 / 5 / 96$ & 3 & 32414 & $B F$ \\
\hline 2839 & REFCON & PC_CH4 & $\mathrm{Pa}$ & CONSTANT & $4.62 E+06$ & $4.62 E+06$ & $0.00 E+00$ & $4.62 E+06$ & $4.62 E+06$ & 2/5/96 & 3 & 32415 & $\mathrm{BF}$ \\
\hline 2838 & REFCON & PC_CO2 & $\mathrm{Pa}$ & CONSTANT & $7.38 \mathrm{E}+06$ & 7.38E+06 & $0.00 \mathrm{E}+00$ & 7.38E+06 & $7.38 E+06$ & 2/5/96 & 3 & 32416 & BF \\
\hline 2837 & REFCON & PC_H2 & $\mathrm{Pa}$ & CONSTANT & $2.05 E+06$ & $2.05 E+06$ & $0.00 E+00$ & $2.05 E+06$ & $2.05 E+06$ & $2 / 5 / 96$ & 3 & 32417 & BF \\
\hline 2868 & REFCON & MW_HNO3 & $\mathrm{kg} / \mathrm{mole}$ & CONSTANT & $6.30 \mathrm{E}-02$ & 6.30E-02 & $0.00 E+00$ & $6.30 \mathrm{E}-02$ & $6.30 \mathrm{E}-02$ & $12 / 4 / 96$ & 5 & $32408^{\circ}$ & $\begin{array}{c}\text { FUCSI } \\
\text { TPIBFINU }\end{array}$ \\
\hline
\end{tabular}


Page intentionally blank

A3-44 
Appendix B1

\section{Parameter Cross Reference} by ID Number

B1-1 
ID Number

\begin{tabular}{|c|c|c|}
\hline Parameter ID & Material & Parameter \\
\hline 2 & AM241 & ATWEIGHT \\
\hline 3 & AM241 & HALFLIFE \\
\hline 4 & AM241 & INVCHD \\
\hline 5 & AM241 & INVRHD \\
\hline 6 & AM243 & HALFLIFE \\
\hline 7 & OPS_AREA & CAP_MOD \\
\hline 8 & OPS_AREA & COMP_RCK \\
\hline 9 & OPS_AREA & PC_MAX \\
\hline 10 & OPS_AREA & PORE_DIS \\
\hline 11 & OPS_AREA & POROSITY \\
\hline 12 & OPS_AREA & PO_MIN \\
\hline 13 & OPS_AREA & PRESSURE \\
\hline 14 & OPS_AREA & PRMX_LOG \\
\hline 15 & OPS_AREA & |PRMY_LOG \\
\hline 16 & OPS_AREA & PRMZ_LOG \\
\hline 19 & OPS_AREA & RELP_MOD \\
\hline 20 & OPS_AREA & SAT_IBRN \\
\hline 21 & OPS_AREA & SAT_RBRN \\
\hline 22 & OPS_AREA & SAT_RGAS \\
\hline 23 & BOREHOLE & CAP_MOD \\
\hline 25 & BOREHOLE & COMP_RCK \\
\hline 26 & BOREHOLE & DIAMMOD \\
\hline 27 & BOREHOLE & DOMEGA \\
\hline 29 & BOREHOLE & PC_MAX \\
\hline 30 & BOREHOLE & PORE_DIS \\
\hline 31 & BOREHOLE & POROSITY \\
\hline 32 & BOREHOLE & |PO_MIN \\
\hline 33 & BOREHOLE & PRESSURE \\
\hline 34 & BOREHOLE & PRMX_LOG \\
\hline 35 & BOREHOLE & PRMY_LOG \\
\hline 36 & BOREHOLE & PRMZ_LOG \\
\hline 38 & BOREHOLE & PTINDEX \\
\hline 40 & BOREHOLE & RELP_MOD \\
\hline 41 & BOREHOLE & SAT_RBRN \\
\hline 42 & BOREHOLE & SAT_RGAS \\
\hline 43 & BRINECST & COMPRES \\
\hline 44 & BRINECST & DNSFLUID \\
\hline 48 & BRINESAL & COMPRES \\
\hline 49 & BRINESAL & DNSFLUID \\
\hline 50 & BRINESAL & REF_PRES \\
\hline 51 & BRINESAL & REF_TEMP \\
\hline 52 & BRINESAL & RSLOPE \\
\hline 55 & BRINESAL & VISCO \\
\hline 57 & BRINESAL & WTF \\
\hline 58 & CASTILER & AREA_FRC \\
\hline 60 & CASTILER & CAP_MOD \\
\hline 61 & CASTILER & COMP_RCK \\
\hline 62 & CASTILER & PC_MAX \\
\hline 63 & CASTILER & PORE_DIS \\
\hline 64 & CASTILER & POROSITY \\
\hline 65 & CASTILER & PO_MIN \\
\hline
\end{tabular}

\begin{tabular}{|c|c|c|}
\hline Parameter ID & Material & Parameter \\
\hline 66 & CASTILER & PRESSURE \\
\hline 67 & CASTILER & PRMX_LOG \\
\hline 68 & CASTILER & PRMY_LOG \\
\hline 69 & CASTILER & PRMZ_LOG \\
\hline 72 & CASTILER & RELP_MOD \\
\hline 73 & CASTILER & SAT_IBRN \\
\hline 74 & CASTILER & ISAT_RBRN \\
\hline 75 & CASTILER & SAT_RGAS \\
\hline 76 & CAVITY_1 & CAP_MOD \\
\hline 77 & CAVITY_1 & COMP_RCK \\
\hline 78 & |CAVITY_1 & PC_MAX \\
\hline 79 & CAVITY_1 & PORE_DIS \\
\hline 80 & CAVITY_1 & POROSITY \\
\hline 81 & CAVITY_1 & PO_MIN \\
\hline 82 & CAVITY_1 & PRESSURE \\
\hline 83 & CAVITY_1 & PRMX_LOG \\
\hline 84 & CAVITY_ 1 & PRMY_LOG \\
\hline 85 & |CAVITY_1 & PRMZ_LOG \\
\hline 88 & CAVITY_1 & RELP_MOD \\
\hline 89 & CAVITY_1 & SAT_RBRN \\
\hline 90 & CAVITY_1 & SAT_RGAS \\
\hline 91 & |CAVITY_2 & CAP_MOD \\
\hline 92 & CAVITY_2 & COMP_RCK \\
\hline 93 & CAVITY_2 & PC_MAX \\
\hline 94 & CAVITY_2 & PORE_DIS \\
\hline 95 & CAVITY_2 & POROSITY \\
\hline 96 & CAVITY_2 & PO_MIN \\
\hline 97 & CAVITY_2 & PRESSURE \\
\hline 98 & CAVITY_2 & PRMX_LOG \\
\hline 99 & CAVITY_2 & PRMY_LOG \\
\hline 100 & CAVITY_2 & PRMZ_LOG \\
\hline 103 & CAVITY_2 & RELP_MOD \\
\hline 104 & CAVITY_2 & SAT_RBRN \\
\hline 105 & CAVITY_2 & SAT_RGAS \\
\hline 106 & CF252 & ATWEIGHT \\
\hline 107 & CF252 & HALFLIFE \\
\hline 108 & CF252 & INVCHD \\
\hline 109 & CF252 & INVRHD \\
\hline 110 & СM244 & ATWEIGHT \\
\hline 111 & CM244 & HALFLIFE \\
\hline 112 & СM244 & INVCHD \\
\hline 113 & CM244 & INVRHD \\
\hline 115 & CM248 & HALFLIFE \\
\hline 116 & CS137 & ATWEIGHT \\
\hline 117 & CS137 & HALFLIFE \\
\hline 118 & CS137 & INVRHD \\
\hline 119 & CULEBRA & CAP_MOD \\
\hline 120 & CULEBRA & COMP_RCK \\
\hline 123 & CULEBRA & FRCTRIDX \\
\hline 137 & CULEBRA & PC_MAX \\
\hline 139 & CULEBRA & PORE_DIS \\
\hline
\end{tabular}


ID Number

\begin{tabular}{|c|c|c|}
\hline Parameter ID & Material & Parameter \\
\hline 140 & CULEBRA & POROSITY \\
\hline 141 & CULEBRA & PO_MIN \\
\hline 142 & CULEBRA & PRESSURE \\
\hline 143 & CULEBRA & PRMX_LOG \\
\hline 144 & CULEBRA & PRMY_LOG \\
\hline 145 & CULEBRA & PRMZ_LOG \\
\hline 148 & CULEBRA & RELP_MOD \\
\hline 149 & CULEBRA & SAT_IBRN \\
\hline 150 & CULEBRA & SAT_RBRN \\
\hline 151 & CULEBRA & SAT_RGAS \\
\hline 153 & DEWYLAKE & CAP_MOD \\
\hline 154 & DEWYLAKE & COMP_RCK \\
\hline 156 & DEWYLAKE & PC_MAX \\
\hline 157 & DEWYLAKE & PORE_DIS \\
\hline 158 & DEWYLAKE & POROSITY \\
\hline 159 & DEWYLAKE & PO_MIN \\
\hline 160 & DEWYLAKE & PRESSURE \\
\hline 161 & DEWYLAKE & PRMX_LOG \\
\hline 162 & DEWYLAKE & PRMY_LOG \\
\hline 163 & DEWYLAKE & PRMZ_LOG \\
\hline 166 & DEWYLAKE & RELP_MOD \\
\hline 167 & DEWYLAKE & SAL_USAT \\
\hline 168 & DEWYLAKE & SAT_IBRN \\
\hline 169 & DEWYLAKE & SAT_RBRN \\
\hline 170 & DEWYLAKE & SAT_RGAS \\
\hline 171 & DRILLMUD & DNSFLUID \\
\hline 172 & DRILLMUD & VISCO \\
\hline 173 & DRILLMUD & YLDSTRSS \\
\hline 174 & DRZ_0 & CAP_MOD \\
\hline 175 & DRZ_0 & COMP_RCK \\
\hline 176 & DRZ_0 & PC_MAX \\
\hline 177 & DRZ_0 & PORE_DIS \\
\hline 178 & DRZ_O & POROSITY \\
\hline 179 & DRZ_O & PO_MIN \\
\hline 181 & DRZ_0 & PRMX_LOG \\
\hline 182 & DRZ_0 & PRMY_LOG \\
\hline 183 & $D R Z$ DO & PRMZ_LOG \\
\hline 186 & DRZ_0 & RELP_MOD \\
\hline 187 & DRZ_0 & SAT_IBRN \\
\hline 188 & DRZ_0 & SAT_RBRN \\
\hline 189 & DRZ_0 & SAT_RGAS \\
\hline 190 & $D R Z$ 1 & CAP_MOD \\
\hline 191 & $D R Z$ D & COMP_RCK \\
\hline 193 & DRZ_1 & PC_MAX \\
\hline 194 & DRZ_1 & PORE_DIS \\
\hline 195 & DRZ_1 & POROSITY \\
\hline 196 & DRZ_1 & PO_MIN \\
\hline 198 & DRZ_1 & PRMX_LOG \\
\hline 199 & DRZ_1 & PRMY_LOG \\
\hline 200 & DRZ_1 & PRMZ_LOG \\
\hline 203 & DRZ_1 & RELP_MOD \\
\hline
\end{tabular}

\begin{tabular}{|c|c|c|}
\hline Parameter ID & Material & Parameter \\
\hline 205 & DRZ 1 & SAT_RBRN \\
\hline 206 & DRZ_1 & SAT_RGAS \\
\hline 207 & EXP_AREA & CAP_MOD \\
\hline 208 & EXP_AREA & COMP_RCK \\
\hline 209 & EXP_AREA & PC_MAX \\
\hline 210 & EXP_AREA & PORE_DIS \\
\hline 211 & EXP_AREA & POROSITY \\
\hline 212 & EXP_AREA & PO_MIN \\
\hline 213 & EXP_AREA & PRESSURE \\
\hline 214 & EXP_AREA & PRMX_LOG \\
\hline 215 & EXP_AREA & PRMY_LOG \\
\hline 216 & EXP_AREA & PRMZ_LOG \\
\hline 219 & EXP_AREA & RELP_MOD \\
\hline 220 & EXP_AREA & SAT_IBRN \\
\hline 221 & EXP_AREA & SAT_RBRN \\
\hline 222 & EXP_AREA & SAT_RGAS \\
\hline 223 & GLOBAL & CLIMTIDX \\
\hline 225 & GLOBAL & TRANSIDX \\
\hline 228 & $\mathrm{H} 2$ & VISCO \\
\hline 229 & IMPERM_Z & CAP_MOD \\
\hline 230 & IMPERM_Z & COMP_RCK \\
\hline 231 & IMPERM_Z & PC_MAX \\
\hline 232 & IMPERM_Z & PORE_DIS \\
\hline 233 & IMPERM_Z & POROSITY \\
\hline 234 & IMPERM_Z & PO_MIN \\
\hline 236 & IMPERM_Z & PRMX_LOG \\
\hline 237 & IMPERM_Z & PRMY_LOG \\
\hline 238 & IMPERM_Z & PRMZ_LOG \\
\hline 241 & IMPERM_Z & RELP_MOD \\
\hline 243 & IMPERM_Z & SAT_RBRN \\
\hline 244 & IMPERM_Z & SAT_RGAS \\
\hline 246 & NP237 & ATWEIGHT \\
\hline 247 & NP237 & HALFLIFE \\
\hline 248 & NP237 & INVCHD \\
\hline 249 & NP237 & INVRHD \\
\hline 250 & PA231 & ATWEIGHT \\
\hline 251 & PA231 & HALFLIFE \\
\hline 252 & PAN_SEAL & CAP_MOD \\
\hline 253 & PAN_SEAL & COMP_RCK \\
\hline 254 & PAN_SEAL & PC_MAX \\
\hline 255 & PAN_SEAL & PORE_DIS \\
\hline 256 & PAN_SEAL & POROSITY \\
\hline 257 & PAN_SEAL & PO_MIN \\
\hline 258 & PAN_SEAL & PRESSURE \\
\hline 259 & PAN_SEAL & PRMX_LOG \\
\hline 260 & PAN_SEAL & PRMY_LOG \\
\hline 261 & PAN_SEAL & PRMZ_LOG \\
\hline 264 & PAN_SEAL & RELP_MOD \\
\hline 265 & PAN_SEAL & SAT_RBRN \\
\hline 266 & PAN_SEAL & SAT_RGAS \\
\hline 282 & PB & LOGSOLM \\
\hline
\end{tabular}


ID Number

\begin{tabular}{|c|c|c|}
\hline Parameter ID & Material & Parameter \\
\hline 283 & PB210 & ATWEIGHT \\
\hline 284 & PB210 & HALFLIFE \\
\hline 285 & PB210 & INVCHD. \\
\hline 286 & PB210 & INVRHD \\
\hline 287 & PM147 & ATWEIGHT \\
\hline 288 & PM147 & HALFLIFE \\
\hline 289 & PM147 & INVRHD \\
\hline 291 & PU238 & ATWEIGHT \\
\hline 292 & PU238 & HALFLIFE \\
\hline 293 & PU238 & INVCHD \\
\hline 294 & PU238 & INVRHD \\
\hline 295 & PU239 & ATWEIGHT \\
\hline 296 & PU239 & HALFLIFE \\
\hline 297 & PU239 & INVCHD \\
\hline 298 & PU239 & INVRHD \\
\hline 299 & PU240 & ATWEIGHT \\
\hline 300 & PU240 & HALFLIFE \\
\hline 301 & PU240 & INVCHD \\
\hline 302 & PU240 & INVRHD \\
\hline 303 & PU241 & ATWEIGHT \\
\hline 304 & PU241 & HALFLIFE \\
\hline 305 & PU241 & INVCHD \\
\hline 306 & PU241 & INVRHD \\
\hline 307 & PU242 & ATWEIGHT \\
\hline 308 & PU242 & HALFLIFE \\
\hline 309 & PU242 & INVCHD \\
\hline 310 & PU242 & INVRHD \\
\hline 311 & PU244 & ATWEIGHT \\
\hline 312 & PU244 & HALFLIFE \\
\hline 313 & RA & LOGSOLM \\
\hline 314 & RA226 & ATWEIGHT \\
\hline 315 & RA226 & HALFLIFE \\
\hline 316 & RA226 & INVCHD \\
\hline 317 & RA226 & INVRHD \\
\hline 318 & RA228 & ATWEIGHT \\
\hline 319 & RA228 & HALFLIFE \\
\hline 336 & SANTAROS & CAP_MOD \\
\hline 337 & SANTAROS & COMP_RCK \\
\hline 339 & SANTAROS & PC_MAX \\
\hline 340 & SANTAROS & PORE_DIS \\
\hline 341 & SANTAROS & POROSITY \\
\hline 342 & SANTAROS & PO_MIN \\
\hline 343 & SANTAROS & PRESSURE \\
\hline 344 & SANTAROS & PRMX_LOG \\
\hline 345 & SANTAROS & PRMY_LOG \\
\hline 346 & SANTAROS & PRMZ_LOG \\
\hline 349 & SANTAROS & RELP_MOD \\
\hline 350 & SANTAROS & SAT_IBRN \\
\hline 351 & SANTAROS & SAT_RBRN \\
\hline 352 & SANTAROS & SAT_RGAS \\
\hline 514 & SM147 & ATWEIGHT \\
\hline
\end{tabular}

\begin{tabular}{|c|c|c|}
\hline Parameter ID & Material & Parameter \\
\hline 515 & SM147 & HALFLIFE \\
\hline 517 & SR90 & HALFLIFE \\
\hline 518 & SR90 & INVRHD \\
\hline 520 & S_ANH_AB & CAP_MOD \\
\hline 521 & S_ANH_AB & COMP_RCK \\
\hline 522 & $S \_A N H \_A B$ & PC_MAX \\
\hline 524 & S_ANH_AB & PF_DELTA \\
\hline 526 & S_ANH_AB & PI_DELTA \\
\hline 527 & S_ANH_AB & PORE_DIS \\
\hline 528 & S_ANH_AB & POROSITY \\
\hline 529 & S_ANH_AB & PO_MIN \\
\hline 531 & S_ANH_AB & PRMX_LOG \\
\hline 532 & S_ANH_AB & PRMY_LOG \\
\hline 533 & S_ANH_AB & PRMZ_LOG \\
\hline 536 & S_ANH_AB & RELP_MOD \\
\hline 537 & S_ANH_AB & SAT_IBRN \\
\hline 538 & S_ANH_AB & SAT_RBRN \\
\hline 539 & S_ANH_AB & SAT_RGAS \\
\hline 540 & S_HALITE & CAP_MOD \\
\hline 541 & S_HALITE & COMP_RCK \\
\hline 542 & S_HALITE & PC_MAX \\
\hline 543 & S_HALITE & PORE_DIS \\
\hline 544 & S_HALITE & POROSITY \\
\hline 545 & S_HALITE & PO_MIN \\
\hline 546 & S_HALITE & PRESSURE \\
\hline 547 & S_HALITE & PRMX_LOG \\
\hline 548 & S_HALITE & PRMY_LOG \\
\hline 549 & S_HALITE & PRMZ_LOG \\
\hline 553 & S_HALITE & RELP_MOD \\
\hline 554 & S_HALITE & SAT_IBRN \\
\hline 555 & S_HALITE & SAT_RBRN \\
\hline 556 & S_HALITE & SAT_RGAS \\
\hline 559 & S_MB138 & CAP_MOD \\
\hline 560 & S_MB138 & COMP_RCK \\
\hline 561 & S_MB138 & PC_MAX \\
\hline 563 & S_MB138 & PF_DELTA \\
\hline 565 & S_MB138 & PI_DELTA \\
\hline 566 & S_MB138 & PORE_DIS \\
\hline 567 & S_MB138 & POROSITY \\
\hline 568 & S_MB138 & PO_MIN \\
\hline 569 & S_MB138 & PRESSURE \\
\hline 570 & S_MB138 & PRMX_LOG \\
\hline 571 & S_MB138 & PRMY_LOG \\
\hline 572 & S_MB138 & PRMZ_LOG \\
\hline 575 & S_MB138 & RELP_MOD \\
\hline 576 & S_MB138 & SAT_IBRN \\
\hline 577 & S_MB138 & SAT_RBRN \\
\hline 578 & $S M B 138$ & SAT_RGAS \\
\hline 579 & S_MB139 & CAP_MOD \\
\hline 580 & S_MB139 & COMP_RCK \\
\hline 582 & S_MB139 & PC_MAX \\
\hline
\end{tabular}


ID Number

\begin{tabular}{|c|c|c|}
\hline Parameter ID & Material & Parameter \\
\hline 586 & S_MB139 & PI_DELTA \\
\hline 587 & S_MB139 & PORE_DIS \\
\hline 588 & S_MB139 & POROSITY \\
\hline 589 & S_MB139 & PO_MIN \\
\hline 590 & S_MB139 & PRESSURE \\
\hline 591 & S_MB139 & PRMX_LOG \\
\hline 592 & S_MB139 & PRMY_LOG \\
\hline 593 & S_MB139 & PRMZ_LOG \\
\hline 596 & S_MB139 & RELP_MOD \\
\hline 597 & S_MB139 & SAT_IBRN \\
\hline 598 & S_MB139 & SAT_RBRN \\
\hline 599 & S_MB139 & SAT_RGAS \\
\hline 603 & TH229 & ATWEIGHT \\
\hline 604 & TH229 & HALFLIFE \\
\hline 605 & TH229 & INVCHD \\
\hline 606 & TH229 & INVRHD \\
\hline 607 & TH230 & ATWEIGHT \\
\hline 608 & TH230 & HALFLIFE \\
\hline 609 & TH230 & INVCHD \\
\hline 610 & TH230 & INVRHD \\
\hline 611 & TH232 & ATWEIGHT \\
\hline 612 & TH232 & HALFLIFE \\
\hline 613 & TH232 & INVCHD \\
\hline 614 & TH232 & INVRHD \\
\hline 632 & U233 & ATWEIGHT \\
\hline 633 & U233 & HALFLIFE \\
\hline 634 & U233 & INVCHD \\
\hline 635 & U233 & INVRHD \\
\hline 636 & U234 & ATWEIGHT \\
\hline 637 & U234 & HALFLIFE \\
\hline 638 & U234 & INVCHD \\
\hline 639 & U234 & INVRHD \\
\hline 640 & U235 & ATWEIGHT \\
\hline 641 & U235 & HALFLIFE \\
\hline 642 & U235 & INVCHD \\
\hline 643 & U235 & INVRHD \\
\hline 644 & U236 & ATWEIGHT \\
\hline 645 & U236 & HALFLIFE \\
\hline 646 & U236 & INVRHD \\
\hline 647 & $\mathrm{U} 238$ & ATWEIGHT \\
\hline 648 & U238 & HALFLIFE \\
\hline 649 & $\mathrm{U} 238$ & INVCHD \\
\hline 650 & U238 & INVRHD \\
\hline 651 & WAS_AREA & ABSROUGH \\
\hline 652 & WAS_AREA & CAP_MOD \\
\hline 653 & WAS_AREA & COMP_RCK \\
\hline 656 & WAS_AREA & GRATMICH \\
\hline 657 & WAS_AREA & GRATMICI \\
\hline 658 & WAS_AREA & PC_MAX \\
\hline 659 & WAS_AREA & PORE_DIS \\
\hline 660 & WAS_AREA & POROSITY \\
\hline
\end{tabular}

\begin{tabular}{|c|c|c|}
\hline Parameter ID & Material & Parameter \\
\hline 661 & WAS_AREA & PO_MIN \\
\hline 662 & WAS_AREA & PRESSURE \\
\hline 663 & WAS_AREA & PRMX_LOG \\
\hline 664 & WAS_AREA & PRMY_LOG \\
\hline 665 & WAS_AREA & PRMZ_LOG \\
\hline 668 & WAS_AREA & RELP_MOD \\
\hline 669 & WAS_AREA & SAT_IBRN \\
\hline 670 & WAS_AREA & SAT_RBRN \\
\hline 671 & WAS_AREA & SAT_RGAS \\
\hline 838 & $\mathrm{CF}$ & LOGSOLM \\
\hline 841 & CS & LOGSOLM \\
\hline 843 & CULEBRA & DNSGRAIN \\
\hline 861 & CULEBRA & FTORT \\
\hline 944 & DRZ_ 0 & MDISP_L \\
\hline 945 & DRZ_0 & MDISP_T \\
\hline 962 & DRZ_0 & TORTUSTY \\
\hline 1167 & PM & LOGSOLM \\
\hline 1659 & SR & LOGSOLM \\
\hline 1661 & S_ANH_AB & DNSGRAIN \\
\hline 1684 & S_ANH_AB & MDISP_L \\
\hline 1685 & S_ANH_AB & MDISP_T \\
\hline 1702 & S_ANH_AB & TORTUSTY \\
\hline 1703 & S_HALITE & DNSGRAIN \\
\hline 1724 & S_HALITE & MDISP_L \\
\hline 1725 & S_HALITE & MDISP_T \\
\hline 1742 & S_HALITE & TORTUSTY \\
\hline 1743 & S_MB138 & DNSGRAIN \\
\hline 1784 & S_MB139 & DNSGRAIN \\
\hline 1992 & WAS_AREA & DIRNCCHW \\
\hline 1993 & WAS_AREA & DIRNCRHW \\
\hline 1994 & WAS_AREA & DNSGRAIN \\
\hline 1995 & WAS_AREA & DPLSCCHW \\
\hline 2037 & CS137 & INVCHD \\
\hline 2038 & PM147 & INVCHD \\
\hline 2039 & SR90 & INVCHD \\
\hline 2040 & WAS_AREA & DIRONCHW \\
\hline 2041 & WAS_AREA & DCELLCHW \\
\hline 2042 & WAS_AREA & DRUBBCHW \\
\hline 2043 & WAS_AREA & DPLASCHW \\
\hline 2044 & WAS_AREA & DIRONRHW \\
\hline 2046 & WAS_AREA & DRUBBRHW \\
\hline 2049 & CAVITY_3 & CAP_MOD \\
\hline 2051 & CAVITY_3 & COMP_RCK \\
\hline 2052 & CAVITY 3 & PORE_DIS \\
\hline 2053 & CAVITY_3 & POROSITY \\
\hline 2054 & CAVITY_3 & PRMX_LOG \\
\hline 2055 & CAVITY_3 & PRMY_LOG \\
\hline 2056 & CAVITY_3 & PRMZ_LOG \\
\hline 2058 & CAVITY_3 & RELP_MOD \\
\hline 2059 & CAVITY_3 & SAT_RGAS \\
\hline 2060 & CAVITY_4 & CAP_MOD \\
\hline
\end{tabular}


ID Number

\begin{tabular}{|c|c|c|}
\hline Parameter ID & Material & Parameter \\
\hline 2062 & ICAVITY_4 & COMP_RCK \\
\hline 2063 & CAVITY_4 & PORE_DIS \\
\hline 2064 & CAVITY_4 & POROSITY \\
\hline 2065 & CAVITY_4 & PRMX_LOG \\
\hline 2066 & CAVITY_4 & PRMY_LOG \\
\hline 2067 & CAVITY 4 & PRMZ_LOG \\
\hline 2069 & CAVITY_4 & RELP_MOD \\
\hline 2070 & CAVITY_4 & SAT_RGAS \\
\hline 2071 & CULEBRA & THICK \\
\hline 2085 & FORTYNIN & CAP_MOD \\
\hline 2087 & FORTYNIN & PORE_DIS \\
\hline 2088 & FORTYNIN & POROSITY \\
\hline 2093 & FORTYNIN & RELP_MOD \\
\hline 2094 & FORTYNIN & SAT_RGAS \\
\hline 2097 & MAGENTA & CAP_MOD \\
\hline 2098 & MAGENTA & PC_MAX \\
\hline 2099 & MAGENTA & PORE_DIS \\
\hline 2100 & MAGENTA & POROSITY \\
\hline 2101 & MAGENTA & PRESSURE \\
\hline 2102 & MAGENTA & PRMX_LOG \\
\hline 2103 & MAGENTA & PRMY_LOG \\
\hline 2104 & MAGENTA & PRMZ_LOG \\
\hline 2106 & MAGENTA & RELP_MOD \\
\hline 2107 & MAGENTA & SAT_RGAS \\
\hline 2109 & MAGENTA & THICK \\
\hline 2110 & REPOSIT & CAP_MOD \\
\hline 2112 & REPOSIT & COMP_RCK \\
\hline 2113 & REPOSIT & DCELLCHW \\
\hline 2114 & REPOSIT & DCELLRHW \\
\hline 2115 & REPOSIT & DIRNCCHW \\
\hline 2116 & REPOSIT & DIRNCRHW \\
\hline 2117 & REPOSIT & DIRONCHW \\
\hline 2118 & REPOSIT & DIRONRHW \\
\hline 2119 & REPOSIT & DPLASCHW \\
\hline 2120 & REPOSIT & DPLASRHW \\
\hline 2121 & REPOSIT & DPLSCCHW \\
\hline 2122 & REPOSIT & DRUBBCHW \\
\hline 2123 & REPOSIT & DRUBBRHW \\
\hline 2127 & REPOSIT & GRATMICH \\
\hline 2128 & REPOSIT & GRATMICI \\
\hline 2129 & REPOSIT & PORE DIS \\
\hline 2130 & REPOSIT & POROSITY \\
\hline 2131 & REPOSIT & PRMX_LOG \\
\hline 2132 & REPOSIT & PRMY_LOG \\
\hline 2133 & REPOSIT & PRMZ_LOG \\
\hline 2135 & REPOSIT & RELP_MOD \\
\hline 2137 & REPOSIT & SAT_RGAS \\
\hline 2141 & REPOSIT & VOLCHW \\
\hline 2142 & REPOSIT & VOLRHW \\
\hline 2158 & S_ANH_AB & DPHIMAX \\
\hline 2159 & S_ANH_AB & KMAXLOG \\
\hline
\end{tabular}

\begin{tabular}{|c|c|c|}
\hline Parameter ID & Material & Parameter \\
\hline 2169 & S_MB138 & DPHIMAX \\
\hline 2170 & S_MB138 & KMAXLOG \\
\hline 2177 & S_MB139 & DPHIMAX \\
\hline 2178 & S_MB139 & KMAXLOG \\
\hline 2180 & S_MB139 & PF_DELTA \\
\hline 2183 & TAMARISK & CAP_MOD \\
\hline 2185 & TAMARISK & PORE_DIS \\
\hline 2186 & TAMARISK & POROSITY \\
\hline 2191 & TAMARISK & RELP_MOD \\
\hline 2192 & TAMARISK & SAT_RGAS \\
\hline 2216 & U236 & INVCHD \\
\hline 2217 & UNNAMED & CAP_MOD \\
\hline 2218 & UNNAMED & COMP_RCK \\
\hline 2219 & UNNAMED & PORE_DIS \\
\hline 2220 & UNNAMED & POROSITY \\
\hline 2225 & UNNAMED & RELP_MOD \\
\hline 2226 & UNNAMED & ISAT_RGAS \\
\hline 2228 & WAS_AREA & DPLSCRHW \\
\hline 2231 & WAS_AREA & SAT_WICK \\
\hline 2232 & WAS_AREA & VOLCHW \\
\hline 2233 & WAS_AREA & VOLRHW \\
\hline 2234 & CAVITY_3 & PC_MAX \\
\hline 2235 & CAVITY_3 & SAT_RBRN \\
\hline 2236 & CAVITY_4 & PC_MAX \\
\hline 2237 & CAVITY_4 & SAT_RBRN \\
\hline 2238 & FORTYNIN & COMP_RCK \\
\hline 2239 & FORTYNIN & PC_MAX \\
\hline 2240 & FORTYNIN & SAT_RBRN \\
\hline 2241 & MAGENTA & SAT_RBRN \\
\hline 2242 & REPOSIT & PC_MAX \\
\hline 2243 & TAMARISK & COMP_RCK \\
\hline 2244 & TAMARISK & PC_MAX \\
\hline 2245 & TAMARISK & SAT_RBRN \\
\hline 2247 & UNNAMED & PC_MAX \\
\hline 2248 & UNNAMED & SAT_RBRN \\
\hline 2254 & BOREHOLE & TAUFAIL \\
\hline 2265 & CM248 & INVCHD \\
\hline 2266 & CM248 & INVRHD \\
\hline 2267 & PA231 & INVCHD \\
\hline 2268 & PA231 & INVRHD \\
\hline 2269 & PU244 & INVCHD \\
\hline 2270 & PU244 & INVRHD \\
\hline 2271 & RA228 & INVCHD \\
\hline 2272 & RA228 & INVRHD \\
\hline 2274 & WAS_AREA & DCELLRHW \\
\hline 2275 & WAS_AREA & DPLASRHW \\
\hline 2276 & ASPHALT & CAP_MOD \\
\hline 2277 & ASPHALT & COMP_RCK \\
\hline 2278 & ASPHALT & PC_MAX \\
\hline 2279 & ASPHALT & PORE_DIS \\
\hline 2280 & ASPHALT & TPOROSITY \\
\hline
\end{tabular}


ID Number

\begin{tabular}{|c|c|c|}
\hline Parameter ID & Material & Parameter \\
\hline 2281 & ASPHALT & PO_MIN \\
\hline 2282 & ASPHALT & PRESSURE \\
\hline 2283 & ASPHALT & PRMX_LOG \\
\hline 2284 & ASPHALT & PRMY_LOG \\
\hline 2285 & ASPHALT & PRMZ_LOG \\
\hline 2289 & ASPHALT & RELP_MOD \\
\hline 2290 & ASPHALT & SAT_IBRN \\
\hline 2291 & ASPHALT & SAT_RBRN \\
\hline 2292 & ASPHALT & SAT_RGAS \\
\hline 2310 & CLAY_BOT & CAP_MOD \\
\hline 2311 & CLAY_BOT & COMP_RCK \\
\hline 2312 & CLAY_BOT & PC_MAX \\
\hline 2313 & CLAY_BOT & PORE_DIS \\
\hline 2314 & CLAY_BOT & POROSITY \\
\hline 2315 & CLAY_BOT & PO_MIN \\
\hline 2316 & CLAY_BOT & PRESSURE \\
\hline 2317 & CLAY_BOT & PRMX_LOG \\
\hline 2318 & CLAY_BOT & PRMY_LOG \\
\hline 2319 & CLAY_BOT & PRMZ_LOG \\
\hline 2323 & CLAY_BOT & RELP_MOD \\
\hline 2324 & CLAY_BOT & SAT_IBRN \\
\hline 2325 & CLAY_BOT & SAT_RBRN \\
\hline 2326 & CLAY_BOT & SAT_RGAS \\
\hline 2327 & $C L \_L / T 1$ & CAP_MOD \\
\hline 2328 & CL_L_T1 & COMP_RCK \\
\hline 2329 & $C L \_L=T 1$ & PC_MAX \\
\hline 2330 & $C L \_L$ T1 & PORE_DIS \\
\hline 2331 & CL_L_T1 & POROSITY \\
\hline 2332 & CL_L_T1 & PO_MIN \\
\hline 2333 & CL_L_T1 & PRESSURE \\
\hline 2334 & $C L L$ LI & PRMX_LOG \\
\hline 2335 & $C L \_L \_T 1$ & PRMY_LOG \\
\hline 2336 & $C L \_L \_T 1$ & PRMZ_LOG \\
\hline 2340 & CL_L_T1 & RELP_MOD \\
\hline 2341 & $C L \_L I 1$ & SAT_IBRN \\
\hline 2342 & $\mathrm{CL}_{2} \mathrm{~L}_{-} \mathrm{T1}$ & SAT_RBRN \\
\hline 2343 & CL_L_T1 & SAT_RGAS \\
\hline 2344 & CL_L_T2 & CAP_MOD \\
\hline 2345 & CL_L_T2 & COMP_RCK \\
\hline 2346 & $C L \_L \_T 2$ & PC_MAX \\
\hline 2347 & CL_L_T2 & PORE_DIS \\
\hline 2348 & CL_L_T2 & POROSITY \\
\hline 2349 & CL_L_T2 & PO_MIN \\
\hline 2351 & CL_L_T2 & PRMX_LOG \\
\hline 2352 & CL_L_T2 & PRMY_LOG \\
\hline 2353 & CL_L_T2 & PRMZ_LOG \\
\hline 2357 & $C L \_L / T 2$ & RELP_MOD \\
\hline 2359 & CL_L_T2 & SAT_RBRN \\
\hline 2360 & $C L L L T 2$ & SAT_RGAS \\
\hline 2361 & CL_L_T3 & CAP_MOD \\
\hline 2362 & CL_L_T3 & ICOMP_RCK \\
\hline
\end{tabular}

\begin{tabular}{|c|c|c|}
\hline Parameter ID & Material & Parameter \\
\hline 2363 & CL_L_T3 & PC_MAX \\
\hline 2364 & CL_L_T3 & PORE_DIS \\
\hline 2365 & CL_L_T3 & POROSITY \\
\hline 2366 & CL_L_T3 & PO_MIN \\
\hline 2368 & CL_L_T3 & PRMX_LOG \\
\hline 2369 & $\mathrm{CL}_{\mathrm{L}} \mathrm{T} 3$ & PRMY_LOG \\
\hline 2370 & CL_L_T3 & PRMZ_LOG \\
\hline 2374 & CL_L_T3 & RELP_MOD \\
\hline 2376 & CL_L_T3 & SAT_RBRN \\
\hline 2377 & CL_L_T3 & SAT_RGAS \\
\hline 2378 & CL_M_T1 & CAP_MOD \\
\hline 2379 & CL_M_T1 & COMP_RCK \\
\hline 2380 & CL_M_T1 & PC_MAX \\
\hline 2381 & CL_M_T1 & PORE_DIS \\
\hline 2382 & CL_M_T1 & POROSITY \\
\hline 2383 & CL_M_T1 & PO_MIN \\
\hline 2384 & CL_M_T1 & PRESSURE \\
\hline 2385 & CL_M_T1 & PRMX_LOG \\
\hline 2386 & CL_M_T1 & PRMY_LOG \\
\hline 2387 & CL_M_T1 & PRMZ_LOG \\
\hline 2391 & CL_M_T1 & RELP_MOD \\
\hline 2392 & CL_M_T1 & SAT_IBRN \\
\hline 2393 & CL_M_T1 & SAT_RBRN \\
\hline 2394 & $C L \_$M_T1 & SAT_RGAS \\
\hline 2395 & CL_M_T2 & CAP_MOD \\
\hline 2396 & CL_M_T2 & COMP_RCK \\
\hline 2397 & CL_M_T2 & PC_MAX \\
\hline 2398 & CL_M_T2 & PORE_DIS \\
\hline 2399 & CL_M_T2 & POROSITY \\
\hline 2400 & $C L \_M \_T 2$ & PO_MIN \\
\hline 2402 & $C L \_M \_T 2$ & PRMX_LOG \\
\hline 2403 & CL_M_T2 & PRMY_LOG \\
\hline 2404 & CL_M_T2 & PRMZ_LOG \\
\hline 2408 & CL_M_T2 & RELP_MOD \\
\hline 2410 & CL_M_T2 & SAT_RBRN \\
\hline 2411 & CL_M_T2 & SAT_RGAS \\
\hline 2412 & CL_M_T3 & CAP_MOD \\
\hline 2413 & $C L \_M \_T 3$ & COMP_RCK \\
\hline 2414 & CL_M_T3 & PC_MAX \\
\hline 2415 & $C L \_M \_T 3$ & PORE_DIS \\
\hline 2416 & CL_M_T3 & POROSITY \\
\hline 2417 & CL_M_T3 & PO_MIN \\
\hline 2419 & CL_M_T3 & PRMX_LOG \\
\hline 2420 & CL_M_T3 & PRMY_LOG \\
\hline 2421 & CL_M_T3 & PRMZ_LOG \\
\hline 2425 & CL_M_T3 & RELP_MOD \\
\hline 2427 & CL_M_T3 & SAT_RBRN \\
\hline 2428 & CL_M_T3 & SAT_RGAS \\
\hline 2429 & CL_M_T4 & CAP_MOD \\
\hline 2430 & CL_M_T4 & COMP_RCK \\
\hline 2431 & CL_M_T4 & PC_MAX \\
\hline
\end{tabular}


ID Number

\begin{tabular}{|c|c|c|}
\hline Parameter ID & Material & Parameter \\
\hline 2432 & CL_M_T4 & PORE_DIS \\
\hline 2433 & CL_M_T4 & POROSITY \\
\hline 2434 & CL_M_T4 & PO_MIN \\
\hline 2436 & CL_M_T4 & PRMX_LOG \\
\hline 2437 & CL_M_T4 & PRMY_LOG \\
\hline 2438 & CL_M_T4 & PRMZ_LOG \\
\hline 2442 & $\mathrm{CL}$ M_T4 & RELP_MOD \\
\hline 2444 & $\mathrm{CL} \_\mathrm{M} \_\mathrm{T} 4$ & SAT_RBRN \\
\hline 2445 & CL_M_T4 & SAT_RGAS \\
\hline 2446 & CL_M_T5 & CAP_MOD \\
\hline 2447 & CL_M_T5 & COMP_RCK \\
\hline 2448 & CL_M_T5 & PC_MAX \\
\hline 2449 & CL_M_T5 & PORE_DIS \\
\hline 2450 & CL_M_T5 & POROSITY \\
\hline 2451 & CL_M_T5 & PO_MIN \\
\hline 2453 & CL_M_T5 & PRMX_LOG \\
\hline 2454 & CL_M_T5 & PRMY_LOG \\
\hline 2455 & CL_M_T5 & PRMZ_LOG \\
\hline 2459 & CL_M_T5 & RELP_MOD \\
\hline 2461 & CL_M_T5 & SAT_RBRN \\
\hline 2462 & CL_M_T5 & SAT_RGAS \\
\hline 2463 & CONC_T1 & CAP_MOD \\
\hline 2464 & CONC_T1 & COMP_RCK \\
\hline 2465 & CONC_T1 & PC_MAX \\
\hline 2466 & CONC_T1 & PORE_DIS \\
\hline 2467 & CONC_T1 & POROSITY \\
\hline 2468 & CONC_T1 & PO_MIN \\
\hline 2469 & CONC_T1 & PRESSURE \\
\hline 2470 & CONC_T1 & PRMX_LOG \\
\hline 2471 & CONC_T1 & PRMY_LOG \\
\hline 2472 & CONC_T1 & PRMZ_LOG \\
\hline 2476 & CONC_T1 & RELP_MOD \\
\hline 2477 & CONC_T1 & SAT_IBRN \\
\hline 2478 & CONC_T1 & SAT_RBRN \\
\hline 2479 & CONC_T1 & SAT_RGAS \\
\hline 2480 & CONC_T2 & CAP_MOD \\
\hline 2481 & CONC_T2 & COMP_RCK \\
\hline 2482 & CONC_T2 & PC_MAX \\
\hline 2483 & CONC_T2 & PORE_DIS \\
\hline 2484 & CONC_T2 & POROSITY \\
\hline 2485 & CONC_T2 & PRESSURE \\
\hline 2486 & CONC_T2 & PRMX_LOG \\
\hline 2487 & CONC_T2 & PRMY_LOG \\
\hline 2488 & CONC_T2 & PRMZ_LOG \\
\hline 2492 & CONC_T2 & RELP_MOD \\
\hline 2493 & CONC_T2 & SAT_IBRN \\
\hline 2494 & CONC_T2 & SAT_RBRN \\
\hline 2495 & CONC_T2 & SAT_RGAS \\
\hline 2496 & EARTH & CAP_MOD \\
\hline 2497 & EARTH & COMP_RCK \\
\hline 2498 & EARTH & PC_MAX \\
\hline
\end{tabular}

\begin{tabular}{|c|c|c|}
\hline Parameter ID & \begin{tabular}{|c|} 
Material \\
\end{tabular} & Parameter \\
\hline 2499 & EARTH & PORE_DIS \\
\hline 2500 & EARTH & POROSITY \\
\hline 2501 & EARTH & PO_MIN \\
\hline 2502 & EARTH & PRESSURE \\
\hline 2503 & EARTH & PRMX_LOG \\
\hline 2504 & EARTH & PRMY_LOG \\
\hline 2505 & EARTH & PRMZ_LOG \\
\hline 2509 & EARTH & RELP_MOD \\
\hline 2510 & EARTH & SAT_IBRN \\
\hline 2511 & EARTH & SAT_RBRN \\
\hline 2512 & EARTH & SAT_RGAS \\
\hline 2513 & SALT_T1 & CAP_MOD \\
\hline 2514 & SALT_T1 & COMP_RCK \\
\hline 2515 & SALT_T1 & PC_MAX \\
\hline 2516 & SALT_T1 & PORE_DIS \\
\hline 2517 & SALT_T1 & POROSITY \\
\hline 2518 & SALT_T1 & PO_MIN \\
\hline 2519 & SALT_T1 & PRESSURE \\
\hline 2526 & SALT_T1 & RELP_MOD \\
\hline 2527 & SALT_T1 & SAT_IBRN \\
\hline 2528 & SALT_T1 & SAT_RBRN \\
\hline 2529 & SALT_T1 & SAT_RGAS \\
\hline 2530 & SALT_T2 & CAP_MOD \\
\hline 2531 & SALT_T2 & COMP_RCK \\
\hline 2532 & SALT_T2 & PC_MAX \\
\hline 2533 & SALT_T2 & PORE_DIS \\
\hline 2534 & SALT_T2 & POROSITY \\
\hline 2535 & SALT_T2 & PO_MIN \\
\hline 2543 & SALT_T2 & RELP_MOD \\
\hline 2545 & SALT_T2 & SAT_RBRN \\
\hline 2546 & SALT_T2 & SAT_RGAS \\
\hline 2547 & SALT_T3 & CAP_MOD \\
\hline 2548 & SALT_T3 & COMP_RCK \\
\hline 2549 & SALT_T3 & PC_MAX \\
\hline 2550 & SALT_T3 & PORE_DIS \\
\hline 2551 & SALT_T3 & POROSITY \\
\hline 2552 & SALT_T3 & PO_MIN \\
\hline 2560 & SALT_T3 & RELP_MOD \\
\hline 2562 & SALT_T3 & SAT_RBRN \\
\hline 2563 & SALT_T3 & SAT_RGAS \\
\hline 2564 & SALT_T4 & CAP_MOD \\
\hline 2565 & SALT_T4 & COMP_RCK \\
\hline 2566 & SALT_T4 & PC_MAX \\
\hline 2567 & SALT_T4 & PORE_DIS \\
\hline 2568 & SALT_T4 & POROSITY \\
\hline 2569 & SALT_T4 & PO_MIN \\
\hline 2577 & SALT_T4 & RELP_MOD \\
\hline 2579 & SALT_T4 & SAT_RBRN \\
\hline 2580 & SALT_T4 & SAT_RGAS \\
\hline 2581 & SALT_T5 & CAP_MOD \\
\hline 2582 & SALT_T5 & COMP_RCK \\
\hline
\end{tabular}


ID Number

\begin{tabular}{|c|c|c|}
\hline Parameter ID & Material & Parameter \\
\hline 2583 & SALT_T5 & PC_MAX \\
\hline 2585 & SALT_T5 & POROSITY \\
\hline 2594 & SALT_T5 & RELP_MOD \\
\hline 2596 & SALT_T5 & SAT_RBRN \\
\hline 2597 & SALT_T5 & SAT_RGAS \\
\hline 2599 & ASPHALT & KPT \\
\hline 2600 & ASPHALT & PCT_A \\
\hline 2601 & ASPHALT & PCT_EXP \\
\hline 2604 & OPS_AREA & KPT \\
\hline 2605 & OPS_AREA & PCT_A \\
\hline 2606 & OPS_AREA & PCT_EXP \\
\hline 2608 & CASTILER & KPT \\
\hline 2609 & CASTILER & PCT_A \\
\hline 2610 & CASTILER & PCT_EXP \\
\hline 2612 & CAVITY_1 & KPT \\
\hline 2613 & CAVITY_1 & PCT_A \\
\hline 2614 & CAVITY_1 $_{1}$ & PCT_EXP \\
\hline 2616 & CAVITY_2 & KPT \\
\hline 2617 & CAVITY_2 & PCT_A \\
\hline 2618 & CAVITY_2 & PCT_EXP \\
\hline 2620 & CAVITY_3 & KPT \\
\hline 2621 & CAVITY 3 & PCT_A \\
\hline 2622 & CAVITY_3 & PCT_EXP \\
\hline 2623 & CAVITY_3 & PO_MIN \\
\hline 2625 & CAVITY_4 & KPT \\
\hline 2626 & CAVITY_4 & PCT_A \\
\hline 2627 & CAVITY_4 & PCT_EXP \\
\hline 2628 & CAVITY_4 & PO_MIN \\
\hline 2636 & CLAY_BOT & KPT \\
\hline 2637 & CLAY_BOT & PCT_A \\
\hline 2638 & CLAY_BOT & PCT_EXP \\
\hline 2641 & CL_L_T1 & KPT \\
\hline 2642 & $C L \_L / T 1$ & PCT_A \\
\hline 2643 & CL_L_T1 & PCT_EXP \\
\hline 2646 & CL_L_T2 & KPT \\
\hline 2647 & CL_L_T2 & PCT_A \\
\hline 2648 & CL_L_T2 & PCT_EXP \\
\hline 2651 & $\mathrm{CL} L \mathrm{~L}$ T3 & KPT \\
\hline 2652 & CL_L_T3 & PCT_A \\
\hline 2653 & $C L \_L T 3$ & PCT_EXP \\
\hline 2656 & CL_M_T1 & KPT \\
\hline 2657 & CL_M_T1 & PCT_A \\
\hline 2658 & CL_M_T1 & PCT_EXP \\
\hline 2661 & $C L \_M \_T 2$ & KPT \\
\hline 2662 & CL_M_T2 & PCT_A \\
\hline 2663 & CL_M_T2 & PCT_EXP \\
\hline 2666 & CL_M_T3 & KPT \\
\hline 2667 & CL_M_T3 & PCT_A \\
\hline 2668 & CL_M_T3 & PCT_EXP \\
\hline 2671 & CL_M_T4 & |КРТ \\
\hline 2672 & CL_M_T4 & |PCT_A \\
\hline
\end{tabular}

\begin{tabular}{|c|c|c|}
\hline Parameter ID & Material & Parameter \\
\hline 2673 & CL_M_T4 & PCT_EXP \\
\hline 2676 & CL_M_T5 & KPT \\
\hline 2677 & CL_M_T5 & PCT_A \\
\hline 2678 & CL_M_T5 & PCT_EXP \\
\hline 2681 & CONC_T1 & KPT \\
\hline 2682 & CONC_T1 & PCT_A \\
\hline 2683 & CONC_T1 & PCT_EXP \\
\hline 2686 & CONC_T2 & KPT \\
\hline 2687 & CONC_T2 & PCT_A \\
\hline 2688 & CONC_T2 & PCT_EXP \\
\hline 2691 & CULEBRA & KPT \\
\hline 2692 & CULEBRA & PCT_A \\
\hline 2693 & CULEBRA & PCT_EXP \\
\hline 2696 & DEWYLAKE & KPT \\
\hline 2697 & DEWYLAKE & PCT_A \\
\hline 2698 & DEWYLAKE & PCT_EXP \\
\hline 2701 & DRZ_0 & KPT \\
\hline 2702 & $D R Z$ D 0 & PCT_A \\
\hline 2703 & $D R Z=0$ & PCT_EXP \\
\hline 2706 & EARTH & KPT \\
\hline 2707 & EARTH & PCT_A \\
\hline 2708 & EARTH & PCT_EXP \\
\hline 2711 & EXP_AREA & KPT \\
\hline 2712 & EXP_AREA & PCT_A \\
\hline 2713 & EXP_AREA & PCT_EXP \\
\hline 2715 & FORTYNIN & KPT \\
\hline 2716 & FORTYNIN & PCT_A \\
\hline 2717 & FORTYNIN & PCT_EXP \\
\hline 2718 & FORTYNIN & PO_MIN \\
\hline 2720 & IMPERM_Z & KPT \\
\hline 2721 & IMPERM_Z & PCT_A \\
\hline 2722 & IMPERM_Z & PCT_EXP \\
\hline 2725 & MAGENTA & KPT \\
\hline 2726 & MAGENTA & PCT_A \\
\hline 2727 & MAGENTA & PCT_EXP \\
\hline 2728 & MAGENTA & PO_MIN \\
\hline 2731 & PAN_SEAL & KPT \\
\hline 2732 & PAN_SEAL & PCT_A \\
\hline 2733 & PAN_SEAL & PCT_EXP \\
\hline 2734 & PAN_SEAL & SAT_IBRN \\
\hline 2736 & REPOSIT & KPT \\
\hline 2737 & REPOSIT & PCT_A \\
\hline 2738 & REPOSIT & PCT_EXP \\
\hline 2739 & REPOSIT & PO_MIN \\
\hline 2740 & REPOSIT & SAT_IBRN \\
\hline 2741 & REPOSIT & SAT_RBRN \\
\hline 2744 & SALT_T1 & KPT \\
\hline 2745 & SALT_T1 & PCT_A \\
\hline 2746 & SALT_T1 & PCT_EXP \\
\hline 2749 & SALT_T2 & KPT \\
\hline 2750 & SALT_T2 & PCT_A \\
\hline
\end{tabular}


ID Number

\begin{tabular}{|c|c|c|}
\hline Parameter ID & Material & Parameter \\
\hline 2751 & SALT_T2 & PCT_EXP \\
\hline 2754 & SALT_T3 & KPT \\
\hline 2755 & SALT_T3 & PCT_A \\
\hline 2756 & SALT_T3 & PCT_EXP \\
\hline 2759 & SALT_T4 & KPT \\
\hline 2760 & SALT_T4 & PCT_A \\
\hline 2761 & SALT_T4 & PCT_EXP \\
\hline 2764 & SALT_T5 & KPT \\
\hline 2765 & SALT_T5 & PCT_A \\
\hline 2766 & SALT_T5 & PCT_EXP \\
\hline 2768 & SANTAROS & KPT \\
\hline 2769 & SANTAROS & PCT_A \\
\hline 2770 & SANTAROS & PCT_EXP \\
\hline 2773 & S_ANH_AB & KPT \\
\hline 2774 & S_ANH_AB & PCT_A \\
\hline 2775 & S_ANH_AB & PCT_EXP \\
\hline 2778 & S_HALITE & KPT \\
\hline 2779 & S_HALITE & PCT_A \\
\hline 2780 & S_HALITE & PCT_EXP \\
\hline 2783 & S_MB138 & KPT \\
\hline 2784 & S_MB138 & PCT_A \\
\hline 2785 & S_MB138 & PCT_EXP \\
\hline 2788 & S_MB139 & KPT \\
\hline 2789 & S_MB139 & PCT_A \\
\hline 2790 & S_MB139 & PCT_EXP \\
\hline 2793 & TAMARISK & KPT \\
\hline 2794 & TAMARISK & PCT_A \\
\hline 2795 & TAMARISK & PCT_EXP \\
\hline 2796 & TAMARISK & PO_MIN \\
\hline 2799 & UNNAMED & KPT \\
\hline 2800 & UNNAMED & PCT_A \\
\hline 2801 & UNNAMED & PCT_EXP \\
\hline 2802 & UNNAMED & PO_MIN \\
\hline 2804 & WAS_AREA & KPT \\
\hline 2805 & WAS_AREA & PCT_A \\
\hline 2806 & WAS_AREA & PCT_EXP \\
\hline 2808 & CONC_T2 & PO_MIN \\
\hline 2809 & SALT_T5 & PORE_DIS \\
\hline 2810 & S_MB138 & IFRX \\
\hline 2811 & S_MB139 & IFRX \\
\hline 2812 & S_ANH_AB & IFRX \\
\hline 2813 & S_MB138 & IFRY \\
\hline 2814 & S_MB139 & IFRY \\
\hline 2815 & S_ANH_AB & IFRY \\
\hline 2816 & S_MB138 & IFRZ \\
\hline 2817 & S_MB139 & IFRZ \\
\hline 2818 & S_ANH_AB & IFRZ \\
\hline 2819 & S_ANH_AB & BKLINK \\
\hline 2820 & S_ANH_AB & EXPKLINK \\
\hline 2823 & WAS_AREA & PROBDEG \\
\hline 2824 & REPOSIT & PROBDEG \\
\hline
\end{tabular}

\begin{tabular}{|c|c|c|}
\hline Parameter ID & Material & Parameter \\
\hline 2825 & REFCON & TC_H2 \\
\hline 2826 & REFCON & TC_CO2 \\
\hline 2827 & REFCON & TC_CH4 \\
\hline 2828 & REFCON & TC_N2 \\
\hline 2829 & REFCON & TC_H2S \\
\hline 2830 & REFCON & TC_O2 \\
\hline 2831 & REFCON & ACF_H2 \\
\hline 2832 & REFCON & ACF_CO2 \\
\hline 2833 & REFCON & ACF_CH4 \\
\hline 2834 & REFCON & ACF_N2 \\
\hline 2835 & REFCON & ACF_H2S \\
\hline 2836 & REFCON & ACF_O2 \\
\hline 2837 & REFCON & PC_H2 \\
\hline 2838 & REFCON & PC_CO2 \\
\hline 2839 & REFCON & $\mathrm{PC} \_\mathrm{CH} 4$ \\
\hline 2840 & REFCON & PC_N2 \\
\hline 2841 & REFCON & PC_H2S \\
\hline 2842 & REFCON & PC_O2 \\
\hline 2858 & REFCON & $\mathrm{MW}$ \\
\hline 2859 & REFCON & $\mathrm{MW}=\mathrm{CO} 2$ \\
\hline 2860 & REFCON & $\mathrm{MW} \_\mathrm{CH} 4$ \\
\hline 2861 & REFCON & MW_N2 \\
\hline 2862 & REFCON & MW_H2S \\
\hline 2863 & REFCON & $M W=02$ \\
\hline 2864 & REFCON & $\mathrm{MW}+\mathrm{H} 2 \mathrm{O}$ \\
\hline 2865 & REFCON & MW_FE \\
\hline 2866 & REFCON & $\mathrm{MW} \_\mathrm{CH} 2 \mathrm{O}$ \\
\hline 2867 & REFCON & MW_H2SO4 \\
\hline 2868 & REFCON & MW_HNO3 \\
\hline 2869 & REFCON & MW_FES2 \\
\hline 2870 & REFCON & MW_FECO3 \\
\hline 2871 & REFCON & MW_FEOH2 \\
\hline 2872 & REFCON & MW_FEOOH \\
\hline 2873 & REFCON & MW_FE3O4 \\
\hline 2874 & REFCON & MW_FES \\
\hline 2875 & REFCON & MW_CAO \\
\hline 2876 & REFCON & $\mathrm{MW}$ _CAOH2 \\
\hline 2877 & REFCON & $\mathrm{MW} \_\mathrm{CACO} 3$ \\
\hline 2878 & REFCON & MW_NACL \\
\hline 2879 & REFCON & BBLG \\
\hline 2880 & REFCON & PASCP \\
\hline 2881 & REFCON & FTM \\
\hline 2882 & REFCON & DARM2 \\
\hline 2883 & REFCON & F3M3 \\
\hline 2884 & REFCON & GTI3 \\
\hline 2885 & REFCON & LBKG \\
\hline 2886 & REFCON & KGLB \\
\hline 2887 & REFCON & DAYSEC \\
\hline 2888 & REFCON & YRSEC \\
\hline 2889 & REFCON & GRAVACC \\
\hline 2890 & REFCON & ATMPA \\
\hline
\end{tabular}


ID Number

\begin{tabular}{|c|c|c|}
\hline Parameter ID & Material & Parameter \\
\hline 2891 & REFCON & RTK \\
\hline 2892 & REFCON & PSIPA \\
\hline 2893 & REFCON & $R$ \\
\hline 2894 & REFCON & OMEGAA \\
\hline 2895 & REFCON & OMEGAB \\
\hline 2896 & REFCON & $\mathrm{PI}$ \\
\hline 2897 & REFCON & AL2 \\
\hline 2898 & STEEL & STOIFX \\
\hline 2899 & FORTYNIN & PRMX_LOG \\
\hline 2900 & FORTYNIN & PRMY_LOG \\
\hline 2901 & FORTYNIN & PRMZ_LOG \\
\hline 2902 & S_MB138 & EXPKLINK \\
\hline 2903 & S_MB139 & EXPKLINK \\
\hline 2904 & S_MB138 & BKLINK \\
\hline 2905 & S_MB139 & BKLINK \\
\hline 2906 & NITRATE & QINIT \\
\hline 2907 & STEEL & CORRMCO2 \\
\hline 2908 & STEEL & CORRWCO2 \\
\hline 2909 & SULFATE & QINIT \\
\hline 2910 & STEEL & HUMCORR \\
\hline 2911 & UNNAMED & PRMX_LOG \\
\hline 2912 & UNNAMED & PRMY_LOG \\
\hline 2913 & UNNAMED & PRMZ_LOG \\
\hline 2914 & TAMARISK & PRMX_LOG \\
\hline 2915 & TAMARISK & PRMY_LOG \\
\hline 2916 & TAMARISK & PRMZ_LOG \\
\hline 2918 & CASTILER & VOLUME \\
\hline 2919 & CL_M_T4 & RSH_AIR \\
\hline 2920 & CL_M_T4 & RSH_SAL \\
\hline 2921 & CL_M_T4 & RSH_WAS \\
\hline 2922 & CL_M_T4 & RSH_EXH \\
\hline 2923 & CL_M_T4 & RADN_DRZ \\
\hline 2924 & CL_M_T5 & RSH_AIR \\
\hline 2925 & CL_M_T5 & RSH_SAL \\
\hline 2926 & CL_M_T5 & RSH_WAS \\
\hline 2927 & CL_M_T5 & RSH_EXH \\
\hline 2928 & $C L \_M \_T 5$ & RADN_DRZ \\
\hline 2929 & ASPHALT & RSH_AIR \\
\hline 2930 & ASPHALT & RSH_SAL \\
\hline 2931 & ASPHALT & RSH_WAS \\
\hline 2932 & ASPHALT & RSH_EXH \\
\hline 2933 & ASPHALT & RADN_DRZ \\
\hline 2934 & SALT_T1 & RSH_AIR \\
\hline 2935 & SALT_T1 & RSH_SAL \\
\hline 2936 & SALT_T1 & RSH_WAS \\
\hline 2937 & SALT_T1 & RSH_EXH \\
\hline 2938 & SALT_T1 & RADN_DRZ \\
\hline 2939 & SALT_T1 & CUMPROB \\
\hline 2940 & SALT_T1 & PMLT_MD \\
\hline 2941 & SALT_T1 & PMLT_LO \\
\hline 2942 & SALT_T1 & PMLT_HI \\
\hline
\end{tabular}

\begin{tabular}{|c|c|c|}
\hline Parameter ID & Material & Parameter \\
\hline 2943 & SALT_T2 & RSH_AIR \\
\hline 2944 & SALT_T2 & RSH_SAL \\
\hline 2945 & SALT_T2 & RSH_WAS \\
\hline 2946 & SALT_T2 & RSH_EXH \\
\hline 2947 & SALT_T2 & RADN_DRZ \\
\hline 2948 & SALT_T2 & PMLT_MD \\
\hline 2949 & SALT_T2 & PMLT_LO \\
\hline 2950 & SALT_T2 & PMLT_HI \\
\hline 2951 & SALT_T3 & RSH_AIR \\
\hline 2952 & SALT_T3 & RSH_SAL \\
\hline 2953 & SALT_T3 & RSH_WAS \\
\hline 2954 & SALT_T3 & RSH_EXH \\
\hline 2955 & SALT_T3 & RADN_DRZ \\
\hline 2956 & SALT_T3 & PMLT_MD \\
\hline 2957 & SALT_T3 & PMLT_LO \\
\hline 2958 & SALT_T3 & PMLT_HI \\
\hline 2959 & SALT_T4 & RSH_AIR \\
\hline 2960 & SALT_T4 & RSH_SAL \\
\hline 2961 & SALT_T4 & RSH_WAS \\
\hline 2962 & SALT_T4 & RSH_EXH \\
\hline 2963 & SALT_T4 & RADN_DRZ \\
\hline 2964 & SALT_T4 & PMLT_MD \\
\hline 2965 & ISALT_T4 & PMLT_LO \\
\hline 2966 & SALT_T4 & PMLT_HI \\
\hline 2967 & SALT_T5 & RSH_AIR \\
\hline 2968 & SALT_T5 & RSH_SAL \\
\hline 2969 & SALT_T5 & RSH_WAS \\
\hline 2970 & SALT_T5 & RSH_EXH \\
\hline 2971 & SALT_T5 & RADN_DRZ \\
\hline 2972 & SALT_T5 & PMLT_MD \\
\hline 2973 & SALT_T5 & PMLT_LO \\
\hline 2974 & SALT_T5 & PMLT_HI \\
\hline 2975 & SALT_T6 & RSH_AIR \\
\hline 2976 & SALT_T6 & RSH_SAL \\
\hline 2977 & SALT_T6 & RSH_WAS \\
\hline 2978 & SALT_T6 & RSH_EXH \\
\hline 2979 & SALT_T6 & RADN_DRZ \\
\hline 2980 & SALT_T6 & PMLT_MD \\
\hline 2981 & SALT_T6 & PMLT_LO \\
\hline 2982 & SALT_T6 & PMLT_HI \\
\hline 2983 & SALT_T6 & CAP_MOD \\
\hline 2984 & SALT_T6 & COMP_RCK \\
\hline 2985 & SALT_T6 & KPT \\
\hline 2986 & SALT_T6 & PC_MAX \\
\hline 2987 & SALT_T6 & PCT_A \\
\hline 2988 & SALT_T6 & PCT_EXP \\
\hline 2989 & SALT_T6 & PORE_DIS \\
\hline 2990 & SALT_T6 & IPOROSITY \\
\hline 2991 & SALT_T6 & RELP_MOD \\
\hline 2992 & SALT_T6 & SAT_RBRN \\
\hline 2993 & SALT_T6 & |SAT_RGAS \\
\hline
\end{tabular}


ID Number

\begin{tabular}{|c|c|c|}
\hline Parameter ID & Material & Parameter \\
\hline 2994 & CELLULS & FBETA \\
\hline 2995 & REPOSIT & DPLSCRHW \\
\hline 2996 & CLAY_RUS & RSH_AIR \\
\hline 2997 & CLAY_RUS & RSH_SAL \\
\hline 2998 & CLAY_RUS & RSH_WAS \\
\hline 2999 & CLAY_RUS & RSH_EXH \\
\hline 3000 & CLAY_RUS & CAP_MOD \\
\hline 3001 & CLAY_RUS & COMP_RCK \\
\hline 3002 & CLAY_RUS & KPT \\
\hline 3003 & CLAY_RUS & PC_MAX \\
\hline 3004 & CLAY_RUS & PCT_A \\
\hline 3005 & CLAY_RUS & PCT_EXP \\
\hline 3006 & CLAY_RUS & PORE_DIS \\
\hline 3007 & CLAY_RUS & POROSITY \\
\hline 3008 & CLAY_RUS & PRESSURE \\
\hline 3009 & CLAY_RUS & PRMX_LOG \\
\hline 3010 & CLAY_RUS & PRMY_LOG \\
\hline 3011 & CLAY_RUS & PRMZ_LOG \\
\hline 3012 & CLAY_RUS & RELP_MOD \\
\hline 3013 & CLAY_RUS & SAT_IBRN \\
\hline 3014 & CLAY_RUS & SAT_RBRN \\
\hline 3015 & CLAY_RUS & SAT_RGAS \\
\hline 3016 & MAGENTA & COMP_RCK \\
\hline 3017 & CL_L_T1 & RSH_AIR \\
\hline 3018 & CL_L_T1 & RSH_SAL \\
\hline 3019 & $C L L L=T 1$ & RSH_WAS \\
\hline 3020 & $C L \_L \_T 1$ & RSH_EXH \\
\hline 3021 & CL_L_T1 & RADN_DRZ \\
\hline 3022 & $C L \_L / T 2$ & RSH_AIR \\
\hline 3023 & CL_L_T2 & RSH_SAL \\
\hline 3024 & $\mathrm{CL}_{\text {L L_T2 }}$ & RSH_WAS \\
\hline 3025 & CL_L_T2 & RSH_EXH \\
\hline 3026 & $C L \_L / T 2$ & RADN_DRZ \\
\hline 3027 & CL_L_T3 & RSH_AIR \\
\hline 3028 & $C L L L=T 3$ & RSH_SAL \\
\hline 3029 & CL_L_T3 & RSH_WAS \\
\hline 3030 & CL_L_T3 & RSH_EXH \\
\hline 3031 & CL_L_T3 & RADN_DRZ \\
\hline 3032 & EARTH & RSH_AIR \\
\hline 3033 & EARTH & RSH_SAL \\
\hline 3034 & EARTH & RSH_WAS \\
\hline 3035 & EARTH & RSH_EXH \\
\hline 3036 & CONC_T1 & RSH_AIR \\
\hline 3037 & CONC_T1 & RSH_SAL \\
\hline 3038 & CONC_T1 & RSH_WAS \\
\hline 3039 & CONC_T1 & RSH_EXH \\
\hline 3040 & CONC_T1 & RADN_DRZ \\
\hline 3041 & CONC_T2 & RSH_AIR \\
\hline 3042 & CONC_T2 & RSH_SAL \\
\hline 3043 & CONC_T2 & RSH_WAS \\
\hline 3044 & CONC_T2 & RSH_EXH \\
\hline
\end{tabular}

\begin{tabular}{|c|c|c|}
\hline Parameter ID & Material & Parameter \\
\hline 3045 & CONC_T2 & RADN_DRZ \\
\hline 3046 & CONC MON & RSH_AIR \\
\hline 3047 & CONC_MON & RSH_SAL \\
\hline 3048 & CONC_MON & RSH_WAS \\
\hline 3049 & CONC_MON & RSH_EXH \\
\hline 3050 & CONC_MON & RADN_DRZ \\
\hline 3051 & CONC_MON & CAP_MOD \\
\hline 3052 & CONC_MON & COMP_RCK \\
\hline 3053 & CONC_MON & KPT \\
\hline 3054 & CONC_MON & PC_MAX \\
\hline 3055 & CONC_MON & PCT_A \\
\hline 3056 & CONC_MON & PCT_EXP \\
\hline 3057 & CONC_MON & PORE_DIS \\
\hline 3058 & CONC_MON & POROSITY \\
\hline 3059 & CONC_MON & PRMX_LOG \\
\hline 3060 & CONC_MON & PRMY_LOG \\
\hline 3061 & CONC_MON & PRMZ_LOG \\
\hline 3062 & CONC MON & RELP_MOD \\
\hline 3063 & CONC_MON & SAT_RBRN \\
\hline 3064 & CONC MON & SAT_RGAS \\
\hline 3065 & $C L_{2} L_{-} T 4$ & RSH_AIR \\
\hline 3066 & $C L_{2} L_{-} T 4$ & RSH_SAL \\
\hline 3067 & CL_L_T4 & RSH_WAS \\
\hline 3068 & CL_L_T4 & RSH_EXH \\
\hline 3069 & CL_L_T4 & RADN_DRZ \\
\hline 3070 & CL_L_T4 & CAP_MOD \\
\hline 3071 & $C L \_L$ T4 & COMP_RCK \\
\hline 3072 & CL_L_T4 & KPT \\
\hline 3073 & CL_L_T4 & PC_MAX \\
\hline 3074 & CL_L_T4 & PCT_A \\
\hline 3075 & $C L=L$ T4 & PCT_EXP \\
\hline 3076 & CL_L_T4 & PORE_DIS \\
\hline 3077 & CL_L_T4 & POROSITY \\
\hline 3078 & $\mathrm{CL} \_$L_T4 & PRMX_LOG \\
\hline 3079 & $C L \_L, T 4$ & PRMY__LOG \\
\hline 3080 & CL_L_T4 & PRMZ_LOG \\
\hline 3081 & CL_L_T4 & RELP_MOD \\
\hline 3082 & $C L \_$L $T 4$ & SAT_RBRN \\
\hline 3083 & $\mathrm{CL}_{2}{ }_{-T 4}$ & SAT_RGAS \\
\hline 3084 & CL_M_T1 & RSH_AIR \\
\hline 3085 & CL_M_T1 & RSH_SAL \\
\hline 3086 & CL_M_T1 & RSH_WAS \\
\hline 3087 & CL_M_T1 & RSH_EXH \\
\hline 3088 & $C_{2} M_{-}{ }_{1}$ & RADN_DRZ \\
\hline 3089 & CL_M_T2 & RSH_AIR \\
\hline 3090 & CL_M_T2 & RSH_SAL \\
\hline 3091 & $C_{2}{ }_{1}$ T2 2 & RSH_WAS \\
\hline 3092 & CL_M_T2 & RSH_EXH \\
\hline 3093 & CL_M_T2 & RADN_DRZ \\
\hline 3094 & CL_M_T3 & RSH_AIR \\
\hline 3095 & CL_M_T3 & RSH_SAL \\
\hline
\end{tabular}


ID Number

\begin{tabular}{|c|c|c|}
\hline Parameter ID & Material & Parameter \\
\hline 3096 & CL_M_T3 & RSH_WAS \\
\hline 3097 & CL_M_T3 & RSH_EXH \\
\hline 3098 & CL_M_T3 & RADN_DRZ \\
\hline 3099 & CAVITY_1 & SAT_IBRN \\
\hline 3100 & CAVITY_2 & SAT_IBRN \\
\hline 3101 & CAVITY_3 & PRESSURE \\
\hline 3102 & CAVITY_3 & SAT_IBRN \\
\hline 3103 & CAVITY_4 & PRESSURE \\
\hline 3104 & CAVITY_4 & SAT_IBRN \\
\hline 3105 & REFCON & VROOM \\
\hline 3106 & REFCON & ASDRUM \\
\hline 3107 & REFCON & VPANLEX \\
\hline 3108 & REFCON & VREPOS \\
\hline 3109 & REFCON & AVOGADRO \\
\hline 3110 & REFCON & ZCINK \\
\hline 3111 & REFCON & CITOBQ \\
\hline 3112 & REFCON & SECYR \\
\hline 3113 & REFCON & ACTCONST \\
\hline 3114 & CONC_MON & PRESSURE \\
\hline 3115 & CONC_MON & SAT_IBRN \\
\hline 3116 & DRZ_1 & KPT \\
\hline 3120 & BOREHOLE & PCT_A \\
\hline 3121 & BOREHOLE & PCT_EXP \\
\hline 3122 & BOREHOLE & KPT \\
\hline 3123 & CL_L_T4 & PO_MIN \\
\hline 3124 & CONC_MON & PO_MIN \\
\hline 3125 & SALT_T5 & PO_MIN \\
\hline 3126 & SALT_T6 & PO_MIN \\
\hline 3128 & DRZ_1 & PCT_A \\
\hline 3129 & DRZ_1 & PCT_EXP \\
\hline 3131 & CLAY_RUS & PO_MIN \\
\hline 3132 & REFCON & DRROOM \\
\hline 3133 & SHFT_DRZ & PRMX_LOG \\
\hline 3134 & BH_OPEN & PRMX_LOG \\
\hline 3135 & BH_OPEN & POROSITY \\
\hline 3136 & BH_OPEN & COMP_RCK \\
\hline 3137 & $\mathrm{BH}$ OPEN & RELP_MOD \\
\hline 3138 & BH_OPEN & CAP_MOD \\
\hline 3139 & BH_OPEN & PC_MAX \\
\hline 3140 & BH_OPEN & SAT_RBRN \\
\hline 3141 & BH_OPEN & SAT_RGAS \\
\hline 3142 & BH_OPEN & PORE_DIS \\
\hline 3143 & BH_OPEN & PO_MIN \\
\hline 3144 & BH_OPEN & KPT \\
\hline 3145 & BH_OPEN & PCT_A \\
\hline 3146 & BH_OPEN & PCT_EXP \\
\hline 3147 & CONC_PLG & POROSITY \\
\hline 3148 & CONC_PLG & COMP_RCK \\
\hline 3149 & CONC_PLG & RELP_MOD \\
\hline 3150 & CONC_PLG & CAP_MOD \\
\hline 3151 & CONC_PLG & PC_MAX \\
\hline
\end{tabular}

\begin{tabular}{|c|c|c|}
\hline Parameter ID & Material & Parameter \\
\hline 3152 & CONC_PLG & SAT_RBRN \\
\hline 3153 & CONC_PLG & SAT_RGAS \\
\hline 3154 & CONC_PLG & PORE_DIS \\
\hline 3155 & CONC_PLG & PO_MIN \\
\hline 3156 & CONC_PLG & KPT \\
\hline 3157 & CONC_PLG & PCT_A \\
\hline 3158 & CONC_PLG & PCT_EXP \\
\hline 3159 & BH_SAND & POROSITY \\
\hline 3160 & BH_SAND & COMP_RCK \\
\hline 3161 & BH_SAND & RELP_MOD \\
\hline 3162 & BH_SAND & CAP_MOD \\
\hline 3163 & BH_SAND & PC_MAX \\
\hline 3164 & BH_SAND & SAT_RBRN \\
\hline 3165 & BH_SAND & SAT_RGAS \\
\hline 3166 & BH_SAND & PORE_DIS \\
\hline 3167 & BH_SAND & PO_MIN \\
\hline 3168 & BH_SAND & KPT \\
\hline 3169 & BH_SAND & PCT_A \\
\hline 3170 & BH_SAND & PCT_EXP \\
\hline 3171 & BH_CREEP & POROSITY \\
\hline 3172 & BH_CREEP & COMP_RCK \\
\hline 3173 & BH_CREEP & RELP_MOD \\
\hline 3174 & BH_CREEP & CAP_MOD \\
\hline 3175 & BH_CREEP & PC_MAX \\
\hline 3176 & BH_CREEP & SAT_RBRN \\
\hline 3177 & BH_CREEP & SAT_RGAS \\
\hline 3178 & BH_CREEP & PORE_DIS \\
\hline 3179 & BH_CREEP & PO_MIN \\
\hline 3180 & BH_CREEP & KPT \\
\hline 3181 & BH_CREEP & PCT_A \\
\hline 3182 & BH_CREEP & PCT_EXP \\
\hline 3183 & BH_CREEP & PRMX_LOG \\
\hline 3184 & BH_SAND & PRMX_LOG \\
\hline 3185 & CONC_PLG & PRMX_LOG \\
\hline 3186 & BH_OPEN & PRMY_LOG \\
\hline 3187 & BH_OPEN & PRMZ_LOG \\
\hline 3188 & BH_CREEP & PRMY_LOG \\
\hline 3189 & BH_CREEP & PRMZ_LOG \\
\hline 3190 & BH_SAND & PRMY_LOG \\
\hline 3191 & BH_SAND & PRMZ_LOG \\
\hline 3192 & CONC_PLG & PRMY_LOG \\
\hline 3193 & CONC_PLG & PRMZ_LOG \\
\hline 3194 & CASTILER & GRIDFLO \\
\hline 3196 & TL207 & ATWEIGHT \\
\hline 3197 & PA234M & ATWEIGHT \\
\hline 3198 & Y90 & ATWEIGHT \\
\hline 3199 & ZR90 & ATWEIGHT \\
\hline 3200 & PB211 & ATWEIGHT \\
\hline 3201 & PB212 & ATWEIGHT \\
\hline 3202 & PB214 & ATWEIGHT \\
\hline 3203 & $\mathrm{PO} 212$ & ATWEIGHT \\
\hline
\end{tabular}


ID Number

\begin{tabular}{|c|c|c|}
\hline Parameter ID & Material & Parameter \\
\hline 3204 & |PO213 & ATWEIGHT \\
\hline 3205 & PO214 & ATWEIGHT \\
\hline 3206 & PO215 & ATWEIGHT \\
\hline 3207 & PO216 & ATWEIGHT \\
\hline 3208 & PO218 & ATWEIGHT \\
\hline 3209 & RA223 & ATWEIGHT \\
\hline 3210 & RA224 & ATWEIGHT \\
\hline 3211 & RA225 & ATWEIGHT \\
\hline 3212 & RN219 & ATWEIGHT \\
\hline 3213 & RN220 & ATWEIGHT \\
\hline 3214 & RN222 & ATWEIGHT \\
\hline 3215 & SR90 & ATWEIGHT \\
\hline 3216 & TH227 & ATWEIGHT \\
\hline 3217 & TH228 & ATWEIGHT \\
\hline 3218 & TH231 & ATWEIGHT \\
\hline 3219 & TH234 & ATWEIGHT \\
\hline 3220 & AC225 & ATWEIGHT \\
\hline 3221 & AC227 & ATWEIGHT \\
\hline 3222 & AC228 & ATWEIGHT \\
\hline 3223 & AM243 & ATWEIGHT \\
\hline 3224 & AT217 & ATWEIGHT \\
\hline 3225 & BA137 & ATWEIGHT \\
\hline 3226 & BA137M & ATWEIGHT \\
\hline 3227 & $\mathrm{~B} 1211$ & ATWEIGHT \\
\hline 3228 & $\mathrm{~B} 1212$ & ATWEIGHT \\
\hline 3229 & $\mathrm{~B} \mid 213$ & ATWEIGHT \\
\hline 3230 & $\mathrm{~B} \mid 214$ & ATWEIGHT \\
\hline 3231 & СM243 & ATWEIGHT \\
\hline 3232 & CM245 & ATWEIGHT \\
\hline 3233 & CM248 & ATWEIGHT \\
\hline 3234 & FR221 & ATWEIGHT \\
\hline 3235 & ND143 & ATWEIGHT \\
\hline 3236 & NP239 & ATWEIGHT \\
\hline 3237 & PA233 & ATWEIGHT \\
\hline 3238 & ASPHALT & DNSGRAIN \\
\hline 3239 & BOREHOLE & INV_AR \\
\hline 3240 & BOREHOLE & ROUGHP \\
\hline 3241 & BOREHOLE & PIPED \\
\hline 3242 & BOREHOLE & COLDIA \\
\hline 3243 & BOREHOLE & L2 \\
\hline 3244 & BOREHOLE & $L 1$ \\
\hline 3245 & BLOWOUT & CEMENT \\
\hline 3246 & BLOWOUT & PARTDIA \\
\hline 3247 & BLOWOUT & RHOS \\
\hline 3248 & BLOWOUT & ROOM \\
\hline 3249 & BLOWOUT & RPANEL \\
\hline 3250 & BLOWOUT & HREPO \\
\hline 3251 & BLOWOUT & PSUF \\
\hline 3252 & BLOWOUT & VISC \\
\hline 3253 & BLOWOUT & RGAS \\
\hline 3254 & BLOWOUT & KGAS \\
\hline
\end{tabular}

\begin{tabular}{|c|c|c|}
\hline Parameter ID & Material & Parameter \\
\hline 3255 & BLOWOUT & FSE \\
\hline 3256 & BLOWOUT & FGE \\
\hline 3257 & BLOWOUT & SUFTEN \\
\hline 3258 & BLOWOUT & TREPO \\
\hline 3259 & BLOWOUT & APORO \\
\hline 3260 & BLOWOUT & INPORO \\
\hline 3261 & BOREHOLE & RHW_AR \\
\hline 3262 & SOLAM3 & SOLSIM \\
\hline 3263 & SOLAM3 & SOLCIM \\
\hline 3264 & SOLPU3 & $\longdiv { \text { SOLCIM } }$ \\
\hline 3265 & SOLPU3 & SOLSIM \\
\hline 3266 & SOLPU4 & SOLSIM \\
\hline 3267 & AC225 & HALFLIFE \\
\hline 3268 & AC227 & HALFLIFE \\
\hline 3269 & AC228 & HALFLIFE \\
\hline 3270 & AT217 & HALFLIFE \\
\hline 3271 & BA137 & HALFLIFE \\
\hline 3272 & BA137M & HALFLIFE \\
\hline 3273 & $\mathrm{~B} 1211$ & HALFLIFE \\
\hline 3274 & $\mathrm{BI} 212$ & HALFLIFE \\
\hline 3275 & $\mathrm{BI} 213$ & HALFLIFE \\
\hline 3276 & $\mathrm{~B} 1214$ & HALFLIFE \\
\hline 3277 & CM243 & HALFLIFE \\
\hline 3278 & CM245 & HALFLIFE \\
\hline 3279 & FR221 & HALFLIFE \\
\hline 3280 & ND143 & HALFLIFE \\
\hline 3281 & NP239 & HALFLIFE \\
\hline 3282 & PA233 & HALFLIFE \\
\hline 3283 & PA234M & HALFLIFE \\
\hline 3284 & PB209 & HALFLIFE \\
\hline 3285 & PB211 & HALFLIFE \\
\hline 3286 & PB212 & HALFLIFE \\
\hline 3287 & PB214 & HALFLIFE \\
\hline 3288 & PO212 & HALFLIFE \\
\hline 3289 & PO213 & HALFLIFE \\
\hline 3290 & PO214 & HALFLIFE \\
\hline 3291 & PO215 & HALFLIFE \\
\hline 3292 & PO216 & HALFLIFE \\
\hline 3293 & PO218 & HALFLIFE \\
\hline 3294 & RA223 & HALFLIFE \\
\hline 3295 & RA224 & HALFLIFE \\
\hline 3296 & RA225 & HALFLIFE \\
\hline 3297 & RN219 & HALFLIFE \\
\hline 3298 & RN220 & HALFLIFE \\
\hline 3299 & RN222 & HALFLIFE \\
\hline 3300 & TH227 & HALFLIFE \\
\hline 3301 & TH228 & HALFLIFE \\
\hline 3302 & TH231 & HALFLIFE \\
\hline 3303 & TH234 & HALFLIFE \\
\hline 3304 & TL207 & HALFLIFE \\
\hline 3305 & Y90 & HALFLIFE \\
\hline
\end{tabular}


ID Number

\begin{tabular}{|c|c|c|}
\hline Parameter ID & Material & Parameter \\
\hline 3306 & ZR90 & HALFLIFE \\
\hline 3307 & U & CONCINT \\
\hline 3308 & $U$ & CAPMIC \\
\hline 3309 & $U$ & PROPMIC \\
\hline 3310 & AM & CONCINT \\
\hline 3311 & $A M$ & PROPMIC \\
\hline 3312 & NP & CONCINT \\
\hline 3313 & NP & CAPMIC \\
\hline 3314 & $\mathrm{NP}$ & PROPMIC \\
\hline 3315 & $\mathrm{PU}$ & CAPMIC \\
\hline 3316 & $\mathrm{PU}$ & CONCINT \\
\hline 3317 & $\mathrm{PU}$ & PROPMIC \\
\hline 3318 & $\mathrm{TH}$ & CAPMIC \\
\hline 3319 & $\mathrm{TH}$ & CONCINT \\
\hline 3320 & $\mathrm{TH}$ & PROPMIC \\
\hline 3321 & AC225 & EPAREL \\
\hline 3322 & AC228 & EPAREL \\
\hline 3323 & AT217 & EPAREL \\
\hline 3324 & BA137 & EPAREL \\
\hline 3325 & BA137M & EPAREL \\
\hline 3326 & $\mathrm{~B} 1211$ & EPAREL \\
\hline 3327 & $\mathrm{~B} \mid 212$ & EPAREL \\
\hline 3328 & $B \mid 213$ & EPAREL \\
\hline 3329 & $\mathrm{~B} 1214$ & EPAREL \\
\hline 3330 & CF252 & EPAREL \\
\hline 3331 & CM244 & EPAREL \\
\hline 3332 & FR221 & EPAREL \\
\hline 3333 & ND143 & EPAREL \\
\hline 3334 & NP239 & EPAREL \\
\hline 3335 & PA233 & EPAREL \\
\hline 3336 & PA234M & EPAREL \\
\hline 3337 & PB209 & EPAREL \\
\hline 3338 & PB211 & EPAREL \\
\hline 3339 & PB212 & EPAREL \\
\hline 3340 & PB214 & EPAREL \\
\hline 3341 & PM147 & EPAREL \\
\hline 3342 & $\mathrm{PO} 212$ & EPAREL \\
\hline 3343 & P0213 & EPAREL \\
\hline 3344 & PO214 & EPAREL \\
\hline 3345 & PO215 & EPAREL \\
\hline 3346 & PO216 & EPAREL \\
\hline 3347 & $\mathrm{PO} 218$ & EPAREL \\
\hline 3348 & PU241 & EPAREL \\
\hline 3349 & RA223 & EPAREL \\
\hline 3350 & RA224 & EPAREL \\
\hline 3351 & RA225 & EPAREL \\
\hline 3352 & RA228 & EPAREL \\
\hline 3353 & RN219 & EPAREL \\
\hline 3354 & RN220 & EPAREL \\
\hline 3355 & RN222 & EPAREL \\
\hline 3356 & TH227 & EPAREL \\
\hline
\end{tabular}

\begin{tabular}{|c|c|c|}
\hline Parameter ID & Material & Parameter \\
\hline 3357 & TH228 & EPAREL \\
\hline 3358 & TH231 & EPAREL \\
\hline 3359 & TH234 & EPAREL \\
\hline 3360 & TL207 & EPAREL \\
\hline 3361 & Y90 & EPAREL \\
\hline 3362 & ZR90 & EPAREL \\
\hline 3363 & AM241 & EPAREL \\
\hline 3364 & $A C 227$ & EPAREL \\
\hline 3365 & AM243 & EPAREL \\
\hline 3366 & CM243 & EPAREL \\
\hline 3367 & CM245 & EPAREL \\
\hline 3368 & CM248 & EPAREL \\
\hline 3369 & CS137 & EPAREL \\
\hline 3370 & NP237 & EPAREL \\
\hline 3371 & PA231 & EPAREL \\
\hline 3372 & PB210 & EPAREL \\
\hline 3373 & PU238 & EPAREL \\
\hline 3374 & PU239 & EPAREL \\
\hline 3375 & PU240 & EPAREL \\
\hline 3376 & PU242 & EPAREL \\
\hline 3377 & PU244 & EPAREL \\
\hline 3378 & RA226 & EPAREL \\
\hline 3379 & SM147 & EPAREL \\
\hline 3380 & SR90 & EPAREL \\
\hline 3381 & TH229 & EPAREL \\
\hline 3382 & TH230 & EPAREL \\
\hline 3383 & TH232 & EPAREL \\
\hline 3384 & U233 & EPAREL \\
\hline 3385 & U234 & EPAREL \\
\hline 3386 & U235 & EPAREL \\
\hline 3387 & U236 & EPAREL \\
\hline 3388 & U238 & EPAREL \\
\hline 3389 & SOLPU4 & SOLCIM \\
\hline 3390 & SOLU4 & SOLSIM \\
\hline 3391 & SOLU6 & SOLSIM \\
\hline 3392 & SOLU6 & SOLCIM \\
\hline 3393 & SOLTH4 & SOLSIM \\
\hline 3402 & SOLMOD3 & SOLCIM \\
\hline 3403 & SOLMOD4 & SOLCIM \\
\hline 3404 & SOLMOD5 & SOLCIM \\
\hline 3405 & SOLMOD6 & SOLCIM \\
\hline 3406 & SOLMOD3 & SOLSIM \\
\hline 3407 & SOLMOD4 & SOLSIM \\
\hline 3408 & SOLMOD5 & SOLSIM \\
\hline 3409 & SOLMOD6 & SOLSIM \\
\hline 3410 & CM243 & INVCHD \\
\hline 3411 & СM243 & INVRHD \\
\hline 3412 & CM245 & INVCHD \\
\hline 3413 & CM245 & INVRHD \\
\hline 3414 & BOREHOLE & WUF \\
\hline 3415 & AM243 & IINVCHD \\
\hline
\end{tabular}


ID Number

\begin{tabular}{|c|c|c|}
\hline Parameter ID & Material & Parameter \\
\hline 3416 & AM243 & INVRHD \\
\hline 3417 & GLOBAL & OXSTAT \\
\hline 3418 & CULEBRA & MEA_STOR \\
\hline 3419 & CULEBRA & MINP_FAC \\
\hline 3420 & BLOWOUT & FCE \\
\hline 3421 & PB209 & ATWEIGHT \\
\hline 3429 & PHUMOX3 & PHUMCIM \\
\hline 3430 & PHUMOX4 & PHUMCIM \\
\hline 3431 & PHUMOX5 & PHUMCIM \\
\hline 3432 & PHUMOX6 & PHUMCIM \\
\hline 3433 & PHUMOX3 & PHUMSIM \\
\hline 3434 & PHUMOX4 & PHUMSIM \\
\hline 3435 & PHUMOX5 & PHUMSIM \\
\hline 3436 & PHUMOX6 & PHUMSIM \\
\hline 3437 & TH & CONCMIN \\
\hline 3438 & $\mathrm{U}$ & CONCMIN \\
\hline 3439 & $\mathrm{NP}$ & CONCMIN \\
\hline 3440 & PU & CONCMIN \\
\hline 3441 & $A M$ & CONCMIN \\
\hline 3442 & $\mathrm{PU}+3$ & MDO \\
\hline 3443 & $P U+4$ & MDO \\
\hline 3444 & $A M+3$ & MDO \\
\hline 3446 & $U+4$ & MDO \\
\hline 3447 & AM & CAPMIC \\
\hline 3448 & $U+6$ & MDO \\
\hline 3449 & $\mathrm{TH}+4$ & MDO \\
\hline 3454 & WAS_AREA & CLOSMOD \\
\hline 3455 & REPOSIT & CLOSMOD \\
\hline 3456 & BLOWOUT & RE_CAST \\
\hline 3457 & $A M$ & CAPHUM \\
\hline 3458 & NP & CAPHUM \\
\hline 3459 & $\mathrm{PU}$ & CAPHUM \\
\hline 3460 & $U$ & CAPHUM \\
\hline 3461 & TH & CAPHUM \\
\hline 3462 & CULEBRA & ETHICK \\
\hline
\end{tabular}

\begin{tabular}{|c|c|c|}
\hline Parameter ID & Material & Parameter \\
\hline 3469 & CULEBRA & SKIN_RES \\
\hline 3470 & BLOWOUT & GAS_MIN \\
\hline 3471 & BLOWOUT & MAXFLOW \\
\hline 3472 & BLOWOUT & MINFLOW \\
\hline 3473 & BLOWOUT & THCK_CAS \\
\hline 3474 & CULEBRA & DTORT \\
\hline 3475 & $U+6$ & MKD_U \\
\hline 3476 & $\mathrm{NP}+5$ & MKD_NP \\
\hline 3477 & $\mathrm{NP}+4$ & MKD_NP \\
\hline 3478 & $\mathrm{TH}+4$ & MKD_TH \\
\hline 3479 & $U+4$ & MKD_U \\
\hline 3480 & $\mathrm{PU}+3$ & MKD_PU \\
\hline 3481 & $\mathrm{PU}+4$ & MKD_PU \\
\hline 3482 & $A M+3$ & MKD_AM \\
\hline 3483 & CULEBRA & DISP_L \\
\hline 3484 & CULEBRA & DISPT_L \\
\hline 3485 & CULEBRA & HMBLKLT \\
\hline 3486 & CULEBRA & DPOROS \\
\hline 3487 & CULEBRA & APOROS \\
\hline 3488 & REFCON & AREA_ZRO \\
\hline 3489 & REFCON & AREA_CH \\
\hline 3490 & REFCON & VOLWP \\
\hline 3491 & GLOBAL & TA \\
\hline 3492 & REFCON & FWW \\
\hline 3493 & GLOBAL & PBRINE \\
\hline 3494 & GLOBAL & LAMBDAD \\
\hline 3495 & GLOBAL & PLGPAT \\
\hline 3496 & REFCON & AREA_RH \\
\hline 3497 & GLOBAL & MINERT \\
\hline 3498 & GLOBAL & TPICM \\
\hline 3499 & GLOBAL & TPICD \\
\hline 3500 & GLOBAL & FPICM \\
\hline 3501 & GLOBAL & FPICD \\
\hline 3502 & REFCON & $\mathrm{HRH}$ \\
\hline 3503 & REFCON & ABERM \\
\hline
\end{tabular}


Appendix B2

\section{Parameter Cross Reference by Material Name}


Material Name

\begin{tabular}{|c|c|c|}
\hline Parameter ID & Material & Parameter \\
\hline 3220 & AC225 & ATWEIGHT \\
\hline 3321 & $A C 225$ & EPAREL \\
\hline 3267 & AC225 & |HALFLIFE \\
\hline 3221 & AC227 & ATWEIGHT \\
\hline 3364 & $A C 227$ & EPAREL \\
\hline 3268 & $A C 227$ & HALFLIFE \\
\hline 3222 & AC228 & ATWEIGHT \\
\hline 3322 & $A C 228$ & EPAREL \\
\hline 3269 & AC228 & HALFLIFE \\
\hline 3457 & $A M$ & CAPHUM \\
\hline 3447 & $A M$ & CAPMIC \\
\hline 3310 & $A M$ & CONCINT \\
\hline 3441 & AM & CONCMIN \\
\hline 3311 & $A M$ & PROPMIC \\
\hline 3444 & $A M+3$ & MDO \\
\hline 3482 & $A M+3$ & MKD_AM \\
\hline 2 & AM241 & ATWEIGHT \\
\hline 3363 & AM241 & EPAREL \\
\hline 3 & AM241 & HALFLIFE \\
\hline 4 & AM241 & INVCHD \\
\hline 5 & AM241 & INVRHD \\
\hline 3223 & AM243 & ATWEIGHT \\
\hline 3365 & AM243 & EPAREL \\
\hline 6 & AM243 & HALFLIFE \\
\hline 3415 & AM243 & INVCHD \\
\hline 3416 & AM243 & INVRHD \\
\hline 2276 & ASPHALT & CAP_MOD \\
\hline 2277 & ASPHALT & COMP_RCK \\
\hline 3238 & ASPHALT & DNSGRAIN \\
\hline 2599 & ASPHALT & KPT \\
\hline 2278 & ASPHALT & PC_MAX \\
\hline 2600 & ASPHALT & PCT_A \\
\hline 2601 & ASPHALT & PCT_EXP \\
\hline 2281 & ASPHALT & PO_MIN \\
\hline 2279 & ASPHALT & PORE_DIS \\
\hline 2280 & ASPHALT & POROSITY \\
\hline 2282 & ASPHALT & PRESSURE \\
\hline 2283 & ASPHALT & PRMX_LOG \\
\hline 2284 & ASPHALT & PRMY_LOG \\
\hline 2285 & ASPHALT & PRMZ_LOG \\
\hline 2933 & ASPHALT & RADN_DRZ \\
\hline 2289 & ASPHALT & RELP_MOD \\
\hline 2929 & ASPHALT & RSH_AIR \\
\hline 2932 & ASPHALT & RSH_EXH \\
\hline 2930 & ASPHALT & RSH_SAL \\
\hline 2931 & ASPHALT & RSH_WAS \\
\hline 2290 & ASPHALT & SAT_IBRN \\
\hline 2291 & ASPHALT & SAT_RBRN \\
\hline 2292 & ASPHALT & SAT_RGAS \\
\hline 3224 & AT217 & ATWEIGHT \\
\hline 3323 & AT217 & EPAREL \\
\hline
\end{tabular}

\begin{tabular}{|c|c|c|}
\hline Parameter ID & Material & Parameter \\
\hline 3270 & AT217 & HALFLIFE \\
\hline 3225 & BA137 & ATWEIGHT \\
\hline 3324 & BA137 & EPAREL \\
\hline 3271 & BA137 & HALFLIFE \\
\hline 3226 & BA137M & ATWEIGHT \\
\hline 3325 & BA137M & EPAREL \\
\hline 3272 & BA137M & HALFLIFE \\
\hline 3174 & BH_CREEP & CAP_MOD \\
\hline 3172 & BH_CREEP & COMP_RCK \\
\hline 3180 & BH_CREEP & KPT \\
\hline 3175 & BH_CREEP & PC_MAX \\
\hline 3181 & BH_CREEP & PCT_A \\
\hline 3182 & BH_CREEP & PCT_EXP \\
\hline 3179 & BH_CREEP & PO_MIN \\
\hline 3178 & BH_CREEP & PORE_DIS \\
\hline 3171 & BH_CREEP & POROSITY \\
\hline 3183 & BH_CREEP & PRMX_LOG \\
\hline 3188 & BH_CREEP & PRMY_LOG \\
\hline 3189 & BH_CREEP & PRMZ_LOG \\
\hline 3173 & BH_CREEP & RELP_MOD \\
\hline 3176 & BH_CREEP & SAT_RBRN \\
\hline 3177 & BH_CREEP & SAT_RGAS \\
\hline 3138 & BH_OPEN & CAP_MOD \\
\hline 3136 & BH_OPEN & COMP_RCK \\
\hline 3144 & BH_OPEN & KPT \\
\hline 3139 & BH_OPEN & PC_MAX \\
\hline 3145 & BH_OPEN & PCT_A \\
\hline 3146 & BH_OPEN * & PCT_EXP \\
\hline 3143 & BH_OPEN & PO_MIN \\
\hline 3142 & BH_OPEN & PORE_DIS \\
\hline 3135 & BH_OPEN & POROSITY \\
\hline 3134 & BH_OPEN & PRMX_LOG \\
\hline 3186 & BH_OPEN & PRMY_LOG \\
\hline 3187 & BH_OPEN & PRMZ_LOG \\
\hline 3137 & BH_OPEN & RELP_MOD \\
\hline 3140 & BH_OPEN & SAT_RBRN \\
\hline 3141 & BH_OPEN & SAT_RGAS \\
\hline 3162 & BH_SAND & CAP_MOD \\
\hline 3160 & BH_SAND & COMP_RCK \\
\hline 3168 & BH_SAND & KPT \\
\hline 3163 & BH_SAND & PC_MAX \\
\hline 3169 & BH_SAND & PCT_A \\
\hline 3170 & BH_SAND & PCT_EXP \\
\hline 3167 & BH_SAND & PO_MIN \\
\hline 3166 & BH_SAND & PORE_DIS \\
\hline 3159 & BH_SAND & POROSITY \\
\hline 3184 & BH_SAND & PRMX_LOG \\
\hline 3190 & BH_SAND & PRMY_LOG \\
\hline 3191 & BH_SAND & PRMZ_LOG \\
\hline 3161 & BH_SAND & RELP_MOD \\
\hline 3164 & BH_SAND & SAT_RBRN \\
\hline
\end{tabular}


Material Name

\begin{tabular}{|c|c|c|}
\hline Parameter ID & Material & Parameter \\
\hline 3165 & BH_SAND & SAT_RGAS \\
\hline 3227 & $B \mid 211$ & ATWEIGHT \\
\hline 3326 & $\mathrm{~B} \mid 211$ & EPAREL \\
\hline 3273 & BI211 & HALFLIFE \\
\hline 3228 & $B 1212$ & ATWEIGHT \\
\hline 3327 & $\mathrm{~B} \mid 212$ & EPAREL \\
\hline 3274 & $B 1212$ & HALFLIFE \\
\hline 3229 & $\mathrm{BI} 213$ & ATWEIGHT \\
\hline 3328 & $\mathrm{~B} \mid 213$ & EPAREL \\
\hline 3275 & $\mathrm{~B} \mid 213$ & HALFLIFE \\
\hline 3230 & $\mathrm{Bl} 214$ & ATWEIGHT \\
\hline 3329 & $B \mid 214$ & EPAREL \\
\hline 3276 & $B 1214$ & HALFLIFE \\
\hline 3259 & BLOWOUT & APORO \\
\hline 3245 & BLOWOUT & CEMENT \\
\hline 3420 & BLOWOUT & FCE \\
\hline 3256 & BLOWOUT & FGE \\
\hline 3255 & BLOWOUT & FSE \\
\hline 3470 & BLOWOUT & GAS_MIN \\
\hline 3250 & BLOWOUT & HREPO \\
\hline 3260 & BLOWOUT & INPORO \\
\hline 3254 & BLOWOUT & KGAS \\
\hline 3471 & BLOWOUT & MAXFLOW \\
\hline 3472 & BLOWOUT & MINFLOW \\
\hline 3246 & BLOWOUT & PARTDIA \\
\hline 3251 & BLOWOUT & PSUF \\
\hline 3456 & BLOWOUT & RE_CAST \\
\hline 3253 & BLOWOUT & RGAS \\
\hline 3247 & BLOWOUT & RHOS \\
\hline 3248 & BLOWOUT & ROOM \\
\hline 3249 & BLOWOUT & RPANEL \\
\hline 3257 & BLOWOUT & SUFTEN \\
\hline 3473 & BLOWOUT & THCK_CAS \\
\hline 3258 & BLOWOUT & TREPO \\
\hline 3252 & BLOWOUT • & VISC \\
\hline 23 & BOREHOLE & CAP_MOD \\
\hline 3242 & BOREHOLE & COLDIA \\
\hline 25 & BOREHOLE & COMP_RCK \\
\hline 26 & BOREHOLE & DIAMMOD \\
\hline 27 & BOREHOLE & DOMEGA \\
\hline 3239 & BOREHOLE & INV_AR \\
\hline 3122 & BOREHOLE & KPT \\
\hline 3244 & BOREHOLE & L1 \\
\hline 3243 & BOREHOLE & L2 \\
\hline 29 & BOREHOLE & PC_MAX \\
\hline 3120 & BOREHOLE & PCT_A \\
\hline 3121 & BOREHOLE & PCT_EXP \\
\hline 3241 & BOREHOLE & PIPED \\
\hline 32 & BOREHOLE & PO_MIN \\
\hline 30 & BOREHOLE & PORE_DIS \\
\hline 31 & BOREHOLE & POROSITY \\
\hline
\end{tabular}

\begin{tabular}{|c|c|c|}
\hline Parameter ID & Material & Parameter \\
\hline 33 & BOREHOLE & PRESSURE \\
\hline 34 & BOREHOLE & PRMX_LOG \\
\hline 35 & BOREHOLE & PRMY_LOG \\
\hline 36 & BOREHOLE & PRMZ_LOG \\
\hline 38 & BOREHOLE & PTINDEX \\
\hline 40 & BOREHOLE & RELP_MOD \\
\hline 3261 & BOREHOLE & RHW_AR \\
\hline 3240 & BOREHOLE & ROUGHP \\
\hline 41 & BOREHOLE & SAT_RBRN \\
\hline 42 & BOREHOLE & SAT_RGAS \\
\hline 2254 & BOREHOLE & TAUFAIL \\
\hline 3414 & BOREHOLE & WUF \\
\hline 43 & BRINECST & COMPRES \\
\hline 44 & BRINECST & DNSFLUID \\
\hline 48 & BRINESAL & COMPRES \\
\hline 49 & BRINESAL & DNSFLUID \\
\hline 50 & BRINESAL & REF_PRES \\
\hline 51 & BRINESAL & REF_TEMP \\
\hline 52 & BRINESAL & RSLOPE \\
\hline 55 & BRINESAL & VISCO \\
\hline 57 & BRINESAL & WTF \\
\hline 58 & CASTILER & AREA_FRC \\
\hline 60 & CASTILER & CAP_MOD \\
\hline 61 & CASTILER & COMP_RCK \\
\hline 3194 & CASTILER & GRIDFLO \\
\hline 2608 & CASTILER & KPT \\
\hline 62 & CASTILER & PC_MAX \\
\hline 2609 & CASTILER & PCT_A \\
\hline 2610 & CASTILER & PCT_EXP \\
\hline 65 & CASTILER & PO_MIN \\
\hline 63 & CASTILER & PORE DIS \\
\hline 64 & CASTILER & POROSITY \\
\hline 66 & CASTILER & PRESSURE \\
\hline 67 & CASTILER & PRMX_LOG \\
\hline 68 & CASTILER & PRMY LOG \\
\hline 69 & CASTILER & PRMZ_LOG \\
\hline 72 & CASTILER & RELP_MOD \\
\hline 73 & CASTILER & SAT_IBRN \\
\hline 74 & CASTILER & SAT_RBRN \\
\hline 75 & CASTILER & SAT_RGAS \\
\hline 2918 & CASTILER & VOLUME \\
\hline 76 & CAVITY_1 & CAP_MOD \\
\hline 77 & CAVITY_1 & COMP_RCK \\
\hline 2612 & CAVITY_1 & KPT \\
\hline 78 & CAVITY_1 & PC_MAX \\
\hline 2613 & CAVITY_1 & PCT_A \\
\hline 2614 & CAVITY_1 & PCT_EXP \\
\hline 81 & CAVITY_1 & PO_MIN \\
\hline 79 & CAVITY_1 & PORE_DIS \\
\hline 80 & CAVITY_1 & POROSITY \\
\hline 82 & CAVITY 1 & PRESSURE \\
\hline
\end{tabular}


Material Name

\begin{tabular}{|c|c|c|}
\hline Parameter ID & Material & Parameter \\
\hline 83 & CAVITY_1 & PRMX_LOG \\
\hline 84 & CAVITY_1 & PRMY_LOG \\
\hline 85 & CAVITY_1 & PRMZ_LOG \\
\hline 88 & CAVITY_1 & RELP_MOD \\
\hline 3099 & CAVITY_1 & SAT_IBRN \\
\hline 89 & CAVITY_1 & SAT_RBRN \\
\hline 90 & CAVITY_1 & SAT_RGAS \\
\hline 91 & CAVITY_2 & CAP_MOD \\
\hline 92 & CAVITY_2 & COMP_RCK \\
\hline 2616 & CAVITY_2 & KPT \\
\hline 93 & CAVITY_2 & PC_MAX \\
\hline 2617 & CAVITY_2 & PCT_A \\
\hline 2618 & CAVITY_2 & PCT_EXP \\
\hline 96 & CAVITY_2 & PO_MIN \\
\hline 94 & CAVITY_2 & IPORE_DIS \\
\hline 95 & CAVITY_2 & POROSITY \\
\hline 97 & CAVITY 22 & PRESSURE \\
\hline 98 & CAVITY_2 & PRMX_LOG \\
\hline 99 & CAVITY_2 & PRMY_LOG \\
\hline 100 & CAVITY_2 & PRMZ_LOG \\
\hline 103 & CAVITY_2 & RELP_MOD \\
\hline 3100 & CAVITY 2 & SAT_IBRN \\
\hline 104 & CAVITY_2 & SAT_RBRN \\
\hline 105 & CAVITY_2 & SAT_RGAS \\
\hline 2049 & CAVITY_3 & CAP_MOD \\
\hline 2051 & CAVITY_3 & COMP_RCK \\
\hline 2620 & CAVITY_3 & KPT \\
\hline 2234 & CAVITY 3 & PC_MAX \\
\hline 2621 & CAVITY_3 & PCT_A \\
\hline 2622 & CAVITY_3 & PCT_EXP \\
\hline 2623 & CAVITY_3 & PO_MIN \\
\hline 2052 & CAVITY_3 & PORE_DIS \\
\hline 2053 & CAVITY_3 & POROSITY \\
\hline 3101 & CAVITY_3 & PRESSURE \\
\hline 2054 & CAVITY_3 & PRMX_LOG \\
\hline 2055 & CAVITY_3 & PRMY_LOG \\
\hline 2056 & CAVITY 3 & PRMZ_LOG \\
\hline 2058 & CAVITY_3 & RELP_MOD \\
\hline 3102 & CAVITY_3 & SAT_IBRN \\
\hline 2235 & CAVITY_3 & SAT_RBRN \\
\hline 2059 & CAVITY 3 & SAT_RGAS \\
\hline 2060 & CAVITY 4 & CAP_MOD \\
\hline 2062 & CAVITY_ 4 & COMP_RCK \\
\hline 2625 & CAVITY_4 & KPT \\
\hline 2236 & CAVITY_4 & PC_MAX \\
\hline 2626 & CAVITY_4 & PCT_A \\
\hline 2627 & CAVITY_4 & PCT_EXP \\
\hline 2628 & CAVITY_4 & PO_MIN \\
\hline 2063 & CAVITY 4 & PORE_DIS \\
\hline 2064 & CAVITY_4 & POROSITY \\
\hline 3103 & CAVITY_4 & PRESSURE \\
\hline
\end{tabular}

\begin{tabular}{|c|c|c|}
\hline Parameter ID & Material & Parameter \\
\hline 2065 & CAVITY_4 & PRMX_LOG \\
\hline 2066 & CAVITY 4 & PRMY_LOG \\
\hline 2067 & CAVITY_4 & PRMZ_LOG \\
\hline 2069 & CAVITY_4 & RELP_MOD \\
\hline 3104 & CAVITY_4 & SAT_IBRN \\
\hline 2237 & CAVITY_4 & SAT_RBRN \\
\hline 2070 & CAVITY_4 & SAT_RGAS \\
\hline 2994 & CELLULS & FBETA \\
\hline 838 & CF & LOGSOLM \\
\hline 106 & CF252 & ATWEIGHT \\
\hline 3330 & CF252 & EPAREL \\
\hline 107 & CF252 & HALFLIFE \\
\hline 108 & CF252 & INVCHD \\
\hline 109 & CF252 & INVRHD \\
\hline 2327 & CL_L_T1 & CAP_MOD \\
\hline 2328 & CL_L_T1 & COMP_RCK \\
\hline 2641 & CL_L_T1 & KPT \\
\hline 2329 & CL_L_T1 & PC_MAX \\
\hline 2642 & CL_L_T1 & PCT_A \\
\hline 2643 & $C L \_L T 1$ & PCT_EXP \\
\hline 2332 & CL_L_T1 & PO_MIN \\
\hline 2330 & CL_L_T1 & PORE_DIS \\
\hline 2331 & CL_L_T1 & POROSITY \\
\hline 2333 & CL_L_T1 & PRESSURE \\
\hline 2334 & CL_L_T1 & PRMX_LOG \\
\hline 2335 & $C L \_$L 1 & PRMY_LOG \\
\hline 2336 & CL_L_T1 & PRMZ_LOG \\
\hline 3021 & CL_L_T1 & RADN_DRZ \\
\hline 2340 & $C L \_L=T 1$ & RELP_MOD \\
\hline 3017 & CL_L_T1 & RSH_AIR \\
\hline 3020 & CL_L_T1 & RSH_EXH \\
\hline 3018 & CL_L_T1 & RSH_SAL \\
\hline 3019 & CL_L_T1 & RSH_WAS \\
\hline 2341 & CL_L_T1 & SAT_IBRN \\
\hline 2342 & CL_L_T1 & SAT_RBRN \\
\hline 2343 & CL_L_T1 & SAT_RGAS \\
\hline 2344 & CL_L_T2 & CAP_MOD \\
\hline 2345 & $\mathrm{CL}_{\mathrm{L}} \mathrm{T}_{\mathrm{T}} \mathrm{2}$ & COMP_RCK \\
\hline 2646 & CL_L_T2 & KPT \\
\hline 2346 & CL_L_T2 & PC_MAX \\
\hline 2647 & CL_L_T2 & PCT_A \\
\hline 2648 & CL_L_T2 & PCT_EXP \\
\hline 2349 & CL_L_T2 & PO_MIN \\
\hline 2347 & $C L \_L-T 2$ & PORE_DIS \\
\hline 2348 & CL_L_T2 & POROSITY \\
\hline 2351 & CL_L_T2 & PRMX_LOG \\
\hline 2352 & CL_L_T2 & PRMY_LOG \\
\hline 2353 & CL_L_T2 & PRMZ_LOG \\
\hline 3026 & CL_L_T2 & RADN_DRZ \\
\hline 2357 & CL_L_T2 & RELP_MOD \\
\hline 3022 & $\mathrm{CL}_{-}{ }_{-T 2}$ & RSH_AIR \\
\hline
\end{tabular}


Material Name

\begin{tabular}{|c|c|c|}
\hline Parameter ID & Material & Parameter \\
\hline 3025 & CL_L_T2 & RSH_EXH \\
\hline 3023 & CL_L_T2 & RSH_SAL \\
\hline 3024 & CL_L_T2 & RSH_WAS \\
\hline 2359 & $C L \_L / T 2$ & SAT_RBRN \\
\hline 2360 & CL_L_T2 & SAT_RGAS \\
\hline 2361 & CL_L_T3 & CAP_MOD \\
\hline 2362 & $\mathrm{CL} L$ T3 & COMP_RCK \\
\hline 2651 & $C L \_L \_T 3$ & KPT \\
\hline 2363 & CL_L_T3 & PC_MAX \\
\hline 2652 & CL_L_T3 & PCT_A \\
\hline 2653 & CL_L_T3 & PCT_EXP \\
\hline 2366 & CL_L_T3 & PO_MIN \\
\hline 2364 & $\mathrm{CL}_{2} \mathrm{~L}_{-} \mathrm{T} 3$ & PORE_DIS \\
\hline 2365 & CL_L_T3 & POROSITY \\
\hline 2368 & CL_L_T3 & PRMX_LOG \\
\hline 2369 & CL_L_T3 & PRMY_LOG \\
\hline 2370 & $\mathrm{CL}_{-} L_{-} T 3$ & PRMZ_LOG \\
\hline 3031 & CL_L_T3 & RADN_DRZ \\
\hline 2374 & $\mathrm{CL}_{2} \mathrm{~L}_{2} \mathrm{~T} 3$ & RELP_MOD \\
\hline 3027 & CL_L_T3 & RSH_AIR \\
\hline 3030 & $C L=L \_T 3$ & RSH_EXH \\
\hline 3028 & $C L \_L / T 3$ & RSH_SAL \\
\hline 3029 & CL_L_T3 & RSH_WAS \\
\hline 2376 & CL_L_T3 & SAT_RBRN \\
\hline 2377 & CL_L_T3 & SAT_RGAS \\
\hline 3070 & CL_L_T4 & CAP_MOD \\
\hline 3071 & CL_L_T4 & COMP_RCK \\
\hline 3072 & CL_L_T4 & KPT \\
\hline 3073 & CL_L_T4 & PC_MAX \\
\hline 3074 & CL_L_T4 & PCT_A \\
\hline 3075 & CL_L_T4 & PCT_EXP \\
\hline 3123 & $C L_{2} L_{-} T 4$ & PO_MIN \\
\hline 3076 & $C L L L=T 4$ & PORE_DIS \\
\hline 3077 & $C L \_L / T 4$ & POROSITY \\
\hline 3078 & CL_L_T4 & PRMX_LOG \\
\hline 3079 & CL_L_T4 & PRMY_LOG \\
\hline 3080 & CL_L_T4 & PRMZ_LOG \\
\hline 3069 & CL_L_T4 & RADN_DRZ \\
\hline 3081 & CL_L_T4 & RELP_MOD \\
\hline 3065 & CL_L_T4 & RSH_AIR \\
\hline 3068 & CL_L_T4 & RSH_EXH \\
\hline 3066 & $\mathrm{CL} \_$L T4 & RSH_SAL \\
\hline 3067 & $C L=L \_T 4$ & RSH_WAS \\
\hline 3082 & $C L=L=T 4$ & SAT_RBRN \\
\hline 3083 & CL_L_T4 & SAT_RGAS \\
\hline 2378 & $C L \_M \_T 1$ & CAP_MOD \\
\hline 2379 & CL_M_T1 & COMP_RCK \\
\hline 2656 & CL_M_T1 & KPT \\
\hline 2380 & CL_M_T1 & PC_MAX \\
\hline 2657 & CL_M_T1 & PCT_A \\
\hline 2658 & CL_M_T1 & PCT_EXP \\
\hline
\end{tabular}

\begin{tabular}{|c|c|c|}
\hline Parameter ID & Material & Parameter \\
\hline 2383 & CL_M_T1 & PO_MIN \\
\hline 2381 & CL_M_T1 & PORE_DIS \\
\hline 2382 & CL_M_T1 & POROSITY \\
\hline 2384 & CL_M_T1 & PRESSURE \\
\hline 2385 & $\mathrm{CL}_{-} \mathrm{M}_{-} \mathrm{T1}$ & PRMX_LOG \\
\hline 2386 & CL_M_T1 & PRMY_LOG \\
\hline 2387 & CL_M_T1 & PRMZ_LOG \\
\hline 3088 & CL_M_T1 & RADN_DRZ \\
\hline 2391 & CL_M_T1 & RELP_MOD \\
\hline 3084 & $\mathrm{CL}_{2} \mathrm{M}_{-} \mathrm{T1}$ & RSH_AIR \\
\hline 3087 & CL_M_T1 & RSH_EXH \\
\hline 3085 & CL_M_T1 & RSH_SAL \\
\hline 3086 & CL_M_T1 & RSH_WAS \\
\hline 2392 & CL_M_T1 & SAT_IBRN \\
\hline 2393 & CL_M_T1 & SAT_RBRN \\
\hline 2394 & CL_M_T1 & SAT_RGAS \\
\hline 2395 & CL_M_T2 & CAP_MOD \\
\hline 2396 & CL_M_T2 & COMP_RCK \\
\hline 2661 & CL_M_T2 & KPT \\
\hline 2397 & CL_M_T2 & PC_MAX \\
\hline 2662 & CL_M_T2 & PCT_A \\
\hline 2663 & $C L \_M-T 2$ & PCT_EXP \\
\hline 2400 & CL_M_T2 & PO_MIN \\
\hline 2398 & CL_M_T2 & PORE_DIS \\
\hline 2399 & $\mathrm{CL} \_\mathrm{M} \_\mathrm{T} 2$ & POROSITY \\
\hline 2402 & CL_M_T2 & PRMX_LOG \\
\hline 2403 & CL_M_T2 & PRMY_LOG \\
\hline 2404 & CL_M_T2 & PRMZ_LOG \\
\hline 3093 & CL_M_T2 & RADN_DRZ \\
\hline 2408 & $\mathrm{CL}_{2} \mathrm{M}_{-} \mathrm{T} 2$ & RELP_MOD \\
\hline 3089 & CL_M_T2 & RSH_AIR \\
\hline 3092 & $\mathrm{CL} \_\mathrm{M}-\mathrm{T2}$ & RSH_EXH \\
\hline 3090 & CL_M_T2 & RSH_SAL \\
\hline 3091 & CL_M_T2 & RSH_WAS \\
\hline 2410 & CL_M_T2 & SAT_RBRN \\
\hline 2411 & CL_M_T2 & SAT_RGAS \\
\hline 2412 & CL_M_T3 & CAP_MOD \\
\hline 2413 & $\mathrm{CL} M$ M T3 & COMP_RCK \\
\hline 2666 & CL_M_T3 & KPT \\
\hline 2414 & CL_M_T3 & PC_MAX \\
\hline 2667 & CL_M_T3 & PCT_A \\
\hline 2668 & CL_M_T3 & PCT_EXP \\
\hline 2417 & CL_M_T3 & PO_MIN \\
\hline 2415 & CL_M_T3 & PORE_DIS \\
\hline 2416 & CL_M_T3 & POROSITY \\
\hline 2419 & CL_M_T3 & PRMX_LOG \\
\hline 2420 & CL_M_T3 & PRMY_LOG \\
\hline 2421 & $C L \_M \_T 3$ & PRMZ_LOG \\
\hline 3098 & CL_M_T3 & RADN_DRZ \\
\hline 2425 & CL_M_T3 & RELP_MOD \\
\hline 3094 & $C L \_M \_T 3$ & RSH_AIR \\
\hline
\end{tabular}


Material Name

\begin{tabular}{|c|c|c|}
\hline Parameter ID & Material & Parameter \\
\hline 3097 & CL_M_T3 & RSH_EXH \\
\hline 3095 & CL_M_T3 & RSH_SAL \\
\hline 3096 & CL_M_T3 & RSH_WAS \\
\hline 2427 & CL_M_T3 & SAT_RBRN \\
\hline 2428 & CL_M_T3 & SAT_RGAS \\
\hline 2429 & CL_M_T4 & CAP_MOD \\
\hline 2430 & CL_M_T4 & COMP_RCK \\
\hline 2671 & CL_M_T4 & KPT \\
\hline 2431 & CL_M_T4 & PC_MAX \\
\hline 2672 & CL_M_T4 & $\overline{P C T} A$ \\
\hline 2673 & CL_M_T4 & PCT_EXP \\
\hline 2434 & CL_M_T4 & PO_MIN \\
\hline 2432 & CL_M_T4 & PORE_DIS \\
\hline 2433 & $C L \_M \_T 4$ & POROSITY \\
\hline 2436 & CL_M_T4 & PRMX_LOG \\
\hline 2437 & CL_M_T4 & PRMY_LOG \\
\hline 2438 & CL_M_T4 & PRMZ_LOG \\
\hline 2923 & CL_M_T4 & RADN_DRZ \\
\hline 2442 & CL_M_T4 & RELP_MOD \\
\hline 2919 & CL_M_T4 & RSH_AIR \\
\hline 2922 & CL_M_T4 & RSH_EXH \\
\hline 2920 & CL_M_T4 & RSH_SAL \\
\hline 2921 & CL_M_T4 & RSH_WAS \\
\hline 2444 & CL_M_T4 & SAT_RBRN \\
\hline 2445 & CL_M_T4 & SAT_RGAS \\
\hline 2446 & CL_M_T5 & CAP_MOD \\
\hline 2447 & CL_M_T5 & COMP_RCK \\
\hline 2676 & CL_M_T5 & KPT \\
\hline 2448 & CL_M_T5 & PC_MAX \\
\hline 2677 & CL_M_T5 & PCT_A \\
\hline 2678 & CL_M_T5 & PCT_EXP \\
\hline 2451 & CL_M_T5 & PO_MIN \\
\hline 2449 & CL_M_T5 & PORE_DIS \\
\hline 2450 & CL_M_T5 & POROSITY \\
\hline 2453 & CL_M_T5 & PRMX_LOG \\
\hline 2454 & CL_M_T5 & PRMY_LOG \\
\hline 2455 & CL_M_T5 & PRMZ_LOG \\
\hline 2928 & CL_M_T5 & RADN_DRZ \\
\hline 2459 & CL_M_T5 & RELP_MOD \\
\hline 2924 & CL_M_T5 & RSH_AIR \\
\hline 2927 & CL_M_T5 & RSH_EXH \\
\hline 2925 & CL_M_T5 & RSH_SAL \\
\hline 2926 & CL_M_T5 & RSH_WAS \\
\hline 2461 & CL_M_T5 & SAT_RBRN \\
\hline 2462 & CL_M_T5 & SAT_RGAS \\
\hline 2310 & CLAY_BOT & CAP_MOD \\
\hline 2311 & CLAY_BOT & COMP_RCK \\
\hline 2636 & CLAY_BOT & KPT \\
\hline 2312 & CLAY_BOT & PC_MAX \\
\hline 2637 & CLAY_BOT & PCT_A \\
\hline 2638 & CLAY_BOT & PCT_EXP \\
\hline
\end{tabular}

\begin{tabular}{|c|c|c|}
\hline Parameter ID & Material & Parameter \\
\hline 2315 & |CLAY_BOT & PO_MIN \\
\hline 2313 & CLAY_BOT & PORE_DIS \\
\hline 2314 & CLAY_BOT & POROSITY \\
\hline 2316 & CLAY_BOT & |PRESSURE \\
\hline 2317 & CLAY_BOT & PRMX_LOG \\
\hline 2318 & CLAY_BOT & PRMY_LOG \\
\hline 2319 & CLAY_BOT & PRMZ_LOG \\
\hline 2323 & CLAY_BOT & RELP_MOD \\
\hline 2324 & CLAY_BOT & SAT_IBRN \\
\hline 2325 & CLAY_BOT & SAT_RBRN \\
\hline 2326 & CLAY_BOT & SAT_RGAS \\
\hline 3000 & CLAY_RUS & CAP_MOD \\
\hline 3001 & CLAY_RUS & COMP_RCK \\
\hline 3002 & CLAY_RUS & KPT \\
\hline 3003 & CLAY_RUS & PC_MAX \\
\hline 3004 & CLAY_RUS & PCT_A \\
\hline 3005 & CLAY_RUS & PCT_EXP \\
\hline 3131 & CLAY_RUS & PO_MIN \\
\hline 3006 & CLAY_RUS & PORE_DIS \\
\hline 3007 & CLAY_RUS & POROSITY \\
\hline 3008 & CLAY_RUS & PRESSURE \\
\hline 3009 & CLAY_RUS & PRMX_LOG \\
\hline 3010 & CLAY_RUS & PRMY_LOG \\
\hline 3011 & CLAY_RUS & PRMZ_LOG \\
\hline 3012 & CLAY_RUS & RELP_MOD \\
\hline 2996 & CLAY_RUS & RSH_AIR \\
\hline 2999 & CLAY_RUS & RSH_EXH \\
\hline 2997 & CLAY_RUS & RSH_SAL \\
\hline 2998 & CLAY_RUS & RSH_WAS \\
\hline 3013 & CLAY_RUS & SAT_IBRN \\
\hline 3014 & CLAY_RUS & SAT_RBRN \\
\hline 3015 & CLAY_RUS & SAT_RGAS \\
\hline 3231 & CM243 & ATWEIGHT \\
\hline 3366 & CM243 & EPAREL \\
\hline 3277 & CM243 & HALFLIFE \\
\hline 3410 & CM243 & INVCHD \\
\hline 3411 & CM243 & INVRHD \\
\hline 110 & CM244 & ATWEIGHT \\
\hline 3331 & CM244 & EPAREL \\
\hline 111 & CM244 & HALFLIFE \\
\hline 112 & CM244 & INVCHD \\
\hline 113 & CM244 & INVRHD \\
\hline 3232 & CM245 & ATWEIGHT \\
\hline 3367 & CM245 & EPAREL \\
\hline 3278 & CM245 & HALFLIFE \\
\hline 3412 & CM245 & INVCHD \\
\hline 3413 & CM245 & INVRHD \\
\hline 3233 & CM248 & ATWEIGHT \\
\hline 3368 & CM248 & EPAREL \\
\hline 115 & CM248 & HALFLIFE \\
\hline 2265 & СM248 & INVCHD \\
\hline
\end{tabular}


Material Name

\begin{tabular}{|c|c|c|}
\hline Parameter ID & Material & Parameter \\
\hline 2266 & CM248 & INVRHD \\
\hline 3051 & CONC_MON & CAP_MOD \\
\hline 3052 & CONC_MON & COMP_RCK \\
\hline 3053 & CONC_MON & KPT \\
\hline 3054 & CONC_MON & PC_MAX \\
\hline 3055 & CONC_MON & PCT_A \\
\hline 3056 & CONC_MON & PCT_EXP \\
\hline 3124 & CONC_MON & PO_MIN \\
\hline 3057 & CONC_MON & PORE_DIS \\
\hline 3058 & CONC_MON & POROSITY \\
\hline 3114 & CONC_MON & PRESSURE \\
\hline 3059 & CONC_MON & PRMX_LOG \\
\hline 3060 & CONC_MON & PRMY_LOG \\
\hline 3061 & CONC_MON & PRMZ_LOG \\
\hline 3050 & CONC_MON & RADN_DRZ \\
\hline 3062 & CONC_MON & RELP_MOD \\
\hline 3046 & CONC_MON & RSH_AIR \\
\hline 3049 & CONC_MON & RSH_EXH \\
\hline 3047 & CONC_MON & RSH_SAL \\
\hline 3048 & CONC_MON & RSH_WAS \\
\hline 3115 & CONC_MON & SAT_IBRN \\
\hline 3063 & CONC_MON & SAT_RBRN \\
\hline 3064 & CONC_MON & SAT_RGAS \\
\hline 3150 & CONC_PLG & CAP_MOD \\
\hline 3148 & CONC_PLG & COMP_RCK \\
\hline 3156 & CONC_PLG & KRT \\
\hline 3151 & CONC_PLG & PC_MAX \\
\hline 3157 & CONC_PLG & PCT_A \\
\hline 3158 & CONC_PLG & PCT_EXP \\
\hline 3155 & CONC_PLG & PO_MIN \\
\hline 3154 & CONC_PLG & PORE_DIS \\
\hline 3147 & CONC_PLG & POROSITY \\
\hline 3185 & CONC_PLG & PRMX_LOG \\
\hline 3192 & CONC_PLG & PRMY_LOG \\
\hline 3193 & CONC_PLG & PRMZ_LOG \\
\hline 3149 & CONC_PLG & RELP_MOD \\
\hline 3152 & CONC_PLG & SAT_RBRN \\
\hline 3153 & CONC_PLG & SAT_RGAS \\
\hline 2463 & CONC_T1 & CAP_MOD \\
\hline 2464 & CONC_T1 & COMP_RCK \\
\hline 2681 & CONC_T1 & KPT \\
\hline 2465 & CONC_T1 & PC_MAX \\
\hline 2682 & CONC_T1 & PCT_A \\
\hline 2683 & CONC_T1 & PCT_EXP \\
\hline 2468 & CONC_T1 & PO_MIN \\
\hline 2466 & CONC_T1 & PORE_DIS \\
\hline 2467 & CONC_T1 & POROSITY \\
\hline 2469 & CONC_T1 & PRESSURE \\
\hline 2470 & CONC_T1 & PRMX_LOG \\
\hline 2471 & CONC_T1 & PRMY_LOG \\
\hline 2472 & CONC_T1 & PRMZ_LOG \\
\hline
\end{tabular}

\begin{tabular}{|c|c|c|}
\hline Parameter ID & Material & Parameter \\
\hline 3040 & CONC_T1 & RADN_DRZ \\
\hline 2476 & CONC_T1 & RELP_MOD \\
\hline 3036 & CONC_T1 & RSH_AIR \\
\hline 3039 & CONC_T1 & RSH_EXH \\
\hline 3037 & CONC_T1 & RSH_SAL \\
\hline 3038 & CONC_T1 & RSH_WAS \\
\hline 2477 & CONC_T1 & SAT_IBRN \\
\hline 2478 & CONC_T1 & SAT_RBRN \\
\hline 2479 & CONC_T1 & SAT_RGAS \\
\hline 2480 & CONC_T2 & CAP_MOD \\
\hline 2481 & CONC_T2 & COMP_RCK \\
\hline 2686 & CONC_T2 & KPT \\
\hline 2482 & CONC_T2 & PC_MAX \\
\hline 2687 & CONC_T2 & PCT_A \\
\hline 2688 & CONC_T2 & PCT_EXP \\
\hline 2808 & CONC_T2 & PO_MIN \\
\hline 2483 & CONC_T2 & PORE_DIS \\
\hline 2484 & CONC_T2 & POROSITY \\
\hline 2485 & CONC_T2 & PRESSURE \\
\hline 2486 & CONC_T2 & PRMX_LOG \\
\hline 2487 & CONC_T2 & PRMY_LOG \\
\hline 2488 & CONC_T2 & PRMZ_LOG \\
\hline 3045 & CONC_T2 & RADN_DRZ \\
\hline 2492 & CONC_T2 & RELP_MOD \\
\hline 3041 & CONC_T2 & RSH_AIR \\
\hline 3044 & CONC_T2 & RSH_EXH \\
\hline 3042 & CONC_T2 & RSH_SAL \\
\hline 3043 & CONC_T2 & RSH_WAS \\
\hline 2493 & CONC_T2 & SAT_IBRN \\
\hline 2494 & CONC_T2 & SAT_RBRN \\
\hline 2495 & CONC_T2 & SAT_RGAS \\
\hline 841 & CS & LOGSOLM \\
\hline 116 & CS137 & ATWEIGHT \\
\hline 3369 & CS137 & EPAREL \\
\hline 117 & CS137 & HALFLIFE \\
\hline 2037 & CS137 & INVCHD \\
\hline 118 & CS137 & INVRHD \\
\hline 3487 & CULEBRA & APOROS \\
\hline 119 & CULEBRA & CAP_MOD \\
\hline 120 & CULEBRA & COMP_RCK \\
\hline 3483 & CULEBRA & DISP_L \\
\hline 3484 & CULEBRA & DISPT_L \\
\hline 843 & CULEBRA & DNSGRAIN \\
\hline 3486 & CULEBRA & DPOROS \\
\hline 3474 & CULEBRA & DTORT \\
\hline 3462 & CULEBRA & ETHICK \\
\hline 123 & CULEBRA & FRCTRIDX \\
\hline 861 & CULEBRA & FTORT \\
\hline 3485 & CULEBRA & HMBLKLT \\
\hline 2691 & CULEBRA & KPT \\
\hline 3418 & CULEBRA & MEA_STOR \\
\hline
\end{tabular}


Material Name

\begin{tabular}{|c|c|c|c|c|c|}
\hline Parameter ID & Material & Parameter & Parameter ID & Material & Parameter \\
\hline 3419 & CULEBRA & MINP_FAC & 183 & DRZ_O & PRMZ_LOG \\
\hline 137 & CULEBRA & PC_MAX & 186 & DRZ_O & RELP_MOD \\
\hline 2692 & CULEBRA & PCT_A & 187 & DRZ_0 & SAT_IBRN \\
\hline 2693 & CULEBRA & PCT_EXP & 188 & DRZ_0 & SAT_RBRN \\
\hline 141 & CULEBRA & PO_MIN & 189 & DRZ_0 & SAT_RGAS \\
\hline 139 & CULEBRA & PORE_DIS & 962 & DRZ_0 & TORTUSTY \\
\hline 140 & CULEBRA & POROSITY & 190 & DRZ_1 & CAP_MOD \\
\hline 142 & CULEBRA & |PRESSURE & 191 & DRZ_1 & COMP_RCK \\
\hline 143 & CULEBRA & PRMX_LOG & 3116 & DRZ_1 & KPT \\
\hline 144 & CULEBRA & PRMY_LOG & 193 & DRZ_1 & PC_MAX \\
\hline 145 & CULEBRA & PRMZ_LOG & 3128 & DRZ_1 & PCT_A \\
\hline 148 & CULEBRA & RELP_MOD & 3129 & $D R Z_{1}$ & PCT_EXP \\
\hline 149 & CULEBRA & SAT_IBRN & 196 & DRZ_1 & PO_MIN \\
\hline 150 & CULEBRA & SAT_RBRN & 194 & $D R Z \_1$ & PORE_DIS \\
\hline 151 & CULEBRA & SAT_RGAS & 195 & $D R Z \_1$ & POROSITY \\
\hline 3469 & CULEBRA & SKIN_RES & 198 & DRZ_1 & PRMX_LOG \\
\hline 2071 & CULEBRA & THICK & 199 & DRZ_1 & PRMY_LOG \\
\hline 153 & DEWYLAKE & CAP_MOD & 200 & DRZ_1 & PRMZ_LOG \\
\hline 154 & DEWYLAKE & COMP_RCK & 203 & $D R Z$ 1 & RELP_MOD \\
\hline 2696 & DEWYLAKE & KPT & 205 & DRZ_1 & SAT_RBRN \\
\hline 156 & DEWYLAKE & PC_MAX & 206 & $D R Z$ _1 & SAT_RGAS \\
\hline 2697 & DEWYLAKE & PCT_A & 2496 & EARTH & CAP_MOD \\
\hline 2698 & DEWYLAKE & PCT_EXP & 2497 & EARTH & COMP_RCK \\
\hline 159 & DEWYLAKE & PO_MIN & 2706 & EARTH & KPT \\
\hline 157 & DEWYLAKE & PORE_DIS & 2498 & EARTH & PC_MAX \\
\hline 158 & DEWYLAKE & POROSITY & 2707 & EARTH & PCT_A \\
\hline 160 & DEWYLAKE & PRESSURE & 2708 & EARTH & PCT_EXP \\
\hline 161 & DEWYLAKE & PRMX_LOG & 2501 & EARTH & PO_MIN \\
\hline 162 & DEWYLAKE & PRMY_LOG & 2499 & EARTH & PORE_DIS \\
\hline 163 & DEWYLAKE & PRMZ_LOG & 2500 & EARTH & POROSITY \\
\hline 166 & DEWYLAKE & RELP_MOD & 2502 & EARTH & PRESSURE \\
\hline 167 & DEWYLAKE & SAL_USAT & 2503 & EARTH & PRMX_LOG \\
\hline 168 & DEWYLAKE & SAT_IBRN & 2504 & EARTH & PRMY_LOG \\
\hline 169 & DEWYLAKE & SAT_RBRN & 2505 & EARTH & PRMZ_LOG \\
\hline 170 & DEWYLAKE & SAT_RGAS & 2509 & EARTH & RELP MOD \\
\hline 171 & DRILLMUD & DNSFLUID & 3032 & EARTH & RSH_AIR \\
\hline 172 & DRILLMUD & VISCO & 3035 & EARTH & RSH_EXH \\
\hline 173 & DRILLMUD & YLDSTRSS & 3033 & EARTH & RSH_SAL \\
\hline 174 & DRZ_0 & CAP_MOD & 3034 & EARTH & RSH_WAS \\
\hline 175 & DRZ_0 & COMP_RCK & 2510 & EARTH & SAT_IBRN \\
\hline 2701 & DRZ_0 & KPT & 2511 & EARTH & SAT_RBRN \\
\hline 944 & DRZ_0 & MDISP_L & 2512 & EARTH & SAT_RGAS \\
\hline 945 & DRZ_0 & MDISP_T & 207 & EXP AREA & CAP MOD \\
\hline 176 & DRZ_0 & PC_MAX & 208 & EXP_AREA & COMP RCK \\
\hline 2702 & DRZ_0 & PCT_A & 2711 & EXP_AREA & KPT \\
\hline 2703 & DRZ_O & PCT_EXP & 209 & EXP_AREA & PC_MAX \\
\hline 179 & DRZ_0 & PO_MIN & 2712 & EXP_AREA & PCT_A \\
\hline 177 & $D R Z=0$ & PORE_DIS & 2713 & EXP_AREA & PCT_EXP \\
\hline 178 & $D R Z=0$ & POROSITY & 212 & EXP_AREA & PO_MIN \\
\hline 181 & DRZ_0 & PRMX_LOG & 210 & EXP_AREA & PORE_DIS \\
\hline 182 & DRZ_o & PRMY_LOG & 211 & EXP_AREA & POROSITY \\
\hline
\end{tabular}


Material Name

\begin{tabular}{|c|c|c|}
\hline Parameter ID & Material & Parameter \\
\hline 213 & EXP_AREA & PRESSURE \\
\hline 214 & EXP_AREA & PRMX_LOG \\
\hline 215 & EXP_AREA & PRMY_LOG \\
\hline 216 & EXP_AREA & PRMZ_LOG \\
\hline 219 & EXP_AREA & RELP_MOD \\
\hline 220 & EXP_AREA & SAT_IBRN \\
\hline 221 & EXP_AREA & SAT_RBRN \\
\hline 222 & EXP_AREA & SAT_RGAS \\
\hline 2085 & FORTYNIN & CAP_MOD \\
\hline 2238 & FORTYNIN & COMP_RCK \\
\hline 2715 & FORTYNIN & KPT \\
\hline 2239 & FORTYNIN & PC_MAX \\
\hline 2716 & FORTYNIN & PCT_A \\
\hline 2717 & FORTYNIN & PCT_EXP \\
\hline 2718 & FORTYNIN & PO_MIN \\
\hline 2087 & FORTYNIN & PORE_DIS \\
\hline 2088 & FORTYNIN & POROSITY \\
\hline 2899 & FORTYNIN & PRMX_LOG \\
\hline 2900 & FORTYNIN & PRMY_LOG \\
\hline 2901 & FORTYNIN & PRMZ_LOG \\
\hline 2093 & FORTYNIN & RELP_MOD \\
\hline 2240 & FORTYNIN & SAT_RBRN \\
\hline 2094 & FORTYNIN & SAT_RGAS \\
\hline 3234 & FR221 & ATWEIGHT \\
\hline 3332 & FR221 & EPAREL \\
\hline 3279 & FR221 & HALFLIFE \\
\hline 223 & GLOBAL & CLIMTIDX \\
\hline 3501 & GLOBAL & FPICD \\
\hline 3500 & GLOBAL & FPICM \\
\hline 3494 & GLOBAL & $\angle A M B D A D$ \\
\hline 3497 & GLOBAL & MINERT \\
\hline 3417 & GLOBAL & OXSTAT \\
\hline 3493 & GLOBAL & PBRINE \\
\hline 3495 & GLOBAL & PLGPAT \\
\hline 3491 & GLOBAL & TA \\
\hline 3499 & GLOBAL & TPICD \\
\hline 3498 & GLOBAL & TPICM \\
\hline 225 & GLOBAL & TRANSIDX \\
\hline 228 & $\mathrm{H} 2$ & VISCO \\
\hline 229 & IMPERM_Z & CAP_MOD \\
\hline 230 & IMPERM_Z & COMP_RCK \\
\hline 2720 & IMPERM_Z & KPT \\
\hline 231 & IMPERM_Z & PC_MAX \\
\hline 2721 & IMPERM_Z & PCT_A \\
\hline 2722 & IMPERM_Z & PCT_EXP \\
\hline 234 & IMPERM_Z & PO_MIN \\
\hline 232 & IMPERM_Z & PORE_DIS \\
\hline 233 & IMPERM_Z & POROSITY \\
\hline 236 & IMPERM_Z & PRMX_LOG \\
\hline 237 & IMPERM_Z & PRMY_LOG \\
\hline 238 & IMPERM_Z & PRMZ_LOG \\
\hline
\end{tabular}

\begin{tabular}{|c|c|c|}
\hline Parameter ID & Material & Parameter \\
\hline 241 & IMPERM_Z & RELP_MOD \\
\hline 243 & IMPERM_Z & SAT_RBRN \\
\hline 244 & IMPERM_Z & SAT_RGAS \\
\hline 2097 & MAGENTA & CAP_MOD \\
\hline 3016 & MAGENTA & COMP_RCK \\
\hline 2725 & MAGENTA & KPT \\
\hline 2098 & MAGENTA & PC_MAX \\
\hline 2726 & MAGENTA & PCT_A \\
\hline 2727 & MAGENTA & PCT_EXP \\
\hline 2728 & MAGENTA & PO_MIN \\
\hline 2099 & MAGENTA & PORE_DIS \\
\hline 2100 & MAGENTA & POROSITY \\
\hline 2101 & MAGENTA & PRESSURE \\
\hline 2102 & MAGENTA & PRMX_LOG \\
\hline 2103 & MAGENTA & PRMY_LOG \\
\hline 2104 & MAGENTA & PRMZ_LOG \\
\hline 2106 & MAGENTA & RELP_MOD \\
\hline 2241 & MAGENTA & SAT_RBRN \\
\hline 2107 & MAGENTA & SAT_RGAS \\
\hline 2109 & MAGENTA & THICK \\
\hline 3235 & ND143 & ATWEIGHT \\
\hline 3333 & ND143 & EPAREL \\
\hline 3280 & ND143 & HALFLIFE \\
\hline 2906 & NITRATE & QINIT \\
\hline 3458 & NP & CAPHUM \\
\hline 3313 & $\mathrm{NP}$ & CAPMIC \\
\hline 3312 & $\mathrm{NP}$ & CONCINT \\
\hline 3439 & $\mathrm{NP}$ & CONCMIN \\
\hline 3314 & $\mathrm{NP}$ & PROPMIC \\
\hline 3477 & $\mathrm{NP}+4$ & MKD_NP \\
\hline 3476 & $\mathrm{NP}+5$ & MKD_NP \\
\hline 246 & NP237 & ATWEIGHT \\
\hline 3370 & NP237 & EPAREL \\
\hline 247 & NP237 & HALFLIFE \\
\hline 248 & NP237 & INVCHD \\
\hline 249 & NP237 & INVRHD \\
\hline 3236 & NP239 & ATWEIGHT \\
\hline 3334 & NP239 & EPAREL \\
\hline 3281 & NP239 & HALFLIFE \\
\hline 7 & OPS_AREA & CAP_MOD \\
\hline 8 & OPS_AREA & COMP_RCK \\
\hline 2604 & OPS_AREA & KPT \\
\hline 9 & OPS_AREA & PC_MAX \\
\hline 2605 & OPS_AREA & PCT_A \\
\hline 2606 & OPS_AREA & PCT_EXP \\
\hline 12 & OPS_AREA & PO_MIN \\
\hline 10 & OPS_AREA & PORE_DIS \\
\hline 11 & OPS_AREA & POROSITY \\
\hline 13 & OPS_AREA & PRESSURE \\
\hline 14 & OPS_AREA & PRMX_LOG \\
\hline 15 & OPS_AREA & PRMY_LOG \\
\hline
\end{tabular}


Material Name

\begin{tabular}{|c|c|c|}
\hline Parameter ID & Material & Parameter \\
\hline 16 & OPS_AREA & PRMZ_LOG \\
\hline 19 & OPS_AREA & RELP_MOD \\
\hline 20. & OPS_AREA & SAT_IBRN \\
\hline 21 & OPS_AREA & SAT_RBRN \\
\hline 22 & OPS_AREA & SAT_RGAS \\
\hline 250 & PA231 & ATWEIGHT \\
\hline 3371 & PA231 & EPAREL \\
\hline 251 & PA231 & HALFLIFE \\
\hline 2267 & PA231 & INVCHD \\
\hline 2268 & PA231 & INVRHD \\
\hline 3237 & PA233 & ATWEIGHT \\
\hline 3335 & PA233 & EPAREL \\
\hline 3282 & PA233 & HALFLIFE \\
\hline 3197 & PA234M & ATWEIGHT \\
\hline 3336 & PA234M & EPAREL \\
\hline 3283 & PA234M & HALFLIFE \\
\hline 252 & PAN_SEAL & CAP_MOD \\
\hline 253 & PAN_SEAL & COMP_RCK \\
\hline 2731 & PAN_SEAL & KPT \\
\hline 254 & PAN_SEAL & PC_MAX \\
\hline 2732 & PAN_SEAL & $\mathrm{PCT} A$ \\
\hline 2733 & PAN_SEAL & PCT_EXP \\
\hline 257 & PAN_SEAL & PO_MIN \\
\hline 255 & PAN_SEAL & PORE_DIS \\
\hline 256 & PAN_SEAL & POROSITY \\
\hline 258 & PAN_SEAL & PRESSURE \\
\hline 259 & PAN_SEAL & PRMX_LOG \\
\hline 260 & PAN_SEAL & PRMY_LOG \\
\hline 261 & PAN_SEAL & PRMZ_LOG \\
\hline 264 & PAN_SEAL & RELP_MOD \\
\hline 2734 & PAN_SEAL & SAT_IBRN \\
\hline 265 & PAN_SEAL & SAT_RBRN \\
\hline 266 & PAN_SEAL & SAT_RGAS \\
\hline 282 & PB & LOGSOLM \\
\hline 3421 & PB209 & ATWEIGHT \\
\hline 3337 & PB209 & EPAREL \\
\hline 3284 & PB209 & HALFLIFE \\
\hline 283 & PB210 & ATWEIGHT \\
\hline 3372 & PB210 & EPAREL \\
\hline 284 & PB210 & HALFLIFE \\
\hline 285 & PB210 & INVCHD \\
\hline 286 & PB210 & INVRHD \\
\hline 3200 & PB211 & ATWEIGHT \\
\hline 3338 & PB211 & EPAREL \\
\hline 3285 & PB211 & HALFLIFE \\
\hline 3201 & PB212 & ATWEIGHT \\
\hline 3339 & PB212 & EPAREL \\
\hline 3286 & PB212 & HALFLIFE \\
\hline 3202 & PB214 & ATWEIGHT \\
\hline 3340 & PB214 & EPAREL \\
\hline 3287 & PB214 & HALFLIFE \\
\hline
\end{tabular}

\begin{tabular}{|c|c|c|}
\hline Parameter ID & Material & Parameter \\
\hline 3429 & PHUMOX3 & PHUMCIM \\
\hline 3433 & PHUMOX3 & PHUMSIM \\
\hline 3430 & PHUMOX4 & PHUMCIM \\
\hline 3434 & PHUMOX4 & PHUMSIM \\
\hline 3431 & PHUMOX5 & PHUMCIM \\
\hline 3435 & PHUMOX5 & PHUMSIM \\
\hline 3432 & PHUMOX6 & PHUMCIM \\
\hline 3436 & PHUMOX6 & PHUMSIM \\
\hline 1167 & $\mathrm{PM}$ & LOGSOLM \\
\hline 287 & PM147 & ATWEIGHT \\
\hline 3341 & PM147 & EPAREL \\
\hline 288 & PM147 & HALFLIFE \\
\hline 2038 & PM147 & INVCHD \\
\hline 289 & PM147 & INVRHD \\
\hline 3203 & PO212 & ATWEIGHT \\
\hline 3342 & PO212 & EPAREL \\
\hline 3288 & $\mathrm{PO} 212$ & HALFLIFE \\
\hline 3204 & $\mathrm{PO} 213$ & ATWEIGHT \\
\hline 3343 & $\mathrm{PO} 213$ & EPAREL \\
\hline 3289 & $\mathrm{PO} 213$ & HALFLIFE \\
\hline 3205 & PO214 & ATWEIGHT \\
\hline 3344 & PO214 & EPAREL \\
\hline 3290 & P0214 & HALFLIFE \\
\hline 3206 & PO215 & ATWEIGHT \\
\hline 3345 & PO215 & EPAREL \\
\hline 3291 & P0215 & HALFLIFE \\
\hline 3207 & P0216 & ATWEIGHT \\
\hline 3346 & PO216 & EPAREL \\
\hline 3292 & PO216 & HALFLIFE \\
\hline 3208 & $\mathrm{PO} 218$ & ATWEIGHT \\
\hline 3347 & PO218 & EPAREL \\
\hline 3293 & PO218 & HALFLIFE \\
\hline 3459 & PU & CAPHUM \\
\hline 3315 & $\mathrm{PU}$ & CAPMIC \\
\hline 3316 & $\mathrm{PU}$ & CONG!NT \\
\hline 3440 & PU & CONCMIN \\
\hline 3317 & PU & PROPMIC \\
\hline 3442 & $\mathrm{PU}+3$ & MDO \\
\hline 3480 & PU+3 & MKD_PU \\
\hline 3443 & PU+4 & MDO \\
\hline 3481 & $\mathrm{PU}+4$ & MKD_PU \\
\hline 291 & PU238 & ATWEIGHT \\
\hline 3373 & PU238 & EPAREL \\
\hline 292 & PU238 & HALFLIFE \\
\hline 293 & PU238 & INVCHD \\
\hline 294 & PU238 & INVRHD \\
\hline 295 & PU239 & ATWEIGHT \\
\hline 3374 & PU239 & EPAREL \\
\hline 296 & PU239 & HALFLIFE \\
\hline 297 & PU239 & INVCHD \\
\hline 298 & PU239 & INVRHD \\
\hline
\end{tabular}


Material Name

\begin{tabular}{|c|c|c|}
\hline Parameter ID & Material & Parameter \\
\hline 299 & PU240 & ATWEIGHT \\
\hline 3375 & PU240 & |EPAREL \\
\hline 300 & PU240 & HALFLIFE \\
\hline 301 & PU240 & INVCHD \\
\hline 302 & PU240 & INVRHD \\
\hline 303 & PU241 & ATWEIGHT \\
\hline 3348 & PU241 & EPAREL \\
\hline 304 & PU241 & HALFLIFE \\
\hline 305 & PU241 & INVCHD \\
\hline 306 & PU241 & INVRHD \\
\hline 307 & PU242 & ATWEIGHT \\
\hline 3376 & PU242 & EPAREL \\
\hline 308 & PU242 & HALFLIFE \\
\hline 309 & PU242 & INVCHD \\
\hline 310 & PU242 & INVRHD \\
\hline 311 & PU244 & ATWEIGHT \\
\hline 3377 & PU244 & EPAREL \\
\hline 312 & PU244 & HALFLIFE \\
\hline 2269 & PU244 & INVCHD \\
\hline 2270 & PU244 & INVRHD \\
\hline 313 & $R A$ & LOGSOLM \\
\hline 3209 & RA223 & ATWEIGHT \\
\hline 3349 & RA223 & EPAREL \\
\hline 3294 & RA223 & HALFLIFE \\
\hline 3210 & RA224 & ATWEIGHT \\
\hline 3350 & RA224 & EPAREL \\
\hline 3295 & RA224 & HALFLIFE \\
\hline 3211 & RA225 & ATWEIGHT \\
\hline 3351 & RA225 & EPAREL \\
\hline 3296 & RA225 & HALFLIFE \\
\hline 314 & RA226 & ATWEIGHT \\
\hline 3378 & RA226 & EPAREL \\
\hline 315 & RA226 & HALFLIFE \\
\hline 316 & RA226 & INVCHD \\
\hline 317 & RA226 & INVRHD \\
\hline 318 & RA228 & ATWEIGHT \\
\hline 3352 & RA228 & EPAREL \\
\hline 319 & RA228 & HALFLIFE \\
\hline 2271 & RA228 & INVCHD \\
\hline 2272 & RA228 & INVRHD \\
\hline 3503 & REFCON & ABERM \\
\hline 2833 & REFCON & $\mathrm{ACF} \_\mathrm{CH} 4$ \\
\hline 2832 & REFCON & ACF_CO2 \\
\hline 2831 & REFCON & ACF_H2 \\
\hline 2835 & REFCON & ACF_H2S \\
\hline 2834 & REFCON & ACF_N2 \\
\hline 2836 & REFCON & ACF_O2 \\
\hline 3113 & REFCON & ACTCONST \\
\hline 2897 & REFCON & AL2 \\
\hline 3489 & REFCON & AREA_CH \\
\hline 3496 & REFCON & AREA_RH \\
\hline
\end{tabular}

\begin{tabular}{|c|c|c|}
\hline Parameter ID & Material & Parameter \\
\hline 3488 & REFCON & AREA_ZRO \\
\hline 3106 & REFCON & ASDRUM \\
\hline 2890 & REFCON & ATMPA \\
\hline 3109 & REFCON & AVOGADRO \\
\hline 2879 & REFCON & BBLG \\
\hline 3111 & REFCON & CITOBQ \\
\hline 2882 & REFCON & DARM2 \\
\hline 2887 & REFCON & DAYSEC \\
\hline 3132 & REFCON & DRROOM \\
\hline 2883 & REFCON & F3M3 \\
\hline 2881 & REFCON & FTM \\
\hline 3492 & REFCON & FWW \\
\hline 2889 & REFCON & GRAVACC \\
\hline 2884 & REFCON & GTI3 \\
\hline 3502 & REFCON & HRH \\
\hline 2886 & REFCON & KGLB \\
\hline 2885 & REFCON & LBKG \\
\hline 2877 & REFCON & MW_CACO3 \\
\hline 2875 & REFCON & MW_CAO \\
\hline 2876 & REFCON & $\mathrm{MW} \_\mathrm{CAOH} 2$ \\
\hline 2866 & REFCON & $\mathrm{MW}$-CH2O \\
\hline 2860 & REFCON & $\mathrm{MW}$-CH4 \\
\hline 2859 & REFCON & $\mathrm{MW} \_\mathrm{CO} 2$ \\
\hline 2865 & REFCON & MW_FE \\
\hline 2873 & REFCON & MW_FE3O4 \\
\hline 2870 & REFCON & MW_FECO3 \\
\hline 2871 & REFCON & MW_FEOH2 \\
\hline 2872 & REFCON & MW_FEOOH \\
\hline 2874 & REFCON & MW_FES \\
\hline 2869 & REFCON & MW_FES2 \\
\hline 2858 & REFCON & MW_H2 \\
\hline 2864 & REFCON & MW_H2O \\
\hline 2862 & REFCON & MW_H2S \\
\hline 2867 & REFCON & $\mathrm{MW} \_\mathrm{H} 2 \mathrm{SO} 4$ \\
\hline 2868 & REFCON & MW_HNO3 \\
\hline 2861 & REFCON & MW_N2 \\
\hline 2878 & REFCON & MW_NACL \\
\hline 2863 & REFCON & MW_O2 \\
\hline 2894 & REFCON & OMEGAA \\
\hline 2895 & REFCON & OMEGAB \\
\hline 2880 & REFCON & PASCP \\
\hline 2839 & REFCON & PC_CH4 \\
\hline 2838 & REFCON & PC_CO2 \\
\hline 2837 & REFCON & PC_H2 \\
\hline 2841 & REFCON & PC_H2S \\
\hline 2840 & REFCON & PC_N2 \\
\hline 2842 & REFCON & PC_O2 \\
\hline 2896 & REFCON & $|P|$ \\
\hline 2892 & REFCON & TPSIPA \\
\hline 2893 & REFCON & $R$ \\
\hline 2891 & REFCON & |RTK \\
\hline
\end{tabular}


Material Name

\begin{tabular}{|c|c|c|}
\hline Parameter ID & Material & Parameter \\
\hline 3112 & REFCON & SECYR \\
\hline 2827 & REFCON & TC_CH4 \\
\hline 2826 & REFCON & TC_CO2 \\
\hline 2825 & REFCON & TC_H2 \\
\hline 2829 & REFCON & TC_H2S \\
\hline 2828 & REFCON & TC_N2 \\
\hline 2830 & REFCON & TC_O2 \\
\hline 3490 & REFCON & VOLWP \\
\hline 3107 & REFCON & VPANLEX \\
\hline 3108 & REFCON & VREPOS \\
\hline 3105 & REFCON & VROOM \\
\hline 2888 & REFCON & YRSEC \\
\hline 3110 & REFCON & ZCINK \\
\hline 2110 & REPOSIT & CAP_MOD \\
\hline 3455 & REPOSIT & CLOSMOD \\
\hline 2112 & REPOSIT & COMP_RCK \\
\hline 2113 & REPOSIT & DCELLCHW \\
\hline 2114 & REPOSIT & DCELLRHW \\
\hline 2115 & REPOSIT & DIRNCCHW \\
\hline 2116 & REPOSIT & DIRNCRHW \\
\hline 2117 & REPOSIT & DIRONCHW \\
\hline 2118 & REPOSIT & DIRONRHW \\
\hline 2119 & REPOSIT & DPLASCHW \\
\hline 2120 & REPOSIT & DPLASRHW \\
\hline 2121 & REPOSIT & DPLSCCHW \\
\hline 2995 & REPOSIT & DPLSCRHW \\
\hline 2122 & REPOSIT & DRUBBCHW \\
\hline 2123 & REPOSIT & DRUBBRHW \\
\hline 2127 & REPOSIT & GRATMICH \\
\hline 2128 & REPOSIT & GRATMICI \\
\hline 2736 & REPOSIT & KPT \\
\hline 2242 & REPOSIT & PC_MAX \\
\hline 2737 & REPOSIT & PCT_A \\
\hline 2738 & REPOSIT & PCT_EXP \\
\hline 2739 & REPOSIT & PO_MIN \\
\hline 2129 & REPOSIT & PORE_DIS \\
\hline 2130 & REPOSIT & POROSITY \\
\hline 2131 & REPOSIT & PRMX_LOG \\
\hline 2132 & REPOSIT & PRMY_LOG \\
\hline 2133 & REPOSIT & PRMZ_LOG \\
\hline 2824 & REPOSIT & PROBDEG \\
\hline 2135 & REPOSIT & RELP_MOD \\
\hline 2740 & REPOSIT & SAT_IBRN \\
\hline 2741 & REPOSIT & SAT_RBRN \\
\hline 2137 & REPOSIT & SAT_RGAS \\
\hline 2141 & REPOSIT & VOLCHW \\
\hline 2142 & REPOSIT & VOLRHW \\
\hline 3212 & RN219 & ATWEIGHT \\
\hline 3353 & RN219 & EPAREL \\
\hline 3297 & RN219 & HALFLIFE \\
\hline 3213 & RN220 & ATWEIGHT \\
\hline
\end{tabular}

\begin{tabular}{|c|c|c|}
\hline Parameter ID & Material & Parameter \\
\hline 3354 & RN220 & EPAREL \\
\hline 3298 & RN220 & HALFLIFE \\
\hline 3214 & RN222 & ATWEIGHT \\
\hline 3355 & RN222 & EPAREL \\
\hline 3299 & RN222 & HALFLIFE \\
\hline 2819 & S_ANH_AB & BKLINK \\
\hline 520 & S_ANH_AB & CAP_MOD \\
\hline 521 & S_ANH_AB & COMP_RCK \\
\hline 1661 & S_ANH_AB & DNSGRAIN \\
\hline 2158 & S_ANH_AB & DPHIMAX \\
\hline 2820 & S_ANH_AB & EXPKLINK \\
\hline 2812 & S_ANH_AB & IFRX \\
\hline 2815 & $S \_A N H \_A B$ & IFRY \\
\hline 2818 & S_ANH_AB & IFRZ \\
\hline 2159 & S_ANH_AB & KMAXLOG \\
\hline 2773 & S_ANH_AB & KPT \\
\hline 1684 & S_ANH_AB & MDISP_L \\
\hline 1685 & S_ANH_AB & MDISP_T \\
\hline 522 & S_ANH_AB & PC_MAX \\
\hline 2774 & S_ANH_AB & PCT_A \\
\hline 2775 & S_ANH_AB & PCT_EXP \\
\hline 524 & S_ANH_AB & PF_DELTA \\
\hline 526 & $S \_A N H \_A B$ & PI_DELTA \\
\hline 529 & S_ANH_AB & PO_MIN \\
\hline 527 & S_ANH_AB & PORE_DIS \\
\hline 528 & S_ANH_AB & POROSITY \\
\hline 531 & S_ANH_AB & PRMX_LOG \\
\hline 532 & S_ANH_AB & PRMY_LOG \\
\hline 533 & S_ANH_AB & PRMZ_LOG \\
\hline 536 & S_ANH_AB & RELP_MOD \\
\hline 537 & S_ANH_AB & SAT_IBRN \\
\hline 538 & S_ANH_AB & SAT_RBRN \\
\hline 539 & $S_{-} \mathrm{ANH}_{-} \mathrm{AB}$ & SAT_RGAS \\
\hline 1702 & S_ANH_AB & TORTUSTY \\
\hline 540 & S_HALITE & CAP_MOD \\
\hline 541 & S_HALITE & COMP_RCK \\
\hline 1703 & S_HALITE & DNSGRAIN \\
\hline 2778 & S_HALITE & KPT \\
\hline 1724 & S_HALITE & MDISP_L \\
\hline 1725 & S_HALITE & MDISP_T \\
\hline 542 & S_HALITE & PC_MAX \\
\hline 2779 & S_HALITE & PCT_A \\
\hline 2780 & S_HALITE & PCT_EXP \\
\hline 545 & S_HALITE & PO_MIN \\
\hline 543 & S_HALITE & PORE_DIS \\
\hline 544 & S_HALITE & POROSITY \\
\hline 546 & S_HALITE & PRESSURE \\
\hline 547 & S_HALITE & PRMX_LOG \\
\hline 548 & S_HALITE & PRMY_LOG \\
\hline 549 & S_HALITE & PRMZ_LOG \\
\hline 553 & S_HALITE & RELP_MOD \\
\hline
\end{tabular}


Material Name

\begin{tabular}{|c|c|c|}
\hline Parameter ID & Material & Parameter \\
\hline 554 & S_HALITE & SAT_IBRN \\
\hline 555 & S_HALITE & SAT_RBRN \\
\hline 556 & S_HALITE & SAT_RGAS \\
\hline 1742 & S_HALITE & TORTUSTY \\
\hline 2904 & S_MB138 & BKLINK \\
\hline 559 & S_MB138 & CAP_MOD \\
\hline 560 & S_MB138 & COMP_RCK \\
\hline 1743 & S_MB138 & DNSGRAIN \\
\hline 2169 & S_MB138 & DPHIMAX \\
\hline 2902 & S_MB138 & EXPKLINK \\
\hline 2810 & S. MB138 & IFRX \\
\hline 2813 & S_MB138 & IFRY \\
\hline 2816 & $S^{i} M B 138$ & IFRZ \\
\hline 2170 & S_MB138 & KMAXLOG \\
\hline 2783 & S_MB138 & KPT \\
\hline 561 & S_MB138 & PC_MAX \\
\hline 2784 & S_MB138 & PCT_A \\
\hline 2785 & S_MB138 & PCT_EXP \\
\hline 563 & S_MB138 & PF_DELTA \\
\hline 565 & S_MB138 & PI_DELTA \\
\hline 568 & S_MB138 & PO_MIN \\
\hline 566 & S_MB138 & PORE_DIS \\
\hline 567 & S_MB138 & POROSITY \\
\hline 569 & S_MB138 & PRESSURE \\
\hline 570 & S_MB138 & PRMX_LOG \\
\hline 571 & S_MB138 & PRMY_LOG \\
\hline 572 & S_MB138 & PRMZ_LOG \\
\hline 575 & S_MB138 & RELP_MOD \\
\hline 576 & S_MB138 & SAT_IBRN \\
\hline 577 & S_MB138 & SAT_RBRN \\
\hline 578 & S_MB138 & SAT_RGAS \\
\hline 2905 & S_MB139 & BKLINK \\
\hline 579 & S_MB139 & CAP_MOD \\
\hline 580 & S_MB139 & COMP_RCK \\
\hline 1784 & S_MB139 & DNSGRAIN \\
\hline 2177 & S_MB139 & DPHIMAX \\
\hline 2903 & S_MB139 & EXPKLINK \\
\hline 2811 & S_MB139 & IFRX \\
\hline 2814 & S_MB139 & IFRY \\
\hline 2817 & S_MB139 & IFRZ \\
\hline 2178 & S_MB139 & KMAXLOG \\
\hline 2788 & S_MB139 & KPT \\
\hline 582 & S_MB139 & PC_MAX \\
\hline 2789 & S_MB139 & |PCT_A \\
\hline 2790 & S_MB139 & PCT_EXP \\
\hline 2180 & S_MB139 & PF_DELTA \\
\hline 586 & S_MB139 & PI_DELTA \\
\hline 589 & S_MB139 & PO_MIN \\
\hline 587 & S_MB139 & PORE_DIS \\
\hline 588 & S_MB139 & POROSITY \\
\hline 590 & S_MB139 & |PRESSURE \\
\hline
\end{tabular}

\begin{tabular}{|c|c|c|}
\hline Parameter ID & Material & Parameter \\
\hline 591 & S_MB139 & PRMX_LOG \\
\hline 592 & S_MB139 & PRMY_LOG \\
\hline 593 & S_MB139 & PRMZ_LOG \\
\hline 596 & S_MB139 & RELP_MOD \\
\hline 597 & S_MB139 & SAT_IBRN \\
\hline 598 & S_MB139 & SAT_RBRN \\
\hline 599 & S_MB139 & SAT_RGAS \\
\hline 2513 & SALT_T1 & CAP_MOD \\
\hline 2514 & SALT_T1 & COMP_RCK \\
\hline 2939 & SALT_T1. & CUMPROB \\
\hline 2744 & SALT_T1 & KPT \\
\hline 2515 & SALT_T1 & PC_MAX \\
\hline 2745 & SALT_T1 & PCT_A \\
\hline 2746 & SALT_T1 & PCT_EXP \\
\hline 2942 & SALT_T1 & PMLT_HI \\
\hline 2941 & SALT_T1 & PMLT_LO \\
\hline 2940 & SALT_T1 & PMLT_MD \\
\hline 2518 & SALT_T1 & PO_MIN \\
\hline 2516 & SALT_T1 & PORE_DIS \\
\hline 2517 & SALT_T1 & POROSITY \\
\hline 2519 & SALT_T1 & PRESSURE \\
\hline 2938 & SALT_T1 & RADN_DRZ \\
\hline 2526 & SALT_T1 & RELP_MOD \\
\hline 2934 & SALT_T1 & RSH_AIR \\
\hline 2937 & SALT_T1 & RSH_EXH \\
\hline 2935 & SALT_T1 & RSH_SAL \\
\hline 2936 & SALT_T1 & RSH_WAS \\
\hline 2527 & SALT_T1 & SAT_IBRN \\
\hline 2528 & SALT_T1 & SAT_RBRN \\
\hline 2529 & SALT_T1 & SAT_RGAS \\
\hline 2530 & SALT_T2 & CAP_MOD \\
\hline 2531 & SALT_T2 & COMP_RCK \\
\hline 2749 & SALT_T2 & KPT \\
\hline 2532 & SALT_T2 & PC_MAX \\
\hline 2750 & SALT_T2 & PCT_A \\
\hline 2751 & SALT_T2 & PCT_EXP \\
\hline 2950 & SALT_T2 & PMLT_HI \\
\hline 2949 & SALT_T2 & PMLT_LO \\
\hline 2948 & SALT_T2 & PMLT_MD \\
\hline 2535 & SALT_T2 & PO_MIN \\
\hline 2533 & SALT_T2 & PORE_DIS \\
\hline 2534 & SALT_T2 & POROSITY \\
\hline 2947 & SALT_T2 & RADN_DRZ \\
\hline 2543 & SALT_T2 & RELP_MOD \\
\hline 2943 & SALT_T2 & RSH_AIR \\
\hline 2946 & SALT_T2 & RSH_EXH \\
\hline 2944 & SALT_T2 & RSH_SAL \\
\hline 2945 & SALT_T2 & RSH_WAS \\
\hline 2545 & SALT_T2 & SAT_RBRN \\
\hline 2546 & SALT_T2 & SAT_RGAS \\
\hline 2547 & SALT_T3 & CAP_MOD \\
\hline
\end{tabular}


Material Name

\begin{tabular}{|c|c|c|}
\hline Parameter ID & Material & Parameter \\
\hline 2548 & SALT_T3 & COMP_RCK \\
\hline 2754 & SALT_T3 & KRPT \\
\hline 2549 & SALT_T3 & PC_MAX \\
\hline 2755 & SALT_T3 & PCT_A \\
\hline 2756 & SALT_T3 & PCT_EXP \\
\hline 2958 & SALT_T3 & PMLT_HI \\
\hline 2957 & SALT_T3 & PMLT_LO \\
\hline 2956 & SALT_T3 & PMLT_MD \\
\hline 2552 & SALT_T3 & PO_MIN \\
\hline 2550 & SALT_T3 & PORE_DIS \\
\hline 2551 & SALT_T3 & POROSITY \\
\hline 2955 & SALT_T3 & RADN_DRZ \\
\hline 2560 & SALT_T3 & RELP_MOD \\
\hline 2951 & SALT_T3 & RSH_AIR \\
\hline 2954 & SALT_T3 & RSH_EXH \\
\hline 2952 & SALT_T3 & RSH_SAL \\
\hline 2953 & SALT_T3 & RSH_WAS \\
\hline 2562 & SALT_T3 & SAT_RBRN \\
\hline 2563 & SALT_T3 & SAT_RGAS \\
\hline 2564 & SALT_T4 & CAP_MOD \\
\hline 2565 & SALT_T4 & COMP_RCK \\
\hline 2759 & SALT_T4 & KPT \\
\hline 2566 & SALT_T4 & PC_MAX \\
\hline 2760 & SALT_T4 & PCT_A \\
\hline 2761 & SALT_T4 & PCT_EXP \\
\hline 2966 & SALT_T4 & PMLT_HI \\
\hline 2965 & SALT_T4 & PMLT_LO \\
\hline 2964 & SALT_T4 & PMLT_MD \\
\hline 2569 & SALT_T4 & PO_MIN \\
\hline 2567 & SALT_T4 & PORE_DIS \\
\hline 2568 & SALT_T4 & POROSITY \\
\hline 2963 & SALT_T4 & RADN_DRZ \\
\hline 2577 & SALT_T4 & RELP_MOD \\
\hline 2959 & SALT_T4 & RSH_AIR \\
\hline 2962 & SALT_T4 & RSH_EXH \\
\hline 2960 & SALT_T4 & RSH_SAL \\
\hline 2961 & SALT_T4 & RSH_WAS \\
\hline 2579 & SALT_T4 & SAT_RBRN \\
\hline 2580 & SALT_T4 & SAT_RGAS \\
\hline 2581 & SALT_T5 & CAP_MOD \\
\hline 2582 & SALT_T5 & COMP_RCK \\
\hline 2764 & SALT_T5 & KPT \\
\hline 2583 & SALT_T5 & PC_MAX \\
\hline 2765 & SALT_T5 & PCT A \\
\hline 2766 & SALT_T5 & PCT_EXP \\
\hline 2974 & SALT_T5 & PMLT_HI \\
\hline 2973 & SALT_T5 & PMLT_LO \\
\hline 2972 & SALT_T5 & PMLT_MD \\
\hline 3125 & SALT_T5 & PO_MIN \\
\hline 2809 & SALT_T5 & PORE_DIS \\
\hline 2585 & SALT_T5 & POROSITY \\
\hline
\end{tabular}

\begin{tabular}{|c|c|c|}
\hline Parameter ID & Material & Parameter \\
\hline 2971 & SALT_T5 & RADN_DRZ \\
\hline 2594 & SALT_T5 & RELP_MOD \\
\hline 2967 & SALT_T5 & RSH_AIR \\
\hline 2970 & SALT_T5 & RSH_EXH \\
\hline 2968 & SALT_T5 & RSH_SAL \\
\hline 2969 & SALT_T5 & RSH_WAS \\
\hline 2596 & SALT_T5 & SAT_RBRN \\
\hline 2597 & SALT_T5 & SAT_RGAS \\
\hline 2983 & SALT_T6 & CAP MOD \\
\hline 2984 & SALT_T6 & COMP_RCK \\
\hline 2985 & SALT_T6 & |KPT \\
\hline 2986 & SALT_T6 & PC_MAX \\
\hline 2987 & SALT_T6 & PCT_A \\
\hline 2988 & SALT_T6 & PCT_EXP \\
\hline 2982 & SALT_T6 & PMLT_HI \\
\hline 2981 & SALT_T6 & PMLT_LO \\
\hline 2980 & SALT_T6 & PMLT_MD \\
\hline 3126 & SALT_T6 & PO_MIN \\
\hline 2989 & SALT_T6 & PORE_DIS \\
\hline 2990 & SALT_T6 & POROSITY \\
\hline 2979 & SALT_T6 & RADN_DRZ \\
\hline 2991 & SALT_T6 & RELP_MOD \\
\hline 2975 & SALT_T6 & RSH_AIR \\
\hline 2978 & SALT_T6 & RSH_EXH \\
\hline 2976 & SALT_T6 & RSH_SAL \\
\hline 2977 & SALT_T6 & RSH_WAS \\
\hline 2992 & SALT_T6 & SAT_RBRN \\
\hline 2993 & SALT_T6 & SAT_RGAS \\
\hline 336 & SANTAROS & CAP_MOD \\
\hline 337 & SANTAROS & COMP_RCK \\
\hline 2768 & SANTAROS & KPT \\
\hline 339 & SANTAROS & PC_MAX \\
\hline 2769 & SANTAROS & PCT_A \\
\hline 2770 & SANTAROS & PCT_EXP \\
\hline 342 & SANTAROS & PO_MIN \\
\hline 340 & SANTAROS & PORE_DIS \\
\hline 341 & SANTAROS & POROSITY \\
\hline 343 & SANTAROS & PRESSURE \\
\hline 344 & SANTAROS & PRMX_LOG \\
\hline 345 & SANTAROS & PRMY_LOG \\
\hline 346 & SANTAROS & PRMZ_LOG \\
\hline 349 & SANTAROS & RELP_MOD \\
\hline 350 & SANTAROS & SAT_IBRN \\
\hline 351 & SANTAROS & SAT_RBRN \\
\hline 352 & SANTAROS & SAT_RGAS \\
\hline 3133 & SHFT_DRZ & PRMX_LOG \\
\hline 514 & SM147 & ATWEIGHT \\
\hline 3379 & SM147 & EPAREL \\
\hline 515 & SM147 & HALFLIFE \\
\hline 3263 & SOLAM3 & SOLCIM \\
\hline 3262 & SOLAM3 & SOLSIM \\
\hline
\end{tabular}


Material Name

\begin{tabular}{|c|c|c|}
\hline Parameter ID & Material & Parameter \\
\hline 3402 & SOLMOD3 & SOLCIM \\
\hline 3406 & SOLMOD3 & SOLSIM \\
\hline 3403 & SOLMOD4 & SOLCIM \\
\hline 3407 & SOLMOD4 & SOLSIM \\
\hline 3404 & SOLMOD5 & SOLCIM \\
\hline 3408 & SOLMOD5 & SOLSIM \\
\hline 3405 & SOLMOD6 & SOLCIM \\
\hline 3409 & SOLMOD6 & SOLSIM \\
\hline 3264 & SOLPU3 & SOLCIM \\
\hline 3265 & SQLPU3 & SOLSIM \\
\hline 3389 & SOLPU4 & SOLCIM \\
\hline 3266 & SOLPU4 & SOLSIM \\
\hline 3393 & SOLTH4 & SOLSIM \\
\hline 3390 & SOLU4 & SOLSIM \\
\hline 3392 & SOLU6 & SOLCIM \\
\hline 3391 & SOLU6 & SOLSIM \\
\hline 1659 & SR & LOGSOLM \\
\hline 3215 & SR90 & ATWEIGHT \\
\hline 3380 & SR90 & EPAREL \\
\hline 517 & SR90 & HALFLIFE \\
\hline 2039 & SR90 & INVCHD \\
\hline 518 & SR90 & INVRHD \\
\hline 2907 & STEEL & CORRMCO2 \\
\hline 2908 & STEEL & CORRWCO2 \\
\hline 2910 & STEEL & HUMCORR \\
\hline 2898 & STEEL & STOIFX \\
\hline 2909 & SULFATE & QINIT \\
\hline 2183 & TAMARISK & CAP_MOD \\
\hline 2243 & TAMARISK & COMP_RCK \\
\hline 2793 & TAMARISK & KPT \\
\hline 2244 & TAMARISK & PC_MAX \\
\hline 2794 & TAMARISK & PCT_A \\
\hline 2795 & TAMARISK & PCT_EXP \\
\hline 2796 & TAMARISK & PO_MIN \\
\hline 2185 & TAMARISK & PORE_DIS \\
\hline 2186 & TAMARISK & POROSITY \\
\hline 2914 & TAMARISK & PRMX_LOG \\
\hline 2915 & TAMARISK & PRMY_LOG \\
\hline 2916 & TAMARISK & PRMZ_LOG \\
\hline 2191 & TAMARISK & RELP_MOD \\
\hline 2245 & TAMARISK & SAT_RBRN \\
\hline 2192 & TAMARISK & SAT_RGAS \\
\hline 3461 & $\mathrm{TH}$ & CAPHUM \\
\hline 3318 & $\mathrm{TH}$ & CAPMIC \\
\hline 3319 & $\mathrm{TH}$ & CONCINT \\
\hline 3437 & TH & CONCMIN \\
\hline 3320 & $\mathrm{TH}$ & PROPMIC \\
\hline 3449 & $\mathrm{TH}+4$ & MDO \\
\hline 3478 & $\mathrm{TH}+4$ & MKD_TH \\
\hline 3216 & TH227 & ATWEIGHT \\
\hline 3356 & TH227 & EPAREL \\
\hline
\end{tabular}

\begin{tabular}{|c|c|c|}
\hline Parameter ID & Material & Parameter \\
\hline 3300 & TH227 & HALFLIFE \\
\hline 3217 & TH228 & ATWEIGHT \\
\hline 3357 & TH228 & EPAREL \\
\hline 3301 & TH228 & HALFLIFE \\
\hline 603 & TH229 & ATWEIGHT \\
\hline 3381 & TH229 & EPAREL \\
\hline 604 & TH229 & HALFLIFE \\
\hline 605 & TH229 & INVCHD \\
\hline 606 & TH229 & INVRHD \\
\hline 607 & TH230 & ATWEIGHT \\
\hline 3382 & TH230 & EPAREL \\
\hline 608 & TH230 & HALFLIFE \\
\hline 609 & TH230 & INVCHD \\
\hline 610 & TH230 & INVRHD \\
\hline 3218 & TH231 & ATWEIGHT \\
\hline 3358 & TH231 & EPAREL \\
\hline 3302 & TH231 & HALFLIFE \\
\hline 611 & TH232 & ATWEIGHT \\
\hline 3383 & TH232 & EPAREL \\
\hline 612 & TH232 & HALFLIFE \\
\hline 613 & TH232 & INVCHD \\
\hline 614 & TH232 & INVRHD \\
\hline 3219 & TH234 & ATWEIGHT \\
\hline 3359 & TH234 & EPAREL \\
\hline 3303 & TH234 & HALFLIFE \\
\hline 3196 & TL207 & ATWEIGHT \\
\hline 3360 & TL207 & EPAREL \\
\hline 3304 & TL207 & HALFLIFE \\
\hline 3460 & $\mathrm{U}$ & CAPHUM \\
\hline 3308 & $U$ & CAPMIC \\
\hline 3307 & $\mathrm{U}$ & CONCINT \\
\hline 3438 & $\mathrm{U}$ & CONCMIN \\
\hline 3309 & $U$ & PROPMIC \\
\hline 3446 & $U+4$ & MDO \\
\hline . 3479 & $\mathrm{U}+4$ & MKD_ل \\
\hline 3448 & $U+6$ & MDO \\
\hline 3475 & $\mathrm{U}+6$ & MKD_U \\
\hline 632 & U233 & ATWEIGHT \\
\hline 3384 & U233 & EPAREL \\
\hline 633 & U233 & HALFLIFE \\
\hline 634 & U233 & INVCHD \\
\hline 635 & U233 & INVRHD \\
\hline 636 & U234 & ATWEIGHT \\
\hline 3385 & U234 & EPAREL \\
\hline 637 & U234 & HALFLIFE \\
\hline 638 & U234 & INVCHD \\
\hline 639 & U234 & INVRHD \\
\hline 640 & U235 & ATWEIGHT \\
\hline 3386 & U235 & EPAREL \\
\hline 641 & U235 & HALFLIFE \\
\hline 642 & U235 & INVCHD \\
\hline
\end{tabular}


Material Name

\begin{tabular}{|c|c|c|}
\hline Parameter ID & Material & Parameter \\
\hline 643 & U235 & INVRHD \\
\hline 644 & U236 & ATWEIGHT \\
\hline 3387 & U236 & EPAREL \\
\hline 645 & U236 & HALFLIFE \\
\hline 2216 & U236 & INVCHD \\
\hline 646 & U236 & INVRHD \\
\hline 647 & U238 & ATWEIGHT \\
\hline 3388 & U238 & EPAREL \\
\hline 648 & U238 & HALFLIFE \\
\hline 649 & U238 & INVCHD \\
\hline 650 & U238 & INVRHD \\
\hline 2217 & UNNAMED & CAP_MOD \\
\hline 2218 & UNNAMED & COMP_RCK \\
\hline 2799 & UNNAMED & KPT \\
\hline 2247 & UNNAMED & PC_MAX \\
\hline 2800 & UNNAMED & PCT_A \\
\hline 2801 & UNNAMED & PCT_EXP \\
\hline 2802 & UNNAMED & PO_MIN \\
\hline 2219 & UNNAMED & PORE_DIS \\
\hline 2220 & UNNAMED & POROSITY \\
\hline 2911 & UNNAMED & PRMX_LOG \\
\hline 2912 & UNNAMED & PRMY_LOG \\
\hline 2913 & UNNAMED & PRMZ_LOG \\
\hline 2225 & UNNAMED & RELP_MOD \\
\hline 2248 & UNNAMED & SAT_RBRN \\
\hline 2226 & UNNAMED & SAT_RGAS \\
\hline 651 & WAS_AREA & ABSROUGH \\
\hline 652 & WAS_AREA & CAP_MOD \\
\hline 3454 & WAS_AREA & CLOSMOD \\
\hline 653 & WAS_AREA & COMP_RCK \\
\hline 2041 & WAS_AREA & DCELLCHW \\
\hline 2274 & WAS_AREA & DCELLRHW \\
\hline 1992 & WAS_AREA & DIRNCCHW \\
\hline 1993 & WAS_AREA & DIRNCRHW \\
\hline 2040 & WAS_AREA & DIRONCHW \\
\hline
\end{tabular}

\begin{tabular}{|c|c|c|}
\hline Parameter ID & Material & Parameter \\
\hline 2044 & WAS_AREA & DIRONRHW \\
\hline 1994 & WAS_AREA & DNSGRAIN \\
\hline 2043 & WAS_AREA & DPLASCHW \\
\hline 2275 & WWAS_AREA & DPLASRHW \\
\hline 1995 & WAS_AREA & DPLSCCHW \\
\hline 2228 & WAS_AREA & DPLSCRHW \\
\hline 2042 & WAS_AREA & DRUBBCHW \\
\hline 2046 & WAS_AREA & DRUBBRHW \\
\hline 656 & WAS AREA & GRATMICH \\
\hline 657 & WAS_AREA & GRATMICI \\
\hline 2804 & WAS_AREA & KPT \\
\hline 658 & WAS_AREA & PC_MAX \\
\hline 2805 & WAS_AREA & PCT_A \\
\hline 2806 & WAS_AREA & PCT_EXP \\
\hline 661 & WAS_AREA & PO_MIN \\
\hline 659 & WAS_AREA & PORE_DIS \\
\hline 660 & WAS_AREA & POROSITY \\
\hline 662 & WAS_AREA & PRESSURE \\
\hline 663 & WAS_AREA & PRMX_LOG \\
\hline 664 & WWAS_AREA & |PRMY_LOG \\
\hline 665 & WAS_AREA & PRMZ_LOG \\
\hline 2823 & WAS_AREA & PROBDEG \\
\hline 668 & WAS_AREA & RELP_MOD \\
\hline 669 & WAS_AREA & SAT_IBRN \\
\hline 670 & WAS_AREA & SAT_RBRN \\
\hline 671 & WAS_AREA & SAT_RGAS \\
\hline 2231 & WAS_AREA & SAT_WICK \\
\hline 2232 & WAS_AREA & VOLCHW \\
\hline 2233 & WAS_AREA & VOLRHW \\
\hline 3198 & Y90 & ATWEIGHT \\
\hline 3361 & Y90 & EPAREL \\
\hline 3305 & $Y 90$ & HALFLIFE \\
\hline 3199 & ZR90 & ATWEIGHT \\
\hline 3362 & ZR90 & EPAREL \\
\hline 3306 & ZR90 & HALFLIFE \\
\hline
\end{tabular}


Appendix B3

Parameter Cross Reference

by Parameter Property Name 
Parameter Property Name

\begin{tabular}{|c|c|c|}
\hline Parameter ID & Material & Parameter \\
\hline 3503 & REFCON & ABERM \\
\hline 651 & WAS_AREA & ABSROUGH \\
\hline 2833 & REFCON & ACF_CH4 \\
\hline 2832 & REFCON & $\mathrm{ACF}{ }_{2} \mathrm{CO} 2$ \\
\hline 2831 & REFCON & ACF_H2 \\
\hline 2835 & REFCON & ACF_H2S \\
\hline 2834 & REFCON & ACF_N2 \\
\hline 2836 & REFCON & ACF_02 \\
\hline 3113 & REFCON & ACTCONST \\
\hline 2897 & REFCON & $\mathrm{AL2}$ \\
\hline 3259 & BLOWOUT & APORO \\
\hline 3487 & CULEBRA & APOROS \\
\hline 3489 & REFCON & AREA_CH \\
\hline 58 & CASTILER & AREA_FRC \\
\hline 3496 & REFCON & AREA_RH \\
\hline 3488 & REFCON & AREA_ZRO \\
\hline 3106 & REFCON & ASDRUM \\
\hline 2890 & REFCON & ATMPA \\
\hline 3220 & $A C 225$ & ATWEIGHT \\
\hline 3221 & AC227 & ATWEIGHT \\
\hline 3222 & AC228 & ATWEIGHT \\
\hline 2 & AM241 & ATWEIGHT \\
\hline 3223 & AM243 & ATWEIGHT \\
\hline 3224 & AT217 & ATWEIGHT \\
\hline 3225 & BA137 & ATWEIGHT \\
\hline 3226 & BA137M & ATWEIGHT \\
\hline 3227 & $B 1211$ & ATWEIGHT \\
\hline 3228 & $\mathrm{~B} \mid 212$ & ATWEIGHT \\
\hline 3229 & $\mathrm{Bl} 213$ & ATWEIGHT \\
\hline 3230 & $\mathrm{~B} 1214$ & ATWEIGHT \\
\hline 106 & CF252 & ATWEIGHT \\
\hline 3231 & CM243 & ATWEIGHT \\
\hline 110 & CM244 & ATWEIGHT \\
\hline 3232 & CM245 & ATWEIGHT \\
\hline 3233 & CM248 & ATWEIGHT \\
\hline 116 & CS137 & ATWEIGHT \\
\hline 3234 & FR221 & ATWEIGHT \\
\hline 3235 & ND143 & ATWEIGHT \\
\hline 246 & NP237 & ATWEIGHT \\
\hline 3236 & NP239 & ATWEIGHT \\
\hline 250 & PA231 & ATWEIGHT \\
\hline 3237 & PA233 & ATWEIGHT \\
\hline 3197 & PA234M & ATWEIGHT \\
\hline 3421 & PB209 & ATWEIGHT \\
\hline 283 & PB210 & ATWEIGHT \\
\hline 3200 & PB211 & ATWEIGHT \\
\hline 3201 & PB212 & ATWEIGHT \\
\hline 3202 & PB214 & ATWEIGHT \\
\hline 287 & PM147 & ATWEIGHT \\
\hline 3203 & $\mathrm{PO} 212$ & ATWEIGHT \\
\hline 3204 & $\mathrm{PO} 213$ & |ATWEIGHT \\
\hline
\end{tabular}

\begin{tabular}{|c|c|c|}
\hline Parameter ID & Material & Parameter \\
\hline 3205 & PO214 & ATWEIGHT \\
\hline 3206 & PO215 & ATWEIGHT \\
\hline 3207 & P0216 & ATWEIGHT \\
\hline 3208 & PO218 & ATWEIGHT \\
\hline 291 & PU238 & ATWEIGHT \\
\hline 295 & PU239 & ATWEIGHT \\
\hline 299 & PU240 & ATWEIGHT \\
\hline 303 & PU241 & ATWEIGHT \\
\hline 307 & PU242 & ATWEIGHT \\
\hline 311 & PU244 & ATWEIGHT \\
\hline 3209 & RA223 & ATWEIGHT \\
\hline 3210 & RA224 & ATWEIGHT \\
\hline 3211 & RA225 & ATWEIGHT \\
\hline 314 & RA226 & ATWEIGHT \\
\hline 318 & RA228 & ATWEIGHT \\
\hline 3212 & RN219 & ATWEIGHT \\
\hline 3213 & RN220 & ATWEIGHT \\
\hline 3214 & RN222 & ATWEIGHT \\
\hline 514 & SM147 & ATWEIGHT \\
\hline 3215 & SR90 & ATWEIGHT \\
\hline 3216 & TH227 & ATWEIGHT \\
\hline 3217 & TH228 & ATWEIGHT \\
\hline 603 & TH229 & ATWEIGHT \\
\hline 607 & TH230 & ATWEIGHT \\
\hline 3218 & TH231 & ATWEIGHT \\
\hline 611 & TH232 & ATWEIGHT \\
\hline 3219 & TH234 & ATWEIGHT \\
\hline 3196 & TL207 & ATWEIGHT \\
\hline 632 & U233 & ATWEIGHT \\
\hline 636 & U234 & ATWEIGHT \\
\hline 640 & $\mathrm{U} 235$ & ATWEIGHT \\
\hline 644 & U236 & ATWEIGHT \\
\hline 647 & $\mathrm{U} 238$ & ATWEIGHT \\
\hline 3198 & Y90 & ATWEIGHT \\
\hline 3199 & ZR90 & ATWEIGHT \\
\hline 3109 & REFCON & AVOGADRO \\
\hline 2879 & REFCON & BBLG \\
\hline 2819 & S_ANH_AB & BKLINK \\
\hline 2904 & S_MB138 & BKLINK \\
\hline 2905 & S_MB139 & BKLINK \\
\hline 2276 & ASPHALT & CAP_MOD \\
\hline 3174 & BH_CREEP & CAP_MOD \\
\hline 3138 & BH_OPEN & CAP_MOD \\
\hline 3162 & BH_SAND & CAP_MOD \\
\hline 23 & BOREHOLE & CAP_MOD \\
\hline 60 & CASTILER & CAP_MOD \\
\hline 76 & CAVITY_1 & CAP_MOD \\
\hline 91 & CAVITY_2 & CAP_MOD \\
\hline 2049 & CAVITY_3 & CAP_MOD \\
\hline 2060 & CAVITY 4 & CAP_MOD \\
\hline 2327 & CL_L_T1 & CAP_MOD \\
\hline
\end{tabular}


Parameter Property Name

\begin{tabular}{|c|c|c|c|c|c|}
\hline Parameter ID & Material & Parameter & Parameter ID & Material & Parameter \\
\hline 2344 & CL_L_T2 & CAP_MOD & 3111 & REFCON & CITOBQ \\
\hline 2361 & CL_L_T3 & CAP_MOD & 223 & GLOBAL & CLIMTIDX \\
\hline 3070 & CL_L_T4 & CAP_MOD & 3455 & REPOSIT & CLOSMOD \\
\hline 2378 & CL_M_T1 & CAP_MOD & 3454 & WAS_AREA & CLOSMOD \\
\hline 2395 & $C_{2} M_{-}{ }_{2} 2$ & CAP_MOD & 3242 & BOREHOLE & COLDIA \\
\hline 2412 & CL_M_T3 & CAP_MOD & 2277 & ASPHALT & COMP_RCK \\
\hline 2429 & CL_M_T4 & CAP_MOD & 3172 & BH_CREEP & COMP_RCK \\
\hline 2446 & CL_M_T5 & CAP_MOD & 3136 & $\mathrm{BH} \_\mathrm{OPEN}$ & COMP_RCK \\
\hline 2310 & CLAY_BOT & CAP_MOD & 3160 & BH_SAND & COMP_RCK \\
\hline 3000 & CLAY_RUS & CAP_MOD & 25 & BOREHOLE & COMP_RCK \\
\hline 3051 & CONC_MON & CAP_MOD & 61 & CASTILER & COMP_RCK \\
\hline 3150 & CONC_PLG & CAP_MOD & 77 & CAVITY_1 & COMP_RCK \\
\hline 2463 & CONC_T1 & CAP_MOD & 92 & CAVITY_2 & COMP_RCK \\
\hline 2480 & CONC_T2 & CAP_MOD & 2051 & CAVITY_3 & COMP_RCK \\
\hline 119 & CULEBRA & CAP_MOD & 2062 & CAVITY_4 & COMP_RCK \\
\hline 153 & DEWYLAKE & CAP_MOD & 2328 & CL_L_T1 & COMP_RCK \\
\hline 174 & DRZ_0 & CAP_MOD & 2345 & CL_L_T2 & COMP_RCK \\
\hline 190 & DRZ_1 & CAP_MOD & 2362 & $C_{2} L_{2} T 3$ & COMP_RCK \\
\hline 2496 & EARTH & CAP_MOD & 3071 & CL_L_T4 & COMP_RCK \\
\hline 207 & EXP_AREA & CAP_MOD & 2379 & CL_M_T1 & COMP_RCK \\
\hline 2085 & FORTYNIN & CAP_MOD & 2396 & CL_M_T2 & COMP_RCK \\
\hline 229 & IMPERM_Z & CAP_MOD & 2413 & CL_M_T3 & COMP_RCK \\
\hline 2097 & MÁGENTA & CAP_MOD & 2430 & CL_M_T4 & COMP_RCK \\
\hline 7 & OPS_AREA & CAP_MOD & 2447 & CL_M_T5 & COMP_RCK \\
\hline 252 & PAN_SEAL & CAP_MOD & 2311 & CLAY_BOT & COMP_RCK \\
\hline 2110 & REPOSIT & CAP_MOD & 3001 & CLAY_RUS & COMP_RCK \\
\hline 520 & S_ANH_AB & CAP_MOD & 3052 & CONC_MON & COMP_RCK \\
\hline 540 & S_HALITE & CAP_MOD & 3148 & CONC_PLG & COMP_RCK \\
\hline 559 & S_MB138 & CAP_MOD & 2464 & CONC_T1 & COMP_RCK \\
\hline 579 & S_MB139 & CAP_MOD & 2481 & CONC_T2 & COMP_RCK \\
\hline 2513 & SALT_T1 & CAP_MOD & 120 & CULEBRA & COMP_RCK \\
\hline 2530 & SALT_T2 & CAP_MOD & 154 & DEWYLAKE & COMP_RCK \\
\hline 2547 & SALT_T3 & CAP_MOD & 175 & DRZ_0 & COMP_RCK \\
\hline 2564 & SALT_T4 & CAP_MOD & 191 & DRZ_1 & COMP_RCK \\
\hline 2581 & SALT_T5 & CAP_MOD & 2497 & EARTH & COMP_RCK \\
\hline 2983 & SALT_T6 & CAP_MOD & 208 & EXP_AREA & COMP_RCK \\
\hline 336 & SANTAROS & CAP_MOD & 2238 & FORTYNIN & COMP_RCK \\
\hline 2183 & TAMARISK & CAP_MOD & 230 & IMPERM_Z & COMP_RCK \\
\hline 2217 & UNNAMED & CAP_MOD & 3016 & MAGENTA & COMP_RCK \\
\hline 652 & WAS_AREA & CAP_MOD & 8 & OPS_AREA & COMP_RCK \\
\hline 3457 & $A M$ & CAPHUM & 253 & PAN_SEAL & COMP_RCK \\
\hline 3458 & NP & CAPHUM & 2112 & REPOSIT & COMP_RCK \\
\hline 3459 & $\mathrm{PU}$ & CAPHUM & 521 & S_ANH_AB & COMP_RCK \\
\hline 3461 & TH & CAPHUM & 541 & S_HALITE & COMP_RCK \\
\hline 3460 & $\mathrm{U}$ & CAPHUM & 560 & S_MB138 & COMP_RCK \\
\hline 3447 & $A M$ & CAPMIC & 580 & S_MB139 & COMP_RCK \\
\hline 3313 & NP & CAPMIC & 2514 & SALT_T1 & COMP_RCK \\
\hline 3315 & PU & CAPMIC & 2531 & SALT_T2 & COMP_RCK \\
\hline 3318 & $\mathrm{TH}$ & CAPMIC & 2548 & SALT_T3 & COMP_RCK \\
\hline 3308 & $\mathrm{U}$ & CAPMIC & 2565 & SALT_T4 & COMP_RCK \\
\hline 3245 & BLOWOUT & CEMENT & 2582 & SALT_T5 & COMP_RCK \\
\hline
\end{tabular}


Parameter Property Name

\begin{tabular}{|c|c|c|}
\hline Parameter ID & Material & Parameter \\
\hline 2984 & SALT_T6 & COMP_RCK \\
\hline 337 & SANTAROS & COMP_RCK \\
\hline 2243 & TAMARISK & COMP_RCK \\
\hline 2218 & UNNAMED & COMP_RCK \\
\hline 653 & WAS_AREA & COMP_RCK \\
\hline 43 & BRINECST & COMPRES \\
\hline 48 & BRINESAL & COMPRES \\
\hline 3310 & AM & CONCINT \\
\hline 3312 & NP & CONCINT \\
\hline 3316 & PU & CONCINT \\
\hline 3319 & $\mathrm{TH}$ & CONCINT \\
\hline 3307 & $\mathrm{U}$ & CONCINT \\
\hline 3441 & $A M$ & CONCMIN \\
\hline 3439 & NP & CONCMIN \\
\hline 3440 & PU & CONCMIN \\
\hline 3437 & $\mathrm{TH}$ & CONCMIN \\
\hline 3438 & $\mathrm{U}$ & CONCMIN \\
\hline 2907 & STEEL & CORRMCO2 \\
\hline 2908 & STEEL & CORRWCO2 \\
\hline 2939 & SALT_T1 & CUMPROB \\
\hline 2882 & REFCON & DARM2 \\
\hline 2887 & REFCON & DAYSEC \\
\hline 2113 & REPOSIT & DCELLCHW \\
\hline 2041 & WAS_AREA & DCELLCHW \\
\hline 2114 & REPOSIT & DCELLRHW \\
\hline 2274 & WAS_AREA & DCELLRHW \\
\hline 26 & BOREHOLE & DIAMMOD \\
\hline 2115 & REPOSIT & DIRNCCHW \\
\hline 1992 & WAS_AREA & DIRNCCHW \\
\hline 2116 & REPOSIT & DIRNCRHW \\
\hline 1993 & WAS_AREA & DIRNCRHW \\
\hline 2117 & REPOSIT & DIRONCHW \\
\hline 2040 & WAS_AREA & DIRONCHW \\
\hline 2118 & REPOSIT & DIRONRHW \\
\hline 2044 & WAS_AREA & DIRONRHW \\
\hline 3483 & CULEBRA & DISP_L \\
\hline 3484 & CULEBRA & DISPT_L \\
\hline 44 & BRINECST & DNSFLUID \\
\hline 49 & BRINESAL & DNSFLUID \\
\hline 171 & DRILLMUD & DNSFLUID \\
\hline 3238 & ASPHALT & DNSGRAIN \\
\hline 843 & CULEBRA & DNSGRAIN \\
\hline 1661 & $S \_A N H \_A B$ & DNSGRAIN \\
\hline 1703 & S_HALITE & DNSGRAIN \\
\hline 1743 & S_MB138 & DNSGRAIN \\
\hline 1784 & S_MB139 & DNSGRAIN \\
\hline 1994 & WAS_AREA & DNSGRAIN \\
\hline 27 & BOREHOLE & DOMEGA \\
\hline 2158 & S_ANH_AB & DPHIMAX \\
\hline 2169 & S_MB138 & DPHIMAX \\
\hline 2177 & S_MB139 & DPHIMAX \\
\hline
\end{tabular}

\begin{tabular}{|c|c|c|}
\hline Parameter ID & Material & Parameter \\
\hline 2119 & REPOSIT & DPLASCHW \\
\hline 2043 & WAS_AREA & DPLASCHW \\
\hline 2120 & REPOSIT & DPLASRHW \\
\hline 2275 & WAS_AREA & DPLASRHW \\
\hline 2121 & REPOSIT & DPLSCCHW \\
\hline 1995 & WAS_AREA & DPLSCCHW \\
\hline 2995 & REPOSIT & DPLSCRHW \\
\hline 2228 & WAS_AREA & DPLSCRHW \\
\hline 3486 & CULEBRA & DPOROS \\
\hline 3132 & REFCON & DRROOM \\
\hline 2122 & REPOSIT & DRUBBCHW \\
\hline 2042 & WAS_AREA & DRUBBCHW \\
\hline 2123 & REPOSIT & DRUBBRHW \\
\hline 2046 & WAS_AREA & DRUBBRHW \\
\hline 3474 & CULEBRA & DTORT \\
\hline 3321 & AC225 & EPAREL \\
\hline 3364 & $A C 227$ & EPAREL \\
\hline 3322 & AC228 & EPAREL \\
\hline 3363 & AM241 & EPAREL \\
\hline 3365 & AM243 & EPAREL \\
\hline 3323 & AT217 & EPAREL \\
\hline 3324 & BA137 & EPAREL \\
\hline 3325 & BA137M & EPAREL \\
\hline 3326 & $\mathrm{~B} 1211$ & EPAREL \\
\hline 3327 & $\mathrm{Bl} 212$ & EPAREL \\
\hline 3328 & $\mathrm{~B} \mid 213$ & EPAREL \\
\hline 3329 & $\mathrm{~B} \mid 214$ & EPAREL \\
\hline 3330 & CF252 & EPAREL \\
\hline 3366 & CM243 & EPAREL \\
\hline 3331 & CM244 & EPAREL \\
\hline 3367 & CM245 & EPAREL \\
\hline 3368 & CM248 & EPAREL \\
\hline 3369 & $\mathrm{CS} 137$ & EPAREL \\
\hline 3332 & FR221 & EPAREL \\
\hline 3333 & ND143 & EPAREL \\
\hline 3370 & NP237 & EPAREL \\
\hline 3334 & NP239 & EPAREL \\
\hline 3371 & PA231 & EPAREL \\
\hline 3335 & PA233 & EPAREL \\
\hline 3336 & PA234M & EPAREL \\
\hline 3337 & PB209 & EPAREL \\
\hline 3372 & PB210 & EPAREL \\
\hline 3338 & PB211 & EPAREL \\
\hline 3339 & PB212 & EPAREL \\
\hline 3340 & PB214 & EPAREL \\
\hline 3341 & PM147 & EPAREL \\
\hline 3342 & PO212 & EPAREL \\
\hline 3343 & $\mathrm{PO213}$ & EPAREL \\
\hline 3344 & P0214 & EPAREL \\
\hline 3345 & PO215 & EPAREL \\
\hline 3346 & PO216 & EPAREL \\
\hline
\end{tabular}


Parameter Property Name

\begin{tabular}{|c|c|c|}
\hline Parameter ID & Material & Parameter \\
\hline 3347 & PO218 & EPAREL \\
\hline 3373 & PU238 & EPAREL \\
\hline 3374 & PU239 & EPAREL \\
\hline 3375 & PU240 & EPAREL \\
\hline 3348 & PU241 & EPAREL \\
\hline 3376 & PU242 & EPAREL \\
\hline 3377 & PU244 & EPAREL \\
\hline 3349 & RA223 & EPAREL \\
\hline 3350 & RA224 & EPAREL \\
\hline 3351 & RA225 & EPAREL \\
\hline 3378 & RA226 & EPAREL \\
\hline 3352 & RA228 & EPAREL \\
\hline 3353 & RN219 & EPAREL \\
\hline 3354 & RN220 & EPAREL \\
\hline 3355 & RN222 & EPAREL \\
\hline 3379 & SM147 & EPAREL \\
\hline 3380 & SR90 & EPAREL \\
\hline 3356 & TH227 & EPAREL \\
\hline 3357 & TH228 & EPAREL \\
\hline 3381 & TH229 & EPAREL \\
\hline 3382 & TH230 & EPAREL \\
\hline 3358 & TH231 & EPAREL \\
\hline 3383 & TH232 & EPAREL \\
\hline 3359 & TH234 & EPAREL \\
\hline 3360 & TL207 & EPAREL \\
\hline 3384 & U233 & EPAREL \\
\hline 3385 & $\mathrm{U} 234$ & EPAREL \\
\hline 3386 & $\mathrm{U} 235$ & EPAREL \\
\hline 3387 & U236 & EPAREL \\
\hline 3388 & U238 & EPAREL \\
\hline 3361 & Y90 & EPAREL \\
\hline 3362 & ZR90 & EPAREL \\
\hline 3462 & CULEBRA & ETHICK \\
\hline 2820 & S_ANH_AB & EXPKLINK \\
\hline 2902 & S_MB138 & EXPKLINK \\
\hline 2903 & S_MB139 & EXPKLINK \\
\hline 2883 & REFCON & F3M3 \\
\hline 2994 & CELLULS & FBETA \\
\hline 3420 & BLOWOUT & FCE \\
\hline 3256 & BLOWOUT & FGE \\
\hline 3501 & GLOBAL & FPICD \\
\hline 3500 & GLOBAL & FPICM \\
\hline 123 & CULEBRA & FRCTRIDX \\
\hline 3255 & BLOWOUT & FSE \\
\hline 2881 & REFCON & FTM \\
\hline 861 & CULEBRA & FTORT \\
\hline 3492 & REFCON & FVW \\
\hline 3470 & BLOWOUT & GAS_MIN \\
\hline 2127 & REPOSIT & GRATMICH \\
\hline 656 & WAS_AREA & GRATMICH \\
\hline 2128 & REPOSIT & GRATMICI \\
\hline
\end{tabular}

\begin{tabular}{|c|c|c|}
\hline Parameter ID & Material & Parameter \\
\hline 657 & WAS_AREA & GRATMICI \\
\hline 2889 & REFCON & GRAVACC \\
\hline 3194 & CASTILER & GRIDFLO \\
\hline 2884 & REFCON & GTI3 \\
\hline 3267 & $A C 225$ & HALFLIFE \\
\hline 3268 & AC227 & HALFLIFE \\
\hline 3269 & AC228 & HALFLIFE \\
\hline 3 & AM241 & HALFLIFE \\
\hline 6 & AM243 & HALFLIFE \\
\hline 3270 & AT217 & HALFLIFE \\
\hline 3271 & BA137 & HALFLIFE \\
\hline 3272 & BA137M & HALFLIFE \\
\hline 3273 & $\mathrm{Bl} 211$ & HALFLIFE \\
\hline 3274 & $\mathrm{~B} 1212$ & HALFLIFE \\
\hline 3275 & $\mathrm{~B} \mid 213$ & HALFLIFE \\
\hline 3276 & $\mathrm{BI} 214$ & HALFLIFE \\
\hline 107 & CF252 & HALFLIFE \\
\hline 3277 & CM243 & HALFLIFE \\
\hline 111 & CM244 & HALFLIFE \\
\hline 3278 & CM245 & HALFLIFE \\
\hline 115 & CM248 & HALFLIFE \\
\hline 117 & CS137 & HALFLIFE \\
\hline 3279 & FR221 & HALFLIFE \\
\hline 3280 & ND143 & HALFLIFE \\
\hline 247 & NP237 & HALFLIFE \\
\hline 3281 & NP239 & HALFLIFE \\
\hline 251 & PA231 & HALFLIFE \\
\hline 3282 & PA233 & HALFLIFE \\
\hline 3283 & PA234M & HALFLIFE \\
\hline 3284 & PB209 & HALFLIFE \\
\hline 284 & PB210 & HALFLIFE \\
\hline 3285 & PB211 & HALFLIFE \\
\hline 3286 & PB212 & HALFLIFE \\
\hline 3287 & PB214 & HALFLIFE \\
\hline 288 & PM147 & HALFLIFE \\
\hline 3288 & PO212 & HALFLIFE \\
\hline 3289 & P0213 & HALFLIFE \\
\hline 3290 & PO214 & HALFLIFE \\
\hline 3291 & $\mathrm{PO} 215$ & HALFLIFE \\
\hline 3292 & PO216 & HALFLIFE \\
\hline 3293 & PO218 & HALFLIFE \\
\hline 292 & PU238 & HALFLIFE \\
\hline 296 & PU239 & HALFLIFE \\
\hline 300 & PU240 & HALFLIFE \\
\hline 304 & PU241 & HALFLIFE \\
\hline 308 & PU242 & HALFLIFE \\
\hline 312 & PU244 & HALFLIFE \\
\hline 3294 & RA223 & HALFLIFE \\
\hline 3295 & RA224 & HALFLIFE \\
\hline 3296 & RA225 & HALFLIFE \\
\hline 315 & RA226 & HALFLIFE \\
\hline
\end{tabular}


Parameter Property Name

\begin{tabular}{|c|c|c|}
\hline Parameter ID & Material & Parameter \\
\hline 319 & RA228 & HALFLIFE \\
\hline 3297 & RN219 & HALFLIFE \\
\hline 3298 & RN220 & HALFLIFE \\
\hline 3299 & RN222 & HALFLIFE \\
\hline 515 & SM147 & HALFLIFE \\
\hline 517 & SR90 & HALFLIFE \\
\hline 3300 & TH227 & HALFLIFE \\
\hline 3301 & TH228 & HALFLIFE \\
\hline 604 & TH229 & HALFLIFE \\
\hline 608 & TH230 & HALFLIFE \\
\hline 3302 & TH231 & |HALFLIFE \\
\hline 612 & TH232 & HALFLIFE \\
\hline 3303 & TH234 & HALFLIFE \\
\hline 3304 & TL207 & |HALFLIFE \\
\hline 633 & U233 & HALFLIFE \\
\hline 637 & $\mathrm{U} 234$ & HALFLIFE \\
\hline 641 & U235 & HALFLIFE \\
\hline 645 & U236 & HALFLIFE \\
\hline 648 & $\mathrm{U} 238$ & HALFLIFE \\
\hline 3305 & Y9D & HALFLIFE \\
\hline 3306 & ZR90 & HALFLIFE \\
\hline 3485 & CULEBRA & IHMBLKLT \\
\hline 3250 & BLOWOUT & HREPO \\
\hline 3502 & REFCON & HRH \\
\hline 2910 & STEEL & HUMCORR \\
\hline 2812 & S_ANH_AB & IFRX \\
\hline 2810 & S_MB138 & IFRX \\
\hline 2811 & S_MB139 & IFRX \\
\hline 2815 & S_ANH_AB & IFRY \\
\hline 2813 & S_MB138 & IFRY \\
\hline 2814 & S_MB139 & IFRY \\
\hline 2818 & S_ANH_AB & IFRZ \\
\hline 2816 & S_MB138 & IFRZ \\
\hline 2817 & S_MB139 & IFRZ \\
\hline 3260 & BLOWOUT & INPORO \\
\hline 3239 & BOREHOLE & INV_AR \\
\hline 4 & AM241 & INVCHD \\
\hline 3415 & AM243 & INVCHD \\
\hline 108 & CF252 & INVCHD \\
\hline 3410 & CM243 & INVCHD \\
\hline 112 & CM244 & INVCHD \\
\hline 3412 & CM245 & INVCHD \\
\hline 2265 & CM248 & INVCHD \\
\hline 2037 & CS137 & INVCHD \\
\hline 248 & NP237 & INVCHD \\
\hline 2267 & PA231 & INVCHD \\
\hline 285 & PB210 & INVCHD \\
\hline 2038 & PM147 & INVCHD \\
\hline 293 & PU238 & INVCHD \\
\hline 297 & PU239 & INVCHD \\
\hline 301 & PU240 & INVCHD \\
\hline
\end{tabular}

\begin{tabular}{|c|c|c|}
\hline Parameter ID & Material & Parameter \\
\hline 305 & PU241 & INVCHD \\
\hline 309 & PU242 & INVCHD \\
\hline 2269 & PU244 & INVCHD \\
\hline 316 & RA226 & INVCHD \\
\hline 2271 & RA228 & INVCHD \\
\hline 2039 & SR90 & INVCHD \\
\hline 605 & TH229 & INVCHD \\
\hline 609 & TH230 & INVCHD \\
\hline 613 & TH232 & INVCHD \\
\hline 634 & U233 & INVCHD \\
\hline 638 & $\mathrm{U} 234$ & INVCHD \\
\hline 642 & U235 & INVCHD \\
\hline 2216 & U236 & INVCHD \\
\hline 649 & U238 & INVCHD \\
\hline 5 & AM241 & INVRHD \\
\hline 3416 & AM243 & INVRHD \\
\hline 109 & CF252 & INVRHD \\
\hline 3411 & CM243 & INVRHD \\
\hline 113 & $\mathrm{CM} 244$ & INVRHD \\
\hline 3413 & CM245 & INVRHD \\
\hline 2266 & CM248 & INVRHD \\
\hline 118 & CS137 & INVRHD \\
\hline 249 & NP237 & INVRHD \\
\hline 2268 & PA231 & INVRHD \\
\hline 286 & PB210 & INVRHD \\
\hline 289 & PM147 & INVRHD \\
\hline 294 & PU238 & INVRHD \\
\hline 298 & PU239 & INVRHD \\
\hline 302 & PU240 & INVRHD \\
\hline 306 & PU241 & INVRHD \\
\hline 310 & PU242 & INVRHD \\
\hline 2270 & PU244 & INVRHD \\
\hline 317 & RA226 & INVRHD \\
\hline 2272 & RA228 & INVRHD \\
\hline 518 & SR90 & INVRHD \\
\hline 606 & TH229 & INVRHD \\
\hline 610 & TH230 & INVRHD \\
\hline 614 & TH232 & INVRHD \\
\hline 635 & U233 & INVRHD \\
\hline 639 & $\mathrm{U} 234$ & INVRHD \\
\hline 643 & U235 & INVRHD \\
\hline 646 & $\mathrm{U} 236$ & INVRHD \\
\hline 650 & U238 & INVRHD \\
\hline 3254 & BLOWOUT & KGAS \\
\hline 2886 & REFCON & KGLB \\
\hline 2159 & S_ANH_AB & KMAXLOG \\
\hline 2170 & S_MB138 & KMAXLOG \\
\hline 2178 & S_MB139 & KMAXLOG \\
\hline 2599 & ASPHALT & KPT \\
\hline 3180 & BH_CREEP & KPT \\
\hline 3144 & BH_OPEN & KPT \\
\hline
\end{tabular}


Parameter Property Name

\begin{tabular}{|c|c|c|}
\hline Parameter ID & Material & Parameter \\
\hline 3168 & BH_SAND & |KPT \\
\hline 3122 & BOREHOLE & KPT \\
\hline 2608 & CASTILER & KPT \\
\hline 2612 & CAVITY_1 & KPT \\
\hline 2616 & CAVITY_2 & KPT \\
\hline 2620 & CAVITY_3 & KPT \\
\hline 2625 & CAVITY_4 & KPT \\
\hline 2641 & $C L \_L / T 1$ & KPT \\
\hline 2646 & CL_L_T2 & KPT \\
\hline 2651 & CL_L_T3 & KPT \\
\hline 3072 & CL_L_T4 & KPT \\
\hline 2656 & CL_M_T1 & KPT \\
\hline 2661 & CL_M_T2 & KPT \\
\hline 2666 & CL_M_T3 & KPT \\
\hline 2671 & CL_M_T4 & KPT \\
\hline 2676 & CL_M_T5 & KPT \\
\hline 2636 & CLAY_BOT & KPT \\
\hline 3002 & CLAY_RUS & KPT \\
\hline 3053 & CONC_MON & KPT \\
\hline 3156 & CONC_PLG & KPT \\
\hline 2681 & CONC_T1 & KPT \\
\hline 2686 & CONC_T2 & KPT \\
\hline 2691 & CÚLEBRA & KPT \\
\hline 2696 & DEWYLAKE & KPT \\
\hline 2701 & DRZ_0 & KPT \\
\hline 3116 & DRZ_1 & KPT \\
\hline 2706 & EARTH & KPT \\
\hline 2711 & EXP_AREA & KPT \\
\hline 2715 & FORTYNIN & KPT \\
\hline 2720 & IMPERM_Z & KPT \\
\hline 2725 & MAGENTA & KPT \\
\hline 2604 & OPS_AREA & KPT \\
\hline 2731 & PAN_SEAL & KPT \\
\hline 2736 & REPOSIT & KPT \\
\hline 2773 & S_ANH_AB & KPT \\
\hline 2778 & S_HALITE & KPT \\
\hline 2783 & S_MB138 & KPT \\
\hline 2788 & S_MB139 & KPT \\
\hline 2744 & SALT_T1 & KPT \\
\hline 2749 & SALT_T2 & KPT \\
\hline 2754 & SALT_T3 & KPT \\
\hline 2759 & SALT_T4 & KPT \\
\hline 2764 & SALT_T5 & KPT \\
\hline 2985 & SALT_T6 & KPT \\
\hline 2768 & SANTAROS & $\widehat{\text { KPT }}$ \\
\hline 2793 & TAMARISK & KPT \\
\hline 2799 & UNNAMED & KPT \\
\hline 2804 & WAS_AREA & $\widehat{\text { KPT }}$ \\
\hline 3244 & BOREHOLE & L1 \\
\hline 3243 & BOREHOLE & $L 2$ \\
\hline 3494 & GLOBAL & LAMBDAD \\
\hline
\end{tabular}

\begin{tabular}{|c|c|c|}
\hline Parameter ID & Material & Parameter \\
\hline 2885 & REFCON & LBKG \\
\hline 838 & CF & LOGSOLM \\
\hline 841 & CS & LOGSOLM \\
\hline 282 & $\overline{\mathrm{PB}}$ & LOGSOLM. \\
\hline 1167 & PM & LOGSOLM \\
\hline 313 & $\mathrm{RA}$ & LOGSOLM \\
\hline 1659 & SR & LOGSOLM \\
\hline 3471 & BLOWOUT & MAXFLOW \\
\hline 3444 & $A M+3$ & MDO \\
\hline 3442 & $\mathrm{PU}+3$ & MDO \\
\hline 3443 & $\mathrm{PU}+4$ & MDO \\
\hline 3449 & $\mathrm{TH}+4$ & MDO \\
\hline 3446 & $\mathrm{U}+4$ & MDO \\
\hline 3448 & $U+6$ & MDO \\
\hline 944 & DRZ_O & MDISP_L \\
\hline 1684 & $S \_A N H \_A B$ & MDISP_L \\
\hline 1724 & S_HALITE & MDISP_L \\
\hline 945 & DRZ_0 & MDISP_T \\
\hline 1685 & $S \_A N H \_A B$ & MDISP_T \\
\hline 1725 & S_HALITE & MDISP_T \\
\hline 3418 & CULEBRA & MEA_STOR \\
\hline 3497 & GLOBAL & MINERT \\
\hline 3472 & BLOWOUT & MINFLOW \\
\hline 3419 & CULEBRA & MINP_FAC \\
\hline 3482 & $A M+3$ & MKD AM \\
\hline 3477 & $N P+4$ & MKD_NP \\
\hline 3476 & $\widehat{N P+5}$ & MKD_NP \\
\hline 3480 & PU+3 & MKD_PU \\
\hline 3481 & $P U+4$ & MKD_PU \\
\hline 3478 & $\mathrm{TH}+4$ & MKD_TH \\
\hline 3479 & $U+4$ & MKD_U \\
\hline 3475 & $U+6$ & MKD_U \\
\hline 2877 & REFCON & MW_CACO3 \\
\hline 2875 & REFCON & MW_CAO \\
\hline 2876 & REFCON & MW_CAOH2 \\
\hline 2866 & REFCON & $\mathrm{MW}$ _CH2O \\
\hline 2860 & REFCON & $\mathrm{MW}$ CH4 \\
\hline 2859 & REFCON & MW_CO2 \\
\hline 2865 & REFCON & MW_FE \\
\hline 2873 & REFCON & MW_FE3O4 \\
\hline 2870 & REFCON & MW_FECO3 \\
\hline 2871 & REFCON & MW_FEOH2 \\
\hline 2872 & REFCON & MW_FEOOH \\
\hline 2874 & REFCON & MW_FES \\
\hline 2869 & REFCON & MW_FES2 \\
\hline 2858 & REFCON & MW_H2 \\
\hline 2864 & REFCON & $\mathrm{MW} \_\mathrm{H} 2 \mathrm{O}$ \\
\hline 2862 & REFCON & $\mathrm{MW} H 2 \mathrm{~S}$ \\
\hline 2867 & REFCON & MW_H2SO4 \\
\hline 2868 & REFCON & MW_HNO3 \\
\hline 2861 & REFCON & MW_N2 \\
\hline
\end{tabular}


Parameter Property Name

\begin{tabular}{|c|c|c|}
\hline Parameter ID & Material & Parameter \\
\hline 2878 & REFCON & MW_NACL \\
\hline 2863 & REFCON & MW_O2 \\
\hline 2894 & REFCON & OMEGAA \\
\hline 2895 & REFCON & OMEGAB \\
\hline 3417 & GLOBAL & OXSTAT \\
\hline 3246 & BLOWOUT & PARTDIA \\
\hline 2880 & REFCON & PASCP \\
\hline 3493 & GLOBAL & PBRINE \\
\hline 2839 & REFCON & PC_CH4 \\
\hline 2838 & REFCON & PC_CO2 \\
\hline 2837 & REFCON & PC_H2 \\
\hline 2841 & REFCON & PC_H2S \\
\hline 2278 & ASPHALT & PC_MAX \\
\hline 3175 & BH_CREEP & PC_MAX \\
\hline 3139 & BH_OPEN & PC_MAX \\
\hline 3163 & BH_SAND & PC_MAX \\
\hline 29 & BOREHOLE & PC_MAX \\
\hline 62 & CASTILER & PC_MAX \\
\hline 78 & CAVITY_1 & PC_MAX \\
\hline 93 & CAVITY_2 & PC_MAX \\
\hline 2234 & CAVITY_3 & PC_MAX \\
\hline 2236 & CAVITY_4 & PC_MAX \\
\hline 2329 & CL_L_T1 & PC_MAX \\
\hline 2346 & CL_L_T2 & PC_MAX \\
\hline 2363 & $C L \_L 33$ & PC_MAX \\
\hline 3073 & $\mathrm{CL} L$ L T4 & PC_MAX \\
\hline 2380 & CL_M_T1 & PC_MAX \\
\hline 2397 & CL_M_T2 & PC_MAX \\
\hline 2414 & $\mathrm{CL} \_\mathrm{M} \_\mathrm{T3}$ & PC_MAX \\
\hline 2431 & CL_M_T4 & PC_MAX \\
\hline 2448 & CL_M_T5 & PC_MAX \\
\hline 2312 & CLAY_BOT & PC_MAX \\
\hline 3003 & CLAY_RUS & PC_MAX \\
\hline 3054 & CONC_MON & PC_MAX \\
\hline 3151 & CONC_PLG & PC_MAX \\
\hline 2465 & CONC_T1 & PC_MAX \\
\hline 2482 & CONC_T2 & PC_MAX \\
\hline 137 & CULEBRA & PC_MAX \\
\hline 156 & DEWYLAKE & $\overline{P C \_M A X}$ \\
\hline 176 & $D R Z$ D & PC_MAX \\
\hline 193 & DRZ_1 & PC_MAX \\
\hline 2498 & EARTH & PC_MAX \\
\hline 209 & EXP_AREA & PC_MAX \\
\hline 2239 & FORTYNIN & PC_MAX \\
\hline 231 & IMPERM_Z & PC_MAX \\
\hline 2098 & MAGENTA & PC_MAX \\
\hline 9 & OPS_AREA & PC_MAX \\
\hline 254 & PAN_SEAL & PC_MAX \\
\hline 2242 & REPOSIT & PC_MAX \\
\hline 522 & S_ANH_AB & PC_MAX \\
\hline 542 & S_HALITE & PC_MAX \\
\hline
\end{tabular}

\begin{tabular}{|c|c|c|}
\hline Parameter ID & Material & Parameter \\
\hline 561 & S_MB138 & PC_MAX \\
\hline 582 & S_MB139 & PC_MAX \\
\hline 2515 & SALT_T1 & PC_MAX \\
\hline 2532 & SALT_T2 & PC_MAX \\
\hline 2549 & SALT_T3 & PC_MAX \\
\hline 2566 & SALT_T4 & PC_MAX \\
\hline 2583 & SALT_T5 & PC_MAX \\
\hline 2986 & SALT_T6 & PC_MAX \\
\hline 339 & SANTAROS & PC_MAX \\
\hline 2244 & TAMARISK & PC_MAX \\
\hline 2247 & IUNNAMED & PC_MAX \\
\hline 658 & WAS_AREA & PC_MAX \\
\hline 2840 & REFCON & PC_N2 \\
\hline 2842 & REFCON & PC_O2 \\
\hline 2600 & ASPHALT & PCT_A \\
\hline 3181 & BH_CREEP & PCT_A \\
\hline 3145 & BH_OPEN & PCT_A \\
\hline 3169 & BH_SAND & PCT_A \\
\hline 3120 & BOREHOLE & PCT_A \\
\hline 2609 & CASTILER & PCT_A \\
\hline 2613 & CAVITY_1 & PCT_A \\
\hline 2617 & CAVITY_2 & PCT_A \\
\hline 2621 & CAVITY_3 & PCT_A \\
\hline 2626 & CAVITY_4 & PCT_A \\
\hline 2642 & CL_L_T1 & PCT_A \\
\hline 2647 & CL_L_T2 & PCT_A \\
\hline 2652 & CL_L_T3 & PCT_A \\
\hline 3074 & CL_L_T4 & PCT_A \\
\hline 2657 & CL_M_T1 & PCT_A \\
\hline 2662 & CL_M_T2 & PCT_A \\
\hline 2667 & CL_M_T3 & PCT_A \\
\hline 2672 & CL_M_T4 & PCT_A \\
\hline 2677 & CL_M_T5 & PCT_A \\
\hline 2637 & CLAY_BOT & PCT_A \\
\hline 3004 & CLAY_RUS & PCT_A \\
\hline 3055 & CONC_MON & PCT_A \\
\hline 3157 & CONC_PLG & PCT_A \\
\hline 2682 & CONC_T1 & PCT_A \\
\hline 2687 & CONC_T2 & PCT_A \\
\hline 2692 & CULEBRA & PCT_A \\
\hline 2697 & DEWYLAKE & PCT_A \\
\hline 2702 & DRZ_0 & PCT_A \\
\hline 3128 & DRZ_1 & PCT_A \\
\hline 2707 & EARTH & PCT_A \\
\hline 2712 & EXP_AREA & PCT_A \\
\hline 2716 & FORTYNIN & PCT_A \\
\hline 2721 & IMPERM_Z & PCT_A \\
\hline 2726 & MAGENTA & PCT_A \\
\hline 2605 & OPS_AREA & PCT_A \\
\hline 2732 & PAN_SEAL & PCT_A \\
\hline 2737 & REPOSIT & PCT_A \\
\hline
\end{tabular}


Parameter Property Name

\begin{tabular}{|c|c|c|}
\hline Parameter ID & Material & Parameter \\
\hline 2774 & S_ANH_AB & PCT_A \\
\hline 2779 & S_HALITE & PCT_A \\
\hline 2784 & S_MB138 & PCT_A \\
\hline 2789 & S_MB139 & PCT_A \\
\hline 2745 & SALT_T1 & PCT_A \\
\hline 2750 & SALT_T2 & PCT_A \\
\hline 2755 & SALT_T3 & PCT_A \\
\hline 2760 & SALT_T4 & PCT_A \\
\hline 2765 & SALT_T5 & PCT_A \\
\hline 2987 & SALT_T6 & PCT_A \\
\hline 2769 & SANTAROS & PCT_A \\
\hline 2794 & TAMARISK & PCT_A \\
\hline 2800 & UNNAMED & PCT_A \\
\hline 2805 & WAS_AREA & PCT_A \\
\hline 2601 & ASPHALT & PCT_EXP \\
\hline 3182 & BH_CREEP & PCT_EXP \\
\hline 3146 & BH_OPEN & PCT_EXP \\
\hline 3170 & BH_SAND & PCT_EXP \\
\hline 3121 & BOREHOLE & PCT_EXP \\
\hline 2610 & CASTILER & PCT_EXP \\
\hline 2614 & CAVITY_1 & PCT_EXP \\
\hline 2618 & CAVITY_2 & PCT_EXP \\
\hline 2622 & CAVITY_3 & PCT_EXP \\
\hline 2627 & CAVITY_4 & PCT_EXP \\
\hline 2643 & CL_L_T1 & PCT_EXP \\
\hline 2648 & $\mathrm{CL} \mathrm{L}_{-} \mathrm{T} 2$ & PCT_EXP \\
\hline 2653 & CL_L_T3 & PCT_EXP \\
\hline 3075 & CL_L_T4 & PCT_EXP \\
\hline 2658 & CL_M_T1 & PCT_EXP \\
\hline 2663 & CL_M_T2 & PCT_EXP \\
\hline 2668 & CL_M_T3 & PCT_EXP \\
\hline 2673 & CL_M_T4 & PCT_EXP \\
\hline 2678 & CL_M_T5 & PCT_EXP \\
\hline 2638 & CLAY_BOT & PCT_EXP \\
\hline 3005 & CLAY_RUS & PCT_EXP \\
\hline 3056 & CONC_MON & PCT_EXP \\
\hline 3158 & CONC_PLG & PCT_EXP \\
\hline 2683 & CONC_T1 & PCT_EXP \\
\hline 2688 & CONC_T2 & PCT_EXP \\
\hline 2693 & CULEBRA & PCT_EXP \\
\hline 2698 & DEWYLAKE & PCT_EXP \\
\hline 2703 & DRZ_0 & PCT_EXP \\
\hline 3129 & DRZ_1 & PCT_EXP \\
\hline 2708 & EARTH & PCT_EXP \\
\hline 2713 & EXP_AREA & PCT_EXP \\
\hline 2717 & FORTYNIN & PCT_EXP \\
\hline 2722 & IMPERM_Z & PCT_EXP \\
\hline 2727 & MAGENTA & PCT_EXP \\
\hline 2606 & OPS_AREA & PCT_EXP \\
\hline 2733 & PAN_SEAL & PCT_EXP \\
\hline 2738 & REPOSIT & PCT_EXP \\
\hline
\end{tabular}

\begin{tabular}{|c|c|c|}
\hline Parameter ID & Material & Parameter \\
\hline 2775 & S_ANH_AB & PCT_EXP \\
\hline 2780 & S_HALITE & PCT_EXP \\
\hline 2785 & S_MB138 & PCT_EXP \\
\hline 2790 & S_MB139 & PCT_EXP \\
\hline 2746 & SALT_T1 & PCT_EXP \\
\hline 2751 & SALT_T2 & PCT_EXP \\
\hline 2756 & SALT_T3 & PCT_EXP \\
\hline 2761 & SALT_T4 & PCT_EXP \\
\hline 2766 & SALT_T5 & PCT_EXP \\
\hline 2988 & SALT_T6 & PCT_EXP \\
\hline 2770 & SANTAROS & PCT_EXP \\
\hline 2795 & TAMARISK & PCT_EXP \\
\hline 2801 & UNNAMED & PCT_EXP \\
\hline 2806 & WAS_AREA & PCT_EXP \\
\hline 524 & S_ANH_AB & PF_DELTA \\
\hline 563 & S_MB138 & PF_DELTA \\
\hline 2180 & S_MB139 & PF_DELTA \\
\hline 3429 & PHUMOX3 & PHUMCIM \\
\hline 3430 & PHUMOX4 & PHUMCIM \\
\hline 3431 & PHUMOX5 & PHUMCIM \\
\hline 3432 & PHUMOX6 & PHUMCIM \\
\hline 3433 & PHUMOX3 & PHUMSIM \\
\hline 3434 & PHUMOX 4 & PHUMSIM \\
\hline 3435 & PHUMOX5 & PHUMSIM \\
\hline 3436 & PHUMOX6 & PHUMSIM \\
\hline 2896 & REFCON & PI \\
\hline 526 & S_ANH_AB & PI_DELTA \\
\hline 565 & S_MB138 & PI_DELTA \\
\hline 586 & S_MB139 & PI_DELTA \\
\hline 3241 & BOREHOLE & PIPED \\
\hline 3495 & GLOBAL & PLGPAT \\
\hline 2942 & SALT_T1 & PMLT_HI \\
\hline 2950 & SALT_T2 & PMLT_HI \\
\hline 2958 & SALT_T3 & PMLT_HI \\
\hline 2966 & SALT_T4 & PMLT_HI \\
\hline 2974 & SALT_T5 & PMLT_HI \\
\hline 2982 & SALT_T6 & PMLT_HI \\
\hline 2941 & SALT_T1 & PMLT_LO \\
\hline 2949 & SALT_T2 & PMLT_LO \\
\hline 2957 & SALT_T3 & PMLT_LO \\
\hline 2965 & SALT_T4 & PMLT_LO \\
\hline 2973 & SALT_T5 & PMLT_LO \\
\hline 2981 & SALT_T6 & PMLT_LO \\
\hline 2940 & SALT_T1 & PMLT_MD \\
\hline 2948 & SALT_T2 & PMLT_MD \\
\hline 2956 & SALT_T3 & PMLT_MD \\
\hline 2964 & SALT_T4 & PMLT_MD \\
\hline 2972 & SALT_T5 & PMLT_MD \\
\hline 2980 & SALT_T6 & PMLT_MD \\
\hline 2281 & ASPHALT & PO_MIN \\
\hline 3179 & BH_CREEP & PO_MIN \\
\hline
\end{tabular}


Parameter Property Name

\begin{tabular}{|c|c|c|}
\hline Parameter ID & Material & Parameter \\
\hline 3143 & BH_OPEN & PO_MIN \\
\hline 3167 & BH_SAND & PO_MIN \\
\hline 32 & BOREHOLE & PO_MIN \\
\hline 65 & CASTILER & PO_MIN \\
\hline 81 & CAVITY_1 & PO_MIN \\
\hline 96 & CAVITY_2 & PO_MIN \\
\hline 2623 & CAVITY_3 & PO_MIN \\
\hline 2628 & CAVITY_4 & PO_MIN \\
\hline 2332 & $C L L I 1$ & PO_MIN \\
\hline 2349 & $\mathrm{CL} \_\mathrm{L}-\mathrm{T} 2$ & PO_MIN \\
\hline 2366 & CL_L_T3 & PO_MIN \\
\hline 3123 & CL_L_T4 & PO_MIN \\
\hline 2383 & CL_M_T1 & PO_MIN \\
\hline 2400 & CL_M_T2 & PO_MIN \\
\hline 2417 & $\mathrm{CL}$ M_T3 & PO_MIN \\
\hline 2434 & CL_M_T4 & PO_MIN \\
\hline 2451 & CL_M_T5 & PO_MIN \\
\hline 2315 & CLAY_BOT & PO_MIN \\
\hline 3131 & CLAY_RUS & PO_MIN \\
\hline 3124 & CONC_MON & PO_MIN \\
\hline 3155 & CONC_PLG & PO_MIN \\
\hline 2468 & CONC_T1 & PO_MIN \\
\hline 2808 & CONC_T2 & PO_MIN \\
\hline 141 & CULEBRA & PO_MIN \\
\hline 159 & DEWYLAKE & PO_MIN \\
\hline 179 & DRZ_o & PO_MIN \\
\hline 196 & DRZ_1 & PO_MIN \\
\hline 2501 & EARTH & PO_MIN \\
\hline 212 & EXP_AREA & PO_MIN \\
\hline 2718 & FORTYNIN & PO_MIN \\
\hline 234 & IMPERM_Z & PO_MIN \\
\hline 2728 & MAGENTA & PO_MIN \\
\hline 12 & OPS_AREA & PO_MIN \\
\hline 257 & PAN_SEAL & PO_MIN \\
\hline 2739 & REPOSIT & PO_MIN \\
\hline 529 & S_ANH_AB & PO_MIN \\
\hline 545 & S_HALITE & PO_MIN \\
\hline 568 & S_MB138 & PO_MIN \\
\hline 589 & S_MB139 & PO_MIN \\
\hline 2518 & SALT_T1 & PO_MIN \\
\hline 2535 & SALT_T2 & PO_MIN \\
\hline 2552 & SALT_T3 & PO_MIN \\
\hline 2569 & SALT_T4 & PO_MIN \\
\hline 3125 & SALT_T5 & PO_MIN \\
\hline 3126 & SALT_T6 & PO_MIN \\
\hline 342 & SANTAROS & PO_MIN \\
\hline 2796 & TAMARISK & PO_MIN \\
\hline 2802 & UNNAMED & PO_MIN \\
\hline 661 & WAS_AREA & PO_MIN \\
\hline 2279 & ASPHALT & PORE_DIS \\
\hline 3178 & BH_CREEP & PORE_DIS \\
\hline
\end{tabular}

\begin{tabular}{|c|c|c|}
\hline Parameter ID & Material & Parameter \\
\hline 3142 & BH_OPEN & PORE_DIS \\
\hline 3166 & BH_SAND & PORE_DIS \\
\hline 30 & BOREHOLE & PORE_DIS \\
\hline 63 & CASTILER & PORE_DIS \\
\hline 79 & CAVITY_1 & PORE_DIS \\
\hline 94 & CAVITY_2 & PORE_DIS \\
\hline 2052 & CAVITY_3 & PORE_DIS \\
\hline 2063 & CAVITY_4 & PORE_DIS \\
\hline 2330 & CL_L_T1 & PORE DIS \\
\hline 2347 & CL_L_T2 & PORE_DIS \\
\hline 2364 & CL_L_T3 & PORE_DIS \\
\hline 3076 & CL_L_T4 & PORE_DIS \\
\hline 2381 & CL_M_T1 & PORE_DIS \\
\hline 2398 & CL_M_T2 & PORE_DIS \\
\hline 2415 & CL_M_T3 & PORE_DIS \\
\hline 2432 & CL_M_T4 & PORE_DIS \\
\hline 2449 & CL_M_T5 & PORE_DIS \\
\hline 2313 & CLAY_BOT & PORE_DIS \\
\hline 3006 & CLAY_RUS & PORE_DIS \\
\hline 3057 & CONC_MON & PORE_DIS \\
\hline 3154 & CONC_PLG & PORE_DIS \\
\hline 2466 & CONC_T1 & PORE_DIS \\
\hline 2483 & CONC_T2 & PORE_DIS \\
\hline 139 & CULEBRA & PORE_DIS \\
\hline 157 & DEWYLAKE & PORE_DIS \\
\hline 177 & $D R Z$ D 0 & PORE_DIS \\
\hline 194 & $D R Z$ 1 & PORE_DIS \\
\hline 2499 & EARTH & PORE_DIS \\
\hline 210 & EXP_AREA & PORE_DIS \\
\hline 2087 & FORTYNIN & PORE_DIS \\
\hline 232 & IMPERM_Z & PORE_DIS \\
\hline 2099 & MAGENTA & PORE_DIS \\
\hline 10 & OPS_AREA & PORE_DIS \\
\hline 255 & PAN_SEAL & PORE_DIS \\
\hline 2129 & REPOSIT & PORE_DIS \\
\hline 527 & S_ANH_AB & PORE_DIS \\
\hline 543 & S_HALITE & PORE_DIS \\
\hline 566 & S_MB138 & PORE_DIS \\
\hline 587 & S_MB139 & PORE_DIS \\
\hline 2516 & SALT_T1 & PORE_DIS \\
\hline 2533 & SALT_T2 & PORE_DIS \\
\hline 2550 & SALT_T3 & PORE_DIS \\
\hline 2567 & SALT_T4 & PORE_DIS \\
\hline 2809 & SALT_T5 & PORE_DIS \\
\hline 2989 & SALT_T6 & PORE DIS \\
\hline 340 & SANTAROS & PORE_DIS \\
\hline 2185 & TAMARISK & PORE_DIS \\
\hline 2219 & UNNAMED & PORE_DIS \\
\hline 659 & WAS_AREA & PORE_DIS \\
\hline 2280 & ASPHALT & POROSITY \\
\hline 3171 & BH_CREEP & POROSITY \\
\hline
\end{tabular}


Parameter Property Name

\begin{tabular}{|c|c|c|}
\hline Parameter ID & Material & Parameter \\
\hline 3135 & BH_OPEN & POROSITY \\
\hline 3159 & BH_SAND & POROSITY \\
\hline 31 & BOREHOLE & POROSITY \\
\hline 64 & CASTILER & POROSITY \\
\hline 80 & CAVITY_1 & POROSITY \\
\hline 95 & CAVITY_2 & POROSITY \\
\hline 2053 & CAVITY_3 & POROSITY \\
\hline 2064 & CAVITY_4 & POROSITY \\
\hline 2331 & CL_L_T1 & POROSITY \\
\hline 2348 & CL_L_T2 & POROSITY \\
\hline 2365 & CL_L_T3 & POROSITY \\
\hline 3077 & $C L \_L \_T 4$ & POROSITY \\
\hline 2382 & CL_M_T1 & POROSITY \\
\hline 2399 & CL_M_T2 & POROSITY \\
\hline 2416 & CL_M_T3 & POROSITY \\
\hline 2433 & CL_M_T4 & POROSITY \\
\hline 2450 & CL_M_T5 & POROSITY \\
\hline 2314 & CLAY_BOT & POROSITY \\
\hline 3007 & CLAY_RUS & POROSITY \\
\hline 3058 & CONC_MON & POROSITY \\
\hline 3147 & CONC_PLG & POROSITY \\
\hline 2467 & CONC_T1 & POROSITY \\
\hline 2484 & CONC_T2 & POROSITY \\
\hline 140 & CULEBRA & POROSITY \\
\hline 158 & DEWYLAKE & POROSITY \\
\hline 178 & DRZ_0 & POROSITY \\
\hline 195 & DRZ_1 & POROSITY \\
\hline 2500 & EARTH & POROSITY \\
\hline 211 & EXP_AREA & POROSITY \\
\hline 2088 & FORTYNIN & POROSITY \\
\hline 233 & IMPERM_Z & POROSITY \\
\hline 2100 & MAGENTA & POROSITY \\
\hline 11 & OPS_AREA & POROSITY \\
\hline 256 & PAN_SEAL & POROSITY \\
\hline 2130 & REPOSIT & POROSITY \\
\hline 528 & S_ANH_AB & POROSITY \\
\hline 544 & S_HALITE & POROSITY \\
\hline 567 & S_MB138 & POROSITY \\
\hline 588 & S_MB139 & POROSITY \\
\hline 2517 & SALT_T1 & POROSITY \\
\hline 2534 & SALT_T2 & POROSITY \\
\hline 2551 & SALT_T3 & POROSITY \\
\hline 2568 & SALT_T4 & POROSITY \\
\hline 2585 & SALT_T5 & POROSITY \\
\hline 2990 & SALT_T6 & POROSITY \\
\hline 341 & SANTAROS & POROSITY \\
\hline 2186 & TAMARISK & POROSITY \\
\hline 2220 & UNNAMED & POROSITY \\
\hline 660 & WAS_AREA & POROSITY \\
\hline 2282 & ASPHALT & PRESSURE \\
\hline 33 & BOREHOLE & PRESSURE \\
\hline
\end{tabular}

\begin{tabular}{|c|c|c|}
\hline Parameter ID & Material & Parameter \\
\hline 66 & CASTILER & PRESSURE \\
\hline 82 & CAVITY_1 & PRESSURE \\
\hline 97 & CAVITY 2 & PRESSURE \\
\hline 3101 & CAVITY_3 & PRESSURE \\
\hline 3103 & CAVITY_4 & PRESSURE \\
\hline 2333 & CL_L_T1 & PRESSURE \\
\hline 2384 & CL_M_T1 & PRESSURE \\
\hline 2316 & CLAY_BOT & PRESSURE \\
\hline 3008 & CLAY_RUS & PRESSURE \\
\hline 3114 & CONC_MON & PRESSURE \\
\hline 2469 & CONC_T1 & PRESSURE \\
\hline 2485 & CONC_T2 & PRESSURE \\
\hline 142 & CULEBRA & PRESSURE \\
\hline 160 & DEWYLAKE & PRESSURE \\
\hline 2502 & EARTH & PRESSURE \\
\hline 213 & EXP_AREA & PRESSURE \\
\hline 2101 & MAGENTA & PRESSURE \\
\hline 13 & OPS_AREA & PRESSURE \\
\hline 258 & PAN_SEAL & PRESSURE \\
\hline 546 & S_HALITE & PRESSURE \\
\hline 569 & S_MB138 & PRESSURE \\
\hline 590 & S_MB139 & PRESSURE \\
\hline 2519 & SALT_T1 & PRESSURE \\
\hline 343 & SANTAROS & PRESSURE \\
\hline 662 & WAS_AREA & PRESSURE \\
\hline 2283 & ASPHALT & PRMX_LOG \\
\hline 3183 & BH_CREEP & PRMX_LOG \\
\hline 3134 & BH_OPEN & PRMX_LOG \\
\hline 3184 & BH_SAND & PRMX_LOG \\
\hline 34 & BOREHOLE & PRMX_LOG \\
\hline 67 & CASTILER & PRMX_LOG \\
\hline 83 & CAVITY_1 & PRMX_LOG \\
\hline 98 & CAVITY_2 & PRMX_LOG \\
\hline 2054 & CAVITY_3 & PRMX_LOG \\
\hline 2065 & CAVITY_4 & PRMX_LOG \\
\hline 2334 & CL_L_T1 & PRMX_LOG \\
\hline 2351 & $C L \perp{ }^{\prime}{ }_{2}$ & PRMX_LOG \\
\hline 2368 & CL_L_T3 & PRMX_LOG \\
\hline 3078 & CL_L_T4 & PRMX_LOG \\
\hline 2385 & CL_M_T1 & PRMX_LOG \\
\hline 2402 & CL_M_T2 & PRMX_LOG \\
\hline 2419 & CL_M_T3 & PRMX_LOG \\
\hline 2436 & CL_M_T4 & PRMX_LOG \\
\hline 2453 & $C L \_M \_T 5$ & PRMX_LOG \\
\hline 2317 & CLAY_BOT & PRMX_LOG \\
\hline 3009 & CLAY_RUS & PRMX_LOG \\
\hline 3059 & CONC_MON & PRMX_LOG \\
\hline 3185 & CONC_PLG & PRMX_LOG \\
\hline 2470 & CONC_T1 & PRMX_LOG \\
\hline 2486 & CONC_T2 & PRMX_LOG \\
\hline 143 & CULEBRA & PRMX_LOG \\
\hline
\end{tabular}


Parameter Property Name

\begin{tabular}{|c|c|c|}
\hline Parameter ID & Material & Parameter \\
\hline 161 & DDEWYLAKE & PRMX_LOG \\
\hline 181 & DRZ_0 & PRMX_LOG \\
\hline 198 & DRZ_1 & PRMX_LOG \\
\hline 2503 & EARTH & PRMX_LOG \\
\hline 214 & EXP_AREA & PRMX_LOG \\
\hline 2899 & FORTYNIN & PRMX_LOG \\
\hline 236 & IMPERM_Z & PRMX_LOG \\
\hline 2102 & MAGENTA & PRMX_LOG \\
\hline 14 & OPS_AREA & PRMX_LOG \\
\hline 259 & PAN_SEAL & PRMX_LOG \\
\hline 2131 & REPOSIT & PRMX_LOG \\
\hline 531 & S_ANH_AB & PRMX_LOG \\
\hline 547 & S_HALITE & PRMX_LOG \\
\hline 570 & S_MB138 & PRMX_LOG \\
\hline 591 & S_MB139 & PRMX_LOG \\
\hline 344 & SANTAROS & PRMX_LOG \\
\hline 3133 & SHFT_DRZ & PRMX_LOG \\
\hline 2914 & TAMARISK & PRMX_LOG \\
\hline 2911 & UNNAMED & PRMX_LOG \\
\hline 663 & WAS_AREA & PRMX_LOG \\
\hline 2284 & ASPHALT & PRMY_LOG \\
\hline 3188 & BH_CREEP & PRMY_LOG \\
\hline 3186 & BH_OPEN & PRMY_LOG \\
\hline 3190 & BH_SAND & PRMY_LOG \\
\hline 35 & BOREHOLE & PRMY_LOG \\
\hline 68 & CASTILER & PRMY_LOG \\
\hline 84 & CAVITY_1 & PRMY_LOG \\
\hline 99 & CAVITY_2 & PRMY_LOG \\
\hline 2055 & CAVITY_3 & PRMY_LOG \\
\hline 2066 & CAVITY_4 & PRMY_LOG \\
\hline 2335 & CL_L_T1 & PRMY_LOG \\
\hline 2352 & CL_L_T2 & PRMY_LOG \\
\hline 2369 & CL_L_T3 & PRMY_LOG \\
\hline 3079 & $C L \_L \_T 4$ & PRMY_LOG \\
\hline 2386 & CL_M_T1 & PRMY_LOG \\
\hline 2403 & $C L=M \_T 2$ & PRMY_LOG \\
\hline 2420 & CL_M_T3 & PRMY_LOG \\
\hline 2437 & CL_M_T4 & PRMY_LOG \\
\hline 2454 & CL_M_T5 & PRMY_LOG \\
\hline 2318 & CLAY_BOT & PRMY_LOG \\
\hline 3010 & CLAY_RUS & PRMY_LOG \\
\hline 3060 & CONC_MON & PRMY_LOG \\
\hline 3192 & CONC_PLG & PRMY_LOG \\
\hline 2471 & CONC_T1 & PRMY_LOG \\
\hline 2487 & CONC_T2 & PRMY_LOG \\
\hline 144 & CULEBRA & PRMY_LOG \\
\hline 162 & DEWYLAKE & PRMY_LOG \\
\hline 182 & DRZ_o & PRMY_LOG \\
\hline 199 & DRZ_1 & PRMY_LOG \\
\hline 2504 & EARTH & PRMY_LOG \\
\hline 215 & EXP_AREA & PRMY_LOG \\
\hline
\end{tabular}

\begin{tabular}{|c|c|c|}
\hline Parameter ID & Material & Parameter \\
\hline 2900 & FORTYNIN & PRMY_LOG \\
\hline 237 & IMPERM_Z & PRMY_LOG \\
\hline 2103 & MAGENTA & PRMY_LOG \\
\hline 15 & OPS_AREA & PRMY_LOG \\
\hline 260 & PAN_SEAL & PRMY_LOG \\
\hline 2132 & REPOSIT & PRMY_LOG \\
\hline 532 & $S$ ANH $A B$ & PRMY LOG \\
\hline 548 & S_HALITE & PRMY_LOG \\
\hline 571 & S_MB138 & PRMY LOG \\
\hline 592 & S_MB139 & PRMY_LOG \\
\hline 345 & SANTAROS & PRMY_LOG \\
\hline 2915 & TAMARISK & PRMY_LOG \\
\hline 2912 & UNNAMED & PRMY_LOG \\
\hline 664 & WAS_AREA & PRMY_LOG \\
\hline 2285 & ASPHALT & PRMZ_LOG \\
\hline 3189 & BH_CREEP & PRMZ_LOG \\
\hline 3187 & BH_OPEN & PRMZ_LOG \\
\hline 3191 & BH_SAND & PRMZ_LOG \\
\hline 36 & BOREHOLE & PRMZ_LOG \\
\hline 69 & CASTILER & PRMZ_LOG \\
\hline 85 & CAVITY_1 & PRMZ_LOG \\
\hline 100 & CAVITY_2 & PRMZ_LOG \\
\hline 2056 & CAVITY_3 & PRMZ_LOG \\
\hline 2067 & CAVITY_4 & PRMZ_LOG \\
\hline 2336 & CL_L_T1 & PRMZ_LOG \\
\hline 2353 & CL_L_T2 & PRMZ_LOG \\
\hline 2370 & CL_L_T3 & PRMZ_LOG \\
\hline 3080 & CL_L_T4 & PRMZ_LOG \\
\hline 2387 & CL_M_T1 & PRMZ_LOG \\
\hline 2404 & CL_M_T2 & PRMZ_LOG \\
\hline 2421 & $C L, M \_T 3$ & PRMZ_LOG \\
\hline 2438 & CL_M_T4 & PRMZ_LOG \\
\hline 2455 & CL_M_T5 & PRMZ_LOG \\
\hline 2319 & CLAY_BOT & PRMZ_LOG \\
\hline 3011 & CLAY_RUS & PRMZ_LOG \\
\hline 3061 & CONC_MON & PRMZ_LOG \\
\hline 3193 & CONC_PLG & PRMZ_LOG \\
\hline 2472 & CONC_T1 & PRMZ_LOG \\
\hline 2488 & CONC_T2 & PRMZ_LOG \\
\hline 145 & CULEBRA & PRMZ_LOG \\
\hline 163 & DEWYLAKE & PRMZ_LOG \\
\hline 183 & DRZ_0 & PRMZ_LOG \\
\hline 200 & DRZ_1 & PRMZ_LOG \\
\hline 2505 & EARTH & PRMZ_LOG \\
\hline 216 & EXP_AREA & PRMZ_LOG \\
\hline 2901 & FORTYNIN & PRMZ_LOG \\
\hline 238 & IMPERM_Z & PRMZ_LOG \\
\hline 2104 & MAGENTA & PRMZ_LOG \\
\hline 16 & OPS_AREA & PRMZ_LOG \\
\hline 261 & PAN_SEAL & PRMZ_LOG \\
\hline 2133 & REPOSIT & PRMZ_LOG \\
\hline
\end{tabular}


Parameter Property Name

\begin{tabular}{|c|c|c|}
\hline Parameter ID & Material & Parameter \\
\hline 533 & S_ANH_AB & PRMZ_LOG \\
\hline 549 & S_HALITE & PRMZ_LOG \\
\hline 572 & S_MB138 & PRMZ_LOG \\
\hline 593 & S_MB139 & PRMZ_LOG \\
\hline 346 & SANTAROS & PRMZ_LOG \\
\hline 2916 & TAMARISK & PRMZ_LOG \\
\hline 2913 & UNNAMED & PRMZ_LOG \\
\hline 665 & WAS_AREA & PRMZ_LOG \\
\hline 2824 & REPOSIT & PROBDEG \\
\hline 2823 & WAS_AREA & PROBDEG \\
\hline 3311 & $A M$ & PROPMIC \\
\hline 3314 & NP & PROPMIC \\
\hline 3317 & $P U$ & PROPMIC \\
\hline 3320 & $\mathrm{TH}$ & PROPMIC \\
\hline 3309 & $U$ & PROPMIC \\
\hline 2892 & REFCON & PSIPA \\
\hline 3251 & BLOWOUT & PSUF \\
\hline 38 & BOREHOLE & PTINDEX \\
\hline 2906 & NITRATE & QINIT \\
\hline 2909 & SULFATE & QINIT \\
\hline 2893 & REFCON & $R$ \\
\hline 2933 & ASPHALT & RADN_DRZ \\
\hline 3021 & CL_L_T1 & RADN_DRZ \\
\hline 3026 & $C L \_L / T 2$ & RADN_DRZ \\
\hline 3031 & $\mathrm{CL} L \mathrm{~L}$ T3 & RADN_DRZ \\
\hline 3069 & CL_L_T4 & RADN_DRZ \\
\hline 3088 & CL_M_T1 & RADN_DRZ \\
\hline 3093 & CL_M_T2 & RADN_DRZ \\
\hline 3098 & CL_M_T3 & RADN_DRZ \\
\hline 2923 & CL_M_T4 & RADN_DRZ \\
\hline 2928 & CL_M_T5 & RADN_DRZ \\
\hline 3050 & CONC_MON & RADN_DRZ \\
\hline 3040 & CONC_T1 & RADN_DRZ \\
\hline 3045 & CONC_T2 & RADN_DRZ \\
\hline 2938 & SALT_T1 & RADN_DRZ \\
\hline 2947 & SALT_T2 & RADN_DRZ \\
\hline 2955 & SALT_T3 & RADN_DRZ \\
\hline 2963 & SALT_T4 & RADN_DRZ \\
\hline 2971 & SALT_T5 & RADN_DRZ \\
\hline 2979 & SALT_T6 & RADN_DRZ \\
\hline 3456 & BLOWOUT & RE_CAST \\
\hline 50 & BRINESAL & REF_PRES \\
\hline 51 & BRINESAL & REF_TEMP \\
\hline 2289 & ASPHALT & RELP_MOD \\
\hline 3173 & BH_CREEP & RELP_MOD \\
\hline 3137 & BH_OPEN & RELP_MOD \\
\hline 3161 & BH_SAND & RELP_MOD \\
\hline 40 & BOREHOLE & RELP_MOD \\
\hline 72 & CASTILER & RELP_MOD \\
\hline 88 & CAVITY_1 & RELP_MOD \\
\hline 103 & CAVITY_2 & RELP_MOD \\
\hline
\end{tabular}

\begin{tabular}{|c|c|c|}
\hline Parameter ID & Material & Parameter \\
\hline 2058 & CAVITY_3 & RELP_MOD \\
\hline 2069 & CAVITY_4 & RELP_MOD \\
\hline 2340 & $C L \_L=T 1$ & RELP_MOD \\
\hline 2357 & $C L_{L} L_{-T 2}$ & RELP_MOD \\
\hline 2374 & CL_L_T3 & RELP_MOD \\
\hline 3081 & $C L \perp T 4$ & RELP_MOD \\
\hline 2391 & CL_M_T1 & RELP_MOD \\
\hline 2408 & $C L \_M$ T2 & RELP_MOD \\
\hline 2425 & CL_M_T3 & RELP_MOD \\
\hline 2442 & CL_M_T4 & RELP_MOD \\
\hline 2459 & CL_M_T5 & RELP_MOD \\
\hline 2323 & CLAY_BOT & RELP_MOD \\
\hline 3012 & CLAY_RUS & RELP_MOD \\
\hline 3062 & CONC_MON & RELP_MOD \\
\hline 3149 & CONC_PLG & RELP_MOD \\
\hline 2476 & CONC_T1 & RELP_MOD \\
\hline 2492 & CONC_T2 & RELP_MOD \\
\hline 148 & CULEBRA & RELP_MOD \\
\hline 166 & DEWYLAKE & RELP_MOD \\
\hline 186 & DRZ_o & RELP_MOD \\
\hline 203 & DRZ_1 & RELP_MOD \\
\hline 2509 & EARTH & RELP_MOD \\
\hline 219 & EXP_AREA & RELP_MOD \\
\hline 2093 & FORTYNIN & RELP_MOD \\
\hline 241 & IMPERM_Z & RELP_MOD \\
\hline 2106 & MAGENTA & RELP_MOD \\
\hline 19 & OPS_AREA & RELP_MOD \\
\hline 264 & PAN_SEAL & RELP_MOD \\
\hline 2135 & REPOSIT & RELP_MOD \\
\hline 536 & S_ANH_AB & RELP_MOD \\
\hline 553 & S_HALITE & RELP_MOD \\
\hline 575 & S_MB138 & RELP_MOD \\
\hline 596 & S_MB139 & RELP_MOD \\
\hline 2526 & SALT_T1 & RELP_MOD \\
\hline 2543 & SALT_T2 & RELP_MOD \\
\hline 2560 & SALT_T3 & RELP_MOD \\
\hline 2577 & SALT_T4 & RELP_MOD \\
\hline 2594 & SALT_T5 & RELP_MOD \\
\hline 2991 & SALT_T6 & RELP_MOD \\
\hline 349 & SANTAROS & RELP_MOD \\
\hline 2191 & TAMARISK & RELP_MOD \\
\hline 2225 & UNNAMED & RELP_MOD \\
\hline 668 & WAS_AREA & RELP_MOD \\
\hline 3253 & BLOWOUT & RGAS \\
\hline 3247 & BLOWOUT & RHOS \\
\hline 3261 & BOREHOLE & RHW_AR \\
\hline 3248 & BLOWOUT & ROOM \\
\hline 3240 & BOREHOLE & ROUGHP \\
\hline 3249 & BLOWOUT & RPANEL \\
\hline 2929 & ASPHALT & RSH_AIR \\
\hline 3017 & CL_L_T1 & RSH_AIR \\
\hline
\end{tabular}


Parameter Property Name

\begin{tabular}{|c|c|c|c|c|c|}
\hline Parameter ID & Material & Parameter & Parameter ID & Material & Parameter \\
\hline 3022 & $C L \_L \_T 2$ & RSH_AIR & 3047 & CONC_MON & RSH_SAL \\
\hline 3027 & CL_L_T3 & RSH_AIR & 3037 & CONC_T1 & RSH_SAL \\
\hline 3065 & $\mathrm{CL}_{2} \mathrm{~L}_{-} \mathrm{T} 4$ & RSH_AIR & 3042 & CONC_T2 & RSH_SAL \\
\hline 3084 & CL_M_T1 & RSH_AIR & 3033 & EARTH & RSH_SAL \\
\hline 3089 & CL_M_T2 & RSH_AIR & 2935 & SALT_T1 & RSH_SAL \\
\hline 3094 & $\mathrm{CL}_{-} \mathrm{M}_{-} \mathrm{T3}$ & RSH_AIR & 2944 & SALT_T2 & RSH_SAL \\
\hline 2919 & CL_M_T4 & RSH_AIR & 2952 & SALT_T3 & RSH_SAL \\
\hline 2924 & $C_{L} M_{-}$T5 & RSH_AIR & 2960 & SALT_T4 & RSH_SAL \\
\hline 2996 & CLAY_RUS & RSH_AIR & 2968 & SALT_T5 & RSH_SAL \\
\hline 3046 & CONC_MON & RSH_AIR & 2976 & SALT_T6 & RSH_SAL \\
\hline 3036 & CONC_T1 & RSH_AIR & 2931 & ASPHALT & RSH_WAS \\
\hline 3041 & CONC_T2 & RSH_AIR & 3019 & $C L L L T 1$ & RSH_WAS \\
\hline 3032 & EARTH & RSH_AIR & 3024 & $C L=L \_T 2$ & RSH_WAS \\
\hline 2934 & SALT_T1 & RSH_AIR & 3029 & CL_L_T3 & RSH_WAS \\
\hline 2943 & SALT_T2 & RSH_AIR & 3067 & CL_L_T4 & RSH_WAS \\
\hline 2951 & SALT_T3 & RSH_AIR & 3086 & $C L \_M \_T 1$ & RSH_WAS \\
\hline 2959 & SALT_T4 & RSH_AIR & 3091 & CL_M_T2 & RSH_WAS \\
\hline 2967 & SALT_T5 & RSH_AIR & 3096 & CL_M_T3 & RSH_WAS \\
\hline 2975 & SALT_T6 & RSH_AIR & 2921 & CL_M_T4 & RSH_WAS \\
\hline 2932 & ASPHALT & RSH_EXH & 2926 & CL_M_T5 & RSH_WAS \\
\hline 3020 & CL_L_T1 & RSH_EXH & 2998 & CLAY_RUS & RSH_WAS \\
\hline 3025 & CL_L_T2 & RSH_EXH & 3048 & CONC_MON & RSH_WAS \\
\hline 3030 & CL_L_T3 & RSH_EXH & 3038 & CONC_T1 & RSH_WAS \\
\hline 3068 & CL_L_T4 & RSH_EXH & 3043 & CONC_T2 & RSH_WAS \\
\hline 3087 & $C L=M \_T 1$ & RSH_EXH & 3034 & EARTH & RSH_WAS \\
\hline 3092 & CL_M_T2 & RSH_EXH & 2936 & SALT_T1 & RSH_WAS \\
\hline 3097 & CL_M_T3 & RSH_EXH & 2945 & SALT_T2 & RSH_WAS \\
\hline 2922 & CL_M_T4 & RSH_EXH & 2953 & SALT_T3 & RSH_WAS \\
\hline 2927 & $C L \_M \_T 5$ & RSH_EXH & 2961 & SALT_T4 & RSH_WAS \\
\hline 2999 & CLAY_RUS & RSH_EXH & 2969 & SALT_T5 & RSH_WAS \\
\hline 3049 & CONC_MON & RSH_EXH & 2977 & SALT_T6 & RSH_WAS \\
\hline 3039 & CONC_T1 & RSH_EXH & 52 & BRINESAL & RSLOPE \\
\hline 3044 & CONC_T2 & RSH_EXH & 2891 & REFCON & RTK \\
\hline 3035 & EARTH & RSH_EXH & 167 & DEWYLAKE & SAL_USAT \\
\hline 2937 & SALT_TI & RSH_EXH & 2290 & ASPHALT & SAT JBRN \\
\hline 2946 & SALT_T2 & RSH_EXH & 73 & CASTILER & SAT_IBRN \\
\hline 2954 & SALT_T3 & RSH_EXH & 3099 & CAVITY_1 & SAT_IBRN \\
\hline 2962 & SALT_T4 & RSH_EXH & 3100 & CAVITY_2 & SAT_IBRN \\
\hline 2970 & SALT_T5 & RSH_EXH & 3102 & CAVITY_3 & SAT_IBRN \\
\hline 2978 & SALT_T6 & RSH_EXH & 3104 & CAVITY_ 4 & SAT_IBRN \\
\hline 2930 & ASPHALT & RSH_SAL & 2341 & $C L \_L$ T1 & SAT_IBRN \\
\hline 3018 & $C L \_L I T 1$ & RSH_SAL & 2392 & $C L \_M \_T 1$ & SAT_IBRN \\
\hline 3023 & $C L \_L T 2$ & RSH_SAL & 2324 & CLAY_BOT & SAT_IBRN \\
\hline 3028 & CL_L_T3 & RSH_SAL & 3013 & CLAY_RUS & SAT_IBRN \\
\hline 3066 & $C L \_L$ T4 & RSH_SAL & 3115 & CONC_MON & SAT_IBRN \\
\hline 3085 & CL_M_T1 & RSH_SAL & 2477 & CONC_T1 & SAT_IBRN \\
\hline 3090 & CL_M_T2 & RSH_SAL & 2493 & CONC_T2 & SAT_IBRN \\
\hline 3095 & CL_M_T3 & RSH_SAL & 149 & CULEBRA & SAT_IBRN \\
\hline 2920 & $\mathrm{CL}_{2} \mathrm{M}_{-} \mathrm{T4}$ & RSH_SAL & 168 & DEWYLAKE & SAT_IBRN \\
\hline 2925 & CL_M_T5 & RSH_SAL & 187 & DRZ_0 & SAT_IBRN \\
\hline 2997 & CLAY_RUS & RSH_SAL & 2510 & EARTH & SAT_IBRN \\
\hline
\end{tabular}


Parameter Property Name

\begin{tabular}{|c|c|c|}
\hline Parameter ID & Material & Parameter \\
\hline 220 & EXP_AREA & SAT_IBRN \\
\hline 20 & OPS_AREA & SAT_IBRN \\
\hline 2734 & PAN_SEAL & SAT IBRN \\
\hline 2740 & REPOSIT & SAT_IBRN \\
\hline 537 & S_ANH_AB & SAT_IBRN \\
\hline 554 & S_HALITE & SAT_IBRN \\
\hline 576 & S_MB138 & SAT_IBRN \\
\hline 597 & S_MB139 & SAT_IBRN \\
\hline 2527 & SALT_T1 & SAT_IBRN \\
\hline 350 & SANTAROS & SAT_IBRN \\
\hline 669 & WAS_AREA & SAT_IBRN \\
\hline 2291 & ASPHALT & SAT_RBRN \\
\hline 3176 & BH_CREEP & SAT_RBRN \\
\hline 3140 & BH_OPEN & SAT_RBRN \\
\hline 3164 & |BH_SAND & SAT_RBRN \\
\hline 41 & BOREHOLE & SAT_RBRN \\
\hline 74 & CASTILER & SAT_RBRN \\
\hline 89 & CAVITY_1 & SAT_RBRN \\
\hline 104 & CAVITY_2 & SAT_RBRN \\
\hline 2235 & CAVITY_3 & SAT_RBRN \\
\hline 2237 & CAVITY 4 & SAT_RBRN \\
\hline 2342 & CL_L_T1 & SAT_RBRN \\
\hline 2359 & CL_L_T2 & SAT_RBRN \\
\hline 2376 & CL_L_T3 & SAT_RBRN \\
\hline 3082 & CL_L_T4 & SAT_RBRN \\
\hline 2393 & $\mathrm{CL}_{2} \mathrm{M}_{-} \mathrm{T} 1$ & SAT_RBRN \\
\hline 2410 & $C L=M-T 2$ & SAT_RBRN \\
\hline 2427 & CL_M_T3 & SAT_RBRN \\
\hline 2444 & CL_M_T4 & SAT_RBRN \\
\hline 2461 & CL_M_T5 & SAT_RBRN \\
\hline 2325 & CLAY_BOT & SAT_RBRN \\
\hline 3014 & CLAY_RUS & SAT_RBRN \\
\hline 3063 & CONC_MON & SAT_RBRN \\
\hline 3152 & CONC PLG & SAT_RBRN \\
\hline 2478 & CONC_T1 & SAT_RBRN \\
\hline 2494 & CONC_T2 & SAT_RBRN \\
\hline 150 & CULEBRA & SAT_RBRN \\
\hline 169 & DEWYLAKE & SAT_RBRN \\
\hline 188 & $D R Z$ D & SAT_RBRN \\
\hline 205 & DRZ_1 & SAT_RBRN \\
\hline 2511 & EARTH & SAT_RBRN \\
\hline 221 & EXP_AREA & SAT_RBRN \\
\hline 2240 & FORTYNIN & SAT_RBRN \\
\hline 243 & IMPERM_Z & SAT_RBRN \\
\hline 2241 & MAGENTA & SAT_RBRN \\
\hline 21 & OPS_AREA & SAT_RBRN \\
\hline 265 & PAN_SEAL & SAT_RBRN \\
\hline 2741 & REPOSIT & SAT_RBRN \\
\hline 538 & S_ANH_AB & SAT_RBRN \\
\hline 555 & S_HALITE & SAT_RBRN \\
\hline 577 & S_MB138 & SAT_RBRN \\
\hline
\end{tabular}

\begin{tabular}{|c|c|c|}
\hline Parameter ID & Material & Parameter \\
\hline 598 & S_MB139 & SAT_RBRN \\
\hline 2528 & SALT_T1 & SAT_RBRN \\
\hline 2545 & $\widehat{\text { SALT_T2 }}$ & SAT_RBRN \\
\hline 2562 & SALT_T3 & SAT_RBRN \\
\hline 2579 & SALT_T4 & SAT_RBRN \\
\hline 2596 & SALT_T5 & SAT_RBRN \\
\hline 2992 & SALT_T6 & SAT_RBRN \\
\hline 351 & SANTAROS & SAT_RBRN \\
\hline 2245 & TAMARISK & SAT_RBRN \\
\hline 2248 & UNNAMED & SAT_RBRN \\
\hline 670 & WAS_AREA & SAT_RBRN \\
\hline 2292 & ASPHALT & SAT_RGAS \\
\hline 3177 & BH_CREEP & SAT_RGAS \\
\hline 3141 & BH_OPEN & SAT_RGAS \\
\hline 3165 & BH_SAND & SAT_RGAS \\
\hline 42 & BOREHOLE & SAT_RGAS \\
\hline 75 & CASTILER & SAT_RGAS \\
\hline 90 & CAVITY_1 & SAT_RGAS \\
\hline 105 & CAVITY_2 & SAT_RGAS \\
\hline 2059 & CAVITY_3 & SAT_RGAS \\
\hline 2070 & |CAVITY_4 & SAT_RGAS \\
\hline 2343 & CL_L_T1 & SAT_RGAS \\
\hline 2360 & CL_L_T2 & SAT_RGAS \\
\hline 2377 & CL_L_T3 & SAT_RGAS \\
\hline 3083 & $C L \_L \_T 4$ & SAT_RGAS \\
\hline 2394 & CL_M_T1 & SAT_RGAS \\
\hline 2411 & CL_M_T2 & SAT_RGAS \\
\hline 2428 & CL_M_T3 & SAT_RGAS \\
\hline 2445 & CL_M_T4 & SAT_RGAS \\
\hline 2462 & CL_M_T5 & SAT_RGAS \\
\hline 2326 & CLAY_BOT & SAT_RGAS \\
\hline 3015 & CLAY_RUS & SAT_RGAS \\
\hline 3064 & CONC_MON & SAT_RGAS \\
\hline 3153 & CONC_PLG & SAT_RGAS \\
\hline 2479 & CONC_T1 & SAT_RGAS \\
\hline 2495 & CONC_T2 & SAT_RGAS \\
\hline 151 & CULEBRA & SAT_RGAS \\
\hline 170 & DEWYLAKE & SAT_RGAS \\
\hline 189 & DRZ_o & SAT_RGAS \\
\hline 206 & DRZ_1 & SAT_RGAS \\
\hline 2512 & EARTH & SAT_RGAS \\
\hline 222 & EXP_AREA & SAT_RGAS \\
\hline 2094 & FORTYNIN & SAT_RGAS \\
\hline 244 & IMPERM_Z & SAT_RGAS \\
\hline 2107 & MAGENTA & SAT_RGAS \\
\hline 22 & OPS_AREA & SAT_RGAS \\
\hline 266 & PAN_SEAL & SAT_RGAS \\
\hline 2137 & REPOSIT & SAT_RGAS \\
\hline 539 & $S \_A N H \_A B$ & SAT_RGAS \\
\hline 556 & S_HALITE & SAT_RGAS \\
\hline 578 & S_MB138 & SAT_RGAS \\
\hline
\end{tabular}


Parameter Property Name

\begin{tabular}{|c|c|c|}
\hline Parameter ID & Material & Parameter \\
\hline 599 & S_MB139 & SAT_RGAS \\
\hline 2529 & SALT_T1 & SAT_RGAS \\
\hline 2546 & SALT_T2 & SAT_RGAS \\
\hline 2563 & SALT_T3 & SAT_RGAS \\
\hline 2580 & SALT_T4 & SAT_RGAS \\
\hline 2597 & SALT_T5 & SAT_RGAS \\
\hline 2993 & SALT_T6 & SAT_RGAS \\
\hline 352 & SANTAROS & SAT_RGAS \\
\hline 2192 & TAMARISK & SAT_RGAS \\
\hline 2226 & UNNAMED & SAT_RGAS \\
\hline 671 & WAS_AREA & SAT_RGAS \\
\hline 2231 & WAS_AREA & SAT_WICK \\
\hline 3112 & REFCON & SECYR \\
\hline 3469 & CULEBRA & SKIN_RES \\
\hline 3263 & SOLAM3 & SOLCIM \\
\hline 3402 & SOLMOD3 & SOLCIM \\
\hline 3403 & SOLMOD4 & SOLCIM \\
\hline 3404 & SOLMOD5 & SOLCIM \\
\hline 3405 & SOLMOD6 & SOLCIM \\
\hline 3264 & SOLPU3 & SOLCIM \\
\hline 3389 & SOLPU4 & SOLCIM \\
\hline 3392 & SOLU6 & SOLCIM \\
\hline 3262 & SOLAM3 & SOLSIM \\
\hline 3406 & SOLMOD3 & SOLSIM \\
\hline 3407 & SOLMOD4 & SOLSIM \\
\hline 3408 & SOLMOD5 & SOLSIM \\
\hline 3409 & SOLMOD6 & SOLSIM \\
\hline 3265 & SOLPU3 & SOLSIM \\
\hline 3266 & SOLPU4 & SOLSIM \\
\hline 3393 & SOLTH4 & SOLSIM \\
\hline 3390 & SOLU4 & SOLSIM \\
\hline 3391 & SOLU6 & SOLSIM \\
\hline 2898 & STEEL & STOIFX \\
\hline 3257 & BLOWOUT & SUFTEN \\
\hline 3491 & GLOBAL & TA \\
\hline
\end{tabular}

\begin{tabular}{|c|c|c|}
\hline Parameter ID & Material & Parameter \\
\hline 2254 & BOREHOLE & TAUFAIL \\
\hline 2827 & REFCON & TC_CH4 \\
\hline 2826 & REFCON & TC_CO2 \\
\hline 2825 & REFCON & TC_H2 \\
\hline 2829 & REFCON & TC_H2S \\
\hline 2828 & REFCON & TC_N2 \\
\hline 2830 & REFCON & TC_O2 \\
\hline 3473 & BLOWOUT & THCK_CAS \\
\hline 2071 & CULEBRA & THICK \\
\hline 2109 & MAGENTA & THICK \\
\hline 962 & DRZ_0 & TORTUSTY \\
\hline 1702 & S_ANH_AB & TORTUSTY \\
\hline 1742 & S_HALITE & TORTUSTY \\
\hline 3499 & GLOBAL & TPICD \\
\hline 3498 & GLOBAL & TPICM \\
\hline 225 & GLOBAL & TRANSIDX \\
\hline 3258 & BLOWOUT & TREPO \\
\hline 3252 & BLOWOUT & VISC \\
\hline 55 & BRINESAL & VISCO \\
\hline 172 & DRILLMUD & VISCO \\
\hline 228 & $\mathrm{H} 2$ & VISCO \\
\hline 2141 & REPOSIT & VOLCHW \\
\hline 2232 & WAS_AREA & VOLCHW \\
\hline 2142 & REPOSIT & VOLRHW \\
\hline 2233 & WAS_AREA & VOLRHW \\
\hline 2918 & CASTILER & VOLUME \\
\hline 3490 & REFCON & VOLWP \\
\hline 3107 & REFCON & VPANLEX \\
\hline 3108 & REFCON & VREPOS \\
\hline 3105 & REFCON & VROOM \\
\hline 57 & BRINESAL & WTF \\
\hline 3414 & BOREHOLE & WUF \\
\hline 173 & DRILLMUD & YLDSTRSS \\
\hline 2888 & REFCON & YRSEC \\
\hline 3110 & REFCON & ZCINK \\
\hline
\end{tabular}




\section{Appendix C}

\section{Parameters Whose Underlying Data Were Qualified by Peer Review}


Parameters Whose Underlying Data Were Qualified by Peer Review

\begin{tabular}{|c|c|c|c|c|}
\hline ID: & HáertáaD: & 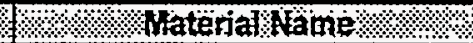 & Páanteterto & Raraméternamo \\
\hline 48 & BRINESAL & Salado Brine & COMPRES & Brine Compressibility \\
\hline 49 & BRINESAL & Salado Brine & DNSFLUID & Brine Density \\
\hline 55 & BRINESAL & Salado Brine & VISCO & $\begin{array}{l}\text { Viscosity of } \mathrm{H} 2 \text { gas at } 27 \text { degrees } \\
\text { Celsius and } 0.101325 \mathrm{MPa}\end{array}$ \\
\hline 57 & BRINESAL & Salado Brine & WTF & Mass fraction of salt in brine \\
\hline 61 & CASTILER & Castile Brine Reservoir & COMP RCK & Bulk Compressibility \\
\hline 64 & CASTILER & Castile Brine Reservoir & POROSITY & Effective porosity \\
\hline 66 & CASTILER & Castile Brine Reservoir & PRESSURE & Brine far-field pore pressure \\
\hline 67 & CASTILER & Castile Brine Reservoir & PRMX_LOG & Log of intrinsic permeability, $X$-direction \\
\hline 68 & CASTILER & Castile Brine Reservoir & PRMY LOG & Log of intrinsic permeability, Y-direction \\
\hline 69 & CASTILER & Castile Brine Reservoir & PRMZ_LOG & Log of intrinsic permeability, Z-direction \\
\hline 2918 & CASTILER & Castile Brine Reservoir & VOLUME & Total Reservoir Volume \\
\hline 843 & CULEBRA & $\begin{array}{l}\text { Culebra member of the Rustler } \\
\text { formation }\end{array}$ & DNSGRAIN & Material Grain Density \\
\hline 2071 & CULEBRA & $\begin{array}{l}\text { Culebra member of the Rustler } \\
\text { formation }\end{array}$ & THICK & Thickness of feature or layer \\
\hline 3487 & CULEBRA & $\begin{array}{l}\text { Culebra member of the Rustler } \\
\text { formation }\end{array}$ & APOROS & Culebra Advective Porosity \\
\hline 3486 & CULEBRA & $\begin{array}{l}\text { Culebra member of the Rustler } \\
\text { formation }\end{array}$ & DPOROS & Diffusive Porosity for Culebra Dolomite \\
\hline 3474 & CULEBRA & $\begin{array}{l}\text { Culebra member of the Rustler } \\
\text { formation }\end{array}$ & DTORT & Diffusive Tortuosity \\
\hline 3474 & CULEBRA & $\begin{array}{l}\text { Culebra member of the Rustler } \\
\text { formation }\end{array}$ & DTORT & Diffusive Tortuosity \\
\hline 3485 & CULEBRA & $\begin{array}{l}\text { Culebra member of the Rustler } \\
\text { formation }\end{array}$ & HMBLKLT & Culebra Half Matrix-Block Length \\
\hline 3418 & CULEBRA & $\begin{array}{l}\text { Culebra member of the Rustler } \\
\text { formation }\end{array}$ & MEA_STOR & Measured Storativity \\
\hline 175 & DRZ_0 & $\begin{array}{l}\text { Disturbed rock zone; time period } \\
-5 \text { to } 0 \text { years }\end{array}$ & COMP_RCK & Bulk Compressibility \\
\hline 191 & DRZ_1 & $\begin{array}{l}\text { Disturbed rock zone; time period } \\
0 \text { to } 10,000 \text { years }\end{array}$ & COMP_RCK & Bulk Compressibility \\
\hline 541 & S_HALITE & Salado halite, intact & COMPRCK & Bulk Compressibility \\
\hline 546 & S.HALITE & Salado halite, intact & PRESSURE & Brine far-field pore pressure \\
\hline 343 & SANTAROS & Santa Rosa Formation & PRESSURE & Brine far-field pore pressure \\
\hline 3263 & SOLAM3 & Solubility Multiplier for $\mathrm{Am}+3$ & SOLCIM & $\begin{array}{l}\text { Solubility Mult. in Castile Brine, Inorganic } \\
\text { Chem Controlled by } \mathrm{Mg}(\mathrm{OH}) 2-\mathrm{MgCO} 3\end{array}$ \\
\hline 3262 & SOLAM3 & Solubility Multiplier for Am+3 & SOLSIM & $\begin{array}{l}\text { Solubility Mult. in Salado Brine, Inorganic } \\
\text { Chem Controlled by } \mathrm{Mg}(\mathrm{OH}) 2-\mathrm{MgCO} 3\end{array}$ \\
\hline 3402 & SOLMOD3 & Oxidation state III model & SOLCIM & $\begin{array}{l}\text { Solubility Mult. in Castile Brine, Inorganic } \\
\text { Chem Controlled by } \mathrm{Mg}(\mathrm{OH}) 2-\mathrm{MgCO} 3 \\
\end{array}$ \\
\hline 3406 & SOLMOD3 & Oxidation state III model & SOLSIM & $\begin{array}{l}\text { Solubility Mult. in Salado Brine, Inorganic } \\
\text { Chem Controlled by } \mathrm{Mg}(\mathrm{OH}) 2-\mathrm{MgCO} 3\end{array}$ \\
\hline 3403 & SOLMOD4 & Oxidation state IV model & SOLCIM & $\begin{array}{l}\text { Solubility Mult. in Castile Brine, Inorganic } \\
\text { Chem Controlled by } \mathrm{Mg}(\mathrm{OH}) 2-\mathrm{MgCO} 3\end{array}$ \\
\hline 3407 & SOLMOD4 & Oxidation state IV model & SOLṢIM & $\begin{array}{l}\text { Solubility Mult. in Salado Brine, Inorganic } \\
\text { Chem Controlled by } \mathrm{Mg}(\mathrm{OH}) 2-\mathrm{MgCO} 3\end{array}$ \\
\hline 3404 & SOLMOD5 & Oxidation state $V$ model & SOLCIM & $\begin{array}{l}\text { Solubility Mult. in Castile Brine, Inorganic } \\
\text { Chem Controlled by } \mathrm{Mg}(\mathrm{OH}) 2-\mathrm{MgCO} 3\end{array}$ \\
\hline 3408 & SOLMOD5 & Oxidation state $V$ model & SOLSIM & $\begin{array}{l}\text { Solubility Mult. in Salado Brine, Inorganic } \\
\text { Chem Controlled by } \mathrm{Mg}(\mathrm{OH}) 2-\mathrm{MgCO} 3\end{array}$ \\
\hline 3405 & SOLMOD6 & Oxidation state VI model & SOLCIM & $\begin{array}{l}\text { Solubility Mult. in Castile Brine, Inorganic } \\
\text { Chem Controlled by } \mathrm{Mg}(\mathrm{OH}) 2-\mathrm{MgCO} 3 \\
\end{array}$ \\
\hline 3264 & SOLPU3 & Solubility Multiplier for Pu+3 & SOLCIM & $\begin{array}{l}\text { Solubility Mult. in Castile Brine, Inorganic } \\
\text { Chem Controlled by } \mathrm{Mg}(\mathrm{OH}) 2-\mathrm{MgCO} 3\end{array}$ \\
\hline
\end{tabular}


Parameters Whose Underlying Data Were Qualified by Peer Review

\begin{tabular}{|c|c|c|c|c|}
\hline \%D: & Material:[D? & 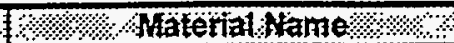 & Pamameterib: & \% \\
\hline 3265 & SOLPU3 & Solubility Multiplier for Pu+3 & SOLSIM & $\begin{array}{l}\text { Solubility Mult. in Salado Brine, Inorganic } \\
\text { Chem Controlled by } \mathrm{Mg}(\mathrm{OH}) 2-\mathrm{MgCO} 3\end{array}$ \\
\hline 3389 & SOLPU4 & Solubility Multiplier for $\mathrm{Pu}+4$ & SOLCIM & $\begin{array}{l}\text { Solubility Mult. in Castile Brine, Inorganic } \\
\text { Chem Controlled by } \mathrm{Mg}(\mathrm{OH}) 2-\mathrm{MgCO} 3\end{array}$ \\
\hline 3266 & SOLPU4 & Solubility Multiplier for Pu+4 & SOLSIM & $\begin{array}{l}\text { Solubility Mult. in Salado Brine, Inorganic } \\
\text { Chem Controlled by } \mathrm{Mg}(\mathrm{OH}) 2-\mathrm{MgCO} 3\end{array}$ \\
\hline 3393 & SOLTH4 & Solubility Multiplier for $\mathrm{Th}+4$ & SOLSIM & $\begin{array}{l}\text { Solubility Mult. in Salado Brine, Inorganic } \\
\text { Chem Controlled by } \mathrm{Mg}(\mathrm{OH}) 2-\mathrm{MgCO} 3\end{array}$ \\
\hline 3390 & SOLU4 & Solubility Multiplier for U+4 & SOLSIM & $\begin{array}{l}\text { Solubility Mult. in Salado Brine, Inorganic } \\
\text { Chem Controlled by } \mathrm{Mg}(\mathrm{OH}) 2-\mathrm{MgCO} 3\end{array}$ \\
\hline 3392 & SOLU6 & Solubility Multiplier for U+6 & SOLCIM & $\begin{array}{l}\text { Solubility Mult. in Castile Brine, Inorganic } \\
\text { Chem Controlled by } \mathrm{Mg}(\mathrm{OH}) 2-\mathrm{MgCO} 3\end{array}$ \\
\hline 3391 & SOLU6 & Solubility Multiplier for U+6 & SOLSIM & $\begin{array}{l}\text { Solubility Mult. in Salado Brine, Inorganic } \\
\text { Chem Controlled by } \mathrm{Mg}(\mathrm{OH}) 2-\mathrm{MgCO} 3\end{array}$ \\
\hline 3409 & SOLMOD6 & Oxidation state VI model & SOLSIM & $\begin{array}{l}\text { Solubility Mult. in Salado Brine, Inorganic } \\
\text { Chem Controlled by } \mathrm{Mg}(\mathrm{OH}) 2-\mathrm{MgCO} 3\end{array}$ \\
\hline 2467 & CONC_T1 & $\begin{array}{l}\text { Concrete column: } 0 \text { to } 400 \\
\text { years }\end{array}$ & POROSITY & Effective porosity \\
\hline 2470 & CONC_T1 & $\begin{array}{l}\text { Concrete column: } 0 \text { to } 400 \\
\text { years }\end{array}$ & PRMX_LOG & Log of intrinsic permeability, X-direction \\
\hline 2471 & CONC_T1 & $\begin{array}{l}\text { Concrete column: } 0 \text { to } 400 \\
\text { years }\end{array}$ & PRMY_LOG & Log of intrinsic permeability, Y-direction \\
\hline 2472 & 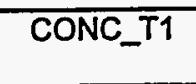 & $\begin{array}{l}\text { Concrete column: } 0 \text { to } 400 \\
\text { years }\end{array}$ & PRMZ_LOG & Log of intrinsic permeability, Z-direction \\
\hline 2484 & CONC_T2 & $\begin{array}{l}\begin{array}{l}\text { Concrete column: } 400 \text { to } 10 \mathrm{~K} \\
\text { years }\end{array} \\
\end{array}$ & POROSITY & Effective porosity \\
\hline 2486 & CONC_T2 & $\begin{array}{l}\text { Concrete column: } 400 \text { to } 10 \mathrm{~K} \\
\text { years }\end{array}$ & PRMX_LOG & Log of intrinsic permeability, X-direction \\
\hline 2487 & CONC_T2 & $\begin{array}{l}\text { Concrete column: } 400 \text { to } 10 \mathrm{~K} \\
\text { years }\end{array}$ & PRMY_LOG & Log of intrinsic permeability, Y-direction \\
\hline 2488 & CONC_T2 & $\begin{array}{l}\text { Concrete column: } 400 \text { to } 10 \mathrm{~K} \\
\text { years }\end{array}$ & PRMZ_LOG & Log of intrinsic permeability, Z-direction \\
\hline 2514 & $\overline{S A L T \_T 1}$ & $\begin{array}{l}\text { Shaft salt column compacted: } \\
\text { time } 0 \text { to } 10 \text { years }\end{array}$ & COMP_RCK & Bulk Compressibility \\
\hline 2942 & $\overline{\text { SALT_T1 }}$ & $\begin{array}{l}\text { Shaft salt column compacted: } \\
\text { time } 0 \text { to } 10 \text { years }\end{array}$ & PMLT_HI & $\begin{array}{l}\text { Log triangular distribution high value for } \\
\text { permeability }\end{array}$ \\
\hline 2941 & $\overline{\text { SALT_T1 }}$ & $\begin{array}{l}\text { Shaft salt column compacted: } \\
\text { time } 0 \text { to } 10 \text { years }\end{array}$ & PMLT_LO & $\begin{array}{l}\text { Log triangular distribution low value for } \\
\text { permeability }\end{array}$ \\
\hline 2940 & SALT_T1 & $\begin{array}{l}\text { Shaft salt column compacted: } \\
\text { time } 0 \text { to } 10 \text { years }\end{array}$ & PMLT_MD & $\begin{array}{l}\text { Log triangular distribution mode for } \\
\text { permeability }\end{array}$ \\
\hline 2531 & $\overline{\text { SALT_T2 }}$ & $\begin{array}{l}\text { Shaft salt column compacted: } \\
\text { time } 10 \text { to } 25 \text { years }\end{array}$ & COMP_RCK & Bulk Compressibility \\
\hline 2950 & SALT_T2 & $\begin{array}{l}\text { Shaft salt column compacted: } \\
\text { time } 10 \text { to } 25 \text { years }\end{array}$ & PMLT_HI & $\begin{array}{l}\text { Log triangular distribution high value for } \\
\text { permeability }\end{array}$ \\
\hline 2949 & SALT_T2 & $\begin{array}{l}\text { Shaft salt column compacted: } \\
\text { time } 10 \text { to } 25 \text { years }\end{array}$ & PMLT_LO & $\begin{array}{l}\text { Log triangular distribution low value for } \\
\text { permeability }\end{array}$ \\
\hline 2948 & $\overline{S A L T \_T 2}$ & $\begin{array}{l}\text { Shaft salt column compacted: } \\
\text { time } 10 \text { to } 25 \text { years }\end{array}$ & PMLT_MD & $\begin{array}{l}\text { Log triangular distribution mode for } \\
\text { permeability }\end{array}$ \\
\hline 2548 & SALT_T3 & $\begin{array}{l}\text { Shaft salt column compacted: } \\
\text { time } 25 \text { to } 50 \text { years }\end{array}$ & COMP_RCK & Bulk Compressibility \\
\hline 2958 & SALT_T3 & $\begin{array}{l}\text { Shaft salt column compacted: } \\
\text { time } 25 \text { to } 50 \text { years }\end{array}$ & PMLT_HI & $\begin{array}{l}\text { Log triangular distribution high value for } \\
\text { permeability }\end{array}$ \\
\hline 2957 & SALT_T3 & $\begin{array}{l}\text { Shaft salt column compacted: } \\
\text { time } 25 \text { to } 50 \text { years }\end{array}$ & PMLT_LO & $\begin{array}{l}\text { Log triangular distribution low value for } \\
\text { permeability }\end{array}$ \\
\hline 2956 & SALT_T3 & $\begin{array}{l}\text { Shaft salt column compacted: } \\
\text { time } 25 \text { to } 50 \text { years }\end{array}$ & PMLT_MD & $\begin{array}{l}\text { Log triangular distribution mode for } \\
\text { permeability }\end{array}$ \\
\hline
\end{tabular}


Parameters Whose Underlying Data Were Qualified by Peer Review

\begin{tabular}{|c|c|c|c|c|}
\hline ID: & Aaterial:1D $\%$ & Nfafetial haine. & Parainetet & Poramétarkame \\
\hline 2565 & SALT_T4 & $\begin{array}{l}\text { Shaft salt column compacted: } \\
\text { time } 50 \text { to } 100 \text { years }\end{array}$ & COMP_RCK & Bulk Compressibility \\
\hline 2966 & SALT_T4 & $\begin{array}{l}\text { Shaft salt column compacted: } \\
\text { time } 50 \text { to } 100 \text { years }\end{array}$ & PMLT_HI & $\begin{array}{l}\text { Log triangular distribution high value for } \\
\text { permeability }\end{array}$ \\
\hline 2965 & $\overline{\text { SALT_T4 }}$ & $\begin{array}{l}\text { Shaft salt column compacted: } \\
\text { time } 50 \text { to } 100 \text { years }\end{array}$ & PMLT_LO & $\begin{array}{l}\text { Log triangular distribution low value for } \\
\text { permeability }\end{array}$ \\
\hline 2964 & SALT_T4 & $\begin{array}{l}\text { Shaft salt column compacted: } \\
\text { time } 50 \text { to } 100 \text { years }\end{array}$ & PMLT_MD & $\begin{array}{l}\text { Log triangular distribution mode for } \\
\text { permeability }\end{array}$ \\
\hline 2582 & SALT_T5 & $\begin{array}{l}\text { Shaft salt column compacted: } \\
\text { time } 100 \text { to } 200 \text { years }\end{array}$ & COMP_RCK & Bulk Compressibility \\
\hline 2974 & SALT_T5 & $\begin{array}{l}\text { Shaft salt column compacted: } \\
\text { time } 100 \text { to } 200 \text { years }\end{array}$ & PMLT_HI & $\begin{array}{l}\text { Log triangular distribution high value for } \\
\text { permeability }\end{array}$ \\
\hline 2973 & SALT_T5 & $\begin{array}{l}\text { Shaft salt column compacted: } \\
\text { time } 100 \text { to } 200 \text { years }\end{array}$ & PMLT_LO & $\begin{array}{l}\text { Log triangular distribution low value for } \\
\text { permeability }\end{array}$ \\
\hline 2972 & SALT_T5 & $\begin{array}{l}\text { Shaft salt column compacted: } \\
\text { time } 100 \text { to } 200 \text { years }\end{array}$ & PMLT_MD & $\begin{array}{l}\text { Log triangular distribution mode for } \\
\text { permeability }\end{array}$ \\
\hline 2984 & SALT_T6 & $\begin{array}{l}\text { Shaft salt column compacted: } \\
\text { time } 200 \text { to } 10 \mathrm{~K} \text { years }\end{array}$ & COMP_RCK & Bulk Compressibility \\
\hline 2982 & SALT_T6 & $\begin{array}{l}\text { Shaft salt column compacted: } \\
\text { time } 200 \text { to } 10 \mathrm{~K} \text { years }\end{array}$ & PMLT_HI & $\begin{array}{l}\text { Log triangular distribution high value for } \\
\text { permeability }\end{array}$ \\
\hline 2981 & SALT_T6 & $\begin{array}{l}\text { Shaft salt column compacted: } \\
\text { time } 200 \text { to } 10 \mathrm{~K} \text { years }\end{array}$ & PMLT_LO & $\begin{array}{l}\text { Log triangular distribution low value for } \\
\text { permeability }\end{array}$ \\
\hline 2980 & $\overline{\text { SALT_T6 }}$ & $\begin{array}{l}\text { Shaft salt column compacted: } \\
\text { time } 200 \text { to } 10 \mathrm{~K} \text { years }\end{array}$ & PMLT_MD & $\begin{array}{l}\text { Log triangular distribution mode for } \\
\text { permeability }\end{array}$ \\
\hline 2334 & $\overline{C L} L_{-} T 1$ & $\begin{array}{l}\text { Lower Salado clay: } 0 \text { to } 10 \\
\text { years }\end{array}$ & PRMX_LOG & Log of intrinsic permeability, X-direction \\
\hline 2335 & CL_L_T1 & $\begin{array}{l}\text { Lower Salado clay: } 0 \text { to } 10 \\
\text { years }\end{array}$ & PRMY_LOG & Log of intrinsic permeability, Y-direction \\
\hline 2336 & $\overline{C L} L_{-} T 1$ & $\begin{array}{l}\text { Lower Salado clay: } 0 \text { to } 10 \\
\text { years }\end{array}$ & PRMZ_LOG & Log of intrinsic permeability, Z-direction \\
\hline 2351 & $\overline{C L} L_{2}$ T2 & $\begin{array}{l}\text { Lower Salado clay: } 10 \text { to } 25 \\
\text { years }\end{array}$ & PRMX_LOG & Log of intrinsic permeability, X-direction \\
\hline 2352 & CL_L_T2 & $\begin{array}{l}\text { Lower Salado clay: } 10 \text { to } 25 \\
\text { years }\end{array}$ & PRMY_LOG & Log of intrinsic permeability, Y-direction \\
\hline 2353 & CL_L_T2 & $\begin{array}{l}\text { Lower Salado clay: } 10 \text { to } 25 \\
\text { years }\end{array}$ & PRMZ_LOG & Log of intrinsic permeability, Z-direction \\
\hline 2368 & CL_L_T3 & $\begin{array}{l}\text { Lower Salado clay: } 25 \text { to } 50 \\
\text { years }\end{array}$ & PRMX_LOG & Log of intrinsic permeability, $X$-direction \\
\hline 2369 & $\overline{C L} L_{2} T 3$ & $\begin{array}{l}\text { Lower Salado clay: } 25 \text { to } 50 \\
\text { years }\end{array}$ & PRMY_LOG & $\begin{array}{c}\text { Log of intrinsic permeability, Y-direction } \\
.-\end{array}$ \\
\hline 2370 & CL_L_T3 & $\begin{array}{l}\text { Lower Salado clay: } 25 \text { to } 50 \\
\text { years }\end{array}$ & PRMZ_LOG & Log of intrinsic permeability, Z-direction \\
\hline 2385 & $\overline{C L}$ M_T1 & $\begin{array}{l}\text { Upper Salado clay: } 0 \text { to } 10 \\
\text { years }\end{array}$ & PRMX_LOG & Log of intrinsic permeability, X-direction \\
\hline 2386 & CL_M_T1 & $\begin{array}{l}\text { Upper Salado clay: } 0 \text { to } 10 \\
\text { years }\end{array}$ & PRMY_LOG & Log of intrinsic permeability, Y-direction \\
\hline 2387 & $\overline{C L}$ M_TI $_{1}$ & $\begin{array}{l}\text { Upper Salado clay: } 0 \text { to } 10 \\
\text { years }\end{array}$ & PRMZ_LOG & Log of intrinsic permeability, Z-direction \\
\hline 2402 & $\overline{C L}$ L_T_T2 $_{2}$ & $\begin{array}{l}\text { Upper Salado clay: } 10 \text { to } 25 \\
\text { years }\end{array}$ & PRMX_LOG & Log of intrinsic permeability, X-direction \\
\hline 2403 & $\overline{C L}$ L_M_T2 $_{2}$ & $\begin{array}{l}\text { Upper Salado clay: } 10 \text { to } 25 \\
\text { years }\end{array}$ & PRMY_LOG & Log of intrinsic permeability, Y-direction \\
\hline 2404 & CL_M_T2 & $\begin{array}{l}\text { Upper Salado clay: } 10 \text { to } 25 \\
\text { years }\end{array}$ & PRMZ_LOG & Log of intrinsic permeability, Z-direction \\
\hline 2419 & $\overline{C L}{ }_{2}{ }_{2}{ }^{3}$ & $\begin{array}{l}\text { Upper Salado clay: } 25 \text { to } 50 \\
\text { years }\end{array}$ & PRMX_LOG & Log of intrinsic permeability, X-direction \\
\hline
\end{tabular}


Parameters Whose Underlying Data Were Qualified by Peer Review

\begin{tabular}{|c|c|c|c|c|}
\hline \%क: & Aaterialib: & \% & ParameteriD & W. Parameter Name: $\quad . \cdots$ \\
\hline 2420 & CL_M_T3 & $\begin{array}{l}\text { Upper Salado clay: } 25 \text { to } 50 \\
\text { years }\end{array}$ & PRMY_LOG & Log of intrinsic permeability, Y-direction \\
\hline 2421 & CL_M_T3 & $\begin{array}{l}\text { Upper Salado clay: } 25 \text { to } 50 \\
\text { years }\end{array}$ & PRMZ_LOG & Log of intrinsic permeability, Z-direction \\
\hline 2436 & $\overline{C L}$ L_T $_{4}$ & $\begin{array}{l}\text { Upper Salado clay: } 50 \text { to } 100 \\
\text { years }\end{array}$ & PRMX_LOG & Log of intrinsic permeability, $X$-direction \\
\hline 2437 & $\overline{C L}$ M_T4 $_{1}$ & $\begin{array}{l}\text { Upper Salado clay: } 50 \text { to } 100 \\
\text { years }\end{array}$ & PRMY_LOG & Log of intrinsic permeability, Y-direction \\
\hline 2438 & CL_M_T4 & $\begin{array}{l}\text { Upper Salado clay: } 50 \text { to } 100 \\
\text { years }\end{array}$ & PRMZ_LOG & Log of intrinsic permeability, Z-direction \\
\hline 2453 & CL_M_T5 & $\begin{array}{l}\text { Upper Salado clay: } 100 \text { to } 10 \mathrm{~K} \\
\text { years }\end{array}$ & PRMX_LOG & Log of intrinsic permeability, X-direction \\
\hline 2454 & CL_M_T5 & $\begin{array}{l}\text { Upper Salado clay: } 100 \text { to } 10 \mathrm{~K} \\
\text { years }\end{array}$ & PRMY_LOG & Log of intrinsic permeability, Y-direction \\
\hline 2455 & CL_M_T5 & $\begin{array}{l}\text { Upper Salado clay: } 100 \text { to } 10 \mathrm{~K} \\
\text { years }\end{array}$ & PRMZ_LOG & Log of intrinsic permeability, Z-direction \\
\hline 2317 & CLAY_BOT & Shaft Bottom Clay & PRMX_LOG & Log of intrinsic permeability, $X$-direction \\
\hline 2318 & CLAY BOT & Shaft Bottom Clay & PRMY LOG & Log of intrinsic permeability, Y-direction \\
\hline 2319 & CLAY BOT & Shaft Bottom Clay & PRMZ_LOG & Log of intrinsic permeability, Z-direction \\
\hline 3009 & CLAY RUS & Clay seals in Rustler formation & PRMX_LOG & Log of intrinsic permeability, X-direction \\
\hline 3010 & CLAY RUS & Clay seals in Rustler formation & PRMY LOG & Log of intrinsic permeability, Y-direction \\
\hline 3011 & CLAY RUS & Clay seals in Rustler formation & PRMZ_LOG & Log of intrinsic permeability, Z-direction \\
\hline 223 & GLOBAL & Information that applies globally & CLIMTIDX & Climate Index \\
\hline 3078 & CL_L_T4 & $\begin{array}{l}\text { Lower Salado clay: } 50 \text { to } 10 \mathrm{~K} \\
\text { years }\end{array}$ & PRMX_LOG & Log of intrinsic permeability, X-direction \\
\hline 3079 & CL_L_T4 & $\begin{array}{l}\text { Lower Salado clay: } 50 \text { to } 10 \mathrm{~K} \\
\text { years }\end{array}$ & PRMY_LOG & Log of intrinsic permeability, Y-direction \\
\hline 3080 & CL_L_T4 & $\begin{array}{l}\text { Lower Salado clay: } 50 \text { to } 10 \mathrm{~K} \\
\text { years }\end{array}$ & PRMZ_LOG & Log of intrinsic permeability, Z-direction \\
\hline 3058 & CONC_MON & ete monolith at & POROSITY & Effective porosity \\
\hline 3059 & CONC_MON & $\begin{array}{l}\text { Degraded concrete monolith at } \\
\text { shaft base }\end{array}$ & PRMX_LOG & Log of intrinsic permeability, $X$-direction \\
\hline 3060 & CONC_MON & $\begin{array}{l}\text { Degraded concrete monolith at } \\
\text { shaft base }\end{array}$ & PRMY_LOG & Log of intrinsic permeability, Y-direction \\
\hline 3061 & CONC_MON & $\begin{array}{l}\text { Degraded concrete monolith at } \\
\text { shaft base }\end{array}$ & PRMZ_LOG & Log of intrinsic permeability, Z-direction \\
\hline 580 & S_MB139 & $\begin{array}{l}\text { Salado marker bed } 139 \text {, intact } \\
\text { and fractured }\end{array}$ & COMP_RCK & Bulk Compressibility \\
\hline 590 & S_MB139 & $\begin{array}{l}\text { Salado marker bed 139, intact } \\
\text { and fractured }\end{array}$ & PRESSURE & Brine far-field pore pressure \\
\hline 142 & CULEBRA & $\begin{array}{l}\text { Culebra member of the Rustler } \\
\text { formation }\end{array}$ & PRESSURE & Brine far-field pore pressure \\
\hline 143 & CULEBRA & $\begin{array}{l}\text { Culebra member of the Rustler } \\
\text { formation }\end{array}$ & PRMX_LOG & Log of intrinsic permeability, $\mathrm{X}$-direction \\
\hline 144 & CULEBRA & $\begin{array}{l}\text { Culebra member of the Rustler } \\
\text { formation }\end{array}$ & PRMY_LOG & Log of intrinsic permeability, Y-direction \\
\hline 145 & CULEBRA & $\begin{array}{l}\text { Culebra member of the Rustler } \\
\text { formation }\end{array}$ & PRMZ_LOG & Log of intrinsic permeability, Z-direction \\
\hline 160 & DEWYLAKE & Dewey Lake Red Beds & PRESSURE & Brine far-field pore pres: \\
\hline 161 & DEWYLAKE & Dewey Lake Red Beds & PRMX_LOG & Log of intrinsic permeability, $X$-direction \\
\hline 162 & & Dewey Lake Red Beds & PRMY LOG & Log of intrinsic permeability, Y-direction \\
\hline 163 & DEWYLAKE & Dewey Lake Red Beds & PRMZ_LOG & Log of intrinsic permeability, Z-direction \\
\hline 2101 & MAGENTA & Magenta Member & PRESSURE & Brine far-field pore pressure \\
\hline 2102 & MAGENTA & Magenta Member & PRMX_LOG & Log of intrinsic permeability, X-direction \\
\hline 2103 & MAGENTA & Magenta Member & PRMY_LOG & Log of intrinsic permeability, Y-direction \\
\hline 2104 & MAGENTA & Magenta Member & PRMZ_LOG & Log of intrinsic permeability, Z-direction \\
\hline
\end{tabular}


Parameters Whose Underlying Data Were Qualified by Peer Review

\begin{tabular}{|c|c|c|c|c|}
\hline tos & Bhatenaleb: & $1 \%$ Materialintame: & Paramete un & Parameter Name: \\
\hline 547 & S_HALITE & Salado halite, intact & PRMX LOG & Log of intrinsic permeability, $X$-direction \\
\hline 548 & S_HALITE & Salado halite, intact & PRMY LOG & Log of intrinsic permeability, Y-direction \\
\hline 549 & S_HALITE & Salado halite, intact & PRMZ LOG & Log of intrinsic permeability, Z-direction \\
\hline 663 & WAS_AREA & $\begin{array}{l}\text { Waste emplacement area and } \\
\text { waste }\end{array}$ & PRMX_LOG & Log of intrinsic permeability, X-direction \\
\hline 664 & WAS_AREA & $\begin{array}{l}\text { Waste emplacement area and } \\
\text { waste }\end{array}$ & PRMY_LOG & Log of intrinsic permeability, Y-direction \\
\hline 665 & WAS_AREA & $\begin{array}{l}\text { Waste emplacement area and } \\
\text { waste }\end{array}$ & PRMZ_LOG & Log of intrinsic permeability, Z-direction \\
\hline 3259 & BLOWOUT & $\begin{array}{l}\text { BRAGFLOW Direct Brine } \\
\text { Releases }\end{array}$ & APORO & Waste permeability in CUTTINGS model \\
\hline 3462 & CULEBRA & $\begin{array}{l}\text { Culebra member of the Rustler } \\
\text { formation }\end{array}$ & ETHICK & Effective Thickness \\
\hline 3499 & GLOBAL & Information that applies globally & TPICD & $\begin{array}{l}\text { Time over which passive institutional } \\
\text { controls reduce rate of drilling }\end{array}$ \\
\hline
\end{tabular}


Appendix D

\section{$T=0$ for SNL Subcontractors}


Page intentionally blank

D-2 
date: November 26, 1997

to: Susan M. Howarth, MS1335

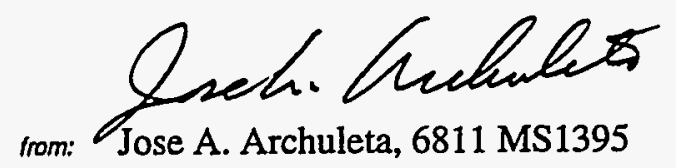

subject: Updated Summary of $\mathrm{T}=0$ Activities

Please use the attached updated $\mathrm{T}=0$ Matrix to supplement Table 5-6 of the Compliance Certification Application (CCA). This matrix summarizes the activities reviewed by the $\mathrm{T}=0$ Process defined by WIPP QAP 20-7, Revision 0, "Establishing $T=0$ for Internal and External Experiment Activity QA Programs" (effective 11/20/96) or its predecessor WIPP Desk Instruction "Establishing $T=0$ for Subcontractor QA Programs" (effective 10/27/95). The $T=0$ dates shown on the matrix match the $\mathrm{T}=0$ dates established on the corresponding checklist developed for each individual activity. All checklists have concurrence from the responsible Principal Investigator (PI) and the WIPP QA Chief.

There are two corrections to Table 5-6. The T=0 date for contract AG-4910, INTERA, is 6/94 and the $T=0$ date for contract $A A-2020, R E / S P E C$, is 8/92. Also shown on the matrix are the WPO numbers which have been assigned to each document and are a quick reference when requesting copies of the checklists from the SWCF.

Please call me at $234-0060$ or Greg Miller at 848-0330 if you have any questions regarding this memo or the attached matrix.

Cc:

MS1395 L. E. Shephard

S. Y. Pickering

J. A. Archuleta

F. D. Hansen

M. K. Knowles

MS1335 M.S. Y. Chu

J. G. Miller

N. J. Linarez-Royce

D. S. Coffey

D. M. Bullock

MS1341 J. T. Holmes

R. C. Moore

M. D. Siegel

H. W. Papenguth

L. H. Brush

D. A. Lucero

MS1322 J. R. Tillerson

A. W. Dennis

MS1324 P. B. Davies

R. L. Beauheim

L. C. Meigs

SWCF-A:1.4.01.5:T=0;QA;COR;Updated Summary of $\mathrm{T}=0$ activities (2) 
T=0 MATRIX (as of 11/11/97)

Page 3 of 3

\begin{tabular}{|c|c|c|c|c|c|c|c|c|}
\hline Item & Acttity & SNL PI/Contractor & $\begin{array}{l}\text { Conitract } \\
\text { No }\end{array}$ & Procedure & Audit & $\begin{array}{l}\text { WPO \# For } \\
\text { Cover Lettér } \\
\text { and Checklist }\end{array}$ & $\mathrm{T}=0 \mathrm{D}$ Date & $\begin{array}{l}\text { Supplement } \\
\text { Meno } \\
\text { Wpo }\end{array}$ \\
\hline 19 & $\begin{array}{l}\text { Series A\&B Re-Testing; Small } \\
\text { Scale Seals Performance Tests }\end{array}$ & Kathy Knowles & Internal & $\begin{array}{l}\text { QAP 20-7 } \\
(11 / 20 / 96)\end{array}$ & IA $95-03$ & 45221 & Nov-93 & N/A \\
\hline 20 & $\begin{array}{l}\text { Culebra Tracer Testing and } \\
\text { Well Program (H19) }\end{array}$ & Rick Beauheim & Internal & $\begin{array}{l}\text { QAP 20-7 } \\
(11 / 20 / 96)\end{array}$ & IA 95-03 & 44763 & Jan-95 & N/A \\
\hline 21 & $\begin{array}{l}\text { Colloid Characterization and } \\
\text { Transport }\end{array}$ & Hans Papenguth & Internal & $\begin{array}{l}\text { QAP 20-7 } \\
(11 / 20 / 96)\end{array}$ & IA $95-03$ & 44761 & Jan-93 & N/A \\
\hline 22 & $\begin{array}{l}\text { Non-Salado Absorption } \\
\text { Studies }\end{array}$ & Larry Brush & Internal & $\begin{array}{l}\text { QAP 20-7 } \\
(11 / 20 / 96)\end{array}$ & IA $95-03$ & 44762 & Jan-93 & N/A \\
\hline 23 & $\begin{array}{l}\text { Geologic and Hydrogeologic } \\
\text { Support and Modeling at the } \\
\text { WIPP Site }\end{array}$ & $\begin{array}{l}\text { Rick Beauheim/INTERA } \\
\text { *Note: The T=0 Process was } \\
\text { superseded by the QED Process } \\
\text { defined by QAP 20-3. }\end{array}$ & $\begin{array}{l}\text { Internal } \\
63-5605\end{array}$ & $\begin{array}{l}\text { QAP 20-7 } \\
(11 / 20 / 96) \\
\text { *See note }\end{array}$ & $\begin{array}{l}\text { EA 94-06 } \\
\text { IA } 95-03\end{array}$ & $\begin{array}{l}43487 \\
45253\end{array}$ & $\begin{array}{c}\text { N/A } \\
\text { * See note }\end{array}$ & $\begin{array}{c}46673 \\
\text { * See note } \\
\text { IRT Review } \\
\text { on DRP } \\
\text { WPO\# 42269 }\end{array}$ \\
\hline 24 & $\begin{array}{l}\text { SSSPT-Series D, Phase } 2 \\
\text { Brine Flow Tests }\end{array}$ & Kathy Knowles & Internal & $\begin{array}{l}\text { QAP 20-7 } \\
(11 / 20 / 96)\end{array}$ & IA $95-03$ & 46159 & Mar-95 & N/A \\
\hline
\end{tabular}


$T=0$ MATRIX (as of 11/11/97)

\begin{tabular}{|c|c|c|c|c|c|c|c|c|}
\hline Item & Activity & SNL PI/Contractor & $\begin{array}{c}\text { Contract } \\
\text { No. }\end{array}$ & Procedure & Audit Basis & $\begin{array}{l}\text { WPO \# For } \\
\text { Cover Letter } \\
\text { and Checklist }\end{array}$ & $\mathrm{T}=0$ Date & $\begin{array}{l}\text { Supplement } \\
\text { Memo } \\
\text { WPO\# }\end{array}$ \\
\hline 10 & Shaft Seals Design & Parsons-Brinkerhoff (Al Dennis) & AG-4909 & $\begin{array}{c}\text { Desk } \\
\text { Instruction } \\
(10 / 27 / 95)\end{array}$ & EA $95-10$ & 41878 & Mar-94 & 44359 \\
\hline 11 & $\begin{array}{l}\text { Fluid Flow Analysis for Shaft } \\
\text { Seal Design }\end{array}$ & INTERA (Al Dennis) & AG-4910 & $\begin{array}{c}\text { Desk } \\
\text { Instruction } \\
\text { (10/27/95) }\end{array}$ & EA 95-11 & 41875 & Jun-94 & N/A \\
\hline 12 & Salado 2-Phase Flow & $\begin{array}{l}\text { Core Laboratories (Susan } \\
\text { Howarth) }\end{array}$ & AF-3945 & $\begin{array}{c}\text { Desk } \\
\text { Instruction } \\
(10 / 27 / 95)\end{array}$ & EA 94-04 & 42351 & May-93 & 44360 \\
\hline 13 & Anhydrite Marker Bed 139 & RE/SPEC Inc (Susan Howarth) & AA-2020 & $\begin{array}{l}\text { QAP 20-7 } \\
(11 / 20 / 96) \\
\end{array}$ & EA 94-05 & 47965 & Aug-92 & $\mathrm{N} / \mathrm{A}$ \\
\hline 14 & Porosity and Permeability & TerraTek Inc (Susan Howarth) & AD-3656 & $\begin{array}{l}\text { QAP 20-7 } \\
(11 / 20 / 96)\end{array}$ & EA 93-04 & 44759 & Feb-93 & N/A \\
\hline 15 & Anoxic Corrosion of Metals & PNL Battelle (Larry Brush) & AJ-1365 & $\begin{array}{l}\text { QAP 20-7 } \\
(11 / 20 / 96) \\
\end{array}$ & EA $95-12$ & 44760 & Feb-94 & $N / A$ \\
\hline 16 & $\begin{array}{l}\text { Retardation of Radionuclide } \\
\text { Transport }\end{array}$ & Dan Lucero & Internal & $\begin{array}{l}\text { QAP 20-7 } \\
(11 / 20 / 96)\end{array}$ & IA $95-03$ & 45221 & Oct-94 & N/A \\
\hline 17 & $\begin{array}{l}\text { DRZ Characterization in Air } \\
\text { Intake Shaft }\end{array}$ & Kathy Knowles & Internal & $\begin{array}{l}\text { QAP 20-7 } \\
(11 / 20 / 96)\end{array}$ & IA $95-03$ & 45221 & Jul-95 & N/A \\
\hline 18 & $\begin{array}{l}\text { DRZ Characterization in } \\
\text { Room D }\end{array}$ & Kathy Knowles & Internal & $\begin{array}{l}\text { QAP 20-7 } \\
(11 / 20 / 96)\end{array}$ & IA $95-03$ & 45221 & Feb-95 & N/A \\
\hline
\end{tabular}


T=0 MATRIX (as of 11/11/97)

J. A. Archuleta, 6811

(505-234-0060)

Page 1 of 3

\begin{tabular}{|c|c|c|c|c|c|c|c|c|}
\hline Item & Activity & SNL PU/Contractor & $\begin{array}{l}\text { Cóntract } \\
\text { Nó. }\end{array}$ & Procedure & Audit & $\begin{array}{l}\text { WPO \# For } \\
\text { Cover Letter } \\
\text { and Checklist }\end{array}$ & $\mathbf{T}=\mathbf{0}$ Dáté & $\begin{array}{l}\text { Supplement } \\
\text { Memo } \\
\text { WPo\# }\end{array}$ \\
\hline 1 & +5 Actinide Solution & $\begin{array}{l}\text { Lawrence Berkely National } \\
\text { Laboratory (Craig Novak) }\end{array}$ & AH-5592 & $\begin{array}{c}\text { Desk } \\
\text { Instruction } \\
(10 / 27 / 95)\end{array}$ & EA 94-07 & 41879 & Sept-93 & 44301 \\
\hline 2 & Bentonite Specification & $\begin{array}{l}\text { University of Nevada Reno } \\
\text { (Frank Hansen) }\end{array}$ & AG-4915 & $\begin{array}{c}\text { Desk } \\
\text { Instruction } \\
(10 / 27 / 95)\end{array}$ & IA 95-01 & $\begin{array}{l}41877 \\
44297\end{array}$ & Jan-95 & 44299 \\
\hline 3 & Oganic Ligand Complexation & $\begin{array}{l}\text { Florida State University } \\
\text { (Bob Moore) }\end{array}$ & AH-5590 & $\begin{array}{c}\text { Desk } \\
\text { Instruction } \\
(10 / 27 / 95)\end{array}$ & EA 95-02 & 41876 & Jul-94 & 44304 \\
\hline 4 & +3 and +4 Actinide Solubility & PNL Battelle (Craig Novak) & AF-3339 & $\begin{array}{c}\text { Desk } \\
\text { Instruction } \\
(10 / 27 / 95)\end{array}$ & EA 95-01 & 41874 & Dec-93 & 44303 \\
\hline 5 & Culebra Tracer Tests & $\begin{array}{l}\text { University of Nevada Las Vegas } \\
\text { (Lucy Meigs) }\end{array}$ & AJ-8745 & $\begin{array}{c}\text { Desk } \\
\text { Instruction } \\
(10 / 27 / 95)\end{array}$ & EA95-04 & $\begin{array}{l}42034 \\
44296\end{array}$ & Apr-95 & 44306 \\
\hline 6 & Actinide Source Term & $\begin{array}{l}\text { Stanford University (Malcolm } \\
\text { Siegel) }\end{array}$ & AG-4979 & $\begin{array}{c}\text { Desk } \\
\text { Instruction } \\
(10 / 27 / 95)\end{array}$ & EA 95-05 & 42035 & Nov-94 & 44307 \\
\hline 7 & $\begin{array}{l}\text { Colloids-Studies of Actinide } \\
\text { Colloids in WIPP Brine }\end{array}$ & $\begin{array}{l}\text { Lawrence Livermore National } \\
\text { Laboratory (Hans Papenguth) }\end{array}$ & $\begin{array}{l}\text { AG-4965 } \\
\text { AF-3341 }\end{array}$ & $\begin{array}{c}\text { Desk } \\
\text { Instruction } \\
(10 / 27 / 95)\end{array}$ & EA $95-13$ & 41873 & Apr-95 & 44358 \\
\hline 8 & Tertiary Creep Studies & RE/SPEC Inc (Al Dennis) & AF-3334 & $\begin{array}{c}\text { Desk } \\
\text { Instruction } \\
(10 / 27 / 95) \\
\end{array}$ & EA 95-06 & 42036 & May-94 & 44357 \\
\hline 9 & Structural and Rock Mechanics & RE/SPEC Inc (Al Dennis) & AG-4911 & $\begin{array}{c}\text { Desk } \\
\text { Instruction } \\
(10 / 27 / 95) \\
\end{array}$ & EA 95-06 & 41872 & Apr-94 & 44357 \\
\hline
\end{tabular}




\section{Appendix E \\ 4B Cross Reference Memorandum}

E-1 


\section{Page intentionally blank}

E-2 


$$
\begin{aligned}
& \text { WPO } 44201 \\
& \text { This will beativelud to } \\
& \text { seren is } 464 \text { form. }
\end{aligned}
$$

cale: March 3. 1997

10

Susan Howarh

rem:

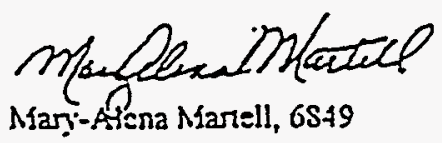

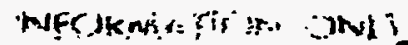

团

Sandia National Laboratories

Operated for the U.S. Department o: Energy by Sandia Corporation

Albuquerque. New Mexico 87155 .

Cross Reference of Categon 4B Parameters to the Analysis Package for the Code utilizing the parameter.

This memo is sent to provide information correlating the $4 B$ parameters listed in the Ingres CCA IVIPP parameter database and the analyses paciage write-up for each code utilizing the parameicr. The analysis packages are:

1) Analysis Package for the Salado Flow Calculations (Task 1) of the Periomance Assessmen Analyses Supporing Uis Compliance Cerification Application WPOت̈ 40514 (BRAGFLO).

2) Analysis Package for the Salado Transpor Calculations (Task 2) of the Performance Assessment Aralysas Supponing the Compliance Cerification Application WPOت̈ 40515 (NUTS. P.ANEL).

3) Analysis Package for the Culebra Flow and Transpon Calculations (Task 3) of the Performance Assessnent Analyses Supporing ule Compliance Cerification Applicauion WPO" 40516 (SECO_FL and SECO_TP),

4) Analysis Package for the BRAGFLO Direct Release Calculations (Task 4) of the Performance Asscssment $\therefore$ inal!ses Supporting the Compliance Cenification Application WPO $40 \$ 20$ and (BRAGFLO_DBR).

5) Analysis Package for the Cuttings and Spalling Calculations (Tasks 5 and 6 ) of the Performance Assessment Analyses Supporting the Compliance Centification Application WPO\# 40521 (CUTTINGS_S).

6) Analysis Package for the CCDF Construction-(Tasks 7)-of the Performance Assessment Analyses Supporing the Compliance Cenification Application WPO $4052+$ (CCDFGF)

The paramelers are separaled in Table 1 (paramelers for BRAGFLO. NUTS, PANEL, CUTTINGS S. BRAGFLO_DBR, SECO_FL and SECO_TP) and Table 2 (parameicrs for CCDFGF). CCDFGF paramelers are distinguished from the other paramelers due to the difference in the naming process used in the analysis

\begin{tabular}{|c|c|c|c|c|c|}
\hline idQ/ & $6 y^{3 d m i l}$ & ingl & 3nulvis hiletp & 4. & \\
\hline 58:AREA FRC & CASTIIER & $4 B$ & SAND92/0700/3 & pg 5-1 & \\
\hline $2276: \mathrm{CAP}$ MOD & ASPHAIT & $4 B$ & 11p040514 & $\operatorname{secl} \sec 1+2.4$ & \\
\hline $2599 \mathrm{KPT}$ & IASPHAII & $A B$ & up040514 & $\sec L 4.2 .4$ & \\
\hline $2278 \mathrm{PC} \mathrm{MAX}$ & ASPHALT & $4 B$ & wpo40514 & sect. 4.2 .4 & \\
\hline $2281: \mathrm{PO} \mathrm{MN}$ & ASPHAIT & $A B$ & 11p0+10514 & $\sec .4 .2 .4$ & \\
\hline 2289IRELP_MOD & ASPHALT & $\{B$. & 13p040514 & $\sec .4 .2 .4$ & \\
\hline
\end{tabular}
package.

Table 1

Exceptional Service in the National Interest 


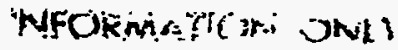

Susan Howarh

\begin{tabular}{|c|c|c|c|c|c|}
\hline 2929 RSH_AlR & ASPHALI & 43 & "1po-40514 & sect. 4.2 .4 & \\
\hline 2932iRSH_EXH & ASPHALT & $4 B$ & 1pot0514 & sect. +.2.t & \\
\hline 2930 RSH_SAI & ASPHLIT & $A B$ & "ipo40514 & sect. 4.2 .4 & \\
\hline 2931 RSH_WAS & ASPHALT & $4 B$ & 11pot0514 & sect 4.2 .4 & \\
\hline 3174 CAP_MOD & BH_CREEP & $A B$ & "pot0514 & sect. 4.2 .4 & \\
\hline 3172 COMP RCK & BH_CREEP & $4 B$ & 11po+0514 & $\sec 4.2 .4$ & \\
\hline $3180 \mathrm{KPT}$ & BH_CREEP & $4 B$ & 1pot0514 & sect. +.2 .4 & \\
\hline B175PC MAX & BH_CREEP & $4 B$ & "pot051t & sect. 4.2.4 & \\
\hline S181:PCT_A & BH_CREEP & $+B$ & "1pot0514 & $\operatorname{sect} 4.2 .4$ & \\
\hline 3182:PCT_EXP & $\mathrm{BH}$ CREEP & $4 B$ & upot0514 & sect. 4.2 .4 & \\
\hline J179:PO_MIN & BH_CREEP & $4 B$ & 11p040514 & sect 4.2 .4 & \\
\hline J178:PORE_DIS & BH_CREEP & $4 B$ & 1po40514 & sect +.2 .4 & \\
\hline B171:POROSITY & BH_CREEP & $f B$ & wpot0514 & $\operatorname{sect}+.2 .4$ & \\
\hline 3173:RELP_MOD & BH_CREEP & $4 B$ & 1pot0514 & $\sec 1.2 .4$ & \\
\hline 3176ISAT_RBRN & BH_CREEP & $4 B$ & "1pot0514 & sect 4.2 .4 & \\
\hline 3177/SAT_RGAS & $\mathrm{BH}=\mathrm{CREE}$ & $4 B$ & "pot0514 & sect. +.2 .4 & \\
\hline $313 S \mathrm{CAP} M O D$ & BH_OPEN & $4 B$ & "po40514 & $\operatorname{sect} 4.2 .4$ & \\
\hline 3156 COMPPRCK & BH_OPEN & $4 B$ & "po-10514 & $\operatorname{sect}+.2 .4$ & \\
\hline $31+4$ KPI & BH_OPEN & $4 B$ & "1potos14 & sect. +.2 .4 & \\
\hline IIS9:PC_MAXX & BH_OPEN & 43 & "pot0514 & sect. 4.2 .4 & \\
\hline I145PCT_A & $\mathrm{BH}$ OPEN & $4 B$ & "1p040514 & sect. 4.2.t & \\
\hline $3146 P C T$ EXP & BH_OPEN & $4 B$ & "po+0514 & sect. 4.2.4 & \\
\hline $3143 \mathrm{PO} M \mathrm{MIN}$ & BH_OPEN & $A B$ & "po40514 & $\operatorname{sect} 4.2 .4$ & \\
\hline 31+2:PORE_DIS & BH_OPEN & $4 B$ & "po40514 & sect +.2.t & \\
\hline 3135:POROSITY & $\mathrm{BH}$ OPEN & $4 B$ & "1p040514 & sect. $\$ .2 .4$ & \\
\hline 3134 PRMA_LOG & $\mathrm{BH}$ OPEN & $4 B$ & 11p040514 & sect. 4.2.4 & \\
\hline ISG PRMY_LOG & BH_OPEN & $4 B$ & 1p040514 & sect. +.2 .4 & \\
\hline 3187 PRNZ_LOG & $\mathrm{BH} \_\mathrm{OPEN}$ & 43 & "p040514 & seet. 4.2 .4 & \\
\hline 3137 RELP_MOD & BH_OPEN & $4 B$ & "p040514 & sect. 4.2 .4 & \\
\hline $31+0$ SAT_RBRN & $\mathrm{BH}$ & 43 & "1p040514 & sect. 4.2 .4 & \\
\hline 3141 SAT RGAS & BH_OPEN & $4 \mathrm{~B}$ & "po40514 & sect. 4.2 .7 & \\
\hline 3162 CAP_MOD & BH_SAND & $4 B$ & "1pot0514 & $\operatorname{sect} 4.2 .4$ & $\because$ \\
\hline 3160 COMP_RCK & BH_SAND & $4 B$ & 1po-405!4 & $\operatorname{sect}+.2 .4$ & \\
\hline $3168 \mathrm{KPT}$ & BH_SAND & $4 B$ & I1po+0514 & sect. +.2.t & \\
\hline $3163 \mathrm{PC}$ MAX & $\mathrm{BH}$ SAND & $4 B$ & "po40514 & sect. 4.2.t & \\
\hline $3169 ! P C A_{\text {A }} \mathrm{A}$ & BH_SAND & $4 B$ & 1pot0514 & $\operatorname{secl}+1.2 .4$ & \\
\hline 3170 PCT EXP & BH_SAND & $4 B$ & 1pot0514 & sect. 4.2 .4 & \\
\hline $3167 \mathrm{PO} \mathrm{MIN}$ & BH_SAND & $4 B$ & "po40514 & sect. 4.2 .4 & \\
\hline 3166 PORE_DIS & $\mathrm{BH}$ SAND & $4 B$ & "pot0514 & sect. +.2 .4 & \\
\hline 3159 POROSITY & $\mathrm{BH}$ SAND & $4 B$ & wpot0514 & sect. +.2 .4 & \\
\hline 3161 RELP_MOD & BH_SAND & $4 B$ & 1pot0514 & sect. 4.2 .4 & \\
\hline $316+$ SAT_RBRN & $\mathrm{BH}$ SAND & $4 B$ & upo40514 & sect. 4.2 .7 & \\
\hline 3165 SAT_RGAS & BH_SAND & $4 B$ & "po40514 & $\operatorname{sect} 4.2 .4$ & \\
\hline 23 CAP MOD & BOREHOLE & $4 B$ & "p 040514 & $\mathrm{pg} 4-142$ & \\
\hline $3122 \mathrm{KPT}$ & BOREHOLE & 48 & "1po40514 & $\mathrm{pg} 4-142$ & \\
\hline $29 \mathrm{PC} M A X$ & BOREHOLE & $4 B$ & spo-40514 & secL 4.2.4 & \\
\hline 3120 PCI $A$ & BOREHOLE & $4 B$ & "po-10514 & sect 4.2 .4 & \\
\hline 3121 PCT_EXP & BOREHOLE & $4 B$ & "po40514 & $\operatorname{sect} .4 .2 .4$ & \\
\hline
\end{tabular}




\begin{tabular}{|c|c|c|c|c|c|}
\hline 32:PO_MIN & BOREHOLE & +3 & "pot0514 & $\mathrm{Pg} 4-1+2$ & \\
\hline 3HPRRA_LOG & BOREHOLE & $\sqrt{3}$ & "pot0514 & $p g+-1+2$ & \\
\hline 35:PRMI_LOG & BOREHOLE & $A B$ & "pot0514 & sect. 4.2.4 & \\
\hline .36 PRMZ_LOG & BOREHOLE & $4 B$ & "pot0514 & $\operatorname{sect} 4.2 .4$ & \\
\hline +0IRELP_MOD & BOREHOLE & $+B$ & ipo40514 & $\mathrm{pg} 4-142$ & \\
\hline SOIREF PRES & IBRINESAI & $4 B$ & 1pot0514 & sect. 4.2.4 & sect. 4.2 .5 \\
\hline 51 REF TEMP & BRNNESAI & $4 B$ & upot0514 & sect. 4.2.t & sect. 4.2.5 \\
\hline GO:CAP_MOD & CASTILER. & $4 B$ & "pot0514 & sect. +.2.t & \\
\hline J194GRDFLO & CASTLER & AB & ipot0514 & sect. 4.2 .4 & \\
\hline 2605 KPT & CASTLLER & $4 B$ & 1po40514 & sect. 4.2.4 & \\
\hline 62) PC_MAX & CASTILER & $i B$ & "po-0517 & sect 4.2 .4 & \\
\hline 2609iPCT_A & CASTLER & $4 \mathrm{~B}$ & 1pot0514 & sect 4.2.7 & \\
\hline 2010 PCT_EXP & CASTILER & $+B$ & 1p040514 & sect 4.2.4 & \\
\hline $65 \mathrm{PO} \mathrm{MIN}$ & C.ASTLER & $4 B$ & ipo40.514 & sect. +.2.t & \\
\hline G3:PORE DIS & CASTLER & $4 B$ & "po40514 & sect. +.2 .4 & \\
\hline 72:RELP_MOD & CASTILER & $+B$ & 11pot051t & sect. 4.2 .4 & \\
\hline 73 SSAT_IBRN & CASTILER & tB & 1pot0514 & $\operatorname{sect} 4.2 .4$ & \\
\hline 74 SAT RBRN & CASTIIER & $\div 3$ & "po-10514 & $\sec 1.4 .2 .4$ & \\
\hline 75 S.AT_RGAS & CASTILER & $4 B$ & "po-10514 & sect +.2 .4 & \\
\hline 76!CAP MOD & CAVITI 1 & $A B$ & "po+0514 & sect +.2 .4 & \\
\hline 77 COMP_RCK & C.AVTTY I & $4 B$ & upo+0514 & sect. 4.2.4 & \\
\hline $2612 \mathrm{~K}$ KI & CAVITY 1 & 43 & "po-10514 & $\operatorname{sect}+1.2 .4$ & \\
\hline $78 \mathrm{PC}$ MAX & CAVITY 1 & $4 B$ & 11pot0514 & SeCL 4.2 .4 & \\
\hline 2613 PCT_A & CAVTYY 1 & $A B$ & 1pot051t & sect. 4.2.4 & \\
\hline 2614 PCT_EXP & CAVITY I & $\$ B$ & "pot0514 & sect 4.2 .4 & \\
\hline SI:PO_MIN & C.AVITY I & 43 & $11 p 0+0514$ & sect. 4.2 .4 & \\
\hline 79 PORE_DIS & CAVITY_1 & 43 & 11p040514 & secl. +.2 .4 & \\
\hline SOPOROSITY & C.AVIT' 1 & 43 & "po-10514 & sect. +.2.4 & \\
\hline S2 PRESSURE & CAVTY_I & $4 B$ & $11 p 040514$ & sect. 4.2.4 & \\
\hline SI:PRUL_LOG & CAVTY 1 & $4 B$ & "po+0514 & sect. 4.2.4 & \\
\hline $8+$ PRMY LOG & CAVTY_1 & $4 B$ & upot0514 & sect 4.2 .4 & \\
\hline 85:PRMZ_LOG & CAVITY 1 & $4 B$ & 11p040514 & sect. 4.2.4 & \\
\hline SSRELP_MOD & CAVITY 1 & $\pm B$ & "1po40514 & sect. 4.2 .4 & \\
\hline 3099 SAT_IBRN & CAVITYI & $4 B$ & "po40514 & sect. +.2.4 & \\
\hline S9:SAT_RBRN & CAVTY 1 & $A B$ & upot0514 & sect. 4.2.4 & \\
\hline 90:SAT_RGAS & CAVITY_I & $A B$ & 1p040517 & sect. 4.2.4 & \\
\hline 91 CAP MOD & CAVTYY 2 & $4 B$ & "pot0514 & sect. 4.2.4 & \\
\hline 92 COMP RCK & CAVITY 2 & $4 B$ & 1po+0514 & sect. 4.2.4 & \\
\hline $2616 \mathrm{KPT}$ & CAVTY 2 & 43 & 1p040514 & sect. 4.2 .4 & \\
\hline $93 \mathrm{PCMAX}$ & CAVITY 2 & $4 B$ & 11p040514 & sect. 4.2 .4 & \\
\hline 2617 PCT_A & CAVITY ? & $4 B$ & "pot0514 & sect. 4.2.4 & \\
\hline 2618:PCT_EXP & CAVIY 2 & $4 B$ & 1pot0514 & sect. 4.2.4 & \\
\hline 96:PO_MIN & CAVITY_ 2 & $4 B$ & 11p010514 & sect. 4.2 .4 & \\
\hline 94:PORE DIS & CAVIY 2 & $4 B$ & "p040514 & $\sec .4 .2 .4$ & \\
\hline 95:POROSITY & CAVITY 2 & $A B$ & "1pot0514 & sect. 7.2 .4 & \\
\hline 97:PRESSURE & CAVTY 2 & 48 & "pot0514 & $\mathrm{sect} 4.2 .4$ & \\
\hline 98:PRMX_LOG: & CAVTY 2 & $4 B$ & "po10514 & sect. 4.2.4 & \\
\hline 99:PRMY LOG & CAVTY? & TB & $11 p 040514$ & sect. 4.2 .4 & \\
\hline
\end{tabular}


NFOEMATICH: ONAT

Susan Howarh

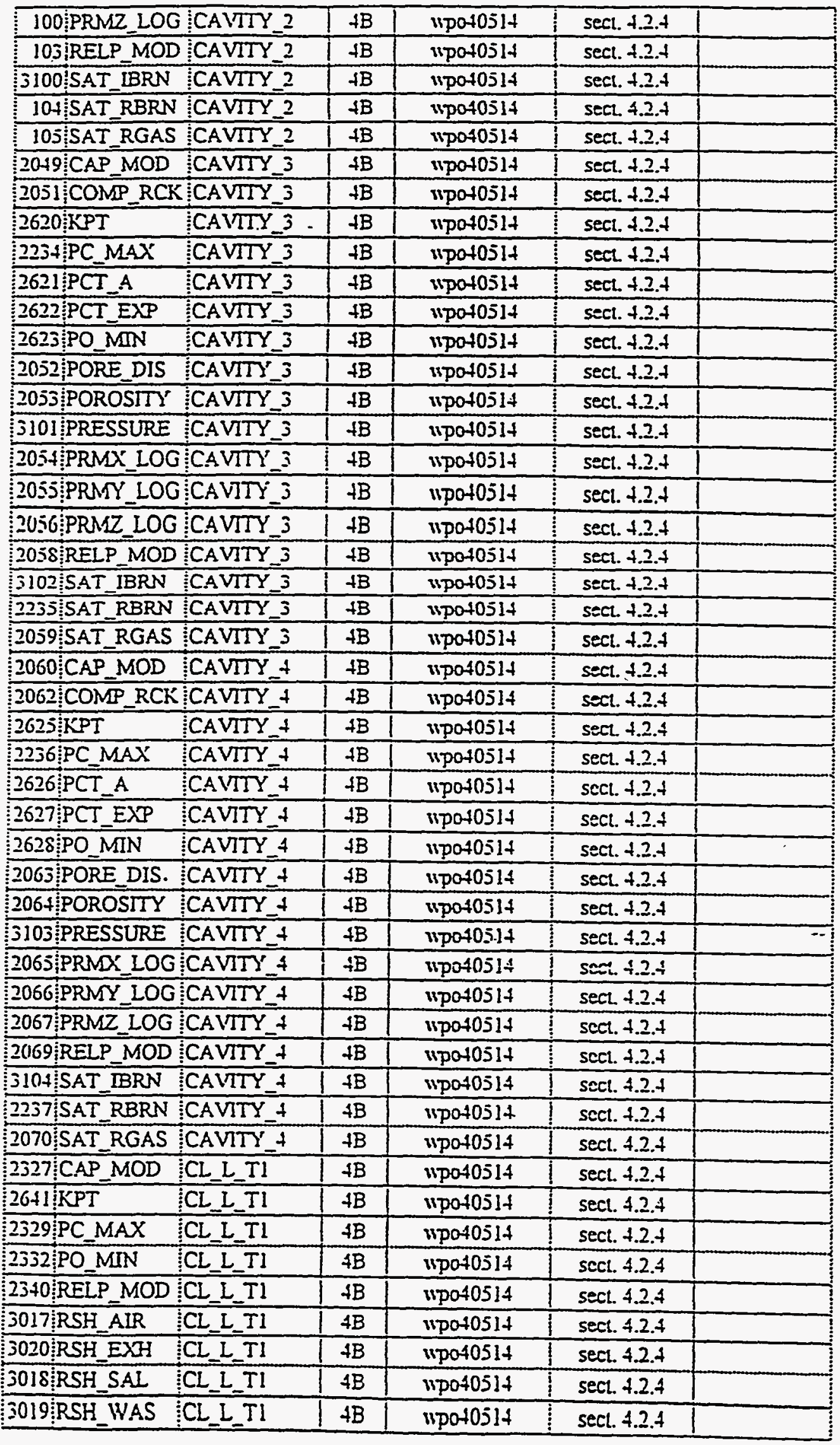




\begin{tabular}{|c|c|c|c|c|}
\hline & $5 \tau^{\circ}+7025$ & tISOtodin & gt & $X \nabla N J d+1+\bar{c}$ \\
\hline & 5ัで5.1005 & $5150+0 d n$ & Et & SINTD \\
\hline & $5 \sigma^{\circ}+1025$ & tisotodin & Et & SI WTD GOW $D V D Z I+C$ \\
\hline & $5 \tau+1005$ & 5IsOtodil & $g_{t}^{+}$ & ZINTD SHM HSUIGOS \\
\hline & เ゙で 700s & tisotod 11 & gt & LIWTD TVSHSY060S \\
\hline & เัน゙ท & 5Isotodil & gt & HXI HSY $260 \mathrm{~S}$ \\
\hline & tit 2005 & tIsotodil & gt & प्गTH HSd:6SOS \\
\hline & 5."t 1005 & tISOtodu & gt & ZIWTJ GOW dTJdsorc \\
\hline & tご์ 2005 & HISOtods & $g_{t}$ & ZINTD NIW OdOOOL \\
\hline & $t \cdot 2+720 s$ & tISOtodil & $g_{t}$ & $X \nabla J J \mathrm{~d} \angle G \subseteq \tau$ \\
\hline & $t \bar{c}+100 s$ & FISOtodil & gt & IdX: 1992 \\
\hline & tでt & tIsotodil & $g_{t}$ & वOW d8J $56 \subseteq \tau$ \\
\hline & $5 \tau+2005$ & tisotodil & gt & SYM HSd:980S \\
\hline & $t c+1005$ & tisotodil & $g t$ & TYS HSUISSOS \\
\hline & $t c t 100 s$ & tisotodn & Et & $\mathrm{HXX}{ }^{-} \mathrm{HS}: \angle 30 \mathrm{~S}$ \\
\hline & $t c t 100 s$ & fISOtodn & $E_{t}^{t}$ & II WTO QTH HSUITSOS \\
\hline - & $5 \tau+2005$ & tisotodn & Et & II'W'TD: GOW DTAYIISL \\
\hline & $t \tau+200 s$ & tisotodu & $g t$ & IINTD: NUWOdSSST \\
\hline & $t i t \cdot 100 s$ & tisotodn & gt & 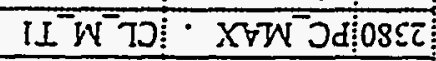 \\
\hline & tct. & 5 Isotod:1 & Et & IINTJ: IdXISSTL \\
\hline & $5 \tau+7005$ & tisotodil & $\varepsilon_{t}^{t}$ & aOW drJSSLSL \\
\hline & tct. 1005 & tIs0todil & Et & SYM HSd: $\angle 90 S$ \\
\hline & $t \tau+.3005$ & tIS050d11 & $\varepsilon t$ & TiSHSd990S \\
\hline & †で・ & tisotodn & gt & HEXI HSA!S90S \\
\hline & $5 \tau+1005$ & tIsotodi1 & gt & IITTJ YTV HSUISOOS \\
\hline & $52+1005$ & tisotod"I & gt & FI'T'TD CON dTERIISOS \\
\hline & tCt 2005 & tIsotodil & $g t$ & HITTD NUWOdISCIS \\
\hline & tนเ & tisotodu & $g_{2}^{\frac{1}{2}}$ & XVIN Jd:LLE \\
\hline & $t \bar{c} t \cos$ & tisotodn & gt & FIT'TD IDAICLOS \\
\hline & t.t. & tIs0todil & $g t$ & II T TJ GOW $d \forall D O O L O S$ \\
\hline & $t \bar{c}+150 s$ & tIsotodil & $g t$ & S8MA HSA 6COS \\
\hline & t.ct joss & tIs0todil & $\varepsilon_{5}$ & THS HSY:STOS \\
\hline & tot. joss & tisotodn & gt & HLI HSUOSOS \\
\hline & tันt 1525 & HISOtodil & at & SIITJ UTVHSU:LOS \\
\hline & $t c t 202 s$ & tIsotodil & gt & SI T TJ GONN dTIRITLSL \\
\hline & tCt 7005 & H Isotodu & gt & SIT T TJ NINOd \\
\hline & $t c t 200 s$ & tisotodil & Et & 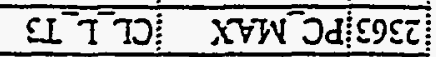 \\
\hline & $t c+2025$ & tIsotodil & Et & IdAIIS9G \\
\hline & tit. & hlsotodil & gt & COW dVJ:19: \\
\hline & tititioss & tisotodil & $g t$ & SYM HSUtFOL \\
\hline & tCt loos & tIs0tod 11 & 85 & TYS HSYSEOS \\
\hline & tてt wos & tisotodil & Et & HXY HSU SCOS \\
\hline & $t \tau+1025$ & tIsotodis & $\mathrm{gt}$ & 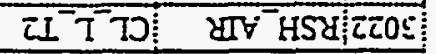 \\
\hline & rit?oas & tisotodil & gt & LI T TD GOW dTIU $\angle \subseteq \subseteq L$ \\
\hline & $F \bar{c}+1005$ & tIsotodn & $g t$ & ZITTD NINOdG $6+C L$ \\
\hline & $t \bar{c}+70=5$ & HISOtodil & gt & 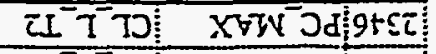 \\
\hline & $t \bar{c}+7025$ & HISOtod"I & gt & II T TJ IST \\
\hline & 505.2025 & $5150 t 0 d 11$ & gt & 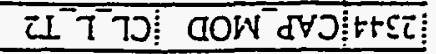 \\
\hline
\end{tabular}




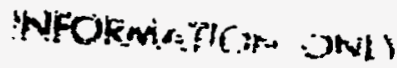

Susan Howarh

$-6-$

March 3. 1007

\begin{tabular}{|c|c|c|c|c|}
\hline 2417 PO_MIN & $\mathrm{CL} M \mathrm{T3}$ & $4 B$ & "pot0514 & secl. 4.2 .4 \\
\hline $2+25$ RELP MOD & $\mathrm{CL} M \mathrm{M}$ & $\mathrm{AB}$ & "pot0514 & $\sec 1.4 .2 .4$ \\
\hline J094RSH_ARR & $\mathrm{CL} M \mathrm{~T} 3$ & $A B$ & 11p040514 & sect. 4.2.t \\
\hline 3097RSH EXH & $\mathrm{CL} M-\mathrm{I} 3$ & $4 B$ & "po40514 & sect. 4.2.4 \\
\hline 3095:RSH_SAL & CL_M T3 & $4 B$ & "po40514 & sect. +.2 .4 \\
\hline 3096 RSH_WAS & CL_M_T3 & $A B$ & vpo40514 & sect. 4.2.4 \\
\hline $2429: C A P \_M O D$ & CL_M_T4 & $+B$ & "pot0514 & secL +.2.t \\
\hline $2671 \mathrm{KPT}$ & $\mathrm{CL} M \mathrm{M}+\ldots$ & $+\mathrm{B}$ & 11pot0514 & sect. +.2.t \\
\hline $2431: P C, M A X$ & CL_M_It & $4 B$ & 1po40514 & sect 4.2 .4 \\
\hline $243+\mathrm{PO}$ MNN & $\mathrm{Cl} M \mathrm{Mt}$ & $4 B$ & "pot0514 & sect. +.2 .4 \\
\hline 2442 RELP_MOD & $\mathrm{CL}_{-} \mathrm{M}_{-} \mathrm{TH}$ & $+\mathrm{B}$ & "1pot0514 & sect. 4.2.4 \\
\hline 2919 RSH_AIR & $\mathrm{CL} M \mathrm{It}$ & $4 B$ & "pot0514 & sect +.2.4 \\
\hline 2922:RSH_EXH & $\mathrm{CL} M \mathrm{TI}$ & $4 B$ & "pot0514 & $\operatorname{sect}+.2 .4$ \\
\hline 2920 RSH_SAI & $\mathrm{CL} M$ It & $4 B$ & 1po+0514 & sect. 4.2.t \\
\hline 2921 RSH_WAS & $\mathrm{CL}=\mathrm{M}$ T4 & $+B$ & "po+0514 & sect. +.2 .4 \\
\hline $2446 \mathrm{CAP}$ MOD & $C L=M_{-} T 5$ & $A B$ & "po40514 & sect. 4.2.t \\
\hline $2676 \mathrm{KPT}$ & $\mathrm{CL} M-15$ & $4 B$ & upot0514 & sect. 4.2.4 \\
\hline $2-4 S \mathrm{PC} M A X$ & $C L=M-T 5$ & $4 B$ & 1pot0514 & sect. 4.2 .4 \\
\hline $2 \div 51 \mathrm{PO} M \mathrm{MNN}$ & $\mathrm{Cl} M$ T T5 & $4 B$ & "po40514 & sect +.2 .4 \\
\hline 2459 RELP_MOD & $C L$ MIS & $+B$ & 11pot0514 & $\sec 1 .+2.4$ \\
\hline $292+$ RSH_AIR & $\mathrm{CL} M \mathrm{TJ}$ & $+B$ & "pot0514 & $\operatorname{sect}+.2 .4$ \\
\hline $2927 \mathrm{RSH}$ EXH & $\mathrm{CL} M \mathrm{MJ}$ & $4 \mathrm{~B}$ & 1pot0514 & $\operatorname{sect}+.2 .4$ \\
\hline $2925 \mathrm{RSH} S \mathrm{SAL}$ & CL M T5 & $4 B$ & I1p040514 & sec1. 4.2 .4 \\
\hline 2926 RSH_WAS & $C L M I S$ & $A B$ & "po40514 & sect. 4.2.4 \\
\hline $2310 \mathrm{CAP}=\mathrm{MOD}$ & CLAY_BOT & $4 B$ & "1pot0514 & sect. +.2 .4 \\
\hline $2311 \mathrm{COMP} R \mathrm{RCK}$ & CLAY_BOT & $A B$ & "pot0514 & sect. 4.2.4 \\
\hline $20056 \mathrm{KPT}$ & CLAY BOT & $A B$ & "pot0514 & sect. +.2 .4 \\
\hline $2312 \mathrm{PC} M A X$ & CLAY_BOT & $A B$ & "1po40514 & sect. 4.2.4 \\
\hline 2657 PCT_A & CLAY_BOT & $4 B$ & 1p040514 & sect. 4.2 .4 \\
\hline 2638 PCT_EXP & $\mathrm{CLAY} \mathrm{BOT}$ & $4 B$ & 11pot0514 & sect. 4.2 .4 \\
\hline $2315 \mathrm{PO} M \mathrm{MN}$ & CLAY BOT & $A B$ & "pot0514 & sect. 4.2 .4 \\
\hline 2316:PRESSURE & CLAY_BOT & $4 B$ & "po40514 & sect. 4.2 .4 \\
\hline 23I3RELP_MOD & $\mathrm{CLAY}^{\prime} \mathrm{BOT}$ & $A B$ & 1pot0514 & sect. 4.2.4 \\
\hline 3000 CAP_MOD & CLAY_RUS & $4 B$ & 11p040514 & sect. 4.2 .4 \\
\hline $3002 \mathrm{KPT}$ & CLAY_RUS & $4 B$ & "po40514 & sect. 4.2.7 \\
\hline 3003 PC MAX & CLAY_RUS & $4 B$ & "p040514 & sect. 4.2.4 \\
\hline 3151:PO_MIN. & CLAY_RUS & $4 B$ & "pot0514 & sect. 4.2 .4 \\
\hline 3012 RELP_MOD & CLAY RUS & $4 B$ & 1po40514 & $\operatorname{sect} .+2.4$ \\
\hline 2996 RSH_AIR & CLAY_RUS & $+B$ & 1 po40514 & sect. 4.2.t \\
\hline 2999 RSH_EXH & CLAY_RUS & $A B$ & 11pot0514 & scct. 4.2.4 \\
\hline 2997 RSH_SAI & CLAY RUS & $4 B$ & 11pot0514 & $\sec .4 .2 .4$ \\
\hline 2998:RSH_IVAS & CLAY_RUS & $4 B$ & 11p040514 & sect. 4.2 .4 \\
\hline 3051 CAP_MOD & CONC_MON & $4 B$ & "po40514 & sect. 4.2.4 \\
\hline $3052 \mathrm{COMP}$ RCK & CONC_MON & $4 B$ & "po10514 & sect. 4.2.7 \\
\hline $3053 \mathrm{KKPT}$ & CONC_MON & $4 \mathrm{~B}$ & "p040514 & sect. 4.2.4 \\
\hline $305+\mathrm{PC}$ MAX & CONC MON & $4 B$ & "pot0514 & sect. 4.2 .4 \\
\hline 212+PO_MN & CONC MON & $A B$ & "po40514 & sect. 4.2 .4 \\
\hline 3062 PEELP_MOD & CONC_MON & $4 B$ & "po40514 & secl. 4.2.4 \\
\hline
\end{tabular}




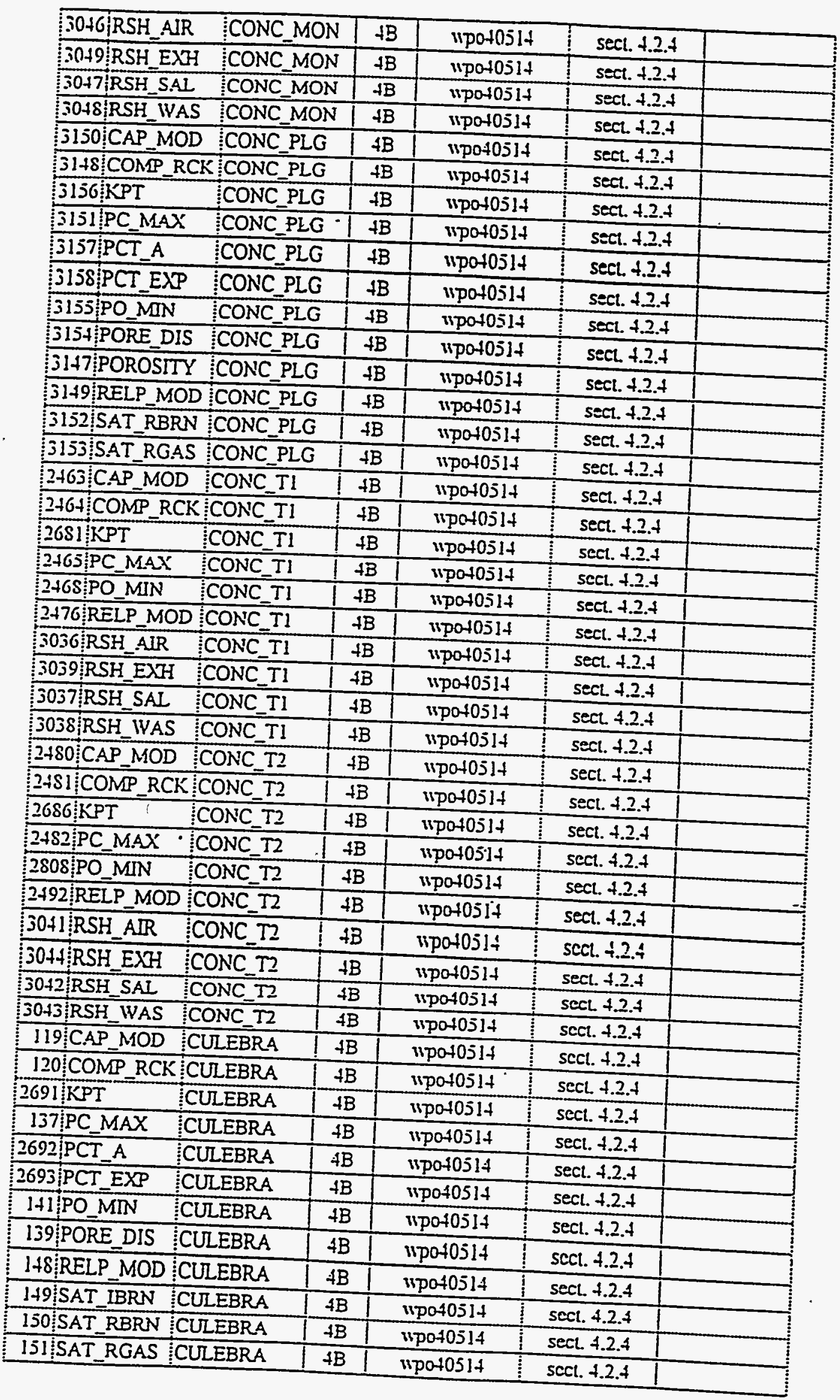




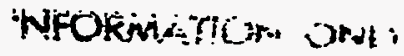

Susan Howarh

\begin{tabular}{|c|c|c|c|c|c|}
\hline $153 \mathrm{CAP} M O D$ & DEWIAKE & $A B$ & "pos0514 & sect. 4.2.t & \\
\hline 154 COMP_RCK & DEWILAKE & $4 B$ & "po4051t & sect. 4.2.4 & \\
\hline $2696 \mathrm{KPT}$ & DEW I AKE & $4 B$ & "1po40514 & sect. 4.2.t & \\
\hline $156 \mathrm{PC}$ MAAX & DEWIAKE & $4 B$ & "pot0514 & $\operatorname{sect} 4.2 .4$ & \\
\hline 2697 PCT_A & DEWILAKE & $A B$ & 11pot0514 & $\sec t .+2.4$ & \\
\hline 269SịCT_EXP & DEWILAKE & $4 B$ & 11pot0514 & sect. 4.2.4 & \\
\hline $159 \mathrm{PO} \mathrm{MIN}$ & DEWILAKE & $+B$ & 1p040514 & sect 4.2 .4 & \\
\hline 157PORE_DIS & DEVILAKE- & AB & "pot0514 & $\sec +.2 .4$ & \\
\hline 166RELP_MOD & DEW LAKE & $4 B$ & "pot0517 & $\sec .4 .2 .4$ & \\
\hline 16SSSAT_IBRN & DEWILAKE & $+B$ & "pot0514 & sect. +.2.4 & \\
\hline 169 SAI_RBRN & DEIVILAKE & 48 & "pot0514 & sect. 4.2 .4 & \\
\hline 170:SAT_RGAS & DEW ILAKE & $4 B$ & "p040514 & $\operatorname{sect} 4.2 .4$ & \\
\hline 174 CAP MOD & DRZ 0 & $4 B$ & "pot0514 & sect +.2 .4 & \\
\hline $175 \mathrm{COMP}_{-} \mathrm{RCK}$ & DRZ 0 & $4 B$ & "pot0514 & sect +.2 .4 & \\
\hline $2701 \mathrm{KPT}$ & $\mathrm{DRZ}=0$ & $\pm B$ & "pot0514 & sect. +.2 .4 & \\
\hline $176 \mathrm{PC} M \mathrm{MA}$ & $\mathrm{DRZ}=0$ & $A B$ & "pot0514 & sect. +.2.t & \\
\hline 2702 :PCT_A & DRZ 0 & $+B$ & 1pot0514 & sect. +.2 .4 & \\
\hline 2703 PCT_EXP & $\mathrm{DRZ} 0$ & $\pm B$ & "pot0514 & sect 4.2 .4 & \\
\hline 179:PO_MIN & $D R Z$ O & $4 B$ & "pot0514 & $\sec .+1.2 .4$ & \\
\hline 177 PORE DIS & $\mathrm{DRZ} 0$ & $A B$ & 1po40514 & sect. 4.2.4 & \\
\hline 17S:POROSITI & DRZ 0 & $4 B$ & "1po40514 & sect 4.2.4 & \\
\hline ISI:PRMX_LOG & DRZ 0 & $A B$ & "p040517 & sect +.2 .4 & \\
\hline 182:PRM & DRZ 0 & $4 B$ & "1pot0514 & sect. 4.2 .4 & \\
\hline IS3:PRMZ_LOG & $D R Z=0$ & $4 B$ & "pot051t & sect. 4.2.4 & \\
\hline ISG RELP_MOD & DRZ 0 & $A B$ & 11pot0514 & sect. +.2.4 & \\
\hline 187 SAT_RBRN & $\mathrm{DRZ} 0$ & 43 & "po40514 & sect. 4.2 .5 & \\
\hline ISS SAT_RBRN & DRZ 0 & +3 & "pot0514 & sect. 4.2.t & \\
\hline IS9SAT_RGAS & DRZ 0 & 43 & 13po40514 & sect. 4.2.4 & \\
\hline $190 \mathrm{CAP}_{1}$ MOD & DRZ_1 & $4 B$ & 1pot0514 & sect. 4.2.4 & \\
\hline $191 \mathrm{COMP}$ RCK & DRZ_1 & 43 & rpo40514 & sect 4.2 .4 & \\
\hline $3116 \mathrm{KPT}$ & DRZ_l & $4 B$ & "1p040514 & sect 4.2 .4 & \\
\hline $193 \mathrm{PC} M A X$ & DRZ_1 & $4 B$ & ipo40514 & sect. 4.2.4 & \\
\hline 3i28SPCT_A & DRZ_1 & $4 B$ & ipot0514 & $\operatorname{sect} 4.2 .4$ & \\
\hline 3129:PCT_EXP & DRZ_ 1 & 43 & 1po40514 & sect. 4.2 .4 & \\
\hline 196:PO_MIN & DRZ_1 & $A B$ & 11pot0514 & sect 4.2.4 & \\
\hline 194 PORE_DIS & DRZ I & $4 B$ & ipot0514 & sect. 4.2.4 & \\
\hline 195:POROSITY & DRZ_ 1 & $A B$ & "pot0514 & sect. 4.2.4 & \\
\hline 19S:PRMX LOG & DRZ 1 & $4 B$ & "pot0514 & sect. 4.2 .4 & \\
\hline 199:PRMY_LOG & DRZ_l & $A B$ & upotos14 & $\operatorname{sect} .4 .2 .4$ & \\
\hline 200:PRMZ_LOG & DRZ_I & $4 B$ & upot0514 & sect. 4.2 .4 & \\
\hline 203 RELP MOD & DRZ_1 & $4 B$ & 11p040514 & sect. 4.2 .4 & \\
\hline 205 SAT_RBRN & DRZ I 1 & $4 B$ & "pot0514 & sect. 4.2 .4 & \\
\hline 206 SAT_RGAS & $D R Z_{1}$ & $4 B$ & "pot0514 & $\operatorname{sect} .4 .2 .4$ & \\
\hline 2496 CAP_MOD & EARTH & $4 B$ & upot0514 & sect. 4.2 .4 & \\
\hline $2706 \mathrm{KPT}$ & EARTH & $4 B$ & "po40514 & sect. 4.2 .4 & \\
\hline $2498 \mathrm{PC} M A \mathrm{AX}$ & EARTH & $4 B$ & "pot0514 & $\operatorname{sect} 4.2 .4$ & \\
\hline $2501 \mathrm{PO} M \mathrm{MNN}$ & EARTH & $4 B$ & "p040514 & sect. 4.2 .4 & \\
\hline 2509 RELP_MOD & EARTH & $4 B$ & "1p040514 & sect. +.2 .4 & \\
\hline
\end{tabular}




\begin{tabular}{|c|c|c|c|c|}
\hline 3032)RSH_AIR & EARTH & $A B$ & Ipo40514 & $\sec 1 .+2.4$ \\
\hline 3035:RSH_EXH & EARTH & $4 B$ & "pot0514 & sect. +.2.4 \\
\hline 3033 RSH_SAL & EARTH & $4 B$ & "po40514 & sect. +.2 .4 \\
\hline $303+$ RSH_WAS & EARTH & $4 B$ & 11po+0514 & sect. +2.4 \\
\hline 207/CAP_MOD & EXP_AREA & $4 B$ & "po40514 & sect. 4.2.4 \\
\hline 208:COMP RCK & EXP_AREA & $A B$ & 1pot0514 & $\operatorname{sect}+.2 .4$ \\
\hline $2711 \mathrm{KPI}$ & EXP_AREA & $A B$ & "1pot0514 & sect. 4.2 .4 \\
\hline 209:PC MAX & EXP_AREA & $A B$ & "po4051t & sect. 4.2.4 \\
\hline 2712 PCT_A & EXP_AREA & $4 B$ & "pot0514 & sect. 4.2 .4 \\
\hline 2713 PCT_EXP & EXP_AREA & $A B$ & 11pot0514 & sect. 4.2.4 \\
\hline $212 \mathrm{PO} \mathrm{MmN}$ & EXP_AREA & $4 B$ & ipot0514 & sect. +.2 .4 \\
\hline 210:PORE_DIS & EXP_AREA & $4 B$ & "pot0514 & sect. 4.2.4 \\
\hline 211 :POROSITY & EXP_AREA & $+B$ & I1pot0514 & seci. +.2 .4 \\
\hline 213:PRESSURE & EXP_AREA & $4 B$ & ipot0514 & sect. +.2.t \\
\hline $21+$ PRAR LOG & EXP_AREA & $4 B$ & ipot0514 & $\mathrm{sect}+.2 .4$ \\
\hline $215 \mathrm{PRM}^{\prime}$ LOG & EXP_AREA & $4 B$ & i3pot0514 & sect. +.2.t \\
\hline 216:PRMZ_LOG & EXP_AREA & $+B$ & 1pot0517 & sect. 4.2.t \\
\hline 219 RELP_MOD & EXP_AREA & $A B$ & 11pot0514 & sect. +.2.t \\
\hline 220 S.AT_LBRN & EXP_AREA & $4 B$ & xpot0514 & sect. 4.2.4 \\
\hline 221 SAT_RBRN & EXP_AREA & $4 B$ & "pot0514 & sect. 4.2.4 \\
\hline 222ISAT RGAS & EXP_AREA & $4 B$ & "po40514 & sect. 4.2 .4 \\
\hline $20 S 5$ C.AP_MOD & FORTYNIN & \&B & "pot051t & sect. 4.2 .4 \\
\hline $2238 \mathrm{SOMP}_{-} \mathrm{RCK}$ & FORTYNIN & $4 B$ & 1pot0514 & sect. 4.2 .4 \\
\hline $2715 \mathrm{KPT}$ & FORTYNIN & $4 B$ & Ipot0514 & sect. 4.2.4 \\
\hline 2239 PC $M A X$ & FORTYNIN & $4 B$ & ipot0514 & sect. +.2.t \\
\hline 2716 PCT_A & FORTININ & $4 B$ & upot0514 & sect. 4.2 .4 \\
\hline 2717:PCT EXP & FORTININ & $4 B$ & 1pot0514 & sect. 4.2.t \\
\hline $2715: \mathrm{PO} M \mathrm{MIN}$ & FORTYNIN & $4 B$ & 1pot0514 & sect. +.2 .4 \\
\hline 20S7PORE DIS & FORTYNIN & $A B$ & ipot0514 & sect. +.2 .4 \\
\hline $2899 \mathrm{PRM} \perp \angle O G$ & FORTININ & $A B$ & "1po+0514 & sect. 4.2 .4 \\
\hline 2900 PRMY LOG & FORTYNIN & $4 B$ & "1po40514 & sect. 4.2.4 \\
\hline $2901 \mathrm{PRR}=2 O G$ & FORTYNIN & $4 B$ & 1pot0517 & sect. 4.2.t \\
\hline 2093:RELP_MOD & FORTININ & $4 B$ & spot0514 & $\operatorname{sect}+2.2 .4$ \\
\hline 2240 SAT_RBRN & FORTYNIN & $4 B$ & "pot0517 & $\operatorname{sect} .4 .2 .4$ \\
\hline $209+$ SAT_RGAS & FORTYNIN & $4 B$ & 1pot0514 & sect. 4.2 .4 \\
\hline 229 CAP_MOD & IMPERM_Z & $4 B$ & "pot0514 & sect. 4.2 .4 \\
\hline 230 COMP RCK & IMPERM Z & $4 B$ & Ipo40514 & sect. 4.2.4 \\
\hline 2720 KPT & IMPERM Z & $4 B$ & $11 p 040514$ & sect. +.2.t \\
\hline $231 \mathrm{PC} M A X$ & IMPERM_Z & $4 B$ & "pot0514 & sect. 4.2 .4 \\
\hline $2721 \mathrm{PCT} A$ & IIMPERM Z & $4 B$ & "po40514 & secl 4.2 .4 \\
\hline $2722 \mathrm{PCI}$ EXP & IMPERM Z Z & $4 B$ & "po40514 & scal. +.2 .4 \\
\hline $234 \mathrm{PO} \mathrm{MIN}$ & IMPERM $Z$ & $4 B$ & ipo-10514 & $\sec 1.4 .2 .4$ \\
\hline 232IPORE_DIS & IMPERM Z Z & $4 B$ & "pot0514 & scci. 4.2.4 \\
\hline 233 POROSITY & IMPERM_Z & $4 B$ & 1p040514 & sect. 4.2.4 \\
\hline 236 PRMX_LOG & IMPERM Z & $A B$ & 11p040514 & $\operatorname{sect} .4 .2 .4$ \\
\hline $237 \mathrm{PRAY} L \mathrm{LOG}$ & IMPERM Z & $A B$ & "po+0514 & sect. 4.2 .4 \\
\hline 238 PRMZ LOG & IMPERM_Z & $A B$ & upo40514 & sect. 4.2 .4 \\
\hline $2+1$ RELP MOD & IMPERM_Z & $A B$ & "po40514 & sect. 4.2.4 \\
\hline
\end{tabular}




\begin{tabular}{|c|c|c|c|c|c|}
\hline 243 SAT RBRN & IMPERM Z & $\$ B$ & "pot0514 & sect. 4.2 .4 & \\
\hline $24+$ SAT_RGAS & IMPERM Z & $4 B$ & "pot0514 & sect. 4.2.4 & \\
\hline 2097 CAP_MOD & MAGENTA & $4 B$ & "pot0514 & sect. 4.2.t & \\
\hline $2725 \mathrm{KPT}$ & MAGENTA & $4 B$ & "pot051t & sect. +.2 .4 & \\
\hline 2098 PC MLAX & MAGENTA & $4 B$ & "pot0514 & secL +.2 .4 & \\
\hline 2726 PCT A & MAGENTA & $4 B$ & 1p040514 & $\sec .+2.4$ & \\
\hline 2727:PCT_EXP & MAGENTA & $4 B$ & "pot0514 & sect. +.2 .4 & \\
\hline 272S:PO_MIN & MAGENTA & $4 B$ & "pot0514 & sect. 4.2.4 & \\
\hline 2099:PORE_DIS & MAGENTA & $4 B$ & 11po+0514 & sect. 4.2.t & \\
\hline 2241 SAT_RBRN & MAGENTA & $4 B$ & upot0514 & sect. +.2.t & \\
\hline 2107 SAT_RGAS & MAGENTA & $4 B$ & "pot0514 & sect. 4.2.t & \\
\hline 7 CAP_MOD & OPS_AREA & $4 B$ & "pot0514 & sect. 4.2.4 & \\
\hline SCOMP RCK & OPS_AREA & $+B$ & "pot0514 & sect. 4.2.t & \\
\hline $260+\mathrm{KPT}$ & OPS_AREA & $A B$ & "1pot051t & sect. 4.2.t & \\
\hline 9:PC MALI & OPS_AREA & $4 B$ & 11pot0514 & sect. +2.4 & \\
\hline 2605PCI_A & OPS_AREA & 43 & 11pot0514 & sect. +.2 .4 & \\
\hline 2606PCT_EXP & OPS_AREA & $4 B$ & 11pot0514 & sect. 4.2.4 & \\
\hline $12 \mathrm{PO} M \mathrm{MN}$ & OPS_AREA & $4 B$ & 1pot0514 & sect. 4.2.4 & \\
\hline I0 PORE_DIS & OPS_AREA & $4 B$ & "pot0514 & sect. 4.2.t & \\
\hline 11 POROSITY & OPS_AREA & $+B$ & "pot0514 & sect. 4.2.4 & \\
\hline 13:PRESSURE & OPS_AREA & $4 B$ & ipo40514 & sect. +.2.4 & \\
\hline 14 PRAD LOG & OPS_AREA & $+B$ & "pot0514 & sect. +.2.t & \\
\hline 15 PRMN_LOG & OPS_AREA & 43 & "pot0514 & sect. 4.2 .4 & \\
\hline 16:PRMZ_LOG & OPS_AREA & +3 & "pot0514 & $\mathrm{sec} .+2.4$ & \\
\hline 19 RELP_MOD & OPS_AREA & $4 B$ & upot0514 & sect. +.2.t & \\
\hline 20 SAT_IBRN & OPS_AREA & $4 B$ & 1pot0514 & sect. +.2.4 & \\
\hline $21: S A T \_R B R N$ & OPS_AREA & 13 & 11p040514 & sect. +2.4 & \\
\hline 22:SAT_RGAS & OPS_AREA & $4 B$ & "1pot0514 & sect. +.2.4 & \\
\hline $252 \mathrm{CAP}$ MOD & PAN_SEAI & $4 B$ & "pot0514 & sect. +.2.t & \\
\hline $253 \mathrm{COMP}=\mathrm{RCK}$ & PAN_SEAL & $\pm B$ & "pot0514 & sect. +.2 .4 & \\
\hline $2731 \mathrm{KPI}$ & PAN_SEAI & $\pm B$ & 11pot0514 & $\operatorname{sect}+.2 .4$ & \\
\hline $254 \mathrm{PC} M A X$ & PAN SEAI & $4 B$ & "pot0514 & sect. +.2 .4 & \\
\hline I7j2PCT_A & PAN_SEAI & $4 B$ & I1poi0514 & sect. +2.4 & \\
\hline 2733 PCT_EXP & PAN_SEAI & $4 B$ & 11pot0514 & sect. +.2.4 & \\
\hline 257:PO_MIN & PAN_SEAL & $4 B$ & "1p010514 & sect. +.2.4 & \\
\hline 25JPORE DIS & PAN SEAL & $4 B$ & upot0514 & secl. +.2.t & \\
\hline 256:POROSITY & PAN_SEAL & $A B$ & "pot0514 & sect. +.2.t & \\
\hline 25S PRRSSURE & PAN_SEAL & $4 B$ & "pot0514 & $\operatorname{secl} .4 .2 .4$ & \\
\hline 259 PRMA _ LOG & PAN_SEAL & $4 B$ & 1pot0514 & sccl. +.2.4 & \\
\hline 260:PRMY LOG & PAN_SEAL & $4 B$ & "pot0514 & sect. 4.2 .4 & \\
\hline 261 PRMZ LOG & PAN_SEAL & $4 B$ & wp040514 & secl. 4.2.4 & \\
\hline 264RELP_MOD & PAN_SEAL & $4 B$ & ipot0514 & sect. 4.2 .4 & \\
\hline 2734 SAT_IBRN & PAN_SEAL & AB & 11pot0514 & sect. 4.2.t & \\
\hline 265 SAT_RBRN & PAN_SEAL & $A B$ & "1po40514 & sect. 4.2 .4 & \\
\hline 266SAT_RGAS & PAN SEAI & $4 B$ & "po40514 & sect. +.2.t & \\
\hline 3106 ASDRUM & REFCON & $A B$ & "pot0514 & sect. $+2 . t$ & \\
\hline 3107 VPANLEX & REFCON & $4 B$ & "po40514 & $\operatorname{sect} .4 .2 .4$ & \\
\hline 3108 VREPOS & REFCON & $4 B$ & "p040514 & sect. 4.2.4 & \\
\hline
\end{tabular}




\begin{tabular}{|c|c|c|c|c|c|}
\hline 3105 VROOM & REFCON & $A B$ & "po40514 & sect. 4.2 .4 & \\
\hline $2110 ; C A P M O D$ & REPOSIT & $A B$ & "po40514 & sect. 4.2.4 & \\
\hline $3+55:$ CLOSMOD & REPOSIT & $\sqrt{B}$ & 1p040514 & sect. 4.2 .4 & \\
\hline $2112 \operatorname{CONP} R C K$ & REPOSIT & $A B$ & "pot0514 & sect 4.2 .4 & \\
\hline $2736 \mathrm{KPT}$ & REPOSIT & $A B$ & 11po40514 & sect. 4.2 .4 & \\
\hline $22+2 P C M A X$ & REPOSIT & $4 B$ & "po-40514 & sect. +.2 .4 & \\
\hline 2737 PCT A & REPOSIT & $4 B$ & "po40514 & secl. +.2 .4 & \\
\hline 273SPCT EXP & REPOSIT & $A B$ & "po+0514 & sect. 4.2 .4 & \\
\hline 2739:PO_MIN & REPOSIT & $4 B$ & wpot0514 & sect. 4.2.t & \\
\hline 2129:PORE_DIS & REPOSII & $A B$ & upot0514 & SecL +.2.t & \\
\hline 2130 POROSITY & REPOSIT & $4 B$ & 1po40514 & secl. 4.2.t & \\
\hline 2 224PROBDEG & REPOSII & $4 B$ & upo40514 & sect. 4.2.4 & \\
\hline 2135 RELP_MOD & REPOSIT & $4 B$ & 1pot0514 & secL +.2.4 & \\
\hline 2740 SAT_IBRN & REPOSIT & $+B$ & "pot0514 & sect. 4.2 .4 & \\
\hline $27+1$ SAT_RBRN & REPOSIT & $4 B$ & 1pot0514 & sect. 4.2.4 & \\
\hline 2137SAI_RGAS & REPOSIT & $A B$ & 11pot0514 & sect. 4.2.4 & \\
\hline 215S:DPHIMLAX & $\mathrm{S} \_\mathrm{ANH} \_\mathrm{AB}$ & $A B$ & upot0514 & sect. 4.2.4 & \\
\hline 2S12IFRX & $\mathrm{SANH} A \mathrm{AB}$ & $+B$ & $1100+0514$ & sect. +.2 .4 & \\
\hline $2 S 15$ IFRY & S_ANH_AB & $4 B$ & upo10514 & sect. 4.2.t & \\
\hline ISISIIFRZ & $S_{-} \mathrm{ANH} \_\mathrm{AB}$ & $4 B$ & upo+0514 & sect. +.2 .4 & \\
\hline $2159: K M A L I O G$ & $S A N A B A B$ & $+B$ & "pot0514 & sect. 4.2 .4 & \\
\hline $2773 \mathrm{KPT}$ & $\mathrm{S}$ ANNH_AB & $4 B$ & "pot0514 & $\mathrm{sect}$. .2 .4 & \\
\hline 524 PF DELTA & $S_{-} \mathrm{ANH} A \mathrm{AB}$ & $4 B$ & 1pot0517 & sect. 4.2 .4 & \\
\hline 526:PI_DELTA & S_ANH_AB & $4 B$ & upotn51t & sect 7.2 .4 & \\
\hline 529:PO_MIN & $S_{\triangle} A N H_{-} A B$ & $4 B$ & 11p0+051t & sect. 4.2 .4 & \\
\hline $540 \mathrm{CAP} M O D$ & S_HALITE & $4 B$ & 1 pot0514 & sec1. +.2 .4 & \\
\hline $2778 \mathrm{KPT}$ & S_HALITE & 43 & "1po40514 & sect. +.2 .4 & \\
\hline I+2PC MAX & SHALITE & 43 & 11p040514 & sect. +.2 .4 & \\
\hline 2779:PCT A & S_HALITE & $4 B$ & 11p0+1051t & $\operatorname{secl} .+.2 .4$ & \\
\hline 2780 PCT EXP & S_HALITE & $4 B$ & 11po+0514 & sect. 4.2 .4 & \\
\hline $545 \mathrm{PO} \mathrm{MIN}$ & SHALITE & $4 B$ & 11p040514 & sect. +.2 .4 & \\
\hline 543 PORE D DIS & S_HAIITE & $A B$ & 1pot0514 & $\sec +2.4$ & \\
\hline 553 RELP_MOD & S_HAIITE & $4 B$ & 1poi0514 & sect +2.2 & \\
\hline 554 SAT_IBRN & S HALITE & $4 B$ & "pot0514 & sect. 4.2.4 & \\
\hline 555 SAT_RBRN & S_HALITE & 48 & "1poto514 & sect. +.2 .4 & \\
\hline 556 SAT_RGAS & S_HALITE & $4 B$ & "po-10514 & sect. 4.2 .4 & \\
\hline 2169 DPHIMAX & $S M B B 138$ & $4 B$ & "spot0514 & sect. +.2.4 & \\
\hline 2510 IFRX & $S M B 158$ & $4 B$ & upot051t & scct. +.2.4 & \\
\hline 2S13 IFRY & 5 MBB 138 & $4 B$ & "pot0517 & scct. +.2 .4 & \\
\hline 2S1GIIFRZ & S_MB138 & $4 B$ & "pot051t & sect. 4.2.4 & \\
\hline $2170 \mathrm{KM} \mathrm{AXIOG}$ & $S M B 138$ & $4 B$ & upot051t & secl. +.2 .4 & \\
\hline $2783 \mathrm{iKPT}$ & $S \_B B 138$ & $A B$ & $11 p 0+0514$ & sect. +.2 .4 & \\
\hline $563 ! \mathrm{PF} D \mathrm{DELTA}$ & S_MB138 & $4 B$ & 11p0+0517 & sect. +.2 .4 & \\
\hline 565:PI_DELTA & S_MB138 & $4 B$ & "pot0517 & sect. 4.2.4 & \\
\hline 568:PO_MIN & S_MB138 & $4 B$ & "po40514 & sect. 4.2.4 & \\
\hline 2177DPHIMAX & S_MB139 & $4 B$ & 1pot0514 & sect. $\$ .2 .4$ & \\
\hline 2SII!IFRX & S_MB 139 & $4 B$ & "pot0514 & sect. 4.2 .4 & \\
\hline 2814 IFRY & SMB139 & $4 B$ & ipot0514 & sect. 4.2.4 & \\
\hline
\end{tabular}




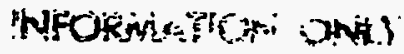

Susan Howarth

$-12-$

March 3. 1997

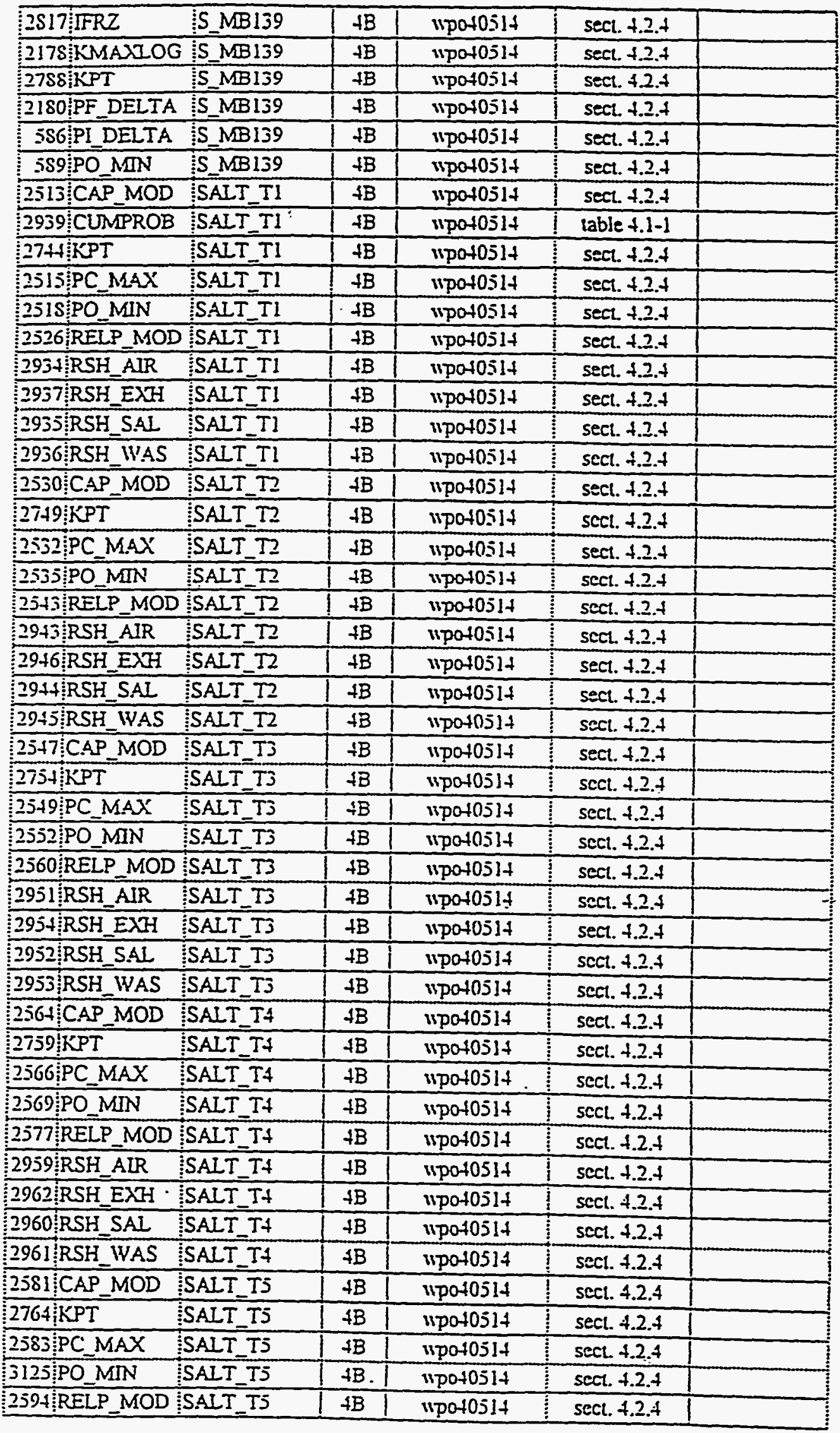

E-14 


\begin{tabular}{|c|c|c|c|c|c|}
\hline 2967RSH_ALR & SALT TS & $4 B$ & 15040514 & sect. 4.2 .4 & \\
\hline 2970 RSH_EXH & SAIT_TJ & $4 B$ & upo40514 & sect. 4.2.t & \\
\hline 2968 RSH_SAL & SALT_T5 & $4 B$ & "1pot0514 & sect. +.2.4 & \\
\hline 2969 RSH_WAS & SAIT_IS & $\pm B$ & 11p040514 & sect. 4.2.7 & \\
\hline $2953 \mathrm{CAP}_{\mathrm{CAOD}}$ & SALT_T6 & $4 B$ & 11pot0514 & sect. 4.2 .4 & \\
\hline 29S5IKPT & SALT_TO & $4 B$ & "pot0514 & sect +.2 .4 & \\
\hline $2956 \mathrm{PC} M A 4 X$ & SALT_TG & $4 B$ & "po40514 & sect. +.2 .4 & \\
\hline $3126 \mathrm{PO} \mathrm{MTN}$ & SALT_TG: & $4 B$ & 1pot051t & sect. 4.2.t & \\
\hline 2991 RELP MOD & SALI T6 & $4 B$ & "pot0514 & sect 4.2 .4 & \\
\hline $2975 \mathrm{RSH}$ AIR & SALT_T6 & $+B$ & "pot0514 & $\sec 1 .+.2 .4$ & \\
\hline $297 S$ RSH EXH & SALT T6 & $4 B$ & "pot0514 & sect 4.2 .4 & \\
\hline 2976RSH_SAL & SAIT T6 & $A B$ & 1pot051t & sect. 4.2 .4 & \\
\hline 2977!RSH_WAS & SALT_T6 & $4 B$ & upot0514 & sect. 4.2 .4 & \\
\hline $336 \mathrm{CAP} M O D$ & SANTAROS & $4 B$ & upot0514 & sect. 4.2.t & \\
\hline 337 COMP RCK & SANTAROS & $4 B$ & upo40514 & sect. +.2 .4 & \\
\hline 276S:KPT & SANISAROS & $A B$ & 11p040514 & sect. 4.2.4 & \\
\hline $539 P C \wedge 14 \lambda$ & SANTAROS & $A B$ & 11p0+0514 & sect. +.2.t & \\
\hline 2769:PCT_A & SANTAROS & $\pm B$ & 11p040514 & $\sec 2+.2 .4$ & \\
\hline 2770 PCT EXP & SANTAROS & $A B$ & "pot0514 & sect. +.2.4 & \\
\hline B+2?:PO_MIN & SANTAROS & $A B$ & 11pot0514 & sect. +.2.4 & \\
\hline 340!PORE_DIS & SANTAROS & $4 B$ & "pot0514 & sect. 4.2.4 & \\
\hline 3+1:POROSITY & SANTAROS & $4 B$ & "pot051t & sect. +.2.4 & \\
\hline $3+4$ PRMR IOG & SANTAROS & $4 B$ & "1pot0517 & sect. +.2 .4 & \\
\hline $3+5$ PRMY LOG & SANTAROS & $4 B$ & "pot0514 & sect. 4.2.4 & \\
\hline $3+6$ PRMZ LOG & SANTAROS & $4 B$ & "pot0514 & sect 4.2 .4 & \\
\hline I-19RELP_MOD & SANTAROS & $4 B$ & "pot0514 & sect. 4.2 .4 & \\
\hline 350 SAT_IBRN & S.ANT.AROS & $A B$ & 1p040514 & sect. 4.2 .4 & \\
\hline 351SSAT_RBRN & SANTAROS & 43 & spot0514 & sect. +.2 .7 & \\
\hline 252 SAT RGAS & SANTAROS & $4 B$ & 1pot0514 & sect. +.2.7 & \\
\hline 2183 C.AP MOD & TAMARISK & AB & 110040514 & sect. 4.2 .7 & \\
\hline $22+3$ COMP_RCK & TAMARISK & $4 B$ & "p040514 & sect. +.2 .4 & \\
\hline 2793 KPT & TAMARISK & $4 B$ & 1pot0514 & sect. 4.2.t & \\
\hline 2244: $\mathrm{PC} M A X$ & TAMARISK & $4 B$ & 11p040517 & sect +.2 .4 & \\
\hline 2794:PCT_A & TAMARISK & $4 B$ & 11pot0514 & sect. 4.2.4 & \\
\hline 2795:PCI_EXP & TAMARISK & $4 B$ & "pot0517 & sect. 4.2.4 & \\
\hline 2796:PO_MIN & TAMARISK & $4 B$ & ipot0514 & $\sec 1.4 .2 .4$ & \\
\hline 2IS5:PORE_DIS & TAMARISK & $A B$ & "pot0514 & sect. 4.2 .4 & \\
\hline 291+PPRMX LOG & TAMARISK & 48 & "pot0514 & sect. +.2 .4 & \\
\hline 2915 PRRMYLLOG & TAMARISK & $4 B$ & "po40514 & sect. 4.2.t & \\
\hline 2916:PRMZ_LOG & TAMARISK & $4 B$ & "po4051t & sect. 4.2.t & \\
\hline 2191 RELP_MOD & TAMARISK & $4 B$ & "pot0514 & sect. +.2.t & \\
\hline 2275 SAT RBRN & TAMARISK & $4 B$ & "pot0514 & $\sec 1.4 .2 .4$ & \\
\hline 2192 SAT_RGAS & TAMARISK & $4 B$ & "pot0514 & sect. +.2 .4 & \\
\hline $2217 \mathrm{CAP}_{\mathrm{O}} \mathrm{MOD}$ & UNNAMED & $4 B$ & 1p040514 & $\operatorname{sect} .4 .2 .4$ & \\
\hline $2218 \mathrm{COMP}$ RCK & UNNAMED & $4 B$ & 11p040514 & $\operatorname{secL}+.2 .4$ & \\
\hline 2799:KPT & UNNAMED & $4 B$ & 110040514 & scel. 4.2 .4 & \\
\hline $22+7$ PC MAX & UNNAMED & $4 B$ & 11p040514 & sect. 4.2 .4 & \\
\hline 2800 PCT_A & UNNAMED & $A B$ & ipot0514 & sect. 4.2 .4 & \\
\hline
\end{tabular}




\begin{tabular}{|c|c|c|c|c|}
\hline & $\angle 1005$ & 1250 todn & gt & IJNËdS:6tEs \\
\hline & $\angle$ LOSS & $1250 t a d 11$ & $g t$ & InOMOTg \\
\hline & $\angle 1005$ & IzSOtodn & gt & InONO78 \\
\hline & $\angle$ DOS & izS0tod"I & gt & O\&OdNIOSTS \\
\hline & $\angle 10 a S$ & izsotod.11 & gt & OdIIAH: $0 \leq \overline{C S}$ \\
\hline & $\angle D O S$ & IISOtod:1 & gt & InOMOTg: \\
\hline & $\angle 1035$ & IIS0todil & Et & InOMOTR: \\
\hline & $\angle 1005$ & IIsotodil & at & InOMOTa: \\
\hline & $\angle I D O S$ & IIsotodil & gt & InOMOTR: INEWIDS StCE \\
\hline & 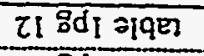 & $0 \widetilde{c}$ otod"I & $g_{t}$ & InOMOTR SYJ XJHI SLTE \\
\hline & 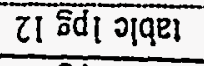 & $.0 \tau s 0 t 0 d 11$ & $g t$ & InOMOTg: ISHJ Jd:OSt? \\
\hline & ¿I รd ग ग१8] & $0 \bar{c} \leq 0+0 d 11$ & Et & InOMOTR MOTINUWVILLE \\
\hline & दा डd ग गq8! & OtSOtod:I & Et & InOMOTR: MOTLXVWNILIS \\
\hline & Zा हd ग गQ! & 0zS0todil & $g t$ & InOMOTg NWN S\$DOLLS \\
\hline & $p-x a d d e$ & $9150+0 d 11$ & $g t$ & TYGOTD XUISNFAL CEL \\
\hline & p-ladde & $9150+0 d 11$ & $\overline{g t}$ & 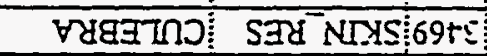 \\
\hline & p-ivdde & 9LSOtod.11 & $\mathrm{gt}$ & IXOLIITS \\
\hline & $s .7025$ & sIsotodn & gt & WTOSDOT:6S9I \\
\hline & 57005 & sIsotodil. & gt & Bd .NTOSDOTEIS \\
\hline & c 1025 & sIsotodn & Et & gd INTOS9OTLST \\
\hline & $\underline{5} 1205$ & SI SOtod:I & $\varepsilon_{t}$ & SD WNTOSDOTItS \\
\hline & s loss & slsotod" & gt & SD WTOSOOT:SES \\
\hline & $\pm \tau+1025$ & HIsotodil & gt & 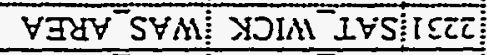 \\
\hline & $5 t+1025$ & 5isotodn & gt & $\forall A Q 7$ SVM SVDY IVS IL9 \\
\hline & tit was & tisotodu & Et & $\forall J Q Y$ SYM NQGY IYS:0L9 \\
\hline & 505 toss & IIsotodil & $g t$ & $\forall \exists Q 8$ SYM: NQAl I $\forall S$ : 699 \\
\hline & titivas & tisotadn & $g t$ & 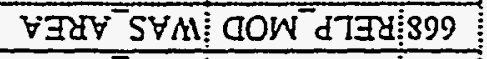 \\
\hline & tit toss & tIsotodil & $\overline{g t}$ & 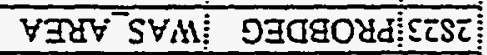 \\
\hline & $t \tau+100 s$ & $515050 d 11$ & gt & 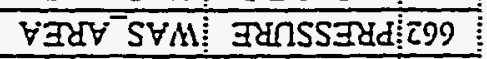 \\
\hline & $5 \cdot \frac{0}{2025}$ & tIsotodil & gt & $\forall \equiv C \forall$ SYM \\
\hline & tit 7005 & 5I50todis & at & SId $\equiv 80 d 6 \leqq 9$ \\
\hline & 5.ct 2025 & tisotodu & gt & $\forall \equiv Q Y Z S \forall M$ \\
\hline & f.t. TJ22 & tisotodu & gt & $d X \Xi I J d 90 S c$ \\
\hline & tit.joss & $5[50$ tod 11 & gt & $\forall \exists C V S \forall M$ \\
\hline & $t \tau+2005$ & tisotodil & $g t$ & $x \nabla J 42 d \beta S 9$ \\
\hline & $+2+20=5$ & risotodu & gt & Y3QV SYM $\quad$ IDXITOSL \\
\hline & tit ioss & tisotodu & gt & $\forall \exists Q V$ SYM XJY dWOJ SSS \\
\hline & $t c t+25 s$ & tIs0todi1 & gt & $\forall \exists Q V$ SYMI GOWSOTD TStE \\
\hline & $5 \tau+20=5$ & 5 ISOtodil & Et & $\forall \exists Q V$ SVM GOW dVJTSO \\
\hline & $t \tau+2025$ & tisotodil & gt & GEIWTINLC! SHOY IVS:9TLL \\
\hline & titivos & 5isotod"1 & gt & - GFWVNNO NQGQ IYSSSTL \\
\hline & $5 \cdot t+2005$ & tisotod"1 & Et & 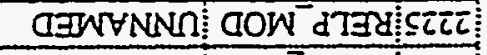 \\
\hline & tit 720s & HIsotodil & $g t$ & वINVNNC DOT ZWRd SI6L \\
\hline & tictivas & tISOtod"I & $g t$ & GINVNNI DOI NWAd \\
\hline & tit 700s & tIsotodil & gt & 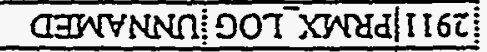 \\
\hline & เで๋ 100s & $5150 t 0 d 11$ & gt & 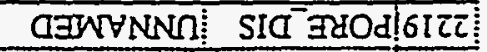 \\
\hline & $50+120 s$ & tisotodil & gt & 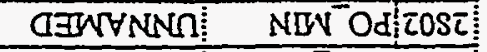 \\
\hline & t⿱宀匕 toos & tIsotodil & at & $\mathrm{dX} \Xi \perp \supset \mathrm{d} ! 108 \bar{c}$ \\
\hline
\end{tabular}




\begin{tabular}{|c|c|c|c|c|c|}
\hline $32+2$, COLDIA & BOREHOLE & 45 & "pot0521 & $\operatorname{sed} 7$ & \\
\hline 26 DIAMMOD & BOREHOLE & +3 & "pot0521 & $\operatorname{sect} 7$ & \\
\hline 27 DOMEGA & BOREHOLE & $4 B$ & 1pot0521 & sect 7 & \\
\hline 3I39IINV_AR & BOREHOLE & $t B$ & "1pot05?1 & $\operatorname{sect} 7$ & \\
\hline $32+4 \mathrm{LI}$ & BOREHOLE & $A B$ & 1p040521 & $\operatorname{sect} 7$ & \\
\hline $32+3: 12$ & BOREHOLE & $4 B$ & "1pot0521 & $\operatorname{sect} 7$ & \\
\hline 3241 PIPED & BOREHOLE & $4 B$ & "po-40521 & $\sec 7$ & \\
\hline 3261:RHN_AR & BOREHOLE & $4 B$ & upo+0521 & $\operatorname{sect} 7$ & \\
\hline 171 DNSFLUID & DRILLMUD & $4 B$ & "pot0521 & $\operatorname{sect} 7$ & \\
\hline $172:$ VISCO & DRRLMUD & $4 B$ & "1po40.21 & $\operatorname{sect} 7$ & \\
\hline 173 ILDSTRSS & DRULLMUD & $4 B$ & "pot0521 & $\sec 7$ & \\
\hline 651:ABSROUGH & WAS AREA & $4 B$ & "pot0521 & $\sec 7$ & \\
\hline
\end{tabular}

The following list of parameters in Table 2 are the nu paramclers neccssar to run CCDFGF. The tables in "Analysis Pacliage for the CCDF Construcion (Tasks 7) of the Performance Assessment Analyscs Supponing the Compliance Cenifiation Application 11POت̈ $40524^{\circ}$ lists a variable name for the parameter id. The jollowing table correlates the Ingres id:idpram:idmtrl name and the variable name used by CCDFGF for the paramelers stored in the WIPP CCA databass.

Tabic 2

\begin{tabular}{|c|c|c|c|c|c|c|c|c|}
\hline $\begin{array}{l}i d: \\
\cdots \\
\cdots \\
3\end{array}$ & idpiamy & miand & mot & aralisis nriteup & $\begin{array}{l}\text { inpuit } \\
\text { variable } \\
\text { name }\end{array}$ & 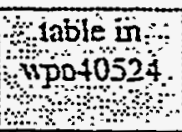 & $\begin{array}{l}\text { inpur tariable } \\
\text { name } \\
\text { name }\end{array}$ & $\begin{array}{l}\text { table in } \\
\text { mpot0524 } \\
\therefore \cdots . .:\end{array}$ \\
\hline 3501 & FPICD & GLOBAL & $A B$ & "pot0s24 & SFDR & $\begin{array}{c}\text { table A1 } 1.42 \\
+3\end{array}$ & SDBV:-4L(16) & whle Ad \\
\hline 3500 & FPICNI & GLOB.LL & $A B$ & "ppo-10524 & SFAIR & 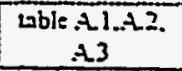 & SDBISU(1S) & wble AtA \\
\hline $349 !$ & & jGLOB.LL & $A B$ & "potos24 & ACTI & $\begin{array}{c}\text { wable }+11 .+22 \\
A 3\end{array}$ & SDBIAL(4) & whl: At \\
\hline $3498:$ & TPJC.I & GLOS.IL & $4 B$ & "pot0s24 & TPICMI & $\begin{array}{c}\text { wable Al. } 22 \\
+3\end{array}$ & SDBl:내(13) & bahle atd \\
\hline 3503 & ABERAI & REFCON & $A B$ & "po40 24 & $\triangle B E R M$ & $\begin{array}{c}\text { able Allat?. } \\
+3\end{array}$ & SDIST:U(18) & Lable otf \\
\hline 3489 & AREA_CH & REFCON & $A B$ & "ppet0s24 & AREA_CII & & SDBV: $44(2)$ & Lable At \\
\hline $3490^{\circ}$ & AREA_RH & jREFCON & $A B$ & "pet0s24 & AREA_RH & & SDBI:AE(11) & whle At \\
\hline 3.88 & APEA_ZRO & REFCON & $A B$ & "potos24 & AREA_ZRO & & SDBV:AL(1) & wable Ad \\
\hline 3497 & FTW & REFCON & $4 B$ & "pot0524 & Fา" & & SDIVALS) & wable At \\
\hline 3507 & IIRH & REFCON & $4 B$ & "Tpe-10524 & HRH & tabis AS & SDBI:AL (17) & tuble Ald \\
\hline 3490 & TOLWP & REFCON & $A B$ & "1potos24 & TOLII' & & SDBL:L4(3) & whle its \\
\hline
\end{tabular}

Disıribution:

MS 132S Hong-Nian Jow, 6848

MS 1328 Martin S. Tiemcy, 6848

MIS 1324 S. Howanh. 6115

MS $1.322 \mathrm{~J}$. Holmes. 6832

IVBS: 1.2.07.1.2:PDD:QA:GENERAL 


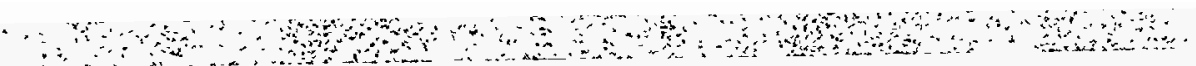

Page intentionally blank

E-18 


\section{Appendix F}

\section{Legacy and Placeholder Parameter}

Cross Reference Memoranda 
Page intentionally blank 
date: June 17, 1996

Abuquerque, New Mesico 87185

10: File

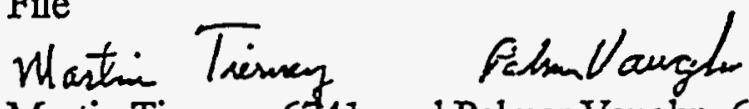

subject Designation of "Legacy Parameters" and "Placeholders" in the WIPP Parameter Database

A "legacy parameter" is a parameter which appeared in the baseline WIPP Parameter Data Base of March 2, 1996 (version desc_0000, values_0000) and was not subsequently modified up to the date of this memo; consequently, Form $464 \mathrm{~s}$ have not been prepared for these parameters, which are identified on Attachment $A$. There are numerous sources for these parameters (e.g. the 1992 PA [SAND92-0700/3] or the SPM2 [SAND95-2017/2]), but the majority of them can be considered as the choice of the PA analyst. All legacy parameters on this list have been checked by the appropriate PA analyst (Palmer Vaughn or his representatives) and have been found to be acceptable as model-configuration parameters (Category 4B).

A "placeholder parameter" is a parameter which, strictly speaking, should be inactive or not used (Category 5) in the WIPP Parameter Database but, to facilitate the correct reading of all parameters for BRAGFLO from the SCMS files, has been allowed to remain in WIPP Parameter Data Base as an inert place holder. A list of placeholder parameters is also shown on Attachment $A$; these parameters have been designated as Category $4 B$ parameters.

Distribution:

MS 1328 Hong-Nian Jow

MS 1328 Amy Johnson

MS 1328 Mary-Alena Martel

WBS: 1.2.07.1.2:PDD:QA:GENERAL 
Attachment A to Memo to File dated 6/17/96

Page 1 of 3

The following parameters are designated "legacy parameters":

\begin{tabular}{|c|c|c|}
\hline id number & idmtrl & idpram \\
\hline 50 & BRINESAL & REF_PRES \\
\hline 51 & BRINESAL & REF_TEMP \\
\hline 60 & CASTILER & CAP_MOD \\
\hline 62 & CASTILER & PC_ $\bar{M} A X$ \\
\hline 2609 & CASTLER & PCI_A \\
\hline 2610 & CASTILER & PCT_EXP \\
\hline 63 & CASTILER & PORE_DIS \\
\hline 72 & CASTILER & RELP_MOD \\
\hline 73 & CASTILER & SAT_IBRN \\
\hline 74 & CASTILER & SAT_RBRN \\
\hline 75 & CASTILER & SAT_RGAS \\
\hline 148 & CULEBRA & RELP_MOD \\
\hline 149 & CULEBRA & SAT_IBRN \\
\hline 154 & DEWYLAKE & COMP_RCK \\
\hline 166 & DEWYLAKE & RELP_MOD \\
\hline 168 & DEWYLAKE & SAT_IBRN \\
\hline 171 & DRILLUD & DNSFLUID \\
\hline 172 & DRILLMUD & VISCO \\
\hline 173 & DRILLMUD & YLDSTRSS \\
\hline 176 & DRZ_0 & PC_MAX \\
\hline 2702 & DRZ_0 & PCT-A \\
\hline 2703 & DRZ_0 & PCT_EXP \\
\hline 181 & $D R Z O 0$ & PRMX_LOG \\
\hline 182 & DRZ_O & PRMY_LOG \\
\hline 183 & DRZ_O & PRMZ_LOG \\
\hline 186 & DRZ_0 & REIP_MOD \\
\hline 187 & DRZ_0 & SAT_īBRN \\
\hline 193 & DRZ_1 & PC_M̄AX \\
\hline 198 & DRZ_1 & PRMX_LOG \\
\hline 199 & DRZ_1 & PRMY_LOG \\
\hline 200 & DRZ_1 & PRMZ_LOG \\
\hline 205 & $\mathrm{DRZ} 1$ & SAT_RBRN \\
\hline 206 & $\mathrm{DRZ}_{-1}$ & SAT_RGAS \\
\hline 209 & EXP_AREA & PC_MAX \\
\hline 2712 & EXP_AREA & PCT_A \\
\hline 2713 & EXP_AREA & PCT_EXP \\
\hline 210 & EXP_AREA & PORE_DIS \\
\hline 213 & EXP_AREA & PRESSURE \\
\hline 219 & EXP_AREA & RELP_MOD \\
\hline 225 & GLOBAL & TRANSIDX \\
\hline 540 & S_HALITE & CAP_MOD \\
\hline 542 & S_HALITE & PC_M MAX \\
\hline $5+3$ & S_HALITE & PORE_DIS \\
\hline 553 & S_HALITE & REIP_MOD \\
\hline 554 & S_HALITE & SAT_IBRN \\
\hline 555 & S_HALITE & SAT_RBRN \\
\hline 556 & S_HALITE & SAT_RGAS \\
\hline
\end{tabular}


The following parameters are designated "legacy parameters":

\begin{tabular}{|c|c|c|}
\hline id number & idmtrl & idpram \\
\hline 337 & SANTAROS & COMP_RCK \\
\hline 341 & SANTAROS & POROSITY \\
\hline 344 & SANTAROS & PRMX_LOG \\
\hline 345 & SANTAROS & PRMY_LOG \\
\hline 346 & SANTAROS & PRMZ_LOG \\
\hline 349 & SANTAROS & RELP_MOD \\
\hline 658 & WAS_AREA & PC_MAX \\
\hline 662 & WAS_AREA & PRĒSSURE \\
\hline 2231 & WAS_AREA & SAT_WICK \\
\hline
\end{tabular}

The following parameters are designated "placeholder parameters":

$\begin{array}{rll}\text { id number } & \text { idmtrl } & \text { idpram } \\ 27 & \text { BOREHOLE } & \text { DOMEGA } \\ 33 & \text { BOREHOLE } & \text { PRESSURE } \\ 34 & \text { BOREHOLE } & \text { PRMX_LOG } \\ 35 & \text { BOREHOLE } & \text { PRMY_LOG } \\ 36 & \text { BOREHOLE } & \text { PRMZ_LOG } \\ 38 & \text { BOREHOLE } & \text { PTINDEX } \\ 2085 & \text { FORTYNIN } & \text { CAP_MOD } \\ 2238 & \text { FORTYNIN } & \text { COMP_RCK } \\ 2239 & \text { FORTYNIN } & \text { PC_MAX } \\ 2716 & \text { FORTYNIN } & \text { PCT_A } \\ 2717 & \text { FORTYNIN } & \text { PCT_EXP } \\ 2087 & \text { FORTYNIN } & \text { PORE_DIS } \\ 2089 & \text { FORTYNIN } & \text { PRMX } \\ 2090 & \text { FORTYNIN } & \text { PRMY_. } \\ 2091 & \text { FORTYNIN } & \text { PRMZ } \\ 2093 & \text { FORTYNIN } & \text { RELP_MOD } \\ 2240 & \text { FORTYNIN } & \text { SAT_RBRN } \\ 2094 & \text { FORTYNIN } & \text { SAT_RGAS } \\ 229 & \text { IMPERM_Z } & \text { CAP_MOD } \\ 230 & \text { IMPERM_Z } & \text { COMP_RCK } \\ 231 & \text { IMPERM_Z } & \text { PC_MAX } \\ 2721 & \text { IMPERM_Z } & \text { PCT_A } \\ 2722 & \text { IMPERM_Z } & \text { PCT_EXP } \\ 233 & \text { IMPERM_Z } & \text { POROSITY } \\ 241 & \text { IMPERM_Z } & \text { RELP_MOD } \\ 243 & \text { IMPERM_Z } & \text { SAT_RBRN } \\ 244 & \text { MPERMZZ } & \text { SAT_RGAS } \\ 2183 & \text { TAMARISK } & \text { CAP_MOD } \\ 2243 & \text { TAMARISK } & \text { COMP_RCK } \\ & & \end{array}$




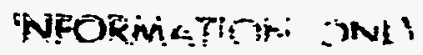

Attachment A to Memo to File dated 6/17/96

Page 3 of 3

The following parameters are designated "placeholder parameters":

$\begin{array}{lll}\text { id number } & \text { idmtrl } & \text { idpram } \\ 2244 & \text { TAMARISK } & \text { PC_MAX } \\ 2794 & \text { TAMARISK } & \text { PCT_A } \\ 2795 & \text { TAMARISK } & \text { PCT_EXP } \\ 2185 & \text { TAMARISK } & \text { PORE_DIS } \\ 2191 & \text { TAMARISK } & \text { REIP_MOD } \\ 2245 & \text { TAMARISK } & \text { SAT_RBRN } \\ 2192 & \text { TAMARISK } & \text { SAT_RGAS } \\ 2217 & \text { UNNAMED } & \text { CAP_MOD } \\ 2218 & \text { UNNAMED } & \text { COMP_RCK } \\ 2247 & \text { UNNAMED } & \text { PC_MAX } \\ 2800 & \text { UNNAMED } & \text { PCT_A } \\ 2801 & \text { UNNAMED } & \text { PCT_EXP } \\ 2219 & \text { UNNAMED } & \text { PORE_DIS } \\ 2225 & \text { UNNAMED } & \text { REIP_MOD } \\ 2248 & \text { UNNAMED } & \text { SAT_RBRN } \\ 2226 & \text { UNNAMED } & \text { SAT_RGAS }\end{array}$



"Legacy Parameters" and "Placeholders" in the WIPP Parameter Database

This memo is sent to clarifi- information in the subject memo and to provide additional information on some Legacy and Placeholder parameters not included in the subject memo. This addenda to WPO 38568 expands the legacy and placeholder parameter list for BRAGFLO and includes legarg parameters used by NUTS. PANEL, CUTTINGS_S, and SECO_TP.

Several clarifications or corrections are necessary for the firs paragraph of the subject memo. The IVIPP Parameter Baseline Data Base of March 2 mas in 1995 rather than 1996. Legacy parameters are parameters which existed in the baseline WIPP Performance Assessment (PA) Database of January 1996 prior to the effective date of QAP 9-2 "Quality Assurance Requirements for the Selection and Documentation of Parameter Values Used in WIPP Performance Assessment". The subject memo states that values were not ..." subsequently modified up to the date of this memo;..." when in fact legacr parameter values are never modified from the original entry and no new information or data is added The phrase -(version desc_0000, values_0000)" should be replaced with "tro tables in WIPP_DB with the baseline being contained in NMVP_DB." In addition, at the time of issuance of the subject memo, 4645 had not been prepared for the parameters listed, however, they were prepared subsequent to the issuance of the memo. All legacy parameters now have $464 \mathrm{~s}$ which contain information regarding sources.

Legacy parameters will now include all parameters that did not have a value change for the CCA Calculation. Therefore, all 'Legacy' parameters will have entry dates placed prior to 1996.

A 'placeholder parameter' is a parameter whose value is not used, but is left in the database to facilitate the correct reading of all parameters for BRAGFLO from the SCMS files. The method by which MATSET retrieves data requires an unchanging sequential ordering of data. Therefore once a parameter is entered into a block of data, it must remain in its original place, whether used or not in order not to alter the sequence of the data as it is read by the MATSET software.

The following list of parameters are separated into tables mith a short heading explaining the use and the potential code. The "Analysis write-up" WPO number is included and may be from either the PA or PI. There are several category ' 1 ' parameters that fall under the new 'Legact" definition. These categor: -1 ' parameters have the PI package WPO listed The June 17, 1996 listing is from the subject memo, and the list of "additional" parameters is being added for completeness as not all were included in the June 17 memo.

\begin{tabular}{|c|c|c|c|c|c|}
\hline \multicolumn{6}{|c|}{ 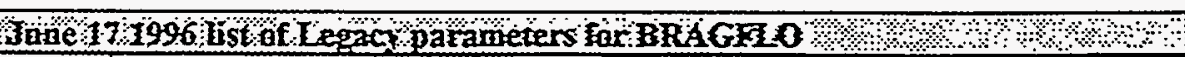 } \\
\hline id & idmtrl & idpram & impt & $\begin{array}{c}\text { Analysis } \\
\text { nriteup/ or }\end{array}$ & $\begin{array}{c}\text { Section } \\
\text { \#/page }\end{array}$ \\
\hline & BRNESAL & REF_PRES & $4 B$ & 1HPO40514 & 4.2 .5 \\
\hline
\end{tabular}

Exceptional Service in the Netional Interest 


\begin{tabular}{|c|c|c|c|c|}
\hline SI!BRAESAL & REF_IEMP & $4 B$ & WPO40514 & 425 \\
\hline 60:CASTILER & CAP_MOD & $4 B$ & HPO40514 & 4.25 \\
\hline 62)CASTIIER & IPC_MAX & 48 & RPO40514 & 4.25 \\
\hline 2609:CASTILER & PCT_A & $4 B$ & KPO40514 & $\$ 4.2 .5$ \\
\hline 2610|CASTILER & PCT_EXP & $4 \mathrm{~B}$ & HPO 40514 & 4.2 .5 \\
\hline 63!CASTILER & PORE_DIS & $4 B$ & WPO40514 & 425 \\
\hline 72|C.ASTILER & RELP_MOD & $4 B$ & WPO40514 & 4.2 .5 \\
\hline 73!CASTILER & SAT_IBRR & $4 B$ & WPO40514 & 4.25 \\
\hline 74/CASTILER & SAT_RBRI & $4 B$ & WPO40514 & 4.2 .5 \\
\hline 75 CASTIIER & SAT_RGAS & $4 B$ & WPO40514 & 42.5 \\
\hline 148 CULEBRA & IRELP_MOD & $4 B$ & KPO40514 & 4.2 .5 \\
\hline 149 CULEBRA & ISAT_IBRA & $4 \mathrm{~B}$ & HPO40514 & 4.25 \\
\hline 154!DEWTLAKE - - & COMP_RCK & $4 \mathrm{AB}$ & 16PO40514 & 42.5 \\
\hline 166!DEKYRAKE & RELP_MOD & $4 \mathrm{~B}$ & KPO40514 & 4.25 \\
\hline 168:DEHTLAKE & SAT_BRR & $4 B$ & HPO40514 & 4.25 \\
\hline $176: \mathrm{DRZ} 0$ & PC_ALAX & $4 B$ & RPO40514 & 4.2 .5 \\
\hline $2702: D R Z 0$ & PCT_A & $4 B$ & HPO40S14 & 425 \\
\hline 2703 DRZ_o & PCT_EXP & $4 B$ & WPO40S14 & 425 \\
\hline 181 DRZ_o & PRIDE_LOG & $4 B$ & HPO-40514 & 4.25 \\
\hline 182:DRZ_0 & PRIR_LOG & $4 B$ & WPO40514 & 425 \\
\hline 183!DRZ_0 & PRIIZ_LOG & $4 \mathrm{~B}$ & HPO40514 & 4.2 .5 \\
\hline 186!DRZ_0 & IRELP_MOD & $4 B$ & |WPO40514 & 3.2 .5 \\
\hline 187:DRZ_0 & ISAT_IBRS & $4 \mathrm{~B}$ & HPOO40514 & 4.2 .5 \\
\hline 193:DRZ_1 & IPC_NLAX & $4 \mathrm{~B}$ & |WPO40514 & 4.2 .5 \\
\hline 198!DRZ_1 & PRIR LOG & $4 B$ & LPO40S14 & 4.25 \\
\hline 199:̣RZ_I & PRMY_LOG & AB & HPO40514 & 42.5 \\
\hline 200 DRZ_I & PRIE_LOG & $4 B$ & WP040514 & 4.25 \\
\hline $205 ! D R Z 1$ & ISAT_RBRI & $4 B$ & HPO40514 & 4.25 \\
\hline 206!̣:DR_I & SAT_RGAS & $4 B$ & HPO40514 & 42.25 \\
\hline 209:ERP_AREA & PC_ALAX & $4 B$ & IIPO40514 & 4.2 .5 \\
\hline 2712:EX_AREA & PPCT_A & 48 & ИPO40514 & 42.5 \\
\hline 2713:ESP_AREA & IPCT_EXP & $4 B$ & 115040514 & 425 \\
\hline 210 EXP_AREA & IPORE_DIS & $4 B$ & WPOS0514 & 4.25 \\
\hline 213:EXP_AREA & PRESSURE & $4 B$ & NTO40514 & 4.25 \\
\hline 219:ESP_AREA & RELP_AOD & $4 \mathrm{~B}$ & |11PO40514 & 4.2 .5 \\
\hline 2085IFORTYNLN & ICAP_MOD & $4 B$ & IKPO40514 & 4.2 .4 \\
\hline 540!S_HALITE & CAP_MOD & $4 \mathrm{~B}$ & 117040514 & 4.2 .5 \\
\hline 542is_HALITE & PC_MLAX & 14B & IिPO40514 & 4.2 .5 \\
\hline 543 S HALTIE & PORE_DIS & $4 B$ & 12PO40514 & 4.25 \\
\hline 553/S_HALITE & RELP_MOD & $4 B$ & 1KP040514 & 42.5 \\
\hline 554!S_HALITE & SAT_IBRN & $4 \mathrm{~B}$ & HPO40514 & 42.5 \\
\hline 555|S_HALITE & SAT_RBRI & $4 B$ & WPO40514 & 4.25 \\
\hline 556|S_HALITE & SAT_RGAS & $4 B$ & KPO\$0514 & 4.2 .5 \\
\hline 337; SALTAROS & CONP_RCK & $4 B$ & WPO40514 & 4.2 .5 \\
\hline 341 SALTAROS & IPOROSTT & $4 B$ & LPO40514 & 4.2 .5 \\
\hline 3+4|SAITAROS & PRAX_LOG & $4 B$ & HPO40514 & 4.2 .5 \\
\hline 345:S.AITAROS & IPRIYYLOG & $4 \mathrm{~B}$ & |11PO40514 & 4.2 .5 \\
\hline 346:SAITAROS & PRAIZLOG & $4 \mathrm{~B}$ & |WPO40514 & 4.2 .5 \\
\hline 349|SALTAROS & RELP_MOD & $4 B$ & WPO40514 & 4.2 .5 \\
\hline 658!WAS_AREA & PC_MLAX & $4 \mathrm{~B}$ & |11P040514 & 425 \\
\hline 662|WAS_AREA & IPRESSURE & $4 B$ & WPO40514 & 4.2 .5 \\
\hline 2231!WAS_AREA & ISAT_WICK & $4 B$ & KPO40514 & 4.2 .5 \\
\hline
\end{tabular}

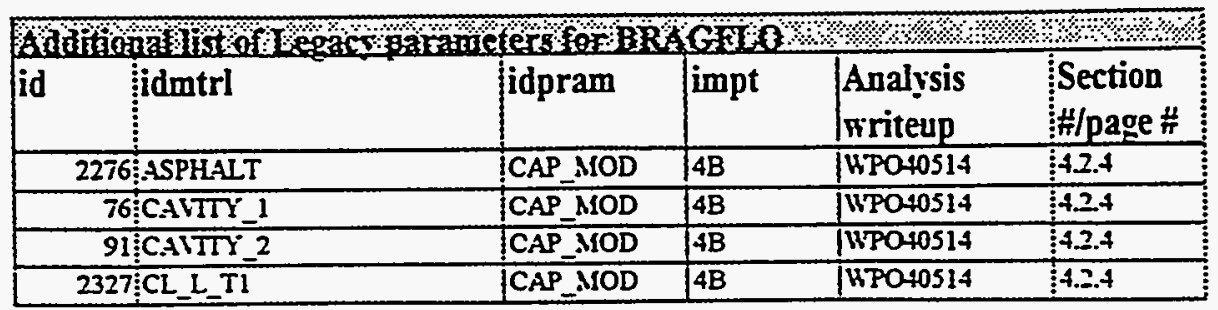




\begin{tabular}{|c|c|c|c|c|}
\hline 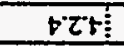 & FIsotodnl & $g+1$ & Goli dTIS: & SIIITSS: 652 \\
\hline 525 & tIsotadnI & gt & Gorर dTad! & $\$ I I T H S: \angle S L$ \\
\hline$\nabla 25$ & tIsotadnI & at) & Gorr d7ज8! & EI ITVS:0952 \\
\hline+25 & IIsotadnI & gt & gotí dTad: & IL'ITHS: \\
\hline tで! & IISOtOdnI & $g t$ & corr टा98! & TFGS $x 7$ ditgr \\
\hline เで! & tisotodnI & $y+$ & 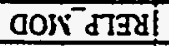 & 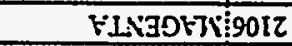 \\
\hline$t \tau 5$ & tisotadrl & $g t$ & Gojर d73d & 109 XFาว:ECEZ \\
\hline$\Delta \tau t$ & tisotadsl & gat & Golर d73d! & SL IN $70: 6 S t 2$ \\
\hline 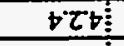 & tisotadyl & gt) & 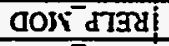 & 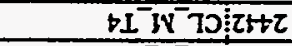 \\
\hline$t \tau t$ & tISOtOdnI & $g$ & gori d734! & {$\left[I^{-} I^{-} 70: 0+\varepsilon \Omega\right.$} \\
\hline $5 \tau t$ & tIsotadin] & $g b$ & Gorr dTId! & $\tau$ XIIAFO:EOI \\
\hline $6 \tau t$ & $\rightarrow[50 t 04 n$ & $g+1$ & ब0ir d73y & 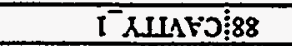 \\
\hline$t \tau t$ & tISOtOLIII & $g+1$ & Gorv d7J8) & LTHHdSY 6822 \\
\hline [Dos-yॉप्यs: & OF90EOLMI & II & DOT ZIZd! & ZI JNO0:88b2 \\
\hline 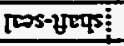 & Ot90EOdMI & I) & 507.urgd & $215 \times 00: 18+2$ \\
\hline [Ds-yers: & Ot90EOLHI & II & Don s. & $210 \times 00: 9812$ \\
\hline tで! & tISOtOLMI & $8+1$ & İ7SSI8d & $Z^{-}$XINFD:L6 \\
\hline$t \tau t !$ & tISOtOLMI & at+1 & I\&7SSI8d! & $\mathrm{I}^{-}$XIUAF) 28 \\
\hline 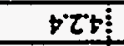 & $\rightarrow[s 0 t 04 n \mid$ & gb & LIISO\&Od! & TVIS NTd:9S2 \\
\hline$t=25$ & tISOtOLM & gb & JIISOYOd! & Z XIIAFJ:56 \\
\hline 825 & $\$$ ISOtOAn & $\mathrm{g}$ & IUSO\&Od! & I' XIИト0:08 \\
\hline $5 \tau \hbar$ & tISOtOdnI & $g+$ & sta IaOd! & $\tau$ XIInY0it6 \\
\hline $5 \tau+$ & t[SOtOLU] & $g+1$ & sta' 380d! & $I^{-}$XIIArJ:6L \\
\hline $5 \tau t$ & IISOtOdMI & gb & Throd & $\tau$ XULAFD 96 \\
\hline $52 t$ & tisotadsI & $g+$ & $d \mathrm{JIO}^{-}$ & 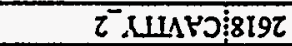 \\
\hline$t \tau t$ & ISOtOdHI & $g+1$ & FIDd! & $\tau$ ZLIAFD:LI9Z \\
\hline 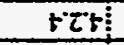 & $t$ IsotodnI & gt & 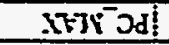 & SLITFS:E8SL \\
\hline $5 \tau 5$ & t5otadnl & $g+1$ & X7FT Od & DIITHS 9952 \\
\hline tでt! & tIsotadyl & gt & XTTK $2 d$ & EIITES $6 F S Z$ \\
\hline$t \tau t !$ & tISOtOdMI & gt & IFTrod! & ZIITHS:ZEST \\
\hline 525 & + tsotadnl & gab & xFTrod! & ILITHS:SISZ \\
\hline $5 \tau+\vdots$ & tISOtOInI & $\mathrm{g}$ & X7FY OA! & IISOdI8: $\tau+\tau \tau$ \\
\hline $5 \tau+$ & tISOtaxyl & gt & XFTY Od! & TYIS NTd:TSS \\
\hline$t c t$ & tisotadyl & get & XFTK $2 \mathrm{~d}$ & FINEDFTV:860Z \\
\hline $5 \tau+$ & tisotadx & gt & X7Tr & HILVI $3: 86+\tau$ \\
\hline $5+5$ & $\pm 150 t a d x \mid$ & gtt & X7TT Od! & IXVLLHבd:95I \\
\hline 525 & tIs0tadnl & gt) & striod! & F्य83720:LEI \\
\hline $5 \tau t$ & tIsotodnl & at) & IFTi $2 \mathrm{~d}$ & $152000 \div 59+2$ \\
\hline$t \tau t$ & t5otadnl & at & 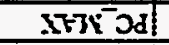 & $\left.\operatorname{LOg}^{-} x+7\right)^{\prime} 21 \Omega 2$ \\
\hline +て5! & tssotodnI & gt) & 177521 & 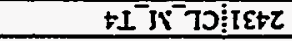 \\
\hline$t \tau t !$ & t5otosyl & grt & XTTS 24 & EIII IJ:5It2 \\
\hline 525 & tisotodul & gt & .ITTर & 21 IN 75: $\angle 6 \varepsilon 2$ \\
\hline 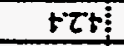 & tisotody & at) & ถรTा 2d & IIIN 70:08E2 \\
\hline $52 b i$ & fisotodul & gb & IFTK 2 & $\tau$ ¿IIIF): \\
\hline$t \tau t$ & tisotadul & gt & IFTर & I-XIII \\
\hline$t \tau t$ & t50tadn & $\mathrm{gt}$ & 37Tर $2 \mathrm{~d}$ & ITHAdST:8LZZ \\
\hline$t \tau t$ & bisotadul & gat & IdXI & 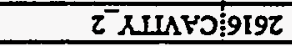 \\
\hline $5 \tau t$ & tIsotadnl & gt & YOX' dरOO| & หJaH dn]:80Z \\
\hline $5 \tau \hbar$ & bisotadyl & gt) & xor droD & = F\&g3710ว:021 \\
\hline$+\tau b !$ & olsotody & at & प्रव वर्ण & $\tau$ IIIAYO 26 \\
\hline $525 \vdots$ & bISOtOdy) & $g b \mid$ & Yर०प d & $I$ IULED:L \\
\hline$t \tau t \vdots$ & tISOtodn] & $\mathrm{gat}$ & CoNrdVOI & SI ITHS:18SL \\
\hline 5 เた & tISOtOAnI & gt & Gorर' dVDI & DIITHS:5952 \\
\hline $5 て 5$ & tsotodnl & gt & GOSN dVD! & EIITFS:LFSL \\
\hline$+\tau t$ & tIsotadyl & gat & gox dVDl & ZIITHS:OESZ \\
\hline 七で! & $+150+0 d 4$ & ar & Gosídus| & IIITHS:EISZ \\
\hline 5 tた & tISOtOdnl & gt & GOKर dVD! & FINGOFTY:L60Z \\
\hline$t \tau t$ & tisotadyl & gt: & dost dVDI & Vdg37กว:6II \\
\hline$t \tau t$ & tisotadxl & gt & aOst dVD & IOA $x \forall 70: 01 \varepsilon 2$ \\
\hline $5 \tau$ & t|sotadyl & gat & Gori dVDI & 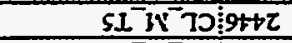 \\
\hline เてt! & tIsotadnl & gt) & Gortidvo! & $7 L^{-} \times-70 \vdots 6262$ \\
\hline
\end{tabular}




\begin{tabular}{|c|c|c|c|c|}
\hline 2510 EARTH & ISAT_IBRS & 1 & LPO30640 & 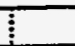 \\
\hline 265:PAN_SEAL & ISAT_RBRT & $\longdiv { 4 B }$ & RPO40514 & 4.24 \\
\hline 266 PAN_SEAL & SAT_RGAS & $4 B$ & APO40514 & 4.2 .4 \\
\hline
\end{tabular}

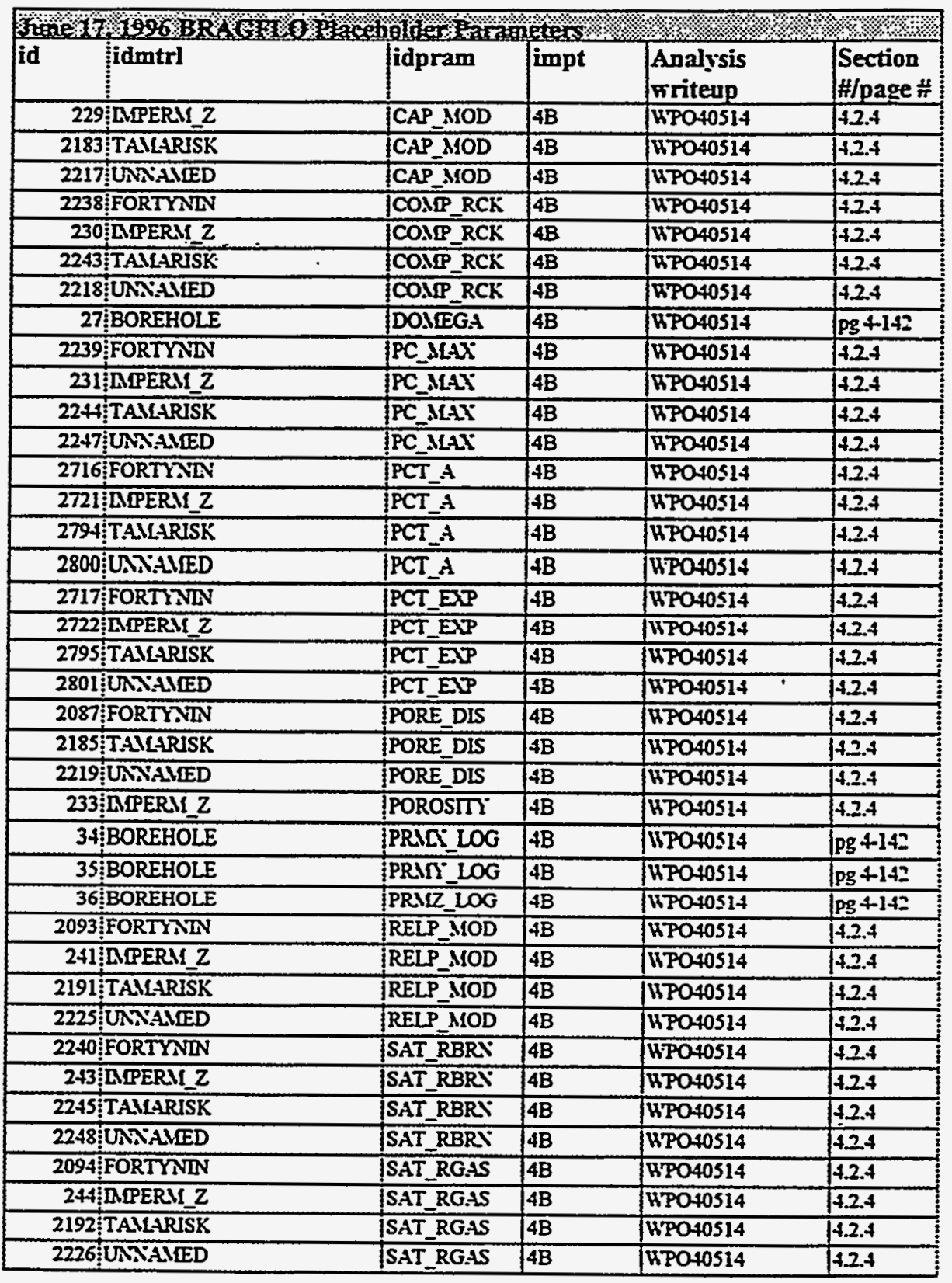

\begin{tabular}{|c|c|c|c|c|c|}
\hline \multicolumn{6}{|c|}{ 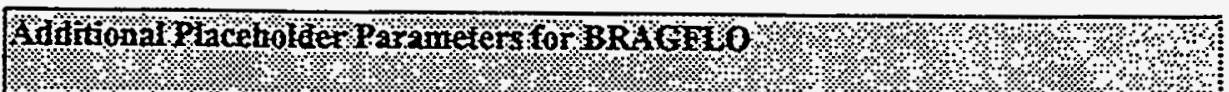 } \\
\hline id & idmtrl & idpram & impt & Analysis & Section \\
\hline & 40:BOREHOLE & RELP MO & & INPO40514 & \\
\hline & $41 \mathrm{BOR}$ & SAT R & & KPO40S14 & $\mathrm{pg} 4142$ \\
\hline & 29:BOREHOLE & PCC ALAT & $14 \mathrm{~B}$ & WPO40514 & $102+14=$ \\
\hline
\end{tabular}

\begin{tabular}{|c|c|c|c|c|c|}
\hline \multicolumn{6}{|c|}{ 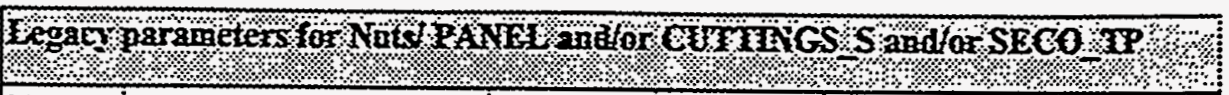 } \\
\hline id & idmtrl & idpram & impt & $\begin{array}{l}\text { Analysis } \\
\text { writeup }\end{array}$ & $\begin{array}{l}\text { Section } \\
\text { iffpage } \frac{\pi}{\pi}\end{array}$ \\
\hline & W.AS AREA & \multicolumn{2}{|c|}{ ABSROCGH $4 \mathrm{~B}$} & |KPO40521 & 38 \\
\hline
\end{tabular}




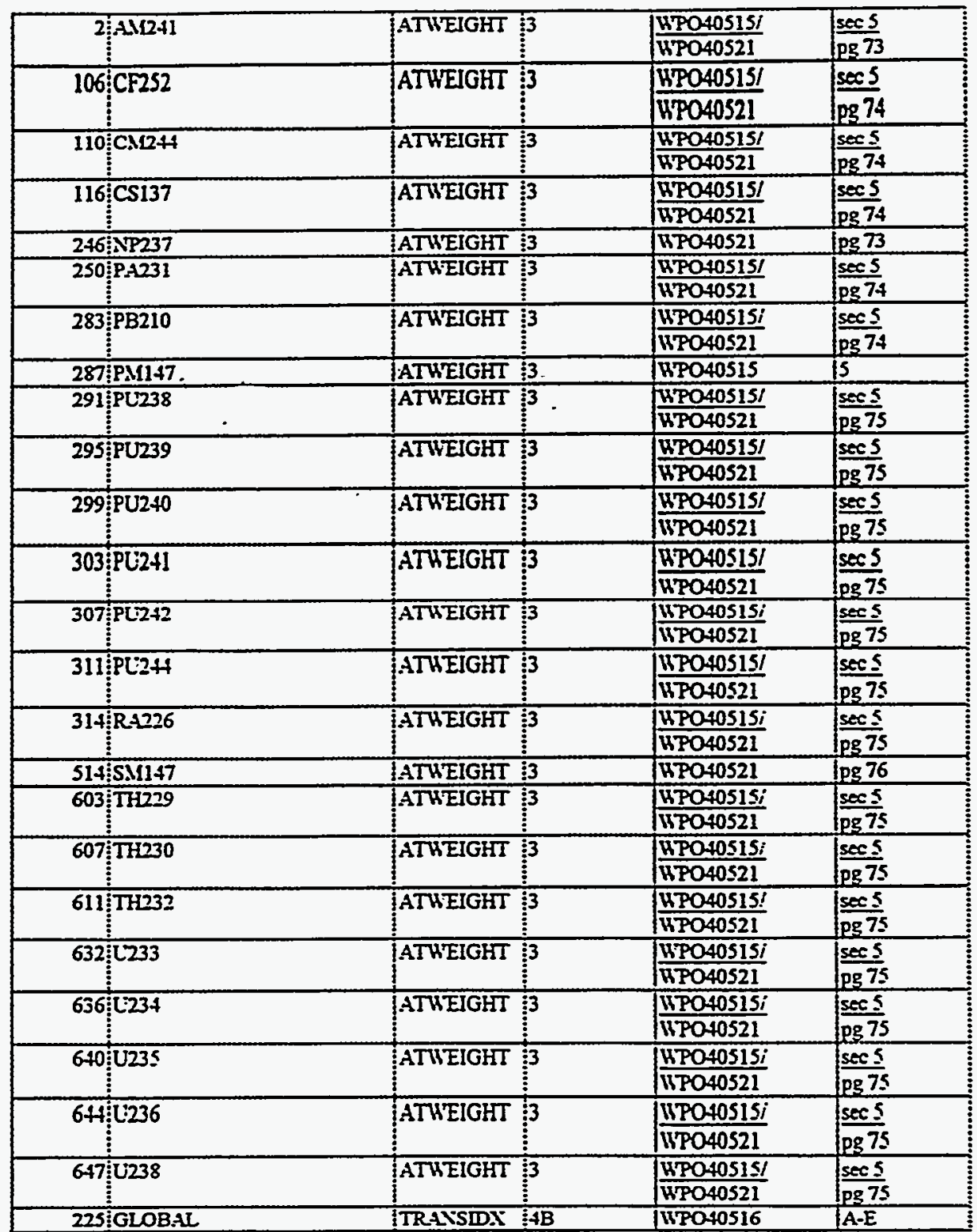

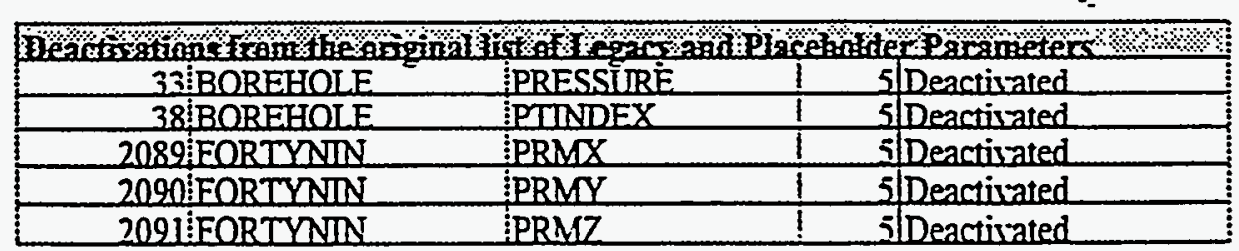

Distribution:

MS 1328 Hong-Nian Jow, 6848

MS 1328 Martin S. Tiemey. 6848

MS $132+$ S. Howarth, 6115

MS 1322 J. Holmes, 6832

WBS: 1.2.0\%.1.2:PDD:QA:GENERAL 


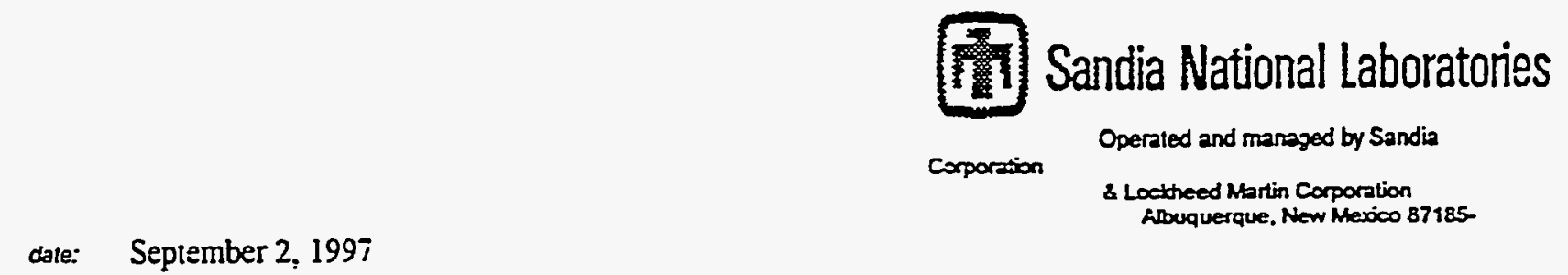

to:

Memo of Record
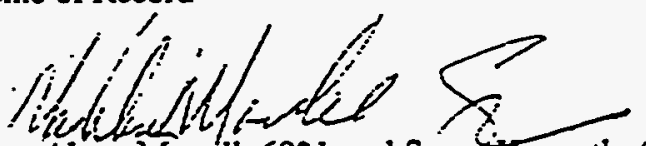

from:

Mari-Alena Martell. 6821 and Susatiriowarth, 6801

Prior to the 1996 CCA Calculation. the QAP 9-2 was not in plaa and the fót Forms for data entri were not required. As a mechanism to provide traceabling of souree information and to provide Regulators uith a complete set of 464 's for all values used in the CCAA 464 's nere completed for the 'legary parameters' and processed through formal channels. This process required tine PTI (Parameter Task Leader) to complete the 464 forms and pass them to the DBA (Database Administrator). The DBA revieried the forms. per QAP 9-4. for completeness, checked information against the controlled database entered any additional information, and submitted complete PRP's (Parameter Record Packages) to the SWCF in accordance with SAI WIPP QAP 17-1. Per QAP 94 page 13 the completed WIPP Parameter Data Entry Form Form 464, was interpreted to mean all sections completed.

An issue has been raised regarding which box to check on the Form fót for "legast" parameter (i.e. major modification, new, error correction or deactivation). WPO $38568^{1}$ (the original designation of 'Legacy Parameters' memo) was used to document the categorization of 'Legary Parameters' as modelconfiguration parameters (categor 4 B). The process for completing UTPP Data Entry Forms for legacy parameters was placed under the major modification category. QAP 9-2 page 10 section 5.3 under procedure "Note 1:The modification process described in this section snould be used for cringes that will not require an impact assessment (e.g. changes io non-numerical fielös.....". The entri- boxes on the top of the form 464 (modification, error correction, net; or dearivation) identifies from the PTL to the DBA the type of action the form represents. Major modification was checked beause the non-numerical field (Category) for the 'Legacy Parameters' required changing to $4 B$ per WPO=38568; no changes were made to parameter values or distributions of values.

Before, the 464's were completed for the 'Legacy. Parameters', the lis of legacy parameters found in the June 17, 1996 "Legacy Memorandum " (Tierney and Vaughn to File, WPO 38568) was sent to Susan Howarth (a Principal Investigator (PI) in Dept. 6115). As the lead for the PI's on the parameter process, she revieued the list with appropriate PI's. Except for the $D R Z_{-} 0$ and $D R Z_{-} 1$ permeabilities there kas no net data available for the listed Legacy. Parameters and therefore the parameters rere logically put into the $4 \mathrm{~B}$ category. The appropriate PI's were involved in documenting the DRZ_0 and DRZ_1 permeabilities. The values used in the CCA were the same as the values used in the $1992 \overline{P A}$ and not based on site specific data; hence, the PI's agreed that these parameters could logically be categorized as $4 B$ 's.

The decision to use the values consisent with the 1992 PA can be traced along lines of reasoning that PA calculations use a constant value for $D R Z$ permeabiling of $-15.0 \log \left(\mathrm{m}^{\wedge} 2\right)$, a value substantiated in the records package FEP:S6 which screened out the need for dymamic (time-rarying) porosity and permeability properies and from UPO35106 DRZ permeability has to be treared as isotropic and 


\section{WFOKHiaglori ONiLY}

assigned a value of $-15.0 \log \left(\mathrm{m}^{\wedge} 2\right)$. The following list of documents trace the project position for the values used for the $D R Z$ permeabilitr.

1) S.A.ND92-0700/3 section 2.3.1 pg. 2-11 and pg. 2-12

2) FEP: S6, "Dinamic Alterations of the Disturbed Rock Zone/Iransition Zone." SWCF:1.1.6.3:PA:NG:TSK:DR2. DR3, DR6. DR7, and S6, Sandia National Laboratories. 1996 WPO38152;

3) Afemo from K.W. Larson to M.S. Tiemey, 3/1/96 "Panel Closure Permeabilities for the 1996 CCA Calculations" WPO35106

4) T.W. Thompson. F.D. Hanson to M. G. Marietta 8/1/96, "Long-term Performance of Panel Closures" WPO 40418

5) Memo: from Kur Larson to Margaret Chu, 10/31/96, "Bringing together information on the Salado DRZ: DRZ Healing, PA Parameter Values, Record Packages, FEP's Modeling , and Scmario Development" WPO44329

6) Kurt Larson to Margaret Chu. 11/27/96, "Bringing together information on the DRZ-Lipdate" WPO42512

The following list of legacy parameters ${ }^{3}$ contains the current categor: and the original categor assigned. The process of assigning categories to parameters began in August. 1995. The process pas incomplete at the sart of Januar: 1996. Once the 464 process ras in place for the CCA Calculation in 1996. the categon was placed on the 464 form, and became an integral part of defining a parameter.

\begin{tabular}{|c|c|c|c|}
\hline idmtrl & idpram & $\begin{array}{l}\text { Category } \\
\text { assigned for } \\
\text { CCA }\end{array}$ & $\begin{array}{l}\text { old caregory as of } \\
\text { Dezmber } 1996\end{array}$ \\
\hline 2 AM241 & ATWEIGHT & 3 & 3 \\
\hline 2276 ASPHALT & CAP_MOD & $4 \mathrm{~B}$ & 4 \\
\hline 2278 ASPHALT & PC_MAX & $4 B$ & 1 \\
\hline 2289 ASPHALT & RELP_MOD & $4 B$ & 4 \\
\hline 27 BOREHOLE & DOMEGA & $4 \mathrm{~B}$ & 1 \\
\hline 29 BOREHOLE & PC_MAX & $4 \mathrm{~B}$ & 1 \\
\hline 34 BOREHOLE & PRMXX_LOG & $4 B$ & 1 \\
\hline 35 BOREHOLE & PRMY_LOG & $4 \mathrm{~B}$ & 1 \\
\hline 36 BOREHOLE & PRMZ_LOG & $4 B$ & 1 \\
\hline 40 BOREHOLE & REIP_MOD & $4 B$ & 4 \\
\hline 41 BOREHOLE & SAT_RBRN & $4 \mathrm{~A}$ & 1 \\
\hline 50 BRINESAL & REF_PRES & $4 B$ & 4 \\
\hline 51 BRINESAL & REF_TEMP & $4 B$ & 4 \\
\hline 60 CASTILER & CAP_MOD & $4 B$ & 4 \\
\hline 62 CASTIEER & PC_MAX & AB & 4 \\
\hline 2609 CASTILER & PCT_A & $4 B$ & \\
\hline 2610 CASTILER & PCT_EXP & $4 B$ & \\
\hline 63 CASTILER & PORE_DIS & $4 B$ & 4 \\
\hline 72 CASTILER & RELP_MOD & $4 B$ & 4 \\
\hline 73 CASTILER & SAT_IBRN & $4 B$ & 4 \\
\hline 74 CASTILER & SAT_RBRN & $4 B$ & 4 \\
\hline 75 CASTILER & SAT_RGAS & $4 B$ & 4 \\
\hline 76 CAVITY_1 & CAP_MOD & $4 B$ & 4 \\
\hline 77 CAVITY_I $^{-}$ & COMP_RCK & $4 B$ & 4 \\
\hline 78 CAVITY_1 & PC_MAX & $4 B$ & 4 \\
\hline 79 CAVITY_1 & PORE_DIS & $4 B$ & 4 \\
\hline
\end{tabular}


WFOKMKATIOP

ON!1

\begin{tabular}{|c|c|c|c|}
\hline 80 CAVITY_I & POROSTYY & 43 & 4 \\
\hline 82 CAVTTY_I $_{1}$ & PRESSLRE & $4 B$ & 4 \\
\hline 88 CAVITY_1 & RELP_MOD & $4 B$ & 4 \\
\hline 91 CAVTTY_? & CAP_M̄OD & $4 B$ & 4 \\
\hline 92 CAVITY_? & COMP_RCK & $4 B$ & 4 \\
\hline 2616 CAVITY_2 & KPT & 43 & \\
\hline 93 CAVITY_2 & PC_MAX & 43 & 4 \\
\hline 2617 CAVITY_2 & PCT_A & 43 & \\
\hline 2618 CAVTTY_2 & PCT_EXP & 43 & \\
\hline 96 CAVITY_2 & PO_MN & 43 & \\
\hline 94 CAVITY_2 & PORE_DIS & 43 & 4 \\
\hline 95 CAVTTY_2 $_{2}$ & POROSITY & $4 B$ & 4 \\
\hline 97 CAVITY_2 & PRESSLRE & 43 & 4 \\
\hline 103 CAVITY_2 & RELP_MOD & 43 & 4 \\
\hline 106 CF252 & ATWEIGHT & 3 & \\
\hline 2327 CL_L_TI & CAP_MOD & 43 & 4 \\
\hline 2340 CL_L_Tl & RELP_MOD & 43 & 4 \\
\hline 2380 CL_M_TI & PC_MAX & 43 & 1 \\
\hline 2397.CL_M_T2 & PC_MAX & 43 & 1 \\
\hline 2414 CL_M_T3 & PC_MAX & 43 & 1 \\
\hline 2429 CL_M_T4 & CAP_MOD & 43 & 4 \\
\hline 2431 CL_M_Tt & PC_MAX & $4 B$ & 1 \\
\hline $2442 \mathrm{CL}_{-} \mathrm{M}_{-} \mathrm{T} 4$ & RELP_AOD & 43 & 4 \\
\hline 2446 CL_M_T5 & CAP_MOD & 43 & 4 \\
\hline 2459 CL_M_T5 & RELP_MOD & 43 & 4 \\
\hline 2310 CLAY_BOT & CAP_MOD & 43 & 4 \\
\hline 2312 CLAY_BOT & PC_MAX & 43 & 1 \\
\hline 2323 CLAY_BOT & RELP_MOD & 43 & 4 \\
\hline $110 \mathrm{CM} 2+4$ & ATWEIGHT & 3 & 3 \\
\hline 2465 CONC_II & PC_MAX & $4 B$ & 1 \\
\hline 2486 CONC_T2 & PRMX_LOG & 1 & 1 \\
\hline 2487 CONC_T2 & PRMY_LOG & 1 & 1 \\
\hline 2488 CONC_T2 & PRMZ_LOG & 1 & 1 \\
\hline 116 CS137 & ATWEIGHT & 3 & 3 \\
\hline 119 CULEBRA & CAP_MOD & $4 B$ & 4 \\
\hline 120 CULEBRA & COMP_RCK & 43 & 1 \\
\hline 137 CULEBRA & PC_MAX & $4 B$ & 1 \\
\hline 148 CULEBRA & RELP_MOD & 43 & 4 \\
\hline 149 CULEBRA & SAT_IBRV & 43 & 1 \\
\hline 154 DEWYLAKE & COMP_RCK & 43 & 1 \\
\hline 156 DEWYLAKE & PC_MAX & $4 B$ & 1 \\
\hline 166 DEWYLAKE & RELP_MOD & $4 B$ & 4 \\
\hline 168 DEWYLAKE & SAT_IBRN & $4 B$ & 4 \\
\hline 176 DRZ_0 & PC_MAX & $4 B$ & 4 \\
\hline 2702 DRZ_0 & PCT_A & $4 B$ & \\
\hline 2703 DRZ_0 & PCT_EXP & $4 B$ & \\
\hline 181 DRZ_0 & PRMX_LOG & $4 B$ & 1 \\
\hline $182 \mathrm{DRZ}=0$ & PRMY_LOG & 43 & 1 \\
\hline $183 \mathrm{DRZ}, 0$ & PRMZ_LOG & +3 & 1 \\
\hline 186 DRZ_0 & RELP_MOD & 43 & \\
\hline
\end{tabular}




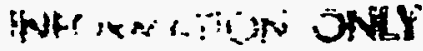

\begin{tabular}{|c|c|c|}
\hline 187 DRZ_0 & SAT_IBRN & $4 B$ \\
\hline 193 DRZ_I & PC_MAX & $4 \mathrm{~B}$ \\
\hline 198 DRZ_1 & PRMCX_LOG & $4 B$ \\
\hline $199 \mathrm{DRZ}_{-} 1$ & PRMY_LOG & $4 B$ \\
\hline $200 \mathrm{DRZ}_{-1}$ & PRMZ_LOG & $4 B$ \\
\hline $205 \mathrm{DRZ} 1$ & SAT_RBRN & $4 B$ \\
\hline $206 \mathrm{DRZ}_{-}^{-1}$ & SAT_RGAS & $4 B$ \\
\hline 2498 EARTH & PC_MAX & $4 B$ \\
\hline 2510 EARTH & SAT_IBRA & 1 \\
\hline 208 EXP_AREA & COMP_RCK & $4 B$ \\
\hline 209 EXP_AREA & PC_MAX & $\pm B$ \\
\hline 2712 EXP_AREA & PCT__A & $4 B$ \\
\hline 2713 EXP_AREA & PCT_EXP & $4 B$ \\
\hline 210 EXP_AREA & PORE_DIS & $A B$ \\
\hline 213 EXP_AREA & PRESSTLRE & $4 B$ \\
\hline 219 EXP_AREA & RELP_MOD & $4 \mathrm{~B}$ \\
\hline 2085 FORTI:NT & CAP_NOD & $\div B$ \\
\hline 2238 FORTYXIF & COMP_RCK & 43 \\
\hline 2239 FORTHZIET & PC_MAEX & 43 \\
\hline 2716 FORTIXIN & PCT_A & $4 B$ \\
\hline 2717 FORTYAIN & PCT_EXP & $4 B$ \\
\hline 2087 FORTYNIN & PORE_DIS & $4 B$ \\
\hline 2093 FORTYALN & RELP_MOD & $4 B$ \\
\hline 2240 FORTYNLN & SAT_RBRN & $4 \mathrm{~B}$ \\
\hline 2094 FORTYNTN & SAT_RGAS & $4 B$ \\
\hline 225 GLOBAL & TRANSDXX & 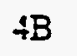 \\
\hline 229 IMPERM_Z & CAP_MOD & $4 B$ \\
\hline 230 IMPERM_Z & COMP_RCK & $4 B$ \\
\hline 231 IMPERM_Z & PC_MAX & $4 B$ \\
\hline 2721 MPERM_Z & PCT'_A & $4 B$ \\
\hline 2722 MPERM_Z & PCT_EXP & $4 B$ \\
\hline 233 IMPERM_Z & POROSTIY & $4 B$ \\
\hline 241 IMPERM_Z & RELP_MOD & $4 \mathrm{~B}$ \\
\hline 243 IMPERM_Z & SAT_RBRN & $4 \mathrm{~B}$ \\
\hline 244 IMPERM_Z & SAT_RGAS & $4 \mathrm{~B}$ \\
\hline 2097 MAGENT̄A & CAP_MOD & $4 \mathrm{~B}$ \\
\hline 2098 MAGENTA & PC_MAX & $4 B$ \\
\hline 2106 MAGENTA & RELP_MOD & $4 A$ \\
\hline 246 NP237 & ATWEIGHT & 3 \\
\hline 250 PA231 & ATWEIGHT & 3 \\
\hline 254 PAN_SEAL & PC_MAX & $4 B$ \\
\hline 256 PAN_SEAL & POROSITY & $4 B$ \\
\hline 264 PAN_SEAL & RELP_MOD & $4 B$ \\
\hline 265 PAN_SEAL & SAT_RBRN & $4 B$ \\
\hline 266 PAN_SEAL & SAT_RGAS & $4 B$ \\
\hline 283 PB210 & ATWEIGHT & 3 \\
\hline 287 PM147 & ATWEIGHT & 3 \\
\hline 291 PU238 & ATWEIGHT & 3 \\
\hline 295 PU239 & ATWEIGIT & 3 \\
\hline 299 PU240 & ATWEIGIT & 3 \\
\hline
\end{tabular}




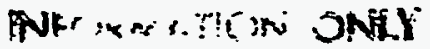

\begin{tabular}{|c|c|c|}
\hline 303 PU2+1 & ATWEIGHT & 3 \\
\hline 307 PU242 & ATWEIGHT & 3 \\
\hline 311 PU244 & ATWEIGHT & 3 \\
\hline 314 RA226 & ATWEIGHT & 3 \\
\hline 2242 REPOST & PC_MAX & $4 B$ \\
\hline 540 S_HAIITE & CAP_MOD & $4 B$ \\
\hline 542 S_HALITE & PC_MAX & $4 B$ \\
\hline 543 S_HAIIIE & PORE_DIS & $\{B$ \\
\hline 553 S_HAITE & RELP_ASOD & $4 B$ \\
\hline 554 S_HALITE & SAT_IBRV & $4 B$ \\
\hline 555 S_HALIIE & SAT_RBRN & $4 B$ \\
\hline 556 S_HALIIE & SAT_RGAS & $4 B$ \\
\hline 2513 SAIT_TI & CAP_MOD & $4 B$ \\
\hline 2515SALT_TI & PC_MAX & $4 B$ \\
\hline 2526 SALT_Tl & RELP_MOD & $4 B$ \\
\hline 2530 SALT_T2 & CAP_MOD & $\{\mathrm{B}$ \\
\hline 2532 SAII_I2 & PC_MAII & 43 \\
\hline 2547 SAIT_Tj & CAP_AOD & $4 B$ \\
\hline 2549 SALT. Ts & PC_MAI & 42 \\
\hline 2560 SALT_T3 & RELP_MOD & $4 B$ \\
\hline 2564 SALT_I4 & CAP_MOD & $4 B$ \\
\hline 2566 SALT_T4 & PC_MAX & 43 \\
\hline 2577 SALT_T4 & RELP_MOD & $4 B$ \\
\hline 2581 SALT_I5 & CAP_MOD & $4 B$ \\
\hline 2583 SALT_T5 & PC_MAX & 4B \\
\hline 2594 SALT_T5 & RELP_MOD & $4 B$ \\
\hline 337 SANTAROS & COMP_RCK & $4 B$ \\
\hline 341 SANTAROS & POROSITI & $4 B$ \\
\hline 344 SANTAROS & PRMIX_LOG & $4 B$ \\
\hline 345 SANTAROS & PRMY_LOG & $4 B$ \\
\hline 346 SANTAROS & PRMZ_LOG & $4 B$ \\
\hline 349 SANTAROS & RELP_MOD & $4 \mathrm{~B}$ \\
\hline 514 SM147 & ATWEIGHT & 3 \\
\hline 2183 TAMARISK & CAP_MOD & $4 \mathrm{~B}$ \\
\hline 2243 TAMARISK & COMP_RCK & $4 B$ \\
\hline 2244 TAMARISK & PC_MAX & $4 B$ \\
\hline 2794 TAMARISK & PCT_A & $4 B$ \\
\hline 2795 TAMIARISK & PCT_EXP & $4 B$ \\
\hline 2185 TAMLARISK & PORE_DIS & $4 B$ \\
\hline 2191 TAMARISK & RELP_MOD & $4 B$ \\
\hline 2245 TAMAARISK & SAT_RBRN & $4 B$ \\
\hline 2192 TAMARISK & SAT_RGAS & $4 B$ \\
\hline 603 TH229 & ATWEIGHT & 3 \\
\hline $607 T H 230$ & ATWEIGHT & 3 \\
\hline 611 TH232 & ATWEIGHT & 3 \\
\hline 632 U233 & ATWEIGHT & 3 \\
\hline 636 U234 & ATWEIGHT & 3 \\
\hline $640 \cup 235$ & ATREIGHT & 3 \\
\hline 644 U236 & ATWEIGHT & 3 \\
\hline 647 U238 & ATWEIGIT & 3 \\
\hline
\end{tabular}




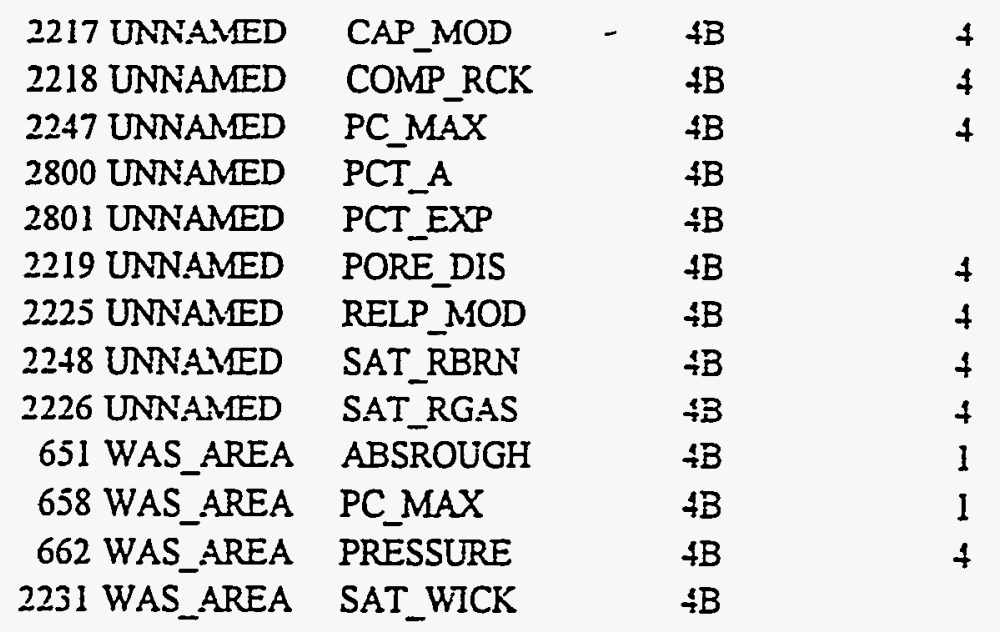

\section{Copy to:}

MS-1341 Lori J. Dotson, 6848

MS-1335 Susan Howarth 6801

MIS-1328 Mary-Alena Marell, 68:9

MS-1328 Marin S. Tierney, 6848

MS-1335 Robert Richards, 6811

SNSS1330:WBS

\footnotetext{
'Memo: Marin Tierney and Palmer Vaughn. June 17, 1996. "Designation of "Legaç Parameters: and "Placeholders" in the WIPP Parameter Database. WPO $\$ 38568$

= Ibid.

${ }^{3}$ Addenda to WPO\#38568 SNL Internal Memo: M. Tierner and P. Vaughn to File, 6/17/96, Designation of Legacy Parameters" and "Placeholders" in the WIPP Parameter Database
} 
Page intentionally blank

F-18 


\section{Appendix G}

Distribution Memoranda

G-1 
Page intentionally blank

G-2 


\section{Sandia National Laboratories}

Albuquerque, New Mexico 87185-1328

date: March 21, 1996

to: Distribution

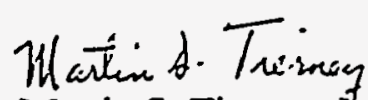

from: Martin S. Tierney, MS-1328 (6741)

subject: Distributions

Attached are discussions for some of the various types of distributions assigned to parameters used for the 1996 CCA Calculation. Each discussion outlines the appropriate use for each distribution and references are included to support its use.

The names of the distributions discussed in this memo are: COISTANT, CUMULATIVE, DELTA, NORMAL, TRIANGULAR, UNIFORM, LOGNORMAL, LOGUNIFORM.

Attachments

Distribution:

MS1324 Rick Beauheim

MS1342 Larry Brush

MS1342 Barry Butcher

MS1324 Tracey Christian-Frear

MS1324 Susan Howarth

MS1324 Al Lappin

MS1328 Amy Johnson

MS1328 Hong-Nian Jow

MS1328 Mary Alena Martell

MS1324 Lucy Meigs

MS1341 Bob Moore

MS1320 Craig Novak

MS1320 Hans Papenguth

MS1320 Malcolm Siegel

MS1341 Yifeng Wang

MS1341 Ruth Weiner

WBS:1.2.07.1.2:PDD:QA:GENERAL 


\section{Cumulative Distribution}

A Cumulative Distribution (also called a Constructed Distribution) is described by a set of $N$ ordered pairs:

$$
\left.\left(x_{1}, 0\right),\left(x_{2}, P_{2}\right),\left(x_{3}, P_{3}\right), \cdots,\left(x_{N}, 1\right) \text { \{i.e. } P_{1}=0 \text { and } P_{N}=1 \text { always }\right\}
$$

where $x_{1}<x_{2}<x_{3}<\cdots<x_{N}$ and $0<P_{2}<P_{3}<\cdots<P_{N-1}<1$

Due to the nature of the data, the probability density function (pdf) for this distribution takes the form :

$$
P(\xi)=\left\{\begin{array}{cl}
0 & \text { if } \xi<x_{1} \\
\frac{P_{n}-P_{n-1}}{x_{n}-x_{n-1}} & \text { if } x_{n-1} \leq \xi \leq x_{n}, \quad n=2,3, \cdots, N \\
0 & \text { if } \xi \geq x_{N}
\end{array}\right.
$$

and so the cumulative distribution function (CDF) takes the form:

$$
P_{n}\{X \leq \xi\} \approx \Pi(\xi)=\left\{\begin{array}{cc}
0 & \text { if } \xi<x_{1} \\
P_{n-1}+\frac{\left(P_{n}-P_{n-1}\right)\left(\xi-x_{n-1}\right)}{\left(x_{n}-x_{n-1}\right)} & \text { if } \frac{x_{n-1} \leq \xi \leq x_{x}}{n=2,3, \cdots, N} \\
1 & \text { if } \xi>x_{N}
\end{array}\right.
$$

Expected Value: $\quad E(X)=\sum_{n=2}^{v}\left(P_{n}-P_{n-1}\right) \frac{\left(x_{n}+x_{n-1}\right)}{2}$

Variance: $\quad V(X)=\sum_{n=2}^{N}\left(P_{n}-P_{n-1}\right) \frac{\left(x_{n}^{2}+x_{n} x_{n-1}+x_{n-1}^{2}\right)}{3}-\{E(X)\}^{2}$

Median: $\quad X_{0.50}=x_{m-1}+\left(x_{m}-x_{m-1}\right) \frac{\left(0.50-P_{m-1}\right)}{\left(P_{m}-P_{m-1}\right)}$ where $P_{m-1} \leq 0.50<P_{m}$.

When use of the cumulative distribution is appropriate:

The cumulative distribution takes its name from the fact that it closely resembles the empirical cumulative distribution function(Blom 1989; pg. 216) obtained by plotting the empirical percentiles of the data set $\left(x_{1}, x_{2}, x_{3}, \ldots, x_{N}\right)$. Usually, the cumulative distribution (in the sense used here) is the result of plotting the subjectively determined percentile points $\left(x_{1}, P_{1}\right),\left(x_{2}, P_{2}\right),\left(x_{3}, P_{3}\right) \ldots$, that arise in a formal elicitation of expert opinion concerning the form of the distribution of the parameter in question. Also, a simple form of the cumulative distribution is appropriately used when the range $[a, c]$ of the parameter is known and the analyst believes that his or her "best estimate" value, $b$, is also the median (or $50^{\text {th }}$ percentile) of the unknown distribution. In this case, the subjectively determined percentile points take the form: $(a, 0.0),(b, 0.5),(c, 1.0)$.

The cumulative distribution is the Maximum Entropy distribution associated with a set of percentile points $\left(x_{1}, P_{1}\right),\left(x_{2}, P_{2}\right), \ldots,\left(x_{N}, P_{N}\right)$, no matter how that set of percentile points is obtained (i.e. independent of whether the points are empirically or subjectively derived). 


\section{WFORMATICIr: Sr:!}

References for Cumulative Distribution:

Blom, Gunnar. 1989. Probability and Statistics: Theory and Applications. New York: SpringerVerlag.

Tiemey, Martin. 1990. Constructing Probability Distributions of Uncertain Variables in Models of the Performance of the Waste Isolation Pilot Plant: The 1990 Performance Simulations. SAND902510. Albuquerque, NM: Sandia National Laboratories 


\section{Logcumulative Distribution}

In this case, the independent variable is $Y$, where $Y=\log X$. As with the cumulative data, this distribution is described by a set of $\mathrm{N}$ ordered pairs:

$$
\left(y_{1}, 0\right),\left(y_{2}, P_{2}\right),\left(y_{3}, P_{3}\right), \cdots,\left(y_{N}, 1\right) \text { \{i.e. } P_{1}=0 \text { and } P_{N}=1 \text { alwan:s }
$$

where $y_{1}<y_{2}<y_{3}<\cdots<y_{N}$ and $0<P_{2}<P_{3}<\cdots<P_{N-1}<1$

Due to the nature of the data, the probability density function (pdf) for this distribution takes the form:

$$
P(\xi)=\left\{\begin{array}{cc}
0 & \text { if } \xi<x_{1} \\
\frac{P_{n}-P_{n-1}}{\ln x_{n}-\ln x_{n-1}} \frac{1}{\xi} & \text { if } x_{n-1} \leq \xi \leq x_{n}, \quad n=2,3, \cdots, N \\
0 & \text { if } \xi \geq x_{N} .
\end{array}\right.
$$

and so the cumulative distribution function (CDF) takes the form:

$$
P_{n}\{X \leq \xi\} \approx \Pi(\xi)=\left\{\begin{array}{cc}
0 & \text { if } \xi<x_{1} \\
P_{n-1}+\frac{\left(P_{n}-P_{n-1}\right)\left(\ln \xi-\ln x_{n-1}\right)}{\left(\ln x_{n}-\ln x_{n-1}\right)} & \text { if } \frac{x_{n-1} \leq \xi \leq x_{x}}{n=2,3, \cdots, N} \\
1 & \text { if } \xi>x_{x}
\end{array}\right.
$$

Expected Value: $E(X)=\sum_{n=2}^{N}\left(P_{n}-P_{n-1}\right) \frac{\left(x_{n}-x_{n-1}\right)}{\ln x_{n}-\ln x_{n-1}}$

Variance: $\quad V(X)=\sum_{n=2}^{N} \frac{1}{2}\left(P_{n}-P_{n-1}\right) \frac{\left(x_{n}^{2}-x_{n-1}^{2}\right)}{\left(\ln x_{n}-\ln x_{n-1}\right)}-\{E(X)\}^{2}$

Median: $\quad X_{0.50}=10^{\wedge}\left\{x_{m-1}+\left(x_{m}-x_{m-1}\right) \frac{\left(0.50-P_{m-1}\right)}{\left(P_{m}-P_{m-1}\right)}\right\}$ where $P_{m-1} \leq 0.50<P_{m}$. 
The Delta Distribution is used to assign probabilities to the elements of some finite set. For example, if the finite set consists of four altemative mathematical models of some phenomena and each model is labelled with one of the integers $\{1,2,3,4\}$, in other words,

$$
M_{1}, M_{2}, M_{3}, M_{4}
$$

then we might assign the vector of probabilities $\left(p_{1}, p_{2}, p_{3}, p_{4}\right)$, where each $p_{1}$ is a number between 0 and 1 and

$$
p_{1}+p_{2}+p_{3}+p_{4}=1
$$

The cumulative distribution function (CDF) associated with this Delta Distribution can be symbolically expressed by

$$
F(x)=\sum_{n=1}^{4} p_{n} u(x-n) .
$$

The graph of this CDF can be visualized as an ascending "staircase" starting at zero level for $x$ less than one, and having "steps" of height $p_{n}$ at the points $x=n, n=1,2,3,4$.

The notion of mean value and variance still apply to a Delta Distribution but the meanings of these quantities may require careful interpretation. For the example of the four altemative mathematical models, the "mean" mathematical model is the linear combination

$$
\bar{M}=\sum_{n=1}^{4} p_{n} M_{n}
$$

and the "variance" of the models is similarly defined:

$$
\Sigma^{2}=\sum_{n=1}^{4} p_{n}\left(\bar{M}-M_{n}\right)^{2}
$$

The notion of median value is usually meaningless for a Delta Distribution. 


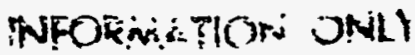

\section{Normal Distribution}

Density function :

$$
f(x)=\frac{1}{\sigma \sqrt{2 \pi}} \exp \left\{\frac{-(x-\mu)^{2}}{2 \sigma^{2}}\right\} \quad-\infty<x<\infty
$$

Distribution function:

$$
F(x)=\int_{-\infty}^{x} f(t) d t \quad-\infty<x<\infty
$$

Expected value and variance: $E(X)=\mu$ and $V(X)=\sigma^{2}$.

This program employs a truncated normal distribution where data is concentrated within an interval (lowrange, hirange). The parameters of the truncated distribution can be expressed as follows:

$$
E(X)=\mu=\frac{\text { (lowrange }+ \text { hirange })}{2} \quad V(X)=\sigma^{2}=\left(\frac{\text { hirange }- \text { lowrange }}{6.18}\right)^{2}
$$

Median $=$ mean $(u)$ and lowrange $=0.01$ quantile, hirange $=0.99$ quantile. The range of the random variable is arbitrarily set to (lowrange, hirange). Alternatively, the expected value $\mu$ and the standard deviation $\sigma$ can be specified by the user of this distribution; in this case, the random variable takes on the range $(-\infty, \infty)$.

When use of the normal distribution is appropriate:

Use of the normal distribution is appropriate whenever it is known that the parameter is the sum of independent, identically-distributed random variables (this is seldom the case in practice) and there are a sufficient number of measurements of the parameter $(N>10)$ to make accurate, unbiassed estimates of the mean $(\mu)$ and variance $\left(\sigma^{2}\right)$.

\section{References:}

Tiemey, Martin. 1990. Constructing Probability Distributions of Uncertain Variables in Models of the Performance of the Waste Isolation Pilot Plant: The 1990 Performance Simulations. SAND902510. Albuquerque, NM: Sandia National Laboratories

Sandia WIPP Project. 1992. Preliminary Pefformance Assessment for the Waste Isolation Pilot Plant, December 1992. Volume 3: Model Parameters. SAND92-0700/3. Albuquerque, NM: Sandia National Laboratories. (see sections 1.2.1 to 1.2.7) 


\section{Trianqular Distribution}

Density Function: $\quad \begin{aligned} f(x) & =\frac{2(x-a)}{(c-a)(b-a)} & & a \leq x \leq b \\ & =\frac{2(c-x)}{(c-a)(c-b)} & & b \leq x \leq c\end{aligned}$

Distribution Function: $F(x)=\frac{(x-a)^{2}}{(c-a)(b-a)} \quad a \leq x \leq b$

$$
=\frac{b-a}{c-a}-\frac{(x+b-2 c)(x-b)}{(c-a)(c-b)} \quad b \leq x \leq c
$$

Expected Value: $\quad E(X)=\frac{a+b+c}{3}$

Variance:

$$
V(X)=\frac{a(a-b)+b(b-c)+c(c-a)}{18}
$$

Median:

$$
\begin{aligned}
X_{0.5} & =a+\sqrt{\frac{(c-a)(b-a)}{2}} & & \text { if } b \geq \frac{a+c}{2} \\
& =c-\sqrt{\frac{(c-b)(c-a)}{2}} & & \text { if } b \leq \frac{a+c}{2}
\end{aligned}
$$

The Triangular Distribution is defined on the range $[a, c]$ and has mode $b$. The mode can equal either of the two boundary values, which may simplify the computations above.

When use of the triangular distribution is appropriate:

Use of the triangular distribution is appropriate when the range, $[a, c]$, of the parameter is known and the analyst believes that his or her "best estimate" value, $b$, is also the mode (or most probable value) of the unknown distribution.

References:

Iman, R.L. and M.J. Shortencarier. 1984. A FORTRAN 77 Program and User's Guide for the Generation of Latin Hypercube and Random Samples for. Use With Computer Models.

SAND83-2365. Albuquerque, NM: Sandia National Laboratories. 


\section{$\underline{\text { Uniform Distribution }}$}

$\begin{array}{llr}\text { Density Function: } & f(x)=\frac{1}{B-A} & A \leq x \leq B \\ \text { Distribution Function: } & F(x)=\frac{x-A}{B-A} & A \leq x \leq B \\ \text { Expected value and variance: } & E(X)=\frac{A+B}{2} & V(X)=\frac{(B-A)^{2}}{12} \\ \text { Median: } & X_{0.5}=\text { mean } & \end{array}$

The program requires the endpoints $A$ and $B$.

When use of the uniform distribution is appropriate:

Use of the uniform distribution is appropriate when all that is known about a parameter is its range $(A, B)$; the uniform distribution is the Maximum Entropy distribution under these circumstances.

References:

Tiemey, Martin. 1990. Constructing Probability Distributions of Uncertain Variables in Models of the Performance of the Waste Isolation Pilot Plant: The 1990 Performance Simulations. SAND902510. Albuquerque, NM: Sandia National Laboratories 


\section{Lognormal Distribution}

If $X \sim N\left(\mu, \sigma^{2}\right)$ and $Y=e^{x}$, then $Y$ has a lognomal distribution.

Density function: $\quad f(y)=\frac{1}{y \sigma \sqrt{2 \pi}} \exp \left\{\frac{-(\ln \mathrm{y}-\mu)^{2}}{2 \sigma^{2}}\right\} \quad \mathrm{y}>0$

Distribution function: $\quad F(x)=\int_{0}^{y} f(t) d t \quad y>0$

Expected value and variance: $E(Y)=\exp \left(\mu+\frac{\sigma^{2}}{2}\right) \quad V(Y)=\exp \left(2 \mu \div \sigma^{2}\right)\left[\exp \left(\sigma^{2}\right)-1\right]$ Median : $\quad X_{0.5}=e^{\mu}$

As with the normal distribution, the lognormal distribution requires lowrange and hirange values. These values are in logarithmic form and are utilized in a nomal distribution to determine a mean $(\mu)$ and a variance $\left(\sigma^{2}\right)$, which in tum are used to identify the expected value and variance for the lognormal distribution. 


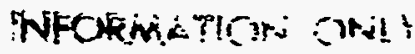

\section{Loguniform Distribution}

If $X$ has a loguniform distribution on the interval from $A$ to $B$ where $B>A>0$, then $Y=\log _{10} X$ has a uniform distribution from $\log _{10} A$ to $\log _{10} B$.

$\begin{array}{lll}\text { Density Function: } & f(x)=\frac{1}{x}(\ln B-\ln A) & \mathrm{A}<\mathrm{x}<\mathrm{B} \\ \text { Distribution Function : } & F(x)=\frac{\ln x-\ln A}{\ln B-\ln A} & \mathrm{~A}<\mathrm{x}<\mathrm{B}\end{array}$

Expected Value: $\quad E(X)=\frac{B-A}{\ln B-\ln A}$

Variance:

$$
V(X)=(B-A)\left[\frac{(\ln B-\ln A)(B+A)-2(B-A)}{2(\ln B-\ln A)^{2}}\right]
$$

Median:

$$
X_{0.5}=\sqrt{A B}
$$

When use of the loguniform distribution is appropriate:

Use of the loguniform distribution is appropriate when all that is known about a parameter is its range $(A, B)$ and $B / A \gg 10$; that is, the range $(A, B)$ spans many orders of magnitude.

\section{References:}

Iman, R.L. and M.J. Shortencarier. 1984. A FORTRAN 77 Program and User's Guide for the Generation of Latin Hypercube and Random Samples for Use With Computer Models.

SAND83-2365. Albuquerque, NM: Sandia National Laboratories. 
date: April 4, 1996

to: Distribution

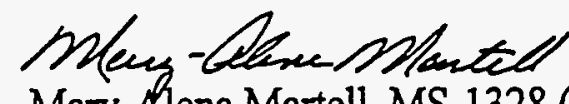

trom: Mary-Alena Martell, MS-1328 (6849)

subject Student-T Distribution

Attached is the discussion for Student-T distribution assigned to parameters used for the 1996 CCA Calculation. The discussion outlines the appropriate use for the distribution and references are included to support its use.
Attachments
Distribution:
MS1324
Rick Beauheim
MS1342 Larry Brush
MS1342 Barry Butcher
MS1324 Tracey Christian-Frear
MS1324 Susan Howarth
MS1324 Al Lappin
MS1328 Amy Johnson
MS1328 Hon-Nian Jow
MS1328 Martin Tierney
MS1324 Lucy Meigs
MS1341 Bob Moore
MS1320 Craig Novak
MS1320 Hans Papenguth
MS1320 Malcolm Siegel
MS1341 Yifeng Wang
MS1341 Ruth Weiner

SWCF:1.2.07.1.2:PDD:QA:GENERAL (WPO 36380) 
The t-Distribution

We want to estimate the unknown mean, $m$, of a distribution, given a limited number of samples of the underlying random variable $x$.

$$
x_{1}, x_{2}, x_{3}, \ldots, x_{n}
$$

We take a Bayesian point of view and regard $m$ as a random variable $M$ whose distribution we want to estimate, given sample statistics mentioned above. Our starting point is [Blom, 1989; pg. 232]

The ratio $(\bar{x}-M) / d$, where $d=s / \sqrt{n}$, is an observation on a random variable that has a t-distribution with n-1 degrees of freediom" (where)

$$
\begin{gathered}
\bar{x}=\frac{1}{n} \sum_{i=1}^{n} x_{i}, \quad \text { the sample mean; } \\
s=\left[\frac{1}{(n-1)} \sum_{i=1}^{n}\left(x_{i}-\bar{x}\right)^{2}\right]^{1 / 2} \text {, the sample standard deviation. }
\end{gathered}
$$

From the above quotation, we are allowed to express $M$ as a liriear transformation

$$
M=\bar{X}+d T
$$

and the RV T is distributed according to a standard $t$-distribution with $k=n-1$ degrees of freedom; the probability density function for the t-distribution has the form:

$$
f(t \mid k)=\frac{1}{k^{1 / 2} B(k / 2,1 / 2)}\left(1+\frac{t^{2}}{k}\right)^{-\frac{(k+1)}{2}},-\infty<t<+\infty
$$

where

$$
B(w, z)=\frac{\Gamma(z) \Gamma(w)}{\Gamma(z+w)}
$$

The CDF corresponding to (1) is therefore

$$
F(t \mid k)=\operatorname{Pr}\{T \leq t\}=\frac{1}{k^{1 / 2} B(k / 2,1 / 2)} \int_{-\infty}^{t}\left(1+\frac{\sigma^{2}}{k}\right)^{-(k+1) / 2} d \sigma
$$

This can be put in "closed form" by introducing the incomplete beta function:

$$
I_{[x]}(a, b)=\frac{1}{B(a, b)} \int_{0}^{x} t^{a-1}(1-t)^{b-1} d t \quad(a, b>0),
$$

and making the following change of variables in Equ. (2): let 


$$
w=\frac{1}{\left(1+s^{2} / k\right)}=\frac{k}{\left(k \div s^{2}\right)}
$$

and first consider the case $t \geq 0$, i.e.,

$$
\begin{aligned}
& F(t \mid k)=\operatorname{Pr}\{T \leq o\}+\int_{0}^{t}\left(1 \div \frac{\sigma^{2}}{k}\right)^{-(k+1) / 2} d \sigma \\
& =\frac{1}{2}+\frac{1}{k^{1 / 2} B(k / 2,1 / 2)} \int_{0}^{t}\left(1 \div \frac{\sigma^{2}}{k}\right)^{-(k+1)=2} d \sigma
\end{aligned}
$$

Under transformation (3); as $\sigma \rightarrow 0, w \rightarrow 1$ and as $\sigma \rightarrow t, w=\frac{k}{\left(k+t^{2}\right)}<1$ Also

$$
\left(1+\frac{\sigma^{2}}{k}\right)^{-(k+1) / 2}=w^{(k-1) 2 z} ; d \sigma=-\frac{k^{1: 2}}{2}(1-w)^{-1.2}{w^{1}}^{-3: 2} d \sigma
$$

Thus

$$
\begin{aligned}
F(t \mid k) & =\frac{1}{2}-\frac{1}{B(k / 2,1 / 2)} \int_{1}^{k /\left(k+z^{2}\right)}{ }_{w^{\prime}}^{(z-1) / 2} \cdot \frac{1}{2}\left(1-w^{2}\right)^{-1 / 2} w^{-3 / 2} d w \\
= & \frac{1}{2}-\frac{1}{2 B(k / 2,1 / 2)} \int_{1}^{k /\left(k+z^{2}\right)}{ }_{w^{\prime}}^{(k-2) / 2}(1-w)^{-12} d w
\end{aligned}
$$

Not quite there! Let $u=1-w[w=1-u], d w=-d u$ and $w=1 \rightarrow u=0 ; w=k /\left(k+t^{2}\right) \rightarrow u=t^{2} /\left(k+t^{2}\right)$; thus

$$
F(t \mid k)=\frac{1}{2}+\frac{1}{2 B(k / 2,1 / 2)} \int_{0}^{\frac{\dot{z}^{2}}{\left(k+\dot{r}^{2}\right)}}(1-u){ }^{(k / 2-1) u-1 / 2} d u
$$

or

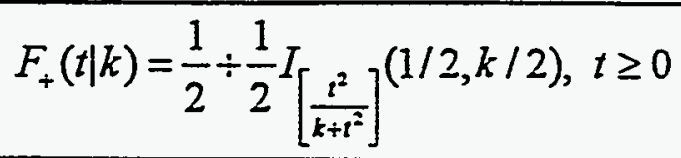

This has the sight behavior, since

$$
I_{[0]}(a, b)=0, I,(a, b)=1 .
$$

Next, assume that $t<0$. Then 


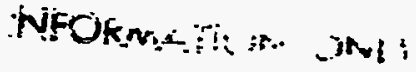

$$
\begin{aligned}
& F_{-}(t \mid k)=F_{+}(t \mid k)-\frac{1}{k^{1 / 2} B(k / 2,1 / 2)} \int_{-1}^{t}\left(1 \div \frac{\sigma^{2}}{k}\right)^{-(k \div 1) / 2} d \sigma \\
& =F_{+}(t \mid k)-A(t \mid k) \\
& \left.=\frac{1}{2}+\frac{1}{2} \mathrm{I}_{\left[\frac{\mathrm{t}^{2}}{k+\mathrm{t}^{2}}\right]}\right]^{(1 / 2, k / 2)-1 \div \mathrm{I}_{\left[\frac{\mathrm{k}}{k+\mathrm{t}^{2}}\right]}(1 / 2, k / 2)}
\end{aligned}
$$

So, upon using the symmetry relation $l_{x}(a, b)=1-l_{1-x}(b, a)$, we have

$$
\mathrm{I}_{\left[\frac{k}{k+t^{2}}\right]}(k / 2,1 / 2)=1-\mathrm{I}_{\left[\frac{\mathrm{t}}{k+t^{2}}\right]}(1 / 2, k / 2)
$$

Therefore

$$
F_{-}(t \mid k)=\frac{1}{2}-\frac{1}{2} I_{\left[\frac{t^{2}}{k+t^{2}}\right]}(1 / 2, k / 2), t<0
$$




\section{Sandia National Laboratories}

Albuquerque, New Mexico 87185-1341

date: April 16, 1996

to:• Distribution

$$
\text { Mestin Tearay }
$$

from: Martin S. Tierney, MS-1328 (6741)

Parameter Task Leader

subject: DEVELOPMENT OF PARAMETER VALUES DISTRIBUTION FUNCTIONS

Attached is a flow chart which shows the development of the parameter values distribution functions as defined in QAP 9-2. This flow chart is meant to supplement the earlier memo dated 3/21/96 to provide a generic description of the parameter development process.

\section{Copy to:}

Rick Beauheim, 6115, MS 1324

Larry Brush, 6748, MS1341

Barry Butcher, 6748, MS1341

Tracy Christian-Frear, 6115, MS1324

Susan Howarth, 6115, MS1324

Al Lappin, 6115, MS1324

Amy Johnson, 6741, MS1328

Hong-Nian Jow, 6741, MS1328

Mary Alena Martell, 6749, MS1328

Lucy Meigs, 6115, MS1324

Bob Moore, 6748, MS1341

Craig Novak, 6748, MS1341

Hans Pappenguth, 6748, MS1320

Malcolm Siegel, 6748, MS1320

Yifeng Wang, 6748, MS1341

Ruth Weiner, 6748, MS1341

¿WCF-A:1.2.07.21.2:PDD;QA:Genernl Flow Chat: 


\section{Development of Parameter Values Distribution Functions}

Solicit Information from Scientists/Investigators Regarding Parameters Needed for the Quantification

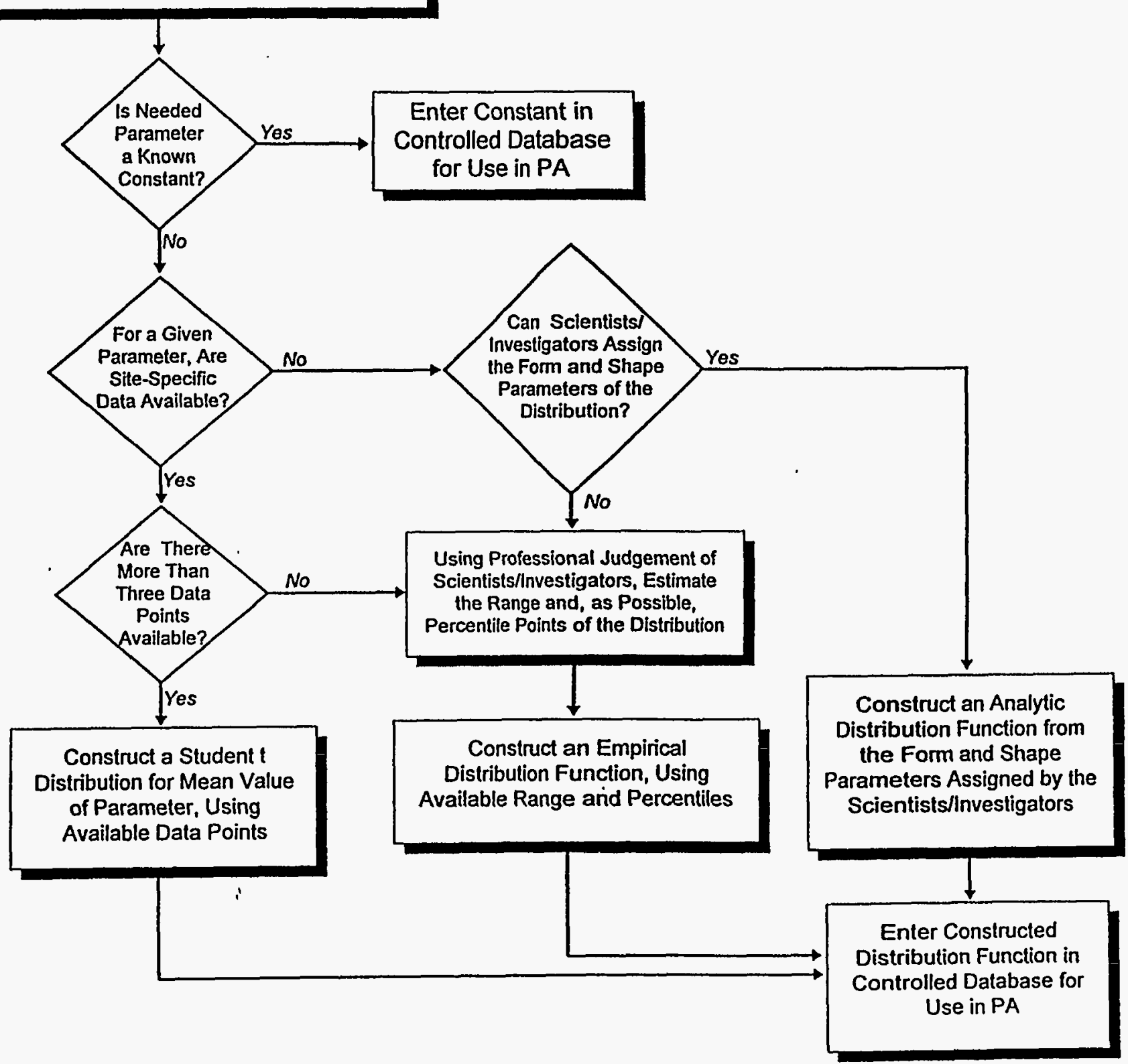


INFORMATION ONLY

date:

to:

from

subjoct

December 13, 1996

\section{Distribution}

Mation Tein

Sandia National Laboratories

Operated for the U.S. Department of Energy by Sandia Corporation

Abuquerque, New Mecico 87185

Martin Tierney, 6848, and Mary-Alena Martell, 6849

\section{On criteria for specifying CONSTANTs in the WIPP PA Parameter Database}

In this memo we state our reasons for choosing constant values for certain parameters appearing in the WIPP PA Parameter Database and give some informal discussion of those reasons. Briefly, a parameter was assigned a constant value for one of three reasons: (1) The quoted or calculated value of the parameter was known to be highly precise, i.e. its coefficient of variation is demonstrably much less than one (see discussion below). (2) Nothing was known about the precision of the quoted value of the parameter but a rational for its choice as a constant was supplied by the PA analyst or a subject-matter expert. (3) The parameter was deemed to be an uncertain parameter and was assigned a probability distribution but was not "sampled" in the 1996 series of sensitivity/uncertainty studies. A constant value (usually the median of the distribution) was assigned to all parameters that had distributions but were not sampled. The choice of almost all Category 3 (and some Category 1 and 4) constants in the PA Parameter Database can be justified by Reason (1). Reason (2) applies to all Category 2 and almost all Category 4 constants. A discussion of the process for choosing the particular parameters that were sampled in the 1996 sensitivity/uncertainty studies is outside the scope of this note; the reader should consult other WIPP Project documents for this information.

The Coefficient of Variation.

A convenient measure of the precision of any experimentally derived number is the coefficient of variation associated with the set of measurements used to estimate the number. Usually, the number to be estimated represents the spatial average or mean value of some physical quantity, e.g. rock porosity or a chemical rate constant (Sandia WTPP Project 1992; Section 1.3.3) but it also may be a fundamental constant that is to be estimated using noisy measurements. The coefficient of variation is the ratio of the sample standard deviation of the set of measurements to the sample mean of the measurements. [Those unfamiliar with statistical terminology should see, for example, Chapter 1 of Smith (1991)]. The smaller the coefficient of variation, the higher the precision. Furthermore, the smaller the coefficient of variation, the more justification for treating a parameter as a constant instead of assigning a probability distribution to it. Most fundamental physical constants, those compiled in longstanding and well-reviewed sources such as the CRC Handbook of Chemistry and Physics, have tiny coefficients of variations of the order of $1.0 \mathrm{E}-10$ to $1.0 \mathrm{E}-06$. Tiny coefficients of variation also occur for those WIPP related, spatially-averaged quantities for which there are numerous small-scale measurements: For example, there are 103 small-scale measurements of matrix porosity of the Culebra Dolomite Member of the Rustler Formation: The average of these measurements (0.151) is an unbiassed estimate of the spatially averaged porosity required by the PA models; the standard deviation of this estimate is the sample standard 
deviation (2.9E-03) divided by the square root of 103 (10.149) or about 2.9E-04. Thus, the coefficient of variation of the estimate of porosity is about $1.9 \mathrm{E}-03$ which is sufficiently small to justify the assignment of a constant porosity equal to 0.151 .

The idea of the coefficient of variation is useful even if the "measurements" are only the results of different person's estimates of the same number, in that case, the coefficient of variation times 100 can be interpreted as relative error. For an example of this, consider the following. Three numbers are given for the Gas Constant for hydrogen: $4116.0,4124.18$, and 4129.72. The sample mean of these numbers is 4123.30 , the sample variance is 47.640 and so the sample standard deviation is 6.9022 ; the coefficient of variation is therefore 6.9022 divided by 4123.30 or 1.6740E-3. The use of any one of these estimates of the Gas Constant would involve an error less than 0.2 percent.

Criteria for imprecisely determined parameters.

How imprecise does a parameter have to be (i.e., how big does the coefficient of variation have to be ) before we must seriously consider assigning that parameter a probability distribution? The answer to this question depends upon the nature of the model in which the parameter appears and whether we have reason to believe that model outputs are sensitive to changes in the parameter's value. No physical constant, even the speed of light in the vacuum, is without imprecision; all are known only to a limited number of significant figures, and the number of significant figures that one uses in an application depends, naturally, upon the precision required of the outputs of that application: For example, constants such as $\pi$ or the speed of light in the vacuum may have to be used with high precision (six or more significant figures) in celestial mechanics, geodesy, or some time-keeping applications; whereas $\pi=3.14$ may be more than adequate in laying out a circular garden plot. WIPP PA models fall into the category of "garden plot layout" applications because of the large uncertainty in WIPP conceptual models and the (usually) clear distinction that can be made between the truly uncertain and the manifestly constant parameters of the mathematical models.

Another situation where precision in the specification of parameter values becomes important is in investigations with intrinsically stochastic mathematical models (Glimm 1991). Models of this type show extreme sensitivity to the values assigned to initial conditions or to certain internal constants, and are sometimes called chaotic. As simple examples of chaotic models, take certain algorithms used to generate pseudorandom numbers on a digital computer (Peitgen, Jurgens and Saupe, 1992; Section 1.5). The input to these algorithms is usually one number called the "seed" and, given a definite value for the seed, the output is a unique and reproducible sequence of numbers between zero and one. Nevertheless, even a tiny change in the "seed" value (say, in the 12th decimal place) ultimately produces a sequence of pseudorandom numbers that is very different from the one produced using the unchanged seed value.

As far as we can tell at this time, none of the the dominating mathematical models used in the WIPP PA are chaotic. Calculations for the 1996 CCA indicate that the total-system performance measure is dominated by a few submodels whose numerical solutions depend smoothly, almost linearly, upon a few critical problem parameters and there are no reasons for believing that this smooth dependence does not extend to non-critical or untested parameters. 
We therefore feel justified in not making any hard and fast rules about when a parameter should be treated as a constant. In general, a parameter whose coefficient of variation is less than 0.1 is treated as a constant; parameters with coefficients of variation of the order of unity or larger are usually treated as random variables and a probability distribution is assigned to them.

References:

Glimm, J. 1991. "Nonlinear and Stochastic Phenomena: The Grand Challenge for Partial Differential Equations," SIAM:Review. Vol.33, no. 4, 626-643.

Peitgen, H.-O., H. Jürgens, and D. Saupe. 1992. Chaos and Fractals: New Frontiers of Science. New York, NY: Springer-Verlag.

Smith, G. 1991. Statistical Reasoning. 3rd ed. Boston, MA: Allyn and Bacon.

Sandia WIPP Project. 1992. Preliminary Performance Assessment for the Waste Isolation Pilot Plant, December 1992. Volume 3: Model Parameters. SAND92-0700/3. Albuquerque, NM: Sandia National Laboratories.

Distribution:

MS1324 Susan Howarth

MS1324 Al Lappin

MS1328 Amy Johnson

MS1328 Hong-Nian Jow

MS1335 Margaret S.Y. Chu

WBS:1.2.07.1.2:PDD:QA:GENERAL

(this memo supplements WPO\#35268) 
Page intentionally blank 


\section{Appendix $\mathrm{H}$}

Sampled Parameters from Appendix PAR 
Parameters Sampled in LHS Code (and parameters to which sampled values were applied)

\begin{tabular}{|c|c|c|c|c|c|c|c|c|c|c|c|c|c|}
\hline Lisis & Id & Material : & Matertal Descripllon & Parameter & Parameter Deseriplion & $\begin{array}{l}\text { Distribuillon } \\
\text { Type }\end{array}$ & Units & Meañ & Median & Low: & fígh & $\begin{array}{l}\text { Standiard } \\
\text { Devlátión. }\end{array}$ & $\begin{array}{l}\text { WPÖ } \\
\text { \# }\end{array}$ \\
\hline 1 & 2907 & STEEL & Generic stecl in waste & $\begin{array}{r}\text { CORRMCO2 } \\
. \\
\end{array}$ & $\begin{array}{l}\begin{array}{l}\text { Inundated corrosion rate } \\
\text { for stcel without } \mathrm{CO} 2 \\
\text { present }\end{array} \\
\end{array}$ & UNIFORM & $\mathbf{m} / \mathbf{s}$ & $7.9370 \mathrm{E}-15$ & $7.9370 \mathrm{E}-15$ & 0 & $1.5870 \mathrm{E}-14$ & 0 & 34357 \\
\hline 2 & 2823 & WAS_AREA & $\begin{array}{l}\text { Waste emplacement } \\
\text { area and waste }\end{array}$ & PROBDEG & $\begin{array}{l}\text { Prob. of plastics \&rubber } \\
\text { biodegradation in event } \\
\text { of significant microblal } \\
\text { gas generation }\end{array}$ & DELTA & NONE & n.a. & n.a. & $\mathbf{0}$ & 2 & n.a. & 34881 \\
\hline (2) & 2824 & REPOSIT & $\begin{array}{l}\text { Repository regions } \\
\text { outside of Panel region }\end{array}$ & PROBDEG & $\begin{array}{l}\text { Prob. of plastics\&rubber } \\
\text { biodegradation in event of } \\
\text { microbial gas generation }\end{array}$ & DELTA & NONE & n.a. & n.a. & 0 & 2 & n.a. & 33264 \\
\hline 3 & 657 & WAS_AREA & $\begin{array}{l}\text { Waste emplacement } \\
\text { area and waste }\end{array}$ & GRATMICI & $\begin{array}{l}\text { Blodegradation rate, } \\
\text { inundated conditions } \\
\end{array}$ & UNIFORM & mol/kg*s & $4.9150 \mathrm{E}-09$ & $4.9150 \mathrm{E}-09$ & $3.1710 \mathrm{E}-10$ & 9.5129E-09 & 0 & 34928 \\
\hline (3) & 2128 & REPOSIT & $\begin{array}{l}\text { Repository regions } \\
\text { outside of Panel region }\end{array}$ & GRATMICI & $\begin{array}{l}\text { Gas production rate, } \\
\text { microbial, inundated } \\
\text { conditions }\end{array}$ & UNIFORM & $\mathrm{mol} / \mathrm{kg}{ }^{*} \mathrm{~s}$ & $4.9150 \mathrm{E}-09$ & $4.9150 \mathrm{E}-09$ & $3.1710 \mathrm{E}-10$ & $9.5129 \mathrm{E}-09$ & 0 & 33235 \\
\hline 4 & 656 & WAS_AREA & $\begin{array}{l}\text { Waste emplacement } \\
\text { area and waste }\end{array}$ & GRATMICH & $\begin{array}{l}\text { Biodegradation rate, } \\
\text { humid conditions relative } \\
\text { to inundated rate }\end{array}$ & UNIFORM & $\mathrm{mol} / \mathrm{kg} * \mathrm{~s}$ & $6.3420 \mathrm{E}-10$ & $6.3420 \mathrm{E}-10$ & 0 & $1.2684 \mathrm{E}-09$ & 0 & 34923 \\
\hline (4) & 2127 & REPOSIT & $\begin{array}{l}\text { Repository regions } \\
\text { outside of Pancl region }\end{array}$ & GRATMICH & $\begin{array}{l}\text { Gas production rate, } \\
\text { microbial, humid } \\
\text { conditions relative to } \\
\text { inundated rate }\end{array}$ & UNIFORM & $\mathrm{mol} / \mathrm{kg} * \mathrm{~s}$ & $6.3420 \mathrm{E}-10$ & $6.3420 \mathrm{E}-10$ & 0 & $1.2684 \mathrm{E}-09$ & 0 & 3.3234 \\
\hline 5 & 2994 & CELLULS & Cellulose & FBETA & $\begin{array}{l}\text { Factor beta for microbial } \\
\text { reaction rates }\end{array}$ & UNIFORM & NONE & $5.0000 \mathrm{E}-01$ & $5.0000 \mathrm{E}-01$ & 0 & $1.0000 \mathrm{E}+00$ & 0.29 & 31826 \\
\hline 6 & 671 & WAS_AREA & $\begin{array}{l}\text { Waste emplacement } \\
\text { area and waste }\end{array}$ & SAT_RGAS & Residual Gas Saturation & UNIFORM & NONE & $7.5000 \mathrm{E}-02$ & $7.5000 \mathrm{E}-02$ & 0 & $1.5000 \mathrm{E}-01$ & 0.04 & 34905 \\
\hline (6) & 2137 & REPOSIT & $\begin{array}{l}\text { Repository regions } \\
\text { outside of Panel region }\end{array}$ & SAT_RGAS & Residual Gas Saturation & UNIFORM & NONE & $7.5000 \mathrm{E}-02$ & $7.5000 \mathrm{E}-02$ & 0 & $1.5000 \mathrm{E}-01$ & 0.04 & 33286 \\
\hline 7 & 670 & WAS_AREA & $\begin{array}{l}\text { Waste emplacement } \\
\text { area and waste }\end{array}$ & SAT_RBRN & $\begin{array}{l}\text { Residual Brine } \\
\text { Saturation } \\
\end{array}$ & UNIFORM & NONE & 2.7600E-01 & $2.7600 \mathrm{E}-01$ & 0 & $5.5200 \mathrm{E}-01$ & 0.16 & 34902 \\
\hline (7) & 2741 & REPOSIT & $\begin{array}{l}\text { Repository regions } \\
\text { outside of Panel region }\end{array}$ & SAT_RBRN & Residual Brine Saturation & UNIFORM & NONE & $2.7600 \mathrm{E}-01$ & $2.7600 \mathrm{E}-01$ & 0 & $5.5200 \mathrm{E}-01$ & 0.16 & 33283 \\
\hline 8 & 2231 & WAS_AREA & $\begin{array}{l}\text { Wastc emplacement } \\
\text { area and waste }\end{array}$ & SAT_WICK & $\begin{array}{l}\text { Index for computing } \\
\text { wicking }\end{array}$ & UNIFORM & NONE & $5.0000 \mathrm{E}-01$ & $5.0000 \mathrm{E}-01$ & $\mathbf{0}$ & $1.0000 E+00$ & 0.29 & 34908 \\
\hline (8) & 2138 & REPOSIT & $\begin{array}{l}\text { Repository regions } \\
\text { outside of Panel region }\end{array}$ & SAT_WICK & $\begin{array}{l}\text { Index for computing } \\
\text { wicking }\end{array}$ & UNIFORM & NONE & $5.0000 \mathrm{E}-01$ & $5.0000 \mathrm{E}-01$ & 0 & $1.0000 \mathrm{E}+00$ & 0.29 & 33289 \\
\hline 9 & 2334 & CL_L_TI & $\begin{array}{l}\text { Lower Salado clay: } 0 \\
\text { to } 10 \text { years }\end{array}$ & $\begin{array}{c}\text { PRMX_LOG } \\
\vdots \\
\end{array}$ & $\begin{array}{l}\text { Log of intrinsic } \\
\text { permeability, X-direction }\end{array}$ & TRIANGULAR & $\log \left(m^{\wedge} 2\right)$ & $-1.8867 E+01$ & $-1.8301 \mathrm{E}+01$ & $-2.1000 \mathrm{E}+01$ & $-1.7301 E+01$ & 0.78 & 31908 \\
\hline (9) & 2335 & CL_L_Tl & $\begin{array}{l}\text { Lower Salado clay: } 0 \text { to } \\
10 \text { years }\end{array}$ & PRMY_LOG & $\begin{array}{l}\text { Log of intrinsic } \\
\text { permeability. Y-direction }\end{array}$ & TRIANGULAR & $\log \left(m^{\wedge} 2\right)$ & $-1.8867 E+01$ & $-1.830 \mid E+01$ & $-2.1000 \mathrm{E}+01$ & $-1.7301 E+01$ & 0.78 & 31909 \\
\hline
\end{tabular}

'For parameters with a triangular distribution, the value provided for the median is actually the mode. 
Parameters Sampled in LHS Code (and parameters to which sampled values were applied) (Continued)

\begin{tabular}{|c|c|c|c|c|c|c|c|c|c|c|c|c|c|}
\hline $\begin{array}{l}\text { LHS } \\
\text { 类 }\end{array}$ & Id & Material & Material Description & Parameler & Parameler Description & $\begin{array}{l}\text { Distribution } \\
\text { Type. }\end{array}$ & Units & Mean & Median ${ }^{1}$ & Low & High & $\begin{array}{l}\text { Standard } \\
\text { Devtation }\end{array}$ & $\begin{array}{l}\text { WPO } \\
\#\end{array}$ \\
\hline (9) & 2336 & CL_L_TI & $\begin{array}{l}\text { Lower Salado clay: } 0 \text { to } \\
10 \text { years }\end{array}$ & PRMZ_LOG & $\begin{array}{l}\text { Log of intrinsic } \\
\text { permeability, Z-direction }\end{array}$ & TRIANGULAR & $\log \left(m^{\wedge} 2\right)$ & $-1.8867 E+01$ & $-1.8301 E+01$ & $-2.1000 \mathrm{E}+01$ & $-1.7301 E+01$ & 0.78 & 31910 \\
\hline (9) & 3009 & CLAY_RUS & $\begin{array}{l}\text { Clay seals in Rustler } \\
\text { formation }\end{array}$ & PRMX_LOG & $\begin{array}{l}\text { Log of intrinsic } \\
\text { permeability, X-direction }\end{array}$ & TRIANGULAR & $\log \left(m^{\wedge} 2\right)$ & $-1.8867 \mathrm{E}+01$ & $-1.8301 E+01$ & $-2.1000 \mathrm{E}+01$ & $-1.7301 E+01$ & 0.78 & 31886 \\
\hline (9) & 3010 & CLAY_RUS & $\begin{array}{l}\text { Clay seals in Rusiler } \\
\text { formation }\end{array}$ & PRMY_LOG & $\begin{array}{l}\text { Log of intrinsic } \\
\text { perneability. Y-direction }\end{array}$ & TRIANGULAR & $\log \left(m^{\wedge} 2\right)$ & $-1.8867 \mathrm{E}+01$ & $-1.8301 E+01$ & $-2.1000 \mathrm{E}+01$ & $-1.7301 E+01$ & 0.78 & 31887 \\
\hline (9) & 3011 & CLAY_RUS & $\begin{array}{l}\text { Clay seals in Rustler } \\
\text { formation }\end{array}$ & PRMZLOG & $\begin{array}{l}\text { Log of intrinsic } \\
\text { permeability, Z-direction }\end{array}$ & TRIANGULAR & $\log \left(m^{\wedge} 2\right)$ & $-1.8867 \mathrm{E}+01$ & $-1.8301 E+01$ & $-2.1000 \mathrm{E}+01$ & $-1.7301 \mathrm{E}+01$ & 0.78 & 31888 \\
\hline (9) & 2351 & CL_L_T2 & $\begin{array}{l}\text { Lower Salado clay: } 10 \text { to } \\
25 \text { years }\end{array}$ & PRMX_LOG & $\begin{array}{l}\text { Log of intrinsic } \\
\text { permeability. X-direction }\end{array}$ & TRIANGULAR & $\log \left(m^{\wedge} 2\right)$ & $-1.8867 \mathrm{E}+01 \mid$ & $-1.8301 E+01$ & $-2.1000 \mathrm{E}+01 \mid$ & $-1.7301 \mathrm{E}+01$ & 0.78 & 31935 \\
\hline (9) & 2352 & CL_L_T2 & $\begin{array}{l}\text { Lower Salado clay: } 10 \text { to } \\
25 \text { years }\end{array}$ & PRMY_LOG & $\begin{array}{l}\text { Log of intrinsic } \\
\text { permenbility, Y-direction }\end{array}$ & TRIANGULAR & $\log \left(m^{\wedge} 2\right)$ & $-1.8867 \mathrm{E}+01$ & $-1.8301 E+01$ & $-2.1000 E+01 \mid$ & $|-1.7301 E+01|$ & 0.78 & 31936 \\
\hline (9) & 2353 & CL_L_T2 & $\begin{array}{l}\text { Lower Salado clay: } 10 \text { to } \\
25 \text { years }\end{array}$ & PRMZ_LOG & $\begin{array}{l}\text { Log of intrinsic } \\
\text { permeability, Z-direction }\end{array}$ & TRIANGULAR & $\log \left(m^{\wedge} 2\right)$ & $-1.8867 \mathrm{E}+01$ & $-1.8301 E+01$ & $-2.1000 E+01$ & $-1.7301 E+01$ & 0.78 & 31937 \\
\hline (9) & 2368 & CL_L_T3 & $\begin{array}{l}\text { Lower Salado clay: } 25 \text { to } \\
50 \text { years }\end{array}$ & PRMX_LOG & $\begin{array}{l}\text { Log of intrinsic } \\
\text { permeability, X-direction }\end{array}$ & TRIANGULAR & $\log \left(m^{\wedge} 2\right)$ & $-1.8867 E+01$ & $-1.8301 E+01$ & $-2.1000 \mathrm{E}+01$ & $-1.7301 E+01$ & 0.78 & 31990 \\
\hline (9) & 2369 & CL_L_T3 & $\begin{array}{l}\text { Lower Salado clay: } 25 \text { to } \\
50 \text { years }\end{array}$ & PRMY_LOG & $\begin{array}{l}\text { Log of intrinsic } \\
\text { permeability, Y-direction }\end{array}$ & TRIANGULAR & $\log \left(m^{\wedge} 2\right)$ & $-1.8867 \mathrm{E}+01$ & $-1.8301 E+01$ & $|-2.1000 \mathrm{E}+01|$ & $-1.7301 E+01 \mid$ & 0.78 & 31992 \\
\hline (9) & 2370 & CL_L_T3 & $\begin{array}{l}\text { Lower Salado clay: } 25 \text { to } \\
\text { 50 years }\end{array}$ & PRMZ_LOG & $\begin{array}{l}\text { Log of intrinsic } \\
\text { permeability, Z-direction }\end{array}$ & TRIANGULAR & $\log \left(m^{\wedge} 2\right)$ & $-1.8867 \mathrm{E}+01$ & $-1.8301 E+01$ & $-2.1000 E+01$ & $-1.7301 \mathrm{E}+01$ & 0.78 & 31993 \\
\hline (9) & 3078 & CL_L_T4 & $\begin{array}{l}\text { Lower Salado clay: } 50 \text { to } \\
\text { loK years }\end{array}$ & PRMX_LOG & $\begin{array}{l}\text { Log of intrinsic } \\
\text { permeability, X-direction }\end{array}$ & TRIANGULAR & $\log \left(m^{\wedge} 2\right)$ & $-1.8867 \mathrm{E}+01$ & $-1.8301 \mathrm{E}+01$ & $-2.1000 \mathrm{E}+01$ & $|-1.7301 \mathrm{E}+01|$ & 0.78 & 32016 \\
\hline (9) & 3079 & CL_L_.T4 & $\begin{array}{l}\text { Lower Salado clay: } 50 \text { to } \\
\text { 10K years. }\end{array}$ & PRMY_LOG & $\begin{array}{l}\text { Log of intrinsic } \\
\text { permeability. Y-direction }\end{array}$ & TRIANGULAR & $\log \left(m^{\wedge} 2\right)$ & $-1.8867 E+01$ & $-1.8301 E+01$ & $-2.1000 E+01 \mid$ & $-1.7301 \mathrm{E}+01$ & 0.78 & 32017 \\
\hline (9) & 3080 & CL_L_T4 & $\begin{array}{l}\text { Lower Salado clay: } 50 \text { to } \\
\text { I0K years }\end{array}$ & PRMZ_LOG & $\begin{array}{l}\text { Log of intrinsic } \\
\text { permeability, Z-direction }\end{array}$ & TRIANGULAR & $\log \left(m^{\wedge} 2\right)$ & $-1.8867 \mathrm{E}+01$ & $-1.8301 E+01$ & $|-2.1000 E+01|$ & $|-1.7301 E+01|$ & 0.78 & 32018 \\
\hline (9) & 2385 & CL_M_TI & $\begin{array}{l}\text { Upper Salado clay: } 0 \text { to } \\
10 \text { years }\end{array}$ & PRMX_LOG & $\begin{array}{l}\text { Log of intrinsic } \\
\text { permeability, X-direction }\end{array}$ & TRIANGULAR & $\log \left(m^{\wedge} 2\right)$ & $-1.8867 \mathrm{E}+01$ & $-1.8301 \mathrm{E}+01$ & $-2.1000 E+01 \mid$ & $-1.7301 E+01 \mid$ & 0.78 & 32048 \\
\hline (9) & 2386 & CL_M_TI & $\begin{array}{l}\text { Upper Salado clay: } 0 \text { to } \\
10 \text { years }\end{array}$ & PRMY_LOG & $\begin{array}{l}\text { Log of intrinsic } \\
\text { permeability. Y-direction }\end{array}$ & TRIANGULAR & $\log \left(m^{\wedge} 2\right)$ & $-1.8867 \mathrm{E}+01$ & $-1.8301 \mathrm{E}+01$ & $-2.1000 E+01 \mid$ & $-1.7301 \mathrm{E}+01$ & 0.78 & 32049 \\
\hline (9) & 2387 & CL_M_TI & $\begin{array}{l}\text { Upper Salado clay: } 0 \text { to } \\
10 \text { years }\end{array}$ & PRMZ_LOG & $\begin{array}{l}\text { Log of intrinsic } \\
\text { permeability, Z-direction }\end{array}$ & TRIANGULAR & $\log \left(m^{\wedge} 2\right)$ & $-1.8867 \mathrm{E}+01$ & $-1.8301 \mathrm{E}+01$ & $|-2.1000 \mathrm{E}+01|$ & $-1.7301 E+01$ & 0.78 & 32050 \\
\hline (9) & 2402 & CL_M_T2 & $\begin{array}{l}\text { Upper Salado clay: } 10 \text { to } \\
25 \text { years }\end{array}$ & PRMX_LOG & $\begin{array}{l}\text { Log of intrinsic } \\
\text { permeability, X-direction }\end{array}$ & TRIANGULAR & $\log \left(m^{\wedge} 2\right)$ & $-1.8867 \mathrm{E}+01$ & $-1.8301 E+01$ & $-2.1000 \mathrm{E}+01$ & $-1.7301 \mathrm{E}+01$ & 0.78 & 32123 \\
\hline (9) & 2403 & CL_M_T2 & $\begin{array}{l}\text { Upper Salado clay: } 10 \text { to } \\
25 \text { years }\end{array}$ & PRMY_LOG & $\begin{array}{l}\text { Log of intrinsic } \\
\text { permeability, Y-direction }\end{array}$ & TRIANGULAR & $\log \left(m^{\wedge} 2\right)$ & $-1.8867 \mathrm{E}+01$ & $-1.8301 E+01$ & $-2.1000 E+01$ & $-1.730 \mid \mathrm{E}+01$ & 0.78 & 32124 \\
\hline (9) & 2404 & CL_M_T2 & $\begin{array}{l}\text { Upper Salado clay: } 10 \text { to } \\
25 \text { years }\end{array}$ & PRMZ_LOG & $\begin{array}{l}\text { Log of intrinsic } \\
\text { permeability, Z-direction }\end{array}$ & TRIANGULAR & $\log \left(m^{\wedge} 2\right)$ & $-1.8867 \mathrm{E}+01$ & $-1.8301 E+01$ & $-2.1000 \mathrm{E}+01$ & $-1.7301 \mathrm{E}+01$ & 0.78 & 32125 \\
\hline (9) & 2419 & CL_M_T3 & $\begin{array}{l}\text { Upper Salado clay: } 25 \text { to } \\
50 \text { years }\end{array}$ & PRMX_LOG & $\begin{array}{l}\text { Log of intrinsic } \\
\text { permeability, X-direction }\end{array}$ & TRIANGULAR & $\log \left(m^{\wedge} 2\right)$ & $-1.8867 \mathrm{E}+0 \mathrm{I}$ & $-1.8301 \mathrm{E}+01$ & $-2.1000 \mathrm{E}+01$ & $-1.7301 E+01$ & 0.78 & 32155 \\
\hline
\end{tabular}

'For parameters with a triangular distribution, the value provided for the median is actually the mode. 
Parameters Sampled in LHS Code (and parameters to which sampled values were applied) (Continued)

\begin{tabular}{|c|c|c|c|c|c|c|c|c|c|c|c|c|c|}
\hline LHS & Idd & Máterial & Mátérial Description & $\begin{array}{l}0 \\
\text { Parnmeter } \\
\end{array}$ & Pärameter Descríptlon: & $\begin{array}{l}\text { Distributlon : } \\
\text { Type }\end{array}$ & Units & Meah & Medlan & Lôn? & High $: \therefore$ & $\begin{array}{l}\text { Standard } \\
\text { Deviation }\end{array}$ & $\begin{array}{l}\text { Wro } \\
\text { W }\end{array}$ \\
\hline (9) & 2420 & CL_M_T3 & $\begin{array}{l}\text { Upper Salado clay: } 25 \text { to } \\
50 \text { years }\end{array}$ & PRMY_LOG & $\begin{array}{l}\text { Log of intrinsic } \\
\text { penneability. Y-direction }\end{array}$ & TRIANGULAR & $\log \left(\mathrm{m}^{\wedge} 2\right)$ & $-1.8867 E+01$ & $-1.830 ! E+0 !$ & $-2.1000 \mathrm{E}+01$ & $-1.7301 E+01$ & 0.78 & 32156 \\
\hline (9) & 2421 & CL_M_T3 & $\begin{array}{l}\text { Upper Salado clay: } 25 \text { to } \\
50 \text { years }\end{array}$ & PRMZ_LOG & $\begin{array}{l}\text { Log of intrinsic } \\
\text { pernneability, Z-direction }\end{array}$ & TRIANGULAR & $\log \left(m^{\wedge} 2\right)$ & $|-1.8867 E+01|$ & $-1.8301 E+01$ & $-2.1000 \mathrm{E}+01$ & $-1.7301 E+01$ & 0.78 & 32157 \\
\hline (9) & 2436 & CL_M_T4 & $\begin{array}{l}\text { Upper Salado clay: } 50 \text { to } \\
100 \text { years }\end{array}$ & PRMX_LOG & $\begin{array}{l}\text { Log of intrinsic } \\
\text { permeability, X-direction }\end{array}$ & TRIANGULAR & $\log \left(m^{\wedge} 2\right)$ & $|-1.8867 E+01|$ & $-1.8301 E+01$ & $-2.1000 \mathrm{E}+01 \mid$ & $-1.7301 E+01$ & 0.78 & 32202 \\
\hline (9) & 2437 & CL_M_T4 & $\begin{array}{l}\text { Upper Salado clay: } 50 \text { to } \\
100 \text { years }\end{array}$ & PRMY_LOG & $\begin{array}{l}\text { Log of intrinsic } \\
\text { permeability. Y-direction }\end{array}$ & TRIANGULAR & $\log \left(m^{\wedge} 2\right)$ & $-1.8867 \mathrm{E}+01$ & $-1.8301 E+01$ & $-2.1000 \mathrm{E}+01 \mid$ & $-1.7301 E+01$ & 0.78 & 32203 \\
\hline (9) & 2438 & CL_M_T4 & $\begin{array}{l}\text { Upper Salado clay: } 50 \text { to } \\
100 \text { years }\end{array}$ & PRMZ_LOG & $\begin{array}{l}\text { Log of intrinsic } \\
\text { permeability.Z-direction }\end{array}$ & TRIANGULAR & $\log \left(m^{\wedge} 2\right)$ & $-1.8867 E+01$ & $-1.8301 E+01$ & $|-2.1000 \mathrm{E}+01|$ & $-1.7301 E+01$ & 0.78 & 32204 \\
\hline (9) & 2453 & CL_M_T5 & $\begin{array}{l}\text { Upper Salado clay: } 100 \\
\text { to 10K years } \\
\end{array}$ & PRMX_LOG & $\begin{array}{l}\text { Log of intrinsic } \\
\text { permeability, X-direction }\end{array}$ & TRIANGULAR & $\log \left(m^{\wedge} 2\right)$ & $-1.8867 E+01$ & $-1.8301 \mathrm{E}+01$ & $-2.1000 \mathrm{E}+01$ & $-1.730 \mid E+01$ & 0.78 & 32239 \\
\hline (9) & 2454 & CL_M_T5 & $\begin{array}{l}\text { Upper Salado clay: } 100 \\
\text { to } 10 \mathrm{~K} \text { years }\end{array}$ & PRMY_LOG & $\begin{array}{l}\text { Log of intrinsic } \\
\text { permeability. Y-direction }\end{array}$ & TRIANGULAR & $\log \left(m^{\wedge} 2\right)$ & $-1.8867 E+01 \mid$ & $-1.8301 E+01$ & $-2.1000 \mathrm{E}+01$ & $-1.7301 E+01$ & 0.78 & 32240 \\
\hline (9) & 2455 & CL_M_T5 & $\begin{array}{l}\text { Upper Salado clay: } 100 \\
\text { to 10K years }\end{array}$ & PRMZ_LOG & $\begin{array}{l}\text { Log of intrinsic } \\
\text { permeability. Z-direction }\end{array}$ & TRIANGULAR & $\log \left(m^{\wedge} 2\right)$ & $-1.8867 E+01$ & $-1.8301 E+01$ & $-2.1000 \mathrm{E}+01 \mid$ & $-1.7301 E+01$ & 0.78 & 32241 \\
\hline (9) & 2317 & CLAY_BOT & Shaft Bottom Clay & PRMX_LOG & $\begin{array}{l}\text { Log of intrinsic } \\
\text { permeability, X-direction }\end{array}$ & TRIANGULAR & $\log \left(m^{\wedge} 2\right)$ & $-1.8867 E+01$ & $-1.8301 \mathrm{E}+01$ & $|-2.1000 \mathrm{E}+01|$ & $-1.7301 E+01$ & 0.78 & 31866 \\
\hline (9) & 2318 & CLAY_BOT & Shaft Bottom Clay & PRMY_LOG & $\begin{array}{l}\text { Log of intrinsic } \\
\text { permeability, Y-direction }\end{array}$ & TRIANGULAR & $\log \left(m^{\wedge} 2\right)$ & $-1.8867 E+01$ & $-1.8301 \mathrm{E}+0 \mathrm{I}$ & $-2.1000 \mathrm{E}+01 \mid$ & $-1.7301 E+01$ & 0.78 & 31867 \\
\hline (9) & 2319 & CLAY_BOT & $\begin{array}{l}\text { Shaft Bottom Clay } \\
\text { Upper Salado clay: } 0 \text { to } \\
10 \text { years }\end{array}$ & PRMZ_LOG & $\begin{array}{l}\text { Log of intrinsic } \\
\text { permeability, Z-direction }\end{array}$ & TRIANGULAR & $\log \left(m^{\wedge} 2\right)$ & $-1.8867 \mathrm{E}+01$ & $-1.8301 \mathrm{E}+01$ & $-2.1000 E+01 \mid$ & $-1.7301 E+01$ & 0.78 & 31868 \\
\hline 10 & 2470 & CONC_T1 & $\begin{array}{l}\text { Concrete column: } 0 \text { to } \\
400 \text { years }\end{array}$ & PRMX_LOG & $\begin{array}{l}\text { Log of intrinsic } \\
\text { permeability, } X \text {-direction }\end{array}$ & TRIANGULAR & $\log \left(m^{\wedge} 2\right)$ & $-1.8816 E+01$ & $-1.8750 \mathrm{E}+01$ & $|-2.0699 E+01|$ & $-1.7000 \mathrm{E}+01$ & 0.76 & 32583 \\
\hline (10) & 2471 & CONC_TI & $\begin{array}{l}\text { Concrete column: } 0 \text { to } \\
400 \text { years }\end{array}$ & PRMY_LOG & $\begin{array}{l}\text { Log of intrinsic } \\
\text { permeability, Y-direction }\end{array}$ & TRIANGULAR & $\log \left(m^{\wedge} 2\right)$ & $-1.8816 E+01$ & $-1.8750 \mathrm{E}+01$ & $-2.0699 E+01 \mid$ & $-1.7000 E+01$ & 0.76 & 32585 \\
\hline (10) & 2472 & CONC_TI & $\begin{array}{l}\text { Concrete column: } 0 \text { to } \\
400 \text { years }\end{array}$ & PRMZ_LOG & $\begin{array}{l}\text { Log of intrinsic } \\
\text { permeability. Z-direction }\end{array}$ & TRIANGULAR & $\log \left(m^{\wedge} 2\right)$ & $-1.8816 E+01$ & $-1.8750 E+01 \mid$ & $-2.0699 \mathrm{E}+01$ & $-1.7000 E+01$ & 0.76 & 32587 \\
\hline 11 & 2283 & ASPHALT & Asphalt column & PRMX_LOG & $\begin{array}{l}\text { Log of intrinsic } \\
\text { permeability, X-direction }\end{array}$ & TRIANGULAR & $\log \left(m^{\wedge} 2\right)$ & $-1.9667 \mathrm{E}+01$ & $-2.0000 E+01$ & $|-2.1000 \mathrm{E}+01|$ & $-1.8000 E+01$ & 0.62 & 31390 \\
\hline (11) & 2284 & ASPHALT & Asphalt column & PRMY_LOG & $\begin{array}{l}\text { Log of intrinsic } \\
\text { permeability, Y-direction }\end{array}$ & TRIANGULAR & $\log \left(m^{\wedge} 2\right)$ & $-1.9667 \mathrm{E}+01$ & $-2.0000 \mathrm{E}+01$ & $-2.1000 \mathrm{E}+01$ & $-1.8000 E+01 \mid$ & 0.62 & 31391 \\
\hline (11) & 2285 & ASPHALT & Asphait column & PRMZ_LQG & $\begin{array}{l}\text { Log of intrinsic } \\
\text { permeability, Z-direction }\end{array}$ & TRIANGULAR & $\log \left(m^{\wedge} 2\right)$ & $-1.9667 E+01$ & $-2.0000 \mathrm{E}+01$ & $-2.1000 \mathrm{E}+01 \mid$ & $-1.8000 \mathrm{E}+01$ & 0.62 & 31392 \\
\hline 12 & 3133 & SHFT_DRZ & $\begin{array}{l}\text { Shaft disturbed Rock } \\
\text { Zone }\end{array}$ & PRMX_LOG & $\begin{array}{l}\text { Log of intrinsic } \\
\text { permeability, } \mathrm{X} \text {-direction }\end{array}$ & TRIANGULAR & $\log \left(m^{\wedge} 2\right)$ & $-1.5333 E+01$ & $-1.5000 \mathrm{E}+01$ & $-1.7000 E+01 \mid$ & $-1.4000 \mathrm{E}+01$ & 0.62 & 36563 \\
\hline 13 & 2939 & SALT_TI & $\begin{array}{l}\text { Shaft salt column } \\
\text { compacted: time } 0 \text { to } \\
10 \text { years }\end{array}$ & CUMPROB & Cumulative Probability & UNIFORM & NONE & $5.0000 \mathrm{E}-01$ & $5.0000 \mathrm{E}-01$ & 0 & $1.0000 \mathrm{E}+00$ & 0.29 & 33361 \\
\hline
\end{tabular}

'For parameters with a triangular distribution, the value provided for the median is actually the mode. 
Parameters Sampled in LHS Code (and parameters to which sampled values were applied) (Continued)

\begin{tabular}{|c|c|c|c|c|c|c|c|c|c|c|c|c|c|}
\hline $\begin{array}{l}\text { LHS } \\
\text { H. }\end{array}$ & $\begin{array}{l}\text { Id } \\
\#\end{array}$ & Material & Material Description & Parameler & Parameter Description & $\begin{array}{l}\text { Distribution: } \\
\text { Type }\end{array}$ & Unils & Mcan & Medlan & Low & High & $\begin{array}{l}\text { Standard } \\
\text { Deviation }\end{array}$ & $\begin{array}{l}\text { WPO } \\
\#\end{array}$ \\
\hline 14 & 2529 & SALT_TI & $\begin{array}{l}\text { Shaft sall column } \\
\text { compacted: time } 0 \text { to } \\
10 \text { years }\end{array}$ & SAT_RGAS & Residual Gas Saturation & UNIFORM & NONE & $2.0000 \mathrm{E}-01$ & $2.0000 \mathrm{E}-01$ & 0 & $4.0000 E-01$ & 0.12 & 33420 \\
\hline (14) & 2546 & SALT_T2 & $\begin{array}{l}\text { Shaft salt column } \\
\text { compacted: time } 10 \text { to } \\
25 \text { years }\end{array}$ & SAT_RGAS & Residual Gas Saturation & UNIFORM & NONE & $2.0000 \mathrm{E}-01$ & $2.0000 \mathrm{E}-01$ & 0 & $4.0000 \mathrm{E}-01$ & 0.12 & 33393 \\
\hline (14) & 2563 & SALT_T3 & $\begin{array}{l}\text { Shaft salt column } \\
\text { compacted: time } 25 \text { to } \\
50 \text { years } \\
\end{array}$ & SAT_RGAS & Residual Gas Saturation & UNIFORM & NONE & $2.0000 \mathrm{E}-01$ & $2.0000 \mathrm{E}-01$ & $\ldots$ & $4.0000 \mathrm{E}-01$ & 0.12 & 33447 \\
\hline (14) & 2580 & SALT_T4 & \begin{tabular}{|l} 
Shaft salt column \\
compacted: time 50 to \\
100 years \\
\end{tabular} & SAT_RGAS & Residual Gas Saturation & UNIFORM & NONE & $2.0000 \mathrm{E}-01$ & $2.0000 \mathrm{E}-01$ & 0 & $4.0000 \mathrm{E}-01$ & 0.12 & 33565 \\
\hline (14) & 2597 & SALT_T5 & \begin{tabular}{|l} 
Shaft salt column \\
compacted: time 100 to \\
200 years
\end{tabular} & SAT_RGAS & Residual Gas Saturation & UNIFORM & NONE & $2.0000 \mathrm{E}-01$ & $2.0000 \mathrm{E}-01$ & 0 & $4.0000 \mathrm{E}-01$ & 0.12 & 33628 \\
\hline (14) & 2993 & SALT_T6 & $\begin{array}{l}\text { Shaft salt column } \\
\text { compacted: time } 200 \text { to } \\
10 \mathrm{~K} \text { years } \\
\end{array}$ & SAT_RGAS & Residual Gas Saturation & UNIFORM & NONE & $2.0000 \mathrm{E}-01$ & $2.0000 \mathrm{E}-01$ & 0 & $4.0000 \mathrm{E} \cdot 01$ & 0.12 & 33484 \\
\hline (14) & 2512 & EARTH & Earthen Fill & SAT_RGAS & Residual Gas Saluration & UNIFORM & NONE & $2.0000 \mathrm{E}-01$ & $2.0000 \mathrm{E}-01$ & of & $4.0000 \mathrm{E}-01$ & 0.12 & 32964 \\
\hline (14) & 3015 & CLAY_RUS & $\begin{array}{l}\text { Clay seals in Rustler } \\
\text { fonnation }\end{array}$ & SAT_RGAS & Residual Gas Saturation & UNIFORM & NONE & $2.0000 \mathrm{E}-01$ & $2.0000 \mathrm{E}-01$ & 0 & $4.0000 \mathrm{E}-01$ & 0.12 & 31896 \\
\hline (14) & 2343 & CL_L_TI & $\begin{array}{l}\text { Lower Salado clay: } 0 \text { to } \\
10 \text { years }\end{array}$ & SAT_RGAS & Residual Gas Saluration & UNIFORM & NONE & $2.0000 \mathrm{E}-01$ & $2.0000 \mathrm{E}-01$ & 0 & $4.0000 \mathrm{E}-01$ & 0.12 & 31922 \\
\hline (14) & 2360 & CL_L_T2 & $\begin{array}{l}\text { Lower Salado clay: } 10 \text { to } \\
25 \text { years }\end{array}$ & SAT_RGAS & Residual Gas Saturation & UNIFORM & NONE & $2.0000 \mathrm{E}-01$ & $2.0000 \mathrm{E}-01$ & 0 & $4.0000 \mathrm{E}-01$ & 0.12 & 31971 \\
\hline (14) & 2377 & CL_L_T3 & $\begin{array}{l}\text { Lower Salado clay: } 25 \text { to } \\
50 \text { years }\end{array}$ & SAT_RGAS & Residual Gas Saturation & UNIFORM & NONE & $2.0000 \mathrm{E}-01$ & $2.0000 \mathrm{E}-01$ & 0 & $4.0000 \mathrm{E}-01$ & 0.12 & 32005 \\
\hline (14) & 3083 & CL_L_T4 & $\begin{array}{l}\text { Lower Salado clay: } 50 \text { to } \\
\text { 10K years } \\
\end{array}$ & SAT_RGAS & Residual Gas Saturation & UNIFORM & NONE & $2.0000 \mathrm{E}-01$ & $2.0000 \mathrm{E}-01$ & 0 & $4.0000 \mathrm{E}-01$ & 0.12 & 32027 \\
\hline (14) & 2394 & CL_M_TI & $\begin{array}{l}\text { Upper Salado clay: } 0 \text { to } \\
10 \text { years } \\
\end{array}$ & SAT_RGAS & Residual Gas Saturation & UNIFORM & NONE & $2.0000 \mathrm{E}-01$ & $2.0000 \mathrm{E}-01$ & 0 & $4.0000 \mathrm{E}-01$ & 0.12 & 32062 \\
\hline (14) & 2411 & CL_M_T2 & $\begin{array}{l}\text { Upper Salado clay: } 10 \text { to } \\
25 \text { years }\end{array}$ & SAT_RGAS & Residual Gas Saturation & UNIFORM & NONE & $2.0000 \mathrm{E}-01$ & $2.0000 \mathrm{E}-01$ & 0 & $4.0000 \mathrm{E}-01$ & 0.12 & 32137 \\
\hline (14) & 2428 & CL_M_T3 & $\begin{array}{l}\text { Upper Salado clay: } 25 \text { to } \\
50 \text { years }\end{array}$ & SAT_RGAS & Residual Gas Saturation & UNIFORM & NONE & $2.0000 \mathrm{E}-01$ & $2.0000 \mathrm{E}-01$ & 0 & $4.0000 \mathrm{E}-01$ & 0.12 & 32169 \\
\hline (14) & 2445 & CL_M_T4 & $\begin{array}{l}\text { Upper Salado clay: } 50 \text { to } \\
100 \text { years } \\
\end{array}$ & SAT_RGAS & Residual Gas Saturation & UNIFORM & NONE & $2.0000 \mathrm{E}-01$ & $2.0000 \mathrm{E}-01$ & 0 & $4.0000 \mathrm{E}-01$ & 0.12 & 32226 \\
\hline (14) & 2462 & CL_M_T5 & $\begin{array}{l}\text { Upper Salado clay: } 100 \\
\text { to } 10 \mathrm{~K} \text { years } \\
\end{array}$ & SAT_RGAS & Residual Gas Saturation & UNIFORM & NONE & $2.0000 \mathrm{E}-01$ & $2.0000 \mathrm{E}-01$ & 0 & $4.0000 \mathrm{E}-01$ & 0.12 & 32255 \\
\hline (14) & 2326 & CLAY BOT & Shaft Bottom Clay & SAT RGAS & Residual Gas Saturation & UNIFORM & NONE & $2.0000 \mathrm{E}-01$ & $2.0000 \mathrm{E}-01$ & ol & $4.0000 \mathrm{E}-01$ & 0.12 & 31875 \\
\hline
\end{tabular}

'For parameters with a triangular distribution, the value provided for the median is actually the mode. 


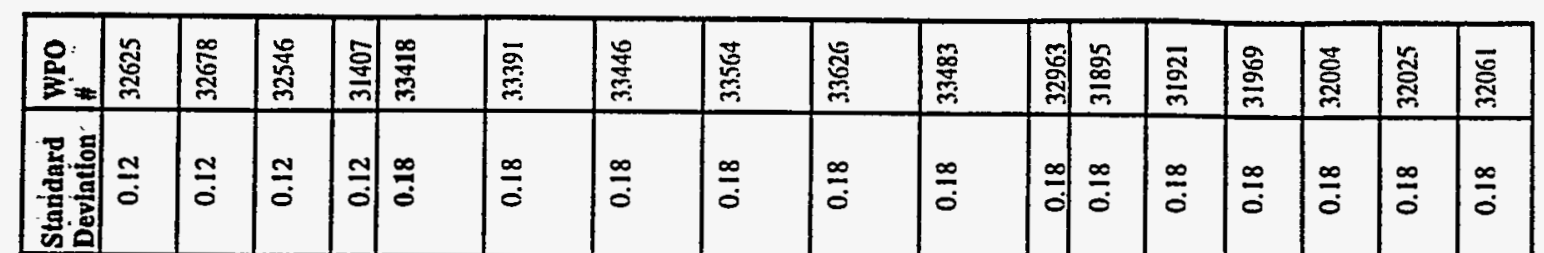

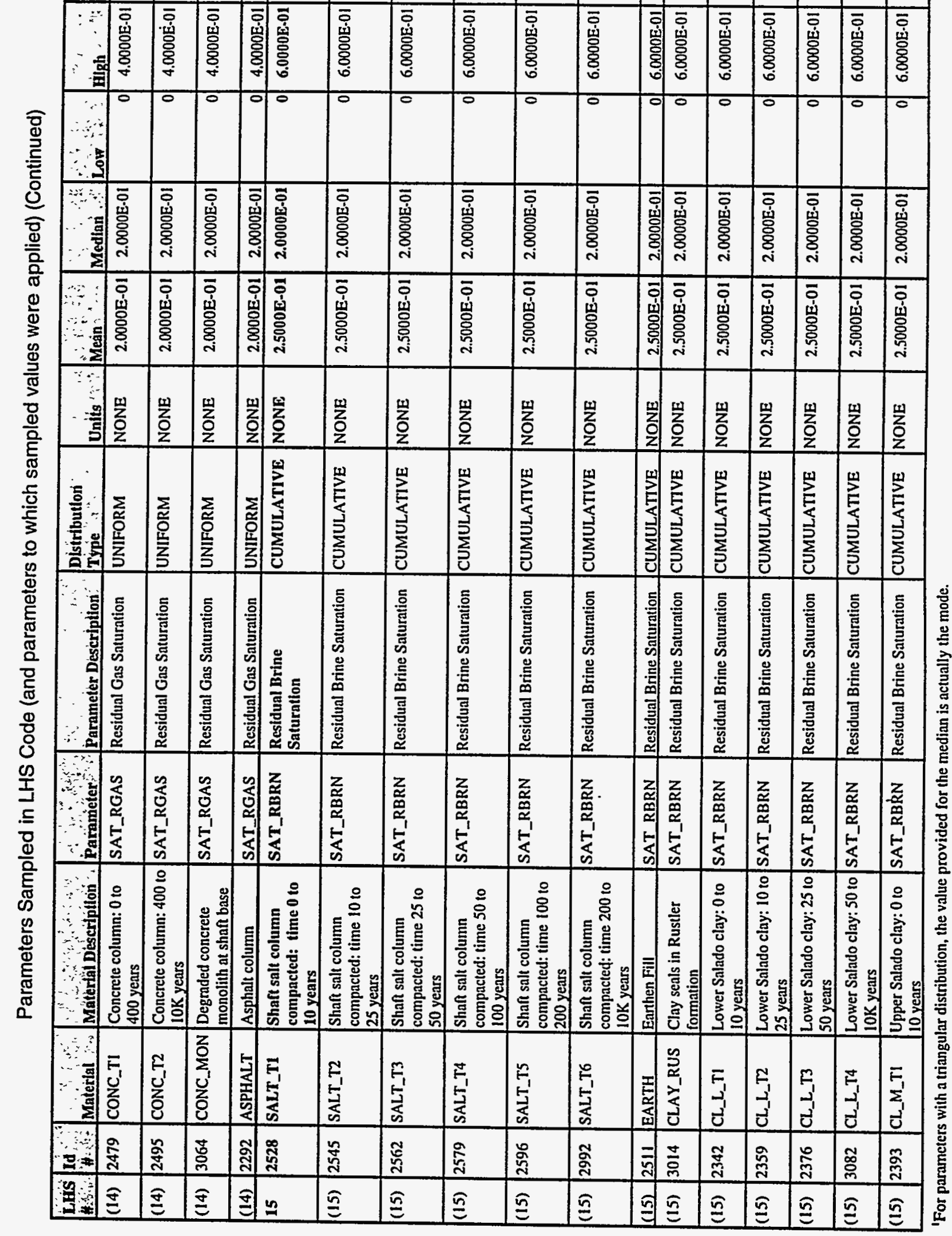


Parameters Sampled in LHS Code (and parameters to which sampled values were applied) (Continued)

\begin{tabular}{|c|c|c|c|c|c|c|c|c|c|c|c|c|c|}
\hline $\begin{array}{l}\text { LHS } \\
\# \\
\end{array}$ & $\begin{array}{l}\mathbf{I d} \\
\mathbf{f}\end{array}$ & Material. & Material Description & Parameter & Parimeter Description & \begin{tabular}{|l} 
Distribution \\
Type
\end{tabular} & Units & Mean & Median & Low: & High & $\begin{array}{l}\text { Standard } \\
\text { Deviation }\end{array}$ & $\begin{array}{l}\text { WPO } \\
4\end{array}$ \\
\hline (15) & 2410 & CL_M_T2 & $\begin{array}{l}\text { Upper Salado clay: } 10 \text { to } \\
25 \text { years }\end{array}$ & SAT_RBRN & Residual Brine Saturation & CUMULATIVE & NONE & $2.5000 \mathrm{E}-01$ & $2.0000 \mathrm{E}-01$ & 0 & $6.0000 \mathrm{E}-01$ & 0.18 & 32136 \\
\hline (15) & 2427 & CL_M_T3 & $\begin{array}{l}\text { Upper Salado clay: } 25 \text { to } \\
50 \text { years }\end{array}$ & SAT_RBRN & Residual Brine Saturation & CUMULATIVE & NONE & $2.5000 \mathrm{E}-01$ & $2.0000 \mathrm{E}-01$ & 0 & $6.0000 \mathrm{E}-01$ & 0.18 & 32168 \\
\hline (15) & 2444 & CL_M_T4 & $\begin{array}{l}\text { Upper Salado clay: } 50 \text { to } \\
100 \text { years }\end{array}$ & SAT_RBRN & Residual Brine Saturation & CUMULATIVE & NONE & $2.5000 \mathrm{E}-01$ & $2.0000 \mathrm{E}-01$ & 0 & $6.0000 \mathrm{E}-01$ & 0.18 & 32225 \\
\hline (15) & 2461 & CL_M_T5 & $\begin{array}{l}\text { Upper Salado clay: } 100 \\
\text { to } 10 \mathrm{~K} \text { years }\end{array}$ & SAT_RBRN & Residual Brine Saturation & CUMULATIVE & NONE & $2.5000 \mathrm{E}-01$ & $2.0000 \mathrm{E}-01$ & 0 & $6.0000 \mathrm{E}-01$ & 0.18 & 32254 \\
\hline (15) & 2325 & CLAY_BOT & Shaft Bottom Clay & SAT_RBRN & Residual Brine Saturation & CUMULATIVE & NONE & $2.5000 \mathrm{E}-01$ & $2.0000 \mathrm{E}-01$ & 0 & $6.0000 \mathrm{E}-01$ & 0.18 & $31874 \mathrm{~A}$ \\
\hline (15) & 2478 & CONC_TI & $\begin{array}{l}\text { Concrete column: } 0 \text { to } \\
400 \text { years }\end{array}$ & SAT_RBRN & Residual Brine Saturation & CUMULATIVE & NONE & $2.5000 \mathrm{E}-01$ & $2.0000 \mathrm{E}-01$ & 0 & $6.0000 \mathrm{E}-01$ & 0.18 & 32623 \\
\hline (15) & 2494 & CONC_T2 & $\begin{array}{l}\text { Concrete column: } 400 \text { to } \\
\text { 10K years }\end{array}$ & SAT_RBRN & Residual Brine Saturation & CUMULATIVE & NONE & $2.5000 \mathrm{E}-01$ & $2.0000 \mathrm{E}-01$ & 0 & $6.0000 \mathrm{E}-01$ & 0.18 & 32676 \\
\hline (15) & 3063 & CONC_MON & $\begin{array}{l}\text { Degraded concrete } \\
\text { monolith at shaft base }\end{array}$ & SAT_RBRN & Residual Brine Saturation & CUMULATIVE & NONE & $2.5000 \mathrm{E}-01$ & $2.0000 \mathrm{E}-01$ & 0 & $6.0000 \mathrm{E}-01$ & 0.18 & 32542 \\
\hline (15) & 2291 & ASPHALT & Asphalt column & SAT_RBRN & Residual Brine Saturation & CUMULATIVE & NONE & $2.5000 \mathrm{E}-01$ & $2.0000 \mathrm{E}-01$ & $\underline{0}$ & $6.0000 \mathrm{E}-01$ & 0.18 & 31405 \\
\hline 16 & 2516 & SALT_T1 & $\begin{array}{l}\text { Shaft salt column } \\
\text { compacted: time } 0 \text { to } \\
10 \text { years }\end{array}$ & PORE_DIS & $\begin{array}{l}\text { Brooks-Corcy pore } \\
\text { distribution parameter }\end{array}$ & CUMULATIVE & NONE & $2.5200 \mathrm{E}+00$ & $9.4000 \mathrm{E}-01$ & $1.1000 \mathrm{E}-01$ & $8.1000 \mathrm{E}+00$ & 2.48 & 33380 \\
\hline (16) & 2550 & SALT_T3 & $\begin{array}{l}\text { Shaft salt column } \\
\text { compacted: time } 25 \text { to } \\
50 \text { years }\end{array}$ & POR_DIS & $\begin{array}{l}\text { Brooks-Corey pore } \\
\text { distribution parameter }\end{array}$ & CUMULATIVE & NONE & $2.5200 E+00$ & $9.4000 \mathrm{E}-01$ & $1.1000 \mathrm{E}-01$ & $8.1000 \mathrm{E}+00$ & 2.48 & 33415 \\
\hline (16) & 2567 & SALT_T4 & $\begin{array}{l}\text { Shaft salt column } \\
\text { compacted: time } 50 \text { to } \\
100 \text { years }\end{array}$ & POR_DIS & $\begin{array}{l}\text { Bronks-Corcy pore } \\
\text { distribution parameter }\end{array}$ & CUMULATIVE & NONE & $2.5200 \mathrm{E}+00$ & $9.4000 \mathrm{E}-01$ & $1.1000 \mathrm{E}-01$ & $8.1000 \mathrm{E}+00$ & 2.48 & 33465 \\
\hline (16) & 2533 & SALT_T2 & $\begin{array}{l}\text { Shaft salt column } \\
\text { compacted: time } 10 \text { to } \\
25 \text { years } \\
\end{array}$ & POR_DIS & $\begin{array}{l}\text { Brooks-Corey pore } \\
\text { distribution parameter }\end{array}$ & CUMULATIVE & NONE & $2.5200 \mathrm{E}+00$ & $9.4000 \mathrm{E}-01$ & $1.1000 \mathrm{E}-01$ & $8.1000 E+00$ & 2.48 & 333.55 \\
\hline (16) & 2809 & SALT_T5 & $\begin{array}{l}\text { Shaft salt column } \\
\text { compacted: time } 100 \text { to } \\
200 \text { years }\end{array}$ & POR_DIS & $\begin{array}{l}\text { Brooks-Corey pore } \\
\text { distribution parameter }\end{array}$ & CUMULATIVE & NONE & $2.5200 \mathrm{E}+00$ & $9.4000 \mathrm{E}-01$ & $1.1000 \mathrm{E}-01$ & $8.1000 E+00$ & 2.48 & 33588 \\
\hline (16) & 2989 & SALT_T6 & $\begin{array}{l}\text { Shaft salt column } \\
\text { compacted: time } 200 \text { to } \\
\text { 10K years }\end{array}$ & POR_DIS & $\begin{array}{l}\text { Brooks-Corcy pore } \\
\text { distribution parameter }\end{array}$ & CUMULATTVE & NONE & $2.5200 \mathrm{E}+00$ & $9.4000 \mathrm{E}-01$ & $1.1000 \mathrm{E}-01$ & $8.1000 \mathrm{E}+00$ & 2.48 & 33655 \\
\hline (16) & 2499 & EARTH & Earthen Fill & POR_DIS & $\begin{array}{l}\text { Brooks-Corey pore } \\
\text { distribution parameter }\end{array}$ & CUMULATIVE & NONE & $2.5200 \mathrm{E}+00$ & $9.4000 \mathrm{E}-01$ & $1.1000 \mathrm{E}-01$ & $8.1000 \mathrm{E}+00$ & 2.48 & 3292.3 \\
\hline (16) & 3006 & CLAY_RUS & $\begin{array}{l}\text { Clay seals in Rustler } \\
\text { formation }\end{array}$ & POR_DIS & \begin{tabular}{|l|}
$\begin{array}{l}\text { Brooks-Corey pore } \\
\text { distribution parameter }\end{array}$ \\
\end{tabular} & CUMULATIVE & NONE & $2.5200 \mathrm{E}+00$ & $9.4000 \mathrm{E}-01$ & $1.1000 \mathrm{E}-01$ & $8.1000 \mathrm{E}+00$ & 2.48 & 31883 \\
\hline (16) & 2330 & CL_L_Tl & $\begin{array}{l}\text { Lower Salado clay: } 0 \text { to } \\
10 \text { years }\end{array}$ & POR_DIS & $\begin{array}{l}\text { Brooks-Corey pore } \\
\text { distribution parameter }\end{array}$ & CUMULATIVE & NONE & $2.5200 \mathrm{E}+00$ & $9.4000 \mathrm{E}-01$ & $1.1000 \mathrm{E}-01$ & $8.1000 \mathrm{E}+00$ & 2.48 & 31904 \\
\hline
\end{tabular}

'For parameters with a triangular distribution, the value provided for the median is actually the mode. 
Parameters Sampled in LHS Code (and parameters to which sampled values were applied) (Continued)

\begin{tabular}{|c|c|c|c|c|c|c|c|c|c|c|c|c|c|}
\hline 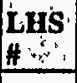 & Id & Material & Matertai Descriptlon & Parameter & Parameter Descriplion & $\begin{array}{l}\text { Distribution } \\
\text { Typé }\end{array}$ & Units $: \vdots$ & Mean & Median & Low & Ḧ̈ghi: $\cdots$ & $\begin{array}{l}\text { Standard } \\
\text { Deviatlon: }\end{array}$ & WPO \\
\hline (16) & 2347 & CL_L_T2 & $\begin{array}{l}\text { Lower Salado clay: } 10 \text { to } \\
25 \text { years }\end{array}$ & POR_DIS & $\begin{array}{l}\text { Brooks-Corey pore } \\
\text { distribution parameter }\end{array}$ & CUMULATIVE & NONE & $2.5200 \mathrm{E}+00$ & $9.4000 \mathrm{E}-01$ & $1.1000 \mathrm{E}-01$ & $8.1000 \mathrm{E}+00$ & 2.48 & 31931 \\
\hline (16) & 2364 & CL_L_T3 & $\begin{array}{l}\text { Lower Salado clay: } 25 \text { to } \\
50 \text { years }\end{array}$ & POR_DIS & $\begin{array}{l}\text { Brooks-Corey pore } \\
\text { distribution parameter }\end{array}$ & CUMULATIVE & NONE & $2.5200 \mathrm{E}+00$ & $9.4000 \mathrm{E}-01$ & $1.1000 \mathrm{E}-01$ & $8.1000 \mathrm{E}+00$ & 2.48 & 31982 \\
\hline (16) & 3076 & CL_L_T4 & $\begin{array}{l}\text { Lower Salado clay: } 50 \text { to } \\
100 \text { years }\end{array}$ & POR_DIS & $\begin{array}{l}\text { Brooks-Corey pore } \\
\text { distribution parameter }\end{array}$ & CUMULATIVE & NONE & $2.5200 \mathrm{E}+00$ & $9.4000 \mathrm{E}-01$ & $1.1000 \mathrm{E}-01$ & $8.1000 \mathrm{E}+00$ & 2.48 & 32013 \\
\hline (16) & 2381 & CL_M_Tl & $\begin{array}{l}\text { Upper Salado clay: } 0 \text { to } \\
10 \text { years }\end{array}$ & POR_DIS & $\begin{array}{l}\text { Brooks-Corey pore } \\
\text { distribution parameter }\end{array}$ & CUMULATIVE & NONE & $2.5200 \mathrm{E}+00$ & $9.4000 \mathrm{E}-01$ & $1.1000 \mathrm{E}-01$ & $8.1000 \mathrm{E}+00$ & 2.48 & 32044 \\
\hline (16) & 2398 & CL_M_T2 & $\begin{array}{l}\text { Upper Salado clay: } 10 \text { to } \\
25 \text { years }\end{array}$ & POR_DIS & $\begin{array}{l}\text { Brooks-Coney pore } \\
\text { distribution parameter }\end{array}$ & CUMULATIVE & NONE & $2.5200 \mathrm{E}+00$ & $9.4000 \mathrm{E}-01$ & $1.1000 \mathrm{E}-01$ & $8.1000 \mathrm{E}+00$ & 2.48 & 32119 \\
\hline (16) & 2415 & CL_M_T3 & $\begin{array}{l}\text { Upper Salado clay: } 25 \text { to } \\
50 \text { years }\end{array}$ & POR_DIS & $\begin{array}{l}\text { Brooks-Corey pore } \\
\text { distribution parameter }\end{array}$ & CUMULATIVE & NONE & $2.5200 \mathrm{E}+00$ & $9.4000 \mathrm{E}-01$ & $1.1000 \mathrm{E}-01$ & $8.1000 \mathrm{E}+00$ & 2.48 & 32146 \\
\hline (16) & 2432 & CL_M_T4 & $\begin{array}{l}\text { Upper Salado clay: } 50 \text { to } \\
100 \text { years }\end{array}$ & POR_DIS & $\begin{array}{l}\text { Brooks-Corey pore } \\
\text { distribution parameter }\end{array}$ & CUMULATIVE & NONE & $2.5200 \mathrm{E}+00$ & $9.4000 \mathrm{E}-01$ & $1.1000 \mathrm{E}-0 \mathrm{I}$ & $8.1000 \mathrm{E}+00$ & 2.48 & 32198 \\
\hline (16) & 2449 & CL_M_T5 & $\begin{array}{l}\text { Upper Salado clay: } 100 \\
\text { to 10K years }\end{array}$ & POR_DIS & $\begin{array}{l}\text { Brooks-Corey pore } \\
\text { distribution parameter }\end{array}$ & CUMULATIVE & NONE & $2.5200 \mathrm{E}+00$ & $9.4000 \mathrm{E}-01$ & $1.1000 \mathrm{E}-01$ & $8.1000 \mathrm{E}+00$ & 2.48 & 32235 \\
\hline (16) & 2313 & CLAY_BOT & Shaft Bottom Clay & POR_DIS & $\begin{array}{l}\text { Brooks-Corey pore } \\
\text { distribution parameter }\end{array}$ & CUMULATIVE & NONE & $2.5200 \mathrm{E}+00$ & $9.4000 \mathrm{E}-01$ & $1.1000 \mathrm{E}-0 \mathrm{I}$ & $8.1000 \mathrm{E}+00$ & 2.48 & 31862 \\
\hline (16) & 2466 & CONC_TI & $\begin{array}{l}\text { Concrete column: } 0 \text { to } \\
400 \text { years }\end{array}$ & POR_DIS & $\begin{array}{l}\text { Brooks-Corey pore } \\
\text { distribution parameter }\end{array}$ & CUMULATIVE & NONE & $2.5200 \mathrm{E}+00$ & $9.4000 \mathrm{E}-01$ & $1.1000 \mathrm{E}-01$ & $8.1000 E+00$ & 2.48 & 32570 \\
\hline (16) & 2466 & CONC_TI & $\begin{array}{l}\text { Concrete column: } 0 \text { to } \\
400 \text { years }\end{array}$ & POR_DIS & $\begin{array}{l}\text { Brooks-Corey pore } \\
\text { distribution parameter }\end{array}$ & CUMULATIVE & NONE & $2.5200 \mathrm{E}+00$ & $9.4000 \mathrm{E}-01$ & $1.1000 \mathrm{E}-01$ & $8.1000 E+00$ & 2.48 & 32570 \\
\hline (16) & 2483 & CONC_T2 & $\begin{array}{l}\text { Concrete column: } 400 \text { to } \\
10 \mathrm{~K} \text { years }\end{array}$ & POR_DIS & $\begin{array}{l}\text { Brooks-Corey pore } \\
\text { distribution parameter }\end{array}$ & CUMULATIVE & NONE & $2.5200 \mathrm{E}+00$ & $9.4000 \mathrm{E}-01$ & $1.1000 \mathrm{E}-01$ & $8.1000 \mathrm{E}+00$ & 2.48 & 32658 \\
\hline (16) & 3057 & CONC_MON & $\begin{array}{l}\text { Degraded concrete } \\
\text { monolith at shaft base } \\
\end{array}$ & POR_DIS & $\begin{array}{l}\text { Brooks-Corey pore } \\
\text { distribution parameter }\end{array}$ & CUMULATIVE & NONE & $2.5200 \mathrm{E}+00$ & $9.4000 \mathrm{E}-01$ & $1.1000 \mathrm{E}-01$ & $8.1000 \mathrm{E}+00$ & 2.48 & 32514 \\
\hline (16) & 2279 & ASPHALT & Asphalt column & POR_DIS & $\begin{array}{l}\text { Brooks-Corey pore } \\
\text { distribution parameter }\end{array}$ & CUMULATIVE & NONE & $2.5200 \mathrm{E}+00$ & $9.4000 \mathrm{E}-01$ & $1.1000 \mathrm{E}-01$ & $8.1000 E+00$ & 2.48 & 31386 \\
\hline 17 & 544 & S_HALITE & Salado halite, intact & POROSITY & Effective porosity & CUMULATIVE & NONE & $1.2800 \mathrm{E}-02$ & $1.0000 \mathrm{E}-02$ & $1.0000 \mathrm{E}-03$ & $3.0000 \mathrm{E}-02$ & 0.01 & 34387 \\
\hline 18 & 547 & S_HALITE & Salado halite, intact & PRMX_LOG & $\begin{array}{l}\text { Log of intrinsic } \\
\text { permeability, X-direction }\end{array}$ & UNIFORM & $\log \left(m^{\wedge} 2\right)$ & $-2.2500 \mathrm{E}+01$ & $-2.2500 \mathrm{E}+01$ & $-2.4000 \mathrm{E}+01$ & $-2.1000 \mathrm{E}+01$ & 0.87 & $34397 \mathrm{~A}$ \\
\hline (18) & 548 & S_HALITE & Salado halite, intact & PRMY_LOG & $\begin{array}{l}\text { Log of intrinsic } \\
\text { permeability. Y-direction }\end{array}$ & UNIFORM & $\log \left(m^{\wedge} 2\right)$ & $-2.2500 \mathrm{E}+01$ & $-2.2500 E+01$ & $-2.4000 \mathrm{E}+01$ & $-2.1000 \mathrm{E}+01$ & 0.87 & $34399 A$ \\
\hline (18) & 549 & S_HALITE & Salado halite, intact & PRMZ_LOG & $\begin{array}{l}\text { Log of intrinsic } \\
\text { permeability, Z-direction }\end{array}$ & UNIFORM & $\log \left(m^{\wedge} 2\right)$ & $-2.2500 \mathrm{E}+01$ & $-2.2500 \mathrm{E}+01$ & $-2.4000 \mathrm{E}+01$ & $-2.1000 \mathrm{E}+01$ & 0.87 & $34401 \mathrm{~A}$ \\
\hline 19 & 541 & S_HALITE & Salado halite, intact & COMP RCK & Bulk Compressibility & UNIFORM & $\mathrm{Pa}^{\wedge}-1$ & 9.7500E-11 & $9.7500 \mathrm{E}-11$ & $2.9400 \mathrm{E}-12$ & $1.9200 \mathrm{E}-10$ & 0 & 34210 \\
\hline 20 & 591 & S_MB139 & $\begin{array}{l}\text { Salado MB139, intact } \\
\text { and fractured }\end{array}$ & PRMX_LOG & $\begin{array}{l}\text { Log of intrinsic } \\
\text { permeability, X-direction }\end{array}$ & STUDENT'S-T & $\log \left(m^{\wedge} 2\right)$ & $-1.8890 \mathrm{E}+01$ & $-1.8890 \mathrm{E}+01$ & $-2.1000 \mathrm{E}+01$ & $-1.7100 \mathrm{E}+01$ & 1.20 & 34865 \\
\hline
\end{tabular}

'For parameters with a triangular distribution, the value provided for the median is actually the mode. 
Parameters Sampled in LHS Code (and parameters to which sampled values were applied) (Continued)

\begin{tabular}{|c|c|c|c|c|c|c|c|c|c|c|c|c|c|}
\hline LHS & Id & Material & Material Description & Parameter & Parameter Descrintion & $\begin{array}{l}\text { Distribution } \\
\text { Trpe }\end{array}$ & Units & Mean & Median & Low & Hiph. & $\begin{array}{l}\text { Standard } \\
\text { Deviation }\end{array}$ & $\begin{array}{l}\text { WPO } \\
\text { \# }\end{array}$ \\
\hline (20) & 592 & S_MB139 & $\begin{array}{l}\text { Salado MBI39, intact } \\
\text { and fractured }\end{array}$ & PRMY_LOG & $\begin{array}{l}\text { Log of intrinsic } \\
\text { permeability, Y-direction }\end{array}$ & STUDENT"S-T & $\log \left(m^{\wedge} 2\right)$ & $-1.8890 E+01$ & $-1.8890 E+01$ & $-2.1000 E+01$ & $-1.7100 \mathrm{E}+01$ & 1.20 & 34866 \\
\hline (20) & 593 & S_MB139 & $\begin{array}{l}\text { Salado MB139, intact } \\
\text { and fractured }\end{array}$ & PRMZ_LOG & $\begin{array}{l}\text { Log of intrinsic } \\
\text { permeability, Z-ditection }\end{array}$ & STUDENT'S-T & $\log \left(m^{\wedge} 2\right)$ & $-1.8890 \mathrm{E}+01$ & $-1.8890 E+01$ & $-2.1000 \mathrm{E}+01$ & $-1.7100 E+01$ & 1.20 & 34868 \\
\hline (20) & 531 & S_ANH_AB & $\begin{array}{l}\text { Salado anhydrite beds a } \\
\& \text { b, intact and fractured }\end{array}$ & PRMX_LOG & $\begin{array}{l}\text { Log of intrinsic } \\
\text { permeability, X-direction }\end{array}$ & STUDENT'S-T & $\log \left(m^{\wedge} 2\right)$ & $-1.8890 \mathrm{E}+01$ & $-1.8890 \mathrm{E}+01$ & $-2.1000 \mathrm{E}+01$ & $-1.7100 E+01$ & 1.20 & 34196 \\
\hline (20) & 532 & S_ANH_AB & $\begin{array}{l}\text { Salado anhydrite beds a } \\
\& \text { b, intact and fractured }\end{array}$ & PRMY_LOG & $\begin{array}{l}\text { Log of intrinsic } \\
\text { permeability. Y-direction }\end{array}$ & STUDENT'S-T & $\log \left(m^{\wedge} 2\right)$ & $-1.8890 \mathrm{E}+01$ & $-1.8890 E+01$ & $-2.1000 \mathrm{E}+01$ & $-1.7100 E+01$ & 1.20 & 34197 \\
\hline (20) & 533 & S_ANH_AB & $\begin{array}{l}\text { Salado anhydrite beds a } \\
\text { \& b, intact and fractured }\end{array}$ & PRMZ_LOG & $\begin{array}{l}\text { Log of intrinsic } \\
\text { permeability, Z-direction }\end{array}$ & STUDENT'S-T & $\log \left(m^{\wedge} 2\right)$ & $-1.8890 \mathrm{E}+01$ & $-1.8890 \mathrm{E}+0 \mathrm{I}$ & $-2.1000 \mathrm{E}+01$ & $-1.7100 \mathrm{E}+01$ & 1.20 & 34198 \\
\hline (20) & 570 & S_MB138 & $\begin{array}{l}\text { Salado MB } 138 \text {, intact } \\
\text { and fractured }\end{array}$ & PRMX_LOG & $\begin{array}{l}\text { Log of intrinsic } \\
\text { permeability. X-direction }\end{array}$ & STUDENT'S-T & $\log \left(m^{\wedge} 2\right)$ & $-1.8890 \mathrm{E}+01$ & $-1.8890 \mathrm{E}+01$ & $-2.1000 \mathrm{E}+01$ & $-1.7100 \mathrm{E}+01$ & 1.20 & 34536 \\
\hline (20) & 571 & S_MB138 & $\begin{array}{l}\text { Salado MB138, intact } \\
\text { and fractured }\end{array}$ & PRMY_LOG & $\begin{array}{l}\text { Log of intrinsic } \\
\text { permeability. Y-direction }\end{array}$ & STUDENT'S-T & $\log \left(m^{\wedge} 2\right)$ & $-1.8890 E+01$ & $-1.8890 \mathrm{E}+01$ & $\mid-2.1000 \mathrm{E}+01$ & $-1.7100 \mathrm{E}+01$ & 1.20 & 34537 \\
\hline (20) & 572 & S_MB138 & $\begin{array}{l}\text { Salado MBI38, intact } \\
\text { and fractured }\end{array}$ & PRMZ_LOG & $\begin{array}{l}\text { Log of intrinsic } \\
\text { permeability, Z-direction }\end{array}$ & STUDENT'S-T & $\log \left(m^{\wedge} 2\right)$ & $-1.8890 \mathrm{E}+01$ & $-1.8890 \mathrm{E}+01$ & $-2.1000 \mathrm{E}+01$ & $-1.7100 \mathrm{E}+01$ & 1.20 & 34538 \\
\hline 21 & 580 & S_MB139 & $\begin{array}{l}\text { Salado MB139, intact } \\
\text { and fractured }\end{array}$ & COMP_RCK & Bulk Compressibility & STUDENT'S-T & $\mathrm{Pa}^{\wedge}-1$ & $8.2630 \mathrm{E}-11$ & $8.2630 \mathrm{E}-11$ & $1.0900 \mathrm{E}-11$ & $2.7500 \mathrm{E}-10$ & 0 & 34574 \\
\hline (21) & 521 & S_ANH_AB & $\begin{array}{l}\text { Salado anhydrite beds a } \\
\& \mathbf{b} \text {, intact and fractured }\end{array}$ & COMP_RCK & Bulk Compressibility & STUDENT'S-T & $\mathrm{Pa}^{\wedge}-1$ & $8.2630 \mathrm{E}-11$ & $8.2630 \mathrm{E}-11$ & $1.0900 \mathrm{E}-11$ & $2.7500 \mathrm{E}-10$ & 0 & 341.35 \\
\hline (21) & 560 & S_MBI38 & $\begin{array}{l}\text { Salado MB } 138 \text {, intact } \\
\text { and fractured }\end{array}$ & COMP_RCK & Bulk Compressibility & STUDENT'S-T & $P a^{\wedge}-1$ & $8.2630 \mathrm{E}-11$ & $8.2630 \mathrm{E}-11$ & $1.0900 \mathrm{E}-11$ & $2.7500 \mathrm{E}-10$ & 0 & 34439 \\
\hline 22 & 596 & S_MB139 & $\begin{array}{l}\text { Salado MB139, intact } \\
\text { and fractured }\end{array}$ & $\begin{array}{c}\text { RELP_MOD } \\
. \\
\end{array}$ & $\begin{array}{l}\text { Model number, relative } \\
\text { permeability model }\end{array}$ & DELTA & NONE & n.a. & n.a. & 1 & 4 & n.a. & 34500 \\
\hline (22) & 536 & S_ANH_AB & $\begin{array}{l}\text { Salado anhydrite beds a } \\
\& \text { b, intact and fractured }\end{array}$ & RELP_MOD & $\begin{array}{l}\text { Model number, relative } \\
\text { permeability model }\end{array}$ & DELTA & NONE & n.a. & n.a. & 1 & 4 & n.a. & 34201 \\
\hline (22) & 575 & S_MBI38 & $\begin{array}{l}\text { Salado MB } 138 \text {, intact } \\
\text { and fractured }\end{array}$ & RELP_MOD & $\begin{array}{l}\text { Model number, relative } \\
\text { perneability model }\end{array}$ & DELTA & NONE & n.a. & n.a. & 1 & 4 & n.a. & 34.542 \\
\hline 23 & 598 & S_MB139 & $\begin{array}{l}\text { Salado MB139, intact } \\
\text { and fractured }\end{array}$ & SAT_RBRN & $\begin{array}{l}\text { Residual Brine } \\
\text { Saturation } \\
\end{array}$ & STUDENT'S-T & NONE & $8.3630 \mathrm{E}-02$ & $8.3630 \mathrm{E}-02$ & $7.8460 \mathrm{E}-03$ & $1.7400 \mathrm{E}-01$ & 0.05 & 34506 \\
\hline (23) & 538 & S_ANH_AB & $\begin{array}{l}\text { Salado anhydrite beds a } \\
\& \text { b. intact and fractured }\end{array}$ & SAT_RBRN & Residual Brine Saturation & STUDENT'S-T & NONE & $8.3630 \mathrm{E}-02$ & $8.3630 \mathrm{E}-02$ & $7.8460 \mathrm{E}-0.3$ & $1.7400 \mathrm{E}-01$ & 0.05 & 34203 \\
\hline (23) & 577 & S_MBI38 & $\begin{array}{l}\text { Salado MB } 138, \text { intact } \\
\text { and fractured }\end{array}$ & SAT_RBRN & Residual Brine Saturation & STUDENT'S-T & NONE & $8.3630 \mathrm{E}-02$ & $8.3630 \mathrm{E}-02$ & $7.8460 \mathrm{E}-03$ & $1.7400 \mathrm{E}-01$ & 0.05 & 34.545 \\
\hline
\end{tabular}

'For paraneters with a triangular distribution, the value provided for the median is actually the mode. 
Parameters Sampled in LHS Code (and parameters to which sampled values were applied) (Continued)

\begin{tabular}{|c|c|c|c|c|c|c|c|c|c|c|c|c|c|}
\hline Lits & $\begin{aligned} & \mathbf{I d} \cdots \\
& \# \cdots \\
&\end{aligned}$ & Materlai & Material Description & Parameter & Parameter Descripllon & $\begin{array}{l}\text { Distribution } \\
\text { Typet; }\end{array}$ & Units & Méan & Médlan: & Low & High & $\begin{array}{l}\text { Standard } \\
\text { Devlation. } \\
\text { Devition }\end{array}$ & WPO \\
\hline 24 & 599 & S_MB139 & $\begin{array}{l}\text { Salado MB139, intact } \\
\text { and fractured }\end{array}$ & SAT_RGAS & Residual Gas Saturation & STUDENT'S-T & NONE & $7.7110 \mathrm{E}-02$ & $7.7110 \mathrm{E}-02$ & 1.3980E-02 & 1.9719E-01 & 0.06 & 34508 \\
\hline (24) & 539 & S_ANH_AB & $\begin{array}{l}\text { Salado anhydrite beds a } \\
\& \text { b, intact and fractured }\end{array}$ & SAT_RGAS & Residual Gas Saturation & STUDENT'S-T & NONE & $7.7110 \mathrm{E}-02$ & 7.7110E-02 & 1.3980E-02 & $1.9719 \mathrm{E}-01$ & 0.06 & 34204 \\
\hline (24) & 578 & S_MB 138 & $\begin{array}{l}\text { Salado MB 138, intact } \\
\text { and fractured }\end{array}$ & SAT_RGAS & Residual Gas Saturation & STUDENT'S-T & NONE & $7.7110 \mathrm{E}-02$ & $7.7110 \mathrm{E}-02$ & $1.3980 \mathrm{E}-02$ & $1.9719 \mathrm{E}-01$ & 0.06 & 34546 \\
\hline 25 & 587 & S_MB139 & $\begin{array}{l}\text { Salado MB139, intact } \\
\text { and fractured }\end{array}$ & PORE_DIS & $\begin{array}{l}\text { Brooks-Corey pore } \\
\text { distribution parameter }\end{array}$ & STUDENT'S-T & NONE & $6.4360 \mathrm{E}-01$ & $6.4360 \mathrm{E}-01$ & 4.9053E-01 & $8.4178 \mathrm{E}-01$ & 0.11 & 34859 \\
\hline (25) & 527 & S_ANH_AB & $\begin{array}{l}\text { Salado anhydrite beds a } \\
\& b \text {, intact and fractured } \\
\end{array}$ & POR_DIS & $\begin{array}{l}\text { Brooks-Cony pore } \\
\text { distribution parameter }\end{array}$ & STUDENT'S-T & NONE & $6.4360 \mathrm{E}-01$ & $6.4360 \mathrm{E}-01$ & $4.9053 \mathrm{E}-01$ & $8.4178 \mathrm{E}-01$ & 0.11 & 34192 \\
\hline (25) & 566 & S_MBI38 & $\begin{array}{l}\text { Salado MB } 138 \text {, intact } \\
\text { and fractured }\end{array}$ & POR_DIS & $\begin{array}{l}\text { Brooks-Corey pore } \\
\text { distribution parameter }\end{array}$ & STUDENT'S-T & NONE & $6.4360 \mathrm{E}-01$ & $6.4360 \mathrm{E}-01$ & $4.9053 \mathrm{E}-01$ & $8.4178 \mathrm{E}-01$ & 0.11 & .34527 \\
\hline 26 & 546 & S_HALITE & Salado halite, intact & PRESSURE & $\begin{array}{l}\text { Brine far-field pore } \\
\text { pressure }\end{array}$ & UNIFORM & $\mathbf{P a}$ & $1.2470 \mathrm{E}+07$ & $1.2470 \mathrm{E}+07$ & $1.1040 E+07$ & $1.3890 \mathrm{E}+07$ & 823000.00 & 34394 \\
\hline 27 & 66 & CASTILER & $\begin{array}{l}\text { Castile Brine } \\
\text { Reservoir } \\
\end{array}$ & PRESSURE & $\begin{array}{l}\text { Brine far-field pore } \\
\text { pressure }\end{array}$ & TRIANGULAR & $\mathbf{P a}$ & $1.3600 E+07$ & $1.2700 \mathrm{E}+07$ & $1.1100 \mathrm{E}+07$ & $1.7000 \mathrm{E}+07$ & 1245700.00 & $31612 A$ \\
\hline 28 & 67 & CASTILER & $\begin{array}{l}\text { Castile Brine } \\
\text { Reservoir }\end{array}$ & PRMX_LOG & $\begin{array}{l}\text { Log of intrinsic } \\
\text { permeability, } X \text {-direction }\end{array}$ & TRIANGULAR & $\log \left(m^{\wedge} 2\right)$ & $-1.2100 \mathrm{E}+01$ & $-1.1800 E+01 \mid$ & $-1.4700 \mathrm{E}+01$ & $-9.8000 E+00$ & 1.01 & 31613 \\
\hline (28) & 68 & CASTILER & Castile Brine Reservoir & PRMY_LOG & $\begin{array}{l}\text { Log of intrinsic } \\
\text { permeability, Y-direction }\end{array}$ & TRIANGULAR & $\log \left(m^{\wedge} 2\right)$ & $-1.2100 \mathrm{E}+01$ & $-1.1800 \mathrm{E}+01$ & $-1.4700 \mathrm{E}+01$ & $-9.8000 \mathrm{E}+00$ & 1.01 & 31614 \\
\hline (28) & 69 & CASTILER & Castile Brine Reservoir & PRMZ_LOG & $\begin{array}{l}\text { Log of intrinsic } \\
\text { penneability, Z-direction }\end{array}$ & TRIANGULAR & $\log \left(m^{\wedge} 2\right)$ & $-1.2100 E+01$ & $-1.1800 \mathrm{E}+01$ & $-1.4700 \mathrm{E}+01$ & $-9.8000 \mathrm{E}+00$ & 1.01 & 31615 \\
\hline 29 & 61 & CASTILER & $\begin{array}{l}\text { Castile Brine } \\
\text { Reservoir }\end{array}$ & COMP_RCK & Bulk Compressibility & TRIANGULAR & $\log \left(\mathrm{Pa}^{\wedge}-1\right)$ & $-9.8000 \mathrm{E}+00$ & $|-1.0000 E+01|$ & $-1.1300 E+01$ & $-8.0000 \mathrm{E}+00$ & 0.68 & 31561 \\
\hline 30 & 3184 & BH_SAND & $\begin{array}{l}\text { Borchole filled with } \\
\text { silty sand }\end{array}$ & PRMX_LOG & $\begin{array}{l}\text { Log of intrinsic } \\
\text { permeability, X-direction }\end{array}$ & UNIFORM & $\log \left(m^{\wedge} 2\right)$ & $-1.2500 \mathrm{E}+01$ & $-1.2500 \mathrm{E}+01$ & $-1.4000 \mathrm{E}+01$ & $-1.1000 E+01$ & 0.87 & 36641 \\
\hline (30) & 3190 & BH_SAND & $\begin{array}{l}\text { Borehole filled with silly } \\
\text { sand }\end{array}$ & PRMY_LOG & $\begin{array}{l}\text { Log of intrinsic } \\
\text { permeability, Y-direction }\end{array}$ & UNIFORM & $\log \left(m^{\wedge} 2\right)$ & $-1.2500 E+01$ & $-1.2500 \mathrm{E}+01$ & $-1.4000 \mathrm{E}+01$ & $-1.1000 \mathrm{E}+01$ & 0.87 & 36654 \\
\hline (30) & 3191 & BH_SAND & $\begin{array}{l}\text { Borehole filled with silty } \\
\text { sand }\end{array}$ & PRMZ_LOG & $\begin{array}{l}\text { Log of intrinsic } \\
\text { permeability.Z-direction }\end{array}$ & UNIFORM & $\log \left(m^{\wedge} 2\right)$ & $-1.2500 \mathrm{E}+01$ & $-1.2500 \mathrm{E}+01$ & $-1.4000 \mathrm{E}+01$ & $-1.1000 \mathrm{E}+01$ & 0.87 & 36655 \\
\hline 31 & 3194 & CASTILER & $\begin{array}{l}\text { Castile Brine } \\
\text { Reservoir } \\
\end{array}$ & GRIDFLO & $\begin{array}{l}\text { Index for selecting a } \\
\text { Brine Pocket }\end{array}$ & DELTA & NONE & n.a. & n.a. & 1 & 32 & n.a. & $36658 \AA$ \\
\hline 32 & 3246 & BLOWOUT & $\begin{array}{l}\text { BRAGFLO Direct } \\
\text { Brine Releases }\end{array}$ & PARTDIA & $\begin{array}{l}\text { Logarithm of waste } \\
\text { particle diameter in } \\
\text { CUTTINGS model }\end{array}$ & LOGUNIFORM & $m$ & $2.3500 \mathrm{E}-02$ & $2.8000 \mathrm{E} \cdot 03$ & 4.0000E-05 & $2.0000 \mathrm{E}-01$ & 0.04 & 37088 \\
\hline
\end{tabular}

'For parameters with a triangular distribution, the value provided for the median is actually the mode. 
Parameters Sampled in LHS Code (and parameters to which sampled values were applied) (Continued)

\begin{tabular}{|c|c|c|c|c|c|c|c|c|c|c|c|c|c|}
\hline $\begin{array}{l}\text { LHS } \\
\#\end{array}$ & Id & Materinl & Material Description & Parameter & Parameler Description & $\begin{array}{l}\text { Distribution } \\
\text { Type }\end{array}$ & Units & Mean & Median & Low & High & $\begin{array}{l}\text { Standard } \\
\text { Devlation }\end{array}$ & $\begin{array}{l}\text { WPO } \\
\#\end{array}$ \\
\hline 33 & 2254 & BOREHOLE & Borehole and Fill & TAUFAIL & $\begin{array}{l}\text { Effective shear strength } \\
\text { for erosion (rfail) }\end{array}$ & UNIFORM & $\mathbf{P a}$ & $5.0300 \mathrm{E}+00$ & $5.0300 \mathrm{E}+00$ & $5.0000 \mathrm{E}-02$ & $1.0000 E+01$ & 2.90 & 31536 \\
\hline 34 & 3419 & CULEBRA & $\begin{array}{l}\text { Culebra member of the } \\
\text { Rustler formation }\end{array}$ & MINP_FAC & $\begin{array}{l}\text { Mining Transmissivity } \\
\text { Multiplier }\end{array}$ & UNIFORM & NONE & $5.0050 \mathrm{E}+02$ & $5.0050 \mathrm{E}+02$ & $1.0000 \mathrm{E}+00$ & $1.0000 \mathrm{E}+03$ & 288.40 & 37666 \\
\hline 35 & 225 & GLOBAL & $\begin{array}{l}\text { Information that } \\
\text { applies globally }\end{array}$ & TRANSIDX & \begin{tabular}{|l} 
Index for selecting \\
realizations of the \\
Transmissivity Ficld
\end{tabular} & UNIFORM & NONE & $5.0000 \mathrm{E}-01$ & $5.0000 \mathrm{E}-01$ & $0.0000 \mathrm{E}+00$ & $1.0000 E+00$ & 0.29 & 33055 \\
\hline 36 & 3262 & SOLAM3 & $\begin{array}{l}\text { Solubility of } \\
\text { Americium in } \\
\text { oxidation state III }\end{array}$ & SOLSIM & $\begin{array}{l}\text { Solubility in Salado } \\
\text { brine, inorganic } \\
\text { chemistry controlled by } \\
\text { the } \mathrm{Mg}(\mathrm{OH}) 2-\mathrm{MgCO} 3\end{array}$ & CUMULATIVE & NONE & $1.8000 \mathrm{E}-01$ & $-9.0000 \mathrm{E}-02$ & $-2.0000 \mathrm{E}+00$ & $1.4000 \mathrm{E}+00$ & 0.37 & $37105 A$ \\
\hline 37 & 3263 & SOLAM3 & $\begin{array}{l}\text { Solubility of } \\
\text { Americium in } \\
\text { oxidation state III }\end{array}$ & SOLCIM & \begin{tabular}{|l} 
Solubility in Castile \\
brine inorganic with \\
chemistry controlled by \\
$\mathrm{Mg}(\mathrm{OH}) 2-\mathrm{MgCO}$
\end{tabular} & CUMULATIVE & NONE & $1.8000 \mathrm{E}-01$ & $-9.0000 \mathrm{E}-02$ & $-2.0000 \mathrm{E}+00$ & $1.4000 \mathrm{E}+00$ & 0.37 & $37106 A$ \\
\hline 38 & 3265 & SOLPU3 & $\begin{array}{l}\text { Solubility of Plutonium } \\
\text { in oxidation state III }\end{array}$ & SOLSIM & $\begin{array}{l}\text { Solubility in Salado } \\
\text { brine, inorganic } \\
\text { chemistry controlled by } \\
\text { the } \mathrm{Mg}(\mathrm{OH}) 2-\mathrm{MgCO} 3\end{array}$ & CUMULATIVE & NONE & $1.8000 \mathrm{E}-01$ & $-9.0000 \mathrm{E}-02$ & $-2.0000 \mathrm{E}+00$ & $1.4000 \mathrm{E}+00$ & 0.37 & $37109 A$ \\
\hline 39 & 3264 & SOLPU3 & $\begin{array}{l}\text { Solubility of Plutonium } \\
\text { in oxidation state III }\end{array}$ & SOLCIM & $\begin{array}{l}\text { Solubility in Castile } \\
\text { brine inorganic with } \\
\text { chemistry controlled by } \\
\mathrm{Mg}(\mathrm{OH}) 2-\mathrm{MgCO}^{\circ}\end{array}$ & CUMULATIVE & NONE & $1.8000 \mathrm{E}-01$ & \begin{tabular}{|l|}
$-9.0000 \mathrm{E}-02$ \\
\end{tabular} & $-2.0000 \mathrm{E}+00$ & $1.4000 \mathrm{E}+00$ & 0.37 & $37108 \AA$ \\
\hline 40 & 3266 & SOLPU4 & $\begin{array}{l}\text { Solubility of Plutonium } \\
\text { in oxidation state IV } \\
\text {. }\end{array}$ & SOLSIM & $\begin{array}{l}\text { Solubility in Salado } \\
\text { brine, inorganic } \\
\text { chemistry controlled by } \\
\text { the } \mathrm{Mg}(\mathrm{OH}) 2 \cdot \mathrm{MgCO} 3 \\
\end{array}$ & CUMULATIVE & NONE & $1.8000 \mathrm{E}-01$ & $\mid-9.0000 \mathrm{E}-02$ & $-2.0000 E+00$ & $1.4000 \mathrm{E}+00$ & 0.37 & $37110 A$ \\
\hline 41 & 3389 & SOLPU4 & $\begin{array}{l}\text { Solubility of Plutonium } \\
\text { in oxidation state IV }\end{array}$ & SOLCIM & \begin{tabular}{|l} 
Solubility in Castile \\
brine inorganic with \\
chemistry controlled by \\
$\mathrm{Mg}(\mathrm{OH}) 2-\mathrm{MgCO3}$
\end{tabular} & CUMULATIVE & NONE & $1.8000 \mathrm{E}-01$ & -9.0000E-02 & $-2.0000 E+00$ & $1.4000 E+00$ & 0.37 & $37111 \mathrm{~A}$ \\
\hline 42 & 3390 & SOLU4 & $\begin{array}{l}\text { Solubility of Uranium } \\
\text { in oxidation state IV }\end{array}$ & SOLSIM & $\begin{array}{l}\text { Solubility in Salado } \\
\text { brine, inorganic } \\
\text { chemistry controlled by } \\
\text { the } \mathrm{Mg}(\mathrm{OH}) 2-\mathrm{MgCO} 3\end{array}$ & CUMULATIVE & NONE & $1.8000 \mathrm{E}-01$ & \begin{tabular}{|l|}
$-9.0000 \mathrm{E}-02$ \\
\end{tabular} & $-2.0000 E+00$ & $1.4000 \mathrm{E}+00$ & 0.37 & $37112 A$ \\
\hline 43 & 3391 & SOLU6 & $\begin{array}{l}\text { Solubility of Uranium } \\
\text { in oxidation state VI }\end{array}$ & SOLSIM & $\begin{array}{l}\text { Solubility in Salado } \\
\text { brine, inorganic } \\
\text { chemistry controlled by } \\
\text { the } \mathrm{Mg}(\mathrm{OH}) 2-\mathrm{MgCO} 3\end{array}$ & CUMULATIVE & NONE & $1.8000 \mathrm{E}-01$ & \begin{tabular}{|l|}
$-9.0000 \mathrm{E}-02$ \\
\end{tabular} & $-2.0000 E+00$ & $1.4000 \mathrm{E}+00$ & 0.37 & $37113 \AA$ \\
\hline
\end{tabular}

'For parameters with a triangular distribution, the value provided for the median is actually the mode. 
Parameters Sampled in LHS Code (and parameters to which sampled values were applied) (Continued)

\begin{tabular}{|c|c|c|c|c|c|c|c|c|c|c|c|c|c|}
\hline Lits & 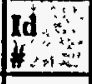 & Mátêrai & Material Description : & Parameler & Paraméter Descriplion & $\begin{array}{l}\text { Distributhon } \\
\text { Type }\end{array}$ & Units :- & Meân & Median' & Low & 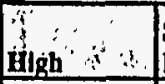 & $\begin{array}{l}\text { Ştandard } \\
\text { Devlatlion: }\end{array}$ & $\begin{array}{l}\text { WPO } \\
\text { \# } \therefore \text {. }\end{array}$ \\
\hline 44 & 3392 & SOLU6 & $\begin{array}{l}\text { Solubility of Uranium } \\
\text { in oxidation state VI }\end{array}$ & SOLCIM & $\begin{array}{l}\text { Solubility in Castile brine } \\
\text { inorganic with chemistry } \\
\text { controlled by } \mathrm{Mg}(\mathrm{OH}) 2 \text {. } \\
\mathrm{MgCO3}\end{array}$ & CUMULATIVE & NONE & $1.8000 \mathrm{E}-01$ & $|-9.0000 \mathrm{E}-02|$ & $-2.0000 \mathrm{E}+00 \mid$ & $1.4000 \mathrm{E}+00$ & 0.37 & 37114A \\
\hline 45 & 3393 & SOLTH4 & $\begin{array}{l}\text { Solubility of Thorium } \\
\text { in oxidation state IV }\end{array}$ & SOLSIM & $\begin{array}{l}\text { Solubility in Salado } \\
\text { brine, inorganic } \\
\text { chemistry controlled by } \\
\text { the } \mathrm{Mg}(\mathrm{OH}) 2-\mathrm{MgCO3}\end{array}$ & CUMULATIVE & NONE & $1.8000 \mathrm{E}-01$ & $|-9.0000 \mathrm{E}-02|$ & $-2.0000 \mathrm{E}+00$ & $1.4000 E+00$ & 0.37 & $37115 A$ \\
\hline 46 & 3429 & PHUMOX3 & $\begin{array}{l}\text { Proportionality } \\
\text { constant with humic } \\
\text { colloids for actinide in } \\
\text { oxidation state III }\end{array}$ & PHUMCIM & $\begin{array}{l}\text { Proportionality constant } \\
\text { of actinides incastile } \\
\text { brine w/ humlc colloids, } \\
\text { inorgan }\end{array}$ & CUMULATIVE & moles/iter & $1.1000 E+00$ & $1.3700 \mathrm{E}+00$ & $6.5000 \mathrm{E}-02$ & $1.6000 \mathrm{E}+00$ & 0.47 & 37683 \\
\hline 47 & 3417 & GLOBAL & $\begin{array}{l}\text { Information that } \\
\text { applies globally }\end{array}$ & OXSTAT & $\begin{array}{l}\text { Index for Oxidation } \\
\text { Solubllities } \\
\end{array}$ & UNIFORM & NONE & $5.0000 \mathrm{E}-01$ & 5.0000E-01 & $0.0000 \mathrm{E}+00$ & $1.0000 E+00$ & 0.29 & 37663 \\
\hline 48 & 223 & GLOBAL & $\begin{array}{l}\text { Information that } \\
\text { applies globally }\end{array}$ & CLIMTIDX & Climate Index & CUMULATIVE & NONE & $1.3100 E+00$ & $1.1700 E+00$ & $1.0000 E+00$ & $2.2500 \mathrm{E}+00$ & 0.35 & 33031 \\
\hline 49 & 3485 & CULEBRA & $\begin{array}{l}\text { Culebra member of the } \\
\text { Rustler formation }\end{array}$ & HMBLKLT & $\begin{array}{l}\text { Culebra Half Matrix- } \\
\text { Block Length }\end{array}$ & UNIFORM & $\mathbf{m}$ & $2.7500 \mathrm{E}-01$ & 2.7500E-01 & $5.0000 E-02$ & $5.0000 \mathrm{E}-01$ & 0.13 & 38356 \\
\hline 50 & 3487 & CULEBRA & $\begin{array}{l}\text { Culebra member of the } \\
\text { Rustler formation }\end{array}$ & APOROS & $\begin{array}{l}\text { Culebra Advective } \\
\text { Porosity }\end{array}$ & LOGUNIFORM & NONE & $2.1000 \mathrm{E}-03$ & $1.0000 \mathrm{E}-03$ & $1.0000 \mathrm{E}-04$ & $1.0000 \mathrm{E}-02$ & 0 & 38358 \\
\hline 51 & 3486 & CULEBRA & $\begin{array}{l}\text { Culebra member of the } \\
\text { Rustler formation }\end{array}$ & DPOROS & $\begin{array}{l}\text { Diffusive Porosity for } \\
\text { Culebra Dolomite }\end{array}$ & CUMULATIVE & NONE & $1.6000 \mathrm{E}-01$ & $1.6000 \mathrm{E}-01$ & $1.0000 \mathrm{E}-01$ & $2.5000 \mathrm{E}-01$ & 0.04 & 38357 \\
\hline 52 & 3475 & $U+6$ & Uranium VI & MKD_U & $\begin{array}{l}\text { Matrix Distribution } \\
\text { Coefficient for Uranium }\end{array}$ & UNIFORM & $m^{\wedge} 3 / k g$ & $1.5000 \mathrm{E}-02$ & $1.5000 \mathrm{E}-02$ & $3.0000 \mathrm{E}-05$ & $3.0000 \mathrm{E}-02$ & 0.01 & 38346 \\
\hline 53 & 3479 & $U+4$ & Uranium IV & MKD_U & $\begin{array}{l}\text { Matrix Distribution } \\
\text { Coefficient for Uranium }\end{array}$ & UNIFORM & $\mathrm{m}^{\wedge} \mathbf{3} / \mathrm{kg}$ & $1.0000 \mathrm{E}+01$ & $1.0000 E+01$ & $9.0000 E-01$ & $2.0000 \mathrm{E}+01$ & 5.50 & 38350 \\
\hline 54 & 3480 & PU+3 & Plutonium III & MKD_PU & $\begin{array}{l}\text { Matrix Distribution } \\
\text { Coefficient for Plutonium }\end{array}$ & UNIFORM & $\mathrm{m}^{\wedge} \mathbf{3} / \mathrm{kg}$ & $2.6000 \mathrm{E}-01$ & $2.6000 \mathrm{E}-01$ & $2.0000 \mathrm{E}-02$ & 5.0000E-01 & 0.14 & 38351 \\
\hline 55 & 3481 & $P U+4$ & Plutonium IV & MKD_PU & $\begin{array}{l}\text { Matrix Distribution } \\
\text { Cocficient for Plutonium } \\
\end{array}$ & UNIFORM & $\mathrm{m}^{\wedge} \mathbf{3} / \mathrm{kg}$ & $1.0000 \mathrm{E}+01$ & $1.0000 \mathrm{E}+01$ & $9.0000 \mathrm{E}-01$ & $2.0000 E+01$ & 5.50 & 38352 \\
\hline 56 & 3478 & $T H+4$ & Thorium IV & MKD_TH & $\begin{array}{l}\text { Matrix Distribution } \\
\text { Coefficient for Thorium }\end{array}$ & UNIFORM & $\mathrm{m}^{\wedge} 3 / \mathrm{kg}$ & $1.0000 \mathrm{E}+01$ & $1.0000 \mathrm{E}+01$ & $9.0000 \mathrm{E}-01$ & $2.0000 \mathrm{E}+01$ & 5.50 & 38349 \\
\hline 57 & 3482 & $A M+3$ & Americium III & MKD_AM & $\begin{array}{l}\text { Matrix Distribution } \\
\text { Coefficient for } \\
\text { Americium } \\
\end{array}$ & UNIFORM & $\mathrm{m}^{\wedge} \mathbf{3} / \mathrm{kg}$ & $2.6000 \mathrm{E}-01$ & $2.6000 \mathrm{E}-0 \mathrm{I}$ & $2.0000 \mathrm{E}-02$ & $5.0000 \mathrm{E}-01$ & 0.14 & 38353 \\
\hline
\end{tabular}

'For parameters with a triangular distribution, the value provided for the median is actually the mode. 


\section{Appendix I \\ Suggested PI Parameter Package Format Outline}

\section{$r$}




\section{Suggested PI Parameter Package Format Outline}

I. Parameter No. (id)

II. Data/Parameter

III. Parameter id (idpram)

IV. Material

V. Material id (idmtrl)

VI. Units

VII. Distribution Information
A. Category
B. Mean
C. Median
D. Standard deviation
E. Maximum
F. Minimum
G. Number of Data Points

VIII. Data Collection and Interpretation Information

A. Data Source Information

1. Data Source

2. Supporting Explanation/Justification for selection in \#l if other than WIPP Observational Data

3. References for selection in \#l above if other than WIPP Observational Data

B. Data Collection (for WIPP Observational Data)

1. Data Collection and Test Method

2. Assumptions Made During Testing

3. Standard Error of Measurement of Tests Performed

4. Form of Raw Data

5. References Related to Data Collection

a. Contract numbers

b. PI Name(s)

c. Title of approved Test Plan under which data was collected

d. SAND Report number

e. Sandia WIPP Central Files (SWCF) file code for non-SAND references

f. SWCF code for; data package(s)

6. QA Status of Data

a. Are all of the data qualified (Yes or No)?

If Yes, answer questions below to identify method of qualification

If No, list those data which are not yet qualified

b. Was data qualified by QAP 20-3 (Yes or No)?

If Yes, for a above, provide SWCF of qualified data package

c. Was the data the subject of audit/surveillance by SNL or DOE

(Yes or No; SNL or DOE)?

If Yes for $\mathrm{c}$ above, provide audit reference number 
C. Interpretation of Data

d. Was the data collected under an SNL-approved QA Program (Yes or No?) If Yes, provide title and approval dates of the QA Program(s)

1. Was the interpretation made by reference to previous work (Yes or No)?

If Yes, provide reference and answer \#3 below.

2. Was the interpretation made by using newly performed calculations (Yes or No)? If Yes, answer questions 3-9 below.

3. Form of Interpreted Data (e.g., histogram, table of interpreted values)/

4. Assumptions made during interpretation

5. Name of Code(s)/Software used to interpret data

6. QA Status of Code(s) used to interpret data. For SNL codes:

a. Was the code qualified under QAP 19-1 (Yes or No)?

b. QAP 9-1 (Yes or No)?

7. References related to data interpretation

a. Contract numbers

b. SAND Report number

c. Sandia WIPP Central Files (SWCF) file code for non-SAND references

d. SWCF code for data package

8. For interpretations made using newly performed calculations provide documentation that you followed the requirements of QAP 9-1 Appendix B.

9. For routine calculations (not using code) did you follow requirements of QAP 9-5 (Yes or No)?

IX. Correlation with other Parameters (List only those not statistically independent of the parameter documented here)

X. Limitations or qualifications for usage of data by Performance Assessment (PA)

XI. Attachments 


\section{Page intentionally blank}




\section{Appendix $\mathrm{J}$}

Example of a Typical PI Parameter Package 
Page intentionally blank 


\title{
PARAMETER PACKAGE MATERIAL FOR:
}

\author{
WPOت-30603.
}

THE MATERIAL WITHIN THIS COVER SHEET IS WHAT HAS BEEN SUBMITIED TO THE SWCF TO DATE FOR THIS WPOH. WHEN THE PARAMETER PACKAGE IS CLOSED, AN AIMS-DATABASE TABLE OF CONTENTS WILL BE GENERATED AND INCLUDED AT THE FRONT OF THE PACKAGE.

TOTAL PAGES: 47 (EXCLUDNG THE COVER SHEET) 
date:

to:

from:

subjoct

October 30, 1996

\section{团 Sandia National laboratories \\ Operated for the U.S. Deportment $\alpha$ Energy by \\ Sandia Corporation}

Albuquerque. Now Mexico 87985 -

Lori J. Dotson, 6822

Martin Tierney, 6848 Wattin Termen

Justification memo for choice of anhydrite permeabilities (MB138, MB139, Anh A\&B)

The assignment of values to the parameters specifying intrinsic, undisturbed permeability of anhydrite units MB 138 and 139 employed two sets of measurements: five (5) measurements of field-scale undisturbed permeability from the work of Paul Domski (WPO \# 31217); and 29 measurements of permeability on laboratory sized samples at a net effective stress of $6 \mathrm{MPa}$ taken from the work of Susan Howarth (WPO \#s 30603, 30605, 30606). The field-scale measurements sampled rock volumes of at least a few cubic meters; the standard errors of measurement were not mentioned by Domski and were assumed to be negligible. Howarth's laboratory-scale measurements were made on rock samples having volumes no more than a few hundred cubic centimeters; standard errors of measurements were again assumed to be small.

It was our intent to follow the WIPP Project's standard procedure for assigning a parameter when WIPP-specific measurements are available (Tiemey 1990, 1994) and use these data to fit a Student-t distribution for the unknown spatial average of the undisturbed anhydrite permeability. However, there was the immediate proble of how to correctly combine the two sets of measurements. Fitting a Student-t distribution requires that we have at least three samples of the random variable that are drawn from the same distribution. Although the latter requirement can be considered to be met by each of the individual data sets (i.e. Domski's or Howarth's), it would not be reasonably met by the data set which results from the pooling of field-scale and lab-scale data; this is because of the fact that, for highly inhomogeneous rocks, the variance of rock properties such as porosity and permeability is inversely proportional to sample size, and the great disparity between between the sample sizes of the field- and labscale measurements is counterindicative of their being drawn from the same distribution (for a short discussion of scaling of material-property parameters, see Section 1.3.3 in Sandia WIPP Project 1992).

The problem of combining the two disparate sets of measurements was first solved by choosing only the field-scale measurements (which were believed by some of us to more accurately reflect the permeability on the scale of the computational mesh) and ignoring the lab-scale measurements. This solution was naturally and rightfully not acceptable to Susan Howarth. After some discussion, we arrived at a second solution that was acceptable to all: take the average of the 29 laboratory-scale measurements and append that average to the field-scale measurements as a sixth point. The resulting data points on which the Student-t distribution for the logarithms of spatially averaged anhydrite permeability are based are listed on the attachment to the Form 464 for parameters No. 591, 592, 593 (WPO\#34865). 
Lori J. Dotson, 6822

Error! No text of specified style in document.

\section{References:}

Sandia WIPP Project. 1992. Preliminary Performance Assessment for the Waste Isolation Pilot Plant, December 1992. Volume 3: Model Parameters. SAND92-0700/3. Albuquerque, NM: Sandia National Laboratories.

Tierney, M.S. 1990. Constructing Probability Distributions of Uncertain Variables in the Models of the Performance of the Waste Isolation Pilot Plant (WIPP). SAND90-2510. Albuquerque, NM: Sandia National Laboratories.

Tierney, M.S. 1994. Using Data and Information to Form Distributions of Model Parameters in Stochastic Simulations of Performance of the Waste Isolation Pilot Plant (WIPP) in Proceedings of PSAM-II Volume 2. An International Conference Devoted to the Advancement of System-Based Methods for the Design and Operation of Technological Systems and Processes. San Diego, California USA. March 20-25, 1994. pp 051-9 through 051-16.

CC:

MS 1328 Mary-Alena Martell

MS 1328 Amy Johnson

WBS: 1.2.07.1.2:PDD:QA:PAETERS DATABASE Salado PKG 
Sandia National Laboratories_

Albuquerque. New Mevico $87: 85$

\author{
to: SWCF-A Records Center \\ SWCF-A:WBS1.2.07.1:PDD:QA:SALADO:PKG13:PRMX_LOG Log of \\ permeability in $\mathrm{x}$ direction/anh \\ from: Susand $6115, \mathrm{M} / \mathrm{S} 1324$ \\ date: April 30, 1996 \\ subject: SALADO PARAMETER PRINCIPAL INVESTIGATOR \\ DOCUMENTATION PACKAGE FOR: Salado Anhydrite Permeability in X- \\ Direction
}

The attached record package contains the permeability of anhydrite from laboratory tests performed on Salado Marker Bed 139 core samples. This record package replaces the existing version in the SWCF dated January 26,1996 . This package contains some information that was not contained in the January 26,1996 package including a spreadsheet verification technical review. There are no changes to the data contained in January 26 , 1996 version and therefore no resulting changes to the PA parameter distribution contained on the 464 form for Salado Anhydrite Permeability.

The parameter information provided in this record package was collected by Principal Investigators for input to the WIPP Data Entry Form and for use by Performance Assessment personnel making parameter estimates. The record package was prepared in accordance with WIPP Quality Assurance Procedure (QAP) 17-1, Rev. 1, WIPP QA Record Source Requirements.

Please call me at (505) 848-0676 if you have any questions. 


\title{
RECORD PACKAGE: \\ SALADO PARAMETERS REQUTRED FOR BRAGFLO
}

\author{
Salado Anhydrite Permeability in X-Direction
}

Purpose:

The data in this package was collected by Principal Investigators for input to the WIPP Data Entry Form and for use by

Performance Assessment personnel making parameter estimates.

Date of Record:

Author/Organization:

Recipient:

File Code:

SWCF-A:WBS1.2.07.1:PDD:QA:SALADO:PKG13:PRMX_LOG Log of permeability in $x$ direction/anh
April 30, 1996

Dr. Susan Howarth

Geohydrology Department 6115, M/S 1324

Sandia National Laboratories

Albuquerque, NM 87185-1324

(505) 848-0676

NWM Records Center 
RECORD PACKAGE:

SALADO PARAMETERS REQUIRED FOR BRAGFLO

PRINCIPAL INVESTIGATOR DOCUMENTATION PACKAGE FOR

Salado Anhydrite Permeability - X-Direction

SWCF-A:WBS1.2.07.1:PDD:QA:SALADO:PKG13:PRMX_LOG Log of permeability in $x$ direction/anh

TABLE OF CONTENTS

\begin{tabular}{|c|c|c|c|}
\hline $\begin{array}{l}\text { Record } \\
\text { Number }\end{array}$ & & Contents & $\begin{array}{l}\text { Number } \\
\text { of Pages }\end{array}$ \\
\hline 1 & $\begin{array}{l}\text { Section } \\
\text { I. } \\
\text { II. } \\
\text { III. } \\
\text { IV. } \\
\text { V. } \\
\text { VI. } \\
\text { VII. } \\
\text { VIII. } \\
\text { IX. } \\
\text { X. } \\
\text { XI. }\end{array}$ & 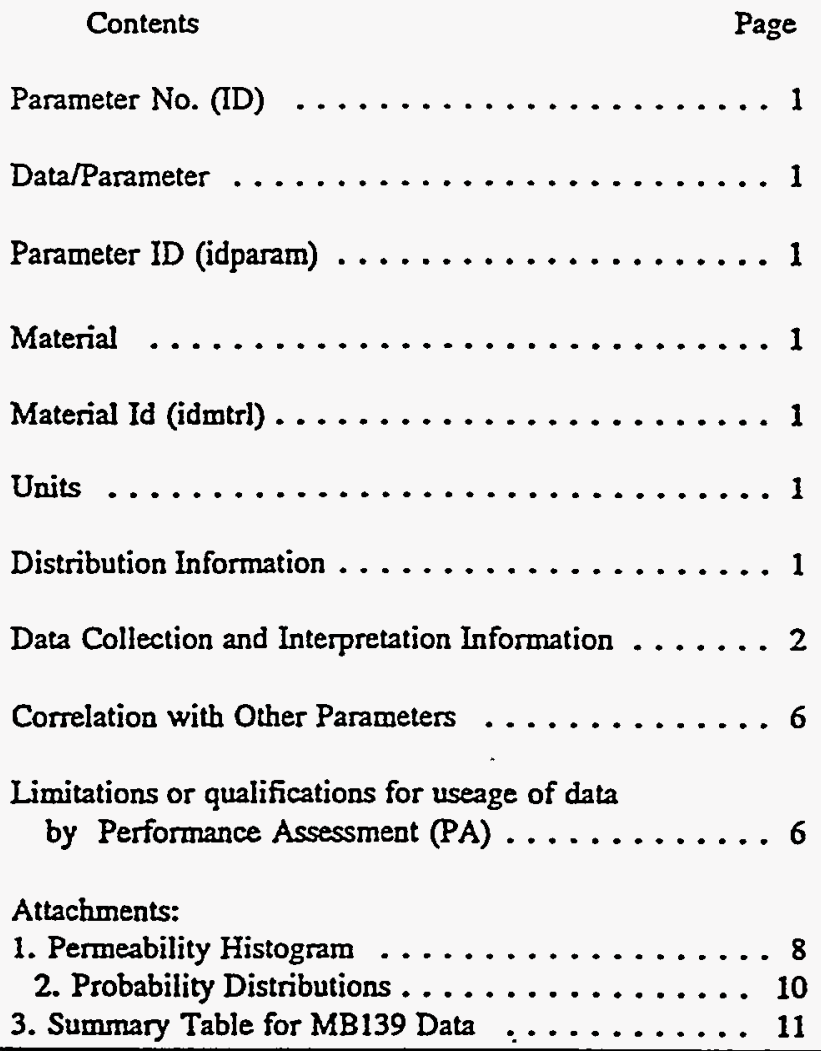 & 11 \\
\hline 2 & \multicolumn{2}{|c|}{ Technical Review of Record Package } & 6 \\
\hline 3 & \multicolumn{2}{|c|}{ Roadmap to Supporting Documentation } & 1 \\
\hline 4 & \multicolumn{2}{|c|}{$\begin{array}{l}\text { Memorandum to PA Parameter Task Leader } \\
\text { (Attachments not included - same as Records } 1 \text { and 2) }\end{array}$} & 1 \\
\hline
\end{tabular}

Total Record Package Pages $=21$ 
Record 1:

Parameter Record Package 
April 29, 1996

Susan Howarth (848-0676)

\section{Salado Data/Parameters}

I. Parameter No. (id) $: 531,570,591$

II. Data/Parameter:

Log of Permeability

Data from Laboratory Tests on Core Specimens approximately 10 to $825 \mathrm{cc}$.

III. Parameter id (idpram):

PRMX_LOG

IV. Material:

Anhydrite

V. Material Id (idmtrl):

S_MB138, S_MB139, S_ANH_AB

VI. Units:

$\mathrm{m}^{2}$ (data are reported as $\log (\mathrm{k})$ )

Distribution Information:

As discussed in SAND94-0472, for simplicity, the results for gas permeability tests performed at 1.6 MPa, 2.0 MPa, and 3.4 MPa are grouped together in this discussion and referred to as the $2 \mathrm{MPa}$ data. Similarly, the $5.6 \mathrm{MPa}$ and $6.0 \mathrm{MPa}$ data are combined and referred to as the $6 \mathrm{MPa}$ data, and the $9.6 \mathrm{MPa}$ and $10.0 \mathrm{MPa}$ data are combined and referred to as the $10 \mathrm{MPa}$ data.

\section{A. Category}

Constructed

B. Mean

$2 \mathrm{MPa}$ net effective stress: $\quad-17.82$

$6 \mathrm{MPa}$ net effective stress $\quad-18.08$

$10 \mathrm{MPa}$ net effective stress: $\quad-18.28$

C. Median

$2 \mathrm{MPa}$ net effective stress: $\quad-17.89$

$6 \mathrm{MPa}$ net effective stress: $\quad-18.24$

$10 \mathrm{MPa}$ net effective stress: $\quad-18.51$ 


\section{Salado Data/Parameters}

D. Std Deviation

$2 \mathrm{MPa}$ net effective stress: $\quad 0.67$

$6 \mathrm{MPa}$ net effective stress: $\quad 0.69$

$10 \mathrm{MPa}$ net effective stress: $\quad 0.83$

E. Maximum

$2 \mathrm{MPa}$ net effective stress: $\quad-15.08$

$6 \mathrm{MPa}$ net effective stress: $\quad-15.52$

$10 \mathrm{MPa}$ net effective stress: $\quad-15.82$

F. Minimum

$2 \mathrm{MPa}$ net effective stress: $\quad-18.84$

$6 \mathrm{MPa}$ net effective stress: $\quad-19.23$

$10 \mathrm{MPa}$ net effective stress: $\quad-19.30$

G. Number of data points

$2 \mathrm{MPa}$ net effective stress: $\quad 31$

$6 \mathrm{MPa}$ net effective stress: $\quad 29$

$10 \mathrm{MPa}$ net effective stress: 22

VIII. Data Collection and Interpretation Information:

A. Data Source Information:

1. Data Source:

WIPP Observational Laboratory-Measured Data

2. Supporting Explanation/Justification for selection in \#1 if other than WIPP Observational Data:

NA

3. References for selection in \#1 above if other than WIPP Observational Data:

NA 


\section{Salado Data/Parameters}

\section{B. Data Collection (for WIPP Observational Data):}

\section{Data Collection and Test Method:}

Single-phase, steady-state, Klinkenberg-corrected gas permeability measurements were performed at three different effective stress states on a total of $31 \mathrm{MB} 139$ cylindrical specimens by three different laboratories. 31 of the specimens produced usable permeability data at one or more of the stress states. The specimens were cut from whole core taken from 6 underground boreholes at the WIPP. Two of the cores were taken outside Room L3 and the other 4 from E140 Drift at the intersection of N1100 Drift.

\section{Assumptions Made During Testing:}

1) The specimens were saturated with the permeate fluid.

2) The experiments were conducted at steady-state

3. Standard Error of Measurement of Tests Performed:

Approximately $10 \%$ error in the permeability measurements

4. Form of Raw Data:

Pressures, temperatures, time, lengths and volumes

5. References Related to Data Collection:

Contract numbers:

Rock Physics Associates (who contracted Core Labs) AF-3945

RE/SPEC AA-2020

TerraTek AD-3656

PI name(s):

Susan Howarth

Title of approved Test Plan under which data was collected:

Test Plan: Two-Phase Flow Laboratory Program for the Waste Isolation Pilot Plant

SAND report number:

SAND94-0472: Porosity, Single-Phase Permeability, Capillary Pressure Data from Preliminary Laboratory Experiments on Selected Samples from Marker Bed 139 at the Waste Isolation Pilot Plant (draft -please see Record 3 for details) 
Salado Data/Parameters

Sandia WIPP Central Files (SWCF) file code for non-SAND references: SWCF-A:WBS1.1.4.1;HYD/TPF

SWCF code for data package(s):

SWCF-A:WBS1.1.4.1;HYD/TPF

\section{QA Status of Data:}

a. Are all of the data qualified (Yes or No?)

If $Y e s$, answer questions below to identify method of qualification.

If No, list those data which are not yet qualified.

Yes

b. Was data qualified by QAP 20-3 (Yes or No?)

If Yes for " $\mathrm{a}$ " above, give SWCF of qualified data package. No

c. Was the data the subject of audit/surveillance by SNL or DOE? (Yes or No; and SNL or DOE?)

If $Y e s$ for " $c$ " above, give audit reference number.

Yes: Core Laboratories Audit 94-04, RE/SPEC audit 94-05, and TerraTek audit 93-04

d. Was the data collected under an SNL approved QA Program? (Yes or No?) If Yes, give title and approval dates of the QA Program(s)

Yes.

Rock Physics Associates:

Test Procedures and Quality Assurance Plan: Porosity, Permeability and Capillary Pressure Measurements in Anhydrite Samples from WIPP. Approved by SNL QA (Susan Pickering) on May 28, 1993.

RE/SPEC:

All work was performed under a Quality Assurance Program that complies with the criteria of the United States Code of Federal Regulations, Title 10, Part 50 (10CFR50), Appendix B. All software meets the requirements stated in SNL Quality Assurance Procedure 19-1 Rev. D, Computer Software Requirements. Quality Assurance requirements were overseen 


\section{Salado Data/Parameters}

by RE/SPEC quality assurance personnel. Work was performed under a single, comprehensive n existing Quality Assurance Plan that was submitted and approved by SNL.

TerraTek:

Quality Assurance Program Plan Contract AD-3656 Task 2: Measurement of Saturation, Porosity, and Permeability and Investigation of CT Core Imaging. Approved by SNL QA (Susan Pickering) on February 16, 1993.

C. Interpretation of Data:

1. Was the interpretation made by reference to previous work (Yes or No?) If yes, give reference and answer \#3 below.

No

2. Was the interpretation made by using newly performed calculations (Yes or No?) If, yes, answer questions 3-9 below. Yes

3. Form of Interpreted Data: (Example: histogram, table of interpreted values)

Histogram, Tabulated (see attachments)

4. Assumptions Made During Interpretation:

1) The specimens were saturated with the permeate fluid.

2) The experiments were conducted at steady-state

5. Name of Code(s)/Software used to Interpret Data:

Microsoft Excel v. 4.0 and KaleidaGraph v. $3.0 .3 \mathrm{~b} 2$

6. QA Status of Code(s) used to Interpret Data: For Sandia Codes:

a. Was the code qualified under QAP 19-1(Yes or No? NA

b. QAP 9-1 (Yes or No?) N/A 
April 29, 1996

Susan Howarth (848-0676)

\section{Salado Data/Parameters}

7. References Related to Data Interpretation:

Contract numbers:

Rock Physics Associates (who contracted Core Laboratories) AF-3945

RE/SPEC AA-2020

TerraTek AD-3656

SAND report number:

SAND94-0472: Porosity, Single-Phase Permeability, Capillary Pressure Data from Preliminary Laboratory Experiments on Selected Samples from Marker Bed 139 at the Waste Isolation Pilot Plant (draft -please see Record 3 for details)

Sandia WIPP Central Files (SWCF) file code for non-SAND references: SWCF-A:WBS1.1.4.1;HYD/TPF

SWCF code for data package(s):

SWCF-A:WBS1.1.4.1;HYD/TPF

8. For interpretations made by using newly performed calculations provide documentation that you followed the requirements of QAP 9-1 Appendix B.

NA

9. For routine calculations (not using code) did you follow requirements of QAP 9-5 (Yes or No?) Yes

IX Correlation with other Parameters (List only those not statistically independent of the parameter documented here): N/A

X. Limitations or qualifications for usage of data by Performance Assessment (PA):

Data was only measured on specimens from MB139 taken from intact rock; permeability is isotropic.

Experimental errors are derived from formal propagation of random and systemic errors. Source of errors include differential pressure transducer accuracy, accuracy of flow rate measurements (volumes and time), caliper accuracy, uncertainty in fluid viscosity, and least

SWCF-A:WBS 1.2.07.1:PDD:QA:SALADO:PKG13:PRMX_LOGLLg of permeability in X direction/anh 6 
Salado Data/Parameters

squares curve fit of data. RE/SPEC reported (see SAND94-0472 (draft -please see Record 3 for details)) an average error of $5.0 \%$ for the gas permeability measurements. TerraTek reported (see SAND94-0472 (draft -please see Record 3 for details)) an average error of 3.8\% for the gas permeability measurements. No error calculations were reported by Rock Physics Associates; it is assumed that the enror in the permeability measurements at Core Laboratories is approximately the same as that at RE/SPEC and TerraTek. Therefore, the error in the permeability measurements is approximately $6 \%$.

\section{Attachments:}

1. Histograms

2. Cumulative frequency plot

3. Tabulated data

Guidance documents are found in SWCF-A:WBS 1.2.07.1:PDD:Salado:CORR:Guidance Documents 


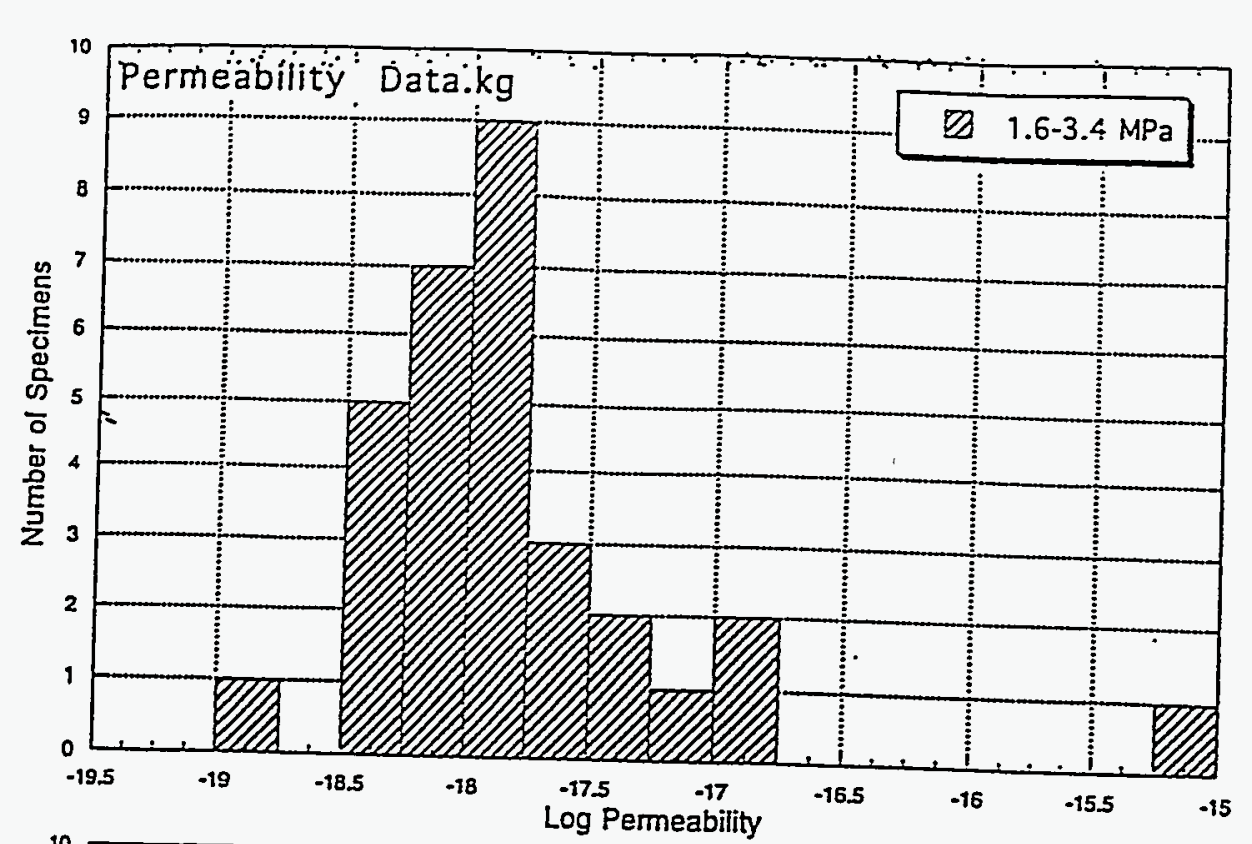

Priachmen
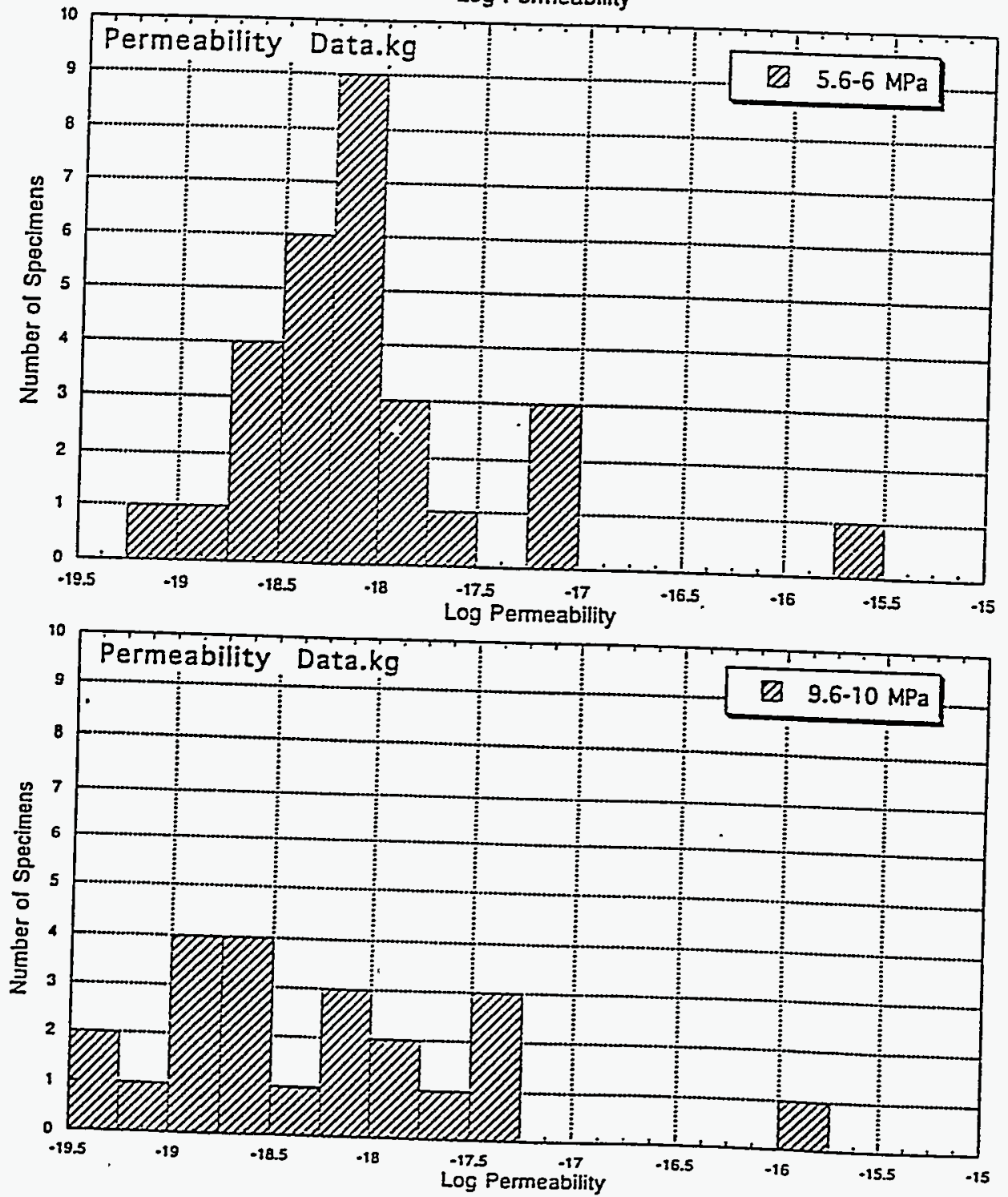


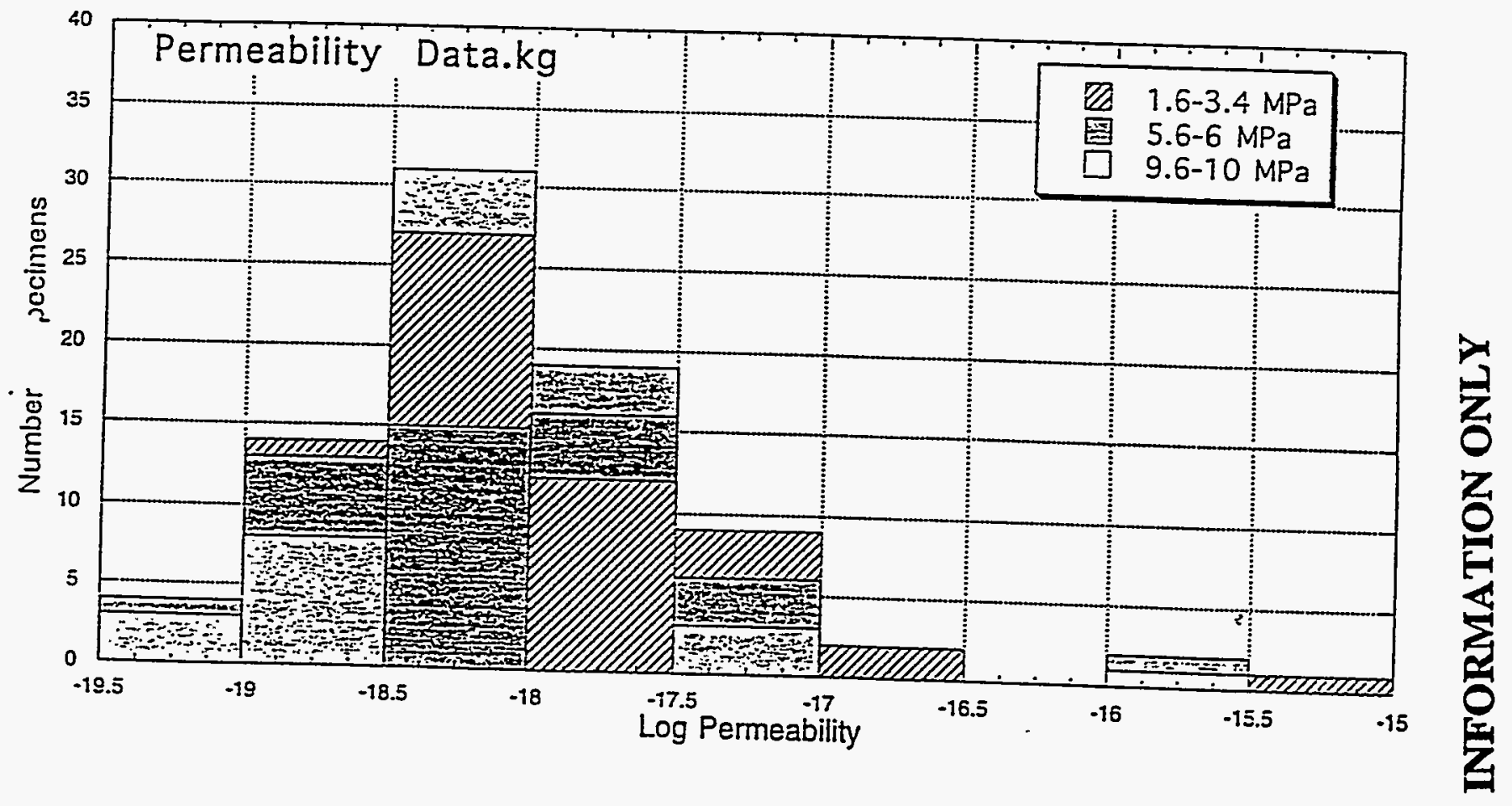


Attreimatiz

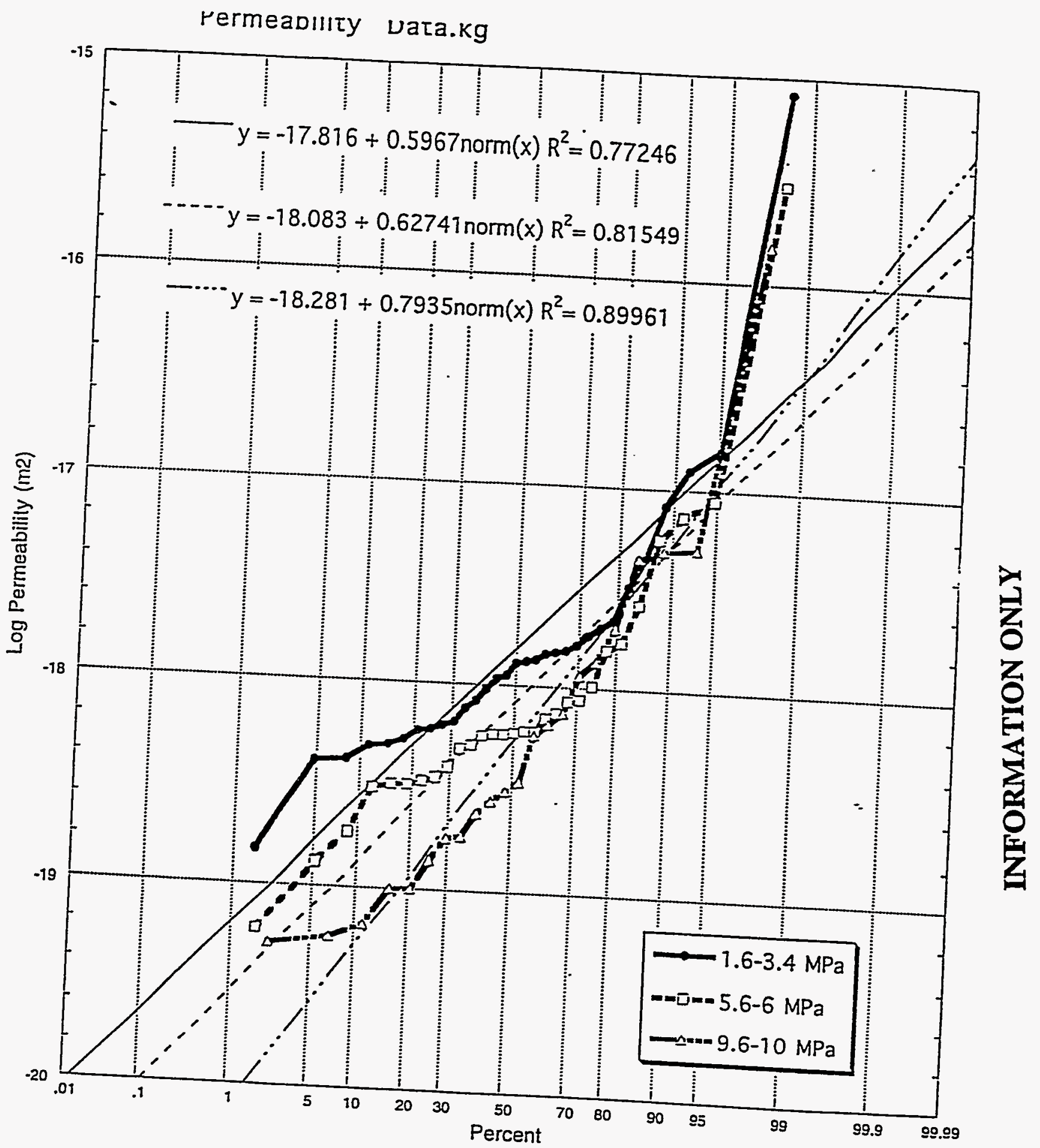


Attachment $x 3$

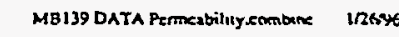

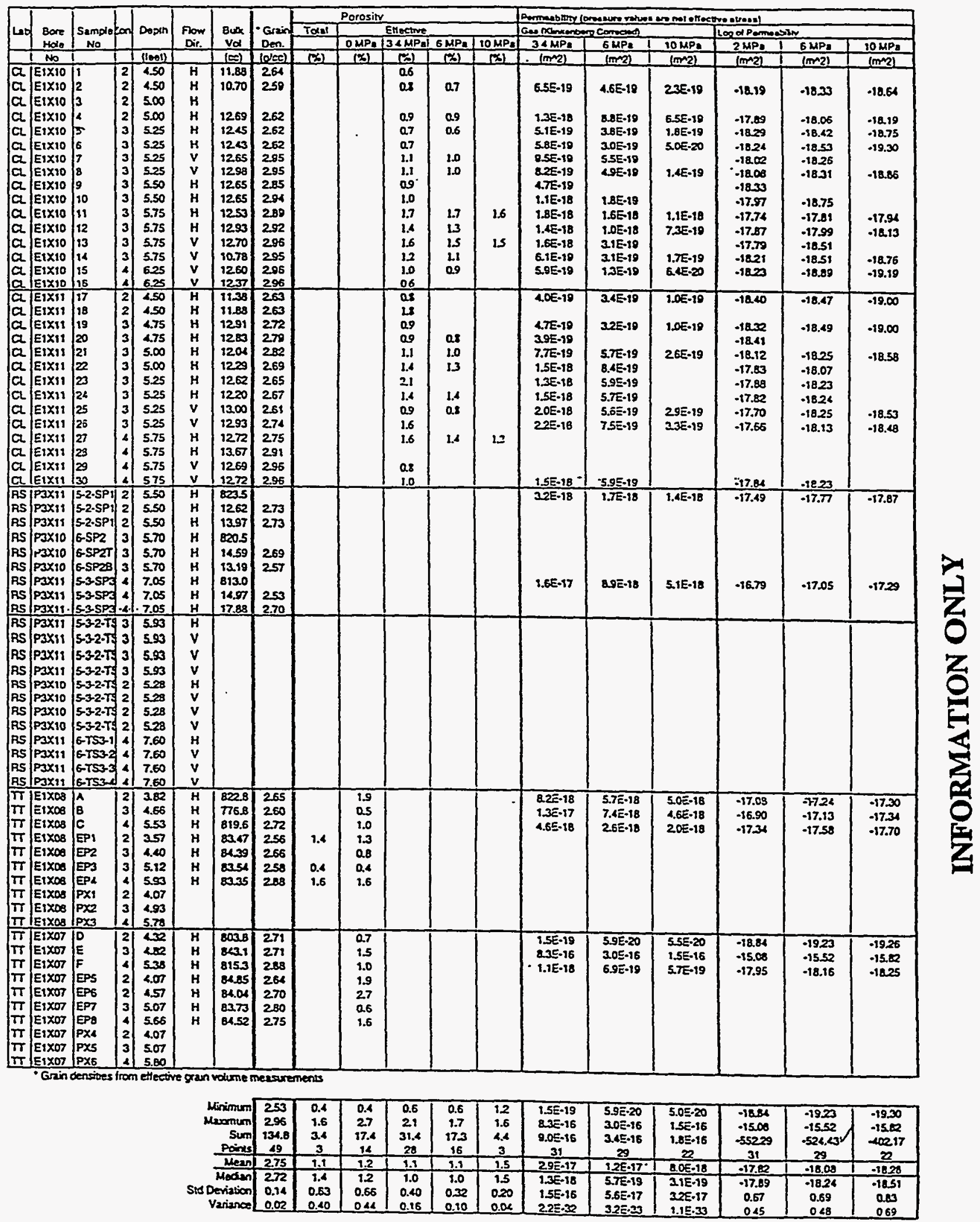


Record 2:

Technical Review of Records Package 


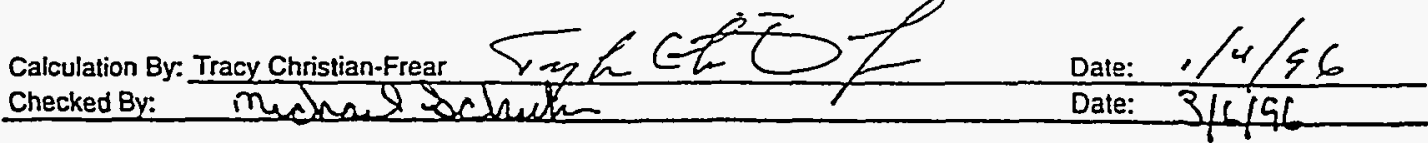

Purpose:

To verify grain density, porosity, permeability and anhydrite content of MB139 data inio a spread sheel Verify Statistical evaluation.

The grain density, porosity and permeability data will be used for parameter input to $P A$.

The grain density, porosity, anhydrite content and permeability data will be used for inpul to SAND94-0472.

Data Description:

Tree laboratories lested specinens of $M B 139$.

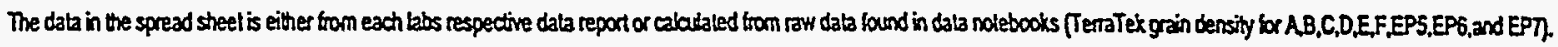
Ditherences do cocar between reported dala and the spread sheel The reasons are:

1) Errors in the data reports found when a check of data was performed. Errors indicated on Errata sheets

2) Round-oft differences (the data on the spread sheet should be within $1 \%$ of the reported value in the data report)

because most of the data entered on the spread sheet are from verification calculations using the raw data

reported in the laboratory notebooks.

Precision:

The data on the spread sheet should be within $1 \%$ of the reported value in the data report (unless the data in the report was wrong)

References:

1 Capillary Pressure Measurements in Anhydrite Samples from MB139
Rock Physics

2 Laboratory Measurements of Fluid Transport Properties for MB139 Anhydrite from the WIPP RESPEC

3 Characterization of MB139 at WIPP

TerraTek

Process:

1) Find data in appropriate data report and check that it was entered onto the data sheet correctly within the precision given.

2) If data is different, check errata sheets to ensure that the data in the report is correct. If there is still a difference, identify the erroneous entry and continue the check.

3) Check that the statistical anatysis is correct for the requested values.

A list of the spreadsheet formulas are given in Attachment ?.

4) Identify any errors to the person who perfomed the calcutation

5) After resolution of any erors, sign, date and indicate items checked.

Results:

(Include a Brief Description of What Was Checked)

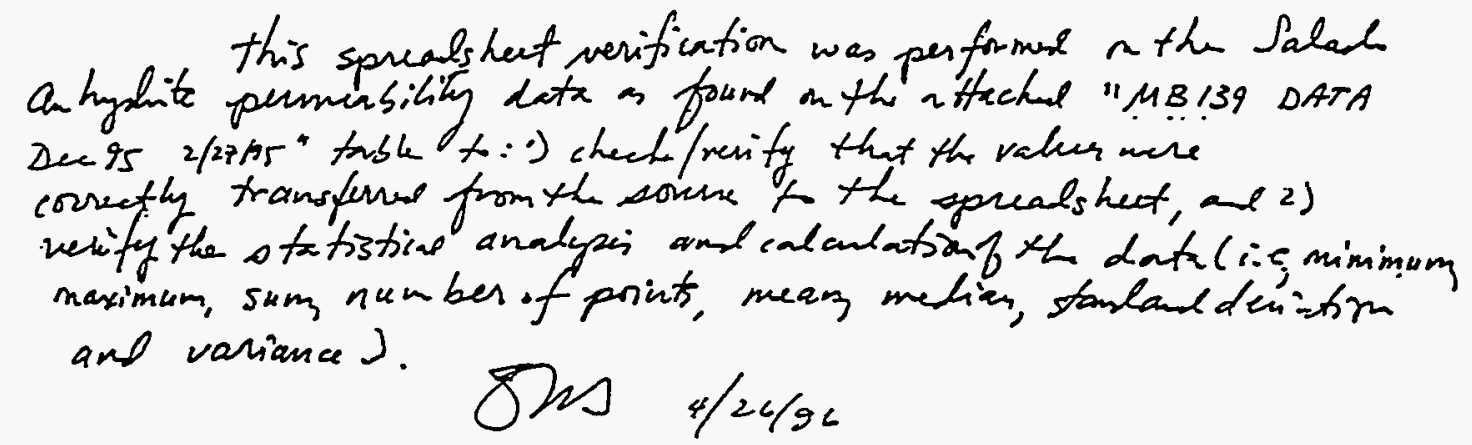


NID139 DATADEC US SETP,

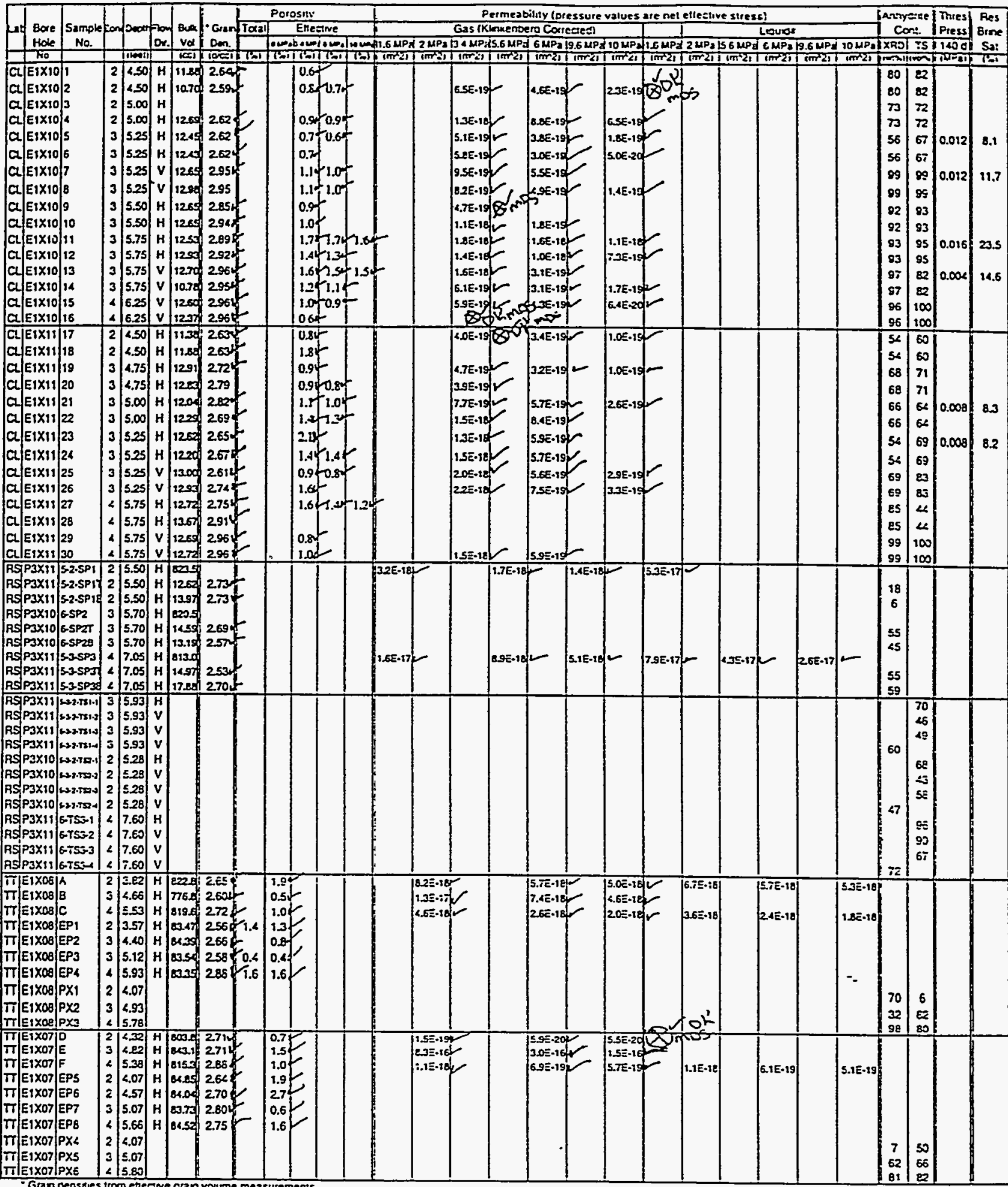

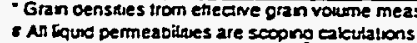

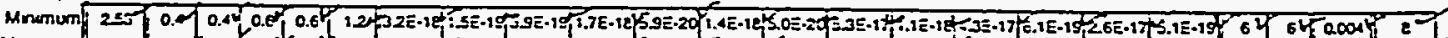

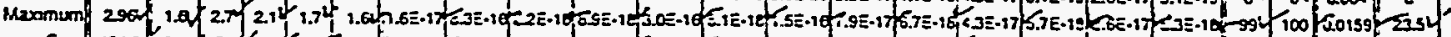

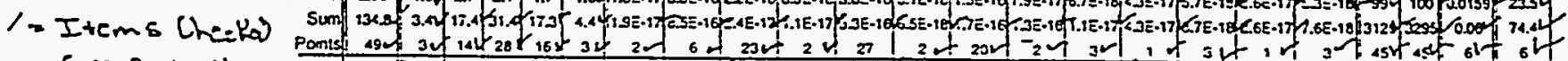

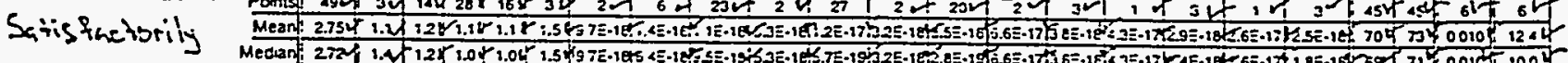

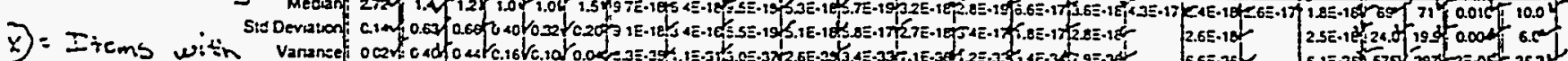
possible eiro:s

Michesel Schuhen $3 / 5 / 96$

All concerens were addresee) to my stifiacion. mis 3/6/gl 
MDI30 DATA Dec 95.fonmula $\quad$ 227/96

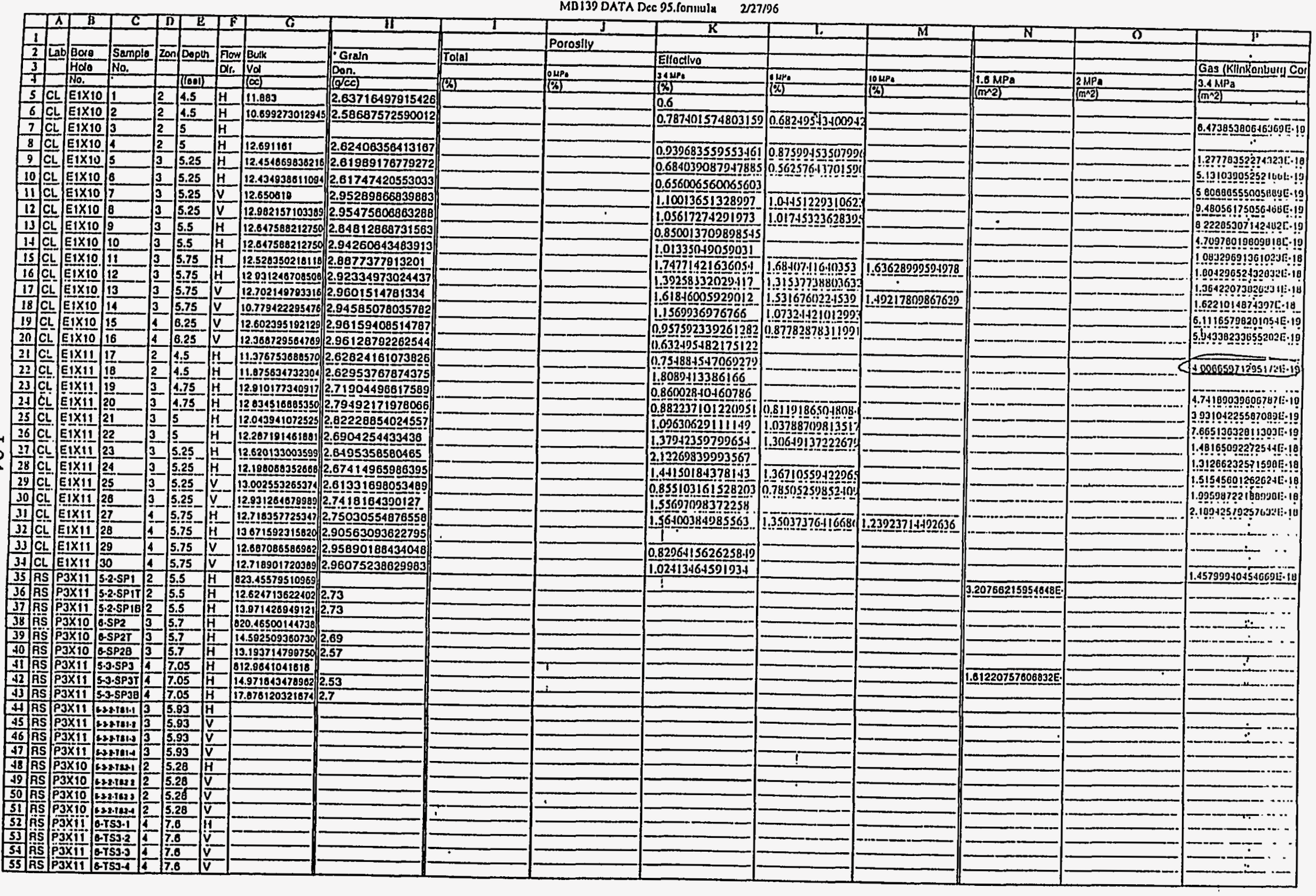

INFORMATION ONLY 


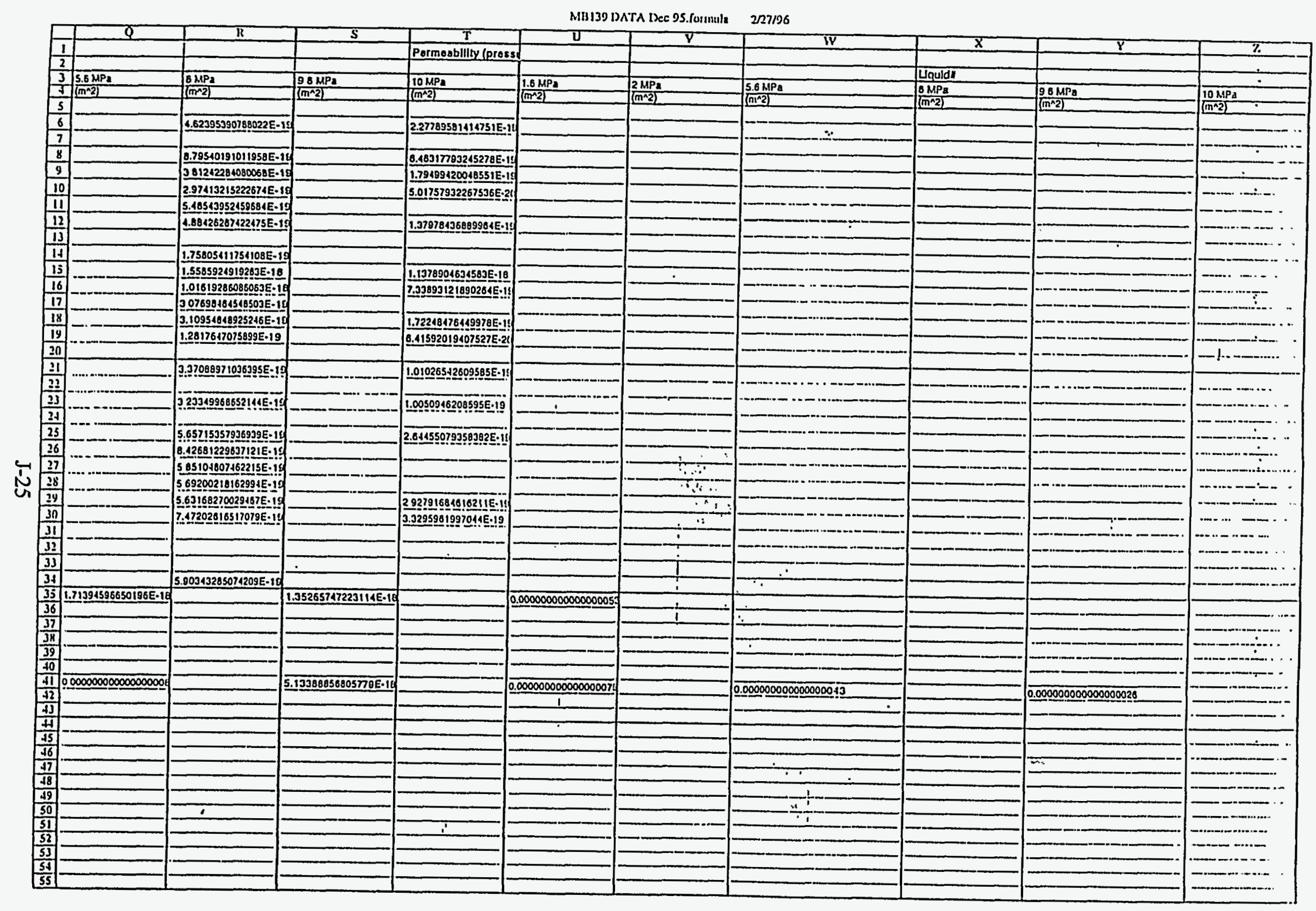

INFORMATION ONLY 


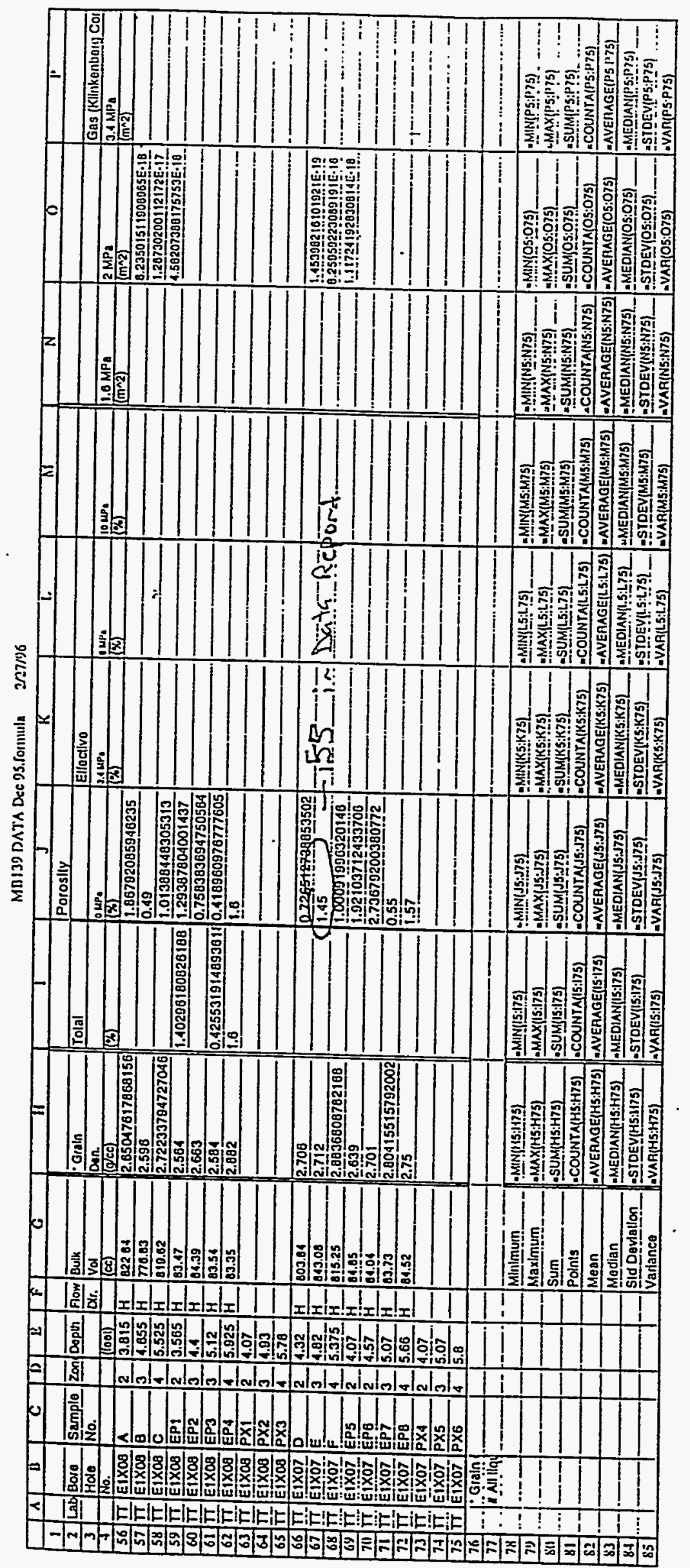


MD139 DATA DeE 95.formula

\begin{tabular}{|c|c|c|c|}
\hline IV & $\bar{x}$ & Y & T. \\
\hline & Llquid" & & \\
\hline $5 B \mathrm{MPa}$ & $8 \mathrm{MPa}$ & $96 \mathrm{MPa}$ & $10 \mathrm{MPa}$ \\
\hline$\left[\left(\frac{\pi}{\wedge} 2\right)\right.$ & 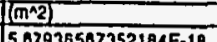 & $\left(\frac{\pi}{1 \times 2}\right)$ & 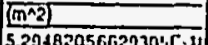 \\
\hline & $3=07936300 / 3521086=-18$ & & 5 \\
\hline & $2.99739024438221 E-18$ & & $1.8100 ! 9223303 \mathrm{E}-1 \mathrm{~g}^{-1}$ \\
\hline & & & \\
\hline & & & -.......... \\
\hline & & & \\
\hline & & & $-\ldots$ \\
\hline & & & \\
\hline & & $\because$ & - \\
\hline & $8.14815399320739 E_{10}$ & & $5.108+96897072856$ \\
\hline & & - & $-\ldots$ \\
\hline & & - & -7 \\
\hline & & $1-1$ & $-1-\ldots$ \\
\hline & & . & ...___............. \\
\hline & & -1 & $\ldots+\cdots$ \\
\hline & & - & - \\
\hline Min(W5:W75) & $\operatorname{MIN}(X 5: \times 75)$ & - All1(Y5:Y75) & $2 \mathrm{SAN}(25: 275)$ \\
\hline 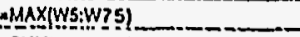 & $-M A \times(X 5: \times 75)$ & MAXIYS:YZISI & $-M A \times(25: 275)$ \\
\hline SUM!W(W5:W7?5) _. & $=5 U M \times 1 \times 5: \times 75$ & -SUMYYS:Y75L & -5UMi(25:275) \\
\hline COUNTAIW5:W75) & COUNTA $(X 5: X 75)$ & CCOUNTA YYS:Y75) & C.OUNTA(Z5-275) \\
\hline AVERAGESW W5:W75) & AVERAGE $(X 5: X 75)$ & AVERAGE $(Y 5: Y 75)$ & UAVERAGR(ZS.7/!I) \\
\hline 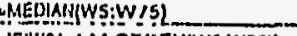 & AMEDIAN!(X5:X75) & MEOIAN(YS;YZST & LMEQIN!I $(25 \div 275)$ \\
\hline 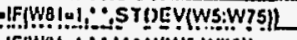 & $\triangle S I D E V(X 5: \times 75)$ & 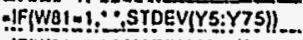 & STTQEVIZS:Z75)" \\
\hline IF(WBiL $1, \because$, VAIU(W5:W73)] & -VAn $(x 5: \times 75)$ & 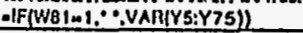 & ivan $2 z 5 \cdot 275$ \\
\hline
\end{tabular}


Record 3:

Roadmap to Supporting Documentation 


\section{RECORD PACKAGE: \\ SALADO PARAMETERS REQUIRED FOR BRAGFLO}

\section{PRINCIPAL INVESTIGATOR DOCUMENTATION PACKAGE FOR Salado Anhydrite Permeability in X-Direction}

Roadmap for Supporting Documentation for Salado Anhydrite Permeability in X-Direction

Anhydrite Permeability data is found in SAND94-0472:

Howarth, S. and T. Christian-Frear: "Porosity, Single-Phase Permeability, and Capillary Pressure Results from Preliminary Laboratory Experiments on Selected Samples from Marker Bed 139 at the Waste Isolation Pilot Plant (WIPP)". Albuquerque, NM: Sandia National Laboratories. SAND94-0472. This data report has been peer, SNL management and WIPP-DOE reviewed and approved. However, due to budget constraints, it has not been printed. Copies of the Camera-ready draft are available from the authors.

The raw data for this parameter was collected under SNL Contracts AA-2020 (RE/SPEC), AF-3945 (Core Laboratories for Rock Physics Associates), and AD-3656 (TerraTek) and is found in the SWCF under Salado Hydrology Laboratory Tests for Porosity and Permeability (and for AF-3945, Capillary Pressure) tests under the following WPO's: WPO $37071 \mathrm{RE} / \mathrm{SPEC}$

WPO 37073 Core Laboratories (for Rock Physics Associates)

WPO 37072 TerraTek

SAND94-0472 contains a summary of the results of laboratory tests performed under these contracts and includes the final report from each contractor as an appendix. This work was performed as part of the Salado Two-Phase Flow Laboratory Program which was WBS 1.1.4.1 through FY95. 
Record 4:

Memorandum to PA Parameter Task Leader

(Attachments not included -- same as Records 1 and 2) 


$$
w 00 * 37614
$$

to: Martin Tiemey, PA Parameter Task Leader

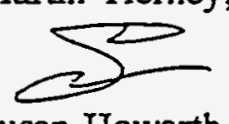

from: $\quad$ Susan Howarth, 6115, M/S 1324

date: April 29, 1996

\section{Sandia National Laboratories}

Albuquerque. New Miexico 87185

subject: SALADO PARAMETER PRINCIPAL INVESTIGATOR DOCUMENTATION PACKAGE FOR: Salado Anhydrite Permeability in XDirection

The attached record package contains the permeability of anhydrite from laboratory tests performed on Salado Marker Bed 139 core samples. This record package replaces the existing version in the SWCF dated January 26,1996 . This package contains some information that was not contained in the January 26, 1996 package including a spreadsheet verification technical review. There are no changes to the data contained in January 26 , 1996 version and therefore no resulting changes to the PA parameter distribution contained on the 464 form for Salado Anhydrite Effective Porosity.

The parameter information provided in this record package was collected by Principal Investigators for input to the WIPP Data Entry Form and for use by Performance Assessment personnel making parameter estimates. The record package was prepared in accordance with WIPP Quality Assurance Procedure (QAP) 17-1, Rev. 1, WIPP QA Record Source Requirements.

SWCF-A:WBS1 2.07.1:PDD:QA:SALADO:PKG13:PRMX_LOG Log of permeability in $x$ direction/anh

The WPO Number for this Parameter Package is 30603

The WPO Numbers for the 464 Form Packages are:

Salado Anhydrite Marker Bed 13834536

Salado Anhydrite Marker Bed 13934865

Salado Anhydrites a \& b $\quad 34196$

The data contained in this report is Qualified as it was taken by subcontractor (to SNL) laboratories under SNL-Approved QA plans. In addition, an SNL QA audit was performed at all of the subcontractor laboratories.

Please call me at (505) 848-0676 if you have any questions.

Attachments:

1. Salado Data/Parameters: Permeability of Anhydrite

2. Technical Review of Record Package 


\section{WP0.39065}

\section{Sandia National Laboratories \\ Operated for the U.S. Deportment of Eneroy by - Sandia Corporation \\ Albuquerque, New Mexico 87185-1324}

date: June 26, 1996

to: Susan Howarth, 6115, M/S 1324

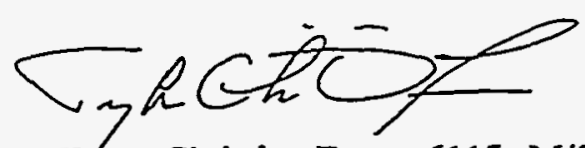

from: Tracy Christian-Frear, 6115, M/S 1324

subject: Parameter Resolution for Salado Anhydrite Permeability

On January 23, 1996, a dispute resolution meeting was held to address differences in opinion between PI's (Susan Howarth, Rick Beauheim, Tracy Christian-Frear, and Paul Domsk) and the Parameter Task Leader (Martin Tiemey) regarding the proposed distributions for Salado anhydrite porosity and permeability. A February 5, 1996 memorandum from Kathy Gaither to Distribution (see Attachment) described the differences in opinion, resolution process, and proposed resolution. The difference in opinion for the anhydrite permeability distribution was that the PTL, citing scaling issues, decided to use only the field permeability test results from Beauheim and Domski to determine the Salado anhydrite permeability distribution, thereby ignoring the laboratory permeability data from Howarth and ChristianFrear.

To resolve the dispute, it was agreed that the laboratory data would be grouped according to the identification number of the borehole from which the core sample was taken. A mean permeability value would be calculated from the samples in each borehole group. The PTL would then use each of these mean values as a single permeability data point equivalent to a single field borehole permeability test value to determine the anhydrite permeability distribution using both the field and laboratory measurements.

In use, however, this process proved to be unworkable because in some cases only one or two laboratory samples were taken from a single borehole and, in other cases, a large number of laboratory samples were taken from a single borehole. With concurrence from the PTL (Martin Tiemey) and Susan Howarth, it was agreed that a single the mean value using all the laboratory data-would be calculated. This single mean value would be used to represent all of the laboratory data. The anhydrite permeability distribution for Salado Marker Beds 138 and 139 and anhydrites " $a$ " and " $b$ " was then determined by the PTL from the single laboratory mean value and the 5 field test permeability points.

\section{Attachment::}

Memorandum Kathy Gaither to Distribution

RE: Salado and Non-Salado Parameters Resolution of Parameter Value(s) on Form \#464 between Principal Investigator (PI)/Scientific Investigator (SI) and Parameters Task Leader (PTL) for Selected idprams/idmtrls) to distribution. February 5, 1996.

$$
S \% 1 C F-A: W B S 1.2 .07 .1: \text { PDD : QA: (PRP-WP0 } 30603)
$$


iv po 32320

\section{Sandia National Laboratories \\ P.O. Box 5800 Dept. 6115 \\ Abuquerque, NM 87185-1324}

Date: February 5, 1996

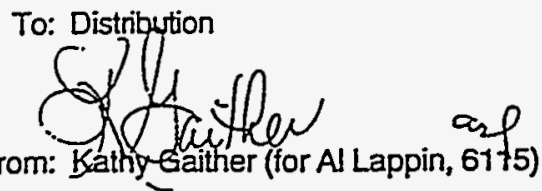

Subject: Salado and Non-Salado Parameters Resolution of Parameter Value(s) on Form $\# 464$ between Principal Investigator (PI)/Scientific Investigator (SI) and Parameters Task Leader (PTL) for Selected idprams/idmtris

This memo is sent to serve two purposes. It describes the process of iniormal resolution of disputed parameter value(s) chosen by the PTL on Form $\# 464$ when the value chosen is not acceptable to the $\mathrm{PI} / \mathrm{SI}(\mathrm{s})$. It also documents the resolution of disputed values for parameters with the following ids: $541,547,548,549,567,570,571,572,588,591,592,593,528,531,532$, and 533 at a meeting held January 23,1996 . This informal process for resolution of disputed values is intended to determine whether agreement can be reached with Management arbitration before resoring to the more formal requirements of QAP 2-10. If, concurrence cannot be reached through informal arbitration, the procedures in QAP 2-10 will be followed.

\section{INFORMAL PROCEDURE FOR DISPUTE RESOLUTION FOR VALUES ON FORM \#464}

The information on the WIPP Data Entry Form (\#464) for a given idprarnidmtri combination is used as input into the controlled database for compliance-level modeling by periormance assessment (PA) personnel. PI/SI sion-off in the "Concurrence" field on the Form signifies agreement between the PI/SI and the Performance Assessment Analyst (PAA) sioniiies that the parameter value(s) on this form are appropriate given currently available information. PISI signature does it imply affirmation of the qualification status of the parameter.

Therefore, the goal of the informal dispute resolution process is to arrive at a value, or range of values, upon which the PTL and the PVSI agree. The PTL chooses the value(s) on Form $\frac{4}{\pi} 464$, and signs the form, and a copy is made and given to the PUSI. The PVSI is asked to either signify concurrence by signing the original of Form \#464 or inform the PTL that there is a disagreement regarding the value(s) chosen. If the interchange between the PTL and PI/SI doesn't result in concurrence an informal dispute resolution session is set up.

Participants in an informal dispute resolution should include the PISI(s), PTL, PA A, PA Management (Hong-Nian Jow, 6741), the WIPP Deputy Project Manager (Margaret Chu, 6801), and the appropriate PI Team Leader or Manager. Others may also attend the meeting. The PTL and $\mathrm{PI} / \mathrm{SI}(\mathrm{s})$ should present their data, justification or rationale, and any supporting documentation regarding the disputed value(s). If the PTL and PVSIs still disagree after the presentations and discussions, the Deputy Project Manager may suggest their solution to the problem or invoke use of the process in QAP 2-10. The PI/SI or PTL may also request use of the process in QAP 2-10, if they do not agree with the solution proposed by the Deputy Project Manager. The PTL for all Form \#464 entries referred to herein is Martin Tiemey, 6741.

The informal dispute resolution process was employed at a meeting held January 23,1996 to resolve Form $\# 464$ values for idpramidmtris listed below.

Exceptional Service in the National Interest

SWCF-A:WBS 1.2.07:PDD:OA:SALADO:CORR:Planning 


$$
\begin{aligned}
& \text { Tran Frept } \\
& \qquad / 23 / 96
\end{aligned}
$$

Difference:

Porosity of Anhydrite Beds. Martin chose a constant value.

Resolution:

Martin will document why he choose a constant value.

Difference:

Permeability of Anhydrite Beds. Martin chose to ignore the laboratory data.

Resolution:

Tracy will group together data from the different sets of boreholes, determine the mean of those data points, and provide Martin with those mean values to be grouped together with the other 5 field data points. A distribution will then be determined based upon the field and the lab data.

Difference:

Permeability of Halite. Martin chose a constant value.

Resolution:

Martin will determine a distribution to be sampled upon

Difference:

Rock Compressibility of Halite. Martin did not include that he will recommend that the rock compressibility and permeability are inversely related.

Resolution:

Martin will include this on the 464 . 


\section{Sandia National Laboratories}

íltucierque. New Mexico Sziss

to: SWCF-A WPO Numbers: $30599,30600,30603,30605,30606,30642$, and 30643

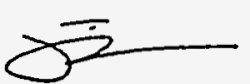

from: Susan Howarth, 6115

date: June 3, 1996

subject: $\quad$ Draft of SAND94-0472 now filed in WPO\# 38019

SWCF-A WPO files 30599, 30600, 30603, 30605, 30606, 30642, and 30643 contain Parameter Records Packages which reference SAND94-0472 as a data source document. SAND94-0472 is a compilation of the results of porosity, permeability and capillary pressure laboratory measurements made at three subcontractor laboratories. The report was peer reviewed/approved in September 1995, SNL WIPP management reviewed/approved in October 1995, and submitted to DOE/CAO for review in October 1995. DOE/CAO approval was received on November 3, 1995.

SAND94-0472 was reviewed and approved by SNL peers (Rick Beauheim, Kurt Larson), SNL management (Peter Davies), SNL WIPP management (Les Shephard), and DOE/CAO (Dick Lark). As appropriate, changes were made to reflect review comments by all reviewers, and the manuscript was sent to Tech Reps, Inc. to be made camera-ready in preparation for publication. However, due to budget constraints, this process was interrupted on April 24, 1996 and the report has not been printed. At the time publication was stopped, the text of the report was camera-ready, but the appendices were not cameraready. It is expected that the report will be published in FY97. Because this report is referenced in a number of Parameter Records Packages which support BRAGFLO CCA calculations, it was submitted to the SWCF in DRAFT form and filed as WPO\#38019.

WPO $\# 38019$ contains three, three-ring binders. One binder contains copies of review and approval forms, Document Review and Comment forms, the April 24, 1996 Memorandum from Les Shephard requesting that publication of this report be postponed, and the review comments from Beauheim, Larson, and Davies. The second binder contains the draft text of SAND94-0472 and is a copy of the camera-ready text. The third binder contains the draft appendices of SAND94-0472 which are not camera-ready at this time. Please note that some of the figures contained in the appendices are black-and-white photocopies of color photographs and may not be of high quality. Currently, the originals of all the figures and photographs can be viewed in my office (BDM Building Room 2226). Once SAND94-0472 is printed, the originals will be forwarded to the SWCF as appropriate.

If you have any questions, please contact me at (505) 848-0676.

Copy to: (without attachments)

M/S 1324 P.B. Davies, 6115

M/S 1324 A.R. Lappin, 6115

M/S 1324 T.L. Christian-Frear, 6115 
Page intentionally blank 


\section{Appendix K}

\section{Example of Roadmap Found in Shaft Seals PI Parameter Package}


Page intentionally blank 


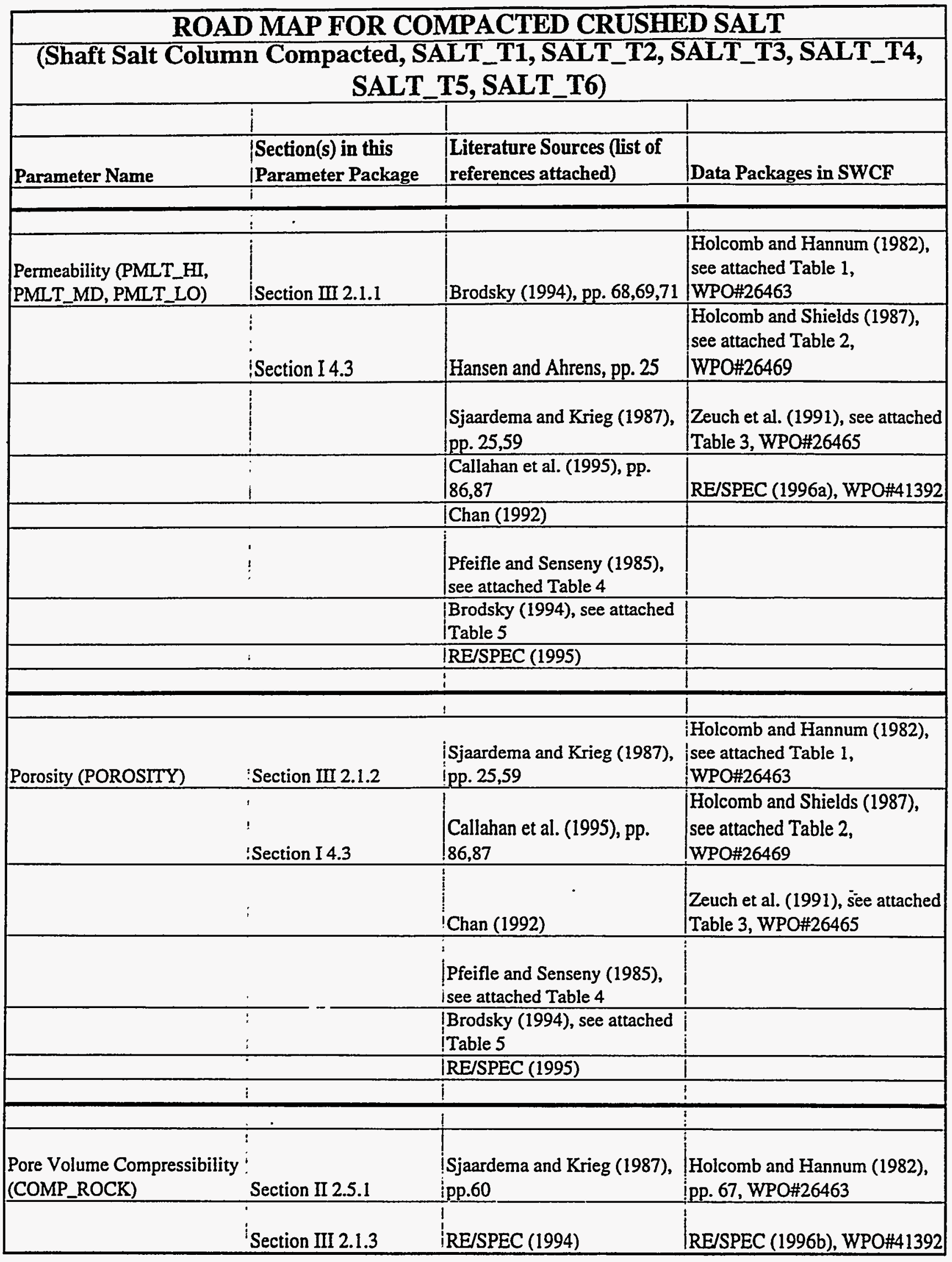




\begin{tabular}{|c|c|c|c|}
\hline & Section I 4.3 & & 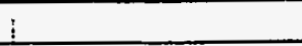 \\
\hline & 1 & & \\
\hline & $!$ & & \\
\hline $\begin{array}{l}\text { Initial Pressure (Initial pore } \\
\text { fluid pressure, pore pressure, } \\
\text { PRESSURE) }\end{array}$ & Section II 2.5.3 & ; & \\
\hline & |Section I 4.3 & & $i$ \\
\hline & 1 & $i$ & $i$ \\
\hline & $\vdots$ & $\dot{\cdot}$ & $!$ \\
\hline $\begin{array}{l}\text { Initial Wetting Phase } \\
\text { Saturation (Initial Brine } \\
\text { Saturation, SAT_IBRN) }\end{array}$ & Section II 2.5.2 & 'Hansen (1995) & No data packages \\
\hline & Section I 4.3 & 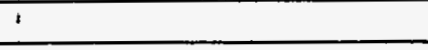 & 1 \\
\hline & $!$ & & $i$ \\
\hline & 1 & & 1 \\
\hline $\begin{array}{l}\text { Threshold Pressure (PCT_A, } \\
\text { PCT_EXP) }\end{array}$ & Section $\amalg$ 2.5.4 & Davies (1991), pp. 25 & INo data packages \\
\hline & |Section I 4.3 & .Pihlajavaara (1991), pp. 47 & 1 \\
\hline & 1 & & $i$ \\
\hline & $!$ & & $!$ \\
\hline $\begin{array}{l}\text { Residual Wetting Phase } \\
\text { Saturation (Residual Brine } \\
\text { Saturation, Residual Liquid } \\
\text { Saturation, SAT_RBRN) }\end{array}$ & Section II 2.5.5 & iTable 6 attached & No data packages \\
\hline & ISection I 4.3 & & 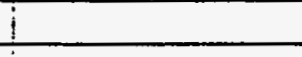 \\
\hline & $\cdot$ & & $\vdots$ \\
\hline & & & $\therefore$ \\
\hline $\begin{array}{l}\text { Residual Non-Wetting Phase } \\
\text { Saturation (Residual Gas } \\
\text { Saturation, SAT_RGAS) }\end{array}$ & ISection II 2.5.6 & Mayer et al. (1992), pp. 173 & No data packages \\
\hline & Section I 4.3 & & $!$ \\
\hline & $:$ & & 1 \\
\hline & $\vdots$ & & $\vdots$ \\
\hline $\begin{array}{l}\text { Pore Size Distribution } \\
\text { (Lambda, POR_DIS) }\end{array}$ & Section II 2.5.7 & Table 7 attached & No data packages \\
\hline & Section I 4.3 & & $:$ \\
\hline
\end{tabular}


Table 1. Cross-Reference Between Crushed Salt Data and Holcomb and Hannum, 1982

\begin{tabular}{|c|c|c|c|}
\hline \multirow{2}{*}{$\begin{array}{l}\text { Test } \\
\text { I.D. }\end{array}$} & \multirow{2}{*}{$\begin{array}{l}\text { Test } \\
\text { Type }\end{array}$} & \multicolumn{2}{|c|}{ Page Numbers in Reference } \\
\hline & & Plots & Tables \\
\hline 08JUN82(M) & \multirow{7}{*}{$\begin{array}{l}\text { Hydrostatic } \\
\text { Compaction }\end{array}$} & 25 & 48,51 \\
\hline 08JUN82 & & 30 & 48,50 \\
\hline 23FEB82(M) & & 26 & $48,52-55$ \\
\hline 25FEB82(M) & & 27 & $48,56-58$ \\
\hline 16MAR82(M) & & 28 & $48,59-62$ \\
\hline 25MAR82(M) & & 29 & $48,63-66$ \\
\hline 24MAY82(M) & & 31 & 48,67 \\
\hline 27JUL82(M) & \multirow{12}{*}{$\begin{array}{l}\text { Hydrostatic } \\
\text { Consolidation }\end{array}$} & 34,35 & $49,68-70$ \\
\hline 04MAY82(M) & & 36 & $49,71-73$ \\
\hline 30APR82(M) & & 37 & $49,74-77$ \\
\hline 07MAY82(M) & & 38 & $49,78-80$ \\
\hline 12MAY82(M) & & 39 & $49,81-83$ \\
\hline 05FEB82(M) & & 40 & $49,84-87$ \\
\hline 09APR82(M) & & 41 & $49,88-91$ \\
\hline 26MAR82(M) & & 42 & $49,92-95$ \\
\hline 02APR82(M) & & 43 & $49,96-99$ \\
\hline 13APR82(M) & & 44 & $49,100-102$ \\
\hline 29JAN82(M) & & 45 & $49,103-105$ \\
\hline 26FEB82(M) & & 46 & $49,105-109$ \\
\hline
\end{tabular}


Table 2. Cross Reference Between Crushed Salt Data and Holcomb and Shields, 1987

\begin{tabular}{|c|c|c|c|}
\hline \multirow{2}{*}{$\begin{array}{l}\text { Test } \\
\text { I.D. }\end{array}$} & \multirow{2}{*}{$\begin{array}{l}\text { Test } \\
\text { Type }\end{array}$} & \multicolumn{2}{|c|}{ Page Numbers in Reference } \\
\hline & & Plots & Tables \\
\hline 27JU61 & \multirow{17}{*}{$\begin{array}{l}\text { Hydrostatic } \\
\text { Consolidation }\end{array}$} & $37-40$ & 29 \\
\hline 23JL51 & & $45-48$ & 29 \\
\hline $14 N V 51$ & & $49-52$ & 29 \\
\hline 25FE61 & & $53-56$ & 29 \\
\hline $10 \mathrm{MY} 51$ & & $57-60$ & 29 \\
\hline 20AU51 & & $61-64$ & 29 \\
\hline $16 \pi 551$ & & $69-72$ & 29 \\
\hline $18 \pi \mathrm{J} 51$ & & $73-76$ & 29 \\
\hline $300 C 51$ & & $77-80$ & 29 \\
\hline 16JA61 & & $81-84$ & 29 \\
\hline 19DC44 & & $85-88$ & 29 \\
\hline 13AU51 & & $89-92$ & 29 \\
\hline $240 C 61-1$ & & $93-96$ & 29 \\
\hline $240 \mathrm{OC} 61-2$ & & $97-100$ & 29 \\
\hline $240 C 61-3$ & & $100-104$ & 29 \\
\hline $240 \mathrm{C} 61-4$ & & $105-108$ & 29 \\
\hline $240 C 61-5$ & & $109-112$ & $29^{-}$ \\
\hline
\end{tabular}


Table 3. Cross-Reference Between Crushed Salt Data and Zeuch et al., 1991

\begin{tabular}{|c|c|c|c|}
\hline \multirow{2}{*}{$\begin{array}{l}\text { Test } \\
\text { I.D. }\end{array}$} & \multirow{2}{*}{$\begin{array}{l}\text { Test } \\
\text { Type }\end{array}$} & \multicolumn{2}{|c|}{ Page Numbers in Reference } \\
\hline & & Plots & Tables \\
\hline $24 \pi \mathrm{J} 71$ & \multirow{6}{*}{$\begin{array}{l}\text { Hydrostatic } \\
\text { Consolidation }\end{array}$} & 38,46 & 28,29 \\
\hline $09 \pi 488$ & & 39,47 & 28,29 \\
\hline 09JU88-2 & & 39,47 & 28,29 \\
\hline 08MR89 & & 40,48 & 28,29 \\
\hline 20SE89 & & 41,49 & 28,29 \\
\hline 18AP89 & & 42,50 & 28,29 \\
\hline $120 C 89$ & \multirow{3}{*}{$\begin{array}{c}\text { Shear } \\
\text { Consolidation }\end{array}$} & $51,52,57-59$ & 30,31 \\
\hline $120 C 89$ & & $51,52,57-59$ & 30,31 \\
\hline $120 C 89$ & & $51,52,57-59$ & 30,31 \\
\hline
\end{tabular}


Table 4. Cross-Reference Between Crushed Salt Data and Pfeifle and Senseny, 1985

\begin{tabular}{|c|c|c|c|}
\hline \multirow{2}{*}{$\begin{array}{l}\text { Test } \\
\text { I.D }\end{array}$} & \multirow{2}{*}{$\begin{array}{l}\text { Test } \\
\text { Type }\end{array}$} & \multicolumn{2}{|c|}{ Page Numbers in Reference } \\
\hline & & Plots & Tables \\
\hline $\mathrm{CS} 1$ & \multirow{10}{*}{$\begin{array}{l}\text { Hydrostatic } \\
\text { Consolidation }\end{array}$} & 24,25 & 23 \\
\hline $\mathrm{CS} 2$ & & 24,25 & 23 \\
\hline $\operatorname{CS} 3$ & & 24,25 & 23 \\
\hline CS4 & & 24,25 & 23 \\
\hline CS5 & & 26,27 & 23 \\
\hline CS6 & & 26,27 & 23 \\
\hline CS7 & & 26,27 & 23 \\
\hline CS8 & & 26,27 & 23 \\
\hline $\operatorname{CS} 9$ & & 31,32 & 23 \\
\hline $\mathrm{CS} 10$ & & 31,32 & 23 \\
\hline
\end{tabular}


Table 5. Cross-Reference Between Crushed Salt Data and Brodsky, 1994

\begin{tabular}{|c|c|c|c|}
\hline \multirow{2}{*}{$\begin{array}{l}\text { Test } \\
\text { I.D. }\end{array}$} & \multirow{2}{*}{$\begin{array}{l}\text { Test } \\
\text { Type }\end{array}$} & \multicolumn{2}{|c|}{ Page Numbers in Reference } \\
\hline & & Plots & Tables \\
\hline $\mathrm{HClA}$ & \multirow{6}{*}{$\begin{array}{l}\text { Hydrostatic } \\
\text { Consolidation }\end{array}$} & $47,51,68$ & $17,46,48$ \\
\hline $\mathrm{HC} 2 \mathrm{~A}$ & & $47,51,68$ & $17,46,48$ \\
\hline $\mathrm{HC} 3 \mathrm{~A}$ & & $47,51,68$ & $17,46,48$ \\
\hline $\mathrm{HC} 4 \mathrm{~A}$ & & $47,51,68$ & $17,46,48$ \\
\hline $\mathrm{HC} 5 \mathrm{~A}$ & & $47,51,68$ & $17,46,48$ \\
\hline HC6A & & $47,51,68$ & $17,46,48$ \\
\hline SC1B & \multirow{9}{*}{$\begin{array}{c}\text { Shear } \\
\text { Consolidation }\end{array}$} & $52,57,60,63,69$ & $18,50,55$ \\
\hline $\mathrm{SC} 2 \mathrm{~A}$ & & $52,57,60,63,69$ & $18,50,55$ \\
\hline $\mathrm{SC} 3 \mathrm{~A}$ & & $52,57,60,63,69$ & $18,50,55$ \\
\hline $\mathrm{SC} 4 \mathrm{~A}$ & & $54,59,62,65,69$ & $18,50,55$ \\
\hline $\mathrm{SC} 5 \mathrm{~A}$ & & $54,59,62,65,69$ & $18,50,55$ \\
\hline SC6A & & $54,59,62,65,69$ & $18,50,55$ \\
\hline SC7A & & $53,58,61,64,69$ & $18,50,55$ \\
\hline SC8A & & $53,58,61,64,69$ & $18,50,55$ \\
\hline SC9B & & $53,58,61,64,69$ & $18,50,55$ \\
\hline
\end{tabular}


Table 6. Literature Values of Residual Liquid Saturations for Geologic Material and Concrete

\begin{tabular}{|c|c|c|}
\hline Material & $\begin{array}{l}\text { Residual } \\
\text { Liquid } \\
\text { Saturation } \\
\end{array}$ & Reference \\
\hline $\begin{array}{l}\text { Consolidated Rock } \\
\text { Berea Sandstone } \\
\text { Hygiene Sandstone } \\
\text { Hygiene Sandstone } \\
\text { Hygie } \\
\end{array}$ & $\begin{array}{l}0.299 \\
0.577 \\
0.572 \\
\end{array}$ & $\begin{array}{l}\text { Brooks and Corey (1964), pg } 27 \\
\text { Brooks and Corey (1964), pg } 27 \\
\text { Lappala et al. (1987),pg 20(4) }\end{array}$ \\
\hline $\begin{array}{l}\text { Sand } \\
\text { Sand } \\
\text { Sand (texture class) } \\
\text { Fine Sand G.E. 13 } \\
\text { Fine Sand G.E. } 13 \\
\text { Volcanic Sand } \\
\text { Del Monte Sand } \\
\text { Fresno Medium Sand } \\
\text { Sandy Porous Media } \\
\text { Unconsolidated Sand } \\
\text { Fragment Fox Hill SS }\end{array}$ & $\begin{array}{l}0.110 \\
0.046 \\
0.186 \\
0.167 \\
0.157 \\
0.081 \\
0.085 \\
0.000 \\
0.146 \\
0.300 \\
\end{array}$ & $\begin{array}{l}\text { Lappala et al. (1987), pg 20(1) } \\
\text { Rawls et al. (1982),pg 1318() } \\
\text { Lappala et al. (1987),pg 20(1) } \\
\text { Brooks and Corey (1964), pg } 25 \\
\text { Brooks and Corey (1964), pg } 25 \\
\text { Lappala et al. (1987), pg } 20^{(1)} \\
\text { Lappala et al. (1987), pg } 20^{(1)} \\
\text { Parker et al. (1987), pg 621 } \\
\text { Lappala et al. (1987), pg 20(1) } \\
\text { Brooks and Corey (1964),pg } 25\end{array}$ \\
\hline $\begin{array}{l}\text { Loamy Sand } \\
\text { Loamy Sand (texture } \\
\text { class) }\end{array}$ & 0.080 & Rawls et al. (1982),pg $1318^{(4)}$ \\
\hline $\begin{array}{l}\text { Sandy Loam } \\
\text { Columbia Sandy Loam } \\
\text { Sandy Loam (texture } \\
\text { class) }\end{array}$ & $\begin{array}{l}0.282 \\
0.091\end{array}$ & $\begin{array}{l}\text { Lappala et al. (1987), pg 20(4) } \\
\text { Rawls et al. (1982),pg } 1318^{(0)}\end{array}$ \\
\hline $\begin{array}{l}\text { Loam } \\
\text { Adelanto Loam } \\
\text { Loam (texture class) }\end{array}$ & $\begin{array}{l}0.374 \\
0.058 \\
\end{array}$ & $\begin{array}{l}\text { Lappala et al. (1987), pg } 21^{(1)} \\
\text { Rawls et al. (1982),pg } 1318^{(1)}\end{array}$ \\
\hline $\begin{array}{l}\text { Silt } \\
\text { Limon Silt }\end{array}$ & 0.009 & Lappala et al. (1987), pg $21^{(0)}$ \\
\hline $\begin{array}{l}\text { Silt Loam } \\
\text { Touchet Silt Loam } \\
\text { Touchet Silt Loam (GE } \\
\text { 3) } \\
\text { Silt Loam (texture } \\
\text { class) }\end{array}$ & $\begin{array}{l}0.337 \\
0.270 \\
0.030\end{array}$ & $\begin{array}{l}\text { Lappala et al (1987), pg 20(1) } \\
\text { Brooks and Corey (1964), pg } 25 \\
\text { Rawls et al. (1982), pg } 1318^{(1)}\end{array}$ \\
\hline $\begin{array}{l}\text { Sandy Clay Loam } \\
\text { Sandy Clay Loam } \\
\text { (texture class) } \\
\end{array}$ & 0.171 & Rawls et al. (1982),pg 1318() \\
\hline $\begin{array}{l}\text { Clay Loam } \\
\text { Clay Loam (texture } \\
\text { class) }\end{array}$ & 0.162 & Rawls et al. (1982), pg 1318(1) \\
\hline
\end{tabular}


Table 6. Literature Values of Residual Liquid Saturations for Geologic Material and Concrete (Continued)

\begin{tabular}{|c|c|c|}
\hline Material & $\begin{array}{l}\text { Residual } \\
\text { Liquid } \\
\text { Saturation } \\
\end{array}$ & Reference \\
\hline $\begin{array}{l}\text { Silty Clay Loam } \\
\text { Silty Clay Loam } \\
\text { (texture class) } \\
\end{array}$ & 0.085 & Rawls et al. (1982), pg $1318^{(1)}$ \\
\hline $\begin{array}{l}\text { Sandy Clay } \\
\text { Sandy Clay (texture } \\
\text { class) }\end{array}$ & 0.253 & Rawls et al. (1982), pg 1318(4) \\
\hline $\begin{array}{l}\text { Silty Clay } \\
\text { Silty Clay (texture } \\
\text { class) }\end{array}$ & 0.117 & Rawls et al. (1982), pg 1318(1) \\
\hline $\begin{array}{l}\text { Clay } \\
\text { Yolo Light Clay } \\
\text { Clayey Porous Media } \\
\text { Clay (texture class) } \\
\end{array}$ & $\begin{array}{l}0.299 \\
0.360 \\
0.189 \\
\end{array}$ & $\begin{array}{l}\text { Lappala et al. (1987), pg } 21^{(4)} \\
\text { Parker et al. (1987), pg } 621 \\
\text { Rawls et al. (1982), pg } 1318^{(4)}\end{array}$ \\
\hline $\begin{array}{l}\text { Fragmented Mixture } \\
\text { Fragmented Mixture }\end{array}$ & 0.276 & Brooks and Corey (1964), pg 25 \\
\hline $\begin{array}{l}\text { Concrete } \\
\text { Normal Concrete }\end{array}$ & $0.300^{(3)}$ & Mayer et al. (1992), pg 173 \\
\hline
\end{tabular}
(l) - Core perpendicular to bedding planes
(B) Core contained no visible bedding planes
(3) Estimated
(4) The indicated reference reports residual water content and porosity from which the residual liquid saturation was calculated. 
Table 7. Literature Values of Lambda for Geologic Material

\begin{tabular}{|c|c|c|}
\hline Material & Lambda $a^{(\Im)}$ & Reference \\
\hline \multicolumn{3}{|l|}{ Consolidated Rock } \\
\hline Berea Sandstone ${ }^{(1)}$ & 3.69 & Brooks and Corey (1964), pg 27 \\
\hline Berea Sandstone & 2.13 & Mualem (1976), page 515 \\
\hline average & 2.91 & calculated $[(3.69+2.13) / 2]$ \\
\hline Hygiene Sandstone $e^{(2)}$ & 4.17 & Brooks and Corey (1964), pg 27 \\
\hline Hygiene Sandstone & 3.78 & Mualem (1976), pg 515 \\
\hline Hygiene Sandstone & $9.4(10.4)$ & van Genuchten (1980), pg $897^{\circ}$ \\
\hline Hygiene Sandstone & 2.9 & Lappala et al. (1987), pg 20 \\
\hline average & 6.75 & calculated $[(4.17+3.78+9.4+2.9) / 4]$ \\
\hline Tottori Sand & 2.157 & Haverkamp and Parlange (1986),pg 331 \\
\hline Preston Sand & 1.653 & Haverkamp and Pariange (1986), pg 331 \\
\hline Gormley Sand & 1.861 & Haverkamp and Pariange (1986), pg 331 \\
\hline Botton Sand & 1.723 & Haverkamp and Parlange (1986), pg 331 \\
\hline \multicolumn{3}{|l|}{ Netherlands Coarse-Textured } \\
\hline topsoils $s^{(3)}$ & $0.59^{(9)}$ & Wosten and van Genuchten (1988), pg 1763 \\
\hline subsoils ${ }^{(3)}$ & $1.01^{(\theta)}$ & Wosten and van Genuchten (1988), pg 1763 \\
\hline \multicolumn{3}{|l|}{ Netherlands Medium-Textured } \\
\hline topsoils(s) & $0.22^{(\theta)}$ & Wosten and van Genuchten (1988), pg 1763 \\
\hline subsoils $s^{(4)}$ & $0.27^{(\bullet)}$ & Wosten and van Genuchten (1988), pg 1763 \\
\hline \multicolumn{3}{|l|}{ Netherlands Fine-Textured } \\
\hline topsoils $s^{(t)}$ & $0.11^{(\theta)}$ & Wosten and van Genuchten (1988), pg 1763 \\
\hline subsoils ${ }^{(4)}$ & $0.12^{(0)}$ & Wosten and van Genuchten (1988), pg 1763 \\
\hline \multicolumn{3}{|l|}{ Lacustrine Sediments } \\
\hline $\begin{array}{l}\text { thinly laminated clay to } \\
\text { silty clay }\end{array}$ & $0.65(1.65)$ & Stephens et al. (1988), pg 62 \\
\hline massive clay to silty clay & $0.66(1.66)$ & Stephens et al. (1988), pg 62 \\
\hline thin bedded silt & $0.80(1.80)$ & Stephens et al. (1988), pg 62 \\
\hline thin bedded clay & $0.90(1.90)$ & Stephens et al. (1988), pg 62 \\
\hline $\begin{array}{l}\text { thin bedded silts, clay and } \\
\text { clayey silt }\end{array}$ & $0.85(1.85)$ & Stephens et al. (1988), pg 62 \\
\hline \multicolumn{3}{|l|}{ Sand } \\
\hline Sand & 1.83 & Mualem (1976), pg 515 \\
\hline Sand & 2.87 & Mualem (1976), pg 515 \\
\hline Sand & 0.84 & Lappala et al. (1987), pg 20 \\
\hline average & 1.85 & calculated $[(1.83+2.87+0.84) / 3]$ \\
\hline Sand (texture class) & 0.694 & Rawls et al. (1982), pg 1318 \\
\hline Beit Dagan Sand & 0.37 & Mualem (1976), pg 515 \\
\hline
\end{tabular}


Table 7. Literature Values of Lambda for Geologic Material (Continued)

\begin{tabular}{|c|c|c|}
\hline Material & Lambda & Reference \\
\hline \multicolumn{3}{|l|}{ Sand (continued) } \\
\hline Fine Sand GE 13 & 1.98 & Mualem (1976), pg 515 \\
\hline Fine Sand GE 13 & 3.7 & Lappala et al (1987), pg 20 \\
\hline $\begin{array}{l}\text { Fine Sand GE } 13 \\
\text { average }\end{array}$ & $\begin{array}{c}3.7 \\
3.13\end{array}$ & $\begin{array}{l}\text { Brooks and Corey }(1964), \mathrm{pg} 25 \\
\text { calculated }[(1.98+3.7+3.7) / 3]\end{array}$ \\
\hline Volcanic Sand & 1.65 & Mualem (1976), pg 515 \\
\hline Volcanic Sand & 2.29 & Brooks and Corey (1964), pg 25 \\
\hline average & 1.97 & calculated $[(1.65+2.29) / 2]$ \\
\hline Volcanic Sand & 1.3 & Mualem (1976), pg 515 \\
\hline Plainfield Sand & 1.45 & Mualem (1976), pg 515 \\
\hline Del Monte Sand & 2.5 & Lappala et al. (1987), pg 20 \\
\hline Fresno Medium Sand & 0.84 & Lappala et al. (1987), pg 20 \\
\hline Molonglo River Sand & 0.96 & Mualem (1976), pg 515 \\
\hline Botany Sand Fraction & 8.35 & Mualem (1976), pg 515 \\
\hline River Sand (screened) & 1.57 & Mualem (1976), pg 515 \\
\hline Gilat Fine Sand & 0.27 & Mualem (1976), pg 515 \\
\hline Rehovot Sand & 0.83 & Mualem (1976), pg 515 \\
\hline Pouder River Sand & 2.92 & Mualem (1976), pg 515 \\
\hline Sable de riviere & 0.92 & Mualem (1976), pg 515 \\
\hline Grenoble Sand 1 & 1.523 & Haverkamp and Parlange (1986), pg 331 \\
\hline Grenoble Sand 2 & 2.309 & Haverkamp and Parlange (1986), pg 331 \\
\hline Grenoble Sand 3 & 1.479 & Haverkamp and Pariange (1986), pg 331 \\
\hline Grenoble Sand 4 & 2.984 & Haverkamp and Parlange (1986), pg 331 \\
\hline Genoble Sand 5 & 1.454 & Haverkamp and Parlange (1986), pg 331 \\
\hline Grenoble Sand 6 & 1.587 & Haverkamp and Parlange (1986), pg 331 \\
\hline Sand Fraction $(150-300 \mu \mathrm{m})$ & 4.94 & Mualem (1976), pg 515 \\
\hline Sand Fraction $(150-300 \mu \mathrm{m})$ & 11.67 & Mualem (1976), pg 515 \\
\hline Sand Fraction (0.5-0.25 mm) & 5.9 & Mualem (1976), pg 515 \\
\hline Sand Fraction (1.0-0.5 mm) & 2.8 & Mualem (1976), pg 515 \\
\hline GENo. 2 Sand & $4.05(5.05)$ & van Genuchten and Nielsen (1985), pg 619 \\
\hline Sandy Porous Media & $0.84(1.84)$ & Parker et al. (1987), pg 621 \\
\hline Unconsolidated Sand & 4.4 & Lappala et al. (1987), pg 20 \\
\hline Fragmented Fox Hill Sandstone & 1.92 & Brooks and Corey (1964), pg 25 \\
\hline Fragmented Fox Hill Sandstone & 2.61 & Mualem (1976), pg 515 \\
\hline average & 2.27 & caiculated $[(1.92+2.61) / 2]$ \\
\hline \multicolumn{3}{|l|}{ Ioamy Sand } \\
\hline Loamy Sand (texture class) & 0.553 & Rawls et al. (1982), pg 1318 \\
\hline
\end{tabular}




\section{Table 7. Literature Values of Lambda for Geologic Material (Continued)}

\begin{tabular}{|c|c|c|}
\hline Material & Lambda $(s)$ & Reference \\
\hline $\begin{array}{l}\text { Sandy Loam } \\
\text { Columbia Sandy Loam } \\
\text { Rubicon Sandy Loam } \\
\text { Gilat Sandy Loam }\end{array}$ & $\begin{array}{l}1.6 \\
2.08 \\
0.44\end{array}$ & $\begin{array}{l}\text { Lappala et al. (1987), pg } 20 \\
\text { Mualem (1976), pg } 515 \\
\text { Mualem (1976), pg } 515\end{array}$ \\
\hline $\begin{array}{l}\text { Norfolk Sandy Loam } 20^{\circ} \mathrm{C} \\
\text { Norfolk Sandy Loam } 44.5^{\circ} \mathrm{C} \\
\text { average }\end{array}$ & $\begin{array}{c}1.403(2.403) \\
1.339(2.339) \\
1.371\end{array}$ & $\begin{array}{l}\text { Hopmans and Overmars (1986), pg } 139 \\
\text { Hopmans and Overmars }(1986), \text { pg } 139 \\
\text { calculated }[(1.403+1.339) / 2]\end{array}$ \\
\hline Sandy Loam (texture class) & 0.378 & Rawls et al. (1982),pg 1318 \\
\hline $\begin{array}{l}\text { Loam } \\
\text { Gueiph Loam } \\
\text { Guelph Loam } \\
\text { average }\end{array}$ & $\begin{array}{l}0.41 \\
1.03(2.03) \\
0.72\end{array}$ & $\begin{array}{l}\text { Mualem }(1976), \text { pg } 515 \\
\text { van Genuchten }(1980), \text { pg } 897 \\
\text { calculated }[(0.41+1.03) / 2]\end{array}$ \\
\hline Sarpy Loam & $0.6(1.60)$ & van Genuchten and Nielsen (1985), pg 619 \\
\hline $\begin{array}{l}\text { Adelanto Loam } \\
\text { Adelanto Loam } \\
\text { average }\end{array}$ & $\begin{array}{l}0.5 \\
0.51 \\
0.51\end{array}$ & $\begin{array}{l}\text { Mualem (1976), pg } 515 \\
\text { Lappala et al }(1987), \mathrm{pg} 21 \\
\text { calculated }[(0.5+0.51) / 2]\end{array}$ \\
\hline $\begin{array}{l}\text { Pachappa Loam } \\
\text { Indio Loam } \\
\text { Loam (texture class) } \\
\end{array}$ & $\begin{array}{c}0.42 \\
0.81 \\
0.252 \\
\end{array}$ & $\begin{array}{l}\text { Mualem (1976), pg } 515 \\
\text { Mualem (1976), pg } 515 \\
\text { Rawls et al. (1982),pg } 1318 \\
\end{array}$ \\
\hline $\begin{array}{l}\text { Silt } \\
\text { Silt Mont Cenis } \\
\text { Limon Silt } \\
\end{array}$ & $\begin{array}{l}0.36 \\
0.22 \\
\end{array}$ & $\begin{array}{l}\text { Mualem (1976), pg } 515 \\
\text { Lappale et al. (1987), pg } 21\end{array}$ \\
\hline $\begin{array}{l}\text { Silt Loam } \\
\text { Touchet Silt Loam } \\
\text { Touchet Silt Loam } \\
\text { Touchet Silt Loam } \\
\text { average }\end{array}$ & $\begin{array}{c}1.71 \\
2.59(3.59) \\
1.7 \\
2.00\end{array}$ & $\begin{array}{l}\text { Mualem (1976), pg } 515 \\
\text { van Genuchten and Nielsen (1985), pg } 619 \\
\text { Lappala et al. }(1987), \text { pg } 20 \\
\text { calculated }[(1.71+2.59+1.70) / 3]\end{array}$ \\
\hline $\begin{array}{l}\text { Touchet Silt Loam (GE 3) } \\
\text { Touchet Silt Loam (GE 3) } \\
\text { Touchet Silt Loam (GE 3) } \\
\text { average }\end{array}$ & $\begin{array}{c}1.89 \\
1.82 \\
6.09(7.09) \\
3.27\end{array}$ & $\begin{array}{l}\text { Mualem (1976), pg 515 } \\
\text { Brooks and Corey (1964), pg } 25 \\
\text { van Genuchten }(1980), \mathrm{pg} 897 \\
\text { calculated }[(1.89+1.82+6.09) / 3]\end{array}$ \\
\hline $\begin{array}{l}\text { Caribou Silt Loam } \\
\text { Grenville Silt Loam } \\
\text { Ida Silt Loam (>15 cm) } \\
\text { Ida Silt Loam (0-15 cm) } \\
\text { Silt Loam GE } 3 \\
\text { Silt Loam (texture class) }\end{array}$ & $\begin{array}{c}0.91 \\
0.34 \\
0.38 \\
0.27 \\
1.06(2.06) \\
0.234 \\
\end{array}$ & $\begin{array}{l}\text { Mualem (1976), pg } 515 \\
\text { Mualem (1976), pg } 515 \\
\text { Mualem (1976),pg } 515 \\
\text { Mualem (1976),pg } 515 \\
\text { van Genuchten (1980), pg } 897 \\
\text { Rawls et al: (1982),pg } 1318 \\
\end{array}$ \\
\hline $\begin{array}{l}\text { Sandy Clay Loam } \\
\text { Sandy Clay Loam (texture class) }\end{array}$ & 0.319 & Rawis et al. (1982),pg 1318 \\
\hline
\end{tabular}


Table 7. Literature Values of Lambda for Geologic Material (Continued)

\begin{tabular}{|c|c|c|}
\hline Material & Lambda ${ }^{(s)}$ & Reference \\
\hline $\begin{array}{l}\text { Clay Loam } \\
\text { Rideau Clay Loam } \\
\text { Clay Loam (texture class) }\end{array}$ & $\begin{array}{r}1.62 \\
0.242 \\
\end{array}$ & $\begin{array}{l}\text { Mualem (1976), pg } 515 \\
\text { Rawis et al. (1982),pg } 1318 \\
\end{array}$ \\
\hline $\begin{array}{l}\text { Silty Clay Loam } \\
\text { Weld Silty Clay Loam } \\
\text { Weld Silty Clay Loam } \\
\text { average }\end{array}$ & $\begin{array}{l}1.52 \\
4.45(5.45) \\
2.99\end{array}$ & $\begin{array}{l}\text { Mualem (1976), pg } 515 \\
\text { van Genuchten and Nielsen (1985), pg } 619 \\
\text { calculated }[(1.52+4.45) / 2]\end{array}$ \\
\hline $\begin{array}{l}\text { Amarillo Silty Clay Loam } \\
\text { Silty Clay Loam (texture class) }\end{array}$ & $\begin{array}{l}2.35 \\
0.177 \\
\end{array}$ & $\begin{array}{l}\text { Mualem (1976), pg } 515 \\
\text { Rawis et al. (1982),pg } 1318 \\
\end{array}$ \\
\hline $\begin{array}{l}\text { Sandy Clay } \\
\text { Pachappa Fine Sandy Clay } \\
\text { Sandy Clay (texture class) }\end{array}$ & $\begin{array}{c}0.44 \\
0.223 \\
\end{array}$ & $\begin{array}{l}\text { Mualem (1976), pg } 515 \\
\text { Rawls et al. (1982),pg } 1318 \\
\end{array}$ \\
\hline $\begin{array}{l}\text { Silty Clay } \\
\text { Shluhot Silty Clay } \\
\text { Silty Clay (texture class) } \\
\end{array}$ & $\begin{array}{c}0.2 \\
0.15 \\
\end{array}$ & $\begin{array}{l}\text { Mualem (1976), pg } 515 \\
\text { Rawls et al. (1982),pg } 1318 \\
\end{array}$ \\
\hline $\begin{array}{l}\text { Clay } \\
\text { Beit Netofa Clay } \\
\text { Beit Netofa Clay } \\
\text { average }\end{array}$ & $\begin{array}{l}0.19 \\
0.17(1.17) \\
0.18\end{array}$ & $\begin{array}{l}\text { Mualem (1976), pg 515 } \\
\text { van Genuchten }(1980), \text { pg } 897 \\
\text { calculated [(0.19+0.17)/2] }\end{array}$ \\
\hline $\begin{array}{l}\text { Yolo Light Clay } \\
\text { Clayey Porous Media } \\
\text { Clay (texture class) } \\
\end{array}$ & $\begin{array}{c}0.25 \\
0.86(1.86) \\
0.165 \\
\end{array}$ & $\begin{array}{l}\text { Lappala et al. (1987), pg } 21 \\
\text { Parker et al. (1987), pg } 621 \\
\text { Rawls et al. (1982),pg } 1318 \\
\end{array}$ \\
\hline $\begin{array}{l}\text { Slate } \\
\text { Slate Dust }\end{array}$ & 5.69 & Mualem (1976), pg 515 \\
\hline $\begin{array}{l}\text { Fragmented Mixture } \\
\text { Fragmented Mixture } \\
\text { Fragmented Mixture } \\
\text { average } \\
\end{array}$ & $\begin{array}{l}2.65 \\
2.89 \\
2.77\end{array}$ & $\begin{array}{l}\text { Mualem (1976), pg 515 } \\
\text { Brooks and Corey }(1964), \mathrm{pg} 25 \\
\text { calculated [(2.65+2.89)/2] }\end{array}$ \\
\hline
\end{tabular}

(1) core perpendicular to bedding planes

(2) core contained no visible bedding planes

(B) average of four values reported in reference

(4) average of three values reported in reference

(5) the number in parentheses is the $n$ value reported in the reference

(9) this value is the average of the original reference values 


\section{Compacted Crushed Salt References}

Brodsky, N. S., 1994. Hydrostatic and Shear Consolidation Tests With Permeability Measurements on Waste Isolation Pilot Plant Crushed Salt, SAND93-7058, prepared by RE/SPEC Inc, Rapid City, SD, for Sandia National Laboratories, Albuquerque, NM.

Brooks, R.H., and A.T. Corey, 1964, Hydraulic Properties of Porous Media. Hydrology Papers, Colorado State University, Fort Collins, CO.

Callahan, G. D., M. C. Loken, L. L. Van Sambeek, R. Chen, T. W. Pfeifle, J. D. Nieland, and F. D. Hansen, 1995. Evaluation of Potential Crushed-Salt Models, SAND95-2143, prepared by RE/SPEC Inc., Rapid City, SD, for Sandia National Laboratories, Albuquerque, NM.

Chan, K. S., Bodner, S. R., Fossum, A. F., and Munson, D. E., 1992. A Constitutive Model for Inelastic Flow and Damage Evolution in Solids Under Triaxial Compression, Mechanics of Materials, Vol. 14, pp. 1-14.

Davies, P.B., 1991, Evaluation of the Role of Threshold Pressure in Controlling Flow of WasteGenerated Gas into Bedded Salt at the Waste Isolation Pilot Plant. SAND90-3246. Sandia National Laboratories, Albuquerque, NM.

Hansen, F.D., 1995, Memorandum to T. Jones, INTERA Inc., November 30, 1995. (WPO\#30640, Section I 4.3.2).

Hansen, F. D. and E. H. Ahrens, 1996. Large-Scale Dynamic Compaction Demonstration Using WIPP Salt: Fielding and Preliminary Results, SAND95-1941, prepared by Sandia National Laboratories, Albuquerque, NM.

Haverkamp, R., and J.Y. Parlange, 1986, Predicting the Water-Retention Curve From ParticleSize Distribution: 1. Sandy Soils Without Organic Matter, Soil Science. Vol. 142, No. 6, pp. 325-339.

Holcomb, D. J. and D. W. Hannum, 1982. Consolidation of Crushed Salt Backfill Under Conditions Appropriate to the WIPP Facility, SAND82-0630, prepared by Sandia National Laboratories, Albuquerque, NM. (WPO \#26463).

Holcomb, D. J. and M. E. Shields, 1987. Hydrostatic Creep of Crushed Salt With Added Water, SAND87-1990, prepared by Sandia National Laboratories, Albuquerque, NM. (WPO \#26469).

Hopmans, J.W., and B. Overmars, 1986, Presentation and Application of an Analytical Model to Describe Soil Hydraulic Properties, Journal of Hydrology. Vol. 87, pp. 135-143.

Lappala, E.G., R.W. Healy, and E.P. Weeks, 1987, Documentation of Computer Program VS2D to Solve the Equations of Fluid Flow in Variably Saturated Porous Media. Water- 
Resources Investigations Report 83-4099. U.S. Geological Survey, Denver, CO.

Mayer, G., F. Jacobs, and F.H. Wittmann, 1992, Experimental Determination and Numerical Simulation of the Permeability of Cementitious Materials, Nuclear Engineering and Design. Vol. 138, pp. 171-177.

Mualem, Y., 1976, New Model for Predicting the Hydraulic Conductivity of Unsaturated Porous Media, Water Resources Research. Vol.12, no. 3, pp. 513-522.

Parker, J.C., R.J. Lenhard, and T. Kuppusamy, 1987, A Parametric Model for Constitutive Properties Governing Multiphase Flow in Porous Media, Water Resources Research. Vol. 23, no. 4, pp. 618-624.

Pfeifle, T. W. and P. E. Senseny, 1985. Permeability and Consolidation of Crushed Salt From the WIPP Site, RSI-0278, prepared by RE/SPEC Inc., Rapid City, SD, for Sandia National Laboratories, Albuquerque, NM. (WPO\#36824)

Pihlajavaara, S.E., 1991, Long-Term Gas Permeability Properties of Concrete in Wet Repository Conditions, Nuclear Engineering and Design. Vol. 129, pp. 41-48.

Rawls, W.J., D.L. Brakensiek, and K.E. Saxton, 1982, Estimation of Soil Water Properties, Transactions of the ASAE, pp. 1316-1328.

RE/SPEC, 1994. Determination of Intact Density of WIPP Salt Specimens, Calculation File 325/3.4/01, prepared by RE/SPEC Inc., Rapid City, SD, for Sandia National Laboratories, Albuquerque, NM. (WPO\#42723)

RE/SPEC, 1995. WIPP Crushed-Salt Database for Constitutive Model Evaluations, Calculation File 325/4/03, prepared by RE/SPEC Inc., Rapid City, SD, for Sandia National Laboratories, Albuquerque, NM. (WPO\#40871)

RE/SPEC, 1996a. Shear Consolidation Testing Performed to Supplement the WIPP CrushedSalt Database, Calculation File 325/12.1/01, prepared by RE/SPEC Inc., Rapid City, SD, for Sandia National Laboratories, Albuquerque, NM. (WPO\#41392)

RE/SPEC, 1996b. Mechanical and Hydrologic Properties Testing of Dynamically-Compacted Crushed Salt, Calculation File 325/12.1/02, prepared by RE/SPEC Inc., Rapid City, SD, for Sandia National Laboratories, Albuquerque, NM. (WPO\#41392)

Sjaardema, G. D. and R. D. Krieg, 1987. A Constitutive Model for the Consolidation of WIPP Crushed Salt and Its Use in Analyses of Backfilled Shaft and Drift Configurations, SAND87-1977, prepared for Sandia National Laboratories, Albuquerque, NM.

Stephens, D.B., M.Unruh, J. Havlena, R.G. Knowlton, Jr., E. Mattson, and W. Cox, 1988, Vadose Zone Characterization of Low-Permeability Sediments Using Field Permeameters, Ground Water Monitoring Review. Vol. 8, pp. 59-66. 
van Genuchten, M.Th., 1980, A Closed-form Equation for Predicting the Hydraulic Conductivity of Unsaturated Soils, Soil Science Society of America Journal. Vol. 44, pp. 892-898.

van Genuchten, M.Th., and D.R. Nielsen, 1985, On Describing and Predicting the Hydraulic Properties of Unsaturated Soils, Annales Geophysicae. Vol. 3, no. 5, pp. 615-628.

Wosten, J.H.M., and M.Th. van Genuchten, 1988, Using Texture and Other Soil Properties to Predict the Unsaturated Soil Hydraulic Functions, Soil Science Society of America Journal. Vol. 52, pp. 1762-1770.

Zeuch, D.H., D.J. Zimmerer, and M.E. Shields, 1991. Interim Report on the Effects of BrineSaturation and Shear Stress on Consolidation of Crushed, Natural Roxk Salt From the Waste Isolation Pilot Plant (WIPP), SAND91-0105, prepared by Sandia National Laboratories, Albuquerque, NM. (WPO\#26465). 


\section{Appendix $L$}

Request Memo Memorandum 


\section{Page intentionally blank}


data:

8: . Memorandum of Record

Irom:

subjoct:

September 16, 1996

Gin Sandia National Laboratories

Operated for the U.S. Department of Energy by

Sandia Corporation

Abuquerque, Now Maxico 87185-6848

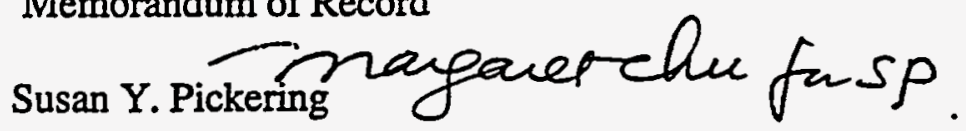

Request, Intended Use and Rational for Major Modifications for Parameter Values Used in Support of the CCA Calculation.

Note: This memorandum supersedes requests memos identified on the Form 464.

The purpose of this memorandum is to provide additional information to support Forms 464 linking parameter values to request memoranda, discussing the intended use of the parameter and providing rationale for why parameter values have been modified. This memo is also intended to demonstrate that the parameters were developed under the auspices of SNL QAP 9-2.

Table 1 provides reference from the parameter types (Category 1 and $4 a$ parameters) to the WPO numbers of the request documentation used in the development of the parameters.

TABLE 1:

\begin{tabular}{|c|c|}
\hline Parameter Types & WVPO Number \\
\hline \multirow[t]{3}{*}{ Salado/Non-Salado Flow } & 35311 \\
\hline & 31035 \\
\hline & 35597 \\
\hline \multirow[t]{2}{*}{ Shaft Seals } & 30640 \\
\hline & 35597 \\
\hline Gas Generation & 30819 \\
\hline Disposal Room & 35697 \\
\hline \multirow{2}{*}{ Dissolved Actinide Retardation } & 35269 \\
\hline & 38231 \\
\hline \multirow[t]{4}{*}{$\begin{array}{l}\text { Colloid Actinide Concentration / } \\
\text { Retardation }\end{array}$} & 36768 \\
\hline & 35135 \\
\hline & 36204 \\
\hline & 36052 \\
\hline \multirow[t]{3}{*}{ Dissolved Species } & $35835^{-}$ \\
\hline & 36053 \\
\hline & 37151 \\
\hline Cuttings & 36301 \\
\hline $\begin{array}{l}\text { Non-Salado Culebra } \\
\text { Transmissivity Zone }\end{array}$ & 35270 \\
\hline Non-Salado Physical Transport & 35270 \\
\hline Category 2 Parameters & 40659 \\
\hline Category 3 Parameters & 40658 \\
\hline
\end{tabular}


It should also be noted that all parameters have been and were intended for use in the Performance Assessment Compliance Certification Application (CCA) as required by CFR 191. These parameters were used for the following analysis tasks as listed in Table 2 (see Analysis Plans for additional discussion). The specific use of the parameter is included in the analysis record package.

TABLE 2:

\begin{tabular}{|l|l|c|}
\hline \multicolumn{2}{|c|}{ CCA Analysis Plan Tille } & 36338 \\
\hline AP AAC & $\begin{array}{l}\text { Salado Calculations (Task 1) of the Performance Assessment Analyses } \\
\text { Supporting the Compliance Certification Application }\end{array}$ & 34798 \\
\hline AP 023 & $\begin{array}{l}\text { Salado Transport Calculations (Task 2) of the Performance Assessment } \\
\text { Analyses Supporting the Compliance Certification Application }\end{array}$ & 37267 \\
\hline AP 019 & $\begin{array}{l}\text { Culebra Flow and Transport Calculations (Task 3) of the Performance } \\
\text { Assessment Analyses Supporting the Compliance Certification } \\
\text { Application }\end{array}$ & 39387 \\
\hline AP 029 & $\begin{array}{l}\text { BRAGFLO Direct Release Calculations (Task 4) of the Performance } \\
\text { Assessment Analyses Supporting the Compliance Certification } \\
\text { Application }\end{array}$ & 37262 \\
\hline AP 016 & $\begin{array}{l}\text { Cuttings Calculations (Task 5) of the Performance Assessment Analyses } \\
\text { Supporting the Compliance Certification Application }\end{array}$ & 37261 \\
\hline AP 015 & $\begin{array}{l}\text { Spallings Calculations (Task 6) of the Performance Assessment Analyses } \\
\text { Supporting the Compliance Certification Application }\end{array}$ \\
\hline
\end{tabular}

It was sometimes necessary to modify parameter values that were documented in the WIPP PA parameter database. Listed below is rationale used for modifying parameter values. Detailed discussions of these parameters are included in Appendix PAR of the CCA and included in the analysis record package.

- New experimental or empirical data

- Changes in the conceptual models

- Model configuration parameters

- New codes were used

- Changes in the parameter categories 
Memorandum of Record 'NFOEMaTICr.i SW IL'-

September 16, 1996

The following signatures indicate concurrence.

Parameter

Task Leader:

6848 Department Manager:

6115 Department Manager:

6832 Department Manager:

6121 Department Manager:
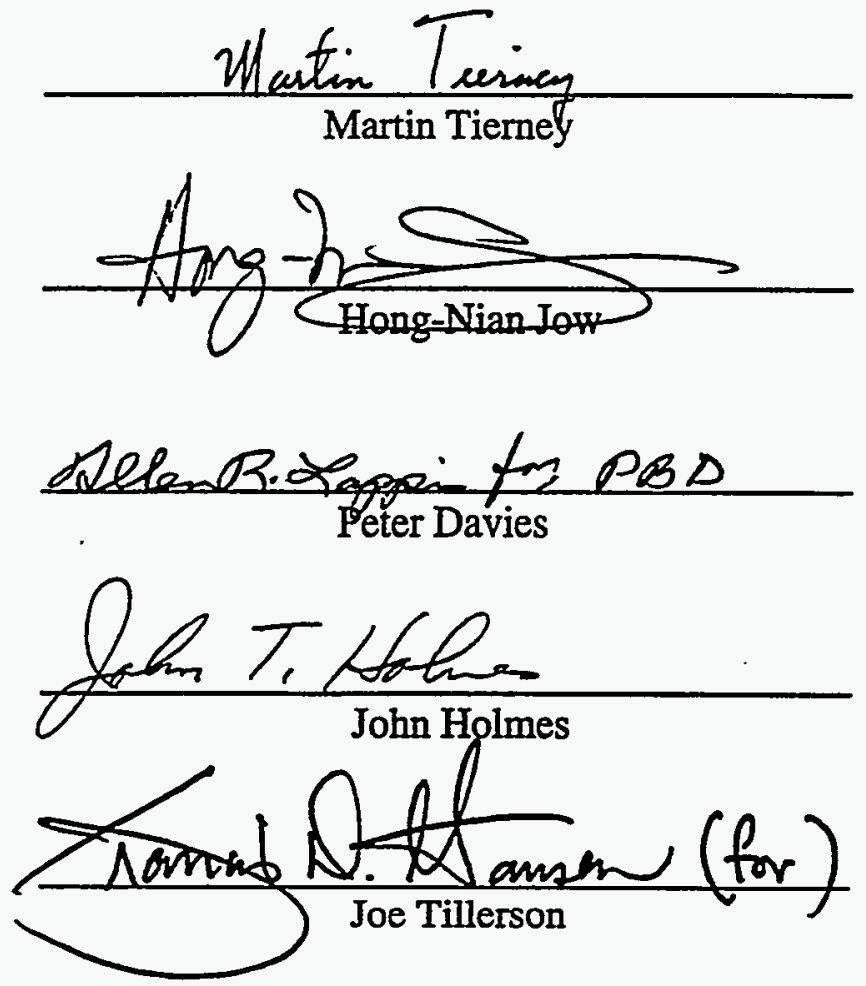
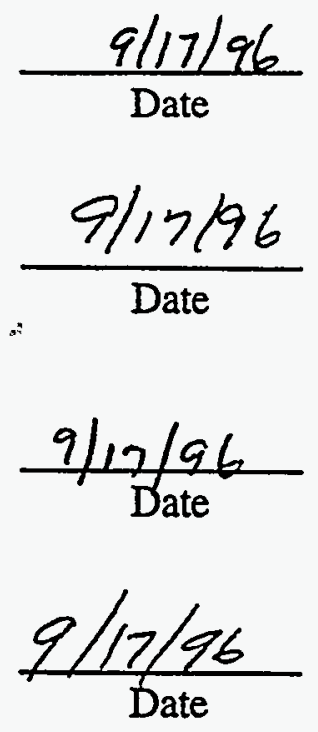

$\frac{9 / 17 / 96}{\text { Date }}$

Distribution:

SNL WIPP Department Managers

M. Fewell, MS-1328 (6849)

M. A. Mantel, MS-1328 (6849)

SWCF-A:1.4.1:AUD:QA:DOE/CAO, S-96-10, CAO Surveillance of Parameters,

GAO CAR 030

SWCF-A:1.2.07.1:QA:PDD: General

L-5 
Appendix M1

Table from EPA Parameter Database: CCA Parameter Listing 
CCA Parameter Listing

\begin{tabular}{|c|c|c|c|c|c|c|c|c|c|c|}
\hline $\begin{array}{l}\text { Pardinter in } \\
\text { Database? } \\
\text { (YN) }\end{array}$ & 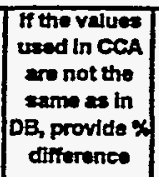 & 10 & Matertal ID & Matterial Name & $\begin{array}{c}\text { Paramoter } \\
\text { to }\end{array}$ & Parameter Nerno & $\begin{array}{l}\text { PRPID } \\
\text { (WPOA) }\end{array}$ & $\begin{array}{c}\text { Dertentry } \\
\text { Deto }\end{array}$ & $\begin{array}{l}\text { to the } 464 \\
\text { convistent } \\
\text { wth the } \\
\text { parameter } \\
\text { in DB? } \\
\text { (Y, NA) }\end{array}$ & $\begin{array}{l}\text { Parameter } \\
\text { Cintegory } \\
\text { (footnote 1) }\end{array}$ \\
\hline $\mathbf{Y}$ & 0.02 & 2 & AM241 & Americium 241 & ATWEIGHT & Atomic Weight in $\mathrm{kg} / \mathrm{mole}$ & 31357 & 02-Nov-95 & $\mathbf{Y}$ & 3 \\
\hline $\bar{Y}$ & 0.02 & 2 & AM241 & Americium 241 & ATWEIGHI & Atomic Weight in kg/mole & 31357 & 02-Nov-95 & $\bar{Y}$ & 3 \\
\hline $\mathbf{Y}$ & 0.02 & 2 & AM241 & Americium 241 & ATWEIGHT & Atomic Weight in $\mathrm{kg} / \mathrm{mole}$ & 31357 & 02-Nov-95 & $\mathbf{Y}$ & 3 \\
\hline $\bar{Y}$ & 100 & 3 & AM241 & Americium 241 & HALFLIFE & Hatilife & 31358 & 16-Apr-96 & $\bar{Y}$ & 3 \\
\hline$Y$ & 100 & 3 & AM241 & Americium 241 & HALFLIFE & Hallifite & 31358 & 16-Apr-96 & $Y$ & 3 \\
\hline$Y$ & 100 & 3 & AM241 & Americium 241 & HALFLIFE & Hattlifo & 31358 & 16-Apr-96 & $Y$ & 3 \\
\hline $\mathbf{Y}$ & & 4 & AM241 & Americium 241 & INVCHD & $\begin{array}{l}\text { Imventory of Contact Handied } \\
\text { Design }\end{array}$ & 31359A & 29-0ct-96 & $\mathbf{Y}$ & 2 \\
\hline $\mathbf{Y}$ & & 4 & AM241 & Americium $24 t$ & INVCHD & $\begin{array}{l}\text { Inventory of Contact Handied } \\
\text { Design }\end{array}$ & 31359A & $29-0 u-96$ & $\mathbf{Y}$ & 2 \\
\hline $\mathbf{Y}$ & & 4 & AM241 & Americium 241 & INVCHD & $\begin{array}{l}\text { Inventory of Contact Handled } \\
\text { Design }\end{array}$ & 31359A & $29-0$ ct-96 & $Y$ & 2 \\
\hline Y & & 4 & AM241 & Americium 241 & INVCHD & $\begin{array}{l}\text { Inventory of Contact Handled } \\
\text { Design }\end{array}$ & 31359A & $29-0 c t-96$ & $\mathbf{Y}$ & 2 \\
\hline $\mathbf{Y}$ & & 5 & AM241 & Americium 241 & |INVRHD & $\begin{array}{l}\text { Inventory of Remote Handled } \\
\text { Design }\end{array}$ & $31360 \mathrm{~A}$ & $29-0 \mathrm{ct}-96$ & $\mathbf{Y}$ & 2 \\
\hline $\mathbf{Y}$ & & 5 & AM241 & Americium 241 & INVRHD & $\begin{array}{l}\text { Inventory of Remote Handied } \\
\text { Design }\end{array}$ & $31360 \mathrm{~A}$ & $29-0 c t-96$ & $\mathbf{Y}$ & 2 \\
\hline Y & & 5 & AM241 & Americium 241 & |INVRHD & $\begin{array}{l}\text { inventory of Remote Handled } \\
\text { Design }\end{array}$ & $31360 \mathrm{~A}$ & $29-0$ ct-96 & $\mathbf{Y}$ & 2 \\
\hline $\mathbf{Y}$ & & 5 & AM241 & Americium 241 & INVRHD & $\begin{array}{l}\text { Inventory of Remote Handled } \\
\text { Design }\end{array}$ & 313604 & 29-0ct-96 & $\mathbf{Y}$ & 2 \\
\hline$Y$ & 100 & 6 & AN243 & Americium 243 & HALFLIFE & Halfife & 31374 & $16-A p r-96$ & $Y$ & 3 \\
\hline $\bar{Y}$ & 100 & 6 & AM243 & Americium 243 & HALFLIFE & Hathfo & 31374 & 16-Apr-96 & $\mathbf{Y}$ & 3 \\
\hline $\mathbf{Y}$ & 100 & 6 & AM243 & Americium 243 & HALFLIFE & Halfifife & 31374 & 16-Apr-96 & $\mathbf{Y}$ & 3 \\
\hline$\gamma$ & & 7 & OPS_AREA & Operations Region & |CAP_MOD & $\begin{array}{l}\text { Model number, capillary pressure } \\
\text { model }\end{array}$ & 32604 & 16-Feb-96 & $\mathbf{Y}$ & 48 \\
\hline$Y$ & & 7 & OPS_AREA & Operations Region & CAP_MOD & $\begin{array}{l}\text { Model number, capillary pressure } \\
\text { model }\end{array}$ & 32604 & 16 -feb-96 & $\mathbf{Y}$ & $4 B$ \\
\hline Y & & 7 & OPS_AREA & Operations Region & CAP_MOD & $\begin{array}{l}\text { Model number, capillary pressure } \\
\text { model }\end{array}$ & 32604 & 16 -Feb-96 & $\mathbf{Y}$ & $4 B$ \\
\hline $\mathbf{Y}$ & & 7 & OPS_AREA & Operations Region & CAP_MOD & $\begin{array}{l}\text { Model number, capillary pressure } \\
\text { model }\end{array}$ & 32604 & 16-Feb-96 & $\mathbf{Y}$ & 48 \\
\hline $\mathbf{Y}$ & & 8 & OPS_AREA & Operations Region & COMP_RCK & Bulk Compressibility & 32606 & 16-Feb-96 & $\mathbf{Y}$ & 48 \\
\hline $\mathbf{Y}$ & & 8 & OPS_AREA & Operations Region & COMP_RCK & Bulk Compressibility & 32606 & 16-Feb-96 & $\mathbf{Y}$ & $4 B$ \\
\hline $\mathbf{Y}$ & & 8 & OPS_AREA & Operations Region & COMP_RCK & Bulk Compressibility & 32606 & 16-Feb-96 & $\mathbf{Y}$ & $4 B$ \\
\hline$Y$ & & 8 & OPS_AREA & Operations Region & COMP_RCK & Bulk Comprossibility & 32605 & 16-Feb-96 & $\mathbf{Y}$ & 48 \\
\hline $\mathbf{Y}$ & & 9 & OPS_AREA & Operations Region & PC_max & $\begin{array}{l}\text { Maximum allowable capillary } \\
\text { pressure }\end{array}$ & 32619 & 16 -Feb-96 & $\mathbf{Y}$ & 48 \\
\hline $\mathbf{Y}$ & & 9 & OPS_AREA & Oporations Region & PC_max & $\begin{array}{l}\text { Maximum allowable capillay } \\
\text { pressure }\end{array}$ & 32619 & 16-Feb-96 & $\mathbf{Y}$ & 48 \\
\hline $\mathbf{Y}$ & & 9 & OPS_AREA & Operations Region & PC_MAX & $\begin{array}{l}\text { Maximum allowable capillary } \\
\text { pressure }\end{array}$ & 32619 & $16-$ Feb-96 & $\mathbf{Y}$ & $4 B$ \\
\hline Y & & 9 & OPS_AREA & Operations Region & PC_max & $\begin{array}{l}\text { Maxmum allowable capillary } \\
\text { pressure }\end{array}$ & 32619 & 16-Feb-96 & $-Y$ & 48 \\
\hline $\mathbf{Y}$ & & 10 & OPS_AREA & Operations Region & PORE_DIS & $\begin{array}{l}\text { Brooks-Corey pore distnbution } \\
\text { parameter }\end{array}$ & 32624 & 16 -Feb-96 & $\mathbf{Y}$ & 48 \\
\hline $\mathbf{Y}$ & & 10 & OPS_AREA & Operations Region & PORE_DIS & $\begin{array}{l}\text { Brooks-Corey pore distrbution } \\
\text { parameter }\end{array}$ & 32624 & 16 -Feb-96 & $\mathbf{Y}$ & 48 \\
\hline$Y$ & & 10 & OPS_AREA & Operations Region & PORE_DIS & $\begin{array}{l}\text { Brooks-Coray pore distribution } \\
\text { parameter }\end{array}$ & 32624 & 16-Feb-96 & $\mathbf{Y}$ & 4B \\
\hline$Y$ & & 10 & OPS_AREA & Operations Rogion & PORE_DIS & $\begin{array}{l}\text { Brooks-Corey pore distribution } \\
\text { paremeter }\end{array}$ & 32624 & 16-Feb-96 & $\mathbf{Y}$ & $4 B$ \\
\hline $\mathbf{Y}$ & & 11 & OPS_AREA & Operations Region & POROSITY & Effoctive porosity & 32626 & 16-Feb-96 & $Y$ & 48 \\
\hline$Y$ & & 11 & OPS_AREA & Operations Region & POROSITY & Effective porosity & 32626 & 16-Fob-96 & $\cdot \mathrm{Y}$ & 48 \\
\hline$Y$ & & 11 & OPS_AREA & Operations Region & POROSITY & Effective porosity & 32626 & 16-Fob-96 & $Y$ & $4 B$ \\
\hline$Y$ & & 11 & OPS_AREA & Operations Region & POROSITY & Effective porosity & 32626 & 16-Feb-96 & $Y$ & $4 B$ \\
\hline$Y$ & & 12 & OPS_AREA & Operations Region & PO_MIN & $\begin{array}{l}\text { Minimum brne pressure for } \\
\text { capillary model KPC=3 }\end{array}$ & 31474 & 16-feb-96 & $\mathbf{Y}$ & $4 B$ \\
\hline$Y$ & & 12 & OPS_AREA & Operations Region & PO_MIN & $\begin{array}{l}\text { Mnimum brine pressure for } \\
\text { capillary model KPC=3 }\end{array}$ & 31474 & 16-Feb-96 & $Y$ & $4 B$ \\
\hline$Y$ & & 12 & OPS_AREA & Operations Region & PO_MIN & $\begin{array}{l}\text { Minimum brine pressure for } \\
\text { capillary model KPC=3 }\end{array}$ & 31474 & 16-Feb-96 & $\mathbf{Y}$ & AB \\
\hline$Y$ & & 12 & OPS_AREA & Operations Region & PO_MIN & $\begin{array}{l}\text { Minimum brine pressure for } \\
\text { capillary model KPC=3 }\end{array}$ & 31474 & 16-Feb-96 & $\mathbf{Y}$ & AB \\
\hline$\gamma$ & & 12 & OPS_AREA & Operations Region & PO_MIN & $\begin{array}{l}\text { Minimum brme pressure for } \\
\text { capillary model } \mathrm{KPC}=3\end{array}$ & 31474 & 16 -Feb-96 & $\mathbf{Y}$ & AB \\
\hline
\end{tabular}


CCA Parameter Listing

\begin{tabular}{|c|c|c|c|c|c|c|c|c|c|c|}
\hline$\overline{10}$ & \begin{tabular}{|c|} 
Ciassifi- \\
cation of \\
Date \\
Category \\
(footnoto 2)
\end{tabular} & $\begin{array}{l}\text { Distribertion } \\
\text { Type }\end{array}$ & Meen & Modian & Mintreum & Moxtinum & Units & Reference ID & $\begin{array}{l}\text { Wea the dinta } \\
\text { developed } \\
\text { under an } \\
\text { NQA-1 } \\
\text { Program? }\end{array}$ & $\begin{array}{c}\text { Which } \\
\text { mothods } \\
\text { wero used to } \\
\text { quallfy } \\
\text { oxdsting } \\
\text { datta? } \\
\text { (footrote 3) }\end{array}$ \\
\hline 2 & 4 & CONSTANT & $2.4105700 E-01$ & $2.4105700 E-01$ & 2.4105700E-01 & $2.4105700 E-01$ & $\mathrm{~kg} / \mathrm{mole}$ & CRC-72 & $Y$ & N/A \\
\hline 2 & 4 & CONSTANT & $2.4105700 E-01$ & 2.4105700E-01 & 24105700E-01 & $2.4105700 \mathrm{E}-01$ & $\mathrm{~kg} / \mathrm{mole}$ & WPO36298 & $\bar{Y}$ & N/A \\
\hline 2 & 4 & CONSTANT & $2.4105700 \mathrm{E}-01$ & $2.4105700 E-01$ & 24105700E-01 & $2.4105700 E-01$ & kg/mole & WP036301 & $\bar{Y}$ & N/A \\
\hline 3 & 4 & CONSTANT & $1.3640000 \mathrm{E}+10$ & $1.3640000 \mathrm{E}+10$ & $1.3540000 \mathrm{E}+10$ & $1.3640000 \mathrm{E}+10$ & 5 & WP036301 & $Y$ & NA \\
\hline 3 & 4 & CONSTANT & $1.3640000 \mathrm{E}+10$ & $1.3640000 \mathrm{E}+10$ & $1.3640000 E+10$ & $1.3640000 \mathrm{E}+10$ & 5 & WP037404 & $Y$ & NA \\
\hline 3 & 4 & CONSTANT & $1.3640000 \mathrm{E}+10$ & $1.3640000 E+10$ & $1.3640000 E+10$ & $1.3640000 \mathrm{E}+10$ & s & WP040434 & $Y$ & N/A \\
\hline 4 & 2 & CONSTANT & $4.7800000 E+05$ & $4.7800000 E+05$ & $4.78000000 E+05$ & $4.78000000+05$ & $\mathrm{Ci}$ & WP037061 & $Y$ & NA \\
\hline 4 & 2 & CONSTANT & $4.7800000 E+05$ & $4.7800000 E+05$ & $4.7800000 E+05$ & $4.7800000 E+05$ & $\mathrm{Ci}$ & WP039260 & $Y$ & N/A \\
\hline 4 & 2 & CONSTANT & $4.7800000 E+05$ & $4.7800000 E+0.5$ & $4.7800000 E+05$ & $4.7800000 E+05$ & $\mathbf{C i}$ & WP041559 & $\mathbf{\gamma}$ & NA \\
\hline 4 & 2 & CONSTANT & $4.7800000 E+05$ & $4.7800000 \mathrm{E}+0.5$ & $4.7800000 E+05$ & $4.7800000 \mathrm{E}+0.5$ & $\mathrm{Ci}$ & WPO41560 & $\mathbf{Y}$ & NA \\
\hline 5 & 2 & CONSTANT & $9.4400000 E+03$ & $9.4400000 \mathrm{E}+03$ & $9.4400000 E+03$ & $9.4400000 E+03$ & $\mathrm{Ci}$ & WP037061 & $\mathbf{Y}$ & NA \\
\hline 5 & 2 & CONSTANT & $9.4400000 \mathrm{E}+03$ & $9.4400000 E+03$ & $9.4400000 E+03$ & $9.4400000 E+03$ & $\mathrm{Ci}$ & WP039260 & $Y$ & N/A \\
\hline 5 & 2 & CONSTANT & $9.4400000 E+03$ & $9.4400000 E+03$ & $9.4400000 E+03$ & $9.4400000 E+03$ & $\mathrm{Ci}$ & WP041559 & $\mathbf{Y}$ & NA \\
\hline 5 & 2 & CONSTANT & $9.4400000 E+03$ & $9.4400000 E+03$ & $9.4400000 \mathrm{E}+03$ & $.9 .4400000 E+03$ & $\mathrm{Ci}$ & WP041560 & $\mathbf{Y}$ & NA \\
\hline 6 & 4 & CONSTANT & $2.3290000 E+11$ & $2.3290000 \mathrm{E}+11$ & $2.3290000 E+11$ & $2.3290000 \mathrm{E}+11$ & s & WP035301 & $\gamma$ & NA \\
\hline 6 & 4 & CONSTANT & $2.3290000 E+11$ & $2.32900000+11$ & $2.3290000 \mathrm{E}+11$ & $23290000 \mathrm{E}+11$ & $\mathbf{s}$ & WP037404 & $\gamma$ & NA \\
\hline 6 & 4 & CONSTANT & $2.3290000 \mathrm{E}+11$ & $2.3290000 E+11$ & $2.3290000 E+11$ & $2.3290000 E+11$ & $\mathbf{s}$ & WPO40434 & $Y$ & N/A \\
\hline 7 & 1.3 & CONSTANT & $1.0000000 E+\infty 0$ & $1.0000000 E+00$ & $1.0000000 E+00$ & $1.0000000 E+\infty 0$ & NONE & WPO35330 & $Y$ & NA \\
\hline 7 & 1,3 & CONSTANT & $1.0000000 E+\infty 0$ & $1.0000000 E+\infty 0$ & $1.0000000 E+00$ & $1.0000000 E+00$ & NONE & WP035597 & $Y$ & N/A \\
\hline 7 & 1,3 & CONSTANT & $1.0000000 E+00$ & $1.0000000 E+\infty 0$ & $1.0000000 E+00$ & $1.0000000 E+00$ & NONE & WP035320 & $Y$ & NA \\
\hline 7 & 1,3 & CONSTANT & $1.0000000 E+\infty 0$ & $1.0000000 E+\infty 0$ & $1.0000000 \mathrm{E}+\infty 0$ & $1.0000000 E+00$ & NONE & WPO40514 & $Y$ & N/A \\
\hline 8 & 1,3 & CONSTANT & $0.0000000 E+00$ & $0.0000000 E+00$ & $0.0000000 E+00$ & $0.0000000 E+00$ & $P a^{\wedge}-1$ & WPO35330 & $Y$ & N/A \\
\hline 8 & 1,3 & CONSTANT & $0.0000000 E+00$ & $0.0000000 E+00$ & $0.0000000 \mathrm{E}+00$ & $0.0000000 E+\infty 0$ & $P^{A}-1$ & WP035597 & $\mathbf{Y}$ & N/A \\
\hline 8 & 1,3 & CONSTANT & $0.0000000 E+00$ & $0.0000000 \mathrm{E}+00$ & $0.0000000 E+\infty 0$ & $0.0000000 E+00$ & $\mathrm{~Pa}^{\wedge}-1$ & WPO36320 & $Y$ & NA \\
\hline 8 & 1,3 & CONSTANT & $0.0000000 E+\infty 0$ & $0.0000000 \mathrm{E}+00$ & $0.0000000 \mathrm{E}+\infty 0$ & $0.0000000 E+00$ & $P a^{\wedge}-1$ & WP040514 & $\mathbf{Y}$ & N/A \\
\hline 9 & 1,3 & CONSTANT & $1.00000000+08$ & $1.00000000+08$ & $1.0000000 \mathrm{E}+08$ & $1.0000000 E+08$ & $\mathrm{~Pa}$ & WP035330 & $Y$ & N/A \\
\hline 9 & 1,3 & CONSTANT & $1.0000000 E+08$ & $1.0000000 E+C 8$ & $1.0000000 E+08$ & $1.0000000 \mathrm{E}+08$ & $\mathrm{~Pa}$ & WP035597 & $\mathbf{Y}$ & N/A \\
\hline $\mathbf{9}$ & 1,3 & CONSTANT & $1.0000000 E+08$ & $1.0000000 E+08$ & $1.0000000 E+08$ & $1.0000000 E+08$ & $\mathbf{P a}$ & WP036320 & $\mathbf{Y}$ & N/A \\
\hline 9 & 1,3 & CONSTANT & $1.0000000 E+08$ & $1.0000000 E+08$ & $1.0000000 \mathrm{E}+08$ & $1.0000000 E+08$ & $\mathrm{~Pa}$ & WPO40514 & $-Y$ & N/A \\
\hline 10 & 1,3 & CONSTANT & $7.0000000 E-01$ & $7.0000000 E-01$ & $7.0000000 E-01$ & $7.0000000 E-01$ & NONE & WP035330 & $\mathbf{Y}$ & NA \\
\hline 10 & 1,3 & CONSTANT & $7.0000000 E-01$ & $7.0000000 E-01$ & $7.0000000 E-01$ & $7.0000000 \mathrm{E}-01$ & NONE & WP035597 & $\mathbf{Y}$ & NA \\
\hline 10 & 1,3 & CONSTANT & $7.0000000 E-01$ & $7.0000000 E-01$ & $7.0000000 E-01$ & $7.0000000 \mathrm{E}-01$ & NONE & WPO36320 & $\mathbf{Y}$ & N/A \\
\hline 10 & 1,3 & CONSTANT & $7.0000000 E-01$ & $7.0000000 E-01$ & $7.0000000 E-01$ & $7.0000000 E-01$ & NONE & WPO40514 & $\mathbf{Y}$ & N/A \\
\hline 11 & 1,3 & CONSTANT & $1.8000000 \mathrm{E}-01$ & $1.8000000 \mathrm{E}-01$ & $1.8000000 E-01$ & $1.8000000 \mathrm{E}-01$ & NONE & WP032281 & $Y$ & N/A \\
\hline 11 & 1,3 & CONSTANT & $1.8000000 \mathrm{E}-01$ & $1.8000000 \mathrm{E}-01$ & $1.8000000 \mathrm{E}-01$ & $1.8000000 \mathrm{E}-01$ & NONE & WPO35330 & $Y$ & NA \\
\hline 11 & 1,3 & CONSTANT & $1.8000000 \mathrm{E}-01$ & $1.8000000 \mathrm{E}-01$ & 1.8000000 E-01 & $1.8000000 E-01$ & NONE & WP035597 & $Y$ & N/A \\
\hline 11 & 1,3 & CONSTANT & $1.8000000 \mathrm{E}-01$ & 1.8000000 E-01 & $1.8000000 E-01$ & $1.8000000 \mathrm{E}-01$ & NONE & WPO40514 & $Y$ & N/A \\
\hline 12 & 1,3 & CONSTANT & $1.0132500 E+05$ & $1.0132500 E+05$ & $1.0132500 E+05$ & $1.0132500 E+05$ & $\mathbf{P a}$ & WP035330 & $\mathbf{Y}$ & NA \\
\hline 12 & 1,3 & CONSTANT & $1.0132500 E+05$ & $1.0132500 \Omega+05$ & $1.0132500 E+05$ & $1.0132500 E+05$ & $\mathrm{~Pa}$ & WP035597 & $\mathbf{Y}$ & NA \\
\hline 12 & 1,3 & CONSTANT & $1.0132500 E+05$ & $1.0132500 E+05$ & $1.0132500 E+05$ & $1.0132500 \mathrm{E}+05$ & $\mathrm{~Pa}$ & WP036051 & $\mathbf{Y}$ & N/A \\
\hline 12 & 1,3 & CONSTANT & $1.0132500 E+05$ & $1.0132500 \mathrm{E}+05$ & 1.0132500E+05 & $1.0132500 E+05$ & $\mathrm{~Pa}$ & WP036320 & $Y$ & N/A \\
\hline 12 & 1,3 & CONSTANT & $1.0132500 E+05$ & $1.0132500 E+05$ & $1.0132500 E+05$ & $1.0132500 E+0.5$ & $\mathrm{~Pa}$ & WP040514 & $\mathbf{Y}$ & N/A \\
\hline
\end{tabular}


CCA Parameter Listing

\begin{tabular}{|c|c|c|c|c|c|c|c|c|c|c|}
\hline $\begin{array}{c}\text { Percmoter in } \\
\text { Databasa? } \\
\text { (YN) }\end{array}$ & \begin{tabular}{c|} 
If the values \\
used in CCAA \\
wo not the \\
samo as in \\
DB, provide $x$ \\
difference
\end{tabular} & ID & Material to & Material Neme & $\begin{array}{c}\text { Parameter } \\
\text { ID. }\end{array}$ & Paramoter Namo & $\begin{array}{l}\text { PRP ID } \\
\text { (MPO *) }\end{array}$ & $\begin{array}{c}\text { Data Entry } \\
\text { Deto }\end{array}$ & $\begin{array}{l}\text { Es the } 464 \\
\text { consiatent } \\
\text { with the } \\
\text { perameter } \\
\text { in DB? } \\
(Y, N A)\end{array}$ & $\begin{array}{l}\text { Parametor } \\
\text { Crtegory } \\
\text { (footrote 1) }\end{array}$ \\
\hline $\mathbf{Y}$ & & 13 & OPS_AREA & Operations Region & PRESSURE & Brine far-field pore pressure & 32629 & $16-F 0 b-96$ & $\mathbf{Y}$ & AB \\
\hline$Y$ & & 13 & OPS_AREA & Operations Region & PRESSURE & Brine far-field pore pressure & 32629 & 16-feb-96 & $\bar{Y}$ & $4 B$ \\
\hline$Y$ & & 13 & OPS_AREA & Operations Region & PRESSURE & Brine far-field pore pressure & 32629 & 16-Fob-96 & $Y$ & 48 \\
\hline$Y$ & & 13 & OPS_AREA & Operations Region & PRESSURE & Brine far-field pore pressure & 32629 & 16 Feb-96 & $\mathbf{Y}$ & 48 \\
\hline $\mathbf{Y}$ & & 14 & OPS_AREA & Operations Region & PRMX_LOG & $\begin{array}{l}\text { Log of intminsic permeabilty, } X \text { - } \\
\text { direction }\end{array}$ & 32630 & 16-Fob-96 & $\mathbf{Y}$ & 4B \\
\hline $\mathbf{Y}$ & & 14 & OPS_AREA & Operations Region & PRMX_LOG & $\begin{array}{l}\text { Log of intrinsic permeability, } X \text { - } \\
\text { direction }\end{array}$ & 32630 & 16 Fob-96 & $\mathbf{Y}$ & AB \\
\hline $\mathbf{Y}$ & & 14 & OPS_AREA & Operations Region & PRMX_LOG & $\begin{array}{l}\text { Log of intrinsic permeability, } X- \\
\text { direction }\end{array}$ & 32630 & 16 feb-96 & $\mathbf{Y}$ & 4B \\
\hline$Y$ & & 14 & OPS_AREA & Operations Region & PRMX_LOG & $\begin{array}{l}\text { Log of intrinste permeabilty, } X- \\
\text { direction }\end{array}$ & 32630 & 16feb-96 & $\mathbf{Y}$ & 4B \\
\hline $\mathbf{Y}$ & & 15 & OPS_AREA & Operations Region & PRMY_LOG & $\begin{array}{l}\text { Log of intrinsic permeabilty, } Y \text { - } \\
\text { direction }\end{array}$ & 32632 & 16-Fob-96 & $\mathbf{Y}$ & AB \\
\hline$Y$ & & 15 & OPS_AREA & Operations Region & PRMY_LOG & $\begin{array}{l}\text { Log of intrinsic permeability, Y- } \\
\text { direction }\end{array}$ & 32632 & 16-Feb-96 & $\mathbf{Y}$ & 4B \\
\hline $\mathbf{Y}$ & & 15 & OPS_AREA & Operations Region & PRAY_LOG & $\begin{array}{l}\text { Log of intrinsic permeability, Y- } \\
\text { direction }\end{array}$ & 32632 & 16-Fob-96 & $\mathbf{Y}$ & $4 B$ \\
\hline $\mathbf{Y}$ & & 15 & OPS_AREA & Operations Region & PRMY_LOG & $\begin{array}{l}\text { Log of intrinsic permeabillty, Y- } \\
\text { direction }\end{array}$ & 32632 & 16-Fab-96 & $Y$ & 48 \\
\hline$Y$ & & 16 & OPS_AREA & Operations Region & PRMZ_LOG & $\begin{array}{l}\text { Log of intrinsic permeability, Z- } \\
\text { direction }\end{array}$ & 32533 & 16-Fob-96 & $\mathbf{Y}$ & 4B \\
\hline$Y$ & & 16 & OPS_AREA & Operations Region & PRMZ_LOG & $\begin{array}{l}\text { Log of intinsic permeability, Z- } \\
\text { direction }\end{array}$ & 32533 & 16 Fob-96 & $\mathbf{Y}$ & 48 \\
\hline $\mathbf{Y}$ & & 16 & OPS_AREA & Operations Region & PRMZ_LOG & $\begin{array}{l}\text { Log of intrinsic permeability, Z- } \\
\text { direction }\end{array}$ & 32633 & 16-Feb-96 & $\mathbf{Y}$ & AB \\
\hline$Y$ & & 16 & OPS_AREA & Operations Region & PRMZ_LOG & $\begin{array}{l}\text { Log of intrinsic permeability, } z \text { - } \\
\text { direction }\end{array}$ & 32633 & $16-F o b-96$ & $Y$ & 4B \\
\hline$Y$ & & 19 & OPS_AREA & Operations Rogion & RELP_MOD & $\begin{array}{l}\text { Model nember, relativo } \\
\text { permeability model }\end{array}$ & 32634 & 16-Fab-96 & $\mathbf{Y}$ & AB \\
\hline $\mathbf{Y}$ & & 19 & OPS_AREA & Operations Region & RELP_MOD & $\begin{array}{l}\text { Model number, relative } \\
\text { permsability model }\end{array}$ & 32634 & 16-feb-96 & $\mathbf{Y}$ & 4B \\
\hline $\mathbf{Y}$ & & 19 & OPS_AREA & Operations Region & RELP_MOD & $\begin{array}{l}\text { Model number, relative } \\
\text { permeability model }\end{array}$ & 32634 & 16-Feb-96 & $\mathbf{Y}$ & AB \\
\hline $\mathbf{Y}$ & & 19 & OPS_AREA & Operations Region & RELP_MOD & $\begin{array}{l}\text { Model number, relative } \\
\text { permeability model }\end{array}$ & 32634 & 16 feb-96 & $\mathbf{Y}$ & $4 B$ \\
\hline$Y$ & & 20 & OPS_AREA & Operations Region & SAT_IBRN & Initial Brine Saturation & 32637 & 16-Feb-96 & $Y$ & $4 B$ \\
\hline$Y$ & & 20 & OPS_AREA & Operations Region & SAT_IBRN & Initial Brine Saturation & 32637 & 16-Fob-96 & $Y$ & 48 \\
\hline$Y$ & & 20 & OPS_AREA & Operations Region & SAT_IBRN & Initial Brine Saturation & 32637 & 16-Fob-96 & $Y$ & 48 \\
\hline$Y$ & & 20 & OPS_AREA & Operations Region & SAT_IBRN & Initial Brine Saturation & 32637 & 16-Feb-96 & $Y$ & 48 \\
\hline$Y$ & & 21 & OPS_AREA & Operations Region & SAT_RBRN & Residual Brine Saturation & 32639 & 16-Fob-96 & $\bar{Y}$ & $4 B$ \\
\hline$Y$ & & 21 & OPS_AREA & Operations Region & SAT_RBRN & Residual Brine Saturation & 32639 & 16-feb-96 & $Y$ & 48 \\
\hline$Y$ & & 21 & OPS_AREA & Operations Region & SAT_RBRN & Residual Brine Saturation & 32639 & 16-Fob-96 & $Y$ & 48 \\
\hline$Y$ & & 21 & OPS_AREA & Operations Region & SAT_RBRN & Residual Brine Saturation & 32639 & 16-Fob-96 & $\mathbf{Y}$ & AB \\
\hline$Y$ & & 22 & OPS_AREA & Operations Region & SAT_RGAS & Residual Gas Saturation & 32641 & 16-Feb-96 & $-Y$ & $4 B$ \\
\hline$Y$ & & 22 & OPS_AREA & Operations Region & SAT_RGAS & Residual Gas Saturation & 32641 & 16-Feb-96 & $Y$ & 48 \\
\hline$Y$ & & 22 & OPS_AREA & Operations Region & SAT_RGAS & Residual Gas Saturation & 32641 & 16-Feb-96 & $Y$ & 48 \\
\hline$Y$ & & 22 & OPS_AREA & Operations Region & SAT_RGAS & Residual Gas Saturation & 32641 & 16-Feb-96 & $\mathbf{Y}$ & 4B \\
\hline $\mathbf{Y}$ & & 23 & BOREHOLE & Borehole and Fill & CAP_MOD & $\begin{array}{l}\text { Model number, capillary pressure } \\
\text { model }\end{array}$ & $31486 \mathrm{~A}$ & 20-Fob-96 & $\mathbf{Y}$ & 4B \\
\hline $\mathbf{Y}$ & & 23 & BOREHOLE & Borohole and Fill & CAP_MOD & $\begin{array}{l}\text { Modol number, capillary pressure } \\
\text { model }\end{array}$ & $31486 \mathrm{~A}$ & $20-F e b-96$ & $\mathbf{Y}$ & 4B \\
\hline $\mathbf{Y}$ & & 23 & BOREHOLE & Borehole and Fill & CAP_MOD & $\begin{array}{l}\text { Model number, capillary pressure } \\
\text { model }\end{array}$ & $31486 \mathrm{~A}$ & 20-Feb-96 & $\mathbf{Y}$ & 48 \\
\hline$Y$ & & 25 & BOREHOLE & Borehole and Fill & COMP_RCK & Bulk Compressibility & 31510 & 20-Fob-96 & $\mathbf{Y}$ & $4 \mathrm{~A}$ \\
\hline$Y$ & & 25 & BOREHOLE & Borehole and Fill & COMP_RCK & Bulk Compressibility & 31510 & 20-Feb-96 & $Y$ & 4A \\
\hline $\mathbf{Y}$ & & 26 & BOREHOLE & Borahole and Fill & DIAMMOD & Modem or aurrent diameter & 31511 & 22-Feb-96 & $\mathbf{Y}$ & $4 B$ \\
\hline$Y$ & & 26 & BOREHOLE & Borehole and Fill & DIAMMOD & Modem or current diameter & 31511 & 22-Fob-96 & $\mathbf{Y}$ & $A B$ \\
\hline$Y$ & & 26 & BOREHOLE & Borehole and Fill & DIAMMOD & Modern or aurrent diameter & 31511 & 22fob-96 & $Y$ & 48 \\
\hline$\underline{Y}$ & & 26 & BOREHOLE & Borehole and Fill & DIAMMOD & Modem or current diameter & 31511 & 22fob-96 & $\mathbf{Y}$ & 48 \\
\hline$Y$ & & 26 & BOREHOLE & Borehole and Fill & DLAMMOD & Modem or current diameter & 31511 & 22fob-96 & $\mathbf{Y}$ & $4 B$ \\
\hline $\mathbf{Y}$ & & 27 & BOREHOLE & Borehole and Fill & DOMEGA & Drill string angular velocity $(0)$ & 31512 & 09-Jan-95 & $Y$ & 48 \\
\hline$Y$ & & 27 & BOREHOLE & Borehole and Fill & DOMEGA & Drill string angular velocity $(0)$ & 31512 & 09-Jan-95 & $Y$ & AB \\
\hline $\mathbf{Y}$ & & 27 & BOREHOLE & Borehole and Fill & DOMEGA & Drill string angular velocity (0) & 31512 & 09-Jan-95 & $\mathbf{Y}$ & AB \\
\hline
\end{tabular}


CCA Parameter Listing

\begin{tabular}{|c|c|c|c|c|c|c|c|c|c|c|}
\hline 10 & \begin{tabular}{|c|} 
Classiff- \\
cation of \\
Data \\
Catogory \\
(footnote 2)
\end{tabular} & $\begin{array}{l}\text { Distribution } \\
\text { Type }\end{array}$ & Meenn & Medlinn & Mlntroem & Madmum & Units & Reference ID & $\begin{array}{l}\text { Wes the dets } \\
\text { developed } \\
\text { under an } \\
\text { NQA-1 } \\
\text { Program? }\end{array}$ & $\begin{array}{l}\text { Which } \\
\text { mothods } \\
\text { mere uavd to } \\
\text { qually } \\
\text { oxisting } \\
\text { deta? } \\
\text { (footnote s) }\end{array}$ \\
\hline 13 & 1,3 & CONSTANT & $1.0132500 E+05$ & $1.0132500 \mathrm{E}+0.5$ & $1.0132500 E+05$ & $1.0132500 \mathrm{E}+0.5$ & $\mathbf{P a}$ & WP035330 & $\bar{Y}$ & $N / A$ \\
\hline 13 & 1,3 & CONSTANT & $1.0132500 \mathrm{E}+05$ & $1.0132500 \mathrm{E}+05$ & $1.0132500 E+05$ & $1.0132500 E+05$ & $\mathrm{~Pa}$ & WP035597 & $\bar{Y}$ & NA \\
\hline 13 & 1.3 & CONSTANT & $1.0132500 \mathrm{E}+05$ & $1.0132500 \mathrm{E}+0.5$ & $1.0132500 E+05$ & $1.0132500 E+05$ & $\mathrm{~Pa}$ & WPO36320 & $Y$ & $N / A$ \\
\hline 13 & 1,3 & CONSTANT & $1.0132500 \mathrm{E}+05$ & $1.0132500 E+05$ & $1.0132500 E+05$ & $1.0132500 \mathrm{E}+0.5$ & $\mathbf{P z}$ & WPO40514 & $\mathbf{Y}$ & NA \\
\hline 14 & 1,3 & CONSTANT & $-1.1000000 E+01$ & $-1.1000000 E+01$ & $-1.1000000 E+01$ & $-1.1000000 E+01$ & $\log \left(m^{\wedge} 2\right)$ & WPO35330 & $Y$ & $N / A$ \\
\hline 14 & 1,3 & CONSTANT & $-1.1000000 E+01$ & $-1.1000000 E+01$ & $-1.1000000 E+01$ & $-1.1000000 E+01$ & $\log \left(m^{\wedge} 2\right)$ & WP035597 & $\mathbf{Y}$ & NA \\
\hline 14 & 1,3 & CONSTANT & $-1.1000000 E+01$ & $-1.1000000 \mathrm{E}+01$ & $-1.1000000 E+01$ & $-1.1000000 E+01$ & $\log \left(m^{\wedge} 2\right)$ & WPO36320 & $Y$ & NA \\
\hline 14 & 1,3 & CONSTANT & $-1.1000000 \mathrm{E}+01$ & $-1.1000000 E+01$ & $-1.1000000 E+01$ & $-1.1000000 E+01$ & $\log \left(m^{\wedge} 2\right)$ & WPO40514 & $\mathbf{Y}$ & $N / A$ \\
\hline 15 & 1,3 & CONSTANT & $-1.1000000 \mathrm{E}+01$ & $-1.1000000 E+01$ & $-1.1000000 E+01$ & $-1.1000000 E+01$ & $\log \left(m^{\wedge} 2\right)$ & WPO35330 & $Y$ & N/A \\
\hline 15 & 1,3 & CONSTANT & $-1.1000000 E+01$ & $-1.1000000 E+01$ & $-1.1000000 E+01$ & $-1.1000000 E+01$ & $\log \left(m^{\wedge} 2\right)$ & WPO35597 & $Y$ & N/A \\
\hline 15 & 1,3 & CONSTANT & $-1.1000000 E+01$ & $-1.1000000 E+01$ & $-1.1000000 E+01$ & $-1.1000000 E+01$ & $\log \left(m^{\wedge} 2\right)$ & WP036320 & $\mathbf{Y}$ & N/A \\
\hline 15 & 1,3 & CONSTANT & $-1.1000000 E+01$ & $-1.1000000 E+01$ & $-1.1000000 E+01$ & $-1.1000000 E+01$ & $\log \left(m^{\wedge} 2\right)$ & WPO40514 & $\mathbf{Y}$ & NA \\
\hline 16 & 1,3 & CONSTANT & $-1.1000000 E+01$ & $-1.1000000 E+01$ & $-1.1000000 E+01$ & $-1.1000000 E+01$ & $\log \left(m^{\wedge} 2\right)$ & WPO35330 & $\mathbf{Y}$ & N/A \\
\hline 16 & 1,3 & CONSTANT & $-1.1000000 E+01$ & $-1.1000000 E+01$ & $-1.1000000 E+01$ & $-1.1000000 E+01$ & $\log \left(m^{\wedge} 2\right)$ & WP035597 & $Y$ & $N A$ \\
\hline 16 & 1,3 & CONSTANT & $-1.1000000 E+01$ & $-1.1000000 \mathrm{E}+01$ & $-1.1000000 E+01$ & $-1.1000000 E+01$ & $\log \left(m^{\wedge} 2\right)$ & WPO36320 & $Y$ & NA \\
\hline 16 & 1,3 & CONSTANT & $-1.1000000 E+01$ & $-1,1000000 E+01$ & $-1.1000000 E+01$ & $-1.1000000 E+01$ & $\log \left(m^{\wedge} 2\right)$ & WPO40514 & $Y$ & NA \\
\hline 19 & 1,3 & CONSTANT & $4.0000000 E+00$ & $4.0000000 \mathrm{E}+\infty 0$ & $4.0000000 E+00$ & $4.0000000 E+\infty 0$ & NONE & WP035330 & $\mathbf{Y}$ & NA \\
\hline 19 & 1,3 & CONSTANT & $4.0000000 E+00$ & $4.0000000 E+\infty 0$ & $4.0000000 \mathrm{E}+00$ & $4.0000000 \mathrm{E}+00$ & NONE & WP035597 & $Y$ & NA \\
\hline 19 & 1,3 & CONSTANT & $4.0000000 \mathrm{E}+00$ & $4.0000000 E+\infty 0$ & $4.0000000 \mathrm{E}+00$ & $4.0000000 \mathrm{E}+00$ & NONE & WP035320 & $\mathbf{Y}$ & $\mathrm{N} / \mathrm{A}$ \\
\hline 19 & 1,3 & CONSTANT & $4.0000000 E+00$ & $4.0000000 E+00$ & $4.0000000 E+\infty 0$ & $4.0000000 E+\infty$ & NONE & WP040514 & $\mathbf{Y}$ & N/A \\
\hline 20 & 1,3 & CONSTANT & $0.0000000 \mathrm{E}+\infty 0$ & $0.0000000 \mathrm{E}+00$ & $0.0000000 \mathrm{E}+00$ & $0.0000000 \mathrm{E}+00$ & NONE & WP035330 & $\mathbf{Y}$ & N/A \\
\hline 20 & 1,3 & CONSTANT & $0.0000000 \mathrm{E}+00$ & $0.0000000 \mathrm{E}+\infty$ & $0.0000000 E+00$ & $0.0000000 \mathrm{E}+00$ & NONE & WP035597 & $\bar{Y}$ & $N / A$ \\
\hline 20 & 1,3 & CONSTANT & $0.0000000 E+00$ & $0.0000000 E+00$ & $0.0000000 E+00$ & $0.0000000 \mathrm{E}+00$ & NONE & WP035320 & $Y$ & NA \\
\hline 20 & 1,3 & CONSTANT & $0.0000000 E+00$ & $0.0000000 E+00$ & $0.0000000 E+00$ & $0.0000000 E+\infty 0$ & NONE & WPO40514 & $\mathbf{Y}$ & N/A \\
\hline 21 & 1,3 & CONSTANT & $0.0000000 E+00$ & $0.0000000 \mathrm{E}+00$ & $0.0000000 E+\infty 0$ & $0.0000000 \mathrm{E}+00$ & NONE & WP035330 & $Y$ & N/A \\
\hline 21 & 1,3 & CONSTANI & $0.0000000 E+\infty 0$ & $0.0000000 \mathrm{E}+00$ & $0.0000000 E+00$ & $0.0000000 E+\infty$ & NONE & WP035597 & $Y$ & N/A \\
\hline 21 & 1,3 & CONSTANT & $0.0000000 E+00$ & $0.0000000 E+00$ & $0.0000000 E+00$ & $0.0000000 E+00$ & NONE & WPO35320 & $Y$ & $N / A$ \\
\hline 21 & 1,3 & CONSTANT & $0.0000000 E+\infty 0$ & $0.0000000 \mathrm{E}+00$ & $0.0000000 E+00$ & $0.0000000 E+00$ & NONE & WPO40514 & $Y$ & $N / A$ \\
\hline 22 & 1,3 & CONSTANT & $0,0000000 \mathrm{E}+00$ & $0.0000000 E+00$ & $0.0000000 \mathrm{E}+\infty 0$ & $0.0000000 E+00$ & NONE & WPO35330 & $-Y$ & $N / A$ \\
\hline 22 & 1,3 & CONSTANT & $0.0000000 \mathrm{E}+00$ & $0.0000000 E+00$ & $0.0000000 E+\infty 0$ & $0.0000000 E+00$ & NONE & WP035597 & $\mathbf{Y}$ & N/A \\
\hline 22 & 1,3 & CONSTANT & $0.0000000 E+00$ & $0.0000000 E+00$ & $0.0000000 E+00$ & $0.0000000 \mathrm{E}+\infty 0$ & NONE & WPO36320 & $Y$ & N/A \\
\hline 22 & 1,3 & CONSTANT & $0.0000000 E+00$ & $0.0000000 E+00$ & $0.0000000 \mathrm{E}+\infty 0$ & $0.0000000 E+00$ & NONE & WP040514 & $Y$ & $N / A$ \\
\hline 23 & Not Used & CONSTANT & $2.0000000 E+00$ & $2.0000000 \mathrm{E}+00$ & $2.0000000 E+00$ & $2.0000000 E+00$ & NONE & WP030640 & $\mathbf{Y}$ & $N / A$ \\
\hline 23 & Nol Used & CONSTANT & $2.0000000 \mathrm{E}+\infty 0$ & $2.0000000 E+\infty$ & $2.0000000 E+\infty 0$ & $2.0000000 E+\infty 0$ & NONE & WP035597 & $\mathbf{Y}$ & NA \\
\hline 23 & Not Usod & CONSTANT & $2.0000000 E+00$ & $2.0000000 E+\infty 0$ & $20000000 E+\infty 0$ & $20000000 E+\infty 0$ & NONE & WPO40514 & $\mathbf{Y}$ & N/A \\
\hline 25 & Not Used & CONSTANT & $2.6400000 \mathrm{E}-09$ & $26400000 \mathrm{E}-09$ & 26400000E-09 & 26400000E-09 & $P a^{n}-1$ & WP030640 & $\mathbf{Y}$ & NA \\
\hline 25 & Not Used & CONSTANT & $2.6400000 \mathrm{E}-09$ & $2.6400000 E-09$ & 26400000E-09 & $26400000 \mathrm{E}-09$ & $P a^{n}-1$ & WP035597 & $Y$ & $N / A$ \\
\hline 26 & Not Used & CONSTANT & $3.1115000 E-01$ & $3.1115000 E-01$ & 3.1115000E-01 & $3.1115000 \mathrm{E}-01$ & $m$ & WP035329 & $\bar{Y}$ & N/A \\
\hline 26 & Not Used & CONSTANT & $3.1115000 E-01$ & $3.1115000 \mathrm{E}-01$ & $3.1115000 E-01$ & $3.1115000 \mathrm{E}-01$ & $\mathbf{m}$ & WP035597 & $Y$ & N/A \\
\hline 26 & Not Used & CONSTANT & $3.1115000 E-01$ & $3.1115000 \varepsilon-01$ & 3.1115000E-01 & $3.1115000 E-01$ & $\mathbf{m}$ & WPO38343 & $Y$ & N/A \\
\hline 26 & Not Used & CONSTANT & $3.1115000 \mathrm{E}-01$ & $3.1145000 E-01$ & $3.1115000 E-01$ & $3.1115000 E-01$ & $m$ & WPO38344 & $Y$ & N/A \\
\hline 26 & Not Used & CONSTANT & $3.1115000 \mathrm{E}-01$ & $3.1115000 \mathrm{E}-01$ & 3.1115000E-01 & $3.1115000 E-01$ & $m$ & WPO40521 & $Y$ & N/A \\
\hline 27 & 3 & CUMULATIVE & $8.6300000 E+\infty 0$ & $7.8000000 \mathrm{E}+\infty 0$ & $4.2000000 \mathrm{E}+\infty 0$ & $2.3000000 E+01$ & $\mathrm{rad} / \mathrm{s}$ & SAND92-0700/3 & $\mathbf{Y}$ & N/A \\
\hline 27 & 3 & CUMULATIVE & $8.6300000 E+00$ & $7.8000000 E+00$ & $4.2000000 E+\infty 0$ & $2.3000000 \mathrm{E}+01$ & $\mathrm{rad} / \mathrm{s}$ & WP035268 & $Y$ & $N A$ \\
\hline 27 & 3 & CUMULATIVE & $8.6300000 E+00$ & $7.8000000 \mathrm{E}+00$ & $4.2000000 \mathrm{E}+00$ & $2.3000000 \mathrm{E}+01$ & $\mathrm{rad} / \mathrm{s}$ & WP035597 & $Y$ & N/A \\
\hline
\end{tabular}


CCA Parameter Listing

\begin{tabular}{|c|c|c|c|c|c|c|c|c|c|c|}
\hline $\begin{array}{c}\text { Parameter in } \\
\text { Databaze? } \\
\text { (YNN) }\end{array}$ & $\begin{array}{c}\text { if the values } \\
\text { used in CaA } \\
\text { are not the } \\
\text { same as in } \\
\text { DB, provide } x \\
\text { difference }\end{array}$ & 10 & Mettial ID & Metorial Namo & $\begin{array}{c}\text { Paramotor } \\
\text { to }\end{array}$ & Perratnotar Name & $\begin{array}{l}\text { PRPD } \\
\text { (WPON) }\end{array}$ & $\begin{array}{c}\text { Dartentry } \\
\text { Dato }\end{array}$ & $\begin{array}{l}\text { to the } 46 \text { a } \\
\text { consistent } \\
\text { wth the } \\
\text { parameter } \\
\text { in DB? } \\
(Y, N \text { ) }\end{array}$ & $\begin{array}{l}\text { Paramiter } \\
\text { Category } \\
\text { (footnots 1) }\end{array}$ \\
\hline $\bar{Y}$ & & 27 & BOREHOLE & Borahole and Fill & DOMEGA & Drill string angular velocity $(0)$ & 31512 & 09-Jan-95 & $\mathbf{Y}$ & 48 \\
\hline$Y$ & & 27 & BOREHOLE & Borehole and Fill & DOMEGA & Drill string angular velocity (0) & 31512 & 09-Jan-95 & $\bar{Y}$ & 48 \\
\hline$Y$ & & 29 & BOREHOLE & Borehole and Fill & PC_max & $\begin{array}{l}\text { Maximum allowrabte capillary } \\
\text { pressure }\end{array}$ & 31514 & 20-feb-96 & $\mathbf{Y}$ & 48 \\
\hline $\mathbf{Y}$ & & 29 & BOREHOLE & Borehole and Fill & PC_MAX & $\begin{array}{l}\text { Maximum aliowablo capilitary } \\
\text { pressure }\end{array}$ & 31514 & 20-Fab-96 & $\mathbf{Y}$ & $4 B$ \\
\hline $\mathbf{Y}$ & & 29 & BOREHOLE & Borehole and Fill & PC_MAX & $\begin{array}{l}\text { Maximum alloweble capiliary } \\
\text { pressure }\end{array}$ & 31514 & 20-Fob-96 & $\mathbf{Y}$ & 48 \\
\hline $\mathbf{Y}$ & & 30 & BOREHOLE & Borahole and Fill & PORE_DIS & $\begin{array}{l}\text { Brooks-Corey pore distribution } \\
\text { parameter }\end{array}$ & 31521 & 20-Fob-96 & $\mathbf{Y}$ & 4A \\
\hline $\mathbf{Y}$ & & 30 & BOREHOLE & Borehole and Fill & PORE_DIS & $\begin{array}{l}\text { Brooks-Corey pore distribution } \\
\text { parameter }\end{array}$ & 31521 & 20-Feb-96 & $\mathbf{Y}$ & $4 \mathrm{~A}$ \\
\hline$Y$ & & 31 & BOREHOLE & Borehole and Fill & POROSITY & Effective porosity & 31522 & 21-Fob-96 & $\mathbf{Y}$ & AA \\
\hline $\mathbf{Y}$ & & 31 & BOREHOLE & Borehole and Fill & POROSITY & Effective porosity & 31522 & 21-Feb-96 & $\bar{Y}$ & $4 \mathrm{~A}$ \\
\hline $\mathbf{Y}$ & & 32 & BOREHOLE & Borehole and Fill & PO_MIN & $\begin{array}{l}\text { Minimum brine pressure for } \\
\text { capillary model KPC }=3\end{array}$ & 31523 & 21+\%b-96 & $\boldsymbol{Y}$ & 4B \\
\hline $\mathbf{Y}$ & & 32 & BOREHOLE & Borahole and Fill & PO_MIN & $\begin{array}{l}\text { Minimum brine pressura for } \\
\text { capillary model KPC }=3\end{array}$ & 31523 & 21-Feb-96 & $\mathbf{Y}$ & 48 \\
\hline$Y$ & & 32 & BOREHOLE & Borehoie and Fill & PO_MIN & $\begin{array}{l}\text { Minimum brine pressure for } \\
\text { capillary model KPC=3 }\end{array}$ & 31523 & 21-Feb-96 & $\mathbf{Y}$ & 48 \\
\hline$Y$ & & 34 & BOREHOLE & Borehole and Fill & PRMX_LOG & $\begin{array}{l}\text { Log of intrinsic perm Bability, } X- \\
\text { direction }\end{array}$ & 31525 & 31-0ct-95 & $\boldsymbol{\gamma}$ & 48 \\
\hline $\mathbf{Y}$ & & 34 & BOREHOLE & Borehole and Fill & PRMX_LOG & $\begin{array}{l}\text { Log of intrinsic permeabilhy, } x \text { - } \\
\text { direction }\end{array}$ & 31525 & 31-0et-95 & $\mathbf{Y}$ & $4 B$ \\
\hline $\mathbf{Y}$ & & 34 & BOREHOLE & Borehole and Fill & PRMX_LOG & $\begin{array}{l}\text { Log of intrinsic permeability, X- } \\
\text { direction }\end{array}$ & 31525 & 31-0ct-95 & $\mathbf{Y}$ & $4 B$ \\
\hline $\mathbf{Y}$ & & 35 & BOREHOLE & Borehole and Fill & PRMY_LOG & $\begin{array}{l}\text { Log of intrinsic permeabilty, Y- } \\
\text { direction }\end{array}$ & 31527 & 31-0ct-95 & $\boldsymbol{\gamma}$ & 48 \\
\hline$Y$ & & 35 & BOREHOLE & Borehole and Fill & PRMY_LOG & $\begin{array}{l}\text { Log of intrinsic permeability, Y- } \\
\text { direction }\end{array}$ & 31527 & 31-0ct-95 & $\mathbf{Y}$ & 48 \\
\hline$Y$ & & 35 & BOREHOLE & Borehole and Fill & PRMY_LOG & $\begin{array}{l}\text { Log of intrinsic permeability, Y- } \\
\text { direction }\end{array}$ & 31527 & 31-0ct-95 & $\mathbf{Y}$ & $4 B$ \\
\hline$Y$ & & 36 & BOREHOLE & Borehole and Fill & PRMZ_LOG & $\begin{array}{l}\text { Log of intrinsic perm rability, Z- } \\
\text { direction }\end{array}$ & 31528 & $31-0$ ct.95 & $\mathbf{Y}$ & $4 B$ \\
\hline$Y$ & & 36 & BOREHOLE & Borehole and Fill & PRMZ_LOG & $\begin{array}{l}\text { Log of intrinsic permeability, Z- } \\
\text { direction }\end{array}$ & 31528 & 31-0ct-95 & $\mathbf{Y}$ & 48 \\
\hline $\mathbf{Y}$ & & 36 & BOREHOLE & Borehole and Fill & PRMZ_LOG & $\begin{array}{l}\text { Log of intrinsic permeability, Z- } \\
\text { direction }\end{array}$ & 31528 & 31-0ct-95 & $\mathbf{Y}$ & $4 B$ \\
\hline$Y$ & & 40 & BOREHOLE & Borahole and Fill & RELP_MOD & $\begin{array}{l}\text { Model number, relative } \\
\text { permeability modal }\end{array}$ & 31532 & $31-0 c t-95$ & $\mathbf{Y}$ & $4 B$ \\
\hline $\mathbf{Y}$ & & 40 & BOREHOLE & Borehole and Fill & RELP_MOD & $\begin{array}{l}\text { Model number, relative } \\
\text { permeability model }\end{array}$ & 31532 & $31-0 c t-95$ & $\mathbf{\gamma}$ & $4 B$ \\
\hline$Y$ & & 40 & BOREHOLE & Borehole and Fill & RELP_MOD & $\begin{array}{l}\begin{array}{l}\text { Model number, relative } \\
\text { permeability model }\end{array} \\
\end{array}$ & 31532 & 31-0ct-95 & $\mathbf{Y}$ & $4 B$ \\
\hline$Y$ & & 41 & BOREHOLE & Borehole and Fill & SAT_RBRN & Residual Brine Saturation & 31533 & 31-0ct-95 & $\mathbf{Y}$ & $4 A$ \\
\hline$Y$ & & 41 & BOREHOLE & Borehole and Fill & SAT_RBRN & Residual Brine Saturation & 31533 & 31-0ct-95 & $Y$ & $4 \mathrm{~A}$ \\
\hline $\bar{Y}$ & & 42 & BOREHOLE & Borehole and Fill & SAT_RGAS & Residual Gas Saturation & 31535 & 20-Fob-96 &..$- Y$ & $4 A$ \\
\hline $\mathbf{Y}$ & & 42 & BOREHOLE & Borehole and Fitl & SAT_RGAS & Residual Gas Saturation & 31535 & 20-Feb-96 & $\mathbf{Y}$ & $4 \mathrm{~A}$ \\
\hline$Y$ & & 48 & BRINESAL & Salado Brine & COMPRES & Bme Compressibility & 31540 & 01-Fob-96 & $Y$ & 1 \\
\hline$Y$ & & 48 & BRINESAL & Salado Brine & COMPRES & Bnne Compressibility & 31540 & 01-Feb-96 & $Y$ & 1 \\
\hline $\bar{Y}$ & & 49 & BRINESAL & Salado Brine & DNSFLUID & Bnne Density & 31541 & 01-Feb-96 & $Y$ & 1 \\
\hline$Y$ & & 49 & BRINESAL & Salado Brino & DNSFLUID & Bnne Density & 31541 & 01-Feb-96 & $Y$ & 1 \\
\hline $\mathbf{Y}$ & & so & BRINESAL & Selado Brine & REF_PRES & Reterence pressure for porosity & 31542 & 02-Nov-95 & $\mathbf{Y}$ & $4 B$ \\
\hline $\mathbf{Y}$ & & 50 & BRINESAL & Salado Arine & REF_PRES & Reference pressure for porosity & 31542 & 02-Nov-95 & $\mathbf{Y}$ & $4 B$ \\
\hline $\mathbf{Y}$ & & 51 & BRINESAL & Salado Brine & REF_TEMP & Reference Temperature & 31543 & O2-Nov-95 & $\mathbf{Y}$ & 48 \\
\hline$Y$ & & 51 & BRINESAL & Salado Brine & REF_TEMP & Reference Temperature & 31543 & O2-Nov-95 & $\bar{Y}$ & 4B \\
\hline$Y$ & & 55 & BRINESAL & Salado Brine & Visco & Viscosity & 31548 & 09-feb-96 & $\bar{Y}$ & 1 \\
\hline$Y$ & & 55 & BRINESAL & Salado Brine & Visco & Viscosity & 31548 & Q9-Fob-96 & $Y$ & 1 \\
\hline$Y$ & & 57 & BRINESAL & Salado Brine & WTF & Mass fraction of salt in brine & 31552 & 20-Feb-96 & $Y$ & 1 \\
\hline$Y$ & & 57 & BRINESAL & Salado Brine & WWrF & Mass fraction of salt in brine & 31552 & 20-Fob-96 & $Y$ & 1 \\
\hline $\mathbf{Y}$ & & 57 & BRINESAL & Salado Brine & WTF & Mass fraction of salt in brine & 31552 & $20-F a b-96$ & $Y$ & 1 \\
\hline $\mathbf{Y}$ & & 60 & CASTILER & Castile Brine Reservoir & CAP_MOD & $\begin{array}{l}\text { Model number, capillary pressure } \\
\text { model }\end{array}$ & 31556 & 02-Nov-95 & $\gamma$ & AB \\
\hline$Y$ & & 60 & CASTILER & Castile Brine Reservoir & CAP_MOD & $\begin{array}{l}\text { Model number, capillary pressure } \\
\text { model }\end{array}$ & 31556 & 02-Nov-95 & $\mathbf{Y}$ & 4B \\
\hline
\end{tabular}


CCA Parameter Listing

\begin{tabular}{|c|c|c|c|c|c|c|c|c|c|c|}
\hline ID & $\begin{array}{c}\text { Classiti- } \\
\text { Catton of } \\
\text { Datta } \\
\text { Cextegory } \\
\text { (footnoto 2) }\end{array}$ & $\begin{array}{l}\text { Distribution } \\
\text { Type }\end{array}$ & Masen & Medien & Minimum & 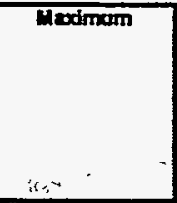 & Units & Reforence 10 & $\begin{array}{l}\text { Was the dath } \\
\text { developed } \\
\text { under an } \\
\text { NOA-1 } \\
\text { Program? - }\end{array}$ & 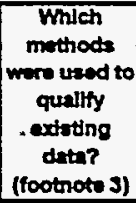 \\
\hline 27 & 3 & CUMULATIVE & $8.6300000 E+00$ & $7.8000000 \mathrm{E}+\infty 0$ & $4.2000000 \mathrm{E}+\infty 0$ & $2.3000000 E+01$ & $\mathrm{rad} / \mathrm{s}$ & WPO38568 & $\mathbf{Y}$ & $N / A$ \\
\hline 27 & 3 & CUMULATIVE & $8.6300000 E+00$ & $7.8000000 \mathrm{E}+\infty 0$ & 4.2000000E+00 & $2.3000000 E+01$ & $\mathrm{rad} / \mathrm{s}$ & WPO40521 & $Y$ & N/A \\
\hline 29 & Not Usod & CONSTANT & $1.0000000 E+08$ & $1.0000000 \mathrm{E}+0.8$ & $1.0000000 E+08$ & $1.0000000 E+08$ & $\mathbf{P a}$ & WPO35597 & $\mathbf{Y}$ & NA \\
\hline 29 & Not Usod & CONSTANT & $1.0000000 E+08$ & $1.0000000 E+08$ & $1.0000000 E+08$ & $1.0000000 E+08$ & $\mathbf{P a}$ & WP038568 & $\mathbf{Y}$ & NA \\
\hline 29 & Not Used & CONSTANT & $1.0000000 \mathrm{E}+08$ & $1.0000000 E+08$ & $1.0000000 E+08$ & $1.0000000 E+08$ & $\mathrm{~Pa}$ & WP040514 & $Y$ & NA \\
\hline 30 & Not Used & CONSTANT & $9.4000000 E-01$ & $9.4000000 E-01$ & $9.4000000 E-01$ & $9.4000000 E-01$ & NONE & WPO30640 & $Y$ & N/A \\
\hline 30 & Not Used & CONSTANT & $9.4000000 \mathrm{E}-01$ & $9.4000000 E-01$ & $9.4000000 \mathrm{E}-01$ & $9.4000000 E-01$ & NONE & WP035597 & $\mathbf{Y}$ & N/A \\
\hline 31 & Not Used & CONSTANT & $5.0000000 \mathrm{E}-02$ & $5.0000000 E-02$ & $5.0000000 \mathrm{E}-02$ & $5.0000000 \mathrm{E}-02$ & NONE & WP030640 & $Y$ & NA \\
\hline 31 & Not Used & CONSTANT & $5.0000000 \mathrm{E}-02$ & $5.0000000 \mathrm{E}-02$ & $5.0000000 \mathrm{E}-02$ & $5.0000000 \mathrm{E}-02$ & NONE & WPO35597 & $Y$ & NA \\
\hline 32 & Not Used & CONSTANT & $1.0132500 E+05$ & $1.0132500 E+05$ & $1.0132500 E+05$ & $1.0132500 E+05$ & $\mathbf{P a}$ & WP035597 & $Y$ & N/A \\
\hline 32 & Not Used & CONSTANT & $1.0132500 E+05$ & $1.0132500 E+05$ & $1.0132500 E+05$ & $1.0132500 E+05$ & $\mathbf{P a}$ & WP036051 & $\mathbf{Y}$ & N/A \\
\hline 32 & Not Used & CONSTANT & $1.0132500 \mathrm{E}+05$ & $1.0132500 \mathrm{E}+05$ & $1.0132500 E+05$ & $1.0132500 E+05$ & $\mathbf{P a}$ & WP040514 & $\mathbf{Y}$ & N/A \\
\hline 34 & Not Used & NORMAL & $-1.2230000 \mathrm{E}+01$ & $-1.2500000 \mathrm{E}+01$ & $-1.4000000 E+01$ & $-1.1000000 E+01$ & $\log \left(m^{\wedge} 2\right)$ & FREEZE-1 & $Y$ & N/A \\
\hline 34 & Not Used & NORMAL & $-1.2230000 E+01$ & $-1.2500000 E+01$ & $-1.4000000 E+01$ & $-1.1000000 E+01$ & $\log \left(m^{\wedge} 2\right)$ & WP038568 & $Y$ & N/A \\
\hline 34 & Not Used & NORMAL & $-1.2230000 \mathrm{E}+01$ & $-1.2500000 E+01$ & $-1.4000000 E+01$ & $-1.1000000 E+01$ & $\log \left(m^{\wedge} 2\right)$ & WPO40514 & $Y$ & N/A \\
\hline 35 & Not Used & NORMAL. & $-1.2230000 \mathrm{E}+01$ & $-1.2500000 \Xi+01$ & $-1.4000000 E+01$ & $-1.1000000 E+01$ & $\log \left(m^{\wedge} 2\right)$ & FREEZE-1 & $Y$ & NA \\
\hline 35 & Not Used & NORMAL & $-1.2230000 E+01$ & $-1.2500000 E+01$ & $-1.4000000 E+01$ & $-1.1000000 E+01$ & $\log \left(m^{\wedge} 2\right)$ & WP033568 & $\gamma$ & NA \\
\hline 35 & Not Used & NORMAL & $-1.2230000 \mathrm{E}+01$ & $-1.2500000 E+01$ & $-1.4000000 E+01$ & $-1.1000000 E+01$ & $\log \left(m^{\wedge} 2\right)$ & WP040514 & $\mathbf{Y}$ & NA \\
\hline 36 & Not Used & NORMAL & $-1.2230000 E+01$ & $-1.2500000 E+01$ & $-1.4000000 \mathrm{E}+01$ & $-1.1000000 E+01$ & $\log \left(m^{\wedge} 2\right)$ & FREEZE-1 & $Y$ & NA \\
\hline 36 & Not Used & NORMAL & $-1.2230000 E+01$ & $-1.2500000 E+01$ & $-1.40000000 E+01$ & $-1.1000000 E+01$ & $\log \left(m^{\wedge} 2\right)$ & WPO38568 & $\mathbf{Y}$ & N/A \\
\hline 36 & Not Used & NORMAL & $-1.2230000 \mathrm{E}+01$ & $-1.2500000 \mathrm{E}+01$ & $-1.4000000 E+01$ & $-1.1000000 E+01$ & $\log \left(m^{\wedge} 2\right)$ & WPO40514 & $Y$ & $N A$ \\
\hline 40 & Not Used & CONSTANT & $4.0000000 E+00$ & $4.0000000 E+00$ & $4.0000000 E+\infty 0$ & $4.0000000 E+00$ & NONE & WP035597 & $Y$ & NA \\
\hline 40 & Not Used & CONSTANT & $4.0000000 E+\infty 0$ & $4.0000000 E+00$ & $4.0000000 \mathrm{E}+\infty 0$ & $4.0000000 E+\infty 0$ & NONE & WP038568 & $\mathbf{Y}$ & N/A \\
\hline 40 & Not Used & CONSTANT & 4.0000000E+00 & $4.0000000 E+00$ & $4.0000000 E+\infty 0$ & $4.0000000 E+\infty 0$ & NONE & WPO40514 & $Y$ & $N A$ \\
\hline 41 & Not Used & CONSTANT & 2.0000000E-01 & $2.0000000 \mathrm{E}-01$ & $2.0000000 \mathrm{E}-01$ & $20000000 E-04$ & NONE & WP030640 & $\mathbf{Y}$ & N/A \\
\hline 41 & Not Used & CONSTANT & $20000000 E-01$ & $2.0000000 \mathrm{E}-01$ & $2.0000000 E-01$ & $2.0000000 E-01$ & NONE & WP035597 & $Y$ & NA \\
\hline 42 & Not Used & CONSTANT & $2.0000000 \mathrm{E}-01$ & $2.0000000 \mathrm{E}-01$ & $2.0000000 \mathrm{E}-01$ & $2.0000000 \mathrm{E}-01$ & NONE & WP030640 & $-Y$ & N/A \\
\hline 42 & Not Used & CONSTANT & $2.0000000 \mathrm{E}-01$ & $2.0000000 E-01$ & $2.0000000 E-01$ & $2.0000000 \mathrm{E}-01$ & NONE & WP035597 & $Y$ & NA \\
\hline 48 & 3,4 & CONSTANT & $3.1000000 E-10$ & 3.1000000 E-10 & $3.1000000 E-10$ & $3.1000000 E-10$ & $\mathrm{~Pa}^{\mathrm{N}-1}$ & WP031174 & $\mathbf{N}$ & 1 \\
\hline 48 & 3,4 & CONSTANT & $3.1000000 \mathrm{E}-10$ & $3.1000000 \mathrm{E}-10$ & $3.1000000 \mathrm{E}-10$ & $3.1000000 \mathrm{E}-10$ & Pan-1 & WPO35597 & $\mathbf{N}$ & 1 \\
\hline 49 & 1 & CONSTANT & $1.2200000 \mathrm{E}+03$ & $1.2200000 \mathrm{E}+03$ & $1.2200000 \mathrm{E}+03$ & $1.2200000 \mathrm{E}+03$ & $\mathrm{~kg} / \mathrm{m}^{\wedge} \mathbf{3}$ & WP031175 & $\mathbf{N}$ & 1 \\
\hline 49 & 1 & CONSTANT & $1.2200000 \mathrm{E}+03$ & $1.2200000 E+03$ & $1.2200000 \mathrm{E}+03$ & $1.2200000 E+03$ & $\mathrm{~kg} / \mathrm{m}^{\wedge} 3$ & WP035597 & $\mathbf{N}$ & 1 \\
\hline 50 & $1,3,5$ & CONSTANT & $1.0132500 E+05$ & $1.0132500 \mathrm{E}+05$ & $1.0132500 E+05$ & $1.0132500 E+05$ & $\mathrm{~Pa}$ & WP038568 & $\mathbf{Y}$ & N/A \\
\hline 50 & $1,3,5$ & CONSTANT & $1.0132500 E+05$ & $1.0132500 \mathrm{E}+05$ & $1.0132500 E+05$ & $1.0132500 E+05$ & $\mathrm{~Pa}$ & WPO405i4 & $\mathbf{Y}$ & NA \\
\hline 51 & $1,3,4$ & CONSTANT & $3.0015000 \mathrm{E}+02$ & $3.0015000 \mathrm{E}+02$ & $3.0015000 \mathrm{E}+02$ & $3.0015000 \mathrm{E}+02$ & $k$ & WP038568 & $\mathbf{Y}$ & N/A \\
\hline 51 & $1,3,4$ & CONSTANT & $3.0015000 E+02$ & $3.0015000 \mathrm{E}+02$ & $3.0015000 \mathrm{E}+02$ & $3.0015000 \mathrm{E}+02$ & $K$ & WPO40514 & $Y$ & N/A \\
\hline 55 & 1 & CONSTANT & $2.1000000 E-03$ & $2.1000000 E-03$ & $2.1000000 E-03$ & $2.1000000 \mathrm{E}-03$ & $\mathrm{~Pa}^{*} 3$ & WP031168 & $N$ & 1 \\
\hline 55 & 1 & CONSTANT & $2.1000000 E-03$ & $2.1000000 E-03$ & $2.1000000 E-03$ & $2.1000000 E-03$ & Pa's & WP035597 & $\mathbf{N}$ & 1 \\
\hline 57 & 1 & STUDENT & $3.2400000 E-01$ & $3.2400000 \mathrm{E}-01$ & $3.0700000 \mathrm{E}-01$ & $3.3200000 \mathrm{E}-01$ & NONE & WP031171 & $Y$ & 1 \\
\hline 57 & 1 & STUDENT & $3.2400000 \mathrm{E}-01$ & $3.2400000 \mathrm{E}-01$ & $3.0700000 E-01$ & $3.3200000 E-01$ & NONE & WP035597 & $Y$ & 1 \\
\hline 57 & 1 & STUDENT & $3.2400000 \mathrm{E}-01$ & $3.2400000 E-01$ & 3.0700000E-01 & 3.3200000E-01 & NONE & WPO36380 & $Y$ & 1 \\
\hline 60 & 3,5 & CONSTANT & $2,0000000 E+00$ & $2.0000000 E+\infty 0$ & $20000000 E+\infty$ & $2.0000000 E+00$ & NONE & WPO38568 & $Y$ & N/A \\
\hline 60 & 3,5 & CONSTANT & $2.0000000 E+00$ & $20000000 E+\infty 0$ & $2.0000000 E+\infty 0$ & $2.0000000 \mathrm{E}+00$ & NONE & WPO40514 & $\mathbf{Y}$ & N/A \\
\hline
\end{tabular}


CCA Parameter Listing

\begin{tabular}{|c|c|c|c|c|c|c|c|c|c|c|}
\hline $\begin{array}{c}\text { Parmeter in } \\
\text { Detabean? } \\
\text { (YNN) }\end{array}$ & \begin{tabular}{|c|} 
ff the values \\
used in CCA \\
are not the \\
same to in \\
DB, provide $x$ \\
difference
\end{tabular} & 10 & Mettorial ID & Material Nerne & $\begin{array}{c}\text { Paramoter } \\
\text {. ID }\end{array}$ & $\begin{array}{c}\text { Parmater Kam } \\
\cdot\end{array}$ & $\begin{array}{c}\text { PRP ID } \\
\text { (WPO I) }\end{array}$ & $\begin{array}{c}\text { Data Entry } \\
\text { Dato }\end{array}$ & 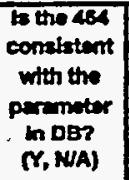 & $\begin{array}{l}\text { Parameter } \\
\text { Category } \\
\text { (footnote 1) }\end{array}$ \\
\hline$Y$ & & 61 & CASTILER & Castile Brine Reservoir & COMP_RCK & Butk Compressibility & 31561 & 11-Apr-96 & $\mathbf{Y}$ & 1 \\
\hline $\mathbf{Y}$ & & 61 & CASTILER & Castile Brine Reservoir & COMP_RCK & Bukk Compressibility & 31561 & 11 Apr-96 & $\mathbf{Y}$ & 1 \\
\hline $\mathbf{Y}$ & & 61 & CASTILER & Castile Brine Reservoir & COMP_RCK & Bulk Compressibilty & 31561 & $11-A p r-96$ & $\mathbf{Y}$ & 1 \\
\hline $\mathbf{Y}$ & & 62 & CASTILER & Cestile Brine Reservoir & PC_MAX & $\begin{array}{l}\text { Maximum allowable capillary } \\
\text { pressure }\end{array}$ & 31607 & O2-Nov-95 & $\mathbf{Y}$ & 48 \\
\hline $\mathbf{Y}$ & & 62 & CASTILER & Castile Brine Reservoir & PC_MAX & $\begin{array}{l}\text { Maximum allowable capillary } \\
\text { pressure }\end{array}$ & 31607 & O2NNov-95 & $Y$ & $4 B$ \\
\hline $\mathbf{Y}$ & & $\boldsymbol{6}$ & CASTILER & Castile Brine Reservoir & PORE_DIS & $\begin{array}{l}\text { Brooks-Coroy pore distribution } \\
\text { parameter }\end{array}$ & 31609 & 02+Nov-95 & $\mathbf{Y}$ & $4 B$ \\
\hline $\mathbf{Y}$ & & 63 & CASTILER & Castile Brine Reservoir & PORE_DIS & $\begin{array}{l}\text { Brooks-Corey pore distribution } \\
\text { parameter }\end{array}$ & 31609 & D2Nov-95 & $\mathbf{Y}$ & $4 B$ \\
\hline $\mathbf{Y}$ & varies & 64 & CASTILER & Castile Brine Reservoir & POROSITY & Effective porosity & 31610 & 31-Jan-96 & $\mathbf{Y}$ & 1 \\
\hline$Y$ & varies & 64 & CASTILER & Cestile Brine Reservoir & POROSTYY & Effective porosity & 31610 & $31-\tan -96$ & $\mathbf{Y}$ & 1 \\
\hline $\mathbf{Y}$ & varies & 64 & CASTILER & Castile Brine Reservoir & POROSIT & Effective porosity & 31610 & 31-Jan-96 & $Y$ & 1 \\
\hline $\mathbf{Y}$ & varies & 64 & CASTILER & Castule Brine Reservoir & POROSIT & Effective porosity & 31610 & 3i-Jan-96 & $\mathbf{Y}$ & 1 \\
\hline $\mathbf{Y}$ & & 65 & CASTILER & Castile Brine Reservoir & PO_MIN & $\begin{array}{l}\text { Minimum brine pressure for } \\
\text { capillery model KPC=3 }\end{array}$ & 31611 & 21-Feb-96 & $\mathbf{Y}$ & 48 \\
\hline $\mathbf{Y}$ & & 65 & CASTILER & Castile Brine Reservoir & PO_MIN & $\begin{array}{l}\text { Minimum brine pressure for } \\
\text { capillary model KP C }=3\end{array}$ & 31611 & 21Fob-96 & $\mathbf{Y}$ & $4 B$ \\
\hline $\boldsymbol{\gamma}$ & & 65 & CASTILER & Castile Brine Reservoir & PO_MIN & $\begin{array}{l}\text { Minimum brine pressure for } \\
\text { capillary model } \mathrm{KPC}=3\end{array}$ & 31611 & 21-Feb-96 & $\mathbf{Y}$ & $4 B$ \\
\hline $\mathbf{Y}$ & & 66 & CASTILER & Castile Brine Reservoir & PRESSURE & Brine far-field pore pressure & $31612 A$ & 21-Mar-96 & $\mathbf{Y}$ & 1 \\
\hline $\mathbf{Y}$ & & 66 & CASTILER & Castile Brine Reservoir & PRESSURE & Brine tar-field pore pressure & $31612 A$ & 21+Mar-96 & $\mathbf{Y}$ & 1 \\
\hline$Y$ & & 66 & CASTILER & Castile Brine Reservoir & PRESSURE & Brine far-field pore pressure & 31612A & 21+Mar-96 & $\mathbf{Y}$ & 1 \\
\hline $\mathbf{Y}$ & & 66 & CASTILER & Castife Brine Reservoir & PRESSURE & Brine far-field pore pressure & 31612A & 21-Mar-96 & $\mathbf{Y}$ & 1 \\
\hline $\mathbf{Y}$ & & 67 & CASTILER & Castile Brine Reservoir & PRMX_LOG & $\begin{array}{l}\text { Log of intrinsic permeability, X- } \\
\text { drection }\end{array}$ & 31613 & 12-Fab-96 & $\mathbf{Y}$ & 1 \\
\hline$Y$ & & 67 & CASTILER & Castile Brine Reservoir & PRMX_LOG & $\begin{array}{l}\text { Log of intrinsic permeability, } X- \\
\text { direction }\end{array}$ & 31613 & 12 feb-96 & $\mathbf{Y}$ & 1 \\
\hline $\mathbf{Y}$ & & 67 & CASTILER & Castile Brine Reservoir & PRMX_LOG & $\begin{array}{l}\text { Log of intrinsic permeabilty, } X- \\
\text { direction }\end{array}$ & 31613 & 12 Feb-96 & $\mathbf{Y}$ & 1 \\
\hline$Y$ & & 68 & CASTILER & Castile Brino Reservoir & PRMY_LOG & $\begin{array}{l}\text { Log of intrinsic permeability, Y- } \\
\text { direction }\end{array}$ & 31614 & 12Feb-96 & $\mathbf{Y}$ & 1 \\
\hline$Y$ & & 68 & CASTILER & Cestile Brine Reservoir & PRMY_LOG & $\begin{array}{l}\text { Log of intrinsic permeability, } Y- \\
\text { direction }\end{array}$ & 31614 & 12feb-96 & $\mathbf{Y}$ & 1 \\
\hline $\mathbf{Y}$ & & 68 & CASTILER & Castile Brine Reservoir & PRMY_LOG & $\begin{array}{l}\text { Log of intunsic permeability, } Y- \\
\text { direction }\end{array}$ & 31614 & 12feb-96 & $\mathbf{Y}$ & 1 \\
\hline $\mathbf{Y}$ & & 69 & CASTILER & Castile Brine Reservoir & PRMZ_LOG & $\begin{array}{l}\text { Log of intrinsic permeabillty, Z- } \\
\text { direction }\end{array}$ & 31615 & 12-Feb-96 & $-Y \gamma$ & 1 \\
\hline $\mathbf{Y}$ & & 69 & CASTILER & Cestile Brino Reservoir & PRMZ_LOG & $\begin{array}{l}\text { Log of intrinsic permeability, Z- } \\
\text { drection }\end{array}$ & 31615 & 12-feb-96 & $\mathbf{Y}$ & 1 \\
\hline $\mathbf{Y}$ & & 69 & CASTILER & Castile Brine Reservoir & PRMZ_LOG & $\begin{array}{l}\text { Log of intrinsic permeability, Z- } \\
\text { direction }\end{array}$ & 31615 & 12Feb-96 & $\mathbf{Y}$ & 1 \\
\hline$Y$ & & 72 & CASTILER & Cestile Brine Reservoir & RELP_MOD & $\begin{array}{l}\text { Model number, relative } \\
\text { permeability model }\end{array}$ & 31619 & 02-Nov-95 & $\mathbf{Y}$ & $4 B$ \\
\hline$Y$ & & 72 & CASTILER & Castile Brine Reservoir & RELP_MOD & $\begin{array}{l}\text { Model number, relative } \\
\text { permeability model }\end{array}$ & 31619 & 02-Nov-95 & $\mathbf{Y}$ & $4 B$ \\
\hline $\mathbf{Y}$ & & 73 & CASTILER & Castile Brine Reservoir & SAT_IBRN & Initial Brine Saturation & 31620 & 02-Nov-95 & $\mathbf{Y}$ & $4 B$ \\
\hline $\mathbf{Y}$ & & 73 & CASTILER & Castile Brine Reservoir & SAT_IBRN & Initial Brino Saturation & 31620 & 02-Nov-95 & $\mathbf{Y}$ & 48 \\
\hline $\mathbf{Y}$ & & 74 & CASTILER & Cestule Brine Reservoir & SAT_RBRN & Residual Brine Saturation & 31621 & 02-Nov-95 & $\mathbf{Y}$ & $4 B$ \\
\hline $\mathbf{Y}$ & & 74 & CASTILER & Cestile Brine Reservoir & SAT_RBRN & Residual Brine Saturation & 31621 & 02-Nov-95 & $\mathbf{Y}$ & 48 \\
\hline $\mathbf{Y}$ & & 75 & CASTILER & Castile Brine Reservoir & SAT_RGAS & Residual Gas Saturation & 31622 & 02-Nov-95 & $\mathbf{Y}$ & $4 B$ \\
\hline $\mathbf{Y}$ & & 75 & CASTILER & Castile Brine Reservoir & SAT_RGAS & Residual Ges Saturation & 31622 & 02-Nov-95 & $\mathbf{Y}$ & $4 B$ \\
\hline $\mathbf{Y}$ & & 76 & CAVITY_1 & Cenity for Waste Areas & CAP_MOD & $\begin{array}{l}\text { Model number, capillary pressure } \\
\text { model }\end{array}$ & 31626 & O2-Nov-95 & $Y$ & $4 B$ \\
\hline
\end{tabular}


CCA Parameter Listing

\begin{tabular}{|c|c|c|c|c|c|c|c|c|c|c|}
\hline 10 & $\begin{array}{c}\text { Claseffie } \\
\text { Cation of } \\
\text { Date } \\
\text { Category } \\
\text { (footnote 2) }\end{array}$ & $\begin{array}{l}\text { Distribution } \\
\text { Type }\end{array}$ & mean - & Median & Mlnimum & Maximum & Unit: & Reference id & $\begin{array}{l}\text { Was the data } \\
\text { developed } \\
\text { under an } \\
\text { NoA-1 } \\
\text { Program? }\end{array}$ & $\begin{array}{c}\text { Which } \\
\text { mothod } \\
\text { woro used to } \\
\text { qually } \\
\text { oxisting } \\
\text { data? } \\
\text { (tootnote 3) }\end{array}$ \\
\hline 61 & 1,4 & TRLANGULAR & $-9.8000000 E+\infty 0$ & $-1.0000000 \mathrm{E}+01$ & $-7.1300000 E+01$ & $-8.0000000 E+\infty$ & $\log \left(P a^{n}-1\right)$ & WP031084 & $\mathbf{N}$ & 1 \\
\hline 61 & 1,4 & TRLANGULAR & $-9.8000000 E+00$ & $-1.0000000 E+01$ & $-1.1300000 E+01$ & $-8.0000000 E+00$ & $\log \left(P a^{n}-1\right)$ & WP035268 & $\mathbf{N}$ & 1 \\
\hline 61 & 1,4 & TRIANGULAR & $\cdot-9.8000000 E+\infty 0 \mid$ & $-1.0000000 E+01$ & $-1.1300000 E+01$ & $-8.0000000 E+\infty$ & $\log \left(P a^{\wedge}-1\right) \mid$ & WP035597 & $N$ & 1 \\
\hline 62 & 3,5 & CONSTANT & $1.0000000 \mathrm{E}+08$ & $1.0000000 \mathrm{E}+08$ & $1.0000000 E+08$ & $1.0000000 E+08$ & $\mathbf{P a}$ & WP038568 & $\mathbf{Y}$ & NAA \\
\hline 62 & 3,5 & CONSTANT & $1,0000000 E+08$ & $1.0000000 \mathrm{E}+08$ & $1.0000000 \mathrm{E}+08$ & $1.0000000 E+08$ & $\mathbf{P a}$ & WP040514 & $\mathbf{Y}$ & NA \\
\hline 63 & $1,3,4$ & CONSTANT & $7.0000000 \mathrm{E}-01$ & $7.0000000 E-01$ & $7.0000000 E-01$ & $7.0000000 \mathrm{E}-01$ & NONE & WP038568 & $\mathbf{Y}$ & NAA \\
\hline 63 & $1,3,4$ & CONSTANT & $7.0000000 E-01$ & $7.0000000 \mathrm{E}-01$ & $7.0000000 E-01$ & $7.0000000 \mathrm{E}-01$ & NONE & WP040514 & $\mathbf{Y}$ & NA \\
\hline 64 & $1,3,5$ & STUDENT & $8.7000000 \mathrm{E}-03$ & $8.7000000 E-03$ & $2.0000000 E-03$ & $1.6000000 E-02$ & - NONE & WP031083 & N & 1 \\
\hline 64 & $1,3,5$ & STUDENT & 8.7000000E-03 & 8.7000000E-03 & $2.0000000 E-03$ & $1.6000000 E-02$ & NONE & WP035597 & $\mathbf{N}$ & 1 \\
\hline 64 & $1,3,5$ & STUDENT & $8.7000000 E-03$ & $8.7000000 \mathrm{E}-03$ & $2.0000000 E-03$ & $1.6000000 \mathrm{E}-02$ & NONE & WP036380 & $\mathbf{N}$ & 1 \\
\hline 64 & $1,3,5$ & STUDENT & $8.7000000 E-03$ & $8.7000000 \mathrm{E}-03$ & 2.0000000E-03 & $1.6000000 \mathrm{E}-02$ & NONE & WPO40434 & $\mathbf{N}$ & 1 \\
\hline 65 & 3,5 & CONSTANT & $1.0132500 E+05$ & $1.0132500 E+0.5$ & $1.0132500 E+05$ & $1.0132500 E+05$ & $\mathrm{~Pa}$ & WP035597 & $\mathbf{Y}$ & N/A \\
\hline 65 & 3,5 & CONSTANT & $1.0132500 E+05$ & $1.0132500 E+0.5$ & $1.0132500 E+05$ & $1.0132500 E+05$ & $\mathrm{~Pa}$ & WP036051 & $\mathbf{Y}$ & N/A \\
\hline 65 & 3,5 & CONSTANT & $1.0132500 E+05$ & $1.0132500 E+05$ & $1.0132500 E+05$ & $1.0132500 E+05$ & $\mathrm{~Pa}$ & WP040514 & $\mathbf{Y}$ & NA \\
\hline 66 & 1 & TRIANGULAR & $1.3600000 E+07$ & 1.2700000E+07 & $1.1100000 E+07$ & $1.7000000 \mathrm{E}+07$ & $\mathrm{~Pa}$ & WP031072 & $\mathbf{N}$ & 1 \\
\hline 66 & 1 & TRLANGULAR & $1.3500000 E+07$ & $1.2700000 E+07$ & $1.1100000 E+07$ & $1.7000000 \mathrm{E}+07$ & $\mathbf{P a}$ & WP035268 & $\mathbf{N}$ & 1 \\
\hline 66 & 1 & TRIANGULAR & $1.3600000 E+07$ & $1.2700000 E+07$ & $1.1100000 E+07$ & $1.7000000 E+07$ & $\mathrm{~Pa}$ & WP035597 & $\mathbf{N}$ & 1 \\
\hline 66 & 1 & TRIANGULAR & $1.3600000 E+07$ & $1.2700000 E+07$ & $1.1100000 E+07$ & $1.7000000 E+07$ & $\mathbf{P a}$ & WP037148 & $\mathbf{N}$ & 1 \\
\hline 67 & 1 & TRIANGULAR & $-1.2100000 E+01$ & $-1.1800000 E+01$ & $-1.4700000 E+01$ & $-9.8000000 E+\infty$ & $\log \left(m^{\wedge} 2\right)$ & WP031070 & $\mathbf{N}$ & 1 \\
\hline 67 & 1 & TRLANGULAR & $-1.2100000 E+01$ & $-1.1800000 E+01$ & $-1.4700000 E+01$ & $-9.8000000 E+00$ & $\log \left(m^{\wedge} 2\right)$ & WP035268 & $\mathbf{N}$ & 1 \\
\hline 67 & 1 & TRIANGULAR & $-1.2100000 E+01$ & $-1.1800000 E+01$ & $-1.4700000 E+01$ & $-9.8000000 E+\infty 0$ & $\log \left(m^{\wedge} 2\right)$ & WP035597 & $\mathbf{N}$ & 1 \\
\hline 68 & 1 & TRLANGULAR & $-1.2100000 E+01$ & $-1.1800000 E+01$ & $-1.4700000 E+01$ & $-9.8000000 E+00$ & $\log \left(m^{\wedge} 2\right)$ & WP031070 & $\mathbf{N}$ & 1 \\
\hline 68 & 1 & TRIANGULAR & $-1.2100000 E+01$ & $-1.1800000 E+01$ & $-1.4700000 E+01$ & $-9.8000000 E+00$ & $\log \left(m^{\wedge} 2\right)$ & WP035268 & $\mathbf{N}$ & 1 \\
\hline 68 & 1 & TRIANGULAR & $-1.2100000 E+01$ & $-1.1800000 E+01$ & $-1.4700000 E+01$ & $-9.8000000 E+00$ & $\log \left(m^{\wedge} 2\right)$ & WP035597 & $\mathbf{N}$ & 1 \\
\hline 69 & 1 & TRIANGULAR & $-1.2100000 E+01$ & $-1.1800000 E+01$ & $-1.4700000 E+01$ & $-9.8000000 E+00$ & $\log \left(m^{\wedge} 2\right)$ & WP031070 & $=-\mathbf{N}$ & 1 \\
\hline 69 & 1 & TRIANGULAR & $-1.2100000 \mathrm{E}+01$ & $-1.1800000 E+01$ & $-1.4700000 E+01$ & $-9.8000000 E+00$ & $\log \left(m^{\wedge} 2\right)$ & WP035268 & $\mathbf{N}$ & 1 \\
\hline 69 & 1 & TRIANGULAR & $-1.2100000 E+01$ & $-1.1800000 E+01$ & $-1.4700000 E+01$ & $-9.8000000 E+\infty 0$ & $\log \left(m^{\wedge} 2\right)$ & WP035597 & $\mathbf{N}$ & 1 \\
\hline 72 & $1,3,4$ & CONSTANT & $4.0000000 E+00$ & $4.0000000 E+\infty$ & $4.0000000 E+00$ & $4.0000000 E+00$ & NONE & WP038568 & $\mathbf{Y}$ & N/A \\
\hline 72 & $1,3,4$ & CONSTANT & $4.0000000 E+00$ & $4.0000000 E+00$ & $4.0000000 E+00$ & $4.0000000 E+\infty 0$ & NONE & WP040514 & $\mathbf{Y}$ & $N / A$ \\
\hline 73 & 1.3 & CONSTANT & $1.0000000 E+\infty 0$ & $1.0000000 E+\infty 0$ & $1.0000000 E+00$ & $1.0000000 E+00$ & NONE & WPO 038568 & $Y$ & N/A \\
\hline 73 & 1,3 & CONSTANT & $1.0000000 E+00$ & $1.0000000 \mathrm{E}+00$ & $1.0000000 E+\infty$ & $1.0000000 \mathrm{E}+00$ & NONE & WPO40514 & $\mathbf{Y}$ & NA \\
\hline 74 & $1,3,4$ & CONSTANT & $2.0000000 E-01$ & $2.0000000 E-01$ & $2.0000000 E-01$ & $2.0000000 E-01$ & NONE & WP038568 & $\mathbf{Y}$ & N/A \\
\hline 74 & $1,3,4$ & CONSTANT & $2.0000000 E-01$ & $2.0000000 E-01$ & $2.0000000 \mathrm{E}-01$ & $2.0000000 E-01$ & NONE & WP040514 & $\mathbf{Y}$ & N/A \\
\hline 75 & $1,3,4$ & CONSTANT & $2.0000000 \mathrm{E}-01$ & $2.0000000 \mathrm{E}-01$ & $2,0000000 E-01$ & $20000000 E-01$ & NONE & WP038568 & $\mathbf{Y}$ & N/A \\
\hline 75 & $1,3,4$ & CONSTANT & $2.0000000 \mathrm{E}-01$ & $2.0000000 E-01$ & $2.0000000 E-01$ & $20000000 E-01$ & NONE & WP040514 & $\mathbf{Y}$ & NA \\
\hline 76 & 3,5 & CONSTANT & $1.0000000 E+\infty$ & $1.0000000 E+\infty$ & $1.0000000 \mathrm{E}+00$ & $1.0000000 \mathrm{E}+\infty 0$ & NONE & $\begin{array}{l}\text { MEMO:VAUGHN } \\
\text { TERNEY-9 }\end{array}$ & $\mathbf{Y}$ & NA \\
\hline
\end{tabular}


CCA Parameter Listing

\begin{tabular}{|c|c|c|c|c|c|c|c|c|c|c|}
\hline $\begin{array}{l}\text { Parameterln } \\
\text { Database? } \\
\text { (MnN) }\end{array}$ & 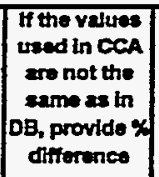 & ID & Materialo & Mrterial Neme & $\begin{array}{l}\text { Parameter } \\
\text { ID }\end{array}$ & Perameter Name & $\begin{array}{l}\text { PRPD } \\
\text { (WPO *) }\end{array}$ & $\begin{array}{c}\text { Data Entry } \\
\text { Date }\end{array}$ & $\begin{array}{l}\text { Is the } 454 \\
\text { consistent } \\
\text { with the } \\
\text { parameter } \\
\text { in DB? } \\
(Y, N A)\end{array}$ & $\begin{array}{l}\text { Parmater } \\
\text { Category } \\
\text { (footnote 1) }\end{array}$ \\
\hline $\mathbf{Y}$ & & 76 & CAVITY_1 & Cavity for Waste Areas & CAP_MOD & $\begin{array}{l}\text { Model number, capillary prossura } \\
\text { model }\end{array}$ & 31626 & 02-Nov-95 & $\mathbf{Y}$ & 48 \\
\hline$Y$ & & 76 & CAVITY_1 & Cavity for Waste Areas & CAP_MOD & $\begin{array}{l}\text { Model mumber, capillary pressure } \\
\text { model }\end{array}$ & 31626 & 02-Nov-95 & $\mathbf{Y}$ & $A B$ \\
\hline $\mathbf{Y}$ & & $\pi$ & CAVITY_1 & Cavity for Wasto Areas & COMP_RCK & Buik Compressibility & 31628 & 02-Nov-95 & $\mathbf{Y}$ & $4 B$ \\
\hline $\mathbf{Y}$ & & $\pi 7$ & CAVITY_1 & Cavity for Waste Areas & COMP_RCK & Bulk Compressibility & 31628 & 02-Nov-95 & $\mathbf{Y}$ & 48 \\
\hline $\mathbf{Y}$ & & 77 & CAVITY_1 & Cavity for Waste Areas & COMP_RCK & Bulk Compressibility & 31628 & 02-Nov-95 & $\mathbf{Y}$ & 48 \\
\hline$Y$ & & 78 & CAVITY_1 & Cavity for Waste Areas & PC_MAX & $\begin{array}{l}\text { Maximum allowable capillary } \\
\text { pressure }\end{array}$ & 31673 & $02-N o v-95$ & $\mathbf{Y}$ & $4 B$ \\
\hline $\mathbf{Y}$ & & 78 & CAVITY_1 & Cavity for Waste Areas & PC_MAX & $\begin{array}{l}\text { Maximum allowable capillary } \\
\text { pressure }\end{array}$ & 31673 & $02-N o v-95$ & $\mathbf{Y}$ & $4 B$ \\
\hline $\mathbf{Y}$ & & 78 & CAVITY_1 & Cavity for Waste Areas & PC_MAX & $\begin{array}{l}\text { Maxmum allowable capillary } \\
\text { pressure }\end{array}$ & 31673 & Q2-Nov-95 & $\mathbf{Y}$ & 48 \\
\hline $\mathbf{Y}$ & & 79 & CAVITY_i & Cavity for Waste Areas & PORE_DIS & $\begin{array}{l}\text { Brooks-Corey porb distribution } \\
\text { parameter }\end{array}$ & 31675 & O2-Nov-95 & $\mathbf{Y}$ & 48 \\
\hline$Y$ & & 79 & CAVITY_1 & Cavity for Waste Areas & PORE_DIS & $\begin{array}{l}\text { Brooks-Corey pore distribution } \\
\text { parameter }\end{array}$ & 31675 & 02-Nov-95 & $\mathbf{Y}$ & 48 \\
\hline$Y$ & & 79 & CAVITY_1 & Cavity for Waste Areas & PORE_DIS & $\begin{array}{l}\text { Brooks-Corey pore distribution } \\
\text { parameter }\end{array}$ & 31675 & 02-Nov-95 & $\mathbf{Y}$ & 48 \\
\hline $\mathbf{Y}$ & & 80 & CAVITY_i & Cavity for Wasto Areas & POROSTTY & Effective porosity & 31677 & 02-Nov-95 & $\mathbf{Y}$ & 48 \\
\hline $\mathbf{Y}$ & & 80 & CAVITY_1 & Cavity for Waste Areas & POROSTr & Effective porosity & 31677 & O2-Nov-95 & $\mathbf{Y}$ & 48 \\
\hline $\mathbf{Y}$ & & 80 & CAVITY_1 & Cavity for Waste Areas & POROSITY & Effective porosity & 31677 & 02-Nov-95 & $\mathbf{Y}$ & $4 B$ \\
\hline $\mathbf{Y}$ & & 84 & CAVTrY_1 & Cavity for Waste Areas & PO_MIN & $\begin{array}{l}\text { Minimum brine pressure for } \\
\text { capillary model KPC=3 }\end{array}$ & 31678 & $21-F a b-96$ & $\mathbf{Y}$ & $4 B$ \\
\hline$Y$ & & 81 & CAVITY_1 & Cavity for Waste Areas & PO_MIN & $\begin{array}{l}\text { Minimum brune pressure for } \\
\text { capillary model KPC=3 }\end{array}$ & 31678 & $21+$ eab-96 & $\boldsymbol{Y}$ & 48 \\
\hline $\mathbf{Y}$ & & 81 & CAVITY_1 & Cavity for Waste Areas & PO_MIN & $\begin{array}{l}\text { Minimum brine pressure for } \\
\text { capillary model KPC=3 }\end{array}$ & 31678 & 21-Feb-96 & $\boldsymbol{Y}$ & $4 B$ \\
\hline $\mathbf{Y}$ & & 82 & CAVITY_1 & Cavity for Waste Areas & PRESSURE & Brine far-field pore pressure & 31679 & 02-Nov-95 & $\mathbf{Y}$ & $4 B$ \\
\hline $\mathbf{Y}$ & & 82 & CAVITY_1 & Cavity for Wasto Areas & PRESSURE & Brine far-field pore pressure & 31679 & 02-Nov-95 & $Y$ & 48 \\
\hline $\mathbf{Y}$ & & 82 & CAVITY_1 & Cavity for Waste Areas & PRESSURE & Brine far-field pore pressure & 31679 & 02-Nov-95 & $\mathbf{Y}$ & 48 \\
\hline $\mathbf{Y}$ & & 83 & CAVITY_1 & Cavity for Waste Areas & PRMX_LOG & $\begin{array}{l}\text { Log of intrinsic permeability, } X \text { - } \\
\text { direction }\end{array}$ & 31680 & 20-Feb-96 & $\mathbf{Y}$ & 48 \\
\hline$Y$ & & 83 & CAVITY_1 & Cavity for Waste Areas & PRMX_LOG & $\begin{array}{l}\text { Log of intrinsic permeability, } X \text { - } \\
\text { direction }\end{array}$ & 39680 & 20-Feb-96 & $\mathbf{Y}$ & 48 \\
\hline $\mathbf{Y}$ & & 83 & CAVITY_1 & Cavity for Waste Areas & PRMX_LOG & $\begin{array}{l}\text { Log of intrinsic permeabilty, } X \text { - } \\
\text { direction }\end{array}$ & 31680 & 20-Feb-95 & $\mathbf{Y}$ & $4 B$ \\
\hline $\mathbf{Y}$ & & 84 & CAVIT__1 & Cavity for Waste Areas & PRMY_LOG & $\begin{array}{l}\text { Log of intrnsic permeability, Y- } \\
\text { direction }\end{array}$ & 39681 & 20-Feb-96 & $Y$ & 48 \\
\hline $\mathbf{Y}$ & & 84 & CAVITY_1 & Cavity for Waste Areas & PRMY_LOG & $\begin{array}{l}\text { Log of intrinsic permeability, Y- } \\
\text { direction }\end{array}$ & 31681 & 20-Feb-96 &.$- y$ & 48 \\
\hline$Y$ & & 84 & CAVITY_1 & Cavity for Waste Areas & PRMY_LOG & $\begin{array}{l}\text { Log of intrinsic permeabilty, } Y_{-} \\
\text {direction }\end{array}$ & 31681 & 20-Feb-95 & $\mathbf{Y}$ & 48 \\
\hline $\mathbf{Y}$ & & 85 & CAVITY_1 & Cavity for Waste Aroas & PRMZ_LOG & $\begin{array}{l}\text { Log of intrinsic permeability, Z- } \\
\text { direction }\end{array}$ & 31682 & 20-Feb-96 & $\mathbf{Y}$ & 48 \\
\hline $\mathbf{Y}$ & & 85 & CAVITY_1 & Cavity for Waste Areas & PRMZ_LOG & $\begin{array}{l}\text { Log of intrinsic permeabilty, 2- } \\
\text { direction }\end{array}$ & 31682 & 20-Feb-96 & $\mathbf{Y}$ & $4 B$ \\
\hline $\mathbf{Y}$ & & 85 & CAVITY_1 & Cavity for Waste Areas & PRMZ_LOG & $\begin{array}{l}\text { Log of intrinsic permeabilty, Z- } \\
\text { droction }\end{array}$ & 31682 & 20-Feb-96 & $\mathbf{Y}$ & 48 \\
\hline $\mathbf{Y}$ & & 88 & CAVITY_1 & Cevity for Waste Areas & RELP_MOD & $\begin{array}{l}\text { Model number, relative } \\
\text { permeability model }\end{array}$ & 31685 & 02-Nov-95 & $\mathbf{Y}$ & 48 \\
\hline $\mathbf{Y}$ & & 88 & CAVITY_1 & Cavity for Waste Areas & RELP_MOD & $\begin{array}{l}\text { Model number, relative } \\
\text { permeability model }\end{array}$ & 31685 & O2-Nov-95 & $\mathbf{Y}$ & 48 \\
\hline $\mathbf{Y}$ & & 88 & CAVITY_1 & Cavity for Waste Areas & RELP_MOD & $\begin{array}{l}\text { Model number, relative } \\
\text { permeability model }\end{array}$ & 31685 & 02-Nov-95 & $\mathbf{Y}$ & $4 B$ \\
\hline $\mathbf{Y}$ & & 89 & CAVITY_1 & Cavity for Waste Areas & SAT_RBRN & Residual Brine Saturation & 31687 & 17-Feb-96 & $\mathbf{Y}$ & $4 \mathrm{~B}$ \\
\hline $\mathbf{Y}$ & & 89 & CAVITY_1 & Cavity for Waste Areas & SAT_RBRN & Residual Brine Saturation & 31687 & 17freb-96 & $\mathbf{Y}$ & $4 B$ \\
\hline $\mathbf{Y}$ & & 89 & CAVITY_1 & Cavity for Waste Areas & SAT_RBRN & Residual Brine Seturation & 31687 & 17freb-96 & $\mathbf{Y}$ & $4 B$ \\
\hline $\mathbf{Y}$ & & 90 & CAVITY_1 & Cavity for Wasle Areas & SAT_RGAS & Residual Gas Saturation & 31688 & 17-Fob-96 & $\mathbf{Y}$ & $4 B$ \\
\hline
\end{tabular}


CCA Parameter Listing

\begin{tabular}{|c|c|c|c|c|c|c|c|c|c|c|}
\hline ID & $\begin{array}{c}\text { Clesedfle } \\
\text { extion of } \\
\text { Data } \\
\text { Cortagory } \\
\text { (footnote 2) }\end{array}$ & $\begin{array}{l}\text { Distribution } \\
\text { Typo }\end{array}$ & $\operatorname{man}$ & Modien & - Mintmem & Meximum & $\begin{array}{l}\text { Unts } \\
\therefore \\
\therefore .\end{array}$ & $\begin{array}{l}\text { Reforenco ID } \\
\therefore \\
\therefore\end{array}$ & $\begin{array}{l}\text { Wes the dati } \\
\text { doweloped } \\
\text { under an } \\
\text { NOA-1 } \\
\text { Progren? }\end{array}$ & $\begin{array}{c}\text { Which } \\
\text { methods } \\
\text { wore used to } \\
\text { qualty } \\
\text { oxtetting } \\
\text { data? } \\
\text { (footnote J) } \\
\end{array}$ \\
\hline 76 & 3,5 & CONSTANT & $1.0000000 \mathrm{E}+00$ & $1.0000000 \varepsilon+00$ & $1.0000000 \mathrm{E}+00$ & $1.0000000 E+\infty 0$ & NONE & WP035597 & $\mathbf{Y}$ & N/A \\
\hline 76 & 3,5 & CONSTANT & $1.0000000 \mathrm{E}+00$ & $1.0000000 \mathrm{E}+00$ & $1.0000000 E+00$ & $1.0000000 E+00$ & NONE & WPO40514 & $Y$ & NA \\
\hline$\pi$ & 3,5 & CONSTANT & $0.0000000 E+00$ & $0.0000000 \mathrm{E}+00$ & $0.0000000 \mathrm{E}+00$ & $0.0000000 E+\infty$ & $P_{a}-1$ & $\begin{array}{c}\text { MEMO:VAUGHNI } \\
\text { TIERNEY-9 } \\
\end{array}$ & $\mathbf{Y}$ & $N / A$ \\
\hline 77 & 3,5 & CONSTANT & $0.0000000 E+00$ & $0.0000000 E+00$ & $0.0000000 E+\infty 0$ & $0.0000000 E+00$ & $P a^{A}-1$ & WP035597 & Y & NA \\
\hline 77 & 3,5 & CONSTANT & $0.0000000 E+\infty 0$ & $0.0000000 E+\infty 0$ & $0.0000000 E+\infty 0$ & $0.0000000 E+00$ & $P q^{n-1}$ & WP040514 & $\mathbf{Y}$ & NA \\
\hline 78 & 3,5 & CONSTANT & $1.0000000 E+08$ & $1.0000000 E+08$ & $1.0000000 E+08$ & $1.0000000 E+08$ & $\mathrm{~Pa}$ & $\begin{array}{c}\text { MEMO:VAUGHNI } \\
\text { TIERNEY-9 }\end{array}$ & $\mathbf{Y}$ & N/A \\
\hline 78 & 3,5 & CONSTANT & $1.0000000 E+08$ & $1.0000000 E+08$ & $1.0000000 E+08$ & $1.0000000 E+08$ & $\mathrm{~Pa}$ & WP035597 & $\mathbf{Y}$ & N/A \\
\hline 78 & 3,5 & CONSTANT & $1.0000000 E+08$ & $1.0000000 E+08$ & $1.0000000 E+08$ & $1.0000000 E+08$ & $\mathrm{~Pa}$ & WP040514 & $\mathbf{Y}$ & N/A \\
\hline 79 & 3,5 & CONSTANT & $7.0000000 E-01$ & $7.0000000 \mathrm{E}-01$ & $7.0000000 E-01$ & $7.0000000 E-01$ & NONE & $\begin{array}{l}\text { MEMO:VAUGHN } \\
\text { TIERNEY-9 }\end{array}$ & $\mathbf{Y}$ & N/A \\
\hline 79 & 3,5 & CONSTANT & $7.0000000 \mathrm{E}-01$ & $7.0000000 E-01$ & $7.0000000 E-01$ & $7.0000000 E-01$ & NONE & WPO35597 & $\mathbf{Y}$ & N/A \\
\hline 79 & 3,5 & CONSTANT & $7.0000000 E-01$ & $7.0000000 E-01$ & $7.0000000 E-01$ & $7.0000000 E-01$ & NONE & WP040514 & $Y$ & NA \\
\hline 80 & 3,5 & CONSTANT & $1.0000000 E+00$ & $1.0000000 E+00$ & $1.0000000 E+\infty$ & $1.0000000 E+\infty 0$ & NONE & $\begin{array}{c}\text { MEMO:VAUGHNI } \\
\text { TIERNEY-9 } \\
\end{array}$ & $\mathbf{Y}$ & N/A \\
\hline 80 & 3,5 & CONSTANT & $1.0000000 \mathrm{E}+00$ & $1.0000000 E+00$ & $1.0000000 E+\infty 0$ & $1.0000000 \mathrm{E}+\infty 0$ & NONE & WPO35597 & $\mathbf{Y}$ & NA \\
\hline 80 & 3,5 & CONSTANT & $1.0000000 E+00$ & $1.0000000 E+\infty 0$ & $1.0000000 E+00$ & $1.0000000 \mathrm{E}+00$ & NONE & WP040514 & $Y$ & NA \\
\hline 89 & 3,5 & CONSTANT & $1.0132500 E+05$ & $1.0132500 E+05$ & $1.0132500 E+05$ & $1.0132500 E+05$ & $\mathbf{P a}$ & WP035597 & $\because Y$ & N/A \\
\hline 81 & 3,5 & CONSTANT & $1.0132500 E+05$ & $1.0132500 E+05$ & $1.0132500 E+05$ & $1.0932500 E+05$ & $\mathbf{P a}$ & WP036051 & $Y$ & NA \\
\hline 81 & 3,5 & CONSTANT & $1.0132500 E+05$ & $1.0132500 E+05$ & $1.0132500 E+05$ & $1.0132500 E+05$ & $\mathrm{~Pa}$ & WPO40514 & $\mathbf{Y}$ & N/A \\
\hline 82 & $1,3,5$ & CONSTANT & $1.0132500 E+05$ & $1.0132500 E+05$ & $1.0132500 E+05$ & $1.0132500 E+05$ & $\mathrm{~Pa}$ & $\begin{array}{l}\text { MEMO:VAUGHN } \\
\text { TERNEY-9 } \\
\end{array}$ & $Y$ & N/A \\
\hline 82 & $1,3,5$ & CONSTANT & $1.0132500 E+0.5$ & $1.0132500 E+05$ & $1.0132500 E+05$ & $1.0132500 E+05$ & $\mathrm{~Pa}$ & WP035597 & $\mathbf{Y}$ & NA \\
\hline 82 & $1,3,5$ & CONSTANT & $1.0132500 E+05$ & $1.0132500 E+0.5$ & $1.0132500 E+05$ & $1.0132500 E+05$ & $\mathrm{~Pa}$ & WP040514 & $Y$ & $N / A$ \\
\hline 83 & 3,5 & CONSTANT & $-1.0000000 E+01$ & $-1.0000000 E+01$ & $-1.0000000 E+01$ & $-1.0000000 \mathrm{E}+01$ & $\log \left(m^{\wedge} 2\right)$ & $\begin{array}{l}\text { MEMO:VAUGHN } \\
\text { TIERNEY-9 }\end{array}$ & $\mathbf{Y}$ & $N / A$ \\
\hline 83 & 3,5 & CONSTANT & $-1.0000000 E+01$ & $-1.0000000 E+01$ & $-1.0000000 E+01$ & $-1.0000000 E+01$ & $\log \left(m^{\wedge} 2\right)$ & WP035597 & $Y$ & N/A \\
\hline 83 & 3,5 & CONSTANT & $-1.0000000 E+01$ & $-1.0000000 E+01$ & $-1.0000000 E+01$ & $-1.00000000+01$ & $\log \left(m^{\wedge} 2\right)$ & WPO40514 & $Y$ & NA \\
\hline 84 & 3,5 & CONSTANT & $-1.0000000 E+01$ & $-1.0000000 E+01$ & $-1.0000000 E+01$ & $-1.0000000 E+01$ & $\log \left(m^{\wedge} 2\right)$ & $\begin{array}{c}\text { MEMO:VAUGHN } \\
\text { TIERNEY-9 } \\
\end{array}$ & $\mathbf{Y}$ & N/A \\
\hline 84 & 3,5 & CONSTANT & $-1.0000000 E+01$ & $-1.0000000 E+01$ & $-1.0000000 E+01$ & $-1,0000000 E+01$ & $\log \left(m^{\wedge} 2\right)$ & WP035597 & $=Y$ & $N / A$ \\
\hline 84 & 3,5 & CONSTANT & $-1.0000000 E+01$ & $-1.0000000 E+01$ & $-1.0000000 \mathrm{E}+01$ & $-1.0000000 E+01$ & $\log \left(m^{\wedge} 2\right)$ & WP040514 & $\mathbf{Y}$ & NA \\
\hline 85 & 3,5 & CONSTANT & $-1.0000000 E+01$ & $-1.0000000 E+01$ & $-1.0000000 E+01$ & $-1.0000000 E+01$ & $\log \left(m^{\wedge} 2\right)$ & $\begin{array}{c}\text { MEMO:VAUGHN } \\
\text { TIERNEY-9 } \\
\end{array}$ & $\gamma$ & N/A \\
\hline 85 & 3,5 & CONSTANT & $-1.0000000 E+01$ & $-1.0000000 E+01$ & $-1.0000000 E+01$ & $-1.0000000 E+01$ & $\log \left(m^{\wedge} 2\right)$ & WP035597 & $\mathbf{Y}$ & NA \\
\hline 85 & 3,5 & CONSTANT & $-1.0000000 E+01$ & $-1.0000000 E+01$ & $-1.0000000 E+01$ & $-1.0000000 E+01$ & $\log \left(m^{\wedge} 2\right)$ & WPO40514 & $\mathbf{Y}$ & N/A \\
\hline 88 & 3,5 & CONSTANT & $4.0000000 E+\infty 0$ & $4.0000000 E+\infty 0$ & $4.0000000 E+\infty$ & $4.0000000 E+\infty$ & NONE & $\begin{array}{c}\text { MEMO:VAUGHN } \\
\text { TIERNEY-9 } \\
\end{array}$ & $\gamma$ & N/A \\
\hline 88 & 3,5 & CONSTANT & $4.0000000 E+\infty 0$ & $4.0000000 E+\infty 0$ & $4.0000000 \mathrm{E}+00$ & $4.0000000 E+00$ & NONE & WP035597 & $\mathbf{Y}$ & NA \\
\hline 88 & 3,5 & CONSTANT & $4.0000000 \mathrm{E}+00$ & $4.0000000 \mathrm{E}+00$ & $4.0000000 E+00$ & $4.0000000 \mathrm{E}+00$ & NONE & WP040514 & $\mathbf{Y}$ & NA \\
\hline 89 & 3,5 & CONSTANT & $0.0000000 E+00$ & $0.0000000 E+00$ & $0.0000000 E+00$ & $0.0000000 \mathrm{E}+\infty$ & NONE & $\begin{array}{c}\text { MEMO:VAUGHN } \\
\text { TIERNEY-9 } \\
\end{array}$ & $Y$ & N/A \\
\hline 89 & 3,5 & CONSTANT & $0.0000000 E+00$ & $0.0000000 E+\infty 0$ & $0.0000000 E+00$ & $0.0000000 E+00$ & NONE & WP035597 & $\mathbf{Y}$ & N/A \\
\hline 89 & 3,5 & CONSTANT & $0.0000000 \mathrm{E}+00$ & $0.0000000 E+00$ & $0.0000000 \mathrm{E}+\infty 0$ & $0.0000000 \mathrm{E}+\infty$ & NONE & WPO40514 & $\mathbf{Y}$ & NA \\
\hline 90 & 3,5 & CONSTANT & $0.0000000 E+\infty 0$ & $0.0000000 E+00$ & $0.0000000 E+\infty 0$ & $0.0000000 \mathrm{E}+\infty 0$ & NONE & $\begin{array}{c}\text { MEMO:VAUGHN } \\
\text { TIERNEY-9 } \\
\end{array}$ & $\mathbf{Y}$ & NA \\
\hline
\end{tabular}


CCA Parameter Listing

\begin{tabular}{|c|c|c|c|c|c|c|c|c|c|c|}
\hline $\begin{array}{c}\text { Parameterin } \\
\text { Detabase? } \\
\text { (YN) }\end{array}$ & \begin{tabular}{|c|} 
If the values \\
used in CCA \\
are not the \\
same ax in \\
DB, provide $x$. \\
differences
\end{tabular} & ID & Meterialo & Mattoria Neme & $\begin{array}{l}\text { Parameter } \\
\text { to } \\
\text {. }\end{array}$ & Pernnater Name & $\begin{array}{l}\text { PRPID } \\
\text { (MPO:) }\end{array}$ & $\begin{array}{c}\text { Dote Entry } \\
\text { Dato }\end{array}$ & $\begin{array}{l}\text { Is tho } 464 \\
\text { conslatent } \\
\text { with the } \\
\text { perameter } \\
\text { in DB? } \\
\left(Y_{1} N \text { N }\right.\end{array}$ & $\begin{array}{l}\text { Paramotar } \\
\text { Category } \\
\text { (footnote 1) }\end{array}$ \\
\hline Y & & 90 & CAVTTY_1 & Cavity for Waste Areas & SAT_RGAS & Residual Gas Saturation & 31688 & 17 feb-96 & $Y$ & 4B \\
\hline$Y$ & . & 90 & CAVITY_1 & Cavity for Waste Areas & SAT_RGAS & Residual Gas Saturation & 31688 & 17 -Feb-96 & $\mathbf{Y}$ & 48 \\
\hline Y & & 91 & CAVTrY_2 & $\begin{array}{l}\text { Cavity for Non-waste } \\
\text { Areas }\end{array}$ & CAP_MOD & $\begin{array}{l}\text { Model number, capillary pressure } \\
\text { model }\end{array}$ & 31692 & 02-Hov.95 & $\mathbf{Y}$ & AB \\
\hline $\mathbf{Y}$ & & 91 & CAVITY_2 & $\begin{array}{l}\text { Cavity for Nontwaste } \\
\text { Areas }\end{array}$ & CAP_MOD & $\begin{array}{l}\text { Model number, capillary pressure } \\
\text { model }\end{array}$ & 31692 & 02-Nov-95 & $\mathbf{Y}$ & 48 \\
\hline $\mathbf{Y}$ & & 91 & CAVTTY_2 & $\begin{array}{l}\text { Cavity for Non-waste } \\
\text { Areas }\end{array}$ & CAP_MOD & $\begin{array}{l}\text { Model number, capillary pressure } \\
\text { model }\end{array}$ & 31692 & 02-Nov-95 & $\gamma$ & AB \\
\hline $\mathbf{Y}$ & & 92 & CAVITY_2 & $\begin{array}{l}\text { Cavity for Non-wasto } \\
\text { Areas }\end{array}$ & COMP_RCK & Butk Compressibility & 31694 & 02-Nov-95 & $\mathbf{Y}$ & 48 \\
\hline$\gamma$ & & 92 & CAVITY_2 & $\begin{array}{l}\text { Cavity for Nortwaste } \\
\text { Areas }\end{array}$ & COMP_RCK & Bulk Compressibility & 31694 & 02-Nov-95 & $\mathbf{Y}$ & $4 B$ \\
\hline $\mathbf{Y}$ & & 92 & CAVITY_2 & $\begin{array}{l}\text { Cavity for Non-waste } \\
\text { Areas }\end{array}$ & COMP_RCK & Bulk Compressibility & 31694 & 02-Nov-95 & $\mathbf{Y}$ & 48 \\
\hline $\mathbf{Y}$ & & 93 & CAVITY_2 & $\begin{array}{l}\text { Cavity for Non-waste } \\
\text { Areas }\end{array}$ & PC_mAX & $\begin{array}{l}\text { Meximum allowable capillary } \\
\text { pressure }\end{array}$ & 31743 & 02-Nov-95 & $\mathbf{Y}$ & 48 \\
\hline$Y$ & & 93 & CAVITY_2 & $\begin{array}{l}\text { Cavity for Non-wasto } \\
\text { Areas }\end{array}$ & PC_MAX & $\begin{array}{l}\text { Maximum allowable capillary } \\
\text { pressure }\end{array}$ & 31743 & 02-Nov-95 & $\mathbf{Y}$ & $4 B$ \\
\hline$Y$ & & 93 & $\mathrm{CAVITY}_{2}$ & $\begin{array}{l}\text { Cavity for Non-waste } \\
\text { Areas }\end{array}$ & PC_MAX & $\begin{array}{l}\text { Maximum allowable copillary } \\
\text { pressure }\end{array}$ & 31743 & 02-Nov-95 & $\mathbf{Y}$ & 4B \\
\hline $\mathbf{Y}$ & & 94 & CAVITY_2 & $\begin{array}{l}\text { Cavity for Non-waste } \\
\text { Areas }\end{array}$ & PORE_DIS & $\begin{array}{l}\text { Brooks-Corey pore distribution } \\
\text { parameter }\end{array}$ & 31746 & O2-Nov-95 & $\mathbf{Y}$ & 48 \\
\hline Y & & 94 & CAVITY_2 & $\begin{array}{l}\text { Cavity for Non-waste } \\
\text { Areas }\end{array}$ & PORE_DIS & $\begin{array}{l}\text { Brooks-Corey pore distribution } \\
\text { parameter }\end{array}$ & 31746 & 02-Nov-95 & $\mathbf{Y}$ & 48 \\
\hline $\mathbf{Y}$ & & 94 & CAVTY_2 & $\begin{array}{l}\text { Cavity for Non-wasto } \\
\text { Areas }\end{array}$ & PORE_DIS & $\begin{array}{l}\text { Brooks-Corey pore distribution } \\
\text { parameter }\end{array}$ & 31746 & O2-Nov-95 & $\mathbf{Y}$ & 48 \\
\hline $\mathbf{Y}$ & & 95 & CAVITY_2 & $\begin{array}{l}\text { Cavity for Non-wasto } \\
\text { Areas }\end{array}$ & POROSTY & Effective porosity & 31747 & O2-Nov-95 & $\mathbf{Y}$ & $4 B$ \\
\hline $\mathbf{Y}$ & & 95 & CAVITY_2 & $\begin{array}{l}\text { Cavity for Non-waste } \\
\text { Areas }\end{array}$ & POROSITY & Effective porosity & 31747 & 02-Nov-95 & $Y$ & 48 \\
\hline $\mathbf{Y}$ & & 95 & CAVITY_2 & $\begin{array}{l}\text { Cavity for Non-waste } \\
\text { Areas }\end{array}$ & POROSITY & Effective porosity & 31747 & 02-Nov-95 & $\mathbf{Y}$ & 48 \\
\hline $\mathbf{Y}$ & & 96 & CAVIrY_2 & $\begin{array}{l}\text { Cavity for Non-wasto } \\
\text { Areas }\end{array}$ & PO_MIN & $\begin{array}{l}\text { Minimum brune pressuro for } \\
\text { capiliary model KPC=3 }\end{array}$ & 31749 & 02-Nov-95 & $\mathbf{Y}$ & 48 \\
\hline $\mathbf{Y}$ & & 96 & CAVITY_2 & $\begin{array}{l}\text { Cavity for Non-waste } \\
\text { Areas }\end{array}$ & PO_MIN & $\begin{array}{l}\text { Minimum brne pressure for } \\
\text { capillary model KPC=3 }\end{array}$ & 31749 & 02-Nov-95 & $\mathbf{Y}$ & $4 B$ \\
\hline$Y$ & & 96 & CAVITY_2 & $\begin{array}{l}\text { Cavity for Non-wasto } \\
\text { Areas }\end{array}$ & PO_MIN & $\begin{array}{l}\text { Minimum brine pressure for } \\
\text { capillary model KPC }=3\end{array}$ & 31749 & 02-Nov-95 & $\mathbf{Y}$ & 48 \\
\hline Y & & 96 & CAVITY_2 & $\begin{array}{l}\text { Cavity for Non-waste } \\
\text { Areas }\end{array}$ & PO_MIN & $\begin{array}{l}\text { Minimum brine pressurs for } \\
\text { capillary model KPC=3 }\end{array}$ & 31749 & 02-Nov-95 & $\mathbf{Y}$ & $4 B$ \\
\hline $\mathbf{Y}$ & & 97 & CAVITY_2 & $\begin{array}{l}\text { Cavity for Non-wasto } \\
\text { Areas }\end{array}$ & PRESSURE & Brine far-field pore pressure & 31750 & 02-Nov-95 & $\mathbf{Y}$ & $4 B$ \\
\hline $\mathbf{Y}$ & & 97 & CAVITY_2 & $\begin{array}{l}\text { Cavity for Non-wasto } \\
\text { Areas }\end{array}$ & PRESSURE & Brine far-field pore pressure & 31750 & 02-Nov-95 & $\mathbf{Y}$ & $4 B$ \\
\hline$Y$ & & 97 & CAVITY_2 & $\begin{array}{l}\text { Cavity for Non-wasto } \\
\text { Areas }\end{array}$ & PRESSURE & Brine farfield pore pressure & 31750 & 02-Nov-95 & $\mathbf{Y}$ & 48 \\
\hline$\gamma$ & & 98 & CAVITY_2 & $\begin{array}{l}\text { Cavity for Non-waste } \\
\text { Areas }\end{array}$ & PRMX_LOG & $\begin{array}{l}\text { Log of intrnsic permeability, X- } \\
\text { direction }\end{array}$ & 31751 & 17 fob-96 &.$- Y$ & 48 \\
\hline$Y$ & & 98 & CAVTTY_2 & $\begin{array}{l}\text { Cavity for Non-waste } \\
\text { Areas }\end{array}$ & PRMX_LOG & $\begin{array}{l}\text { Log of intsinsic permeability, X- } \\
\text { direction }\end{array}$ & 31751 & 17 feb-96 & $\mathbf{Y}$ & $4 B$ \\
\hline$Y$ & & 98 & CAVTY_2 & $\begin{array}{l}\text { Cavity for Non-wasto } \\
\text { Areas }\end{array}$ & PRMX_LOG & $\begin{array}{l}\text { Log of intrnsic permeabiltty, X- } \\
\text { direction }\end{array}$ & 31751 & 17 feb-96 & $Y$ & 48 \\
\hline$Y$ & & 99 & CAVITY_2 & $\begin{array}{l}\text { Cavity for Non-wasto } \\
\text { Aroas }\end{array}$ & PRMY_LOG & $\begin{array}{l}\text { Log of intrinsic permeability, Y- } \\
\text { direction }\end{array}$ & 31752 & 17 fob-96 & $\mathbf{Y}$ & $4 B$ \\
\hline$\gamma$ & & 99 & CAVITY_2 & $\begin{array}{l}\text { Cavity for Non-wasto } \\
\text { Aroas }\end{array}$ & PRMY_LOG & $\begin{array}{l}\text { Log of intrinsic permeabiltty, Y- } \\
\text { direction }\end{array}$ & 31752 & $17-f 6 b-96$ & $\mathbf{Y}$ & $4 B$ \\
\hline$Y$ & & 99 & CAVITY_2 & $\begin{array}{l}\text { Cavity for Non-waste } \\
\text { Areas }\end{array}$ & PRMY_LOG & $\begin{array}{l}\text { Log of ntrmsic permeability, Y- } \\
\text { diroction }\end{array}$ & 31752 & 17f-Fob-96 & $\mathbf{Y}$ & 4B \\
\hline$Y$ & & 100 & CAVITY_2 & $\begin{array}{l}\text { Cavity for Non-wasto } \\
\text { Areas }\end{array}$ & PRMZ_LOG & $\begin{array}{l}\text { Log of intrnusic permeabilty, Z- } \\
\text { direction }\end{array}$ & 31754 & 17 Feb-96 & $\mathbf{Y}$ & AB \\
\hline Y & & 100 & CAVTTY_2 & $\begin{array}{l}\text { Cavity for Norn-waste } \\
\text { Areas }\end{array}$ & PRMZ_LOG & $\begin{array}{l}\text { Log of minnssk permeabilty, Z- } \\
\text { direction }\end{array}$ & 31754 & 17 fob-96 & $Y$ & 48 \\
\hline$Y$ & & 100 & CAVITY_2 & $\begin{array}{l}\text { Cavity for Nortwaste } \\
\text { Areas }\end{array}$ & PRMZ_LOG & $\begin{array}{l}\text { Log of minnsic permeability, Z- } \\
\text { direction }\end{array}$ & 31754 & 17.Fob-96 & $\mathbf{Y}$ & AB \\
\hline$Y$ & & 103 & CAVITY_2 & $\begin{array}{l}\text { Cavity for Non-wasto } \\
\text { Areas }\end{array}$ & RELP_MOD & $\begin{array}{l}\text { Model number, relative } \\
\text { permeability mode! }\end{array}$ & 31759 & 02-Nov-95 & $\mathbf{Y}$ & 4B \\
\hline $\mathbf{Y}$ & & 103 & CAVITY_2 & $\begin{array}{l}\text { Cavity for Non-wasto } \\
\text { Areas }\end{array}$ & RELP_MOD & $\begin{array}{l}\text { Model number, nolativo } \\
\text { permeability model }\end{array}$ & 31759 & C2-Nov-95 & $\mathbf{Y}$ & AB \\
\hline $\mathbf{Y}$ & & 103 & CAVITY_2 & $\begin{array}{l}\text { Cavity for Non-wasto } \\
\text { Areas }\end{array}$ & RELP_MOD & $\begin{array}{l}\text { Model number, relativo } \\
\text { permeability model }\end{array}$ & 31759 & 02-Nov-95 & $\mathbf{Y}$ & 48 \\
\hline
\end{tabular}


CCA Parameter Listing

\begin{tabular}{|c|c|c|c|c|c|c|c|c|c|c|}
\hline tD & \begin{tabular}{c|} 
Clessith- \\
cation of \\
Data \\
Cattegory \\
(footnote 2)
\end{tabular} & $\begin{array}{l}\text { Distribution } \\
\text { Typo }\end{array}$ & Mean & Modien & Minimum & Mextrum & Units & Reference id & $\begin{array}{l}\text { Was the dista } \\
\text { developed } \\
\text { under en } \\
\text { NQA-1 } \\
\text { Progrem?. }\end{array}$ & $\begin{array}{c}\text { Which } \\
\text { mothods } \\
\text { were ured to } \\
\text { qually } \\
\text { exdeting } \\
\text { data? } \\
\text { (footnote 3) }\end{array}$ \\
\hline 90 & 3,5 & CONSTANT & $0.0000000 \mathrm{E}+00$ & $0.0000000 E+\infty 0$ & $0.0000000 E+00$ & $0.0000000 E+\infty 0$ & NONE & WPO35597 & $\mathbf{Y}$ & N/A \\
\hline 90 & 3,5 & CONSTANT & $0.0000000 E+00$ & $0.0000000 E+00$ & $0.0000000 E+00$ & $0.0000000 \mathrm{E}+00$ & NONE & WP040514 & $\mathbf{Y}$ & N/A \\
\hline 91 & 1,3 & CONSTANT & $1.0000000 \mathrm{E}+00$ & $1.0000000 \mathrm{E}+\infty 0$ & $1.0000000 \mathrm{E}+00$ & $1.0000000 E+\infty$ & NONE & $\begin{array}{l}\text { MEMO:VAUGHN } \\
\text { TERNEY-9 }\end{array}$ & $Y$ & NA \\
\hline 91 & 1,3 & CONSTANT & $1.0000000 E+\infty 0$ & $1.0000000 \mathrm{E}+00$ & $1.0000000 E+00$ & $1.0000000 E+00$ & NONE & WP035597 & $\mathbf{Y}$ & NA \\
\hline 91 & 1,3 & CONSTANT & $1.0000000 \mathrm{E}+\infty 0$ & $1.0000000 \mathrm{E}+\infty 0$ & $1.0000000 E+\infty 0$ & $1.0000000 E+\infty 0$ & NONE & WPO40514 & $\mathbf{Y}$ & N/A \\
\hline 92 & 1,3 & CONSTANT & $0.0000000 E+\infty 0$ & $0.0000000 E+00$ & $0.0000000 E+00$ & $0.0000000 E+00$ & $P a^{n-1}$ & $\begin{array}{l}\text { MEMO:VAUGHN } \\
\text { TIERNEY-9 }\end{array}$ & $\mathbf{Y}$ & N/A \\
\hline 92 & 1,3 & CONSTANT & $0.0000000 E+\infty$ & $0.0000000 E+\infty 0$ & $0.0000000 E+\infty 0$ & $0.0000000 E+00$ & $\mathrm{~Pa}^{-1}$ & WP035597 & $-Y$ & N/A \\
\hline 92 & 1,3 & CONSTANT & $0.0000000 \mathrm{E}+00$ & $0.0000000 E+\infty 0$ & $0.0000000 E+00$ & $0.0000000 E+00$ & $P a^{x}-1$ & WP040514 & $\mathbf{Y}$ & N/A \\
\hline 93 & 1,3 & CONSTANT & $1.0000000 E+08$ & $1.0000000 E+08$ & $1.0000000 E+08$ & $1.0000000 \mathrm{E}+08$ & $\mathrm{~Pa}$ & $\begin{array}{l}\text { MEMO:VAUGHN } \\
\text { TIERNEY-9 }\end{array}$ & $\mathbf{Y}$ & NA \\
\hline 93 & 1,3 & CONSTANT & $1.0000000 E+08$ & $1.0000000 E+08$ & $1.0000000 E+08$ & $1.0000000 E+08$ & $\mathrm{~Pa}$ & WPO35597 & $\mathbf{Y}$ & NA \\
\hline 93 & 1,3 & CONSTANT & $1.0000000 E+08$ & $1.0000000 E+08$ & $1.0000000 \mathrm{E}+08$ & $1.0000000 \mathrm{E}+08$ & $\mathrm{~Pa}$ & WP040514 & $\mathbf{Y}$ & N/A \\
\hline 94 & 1,3 & CONSTANT & $7.0000000 E-01$ & $7.0000000 E-01$ & $7.0000000 E-01$ & $7.0000000 E-01$ & NONE & $\begin{array}{l}\text { MEMO:VAUGHN } \\
\text { TERNEY-9 }\end{array}$ & $\mathbf{Y}$ & NA \\
\hline 94 & 1,3 & CONSTANT & $7.0000000 E-01$ & $7.0000000 E-01$ & $7.0000000 E-01$ & $7.0000000 E-01$ & NONE & WP035597 & $Y$ & N/A \\
\hline 94 & 1,3 & CONSTANT & $7,0000000 E-01$ & $7.0000000 E-01$ & $7.0000000 E-01$ & $7.0000000 E-01$ & NONE & WPO40514 & $\mathbf{Y}$ & N/A \\
\hline 95 & 1,3 & CONSTANT & $1.0000000 E+00$ & $1.0000000 E+00$ & $1.0000000 E+00$ & $1.0000000 \mathrm{E}+\infty 0$ & NONE & $\begin{array}{c}\text { MEMO:VAUGHN } \\
\text { TIERNEY-9 }\end{array}$ & $\mathbf{Y}$ & N/A \\
\hline 95 & 1.3 & CONSTANT & $1.0000000 E+00$ & $1.0000000 E+00$ & $1.0000000 E+\infty$ & $1,0000000 E+\infty 0$ & NONE & WPO35597 & $\mathbf{Y}$ & N/A \\
\hline 95 & 1,3 & CONSTANT & $1.0000000 E+00$ & $1.0000000 E+\infty 0$ & $1.0000000 E+\infty 0$ & $1.0000000 \mathrm{E}+00$ & NONE & WPO40514 & $\mathbf{Y}$ & N/A \\
\hline 96 & 1,3 & CONSTANT & $1.0132500 E+05$ & $1.0132500 E+05$ & $1.0132500 E+05$ & $1.0132500 E+05$ & $\mathrm{~Pa}$ & $\begin{array}{c}\text { MEMO:VAUGHN } \\
\text { TIERNEY-9 }\end{array}$ & $\mathbf{Y}$ & NA \\
\hline 96 & 1.3 & CONSTANT & $1.0132500 E+05$ & $1.0132500 E+05$ & $1.0132500 E+05$ & $1.0132500 E+0.5$ & $\mathrm{~Pa}$ & WPO35597 & $\mathbf{Y}$ & NA \\
\hline 96 & 1,3 & CONSTANT & $1.0132500 E+05$ & $1.0132500 E+05$ & $1.0132500 \mathrm{E}+05$ & $1.0132500 \mathrm{E}+05$ & $\mathrm{~Pa}$ & WP036051 & $Y$ & NA \\
\hline 96 & 1,3 & CONSTANT & $1.0132500 E+05$ & $1.0132500 E+05$ & $1.0132500 E+05$ & $1.0132500 E+05$ & $\mathrm{~Pa}$ & WPO40514 & $\mathbf{Y}$ & N/A \\
\hline 97 & 1,3 & CONSTANT & $1.0132500 E+05$ & $1.0132500 E+05$ & $1.0132500 E+05$ & $1.0132500 E+05$ & $\mathrm{~Pa}$ & $\begin{array}{c}\text { MEMO:VAUGHN } \\
\text { TIERNEY-9 }\end{array}$ & $\mathbf{Y}$ & NA \\
\hline 97 & 1,3 & CONSTANT & $1.0132500 E+05$ & $1.0132500 E+05$ & $1.0132500 E+05$ & $1.0132500 E+05$ & $\mathrm{~Pa}$ & WP035597 & $\mathbf{Y}$ & N/A \\
\hline 97 & 1,3 & CONSTANT & $1.0132500 E+05$ & $1.0132500 E+05$ & $1.0132500 E+05$ & $1.0132500 E+0.5$ & $\mathbf{P a}$ & WP0 040514 & $\mathbf{Y}$ & NA \\
\hline 98 & 1,3 & CONSTANT & $-1.0000000 E+01$ & $-1.0000000 E+01$ & $-1.0000000 E+01$ & $-1.0000000 E+01$ & $\log \left(m^{\wedge} 2\right)$ & $\begin{array}{c}\text { MEMO:VAUGHN } \\
\text { TIERNEY.9 }\end{array}$ &.$- Y$ & NA \\
\hline 98 & 1,3 & CONSTANT & $-1.0000000 E+01$ & $-1.0000000 E+01$ & $-1.0000000 E+01$ & $-1.0000000 \mathrm{E}+01$ & $\log \left(m^{\wedge} 2\right)$ & WP035597 & $Y$ & NAA \\
\hline 98 & 1,3 & CONSTANT & $-1.0000000 E+01$ & $-1.0000000 E+01$ & $-1.0000000 E+01$ & $-1.0000000 E+01$ & $\log \left(m^{\wedge} 2\right)$ & WPO40514 & $\mathbf{Y}$ & N/A \\
\hline 99 & 1,3 & CONSTANT & $-1.0000000 E+01$ & $-1.0000000 E+01$ & $-1.0000000 E+01$ & $-1.0000000 E+01$ & $\log \left(m^{\wedge} 2\right)$ & $\begin{array}{c}\text { MEMO:VAUGHN } \\
\text { TIERNEY.9 }\end{array}$ & $\mathbf{Y}$ & NA \\
\hline 99 & 1,3 & CONSTANT & $-1.0000000 E+01$ & $-1.0000000 E+01$ & $-1.0000000 E+01$ & $-1.0000000 E+01$ & $\log \left(m^{\wedge} 2\right)$ & WP035597 & $\mathbf{Y}$ & N/A \\
\hline 99 & 1,3 & CONSTANT & $-1.0000000 E+01$ & $-1.0000000 E+01$ & $-1.0000000 E+01$ & $-1.0000000 E+01$ & $\log \left(m^{\wedge} 2\right)$ & WPO40514 & $\mathbf{Y}$ & NA \\
\hline 100 & 1,3 & CONSTANT & $-1.0000000 E+01$ & $-1.0000000 E+01$ & $-1.0000000 E+01$ & $-1.0000000 E+01$ & $\log \left(m^{\wedge} 2\right)$ & $\begin{array}{l}\text { MEMO:VAUGHNI } \\
\text { TIERNEY-9 }\end{array}$ & $\mathbf{Y}$ & NA \\
\hline 100 & 1,3 & CONSTANT & $-1.0000000 \mathrm{E}+01$ & $-1.0000000 E+01$ & $-1.0000000 \mathrm{E}+01$ & $-1.0000000 E+08$ & $\log \left(m^{\wedge} 2\right)$ & WP035597 & $\mathbf{Y}$ & N/A \\
\hline 100 & 1,3 & CONSTANT & $-1.0000000 E+01$ & $-1.0000000 E+01$ & $-1.0000000 E+01$ & $-1.0000000 \mathrm{E}+01$ & $\log \left(m^{\wedge} 2\right)$ & WPO40514 & $Y$ & N/A \\
\hline 103 & 1,3 & CONSTANT & $4.0000000=+00$ & $4.0000000 \mathrm{E}+00$ & $4.0000000 E+00$ & $4.0000000 E+00$ & NONE & $\begin{array}{l}\text { MEMO:VAUGHN } \\
\text { TERNEY-9 }\end{array}$ & $\gamma$ & NA \\
\hline 103 & 1,3 & CONSTANT & $4.0000000 \mathrm{E}+00$ & $4.0000000 \mathrm{E}+00$ & $4.0000000 E+\infty 0$ & $4.0000000 E+00$ & NONE & WP035597 & $\mathbf{Y}$ & NA \\
\hline 103 & 1,3 & CONSTANT & $4.0000000 E++00$ & $4.0000000 E+00$ & $4.0000000 E+\infty$ & $4.0000000 \mathrm{E}+\infty 0$ & NONE & WPO40514 & $\mathbf{Y}$ & NA \\
\hline
\end{tabular}


CCA Parameter Listing

\begin{tabular}{|c|c|c|c|c|c|c|c|c|c|c|}
\hline $\begin{array}{c}\text { Parametar in } \\
\text { Database? } \\
\text { (rnN) } \\
\end{array}$ & $\begin{array}{l}\text { th the values } \\
\text { urad in cea } \\
\text { are not the } \\
\text { same ns in } \\
\text { DB, provide } x \\
\text { difference }\end{array}$ & D & Material ID & Materlal Name & $\begin{array}{c}\text { Peramoter } \\
\text { to }\end{array}$ & Parmoneter Name & $\begin{array}{l}\text { PRPID } \\
\text { (WPOA) }\end{array}$ & $\begin{array}{c}\text { Deta Entry } \\
\text { Deto }\end{array}$ & 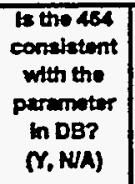 & $\begin{array}{l}\text { Perameter } \\
\text { Catagory } \\
\text { (footrota 1) }\end{array}$ \\
\hline Y & & 104 & CAVITY_2 & $\begin{array}{l}\text { Cavity for Non-waste } \\
\text { Areas }\end{array}$ & SAT_RBRN & Residual Brine Saturation & 31761 & 17-Fob-96 & $\mathbf{Y}$ & 4B \\
\hline$Y$ & & 104 & CAVTY_2 & $\begin{array}{l}\text { Cavity for Non-waste } \\
\text { Areas }\end{array}$ & SAT_RBRN & Residual Brine Saturation & 31761 & $\mid 17$-Feb-96 & $\mathbf{Y}$ & 48 \\
\hline $\mathbf{Y}$ & & 104 & CAVITY_2 & $\begin{array}{l}\text { Cavity for Non-waste } \\
\text { Areas }\end{array}$ & SAT_RBRN & Residual Brine Saturation & 31761 & 17 Fob-96 & $Y$ & $4 B$ \\
\hline$\gamma$ & & 105 & CAVITY_2 & $\begin{array}{l}\text { Cavity for Non-wasto } \\
\text { Aneas }\end{array}$ & SAT_RGAS & Residual Gas Saturation & 31763 & 17-Fob-96 & $\mathbf{Y}$ & 4B \\
\hline $\mathbf{Y}$ & & 105 & CAVITY_2 & $\begin{array}{l}\text { Cavity for Non-waste } \\
\text { Areas }\end{array}$ & SAT_RGAS & Residual Gas Saturation & 31763 & 17-Feb-96 & $\mathbf{Y}$ & 48 \\
\hline $\mathbf{Y}$ & & 105 & CAVITY_2 & $\begin{array}{l}\text { Cavity for Non-wasto } \\
\text { Areas }\end{array}$ & SAT_RGAS & Residual Gas Saturation & 31763 & 17 feb-96 & $\mathbf{Y}$ & $4 B$ \\
\hline$Y$ & 0.03 & 106 & CF252 & Califomium 252 & ATWEIGHT & Atomic Waight in kg/mole & 31828 & 02-Nov-95 & $Y$ & 3 \\
\hline$Y$ & 0.03 & 106 & CF252 & Califomium 252 & ATWEIGHT & Atomic Weight in $\mathrm{kg} / \mathrm{mol}$ & 31828 & 02-Nov-95 & $\mathbf{Y}$ & 3 \\
\hline$Y$ & 0.03 & 106 & CF252 & Celffomium 252 & ATWEIGHT & Atomic Weight in $\mathrm{kg} / \mathrm{mole}$ & 31828 & O2-Nov-95 & $\mathbf{Y}$ & 3 \\
\hline$Y$ & 100 & 107 & CF252 & Californium 252 & HALFLIFE & Halfifife & 31829 & 16-Apr-96 & $\mathbf{Y}$ & 3 \\
\hline$Y$ & 100 & 107 & CF252 & Californium 252 & HALFLIFE & Halfife & 31829 & 16-Apr.96 & $Y$ & 3 \\
\hline$Y$ & 100 & 107 & CF252 & Californium 252 & HALFLIFE & Haiflife & 31829 & 16-Apr-96 & $Y$ & 3 \\
\hline$Y$ & & 108 & CF252 & Calffornium 252 & INVCHD & $\begin{array}{l}\text { Inventory of Contact Handlod } \\
\text { Design }\end{array}$ & $31830 \mathrm{~A}$ & 29-0ct-96 & $\mathbf{Y}$ & 2 \\
\hline $\mathbf{Y}$ & & 108 & CF252 & Californium 252 & INVCHD & $\begin{array}{l}\text { Inventory of Contact Handled } \\
\text { Design }\end{array}$ & $31830 A$ & $29-0 \mathrm{ct}-96$ & $\mathbf{Y}$ & 2 \\
\hline$Y$ & & 108 & CF252 & Calfifornium 252 & INVCHD & $\begin{array}{l}\text { Inventory of Contact Handied } \\
\text { Design }\end{array}$ & $31830 \mathrm{~A}$ & 29-0et-96 & $\mathbf{Y}$ & 2 \\
\hline $\mathbf{Y}$ & & 108 & CF252 & Calfromium 252 & INVCHD & $\begin{array}{l}\text { Inventory of Contact Handied } \\
\text { Design }\end{array}$ & $31830 \mathrm{~A}$ & $29-0 c t-96$ & $\mathbf{Y}$ & 2 \\
\hline $\mathbf{Y}$ & & 109 & CF252 & Californium 252 & IINVRHD & $\begin{array}{l}\text { Inventory of Remote Handled. } \\
\text { Design }\end{array}$ & 31831A & $29-0 \mathrm{~d}-96$ & $\mathbf{Y}$ & 2 \\
\hline $\mathbf{Y}$ & & 109 & CF252 & Californium 252 & |INVRHD & $\begin{array}{l}\text { Inventory of Remote Handled } \\
\text { Design }\end{array}$ & 31831A & 29-0ct-96 & $\mathbf{Y}$ & 2 \\
\hline $\mathbf{Y}$ & & 109 & CF252 & Californium 252 & IINVRHD & $\begin{array}{l}\text { Imventory of Remote Handled } \\
\text { Design }\end{array}$ & $31831 \mathrm{~A}$ & 29-0ct-96 & $\mathbf{Y}$ & 2 \\
\hline $\mathbf{Y}$ & & 109 & CF252 & Californium 252 & INVRHD & $\begin{array}{l}\text { Inventory of Remote Handled } \\
\text { Design }\end{array}$ & 31831A & 29-0ct-96 & $\mathbf{Y}$ & 2 \\
\hline $\mathbf{Y}$ & 0.03 & 110 & CM244 & Curium 244 & ATWEIGHT & Atomic Weight in $\mathrm{kg} / \mathrm{mole}$ & 32331 & 02-Nov-95 & $Y$ & 3 \\
\hline$Y$ & 0.03 & 110 & CM244 & Curium 244 & ATWEIGHT & Atornic Weight in $\mathrm{kg} / \mathrm{mol}$ & 32331 & 02-Nov-95 & $Y$ & 3 \\
\hline$Y$ & 0.03 & 110 & CM244 & Curium 244 & ATWEIGHT & Atomic Waight in kg/mole & 32331 & 02-Nov-95 & $Y$ & 3 \\
\hline$Y$ & 100 & 111 & CM244 & Curium 244 & HALFLIFE & Halfifie & 32495 & 16-Apr-96 & $Y$ & 3 \\
\hline$Y$ & 100 & 111 & CM244 & Curiem 244 & HALFLIFE & Haltifito & 32495 & 16-Apr-96 & $Y$ & 3 \\
\hline $\mathbf{Y}$ & 100 & 119 & CM244 & Curium 244 & HALFLIFE & Halfifife & 32495 & $16-A p r-96$ & $Y$ & 3 \\
\hline $\mathbf{Y}$ & & 112 & CM244 & Curium 244 & INVCHD & $\begin{array}{l}\text { Inventory of Contact Handled } \\
\text { Design }\end{array}$ & $32496 \mathrm{~A}$ & $29-0 c t-96$ & $\mathbf{Y}$ & 2 \\
\hline $\mathbf{Y}$ & & 112 & СM244 & Curium 244 & INVCHD & $\begin{array}{l}\text { Inventory of Contact Handled } \\
\text { Design }\end{array}$ & $32496 \mathrm{~A}$ & 29-0ct-96 & $\mathbf{Y}$ & 2 \\
\hline $\mathbf{Y}$ & & 112 & CM244 & Curium 244 & INVCHD & $\begin{array}{l}\text { Inventory of Contact Handled } \\
\text { Design }\end{array}$ & $32496 A$ & 29-0ct.96 & $=Y$ & 2 \\
\hline$Y$ & & 112 & CM244 & Curium 244 & INVCHD & $\begin{array}{l}\text { Imventory of Contact Handled } \\
\text { Desıgn }\end{array}$ & $32496 \mathrm{~A}$ & $29-0 c t-96$ & $\mathbf{Y}$ & 2 \\
\hline $\mathbf{Y}$ & & 113 & CM244 & Curium 244 & INVRHD & $\begin{array}{l}\text { Inventory of Remote Handled } \\
\text { Design }\end{array}$ & $32497 \mathrm{~A}$ & $29-0 c t-96$ & $\mathbf{Y}$ & 2 \\
\hline$Y$ & & 113 & CM244 & Curiem 244 & INVRHD & $\begin{array}{l}\text { Inventory of Remote Handled } \\
\text { Design }\end{array}$ & $32497 A$ & 29-0ct-96 & $\mathbf{Y}$ & 2 \\
\hline $\mathbf{Y}$ & & 113 & CM244 & Curium 244 & INVRHD & $\begin{array}{l}\text { Imventory of Remote Handled } \\
\text { Design }\end{array}$ & $32497 A$ & 29-0ct-96 & $\mathbf{Y}$ & 2 \\
\hline$Y$ & & 113 & CM244 & Curium 244 & INVRHD & $\begin{array}{l}\text { Inventory of Remote Handled } \\
\text { Design }\end{array}$ & $32497 \mathrm{~A}$ & 29-0ct-96 & $\mathbf{Y}$ & 2 \\
\hline$Y$ & 100 & 115 & . CM248 & Curium 248 & HALFLIFE & Halfifie & 32499 & 16-Apr-96 & $Y$ & 3 \\
\hline$Y$ & 100 & 115 & CM248 & Curium 248 & HALFLIFE & Hatfife & 32499 & 16-Apr-96 & $\mathbf{Y}$ & 3 \\
\hline$Y$ & 100 & 115 & CM248 & Curium 248 & HALFLIFE & Halfifie & 32499 & 16-Apr-96 & $Y$ & 3 \\
\hline$Y$ & 0.07 & 116 & CS137 & Cesium 137 & ATWEIGHT & Atomic Weight in $\mathrm{kg} / \mathrm{mole}$ & 32332 & 02-Nov-95 & $Y$ & 3 \\
\hline$Y$ & 0.07 & 116 & CS137 & Cesium 137 & ATWEIGHT & Atomic Weight in $\mathrm{kg} / \mathrm{mol}$ & 32332 & 02-Nov-95 & $Y$ & 3 \\
\hline$Y$ & 0.07 & 116 & CS137 & Cesium 137 & ATWEIGHT & Atomic Weight in kg/moto & 32332 & 02-Nov-95 & $Y$ & 3 \\
\hline$Y$ & 100 & 117 & CS137 & Cesium 137 & HALFLIFE & Halnifo & 32682 & 16-Apr-96 & $Y$ & 3 \\
\hline$Y$ & 100 & 197 & Cs137 & Cesium 137 & HALFLIFE & Halnifo & 32682 & 16-Apr-96 & $Y$ & 3 \\
\hline$Y$ & 100 & 117 & CS137 & Cosium 137 & HALFLIFE & Hatlife & 32682 & 16-Apr-96 & $Y$ & 3 \\
\hline
\end{tabular}


CCA Parameter Listing

\begin{tabular}{|c|c|c|c|c|c|c|c|c|c|c|}
\hline ID & 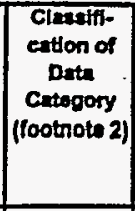 & $\begin{array}{l}\text { Distribution } \\
\text { Type }\end{array}$ & Man & Medien & ${ }^{\circ}$ & Mncroum & Units & Reference ID & $\begin{array}{l}\text { Was the dinta } \\
\text { doreloped } \\
\text { under an } \\
\text { NoA-1 } \\
\text { Proprem? }\end{array}$ & $\begin{array}{l}\text { Which } \\
\text { methods } \\
\text { were used to } \\
\text { quallfy } \\
\text { oxdating } \\
\text { data? } \\
\text { (footnots 3) }\end{array}$ \\
\hline 104 & 1,3 & CONSTANT & $0.0000000 E+\infty 0$ & $0.0000000 E+00$ & $0.0000000 E+\infty 0$ & $0.0000000 \mathrm{E}+\infty$ & NONE & $\begin{array}{c}\text { MEMO:VAUGHN } \\
\text { TIERNEY-9 }\end{array}$ & $\mathbf{Y}$ & N/A \\
\hline 104 & 1.3 & CONSTANT & $0.0000000 E+00$ & $0.0000000 E+00$ & $0.0000000 \mathrm{E}+00$ & $0.0000000 E+\infty 0$ & NONE & WP035597 & $\mathbf{Y}$ & NA \\
\hline 104 & 1,3 & CONSTANT & $0.0000000 \mathrm{E}+\infty$ & $0.0000000 E+00$ & $0.0000000 \mathrm{E}+00$ & $0.00000000 E+00$ & NONE & WPO40514 & $\mathbf{Y}$ & NA \\
\hline 105 & 1,3 & CONSTANT & $0.0000000 E+00$ & $0.0000000 \mathrm{E}+\infty 0$ & $0.0000000 \mathrm{E}+\infty 0$ & $0.0000000 E+\infty 0$ & NONE & $\begin{array}{l}\text { MEMO:VAUGHN } \\
\text { TIERNEY-9 }\end{array}$ & $\mathbf{Y}$ & N/A \\
\hline 105 & 1,3 & CONSTANT & $0.0000000 E+00$ & $0.0000000 \mathrm{E}+00$ & $0.0000000 E+00$ & $0.0000000 E+\infty 0$ & NONE & WP035597 & $\mathbf{Y}$ & NA \\
\hline 105 & 1,3 & CONSTANT. & $0.0000000 E+00$ & $0.0000000 E+\infty$ & $0.0000000 E+00$ & $0.0000000 E+\infty 0$ & NONE & WPO40514 & $Y$ & NA \\
\hline 106 & 4 & CONSTANT & $2.5208200 \mathrm{E}-01$ & $25203200 \mathrm{E}-01$ & $2.5208200 E-01$ & $2.5208200 \mathrm{E}-01$ & $\mathrm{~kg} / \mathrm{mole}$ & CRC- 72 & $Y$ & N/A \\
\hline 106 & 4 & CONSTANT & $2.5208200 \mathrm{E}-01$ & 2.5208200E-01 & $2.5208200 E-01$ & $2.5208200 E-01$ & $\mathrm{~kg} / \mathrm{molo}$ & WP036298 & $\mathbf{Y}$ & $N / A$ \\
\hline 106 & 4 & CONSTANT & $2.5208200 \mathrm{E}-01$ & 25208200E-01 & $25208200 E-01$ & 2.5208200E-01 & $\mathrm{kg}$ /mole & WP036301 & $Y$ & NA \\
\hline 107 & 4 & CONSTANT & $8.3250000 E+07$ & $8.3250000 E+07$ & $8.3250000 E+07$ & $8.3250000 E+07$ & 5 & WPO36301 & $Y$ & NA \\
\hline 107 & 4 & CONSTANT & $8.3250000 E+07$ & $8.3250000 \mathrm{E}+07$ & $-8.3250000 E+07$ & $.8 .3250000 \mathrm{E}+07$ & 8 & WPO37404 & $\bar{Y}$ & NA \\
\hline 107 & 4 & CONSTANT & $8.3250000 E+07$ & $8.3250000 E+07$ & $8.3250000 \mathrm{E}+07$ & $8.3250000 \mathrm{E}+07$ & 5 & WPO40434 & $Y$ & NA \\
\hline 108 & 2 & CONSTANT & $1.1200000 E-04$ & $1.1200000 \mathrm{E}-04$ & $1.1200000 \mathrm{E}-04$ & $1.1200000 E-04$ & $\mathbf{C i}$ & WP037061 & Y & N/A \\
\hline 108 & 2 & CONSTANT & $1.1200000 E-04$ & $1.1200000 \mathrm{E}-04$ & $1.1200000 E-04$ & $1.1200000 E-04$ & $\mathrm{Ci}$ & WP039260 & $\mathbf{Y}$ & N/A \\
\hline 108 & 2 & CONSTANT & $1.1200000 E-04$ & $1.1200000 E-04$ & $1.1200000 \mathrm{E}-04$ & $1.1200000 E-04$ & $\mathbf{C i}$ & WPO\$1559 & $\mathbf{Y}$ & $N / A$ \\
\hline 108 & 2 & CONSTANT & 1.1200000E-04 & $1.1200000 E-04$ & 1.1200000E-04 & $1.1200000 E-04$ & $\mathbf{C i}$ & WPO41560 & $Y$ & NA \\
\hline 109 & 2 & CONSTANT & $5,9600000 E-05$ & $5.9600000 \mathrm{E}-05$ & $5.9600000 \mathrm{E}-0.5$ & $5.9600000 E-05$ & $\mathrm{Ci}$ & WP037061 & $Y$ & $N A$ \\
\hline 109 & 2 & CONSTANT & $5.9600000 E-05$ & $5.9600000 E-05$ & $5.9600000 E-05$ & $5.9600000 E-05$ & $\mathrm{Ci}$ & WP039260 & $\mathbf{Y}$ & NA \\
\hline 109 & 2 & CONSTANT & $5.9600000 E-05$ & $5.9600000 E-05$ & $5.9600000 \mathrm{E}-0.05$ & $5.9600000 E-05$ & Ci & WP041559 & $Y$ & NA \\
\hline 109 & 2 & CONSTANT & $5.9600000 E-05$ & $5.9600000 E-05$ & $5.9600000 E-05$ & $5.9600000 E-05$ & $\mathrm{Ci}$ & WPO41560 & $Y$ & N/A \\
\hline 110 & 4 & CONSTANT & $2.4405300 E-01$ & $2.4406300 \mathrm{E}-01$ & $2.4405300 \mathrm{E}-01$ & 2.4405300E-01 & $\mathrm{kg}$ mole & CRC-72 & $Y$ & $N / A$ \\
\hline 110 & 4 & CONSTANT & $2.4406300 E-01$ & $2.4406300 \mathrm{E}-01$ & $2.4405300 E-01$ & $2.4406300 \mathrm{E}-01$ & $\mathrm{~kg} / \mathrm{mole}$ & WPO36298 & $Y$ & N/A \\
\hline 110 & 4 & CONSTANT & $24406300 E-01$ & $2.4406300 \mathrm{E}-01$ & $2.4405300 E-01$ & $2.4406300 \mathrm{E}-01$ & $\mathrm{~kg} / \mathrm{mole}$ & WP036301 & $Y$ & N/A \\
\hline 111 & 4 & CONSTANT & $5.7150000 \mathrm{E}+08$ & $5.7150000 E+08$ & $5.7150000 E+08$ & $5.7150000 E+08$ & $s$ & WP036301 & $Y$ & NA \\
\hline 111 & 4 & CONSTANT & $5.7150000 E+08$ & $5.7150000 \mathrm{E}+08$ & $5.7150000 \mathrm{E}+08$ & $5.7150000 \mathrm{E}+08$ & $s$ & WP037404 & $Y$ & NA \\
\hline 111 & 4 & CONSTANT & $5.7150000 \mathrm{E}+08$ & $5.7150000 E+08$ & $5.7150000 E+08$ & $5.7150000 E+08$ & s & WPO40434 & $Y$ & N/A \\
\hline 112 & 2 & CONSTANT & $7.3600000 E+03$ & $7.3600000 E+03$ & $7.3600000 E+03$ & $7.3600000 E+03$ & $\mathbf{C i}$ & WP037061 & $Y$ & NA \\
\hline 112 & 2 & CONSTANT & $7.3600000 E+03$ & $7.3600000 E+03$ & $7.3600000 E+03$ & $7.3600000 E+03$ & $\mathrm{Ci}$ & WP039260 & $\mathbf{Y}$ & NA \\
\hline 112 & 2 & CONSTANT & $7.3600000 E+03$ & $7.3600000 E+03$ & $7.3600000 E+03$ & $7.3600000 \mathrm{E}+03$ & $\mathrm{Ci}$ & WP041559 & $-y$ & NA \\
\hline 112 & 2 & CONSTANT & $7.3600000 E+03$ & $7.3600000 E+03$ & $7.3600000 \mathrm{E}+03$ & $7.3600000 \mathrm{E}+03$ & $\mathrm{Ci}$ & WPO41560 & $\mathbf{Y}$ & NA \\
\hline 113 & 2 & CONSTANT & $7.3600000 \mathrm{E}+01$ & $7.3600000 E+01$ & $7.3600000 \mathrm{E}+01$ & $7.3600000 E+01$ & $\mathbf{C i}$ & WP037061 & $\mathbf{Y}$ & NA \\
\hline 113 & 2 & CONSTANT & $7.3600000 E+01$ & $7.3600000 \mathrm{E}+01$ & $7.3600000 E+01$ & $7.3600000 E+01$ & $\mathrm{Ci}$ & WP039260 & $\mathbf{Y}$ & N/A \\
\hline 113 & 2 & CONSTANT & $7.3600000 \mathrm{E}+01$ & $7.3600000 E+01$ & $7.36000000+01$ & $7.3600000 E+01$ & $\mathrm{Ci}$ & WPO41559 & $\mathbf{Y}$ & NA \\
\hline 113 & 2 & CONSTANT & $7.3600000 E+01$ & $7.3600000 E+01$ & $7.3600000 E+01$ & $7.3600000 E+01$ & $\mathbf{C i}$ & WPO41560 & $\mathbf{Y}$ & N/A \\
\hline 115 & 4 & CONSTANT & $1.0700000 \mathrm{E}+13$ & $1.0700000 \mathrm{E}+13$ & $1.0700000 \mathrm{E}+13$ & $1.0700000 \mathrm{E}+13$ & $s$ & WP035301 & $Y$ & NA \\
\hline 115 & 4 & CONSTANT & $1.0700000 \mathrm{E}+13$ & $1.0700000 \mathrm{E}+13$ & $1.0700000 \mathrm{E}+13$ & $1.0700000 E+13$ & 8 & WPO37404 & $Y$ & N/A \\
\hline 115 & 4 & CONSTANT & $1.0700000 \mathrm{E}+13$ & $1.0700000 \mathrm{E}+13$ & $1.0700000 \mathrm{E}+13$ & $1.0700000 \mathrm{E}+13$ & s & WPO40434 & $Y$ & NA \\
\hline 116 & 4 & CONSTANT & $1.3690700 \mathrm{E}-01$ & $1.3690700 E-01$ & 1.3690700E-01 & $1.3690700 \mathrm{E}-01$ & $\mathrm{~kg} / \mathrm{mole}$ & CRC-72 & $Y$ & N/A \\
\hline 116 & 4 & CONSTANT & $1.3690700 E-01$ & $1.3690700 E-01$ & 1.3690700E-01 & $1.3690700 E-01$ & $\mathrm{~kg} / \mathrm{mole}$ & WP036298 & $Y$ & N/A \\
\hline 116 & 4 & CONSTANT & 1.3690700E-01 & 1.3690700E-01 & 1.3690700E-01 & 1.3690700E-01 & $\mathrm{kg} / \mathrm{mole}$ & WPO36301 & $\bar{Y}$ & $\mathrm{NA}$ \\
\hline 117 & 4 & CONSTANT & $9.4670000 E+08$ & $9.4670000 \mathrm{E}+08$ & $9.4670000 E+08$ & $9.4570000 \mathrm{E}+08$ & 8 & WP036301 & $\bar{Y}$ & N/A \\
\hline 117 & 4 & CONSTANT & $9.4670000 \mathrm{E}+08$ & $9.4670000 E+08$ & $9.4670000 E+08$ & $9.4670000 \mathrm{E}+08$ & 5 & WP037404 & $Y$ & NA \\
\hline 117 & 4 & CONSTANT & $9.4670000 E+08$ & $9.4670000 E+08$ & $9.4670000 \equiv+08$ & $9.4670000 E+08$ & s & WPO40434 & $Y$ & NA \\
\hline
\end{tabular}


CCA Parameter Listing

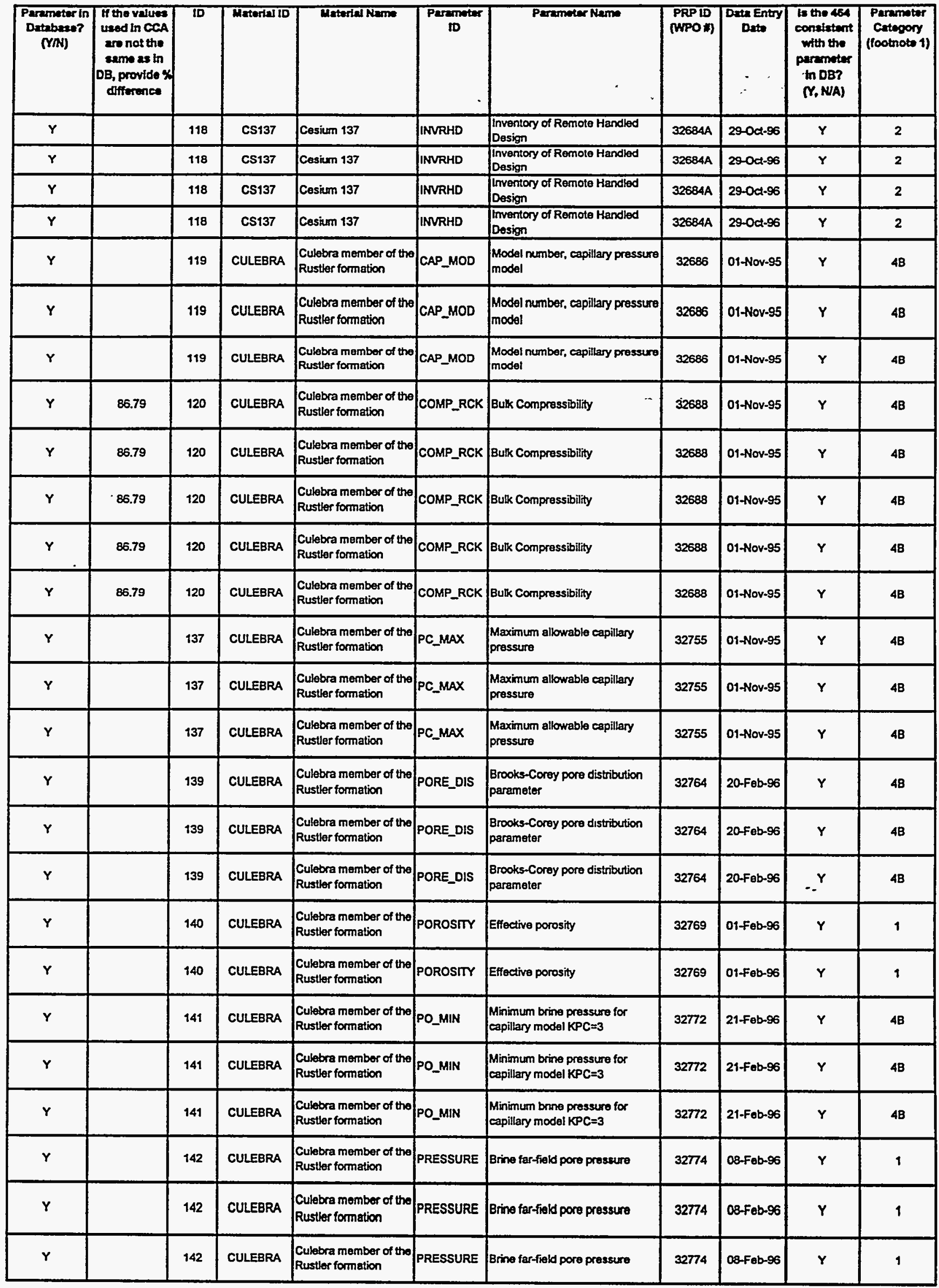


CCA Parameter Listing

\begin{tabular}{|c|c|c|c|c|c|c|c|c|c|c|}
\hline ID & $\begin{array}{c}\text { Clessifi- } \\
\text { cation of } \\
\text { Data } \\
\text { Category } \\
\text { (lootnote 2) }\end{array}$ & $\begin{array}{l}\text { Distribution } \\
\text { Type }\end{array}$ & thesn & - Modian & . & $\begin{array}{l}\text { Modmum } \\
\text { i }\end{array}$ & Units & Reference ID & $\begin{array}{l}\text { Wes the data } \\
\text { dernloped } \\
\text { under an } \\
\text { NOA-1 } \\
\text { Progrem? }\end{array}$ & $\begin{array}{c}\text { Which } \\
\text { mothods } \\
\text { more used to } \\
\text { qualify } \\
\text { 1. exdsting } \\
\text { diata? } \\
\text { (tootnote 3) }\end{array}$ \\
\hline 118 & 2 & CONSTANT & $8.9800000 E+04$ & $8.9800000 E+04$ & $8.5800000 E+04$ & $8.9800000 E+04$ & $\mathbf{C i}$ & WP037051 & $\mathbf{Y}$ & NA \\
\hline 118 & 2 & CONSTANT & $8.9800000 \mathrm{E}+04$ & $8.9800000 E+04$ & $8.9800000 E+04$ & $8.9800000 E+04$ & $\mathbf{C i}$ & WPO39260 & $\mathbf{Y}$ & N/A \\
\hline 118 & 2 & CONSTANT & $6.9800000 E+04$ & $8.9800000 E+04$ & $8.9800000 E+04$ & $8.9800000 E+04$ & $\mathbf{C i}$ & WPO41559 & $\mathbf{Y}$ & NA \\
\hline 118 & 2 & CONSTANT & $8.9800000 \mathrm{E}+04$ & $8.9800000 E+04$ & $8.9800000 E+04$ & $8.9800000 E+04$ & $\mathbf{C i}$ & WPO41560 & $\mathbf{Y}$ & NA \\
\hline 119 & $1,3,4$ & CONSTANT & $2.0000000 E+\infty 0$ & $2.0000000 E+00$ & $2.0000000 E+\infty$ & $2.0000000 E+\infty 0$ & NONE & $\begin{array}{c}\text { WP031041 } \\
i_{2} \\
\end{array}$ & $\mathbf{Y}$ & N/A \\
\hline 119 & $1,3,4$ & CONSTANT & $2.0000000 E+00$ & $2.0000000 E+00$ & $2.0000000 E+\infty 0$ & $2.0000000 E+00$ & NONE & WP035597 & $\mathbf{Y}$ & N/A \\
\hline 119 & $1,3,4$ & CONSTANT & $2.0000000 E+C 0$ & $2.0000000 E+00$ & $2.0000000 E+00$ & $2.0000000 E+\infty 0$ & NONE & WPO40514 & $\mathbf{Y}$ & $N A$ \\
\hline 120 & 4 & CONSTANT & $1.0000000 E-10$ & $1.0000000 E-10$ & $1.0000000 \mathrm{E}-10$ & $1.0000000 \mathrm{E}-10$ & Pan-1 & FREEZE-1 & Y\&N & 1 \\
\hline 120 & 4 & CONSTANT & $1.0000000 \mathrm{E}-10$ & $1.0000000 \mathrm{E}-10$ & $1.0000000 \mathrm{E}-10$ & $1.0000000 \mathrm{E}-10$ & $P a^{n}-1$ & WP035597 & Y\&N & 1 \\
\hline 120 & 4 & CONSTANT & $1.0000000 E-10$ & $1.0000000 \mathrm{E}-10$ & $1.0000000 \mathrm{E}-10$ & $1.0000000 E-10$ & $P a^{n}-1$ & WP038568 & Y\&N & 1 \\
\hline 120 & 4 & CONSTANT & 1.0000000 E- 10 & $1.0000000 E-10$ & $1.0000000 E-10$ & $1.0000000 \mathrm{E}-10$ & $P a^{n}-1$ & WPO40434 & Y\&N & 1 \\
\hline 120 & 4 & CONSTANT & $1.0000000 E-10$ & $1.0000000 E-10$ & $1.0000000 \mathrm{E}-10$ & $1.0000000 E-10$ & $P a^{n-1}$ & WPO40514 & Y\&N & 1 \\
\hline 137 & 3,5 & CONSTANT & $1.0000000 E+08$ & $1.0000000 E+08$ & $1.0000000 E+08$ & $1.0000000 E+08$ & $\mathbf{P a}$ & WP031041 & $\mathbf{Y}$ & NAA \\
\hline 137 & 3,5 & CONSTANT & $1.0000000 E+08$ & $1.0000000 E+08$ & $1.0000000 E+08$ & $1.0000000 \mathrm{E}+08$ & $\mathrm{~Pa}$ & WP035597 & $\mathbf{Y}$ & NA \\
\hline 137 & 3,5 & CONSTANT & $1.0000000 E+08$ & $1.0000000 E+08$ & $1.0000000 E+08$ & $1.0000000 \mathrm{E}+08$ & $\mathbf{P a}$ & WP040514 & $\boldsymbol{Y}$ & N/A \\
\hline 139 & $1,3,4$ & CONSTANT & $6.4360000 E-01$ & $6.4360000 E-01$ & $6.4360000 E-01$ & $6.4360000 E-01$ & NONE & WP031041 & $\mathbf{Y}$ & N/A \\
\hline 139 & $1,3,4$ & CONSTANT & $6.4360000 E-01$ & $6.4360000 E-01$ & $6.4360000 E-01$ & $6.4360000 E-01$ & NONE & WP035597 & $\mathbf{Y}$ & NAA \\
\hline 139 & $1,3,4$ & CONSTANT & $6.4360000 E-01$ & $6.4360000 \mathrm{E}-01$ & $6.4360000 \mathrm{E}-01$ & $6.4360000 E-01$ & NONE & WPO40514 & $-Y$ & NAA \\
\hline 140 & 1 & CONSTANT & $1,5100000 E-01$ & $1.5100000 E-01$ & $1.5100000 E-01$ & $1.5100000 E-01$ & NONE & WP030608 & Y\&N & 1 \\
\hline 140 & 1 & CONSTANT & $1.5100000 \mathrm{E}-01$ & $1.5100000 \mathrm{E}-01$ & $1.5100000 \mathrm{E}-01$ & $1.5100000 E-01$ & NONE & WP035597 & YRN & 1 \\
\hline 141 & 3,5 & CONSTANT & $1.0132500 E+05$ & $1.0132500 E+05$ & $1.0132500 E+05$ & $1.0132500 E+05$ & $\mathbf{P a}$ & WP035597 & $\mathbf{Y}$ & NA \\
\hline 141 & 3,5 & CONSTANT & $1.0132500 E+05$ & $1.0132500 E+0.5$ & $1.0132500 E+05$ & $1.0132500 \mathrm{E}+05$ & $\mathrm{~Pa}$ & WP036051 & $\mathbf{Y}$ & N/A \\
\hline 141 & 3,5 & CONSTANT & $1.0132500 \mathrm{E}+05$ & $1.0132500 E+05$ & $1.0132500 E+05$ & $1.0132500 E+05$ & $\mathrm{~Pa}$ & WPO40514 & $\mathbf{Y}$ & NA \\
\hline 142 & 1 & CONSTANT & $8.2200000 E+05$ & $8.2200000 \mathrm{E}+0.5$ & $8.2200000 E+05$ & $8.2200000 E+05$ & $\mathrm{~Pa}$ & WPO30713 & $\mathbf{N}$ & 1 \\
\hline 142 & 1 & CONSTANT & $8.2200000 E+05$ & $8.2200000 E+05$ & $8.2200000 E+05$ & $8.2200000 E+05$ & $\mathbf{P a}$ & WP032290 & $\mathbf{N}$ & 1 \\
\hline 142 & 1 & CONSTANT & $8.2200000 \mathrm{E}+05$ & $8.2200000 E+05$ & $8.2200000 E+05$ & $8.2200000 E+05$ & $\mathbf{P a}$ & WP035597 & N & 1 \\
\hline
\end{tabular}


CCA Parameter Listing

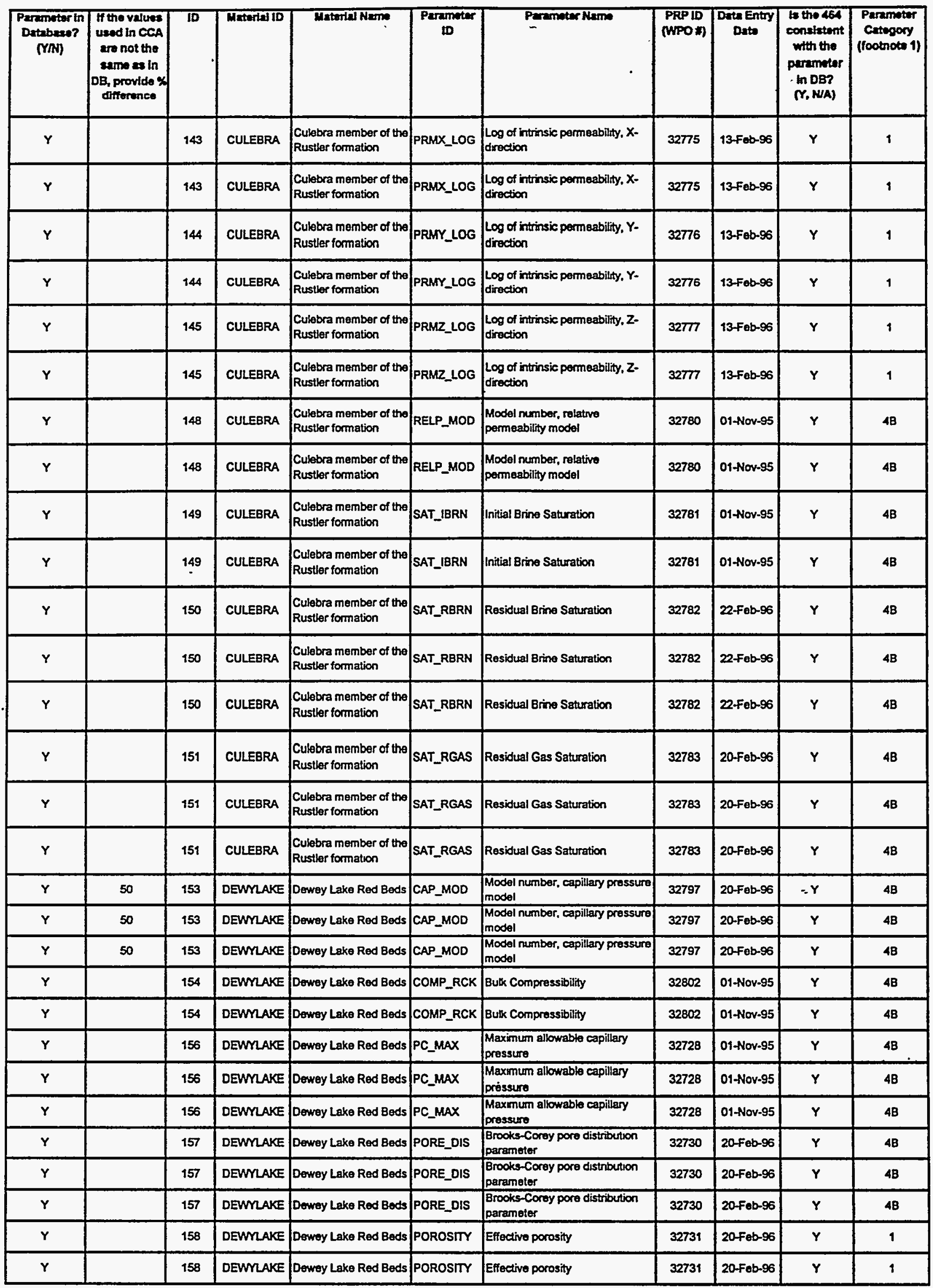


CCA Parameter Listing

\begin{tabular}{|c|c|c|c|c|c|c|c|c|c|c|}
\hline 10 & $\begin{array}{c}\text { Clasistr- } \\
\text { casion of } \\
\text { Dath } \\
\text { Cattegory } \\
\text { (footnoto 2) }\end{array}$ & $\begin{array}{l}\text { Distribusion } \\
\text { Type }\end{array}$ & man & modien & Mlntrmum & Maxdrimers & Unt: & Rotermea id & $\begin{array}{l}\text { Was the detal } \\
\text { doweloped } \\
\text { under an } \\
\text { NaA-1 } \\
\text { Program? }\end{array}$ & $\begin{array}{l}\text { Which } \\
\text { mothods } \\
\text { more used to } \\
\text { quality } \\
\text { oxtating } \\
\text { data? } \\
\text { (footnot J) }\end{array}$ \\
\hline 143 & 1 & CONSTANT & $-1.3678000 E+01$ & $-1.3678000 E+01$ & $-1.3678000 E+01$ & $-1.3678000 E+01$ & $\log \left(m^{\wedge} 2\right)$ & WP035266 & N & 1 \\
\hline 143 & 1 & CONSTANT & $-1.3678000 E+01$ & $-1.3678000 \mathrm{E}+01$ & $-1.3678000 \mathrm{E}+01$ & $-1.3678000 E+01$ & $\log \left(m^{\wedge} 2\right)$ & WP035597 & N & 1 \\
\hline 144 & 1 & CONSTANT & $-1.3678000 E+01$ & $-1.3678000 E+01$ & $-1.3678000 E+01$ & $-1.3678000 E+01$ & $\log \left(m^{\wedge} 2\right)$ & WP035265 & $\mathbf{N}$ & 1 \\
\hline 144 & 1 & CONSTANT & $-1.3678000 E+01$ & $-1.3678000 E+01$ & $-1.3678000 E+01$ & $-1.3678000 E+01$ & $\log \left(m^{\wedge} 2\right)$ & WP035597 & $\mathbf{N}$ & 1 \\
\hline 145 & 1 & CONSTANT & $-1.3678000 E+01$ & $-1.3678000 E+01$ & $-1.3678000 E+01$ & $-1.3678000 E+01$ & $\log \left(m^{\wedge} 2\right)$ & WPO35265 & N & 1 \\
\hline 145 & 1 & CONSTANT & $-1.3678000 E+01$ & $-1.3678000 E+01$ & $-1.3678000 E+01$ & $-1.3578000 E+01$ & $\log \left(m^{\wedge} 2\right)$ & WP035597 & N & 1 \\
\hline 148 & $1,3,4$ & CONSTANT & $4.0000000 \mathrm{E}+00$ & $4.0000000 E+\infty 0$ & $4.0000000 E+00$ & $4.0000000 \mathrm{E}+\infty 0$ & NONE & WP038568 & $\mathbf{Y}$ & N/A \\
\hline 148 & $1,3,4$ & CONSTANT & 4.0000000E +00 & $4.0000000 E+\infty 0$ & $4.0000000 \mathrm{E}+\infty 0$ & $4.0000000 \mathrm{E}+00$ & NONE & WP040514 & $Y$ & NA \\
\hline 149 & $1,3,4$ & CONSTANT & $1.0000000 E+\infty 0$ & $1.0000000 E+\infty 0$ & $1.0000000 E+\infty$ & $1.0000000 E+\infty 0$ & NONE & WPO39568 & $\mathbf{Y}$ & NA \\
\hline 149 & $1,3,4$ & CONSTANT & $1.0000000 E+00$ & $1.0000000 E+\infty 0$ & $1.0000000 E+\infty 0$ & $1.0000000 E+\infty 0$ & NONE & WP040514 & $\mathbf{Y}$ & NA \\
\hline 150 & $1,3,4$ & CONSTANT & $8.3630000 E-02$ & $8.3630000 E-02$ & $8.3630000 E-02$ & $8.3630000 E-02$ & NONE & WP031041 & $\mathbf{Y}$ & N/A \\
\hline 150 & $1,3,4$ & CONSTANT & $8.3630000 E-02$ & $8.3630000 E-02$ & $8.3630000 E-02$ & $8.3630000 E-02$ & NONE & WP035597 & $Y$ & NA \\
\hline 150 & $1,3,4$ & CONSTANT & $8.3630000 \mathrm{E}-02$ & $8.3630000 E-02$ & $8.3630000 E-02$ & $8.3630000 E-02$ & NONE & WP040514 & $\mathbf{Y}$ & NA \\
\hline 151 & $1,3,4$ & CONSTANT & $7.7110000 E-02$ & $7.7110000 E-02$ & $7.7190000 \mathrm{E}-02$ & $7.7110000 E-02$ & NONE & WP031041 & $Y$ & N/A \\
\hline 151 & $1,3,4$ & CONSTANT & $7.7110000 \mathrm{E}-02$ & $7.7110000 \mathrm{E}-02$ & $7.7110000 \mathrm{E}-02$ & $7.7110000 \mathrm{E}-02$ & NONE & WP035597 & $\mathbf{Y}$ & N/A \\
\hline 151 & $1,3,4$ & CONSTANT & $7.7110000 \mathrm{E}-02$ & $7.7110000 E-02$ & $7.7110000 E-02$ & $7.7110000 \mathrm{E}-02$ & NONE & WPO40514 & $Y$ & N/A \\
\hline 953 & $1,3,4$ & CONSTANT & $2.0000000 E+\infty 0$ & $2.0000000 E+\infty 0$ & $2.0000000 E+\infty$ & $2.0000000 E+\infty 0$ & NONE & WP031041 & -Y & N/A \\
\hline 153 & $1,3,4$ & CONSTANT & $2.0000000 E+\infty$ & $2.0000000 E+\infty 0$ & $2.0000000 E+\infty 0$ & $2.0000000 E+\infty 0$ & NONE & WP035597 & $Y$ & N/A \\
\hline 153 & $1,3,4$ & CONSTANT & $2.0000000 E+00$ & $2.0000000 E+\infty 0$ & $2.0000000 E+\infty 0$ & $2.0000000 E+00$ & NONE & WPO40514 & $Y$ & NA \\
\hline 154 & $1,3,4$ & CONSTANT & $1.0000000 \mathrm{E}-08$ & $1.0000000 E-08$ & $1.0000000 E-08$ & $1.0000000 E-08$ & $P a^{n}-1$ & WP038568 & $\mathbf{Y}$ & NA \\
\hline 154 & $1,3,4$ & CONSTANT & $1.0000000 \mathrm{E}-08$ & $1.0000000 E-08$ & $1.0000000 E-08$ & $1.0000000 E-08$ & $P a^{a}=1$ & WP040514 & $Y$ & N/A \\
\hline 156 & 3,5 & CONSTANT & $1.0000000 \mathrm{E}+08$ & $1.0000000 E+08$ & $1.0000000 E+08$ & $1.0000000 E+08$ & $\mathbf{P a}$ & WP031041 & $\mathbf{Y}$ & N/A \\
\hline 156 & 3,5 & CONSTANT & $1.0000000 \mathrm{E}+08$ & $1.0000000 E+08$ & $1.0000000 E+08$ & $1.0000000 E+08$ & $\mathbf{P a}$ & WP035597 & $\mathbf{Y}$ & NA \\
\hline 156 & 3,5 & CONSTANT & $1.0000000 \mathrm{E}+08$ & $1.0000000 E+08$ & $1.0000000 E+08$ & 1.0000000E+08 & $\mathrm{Pa}$ & WP040514 & $Y$ & NA \\
\hline 157 & $1,3,4$ & CONSTANT & $6.4360000 E-01$ & $6.4360000 E-01$ & $6.4360000 E-01$ & $6.4360000 \mathrm{E}-01$ & NONE & WP031041 & $Y$ & N/A \\
\hline 157 & $1,3,4$ & CONSTANT & $6.4360000 E-01$ & $6.4360000 E-01$ & $6.4360000 E-01$ & $6.4360000 \mathrm{E}-01$ & NONE & WP035597 & $Y$ & NA \\
\hline 157 & $1,3,4$ & CONSTANT & $6.4360000 E-01$ & $6.4360000 E-01$ & $6.4360000 E-01$ & 6.4360000E-01 & NONE & WP040514 & $\mathbf{Y}$ & N/A \\
\hline 158 & 1 & STUDENT & $1.4300000 E-01$ & $1.4300000 E-01$ & $3.5000000 E-02$ & $2.4800000 E-01$ & NONE & WP030608 & $\gamma$ & N/A \\
\hline 158 & 1 & STUDENT & $1.4300000 E-01$ & $1.4300000 \mathrm{E}-01$ & $3.5000000 \mathrm{E}-02$ & $2.4800000 \mathrm{E}-01$ & NONE & WP035597 & $\mathbf{Y}$ & N/A \\
\hline
\end{tabular}


CCA Parameter Listing

\begin{tabular}{|c|c|c|c|c|c|c|c|c|c|c|}
\hline $\begin{array}{c}\text { Parametor in } \\
\text { Databuce? } \\
\text { (rnN) } \\
-\end{array}$ & 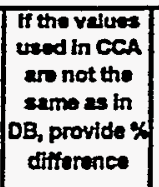 & ID & Meterialio & Matarial Neme & $\begin{array}{c}\text { Paramoter } \\
\text { to }\end{array}$ & Parameter Kamo & $\begin{array}{l}\text { PRPID } \\
\text { (MPO : })\end{array}$ & $\begin{array}{c}\text { Date Entry } \\
\text { Dato }\end{array}$ & $\begin{array}{l}\text { Is the AGA } \\
\text { conalstent } \\
\text { wth the } \\
\text { parameter } \\
\text { in DB? } \\
(Y, N A)\end{array}$ & $\begin{array}{l}\text { Parameter } \\
\text { Catiogory } \\
\text { (footnote 1) }\end{array}$ \\
\hline$Y$ & & 158 & DEWMLAKE & Dowey Lake Rod Bads & POROSTY & Effective porosity & 32731 & 20-Fob-96 & $\mathbf{Y}$ & 1 \\
\hline $\mathbf{Y}$ & & 159 & DEWMLAKE & Dowroy Lake Red Bods & PO_MIN & $\begin{array}{l}\text { Minamum brene pressure for } \\
\text { capillary model KPC=3 }\end{array}$ & 32732 & 21Fob-96 & $\boldsymbol{\gamma}$ & AB \\
\hline$Y$ & & 159 & DEWMLAKE & Dewrey Lake Red Beds & PO_MIN & $\begin{array}{l}\text { Minimum brine pressure for } \\
\text { capillary model KPC=3 }\end{array}$ & 32732 & 21-Fob-96 & $\mathbf{Y}$ & $A B$ \\
\hline Y & & 159 & DEWYLAKE & Dewey Lake Red Beds & PO_MIN & $\begin{array}{l}\text { Minimum brine pressure for } \\
\text { capillary model KPC=3 }\end{array}$ & 32732 & $21+a b-96$ & $\mathbf{Y}$ & 4B \\
\hline$Y$ & & 160 & DEWYLAKE & Dowry Lake Rod Bods & PRESSURE & Brine far-field pore pressure & 32733 & osfreb-96 & Y & 1 \\
\hline$Y$ & ' & 160 & DEWMLAKE & Dewey Lake Rod Bods & PRESSURE & Brine fer-field pore pressure & 32733 & 08-Feb-96 & $\mathbf{Y}$ & 1 \\
\hline$Y$ & & 160 & DEWMLAKE & Dewey Lake Red Beds & PRESSURE & Brine far-field pore pressure & 32733 & 08-Fob-96 & $\mathbf{Y}$ & 1 \\
\hline $\mathbf{Y}$ & & 161 & DEWVLAKE & Dewoy Lake Red Beds & PRMX_LOG & $\begin{array}{l}\text { Log of intrinsic permeabilty, } x- \\
\text { direction }\end{array}$ & 32734 & 22Fob-96 & $\mathbf{Y}$ & 1 \\
\hline $\mathbf{Y}$ & & 161 & DEWMLAKE & Dowey Lake Red Beds & PRMX_LOG & $\begin{array}{l}\text { Log of intrinsic permeability, } X- \\
\text { direction }\end{array}$ & 32734 & 22 Fab-96 & $\mathbf{Y}$ & 1 \\
\hline $\mathbf{Y}$ & & 161 & DEWYLAKE & Dewry Lake Rod Beds & PRMX_LOG & $\begin{array}{l}\text { Log of intrinsic permeability, } X \text { - } \\
\text { direction }\end{array}$ & 32734 & 22-Fab-96 & $\mathbf{Y}$ & 1 \\
\hline$\gamma$ & & 162 & DEWMLAKE & Dewey Lake Red Beds & PRMY_LOG & $\begin{array}{l}\text { Log of intrinsic permeability, Y- } \\
\text { diroction }\end{array}$ & 32735 & 22 Feb-96 & $\mathbf{Y}$ & 1 \\
\hline$Y$ & & 162 & DEWMLAKE & Dewrey Lake Red Bods & PRMY_LOG & $\begin{array}{l}\text { Log of intrinsic permeability, Y- } \\
\text { direction }\end{array}$ & 32735 & 22Fob-96 & Y & 1 \\
\hline $\mathbf{Y}$ & & 162 & DEWTLAKE & Dewey Lake Red Beds & PRMY_LOG & $\begin{array}{l}\text { Log of intrinsic permeability, Y- } \\
\text { direction }\end{array}$ & 32735 & 22-Fob-96 & $\mathbf{Y}$ & 1 \\
\hline$\gamma$ & & 163 & DEWMLAKE & Dewey Lake Red Beds & PRMZ_LOG & $\begin{array}{l}\text { Log of intrinsic permeability, z- } \\
\text { direction }\end{array}$ & 32736 & $22-F o b-96$ & $Y$ & 1 \\
\hline$Y$ & & 163 & DEWYLAKE & Dewrey Lake Red Beds & PRMZ_LOG & $\begin{array}{l}\text { Log of intrinsic permeability, Z- } \\
\text { direction }\end{array}$ & 32736 & 22-Fab-96 & $\boldsymbol{Y}$ & 1 \\
\hline $\mathbf{Y}$ & & 163 & DEWYLAKE & Dewrey Lake Red Beds & PRMZ_LOG & $\begin{array}{l}\text { Log of intunsic permeability, Z- } \\
\text { direction }\end{array}$ & 32736 & 22 Feb-96 & $\mathbf{Y}$ & 1 \\
\hline $\mathbf{Y}$ & & 166 & DEWILAKE & Dewey Lake Red Bods & RELP_MOD & $\begin{array}{l}\text { Model number, relative } \\
\text { permeability model }\end{array}$ & 32739 & 01-Nov-95 & $\mathbf{Y}$ & 48 \\
\hline$Y$ & & 166 & DEWYLAKE & Dewey Lake Rod Beds & RELP_MOD & $\begin{array}{l}\text { Model number, relative } \\
\text { permbability model }\end{array}$ & 32739 & O1-Nov-95 & $Y$ & 4B \\
\hline$Y$ & & 167 & DEWYLAKE & Dewey Lake Red Beds & SAL_USAT & $\begin{array}{l}\text { Average saturation, unsaturated } \\
\text { zones }\end{array}$ & 32740 & 16-Apr-97 & $\mathbf{Y}$ & $4 B$ \\
\hline$\gamma$ & & 168 & DEWMAKE & Denwey Lake Rod Beds & SAT_IBRN & Initial Brine Saturation & 32741 & 01-Nov-95 & $\mathbf{Y}$ & $4 B$ \\
\hline$Y$ & & 168 & DEWMLAKE & Dewey Lake Red Beds & SAT_IBRN & Initial Brine Saturation & 32741 & 01-Nov-95 & $Y$ & 4B \\
\hline$Y$ & & 169 & DEWMAKE & Dewey Lake Red Bods & SAT_RBRN & Residual Brine Saturation & 32742 & 22-Feb-96 & $Y$ & 4B \\
\hline $\mathbf{Y}$ & & 169 & DEWYLAKE & Denrey Lake Red Beds & SAT_RBRN & Residual Brine Saturation & 32742 & 22-Fob-96 & $Y$ & 48 \\
\hline $\mathbf{Y}$ & & 169 & DEWMLAKE & Dewey Lake Red Beds & SAT_RBRN & Residual Brine Saturation & 32742 & 22-Fob-96 & $Y$ & 4B \\
\hline$Y$ & & 170 & DEWMLAKE & Dewey Lake Red Bods & SAT_RGAS & Residual Gas Saturation & 32743 & 20-Fob-96 & $-Y Y$ & 4B \\
\hline$Y$ & & 170 & DEWMLAKE & Dewey Lake Red Beds & SAT_RGAS & Residual Gas Saturation & 32743 & 20-Fob-96 & $Y$ & $4 B$ \\
\hline$Y$ & & 170 & DEWYLAKE & Dewry Lake Red Bods & SAT_RGAS & Residual Gas Saturation & 32743 & 20-Feb-96 & Y & $4 B$ \\
\hline $\mathbf{Y}$ & & 171 & DRILLMUD & Drilling Mud & DNSFLUID & Brine Density & 32748 & 09-Jan-97 & $\mathbf{Y}$ & 48 \\
\hline$Y$ & & 171 & DRILUMUD & Drilling Mud & DNSFLUID & Brine Density & 32748 & 09-Jan-97 & $Y$ & $4 B$ \\
\hline $\bar{Y}$ & & 171 & DRILLMUD & Drilling Mud & DNSFLUID & Brine Density & 32748 & 09-Jan-97 & $Y$ & 4B \\
\hline$Y$ & & 171 & DRILLMUD & Drilling Mud & DNSFLUID & Brine Density & 32748 & 09-Jan-97 & $\mathbf{Y}$ & AB \\
\hline$Y$ & & 171 & DRILMUD & Dritling Mud & DNSFLUID & Brine Density & 32748 & 0Q-Jan-97 & $Y$ & AB \\
\hline$Y$ & & 172 & DRILMUD & Orilling Mud & visco & Viscosity & 32750 & 09-Jan-97 & $Y$ & $4 B$ \\
\hline$Y$ & & 172 & DRILLMUD & Drilling Mus & Visco & Viscosity & 32750 & 09-Jan-97 & $Y$ & $4 B$ \\
\hline$Y$ & & 172 & DRILLMUD & Drilling Mud & Jisco & Viscosity & 32750 & 09-Jan-97 & $Y$ & 48 \\
\hline$Y$ & & 172 & DRILLMUD & Drilling Mud & visco & Viscosity & 32750 & O9-Jan-97 & $Y$ & 48 \\
\hline $\mathbf{Y}$ & & 472 & DRILLMUD & Drilling Mus & |Visco & Viscosity & 32750 & 09-Jan-97 & $Y$ & 48 \\
\hline$Y$ & & 173 & DRILLMUD & Drilling Mud & IVLSTRSS & Yield Stress Point & 32751 & 09-Jan-97 & $Y$ & $A B$ \\
\hline$Y$ & & 173 & DRILLMUD & Drilling Mud & InDSTRSS & Yreld Stress Point & 32751 & 09-Jan-97 & $Y$ & 48 \\
\hline$Y$ & & 173 & DRILMUD & Drilling Mud & IRDSTRSS & Yreld Stress Point & 32751 & 09-Jar-97 & $Y$ & $4 B$ \\
\hline $\mathbf{Y}$ & & 173 & DRILLMUD & Drilling Mud & YLDSTRSS & Yield Stress Point & 32751 & 09-Jan-97 & $\mathbf{Y}$ & 48 \\
\hline
\end{tabular}


CCA Parameter Listing

\begin{tabular}{|c|c|c|c|c|c|c|c|c|c|c|}
\hline ID & \begin{tabular}{|c|} 
Ciassifi- \\
Cation of \\
Data \\
Cutegory \\
(footnote 2)
\end{tabular} & $\begin{array}{l}\text { Dlstriburtion } \\
\text { Typo }\end{array}$ & Masn & Medlen & Mintroum & i & Unit: & Reference ID & $\begin{array}{l}\text { Was the data } \\
\text { developed } \\
\text { under an } \\
\text { NOA-4 } \\
\text { Program? }\end{array}$ & $\begin{array}{c}\text { Which } \\
\text { method } \\
\text { wore usid to } \\
\text { qually } \\
\text { existing } \\
\text { duta? } \\
\text { (footnote 9) }\end{array}$ \\
\hline 158 & 1 & STUDENT & $1.4300000 E-01$ & $1.4300000 E-01$ & $3.5000000 E-02$ & $2.4800000 E-01$ & NONE & WPO35380 & $\mathbf{Y}$ & NAA \\
\hline 159 & 3,5 & CONSTANT & $1.0132500 \mathrm{E}+05$ & $1.0132500 E+05$ & $1.0132500 E+05$ & $1.0132500 E+05$ & $\mathrm{~Pa}$ & WPO35597 & $Y$ & NA \\
\hline 159 & 3,5 & CONSTANT & $1,0132500 E+05$ & $1.0132500 E+05$ & $1.0132500 E+05$ & $1.0132500 E+05$ & $\mathrm{~Pa}$ & WP036051 & $Y$ & $N / A$ \\
\hline 159 & 3,5 & CONSTANT & $1.0132500 E+05$ & $1.0132500 E+05$ & $1.0132500 E+05$ & $1.0132500 E+05$ & $\mathbf{P a}$ & WPO40514 & $\mathbf{Y}$ & N/A \\
\hline 160 & 3,5 & CONSTANT & $1.0132500 E+05$ & $1.0132500 \mathrm{E}+05$ & $1.0132500 E+05$ & $1.0132500 E+05$ & $\mathbf{P a}$ & WP030713 & $\mathbf{Y}$ & 1 \\
\hline 160 & 3,5 & CONSTANT & $1.0132500 E+05$ & $1.0132500 \mathrm{E}+05$ & $1.0132500 E+0.5$ & $1.0132500 E+05$ & $\mathrm{~Pa}$ & WP032290 & $\mathbf{Y}$ & 1 \\
\hline 160 & 3,5 & CONSTANT & $1.0132500 \mathrm{E}+05$ & $4.0132500 E+05$ & $1.0132500 E+0.5$ & $1.0132500 E+05$ & $\mathbf{P a}$ & WP035597 & $Y$ & 1 \\
\hline 961 & 1 & CONSTANT & $-1.6300000 \mathrm{E}+01$ & $-1.6300000 E+01$ & $-1.6300000 E+01$ & $-1.6300000 E+01$ & $\log \left(m^{\wedge} 2\right)$ & $\begin{array}{c}\text { MEMO:VAUGHN } \\
\text { TIERNEY-10 }\end{array}$ & $\mathbf{Y}$ & 1 \\
\hline 161 & 1 & CONSTANT & $-1.6300000 E+01$ & $-1.6300000 E+01$ & $-1.6300000 E+01$ & $-1.6300000 E+01$ & $\log \left(m^{\wedge} 2\right)$ & WP030607 & $\mathbf{Y}$ & 1 \\
\hline 161 & 1 & CONSTANT & $-1.6300000 E+01$ & $-1.6300000 \mathrm{E}+01$ & $-1.6300000 E+01$ & $-1.6300000 E+01$ & $\log \left(m^{\wedge} 2\right)$ & WP035597 & $\mathbf{Y}$ & 1 \\
\hline 162 & 1 & CONSTANT & $-1.6300000 E+01$ & $-1.6300000 E+01$ & $-1.6300000 \mathrm{E}+01$ & $-1.6300000 E+01$ & $\log \left(m^{\wedge} 2\right)$ & $\begin{array}{l}\text { MEMO:VAUGHNW } \\
\text { TIERNEY-10 }\end{array}$ & $\mathbf{Y}$ & 1 \\
\hline 162 & 1 & CONSTANT & $-1.6300000 E+01$ & $-1.6300000 E+01$ & $-1.6300000 E+01$ & $-1.6300000 E+01$ & $\log \left(m^{\wedge} 2\right)$ & WP030607 & $\mathbf{Y}$ & 1 \\
\hline 162 & 1 & CONSTANT & $-1.6300000 E+01$ & $-1.6300000 \mathrm{E}+01$ & $-1.6300000 E+01$ & $-1.6300000 E+01$ & $\log \left(m^{\wedge} 2\right)$ & WP035597 & $Y$ & 1 \\
\hline 163 & 1 & CONSTANT & $-1.6300000 E+01$ & $-1.6300000 E+01$ & $-1.6300000 E+01$ & $-1.6300000 E+01$ & $\log \left(m^{\wedge} 2\right)$ & $\begin{array}{l}\text { MEMO:VAUGHN } \\
\text { TIERNEY-10 }\end{array}$ & $\mathbf{Y}$ & 1 \\
\hline 163 & 1 & CONSTANT & $-1.6300000 E+01$ & $-1.6300000 E+01$ & $-1.6300000 \mathrm{E}+01$ & $-1.6300000 E+01$ & $\log \left(m^{\wedge} 2\right)$ & WP030607 & $\mathbf{Y}$ & 1 \\
\hline 163 & 1 & CONSTANT & $-1.6300000 E+01$ & $-1.6300000 \mathrm{E}+01$ & $-1.6300000 E+01$ & $-1.6300000 E+01$ & $\log \left(m^{\wedge} z\right)$ & WP035597 & $Y$ & 1 \\
\hline 166 & $1,3,4$ & CONSTANT & $4.0000000 E+00$ & $4.0000000 E+00$ & $4.0000000 E+00$ & $4.0000000 \mathrm{E}+\infty 0$ & NONE & WPO38568 & $Y$ & N/A \\
\hline 166 & $1,3,4$ & CONSTANT & $4.0000000 E+00$ & $4.0000000 E+00$ & $4.0000000 E+00$ & $4.0000000 \mathrm{E}+\infty 0$ & NONE & WPO40514 & $\mathbf{Y}$ & N/A \\
\hline 167 & $1,3,4$ & CONSTANT & $8.3600000 E-02$ & $8.3600000 E-02$ & $8.3600000 E-02$ & $8.3600000 E-02$ & NONE & NONE & $\mathbf{Y}$ & N/A \\
\hline 168 & $1,3,4$ & CONSTANT & $1.0000000 \mathrm{E}+\infty 0$ & $1.0000000 E+\infty 0$ & $1.0000000 E+\infty$ & $1.0000000 E+00$ & NONE & WP038568 & $Y$ & NA \\
\hline 168 & $1,3,4$ & CONSTANT & $1.0000000 \mathrm{E}+00$ & $1.0000000 E+\infty 0$ & $1.0000000 E+00$ & $1.0000000 \mathrm{E}+\infty 0$ & NONE & WPO40514 & $Y$ & NA \\
\hline 169 & $1,3,4$ & CONSTANT & $8.3630000 E-02$ & $8.3630000 E-02$ & $8.3530000 E-02$ & $8.3530000 E-02$ & NONE & WP031041 & $\mathbf{Y}$ & N/A \\
\hline 169 & $1,3,4$ & CONSTANT & $8.3630000 E-02$ & $8.3630000 E-02$ & $8.3630000 E-02$ & $8.3530000 E-02$ & NONE & WP035597 & $\mathbf{Y}$ & NA \\
\hline 169 & $1,3,4$ & CONSTANT & $8.3630000 E-02$ & $8.3630000 E-02$ & $8.3530000 E-02$ & $8.3530000 E-02$ & NONE & WP040514 & $Y$ & N/A \\
\hline 170 & $1,3,4$ & CONSTANT & 7.7110000E-02 & $7.7110000 E-02$ & $7.7110000 E-02$ & $7.7410000 E-02$ & NONE & WP031041 &.$- Y$ & N/A \\
\hline 170 & $1,3,4$ & CONSTANT & $7.7110000 E-02$ & $7.7110000 E-02$ & $7.7110000 E-02$ & $7.7110000 E-02$ & NONE & WP035597 & $Y$ & N/A \\
\hline 170 & $1,3,4$ & CONSTANT & $7.7110000 E-02$ & $7.7110000 E-02$ & 7.7110000 E- 02 & $7.7110000 E-02$ & NONE & WP040514 & $Y$ & N/A \\
\hline 171 & 3,5 & CUMULATIVE & $1.2100000 \mathrm{E}+03$ & $1.2100000 \mathrm{E}+03$ & $1.1400000 \mathrm{E}+03$ & $1.3800000 \mathrm{E}+03$ & $\mathrm{~kg} / \mathrm{m}^{\wedge} 3$ & SAND92-070013 & $\bar{Y}$ & N/A \\
\hline 171 & 3,5 & CUMULATIVE & $1.2100000 E+03$ & $1.2100000 E+03$ & $1.1400000 E+03$ & $1.3800000 \mathrm{E}+03$ & $\mathrm{~kg} / \mathrm{m}^{\wedge} 3$ & WP035268 & $\mathbf{Y}$ & N/A \\
\hline 171 & 3,5 & CUMULATIVE & $1.2100000 E+03$ & $1.2100000 E+03$ & $1.1400000 \mathrm{E}+03$ & $1.3800000 E+03$ & $\mathrm{~kg} / \mathrm{m}^{\wedge} 3$ & WPO35597 & $Y$ & N/A \\
\hline 171 & 3,5 & CUMULATIVE & $1.2100000 E+03$ & $1.2100000 \mathrm{E}+03$ & $1.1400000 E+03$ & $1.3800000 E+03$ & $\mathrm{~kg} / \mathrm{m}^{\wedge} 3$ & WPO38558 & $Y$ & $N / A$ \\
\hline 171 & 3,5 & CUMULATIVE & $1.2100000 E+03$ & $1.2100000 E+03$ & $1.1400000 \mathrm{E}+03$ & $1.3800000 \mathrm{E}+03$ & $\mathrm{~kg} / \mathrm{m}^{\wedge 3}$ & WPO40521 & $\bar{Y}$ & N/A \\
\hline 172 & 3,5 & CUMULATIVE & $1.1000000 \mathrm{E}-02$ & $9.1700000 E-03$ & $5.00000000 \mathrm{E}-03$ & $3.0000000 E-02$ & Pa's & SAND92-0700/3 & $\bar{Y}$ & N/A \\
\hline 172 & 3,5 & CUMULATIVE & $1.1000000 \mathrm{E}-02$ & $9.1700000 E-03$ & $5.0000000 E-03$ & $3.0000000 E-02$ & Pa's & WP035268 & $\bar{Y}$ & N/A \\
\hline 172 & 3,5 & CUMULATINE & $1.1000000 E-02$ & $9.1700000 E-03$ & $5.0000000 \mathrm{E}-03$ & $3.0000000 \mathrm{E}-02$ & Pa's & WP035597 & $Y$ & N/A \\
\hline 172 & 3,5 & CUMULATIVE & $1.1000000 E-02$ & $9.1700000 E-03$ & $5.0000000 E-03$ & $3.0000000 E-02$ & Pa*s & WPO38568 & $\bar{Y}$ & N/A \\
\hline 172 & 3,5 & CUMULATIVE & $1.1000000 \mathrm{E}-02$ & $9.1700000 \mathrm{E}-03$ & $5.0000000 \mathrm{E}-03$ & $3.0000000 \mathrm{E}-02$ & Pa's & WP040521 & $\bar{Y}$ & N/A \\
\hline 173 & 3,5 & CUMULATNE & $5.9800000 \mathrm{E}+00$ & $4.4000000 \mathrm{E}+00$ & $2.4000000 \mathrm{E}+\infty 0$ & $1.9200000 \mathrm{E}+01$ & $\mathrm{~Pa}$ & SAND92-070013 & $\bar{Y}$ & NA \\
\hline 173 & 3,5 & CUMULATNE & $5.9800000 \mathrm{E}+00$ & $4.4000000 E+00$ & $2.4000000 E+\infty 0$ & $1.9200000 E+01$ & $\mathrm{~Pa}$ & WP035268 & $\bar{Y}$ & NA \\
\hline 173 & 3.5 & CUMULATIVE & $5.9800000 E+00$ & $4.4000000 E+00$ & $24000000 E+00$ & $1.9200000 E+01$ & $\overline{\mathrm{Pa}}$ & WP035597 & $\bar{Y}$ & NA \\
\hline 173 & 3,5 & CUMULATIVE & $5.9800000 \mathrm{E}+00$ & $4.4000000 E+00$ & $2.4000000 \mathrm{E}+00$ & $1.9200000 \mathrm{E}+01$ & $\mathbf{P a}$ & WP038568 & $\mathbf{Y}$ & NAA \\
\hline
\end{tabular}


CCA Parameter Listing

\begin{tabular}{|c|c|c|c|c|c|c|c|c|c|c|}
\hline $\begin{array}{c}\text { Parameter in } \\
\text { Datubaee? } \\
\text { (YNN) }\end{array}$ & $\begin{array}{l}\text { if the values } \\
\text { used in CCA } \\
\text { are not the } \\
\text { same as in } \\
\text { DB, provide } \\
\text { difference }\end{array}$ & tD & Material ID & Material heme & $\begin{array}{c}\text { Parameter } \\
\text { is }\end{array}$ & Perameter Nams & $\begin{array}{l}\text { PRP ID } \\
\text { (MPO * *) }\end{array}$ & $\begin{array}{c}\text { Dita Entry } \\
\text { Date }\end{array}$ & $\begin{array}{l}\text { Is the } 464 \\
\text { consistent } \\
\text { with the } \\
\text { parameter } \\
\text { in DB? } \\
(Y, N \text { N) }\end{array}$ & $\begin{array}{l}\text { Paramatar } \\
\text { Category } \\
\text { (footnota 1) }\end{array}$ \\
\hline$Y$ & & 173 & DRILLMUD & Drilling Mud & YLDSTRSS & Yield Stress Point & 32751 & 09-Jan-97 & $\mathbf{Y}$ & $4 B$ \\
\hline $\mathbf{Y}$ & & 174 & DRZ_0 & $\begin{array}{l}\text { Disturbed rock zone; } \\
\text { tume period }-5 \text { to } 0 \\
\text { years }\end{array}$ & CAP_MOD & $\begin{array}{l}\text { Model number, capillary pressuro } \\
\text { model }\end{array}$ & 32754 & 12-Feb-96 & $\mathbf{Y}$ & 48 \\
\hline $\mathbf{Y}$ & & 174 & DRZ_O & $\begin{array}{l}\text { Disturbed rock zone; } \\
\text { time period }-5 \text { to } 0 \\
\text { years }\end{array}$ & CAP_MOD & $\begin{array}{l}\text { Model number, capillary pressure } \\
\text { model }\end{array}$ & 32754 & 12 Fob- 96 & $\mathbf{Y}$ & 48 \\
\hline $\mathbf{Y}$ & & 174 & DRZ_O & $\begin{array}{l}\text { Disturbed rock zone; } \\
\text { time period } 5 \text { to } 0 \\
\text { years }\end{array}$ & CAP_MOD & $\begin{array}{l}\text { Model number, capillary pressure } \\
\text { model }\end{array}$ & 32754 & $12+F a b-96$ & $\mathbf{Y}$ & $4 B$ \\
\hline $\mathbf{Y}$ & & 175 & DRZ_o & $\begin{array}{l}\text { Disturbed rock zone; } \\
\text { time period -5 to } 0 \\
\text { years }\end{array}$ & COMP_RCK & Buk Compressibility & 32758 & 12Fob-96 & $\mathbf{Y}$ & $4 B$ \\
\hline $\mathbf{Y}$ & & 175 & $D R Z$ O & $\begin{array}{l}\text { Disturbed rock zone; } \\
\text { time period }-5 \text { to } 0 \\
\text { years }\end{array}$ & COMP_RCK & Bulk Compressibility & 32758 & 12Feb-96 & $\mathbf{Y}$ & $4 B$ \\
\hline $\mathbf{Y}$ & & 175 & DRZ_o & $\begin{array}{l}\text { Disturbed rock zone; } \\
\text { time period }-5 \text { to } 0 \\
\text { years }\end{array}$ & COMP_RCK & Bulk Compressibility & 32758 & 12Fob-96 & $Y$ & $4 B$ \\
\hline $\mathbf{Y}$ & & 176 & $D R Z$ _o & $\begin{array}{l}\text { Disturbed rock zone; } \\
\text { time period }-5 \text { to } 0 \\
\text { years }\end{array}$ & PC_MAX & $\begin{array}{l}\text { Maximum allowable capillary } \\
\text { pressure }\end{array}$ & 32834 & 01-Nov-95 & $\mathbf{Y}$ & AB \\
\hline $\mathbf{Y}$ & & 176 & $D R Z$ O & $\begin{array}{l}\text { Distubed rock zone; } \\
\text { time period }-5 \text { to } 0 \\
\text { years }\end{array}$ & PC_MAX & $\begin{array}{l}\text { Maximum allowable capillary } \\
\text { pressure }\end{array}$ & 32834 & 01-Nov-95 & $\mathbf{Y}$ & AB \\
\hline $\mathbf{Y}$ & & 177 & DRZ_O & $\begin{array}{l}\text { Distubed rock zone; } \\
\text { time period -5 to } 0 \\
\text { years }\end{array}$ & PORE_DIS & $\begin{array}{l}\text { Brooks-Corey pore distribution } \\
\text { parameter }\end{array}$ & 32837 & 12Fab-96 & $\mathbf{Y}$ & $4 B$ \\
\hline $\mathbf{Y}$ & & 177 & DRZ_O & $\begin{array}{l}\text { Disturbed rock zone; } \\
\text { time period -5 to } 0 \\
\text { years }\end{array}$ & PORE_DIS & $\begin{array}{l}\text { Brooks-Corey pore distribution } \\
\text { parameter }\end{array}$ & 32837 & 12feb-96 & $\mathbf{Y}$ & AB \\
\hline $\mathbf{Y}$ & & 177 & DRZ_O & $\begin{array}{l}\text { Distubed rock zone; } \\
\text { time period }-5 \text { to } 0 \\
\text { years }\end{array}$ & PORE_DIS & $\begin{array}{l}\text { Brooks-Corey pore distribution } \\
\text { parameter }\end{array}$ & 32837 & 12-Feb-96 & $Y$ & $4 B$ \\
\hline $\mathbf{Y}$ & varies & 178 & DRZ_O & $\begin{array}{l}\text { Disturbed rock zone; } \\
\text { time period }-5 \text { to } 0 \\
\text { years }\end{array}$ & POROSITY & Effective porosity & 32839 & 12-Feb-96 & $\mathbf{Y}$ & $4 B$ \\
\hline $\mathbf{Y}$ & varies & 178 & DRZ_O & $\begin{array}{l}\text { Disturbed rock zone; } \\
\text { time period }-5 \text { to } 0 \\
\text { years }\end{array}$ & POROSITY & Effective porosity & 32839 & 12-Feb-96 & $\mathbf{Y}$ & 48 \\
\hline $\mathbf{Y}$ & varies & 178 & $D R Z_{-} 0$ & $\begin{array}{l}\text { Disturbed rock zone; } \\
\text { time period }-5 \text { to } 0 \\
\text { years }\end{array}$ & POROSITY & Effective porosity & 32839 & 12-Feb-96 & $\mathbf{Y}$ & $4 B$ \\
\hline $\mathbf{Y}$ & varies & 178 & $D R Z$ OO & $\begin{array}{l}\text { Disturbed rock zone; } \\
\text { time period }-5 \text { to } 0 \\
\text { years }\end{array}$ & POROSITY & Effective porosity & 32839 & 12-Feb-96 & $\mathbf{Y}$ & $4 B$ \\
\hline $\mathbf{Y}$ & varies & 178 & DRZ_O & $\begin{array}{l}\text { Disturbed rock zone; } \\
\text { time period -5 to } 0 \\
\text { years }\end{array}$ & POROSITY & Effective porosity & 32839 & 12feb-96 & $\mathbf{Y}$ & 48 \\
\hline $\mathbf{Y}$ & varies & 178 & $D R Z$ O & $\begin{array}{l}\text { Distubed rock zone; } \\
\text { time period }-5 \text { to } 0 \\
\text { years }\end{array}$ & POROSITY & Effective porosity & 32839 & 12-Fob-96 & $\mathbf{Y}$ & $4 B$ \\
\hline $\mathbf{Y}$ & & 179 & $D R Z$ O & $\begin{array}{l}\text { Disturted rock zone; } \\
\text { time period }-5 \text { to } 0 \\
\text { years }\end{array}$ & PO_MIN & $\begin{array}{l}\text { Minimum brine pressure for } \\
\text { capillary model } \mathrm{KPC}=3\end{array}$ & 32844 & $21-$ eb-96 & $\mathbf{Y}$ & $4 B$ \\
\hline $\mathbf{Y}$ & & 179 & DRZ_O & $\begin{array}{l}\text { Disturbed rock zone; } \\
\text { time period }-5 \text { to } 0 \\
\text { years }\end{array}$ & PO_MIN & $\begin{array}{l}\text { Minimum brine pressure for } \\
\text { capillary model KPC=3 }\end{array}$ & 32844 & 21-Fob-96 & $\mathbf{Y}$ & $4 B$ \\
\hline $\mathbf{Y}$ & & 179 & $D R Z \_0$ & $\begin{array}{l}\text { Disturbed rock zone; } \\
\text { time period }-5 \text { to } 0 \\
\text { years }\end{array}$ & PO_MIN & $\begin{array}{l}\text { Minimum brine pressure for } \\
\text { capiliary model KPC=3 }\end{array}$ & 32844 & 21-Feb-96 & $\cdot \boldsymbol{Y}$ & $4 B$ \\
\hline $\mathbf{Y}$ & & 181 & $D R Z_{-} 0$ & $\begin{array}{l}\text { Disturbed rock zone; } \\
\text { time period }-5 \text { to } 0 \\
\text { years }\end{array}$ & PRMX_LOG & $\begin{array}{l}\text { Log of intrinsic permeabilty, } X \text { - } \\
\text { direction }\end{array}$ & 32847 & 01-Nov-95 & $\mathbf{Y}$ & $A B$ \\
\hline$Y$ & & 181 & DRZ_o & $\begin{array}{l}\text { Disturbed rock zone; } \\
\text { time period } 5 \text { to } 0 \\
\text { years }\end{array}$ & PRMX_LOG & $\begin{array}{l}\text { Log of intrinsic permeability, } X \text { - } \\
\text { direction }\end{array}$ & 32847 & 01-Nov-95 & $\mathbf{Y}$ & $4 B$ \\
\hline $\mathbf{Y}$ & & 181 & DRZ_o & $\begin{array}{l}\text { Disturbed rock zone; } \\
\text { time period }-5 \text { to } 0 \\
\text { years }\end{array}$ & PRMX_LOG & $\begin{array}{l}\text { Log of intrinsic permeability, X- } \\
\text { diroction }\end{array}$ & 32847 & 01-Nov-95 & $\mathbf{Y}$ & $4 B$ \\
\hline $\mathbf{Y}$ & & 181 & DRZ_O & $\begin{array}{l}\text { Disturbed rock zone: } \\
\text { time period -5 to } 0 \\
\text { years }\end{array}$ & PRMX_LOG & $\begin{array}{l}\text { Log of intrinsic permeability, } X \text { - } \\
\text { direction }\end{array}$ & 32847 & 01-Nov-95 & $\mathbf{Y}$ & $4 B$ \\
\hline
\end{tabular}


CCA Parameter Listing

\begin{tabular}{|c|c|c|c|c|c|c|c|c|c|c|}
\hline 10 & $\begin{array}{c}\text { Clazsin- } \\
\text { cattion of } \\
\text { Datt } \\
\text { Cattegory } \\
\text { (footrote 2) }\end{array}$ & $\begin{array}{l}\text { Diatibution } \\
\text { Typo }\end{array}$ & $\operatorname{man}$ & Modian & Minimum & maxisum & 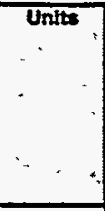 & Rererence ID & $\begin{array}{l}\text { Was the data } \\
\text { doveloped } \\
\text { under an } \\
\text { NOA-1 } \\
\text { Program? }\end{array}$ & \begin{tabular}{|c|} 
Which \\
mothods \\
wero used to \\
qually \\
oxisting \\
detta? \\
(footnote 3) \\
\end{tabular} \\
\hline 173 & 3,5 & CUMULATIVE & $5.9800000 E+00$ & $4.4000000 E+00$ & $2.4000000 E+00$ & $1.9200000 \mathrm{E}+01$ & $\mathbf{P a}$ & WPO40521 & $\mathbf{Y}$ & NA \\
\hline 174 & 3,5 & CONSTANT & $1.0000000 E+\infty 0$ & $1.0000000 E+00$ & $1.0000000 E+\infty 0$ & $1.0000000 E+\infty 0$ & NONE & WP032288 & $\mathbf{Y}$ & NA \\
\hline 174 & 3,5 & CONSTANT & $1.0000000 E+00$ & $1.0000000 E+00$ & $1.0000000 E+00$ & $1.0000000 \mathrm{E}+00$ & NONE & WP035597 & $\mathbf{Y}$ & NA \\
\hline 174 & 3,5 & CONSTANT & $1.0000000 \mathrm{E}+00$ & $1.0000000 E+\infty 0$ & $1.0000000 \mathrm{E}+\infty 0$ & $1.0000000 \mathrm{E}+00$ & NONE & WPO40514 & $\mathbf{Y}$ & NA \\
\hline 175 & $1,3,4$ & CONSTANT & $7.4100000 E-10$ & $7.4100000 E-10$ & $7.4100000 E-10$ & $7.4100000 E-10$ & $P a^{A}-1$ & WP032288 & $Y$ & 1 \\
\hline 175 & $1,3,4$ & CONSTANT & $7.4100000 E-10$ & $7.4100000 E-10$ & $7.4100000 E-10$ & $7.4100000 E-10$ & Pal1 & WP035597 & $\mathbf{Y}$ & 1 \\
\hline 175 & $1,3,4$ & CONSTANT & $7.4100000 E-10$ & $7.4100000 E-10$ & $7.4100000 E-10$ & $7.4100000 E-10$ & $\mathrm{Par}-1$. & WPO40514 & $\mathbf{Y}$ & 1 \\
\hline 176 & 3,5 & CONSTANT & $1.0000000 E+08$ & $1.0000000 E+08$ & $1.0000000 E+08$ & $1.0000000 \mathrm{E}+08$ & $\mathbf{P a}$ & WP038568 & $\mathbf{Y}$ & NA \\
\hline 176 & 3,5 & CONSTANT & $1.0000000 E+08$ & $1.0000000 E+08$ & $1.0000000 E+08$ & $1.0000000 E+08$ & $\mathbf{P a}$ & WP040514 & $\gamma$ & N/A \\
\hline 177 & $1,3,4$ & CONSTANT & $7.0000000 E-01$ & 7.0000000E-01 & $7.0000000 E-01$ & $7.0000000 E-01$ & NONE & WP032288 & $\mathbf{Y}$ & NA \\
\hline 177 & $1,3,4$ & CONSTANT & $7.0000000 E-01$ & $7.0000000 E-01$ & $7.0000000 E-01$ & 7.0000000E-01 & NONE & WP035597 & $\mathbf{Y}$ & N/A \\
\hline 177 & $1,3,4$ & CONSTANT & $7.0000000 E-01$ & $7.0000000 E-01$ & $7.0000000 E-01$ & $7.0000000 E-01$ & NONE & WPO40514 & $Y$ & N/A \\
\hline 178 & $1,3,4$ & CUMULATIVE & $1.5650000 E-02$ & $1.2900000 \mathrm{E}-02$ & $3.9000000 \mathrm{E}-03$ & $3.2900000 E-02$ & NONE & WP032288 & $Y$ & NA \\
\hline 178 & $1,3,4$ & CUMULATIVE & $1.5650000 E-02$ & 1.2900000E-02 & $3.9000000 E-03$ & $3.2900000 E-02$ & NONE & WP035268 & $\mathbf{Y}$ & NA \\
\hline 178 & $1,3,4$ & CUMULATIVE & $1.5650000 E-02$ & $1.2900000 \mathrm{E}-02$ & $3.9000000 E-03$ & $3.2900000 \mathrm{E}-02$ & NONE & WP035597 & $\mathbf{Y}$ & N/A \\
\hline 178 & $1,3,4$ & CUMULATIVE & $1.5650000 E-02$ & $1.2900000 E-02$ & $3.9000000 E-03$ & $3.2900000 \mathrm{E}-02$ & NONE & WPO40434 & $Y$ & NA \\
\hline 178 & $1,3,4$ & CUMULATINE & $1.5650000 E-02$ & $1.2900000 E-02$ & $3.9000000 E-03$ & $3.2900000 E-02$ & NONE & WP040514 & $\mathbf{Y}$ & N/A \\
\hline 178 & $1,3,4$ & CUMULATIVE & $1.5650000 E-02$ & $1.2900000 E-02$ & $3.9000000 E-03$ & $3.2900000 \mathrm{E}-02$ & NONE & WP042257 & $\mathbf{Y}$ & N/A \\
\hline 179 & 3,5 & CONSTANT & $1.0132500 E+05$ & $1.0132500 E+05$ & $1.0132500 E+05$ & $1.0132500 E+05$ & $\mathbf{P a}$ & WP035597 & $\mathbf{Y}$ & NA \\
\hline 179 & 3,5 & CONSTANT & $1.0132500 E+05$ & $1.0132500 E+05$ & $1.0132500 E+05$ & $1.0132500 E+05$ & $\mathbf{P a}$ & WP036051 & $\mathbf{Y}$ & N/A \\
\hline 179 & 3,5 & CONSTANT & $1.0132500 E+0.5$ & $1.0132500 E+05$ & $1.0132500 E+05$ & $1.0132500 E+05$ & $\mathrm{~Pa}$ & WPO40514 & $\mathbf{Y}$ & NA \\
\hline 181 & 1 & CONSTANT & $-1.7000000 E+01$ & $-1.7000000 \mathrm{E}+01$ & $-1.7000000 \mathrm{E}+01$ & $-1.7000000 E+01$ & $\log \left(m^{\wedge} 2\right)$ & WPO22443 & $\mathbf{Y}$ & NA \\
\hline 181 & 1 & CONSTANT & $-1.7000000 E+01$ & $-1.7000000 E+01$ & $-1.7000000 E+01$ & $-1.7000000 E+01$ & $\log \left(m^{\wedge} 2\right)$ & WP030994 & $Y$ & NA \\
\hline 181 & 1 & CONSTANT & $-1.7000000 \mathrm{E}+01$ & $-1.7000000 E+01$ & $-1.7000000 E+01$ & $-1.7000000 E+01$ & $\log \left(m^{\wedge} 2\right)$ & WP032038 & $\mathbf{Y}$ & NA \\
\hline 181 & 1 & CONSTANT & $-1.7000000 E+01$ & $-1.7000000 E+01$ & $-1.7000000 E+01$ & $-1.7000000 E+01$ & $\log \left(m^{\wedge} \mathbf{2}\right)$ & WP032288 & $\mathbf{Y}$ & N/A \\
\hline
\end{tabular}


CCA Parameter Listing

\begin{tabular}{|c|c|c|c|c|c|c|c|c|c|c|}
\hline $\begin{array}{c}\text { Paramitartin } \\
\text { Databewe? } \\
\text { (Y/N) }\end{array}$ & $\begin{array}{l}\text { ff the values } \\
\text { used in CeA } \\
\text { are not the } \\
\text { same ine in } \\
\text { DB, provide } x \\
\text { dffierence }\end{array}$ & ID & Metorial ID & Mntarial Name & $\begin{array}{c}\text { Parmeter } \\
\text { to }\end{array}$ & Parameter Namb & $\begin{array}{l}\text { PRP ID } \\
\text { (WPO in) }\end{array}$ & $\begin{array}{c}\text { Deta Entry } \\
\text { Dats }\end{array}$ & $\begin{array}{l}\text { bo the } 4 \mathrm{~A} \\
\text { conatetent } \\
\text { with the } \\
\text { permeter } \\
\text { in DE? } \\
(\mathrm{N}, \mathrm{NA})\end{array}$ & $\begin{array}{l}\text { Parmiter } \\
\text { Centugory } \\
\text { (footnote 1) }\end{array}$ \\
\hline $\mathbf{Y}$ & & 181 & DRZ_o & $\begin{array}{l}\text { Disturbed rock zone; } \\
\text { time period }-5 \text { to } 0 \\
\text { years }\end{array}$ & PRMX_LOG & $\begin{array}{l}\text { Log of intrinsic permeability, } X \text { - } \\
\text { direction }\end{array}$ & 32847 & 01-Nov-95 & $\mathbf{Y}$ & 4B \\
\hline $\mathbf{Y}$ & & 181 & DRZ_O & $\begin{array}{l}\text { Disturbed rock zone; } \\
\text { time period }-5 \text { to } 0 \\
\text { years }\end{array}$ & PRMX_LOG & $\begin{array}{l}\text { Log of intrinsic permeability, } x \text { - } \\
\text { drection }\end{array}$ & 32847 & 01-Nov-95 & $\mathbf{Y}$ & 48 \\
\hline $\mathbf{Y}$ & & 182 & DRZ_O & $\begin{array}{l}\text { Disturbed rock zone; } \\
\text { time period }-5 \text { to } 0 \\
\text { years }\end{array}$ & PRMY_LOG & $\begin{array}{l}\text { Log of intrinsic permeability, Y- } \\
\text { direction }\end{array}$ & 32849 & 01 Nov-95 & $\mathbf{Y}$ & $4 B$ \\
\hline $\mathbf{Y}$ & & 182 & $D R Z \_0$ & $\begin{array}{l}\text { Disturbed rock zone; } \\
\text { time period }-5 \text { to } 0 \\
\text { years }\end{array}$ & PRMY_LOG & $\begin{array}{l}\text { Log of intrinsic permeability, Y- } \\
\text { direction }\end{array}$ & 32849 & 01-Nov-95 & $\mathbf{Y}$ & 48 \\
\hline $\mathbf{Y}$ & & 182 & DRZ_o & $\begin{array}{l}\text { Disturbed rock zone; } \\
\text { time period }-5 \text { to } 0 \\
\text { years }\end{array}$ & PRMY_LOG & $\begin{array}{l}\text { Log of intrinsic permeability. Y- } \\
\text { drection }\end{array}$ & 32849 & OT-Nov-95 & $\mathbf{Y}$ & $4 B$ \\
\hline $\mathbf{Y}$ & & 182 & DRZ_o & $\begin{array}{l}\text { Disturbed rock zone; } \\
\text { time period }-5 \text { to } 0 \\
\text { years }\end{array}$ & PRMY_LOG & $\begin{array}{l}\text { Log of intrinsic permeability, } Y \text {. } \\
\text { diroction }\end{array}$ & 32849 & 01-Nov-95 & $\mathbf{Y}$ & 48 \\
\hline $\mathbf{Y}$ & & 182 & DRZ_o & $\begin{array}{l}\text { Disturbed rock zone; } \\
\text { time period }-5 \text { to } 0 \\
\text { years }\end{array}$ & PRMY_LOG & $\begin{array}{l}\text { Log of intrinsic permeability, Y- } \\
\text { dirbction . }\end{array}$ & 32849 & 01 Nov-95 & $\mathbf{Y}$ & 48 \\
\hline $\mathbf{Y}$ & & 182 & DRZ_o & $\begin{array}{l}\text { Disturbed rock zone; } \\
\text { time period }-5 \text { to } 0 \\
\text { years }\end{array}$ & PRMY_LOG & $\begin{array}{l}\text { Log of intrinsic permeabilty, } Y \text { - } \\
\text { direction }\end{array}$ & 32849 & 01-Nov-95 & $\mathbf{Y}$ & $4 B$ \\
\hline $\mathbf{Y}$ & & 183 & DRZ_o & $\begin{array}{l}\text { Disturbed rock zone; } \\
\text { time period }-5 \text { to } 0 \\
\text { years }\end{array}$ & PRMZ_LOG & $\begin{array}{l}\text { Log of intrinsic permeability, } z \text { - } \\
\text { direction }\end{array}$ & 32850 & 01-Nov-95 & $\mathbf{Y}$ & $4 B$ \\
\hline $\mathbf{Y}$ & & 183 & $D R Z$ O & $\begin{array}{l}\text { Disturbed rock zone; } \\
\text { time period }-5 \text { to } 0 \\
\text { years }\end{array}$ & PRMZ_LOG & $\begin{array}{l}\text { Log of intrinsic permeability, Z- } \\
\text { direction }\end{array}$ & 32850 & 01-Nov-95 & $\mathbf{Y}$ & 4B \\
\hline $\mathbf{Y}$ & . & 183 & DRZ_o & $\begin{array}{l}\text { Disturbed rock zone: } \\
\text { time period }-5 \text { to } 0 \\
\text { years }\end{array}$ & PRMZ_LOG & $\begin{array}{l}\text { Log of intrinsic permeability, Z- } \\
\text { direction }\end{array}$ & 32850 & $04-N o v-95$ & $\mathbf{Y}$ & $4 B$ \\
\hline $\mathbf{Y}$ & & 183 & DRZ_O & $\begin{array}{l}\text { Disturbed rock zone; } \\
\text { time period }-5 \text { to } 0 \\
\text { years }\end{array}$ & PRMZ_LOG & $\begin{array}{l}\text { Log of intrinsic permeability, z- } \\
\text { direction }\end{array}$ & 32850 & 01-Nov-95 & $\mathbf{Y}$ & $4 B$ \\
\hline $\mathbf{Y}$ & & 183 & DRZ_o & $\begin{array}{l}\text { Disturbed rock zone; } \\
\text { time period }-5 \text { to } 0 \\
\text { years }\end{array}$ & PRMZ_LOG & $\begin{array}{l}\text { Log of intrinsic permeability, Z- } \\
\text { direction }\end{array}$ & 32850 & 01-Nov-95 & $\mathbf{Y}$ & $4 B$ \\
\hline $\mathbf{Y}$ & & 183 & DRZ_o & $\begin{array}{l}\text { Disturbed rock zone; } \\
\text { time period - } 5 \text { to } 0 \\
\text { years }\end{array}$ & PRMZ_LOG & $\begin{array}{l}\text { Log of intrinsic permeability, Z- } \\
\text { direction }\end{array}$ & 32850 & 01-Nov-95 & $\mathbf{Y}$ & $4 B$ \\
\hline $\mathbf{Y}$ & & 186 & DRZ_O & $\begin{array}{l}\text { Disturbed rock zone; } \\
\text { time period }-5 \text { to } 0 \\
\text { years }\end{array}$ & RELP_MOD & $\begin{array}{l}\text { Model number, rolative } \\
\text { permeability model }\end{array}$ & 32856 & 01-Nov-95 & $\mathbf{Y}$ & $4 B$ \\
\hline $\mathbf{Y}$ & & 186 & DRZ_o & $\begin{array}{l}\text { Disturbed rock zone; } \\
\text { time period }-5 \text { to } 0 \\
\text { years }\end{array}$ & RELP_MOD & $\begin{array}{l}\text { Model mumber, relative } \\
\text { permeabilty model }\end{array}$ & 32856 & 01-Nov-95 & $\mathbf{Y}$ & 4B \\
\hline $\mathbf{Y}$ & & 187 & DRZ_O & $\begin{array}{l}\text { Disturbed rock zone; } \\
\text { time period }-5 \text { to } 0 \\
\text { years }\end{array}$ & SAT_IBRN & Initial Brine Saturation & 32857 & 01-Nov-95 & $-\gamma$ & $4 B$ \\
\hline $\mathbf{Y}$ & & 187 & DRZ_O & $\begin{array}{l}\text { Disturbed rock zone; } \\
\text { time period }-5 \text { to } 0 \\
\text { years }\end{array}$ & SAT_IBRN & Initial Brine Saturation & 32857 & $01-N o v-95$ & $\mathbf{Y}$ & $4 B$ \\
\hline $\mathbf{Y}$ & & 187 & $D R Z 0$ & $\begin{array}{l}\text { Disturbed rock zone; } \\
\text { time period }-5 \text { to } 0 \\
\text { years }\end{array}$ & SAT_IBRN & Initial Brine Saluration & 32857 & 01-Nov-95 & $\mathbf{Y}$ & 4B \\
\hline $\mathbf{Y}$ & & 188 & DRZ_O & $\begin{array}{l}\text { Disturbed rock zane; } \\
\text { time period }-5 \text { to } 0 \\
\text { years }\end{array}$ & SAT_RBRN & Residual Brine Saturation & 32860 & 12 Fob-96 & $\mathbf{Y}$ & $4 B$ \\
\hline $\mathbf{Y}$ & & 188 & DRZ_O & $\begin{array}{l}\text { Disturbed rock zone; } \\
\text { time period }-5 \text { to } 0 \\
\text { years }\end{array}$ & SAT_RBRN & Residual Brine Saturation & 32860 & 12-Feb-96 & $\mathbf{Y}$ & $4 B$ \\
\hline $\mathbf{Y}$ & & 188 & DRZ_O & $\begin{array}{l}\text { Disturbed rock zone: } \\
\text { time period }-5 \text { to } 0 \\
\text { years }\end{array}$ & SAT_RBRN & Residual Brine Saturation & 32860 & 12feb-96 & $\mathbf{Y}$ & $4 \mathrm{~B}$ \\
\hline $\mathbf{Y}$ & & 189 & DRZ_O & $\begin{array}{l}\text { Disturbed rock zone; } \\
\text { time period }-5 \text { to } 0 \\
\text { years }\end{array}$ & SAT_RGAS & Residual Gas Saturation & 32862 & 12-Fob-96 & $\mathbf{Y}$ & $4 B$ \\
\hline $\mathbf{Y}$ & & 189 & DRZ_O & $\begin{array}{l}\text { Disturbed rock zone; } \\
\text { time period }-5 \text { to } 0 \\
\text { years }\end{array}$ & SAT_RGAS & Resicual Gas Saturation & 32852 & 12 feb-s6 & $\mathbf{Y}$ & 48 \\
\hline
\end{tabular}


CCA Parameter Listing

\begin{tabular}{|c|c|c|c|c|c|c|c|c|c|c|}
\hline 10 & $\begin{array}{c}\text { Classfif- } \\
\text { cation of } \\
\text { Data } \\
\text { Category } \\
\text { (tootnoto 2) }\end{array}$ & $\begin{array}{c}\text { Dlatribution } \\
\text { Type }\end{array}$ & Mean & Median & $\begin{array}{l}\text { Minimum } \\
-\end{array}$ & Maxdmum & Units & $\begin{array}{l}\text { Roterence ID } \\
\ldots\end{array}$ & $\begin{array}{l}\text { Was the dita } \\
\text { developed } \\
\text { under an } \\
\text { NoA-1 } \\
\text { Program? }\end{array}$ & $\begin{array}{l}\text { Which } \\
\text { mithods } \\
\text { whe used to } \\
\text { qually } \\
\text { exdsting } \\
\text { dinta? } \\
\text { (footnote 3) }\end{array}$ \\
\hline 181 & 1 & CONSTANT & $-1,7000000 E+01$ & $-1.7000000 E+01$ & $-1.7000000 E+01$ & $-1.7000000 E+01$ & $\log \left(m^{\wedge} 2\right)$ & WP038568 & $\mathbf{Y}$ & N/A \\
\hline 181 & 1 & CONSTANT & $-1,7000000 \mathrm{E}+01$ & $-1.7000000 E+01$ & $-1.7000000 \mathrm{E}+01$ & $-1.7000000 E+01$ & $\log \left(m^{\wedge} 2\right)$ & WPO40514 & $Y$ & NA \\
\hline 182 & 1 & CONSTANT & $-1.7000000 E+01$ & $-1.7000000 E+01$ & $-1.7000000 E+01$ & $-1.7000000 E+01$ & $\log \left(m^{\wedge} 2\right)$ & WPO22443 & $\mathbf{Y}$ & N/A \\
\hline 182 & 1 & CONSTANT & $-1.7000000 E+01$ & $-1.7000000 E+01$ & $-1.7000000 E+01$ & $-1.7000000 E+01$ & $\log \left(m^{\wedge} 2\right)$ & $\begin{array}{c}\text { WP030994 } \\
1\end{array}$ & $\mathbf{Y}$ & N/A \\
\hline 182 & 1 & CONSTANT & $-1.7000000 E+01$ & $-1.7000000 \mathrm{E}+01$ & $-1.7000000 E+01$ & $-1.7000000 E+01$ & $\log \left(m^{\wedge} 2\right)$ & WP032038 & $\mathbf{Y}$ & N/A \\
\hline 182 & 1 & CONSTANT & $-1.7000000 E+01$ & $-1.7000000 E+01$ & $-1.7000000 E+01$ & $-1.7000000 E+01$ & $\log \left(m^{\wedge} 2\right)$ & WP032288 & $\mathbf{Y}$ & NA \\
\hline 182 & 1 & CONSTANT & $-1.7000000 E+01$ & $-1.7000000 E+01$ & $-1.7000000 E+01$ & $-1.7000000 E+01$ & $\log \left(m^{\wedge} 2\right)$ & WP038568 & $\mathbf{Y}$ & N/A \\
\hline 182 & 1 & CONSTANT & $-1.7000000 E+01$ & $-1.7000000 E+01$ & $-1.7000000 E+01$ & $-1.7000000 E+01$ & $\log \left(m^{\wedge} 2\right)$ & WP040514 & $\mathbf{Y}$ & N/A \\
\hline 183 & 1 & CONSTANT & $-1.7000000 E+01$ & $-1.7000000 E+01$ & $-1.7000000 E+01$ & $-1.7000000 E+01$ & $\log \left(m^{\wedge} 2\right)$ & WPO22443 & $\mathbf{Y}$ & N/A \\
\hline 183 & 1 & CONSTANT & $-1.7000000 E+01$ & $-1.7000000 \mathrm{E}+01$ & $-1.7000000 E+01$ & $-1.7000000 E+01$ & $\log \left(m^{\wedge} 2\right)$ & WP030994 & $\mathbf{Y}$ & N/A \\
\hline 183 & 1 & CONSTANT & $-1.7000000 E+01$ & $-1.7000000 E+01$ & $-1.7000000 E+01$ & $-1.7000000 E+01$ & $\log \left(m^{\wedge} 2\right)$ & WP032038 & $\mathbf{Y}$ & N/A \\
\hline 183 & 1 & CONSTANT & $-1.7000000 E+01$ & $-1.7000000 E+01$ & $-1.7000000 E+01$ & $-1.7000000 E+01$ & $\log \left(m^{\wedge} 2\right)$ & WP032288 & $\mathbf{Y}$ & NA \\
\hline 183 & 1 & CONSTANT & $-1.7000000 \mathrm{E}+01$ & $-1.7000000 E+01$ & $-1.7000000 E+01$ & $-1.7000000 E+01$ & $\log \left(m^{\wedge} 2\right)$ & WP038568 & $Y$ & NA \\
\hline 183 & 1 & CONSTANT & $-1.7000000 E+01$ & $-1.7000000 E+01$ & $-1.7000000 E+01$ & $-1.7000000 E+01$ & $\log \left(m^{\wedge} 2\right)$ & WP040514 & $\mathbf{Y}$ & N/A \\
\hline 186 & $1,3,4$ & DELTA & $4.0000000 E+\infty 0$ & $4.0000000 E+\infty 0$ & $1.0000000 E+00$ & $4.0000000 E+00$ & NONE & WPO38568 & $\mathbf{Y}$ & NA \\
\hline 186 & $1,3,4$ & DELTA & $4.0000000 E+\infty 0$ & $4.0000000 E+00$ & $1.0000000 E+\infty 0$ & $4.0000000 E+00$ & NONE & WP040514 & $\mathbf{Y}$ & N/A \\
\hline 187 & $1,3,5$ & CONSTANT & $1.0000000 E+00$ & $1.0000000 E+\infty 0$ & $1.0000000 E+00$ & $1.0000000 \mathrm{E}+\infty 0$ & NONE & WPO32288 & $" Y$ & N/A \\
\hline 187 & $1,3,5$ & CONSTANT & $1.0000000 \mathrm{E}+\infty$ & $1.0000000 E+\infty$ & $1.0000000 E+\infty$ & $1.0000000 E+00$ & NONE & WP038568 & $\mathbf{Y}$ & $N / A$ \\
\hline 187 & $1,3,5$ & CONSTANT & $1.0000000 \mathrm{E}+\infty 0$ & $1.0000000 E+\infty$ & $1.0000000 E+\infty$ & $1.0000000 E+\infty 0$ & NONE & WPO40514 & $Y$ & N/A \\
\hline 188 & 3,5 & CONSTANT & $0.0000000 E+\infty 0$ & $0.0000000 E+\infty 0$ & $0.0000000 E+\infty$ & $0.0000000 E+00$ & NONE & WPO32288 & $\mathbf{Y}$ & N/A \\
\hline 188 & 3,5 & CONSTANT & $0.0000000 E+00$ & $0.0000000 E+00$ & $0.0000000 E+\infty$ & $0.0000000 E+00$ & NONE & WP035597 & $\mathbf{Y}$ & N/A \\
\hline 188 & 3,5 & CONSTANT & $0.0000000 E+\infty 0$ & $0.0000000 E+\infty 0$ & $0.0000000 E+\infty 0$ & $0.0000000 E+00$ & NONE & WP040514 & $\mathbf{Y}$ & NA \\
\hline 189 & 3,5 & CONSTANT & $0.0000000 \mathrm{E}+\infty$ & $0.0000000 E+00$ & $0.0000000 E+00$ & $0.0000000 E+\infty 0$ & NONE & WP032288 & $\mathbf{Y}$ & NA \\
\hline 189 & 3,5 & CONSTANT & $0.0000000 E+\infty$ & $0.0000000 E+\infty 0$ & $0.0000000 E+00$ & $0.0000000 E+\infty 0$ & NONE & WP035597 & $\mathbf{Y}$ & N/A \\
\hline
\end{tabular}


CCA Parameter Listing

\begin{tabular}{|c|c|c|c|c|c|c|c|c|c|c|}
\hline $\begin{array}{c}\text { Paramoterin } \\
\text { Databuae? } \\
\text { (YN) }\end{array}$ & \begin{tabular}{|l|} 
If the values \\
used in CoAA \\
are not the \\
eame as in \\
DB, provide *A \\
difference
\end{tabular} & ID & Matorial ID & Unterial Name & $\begin{array}{l}\text { Parameter } \\
\text { iD }\end{array}$ & Partametar Natre & $\begin{array}{l}\text { PRPID } \\
\text { (HPO = ) }\end{array}$ & $\begin{array}{c}\text { Data Enty } \\
\text { Dato }\end{array}$ & $\begin{array}{l}\text { Is the DSA } \\
\text { consistent } \\
\text { wtth the } \\
\text { peramoter } \\
\text { in DB? } \\
(X, N) A]\end{array}$ & $\begin{array}{l}\text { Perameter } \\
\text { Category } \\
\text { (footnote 1) }\end{array}$ \\
\hline$Y$ & & 189 & DRz_o & $\begin{array}{l}\text { Disturbed rock zone; } \\
\text { time period }-5 \text { to } 0 \\
\text { years }\end{array}$ & SAT_RGAS & Resictual Gas Saturation & 32862 & 12 Fob-96 & $\mathbf{Y}$ & AB \\
\hline $\mathbf{Y}$ & $0.0 \mathrm{hw}$ & 190 & DRZ_1 & $\begin{array}{l}\text { Disturbed rock zone; } \\
\text { time period o to } 1000 \\
\text { years }\end{array}$ & CAP_MOD & $\begin{array}{l}\text { Model number, capillary prassuro } \\
\text { model }\end{array}$ & 32869 & 12-feb-96 & $\mathbf{Y}$ & 48 \\
\hline $\mathbf{Y}$ & $0.0 \mathrm{mw}$ & 190 & DRZ_1 & $\begin{array}{l}\text { Disturbed rock zone; } \\
\text { time period } 0 \text { to } 1000 \\
\text { years }\end{array}$ & CAP_MOD & $\begin{array}{l}\text { Model number, capillary pressure } \\
\text { model }\end{array}$ & 32869 & 12 Feb-96 & $\mathbf{Y}$ & 48 \\
\hline $\mathbf{Y}$ & $0.0 \mathrm{mw}$ & 190 & DRZ_1 & $\begin{array}{l}\text { Distubed rock zone; } \\
\text { time period } 0 \text { to } 1000 \\
\text { years }\end{array}$ & CAP_MOD & $\begin{array}{l}\text { Model number, capillary pressure } \\
\text { model }\end{array}$ & 32869 & $12+$ eb-96 & $\mathbf{Y}$ & $4 B$ \\
\hline $\mathbf{Y}$ & & 191 & DRZ_1 & $\begin{array}{l}\text { Disturbed rock zone; } \\
\text { time period } 0 \text { to } 1000 \\
\text { years }\end{array}$ & COMP_RCK & Bulk Compressibility & 32871 & 12-feb-96 & $\mathbf{Y}$ & 48 \\
\hline $\mathbf{Y}$ & & 191 & DRZ_1 & $\begin{array}{l}\text { Disturbed rock zone; } \\
\text { time period } 0 \text { to } 1000 \\
\text { years }\end{array}$ & COMP_RCK & Bulk Compressibitity & 32871 & $12-F a b-96$ & $\mathbf{Y}$ & 48 \\
\hline$Y$ & & 191 & DRZ_1 & $\begin{array}{l}\text { Disturbed rock zone; } \\
\text { time period o to } 1000 \\
\text { years }\end{array}$ & COMP_RCK & Buk Compressibility & 32871 & 12-Feb-96 & $\mathbf{Y}$ & $4 B$ \\
\hline$Y$ & & 193 & DRZ_1 & $\begin{array}{l}\text { Disturbed rock zone; } \\
\text { time period } 0 \text { to } 1000 \\
\text { years }\end{array}$ & PC_MAX & $\begin{array}{l}\text { Maximum allowable capillary } \\
\text { pressure }\end{array}$ & 32899 & 01-Nov-95 & $\mathbf{Y}$ & 48 \\
\hline $\mathbf{Y}$ & & 193 & DRZ_1 & $\begin{array}{l}\text { Disturbed rock zone; } \\
\text { time period } 0 \text { to } 1000 \\
\text { years }\end{array}$ & PC_MAX & $\begin{array}{l}\text { Maximum allowrable capillary } \\
\text { pressure }\end{array}$ & 32899 & 01-Nov-95 & $\mathbf{Y}$ & 48 \\
\hline$Y$ & & 194 & DRZ_1 & $\begin{array}{l}\text { Disturbed rock zone; } \\
\text { time period O to } 1000 \\
\text { years }\end{array}$ & PORE_DIS & $\begin{array}{l}\text { Brooks-Corey pore distribution } \\
\text { parameter }\end{array}$ & 32901 & 12 Fob-96 & $\mathbf{Y}$ & $4 B$ \\
\hline $\mathbf{Y}$ & & 194 & DRZ_1 & $\begin{array}{l}\text { Disturbed rock zone; } \\
\text { time period O to } 1000 \\
\text { years }\end{array}$ & PORE_DIS & $\begin{array}{l}\text { Brooks-Corey pore distribution } \\
\text { paramster }\end{array}$ & 32901 & 12 -Fob-96 & $\mathbf{Y}$ & $4 B$ \\
\hline $\mathbf{Y}$ & & 194 & DRZ_1 & $\begin{array}{l}\text { Disturbed rock zone; } \\
\text { time period } 0 \text { to } 1000 \\
\text { years }\end{array}$ & PORE_DIS & $\begin{array}{l}\text { Brooks-Corey pore distribution } \\
\text { parameter }\end{array}$ & 32901 & 12 Feb-96 & $\mathbf{Y}$ & 48 \\
\hline$Y$ & varies & 195 & DRZ_1 & $\begin{array}{l}\text { Disturbed rock zone; } \\
\text { time period } 0 \text { to } 1000 \\
\text { years }\end{array}$ & POROSITY & Effective porosity & 32902 & 12-feb-96 & $\mathbf{Y}$ & 48 \\
\hline $\mathbf{Y}$ & varies & 195 & DRZ_1 & $\begin{array}{l}\text { Disturbed rock zone; } \\
\text { time period } 0 \text { to } 1000 \\
\text { years }\end{array}$ & POROSITY & Effective porosity & 32902 & $12 \mathrm{Feb}-96$ & $\mathbf{Y}$ & $4 B$ \\
\hline$Y$ & varies & 195 & DRZ_1 & $\begin{array}{l}\text { Disturbed rock zone; } \\
\text { time period } 0 \text { to } 1000 \\
\text { years }\end{array}$ & POROSITY & Effective porosity & 32902 & 12-Fob-96 & $\mathbf{Y}$ & $4 B$ \\
\hline $\mathbf{Y}$ & varies & 195 & DRZ_1 & $\begin{array}{l}\text { Disturbed rock zone; } \\
\text { time period } 0 \text { to } 1000 \\
\text { years }\end{array}$ & POROSTYY & Effective porosity & 32902 & 12-Fob-96 & $Y$ & 48 \\
\hline $\mathbf{Y}$ & varies & 195 & DRZ_1 & $\begin{array}{l}\text { Disturbed rock zone; } \\
\text { time period } 0 \text { to } 1000 \\
\text { years }\end{array}$ & POROSITY & Effective porosity & 32902 & 12 feb-96 & ${ }^{-} \gamma$ & $4 B$ \\
\hline $\mathbf{Y}$ & varies & 195 & DRZ_1 & $\begin{array}{l}\text { Disturbed rock zone; } \\
\text { time period } 0 \text { to } 1000 \\
\text { years }\end{array}$ & POROSTTY & Effective porosity & 32902 & 12-Feb-96 & $\mathbf{Y}$ & $4 B$ \\
\hline $\mathbf{Y}$ & & 196 & DRZ_1 & $\begin{array}{l}\text { Disturbed rock zone; } \\
\text { time period o to } 1000 \\
\text { years }\end{array}$ & PO_MIN & $\begin{array}{l}\text { Mrnimum brine pressure for } \\
\text { capillary model KPC }=3\end{array}$ & 32903 & 21-Feb-96 & $\mathbf{Y}$ & $4 B$ \\
\hline $\mathbf{Y}$ & & 196 & DRZ_1 & $\begin{array}{l}\text { Disturbed rock zone: } \\
\text { time period } 0 \text { to } 1000 \\
\text { years }\end{array}$ & PO_MIN & $\begin{array}{l}\text { Minmum brine pressure for } \\
\text { capillary model KPC=3 }\end{array}$ & 32903 & 21-Fob-96 & $Y$ & AB \\
\hline $\mathbf{Y}$ & & 196 & DRZ_1 & $\begin{array}{l}\text { Disturbed rock zone; } \\
\text { time period O to } 1000 \\
\text { years }\end{array}$ & PO_MIN & $\begin{array}{l}\text { Minimum brine pressure for } \\
\text { capillary model } \mathrm{KPC}=3\end{array}$ & 32903 & 21-Fob-96 & $\mathbf{Y}$ & $4 B$ \\
\hline $\mathbf{Y}$ & & 198 & DRZ_1 & $\begin{array}{l}\text { Disturbed rock zone; } \\
\text { time period o to } 1000 \\
\text { years }\end{array}$ & PRMX_LOG & $\begin{array}{l}\text { Log of intrinsic permeabilty, } X \text { - } \\
\text { drection }\end{array}$ & 32905 & 01-Nov-95 & $\mathbf{Y}$ & 48 \\
\hline $\mathbf{Y}$ & & 198 & DRZ_1 & $\begin{array}{l}\text { Disturbed rock zone; } \\
\text { time period } 0 \text { to } 1000 \\
\text { years }\end{array}$ & PRMX_LOG & $\begin{array}{l}\text { Log of intrinsic permeability, } X \text { - } \\
\text { direction }\end{array}$ & 32905 & 01-Nov-95 & $Y$ & $4 B$ \\
\hline $\mathbf{Y}$ & & 198 & DRZ_I & $\begin{array}{l}\text { Disturbed rock zone; } \\
\text { time period } 0 \text { to } 1000 \\
\text { years }\end{array}$ & PRMX_LOG & $\begin{array}{l}\text { Log of intrinsic permeability, } X \text { - } \\
\text { direction }\end{array}$ & 32905 & 01-Nov-95 & $\mathbf{Y}$ & 48 \\
\hline
\end{tabular}


CCA Parameter Listing

\begin{tabular}{|c|c|c|c|c|c|c|c|c|c|c|}
\hline ID & \begin{tabular}{|c|} 
Clasednt- \\
cation of \\
Data \\
Catogory- \\
(footrote 2)
\end{tabular} & $\begin{array}{l}\text { Detribution } \\
\text { Type }\end{array}$ & Mean & median & Mintroum & Maximum & Units & Roference 10 & $\begin{array}{l}\text { Wos the date } \\
\text { demoloped } \\
\text { under an } \\
\text { NOA.1 } \\
\text { Program? }\end{array}$ & $\begin{array}{c}\text { Which } \\
\text { mothods } \\
\text { wore used to } \\
\text { quallfy } \\
\text { existing } \\
\text { data? } \\
\text { (footnote 3) }\end{array}$ \\
\hline 189 & 3,5 & CONSTANT & $0.0000000 E+\infty 0$ & $0.0000000 E+\infty$ & $0.0000000 E+\infty 0$ & $0.0000000 E+\infty 0$ & NONE & WP040514 & $\mathbf{Y}$ & NA \\
\hline 190 & 3,5 & CONSTANT & $1.0000000 E+\infty$ & $1.0000000 E+\infty 0$ & $1.0000000 E+\infty 0$ & $1.0000000 E+\infty 0$ & NONE & WP032288 & $Y$ & NA \\
\hline 190 & 3,5 & CONSTANT & $1.0000000 \mathrm{E}+00$ & $1.0000000 E+\infty 0$ & $1.0000000 E+\infty 0$ & $1.0000000 E+\infty 0$ & NONE & WP035597 & $Y$ & N/A \\
\hline 190 & 3,5 & CONSTANT & $1.0000000 E+\infty 0$ & $1.0000000 E+\infty 0$ & $1.0000000 E+00$ & $1.0000000 E+\infty 0$ & NONE & WPO40514 & $Y$ & N/A \\
\hline 191 & $1,3,4$ & CONSTANT & $7.4100000 \mathrm{E}-10$ & $7.4100000 \mathrm{E}-10$ & $7.4100000 E-10$ & $7.4100000 E-10$ & $P a^{n}-1$ & WP032288 & $\mathbf{Y}$ & 1 \\
\hline 191 & $1,3,4$ & CONSTANT & $7.4100000 E-10$ & $7.4100000 \mathrm{E}-10$ & $7.4100000 E-10$ & $7.4100000=-10$ & $P a^{n-1}$ & WP035597 & $\mathbf{Y}$ & 1 \\
\hline 191 & $1,3,4$ & CONSTANT & $7.4100000 E-10$ & $7.4100000 \mathrm{E}-10$ & $7.4100000 E-10$ & $7.4100000 E-10$ & Pan-1 & WP040514 & $Y$ & 1 \\
\hline 193 & 3,5 & CONSTANT & $1.0000000 E+08$ & $1.0000000 E+08$ & $1.0000000 E+08$ & $1.0000000 \mathrm{E}+08$ & $\mathrm{~Pa}$ & WP038568 & Y & NA \\
\hline 193 & 3,5 & CONSTANT & $1.0000000 E+08$ & $1.0000000 E+08$ & $1.0000000 E+08$ & $1.0000000 E+08$ & $\mathbf{P a}$ & WPO40514 & $Y$ & N/A \\
\hline 194 & 3,5 & CONSTANT & $7.0000000 E-01$ & $7.0000000 E-01$ & $7.0000000 E-01$ & $7.0000000 \mathrm{E}-01$ & NONE & WPO32288 & $Y$ & NA \\
\hline 194 & 3,5 & CONSTANT & $7.0000000 E-01$ & $7.0000000 E-01$ & $7.0000000 E-01$ & $7.0000000 E-01$ & NONE & WP035597 & $Y$ & NA \\
\hline 194 & 3,5 & CONSTANT & $7.0000000 E-01$ & $7.0000000 E-01$ & $7.0000000 E-01$ & $7.0000000 E-01$ & NONE & WPO40514 & $\mathbf{Y}$ & NA \\
\hline 195 & $1,3,4$ & CUMULATIVE & $1.5650000 E-02$ & $1.2900000 \mathrm{E}-02$ & $3.9000000 E-03$ & $3.2900000 \mathrm{E}-02$ & NONE & WP032288 & $\gamma$ & $N A$ \\
\hline 195 & $1,3,4$ & CUMULATIVE & $1.5650000 E-02$ & $1.2900000 \mathrm{E}-02$ & $3.9000000 E-03$ & $3.2900000 \mathrm{E}-02$ & NONE & WP035268 & $\mathbf{Y}$ & N/A \\
\hline 195 & $1,3,4$ & CUMULATIVE & $1.5650000 E-02$ & $1.2900000 \mathrm{E}-02$ & $3.9000000 E-03$ & $3.2900000 E-02$ & NONE & WP035597 & $Y$ & NA \\
\hline 195 & $1,3,4$ & CUMULATIVE & $1.5650000 E-02$ & $1.2900000 \mathrm{E}-02$ & $3.9000000 E-03$ & $3.2900000 E-02$ & NONE & WPO40434 & $\gamma$ & NA \\
\hline 195 & $1,3,4$ & CUMULATIVE & $1.5650000 E-02$ & $1.2900000 E-02$ & $3.9000000 E-03$ & $3.2900000 E-02$ & NONE & WPO40514 & 'r & NA \\
\hline 195 & $1,3,4$ & CUMULATINE & $1.5650000 \mathrm{E}-02$ & $1.2900000 E-02$ & $3.9000000 E-03$ & $3.2900000 E-02$ & NONE & WP042257 & $Y$ & NAA \\
\hline 196 & 3,5 & CONSTANT & $1.0132500 E+05$ & $1.0132500 E+0.5$ & $1.0132500 E+05$ & $1.0132500 E+05$ & $\mathrm{~Pa}$ & WP035268 & $\mathbf{Y}$ & N/A \\
\hline 196 & 3,5 & CONSTANT & $1.0132500 E+05$ & $1.0132500 E+05$ & $1.0132500 E+05$ & $1.0132500 E+05$ & $\mathrm{~Pa}$ & WP036051 & $\gamma$ & NA \\
\hline 196 & 3,5 & CONSTANT & $1.0132500 E+05$ & $1.0132500 E+05$ & $1.0132500 E+05$ & $1.0132500 E+05$ & $\mathrm{~Pa}$ & WP040514 & $Y$ & N/A \\
\hline 198 & 3,5 & CONSTANT & $-1.5000000 E+01$ & $-1.5000000 E+01$ & $-1.5000000 E+01$ & $-1.5000000 E+01$ & $\log \left(m^{\wedge} 2\right)$ & WP022443 & $\mathbf{Y}$ & N/A \\
\hline 198 & 3,5 & CONSTANT & $-1.5000000 E+01$ & $-1.5000000 E+01$ & $-1.5000000 E+01$ & $-1.5000000 E+01$ & $\log \left(m^{\wedge} 2\right)$ & WP030994 & $\mathbf{Y}$ & N/A \\
\hline 198 & 3,5 & CONSTANT & $-1.5000000 E+01$ & $-1.5000000 \mathrm{E}+01$ & $-1.5000000 E+01$ & $-1.5000000 E+01$ & $\log \left(m^{\wedge} 2\right)$ & WP032288 & Y & N/A \\
\hline
\end{tabular}


CCA Parameter Listing

\begin{tabular}{|c|c|c|c|c|c|c|c|c|c|c|}
\hline $\begin{array}{c}\text { Parameter in } \\
\text { Databses? } \\
\text { (YnN) }\end{array}$ & \begin{tabular}{|c|} 
If the values \\
used In CCA \\
are not the \\
same ses in \\
DB, provide X/ \\
difference
\end{tabular} & 10 & Material ID & Materlal Namo & $\begin{array}{c}\text { Parameter } \\
\text { tD }\end{array}$ & Parameter Rime & $\begin{array}{l}\text { PRPDD } \\
\text { (MPO })\end{array}$ & $\begin{array}{c}\text { Detan Entry } \\
\text { Date }\end{array}$ & $\begin{array}{l}\text { is the } 4 G 4 \\
\text { consistent } \\
\text { with the } \\
\text { perameter } \\
\text { in OB? } \\
(\mathrm{N}, \mathrm{NA})\end{array}$ & $\begin{array}{l}\text { Paramoter } \\
\text { Cetegory } \\
\text { (footnote 1) }\end{array}$ \\
\hline $\mathbf{Y}$ & & 198 & DRZ_1 & $\begin{array}{l}\text { Disturbed rock zone; } \\
\text { time period } 0 \text { to } 1000 \\
\text { years }\end{array}$ & PRMX_LOG & $\begin{array}{l}\text { Log of intrinsic permeability, } X- \\
\text { direction }\end{array}$ & 32905 & $01-$ Nov-95 & $\mathbf{Y}$ & 48 \\
\hline $\mathbf{Y}$ & & 198 & DRZ_I & $\begin{array}{l}\text { Disturbed rock zone; } \\
\text { time period } 0 \text { to } 1000 \\
\text { years }\end{array}$ & PRMX_LOG & $\begin{array}{l}\text { Log of intrinsic permeability, } X \text { - } \\
\text { direction }\end{array}$ & 32905 & 01+Nov-95 & $\mathbf{Y}$ & $4 B$ \\
\hline $\mathbf{Y}$ & & 199 & DRZ_1 & $\begin{array}{l}\text { Disturbed rock zone; } \\
\text { time period } 0 \text { to } 1000 \\
\text { years }\end{array}$ & PRMY_LOG & $\begin{array}{l}\text { Log of intrinsic permeability, Y- } \\
\text { direction }\end{array}$ & 32906 & Oi-Nov-95 & $\mathbf{Y}$ & 48 \\
\hline $\mathbf{Y}$ & & 199 & DRZ_1 & $\begin{array}{l}\text { Disturbed rock zone; } \\
\text { time period } 0 \text { to } 1000 \\
\text { years }\end{array}$ & PRMY_LOG & $\begin{array}{l}\text { Log of intrinsic permeability, } Y \text { - } \\
\text { direction }\end{array}$ & 32906 & 01-Nov-95 & $\mathbf{Y}$ & $4 B$ \\
\hline $\mathbf{Y}$ & & 199 & DRZ_1 & $\begin{array}{l}\text { Disturbed rock zone; } \\
\text { time period } 0 \text { to } 1000 \\
\text { years }\end{array}$ & PRMYYLOG & $\begin{array}{l}\text { Log of intrinsic perm aability, } Y \text { - } \\
\text { direction }\end{array}$ & 32906 & 01-Nov-95 & $\mathbf{Y}$ & $4 B$ \\
\hline $\mathbf{Y}$ & & 199 & DRZ_1 & $\begin{array}{l}\text { Disturbed rock zone; } \\
\text { tine period O to } 1000 \\
\text { years }\end{array}$ & PRMY_LOG & $\begin{array}{l}\text { Log of intrinsic permaabilty, Y- } \\
\text { drection }\end{array}$ & 32906 & 01-Nov-95 & $\mathbf{Y}$ & 48 \\
\hline $\mathbf{Y}$ & & 199 & DRZ_1 & $\begin{array}{l}\text { Disturbed rock zone; } \\
\text { time period } 0 \text { to } 1000 \\
\text { years }\end{array}$ & PRMY_LOG & $\begin{array}{l}\text { Log of intrinsic permaability, Y- } \\
\text { direction }\end{array}$ & 32905 & 01-Nov-95 & $\mathbf{Y}$ & $4 B$ \\
\hline $\mathbf{Y}$ & & 200 & DRZ_1 & $\begin{array}{l}\text { Disturbed rock zone; } \\
\text { time period } 0 \text { to } 1000 \\
\text { years }\end{array}$ & PRMZ_LOG & $\begin{array}{l}\text { Log of intrinsic permeability. Z- } \\
\text { direction }\end{array}$ & 32907 & 01-Nor-95 & $\mathbf{Y}$ & $4 B$ \\
\hline $\mathbf{Y}$ & & 200 & DRZ_1 & $\begin{array}{l}\text { Disturbed rock zone; } \\
\text { time period } 0 \text { to } 1000 \\
\text { years }\end{array}$ & PRMZ_LOG & $\begin{array}{l}\text { Log of intrinsic permsability, Z- } \\
\text { direction }\end{array}$ & 32907 & 01-Nov-95 & $\mathbf{Y}$ & $4 B$ \\
\hline$Y$ & & 200 & DRZ_1 & $\begin{array}{l}\text { Disturbed rock zone; } \\
\text { time period } 0 \text { to } 1000 \\
\text { years }\end{array}$ & PRMZ_LOG & $\begin{array}{l}\text { Log of intrinsic permeability, } z \text { - } \\
\text { direction }\end{array}$ & 32907 & 01-Nov-95 & $\mathbf{Y}$ & $4 B$ \\
\hline $\mathbf{Y}$ & & 200 & DRZ_1 & $\begin{array}{l}\text { Disturbed rock zone; } \\
\text { time period } 0 \text { to } 1000 \\
\text { years }\end{array}$ & PRMZ_LOG & $\begin{array}{l}\text { Log of intrinsic permeability, Z- } \\
\text { direction }\end{array}$ & 32907 & 01-Nov-95 & $\mathbf{Y}$ & AB \\
\hline$Y$ & & 200 & DRZ_1 & $\begin{array}{l}\text { Disturbed rock zoner, } \\
\text { time period } 0 \text { to } 1000 \\
\text { years }\end{array}$ & PRMZ_LOG & $\begin{array}{l}\text { Log of intrinsic permeability, Z- } \\
\text { direction }\end{array}$ & 32907 & 01 Nov.95 & $\mathbf{Y}$ & 48 \\
\hline $\mathbf{Y}$ & & 203 & DRZ_1 & $\begin{array}{l}\text { Disturbed rock zone; } \\
\text { time period } 0 \text { to } 1000 \\
\text { years }\end{array}$ & RELP_MOD & $\begin{array}{l}\text { Model number, relative } \\
\text { permeability model }\end{array}$ & 32910 & 12-Fob-96 & $\mathbf{Y}$ & $A B$ \\
\hline $\mathbf{Y}$ & & 203 & $0 R Z \_1$ & $\begin{array}{l}\text { Disturbed rock zone: } \\
\text { time period } 0 \text { to } 1000 \\
\text { years }\end{array}$ & RELP_MOD & $\begin{array}{l}\text { Model number, relative } \\
\text { permeability model }\end{array}$ & 32910 & 12feb-96 & $\mathbf{Y}$ & $4 B$ \\
\hline $\mathbf{Y}$ & & 203 & DRZ_1 & $\begin{array}{l}\text { Disturbed rock zone; } \\
\text { time period } 0 \text { to } 1000 \\
\text { years }\end{array}$ & RELP_MOD & $\begin{array}{l}\text { Model number, relative } \\
\text { permeability model }\end{array}$ & 32910 & 12-Feb-96 & $\mathbf{Y}$ & 48 \\
\hline $\mathbf{Y}$ & & 203 & DRZ_1 & $\begin{array}{l}\text { Disturbed rock zone: } \\
\text { time period } 0 \text { to } 1000 \\
\text { years }\end{array}$ & RELP_MOD & $\begin{array}{l}\text { Model number, relative } \\
\text { permeability model }\end{array}$ & 32910 & 12 fob-96 & $\mathbf{Y}$ & $4 B$ \\
\hline $\mathbf{Y}$ & & 203 & DRZ_1 & $\begin{array}{l}\text { Disturbed rock zone; } \\
\text { time period } 0 \text { to } 1000 \\
\text { years }\end{array}$ & RELP_MOD & $\begin{array}{l}\text { Model number, relative } \\
\text { permeability model }\end{array}$ & 32910 & 12-Feb-96 & $=\mathbf{Y}$ & $4 B$ \\
\hline $\mathbf{Y}$ & & 205 & DRZ_I & $\begin{array}{l}\text { Disturbed rock zone: } \\
\text { time period } 0 \text { to } 1000 \\
\text { years }\end{array}$ & SAT_RBRN & Residual Brine Saturation & 32912 & 01-Nov-95 & $\mathbf{Y}$ & 48 \\
\hline $\mathbf{Y}$ & & 205 & DRZ_1 & $\begin{array}{l}\text { Disturbed rock zone; } \\
\text { time period } 0 \text { to } 1000 \\
\text { years }\end{array}$ & SAT_RBRN & Residual Brine Saturation & 32912 & 01-Nov-95 & $\mathbf{Y}$ & $4 B$ \\
\hline $\mathbf{Y}$ & & 205 & DRZ_1 & $\begin{array}{l}\text { Disturbed rock zone; } \\
\text { time period } 0 \text { to } 1000 \\
\text { years }\end{array}$ & SAT_RBRN & Residual Brine Saturation & 32912 & 01-Nov-95 & $\mathbf{Y}$ & $4 B$ \\
\hline $\mathbf{Y}$ & & 206 & DRZ_1 & $\begin{array}{l}\text { Disturbed rock zone; } \\
\text { time period } 0 \text { to } 1000 \\
\text { years }\end{array}$ & SAT_RGAS & Residual Gas Saturation & 32913 & 01-Nov.95 & $\mathbf{Y}$ & $4 B$ \\
\hline $\mathbf{Y}$ & & 206 & DRZ_1 & $\begin{array}{l}\text { Disturbed rook zone; } \\
\text { time period } 0 \text { to } 1000 \\
\text { years }\end{array}$ & SAT_RGAS & Residual Gas Saturation & 32913 & 01-Nov-95 & $\mathbf{Y}$ & $4 B$ \\
\hline $\mathbf{Y}$ & & 206 & $D R Z$ 1 & $\begin{array}{l}\text { Disturbed rock zone; } \\
\text { time period } 0 \text { to } 1000 \\
\text { years }\end{array}$ & SAT_RGAS & Residual Gas Saturation & 32913 & 01-Nov-95 & $\mathbf{Y}$ & $4 B$ \\
\hline $\mathbf{Y}$ & & 207 & EXP_AREA & Experimental Area & CAP_MOD & $\begin{array}{l}\text { Model number, capiltary pressure } \\
\text { model }\end{array}$ & 32969 & 17 feb-96 & $\mathbf{Y}$ & $4 B$ \\
\hline $\mathbf{Y}$ & & 207 & EXP_AREA & Experimental Area & CAP_MOD & $\begin{array}{l}\text { Model number, captlary pressure } \\
\text { model }\end{array}$ & 32969 & 17 -Feb-96 & $\mathbf{Y}$ & $4 B$ \\
\hline
\end{tabular}


CCA Parameter Listing

\begin{tabular}{|c|c|c|c|c|c|c|c|c|c|c|}
\hline 10 & $\begin{array}{l}\text { Classfiti- } \\
\text { cation of } \\
\text { Data } \\
\text { Cattogory } \\
\text { (footnote 2) }\end{array}$ & $\begin{array}{l}\text { Distriburtion } \\
\text { Type }\end{array}$ & masen & Median & Minimum & Maximum & Units & Reference ID & $\begin{array}{l}\text { Was the data } \\
\text { developod } \\
\text { under an } \\
\text { NOA-1 } \\
\text { Progran? }\end{array}$ & $\begin{array}{c}\text { Which } \\
\text { mothods } \\
\text { wore used to } \\
\text { quallyy } \\
\text { oxdsting } \\
\text { data? } \\
\text { (footnote 3) }\end{array}$ \\
\hline 198 & 3,5 & CONSTANT & $-1.5000000 E+01$ & $-1.5000000 E+01$ & $-1.5000000 \mathrm{E}+01$ & $-1.5000000 E+01$ & $\log \left(m^{\wedge} 2\right)$ & WPO38568 & $Y$ & N/A \\
\hline 198 & 3,5 & CONSTANT & $-1.5000000 E+01$ & $-1.5000000 E+01$ & $-1.5000000 E+01$ & $-1.5000000 E+01$ & $\log \left(m^{\wedge} 2\right)$ & WPO40514 & $Y$ & NA \\
\hline 199 & 3,5 & CONSTANT & $-1.5000000 E+01$ & $-1.5000000 E+01$ & $-1.5000000 E+01$ & $-1.5000000 E+01$ & $\log \left(m^{\wedge} 2\right)$ & WPO22443 & $\mathbf{Y}$ & NAA \\
\hline 199 & 3,5 & CONSTANT & $-1.5000000 E+01$ & $-1.5000000 \mathrm{E}+01$ & $-1.5000000 E+01$ & $-1.5000000 E+01$ & $\log \left(m^{\wedge} 2\right)$ & WP030994 & $\gamma$ & N/A \\
\hline 199 & 3,5 & CONSTANT & $-1.5000000 E+01$ & $-1.5000000 E+01$ & $-1.5000000 \mathrm{E}+01$ & $-1.5000000 E+0 t$ & $\log \left(m^{\wedge} 2\right)$ & WP032288 & $Y$ & N/A \\
\hline 199 & 3,5 & CONSTANT & $-1.5000000 E+01$ & $-1.5000000 E+01$ & $-1.5000000 E+01$ & $-1.5000000 \mathrm{E}+01$ & $\log \left(m^{\wedge} 2\right)$ & WP038568 & $\gamma$ & N/A \\
\hline 199 & 3,5 & CONSTANT & $-1.5000000 E+01$ & $-1.5000000 E+01$ & $-1.5000000 E+01$ & $-1.5000000 \equiv+01$ & $\log \left(m^{\wedge} 2\right)$ & WPO40514 & $Y$ & NA \\
\hline 200 & 3,5 & CONSTANT & $-1.5000000 E+01$ & $-1.5000000 E+01$ & $-1.5000000 \mathrm{E}+01$ & $-1.5000000 E+01$ & $\log \left(m^{\wedge} 2\right)$ & WPO22443 & $Y$ & N/A \\
\hline 200 & 3,5 & CONSTANT & $-1.5000000 E+01$ & $-1.5000000 E+01$ & $-1.5000000 E+01$ & $-1.5000000 E+01$ & $\log \left(m^{\wedge} 2\right)$ & WP030994 & $\mathbf{Y}$ & N/A \\
\hline 200 & 3,5 & CONSTANT & $-1.5000000 E+01$ & $-1.5000000 E+01$ & $-1.5000000 E+01$ & $-1.5000000 E+01$ & $\log \left(m^{\wedge} 2\right)$ & WP032288 & $\mathbf{Y}$ & NA \\
\hline 200 & 3,5 & CONSTANT & $-1.5000000 E+01$ & $-1.5000000 E+01$ & $-1.5000000 E+01$ & $-1.5000000 E+01$ & $\log \left(m^{\wedge} 2\right)$ & WPO38568 & $Y$ & NA \\
\hline 200 & 3,5 & CONSTANT & $-1.5000000 E+01$ & $-1.5000000 E+01$ & $-1.5000000 E+01$ & $-1.5000000 E+01$ & $\log \left(m^{\wedge} 2\right)$ & WP040514 & $\boldsymbol{\gamma}$ & N/A \\
\hline 203 & $1,3,4$ & DELTA & $4.0000000 \mathrm{E}+00$ & $4.0000000 \mathrm{E}+00$ & $1.0000000 E+\infty 0$ & $4.0000000 E+\infty 0$ & NONE & WP032288 & $Y$ & N/A \\
\hline 203 & $1,3,4$ & DELTA & $4.0000000 \mathrm{E}+\infty 0$ & $4.0000000 E+00$ & $1.0000000 E+00$ & $4.0000000 E+00$ & NONE & WP035268 & $\mathbf{Y}$ & NA \\
\hline 203 & $1,3,4$ & DELTA & $4.0000000 E+\infty 0$ & $4.0000000 E+\infty 0$ & $1.0000000 E+\infty 0$ & $4.0000000 E+\infty 0$ & NONE & WP035597 & $\mathbf{Y}$ & NA \\
\hline 203 & $1,3,4$ & DELTA & $4.0000000 E+\infty 0$ & $4.0000000 E+00$ & $1.0000000 E+00$ & $4.0000000 E+00$ & NONE & WPO37970 & $Y$ & N/A \\
\hline 203 & $1,3,4$ & DELTA & $4.0000000 E+00$ & $4.0000000 \mathrm{E}+00$ & $1.0000000 E+\infty 0$ & $4.0000000 \mathrm{E}+00$ & NONE & WPO40514 & "Y & N/A \\
\hline 205 & 3,5 & CONSTANT & $0.0000000 E+00$ & $0.0000000 E+\infty 0$ & $0.0000000 E+\infty 0$ & $0.0000000 E+00$ & NONE & WP032288 & $\mathbf{Y}$ & N/A \\
\hline 205 & 3,5 & CONSTANT & $0.0000000 E+00$ & $0.0000000 E+00$ & $0.0000000 \mathrm{E}+00$ & $0.0000000 E+00$ & NONE & WP038568 & $\mathbf{Y}$ & NA \\
\hline 205 & 3,5 & CONSTANT & $0.0000000 E+00$ & $0.0000000 \mathrm{E}+00$ & $0.0000000 E+\infty 0$ & $0.0000000 E+00$ & NONE & WPO40514 & $\mathbf{Y}$ & NA \\
\hline 206 & 3,5 & CONSTANT & $0.0000000 E+\infty 0$ & $0.0000000 \mathrm{E}+00$ & $0.0000000 E+00$ & $0.0000000 E+\infty 0$ & NONE & WPO32288 & $Y$ & N/A \\
\hline 206 & 3,5 & CONSTANT & $0.0000000 E+\infty 0$ & $0.0000000 E+00$ & $0.0000000 E+00$ & $0.0000000 E+\infty 0$ & NONE & WP038568 & $Y$ & NA \\
\hline 206 & 3,5 & CONSTANT & $0.0000000 E+\infty 0$ & $0.00000000+00$ & $0.0000000 \mathrm{E}+00$ & $0.0000000 E+00$ & NONE & WPO40514 & $Y$ & N/A \\
\hline 207 & 3,5 & CONSTANT & $1.0000000 E+\infty 0$ & $1.0000000 E+00$ & $1.0000000 E+00$ & $1.0000000 \mathrm{E}+00$ & NONE & WP035597 & $\mathbf{Y}$ & N/A \\
\hline 207 & 3,5 & CONSTANT & $1.0000000 E+\infty 0$ & $1.0000000 E+\infty 0$ & $1.0000000 E+00$ & $1.0000000 E+\infty$ & NONE & WP036320 & $\mathbf{Y}$ & NA \\
\hline
\end{tabular}


CCA Parameter Listing

\begin{tabular}{|c|c|c|c|c|c|c|c|c|c|c|}
\hline $\begin{array}{c}\text { Parameter in } \\
\text { Databese? } \\
\text { (YN) }\end{array}$ & 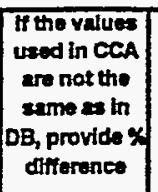 & 10 & Materialo & $\begin{array}{r}\text { Haterial Neme } \\
n-1\end{array}$ & $\begin{array}{c}\text { Parametor } \\
\text { to }\end{array}$ & Parametor Name & $\begin{array}{c}\text { PRP ID } \\
\text { (WPO *) } \\
\because \\
\vdots\end{array}$ & \begin{tabular}{|c|} 
Drta Entry \\
Dats
\end{tabular} & $\begin{array}{l}\text { to the } 464 \\
\text { consistent } \\
\text { with the } \\
\text { perameter } \\
\text { in DB? } \\
(X, N A)\end{array}$ & $\begin{array}{l}\text { Parametor } \\
\text { Category } \\
\text { (footnote 1) }\end{array}$ \\
\hline$Y$ & & 207 & EXP_AREA & Expenmental Area & CAP_MOD & $\begin{array}{l}\text { Model number, capillary pressure } \\
\text { model }\end{array}$ & 32969 & 17freb-96 & $\mathbf{Y}$ & 4B \\
\hline$Y$ & & 208 & EXP_AREA & Expermental Area & COMP_RCK & Bulk Compressibillty & 32972 & 02-Nov-95 & $Y$ & 48 \\
\hline $\bar{Y}$ & & 208 & EXP_AREA & Experimental Area & COMP_RCK & Bulk Compressibility & 32972 & 02-Nov-95 & $\bar{Y}$ & 48 \\
\hline$Y$ & & 208 & EXP_AREA & Experinental Area & COMP_RCK & Bulk Comprossibilty & 32972 & O2-Nov-95 & $Y$ & $\overline{A B}$ \\
\hline $\mathbf{Y}$ & & 209 & EXP_AREA & Experimental Area & PC_max & $\begin{array}{l}\text { Maximum allowabto capillary } \\
\text { pressure }\end{array}$ & 33040 & 02-Nov-95 & $\mathbf{Y}$ & 4B \\
\hline $\boldsymbol{Y}$ & & 209 & EXP_AREA & Experimental Area & PC_maX & $\begin{array}{l}\text { Maxmum allowablo capillary } \\
\text { pressure }\end{array}$ & 33040 & O2-Nov-95 & $Y$ & 4B \\
\hline $\mathbf{Y}$ & & 210 & EXP_AREA & Experimental Area & PORE_DIS & $\begin{array}{l}\text { Brooks-Corey pore distribution } \\
\text { parameter }\end{array}$ & 33045 & 02-Nov-95 & $\mathbf{Y}$ & $4 B$ \\
\hline $\mathbf{Y}$ & & 210 & EXP_AREA & Experimental Area & PORE_DIS & $\begin{array}{l}\text { Brooks-Corey pore distribution } \\
\text { parameter }\end{array}$ & 33045 & 02-Nov-95 & $\mathbf{Y}$ & $4 B$ \\
\hline$Y$ & & 211 & EXP_AREA & Experinental Araa & POROSITY & Effective porosity & 32945 & 17-Fab-96 & $\mathbf{Y}$ & $\mathbf{A B}$ \\
\hline $\bar{Y}$ & & 211 & EXP_AREA & Experimental Area & POROSTY & Effective porosity & 32945 & 17-Feb-96 & $Y$ & 48 \\
\hline $\bar{Y}$ & & 211 & EXP_AREA & Experimental Area & POROSITY & Effective porosity & 32945 & 17-Feb-96 & $\mathbf{Y}$ & 4B \\
\hline $\mathbf{Y}$ & & 212 & EXP_AREA & Experimental Area & PO_MIN & $\begin{array}{l}\text { Minimum brine prossure for } \\
\text { capillary model KPC=3 }\end{array}$ & 32946 & 21 feb-96 & $\mathbf{Y}$ & $4 B$ \\
\hline $\mathbf{Y}$ & & 212 & EXP_AREA & Experimental Area & PO_MIN & $\begin{array}{l}\text { Minimum brine pressure for } \\
\text { capillary model KPC=3 }\end{array}$ & 32946 & 21-Feb-96 & $\mathbf{Y}$ & $4 B$ \\
\hline$Y$ & & 212 & EXP_AREA & Experimental Area & PO_MIN & $\begin{array}{l}\text { Minimum brine pressure for } \\
\text { capillary model } \mathrm{KPC}=3\end{array}$ & 32946 & 21 Fob-96 & $Y$ & $4 B$ \\
\hline $\mathbf{Y}$ & & 212 & EXP_AREA & Experimental Area & PO_MIN & $\begin{array}{l}\text { Minimum brine pressure for } \\
\text { Capiltary model KPC=3 }\end{array}$ & 32946 & 21 fob-96 & $\mathbf{Y}$ & $4 B$ \\
\hline $\bar{Y}$ & & 213 & EXP_AREA & Experimental Area & PRESSURE & Brine far-field pore pressure & 32948 & 02-Nov-95 & $\bar{Y}$ & $4 B$ \\
\hline $\bar{Y}$ & & 213 & EXP_AREA & Experimental Area & PRESSURE & Brine far-field pore pressure & 32948 & 02-Nov-95 & $\bar{Y}$ & $4 B$ \\
\hline $\mathbf{Y}$ & & 214 & EXP_AREA & Experimental Area & PRMX_LOG & $\begin{array}{l}\text { Log of intmsic permeability, } X- \\
\text { direction }\end{array}$ & 32951 & 17 Feb-96 & $\mathbf{Y}$ & 48 \\
\hline $\mathbf{Y}$ & & 214 & EXP_AREA & Experimental Area & PRMX_LOG & $\begin{array}{l}\text { Log of intrinsic permeability, } X \text { - } \\
\text { direction }\end{array}$ & 32951 & $17-F e b-96$ & $Y$ & 48 \\
\hline $\mathbf{Y}$ & & 214 & EXP_AREA & Experimental Area & PRMX_LOG & $\begin{array}{l}\text { Log of intrinsic permeability, X- } \\
\text { direction }\end{array}$ & 32951 & 17fob-96 & $Y$ & $4 B$ \\
\hline$Y$ & & 215 & EXP_AREA & Experimental Area & PRMY_LOG & $\begin{array}{l}\text { Log of intrinsic permesbility, Y- } \\
\text { direction }\end{array}$ & 32953 & 17-feb-96 & $\mathbf{Y}$ & AB \\
\hline $\mathbf{Y}$ & & 215 & EXP_AREA & Experimental Area & PRMY_LOG & $\begin{array}{l}\text { Log of intrinsic permeabilty, Y- } \\
\text { direction }\end{array}$ & 32953 & 17 -Feb-96 & $\mathbf{Y}$ & $4 B$ \\
\hline $\mathbf{Y}$ & & 215 & EXP_AREA & Experimental Area & PRMY_LOG & $\begin{array}{l}\text { Log of intrinsic permeability, Y- } \\
\text { direction }\end{array}$ & 32953 & 17-Feb-96 & $\mathbf{Y}$ & AB \\
\hline $\boldsymbol{Y}$ & & 216 & EXP_AREA & Experimental Area & PRMZ_LOG & $\begin{array}{l}\text { Log of ntrnnsic permeabilty, Z- } \\
\text { direction }\end{array}$ & 32955 & 17-feb-96 & $\mathbf{Y}$ & $4 B$ \\
\hline$Y$ & & 216 & EXP_AREA & Experimental Area & PRMZ_LOG & $\begin{array}{l}\text { Log of intrinsic permeabilty, Z- } \\
\text { drection }\end{array}$ & 32955 & 17 -Fob-96 & $\mathbf{Y}$ & 48 \\
\hline $\mathbf{Y}$ & & 216 & EXP_AREA & Experimental Area & PRMZ_LOG & $\begin{array}{l}\text { Log of intrinsic permsability, Z- } \\
\text { direction }\end{array}$ & 32955 & 17-Fob-96 & $\mathbf{Y}$ & $4 B$ \\
\hline $\mathbf{Y}$ & & 219 & EXP_AREA & Experimental Area & RELP_MOD & $\begin{array}{l}\text { Model number, relative } \\
\text { permeability model }\end{array}$ & 32962 & 02-Nov-95 & $\mathbf{Y}$ & 48 \\
\hline $\mathbf{Y}$ & & 219 & EXP_AREA & Experimontal Area & RELP_MOD & $\begin{array}{l}\text { Model number, relative } \\
\text { permesbility model }\end{array}$ & 32962 & 02-Nov-95 & $\mathbf{Y}$ & 48 \\
\hline $\bar{Y}$ & & 220 & EXP_AREA & Expermental Area & SAT_IBRN & Intial Brme Saturation & 32965 & 17feb-96 & $\mathbf{Y}$ & $4 B$ \\
\hline $\bar{Y}$ & & 220 & EXP_AREA & Experimental Area & SAT_IBRN & Initual Brme Saturation & 32965 & 17-feb-96 & $Y$ & 4B \\
\hline $\bar{Y}$ & & 220 & EXP_AREA & Expenmental Area & SAT_IBRN & Intial Bme Saturation & 32965 & 17-Feb-96 & $\bar{Y}$ & 48 \\
\hline$Y$ & & 221 & EXP_AREA & Experimental Area & SAT_RBRN & Resichual Brine Saturation & 32967 & 17-Feb-96 & $\bar{Y}$ & 48 \\
\hline$Y$ & & 221 & EXP_AREA & Experimental Area & SAT_RBRN & Residual Brine Saturation & 32967 & 17-Feb-96 & $Y$ & $4 B$ \\
\hline $\bar{Y}$ & & 221 & EXP_AREA & Exporimental Area & SAT_RBRN & Residual Brine Saturation & 32967 & 17-Feb-96 & $\mathbf{Y}$ & $4 B$ \\
\hline$Y$ & & 222 & EXP_AREA & Expermental Arsa & SAT_RGAS & Residual Gas Saturation & 32968 & $17 f \circ b-96$ & $\mathbf{Y}$ & $4 B$ \\
\hline$Y$ & & 222 & EXP_AREA & Experimental Area & SAT_RGAS & Residual Gas Saturation & 32968 & 17 Fob- 96 & $Y$ & 48 \\
\hline$Y$ & & 222 & EXP_AREA & Experimental Area & SAT_RGAS & Residual Gas Saturation & 32968 & 17fab-96 & $\mathbf{Y}$ & $4 B$ \\
\hline$\gamma$ & & 223 & GLOBAL & \begin{tabular}{|l|} 
Information that applres \\
globally
\end{tabular} & CLIMTIDX & Climate index & 33031 & 01-May-96 & $\mathbf{Y}$ & 1 \\
\hline $\mathbf{Y}$ & & 223 & GLOBAL & $\begin{array}{l}\text { Information that applies } \\
\text { globally }\end{array}$ & CLIMTIDX & Climato index & 33031 & 01-May-96 & $\mathbf{Y}$ & 1 \\
\hline$Y$ & & 223 & GLOBAL & $\begin{array}{l}\text { Information that applies } \\
\text { globally }\end{array}$ & CLIMTIDX & Climato Index & 33031 & 01+May-96 & $\mathbf{Y}$ & 1 \\
\hline$Y$ & & 225 & GLOBAL & $\begin{array}{l}\text { Information that applies } \\
\text { globally }\end{array}$ & TRANSIDX & $\begin{array}{l}\text { Index for selectung realizations of } \\
\text { the Transmissivity Field }\end{array}$ & 33055 & O2-Nov-95 & $\mathbf{Y}$ & 48 \\
\hline $\mathbf{Y}$ & & 225 & GLOBAL & $\begin{array}{l}\text { Information that applies } \\
\text { globally }\end{array}$ & TRANSIDX & $\begin{array}{l}\text { Index for selecting realizations of } \\
\text { the Transmissivity Field }\end{array}$ & 33055 & 02-Nov-95 & $Y$ & $4 B$ \\
\hline
\end{tabular}


CCA Parameter Listing

\begin{tabular}{|c|c|c|c|c|c|c|c|c|c|c|}
\hline 10. & 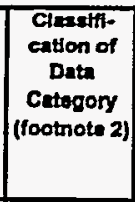 & $\begin{array}{l}\text { Diteribution } \\
\text { Type }\end{array}$ & Mean & Median & Mindmen & Madmum & $\begin{array}{c}\text { Units } \\
\cdot .\end{array}$ & Reforances id & 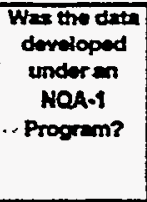 & $\begin{array}{l}\text { Which } \\
\text { methoot } \\
\text { wars uned to } \\
\text { qually } \\
\text { exteting } \\
\text { dita? } \\
\text { (footnote 3) }\end{array}$ \\
\hline 207 & 3,5 & CONSTANT & $1.0000000 E+\infty 0$ & $1.0000000 E+\infty 0$ & $1.0000000 E+\infty 0$ & $1.0000000 \mathrm{E}+\infty 0$ & NONE & WPO40514 & $\mathbf{Y}$ & NA \\
\hline 208 & 3,5 & CONSTANT & $0.0000000 \mathrm{E}+00$ & $0.0000000 E+\infty 0$ & $0.0000000 \mathrm{E}+\infty 0$ & $0.0000000 E+00$ & $\mathrm{~Pa}^{\wedge}-1$ & WP035597 & $\mathbf{Y}$ & NA \\
\hline 208 & 3,5 & CONSTANT & $0.0000000 E+00$ & $0.0000000 E+00$ & $0.0000000 E+00$ & $0.0000000 \mathrm{E}+00$ & $P a^{n}-1$ & WP035320 & $Y$ & NA \\
\hline 208 & 3,5 & CONSTANT & $0.0000000 E+00$ & $0.0000000 E+00$ & $0.0000000 E+\infty 0$ & $0.0000000 \mathrm{E}+00$ & $P a^{n}-1$ & WPO40514 & $Y$ & N/A \\
\hline 209 & 3,5 & CONSTANT & $1.0000000 E+08$ & $1.0000000 \mathrm{E}+08$ & $1.0000000 E+08$ & $1.0000000 \mathrm{E}+08$ & $\mathbf{P a}$ & WP038568 & $\mathbf{Y}$ & N/A \\
\hline 209 & 3,5 & CONSTANT & $1.0000000 E+08$ & $1.0000000 E+08$ & $1,0000000 \mathrm{E}+08$ & $1.0000000 E+08$ & $\mathbf{P a}$ & WPO40514 & $Y$ & NA \\
\hline 210 & 3,5 & CONSTANT & $7.0000000 E-01$ & $7.0000000 E-01$ & 7.0000000E-01 & $7.0000000 E-01$ & NONE & WP0385698 & $\mathbf{Y}$ & NA \\
\hline 210 & 3,5 & CONSTANT & $7.0000000 E-01$ & $7.0000000 E-01$ & $7.0000000 E-01$ & $7.0000000 E-01$ & NONE & WP040514 & $\mathbf{Y}$ & NA \\
\hline 211 & $1,3,4$ & CONSTANT & $1.8000000 \mathrm{E}-01$ & $1.8000000 \mathrm{E}-01$ & $1.8000000 E-01$ & $1.8000000 E-01$ & NONE & WP032281 & $\mathbf{Y}$ & NA \\
\hline 211 & $1,3,4$ & CONSTANT & $1.8000000 \mathrm{E}-01$ & $1.8000000 E-01$ & $1.8000000 \mathrm{E}-01$ & $1.8000000 \mathrm{E}-01$ & NONE & WP035597 & $Y$ & N/A \\
\hline 211 & $1,3,4$ & CONSTANT & $1.8000000 E-01$ & $1.8000000 \mathrm{E}-01$ & $1.8000000 \mathrm{E}-01$ & $1.8000000 \mathrm{E}-01$ & NONE & WPO40514 & $Y$ & N/A \\
\hline 212 & 3,5 & CONSTANT & $1.0132500 E+05$ & $1.0132500 E+05$ & $1.0132500 E+05$ & $1.0132500 E+05$ & $\mathrm{~Pa}$ & WP035597 & $Y$ & NA \\
\hline 212 & 3,5 & CONSTANT & $1.0132500 E+05$ & $1.0132500 E+05$ & $1.0132500 E+05$ & $1.0132500 E+05$ & $\mathbf{P a}$ & WP035051 & $\mathbf{Y}$ & N/A \\
\hline 212 & 3,5 & CONSTANT & $1.0132500 \mathrm{E}+05$ & $1.0132500 E+05$ & $1.0132500 E+05$ & $1.0132500 E+05$ & $\mathrm{~Pa}$ & WPO3 6320 & $\mathbf{Y}$ & NA \\
\hline 212 & 3,5 & CONSTANT & $1.0132500 E+05$ & $1.0132500 E+05$ & $1.0132500 E+05$ & $1.0132500 E+05$ & $\mathbf{P a}$ & WPO40514 & $\mathbf{Y}$ & N/A \\
\hline 213 & $1,3,4$ & CONSTANT & $1.0132500 E+05$ & $1.0132500 E+05$ & $1.0132500 \mathrm{E}+05$ & $1.0132500 E+05$ & $\mathbf{P a}$ & WP038568 & $\mathbf{Y}$ & N/A \\
\hline 213 & $1,3,4$ & CONSTANT & $1.0132500 E+05$ & $1.0132500 E+05$ & $1.0132500 E+05$ & $1.0132500 \mathrm{E}+05$ & $\mathrm{~Pa}$ & WP040514 & $Y$ & N/A \\
\hline 214 & 3,5 & CONSTANT & $-1.1000000 E+01$ & $-1.1000000 E+01$ & $-1.1000000 E+01$ & $-1.1000000 E+01$ & $\log \left(m^{\wedge} 2\right)$ & WP035597 & $\mathbf{Y}$ & NA \\
\hline 214 & 3,5 & CONSTANT & $-1.1000000 E+01$ & $-1.1000000 E+01$ & $-1.1000000 E+01$ & $-1.1000000 E+01$ & $\log \left(m^{\wedge} 2\right)$ & WPO36320 & $\mathbf{Y}$ & N/A \\
\hline 214 & 3,5 & CONSTANT & $-1.1000000 E+01$ & $-1.1000000 E+01$ & $-1.1000000 E+01$ & $-1.1000000 E+01$ & $\log \left(m^{\wedge} 2\right)$ & WPO40514 & $\mathbf{Y}$ & NA \\
\hline 215 & 3,5 & CONSTANT & $-1.1000000 \mathrm{E}+01$ & $-1.1000000 E+01$ & $-1.1000000 \mathrm{E}+01$ & $-1.1000000 E+01$ & $\log \left(m^{\wedge} 2\right)$ & WP035597 & $\mathbf{Y}$ & NA \\
\hline 215 & 3,5 & CONSTANT & $-1.1000000 E+01$ & $-1.1000000 E+01$ & $-1.1000000 E+01$ & $-1.1000000 E+01$ & $\log \left(m^{\wedge} 2\right)$ & WP036320 & $\mathbf{Y}$ & N/A \\
\hline 215 & 3,5 & CONSTANT & $-1.1000000 E+01$ & $-1.1000000 E+01$ & $-1.1000000 E+01$ & $-1.1000000 E+01$ & $\log \left(m^{\wedge} 2\right)$ & WP040514 & $\mathbf{Y}$ & N/A \\
\hline 216 & 3,5 & CONSTANT & $-1.1000000 \mathrm{E}+01$ & $-1.1000000 E+01$ & $-1.1000000 E+01$ & $-1.1000000 E+01$ & $\log \left(m^{\wedge} 2\right)$ & WP035597 & $Y$ & N/A \\
\hline 216 & 3.5 & CONSTANT & $-1.1000000 E+01$ & $-1.1000000 E+01$ & $-1.1000000 E+01$ & $-1.1000000 E+01$ & $\log \left(m^{\wedge} 2\right)$ & WP035320 & $Y$ & N/A \\
\hline 216 & 3,5 & CONSTANT & $-1.1000000 \mathrm{E}+01$ & $-1.1000000 E+01$ & $-1.1000000 E+01$ & $-1.1000000 E+01$ & $\log \left(m^{\wedge} 2\right)$ & WP040514 & $\gamma$ & NA \\
\hline 219 & 3,5 & CONSTANT & $4.0000000 E+00$ & $4.0000000 E+\infty 0$ & $4.0000000 \mathrm{E}+\infty 0$ & $4.0000000 E+\infty 0$ & NONE & WP038568 & $\mathbf{Y}$ & NA \\
\hline 219 & 3,5 & CONSTANT & $4.0000000 \mathrm{E}+00$ & $4.0000000 E+\infty 0$ & $4.0000000 E+\infty 0$ & $4.0000000 E+00$ & NONE & WP040514 & $Y$ & NA \\
\hline 220 & 3.5 & CONSTANT & $0.0000000 E+00$ & $0.0000000 E+00$ & $0.0000000 E+\infty 0$ & $0.0000000 E+\infty 0$ & NONE & WP035597 & $Y$ & NA \\
\hline 220 & 3,5 & CONSTANT & $0.0000000 E+\infty 0$ & $0.0000000 E+00$ & $0.0000000 E+00$ & $0.0000000 E+00$ & NONE & WP036320 & $\mathbf{Y}$ & N/A \\
\hline 220 & 3,5 & CONSTANT & $0.0000000 \mathrm{E}+\infty$ & $0.0000000 E+00$ & $0.0000000 \mathrm{E}+\infty 0$ & $0.0000000 E+00$ & NONE & WP040514 & $Y$ & N/A \\
\hline 221 & 3,5 & CONSTANT & $0.0000000 \mathrm{E}+00$ & $0.0000000 \mathrm{E}+00$ & $0.0000000 \mathrm{E}+00$ & $0.0000000 \mathrm{E}+00$ & NONE & WP035597 & $Y$ & NA \\
\hline 221 & 3,5 & CONSTANT & $0.0000000 \mathrm{E}+00$ & $0.0000000 \mathrm{E}+00$ & $0.0000000 \mathrm{E}+00$ & $0.0000000 \mathrm{E}+00$ & NONE & WPO36320 & $Y$ & NA \\
\hline 221 & 3,5 & CONSTANT & $0.0000000 E+\infty$ & $0.0000000 E+00$ & $0.0000000 \mathrm{E}+\infty 0$ & $0.0000000 \mathrm{E}+00$ & NONE & WP040514 & $Y$ & NA \\
\hline 222 & 3,5 & CONSTANT & $0.0000000 \mathrm{E}+00$ & $0.0000000 E+00$ & $0.0000000 E+\infty$ & $0.0000000 E+00$ & NONE & WP035597 & $\mathbf{Y}$ & N/A \\
\hline 222 & 3.5 & CONSTANT & $0.0000000 \mathrm{E}+00$ & $0.0000000 E+00$ & $0.0000000 \mathrm{E}+\infty 0$ & $0.0000000 \mathrm{E}+00$ & NONE & WPO36320 & $\bar{Y}$ & N/A \\
\hline 222 & 3,5 & CONSTANT & $0.0000000 \mathrm{E}+00$ & $0.0000000 \mathrm{E}+\infty 0$ & $0.0000000 \mathrm{E}+00$ & $0.0000000 \mathrm{E}+\infty$ & NONE & WPO40514 & $Y$ & N/A \\
\hline 223 & $1,3,4,5$ & CUMULATIVE & $1.3100000 E+00$ & $1.1700000 E+\infty 0$ & $1.0000000 E+00$ & $2.2500000 E+\infty 0$ & NONE & WP035268 & N & 1 \\
\hline 223 & $1,3,4,5$ & CUMULATIVE & $1.3100000 \mathrm{E}+\infty 0$ & $1.1700000 E+00$ & $1.0000000 \mathrm{E}+\infty 0$ & $2.2500000 \mathrm{E}+00$ & NONE & WP035597 & $\mathbf{N}$ & 1 \\
\hline 223 & $1,3,4,5$ & CUMULATINE & $1.3100000 E+\infty 0$ & $1.1700000 E+00$ & $1.0000000 E+\infty$ & $2.2500000 \mathrm{E}+00$ & NONE & WP037465 & $\mathbf{N}$ & 1 \\
\hline 225 & $1,3,4$ & UNIFORM & $5.0000000 E-01$ & $5.0000000 E-01$ & $0.0000000 E+\infty 0$ & $1.0000000 \mathrm{E}+00$ & NONE & SAND92-0700/3 & YZN & 1 \\
\hline 225 & $1,3,4$ & UNIFORM & $5.0000000 E-01$ & $5.0000000 E-01$ & $0.0000000 E+\infty 0$ & $1.0000000 \mathrm{E}+00$ & NONE & WPO35193 & Y8N & 1 \\
\hline
\end{tabular}


CCA Parameter Listing

\begin{tabular}{|c|c|c|c|c|c|c|c|c|c|c|}
\hline $\begin{array}{c}\text { Paramotar in } \\
\text { Drtabese? } \\
\text { (YN) }\end{array}$ & 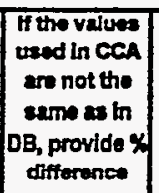 & ID & Meterial io & Matarial Namo & $\begin{array}{c}\text { Parametex } \\
\text { ID }\end{array}$ & Parameter Name & $\begin{array}{l}\text { PRPID } \\
\text { (MPON) }\end{array}$ & $\begin{array}{c}\text { Dexta Entry } \\
\text { Dato }\end{array}$ & 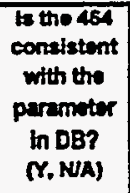 & $\begin{array}{l}\text { Paramoter } \\
\text { Category } \\
\text { (footrote 1) }\end{array}$ \\
\hline$Y$ & & 225 & GLOBAL & $\begin{array}{l}\text { Information that applies } \\
\text { globally }\end{array}$ & TRANSIDX & $\begin{array}{l}\text { Index for selecting realzations of } \\
\text { the Transmissivity Field }\end{array}$ & 33055 & 02-Nov-95 & $\mathbf{Y}$ & AB \\
\hline$Y$ & & 225 & GLOBAL & $\begin{array}{l}\text { Information that applias } \\
\text { globally }\end{array}$ & TRANSIDX & $\begin{array}{l}\text { Index for selocing reatrations of } \\
\text { the Transmissivity Field }\end{array}$ & 33055 & 02-Nov-95 & $\mathbf{Y}$ & AB \\
\hline $\mathbf{Y}^{\circ}$ & & 228 & $\mathrm{H}_{2}$ & Hydrogen Gas & visco & Viscosity & $32334 \mathrm{~A}$ & 14-Mar-96 & $Y$ & 3 \\
\hline$Y$ & & 228 & $\mathrm{H} 2$ & Hydrogen Gas & visco & Viscosity & $32334 \mathrm{~A}$ & 14-Mar-96 & $Y$ & 3 \\
\hline$Y$ & & 229 & IMPERM_Z & Impermesble Zones & CAP_MOD & $\begin{array}{l}\text { Model number, capilary pressure } \\
\text { model }\end{array}$ & 33059 & 02-Nov-95 & $\mathbf{Y}$ & 4B \\
\hline$Y$ & & 229 & IMPERM_Z & Impermeable Zones & CAP_MOD & $\begin{array}{l}\text { Model number, capillary pressure } \\
\text { model }\end{array}$ & 33059 & 02-Nov-95 & $\mathbf{Y}$ & $4 B$ \\
\hline$Y$ & & 230 & IMPERM_Z & Impermeable Zones & COMP_RCK & Bulk Compressibility & 33064 & 02-Nov-95 & $Y$ & 48 \\
\hline $\bar{Y}$ & & 230 & IMPERM_Z & Impermeable Zones & COMP_RCK & Bulk Compressibility & 33064 & 02-Nov-95 & $Y$ & $4 B$ \\
\hline$Y$ & & 231 & IMPERM_Z & Impermeable Zones & PC_MAX & $\begin{array}{l}\text { Maximum allowable capillary } \\
\text { pressure }\end{array}$ & 33177 & 02-Nov-95 & $\mathbf{Y}$ & $4 B$ \\
\hline$Y$ & & 231 & IMPERM_Z & Impormeable Zones & PC_MAX & $\begin{array}{l}\text { Maximum allowable capillary } \\
\text { pressure }\end{array}$ & 33177 & 02-Nov-95 & $\mathbf{Y}$ & 4B \\
\hline $\mathbf{Y}$ & & 232 & IMPERM_Z & |lmpermaable Zones & PORE_DIS & $\begin{array}{l}\text { Brooks-Corey pore distribution } \\
\text { parameter }\end{array}$ & 33185 & 27-Fab-96 & $\mathbf{Y}$ & AB \\
\hline $\mathbf{Y}$ & & 232 & IMPERM_Z & Impermeable Zones & PORE_DIS & $\begin{array}{l}\text { Brooks-Corey pore distribution } \\
\text { parameter }\end{array}$ & 33185 & 27 feb-9s & $Y$ & 48 \\
\hline $\mathbf{Y}$ & & 232 & IMPERM_Z & Impermeable Zones & PORE_DIS & $\begin{array}{l}\text { Brooks-Corey pore distribution } \\
\text { parameter }\end{array}$ & 33185 & 27-Feb-96 & $\mathbf{Y}$ & $4 B$ \\
\hline $\mathbf{Y}$ & & 233 & IMPERM_Z & Impermeable Zones & POROSTY & Effective porosity & 33188 & 02-Nov-95 & $Y$ & 48 \\
\hline$Y$ & & 233 & IMPERM_Z & Impermeable Zones & POROSTY & Effective porosity & 33188 & 02-Nov-95 & $Y$ & 4B \\
\hline$Y$ & & 234 & IMPERM_Z & Impermeabłe Zones & PO_MIN & $\begin{array}{l}\text { Minimum brine pressure for } \\
\text { capillary model KPC }=3\end{array}$ & 33191 & $21+8 \mathrm{~b}-96$ & $\mathbf{Y}$ & 4B \\
\hline$Y$ & & 234 & IMPERM_Z & Impermeable Zones & PO_MIN & $\begin{array}{l}\text { Minimum brine pressure for } \\
\text { capillary model KPC }=3\end{array}$ & 33191 & $21-\mathrm{feb}-96$ & $\mathbf{Y}$ & $4 B$ \\
\hline $\mathbf{Y}$ & & 234 & IMPERM_Z & Impermeable Zones & PO_MIN & $\begin{array}{l}\text { Minimium brine pressure for } \\
\text { capillary model KPC=3 }\end{array}$ & 33191 & 21-Fob-96 & $\mathbf{Y}$ & 4B \\
\hline $\mathbf{Y}$ & & 236 & IMPERM_Z & Impermeable Zones & PRMX_LOG & $\begin{array}{l}\text { Log of intrinsic permeability, X- } \\
\text { direction }\end{array}$ & 33205 & 22fob-96 & $\mathbf{Y}$ & 4B \\
\hline $\mathbf{Y}$ & & 236 & IMPERM_Z & impermeable Zones & PRMX_LOG & $\begin{array}{l}\text { Log of intrinsic permeability, X- } \\
\text { direction }\end{array}$ & 33205 & 22-Fob-96 & $\mathbf{Y}$ & 4B \\
\hline$Y$ & & 237 & IMPERM_Z & Impermeable Zones & PRMY_LOG & $\begin{array}{l}\text { Log of intrinsic permeability, } Y \text { - } \\
\text { direction }\end{array}$ & 33209 & 22-Fab-96 & $\mathbf{Y}$ & 4B \\
\hline $\mathbf{Y}$ & & 237 & IMPERM_Z & Impermeable Zones & PRMY_LOG & $\begin{array}{l}\text { Log of intrinsic permeabilty, } Y \text { - } \\
\text { direction }\end{array}$ & 33209 & 22Fab-96 & $Y$ & 48 \\
\hline $\mathbf{Y}$ & & 238 & IMPERM_Z & Impermeable Zones & PRMZ_LOG & $\begin{array}{l}\text { Log of intrinsic permeabilty, Z- } \\
\text { direction }\end{array}$ & 33214 & 22fob-96 & $\mathbf{Y}$ & $4 B$ \\
\hline$Y$ & & 238 & IMPERM_Z & Impermeable Zones & PRMZ_LOG & $\begin{array}{l}\text { Log of intrinsic permeability, Z- } \\
\text { direction }\end{array}$ & 33214 & 22feb-96 & $\mathbf{Y}$ & 4B \\
\hline $\mathbf{Y}$ & & 241 & IMPERM_Z & Impermeable Zones & RELP_MOD & $\begin{array}{l}\text { Model number, relativa } \\
\text { permeability model }\end{array}$ & 33222 & 02-Nov-95 & $Y$ & $4 B$ \\
\hline Y & & 241 & IMPERM_Z & Impermeable Zones & RELP_MOD & $\begin{array}{l}\text { Model number, relative } \\
\text { permeability model }\end{array}$ & 33220 & 02-Nov-95 & $\mathbf{Y}$ & $4 B$ \\
\hline$Y$ & & 243 & IMPERM_Z & Impermeable Zones & SAT_RBRN & Residual Brine Saturation & 33226 & 02-Nov-95 & $-Y$ & 48 \\
\hline$Y$ & & 243 & IMPERM_Z & Impermeable Zones & SAT_RBRN & Residual Brine Saturation & 33226 & 02-Nov-95 & $Y$ & 4B \\
\hline $\mathbf{Y}$ & & 244 & IMPERM_Z & Impermeable Zones & SAT_RGAS & Residual Gas Saturation & 33230 & O2-Nov-95 & $Y$ & $4 B$ \\
\hline$Y$ & & 244 & IMPERM_Z & impermeable Zones & SAT_RGAS & Residual Gas Saturation & 33230 & 02-Nov-95 & $Y$ & $4 B$ \\
\hline$Y$ & 0.02 & 246 & NP237 & Neptunium 237 & ATWEIGHT & Atomic Weight in kg/mole & 32336 & 02-Nov-95 & $\bar{Y}$ & 3 \\
\hline$Y$ & 0.02 & 246 & NP237 & Neptunium 237 & ATWEIGHT & Atomic Weight in $\mathrm{kg} / \mathrm{mole}$ & 32336 & 02-Nov-95 & $Y$ & 3 \\
\hline$Y$ & 0.02 & 246 & NP237 & Nepturium 237 & ATWEIGHT & Atomic Weight in $\mathrm{kg} / \mathrm{mole}$ & 32336 & 02-Nov-95 & $y$ & 3 \\
\hline$Y$ & 100 & 247 & NP237 & Neptunium 237 & HALFLIFE & Haltifo & 32579 & 16-Apr-96 & $Y$ & 3 \\
\hline$Y$ & 100 & 247 & NP237 & Noptunium 237 & HALFLIFE & Hathifo & 32579 & 16-Apr-86 & $\bar{Y}$ & 3 \\
\hline$\gamma$ & 100 & 247 & NP237 & Noptunium 237 & HALFLIFE & Halnife & 32579 & $16-A p r-96$ & $\mathbf{Y}$ & 3 \\
\hline $\mathbf{Y}$ & & 248 & NP237 & Neptunium 237 & INVCHD & $\begin{array}{l}\text { Imventory of Contact Handled } \\
\text { Design }\end{array}$ & $32584 \mathrm{~A}$ & $29-0 c t-96$ & $\mathbf{Y}$ & 2 \\
\hline$Y$ & & 248 & NP237 & Neptunium 237 & INVCHD & $\begin{array}{l}\text { Inventory of Contact Handled } \\
\text { Design }\end{array}$ & $32584 A$ & $29-0 c t-96$ & $Y$ & 2 \\
\hline$\gamma$ & & 248 & NP237 & Neptunium 237 & INVCHD & $\begin{array}{l}\text { Inventory of Contact Handled } \\
\text { Design }\end{array}$ & $32584 \mathrm{~A}$ & 29-0ct-96 & $\mathbf{Y}$ & 2 \\
\hline$Y$ & & 248 & NP237 & Neptunium 237 & INVCHD & $\begin{array}{l}\text { Inventory of Contact Handled } \\
\text { Design }\end{array}$ & $32584 \mathrm{~A}$ & 29-0ct-96 & $Y$ & 2 \\
\hline$Y$ & & 249 & NP237 & Neptunium 237 & INVRHD & $\begin{array}{l}\text { Inventory of Remote Handled } \\
\text { Design }\end{array}$ & $32593 A$ & 29-0ct-96 & $\mathbf{Y}$ & 2 \\
\hline$Y$ & & 249 & NP237 & Neptunium 237 & INURHD & $\begin{array}{l}\text { Inventory of Remote Handled } \\
\text { Design }\end{array}$ & $32593 A$ & $29-0 \mathrm{ct}-96$ & $\mathbf{Y}$ & 2 \\
\hline
\end{tabular}


CCA Parameter Listing

\begin{tabular}{|c|c|c|c|c|c|c|c|c|c|c|}
\hline 10 & $\begin{array}{c}\text { Clasedfi. } \\
\text { catton of } \\
\text { Datu } \\
\text { Catugory } \\
\text { (footnote 2) }\end{array}$ & $\begin{array}{l}\text { Dutubution } \\
\text { Type }\end{array}$ & $\operatorname{man}$ & Medien & Mintmenn & Moxinum & Ints & Reforence 10 & $\begin{array}{l}\text { Wes the date } \\
\text { doveloped } \\
\text { under in } \\
\text { NQA-1 } \\
\text { Program? }\end{array}$ & $\begin{array}{c}\text { Which } \\
\text { methocts } \\
\text { were used to } \\
\text { qually } \\
\text { oxteting } \\
\text { datin? } \\
\text { (footnote 3) }\end{array}$ \\
\hline 225 & $1,3,4$ & UNIFORM & $5.0000000 \mathrm{E}-01$ & $5.0000000 E-01$ & $0.0000000 \mathrm{E}+00$ & $1.0000000 \mathrm{E}+\infty 0$ & NONE & WPO35270 & Y\&N & 1 \\
\hline 225 & $1,3,4$ & UNIFORM & $5.00000000-01$ & $5.0000000 E-01$ & $0.0000000 E+\infty 0$ & $1.0000000 E+\infty 0$ & NONE & WPO40516 & Y8N & 1 \\
\hline 228 & 4 & CONSTANT & $8.9338900 E-06$ & $8.9338900 \mathrm{E}-06$ & $8.9338900 E-06$ & $8.9338900 E-06$ & Pa's & WP030641 & $\mathbf{Y}$ & NA \\
\hline 228 & 4 & CONSTANT & $8.9338900 E-06$ & $8.9338900 E-06$ & 8.9338900E-06 & 8.9338900E-06 & Pa's & WP035298 & $\mathbf{Y}$ & N/A \\
\hline 229 & 3,5 & CONSTANT & $1.0000000 E+\infty 0$ & $1.0000000 E+\infty 0$ & $1.0000000 E+\infty 0$ & $1.0000000 E+\infty$ & NONE & WP038558 & $Y$ & NA \\
\hline 229 & 3,5 & CONSTANT & $1.0000000 E+\infty 0$ & $1.0000000 E+\infty 0$ & $1.0000000 E+\infty 0$ & $1.0000000 E+00$ & NONE & WP040514, & $\mathbf{Y}$ & N/A \\
\hline 230 & 3,5 & CONSTANT & $0.0000000 E+00$ & $0.0000000 E+00$ & $0.0000000 E+00$ & $0.0000000 E+\infty$ & $P_{a n-1}$ & WPO38568 & $Y$ & N/A \\
\hline 230 & 3,5 & CONSTANT & $0.0000000 E+\infty 0$ & $0.0000000 E+00$ & $0.0000000 E+00$ & $0.0000000 \mathrm{E}+\infty 0$ & $P_{a}-1$ & WP040514 & $Y$ & NA \\
\hline 231 & 3,5 & CONSTANT & $1.0000000 E+08$ & $1.0000000 E+08$ & $1.0000000 E+08$ & $1.0000000 E+08$ & $\mathrm{~Pa}$ & WP038568 & $\mathbf{Y}$ & N/A \\
\hline 231 & 3,5 & CONSTANT & $1.0000000 \mathrm{E}+08$ & $1.0000000 E+08$ & $1.0000000 E+08$ & $1.0000000 E+08$ & $\mathbf{P a}$ & WP040514 & $\gamma$ & N/A \\
\hline 232 & 3,5 & CONSTANT & $7.0000000 E-01$ & $7.0000000 \mathrm{E}-09$ & $7.0000000 E-01$ & $7.0000000 E-01$ & NONE & WP035597 & $\mathbf{Y}$ & N/A \\
\hline 232 & 3,5 & CONSTANT & $7.0000000 E-01$ & $7.0000000 E-01$ & $7.0000000 \mathrm{E}-01$ & $7.0000000 E-01$ & NONE & WP036203 & $\mathbf{Y}$ & NA \\
\hline 232 & 3,5 & CONSTANT & $7.0000000 E-01$ & $7.0000000 E-01$ & $7.0000000 E-01$ & $7.0000000 \mathrm{E}-01$ & NONE & WPO40514 & $Y$ & NA \\
\hline 233 & 3.5 & CONSTANT & $5.0000000 \mathrm{E}-03$ & $5.0000000 E-03$ & $5.0000000 \mathrm{E}-03$ & $5.0000000 E-03$ & NONE & WP038568 & $Y$ & N/A \\
\hline 233 & 3,5 & CONSTANT & $5,0000000 \mathrm{E}-03$ & $5.0000000 \mathrm{E}-03$ & $5.0000000 \mathrm{E}-03$ & $5.0000000 \mathrm{E}-03$ & NONE & WPO40514 & $Y$ & N/A \\
\hline 234 & 3,5 & CONSTANT & $1.0132500 E+05$ & $1.0132500 E+05$ & $1.0132500 E+05$ & $1.0132500 E+05$ & $\mathrm{~Pa}$ & WP035597 & $Y$ & N/A \\
\hline 234 & 3,5 & CONSTANT & $1,0132500 E+05$ & $1.0132500 E+05$ & $1.0132500 E+05$ & $1.0132500 E+05$ & $\mathbf{P a}$ & WP036051 & $\mathbf{Y}$ & N/A \\
\hline 234 & 3,5 & CONSTANT & $1.0132500 E+05$ & $1.0132500 E+05$ & $1.0132500 \mathrm{E}+0 \mathrm{~S}$ & $1.0132500 E+05$ & $\mathbf{P a}$ & WP040514 & $\mathbf{Y}$ & N/A \\
\hline 236 & 3,5 & CONSTANT & $-3.5000000 \mathrm{E}+01$ & $-3.5000000 E+01$ & $-3.5000000 E+01$ & $-3.5000000 E+01$ & $\log \left(m^{\wedge} 2\right)$ & WP038333 & $\mathbf{Y}$ & NA \\
\hline 236 & 3,5 & CONSTANT & $-3.5000000 E+01$ & $3.5000000 E+01$ & $-3.5000000 \mathrm{E}+01$ & $-3.5000000 E+01$ & $\log \left(m^{\wedge} 2\right)$ & WP040514 & $Y$ & N/A \\
\hline 237 & 3,5 & CONSTANT & $-3.5000000 E+01$ & $-3.5000000 E+01$ & $-3.5000000 \mathrm{E}+01$ & $-3.5000000 E+01$ & $\log \left(m^{\wedge} 2\right)$ & WP038333 & $\mathbf{Y}$ & NA \\
\hline 237 & 3,5 & CONSTANT & $-3.5000000 E+01$ & $-3.5000000 E+01$ & $-3.5000000 E+01$ & $-3.5000000 E+01$ & $\log \left(m^{\wedge} 2\right)$ & WPO40514 & $\mathbf{\gamma}$ & N/A \\
\hline 238 & 3,5 & CONSTANT & $-3.5000000 E+01$ & $-3.5000000 \mathrm{E}+01$ & $-3.5000000 \mathrm{E}+01$ & $-3.5000000 E+01$ & $\log \left(m^{\wedge} 2\right)$ & WPO38333 & $\mathbf{Y}$ & N/A \\
\hline 238 & 3,5 & CONSTANT & $-3.5000000 E+01$ & $-3.5000000 E+01$ & $-3.5000000 E E+01$ & $-3.5000000 E+01$ & $\log \left(m^{\wedge} 2\right)$ & WP040514 & $\mathbf{Y}$ & N/A \\
\hline 241 & 3,5 & CONSTANT & $4.0000000 E+\infty 0$ & $4.0000000 E+00$ & $4.0000000 \mathrm{E}+\infty 0$ & $4.0000000 \mathrm{E}+00$ & NONE & WPO38568 & $\mathbf{Y}$ & N/A \\
\hline 241 & 3,5 & CONSTANT & $4.0000000 E+00$ & $4.0000000 E+\infty 0$ & $4.0000000 \mathrm{E}+\infty 0$ & $4.0000000 E+\infty 0$ & NONE & WPO40514 & $\mathbf{Y}$ & N/A \\
\hline 243 & 3,5 & CONSTANT & $0.0000000 E+00$ & $0.0000000 E+\infty 0$ & $0.0000000 E+00$ & $0.0000000 E+\infty$ & NONE & WP038568 & $\boldsymbol{-}_{-Y}$ & N/A \\
\hline 243 & 3,5 & CONSTANT & $0.0000000 E+\infty 0$ & $0.0000000 E+\infty$ & $0.0000000 E+00$ & $0.0000000 E+\infty 0$ & NONE & WP040514 & $\mathbf{Y}$ & NA \\
\hline 244 & 3,5 & CONSTANT & $0.0000000 E+\infty 0$ & $0.0000000 E+\infty 0$ & $0.0000000 E+00$ & $0.0000000 E+\infty 0$ & NONE & WP038568 & $Y$ & N/A \\
\hline 244 & 3,5 & CONSTANT & $0.0000000 \mathrm{E}+00$ & $0.0000000 E+\infty 0$ & $0.0000000 E+\infty 0$ & $0.0000000 \mathrm{E}+00$ & NONE & WP040514 & $Y$ & N/A \\
\hline 246 & 4 & CONSTANT & $2.3704800 E-01$ & $2.3704600 \mathrm{E}-01$ & $2.3704800 E-01$ & 23704800E-01 & $\mathrm{kg} /$ molo & CRC-72 & $\mathbf{Y}$ & N/A \\
\hline 246 & 4 & CONSTANT & $2.3704800 E-01$ & $2.3704800 E-01$ & 2.3704800E-01 & 2.3704800E-01 & $\mathrm{kg} / \mathrm{mole}$ & WPO35298 & $Y$ & N/A \\
\hline 246 & 4 & CONSTANT & $2.3704800 \mathrm{E}-01$ & $2.3704800 \mathrm{E}-01$ & $2.3704800 E-01$ & $2.3704800 E-01$ & $\mathrm{~kg} / \mathrm{mol}$ & WP036301 & $Y$ & NA \\
\hline 247 & 4 & CONSTANT & $6.7530000 E+13$ & $6.7530000 \mathrm{E}+13$ & $6.7530000 E+13$ & $6.7530000 \mathrm{E}+13$ & $s$ & WP036301 & $\bar{Y}$ & N/A \\
\hline 247 & 4 & CONSTANT & $6.7530000 E+13$ & $6.7530000 \mathrm{E}+13$ & $6.7530000 \mathrm{E}+13$ & $6.7530000 \mathrm{E}+13$ & 5 & WP037404 & $Y$ & N/A \\
\hline 247 & 4 & CONSTANT & $6.7530000 \mathrm{E}+13$ & $6.7530000 E+13$ & $6.7530000 E+13$ & $6.7530000 \mathrm{E}+13$ & $\mathbf{s}$ & WPO40434 & $\mathbf{Y}$ & N/A \\
\hline 248 & 2 & CONSTANT & $6.19000000 E+01$ & $6.1900000 E+01$ & $6.1900000 E+01$ & $6.1900000 E+01$ & $\mathrm{Ci}$ & WP037061 & 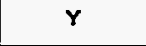 & N/A \\
\hline 248 & 2 & CONSTANT & $6.1900000 E+01$ & $6.1900000 E+01$ & $6.1900000 \mathrm{E}+01$ & $6.1900000 E+01$ & $\mathrm{Ci}$ & WP039260 & $\gamma$ & N/A \\
\hline 248 & 2 & CONSTANT & $6.1900000 E+01$ & $6.1900000 E+01$ & $6.1900000 E+01$ & $6.1900000 E+01$ & $\mathrm{Ci}$ & WPO41559 & $Y$ & NA \\
\hline 248 & 2 & CONSTANT & $6.1900000 E+01$ & $6.1900000 E+01$ & $6.1900000 E+01$ & $6.1900000 E+01$ & Ci & WPO41560 & $Y$ & N/A \\
\hline 249 & 2 & CONSTANT & $2.9500000 \mathrm{E}+00$ & $2.9500000 \mathrm{E}+00$ & $2.9500000 E+\infty$ & $29500000 \mathrm{E}+\infty 0$ & $\mathrm{Ci}$ & WP037061 & $Y$ & N/A \\
\hline 249 & 2 & CONSTANT & $2.9500000 E+\infty 0$ & $29500000 E+00$ & $2.9500000 E+\infty 0$ & $2.9500000 E+\infty 0$ & $\mathrm{Ci}$ & WP039260 & $\mathbf{Y}$ & N/A \\
\hline
\end{tabular}


CCA Parameter Listing

\begin{tabular}{|c|c|c|c|c|c|c|c|c|c|c|}
\hline $\begin{array}{l}\text { Paramotarth } \\
\text { Databato? } \\
\text { (YWN) }\end{array}$ & \begin{tabular}{|c|} 
Hitho values \\
used in CCA \\
are not the \\
eame es in \\
DB, provide $x$ \\
difference
\end{tabular} & ID & Material to & Matoria Name & $\begin{array}{c}\text { Paramoter } \\
\text { DD }\end{array}$ & Paramoter Narmo & $\begin{array}{l}\text { PRP ID } \\
\text { (WPO } 1 \text { I }\end{array}$ & $\begin{array}{c}\text { Deta Entry } \\
\text { Date }\end{array}$ & $\begin{array}{l}\text { Is the } 484 \\
\text { conalatent } \\
\text { wth the } \\
\text { parameter } \\
\text { in DB? } \\
(N, N A)\end{array}$ & $\begin{array}{l}\text { Paremoter } \\
\text { Category } \\
\text { (footnote 1) }\end{array}$ \\
\hline $\mathbf{Y}$ & & 249 & NP237 & Neptunium 237 & INVRHD & $\begin{array}{l}\text { Inventory of Remole Handled } \\
\text { Design }\end{array}$ & $32593 A$ & $29-0 c t-96$ & $\mathbf{Y}$ & 2 \\
\hline$\gamma$ & & 249 & NP237 & Neptunium 237 & INVRHD & $\begin{array}{l}\text { Imventory of Remote Handled } \\
\text { Design }\end{array}$ & $32593 A$ & $29-0 c t-96$ & $\gamma$ & 2 \\
\hline$Y$ & 0.02 & 250 & PA231 & Protactinium 231 & ATWEIGHT & Atomic Weight in $\mathrm{kg} / \mathrm{molo}$ & 32337 & 02-Nov-95 & $Y$ & 3 \\
\hline$Y$ & 0.02 & 250 & PA231 & Protactinium 231 & ATWEIGHT & Atomic Weight in kg/mole & 32337 & 02-Nov-95 & $Y$ & 3 \\
\hline$Y$ & 0.02 & 250 & PA231 & Protactinium 231 & ATWEIGHT & Atomic Weight in $\mathrm{kg} / \mathrm{mole}$ & 32337 & O2-Nov-95 & $\mathbf{Y}$ & 3 \\
\hline $\bar{Y}$ & 100 & 251 & PA231 & Protactinium 231 & HALFLIFE & Hattife & 32929 & 16-Apr-96 & $\bar{Y}$ & 3 \\
\hline$Y$ & 100 & 251 & PA231 & Protactinium 231 & HALFLIFE & Halfife & 32929 & 16-Apr-96 & $Y$ & 3 \\
\hline$Y$ & 100 & 251 & PA231 & Protactinium 231 & HALFLIFE & Halflife & 32929 & 16-Apr-96 & $Y$ & 3 \\
\hline $\mathbf{Y}$ & $50.00 \mathrm{mw}$ & 252 & PAN_SEAL & Panel Seal & CAP_MOD & $\begin{array}{l}\text { Model number, capillary pressure } \\
\text { model }\end{array}$ & 32933 & 20-Fob-96 & $\mathbf{Y}$ & 4B \\
\hline $\mathbf{Y}$ & $50.00 \mathrm{mw}$ & 252 & PAN_SEAL & Panel Seal & CAP_MOD & $\begin{array}{l}\text { Model number, capillary pressure } \\
\text { model }\end{array}$ & 32933 & 20-feb-96 & $\mathbf{Y}$ & 4B \\
\hline$Y$ & $50.00 \mathrm{mw}$ & 252 & PAN_SEAL & Panel Seal & CAP_MOD & $\begin{array}{l}\text { Model number, capillary prossure } \\
\text { model }\end{array}$ & 32933 & 20-Fob-96 & $\mathbf{Y}$ & AB \\
\hline$Y$ & & 253 & PAN_SEAL & Panel Seal & COMP_RCK & Bulk Compressibitity & 32935 & 20-Fob-96 & $\mathbf{Y}$ & AB \\
\hline $\mathbf{Y}$ & & 253 & PAN_SEAL & Panel Seal & COMP_RCK & Bulk Compressibility & 32935 & $20-F 6 b-96$ & $\bar{Y}$ & 4B \\
\hline $\bar{Y}$ & & 253 & PAN_SEAL & Panol Seal & COMP_RCK & Bulk Compressibility & 32935 & 20-Feb-96 & $Y$ & 4B \\
\hline$Y$ & & 254 & PAN_SEAL & Panel Seal & PC_maX & $\begin{array}{l}\text { Maximum allowable capillary } \\
\text { pressure }\end{array}$ & 33109 & 02-Nov-95 & $\mathbf{Y}$ & AB \\
\hline $\mathbf{Y}$ & & 254 & PAN_SEAL & Panel Seal & PC_max & $\begin{array}{l}\text { Maximum allowable capillary } \\
\text { pressure }\end{array}$ & 33109 & 02-Nov-95 & $\mathbf{Y}$ & 48 \\
\hline $\mathbf{Y}$ & & 254 & PAN_SEAL & Panel Seal & PC_MAX & $\begin{array}{l}\text { Maximum allowable capillary } \\
\text { pressure }\end{array}$ & 33109 & 02-Nov-95 & $\gamma$ & $4 B$ \\
\hline$Y$ & & 255 & PAN_SEAL & Panel Seal & PORE_DIS & $\begin{array}{l}\text { Brooks-Corey pore distribution } \\
\text { parameter }\end{array}$ & 33112 & $20-F a b-96$ & $\mathbf{Y}$ & 48 \\
\hline$Y$ & & 255 & PAN_SEAL & Panel Seal & PORE_DIS & $\begin{array}{l}\text { Brooks-Corey pore distribution } \\
\text { parameter }\end{array}$ & 33112 & 20-fab-96 & $\mathbf{Y}$ & 48 \\
\hline$Y$ & & 255 & PAN_SEAL & Panel Seal & PORE_DIS & $\begin{array}{l}\text { Brooks-Corey pore distribution } \\
\text { parameter }\end{array}$ & 33112 & 20-Feb-96 & $\mathbf{Y}$ & 48 \\
\hline$Y$ & & 256 & PAN_SEAL & Panel Sea! & POROSITY & Effective porosity & 33113 & 02-Nov-95 & $Y$ & $4 B$ \\
\hline $\mathbf{Y}$ & & 256 & PAN_SEAL & Panel Sea! & POROSITY & Effective porosity & 33113 & 02-Nov-95 & $Y$ & 48 \\
\hline $\bar{Y}$ & & 256 & PAN_SEAL & Panel Seal & POROSTY & Effective porosity & 33113 & 02-Nov-95 & $Y$ & 4B \\
\hline$Y$ & & 257 & PAN_SEAL & Panel Seal & PO_MIN & $\begin{array}{l}\text { Minutnum brine pressure for } \\
\text { capillary modal } \mathrm{KPC}=3\end{array}$ & 33115 & 21Fob-96 & $\mathbf{Y}$ & $4 B$ \\
\hline$Y$ & & 257 & PAN_SEAL & Panol Seal & PO_MIN & $\begin{array}{l}\text { Munimum brine pressuro for } \\
\text { capillary model KPC }=3\end{array}$ & 33115 & 21-Fab-96 & $\mathbf{Y}$ & 4B \\
\hline $\mathbf{Y}$ & & 257 & PAN_SEAL & Panel Seal & PO_MIN & $\begin{array}{l}\text { Minimum brine pressure for } \\
\text { capillary model KPC }=3\end{array}$ & 33115 & 21feb-96 & $\mathbf{Y}$ & $4 B$ \\
\hline$Y$ & & 258 & PAN_SEAL & Panel Seal & PRESSURE & Brine far-field pore pressure & 33118 & 06-Fob-96 & $\mathbf{Y}$ & 48 \\
\hline $\mathbf{Y}$ & & 258 & PAN_SEAL & Panel Seal & PRESSURE & Brine far-field pore pressure & 33118 & 06-Fob-96 & $Y$ & AB \\
\hline $\mathbf{Y}$ & & 258 & PAN_SEAL & Panel Seal & PRESSURE & Brine far-field pore pressure & 33118 & 06 -Fab-96 & $\bar{Y}$ & 4B \\
\hline $\mathbf{Y}$ & & 259 & PAN_SEAL & Panel Seal & PRMX_LOG & $\begin{array}{l}\text { Log of intrinsic permeability, } X- \\
\text { direction }\end{array}$ & 33120 & 20-Fob-96 & $\mathbf{Y}$ & 4B \\
\hline$Y$ & & 259 & PAN_SEAL & Panel Seal & PRMX_LOG & $\begin{array}{l}\text { Log of intrinsic permeability, X- } \\
\text { direction }\end{array}$ & 33120 & 20-Fob-96 & $\mathbf{Y}$ & $4 B$ \\
\hline$Y$ & & 259 & PAN_SEAL & Panel Seal & PRMX_LOG & $\begin{array}{l}\text { Log of intrnsic permeability, X- } \\
\text { direction }\end{array}$ & 33120 & 20-Fob-96 & $Y$ & 48 \\
\hline$Y$ & & 260 & PAN_SEAL & Panel Seal & PRMY_LOG & $\begin{array}{l}\text { Log of intrinsic permeabilty, Y- } \\
\text { direction }\end{array}$ & 33122 & 20-Feb-9s & $\mathbf{Y}$ & 4B \\
\hline$Y$ & & 260 & PAN_SEAL & Panel Seal & PRMY_LOG & $\begin{array}{l}\text { Log of intrinsic permeability, Y- } \\
\text { direction }\end{array}$ & 33122 & 20-Feb-96 & $\mathbf{Y}$ & 4B \\
\hline $\mathbf{Y}$ & & 260 & PAN_SEAL & Panel Seal & PRMY_LOG & $\begin{array}{l}\text { Log of intrinsic permeability, } Y \text { - } \\
\text { direction }\end{array}$ & 33122 & 20-Fab-96 & $Y$ & $4 B$ \\
\hline$Y$ & & 261 & PAN_SEAL & Panel Saal & PRMZ_LOG & $\begin{array}{l}\text { Log of intrinsic permeabilty, Z- } \\
\text { direction }\end{array}$ & 33123 & 20-Fob-96 & $Y$ & 4B \\
\hline $\mathbf{Y}$ & & 261 & PAN_SEAL & Panel Seal & PRMZ_LOG & $\begin{array}{l}\text { Log of intrinsic permeability, z- } \\
\text { direction }\end{array}$ & 33123 & 20-Fob-96 & $\mathbf{Y}$ & 4B \\
\hline$Y$ & & 261 & PAN_SEAL & Panel Seal & PRMZ_LOG & $\begin{array}{l}\text { Log of intrnsic perm eabiltty, Z- } \\
\text { direction }\end{array}$ & 33123 & 20-fob-96 & $\mathbf{Y}$ & AB \\
\hline $\mathbf{Y}$ & & 264 & PAN_SEAL & Panel Seal & RELP_MOD & $\begin{array}{l}\text { Model number, rolative } \\
\text { permeability model }\end{array}$ & 33131 & O2-Nov-95 & $\mathbf{Y}$ & 4B \\
\hline$Y$ & & 264 & PAN_SEAL & Panel Seal & RELP_MOD & $\begin{array}{l}\text { Model number, relative } \\
\text { permeability model }\end{array}$ & 33131 & 02-Nov-95 & $\mathbf{Y}$ & 4B \\
\hline
\end{tabular}


CCA Parameter Listing

\begin{tabular}{|c|c|c|c|c|c|c|c|c|c|c|}
\hline DD & $\begin{array}{c}\text { Cinatifi- } \\
\text { Cation of } \\
\text { Dath } \\
\text { Crtegory } \\
\text { (footnote 2) }\end{array}$ & $\begin{array}{c}\text { Deatribution } \\
\text { Type }\end{array}$ & Mean & Median & Minimutrm & . & Units & $\begin{array}{c}\text { Referenco ID } \\
\ldots\end{array}$ & $\begin{array}{l}\text { Was the data } \\
\text { doveloped } \\
\text { under an } \\
\text { NOA-1 } \\
\text { Program? }\end{array}$ & $\begin{array}{c}\text { Wrich } \\
\text { mothoch } \\
\text { werv used to } \\
\text { qually } \\
\text { existing } \\
\text { dinta? } \\
\text { (footnote s) }\end{array}$ \\
\hline 249 & 2 & CONSTANT & $2.9500000 E+\infty 0$ & $2.9500000 \mathrm{E}+\infty 0$ & $2.9500000 E+\infty$ & $2.9500000 E+\infty 0$ & $\mathrm{Ci}$ & WP041559 & $\mathbf{Y}$ & NA \\
\hline 249 & 2 & CONSTANT & $2.9500000 E+00$ & $2.9500000 \mathrm{E}+00$ & $2.9500000 E+\infty 0$ & $2.9500000 \mathrm{E}+\infty 0$ & $\mathrm{Ci}$ & WPO41560 & $Y$ & NA \\
\hline 250 & 4 & CONSTANT & $2.3103600 \mathrm{E}-01$ & $2.3103600 E-01$ & 2.3103600E-01 & $2.3103600 E-01$ & kglmole & CRC-72 & $Y$ & NA \\
\hline 250 & 4 & CONSTANT & 2.3103600E-01 & 2.3103600E-01 & 2.3103600E-01 & $23103600 E-01$ & kg'mole & WP036298 & $Y$ & NA \\
\hline 250 & 4 & CONSTANT & $2.3103600 E-01$ & 2.3103600E-01 & $2.3103600 E-01$ & $2.3103600 E-01$ & kg/mole & WP035301 & $\mathbf{Y}$ & NA \\
\hline 251 & 4 & CONSTANT & $1.0340000 E+12$ & $1.0340000 E+12$ & $1.0340000 E+12$ & $1.0340000 \mathrm{E}+12$ & 5 & WP036301 & $Y$ & NA \\
\hline 251 & 4 & CONSTANT & $1.0340000 \mathrm{E}+12$ & 1.0340000E+12 & $1.0340000 E+12$ & $1.0340000 \mathrm{E}+12$ & $\mathbf{s}$ & WP037404 & $Y$ & NA \\
\hline 251 & 4 & CONSTANT & $1.0340000 E+12$ & $1.0340000 E+12$ & $1.0340000 E+12$ & $1.0340000 E+12$ & $s$ & WPO40434 & $\mathbf{Y}$ & NA \\
\hline 252 & 1,3 & CONSTANT & $2.0000000 E+00$ & $2.0000000 E+00$ & $-2.0000000 E+00$ & $2.0000000 E+\infty 0$ & NONE & $\begin{array}{c}\text { VAUGHN: } \\
\text { WORKSHEET-4 } \\
\end{array}$ & $\mathbf{Y}$ & NA \\
\hline 252 & 1,3 & CONSTANT & $2.0000000 \mathrm{E}+00$ & $2.0000000 E+00$ & $2.0000000 E+\infty 0$ & $2.0000000 E+\infty 0$ & NONE & WP035597 & $\mathbf{Y}$ & N/A \\
\hline 252 & 1,3 & CONSTANT & $2.0000000 E+00$ & $2.0000000 E+00$ & $2.0000000 E+\infty 0$ & $2.0000000 E+00$ & NONE & WP040514 & $Y$ & N/A \\
\hline 253 & 1,3 & CONSTANT & $2.6400000 \mathrm{E}-09$ & $2.6400000 E-09$ & $2.6400000 E-09$ & $2.6400000 E-09$ & $P a^{n}-1$ & $\begin{array}{c}\text { VAUGHN: } \\
\text { WORKSHEET-4 }\end{array}$ & $\mathbf{Y}$ & N/A \\
\hline 253 & 1,3 & CONSTANT & $2.6400000 \mathrm{E}-09$ & $26400000 \mathrm{E}-09$ & $26400000 E-09$ & $2.6400000 E-09$ & $P a^{\wedge}+1$ & WP035597 & $Y$ & N/A \\
\hline 253 & 1,3 & CONSTANT & $2.6400000 \mathrm{E}-09$ & $2.6400000 \mathrm{E}-09$ & $2.6400000 \mathrm{E}-09$ & $2.6400000 \mathrm{E}-09$ & $P a^{n}-1$ & WP040514 & $Y$ & N/A \\
\hline 254 & 1.3 & CONSTANT & $1.0000000 E+08$ & $1.0000000 E+08$ & $1.0000000 E+08$ & $1.0000000 E+08$ & $\mathbf{P a}$ & $\begin{array}{c}\text { VAUGHN: } \\
\text { WORKSHEET-4 } \\
\end{array}$ & $\mathbf{Y}$ & N/A \\
\hline 254 & 1,3 & CONSTANT & $1.0000000 \mathrm{E}+08$ & $1.0000000 E+08$ & $1.0000000 \mathrm{E}+08$ & $1.0000000 E+08$ & $\mathbf{P a}$ & WP035597 & $\boldsymbol{\gamma}$ & NA \\
\hline 254 & 1,3 & CONSTANT & $1.0000000 E+08$ & $1.0000000 E+08$ & $1.0000000 E+08$ & $1.0000000 \mathrm{E}+08$ & $\mathrm{~Pa}$ & WP040514 & $\mathbf{Y}$ & NA \\
\hline 255 & 1,3 & CONSTANT & $9.4000000 E-01$ & $9.4000000 E-01$ & $9.4000000 E-01$ & $9.4000000 E-01$ & NONE & $\begin{array}{c}\text { VAUGHN: } \\
\text { WORKSHEET-4 } \\
\end{array}$ & $Y$ & NA \\
\hline 255 & 1,3 & CONSTANT & $9.4000000 E-01$ & $9.4000000 E-01$ & $9.4000000 E-01$ & $9.4000000 E-01$ & NONE & WP035597 & $\mathbf{Y}$ & NA \\
\hline 255 & 1,3 & CONSTANT & $9.4000000 E-01$ & $9.4000000 \mathrm{E}-01$ & $9.4000000 E-01$ & $9.4000000 E-01$ & NONE & WPO40514 & $\mathbf{Y}$ & N/A \\
\hline 256 & 3,5 & CONSTANT & $7.5000000 \mathrm{E}-02$ & $7.5000000 \mathrm{E}-02$ & $7.5000000 \mathrm{E}-02$ & $7.5000000 E-02$ & NONE & $\begin{array}{c}\text { VAUGHN: } \\
\text { WORKSHEET-1 } \\
\end{array}$ & $\mathbf{Y}$ & NA \\
\hline 256 & 3,5 & CONSTANT & $7.5000000 \mathrm{E}-02$ & $7.5000000 E-02$ & $7.5000000 E-02$ & $7.5000000 E-02$ & NONE & WP035597 & $Y$ & N/A \\
\hline 256 & 3,5 & CONSTANT & $7.5000000 \mathrm{E}-02$ & $7.5000000 \mathrm{E}-02$ & $7.5000000 \mathrm{E}-02$ & $7.5000000 E-02$ & NONE & WP040514 & $Y$ & N/A \\
\hline 257 & 1,3 & CONSTANT & $1.0132500 E+05$ & $1.0132500 E+05$ & $1.0132500 E+05$ & $1.0132500 E+05$ & $\mathrm{~Pa}$ & WP035597 & $\mathbf{Y}$ & NA \\
\hline 257 & 1,3 & CONSTANT & $1.0132500 E+05$ & $1.0132500 E+05$ & $1.0132500 E+05$ & $1.0132500 E+05$ & $\mathbf{P a}$ & WP036051 & $Y$ & NA \\
\hline 257 & 1.3 & CONSTANT & $1.0132500 E+05$ & $1.0132500 E+05$ & $1.0132500 E+05$ & $1.0132500 E+05$ & $\mathrm{~Pa}$ & WPO40514 & $Y$ & NA \\
\hline 258 & 1,3 & CONSTANT & $1.0132500 E+05$ & $1.0132500 E+05$ & $1.0132500 E+05$ & $1.0132500 E+05$ & $\mathbf{P a}$ & $\begin{array}{c}\text { VAUGHN: } \\
\text { WORKSHEET-1 }\end{array}$ & $\mathbf{Y}$ & N/A \\
\hline 258 & 1.3 & CONSTANT & $1.0132500 E+05$ & $1.0132500 \mathrm{E}+0 \mathrm{~S}$ & $1.0132500 E+05$ & $1.0132500 E+0.5$ & $\mathrm{~Pa}$ & WP035597 & $Y$ & NA \\
\hline 258 & 1,3 & CONSTANT & $1.0132500 E+05$ & $1.0132500 E+05$ & $1.0132500 E+05$ & $1.0132500 E+05$ & $\mathrm{~Pa}$ & WPO40514 & $Y$ & NA \\
\hline 259 & 1.3 & CONSTANT & $-1.5000000 E+01$ & $-1.5000000 E+01$ & $-1.5000000 E+01$ & $-1.5000000 E+01$ & $\log \left(m^{\wedge} 2\right)$ & $\begin{array}{c}\text { VAUGHN: } \\
\text { WORKSHEET-4 } \\
\end{array}$ & $Y$ & N/A \\
\hline 259 & 1,3 & CONSTANT & $-1.5000000 E+01$ & $-1.5000000 E+01$ & $-1.5000000 E+01$ & $-1.5000000 E+01$ & $\log \left(m^{\wedge} 2\right)$ & WP035597 & $Y$ & NA \\
\hline 259 & 1,3 & CONSTANT & $-1.5000000 E+01$ & $-1.5000000 E+01$ & $-1.50000000 E+01$ & $-1.5000000 E+01$ & $\log \left(m^{\wedge} 2\right)$ & WP040514 & $\mathbf{Y}$ & N/A \\
\hline 260 & 1,3 & CONSTANT & $-1,5000000 E+01$ & $-1.5000000 E+01$ & $-1.5000000 E+01$ & $-1.5000000 E+01$ & $\log \left(m^{\wedge} 2\right)$ & $\begin{array}{c}\text { VAUGHN: } \\
\text { WORKSHEET } 4 \\
\end{array}$ & $Y$ & NA \\
\hline 260 & 1,3 & CONSTANT & $-1.5000000 \mathrm{E}+01$ & $-1.5000000 E+01$ & $-1.5000000 E+01$ & $-1.5000000 E+01$ & $\log \left(m^{\wedge} 2\right)$ & WP035597 & $Y$ & NA \\
\hline 260 & 1,3 & CONSTANT & $-1.5000000 \mathrm{E}+01$ & $-1.5000000 E+01$ & $-1.5000000 \mathrm{E}+01$ & $-1.5000000 E+01$ & $\log \left(m^{\wedge} 2\right)$ & WP040514 & $Y$ & NA \\
\hline 261 & 1,3 & CONSTANT & $-1,5000000 E+01$ & $-1.5000000 E+01$ & $-\$ .5000000 E+01$ & $-1.5000000 E+01$ & $\log \left(m^{\wedge} 2\right)$ & $\begin{array}{c}\text { VAUGHN: } \\
\text { WORKSHEET-4 }\end{array}$ & $Y$ & NA \\
\hline 261 & 1,3 & CONSTANT & $-1.5000000 E+01$ & $-1.5000000 E+01$ & $-1.5000000 E+01$ & $-1.5000000 \mathrm{E}+01$ & $\log \left(m^{\wedge} 2\right)$ & WP035597 & $Y$ & N/A \\
\hline 261 & 1,3 & CONSTANT & $-1.5000000 E+01$ & $-1.5000000 \mathrm{E}+01$ & $-1.5000000 E+01$ & $-1.5000000 E+01$ & $\log \left(m^{\wedge} 2\right)$ & WPO40514 & $Y$ & NA \\
\hline 264 & 1,3 & CONSTANT & $4.0000000 E+00$ & $4.0000000 E+\infty 0$ & $4.0000000 E+\infty 0$ & $4.0000000 E+\infty 0$ & NONE & $\begin{array}{c}\text { VAUGHN: } \\
\text { WORKSHEET-4 }\end{array}$ & $\mathbf{Y}$ & NA \\
\hline 264 & 1,3 & CONSTANT & $4.0000000 \mathrm{E}+00$ & $4.0000000 \mathrm{E}+\infty 0$ & $4.0000000 \mathrm{E}+00$ & $4.0000000 \mathrm{E}+00$ & NONE & WP035597 & $\gamma$ & NA \\
\hline
\end{tabular}


CCA Parameter Listing

\begin{tabular}{|c|c|c|c|c|c|c|c|c|c|c|}
\hline $\begin{array}{c}\text { Paramoter in } \\
\text { Databaee? } \\
\text { (WN) }\end{array}$ & $\begin{array}{l}\text { If the values } \\
\text { uned in CCA } \\
\text { ore not the } \\
\text { eame as in } \\
\text { DB, provide } \% \\
\text { difference }\end{array}$ & ID & Matertal ID & Waterial Name & $\begin{array}{l}\text { Paramoter } \\
\text { ID }\end{array}$ & Parmeter Kam & $\begin{array}{l}\text { PRPID } \\
\text { (NPO }=1\end{array}$ & $\begin{array}{c}\text { Data Entry } \\
\text { Dets }\end{array}$ & $\begin{array}{l}\text { Is the } 464 \\
\text { consictent } \\
\text { with the } \\
\text { parameter } \\
\text { in DBP } \\
(Y, N \text { N) }\end{array}$ & $\begin{array}{l}\text { Paramoter } \\
\text { Cutegory } \\
\text { (footnote 1) }\end{array}$ \\
\hline $\mathbf{Y}$ & & 264 & PAN_SEAL & Panel Seal & RELP_MOD & $\begin{array}{l}\text { Model number, relatrve } \\
\text { permeability model }\end{array}$ & 33131 & 02-Nov-95 & $\mathbf{Y}$ & $4 B$ \\
\hline $\mathbf{Y}$ & & 265 & PAN_SEAL & Panel Seal & SAT_RBRN & Residual Brine Saturation & 33139 & 02-Noy-95 & $\mathbf{Y}$ & $A B$ \\
\hline $\mathbf{Y}$ & & 265 & PAN_SEAL & Panel Seal & SAT_RBRN & Residual Brine Saturation & 33139 & 02 Nov-95 & $\mathbf{Y}$ & $4 B$ \\
\hline $\mathbf{Y}$ & & 265 & PAN_SEAL & Panel Seal & SAT_RBRN & Residual Brine Saturation & 33139 & 02Nox-95 & $\mathbf{Y}$ & 48 \\
\hline $\mathbf{Y}$ & & 266 & PAN_SEAL & Panel Seal & SAT_RGAS & Resicual Gas Saturation & 33141 & 02-Nov-95 & $\mathbf{Y}$ & $4 B$ \\
\hline $\mathbf{Y}$ & & 266 & PAN_SEAL & Panel Seal & SAT_RGAS & Residual Gas Saturation & 33141 & O2-Nov.95 & $\mathbf{Y}$ & 4B \\
\hline$Y$ & & 266 & PAN_SEAL & Panel Seal & SAT_RGAS & Residual Gas Saturation & 33141 & 02-Nov-95 & $Y$ & $4 B$ \\
\hline $\mathbf{Y}$ & & 282 & PB & Lead & LOGSOLM & Log of the Radionudide Solubility & 33213 & 13-May-96 & $\mathbf{Y}$ & $4 B$ \\
\hline $\mathbf{Y}$ & & 282 & PB & Lead & LOGSOLM & Log of the Radionuclide Solubility & 33213 & 13-May-96 & $\mathbf{Y}$ & 48 \\
\hline $\mathbf{Y}$ & & 282 & PB & Lead & LOGSOLM & Log of the Radionuclide Solubility & 33213 & $13-M a y-96$ & $\mathbf{Y}$ & 48 \\
\hline $\mathbf{Y}$ & 0.01 & 283 & PB210 & Lead 210 & ATWEIGHT - & Atomic Waight in kg/mole & 32338 & O2-Nov-95 & $\mathbf{Y}$ & 3 \\
\hline $\mathbf{Y}$ & 0.01 & 283 & PB210 & Lead 210 & ATWEIGHT & Atomic Wejght in kg/mole & 32338 & 02-Nov-95 & $\mathbf{Y}$ & 3 \\
\hline $\mathbf{Y}$ & 0.01 & 283 & PB210 & Lead 210 & ATWEIGHT & Atomic Weight in $\mathrm{kg} / \mathrm{mole}$ & 32338 & 02-Nov-95 & $\mathbf{Y}$ & 3 \\
\hline $\mathbf{Y}$ & 100 & 284 & PB210 & Lead 210 & HALFLIFE & Halfifife & 33218 & 16-Apr-96 & $\mathbf{Y}$ & 3 \\
\hline $\mathbf{Y}$ & 100 & 284 & PB210 & Lead 210 & HALFLIFE & Halfifo & 33218 & 16-Apr-96 & $Y$ & 3 \\
\hline $\mathbf{Y}$ & 100 & 284 & PB210 & Lead 210 & HALFLIFE & Hatfifo & 33218 & 16-Apr-96 & $\mathbf{Y}$ & 3 \\
\hline $\mathbf{Y}$ & & 285 & PB210 & Lead 210 & INVCHD & $\begin{array}{l}\text { Inventory of Contact Handled } \\
\text { Design }\end{array}$ & $33221 \mathrm{~A}$ & $29-0 \mathrm{ct}-96$ & $Y$ & 2 \\
\hline$Y$ & & 285 & PB210 & Lead 210 & INVCHD & $\begin{array}{l}\text { Inventory of Contact Handled } \\
\text { Design }\end{array}$ & $33221 \mathrm{~A}$ & $29-0 \mathrm{ct}-96$ & $\mathbf{Y}$ & 2 \\
\hline $\mathbf{Y}$ & & 285 & PB210 & Lead 210 & INVCHD & $\begin{array}{l}\text { Inventory of Contact Handled } \\
\text { Design }\end{array}$ & $33221 \mathrm{~A}$ & $29-0 \mathrm{ct}-96$ & $\mathbf{Y}$ & 2 \\
\hline $\mathbf{Y}$ & & 286 & PB210 & Lead 290 & INVRHD & $\begin{array}{l}\text { Inventory of Remote Handled } \\
\text { Design }\end{array}$ & $33223 A$ & $29-0 \mathrm{ct}-96$ & $\mathbf{Y}$ & 2 \\
\hline $\mathbf{Y}$ & & 286 & PB210 & Lead 210 & INVRHD & $\begin{array}{l}\text { Inventory of Remote Handled } \\
\text { Design }\end{array}$ & $33223 A$ & $29-0$ et-96 & $\mathbf{Y}$ & 2 \\
\hline $\mathbf{Y}$ & & 286 & PB210 & Lead 210 & INVRHD & $\begin{array}{l}\text { Inventory of Remoto Handled } \\
\text { Design }\end{array}$ & $33223 \mathrm{~A}$ & $29-0 \mathrm{ct}-96$ & $\mathbf{Y}$ & 2 \\
\hline $\mathbf{Y}$ & 0.06 & 287 & PM147 & Promethium 147 & ATWEIGHT & Atomic Weight in $\mathrm{kg} / \mathrm{mole}$ & 32339 & 02-Nov-95 & $\mathbf{Y}$ & 3 \\
\hline$Y$ & 0.06 & 287 & PM147 & Promethiem 147 & ATWEIGHT & Atomic Weight in $\mathrm{kg} / \mathrm{mole}$ & 32339 & O2-Nov-95 & $\mathbf{Y}$ & 3 \\
\hline $\mathbf{Y}$ & 0.06 & 287 & PM147 & Promethium 147 & ATWEIGHT & Alomic Weight in $\mathrm{kg} / \mathrm{mole}$ & 32339 & 02-Nov-95 & $Y$ & 3 \\
\hline$Y$ & 100 & 288 & PM147 & Promethium 147 & HALFLIFE & Haltilifo & 33231 & 16-Apr-96 & $\mathbf{Y}$ & 3 \\
\hline $\mathbf{Y}$ & 100 & 288 & PM147 & Promethium 147 & HALFLIFE & Hahlife & 33231 & 16-Apr-96 & $Y$ & 3 \\
\hline $\mathbf{Y}$ & 100 & 288 & PM147 & Promethium 147 & HALFLIFE & Habfifife & 33231 & 16-Apr-96 & $\mathbf{Y}$ & 3 \\
\hline $\mathbf{Y}$ & & 289 & PM147 & Promethium 147 & INVRHD & $\begin{array}{l}\text { Inventory of Remote Handled } \\
\text { Design }\end{array}$ & $33236 \mathrm{~A}$ & $29-0 \mathrm{ct}-96$ & $\mathbf{Y}$ & 2 \\
\hline $\mathbf{Y}$ & & 289 & PM147 & Promethivm 147 & INVRHD & $\begin{array}{l}\text { Imventory of Remote Handled } \\
\text { Design }\end{array}$ & $33236 \mathrm{~A}$ & 29-0ct-96 & $-Y$ & 2 \\
\hline $\mathbf{Y}$ & & 289 & PMi47 & Promethium 147 & INVRHD & $\begin{array}{l}\text { Inventory of Remote Handled } \\
\text { Design }\end{array}$ & $33236 \mathrm{~A}$ & $29-0 \mathrm{ct}-96$ & $\mathbf{Y}$ & 2 \\
\hline $\mathbf{Y}$ & 0.02 & 291 & PU238 & Phitonium 238 & ATWEIGHT & Atomic Weight in $\mathrm{kg} /$ mole & 32340 & 02-Nov-95 & $\mathbf{Y}$ & 3 \\
\hline $\mathbf{Y}$ & 0.02 & 291 & PU238 & Phtonium 238 & ATWEIGHT & Atomic Weight in $\mathrm{kg} / \mathrm{mol}^{\circ}$ & 32340 & 02-Nov-95 & $\mathbf{Y}$ & 3 \\
\hline $\mathbf{Y}$ & 0.02 & 291 & PU238 & Phtonium 238 & ATWEIGHT & Atomic Weight in kg/mole & 32340 & 02-Nov-95 & $\mathbf{Y}$ & 3 \\
\hline $\mathbf{Y}$ & 100 & 292 & PU238 & Phutonium 238 & HALFLIFE & Halfife & 33245 & 16-Apr-96 & $Y$ & 3 \\
\hline $\mathbf{Y}$ & 100 & 292 & PU238 & Phutonium 238 & HALFLIFE & Halifite & 33245 & 16-Apr-96 & $\mathbf{Y}$ & 3 \\
\hline $\mathbf{Y}$ & 100 & 292 & PU238 & Plutonium 238 & HALFLIFE & Haltife & 33245 & 16 -Apr-96 & $\mathbf{Y}$ & 3 \\
\hline $\mathbf{Y}$ & & 293 & PU238 & Plutonium 238 & INVCHD & $\begin{array}{l}\text { Inventory of Contect Handled } \\
\text { Design }\end{array}$ & $33247 A$ & $29-0 \mathrm{ct} \cdot 96$ & $\mathbf{Y}$ & 2 \\
\hline $\mathbf{Y}$ & & 293 & PU238 & Plutonium 238 & INVCHD & $\begin{array}{l}\text { Inventory of Contact Handled } \\
\text { Design }\end{array}$ & $33247 A$ & $29-0 c t .96$ & $Y$ & 2 \\
\hline $\mathbf{Y}$ & & 293 & Puzz3 & Plutonium 238 & INVCHD & $\begin{array}{l}\text { Inventory of Contact Handled } \\
\text { Design }\end{array}$ & $33247 A$ & $29-0 c t-96$ & $\mathbf{Y}$ & 2 \\
\hline $\mathbf{Y}$ & & 293 & PU238 & Phutonium 238 & INVCHD & $\begin{array}{l}\text { Inventory of Contact Handled } \\
\text { Design }\end{array}$ & $33247 A$ & $29-0 c t-96$ & $\mathbf{Y}$ & 2 \\
\hline $\mathbf{Y}$ & & 294 & PU238 & Phutonium 238 & INVRHD & $\begin{array}{l}\text { Inventory of Remote Handled } \\
\text { Design }\end{array}$ & $33251 \mathrm{~A}$ & $29-0 \mathrm{ct}-96$ & $\mathbf{Y}$ & 2 \\
\hline $\mathbf{Y}$ & & 294 & PU238 & Piutonium 238 & INVRHD & $\begin{array}{l}\text { Imventory of Remote Handled } \\
\text { Design }\end{array}$ & $33251 A$ & $29-0 \mathrm{ct}-96$ & $\mathbf{Y}$ & 2 \\
\hline
\end{tabular}


CCA Parameter Listing

\begin{tabular}{|c|c|c|c|c|c|c|c|c|c|c|}
\hline 10 & $\begin{array}{l}\text { Clessfint- } \\
\text { cation of } \\
\text { Dath } \\
\text { Category } \\
\text { (footnote 2) }\end{array}$ & $\begin{array}{l}\text { Distribution } \\
\text { Type }\end{array}$ & man & Median & Minimum & Maximum & Units & Reforence ID & $\begin{array}{l}\text { Wes the dete } \\
\text { dereloped } \\
\text { under an } \\
\text { NOA-1 } \\
\text { Progrem? }\end{array}$ & $\begin{array}{c}\text { Which } \\
\text { mothods } \\
\text { were used to } \\
\text { qually } \\
\text { extatting } \\
\text { drta? } \\
\text { (footrote s) }\end{array}$ \\
\hline 264 & 1,3 & CONSTANT & $4.0000000 E+\infty 0$ & $4.0000000 \mathrm{E}+00$ & $4.0000000 \mathrm{E}+00$ & $4.0000000 \mathrm{E}+00$ & NONE & WP040514 & $Y$ & N/A \\
\hline 265 & 1,3 & CONSTANT & $2.0000000 E-01$ & $2.0000000 E-01$ & $2.0000000 E-01$ & $20000000 E-01$ & NONE & $\begin{array}{c}\text { VAUGHN: } \\
\text { WORKSHEET A }\end{array}$ & $\mathbf{Y}$ & NA \\
\hline 265 & 1,3 & CONSTANT & $2.0000000 \mathrm{E}-01$ & $2.0000000 \mathrm{E}-01$ & $2.0000000 \mathrm{E}-01$ & $20000000 E-01$ & NONE & WP035597 & $\mathbf{Y}$ & N/A \\
\hline 265 & 1,3 & CONSTANT & $2.0000000 \mathrm{E}-01$ & $2.0000000 E-01$ & $20000000 E-01$ & $2.0000000 E-01$ & NONE & WP040514 & $\mathbf{Y}$ & N/A \\
\hline 266 & 1,3 & CONSTANT & $2.0000000 E-01$ & $2.0000000 \mathrm{E}-01$ & $2.0000000 E-01$ & $20000000 E-01$ & NONE & $\begin{array}{c}\text { VAUGHN: } \\
\text { WORKSHEET-4 }\end{array}$ & $\mathbf{Y}$ & NA \\
\hline 266 & 1,3 & CONSTANT & $2.0000000 E-01$ & 20000000E-01 & $2.0000000 E-01$ & $2.0000000 E-01$ & NONE & WP035597 & $\mathbf{Y}$ & NA \\
\hline 266 & 1,3 & CONSTANT & $2.0000000 E-01$ & $2.0000000 \mathrm{E}-01$ & $20000000 E-01$ & $2,0000000 E-01$ & NONE & WPO40514. & $\bar{Y}$ & NA \\
\hline 282 & Not Used & CONSTANT & $0.0000000 E+00$ & $0.0000000 E+00$ & $0.0000000 E+00$ & $0.0000000 E+00$ & $\log$ (moless) & SAND92-0479 & $\mathbf{Y}$ & NA \\
\hline 282 & Not Used & CONSTANT & $0.0000000 \mathrm{E}+00$ & $0.0000000 \mathrm{E}+\infty 0$ & $0.0000000 \mathrm{E}+00$ & $0.0000000 E+00$ & $\log ($ molesn) & WP037401 & $Y$ & NA \\
\hline 282 & Not Used & CONSTANT & $0.0000000 E+00$ & $0.0000000 E+00$ & $0.0000000 E+00$ & $0.0000000 E+00$ & $\log ($ moles $n)$ & WP040515 & $\mathbf{Y}$ & NA \\
\hline 283 & 4 & CONSTANT & $2.0998400 E-01$ & 2.0998400E-01 & $2.0998400 E-01$ & 2.0998400E-01 & kg/mole & CRC-72 & $\mathbf{Y}$ & N/A \\
\hline 283 & 4 & CONSTANT & 2.0998400E-01 & 2.0998400E-01 & 20998400E-01 & $2.0998400 E-01$ & $\mathrm{~kg} /$ mote & WP036298 & $\mathbf{Y}$ & N/A \\
\hline 283 & 4 & CONSTANT & $2.0998400 E-01$ & $2.0998400 \mathrm{E}-01$ & 2.0998400E-01 & 2.0998400E-01 & $\mathrm{kg} /$ molo & WP036301 & $\mathbf{Y}$ & N/A \\
\hline 284 & 4 & CONSTANT & $7.0370000 E+08$ & $7.0370000 E+08$ & $7.0370000 E+08$ & $7.0370000 E+08$ & s & WP036301 & $Y$ & NA \\
\hline 284 & 4 & CONSTANT & $7.0370000 E+08$ & $7.0370000 E+08$ & $7.0370000 \mathrm{E}+08$ & $7.0370000 E+08$ & $\mathbf{s}$ & WPO37404 & $\mathbf{Y}$ & NA \\
\hline 284 & 4 & CONSTANT & $7.0370000 E+08$ & $7.0370000 E+08$ & $7.0370000 E+08$ & $7.0370000 E+08$ & $\mathbf{s}$ & WPO40434 & $Y$ & N/A \\
\hline 285 & 2 & CONSTANT & $8.7500000 E+00$ & $8.7500000 E+00$ & $8.7500000 E+\infty 0$ & $8.7500000 E+\infty 0$ & Ci & WP037061 & $\mathbf{Y}$ & N/A \\
\hline 285 & 2 & CONSTANT & $8.7500000 E+\infty 0$ & $8.7500000 E+\infty 0$ & $8.7500000 E+00$ & $8.7500000 E+\infty 0$ & Ci & WPO41559 & $Y$ & N/A \\
\hline 285 & 2 & CONSTANT & $8.7500000 E+\infty 0$ & $8.7500000 E+00$ & $8.7500000 E+00$ & $8.7500000 E+\infty 0$ & $\mathrm{Ci}$ & WPO41550 & $Y$ & NA \\
\hline 286 & 2 & CONSTANT & $1.1400000 E-04$ & $1.1400000 E-04$ & $1.1400000 E-04$ & $1.1400000 E-04$ & $\mathbf{C i}$ & WP037061 & $\mathbf{Y}$ & N/A \\
\hline 286 & 2 & CONSTANT & $1.1400000 E-04$ & $1.1400000 E-04$ & $1.1400000 E-04$ & $1.1400000 E-04$ & $\mathrm{Cl}$ & WPO41559 & $Y$ & NA \\
\hline 286 & 2 & CONSTANT & $1.1400000 E-04$ & $1.1400000 E-04$ & $1.1400000 E-04$ & $1.1400000 E-04$ & $\mathrm{Ci}$ & WP041560 & $\mathbf{Y}$ & NA \\
\hline 287 & 4 & CONSTANT & 1.4691500E-01 & $1.4691500 E-01$ & 1.4691500E-01 & 1.4691500E-01 & $\mathrm{kg} / \mathrm{mole}$ & CRC-72 & $\mathbf{Y}$ & NA \\
\hline 287 & 4 & CONSTANT & 1.4691500E-01 & $1.4691500 \mathrm{E}-01$ & 1.4691500E-01 & 1.4691500E-01 & $\mathrm{kg} / \mathrm{mole}$ & WPO36298 & $Y$ & N/A \\
\hline 287 & 4 & CONSTANT & $1.4691500 E-01$ & $1.4691500 E-01$ & $1.4691500 E-01$ & $1.4691500 E-01$ & $\mathrm{~kg} / \mathrm{mole}$ & WPO36301 & $\mathbf{Y}$ & NA \\
\hline 288 & 4 & CONSTANT & $8.2790000 E+07$ & $8.2750000 E+07$ & 8.2790000E+07 & $8.2790000 E+07$ & s & WP036301 & $Y$ & N/A \\
\hline 288 & 4 & CONSTANT & $8.2790000 E+07$ & $8.2790000 \mathrm{E}+07$ & $8.2790000 E+07$ & $8.2790000 E+07$ & $\mathbf{s}$ & WP037404 & $Y$ & N/A \\
\hline 288 & 4 & CONSTANT & $8.2790000 E+07$ & $8.2790000 E+07$ & $8.2790000 E+07$ & 8.2790000E+07 & $s$ & WP040434 & $Y$ & N/A \\
\hline 289 & 2 & CONSTANT & $4.6700000 E-04$ & 4.6700000E-04 & $4.6700000 E-04$ & 4.6700000E-04 & $\mathrm{Ci}$ & WP037061 & $\mathbf{Y}$ & N/A \\
\hline 289 & 2 & CONSTANT & $4.6700000 E-04$ & 4.6700000E-04 & $4.6700000 E-04$ & $4.6700000 E-04$ & $\mathrm{Ci}$ & WP041559 & $-\underline{Y}$ & NA \\
\hline 289 & 2 & CONSTANT & $4.6700000 E-04$ & $4.6700000 E-04$ & $46700000 E-04$ & $4.6700000 E-04$ & $\mathbf{C i}$ & WPO41560 & $\mathbf{Y}$ & N/A \\
\hline 291 & 4 & CONSTANT & $2.3805000 E-01$ & $2.3805000 E-01$ & $2.3805000 E-01$ & $2.3805000 E-01$ & $\mathrm{~kg} /$ mole & CRC-72 & $\mathbf{Y}$ & N/A \\
\hline 291 & 4 & CONSTANT & $2.3805000 E-01$ & $2.3805000 E-01$ & $2.3805000 E-01$ & $2.3805000 E-01$ & $\mathrm{~kg} / \mathrm{mol}$ & WPO36298 & $Y$ & N/A \\
\hline 291 & 4 & CONSTANT & $2.3805000 \mathrm{E}-01$ & $2.3805000 E-01$ & $2.3805000 E-01$ & $2.3805000 \mathrm{E}-01$ & $\mathrm{~kg} / \mathrm{mole}$ & WPO36301 & $Y$ & N/A \\
\hline 292 & 4 & CONSTANT & $2.7690000 \mathrm{E}+09$ & $2.7690000 \mathrm{E}+09$ & $2.7690000 E+09$ & $2.7690000 E+09$ & $\mathbf{s}$ & WPO36301 & $\mathbf{Y}$ & N/A \\
\hline 292 & 4 & CONSTANT & $2.7690000 \mathrm{E}+09$ & $27690000 E+09$ & $2.7690000 E+09$ & $2.7690000 E+09$ & $\mathbf{s}$ & WPO37404 & $Y$ & N/A \\
\hline 292 & 4 & CONSTANT & $2.7690000 \mathrm{E}+09$ & $2.7690000 E+09$ & $2.7690000 E+09$ & $2.7690000 E+09$ & $\mathbf{s}$ & WP040434 & $\mathbf{Y}$ & N/A \\
\hline 293 & 2 & CONSTANT & $1.9300000 \mathrm{E}+06$ & $1.9300000 E+06$ & $1.9300000 E+06$ & $1.9300000 \mathrm{E}+06$ & $\mathbf{C i}$ & WP037061 & $\mathbf{Y}$ & N/A \\
\hline 293 & 2 & CONSTANT & $1.9300000 \mathrm{E}+06$ & $1.9300000 E+06$ & $1.9300000 E+06$ & $1.9300000 E+06$ & $\mathrm{Ci}$ & WP039260 & $\mathbf{Y}$ & NA \\
\hline 293 & 2 & CONSTANT & $1.9300000 \mathrm{E}+06$ & $1.9300000 E+06$ & $1.9300000 E+06$ & $1.9300000 E+06$ & $\mathbf{C i}$ & WP041559 & $\mathbf{Y}$ & NA \\
\hline 293 & 2 & CONSTANT & $1.9300000 E+06$ & $1.9300000 E+06$ & $1.9300000 E+06$ & $1.9300000 E+06$ & $\mathbf{C i}$ & WP041560 & $\mathbf{Y}$ & N/A \\
\hline 294 & 2 & CONSTANT & $1.0800000 E+03$ & $1.0800000 E+03$ & $1.0800000 E+03$ & $1.0800000 E+03$ & $\mathbf{C i}$ & WP037061 & $\mathbf{Y}$ & NA \\
\hline 294 & 2 & CONSTANT & $1.0800000 E+03$ & $1.0800000 E+03$ & $1.0800000 E+03$ & $1.0800000 E+03$ & $\mathrm{Ci}$ & WPO39260 & $\mathbf{Y}$ & N/A \\
\hline
\end{tabular}


CCA Parameter Listing

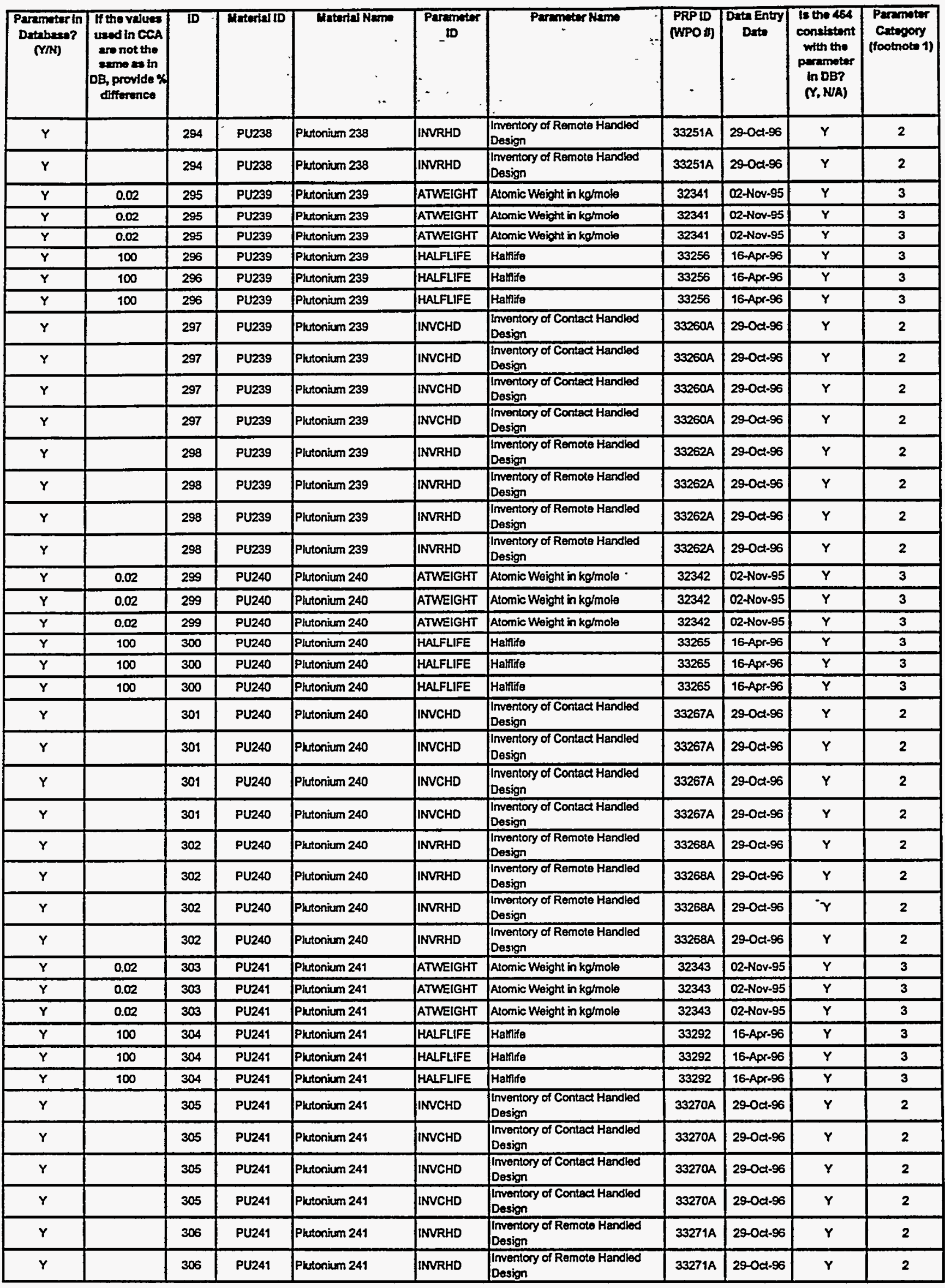


CCA Parameter Listing

\begin{tabular}{|c|c|c|c|c|c|c|c|c|c|c|}
\hline ID & $\begin{array}{c}\text { Classiff- } \\
\text { eation of } \\
\text { Date } \\
\text { Category } \\
\text { (footnote 2) }\end{array}$ & $\begin{array}{l}\text { Dlatribution } \\
\text { Type }\end{array}$ & Moen & Medien & Mlnimum & Maximum & Units & \begin{tabular}{c} 
Reference ID \\
$\because-$ \\
\hdashline
\end{tabular} & $\begin{array}{l}\text { Wes the dath } \\
\text { doviloped } \\
\text { undor an } \\
\text { HOA-1 } \\
\text { Program? }\end{array}$ & $\begin{array}{c}\text { Which } \\
\text { mothods } \\
\text { woro used to } \\
\text { qually } \\
\text { existing } \\
\text { detta? } \\
\text { (footnote 3) } \\
\end{array}$ \\
\hline 294 & 2 & CONSTANT & $1.0300000 E+03$ & $1.0800000 E+03$ & $1.0800000 E+03$ & $1.0800000 E+03$ & $\mathrm{Ci}$ & WPO41559 & $\mathbf{Y}$ & NA \\
\hline 294 & 2 & CONSTANT & $1.0800000 E+03$ & $1.0800000 E+03$ & $1.0800000 \mathrm{E}+03$ & $1.0800000 E+03$ & $\mathrm{Ci}$ & WP041560 & $\mathbf{Y}$ & $N / A$ \\
\hline 295 & 4 & CONSTANT & 2.3905200E-01 & $2.3905200 E-01$ & 23905200E-01 & 23905200E-01 & $\mathrm{kg}$ mole & CRC-72 & $Y$ & N/A \\
\hline 295 & 4 & CONSTANT & $2.3905200 E-01$ & $2.3905200 E-01$ & 23905200E-01 & $2.3905200 \mathrm{E}-01$ & $\mathrm{~kg} /$ molo & WP035298 & $\mathbf{Y}$ & N/A \\
\hline 295 & 4 & CONSTANT & 2.3905200E-01 & 2.3905200E-01 & $23905200 \mathrm{E}-01$ & $2.3905200 E-01$ & $\mathrm{~kg} / \mathrm{mole}$ & WP035301 & $Y$ & N/A \\
\hline 296 & 4 & CONSTANT & $7.5940000 \mathrm{E}+11$ & $7.5940000 \mathrm{E}+11$ & $7.5940000 E+11$ & $7.5940000 E+11$ & 5 & WP036301 & $Y$ & N/A \\
\hline 296 & 4 & CONSTANT & $7.5940000 \mathrm{E}+11$ & $7.5940000 \mathrm{E}+11$ & $7.5940000 \mathrm{E}+11$ & $7.5940000 E+11$ & 5 & WP037404 & $Y$ & N/A \\
\hline 296 & 4 & CONSTANT & $7.5940000 \mathrm{E}+11$ & $7.5940000 \mathrm{E}+11$ & $7.5940000 \mathrm{E}+11$ & $7.5940000 \mathrm{E}+11$ & $\mathbf{s}$ & WPO40434 & $Y$ & $N / A$ \\
\hline 297 & 2 & CONSTANT & $7.8500000 \mathrm{E}+05$ & $7.8500000 E+05$ & $7.8500000 \mathrm{E}+0.5$ & $7.8500000 E+05$ & $\mathbf{C i}$ & WP037061 & $Y$ & NA \\
\hline 297 & 2 & CONSTANT & $7.8500000 E+05$ & $7.8500000 E+0.5$ & $7.8500000 E+05$ & $7.8500000 E+05$ & $\mathbf{C i}$ & WP039260 & $Y$ & NA \\
\hline 297 & 2 & CONSTANT & $7.8500000 E+05$ & $7.8500000 E+05$ & $7.8500000 E+05$ & $7.8500000 E+05$ & $\mathrm{Ci}$ & WP041559 & $Y$ & N/A \\
\hline 297 & 2 & CONSTANT & $7.8500000 E+0.5$ & $7.8500000 E+05$ & $7.8500000 E+05$ & $7.8500000 E+05$ & $\mathrm{Ci}$ & WPO41560 & $Y$ & N/A \\
\hline 298 & 2 & CONSTANT & $1.03000000 E+04$ & $1.0300000 E+04$ & $1.0300000 E+04$ & $1.0300000 E+04$ & $\mathrm{Ci}$ & WP037061 & $Y$ & NA \\
\hline 298 & 2 & CONSTANT & $1.03000000+04$ & $1.0300000 E+04$ & $1.0300000 E+04$ & $1.0300000 E+04$ & $\mathbf{C i}$ & WP039260 & $Y$ & N/A \\
\hline 298 & 2 & CONSTANT & $1.0300000 \mathrm{E}+04$ & $1.0300000 E+04$ & $1.0300000 E+04$ & $1.0300000 E+04$ & $\mathrm{Ci}$ & WP041559 & $\mathbf{Y}$ & N/A \\
\hline 298 & 2 & CONSTANT & $1.03000000 E+04$ & $1.03000000 E+04$ & $1.0300000 E+04$ & $1.0300000 E+04$ & $\mathrm{Ci}$ & WPO41560 & $\mathbf{Y}$ & NA \\
\hline 299 & 4 & CONSTANT & 2.4005400E-01 & 2.4005400E-01 & $2.4005400 \mathrm{E}-01$ & $2.4005400 \mathrm{E}-01$ & kg/mole & CRC-72 & $Y$ & NA \\
\hline 299 & 4 & CONSTANT & 2.4005400E-01 & 2.4005400E-01 & $2.4005400 \mathrm{E}-01$ & $2.4005400 E-01$ & $\mathrm{~kg} /$ mote & WP036298 & $Y$ & NA \\
\hline 299 & 4 & CONSTANT & 24005400E-01 & $2.4005400 E-01$ & $24005400 E-01$ & $2.4005400 E-01$ & $\mathrm{~kg} / \mathrm{mole}$ & WP036301 & $\begin{array}{r}Y \\
\end{array}$ & N/A \\
\hline 300 & 4 & CONSTANT & $2.0630000 \mathrm{E}+11$ & $20630000 \mathrm{E}+11$ & $2.0630000 \mathrm{E}+11$ & $2.0630000 \mathrm{E}+11$ & $\mathbf{s}$ & WPO36301 & $Y$ & N/A \\
\hline 300 & 4 & CONSTANT & $2.0630000 \mathrm{E}+11$ & $2.0630000 \mathrm{E}+11$ & $20630000 E+11$ & $2.0630000 E+11$ & s & WP037404 & $Y$ & N/A \\
\hline 300 & 4 & CONSTANT & $2.0630000 \mathrm{E}+11$ & $2.0630000 E+11$ & $2.0630000 E+11$ & $2.0630000 \mathrm{E}+11$ & s & WPO40434 & $Y$ & NA \\
\hline 301 & 2 & CONSTANT & $2.0900000 E+05$ & $2.0900000 E+05$ & $2.0900000 E+05$ & $2.0900000 E+05$ & $\mathbf{C i}$ & WP037061 & $\mathbf{Y}$ & N/A \\
\hline 301 & 2 & CONSTANT & $2.0900000 E+05$ & $2.0900000 E+05$ & $2.0900000 E+05$ & $2.0900000 E+05$ & $\mathbf{C i}$ & WP039260 & $\mathbf{Y}$ & N/A \\
\hline 301 & 2 & CONSTANT & $2.0900000 E+05$ & $2.0900000 E+05$ & $2.09000000+05$ & $2,0900000 \mathrm{E}+05$ & $\mathbf{C i}$ & WPO41559 & $\mathbf{Y}$ & NA \\
\hline 301 & 2 & CONSTANT & $2.0900000 E+0.5$ & $2.0900000 E+05$ & $2.0900000 \mathrm{E}+0.5$ & $2.0900000 E+05$ & $\mathbf{C i}$ & WP041560 & $\mathbf{Y}$ & N/A \\
\hline 302 & 2 & CONSTANT & $5.0500000 E+03$ & $5.0500000 \mathrm{E}+03$ & $5.0500000 E+03$ & $.5 .0500000 E+03$ & $\mathbf{C i}$ & WP037061 & $Y$ & N/A \\
\hline 302 & 2 & CONSTANT & $5.0500000 E+03$ & $5.0500000 E+03$ & $5.0500000 E+03$ & $5.0500000 E+03$ & $\mathbf{C i}$ & WPO39260 & $\mathbf{Y}$ & N/A \\
\hline 302 & 2 & CONSTANT & $5.0500000 E+03$ & $5.0500000 E+03$ & $5.0500000 E+03$ & $5.0500000 E+03$ & $\mathbf{C i}$ & WPO41559 & 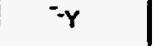 & N/A \\
\hline 302 & 2 & CONSTANT & $5.0500000 E+03$ & $5.0500000 E+03$ & $5.0500000 E+03$ & $5.0500000 E+03$ & $\mathbf{C i}$ & WPO41560 & $\mathbf{Y}$ & NA \\
\hline 303 & 4 & CONSTANT & $2.4105700 E-01$ & 2.4105700E-01 & 2.4105700E-01 & $2.4105700 E-01$ & $\mathrm{~kg} /$ molo & CRC-72 & $\mathbf{Y}$ & N/A \\
\hline 303 & 4 & CONSTANT & $2.4105700 \mathrm{E}-01$ & 24105700E-01 & $2.4105700 E-01$ & $2.4105700 E-01$ & $\mathrm{~kg} / \mathrm{mol}$ & WPO36298 & $Y$ & N/A \\
\hline 303 & 4 & CONSTANT & 2.4105700E-01 & $2.4105700 \mathrm{E}-01$ & $2.4105700 E-01$ & $2.4105700 E-01$ & kg/mole & WP036301 & $Y$ & N/A \\
\hline 304 & 4 & CONSTANT & $4.5440000 \mathrm{E}+08$ & $4.5440000 \mathrm{E}+08$ & $4.5440000 E+08$ & $4.5440000 \mathrm{E}+08$ & 3 & WPO36301 & $Y$ & N/A \\
\hline 304 & 4 & CONSTANT & $4.5440000 \mathrm{E}+08$ & $4.5440000 E+08$ & $4.5440000 E+08$ & $4.5440000 \mathrm{E}+08$ & $s$ & WPO37404 & $Y$ & NA \\
\hline 304 & 4 & CONSTANT & $4.5440000 \mathrm{E}+08$ & $4.5440000 \mathrm{E}+08$ & $4.5440000 E+08$ & $4.5440000 E+08$ & $s$ & WPO40434 & $Y$ & N/A \\
\hline 305 & 2 & CONSTANT & $3.7100000 E+05$ & $3.7100000 E+05$ & $3.7400000 E+05$ & $3.7100000 \mathrm{E}+05$ & $\mathbf{C i}$ & WP037061 & $\mathbf{Y}$ & $N / A$ \\
\hline 305 & 2 & CONSTANT & $3.7100000 E+05$ & $3.7100000 E+05$ & $3.7100000 E+05$ & $3.7100000 E+05$ & $\mathbf{C i}$ & WP039260 & $\mathbf{Y}$ & NA \\
\hline 305 & 2 & CONSTANT & $3.7100000 E+05$ & $3.7100000 E+05$ & $3.7100000 E+05$ & $3.7100000 E+05$ & $\mathbf{C i}$ & WP041559 & $\mathbf{Y}$ & NA \\
\hline 305 & 2 & CONSTANT & $3.7100000 \mathrm{E}+05$ & $3.7100000 \mathrm{E}+05$ & $3.7100000 E+05$ & $3.7100000 E+05$ & $\mathbf{C i}$ & WPO41560 & $\mathbf{Y}$ & NA \\
\hline 306 & 2 & CONSTANT & $2.28000000 E+04$ & $2.2800000 \mathrm{E}+04$ & $2.2800000 E+0.4$ & $2.2800000 \mathrm{E}+04$ & $\mathbf{C i}$ & WP037061 & $\mathbf{Y}$ & N/A \\
\hline 306 & 2 & CONSTANT & $2.2800000 E+04$ & $2.2800000 E+04$ & $2.2800000 E+04$ & $2.2800000 E+0.4$ & Ci & WP039260 & $\mathbf{Y}$ & N/A \\
\hline
\end{tabular}


CCA Parameter Listing

\begin{tabular}{|c|c|c|c|c|c|c|c|c|c|c|}
\hline $\begin{array}{c}\text { Parameterin } \\
\text { Database? } \\
\text { (YN) }\end{array}$ & \begin{tabular}{|l|} 
If the values \\
used in cead \\
ese not the \\
seme as in \\
DB, provide $x$ \\
offference
\end{tabular} & ID & Meterial ID & Taterlal Aamo & $\begin{array}{l}\text { Parametor } \\
\text { ID }\end{array}$ & Parander Kame & $\begin{array}{l}\text { PRPID } \\
\text { (MPO DI) }\end{array}$ & $\begin{array}{c}\text { Datn Entry } \\
\text { Date }\end{array}$ & $\begin{array}{l}\text { Is the } 454 \\
\text { consalstent } \\
\text { with the } \\
\text { prrameter } \\
\text { In DB? } \\
(Y, N A)\end{array}$ & $\begin{array}{l}\text { Parameter } \\
\text { Crtegory } \\
\text { (footnote 1) }\end{array}$ \\
\hline$Y$ & & 306 & PU241 & Phtonium 241 & INVRHD & $\begin{array}{l}\text { Inventory of Remole Handlod } \\
\text { Design }\end{array}$ & $33271 \mathrm{~A}$ & $29-0 \mathrm{ct}-96$ & $Y$ & 2 \\
\hline Y & & 306 & PU241 & Phtonium 241 & INVRHD & $\begin{array}{l}\text { Inventory of Remote Handled } \\
\text { Design }\end{array}$ & $33271 A$ & $29-0 \mathrm{ct}-96$ & Y & 2 \\
\hline$Y$ & 0.02 & 307 & PU242 & Phitonium 242 & ATWEIGHT & Alomic Weight in $\mathrm{kg} /$ mole & 32344 & 02-Nov-95 & $Y$ & 3 \\
\hline$Y$ & 0.02 & 307 & PU242 & Phroniven 242 & ATWEIGHT & Atomic Weight in $\mathrm{kg} / \mathrm{mol}^{2}$ & 32344 & 02-Nov-95 & $Y$ & 3 \\
\hline$Y$ & 0.02 & 307 & PU242 & Phitoniem 242 & ATWEIGHT & Atomic Woight in $\mathrm{kg} / \mathrm{mole}$ & 32344 & O2-Nov-95 & $\bar{Y}$ & 3 \\
\hline $\bar{Y}$ & 100 & 308 & PU242 & Plutonium 242 & HALFLIFE & Hahlifi & 33295 & 16-Apr-96 & $Y$ & 3 \\
\hline$Y$ & 100 & 308 & PU242 & Phutonium 242 & HALFLIFE & Halfitifo & 33295 & 16-Apr-96 & $Y$ & 3 \\
\hline$Y$ & 100 & 308 & PU242 & Phutonium 242 & HALFLIFE & Halfifie & 33295 & 16-Apr-96 & $\gamma$ & 3 \\
\hline $\mathbf{Y}$ & & 309 & PU242 & Phutonium 242 & IINVCHD & $\begin{array}{l}\text { Inventory of Contact Handled } \\
\text { Design }\end{array}$ & 332744 & 29-0ct-96 & $\cdot \mathbf{Y}$ & 2 \\
\hline $\mathbf{Y}$ & & 309 & PU242 & Prutonium 242 & INVCHD & $\begin{array}{l}\text { Inventory of Contact Handled } \\
\text { Design }\end{array}$ & $33274 \mathrm{~A}$ & $29-0 \mathrm{ct}-96$ & $\mathbf{Y}$ & 2 \\
\hline$Y$ & & 309 & PU242 & Plutonium 242 & INVCHD & $\begin{array}{l}\text { Inventory of Contact Handled } \\
\text { Design }\end{array}$ & $33274 A$ & $29-0 d t-96$ & $\mathbf{Y}$ & 2 \\
\hline $\mathbf{Y}$ & & 310 & PU242 & Plutonium 242 & INVRHD & $\begin{array}{l}\text { Imventory of Remote Handled } \\
\text { Design }\end{array}$ & $33272 A$ & $29-0 c t-96$ & $\mathbf{Y}$ & 2 \\
\hline$Y$ & & 310 & PU242 & Plutonium 242 & INVRHD & $\begin{array}{l}\text { Inventory of Remote Handled } \\
\text { Design }\end{array}$ & $33272 A$ & 29-0ct-96 & $\mathbf{Y}$ & 2 \\
\hline $\mathbf{Y}$ & & 310 & PU242 & Plutonium 242 & INVRHD & \begin{tabular}{|l} 
Inventory of Remote Handled \\
Design \\
\end{tabular} & $33272 A$ & $29-0 c t-96$ & $\mathbf{Y}$ & 2 \\
\hline $\bar{Y}$ & 0.03 & 311 & PU244 & Plutonium 244 & ATWEIGHT & Atomic Weight in kg/mole & 32345 & 02-Nov-95 & $Y$ & 3 \\
\hline$Y$ & 0.03 & 311 & PU244 & Phetonium 244 & ATWEIGHT & Atomic Woight in kg/mole & 32345 & 02-Nov-95 & $Y$ & 3 \\
\hline $\bar{Y}$ & 0.03 & 391 & PU244 & Plutonium 244 & ATWEIGHT & Atomic Weight in kg/moles & 32345 & 02-Nov-95 & $\bar{Y}$ & 3 \\
\hline$Y$ & 100 & 312 & PU244 & Plutonium 244 & HALFLIFE & Halfife & 33297 & 16-Apr-96 & $Y$ & 3 \\
\hline$Y$ & 100 & 312 & PU244 & Plutonium 244 & HALFLIFE & Halfife & 33297 & 16 -Apr-96 & $Y$ & 3 \\
\hline$Y$ & 100 & 312 & PU244 & Plutonium 244 & HALFLIFE & Halfifie & 33297 & 16-Apr-96 & $\bar{Y}$ & 3 \\
\hline$Y$ & & 313 & RA & Radium & LOGSOLM & Log of the Radionuclide Solubility & 33455 & $01+$ May-96 & $Y$ & $A B$ \\
\hline$Y$ & & 313 & RA & Radium & LOGSOLM & Log of the Radionuclide Solubility & 33455 & $01+$ May-96 & $\mathbf{Y}$ & 48 \\
\hline$Y$ & 0.01 & 314 & RA226 & Radum 226 & ATWEIGHT & Atomic Woight in kg/mole & 32347 & 02-Nov-95 & $Y$ & 3 \\
\hline$y$ & 0.01 & 314 & RA226 & Radium 226 & ATWEIGHT & Atornic Waight in $\mathrm{kg} / \mathrm{mola}$ & 32347 & 02-Nov-95 & $Y$ & 3 \\
\hline$Y$ & 0.01 & 314 & RA226 & Radium 226 & ATWEIGHT & Atornic Weight in $\mathrm{kg} / \mathrm{mole}$ & 32347 & 02-Nov-95 & $\mathbf{Y}$ & 3 \\
\hline$Y$ & 100 & 315 & RA226 & Radium 226 & HALFLIFE & Halfife & 33458 & 16-Apr-96 & $Y$ & 3 \\
\hline$Y$ & 100 & 315 & RA226 & Radium 226 & HALFLIFE & Hahflife & 33458 & 16-Apr-96 & $\bar{Y}$ & 3 \\
\hline $\bar{Y}$ & 100 & 315 & RA226 & Radium 226 & HALFLIFE & Hahthro & 33458 & 16-Apr-96 & $\bar{Y}$ & 3 \\
\hline$Y$ & & 316 & RA226 & Radium 226 & INVCHD & $\begin{array}{l}\text { Inventory of Contact Handled } \\
\text { Design }\end{array}$ & $33462 A$ & $29-0 c t-96$ & $\mathbf{Y}$ & 2 \\
\hline $\mathbf{Y}$ & & 316 & RA226 & Radium 226 & INVCHD & $\begin{array}{l}\text { Inventory of Contact Handled } \\
\text { Design }\end{array}$ & $33462 A$ & $29-0 a t-96$ & $\mathbf{Y}$ & 2 \\
\hline $\mathbf{Y}$ & & 316 & RA226 & Radium 226 & INVCHD & $\begin{array}{l}\text { Inventory of Contect Handled } \\
\text { Design }\end{array}$ & $33462 A$ & 29-0ct-96 & $\bar{Y}$ & 2 \\
\hline$Y$ & & 316 & RA226 & Radium 226 & INVCHD & $\begin{array}{l}\text { Inventory of Contact Handled } \\
\text { Design }\end{array}$ & $33462 A$ & $29-0 c t-96$ & $\mathbf{Y}$ & 2 \\
\hline$Y$ & & 317 & RA226 & Radium 226 & INVRHD & $\begin{array}{l}\text { Inventory of Remote Handled } \\
\text { Design }\end{array}$ & $33464 A$ & $29-0 c t-96$ & $Y$ & 2 \\
\hline$Y$ & & 317 & RA226 & Radium 226 & INVRHD & $\begin{array}{l}\text { Inventory of Remote Handled } \\
\text { Design }\end{array}$ & $33464 \mathrm{~A}$ & $29-0 c t-96$ & $\mathbf{Y}$ & 2 \\
\hline $\mathbf{Y}$ & & 317 & RA226 & Radium 226 & INVRHD & $\begin{array}{l}\text { Inventory of Remote Handled } \\
\text { Design }\end{array}$ & $33464 \mathrm{~A}$ & $29-0 \mathrm{ct}-96$ & 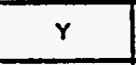 & 2 \\
\hline$Y$ & & 317 & RA226 & Redium 226 & INVRHD & $\begin{array}{l}\text { inventory of Remote Handled } \\
\text { Design }\end{array}$ & $33464 \mathrm{~A}$ & 29-0ct-96 & $\mathbf{Y}$ & 2 \\
\hline$Y$ & 0.01 & 318 & RA228 & Radium 228 & ATWEIGHT & Atomic Weight in $\mathrm{kg} / \mathrm{mole}$ & 32348 & $12+$ eb-96 & $Y$ & 3 \\
\hline $\bar{Y}$ & 0.01 & 318 & RA228 & Radium 228 & ATWEIGHT & Atomic Weight in $\mathrm{kg} /$ mole & 32348 & 12-Fob-96 & $Y$ & 3 \\
\hline$Y$ & 0.01 & 318 & RA228 & Radum 228 & ATWEIGHT & Atomic Weight in $\mathrm{kg} / \mathrm{mol}$ & 32348 & 12-Fob-96 & $Y$ & 3 \\
\hline$Y$ & 100 & 319 & RA228 & Redium 228 & HALFLIFE & Hatflife & 33468 & 16-Apr-96 & $Y$ & 3 \\
\hline$Y$ & 100 & 319 & RA228 & Radium 228 & HALFLIFE & Halfifí & 33468 & 16-Apr-96 & $Y$ & 3 \\
\hline$Y$ & 100 & 319 & RA228 & Radium 228 & HALFLIFE & Hainfife & 33468 & 16-Apr-96 & $Y$ & 3 \\
\hline $\mathbf{Y}$ & & 336 & SANTAROS & Santa Rosa Formation & CAP_MOD & $\begin{array}{l}\text { Model number, capillary pressure } \\
\text { model }\end{array}$ & 33485 & 22-Fob-96 & $\mathbf{Y}$ & AB \\
\hline$Y$ & & 336 & SANTAROS & Santa Rosa Formation & CAP_MOD & $\begin{array}{l}\text { Model number, capillary pressure } \\
\text { model }\end{array}$ & 33485 & 22-Fob-96 & $\mathbf{Y}$ & 4B \\
\hline
\end{tabular}


CCA Parameter Listing

\begin{tabular}{|c|c|c|c|c|c|c|c|c|c|c|}
\hline DD & \begin{tabular}{c|} 
Clunstif- \\
calton of \\
Data \\
Category \\
(footnote 2)
\end{tabular} & $\begin{array}{l}\text { Distributton } \\
\text { Type }\end{array}$ & maxn & Medien & Mintrom & Moxinum & Units & Referones id & $\begin{array}{l}\text { Was the dith } \\
\text { doveloped } \\
\text { under an } \\
\text { NaA-1 } \\
\text { Progrem? }\end{array}$ & $\begin{array}{c}\text { Which } \\
\text { mothods } \\
\text { were used to } \\
\text { quality } \\
\text {-exdsting } \\
\text { deta? } \\
\text { (footnote 3) }\end{array}$ \\
\hline 306 & 2 & CONSTANT & $2.2800000 E+04$ & $2.2800000 E+04$ & $2.2800000 E+04$ & $2.2800000 E+04$ & $\mathbf{C i}$ & WP041559 & $\mathbf{Y}$ & NA \\
\hline 306 & 2 & CONSTANT & $2.2800000 E+04$ & $2.2800000 E+04$ & $2.2800000 E+04$ & $22800000 E+04$ & $\mathbf{C i}$ & WP041560 & $\mathbf{Y}$ & N/A \\
\hline 307 & 4 & CONSTANT & $2.4205900 \mathrm{E}-01$ & $2.4205900 E-01$ & $2.4205900 E-01$ & $2.4205900 E-01$ & kg/mote & CRC-72 & $Y$ & NA \\
\hline 307 & 4 & CONSTANT & $2.4205900 E-01$ & 2.4205900E-01 & 2.4205900E-01 & 2.4205900E-01 & $\mathrm{kg} / \mathrm{mote}$ & WPO36298 & $Y$ & $N A$ \\
\hline 307 & 4 & CONSTANT & $2.4205900 E-01$ & $2.4205900 E-01$ & 2.4205900E-01 & 2.4205900E-01 & kg/molo & WP036301 & $Y$ & NA \\
\hline 308 & 4 & CONSTANT & $1.2210000 \mathrm{E}+13$ & $1.2210000 \mathrm{E}+13$ & $1.2210000 \mathrm{E}+13$ & $1.2210000 E+13$ & $\mathbf{s}$ & WP036301 & $Y$ & N/A \\
\hline 308 & 4 & CONSTANT & $1.2210000 \mathrm{E}+13$ & $1.2210000 \mathrm{E}+13$ & $1.2210000 \mathrm{E}+13$ & $1.2210000 \mathrm{E}+13$ & s & WP037404 & $Y$ & NA \\
\hline 308 & 4 & CONSTANT & $1.2210000 E+13$ & $1.2290000 E+13$ & $1.2210000 E+13$ & $1.2210000 E+13$ & $\mathbf{s}$ & WP040434 & $\mathbf{Y}$ & N/A \\
\hline 309 & 2 & CONSTANT & $1.1700000 E+03$ & $1.1700000 E+03$ & $1.1700000 \mathrm{E}+03$ & $1.1700000 E+03$ & Ci. & WP037051 & $\mathbf{Y}$ & NA \\
\hline 309 & 2 & CONSTANT & $1.1700000 E+03$ & $1.1700000 E+03$ & $1.1700000 E+03$ & $1.1700000 E+03$ & Ci & WPO41559 & $\mathbf{Y}$ & NA \\
\hline 309 & 2 & CONSTANT & $1.1700000 E+03$ & $1.1700000 E+03$ & $1.1700000 E+03$ & $1.1700000 E+03$ & Ci & WP041560 & $\mathbf{Y}$ & N/A \\
\hline 310 & 2 & CONSTANT & $1.5000000 E-01$ & $1.5000000 E-01$ & $1.5000000 \mathrm{E}-01$ & $1.5000000 E-01$ & $\mathrm{Ci}$ & WP037061 & $Y$ & NA \\
\hline 310 & 2 & CONSTANT & $1.5000000 E-01$ & $1.5000000 E-01$ & $1.5000000 E-01$ & $1.5000000 E-01$ & $\mathrm{Ci}$ & WPO41559 & $\mathbf{Y}$ & N/A \\
\hline 310 & 2 & CONSTANT & $1.5000000 \mathrm{E}-01$ & $1.5000000 E-01$ & $1.5000000 E-01$ & $1.5000000 E-01$ & $\mathrm{Ci}$ & WP041560 & $Y$ & NA \\
\hline 311 & 4 & CONSTANT & 2.4406400E-01 & 2.4406400E-01 & 2.4406400E-01 & $2.4406400 E-01$ & $\mathrm{~kg} / \mathrm{mole}$ & CRC-72 & $Y$ & $N / A$ \\
\hline 311 & 4 & CONSTANT & 2.4406400E-01 & 2.4406400E-01 & 2.4406400E-01 & 2.4406400E-01 & $\mathrm{kg} / \mathrm{mole}$ & WP036298 & $Y$ & NA \\
\hline 311 & 4 & CONSTANT & $2.4406400 E-01$ & $2.4406400 \mathrm{E}-01$ & 2.4406400E-01 & $2.4406400 E-01$ & $\mathrm{~kg} / \mathrm{mole}$ & WP035301 & $Y$ & NA \\
\hline 312 & 4 & CONSTANT & $2.6070000 E+15$ & $2.6070000 E+15$ & $2.6070000 E+15$ & $26070000 E+15$ & $\mathbf{s}$ & WP036301 & $Y$ & NA \\
\hline 312 & 4 & CONSTANT & $2.6070000 E+15$ & $2.6070000 \mathrm{E}+15$ & $2.6070000 \mathrm{E}+15$ & $2.6070000 E+15$ & $\mathbf{s}$ & WP037404 & $Y$ & N/A \\
\hline 312 & 4 & CONSTANT & $2.6070000 \mathrm{E}+15$ & $2.6070000 E+15$ & $2.6070000 E+15$ & $2.6070000 E+15$ & $\mathbf{s}$ & WPO40434 & $Y$ & NA \\
\hline 313 & Not Used & CONSTANT & $0.0000000 E+00$ & $0.0000000 E+00$ & $0.0000000 E+00$ & $0.0000000 E+00$ & $\log ($ moles $n)$ & WP035724 & $Y$ & N/A \\
\hline 313 & Not Used & CONSTANT & $0.0000000 \mathrm{E}+00$ & $0.0000000 E+00$ & $0.0000000 E+00$ & $0.0000000 E+\infty 0$ & $\log ($ moles $n)$ & WP040515 & $\mathbf{Y}$ & N/A \\
\hline 314 & 4 & CONSTANT & $2.2602500 E-01$ & 2.2602500E-01 & $2.2602500 \mathrm{E}-01$ & $2.2602500 E-01$ & $\mathrm{~kg} / \mathrm{mole}$ & CRC-72 & $Y$ & $N A$ \\
\hline 314 & 4 & CONSTANT & 2.2602500E-01 & $2.2602500 E-01$ & $2.2602500 E-01$ & $2.2602500 E-01$ & $\mathrm{~kg} /$ mole & WP035298 & $Y$ & N/A \\
\hline 314 & 4 & CONSTANT & $2.2602500 \mathrm{E}-01$ & $2.2602500 E-01$ & $2.2602500 \mathrm{E}-01$ & 2.2602500E-01 & kg/mole & WPO36301 & $\bar{Y}$ & N/A \\
\hline 315 & 4 & CONSTANT & $5.0490000 \mathrm{E}+10$ & $5.0490000 E+10$ & $5.0490000 E+10$ & $5.0490000 \mathrm{E}+10$ & $\mathbf{s}$ & WPO35301 & $Y$ & N/A \\
\hline 315 & 4 & CONSTANT & $5.0490000 \mathrm{E}+10$ & $5.0490000 \mathrm{E}+10$ & $5.0490000 \mathrm{E}+10$ & $5.0490000 \mathrm{E}+10$ & $s$ & WP037404 & $Y$ & N/A \\
\hline 315 & 4 & CONSTANT & $5.0490000 \mathrm{E}+10$ & $5.0490000 \mathrm{E}+10$ & $5.0490000 \mathrm{E}+10$ & $5.0490000 E+10$ & $s$ & WPO40434 & $\mathbf{Y}$ & NA \\
\hline 396 & 2 & CONSTANT & $1.1400000 E+01$ & $1.1400000 E+01$ & $1.1400000 E+01$ & $1.1400000 E+01$ & $\mathrm{Ci}$ & WP037061 & $\mathbf{Y}$ & NA \\
\hline 316 & 2 & CONSTANT & $1.1400000 E+01$ & $1.1400000 E+01$ & $1.1400000 \mathrm{E}+01$ & $1.1400000 E+01$ & $\mathrm{Ci}$ & WP039260 & $Y$ & N/A \\
\hline 316 & 2 & CONSTANT & $1.1400000 E+01$ & $1.1400000 E+01$ & $1.1400000 E+01$ & $1.1400000 E+01$ & $\mathrm{Ci}$ & WPO41559 & $\bar{Y}$ & N/A \\
\hline 316 & 2 & CONSTANT & $1.1400000 E+01$ & $1.1400000 E+01$ & $1.1400000 \mathrm{E}+01$ & $1.1400000 \mathrm{E}+01$ & $\mathrm{Ci}$ & WPO41560 & $Y$ & N/A \\
\hline 317 & 2 & CONSTANT & $2.7900000 E-04$ & $27900000 E-04$ & $27900000 E-04$ & $2.7900000 E-04$ & $\mathrm{Ci}$ & WP037061 & $\mathbf{Y}$ & N/A \\
\hline 317 & 2 & CONSTANT & $2.7900000 E-04$ & $2.7900000 E-04$ & $2.7900000 E-04$ & $2.7900000 E-04$ & $\mathrm{Ci}$ & WP039260 & $\mathbf{Y}$ & NA \\
\hline 317 & 2 & CONSTANT & $2.7900000 \mathrm{E}-04$ & $2.7900000 E-04$ & $2.7900000 E-04$ & $2.7900000 E-04$ & $\mathrm{Ci}$ & WPO41559 & $\mathbf{Y}$ & $N / A$ \\
\hline 317 & 2 & CONSTANT & $2.7900000 E-04$ & $2.7900000 E-04$ & $2.7900000 E-04$ & $2.7900000 E-04$ & $\mathrm{Ci}$ & WPO41560 & $\mathbf{Y}$ & NA \\
\hline 318 & 4 & CONSTANT & $2.2803900 E-01$ & $2.2803100 \mathrm{E}-01$ & 2.2803100E-01 & $2.2803100 E-01$ & $\mathrm{~kg} / \mathrm{mole}$ & $\mathrm{CRC}-72$ & $Y$ & NA \\
\hline 318 & 4 & CONSTANT & $2.2803100 E-01$ & 2.2803100E-01 & $22803100 E-01$ & 2.2803100E-01 & $\mathrm{kg} / \mathrm{mole}$ & WP036298 & $Y$ & N/A \\
\hline 318 & 4 & CONSTANT & 2.2803100E-01 & $2.2803100 E-01$ & 2.2803100E-01 & 2.2803100E-01 & $\mathrm{kg} /$ mole & WP036301 & $\mathbf{Y}$ & NA \\
\hline 319 & 4 & CONSTANT & $2.1143000 E+08$ & $2.1143000 E+08$ & $2.1143000 E+08$ & $2.1143000 \mathrm{E}+08$ & $s$ & WP036301 & $Y$ & NA \\
\hline 319 & 4 & CONSTANT & $2.1143000 E+\infty 8$ & $2.1143000 E+08$ & $2.1143000 E+08$ & $2.1143000 E+08$ & $\mathbf{s}$ & WP037404 & $Y$ & N/A \\
\hline 319 & 4 & CONSTANT & $2.1143000 E+08$ & $2.1143000 E+08$ & $2.1143000 E+08$ & $2.1143000 \mathrm{E}+08$ & 5 & WPO 40434 & $Y$ & N/A \\
\hline 336 & 1,3 & CONSTANT & $1.0000000 E+\infty 0$ & $1.0000000 \mathrm{E}+\infty 0$ & $1.0000000 E+\infty 0$ & $1.0000000 E+\infty 0$ & NONE & WP031041 & $Y$ & NA \\
\hline 336 & 1,3 & CONSTANT & $1.0000000 E+\infty 0$ & $1.0000000 E+\infty 0$ & $1.0000000 E+\infty 0$ & $1.0000000 E+00$ & NONE & WP035597 & $\mathbf{Y}$ & NA \\
\hline
\end{tabular}


CCA Parameter Listing

\begin{tabular}{|c|c|c|c|c|c|c|c|c|c|c|}
\hline $\begin{array}{c}\text { Parameter in } \\
\text { Detabese? } \\
\text { (YNN) }\end{array}$ & 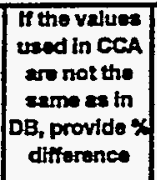 & TD & Material ID & Matesist Name & $\begin{array}{l}\text { Parcmeter } \\
\text { ID }\end{array}$ & Peremeter Name & $\begin{array}{c}\text { PRPD } \\
\text { (KPOD }\end{array}$ & $\begin{array}{c}\text { Data Entry } \\
\text { Dato }\end{array}$ & $\begin{array}{l}\text { Is tho } 464 \\
\text { consietent } \\
\text { with the } \\
\text { parnmeter } \\
\text { in DB? } \\
(\mathrm{N}, \mathrm{N} / \mathrm{A})\end{array}$ & $\begin{array}{l}\text { Paramoter } \\
\text { Cutegory } \\
\text { (footnote 1) }\end{array}$ \\
\hline $\mathbf{Y}$ & & 336 & SANTAROS & Santa Rosa Formation & CAP_MOD & $\begin{array}{l}\text { Model number, capillary pressure } \\
\text { model }\end{array}$ & 33485 & $22+50 b-96$ & $\mathbf{Y}$ & $4 B$ \\
\hline $\mathbf{Y}$ & & 336 & SANTAROS & Santa Rosa Formation & CAP_MOD & $\begin{array}{l}\text { Model number, capillary pressure } \\
\text { model }\end{array}$ & 33485 & 22freb-96 & $\mathbf{Y}$ & 48 \\
\hline $\mathbf{Y}$ & & 337 & SANTAROS & Santa Rosa Formation & COMP_RCK & Bux Compressibility & 33487 & 01-Nov-95 & $\mathbf{Y}$ & 48 \\
\hline $\mathbf{Y}$ & & 337 & SANTAROS & Santa Rosa Formation & COMP_RCK & Buk Compressibility & 33487 & 01-Nov-95 & $\mathbf{Y}$ & $4 B$ \\
\hline $\mathbf{Y}$ & & 339 & SANTAROS & Santa Rosa Formation & PC_max & $\begin{array}{l}\text { Maximum allowable capillary } \\
\text { pressure }\end{array}$ & 33530 & 20-Fob-96 & $\mathbf{Y}$ & $A B$ \\
\hline $\mathbf{Y}$ & & 339 & SANTAROS & Senta Rose Formation & PC_max & $\begin{array}{l}\text { Maximum allowable capillary } \\
\text { pressure }\end{array}$ & 33530 & 20Fob-96 & $\mathbf{Y}$ & AB \\
\hline $\mathbf{Y}$ & & 339 & SANTAROS & Santa Rosa Formation & PC_MAX & $\begin{array}{l}\text { Maximum allowable capillary } \\
\text { pressure }\end{array}$ & 33530 & $20 F=b-96$ & $\mathbf{Y}$ & $4 B$ \\
\hline $\mathbf{Y}$ & & 340 & SANTAROS & Santa Rosa Formation & PORE_DIS & $\begin{array}{l}\text { Brocks-Corey pore distribution } \\
\text { parameter }\end{array}$ & 33538 & $20-F a b-96$ & $\mathbf{Y}$ & $4 B$ \\
\hline $\mathbf{Y}$ & & 340 & SANTAROS & Santa Rosa Formation & PORE_DIS & $\begin{array}{l}\text { Brooks-Coroy pora distribution } \\
\text { parameter }\end{array}$ & 33538 & 20-Fob-96 & $\mathbf{Y}$ & $4 \mathrm{~B}$ \\
\hline $\mathbf{Y}$ & & 340 & SANTAROS & Santa Rosa Formation & PORE_DIS & $\begin{array}{l}\text { Brooks-Corey pora distribution } \\
\text { parameter }\end{array}$ & 33538 & 20-Feb-96 & $\mathbf{Y}$ & 48 \\
\hline $\mathbf{Y}$ & & 341 & SANTAROS & Santa Rosa Formation & POROSITY & Effective porosity & 33542 & 01-Nov-95 & $\mathbf{Y}$ & $4 B$ \\
\hline $\mathbf{Y}$ & & 341 & SANTAROS & Santa Rosa Formation & POROSTYY & Effective porosity & 33542 & 01-Nov-95 & $\mathbf{Y}$ & $4 B$ \\
\hline $\mathbf{Y}$ & & 341 & SANTAROS & Santa Rosa Formation & POROSTIY & Effective porosity & 33542 & 01-Nov-95 & $\mathbf{Y}$ & 48 \\
\hline $\mathbf{Y}$ & & 342 & SANTAROS & Santa Rosa Formation & PO_MIN & $\begin{array}{l}\text { Minimum brine pressure for } \\
\text { capillary model KPC=3 }\end{array}$ & 33543 & 21-Fob-96 & $\mathbf{Y}$ & 48 \\
\hline $\mathbf{Y}$ & & 342 & SANTAROS & Santa Rosa Formation & PO_MIN & $\begin{array}{l}\text { Minimum brine pressure for } \\
\text { capillary model KPC=3 }\end{array}$ & 33543 & 21-Feb-S6 & $\mathbf{Y}$ & 48 \\
\hline $\mathbf{Y}$ & & 342 & SANTAROS & Santa Rosa Formation & PO_MIN & $\begin{array}{l}\text { Minimum brine pressure for } \\
\text { apillary model KPC=3 }\end{array}$ & 33543 & 21-Fob-96 & $\mathbf{Y}$ & $4 B$ \\
\hline $\mathbf{Y}$ & & 343 & SANTAROS & Santa Rosa Formation & PRESSURE & Brine fer-field pore pressure & 33544 & 22 feb-96 & $\mathbf{Y}$ & 1 \\
\hline $\mathbf{Y}$ & & 343 & SANTAROS & Santa Rosa Formation & PRESSURE & Brine far-field pore pressure & 33544 & 22Feb-96 & $\mathbf{Y}$ & 1 \\
\hline $\mathbf{Y}$ & & 343 & SANTAROS & Santa Rosa Formation & PRESSURE & Brine far-field pore pressure & 33544 & 22feb-96 & $\mathbf{Y}$ & 1 \\
\hline $\mathbf{Y}$. & & 344 & SANTAROS & Santa Rosa Formation & PRMX_LOG & $\begin{array}{l}\text { Log of intmsic permeability, } X- \\
\text { diroction }\end{array}$ & 33545 & $01-$ Nov-95 & $Y$ & $4 B$ \\
\hline $\mathbf{Y}$ & & 344 & SANTAROS & Santa Rosa Formation & PRMX_LOG & $\begin{array}{l}\text { Log of intrinsic permeability. } X \text { - } \\
\text { direction }\end{array}$ & 33545 & 01-Nov-95 & $\mathbf{Y}$ & $4 B$ \\
\hline $\mathbf{Y}$ & & 345 & SANTAROS & Santa Rosa Fomation & PRMY_LOG & $\begin{array}{l}\text { Log of intrinsic permeability, Y- } \\
\text { direction }\end{array}$ & 33546 & 01-Nov-95 & $\mathbf{Y}$ & $4 B$ \\
\hline $\mathbf{Y}$ & & 345 & SANTAROS & Santa Rosa Formation & PRMY_LOG & $\begin{array}{l}\text { Log of intrinsic permeability, } Y \text { - } \\
\text { direction }\end{array}$ & 33546 & 01-Nov-95 & $\mathbf{Y}$ & $4 B$ \\
\hline $\mathbf{Y}$ & & 346 & SANTAROS & Senta Rosa Formation & PRMZ_LOG & $\begin{array}{l}\text { Log of intronsic permeability, Z- } \\
\text { direction }\end{array}$ & 33547 & 01-Nov-95 & $\mathbf{Y}$ & $4 B$ \\
\hline $\mathbf{Y}$ & & 346 & SANTAROS & Santa Rosa Formation & PRMZ_LOG & $\begin{array}{l}\text { Log of intrinsic permeability, Z- } \\
\text { drection }\end{array}$ & 33547 & 01-Nov-95 &.$- Y$ & $4 B$ \\
\hline $\mathbf{Y}$ & & 349 & SANTAROS & Santa Rosa Formation & RELP_MOD & $\begin{array}{l}\text { Model number, relative } \\
\text { pormeability model }\end{array}$ & 33550 & 01-Nov-95 & $\mathbf{Y}$ & $4 B$ \\
\hline $\mathbf{Y}$ & & 349 & SANTAROS & Santa Rosa Formation & RELP_MOD & $\begin{array}{l}\text { Model mumber, relative } \\
\text { permeability model }\end{array}$ & 33550 & 01-Nov-95 & $\mathbf{Y}$ & $4 B$ \\
\hline $\mathbf{Y}$ & & 350 & SANTAROS & Sante Rosa Formation & SAT_IBRN & Initual Brine Saturation & 33552 & 22feb-96 & $\mathbf{Y}$ & 48 \\
\hline $\mathbf{Y}$ & & 350 & SANTAROS & Santa Rosa Formation & SAT_IBRN & Initual Brine Saturation & 33552 & 22-Fab-96 & $\mathbf{Y}$ & 4B \\
\hline $\mathbf{Y}$ & & 350 & SANTAROS & Santa Rosa Formation & SAT_IBRN & Initial Brine Saturation & 33552 & 22-fob-96 & $\mathbf{Y}$ & $4 B$ \\
\hline $\mathbf{Y}$ & & 351 & SANTAROS & Santa Rosa Formation & SAT_RBRN & Residual Brine Saturation & 33554 & 22feb-96 & $\mathbf{Y}$ & 48 \\
\hline $\mathbf{Y}$ & & 351 & SANTAROS & Santa Rosa Formation & SAT_RBRN & Residual Brino Saturation & 33554 & 22-Feb-96 & $\mathbf{Y}$ & $4 B$ \\
\hline $\mathbf{Y}$ & & 351 & SANTAROS & Santa Rosa Formation & SAT_RBRN & Residual Brine Saturation & 33554 & $22+F a b-96$ & $Y$ & $4 B$ \\
\hline $\mathbf{Y}$ & & 351 & SANTAROS & Santa Rosa Formation & SAT_RBRN & Residual Brine Saturation & 33554 & 22-Fob-96 & $\mathbf{Y}$ & AB \\
\hline $\mathbf{Y}$ & & 352 & SANTAROS & Santa Rosa Formation & SAT_RGAS & Residual Gas Saturation & 33557 & 20-Fob-96 & $\mathbf{Y}$ & $4 B$ \\
\hline $\mathbf{Y}$ & & 352 & SANTAROS & Santa Rosa Formation & SAT_RGAS & Residual Gas Saturation & 33557 & 20-Feb-96 & $\mathbf{Y}$ & $4 B$ \\
\hline
\end{tabular}


CCA Parameter Listing

\begin{tabular}{|c|c|c|c|c|c|c|c|c|c|c|}
\hline ID & $\begin{array}{c}\text { Clessiff- } \\
\text { cution of } \\
\text { Data } \\
\text { Category } \\
\text { (footnote 2) }\end{array}$ & $\begin{array}{l}\text { Distribution } \\
\text { Type }\end{array}$ & Mean & Median & Minimum & Modmum = & Units & + & $\begin{array}{l}\text { Was the date } \\
\text { developed } \\
\text { under en } \\
\text { NoA-1 } \\
\text { "Program? }\end{array}$ & $\begin{array}{c}\text { Which } \\
\text { mothods } \\
\text { ware used to } \\
\text { quallfy } \\
\text { exdsting } \\
\text { data? } \\
\text { (footnote J) }\end{array}$ \\
\hline 336 & 1,3 & CONSTANT & $1.0000000 E+00$ & $1.0000000 E+\infty 0$ & $1.0000000 E+\infty 0$ & $1.0000000 E+\infty$ & NONE & WP040514 & $\mathbf{Y}$ & NA \\
\hline 336 & 1,3 & CONSTANT & $1.0000000 E+\infty 0$ & $1.0000000 E+\infty$ & $1.0000000 E+00$ & $1.0000000 E+\infty$ & NONE & WS_PALMER & $Y$ & NA \\
\hline 337 & 1,3 & CONSTANT & $1,0000000 \mathrm{E}-08$ & $1.0000000 E-08$ & $1.0000000 E-08$ & $1.0000000 \mathrm{E}-08$ & $P a^{n}-1$ & WP038568 & $Y$ & NA \\
\hline 337 & 1,3 & CONSTANT & $1.0000000 E-08$ & $1.0000000 \mathrm{E}-08$ & $1.0000000 \mathrm{E}-08$ & $1.0000000 \mathrm{E}-08$ & $P a^{A}-1$ & WP040514 & $\mathbf{Y}$ & $N / A$ \\
\hline 339 & 1,3 & CONSTANT & $1.0000000 E+08$ & $1.0000000 E+08$ & $1.0000000 E+08$ & $1.0000000 E+08$ & $\mathrm{~Pa}$ & WPO31041 & $\mathbf{Y}$ & NA \\
\hline 339 & 1,3 & CONSTANT & $1.0000000 E+08$ & $1.0000000 E+08$ & $1.0000000 E+08$ & $1.0000000 \mathrm{E}+08$ & $\mathbf{P a}$ & WP035597 & $\mathbf{Y}$ & NA \\
\hline 339 & 1,3 & CONSTANT & $1.0000000 E+08$ & $1.0000000 E+08$ & $1.0000000 E+08$ & $1.0000000 E+08$ & $\mathbf{P a}$ & WPO40514 & $\mathbf{Y}$ & $N / A$ \\
\hline 340 & 1,3 & CONSTANT & $6.4360000 E-01$ & $6.4360000 \mathrm{E}-01$ & $6.4360000 E-01$ & $6.4360000 \mathrm{E}-01$ & NONE & WP031041 & $\mathbf{Y}$ & $N / A$ \\
\hline 340 & 1,3 & CONSTANT & $6.4360000 \mathrm{E}-01$ & $6.4360000 E-01$ & $6.4360000 \mathrm{E}-01$ & $6.4360000 E-01$ & NONE & WP035597 & $\mathbf{Y}$ & NA \\
\hline 340 & 1,3 & CONSTANT & $6.4360000 E-01$ & $6.4360000 \mathrm{E}-01$ & 6.4360000E-01 & $6.4360000 \mathrm{E}-01$ & NONE & WPO40514 & $\mathbf{Y}$ & N/A \\
\hline 341 & $1,3,4$ & CONSTANT & $1.7500000 \mathrm{E}-01$ & $1.7500000 E-01$ & $1.7500000 \mathrm{E}-01$ & $1.7500000 E-01$ & NONE & FREEZE-1 & $Y$ & NA \\
\hline 341 & $1,3,4$ & CONSTANT & $1.7500000 E-01$ & $1.7500000 \mathrm{E}-01$ & 1.7500000 E-01 & $1.7500000 \mathrm{E}-01$ & NONE & WP040511 & $\mathbf{Y}$ & N/A \\
\hline 341 & $1,3,4$ & CONSTANT & $1.7500000 \mathrm{E}-01$ & $1.7500000 \mathrm{E}-01$ & $1.7500000 \mathrm{E}-01$ & $1.7500000 \mathrm{E}-01$ & NONE & WP040514 & $\mathbf{Y}$ & $N / A$ \\
\hline 342 & 1,3 & CONSTANT & $1.0132500 \mathrm{E}+05$ & $1.0132500 E+05$ & $1.0132500 E+05$ & $1.0132500 E+05$ & $\mathbf{P a}$ & WP035597 & $\mathbf{Y}$ & NAA \\
\hline 342 & 1,3 & CONSTANT & $1.0132500 E+05$ & $1.0132500 E+05$ & $1.0132500 E+05$ & $1.0132500 E+0.5$ & $\mathrm{~Pa}$ & WP036051 & $\mathbf{Y}$ & N/A \\
\hline 342 & 1,3 & CONSTANT & $1.0132500 E+05$ & $1.0132500 E+05$ & $1.0132500 E+05$ & $1.0132500 E+05$ & $\mathbf{P a}$ & WP040514 & $\mathbf{Y}$ & N/A \\
\hline 343 & 1 & CONSTANT & $1.0132500 E+05$ & $1.0132500 E+0.5$ & $1.0132500 E+05$ & $1.0132500 E+05$ & $P a$ & WP030713 & $\mathbf{Y}$ & 1 \\
\hline 343 & 9 & CONSTANT & $1.0132500 E+05$ & $1.0132500 E+05$ & $1.0132500 E+0.5$ & $1.0132500 E+0.5$ & $\mathbf{P a}$ & WP032290 & $\mathbf{Y}$ & 1 \\
\hline 343 & 1 & CONSTANT & $1.0132500 E+05$ & $1.0132500 E+05$ & $1.0132500 E+0.5$ & $1.0132500 E+05$ & $\mathrm{~Pa}$ & WP035597 & $\mathbf{Y}$ & 1 \\
\hline 344 & $1,3,4$ & CONSTANT & $-1,0000000 E+01$ & $-1.0000000 E+01$ & $-1.0000000 E+01$ & $-1.0000000 E+01$ & $\log \left(m^{\wedge} 2\right)$ & WP038568 & $Y$ & N/A \\
\hline 344 & $1,3,4$ & CONSTANT & $-1.0000000 E+01$ & $-1.0000000 E+01$ & $-1.0000000 E+01$ & $-1.0000000 E+01$ & $\log \left(m^{\wedge} 2\right)$ & WP040514 & $\mathbf{Y}$ & N/A \\
\hline 345 & $1,3,4$ & CONSTANT & $-1.0000000 E+01$ & $-1.0000000 \mathrm{E}+01$ & $-1.0000000 E+01$ & $-1.0000000 E+01$ & $\log \left(m^{\wedge} 2\right)$ & WPO38568 & $\mathbf{Y}$ & N/A \\
\hline 345 & $1,3,4$ & CONSTANT & $-1.0000000 E+01$ & $-1.0000000 E+01$ & $-1.0000000 E+01$ & $-1.0000000 E+01$ & $\log \left(m^{\wedge} 2\right)$ & WP040514 & $Y$ & NA \\
\hline 346 & $1,3,4$ & CONSTANT & $-1.0000000 E+01$ & $-1.0000000 E+01$ & $-1.0000000 E+01$ & $-1.0000000 E+01$ & $\log \left(m^{\wedge} 2\right)$ & WP038568 & $Y$ & NA \\
\hline 346 & $1,3,4$ & CONSTANT & $-1.0000000 E+01$ & $-1.0000000 E+01$ & $-1.0000000 E+01$ & $-1.0000000 E+01$ & $\log \left(m^{\wedge} 2\right)$ & WP040514 & $-\not$ & N/A \\
\hline 349 & 1,3 & CONSTANT & $4,0000000 E+00$ & $4.0000000 E+\infty$ & $4.0000000 E+\infty 0$ & $4.0000000 E+\infty 0$ & NONE & WPO38568 & $\mathbf{Y}$ & NA \\
\hline 349 & 1,3 & CONSTANT & $4.0000000 E+00$ & $4.0000000 E+\infty$ & $4.0000000 E+\infty 0$ & $4.0000000 E+00$ & NONE & WP040514 & $\mathbf{Y}$ & NA \\
\hline 350 & 1,3 & CONSTANT & $8.3630000 E-02$ & $8.3630000 E-02$ & $8.3530000 \mathrm{E}-02$ & $8.3630000 E-02$ & NONE & WP035597 & $Y$ & NA \\
\hline 350 & 1,3 & CONSTANT & 8.3630000E-02 & $8.3630000 E-02$ & $8.3630000 \varepsilon-02$ & $8.3630000 E-02$ & NONE & WPO40514 & $Y$ & N/A \\
\hline 350 & 1,3 & CONSTANT & $8.3630000 E-02$ & $8.3630000 E-02$ & $8.3630000 E-02$ & $8.3630000 E-02$ & NONE & WS_PALMER & $\mathbf{Y}$ & NA \\
\hline 351 & 1,3 & CONSTANT & $8.3630000 E-02$ & $8.3630000 E-02$ & $8.3630000 E-02$ & $8.3530000 E-02$ & NONE & WP031041 & $\mathbf{Y}$ & NA \\
\hline 351 & 1,3 & CONSTANT & 8.3630000E-02 & $8.3630000 E-02$ & $8.3630000 E-02$ & $8.3630000 E-02$ & NONE & WP035597 & $Y$ & NA \\
\hline 351 & 1,3 & CONSTANT & 8.3630000E-02 & $8.3630000 \mathrm{E}-02$ & $8.3630000=-02$ & $8.3630000 E-02$ & NONE & WPO40514 & $\mathbf{Y}$ & NAA \\
\hline 351 & 1,3 & CONSTANT & $8.3630000 E-02$ & $8.3630000 E-02$ & $8.3630000 E-02$ & $8.3630000 E-02$ & NONE & WS_PALMER & $\mathbf{Y}$ & N/A \\
\hline 352 & 1,3 & CONSTANT & $7.7110000 E-02$ & $7.7110000 E-02$ & $7.7110000 \mathrm{E}-02$ & $7.7110000 E-02$ & NONE & WP031041 & $Y$ & NA \\
\hline 352 & 1,3 & CONSTANT & 7.7110000 E-02 & $7.7110000 E-02$ & $7.7110000 \mathrm{E}-02$ & $7.7110000 E-02$ & NONE & WP035597 & $\mathbf{Y}$ & N/A \\
\hline
\end{tabular}


CCA Parameter Listing

\begin{tabular}{|c|c|c|c|c|c|c|c|c|c|c|}
\hline $\begin{array}{c}\text { Paramotar in } \\
\text { Database? } \\
\text { (NIN) } \\
\end{array}$ & \begin{tabular}{|l|} 
If the values \\
used in Ceca \\
are not the \\
ome as in \\
oB, prowde \\
difference
\end{tabular} & 1D & Materalio & Matorial Name & $\begin{array}{c}\text { Paramater } \\
\text { ID }\end{array}$ & Paranitar Name & $\begin{array}{l}\text { PRP DD } \\
\text { MPO If }\end{array}$ & $\begin{array}{c}\text { Data Entry } \\
\text { Date }\end{array}$ & $\begin{array}{l}\text { is the } 464 \\
\text { convilutent } \\
\text { with the } \\
\text { purrmoter } \\
\text { in DB? } \\
\left(M_{0} \text { NA) }\right.\end{array}$ & $\begin{array}{l}\text { Parmater } \\
\text { Category } \\
\text { (footnots 1) }\end{array}$ \\
\hline $\mathbf{Y}$ & & 352 & SANTAROS & Santa Rosa Formation & SAT_RGAS & Residual Gas Saturation & 33557 & 20 feb-96 & $\mathbf{Y}$ & $4 B$ \\
\hline $\mathbf{Y}$ & & 352 & SANTAROS & Santa Rosa Formation & SAT_RGAS & Residual Gas Saturation & 33557 , & 20-Feb-96 & $\mathbf{Y}$ & 48 \\
\hline $\mathbf{Y}$ & 0.06 & 514 & SM147 & Samarum 147 & ATWEIGHT & Atomic Weight in $\mathrm{kg} / \mathrm{mole}$ & 32455 & 02-Nov-95 & $\mathbf{Y}$ & 3 \\
\hline $\mathbf{Y}$ & 0.06 & 514 & SM147 & Samarium 147 & ATWEIGHT & Atomic Weight in $\mathrm{kg} / \mathrm{mole}$ & 32455 & O2Nov-95 & $\mathbf{Y}$ & 3 \\
\hline $\mathbf{Y}$ & 0.06 & 514 & SM147 & Samarium 147 & ATWEIGHT & Atomic Weight in $\mathrm{kg} / \mathrm{mole}$ & 32455 & 02-Nov-95 & $\mathbf{Y}$ & $3 \cdot$ \\
\hline $\mathbf{Y}$ & 100 & 515 & SM147 & Samarium 147 & HALFLIFE & Hathife & 34350 & 16-Apr-96 & $Y$ & 3 \\
\hline $\mathbf{Y}$ & 100 & 515 & SM147 & Samarium 147 & HALFLIFE & Halifitio & 34350 & $16-A p r+96$ & $\mathbf{Y}$ & 3 \\
\hline$Y$ & 100 & 515 & SMi47 & Samarium 147 & HALFLIFE & Halfifife & 34350 & 16-Apr-96 & $\mathbf{Y}$ & 3 \\
\hline $\mathbf{Y}$ & 100 & 517 & SR90 & Strontium 90 & HALFLIFE & Halfife & 34353 & 16 -Apr-86 & $Y$ & 3 \\
\hline $\mathbf{Y}$ & 100 & 517 & SR90 & Strontium 90 & HALFLIFE & Halffife & 34353 & 16-Apr-96 & $\mathbf{Y}$ & 3 \\
\hline$Y$ & 100 & 517 & SR90 & Strontium 90 & HALFLIFE & Halnifo & 34353 & 16-Apr-96 & $\mathbf{Y}$ & 3 \\
\hline $\mathbf{Y}$ & & 518 & SR90 & Strontium 90 & INVRHD & $\begin{array}{l}\text { Inventory of Remote Handled } \\
\text { Design }\end{array}$ & $34355 A$ & $29-0 c t-96$ & $\mathbf{Y}$ & 2 \\
\hline $\mathbf{Y}$ & & 518 & SR90 & Strontium 90 & INVRHD & $\begin{array}{l}\text { Inventory of Remole Handled } \\
\text { Design }\end{array}$ & $34355 A$ & $29-0 c t-96$ & $\mathbf{Y}$ & 2 \\
\hline $\mathbf{Y}$ & & 518 & SR90 & Strontium 90 & INVRHD & $\begin{array}{l}\text { Inventory of Remole Handled } \\
\text { Design }\end{array}$ & $34355 A$ & $29-0 c t-96$ & $\mathbf{Y}$ & 2 \\
\hline $\mathbf{Y}$ & & 518 & SR9O & Strontium 90 & INVRHD & $\begin{array}{l}\text { Inventory of Remote Handled } \\
\text { Design }\end{array}$ & $34355 A$ & $29-0 \mathrm{ct}-96$ & $\mathbf{Y}$ & 2 \\
\hline $\mathbf{Y}$ & & 520 & S_ANH_AB & $\begin{array}{l}\text { Salado anhydrite beds } \\
\text { A and B, intact and } \\
\text { fractured }\end{array}$ & CAP_MOD & $\begin{array}{l}\text { Model number, capillary pressure } \\
\text { model }\end{array}$ & 34130 & 08-Feb-9s & $\mathbf{Y}$ & AA \\
\hline $\mathbf{Y}$ & & 520 & S_ANH_AB & $\begin{array}{l}\text { Salado anhydrite beds } \\
\text { A and B, intact and } \\
\text { fractured }\end{array}$ & CAP_MOD & $\begin{array}{l}\text { Model number, capillary pressure } \\
\text { model }\end{array}$ & 34130 & OS-Feb-96 & $\mathbf{Y}$ & $4 A$ \\
\hline $\mathbf{Y}$ & & 521 & S_ANH_AB & $\begin{array}{l}\text { Salado anhydinte beds } \\
\text { A and B, intact and } \\
\text { fractured }\end{array}$ & COMP_RCK & Bulk Compressibility & 34135 & 14 Feb-96 & $Y$ & $4 A$ \\
\hline $\mathbf{Y}$ & & 521 & S_ANH_AB & $\begin{array}{l}\text { Salado anhydrite beds } \\
\text { A and } B \text {, intact and } \\
\text { fractured }\end{array}$ & COMP_RCK & Bulk Compressibility & 34135 & 14Feb-96 & $\mathbf{Y}$ & $4 A$ \\
\hline $\mathbf{Y}$ & & 521 & S_ANH_AB & $\begin{array}{l}\text { Salado anhydrite beds } \\
\text { A and } B \text {, intact and } \\
\text { fractured }\end{array}$ & COMP_RCK & Bulk Compressibility & 34135 & 14-Feb-96 & $\mathbf{Y}$ & $4 A$ \\
\hline $\mathbf{Y}$ & & 522 & S_ANH_AB & $\begin{array}{l}\text { Salado anhydrite beds } \\
\text { A and B, intact and } \\
\text { fractured }\end{array}$ & PC_MAX & $\begin{array}{l}\text { Maximum allowable capillary } \\
\text { pressure }\end{array}$ & 34185 & 11 feb-96 & $\mathbf{Y}$ & $4 A$ \\
\hline $\mathbf{Y}$ & & 522 & S_ANH_AB & $\begin{array}{l}\text { Salado anhydrite beds } \\
\text { A and } B \text {, intact and } \\
\text { fractured }\end{array}$ & PC_MAX & $\begin{array}{l}\text { Maximum allowable capillary } \\
\text { pressure }\end{array}$ & 34185 & 11 Fob-96 & $\mathbf{Y}$ & $4 A$ \\
\hline $\mathbf{Y}$ & & 524 & S_ANH_AB & $\begin{array}{l}\text { Salado antydirite beds } \\
\text { A and B, intact and } \\
\text { fractured }\end{array}$ & PF_DELTA & $\begin{array}{l}\text { Incremental pressure for full } \\
\text { fracture development }\end{array}$ & 34187 & 22Feb-96 & $Y$ & $4 B$ \\
\hline$Y$ & & 524 & S_ANH_AB & $\begin{array}{l}\text { Salado anhydrite beds } \\
A \text { and } B \text {, intact and } \\
\text { fractured }\end{array}$ & PF_DELTA & $\begin{array}{l}\text { incremental pressure for full } \\
\text { fracture development }\end{array}$ & 34187 & 22-Fab-96 & $\bar{Y}$ & $4 B$ \\
\hline $\mathbf{Y}$ & & 524 & S_ANH_AB & $\begin{array}{l}\text { Salado anhydinte beds } \\
A \text { and } B \text {, intect and } \\
\text { fractured }\end{array}$ & PF_DELTA & $\begin{array}{l}\text { Incremental pressure for full } \\
\text { fracture development }\end{array}$ & 34187 & 22-Fob-96 & $\mathbf{Y}$ & $4 B$ \\
\hline $\mathbf{Y}$ & & 524 & S_ANH_AB & $\begin{array}{l}\text { Salado anhydrite beds } \\
\text { A and B, intact and } \\
\text { fractured }\end{array}$ & PF_DELTA & $\begin{array}{l}\text { Incremental pressure for full } \\
\text { fracture development }\end{array}$ & 34187 & 22-Fob-96 & $\mathbf{Y}$ & $4 B$ \\
\hline $\mathbf{Y}$ & & 526 & S_ANH_AB & $\begin{array}{l}\text { Salado anhydnte beds } \\
\text { A and B, intact and } \\
\text { tractured }\end{array}$ & PI_DELTA & $\begin{array}{l}\text { Fracture initiation pressure } \\
\text { increment }\end{array}$ & 34190 & 22-Feb-96 & $\mathbf{Y}$ & $4 B$ \\
\hline$Y$ & & 526 & S_ANH_AB & $\begin{array}{l}\text { Salado anhydrite beds } \\
A \text { and } B \text {, intact and } \\
\text { fractured }\end{array}$ & PI_DELTA & $\begin{array}{l}\text { Fracture initiation pressure } \\
\text { increment }\end{array}$ & 34190 & 22-Feb-96 & $\mathbf{Y}$ & $4 B$ \\
\hline $\mathbf{Y}$ & & 526 & S_ANH_AB & $\begin{array}{l}\text { Salado anhydrite beds } \\
A \text { and } B \text {, intact and } \\
\text { tractured }\end{array}$ & PI_DELTA & $\begin{array}{l}\text { Fracture initiation pressure } \\
\text { increment }\end{array}$ & 34190 & 22-Feb-96 & $\mathbf{Y}$ & $4 B$ \\
\hline $\mathbf{Y}$ & & 526 & S_ANH_AB & $\begin{array}{l}\text { Salado anhydrto beds } \\
A \text { and } B \text {, intect and } \\
\text { fractured }\end{array}$ & PI_DELTA & $\begin{array}{l}\text { Fracture initiation pressure } \\
\text { increment }\end{array}$ & 34190 & 22-Fob-96 & $\mathbf{Y}$ & 4B \\
\hline$Y$ & & 527 & S_ANH_AB & $\begin{array}{l}\text { Salado anhydite beds } \\
A \text { and } B \text {, intact and } \\
\text { fractured }\end{array}$ & PORE_DIS & $\begin{array}{l}\text { Brooks-Corby pore distribution } \\
\text { parameter }\end{array}$ & 34192 & 22feb-96 & $\mathbf{Y}$ & $4 A$ \\
\hline
\end{tabular}


CCA Parameter Listing

\begin{tabular}{|c|c|c|c|c|c|c|c|c|c|c|}
\hline ID & $\begin{array}{c}\text { Claseffi- } \\
\text { cation of } \\
\text { Dath } \\
\text { Cattogory } \\
\text { (footrote 2) }\end{array}$ & $\begin{array}{l}\text { Distribution } \\
\text { Typo }\end{array}$ & $\operatorname{man}$ & medien & Minimum & Maximum & Units & Reformen id & $\begin{array}{l}\text { Was the derta } \\
\text { developed } \\
\text { under an } \\
\text { NOA-1 } \\
\text { Progrum? }\end{array}$ & \begin{tabular}{|c|} 
Which \\
mothods \\
wore used to \\
quility \\
existing \\
ditte? \\
(footnote 3)
\end{tabular} \\
\hline 352 & 1,3 & CONSTANT & $7.7110000 E-02$ & 7.7110000E-02 & $7.7110000 E-02$ & $7.7110000 E-02$ & NONE & WP040514 & $Y$ & N/A \\
\hline 352 & 1,3 & CONSTANT & $7.7110000 E-02$ & $7.7110000 E-02$ & $7.7110000 E-02$ & $7.7110000 E-02$ & NONE & WS_PALMER & $\mathbf{Y}$ & NA \\
\hline 514 & 4 & CONSTANT & $1.4691500 \mathrm{E}-01$ & $1.4691500 \mathrm{E}-01$ & 1.4691500E-01 & 1.4691500E-01 & $\mathrm{kg} / \mathrm{mole}$ & CRC-72 & $\mathbf{Y}$ & NA \\
\hline 514 & 4 & CONSTANT & $1.4691500 E-01$ & $1.4691500 \mathrm{E}-01$ & 1.4691500E-01 & 1.4691500E-01 & $\mathrm{kg} / \mathrm{mole}$ & WP036298 & $\mathbf{Y}$ & N/A \\
\hline 514 & 4 & CONSTANT & $1.4691500 \mathrm{E}-01$ & $1.4691500 E-01$ & 1.4691500E-01 & $1.4691500 \mathrm{E}-01$ & $\mathrm{~kg} / \mathrm{mole}$ & WPO36301 & $\mathbf{Y}$ & N/A \\
\hline 515 & 4 & CONSTANT & $3.3770000 \mathrm{E}+18$ & $3.3770000 \mathrm{E}+18$ & $3.3770000 \mathrm{E}+18$ & $3.3770000 \mathrm{E}+18$ & s & WPO36301 & $Y$ & NA \\
\hline 515 & 4 & CONSTANT & $3.3770000 \mathrm{E}+18$ & $3.3770000 \mathrm{E}+18$ & $3.3770000 \mathrm{E}+18$ & $3.3770000 E+18$ & $s$ & WP037404 & $\mathbf{Y}$ & NA \\
\hline 515 & 4 & CONSTANT & $3.3770000 \mathrm{E}+18$ & $3.3770000 E+18$ & $3.3770000 \mathrm{E}+18$ & $3.3770000 \mathrm{E}+18$ & s & WPO40434 & $Y$ & NA \\
\hline 517 & 4 & CONSTANT & $9.1900000 E+08$ & $9.1900000 E+08$ & $9.1900000 E+08$ & $9.1900000 \mathrm{E}+08$ & $\mathbf{s}$ & WP036301 & $\mathbf{Y}$ & N/A \\
\hline 517 & 4 & CONSTANT & $9.1900000 \mathrm{E}+08$ & $9.1900000 \mathrm{E}+08$ & $9.1900000 \mathrm{E}+08$ & $9.1900000 \mathrm{E}+08$ & $s$ & WPO37404 & $Y$ & N/A \\
\hline 517 & 4 & CONSTANT & $9.1900000 \mathrm{E}+08$ & $9.1900000 E+08$ & $9.1900000 E+08$ & $9.1900000 \mathrm{E}+08$ & s & WPO40434 & $\bar{Y}$ & NA \\
\hline 518 & 2 & CONSTANT & $8.4500000 E+04$ & $8.4500000 E+04$ & $8.4500000 E+04$ & $8.4500000 E+04$ & $\mathrm{Ci}$ & WP037061 & $\mathbf{Y}$ & NA \\
\hline 518 & 2 & CONSTANT & $8.4500000 E+0.4$ & $8.4500000 E+04$ & $8.4500000 E+04$ & $8.45000000+04$ & $\mathbf{C i}$ & WP039260 & $\mathbf{Y}$ & NA \\
\hline 518 & 2 & CONSTANT & $8.4500000 E+04$ & $8.4500000 E+04$ & $8.4500000 E+04$ & $8.4500000 E+04$ & $\mathrm{Ci}$ & WP041559 & $\mathbf{Y}$ & N/A \\
\hline 598 & 2 & CONSTANT & $8,4500000 E+04$ & $8.4500000 E+04$ & $8.4500000 E+04$ & $8.4500000 E+04$ & $\mathrm{Ci}$ & WPO41560 & $\mathbf{Y}$ & NA \\
\hline 520 & 1,3 & CONSTANT & $2.0000000 E+00$ & $2.0000000 \mathrm{E}+00$ & $2.0000000 E+\infty$ & $2.0000000 E+\infty 0$ & NONE & WP030643 & $\mathbf{Y}$ & N/A \\
\hline 520 & 1,3 & CONSTANT & $2.0000000 E+00$ & $2.0000000 E+\infty 0$ & $2,0000000 E+\infty 0$ & $2.0000000 E+\infty 0$ & NONE & WP035597* & $\mathbf{Y}$ & N/A \\
\hline 521 & 1,3 & STUDENT & $8.2630000 E-11$ & $8.2630000 E-11$ & $1.0900000 \mathrm{E}-11$ & $2.7500000 E-10$ & $P a^{n-1}$ & WP031186 & $\mathbf{Y}$ & N/A \\
\hline 521 & 1,3 & STUDENT & $8.2630000 E-11$ & $8.2630000 E-11$ & $1.0900000 E-11$ & $2.7500000 E-10$ & $P^{a}-1$ & WP035597 & $\mathbf{Y}$ & N/A \\
\hline 521 & 1,3 & STUDENT & $8.2630000 E-11$ & $8.2630000 E-11$ & $1.0900000 \mathrm{E}-11$ & $2.7500000 E-10$ & $\mathrm{~Pa}^{n-1}$ & WPO36380 & $\mathbf{Y}$ & N/A \\
\hline 522 & 1,3 & CONSTANT & $1.0000000 E+08$ & $1.0000000 E+08$ & $1.0000000 E+08$ & $1.0000000 E+08$ & $\mathbf{P a}$ & WP030643 & $\mathbf{Y}$ & N/A \\
\hline 522 & 1,3 & CONSTANT & $1.0000000 E+08$ & $1.0000000 E+08$ & $1.0000000 E+08$ & $1.0000000 E+08$ & $\mathbf{P a}$ & WP035597 & $Y$ & N/A \\
\hline 524 & 1,3 & CONSTANT & $3.8000000 E+06$ & $3.8000000 \mathrm{E}+06$ & $3.8000000 \mathrm{E}+06$ & $3.8000000 E+06$ & $\mathbf{P a}$ & WP035597 & $\mathbf{Y}$ & N/A \\
\hline 524 & 1,3 & CONSTANT & $3.8000000 \mathrm{E}+06$ & $3.8000000 E+06$ & $3.8000000 E+06$ & $3.8000000 E+06$ & $\mathbf{P a}$ & WP038274 & $\bar{Y}_{Y}$ & N/A \\
\hline 524 & 1,3 & CONSTANT & $3.8000000 E+06$ & $3.8000000 \mathrm{E}+06$ & $3.8000000 E+06$ & $3.8000000 E+06$ & $\mathrm{~Pa}$ & WP038569 & $\mathbf{Y}$ & NA \\
\hline 524 & 1,3 & CONSTANT & $3.8000000 E+08$ & $3.8000000 E+06$ & $3.8000000 E+06$ & $3.8000000 E+06$ & $\mathrm{~Pa}$ & WPO40514 & $Y$ & NA \\
\hline 526 & 1,3 & CONSTANT & $2.0000000 \mathrm{E}+05$ & $2.0000000 \mathrm{E}+0.5$ & $2.0000000 E+05$ & $2.0000000 E+05$ & $\mathbf{P a}$ & WP035597 & $\mathbf{Y}$ & N/A \\
\hline 526 & 1,3 & CONSTANT & $2.0000000 E+05$ & $2.0000000 E+05$ & $2.0000000 E+05$ & $2.0000000 E+05$ & $\mathrm{~Pa}$ & WP038274 & $\mathbf{Y}$ & N/A \\
\hline 526 & 1,3 & CONSTANT & $2.0000000 E+05$ & $2.00000000+05$ & $2.00000000+05$ & $2.0000000 E+05$ & $\mathrm{~Pa}$ & WP038569 & $Y$ & N/A \\
\hline 526 & 1,3 & CONSTANT & $2.0000000 E+05$ & $2.0000000 E+05$ & $2.0000000 \mathrm{E}+05$ & $2.0000000 E+05$ & $\mathbf{P a}$ & WP040514 & $\mathbf{Y}$ & N/A \\
\hline 527 & 1,3 & STUDENT & $6.4360000 E-01$ & $6.4360000 E-01$ & 4.9053000E-01 & 8.4178000E-01 & NONE & WP030643 & $\mathbf{Y}$ & NA \\
\hline
\end{tabular}


CCA Parameter Listing

\begin{tabular}{|c|c|c|c|c|c|c|c|c|c|c|}
\hline $\begin{array}{c}\text { Paramet in } \\
\text { Databese? } \\
\text { (YN) }\end{array}$ & $\begin{array}{l}\text { ft tho values } \\
\text { used in CCAA } \\
\text { are not the } \\
\text { same as in } \\
\text { DB, provide } x \\
\text { difference }\end{array}$ & ID & Material ID & Material Name & $\begin{array}{l}\text { Paramotior } \\
\text { ID }\end{array}$ & Pramoter Namo & $\begin{array}{c}\text { PRPID } \\
\text { (MPOA) } \\
\cdots \\
\vdots: \\
\vdots:\end{array}$ & Dates Entry & $\begin{array}{c}\text { is the } 464 \\
\text { consistent } \\
\text { with the } \\
\text { perrmeter } \\
\text { in DB? } \\
(Y, N / A)\end{array}$ & $\begin{array}{l}\text { Perametor } \\
\text { Cattegory } \\
\text { (footrote f) }\end{array}$ \\
\hline$Y$ & & 527 & S_ANH_AB & $\begin{array}{l}\text { Salado anhydrte beds } \\
\text { A and B, intact and } \\
\text { fractured }\end{array}$ & PORE_DIS & $\begin{array}{l}\text { Brooks-Corey pore distribution } \\
\text { parameter }\end{array}$ & 34192 & 22-Feb-96 & $\mathbf{Y}$ & $\mathbb{A A}$ \\
\hline$\gamma$ & & 527 & S_ANH_AB & $\begin{array}{l}\text { Salado andydinte beds } \\
\text { A and B, intact and } \\
\text { fractured }\end{array}$ & PORE_DIS & $\begin{array}{l}\text { Brooks-Coroy pore distribution } \\
\text { parameter }\end{array}$ & 34192 & 22-Fob-96 & $\mathbf{Y}$ & 44 \\
\hline$Y$ & & 528 & S_ANH_AB & $\begin{array}{l}\text { Salado anhydrte beds } \\
\text { A and B, intact and } \\
\text { fractured }\end{array}$ & POROSTY & Effective porosity & 34193 & 21-Fab-96 & $\mathbf{Y}$ & $\mathbb{A A}$ \\
\hline$Y$ & & 528 & S_ANH_AB & $\begin{array}{l}\text { Salado anhydrite bods } \\
\mathrm{A} \text { and } \mathrm{B} \text {, intact and } \\
\text { fractured }\end{array}$ & POROSITY & Effective porosity & 34193 & 21-Fob-96 & $\mathbf{Y}$ & $4 A$ \\
\hline $\mathbf{Y}$ & & 528 & S_ANH_AB & $\begin{array}{l}\text { Salado anhydrito beds } \\
\text { A and B, intact and } \\
\text { fractured }\end{array}$ & POROSTY & Effective porasity & 34193 & 21 fob-96 & $\gamma$ & $4 A$ \\
\hline $\mathbf{Y}$ & & 529 & S_ANH_AB & $\begin{array}{l}\text { Salado anhydrite beds } \\
\text { A and } B \text {, intact and } \\
\text { fractured }\end{array}$ & PO_MIN & $\begin{array}{l}\text { Minimum brine pressure for } \\
\text { capillary model KPC=3 }\end{array}$ & 34194 & 21-Feb-96 & $\mathbf{Y}$ & 48 \\
\hline$Y$ & & 529 & S_ANH_AB & $\begin{array}{l}\text { Salado anhydrite beds } \\
\text { A and } B \text {, intact and } \\
\text { fractured }\end{array}$ & PO_MIN & $\begin{array}{l}\text { Minimum brine pressurs for } \\
\text { capillary model KPC=3 }\end{array}$ & 34194 & 21-Fob-96 & $\mathbf{Y}$ & 48 \\
\hline $\mathbf{Y}$ & & 529 & $S_{-}$ANH_AB & \begin{tabular}{|l|} 
Salado anhydrite beds \\
A and $B$, intact and \\
fractured
\end{tabular} & PO_MIN & $\begin{array}{l}\text { Minimum brine pressure for } \\
\text { capillary model KPC=3 }\end{array}$ & 34194 & $21-F a b-96$ & $\gamma$ & $4 B$ \\
\hline $\mathbf{Y}$ & & 531 & S_ANH_AB & $\begin{array}{l}\text { Salado anhydrite bods } \\
\text { A and } B \text {, intact and } \\
\text { fractured }\end{array}$ & PRMX_LOG & $\begin{array}{l}\log \text { of intrinsic permeability, } X \text { - } \\
\text { direction }\end{array}$ & 34196 & 11-Fab-96 & $\mathbf{Y}$ & $4 A$ \\
\hline$Y$ & & 531 & S_ANH_AB & $\begin{array}{l}\text { Salado anhydofite bods } \\
\text { A and } B \text {, intact and } \\
\text { fractured }\end{array}$ & PRMX_LOG & $\begin{array}{l}\text { Log of intrinsic permeability, } x \text { - } \\
\text { direction }\end{array}$ & 34196 & 11-Fob-96 & $Y$ & $4 A$ \\
\hline $\mathbf{Y}$ & & 531 & S_ANH_AB & $\begin{array}{l}\text { Salado anhydrite beds } \\
\text { A and } B \text {, intact and } \\
\text { fractured }\end{array}$ & PRMX_LOG & $\begin{array}{l}\text { Log of intrinsic permeability, } X \text { - } \\
\text { direction }\end{array}$ & 34196 & 11 -Fob-96 & $\mathbf{Y}$ & $4 \mathrm{~A}$ \\
\hline$Y$ & & 531 & S_ANH_AB & $\begin{array}{l}\text { Salado anthydrite beds } \\
\text { A and B, intact and } \\
\text { fractured }\end{array}$ & PRMX_LOG & $\begin{array}{l}\text { Log of intrinsic permeability, } x \text { - } \\
\text { direction }\end{array}$ & 34196 & 11 Fob-96 & $Y$ & $4 \mathrm{~A}$ \\
\hline$Y$ & & 531 & S_ANH_AB & $\begin{array}{l}\text { Salado anhydrito bods } \\
\text { A and B, intact and } \\
\text { fractured }\end{array}$ & PRMX_LOG & $\begin{array}{l}\text { Log of intrinsic permeability, } X- \\
\text { direction }\end{array}$ & 34196 & $11-F a b-96$ & $Y$ & $4 A$ \\
\hline$\gamma$ & & 531 & S_ANH_AB & $\begin{array}{l}\text { Salado anhydrite bods } \\
\text { A and B, intact and } \\
\text { fractured }\end{array}$ & PRMX_LOG & $\begin{array}{l}\text { Log of intrinsic permeability, X- } \\
\text { direction }\end{array}$ & 34196 & $11-f a b-96$ & $\mathbf{Y}$ & $4 A$ \\
\hline$Y$ & & 532 & S_ANH_AB & $\begin{array}{l}\text { Salado antydrite bods } \\
\text { A and } B \text {, intact and } \\
\text { fracturod }\end{array}$ & PRMY_LOG & $\begin{array}{l}\text { Log of intrinsic permeability. Y- } \\
\text { direction }\end{array}$ & 34197 & 11-Fab-96 & $\mathbf{Y}$ & $4 A$ \\
\hline $\mathbf{Y}$ & & 532 & S_ANH_AB & $\begin{array}{l}\text { Salado anhydrite bods } \\
\text { A and } B \text {, intact and } \\
\text { fractured }\end{array}$ & PRMY_LOG & $\begin{array}{l}\text { Log of intrinsic pormeability. Y. } \\
\text { direction }\end{array}$ & 34197 & 11 fab-96 & $Y$ & $4 A$ \\
\hline$Y$ & & 532 & S_ANH_AB & $\begin{array}{l}\text { Salado anhydrite beds } \\
\text { A and B, intact and } \\
\text { fractured }\end{array}$ & PRMY_LOG & $\begin{array}{l}\text { Log of intrinsic permeability, Y- } \\
\text { direction }\end{array}$ & 34497 & 11-Fob-96 & $" Y$ & $4 A$ \\
\hline$Y$ & & 532 & S_ANH_AB & $\begin{array}{l}\text { Salado anhydrite beds } \\
\text { A and } \mathrm{B} \text {, intact and } \\
\text { fractured }\end{array}$ & PRMY_LOG & $\begin{array}{l}\text { Log of intrinsic permeabillty, Y- } \\
\text { drection }\end{array}$ & 34197 & 11-Feb-96 & $Y$ & $4 A$ \\
\hline$Y$ & & 532 & S_ANH_AB & $\begin{array}{l}\text { Salado anhydinte bods } \\
\text { A and } B \text {, intact and } \\
\text { fractured }\end{array}$ & PRMY_LOG & $\begin{array}{l}\text { Log of intrinsic permeability. } Y \text { - } \\
\text { direction }\end{array}$ & 34197 & 11 Feb-96 & $\mathbf{Y}$ & $4 A$ \\
\hline$Y$ & & 532 & S_ANH_AB & $\begin{array}{l}\text { Salado anhydrito bods } \\
\text { A and } 8 \text {, intact and } \\
\text { fracturod }\end{array}$ & PRMY_LOG & $\begin{array}{l}\text { Log of intrinsic permeability, Y- } \\
\text { direction }\end{array}$ & 34197 & 11-Feb-96 & $Y$ & $\mathbf{A A}$ \\
\hline $\mathbf{Y}$ & & 533 & S_ANH_AB & $\begin{array}{l}\text { Salado antydrite bods } \\
\text { A and B, intact and } \\
\text { fractured }\end{array}$ & PRMI_LOG & $\begin{array}{l}\text { Log of intrinsic permeabillty, Z- } \\
\text { direction }\end{array}$ & 34198 & 11Feb-96: & $\mathbf{Y}$ & $\mathbb{A A}$ \\
\hline$Y$ & & 533 & S_ANH_AB & $\begin{array}{l}\text { Salado anhydrite bods } \\
\text { A and B, intact and } \\
\text { fractured }\end{array}$ & PRMZ_LOG & $\begin{array}{l}\text { Log of intrinsic permeability, Z- } \\
\text { direction }\end{array}$ & 34198 & 11Fob-96 & $\mathbf{Y}$ & $\mathbb{A A}$ \\
\hline$Y$ & & 533 & S_ANH_AB & $\begin{array}{l}\text { Salado antydrife beds } \\
\text { A and B, intact and } \\
\text { fractured }\end{array}$ & PRMZ_LOG & $\begin{array}{l}\text { Log of intrinsic permeability, Z- } \\
\text { direction }\end{array}$ & 34198 & 11-Fob-96 & $Y$ & 4A \\
\hline$Y$ & & 533 & S_ANH_AB & $\begin{array}{l}\text { Salado anhydrte bods } \\
\text { A and B, intact and } \\
\text { fractured }\end{array}$ & PRMZ_LOG & $\begin{array}{l}\text { Log of intrinsic permeability, } z \text { - } \\
\text { direction }\end{array}$ & 34198 & 11fob-9s & $\mathbf{Y}$ & AA \\
\hline
\end{tabular}


CCA Parameter Listing

\begin{tabular}{|c|c|c|c|c|c|c|c|c|c|c|}
\hline ID. & \begin{tabular}{|c|} 
Cineseffi- \\
Cation of \\
Date \\
Category \\
(footnote 2)
\end{tabular} & $\begin{array}{l}\text { Distribution } \\
\text { Typo }\end{array}$ & Moan & Modien & nintmentn & Anodram & Units & Reformen id & $\begin{array}{l}\text { Was the dint } \\
\text { doreloped } \\
\text { under an } \\
\text { NOA-1 } \\
\text { Program? }\end{array}$ & $\begin{array}{c}\text { Which } \\
\text { mothods } \\
\text { wore ured to } \\
\text { quillfy } \\
\text { extatting } \\
\text { dita? } \\
\text { (footnote 3) }\end{array}$ \\
\hline 527 & 1,3 & STUDENT & $6,4360000 E-01$ & $6.4360000 \mathrm{E}-01$ & 4.9053000E-01 & $8.4178000 E-01$ & NONE & WPO35597 & $Y$ & NA \\
\hline 527 & 1,3 & STUDENT & $6.4360000 \mathrm{E}-01$ & $6.4360000 E-01$ & 4.9053000E-01 & 8.4178000E-01 & NONE & WPO35380 & $\mathbf{Y}$ & NA \\
\hline 528 & 1,3 & STUDENT & $1.1000000 E-02$ & $1.1000000 E-02$ & $6.0000000=-03$ & $1.7000000 E-02$ & NONE & WP030600 & $\mathbf{Y}$ & NA \\
\hline 528 & 1,3 & STUDENT & $1,1000000 E-02$ & $1.1000000 E-02$ & $6.0000000 E-03$ & $1.7000000=-02$ & NONE & $\begin{array}{c}\text { WP035597 } \\
\text { W }\end{array}$ & $\mathbf{Y}$ & N/A \\
\hline 528 & 1,3 & STUDENT & $1.1000000 E-02$ & $1.1000000 E-02$ & $6.0000000 E-03$ & $1.7000000 \mathrm{E}-02$ & NONE & WPO36380 & $\mathbf{Y}$ & NA \\
\hline 529 & 1,3 & CONSTANT & $1.0132500 E+05$ & $1.0132500 E+05$ & $1.0132500 E+05$ & $1.0132500 E+05$ & $\mathbf{P a}$ & WP035597 & $\mathbf{Y}$ & N/A \\
\hline 529 & 1,3 & CONSTANT & $1.0132500 E+05$ & $1.0132500 E+05$ & $1.0132500 E+05$ & $1.0132500 E+05$ & $\mathrm{~Pa}$ & WP036051 & $\mathbf{Y}$ & N/A \\
\hline 529 & 1,3 & CONSTANT & $1.0132500 E+05$ & $1.0132500 E+05$ & $1.0132500 E+0.5$ & $1.0132500 E+05$ & $\mathbf{P a}$ & WP040514 & $Y$ & NA \\
\hline 531 & 1,3 & STUDENT & $-1.8890000 E+01$ & $-1.8890000 E+01$ & $-21000000 E+01$ & $-1.7100000 E+01$ & $\log \left(m^{\wedge} 2\right)$ & WP030603 & $Y$ & NA \\
\hline 531 & 1,3 & STUDENT. & $-1.8890000 E+01$ & $-1.8890000 E+01$ & $-2.1000000 E+01$ & $-1.7100000 E+01$ & $\log \left(m^{\wedge} 2\right)$ & WP031217 & $\mathbf{Y}$ & NA \\
\hline 531 & 1,3 & STUDENT & $-1.8890000 E+01$ & $-1.8890000 E+01$ & $-2.1000000 E+01$ & $-1.7100000 E+01$ & $\log \left(m^{\wedge} 2\right)$ & WP035597 & $Y$ & N/A \\
\hline 531 & 1,3 & STUDENT & $-1.8890000 E+01$ & $-1.88900000+01$ & $-2.1000000 E+01$ & $-1.7100000 E+01$ & $\log \left(m^{\wedge} 2\right)$ & WP036380 & $\mathbf{Y}$ & NA \\
\hline 531 & 1,3 & STUDENT & $-1.8890000 E+01$ & $-1.8890000 E+01$ & $-2.1000000 E+01$ & $-1.7100000 E+01$ & $\log \left(m^{\wedge} 2\right)$ & WP039065 & $\mathbf{Y}$ & NA \\
\hline 531 & 1,3 & STUDENT & $-1.8890000 \mathrm{E}+01$ & $-1.8890000 \mathrm{E}+01$ & $-2.1000000 E+01$ & $-1.7100000 E+01$ & $\log \left(\pi^{\wedge} 2\right)$ & WP039178 & $\mathbf{Y}$ & N/A \\
\hline 532 & 1,3 & STUDENT & $-1.8890000 \mathrm{E}+01$ & $-1.8890000 E+01$ & $-2.1000000 E+01$ & $-1.7100000 \mathrm{E}+01$ & $\log \left(m^{\wedge} 2\right)$ & WP030605 & $Y$ & NA \\
\hline 532 & 1,3 & STUDENT & $-1.8890000 E+01$ & $-1.8890000 \mathrm{E}+01$ & $-2.1000000 E+01$ & $-1.7100000 E+01$ & $\log \left(m^{\wedge} 2\right)$ & WP031217 & $\mathbf{Y}$ & N/A \\
\hline 532 & 1,3 & STUDENT & $-1.8890000 E+01$ & $-1.8890000 E+01$ & $-2.1000000 \mathrm{E}+01$ & $-1.7100000 E+01$ & $\log \left(m^{\wedge} 2\right)$ & WP035597 & -4 & N/A \\
\hline 532 & 1,3 & STUDENT & $-1.8890000 E+01$ & $-1.8890000 E+01$ & $-2.1000000 E+01$ & $-1.7100000 E+01$ & $\log \left(m^{\wedge} 2\right)$ & WP036380 & Y & N/A \\
\hline 532 & 1,3 & STUDENT & $-1.8890000 E+01$ & $-1.8890000 E+01$ & $-2.1000000 E+01$ & $-1.7100000 E+01$ & $\log \left(m^{\wedge} 2\right)$ & WP039065 & Y & $\dot{N} / A$ \\
\hline 532 & 1,3 & STUDENT & $-1.8890000 E+01$ & $-1.8890000 E+01$ & $-2.1000000 E+01$ & $-1.7100000 E+0 t$ & $\log \left(m^{\wedge} 2\right)$ & WP039178 & Y & N/A \\
\hline 533 & 1,3 & STUDENT & $-1.8890000 E+01$ & $-1.8890000 E+01$ & $-2.1000000 E+01$ & $-1.7100000 E+01$ & $\log \left(m^{\wedge} 2\right)$ & WP030606 & $\mathbf{Y}$ & N/A \\
\hline 533 & 1,3 & STUDENT & $-1.8890000 E+01$ & $-1.8890000 E+01$ & $-2.1000000 E+01$ & $-1.7100000 E+01$ & $\log \left(m^{\wedge} 2\right)$ & WP031217 & $Y$ & N/A \\
\hline 533 & 1,3 & STUDENT & $-1.8890000 E+01$ & $-1.8890000 E+01$ & $-2.1000000 E+01$ & $-1.7100000 E+01$ & $\log \left(m^{\wedge} 2\right)$ & WP035597 & $\mathbf{Y}$ & NA \\
\hline 533 & 1,3 & STUDENT & $-1.8890000 E+01$ & $-1.8890000 E+01$ & $-2,1000000 E+01$ & $-1.7100000 E+01$ & $\log \left(m^{\wedge} 2\right)$ & WPO36380 & $\mathbf{Y}$ & NA \\
\hline
\end{tabular}


CCA Parameter Listing

\begin{tabular}{|c|c|c|c|c|c|c|c|c|c|c|}
\hline $\begin{array}{l}\text { Paramith } \\
\text { Databese? } \\
\text { (NAN) }\end{array}$ & $\begin{array}{l}\text { Hthe values| } \\
\text { used in CCAA } \\
\text { are not the } \\
\text { same as in } \\
\text { DB, provide } x \\
\text { differonce }\end{array}$ & DI & Metortad ID & 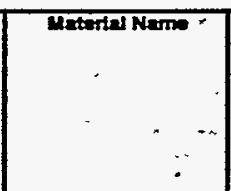 & $\begin{array}{c}\text { Paranetor } \\
\text { to }\end{array}$ & 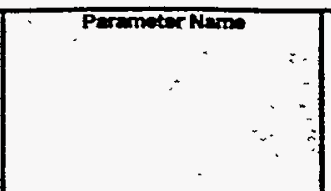 & $\begin{array}{l}\text { PRP ID } \\
\text { (MPO : })\end{array}$ & $\begin{array}{c}\text { Det Enty } \\
\text { Date }\end{array}$ & $\begin{array}{l}\text { fo the } 454 \\
\text { consiatert } \\
\text { whth the } \\
\text { perameter } \\
\text { 'In DB? } \\
\text { (Y.NA) }\end{array}$ & $\begin{array}{l}\text { Paramatar } \\
\text { Category } \\
\text { (footnote 1) }\end{array}$ \\
\hline $\mathbf{Y}$ & & 533 & S_ANH_AB & $\begin{array}{l}\text { Salado anhydrite bods } \\
\text { A and B, intact and } \\
\text { fractured }\end{array}$ & PRMZ_LOG & $\begin{array}{l}\text { Log of intrinsic permeabilty, Z- } \\
\text { direction }\end{array}$ & 34998 & 11 fob-96 & $\mathbf{Y}$ & $\mathbb{A A}$ \\
\hline $\mathbf{Y}$ & & 533 & S_ANH_AB & $\begin{array}{l}\text { Salado anhydrte bods } \\
A \text { and } B \text {, intect and } \\
\text { frectured }\end{array}$ & PRMZ_LOG & $\begin{array}{l}\text { Log of intrinsic permesbility, Z- } \\
\text { direction }\end{array}$ & 34198 & $11+$ eb-96 & $\mathbf{Y}$ & $\mathbb{A A}$ \\
\hline $\mathbf{Y}$ & & 536 & S_ANH_AB & $\begin{array}{l}\text { Salado anhydrite beds } \\
A \text { and } B \text {, intact and } \\
\text { fractured }\end{array}$ & RELP_MOD & $\begin{array}{l}\text { Model number, relativo } \\
\text { permeability model }\end{array}$ & 34209 & $08-F 0 b-96$ & Y & $\mathbb{A A}$ \\
\hline $\mathbf{Y}$ & & 536 & S_ANH_AB & $\begin{array}{l}\text { Salado anhydrite beds } \\
\text { A and B, intact and } \\
\text { fractured }\end{array}$ & RELP_MOD & $\begin{array}{l}\text { Model mumber, relative } \\
\text { permeability model }\end{array}$ & 34201 & as-Feb-96 & $\mathbf{Y}$ & $4 A$ \\
\hline$Y$ & & 535 & S_ANH_AB & $\begin{array}{l}\text { Salado anhydrito beds } \\
\text { A and } B \text {, intact and } \\
\text { fractured }\end{array}$ & RELP_MOD & $\begin{array}{l}\text { Model number, relative } \\
\text { permesbility model }\end{array}$ & 34201 & De-Fob-96 & $\mathbf{Y}$ & $4 A$ \\
\hline $\mathbf{Y}$ & & 537 & S_ANH_AB & $\begin{array}{l}\text { Salado antydrite beds } \\
\text { A and B, intact and } \\
\text { fractured }\end{array}$ & SAT_IBRN & Initial Brine Saturation & 34202 & $08-5 b-96$ & $Y$ & $4 \mathrm{~A}$ \\
\hline Y & & 537 & S_ANH_AB & $\begin{array}{l}\text { Salado anhydrito beds } \\
A \text { and } B \text {, intact and } \\
\text { fractured }\end{array}$ & SAT_IBRN & Initial Brine Saturation & 34202 & 08-Fob-96 & $\mathbf{Y}$ & $4 A$ \\
\hline $\mathbf{Y}$ & & 538 & S_ANH_AB & $\begin{array}{l}\text { Salado anhydrite beds } \\
\text { A and } B \text {, intact and } \\
\text { fractured }\end{array}$ & SAT_RBRN & Residual Brine Saturation & 34203 & 21-Hov-96 & $\mathbf{Y}$ & $4 A$ \\
\hline $\mathbf{Y}$ & & 538 & S_ANH_AB & $\begin{array}{l}\text { Salado anhydrite bods } \\
\text { A and B, intact and } \\
\text { fractured }\end{array}$ & SAT_RBRN & Residual Brine Saturation & 34203 & 21-Nov-96 & $\mathbf{Y}$ & $4 A$ \\
\hline Y & & 538 & S_ANH_AB & $\begin{array}{l}\text { Salado anhydrito bods } \\
\text { A and B, intact and } \\
\text { fractured }\end{array}$ & SAT_RBRN & Residual Brine Saturation & 34203 & 21-Nov-96 & $\mathbf{Y}$ & $4 A$ \\
\hline $\mathbf{Y}$ & & 539 & S_ANH_AB & $\begin{array}{l}\text { Salado anhydrite beds } \\
\text { A and B, intect and } \\
\text { fractured }\end{array}$ & SAT_RGAS & Residual Gas Saturation & 34204 & 19fob-96 & $\mathbf{Y}$ & 4A \\
\hline $\mathbf{Y}$ & & 539 & S_ANH_AB & $\begin{array}{l}\text { Salado anhydrite beds } \\
\text { A and } B \text {, intact and } \\
\text { fractured }\end{array}$ & SAT_RGAS & Residual Gas Saturation & 34204 & 19 Feb-96 & $\mathbf{Y}$ & $4 \mathrm{~A}$ \\
\hline $\mathbf{Y}$ & & 539 & S_ANH_AB & $\begin{array}{l}\text { Salado anhydrite bods } \\
A \text { and } B \text {, intact and } \\
\text { fractured }\end{array}$ & SAT_RGAS & Residual Gas Saturation & 34204 & 19-Feb-96 & $\mathbf{Y}$ & $4 A$ \\
\hline $\mathbf{Y}$ & $100.00 \mathrm{hw}$ & 540 & S_HALTTE & Salado halite, intact & CAP_MOD & $\begin{array}{l}\text { Model number, capillary pressure } \\
\text { model }\end{array}$ & 34208 & 07-Nov-95 & $\mathbf{Y}$ & AB \\
\hline $\mathbf{Y}$ & $100.00 \mathrm{hw}$ & 540 & S_HALITE & Salado halite, intect & CAP_MOD & $\begin{array}{l}\text { Model number, capiliary pressure } \\
\text { model }\end{array}$ & 34208 & 01-Nov-95 & $\mathbf{Y}$ & AB \\
\hline$\gamma$ & & 541 & S_HALITE & Salado halte, intact & COMP_RCK & Bulk Compressibility & 34210 & 01 feb-96 & $Y$ & 1 \\
\hline $\mathbf{Y}$ & & 541 & S_HALTTE & Salado halite, intact & COMP_RCK & Bullk Compressibility & 34210 & 01-Feb-96 & $\bar{Y}$ & 1 \\
\hline$Y$ & & 541 & S_HALITE & Salado halte, intact & COMP_RCK & Bulk Compressibility & 34210 & 01-Fab-96 & $Y$ & 1 \\
\hline$Y$ & & 541 & S_HALITE & Salado halite, intact & COMP_RCK & Bulk Comprossibility & 34210 & 01-Fob-96 & $\mathbf{Y}$ & 1 \\
\hline Y & & 542 & S_HALITE & Salado halte, intact & PC_max & $\begin{array}{l}\text { Meximum allowable capillary } \\
\text { pressure }\end{array}$ & 34379 & 01-Nov-95 & $=y$ & AB \\
\hline $\mathbf{Y}$ & & 542 & S_HALITE & Salado halite, intact & PC_maX & $\begin{array}{l}\text { Maximum allowable capillary } \\
\text { pressuro }\end{array}$ & 34379 & 01-Nov-95 & $\mathbf{Y}$ & 4B \\
\hline $\mathbf{Y}$ & & 543 & S_HALTE & Salado halite, intact & PORE_DIS & $\begin{array}{l}\text { Brooks-Coroy pore distrabution } \\
\text { parameter }\end{array}$ & 34385 & 01-Nov-95 & $\mathbf{Y}$ & 4B \\
\hline Y & & 543 & S_HALTE & Salado haite, intact & PORE_DIS & $\begin{array}{l}\text { Brooks-Coroy poro distribution } \\
\text { paramater }\end{array}$ & 34385 & 01-Nov-95 & $\mathbf{Y}$ & AB \\
\hline $\mathbf{Y}$ & & 544 & S_HALTE & Salado hailte, intect & POROSTY & Efrective porosity & 34387 & 01-Fab-96 & $\mathbf{Y}$ & 1 \\
\hline$Y$ & & 544 & S_HALITE & Salado halite, intect & POROSTY & Effective porosity & 34387 & 01-Fab-96 & $\mathbf{Y}$ & 1 \\
\hline$Y$ & & 544 & S_HALITE & Salado halte, intact & POROSTTY & Effective porosity & 34387 & 01Fob-96 & $\because \mathbf{Y}$ & 1 \\
\hline $\mathbf{Y}$ & & 545 & S_HALITE & Salado halite, intact & PO_MIN & $\begin{array}{l}\text { Minimum brine pressure for } \\
\text { capillary model KPC=3 }\end{array}$ & 34391 & 21-Feb-96 & $\mathbf{Y}$ & AB \\
\hline $\mathbf{Y}$ & & 545 & S_HALITE & Salado halite, intact & PO_MIN & $\begin{array}{l}\text { Minimum brine pressure for } \\
\text { capillary model } \mathrm{KPC}=3\end{array}$ & 34391 & $21-F \circ b-96$ & $\mathbf{Y}$ & $A B$ \\
\hline $\mathbf{Y}$ & & 545 & S_HALTTE & Salado halite, intact & PO_MIN & $\begin{array}{l}\text { Minmum brne pressure for } \\
\text { capillary model KPC=3 }\end{array}$ & 34391 & 21-Fob-96 & $\mathbf{Y}$ & 18 \\
\hline $\mathbf{Y}$ & & 546 & S_HALTTE & Salado halte, intact & PRESSURE & Brine far-field pore pressure & 34394 & a8-feb-96 & $\mathbf{Y}$ & 1 \\
\hline $\mathbf{Y}$ & & 546 & S_HALITE & Salado halite, intact & PRESSURE & Brino far-field pore pressure & 34394 & 08-feb-96 & $\mathbf{Y}$ & 1 \\
\hline $\mathbf{Y}$ & & 546 & S_HALITE & Salado halite, intact & PRESSURE & Brine far-fiold pore pressurio & 34394 & 08-Fob-96 & $\overline{\mathbf{Y}}$ & 1 \\
\hline $\mathbf{Y}$ & & 547 & S_HALTE & Salado halite, intact & PRMX_LOG & $\begin{array}{l}\text { Log of intrinsic pormeability, } X \text { - } \\
\text { direction }\end{array}$ & 34397A & 06-Mar-96 & $\mathbf{Y}$ & 1 \\
\hline
\end{tabular}


CCA Parameter Listing

\begin{tabular}{|c|c|c|c|c|c|c|c|c|c|c|}
\hline ID & \begin{tabular}{c|} 
Clessiff- \\
cation of \\
Data \\
Category \\
(footnoto 2)
\end{tabular} & $\begin{array}{l}\text { Odstriburtion } \\
\text { Type }\end{array}$ & Man & Median & Sinfmum & Moximum & Units & $\begin{array}{l}\text { Reference to } \\
\cdots \\
\end{array}$ & $\begin{array}{l}\text { Wes the dat } \\
\text { developed } \\
\text { under an } \\
\text { NOA-1 } \\
\text { Progrem? }\end{array}$ & $\begin{array}{c}\text { Which } \\
\text { mothode } \\
\text { were used to } \\
\text { qually } \\
\text { oxtrating } \\
\text { data? } \\
\text { (footnote J) }\end{array}$ \\
\hline 533 & 1,3 & STUDENT & $-1.8890000 E+01$ & $-1.8890000 E+01$ & $-2.1000000 E+01$ & $-1.7100000 E+01$ & $\log \left(m^{\wedge} 2\right)$ & WP039065 & $\boldsymbol{Y}$ & N/A \\
\hline 533 & 1,3 & STUDENT & $-1.8890000 E+01$ & $-1.8890000 \mathrm{E}+01$ & $-2.1000000 E+01$ & $-1.7100000 E+01$ & $\log \left(m^{\wedge} 2\right)$ & WP039178 & $Y$ & N/A \\
\hline 536 & 1,3 & DELTA & $4.0000000 E+\infty 0$ & $4.0000000 E+00$ & $1.0000000 E+00$ & $4.0000000 E+\infty 0$ & NONE & WP030643 & $\mathbf{Y}$ & N/A \\
\hline 536 & 1,3 & DELTA & $4.0000000 E+\infty 0$ & $4.0000000 E+\infty 0$ & $1.0000000 \varepsilon+00$ & $4.0000000 \mathrm{E}+00$ & NONE & WP0352̇́8 & $\mathbf{Y}$ & NA \\
\hline 536 & 1,3 & DELTA & $4.0000000 E+00$ & $4.0000000 E+00$ & $1.0000000 E+\infty 0$ & $4.0000000 E+00$ & NONE & WP035597 & $Y$ & NA \\
\hline 537 & 1,3 & CONSTANT & $10000000 \mathrm{E}+\infty$ & $1.0000000 E+00$ & $1.0000000 \mathrm{E}+\infty 0$ & $1.0000000 E+\infty 0$ & NONE & WP030643 & $Y$ & N/A \\
\hline 537 & 1,3 & CONSTANT & $1.0000000 E+00$ & $1.0000000 E+00$ & $1.0000000 E+00$ & $1.0000000 \mathrm{E}+\infty 0$ & NONE & WP035597 & $\mathbf{Y}$ & N/A \\
\hline 538 & 1,3 & STUDENT & $8,3620000 E-02$ & $8.3620000 \mathrm{E}-02$ & $7.7846000 \mathrm{E}-03$ & 1.7401000E-01 & NONE & WP030643 & $Y$ & N/A \\
\hline 538 & 1,3 & STUDENT & $8.3620000 E-02$ & $8.3620000 E-02$ & $7.7846000 \mathrm{E}-03$ & $1.7401000 E-01$ & NONE & WP035597 & $\mathbf{Y}$ & N/A \\
\hline 538 & 1,3 & STUDENT. & $8.3620000 \mathrm{E}-02$ & $8.3620000 \mathrm{E}-02$ & $7.7846000 E-03$ & $1.7401000 \mathrm{E}-01$ & NONE & WPO36380 . & $Y$ & NA \\
\hline 539 & 1,3 & STUDENT & $7.7110000 E-02$ & $7.7110000 E-02$ & $1.3980000 E-02$ & $1.9719000 E-01$ & NONE & WP030643 & $\mathbf{Y}$ & NA \\
\hline 539 & 1,3 & STUDENT & $7.7110000 E-02$ & $7.7110000 E-02$ & $1.3980000 E-02$ & $1.9719000 E-01$ & NONE & WP035597 & $\mathbf{Y}$ & NA \\
\hline 539 & 1,3 & STUDENT & $7.7110000 E-02$ & $7.7110000 E-02$ & $1.3980000 E-02$ & $1.9719000 E-01$ & NONE & WPO35380 & $\mathbf{Y}$ & N/A \\
\hline 540 & 1.3 & CONSTANT & $2.0000000 \mathrm{E}+\infty 0$ & $2.0000000 E+\infty 0$ & $2.0000000 \mathrm{E}+\infty 0$ & $2,0000000 \mathrm{E}+\infty 0$ & NONE & WPO38568 & $Y$ & $N / A$ \\
\hline 540 & 1,3 & CONSTANT & $2.0000000 \mathrm{E}+\infty 0$ & $2.0000000 E+00$ & $2.0000000 \mathrm{E}+\infty 0$ & $2.0000000 E+\infty 0$ & NONE & WPO40514 & $y$ & N/A \\
\hline 541 & $1,3,5$ & UNIFORM & $9.7500000 \mathrm{E}-11$ & $9.7500000 \mathrm{E}-11$ & $2.9400000 \mathrm{E}-12$ & $1.9200000 E-10$ & $P a^{n}-1$ & WP030598 & $\mathbf{Y}$ & 1 \\
\hline 541 & $1,3,5$ & UNIFORM & $9.7500000 \mathrm{E}-11$ & $9.7500000 \mathrm{E}-11$ & $2.9400000 \mathrm{E}-12$ & $1.9200000 \mathrm{E}-10$ & $P a^{\wedge}-1$ & WPO31220 & $Y$ & 1 \\
\hline 541 & $1,3,5$ & UNIFORM & $9.7500000 \mathrm{E}-11$ & $9.7500000 \mathrm{E}-11$ & $2.9400000 E-12$ & $1.9200000 E-10$ & $P a^{n}-1$ & WP035268 & $\mathbf{Y}$ & 1 \\
\hline 541 & $1,3,5$ & UNIFORM & $9.7500000 E-11$ & $9.7500000 \mathrm{E}-11$ & $2.9400000 \mathrm{E}-12$ & $1.9200000 \mathrm{E}-10$ & $\mathrm{~Pa}^{\mathrm{N}-1}$ & WPO35597 & $\bar{Y}$ & 1 \\
\hline 542 & 3,5 & CONSTANT & $1.00000000 E+08$ & $1.0000000 E+08$ & $1.0000000 \mathrm{E}+08$ & $1.0000000 E+08$ & $\mathrm{~Pa}$ & WP038568 & $=y$ & N/A \\
\hline 542 & 3,5 & CONSTANT & $1.0000000 E+08$ & $1.0000000 E+08$ & $1.0000000 E+08$ & $1.0000000 \mathrm{E}+08$ & $\mathrm{~Pa}$ & WPO40514 & $Y$ & N/A \\
\hline 543 & $1,3,4$ & CUMULATIVE & $2.9000000 \mathrm{E}+00$ & $7.0000000 E-01$ & $2.0000000 E-01$ & $1.0000000 E+01$ & NONE & WPO38568 & $\mathbf{Y}$ & NA \\
\hline 543 & $1,3,4$ & CUMULATIVE & $29000000 \mathrm{E}+00$ & 7.0000000E-01 & $2.0000000 E-01$ & $1.0000000 E+01$ & NONE & WP040514 & $\mathbf{Y}$ & N/A \\
\hline 544 & $1,3,4$ & CUMULATTVE & $1.2800000 \mathrm{E}-02$ & $1.0000000 E-02$ & $1.0000000 \mathrm{E}-03$ & $3.0000000 E-02$ & NONE & WP030601 & $\bar{N}$ & 1 \\
\hline 544 & $1,3,4$ & CUMULATIVE & $1.2800000 E-02$ & $1.0000000 \mathrm{E}-02$ & $1.0000000 E-03$ & $3.0000000 E-02$ & NONE & WP035258 & $\mathbf{N}$ & 1 \\
\hline 544 & $1,3,4$ & CUMULATIVE & $1.2800000 \mathrm{E}-02$ & $1.0000000 E-02$ & $1.0000000 \mathrm{E}-03$ & $3.0000000 E-02$ & NONE & WP035597 & $\mathbf{N}$ & 1 \\
\hline 545 & 3,5 & CONSTANT & $1.0132500 E+05$ & $1.0132500 E+05$ & $1.0132500 E+05$ & $1.0132500 E+05$ & $\mathbf{P a}$ & WP035597 & $\mathbf{Y}$ & N/A \\
\hline 545 & 3,5 & CONSTANT & $1.0132500 E+05$ & $1.0132500 E+05$ & $1.0132500 \mathrm{E}+0.5$ & $1.0132500 E+05$ & $\mathrm{~Pa}$ & WP036051 & $Y$ & NA \\
\hline 545 & 3,5 & CONSTANT & $1.0132500 \mathrm{E}+05$ & $1.0132500 E+05$ & $1.0132500 E+05$ & $1.0132500 E+05$ & $\mathbf{P a}$ & WPO40514 & $\mathbf{Y}$ & NA \\
\hline 546 & 1 & UNIFORM & $1.2470000 \mathrm{E}+07$ & $1.2470000 E+07$ & $1.1040000 \mathrm{E}+07$ & $1.3890000 \mathrm{E}+07$ & $\mathrm{~Pa}$ & WPO31221 & Y\&N & 1 \\
\hline 546 & 1 & UNIFORM & $1.2470000 E+07$ & $1.2470000 E+07$ & $1.10400000 \mathrm{E}+07$ & $1.3890000 E+07$ & $\mathrm{~Pa}$ & WP035268 & Y\&N & 1 \\
\hline 546 & 1 & UNIFORM & 1.2470000E+07 & $1.2470000 E+07$ & $1.1040000 E+07$ & $1.3890000 E+07$ & $\mathrm{~Pa}$ & WP035597 & Y\&N & 1 \\
\hline 547 & 1.3 & UNIFORM & $-2.2500000 \mathrm{E}+01$ & $-2.2500000 \mathrm{E}+01$ & $-2.4000000 E+01$ & $-2,1000000 E+01$ & $\log \left(m^{\wedge} 2\right)$ & WP030721 & Y\&N & 1 \\
\hline
\end{tabular}


CCA Parameter Listing

\begin{tabular}{|c|c|c|c|c|c|c|c|c|c|c|}
\hline $\begin{array}{c}\text { Paramoter in } \\
\text { Databuse? } \\
\text { (YNN) } \\
\end{array}$ & \begin{tabular}{|c|} 
If the values \\
uned in CCA \\
are not the \\
same as in \\
OB, provide $x$ \\
diffierence
\end{tabular} & $\overline{10}$ & Materalio & Matarial Name & $\begin{array}{l}\text { Parametar } \\
\text { ID }\end{array}$ & $\begin{array}{l}\text { Parmmoter Neme } \\
\ldots\end{array}$ & $\begin{array}{l}\text { PRPID } \\
\text { (MPO \#) }\end{array}$ & $\begin{array}{c}\text { Date Entry } \\
\text { Date }\end{array}$ & 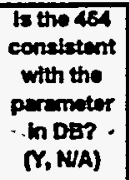 & $\begin{array}{l}\text { Paramoter } \\
\text { Category } \\
\text { (tootnote 1) }\end{array}$ \\
\hline$Y$ & & 547 & S_HALTTE & Salado halite, intact & PRMX_LOG & $\begin{array}{l}\text { Log of intrinste permeabillty, } X- \\
\text { direction }\end{array}$ & 34397A & 06-Mar-96 & $\mathbf{Y}$ & 1 \\
\hline $\mathbf{Y}$ & & 547 & S_HALTTE & Salado halite, intact & PRMX_LOG & $\begin{array}{l}\text { Log of intrinsic permeabilty, } X- \\
\text { direction }\end{array}$ & 34397A & 06-Mar-96 & $\mathbf{Y}$ & 1 \\
\hline $\mathbf{Y}$ & & 547 & S_HALITE & Salado halite, intact & PRMX_LOG & $\begin{array}{l}\text { Log of intrinstc permeability, } X- \\
\text { direction }\end{array}$ & $34397 A$ & OG-Mar-96 & $\mathbf{Y}$ & 1 \\
\hline$Y$ & & 547 & S_HALITE & Salado halite, intact & PRMX_LOG & $\begin{array}{l}\text { Log of intrinsic permeability, } X- \\
\text { direction }\end{array}$ & 34397A & 06-Mar-96 & $Y$ & 1 \\
\hline $\mathbf{Y}$ & & 548 & S_HALITE & Salado halite, intact & PRMY_LOG & $\begin{array}{l}\text { Log of intrinsic permeability, Y- } \\
\text { direction }\end{array}$ & $34399 A$ & 06+Mar-96 & $\mathbf{Y}$ & 1 \\
\hline $\mathbf{Y}$ & & 548 & S_HALITE & Salado halte, intact & PRMY_LOG & $\begin{array}{l}\text { Log of intrinsic permeability, Y- } \\
\text { direction }\end{array}$ & $34399 A$ & 06 -Mar-96 & $\mathbf{Y}$ & 1 \\
\hline $\mathbf{Y}$ & & 548 & S_HALITE & Salado halite, intact & PRMY_LOG & $\begin{array}{l}\text { Log of intrinsic permeabilty, Y- } \\
\text { direction }\end{array}$ & $34399 A$ & $06+19 a-96$ & $\mathbf{Y}$ & 1 \\
\hline $\mathbf{Y}$ & & 548 & S_HALITE & Salado halite, intact & PRMY_LOG & $\begin{array}{l}\text { Log of intrinsic permeability, } Y \text { - } \\
\text { direction }\end{array}$ & $34399 A$ & 06-Mar-96 & $\mathbf{Y}$ & 1 \\
\hline $\mathbf{Y}$ & & 548 & S_hALTE & Salado halite, intact & PRMY_LOG & $\begin{array}{l}\text { Log of intrinsic perm } 8 \text { ability, } Y \text { - } \\
\text { direction }\end{array}$ & $34399 A$ & OS-Mar-96 & $Y$ & 1 \\
\hline $\mathbf{Y}$ & & 549 & S_HALTE & Salado halite, intset & PRMZ_LOG & $\begin{array}{l}\text { Log of intrinsic permeabilty, Z- } \\
\text { direction }\end{array}$ & $34401 A$ & OG-Mar-96 & $\mathbf{Y}$ & 1 \\
\hline$Y$ & & 549 & S_HALITE & Salado halite, intact & PRMZ_LOG & $\begin{array}{l}\text { Log of intrinsic permeability, Z- } \\
\text { diroction }\end{array}$ & $34401 A$ & Os-Mar-96 & $\mathbf{Y}$ & 1 \\
\hline $\mathbf{Y}$ & & 549 & S_HALITE & Salado halite, intect & PRMZ_LOG & $\begin{array}{l}\text { Log of intrinsic permeability, Z- } \\
\text { diroction }\end{array}$ & $34401 A$ & Os-Mar-96 & Y & 1 \\
\hline$Y$ & & 549 & S_HALITE & Salado halite, intact & PRMZ_LOG & $\begin{array}{l}\text { Log of intrinsic permeability, Z- } \\
\text { direction }\end{array}$ & $34401 A$ & 06-Mar-96 & $\mathbf{Y}$ & 1 \\
\hline$Y$ & & 549 & S_HALITE & Salado halite, intact & PRMZ_LOG & $\begin{array}{l}\text { Log of intrinsic permeabillty, Z- } \\
\text { direction }\end{array}$ & $34401 A$ & 06-Mar-96 & Y & 1 \\
\hline$Y$ & & 553 & S_HALITE & Salado halite, intact & RELP_MOD & $\begin{array}{l}\text { Model number, relative } \\
\text { permeability model }\end{array}$ & 34412 & 01-Nov-95 & $\mathbf{Y}$ & 48 \\
\hline $\mathbf{Y}$ & & 553 & S_HALTE & Salado halite, nintact & RELP_MOD & $\begin{array}{l}\text { Model number, relative } \\
\text { permeability model }\end{array}$ & 34412 & 01-Nov-95 & $\mathbf{Y}$ & $4 B$ \\
\hline $\bar{Y}$ & & 554 & S_HALITE & Salado halite, intect & SAT_IBRN & Initial Brine Saturation & 34416 & 01-Nov-95 & $Y$ & 4B \\
\hline $\bar{Y}$ & & 554 & S_HALITE & Salado halite, intact & SAT_IBRN & Initial Brise Saturation & 34416 & 01-Nov-95 & $\bar{Y}$ & $4 B$ \\
\hline$Y$ & & 555 & S_HALITE & Salado halite, intact & SAT_RBRN & Residual Brine Saturation & 34418 & 01-Nov-95 & $Y$ & $4 \mathrm{~B}$ \\
\hline $\bar{Y}$ & & 555 & S_HALITE & Salado halte, intact & SAT_RBRN & Resichual Brine Saturation & 34418 & 01-Nov-95 & $Y$ & $4 B$ \\
\hline $\bar{Y}$ & & 556 & S_HALITE & Salado halite, intact & SAT_RGAS & Residual Gas Saturation & 34420 & 01-Nov-95 & $Y$ & $4 B$ \\
\hline $\mathrm{Y}$ & & 556 & S_HALITE & Salado halite, intact & SAT_RGAS & Residual Gas Saturation & 34420 & 01-Nov-95 & $\bar{Y}$ & $4 B$ \\
\hline $\mathbf{Y}$ & & 559 & S_MB138 & $\begin{array}{l}\text { Salado marker bed } \\
\text { 138, intact and } \\
\text { fractured }\end{array}$ & CAP_MOD & $\begin{array}{l}\text { Model number, capiltary pressure } \\
\text { model }\end{array}$ & $34430 \mathrm{~A}$ & 11-fob-96 & $\mathbf{Y}$ & $4 A$ \\
\hline $\mathbf{Y}$ & & 559 & S_MB138 & $\begin{array}{l}\text { Salado marker bed } \\
\text { 138, intact and } \\
\text { fractured }\end{array}$ & CAP_MOD & $\begin{array}{l}\text { Model number, capillary pressure } \\
\text { model }\end{array}$ & $34430 \mathrm{~A}$ & 11-fob-95 & $\checkmark$ & $4 A$ \\
\hline $\mathbf{Y}$ & & 560 & S_MB138 & $\begin{array}{l}\text { Salado marker bod } \\
\text { 138, intact and } \\
\text { fractured }\end{array}$ & COMP_RCK & Bulk Compressibility & 34439 & 14feb-96 & $Y$ & $4 A$ \\
\hline $\mathbf{Y}$ & & 560 & S_MB138 & $\begin{array}{l}\text { Salado marker bed } \\
138 \text {, intact and } \\
\text { fractured }\end{array}$ & COMP_RCK & Bulk Compressibility & 34439 & 14-Feb-96 & $\mathbf{Y}$ & $4 A$ \\
\hline $\mathbf{Y}$ & & 560 & S_MB138 & $\begin{array}{l}\text { Salado marker bed } \\
138 \text {, intact and } \\
\text { fractured }\end{array}$ & COMP_RCK & Bulx Compressibility & 34439 & 14-Feb-96 & $\mathbf{Y}$ & $4 A$ \\
\hline $\mathbf{Y}$ & & 561 & S_MB138 & $\begin{array}{l}\text { Salado marker bed } \\
138 \text {, intsct and } \\
\text { fractured }\end{array}$ & PC_max & $\begin{array}{l}\text { Maxmum allowable capillary } \\
\text { pressuro }\end{array}$ & $34512 A$ & 01-Feb-96 & $\mathbf{Y}$ & $4 A$ \\
\hline$Y$ & & $561^{\circ}$ & S_MB138 & $\begin{array}{l}\text { Salado marker bed } \\
\text { 138, intact and } \\
\text { frectured }\end{array}$ & PC_maX & $\begin{array}{l}\text { Maximum allowable capillary } \\
\text { pressure }\end{array}$ & $34512 A$ & 01-Feb-96 & $\mathbf{Y}$ & $\mathbb{A A}$ \\
\hline$Y$ & & 563 & S_MB138 & $\begin{array}{l}\text { Salado marker bed } \\
\text { 138, intact and } \\
\text { fractured }\end{array}$ & PF_DELTA & $\begin{array}{l}\text { Incremental pressure for full } \\
\text { fracture development }\end{array}$ & 34516 & 22-Fob-96 & $\mathbf{Y}$ & $A B$ \\
\hline$Y$ & & 563 & S_MB138 & $\begin{array}{l}\text { Salado marker bed } \\
\text { 138, intact and } \\
\text { fractured }\end{array}$ & PF_DELTA & $\begin{array}{l}\text { incremental prossure for full } \\
\text { fracture development }\end{array}$ & 34516 & 22-Fob-96 & $\mathbf{Y}$ & $A B$ \\
\hline $\mathbf{Y}$ & & 563 & S_MB138 & $\begin{array}{l}\text { Salado marker bed } \\
138 \text {, intact and } \\
\text { fractured }\end{array}$ & PF_DELTA & $\begin{array}{l}\text { incremental prossure for full } \\
\text { iracture development }\end{array}$ & 34516 & 22-Feb-96 & $\mathbf{Y}$ & 48 \\
\hline $\mathbf{Y}$ & & 565 & S_MB138 & $\begin{array}{l}\text { Salado marker bod } \\
138 \text {, intact and } \\
\text { fractured }\end{array}$ & PI_DELTA & $\begin{array}{l}\text { Fracture initiation pressure } \\
\text { increment }\end{array}$ & 34523 & 22+*b-96 & $\mathbf{Y}$ & 4B \\
\hline
\end{tabular}


CCA Parameter Listing

\begin{tabular}{|c|c|c|c|c|c|c|c|c|c|c|}
\hline ID & \begin{tabular}{|c|} 
Canastri- \\
Cation of \\
Data \\
Cantegory \\
(footnote 2)
\end{tabular} & $\begin{array}{l}\text { Distribution } \\
\text { Type }\end{array}$ & $\begin{array}{c}\text { mean } \\
\text {. }\end{array}$ & Mecian & Mlnineum & Maxdmum & Units & Ruference iD & $\begin{array}{l}\text { Was the data } \\
\text { developod } \\
\text { under en } \\
\text { NOA-1 } \\
\text { Progrem? }\end{array}$ & $\begin{array}{c}\text { Which } \\
\text { methocks } \\
\text { wore used to } \\
\text { qually } \\
\text { exdsting } \\
\text { deta? } \\
\text { (footnote 3) }\end{array}$ \\
\hline 547 & 1,3 & UNIFORM & $-22500000 E+01$ & $-2.2500000 E+01$ & $-2.4000000 E+01$ & $-2.1000000 E+01$ & $\log \left(m^{\wedge} 2\right)$ & WPO31218 & YEN & 1 \\
\hline 547 & 1,3 & UNIFORM & $-2.2500000 \mathrm{E}+01$ & $-22500000 E+01$ & $-2.4000000 E+01$ & $-2.1000000 E+01$ & $\log \left(m^{\wedge} 2\right)$ & WP035268 & YEN & 1 \\
\hline 547 & 1,3 & UNIFORM & $-2.2500000 E+01$ & $-2.2500000 E+01$ & $-2.4000000 E+01$ & $-2.1000000 E+01$ & $\log \left(m^{\wedge} 2\right)$ & WP035597 & Y8N & 1 \\
\hline 547 & 1,3 & UNIFORM & $-2.2500000 E+01$ & $-2.2500000 E+01$ & $-2.4000000 E+01$ & $-2.1000000 E+01$ & $\log \left(m^{\wedge} 2\right)$ & WP036772 & Y8N & 1 \\
\hline 548 & 1,3 & UNIFORM & $-2.2500000 E+04$ & $-2.2500000 \mathrm{E}+01$ & $-24000000 E+01$ & $-2.1000000 E+01$ & $\log \left(m^{\wedge} 2\right)$ & WP030721 & YEN & 1 \\
\hline 548 & 1,3 & UNIFORM & $-2.2500000 E+01$ & $-2.2500000 E+01$ & $-2.4000000 E+01$ & $-2.1000000 E+01$ & $\log \left(m^{\wedge} 2\right)$ & WP031218 & YZN & 1 \\
\hline 548 & 1,3 & UNIFORM & $-2.2500000 \mathrm{E}+01$ & $-2.2500000 E+01$ & $-2.4000000 E+01$ & $-2.1000000 E+01$ & $\log \left(m^{\wedge} 2\right)$ & WP035268 & Y\&N & 1 \\
\hline 548 & 1,3 & UNIFORM & $-2.2500000 E+01$ & $-2.2500000 E+01$ & $-2.4000000 E+01$ & $-2.1000000 E+01$ & $\log \left(m^{\wedge} 2\right)$ & WP035597 & Y\&N & 1 \\
\hline 548 & 1,3 & UNIFORM & $-2.2500000 E+01$ & $-2.2500000 E+01$ & $-2.4000000 \mathrm{E}+01$ & $-2.1000000 E+01$ & $\log \left(m^{\wedge} 2\right)$ & WP036772 & YEN & 1 \\
\hline 549 & 1.3 & UNIFORM & $-2.2500000 E+01$ & $-2.2500000 E+01$ & $-2.4000000 E+01$ & $-2.1000000 E+01$ & $\log \left(m^{\wedge} 2\right)$ & WP030721 & Y8N & 1 \\
\hline 549 & 1,3 & UNIFORM & $-2.2500000 E+01$ & $-2.2500000 E+01$ & $-2.4000000 E+01$ & $-2.1000000 E+01$ & $\log \left(m^{\wedge} 2\right)$ & WP031218 & YEN & 1 \\
\hline 549 & 1,3 & UNIFORM & $-2.2500000 E+01$ & $-2.2500000 \mathrm{E}+01$ & $-2.4000000 E+01$ & $-2,1000000 E+01$ & $\log \left(m^{\wedge} 2\right)$ & WPO35268 & Y\&N & 1 \\
\hline 549 & 1,3 & UNIFORM & $-2.2500000 \mathrm{E}+01$ & $-2.2500000 E+01$ & $-2.4000000 E+01$ & $-2.1000000 E+01$ & $\log \left(m^{\wedge} 2\right)$ & WP035597 & YEN & 1 \\
\hline 549 & 1,3 & UNIFORM & $-2.2500000 E+01$ & $-2.2500000 E+01$ & $-2.4000000 E+01$ & $-2.1000000 E+01$ & $\log \left(m^{\wedge} 2\right)$ & WP036772 & Y8N & 1 \\
\hline 553 & $1,3,4$ & DELTA . & $4.0000000 E+00$ & $4.0000000 \mathrm{E}+00$ & $1.0000000 E+\infty 0$ & $4.0000000 E+00$ & NONE & WP038568 & $\mathbf{Y}$ & N/A \\
\hline 553 & $1,3,4$ & DELTA & $4.00000000+\infty 0$ & $4.00000000+00$ & $1.0000000 E+\infty 0$ & $4.0000000 E+00$ & NONE & WP040514 & $Y$ & NA \\
\hline 554 & 1,3 & CONSTANT & $1.0000000 E+00$ & $1.0000000 \mathrm{E}+\infty 0$ & $1.0000000 \mathrm{E}+00$ & $1.0000000 \mathrm{E}+00$ & NONE & WPO38568 & $\bar{Y}$ & N/A \\
\hline 554 & 1,3 & CONSTANT & $1.0000000 \mathrm{E}+00$ & $1.0000000 E+\infty 0$ & $1.0000000 \mathrm{E}+00$ & $1.0000000 \mathrm{E}+00$ & NONE & WPO40514 & $\mathbf{Y}$ & N/A \\
\hline 555 & $1,3,4$ & UNIFORM & $3.0000000 E-01$ & $3.0000000 E-01$ & $0.0000000 E+00$ & $6.0000000 E-01$ & NONE & WP038568 & $\bar{Y}$ & N/A \\
\hline 555 & $1,3,4$ & UNIFORM & $3.0000000 \mathrm{E}-01$ & $3.0000000 E-01$ & $0.0000000 \mathrm{E}+00$ & $6.0000000 E-01$ & NONE & WP040514 & $\mathbf{Y}$ & NA \\
\hline 556 & $1,3,4$ & UNIFORM & 2.0000000 E- 01 & $2.0000000 \mathrm{E}-01$ & $0.0000000 E+00$ & $4.0000000 \mathrm{E}-01$ & NONE & WPO38568 & $\bar{Y}$ & NAA \\
\hline 556 & $1,3,4$ & UNIFORM & $2.0000000 E-01$ & $2.0000000 \mathrm{E}-01$ & $0.0000000 \mathrm{E}+00$ & $4.0000000 \mathrm{E}-01$ & NONE & WPO40514 & $Y$ & N/A \\
\hline 559 & 1,3 & CONSTANT & $2.0000000 E+00$ & $2.0000000 \mathrm{E}+\infty 0$ & $2.0000000 E+\infty 0$ & $2.0000000 \mathrm{E}+00$ & NONE & WP030643 & $\mathbf{Y}$ & NA \\
\hline 659 & 1,3 & CONSTANT & $2.0000000 E+00$ & $2.00000000+\infty$ & $2.0000000 \mathrm{E}+00$ & $2.0000000 \mathrm{E}+00$ & NONE & WP035597 & $\mathbf{Y}$ & NA \\
\hline 560 & 1,3 & STUDENT & $8.2630000 E-11$ & $8.2630000 E-11$ & $1.0900000 \mathrm{E}-11$ & $2.7500000 \mathrm{E}-10$ & $P a^{\wedge}-1$ & WP031186 & $\mathbf{Y}$ & N/A \\
\hline 560 & 1,3 & STUDENT & $8.2630000 E-11$ & $8.2630000 E-11$ & $1.0900000 E-11$ & $2.7500000 \mathrm{E}-10$ & $P a^{n}-1$ & WP035597 & $Y$ & N/A \\
\hline 560 & 1,3 & STUDENT & $8.2630000 E-11$ & $8.2630000 E-11$ & $1.0900000 \mathrm{E}-11$ & $2.7500000 \mathrm{E}-10$ & $P a^{N}-1$ & WP036380 & $\mathbf{Y}$ & NA \\
\hline 561 & 1.3 & CONSTANT & $1.0000000 \mathrm{E}+08$ & $1.0000000 E+08$ & $1.0000000 \mathrm{E}+08$ & $1.0000000 E+08$ & $\mathbf{P a}$ & WPO30643 & $\mathbf{Y}$ & NA \\
\hline 561 & 1,3 & CONSTANT & $1.0000000 E+08$ & $1.0000000 E+08$ & $1.0000000 E+08$ & $1.0000000 \mathrm{E}+08$ & $\mathrm{~Pa}$ & WP035597 & $Y$ & N/A \\
\hline 563 & 1,3 & CONSTANT & $3.8000000 \mathrm{E}+06$ & $3.8000000 \mathrm{E}+06$ & $3.8000000 \mathrm{E}+06$ & $3.8000000 \mathrm{E}+06$ & $\mathbf{P a}$ & WP035597 & $\mathbf{Y}$ & N/A \\
\hline 563 & 1,3 & CONSTANT & $3.80000000 E+\infty 6$ & $3.8000000 \mathrm{E}+06$ & $3.8000000 E+06$ & $3.8000000 E+06$ & $\mathbf{P a}$ & WPO38274 & $\mathbf{Y}$ & N/A \\
\hline 563 & 1,3 & CONSTANT & $3.8000000 \mathrm{E}+06$ & $3.8000000 E+05$ & $3.8000000 \mathrm{E}+06$ & $3.6000000 \mathrm{E}+06$ & $\mathbf{P a}$ & WP040514 & $\mathbf{Y}$ & NA \\
\hline 565 & 1,3 & CONSTANT & $2.0000000 E+05$ & $2.0000000 E+05$ & $20000000 E+05$ & $2.0000000 E+05$ & $\mathbf{P a}$ & WP035597 & $\mathbf{Y}$ & N/A \\
\hline
\end{tabular}


CCA Parameter Listing

\begin{tabular}{|c|c|c|c|c|c|c|c|c|c|c|}
\hline $\begin{array}{l}\text { Parameter in } \\
\text { Datubaze? } \\
\text { (YNN) }\end{array}$ & $\begin{array}{c}\text { tf the values } \\
\text { ueed in CCA } \\
\text { one not the } \\
\text { same tes in } \\
\mathrm{OB}, \text { provide } x \\
\text { difference }\end{array}$ & 10: & Matorial to & $\begin{array}{l}\text { Haterial Name } \\
\text {, }\end{array}$ & $\begin{array}{c}\text { Parmetar } \\
\text { it }\end{array}$ & 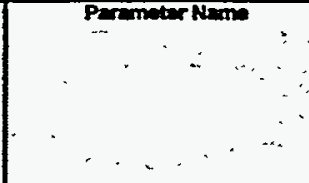 & $\begin{array}{l}\text { PRP D } \\
\text { WPO } \#) \\
\therefore \\
\therefore\end{array}$ & $\begin{array}{c}\text { Dete Entry } \\
\text { Dete }\end{array}$ & $\begin{array}{l}\text { fo the } 484 \\
\text { consalstent } \\
\text { with the } \\
\text { purameter } \\
\text { in DB? } \\
(Y, N A)\end{array}$ & $\begin{array}{l}\text { Parametar } \\
\text { Category } \\
\text { (footnote 1) }\end{array}$ \\
\hline $\mathbf{Y}$ & & 565 & S_MB138 & $\begin{array}{l}\text { Salado marker bed } \\
138 \text {, intact and } \\
\text { fractured }\end{array}$ & PI_DELTA & $\begin{array}{l}\text { Fracture initiation pressure } \\
\text { increment }\end{array}$ & 34523 & 22fob-96 & $\mathbf{Y}$ & 48 \\
\hline $\mathbf{Y}$ & & 565 & S_MB138 & $\begin{array}{l}\text { Salado marker bed } \\
\text { 138, intect and } \\
\text { fractured }\end{array}$ & PI_DELTA & $\begin{array}{l}\text { Fracture initiation pressure } \\
\text { increment }\end{array}$ & 34523 & 22-Fob-96 & $\mathbf{Y}$ & 48 \\
\hline $\mathbf{Y}$ & & 565 & S_MB138 & $\begin{array}{l}\text { Salado marker bed } \\
138 \text {, intact and } \\
\text { fractured }\end{array}$ & PI_DELTA & $\begin{array}{l}\text { Fracture initiation prossure } \\
\text { increment }\end{array}$ & 34523 & 22Feb-96 & $\mathbf{Y}$ & 48 \\
\hline $\mathbf{Y}$ & & 566 & S_MB138 & $\begin{array}{l}\text { Salado marker bed } \\
138 \text {, intact and } \\
\text { fractured }\end{array}$ & PORE_DIS & $\begin{array}{l}\text { Brooks-Corey pore distribution } \\
\text { parameter }\end{array}$ & 34527 & 22-Fob-96 & $\mathbf{Y}$ & $4 A$ \\
\hline $\mathbf{Y}$ & & 566 & S_MB138 & $\begin{array}{l}\text { Salado marker bed } \\
138 \text {, intact and } \\
\text { fractured }\end{array}$ & PORE_DIS & $\begin{array}{l}\text { Brooks-Corey pore distribution } \\
\text { paramater }\end{array}$ & 34527 & 22freb-96 & $\mathbf{Y}$ & $4 A$ \\
\hline $\mathbf{Y}$ & & 566 & S_MB13B & $\begin{array}{l}\text { Salado marker bed } \\
\text { 138, intact and } \\
\text { fractured }\end{array}$ & PORE_DIS & $\begin{array}{l}\text { Brooks-Corey pore distribution } \\
\text { parameter }\end{array}$ & 34527 & 22Fob-96 & $\mathbf{Y}$ & $4 A$ \\
\hline $\mathbf{Y}$ & & 567 & S_MB138 & $\begin{array}{l}\text { Salado marker bed } \\
138 \text {, intact and } \\
\text { fractured }\end{array}$ & POROSITY & Effective porosity & 34530 & 21 Feb-96 & $Y$ & AA \\
\hline $\mathbf{Y}$ & & 567 & S_MB138 & $\begin{array}{l}\text { Salado marker bed } \\
138 \text {, intact and } \\
\text { fractured }\end{array}$ & POROSITY & Effective porosity & 34530 & $21+$ feb-96 & $\mathbf{Y}$ & $4 A$ \\
\hline $\mathbf{Y}$ & & 567 & S_MB138 & $\begin{array}{l}\text { Salado marker bed } \\
138, \text { intact and } \\
\text { fractured }\end{array}$ & POROSITY & Effective porosity & 34530 & 21-Fob-96 & $\mathbf{Y}$ & $4 A$ \\
\hline $\mathbf{Y}$ & & 568 & S_MB138 & $\begin{array}{l}\text { Salado marker bed } \\
138 \text {, intact and } \\
\text { fractured }\end{array}$ & PO_MIN & $\begin{array}{l}\text { Minimum brine pressure for } \\
\text { capillery model KPC=3 }\end{array}$ & 34531 & $21+{ }_{0 b}-96$ & $\mathbf{Y}$ & AB \\
\hline $\mathbf{Y}$ & & 568 & S_MB138 & $\begin{array}{l}\text { Salado marker bed } \\
138, \text { intact and } \\
\text { fractured }\end{array}$ & PO_MIN & $\begin{array}{l}\text { Minimum brine prossere for } \\
\text { capillary model KPC=3 }\end{array}$ & 34531 & 21-Fob-96 & $Y$ & $4 B$ \\
\hline $\mathbf{Y}$ & & 568 & S_MB138 & $\begin{array}{l}\text { Salado marker bed } \\
138 \text {, intact and } \\
\text { fractured }\end{array}$ & PO_MIN & $\begin{array}{l}\text { Minimum brine prossure for } \\
\text { capillary model KPC=3 }\end{array}$ & 34531 & $21-50 \mathrm{~b}-96$ & $\mathbf{Y}$ & AB \\
\hline $\mathbf{Y}$ & varies & 569 & S_MB138 & $\begin{array}{l}\text { Salado marker bed } \\
138 \text {, intact and } \\
\text { fracturod }\end{array}$ & PRESSURE & Brine far-field pore pressure & 34532 & 19 Frob-96 & $\mathbf{Y}$ & $4 A$ \\
\hline $\mathbf{Y}$ & varies & 569 & S_MB138 & $\begin{array}{l}\text { Salado marker bed } \\
138, \text { intact and } \\
\text { fractured }\end{array}$ & PRESSURE & Brine tar-field pore pressure & 34532 & 19-Feb-96 & $\mathbf{Y}$ & AA \\
\hline $\mathbf{Y}$ & varies & 569 & S_MB138 & $\begin{array}{l}\text { Salado marker bed } \\
\text { 138, intact and } \\
\text { fractured }\end{array}$ & PRESSURE & Brine far-field pore pressure & 34532 & 19-Fub-96 & $\mathbf{Y}$ & $4 A$ \\
\hline $\mathbf{Y}$ & varies & 569 & S_M8138 & $\begin{array}{l}\text { Salado marker bod } \\
138, \text { intact and } \\
\text { frectured }\end{array}$ & PRESSURE & Brine far-field pore pressure & 34532 & 19-Fob-96 & $\mathbf{Y}$ & $4 A$ \\
\hline $\mathbf{Y}$ & & 570 & S_MB138 & $\begin{array}{l}\text { Salado marker bed } \\
\text { 138, intect and } \\
\text { fractured }\end{array}$ & PRMX_LOG & $\begin{array}{l}\text { Log of intrinsic permeability, } X \text { - } \\
\text { direction }\end{array}$ & 34536 & 11 feb-96 & $\bar{Y}$ & $4 A$ \\
\hline $\mathbf{Y}$ & & 570 & S_MB138 & $\begin{array}{l}\text { Salado marker bed } \\
138 \text {, intact and } \\
\text { fractured }\end{array}$ & PRMX_LOG & $\begin{array}{l}\text { Log of intrinsic permeability, } X \text { - } \\
\text { direction }\end{array}$ & 34536 & $11-$ Feb-96 & $\mathbf{Y}$ & $4 A$ \\
\hline $\mathbf{Y}$ & & 570 & S_MB138 & $\begin{array}{l}\text { Salado marker bed } \\
138 \text {, intact and } \\
\text { fractured }\end{array}$ & PRMX_LOG & $\begin{array}{l}\text { Log of intrinsic permeability, } x \text { - } \\
\text { direction }\end{array}$ & 34536 & 11 Fob-96 & $\mathbf{Y}$ & $4 A$ \\
\hline $\mathbf{Y}$ & & 570 & S_MB138 & $\begin{array}{l}\text { Salado marker bed } \\
138 \text {, intact and } \\
\text { fractured }\end{array}$ & PRMX_LOG & $\begin{array}{l}\text { Log of intrinsic permeability, } X \text { - } \\
\text { direction }\end{array}$ & 34536 & 11-Fab-96 & $\mathbf{Y}$ & $4 A$ \\
\hline $\mathbf{Y}$ & & 570 & S_MB138 & $\begin{array}{l}\text { Salsdo marker bed } \\
138 \text {, intact and } \\
\text { fractured }\end{array}$ & PRMX_LOG & $\begin{array}{l}\text { Log of intrnsic permeability, } X \text { - } \\
\text { direction }\end{array}$ & 34536 & 11 Fob-96 & $\mathbf{Y}$ & $4 A$ \\
\hline$Y$ & & 570 & S_MB138 & $\begin{array}{l}\text { Salado markor bed } \\
\text { 138, intact and } \\
\text { fractured }\end{array}$ & PRMX_LOG & $\begin{array}{l}\text { Log of intrinsic permeability, } X \text { - } \\
\text { direction }\end{array}$ & 34536 & 11 Feb-96 & $\mathbf{Y}$ & AA \\
\hline $\mathbf{Y}$ & & 571 & S_MB138 & $\begin{array}{l}\text { Salado marker bed } \\
138 \text {, intact and } \\
\text { fractured }\end{array}$ & PRHY_LOG & $\begin{array}{l}\text { Log of intrinsic permeability, Y- } \\
\text { direction }\end{array}$ & 34537 & 11-Fob-96 & $\mathbf{Y}$ & $\mathbf{A A}$ \\
\hline $\mathbf{Y}$ & & 571 & S_MB138 & $\begin{array}{l}\text { Salado marker bed } \\
\text { 138, intact and } \\
\text { fractured }\end{array}$ & PRMY_LOG & $\begin{array}{l}\text { Log of intrinsic permeability, } Y \text { - } \\
\text { direction }\end{array}$ & 34537 & 11Fob-96 & $\mathbf{Y}$ & $4 A$ \\
\hline
\end{tabular}


CCA Parameter Listing

\begin{tabular}{|c|c|c|c|c|c|c|c|c|c|c|}
\hline ID & \begin{tabular}{c|} 
Clesesfle- \\
cation of \\
Datz \\
Cattogory \\
(footnoto 2)
\end{tabular} & $\begin{array}{l}\text { Dlatribution } \\
\text { Type }\end{array}$ & Mean & Median & Minimum & Moximum & $\begin{array}{l}\text { Units } \\
-\quad .\end{array}$ & . & $\begin{array}{l}\text { Wes the data } \\
\text { doweloped } \\
\text { underen } \\
\text { NaA-1 } \\
\text { Progrem? }\end{array}$ & $\begin{array}{l}\text { Which } \\
\text { methods } \\
\text { were used to } \\
\text { qually } \\
\text { oxdeting } \\
\text { data? } \\
\text { (footnote 3) }\end{array}$ \\
\hline 565 & 1.3 & CONSTANT & $2.0000000 E+05$ & $20000000 E+0.5$ & $2.0000000 E+05$ & $2.0000000 E+05$ & $\mathbf{P a}$ & WP038274 & $\mathbf{Y}$ & NA \\
\hline 565 & 1,3 & CONSTANT & $2.0000000 E+05$ & $2.0000000 E+05$ & $2.0000000 E+05$ & $2.0000000 E+05$ & $\mathbf{P a}$ & WP038569 & $\mathbf{Y}$ & NA \\
\hline 565 & 1,3 & CONSTANT & $2.0000000 E+05$ & $2,0000000 E+05$ & $2.0000000 E+05$ & $2.0000000 E+05$ & $\mathrm{~Pa}$ & WP040514 & $\mathbf{Y}$ & NA \\
\hline 566 & 1,5 & STUDENT & $6.4360000 E-01$ & $6.4360000 E-01$ & 4.9053000E-01 & $8.4178000 E-01$ & NONE & WPO30643 & $\mathbf{Y}$ & NA \\
\hline 566 & 1,5 & STUDENT & $6.4360000 E-01$ & $6.4360000 E-01$ & 4.9053000E-01 & $8.4178000 E-01$ & NONE & WP035597 & $\mathbf{Y}$ & N/A \\
\hline 566 & 1,5 & STUDENT & $6.4360000 E-01$ & $6.4360000 E-01$ & 4.9053000E-01 & 8.4178000E-01 & NONE & WPO36380 & Y & N/A \\
\hline 567 & 1,3 & STUDENT & $1.1000000 \mathrm{E}-02$ & $1.1000000 E-02$ & $6.0000000 E-03$ & $1.7000000 \mathrm{E}-02$ & NONE & WPO30600 & $\mathbf{Y}$ & NA \\
\hline 567 & 1,3 & STUDENT & $1.1000000 \mathrm{E}-02$ & $1.1000000 E-02$ & $6.0000000 E-03$ & $1.7000000 E-02$ & NONE & WP035597 & $\mathbf{Y}$ & NA \\
\hline 567 & 1.3 & STUDENT & $1.1000000 \mathrm{E}-02$ & $1.1000000 E-02$ & $6.0000000 E-03$ & $1.7000000 \mathrm{E}-02$ & NONE & WP036380 & $\mathbf{Y}$ & NA \\
\hline 568 & 1,3 & CONSTANT & $1.0132500 E+05$ & $1.0132500 E+05$ & $1.0132500 E+05$ & $1.0132500 E+05$ & $\mathbf{P a}$ & WP035597 & $\mathbf{Y}$ & N/A \\
\hline 568 & 1,3 & CONSTANT & $1.0132500 E+05$ & $1.0132500 E+05$ & $1.0132500 E+05$ & $1.0132500 E+05$ & $\mathrm{~Pa}$ & WP036051 & $Y$ & $N / A$ \\
\hline 568 & 1,3 & CONSTANT & $1.0132500 E+05$ & $1.0132500 E+05$ & $1.0132500 E+05$ & $1.0132500 E+05$ & $\mathrm{~Pa}$ & WP040514 & $\mathbf{Y}$ & NA \\
\hline 569 & 1,3 & STUDENT & $1.1630000 E+07$ & $1.1630000 \mathrm{E}+07$ & $9.3800000 E+06$ & $1.2940000 E+07$ & $\mathrm{~Pa}$ & WP031185 & $Y$ & NA \\
\hline 569 & 1,3 & STUDENT & 1.1630000E+07 & $8.1630000 E+07$ & $9.3800000 E+06$ & $1.2940000 E+07$ & $\mathrm{~Pa}$ & WP035597 & $\mathbf{Y}$ & N/A \\
\hline 569 & 1,3 & STUDENT & $1.1630000 \mathrm{E}+07$ & $1.1630000 E+07$ & $9.3800000 E+06$ & $1.2940000 E+07$ & $\mathrm{~Pa}$ & WPO36380 & $\mathbf{Y}$ & NA \\
\hline 569 & 1,3 & STUDENT & $1,1630000 \mathrm{E}+07$ & $1.1630000 \mathrm{E}+07$ & $9.3800000 E+06$ & 1.2940000E+07 & $\mathrm{Pa}$ & WPO40434 & $\mathbf{Y}$ & N/A \\
\hline 570 & 1,3 & STUDENT & $-1.8890000 \mathrm{E}+01$ & $-1.8890000 \mathrm{E}+01$ & $-2.1000000 E+01$ & $-1.7100000 E+01$ & $\log \left(m^{\wedge} 2\right)$ & WP030603 & ' $Y$ & NA \\
\hline 570 & 1,3 & STUDENT & $-1.8890000 E+01$ & $-1.8890000 E+01$ & $-2.1000000 E+01$ & $-1.7100000 \mathrm{E}+01$ & $\log \left(m^{\wedge} 2\right)$ & WP031217 & $\mathbf{Y}$ & N/A \\
\hline 570 & 1,3 & STUDENT & $-1.8890000 E+01$ & $-1.8890000 E+01$ & $-2.1000000 E+01$ & $-1.7100000 E+01$ & $\log \left(m^{n} 2\right)$ & WP035597 & $Y$ & NA \\
\hline 570 & 1,3 & STUDENT & $-1.8890000 E+01$ & $-1.8890000 E+01$ & $-2.1000000 E+01$ & $-1.7100000 E+01$ & $\log \left(m^{\wedge} 2\right)$ & WP036380 & $\mathbf{Y}$ & N/A \\
\hline 570 & 1,3 & STUDENT & $-1.8890000 E+01$ & $-1.8890000 E+01$ & $-2.1000000 E+01$ & $-1.7100000 E+01$ & $\log \left(m^{\wedge} 2\right)$ & WP039065 & $Y$ & N/A \\
\hline 570 & 1,3 & STUDENT & $-1.8890000 E+01$ & $-1.8890000 E+01$ & $-2.1000000 E+0 t$ & $-1.7100000 E+01$ & $\log \left(m^{\wedge} 2\right)$ & WPO39178 & $Y$ & NA \\
\hline 571 & 1,3 & STUDENT & $-1.8890000 E+01$ & $-1.8890000 \mathrm{E}+01$ & $-2,1000000 E+01$ & $-1.7900000 E+01$ & $\log \left(m^{\wedge} 2\right)$ & WP030505 & $\mathbf{Y}$ & NA \\
\hline 571 & 1,3 & STUDENT & $-1.8890000 E+01$ & $-1.8890000 E+01$ & $-2.1000000 E+01$ & $-1.7100000 E+01$ & $\log \left(m^{\wedge} 2\right)$ & WP031217 & $\mathbf{Y}$ & N/A \\
\hline
\end{tabular}


CCA Parameter Listing

\begin{tabular}{|c|c|c|c|c|c|c|c|c|c|c|}
\hline $\begin{array}{c}\text { Parametor in } \\
\text { Datubase? } \\
\text { (YnN) }\end{array}$ & \begin{tabular}{|l|} 
if the values \\
uned in CCA \\
are not the \\
same as in \\
DB, provide XX \\
difference
\end{tabular} & ID & $\begin{array}{c}\text { Material io } \\
. \\
.\end{array}$ & $\begin{array}{l}\text { Materid Kams. } \\
\text {. }\end{array}$ & Parameter & 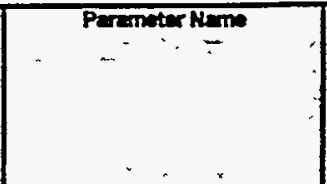 & 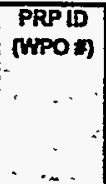 & 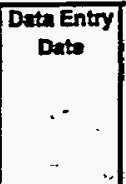 & $\begin{array}{l}\text { Es the 464 } \\
\text { consistent } \\
\text { wth the } \\
\text { perameter } \\
\text { in DB? } \\
(T, N A)\end{array}$ & $\begin{array}{l}\text { Parameter } \\
\text { Citegory } \\
\text { (footrote 1) }\end{array}$ \\
\hline$Y$ & & 571 & S_MB138 & $\begin{array}{l}\text { Salado marker bed } \\
138, \text { intact and } \\
\text { fractured }\end{array}$ & PRAY_LOG & $\begin{array}{l}\text { Log of intrinsic permeability, Y- } \\
\text { direction }\end{array}$ & 34537 & 11Fob-96 & $\mathbf{Y}$ & 4A \\
\hline $\mathbf{Y}$ & & 571 & S_MB138 & $\begin{array}{l}\text { Salado marker bed } \\
138, \text { intact and } \\
\text { fractured }\end{array}$ & PRMY_LOG & $\begin{array}{l}\text { Log of intrinsic permeability, } Y \text { - } \\
\text { direction }\end{array}$ & 34537 & 11 Feb-96 & $\mathbf{Y}$ & $4 A$ \\
\hline $\mathbf{Y}$ & & 571 & S_MB138 & $\begin{array}{l}\text { Salado marker bed } \\
138, \text { intact and } \\
\text { fractured }\end{array}$ & PRMY_LOG & $\begin{array}{l}\text { Log of intrinsic permeability, Y- } \\
\text { direction }\end{array}$ & 34537 & 11 feb-96 & $\mathbf{Y}$ & AA \\
\hline $\mathbf{Y}$ & & 571 & S_MB138 & $\begin{array}{l}\text { Salado marker bed } \\
\text { 138, intact and } \\
\text { fractured }\end{array}$ & PRMY_LOG & $\begin{array}{l}\text { Log of intrinsic permeability, } Y \text { - } \\
\text { diroction }\end{array}$ & 34537 & 11 fob-96 & $\mathbf{Y}$ & $4 A$ \\
\hline $\mathbf{Y}$ & & 572 & S_MB138 & $\begin{array}{l}\text { Salado marker bed } \\
\text { 138, intact and } \\
\text { fractured }\end{array}$ & PRMZ_LOG & $\begin{array}{l}\text { Log of intrinsic permeability, Z- } \\
\text { direction }\end{array}$ & 34538 & 11 Fob-96 & $\mathbf{Y}$ & $4 A$ \\
\hline $\mathbf{Y}$ & & 572 & S_MB138 & $\begin{array}{l}\text { Salado marker bod } \\
138 \text {, intact and } \\
\text { fractured }\end{array}$ & PRMZ_LOG & $\begin{array}{l}\text { Log of intrinsic permeability, Z- } \\
\text { direction }\end{array}$ & 34538 & 11Feb-96 & $Y$ & AA \\
\hline $\mathbf{Y}$ & & 572 & S_MB138 & $\begin{array}{l}\text { Salado marker bed } \\
138 \text {, intact and } \\
\text { fractured }\end{array}$ & PRMZ_LOG & $\begin{array}{l}\text { Log of intrinsic permeability, } z \text { - } \\
\text { direction }\end{array}$ & 34538 & 11 feb-96 & $\mathbf{Y}$ & $4 A$ \\
\hline $\mathbf{Y}$ & & 572 & S_MB138 & $\begin{array}{l}\text { Salado marker bed } \\
138, \text { intact and } \\
\text { fractured }\end{array}$ & PRMZ_LOG & $\begin{array}{l}\text { Log of intrinsic permeability, Z- } \\
\text { direction }\end{array}$ & 34538 & 11-Fob-96 & $\mathbf{Y}$ & $4 A$ \\
\hline $\mathbf{Y}$ & & 572 & S_MB138 & $\begin{array}{l}\text { Salado marker bed } \\
138 \text {, intact and } \\
\text { fractured }\end{array}$ & PRMZ_LOG & $\begin{array}{l}\text { Log of intrinsic permeability, Z- } \\
\text { direction }\end{array}$ & 34538 & 11-Feb-96 & $\mathbf{Y}$ & 4A \\
\hline $\mathbf{Y}$ & & 572 & S_MB138 & $\begin{array}{l}\text { Salado marker bed } \\
138, \text { intact and } \\
\text { fractured }\end{array}$ & PRMZ_LOG & $\begin{array}{l}\text { Log of intrinsic permeability, } z \text { - } \\
\text { direction . }\end{array}$ & 34538 & 11-Feb-96 & $\mathbf{Y}$ & $4 A$ \\
\hline $\mathbf{Y}$ & & 575 & S_MB138 & $\begin{array}{l}\text { Salado marker bed } \\
\text { 138, intact and } \\
\text { fractured }\end{array}$ & RELP_MOD & $\begin{array}{l}\text { Model number, relative } \\
\text { permeability model }\end{array}$ & 34542A & 08-Feb-96 & $\mathbf{Y}$ & $4 A$ \\
\hline$Y$ & & 575 & S_MB138 & $\begin{array}{l}\text { Salado marker bed } \\
138 \text {, intact and } \\
\text { fractured }\end{array}$ & RELP_MOD & $\begin{array}{l}\text { Model number, relative } \\
\text { permeability model }\end{array}$ & 34542A & 08-Feb-96 & $\mathbf{Y}$ & $4 A$ \\
\hline $\mathbf{Y}$ & & 575 & S_MB138 & $\begin{array}{l}\text { Salado marker bed } \\
138 \text {, intact and } \\
\text { fractured }\end{array}$ & RELP_MOD & $\begin{array}{l}\text { Model number, relative } \\
\text { permeability model }\end{array}$ & $34542 A$ & 08feb-9s & $\mathbf{Y}$ & $4 A$ \\
\hline $\mathbf{Y}$ & & 576 & S_MB138 & $\begin{array}{l}\text { Salado marker bed } \\
138 \text {, intact and } \\
\text { fractured }\end{array}$ & SAT_IBRN & Initial Brine Saturation & 34544 & 11-Feb-96 & $\mathbf{Y}$ & 1 \\
\hline$Y$ & & 576 & S_MB138 & $\begin{array}{l}\text { Salado marker bed } \\
138 \text {, intact and } \\
\text { fractured }\end{array}$ & SAT_IBRN & Initial Brine Saturation & 34544 & 11 fob-96 & $\mathbf{Y}$ & 1 \\
\hline $\mathbf{Y}$ & & 577 & S_MB138 & $\begin{array}{l}\text { Salado marker bed } \\
138 \text {, intact and } \\
\text { fractured }\end{array}$ & SAT_RBRN & Residual Brine Saturation & 34545 & 21-Nov-96 & $\mathbf{Y}$ & $4 A$ \\
\hline$Y$ & & 577 & S_MB138 & $\begin{array}{l}\text { Salado marker bed } \\
138 \text {, intact and } \\
\text { fractured }\end{array}$ & SAT_RBRN & Residual Brine Saturation & 34545 & 21-Nov-96 & ${ }^{-} Y$ & $4 A$ \\
\hline $\mathbf{Y}$ & & 577 & S_MB138 & $\begin{array}{l}\text { Salado markor bod } \\
\text { 138, intact and } \\
\text { fractured }\end{array}$ & SAT_RBRN & Residual Brine Saturation & 34545 & 21-Nov-96 & $\mathbf{Y}$ & $4 A$ \\
\hline $\mathbf{Y}$ & & 578 & S_MB138 & $\begin{array}{l}\text { Saledo marker bod } \\
138 \text {, intect and } \\
\text { fractured }\end{array}$ & SAT_RGAS & Residual Gas Saturation & 34546 & 22-Feb-96 & $\mathbf{Y}$ & $4 A$ \\
\hline $\mathbf{Y}$ & & 578 & S_MB138 & $\begin{array}{l}\text { Salado marker bed } \\
138 \text {, intact and } \\
\text { fractured }\end{array}$ & SAT_RGAS & Rosidual Gas Saturation & 34545 & 22-fob-96 & $\mathbf{Y}$ & $4 A$ \\
\hline $\mathbf{Y}$ & & 578 & S_MB138 & $\begin{array}{l}\text { Salado marker bed } \\
138 \text {, intect and } \\
\text { fractured }\end{array}$ & SAT_RGAS & Residual Gas Saturation & 34546 & 22feb-96 & $\mathbf{Y}$ & $4 A$ \\
\hline $\mathbf{Y}$ & & 579 & S_MB139 & $\begin{array}{l}\text { Salado marker bed } \\
139, \text { intact and } \\
\text { fractured }\end{array}$ & CAP_MOD & $\begin{array}{l}\text { Model number, capillary pressure } \\
\text { model }\end{array}$ & 34559 & 11Fab-96 & $\mathbf{Y}$ & 1 \\
\hline $\mathbf{Y}$ & & 579 & S_MB139 & $\begin{array}{l}\text { Salado marker bed } \\
\text { 139, intact and } \\
\text { fractured }\end{array}$ & CAP_MOD & $\begin{array}{l}\text { Model number, capillary pressure } \\
\text { model }\end{array}$ & 34559 & 11 fob-96 & $\mathbf{Y}$ & 1 \\
\hline $\mathbf{Y}$ & & 580 & S_MB139 & $\begin{array}{l}\text { Salado marker bed } \\
139 \text {, intect and } \\
\text { fractured }\end{array}$ & COMP_RCK & Bulk Compressibility & 34574 & 14Fob-96 & $\mathbf{Y}$ & 1 \\
\hline
\end{tabular}


CCA Parameter Listing

\begin{tabular}{|c|c|c|c|c|c|c|c|c|c|c|}
\hline 10 & $\begin{array}{c}\text { Clasefin- } \\
\text { Cation of } \\
\text { Data } \\
\text { Category } \\
\text { (footnote 2) }\end{array}$ & $\begin{array}{l}\text { Distribution } \\
\text { Type }\end{array}$ & Man & Median & Mlnlmum & Modinum & Units & Reference ID & $\begin{array}{l}\text { Was the data } \\
\text { developed } \\
\text { under an } \\
\text { NeA-1 } \\
\text { Program? }\end{array}$ & \begin{tabular}{|c|} 
Which \\
mothod \\
more used to \\
quallty \\
exdsting \\
data? \\
(footnote 3)
\end{tabular} \\
\hline 571 & 1,3 & STUDENT & $-1.8890000 E+01$ & $-1.8890000 \mathrm{E}+01$ & $-2.1000000 E+01$ & $-1.7100000 E+01$ & $\log \left(m^{\wedge} 2\right)$ & WP035597 & $\mathbf{Y}$ & NA \\
\hline 571 & 1,3 & STUDENT & $-1.8890000 E+01$ & $-1.8890000 E+01$ & $-2.1000000 E+01$ & $-1.7100000 E+01$ & $\log \left(m^{\wedge} 2\right)$ & WPO36380 & $\mathbf{Y}$ & NA \\
\hline 571 & 1,3 & STUDENT & $-1.8890000 E+01$ & $-1.8890000 E+01$ & $-2.1000000 E+01$ & $-1.7100000 E+01$ & $\log \left(m^{\wedge} 2\right)$ & WP039065 & $\mathbf{Y}$ & NAA \\
\hline 571 & 1,3 & STUDENT & $-1.8890000 E+01$ & $-1.8890000 E+01$ & $-2.1000000 E+01$ & $-1.7100000 E+01$ & $\log \left(m^{\wedge} 2\right)$ & WP039178 & $\mathbf{Y}$ & NA \\
\hline 572 & 1,3 & STUDENT & $-1.8890000 E+01$ & $-1.8890000 E+01$ & $-2.1000000 \mathrm{E}+01$ & $-1.7100000 E+01$ & $\log \left(m^{\wedge} 2\right)$ & WP030506 & $\mathbf{Y}$ & N/A \\
\hline 572 & 1,3 & STUDENT & $-1.8890000 E+01$ & $-1.8890000 E+01$ & $-2.1000000 E+01$ & $-1.7100000 E+01$ & $\log \left(m^{\wedge} 2\right)$ & WP031217 & $\mathbf{Y}$ & N/A \\
\hline 572 & 1,3 & STUDENT & $-1.8890000 E+01$ & $-1.8890000 E+01$ & $-2.1000000 E+01$ & $-1.7100000 \mathrm{E}+01$ & $\log \left(m^{\wedge} 2\right)$ & WP035597 & $\mathbf{Y}$ & NA \\
\hline 572 & 1,3 & STUDENT & $-1.8890000 E+01$ & $-1.8890000 E+01$ & $-2.1000000 E+01$ & $-1.7100000 E+01$ & $\log \left(m^{\wedge} 2\right)$ & WP036380 & $\mathbf{Y}$ & NA \\
\hline 572 & 1,3 & STUDENT & $-1.8890000 E+01$ & $-1.8890000 E+01$ & $-21000000 E+01$ & $-1.7100000 E+01$ & $\log \left(m^{\wedge} 2\right)$ & WP039065 & $\mathbf{Y}$ & NA \\
\hline 572 & 1,3 & STUDENT. & $-1.8890000 E+01$ & $-1.8890000 E+01$ & $-2.1000000 E+01$ & $-1.7100000 E+01$ & $\log \left(m^{\wedge} 2\right)$ & WP039178 & $\mathbf{Y}$ & N/A \\
\hline 575 & 1,3 & DELTA & $4.0000000 E+\infty 0$ & $4.0000000 E+00$ & $1.0000000 \mathrm{E}+00$ & $4.0000000 E+00$ & NONE & WPO30643 & $Y$ & NA \\
\hline 575 & 1,3 & DELTA & $4.0000000 E+00$ & $4.0000000 E+\infty 0$ & $1.0000000 E+00$ & $4.0000000 E+\infty 0$ & NONE & WP035268 & $\mathbf{Y}$ & N/A \\
\hline 575 & 1,3 & DELTA & $4.0000000 \mathrm{E}+\infty 0$ & $4.0000000 E+\infty 0$ & $1.0000000 E+\infty 0$ & $4.0000000 E+\infty 0$ & NYONE & WP035597 & $\mathbf{Y}$ & N/A \\
\hline 576 & 1,5 & CONSTANT & $1.0000000 \mathrm{E}+\infty 0$ & $1.0000000 E+\infty 0$ & $1.0000000 E+\infty 0$ & $1.0000000 E+\infty 0$ & NONE & WP030643 & $\mathbf{Y}$ & N/A \\
\hline 576 & 1,5 & CONSTANT & $1.0000000 E+00$ & $1.0000000 E+00$ & $1.0000000 E+00$ & $1.0000000 E+\infty 0$ & NONE & WP035597 & $\mathbf{Y}$ & N/A \\
\hline 577 & 1,3 & STUDENT & $8.3620000 E-02$ & $8.3620000 E-02$ & $7.7846000 \mathrm{E}-03$ & $1.7401000 \mathrm{E}-01$ & NONE & WP030643 & $\mathbf{Y}$ & NA \\
\hline 577 & 1,3 & STUDENT & $8.3620000 E-02$ & $8.3620000 E-02$ & $7.7846000 \mathrm{E}-03$ & $1.7401000 E-01$ & NONE & WPO35597 & $-\mathbf{Y}$ & NA \\
\hline 577 & 1,3 & STUDENT & $8.3620000 \mathrm{E}-02$ & $8.3620000 \mathrm{E}-02$ & $7.7846000 E-03$ & $1.7401000 \mathrm{E}-01$ & NONE & WP036380 & $\mathbf{Y}$ & N/A \\
\hline 578 & 1.5 & STUDENT & $7.7110000 E-02$ & $7.7110000 \mathrm{E}-02$ & $1.3980000 E-02$ & $1.9719000 E-01$ & NONE & WPO30643 & $\mathbf{Y}$ & NA \\
\hline 578 & 1,5 & STUDENT & $7.7110000 \mathrm{E}-02$ & $7.7110000 E-02$ & $1.3980000 \mathrm{E}-02$ & $1.9719000 E-01$ & NONE & WPO35597 & $\mathbf{Y}$ & N/A \\
\hline 578 & 1,5 & STUDENT & $7.7110000 \mathrm{E}-02$ & $7.7110000 E-02$ & $1.3980000 E-02$ & 1.9719000 E-01 & NONE & WPO36380 & $\mathbf{Y}$ & N/A \\
\hline 579 & 1,5 & CONSTANT & $20000000 E+00$ & $2.0000000 E+\infty$ & $2.0000000 E+\infty 0$ & $2.0000000 E+00$ & NONE & WP030643 & $\mathbf{Y}$ & N/A \\
\hline 579 & 1,5 & CONSTANT & $2.0000000 \mathrm{E}+\infty 0$ & $2.0000000 E+00$ & $2.0000000 E+\infty 0$ & $2.0000000 E+\infty$ & NONE & WP035597 & $\mathbf{Y}$ & N/A \\
\hline 580 & 1 & STUDENT & $8.2630000 \mathrm{E}-11$ & $8.2630000 E-11$ & $1.0900000 \mathrm{E}-11$ & $2.7500000 \mathrm{E}-10$ & $P a^{n}-1$ & WP031186 & Y\&N & 1 \\
\hline
\end{tabular}


CCA Parameter Listing

\begin{tabular}{|c|c|c|c|c|c|c|c|c|c|c|}
\hline $\begin{array}{c}\text { Parametar in } \\
\text { Dathbase? } \\
\text { (YnN) }\end{array}$ & $\begin{array}{l}\text { Hf the values } \\
\text { ueed in CCA } \\
\text { are not the } \\
\text { seme as in } \\
\text { DB, provide } \% \\
\text { difference }\end{array}$ & ID & Maturial ID & Matura Nam & $\begin{array}{c}\text { Paramoter. } \\
\text { ID }\end{array}$ & Parameter Name & $\begin{array}{l}\text { PRP ID } \\
\text { (WPO\#) }\end{array}$ & $\begin{array}{c}\text { Dette Entry } \\
\text { Date }\end{array}$ & $\begin{array}{l}\text { o the } 464 \\
\text { consistent } \\
\text { whth the } \\
\text { parmimeter } \\
\text { in DB? } \\
(Y, N A)\end{array}$ & $\begin{array}{l}\text { Parmmatir } \\
\text { Cetagory } \\
\text { (footnote 1) }\end{array}$ \\
\hline $\mathbf{Y}$ & & 580 & S_MB139 & $\begin{array}{l}\text { Salado marker bed } \\
139, \text { intact and } \\
\text { fractured }\end{array}$ & COMP_RCK & Bufk Compressibility & 34574 & 14 Feb-96 & $\mathbf{Y}$ & 1 \\
\hline $\mathbf{Y}$ & & 580 & S_MB139 & $\begin{array}{l}\text { Salado marker bed } \\
139 \text {, intact and } \\
\text { fractured }\end{array}$ & COMP_RCK & Bulk Compressibility & 34574 & 14-Feb-96 & $\mathbf{Y}$ & 1 \\
\hline $\mathbf{Y}$ & & 582 & S_MB139 & $\begin{array}{l}\text { Salado marker bed } \\
139 \text {, intact and } \\
\text { fractured }\end{array}$ & PC_MAX & $\begin{array}{l}\text { Maximum allowable capillary } \\
\text { pressure }\end{array}$ & 34848 & 01-Fob-96 & $\mathbf{Y}$ & 1 \\
\hline $\mathbf{Y}$ & & 582 & S_MB139 & $\begin{array}{l}\text { Salado marker bed } \\
139 \text {, intact and } \\
\text { fractured }\end{array}$ & PC_MAX & $\begin{array}{l}\text { Maximum allowable capillary } \\
\text { pressure }\end{array}$ & 34848 & 01-Feb-96 & $\mathbf{Y}$ & 1 \\
\hline $\mathbf{Y}$ & & 586 & S_MB139 & $\begin{array}{l}\text { Salado marker bed } \\
\text { 139, intact and } \\
\text { fractured }\end{array}$ & PI_DELTA & $\begin{array}{l}\text { Fracture initiation pressure } \\
\text { increment }\end{array}$ & 34856 & 22fob-96 & $\mathbf{Y}$ & $4 B$ \\
\hline $\mathbf{Y}$ & & 586 & S_MB139 & $\begin{array}{l}\text { Salado marker bed } \\
\text { 139, intact and } \\
\text { frectured }\end{array}$ & PI_DELTA & $\begin{array}{l}\text { Frecture initiation pressure } \\
\text { increment }\end{array}$ & 34856 & 22f-Fob-96 & $\mathbf{Y}$ & $4 B$ \\
\hline $\mathbf{Y}$ & & 586 & S_MB139 & $\begin{array}{l}\text { Salado masker bed } \\
\text { 139, intact and } \\
\text { fractured }\end{array}$ & PI_DELTA & $\begin{array}{l}\text { Frecture initiation pressure } \\
\text { increment }\end{array}$ & 34856 & 22-Feb-96 & $\mathbf{Y}$ & $4 B$ \\
\hline $\mathbf{Y}$ & & 586 & S_MB139 & $\begin{array}{l}\text { Salado marker bed } \\
139, \text { intact and } \\
\text { fractured }\end{array}$ & PI_DELTA & $\begin{array}{l}\text { Fracture initiation pressure } \\
\text { increment }\end{array}$ & 34856 & 22-Feb-96 & $\mathbf{Y}$ & $4 B$ \\
\hline $\mathbf{Y}$ & . & 587 & S_MB139 & $\begin{array}{l}\text { Salado marker bed } \\
139, \text { intact and } \\
\text { fractured }\end{array}$ & PORE_DIS & $\begin{array}{l}\text { Brooks-Corty pore distribution } \\
\text { parameter }\end{array}$ & 34859 & 22F́b-96 & $\mathbf{Y}$ & 1 \\
\hline $\mathbf{Y}$ & & 587 & S_MB139 & $\begin{array}{l}\text { Salado marker bed } \\
139, \text { intect and } \\
\text { fractured }\end{array}$ & PORE_DIS & $\begin{array}{l}\text { Brooks-Corey pore distribution } \\
\text { parameter }\end{array}$ & 34859 & 22Fab-96 & $\mathbf{Y}$ & 1 \\
\hline $\mathbf{Y}$ & & 587 & S_MB139 & $\begin{array}{l}\text { Salado marker bod } \\
139 \text {, intact and } \\
\text { fractured }\end{array}$ & PORE_DIS & $\begin{array}{l}\text { Brooks-Corey pore distribution } \\
\text { parameter }\end{array}$ & 34859 & 22-Fab-96 & $\mathbf{Y}$ & 1 \\
\hline $\mathbf{Y}$ & & 588 & S_MB139 & $\begin{array}{l}\text { Salado marker bed } \\
139, \text { intact and } \\
\text { fractured }\end{array}$ & POROSITY & Effective porosity & 34860 & 22-Apr-97 & $\mathbf{Y}$ & 1 \\
\hline $\mathbf{Y}$ & & 588 & S_MB139 & $\begin{array}{l}\text { Salado marker bed } \\
139, \text { intact and } \\
\text { fractured }\end{array}$ & POROSTY & Effective porosity & 34860 & 22-Apr-97 & $\mathbf{Y}$ & 1 \\
\hline $\mathbf{Y}$ & & 588 & S_MB139 & $\begin{array}{l}\text { Salado marker bed } \\
\text { 139, intact and } \\
\text { fractured }\end{array}$ & POROSITY & Effective porosity & 34860 & 22-Apr-97 & $\mathbf{Y}$ & 1 \\
\hline $\mathbf{Y}$ & & 589 & S_MB139 & $\begin{array}{l}\text { Salado marker bed } \\
139 \text {, intact and } \\
\text { fractured }\end{array}$ & PO_MIN & $\begin{array}{l}\text { Minimum brine pressure for } \\
\text { capillary model KPC=3 }\end{array}$ & 34852 & 21fob-96 & $\mathbf{Y}$ & 4B \\
\hline $\mathbf{Y}$ & & 589 & S_MB139 & $\begin{array}{l}\text { Salado marker bed } \\
139, \text { intact and } \\
\text { fractured }\end{array}$ & PO_MIN & $\begin{array}{l}\text { Minimum brine pressure for } \\
\text { capiltary model KPC=3 }\end{array}$ & 34862 & 21-Fob-96 & $\mathbf{Y}$ & 4B \\
\hline $\mathbf{Y}$ & & 589 & S_MB139 & $\begin{array}{l}\text { Salado marker bod } \\
139, \text { intact and } \\
\text { fractured }\end{array}$ & PO_MIN & $\begin{array}{l}\text { Minimum brine pressure for } \\
\text { capillary model KPC=3 }\end{array}$ & 34862 & 21-Feb-96 & ${ }^{-} \mathbf{Y}$ & 48 \\
\hline $\mathbf{Y}$ & varies & 590 & S_MB139 & $\begin{array}{l}\text { Salado marker bed } \\
139 \text {, intact and } \\
\text { frectured }\end{array}$ & PRESSURE & Brine far-field pore pressure & 34863 & 17-Sep-96 & $\mathbf{Y}$ & 1 \\
\hline$Y$ & varies & 590 & S_MB139 & $\begin{array}{l}\text { Salado marker bed } \\
139 \text {, intact and } \\
\text { fractured }\end{array}$ & PRESSURE & Brine far-field pore presstre & 34863 & 17-Sep-96 & $\mathbf{Y}$ & 1 \\
\hline $\mathbf{Y}$ & varies & 590 & S_MB139 & $\begin{array}{l}\text { Salado marker bed } \\
139, \text { intact and } \\
\text { fractured }\end{array}$ & PRESSURE & Brine far-field pore pressure & 34863 & 17-Sep-96 & $\mathbf{Y}$ & 1 \\
\hline$Y$ & varies & 590 & S_MB139 & $\begin{array}{l}\text { Salado marker bed } \\
139 \text {, intect and } \\
\text { fractured }\end{array}$ & PRESSURE & Brine fartfield pore pressure & 34863 & 17-Sep-9s & $\mathbf{Y}$ & 1 \\
\hline $\mathbf{Y}$ & & 591 & S_MB139 & $\begin{array}{l}\text { Salado marker bed } \\
139, \text { intact and } \\
\text { fractured }\end{array}$ & PRMX_LOG & $\begin{array}{l}\text { Log of intrinsic permeability, } x \text { - } \\
\text { direction }\end{array}$ & 34865 & $01-F e b-96$ & $\mathbf{Y}$ & 1 \\
\hline $\mathbf{Y}$ & & 591 & S_MB139 & $\begin{array}{l}\text { Salado marker bed } \\
139, \text { intect and } \\
\text { fractured }\end{array}$ & PRMX_LOG & $\begin{array}{l}\text { Log of intrinsic permeability, } X \text { - } \\
\text { direction }\end{array}$ & 34865 & 01-Feb-96 & $\mathbf{Y}$ & 1 \\
\hline $\mathbf{Y}$ & & 591 & S_MB139 & $\begin{array}{l}\text { Salado marker bed } \\
139, \text { intact and } \\
\text { fractured }\end{array}$ & PRMX_LOG & $\begin{array}{l}\text { Log of intrinsic permeability, } X \text { - } \\
\text { direction }\end{array}$ & 34865 & $01-$ feb-96 & $\mathbf{Y}$ & 1 \\
\hline
\end{tabular}


CCA Parameter Listing

\begin{tabular}{|c|c|c|c|c|c|c|c|c|c|c|}
\hline ID & \begin{tabular}{|c|} 
Ciaseffi. \\
cation of \\
Data \\
Catogory \\
(footnote 2)
\end{tabular} & $\begin{array}{l}\text { Distribution } \\
\text { Type. }\end{array}$ & Mo2n & Median & Mintmem & Maxkmum & $\begin{array}{l}\text { Unit: } \\
\text {. }\end{array}$ & Retorence ID & $\begin{array}{l}\text { Wez the dett } \\
\text { doveloped } \\
\text { under an } \\
\text { NQA-1 } \\
\text { Program? }\end{array}$ & $\begin{array}{c}\text { Which } \\
\text { mothods } \\
\text { wore used to } \\
\text { quallyy } \\
\text { existing } \\
\text { data? } \\
\text { (footnote 3) }\end{array}$ \\
\hline 580 & 1 & STUDENT & $8.2630000 E-11$ & $8.2630000 E-11$ & $1.0900000 \mathrm{E}-11$ & $2.7500000 E-10$ & $P a^{\wedge}-1$ & WPO35597 & YSN & 1 \\
\hline 580 & 1 & STUDENT & $8.2630000 E-11$ & $8.2630000 E-11$ & $1.0900000 E-11$ & $2.7500000 E-10$ & $P a^{n-1}$ & WP035380 & YEN & 1 \\
\hline 582 & 1,5 & CONSTANT & $1.0000000 E+08$ & $1.0000000 E+08$ & $1.0000000 \mathrm{E}+08$ & $1.0000000 \mathrm{E}+08$ & $\mathbf{P a}$ & WP030643 & $\mathbf{Y}$ & N/A \\
\hline 582 & 1,5 & CONSTANT & $1.0000000 E+08$ & $1.0000000 E+08$ & $1.0000000 E+08$ & $1.0000000 E+08$ & $\mathbf{P a}$ & WP035597 & $\mathbf{Y}$ & NA \\
\hline 586 & 3,5 & CONSTANT & $2.0000000 E+05$ & $2.0000000 E+05$ & $2.0000000 E+0.5$ & $2.0000000 E+05$ & $\mathrm{~Pa}$ & WP035597 & $\mathbf{Y}$ & NA \\
\hline 586 & 3,5 & CONSTANT & $2.0000000 E+05$ & $2.0000000 E+05$ & $2.0000000 E+05$ & $20000000 E+05$ & $\mathrm{~Pa}$ & WP038274 & $\gamma$ & NA \\
\hline 586 & 3,5 & CONSTANT & $2.0000000 E+05$ & $2.0000000 E+05$ & $2.0000000 E+05$ & $2.0000000 E+05$ & $\mathrm{~Pa}$ & WP038559 & $\mathbf{Y}$ & N/A \\
\hline 586 & 3,5 & CONSTANT & $2.0000000 E+05$ & $2.0000000 E+05$ & $2.0000000 E+05$ & $2.0000000 E+05$ & $\mathbf{P a}$ & WPO40514 & $Y$ & N/A \\
\hline 587 & 1,5 & STUDENT & $6.4360000 E-01$ & $6.4360000 E-01$ & 4.9053000E-01 & $8.4178000 E-01$ & NONE & WP030643 & $\mathbf{Y}$ & NA \\
\hline 587 & 1,5 & STUDENT & $6.4360000 E-01$ & $6.4360000 E-01$ & $4.9053000 E-01$ & $8.4178000 E-01$ & NONE & WP035597 & $Y$ & N/A \\
\hline 587 & 1,5 & STUDENT & $6.4360000 E-01$ & $6.4360000 E-01$ & $4.9053000 E-01$ & $8.4178000 E-01$ & NONE & WPO36380 & $Y$ & N/A \\
\hline 588 & $1,3,4$ & STUDENT & $1.1000000 E-02$ & $1.1000000 E-02$ & $6.0000000 \mathrm{E}-03$ & $1.7000000 E-02$ & NONE & WP030600 & $\mathbf{Y}$ & N/A \\
\hline 588 & $1,3,4$ & STUDENT & $1.1000000 E-02$ & $1.1000000 E-02$ & $6.0000000 E-03$ & $1.7000000 E-02$ & NONE & WPO35597 & $\mathbf{Y}$ & NA \\
\hline 588 & $1,3,4$ & STUDENT & $1.1000000 E-02$ & $1.1000000 E-02$ & $6.0000000 E-03$ & $1.7000000 \mathrm{E}-02$ & NONE & WP036380 & $\mathbf{Y}$ & NA \\
\hline 589 & 3,5 & CONSTANT & $1.0132500 E+05$ & $1.0132500 \mathrm{E}+05$ & $1.0132500 E+05$ & $1.0132500 E+05$ & $\mathbf{P a}$ & WP035597 & $Y$ & N/A \\
\hline 589 & 3,5 & CONSTANT & $1.0132500 E+05$ & $1.0132500 E+05$ & $1.0132500 E+05$ & $1.0132500 E+05$ & $\mathbf{P a}$ & WP036051 & $\mathbf{Y}$ & N/A \\
\hline 589 & 3,5 & CONSTANT & $1.0132500 E+05$ & $1.0132500 E+05$ & $1.0132500 E+05$ & $1.0132500 E+05$ & $\mathbf{P a}$ & WPO40514 & $\gamma$ & N/A \\
\hline 590 & 1 & STUDENT & $1.1630000 \mathrm{E}+07$ & $1.1630000 E+07$ & $9.3800000 E+06$ & $1.2940000 \mathrm{E}+07$ & $\mathrm{~Pa}$ & WP031185 & Y\&N & 1 \\
\hline 590 & 1 & STUDENT & $1.1630000 E+07$ & $1.1630000 E+07$ & $9.3800000 E+06$ & $1.2940000 E+07$ & $\mathbf{P a}$ & WP035597 & Y8N & 1 \\
\hline 590 & 1 & STUDENT & $1.1630000 \mathrm{E}+07$ & $1.1630000 E+07$ & $9.3800000 E+06$ & $1.2940000 E+07$ & $\mathrm{~Pa}$ & WP036380 & YaN & 1 \\
\hline 590 & 1 & STUDENT & $1.1630000 E+07$ & $1.1630000 E+07$ & $9.3800000 E+06$ & $1.2940000 E+07$ & $\mathbf{P a}$ & WP040434 & YEN & 1 \\
\hline 591 & 1 & STUDENT & $-1,8890000 E+01$ & $-1.8890000 E+01$ & $-2.1000000 E+01$ & $-1.7100000 E+01$ & $\log \left(m^{\wedge} 2\right)$ & WP030603 & $Y$ & NA \\
\hline 591 & 1 & STUDENT & $-1.8890000 \mathrm{E}+01$ & $-1.8890000 E+01$ & $-2.1000000 E+01$ & $-1.7100000 E+01$ & $\log \left(m^{\wedge} 2\right)$ & WPO31217 & $\mathbf{Y}$ & NA \\
\hline 591 & 1 & STUDENT & $-1.8890000 E+01$ & $-1.8890000 E+01$ & $-2.1000000 \mathrm{E}+01$ & $-1.7100000 E+01$ & $\log \left(m^{\wedge} 2\right)$ & WP035597 & $\mathbf{Y}$ & NA \\
\hline
\end{tabular}


CCA Parameter Listing

\begin{tabular}{|c|c|c|c|c|c|c|c|c|c|c|}
\hline $\begin{array}{c}\text { Parametor in } \\
\text { Database? } \\
\text { (YN) }\end{array}$ & $\begin{array}{l}\text { if the values } \\
\text { used in CCA } \\
\text { are not the } \\
\text { same es in } \\
\text { o8, provide } x \\
\text { difference }\end{array}$ & ID & Material ID & Materia Nam & $\begin{array}{c}\text { Perameter } \\
\text { to }\end{array}$ & Paramoter Nam & $\begin{array}{l}\text { PRP ID } \\
\text { (MPO D) }\end{array}$ & $\begin{array}{c}\text { Deta Entry } \\
\text { Dato }\end{array}$ & $\begin{array}{l}\text { is the } 464 \\
\text { consietent } \\
\text { with the } \\
\text { parnmeter } \\
\text { in DB? } \\
(Y, N \text { NA) }\end{array}$ & $\begin{array}{l}\text { Parameter } \\
\text { Categony } \\
\text { (footnote 1) }\end{array}$ \\
\hline $\mathbf{Y}$ & & 591 & S_MB139 & $\begin{array}{l}\text { Salado marker bed } \\
139, \text { intact and } \\
\text { fractured }\end{array}$ & PRMX_LOG & $\begin{array}{l}\text { Log of intrinsic permeability, } X \text { - } \\
\text { direction }\end{array}$ & 34865 & 01 fob-96 & $\mathbf{Y}$ & 1 \\
\hline $\mathbf{Y}$ & & 591 & S_MB139 & $\begin{array}{l}\text { Salado marker bed } \\
139, \text { intact and } \\
\text { fractured }\end{array}$ & PRMX_LOG & $\begin{array}{l}\text { Log of intrinsic permeability, } X \text { - } \\
\text { direction }\end{array}$ & 34865 & 01 fob-96 & $\mathbf{Y}$ & 9 \\
\hline $\mathbf{Y}$ & & 591 & S_MB139 & $\begin{array}{l}\text { Salado marker bed } \\
139, \text { intect and } \\
\text { fractured }\end{array}$ & PRMX_LOG & $\begin{array}{l}\text { Log of intrinsic permeability, } x \text { - } \\
\text { direction }\end{array}$ & 34865 & 01 fob-96 & $Y$ & 1 \\
\hline $\mathbf{Y}$ & & 592 & S_MB139 & $\begin{array}{l}\text { Salado marker bed } \\
139 \text {, intact and } \\
\text { fractured }\end{array}$ & PRMY_LOG & $\begin{array}{l}\text { Log of intrinsic permeability, Y- } \\
\text { direction }\end{array}$ & 34866 & 01-Fob-96 & $\mathbf{Y}$ & 1 \\
\hline$Y$ & & 592 & S_MB139 & $\begin{array}{l}\text { Salado marker bed } \\
139, \text { intact and } \\
\text { frectured }\end{array}$ & PRMY_LOG & $\begin{array}{l}\text { Log of intrinsic permeability, Y- } \\
\text { direction }\end{array}$ & 34866 & $01+F \ddot{b}-96$ & $\mathbf{Y}$ & 1 \\
\hline $\mathbf{Y}$ & & 592 & S_MB139 & $\begin{array}{l}\text { Salado marker bed } \\
139, \text { intact and } \\
\text { frachured }\end{array}$ & PRMY_LOG & $\begin{array}{l}\text { Log of intrinsic permeability, Y- } \\
\text { direction }\end{array}$ & 34866 & 01 Feb-96 & $\mathbf{Y}$ & 9 \\
\hline $\mathbf{Y}$ & & 592 & S_MB139 & $\begin{array}{l}\text { Salado marker bed } \\
\text { 139, intact and } \\
\text { fractured }\end{array}$ & PRMY_LOG & $\begin{array}{l}\text { Log of intrinsic permeability, Y- } \\
\text { direction }\end{array}$ & 34866 & 01feb-96 & $\mathbf{Y}$ & 1 \\
\hline $\mathbf{Y}$ & & 592 & S_MB139 & $\begin{array}{l}\text { Salado marker bed } \\
\text { 139, intact and } \\
\text { fractured }\end{array}$ & PRMY_LOG & $\begin{array}{l}\text { Log of intrinsic permeability, Y- } \\
\text { direction }\end{array}$ & 34866 & 01 feb-96 & $\mathbf{Y}$ & 1 \\
\hline $\mathbf{Y}$ & & 592 & S_MB139 & $\begin{array}{l}\text { Salado marker bed } \\
139, \text { intact and } \\
\text { fractured }\end{array}$ & PRMY_LOG & $\begin{array}{l}\text { Log of intrinsic permbability, } Y \text { - } \\
\text { direction }\end{array}$ & 34866 & 01feb-96 & $\mathbf{Y}$ & 1 \\
\hline $\mathbf{Y}$ & & 593 & S_MB139 & $\begin{array}{l}\text { Salado marker bed } \\
\text { 139, intact and } \\
\text { fractured }\end{array}$ & PRMIZ_LOG & $\begin{array}{l}\text { Log of intrinsic permeability, Z- } \\
\text { direction }\end{array}$ & 34868 & $01-F o b-96$ & $\mathbf{Y}$ & 1 \\
\hline $\mathbf{Y}$ & & 593 & S_MB139 & $\begin{array}{l}\text { Salado marker bed } \\
139 \text {, intact and } \\
\text { fractured }\end{array}$ & PRMZ_LOG & $\begin{array}{l}\text { Log of intrinsic permeability, Z- } \\
\text { direction }\end{array}$ & 34868 & 01-Fob-96 & $\mathbf{Y}$ & 1 \\
\hline $\mathbf{Y}$ & & 593 & S_MB139 & $\begin{array}{l}\text { Salado marker bed } \\
139, \text { intact and } \\
\text { fractured }\end{array}$ & PRMZ_LOG & $\begin{array}{l}\text { Log of intrinsic permeability, z- } \\
\text { direction }\end{array}$ & 34868 & 01 feb-96 & $\mathbf{Y}$ & 1 \\
\hline $\mathbf{Y}$ & & 593 & S_MB139 & $\begin{array}{l}\text { Salado marker bed } \\
139, \text { intact and } \\
\text { fractured }\end{array}$ & PRMZ_LOG & $\begin{array}{l}\text { Log of intrinsic permeability, Z- } \\
\text { direction }\end{array}$ & 34868 & $01-F a b-96$ & $\mathbf{Y}$ & 1 \\
\hline $\mathbf{Y}$ & & 593 & S_MB139 & $\begin{array}{l}\text { Salado marker bed } \\
139, \text { intact and } \\
\text { fractured }\end{array}$ & PRMZ_LOG & $\begin{array}{l}\log \text { of intrinsic permeability, } 2- \\
\text { direction }\end{array}$ & 34868 & 01-Fob-96 & $\mathbf{Y}$ & 1 \\
\hline $\mathbf{Y}$ & & 593 & S_MB139 & $\begin{array}{l}\text { Salado marker bed } \\
139 \text {, intact and } \\
\text { fractured }\end{array}$ & PRMZ_LOG & $\begin{array}{l}\text { Log of intrinsic permeabilty, z- } \\
\text { direction }\end{array}$ & 34868 & $01-f a b-96$ & $\mathbf{Y}$ & 1 \\
\hline $\mathbf{Y}$ & & 596 & S_MB139 & $\begin{array}{l}\text { Salado marker bed } \\
\text { 139. intact and } \\
\text { fractured }\end{array}$ & RELP_MOD & $\begin{array}{l}\text { Model number, relative } \\
\text { permeability model }\end{array}$ & 34500 & o8-Feb-96 & $\mathbf{Y}$ & 1 \\
\hline $\mathbf{Y}$ & & 596 & S_MB139 & $\begin{array}{l}\text { Salado marker bed } \\
139, \text { intact and } \\
\text { fractured }\end{array}$ & RELP_MOD & $\begin{array}{l}\text { Model number, relative } \\
\text { permeability model }\end{array}$ & 34500 & 08-Fob-96 & ${ }^{*} \mathbf{Y}$ & 1 \\
\hline $\mathbf{Y}$ & & 596 & S_MB139 & $\begin{array}{l}\text { Salado marker bed } \\
\text { 139, intact and } \\
\text { fractured }\end{array}$ & RELP_MOD & $\begin{array}{l}\text { Model number, relative } \\
\text { permeability model }\end{array}$ & 34500 & 08-Fob-96 & $\mathbf{Y}$ & 1 \\
\hline $\mathbf{Y}$ & & 597 & S_MB139 & $\begin{array}{l}\text { Salado marker bed } \\
139 \text {, intact and } \\
\text { fractured }\end{array}$ & SAT_JBRN & Initial Brine Saturation & 34503 & $11-f 0 b-96$ & $\mathbf{Y}$ & 1 \\
\hline $\mathbf{Y}$ & & 597 & S_MB139 & $\begin{array}{l}\text { Salado merker bed } \\
139 \text {, intact and } \\
\text { fractured }\end{array}$ & SAT_IBRN & Initial Brine Saturation & 34503 & 11 Fob-96 & $\mathbf{Y}$ & 1 \\
\hline $\mathbf{Y}$ & & 598 & S_MB139 & $\begin{array}{l}\text { Salado marker bed } \\
139 \text {, intact and } \\
\text { fractured }\end{array}$ & SAT_RBRN & Residual Brine Saturation & 34506 & 21-Nov-96 & $\boldsymbol{Y}$ & 1 \\
\hline $\mathbf{Y}$ & & 598 & S_MB139 & $\begin{array}{l}\text { Salado marker bed } \\
\text { 139, intact and } \\
\text { fractured }\end{array}$ & SAT_RBRN & Residual Brine Saturation & 34506 & 21-Nov-96 & $\mathbf{Y}$ & 1 \\
\hline $\mathbf{Y}$ & & 598 & S_MB139 & $\begin{array}{l}\text { Salado marker bed } \\
139 \text {, intact and } \\
\text { fractured }\end{array}$ & SAT_RBRN & Residual Brino Saturation & 34506 & 21-Nov-96 & $\mathbf{Y}$ & 1 \\
\hline $\mathbf{Y}$ & & 599 & S_MB139 & $\begin{array}{l}\text { Salado marker bed } \\
\text { 139, intact and } \\
\text { fractured }\end{array}$ & SAT_RGAS & Residual Gas Saturation & 34508 & 22Fob-96 & $\mathbf{Y}$ & 1 \\
\hline
\end{tabular}


CCA Parameter Listing

\begin{tabular}{|c|c|c|c|c|c|c|c|c|c|c|}
\hline ID & \begin{tabular}{|c|} 
Clasesfi- \\
cortion of \\
Dath \\
Cattegory \\
(footnoto 2)
\end{tabular} & $\begin{array}{l}\text { Distribution } \\
\text { Type }\end{array}$ & moan & Modien & Mlntmentm & Maximum & Units & Reference ID & $\begin{array}{l}\text { Was the data } \\
\text { doveloped } \\
\text { Under an } \\
\text { NQA-1 } \\
\text { Program? }\end{array}$ & $\begin{array}{c}\text { Which } \\
\text { methods } \\
\text { were used to } \\
\text { qually } \\
\text { oxisting } \\
\text { deta? } \\
\text { (footnote 3) }\end{array}$ \\
\hline 591 & 1 & STUDENT & $-1.8890000 \mathrm{E}+01$ & $-1.8890000 E+01$ & $-21000000 E+01$ & $-1.7100000 E+01$ & $\log \left(m^{\wedge} 2\right)$ & WP036380 & $\mathbf{Y}$ & NA \\
\hline 591 & 1 & STUDENT & $-1.8890000 E+01$ & $-1.8890000 \mathrm{E}+01$ & $-2.1000000 E+01$ & $-1.7100000 E+01$ & $\log \left(m^{\wedge} 2\right)$ & WP039065 & $\mathbf{Y}$ & N/A \\
\hline 591 & 1 & STUDENT & $-1.8890000 E+01$ & $-1.8890000 \mathrm{E}+01$ & $-2.1000000 E+01$ & $-1.7100000 E+01$ & $\log \left(m^{\wedge} 2\right)$ & WP039178 & $\mathbf{Y}$ & N/A \\
\hline 592 & 1 & STUDENT & $-1.8890000 E+01$ & $-1.8890000 E+01$ & $-2.1000000 \mathrm{E}+01$ & $-1.7100000 E+01$ & $\log \left(m^{\wedge} 2\right)$ & WP030605 & Y & $N / A$ \\
\hline 592 & 1 & STUDENT & $-1.8890000 E+01$ & $-1.8890000 E+01$ & $-2.1000000 E+01$ & $-1.7100000 E+01$ & $\log \left(m^{\wedge} 2\right)$ & WP031217 & $\mathbf{Y}$ & N/A \\
\hline 592 & 1 & STUDENT & $-1.8890000 E+01$ & $-1.8890000 E+01$ & $-2.1000000 E+01$ & $-1.7100000 E+01$ & $\log \left(m^{\wedge} 2\right)$ & WPO35597 & $Y$ & NA \\
\hline 592 & 1 & STUDENT & $-1.8890000 E+01$ & $-1.8890000 E+01$ & $-2.1000000 E+01$ & $-1.7100000 E+01$ & $\log \left(m^{\wedge} 2\right)$ & WP036380 & $\mathbf{Y}$ & NA \\
\hline 592 & 1 & STUDENT & $-1.8890000 E+01$ & $-1.8890000 E+01$ & $-2.1000000 E+01$ & $-1.7100000 E+01$ & $\log \left(m^{\wedge} 2\right)$ & WP039065 & $\mathbf{Y}$ & NA \\
\hline 592 & 1 & STUDENT & $-1.8890000 E+01$ & $-1.8890000 E+01$ & $-2.1000000 E+01$ & $-1.7100000 E+01$ & $\log \left(m^{\wedge} 2\right)$ & WP039178 & $\mathbf{Y}$ & N/A \\
\hline 593 & 1 & STUDENT & $-1.8890000 E+01$ & $-1.8890000 E+01$ & $-2.1000000 E+01$ & $-1.7100000 E+01$ & $\log \left(m^{\wedge} 2\right)$ & WP030606 & $\mathbf{Y}$ & NA \\
\hline 593 & 1 & STUDENT & $-1.8890000 E+01$ & $-1.8890000 E+01$ & $-2.1000000 E+01$ & $-1.7100000 E+01$ & $\log \left(m^{\wedge} 2\right)$ & WPO31217 & Y & NA \\
\hline 593 & 1 & STUDENT & $-1.8890000 \mathrm{E}+01$ & $-1.8890000 \mathrm{E}+01$ & $-2.1000000 E+01$ & $-1.7100000 E+01$ & $\log \left(m^{\wedge} 2\right)$ & WP035597 & $Y$ & N/A \\
\hline 593 & 1 & STUDENT & $-1.8890000 E+01$ & $-1.8890000 E+01$ & $-2.1000000 E+01$ & $-1.7100000 E+01$ & $\log \left(m^{\wedge} 2\right)$ & WP036380 & $\mathbf{Y}$ & NA \\
\hline 593 & 1 & STUDENT & $-1.8890000 E+01$ & $-1.8890000 E+01$ & $-2.1000000 E+01$ & $-1.7100000 E+01$ & $\log \left(m^{\wedge} 2\right)$ & WP039065 & $\mathbf{Y}$ & NA \\
\hline 593 & 1 & STUDENT & $-1.8890000 \mathrm{E}+01$ & $-1.8890000 E+01$ & $-2.1000000 E+01$ & $-1.7100000 E+01$ & $\log \left(m^{\wedge} 2\right)$ & WP039178 & $\mathbf{Y}$ & NA \\
\hline 596 & 1,5 & DELTA & $4,0000000 E+\infty 0$ & $4.0000000 E+\infty 0$ & $1.0000000 E+00$ & $4.0000000 E+\infty 0$ & NONE & WP030643 & $\mathbf{Y}$ & N/A \\
\hline 596 & 1,5 & DELTA & $4.0000000 E+\infty 0$ & $4.0000000 E+00$ & $1.0000000 E+00$ & $4.0000000 E+\infty 0$ & NONE & WP035268 & ${ }^{-} \boldsymbol{Y}$ & N/A \\
\hline 596 & 1,5 & DELTA & $4.0000000 E+\infty 0$ & $4.0000000 E+00$ & $1.0000000 \mathrm{E}+00$ & $4.0000000 E+\infty 0$ & NONE & WP035597 & $\mathbf{Y}$ & NA \\
\hline 597 & 1,5 & CONSTANT & $1.0000000 E+\infty 0$ & $1.0000000 E+00$ & $1.0000000 E+00$ & $1.0000000 E+00$ & NONE & WP030643 & $\mathbf{Y}$ & N/A \\
\hline 597 & 1.5 & CONSTANT & $1.0000000 \mathrm{E}+00$ & $1.0000000 \mathrm{E}+\infty 0$ & $1.0000000 E+\infty 0$ & $1.0000000 E+\infty$ & NONE & WP035597 & $\mathbf{Y}$ & N/A \\
\hline 598 & 1,5 & STUDENT & $8.3620000 E-02$ & $8.3520000 E-02$ & $7.7845000 E-03$ & $1.7401000 E-01$ & NONE & WPO30643 & $\mathbf{Y}$ & NA \\
\hline 598 & 1,5 & STUDENT & $8.3620000 E-02$ & B.3620000E-02 & $7.7846000 \mathrm{E}-03$ & $1.7401000 E-01$ & NONE & WP035597 & $\mathbf{Y}$ & N/A \\
\hline 598 & 1,5 & STUDENT & $8.3620000 E-02$ & 8.3620000E-02 & $7.7846000 \mathrm{E}-03$ & $1.7401000 \mathrm{E}-01$ & NONE & WP035380 & Y & NA \\
\hline 599 & 1,5 & STUDENT & $7.7110000 E-02$ & $7.7110000 E-02$ & $1.3980000 E-02$ & 1.9719000E-01 & NONE & WP030643 & $\mathbf{Y}$ & NA \\
\hline
\end{tabular}


CCA Parameter Listing

\begin{tabular}{|c|c|c|c|c|c|c|c|c|c|c|}
\hline $\begin{array}{c}\text { Parameter in } \\
\text { Databaso? } \\
\text { (YNN) }\end{array}$ & \begin{tabular}{l|} 
tf the valuos \\
ured in CCA \\
are not the \\
same as in \\
DB, provide $x$ \\
ofference
\end{tabular} & 10 & Matental ID & Matorlal Name & $\begin{array}{l}\text { Paramoter } \\
\text { to }\end{array}$ & Paramotor Name & $\begin{array}{l}\text { PRPID } \\
\text { (WPON) }\end{array}$ & $\begin{array}{l}\text { Detan Entry } \\
\text { Dete }\end{array}$ & $\begin{array}{l}\text { Is the } 464 \\
\text { consiatent } \\
\text { with the } \\
\text { perameter } \\
\text { in DB? } \\
(\mathrm{N}, \mathrm{NA})\end{array}$ & $\begin{array}{l}\text { Parnmoter } \\
\text { Cutegory } \\
\text { (footnote 1) }\end{array}$ \\
\hline$Y$ & & 599 & S_MB139 & $\begin{array}{l}\text { Salado marker bed } \\
\text { 139, intact and } \\
\text { fractured }\end{array}$ & SAT_RGAS & Residual Gas Saturation & 34508 & $22-500-96$ & $\mathbf{Y}$ & 1 \\
\hline $\mathbf{r}$ & & 599 & S_MB139 & $\begin{array}{l}\text { Salado marker bed } \\
\text { 139, intact and } \\
\text { fractured }\end{array}$ & SAT_RGAS & Residual Gas Saturation & 34508 & 22 Feb-96 & $\mathbf{Y}$ & 1 \\
\hline$Y$ & 0.01 & 603 & TH229 & Thorium 229 & ATWEIGHT & Atomic Weight in $\mathrm{kg} /$ moto & 34594 & O2-Nov-95 & $Y$ & 3 \\
\hline$Y$ & 0.01 & 603 & TH229 & Thorium 229 & ATWEIGHT & Atomic Weight in $\mathrm{kg} / \mathrm{mole}$ & 34594 & 02 -Nov-95 & $\mathbf{Y}$ & 3 \\
\hline$Y$ & 0.01 & 603 & TH1229 & Thorium 229 & ATWEIGHT & Atomic Woight in $\mathrm{kg} / \mathrm{mol}$ & 34594 & 02-Nov-95 & $Y$ & 3 \\
\hline $\mathbf{Y}$ & 100 & 604 & TH229 & Thorium 229 & HALFLIFE & Halfifite & 34595 & 18-Apr-96 & $\mathbf{Y}$ & 3 \\
\hline$Y$ & 100 & 604 & TH229 & Thorium 229 & HALFLIFE & Halfife & 34595 & 18-Apr-96 & $\mathbf{Y}$ & 3 \\
\hline$Y$ & 100 & 604 & TH1229 & Thoriem 229 & HALFLIFE & Halfife & 34595 & 18-Apr-96 & $\mathbf{Y}$ & 3 \\
\hline $\mathbf{Y}$ & & 605 & $T+229$ & Thorium 229 & INVCHD & $\begin{array}{l}\text { Inventory of Contact Handled } \\
\text { Design }\end{array}$ & $34596 \mathrm{~A}$ & $29-0 c t-96$ & $\mathbf{Y}$ & 2 \\
\hline $\mathbf{Y}$ & & 605 & TH229 & Thorium 229 & INVCHD & $\begin{array}{l}\text { Inventory of Contad Handiod } \\
\text { Design }\end{array}$ & $34596 \mathrm{~A}$ & $29-0 \mathrm{ct}-96$ & $\mathbf{Y}$ & 2 \\
\hline $\mathbf{Y}$ & & 605 & TH229 & Thorium 229 & INVCHD & $\begin{array}{l}\text { Inventory of Contact Handied } \\
\text { Design }\end{array}$ & $34596 \mathrm{~A}$ & 29-0ct.96 & $\mathbf{Y}$ & 2 \\
\hline$Y$ & & 605 & TH229 & Thorium 229 & INVCHD & $\begin{array}{l}\text { Inventory of Contact Handled } \\
\text { Design }\end{array}$ & $34596 \mathrm{~A}$ & 29-0ct-96 & $\mathbf{Y}$ & 2 \\
\hline $\mathbf{Y}$ & & 606 & TH229 & Thorium 229 & INVRHD & $\begin{array}{l}\text { Inventory of Remole Handled } \\
\text { Design }\end{array}$ & 34597A & 29-0ct-96 & $\mathbf{Y}$ & 2 \\
\hline$Y$ & & 606 & $T H 1229$ & Thorium 229 & INVRHD & $\begin{array}{l}\text { Inventory of Remote Handled } \\
\text { Design }\end{array}$ & 34597A & $29-0 \mathrm{ct}-96$ & $\mathbf{Y}$ & 2 \\
\hline$Y$ & & 606 & TH1229 & Thorium 229 & IINVRHD & $\begin{array}{l}\text { Inventory of Remole Handled } \\
\text { Design }\end{array}$ & 34597A & $29-0 \mathrm{ct}-96$ & $Y$ & 2 \\
\hline $\mathbf{Y}$ & & 606 & TH1229 & Thorium 229 & |INVRHD & $\begin{array}{l}\text { Inventory of Remote Handled } \\
\text { Design }\end{array}$ & $34597 \mathrm{~A}$ & $29-0$ ct-96 & Y & 2 \\
\hline$Y$ & 0.01 & 607 & TH230 & Thorium 230 & ATWEIGHT & Atornic Weight in kg/mole & 34600 & 02-Nov-95 & $Y$ & 3 \\
\hline$Y$ & 0.01 & 607 & TH230 & Thorium 230 & ATWEIGHT & Atomic Weight in $\mathrm{kg} /$ mole & 34600 & 02-Nov-95 & $Y$ & 3 \\
\hline$Y$ & 0.01 & 607 & $\pi \mathrm{H} 230$ & Thorium 230 & ATWEIGHT & Atomic Woight in $\mathrm{kg} / \mathrm{mol}$ & 34600 & 02-Nov-95 & $Y$ & 3 \\
\hline$Y$ & 100 & 608 & TH230 & Thorium 230 & HALFLIFE & Halfififo & 34601 & 16-Apr-96 & $Y$ & 3 \\
\hline $\mathbf{Y}$ & 100 & 608 & TH1230 & Thorium 230 & HALFLIFE & Habfifo & 34601 & 16-Apr-96 & $Y$ & 3 \\
\hline$Y$ & 100 & 608 & TH1230 & Thorium 230 & HALFLIFE & Halfifo & 34601 & 16-Apr-96 & $\bar{Y}$ & 3 \\
\hline $\mathbf{Y}$ & & 609 & $T H 230$ & Thorum 230 & INVCHD & $\begin{array}{l}\text { Inventory of Contact Handled } \\
\text { Design }\end{array}$ & $34602 \mathrm{~A}$ & 29-0et-96 & $\mathbf{Y}$ & 2 \\
\hline$Y$ & & 609 & TH230 & Thoriem 230 & INVCHD & $\begin{array}{l}\text { Inventory of Contact Handled } \\
\text { Design }\end{array}$ & $34602 \mathrm{~A}$ & 29-0ct-96 & $\mathbf{Y}$ & 2 \\
\hline$Y$ & & 609 & $T+230$ & Therium 230 & INVCHD & $\begin{array}{l}\text { Inventory of Contact Handled } \\
\text { Design }\end{array}$ & $34602 \mathrm{~A}$ & $29-0$ dt-96 & $\mathbf{Y}$ & 2 \\
\hline $\mathbf{Y}$ & & 609 & TH230 & Thorium 230 & INVCHD & $\begin{array}{l}\text { Inventory of Contact Handled } \\
\text { Design }\end{array}$ & $34602 \mathrm{~A}$ & 29-0ect-96 & $Y$ & 2 \\
\hline$Y$ & & 610 & TH230 & Thoriun 230 & INVRHD & $\begin{array}{l}\text { Inventory of Remole Handled } \\
\text { Design }\end{array}$ & $34603 A$ & 29-0ct-96 & $Y$ & 2 \\
\hline $\mathbf{Y}$ & & 610 & $T H 1230$ & Thorium 230 & INVRHD & $\begin{array}{l}\text { Inventory of Remote Handled } \\
\text { Design }\end{array}$ & $34603 \mathrm{~A}$ & 29-0ct-96 & $-\bar{Y}$ & 2 \\
\hline$Y$ & & 610 & TH1230 & Thorium 230 & |INVRHD & $\begin{array}{l}\text { Imventory of Remote Handled } \\
\text { Design }\end{array}$ & $34603 A$ & $29-0 \mathrm{et}-96$ & $Y$ & 2 \\
\hline $\mathbf{Y}$ & & 610 & TH230 & Thorium 230 & INVRHD & $\begin{array}{l}\text { Inventory of Remote Handied } \\
\text { Design }\end{array}$ & $34603 \mathrm{~A}$ & $29-0 \mathrm{ct}-96$ & $\mathbf{Y}$ & 2 \\
\hline$Y$ & 0.02 & 611 & TH232 & Thorium 232 & ATWEIGHT & Atomic Weight in $\mathrm{kg} / \mathrm{mote}$ & 32458 & 02-Nov-95 & $Y$ & 3 \\
\hline$Y$ & 0.02 & 611 & TH232 & Thorium 232 & ATWEIGHT & Atomic Weight in $\mathrm{kg} / \mathrm{mole}$ & 32458 & 02-Nov-95 & $y$ & 3 \\
\hline$Y$ & 0.02 & 611 & TH1232 & Thorium 232 & ATWEIGHT & Atomic Weight in $\mathrm{kg} / \mathrm{mote}$ & 32458 & 02-Nov-95 & $Y$ & 3 \\
\hline$Y$ & 100 & 612 & $T+232$ & Thorium 232 & HALFLIFE & Hamife & 34605 & 16-Apr-96 & $Y$ & 3 \\
\hline$Y$ & 100 & 612 & TH232 & Thorium 232 & HALFLIFE & Halfife & 34605 & 16-Apr-96 & $Y$ & 3 \\
\hline$Y$ & 100 & 612 & TH232 & Thorium 232 & HALFLIFE & Halffifo & 34605 & 16-Apr-96 & $\bar{Y}$ & 3 \\
\hline $\mathbf{Y}$ & & 613 & TH1232 & Thorium 232 & INVCHD & $\begin{array}{l}\text { Inventory of Contact Handled } \\
\text { Design }\end{array}$ & $34606 \mathrm{~A}$ & 29-0ct-96 & $\mathbf{Y}$ & 2 \\
\hline$Y$ & & 613 & TH232 & Thorium 232 & INVCHD & $\begin{array}{l}\text { Inventory of Contact Handlod } \\
\text { Design }\end{array}$ & $34606 \mathrm{~A}$ & 29-0ct-96 & $Y$ & 2 \\
\hline $\mathbf{Y}$ & & 613 & TH232 & Thorium 232 & INVCHD & $\begin{array}{l}\text { Imventory of Contact Handled } \\
\text { Design }\end{array}$ & $34606 \mathrm{~A}$ & $29-0 \mathrm{ct}-96$ & $\mathbf{Y}$ & 2 \\
\hline $\mathbf{Y}$ & & 613 & TH232 & Thorium 232 & INVCHD & $\begin{array}{l}\text { Inventory of Contact Handled } \\
\text { Design }\end{array}$ & $34606 \mathrm{~A}$ & 29-0d-96 & $\mathbf{Y}$ & 2 \\
\hline$Y$ & & 614 & TH232 & Thorium 232 & IINVRHD & $\begin{array}{l}\text { Inventory of Remote Handled } \\
\text { Design }\end{array}$ & $34607 A$ & $29-0$ ct-96 & $\mathbf{Y}$ & 2 \\
\hline
\end{tabular}


CCA Parameter Listing

\begin{tabular}{|c|c|c|c|c|c|c|c|c|c|c|}
\hline ID & \begin{tabular}{|c|} 
Classifi- \\
cation of \\
Data \\
Category \\
(footnote 2)
\end{tabular} & $\begin{array}{c}\text { Distribution } \\
\text { Type }\end{array}$ & Mean & Medien & Minimum & Maximum & Units & Roference ID & $\begin{array}{l}\text { Was the dita } \\
\text { doviloped } \\
\text { under an } \\
\text { NaA-t } \\
\text { - Program? }\end{array}$ & $\begin{array}{c}\text { Which } \\
\text { methods } \\
\text { werv used to } \\
\text { qually } \\
\text { exdsting } \\
\text { data? } \\
\text { (footnoto 3) }\end{array}$ \\
\hline 599 & 1,5 & STUDENT & 7.7110000E-02 & $7.7110000 E-02$ & $1.3980000 E-02$ & $1.9719000 E-01$ & NONE & WPO35597 & $\mathbf{Y}$ & N/A \\
\hline 599 & 1,5 & STUDENT & $7.7110000 \mathrm{E}-02$ & $7.7110000 E-02$ & $1.3980000 E-02$ & $1.9719000 E-01$ & NONE & WP035380 & $\mathbf{Y}$ & NA \\
\hline 603 & 4 & CONSTANT & 22903200E-01 & $2.2903200 E-01$ & 22903200E-01 & 22903200E-01 & $\mathrm{kg} / \mathrm{mole}$ & CRC-72 & $\mathbf{Y}$ & NA \\
\hline 603 & 4 & CONSTANT & $2.2903200 \mathrm{E}-01$ & 2.2903200E-01 & 2.2903200E-01 & 2.2903200E-01 & $\mathrm{kg} / \mathrm{mole}$ & WP036298 & $Y$ & NA \\
\hline 603 & 4 & CONSTANT & 2.2903200E-01 & $2.2903200 E-01$ & 2.2903200E-01 & $2.2903200 E-01$ & $\mathrm{~kg}$ mole & WP036301 & $Y$ & N/A \\
\hline 604 & 4 & CONSTANT & $2.3160000 E+11$ & $2.3160000 E+11$ & $2.3160000 E+11$ & $2.3160000 E+11$ & 5 & WP036301 & $Y$ & N/A \\
\hline 604 & 4 & CONSTANT & $2.3160000 E+11$ & $2.3160000 E+11$ & $23160000 E+11$ & $2.3160000 E+11$ & $\mathbf{s}$ & WP037404 & $\mathbf{Y}$ & $N / A$ \\
\hline 604 & 4 & CONSTANT & $2.3160000 E+11$ & $23160000 E+11$ & $2.3160000 E+11$ & $2.3160000 E+11$ & s & WPO40434 & $Y$ & N/A \\
\hline 605 & 2 & CONSTANT & $9.2900000 E+00$ & $9.2900000 E+\infty 0$ & $9.2900000 E+00$ & $9.2900000 \mathrm{E}+\infty 0$ & Ci & WP037061 & $Y$ & N/A \\
\hline 605 & 2 & CONSTANT & $9.2900000 \mathrm{E}+\infty 0$ & $9.2900000 E+00$ & $9.2900000 \mathrm{E}+00$ & $9.2900000 E+00$ & $\mathbf{C i}$ & WPO39260 & $Y$ & NA \\
\hline 605 & 2 & CONSTANT & $9.2900000 E+00$ & $9.2900000 \mathrm{E}+00$ & $9.2900000 \mathrm{E}+00$ & $9.2900000 E+00$ & Ci & WP041559 & $\mathbf{Y}$ & N/A \\
\hline 605 & 2 & CONSTANT & $9.2900000 \mathrm{E}+00$ & $9.2900000 E+00$ & $9.2900000 E+00$ & $9.2900000 \mathrm{E}+\infty 0$ & $\mathbf{C i}$ & WPO41560 & $\mathbf{Y}$ & NA \\
\hline 606 & 2 & CONSTANT & $6.8300000 E-01$ & $6.8300000 E-01$ & $6.8300000 E-01$ & $6.8300000 E-01$ & $\mathrm{Ci}$ & WP037061 & $Y$ & $N A$ \\
\hline 606 & 2 & CONSTANT & $6.8300000 E-01$ & $6.8300000 E-01$ & $6.8300000 E-01$ & $6.8300000 E-01$ & $\mathrm{Ci}$ & WP039260 & $Y$ & N/A \\
\hline 605 & 2 & CONSTANT & $6.8300000 E-01$ & $6.8300000 E-01$ & $6.8300000 E-01$ & $6.8300000 E-01$ & $\mathbf{C i}$ & WP041559 & $\mathbf{Y}$ & N/A \\
\hline 606 & 2 & CONSTANT & $6.8300000 E-01$ & $6.8300000 E-01$ & $6.8300000 E-01$ & $6.8300000 E-01$ & $\mathrm{Ci}$ & WP041560 & $\mathbf{Y}$ & N/A \\
\hline 607 & 4 & CONSTANT & $2.3003300 E-01$ & $2.3003300 E-01$ & $23003300 E-01$ & $2,3003300 E-01$ & $\mathrm{~kg} / \mathrm{mole}$ & CRC-72 & $Y$ & NAA \\
\hline 607 & 4 & CONSTANT & $2.3003300 E-01$ & $2.3003300 E-01$ & $2.3003300 E-01$ & $23003300 E-01$ & $\mathrm{~kg} / \mathrm{mole}$ & WP036298 & $\mathbf{Y}$ & N/A \\
\hline 607 & 4 & CONSTANT & $2.3003300 E-01$ & $2.3003300 E-01$ & 23003300E-01 & 2.3003300E-01 & kg/mole & WP035301 & $\mathbf{Y}$ & N/A \\
\hline 608 & 4 & CONSTANT & $2.4300000 E+12$ & $2.4300000 E+12$ & $2.4300000 E+12$ & $2.4300000 E+12$ & s & WP036301 & $Y$ & N/A \\
\hline 608 & 4 & CONSTANT & $2.4300000 E+12$ & $2.4300000 E+12$ & $2.4300000 E+12$ & $2.4300000 \mathrm{E}+12$ & $\mathbf{s}$ & WPO37404 & $Y$ & $N / A$ \\
\hline 608 & 4 & CONSTANT & $2.4300000 \mathrm{E}+12$ & $2.4300000 \mathrm{E}+12$ & $2.4300000 E+12$ & $24300000 E+12$ & s & WPO40434 & $Y$ & N/A \\
\hline 609 & 2 & CONSTANT & 2.8300000E-01 & $2.8300000 E-01$ & $2.8300000 E-01$ & $28300000 E-01$ & $\mathbf{C i}$ & WP037061 & $\mathbf{Y}$ & N/A \\
\hline 609 & 2 & CONSTANT & $2.8300000 E-01$ & $2.8300000 E-01$ & $2.8300000 E-01$ & $2.8300000 E-01$ & $\mathrm{Ci}$ & WP039260 & $\mathbf{Y}$ & N/A \\
\hline 609 & 2 & CONSTANT & $2.8300000 E-01$ & $2.8300000 \mathrm{E}-01$ & $2.8300000 E-01$ & $2.8300000 E-01$ & $\mathrm{Ci}$ & WPO41559 & $\mathbf{Y}$ & $N / A$ \\
\hline 609 & 2 & CONSTANT & $2.8300000 \mathrm{E}-01$ & 2.8300000E-01 & 2.8300000E-01 & $2.8300000 E-01$ & $\mathrm{Ci}$ & WP041550 & $\mathbf{Y}$ & N/A \\
\hline 610 & 2 & CONSTANT & $2.2200000 E-02$ & $2.2200000 E-02$ & $2.2200000 E-02$ & $2.2200000 \mathrm{E}-02$ & $\mathbf{C i}$ & WP037061 & $Y$ & $N / A$ \\
\hline 610 & 2 & CONSTANT & $2.2200000 E-02$ & $2.2200000 \mathrm{E}-02$ & $2.2200000 E-02$ & $2.2200000 \mathrm{E}-02$ & $\mathbf{C i}$ & WPO39260 & ${ }^{-} \mathbf{Y}$ & NA \\
\hline 610 & 2 & CONSTANT & $2.2200000 E-02$ & $2.2200000 E-02$ & $2.2200000 \mathrm{E}-02$ & $2.2200000 \mathrm{E}-02$ & $\mathrm{Ci}$ & WP041559 & $\mathbf{Y}$ & N/A \\
\hline 610 & 2 & CONSTANT & $2.2200000 E-02$ & $2.2200000 E-02$ & $2.2200000 \mathrm{E}-02$ & $2.2200000 E-02$ & $\mathrm{Ci}$ & WP041560 & $\mathbf{Y}$ & NA \\
\hline 611 & 4 & CONSTANT & $2.3203800 E-01$ & $2.3203800 E-01$ & $2.3203800 E-01$ & $2.3203800 E-01$ & $\mathrm{~kg} / \mathrm{mole}$ & CRC-72 & $Y$ & NAA \\
\hline 611 & 4 & CONSTANT & $2.3203800 E-01$ & $2.3203800 E-01$ & $2.3203800 E-01$ & $2.3203800 E-01$ & $\mathrm{~kg} / \mathrm{mole}$ & WP036298 & $Y$ & N/A \\
\hline 611 & 4 & CONSTANT & $2.3203800 E-01$ & $2.3203800 \mathrm{E}-01$ & $2.3203800 \mathrm{E}-01$ & $2.3203800 \mathrm{E}-01$ & $\mathrm{~kg} / \mathrm{mole}$ & WPO35301 & $Y$ & N/A \\
\hline 612 & 4 & CONSTANT & $44340000 E+17$ & $4.4340000 \mathrm{E}+17$ & $4.4340000 E+17$ & $4.4340000 \mathrm{E}+17$ & 8 & WP036301 & $Y$ & N/A \\
\hline 612 & 4 & CONSTANT & $4.4340000 \mathrm{E}+17$ & $4.4340000 E+17$ & $4.4340000 E+17$ & $4.4340000 E+17$ & $\mathbf{s}$ & WPO37404 & $Y$ & N/A \\
\hline 612 & 4 & CONSTANT & $4.4340000 \mathrm{E}+17$ & $4.4340000 \mathrm{E}+17$ & $4.4340000 \mathrm{E}+17$ & $4.4340000 \mathrm{E}+17$ & $s$ & WPO40434 & $Y$ & NA \\
\hline 613 & 2 & CONSTANT & $9.1400000 E-01$ & $9.1400000 E-01$ & $9.1400000 E-01$ & $9.1400000 E-01$ & Ci & WP037061 & $\mathbf{Y}$ & N/A \\
\hline 613 & 2 & CONSTANT & $9.1400000 E-01$ & $9.1400000 E-01$ & $9.1400000 E-01$ & $9.1400000 \mathrm{E}-01$ & $\mathbf{C i}$ & WP039260 & Y & NA \\
\hline 613 & 2 & CONSTANT & $9.1400000 E-01$ & $9.1400000 E-01$ & $9.1400000 E-01$ & $9.1400000 E-01$ & $\mathrm{Ci}$ & WP041559 & $\mathbf{Y}$ & NA \\
\hline 613 & 2 & CONSTANT & $9.1400000 E-01$ & $9.1400000 E-01$ & $9.1400000 E-01$ & $9.1400000 \mathrm{E}-01$ & $\mathbf{C i}$ & WPO41560 & $Y$ & NA \\
\hline 614 & 2 & CONSTANT & $9.2600000 E-02$ & $9.2600000 E-02$ & $9.2600000 \mathrm{E}-02$ & $9.2600000 \mathrm{E}-02$ & $\mathbf{C i}$ & WP037061 & $\mathbf{Y}$ & N/A \\
\hline
\end{tabular}


CCA Parameter Listing

\begin{tabular}{|c|c|c|c|c|c|c|c|c|c|c|}
\hline $\begin{array}{c}\text { Parametar to } \\
\text { Databese? } \\
\text { (YN) }\end{array}$ & 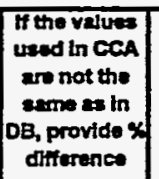 & ID & Material Io & Material Name & $\begin{array}{l}\text { Parwmeter } \\
\text { tD }\end{array}$ & Parametar Name & $\begin{array}{l}\text { PRP ID } \\
\text { (MPO *) }\end{array}$ & $\begin{array}{l}\text { Data Entry } \\
\text { Dato }\end{array}$ & 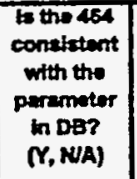 & $\begin{array}{l}\text { Parameter } \\
\text { Category } \\
\text { (footnote 1) }\end{array}$ \\
\hline $\mathbf{Y}$ & & 614 & TH1232 & Thorium 232 & INVRHD & $\begin{array}{l}\text { imventory of Remote Handled } \\
\text { Design }\end{array}$ & $34607 \mathrm{~A}$ & $29-0 c t-96$ & $\mathbf{Y}$ & 2 \\
\hline Y & & 614 & TH232 & Thorium 232 & |INVRHD & $\begin{array}{l}\text { Inventory of Remole Handied } \\
\text { Design }\end{array}$ & 34507A & $29-0 \mathrm{ct}-96$ & Y & 2 \\
\hline$Y$ & & 614 & $T H 1232$ & Thorium 232 & INVRHD & $\begin{array}{l}\text { Inventory of Remote Handied } \\
\text { Design }\end{array}$ & $34607 A$ & 29-0ct-96 & Y & 2 \\
\hline $\bar{Y}$ & 0.02 & 632 & $\mathbf{U} 233$ & Uranium 233 & ATWEIGHT & Atomic Weight in $\mathrm{kg} / \mathrm{mole}$ & 32459 & 02-Nov-95 & $Y$ & 3 \\
\hline $\bar{Y}$ & 0.02 & 632 & $\mathrm{U} 233$ & Uranium 233 & ATWEIGHT & Atomic Weight in kg/mole & 32459 & 02-Nov-95 & $Y$ & 3 \\
\hline $\bar{Y}$ & 0.02 & 632 & $\mathrm{U} 233$ & Uranium 233 & ATWEIGHT & Atomic Weight in kg/mole & 32459 & 02-Nov-95 & $Y$ & 3 \\
\hline $\bar{Y}$ & 100 & 633 & U233 & Utanium 233 & HALFLIFE & Hallife & 34662 & 16-Apr-96 & $Y$ & 3 \\
\hline$Y$ & 100 & 633 & $\mathrm{U} 233$ & Uranium 233 & HALFLIFE & Halfife & 34662 & 16-Apr-96 & $y$ & 3 \\
\hline$Y$ & 100 & 633 & U233 & Uranium 233 & HALFLIFE & Habfifie & 34662 & 16-Apr-96 & $\bar{Y}$ & 3 \\
\hline $\mathbf{Y}$ & & 634 & U233 & Uranium 233 & INVCHD & $\begin{array}{l}\text { inventory of Contact Handled } \\
\text { Design }\end{array}$ & $34663 A$ & 29-0ct-95 & $\mathbf{Y}$ & 2 \\
\hline $\mathbf{r}$ & & 634 & U233 & Uranium 233 & INVCHD & $\begin{array}{l}\text { Inventory of Contact Handied } \\
\text { Design }\end{array}$ & $34653 \mathrm{~A}$ & $29-0 d-96$ & $\mathbf{Y}$ & 2 \\
\hline $\mathbf{Y}$ & & 634 & U233 & Uranium 233 & INVCHD & $\begin{array}{l}\text { Inventory of Contact Handled } \\
\text { Design }\end{array}$ & 346634 & 29-0ct-96 & $\mathbf{Y}$ & 2 \\
\hline $\mathbf{Y}$ & & 634 & U233 & Uranium 233 & INVCHD & $\begin{array}{l}\text { Inventory of Contact Handled } \\
\text { Design }\end{array}$ & 346534 & $29-0$ ct.96 & $\mathbf{Y}$ & 2 \\
\hline $\mathbf{Y}$ & & 635 & U233 & Uranium 233 & INMRHD & $\begin{array}{l}\text { Inventory of Remote Handled } \\
\text { Design }\end{array}$ & $34664 A$ & $29-0 c t-96$ & $\mathbf{Y}$ & 2 \\
\hline $\mathbf{Y}$ & & 635 & U233 & Uranium 233 & INVRHD & $\begin{array}{l}\text { Inventory of Remote Handied } \\
\text { Design }\end{array}$ & $34664 \mathrm{~A}$ & $29-0 c t-96$ & $Y$ & 2 \\
\hline $\mathbf{Y}$ & & 635 & U233 & Uranium 233 & INVRHD & $\begin{array}{l}\text { Inventory of Remote Handled } \\
\text { Design }\end{array}$ & 34664A & $29-0 \mathrm{ct}-96$ & $\mathbf{Y}$ & 2 \\
\hline Y & & 635 & U233 & Uranium 233 & INVRHD & $\begin{array}{l}\text { Inventory of Remote Handied } \\
\text { Design }\end{array}$ & $34664 \mathrm{~A}$ & 29-0ct-96 & $\mathbf{Y}$ & 2 \\
\hline$Y$ & 0.02 & 636 & $\mathrm{U} 234$ & Uranium 234 & ATWEIGHT & Atomic Waight in kgimole & 32460 & 02-Nov-95 & $Y$ & 3 \\
\hline $\mathbf{Y}$ & 0.02 & 636 & $\mathbf{U 2 3 4}$ & Uranium 234 & ATWEIGHT & Atomic Weight in $\mathrm{kg} / \mathrm{mole}$ & 32460 & 02-Nov-95 & $\mathbf{Y}$ & 3 \\
\hline$Y$ & 0.02 & 635 & $\mathrm{U} 234$ & Uranium 234 & ATWEIGHT & Atomic Woight in $\mathrm{kg} / \mathrm{mole}$ & 32460 & 02-Nov-95 & $\bar{Y}$ & 3 \\
\hline$Y$ & 100 & 637 & $\mathrm{U} 234$ & Uranium 234 & HALFLIFE & Habfife & 34667 & 16-Apr-96 & $\mathbf{Y}$ & 3 \\
\hline$r$ & 100 & 637 & U234 & Uranium 234 & HALFLIFE & Halfife & 34667 & 16-Apr-96 & $Y$ & 3 \\
\hline $\bar{Y}$ & 100 & 637 & U234 & Uranium 234 & HALFLIFE & Haitlife & 34667 & 16-Apr-96 & $\mathbf{Y}$ & 3 \\
\hline $\mathbf{Y}$ & & 638 & U234 & Uranium 234 & INVCHD & \begin{tabular}{|l} 
Inventory of Contad Handled \\
Design \\
\end{tabular} & $34668 \mathrm{~A}$ & $29-0 c t-96$ & $\mathbf{Y}$ & 2 \\
\hline $\mathbf{Y}$ & & 638 & U234 & Uranium 234 & INVCHD & $\begin{array}{l}\text { Inventory of Contact Handiad } \\
\text { Design }\end{array}$ & $34668 \mathrm{~A}$ & $29-0 c t-96$ & $Y$ & 2 \\
\hline $\mathbf{Y}$ & & 638 & U234 & Uranium 234 & INVCHD & $\begin{array}{l}\text { Inventory of Contact Handled } \\
\text { Design }\end{array}$ & $34668 A$ & 29-0ct-96 & $\mathbf{Y}$ & 2 \\
\hline $\mathbf{Y}$ & & 638 & U234 & Uranium 234 & INVCHD & $\begin{array}{l}\text { Inventory of Contact Handled } \\
\text { Design }\end{array}$ & $34668 \mathrm{~A}$ & $29-0 c t-96$ & $\mathbf{Y}$ & 2 \\
\hline $\mathbf{Y}$ & & 639 & U234 & Uranium 234 & INVRHD & $\begin{array}{l}\text { Inventory of Remote Handted } \\
\text { Design }\end{array}$ & $34669 \mathrm{~A}$ & $29-0 c t-96$ & $\mathbf{Y}$ & 2 \\
\hline $\mathbf{Y}$ & & 639 & U234 & Uranium 234 & INVRHD & $\begin{array}{l}\text { Inventory of Remote Handled } \\
\text { Design }\end{array}$ & $34669 \mathrm{~A}$ & $29-0 c t-96$ & $Y$ & 2 \\
\hline $\mathbf{Y}$ & & 639 & U234 & Uranium 234 & INVRHD & $\begin{array}{l}\text { Inventory of Remote Handled } \\
\text { Design }\end{array}$ & $34669 \mathrm{~A}$ & $29-0$ ct-96 & $\mathbf{Y}$ & 2 \\
\hline $\mathbf{Y}$ & & 639 & U234 & Uranium 234 & INVRHD & $\begin{array}{l}\text { Imventory of Remote Handled } \\
\text { Design }\end{array}$ & $34669 \mathrm{~A}$ & $29-0 c t-96$ & $Y$ & 2 \\
\hline$Y$ & 0.02 & 640 & $\mathbf{U} 235$ & Uranium 235 & ATWEIGHT & Alomic Weight in kg/mole & 32461 & 02-Nov.95 & $Y$ & 3 \\
\hline$Y$ & 0.02 & 640 & $\mathrm{U} 235$ & Uranium 235 & ATWEIGHT & Alomic Weight in kg/mole & 32461 & 02-Nov-95 & $Y$ & 3 \\
\hline$Y$ & 0.02 & 640 & U235 & Uranium 235 & ATWEIGHT & Atomic Weight in $\mathrm{kg} / \mathrm{mole}$ & 32461 & 02-Nov-95 & $\bar{Y}$ & 3 \\
\hline$Y$ & 100 & 641 & $\mathbf{L 2 3 5}$ & Uranium 235 & HALFLIFE & Hallife & 34671 & 16-Apr-96 & $\underline{Y}$ & 3 \\
\hline$Y$ & 100 & 641 & $\mathrm{U} 235$ & Uranium 235 & HALFLIFE & Hainfe & 34671 & 16-Apr-96 & $Y$ & 3 \\
\hline $\bar{Y}$ & 100 & 641 & U235 & Uranium 235 & HALFLIFE & Hallife & 34671 & $16-A p r-96$ & $Y$ & 3 \\
\hline$Y$ & & 642 & U235 & Uranium 235 & INVCHD & $\begin{array}{l}\text { Inventory of Contact Handied } \\
\text { Design }\end{array}$ & $34672 A$ & $29-0 \mathrm{ct}-96$ & $Y$ & 2 \\
\hline$\gamma$ & & 642 & U235 & Uranium 235 & INVCHD & $\begin{array}{l}\text { Inventory of Contact Handled } \\
\text { Design }\end{array}$ & $34672 A$ & $29-0 c t-96$ & $\mathbf{Y}$ & 2 \\
\hline$Y$ & & 642 & U235 & Uranium 235 & INVCHD & $\begin{array}{l}\text { Inventory of Conlact Handied } \\
\text { Design }\end{array}$ & $34672 A$ & 290 ct-96 & $\mathbf{Y}$ & 2 \\
\hline $\mathbf{Y}$ & & 642 & U235 & Uranium 235 & INVCHD & $\begin{array}{l}\text { Imventory of Contact Handled } \\
\text { Design }\end{array}$ & $34672 A$ & $29-0 c t-96$ & $\mathbf{Y}$ & 2 \\
\hline$Y$ & & 643 & U235 & Uranium 235 & INVRHD & $\begin{array}{l}\text { Inventory of Remote Handled } \\
\text { Design }\end{array}$ & $34674 A$ & $29-0 c t-96$ & $\mathbf{Y}$ & 2 \\
\hline
\end{tabular}


CCA Parameter Listing

\begin{tabular}{|c|c|c|c|c|c|c|c|c|c|c|}
\hline ID & $\begin{array}{l}\text { Claseffi- } \\
\text { eation of } \\
\text { Data } \\
\text { Category } \\
\text { (footnote 2) }\end{array}$ & $\begin{array}{c}\text { Ditulibution } \\
\text { Type }\end{array}$ & Man & Medien & $\begin{array}{l}\text { Minimum } \\
\text { \& }\end{array}$ & Maximum & Unit: & Refierence ID & $\begin{array}{l}\text { Was the data } \\
\text { developed } \\
\text { under an } \\
\text { NeA-1 } \\
\text { Program? }\end{array}$ & $\begin{array}{c}\text { Which } \\
\text { mothods } \\
\text { were used to } \\
\text { qually } \\
\text { existing } \\
\text { datin? } \\
\text { (footnote 3) }\end{array}$ \\
\hline 614 & 2 & CONSTANT & $9.2600000 \mathrm{E}-02$ & $9.2600000 E-02$ & $9.2600000 E-02$ & $9.2600000 \mathrm{E}-02$ & $\mathrm{Ci}$ & WP039260 & $\mathbf{Y}$ & NA \\
\hline 614 & 2 & CONSTANT & $9.2600000 E-02$ & $9.2600000 \mathrm{E}-02$ & $9.2600000 \mathrm{E}-02$ & $9.2600000 E-02$ & $\mathrm{Ci}$ & WPO41559 & $\mathbf{Y}$ & $N / A$ \\
\hline 614 & 2 & CONSTANT & $9.2600000 \mathrm{E}-02$ & $9.2600000 \mathrm{E}-02$ & $9.2600000 E-02$ & $9.2600000 \mathrm{E}-02$ & $\mathrm{Ci}$ & WP041560 & $\mathbf{Y}$ & N/A \\
\hline 632 & 4 & CONSTANT & $2.3304000 E-01$ & 2.3304000E-01 & $2.3304000 E-01$ & $2.3304000 E-01$ & $\mathrm{~kg} / \mathrm{mole}$ & CRC-72 & $\mathbf{Y}$ & $N / A$ \\
\hline 632 & 4 & CONSTANT & $2.3304000 E-01$ & 2.3304000E-01 & $2.3304000 E-01$ & $2.3304000 E-01$ & kgimole & WP035298 & $Y$ & $N / A$ \\
\hline 632 & 4 & CONSTANT & $2.3304000 E-01$ & $2.3304000 E-01$ & $2.3304000 E-01$ & $2.3304000 E-01$ & $\mathrm{~kg} / \mathrm{mole}$ & WP035301 & $\mathbf{Y}$ & N/A \\
\hline 633 & 4 & CONSTANT & $5.0020000 E+12$ & $5.0020000 E+12$ & $5.0020000 E+12$ & $5.0020000 E+12$ & s & WP036301 & $\mathbf{Y}$ & $N A$ \\
\hline 633 & 4 & CONSTANT & $5.0020000 \mathrm{E}+12$ & $5.0020000 E+12$ & $5.0020000 E+12$ & $5.0020000 \mathrm{E}+12$ & s & WP037404 & $Y$ & $N / A$ \\
\hline 633 & 4 & CONSTANT & $5.0020000 E+12$ & $5.0020000 E+12$ & $5.0020000 \mathrm{E}+12$ & $5.0020000 \mathrm{E}+12$ & $s$ & WPO40434 & $Y$ & $N A$ \\
\hline 634 & 2 & CONSTANT & $1.7900000 E+03$ & $1.7900000 E+03$ & $1.7900000 E+03$ & $1.7900000 \mathrm{E}+00$ & $\mathbf{C i}$ & WP037061 & $Y$ & N/A \\
\hline 634 & 2 & CONSTANT & $1.7900000 E+03$ & $1.7900000 \mathrm{E}+03$ & $1.7900000 E+03$ & $1.7900000 \mathrm{E}+03$ & $\mathrm{Ci}$ & WP039260 & $Y$ & NA \\
\hline 634 & 2 & CONSTANT & $1.7900000 E+03$ & $1.7900000 \mathrm{E}+03$ & $1.7900000 E+03$ & $1.7900000 \mathrm{E}+03$ & $\mathbf{C i}$ & WP041559 & $\mathbf{Y}$ & NA \\
\hline 634 & 2 & CONSTANT & $1.7900000 E+03$ & $1.7900000 E+03$ & $1.7900000 E+03$ & $1.7900000 \mathrm{E}+03$ & $\mathrm{Ci}$ & WP041560 & $Y$ & $N / A$ \\
\hline 635 & 2 & CONSTANT & $1.5800000 E+02$ & $1.5800000 E+02$ & $1.5800000 E+02$ & $1.5800000 \mathrm{E}+02$ & $\mathrm{Ci}$ & WP037061 & $\mathbf{Y}$ & N/A \\
\hline 635 & 2 & CONSTANT & $1.5800000 E+02$ & $1.5800000 E+02$ & $1.5800000 E+02$ & $1.5800000 E+02$ & $\mathrm{Ci}$ & WP039260 & $Y$ & NA \\
\hline 635 & 2 & CONSTANT & $1.5800000 \mathrm{E}+02$ & $1.5800000 E+02$ & $1.5800000 \mathrm{E}+02$ & $1.5800000 E+02$ & $\mathrm{Ci}$ & WPO41559 & $Y$ & N/A \\
\hline 635 & 2 & CONSTANT & $1.5800000 \mathrm{E}+02$ & $1.5800000 E+02$ & $1.5800000 E+02$ & $1.5800000 \mathrm{E}+02$ & $\mathrm{Ci}$ & WP041560 & $Y$ & NAA \\
\hline 636 & 4 & CONSTANT & 2.3404100E-01 & 2.3404100E-01 & 2.3404100E-01 & 2.3404100E-01 & $\mathrm{kg} / \mathrm{mole}$ & CRC-72 & $Y$ & $N / A$ \\
\hline 636 & 4 & CONSTANT & 2.3404100E-01 & 2.3404100E-01 & 2.3404100E-01 & $23404100 \mathrm{E}-01$ & $\mathrm{~kg} / \mathrm{mole}$ & WPO36298 & $Y$ & N/A \\
\hline 636 & 4 & CONSTANT & $2.3404100 E-01$ & 2.3404100E-01 & 2.3404100E-01 & $2.3404100 E-01$ & $\mathrm{~kg} / \mathrm{mole}$ & WP035301 & $\bar{Y}$ & N/A \\
\hline 637 & 4 & CONSTANT & $7.7160000 \mathrm{E}+12$ & $7.7160000 \mathrm{E}+12$ & $7.7160000 \mathrm{E}+12$ & $7.7160000 E+12$ & $\mathbf{s}$ & WP036301 & $\mathbf{Y}$ & N/A \\
\hline 637 & 4 & CONSTANT & $7.7160000 E+12$ & $7.7160000 E+12$ & $7.7160000 \mathrm{E}+12$ & $7.7160000 \mathrm{E}+12$ & $\mathbf{s}$ & WP037404 & $Y$ & N/A \\
\hline 637 & 4 & CONSTANT & $7.7160000 \mathrm{E}+12$ & $7.7160000 \mathrm{E}+12$ & $7.7160000 \mathrm{E}+12$ & $7.7160000 \mathrm{E}+12$ & s & WP040434 & $Y$ & N/A \\
\hline 638 & 2 & CONSTANT & $7.0800000 E+02$ & $7.0800000 E+02$ & $7.0800000 \mathrm{E}+02$ & $7.0800000 E+02$ & $\mathrm{Ci}$ & WP037061 & $\mathbf{Y}$ & NA \\
\hline 638 & 2 & CONSTANT & $7.0800000 E+02$ & $7.0800000 \mathrm{E}+02$ & $7.0800000 E+02$ & $7.0800000 E+02$ & $\mathrm{Ci}$ & WP039260 & $Y$ & N/A \\
\hline 638 & 2 & CONSTANT & $7.0800000 E+02$ & $7.0800000 \mathrm{E}+02$ & $7.0800000 E+02$ & $7.0800000 \mathrm{E}+02$ & $\mathrm{Ci}$ & WP041559 & $\mathbf{Y}$ & NA \\
\hline 638 & 2 & CONSTANT & $7.0800000 E+02$ & $7.0800000 E+02$ & $7.0800000 \mathrm{E}+02$ & $7.0800000 E+02$ & $\mathrm{Ci}$ & WP041560 & $Y$ & $N / A$ \\
\hline 639 & 2 & CONSTANT & $4.2900000 E+01$ & $4.2900000 E+01$ & 4.2900000E+01 & $4.2900000 E+01$ & $\mathrm{Ci}$ & WP037061 & $Y$ & N/A \\
\hline 639 & 2 & CONSTANT & $4.2900000 E+01$ & $4.2900000 E+01$ & $4.2900000 E+01$ & $4.2900000 E+01$ & $\mathrm{Ci}$ & WP039260 & $Y$ & NAA \\
\hline 639 & 2 & CONSTANT & $4.2900000 E+01$ & 4.2900000E+01 & $4.2900000 \mathrm{E}+01$ & $4.2900000 \mathrm{E}+01$ & $\mathrm{Ci}$ & WP041559 & $\mathbf{Y}$ & $\mathbf{N} / \mathbf{A}$ \\
\hline 639 & 2 & CONSTANT & $4.2900000 E+01$ & $4.2900000 \mathrm{E}+01$ & $4.2900000 E+01$ & $4.2900000 \mathrm{E}+01$ & $\mathrm{Ci}$ & WP041560 & $Y$ & N/A \\
\hline 640 & 4 & CONSTANT & $2.3504400 E-01$ & $2.3504400 E-01$ & $2.3504400 E-01$ & $2.3504400 E-01$ & $\mathrm{~kg} /$ mole & CRC-72 & $Y$ & NA \\
\hline 640 & 4 & CONSTANT & $2.3504400 E-01$ & $2.3504400 E-01$ & $2.3504400 \mathrm{E}-01$ & $2.3504400 E-01$ & $\mathrm{~kg} /$ mole & WP036298 & $Y$ & N/A \\
\hline 640 & 4 & CONSTANT & $2.3504400 E-01$ & $2.3504400 \mathrm{E}-01$ & $2.3504400 E-01$ & $2.3504400 E-01$ & $\mathrm{~kg} / \mathrm{mole}$ & WPO36301 & $Y$ & N/A \\
\hline 641 & 4 & CONSTANT & $2.2210000 \mathrm{E}+16$ & $2.2210000 \mathrm{E}+16$ & $2.2210000 E+16$ & $2.2210000 E+16$ & s & WPO36301 & $Y$ & N/A \\
\hline 641 & 4 & CONSTANT & $2.2210000 \mathrm{E}+16$ & $2.2210000 \mathrm{E}+16$ & $2.2210000 E+16$ & $2.2210000 E+16$ & $s$ & WP037404 & $Y$ & N/A \\
\hline 641 & 4 & CONSTANT & $2.2210000 E+16$ & $2.2210000 \mathrm{E}+16$ & $2.2210000 \mathrm{E}+16$ & $2.2210000 E+16$ & s & WPO40434 & $Y$ & N/A \\
\hline 642 & 2 & CONSTANT & $1.2800000 E+01$ & $1.2800000 \mathrm{E}+01$ & $1.2800000 E+01$ & 1.2800000E+01 & $\mathbf{C i}$ & WP037061 & $\mathbf{Y}$ & NAA \\
\hline 642 & 2 & CONSTANT & $1.2800000 E+01$ & $1.2800000 E+01$ & $1.2800000 E+01$ & $1.2800000 E+01$ & Ci & WP039250 & $\mathbf{Y}$ & N/A \\
\hline 642 & 2 & CONSTANT & $1.2800000 E+01$ & $1.2800000 \mathrm{E}+01$ & $1.2800000 \mathrm{E}+01$ & $1.2800000 E+01$ & $\mathbf{C i}$ & WP041559 & $\mathbf{Y}$ & NAA \\
\hline 642 & 2 & CONSTANT & $1.2800000 E+01$ & $1.2800000 E+01$ & $1.2800000 E+01$ & $1.2800000 E+01$ & $\mathbf{C i}$ & WP041560 & $\mathbf{Y}$ & N/A \\
\hline 643 & 2 & CONSTANT & $4.6300000 E+00$ & $4.6300000 E+00$ & $4.6300000 E+\infty 0$ & $4.6300000 E+\infty 0$ & Ci & WP037061 & $\mathbf{Y}$ & N/A \\
\hline
\end{tabular}


CCA Parameter Listing

\begin{tabular}{|c|c|c|c|c|c|c|c|c|c|c|}
\hline $\begin{array}{c}\text { Parameter in } \\
\text { Databaeo? } \\
\text { (YnN) }\end{array}$ & 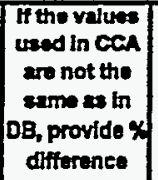 & tD & Maturial ID & Materlal Name & $\begin{array}{l}\text { Parameter } \\
\text { to }\end{array}$ & Parmater Narm & $\begin{array}{l}\text { PRP ID } \\
\text { (WPO })\end{array}$ & $\begin{array}{c}\text { Data Entry } \\
\text { Date }\end{array}$ & $\begin{array}{l}\text { Is the } 464 \\
\text { consistent } \\
\text { with the } \\
\text { parameter } \\
\text { in DB? } \\
(Y, N A)\end{array}$ & $\begin{array}{l}\text { Paramiater } \\
\text { Category } \\
\text { (footnote 1) }\end{array}$ \\
\hline $\mathbf{Y}$ & & 643 & U235 & Uranium 235 & INVRHD & $\begin{array}{l}\text { Inventory of Remote Handled } \\
\text { Design }\end{array}$ & $34674 A$ & $29-0 c t-96$ & $\mathbf{Y}$ & 2 \\
\hline $\mathbf{Y}$ & & 643 & U235 & Uranium 235 & INVRHD & $\begin{array}{l}\text { Imventory of Remote Handied } \\
\text { Design }\end{array}$ & $34674 A$ & $29-0 \mathrm{ct}-56$ & $\mathbf{Y}$ & 2 \\
\hline$Y$ & & 643 & U235 & Uranium 235 & INVRHD & $\begin{array}{l}\text { Inventory of Remote Handled } \\
\text { Design }\end{array}$ & $34674 A$ & $29-0 c t-96$ & $\mathbf{Y}$ & 2 \\
\hline $\mathbf{Y}$ & 0.02 & 644 & U236 & Uranium 236 & ATWEIGHT & Atomic Woight in kghmolo & 32462 & O2-Nov-95 & $\mathbf{Y}$ & 3 \\
\hline $\mathbf{Y}$ & 0.02 & 644 & U236 & Uranium 236 & ATWEIGHT & Atomic Weight in Kolmolo & 32462 & Q2-Nov-95 & $\mathbf{Y}$ & 3 \\
\hline$Y$ & 0.02 & 644 & U236 & Uranium 236 & ATWEIGHT & Atomic Weight in $\mathrm{kg} / \mathrm{mole}$ & 32462 & 02-Nov-95 & $\mathbf{Y}$ & 3 \\
\hline$Y$ & 100 & 645 & U236 & Uranium 236 & HALFLIFE & Hahifie & 34676 & 16 -Apr-96 & $\mathbf{Y}$ & 3 \\
\hline $\mathbf{Y}$ & 100 & 645 & U236 & Uranium 236 & HALFLIFE & Halfife & 34676 & 16 -Apr-96 & $\mathbf{Y}$ & 3 \\
\hline $\mathbf{Y}$ & 100 & 645 & U236 & Uranium 236 & HALFLIFE & Halfife & 34676 & 16 -Apr-96 & $\mathbf{Y}$ & 3 \\
\hline$\gamma$ & & 646 & U236 & Uranium 236 & INVRHD & $\begin{array}{l}\text { Inventory of Remote Handled } \\
\text { Design }\end{array}$ & $34678 \mathrm{~A}$ & $29-0 \mathrm{ct}-96$ & $\mathbf{Y}$ & 2 \\
\hline $\mathbf{Y}$ & & 646 & U236 & Uranium 236 & INVRHD & $\begin{array}{l}\text { Inventory of Remote Handled } \\
\text { Design }\end{array}$ & 34678A & $29-0$ ct-96 & $\mathbf{Y}$ & 2 \\
\hline $\mathbf{Y}$ & & 646 & U236 & Uranium 236 & INVRHD & $\begin{array}{l}\text { Imventory of Remote Handled } \\
\text { Design }\end{array}$ & $34678 A$ & $29-0 \mathrm{ct}-96$ & $\mathbf{Y}$ & 2 \\
\hline $\mathbf{Y}$ & & 646 & U236 & Uranium 236 & INVRHD & $\begin{array}{l}\text { Inventory of Remole Handled } \\
\text { Design }\end{array}$ & 346784 & 29-0ct-96 & $\mathbf{Y}$ & 2 \\
\hline $\mathbf{Y}$ & 0.02 & 647 & U238 & Uranium 238 & ATWEIGHT & Atomic Woight in $\mathrm{kg} / \mathrm{mole}$ & 32463 & 02-Nov-95 & $\mathbf{Y}$ & 3 \\
\hline$Y$ & 0.02 & 647 & U238 & Uranium 238 & ATWEIGHT & Atomic Weight in $\mathrm{kg} / \mathrm{mole}$ & 32463 & 02-Nov-95 & $\mathbf{Y}$ & 3 \\
\hline $\mathbf{Y}$ & 0.02 & 647 & U238 & Uranium 238 & ATWEIGHT & Atomic Weight in $\mathrm{kg} / \mathrm{mole}$ & 32463 & 02-Nov-95 & $\mathbf{Y}$ & 3 \\
\hline$Y$ & 100 & 648 & U238 & Uranium 238 & HALFLIFE & Halfife & 34680 & 16-Apr-96 & $Y$ & 3 \\
\hline $\mathbf{Y}$ & 100 & 648 & U238 & Uranium 238 & HALFLIFE & Halfifie & 34680 & 16-Apr-96 & $\mathbf{Y}$ & 3 \\
\hline $\mathbf{Y}$ & 100 & 648 & U238 & Uranium 238 & HALFLIFE & Hatflifo & 34580 & 16-Apr-96 & $\mathbf{Y}$ & 3 \\
\hline$Y$ & & 649 & U238 & Uranium 238 & INVCHD & $\begin{array}{l}\text { Inventory of Contact Handled } \\
\text { Design }\end{array}$ & $34681 \mathrm{~A}$ & 29-0ct-96 & $\mathbf{Y}$ & 2 \\
\hline$Y$ & & 649 & U238 & Uranium 238 & INVCHD & $\begin{array}{l}\text { Inventory of Contact Handled } \\
\text { Design }\end{array}$ & $34681 A$ & $29-0$ ct.96 & $\mathbf{Y}$ & 2 \\
\hline $\mathbf{Y}$ & & 649 & U238 & Uranium 238 & INVCHD & $\begin{array}{l}\text { Inventory of Contact Handled } \\
\text { Design }\end{array}$ & $34681 \mathrm{~A}$ & 29-0ct-96 & $\mathbf{Y}$ & 2 \\
\hline $\mathbf{Y}$ & & 649 & U238 & Uranitum 238 & INVCHD & $\begin{array}{l}\text { Inventory of Contact Handled } \\
\text { Design }\end{array}$ & $34681 \mathrm{~A}$ & 29-0at-96 & $\mathbf{Y}$ & 2 \\
\hline $\mathbf{Y}$ & & 650 & U238 & Uranium 238 & INVRHD & $\begin{array}{l}\text { Inventory of Remote Handjed } \\
\text { Design }\end{array}$ & $34682 A$ & $29-0 \mathrm{ct}-96$ & $\mathbf{Y}$ & 2 \\
\hline $\mathbf{Y}$ & & 650 & U238 & Uranitum 238 & INVRHD & $\begin{array}{l}\text { Inventory of Remote Handled } \\
\text { Design }\end{array}$ & $34682 A$ & $29-0 \mathrm{et}-96$ & $\mathbf{Y}$ & 2 \\
\hline $\mathbf{Y}$ & & 650 & U238 & Uranium 238 & INVRHD & $\begin{array}{l}\text { Imventory of Remote Handled } \\
\text { Design }\end{array}$ & $34682 A$ & $29-0 c t-96$ & $\mathbf{Y}$. & 2 \\
\hline $\mathbf{Y}$ & & 650 & U238 & Uranium 238 & INVRHD & $\begin{array}{l}\text { Inventory of Remote Handled } \\
\text { Design }\end{array}$ & $34682 A$ & 29-0ct-96 & $\mathbf{Y}$ & 2 \\
\hline $\mathbf{Y}$ & & 651 & WAS_AREA & $\begin{array}{l}\text { Waste emplacement } \\
\text { area and waste }\end{array}$ & ABSROUGH & Absolute roughness of material & 34980 & 14-Nov-95 & $\mathbf{Y}$ & 48 \\
\hline$Y$ & & 651 & WAS_AREA & $\begin{array}{l}\text { Waste emplacement } \\
\text { area and waste }\end{array}$ & ABSROUGH & Absolute roughness of material & 34980 & 14-Nov-95 & $=\mathbf{Y}$ & $4 B$ \\
\hline $\mathbf{Y}$ & & 651 & WAS_AREA & $\begin{array}{l}\text { Waste emplacement } \\
\text { area and waste }\end{array}$ & ABSROUGH & Absolute roughness of material & 34980 & 14-Nov-95 & $\mathbf{Y}$ & 4B \\
\hline $\mathbf{Y}$ & & 651 & WAS_AREA & $\begin{array}{l}\text { Waste emplacement } \\
\text { area and waste }\end{array}$ & ABSROUGH & Absoikte roughness of material & 34980 & 14-Nov-95 & $\mathbf{Y}$ & $4 B$ \\
\hline $\mathbf{Y}$ & & 652 & WAS_AREA & $\begin{array}{l}\text { Waste emplacement } \\
\text { area and waste }\end{array}$ & CAP_MOD & $\begin{array}{l}\text { Model number, capillary pressuro } \\
\text { model }\end{array}$ & 34982 & 08-Feb-96 & $\mathbf{Y}$ & $4 B$ \\
\hline $\mathbf{Y}$ & & 652 & WAS_AREA & $\begin{array}{l}\text { Waste emplacement } \\
\text { area and waste }\end{array}$ & CAP_MOD & $\begin{array}{l}\text { Model number, capillary pressure } \\
\text { model }\end{array}$ & 34982 & Q8-Fob-96 & $\mathbf{Y}$ & $4 B$ \\
\hline$Y$ & & 652 & WAS_AREA & $\begin{array}{l}\text { Waste emplacement } \\
\text { area and waste }\end{array}$ & CAP_MOD & $\begin{array}{l}\text { Model number, cepillary pressure } \\
\text { model }\end{array}$ & 34982 & Q8-Fab-96 & $\mathbf{Y}$ & 48 \\
\hline $\mathbf{Y}$ & & 653 & WAS_AREA & $\begin{array}{l}\text { Wasto emplacement } \\
\text { area and waste }\end{array}$ & COMP_RCK & Bulk Compressibility & 34987 & O8Fob-96 & $\mathbf{Y}$ & $4 B$ \\
\hline $\mathbf{Y}$ & & 653 & WAS_AREA & $\begin{array}{l}\text { Waste emplacement } \\
\text { Erea and waste }\end{array}$ & COMP_RCK & Bulk Compressibility & 34987 & 08-Fob-96 & $\mathbf{Y}$ & 48 \\
\hline $\mathbf{Y}$ & & 653 & WAS_AREA & $\begin{array}{l}\text { Waste emplacernent } \\
\text { area and waste }\end{array}$ & COMP_RCK & Bulk Compressibility & 34987 & 08-Fob-96 & $\mathbf{Y}$ & 48 \\
\hline $\mathbf{Y}$ & & 656 & WAS_AREA & $\begin{array}{l}\text { Waste emplacoment } \\
\text { area and waste }\end{array}$ & GRATMICH & $\begin{array}{l}\text { Gas Production Rate, Microbial, } \\
\text { Humid Conditions Relative to } \\
\text { Inundated Rate }\end{array}$ & 34923 & 08 Feb-96 & $\mathbf{Y}$ & 1 \\
\hline$Y$ & & 656 & WAS_AREA & $\begin{array}{l}\text { Waste emplacement } \\
\text { area and waste }\end{array}$ & GRATMICH & $\begin{array}{l}\text { Ges Production Rate, Microbial, } \\
\text { Humid Conditions Relative to } \\
\text { Inundated Rate }\end{array}$ & 34923 & 08Feb-s6 & $\mathbf{Y}$ & 1 \\
\hline
\end{tabular}


CCA Parameter Listing

\begin{tabular}{|c|c|c|c|c|c|c|c|c|c|c|}
\hline ID & \begin{tabular}{|c|} 
Claedfi- \\
cation of \\
Date \\
Category \\
(footnote 2)
\end{tabular} & $\begin{array}{l}\text { Distribution } \\
\text { Type }\end{array}$ & Mean & Modian & Minimum & Mexdmum & Units & Reference ID & $\begin{array}{l}\text { Wes the dinta } \\
\text { dervioped } \\
\text { under an } \\
\text { NoA-1 } \\
\text { Program? }\end{array}$ & $\begin{array}{c}\text { Which } \\
\text { methods } \\
\text { wore used to } \\
\text { qually } \\
\text { existing } \\
\text { data? } \\
\text { (footnote 3) }\end{array}$ \\
\hline 643 & 2 & CONSTANT & $4.6300000 E+\infty 0$ & $4.6300000 E+\infty 0$ & $4 . \dot{6} 300000 E+00$ & $4.6300000 E+\infty 0$ & $\mathrm{Ci}$ & WPO39260 & $\mathbf{Y}$ & N/A \\
\hline 643 & 2 & CONSTANT & $4.6300000 E+00$ & $4.6300000 E+\infty 0$ & $4.6300000 E+\infty 0$ & $4.6300000 E+\infty 0$ & $\mathbf{C i}$ & WPO41559 & $\mathbf{Y}$ & NA \\
\hline 643 & 2 & CONSTANT & $4.6300000 E+\infty 0$ & $4.6300000 E+\infty 0$ & $4.6300000 \mathrm{E}+00$ & $4.6300000 \mathrm{E}+00$ & Ci & WPOA1550 & $Y$ & NA \\
\hline 644 & 4 & CONSTANT & $2.3604600 E-01$ & $2.3604600 E-01$ & $23604600 E-01$ & $23604600 E-01$ & kg/mole & CRC-72 & $\bar{Y}$ & NA \\
\hline 644 & 4 & CONSTANT & $2.3604600 E-01$ & $2.3604600 \mathrm{E}-01$ & $2.3604600 E-01$ & $2.3604600 E-01$ & $\mathrm{~kg} / \mathrm{mol}$ & WP036298 & $\bar{Y}$ & N/A \\
\hline 644 & 4 & CONSTANT & $2.3604600 \mathrm{E}-01$ & $2.3604600 \mathrm{E}-01$ & $2.3604600 E-01$ & $2.3604600 E-01$ & $\mathrm{~kg} / \mathrm{mole}$ & WP036301 & $Y$ & N/A \\
\hline 645 & 4 & CONSTANT & $7.3890000 E+14$ & $7.3890000 E+14$ & $7.3890000 E+14$ & $7.3890000 \mathrm{E}+114$ & 5 & WP036301 & $Y$ & NA \\
\hline 645 & 4 & CONSTANT & $7.3890000 \mathrm{E}+14$ & $7.3890000 E+14$ & $7.3890000 E+14$ & $7.3890000 E+114$ & $s$ & WPO37404 & $\mathbf{Y}$ & NA \\
\hline 645 & 4 & CONSTANT & $7.3890000 E+14$ & $7.3890000 E+14$ & $7.3850000 \mathrm{E}+14$ & $7.3890000 E+114$ & s & WPO40434 & $Y$ & NA \\
\hline 646 & 2 & CONSTANT & $1.0300000 E-01$ & $1.0300000 E-01$ & $1.0300000 E-01$ & $1.0300000 E-01$ & $\mathrm{Ci}$ & WP037061 & $\mathbf{Y}$ & N/A \\
\hline 646 & 2 & CONSTANT & $1.0300000 E-01$ & $1.0300000 E-01$ & $1.0300000 E-01$ & $1.0300000 E-01$ & $\mathrm{Ci}$ & WP039260 & $\mathbf{Y}$ & N/A \\
\hline 646 & 2 & CONSTANT & $1.0300000 E-01$ & $1.0300000 \mathrm{E}-01$ & $1.0300000=-01$ & $1.0300000 E-01$ & $\mathrm{Ci}$ & WPO41559 & $Y$ & N/A \\
\hline 646 & 2 & CONSTANT & $1.0300000 E-01$ & $1.0300000 E-01$ & $1.0300000=-01$ & $1.0300000 E-01$ & $\mathrm{Ci}$ & WP041560 & $\gamma$ & N/A \\
\hline 647 & 4 & CONSTANT & $2.3805100 E-01$ & $2.3805100 E-01$ & 2.3805100E-01 & $2.3805100 E-01$ & $\mathrm{~kg} / \mathrm{mole}$ & CRC-72 & $\mathbf{Y}$ & NA \\
\hline 647 & 4 & CONSTANT & 2.3805100E-01 & $2.3805100 E-01$ & 23805100E-01 & 23805100E-01 & $\mathrm{kg} / \mathrm{mole}$ & WP036298 & $\mathbf{Y}$ & NA \\
\hline 647 & 4 & CONSTANT & $2.3805100 E-01$ & $2.3805100 E-01$ & $2.3805100 E-01$ & $2.3805100 E-01$ & $\mathrm{~kg} / \mathrm{mol}$ & WP036301 & $Y$ & N/A \\
\hline 648 & 4 & CONSTANT & $1.4100000 E+17$ & $1.4100000 E+17$ & $1.4100000 E+17$ & $1.4100000 E+17$ & s & WPO36301 & $\mathbf{Y}$ & N/A \\
\hline 648 & 4 & CONSTANT & $1.4100000 E+17$ & $1.4100000 E+17$ & $1.4100000 \mathrm{E}+17$ & $1.4100000 \mathrm{E}+17$ & $s$ & WPO37404 & $Y$ & NA \\
\hline 648 & 4 & CONSTANT & $1.4100000 \mathrm{E}+17$ & $1.4100000 E+17$ & $1.4100000 \mathrm{E}+17$ & $1.4100000 E+17$ & $\mathbf{s}$ & WPO40434 & $\mathbf{Y}$ & N/A \\
\hline 649 & 2 & CONSTANT & $3.9600000 E+01$ & $3.9600000 E+01$ & $3.9600000 E+01$ & $3.9600000 E+01$ & $\mathrm{Ci}$ & WP037061 & $Y$ & N/A \\
\hline 649 & 2 & CONSTANT & $3.9600000 E+01$ & $3.9600000 \mathrm{E}+01$ & $3.9600000 \mathrm{E}+01$ & $3.9600000 E+01$ & $\mathbf{C i}$ & WP039260 & $\mathbf{Y}$ & NA \\
\hline 649 & 2 & CONSTANT & $3.9600000 \mathrm{E}+01$ & $3.9600000 E+01$ & $3.9600000 E+01$ & $3.9600000 E+01$ & $\mathrm{Ci}$ & WPO41559 & $\mathbf{Y}$ & N/A \\
\hline 649 & 2 & CONSTANT & $3.9600000 E+01$ & $3.9600000 E+01$ & $3.9600000 \mathrm{E}+01$ & $3.960,0000 E+01$ & $\mathrm{Ci}$ & WP041560 & $Y$ & N/A \\
\hline 650 & 2 & CONSTANT & $1.0500000 E+01$ & $1.0500000 E+01$ & $1.0500000 E+01$ & $1.0500000 \mathrm{E}+01$ & $\mathrm{Ci}$ & WP037061 & $\mathbf{Y}$ & N/A \\
\hline 650 & 2 & CONSTANT & $1.0500000 E+01$ & $1.0500000 E+01$ & $1.0500000 \mathrm{E}+01$ & $1.0500000 E+01$ & $\mathrm{Ci}$ & WP039260 & $Y$ & N/A \\
\hline 650 & 2 & CONSTANT & $1.0500000 E+01$ & $1.0500000 E+01$ & $1.0500000 E+01$ & $1.0500000 \mathrm{E}+01$ & $\mathbf{C i}$ & WPO41559 & $\mathbf{Y}$ & N/A \\
\hline 650 & 2 & CONSTANT & $1.0500000 \mathrm{E}+01$ & $1.0500000 \mathrm{E}+01$ & $1.0500000 E+01$ & $1.0500000 E+01$ & $\mathrm{Ci}$ & WP041560 & $Y$ & N/A \\
\hline 651 & 4,5 & UNIFORM & $2.5000000 E-02$ & $2.5000000 E-02$ & $1.0000000 E-02$ & $4.0000000 E-02$ & $m$ & STREETER-1 & $Y$ & NA \\
\hline 651 & 4,5 & UNIFORM & $2.5000000 E-02$ & $2.5000000 E-02$ & $1.0000000 E-02$ & $4.0000000 E-02$ & $\mathbf{m}$ & WP035597 & $" \gamma$ & N/A \\
\hline 651 & 4,5 & UNIFORM & $2.5000000 E-02$ & $2.5000000 \mathrm{E}-02$ & $1.0000000 \mathrm{E}-02$ & $4.0000000 \mathrm{E}-02$ & $\mathbf{m}$ & WP038568 & $\mathbf{Y}$ & N/A \\
\hline 651 & 4,5 & UNIFORM & $2.5000000 \mathrm{E}-02$ & $2.5000000 \mathrm{E}-02$ & $1.0000000 \mathrm{E}-02$ & $4.0000000 E-02$ & $\mathbf{m}$ & WPO 040521 & $\mathbf{Y}$ & N/A \\
\hline 652 & 1,3 & CONSTANT & $1.0000000 E+00$ & $1.0000000 E+00$ & $1.0000000 E+\infty 0$ & $1.0000000 \mathrm{E}+00$ & NONE & WP035597 & $\mathbf{Y}$ & NA \\
\hline 652 & 1,3 & CONSTANT & $1.0000000 E+\infty 0$ & $1.0000000 E+\infty 0$ & $1.0000000 E+00$ & $1.0000000 \mathrm{E}+\infty 0$ & NONE & WPO40514 & $Y$ & NA \\
\hline 652 & 1,3 & CONSTANT & $1.0000000 E+00$ & $1.0000000 E+\infty$ & $1.0000000 E+00$ & $1.0000000 \mathrm{E}+00$ & NONE & WPO42681 & $\mathbf{Y}$ & N/A \\
\hline 653 & 1,3 & CONSTANT & $0.0000000 E+\infty 0$ & $0.0000000 E+00$ & $0.0000000 E+\infty 0$ & $0.0000000 E+\infty$ & $P a^{\wedge}-1$ & WP035597 & $\mathbf{Y}$ & N/A \\
\hline 653 & 1,3 & CONSTANT & $0.0000000 E+00$ & $0.0000000 \mathrm{E}+00$ & $0.0000000 E+\infty 0$ & $0.0000000 E+00$ & $P a^{n-1}$ & WPO40514 & $Y$ & N/A \\
\hline 653 & 1,3 & CONSTANT & $0.0000000 E+\infty 0$ & $0.0000000 E+00$ & $0.0000000 E+\infty 0$ & $0.0000000 E+\infty$ & $P g^{n}-1$ & WPO42681 & $Y$ & N/A \\
\hline 656 & 1 & UNIFORM & $6.3420000 E-10$ & $6.3420000 E-10$ & $0.0000000 \mathrm{E}+00$ & 1.2684000E-09 & molkg's & WP030819 & YơN & 4 \\
\hline 656 & 1 & UNIFORM & $6.3420000 E-10$ & $6.3420000 E-10$ & $0.0000000 E+\infty 0$ & $1.2684000 E-09$ & molkg*s & WP035268 & YaN & 4 \\
\hline
\end{tabular}


CCA Parameter Listing

\begin{tabular}{|c|c|c|c|c|c|c|c|c|c|c|}
\hline $\begin{array}{c}\text { Parameter In } \\
\text { Databese? } \\
\text { (YNN) }\end{array}$ & \begin{tabular}{|l|} 
If the values \\
used in CCA \\
are not the \\
samo as in \\
DB, provido $x$ \\
differance
\end{tabular} & ID & Material ID & Material Nams & $\begin{array}{l}\text { Parameter } \\
\text { ID }\end{array}$ & Parameter Nams & $\begin{array}{l}\text { PRP DO } \\
\text { (WPO E) }\end{array}$ & $\begin{array}{c}\text { Dext Entry } \\
\text { Dete }\end{array}$ & $\begin{array}{l}\text { Is the 464 } \\
\text { conalatent } \\
\text { with the } \\
\text { purameter } \\
\text { in DB? } \\
(N, N A)\end{array}$ & $\begin{array}{l}\text { Paramoter } \\
\text { Category } \\
\text { (footnote 1) }\end{array}$ \\
\hline $\mathbf{Y}$ & & 656 & WAS_AREA & $\begin{array}{l}\text { Waste emplacement } \\
\text { area and waste }\end{array}$ & GRATMICH & $\begin{array}{l}\text { Gas Production Rate, Mrrobial, } \\
\text { Himid Conditions Relative to } \\
\text { Inundated Rate }\end{array}$ & 34923 & 08-Feb-96 & $\mathbf{Y}$ & 1 \\
\hline $\mathbf{Y}$ & & 657 & WAS_AREA & $\begin{array}{l}\text { Waste emplacement } \\
\text { area and waste }\end{array}$ & GRATMICI & $\begin{array}{l}\text { Gas production rate, mucrobial, } \\
\text { inundated conditions }\end{array}$ & 34928 & 08fab-96 & $\mathbf{Y}$ & 1 \\
\hline $\mathbf{Y}$ & & 657 & WAS_AREA & $\begin{array}{l}\text { Waste emplacoment } \\
\text { area and waste }\end{array}$ & GRATMICI & $\begin{array}{l}\text { Gas production rate, microbial, } \\
\text { inundated conditions }\end{array}$ & 34928 & 08-Feb-96 & $\mathbf{Y}$ & 1 \\
\hline $\mathbf{Y}$ & & 657 & WAS_AREA & $\begin{array}{l}\text { Waste emplacement } \\
\text { area and waste }\end{array}$ & GRATMICI & $\begin{array}{l}\text { Gas production rate, microbial, } \\
\text { inendated conditions }\end{array}$ & 34928 & 08-Fab-96 & $\mathbf{Y}$ & 1 \\
\hline$Y$ & & 658 & WAS_AREA & $\begin{array}{l}\text { Wasto emplacement } \\
\text { area and waste }\end{array}$ & PC_MAX & $\begin{array}{l}\text { Maximum allowable capillary } \\
\text { pressure }\end{array}$ & 34986 & 02-Nov-95 & $\mathbf{Y}$ & $4 B$ \\
\hline $\mathbf{Y}$ & & 658 & WAS_AREA & $\begin{array}{l}\text { Waste emplacement } \\
\text { area and waste }\end{array}$ & PC_MAX & $\begin{array}{l}\text { Maximum allowable capillary } \\
\text { pressure }\end{array}$ & 34986 & 02-Nov-95 & $\mathbf{Y}$ & 48 \\
\hline$Y$ & & 659 & WAS_AREA & $\begin{array}{l}\text { Wasto emplacement } \\
\text { area and waste }\end{array}$ & PORE_DIS & $\begin{array}{l}\text { Brooks-Corey pore distribution } \\
\text { parameter }\end{array}$ & 34989 & 22fob-96 & $\mathbf{Y}$ & 48 \\
\hline $\mathbf{Y}$ & & 659 & WAS_AREA & $\begin{array}{l}\text { Waste emplacement } \\
\text { area and waste }\end{array}$ & PORE_DIS & $\begin{array}{l}\text { Brooks-Corey pore distribution } \\
\text { parameter }\end{array}$ & 34989 & 22-Feb-96 & $\mathbf{Y}$ & $4 B$ \\
\hline $\mathbf{Y}$ & & 659 & WAS_AREA & $\begin{array}{l}\text { Waste emplacement } \\
\text { area and wasto }\end{array}$ & PORE_DIS & $\begin{array}{l}\text { Brooks-Corey pore distribution } \\
\text { parameter }\end{array}$ & 34989 & 22-Fob-96 & $\mathbf{Y}$ & 48 \\
\hline$Y$ & & 659 & WAS_AREA & $\begin{array}{l}\text { Waste emplacoment } \\
\text { area and waste }\end{array}$ & PORE_DIS & $\begin{array}{l}\text { Brooks-Corey pore distribution } \\
\text { parameler }\end{array}$ & 34989 & 22 Feb-96 & $\mathbf{Y}$ & 48 \\
\hline $\mathbf{Y}$ & & 660 & WAS_AREA & $\begin{array}{l}\text { Waste emplacoment } \\
\text { area and waste }\end{array}$ & POROSTYY & Effective porosity & 34874 & 08-Fob-9s & $\mathbf{Y}$ & $4 B$ \\
\hline$Y$ & & 660 & WAS_AREA & $\begin{array}{l}\text { Wasto emplacement } \\
\text { area and waste }\end{array}$ & POROSITY & Effective porosity & 34874 & 08-Fob-96 & $\mathbf{Y}$ & $4 B$ \\
\hline$Y$ & & 660 & WAS_AREA & $\begin{array}{l}\text { Wasto emplacement } \\
\text { area and waste }\end{array}$ & POROSITY & Effective porosity & 34874 & $08-F 0 b-96$ & $\mathbf{Y}$ & $4 B$ \\
\hline $\mathbf{Y}$ & & 661 & WAS_AREA & $\begin{array}{l}\text { Waste emplacement } \\
\text { area and waste }\end{array}$ & PO_MIN & $\begin{array}{l}\text { Minimum brine pressure for } \\
\text { capillary model } \mathrm{KPC}=3\end{array}$ & 34875 & 21-Fob-96 & $\mathbf{Y}$ & 48 \\
\hline $\mathbf{Y}$ & & 661 & WAS_AREA & $\begin{array}{l}\text { Waste emplacement } \\
\text { area and waste }\end{array}$ & PO_MIN & $\begin{array}{l}\text { Minimum brine pressure for } \\
\text { capillary model KPC=3 }\end{array}$ & 34875 & $21-F a b-96$ & $\mathbf{Y}$ & $4 B$ \\
\hline $\mathbf{Y}$ & & 661 & WAS_AREA & $\begin{array}{l}\text { Waste emplacement } \\
\text { area and waste }\end{array}$ & PO_MIN & $\begin{array}{l}\text { Minimum brune pressure for } \\
\text { capillary model } K P C=3\end{array}$ & 34875 & 21-Feb-96 & $\mathbf{Y}$ & $4 B$ \\
\hline $\mathbf{Y}$ & & 662 & WAS_AREA & $\begin{array}{l}\text { Waste emplacement } \\
\text { area and waste }\end{array}$ & PRESSURE & Brine far-field pore pressure & 34876 & 02-Nov-95 & $\mathbf{Y}$ & 48 \\
\hline $\mathbf{Y}$ & & 662 & WAS_AREA & $\begin{array}{l}\text { Waste emplacement } \\
\text { area and waste }\end{array}$ & PRESSURE & Brine far-field pore pressure & 34876 & 02-Nov-95 & $\mathbf{Y}$ & 48 \\
\hline $\mathbf{Y}$ & & 663 & WAS_AREA & $\begin{array}{l}\text { Waste emplacoment } \\
\text { area and waste }\end{array}$ & PRMX_LOG & $\begin{array}{l}\text { Log of intrinsic permeabilty, } X \text { - } \\
\text { direction }\end{array}$ & 34877 & 08-Fab-96 & $\mathbf{Y}$ & 1 \\
\hline $\mathbf{Y}$ & & 663 & WAS_AREA & $\begin{array}{l}\text { Waste emplacement } \\
\text { area and waste }\end{array}$ & PRMX_LOG & $\begin{array}{l}\text { Log of intrinsic permeability, } X \text { - } \\
\text { direction }\end{array}$ & 34877 & D8-Feb-96 & $\mathbf{Y}$ & 1 \\
\hline $\mathbf{Y}$ & & 663 & WAS_AREA & $\begin{array}{l}\text { Waste emplacement } \\
\text { area and waste }\end{array}$ & PRMX_LOG & $\begin{array}{l}\text { Log of intrinsic permeability, } X \text { - } \\
\text { direction }\end{array}$ & 34877 & ob-Fob-96 & $\mathbf{Y}$ & 1 \\
\hline $\mathbf{Y}$ & & 663 & WAS_AREA & $\begin{array}{l}\text { Waste emplacement } \\
\text { area and waste }\end{array}$ & PRMX_LOG & $\begin{array}{l}\text { Log of intrnsic permeability, X- } \\
\text { dureation }\end{array}$ & 34877 & 08-Feb-96 & $\mathbf{Y}$ & 1 \\
\hline$Y$ & & 664 & WAS_AREA & $\begin{array}{l}\text { Waste emplacement } \\
\text { area and waste }\end{array}$ & PRMY_LOG & $\begin{array}{l}\text { Log of intnnsic permeability, } Y_{-} \\
\text {direction }\end{array}$ & 34878 & D8-Fob-96 &.$- Y$ & 1 \\
\hline $\mathbf{Y}$ & & 664 & WAS_AREA & $\begin{array}{l}\text { Waste emplacement } \\
\text { area and waste }\end{array}$ & PRMY_LOG & $\begin{array}{l}\text { Log of intrinsic permeability, Y- } \\
\text { direction }\end{array}$ & 34878 & $08-F a b-96$ & $Y$ & 1 \\
\hline $\mathbf{Y}$ & & 664 & WAS_AREA & $\begin{array}{l}\text { Waste emplacement } \\
\text { area and waste }\end{array}$ & PRMY_LOG & $\begin{array}{l}\text { Log of ntrinsic permeability. Y- } \\
\text { direction }\end{array}$ & 34878 & 08fab-96 & $\mathbf{Y}$ & 1 ; \\
\hline$Y$ & & 664 & WAS_AREA & $\begin{array}{l}\text { Waste emplacement } \\
\text { area and waste }\end{array}$ & PRMY_LOG & $\begin{array}{l}\text { Log of nitrinsic permeability, } Y \text { - } \\
\text { drection }\end{array}$ & 34878 & 08-Feb-96 & $\mathbf{Y}$ & 1 \\
\hline$Y$ & & 665 & WAS_AREA & $\begin{array}{l}\text { Waste emplacement } \\
\text { area and waste }\end{array}$ & PRMZ_LOG & $\begin{array}{l}\text { Log of intrusic permeability, Z- } \\
\text { drection }\end{array}$ & 34879 & 08 -Feb-96 & $\mathbf{Y}$ & 1 \\
\hline$Y$ & & 665 & WAS_AREA & $\begin{array}{l}\text { Waste emplacement } \\
\text { grea and waste }\end{array}$ & PRMZ_LOG & $\begin{array}{l}\text { Log of untrinsıc permeability, Z- } \\
\text { direction }\end{array}$ & 34879 & $08-F a b-96$ & $\mathbf{Y}$ & 1 \\
\hline $\mathbf{Y}$ & & 665 & WAS_AREA & $\begin{array}{l}\text { Waste emplacement } \\
\text { erea and waste }\end{array}$ & PRMZ_LOG & $\begin{array}{l}\text { Log of intrinsic permeability. Z- } \\
\text { drection }\end{array}$ & 34879 & 08-Fob-96 & $\mathbf{Y}$ & 1 \\
\hline $\mathbf{Y}$ & & 665 & WAS_AREA & $\begin{array}{l}\text { Waste emplacement } \\
\text { area and waste }\end{array}$ & PRMZ_LOG & $\begin{array}{l}\text { Log of intrnsic permeability, Z- } \\
\text { direction }\end{array}$ & 34879 & 08 Fab-96 & $\mathbf{Y}$ & 1 \\
\hline $\mathbf{Y}$ & & 688 & WAS_AREA & $\begin{array}{l}\text { Waste emplacement } \\
\text { area and wasto } \\
\end{array}$ & RELP_MOD & $\begin{array}{l}\text { Model number, relative } \\
\text { permeability model }\end{array}$ & 34890 & 08-Fab-96 & $\mathbf{Y}$ & $4 B$ \\
\hline $\mathbf{Y}$ & & 668 & WAS_AREA & $\begin{array}{l}\text { Waste emplacement } \\
\text { area and waste }\end{array}$ & RELP_MOD & $\begin{array}{l}\text { Model number, relative } \\
\text { permeability model }\end{array}$ & 34890 & 08-Feb-96 & $\mathbf{Y}$ & $4 B$ \\
\hline $\mathbf{Y}$ & & 668 & WAS_AREA & $\begin{array}{l}\text { Waste emplacement } \\
\text { area and waste }\end{array}$ & RELP_MOD & $\begin{array}{l}\text { Model number, rolative } \\
\text { permeability model }\end{array}$ & 34890 & 08-Fab-96 & $\mathbf{Y}$ & $4 B$ \\
\hline$Y$ & & 669 & WAS_AREA & $\begin{array}{l}\text { Waste emplacement } \\
\text { area and waste }\end{array}$ & SAT_IBRN & Initial Brine Saturation & 34894 & 20-Feb-96 & $\mathbf{Y}$ & $4 B$ \\
\hline
\end{tabular}


CCA Parameter Listing

\begin{tabular}{|c|c|c|c|c|c|c|c|c|c|c|}
\hline ID & \begin{tabular}{|c|} 
Clastifi- \\
cation of \\
Data \\
Catogory \\
(footnote 2)
\end{tabular} & $\begin{array}{l}\text { Distribution } \\
\text { Type }\end{array}$ & Man & Medien & Mlntroum & Maximum & Units & Reference ID & $\begin{array}{l}\text { Wea the datta } \\
\text { dowloped } \\
\text { under an } \\
\text { NOA-1 } \\
\text { Program? }\end{array}$ & $\begin{array}{c}\text { Which } \\
\text { mothods } \\
\text { worv used to } \\
\text { qualify } \\
\text { oxiating } \\
\text { datu? } \\
\text { (footnote 3) }\end{array}$ \\
\hline 656 & 1 & UNIFORM & 6.3420000E-10 & 6.3420000E-10 & $0.0000000 E+00$ & $1.2684000 E-09$ & molkg"s & WPO35597 & Y\&N & 4 \\
\hline 657 & 1 & UNIFORM & 4.9150000E-09 & 4.9150000E-09 & 3.1710000E-10 & $9.5129000 \mathrm{E}-09$ & molke's & WP030819 & Y8N & 4 \\
\hline 657 & 1 & UNIFORM & $4.9150000 E-09$ & $4.9150000 E-09$ & $3.1710000 E-10$ & $9.5129000 E-09$ & molkg"s & WP035268 & Y\&N & 4 \\
\hline 657 & 1 & UNIFORM & $4,9150000 E-09$ & 4.9150000E-09 & $3.1710000 E-10$ & $9.5129000 \mathrm{E}-09$ & molkg"s & WP035597 & Y $8 \mathrm{~N}$ & 4 \\
\hline 658 & 1,3 & CONSTANT & $1.0000000 E+08$ & $1.0000000 E+08$ & $1.0000000 E+08$ & $1.0000000 E+08$ & $\mathbf{P a}$ & WP038568 & $\mathbf{Y}$ & N/A \\
\hline 658 & 1,3 & CONSTANT & $1.0000000 E+08$ & $1.0000000 E+08$ & $1.0000000 E+08$ & $1.0000000 E+08$ & $\mathrm{~Pa}$ & WPO40514 & $Y$ & NA \\
\hline 659 & 1,3 & CUMULATIVE & $3.2500000 E+00$ & $2.8900000 E+00$ & $1.4400000 E+C 0$ & $5.7800000 E+\infty 0$ & NONE & WP030921 & $\mathbf{Y}$ & N/A \\
\hline 659 & 1,3 & CUMULATIVE & $3.2500000 E+\infty 0$ & $2.8900000 E+C 0$ & $1.4400000 E+00$ & $5.7800000 E+00$ & NONE & WP035268 & $\mathbf{Y}$ & N/A \\
\hline 659 & 1,3 & CUMULATIVE & $3.2500000 E+\infty 0$ & $2.8900000 E+00$ & $1.4400000 E+00$ & $5.7800000 E+\infty 0$ & NONE & WP035597 & $\mathbf{Y}$ & N/A \\
\hline 659 & 1,3 & CUMULATINE & $3.2500000 \mathrm{E}+\infty 0$ & $2.8900000 E+00$ & $1.4400000 E+00$ & $5.7800000 E+\infty 0$ & NONE & WPO40514 & $Y$ & N/A \\
\hline 660 & 1,3 & CONSTANT & $8.4800000 E-01$ & $8.4800000 E-01$ & $8.4800000 E-01$ & $8.4800000 E-01$ & NONE & WP035597 & $\mathbf{Y}$ & NA \\
\hline 660 & 1,3 & CONSTANT & $8.4800000 E-01$ & $8.4800000 E-01$ & $8.4800000 E-01$ & $8.4800000 E-01$ & NONE & WP040514 & $\mathbf{Y}$ & N/A \\
\hline 660 & 1,3 & CONSTANT & $8.4800000 E-01$ & $8.4800000 \mathrm{E}-01$ & $8.4800000 E-01$ & $8.4800000 E-01$ & NONE & WPO42681 & $Y$ & N/A \\
\hline 661 & 1.3 & CONSTANT & $1.0132500 E+05$ & $1.0132500 E+05$ & $1.0132500 E+05$ & $1.0132500 E+05$ & $\mathbf{P a}$ & WP035597 & $\mathbf{Y}$ & NA \\
\hline 661 & 1,3 & CONSTANT & $1.0132500 E+05$ & $1.0132500 E+05$ & $1.0132500 E+05$ & $1.0132500 E+05$ & $\mathbf{P a}$ & WP036051 & $Y$ & $N A$ \\
\hline 661 & 1,3 & CONSTANT & $1.0132500 E+05$ & $1.0132500 E+05$ & $1.0132500 E+05$ & $1.0132500 E+05$ & $\mathbf{P a}$ & WP040514 & $\mathbf{Y}$ & N/A \\
\hline 662 & 1,3 & CONSTANT & $1.0132500 E+05$ & $1.0132500 E+05$ & $1.0132500 E+05$ & $1.0132500 E+05$ & $\mathbf{P a}$ & WP038568 & $\mathbf{Y}$ & $N / A$ \\
\hline 662 & 1,3 & CONSTANT & $1.0132500 E+05$ & $1.0132500 E+05$ & $1.0132500 E+05$ & $1.0132500 \mathrm{E}+05$ & $\mathbf{P a}$ & WP040514 & $\mathbf{Y}$ & N/A \\
\hline 663 & 1 & CONSTANT & $-1.2769000 E+01$ & $-1.2769000 E+01$ & $-1.2769000 E+01$ & $-1.2769000 E+01$ & $\log \left(m^{\wedge} 2\right)$ & WP030921 & N & 1 \\
\hline 663 & 1 & CONSTANT & $-1.2769000 E+01$ & $-1.2769000 \mathrm{E}+01$ & $-1.2769000 E+01$ & $-1.2769000 E+01$ & $\log \left(m^{\wedge} 2\right)$ & WP030925 & $\mathbf{N}$ & 1 \\
\hline 663 & 1 & CONSTANT & $-1.2769000 E+01$ & $-1.2769000 E+01$ & $-1.2769000 \mathrm{E}+01$ & $-1.2769000 E+01$ & $\log \left(m^{\wedge} 2\right)$ & WP035597 & $\mathbf{N}$ & 1 \\
\hline 663 & 1 & CONSTANT & $-1.2769000 E+01$ & $-1.2769000 \mathrm{E}+01$ & $-1.2769000 E+01$ & $-1.2769000 E+01$ & $\log \left(m^{\wedge} 2\right)$ & WPO-42681 & $\mathbf{N}$ & 1 \\
\hline 664 & 1 & CONSTANT & $-1.2769000 E+01$ & $-1.2769000 \mathrm{E}+01$ & $-1.2769000 \mathrm{E}+01$ & $-1.2769000 E+01$ & $\log \left(m^{\wedge} 2\right)$ & WP030921 & $-\Delta$ & 1 \\
\hline 664 & 1 & CONSTANT & $-1.2769000 E+01$ & $-1.2769000 \mathrm{E}+01$ & $-1.2769000 \mathrm{E}+01$ & $-1.2769000 E+01$ & $\log \left(m^{\wedge} 2\right)$ & WP030925 & N & 1 \\
\hline 664 & 1 & CONSTANT & $-1.2769000 E+01$ & $-1.2769000 E+01$ & $-1.2769000 E+01$ & $-1.2769000 E+01$ & $\log \left(m^{\wedge} 2\right)$ & WP035597 & N & 1 \\
\hline 664 & 1 & CONSTANT & $-1.2769000 E+01$ & $-1.2769000 E+01$ & $-1.2769000 E+01$ & $-1.2769000 E+01$ & $\log \left(m^{\wedge} 2\right)$ & WPO42681 & $\mathbf{N}$ & 1 \\
\hline 665 & 1 & CONSTANT & $-1.2769000 E+01$ & $-1.2769000 E+01$ & $-1.2769000 E+01$ & $-1.2769000 E+01$ & $\log \left(m^{\wedge} 2\right)$ & WP030921 & $N$ & 1 \\
\hline 665 & 1 & CONSTANT & $-1.2769000 \mathrm{E}+01$ & $-1.2769000 E+01$ & $-1.2769000 E+01$ & $-1.2769000 \mathrm{E}+01$ & $\log \left(m^{\wedge} 2\right)$ & WP030925 & N & 1 \\
\hline 665 & 1 & CONSTANT & $-1.2769000 \mathrm{E}+01$ & $-1.2769000 E+01$ & $-1.2769000 E+01$ & $-1.2769000 E+01$ & $\log \left(m^{\wedge} z\right)$ & WP035597 & $\mathbf{N}$ & 1 \\
\hline 665 & 1 & CONSTANT & $-1.2769000 E+01$ & $-1.2769000 E+01$ & $-1.2769000 E+01$ & $-1.2769000 E+01$ & $\log \left(m^{\wedge} 2\right)$ & WPO42681 & N & 1 \\
\hline 668 & 1,3 & CONSTANT & $4.0000000 \mathrm{E}+\infty 0$ & $4.0000000 \mathrm{E}+00$ & $4.00000000 \mathrm{E}+00$ & $4.0000000 E+00$ & NONE & WPO35597 & $\mathbf{Y}$ & NA \\
\hline 668 & 1,3 & CONSTANT & $4.0000000 E+\infty 0$ & $4.0000000 \mathrm{E}+\infty 0$ & $4.0000000 E+00$ & $4.0000000 E+00$ & NONE & WP040514 & $\mathbf{Y}$ & NA \\
\hline 668 & 1,3 & CONSTANT & $4.0000000 E+\infty 0$ & $4.0000000 E+00$ & $4.0000000 E+00$ & $4.0000000 E+00$ & NONE & WPO42681 & $\mathbf{Y}$ & NA \\
\hline 669 & 1,3 & CONSTANT & $1.5000000 E-02$ & $1.5000000 E-02$ & $1.5000000 E-02$ & $1.5000000 E-02$ & NONE & WP030925 & $\mathbf{Y}$ & N/A \\
\hline
\end{tabular}


CCA Parameter Listing

\begin{tabular}{|c|c|c|c|c|c|c|c|c|c|c|}
\hline $\begin{array}{c}\text { Parrmeterin } \\
\text { Databeae? } \\
\text { (YNN) }\end{array}$ & $\begin{array}{l}\text { If the values } \\
\text { used in ceA } \\
\text { are not the } \\
\text { same as in } \\
\text { DB, provide } \% \\
\text { difference }\end{array}$ & ID & Material ID & Mataria Natre & $\begin{array}{c}\text { Paramotor } \\
\text { ID }\end{array}$ & Paramoter Name & $\begin{array}{c}\text { PRP DD } \\
\text { (NPO) }\end{array}$ & $\begin{array}{c}\text { Data Entry } \\
\text { Dato }\end{array}$ & $\begin{array}{l}\text { Is the } 464 \\
\text { consiatent } \\
\text { wth the } \\
\text { parameter } \\
\text { in DB? } \\
\text { } \mathrm{N}, \text { NA) }\end{array}$ & $\begin{array}{l}\text { Paramoter } \\
\text { Category } \\
\text { (lootnot: 1) }\end{array}$ \\
\hline $\mathbf{Y}$ & & 669 & WAS_AREA & $\begin{array}{l}\text { Waste emplacement } \\
\text { area and waste }\end{array}$ & SAT_IBRN & Initial Brine Saturation & 34894 & 20-Feb-96 & $\mathbf{Y}$ & 48 \\
\hline $\mathbf{Y}$ & & 669 & WAS_AREA & $\begin{array}{l}\text { Waste emplacoment } \\
\text { area and waste }\end{array}$ & SAT_IBRN & Initial Brine Saturation & 34894 & 20-Fob-96 & $\mathbf{Y}$ & $4 B$ \\
\hline $\mathbf{Y}$ & & 669 & WAS_AREA & $\begin{array}{l}\text { Weste emplacement } \\
\text { aroa and wasto }\end{array}$ & SAT_IBRN & Initial Brine Saturation & 34894 & $20-F a b-96$ & $\mathbf{Y}$ & $4 B$ \\
\hline $\mathbf{Y}$ & & 670 & WAS_AREA & $\begin{array}{l}\text { Waste emplacement } \\
\text { area and waste }\end{array}$ & SAT_RBRN & Residual Brine Saturation & 34902 & 21 Fob-96 & $\mathbf{Y}$ & $4 B$ \\
\hline $\mathbf{Y}$ & & 670 & WAS_AREA & $\begin{array}{l}\text { Waste emplacement } \\
\text { area and waste }\end{array}$ & SAT_RBRN & Residual Brine Saturation & 34902 & 21-Fob-96 & $\mathbf{Y}$ & $4 B$ \\
\hline $\mathbf{Y}$ & & 670 & WAS_AREA & $\begin{array}{l}\text { Wasto emplacement } \\
\text { area and waste }\end{array}$ & SAT_RBRN & Residual Brine Saturation & 34902 & $21+$ ab-96 & $\mathbf{Y}$ & $4 B$ \\
\hline $\mathbf{Y}$ & & 670 & WAS_AREA & $\begin{array}{l}\text { Waste emplacement } \\
\text { area and waste }\end{array}$ & SAT_RBRN & Residual Brine Saturation & 34902 & $21+F o b-96$ & $\mathbf{Y}$ & $4 B$ \\
\hline $\mathbf{Y}$ & & 671 & WAS_AREA & $\begin{array}{l}\text { Waste emplacement } \\
\text { area and waste }\end{array}$ & SAT_RGAS & Residual Gas Saturation & 34905 & 22-Fob-96 & $\mathbf{Y}$ & 48 \\
\hline $\mathbf{Y}$ & & 671 & WAS_AREA & $\begin{array}{l}\text { Waste emplacement } \\
\text { area and waste }\end{array}$ & SAT_RGAS & Residual Gas Saturation & 34905 & 22Fab-96 & $\mathbf{Y}$ & 48 \\
\hline $\mathbf{Y}$ & & 671 & WAS_AREA & $\begin{array}{l}\text { Waste emplacement } \\
\text { area and waste }\end{array}$ & SAT_RGAS & Residual Gas Saturation & 34905 & 22Feb-96 & $\mathbf{Y}$ & 48 \\
\hline $\mathbf{Y}$ & & 671 & WAS_AREA & $\begin{array}{l}\text { Waste emplacement } \\
\text { area and waste }\end{array}$ & SAT_RGAS & Residual Gas Saturation & 34905 & 22-Feb-96 & $\mathbf{Y}$ & 48 \\
\hline $\mathbf{Y}$ & & 671 & WAS_AREA & $\begin{array}{l}\text { Waste emplacement } \\
\text { area and waste }\end{array}$ & SAT_RGAS & Residual Gas Saturation & 34905 & 22-Fob-96 & $\mathbf{Y}$ & $4 B$ \\
\hline $\mathbf{Y}$ & & 838 & CF & Califorinum & LOGSOLM & Log of the Radionuclide Solubility & 31827 & 01-May-96 & $\mathbf{Y}$ & $4 B$ \\
\hline$Y$ & & 838 & CF & Califorinum & LOGSOLM & Log of the Radionuclide Solubility| & 31827 & 01 May-96 & $\mathbf{Y}$ & $4 B$ \\
\hline $\mathbf{Y}$ & & 841 & cs & Cesium & LOGSOLM & Log of the Radionuctide Solubility & $\cdot 32680$ & 01-May-96 & $\mathbf{Y}$ & 48 \\
\hline$Y$ & & 841 & cs & Cesium & LOGSOLM & L $L o g$ of the Radionuctide Solubility] & 32680 & 01-May-96 & $\mathbf{Y}$ & $\mathbf{4 B}$ \\
\hline $\mathbf{Y}$ & 11.35 & 843 & CULEBRA & $\begin{array}{l}\text { Culebra member of the } \\
\text { Rustler formation }\end{array}$ & DNSGRAIN & Material Grain Density & 32689 & 10-Jun-96 & $\mathbf{Y}$ & 1 \\
\hline $\mathbf{Y}$ & 11.35 & 843 & CULEBRA & $\begin{array}{l}\text { Culebra member of the } \\
\text { Rustler formation }\end{array}$ & DNSGRAIN & Material Grain Density & 32689 & 10-Jun-96 & $\mathbf{Y}$ & 1 \\
\hline $\mathbf{Y}$ & 11.35 & 843 & CULEBRA & $\begin{array}{l}\text { Culebra member of the } \\
\text { Rustler formation }\end{array}$ & DNSGRAIN & Material Grain Density & 32689 & $10-J u n-96$ & $\mathbf{Y}$ & 1 \\
\hline $\mathbf{Y}$ & 11.35 & 843 & CULEBRA & $\begin{array}{l}\text { Culebra member of the } \\
\text { Rustler formation }\end{array}$ & DNSGRAIN & Material Grain Density & 32689 & 10-Jur-95 & $\mathbf{Y}$ & 1 \\
\hline $\mathbf{Y}$ & & 851 & CULEBRA & $\begin{array}{l}\text { Culebra member of the } \\
\text { Rustler formation }\end{array}$ & FTORT & Fracture Tortuosity & 32541 & 09-May-96 & $\mathbf{Y}$ & $4 B$ \\
\hline $\mathbf{Y}$ & & 861 & CULEBRA & $\begin{array}{l}\text { Culebra member of the } \\
\text { Rustler formation }\end{array}$ & FTORT & Fracture Tortuosity & 32541 & 09-May-96 & $-Y$ & 48 \\
\hline $\mathbf{Y}$ & & 1659 & SR & Strontium & LOGSOLM & Log of the Radionuclide Solubility & 34352 & 01-May-96 & $\mathbf{Y}$ & 48 \\
\hline $\mathbf{Y}$ & & 1659 & SR & Strontium & LOGSOLM & Log of the Radionuclide Solubility & 34352 & 01-May-96 & $\mathbf{Y}$ & 48 \\
\hline $\mathbf{Y}$ & & 1661 & S_ANH_AB & $\begin{array}{l}\text { Salado anhydrite bods } \\
A \text { and } B \text {, intact and } \\
\text { fractured }\end{array}$ & DNSGRAIN & Material Grain Density & 34137 & 12 feb-96 & $\mathbf{Y}$ & $4 A$ \\
\hline $\mathbf{Y}$ & & 1661 & S_ANH_AB & $\begin{array}{l}\text { Salado anhydrite bods } \\
\text { A and B, intact and } \\
\text { fractured }\end{array}$ & DNSGRAIN & Material Grain Density & 34137 & 12-Fab-96 & $\mathbf{Y}$ & $4 A$ \\
\hline $\mathbf{Y}$ & & 1743 & S_MB138 & $\begin{array}{l}\text { Salado marker bed } \\
138 \text {, intact and } \\
\text { fractured }\end{array}$ & DNSGRAIN & Material Grain Density & 34441 & $01-F_{\theta b-96}$ & $\mathbf{Y}$ & 1 \\
\hline $\mathbf{Y}$ & & 1743 & S_MB138 & $\begin{array}{l}\text { Salado marker bed } \\
138 \text {, intact and } \\
\text { fractured }\end{array}$ & DNSGRAIN & Material Grain Density & 34441 & $01-F_{0 b}-96$ & $\mathbf{Y}$ & 1 \\
\hline $\mathbf{Y}$ & & 1784 & S_MB139 & $\begin{array}{l}\text { Salado marker bed } \\
139, \text { intact and } \\
\text { frectured }\end{array}$ & ONSGRAIN & Material Grain Density & 34579 & 01-Feb-96 & $\mathbf{Y}$ & 1 \\
\hline $\mathbf{Y}$ & & 1784 & S_MB139 & $\begin{array}{l}\text { Salado marker bed } \\
139, \text { intact and } \\
\text { fractured }\end{array}$ & DNSGRAIN & Material Grain Density & 34579 & 01-Fob-96 & $\mathbf{Y}$ & 1 \\
\hline $\mathbf{Y}$ & & 1992 & WAS_AREA & $\begin{array}{l}\text { Wasto emplacement } \\
\text { area and waste }\end{array}$ & DIRNCCHW & $\begin{array}{l}\text { Bulk density of ron containers, } \\
\text { CH waste }\end{array}$ & 32466 & 13-Feb-96 & $\mathbf{Y}$ & 2 \\
\hline
\end{tabular}


CCA Parameter Listing

\begin{tabular}{|c|c|c|c|c|c|c|c|c|c|c|}
\hline ID & \begin{tabular}{|c|} 
Clasedrif \\
cation of \\
Date \\
Category \\
(footnote 2)
\end{tabular} & $\begin{array}{l}\text { Distribution } \\
\text { Type }\end{array}$ & Ment & Median & Minimum & Mnoximum & Units & Ruference id & $\begin{array}{l}\text { Wes the date } \\
\text { dervioped } \\
\text { tnder an } \\
\text { NoA-1 } \\
\text { Program? }\end{array}$ & \begin{tabular}{|c|} 
Which \\
mothoods \\
were used to \\
quallfy \\
edsting \\
data? \\
(foothote 3)
\end{tabular} \\
\hline 669 & 1,3 & CONSTANT & $1.5000000 \mathrm{E}-02$ & $1.5000000 E-02$ & $1.5000000 \mathrm{E}-02$ & $1.5000000 E-02$ & NONE & WP035183 & $\mathbf{Y}$ & NA \\
\hline 669 & 1,3 & CONSTANT & $1.5000000 E-02$ & $1.5000000 E-02$ & $1.5000000 E-02$ & $1.5000000 E-02$ & NONE & WP035597 & $Y$ & N/A \\
\hline 669 & 1,3 & CONSTANT & $1.5000000 \mathrm{E}-02$ & $1.5000000 E-02$ & $1.5000000 E-02$ & $1.5000000 E-02$ & NONE & WP040514 & $\mathbf{Y}$ & N/A \\
\hline 670 & 1,3 & UNIFORM & $2.7600000 E-01$ & $2.7600000 E-01$ & $0.0000000 E+\infty 0$ & $5.5200000 E-01$ & NONE & $\begin{array}{c}\text { MEMO:VAUGHN } \\
\text { TIERNEY-11 }\end{array}$ & $\mathbf{Y}$ & NA \\
\hline 670 & 1,3 & UNIFORM & $2.7600000 E-01$ & $2.7600000 E-01$ & $0.0000000 E+\infty$ & $5.5200000 E-01$ & NONE & WP035268 & $\gamma$ & N/A \\
\hline 670 & 1,3 & UNIFORM & $2.7600000 E-01$ & $2.7600000 E-01$ & $0.0000000 \mathrm{E}+00$ & $5.5200000 E-01$ & NONE & WP035597 & $Y$ & NA \\
\hline 670 & 1,3 & UNIFORM & $27600000 E-01$ & $2.7600000 E-01$ & $0.0000000 E+\infty$ & $5.5200000 E-01$ & NONE & WP040514 & $Y$ & N/A \\
\hline 679 & 1,3 & UNIFORM & $7.5000000 E-02$ & $7.5000000 E-02$ & $0.0000000 E+\infty 0$ & $1.5000000 E-01$ & NONE & WP035268 & $\mathbf{Y}$ & N/A \\
\hline 671 & 1,3 & UNIFORM & $7.5000000 E-02$ & $7.5000000 E-02$ & $0.0000000 E+00$ & $1.5000000 E-01$ & NONE & WP038769 & $Y$ & N/A \\
\hline 671 & 1,3 & UNIFORM & $7.5000000 \mathrm{E}-02$ & $7.5000000 \mathrm{E}-02$ & $0.0000000 E+00$ & $1.5000000 E-01$ & NONE & WPO40432 & $Y$ & NA \\
\hline 671 & 1,3 & UNIFORM & $7.5000000 E-02$ & $7.5000000 E-02$ & $0.0000000 E+00$ & $1.5000000 E-01$ & NONE & WP040514 & $\mathbf{Y}$ & N/A \\
\hline 671 & 1,3 & UNIFORM & 7.5000000E-02 & $7.5000000 \mathrm{E}-02$ & $0.0000000 E+00$ & $1.5000000 E-01$ & NONE & WPO42681 & $\mathbf{Y}$ & NA \\
\hline 838 & Not Used & CONSTANT & $0.0000000 E+00$ & $0.0000000 \mathrm{E}+00$ & $0.0000000 E+00$ & $0.0000000 E+00$ & $\log$ (moless) & WP036724 & $\mathbf{Y}$ & NA \\
\hline 838 & Not Used & CONSTANT & $0,0000000 E+00$ & $0.0000000 E+00$ & $0.0000000 E+00$ & $0.0000000 E+00$ & $\log$ (moles) & WP040515 & $Y$ & NA \\
\hline 841 & Not Used & CONSTANT & $0.0000000 E+00$ & $0.0000000 E+\infty$ & $0.0000000 E+\infty 0$ & $0.0000000 E+00$ & $\log ($ motasn) & WP036724 & $\mathbf{Y}$ & NA \\
\hline 841 & Not Usod & CONSTANT & $0.0000000 E+\infty 0$ & $0.0000000 \mathrm{E}+\infty 0$ & $0.0000000 E+00$ & $0.0000000 E+\infty 0$ & $\log ($ molosn $)$ & WPO40515 & $Y$ & N/A \\
\hline 843 & 1 & CONSTANT & $2.8200000 \mathrm{E}+03$ & $2.8200000 E+03$ & $2.8200000 E+03$ & $2.8200000 E+03$ & $\mathrm{~kg} / \mathrm{m}^{\wedge} 3$ & WP035597 & $Y$ & 1 \\
\hline 643 & 1 & CONSTANT & $2.8200000 E+03$ & $2.8200000 E+03$ & $2.8200000 E+03$ & $2.8200000 E+03$ & $\mathrm{~kg} / \mathrm{m}^{\wedge} 3$ & WP037232 & $Y$ & 1 \\
\hline 843 & 1 & CONSTANT & $2.8200000 E+03$ & $2.8200000 E+03$ & $2.8200000 \mathrm{E}+03$ & $2.8200000 E+03$ & $\mathrm{~kg} / \mathrm{m}^{\wedge} 3$ & WP037841 & $\mathbf{Y}$ & 1 \\
\hline 843 & 1 & CONSTANT & $2.8200000 E+03$ & $2.8200000 E+03$ & $28200000 E+03$ & $2.8200000 E+03$ & $\mathrm{~kg} / \mathrm{m}^{\wedge} 3$ & WPO40434 & $Y$ & 1 \\
\hline 861 & 3,4 & CONSTANT & $1.0000000 E+\infty 0$ & $1.0000000 E+\infty 0$ & $1.0000000 E+00$ & $1.0000000 E+00$ & NONE & WP039365 & $\mathbf{Y}$ & NA \\
\hline 861 & 3.4 & CONSTANT & $1.0000000 \mathrm{E}+00$ & $1.0000000 E+00$ & $1.0000000 E+\infty$ & $1.0000000 E+00$ & NONE & WP040516 & $-Y$ & N/A \\
\hline 1659 & Not Used & CONSTANT & $0.0000000 E+00$ & $0.0000000 E+\infty$ & $0.0000000 \mathrm{E}+00$ & $0.0000000 E+00$ & $\log ($ moles $n)$ & WPO36724 & $\mathbf{Y}$ & NA \\
\hline 1659 & Not Used & CONSTANT & $0.0000000 E+00$ & $0.0000000 E+00$ & $0.0000000 E+00$ & $0.0000000 E+00$ & $\log ($ moles $n)$ & WP040515 & $Y$ & N/A \\
\hline 1661 & 1 & CONSTANT & $2.7500000 E+03$ & $2.7500000 \mathrm{E}+03$ & $2.7500000 E+03$ & $2.7500000 E+03$ & $\mathrm{~kg} / \mathrm{m}^{\wedge} \mathrm{3}$ & WP030599 & Y & N/A \\
\hline 1661 & 1 & CONSTANT & $2.7500000 E+03$ & $2.7500000 E+03$ & $2.7500000 \mathrm{E}+03$ & $2.7500000 E+03$ & $\mathrm{~kg} / \mathrm{m}^{\wedge} 3$ & WP035597 & $Y$ & N/A \\
\hline 1743 & 1 & CONSTANT & $2.7500000 \mathrm{E}+03$ & $2.7500000 E+03$ & $2.7500000 E+03$ & $2.7500000 E+03$ & $\mathrm{~kg} / \mathrm{m}^{\wedge} 3$ & WP030599 & $\mathbf{Y}$ & N/A \\
\hline 1743 & 1 & CONSTANT & $2.7500000 E+03$ & $2.7500000 E+03$ & $2.7500000 E+03$ & $2.7500000 \mathrm{E}+03$ & $\mathrm{~kg} / \mathrm{m}^{\wedge} 3$ & WP035597 & $Y$ & N/A \\
\hline 1784 & 1 & CONSTANT & $2.7500000 E+03$ & $2.7500000 E+03$ & $2.7500000 E+03$ & $2.7500000 E+03$ & $\mathrm{~kg} / \mathrm{m}^{\wedge} 3$ & WP030599 & $Y$ & NA \\
\hline 1784 & 1 & CONSTANT & $2.7500000 E+03$ & $2.7500000 E+03$ & $2.7500000 E+03$ & $2.7500000 E+03$ & $\mathrm{~kg} / \mathrm{m}^{\wedge} 3$ & WP035597 & $\mathbf{Y}$ & N/A \\
\hline 1992 & 2 & CONSTANT & $1.3900000 \mathrm{E}+02$ & $1.3900000 \mathrm{E}+02$ & $1.3900000 E+02$ & $1.3900000 E+02$ & $\mathrm{~kg} / \mathrm{m}^{\wedge} 3$ & WPO32328 & $\mathbf{Y}$ & N/A \\
\hline
\end{tabular}


CCA Parameter Listing

\begin{tabular}{|c|c|c|c|c|c|c|c|c|c|c|}
\hline $\begin{array}{c}\text { Parameter In } \\
\text { Drtabuen? } \\
\text { (YN) }\end{array}$ & $\begin{array}{l}\text { Ht the values } \\
\text { ured in CCA } \\
\text { aro not the } \\
\text { same as in } \\
\text { DB, provide } 4 x \\
\text { difference }\end{array}$ & D & Metertalio & Meteris Name & $\begin{array}{c}\text { Parametar } \\
\text { ID }\end{array}$ & Parameter Neme & $\begin{array}{l}\text { PRPID } \\
\text { (WPOA) }\end{array}$ & $\begin{array}{c}\text { Drtie Entry } \\
\text { Dato }\end{array}$ & $\begin{array}{l}\text { to the dSA } \\
\text { consiatent } \\
\text { whth the } \\
\text { peramoter } \\
\text { in DB? } \\
(\mathrm{Y}, \mathrm{NA})\end{array}$ & $\begin{array}{c}\text { Parametar } \\
\text { Category } \\
\text { (footnote 1) }\end{array}$ \\
\hline $\mathbf{Y}$ & & 1992 & WAS_AREA & $\begin{array}{l}\text { Waste emplacement } \\
\text { area and waste }\end{array}$ & DIRNCCHW & $\begin{array}{l}\text { Bulk densty of iron contaners, } \\
\mathrm{CH} \text { waste }\end{array}$ & 32486 & 13-Fob-96 & $\mathbf{Y}$ & 2 \\
\hline$Y$ & & 1993 & WAS_AREA & $\begin{array}{l}\text { Wasto emplacement } \\
\text { area and waste }\end{array}$ & DIRNCRHW & $\begin{array}{l}\text { Bulk density of iron containers, } \\
\text { RH waste }\end{array}$ & 32467 & $13-F a b-96$ & $\mathbf{Y}$ & 2 \\
\hline$\gamma$ & & 1993 & WAS_AREA & $\begin{array}{l}\text { Waste emplacoment } \\
\text { area and waste }\end{array}$ & DIRNCRHW & $\begin{array}{l}\text { Bulk density of iron containers. } \\
\text { RH waste }\end{array}$ & 32467 & 13-feb-96 & $Y$ & 2 \\
\hline $\mathbf{Y}$ & & 1993 & WAS_AREA & $\begin{array}{l}\text { Waste emplacement } \\
\text { area and waste }\end{array}$ & DIRNCRHW & $\begin{array}{l}\text { Bulk density of iron containers, } \\
\text { RH waste }\end{array}$ & 32467 & 13-Feb-96 & $\mathbf{Y}$ & 2 \\
\hline $\mathbf{Y}$ & & 1995 & WAS_AREA & $\begin{array}{l}\text { Waste emplacement } \\
\text { area and wasto }\end{array}$ & DPLSCCHW & $\begin{array}{l}\text { Bullk density of plastic Iners, CH } \\
\text { wasto }\end{array}$ & 32478 & 13-Fob-96 & $\mathbf{Y}$ & 2 \\
\hline $\mathbf{Y}$ & & 1995 & WAS_AREA & $\begin{array}{l}\text { Waste emplacement } \\
\text { area and waste }\end{array}$ & DPLSCCHW & $\begin{array}{l}\text { Bullk density of plastic liners, } \mathrm{CH} \\
\text { waste }\end{array}$ & 32478 & $13-F 0 b-96$ & $\mathbf{Y}$ & 2 \\
\hline $\mathbf{Y}$ & & 2037 & $\operatorname{cs} 137$ & Cesium 137 & INVCHD & $\begin{array}{l}\text { Inventory of Contact Handied } \\
\text { Design }\end{array}$ & 32683A & 29-0ct-96 & $\mathbf{Y}$ & 2 \\
\hline $\mathbf{Y}$ & & 2037 & CS137 & Cesium 137 & INVCHD & $\begin{array}{l}\text { Inventory of Contact Handled } \\
\text { Design }\end{array}$ & $32683 \mathrm{~A}$ & 29-0et-95 & $\mathbf{Y}$ & 2 \\
\hline $\mathbf{Y}$ & & 2037 & $\operatorname{cs} 137$ & Cesium 137 & INVCHD & $\begin{array}{l}\text { Inventory of Contact Handled } \\
\text { Design }\end{array}$ & $32683 \mathrm{~A}$ & $29-0 c t-96$ & $\mathbf{Y}$ & 2 \\
\hline $\mathbf{Y}$ & & 2037 & $\operatorname{Cs} 137$ & Casium 137 & INVCHD & $\begin{array}{l}\text { Inventory of Contact Handled } \\
\text { Design }\end{array}$ & $32683 A$ & 29-0ct-96 & $\mathbf{Y}$ & 2 \\
\hline $\mathbf{Y}$ & & 2038 & PM147 & Promethium 147 & INVCHD & $\begin{array}{l}\text { Irventory of Contact Handled } \\
\text { Design }\end{array}$ & $33233 \mathrm{~A}$ & 29-0ct-96 & $\mathbf{Y}$ & 2 \\
\hline $\mathbf{Y}$ & & 2038 & PM147 & Promethium 147 & INVCHD & $\begin{array}{l}\text { Inventory of Contact Handled } \\
\text { Design }\end{array}$ & $33233 A$ & $29-0 c t-96$ & $\mathbf{Y}$ & 2 \\
\hline Y & & 2038 & PM147 & Promethium 147 & INVCHD & $\begin{array}{l}\text { Inventory of Contact Handled } \\
\text { Design }\end{array}$ & $33233 A$ & $29-0 a t-96$ & Y & 2 \\
\hline $\mathbf{Y}$ & & 2039 & SR9O & Strontium 90 & INVCHD & $\begin{array}{l}\text { Inventory of Contact Handled } \\
\text { Design } \\
\end{array}$ & $34354 \mathrm{~A}$ & $29-0 c t-96$ & $\mathbf{Y}$ & 2 \\
\hline$Y$ & & 2039 & SR9O & Strontium 90 & INVCHD & $\begin{array}{l}\text { Inventory of Contact Handled } \\
\text { Design }\end{array}$ & $34354 A$ & 29-0ct-96 & $\mathbf{Y}$ & 2 \\
\hline $\mathbf{Y}$ & & 2039 & SR90 & Strontium 90 & INVCHD & $\begin{array}{l}\text { Imventory of Contact Handled } \\
\text { Design }\end{array}$ & $34354 A$ & $29-0 c t-96$ & $\mathbf{Y}$ & 2 \\
\hline $\mathbf{Y}$ & & 2039 & SR9O & Strontivem 90 & INVCHD & $\begin{array}{l}\text { Inventory of Contact Handled } \\
\text { Design }\end{array}$ & $34354 \mathrm{~A}$ & 29-0ct-96 & $\mathbf{Y}$ & 2 \\
\hline Y & & 2040 & WAS_AREA & $\begin{array}{l}\text { Waste emplacement } \\
\text { area and waste }\end{array}$ & DIRONCHW & $\begin{array}{l}\text { Average density of iron-based } \\
\text { material in } \mathrm{CH} \text { waste }\end{array}$ & 32468 & 13-Feb-96 & $\mathbf{Y}$ & 2 \\
\hline$Y$ & & 2040 & WAS_AREA & $\begin{array}{l}\text { Waste emplacement } \\
\text { area and waste }\end{array}$ & DIRONCHW & $\begin{array}{l}\text { Average density of iron-based } \\
\text { material in } \mathrm{CH} \text { waste }\end{array}$ & 32458 & 13-feb-96 & $\mathbf{Y}$ & 2 \\
\hline$Y$ & & 2041 & WAS_AREA & $\begin{array}{l}\text { Waste emplacement } \\
\text { area and waste }\end{array}$ & DCELLCHW & $\begin{array}{l}\text { Average density of cellulosics in } \\
\text { CH waste }\end{array}$ & 32454 & 13-feb-96 & $Y$ & 2 \\
\hline Y & & 2041 & WAS_AREA & $\begin{array}{l}\text { Waste emplacement } \\
\text { area and waste }\end{array}$ & DCELLCHW & $\begin{array}{l}\text { Average density of cellulosics in } \\
\text { CH waste }\end{array}$ & 32464 & 13-Feb-96 & $\mathbf{Y}$ & 2 \\
\hline $\mathbf{Y}$ & & 2042 & WAS_AREA & $\begin{array}{l}\text { Waste emplacement } \\
\text { area and waste }\end{array}$ & DRUBBCHW & $\begin{array}{l}\text { Average density of rubber in CH } \\
\text { waste }\end{array}$ & 32481 & 13-Feb-96 & $\mathbf{Y}$ & 2 \\
\hline $\mathbf{Y}$ & & 2042 & WAS_AREA & $\begin{array}{l}\text { Waste emplacement } \\
\text { area and wasto }\end{array}$ & DRUBBCHW & $\begin{array}{l}\text { Average density of rubber in } \mathrm{CH} \\
\text { weste }\end{array}$ & 32481 & 13-Feb-96 & Y & 2 \\
\hline Y & & 2043 & WAS_AREA & $\begin{array}{l}\text { Waste emplacement } \\
\text { area and waste }\end{array}$ & DPLASCHW & $\begin{array}{l}\text { Average density of plastics in } \mathrm{CH} \\
\text { waste }\end{array}$ & 32474 & 13 -Feb-96 & $\mathbf{Y}$ & 2 \\
\hline $\mathbf{Y}$ & & 2043 & WAS_AREA & $\begin{array}{l}\text { Waste emplacoment } \\
\text { area and waste }\end{array}$ & DPLASCHW & $\begin{array}{l}\text { Average density of plasties in } \mathrm{CH} \\
\text { waste }\end{array}$ & 32474 & 13-Feb-96 & $-X$ & 2 \\
\hline $\mathbf{Y}$ & & 2044 & WAS_AREA & $\begin{array}{l}\text { Waste emplacement } \\
\text { area and waste }\end{array}$ & DIRONRHW & \begin{tabular}{|l|} 
Average density of iron-based \\
material in RH waste
\end{tabular} & 32469 & 13-Feb-96 & $\mathbf{Y}$ & 2 \\
\hline $\mathbf{Y}$ & & 2044 & WAS_AREA & $\begin{array}{l}\text { Waste emplacement } \\
\text { area and waste }\end{array}$ & DIRONRHW & $\begin{array}{l}\text { Average density of iron-based } \\
\text { material in RH waste }\end{array}$ & 32469 & 13-Feb-96 & $\mathbf{Y}$ & 2 \\
\hline$Y$ & & 2046 & WAS_AREA & $\begin{array}{l}\text { Wasto emplacement } \\
\text { area and waste }\end{array}$ & DRUBBRHW & $\begin{array}{l}\text { Average density of nubber in RH } \\
\text { waste }\end{array}$ & 32483 & 13-Feb-96 & $Y$ & 2 \\
\hline $\mathbf{Y}$ & & 2046 & WAS_AREA & $\begin{array}{l}\text { Wasto emplacement } \\
\text { area and waste }\end{array}$ & DRUBBRHW & $\begin{array}{l}\text { Average density of rubber in RH } \\
\text { waste }\end{array}$ & 32483 & 13-Feb-96 & $Y$ & 2 \\
\hline$Y$ & & 2049 & CAVITY_3 & Cavity for Shaft & CAP_MOD & $\begin{array}{l}\text { Model number, capillary pressure } \\
\text { model }\end{array}$ & 31767 & 17 -feb-96 & $\mathbf{Y}$ & 4B \\
\hline Y & & 2049 & CAVITY_3 & Cavity for Shaft & CAP_MOD & $\begin{array}{l}\text { Model number, capiliary pressure } \\
\text { modal }\end{array}$ & 31767 & 17 Feb-96 & $\mathbf{Y}$ & $4 B$ \\
\hline $\mathbf{Y}$ & & 2049 & CAVTTY_3 & Cavity for Shaft & CAP_MOD & $\begin{array}{l}\text { Model number, capillary pressure } \\
\text { model }\end{array}$ & 31767 & 17 Feb-96 & $\mathbf{Y}$ & 48 \\
\hline Y & & 2051 & CAVITY_3 & Cavity for Shaft & COMP_RCK & Bulk Compressibilty & 31769 & 17-feb-96 & $Y$ & $4 B$ \\
\hline$Y$ & & 2051 & CAVIT__3 & Cavity for Shaft & COMP_RCK & Bulk Compressibilty & 31769 & 17-Feb-96 & $Y$ & 48 \\
\hline$Y$ & & 2051 & CAVITY_3 & Cavity for Shaf & COMP_RCK & Bulk Compressibility & 31769 & 17 feb-96 & $\mathbf{Y}$ & $4 B$ \\
\hline Y & & 2052 & CAVITY_3 & Cavity for Shaft & PORE_DIS & $\begin{array}{l}\text { Brooks-Corby pore distribution } \\
\text { parameter }\end{array}$ & 31777 & 17feb-96 & $\mathbf{Y}$ & 4B \\
\hline $\mathbf{Y}$ & & 2052 & CAVITY_3 & Cavity for Shaft & PORE_DIS & $\begin{array}{l}\text { Brooks-Coray pore distribution } \\
\text { parameter }\end{array}$ & 31777 & 17-Feb-96 & $\mathbf{Y}$ & 48 \\
\hline
\end{tabular}


CCA Parameter Listing

\begin{tabular}{|c|c|c|c|c|c|c|c|c|c|c|}
\hline DD & $\begin{array}{c}\text { Clacelfi- } \\
\text { cation of } \\
\text { Data } \\
\text { Cattegory } \\
\text { (footnote 2) }\end{array}$ & $\begin{array}{l}\text { Distribution } \\
\text { Type }\end{array}$ & Mean & Median & Mintroum & -Mexirnum & Unit & Roforence id & $\begin{array}{l}\text { Wes the ditt } \\
\text { deviloped } \\
\text { undor } \\
\text { NOA-s } \\
\text { Program? } \\
\text {. }\end{array}$ & $\begin{array}{c}\text { Which } \\
\text { mothod } \\
\text { wore unod to } \\
\text { qually } \\
\text { oxisting } \\
\text { data? } \\
\text { (footnoto 3) }\end{array}$ \\
\hline 1992 & 2 & CONSTANT & $1.3900000 E+02$ & $1.3900000 E+02$ & $1.3900000 E+02$ & $1.3900000 \mathrm{E}+02$ & $\mathrm{~kg} / \mathrm{m}^{\wedge} 3$ & WP035048 & $\mathbf{Y}$ & NA \\
\hline 1993 & 2 & CONSTANT & $2.5910000 E+03$ & $2.5910000 \mathrm{E}+03$ & $2.5910000 E+03$ & $2.5910000 E+03$ & $\mathrm{~kg} / \mathrm{m}^{\wedge} 3$ & WPO32328 & $Y$ & NA \\
\hline 1993 & 2 & CONSTANT & $2.5910000 E+03$ & $2.5910000 E+03$ & $2.5910000 \mathrm{E}+03$ & $2.5910000 E+03$ & $\mathrm{~kg} / \mathrm{m}^{\wedge} \mathrm{3}$ & WPO36048 & $\mathbf{Y}$ & N/A \\
\hline 1993 & 2 & CONSTANT & $2.5910000 \mathrm{E}+03$ & $2.5910000 E+03$ & $2.5910000 E+03$ & $2.5910000 E+03$ & $\mathrm{~kg} / \mathrm{m}^{\wedge} 3$ & WP038449 & $Y$ & NA \\
\hline 1995 & 2 & CONSTANT & $26000000 E+01$ & $26000000 E+09$ & $2.6000000 E+01$ & $2.6000000 E+01$ & $\mathrm{~kg} / \mathrm{m}^{\wedge 3}$ & WP032328 & $\mathbf{Y}$ & N/A \\
\hline 1995 & 2 & CONSTANT & $2.6000000 E+01$ & $2.6000000 E+01$ & $26000000 E+01$ & $2.6000000 E+01$ & $\mathrm{~kg} / \mathrm{m}^{\wedge} 3$ & WPO36048 & $Y$ & N/A \\
\hline 2037 & 2 & CONSTANT & $3.3500000 E+03$ & $3.3500000 E+03$ & $3.3500000 E+03$ & $3.3500000 E+03$ & $\mathbf{C i}$ & WP037061 & $Y$ & N/A \\
\hline 2037 & 2 & CONSTANT & $3.3500000 \mathrm{E}+03$ & $3.3500000 E+03$ & $3.3500000 E+03$ & $3.3500000 E+03$ & $\mathrm{Ci}$ & WP039260 & $Y$ & NA \\
\hline 2037 & 2 & CONSTANT & $3.3500000 E+03$ & $3.3500000 E+03$ & $3.3500000 \mathrm{E}+03$ & $3.3500000 E+03$ & $\mathrm{Ci}$ & WPO41559 & $Y$ & NA \\
\hline 2037 & 2 & CONSTANT & $3.3500000 E+03$ & $3.3500000 E+03$ & $3.3500000 E+03$ & $3.3500000 E+03$ & $\mathrm{Ci}$ & WPO41560 & $Y$ & N/A \\
\hline 2038 & 2 & CONSTANT & $3.4300000 E-04$ & $3.4300000 E-04$ & $3.4300000 E-04$ & $3.4300000 E-04$ & Ci & WP037061 & $\mathbf{Y}$ & NA \\
\hline 2038 & 2 & CONSTANT & $3.4300000 E-04$ & $3.4300000 \mathrm{E}-0.4$ & $3.4300000 E-04$ & $3.4300000 E-04$ & $\mathrm{Ci}$ & WP041559 & $Y$ & NA \\
\hline 2038 & 2 & CONSTANT & $3.4300000 E-04$ & $3.4300000 E-04$ & $3.4300000 \mathrm{E}-04$ & $3.4300000 E-04$ & $\mathbf{C i}$ & WPO41550 & $\mathbf{Y}$ & NA \\
\hline 2039 & 2 & CONSTANT & $2.7700000 E+03$ & $2.7700000 E+03$ & $2.7700000 \mathrm{E}+03$ & $2.7700000 E+03$ & $\mathbf{C i}$ & WP037061 & $Y$ & NA \\
\hline 2039 & 2 & CONSTANT & $2.7700000 E+03$ & $2.7700000 E+03$ & $27700000 E+03$ & $2.7700000 \mathrm{E}+03$ & $\mathbf{C i}$ & WP039250" & $\mathbf{Y}$ & N/A \\
\hline 2039 & 2 & CONSTANT & $2.7700000 E+03$ & $2.7700000 E+03$ & $27700000 \mathrm{E}+03$ & $2.7700000 E+03$ & $\mathbf{C i}$ & WP041559 & $\mathbf{Y}$ & NA \\
\hline 2039 & 2 & CONSTANT & $2.7700000 E+03$ & $2.7700000 E+03$ & $2.7700000 E+03$ & $2.7700000 E+03$ & $\mathbf{C i}$ & WP041560 & $\mathbf{Y}$ & N/A \\
\hline 2040 & 2 & CONSTANT & $1.7000000 \mathrm{E}+02$ & $1.7000000 E+02$ & $1.7000000 \mathrm{E}+02$ & $1.7000000 E+02$ & $\mathrm{~kg} / \mathrm{m}^{\wedge} 3$ & WP032328 & $\mathbf{Y}$ & NA \\
\hline 2040 & 2 & CONSTANT & $1.7000000 E+02$ & $1.7000000 E+02$ & $1.7000000 \mathrm{E}+02$ & $1.7000000 E+02$ & $\mathrm{~kg} / \mathrm{m}^{\wedge} 3$ & WPO35048 & $\mathbf{Y}$ & NA \\
\hline 2041 & 2 & CONSTANT & 5.4000000E +01 & $5.4000000 E+01$ & $5.4000000 E+01$ & $5.4000000 E+01$ & $\mathrm{~kg} / \mathrm{m}^{\wedge} 3$ & WPO32328 & $Y$ & N/A \\
\hline 2041 & 2 & CONSTANT & $5.4000000 \mathrm{E}+01$ & $5.4000000 E+01$ & $5.4000000 \mathrm{E}+01$ & $5.4000000 E+01$ & $\mathrm{~kg} / \mathrm{m}^{\wedge} 3$ & WPO36048 & $\mathbf{Y}$ & N/A \\
\hline 2042 & 2 & CONSTANT & $1.0000000 E+01$ & $1.0000000 E+01$ & $1.0000000 E+01$ & $1.0000000 E+01$ & $\mathrm{~kg} / \mathrm{m}^{\wedge} 3$ & WPO32328 & $\mathbf{Y}$ & N/A \\
\hline 2042 & 2 & CONSTANT & 1.0000000E+01 & $1.0000000 E+01$ & $1.0000000 E+01$ & $1.0000000 E+01$ & $\mathrm{~kg} / \mathrm{m}^{\wedge} 3$ & WPO36048 & $\mathbf{Y}$ & N/A \\
\hline 2043 & 2 & CONSTANT & $3.4000000 E+01$ & $3.4000000 E+01$ & $3.4000000 E+01$ & $3.4000000 E+01$ & $\mathrm{~kg} / \mathrm{m}^{\wedge} 3$ & WPO32328 & $Y$ & N/A \\
\hline 2043 & 2 & CONSTANT & $3.4000000 E+01$ & $3.4000000 E+01$ & $3.4000000 E+01$ & $3.4000000 E+01$ & $\mathrm{~kg} / \mathrm{m}^{\wedge} 3$ & WP036048 & $-y$ & N/A \\
\hline 2044 & 2 & CONSTANT & $1.0000000 E+02$ & $1.0000000 E+02$ & $1.0000000 E+02$ & $1.0000000 E+02$ & $\mathrm{~kg} / \mathrm{m}^{\wedge} 3$ & WPO32328 & $Y$ & NA \\
\hline 2044 & 2 & CONSTANT & $1.0000000 E+02$ & $1.0000000 E+02$ & $1.0000000 E+02$ & $1.0000000 E+02$ & $\mathrm{~kg} / \mathrm{m}^{\wedge} \mathrm{3}$ & WPO36048 & $\mathbf{Y}$ & N/A \\
\hline 2046 & 2 & CONSTANT & $3.3000000 E+\infty 0$ & $3.3000000 E+00$ & $3.3000000 E+00$ & $3.3000000 E+00$ & $\mathrm{~kg} / \mathrm{m}^{\wedge} 3$ & WPO32328 & $Y$ & NA \\
\hline 2046 & 2 & CONSTANT & $3.3000000 \mathrm{E}+00$ & $3.3000000 E+00$ & $3.3000000 E+\infty 0$ & $3.3000000 E+00$ & $\mathrm{~kg} / \mathrm{m}^{\wedge} 3$ & WPO35048 & $\mathbf{Y}$ & N/A \\
\hline 2049 & 1,3 & CONSTANT & $1.0000000 E+\infty 0$ & $1.0000000 E+\infty$ & $1.0000000 E+\infty 0$ & $1.0000000 \mathrm{E}+00$ & NONE & $\begin{array}{c}\text { MEMO:VAUGHNI } \\
\text { TIERNEY-9 }\end{array}$ & $\mathbf{Y}$ & NA \\
\hline 2049 & 1,3 & CONSTANT & $1.0000000 E+\infty 0$ & $1.0000000 E+\infty 0$ & $1.0000000 E+00$ & $1.0000000 E+\infty 0$ & NONE & WP035597 & $\mathbf{Y}$ & N/A \\
\hline 2049 & 1,3 & CONSTANT & $1.0000000 E+\infty 0$ & $1.0000000 E+00$ & $1.0000000 E+00$ & $1.0000000 \mathrm{E}+\infty 0$ & NONE & WP040514 & $\mathbf{Y}$ & N/A \\
\hline 2051 & 1.3 & CONSTANT & $0.0000000 \mathrm{E}+00$ & $0.0000000 E+\infty 0$ & $0.0000000 E+\infty$ & $0.0000000 \mathrm{E}+\infty 0$ & $P a^{n}-1$ & $\begin{array}{l}\text { MEMO:VAUGHN } \\
\text { TIERNEY-9 }\end{array}$ & $\mathbf{Y}$ & N/A \\
\hline 2051 & 1,3 & CONSTANT & $0.0000000 \equiv+00$ & $0.0000000 E+\infty$ & $0.0000000 \mathrm{E}+00$ & $0.0000000 E+\infty 0$ & $\mathrm{~Pa}^{\wedge}-1$ & WP035597 & $Y$ & N/A \\
\hline 2051 & 1,3 & CONSTANT & $0.0000000 E+00$ & $0.0000000 \mathrm{E}+00$ & $0.0000000 E+00$ & $0.0000000 E+00$ & $P a^{n-1}$ & WPO40514 & $\bar{Y}$ & N/A \\
\hline 2052 & 1,3 & CONSTANT & $7.0000000 E-01$ & $7.0000000 E-01$ & 7.0000000E-01 & $7.0000000 E-01$ & NONE & $\begin{array}{l}\text { MEMO:VAUGHN } \\
\text { TIERNEY-9 }\end{array}$ & $\mathbf{Y}$ & NA \\
\hline 2052 & 1,3 & CONSTANT & $7.0000000 E-01$ & $7.0000000 E-01$ & $7.0000000 E-01$ & $7.0000000 \mathrm{E}-01$ & NONE & WPO35597 & $Y$ & NA \\
\hline
\end{tabular}


CCA Parameter Listing

\begin{tabular}{|c|c|c|c|c|c|c|c|c|c|c|}
\hline $\begin{array}{c}\text { Parmiter in } \\
\text { Database? } \\
\text { (Noi) }\end{array}$ & $\begin{array}{l}\text { If the values } \\
\text { used in cCA } \\
\text { ore not the } \\
\text { same in in } \\
\text { oB, provide } \\
\text { difference }\end{array}$ & ID & Material ID & Materlal Name & $\begin{array}{l}\text { Parameter } \\
\text { in }\end{array}$ & Permeter Nem & $\begin{array}{l}\text { PRPD DD } \\
\text { (WPO *) }\end{array}$ & $\begin{array}{c}\text { Denta Entry } \\
\text { Dats }\end{array}$ & $\begin{array}{l}\text { is the } 464 \\
\text { convalstent } \\
\text { with the } \\
\text { peremeter } \\
\text { in DB? } \\
(Y, N A)\end{array}$ & $\begin{array}{l}\text { Paramater } \\
\text { Category } \\
\text { (footnote 1) }\end{array}$ \\
\hline $\mathbf{Y}$ & & 2052 & CAVITY_3 & Cavity for Sheft & PORE_DIS & $\begin{array}{l}\text { Brooks-Corey pore distribution } \\
\text { parameter }\end{array}$ & 31777 & 17 Fob-96 & $\boldsymbol{Y}$ & 48 \\
\hline $\mathbf{Y}$ & & 2053 & CAVITY_3 & Cavity for Shaft & POROSITY & Effective porosity & 31778 & 17Fob-96. & $\mathbf{Y}$ & $4 B$ \\
\hline $\mathbf{Y}$ & & 2053 & CAVITY_3 & Cavity for Shatt & POROSTY & Effective porosity & 31778 & 17 Fab-96 & $\mathbf{Y}$ & $4 B$ \\
\hline$Y$ & & 2053 & CAVITY_ 3 & Cavity for Shaft & POROSITY & Effective porosity & 31778 & 17 feb-96 & $\mathbf{Y}$ & 48 \\
\hline $\mathbf{Y}$ & & 2054 & CAVITY_3 & Cavity for Shaft & PRMX_LOG & $\begin{array}{l}\text { Log of intrinsic permeability. X- } \\
\text { direction }\end{array}$ & 31781 & 20-Fab-96 & $\mathbf{Y}$ & $4 B$ \\
\hline $\mathbf{Y}$ & & 2054 & CAVITY_3 & Cavity for Shaft & PRMX_LOG & $\begin{array}{l}\text { Log of intrinsic permeability, } X- \\
\text { direction }\end{array}$ & 31781 & 20 feb-96 & $\mathbf{Y}$ & $4 B$ \\
\hline $\mathbf{Y}$ & & 2054 & CAVTrY_3 & Cavity for Shaft & PRMX_LOG & $\begin{array}{l}\text { Log of intrunsic permeability, } X \text { - } \\
\text { direction }\end{array}$ & 31781 & 20-Feb-96 & $\mathbf{Y}$ & $4 B$ \\
\hline $\mathbf{Y}$ & & 2055 & CAVITY_3 & Cavity for Shaft & PRMY_LOG & $\begin{array}{l}\text { Log of intrinsic permeability, Y- } \\
\text { direction }\end{array}$ & 31782 & 20-Feb-96 & $\mathbf{Y}$ & $4 B$ \\
\hline $\mathbf{Y}$ & & 2055 & CAVITY_3 & Cavity for Shaft & PRMY_LOG & $\begin{array}{l}\text { Log of intrinsic permeability, Y- } \\
\text { direction }\end{array}$ & 31782 & 20-Fob-96 & $Y$ & $4 B$ \\
\hline $\mathbf{Y}$ & & 2055 & CAVITY_3 & Cavity for Shat & PREY_LOG. & $\begin{array}{l}\text { Log of intrinsic permeability, } Y \text { - } \\
\text { direction. }\end{array}$ & 31782 & 20 Fob-96 & $\mathbf{Y}$ & $4 B$ \\
\hline $\mathbf{Y}$ & & 2056 & CAVITY_3 & Cavity for Shaft & PRMZ_LOG & $\begin{array}{l}\text { Log of intrinsic permeability, Z- } \\
\text { direction }\end{array}$ & 31783 & 20 Feb-96 & $Y$ & $4 B$ \\
\hline $\mathbf{Y}$ & & 2056 & CAVITY_3 & Cavity for Shaft & PRMZ_LOG & $\begin{array}{l}\text { Log of intrinsic permeability, Z- } \\
\text { direction }\end{array}$ & 31783 & 20 -Feb-96 & $\mathbf{Y}$ & $\mathbf{A B}$ \\
\hline $\mathbf{Y}$ & & 2056 & CAVITY_3 & Cavity for Shaft & PRMZ_LOG & $\begin{array}{l}\text { Log of intrinsic permeability, Z- } \\
\text { direction }\end{array}$ & 31783 & 20Feb-96 & $\mathbf{Y}$ & 4B \\
\hline $\mathbf{Y}$ & & 2058 & CAVITY_3 & Cavity for Shaft & RELP_MOD & $\begin{array}{l}\text { Model number, relative } \\
\text { permeability model }\end{array}$ & 31785 & 17Feb-96 & $Y$ & AB \\
\hline $\mathbf{Y}$ & & 2058 & CAVITY_3 & Cavity for Shatt & RELP_MOD & $\begin{array}{l}\text { Model number, relatrvo } \\
\text { permeability model }\end{array}$ & 31785 & 17 Fob-96 & $\mathbf{Y}$ & 4B \\
\hline $\mathbf{Y}$ & & 2058 & CAVITY_3 & Cavity for Shaft & RELP_MOD & $\begin{array}{l}\text { Model number, relative } \\
\text { permeability model }\end{array}$ & 31785 & $17-F_{0 b-96}$ & $Y$ & $4 B$ \\
\hline $\mathbf{Y}$ & & 2059 & CAVITY_3 & Cavity for Shaft & SAT_RGAS & Residual Gas Saturation & 31789 & $17-F a b-96$ & $\mathbf{Y}$ & $4 B$ \\
\hline$Y$ & & 2059 & CAVITY_3 & Cavity for Shatt & SAT_RGAS & Residual Gas Saturation & 31789 & 17 Fob-96 & $Y$ & 48 \\
\hline $\mathbf{Y}$ & & 2059 & CAVIrr_3 & Cavity for Shaft & SAT_RGAS & Residual Gas Saturation & 31789 & 17-Fob-96 & $Y$ & 48 \\
\hline $\mathbf{Y}$ & & 2060 & CAVITY_4 & Cavity for Borehole & CAP_MOD & $\begin{array}{l}\text { Model number, capillary pressure } \\
\text { model }\end{array}$ & 31791 & $17-F a b-96$ & $\mathbf{Y}$ & $4 B$ \\
\hline $\mathbf{Y}$ & & 2060 & CAVITY_4 & Cavity for Borehole & CAP_MOD & $\begin{array}{l}\text { Model number, capillary pressure } \\
\text { model }\end{array}$ & 31791 & 17 fob-96 & $Y$ & $4 B$ \\
\hline $\mathbf{Y}$ & & 2060 & CAVITY_4 & Cavity for Borehole & CAP_MOD & $\begin{array}{l}\text { Model number, capillary pressure } \\
\text { model }\end{array}$ & 31791 & 17-Feb-96 & $\mathbf{Y}$ & 48 \\
\hline $\mathbf{Y}$ & & 2062 & CAVITY_4 & Cavity for Borehale & COMP_RCK & Bulk Compressibility & 31794 & 17 Feb-96 & $\mathbf{Y}$ & 48 \\
\hline $\mathbf{Y}$ & & 2052 & CAVITY_4 & Cavity for Borehole & COMP_RCK & Bulk Compressibility & 31794 & 17-Feb-95 & $\mathbf{Y}$ & 4B \\
\hline $\mathbf{Y}$ & & 2052 & CAVITY_4 & Cavity for Borehole & COMP_RCK & Bulk Compressibility & 31794 & 17-feb-95 & $\mathbf{Y}$ & $4 B$ \\
\hline $\mathbf{Y}$ & & 2063 & CAVTrY_4 & Cavity for Borehole & PORE_DIS & $\begin{array}{l}\text { Brooks-Corey pore distribution } \\
\text { parameter }\end{array}$ & 31801 & 17fob-95 & $Y$ & 48 \\
\hline$Y$ & & 2063 & CAVITY_4 & Cavity for Borehole & PORE_DIS & $\begin{array}{l}\text { Brooks-Corey pore distribution } \\
\text { parameter }\end{array}$ & 31801 & 17-Feb-96 & $--Y$ & $4 B$ \\
\hline $\mathbf{Y}$ & & 2063 & CAVITY_4 & Cavity for Borehole & PORE_DIS & $\begin{array}{l}\text { Brooks-Corey pore distribution } \\
\text { parameter }\end{array}$ & 31801 & 17-Feb-96 & $\mathbf{Y}$ & $4 B$ \\
\hline $\mathbf{Y}$ & & 2054 & CAVITY_4 & Cavity for Borehole & POROSTYY & Efrective porosity & 31802 & 17-Fob-95 & $\mathbf{Y}$ & 4B \\
\hline $\mathbf{Y}$ & & 2054 & CAVITY_4 & Cavity for Borehole & POROSTY & Effective porosity & 31802 & 17 fob-96 & $\mathbf{Y}$ & 48 \\
\hline $\mathbf{Y}$ & & 2064 & CAVITY_4 & Cavity for Borehole & POROSITY & Effective porosity & 31802 & 17feb-96 & $Y$ & 48 \\
\hline $\mathbf{Y}$ & & 2065 & CAVITY 4 & Cavity for Borehole & PRMX_LOG & $\begin{array}{l}\text { Log of intrinsic permeability, } X- \\
\text { direction }\end{array}$ & 31817 & $20-F e b-96$ & $Y$ & $A B$ \\
\hline $\mathbf{Y}$ & & 2065 & CAVITY_4 & Cavity for Borehole & PRMX_LOG & $\begin{array}{l}\text { Log of intrinsic permeability. } X \text { - } \\
\text { direction }\end{array}$ & 31817 . & 20-Fob-96 & $\mathbf{Y}$ & $4 B$ \\
\hline $\mathbf{Y}$ & & 2065 & CAVITY_4 & Cavity for Borehole & PRMX_LOG & $\begin{array}{l}\text { Log of intrinsic permeability, } X- \\
\text { direction }\end{array}$ & 31817 & 20-Fob-96 & $\mathbf{Y}$ & $A B$ \\
\hline $\mathbf{Y}$ & & 2066 & CAVITY_4 & Cavity for Borehole & PRMY_LOG & $\begin{array}{l}\text { Log of intrinsic permeability, Y. } \\
\text { direction }\end{array}$ & 31818 & 20-Fob-96 & $\mathbf{Y}$ & AB \\
\hline $\mathbf{Y}$ & & 2066 & CAVITY_4 & Cavity for Borehole & PRMY_LOG & $\begin{array}{l}\text { Log of intrnssic permeability, Y- } \\
\text { direction }\end{array}$ & 31818 & $20-50 b-96$ & $\mathbf{Y}$ & $4 B$ \\
\hline $\mathbf{Y}$ & & 2066 & CAVITY_4 & Cavity for Borehole & PRMY_LOG & $\begin{array}{l}\text { Log of intrinsic permeability, Y- } \\
\text { direction }\end{array}$ & 31818 & 20-Fob-96 & $\mathbf{Y}$ & 48 \\
\hline $\mathbf{Y}$ & & 2067 & CAVITY_4 & Cavity for Borehole & PRMZ_LOG & $\begin{array}{l}\text { Log of intrinsic permeabiluty. Z- } \\
\text { drection }\end{array}$ & 31819 & 20Fob-96 & $\mathbf{Y}$ & 48 \\
\hline $\mathbf{Y}$ & & 2067 & CAVITY_4 & Cavity for Borehole & PRMZ_LOG & $\begin{array}{l}\text { Log of intrinsic permeability, } \mathrm{Z} \text { - } \\
\text { direction }\end{array}$ & 31819 & $20-F$ b- 96 & $\mathbf{Y}$ & $4 B$ \\
\hline
\end{tabular}


CCA Parameter Listing

\begin{tabular}{|c|c|c|c|c|c|c|c|c|c|c|}
\hline ID & \begin{tabular}{|c|} 
Clasedfi- \\
cation of \\
Data \\
Category \\
(footnote 2)
\end{tabular} & $\begin{array}{l}\text { Dietribution } \\
\text { Typo }\end{array}$ & Mean & Median & Minimum & Meximum & Units & Roference ID & $\begin{array}{l}\text { Wes the data } \\
\text { developed } \\
\text { under an } \\
\text { NOA-1 } \\
\text { Program? }\end{array}$ & $\begin{array}{c}\text { Which } \\
\text { methods } \\
\text { wero used to } \\
\text { quality } \\
\text { oxisting } \\
\text { ditta? } \\
\text { (footnote 3) }\end{array}$ \\
\hline 2052 & 1,3 & CONSTANT & $7.0000000 E-01$ & $7.0000000 E-01$ & 7.0000000E-01 & $7.0000000 \mathrm{E}-01$ & NONE & WPO40514 & $Y$ & NA \\
\hline 2053 & 1,3 & CONSTANT & $1.0000000 E+\infty$ & $1.0000000 \mathrm{E}+00$ & $1.0000000 \mathrm{E}+00$ & $1.0000000 \mathrm{E}+\infty 0$ & NONE & $\begin{array}{c}\text { MEMO:VAUGHNI } \\
\text { TIERNEY-9 }\end{array}$ & $\mathbf{Y}$ & N/A \\
\hline 2053 & 1,3 & CONSTANT & $1.0000000 \mathrm{E}+00$ & $1.0000000 E+00$ & $1.0000000 \mathrm{E}+00$ & $1.0000000 \mathrm{E}+00$ & NONE & WP035597 & $Y$ & N/A \\
\hline 2053 & 1,3 & CONSTANT & $1.0000000 E+00$ & $1.0000000 \mathrm{E}+00$ & $1.0000000 E+\infty$ & $1.0000000 E+00$ & NONE & WPO40514 & $Y$ & NA \\
\hline 2054 & 1,3 & CONSTANT & $-1.0000000 E+01$ & $-1.0000000 E+01$ & $-1.0000000 E+01$ & $-1.0000000 E+01$ & $\log \left(m^{\wedge} 2\right)$ & $\begin{array}{l}\text { MEMO:VAUGHN } \\
\text { TIERNEY-9 }\end{array}$ & $\mathbf{Y}$ & N/A \\
\hline 2054 & 1,3 & CONSTANT & $-1.0000000 E+01$ & $-1.0000000 \mathrm{E}+01$ & $-1.0000000 E+01$ & $-1.0000000 E+01$ & $\log \left(m^{\wedge} 2\right)$ & WP035597 & $Y$ & N/A \\
\hline 2054 & 1,3 & CONSTANT & $-1.0000000 E+01$ & $-1.0000000 E+01$ & $-1.0000000 \mathrm{E}+01$ & $-1.0000000 E+01$ & $\log \left(m^{\wedge} 2\right)$ & WPO40514 & $Y$ & N/A \\
\hline 2055 & 1.3 & CONSTANT & $-1.0000000 E+01$ & $-1.0000000 E+01$ & $-1.0000000 E+01$ & $-1.0000000 E+01$ & $\log \left(m^{\wedge} 2\right)$ & $\begin{array}{c}\text { MEMO:VAUGHN } \\
\text { TIERNEY-9 } \\
\end{array}$ & $\mathbf{Y}$ & N/A \\
\hline 2055 & 1,3 & CONSTANT & $-1.0000000 E+01$ & $-1.0000000 E+01$ & $-1.0000000 E+01$ & $-1.0000000 E+01$ & $\log \left(m^{\wedge} 2\right)$ & WP035597 & $\mathbf{Y}$ & NAA \\
\hline 2055 & 1,3 & CONSTANT & $-1.0000000 E+01$ & $-1.0000000 E+01$ & $-1.0000000 E+01$ & $-1.0000000 E+01$ & $\log \left(m^{\wedge} 2\right)$ & WPO40514 & $\mathbf{Y}$ & N/A \\
\hline 2056 & 1,3 & CONSTANT & $-1.0000000 E+01$ & $-1.0000000 E+01$ & $-1.0000000 \mathrm{E}+01$ & $-1.0000000 E+01$ & $\log \left(m^{\wedge} 2\right)$ & $\begin{array}{c}\text { MEMO:VAUGHN } \\
\text { TIERNEY-9 } \\
\end{array}$ & $\mathbf{Y}$ & N/A \\
\hline 2056 & 9,3 & CONSTANT & $-1.0000000 E+01$ & $-1.0000000 E+01$ & $-1.0000000 E+01$ & $-1.0000000 E+01$ & $\log \left(m^{\wedge} 2\right)$ & WP035597 & $Y$ & NA \\
\hline 2056 & 1,3 & CONSTANT & $-1.0000000 E+01$ & $-1.0000000 E+01$ & $-1.0000000 E+01$ & $-1.0000000 E+01$ & $\log \left(m^{\wedge} 2\right)$ & WP040514 & $\mathbf{Y}$ & NA \\
\hline 2058 & 1,3 & CONSTANT & $4.0000000 \mathrm{E}+00$ & $4.0000000 E+\infty 0$ & $4.0000000 E+\infty 0$ & 4.0000000E +00 & NONE & $\begin{array}{c}\text { MEMO:VAUGHNI } \\
\text { TIERNEY-9 } \\
\end{array}$ & $\mathbf{Y}$ & N/A \\
\hline 2058 & 1,3 & CONSTANT & $4.0000000 E+\infty 0$ & $4.0000000 \mathrm{E}+00$ & $4.0000000 E+\infty 0$ & $4.0000000 E+\infty 0$ & NONE & WP035597 & $\mathbf{Y}$ & NA \\
\hline 2058 & 1,3 & CONSTANT & $4.0000000 E+00$ & $4.0000000 E+00$ & $4.0000000 E+00$ & $4.0000000 E+\infty 0$ & NONE & WP040514 & $Y$ & N/A \\
\hline 2059 & 1,3 & CONSTANT & $0.0000000 E+00$ & $0.0000000 E+\infty 0$ & $0.0000000 \mathrm{E}+\infty 0$ & $0.0000000 E+\infty 0$ & NONE & $\begin{array}{c}\text { MEMO:VAUGHNI } \\
\text { TIERNEY-9 }\end{array}$ & $Y$ & N/A \\
\hline 2059 & 1,3 & CONSTANT & $0.0000000 \mathrm{E}+00$ & $0.0000000 \mathrm{E}+00$ & $0.00000000+00$ & $0.0000000 \mathrm{E}+00$ & NONE & WP035597 & $Y$ & NA \\
\hline 2059 & 1,3 & CONSTANT & $0.0000000 E+00$ & $0.0000000 \mathrm{E}+00$ & $0.0000000 E+00$ & $0.0000000 E+00$ & NONE & WPO40514 & $Y$ & N/A \\
\hline 2060 & 1,3 & CONSTANT & $1.0000000 E+00$ & $1.0000000 E+\infty 0$ & $1.0000000 E+\infty$ & $1.0000000 E+00$ & NONE & $\begin{array}{c}\text { MEMO:VAUGHN } \\
\text { TIERNEY-9 } \\
\end{array}$ & $\mathbf{Y}$ & NA \\
\hline 2060 & 1,3 & CONSTANT & $1.0000000 \mathrm{E}+00$ & $1.0000000 E+00$ & $1.0000000 E+\infty$ & $1.0000000 E+00$ & NONE & WP035597 & $Y$ & N/A \\
\hline 2060 & 1,3 & CONSTANT & $1.0000000 \mathrm{E}+\infty 0$ & $1.0000000 E+00$ & $1.0000000 E+00$ & $1.0000000 E+\infty 0$ & NONE & WP040514 & $\mathbf{Y}$ & NA \\
\hline 2062 & 1,3 & CONSTANT & $0.0000000 E+00$ & $0.0000000 E+00$ & $0.0000000 E+\infty 0$ & $0.0000000 \mathrm{E}+00$ & $P a^{\wedge}-1$ & $\begin{array}{l}\text { MEMO:VAUGHN } \\
\text { TIERNEY-9 }\end{array}$ & $\mathbf{Y}$ & N/A \\
\hline 2062 & 1,3 & CONSTANT & $0.0000000 E+00$ & $0.0000000 E+00$ & $0.0000000 \mathrm{E}+00$ & $0.0000000 \mathrm{E}+00$ & $P a^{A}-1$ & WPO35597 & $Y$ & NA \\
\hline 2062 & 1,3 & CONSTANT & $0.0000000 \mathrm{E}+00$ & $0.0000000 \mathrm{E}+00$ & $0.0000000 E+00$ & $0.0000000 E+\infty 0$ & $\mathrm{~Pa} a^{\wedge}-1$ & WP040514 & $Y$ & N/A \\
\hline 2063 & 1,3 & CONSTANT & $7.0000000 E-01$ & $7.0000000 E-01$ & $7.0000000 E-01$ & $7.0000000 E-01$ & NONE & $\begin{array}{c}\text { MEMO:VAUGHN } \\
\text { TIERNEY-9 }\end{array}$ & $\mathbf{Y}$ & N/A \\
\hline 2063 & 1,3 & CONSTANT & $7.0000000 E-01$ & $7.0000000 \mathrm{E}-01$ & $7.0000000 E-01$ & $7.0000000 E-01$ & NONE & WP035597 & $\cdot \gamma$ & $N / A$ \\
\hline 2063 & 1,3 & CONSTANT & $7.0000000 E-01$ & 7.0000000E-01 & $7.0000000 E-01$ & $7.0000000 E-01$ & NONE & WPO40514 & $\mathbf{Y}$ & N/A \\
\hline 2064 & 1,3 & CONSTANT & $1.0000000 E+\infty 0$ & $1.0000000 E+00$ & $1.0000000 E+\infty$ & $1.0000000 E+\infty 0$ & NONE & $\begin{array}{c}\text { MEMO:VAUGHN/ } \\
\text { TIERNEY-9 } \\
\end{array}$ & $Y$ & N/A \\
\hline 2064 & 1.3 & CONSTANT & $1.0000000 \mathrm{E}+00$ & $1.0000000 \mathrm{E}+\infty 0$ & $1.0000000 \mathrm{E}+\infty 0$ & $1.0000000 \mathrm{E}+00$ & NONE & WPO35597 & $Y$ & NA \\
\hline 2064 & 1,3 & CONSTANT & $1.0000000 \mathrm{E}+00$ & $1.0000000 \mathrm{E}+\infty$ & $1.0000000 \mathrm{E}+00$ & $1.0000000 \mathrm{E}+00$ & NONE & WP040514 & $\bar{Y}$ & N/A \\
\hline 2065 & 1,3 & CONSTANT & $-1.0000000 E+01$ & $-1.0000000 E+01$ & $-1.0000000 E+01$ & $-1.0000000 E+01$ & $\log \left(m^{\wedge} 2\right)$ & $\begin{array}{c}\text { MEMO:VAUGHNI } \\
\text { TIERNEY-9 } \\
\end{array}$ & $\mathbf{Y}$ & NA \\
\hline 2065 & 1,3 & CONSTANT & $-1.0000000 E+01$ & $-1.0000000 E+01$ & $-1.0000000 E+01$ & $-1.0000000 E+01$ & $\log \left(m^{\wedge} 2\right)$ & WP035597 & $\mathbf{Y}$ & N/A \\
\hline 2065 & 1,3 & CONSTANT & $-1.0000000 E+01$ & $-1.0000000 E+01$ & $-1.0000000 \mathrm{E}+01$ & $-1.0000000 \mathrm{E}+01$ & $\log \left(m^{\wedge} 2\right)$ & WP040514 & $\mathbf{Y}$ & N/A \\
\hline 2066 & 1,3 & CONSTANT & $-1.0000000 E+01$ & $-1.0000000 E+01$ & $-1.0000000 E+01$ & $-1.0000000 E+01$ & $\log \left(m^{\wedge} 2\right)$ & $\begin{array}{c}\text { MEMO:VAUGHNI } \\
\text { TIERNEY-9 } \\
\end{array}$ & $Y$ & N/A \\
\hline 2066 & 1,3 & CONSTANT & $-1.0000000 E+01$ & $-1.0000000 \mathrm{E}+01$ & $-1.0000000 \mathrm{E}+01$ & $-1.0000000 E+01$ & $\log \left(m^{\wedge} 2\right)$ & WP035597 & $\mathbf{Y}$ & N/A \\
\hline 2066 & 1,3 & CONSTANT & $-1.0000000 E+01$ & $-1.0000000 \mathrm{E}+01$ & $-1.0000000 E+01$ & $-1.0000000 E+01$ & $\log \left(m^{\wedge} 2\right)$ & WP040514 & $\mathbf{Y}$ & N/A \\
\hline 2067 & 1,3 & CONSTANT & $-1.0000000 \mathrm{E}+01$ & $-1.0000000 \mathrm{E}+01$ & $-1.0000000 E+01$ & $-1.0000000 E+01$ & $\log \left(m^{\wedge} 2\right)$ & $\begin{array}{c}\text { MEMO:VAUGHN } \\
\text { TIERNEY-9 } \\
\end{array}$ & $Y$ & NA \\
\hline 2067 & 1,3 & CONSTANT & $-1.0000000 E+01$ & $-1.0000000 \mathrm{E}+01$ & $-1.0000000 E+01$ & $-1.0000000 E+01$ & $\log \left(m^{\wedge} 2\right)$ & WP035597 & $\mathbf{Y}$ & NA \\
\hline
\end{tabular}


CCA Parameter Listing

\begin{tabular}{|c|c|c|c|c|c|c|c|c|c|c|}
\hline $\begin{array}{c}\text { Paramoter in } \\
\text { Databaze? } \\
\text { (YNN) }\end{array}$ & $\begin{array}{c}\text { If the values } \\
\text { ured in CCA } \\
\text { are not the } \\
\text { same as in } \\
\text { DB, provide * } \\
\text { difference }\end{array}$ & ID & Material ID & Waterial Namo & $\begin{array}{c}\text { Parameter } \\
\text { ID }\end{array}$ & Paramoter Kamo & $\begin{array}{l}\text { PRPID } \\
\text { (NPO }: \text { ) }\end{array}$ & $\begin{array}{c}\text { Data Entry } \\
\text { Date }\end{array}$ & $\begin{array}{l}\text { Is the } 464 \\
\text { corraktent } \\
\text { with the } \\
\text { parameter } \\
\text { in OB? } \\
(X, N A)\end{array}$ & $\begin{array}{l}\text { Parnmoter } \\
\text { Category } \\
\text { (footnote 1) }\end{array}$ \\
\hline$Y$ & & 2067 & CAVITY_ 4 & Cavity for Borehole & PRMZ_LOG & $\begin{array}{l}\text { Log of intrnsic permeabilty, Z- } \\
\text { diredion }\end{array}$ & 31819 & 20-Feb-96 & $\mathbf{Y}$ & AB \\
\hline$\gamma$ & & 2069 & CAVITY_4 & Cavity for Borehole & RELP_MOD & $\begin{array}{l}\text { Model number, rolative } \\
\text { permsabilty model }\end{array}$ & 31821 & 17-Feb-96 & $\mathbf{Y}$ & 48 \\
\hline $\mathbf{Y}$ & & 2069 & CAVITY_4 & Cavity for Borehole & RELP_MOD & $\begin{array}{l}\text { Model number, relative } \\
\text { permeability model }\end{array}$ & 31821 & 17fob-96 & $\mathbf{Y}$ & 48 \\
\hline $\mathbf{Y}$ & & 2069 & CAVTTY_4 & Cavity for Borehole & RELP_MOD & $\begin{array}{l}\text { Model number, relative } \\
\text { permeability model }\end{array}$ & 31821 & 17.Feb-96 & $\gamma$ & 48 \\
\hline$Y$ & & 2070 & CAVITY_4 & Cavity for Borehole & SAT_RGAS & Residual Gas Saturation & 31824 & 17-feb-9s & $\mathbf{Y}$ & 48 \\
\hline $\bar{Y}$ & & 2070 & CAVITY_4 & Cavity for Bonehole & SAT_RGAS & Residual Gas Saturation & 31824 & 17-Fob-96 & $Y$ & 48 \\
\hline$Y$ & & 2070 & CAVITY_4 & Cavity for Borehole & SAT_RGAS & Residual Gas Saturation & 31824 & 17-feb-96 & $\gamma$ & $4 B$ \\
\hline $\mathbf{Y}$ & & 2071 & CULEBRA & $\begin{array}{l}\text { Culebra member of the } \\
\text { Rustler formation }\end{array}$ & THICK & Thickness of feature or layer & 32790 & 01-May-96 & $\mathbf{Y}$ & 1 \\
\hline $\mathbf{Y}$ & & 2085 & FORTYNIN & Forty Niner Member & CAP_MOD & $\begin{array}{l}\text { Model number, capillary pressure } \\
\text { model }\end{array}$ & 32975 & 01-Nov-95 & $Y$ & AB \\
\hline$Y$ & & 2085 & FORTMNIN & Forty Niner Momber & CAP_MOD & $\begin{array}{l}\text { Model number, capillary pressure } \\
\text { model }\end{array}$ & 32975 & 01-Nov-95 & $\mathbf{Y}$ & 48 \\
\hline$\gamma$ & & 2087 & FORTYNIN & Forty Niner Member & PORE_DIS & $\begin{array}{l}\text { Brooks-Corey pora distribution } \\
\text { parameter }\end{array}$ & 32991 & O4-Nov-95 & $\mathbf{Y}$ & 48 \\
\hline Y & & 2087 & FORTMNIN & Forty Niner Member & PORE_DIS & $\begin{array}{l}\text { Brooks-Corey pore distribution } \\
\text { parameter }\end{array}$ & 32991 & 01-Nov-95 & $Y$ & 48 \\
\hline$Y$ & & 2088 & FORTYNIN & Forty Niner Member & POROSITY & Effective porosity & 32995 & 20-Feb-96 & $Y$ & 1 \\
\hline$Y$ & & 2088 & FORTYNIN & Forty Niner Member & POROSITY & Effective porosity & 32995 & 20-Fob-96 & $Y$ & 1 \\
\hline$Y$ & & 2088 & FORTYNIN & Forty Niner Member & POROSITY & Effective porosity & 32995 & 20-Feb-96 & $Y$ & 1 \\
\hline$Y$ & & 2093 & FORTYNIN & Forty Niner Mamber & RELP_MOD & $\begin{array}{l}\text { Model number, relative } \\
\text { permeability model }\end{array}$ & 33019 & 01-Nov-95 & $\mathbf{Y}$ & AB \\
\hline $\mathbf{Y}$ & & 2093 & FORTYNIN & Forty Niner Member & RELP_MOD & $\begin{array}{l}\text { Model number, relative } \\
\text { permeability model }\end{array}$ & 33019 & 01-Nov-95 & Y & $A B$ \\
\hline$Y$ & & 2094 & FORTMNIN & Forty Niner Mermber & SAT_RGAS & Residual Gas Saturation & 33024 & 01-Nov-95 & $Y$ & $4 \mathrm{~B}$ \\
\hline $\mathbf{Y}$ & & 2094 & FORTYNIN & Forty Niner Member & SAT_RGAS & Residual Gas Saturation & 33024 & 01-Nov-95 & $Y$ & $4 B$ \\
\hline $\mathbf{Y}$ & & 2097 & MAGENTA & Magenta Member & CAP_MOD & $\begin{array}{l}\text { Model number, capiliary pressure } \\
\text { model }\end{array}$ & 33239 & 0i-Nov-95 & $\mathbf{Y}$ & 4B \\
\hline $\mathbf{Y}$ & & 2097 & MAGENTA & Magenta Member & CAP_MOD & $\begin{array}{l}\text { Model number, capillery pressure } \\
\text { model }\end{array}$ & 33239 & Oi-Nov-95 & $\mathbf{Y}$ & 48 \\
\hline $\mathbf{Y}$ & & 2097 & MAGENTA & Magenta Member & CAP_MOD & $\begin{array}{l}\text { Model number, capillary pressure } \\
\text { model }\end{array}$ & 33239 & 01-Nov-95 & $\mathbf{Y}$ & 48 \\
\hline $\mathbf{Y}$ & & 2098 & MAGENTA & Magenta Member & PC_maX & $\begin{array}{l}\text { Maximum allowable capillary } \\
\text { pressure }\end{array}$ & 33263 & 01-Nov-95 & $\gamma$ & $A B$ \\
\hline $\mathbf{Y}$ & & 2098 & MAGENTA & Magenta Member & PC_MAX & $\begin{array}{l}\text { Maxtmum allowable capillary } \\
\text { pressure }\end{array}$ & 33263 & 01-Nov-95 & $\mathbf{Y}$ & $4 B$ \\
\hline $\mathbf{Y}$ & & 2098 & MAGENTA & Magenta Member & PC_MAX & $\begin{array}{l}\text { Maxumum allowable capillary } \\
\text { pressure }\end{array}$ & 33263 & 01-Nov-95 & $Y$ & 4B \\
\hline$Y$ & & 2099 & MAGENTA & Magenta Member & PORE_DIS & $\begin{array}{l}\text { Brooks-Corey pore distribution } \\
\text { parameter }\end{array}$ & 32493 & 22-Feb-96 & $\mathbf{Y}$ & AB \\
\hline$Y$ & & 2099 & MAGENTA & Magenta Member & PORE_DIS & $\begin{array}{l}\text { Brooks-Corey pore distribution } \\
\text { parameter }\end{array}$ & 32493 & 22-Feb-96 & $y$ & 48 \\
\hline $\mathbf{Y}$ & & 2099 & MAGENTA & Magenta Member & PORE_DIS & $\begin{array}{l}\text { Brooks-Corey pore distriburtion } \\
\text { parameter }\end{array}$ & 32493 & 22-Fob-96 & $\mathbf{Y}$ & AB \\
\hline$Y$ & & 2100 & MAGENTA & Magenta Member & POROSITY & Effoctive porosity & 32531 & 06-Feb-96 & $\mathbf{Y}$ & 1 \\
\hline$Y$ & & 2100 & MAGENTA & Magenta Member & POROSITY & Effective porosity & 32531 & $06-$ Feb-96 & $\bar{Y}$ & 1 \\
\hline$Y$ & & 2100 & MAGENTA & Magenta Member & POROSITY & Effective porosity & 32531 & 06-Fab-96 & $Y$ & 1 \\
\hline$Y$ & & 2101 & MAGENTA & Magenta Member & PRESSURE & Bnne far-field pore pressure & 32539 & 08-feb-96 & $Y$ & 1 \\
\hline$Y$ & & 2101 & MAGENTA & Magenta Member & PRESSURE & Brne far-field pore pressure & 32539 & OB-Feb-96 & $Y$ & 1 \\
\hline$Y$ & & 2101 & MAGENTA & Magenta Member & PRESSURE & Brine far-field pore pressure - & 32539 & 09-Feb-96 & $Y$ & 1 \\
\hline $\mathbf{Y}$ & & 2102 & MAGENTA & Magenta Member & PRMX_LOG & $\begin{array}{l}\text { Log of ntrmsic permeabilty, } X- \\
\text { direction }\end{array}$ & 32545 & 13-Feb-96 & $\mathbf{Y}$ & 1 \\
\hline$Y$ & & 2102 & MAGENTA & Magenta Member & PRMX_LOG & $\begin{array}{l}\text { Log of intrinsic permeability, } X \text { - } \\
\text { direction }\end{array}$ & 32545 & $13-F e b-96$ & $\mathbf{Y}$ & 1 \\
\hline$Y$ & & 2103 & MAGENTA & Magenta Member & PRMY_LOG & $\begin{array}{l}\text { Log of intrinsic permeability. Y- } \\
\text { direction }\end{array}$ & 32547 & 13-Feb-96 & $\mathbf{r}$ & 1 \\
\hline $\mathbf{Y}$ & & 2103 & MAGENTA & Magenta Member & PRMY_LOG & $\begin{array}{l}\text { Log of intrnsic permeability, Y- } \\
\text { direction }\end{array}$ & 32547 & 13-feb-96 & $\mathbf{Y}$ & 1 \\
\hline $\mathbf{Y}$ & & 2104 & MAGENTA & Magenta Member & PRMZ_LOG & $\begin{array}{l}\text { Log of intrinsic perm oablity, Z- } \\
\text { direction }\end{array}$ & 32550 & 13-Feb-96 & $\mathbf{Y}$ & 1 \\
\hline$Y$ & & 2104 & MAGENTA & Magenta Member & PRMZ_LOG & $\begin{array}{l}\text { Log of mtrnnsic permeabilty, 2- } \\
\text { direction }\end{array}$ & 32550 & 13-Feb-96 & $Y$ & 1 \\
\hline
\end{tabular}


CCA Parameter Listing

\begin{tabular}{|c|c|c|c|c|c|c|c|c|c|c|}
\hline ID & \begin{tabular}{|c|} 
Classin- \\
Cation of \\
Data \\
Category \\
(footnoto 2)
\end{tabular} & $\begin{array}{l}\text { Distribution } \\
\text { Type }\end{array}$ & Mean & Median & Mintmum & Meximum & Units & Reforence ID & $\begin{array}{l}\text { Was the date } \\
\text { doveloped } \\
\text { under an } \\
\text { NQA-1 } \\
\text { Program? }\end{array}$ & $\begin{array}{c}\text { Which } \\
\text { methods } \\
\text { were used to } \\
\text { qually } \\
\text { existing } \\
\text { data? } \\
\text { (footnoto 3) }\end{array}$ \\
\hline 2067 & 1,3 & CONSTANT & $-1.0000000 E+01$ & $-1.0000000 E+01$ & $-1.0000000 \mathrm{E}+01$ & $-1.0000000 \mathrm{E}+01$ & $\log \left(m^{\wedge} 2\right)$ & WP040514 & $\mathbf{Y}$ & N/A \\
\hline 2069 & 1,3 & CONSTANT & $4.0000000 E+\infty 0$ & $4.0000000 E+00$ & $4.0000000 E+00$ & $4.0000000 E+00$ & NONE & $\begin{array}{l}\text { MEMO:VAUGHN } \\
\text { TIERNEY-9 }\end{array}$ & $\mathbf{Y}$ & $N / A$ \\
\hline 2069 & 1,3 & CONSTANT & $4.0000000 E+00$ & $4.0000000 E+00$ & $4.0000000 \mathrm{E}+00$ & $4.0000000 E+00$ & NONE & WPO35597 & $Y$ & N/A \\
\hline 2069 & 1,3 & CONSTANT & $4.0000000 E+00$ & $4.0000000 E+00$ & $4.0000000 E+00$ & $4.0000000 E+00$ & NONE & WP040514 & $Y$ & NA \\
\hline 2070 & 1.3 & CONSTANT & $0.0000000 E+00$ & $0.0000000 E+00$ & $0.0000000 \mathrm{E}+00$ & $0.0000000 E+\infty 0$ & NONE & $\begin{array}{l}\text { MEMO:VAUGHN } \\
\text { TIERNEY-9 }\end{array}$ & $\mathbf{Y}$ & N/A \\
\hline 2070 & 1,3 & CONSTANT & $0.0000000 \mathrm{E}+00$ & $0.0000000 E+\infty$ & $0.0000000 E+00$ & $0.0000000 \mathrm{E}+00$ & NONE & WP035597 & $Y$ & $N / A$ \\
\hline 2070 & 1,3 & CONSTANT & $0.0000000 E+00$ & $0.0000000 E+00$ & $0.0000000 E+00$ & $0.0000000 E+00$ & NONE & WPO40514 & $Y$ & NA \\
\hline 2071 & 1 & CONSTANT & $7.7500000 E+\infty 0$ & $7.7500000 \mathrm{E}+00$ & $7.7500000 E+\infty 0$ & $7.7500000 \mathrm{E}+00$ & $\mathbf{m}$ & WPO36455 & YEN & 1 \\
\hline 2085 & 1,3 & CONSTANT & $1.0000000 E+00$ & $1.0000000 E+00$ & $1.0000000 E+00$ & $1.0000000 E+\infty$ & NONE & WPO38568 & $Y$ & N/A \\
\hline 2085 & 1,3 & CONSTANT & $1.0000000 \mathrm{E}+00$ & $1.0000000 E+00$ & $1.0000000 E+00$ & $1.0000000 E+\infty 0$ & NONE & WP040514 & $Y$ & N/A \\
\hline 2087 & 1,3 & CONSTANT & $7.0000000 E-01$ & $7.0000000 E-01$ & $7.0000000 E-01$ & $7.0000000 E-01$ & NONE & WPO38568 & $\mathbf{Y}$ & N/A \\
\hline 2087 & 1,3 & CONSTANT & 7.0000000E-01 & 7.0000000E-01 & $7.0000000 E-01$ & 7.0000000E-01 & NONE & WP040514 & $Y$ & NA \\
\hline 2088 & 1 & STUDENT & $8.2000000 \mathrm{E}-02$ & $8.2000000 E-02$ & $0.0000000 \mathrm{E}+\infty 0$ & $2.4000000 E-01$ & NONE & WP030608 & $Y$ & N/A \\
\hline 2088 & 1 & STUDENT & $8.2000000 \mathrm{E}-02$ & $8.2000000 \mathrm{E}-02$ & $0.0000000 \mathrm{E}+00$ & $2.4000000 E-01$ & NONE & WP035597 & $Y$ & N/A \\
\hline 2088 & 1 & STUDENT & $8.2000000 \mathrm{E}-02$ & $8.2000000 E-02$ & $0.0000000 E+00$ & $2.4000000 E-01$ & NONE & WPO36380 & $Y$ & N/A \\
\hline 2093 & 1,3 & CONSTANT & 4. $0000000 \mathrm{E}+00$ & $4.0000000 E+\infty 0$ & $4.0000000 \mathrm{E}+00$ & $4.0000000 E+\infty 0$ & NONE & WPO38568 & $Y$ & NAA \\
\hline 2093 & 1,3 & CONSTANT & $4.0000000 E+\infty 0$ & $4.0000000 E+\infty 0$ & $4.0000000 E+00$ & $4.0000000 \mathrm{E}+00$ & NONE & WP040514 & $\mathbf{Y}$ & N/A \\
\hline 2094 & 1,3 & CONSTANT & $2.0000000 \mathrm{E}-01$ & $2.0000000 E-01$ & $2.0000000 E-01$ & $2.0000000 E-01$ & NONE & WP038568 & $Y$ & N/A \\
\hline 2094 & 1,3 & CONSTANT & $2.0000000 \mathrm{E}-01$ & $2.0000000 \mathrm{E}-01$ & $2.0000000 E-01$ & $2.0000000 \mathrm{E}-01$ & NONE & WPO40514 & $Y$ & N/A \\
\hline 2097 & $1,3,4$ & CONSTANT & $2.0000000 E+\infty 0$ & $2.0000000 E+00$ & $2.0000000 \mathrm{E}+00$ & $20000000 E+\infty 0$ & NONE & WP031041 & $\mathbf{Y}$ & N/A \\
\hline 2097 & $1,3,4$ & CONSTANT & $2.0000000 \mathrm{E}+\infty 0$ & $2.0000000 E+00$ & $2.0000000 E+\infty 0$ & $2.0000000 E+00$ & NONE & WP035597 & Y & N/A \\
\hline 2097 & $1,3,4$ & CONSTANT & $2.0000000 E+00$ & $2.0000000 E+00$ & $2.0000000 E+00$ & $2.0000000 E+00$ & NONE & WP040514 & $Y$ & $N A$ \\
\hline 2098 & 1,3 & CONSTANT & $1.0000000 \mathrm{E}+08$ & $1.0000000 E+08$ & $1.0000000 E+08$ & $1.0000000 E+08$ & $\mathrm{~Pa}$ & WP031041 & $Y$ & NA \\
\hline 2098 & 1,3 & CONSTANT & $1.0000000 \mathrm{E}+08$ & $1.0000000 E+08$ & $1.0000000 E+08$ & $1.0000000 E+08$ & $\mathrm{~Pa}$ & WP035597 & $\mathbf{Y}$ & N/A \\
\hline 2098 & 1,3 & CONSTANT & $1.0000000 \mathrm{E}+08$ & $1.0000000 E+08$ & $1.0000000 E+\infty 8$ & $1.0000000 E+08$ & $\mathbf{P a}$ & WPO40514 & $Y$ & N/A \\
\hline 2099 & $1,3,4$ & CONSTANT & $6.4360000 \mathrm{E}-01$ & $6.4360000 \mathrm{E}-01$ & $6.4360000 E-01$ & $6.4360000 E-01$ & NONE & WP031041 & $\mathbf{Y}$ & N/A \\
\hline 2099 & $1,3,4$ & CONSTANT & $6.4360000 E-01$ & $6.4360000 E-01$ & $6.4360000 E-01$ & $6.4360000 E-01$ & NONE & WP035597 & $Y$ & NA \\
\hline 2099 & $1,3,4$ & CONSTANT & $6.4360000 E-01$ & $6.4360000 E-01$ & $6.4360000 E-01$ & $6.4360000 E-01$ & NONE & WP040514 & Y & N/A \\
\hline 2100 & 1 & STUDENT & $1.3800000 \mathrm{E}-01$ & $1.3800000 E-01$ & $2.7000000 \mathrm{E}-02$ & $2.5200000 E-01$ & NONE & WP030608 & $Y$ & N/A \\
\hline 2100 & 1 & STUDENT & 1.3800000E-01 & $1.3800000 \mathrm{E}-01$ & 2.7000000 E-02 & $2.5200000 \mathrm{E}-01$ & NONE & WP035597 & $Y$ & N/A \\
\hline 2100 & 1 & STUDENT & $1.3800000 \mathrm{E}-01$ & $1.3800000 E-01$ & $2.7000000 E-02$ & $2.5200000 \mathrm{E}-01$ & NONE & WP036380 & $Y$ & NA \\
\hline 2101 & 1 & CONSTANT & $9.1700000 E+05$ & $9.1700000 \mathrm{E}+05$ & $9.1700000 E+05$ & $9.1700000 E+05$ & $\mathrm{~Pa}$ & WP030713 & $\mathbf{N}$ & 1 \\
\hline 2101 & 1 & CONSTANT & $9.1700000 \mathrm{E}+05$ & $9.1700000 \mathrm{E}+05$ & $9.1700000 \mathrm{E}+05$ & $9.1700000 E+05$ & $\mathrm{~Pa}$ & WP032290 & $N$ & 1 \\
\hline 2101 & 1 & CONSTANT & $9.1700000 E+05$ & $9.1700000 \mathrm{E}+05$ & $9.1700000 \mathrm{E}+05$ & $9.1700000 E+05$ & $\mathrm{~Pa} \cdot$ & WP035597 & $\mathbf{N}$ & 1 \\
\hline 2102 & 1 & CONSTANT & $-1.5200000 E+01$ & $-1.5200000 E+01$ & $-1.5200000 \mathrm{E}+01$ & $-1.5200000 E+01$ & $\log \left(m^{\wedge} 2\right)$ & WP030607 & N & 1 \\
\hline 2102 & 1 & CONSTANT & $-1.5200000 \mathrm{E}+01$ & $-1.5200000 \mathrm{E}+01$ & $-1.5200000 E+01$ & $-1.5200000 E+01$ & $\log \left(m^{\wedge} 2\right)$ & WP035597 & $\mathbf{N}$ & 1 \\
\hline 2103 & 1 & CONSTANT & $-1.5200000 E+01$ & $-1.5200000 E+01$ & $-1.5200000 E+01$ & $-1.5200000 E+01$ & $\log \left(m^{\wedge} 2\right)$ & WP030607 & $N$ & 1 \\
\hline 2103 & 1 & CONSTANT & $-1.5200000 E+01$ & $-1.5200000 E+01$ & $-1.5200000 E+01$ & $-1.5200000 E+01$ & $\log \left(m^{\wedge} 2\right)$ & WP035597 & N & 1 \\
\hline 2104 & 1 & CONSTANT & $-1.5200000 E+01$ & $-1.52000000 E+01$ & $-1.5200000 E+01$ & $-1.5200000 E+01$ & $\log \left(m^{\wedge} 2\right)$ & WP030607 & $\mathbf{N}$ & 1 \\
\hline 2104 & 1 & CONSTANT & $-1.5200000 E+01$ & $-1.5200000 E+01$ & $-1.5200000 \mathrm{E}+01$ & $-1.5200000 \mathrm{E}+01$ & $\log \left(m^{\wedge} 2\right)$ & WP035597 & $\mathbf{N}$ & 1 \\
\hline
\end{tabular}


CCA Parameter Listing

\begin{tabular}{|c|c|c|c|c|c|c|c|c|c|c|}
\hline $\begin{array}{c}\text { Parametar In } \\
\text { Databese? } \\
\text { (YN) }\end{array}$ & $\begin{array}{l}\text { If the values } \\
\text { used in CCA } \\
\text { are not the } \\
\text { same as in } \\
\text { DB, provide } x \\
\text { dfference }\end{array}$ & ID & Material to & Materia Name & $\begin{array}{l}\text { Parameter } \\
\text { iD }\end{array}$ & Parameter Hame & $\begin{array}{l}\text { PRP ID } \\
\text { (WPO है) }\end{array}$ & $\begin{array}{c}\text { Data Entry } \\
\text { Date }\end{array}$ & $\begin{array}{l}\text { Is the } 464 \\
\text { consiatent } \\
\text { with the } \\
\text { parameter } \\
\text { in DBY } \\
\text { Y, NAS }\end{array}$ & $\begin{array}{l}\text { Parameter } \\
\text { Catogory } \\
\text { (footnote 1) }\end{array}$ \\
\hline $\mathbf{Y}$ & & 2106 & MAGENTA & Magenta Member & RELP_MOD & $\begin{array}{l}\text { Model number, relative } \\
\text { permeability model }\end{array}$ & 32557 & $01-$ Nov-95 & $\mathbf{Y}$ & $4 A$ \\
\hline $\mathbf{Y}$ & & 2107 & MAGENTA & Magenta Member & SAT_RGAS & Residual Gas Saturation & 32562 & 20-Feb-96 & $\mathbf{Y}$ & 48 \\
\hline $\mathbf{Y}$ & & 2107 & MAGENTA & Magenta Member & SAT_RGAS & Resichual Gas Saturation & 32562 & 20-Feb-96 & $\mathbf{Y}$ & $4 B$ \\
\hline $\mathbf{Y}$ & & 2107 & MAGENTA & Magenta Member & SAT_RGAS & Residual Gas Saturation & 32562 & 20-Fob-96 & $Y$ & $4 B$ \\
\hline $\mathbf{Y}$ & & 2110 & REPOST & \begin{tabular}{|l|} 
Repository regions \\
outside of Panel region
\end{tabular} & CAP_MOD & $\begin{array}{l}\text { Model number, capillary pressure } \\
\text { model }\end{array}$ & 33285 & a8-Fob-96 & $\mathbf{Y}$ & AB \\
\hline $\mathbf{Y}$ & & 2110 & REPOSTT & $\begin{array}{l}\text { Repository regions } \\
\text { outside of Panel region }\end{array}$ & CAP_MOD & $\begin{array}{l}\text { Model number, capillary pressure } \\
\text { model }\end{array}$ & 33285 & 08-Feb-96 & $\mathbf{Y}$ & $4 B$ \\
\hline$Y$ & & 2110 & REPOSIT & $\begin{array}{l}\text { Repository regions } \\
\text { outside of Panel region }\end{array}$ & CAP_MOD & $\begin{array}{l}\text { Model number, capillary pressure } \\
\text { model }\end{array}$ & 33285 & 08-Fob-96 & $\mathbf{Y}$ & $4 B$ \\
\hline $\mathbf{Y}$ & & 2112 & REPOST & $\begin{array}{l}\text { Repository regions } \\
\text { outside of Panel region }\end{array}$ & COMP_RCK & Bulk Compressibility & 33290 & 08-Feb-96 & $\mathbf{Y}$ & $4 B$ \\
\hline$Y$ & & 2112 & REPOSIT & $\begin{array}{l}\text { Repository regions } \\
\text { outside of Panel region }\end{array}$ & COMP_RCK & Bulk Compressibility & 33290 & as-fab-96 & $\mathbf{Y}$ & $4 B$ \\
\hline $\mathbf{Y}$ & & 2112 & REPOSIT & $\begin{array}{l}\text { Repository regions } \\
\text { outside of Panel region }\end{array}$ & COMP_RCK & Butk Compressibility & 33290 & as-Feb-96 & $\mathbf{Y}$ & $4 \mathrm{~B}$ \\
\hline $\mathbf{Y}$ & & 2113 & REPOST & $\begin{array}{l}\text { Repository regions } \\
\text { outside of Panel region }\end{array}$ & DCELLCHW & $\begin{array}{l}\text { Average density of cellulosics in } \\
\text { CH waste }\end{array}$ & 33298 & 13-feb-96 & $\mathbf{Y}$ & 2 \\
\hline $\mathbf{Y}$ & & 2114 & REPOST & $\begin{array}{l}\text { Repository regions } \\
\text { outside of Panel region }\end{array}$ & DCELLRHW & $\begin{array}{l}\text { Average density of collulosics in } \\
\text { RH waste }\end{array}$ & 32436 & 13-Feb-95 & $\mathbf{Y}$ & 2 \\
\hline $\mathbf{Y}$ & & 2114 & REPOSIT & $\begin{array}{l}\text { Repository regions } \\
\text { outside of Panel region }\end{array}$ & DCEULRHW & $\begin{array}{l}\text { Average density of cellulosics in } \\
\text { RH waste }\end{array}$ & 32436 & 13-Feb-96 & $\mathbf{Y}$ & 2 \\
\hline $\mathbf{Y}$ & & 2115 & REPOSIT & $\begin{array}{l}\text { Repository regions } \\
\text { cutside of Panel region }\end{array}$ & DIRNCCHW & $\begin{array}{l}\text { Bulk density of iron containers. } \\
\text { CH waste }\end{array}$ & 32439 & 13-Feb-96 & $\mathbf{Y}$ & 2 \\
\hline$Y$ & & 2115 & REPOSIT & $\begin{array}{l}\text { Repository regions } \\
\text { outside of Panel region }\end{array}$ & DIRNCCHW & $\begin{array}{l}\text { Bulk density of iron containers, } \\
\text { CH waste }\end{array}$ & 32439 & 13-Feb-95 & $\mathbf{Y}$ & 2 \\
\hline$Y$ & & 2116 & REPOSIT & $\begin{array}{l}\text { Repository regions } \\
\text { outside of Panel region }\end{array}$ & DIRNCRHW & $\begin{array}{l}\text { Bulk density of iron containers, } \\
\text { RH waste }\end{array}$ & 32440 & 13-Feb-96 & $\mathbf{Y}$ & 2 \\
\hline $\mathbf{Y}$ & & 2116 & REPOST & $\begin{array}{l}\text { Repository regions } \\
\text { outside of Panel region }\end{array}$ & DIRNCRHW & $\begin{array}{l}\text { Bulk density of iron containers, } \\
\text { RH waste }\end{array}$ & 32440 & $13-F e b-9 s$ & $\mathbf{Y}$ & 2 \\
\hline $\mathbf{Y}$ & & 2116 & REPOST & $\begin{array}{l}\text { Repository regions } \\
\text { outside of Panel region }\end{array}$ & DIRNCRHW & $\begin{array}{l}\text { Bulk density of iron containers, } \\
\text { RH waste }\end{array}$ & 32440 & 13-feb-96 & $\mathbf{Y}$ & 2 \\
\hline $\mathbf{Y}$ & & 2117 & REPOST & $\begin{array}{l}\text { Repository regions } \\
\text { outside of Panel region }\end{array}$ & DIRONCHW & $\begin{array}{l}\text { Average density of iron-based } \\
\text { material in } \mathrm{CH} \text { waste }\end{array}$ & 32442 & 13-Fob-96 & $=Y$ & 2 \\
\hline $\mathbf{Y}$ & & 2117 & REPOSIT & $\begin{array}{l}\text { Repository regions } \\
\text { outside of Panel region }\end{array}$ & DIRONCHW & $\begin{array}{l}\text { Average density of iron-based } \\
\text { meterial in } \mathrm{CH} \text { waste }\end{array}$ & 32442 & 13-Feb-96 & $\mathbf{Y}$ & 2 \\
\hline $\mathbf{Y}$ & & 2118 & REPOST & $\begin{array}{l}\text { Repository regions } \\
\text { outside of Panel region }\end{array}$ & DIRONRHW & $\begin{array}{l}\text { Average density of iron-based } \\
\text { material in RH wasto }\end{array}$ & 32443 & 13-Feb-96 & $\mathbf{Y}$ & 2 \\
\hline $\mathbf{Y}$ & & 2118 & REPOSIT & $\begin{array}{l}\text { Repository regions } \\
\text { outside of Panel region }\end{array}$ & DIRONRHW & $\begin{array}{l}\text { Average density of iron-based } \\
\text { material in RH waste }\end{array}$ & 32443 & 13-Feb-96 & $\mathbf{Y}$ & 2 \\
\hline $\mathbf{Y}$ & & 2119 & REPOST & $\begin{array}{l}\text { Repository regions } \\
\text { outside of Panel region }\end{array}$ & DPLASCHW & $\begin{array}{l}\text { Average density of plastics in } \mathrm{CH} \\
\text { waste }\end{array}$ & 32444 & $13-f e b-96$ & $\mathbf{Y}$ & 2 \\
\hline $\mathbf{Y}$ & & 2119 & REPOST & $\begin{array}{l}\text { Repository regions } \\
\text { outside of Panel region }\end{array}$ & DPLASCHW & $\begin{array}{l}\text { Average density of plastics in } \mathrm{CH} \\
\text { waste }\end{array}$ & 32444 & 13-Feb-96 & $\mathbf{Y}$ & 2 \\
\hline$Y$ & & 2120 & REPOSIT & $\begin{array}{l}\text { Repository regions } \\
\text { outside of Panel region }\end{array}$ & DPLASRHW & $\begin{array}{l}\text { Average density of plastics in } \mathrm{CH} \\
\text { waste }\end{array}$ & 32446 & 13-feb-96 & $\mathbf{Y}$ & 2 \\
\hline $\mathbf{Y}$ & & 2120 & REPOST & $\begin{array}{l}\text { Repository regions } \\
\text { outside of Panel region }\end{array}$ & DPLASRHW & $\begin{array}{l}\text { Average density of plasties in } \mathrm{CH} \\
\text { waste }\end{array}$ & 32446 & 13-Feb-96 & $Y$ & 2 \\
\hline
\end{tabular}


CCA Parameter Listing

\begin{tabular}{|c|c|c|c|c|c|c|c|c|c|c|}
\hline ID & \begin{tabular}{|c|} 
Classint- \\
cation of \\
Data \\
Catogory \\
(footnote 2)
\end{tabular} & $\begin{array}{l}\text { Distribution } \\
\text { Type }\end{array}$ & Ma:n & Median & Minimum & Meximum & Units & Reforence iD & $\begin{array}{l}\text { Was the data } \\
\text { developed } \\
\text { under an } \\
\text { NQA-1 } \\
\text { Program? }\end{array}$ & $\begin{array}{c}\text { Which } \\
\text { methods } \\
\text { were used to } \\
\text { qually } \\
\text { existing } \\
\text { data? } \\
\text { (footnote 3) }\end{array}$ \\
\hline 2106 & $1,3,4$ & CONSTANT & $4.0000000 E+00$ & $4.0000000 E+\infty 0$ & $4.0000000 \mathrm{E}+00$ & $4.0000000 E+00$ & NONE & WP031041 & $Y$ & NA \\
\hline 2107 & $1,3,4$ & CONSTANT & $7.7110000 \mathrm{E}-02$ & $7.7110000 E-02$ & $7.7110000 E-02$ & $7.7110000 E-02$ & NONE & WP031041 & $\mathbf{Y}$ & N/A \\
\hline 2107 & $1,3,4$ & CONSTANT & $7.7110000 \mathrm{E}-02$ & $7.7110000 \mathrm{E}-02$ & $7.7110000=02$ & $7.7110000 E-02$ & NONE & WP035597 & $Y$ & NA \\
\hline 2107 & $1,3,4$ & CONSTANT & $7.7110000 \mathrm{E}-02$ & $7.7110000 E-02$ & $7.7110000 E-02$ & $7.7110000 E-02$ & NONE & WPO40514 & $Y$ & N/A \\
\hline 2110 & 3,5 & CONSTANT & $1.0000000 E+\infty 0$ & $1.0000000 E+\infty 0$ & $1.0000000 \mathrm{E}+\infty 0$ & $1.0000000 \mathrm{E}+\infty 0$ & NONE & WPO35597 & $\mathbf{Y}$ & N/A \\
\hline 2110 & 3,5 & CONSTANT & $1.0000000 E+00$ & $1.0000000 E+\infty 0$ & $1.0000000 E+00$ & $1.0000000 E+00$ & NONE & WPO40514 & $\mathbf{Y}$ & NA \\
\hline 2110 & 3,5 & CONSTANT & $1.0000000 E+\infty 0$ & $1.0000000 \mathrm{E}+\infty 0$ & $1.0000000 E+\infty 0$ & $1.0000000 \mathrm{E}+00$ & NONE & WP042681 & $\mathbf{Y}$ & NA \\
\hline 2112 & 3,5 & CONSTANT & $0.0000000 E+00$ & $0.0000000 E+00$ & $0.0000000 E+00$ & $0.0000000 \mathrm{E}+\infty$ & $P a^{n}-1$ & WP035597 & $\mathbf{Y}$ & N/A \\
\hline 2112 & 3,5 & CONSTANT & $0.0000000 E+\infty 0$ & $0.0000000 E+\infty$ & $0.0000000 E+00$ & $0.0000000 E+\infty 0$ & $P a^{2}-1$ & WP040514 & $\mathbf{Y}$ & N/A \\
\hline 2112 & 3,5 & CONSTANT & $0.0000000 E+00$ & $0.0000000 E+00$ & $0.0000000 E+00$ & $0.0000000 \mathrm{E}+\infty 0$ & $P a^{r}-1$ & WP0 02681 & $\mathbf{Y}$ & N/A \\
\hline 2113 & 2 & CONSTANT & $5.4000000 E+01$ & $5.4000000 E+01$ & $5.4000000 E+01$ & $5.4000000 E+01$ & $\mathrm{~kg} / \mathrm{m}^{\wedge} 3$ & WPO32328 & $\mathbf{Y}$ & N/A \\
\hline 2114 & 2 & CONSTANT & $1.7000000 E+01$ & $1.7000000 E+01$ & $1.7000000 E+01$ & $1.7000000 E+01$ & $\mathrm{~kg} / \mathrm{m}^{\wedge} 3$ & WPO32328 & $\mathbf{Y}$ & N/A \\
\hline 2114 & 2 & CONSTANT & $1.7000000 \mathrm{E}+01$ & $1.7000000 E+01$ & $1.7000000 E+01$ & $1.7000000 E+01$ & $\mathrm{~kg} / \mathrm{m}^{\wedge} 3$ & WP035048 & $\mathbf{Y}$ & N/A \\
\hline 2115 & 2 & CONSTANT & $1.3900000 \mathrm{E}+02$ & $1.3900000 E+02$ & $1.39000000 E+02$ & $1.3900000 E+02$ & $\mathrm{~kg} / \mathrm{m}^{\wedge} 3$ & WPO32328 & $\mathbf{Y}$ & NA \\
\hline 2115 & 2 & CONSTANT & $1.3900000 E+02$ & $1.3900000 \mathrm{E}+02$ & $1.3900000 E+02$ & $1.3900000 \mathrm{E}+02$ & $\mathrm{~kg} / \mathrm{m}^{\wedge} \mathrm{3}$ & WPO36048 & $\mathbf{Y}$ & N/A \\
\hline 2116 & 2 & CONSTANT & $2.5910000 E+03$ & $2.5910000 E+03$ & $2.5910000 E+03$ & $2.5910000 E+03$ & $\mathrm{~kg} / \mathrm{m}^{\wedge} 3$ & WPO32328 & $Y$ & NA \\
\hline 2116 & 2 & CONSTANT & $2.5910000 E+03$ & $25910000 E+03$ & $2.5910000 E+03$ & $2.5910000 E+03$ & $\mathrm{~kg} / \mathrm{m}^{\wedge} 3$ & WP036048 & $\mathbf{Y}$ & N/A \\
\hline 2116 & 2 & CONSTANT & $2.5910000 E+03$ & $2.5910000 E+03$ & $2.5910000 E+03$ & $2.5910000 E+03$ & $\mathrm{~kg} / \mathrm{m}^{\wedge} 3$ & WP038449 & $\mathbf{Y}$ & N/A \\
\hline 2117 & 2 & CONSTANT & $1.7000000 \mathrm{E}+02$ & $1.7000000 E+02$ & $1.7000000 \mathrm{E}+02$ & $1.7000000 \mathrm{E}+02$ & $\mathrm{~kg} / \mathrm{m}^{\wedge} 3$ & WP032328 & $Y$ & N/A \\
\hline 2117 & 2 & CONSTANT & $1.7000000 E+02$ & $1.7000000 E+02$ & $1.7000000 \mathrm{E}+02$ & $1.7000000 E+02$ & $\mathrm{~kg} / \mathrm{m}^{\wedge} 3$ & WP036048 & $\mathbf{Y}$ & N/A \\
\hline 2118 & 2 & CONSTANT & $1.0000000 E+02$ & $1.0000000 E+02$ & $1.0000000 E+02$ & $1.0000000 E+02$ & $\mathrm{~kg} / \mathrm{m}^{\wedge} \mathbf{3}$ & WP032328 & $\mathbf{Y}$ & N/A \\
\hline 2118 & 2 & CONSTANT & $1.0000000 E+02$ & $1.0000000 E+02$ & $1.0000000 E+02$ & $1.0000000 E+02$ & $\mathrm{~kg} / \mathrm{m}^{\wedge} 3$ & WP036048 & $\mathbf{Y}$ & N/A \\
\hline 2119 & 2 & CONSTANT & $3.4000000 E+01$ & $3.4000000 E+01$ & $3.4000000 E+01$ & $3.4000000 \mathrm{E}+01$ & $\mathrm{~kg} / \mathrm{m}^{\wedge} 3$ & WPO32328 & $Y$ & NA \\
\hline 2119 & 2 & CONSTANT & $3.4000000 E+01$ & $3.4000000 E+01$ & $3.4000000 E+01$ & $3.4000000 \mathrm{E}+01$ & $\mathrm{~kg} / \mathrm{m}^{\wedge} 3$ & WPO35048 & $\mathbf{Y}$ & NA \\
\hline 2120 & 2 & CONSTANT & $1.5000000 E+01$ & $1.5000000 E+01$ & $1.5000000 E+01$ & $1.5000000 E+01$ & $\mathrm{~kg} / \mathrm{m}^{\wedge} \mathrm{3}$ & WP032328 & $Y$ & N/A \\
\hline 2120 & 2 & CONSTANT & $1.5000000 E+01$ & $1.5000000 E+01$ & $1.5000000 \mathrm{E}+01$ & $1.5000000 E+01$ & $\mathrm{~kg} / \mathrm{m}^{\wedge} 3$ & WP036048 & $Y$ & N/A \\
\hline
\end{tabular}


CCA Parameter Listing

\begin{tabular}{|c|c|c|c|c|c|c|c|c|c|c|}
\hline $\begin{array}{l}\text { Parameter in } \\
\text { Database? } \\
\text { (YN) }\end{array}$ & \begin{tabular}{|} 
ff the values \\
ured in CCA \\
are not the \\
seme as in \\
DB, provide $x$ \\
difference
\end{tabular} & 10 & Material io & Materia Name & $\begin{array}{l}\text { Parameter } \\
\text { ID }\end{array}$ & Perameter Nama & $\begin{array}{l}\text { PRP ID } \\
\text { (WPO }\end{array}$ & $\begin{array}{l}\text { Data Entry } \\
\text { Date }\end{array}$ & $\begin{array}{l}\text { Is the } 464 \\
\text { consistent } \\
\text { with the } \\
\text { perameter } \\
\text { in OB? } \\
\left(Y_{\text {, NA) }}\right.\end{array}$ & $\begin{array}{l}\text { Parmoter } \\
\text { Category } \\
\text { (footnote 1) }\end{array}$ \\
\hline $\mathbf{Y}$ & & 2121 & REPOSIT & $\begin{array}{l}\text { Repository regions } \\
\text { outside of Panel region }\end{array}$ & DPLSCCHW & $\begin{array}{l}\text { Bulk density of plastic liners, } \mathrm{CH} \\
\text { waste }\end{array}$ & 32447 & 13-Feb-96 & $\mathbf{Y}$ & 2 \\
\hline $\mathbf{Y}$ & & 2121 & REPOSIT & $\begin{array}{l}\text { Repository regions } \\
\text { outside of Panel region }\end{array}$ & DPLSCCHW & $\begin{array}{l}\text { Bulk density of plastic tiners, } \mathrm{CH} \\
\text { waste }\end{array}$ & 32447 & 13-Fob-96 & $\mathbf{Y}$ & 2 \\
\hline $\mathbf{Y}$ & & 2122 & REPOST & $\begin{array}{l}\text { Repository regions } \\
\text { outside of Panel region }\end{array}$ & DRUBBCHW & $\begin{array}{l}\text { Average density of nubber in } \mathrm{CH} \\
\text { waste }\end{array}$ & 32450 & 13 -Fob-96 & $\mathbf{Y}$ & 2 \\
\hline $\mathbf{Y}$ & & 2122 & REPOSIT & $\begin{array}{l}\text { Repository regions } \\
\text { Outside of Panel region }\end{array}$ & DRUBBCHW & $\begin{array}{l}\text { Average density of rubber in CH } \\
\text { waste }\end{array}$ & 32450 & 13 Fob-96 & $\mathbf{Y}$ & 2 \\
\hline $\mathbf{Y}$ & & 2123 & REPOST & $\begin{array}{l}\text { Repository regions } \\
\text { outside of Panel region }\end{array}$ & DRUBBRHW & $\begin{array}{l}\text { Average density of nubber in RH } \\
\text { waste }\end{array}$ & 32451 & 13-Feb-96 & $\mathbf{Y}$ & 2 \\
\hline $\mathbf{Y}$ & & 2123 & REPOSTT & $\begin{array}{l}\text { Repository regions } \\
\text { outside of Panal region }\end{array}$ & DRUBBRHW & $\begin{array}{l}\text { Average density of nubber in RH } \\
\text { waste }\end{array}$ & 32451 & 13-Feb-96 & $\mathbf{Y}$ & 2 \\
\hline $\mathbf{Y}$ & & 2127 & REPOSIT & $\begin{array}{l}\text { Repository regions } \\
\text { outside of Panel region }\end{array}$ & GRATMICH & $\begin{array}{l}\text { Gas Production Rate, Microbial, } \\
\text { Humid Conditions Relative to } \\
\text { Inundated Rate }\end{array}$ & 33234 & O8-Feb-96 & $\mathbf{Y}$ & 1 \\
\hline$Y$ & & 2127 & REPOST & $\begin{array}{l}\text { Repository regions } \\
\text { outside of Panel region }\end{array}$ & GRATMICH & $\begin{array}{l}\text { Gas Production Rate, Microbial, } \\
\text { Hemid Conditions Relative to } \\
\text { Inundated Rate }\end{array}$ & 33234 & 08-feb-96 & $\mathbf{Y}$ & 1 \\
\hline $\mathbf{Y}$ & & 2127 & REPOST & $\left|\begin{array}{l}\text { Repository regions } \\
\text { outside of Panel region }\end{array}\right|$ & GRATMICH & $\begin{array}{l}\text { Gas Production Rate, Microbial, } \\
\text { Humid Conditions Relative to } \\
\text { inumdated Rate }\end{array}$ & 33234 & 08-Feb-96 & $\mathbf{Y}$ & 1 \\
\hline$Y$ & & 2128 & REPOSIT & $\begin{array}{l}\text { Repository regions } \\
\text { outside of Panel region }\end{array}$ & GRATMICI & $\begin{array}{l}\text { Gas production rate, microbial, } \\
\text { inundated conditions. }\end{array}$ & 33235 & 08-Feb-96 & $\mathbf{Y}$ & 1 \\
\hline $\mathbf{Y}$ & & 2128 & REPOST & $\begin{array}{l}\text { Repository regions } \\
\text { outside of Panel region }\end{array}$ & GRATMICI & $\begin{array}{l}\text { Gas production rate, microbial, } \\
\text { inundated conditions }\end{array}$ & 33235 & 08-Feb-96 & $\mathbf{Y}$ & 1 \\
\hline $\mathbf{Y}$ & & 2128 & REPOST & $\begin{array}{l}\text { Repository regions } \\
\text { Outside of Panel region }\end{array}$ & GRATMICI & $\begin{array}{l}\text { Gas production rate, microbial, } \\
\text { inundated conditions }\end{array}$ & 33235 & 08-Feb-96 & $\mathbf{Y}$ & 1 \\
\hline $\mathbf{Y}$ & & 2129 & REPOST & $\begin{array}{l}\text { Repository regions } \\
\text { outside of Panel region }\end{array}$ & PORE_DIS & $\begin{array}{l}\text { Brooks-Corey pore distribution } \\
\text { parameter }\end{array}$ & 33248 & 21-Feb-96 & $\mathbf{Y}$ & $4 B$ \\
\hline $\mathbf{Y}$ & & 2129 & REPOSIT & $\begin{array}{l}\text { Repository regions } \\
\text { outside of Panel region }\end{array}$ & PORE_DIS & $\begin{array}{l}\text { Brocks-Corey pore distribution } \\
\text { parameter }\end{array}$ & 33248 & 21-Feb-96 & $\mathbf{Y}$ & $4 B$ \\
\hline$Y$ & & 2129 & REPOST & $\begin{array}{l}\text { Repository regions } \\
\text { outside of Panel region }\end{array}$ & PORE_DIS & $\begin{array}{l}\text { Brooks-Corey pora distribution } \\
\text { parameter }\end{array}$ & 33248 & 21-Fob-96 & $\mathbf{Y}$ & AB \\
\hline $\mathbf{Y}$ & & 2129 & REPOSTT & $\begin{array}{l}\text { Repository regions } \\
\text { outside of Panel region }\end{array}$ & PORE_DIS & $\begin{array}{l}\text { Brocks-Corey pore distribution } \\
\text { parameter }\end{array}$ & 33248 & 21-Feb-96 & $\mathbf{Y}$ & 48 \\
\hline $\mathbf{Y}$ & & 2130 & REPOST & $\begin{array}{l}\text { Repository regrons } \\
\text { outside of Panel region }\end{array}$ & POROSTY & Effective porosity & 33250 & 08-Feb-96 & $\gamma$ & $4 B$ \\
\hline $\mathbf{Y}$ & & 2130 & REPOST & $\begin{array}{l}\text { Repository regions } \\
\text { outside of Panel region }\end{array}$ & POROSITY & Effective porosity & 33250 & O8-Feb-96 & $\mathbf{Y}$ & AB \\
\hline$Y$ & & 2130 & REPOSI & $\begin{array}{l}\text { Repository regions } \\
\text { outside of Panel region }\end{array}$ & POROSITY & Effective porosity & 33250 & $08-F 0 b-96$ & $\mathbf{Y}$ & $4 B$ \\
\hline $\mathbf{Y}$ & & 2131 & REPOST & $\begin{array}{l}\text { Repository regions } \\
\text { outside of Panel region }\end{array}$ & PRMX_LOG & $\begin{array}{l}\text { Log of intrinsic permeability, } X \text { - } \\
\text { direction }\end{array}$ & 33255 & $08-F e b-96$ & $\mathbf{Y}$ & $4 A$ \\
\hline $\mathbf{Y}$ & & 2131 & REPOSIT & $\begin{array}{l}\text { Repository regions } \\
\text { outside of Panel region }\end{array}$ & PRMX_LOG & $\begin{array}{l}\text { Log of intrinsic permeabilty, } X \text { - } \\
\text { direction }\end{array}$ & 33255 & 08-Fab-96 & $\mathbf{Y}$ & $4 A$ \\
\hline $\mathbf{Y}$ & & 2131 & REPOSIT & $\begin{array}{l}\text { Repository regions } \\
\text { outside of Panel region }\end{array}$ & PRMX_LOG & $\begin{array}{l}\text { Log of intrinsic permeability, } X \text { - } \\
\text { direction }\end{array}$ & 33255 & 08-Feb-96 & $\mathbf{Y}$ & $4 A$ \\
\hline $\mathbf{Y}$ & & 2132 & REPOSIT & $\begin{array}{l}\text { Repository regions } \\
\text { outside of Panel region }\end{array}$ & PRMY_LOG & $\begin{array}{l}\text { Log of intrinsic permeability, Y- } \\
\text { direction }\end{array}$ & 33258 & $08-F a b-96$ & $\mathbf{Y}$ & $4 A$ \\
\hline $\mathbf{Y}$ & & 2132 & REPOST & $\begin{array}{l}\text { Repository regions } \\
\text { outside of Panel region }\end{array}$ & PRMY_LOG & $\begin{array}{l}\text { Log of intrinsic permeability, } Y \text { - } \\
\text { direction }\end{array}$ & 33258 & 08-Feb-96 & $\mathbf{Y}$ & $4 A$ \\
\hline
\end{tabular}


CCA Parameter Listing

\begin{tabular}{|c|c|c|c|c|c|c|c|c|c|c|}
\hline ID & $\begin{array}{c}\text { Ciessitf- } \\
\text { cantion of } \\
\text { Datta } \\
\text { Cattogory } \\
\text { (footnote 2) }\end{array}$ & $\begin{array}{l}\text { Distribution } \\
\text { Type }\end{array}$ & Mean & Modian & Minimum & Maxtriom & Units & Reforence id & $\begin{array}{l}\text { Was the dinta } \\
\text { developed } \\
\text { under an } \\
\text { NOA-1 } \\
\text { Program? }\end{array}$ & $\begin{array}{c}\text { Which } \\
\text { mothods } \\
\text { woro used to } \\
\text { quallfy } \\
\text { exdsting } \\
\text { deta? } \\
\text { (footnote 3) }\end{array}$ \\
\hline 2121 & 2 & CONSTANT & $2.6000000 E+01$ & $2.6000000 \mathrm{E}+01$ & $2.6000000 E+01$ & $2.6000000 \mathrm{E}+01$ & $\mathrm{~kg} / \mathrm{m}^{\wedge} 3$ & WP032328 & $\gamma$ & N/A \\
\hline 2121 & 2 & CONSTANT & $2.6000000 E+01$ & $26000000 E+01$ & $2.6000000 E+01$ & $26000000 E+01$ & $\mathrm{~kg} / \mathrm{m}^{\wedge} \mathrm{3}$ & WP035048 & $Y$ & N/A \\
\hline 2122 & 2 & CONSTANT & $1.0000000 E+01$ & $1.0000000 E+01$ & $1.0000000 E+01$ & $1.0000000 E+01$ & $\mathrm{~kg} / \mathrm{m}^{\wedge} 3$ & WP032328 & $\mathbf{Y}$ & N/A \\
\hline 2122 & 2 & CONSTANT & $1.0000000 E+01$ & $1.0000000 E+01$ & $1.0000000 E+01$ & $1.0000000 E+01$ & $\mathrm{~kg} / \mathrm{m}^{\wedge} \mathrm{3}$ & WP036048 & $Y$ & N/A \\
\hline 2123 & 2 & CONSTANT & $3.3000000 E+00$ & $3.3000000 E+\infty 0$ & $3.3000000 E+00$ & $3.3000000 E+00$ & $\mathrm{~kg} / \mathrm{m}^{\wedge} 3$ & WP032328 & $\mathbf{Y}$ & N/A \\
\hline 2123 & 2 & CONSTANT & $3.3000000 E+00$ & $3.3000000 E+00$ & $3.3000000 \mathrm{E}+00$ & $3.3000000 E+\infty 0$ & $\mathrm{~kg} / \mathrm{m}^{\wedge} 3$ & WPO36048 & $Y$ & NA \\
\hline 2127 & 1 & UNIFORM & $6.3420000 E-10$ & $6.3420000 E-10$ & $0.0000000 E+00$ & $1.2684000 E-09$ & molkg"s & WP030819 & Y8N & 4 \\
\hline 2127 & 1 & UNIFORM & $6.3420000 \mathrm{E}-10$ & $6.3420000 \mathrm{E}-10$ & $0.0000000 E+00$ & $1.2684000 \mathrm{E}-09$ & molkg's & WPO35268 & Y\&N & 4 \\
\hline 2127 & 1 & UNIFORM & $6.3420000 \mathrm{E}-10$ & $6.3420000 E-10$ & $0.0000000 E+00$ & 1.2684000E- 09 & molkg's & WP035597 & Y\&N & 4 \\
\hline 2128 & 1 & UNIFORM & 4.9150000E-09 & 4.9150000E-09 & $3.1710000 \mathrm{E}-10$ & $9.5129000 \mathrm{E}-09$ & molkg's & WP030819 & Y\&N & 4 \\
\hline 2128 & 1 & UNIFORM & 4.9150000E-09 & $4.9150000 E-09$ & $3.1710000 \mathrm{E}-10$ & $9.5129000 E-09$ & molkg"s & WPO35268 & Y\&N & 4 \\
\hline 2128 & 1 & UNIFORM & $4.9150000 E-09$ & 4.9150000E-09 & $3.1710000 E-10$ & $9.5129000 \mathrm{E}-09$ & molkg"s & WP035597 & Y\&N & 4 \\
\hline 2129 & $1,3,4$ & CUMULATIVE & $3.2500000 E+\infty 0$ & $2.8900000 E+00$ & $1.4400000 E+\infty 0$ & $5.7800000 E+\infty 0$ & NONE & WPO30921 & $\mathbf{Y}$ & N/A \\
\hline 2129 & $1,3,4$ & CUMULATIVE & $3.2500000 \mathrm{E}+00$ & $2.8900000 \mathrm{E}+\infty 0$ & $1.4400000 E+\infty 0$ & $5.7800000 E+\infty 0$ & NONE & WP035268 & $\mathbf{Y}$ & N/A \\
\hline 2129 & $1,3,4$ & CUMULATNE & $3.2500000 E+\infty 0$ & $2.8900000 E+\infty 0$ & $1.4400000 E+\infty 0$ & $5.7800000 \mathrm{E}+\infty 0$ & NONE & WP035597 & $\mathbf{Y}$ & N/A \\
\hline 2129 & $1,3,4$ & CUMULATNE & $3.2500000 E+\infty 0$ & $2.8900000 E+\infty 0$ & $1.4400000 E+\infty 0$ & $5.7800000 E+00$ & NONE & WP040514 & $Y$ & N/A \\
\hline 2130 & 1,3 & CONSTANT & $8.4800000 E-01$ & $8.4800000=-01$ & $8.4800000 \mathrm{E}-01$ & $8.48000000-01$ & NONE & WP035597 & $" Y$ & N/A \\
\hline 2130 & 1.3 & CONSTANT & $8.4800000 E-01$ & $8.4800000 E-01$ & $8.4800000 E-01$ & $8.4800000 E-01$ & NONE & WPO40514 & $\mathbf{Y}$ & N/A \\
\hline 2130 & 1,3 & CONSTANT & $8.4800000 E-01$ & $8.4800000 E-01$ & $8.4800000 E-01$ & $8.4800000 E-01$ & NONE & WPO42681 & $\mathbf{Y}$ & N/A \\
\hline 2131 & 1,3 & CONSTANT & $-1.2769000 \mathrm{E}+01$ & $-1.2769000 E+01$ & $-1.2769000 \mathrm{E}+01$ & $-1.2769000 E+01$ & $\log \left(m^{\wedge} 2\right)$ & WP030921 & $\mathbf{Y}$ & N/A \\
\hline 2131 & 1,3 & CONSTANT & $-1.2769000 E+01$ & $-1.2769000 E+01$ & $-1.2769000 E+01$ & $-1.2769000 E+01$ & $\log \left(m^{\wedge} 2\right)$ & WP035597 & $Y$ & N/A \\
\hline 2131 & 1,3 & CONSTANT & $-1.2769000 E+01$ & $-1.2769000 E+01$ & $-1.2769000 E+01$ & $-1.2769000 E+01$ & $\log \left(m^{\wedge} 2\right)$ & UPO42681 & $Y$ & N/A \\
\hline 2132 & 1,3 & CONSTANT & $-1.2769000 E+01$ & $-1.2769000 E+01$ & $-1.2769000 E+01$ & $-1.2769000 \mathrm{E}+01$ & $\log \left(m^{\wedge} 2\right)$ & WPO30921 & $\gamma$ & N/A \\
\hline 2132 & 1,3 & CONSTANT & $-1.2769000 E+01$ & $-1.2769000 E+01$ & $-1.2769000 E+01$ & $-1.2769000 \mathrm{E}+01$ & $\log \left(m^{\wedge} 2\right)$ & WP035597 & $Y$ & N/A \\
\hline
\end{tabular}


CCA Parameter Listing

\begin{tabular}{|c|c|c|c|c|c|c|c|c|c|c|}
\hline $\begin{array}{c}\text { Parameter in } \\
\text { Database? } \\
\text { (YN) }\end{array}$ & $\begin{array}{l}\text { If the values } \\
\text { used in CCA } \\
\text { are not the } \\
\text { same as in } \\
\text { DB, provide } x \\
\text { difference }\end{array}$ & DD & Material ID & Material Nome & $\begin{array}{c}\text { Parametar } \\
\text { ID }\end{array}$ & Parameter Kams & $\begin{array}{c}\text { PRP ID } \\
\text { (MPO E) }\end{array}$ & $\begin{array}{c}\text { Data Entry } \\
\text { Date }\end{array}$ & $\begin{array}{l}\text { Is the } 464 \\
\text { consictent } \\
\text { with the } \\
\text { peremeter } \\
\text { in DB? } \\
(Y, N A)\end{array}$ & $\begin{array}{l}\text { Parameter } \\
\text { Category } \\
\text { (footnote 1) }\end{array}$ \\
\hline $\mathbf{Y}$ & & 2132 & REPOST & $\begin{array}{l}\text { Repository regions } \\
\text { outside of Panel region }\end{array}$ & PRMY_LOG & $\begin{array}{l}\text { Log of intrinsic permeability, Y- } \\
\text { direction }\end{array}$ & 33258 & asfob-96 & $\mathbf{Y}$ & $4 A$ \\
\hline $\mathbf{Y}$ & & 2133 & REPOSIT & $\begin{array}{l}\text { Repository regions } \\
\text { outside of Panel region }\end{array}$ & PRMZ_LOG & $\begin{array}{l}\text { Log of intrinsic permeabillty. Z- } \\
\text { direction }\end{array}$ & 33259 & 08-Fob-96 & $\mathbf{Y}$ & $4 A$ \\
\hline$Y$ & & 2133 & REPOST & $\begin{array}{l}\text { Repository regions } \\
\text { outside of Panel region }\end{array}$ & PRMZ_LOG & $\begin{array}{l}\text { Log of intrinsic permeability, Z- } \\
\text { direction }\end{array}$ & 33259 & 08-Feb-96 & $\mathbf{Y}$ & $4 A$ \\
\hline $\mathbf{Y}$ & & 2133 & REPOSIT & $\begin{array}{l}\text { Repository regions } \\
\text { outside of Panel region }\end{array}$ & PRMZ_LOG & $\begin{array}{l}\text { Log of intrinsic permeability, Z- } \\
\text { direction }\end{array}$ & 33259 & os-Feb-9s & $\mathbf{Y}$ & $4 A$ \\
\hline$Y$ & & 2135 & REPOSIT & $\begin{array}{l}\text { Repository regions } \\
\text { outside of Panel region }\end{array}$ & RELP_MOD & $\begin{array}{l}\text { Model number, relative } \\
\text { permeability model }\end{array}$ & 33275 & 08-Feb-96: & $\mathbf{Y}$ & AB \\
\hline$Y$ & & 2135 & REPOST & $\begin{array}{l}\text { Repository regions } \\
\text { outside of Panel region }\end{array}$ & RELP_MOD & $\begin{array}{l}\text { Model number, relative } \\
\text { permeability model }\end{array}$ & 33275 & 08-Feb-96 & $Y$ & $4 B$ \\
\hline $\mathbf{Y}$ & & 2135 & REPOSIT & $\begin{array}{l}\text { Repository regions } \\
\text { outside of Panel region }\end{array}$ & RELP_MOD & $\begin{array}{l}\text { Model number, relative } \\
\text { permeability model }\end{array}$ & 33275 & 08-Feb-96 & $\mathbf{Y}$ & $4 B$ \\
\hline $\mathbf{Y}$ & & 2137 & REPOSIT & $\begin{array}{l}\text { Repository regions } \\
\text { outside of Panel region }\end{array}$ & SAT_RGAS & Residual Gas Saturation & 33286 & 22-Fab-96 & $\mathbf{Y}$ & $4 B$ \\
\hline $\mathbf{Y}$ & & 2137 & REPOST & $\begin{array}{l}\text { Repository regions } \\
\text { outside of Panel region }\end{array}$ & SAT_RGAS & Residual Gas Saturation & 33286 & 22-Feb-96 & $\mathbf{Y}$ & $4 B$ \\
\hline $\mathbf{Y}$ & & 2137 & REPOST & $\begin{array}{l}\text { Repository regions } \\
\text { outside of Panel region }\end{array}$ & SAT_RGAS & Residual Gas Saturation & 33286 & 22-Fob-96 & $\mathbf{Y}$ & 48 \\
\hline $\mathbf{Y}$ & & 2137 & REPOSTT & $\begin{array}{l}\text { Repository regions } \\
\text { outside of Panei region }\end{array}$ & SAT_RGAS & Residual Gas Saturation & 33286 & 22-Feb-96 & $\mathbf{Y}$ & 48 \\
\hline $\mathbf{Y}$ & & 2141 & REPOSTT & $\begin{array}{l}\text { Repository regions } \\
\text { outside of Panel region }\end{array}$ & VOLCHW & BIR total volume of $\mathrm{CH}$ waste & 32452 & 13-Feb-96 & $\mathbf{Y}$ & 2 \\
\hline $\mathbf{Y}$ & & 2141 & REPOSIT & $\begin{array}{l}\text { Repository regions } \\
\text { outside of Panel region }\end{array}$ & VOLCHW & BIR total volume of $\mathrm{CH}$ waste & 32452 & 13-Feb-96 & $\mathbf{Y}$ & 2 \\
\hline $\mathbf{Y}$ & & 2142 & REPOSIT & $\begin{array}{l}\text { Repository regions } \\
\text { outside of Panel region }\end{array}$ & VOLRHW & BIR total volume of RH waste & 32453 & 13-Feb-96 & $\mathbf{Y}$ & 2 \\
\hline$Y$ & & 2142 & REPOSIT & $\begin{array}{l}\text { Repository regions } \\
\text { outside of Panel region }\end{array}$ & VOLRHW & BIR total volume of RH wasto & 32453 & 13-Fob-96 & $\mathbf{Y}$ & 2 \\
\hline $\mathbf{Y}$ & & 2158 & S_ANH_AB & $\begin{array}{l}\text { Salado anhydrite beds } \\
\text { A and } B \text {, intact and } \\
\text { fractured }\end{array}$ & DPHIMAX & $\begin{array}{l}\text { Incremental increase in porosity } \\
\text { relative to intact conditions }\end{array}$ & 34138 & 22-Feb-96 & $\mathbf{Y}$ & 48 \\
\hline$Y$ & & 2158 & S_ANH_AB & $\begin{array}{l}\text { Salado anhydrite beds } \\
A \text { and } B \text {, intact and } \\
\text { fractured }\end{array}$ & DPHIMAX & $\begin{array}{l}\text { Incremental increase in porosity } \\
\text { relative to intact conditions }\end{array}$ & 34138 & 22-Fob-96 & $\bar{Y}$ & $4 B$ \\
\hline $\mathbf{Y}$ & & 2158 & S_ANH_AB & $\begin{array}{l}\text { Salado anhydinte beds } \\
\text { A and } B \text {, intact and } \\
\text { fractured }\end{array}$ & DPHIMAX & $\begin{array}{l}\text { Ineremental increase in porosity } \\
\text { relative to intact conditions }\end{array}$ & 34138 & 22fob-96 & $Y$ & $4 \mathrm{~B}$ \\
\hline$Y$ & & 2159 & S_ANH_AB & $\begin{array}{l}\text { Salado anhydrite beds } \\
A \text { and } B \text {, intact and } \\
\text { fractured }\end{array}$ & KMAXLOG & $\begin{array}{l}\text { Log of Maximum Permeability in } \\
\text { Altered Anhydrite Flow Model } \\
\text { Anhydrites }\end{array}$ & 34163 & 22-Fab-96 & $Y$ & $4 B$ \\
\hline $\mathbf{Y}$ & & 2159 & S_ANH_AB & $\begin{array}{l}\text { Salado anhydnte beds } \\
\text { A and B, intact and } \\
\text { fractured }\end{array}$ & KMAXLOG & $\begin{array}{l}\text { Log of Maximum Permeability in } \\
\text { Altered Anhydrite Flow Model } \\
\text { Anhydrites }\end{array}$ & 34163 & 22-Fob-96 & $\mathbf{Y}$ & $4 B$ \\
\hline$Y$ & & 2159 & S_ANH_AB & $\begin{array}{l}\text { Salado anhydrito beds } \\
\text { A and B, intact and } \\
\text { fracturod }\end{array}$ & KMAXIOG & $\begin{array}{l}\text { Log of Maximum Permeability in } \\
\text { Altered Anhydrite Flow Model } \\
\text { Antydrites }\end{array}$ & 34163 & 22-Feb-96 & $\mathbf{Y}$ & $4 B$ \\
\hline $\mathbf{Y}$ & & 2159 & S_ANH_AB & $\begin{array}{l}\text { Salado anhyditie beds } \\
A \text { and } B \text {, intact and } \\
\text { fractured }\end{array}$ & KMAXLOG & $\begin{array}{l}\text { Log of Maximum Permeability in } \\
\text { Altered Anhydrite Flow Model } \\
\text { Anhydrtes }\end{array}$ & 34163 & 22-Feb-96 & $Y$ & $4 B$ \\
\hline$Y$ & & 2159 & S_ANH_AB & $\begin{array}{l}\text { Salado anhydrite beds } \\
\text { A and } \mathrm{B} \text {, intact and } \\
\text { fractured }\end{array}$ & KMAXLOG & $\begin{array}{l}\text { Log of Maximum Permeability in } \\
\text { Altered Anhydrite Flow Model } \\
\text { Anhydrites }\end{array}$ & 34163 & 22-Feb-96 & $\mathbf{Y}$ & $4 B$ \\
\hline$Y$ & & 2169 & S_MB138 & $\begin{array}{l}\text { Salado marker bed } \\
138 \text {, intact and } \\
\text { fractured }\end{array}$ & DPHIMAX & $\begin{array}{l}\text { Incremental increase in porosity } \\
\text { relative to intact condtions }\end{array}$ & 34442 & 22.Fob-96 & $Y$ & $A B$ \\
\hline
\end{tabular}


CCA Parameter Listing

\begin{tabular}{|c|c|c|c|c|c|c|c|c|c|c|}
\hline ID & 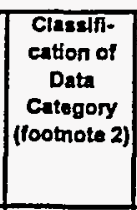 & $\begin{array}{c}\text { Distribution } \\
\text { Type }\end{array}$ & Maan & Medien & Minimum & Meximum & Units & Reforence id & $\begin{array}{l}\text { Was the data } \\
\text { developed } \\
\text { under an } \\
\text { NQA-1 } \\
\text { Progrem? }\end{array}$ & $\begin{array}{c}\text { Which } \\
\text { methods } \\
\text { wore used to } \\
\text { qualify } \\
\text { oxisting } \\
\text { data? } \\
\text { (footnote 3) }\end{array}$ \\
\hline 2132 & 1,3 & CONSTANT & $-1.2769000 E+01$ & $-1.2769000 E+01$ & $-1.2769000 E+01$ & $-9.2769000 E+01$ & $\log \left(m^{\wedge} 2\right)$ & WPO42681 & $\mathbf{Y}$ & NA \\
\hline 2133 & 1,3 & CONSTANT & $-1.2769000 E+01$ & $-1.2769000 E+01$ & $-1.2769000 E+01$ & $-1.2769000 E+01$ & $\log \left(m^{\wedge} 2\right)$ & WP030921 & $Y$ & N/A \\
\hline 2133 & 1,3 & CONSTANT & $-1,2769000 \mathrm{E}+01$ & $-1.2769000 E+01$ & $-1.2769000 \mathrm{E}+01$ & $-1.2769000 E+0\}$ & $\log \left(m^{\wedge} 2\right)$ & WP035597 & $Y$ & N/A \\
\hline 2133 & 1,3 & CONSTANT & $-1.2769000 E+01$ & $-1.2769000 E+01$ & $-1.2769000 E+08$ & $-1.2769000 E+01$ & $\log \left(m^{\wedge} 2\right)$ & WP04268̀1 & $\mathbf{Y}$ & NA \\
\hline 2135 & $1,3,4$ & CONSTANT & $4.0000000 \mathrm{E}+00$ & $4.0000000 E+00$ & 4.0000000E +00 & $4.0000000 \mathrm{E}+\infty$ & NONE & WP035597 & $\mathbf{Y}$ & N/A \\
\hline 2135 & $1,3,4$ & CONSTANT & $4.0000000 E+\infty 0$ & $4.0000000 E+00$ & $4.0000000 E+00$ & $4.0000000 \mathrm{E}+\infty 0$ & NONE & WPO40514 & $\mathbf{Y}$ & N/A \\
\hline 2135 & $1,3,4$ & CONSTANT & $4.0000000 E+00$ & $4.0000000 \mathrm{E}+00$ & $4.0000000 \mathrm{E}+00$ & $4.0000000 E+00$ & NONE & WP042681 & $Y$ & N/A \\
\hline 2137 & $1,3,4$ & UNIFORM & $7.5000000 \mathrm{E}-02$ & $7.5000000 \mathrm{E}-02$ & $0.0000000 \mathrm{E}+\infty 0$ & $1.5000000 E-01$ & NONE & WP035268 & $Y$ & N/A \\
\hline 2137 & $1,3,4$ & UNIFORM & $7.5000000 \mathrm{E}-02$ & $7.5000000 \mathrm{E}-02$ & $0.0000000 E+00$ & $1.5000000 \mathrm{E}-01$ & NONE & WP035597 & $\mathbf{Y}$ & N/A \\
\hline 2137 & $1,3,4$ & UNIFORM. & $7.5000000 E-02$ & $7.5000000 E-02$ & $0.0000000 E+C 0$ & $1.5000000 \mathrm{E}-01$ & NONE & WPO40514 & $\mathbf{Y}$ & NA \\
\hline 2137 & $1,3,4$ & UNIFORM & $7.5000000 \mathrm{E}-02$ & $7.5000000 \mathrm{E}-02$ & $0.0000000 E+\infty 0$ & $1.5000000 \mathrm{E}-01$ & NONE & WPO42681 & $Y$ & N/A \\
\hline 2141 & 2 & CONSTANT & 1.6900000E+05 & 1.6900000E+05 & $1.6900000 E+05$ & $1.6900000 E+05$ & $m^{\wedge} 3$ & WPO32328 & $\mathbf{Y}$ & N/A \\
\hline 2141 & 2 & CONSTANT & 1.6900000E+05 & 1.6900000E+0.5 & 1. $.6900000 \mathrm{E}+05$ & 1.6900000E+05 & $m^{\wedge} 3$ & WPO36048 & $\mathbf{Y}$ & N/A \\
\hline 2142 & 2 & CONSTANT & $7.0800000 E+03$ & $7.0800000 E+03$ & $7.0800000 E+03$ & $7.0800000 E+03$ & $m^{\wedge} 3$ & WPO32328 & $\mathbf{Y}$ & N/A \\
\hline 2142 & 2 & CONSTANT & $7.0800000 \mathrm{E}+03$ & $7.0800000 E+03$ & $7.0800000 E+03$ & $7.0800000 E+03$ & $m^{\wedge} 3$ & WPO36048 & $\mathbf{Y}$ & NA \\
\hline 2158 & 1,3 & CONSTANT & $2.3900000 \mathrm{E}-01$ & $2.3900000 E-01$ & $2.3900000 E-01$ & $2.3900000 E-01$ & NONE & WP035597 & $Y$ & N/A \\
\hline 2158 & 1,3 & CONSTANT & 2.3900000E-01 & $2.3900000 E-01$ & $2.3900000 \mathrm{E}-01$ & $2.3900000 \mathrm{E}-01$ & NONE & WP038274 & $=y$ & N/A \\
\hline 2158 & 1,3 & CONSTANT & $2.3900000 E-01$ & $2.3900000 E-01$ & $2.3900000 \mathrm{E}-01$ & $2.3900000 E-01$ & NONE & WP040514 & $Y$ & N/A \\
\hline 2159 & 1,3 & CONSTANT & $-9.0000000 E+00$ & $-9.0000000 E+00$ & $-9.0000000 \mathrm{E}+00$ & $-9.0000000 E+00$ & $\log \left(m^{\wedge} 2\right)$ & WP035597 & $\mathbf{Y}$ & N/A \\
\hline 2159 & 1,3 & CONSTANT & $-9.0000000 E+\infty 0$ & $-50000000 E+00$ & $-9.0000000 E+00$ & $-9.0000000 E+\infty 0$ & $\log \left(m^{\wedge} 2\right)$ & WP036205 & $Y$ & N/A \\
\hline 2159 & 1,3 & CONSTANT & $-9.0000000 \mathrm{E}+00$ & $-9.0000000 E+00$ & $-9.0000000 E+\infty 0$ & $-9.0000000 E+00$ & $\log \left(m^{\wedge} 2\right)$ & WPO38274 & $Y$ & N/A \\
\hline 2159 & 1,3 & CONSTANT & $-9.0000000 E+\infty$ & $-9.0000000 \mathrm{E}+00$ & $-9.0000000 E+00$ & $-9.0000000 \mathrm{E}+00$ & $\log \left(m^{\wedge} 2\right)$ & WP038569 & $Y$ & N/A \\
\hline 2159 & 9,3 & CONSTANT & $-9.0000000 E+00$ & $-9.0000000 E+\infty 0$ & $-9.0000000 \mathrm{E}+00$ & $-9.0000000 \mathrm{E}+00$ & $\log \left(m^{\wedge} 2\right)$ & WPO40514 & $\mathbf{Y}$ & N/A \\
\hline 2169 & 1,3 & CONSTANT & $3.9000000 E-02$ & $3.9000000 E-02$ & $3.9000000 \mathrm{E}-02$ & $3.9000000 E-02$ & NONE & WPO35597 & $\gamma$ & N/A \\
\hline
\end{tabular}


CCA Parameter Listing

\begin{tabular}{|c|c|c|c|c|c|c|c|c|c|c|}
\hline $\begin{array}{c}\text { Parnmeter in } \\
\text { Databese? } \\
\text { (YN) }\end{array}$ & $\begin{array}{l}\text { If the values } \\
\text { used in CCA } \\
\text { are not the } \\
\text { same as in } \\
\text { DB, provide } 4 \\
\text { difference }\end{array}$ & ID & Material ID & Material Kame & $\begin{array}{l}\text { Parameter } \\
\text { ID }\end{array}$ & Parameter Name & $\begin{array}{l}\text { PRP 10 } \\
\text { (WPOA) }\end{array}$ & $\begin{array}{c}\text { Data Entry } \\
\text { Date }\end{array}$ & $\begin{array}{l}\text { Is the } 464 \\
\text { consistent } \\
\text { with the } \\
\text { parameter } \\
\text { in DB? } \\
(Y, N A)\end{array}$ & $\begin{array}{l}\text { Parameter } \\
\text { Category } \\
\text { (footnote 1) }\end{array}$ \\
\hline $\mathbf{Y}$ & & 2169 & S_MB138 & $\begin{array}{l}\text { Salado marker bed } \\
138, \text { intact and } \\
\text { fractured }\end{array}$ & DPHIMAX & $\begin{array}{l}\text { Incremental increase in porosity } \\
\text { relative to intact conditions }\end{array}$ & 34442 & 22Feb-96 & $\mathbf{Y}$ & $4 B$ \\
\hline $\mathbf{Y}$ & & 2169 & S_MB138 & $\begin{array}{l}\text { Salado marker bed } \\
138 \text {, intact and } \\
\text { fractured }\end{array}$ & DPHIMAX & $\begin{array}{l}\text { incremental increase in porosity } \\
\text { relative to intact conditions }\end{array}$ & 34442 & $22+$ rab-96 & $\mathbf{Y}$ & 48 \\
\hline$Y$ & & 2170 & S_MB138 & $\begin{array}{l}\text { Salado marker bed } \\
138, \text { intact and } \\
\text { fractured }\end{array}$ & KMAXLLOG & $\begin{array}{l}\text { Log of Maximum Permeability in } \\
\text { Antered Anhydrite Flow Modol } \\
\text { Anhydrites }\end{array}$ & 34476 & 22-Feb-96 & $\mathbf{Y}$ & $4 B$ \\
\hline$Y$ & & 2170 & S_MB138 & $\begin{array}{l}\text { Salado makker bod } \\
138 \text {, intact and } \\
\text { fractured }\end{array}$ & KMAXLOOG & $\begin{array}{l}\text { Log of Maximum Permeability in } \\
\text { Altered Anhydrite Flow Model } \\
\text { Anhydrites }\end{array}$ & 34476 & 22-Feb-96 & $\mathbf{Y}$ & $4 B$ \\
\hline $\mathbf{Y}$ & & 2170 & S_MB138 & $\begin{array}{l}\text { Salado marker bed } \\
138, \text { intact and } \\
\text { fractured }\end{array}$ & KONAXLOG & $\begin{array}{l}\text { Log of Maximum Permeability in } \\
\text { Altered Anhydrite Flow Model } \\
\text { Anhydrites }\end{array}$ & 34476 & 22-Fob-96 & $\mathbf{Y}$ & $4 B$ \\
\hline $\mathbf{Y}$ & & 2170 & S_MB138 & $\begin{array}{l}\text { Salado marker bed } \\
138, \text { intact and } \\
\text { fractured }\end{array}$ & KMAXLOG & $\begin{array}{l}\text { Log of Maximum Permeability in } \\
\text { Altered Anhydrite Flow Model } \\
\text { Anhydrites }\end{array}$ & 34476 & 22-Fob-96 & $\mathbf{Y}$ & $4 B$ \\
\hline $\mathbf{Y}$ & & 2177 & S_MB139 & $\begin{array}{l}\text { Salado marker bed } \\
\text { 139, intact and } \\
\text { fractured }\end{array}$ & DPHIMAX & $\begin{array}{l}\text { Incremental increase in porosity } \\
\text { relative to intact conditions }\end{array}$ & 34582 & 22-Fob-95 & $\mathbf{Y}$ & 49 \\
\hline $\mathbf{Y}$ & & 2177 & S_MB139 & $\begin{array}{l}\text { Salado marker bed } \\
\text { 139, intact and } \\
\text { fractured }\end{array}$ & DPHIMAX & $\begin{array}{l}\text { Incremental increase in porosity } \\
\text { relative to intact conditions }\end{array}$ & 34582 & 22-Feb-96 & $\mathbf{Y}$ & $4 B$ \\
\hline $\mathbf{Y}$ & & 2177 & S_MB139 & $\begin{array}{l}\text { Salado marker bed } \\
139, \text { intact and } \\
\text { fractured }\end{array}$ & DPHIMAX & $\begin{array}{l}\text { Incremental increase in porosity } \\
\text { relative to intact conditions }\end{array}$ & 34582 & $22+a b-96$ & $\mathbf{Y}$ & $4 B$ \\
\hline$Y$ & & 2178 & S_MB139 & $\begin{array}{l}\text { Salado marker bed } \\
139, \text { intact and } \\
\text { fractured }\end{array}$ & KMAXLOG & $\begin{array}{l}\text { Log of Maxmum Permeability in } \\
\text { Altered Anhydrite Flow Model } \\
\text { Antydrites }\end{array}$ & 34823 & 22Fob-96 & $\mathbf{Y}$ & $4 B$ \\
\hline $\mathbf{Y}$ & & 2178 & S_MB139 & $\begin{array}{l}\text { Salado marker bed } \\
139, \text { intact and } \\
\text { fractured }\end{array}$ & NMAXLOG & $\begin{array}{l}\text { Log of Maximum Permeability in } \\
\text { Altered Anhydrite Flow Model } \\
\text { Anhydrites }\end{array}$ & 34823 & 22-Feb-96 & $\mathbf{Y}$ & $4 B$ \\
\hline $\mathbf{Y}$ & & 2178 & S_MB139 & $\begin{array}{l}\text { Salado marker bod } \\
139, \text { intact and } \\
\text { fractured }\end{array}$ & KMNAXLOG & $\begin{array}{l}\text { Log of Maximum Permeability in } \\
\text { Altered Antydrite Flow Model } \\
\text { Anhydrites }\end{array}$ & 34823 & 22-Fob-96 & $\mathbf{Y}$ & $4 B$ \\
\hline $\mathbf{Y}$ & & 2178 & S_MB139 & $\begin{array}{l}\text { Salado marker bed } \\
139, \text { intact and } \\
\text { fractured }\end{array}$ & KMAXLOG & $\begin{array}{l}\text { Log of Maximum Permeabilty in } \\
\text { Altered Anhydrite Flow Model } \\
\text { Anhydrites }\end{array}$ & 34823 & 22-Fob-96 & $\mathbf{Y}$ & $4 B$ \\
\hline $\mathbf{Y}$ & & 2178 & S_MB139 & $\begin{array}{l}\text { Salado marker bed } \\
139, \text { intact and } \\
\text { fractured }\end{array}$ & KMAXLOG & $\begin{array}{l}\text { Log of Maximum Permeability in } \\
\text { Altered Anhydrite Flow Model } \\
\text { Anhydrites }\end{array}$ & 34823 & $22+$ ob-96 & $\mathbf{Y}$ & $4 B$ \\
\hline $\mathbf{Y}$ & & 2180 & S_MB139 & $\begin{array}{l}\text { Salado marker bed } \\
\text { 139, intact and } \\
\text { fractured }\end{array}$ & PF_DELTA & $\begin{array}{l}\text { Increm ental pressure for full } \\
\text { fracture development }\end{array}$ & 34850 & 22-Fob-96 & $\mathbf{Y}$ & $4 B$ \\
\hline $\mathbf{Y}$ & & 2180 & S_MB139 & $\begin{array}{l}\text { Salado marker bed } \\
\text { 139, intact and } \\
\text { fractured }\end{array}$ & PF_DELTA & $\begin{array}{l}\text { Incremental pressure for full } \\
\text { fracture development }\end{array}$ & 34850 & 22-Feb-96 & $\mathbf{Y}$ & $4 B$ \\
\hline $\mathbf{Y}$ & & 2180 & S_MB139 & $\begin{array}{l}\text { Salado marker bed } \\
139, \text { intact and } \\
\text { fractured }\end{array}$ & PF_DELTA & $\begin{array}{l}\text { Incremental pressure for full } \\
\text { fracture development }\end{array}$ & 34850 & 22-feb-96 & $Y$ & $4 B$ \\
\hline $\mathbf{Y}$ & & 2180 & S_MB139 & $\begin{array}{l}\text { Salado marker bed } \\
139, \text { intact and } \\
\text { fractured }\end{array}$ & PF_DELTA & $\begin{array}{l}\text { Incremental pressure for full } \\
\text { fracture devalopment }\end{array}$ & 34850 & 22-Feb-96 & $\mathbf{Y}$ & $A B$ \\
\hline $\mathbf{Y}$ & & 2183 & TAMARISK & Tamarisk Member & CAP_MOD & $\begin{array}{l}\text { Model number, capillary pressure } \\
\text { model }\end{array}$ & 34524 & 01 -Nov-95 & $\mathbf{Y}$ & $4 B$ \\
\hline $\mathbf{Y}$ & & 2183 & TAMARISK & Tamarisk Member & CAP_MOD & $\begin{array}{l}\text { Model number, capillary pressure } \\
\text { model }\end{array}$ & 34524 & 01-Nov-95 & $\mathbf{Y}$ & $4 B$ \\
\hline $\mathbf{Y}$ & & 2185 & TAMARISK & Tamarisk Member & PORE_DIS & $\begin{array}{l}\text { Brooks-Corey pore distribution } \\
\text { parameter }\end{array}$ & 34565 & 01-Nov-95 & $\mathbf{Y}$ & 48 \\
\hline$Y$ & & 2185 & TAMARISK & Tamarisk Member & PORE_DIS & $\begin{array}{l}\text { Brooks-Corey pore distribution } \\
\text { parameter }\end{array}$ & 34565 & 01-Nov-95 & $\mathbf{Y}$ & $4 B$ \\
\hline$Y$ & & 2185 & TAMARISK & Tamarisk Member & POROSTYY & Effective porosity & 34568 & $20-f 0 b-96$ & $Y$ & 1 \\
\hline $\mathbf{Y}$ & & 2186 & TAMARISK & Tamarisk Member & POROSITY & Effective porosity & 34568 & 20-Feb-96 & $\mathbf{Y}$ & 1 \\
\hline $\mathbf{Y}$ & & 2186 & TAMARISK & Tamarisk Member & POROSITY & Effective porosity & 34568 & 20 Feb-96 & $\gamma$ & 1 \\
\hline $\mathbf{Y}$ & & 2191 & TAMARISK & Tamarisk Member & RELP_MOD & $\begin{array}{l}\text { Model number, relatrve } \\
\text { pormeability model }\end{array}$ & 34588 & 01-Nov-95 & $\mathbf{Y}$ & $4 B$ \\
\hline $\mathbf{Y}$ & & 2191 & TAMARISK & Tamarisk Member & RELP_MOD & $\begin{array}{l}\text { Model number, relative } \\
\text { permeability model }\end{array}$ & 34588 & 01-Nov-95 & $\mathbf{Y}$ & $4 B$ \\
\hline$Y$ & & 2192 & TAMARISK & Tamarisk Member & SAT_RGAS & Residual Gas Saturation & 34591 & 01-Nov-95 & $\mathbf{Y}$ & $4 B$ \\
\hline $\mathbf{Y}$ & & 2192 & TAMARISK & Tamarisk Member & SAT_RGAS & Residual Gas Saturation & 34591 & 01-Nov-95 & $\mathbf{Y}$ & AB \\
\hline
\end{tabular}


CCA Parameter Listing

\begin{tabular}{|c|c|c|c|c|c|c|c|c|c|c|}
\hline 10 & $\begin{array}{c}\text { Classifi- } \\
\text { calton of } \\
\text { Dath } \\
\text { Catogory } \\
\text { (footnote 2) }\end{array}$ & $\begin{array}{l}\text { Distribution } \\
\text { Type }\end{array}$ & $\operatorname{man}$ & Modian & Mintmum & M्रeximem & Units & Roference ID & $\begin{array}{l}\text { Was tho dath } \\
\text { doveloped } \\
\text { under an } \\
\text { NeA-1 } \\
\text { Program? }\end{array}$ & $\begin{array}{c}\text { Whilen } \\
\text { methoos } \\
\text { wore used to } \\
\text { qualify } \\
\text { oxisting } \\
\text { dxta? } \\
\text { (footrote 3) }\end{array}$ \\
\hline 2169 & 1,3 & CONSTANT & $3.9000000 E-02$ & $3.9000000 E-02$ & $3.9000000 E-02$ & $3.9000000 E-02$ & NONE & WP038274 & $\mathbf{Y}$ & NA \\
\hline 2169 & 1,3 & CONSTANT & $3.9000000 E-02$ & $3.9000000 E-02$ & $3.9000000 E-02$ & $3.9000000 E-02$ & NONE & WPO40514 & $\mathbf{Y}$ & NA \\
\hline 2170 & 1,3 & CONSTANT & $-9.0000000 \mathrm{E}+00$ & $-9.0000000 \mathrm{E}+\infty 0$ & $-9.0000000 E+\infty 0$ & $-9.0000000 E+00$ & $\log \left(m^{\wedge} 2\right)$ & WP035597 & $\boldsymbol{\gamma}$ & NA \\
\hline 2170 & 1.3 & CONSTANT & $-9.0000000 \mathrm{E}+\infty 0$ & $-9.0000000 E+\infty 0$ & $-9.0000000 E+\infty 0$ & $-9.0000000 E+\infty 0$ & $\log \left(m^{\wedge} 2\right)$ & WPO38274 & $Y$ & N/A \\
\hline 2170 & 1,3 & CONSTANT & $-9.0000000 \mathrm{E}+00$ & $-9.0000000 E+\infty 0$ & $-9.0000000 E+\infty 0$ & $-9.0000000 E+00$ & $\log \left(m^{\wedge} 2\right)$ & WP038569 & $\mathbf{Y}$ & N/A \\
\hline 2170 & 1,3 & CONSTANT & $-9.0000000 E+00$ & $-9.0000000 E+\infty 0$ & $-9.0000000 E+\infty 0$ & $-9.0000000 E+00$ & $\log \left(m^{\wedge} 2\right)$ & WP040514 & $Y$ & NA \\
\hline 2177 & 3,5 & CONSTANT & $3.9000000 E-02$ & $3.9000000 E-02$ & $3.9000000 E-02$ & $3.9000000 E-02$ & NONE & WP035597 & $\mathbf{Y}$ & N/A \\
\hline 2177 & 3,5 & CONSTANT & $3.9000000 E-02$ & $3.9000000 E-02$ & $3.9000000 \mathrm{E}-02$ & $3.9000000 E-02$ & NONE & WPO38274 & $\mathbf{Y}$ & N/A \\
\hline 2177 & 3,5 & CONSTANT & $3.9000000 E-02$ & $3.9000000 E-02$ & $3.9000000 E-02$ & $3.9000000 E-02$ & NONE & WP040514 & $\mathbf{Y}$ & $N A$ \\
\hline 2178 & 3,5 & CONSTANT & $-9.0000000 E+00$ & $-9.0000000 E+00$ & $-9.0000000 \mathrm{E}+\infty 0$ & $-9.0000000 E+00$ & $\log \left(m^{\wedge} 2\right)$ & WP035597 & $\mathbf{Y}$ & N/A \\
\hline 2178 & 3,5 & CONSTANT & $-9.0000000 \mathrm{E}+00$ & $-9.0000000 E+\infty 0$ & $-9.0000000 E+\infty 0$ & $-9.0000000 \mathrm{E}+00$ & $\log \left(m^{\wedge} 2\right)$ & WP036205 & $Y$ & N/A \\
\hline 2178 & 3,5 & CONSTANT & $-9.0000000 \mathrm{E}+00$ & $-9.0000000 \mathrm{E}+00$ & $-9.0000000 E+00$ & $-9.0000000 E+00$ & $\log \left(m^{\wedge} 2\right)$ & WP038274 & $\mathbf{Y}$ & N/A \\
\hline 2178 & 3,5 & CONSTANT & $-9.0000000 E+00$ & $-9.0000000 \mathrm{E}+00$ & $-9.0000000 E+00$ & $-9.0000000 E+00$ & $\log \left(m^{\wedge} 2\right)$ & WP038569 & $\mathbf{Y}$ & NA \\
\hline 2178 & 3,5 & CONSTANT & $-9.0000000 \mathrm{E}+00$ & $-9.0000000 E+00$ & $-9.0000000 E+\infty 0$ & $-9.0000000 E+\infty 0$ & $\log \left(m^{\wedge} 2\right)$ & WP040514 & $Y$ & NA \\
\hline 2180 & 3,5 & CONSTANT & $3.8000000 E+06$ & $3.8000000 E+06$ & $3.8000000 \mathrm{E}+06$ & $3.8000000 \mathrm{E}+06$ & $\mathbf{P a}$ & WPO35597 & $\mathbf{Y}$ & N/A \\
\hline 2180 & 3,5 & CONSTANT & $3.8000000 \mathrm{E}+06$ & $3.8000000 E+06$ & $3.8000000 E+06$ & $3.8000000 E+06$ & $\mathrm{~Pa}$ & WP038274 & $\mathbf{Y}$ & N/A \\
\hline 2180 & 3,5 & CONSTANT & $3.8000000 E+06$ & $3.8000000 \mathrm{E}+06$ & $3.8000000 \mathrm{E}+06$ & $3.8000000 E+06$ & $\mathrm{~Pa}$ & WP038569 & $" Y Y$ & NA \\
\hline 2180 & 3,5 & CONSTANT & $3.8000000 E+06$ & $3.8000000 E+06$ & $3.8000000 E+06$ & $3.8000000 E+06$ & $\mathbf{P a}$ & WP040514 & $\mathbf{Y}$ & N/A \\
\hline 2183 & 1,3 & CONSTANT & $1.0000000 E+\infty 0$ & $1.0000000 E+\infty$ & $1.0000000 E+\infty 0$ & $1.0000000 E+\infty 0$ & NONE & WP038568 & $\mathbf{Y}$ & NA \\
\hline 2183 & 1,3 & CONSTANT & $1.0000000 \mathrm{E}+00$ & $1.0000000 E+00$ & $1.0000000 E+00$ & $1.0000000 \mathrm{E}+00$ & NONE & WP040514 & $\boldsymbol{\gamma}$ & NA \\
\hline 2185 & 1,3 & CONSTANT & $7.0000000 E-01$ & $7.0000000 E-01$ & $7.0000000 E-01$ & $7.0000000 E-01$ & NONE & WP038568 & $\mathbf{Y}$ & NA \\
\hline 2185 & 1,3 & CONSTANT & $7.0000000 E-01$ & $7.0000000 E-01$ & $7.0000000=-01$ & $7.0000000 E-01$ & NONE & WPO40514 & $\mathbf{Y}$ & N/A \\
\hline 2186 & 1 & STUDENT & $6.4000000 E-02$ & $6.4000000 \mathrm{E}-02$ & $0.0000000 E+\infty$ & $2.1700000 E-01$ & NONE & WPO30608 & $\bar{Y}$ & N/A \\
\hline 2186 & 1 & STUDENT & $6.4000000 E-02$ & $6.4000000 E-02$ & $0.0000000 E+\infty 0$ & $2.1700000 E-01$ & NONE & WP035597 & $\mathbf{Y}$ & N/A \\
\hline 2186 & 1 & STUDENT & $6.4000000 E-02$ & $6.4000000 E-02$ & $0.0000000 \mathrm{E}+00$ & $2.1700000 E-01$ & NONE & WPO36380 & $\mathbf{Y}$ & N/A \\
\hline 2191 & 1.3 & CONSTANT & $4.0000000 \mathrm{E}+\infty 0$ & $4.0000000 E+\infty 0$ & $4.0000000 \mathrm{E}+\infty 0$ & $4.0000000 E+00$ & NONE & WP038568 & $\mathbf{Y}$ & N/A \\
\hline 2191 & 1.3 & CONSTANT & $4.0000000 E+00$ & $4.0000000 E+00$ & $4.0000000 E+\infty 0$ & $4.0000000 E+00$ & NONE & WP040514 & $\mathbf{Y}$ & NA \\
\hline 2192 & 1,3 & CONSTANT & $20000000 E-01$ & $2.0000000 E-01$ & $2.0000000 E-01$ & $2.0000000 E-01$ & NONE & WP038568 & $\mathbf{Y}$ & N/A \\
\hline 2192 & 1,3 & CONSTANT & $2.0000000 E-01$ & $20000000 \mathrm{E}-01$ & $2.0000000 \mathrm{E}-01$ & $2.0000000 E-01$ & NONE & WPO40514 & $\mathbf{Y}$ & N/A \\
\hline
\end{tabular}


CCA Parameter Listing

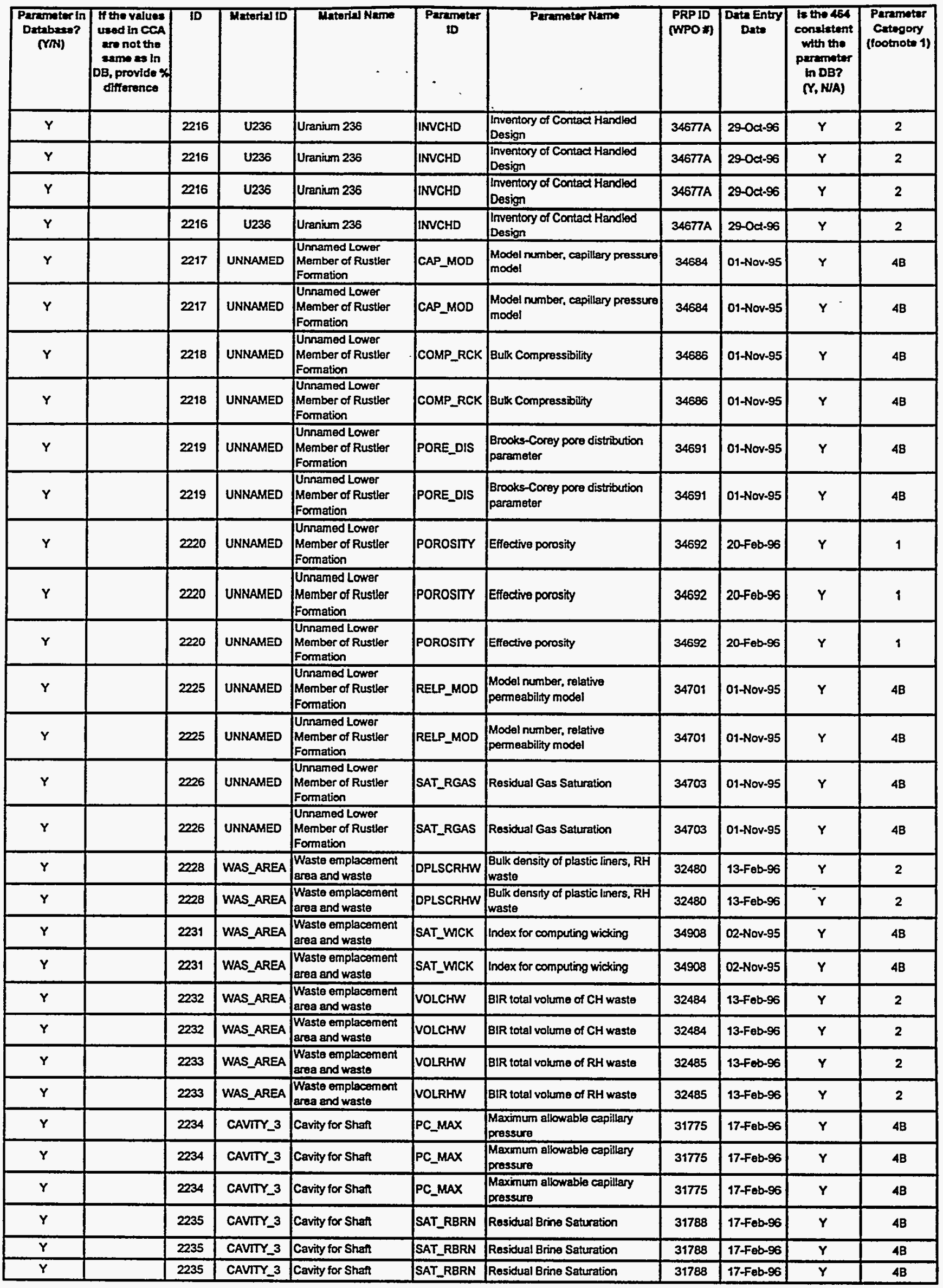


CCA Parameter Listing

\begin{tabular}{|c|c|c|c|c|c|c|c|c|c|c|}
\hline ID & $\begin{array}{c}\text { Classint- } \\
\text { cation of } \\
\text { Data } \\
\text { Catogory } \\
\text { (footnoto 2) }\end{array}$ & $\begin{array}{l}\text { Diatribution } \\
\text { Type }\end{array}$ & Mean & Medien & Minimum & Maximum & Units & Referenco ID & $\begin{array}{l}\text { Was the data } \\
\text { doveloped } \\
\text { under en } \\
\text { NOA-1 } \\
\text { Program? }\end{array}$ & \begin{tabular}{|c|} 
Which \\
mothods \\
wero usod to \\
qually \\
extating \\
data? \\
(footnote J)
\end{tabular} \\
\hline 2216 & 2 & CONSTANT & $5.6900000 E-01$ & $5.6900000 E-01$ & $5.6900000 E-01$ & $5.6900000 \mathrm{E}-01$ & $\mathbf{C i}$ & WP037061 & $\mathbf{Y}$ & N/A \\
\hline 2216 & 2 & CONSTANT & $5.6900000 E-01$ & 5,6900000 E-01 & $5.6900000 E-01$ & $5.6900000 \mathrm{E}-01$ & Ci & WPO39260 & $Y$ & NA \\
\hline 2216 & 2 & CONSTANT & $5,6900000 E-01$ & 5.6900000E-01 & 5.6900000E-01 & 5.6900000E-01 & $\mathrm{Ci}$ & WPO41559 & $\mathbf{Y}$ & N/A \\
\hline 2216 & 2 & CONSTANT & $5.6900000 E-01$ & $5.6900000 E-01$ & $5.6900000 E-01$ & 5.69000000E-01 & $\mathrm{Ci}$ & WPO41560 & $\mathbf{Y}$ & NA \\
\hline 2217 & 1,3 & CONSTANT & $1.0000000 E+\infty 0$ & $8.0000000 \mathrm{E}+00$ & $1.0000000 E+\infty 0$ & $1.0000000 E+\infty$ & NONE & WP038568 & $\mathbf{Y}$ & NA \\
\hline 2217 & 1,3 & CONSTANT & $1.0000000 E+00$ & $.1 .0000000 \mathrm{E}+00$ & $1.0000000 E+\infty 0$ & $1.0000000 \mathrm{E}+\infty 0$ & NONE & WP040514 & $Y$ & NA \\
\hline 2218 & 1,3 & CONSTANT & $0.0000000 E+00$ & $0.0000000 E+00$ & $0.0000000 E+00$ & $0.0000000 E+00$ & $P a^{x}-1$ & WP038568 & $Y$ & N/A \\
\hline 2218 & 1,3 & CONSTANT & $0.0000000 E+00$ & $0,0000000 E+00$ & $0.0000000 E+00$ & $0.0000000 \mathrm{E}+00$ & Pon-1 & WP040514 & $\mathbf{Y}$ & NA \\
\hline 2219 & 1,3 & CONSTANT & $7.0000000 E-01$ & $7.0000000 E-01$ & 7.0000000E-01 & $7.0000000 E-01$ & NONE & WP038568 & $\mathbf{Y}$ & N/A \\
\hline 2219 & 1,3 & CONSTANT & $7.0000000 E-01$ & $7.0000000 E-01$ & $7.0000000 E-01$ & 7.0000000E-01 & NONE & WP040514 & $\mathbf{Y}$ & N/A \\
\hline 2220 & 1 & STUDENT & $1.8100000 E-01$ & $1.8100000 E-01$ & $2.0000000 E-03$ & $2.7300000 E-01$ & NONE & WP030608 & $Y$ & N/A \\
\hline 2220 & 1 & STUDENT & $1.8100000 E-01$ & $1.8100000 E-01$ & $2.0000000 E-03$ & $2.7300000 E-01$ & NONE & WP035597 & $\mathbf{Y}$ & N/A \\
\hline 2220 & 1 & STUDENT & $1.8100000 E-01$ & $1.8100000 E-01$ & $2.0000000 E-03$ & $2.7300000 E-01$ & NONE & WP036380 & $\mathbf{Y}$ & NA \\
\hline 2225 & 1,3 & CONSTANT & $4.0000000 E+00$ & $4.0000000 E+00$ & $4.0000000 E+00$ & $4.0000000 E+00$ & NONE & WP038558 & $\mathbf{Y}$ & N/A. \\
\hline 2225 & 1,3 & CONSTANT & $4.0000000 E+00$ & $4.0000000 E+00$ & $4.0000000 E+00$ & $4.0000000 E+00$ & NONE & WP040514 & $Y$ & N/A \\
\hline 2226 & 1,3 & CONSTANT & $2.0000000 \mathrm{E}-01$ & $2.0000000 E-01$ & $2.0000000 E-01$ & $2.0000000 E-01$ & NONE & WP038568 & $Y$ & N/A \\
\hline 2226 & 1,3 & CONSTANT & $2.0000000 E-01$ & $2.0000000 E-01$ & $2.0000000 \mathrm{E}-01$ & $2.0000000 E-01$ & NONE & WPO40514 & $\mathbf{Y}$ & N/A \\
\hline 2228 & 2 & CONSTANT & $3.1000000 E+00$ & $3.1000000 E+00$ & $3.1000000 E+\infty 0$ & $3.1000000 \mathrm{E}+\infty 0$ & $\mathrm{~kg} / \mathrm{m}^{\wedge} \mathbf{3}$ & WP032328 & $\mathbf{Y}$ & NA \\
\hline 2228 & 2 & CONSTANT & $3.1000000 \mathrm{E}+\infty 0$ & $3.1000000 E+\infty 0$ & $3.1000000 E+\infty 0$ & $3.1000000 E+\infty 0$ & $\mathrm{~kg} / \mathrm{m}^{\wedge} 3$ & WPO36048 & $Y$ & N/A \\
\hline 2231 & 1,3 & UNIFORM & $5.0000000 E-01$ & $5.0000000 E-01$ & $0.0000000 \mathrm{E}+00$ & $1.0000000 E+\infty 0$ & NONE & WPO38568 & $Y$ & N/A \\
\hline 2231 & 1,3 & UNIFORM & $5.0000000 E-01$ & $5.0000000 \mathrm{E}-01$ & $0.0000000 \mathrm{E}+\infty$ & $1.0000000 E+\infty 0$ & NONE & WP040514 & $\mathbf{Y}$ & NA \\
\hline 2232 & 2 & CONSTANT & $1.6900000 E+05$ & $1.6900000 E+05$ & $1.6900000 E+05$ & $1.6900000 E+05$ & $m^{\wedge} 3$ & WPO32328 & $\mathbf{Y}$ & NA \\
\hline 2232 & 2 & CONSTANT & $1.6900000 E+05$ & $1.6900000 \mathrm{E}+05$ & $1.6900000 E+05$ & $1.6900000 \mathrm{E}+05$ & $m^{\wedge} 3$ & WP036048 & $\mathbf{Y}$ & NA \\
\hline 2233 & 2 & CONSTANT & $7.08000000+03$ & $7.0800000 E+03$ & $7.0800000 E+03$ & $7.0800000 E+03$ & $m^{\wedge} 3$ & WPO32328 & $\mathbf{Y}$ & $-N A$ \\
\hline 2233 & 2 & CONSTANT & $7,0800000 E+03$ & $7.0800000 E+03$ & $7.0800000 \mathrm{E}+03$ & $7.0800000 E+03$ & $m^{\wedge} 3$ & WPO36048 & $Y$ & NA \\
\hline 2234 & 1.3 & CONSTANT & $1.0000000 E+08$ & $1.0000000 E+08$ & $1.0000000 \mathrm{E}+08$ & $1.0000000 E+08$ & $\mathrm{~Pa}$ & $\begin{array}{l}\text { MEMO:VAUGHNI } \\
\text { TIERNEY-9 }\end{array}$ & $\mathbf{Y}$ & N/A \\
\hline 2234 & 1,3 & CONSTANT & $1.0000000 E+08$ & $1.0000000 E+08$ & $1.0000000 E+08$ & $1.0000000 E+08$ & $\mathbf{P a}$ & WP035597 & $\mathbf{Y}$ & N/A \\
\hline 2234 & 1,3 & CONSTANT & $1.0000000 E+08$ & $1.0000000 E+08$ & $1.0000000 E+08$ & $1.0000000 E+08$ & $\mathrm{~Pa}$ & WP040514 & $\mathbf{Y}$ & NA \\
\hline 2235 & 1.3 & CONSTANT & $0.0000000 E+00$ & $0.0000000 E+\infty$ & $0.0000000 E+\infty$ & $0.0000000 E+\infty$ & NONE & $\begin{array}{l}\text { MEMO:VAUGHN } \\
\text { TIERNEY-9 }\end{array}$ & $Y$ & NA \\
\hline 2235 & 1,3 & CONSTANT & $0.0000000 E+00$ & $0.0000000 E+00$ & $0.0000000 E+\infty 0$ & $0.0000000 E+00$ & NONE & WP035597 & $\mathbf{Y}$ & N/A \\
\hline 2235 & 1,3 & CONSTANT & $0.0000000 \mathrm{E}+00$ & $0.0000000 \mathrm{E}+00$ & $0.0000000 \mathrm{E}+\infty 0$ & $0.0000000 E+00$ & NONE & WP040514 & $\mathbf{Y}$ & N/A \\
\hline
\end{tabular}


CCA Parameter Listing

\begin{tabular}{|c|c|c|c|c|c|c|c|c|c|c|}
\hline $\begin{array}{l}\text { Parameter in } \\
\text { Datubase? } \\
\text { (YN) }\end{array}$ & $\begin{array}{l}\text { H the values } \\
\text { used in CCA } \\
\text { are not the } \\
\text { same as in } \\
\text { DB, provide } x \\
\text { difference }\end{array}$ & ID & Material ID & Material Nam & $\begin{array}{l}\text { Parameter } \\
\text { tD }\end{array}$ & Parameter Namo & $\begin{array}{l}\text { PRP ID } \\
\text { (WPOW) }\end{array}$ & $\begin{array}{l}\text { Date Entry } \\
\text { Date }\end{array}$ & $\begin{array}{l}\text { Is the } 484 \\
\text { consiatent } \\
\text { with the } \\
\text { peramoter } \\
\text { in DB? } \\
\left(N_{1} \text { NA) }\right.\end{array}$ & $\begin{array}{c}\text { Parmotier } \\
\text { Catepory } \\
\text { (footnote } 1 \text { ) }\end{array}$ \\
\hline $\mathbf{Y}$ & & 2236 & CAVITY_4 & Cavity for Borehole & PC_MAX & $\begin{array}{l}\text { Maxmim allowable capiliary } \\
\text { pressure }\end{array}$ & 31800 & 17.Fob-96 & $\mathbf{Y}$ & 48 \\
\hline $\mathbf{Y}$ & & 2236 & CAVITY_4 & Cavity for Borehole & PC_MAX & $\begin{array}{l}\text { Maxunum allowable capillary } \\
\text { pressure }\end{array}$ & 31800 & 17 -Feb-96 & $\mathbf{Y}$ & 48 \\
\hline $\mathbf{Y}$ & & 2236 & CAVTTY_4 & Cavity for Borehole & PC_MAX & $\begin{array}{l}\text { Maximum allowable capillary } \\
\text { pressure }\end{array}$ & 31800 & $17-F e b-96$ & $\mathbf{Y}$ & $4 B$ \\
\hline $\mathbf{Y}$ & & 2237 & CAVTIY_4 & Cavity for Borehole & SAT_RBRN & Residual Brine Saturation & 31823 & 17 Fob-96 & $\mathbf{Y}$ & 4B \\
\hline $\mathbf{Y}$ & & 2237 & CAVITY_4 & Cavity for Borehote & SAT_RBRN & Residual Brine Saturation & 31823 & 17 Fab-96 & $\mathbf{Y}$ & $4 B$ \\
\hline $\mathbf{Y}$ & & 2237 & CAVITY_4 & Cavity for Borehole & SAT_RBRN & Residual Brine Saturation & 31823 & 17 Feb-96 & $\mathbf{Y}$ & $4 B$ \\
\hline $\mathbf{Y}$ & & 2238 & FORTYNIN & Forty Niner Member & COMP_RCK & Bulk Compressibility & 32979 & 01-Nov-95 & $\mathbf{Y}$ & $4 B$ \\
\hline $\mathbf{Y}$ & & 2239 & FORTYNIN & Forty Niner Member & PC_MAX & $\begin{array}{l}\text { Maxinum allowable capilary } \\
\text { pressure }\end{array}$ & 32989 & 01+Nov-95 & $\mathbf{Y}$ & 48 \\
\hline $\mathbf{Y}$ & & 2239 & FORTYNIN & Forty Niner Member & PC_MAX & $\begin{array}{l}\text { Maximum allowable capillary } \\
\text { pressure }\end{array}$ & 32989 & 01-Nov-95 & $\mathbf{Y}$ & $4 B$ \\
\hline $\mathbf{Y}$ & & 2239 & FORTYNIN & Forty Niner Member & PC_MAX & $\begin{array}{l}\text { Maximum allowable capillary } \\
\text { pressure }\end{array}$ & 32989 & 01-Nov-95 & $\mathbf{Y}$ & $4 B$ \\
\hline $\mathbf{Y}$ & & 2240 & FORTMNIN & Forty Niner Member & SAT_RBRN & Residual Brine Saturation & 33022 & 01-Nov-95 & $\mathbf{Y}$ & 48 \\
\hline $\mathbf{Y}$ & & 2240 & FORTYNIN & Forty Niner Member & SAT_RBRN & Residual Brine Saturation & 33022 & O1-Nov-95 & $\mathbf{Y}$ & AB \\
\hline $\mathbf{Y}$ & & 2241 & MAGENTA & Magenta Member & SAT_RBRN & Residual Brine Saturation & 32560 & 22 Feb-96 & $\mathbf{Y}$ & $4 B$ \\
\hline $\mathbf{Y}$ & & 2241 & MAGENTA & Magenta Member & SAT_RBRN & Residual Brine Saturation & 32560 & 22-Feb-96 & $\mathbf{Y}$ & $4 B$ \\
\hline $\mathbf{Y}$ & & 2241 & MAGENTA & Magenta Momber & SAT_RBRN & Residual Brine Saturation & 32550 & 22-Fab-96 & $\mathbf{Y}$ & $4 \mathrm{~B}$ \\
\hline $\mathbf{Y}$ & & 2242 & REPOSIT & $\begin{array}{l}\text { Repository regions } \\
\text { outside of Panel region }\end{array}$ & PC_MAX & $\begin{array}{l}\text { Maximum allowable capillary } \\
\text { pressure }\end{array}$ & 33246 & 01-Nov-95 & $\mathbf{Y}$ & 48 \\
\hline $\mathbf{Y}$ & & 2242 & REPOSIT & $\begin{array}{l}\text { Repository regions } \\
\text { outside of Panel region }\end{array}$ & PC_MAX & $\begin{array}{l}\text { Maximum allowable capillary } \\
\text { pressure }\end{array}$ & 33246 & 01-Nov-95 & $\mathbf{Y}$ & 48 \\
\hline$Y$ & & 2243 & TAMARISK & Tamarisk Member & COMP_RCK & Bulk Compressibility & 34529 & 01-Nov-95 & $\mathbf{Y}$ & 4B \\
\hline $\mathbf{Y}$ & & 2244 & TAMARISK & Tamarisk Member & PC_MAX & $\begin{array}{l}\text { Maximum allowable capillary } \\
\text { pressure }\end{array}$ & 34563 & 01-Nov-95 & $\mathbf{Y}$ & 48 \\
\hline $\mathbf{Y}$ & & 2244 & TAMARISK & Tamarisk Member & PC_MAX & $\begin{array}{l}\text { Maximum allowable capillary } \\
\text { pressure }\end{array}$ & 34563 & O1-Nov-95 & $\mathbf{Y}$ & AB \\
\hline $\mathbf{Y}$ & & 2245 & TAMARISK & Tamarisk Member & SAT_RBRN & Residual Brine Saturation & 34589 & 01-Nov-95 & $\mathbf{Y}$ & AB \\
\hline $\mathbf{Y}$ & & 2245 & TAMARISK & Tamarisk Member & SAT_RBRN & Residual Brine Saturation & 34589 & O1-Nov-95 & $Y$ & AB \\
\hline $\mathbf{Y}$ & & 2247 & UNNAMED & $\begin{array}{l}\text { Unnamed Lower } \\
\text { Member of Rustler } \\
\text { Formation }\end{array}$ & PC_MAX & $\begin{array}{l}\text { Maximum allowable capillary } \\
\text { pressure }\end{array}$ & 34690 & 01-Nov-95 & $\mathbf{Y}$ & 4B \\
\hline$Y$ & & 2247 & UNNAMED & $\begin{array}{l}\text { Unnamed Lower } \\
\text { Member of Rustler } \\
\text { Formation }\end{array}$ & PC_MAX & $\begin{array}{l}\text { Maximum allowable capillary } \\
\text { pressure }\end{array}$ & 34690 & 01-Nov-95 & $\mathbf{Y}$ & AB \\
\hline $\mathbf{Y}$ & & 2248 & UNNAMED & $\begin{array}{l}\text { Unnamed Lower } \\
\text { Member of Rustler } \\
\text { Formation }\end{array}$ & SAT_RBRN & Residual Brine Saturation & 34702 & 01-Nov-95 & $\because-Y$ & $4 B$ \\
\hline $\mathbf{Y}$ & & 2248 & UNNAMED & $\begin{array}{l}\text { Unnamed Lower } \\
\text { Member of Rustler } \\
\text { Formation }\end{array}$ & SAT_RBRN & Residual Brine Saturation & 34702 & 01-Nov-95 & $\mathbf{Y}$ & 48 \\
\hline $\mathbf{Y}$ & & 2254 & BOREHOLE & Borehole and Fill & TAUFAIL & $\begin{array}{l}\text { Effective shear strength for } \\
\text { erosion (nail) }\end{array}$ & 31536 & 25-Apr-96 & $\mathbf{Y}$ & 1 \\
\hline $\mathbf{Y}$ & & 2254 & BOREHOLE & Borehole and Fill & TAUFAIL. & $\begin{array}{l}\text { Eflectrve shear strength for } \\
\text { erosion (fail) }\end{array}$ & 31536 & 25-Apr-96 & $\mathbf{Y}$ & 1 \\
\hline $\mathbf{Y}$ & & 2254 & BOREHOLE & Borehole and Fill & TAUFAIL & $\begin{array}{l}\text { Effectrve shear strength for } \\
\text { erosion (tail) }\end{array}$ & 31536 & 25-Apr-96 & $\mathbf{Y}$ & 9 \\
\hline $\mathbf{Y}$ & & 2254 & BOREHOLE & Boretole and Fill & TAUFAIL & $\begin{array}{l}\text { Effectrve shear strength for } \\
\text { erosion (rfail) }\end{array}$ & 31536 & 25-Apr-96 & $\mathbf{Y}$ & 1 \\
\hline $\mathbf{Y}$ & & 2265 & CM248 & Curium 248 & INVCHD & $\begin{array}{l}\text { Inventory of Contact Handled } \\
\text { Design }\end{array}$ & $32500 \mathrm{~A}$ & $29-0$ ct-96 & $\mathbf{Y}$ & 2 \\
\hline $\mathbf{Y}$ & & 2265 & CM248 & Curium 248 & INVCHD & $\begin{array}{l}\text { Inventory of Contact Handled } \\
\text { Design }\end{array}$ & $32500 \mathrm{~A}$ & $29-0 \mathrm{ct}-96$ & $\mathbf{Y}$ & 2 \\
\hline $\mathbf{Y}$ & & 2265 & CM248 & Curium 248 & INVCHD & $\begin{array}{l}\text { Inventory of Contact Handled } \\
\text { Design }\end{array}$ & $32500 \mathrm{~A}$ & 29-0ct-96 & $\mathbf{Y}$ & 2 \\
\hline $\mathbf{Y}$ & & 2265 & CM248 & Curium 248 & INVCHD & $\begin{array}{l}\text { Inventory of Contact Handled } \\
\text { Design }\end{array}$ & $32500 \mathrm{~A}$ & 29-0ct-96 & $\mathbf{Y}$ & 2 \\
\hline
\end{tabular}


CCA Parameter Listing

\begin{tabular}{|c|c|c|c|c|c|c|c|c|c|c|}
\hline ID & \begin{tabular}{|c|} 
Classifit- \\
Cation of \\
Data \\
Cattogory \\
(footnoto 2)
\end{tabular} & $\begin{array}{l}\text { Distribution } \\
\text { Typo }\end{array}$ & Hean & Mrallen & MInimum & Maximum & Units & Roference id & $\begin{array}{l}\text { Was the data } \\
\text { doveloped } \\
\text { under an } \\
\text { NoA-1 } \\
\text { Propram? }\end{array}$ & $\begin{array}{c}\text { Which } \\
\text { methods } \\
\text { were used to } \\
\text { qually } \\
\text { extating } \\
\text { data? } \\
\text { (footnote 3) }\end{array}$ \\
\hline 2236 & 1,3 & CONSTANT & $1.0000000 E+08$ & $1.0000000 E+08$ & $1.0000000 E+08$ & $1.0000000 E+08$ & $\mathrm{~Pa}$ & $\begin{array}{c}\text { MEMO:VAUGHN } \\
\text { TIERNEY-9 }\end{array}$ & $\mathbf{Y}$ & N/A \\
\hline 2236 & 1,3 & CONSTANT & $1.0000000 E+08$ & $1.0000000 E+08$ & $1.0000000 E+08$ & $1.0000000 \mathrm{E}+08$ & $\mathrm{~Pa}$ & WP035597 & $Y$ & N/A \\
\hline 2236 & 1,3 & CONSTANT & $1.0000000 E+08$ & $1.0000000 E+08$ & $1.0000000 E+08$ & $1.0000000 E+08$ & $\mathbf{P a}$ & WP040514 & $\mathbf{Y}$ & N/A \\
\hline 2237 & 1,3 & CONSTANT & $0.0000000 E+00$ & $0.0000000 E+00$ & $0.0000000 E+\infty 0$ & $0.0000000 E+00$ & NONE & $\begin{array}{c}\text { MEMO:VAUGHN } \\
\text { TIERNEY-9 }\end{array}$ & $\mathbf{Y}$ & NA \\
\hline 2237 & 1,3 & CONSTANT & $0.0000000 E+\infty 0$ & $0.0000000 \mathrm{E}+00$ & $0.0000000 E+00$ & $0.0000000 E+\infty 0$ & NONE & WP035597 & $\mathbf{Y}$ & NA \\
\hline 2237 & 1,3 & CONSTANT & $0.0000000 E+\infty$ & $0.0000000 E+00$ & $0.0000000 \mathrm{E}+00$ & $0.0000000 E+00$ & NONE & WPO40514 & $Y$ & N/A \\
\hline 2238 & 1,3 & CONSTANT & $0.0000000 E+00$ & $0.0000000 \mathrm{E}+00$ & $0.0000000 E+00$ & $0.0000000 E+00$ & $P x^{n}-1$ & WP038568 & $Y$ & N/A \\
\hline 2238 & 1,3 & CONSTANT & $0.0000000 E+00$ & $0.0000000 \mathrm{E}+\infty 0$ & $0.0000000 \mathrm{E}+00$ & $0.0000000 E+00$ & $P a r-1$ & WP040514 & $\mathbf{Y}$ & N/A \\
\hline 2239 & 1,3 & CONSTANT & $1.0000000 E+08$ & $1.0000000 E+08$ & $1.0000000 \mathrm{E}+08$ & $1.0000000 E+08$ & $\mathrm{~Pa}$ & WP031041 & $\mathbf{Y}$ & N/A \\
\hline 2239 & 1,3 & CONSTANT & $1.0000000 E+08$ & $1.0000000 E+08$ & $1.0000000 E+08$ & $1.0000000 E+08$ & $\mathrm{~Pa}$ & WP038568 & $\mathbf{Y}$ & N/A \\
\hline 2239 & 1,3 & CONSTANT & $1.0000000 E+08$ & $1.0000000 E+08$ & $1.0000000 E+08$ & $1.0000000 E+08$ & $\mathrm{~Pa}$ & WPO40514 & $\mathbf{Y}$ & NA \\
\hline 2240 & 1,3 & CONSTANT & $2.0000000 E-01$ & 2.0000000E-01 & 2,0000000E-01 & 20000000E-01 & NONE & WPO38568 & $Y$ & NA \\
\hline 2240 & 1,3 & CONSTANT & $2.0000000 E-01$ & $2.0000000 \mathrm{E}-01$ & $2.0000000 \mathrm{E}-01$ & $2.0000000 \mathrm{E}-01$ & NONE & WPO40514 & $Y$ & N/A \\
\hline 2241 & $1,3,4$ & CONSTANT & $8.3630000 E-02$ & $8.3630000 E-02$ & $8.3630000 E-02$ & $8.3630000 E-02$ & NONE & WP031041 & $Y$ & N/A \\
\hline 2241 & $1,3,4$ & CONSTANT & $8.3630000 E-02$ & $8.3630000 \mathrm{E}-02$ & $8.3630000 \mathrm{E}-02$ & $8.3630000 \mathrm{E}-02$ & NONE & WP035597 & $y$ & N/A \\
\hline 2241 & $1,3,4$ & CONSTANT & $8.3630000 E-02$ & $8.3630000 E-02$ & $8.3630000 \mathrm{E}-02$ & $8.3630000 E-02$ & NONE & WPO40514 & $Y$ & NA \\
\hline 2242 & 3,5 & CONSTANT & $1.0000000 E+08$ & $1.0000000 \mathrm{E}+08$ & $1.0000000 E+08$ & $1.0000000 E+08$ & $\mathrm{~Pa}$ & WP037970 & $\mathbf{Y}$ & NA \\
\hline 2242 & 3,5 & CONSTANT & $1.0000000 E+08$ & $1.0000000 E+08$ & $1.0000000 \mathrm{E}+08$ & $1.0000000 E+08$ & $\mathbf{P a}$ & WPO40514 & $Y$ & N/A \\
\hline 2243 & 1,3 & CONSTANT & $0.0000000 \mathrm{E}+00$ & $0.0000000 E+00$ & $0.00000000+\infty 0$ & $0.0000000 E+\infty 0$ & $\mathrm{~Pa}^{n}-\mathbf{9}$ & WP038568 & $Y$ & N/A \\
\hline 2243 & 1,3 & CONSTANT & $0.0000000 E+00$ & $0.0000000 E+00$ & $0.0000000 E+00$ & $0.0000000 E+00$ & $P a^{n}-1$ & WP040514 & $Y$ & NA \\
\hline 2244 & 1,3 & CONSTANT & $1.0000000 E+08$ & $1.0000000 \mathrm{E}+08$ & $1.0000000 E+08$ & $1.0000000 E+08$ & $\mathrm{~Pa}$ & WP031041 & $Y$ & N/A \\
\hline 2244 & 1,3 & CONSTANT & $1.0000000 E+08$ & $1.0000000 E+08$ & $1.0000000 E+08$ & $1.0000000 E+08$ & $\mathbf{P a}$ & WP040514 & $\mathbf{Y}$ & N/A \\
\hline 2245 & 1,3 & CONSTANT & $2.0000000 \mathrm{E}-01$ & $2.0000000 E-01$ & $2.0000000 E-01$ & $2.0000000 \mathrm{E}-01$ & NONE & WP038568 & $Y$ & N/A \\
\hline 2245 & 1,3 & CONSTANT & $2.0000000 \mathrm{E}-01$ & $2.0000000 E-01$ & $2.0000000 \mathrm{E}-01$ & $2.0000000 E-01$ & NONE & WP040514 & $Y$ & N/A \\
\hline 2247 & 1,3 & CONSTANT & $1.0000000 E+08$ & $1.0000000 E+08$ & $1.0000000 E+08$ & $1.0000000 E+08$ & $\mathbf{P a}$ & WP038568 & $Y$ & N/A \\
\hline 2247 & 1,3 & CONSTANT & $1.0000000 E+08$ & $1.0000000 E+08$ & $1.0000000 E+08$ & $1.0000000 E+08$ & $\mathbf{P a}$ & WP040514 & $Y$ & $N / A$ \\
\hline 2248 & 1,3 & CONSTANT & $2.0000000 E-01$ & $2.0000000 E-01$ & $2.0000000 E-01$ & $2.0000000 \mathrm{E}-01$ & NONE & WPO38568 & $=-Y$ & N/A \\
\hline 2248 & 1,3 & CONSTANT & $2.0000000 E-01$ & $2.0000000 E-01$ & $2.0000000 E-01$ & $2.0000000 E-01$ & NONE & WP040514 & $\mathbf{Y}$ & $N / A$ \\
\hline 2254 & $3,4,5$ & UNIFORM & $5.0300000 E+00$ & $5.0300000 E+00$ & $5.0000000 E-02$ & $1.0000000 E+01$ & $\mathrm{~Pa}$ & $\begin{array}{c}\text { HYDRAULICSI } \\
\text { ASCE-370 } \\
\end{array}$ & $\mathbf{Y}$ & NA \\
\hline 2254 & $3,4,5$ & UNIFORM & $5.0300000 E+\infty 0$ & $5.0300000 E+00$ & 5.0000000E-02 & $1.0000000 E+01$ & $\mathbf{P a}$ & WP035268 & $\mathbf{Y}$ & N/A \\
\hline 2254 & $3,4,5$ & UNIFORM & $5.0300000 \mathrm{E}+00$ & $5.0300000 E+00$ & $5.0000000 E-02$ & $1.0000000 E+01$ & $\mathrm{~Pa}$ & WP035695 & $Y_{\bullet}$ & NA \\
\hline 2254 & $3,4,5$ & UNIFORM & $5.0300000 E+00$ & $5.0300000 E+00$ & $5.0000000 E-02$ & $1.0000000 E+01$ & $\mathrm{~Pa}$ & WP036766 & $Y$ & NA \\
\hline 2265 & 2 & CONSTANT & $3.6900000 E-02$ & $3.6900000 E-02$ & 3.6900000E-02 & $3.6900000 E-02$ & $\mathrm{Ci}$ & WP037061 & $\mathbf{Y}$ & N/A \\
\hline 2265 & 2 & CONSTANT & $3.6900000 E-02$ & $3.6900000 E-02$ & $3.6900000 E-02$ & $3.6900000 E-02$ & $\mathrm{Ci}$ & WP039260 & $Y$ & N/A \\
\hline 2265 & 2 & CONSTANT & $3.6900000 E-02$ & $3.6900000 \mathrm{E}-02$ & $3.6900000 E-02$ & $3.6900000 \mathrm{E}-02$ & $\mathrm{Ci}$ & WPO41559 & $\mathbf{Y}$ & NA \\
\hline 2265 & 2 & CONSTANT & $3.6900000 E-02$ & $3.6900000 \mathrm{E}-02$ & $3.6900000 E-02$ & $3.6900000 \mathrm{E}-02$ & $\mathrm{Ci}$ & WP041560 & $\mathbf{Y}$ & N/A \\
\hline 2266 & 2 & CONSTANT & $2.1500000 E-04$ & $2.1500000 E-04$ & $2.1500000 E-04$ & $2.1500000 E-04$ & $\mathrm{Ci}$ & WP037061 & $\mathbf{Y}$ & NA \\
\hline 2266 & 2 & CONSTANT & $2.1500000 E-04$ & $2.1500000 E-04$ & $2.1500000 E-04$ & $2.1500000 E-04$ & $\mathrm{Ci}$ & WP039260 & $\mathbf{Y}$ & N/A \\
\hline
\end{tabular}


CCA Parameter Listing

\begin{tabular}{|c|c|c|c|c|c|c|c|c|c|c|}
\hline $\begin{array}{c}\text { Peremeter In } \\
\text { Detnbise? } \\
\text { (N/N) } \\
\end{array}$ & $\begin{array}{l}\text { fit the values } \\
\text { used in CCA } \\
\text { are not the } \\
\text { same as in } \\
\text { DB, provide } x \\
\text { difference }\end{array}$ & ID & Material Io & Material Name & $\begin{array}{l}\text { Paramerter } \\
\text { iD }\end{array}$ & Parameter Nam & $\begin{array}{c}\text { PRP ID } \\
\text { (NPO i) }\end{array}$ & $\begin{array}{c}\text { Data Entry } \\
\text { Dato } \\
\end{array}$ & $\begin{array}{l}\text { Is the } 464 \\
\text { consistent } \\
\text { with the } \\
\text { paremeter } \\
\text { in } \mathrm{DBP} \\
(\mathrm{Y}, \mathrm{NA})\end{array}$ & $\begin{array}{l}\text { Paramatar } \\
\text { Category } \\
\text { (footnote } 1 \text { ) }\end{array}$ \\
\hline$Y$ & & 2266 & $\mathrm{CM} 248$ & Curium 248 & INVRHD & $\begin{array}{l}\text { Inventory of Remote Handled } \\
\text { Design }\end{array}$ & $32501 A$ & $29-0 c t-96$ & $\mathbf{Y}$ & 2 \\
\hline $\mathbf{Y}$ & & 2266 & $\mathrm{CM} 248$ & Curium 248 & INVRHD & $\begin{array}{l}\text { Inventory of Remote Handled } \\
\text { Design }\end{array}$ & $32501 \mathrm{~A}$ & $29-0 a t-96$ & $\mathbf{Y}$ & 2 \\
\hline $\mathbf{Y}$ & & 2267 & PA231 & Protactinium 231 & INVCHD & $\begin{array}{l}\text { Inventory of Contact Handled } \\
\text { Design }\end{array}$ & $32930 \mathrm{~A}$ & 29-0ct-95 & $\mathbf{Y}$ & 2 \\
\hline $\mathbf{Y}$ & & 2267 & PA231 & Protactinium 231 & INVCHD & $\begin{array}{l}\text { Imventory of Contact Handied } \\
\text { Design }\end{array}$ & $32930 \mathrm{~A}$ & $29-0 \mathrm{ct}-96$ & $\mathbf{Y}$ & 2 \\
\hline $\mathbf{Y}$ & & 2267 & PA231 & Protactinium 231 & INVCHD & $\begin{array}{l}\text { Inventory of Contact Handled } \\
\text { Design }\end{array}$ & $32930 \mathrm{~A}$ & $29-0 c t-96$ & $\mathbf{Y}$ & 2 \\
\hline $\mathbf{Y}$ & & 2267 & PA231 & Protactinium 231 & INVCHD & $\begin{array}{l}\text { Inventory of Contact Handled } \\
\text { Design }\end{array}$ & $32930 A$ & 29-0ct-96 & $\mathbf{Y}$ & 2 \\
\hline $\mathbf{Y}$ & & 2268 & PA231 & Protactinium 231 & INVRHD & $\begin{array}{l}\text { Inventory of Remote Handled } \\
\text { Design }\end{array}$ & $32931 \mathrm{~A}$ & $29-0 \mathrm{ct}-96$ & $\mathbf{Y}$ & 2 \\
\hline $\mathbf{Y}$ & & 2268 & PA231 & Protactinium 231 & INVRHD & $\begin{array}{l}\text { Inventory of Remote Handled. } \\
\text { Design }\end{array}$ & $32931 A$ & $29-0 \mathrm{ct}-96$ & $\mathbf{Y}$ & 2 \\
\hline $\mathbf{Y}$ & & 2268 & PA231 & Protactinium 231 & INVRHD & $\begin{array}{l}\text { Inventory of Remote Handied } \\
\text { Design }\end{array}$ & $32931 \mathrm{~A}$ & 29-0ct-96 & $\mathbf{Y}$ & 2 \\
\hline $\mathbf{Y}$ & & 2268 & PA231 & Protactinium 231 & INVRHD & $\begin{array}{l}\text { Inventory of Remote Handled } \\
\text { Design }\end{array}$ & $32931 A$ & $29-0 \mathrm{ct}-96$ & $\mathbf{Y}$ & 2 \\
\hline $\mathbf{Y}$ & & 2269 & PU244 & Plutonium 244 & INVCHD & $\begin{array}{l}\text { Inventory of Contact Handled } \\
\text { Design }\end{array}$ & $33450 A$ & $290 \mathrm{ct}-96$ & $\mathbf{Y}$ & 2 \\
\hline $\mathbf{Y}$ & & 2269 & PU244 & Plutonium 244 & INVCHD & $\begin{array}{l}\text { Inventory of Contact Handled } \\
\text { Design }\end{array}$ & $33450 \mathrm{~A}$ & $29-0 \mathrm{ct}-96$ & $\mathbf{Y}$ & 2 \\
\hline$Y$ & & 2269 & PU244 & Phutonium 244 & INVCHD & $\begin{array}{l}\text { Inventory of Contact Handled } \\
\text { Design }\end{array}$ & $33450 \mathrm{~A}$ & $29-0 \mathrm{ct}-95$ & $\mathbf{Y}$ & 2 \\
\hline$Y$ & & 2270 & PU244 & Plutonium 244 & INVRHD & $\begin{array}{l}\text { Inventory of Remote Handled } \\
\text { Design }\end{array}$ & $33452 A$ & $29-0 c t-96$ & $\mathbf{Y}$ & 2 \\
\hline $\mathbf{Y}$ & & 2270 & PU244 & Plutonium 244 & INVRHD & $\begin{array}{l}\text { Inventory of Remote Handled } \\
\text { Design. }\end{array}$ & $33452 A$ & $29-0 c t-96$ & $\mathbf{x}$ & 2 \\
\hline $\mathbf{Y}$ & & 2270 & PU244 & Plutonium 244 & INVRHD & $\begin{array}{l}\text { Inventory of Remote Handled } \\
\text { Design }\end{array}$ & $33452 A$ & $29-0 c t-96$ & $\mathbf{Y}$ & 2 \\
\hline $\mathbf{Y}$ & & 2271 & RA228 & Radium 228 & INVCHD & $\begin{array}{l}\text { Inventory of Contect Handled } \\
\text { Design }\end{array}$ & 334704 & $29-0 \mathrm{ct}-96$ & $\mathbf{Y}$ & 2 \\
\hline $\mathbf{Y}$ & & 2271 & RA228 & Radium 228 & INVCHD & $\begin{array}{l}\text { Imventory of Contact Handled } \\
\text { Design }\end{array}$ & $33470 A$ & $29-0 c t-96$ & $\mathbf{Y}$ & 2 \\
\hline $\mathbf{Y}$ & & 2271 & RA228 & Radium 228 & INVCHD & $\begin{array}{l}\text { Imventory of Contact Handied } \\
\text { Design }\end{array}$ & $33470 A$ & $29-0$ ct.96 & $\mathbf{Y}$ & 2 \\
\hline $\mathbf{Y}$ & & 2272 & RA228 & Radium 228 & INVRHD & $\begin{array}{l}\text { Inventory of Remote Handled } \\
\text { Design }\end{array}$ & $36968 A$ & 29-0ct-96 & $\mathbf{Y}$ & 2 \\
\hline$Y$ & & 2272 & RA228 & Radium 228 & INVRHD & $\begin{array}{l}\text { Inventory of Remote Handied } \\
\text { Design }\end{array}$ & $36968 \mathrm{~A}$ & $29-0 \mathrm{ct}-95$ & $\mathbf{Y}$ & 2 \\
\hline$Y$ & & 2272 & RA228 & Radium 228 & INVRHD & $\begin{array}{l}\text { Inventory of Remote Handled } \\
\text { Design }\end{array}$ & $36968 \mathrm{~A}$ & $29-0 c t-96$ & $\mathbf{Y}$ & 2 \\
\hline $\mathbf{Y}$ & & 2274 & WAS_AREA & $\begin{array}{l}\text { Waste emplacement } \\
\text { area and waste }\end{array}$ & DCELLRHW & $\begin{array}{l}\text { Average density of collulosics in } \\
\text { RH waste }\end{array}$ & 32465 & 13 Feb-96 & $\mathbf{Y}$ & 2 \\
\hline $\mathbf{Y}$ & & 2274 & WAS_AREA & $\begin{array}{l}\text { Waste emplacement } \\
\text { area and waste }\end{array}$ & DCELLRHW & $\begin{array}{l}\text { Average density of cellulosics in } \\
\text { RH waste }\end{array}$ & 32465 & 13-Feb-96 & $\mathbf{Y}$ & 2 \\
\hline $\mathbf{Y}$ & & 2275 & WAS_AREA & $\begin{array}{l}\text { Waste emplacement } \\
\text { area and waste }\end{array}$ & DPLASRHW & $\begin{array}{l}\text { Average density of plasties in } \mathrm{CH} \\
\text { waste }\end{array}$ & 32476 & 13 Feb-96 & $=Y$ & 2 \\
\hline $\mathbf{Y}$ & & 2275 & WAS_AREA & $\begin{array}{l}\text { Waste emplacoment } \\
\text { area and waste }\end{array}$ & DPLASRHW & \begin{tabular}{|l|}
$\begin{array}{l}\text { Average density of plastics in } \mathrm{CH} \\
\text { waste }\end{array}$ \\
\end{tabular} & 32476 & 13-Feb-96 & $\mathbf{Y}$ & 2 \\
\hline $\boldsymbol{Y}$ & 50 & 2276 & ASPHALT & Asphalt column & CAP_MOD & $\begin{array}{l}\text { Model number, capillary pressure } \\
\text { model }\end{array}$ & $31375 A$ & O3-Nov-95 & $\mathbf{Y}$ & $4 B$ \\
\hline $\mathbf{Y}$ & 50 & 2276 & ASPHALT & Asphatt column & CAP_MOD & $\begin{array}{l}\text { Model number, capillary pressure } \\
\text { mode! }\end{array}$ & $31375 A$ & 03-Nov-95 & $\mathbf{Y}$ & 48 \\
\hline $\mathbf{Y}$ & 50 & 2276 & ASPHALT & Asphalt column & CAP_MOD & $\begin{array}{l}\text { Model number, capillary pressure } \\
\text { model }\end{array}$ & $31375 A$ & 03-Nov-95 & $\mathbf{Y}$ & $4 B$ \\
\hline $\mathbf{Y}$ & 50 & 2276 & ASPHALT & Asphatt column & CAP_MOD & $\begin{array}{l}\text { Model number, capillary pressure } \\
\text { [model }\end{array}$ & $31375 A$ & 03-Nov-95 & $\mathbf{Y}$ & 48 \\
\hline $\boldsymbol{Y}$ & 50 & 2276 & ASPHALT & Asphalt column & CAP_MOD & $\begin{array}{l}\text { Model number, capillary pressure } \\
\text { model }\end{array}$ & 31375A & 03-Nov-95 & $\mathbf{Y}$ & $4 B$ \\
\hline $\mathbf{Y}$ & & 2277 & ASPHALT & Asphalt column & COMP_RCK & Bulk Compressibility & 31380 & 13-Fob-96 & $Y$ & 1 \\
\hline $\mathbf{Y}$ & & 2277 & ASPHALT & Asphalt column & COMP_RCK & Bulk Compressibilty & 31380 & 13-Fob-96 & $Y$ & 1 \\
\hline $\mathbf{Y}$ & & 2277 & ASPHALT & Asphalt column & COMP_RCK & Bulk Compressibility & 31380 & $13-F a b-96$ & $\mathbf{Y}$ & 1 \\
\hline $\mathbf{Y}$ & & 2278 & ASPHALT & Asphalt column & PC_MAX & $\begin{array}{l}\text { Maximum allowable capillary } \\
\text { pressure }\end{array}$ & 31385A & 03-Nov-95 & $\mathbf{Y}$ & $4 B$ \\
\hline $\mathbf{Y}$ & & 2278 & ASPHALT & Asphalt cohumn & PC_MAX & $\begin{array}{l}\text { Maximum allowable capillary } \\
\text { pressure }\end{array}$ & $31385 \mathrm{~A}$ & 03-Nov-95 & $\mathbf{Y}$ & $A B$ \\
\hline $\mathbf{Y}$ & & 2278 & ASPHALT & Asphalt column & PC_MAX & $\begin{array}{l}\text { Maximum allowable cepillary } \\
\text { pressure }\end{array}$ & $31385 A$ & 03-Nov-95 & $Y$ & 48 \\
\hline
\end{tabular}


CCA Parameter Listing

\begin{tabular}{|c|c|c|c|c|c|c|c|c|c|c|}
\hline ID & $\begin{array}{c}\text { Cinselfi- } \\
\text { cation of } \\
\text { Data } \\
\text { Cantegory } \\
\text { (footnote 2) }\end{array}$ & $\begin{array}{l}\text { Dlatrfburtion } \\
\text { Type }\end{array}$ & Matn & Modian & Minimum & Meximum & Unltz & Reterence iD & $\begin{array}{l}\text { Was the dinta } \\
\text { doveloped } \\
\text { under an } \\
\text { NOA-1 } \\
\text { Progrem? }\end{array}$ & $\begin{array}{c}\text { Which } \\
\text { methods } \\
\text { were used to } \\
\text { qually } \\
\text { existing } \\
\text { dita? } \\
\text { (footnote 3) }\end{array}$ \\
\hline 2266 & 2 & CONSTANT & $2.1500000 E-04$ & $2.1500000 E-04$ & $21500000 E-04$ & $2.1500000 \mathrm{E}-0.4$ & Ci & WPO41559 & $Y$ & N/A \\
\hline 2266 & 2 & CONSTANT & $2.1500000 E-04$ & $2.1500000 E-04$ & $2.1500000 E-04$ & $2.1500000 \mathrm{E}-04$ & Ci & WPO41560 & $\mathbf{Y}$ & NA \\
\hline 2267 & 2 & CONSTANT & 4.6200000E-01 & 4.6200000E- 01 & 4.6200000E-01 & 4.62000000E-01 & $\mathrm{Ci}$ & WP037061 & $\mathbf{Y}$ & NA \\
\hline 2267 & 2 & CONSTANT & 4.6200000E-01 & 4.6200000E-01 & 4.6200000E-01 & 4.6200000E-01 & $\mathbf{C i}$ & WPO39260 & $\mathbf{Y}$ & NA \\
\hline 2267 & 2 & CONSTANT & $4.6200000 E-01$ & $4.6200000 \mathrm{E}-01$ & 4.6200000E-01 & $4.6200000 E-01$ & $\mathbf{C i}$ & WPO41559 & $Y$ & N/A \\
\hline 2267 & 2 & CONSTANT & 4.6200000E-01 & 4.6200000E-01 & 4.6200000E-01 & 4.6200000 E-01 & $\mathrm{Ci}$ & WPO41560 & $Y$ & NA \\
\hline 2268 & 2 & CONSTANT & $5.6300000 E-03$ & $5.6300000 E-03$ & $5.6300000 E-03$ & $5.6300000 \mathrm{E}-03$ & Ci & WP037061 & $\mathbf{Y}$ & N/A \\
\hline 2268 & 2 & CONSTANT & $5.6300000 \mathrm{E}-03$ & $5.6300000 \mathrm{E}-03$ & $5.6300000 \mathrm{E}-03$ & $5.6300000 \mathrm{E}-03$ & $\mathrm{Ci}$ & WP039250 & $Y$ & N/A \\
\hline 2268 & 2 & CONSTANT & $5.6300000 E-03$ & 5.6300000E-03 & $5.6300000 E-03$ & $5.6300000 E-03$ & $\mathbf{C i}$ & WPO41559 & $\mathbf{Y}$ & N/A \\
\hline 2268 & 2 & CONSTANT & $5.6300000 \mathrm{E}-03$ & $5.6300000 \mathrm{E}-03$ & $5.6300000 E-03$ & $5.6300000 \mathrm{E}-03$ & $\mathrm{Ci}$ & WPO41560 & $\mathbf{Y}$ & N/A \\
\hline 2269 & 2 & CONSTANT & $1.5100000 E-06$ & 1.5100000E-06 & $1.5100000 \mathrm{E}-06$ & $1.5100000 E-06$ & Ci & WP037051 & $Y$ & N/A \\
\hline 2269 & 2 & CONSTANT & $1.5100000 E-06$ & $1.5100000 E-06$ & $1.5100000 E-06$ & $1.5100000 E-06$ & $\mathbf{C i}$ & WPO41559 & $\mathbf{Y}$ & NA \\
\hline 2269 & 2 & CONSTANT & $1.5100000 E-06$ & $1.5100000 E-06$ & $1.5100000 \mathrm{E}-06$ & $1.5100000 E-06$ & $\mathrm{Ci}$ & WP041560 & $Y$ & $N / A$ \\
\hline 2270 & 2 & CONSTANT & $8.5000000 E-11$ & $8.5000000 E-11$ & $8.5000000 E-11$ & $8.5000000 E-11$ & $\mathrm{Ci}$ & WP037051 & $\mathbf{Y}$ & NA \\
\hline 2270 & 2 & CONSTANT & $8.5000000 E-11$ & $8.5000000 E-11$ & $8.5000000 E-11$ & $8.5000000 E-11$ & $\mathrm{Ci}$ & WP041559 & $\mathbf{Y}$ & NA \\
\hline 2270 & 2 & CONSTANT & $8.5000000 E-11$ & $8.5000000 E-11$ & $8.5000000 E-11$ & $8.5000000 E-11$ & $\mathrm{Ci}$ & WPO41560 & $Y$ & NA \\
\hline 2271 & 2 & CONSTANT & $9.1000000 E-01$ & $9.1000000 E-01$ & $9.1000000 E-01$ & $9.1000000 E-01$ & $\mathrm{Ci}$ & WP037061 & $Y$ & NA \\
\hline 2271 & 2 & CONSTANT & $9.1000000 E-01$ & $9.1000000 E-01$ & $9.1000000 E-01$ & $9.1000000 E-01$ & $\mathrm{Ci}$ & WPO41559 & $Y$ & N/A \\
\hline 2271 & 2 & CONSTANT & $9.1000000 E-01$ & $9.1000000 E-01$ & $9.1000000 E-01$ & $9.1000000 E-01$ & $\mathrm{Ci}$ & WP041560 & $\mathbf{Y}$ & N/A \\
\hline 2272 & 2 & CONSTANT & $9.2300000 E-02$ & $9.2300000 E-02$ & $9.2300000 \mathrm{E}-02$ & $9.2300000 E-02$ & $\mathrm{Ci}$ & WP037061 & $\mathbf{Y}$ & N/A \\
\hline 2272 & 2 & CONSTANT & $9.2300000 \mathrm{E}-02$ & $-9.2300000 \mathrm{E}-02$ & $9.2300000 E-02$ & $9.2300000 E-02$ & $\mathrm{Ci}$ & WPO-41559 & $Y$ & NA \\
\hline 2272 & 2 & CONSTANT & $9,2300000 E-02$ & 9.2300000E-02 & $9.2300000 \mathrm{E}-02$ & $9.2300000 \mathrm{E}-02$ & $\mathbf{C i}$ & WPO41560 & $Y$ & NA \\
\hline 2274 & 2 & CONSTANT & $1.7000000 E+01$ & $1.7000000 \mathrm{E}+01$ & $1.7000000 E+01$ & $1.7000000 E+01$ & $\mathrm{~kg} / \mathrm{m}^{\wedge} 3$ & WPO32328 & $Y$ & N/A \\
\hline 2274 & 2 & CONSTANT & $1.7000000 E+01$ & $1.7000000 E+01$ & $1.7000000 E+01$ & $1.7000000 E+01$ & $\mathrm{~kg} / \mathrm{m}^{\wedge} 3$ & WPO35048 & $\mathbf{Y}$ & N/A \\
\hline 2275 & 2 & CONSTANT & $1.5000000 E+01$ & $1.5000000 \mathrm{E}+01$ & $1.5000000 E+01$ & $1: 5000000 E+01$ & $\mathrm{~kg} / \mathrm{m}^{\wedge} 3$ & WP032328 & $-y$ & N/A \\
\hline 2275 & 2 & CONSTANT & $1.5000000 E+01$ & $1.5000000 E+01$ & $1.5000000 E+01$ & $1.5000000 E+01$ & $\mathrm{~kg} / \mathrm{m}^{\wedge} 3$ & WP035048 & $\mathbf{Y}$ & NA \\
\hline 2276 & 3,5 & CONSTANT & $1.0000000 E+\infty$ & $1.0000000 \mathrm{E}+00$ & $1.0000000 \mathrm{E}+00$ & $1.0000000 E+\infty 0$ & NONE & WPO30640 & $Y$ & NA \\
\hline 2276 & 3.5 & CONSTANT & $1.0000000 E+\infty 0$ & $1.0000000 E+\infty 0$ & $1.0000000 E+00$ & $1.0000000 E+\infty 0$ & NONE & WP030994 & $\mathbf{Y}$ & NA \\
\hline 2276 & 3,5 & CONSTANT & $1.0000000 E+\infty 0$ & $1.0000000 \mathrm{E}+\infty 0$ & $1.0000000 \mathrm{E}+00$ & $1.0000000 E+\infty 0$ & NONE & WP035597 & $\mathbf{Y}$ & N/A \\
\hline 2276 & 3,5 & CONSTANT & $1.0000000 E+\infty 0$ & $1.0000000 E+\infty$ & $1.00000000+00$ & $1.0000000 \varepsilon+\infty$ & NONE & WPO40486 & $Y$ & N/A \\
\hline 2276 & 3,5 & CONSTANT & $1.0000000 E+00$ & $1.0000000 \mathrm{E}+00$ & $1.0000000 E+00$ & $1.0000000 E+00$ & NONE & WPO40514 & $\mathbf{Y}$ & N/A \\
\hline 2277 & 3,4 & CONSTANT & $2.9700000 E-08$ & $2.9700000 \mathrm{E}-08$ & 2.9700000E-08 & $2.9700000 E-08$ & $\mathrm{~Pa}^{n}-1$ & WPO30640 & $Y$ & $\mathrm{~N} / \mathrm{A}$ \\
\hline 2277 & 3,4 & CONSTANT & $2.9700000 \mathrm{E}-08$ & $2.9700000 \mathrm{E}-08$ & $2.9700000 E-08$ & $2.9700000 \mathrm{E}-08$ & $P a^{x}-1$ & WP030994 & $Y$ & NA \\
\hline 2277 & 3,4 & CONSTANT & $2.9700000 E-08$ & $2.9700000 E-08$ & $2.9700000 E-08$ & 29700000E-08 & $P a^{n}-1$ & WPO35597 & $\mathbf{Y}$ & N/A \\
\hline 2278 & 3,5 & CONSTANT & $1.0000000 E+08$ & $1.0000000 E+08$ & $1.0000000 E+08$ & $1.0000000 E+08$ & $\mathrm{~Pa}$ & WP030640 & $Y$ & N/A \\
\hline 2278 & 3,5 & CONSTANT & $1.0000000 \mathrm{E}+08$ & $1.0000000 E+08$ & $1.0000000 \mathrm{E}+08$ & $1.0000000 E+08$ & $\mathrm{~Pa}$ & WP030994 & $Y$ & NA \\
\hline 2278 & 3,5 & CONSTANT & $1.0000000 E+08$ & $1.0000000 E+08$ & $1.0000000 E+08$ & $1.0000000 \mathrm{E}+08$ & $\mathbf{P a}$ & WP035597 & $\mathbf{Y}$ & NA \\
\hline
\end{tabular}


CCA Parameter Listing

\begin{tabular}{|c|c|c|c|c|c|c|c|c|c|c|}
\hline $\begin{array}{c}\text { Paramoter In } \\
\text { Databaso? } \\
\text { (YNN) }\end{array}$ & \begin{tabular}{|c|} 
If the values \\
used in CCA \\
are not the \\
same sas in \\
oB, provide $\%$ \\
dffierence
\end{tabular} & ID & Material ID & Material Namb & $\begin{array}{c}\text { Parameter } \\
\text { ID }\end{array}$ & Parameter Nam & $\begin{array}{l}\text { PRP ID } \\
\text { (NPO *) }\end{array}$ & $\begin{array}{c}\text { Datm Entry } \\
\text { Dato }\end{array}$ & $\begin{array}{l}\text { Is the dBA } \\
\text { consietent } \\
\text { with the } \\
\text { parameter } \\
\text { in DB? } \\
\left(N_{1}, N A\right)\end{array}$ & $\begin{array}{l}\text { Parmeter } \\
\text { Citegory } \\
\text { (footnote 1) }\end{array}$ \\
\hline $\mathbf{Y}$ & & 2278 & ASPHALT & Asphalt column & PC_MAX & $\begin{array}{l}\text { Maximum allowable capillary } \\
\text { pressure }\end{array}$ & $31385 \mathrm{~A}$ & 03-Nov-95 & $Y$ & 48 \\
\hline $\mathbf{Y}$ & & 2278 & ASPHALT & Asphalt column & PC_MAX & $\begin{array}{l}\text { Maxumum allowable capillary } \\
\text { pressure }\end{array}$ & 31385A & 03-Nov-95 & $\mathbf{Y}$ & $4 \mathrm{~B}$ \\
\hline $\mathbf{Y}$ & varies & 2279 & ASPHALT & Asphatt column & PORE_DIS & $\begin{array}{l}\text { Brooks-Corey pore distribution } \\
\text { parameter }\end{array}$ & 31386 & 21 feb-96 & $\mathbf{Y}$ & 1 \\
\hline$Y$ & varies & 2279 & ASPHALT & Asphalt column & PORE_DIS & $\begin{array}{l}\text { Brooks-Corby pore distribution } \\
\text { parameter }\end{array}$ & 31386 & 21 -Fob-96 & $\mathbf{Y}$ & 1 \\
\hline$Y$ & varies & 2279 & ASPHALT & Asphalt column & PORE_DIS & $\begin{array}{l}\text { Brooks-Corey pore distrbution } \\
\text { parameter }\end{array}$ & 31386 & 21-Feb-96 & $\mathbf{Y}$ & 1 \\
\hline $\mathbf{Y}$ & varies & 2279 & ASPHALT & Asphatt column & PORE_DIS & $\begin{array}{l}\text { Brooks-Corey pore distribution } \\
\text { parameter }\end{array}$ & 31386 & 21-Feb-96 & $\mathbf{Y}$ & 1 \\
\hline $\mathbf{Y}$ & varies & 2279 & ASPHALT & Asphalt column & PORE_DIS & $\begin{array}{l}\text { Brooks-Corey pore distribution } \\
\text { parameter }\end{array}$ & 31386 & 21-Feb-95 & $\mathbf{Y}$ & 1 \\
\hline $\mathbf{Y}$ & varies & 2279 & ASPHALT & Asphatt column & PORE_DIS & $\begin{array}{l}\text { Brooks-Corey pore distribution } \\
\text { parameter }\end{array}$ & 31385 & 21-Feb-96 & $\mathbf{Y}$ & 1 \\
\hline$Y$ & & 2280 & ASPHALT & Asphalt column & POROSITY & Effective porasity & 31387 & 13-Fob-95 & $\mathbf{Y}$ & 1 \\
\hline$Y$ & & 2280 & ASPHALT & Asphalt column & POROSITY & Effective porosity & 31387 & 13 Feb-96 & $\mathbf{Y}$ & 1 \\
\hline $\mathbf{Y}$ & & 2280 & ASPHALT & Asphalt column & POROSITY & Effective porosity & 31387 & 13 feb-96 & $\mathbf{Y}$ & 1 \\
\hline $\mathbf{Y}$ & & 2281 & ASPHALT & Asphalt column & PO_MIN & $\begin{array}{l}\text { Minimum brine pressure for } \\
\text { capillary model KPC }=3\end{array}$ & 31388 & $21-F e b-96$ & $\mathbf{Y}$ & $4 B$ \\
\hline$Y$ & & 2281 & ASPHALT & Asphalt column & PO_MIN & $\begin{array}{l}\text { Minimum brine pressure for } \\
\text { capillary model KPC=3 }\end{array}$ & 31388 & $21-F e b-96$ & $\mathbf{Y}$ & AB \\
\hline$Y$ & & 2281 & ASPHALT & Asphalt column & PO_MIN & $\begin{array}{l}\text { Minimum brine pressure for } \\
\text { capillary model KPC=3 }\end{array}$ & 31388 & $21+F e b-96$ & $\mathbf{Y}$ & 48 \\
\hline$Y$ & & 2282 & ASPHALT & Asphalt column & PRESSURE & Brine far-field pore pressure & 31389 & 20-Feb-96 & $\mathbf{Y}$ & 1 \\
\hline$Y$ & & 2282 & ASPHALT & Asphalt column & PRESSURE & Brine far-field pore pressure & 31389 & 20-Feb-96 & $\bar{Y}$ & 1 \\
\hline $\mathbf{Y}$ & varies & 2283 & ASPHALT & Asphalt column & PRMX_LOG & $\begin{array}{l}\text { Log of intrinsic permeability, X- } \\
\text { direction }\end{array}$ & 31390 & 13 feb-96 & $\mathbf{Y}$ & 1 \\
\hline $\mathbf{Y}$ & varies & 2283 & ASPHALT & Asphalt column & PRMX_LOG & $\begin{array}{l}\text { Log of intrinsic permeability, } X- \\
\text { direction }\end{array}$ & 31390 & 13-Feb-96 & $Y$ & 1 \\
\hline $\mathbf{Y}$ & varies & 2283 & ASPHALT & Asphait column & PRMX_LOG & $\begin{array}{l}\text { Log of intrinsic permeability, } X \text { - } \\
\text { direction }\end{array}$ & 31390 & 13-feb-96 & $\mathbf{Y}$ & 1 \\
\hline $\mathbf{Y}$ & varies & 2283 & ASPHALT & Asphalt column & PRMX_LOG & $\begin{array}{l}\text { Log of intrinsic permeability, X- } \\
\text { direction }\end{array}$ & 31390 & 13-Fob-96 & $\mathbf{Y}$ & 1 \\
\hline $\mathbf{Y}$ & vanes & 2283 & ASPHALT & Asphatt column & PRMX_LOG & $\begin{array}{l}\text { Log of intrinsic permeability, X- } \\
\text { direction }\end{array}$ & 31390 & 13-Feb-96 & $\mathbf{Y}$ & 1 \\
\hline $\mathbf{Y}$ & & 2284 & ASPHALT & Asphalt column & PRMY_LOG & $\begin{array}{l}\text { Log of intrinsic permeability, Y- } \\
\text { direction }\end{array}$ & 31391 & 13-Feb-95 & $\mathbf{Y}$ & 1 \\
\hline $\mathbf{Y}$ & & 2284 & ASPHALT & Asphalt column & PRMY_LOG & $\begin{array}{l}\text { Log of intrnsic permeability, Y- } \\
\text { direction }\end{array}$ & 31391 & 13-Fob-96 & $\mathbf{Y}$ & 1 \\
\hline $\mathbf{Y}$ & & 2284 & ASPHALT & Asphatt column & PRMY_LOG & $\begin{array}{l}\text { Log of intrunsic permeability, Y- } \\
\text { direction }\end{array}$ & 39391 & 13-Feb-96 & $\mathbf{Y}$ & 1 \\
\hline$Y$ & & 2284 & ASPHALT & Asphalt column & PRMY_LOG & $\begin{array}{l}\text { Log of intrnsic permeabildy, Y- } \\
\text { direction }\end{array}$ & 31391 & 13-Feb-96 & $\mathbf{Y}$ & 1 \\
\hline $\mathbf{Y}$ & & 2284 & ASPHALT & Asphalt column & PRMY_LOG & $\begin{array}{l}\text { Log of intrinsic permeability, Y- } \\
\text { drection }\end{array}$ & 31391 & 13-Fob-96 & $Y$ & 1 \\
\hline $\mathbf{Y}$ & & 2285 & ASPHALT & Asphalt column & PRMZ_LOG & $\begin{array}{l}\text { Log of intrinsic permeability, Z- } \\
\text { drection }\end{array}$ & 31392 & 13-Feb-96 & $Y$ & 1 \\
\hline $\mathbf{Y}$ & & 2285 & ASPHALT & Asphatt column & PRMZ_LOG & $\begin{array}{l}\text { Log of intrunsic permeability, Z- } \\
\text { drection }\end{array}$ & 31392 & 13-Feb-96 & $\mathbf{Y}$ & 1 \\
\hline $\mathbf{Y}$ & & 2285 & ASPHALT & Asphalt column & PRMZ_LOG & $\begin{array}{l}\text { Log of untrins ic permeability, Z- } \\
\text { durection }\end{array}$ & 31392 & 13-Feb-95 & $Y$ & 1 \\
\hline $\mathbf{Y}$ & & 2285 & ASPHALT & Asphalt column & PRMZ_LOG & $\begin{array}{l}\text { Log of minnsic permeability, Z- } \\
\text { direction }\end{array}$ & 31392 & 13-Fob-96 & $\mathbf{Y}$ & 1 \\
\hline $\mathbf{Y}$ & & 2285 & ASPHALT & Asphalt column & PRMZ_LOG & $\begin{array}{l}\text { Log of intrunsic permeability, Z- } \\
\text { drection }\end{array}$ & 31392 & 13-Feb-96 & $\mathbf{Y}$ & 1 \\
\hline $\mathbf{Y}$ & & 2289 & ASPHALT & Asphalt column & RELP_MOD & $\begin{array}{l}\text { Model number, relative } \\
\text { permeability model }\end{array}$ & 31397A & 03-Nov-95 & $\cdot \boldsymbol{Y}$ & $4 B$ \\
\hline $\mathbf{Y}$ & & 2289 & ASPHALT & Asphalt column & RELP_MOD & $\begin{array}{l}\text { Model number, relative } \\
\text { permeability model }\end{array}$ & $31397 A$ & 03-Nov-95 & $\mathbf{Y}$ & $A B$ \\
\hline $\mathbf{Y}$ & & 2289 & ASPHALT & Asphalt column & RELP_MOD & $\begin{array}{l}\text { Model number, relative } \\
\text { permeability model }\end{array}$ & 31397A & $03+$ Nov-95 & $\mathbf{Y}$ & $4 B$ \\
\hline $\mathbf{Y}$ & & 2289 & ASPHALT & Asphalt column & RELP_MOD & $\begin{array}{l}\text { Model number, relative } \\
\text { permeability model }\end{array}$ & 31397A & Q3-Nov-95 & $\mathbf{Y}$ & $4 B$ \\
\hline $\mathbf{Y}$ & & 2289 & ASPHALT & Asphalt column & RELP_MOD & $\begin{array}{l}\text { Model number, relative } \\
\text { permeability model }\end{array}$ & 31397A & 03-Nov-95 & $\mathbf{Y}$ & $4 B$ \\
\hline $\mathbf{Y}$ & & 2290 & ASPHALT & Asphalt column & SAT_IBRN & Initial Brine Saturation & 31403 & 13-Fob-96 & $\mathbf{Y}$ & 1 \\
\hline $\mathbf{Y}$ & & 2290 & ASPHALT & Asphalt column & SAT_IBRN & Initial Brine Saturation & 31403 & 13-Feb-96 & $Y$ & 1 \\
\hline $\mathbf{Y}$ & & 2290 & ASPHALT & Asphalt column & SAT_IBRN & Initial Brine Saturation & 31403 & 13-Fab-96 & $\mathbf{Y}$ & 1 \\
\hline
\end{tabular}


CCA Parameter Listing

\begin{tabular}{|c|c|c|c|c|c|c|c|c|c|c|}
\hline 10 & $\begin{array}{c}\text { Clasenfi- } \\
\text { cation of } \\
\text { Data } \\
\text { Category } \\
\text { (footnote 2) }\end{array}$ & $\begin{array}{c}\text { Distrlbution } \\
\text { Type }\end{array}$ & Mesn & Median & Minimum & Meximum & Units & Reference ID & $\begin{array}{l}\text { Was the data } \\
\text { developed } \\
\text { under an } \\
\text { NOA-1 } \\
\text { Program? }\end{array}$ & $\begin{array}{c}\text { Which } \\
\text { methods } \\
\text { wore used to } \\
\text { qually } \\
\text { exdsting } \\
\text { data? } \\
\text { (footnota 3) }\end{array}$ \\
\hline 2278 & 3,5 & CONSTANT & $1.0000000 \mathrm{E}+08$ & $1.0000000 E+08$ & $1.0000000 E+08$ & $1.0000000 E+08$ & $\mathrm{~Pa}$ & WP040486 & $\mathbf{Y}$ & NA \\
\hline 2278 & 3,5 & CONSTANT & $1.0000000 E+08$ & $1.0000000 E+08$ & $1.0000000 E+08$ & $1.0000000 E+08$ & $\mathrm{~Pa}$ & WPO40514 & $\mathbf{Y}$ & $N / A$ \\
\hline 2279 & 3,4 & CUMULATIVE & $2.5200000 E+00$ & $9.4000000 E-01$ & $1.1000000 \mathrm{E}-01$ & $8.1000000 E+\infty 0$ & NONE & WP030640 & $Y$ & $N A$ \\
\hline 2279 & 3,4 & CUMULATINE & $2.5200000 E+\infty$ & $9.4000000 E-01$ & $1.1000000 E-01$ & $8.1000000 E+\infty$ & NONE & WPO30994 & $\mathbf{Y}$ & N/A \\
\hline 2279 & 3,4 & CUMULATIVE & $2.5200000 E+\infty 0$ & $9.4000000 \mathrm{E}-01$ & $1.1000000 E-01$ & $8.1000000 E+\infty 0$ & NONE & WP032287 & $Y$ & NA \\
\hline 2279 & 3,4 & CUMULATNE & $2.5200000 E+\infty 0$ & $9.4000000 \mathrm{E}-01$ & $1.1000000 E-01$ & $8.1000000 E+00$ & NONE & WP035268 & $\mathbf{Y}$ & N/A \\
\hline 2279 & 3,4 & CUMULATNE & $2.5200000 E+\infty 0$ & 9.4000000E-01 & $1.1000000 E-01$ & $8.1000000 E+\infty 0$ & NONE & WPO41558 & $Y$ & $N / A$ \\
\hline 2280 & 1,3 & CONSTANT & $1.0000000 \mathrm{E}-02$ & $1.0000000 \mathrm{E}-02$ & $1.0000000 \mathrm{E}-02$ & $1.0000000 \mathrm{E}-02$ & $m^{\wedge} 3 / m^{\wedge} 3$ & WPO30640 & $Y$ & $N / A$ \\
\hline $22 B O$ & 1,3 & CONSTANT & $1.0000000 \mathrm{E}-02$ & $1.0000000 E-02$ & $1.0000000 \mathrm{E}-02$ & $1.0000000 E-02$ & $m^{\wedge} 3 / m^{\wedge} 3$ & WP030994 & $Y$ & NA \\
\hline 2280 & 1,3 & CONSTANT & $1.0000000 E-02$ & $1.0000000 \mathrm{E}-02$ & $1.0000000 E-02$ & $1.0000000 E-02$ & $m^{\wedge} 3 / m^{\wedge} 3$ & WP035597 & $\mathbf{Y}$ & N/A \\
\hline 2281 & 3,5 & CONSTANT & $1.0132500 E+05$ & $1.0132500 E+05$ & $1.0132500 E+05$ & $1.0132500 E+05$ & $\mathbf{P a}$ & WP035597 & $\mathbf{Y}$ & N/A \\
\hline 2281 & 3,5 & CONSTANT & $1.0132500 E+05$ & $1.0132500 E+05$ & $1.0132500 E+05$ & $1.0132500 E+05$ & $\mathrm{~Pa}$ & WP035051 & $\mathbf{Y}$ & NA \\
\hline 2281 & 3,5 & CONSTANT & $1.0132500 E+05$ & $1.0132500 \mathrm{E}+05$ & $1.0132500 E+05$ & $1.0132500 E+05$ & $\mathbf{P a}$ & WP040514 & $Y$ & N/A \\
\hline 2282 & 3,5 & CONSTANT & $1.0132500 E+05$ & $1.0132500 E+05$ & $1.0132500 \mathrm{E}+05$ & $1.0132500 E+05$ & $\mathbf{P a}$ & WPO35597 & $Y$ & N/A \\
\hline 2282 & 3,5 & CONSTANT & $1.0132500 E+05$ & $1.0132500 E+05$ & $1.0132500 E+05$ & $1.0132500 E+05$ & $\mathbf{P a}$ & WP036058 & $\mathbf{Y}$ & N/A \\
\hline 2283 & 3,4 & TRIANGULAR & $-1.9657000 E+01$ & $-2.0000000 E+01$ & $-2.1000000 E+01$ & $-1.8000000 E+01$ & $\log \left(m^{\wedge} 2\right)$ & WP030640 & $\mathbf{Y}$ & N/A \\
\hline 2283 & 3,4 & TRIANGULAR & $-1.9667000 E+01$ & $-2.0000000 E+01$ & $-2.1000000 E+01$ & $-1.8000000 E+01$ & $\log \left(m^{\wedge} 2\right)$ & WP035268 & $\mathbf{Y}$ & N/A \\
\hline 2283 & 3,4 & TRIANGULAR & $-1.9667000 E+01$ & $-2.0000000 E+01$ & $-2.1000000 E+01$ & $-1.8000000 E+01$ & $\log \left(m^{\wedge} 2\right)$ & WP035597 & $\mathbf{Y}$ & NA \\
\hline 2283 & 3,4 & TRLANGULAR & $-1.9667000 E+01$ & $-2.0000000 \mathrm{E}+01$ & $-2.1000000 E+01$ & $-1.8000000 E+01$ & $\log \left(m^{\wedge} 2\right)$ & WP041558 & $Y$ & $N A$ \\
\hline 2284 & 3,4 & TRLANGULAR & $-1.9667000 E+01$ & $-2.0000000 E+01$ & $-2.1000000 E+01$ & $-1.8000000 E+01$ & $\log \left(m^{\wedge} 2\right)$ & WP030640 & $\mathbf{Y}$ & N/A \\
\hline 2284 & 3,4 & TRIANGULAR & $-1.9667000 E+01$ & $-2.0000000 E+01$ & $-2.1000000 E+01$ & $-1.8000000 E+01$ & $\log \left(m^{\wedge} 2\right)$ & WP030994 & $Y$ & NA \\
\hline 2284 & 3,4 & TRIANGULAR & $-1.9667000 E+01$ & $-2.0000000 E+01$ & $-2.1000000 E+01$ & $-1.8000000 E+01$ & $\log \left(m^{\wedge} 2\right)$ & WPO35268 & $Y$ & N/A \\
\hline 2284 & 3,4 & TRLANGULAR & $-1.9667000 E+01$ & $-2.0000000 E+01$ & $-2.1000000 E+01$ & $-1.8000000 E+01$ & $\log \left(m^{\wedge} 2\right)$ & WP035597 & $\mathbf{Y}$ & NAA \\
\hline 2284 & 3,4 & TRIANGULAR & $-1.9667000 E+01$ & $-2.0000000 E+01$ & $-2.1000000 E+01$ & $-1.8000000 E+01$ & $\log \left(m^{\wedge} 2\right)$ & WPO41558 & $\mathbf{Y}$ & N/A \\
\hline 2285 & 3,4 & TRLANGULAR & $-1.9667000 E+01$ & $-2.0000000 E+01$ & $-2.1000000 E+01$ & $-1.8000000 E+01$ & $\log \left(m^{\wedge} 2\right)$ & WP030640 & $\bar{Y}$ & N/A \\
\hline 2285 & 3,4 & TRIANGULAR & $-1.9667000 E+01$ & $-2.0000000 E+01$ & $-2.1000000 E+01$ & $-1.8000000 E+01$ & $\log \left(m^{\wedge} 2\right)$ & WP030994 & $\mathbf{Y}$ & N/A \\
\hline 2285 & 3,4 & TRIANGULAR & $-1.9667000 E+01$ & $-2.0000000 E+01$ & $-2.1000000 E+01$ & $-1.8000000 E+01$ & $\log \left(m^{\wedge} 2\right)$ & WP035268 & $\mathbf{Y}$ & N/A \\
\hline 2285 & 3,4 & TRIANGULAR & $-1.9667000 E+01$ & $-2.0000000 E+01$ & $-2.1000000 E+01$ & $-1.8000000 E+01$ & $\log \left(m^{\wedge} 2\right)$ & WP035597 & $\mathbf{Y}$ & NA \\
\hline 2285 & 3,4 & TRLANGULAR & $-1.9667000 E+01$ & $-2.0000000 \mathrm{E}+01$ & $-2.1000000 E+01$ & $-1.8000000 E+01$ & $\log \left(m^{\wedge} 2\right)$ & WPO41558 & $\mathbf{Y}$ & N/A \\
\hline 2289 & 3,5 & CONSTANT & $4.0000000 \mathrm{E}+00$ & $4.0000000 E+00$ & $4.0000000 E+00$ & $4.0000000 E+00$ & NONE & WP030540 & $\mathbf{\gamma}$ & NA \\
\hline 2289 & 3,5 & CONSTANT & $4.0000000 E+\infty 0$ & $4.0000000 E+00$ & 4.0000000E+00 & $4.0000000 E+00$ & NONE & WP030994 & $\mathbf{Y}$ & N/A \\
\hline 2289 & 3,5 & CONSTANT & $4.0000000 E+00$ & $4.0000000 E+\infty 0$ & $4.0000000 E+\infty 0$ & $4.0000000 \mathrm{E}+00$ & NONE & WP035597 & $Y$ & $N / A$ \\
\hline 2289 & 3,5 & CONSTANT & $4.0000000 E+00$ & $4.0000000 \mathrm{E}+00$ & $4.0000000 E+\infty$ & $4.0000000 E+00$ & NONE & WP040486 & $Y$ & N/A \\
\hline 2289 & 3,5 & CONSTANT & $4.0000000 E+\infty$ & $4.0000000 E+00$ & $4.0000000 E+\infty 0$ & $4.0000000 E+00$ & NONE & WP040514 & $\mathbf{Y}$ & N/A \\
\hline
\end{tabular}


CCA Parameter Listing

\begin{tabular}{|c|c|c|c|c|c|c|c|c|c|c|}
\hline $\begin{array}{c}\text { Parnmetor in } \\
\text { Databaes? } \\
\text { (YNN) }\end{array}$ & 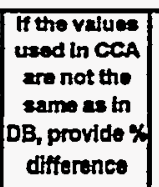 & ID & Materialo & Msterial Neme & $\begin{array}{c}\text { Parameter } \\
\text { to }\end{array}$ & Paramoter Name & $\begin{array}{l}\text { PRP ID } \\
\text { (MPO * })\end{array}$ & $\begin{array}{c}\text { Deta Entry } \\
\text { Detre }\end{array}$ & $\begin{array}{c}\text { Is the } 464 \\
\text { consiztent } \\
\text { with the } \\
\text { prameter } \\
- \text { in DB? } \\
(Y, N A)\end{array}$ & $\begin{array}{l}\text { Paramater } \\
\text { Category } \\
\text { (footrota 1) }\end{array}$ \\
\hline $\bar{Y}$ & 100 & 2291 & ASPHALT & Asphalt colkmn & SAT_RBRN & Residual Brme Saturation & 31405 & 13 -Feb-96 & $\mathbf{Y}$ & 1 \\
\hline $\bar{Y}$ & 100 & 2291 & ASPHALT & Asphatt colkmn & SAT_RBRN & Residual Bnre Saturation & 31405 & 13-feb-96 & $\bar{Y}$ & 1 \\
\hline $\bar{Y}$ & 100 & 2291 & ASPHALT & Asphalt column & SAT_RBRN & Residual Brine Saturation & 31405 & 13-Feb-96 & $\bar{Y}$ & 1 \\
\hline $\bar{Y}$ & 100 & 2291 & ASPHALT & Asphalt column & SAT_RBRN & Residual Brine Saturation & 31405 & 13-Fub-96 & $\bar{Y}$ & 1 \\
\hline $\bar{Y}$ & 100 & 2291 & ASPHALT & Asphalt column & SAT_RBRN & Residual Brine Saturation & 31405 & 13-Feb-96 & $\bar{Y}$ & 1 \\
\hline $\bar{Y}$ & 100 & 2291 & ASPHALT & Asphalt column & SAT_RBRN & Residual Brine Saturation & 31405 & 13-Feb-96 & $\bar{Y}$ & 1 \\
\hline $\bar{Y}$ & 100 & 2292 & ASPHALT & Asphalt column & SAT_RGAS & Residual Gas Saturation & 31407 & 13-feb-96 & $\bar{Y}$ & 1 \\
\hline $\bar{Y}$ & 100 & 2292 & ASPHALT & Asphatt column & SAT_RGAS & Residual Gas Saturation & 31407 & 13-Feb-96 & $\bar{Y}$ & 1 \\
\hline $\bar{Y}$ & 100 & 2292 & ASPHALT & Asphalt column & SAT_RGAS & Residual Gas Saturation & 31407 & 13-fob-96 & $\bar{Y}$ & 1 \\
\hline $\bar{Y}$ & 100 & 2292 & ASPHALT & Asphalt column & SAT_RGAS & Residual Gas Saturation & 31407 & 13-Feb-96 & $Y$ & 1 \\
\hline $\bar{Y}$ & 100 & 2292 & ASPHALT & Asphalt column & SAT_RGAS & \begin{tabular}{|l|} 
Residual Gas Saturation \\
\end{tabular} & 31407 & 13-feb-96 & $\bar{Y}$ & 1 \\
\hline$Y$ & 50 & 2310 & CLAY_BOT & Shaf Bottom Clay & CAP_MOD & $\begin{array}{l}\text { Model number, capillary pressure } \\
\text { model }\end{array}$ & 31855 & O3-Nov-95 & Y & $4 B$ \\
\hline$Y$ & 50 & 2310 & CLAY_BOT & Shaft Bottom Clay & CAP_MOD & $\begin{array}{l}\text { Model number, capillary pressure } \\
\text { model }\end{array}$ & 31855 & 03-Nov-95 & $\mathbf{Y}$ & 4B \\
\hline$Y$ & 50 & 2310 & CLAY_BOT & Shaft Bottom Clay & CAP_MOD & $\begin{array}{l}\text { Model number, capillary pressure } \\
\text { model }\end{array}$ & 31855 & O3-Nov-95 & Y & 4B \\
\hline$Y$ & 50 & 2310 & CLAY_BOT & Shaft Bottom Clay & CAP_MOD & $\begin{array}{l}\text { Model number, capillary pressure } \\
\text { model }\end{array}$ & 31855 & 03-Nov-95 & Y & $4 B$ \\
\hline$Y$ & 50 & 2310 & CLAY_BOT & Shaft Bottom Clay & CAP_MOD & $\begin{array}{l}\text { Model number, capillary pressure } \\
\text { model }\end{array}$ & 31855 & 03-Nov-95 & Y & $4 B$ \\
\hline$Y$ & & 2311 & CLAY_BOT & Shaft Bottom Clay & COMP_RCK & \begin{tabular}{|l|l|} 
Bulk Compressibility \\
\end{tabular} & 31857 & 21-Feb-96 & $\bar{Y}$ & 48 \\
\hline $\bar{Y}$ & & 2311 & CLAY_BOT & Shaft Bottom Clay & COMP_RCK & Bulk Compressibility & 31857 & 21-Feb-96 & $\bar{Y}$ & $4 B$ \\
\hline $\bar{Y}$ & & 2311 & CLAY_BOT & Shaft Bottom Clay & COMP_RCK & Bulk Comprossibilty & 31857 & 21-Fab-96 & $\bar{Y}$ & 48 \\
\hline $\bar{Y}$ & & 2311 & CLAY_BOT & Shaff Bottom Clay & COMP_RCK & Bulk Compressibility & 31857 & 21-Fob-96 & $\bar{Y}$ & $4 B$ \\
\hline $\mathbf{Y}$ & & 2312 & CLAY_BOT & Shaft Bottom Clay & PC_MAX & $\begin{array}{l}\text { Maximum allowable capillary } \\
\text { pressure }\end{array}$ & $31861 \mathrm{~A}$ & 03-Nov-95 & $\mathbf{Y}$ & 4B \\
\hline $\mathbf{Y}$ & & 2312 & CLAY_BOT & Shaft Bottom Clay & PC_maX & $\begin{array}{l}\text { Maximum allowable capillary } \\
\text { prossure }\end{array}$ & $31861 A$ & 03-Nov-95 & $\mathbf{Y}$ & $4 B$ \\
\hline$Y$ & & 2312 & CLAY_BOT & Shaft Bottom Clay & PC_MAX & $\begin{array}{l}\text { Maximum allowable capillary } \\
\text { prossure }\end{array}$ & $31861 \mathrm{~A}$ & 03-Nov-95 & Y & 4B \\
\hline$Y$ & & 2312 & CLAY_BOT & Shaft Bottom Clay & PC_max & $\begin{array}{l}\text { Maximum allowable capillary } \\
\text { pressure }\end{array}$ & $31861 A$ & 03-Nov-95 & $\mathbf{Y}$ & $4 B$ \\
\hline$Y$ & & 2312 & CLAY_BOT & Shath Bottom Clay & PC_MAX & $\begin{array}{l}\text { Maximum a!lowable capillary } \\
\text { pressure }\end{array}$ & $31861 A$ & 03-Nov-95 & $\mathbf{Y}$ & 48 \\
\hline$Y$ & & 2312 & CLAY_BOT & Shaft Bottom Clay & PC_maX & $\begin{array}{l}\text { Maxumum allowable capillary } \\
\text { pressure }\end{array}$ & $31861 A$ & 03-Nov-95 & $\mathbf{r}$ & $4 B$ \\
\hline $\mathbf{Y}$ & varies & 2313 & CLAY_BOT & Shaft Bottom Clay & PORE_DIS & $\begin{array}{l}\text { Brooks-Corey pore distribution } \\
\text { parameter }\end{array}$ & 31862 & 26-Feb-96 & $\mathbf{Y}$ & $4 A$ \\
\hline$Y$ & varies & 2313 & CLAY_BOT & Shaft Bottom Clay & PORE_DIS & $\begin{array}{l}\text { Brooks-Corey pora distribution } \\
\text { parameter }\end{array}$ & 31852 & 26-Feb-96 & $\mathbf{Y}$ & $4 A$ \\
\hline $\mathbf{Y}$ & varies & 2313 & CLAY_BOT & Shaft Bottom Clay & PORE_DIS & $\begin{array}{l}\text { Brooks-Coray pore distrbution } \\
\text { parameter }\end{array}$ & 31862 & 26-Feb-96 & $\gamma$ & $4 A$ \\
\hline Y & varies & 2313 & CLAY_BOT & Shaft Bottom Clay & PORE_DIS & $\begin{array}{l}\text { Brooks-Corey pore distribution } \\
\text { parameter }\end{array}$ & 31862 & 26-Fob-96 & $=Y$ & $4 A$ \\
\hline$Y$ & varies & 2313 & CLAY_BOT & Shaft Bottom Clay & PORE_DIS & $\begin{array}{l}\text { Brooks-Corey pore distribution } \\
\text { parameter }\end{array}$ & 31862 & 26-Feb-96 & $\mathbf{Y}$ & $4 A$ \\
\hline $\mathbf{Y}$ & varios & 2313 & CLAY_BOT & Shaff Bottom Clay & PORE_DIS & $\begin{array}{l}\text { Brooks-Corey pora distribution } \\
\text { parameter }\end{array}$ & 31862 & $26-F o b-96$ & $\mathbf{Y}$ & $4 \mathrm{~A}$ \\
\hline $\mathbf{Y}$ & varies & 2313 & CLAY_BOT & Shaft Bottom Clay & PORE_DIS & $\begin{array}{l}\text { Brooks-Corry pore distribution } \\
\text { parameter }\end{array}$ & 31862 & 26-Fob-96 & $\mathbf{Y}$ & $4 A$ \\
\hline $\bar{Y}$ & & 2314 & CLAY_BOT & Shaft Bottom Clay & POROSIT & Effective porosity & 31863 & 21-Fob-96 & $\mathbf{Y}$ & $4 A$ \\
\hline $\bar{Y}$ & & 2314 & CLAY_BOT & Shaft Bottom Clay & POROSITY & Effective porosity & 31863 & 21-Fab-96 & $Y$ & $4 A$ \\
\hline $\bar{Y}$ & & 2314 & CLAY_BOT & Shaft Bottom Clay & POROSITY & Effective porosity & 31863 & 21fab-96 & $Y$ & $4 \mathrm{~A}$ \\
\hline $\mathbf{Y}$ & & 2314 & CLAY_BOT & Shaft Bottom Clay & POROSITY & Effective porosity & 31863 & $21+8 b-96$ & $\bar{Y}$ & AA \\
\hline $\bar{Y}$ & & 2314 & CLAY_BOT & Shaft Bottom Clay & POROSTTY & Effective porosity & 31863 & 21-Feb-96 & $\mathbf{Y}$ & $4 A$ \\
\hline Y & & 2315 & CLAY_BOT & Shaft Bottom Clay & PO_MIN & $\begin{array}{l}\text { Minimum brine pressure for } \\
\text { capillary model KPC }=3\end{array}$ & 31864 & 21-Feb-96 & $Y$ & $4 B$ \\
\hline $\mathbf{Y}$ & & 2315 & CLAY_BOT & Shaft Bottom Clay & PO_MIN & $\begin{array}{l}\text { Manimum brine pressure for } \\
\text { capillary model KPC=3 }\end{array}$ & 31864 & 21-Feb-96 & $\mathbf{Y}$ & 48 \\
\hline $\mathbf{Y}$ & & 2315 & CLAY_BOT & Shaft Bottom Clay & PO_MIN & $\begin{array}{l}\text { Minimum brine pressure for } \\
\text { Capillary model KPC=3 }\end{array}$ & 31864 & 21-Feb-96 & $\mathbf{Y}$ & 48 \\
\hline$Y$ & & 2316 & CLAY_BOT & Shaft Bottom Clay & PRESSURE & Bnne far-field pore pressure & 31865 & 21-Fab-96 & $\mathbf{Y}$ & $4 B$ \\
\hline$Y$ & & 2316 & CLAY_BOT & Shat Bottom Clay ${ }^{\circ}$ & PRESSURE & Brine far-field pore pressure & 31865 & 21-feb-96 & $\mathbf{Y}$ & $4 B$ \\
\hline $\bar{Y}$ & & 2316 & CLAY_BOT & Shaft Bottom Clay & PRESSURE & Brine far-field pore pressure & 31865 & 21 feb-96 & $Y$ & 48 \\
\hline
\end{tabular}


CCA Parameter Listing

\begin{tabular}{|c|c|c|c|c|c|c|c|c|c|c|}
\hline ID & $\begin{array}{c}\text { Classiff- } \\
\text { cation of } \\
\text { Datt } \\
\text { Category } \\
\text { (Iootnote 2) }\end{array}$ & $\begin{array}{l}\text { Diatribution } \\
\text { Type }\end{array}$ & Man & Median & Minimem & Moximerm & Units & Reference ID & $\begin{array}{c}\text { Was the dinta } \\
\text { developed } \\
\text { under an } \\
\text { NOA-1 } \\
\text { Program? }\end{array}$ & $\begin{array}{c}\text { Which } \\
\text { mothods } \\
\text { wore used to } \\
\text { quallfy } \\
\text { existing } \\
\text { datt? } \\
\text { (footnots 3) }\end{array}$ \\
\hline 2291 & 3,4 & CUMULATIVE & $2.5000000 \mathrm{E}-01$ & $2.0000000 E-01$ & $0.0000000 E+\infty 0$ & $6.0000000 E-01$ & NONE & WP030540 & $Y$ & NA \\
\hline 2291 & 3,4 & CUMULATIVE & $2.5000000 \mathrm{E}-01$ & $2.0000000 E-01$ & $0.0000000 \mathrm{E}+\infty 0$ & $6.0000000 E=01$ & NONE & WP030994 & $Y$ & NA \\
\hline 2291 & 3,4 & CUMULATIVE & $2.5000000 \mathrm{E}-01$ & $2.0000000 \mathrm{E}-01$ & $0.0000000 E+00$ & $6.0000000 E-01$ & NONE & WPO35268 & $Y$ & NA \\
\hline 2291 & 3,4 & CUMULATNE & $2.5000000 E-01$ & $2.0000000 \mathrm{E}-01$ & $0.0000000 \equiv+00$ & $6.0000000 E-01$ & NONE & WP035597 & $Y$ & NA \\
\hline 2291 & 3.4 & CUMULATIVE & $2.5000000 E-01$ & 2.0000000E-01 & $0.0000000 E+00$ & $6.0000000 E-01$ & NONE & WP041558 & $\mathbf{Y}$ & NA \\
\hline 2291 & 3.4 & CUMULATNE & $2.5000000 \mathrm{E}-01$ & $2.0000000 E-01$ & $0.0000000 E+\infty 0$ & $6.0000000 \mathrm{E}-01$ & NONE & WPO42751 & $Y$ & N/A \\
\hline 2292 & 3,4 & UNIFORM & $2.0000000 \mathrm{E}-01$ & $2.0000000 \mathrm{E}-01$ & $0.0000000 E+\infty$ & 4.0000000E-01 & NONE & WP030640 & $Y$ & N/A \\
\hline 2292 & 3,4 & UNIFORM & $2.0000000 \mathrm{E}-01$ & $2.0000000 \mathrm{E}-01$ & $0.0000000 \mathrm{E}+00$ & 4.0000000E-01 & NONE & WP030994 & $Y$ & N/A \\
\hline 2292 & 3,4 & UNIFORM & $20000000 E-01$ & $20000000 E-01$ & $0.0000000 \mathrm{E}+00$ & $4.0000000 \mathrm{E}-01$ & NONE & WP035268 & $Y$ & N/A \\
\hline 2292 & 3.4 & UNIFORM & $2.0000000 \mathrm{E}-01$ & 20000000E-01 & $0.0000000 \mathrm{E}+00$ & 4.0000000E-01 & NONE & WP035597 & $Y$ & $\mathrm{~N} / \mathrm{A}$ \\
\hline 2292 & 3,4 & UNIFORM & 2.0000000E-01 & 2.0000000E-01 & $0.0000000 E+\infty 0$ & $4.0000000 E-01$ & NONE & WPO41558 & $Y$ & NA \\
\hline 2310 & $1,3,4$ & CONSTANT & $2,0000000 E+\infty 0$ & $2.0000000 E+\infty 0$ & $2.0000000 E+\infty 0$ & $2.0000000 E+00$ & NONE & WP030994 & $Y$ & NA \\
\hline 2310 & $1,3,4$ & CONSTANT & $2.0000000 E+00$ & $2.0000000 \mathrm{E}+00$ & $2.0000000 E+\infty 0$ & $2.0000000 E+00$ & NONE & WP035267 & $Y$ & N/A \\
\hline 2310 & $1,3,4$ & CONSTANT & $2.0000000 E+00$ & $2.0000000 \mathrm{E}+00$ & $2.0000000 \mathrm{E}+00$ & $2.0000000 E+00$ & NONE & WP035597 & $Y$ & N/A \\
\hline 2310 & $1,3,4$ & CONSTANT & $2.0000000 E+\infty 0$ & $2.0000000 E+\infty$ & $2.0000000 E+\infty 0$ & $2.0000000 E+00$ & NONE & WP036383 & $Y$ & N/A \\
\hline 2310 & $1,3,4$ & CONSTANT & $2.0000000 E+00$ & $2.0000000 E+\infty 0$ & $2.0000000 E+\infty 0$ & $2.0000000 E+00$ & NONE & WPO40514 & $Y$ & N/A \\
\hline 2311 & $1,3,4$ & CONSTANT & $1.5900000 \mathrm{E}-09$ & $1.5900000 \mathrm{E}-09$ & $1.5900000 \mathrm{E}-09$ & $1.5900000 \mathrm{E}-09$ & $\mathrm{~Pa}^{n}-1$ & WP035267 & $Y$ & $N / A$ \\
\hline 2311 & $1,3.4$ & CONSTANT & 1.5900000 E-09 & $1.5900000 \mathrm{E}-09$ & $1.5900000 \mathrm{E}-09$ & $1.5900000 \mathrm{E}-09$ & $P a^{n}-1$ & WP035597 & $Y$ & $N / A$ \\
\hline 2311 & $1,3,4$ & CONSTANT & $1.5900000 E-09$ & $1.5900000 \mathrm{E}-09$ & $1.5900000 \mathrm{E}-09$ & $1.5900000 \mathrm{E}-09$ & $\mathrm{~Pa}^{\wedge}-1$ & WP036050 & $Y$ & N/A \\
\hline 2311 & $1,3,4$ & CONSTANT & $1.5900000 \mathrm{E}-09$ & $1.5900000 \mathrm{E}-09$ & $1.5900000 \mathrm{E}-09$ & $1.5900000 \mathrm{E}-09$ & $P a^{n}-1$ & WPO40514 & $Y$ & NA \\
\hline 2312 & 1.3 & CONSTANT & $1.0000000 E+08$ & $1.0000000 E+08$ & $1.0000000 E+08$ & $1.0000000 E+08$ & $\mathbf{P a}$ & WP030994 & $\mathbf{Y}$ & N/A \\
\hline 2312 & 1,3 & CONSTANT & $1.0000000 \mathrm{E}+08$ & $1.0000000 E+08$ & $1.0000000 E+08$ & $1.0000000 E+08$ & $\mathbf{P a}$ & WP035267 & $\mathbf{Y}$ & N/A \\
\hline 2312 & 1,3 & CONSTANT & $1.0000000 E+08$ & $1.0000000 E+08$ & $1.0000000 \mathrm{E}+08$ & $1.0000000 \mathrm{E}+08$ & $\mathrm{~Pa}$ & WP035597 & $\mathbf{Y}$ & NA \\
\hline 2312 & 1,3 & CONSTANT & $1.0000000 E+08$ & $1.0000000 E+08$ & $1.0000000 E+08$ & $1.0000000 E+08$ & $\mathrm{~Pa}$ & WP036383 & $Y$ & N/A \\
\hline 2312 & 1,3 & CONSTANT & $1.0000000 E+08$ & $1.0000000 E+08$ & $1.0000000 E+08$ & $1.0000000 \mathrm{E}+08$ & $\mathbf{P a}$ & WPO40486 & $Y$ & N/A \\
\hline 2312 & 1,3 & CONSTANT & $1.0000000 E+08$ & $1.0000000 E+08$ & $1.0000000 E+08$ & $1.0000000 E+08$ & $\mathrm{~Pa}$ & WPO40514 & $\mathbf{Y}$ & NA \\
\hline 2313 & $1,3,4$ & CUMULATIVE & $2.5200000 E+\infty 0$ & $9.4000000 E-01$ & $1.1000000 \mathrm{E}-01$ & $8.1000000 E+00$ & NONE & WP030994 & $\mathbf{Y}$ & N/A \\
\hline 2313 & $1,3,4$ & CUMULATIVE & $2.5200000 E+\infty 0$ & $9.4000000 E-01$ & $1.1000000 E-01$ & $8.1000000 E+\infty 0$ & NONE & WP035267 & $\mathbf{Y}$ & N/A \\
\hline 2313 & $1,3,4$ & CUMULATIVE & $2.5200000 E+\infty 0$ & $9.4000000 E-01$ & $1.1000000 E-01$ & $8.1000000 E+00$ & NONE & WP035268 & $\mathbf{Y}$ & N/A \\
\hline 2313 & $1,3,4$ & CUMULATIVE & $2.5200000 E+\infty 0$ & $9.4000000 \mathrm{E}-01$ & 1.1000000E-01 & $8.1000000 E+\infty 00$ & NONE & WP035597 & $Y$ & N/A \\
\hline 2313 & $1,3,4$ & CUMULATINE & $2.5200000 \mathrm{E}+00$ & $9.4000000 E-01$ & $1.1000000 E-01$ & $8.1000000 E+00$ & NONE & WP036383 & $\mathbf{Y}$ & NA \\
\hline 2313 & $1,3.4$ & CUMULATNE & $2.5200000 E+00$ & $9.4000000 E-01$ & $1.1000000 E-01$ & $8.1000000 E+00$ & NONE & WP041558 & $Y$ & N/A \\
\hline 2313 & $1,3,4$ & CUMULATNE & $2.5200000 \mathrm{E}+\infty 0$ & $9.4000000 E-01$ & $1.1000000 E-01$ & $8.1000000 E+\infty 0$ & NONE & WPO42750 & $\mathbf{Y}$ & N/A \\
\hline 2314 & $1,3,4$ & CONSTANT & $2.4000000 \mathrm{E}-01$ & $2.4000000 E-01$ & $2.4000000 \mathrm{E}-01$ & 2.4000000 E-01 & $m^{\wedge} 3 / m^{\wedge} 3$ & WPO30640 & $Y$ & N/A \\
\hline 2314 & $1,3,4$ & CONSTANT & $2.4000000 E-01$ & 2.4000000E-01 & $2.4000000 \mathrm{E}-01$ & $2.4000000 \mathrm{E}-01$ & $m^{\wedge} 3 / m^{\wedge} 3$ & WP030994 & $Y$ & $N / A$ \\
\hline 2314 & $1,3,4$ & CONSTANT & $2.4000000 \mathrm{E}-01$ & 24000000E-01 & $2.4000000 \mathrm{E}-01$ & $2.4000000 E-01$ & $m^{\wedge} 3 / m^{\wedge} 3$ & WP035267 & $Y$ & NA \\
\hline 2314 & $1,3,4$ & CONSTANT & 2.4000000E-01 & $2.4000000 E-01$ & $2.4000000 \mathrm{E}-01$ & $2.4000000 E-01$ & $m^{\wedge} 3 / m^{\wedge} 3$ & WPO35597 & $Y$ & N/A \\
\hline 2314 & $1,3,4$ & CONSTANT & $2.4000000 \mathrm{E}-01$ & 2.4000000E-01 & $2.4000000 \mathrm{E}-01$ & $2.4000000 \mathrm{E}-01$ & $m^{\wedge} 3 / m^{\wedge} 3$ & WPO36383 & $Y$ & N/A \\
\hline 2315 & 1,3 & CONSTANT & $1.0132500 E+05$ & $1.0132500 E+05$ & $1.0132500 E+05$ & $1.0132500 E+0.5$ & $\mathrm{~Pa}$ & WP035597 & $Y$ & N/A \\
\hline 2315 & 1,3 & CONSTANT & $1.0132500 E+05$ & $1.0132500 E+05$ & $1.0132500 E+05$ & $1.0132500 E+0.5$ & $\mathrm{~Pa}$ & WP036051 & $Y$ & N/A \\
\hline 2315 & 1,3 & CONSTANT & $1.0132500 E+05$ & $1.0132500 E+05$ & $1.0132500 E+05$ & $1.0132500 E+05$ & $\mathrm{~Pa}$ & WP040514 & $\mathbf{Y}$ & $N / A$ \\
\hline 2316 & 3,5 & CONSTANT & $1.0132500 E+05$ & $1.0132500 E+05$ & $1.0132500 E+05$ & $1.0132500 E+05$ & $\mathrm{~Pa}$ & WP030994 & $\mathbf{Y}$ & NA \\
\hline 2316 & 3,5 & CONSTANT & $1.0132500 E+05$ & $1.0132500 E+0.5$ & $1.0132500 E+0.5$ & $1.0132500 E+05$ & $\mathrm{~Pa}$ & WP035597 & $Y$ & N/A \\
\hline 2316 & 3,5 & CONSTANT & $1.0132500 E+05$ & $1.0132500 E+05$ & $1.0132500 \mathrm{E}+05$ & $1.0132500 \mathrm{E}+05$ & $\mathrm{~Pa}$ & WPO40514 & $\mathbf{Y}$ & N/A \\
\hline
\end{tabular}


CCA Parameter Listing

\begin{tabular}{|c|c|c|c|c|c|c|c|c|c|c|}
\hline $\begin{array}{c}\text { Paramater in } \\
\text { Database? } \\
\text { (YNN) }\end{array}$ & \begin{tabular}{l|} 
the values \\
urod in CCAA \\
are not the \\
same sos in \\
DB, provide $\%$. \\
difference
\end{tabular} & ID & Material ID & Waterial Namo & $\begin{array}{c}\text { Parameter } \\
\text { to }\end{array}$ & Paramoter Namo & $\begin{array}{l}\text { PRP ID } \\
\text { (WPO \#) }\end{array}$ & $\begin{array}{c}\text { Datin Entry } \\
\text { Dat: }\end{array}$ & $\begin{array}{l}\text { to the } 464 \\
\text { consistent } \\
\text { wth the } \\
\text { parameter } \\
\text { in DB? } \\
(\mathrm{N}, \mathrm{NA})\end{array}$ & $\begin{array}{l}\text { Paramater } \\
\text { Cintegory } \\
\text { (lootnote 1) }\end{array}$ \\
\hline $\mathbf{Y}$ & varres & 2317 & CLAY_BOT & Shaft Bottom Clay & PRMX_LOG & $\begin{array}{l}\text { Log of intrinsic permeablity, } x \text { - } \\
\text { diraction }\end{array}$ & 31866 & 21-feb-96 & $\mathbf{Y}$ & 1 \\
\hline $\mathbf{Y}$ & varies & 2317 & CLAY_BOT & Shaft Bottom Clay & PRMX_LOG & $\begin{array}{l}\text { Log of intrinsic permeability, } X- \\
\text { direction }\end{array}$ & 31866 & 21-Fob-96 & $Y$ & 1 \\
\hline$Y$ & varies & 2317 & CLAY_BOT & Shaft Bottom Clay & PRMX_LOG & $\begin{array}{l}\text { Log of intrinsic permeability, } X- \\
\text { difection }\end{array}$ & 31866 & 21-Feb-96 & $Y$ & 1 \\
\hline$\gamma$ & varies & 2317 & CLAY_BOT & Shaft Bottom Clay & PRMX_LOG & $\begin{array}{l}\text { Log of intrinsic permeability, } X- \\
\text { direction }\end{array}$ & 31865 & 21.Fab-96 & $\mathbf{Y}$ & 1 \\
\hline$Y$ & varies & 2317 & CLAY_BOT & Shaft Bottom Clay & PRMX_LOG & $\begin{array}{l}\text { Log of intrinsk permeability, } x \text { - } \\
\text { direction }\end{array}$ & 31866 & 21-Feb-96 & $\mathbf{Y}$ & 1 \\
\hline$Y$ & varies & 2317 & CLAY_BOT & Shaft Bottom Clay & PRMX_LOG & $\begin{array}{l}\text { Log of intrinsic permeabilty, X- } \\
\text { durection }\end{array}$ & 31866 & 21-Fob-96 & $\mathbf{Y}$ & 1 \\
\hline$Y$ & & 2318 & CLAY_BOT & Shaft Bottom Clay & PRMY_LOG & $\begin{array}{l}\text { Log of intrinsic permeability, Y- } \\
\text { direction }\end{array}$ & 31867 & 21-Fob-96 & $Y$ & 1 \\
\hline $\mathbf{Y}$ & & 2318 & CLAY_BOT & Shaft Bottom Clay & PRMY_LOG & $\begin{array}{l}\text { Log of intrnssc permeability, Y. } \\
\text { direction }\end{array}$ & 31867 & $21-F 0 b-96$ & $Y$ & 1 \\
\hline$Y$ & & 2318 & CLAY_BOT & Shaft Bottom Clay & PRMY_LOG & $\begin{array}{l}\text { Log of intrinsic permeability, } Y- \\
\text { direction }\end{array}$ & 31867 & 21-Fob-96 & $Y$ & 1 \\
\hline$Y$ & & 2318 & CLAY_BOT & Shaft Bottom Clay & PRMY_LOG & $\begin{array}{l}\text { Log of intrinsic permeability, Y- } \\
\text { direction }\end{array}$ & 31867 & $21-7 a b-96$ & $\mathbf{Y}$ & 1 \\
\hline$Y$ & & 2318 & CLAY_BOT & Shaft Bottom Clay & PRMY_LOG & $\begin{array}{l}\text { Log of intinsic permeability, } Y \text { - } \\
\text { direction }\end{array}$ & 31867 & 21Fab-96 & $\mathbf{Y}$ & 1 \\
\hline$Y$ & & 2318 & CLAY_BOT & Shaft Bottom Clay & PRMY_LOG & $\begin{array}{l}\text { Log of intrinsic permeability, Y- } \\
\text { direction }\end{array}$ & 31867 & 21-Feb-96 & $\mathbf{Y}$ & 1 \\
\hline$Y$ & & 2319 & CLAY_BOT & Shaft Bottom Clay & PRMZ_LOG & $\begin{array}{l}\text { Log of intrnssic permeability, Z- } \\
\text { direction }\end{array}$ & 31858 & 21-Fob-96 & $Y$ & 1 \\
\hline $\mathbf{Y}$ & & 2319 & CLAY_BOT & Shaft Bottom Clay & PRMZ_LOG & $\begin{array}{l}\text { Log of intrinsic permeability, Z- } \\
\text { direction }\end{array}$ & 31868 & 21-Fab-96 & $Y$ & 1 \\
\hline$Y$ & & 2349 & CLAY_BOT & Shaf Bottom Clay & PRMZ_LOG & $\begin{array}{l}\text { Log of intrinssic permeabulity, Z- } \\
\text { direction }\end{array}$ & 31868 & 21Feb-96 & $\mathbf{Y}$ & 1 \\
\hline$Y$ & & 2319 & CLAY_BOT & Shaft Bottom Clay & PRMZ_LOG & $\begin{array}{l}\text { Log of intrinsic permeability, Z- } \\
\text { direction }\end{array}$ & 31868 & 21-Fab-96 & $\mathbf{Y}$ & 1 \\
\hline$Y$ & & 2319 & CLAY_BOT & Shaft Bottom Clay & PRMZ_LOG & $\begin{array}{l}\text { Log of intrinsic permeability, Z- } \\
\text { direction }\end{array}$ & 31858 & 21-Fob-96 & $Y$ & 1 \\
\hline$Y$ & & 2319 & CLAY_BOT & Shat Bottom Clay & PRMZ_LOG & $\begin{array}{l}\text { Log of intrinsic permeabillty, Z- } \\
\text { direction }\end{array}$ & 31868 & 21feb-96 & $\mathbf{Y}$ & 1 \\
\hline $\mathbf{Y}$ & & 2323 & CLAY_BOT & Shaft Bottom Clay & RELP_MOD & $\begin{array}{l}\text { Model number, relative } \\
\text { permeability model }\end{array}$ & 31872 & 03-Nov-95 & $\mathbf{Y}$ & 48 \\
\hline$Y$ & & 2323 & CLAY_BOT & Shaft Bottom Clay & RELP_MOD & $\begin{array}{l}\text { Model number, relative } \\
\text { permeability model }\end{array}$ & 31872 & O3-Nov-95 & $\gamma$ & $4 B$ \\
\hline$Y$ & & 2323 & CLAY_BOT & Shaft Bottom Clay & RELP_MOD & $\begin{array}{l}\text { Model mumber, relative } \\
\text { permeability model }\end{array}$ & 39872 & 03-Nov-95 & $Y$ & $4 B$ \\
\hline$Y$ & & 2323 & CLAY_BOT & Shaft Bottom Clay & RELP_MOD & $\begin{array}{l}\text { Model number, relative } \\
\text { permeability model }\end{array}$ & 31872 & O3-Nov-95 & $Y$ & $4 B$ \\
\hline$Y$ & & 2323 & CLAY_BOT & Shaft Bottom Clay & RELP_MOD & $\begin{array}{l}\text { Model number, relative } \\
\text { permeability model }\end{array}$ & 31872 & 03-Nov-95 & $\gamma$ & $4 B$ \\
\hline$Y$ & -26.58 & 2324 & CLAY_BOT & Shaft Bottom Clay & SAT_IBRN & Initial Brine Saturation & 31873 & 21-Feb-95 & $Y$ & $4 A$ \\
\hline$Y$ & -26.58 & 2324 & CLAY_BOT & Shaf Bottom Clay & SAT_IBRN & Initial Bnne Saturation & 31873 & 21. Feb-96 & $Y$ & $4 A$ \\
\hline$Y$ & -26.58 & 2324 & CLAY_BOT & Shaft Bottom Clay & SAT_IBRN & Initial Brine Saluration & 31873 & 21-Feb-95 & $Y$ & $4 A$ \\
\hline$Y$ & -26.58 & 2324 & CLAY_BOT & Shaft Bottom Clay & SAT_IBRN & Initial Brine Saturation & 31873 & 21-Feb-96 & $Y$ & $4 A$ \\
\hline$Y$ & -26.58 & 2324 & CLAY_BOT & Shaf Bottom Clay & SAT_IBRN & Initial Brine Saturation & 31873 & 21-Feb-96 & $Y$ & $4 A$ \\
\hline$Y$ & -26.58 & 2324 & CLAY_BOT & Shaft Bottom Clay & SAT_IBRN & Initial Brine Saturation & 31873 & 21-Feb-96 & $\bar{Y}$ & $4 \mathrm{~A}$ \\
\hline $\mathbf{Y}$ & 100 & 2325 & CLAY_BOT & Shaft Bottom Clay & SAT_RBRN & Residual Brine Saturation & $31874 A$ & 12-Apr-96 & $\mathbf{Y}$ & 4A \\
\hline$Y$ & 100 & 2325 & CLAY_BOT & Shaft Bottom Clay & SAT_RBRN & Residual Brine Saluration & $31874 \mathrm{~A}$ & 12-Apr-96 & $\bar{Y}$ & AA \\
\hline$Y$ & 100 & 2325 & CLAY_BOT & Shaft Bottom Clay & SAT_RBRN & Residual Brine Saturation & $31874 A$ & 12-Apr-95 & $Y$ & $4 \mathrm{~A}$ \\
\hline $\bar{Y}$ & 100 & 2325 & CLAY_BOT & Shaft Bottom Clay & SAT_RBRN & Residual Brine Saturation & $31874 \mathrm{~A}$ & 12-Apr-96 & $\bar{Y}$ & $\overline{4 A}$ \\
\hline $\mathbf{Y}$ & 100 & 2325 & CLAY_BOT & Shan Bottom Clay & SAT_RBRN & Residual Brine Saturation & $31874 \mathrm{~A}$ & 12-Apr-96 & $\mathbf{Y}$ & $4 A$ \\
\hline$Y$ & 100 & 2326 & CLAY_8OT & Shaft Bottom Clay & SAT_RGAS & Residual Ges Saturation & 31875 & 21-Feb-96 & $Y$ & 1 \\
\hline $\mathbf{Y}$ & 100 & 2325 & CLAY_BOT & Shaft Bottom Clay & SAT_RGAS & Residual Gas Saturation & 31875 & 21-Fob-96 & $\gamma$ & 1 \\
\hline $\bar{Y}$ & 100 & 2326 & CLAY_BOT & Shaft Bottom Clay & SAT_RGAS & Residual Gas Saturation & 31875 & 21-Feb-96 & $\mathbf{Y}$ & 1 \\
\hline$Y$ & 100 & 2326 & CLAY_BOT & Shat Bottom Clay & SAT_RGAS & Residual Gas Saturation & 31875 & 21-Feb-96 & $Y$ & 1 \\
\hline$Y$ & 100 & 2326 & CLAY_BOT & Shat Bottom Clay & SAT_RGAS & Residual Gas Saturation & 31875 & 21 feb-96 & $Y$ & 1 \\
\hline$Y$ & 100 & 2326 & CLAY_BOT & Shaft Bottom Clay & SAT_RGAS & Residual Gas Saturation & 31875 & 21 Fob-96 & $\bar{Y}$ & 1 \\
\hline$Y$ & 50 & 2327 & CL_L_T1 & $\begin{array}{l}\text { Lower Salado clay.0 to } \\
10 \text { years }\end{array}$ & CAP_MOD & $\begin{array}{l}\text { Model number, capillary pressure } \\
\text { model }\end{array}$ & $31897 \mathrm{~A}$ & 02-Nov-95 & $Y$ & $4 B$ \\
\hline $\mathbf{Y}$ & 50 & 2327 & CL_L_TI & $\begin{array}{l}\text { Lower Salado clay:0 to } \\
10 \text { years }\end{array}$ & CAP_MOD & $\begin{array}{l}\text { Model number, capillary pressure } \\
\text { model }\end{array}$ & 31897A & 02-Nov-95 & $\mathbf{Y}$ & 4B \\
\hline
\end{tabular}


CCA Parameter Listing

\begin{tabular}{|c|c|c|c|c|c|c|c|c|c|c|}
\hline ID & \begin{tabular}{|c|} 
Cinssifi- \\
cation of \\
Data \\
Category \\
(footnote 2)
\end{tabular} & $\begin{array}{l}\text { Distribution } \\
\text { Type }\end{array}$ & Mean & Median & Mintmum & Maxdinem & Units & Reforenco id & $\begin{array}{l}\text { Was the data } \\
\text { developed } \\
\text { under an } \\
\text { NOA-1 } \\
\text { Program? }\end{array}$ & $\begin{array}{c}\text { Which } \\
\text { methods } \\
\text { were used to } \\
\text { qually } \\
\text { oxisting } \\
\text { dettin? } \\
\text { (footnote s) }\end{array}$ \\
\hline 2317 & $1,3,4$ & TRIANGULAR & $-1.8867000 E+01$ & $-1.8301000 E+01$ & $-2.1000000 E+01$ & $-1.7301000 E+01$ & $\log \left(m^{\wedge} 2\right)$ & WP030994 & YEN & 1 \\
\hline 2317 & $1,3,4$ & TRIANGULAR & $-1.8857000 \mathrm{E}+01$ & $-1.8301000 E+01$ & $-2.1000000 E+01$ & $-1.7301000 E+01$ & $\log \left(m^{\wedge} 2\right)$ & WP035267 & Y8N & 9 \\
\hline 2317 & $1,3,4$ & TRLANGULAR & $-1.8867000 E+01$ & $-1.8301000 E+01$ & $-2.1000000 E+01$ & $-1.7301000 E+01$ & $\log \left(m^{\wedge} 2\right)$ & WPO35268 & YEN & 1 \\
\hline 2317 & $1,3,4$ & TRLANGULAR & $-1.8857000 E+01$ & $-1.8301000 E+01$ & $-2.1000000 E+01$ & $-1.7301000 E+01$ & $\log \left(m^{\wedge} 2\right)$ & WPO35597 & YEN & 1 \\
\hline 2317 & $1,3,4$ & TRIANGULAR & $-1.8867000 E+01$ & $-1.8301000 E+01$ & $-2.1000000 \mathrm{E}+01$ & $-1.7301000 \mathrm{E}+01$ & $\log \left(m^{\wedge} 2\right)$ & WPO36383 & YEN & 1 \\
\hline 2317 & $1,3,4$ & TRIANGULAR & $-1.8867000 E+01$ & $-1.8301000 E+01$ & $-2.1000000 E+01$ & $-1.7301000 E+01$ & $\log \left(m^{\wedge} 2\right)$ & WP041558 & Y8N & 1 \\
\hline 2318 & $1,3,4$ & TRIANGULAR & $-1.8867000 E+01$ & $-1.8301000 E+01$ & $-2.1000000 \mathrm{E}+01$ & $-1.7301000 \mathrm{E}+01$ & $\log \left(m^{\wedge} 2\right)$ & WPO30994 & Y\&N & 1 \\
\hline 2318 & $1,3,4$ & TRIANGULAR & $-1.8867000 E+01$ & $-1.8301000 E+01$ & $-2.1000000 \mathrm{E}+01$ & $-1.7301000 E+01$ & $\log \left(m^{\wedge} 2\right)$ & WP035257 & Y\&N & 1 \\
\hline 2318 & $1,3,4$ & TRIANGULAR & $-1.8867000 \mathrm{E}+01$ & $-1.8301000 E+01$ & $-2.1000000 E+01$ & $-1.7301000 E+01$ & $\log \left(m^{\wedge} 2\right)$ & WP035268 & Y\&N & 1 \\
\hline 2318 & $1,3,4$ & TRIANGULAR & $-1.8867000 \mathrm{E}+01$ & $-1.8301000 E+01$ & $-2.1000000 E+01$ & $-1.7301000 E+01$ & $\log \left(m^{\wedge} 2\right)$ & WP035597 & YEN & 1 \\
\hline 2318 & $1,3,4$ & TRIANGULAR & $-1.8867000 \mathrm{E}+01$ & $-1.8301000 E+01$ & $-2.1000000 \mathrm{E}+01$ & $-1.7301000 E+01$ & $\log \left(m^{\wedge} 2\right)$ & WPO36383 & YEN & 1 \\
\hline 2318 & $1,3,4$ & TRIANGULAR & $-1.8867000 E+01$ & $-1.830+000 E+01$ & $-2.1000000 E+01$ & $-1.7301000 E+01$ & $\log \left(m^{\wedge} 2\right)$ & WPO41558 & Y\&N & 1 \\
\hline 2319 & $1,3,4$ & TRLANGULAR & $-1.8867000 E+01$ & $-1.8301000 E+01$ & $-2.1000000 E+01$ & $-1.7301000 E+01$ & $\log \left(m^{\wedge} 2\right)$ & WP030994 & Y\&N & 1 \\
\hline 2319 & $1,3,4$ & TRIANGULAR & $-1.8867000 E+01$ & $-1.8301000 E+01$ & $-2.1000000 E+01$ & $-1.7301000 E+01$ & $\log \left(m^{\wedge} 2\right)$ & WP035267 & Y\&N & 1 \\
\hline 2319 & $1,3,4$ & TRIANGULAR & $-1.8867000 E+01$ & $-1.8301000 E+01$ & $-2.1000000 E+01$ & $-1.7301000 E+01$ & $\log \left(m^{\wedge} 2\right)$ & WP035268 & Y\&N & 1 \\
\hline 2319 & $1,3,4$ & TRLANGULAR & $-1.8867000 E+01$ & $-1.8301000 E+01$ & $-2.1000000 E+01$ & $-1.7301000 E+01$ & $\log \left(m^{\wedge} 2\right)$ & WPO35597 & Y\&N & 1 \\
\hline 2319 & $1,3,4$ & TRIANGULAR & $-1.8867000 E+01$ & $-1.8301000 E+01$ & $-2.1000000 E+01$ & $-1.7301000 E+01$ & $\log \left(m^{\wedge} 2\right)$ & WPO36383 & Y\&N & 1 \\
\hline 2319 & $1,3,4$ & TRIANGULAR & $-1,8867000 \mathrm{E}+01$ & $-1.8301000 E+01$ & $-2.1000000 E+01$ & $-1.7301000 E+01$ & $\log \left(m^{\wedge} 2\right)$ & WP041558 & Y8N & 1 \\
\hline 2323 & $1,3,4$ & CONSTANT & $4.0000000 \mathrm{E}+00$ & $4.0000000 E+00$ & $4.0000000 \mathrm{E}+00$ & $4.0000000 E+\infty$ & NONE & WP030994 & $\mathbf{Y}$ & NA \\
\hline 2323 & $1,3,4$ & CONSTANT & $4.0000000 E+00$ & $4.0000000 E+00$ & $4.0000000 E+00$ & $4.0000000 E+\infty 0$ & NONE & WP035267 & $Y$ & N/A \\
\hline 2323 & $1,3,4$ & CONSTANT & $4.0000000 E+\infty 0$ & 4.0000000E+00 & $4.0000000 \mathrm{E}+00$ & $4.0000000 E+00$ & NONE & WP035597 & $\mathbf{Y}$ & N/A \\
\hline 2323 & $1,3,4$ & CONSTANT & $4.0000000 E+\infty 0$ & $4.0000000 E+\infty 0$ & $4.0000000 E+\infty 0$ & $4.0000000 E+\infty$ & NONE & WP036383 & $\mathbf{Y}$ & N/A \\
\hline 2323 & $1,3,4$ & CONSTANT & $4.0000000 E+00$ & $4.0000000 \mathrm{E}+00$ & $4.0000000 E+00$ & $4.0000000 E+\infty 0$ & NONE & WP040514 & $\mathbf{Y}$ & N/A \\
\hline 2324 & $1,3,4$ & CONSTANT & $7.9000000 E-01$ & $7.9000000 \mathrm{E}-01$ & $7.9000000 E-01$ & $7.9000000 \mathrm{E}-01$ & NONE & WP030640 & $\mathbf{Y}$ & N/A \\
\hline 2324 & $1,3,4$ & CONSTANT & $7.9000000 E-01$ & $7.9000000 \mathrm{E}-01$ & $7.9000000 E-01$ & $7.9000000 E-01$ & NONE & WPO30994 & $\bar{Y}$ & N/A \\
\hline 2324 & $1,3,4$ & CONSTANT & $7.9000000 E-01$ & $7.9000000 E-01$ & $7.9000000 \mathrm{E}-01$ & $7.9000000 E-01$ & NONE & WPO35267 & - Y & N/A \\
\hline 2324 & $1,3,4$ & CONSTANT & $7.9000000 \mathrm{E}-01$ & $7.9000000 E-01$ & $7.9000000 \mathrm{E}-01$ & $7.9000000 \mathrm{E}-01$ & NONE & WP035597 & $Y$ & N/A \\
\hline 2324 & $1,3,4$ & CONSTANT & $7.9000000 \mathrm{E}-01$ & $7.9000000 \mathrm{E}-01$ & $7.9000000 E-01$ & $7.9000000 E-01$ & NONE & WPO36050 & $Y$ & N/A \\
\hline 2324 & $1,3,4$ & CONSTANT & $7.9000000 E-01$ & $7.9000000 E-01$ & $7.9000000 E-01$ & $7.9000000 \mathrm{E}-01$ & NONE & WPO40434 & $\mathbf{Y}$ & N/A \\
\hline 2325 & $1,3,4$ & CUMULATINE & $2.5000000 E-01$ & $2.0000000 \mathrm{E}-01$ & $0.0000000 \mathrm{E}+00$ & $6.0000000 \mathrm{E}-01$ & NONE & WP030994 & $\mathbf{Y}$ & N/A \\
\hline 2325 & $1,3,4$ & CUMULATME & $2.5000000 E-01$ & 20000000E-01 & $0.0000000 \mathrm{E}+00$ & $6.0000000 \mathrm{E}-01$ & NONE & WP035267 & $\bar{Y}$ & N/A \\
\hline 2325 & $1,3,4$ & CUMULATIVE & $2.5000000 E-01$ & $2.0000000 E-01$ & $0.0000000 E+00$ & $6.0000000 E-01$ & NONE & WP035268 & $\mathbf{Y}$ & N/A \\
\hline 2325 & $1,3,4$ & CUMULATME & $2.5000000 E-01$ & $2.0000000 \mathrm{E}-01$ & $0.00000000+00$ & $6.0000000 E-01$ & NONE & WP035597 & $\mathbf{Y}$ & N/A \\
\hline 2325 & $1,3,4$ & CUMULATIVE & 25000000E-01 & $2.0000000 E-01$ & $0.0000000 \mathrm{E}+00$ & $6.0000000 E-01$ & NONE & WP041558 & $Y$ & N/A \\
\hline 2326 & 3,4 & UNIFORM & $2.0000000 \mathrm{E}-01$ & $2.0000000 E-01$ & $0.0000000 \mathrm{E}+00$ & $4.0000000 \mathrm{E}-01$ & NONE & WP030994 & $Y$ & NA \\
\hline 2326 & 3,4 & UNIFORM & $2.0000000 \mathrm{E}-01$ & $2.0000000 \mathrm{E}-01$ & $0.0000000 E+00$ & $4.0000000 E-01$ & NONE & WP035267 & $Y$ & N/A \\
\hline 2326 & 3,4 & UNIFORM & $2.0000000 \mathrm{E}-01$ & $20000000 E-01$ & $0.0000000 E+00$ & $4.0000000 E-01$ & NONE & WP035268 & $\mathbf{Y}$ & N/A \\
\hline 2326 & 3,4 & UNIFORM & $2.0000000 E-01$ & $2.0000000 E-01$ & $0.0000000 E+00$ & $4.0000000 E-01$ & NONE & WP035597 & $\mathbf{Y}$ & N/A \\
\hline 2326 & 3,4 & UNIFORM & $2.0000000 E-01$ & $2.0000000 \mathrm{E}-01$ & $0.0000000 \mathrm{E}+\infty 0$ & $4.0000000 E-01$ & NONE & WP036383 & $\mathbf{Y}$ & N/A \\
\hline 2326 & 3,4 & UNIFORM & $2.0000000 E-01$ & $2.0000000 E-01$ & $0.0000000 E+\infty 0$ & $4.0000000 E-01$ & NONE & WP041558 & $\mathbf{Y}$ & N/A \\
\hline 2327 & $1,3,4$ & CONSTANT & $2.0000000 E+\infty 0$ & $2.0000000 \mathrm{E}+00$ & $2.0000000 E+\infty 0$ & $2.0000000 \mathrm{E}+00$ & NONE & WP030640 & $\mathbf{Y}$ & N/A \\
\hline 2327 & $1,3,4$ & CONSTANT & $2.0000000 E+\infty 0$ & $2.0000000 E+\infty 0$ & $2.0000000 E+00$ & $20000000 E+00$ & NONE & WP030994 & $\gamma$ & NA \\
\hline
\end{tabular}


CCA Parameter Listing

\begin{tabular}{|c|c|c|c|c|c|c|c|c|c|c|}
\hline $\begin{array}{c}\text { Parametar in } \\
\text { Datubean? } \\
\text { (YNN) }\end{array}$ & \begin{tabular}{|c|} 
if the values \\
used in CCA \\
are not the \\
same sus in \\
DB, provide $\%$ \\
difference
\end{tabular} & 10 & Metarial ID & Mnterial Name & $\begin{array}{l}\text { Parameter } \\
\text { ID }\end{array}$ & Paramoter Nam & $\begin{array}{l}\text { PRPID } \\
\text { (WPO A) }\end{array}$ & $\begin{array}{c}\text { Data Entry } \\
\text { Dite }\end{array}$ & $\begin{array}{l}\text { is the 46A } \\
\text { consistent } \\
\text { with the } \\
\text { parameter } \\
\text { tn DB? } \\
\left(Y_{1} \text { NA) }\right.\end{array}$ & $\begin{array}{l}\text { Parameter } \\
\text { Cetegory } \\
\text { (footnote 1) }\end{array}$ \\
\hline $\mathbf{Y}$ & 50 & 2327 & CL_L_TI & $\begin{array}{l}\text { Lower Salado clay.0 to } \\
10 \text { years }\end{array}$ & CAP_MOD & $\begin{array}{l}\text { Model number, capillary pressure } \\
\text { model }\end{array}$ & $31897 A$ & 02-Nov-95 & $\mathbf{Y}$ & $A B$ \\
\hline $\mathbf{Y}$ & 50 & 2327 & CL_L_TI & $\begin{array}{l}\text { Lower Salado clay.0 to } \\
\text { to years }\end{array}$ & CAP_MOD & $\begin{array}{l}\text { Model number. capillary pressure } \\
\text { model }\end{array}$ & 31897A & 02-Nov-95 & $\mathbf{Y}$ & 48 \\
\hline $\mathbf{Y}$ & 50 & 2327 & CL_L_TI & $\begin{array}{l}\text { Lower Salado clay:0 to } \\
10 \text { years }\end{array}$ & CAP_MOD & $\begin{array}{l}\text { Model mumber, capillary pressure } \\
\text { model }\end{array}$ & $31897 A$ & 02-Nov-95 & $\mathbf{Y}$ & $4 B$ \\
\hline $\mathbf{Y}$ & & 2328 & CL_L_TI & $\begin{array}{l}\text { Lower Salado clay.0 to } \\
10 \text { years }\end{array}$ & COMP_RCK & Bulk Compressibility & 31899 & 13-Feb-96 & $\mathbf{Y}$ & 1 \\
\hline$Y$ & & 2328 & CL_L_T1 & $\begin{array}{l}\text { Lower Salado clay:0 to } \\
10 \text { years }\end{array}$ & COMP_RCK & Buik Compressibility & 31899 & 13-Feb-96 & $\mathbf{Y}$ & 1 \\
\hline $\mathbf{Y}$ & & 2328 & CL_L_T1 & $\begin{array}{l}\text { Lower Salado clay.0 to } \\
10 \text { years }\end{array}$ & COMP_RCK & Bulk Compressibility & 31899 & 13-Fob-96 & $\mathbf{Y}$ & 1 \\
\hline $\mathbf{Y}$ & & 2329 & CL_L_T1 & $\begin{array}{l}\text { Lower Salado clay:0 to } \\
10 \text { years }\end{array}$ & PC_MAX & $\begin{array}{l}\text { Maximum allowable capillary } \\
\text { pressure }\end{array}$ & $31903 \mathrm{~A}$ & 13-Feb-96 & $\mathbf{Y}$ & $4 B$ \\
\hline $\mathbf{Y}$ & & 2329 & CL_L_T1 & $\begin{array}{l}\text { Lower Salado clay.0 to } \\
10 \text { years }\end{array}$ & PC_MAX & $\begin{array}{l}\text { Maximum allowable capillary } \\
\text { pressure }\end{array}$ & $31903 A$ & 13-Feb-96 & $\mathbf{Y}$ & $4 B$ \\
\hline $\mathbf{Y}$ & & 2329 & CL_L_T1 & \begin{tabular}{|l|} 
Lower Salado clay.0 to \\
10 years
\end{tabular} & PC_MAX & $\begin{array}{l}\text { Maximum allowable capillary } \\
\text { pressure }\end{array}$ & $31903 A$ & 13 Feb-96 & $\mathbf{Y}$ & $4 B$ \\
\hline $\mathbf{Y}$ & & 2329 & CL_L_T1 & $\begin{array}{l}\text { Lower Salado clay. } 0 \text { to } \\
10 \text { years }\end{array}$ & PC_MAX & $\begin{array}{l}\text { Maxmum allowable capillary } \\
\text { pressure }\end{array}$ & $31903 A$ & 13-Feb-96 & $\mathbf{Y}$ & 48 \\
\hline$Y$ & & 2329 & CL_L_T1 & $\begin{array}{l}\text { Lower Salado clay. } 0 \text { to } \\
10 \text { years }\end{array}$ & PC_MAX & $\begin{array}{l}\text { Maximum allowable capillary } \\
\text { pressure }\end{array}$ & $31903 A$ & 13-Feb-96 & $\mathbf{Y}$ & 48 \\
\hline$Y$ & varies & 2330 & CL_L_T1 & $\begin{array}{l}\text { Lower Salado clay.0 to } \\
10 \text { years }\end{array}$ & PORE_DIS & $\begin{array}{l}\text { Brooks-Corey pore distubution } \\
\text { parameter }\end{array}$ & 31904 & 20-Feb-96 & $\mathbf{Y}$ & 1 \\
\hline$Y$ & varies & 2330 & CL_L_T1 & $\begin{array}{l}\text { Lower Salado clay.0 to } \\
10 \text { years }\end{array}$ & PORE_DIS & $\begin{array}{l}\text { Brooks-Corey pore distribution } \\
\text { parameter }\end{array}$ & 31904 & $20-F a b-96$ & $\mathbf{Y}$ & 1 \\
\hline$Y$ & varies & 2330 & CL_L_T1 & $\begin{array}{l}\text { Lower Salado clay:0 to } \\
10 \text { years }\end{array}$ & PORE_DIS & $\begin{array}{l}\text { Brooks-Corey pore distribution } \\
\text { parameter }\end{array}$ & 31904 & 20-Fob-96 & $\mathbf{Y}$ & 1 \\
\hline $\mathbf{Y}$ & varies & 2330 & CL_L_TI & $\begin{array}{l}\text { Lower Salado clay.0 to } \\
10 \text { years }\end{array}$ & PORE_DIS & $\begin{array}{l}\text { Brooks-Corey pore distritution } \\
\text { parameter }\end{array}$ & 31904 & 20-Fob-96 & $Y$ & 1 \\
\hline$Y$ & varies & 2330 & CL_L_T1 & $\begin{array}{l}\text { Lower Salado clay.0 to } \\
10 \text { years }\end{array}$ & PORE_DIS & $\begin{array}{l}\text { Brooks-Corey pore distribution } \\
\text { parameter }\end{array}$ & 31904 & 20-Fob-96 & $\mathbf{Y}$ & 1 \\
\hline $\mathbf{Y}$ & varies & 2330 & CL_L_T1 & $\begin{array}{l}\text { Lower Salado clay.0 to } \\
10 \text { years }\end{array}$ & PORE_DIS & $\begin{array}{l}\text { Brooks-Corey pore distribution } \\
\text { parameter }\end{array}$ & 31904 & $20-F a b-96$ & $Y$ & 1 \\
\hline $\mathbf{Y}$ & & 2331 & CL_L_T1 & $\begin{array}{l}\text { Lower Salado clay.0 to } \\
10 \text { years }\end{array}$ & POROSTY & Effective porosity & 31905 & 13-Fob-96 & $\mathbf{Y}$ & 1 \\
\hline $\mathbf{Y}$ & & 2331 & CL_L_T1 & $\begin{array}{l}\text { Lower Salado clay:0 to } \\
10 \text { years }\end{array}$ & POROSTr & Effective porosity & 31905 & 13-Feb-96 & $\mathbf{Y}$ & 1 \\
\hline $\mathbf{Y}$ & & 2331 & CL_L_T1 & $\begin{array}{l}\text { Lower Salado clay:0 to } \\
10 \text { years }\end{array}$ & POROSTY & Effective porosity & 31905 & 13-Feb-96 & $\mathbf{Y}$ & 1 \\
\hline $\mathbf{Y}$ & & 2332 & CL_L_T1 & $\begin{array}{l}\text { Lower Salado clay.0 to } \\
10 \text { years }\end{array}$ & PO_MIN & $\begin{array}{l}\text { Minimum brine pressure for } \\
\text { capillary model KPC }=3\end{array}$ & 31906 & 21-Feb-96 & $\mathbf{Y}$ & $4 B$ \\
\hline $\mathbf{Y}$ & & 2332 & CL_L_T1 & $\begin{array}{l}\text { Lower Salado clay.0 to } \\
10 \text { years }\end{array}$ & PO_MIN & $\begin{array}{l}\text { Minimum brine pressure for } \\
\text { capillary model KPC }=3\end{array}$ & 31906 & 21-Feb-96 & $Y$ & $4 B$ \\
\hline$Y$ & & 2332 & CL_L_T1 & $\begin{array}{l}\text { Lower Salado clay.0 to } \\
10 \text { years }\end{array}$ & PO_MIN & $\begin{array}{l}\text { Minimum brine pressure for } \\
\text { capillary model KPC=3 }\end{array}$ & 31906 & 21-Feb-96 & $Y$ & 4B \\
\hline$Y$ & & 2333 & CL_L_T1 & $\begin{array}{l}\text { Lower Salado clay.0 to } \\
10 \text { years }\end{array}$ & PRESSURE & Brine far-field pore pressure & 31907 & 20-Fob-96 & $\mathbf{Y}$ & 1 \\
\hline $\mathbf{Y}$ & & 2333 & 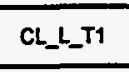 & $\begin{array}{l}\text { Lower Salado clay.0 to } \\
10 \text { years }\end{array}$ & PRESSURE & Brine far-field pore pressure & 31907 & 20-Feb-96 & $-\because Y$ & 1 \\
\hline $\mathbf{Y}$ & varies & 2334 & CL_L_T1 & $\begin{array}{l}\text { Lower Salado clay:0 to } \\
\text { to years }\end{array}$ & PRMX_LOG & $\begin{array}{l}\text { Log of intansic permeabilty, } X \text { - } \\
\text { direction }\end{array}$ & 31908 & 13-feb-96 & $Y$ & 1 \\
\hline $\mathbf{Y}$ & varies & 2334 & CL_L_T1 & $\begin{array}{l}\text { Lower Salado clay:0 to } \\
10 \text { years }\end{array}$ & PRMX_LOG & $\begin{array}{l}\text { Log of intrinsic permeability, } X- \\
\text { direction }\end{array}$ & 31908 & 13-Fob-96 & $\mathbf{Y}$ & 1 \\
\hline $\mathbf{Y}$ & varies & 2334 & CL_L_TI & $\begin{array}{l}\text { Lower Salado clay. } 0 \text { to } \\
10 \text { yoars }\end{array}$ & PRMX_LOG & $\begin{array}{l}\text { Log of intrinsic permeability, } X \text { - } \\
\text { drection }\end{array}$ & 31908 & 13-Feb-96 & $\mathbf{Y}$ & 1 \\
\hline $\mathbf{Y}$ & varies & 2334 & CL_L_TI & $\begin{array}{l}\text { Lower Salado clay.0 to } \\
10 \text { years }\end{array}$ & PRMX_LOG & $\begin{array}{l}\text { Log of intrinsic permeability, } X \text { - } \\
\text { direction }\end{array}$ & 31908 & 13-Feb-96 & $\mathbf{Y}$ & 1 \\
\hline$Y$ & varies & 2334 & CL_L_T1 & $\begin{array}{l}\text { Lower Salado clay.0 to } \\
10 \text { years }\end{array}$ & PRMX_LOG & $\begin{array}{l}\text { Log of intrnsic permesbility, } X \text { - } \\
\text { direction }\end{array}$ & 31908 & 13-Fob-96 & $\mathbf{Y}$ & 1 \\
\hline $\mathbf{Y}$ & & 2335 & CL_L_T1 & $\begin{array}{l}\text { Lower Salado clay.0 to } \\
10 \text { years }\end{array}$ & PRMY_LOG & $\begin{array}{l}\text { Log of intrinsic permeability, Y- } \\
\text { direction }\end{array}$ & 31909 & $13-F e b-96$ & $Y$ & 1 \\
\hline$Y$ & & 2335 & CL_L_T1 & $\begin{array}{l}\text { Lower Salado clay.0 to } \\
\text { to years }\end{array}$ & PRMY_LOG & $\begin{array}{l}\text { Log of intrinsic permeability, } Y \text { - } \\
\text { direction }\end{array}$ & 31909 & 13-Feb-96 & $\mathbf{Y}$ & 1 \\
\hline $\mathbf{Y}$ & & 2335 & CL_L_Tt & $\begin{array}{l}\text { Lower Salado clay.0 to } \\
10 \text { years }\end{array}$ & PRMY_LOG & $\begin{array}{l}\text { Log of intrinsic permeability, Y- } \\
\text { direction }\end{array}$ & 31909 & 13-Feb-96 & $\mathbf{Y}$ & 1 \\
\hline $\mathbf{Y}$ & & 2335 & CL_L_T1 & $\begin{array}{l}\text { Lower Salado clay.0 to } \\
10 \text { years }\end{array}$ & PRMY_LOG & $\begin{array}{l}\text { Log of intrinsic permeability, } Y \text { - } \\
\text { direction }\end{array}$ & 31909 & 13-Fob-96 & $\mathbf{Y}$ & 1 \\
\hline$Y$ & & 2335 & CL_L_T1 & $\begin{array}{l}\text { Lower Salado clay:0 to } \\
10 \text { years }\end{array}$ & PRMY_LOG & $\begin{array}{l}\text { Log of intrinsk permeability, Y- } \\
\text { direction }\end{array}$ & 31909 & 13 feb-96 & $\mathbf{Y}$ & 1 \\
\hline $\mathbf{Y}$ & & 2336 & CL_L_T1 & $\begin{array}{l}\text { Lower Salado clay.0 to } \\
10 \text { years }\end{array}$ & PRMZ_LOG & $\begin{array}{l}\text { Log of intrinsic permeability, Z- } \\
\text { direction }\end{array}$ & 31910 & 13 Fab-96 & $Y$ & 1 \\
\hline
\end{tabular}


CCA Parameter Listing

\begin{tabular}{|c|c|c|c|c|c|c|c|c|c|c|}
\hline ID & \begin{tabular}{|c|} 
Classfifi- \\
cation of \\
Data \\
Category \\
(footnoto 2)
\end{tabular} & $\begin{array}{c}\text { Distribution } \\
\text { Typo }\end{array}$ & Moan & Medien & Mintroum & Meximum & Units & Roference iD & $\begin{array}{l}\text { Was the dath } \\
\text { derveloped } \\
\text { under an } \\
\text { NQA-1 } \\
\text { Progrem? }\end{array}$ & $\begin{array}{c}\text { Which } \\
\text { methods } \\
\text { wort used to } \\
\text { qually } \\
\text { extsting } \\
\text { datt? } \\
\text { (footnote 3) }\end{array}$ \\
\hline 2327 & $1,3,4$ & CONSTANT & $2.0000000 E+00$ & $2.0000000 E+00$ & $2.0000000 E+00$ & $2.0000000 E+\infty$ & NONE & WP035597 & $\mathbf{Y}$ & NA \\
\hline 2327 & $1,3,4$ & CONSTANT & $2.0000000 \mathrm{E}+00$ & $2.0000000 E+\infty 0$ & $2.0000000 \mathrm{E}+\infty 0$ & $2.0000000 \mathrm{E}+\infty 0$ & NONE & WP040486 & $Y$ & N/A \\
\hline 2327 & $1,3,4$ & CONSTANT & $2.0000000 E+\infty 0$ & $2.0000000 E+\infty 0$ & $20000000 E+\infty 0$ & $20000000 E+\infty$ & NONE & WPO40514 & $\gamma$ & N/A \\
\hline 2328 & $1,3,4$ & CONSTANT & $1.5900000 \mathrm{E}-09$ & $1.5900000 \mathrm{E}-09$ & $1.5900000 E-09$ & $1.5900000 \mathrm{E}-09$ & $P a^{n}-1$ & WPO30640 & $Y$ & NA \\
\hline 2328 & $1,3,4$ & CONSTANT & 1.5900000E-09 & $1.5900000 \mathrm{E}-09$ & $1.5900000 \mathrm{E}-09$ & $1.5900000 E-09$ & $P a^{\wedge}-1$ & WP030994 & $\mathbf{Y}$ & NA \\
\hline 2328 & $1,3,4$ & CONSTANT & $1.5900000 \mathrm{E}-09$ & $1.5900000 \mathrm{E}-09$ & $1.5900000 \mathrm{E}-09$ & $1.5900000 \mathrm{E}-09$ & $P a^{n}-1$ & WPO35597 & $Y$ & NA \\
\hline 2329 & 3,5 & CONSTANT & $1.0000000 E+08$ & $1.0000000 E+08$ & $1.0000000 E+08$ & $1.0000000 E+08$ & $\mathbf{P a}$ & WPO30640 & $\mathbf{Y}$ & N/A \\
\hline 2329 & 3,5 & CONSTANT & $1.0000000 E+08$ & $1.0000000 E+08$ & $1.0000000 \mathrm{E}+08$ & $1.0000000 E+08$ & $\mathrm{~Pa}$ & WP030994 & $\mathbf{Y}$ & N/A \\
\hline 2329 & 3,5 & CONSTANT & $1.0000000 E+08$ & $1.0000000 E+08$ & $1.0000000 \mathrm{E}+08$ & $1.0000000 \mathrm{E}+08$ & $\mathbf{P a}$ & WP035597 & $Y$ & N/A \\
\hline 2329 & 3,5 & CONSTANT & $1.0000000 E+08$ & $1.0000000 E+08$ & $1.0000000 E+08$ & $1.0000000 E+08$ & $\mathbf{P a}$ & WP040486 & $\mathbf{Y}$ & N/A \\
\hline 2329 & 3,5 & CONSTANT & $1.0000000 \mathrm{E}+08$ & $1.0000000 E+08$ & $1.0000000 \mathrm{E}+08$ & $1.0000000 E+08$ & $\mathbf{P a}$ & WP040514 & $\mathbf{Y}$ & N/A \\
\hline 2330 & 3,4 & CUMULATIVE & $2.5200000 \mathrm{E}+00$ & $9.4000000 E-01$ & $1.1000000 \mathrm{E}-01$ & $8.1000000 E+\infty 0$ & NONE & WPO30540 & $\mathbf{Y}$ & N/A \\
\hline 2330 & 3,4 & CUMULATIVE & $2.5200000 E+\infty 0$ & 9.4000000E-01 & $1.1000000 \mathrm{E}-01$ & $8.1000000 E+00$ & NONE & WP030994 & $\mathbf{Y}$ & N/A \\
\hline 2330 & 3,4 & CUMULATIVE & $2.5200000 E+\infty 0$ & $9.4000000 E-01$ & $1.1000000 \mathrm{E}-01$ & $8.1000000 E+\infty 0$ & NONE & WPO32287 & $\mathbf{Y}$ & NA \\
\hline 2330 & 3,4 & CUMULATINE & $2.5200000 E+00$ & $9.4000000 E-09$ & $1.1000000 E-01$ & $8.1000000 E+00$ & NONE & WP035268 & $\mathbf{Y}$ & N/A \\
\hline 2330 & 3,4 & CUMULATIVE & $2.5200000 E+\infty 0$ & $9.4000000 E-01$ & $1.1000000 \mathrm{E}-01$ & $8.1000000 E+00$ & NONE & WP035597 & $\mathbf{Y}$ & NA \\
\hline 2330 & 3,4 & CUMULATIVE & $2.5200000 E+\infty 0$ & $9.4000000 E-01$ & $1.1000000 \mathrm{E}-01$ & $8.1000000 E+\infty 0$ & NONE & WPO41558 & $\gamma$ & N/A \\
\hline 2331 & $1,3,4$ & CONSTANT & $2.4000000 E-01$ & $2.4000000 \mathrm{E}-01$ & $2.4000000 \mathrm{E}-01$ & $2.4000000 E-01$ & $m^{\wedge} 3 / m^{\wedge} 3$ & WP030540 & $\mathbf{Y}$ & NA \\
\hline 2331 & $1,3,4$ & CONSTANT & $2.4000000 E-01$ & $2.4000000 E-01$ & 2.4000000E-01 & $2.4000000 E-01$ & $m^{\wedge} 3 / m^{\wedge} 3$ & WP030994 & $Y$ & N/A \\
\hline 2331 & $1,3,4$ & CONSTANT & $2.4000000 \mathrm{E}-01$ & $2.4000000 E-01$ & $24000000 E-01$ & $2.4000000 E-01$ & $m^{\wedge} 3 / m^{\wedge} 3$ & WP035597 & $\mathbf{Y}$ & N/A \\
\hline 2332 & 3,5 & CONSTANT & $1.0132500 E+05$ & $1.0132500 E+05$ & $1.0132500 E+05$ & $1.0132500 E+05$ & $\mathbf{P a}$ & WP035597 & $\mathbf{Y}$ & N/A \\
\hline 2332 & 3,5 & CONSTANT & $1.0132500 \mathrm{E}+05$ & $1.0132500 E+05$ & $1.0132500 \mathrm{E}+0.5$ & $1.0132500 E+05$ & $\mathrm{~Pa}$ & WPO36051 & $Y$ & N/A \\
\hline 2332 & 3,5 & CONSTANT & $1,0132500 E+05$ & $1.0132500 E+0.5$ & $1.0132500 \mathrm{E}+05$ & $1.0132500 E+05$ & $\mathrm{~Pa}$ & WP040514 & $Y$ & NA \\
\hline 2333 & 3,5 & CONSTANT & $1.0132500 E+05$ & $1.0132500 E+05$ & 1.0132500E+0.5 & $1.0132500 E+05$ & $\mathrm{~Pa}$ & WP035597 & $Y$ & N/A \\
\hline 2333 & 3,5 & CONSTANT & $1.0132500 E+05$ & $1.0132500 E+05$ & $1.0132500 E+05$ & $1.0132500 E+05$ & $\mathrm{~Pa}$ & WP036058 & $-Y$ & NA \\
\hline 2334 & $1,3,4$ & TRIANGULAR & $-1.8867000 E+01$ & $-1.8301000 E+01$ & $-2.1000000 E+01$ & $-1.7301000 E+09$ & $\log \left(m^{\wedge} 2\right)$ & WP030640 & Y\&N & 1 \\
\hline 2334 & $1,3,4$ & TRLANGULAR & $-1.8867000 \mathrm{E}+01$ & $-1.8301000 E+01$ & $-2.1000000 E+01$ & $-1.7301000 E+01$ & $\log \left(m^{\wedge} 2\right)$ & WP030994 & YEN & 1 \\
\hline 2334 & $1,3,4$ & TRIANGULAR & $-1.8857000 E+01$ & $-1.8301000 E+01$ & $-2.1000000 E+01$ & $-1.7301000 E+01$ & $\log \left(m^{\wedge} 2\right)$ & WP035268 & Y\&N & 1 \\
\hline 2334 & $1,3,4$ & TRIANGULAR & $-1,8867000 E+01$ & $-1.8301000 E+01$ & $-2.1000000 E+01$ & $-1.7301000 E+01$ & $\log \left(m^{\wedge} 2\right)$ & WP035597 & Y\&N & 1 \\
\hline 2334 & $1,3,4$ & TRLANGULAR & $-1.8867000 \mathrm{E}+01$ & $-1.8301000 \mathrm{E}+01$ & $-2.1000000 E+01$ & $-1.7301000 E+01$ & $\log \left(m^{\wedge} 2\right)$ & WPO41558 & Y8N & 1 \\
\hline 2335 & $1,3,4$ & TRLANGULAR & $-1.8867000 \mathrm{E}+01$ & $-1.8301000 E+01$ & $-2.1000000 E+01$ & $-1.7301000 E+01$ & $\log \left(m^{\wedge} 2\right)$ & WPO30640 & YRN & 1. \\
\hline 2335 & $1,3,4$ & TRIANGULAR & $-1.8867000 E+01$ & $-1.8301000 E+01$ & $-2.1000000 \mathrm{E}+01$ & $-1.7301000 E+01$ & $\log \left(m^{\wedge} 2\right)$ & WP030994 & Y\&N & 1 \\
\hline 2335 & $1,3,4$ & TRIANGULAR & $-1.8857000 E+01$ & $-1.8301000 E+01$ & $-2.1000000 E+01$ & $-1.7301000 \mathrm{E}+01$ & $\log \left(m^{\wedge} 2\right)$ & WP035268 & YRN & 1 \\
\hline 2335 & $1,3,4$ & TRLANGULAR & $-1.8857000 \Omega+01$ & $-1.8301000 E+01$ & $-2.1000000 E+01$ & $-1.7301000 E+01$ & $\log \left(m^{\wedge} 2\right)$ & WP035597 & Y\&N & 1 \\
\hline 2335 & $1,3,4$ & TRLANGULAR & $-1.8867000 E+01$ & $-1.8301000 E+01$ & $-2.1000000 E+01$ & $-1.7301000 E+01$ & $\log \left(m^{\wedge} 2\right)$ & WP041558 & Y $8 \mathrm{~N}$ & 1 \\
\hline 2336 & $1,3,4$ & TRLANGULAR & $-1.8857000 E+01$ & $-1.8301000 E+01$ & $-2.1000000 E+01$ & $-1.7301000 E+01$ & $\log \left(m^{\wedge} 2\right)$ & WP030640 & Y\&N & 1 \\
\hline
\end{tabular}


CCA Parameter Listing

\begin{tabular}{|c|c|c|c|c|c|c|c|c|c|c|}
\hline $\begin{array}{c}\text { Parameter in } \\
\text { Database? } \\
\text { (Yn) }\end{array}$ & $\begin{array}{l}\text { If the values } \\
\text { uned in cca } \\
\text { aro not the } \\
\text { amo as in } \\
\text { oB, provide \% } \\
\text { dfference }\end{array}$ & tD & Mnterial ID & Hatorial Namo & $\begin{array}{c}\text { Parameter } \\
\text { ID }\end{array}$ & Parameter Name & $\begin{array}{l}\text { PRP DD } \\
\text { (MPO I) }\end{array}$ & $\begin{array}{c}\text { Deta Entry } \\
\text { Dat }\end{array}$ & $\begin{array}{l}\text { Is the } 464 \\
\text { consistent } \\
\text { with the } \\
\text { perameter } \\
\text { in DB? } \\
\text { (Y.NA) }\end{array}$ & $\begin{array}{l}\text { Peramoter } \\
\text { Cetegory } \\
\text { (footnote 1) }\end{array}$ \\
\hline $\mathbf{Y}$ & & 2336 & CL_L_II & $\begin{array}{l}\text { Lower Sakado clay.0 to } \\
10 \text { years }\end{array}$ & PRMZ_LOG & $\begin{array}{l}\text { Log of intrinsic permeability, } 2 \text { - } \\
\text { direction }\end{array}$ & 31910 & 13-Feb-96 & $\mathbf{Y}$ & 1 \\
\hline $\mathbf{Y}$ & & 2336 & CL_L_T1 & $\begin{array}{l}\text { Lower Salado clay:0 to } \\
10 \text { years }\end{array}$ & PRMZ_LOG & $\begin{array}{l}\text { Log of intrinsic pemeability, Z- } \\
\text { direction }\end{array}$ & 31910 & $13-5 e b-96$ & $\mathbf{Y}$ & 1 \\
\hline $\mathbf{Y}$ & & 2336 & CL_L_Ts & $\begin{array}{l}\text { Lower Salado clay.0 to } \\
10 \text { years }\end{array}$ & PRMZ_LOG & $\begin{array}{l}\text { Log of intrinsic permeability, Z- } \\
\text { direction }\end{array}$ & 31910 & 13-Fob-96 & $\mathbf{Y}$ & 1 \\
\hline $\mathbf{Y}$ & & 2335 & $C L_{-} L_{-} T 1$ & $\begin{array}{l}\text { Lower Salado clay.0 to } \\
10 \text { years }\end{array}$ & PRMZ_LOG & $\begin{array}{l}\text { Log of intrnsic permeability, Z- } \\
\text { direction }\end{array}$ & 31910 & 13-Fob-96 & $\mathbf{Y}$ & 1 \\
\hline$Y$ & & 2340 & CL_L_T1 & $\begin{array}{l}\text { Lower Salado clay.0 to } \\
10 \text { years }\end{array}$ & RELP_MOD & $\begin{array}{l}\text { Model number, relative } \\
\text { permeability model }\end{array}$ & $31915 A$ & $26-0$ ct-95 & $\mathbf{Y}$ & 48 \\
\hline $\mathbf{Y}$ & & 2340 & $C L L_{-} T 1$ & $\begin{array}{l}\text { Lower Salado clay.0 to } \\
10 \text { years }\end{array}$ & RELP_MOD & $\begin{array}{l}\text { Model number, relative } \\
\text { permeability model }\end{array}$ & $31915 A$ & $26-0 \mathrm{ct}-95$ & $\mathbf{Y}$ & 4B \\
\hline $\mathbf{Y}$ & . & 2340 & CL_L_T1 & $\begin{array}{l}\text { Lower Salado clay.0 to } \\
10 \text { years }\end{array}$ & RELP_MOD & $\begin{array}{l}\text { Model number, relative } \\
\text { permeability model }\end{array}$ & $31915 A$ & 26-0ct-95 & $\mathbf{Y}$ & 48 \\
\hline$Y$ & & 2340 & CL_L_TI & $\begin{array}{l}\text { Lower Salado clay:0 to } \\
10 \text { years }\end{array}$ & RELP_MOD & $\begin{array}{l}\text { Model number, relative } \\
\text { permeability model }\end{array}$ & 31915A & $26-0 c t-95$ & $\mathbf{Y}$ & $4 B$ \\
\hline $\mathbf{Y}$ & & 2340 & CL_L_T1 & $\begin{array}{l}\text { Lower Salado clay.0 to } \\
10 \text { years }\end{array}$ & RELP_MOD & $\begin{array}{l}\text { Model number, relative } \\
\text { permeability model }\end{array}$ & $31915 A$ & 26-0ct-95 & $Y$ & $4 \mathrm{~B}$ \\
\hline $\mathbf{Y}$ & -26.58 & 2341 & CL_L_T1 & $\begin{array}{l}\text { Lower Salado clay:0 to } \\
10 \text { years }\end{array}$ & SAT_IBRN & Initial Brine Saturation & 31920 & 13Feb-96 & $\mathbf{Y}$ & 1 \\
\hline$Y$ & -26.58 & 2341 & CL_L_T1 & $\begin{array}{l}\text { Lower Salado clay. } 0 \text { to } \\
10 \text { years }\end{array}$ & SAT_IBRN & Initial Brine Saturation & 31920 & 13-Fab-96 & $\mathbf{Y}$ & 1 \\
\hline $\mathbf{Y}$ & -26.58 & 2341 & CL_L_T1 & $\begin{array}{l}\text { Lower Salado clay.0 to } \\
10 \text { years }\end{array}$ & SAT_IBRN & Initial Brine Saturation & 31920 & 13-Fab-96 & $\mathbf{Y}$ & 1 \\
\hline$Y$ & -26.58 & 2341 & CL_L_T1 & $\begin{array}{l}\text { Lower Salado clay.0 to } \\
10 \text { years }\end{array}$ & SAT_IBRN & Initial Brine Saturation & 31920 & 13-Fab-96 & $\mathbf{Y}$ & 1 \\
\hline $\mathbf{Y}$ & 100 & 2342 & CL_L_T1 & $\begin{array}{l}\text { Lower Salado clay:0 to } \\
10 \text { years }\end{array}$ & SAT_RBRN & Residual Brine Saturation & 31921 & 13-Fab-S6 & $\mathbf{Y}$ & 1 \\
\hline $\mathbf{Y}$ & 100 & 2342 & CL_L_TI & $\begin{array}{l}\text { Lower Salado clay:0 to } \\
10 \text { years }\end{array}$ & SAT_RBRN & Residual Brine Saturation & 31921 & 13-Fob-96 & $\mathbf{Y}$ & 1 \\
\hline $\mathbf{Y}$ & 100 & 2342 & CL_L_T1 & $\begin{array}{l}\text { Lower Salado clay.0 to } \\
10 \text { years }\end{array}$ & SAT_RBRN & Residual Brine Saturation & 31921 & 13-Feb-96 & $Y$ & 1 \\
\hline $\mathbf{Y}$ & 100 & 2342 & CL_L_TI & $\begin{array}{l}\text { Lower Salado clay.0 to } \\
10 \text { years }\end{array}$ & SAT_RBRN & Residual Brine Saturation & 31921 & 13-Feb-96 & $\mathbf{Y}$ & 1 \\
\hline $\mathbf{Y}$ & 100 & 2342 & CL_L_T1 & $\begin{array}{l}\text { Lower Salado clay:0 to } \\
10 \text { years }\end{array}$ & SAT_RBRN & Residual Brine Saturation & 31921 & 13-Feb-96 & $\mathbf{Y}$ & 1 \\
\hline $\mathbf{Y}$ & 100 & 2342 & CL_L_T1 & $\begin{array}{l}\text { Lower Salado clay:0 to } \\
10 \text { years }\end{array}$ & SAT_RBRN & Residual Brine Saturation & 31921 & 13-Feb-96 & $\mathbf{Y}$ & 1 \\
\hline $\mathbf{Y}$ & 100 & 2343 & CL_L_T1 & $\begin{array}{l}\text { Lower Salado clay. } 0 \text { to } \\
10 \text { years }\end{array}$ & SAT_RGAS & Residual Gas Saturation & 31922 & 13-Fob-95 & $\mathbf{Y}$ & 1 \\
\hline $\mathbf{Y}$ & 100 & 2343 & CL_L_T1 & $\begin{array}{l}\text { Lowrer Salado clay.0 to } \\
\text { to years }\end{array}$ & SAT_RGAS & Residual Gas Saturation & 31922 & 13-Feb-96 & $\mathbf{Y}$ & 1 \\
\hline $\mathbf{Y}$ & 100 & 2343 & CL_L_T1 & $\begin{array}{l}\text { Lower Salado clay:0 to } \\
10 \text { years }\end{array}$ & SAT_RGAS & Residual Gas Saturation & 31922 & 13-Fob-96 & $\mathbf{Y}$ & 1 \\
\hline $\mathbf{Y}$ & 100 & 2343 & CL_L_T9 & $\begin{array}{l}\text { Lower Salado clay.0 to } \\
10 \text { years }\end{array}$ & SAT_RGAS & Residual Gas Saturation & 31922 & 13-Fob-96 & $\mathbf{Y}$ & 1 \\
\hline $\mathbf{Y}$ & 100 & 2343 & CL_L_T1 & \begin{tabular}{|l|} 
Lower Salado clay.0 to \\
10 years
\end{tabular} & SAT_RGAS & Residual Gas Saturation & 31922 & 13-Fob-96 & $\mathbf{Y}$ & 1 \\
\hline $\mathbf{Y}$ & 50 & 2344 & $C L_{-} L_{-} T 2$ & $\begin{array}{l}\text { Lower Salado clay. } 10 \\
\text { to } 25 \text { years }\end{array}$ & CAP_MOD & $\begin{array}{l}\text { Model number, capillary pressuro } \\
\text { model }\end{array}$ & 31924A & 14-Fob-96 & $--Y$ & 48 \\
\hline $\mathbf{Y}$ & 50 & 2344 & CL_L_T2 & $\begin{array}{l}\text { Lower Salado clay: } 10 \\
\text { lo } 25 \text { years }\end{array}$ & CAP_MOD & $\begin{array}{l}\text { Model number, capillary pressure } \\
\text { model }\end{array}$ & $31924 A$ & 14 Feb-96 & $\mathbf{Y}$ & $4 B$ \\
\hline $\mathbf{Y}$ & 50 & 2344 & CL_L_T2 & $\begin{array}{l}\text { Lower Salado clay: } 10 \\
\text { to } 25 \text { years }\end{array}$ & CAP_MOD & $\begin{array}{l}\text { Model number, capiliary pressure } \\
\text { model }\end{array}$ & $31924 A$ & 14-Fob-96 & $\mathbf{Y}$ & $4 B$ \\
\hline $\mathbf{Y}$ & 50 & 2344 & CL_L_T2 & $\begin{array}{l}\text { Lower Salado clay: } 10 \\
\text { to } 25 \text { years }\end{array}$ & CAP_MOD & $\begin{array}{l}\text { Model number, capillary pressure } \\
\text { model }\end{array}$ & 31924A & $14-F e b-96$ & $\mathbf{Y}$ & $4 B$ \\
\hline $\mathbf{Y}$ & 50 & 2344 & CL_L_T2 & $\begin{array}{l}\text { Lower Salado clay: } 10 \\
\text { to } 25 \text { years }\end{array}$ & CAP_MOD & $\begin{array}{l}\text { Model number, capillary pressure } \\
\text { model }\end{array}$ & $31924 A$ & 14-Fob-96 & $\mathbf{Y}$ & $\mathbf{A B}$ \\
\hline$Y$ & & 2345 & CL_L_T2 & $\begin{array}{l}\text { Lower Salado clay: } 10 \\
\text { to } 25 \text { years } \\
\end{array}$ & COMP_RCK & Bulk Compressibility & 31926 & 14-Fab-96 & $\mathbf{Y}$ & 1 \\
\hline $\mathbf{Y}$ & & 2345 & $C L \_L 2$ & $\begin{array}{l}\text { Lower Salado clay: } 10 \\
\text { to } 25 \text { years }\end{array}$ & COMP_RCK & Bulk Compressibility & 31926 & 14Fob-96 & $\mathbf{Y}$ & 1 \\
\hline $\mathbf{Y}$ & & 2345 & CL_L_T2 & $\begin{array}{l}\text { Lower Salado clay: } 10 \\
\text { to } 25 \text { years }\end{array}$ & COMP_RCK & Bulk Compressibility & 31926 & 14Feb-96 & $\mathbf{Y}$ & 1 \\
\hline $\mathbf{Y}$ & & 2346 & CL_L_T2 & $\begin{array}{l}\text { Lower Salado clay. } 10 \\
\text { to } 25 \text { years }\end{array}$ & PC_MAX & $\begin{array}{l}\text { Maxmum allowable capillary } \\
\text { pressure }\end{array}$ & $31930 \mathrm{~A}$ & $14-F a b-96$ & $\mathbf{Y}$ & AB \\
\hline $\mathbf{Y}$ & & 2346 & CL_L_T2 & $\begin{array}{l}\text { Lower Salado clay: } 10 \\
\text { to } 25 \text { years }\end{array}$ & PC_MAX & $\begin{array}{l}\text { Maximum alfowable capillary } \\
\text { pressure }\end{array}$ & $31930 \mathrm{~A}$ & 14-Feb-96 & $\mathbf{Y}$ & $4 B$ \\
\hline $\mathbf{Y}$ & & 2346 & $C L_{-} L_{-} T_{2}$ & $\begin{array}{l}\text { Lower Salado clay: } 10 \\
\text { to } 25 \text { years }\end{array}$ & PC_MAX & $\begin{array}{l}\text { Maximum allowable capillary } \\
\text { pressure }\end{array}$ & $31930 A$ & 14-Feb-96 & $\mathbf{Y}$ & 48 \\
\hline $\mathbf{Y}$ & & 2346 & CL_L_T2 & $\begin{array}{l}\text { Lower Salaco clay: } 10 \\
\text { to } 25 \text { years }\end{array}$ & PC_MAX & $\begin{array}{l}\text { Maximum allowable capillary } \\
\text { pressure }\end{array}$ & 31930A & 14-Feb-96 & $\mathbf{Y}$ & $4 B$ \\
\hline
\end{tabular}


CCA Parameter Listing

\begin{tabular}{|c|c|c|c|c|c|c|c|c|c|c|}
\hline ID & $\begin{array}{c}\text { Cleselfi- } \\
\text { cation of } \\
\text { Data } \\
\text { Category } \\
\text { (footnote 2) }\end{array}$ & $\begin{array}{c}\text { Distribution } \\
\text { Type }\end{array}$ & Mean & Median & Dinimum & Maximum & Unit: & Reforence ID & $\begin{array}{l}\text { Was the data } \\
\text { developed } \\
\text { under an } \\
\text { NQA-1 } \\
\text { Program? }\end{array}$ & $\begin{array}{c}\text { Whlch } \\
\text { methods } \\
\text { were used to } \\
\text { quality } \\
\text { cxisting } \\
\text { data? } \\
\text { (footnota 3) }\end{array}$ \\
\hline 2336 & $1,3,4$ & TRIANGULAR & $-1.8867000 E+01$ & $-1.8301000 E+01$ & $-2.1000000 \mathrm{E}+01$ & $-1.7301000 E+01$ & $\log \left(m^{\wedge} 2\right)$ & WP030994 & Y\&N & 1 \\
\hline 2336 & $1,3,4$ & TRIANGULAR & $-1.8867000 E+01$ & $-1.8301000 E+01$ & $-2.1000000 E+01$ & $-7.7301000 E+01$ & $\log \left(m^{\wedge} 2\right)$ & WP035268 & Y\&N & 1 \\
\hline 2336 & $1,3,4$ & TRIANGULAR & $-1.8867000 E+01$ & $-1.8301000 E+01$ & $-2.1000000 E+01$ & $-9.7301000 E+01$ & $\log \left(m^{\wedge} 2\right)$ & WP035597 & Y\&N & 1 \\
\hline 2336 & $1,3,4$ & TRIANGULAR & $-1.8867000 E+01$ & $-1.8301000 E+01$ & $-2.1000000 E+01$ & $-1.7301000 E+01$ & $\log \left(m^{\wedge} 2\right)$ & WPO41558 & YEN & 1 \\
\hline 2340 & $1,3,4$ & CONSTANT & $4.0000000 E+00$ & $4.0000000 E+\infty 0$ & $4.0000000 E+00$ & $4.0000000 E+00$ & NONE & WPO30640 & $\mathbf{Y}$ & NA \\
\hline 2340 & $1,3,4$ & CONSTANT & $4.0000000 \mathrm{E}+\infty 0$ & $4.0000000 E+00$ & $4.0000000 E+00$ & $4.0000000 E+\infty 0$ & NONE & WPo30g94 & $\mathbf{Y}$ & $N / A$ \\
\hline 2340 & $1,3,4$ & CONSTANT & $4.0000000 E+00$ & $4.0000000 \mathrm{E}+\infty 0$ & $4.0000000 E+00$ & $4.0000000 E+00$ & NONE & WP035597 & $\mathbf{Y}$ & N/A \\
\hline 2340 & $1,3,4$ & CONSTANT & $4.0000000 \mathrm{E}+00$ & $4.0000000 E+00$ & $4.0000000 E+00$ & $4.0000000 E+\infty 0$ & NONE & WPO40486 & $\mathbf{Y}$ & NA \\
\hline 2340 & $1,3,4$ & CONSTANT & $4.0000000 \mathrm{E}+00$ & $4.0000000 \mathrm{E}+00$ & $4.0000000 E+00$ & $4.0000000 E+00$ & NONE & WP040514 & $\mathbf{Y}$ & N/A \\
\hline 2341 & $1,3,4$ & CONSTANT & $7.9000000 E-01$ & $7.9000000 E-01$ & 7.9000000E-01 & $7.9000000 E-01$ & NONE & WPO30540 & $\mathbf{Y}$ & NA \\
\hline 2341 & $1,3,4$ & CONSTANT & $7.9000000 \mathrm{E}-01$ & $7.9000000 \mathrm{E}-01$ & $7.9000000 \mathrm{E}-01$ & $7.9000000 \mathrm{E}-01$ & NONE & WP030994 & $\mathbf{Y}$ & $N / A$ \\
\hline 2341 & $1,3,4$ & CONSTANT & $7.9000000 \mathrm{E}-01$ & $7.9000000 \mathrm{E}-09$ & 7.9000000E-01 & $7.9000000 \mathrm{E}-01$ & NONE & WP035597 & $\mathbf{Y}$ & N/A \\
\hline 2341 & $1,3,4$ & CONSTANT & $7.9000000 \mathrm{E}-01$ & $7.9000000 E-01$ & $7.9000000 E-01$ & $7.9000000 E-01$ & NONE & WP040434 & $\mathbf{Y}$ & N/A \\
\hline 2342 & 3,4 & CUMULATIVE & $2.5000000 E-01$ & $2.0000000 E-01$ & $0.0000000 E+00$ & 6.0000000E-01 & NONE & WP030640 & $\mathbf{Y}$ & N/A \\
\hline 2342 & 3,4 & CUMULATIVE & $2.5000000 E-01$ & $2.0000000 E-01$ & $0.0000000 E+00$ & $6.0000000 E-01$ & NONE & WP030994 & $\mathbf{Y}$ & N/A \\
\hline 2342 & 3,4 & CUMULATIVE & $2.5000000 \mathrm{E}-01$ & $2.0000000 \mathrm{E}-01$ & $0.0000000 E+\infty 0$ & $6.0000000 \mathrm{E}-01$ & NONE & WP035258 & $Y$ & N/A \\
\hline 2342 & 3,4 & CUMULATIVE & $2.5000000 \mathrm{E}-01$ & $2.0000000 E-01$ & $0.0000000 E+00$ & $6.0000000 E-01$ & NONE & WPO35597 & $\mathbf{Y}$ & $N / A$ \\
\hline 2342 & 3,4 & CUMULATNE & $2.5000000 E-01$ & $2.0000000 E-01$ & $0.0000000 E+\infty$ & $6.0000000 E-01$ & NONE & WP041558 & $Y$ & NA \\
\hline 2342 & 3,4 & CUMULATIVE & $2.5000000 E-01$ & $2.0000000 E-01$ & $0.0000000 E+\infty 0$ & $6.0000000 E-01$ & NONE & WPO42751 & $\mathbf{Y}$ & N/A \\
\hline 2343 & 3,4 & UNIFORM & $2.0000000 E-01$ & $2.0000000 E-01$ & $0.0000000 E+\infty 0$ & $4.0000000 E-01$ & NONE & WPO30540 & $\mathbf{Y}$ & N/A \\
\hline 2343 & 3,4 & UNIFORM & $2.0000000 E-01$ & $2.0000000 E-01$ & $0.0000000 E+\infty 0$ & $4.0000000 E-01$ & NONE & WP030994 & $\mathbf{Y}$ & NA \\
\hline 2343 & 3,4 & UNIFORM & $2.0000000 \mathrm{E}-01$ & $2.0000000 E-01$ & $0.0000000 E+\infty 0$ & $4.0000000 E-01$ & NONE & WP035268 & $\mathbf{Y}$ & N/A \\
\hline 2343 & 3,4 & UNIFORM & $2.0000000 \mathrm{E}-01$ & $2.0000000 E-01$ & $0.0000000 E+\infty$ & $4.0000000 E-01$ & NONE & WPO35597 & $\mathbf{Y}$ & N/A \\
\hline 2343 & 3,4 & UNIFORM & $2.0000000 \mathrm{E}-01$ & $2.0000000 E-01$ & $0.0000000 E+\infty 0$ & $4.0000000 \mathrm{E}-01$ & NONE & WPO41558 & $\mathbf{Y}$ & N/A \\
\hline 2344 & $1,3,4$ & CONSTANT & $2.0000000 E+00$ & $2.0000000 E+\infty 0$ & $2.0000000 E+\infty 0$ & $2.0000000 E+00$ & NONE & WP030540 &.$- Y$ & NA \\
\hline 2344 & $1,3,4$ & CONSTANT & $2.0000000 \mathrm{E}+00$ & $2.0000000 E+\infty 0$ & $2.0000000 E+\infty$ & $2.0000000 E+00$ & NONE & WP030994 & $\mathbf{Y}$ & N/A \\
\hline 2344 & $1,3,4$ & CONSTANT & $2.0000000 E+\infty 0$ & $2.0000000 E+\infty 0$ & $2.0000000 E+\infty 0$ & $2.0000000 E+00$ & NONE & WP035597 & $\mathbf{Y}$ & N/A \\
\hline 2344 & $1,3,4$ & CONSTANT & $2.0000000 E+00$ & $2.0000000 E+\infty 0$ & $2.0000000 E+\infty 0$ & $2.0000000 E+00$ & NONE & WP040486 & $Y$ & N/A \\
\hline 2344 & $1,3,4$ & CONSTANT & $2.0000000 E+\infty 0$ & $2.0000000 E+\infty 0$ & $2.0000000 E+00$ & $2.0000000 E+\infty 0$ & NONE & WP040514 & $\mathbf{Y}$ & N/A \\
\hline 2345 & $1,3,4$ & CONSTANT & $1.5900000 E-09$ & $1.5900000 E-09$ & $1.5900000 E-09$ & $1.5900000 E-09$ & $P a^{n}-1$ & WP030540 & $\mathbf{Y}$ & NA \\
\hline 2345 & $1,3,4$ & CONSTANT & $1.5900000 E-09$ & $1.5900000 E-09$ & $1.5900000 \mathrm{E}-09$ & $1.5900000 \mathrm{E}-09$ & $P a^{n}-1$ & WP030994 & $\mathbf{Y}$ & N/A \\
\hline 2345 & $1,3,4$ & CONSTANT & $1.5900000 E-09$ & $1.5900000 \mathrm{E}-09$ & $1.5900000 \mathrm{E}-09$ & $1.5900000 \mathrm{E}-09$ & $P a^{n}-1$ & WP035597 & $Y$ & N/A \\
\hline 2346 & 1,3 & CONSTANT & $1.0000000 E+08$ & $1.0000000 E+08$ & $1.0000000 E+08$ & $1.0000000 E+08$ & $\mathbf{P a}$ & WP030540 & $\mathbf{Y}$ & NA \\
\hline 2346 & 1,3 & CONSTANT & $1.0000000 E+08$ & $1.0000000 E+08$ & $1.0000000 E+08$ & $1.0000000 E+08$ & $\mathbf{P a}$ & WP030994 & $\mathbf{Y}$ & N/A \\
\hline 2346 & 1,3 & CONSTANT & $1.0000000 E+08$ & $1.0000000 \mathrm{E}+08$ & $1.0000000 E+08$ & $1.0000000 E+08$ & $\mathbf{P a}$ & WP035597 & $\mathbf{Y}$ & $N A$ \\
\hline 2346 & 1,3 & CONSTANT & $1.0000000 \mathrm{E}+08$ & $1.0000000 E+08$ & $1.0000000 E+08$ & $1.0000000 E+08$ & $\mathrm{~Pa}$ & WPO40486 & $\mathbf{Y}$ & N/A \\
\hline
\end{tabular}


CCA Parameter Listing

\begin{tabular}{|c|c|c|c|c|c|c|c|c|c|c|}
\hline $\begin{array}{c}\text { Parameter In } \\
\text { Database? } \\
\text { (YNN) }\end{array}$ & $\begin{array}{c}\text { H the valuez } \\
\text { usod in CCA } \\
\text { are not the } \\
\text { same as in } \\
\text { oB, provide } x \\
\text { difference }\end{array}$ & iD & Matarial is & Material Nambe & $\begin{array}{c}\text { Parameter } \\
\text { DD }\end{array}$ & Parameter Neme & $\begin{array}{l}\text { PRPD } \\
\text { (WPO*) }\end{array}$ & $\begin{array}{c}\text { Datan Entry } \\
\text { Date }\end{array}$ & $\begin{array}{l}\text { to the } 464 \\
\text { consistent } \\
\text { with the } \\
\text { parameter } \\
\text { in OB? } \\
\Gamma, N \text { A }\end{array}$ & \begin{tabular}{|} 
Paramiter \\
Category \\
(10otnota 1)
\end{tabular} \\
\hline$Y$ & & 2346 & CL_L_T2 & $\begin{array}{l}\text { Lower Salado clay: } 10 \\
\text { to } 25 \text { years }\end{array}$ & PC_MAX & $\begin{array}{l}\text { Maxmum allowable capillary } \\
\text { pressure }\end{array}$ & $31930 A$ & 14-Feb-96 & $\mathbf{Y}$ & 48 \\
\hline $\mathbf{Y}$ & varies & 2347 & CL_L_T2 & $\begin{array}{l}\text { Lower Salado clay: } 10 \\
\text { to } 25 \text { years }\end{array}$ & PORE_DIS & $\begin{array}{l}\text { Brooks-Corey pore distribution } \\
\text { parameter }\end{array}$ & 31931 & 20-Fob-96 & $\mathbf{Y}$ & 1 \\
\hline $\mathbf{Y}$ & varios & 2347 & CL_L_T2 & $\begin{array}{l}\text { Lower Salado clay. } 10 \\
\text { to } 25 \text { yaars }\end{array}$ & PORE_DIS & $\begin{array}{l}\text { Brooks-Corey pore distribution } \\
\text { parameter }\end{array}$ & 31931 & 20-feb-96 & $\mathbf{Y}$ & 1 \\
\hline $\mathbf{Y}$ & varies & 2347 & $C L L_{-} T 2$ & $\begin{array}{l}\text { Lower Salado clay.10 } \\
\text { to } 25 \text { years }\end{array}$ & PORE_DIS & $\begin{array}{l}\text { Brooks-Corey pore distribution } \\
\text { parameter }\end{array}$ & 31931 & 20-Fob-96 & $\mathbf{r}$ & 1 \\
\hline $\mathbf{Y}$ & varios & 2347 & $C L_{-} L_{-} T 2$ & $\begin{array}{l}\text { Lower Salado clay: } 10 \\
\text { to } 25 \text { years }\end{array}$ & PORE_DIS & $\begin{array}{l}\text { Brooks-Corey pora distribution } \\
\text { parameter }\end{array}$ & 31931 & 20-fob-96 & $Y$ & 1 \\
\hline $\mathbf{Y}$ & varies & 2347 & CL_L_T2 & $\begin{array}{l}\text { Lower Salado clay: } 10 \\
\text { to } 25 \text { years }\end{array}$ & PORE_DIS & $\begin{array}{l}\text { Brooks-Corey pore distribution } \\
\text { parameter }\end{array}$ & 31931 & 20-Fob-96 & $Y$ & 1 \\
\hline $\mathbf{Y}$ & varies & 2347 & CL_L_T2 & $\begin{array}{l}\text { Lower Salado clay: } 10 \\
\text { to } 25 \text { years }\end{array}$ & PORE_DIS & $\begin{array}{l}\text { Brooks-Corey pore distribution } \\
\text { parameler }\end{array}$ & 31931 & 20-Fob-96 & $\mathbf{Y}$ & 1 \\
\hline $\mathbf{Y}$ & & 2348 & CL_L_T2 & $\begin{array}{l}\text { Lower Salado clay: } 10 \\
\text { to } 25 \text { years }\end{array}$ & POROSITY & Effective porosity & 31932 & 14-Feb-96 & $Y$ & 1 \\
\hline$Y$ & & 2348 & CL_L_T2 & $\begin{array}{l}\text { Lower Salado clay: } 10 \\
\text { to } 25 \text { years }\end{array}$ & POROSTTY & Effective porosity & 31932 & 14-Feb-96 & $Y$ & 1 \\
\hline Y & & 2348 & $C L_{-} L_{-} T 2$ & $\begin{array}{l}\text { Lower Salado clay: } 10 \\
\text { to } 25 \text { years }\end{array}$ & POROSTTY & Effective porosity & 31932 & 14 feb-96 & $\mathbf{Y}$ & 1 \\
\hline$Y$ & & 2349 & CL_L_T2 & $\begin{array}{l}\text { Lower Salado clay: } 10 \\
\text { to } 25 \text { years } \\
\end{array}$ & PO_MIN & $\begin{array}{l}\text { Minimum brine pressure for } \\
\text { capillary modal } \mathrm{KPC}=3\end{array}$ & 31933 & 21-fob-96 & $Y$ & $4 B$ \\
\hline$Y$ & & 2349 & CL_L_T2 & $\begin{array}{l}\text { Lower Salado clay. } 10 \\
\text { to } 25 \text { years }\end{array}$ & PO_MIN & $\begin{array}{l}\text { Minimum brine pressure for } \\
\text { capillary model KPC=3 }\end{array}$ & 31933 & 21-fob-96 & $\mathbf{Y}$ & $4 B$ \\
\hline$Y$ & & 2349 & $C L_{-} L_{-} T 2$ & $\begin{array}{l}\text { Lower Salado clay: } 10 \\
\text { 10 } 25 \text { years }\end{array}$ & PO_MIN & $\begin{array}{l}\text { Minimum brine pressure for } \\
\text { capillary model KPC=3 }\end{array}$ & 31933 & 21.Feb-96 & $\mathbf{Y}$ & $4 B$ \\
\hline$Y$ & varies & 2351 & CL_L_T2 & $\begin{array}{l}\text { Lower Salado clay: } 10 \\
\text { to } 25 \text { years }\end{array}$ & PRMX_LOG & $\begin{array}{l}\text { Log of intrnsic permeabilty, X- } \\
\text { direction }\end{array}$ & 31935 & 14Feb-96 & $\mathbf{Y}$ & 1 \\
\hline$Y$ & varies & 2351 & CL_L_T2 & $\begin{array}{l}\text { Lower Salado chay: } 10 \\
\text { to } 25 \text { years }\end{array}$ & PRMX_LOG & $\begin{array}{l}\text { Log of intrnsic permeability, X- } \\
\text { direction }\end{array}$ & 31935 & 14.Feb-96 & $\mathbf{Y}$ & 1 \\
\hline Y & varies & 2351 & CL_L_T2 & $\begin{array}{l}\text { Lower Salado clay: } 10 \\
1025 \text { years }\end{array}$ & PRMX_LOG & $\begin{array}{l}\text { Log of intrinsic permeability, } X \text { - } \\
\text { direction }\end{array}$ & 31935 & 14-Fob-96 & $\mathbf{Y}$ & 1 \\
\hline$Y$ & vanes & 2351 & CL_L_T2 & $\begin{array}{l}\text { Lower Salado clay: } 10 \\
\text { to } 25 \text { years }\end{array}$ & PRMX_LOG & $\begin{array}{l}\text { Log of intrinsic permeability, } X- \\
\text { direction }\end{array}$ & 31935 & 14-Feb-96 & $Y$ & 1 \\
\hline$Y$ & varies & 2351 & CL_L_T2 & $\begin{array}{l}\text { Lower Salado clay: } 10 \\
\text { to } 25 \text { years }\end{array}$ & PRMX_LOG & $\begin{array}{l}\text { Log of intnnsic permeabilthy, } X \text { - } \\
\text { direction }\end{array}$ & 31935 & 14-Feb-9s & $\mathbf{Y}$ & 1 \\
\hline$Y$ & & 2352 & $\mathrm{CL}_{-} \mathrm{L}_{-} \mathrm{T} 2$ & $\begin{array}{l}\text { Lower Salado clay: } 10 \\
\text { to } 25 \text { years }\end{array}$ & PRMY_LOG & $\begin{array}{l}\text { Log of intrinsic permeabillty, Y- } \\
\text { direction }\end{array}$ & 31936 & 14Feb-96 & $Y$ & 1 \\
\hline Y & & 2352 & $C L_{-} L_{-} T 2$ & $\begin{array}{l}\text { Lower Salado clay: } 10 \\
\text { to } 25 \text { years }\end{array}$ & PRMY_LOG & $\begin{array}{l}\text { Log of intnnsic permeabilty, Y- } \\
\text { direction }\end{array}$ & 31936 & 14-Feb-96 & $Y$ & 1 \\
\hline $\mathbf{Y}$ & & 2352 & CL_L_T2 & $\begin{array}{l}\text { Lower Salado clay: } 10 \\
\text { to } 25 \text { years }\end{array}$ & PRMY_LOG & $\begin{array}{l}\text { Log of intrinsic permeabillty, Y- } \\
\text { direction }\end{array}$ & 31936 & 14-Feb-96 & $\mathbf{Y}$ & 1 \\
\hline$Y$ & & 2352 & $C L_{-} L_{-} T 2$ & $\begin{array}{l}\text { Lower Salado clay: } 10 \\
\text { to } 25 \text { years }\end{array}$ & PRMY_LOG & $\begin{array}{l}\text { Log of intrinsic permeabillty, } Y \text { - } \\
\text { direction }\end{array}$ & 31936 & 14-Fab-96 & $\mathbf{Y}$ & 1 \\
\hline $\mathbf{Y}$ & & 2352 & $C L \_L / T 2$ & $\begin{array}{l}\text { Lower Salado clay. } 10 \\
\text { to } 25 \text { years }\end{array}$ & PRMY_LOG & $\begin{array}{l}\text { Log of intrmsic permeability, Y- } \\
\text { direction }\end{array}$ & 31936 & 14-Feb-96 & $\mathbf{Y}$ & 1 \\
\hline$\gamma$ & & 2353 & CL_L_T2 & \begin{tabular}{|l|} 
Lower Salado clay. 10 \\
1025 years
\end{tabular} & PRMZ_LOG & $\begin{array}{l}\text { Log of intrinsic permeabillty, Z- } \\
\text { direction }\end{array}$ & 31937 & 14-Feb-96 & $\mathbf{Y}$ & 1 \\
\hline$Y$ & & 2353 & CL_L_T2 & $\begin{array}{l}\text { Lower Salado clay: } 10 \\
\text { 10 } 25 \text { years }\end{array}$ & PRMZ_LOG & $\begin{array}{l}\text { Log of intrinsie permeabilty, 2- } \\
\text { direction }\end{array}$ & 31937 & 14-Fob-96 & $--Y$ & 1 \\
\hline $\mathbf{Y}$ & & 2353 & CL_L_T2 & $\begin{array}{l}\text { Lower Salado clay: } 10 \\
\text { to } 25 \text { years }\end{array}$ & PRMZ_LOG & $\begin{array}{l}\text { Log of intrinsic permeabiltty, Z- } \\
\text { direction }\end{array}$ & 31937 & 14-Fob-96 & $Y$ & 1 \\
\hline $\mathbf{Y}$ & & 2353 & $C L$ L_T2 & \begin{tabular}{|l|} 
Lower Salado clay: 10 \\
to 25 years \\
\end{tabular} & PRMZ_LOG & $\begin{array}{l}\text { Log of intrnsic permeabilty, Z- } \\
\text { disection }\end{array}$ & 31937 & 14-Fob-96 & $\mathbf{Y}$ & 1 \\
\hline$\gamma$ & & 2353 & CL_L_T2 & $\begin{array}{l}\text { Lower Salado clay: } 10 \\
\text { to } 25 \text { years }\end{array}$ & PRMZ_LOG & $\begin{array}{l}\text { Log of intrinsic permeability, Z- } \\
\text { direction }\end{array}$ & 31937 & 14-Feb-96 & $\mathbf{Y}$ & 1 \\
\hline$Y$ & & 2357 & CL_L_T2 & $\begin{array}{l}\text { Lower Salado clay: } 10 \\
1025 \text { years }\end{array}$ & RELP_MOD & \begin{tabular}{|l|} 
Model number, relative \\
permeability model
\end{tabular} & $31962 A$ & 14 Feb-96 & $Y$ & 4B \\
\hline $\mathbf{Y}$ & & 2357 & $C L_{-} L_{-} T 2$ & $\begin{array}{l}\text { Lower Salado clay: } 10 \\
\text { to } 25 \text { years }\end{array}$ & RELP_MOD & \begin{tabular}{|l} 
Model number, relative \\
permeability model
\end{tabular} & $31962 A$ & 14-Feb-96 & $Y$ & 48 \\
\hline$Y$ & & 2357 & $\mathrm{CL}_{-} \mathrm{L}_{-} \mathrm{T} 2$ & \begin{tabular}{|l|} 
Lower Salaco clay: 10 \\
10 25 years
\end{tabular} & RELP_MOD & $\begin{array}{l}\text { Model number, relative } \\
\text { permeability model }\end{array}$ & $31962 A$ & 14 Feb-96 & $\mathbf{Y}$ & $4 B$ \\
\hline $\mathbf{Y}$ & & 2357 & CL_L_T2 & $\begin{array}{l}\text { Lower Salado clay } 10 \\
\text { to } 25 \text { years }\end{array}$ & RELP_MOD & $\begin{array}{l}\text { Model number, relative } \\
\text { permeability model }\end{array}$ & $31962 A$ & 14-Fob-96 & Y & 48 \\
\hline $\mathbf{Y}$ & & 2357 & CL_L_T2 & $\begin{array}{l}\text { Lower Salado clay: } 10 \\
\text { to } 25 \text { years }\end{array}$ & RELP_MOD & $\begin{array}{l}\text { Model number, relative } \\
\text { permeability model }\end{array}$ & $31962 A$ & $14-F e b-96$ & $\mathbf{Y}$ & 48 \\
\hline $\mathbf{Y}$ & 100 & 2359 & CL_L_T2 & $\begin{array}{l}\text { Lower Salado clay: } 10 \\
1025 \text { years }\end{array}$ & SAT_RBRN & Residual Brine Saturation & 31969 & $14-F e b-96$ & $Y$ & 1 \\
\hline $\mathbf{Y}$ & 100 & 2359 & CL_L_T2 & $\begin{array}{l}\text { Lower Salado clay: } 10 \\
\text { to } 25 \text { years }\end{array}$ & SAT_RBRN & Residual Brine Saturation & 31969 & 14feb-96 & $Y$ & 1 \\
\hline $\mathbf{Y}$ & 100 & 2359 & CL_L_T2 & $\begin{array}{l}\text { Lower Salado clay: } 10 \\
1025 \text { years }\end{array}$ & SAT_RBRN & Residual Brize Saturation & 31969 & $14-F a b-96$ & $\mathbf{Y}$ & 1 \\
\hline
\end{tabular}


CCA Parameter Listing

\begin{tabular}{|c|c|c|c|c|c|c|c|c|c|c|}
\hline ID & $\begin{array}{c}\text { Crmetin- } \\
\text { Catton of } \\
\text { Deta } \\
\text { Category } \\
\text { (lootrote 2) }\end{array}$ & $\begin{array}{c}\text { Dintarloution } \\
\text { Type }\end{array}$ & man & Medien & Minimen & Madron & Units & Reference ID & $\begin{array}{l}\text { Was the data } \\
\text { domiloped } \\
\text { underen } \\
\text { NeA-1 } \\
\text { Progren? }\end{array}$ & 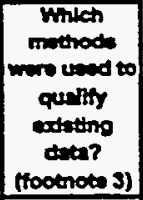 \\
\hline 2346 & 1,3 & CONSTANT & $1.0000000 E+08$ & $1.0000000 E+08$ & $1.0000000 E+08$ & $1.0000000 E+08$ & Pa & UPO40514 & $\mathbf{Y}$ & NA \\
\hline 2347 & 3,4 & CUMULATIVE & $2.5200000 E+\infty$ & $9.4000000 \mathrm{E}-01$ & $1.1000000 E-01$ & $8.1000000 E+\infty 0$ & NONE & WP030540 & $\mathbf{Y}$ & $N A$ \\
\hline 2347 & 3,4 & CUMULATIVE & $2.5200000 E+\infty 0$ & $9.4000000 E-01$ & $1.1000000 E-01$ & $8.1000000 E+\infty$ & NONE & WP030994 & $\mathbf{Y}$ & $N_{A}$ \\
\hline 2347 & 3,4 & CUMULATIVE & $2.5200000 E+\infty 0$ & $9.4000000 E-01$ & $1.1000000 E-01$ & $8.1000000 E+00$ & NONE & WP032287 & $Y$ & NA \\
\hline 2347 & 3,4 & CUMULATIVE & $2.5200000 E+\infty 0$ & $9.4000000 \mathrm{E}-01$ & 1.1000000 E-O1 & $8.1000000 E+\infty 0$ & NONE & WP035268 & $\mathbf{Y}$ & NA \\
\hline 2347 & 3,4 & CUMULATIVE & $25200000 E+\infty 0$ & $9.4000000 E-01$ & $1.1000000 E-01$ & $8.1000000 E+\infty$ & NONE & WP035597 & $\mathbf{Y}$ & N/A \\
\hline 2347 & 3,4 & CUMULATIVE & $2.5200000 E+\infty$ & $9.4000000 E-01$ & $1.1000000 E-01$ & 8. $1000000 E+\infty 0$ & NONE & WPO41558 & $\mathbf{Y}$ & NA \\
\hline 2348 & $1,3,4$ & CONSTANT & $2.4000000 E-01$ & $2.4000000 E-01$ & 2.4000000E-01 & $2.4000000 E-01$ & $m^{\wedge} 3 m^{\wedge} 3$ & WP030640 & $\mathbf{Y}$ & N/A \\
\hline 2348 & $1,3,4$ & CONSTANT & $24000000 E-01$ & 2.4000000E-01 & $2.4000000 E-01$ & 2.4000000E-01 & $m^{\wedge} 3 m^{\wedge}{ }^{\wedge}$ & WP030994 & $Y$ & NA \\
\hline 2348 & $1,3,4$ & CONSTANT & $2.4000000 E-01$ & 2.4000000E-01 & $2.4000000 E-01$ & $2.4000000 E-01$ & $m^{\wedge} 3 m^{n} 3$ & WP035597 & $\mathbf{Y}$ & NA \\
\hline 2349 & 1.3 & CONSTANT & $1.0132500 E+05$ & $1.0132500 E+05$ & $1.0132500 E+05$ & $1.0132500 E+05$ & $\mathrm{~Pa}$ & WP035597 & $\mathbf{Y}$ & $N A$ \\
\hline 2349 & 1,3 & CONSTANT & $1.0132500 E+05$ & $1.0132500 E+05$ & $1.0132500 E+05$ & $1.0132500 E+05$ & $\mathbf{P a}$ & WP036051 & $\mathbf{Y}$ & NA \\
\hline 2349 & 1,3 & CONSTANT & $1.0132500 E+05$ & $1.0132500 \mathrm{E}+05$ & $1.0132500 E+05$ & $1.0132500 E+05$ & $\mathrm{~Pa}$ & WP040514 & $\mathbf{Y}$ & NA \\
\hline 2351 & $1,3,4$ & TRLANGULAR & $-1.8867000 E+01$ & $-1.8301000 \mathrm{E}+01$ & $-2.1000000 E+01$ & $-1.7301000 E+01$ & $\log \left(m^{n} 2\right)$ & WP030640 & YEN & 1 \\
\hline 2351 & $1,3,4$ & TRLANGULAR & $-1.8867000 E+01$ & $-1.8301000 \mathrm{E}+01$ & $-2.1000000 E+01$ & $-1.7301000 E+01$ & $\log \left(m^{n} 2\right)$ & wposos94 & YaN & 1 \\
\hline 2351 & $1,3,4$ & TRLANGULAR & $-1.8867000 E+01$ & $-1.8301000 E+01$ & $-2.1000000 E+01$ & $-1.7301000 \Sigma+01$ & $\log \left(m^{\wedge} 2\right)$ & WP035268 & Y\&N & 1 \\
\hline 2351 & $1,3,4$ & TRLANGULAR & $-1.8857000 E+01$ & $-1.8301000 E+01$ & $-2.1000000 E+01$ & $-1.7301000 E+01$ & $\log \left(m^{n} 2\right)$ & WP035597 & Y\&N & 1 \\
\hline 2351 & $1,3,4$ & TRLANGULAR & $-1.8867000 E+01$ & $-1.8301000 E+01$ & $-2.1000000 E+01$ & $-1.7301000 E+01$ & $\log \left(m^{\wedge} 2\right)$ & WPO41558 & YaN & 1 \\
\hline 2352 & $1,3,4$ & TRIANGULAR & $-1.8857000 E+01$ & $-1.8301000 E+01$ & $-2.1000000 E+01$ & $-1.7301000 E+01$ & $\cos \left(m^{n} 2\right)$ & WP030640 & YaN & 1 \\
\hline 2352 & $1,3,4$ & TRLANGULAR & $-1.8867000 E+01$ & $-1.8301000 E+01$ & $-2.1000000 E+01$ & $-1.7301000 E+01$ & $\log \left(m^{\wedge} 2\right)$ & WP030994 & YaN & 1 \\
\hline 2352 & $1,3,4$ & TRLANGULAR & $-1.8867000 E+01$ & $-1.8301000 E+01$ & $-2.1000000 E+01$ & $-1.7301000 E+01$ & $\log \left(m^{\wedge} 2\right)$ & WP035268 & YaN & 1 \\
\hline 2352 & $1,3,4$ & TRLANGULAR & $-1.8867000 E+01$ & $-1.8301000 E+01$ & $-2.1000000 E+01$ & $-1.7301000 E+01$ & $\log \left(m^{\wedge} 2\right)$ & WP035597 & Y\&N & 1 \\
\hline 2352 & $1,3,4$ & TRIANGULAR & $-1.8867000 E+01$ & $-1.8301000 E+01$ & $-2.1000000 E+01$ & $-1.7301000 E+01$ & $\log \left(m^{\wedge} 2\right)$ & WPO41558 & Y $8 N$ & 1 \\
\hline 2353 & $1,3,4$ & TRLANGULAR & $-1,8867000 E+01$ & $-1.8301000 E+01$ & $-2.1000000 E+01$ & $-1.7301000 E+01$ & $\log \left(m^{n} 2\right)$ & WP030640 & Y\&N & 1 \\
\hline 2353 & $1,3,4$ & TRLANGULAR & $-1.8867000 E+01$ & $-1.8301000 E+01$ & $-2.1000000 E+01$ & $-1.7301000 E+01$ & $\log \left(m^{\wedge} 2\right)$ & WP030994 & ran & 1 \\
\hline 2353 & $1,3,4$ & TRLANGULAR & $-1.8867000 E+01$ & $-1.8301000 E+01$ & $-2.1000000 E+01$ & $-1.7301000 E+01$ & $\log \left(m^{\wedge} 2\right)$ & WP035268 & YaN & 1 \\
\hline 2353 & $1,3,4$ & TRIANGULAR & $-1.8867000 E+01$ & $-1.8301000 E+01$ & $-2.1000000 E+01$ & $-1.7301000 E+01$ & $\log \left(m^{\wedge} 2\right)$ & WP035597 & YaN & 1 \\
\hline 2353 & $1,3,4$ & TRLANGULAR & $-1.8867000 E+01$ & $-1.8301000 E+01$ & $-2.1000000 E+01$ & $-1.7301000 E+01$ & $\log \left(m^{\wedge} 2\right)$ & WPO41558 & YaN & 1 \\
\hline 2357 & $1,3,4$ & CONSTANT & $4.0000000 E+\infty$ & $4.0000000 E+\infty$ & $4.0000000 \mathrm{E}+\infty 0$ & $4.0000000 E+\infty 0$ & NONE & WP030640 & $\mathbf{Y}$ & N/A \\
\hline 2357 & $1,3,4$ & CONSTANT & $4.0000000 \mathrm{E}+\infty 0$ & $4.0000000 E+\infty$ & $4.0000000 \mathrm{E}+\infty$ & $4.0000000 E+\infty$ & NONE & WP030994 & $\mathbf{Y}$ & NA \\
\hline 2357 & $1,3,4$ & CONSTANT & $4.0000000 E+\infty 0$ & $4.0000000 E+\infty$ & $4.00000000 E+\infty 0$ & $4.0000000 E+\infty$ & NONE & WP035597 & $\mathbf{Y}$ & NA \\
\hline 2357 & $1,3,4$ & CONSTANT & $4.0000000 E+00$ & $4.0000000 E+\infty 0$ & $4.0000000 E+00$ & $4.0000000 \mathrm{E}+\infty 0$ & NONE & WP040486 & $\mathbf{Y}$ & $N A$ \\
\hline 2357 & $1,3,4$ & CONSTANT & $4.0000000 E+\infty$ & $4.0000000 E+\infty$ & $4.0000000 E+00$ & $4.0000000 E+\infty$ & NONE & WP040514 & $\mathbf{Y}$ & $N / A$ \\
\hline 2359 & 3,4 & CUMULATIVE & $2.5000000 E-01$ & $2.0000000 E-01$ & $0.0000000 E+\infty$ & $6.0000000 E-01$ & NONE & WP030640 & $\mathbf{Y}$ & N/A \\
\hline 2359 & 3,4 & CUMULATIVE & 2.5000000E-01 & $2.0000000 E-01$ & $0.0000000 E+\infty 0$ & $6.0000000 E-01$ & NONE & WP030994 & $\mathbf{Y}$ & NA \\
\hline 2359 & 3,4 & CUMULATIVE & $2.5000000 E-01$ & 2.0000000 E- 01 & $0.0000000 E+\infty 0$ & $6.0000000 E-01$ & NONE & WP035268 & $Y$ & NA \\
\hline
\end{tabular}


CCA Parameter-Listing

\begin{tabular}{|c|c|c|c|c|c|c|c|c|c|c|}
\hline 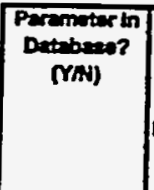 & 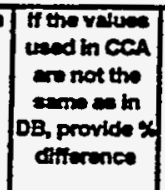 & Do & Maturivio & Mrtaren Nam & ${ }_{\mathbb{1 0}}^{\text {Permon }}$ & Parnotir Momo & $\begin{array}{l}\text { PRP ID } \\
\text { (MPO \#) }\end{array}$ & $\begin{array}{c}\text { Dententry } \\
\text { Dewe }\end{array}$ & $\begin{array}{l}\text { to the } 464 \\
\text { conzalatent } \\
\text { with the } \\
\text { permeter } \\
\text { in DB? } \\
\text { M, NUA) }\end{array}$ & $\begin{array}{l}\text { Parmomoter } \\
\text { Cutwory } \\
\text { (footiote 1) }\end{array}$ \\
\hline $\mathbf{Y}$ & 100 & 2359 & CL_L_T2 & $\begin{array}{l}\text { Lower Selado clay. } 10 \\
\text { to } 25 \text { years }\end{array}$ & SAT_RBRN & Residual Brino Seturation & 31969 & $14 f-b-56$ & $\mathbf{Y}$ & 1 \\
\hline$Y$ & 100 & 2359 & CL_L_T2 & $\begin{array}{l}\text { Lower Salad ctay: } 10 \\
\text { to } 25 \text { vears }\end{array}$ & SAT_RBRN & Residial Brre Seturation & 31969 & $14 f-0006$ & $\mathbf{Y}$ & 1 \\
\hline $\mathbf{Y}$ & 100 & 2359 & CL_L_T2 & $\begin{array}{l}\text { Lower Salaco clay: } 10 \\
\text { to } 25 \text { yeers }\end{array}$ & SAT_RBRM & Rescidual Brine Seturation & 31969 & $14-\infty b-95$ & $\mathbf{Y}$ & 1 \\
\hline $\mathbf{Y}$ & 100 & 2360 & $C L_{-} L_{-} T 2$ & $\begin{array}{l}\text { Lower Stado ctry:10 } \\
\text { to } 25 \text { years }\end{array}$ & SAT_REAS & Revidual Ges Suturation & 31971 & $\mid 14$ fab-96 & $\mathbf{Y}$ & 1 \\
\hline $\mathbf{Y}$ & 100 & 2360 & $C L L_{-} T 2$ & $\begin{array}{l}\text { Lower Saludo clay. } 10 \\
\text { to } 25 \text { years }\end{array}$ & SAT_RGAS & Residusal Gus Sturation & 31971 & $14+40-96$ & $\mathbf{Y}$ & 1 \\
\hline Y & 100 & 2360 & $C L_{-} L_{-} T 2$ & $\begin{array}{l}\text { Lowor Salaso clay: } 10 \\
\text { to } 25 \text { years }\end{array}$ & SAT_RGAS & Residual Gas Saturation & 31971 & $14 F-b-96$ & $\mathbf{Y}$ & 1 \\
\hline$Y$ & 100 & 2350 & $C L_{-} L_{-} T 2$ & $\begin{array}{l}\text { Lowor Salado clay: } 10 \text {. } \\
\text { to } 25 \text { years }\end{array}$ & SAT_RGAS & Resicual Ges Seturation & 31971 & $14 F=0-86$ & $\mathbf{Y}$ & 1 \\
\hline $\mathbf{Y}$ & 100 & 2380 & CL_L_T2 & $\begin{array}{l}\text { Lower Salaco clay: } 10 \\
1025 \text { years }\end{array}$ & SAT_RGAS & Residual Gas Seturation & 31971 & $14-F a b-96$ & $Y$ & 1 \\
\hline$Y$ & 50 & 2361 & $C L_{-} L_{-} T 3$ & $\begin{array}{l}\text { Lower Saloco clay.25 } \\
\text { to } 50 \text { years }\end{array}$ & CAP_MOD & $\begin{array}{l}\text { Hodel number, capillery pressurno } \\
\text { model }\end{array}$ & $31974 A$ & $14+\infty b-96$ & $\mathbf{Y}$ & 48 \\
\hline $\mathbf{Y}$ & 50 & 2361 & CL_L_T3 & $\begin{array}{l}\text { Lowor Selado clay:25 } \\
\text { to } 50 \text { years }\end{array}$ & CAP_MOD & $\begin{array}{l}\text { Model number, capillery pressure } \\
\text { modol }\end{array}$ & 319744 & 14 Feo-9s & $\mathbf{Y}$ & 48 \\
\hline $\mathbf{Y}$ & 50 & 2361 & CL_L_T3 & $\begin{array}{l}\text { Lower Sabado clay.25 } \\
\text { to } 50 \text { years }\end{array}$ & CAP_MOD & $\begin{array}{l}\text { Model number, capillary pressure } \\
\text { model }\end{array}$ & $31974 a$ & 14Feb-96 & $\mathbf{Y}$ & 48 \\
\hline$Y$ & 50 & 2361 & CL_L_T3 & $\begin{array}{l}\text { Lower Salado clay: } 25 \\
\text { to } 50 \text { years } \\
\end{array}$ & CAP_MOD & $\begin{array}{l}\text { Modol number, capilley pressure } \\
\text { model }\end{array}$ & $31974 A$ & $14 F \infty b-96$ & $\gamma$ & $4 B$ \\
\hline $\mathbf{Y}$ & 50 & 2361 & CL_L_T3 & $\begin{array}{l}\text { Lower Salado clay.25 } \\
\text { to } 50 \text { years }\end{array}$ & CAP_MOD & $\begin{array}{l}\text { Model number, capilimy pressuro } \\
\text { modol }\end{array}$ & 319744 & $14 F-b-96$ & $\mathbf{Y}$ & 48 \\
\hline $\mathbf{Y}$ & & 2362 & $C L_{2} L_{-} T 3$ & $\begin{array}{l}\text { Lower Salado clay:25 } \\
\text { to } 50 \text { years }\end{array}$ & COMP_RCK & Buk Compressibility & 31976 & $14 F=b-96$ & $\mathbf{Y}$ & 1 \\
\hline$\gamma$ & & 2362 & CL_L_T3 & $\begin{array}{l}\text { Lower Sabodo diay:25 } \\
\text { to } 50 \text { years }\end{array}$ & COMP_RCK & Butk Comprossibility & 31976 & 14-Fob-96 & $\mathbf{Y}$ & 1 \\
\hline$Y$ & & 2362 & CL_L_T3 & $\begin{array}{l}\text { Lowor Selado clay.25 } \\
\text { to } 50 \text { years }\end{array}$ & COMP_RCK & Buk Compressibility & 31976 & $14 F=0-96$ & $\mathbf{Y}$ & 1 \\
\hline$Y$ & & 2363 & CL_L_T3 & $\begin{array}{l}\text { Lower Salado chay } 25 \\
\text { to } 50 \text { years }\end{array}$ & PC_max & $\begin{array}{l}\text { Maximum allowable capiliary } \\
\text { pressure }\end{array}$ & $31981 A$ & $14 f a b-96$ & $\mathbf{Y}$ & $A B$ \\
\hline $\mathbf{Y}$ & & 2363 & CL_L_T3 & $\begin{array}{l}\text { Lower Salado clay:25 } \\
\text { to } 50 \text { years }\end{array}$ & PC_MAX & $\begin{array}{l}\text { Maxmum allowabte capillary } \\
\text { prossuro }\end{array}$ & $31981 A$ & 14 fed-96 & $\mathbf{Y}$ & 18 \\
\hline Y & & 2363 & CL_L_T3 & $\begin{array}{l}\text { Lower Salado day.25 } \\
\text { to } 50 \text { years }\end{array}$ & PC_max & $\begin{array}{l}\text { Mexumum allowable capillary } \\
\text { pressuro }\end{array}$ & 31981A & 14 fab. 56 & $\mathbf{Y}$ & AB \\
\hline $\mathbf{Y}$ & & 2363 & CL_L_T3 & $\begin{array}{l}\text { Lower Salado day.25 } \\
\text { to } 50 \text { years }\end{array}$ & PC_max & $\begin{array}{l}\text { Maximum allowable capillary } \\
\text { pressure }\end{array}$ & $31981 A$ & 14fob-96 & $\mathbf{Y}$ & $4 B$ \\
\hline$Y$ & & 2363 & CL_L_T3 & \begin{tabular}{|l|} 
Lower Salaso chay 25 \\
to 50 years
\end{tabular} & PC_max & $\begin{array}{l}\text { Meximum allowable capillary } \\
\text { pressure }\end{array}$ & $31981 A$ & 14-Fob-96 & $\mathbf{Y}$ & $A B$ \\
\hline$Y$ & varies & 2364 & CL_L_T3 & $\begin{array}{l}\text { Lower Saledo diny.25 } \\
\text { to } 50 \text { yaers }\end{array}$ & PORE_DIS & $\begin{array}{l}\text { Brooke-Coroy poro distribution } \\
\text { perameter }\end{array}$ & 31982 & $20-f a b-96$ & $\mathbf{Y}$ & 1 \\
\hline$Y$ & varies & 2364 & CL_L_T3 & $\begin{array}{l}\text { Lower Salidos etry:25 } \\
\text { to } 50 \text { years }\end{array}$ & PORE_DIS & $\begin{array}{l}\text { Brooks-Corey pore distroution } \\
\text { parameter }\end{array}$ & 31982 & 20 Feb-96 & $\mathbf{Y}$ & 1 \\
\hline$Y$ & varies & 2364 & CL_L_T3 & $\begin{array}{l}\text { Lower Seledo day.25 } \\
\text { to } 50 \text { years }\end{array}$ & PORE_OIS & $\begin{array}{l}\text { Brooks-Coroy pore distrioution } \\
\text { parameter }\end{array}$ & 31982 & 20-Fob-96 & $\mathbf{Y}$ & 1 \\
\hline$Y$ & varies & 2364 & $C L_{-} L_{-} T 3$ & $\begin{array}{l}\text { Lower Saloco elay:25 } \\
\text { to } 50 \text { years }\end{array}$ & PORE_DIS & $\begin{array}{l}\text { Brooks-Corey pore distribution } \\
\text { percumetor }\end{array}$ & 31982 & $20-50 b-96$ & $-y$ & 1 \\
\hline$\gamma$ & varies & 2364 & CL_L_T3 & $\begin{array}{l}\text { Lower Saloob clay.25 } \\
\text { to } 50 \text { years }\end{array}$ & PORE_DIS & $\begin{array}{l}\text { Brooks-Corey pore distribution } \\
\text { paremetor }\end{array}$ & 31982 & $20-f e b-96$ & $\mathbf{Y}$ & 1 \\
\hline $\mathbf{Y}$ & verios & 2364 & CL_L_T3 & $\begin{array}{l}\text { Lower Subado day:25 } \\
\text { to } 50 \text { year }\end{array}$ & PORE_DIS & $\begin{array}{l}\text { Brooke-Corvy pore distribution } \\
\text { parnmetor }\end{array}$ & 31982 & $20-F a b-96$ & $\mathbf{Y}$ & 1 \\
\hline $\mathbf{Y}$ & & 2365 & CL_L_T3 & $\begin{array}{l}\text { Lower Saledo clay. } 25 \\
\text { to } 50 \text { years }\end{array}$ & POROSIT & Effective porasity & 31984 & $14 F 06-96$ & $\mathbf{Y}$ & 1 \\
\hline $\mathbf{Y}$ & & 2365 & $C C_{-} L_{-} T 3$ & $\begin{array}{l}\text { Lower Saludo ctay } 25 \\
\text { to } 50 \text { yasers }\end{array}$ & POROSTYY & Efroctive porosity & 31984 & $14 f-b-96$ & $\mathbf{Y}$ & 1 \\
\hline $\mathbf{Y}$ & & 2365 & $C L_{-} L_{-} T 3$ & $\begin{array}{l}\text { Lower Seledol etr.25 } \\
\text { to } 50 \text { years }\end{array}$ & POROSTYY & Effective porosity & 31884 & 14 fab-96 & $\mathbf{Y}$ & 1 \\
\hline$Y$ & & 2366 & CL_L_T3 & $\begin{array}{l}\text { Lower Selecos clay.25 } \\
\text { to } 50 \text { years }\end{array}$ & PO_MIN & $\begin{array}{l}\text { Mnimum brime pressure for } \\
\text { capitlary model KPC }=3\end{array}$ & 31885 & $21+c b-96$ & $\mathbf{Y}$ & 48 \\
\hline $\mathbf{Y}$ & & 2366 & $C L \_L / T 3$ & $\begin{array}{l}\text { Low Saledo clay.25 } \\
\text { to } 50 \text { years }\end{array}$ & PO_MIN & $\begin{array}{l}\text { Mrmum brine pressure for } \\
\text { capillary model KPC=3 }\end{array}$ & 31985 & 21 fob- 86 & $\mathbf{Y}$ & 48 \\
\hline$r$ & & 2366 & $C L_{-} L_{-} T 3$ & $\begin{array}{l}\text { Lover Seledo clay:25 } \\
\text { to } 50 \text { years }\end{array}$ & PO_MIN & $\begin{array}{l}\text { Mrnmum brme pressure for } \\
\text { capiliery model KP C }=3\end{array}$ & 31895 & 21-Fob-95 & $\mathbf{Y}$ & $4 B$ \\
\hline$Y$ & varies & 2368 & $C L_{-} L_{-} T 3$ & $\begin{array}{l}\text { Lower Saludo ciay.25 } \\
\text { to } 50 \text { yeans }\end{array}$ & PRMX_LOG & $\begin{array}{l}\text { Los of mbmsxe permeability, } x \text { - } \\
\text { dincetion }\end{array}$ & 31990 & 14 Feb-96 & $\mathbf{Y}$ & 1 \\
\hline$Y$ & varias & 2368 & CL_L_T3 & $\begin{array}{l}\text { Lomer Seladoo eny.25 } \\
\text { to } 50 \text { yenes }\end{array}$ & PRMX_LOG & 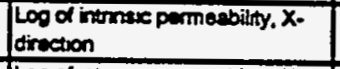 & 31990 & $14 F 00-28$ & $\mathbf{Y}$ & 1 \\
\hline$Y$ & varies & 2368 & CL_L_T3 & $\begin{array}{l}\text { Lower Selodo etry:25 } \\
\text { to } 50 \text { years }\end{array}$ & PRMX_LOG & $\begin{array}{l}\text { Log of mitnse permeabilty, X- } \\
\text { direction }\end{array}$ & 31990 & 14-Fab-s6 & $Y$ & 1 \\
\hline
\end{tabular}


CCA Parameter-Listing

\begin{tabular}{|c|c|c|c|c|c|c|c|c|c|c|}
\hline ID & $\begin{array}{c}\text { Cieseth- } \\
\text { cation of } \\
\text { Orta } \\
\text { Category } \\
\text { (footriote 2) }\end{array}$ & $\begin{array}{c}\text { Dietribution } \\
\text { Type }\end{array}$ & Man & Medien & Mindmum & Dodmura & Unite & Reternese id & $\begin{array}{l}\text { Wes the data } \\
\text { dumloped } \\
\text { underen } \\
\text { NoA-1 } \\
\text { Progrem? }\end{array}$ & $\begin{array}{c}\text { Which } \\
\text { mothods } \\
\text { more used to } \\
\text { quethy } \\
\text { oxisting } \\
\text { dita? } \\
\text { (tootnote 3) }\end{array}$ \\
\hline 2359 & 3,4 & CUMULATINE & 2.5000000E-01 & $2.0000000 E-01$ & 0.0000000 E $+\infty$ & $6.0000000 E-01$ & NONE & WP035597 & $\mathbf{Y}$ & NA \\
\hline 2359 & 3.4 & CUMULATIVE & $25000000 E-01$ & $2.0000000 \mathrm{E}-01$ & $0.0000000 E+\infty$ & 6.0000000E-01 & NONE & WPO41558 & $\mathbf{Y}$ & NA \\
\hline 2359 & 3,4 & CUMULATIVE & $2.5000000 E-01$ & $2.0000000=01$ & $0.0000000 E+\infty 0$ & 6.0000000E-01 & NONE & WPO42751 & $\mathbf{Y}$ & NA \\
\hline 2360 & 3,4 & UNIFORM & $2.0000000 E-01$ & $2,0000000 E-01$ & $0.0000000 E+\infty 0$ & 4.0000000E-08 & NONE & WP030640 & $\mathbf{Y}$ & NA \\
\hline 2360 & 3,4 & UNIFORM & 2.0000000E-01 & $2.0000000=-01$ & $0.0000000 E+\infty$ & 4.0000000E-01 & NONE & WP030994 & $\mathbf{Y}$ & NA \\
\hline 2360 & 3.4 & UNIFORM & $2.0000000 E-01$ & $20000000 E-01$ & $0.0000000 \approx+\infty$ & $4.0000000 E-01$ & NONE & WP035268 & $\mathbf{Y}$ & NA \\
\hline 2360 & 3,4 & UNIFORM & $2.0000000 E-01$ & 20000000E-01 & - $0.0000000 \leq+\infty 0$ & $4.0000000 E-01$ & NONE & WP035597 & $\mathbf{Y}$ & NA \\
\hline 2360 & 3,4 & UNIFORM & $2.0000000 E-01$ & 20000000E-01 & $0.0000000=+\infty$ & $4.0000000 E-01$ & NONE. & WPOS1558 & $\mathbf{Y}$ & N/A \\
\hline 2361 & $1,3,4$ & CONSTANT & $2.0000000 E+\infty 0$ & $2,0000000 E+\infty$ & $20000000 E+\infty 0$ & $2.0000000 E+\infty 0$ & NONE & WP030640 & $\mathbf{Y}$ & NA \\
\hline 2361 & $1,3,4$ & CONSTANT & $20000000 E+00$ & $20000000 E+\infty$ & $20000000 E+\infty 0$ & $2.0000000 E+\infty$ & NONE & WP030994 & $\mathbf{Y}$ & N/A \\
\hline 2361 & $1,3,4$ & CONSTANT & $2.0000000 E+00$ & $2.0000000 E+00$ & $2.0000000 E+\infty 0$ & $2,0000000 E+\infty$ & NONE & WP035597 & $\mathbf{Y}$ & NA \\
\hline 2361 & $1,3,4$ & CONSTANT & $2.0000000 E+\infty 0$ & $2.0000000 E+\infty 0$ & $20000000 \leqslant+\infty$ & $2,0000000 E+\infty 0$ & NONE & WPO40485 & $Y$ & $N / A$ \\
\hline 2361 & $1,3,4$ & CONSTANT & $2.0000000 E+\infty 0$ & $2,0000000 E+\infty 0$ & $2.0000000 E+\infty$ & $2.0000000 E+\infty$ & NONE & WP040514 & $\mathbf{Y}$ & NA \\
\hline 2362 & $1,3,4$ & CONSTANT & $1.5900000 E-09$ & $1.5900000 \mathrm{E}-09$ & $1.5900000 E-09$ & $1.5900000 \mathrm{E}-09$ & Pan-1 & WPOSOS40 & $\mathbf{Y}$ & NAA \\
\hline 2362 & $1,3,4$ & CONSTANT & $1.5900000 E-09$ & $1.5900000 \mathrm{E}-09$ & $1.5900000 E-09$ & $1.5900000 E-09$ & $P x^{n}-9$ & WPO30994 & $\mathbf{Y}$ & NA \\
\hline 2362 & $1,3,4$ & CONSTANT & $1.5900000 E-09$ & $1.5900000 E-09$ & $1.5900000 E-09$ & $1.5900000 E-09$ & $P a^{n}-1$ & WP035597 & $\mathbf{Y}$ & $N A$ \\
\hline 2363 & 1,3 & CONSTANT & $1.0000000 E+08$ & $1.0000000 E+08$ & $1.0000000 E+\infty 8$ & $1.0000000 E+09$ & Pa & WP030640 & $Y$ & N/A \\
\hline 2363 & 1,3 & CONSTANT & $1.0000000 E+08$ & $1.0000000 E+08$ & $1.0000000 E+00$ & $1.0000000 E+08$ & Pa & WP030994 & $\mathbf{Y}$ & NA \\
\hline 2363 & 1,3 & CONSTANT & $1.0000000 E+08$ & $1.0000000 E+08$ & $1.0000000 E+08$ & $1.0000000 E+08$ & Pa & WP035597 & $Y$ & NA \\
\hline 2363 & 1,3 & CONSTANT & $1.0000000 E+08$ & $1.0000000 E+08$ & $1.0000000 \equiv+08$ & $1.0000000 E+08$ & $\mathbf{P a}$ & WP040486 & $\mathbf{Y}$ & NA \\
\hline 2363 & 1,3 & CONSTANT & $1.0000000 E+08$ & $1.0000000 E+08$ & $9.0000000 E+08$ & $1.0000000 E+08$ & $\mathbf{P a}$ & WP040514 & $\mathbf{Y}$ & NA \\
\hline 2364 & 3,4 & CUMULATINE & $2.5200000 E+\infty 0$ & $9.4000000 E-01$ & $1.1000000 E-01$ & $8.1000000 \mathrm{E}+00$ & NONE & WP030640 & $\mathbf{Y}$ & NA \\
\hline 2364 & 3,4 & CUMULATINE & $25200000 E+\infty 0$ & $9.4000000 E-01$ & $1.1000000 E-01$ & $8.1000000 E+\infty 0$ & NONE & WP030994 & $\mathbf{Y}$ & NA \\
\hline 2364 & 3,4 & CUMULATINE & $2.5200000 E+\infty$ & 9.4000000E-01 & $1.1000000 E-01$ & $8.1000000 E+\infty$ & NONE & WP032287 & $\mathbf{Y}$ & N/A \\
\hline 2364 & 3,4 & CUMULATIVE & $2.5200000 E+\infty$ & $9.4000000 E-01$ & $1.1000000 E-01$ & $8.1000000 E+00$ & NONE & WP035268 &.$- Y$ & NA \\
\hline 2364 & 3,4 & CUMULATNE & $2.5200000 E+\infty$ & $9.4000000 E-01$ & $1.1000000 E-01$ & $8.1000000 E+\infty 0$ & NONE & WP035597 & $\mathbf{Y}$ & NA \\
\hline 2364 & 3,4 & CUMULATIVE & $2.5200000 E+\infty$ & $9.4000000 E-01$ & $1.1000000 \mathrm{E}-01$ & $8.1000000 E+00$ & NONE & WPO41558 & $\mathbf{Y}$ & NAA \\
\hline 2365 & $1,3.4$ & CONSTANT & 2.4000000E-01 & $2.4000000 E-01$ & $2.4000000 E-01$ & $2.4000000 E-01$ & $m^{\wedge} 3 m^{\prime \wedge} 3$ & WP030640 & $\mathbf{Y}$ & $N A$ \\
\hline 2365 & $1,3,4$ & CONSTANT & 2.4000000E-01 & $2.4000000 E-01$ & $2.4000000 E-01$ & $2.4000000 E-01$ & $m^{\wedge} 3 m^{\wedge} 3$ & WP030994 & $\mathbf{Y}$ & NA \\
\hline 2365 & $1,3,4$ & CONSTANT & 2.4000000E-01 & $2.4000000 E-01$ & $2.4000000 E-01$ & 2.4000000E-01 & $m^{\wedge} 3 m^{\wedge} \beta$ & WP035597 & $\mathbf{Y}$ & $N / A$ \\
\hline 2366 & 1,3 & CONSTANT & $1.0132500 E+05$ & $1.0132500 E+05$ & $1.0132500 E+05$ & $1.0132500 E+05$ & $\mathbf{P a}$ & WP035597 & $\mathbf{Y}$ & N/A \\
\hline 2366 & 1,3 & CONSTANT & $1.0132500 E+05$ & $1.0132500 E+05$ & $1.0132500 E+05$ & $1.0132500 E+05$ & $\mathrm{~Pa}$ & WPO36051 & $\mathbf{Y}$ & NA \\
\hline 2366 & 1,3 & CONSTANT & $1.0132500 E+05$ & $1.0132500 E+05$ & $1.0132500 E+05$ & $1.0132500 E+05$ & $\mathbf{P a}$ & WP040514 & $\mathbf{Y}$ & NA \\
\hline 2368 & $1,3,4$ & TRIANGULAR & $-1,8867000 E+01$ & $-1.6301000 E+01$ & $-2.1000000 E+01$ & $-1.7301000 E+01$ & $\log \left(m^{\wedge} 2\right)$ & WP030640 & YEN & 1 \\
\hline 2368 & $1,3,4$ & TRIANGULAR & $-1.8867000 E+01$ & $-1.8301000 E+01$ & $-2.1000000 E+01$ & $-1.7301000 E+01$ & $\log \left(m^{\wedge} 2\right)$ & WP030994 & YaN & 1 \\
\hline 2368 & $1,3,4$ & TRLANGULAR & $-1,8867000 E+01$ & $-1.8301000 E+01$ & $-2.1000000 E+01$ & $-1.7301000 E+01$ & $\log \left(m^{\wedge} 2\right)$ & WP035268 & YEN & 1 \\
\hline
\end{tabular}


CCA Parameter Listing -

\begin{tabular}{|c|c|c|c|c|c|c|c|c|c|c|}
\hline 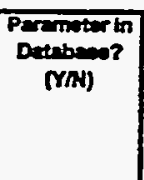 & 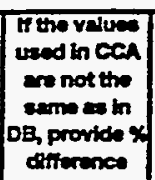 & ID & metarial id & Weterta Name & $\begin{array}{c}\text { Parmoter } \\
\text { to }\end{array}$ & 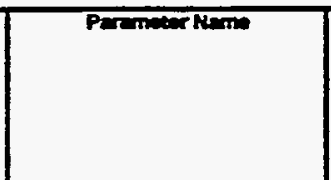 & $\begin{array}{l}\text { PRPD DO } \\
\text { MPO }\end{array}$ & $\begin{array}{c}\text { Detantry } \\
\text { Deto }\end{array}$ & 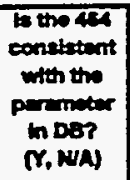 & $\begin{array}{l}\text { Perwinter } \\
\text { Cetegory } \\
\text { (footiote 1) }\end{array}$ \\
\hline$Y$ & varies & 2368 & CL_L_T3 & $\begin{array}{l}\text { Lower Salado clay:25 } \\
\text { to } 50 \text { years }\end{array}$ & PRHX_LOG & $\begin{array}{l}\text { Log of mtrinsx permasbibty, X- } \\
\text { direction }\end{array}$ & 31990 & $14 f 00-96$ & $\mathbf{Y}$ & 1 \\
\hline $\mathbf{Y}$ & varies & 2368 & CL_L_T3 & $\begin{array}{l}\text { Lower Satodo diay.25 } \\
\text { to } 50 \text { yours }\end{array}$ & PRMX_LOG & $\begin{array}{l}\text { Log of nirnsx permenbility, } X \text { - } \\
\text { dinuction }\end{array}$ & 31990 & $14+00-05$ & $\mathbf{Y}$ & 1 \\
\hline$Y$ & & 2369 & CL_L_T3 & $\begin{array}{l}\text { Lower Seleco clay:25 } \\
\text { to } 50 \text { years }\end{array}$ & PRIY_LOG & $\begin{array}{l}\text { Log of ntrmsxe permenabihy. Y- } \\
\text { direction }\end{array}$ & 31992 & 14Feb-96 & $\mathbf{Y}$ & 1 \\
\hline $\mathbf{Y}$ & & 2369 & CL_L_T3 & $\begin{array}{l}\text { Lower Saledo day.25 } \\
\text { to } 50 \text { years }\end{array}$ & PRMY_LOG & $\begin{array}{l}\text { Log of intrinsic permasbilty, Y- } \\
\text { direction }\end{array}$ & 31992 & $147 c b-96$ & $\mathbf{Y}$ & 1 \\
\hline $\mathbf{Y}$ & & 2369 & CL_L_T3 & $\begin{array}{l}\text { Lowor Selaco diay:25 } \\
\text { to } 50 \text { years }\end{array}$ & PRMY_LOG & $\begin{array}{l}\text { Loos of ntrons } x \text { permoabulty, Y- } \\
\text { direction }\end{array}$ & 31892 & 14Feb-96 & $\mathbf{Y}$ & 1 \\
\hline $\mathbf{Y}$ & & 2369 & CL_L_T3 & $\begin{array}{l}\text { Lawer } 5 \text { staco ctay. } 25 \\
\text { to } 50 \text { years }\end{array}$ & PRMY_LOG & $\begin{array}{l}\text { Loo of ntrmsse permosebility, Y- } \\
\text { drection }\end{array}$ & 31992 & 14 Fab-96 & $\mathbf{Y}$ & 1 \\
\hline $\mathbf{Y}$ & & 2369 & CL_L_T3 & $\begin{array}{l}\text { Lowor Salaco day:25 } \\
\text { to } 50 \text { years }\end{array}$ & PRMY_LOG & $\begin{array}{l}\text { Log of mitresse permeability, Y- } \\
\text { drection }\end{array}$ & 31892 & $14 F a b-96$ & $\mathbf{Y}$ & 1 \\
\hline$\gamma$ & & 2370 & CL_L_T3 & $\begin{array}{l}\text { Lower Saladoc day.25 } \\
\text { to } 50 \text { yoars }\end{array}$ & PRMZ_LOG & $\begin{array}{l}\text { Log of intronste permesbility, 2- } \\
\text { drection }\end{array}$ & 31993 & 14Feb-96 & $\mathbf{Y}$ & 1 \\
\hline $\mathbf{Y}$ & & 2370 & CL_L_T3 & $\begin{array}{l}\text { Lower Salado clay:25 } \\
\text { to } 50 \text { years }\end{array}$ & PRMZ_LOG & $\begin{array}{l}\text { Log of intmsac permeability, Z- } \\
\text { direction }\end{array}$ & 39993 & $14 f 0 b-96$ & $Y$ & 1 \\
\hline $\mathbf{Y}$ & & 2370 & CL_L_T3 & $\begin{array}{l}\text { Lowor Salado ciny. } 25 \\
\text { to } 50 \text { years }\end{array}$ & PFUI_LOG & $\begin{array}{l}\text { Log of intonsic permesbility, Z- } \\
\text { direction }\end{array}$ & 31993 & 14 fab-96 & $\mathbf{Y}$ & 1 \\
\hline $\mathbf{Y}$ & & 2370 & CL_L_T3 & $\begin{array}{l}\text { Lower Salado clay } 25 \\
\text { to } 50 \text { years }\end{array}$ & PRMZ_LOG & $\begin{array}{l}\text { Log of ntrmsx permesbilty. Z- } \\
\text { direction }\end{array}$ & 31993 & $14 F \infty 0-96$ & $\mathbf{Y}$ & 1 \\
\hline $\mathbf{Y}$ & & 2370 & CL_L_T3 & $\begin{array}{l}\text { Lower Salado clay.25 } \\
\text { to } 50 \text { years }\end{array}$ & PRMZ_LOG & $\begin{array}{l}\text { Log of mimsac permestitity, Z- } \\
\text { droction }\end{array}$ & 31993 & $14 F e b-96$ & $\mathbf{r}$ & 1 \\
\hline $\mathbf{Y}$ & & 2374 & CL_L_T3 & $\begin{array}{l}\text { Lower Salado clay:25 } \\
\text { to } 50 \text { years }\end{array}$ & RELP_MOD & \begin{tabular}{|l|} 
Mocol number, relativer \\
Dermeabilty model
\end{tabular} & 31998A & 14 feb-96 & $\mathbf{Y}$ & 48 \\
\hline $\mathbf{Y}$ & & 2374 & CL_L_T3 & $\begin{array}{l}\text { Lower Salado clay:25 } \\
\text { to } 50 \text { years }\end{array}$ & RELP_MOD & $\begin{array}{l}\text { Modol number, relative } \\
\text { permosbility model }\end{array}$ & $31998 A$ & 14fob-96 & $\mathbf{Y}$ & 48 \\
\hline $\mathbf{Y}$ & & 2374 & $C L_{-} L_{-} T 3$ & $\begin{array}{l}\text { Lower Salaco clay: } 25 \\
\text { to } 50 \text { years }\end{array}$ & RELP_MOO & $\begin{array}{l}\text { Model number, rolative } \\
\text { permeability mode! }\end{array}$ & $31998 A$ & $14 f a b-96$ & $\mathbf{Y}$ & AB \\
\hline $\mathbf{Y}$ & & 2374 & CL_L_T3 & $\begin{array}{l}\text { Lower Salado day.25 } \\
\text { to } 50 \text { yesss }\end{array}$ & RELP_MOD & $\begin{array}{l}\text { Model number, relative } \\
\text { permesbilty model }\end{array}$ & 31998A & $14 F \cos 9$ & $\mathbf{Y}$ & 48 \\
\hline $\mathbf{Y}$ & & 2374 & CL_L_T3 & $\begin{array}{l}\text { Lower Salado clay:25 } \\
1050 \text { years }\end{array}$ & RELP_MOD & $\begin{array}{l}\text { Model number. relative } \\
\text { pormoabulity modol }\end{array}$ & $31998 A$ & 14 fab-96 & $\mathbf{Y}$ & 48 \\
\hline $\mathbf{Y}$ & 100 & 2376 & CL_L_T3 & $\begin{array}{l}\text { Lower Salaco clay:25 } \\
\text { to } 50 \text { yoars }\end{array}$ & SAT_RBRN & Resscusi Bmo Serturation & 32004 & 14 fob-96 & $\mathbf{Y}$ & 1 \\
\hline $\mathbf{Y}$ & 100 & 2376 & CL_L_T3 & $\begin{array}{l}\text { Lower Satado clay:25 } \\
\text { to } 50 \text { yoars }\end{array}$ & SAT_RBRN & Residul Bmo Suturation & 32004 & 14Fab-96 & $\mathbf{Y}$ & 1 \\
\hline$Y$ & 100 & 2376 & CL_L_T3 & $\begin{array}{l}\text { Lower Salaco clay.25 } \\
\text { to } 50 \text { yoars }\end{array}$ & SAT_RBRH & Resioul Bmo Seduration & 32004 & 14F-b-96 & $\mathbf{Y}$ & 1 \\
\hline$Y$ & 100 & 2376 & CL_L_T3 & $\begin{array}{l}\text { Lower Seleso day. } 25 \\
\text { to } 50 \text { years }\end{array}$ & SAT_RBAN & Resoular Bron Seluration & 32004 & 14-Fob-96 & $\mathbf{Y}$ & 1 \\
\hline$Y$ & 100 & 2376 & CL_L_T3 & 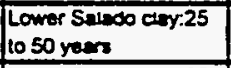 & SAT_RBAN & Restow Bmo Secaration & 32004 & 14 fob-96 & $\mathbf{Y}$ & 1 \\
\hline $\mathbf{Y}$ & 100 & 2376 & CL_L_T3 & $\begin{array}{l}\text { Lowor Seleco any } 25 \\
\text { to } 50 \text { yours }\end{array}$ & SAT_RBAN & Reacued ome Smantion & 32004 & 14 fob-96 & $\mathbf{Y}$ & 1 \\
\hline$Y$ & 100 & 2377 & CL_L_T3 & $\begin{array}{l}\text { Lower Suscos any } 25 \\
\text { to } 50 \text { years }\end{array}$ & SAT_RGAS & Resar Gen Semretion & 32005 & $14 F a b-96$ & $\mathbf{Y}$ & 1 \\
\hline $\mathbf{Y}$ & 100 & 2377 & CL_L_T3 & $\begin{array}{l}\text { Lower } 594000 \text { any } 25 \\
1050 \text { yaurs }\end{array}$ & sat_nces & Renaw Gen searetion & 32005 & 14 fob-9s & $=Y$ & 1 \\
\hline $\mathbf{Y}$ & 100 & 2377 & CL_L_T3 & $\begin{array}{l}\text { Lowor Saleco an } 25 \\
1050 \text { yeurs }\end{array}$ & sar_nous & Renam Gen searmion & 32005 & 14-Fab-96 & $\mathbf{Y}$ & 1 \\
\hline $\mathbf{Y}$ & 100 & $23 \pi$ & CL_L_T3 & $\begin{array}{l}\text { Lower Saluos any } 25 \\
\text { to } 50 \text { yaens }\end{array}$ & sar_ecas & Reman Gen semretion & 32005 & 14 Feb-96 & $\mathbf{Y}$ & 1 \\
\hline $\mathbf{Y}$ & 100 & 2377 & $C L_{-} L_{-} T 3$ & $\begin{array}{l}\text { Lowor Selecos any } 25 \\
1050 \text { yeen }\end{array}$ & Sar_acas & Renaw con semreton & 32005 & $14+$ ab-96 & $\mathbf{Y}$ & 1 \\
\hline $\mathbf{Y}$ & 50 & 2378 & $C L_{-} M_{-} T_{1}$ & $\begin{array}{l}\text { Upper Salucos cay } 0 \text { of } \\
10 \text { years }\end{array}$ & $c_{0}=\infty$ & 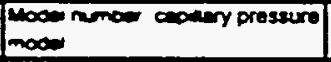 & $32028 \mathrm{~A}$ & 15 fob-96 & $\mathbf{Y}$ & 4B \\
\hline $\mathbf{Y}$ & 50 & 2378 & $C L_{-} M_{-} T H$ & 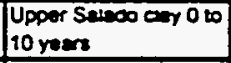 & $\omega_{2}, m \infty$ & 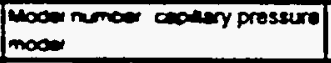 & $32028 \mathrm{~A}$ & $15+a b-96$ & $\mathbf{Y}$ & AB \\
\hline $\mathbf{Y}$ & 50 & 2378 & CL_M_TI & $\begin{array}{l}\text { Uppor Selecos any } 0 \text { to } \\
10 \text { years }\end{array}$ & Cas.mo & 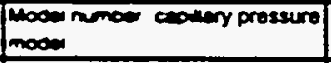 & 320284 & 15-Fob-96 & $Y$ & $A B$ \\
\hline$Y$ & so & 2378 & CL_M_TI & $\begin{array}{l}\text { Uppor Saseo cary } 0 \text { to } \\
10 \text { years }\end{array}$ & $\cos . \infty 0$ & 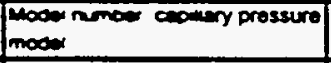 & $32028 A$ & 15 fob-96 & $\mathbf{Y}$ & AB \\
\hline $\mathbf{Y}$ & so & 2378 & $C_{L} M_{-} T I$ & $\begin{array}{l}\text { Uppor Sereco ciny } 0 \text { lo } \\
10 \text { yeess }\end{array}$ & CN_.100 & 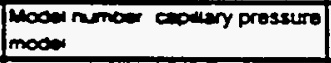 & 320284 & 15-Fob-96 & $\mathbf{Y}$ & AB \\
\hline $\mathbf{Y}$ & & 2379 & $C L_{-} M_{-} T 1$ & $\begin{array}{l}\text { Uppor Selecos any } 0 \text { to } \\
10 \text { yeess }\end{array}$ & cons_nex & Oux Comormenty & 32039 & 15-Feb-96 & $\mathbf{Y}$ & 1 \\
\hline $\mathbf{Y}$ & & 2379 & CL_M_TI & $\begin{array}{l}\text { Upoor Seisco ony } 0 \text { bo } \\
10 \text { yean }\end{array}$ & con_mex & Buc Comoremeroury & 32039 & $15-F=b-86$ & $\mathbf{Y}$ & 1 \\
\hline $\mathbf{Y}$ & & 2379 & CL_M_TI & $\begin{array}{l}\text { Uppor Sercos cioy } 0 \text { to } \\
10 \text { veers }\end{array}$ & Con_Rcx & fust Comormenotiny & 32039 & $15-a b-96$ & $Y$ & 1 \\
\hline
\end{tabular}


CCA Parameter Listing

\begin{tabular}{|c|c|c|c|c|c|c|c|c|c|c|}
\hline DD & 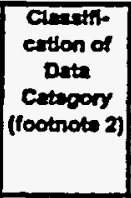 & $\begin{array}{l}\text { Dotstribertion } \\
\text { Type }\end{array}$ & $\operatorname{Men}$ & Mecten & Mtringum & Madminm & Units & Reference id & $\begin{array}{l}\text { Wes tho dind } \\
\text { doveloped } \\
\text { underen } \\
\text { Non-1 } \\
\text { Progren? }\end{array}$ & 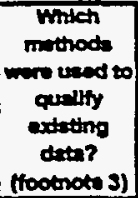 \\
\hline 2368 & $1,3,4$ & TRLANGULAR & $-1.8867000 E+01$ & $-1.8301000 E+01$ & $-2,1000000 E+01$ & $-1.7301000 E+01$ & $\log \left(m^{\wedge} 2\right)$ & WP035597 & YEN & 1 \\
\hline 2368 & $1,3,4$ & TRLANGULAR & $-1.8857000 E+01$ & $-1.8301000 E+01$ & $-21000000 E+01$ & $-1.7301000 E+01$ & $\log \left(m^{\wedge} 2\right)$ & WPOA1558 & Y\&N & 1 \\
\hline 2369 & $1,3,4$ & TRLANGULAR & $-1.8867000 E+01$ & $-1.6301000 E+01$ & $-2.1000000 E+01$ & $-1.7301000 \leq+01$ & $\log \left(m^{\wedge} 2\right)$ & WPO30640 & YaN & 1 \\
\hline 2369 & $1,3,4$ & TRLANGULAR & $-1.8867000 E+01$ & $-1.8301000 E+01$ & $-2.1000000 E+01$ & $-1.7301000 E+01$ & $\log (m-2)$ & WP030994 & Yan & 9 \\
\hline 2369 & $1,3,4$ & TRUNGULAR & $-1,8867000 E+01$ & $-1.83010005+01$ & $-2.1000000 E+01$ & $-1.7301000 E+01$ & $\log \left(m^{\wedge} 2\right)$ & UP035268 & Y\&N & 1 \\
\hline 2369 & $1,3,4$ & TRLANGULAR & $-1.8867000 E+01$ & $-1.8301000 E+01$ & $-2.1000000 E+01$ & $-1.7301000 E+01$ & $\log \left(m^{\wedge} 2\right)$ & WP035597. & YaN & 1 \\
\hline 2369 & $1,3,4$ & TRLANGULAR & $-1.8867000 \mathrm{E}+01$ & $-1.8301000 E+01$ & $-2.1000000 E+01$ & $-1.7301000 E+01$ & $\log \left(m^{2} 2\right)$ & WPO41558 & YaN & 1 \\
\hline 2370 & $1,3,4$ & TRLANGULAR & $-1.8867000 E+01$ & $-1.8301000 E+01$ & $-2.1000000 E+01$ & $-1.7301000 E+01$ & $\log \left(m^{n-2}\right)$ & "WPO30540" & YaN & 1 \\
\hline 2370 & $1,3,4$ & TRLANGULAR & $-1.8867000 E+01$ & $-1.8301000 E+01$ & $-2.1000000 E+0 \mathrm{r}$ & $-1.7301000 E+01$ & $\log \left(m^{\wedge} 2\right)$ & WP030994 & YEN & 1 \\
\hline 2370 & $1,3,4$ & TRLANGULAR & $-1.8867000 E+01$ & $-1.8301000 E+01$ & $-2.1000000 E+01$ & $-1.7301000 E+01$ & $\log \left(m^{n} 2\right)$ & WP035268 & Y\&N & 1 \\
\hline 2370 & $1,3,4$ & TRLANGULAR & $-1.8857000 E+01$ & $-1.8301000 E+01$ & $-2.1000000 E+01$ & $-1.7301000 E+01$ & $\log \left(m^{\wedge} 2\right)$ & WP035597 & Y\&N & 1 \\
\hline 2370 & $1,3,4$ & TRLANGULAR & $-1.8867000 E+01$ & $-1.8301000 E+01$ & $-21000000 E+01$ & $-1.7301000 E+01$ & $\log \left(m^{\wedge} 2\right)$ & WPO41558 & YoN & 1 \\
\hline 2374 & $1,3,4$ & CONSTANT & $4.0000000 E+\infty)$ & $4.0000000 E+00$ & $4.0000000 E+\infty 0$ & $4.00000000=+00$ & NONE & WP030640 & $\mathbf{Y}$ & NA \\
\hline 2374 & $1,3,4$ & CONSTANT & $4.0000000 E+\infty$ & $4.0000000 E+\infty$ & $4.0000000 E+\infty$ & $4.00000000+\infty 0$ & NONE & WP030994 & $\mathbf{Y}$ & NA \\
\hline 2374 & $1,3,4$ & CONSTANT & $4.0000000 E+\infty$ & $4.0000000 E+\infty 0$ & $4.0000000 E+\infty$ & $4.0000000 \mathrm{E}+00$ & NONE & WP035597 “. & $\cdot \boldsymbol{Y}$ & NA \\
\hline 2374 & $1,3,4$ & CONSTANT & $4.00000000 E+\infty$ & $4.0000000 E+\infty 0$ & $4.0000000 E+\infty 0$ & $4.0000000 E+\infty 0$ & NONE & WPO40485 & $\mathbf{Y}$ & NA \\
\hline 2374 & $1,3,4$ & CONSTANT & $4.0000000 E+\infty 0$ & $4.0000000 E+\infty 0$ & $4.0000000 \mathrm{E}+\infty 0$ & $4.0000000 E+\infty 0$ & NONE & WP040514 & $\mathbf{Y}$ & N/A \\
\hline 2376 & 3,4 & CUMULATIVE & $2.5000000 \mathrm{E}-01$ & $2,0000000 E-01$ & $0.0000000 E+\infty$ & $6.0000000 \mathrm{E}-01$ & NONE & WP030640 & $\mathbf{Y}$ & N/A \\
\hline 2376 & 3,4 & CUMULATIVE & $2.5000000 \varepsilon-01$ & $2.0000000 E-01$ & $0.0000000 E+00$ & $6.0000000 E-01$ & NONE & WP030994 & $\mathbf{Y}$ & NA \\
\hline 2376 & 3,4 & CUMULATINE & $2.5000000 E-01$ & $2.0000000 E-01$ & $0.0000000 E+\infty$ & $6.0000000 E-01$ & NONE & UP035268 & $\mathbf{Y}$ & NA \\
\hline 2376 & 3,4 & CUMULATNE & $2.5000000 E-01$ & $20000000 E-01$ & $0.0000000 E+\infty 0$ & $6.0000000 E-01$ & NONE & WP035597 & $\mathbf{Y}$ & NA \\
\hline 2376 & 3,4 & CUMULATIVE & $2.5000000 E-01$ & $2.0000000 E-01$ & $0.0000000 E+\infty 0$ & $6.0000000 E-01$ & NONE & WPO41558 & $\mathbf{Y}$ & N/A \\
\hline 2376 & 3,4 & CUMULATINE & $2.5000000 E-01$ & 2.0000000E-01 & $0.0000000 E+\infty$ & $6.0000000 E-01$ & NONE & WPO42751 & $\mathbf{Y}$ & NA \\
\hline 2377 & 3,4 & UNIFORM & $2.0000000 E-01$ & $2.00000000-01$ & $0.0000000 E+\infty 0$ & $4.0000000 E-01$ & NONE & WP030640 & $\mathbf{Y}$ & NA \\
\hline 2377 & 3,4 & UNIFORM & $2.0000000 \mathrm{E}-01$ & $20000000 E-01$ & $0.0000000 E+\infty 0$ & $4.0000000 \mathrm{E}-01$ & NONE & WP030994 & $--Y$ & $\mathbf{N A}$ \\
\hline 2377 & 3,4 & UNIFORM & $200000000-01$ & $20000000 E-01$ & $0.0000000 E+\infty$ & $4.0000000 \mathrm{E}-01$ & NONE & WP035268 & $\mathbf{Y}$ & NA \\
\hline 2377 & 3,4 & UNIFORM & $2.0000000 E-01$ & $20000000 E-01$ & $0.0000000 E+\infty 0$ & $4.00000000-01$ & NONE & WP035597 & $\mathbf{Y}$ & NA \\
\hline 2377 & 3,4 & UNIFORM & $2.0000000 E-01$ & $2,0000000 E-01$ & $0.0000000 E+\infty$ & $4.0000000 E-01$ & NONE & WPO41558 & $\mathbf{Y}$ & N/A \\
\hline 2378 & $1,3,4$ & CONSTANT & $2.0000000 E+\infty 0$ & $200000000+\infty$ & $2.0000000 E+\infty 0$ & $2.0000000 E+\infty$ & MONE & WP030540 & $\mathbf{Y}$ & NAA \\
\hline 2378 & $1,3,4$ & CONSTANT & $20000000 E+\infty 0$ & $20000000 E+\infty 0$ & $2.0000000 E+\infty 0$ & $2.0000000 E+\infty$ & NONE & WP030994 & $\mathbf{Y}$ & $N / A$ \\
\hline 2378 & $1,3,4$ & CONSTANT & $2.0000000 E+\infty 0$ & $2,0000000 E+\infty 0$ & $2.0000000 E+\infty 0$ & $2.0000000 E+\infty$ & NONE & WP035597 & $\mathbf{Y}$ & $N / A$ \\
\hline 2378 & $1,3,4$ & CONSTANT & $20000000 E+\infty 0$ & $20000000 E+\infty$ & $2,0000000 E+\infty$ & $2,0000000 E+\infty$ & NONE & WP040486 & $\mathbf{Y}$ & NA \\
\hline 2378 & $1.3,4$ & CONSTANT & $2,0000000 E+\infty 0$ & $2.0000000 E+\infty 0$ & $200000000+\infty$ & $2.0000000 E+\infty 0$ & NONE & WP040514 & $\mathbf{Y}$ & N/A \\
\hline 2379 & $1,3,4$ & CONSTANT & $1.8100000 E-09$ & $1.8100000 E-09$ & $1.8100000 E-O 9$ & $1.8100000 E-09$ & $P x^{n}-1$ & WP030540 & $\mathbf{Y}$ & NA \\
\hline 2379 & $1,3,4$ & CONSTANT & $1.8100000=-\infty$ & $1.8100000 E-09$ & $1.8100000 \mathrm{E}-09$ & $1.8100000=-09$ & $P=x-1$ & WP030994 & $\mathbf{Y}$ & NA \\
\hline 2379 & $1,3,4$ & CONSTANT & $1.8100000 E-09$ & $1.8100000 E-09$ & $1.8100000 E-\infty 9$ & $1.8100000 \mathrm{E}-09$ & $P a^{n-1}$ & WP035597 & $\mathbf{Y}$ & N/A \\
\hline
\end{tabular}


CCA Parameter Listing

\begin{tabular}{|c|c|c|c|c|c|c|c|c|c|c|}
\hline $\begin{array}{c}\text { Permoter in } \\
\text { Databee? } \\
\text { (N/N) }\end{array}$ & 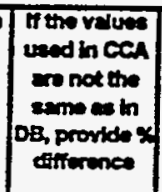 & 10 & Meneral io & Mrtarded $\mathrm{Nem}$ & $\begin{array}{l}\text { Pernotir } \\
\text { D }\end{array}$ & Peramater Marts & $\begin{array}{l}\text { PAPDD } \\
\text { MPPOA }\end{array}$ & $\begin{array}{c}\text { Dententy } \\
\text { Dew }\end{array}$ & 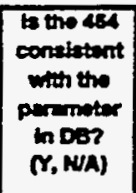 & 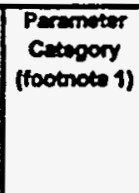 \\
\hline$Y$ & & 2380 & CL_M_TI & $\begin{array}{l}\text { Uppor Salado cay.0 to } \\
10 \text { years }\end{array}$ & PC_max & $\begin{array}{l}\text { Maxmum allowablo capilany } \\
\text { pressure }\end{array}$ & $32043 A$ & OSANO-95 & $\mathbf{Y}$ & 48 \\
\hline$Y$ & & 2380 & CL_M_TI & $\begin{array}{l}\text { Woper Seboco day:0 to } \\
\text { 10 years }\end{array}$ & PC_max & $\begin{array}{l}\text { Maxumum allowablo capilloy } \\
\text { prossure }\end{array}$ & $32043 A$ & OS-Nov-95 & $\mathbf{Y}$ & 48 \\
\hline $\mathbf{Y}$ & & 2380 & CL_M_T1 & $\begin{array}{l}\text { Uppor Saledo chy.0 to } \\
10 \text { yems }\end{array}$ & PC_max & $\begin{array}{l}\text { Maxumum allowablo coputiony } \\
\text { prosesure }\end{array}$ & 320434 & COANON-95 & $\mathbf{Y}$ & 48 \\
\hline$Y$ & & 2380 & CL_M_TI & $\begin{array}{l}\text { Uppoer Salioso ctry.0 to } \\
\text { 10 years }\end{array}$ & PC_max & $\begin{array}{l}\text { Maximum allowablo copittary } \\
\text { prossure }\end{array}$ & $32043 n$ & OBHON-85 & $\mathbf{Y}$ & AB \\
\hline$Y$ & & 2300 & CL_M_T1 & $\begin{array}{l}\text { Upper Salood ciay.0 to } \\
10 \text { years }\end{array}$ & PC_max & $\begin{array}{l}\text { Maxmum allowablo capillory } \\
\text { pressure }\end{array}$ & $32043 A$ & Cos-noves & $\mathbf{Y}$ & 48 \\
\hline $\mathbf{Y}$ & veries & 2389 & CL_M_T1 & $\begin{array}{l}\text { Upper Salado clay.0 to } \\
\text { 10 years }\end{array}$ & PORE_DIS & $\begin{array}{l}\text { Brooks-Corvy pore distrbution } \\
\text { parmeter }\end{array}$ & 32044 & $20 \mp \infty b-96$ & $\mathbf{Y}$ & 1 \\
\hline$Y$ & varies & 2381 & CL_M_T1 & $\begin{array}{l}\text { Upper Salsoco day.0 to } \\
10 \text { years }\end{array}$ & PORE_DIS & $\begin{array}{l}\text { Brooken Corey pore distribution. } \\
\text { peremeter }\end{array}$ & 32044 & $20+\ldots b-96$ & $\mathbf{Y}$ & 1 \\
\hline $\mathbf{Y}$ & varies & 2381 & CL_M_TI & $\begin{array}{l}\text { Upper Selado doy.0 to } \\
10 \text { years }\end{array}$ & PORE_DIS & $\begin{array}{l}\text { Brocks-Corey pore distribution } \\
\text { paremeter }\end{array}$ & 3204 & $20+00-96$ & $\mathbf{Y}$ & 1 \\
\hline $\mathbf{Y}$ & veries & 2381 & $C L_{-} M_{-} T t$ & $\begin{array}{l}\text { Uppor Salado clay.0 to } \\
10 \text { vears }\end{array}$ & PORE_DIS & $\begin{array}{l}\text { Brooks-Corey pore distribution } \\
\text { parameter }\end{array}$ & 32044 & $20+0 b-96$ & $\mathbf{Y}$ & 1 \\
\hline$Y$ & veries & 2381 & CL_M_TI & $\begin{array}{l}\text { Upper Saludo doy.0 to } \\
\text { to years }\end{array}$ & PORE_DIS & $\begin{array}{l}\text { Brooks-Corey pore distribution } \\
\text { paramoter }\end{array}$ & 32044 & $20+706-96$ & $\mathbf{Y}$ & 1 \\
\hline $\mathbf{Y}$ & varies & 2381 & CL_M_T1 & $\begin{array}{l}\text { Upper Selaco clay. } 0 \text { to } \\
10 \text { years }\end{array}$ & PORE_OIS & $\begin{array}{l}\text { Brooks-Corcy pore distribition } \\
\text { parameter }\end{array}$ & 32044 & $20+50-96$ & $\mathbf{Y}$ & 1 \\
\hline $\mathbf{Y}$ & & 2382 & CL_M_TI & $\begin{array}{l}\text { Upper Salado clay.0 to } \\
10 \text { years }\end{array}$ & POROSTr & Effective porosity & 32045 & 15-ab-96 & $\mathbf{Y}$ & 1 \\
\hline$Y$ & & 2382 & CL_M_TI & $\begin{array}{l}\text { Uppoer Salado clay:0 to } \\
10 \text { years }\end{array}$ & POROSTY & Efrective porosity & 32045 & 15Feb-96 & $\mathbf{Y}$ & 1 \\
\hline$Y$ & & 2382 & $C L_{-} M_{-} T_{1}$ & $\begin{array}{l}\text { Upper Salowo clay. } 0 \text { to } \\
10 \text { years }\end{array}$ & POROSITY & Efriective porosity & 32045 & $15 f a b-96$ & $\mathbf{Y}$ & 1 \\
\hline $\mathbf{Y}$ & & 2383 & CL_M_TI & $\begin{array}{l}\text { Upper Salado clay.0 to } \\
10 \text { years }\end{array}$ & PO_MIN & $\begin{array}{l}\text { Minimum brine pressure for } \\
\text { capiliary model KPC }=3\end{array}$ & 32046 & $21+50 b-96$ & $\mathbf{Y}$ & 48 \\
\hline $\mathbf{Y}$ & & 2383 & CL_M_TI & $\begin{array}{l}\text { Uppor Solado clay.0 to } \\
\text { 10 years }\end{array}$ & PO_MIN & $\begin{array}{l}\text { Mnmum brno pressero for } \\
\text { capilary model KPC=3 }\end{array}$ & 32046 & $21+\omega_{0}-96$ & $\mathbf{Y}$ & AB \\
\hline $\mathbf{Y}$ & & 2383 & CL_M_T1 & $\begin{array}{l}\text { Uppor Salado day.0 to } \\
10 \text { yoars }\end{array}$ & PO_MIN & 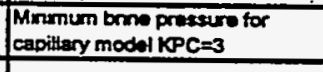 & 32046 & 21-Fab-96 & $\mathbf{Y}$ & 48 \\
\hline$\gamma$ & & 2384 & CL_M_T1 & $\begin{array}{l}\begin{array}{l}\text { Uppor Saloco clay.0 to } \\
10 \text { yoars }\end{array} \\
\end{array}$ & PRESSURE & Brine for-fietd pore prossure & 32047 & $20-F a b-96$ & $\mathbf{Y}$ & 1 \\
\hline $\mathbf{Y}$ & & 2384 & CL_M_T1 & $\begin{array}{l}\text { Upper Salado clay.0 to } \\
10 \text { yoars }\end{array}$ & PRESSURE & Brino fer-field pore prossure & 32047 & 20-Fob-96 & $\mathbf{Y}$ & 1 \\
\hline$Y$ & varies & 2385 & $C L_{-} M_{-} T I$ & $\begin{array}{l}\text { Upper Salado clay.0 to } \\
10 \text { years }\end{array}$ & PRHX_LOG & $\begin{array}{l}\text { Log of intinsic permeability, } X- \\
\text { direction }\end{array}$ & 32048 & 15F-b-96 & $\mathbf{Y}$ & 1 \\
\hline$Y$ & varios & 2385 & CL_M_T1 & $\begin{array}{l}\text { Upper Salado day.0 to } \\
10 \text { years }\end{array}$ & PRMX_LOG & $\begin{array}{l}\text { Log of intonssc permesbility, } X- \\
\text { diroction }\end{array}$ & 32048 & $15-F e b-96$ & $\mathbf{Y}$ & 1 \\
\hline $\mathbf{Y}$ & varies & 2385 & CL_M_TI & $\begin{array}{l}\text { Uppor Salado clay.0 to } \\
10 \text { yeurs }\end{array}$ & PRMX_LOG & $\begin{array}{l}\text { Log of intrnasic permeability, } X \text { - } \\
\text { direction }\end{array}$ & 32048 & $15-F 0 b-96$ & $Y$ & 1 \\
\hline$Y$ & varies & 2385 & CL_M_Ti & $\begin{array}{l}\text { Upper Salado clay.0 to } \\
10 \text { years }\end{array}$ & PRMX_LOG & $\begin{array}{l}\text { Log } \alpha \text { intmans permeabillty, } X- \\
\text { direction }\end{array}$ & 32048 & 15-reb-96 & $\mathbf{Y}$ & 1 \\
\hline $\mathbf{Y}$ & varies & 2385 & CL_M_T1 & $\begin{array}{l}\text { Upper Salado clay. } 0 \text { to } \\
10 \text { yoars }\end{array}$ & PRMX_LOG & $\begin{array}{l}\text { Log of intrinsic permestility, } x \text { - } \\
\text { direction }\end{array}$ & 32048 & 15-Fob-96 & $Y$ & 1 \\
\hline $\mathbf{Y}$ & & 2386 & $C L \_M_{-} T I$ & $\begin{array}{l}\text { Upper Salado chay.0 to } \\
10 \text { years }\end{array}$ & PRMY_LOG & $\begin{array}{l}\text { Log o intrmax permeabilty, Y- } \\
\text { direction }\end{array}$ & 32049 & 15 -feb-96 & $-y$ & 1 \\
\hline $\mathbf{Y}$ & & 2386 & CL_M_TI & $\begin{array}{l}\text { Upper Salado clay.0 to } \\
10 \text { yours }\end{array}$ & PRMY_LOG & $\begin{array}{l}\text { Los of ntminse permesbility, Y- } \\
\text { direction }\end{array}$ & 32049 & 15-Fob-96 & $\mathbf{Y}$ & 1 \\
\hline $\mathbf{Y}$ & & 2386 & CL_M_T1 & $\begin{array}{l}\text { Upper Seledo clay.0 to } \\
\text { to year }\end{array}$ & PRMY_LOG & $\begin{array}{l}\text { Log of minnsic permesbilty, Y- } \\
\text { druetion }\end{array}$ & 32049 & $15 F a b-66$ & $Y$ & 1 \\
\hline$Y$ & & 2380 & CL_H_T1 & $\begin{array}{l}\text { Upper Saledo clay.0 to } \\
10 \text { years }\end{array}$ & PRMY_LOG & $\begin{array}{l}\text { Log of intrinsx permeabilty, Y. } \\
\text { direction }\end{array}$ & 32049 & 15-Fab-96 & $Y$ & 1 \\
\hline$Y$ & & 2306 & CL_M_TI & $\begin{array}{l}\text { Upper Salecto clay:0 to } \\
10 \text { yoars }\end{array}$ & PRMY_LOG & $\begin{array}{l}\text { Log of minex permasility, Y- } \\
\text { direction }\end{array}$ & 32049 & $15 F \infty 0-96$ & $Y$ & 1 \\
\hline $\mathbf{Y}$ & & 2387 & CL_M_TI & $\begin{array}{l}\text { Upper Salodo clay.0 to } \\
10 \text { years }\end{array}$ & PRMZ_LOG & $\begin{array}{l}\text { Log of intontic pmomesbilty, 2- } \\
\text { direction }\end{array}$ & 32050 & $15+=b-96$ & $\mathbf{Y}$ & 1 \\
\hline$Y$ & & 2387 & $C L_{-} M_{-} T_{1}$ & $\begin{array}{l}\text { Uppoer Selado clay.0 to } \\
\text { to years }\end{array}$ & PRMZ_LOG & $\begin{array}{l}\text { Log of intrmace permanabithy, 2- } \\
\text { direction }\end{array}$ & 32050 & 15-Fab-96 & $Y$ & 1 \\
\hline $\mathbf{Y}$ & & 2387 & CL_M_T1 & $\begin{array}{l}\text { Uppor Salado city. } 0 \text { to } \\
10 \text { years }\end{array}$ & PRMZ_LOG & $\begin{array}{l}\text { Log of mimsix permesbilty, 2- } \\
\text { drection }\end{array}$ & 32050 & 15-Feb-96 & $\mathbf{Y}$ & 1 \\
\hline $\mathbf{Y}$ & & 2387 & CL_M_TI & $\begin{array}{l}\text { Uppoer Seledo dey.0 to } \\
10 \text { years }\end{array}$ & PRMZ_LOG & $\begin{array}{l}\text { Log } \alpha \text { intinsax pemmability, } 2 \text { - } \\
\text { dinection }\end{array}$ & 32050 & 15-Feb-96 & $Y$ & 1 \\
\hline $\mathbf{Y}$ & & 2387 & CL_M_TI & $\begin{array}{l}\text { Upper Salado elsy.0 to } \\
10 \text { years }\end{array}$ & PRMZ_LOG & $\begin{array}{l}\text { Log of intrnax pormasbildy, } 2 \text { - } \\
\text { diredion }\end{array}$ & 32050 & 15-Fab-96 & $\mathbf{Y}$ & 1 \\
\hline $\mathbf{Y}$ & & 2391 & CL_M_TI & $\begin{array}{l}\text { Upper Seleco day.0 to } \\
10 \text { yame }\end{array}$ & RELP_MOD & $\begin{array}{l}\text { Model number, raletive } \\
\text { permeability model }\end{array}$ & $32055 A$ & $15-f e b-96$ & $Y$ & $A B$ \\
\hline$Y$ & & 2391 & $C L M_{-} M_{-} T I$ & $\begin{array}{l}\text { Uppoer Silado clay:0 to } \\
10 \text { years }\end{array}$ & RELP_MOD & $\begin{array}{l}\text { Model number, relitise } \\
\text { permeanbility model }\end{array}$ & $32055 A$ & $15-f a b-96$ & $\mathbf{Y}$ & 48 \\
\hline
\end{tabular}


CCA.Parameter-Listing

\begin{tabular}{|c|c|c|c|c|c|c|c|c|c|c|}
\hline DD & 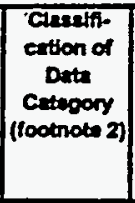 & $\begin{array}{l}\text { Datribution } \\
\text { Type }\end{array}$ & $\operatorname{man}$ & Medien & Mintrimen & Mnodrouses & Unats & Referexces 10 & $\begin{array}{l}\text { Wha the dint? } \\
\text { demeloped } \\
\text { under an } \\
\text { Nol-1 } \\
\text { Proprum? }\end{array}$ & 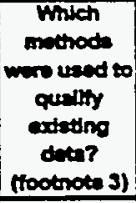 \\
\hline 2380 & 1,3 & CONSTANT & $1.0000000 E+08$ & $1.0000000 E+08$ & $1.0000000 E+08$ & $1.0000000 E+08$ & $\mathrm{~Pa}$ & WP030640 & $Y$ & NA \\
\hline 2380 & 1,3 & CONSTANT & $1.0000000 E+08$ & $1.0000000 E+08$ & $1.0000000 E+06$ & $1.0000000 E+08$ & P\& & WP030994 & $\mathbf{Y}$ & NA \\
\hline 2380 & 1,3 & CONSTANT & $1.0000000 E+08$ & $1.0000000 E+08$ & $1.0000000 E+08$ & $1.0000000 E+08$ & Pa & WP035597 & $Y$ & NA \\
\hline 2380 & 1,3 & CONSTANT & $1.0000000 \mathrm{E}+08$ & $1.0000000 E+08$ & $1.0000000 E+08$ & $1.0000000 E+08$ & Pa & WPO40485 & $\mathbf{Y}$ & NA \\
\hline 2380 & 1,3 & CONSTANT & $1.0000000 E+08$ & $1.0000000 E+08$ & $1.0000000 E+06$ & $1.0000000 E+\infty 8$ & Pa & WP040514 & $\mathbf{Y}$ & NA \\
\hline 2381 & 3,4 & CUMULATTVE & $2.5200000 E+\infty 0$ & $9.4000000 E-01$ & $1.1000000 E-01$ & $8.1000000 E+\infty$ & NONE & WP030640 & $\mathbf{Y}$ & NA \\
\hline 2381 & 3,4 & CUMULATNE & $2.5200000 E+00$ & $9.4000000 E-01$ & $1.1000000 E-01$ & $8.1000000 E+\infty$ & NONE & WP030994. & $\mathbf{Y}$ & N/A \\
\hline 2381 & 3,4 & CUMULATNE & $2.5200000 \mathrm{E}+00$ & $9.4000000 E-01$ & $1.1000000 E-01$ & $8.1000000 E+\infty$ & NONE & WP032287 & $\mathbf{Y}$ & NA \\
\hline 2381 & 3,4 & CUMULATINE & $2.5200000 E+\infty 0$ & $9.4000000 E-01$ & $-1.1000000 \mathrm{E}-01$ & $8.1000000 E+\infty$ & NONE & WP035868 & $\mathbf{Y}$ & NA \\
\hline 2381 & 3,4 & CUMULATINE & $2.5200000 E+\infty$ & $9.4000000 \mathrm{E}-\mathrm{O}$ & $1.1000000 E-01$ & $8.1000000 E+\infty$ & NONE & WP035597 & $\mathbf{Y}$ & N/A \\
\hline 2381 & 3.4 & CUMULATNE & $2.5200000 E+\infty 0$ & $9.4000000 E-01$ & 1.1000000E-01 & $8.1000000 E+\infty 0$ & NONE & WPO41558 & $\mathbf{Y}$ & NA \\
\hline 2382 & $1,3,4$ & CONSTANT & $2.4000000 E-01$ & $2.4000000 E-01$ & $2.4000000 E-01$ & $2.4000000 E-01$ & $m^{\wedge} 3 m^{\wedge} 3$ & WPO30640 & $\boldsymbol{\gamma}$ & NA \\
\hline 2382 & $1,3,4$ & CONSTANT & $2.4000000 E-01$ & $2.4000000 \mathrm{E}-01$ & $2.4000000 E-01$ & $2,4000000 E-01$ & $m^{\wedge} 3 m^{\wedge} \mathbf{3}$ & WP030994 & $\mathbf{Y}$ & NA \\
\hline 2382 & $1,3,4$ & CONSTANT & $2.4000000 E-01$ & $2.4000000 E-01$ & 2.4000000E-01 & $2.4000000 E-01$ & $m^{\wedge} 3 / m^{\wedge} \mathbf{3}$ & WP035597 & $\mathbf{Y}$ & NA \\
\hline 2383 & 1,3 & CONSTANT & $1.0132500 E+05$ & $1.0132500 E+05$ & $1.0132500 E+05$ & $1.0132500 E+05$ & $P a$ & WP035597 & $\mathbf{Y}$ & NA \\
\hline 2383 & 1,3 & CONSTANT & $1.0132500 E+05$ & $1.0132500 E+05$ & $1.0132500 E+05$ & $1.0132500 E+05$ & $P=$ & WP035051 & $\mathbf{Y}$ & NA \\
\hline 2383 & 1,3 & CONSTANT & $1.0132500 E+05$ & $1.0132500 E+05$ & $1.0132500 E+05$ & $1.0132500 E+05$ & $\mathrm{~Pa}$ & WPO40514 & $\mathbf{Y}$ & NA \\
\hline 2384 & 3,5 & CONSTANT & $1.0132500 E+05$ & $1.0132500 E+05$ & $1.0132500 E+05$ & $1.0132500 E+05$ & Pa & WP035597 & $\mathbf{Y}$ & NA \\
\hline 2384 & 3.5 & CONSTANT & $1.0132500 E+05$ & $1.0132500 E+05$ & $1.0132500 E+05$ & $1.0132500 E+05$ & $\mathrm{~Pa}$ & WP036058 & $Y$ & NA \\
\hline 2385 & $1,3,4$ & TRIANGULAR & $-1.8867000 E+01$ & $-1.8301000 E+01$ & $-2.1000000 E+01$ & $-1.7301000 E+01$ & $\log \left(m^{\wedge} 2\right)$ & WPO30640 & Y8N & 1 \\
\hline 2385 & $1,3,4$ & TRLANGULAR & $-1.8867000 E+01$ & $-1.8301000 E+01$ & $-2.1000000 E+01$ & $-1.7301000 E+01$ & $\log \left(m^{\wedge} 2\right)$ & WP030994 & YAN & 1 \\
\hline 2385 & $1,3,4$ & TRIANGULAR & $-1.8867000 E+01$ & $-1.8301000 E+01$ & $-2.1000000 E+01$ & $-1.7301000 E+01$ & $\log \left(m^{\wedge} 2\right)$ & WP035268 & YEN & 1 \\
\hline 2385 & $1,3,4$ & TRLANGULAR & $-1.8867000 E+01$ & $-1.8301000 E+01$ & $-2.1000000 E+01$ & $-1.7301000 E+01$ & $\log \left(m^{\wedge} 2\right)$ & WP035597 & YEN & 1 \\
\hline 2385 & $1,3,4$ & TRLANGULAR & $-1.8867000 \mathrm{E}+01$ & $-1.8301000 E+01$ & $-2.1000000 E+01$ & $-1.7301000 E+01$ & $\log \left(m^{\wedge} 2\right)$ & WPO41558 & YEN & 1 \\
\hline 2386 & $1,3,4$ & TRLANGULAR & $-1.8867000 \mathrm{E}+01$ & $-1.8301000 E+01$ & $-2.1000000 E+01$ & $-1.7301000 E+01$ & $\log \left(\pi^{\wedge} 2\right)$ & WP030640 & YEN & 1 \\
\hline 2386 & $1,3,4$ & TRIANGULAR & $-1.8867000 E+01$ & $-1.8301000 E+01$ & $-2.1000000 E+01$ & $-1.7301000 E+01$ & $\log \left(m^{\wedge} 2\right)$ & WP030994 & YEN & 1 \\
\hline 2386 & $1,3,4$ & TRIANGULAR & $-1.8867000 E+01$ & $-1.8301000 E+01$ & $-21000000 E+01$ & $-1.7301000 E+01$ & $\log \left(m^{\wedge} 2\right)$ & WP035268 & YEN & 1 \\
\hline 2386 & $1,3,4$ & TRLANGULAR & $-1.8867000 E+01$ & $-1.8301000 E+01$ & $-2.1000000 E+01$ & $-1.7301000 E+01$ & $\log \left(m^{\wedge} 2\right)$ & WP035597 & YEN & 1 \\
\hline 2386 & $1,3,4$ & TRLANGULAR & $-1.8867000 E+01$ & $-1.8301000 E+01$ & $-2.1000000 E+01$ & $-1.7301000 E+01$ & $\log \left(m^{\wedge} 2\right)$ & WPO41558 & Y\&N & 1 \\
\hline 2387 & $1,3,4$ & TRIANGULAR & $-1.8867000 E+01$ & $-1.8301000 E+01$ & $-2.1000000 E+01$ & $-1.7301000 E+01$ & $\log \left(m^{\wedge} 2\right)$ & WP030640 & Y\&N & 1 \\
\hline 2387 & $1,3,4$ & TRLANGULAR & $-1.8867000 E+01$ & $-1.8301000 E+01$ & $-2.1000000 E+01$ & $-1.7301000 E+01$ & $\log \left(m^{\wedge} 2\right)$ & WP030994 & Y8N & 1 \\
\hline 2387 & $1,3,4$ & TRIANGULAR & $-1.8867000 E+01$ & $-1.8301000 E+01$ & $-2.1000000 E+01$ & $-1.7301000 E+01$ & $\log \left(m^{\wedge} 2\right)$ & WP035268 & YEN & 1 \\
\hline 2387 & $1,3,4$ & TRIANGULAR & $-1.8867000 E+01$ & $-1.8301000 E+01$ & $-2.1000000 E+01$ & $-1.7301000 E+01$ & $\log \left(m^{\wedge} 2\right)$ & WP035597 & Y\&N & 1 \\
\hline 2387 & $1,3,4$ & TRLANGULAR & $-1.8867000 E+01$ & $-1.8301000 E+01$ & $-2.1000000 E+01$ & $-1.7301000 E+01$ & $\log \left(m^{\wedge} 2\right)$ & WPOS1558 & YSN & 1 \\
\hline 2391 & $1,3,4$ & CONSTANT & $1.0000000 E+00$ & 4.0000000E+00 & $4.0000000 E+\infty$ & $4.0000000 E+\infty 0$ & NONE & WP030640 & $\mathbf{Y}$ & NA \\
\hline 2391 & $1,3,4$ & CONSTANT & $4.0000000 E+\infty 00$ & $4,00000000 E+\infty 0$ & $4.0000000 E+\infty 0$ & $4.0000000 \mathrm{E}+\infty 0$ & NONE & WPO30994 & $\mathbf{Y}$ & N/A \\
\hline
\end{tabular}


CCA Parameter Listing

\begin{tabular}{|c|c|c|c|c|c|c|c|c|c|c|}
\hline 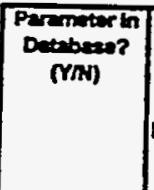 & 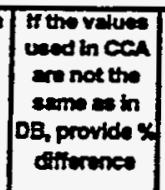 & DD & Deterta ID & Meterial Mam & $\begin{array}{c}\text { Peremberar } \\
\text { D }\end{array}$ & Permover Kord & $\begin{array}{l}\text { PRP ID } \\
\text { MPOS }\end{array}$ & $\begin{array}{c}\text { Den Entry } \\
\text { Dexte }\end{array}$ & 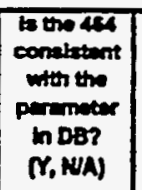 & $\begin{array}{l}\text { Paremoter } \\
\text { Catugory } \\
\text { (footnote 1) }\end{array}$ \\
\hline $\mathbf{Y}$ & & 2391 & $C L_{-} M_{-} T_{1}$ & $\begin{array}{l}\text { Uppor Salado ciry:0 to } \\
10 \text { years } \\
\end{array}$ & REEP_MOD & $\begin{array}{l}\text { Model nember, relative } \\
\text { permeability mocol }\end{array}$ & 320554 & 15 fab-96 & $\mathbf{Y}$ & 48 \\
\hline $\mathbf{Y}$ & & 2391 & CL_M_TS & $\begin{array}{l}\text { Uppor Salado ctay.0 to } \\
10 \text { yours }\end{array}$ & RELP_MOD & $\begin{array}{l}\text { Model number. rolistive } \\
\text { pormesbility model }\end{array}$ & $32055 A$ & $15-F \infty-96$ & $\mathbf{Y}$ & 48 \\
\hline $\mathbf{Y}$ & & 2391 & $C L_{-} M_{-} T 1$ & $\begin{array}{l}\text { Upper Seledo clyy.0 to } \\
10 \text { yesers }\end{array}$ & RELP_MOD & $\begin{array}{l}\text { Modol nimber, relativo } \\
\text { permuability model }\end{array}$ & $32055 A$ & $15-10-96$ & $\mathbf{Y}$ & 48 \\
\hline $\mathbf{Y}$ & -26.58 & 2392 & CL_M_TI & $\begin{array}{l}\text { Uppor Selado chy.0 to } \\
10 \text { years }\end{array}$ & SAT_IBRN & |rritial Brine Saturation & 32060 & $15 f a b-96$ & $\mathbf{Y}$ & 1 \\
\hline Y & -26.58 & 2392 & CL_M_TI & $\begin{array}{l}\text { Upper Salado ciay.0 to } \\
\text { 10 years }\end{array}$ & SAT_IBRN & Initive Brine Seruration & 32060 & 15 Fab-96 & $\mathbf{\gamma}$ & 1 \\
\hline Y & -26.58 & 2392 & CL_M_TI & $\begin{array}{l}\begin{array}{l}\text { Uppor Salacio chay.0 to } \\
10 \text { years }\end{array} \\
\end{array}$ & SAT_IBRN & Initial Brino Saturation & 32060 & $15+a b-96$ & $\mathbf{r}$ & 1 \\
\hline$Y$ & -26.58 & 2392 & CL_M_TI & $\begin{array}{l}\text { Uppor Selaco cloy.0 to } \\
\text { to years }\end{array}$ & SAT_IBRN & Intitial Brimo Saturation & 32060 & 15 fab-S6 & $\mathbf{Y}$ & 1 \\
\hline $\mathbf{Y}$ & 100 & 2393 & CL_M_Tt & $\begin{array}{l}\text { Upper Salaco clay.0 to } \\
10 \text { years }\end{array}$ & SAT_RBRN & Residual Brine Seturation & 32061 & 15 feb-96 & $\mathbf{Y}$ & 1 \\
\hline $\mathbf{Y}$ & 100 & 2393 & $C_{2} M_{-} T_{1}$ & $\begin{array}{l}\text { Upper Salado ctoy.0 to } \\
10 \text { years }\end{array}$ & SAT_RBRN & Residual Brino Seturation & 32061 & $15+\cos 96$ & $\mathbf{Y}$ & 1 \\
\hline$Y$ & 100 & 2393 & $C L_{2} M_{-} T I$ & \begin{tabular}{|l} 
Uppor Salaco ctay.0 to \\
10 years
\end{tabular} & SAT_RBRN & Resictual Brione Saturation & 32061 & 15 feb-96 & $\mathbf{Y}$ & 1 \\
\hline $\mathbf{Y}$ & 100 & 2393 & $C L_{-} M_{-} T_{1}$ & $\begin{array}{l}\text { Upper Salado clay.0 to } \\
10 \text { yoars }\end{array}$ & SAT_RBRN & Residual Brine Ssturation & 32061 & 15-Feb-96 & $\mathbf{Y}$ & 1 \\
\hline $\mathbf{Y}$ & 100 & 2393 & $C L_{-} M_{-} T_{1}$ & $\begin{array}{l}\text { Upper Salado ctay:0 to } \\
10 \text { years }\end{array}$ & SAT_RBRN & Residual Brino Saturation & 32061 & 15-Feb-96 & $\mathbf{Y}$ & 1 \\
\hline $\mathbf{Y}$ & 100 & 2393 & $C L_{2} M_{-} T 1$ & $\begin{array}{l}\text { Uppor Salado ctay.0 to } \\
\text { to years }\end{array}$ & SAT_RBRN & Residual Brino Saturetion & 32061 & $15+500.96$ & $\mathbf{Y}$ & 1 \\
\hline $\mathbf{Y}$ & 100 & 2394 & $C L_{-} M_{-} T 9$ & $\begin{array}{l}\text { Uppor Salado clay.0 to } \\
10 \text { years }\end{array}$ & SAT_RGAS & Resicual Gas Saturation & 32052 & 15 Feb-96 & $\mathbf{Y}$ & 1 \\
\hline $\mathbf{Y}$ & 100 & 2354 & CL_M_T1 & $\begin{array}{l}\text { Upper Salado clay.0 to } \\
10 \text { years }\end{array}$ & SAT_RGAS & Residusa Gas Saturation & 32062 & $15+c a b-96$ & $Y$ & 1 \\
\hline $\mathbf{Y}$ & 100 & 2394 & CL_M_TI & $\begin{array}{l}\text { Upper Salado clay.0 to } \\
10 \text { years }\end{array}$ & SAT_RGAS & Residusal Gas Saturation & 32052 & $15-F c b-96$ & $Y$ & 1 \\
\hline $\mathbf{Y}$ & 100 & 2394 & CL_M_T1 & $\begin{array}{l}\text { Upper Salado clay.0 to } \\
10 \text { years }\end{array}$ & SAT_RGAS & Residuel Ges Saturetion & 32002 & 15 Feb-96 & $\mathbf{Y}$ & 1 \\
\hline $\mathbf{Y}$ & 100 & 2394 & CL_M_TI & $\begin{array}{l}\text { Uppor Salado clay:0 to } \\
10 \text { yoars }\end{array}$ & SAT_RGAS & Residual Ges Saturation & 32062 & 15-Fab-96 & $\mathbf{Y}$ & 1 \\
\hline $\mathbf{Y}$ & 50 & 2395 & CL_M_T2 & $\begin{array}{l}\text { Uppor Salado clay.10 } \\
\text { to } 25 \text { years }\end{array}$ & CAP_MOD & $\begin{array}{l}\text { Model number, capillay pressuro } \\
\text { model }\end{array}$ & 320644 & $15-F a b-96$ & $\mathbf{Y}$ & 48 \\
\hline $\mathbf{Y}$ & 50 & 2395 & $C L \_M I T 2$ & $\begin{array}{l}\text { Uppor Sataco clay: } 10 \\
\text { to } 25 \text { years }\end{array}$ & CAP_MOD & $\begin{array}{l}\text { Mocel number, capillary pressuro } \\
\text { model }\end{array}$ & $32064 \mathrm{~A}$ & 15-Fob-96 & $\mathbf{Y}$ & 48 \\
\hline$Y$ & 50 & 2395 & $C L \_M_{-} T_{2}$ & $\begin{array}{l}\text { Upper Salado day:10 } \\
\text { to } 25 \text { years }\end{array}$ & CAP_MOD & $\begin{array}{l}\text { Model number, capillary prossuro } \\
\text { modol }\end{array}$ & $3206 \mathrm{AA}$ & $15-F a b-96$ & $\mathbf{Y}$ & 48 \\
\hline$\gamma$ & 50 & 2395 & $C L / M \_T 2$ & $\begin{array}{l}\text { Upper Sataco clay: } 10 \\
\text { to } 25 \text { years }\end{array}$ & CAP_MOD & $\begin{array}{l}\text { Model number, capillary pressure } \\
\text { model }\end{array}$ & 320644 & 15-Feb-96 & $\mathbf{Y}$ & 18 \\
\hline $\mathbf{Y}$ & 50 & 2395 & $C L \_M \_I 2$ & $\begin{array}{l}\text { Upper Salado clay: } 10 \\
\text { to } 25 \text { years }\end{array}$ & CAP_MOD & $\begin{array}{l}\text { Model number, capiliery pressure } \\
\text { model }\end{array}$ & $32064 A$ & $15+6 b-96$ & $\mathbf{Y}$ & 48 \\
\hline $\mathbf{Y}$ & & 2396 & $C L_{-} M_{-} T 2$ & $\begin{array}{l}\text { Upper Salado clay. } 10 \\
\text { to } 25 \text { years }\end{array}$ & COMP_RCK & Buk Compressibilty & 32006 & $15-F=b-96$ & $\mathbf{Y}$ & 1 \\
\hline $\mathbf{Y}$ & & 2395 & $C L \_M \_T 2$ & $\begin{array}{l}\text { Upper Salado clay: } 10 \\
\text { to } 25 \text { yoars }\end{array}$ & COMP_RCK & Buk Comprossibility & 32066 & 15-fab-96 &.$- Y$ & 1 \\
\hline $\mathbf{Y}$ & & 2396 & $C L \_H_{-} T 2$ & $\begin{array}{l}\text { Uppor Salado clay. } 10 \\
\text { to } 25 \text { yoarz }\end{array}$ & COMP_RCK & Bulk Comprossibilty & 32066 & 15-feb-96 & $\mathbf{Y}$ & 1 \\
\hline $\mathbf{Y}$ & & 2397 & $C_{1} M_{-} T 2$ & $\begin{array}{l}\text { Upper Salado diry. } 10 \\
\text { to } 25 \text { years }\end{array}$ & PC_max & $\begin{array}{l}\text { Maxinum allowable capillary } \\
\text { pressure }\end{array}$ & 321184 & $26-0$ ct-95 & $\mathbf{Y}$ & 48 \\
\hline $\mathbf{Y}$ & & 2397 & CL_M_T2 & $\begin{array}{l}\text { Upper Salado clay: } 10 \\
\text { to } 25 \text { years }\end{array}$ & PC_MAX & $\begin{array}{l}\text { Maxmum allowable capillary } \\
\text { prossure }\end{array}$ & 321184 & 26-0ct-95 & $\mathbf{Y}$ & $A B$ \\
\hline $\mathbf{Y}$ & & 2397 & CL_M_T2 & $\begin{array}{l}\text { Upper Salaco clay: } 10 \\
\text { to } 25 \text { years }\end{array}$ & PC_max & $\begin{array}{l}\text { Maxmum allowable capilimy } \\
\text { pressure }\end{array}$ & 321184 & 26-0et-95 & $\mathbf{Y}$ & 48 \\
\hline$Y$ & & 2397 & CL_M_T2 & $\begin{array}{l}\text { Uppor Salado cigy:10 } \\
\text { lo } 25 \text { years }\end{array}$ & $P C_{\text {Imax }}$ & $\begin{array}{l}\text { Mexmum alloweblo ceapillory } \\
\text { pressure }\end{array}$ & 321184 & 26-0ct-95 & $\mathbf{Y}$ & 48 \\
\hline $\mathbf{Y}$ & & 2397 & $\mathrm{CL}_{-} \mathrm{M}_{\mathrm{T}} \mathrm{T2}$ & $\begin{array}{l}\text { Upper Selado clay.10 } \\
\text { to } 25 \text { yours }\end{array}$ & PC_max & $\begin{array}{l}\text { Maxminn allownble cepillary } \\
\text { prossure }\end{array}$ & 321184 & 26-0ct-95 & $\mathbf{Y}$ & $4 B$ \\
\hline $\mathbf{Y}$ & veries & 2398 & CL_M_T2 & $\begin{array}{l}\text { Uppor Saledo diny.10 } \\
1025 \text { years }\end{array}$ & PORE_O1S & $\begin{array}{l}\text { Brooks-Corey pore distroution } \\
\text { parametor }\end{array}$ & 32119 & $20-F 0 b-96$ & $\mathbf{Y}$ & 1 \\
\hline$Y$ & varios & 2398 & CL_M_T2 & $\begin{array}{l}\text { Upper Seleodo day. } 10 \\
\text { to } 25 \text { years }\end{array}$ & PORE_DIS & $\begin{array}{l}\text { Brooks-Coroy poro distribution } \\
\text { partemeter }\end{array}$ & 32119 & 20-Feb-96 & $\mathbf{Y}$ & 1 \\
\hline$Y$ & varies & 2398 & CL_M_T2 & $\begin{array}{l}\text { Upper Salodo day.10 } \\
\text { to } 25 \text { years }\end{array}$ & PORE_DIS & $\begin{array}{l}\text { Brocks-Corey pore distroustion } \\
\text { paremetor }\end{array}$ & 32119 & $20-f a b-96$ & $Y$ & 1 \\
\hline$Y$ & varies & 2398 & $C L_{-} M_{-} T 2$ & $\begin{array}{l}\text { Uppoer Salado cty.10 } \\
\text { to } 25 \text { years }\end{array}$ & PORE_DIS & $\begin{array}{l}\text { Brooks-Corey pore destroction } \\
\text { pernemeter }\end{array}$ & 32119 & $20-F \infty b-96$ & $\mathbf{Y}$ & 1 \\
\hline $\mathbf{Y}$ & varies & 2398 & $C L_{-} M_{-} T 2$ & $\begin{array}{l}\text { Uppor Seledo day. } 10 \\
\text { to } 25 \text { years }\end{array}$ & PORE_DIS & $\begin{array}{l}\text { Brocks-Corey pore distroution } \\
\text { peremeter }\end{array}$ & 32119 & $20-500-86$ & $r$ & 1 \\
\hline
\end{tabular}


CCA Parameter-Listing

\begin{tabular}{|c|c|c|c|c|c|c|c|c|c|c|}
\hline 10 & $\begin{array}{c}\text { Crastif } \\
\text { catson of } \\
\text { Ditt } \\
\text { Cutepory } \\
\text { (lootnotio 2) } \\
.\end{array}$ & $\begin{array}{c}\text { Diatribution } \\
\text { Type }\end{array}$ & Man & Medin & Mhatman & Inocionsen & Loth & Refernestid & $\begin{array}{l}\text { Was the datt } \\
\text { dewloped } \\
\text { conder en } \\
\text { Nel-1 } \\
\text { Frogrem? }\end{array}$ & 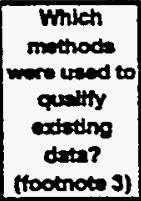 \\
\hline 2391 & $1,3,4$ & CONSTANT & $4.0000000 E+\infty$ & $4.0000000 E+\infty$ & $4.0000000 \bar{E}+\infty$ & $4.0000000 E+00$ & NONE & WP035597 & $\mathbf{Y}$ & NA \\
\hline 2391 & $1,3,4$ & CONSTANT & $4,0000000 E+00$ & $4.0000000 E+00$ & $1,0000000 E+\infty 0$ & $4.0000000 E+00$ & NONE & MP040486 & $Y$ & NA \\
\hline 2391 & $1,3,4$ & CONSTANT & $4.0000000 E+00$ & $4.0000000 E+00$ & $4.0000000 E+\infty$ & $4.00000000+\infty 0$ & NOAE & WPO40514 & $\mathbf{Y}$ & NHA \\
\hline 2392 & $1,3,4$ & CONSTANT & $7.9000000 E-01$ & 7.9000000E-01 & $7.9000000 E-01$ & $7.9000000 E-01$ & NONE & WP030540 & $\mathbf{Y}$ & NA \\
\hline 2392 & $1,3,4$ & CONSTANT & $7.9000000 E-01$ & $7.9000000 E-01$ & $7.9000000 E-01$ & $7.9000000 \mathrm{E}-01$ & NONE & WP030994 & $\mathbf{Y}$ & NA \\
\hline 2392 & $1,3,4$ & CONSTANT & $7.9000000 E-01$ & 7.9000000E-01 & $7.9000000 E-01$ & $7.9000000 E-01$ & NONE & WP035597 & $\mathbf{Y}$ & NAA \\
\hline 2392 & $1,3,4$ & CONSTANT & $7.9000000 E-01$ & 7.9000000E-01 & $7.9000000 E-01$ & $7.9000000 E-01$ & NONE & WPOSOL34 & $Y$ & N/A \\
\hline 2393 & 3,4 & CUMULATME & $2.5000000 E-01$ & $2.0000000 E-01$ & $0.0000000 E+\infty$ & $6.0000000 E-01$ & NONE & WPO30640 & $\mathbf{Y}$ & NA \\
\hline 2393 & 3,4 & CUMULATTVE & $25000000 E-01$ & $2.0000000 E-01$ & $0.0000000 E+\infty$ & $6.0000000 E-01$ & NONE & WP030994 & $\mathbf{Y}$ & NA \\
\hline 2393 & 3,4 & CUMULATIVE & $25000000 E-01$ & $20000000 E-01$ & $0.0000000 E+\infty$ & $6.00000005-01$ & NONE & WP035268 & $\mathbf{Y}$ & NA \\
\hline 2393 & 3,4 & CUMULATINE & $2.5000000 E-01$ & 2,0000000E-01 & $0.0000000 E+\infty 00$ & $6.0000000 E-01$ & NONE & WP035597 & $\mathbf{Y}$ & NA \\
\hline 2393 & 3,4 & CUMULATIVE & $2.5000000 E-01$ & $2.0000000 E-01$ & $0.0000000 E+\infty$ & $6.0000000 E-01$ & NONE & WPOA1558 & $\mathbf{Y}$ & NA \\
\hline 2393 & 3,4 & CUMULATNE & $25000000 E-01$ & $20000000 E-01$ & $0.0000000 E+\infty$ & $6.0000000 E-01$ & NONE & WP042751 & $\mathbf{Y}$ & NA \\
\hline 2394 & 3,4 & UNIFORM & $2.0000000 E-01$ & $2.0000000 E-01$ & $0.0000000 E+\infty$ & $4.0000000 E-01$ & NONE & WPO30540 & $\mathbf{Y}$ & NA \\
\hline 2394 & 3,4 & UNIFORM: & $2.0000000 E-01$ & $2.0000000 E-01$ & $0.0000000 E+\infty$ & $4.0000000 E-01$ & NONE & WP030994 & $\mathbf{Y}$ & NA \\
\hline 2394 & 3,4 & UNIFORM & $2,0000000 E-01$ & $2.0000000 E-01$ & $0.0000000 E+00$ & $4.0000000 E-01$ & NONE & WP035268 & $\mathbf{Y}$ & N/A \\
\hline 2394 & 3.4 & UNIFORM & $2.0000000 E-01$ & $2.0000000 E-01$ & $0.0000000 E+\infty$ & $4.0000000 \mathrm{E}-01$ & NONE & WP035597 & $\mathbf{Y}$ & N/A \\
\hline 2394 & 3,4 & UNIFORM & $20000000 E-01$ & $2.0000000 E-01$ & $0.0000000 E+\infty$ & $4.0000000 E-01$ & NONE & WPO41558 & $\mathbf{Y}$ & NA \\
\hline 2395 & $1,3,4$ & CONSTANT & $2.0000000 E+00$ & $2.0000000 E+\infty$ & $2.0000000 E+\infty 0$ & $2.0000000 \mathrm{E}+\infty 0$ & NONE & WP030640 & $\mathbf{Y}$ & NA \\
\hline 2395 & $1,3,4$ & CONSTANT & $2.0000000 \mathrm{E}+00$ & $2.0000000 \mathrm{E}+\infty 0$ & $2.0000000 E+\infty 0$ & $2.0000000 E+\infty$ & NONE & WP030994 & $\mathbf{Y}$ & N/A \\
\hline 2395 & $1,3,4$ & CONSTANT & $20000000 E+00$ & $2.0000000 \mathrm{E}+\infty$ & $2,0000000 E+00$ & $2.0000000 \mathrm{E}+\infty$ & NONE & WP035597 & $\mathbf{Y}$ & NA \\
\hline 2395 & $1,3,4$ & CONSTANT & $20000000 E+\infty 0$ & $2.0000000 E+\infty 0$ & $20000000 E+\infty$ & $2.0000000 E+\infty 0$ & NONE & WPO40486 & $\mathbf{Y}$ & $N A$ \\
\hline 2395 & $1,3,4$ & CONSTANT & $2.0000000 E+\infty 0$ & $2.0000000 E+\infty$ & $2.0000000 E+\infty 0$ & $2.0000000 E+\infty$ & NONE & WP040514 & $\mathbf{Y}$ & NA \\
\hline 2396 & $1,3,4$ & CONSTANT & $1.8100000 E-09$ & $1.8100000 E-09$ & $1.8100000 E-09$ & 1.8100000E-09 & Pan-1 & WP030640 & $\mathbf{Y}$ & NA \\
\hline 2396 & $1,3,4$ & CONSTANT & $1.8100000 \mathrm{E}-09$ & $1.8100000 E-09$ & $1.8100000 E-09$ & $1.8100000 E-09$ & $P a^{n}-1$ & WP030994 &.$- Y$ & N/A \\
\hline 2396 & $1,3,4$ & CONSTANT & $1.8100000 E-09$ & $1.8100000 E-09$ & $1.8100000 E-09$ & $1.8100000 \mathrm{E}-09$ & $P a^{n}-1$ & WP035597 & $\mathbf{Y}$ & NA \\
\hline 2397 & 1,3 & CONSTANT & $1.0000000 E+08$ & $1.0000000 E+08$ & $1.0000000 E+06$ & $1.0000000 E+08$ & $\mathbf{P a}$ & WP030540 & $\mathbf{Y}$ & N/A \\
\hline 2397 & 1.3 & CONSTANT & $1.0000000 E+08$ & $1.0000000 E+08$ & $1.0000000 E+08$ & $1.0000000 \mathrm{E}+08$ & Pa & WP030994 & $\mathbf{Y}$ & N/A \\
\hline 2397 & 9,3 & CONSTANT & $1.0000000 E+08$ & $1.0000000 E+08$ & $1.0000000 E+\infty 6$ & $1.0000000 E+08$ & Pะ & WP035597 & $\mathbf{Y}$ & $N / A$ \\
\hline 2397 & 1,3 & CONSTANT & $1.0000000 E+00$ & $1.0000000 E+08$ & $1.0000000 E+08$ & $1.0000000 E+08$ & $\mathrm{~Pa}$ & WPO40486 & $\mathbf{Y}$ & NA \\
\hline 2397 & 1,3 & CONSTANT & $1.0000000 E+\infty 8$ & $1.0000000 E+08$ & $1.0000000 E+\infty 8$ & $1.0000000 E+08$ & $P=$ & WPO40514 & $\mathbf{Y}$ & N/A \\
\hline 2398 & 3,4 & CUMULATINE & $2.5200000 E+\infty 0$ & 9.4000000E-01 & $1.1000000 E-01$ & $8.1000000 E+\infty$ & NONE & WP030540 & $\mathbf{Y}$ & NA \\
\hline 2398 & 3,4 & CUMULATINE & $2.5200000 E+00$ & $9.4000000 E-01$ & $1.1000000 E-01$ & $8.1000000 E+\infty$ & NONE & WP030994 & $Y$ & NA \\
\hline 2398 & 3,4 & CUMULATME & $2.5200000 E+\infty 0$ & $9.4000000 E-01$ & 1.1000000E-01 & $8.1000000 E+\infty 0$ & NONE & WP032287 & $Y$ & NA \\
\hline 2398 & 3,4 & CUMULATIVE & $2.5200000 E+\infty 0$ & $9.4000000 E-01$ & $1.1000000 E-01$ & $8.1000000 E+00$ & NONE & WP035268 & $\mathbf{Y}$ & $N A$ \\
\hline 2398 & 3.4 & CUMULATINE & $2.5200000 E+00$ & $9.4000000=-01$ & $1.1000000 \equiv-01$ & $8.1000000 E+\infty 0$ & NONE & WP035597 & $\mathbf{Y}$ & NA \\
\hline
\end{tabular}


CCA Parameter Listing

\begin{tabular}{|c|c|c|c|c|c|c|c|c|c|c|}
\hline 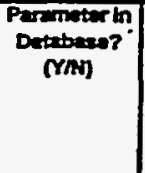 & 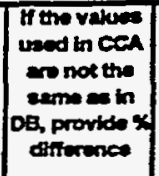 & ID & Matertat & Moterlat Atam & Pandin & Permation & $\begin{array}{l}\text { PRP DD } \\
\text { (MPOA) }\end{array}$ & Defa Entry & 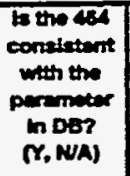 & $\begin{array}{c}\text { Parmoter } \\
\text { Cotocony } \\
\text { (footiote } 1 \text { ) }\end{array}$ \\
\hline $\mathbf{Y}$ & varies & 2398 & $C L_{-} M_{-} T_{2}$ & $\begin{array}{l}\text { Upper Salado ctay: } 10 \\
\text { to } 25 \text { years }\end{array}$ & PORE_DIS & $\begin{array}{l}\text { Erooks-Corey pore dastribution } \\
\text { parameter }\end{array}$ & 32119 & $20+50-56$ & $\mathbf{Y}$ & 1 \\
\hline $\mathbf{Y}$ & & 2399 & $C L / M+T 2$ & $\begin{array}{l}\text { Upper Salsco ctay: } 10 \\
\text { to } 25 \text { yasus }\end{array}$ & POROSITY & Effective porcasty & 32120 & 15 F cb-96 & $\mathbf{Y}$ & 1 \\
\hline $\mathbf{Y}$ & & 2399 & $C L_{-} M_{-} T_{2}$ & $\begin{array}{l}\text { Upper Seledo ctry: } 10 \\
\text { to } 25 \text { years }\end{array}$ & POROSTY & Effective porosity & 32120 & $15+500-86$ & $\mathbf{Y}$ & 1 \\
\hline $\mathbf{Y}$ & & 2399 & CL_M_T2 & $\begin{array}{l}\text { Upper Salado day.10 } \\
\text { to } 25 \text { yeers }\end{array}$ & POROSTY & Effective porosity & 32120 & $15-60-96$ & $\mathbf{Y}$ & 1 \\
\hline $\mathbf{Y}$ & & 2400 & CL_M_T2 & $\begin{array}{l}\text { Upper Salsco clay:10 } \\
\text { to } 25 \text { yoers }\end{array}$ & PO_MIN & 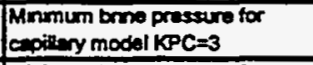 & 32121 & 21 feb-os & $\mathbf{Y}$ & 48 \\
\hline $\mathbf{Y}$ & & 2400 & $C L \_M \_T 2$ & $\begin{array}{l}\text { Upper Salodo day. } 10 \\
\text { to } 25 \text { years }\end{array}$ & PO_MIN & $\begin{array}{l}\text { Minimum brim proseure for } \\
\text { capiliery model } K P C=3\end{array}$ & 32121 & 21feb-s6 & $\mathbf{Y}$ & 48 \\
\hline $\mathbf{Y}$ & & 2400 & $C L \_M_{-} T 2$ & $\begin{array}{l}\text { Uppor Saledo ciny: } 10 \\
\text { to } 25 \text { years }\end{array}$ & PO_MIN & $\begin{array}{l}\text { Minmum brine prosecte for } \\
\text { capilley model } \mathrm{KPC}=3\end{array}$ & 32121 & $21-F a b-96$ & $\mathbf{Y}$ & 48 \\
\hline $\mathbf{Y}$ & vares & 2402 & $C L_{-} M_{-} T 2$ & $\begin{array}{l}\text { Uppor Salodo ciay: } 10 \\
\text { to } 25 \text { years }\end{array}$ & PRMX_LOG & $\begin{array}{l}\text { Log of intminse pacmasbitty. } X \text { - } \\
\text { diroction }\end{array}$ & 32123 & 15fab-96 & $\mathbf{Y}$ & 1 \\
\hline $\mathbf{Y}$ & varies & 2402 & $C_{L} M_{-} T_{2}$ & $\begin{array}{l}\text { Upper Sulado clay: } 10 \\
\text { to } 25 \text { yeare }\end{array}$ & PRAX_LOG & $\begin{array}{l}\text { Log of intrinsic permesbility, } X \text { - } \\
\text { direction }\end{array}$ & 32123 & $15+60-96$ & $\mathbf{Y}$ & 1 \\
\hline $\mathbf{Y}$ & vanes & 24002 & $C_{2} M_{-} T 2$ & $\begin{array}{l}\text { Upper Salacos clay: } 10 \\
\text { to } 25 \text { yeers }\end{array}$ & PRMX_LOG & $\begin{array}{l}\text { Log of intrassic pormeability, } X \text { - } \\
\text { drection }\end{array}$ & 32123 & $15-F a b-96$ & $\mathbf{Y}$ & 1 \\
\hline $\mathbf{Y}$ & varies & 2402 & $C L \_M-T 2$ & $\begin{array}{l}\text { Upper Salado clay. } 10 \\
\text { to } 25 \text { years }\end{array}$ & PRMX_LOG & $\begin{array}{l}\text { Los of indonsic pecmeabitity, } X \text { - } \\
\text { drection }\end{array}$ & 32123 & 15-Feb-96 & $\mathbf{Y}$ & 1 \\
\hline $\mathbf{Y}$ & varies & 2402 & CL_M_T2 & $\begin{array}{l}\text { Upper Salado clay: } 10 \\
\text { to } 25 \text { years }\end{array}$ & FRMX_LOG & $\begin{array}{l}\text { Log of intrinsic pormasbilty, } X \text { - } \\
\text { ofrection }\end{array}$ & 32123 & 15Feb-96 & $\mathbf{Y}$ & 1 \\
\hline $\mathbf{Y}$ & & 2403 & $C L \_M \_T 2$ & $\begin{array}{l}\text { Upper Saledo clay: } 10 \\
\text { to } 25 \text { years }\end{array}$ & PRMY_LOS & $\begin{array}{l}\text { Log of intrinsic permesbility, Y- } \\
\text { direction }\end{array}$ & 32124 & $15+F<-96$ & $\mathbf{Y}$ & 1 \\
\hline $\mathbf{Y}$ & & 2403 & CL_M_T2 & $\begin{array}{l}\text { Upper Seledo day:10 } \\
\text { to } 25 \text { years }\end{array}$ & PRAYY_LOG & $\begin{array}{l}\text { Log of intrinas perm esbility. Y- } \\
\text { direction }\end{array}$ & 32124 & 15-Fub-86 & $\mathbf{Y}$ & 1 \\
\hline $\mathbf{Y}$ & & 2403 & CL_M_T2 & $\begin{array}{l}\text { Upper Salado cty: } 10 \\
\text { to } 25 \text { years }\end{array}$ & PRMY_LOG & $\begin{array}{l}\text { Log of intrinsere permastidy, Y- } \\
\text { direction }\end{array}$ & 32124 & $15+F c b-96$ & $\mathbf{Y}$ & 1 \\
\hline $\mathbf{Y}$ & & 2403 & CL_M_T2 & $\begin{array}{l}\text { Uppor Salado clay:10 } \\
\text { to } 25 \text { years }\end{array}$ & PRMY_LOG & $\begin{array}{l}\text { Log of intrinse pexmesbilty, Y. } \\
\text { direction }\end{array}$ & 32124 & 15-Fob-96 & $\mathbf{Y}$ & 1 \\
\hline $\mathbf{Y}$ & & 2403 & $C L_{-} M_{-} T 2$ & $\begin{array}{l}\text { Upper Salado clay.10 } \\
\text { to } 25 \text { years }\end{array}$ & PRMY_LOG & $\begin{array}{l}\text { Log of intronsic pormesbilty, Y- } \\
\text { diroction }\end{array}$ & 32124 & 15-Feb-96 & $\mathbf{Y}$ & 1 \\
\hline $\mathbf{Y}$ & & 2404 & CL_M_T2 & $\begin{array}{l}\text { Upper Salado clay:10 } \\
\text { to } 25 \text { years } \\
\end{array}$ & PRMZ_LOG & $\begin{array}{l}\text { Log of intensic permasbilty, Z- } \\
\text { direction }\end{array}$ & 32125 & $15+a b-96$ & $Y$ & 1 \\
\hline$Y$ & & 2404 & $C L_{-} M_{-} T 2$ & $\begin{array}{l}\text { Uppor Salado clay:10 } \\
\text { to } 25 \text { yeers }\end{array}$ & PRMZ_LOG & $\begin{array}{l}\text { Los of intensse permesbility, Z- } \\
\text { drection }\end{array}$ & 32125 & $15-\cos -96$ & $\mathbf{Y}$ & 1 \\
\hline$Y$ & & 2404 & CL_M_T2 & $\begin{array}{l}\text { Uppor Salado ciry:10 } \\
\text { to } 25 \text { yers }\end{array}$ & PRMZ_LOG & $\begin{array}{l}\text { Los of intrnsk permasbilty, z- } \\
\text { drection }\end{array}$ & 32125 & $15+50 b-96$ & $\mathbf{Y}$ & 1 \\
\hline $\mathbf{Y}$ & & 2404 & CL_M_T2 & $\begin{array}{l}\text { Upper Salodo clay. } 10 \\
\text { to } 25 \text { years }\end{array}$ & PRMIZLOG & $\begin{array}{l}\text { Log of intrinsic pormestidty. Z- } \\
\text { direction }\end{array}$ & 32125 & 15 feb-96 & $\mathbf{Y}$ & 1 \\
\hline$\gamma$ & & 2404 & $C L \_M_{-} T 2$ & $\begin{array}{l}\text { Upper Salado clay:10 } \\
\text { to } 25 \text { years }\end{array}$ & PRME_LOG & $\begin{array}{l}\text { Log of intrinsic permesbility. Z- } \\
\text { direction }\end{array}$ & 32125 & $15+5=b-96$ & $\mathbf{Y}$ & 1 \\
\hline $\mathbf{Y}$ & & 2408 & CL_M_T2 & $\begin{array}{l}\text { Upper Salado clay. } 10 \\
\text { to } 25 \text { years }\end{array}$ & RELP_MOD & $\begin{array}{l}\text { Model number, reletive } \\
\text { permeability model }\end{array}$ & 321304 & 15 Feb-96 & $\mathbf{Y}$ & $4 B$ \\
\hline $\mathbf{Y}$ & & 2408 & CL_M_T2 & $\begin{array}{l}\text { Upper Salado clay:10 } \\
\text { to } 25 \text { year }\end{array}$ & RELP_MOD & $\begin{array}{l}\text { Model number, relative } \\
\text { permeability model }\end{array}$ & $32130 \mathrm{~A}$ & 15-Fab-96 & $\mathbf{Y}$ & $4 B$ \\
\hline $\mathbf{Y}$ & & 2408 & $C_{2} M_{-} T 2$ & $\begin{array}{l}\text { Upper Salodo clyy:10 } \\
\text { to } 25 \text { years }\end{array}$ & RELP_MOD & $\begin{array}{l}\text { Model number, rolutive } \\
\text { permeability model }\end{array}$ & 321304 & 15-Fob-96 & $-\boldsymbol{Y}$ & 48 \\
\hline $\mathbf{Y}$ & & 2408 & $\mathrm{CL}_{-} \mathrm{M}_{-} \mathrm{T} 2$ & $\begin{array}{l}\text { Upper Saledo clay: } 10 \\
\text { to } 25 \text { years }\end{array}$ & RELP_MOD & $\begin{array}{l}\text { Model number, relatuve } \\
\text { permeability model }\end{array}$ & 321304 & $15-F b-96$ & $\mathbf{Y}$ & $4 B$ \\
\hline $\mathbf{Y}$ & & 2408 & CL_M_T2 & $\begin{array}{l}\text { Upper Saludo cisy: } 10 \\
\text { to } 25 \text { yours }\end{array}$ & RELP_MOD & $\begin{array}{l}\text { Model number, relative } \\
\text { permeability model }\end{array}$ & $32130 \mathrm{~A}$ & $15+$ ob-96 & $\mathbf{Y}$ & 48 \\
\hline $\mathbf{Y}$ & 100 & 2410 & $C L+H_{-} T 2$ & $\begin{array}{l}\text { Upper Saledo day: } 10 \\
\text { to } 25 \text { years }\end{array}$ & SAT_RBRN & Resicusal Brine Saturation & 32136 & $15-F a b-96$ & $\mathbf{Y}$ & 1 \\
\hline $\mathbf{Y}$ & 100 & 2410 & CL_M_T2 & $\begin{array}{l}\text { Upper Seledo clay: } 10 \\
\text { to } 25 \text { years }\end{array}$ & SAT_RBRN & Residual Brine Saturation & 32136 & 15-fob-96 & $\mathbf{Y}$ & 1 \\
\hline $\mathbf{Y}$ & 100 & 2410 & CL_M_T2 & $\begin{array}{l}\text { Upper Salado ciay.10 } \\
\text { to } 25 \text { years }\end{array}$ & SAT_RBRN & Resicual Brine Saturation & 32136 & 15-Fob-96 & $\mathbf{Y}$ & 1 \\
\hline $\mathbf{Y}$ & 100 & 2410 & CL_M_T2 & $\begin{array}{l}\text { Upper Selado ding.10 } \\
\text { to } 25 \text { yeers }\end{array}$ & SAT_RBRN & Residual Brine Seturation & 32136 & $15-F a b-96$ & $\mathbf{Y}$ & 1 \\
\hline $\mathbf{Y}$ & 100 & 2410 & CL_M_T2 & $\begin{array}{l}\text { Upper Seleco ctry.10 } \\
\text { to } 25 \text { yeare }\end{array}$ & SAT_RBRN & Resictual Brino Seturation & 32136 & $15-F a b-96$ & $\mathbf{Y}$ & 1 \\
\hline $\mathbf{Y}$ & 100 & 2410 & $C L \_M-T 2$ & $\begin{array}{l}\text { Upper Salado doy: } 10 \\
\text { to } 25 \text { years }\end{array}$ & SAT_RBRH & Residusal Brina Saturation & 32136 & $15+$ co-96 & $\mathbf{Y}$ & 1 \\
\hline $\mathbf{Y}$ & 100 & 2411 & $C L \_M \_T 2$ & $\begin{array}{l}\text { Upper Salado clay: } 10 \\
\text { to } 25 \text { yeare }\end{array}$ & SAT_RGAS & Residud Ges Suturation & 32137 & 15-feb-96 & $\mathbf{Y}$ & 1 \\
\hline $\mathbf{Y}$ & 100 & 2411 & CL_M_T2 & $\begin{array}{l}\text { Upper Seledo day:10 } \\
\text { to } 25 \text { years }\end{array}$ & SAT_RGAS & Residual Ges Semration & 32137 & 15-Fab-96 & $\mathbf{Y}$ & 1 \\
\hline $\mathbf{Y}$ & 100 & 2411 & $C L \_M \_T 2$ & $\begin{array}{l}\text { Upper Saludo ciry:10 } \\
\text { to } 25 \text { years }\end{array}$ & SAT_RGAS & Resideal Ges Serturation & 32137 & $15+a b-96$ & $\mathbf{Y}$ & 4 \\
\hline
\end{tabular}


CCA-Parameter-Listing

\begin{tabular}{|c|c|c|c|c|c|c|c|c|c|c|}
\hline to & $\begin{array}{l}\text { Cinestit- } \\
\text { catton of } \\
\text { Deta } \\
\text { Cetegory } \\
\text { (footnote 2) }\end{array}$ & $\begin{array}{l}\text { Distribution } \\
\text { Type }\end{array}$ & Man & Medien & Minionem & Maximin & Tnits & Refermen DD & $\begin{array}{l}\text { Wes the dith } \\
\text { dencloped } \\
\text { underen } \\
\text { Nol-1 } \\
\text { Progrem? }\end{array}$ & $\begin{array}{l}\text { Which } \\
\text { methods } \\
\text { morv ueed to } \\
\text { quithy } \\
\text { exdeting } \\
\text { duth? } \\
\text { (footnotis 3) }\end{array}$ \\
\hline 2398 & 3,4 & CUMULATINE & $2.5200000 E+\infty$ & $9.4000000 E-01$ & $1.1000000 E-01$ & $8.1000000 E+\infty 0$ & NONE & WPOS1558 & $\mathbf{Y}$ & NA \\
\hline 2399 & $1,3,4$ & CONSTANT & $2.4000000 E-01$ & 2.4000000E-01 & $2.4000000 E-01$ & 2.4000000E-01 & $m^{\wedge} 3 / m^{\wedge} 3$ & MPO30640 & $\mathbf{Y}$ & N/A \\
\hline 2399 & $1,3,4$ & CONSTANT & 2.4000000E-01 & $2.4000000 E-01$ & 24000000E-01 & $24000000 E-01$ & $m^{\wedge} 3 m^{\wedge} 3$ & WP0s0994 & $\mathbf{Y}$ & NA \\
\hline 2399 & $1,3,4$ & CONSTANT & $2.4000000 E-01$ & $2.4000000 E-01$ & $24000000 E-01$ & 2.4000000E-01 & $m^{\wedge} 3 / m^{n} 3$ & WP035597 & $\mathbf{Y}$ & NA \\
\hline 2400 & 1,3 & CONSTANT & $1.0132500 E+05$ & $1.0132500 E+05$ & $1.0132500 E+05$ & $1.0132500 E+05$ & Pa & WP035597 & $\mathbf{Y}$ & NA \\
\hline 2400 & 1,3 & CONSTANT & $1.0132500 E+05$ & $1.0132500 E+05$ & $1.0132500 E+05$ & $1.0132500 E+05$ & $\mathrm{~Pa}$ & ${\text { WPO } 030051_{1}}_{1}$ & $\mathbf{Y}$ & NA \\
\hline 2400 & 1,3 & CONSTANT & $1.0132500 E+05$ & $1.0132500 \mathrm{E}+0.5$ & $1.0132500 E+05$ & $1.0132500 \mathrm{E}+0.5$ & Pa & WPO40514 & $\mathbf{Y}$ & NA \\
\hline 2402 & $1,3,4$ & TRLANGULAR & $-1.8857000 E+01$ & $-1.8301000 E+01$ & $-2.1000000 E+01$ & $-1.7301000 E+01$ & $\log \left(m^{n} 2\right)$ & WP030s40 & YEN & 1 \\
\hline 2402 & $1,3,4$ & TRLANGULAR & $-1.8857000 E+01$ & $-1.8301000 E+01$ & $-2.1000000 E+01$ & $-1.7301000 E+01$ & $\log \left(m^{\wedge} 2\right)$ & WP030994 & Y\&N & 1 \\
\hline 2402 & $1,3,4$ & TRLANGULAR & $-1.8857000 E+01$ & $-1.8301000 E+01$ & $-2.1000000 E+01$ & $-1.7301000 E+01$ & $\log \left(m^{n} 2\right)$ & WP035268 & YaN & 1 \\
\hline 2402 & $1,3,4$ & TRLANGULAR & $-1.8857000 E+01$ & $-1.8301000 E+01$ & $-2.1000000 E+01$ & $-1.7301000 E+01$ & $\log \left(m^{\wedge} 2\right)$ & WPO35597 & Y\&N & 1 \\
\hline 2402 & $1,3,4$ & TRLANGULAR & $-1.8867000 E+01$ & $-1.8301000 E+01$ & $-2.1000000 E+01$ & $-1.7301000 E+01$ & $\log \left(m^{\wedge} 2\right)$ & WP041558 & Y\&N & 1 \\
\hline 2403 & $1,3,4$ & TRIANGULAR & $-1.8867000 E+01$ & $-1.8301000 E+01$ & $-2.1000000 E+01$ & $-1.7301000 E+01$ & $\log \left(m^{\wedge} 2\right)$ & WP030540 & YaN & 1 \\
\hline 2403 & $\$, 3,4$ & TRLANGULAR & $-1.8867000 E+01$ & $-1.8301000 E+01$ & $-2.1000000 E+01$ & $-1.7301000 E+01$ & $\log \left(m^{\wedge} 2\right)$ & WP030994 & YaN & 1 \\
\hline 2403 & $1,3,4$ & TRLANGULAR & $-1.8867000 E+01$ & $-1.8301000 E+01$ & $-2.1000000 E+01$ & $-1.7301000 E+01$ & $\log \left(m^{\wedge} 2\right)$ & WP035268 & YaN & 1 \\
\hline 2403 & $1,3,4$ & TRLANGULAR & $-1.8867000 E+01$ & $-1.8301000 E+01$ & $-2.1000000 E+01$ & $-17301000 E+01$ & $\cos \left(m^{\wedge} 2\right)$ & WPO35597 & YaN & 1 \\
\hline 2403 & $1,3,4$ & TRIANGULAR & $-1.8867000 \mathrm{E}+01$ & $-1.8301000 E+01$ & $-21000000 E+01$ & $-17301000 E+01$ & $\log \left(m^{\wedge} 2\right)$ & WP041558 & YaN & 1 \\
\hline 2404 & $1,3,4$ & TRIANGULAR & $-1.8867000 E+01$ & $-1.8301000 E+01$ & $-21000000 E+01$ & $-17301000 E+01$ & $\log \left(m^{\wedge} 2\right)$ & WP030540 & YaN & 1 \\
\hline 2404 & $1,3,4$ & TRIANGULAR & $-1.8867000 E+01$ & $-18301000 E+01$ & $-21000000 E+01$ & $-17301000 E+01$ & $\log \left(m^{\wedge} 2\right)$ & WP030994 & Y\&N & 1 \\
\hline 2404 & $1,3,4$ & TRLANGULAR & $-1.8867000 E+01$ & $-1,8301000 E+01$ & $-21000000<+01$ & - $7301000 E+01$ & $\log \left(m^{n} 2\right)$ & WP035268 & Y\&N & 1 \\
\hline 2404 & $1,3,4$ & TRIANGULAR & $-1.8867000 E+01$ & $-18301000 E+01$ & $-21000000 E+01$ & $-17301000 E+01$ & $\log \left(m^{\wedge} 2\right)$ & WP035597 & Y\&N & 1 \\
\hline 2404 & $1,3,4$ & TRIANGULAR & $-1.8867000 E+01$ & $-1.8301000 E+01$ & $-21000000 E+01$ & $-17301000 E+01$ & $\log \left(m^{\wedge} 2\right)$ & WPO41558 & Y $8 N$ & 1 \\
\hline 2408 & $1,3,4$ & CONSTANT & $4.0000000 E+00$ & $40000000 E+\infty$ & $40000000 \times+\infty$ & $4000000<+\infty$ & NONE & WP030640 & $\mathbf{Y}$ & NA \\
\hline 2408 & $1,3,4$ & CONSTANT & $4.0000000 E+\infty 0$ & $40000000 E+\infty$ & $4000000 \times-\infty$ & $4000000 \times+\infty$ & NONE & WP030994 & $\mathbf{Y}$ & $N / A$ \\
\hline 2408 & $1,3,4$ & CONSTANT & $4.0000000 \mathrm{E}+\infty 0$ & $40000000 E+\infty$ & $4 \infty \infty 0000 \in+\infty$ & $400000006+\infty$ & NONE & WP035597 & $-x$ & NA \\
\hline 2408 & $1,3,4$ & CONSTANT & $4.0000000 E+\infty 0$ & $40000000<\cdot \infty$ & $4000000<-\infty$ & $\Delta \infty 0000<<+\infty$ & NONE & WPO40486 & $\mathbf{Y}$ & NA \\
\hline 2408 & $1,3,4$ & CONSTANT & $4.0000000 E+\infty$ & $40000000 E+\infty$ & $4 \infty 00000<-\infty$ & $4000000<-\infty$ & NONE & WPO40514 & $\mathbf{Y}$ & NAA \\
\hline 2410 & 3,4 & CUMULATINE & $2.5000000 E-01$ & $20000000 E-01$ & $00000000<+\infty$ & $6 \infty \infty 0000$ - 01 & NONE & WP030640 & $\mathbf{Y}$ & NA \\
\hline 2410 & 3,4 & CUMULATME & $2.5000000 E-01$ & $20000000 E-01$ & $00000000<-\infty$ & $60000000 E-01$ & NONE & WP030994 & $\mathbf{Y}$ & NA \\
\hline 2410 & 3,4 & CUMULATIVE & $2.5000000 E-01$ & $20000000 E-01$ & $0,000000<-\infty$ & 60000000.01 & NONE & MP035268 & $\mathbf{Y}$ & NA \\
\hline 2410 & 3,4 & CUMULATIVE & $2.5000000 E-01$ & $20000000 E-0 ?$ & $0,000000<-\infty$ & $60000000 \in-01$ & NONE & WP035597 & $\mathbf{Y}$ & NA \\
\hline 2410 & 3,4 & CUMULATINE & $2.5000000 E-01$ & $20000000 E-01$ & $0,0000000<-00$ & $60000000 E-01$ & NONE & WPO41558 & $\mathbf{Y}$ & N/A \\
\hline 2410 & 3,4 & CUMULATINE & $2.5000000 E-01$ & $20000000 E-01$ & $00000000 \times+\infty$ & $60000000 E-01$ & NONE & WPO42751 & $\mathbf{Y}$ & N/A \\
\hline 2411 & 3,4 & UNIFORM & $2.0000000 E-01$ & $20000000 E-01$ & $0,0000000=0 \infty$ & $\triangle 0000000-01$ & NONE & WPO30640 & $Y$ & NA \\
\hline 2411 & 3,4 & UNIFORM & $2.0000000 E-09$ & $20000000 E-01$ & $00000000=+\infty$ & $40000000 E-01$ & NONE & WP030994 & $\mathbf{Y}$ & NA \\
\hline 2411 & 3,4 & UMIFORM & $2.0000000 E-01$ & $2.0000000 E-01$ & $00000000=40$ & $40000000 E-01$ & NONE & WP035268 & $Y$ & $\boldsymbol{N A}$ \\
\hline
\end{tabular}


CCA Parameter Listing

\begin{tabular}{|c|c|c|c|c|c|c|c|c|c|c|}
\hline $\begin{array}{c}\text { Paremeter in } \\
\text { Detabeas? } \\
\text { (YNN) }\end{array}$ & 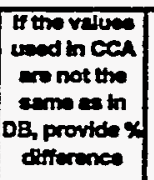 & ID & Metara to & Mara Nam & Peramatar & Parmeter Narts & $\begin{array}{l}\text { FRPD } \\
\text { Mrot }\end{array}$ & Denentry & 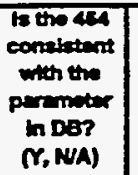 & $\begin{array}{l}\text { Parmitar } \\
\text { Centegory } \\
\text { (footiota 1) }\end{array}$ \\
\hline $\mathbf{Y}$ & 100 & 2411 & CL_M_T2 & $\begin{array}{l}\text { Upper Salado clay: } 10 \\
\text { to } 25 \text { years }\end{array}$ & SAT_RGAS & Residual Ges Suturation & 32137 & 15Fab-96 & $\mathbf{Y}$ & 1 \\
\hline $\mathbf{Y}$ & 100 & 2411 & CL_M_T2 & $\begin{array}{l}\text { Upper Satado clay: } 10 \\
\text { to } 25 \text { years }\end{array}$ & SAT_RGAS & Residual Ges Seturation & 32137 & 15 Febse & $\mathbf{Y}$ & 1 \\
\hline $\mathbf{Y}$ & 50 & 2412 & CL_M_T3 & $\begin{array}{l}\text { Upper Soledo ctay.25 } \\
\text { to } 50 \text { yeers }\end{array}$ & CAP_MOD & $\begin{array}{l}\text { Model number, capillary pressure } \\
\text { model }\end{array}$ & 321394 & $15+a b-96$ & $\mathbf{Y}$ & 48 \\
\hline $\mathbf{Y}$ & 50 & 2412 & CL_M_T3 & $\begin{array}{l}\text { Upper Salado clay. } 25 \\
\text { to } 50 \text { years }\end{array}$ & CAP_MOD & $\begin{array}{l}\text { Model number, capillay pressure } \\
\text { model }\end{array}$ & $32139 \mathrm{~A}$ & 15-Fab-9s & $\mathbf{Y}$ & 48 \\
\hline $\mathbf{Y}$ & 50 & 2412 & CL_M_T3 & $\begin{array}{l}\text { Upper Solicos clay.25 } \\
\text { to } 50 \text { years }\end{array}$ & CAP_MOD & $\begin{array}{l}\text { Hodel iumber, capillary pressure } \\
\text { modil }\end{array}$ & $32139 A$ & $15+500-96$ & $\mathbf{Y}$ & 48 \\
\hline $\mathbf{Y}$ & 50 & 2412 & CL_M_T3 & $\begin{array}{l}\text { Upper Saledo ctay.25 } \\
\text { to } 50 \text { years }\end{array}$ & CAP_MOD & $\begin{array}{l}\text { Hodel mumber, capillay pressure } \\
\text { model }\end{array}$ & $32139 A$ & $15+a b-96$ & $\mathbf{Y}$ & 48 \\
\hline $\mathbf{Y}$ & 50 & 2412 & CL_M_T3 & $\begin{array}{l}\text { Upper Saledo day } 25 \\
\text { to } 50 \text { years }\end{array}$ & CAP_MOD & $\begin{array}{l}\text { Modal number, copiliary prosere } \\
\text { model }\end{array}$ & 321394 & $15 f a b-96$ & $\mathbf{Y}$ & $4 B$ \\
\hline $\mathbf{Y}$ & & 2413 & CL_M_T3 & $\begin{array}{l}\text { Uppor Saledo clay.25. } \\
\text { to } 50 \text { years }\end{array}$ & COMP_RCK & Bulk Compressibitity & 32141 & $15-F=b-96$ & $\mathbf{Y}$ & 1 \\
\hline $\mathbf{Y}$ & & 2413 & CL_M_T3 & $\begin{array}{l}\text { Upper Salado elay.25 } \\
\text { to } 50 \text { years }\end{array}$ & COMP_RCK & Bulk Compressibitity & 32149 & 15-Feb-96 & $\mathbf{Y}$ & 1 \\
\hline $\mathbf{Y}$ & & 2413 & CL_M_T3 & $\begin{array}{l}\text { Upper Salado day:25 } \\
\text { to } 50 \text { years }\end{array}$ & COMP_RCK & Bukk Compressibitity & 32141 & 15-Feb-96 & $\mathbf{Y}$ & 1 \\
\hline $\mathbf{Y}$ & & 2414 & CL_M_T3 & $\begin{array}{l}\text { Upper Salado day: } 25 \\
\text { to } 50 \text { years }\end{array}$ & PC_MAX & $\begin{array}{l}\text { Mexmim allowable capillary } \\
\text { pressure }\end{array}$ & $32145 A$ & $26-004-95$ & $\mathbf{Y}$ & $4 B$ \\
\hline $\mathbf{Y}$ & & 2414 & $C L_{2} M_{-} T_{3}$ & $\begin{array}{l}\text { Uppor Salado clay } 25 \\
\text { to } 50 \text { years }\end{array}$ & PC_MAX & $\begin{array}{l}\text { Mexmum allowable cepillery } \\
\text { pressure }\end{array}$ & $32145 \mathrm{~A}$ & $26-0 c t-95$ & $\mathbf{Y}$ & $A B$ \\
\hline $\mathbf{Y}$ & & 2414 & CL_M_T3 & $\begin{array}{l}\text { Upper Salado ctay.25 } \\
\text { to } 50 \text { years }\end{array}$ & PC_MAX & $\begin{array}{l}\text { Maxumum allowable capillary } \\
\text { pressure }\end{array}$ & $32145 A$ & $26-004-95$ & $\mathbf{Y}$ & $4 B$ \\
\hline $\mathbf{Y}$ & & 2414 & $C L \_M_{-} T 3$ & $\begin{array}{l}\text { Upper Salado clay:25 } \\
\text { to } 50 \text { years }\end{array}$ & PC_MAX & $\begin{array}{l}\text { Maximum allowabla capillary } \\
\text { pressure }\end{array}$ & $32145 A$ & 26-0ct-95 & $\mathbf{Y}$ & $4 B$ \\
\hline $\mathbf{Y}$ & & 2444 & $C L_{-} M_{-} T_{3}$ & $\begin{array}{l}\text { Uppor Salado clay. } 25 \\
\text { to } 50 \text { yaars }\end{array}$ & PC_MAX & $\begin{array}{l}\text { Maxumum alloweble capillary . } \\
\text { pressure }\end{array}$ & $32145 A$ & 26-0at-85 & $\mathbf{Y}$ & 48 \\
\hline $\mathbf{Y}$ & varies & 2415 & $C L_{-} M_{-} T 3$ & $\begin{array}{l}\text { Upper Salado clay.25 } \\
\text { to } 50 \text { years }\end{array}$ & PORE_DIS & $\begin{array}{l}\text { Brooks-Corey pore distribution } \\
\text { parameter }\end{array}$ & 32146 & 20-Feb-96 & $\mathbf{Y}$ & 1 \\
\hline $\mathbf{Y}$ & varies & 2415 & CL_M_T3 & $\begin{array}{l}\text { Upper Salado clay.25 } \\
\text { to } 50 \text { years }\end{array}$ & PORE_DIS & $\begin{array}{l}\text { Brooks-Corey pore distribution } \\
\text { parameter }\end{array}$ & 32146 & $20-50 b-96$ & $\mathbf{Y}$ & 1 \\
\hline $\mathbf{Y}$ & varies & 2415 & $C L \_M \_T 3$ & $\begin{array}{l}\text { Upper Salado ctay:25 } \\
\text { to } 50 \text { years }\end{array}$ & PORE_DIS & $\begin{array}{l}\text { Brooks-Corey pore distribution } \\
\text { parameter }\end{array}$ & 32146 & 20-Feb-96 & $Y$ & 1 \\
\hline $\mathbf{Y}$ & varies & 2415 & $C C_{-} M_{-} \mathrm{T}$ & $\begin{array}{l}\text { Upper Salado clay. } 25 \\
\text { to } 50 \text { yoars }\end{array}$ & PORE_DIS & $\begin{array}{l}\text { Brooks-Corey pore distnbution } \\
\text { perameter }\end{array}$ & 32146 & 20-Fob-96 & $\mathbf{Y}$ & 1 \\
\hline $\mathbf{Y}$ & varios & 2415 & CL_M_T3 & $\begin{array}{l}\text { Upper Salado clay.25 } \\
\text { to } 50 \text { yoars }\end{array}$ & PORE_DIS & $\begin{array}{l}\text { Brooks-Corey pore distribution } \\
\text { parameter }\end{array}$ & 32146 & $20-f e b-96$ & $\mathbf{Y}$ & 1 \\
\hline $\mathbf{Y}$ & varies & 2415 & CL_M_T3 & $\begin{array}{l}\text { Upper Salado clay.25 } \\
\text { to } 50 \text { years }\end{array}$ & PORE_DIS & $\begin{array}{l}\text { Brooks-Corey pore distritoution } \\
\text { parameter }\end{array}$ & 32146 & 20-Feb-96 & $\mathbf{Y}$ & 1 \\
\hline $\mathbf{Y}$ & & 2416 & CL_M_T3 & $\begin{array}{l}\text { Uppor Salado clay. } 25 \\
\text { to } 50 \text { years }\end{array}$ & POROSTIY & Effective porostity & 32148 & 15-Feb-96 & $\mathbf{Y}$ & 1 \\
\hline $\mathbf{Y}$ & & 2416 & CL_M_T3 & $\begin{array}{l}\text { Upper Salado clay: } 25 \\
\text { to } 50 \text { years }\end{array}$ & POROSTrY & Effective porosity & 32148 & 15feb-9s & $\mathbf{Y}$ & 1 \\
\hline $\mathbf{Y}$ & & 2416 & CL_M_T3 & $\begin{array}{l}\text { Upper Sulado ctoy:.25 } \\
\text { to } 50 \text { years }\end{array}$ & POROSTr & Efiectivo porosty & 32148 & 15-Feb-96 & $Y$ & 1 \\
\hline $\mathbf{Y}$ & & 2417 & CL_M_T3 & $\begin{array}{l}\text { Upper Salado ciry.25 } \\
\text { to } 50 \text { years }\end{array}$ & PO_MIN & $\begin{array}{l}\text { Mrnumum onme pressure for } \\
\text { Capllary model KPC=3 }\end{array}$ & 32150 & 21 Fob-96 & $-Y Y$ & $4 B$ \\
\hline $\mathbf{Y}$ & & 2417 & CL_H_T3 & $\begin{array}{l}\text { Upper Saledo day.25 } \\
\text { to } 50 \text { years }\end{array}$ & PO_MIN & $\begin{array}{l}\text { Minmum brea pressuro for } \\
\text { capliery modal } \mathrm{KPC}=3\end{array}$ & 32150 & 21-Fob-96 & $\mathbf{Y}$ & $A B$ \\
\hline $\mathbf{Y}$ & & 2417 & CL_M_T3 & $\begin{array}{l}\text { Upper Salsob cay. } 25 \\
\text { to } 50 \text { years }\end{array}$ & PO_MIN & $\begin{array}{l}\text { Mnmum orre pressure for } \\
\text { ceptliary modol KPC }=3\end{array}$ & 32150 & 21-Feb-96 & $Y$ & $4 B$ \\
\hline $\mathbf{Y}$ & varies & 2419 & CL_M_T3 & $\begin{array}{l}\text { Uppor } 5 a l a d o \text { clay. } 25 \\
\text { to } 50 \text { years }\end{array}$ & PRMX_LOG & $\begin{array}{l}\text { Log of monsic permeabulity, } X \text { - } \\
\text { drection }\end{array}$ & 32155 & 15Feb-96 & $\mathbf{Y}$ & 1 \\
\hline$Y$ & varies & 2419 & CL_M_T3 & $\begin{array}{l}\text { Upper Saledo cliy. } 25 \\
\text { to } 50 \text { years }\end{array}$ & PRMX_LOG & $\begin{array}{l}\text { Lo of mitrinac permesbilty, } X- \\
\text { orection }\end{array}$ & 32155 & 15-Fab-95 & $\mathbf{Y}$ & 1 \\
\hline $\mathbf{Y}$ & varies & 2419 & CL_M_T3 & $\begin{array}{l}\text { Uppor Seledo dey. } 25 \\
\text { to } 50 \text { yeers }\end{array}$ & PRMX_LOG & $\begin{array}{l}\text { Log of monsic permesbilty, } X \text { - } \\
\text { druction }\end{array}$ & 32155 & 15 feb-96 & $\mathbf{Y}$ & 1 \\
\hline $\mathbf{Y}$ & varies & 2419 & $C_{2} M_{-} T 3$ & $\begin{array}{l}\text { Upper Saledo dey. } 25 \\
\text { to } 50 \text { years }\end{array}$ & PRMX_LOG & $\begin{array}{l}\text { Log of mitriac permeabitty, } X \text { - } \\
\text { direction }\end{array}$ & 32155 & 15-Fob-96 & $\mathbf{Y}$ & 1 \\
\hline $\mathbf{Y}$ & varies & 2419 & CL_M_T3 & $\begin{array}{l}\text { Upper Salido clay.25 } \\
\text { to } 50 \text { years }\end{array}$ & PRMX_LOG & $\begin{array}{l}\text { Lo of ntmax permesbulity, } X- \\
\text { direction }\end{array}$ & 32155 & 15-Fob-96 & $\mathbf{Y}$ & 1 \\
\hline $\mathbf{Y}$ & & 2420 & $C_{2} M_{-} T_{3}$ & $\begin{array}{l}\text { Uppor Saledo clay.25 } \\
\text { to } 50 \text { yours }\end{array}$ & PRMY_LOG & $\begin{array}{l}\text { Log of mitrinsic permeability, Y- } \\
\text { drection }\end{array}$ & 32156 & 15-Fob-96 & $\mathbf{Y}$ & 1 \\
\hline $\mathbf{Y}$ & & 2420 & CL_M_T3 & $\begin{array}{l}\text { Upper Salado ctay.25 } \\
\text { to } 50 \text { years }\end{array}$ & PRMY_LOG & $\begin{array}{l}\text { Log of mitrinse permeability, Y- } \\
\text { direction }\end{array}$ & 32156 & $15 f a b-96$ & $\mathbf{Y}$ & 1 \\
\hline $\mathbf{Y}$ & & 2420 & CL_M_T3 & $\begin{array}{l}\text { Uppor Saledo dey:25 } \\
\text { to } 50 \text { year }\end{array}$ & PRMY_LOG & $\begin{array}{l}\text { Log of minnace permescilly. Y- } \\
\text { drection }\end{array}$ & 32156 & $15+5 b-96$ & $\mathbf{Y}$ & 1 \\
\hline$Y$ & & 2420 & CL_M_T3 & $\begin{array}{l}\text { Upper Saledo diy. } 25 \\
\text { to } 50 \text { yeers }\end{array}$ & PRMYLOG & $\begin{array}{l}\text { Log of intriasc permesbildy, } Y \text { - } \\
\text { direction }\end{array}$ & 32156 & 15-Fob-96 & $\mathbf{Y}$ & 1 \\
\hline
\end{tabular}


CCA Parameter Listing

\begin{tabular}{|c|c|c|c|c|c|c|c|c|c|c|}
\hline W & 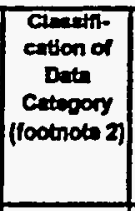 & $\begin{array}{l}\text { Dintributson } \\
\text { Type }\end{array}$ & $\operatorname{men}$ & Wraten & Thimen & Meriment & Uat & Referime to & 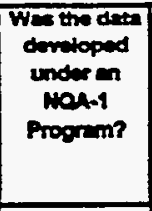 & 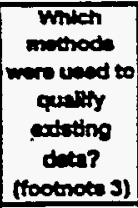 \\
\hline 2411 & 3,4 & UNIFORM & $20000000 E-01$ & $20000000 E-01$ & $0.00000000+\infty$ & 4.0000000E-01 & NONE & WP035597 & $\mathbf{Y}$ & NA \\
\hline 2411 & 3,4 & UNIFORM & $20000000 E-01$ & $2.0000000 E-01$ & $0.00000000=+\infty$ & $4.0000000 E-01$ & NONE & WPO41558 & $\mathbf{Y}$ & NA \\
\hline 2412 & $1,3,4$ & CONSTANT & $20000000 E+\infty 0$ & $2,0000000 E+\infty$ & $2.0000000 \mathrm{E}+\infty 0$ & $200000000+\infty$ & NONE & WP030640 & $\mathbf{Y}$ & N/A \\
\hline 2412 & $1,3,4$ & CONSTANT & $2.0000000 E+\infty 0$ & $20000000 E+\infty$ & $20000000 E+00$ & $20000000 E+\infty$ & NONE & WP030994 & $\gamma$ & NA \\
\hline 2112 & $1,3,4$ & CONSTANT & $20000000 E+\infty 0$ & $2.0000000 E+\infty 0$ & $2,0000000 E+\infty$ & $20000000 \mathrm{E}+\infty$ & NONE & WP035597 & $\mathbf{Y}$ & NA \\
\hline 2412 & $1,3,4$ & CONSTANT & $20000000 E+\infty$ & $2.0000000 E+\infty$ & $2.0000000 E+\infty$ & $20000000 E+\infty$ & NONE & WPO40485; & $\mathbf{Y}$ & NA \\
\hline 2412 & $1,3,4$ & CONSTANT & $2.0000000 \mathrm{E}+\infty$ & $2.0000000 E+\infty$ & $2.0000000 E+\infty 0$ & $2,0000000 E+\infty$ & NONE & WPO40514 & $\mathbf{Y}$ & N/A \\
\hline 2413 & $1,3,4$ & CONSTANT & $1.8100000 E-09$ & $1.8100000 E-09$ & $1.8100000 E-09$ & $1.8100000 E-09$ & $P \geq-1$ & WP030640 & $\mathbf{Y}$ & NA \\
\hline 2413 & $1,3,4$ & CONSTANT & $1.8100000=-09$ & $1.8100000 E-09$ & $1.8100000 E-09$ & $1.8100000=-09$ & $P_{x-1}$ & WP030994 & $\mathbf{Y}$ & NA \\
\hline 2413 & $1,3,4$ & CONSTANT & $1.8100000 E-09$ & $1.8100000 E-09$ & $1.8100000 \mathrm{E}-09$ & $1.8100000 E-09$ & Par-1 & WP035597 & $\mathbf{Y}$ & NA \\
\hline 2414 & 1,3 & CONSTANT & $1.00000000 E+08$ & $1.0000000 \equiv+08$ & $1.0000000 E+08$ & $1.0000000=+08$ & $\mathrm{~Pa}$ & WP030640 & $\mathbf{Y}$ & NA \\
\hline 2414 & 1,3 & CONSTANT & $1.0000000 \mathrm{E}+08$ & $1.0000000 E+08$ & $1.0000000 E+08$ & $1.0000000 E+08$ & Pa & WP030994 & $\mathbf{Y}$ & NA \\
\hline 2414 & 1,3 & CONSTANT & $1.0000000 E+00$ & $1.0000000 E+08$ & $1.0000000 E+00$ & $1.0000000 E+09$ & Pa & WP035597 & $\mathbf{Y}$ & NA \\
\hline 2414 & 1,3 & CONSTANT & $1.0000000 E+08$ & $1.0000000 E+08$ & $1.0000000 E+00$ & $1.0000000 \mathrm{E}+08$ & $\mathrm{~Pa}$ & WPO40485 & $\mathbf{Y}$ & NA \\
\hline 2414 & 1,3 & CONSTANT & $1.0000000 \mathrm{E}+08$ & $1.0000000 \mathrm{E}+08$ & $1.0000000 E+08$ & $1.0000000 \mathrm{E}+00$ & $\cdot \mathbf{P a}$ & WPO40514 & $\mathbf{Y}$ & NA \\
\hline 2415 & 3,4 & CUMULATIVE & $2.5200000 E+\infty$ & 9.4000000E-01 & $1.1000000 E-01$ & $8.1000000 E+\infty$ & NONE & WP030640 & $\mathbf{Y}$ & NA \\
\hline 2415 & 3,4 & CUMULATIVE & $252000000+00$ & $9.4000000 E-01$ & $1.1000000 E-01$ & $8.1000000 E+00$ & NONE & WP030994 & $\mathbf{Y}$ & NA \\
\hline 2415 & 3,4 & CUMULATNE & $252000000+00$ & $9.4000000 E-01$ & $1.1000000 E-01$ & $8.1000000 E+\infty$ & NONE & WPO32287 & $\mathbf{Y}$ & NA \\
\hline 2415 & 3,4 & CUMULATIVE & $2.5200000 \mathrm{E}+\infty$ & $9.4000000 E-01$ & $1.1000000 E-01$ & $8.1000000 E+00$ & NONE & WP035268 & $\mathbf{Y}$ & NA \\
\hline 2415 & 3,4 & CUMULATINE & $2.5200000 E+\infty$ & $9.4000000 \mathrm{E}-01$ & $1.1000000 E-01$ & $8.1000000 E+\infty 0$ & NONE & WP035597 & $Y$ & $N / A$ \\
\hline 2415 & 3,4 & CUMULATNE & $2.5200000 E+\infty 0$ & $9.4000000 E-01$ & $1.1000000 E-01$ & $8.1000000 \leq+\infty$ & NONE & WP041558 & $\mathbf{Y}$ & NA \\
\hline 2416 & $1,3,4$ & CONSTANT & $2.4000000 E-01$ & 24000000E-01 & 2.4000000 E-01 & 2.4000000E-01 & $m^{\wedge} 3 / m^{\wedge} 3$ & WPO30640 & $\mathbf{Y}$ & NA \\
\hline 2416 & $1,3,4$ & CONSTANT & $24000000 E-01$ & 2.4000000E-01 & $2.4000000 E-01$ & $24000000 \mathrm{E}-01$ & $m^{\wedge} 3 / m^{\wedge} 3$ & WPO30994 & $\mathbf{Y}$ & NA \\
\hline 2416 & $1,3,4$ & CONSTANT & $2.4000000 E-01$ & 2.4000000E-01 & $2.4000000 E-01$ & $2,4000000=-01$ & $m^{\wedge} 3 / m^{\wedge} 3$ & WP035597 & $\mathbf{Y}$ & NA \\
\hline 2417 & 1,3 & CONSTANT & $1.0132500 E+05$ & $1.0132500 \mathrm{E}+05$ & $1.0132500 E+05$ & $1.0132500 \varepsilon+05$ & $\mathrm{~Pa}$ & WP035597 & $-y$ & NA \\
\hline 2417 & 1,3 & CONSTANT & $1.0132500 E+05$ & $1.0132500 E+05$ & $1.0132500 E+05$ & $1.0132500 E+05$ & Po & WP036051 & $Y$ & NA \\
\hline 2417 & 1,3 & CONSTANT & $1.0132500 E+05$ & $1.0132500 E+05$ & $1.0132500 E+05$ & $1.0132500=+05$ & $\mathbf{P a}$ & WP040514 & $\mathbf{Y}$ & NA \\
\hline 2419 & $1,3,4$ & TRIANGULAR & $-1.8867000 E+01$ & $-1.8301000 E+01$ & $-2.1000000 E+01$ & $-1.7301000 E+01$ & $\log \left(m^{\alpha} 2\right)$ & WP030640 & YEN & 1 \\
\hline 2419 & $1,3,4$ & TRLANGULAR & $-1.8867000 E+01$ & $-1,8301000 E+01$ & $-21000000 E+01$ & $-1.7301000 E+01$ & $\log \left(m^{\wedge} 2\right)$ & WPO30994 & YEN & 1 \\
\hline 2419 & $1,3,4$ & TRIANGULAR & $-1.8867000 E+01$ & $-1.8301000 E+01$ & $-21000000 E+01$ & $-1.7301000 E+01$ & $\log \left(m^{\wedge} 2\right)$ & WP035268 & Y8N & 1 \\
\hline 2419 & $1,3,4$ & TRLANGULAR & $-1.8867000 E+01$ & $-1.8301000 E+01$ & $-2.1000000 E+01$ & $-1.7301000 \mathrm{E}+01$ & $\log \left(m^{\wedge} 2\right)$ & WP035597 & Y8N & 1 \\
\hline 2419 & $1,3,4$ & TRLANGULAR & $-1.8867000 E+01$ & $-1.8301000 \mathrm{E}+01$ & $-2.1000000 E+01$ & $-1.7301000 E+01$ & $\log \left(m^{\wedge} 2\right)$ & WPO41558 & Yan & 1 \\
\hline 2420 & $1,3,4$ & TRLANGULAR & $-1.8867000 E+01$ & $-1.8301000 E+01$ & $-2.1000000 E+01$ & $-1.7301000 E+01$ & $\log \left(m^{\wedge} 2\right)$ & WP030540 & Y\&N & 1 \\
\hline 2420 & $1,3,4$ & TRLANGULAR & $-1.8867000 E+01$ & $-1.8301000 E+01$ & $-2.1000000 E+01$ & $-1.7301000 E+01$ & $\log \left(m^{\wedge} 2\right)$ & WP030994 & Y\&N & 1 \\
\hline 2420 & $1,3,4$ & TRLANGULAR & $-1,8867000 E+01$ & $-1.8301000 E+01$ & $-2.1000000 E+01$ & $-1.7301000 E+01$ & $\log \left(m^{\wedge} 2\right)$ & WP035268 & Y\&N & 1 \\
\hline 2420 & $1,3,4$ & TRLANGULAR & $-1.8867000 E+01$ & $-1.6301000 E+01$ & $-2.1000000 E+01$ & $-1.7301000 E+01$ & $\log \left(m^{2} 2\right)$ & WP035597 & YEN & 1 \\
\hline
\end{tabular}


CCA Parameter Listing

\begin{tabular}{|c|c|c|c|c|c|c|c|c|c|c|}
\hline $\begin{array}{l}\text { Parameter in } \\
\text { Detubeae? } \\
\text { (YnN) }\end{array}$ & 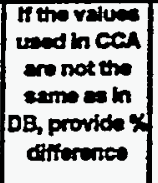 & 10 & Mtariato & Matada Nomo & Pernemerer & Parrietar Nians & $\begin{array}{l}\text { PRPD } \\
\text { (NPO) }\end{array}$ & $\begin{array}{c}\text { Decenty } \\
\text { Dets }\end{array}$ & 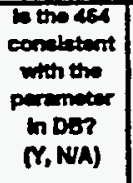 & 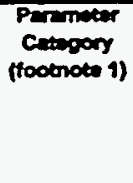 \\
\hline $\mathbf{Y}$ & & 2420 & CL_M_T3 & $\begin{array}{l}\text { Uppoer Salado ctay:25 } \\
\text { to } 50 \text { years }\end{array}$ & PRMY_LOS & $\begin{array}{l}\text { Log of minnsic permesbilty, } Y \text { - } \\
\text { drection }\end{array}$ & 32156 & 15-Fab-96 & $\mathbf{Y}$ & 1 \\
\hline $\mathbf{Y}$ & & 2421 & CL_M_T3 & $\begin{array}{l}\text { Upper Soledo chy.25 } \\
\text { to } 50 \text { years }\end{array}$ & PRMZ_LOG & $\begin{array}{l}\text { Log of inmsic permeability, Z- } \\
\text { direction }\end{array}$ & 32157 & 15Fab-96 & $\mathbf{Y}$ & 1 \\
\hline $\mathbf{Y}$ & & 2421 & $C L_{-} M_{-} T_{3}$ & $\begin{array}{l}\text { Upper Saledo clay } 25 \\
\text { to } 50 \text { years }\end{array}$ & PRMZ_LOG & $\begin{array}{l}\text { Log of intrinsic permasidty, Z- } \\
\text { direction }\end{array}$ & 32157 & 15-Fab-96 & $\mathbf{Y}$ & 1 \\
\hline $\mathbf{Y}$ & & 2421 & $C_{L} M_{-} T_{3}$ & $\begin{array}{l}\text { Upper Salado ctay } 25 \\
\text { to } 50 \text { years }\end{array}$ & PRMZ_LOG & $\begin{array}{l}\text { Log of intrinax permasbilty, Z- } \\
\text { direction }\end{array}$ & 32157 & $15-f a b-86$ & $\mathbf{Y}$ & 1 \\
\hline $\mathbf{Y}$ & & 2421 & CL_M_T3 & $\begin{array}{l}\text { Upper Salado dey.25 } \\
\text { to } 50 \text { years }\end{array}$ & PRMZ_LOG & $\begin{array}{l}\text { Log of minnax permeability, Z- } \\
\text { direction }\end{array}$ & 32157 & 15-Fob-86 & $\mathbf{Y}$ & 1 \\
\hline $\mathbf{Y}$ & & 2421 & CL_M_T3 & $\begin{array}{l}\text { Upper Salado clay } 25 \\
\text { to } 50 \text { years }\end{array}$ & PRMZ_LOG & $\begin{array}{l}\text { Log of intrinsx permeability, Z- } \\
\text { droction }\end{array}$ & 32157 & 15Fabse & $\mathbf{Y}$ & 1 \\
\hline $\mathbf{Y}$ & & 2425 & 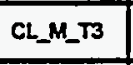 & $\begin{array}{l}\text { Uppor Salado ctay.25 } \\
\text { to } 50 \text { years }\end{array}$ & RELP_MOD & $\begin{array}{l}\text { Model number, relative } \\
\text { permeability model }\end{array}$ & 321624 & 15-Fab-96 & $\mathbf{Y}$ & $4 B$ \\
\hline $\mathbf{Y}$ & & 2425 & CL_M_T3 & $\begin{array}{l}\text { Upper Salodo ciay.25 } \\
\text { to } 50 \text { years }\end{array}$ & RELP_MOD & $\begin{array}{l}\text { Model number, relettre } \\
\text { permeability model }\end{array}$ & $32102 A$ & 15 Fab-s6 & $\mathbf{Y}$ & 48 \\
\hline $\mathbf{Y}$ & & 2425 & CL_M_T3 & $\begin{array}{l}\text { Upper Salado clay.25 } \\
\text { to } 50 \text { years }\end{array}$ & RELP_MOD & $\begin{array}{l}\text { Model number, rolitive } \\
\text { permaabirty model }\end{array}$ & 321624 & $15+F a b-56$ & $\mathbf{Y}$ & $4 B$ \\
\hline $\mathbf{Y}$ & & 2425 & Cl_M_T3 & $\begin{array}{l}\text { Upper Salado ctay } 25 \\
\text { to } 50 \text { years }\end{array}$ & RELP_MOD & $\begin{array}{l}\text { Model number, reletsm } \\
\text { permeability modal }\end{array}$ & $32102 A$ & $15+a b-96$ & $\mathbf{Y}$ & 48 \\
\hline$Y$ & & 2425 & CL_M_T3 & $\begin{array}{l}\text { Upper Salado ctay } 25 \\
\text { to } 50 \text { years }\end{array}$ & RELP_MOD & $\begin{array}{l}\text { Model number, relative } \\
\text { permeability model }\end{array}$ & $32162 A$ & 15-fab-96 & $\mathbf{Y}$ & $4 B$ \\
\hline $\mathbf{Y}$ & 100 & 2427 & CL_M_T3 & $\begin{array}{l}\text { Upper Salado ctay.25 } \\
\text { to } 50 \text { years }\end{array}$ & SAT_RBRH & Residusal Brina Saturation & 32168 & $15+f a b-96$ & $\mathbf{Y}$ & 1 \\
\hline $\mathbf{Y}$ & 100 & 2427 & CL_H_T3 & $\begin{array}{l}\text { Upper Salado clay.25 } \\
\text { to } 50 \text { yoars }\end{array}$ & SAT_RBRN & Resicual Brine Saturation & 32168 & 15-Fab-96 & $\mathbf{Y}$ & 1 \\
\hline $\mathbf{Y}$ & 100 & 2427 & $C L_{2} M$ IS & $\begin{array}{l}\text { Upper Salado clay.25 } \\
\text { to } 50 \text { years }\end{array}$ & SAT_RBRN & Residual Brina Seturation & 32168 & $15-a b-65$ & $\mathbf{Y}$ & 1 \\
\hline$Y$ & 100 & 2427 & $C L \_M \_T 3$ & $\begin{array}{l}\text { Upper Salado clay.25 } \\
\text { to } 50 \text { years }\end{array}$ & SAT_RBRN & Resictued Brine Seturation & 32168 & $15 f \circ b-96$ & $\mathbf{Y}$ & 1 \\
\hline $\mathbf{Y}$ & 100 & 2427 & CL_M_T3 & $\begin{array}{l}\text { Upper Solado clay.25 } \\
\text { to } 50 \text { yoars }\end{array}$ & SAT_RBRN & Residual Brine Seturation & 32168 & 15 Fab-96 & $\mathbf{Y}$ & 1 \\
\hline $\mathbf{Y}$ & 100 & 2427 & CL_M_T3 & $\begin{array}{l}\text { Upper Salado clay.25 } \\
\text { to } 50 \text { years }\end{array}$ & SAT_RBRN & Residual Brino Saturation & 32168 & 15 feb-96 & $\mathbf{Y}$ & 1 \\
\hline $\mathbf{Y}$ & 100 & 2428 & CL_M_T3 & $\begin{array}{l}\text { Upper Salado clay:25 } \\
\text { to } 50 \text { years }\end{array}$ & SAT_RGAS & Residual Ges Saturation & 32169 & $15-F a b-96$ & $\mathbf{Y}$ & 1 \\
\hline $\mathbf{Y}$ & 100 & 2428 & CL_M_T3 & $\begin{array}{l}\text { Upper Salado day: } 25 \\
\text { to } 50 \text { years }\end{array}$ & SAT_RGAS & Resicual Ges Suturation & 32169 & 15-feb-96 & $\mathbf{Y}$ & 1 \\
\hline $\mathbf{Y}$ & 100 & 2428 & CL_M_T3 & $\begin{array}{l}\text { Upper Salodo ctoy.25 } \\
\text { to } 50 \text { years }\end{array}$ & SAT_RGAS & Residual Gas Seturation & 32169 & 15-Feb-96 & $\mathbf{Y}$ & 1 \\
\hline$Y$ & 100 & 2428 & CL_M_T3 & $\begin{array}{l}\text { Upper Salado clay:25 } \\
\text { to } 50 \text { yoars }\end{array}$ & SAT_RGAS & Residual Gas Semuration & 32169 & 15 feb-96 & $\mathbf{Y}$ & 9 \\
\hline$Y$ & 100 & 2428 & CL_M_T3 & $\begin{array}{l}\text { Upper Salado ctay.25 } \\
1050 \text { years }\end{array}$ & SAT_RGAS & Residual Gas Suturation & 32169 & 15-fab-96 & $\mathbf{Y}$ & 1 \\
\hline $\mathbf{Y}$ & 50 & 2429 & CL_M_T4 & $\begin{array}{l}\text { Upper Salado clay.50 } \\
\text { to } 100 \text { years }\end{array}$ & CAP_MOD & $\begin{array}{l}\text { Modal number, capillary pressure } \\
\text { modol }\end{array}$ & $32191 A$ & 02-Nov-95 & $\mathbf{Y}$ & $4 B$ \\
\hline$Y$ & 50 & 2429 & CL_M_T4 & $\begin{array}{l}\text { Upper Salaco clay.50 } \\
\text { to } 100 \text { years }\end{array}$ & CAP_MOD & $\begin{array}{l}\text { Model number, capillary pressirre } \\
\text { model }\end{array}$ & 32191A & C2-Nov-95 & $\mathbf{Y}$ & $\triangle B$ \\
\hline $\mathbf{Y}$ & 50 & 2429 & CL_M_T4 & $\begin{array}{l}\text { Upper Salado day. } 50 \\
\text { to } 100 \text { years }\end{array}$ & CAP_MOD & $\begin{array}{l}\text { Model number, capillery pressure } \\
\text { model }\end{array}$ & $32191 \mathrm{~A}$ & C2+Nov-95 & $=Y$ & 48 \\
\hline $\mathbf{Y}$ & 50 & 2429 & CL_M_T4 & $\begin{array}{l}\text { Upper Salado ctay.50 } \\
\text { to } 100 \text { years }\end{array}$ & CAP_MOD & $\begin{array}{l}\text { Model number, capillay pressure } \\
\text { model }\end{array}$ & $32191 A$ & O2-Nov-95 & $\mathbf{Y}$ & $4 B$ \\
\hline $\mathbf{Y}$ & 50 & 2429 & CL_M_T4 & $\begin{array}{l}\text { Upper Salado elay.50 } \\
\text { to } 100 \text { years }\end{array}$ & CAP_MOD & $\begin{array}{l}\text { Model mumber, capillary pressure } \\
\text { model }\end{array}$ & $32191 A$ & 02+Nov-95 & $\mathbf{Y}$ & $A B$ \\
\hline $\mathbf{Y}$ & & 2430 & CL_M_T4 & $\begin{array}{l}\text { Upper Salado dey.50 } \\
\text { to } 100 \text { yoars }\end{array}$ & COMP_RCK & Butk Compressibility & 32193 & 13-Feb-96 & $\mathbf{Y}$ & 1 \\
\hline $\mathbf{Y}$ & & 2430 & CL_M_T4 & $\begin{array}{l}\text { Upper Selecos dey.50 } \\
\text { to } 100 \text { years }\end{array}$ & COMP_RCK & Buk Compressibility & 32193 & 13-Fob-96 & $\mathbf{Y}$ & 1 \\
\hline $\mathbf{Y}$ & & 2430 & CL_M_T4 & $\begin{array}{l}\text { Upper Salado clay.50 } \\
\text { to } 100 \text { years }\end{array}$ & COMP_RCK & Buk Compressibility & 32193 & 13 Fab-96 & $\mathbf{Y}$ & 1 \\
\hline $\mathbf{Y}$ & & 2431 & CL_M_T4 & $\begin{array}{l}\text { Upper Selado ctoy.50 } \\
\text { to } 100 \text { yeers }\end{array}$ & PC_MAX & $\begin{array}{l}\text { Maxmum allowable capillary } \\
\text { pressure }\end{array}$ & $32187 A$ & $26-00 t-95$ & $\mathbf{Y}$ & 48 \\
\hline $\mathbf{Y}$ & & 2431 & CL_M_T4 & $\begin{array}{l}\text { Upper Selado cioy.50 } \\
\text { to } 100 \text { years }\end{array}$ & PC_max & $\begin{array}{l}\text { Mexumum allowable capillary } \\
\text { pressure }\end{array}$ & 32197A & 26-0at-95 & $\mathbf{Y}$ & 48 \\
\hline $\mathbf{Y}$ & & 2431 & CL_M_TA & $\begin{array}{l}\text { Uppar Saledo clay.50 } \\
\text { to } 100 \text { years }\end{array}$ & PC_MAX & $\begin{array}{l}\text { Maxmum allowable capillary } \\
\text { preasure }\end{array}$ & $32197 A$ & $26-0 c t-95$ & $\mathbf{Y}$ & 48 \\
\hline $\mathbf{Y}$ & & 2431 & CL_M_TA & $\begin{array}{l}\text { Upper Saledo clay.50 } \\
\text { to } 100 \text { years }\end{array}$ & PC_MAX & $\begin{array}{l}\text { Maumum allowable capiltary } \\
\text { pressure }\end{array}$ & 32197A & $26-0 c t-95$ & $\mathbf{Y}$ & 48 \\
\hline $\mathbf{Y}$ & & 2431 & CL_M_T4 & $\begin{array}{l}\text { Upper Saledo clay.50 } \\
\text { to } 100 \text { years }\end{array}$ & PC_MNX & $\begin{array}{l}\text { Mascmum allowablo cespillary } \\
\text { presture }\end{array}$ & $32197 A$ & $26-0<-95$ & $\mathbf{Y}$ & $4 B$ \\
\hline $\mathbf{Y}$ & vanes & 2432 & CL_M_T4 & $\begin{array}{l}\text { Upper Selodo ctay.50 } \\
\text { to } 100 \text { years }\end{array}$ & PORE_DIS & $\begin{array}{l}\text { Brooks-Corry pore dutribution } \\
\text { Deremeter }\end{array}$ & 32198 & 20-Fab-96 & $\mathbf{Y}$ & 1 \\
\hline
\end{tabular}


CCA Parameter Listing --

\begin{tabular}{|c|c|c|c|c|c|c|c|c|c|c|}
\hline 10 & 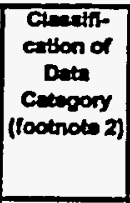 & $\begin{array}{l}\text { Dlotribution } \\
\text { Type }\end{array}$ & Men & Medim & Minimum & Modtrien & Units & Refermato to & $\begin{array}{l}\text { Wes the dat } \\
\text { demloped } \\
\text { under en } \\
\text { not-1 } \\
\text { Progrem? }\end{array}$ & 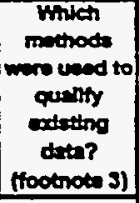 \\
\hline 2420 & $1,3,4$ & TRLANGULAR & $-1.8857000 E+01$ & $-1.8301000 E+01$ & $-2.1000000 E+01$ & $-1.7301000 E+01$ & $\log \left(m^{\wedge} 2\right)$ & WPO41558 & YaN & 1 \\
\hline 2421 & $1,3,4$ & TRLANGULAR & $-1.8857000 E+01$ & $-1.8301000 E+01$ & $-2.1000000 E+01$ & $-9.7301000 E+01$ & $\log \left(m^{\wedge} 2\right)$ & WP030S40 & YEN & 1 \\
\hline 2421 & $1,3,4$ & TRLANGULAR & $-1.8867000 E+01$ & $-1.8301000 E+01$ & $-2,1000000 E+01$ & $-9.7301000 E+01$ & $\log \left(m^{n} 2\right)$ & WP030994 & Y\&N & 1 \\
\hline 2421 & $1,3,4$ & TRLANGULAR & $-1.8867000 E+01$ & $-1.8301000 E+01$ & $-21000000 E+01$ & $-1.7301000 E+01$ & $\log \left(m^{\wedge} 2\right)$ & WP035268 & YaN & 1 \\
\hline 2421 & $1,3,4$ & TRLANGULAR & $-1.8867000 E+01$ & $-1.8301000 E+01$ & $-2.1000000 E+01$ & $-1.73010005+01$ & $\log \left(m^{\wedge} 2\right)$ & WP035597 & YaN & 1 \\
\hline 2421 & $1,3,4$ & TRLANGULAR & $-1.8867000 E+01$ & $-1.8301000 E+01$ & $-2.1000000 E+01$ & $-1.7301000 E+01$ & $\log \left(m^{\wedge} 2\right)$ & WPO41558 & YaN & 1 \\
\hline 2425 & $1,3,4$ & CONSTANT & $4.0000000 E+\infty 0$ & $-4.0000000 E+\infty 0$ & $4.0000000 E+\infty$ & $4.0000000 E+\infty$ & NONE & WP030640 & $\cdot \mathbf{Y}$ & NA \\
\hline 2425 & $1,3,4$ & CONSTANT & $4.0000000 E+\infty$ & $4.0000000 E+\infty$ & $4.0000000 E+00$ & $4.0000000 E+\infty$ & NONE: & WP030994 & $\mathbf{Y}$ & N/A \\
\hline 2425 & $1,3,4$ & CONSTANT & $4.0000000 E+\infty 0$ & $4.0000000 E+\infty$ & $4.0000000 E+\infty 0$ & $1.0000000 E+\infty)$ & NONE & MP035597" & $\mathbf{Y}$ & NA \\
\hline 2425 & $1,3,4$ & CONSTANT & $4.0000000 E+\infty 0$ & $4.0000000 E+\infty$ & $4.0000000=+\infty 0$ & $4.0000000 E+\infty 0$ & NONE & WP040485 & $\mathbf{Y}$ & NA \\
\hline 2425 & $1,3,4$ & CONSTANT & $4.00000000 E+\infty 0$ & $4.0000000 E+00$ & $4.0000000 E+\infty 0$ & $4.0000000 E+\infty 0$ & NONE & MPO40514 & $\mathbf{Y}$ & NA \\
\hline 2427 & 3,4 & CUMULATNE & $2.5000000 E-01$ & $2,0000000 E-01$ & $0.0000000 E+\infty 0$ & $6.0000000 E-01$ & NONE & WP030640 & $\mathbf{Y}$ & N/A \\
\hline 2427 & 3,4 & CUMULATIVE & 2.5000000E-01 & $2.0000000 E-01$ & $0.0000000 E+\infty$ & 6.0000000E-01 & NONE & WP030994 & $\mathbf{Y}$ & NAA \\
\hline 2427 & 3,4 & CUMULATIVE & $2.5000000 E-01$ & $2,0000000 E-01$ & $0.0000000 E+00$ & $6.0000000 E-01$ & NONE & WP035268 & $\mathbf{Y}$ & N/A \\
\hline 2427 & 3,4 & CUMULATME & 2.5000000E-01 & $2,0000000 E-01$ & $0.0000000 E+\infty$ & $6.0000000 E-01$ & MONE & WP035597 & $\mathbf{Y}$ & N/A \\
\hline 2427 & 3,4 & CUMULATINE & $2.5000000 E-01$ & $20000000 E-01$ & $0.0000000 E+\infty 0$ & $6.0000000 E-01$ & NONE & WP041558 & $\mathbf{Y}$ & N/A \\
\hline 2427 & 3,4 & CUMULATINE & $2.5000000 E-01$ & $20000000 E-01$ & $0.0000000 E+00$ & $6.0000000 E-01$ & NONE & WPO42751 & $\mathbf{Y}$ & NA \\
\hline 2428 & 3,4 & UNIFORM & $2,0000000 E-01$ & $2,0000000 E-01$ & $0.0000000 E+\infty$ & $4.0000000 E-01$ & NONE & WPO30640 & $\mathbf{Y}$ & NA \\
\hline 2428 & 3,4 & UNIFORM & $2.0000000 E-01$ & $2.0000000 E-01$ & $0.0000000 E+\infty$ & $4.0000000 E-01$ & MONE & WP030994 & $\mathbf{Y}$ & NA \\
\hline 2428 & 3,4 & UNIFORM & $2,0000000 E-01$ & $2.0000000 E-01$ & $0.0000000 E+\infty 0$ & $4.0000000 E-01$ & NONE & WP035268 & $\mathbf{Y}$ & $N / A$ \\
\hline 2428 & 3,4 & UNIFORM & $20000000 E-01$ & $2.0000000 \mathrm{E}-01$ & $0.0000000 E+\infty$ & $4.0000000 E-01$ & NONE & WP035597 & $\mathbf{Y}$ & NA \\
\hline 2428 & 3,4 & UNIFORM & $2,0000000 E-01$ & $2.0000000 E-01$ & $0.0000000 E+\infty$ & $4.0000000 E-01$ & NONE & WPO41558 & $\mathbf{Y}$ & NA \\
\hline 2429 & $1,3,4$ & CONSTANT & $2.0000000 E+00$ & $2.0000000 E+\infty$ & $2.0000000 E+\infty 0$ & $2,0000000 E+\infty$ & NONE & WP030540 & $\mathbf{Y}$ & N/A \\
\hline 2429 & $1,3,4$ & CONSTANT & $20000000 E+\infty$ & $2.0000000 E+\infty$ & $2.0000000 E+\infty$ & $2.00000000+\infty$ & NONE & WPO30994 & $\mathbf{Y}$ & NA \\
\hline 2429 & $1,3,4$ & CONSTANT & $2.0000000 \leq+\infty 0$ & $2.0000000 E+\infty 0$ & $2.0000000 E+\infty 0$ & $2.0000000 E+\infty$ & NONE & WP035597 & $-y$ & NAA \\
\hline 2429 & $1,3,4$ & CONSTANT & $2,0000000 E+\infty$ & $2,0000000 E+\infty$ & $2.0000000 E+\infty$ & $2.0000000 E+\infty 0$ & NONE & WPO40486 & $\mathbf{Y}$ & NAA \\
\hline 2429 & $1,3,4$ & CONSTANT & $2.0000000 E+\infty$ & $2.0000000 E+\infty$ & $2.0000000 E+00$ & $2,0000000 E+\infty$ & NONE & WPO40514 & $\mathbf{Y}$ & N/A \\
\hline 2430 & $1,3,4$ & CONSTANT & $1.8100000=-09$ & $1.8100000 E-09$ & $1.8100000 E-09$ & $1.8100000 E-09$ & $P e^{n}-1$ & WPO30640 & $\mathbf{Y}$ & $N / A$ \\
\hline 2430 & $1,3,4$ & CONSTANT & $1.8100000 E-09$ & $1.8100000 E-09$ & $1.8100000 E-09$ & $1.8100000 E-09$ & $P \mathbf{a}^{n}+1$ & WP030994 & $\mathbf{Y}$ & NA \\
\hline 2430 & $1,3,4$ & CONSTANT & $1.8100000 E-09$ & $1.8100000 E-09$ & $1.8100000 E-09$ & $1.8100000 E-09$ & $P e^{n}-1$ & WP035597 & $\mathbf{Y}$ & NA \\
\hline 2431 & $.1,3$ & CONSTANT & $1.0000000 E+08$ & $1.0000000 E+08$ & $1.0000000 E+06$ & $1.0000000 E+08$ & $\mathrm{~Pa}$ & WP030640 & $\mathbf{Y}$ & NAA \\
\hline 2431 & 1,3 & CONSTANT & $1.0000000 E+08$ & $1.0000000 E+08$ & $1.0000000 E+08$ & $1.0000000 E+08$ & $\mathbf{P a}$ & WP030994 & $\mathbf{Y}$ & $N / A$ \\
\hline 2431 & 1,3 & CONSTANT & $1.0000000 E+\infty 0$ & $1.0000000 E+08$ & $1.0000000 E+08$ & $1.0000000 E+08$ & $\mathbf{P a}$ & WP035597 & $\mathbf{Y}$ & NA \\
\hline 2431 & 1,3 & CONSTANT & $1.0000000 E+\infty$ & $1.0000000 E+08$ & $1.0000000 E+08$ & $1.0000000 E+08$ & $\mathrm{~Pa}$ & WPO40485 & $\mathbf{Y}$ & $\mathbf{N A}$ \\
\hline 2431 & 1,3 & CONSTANT & $1.0000000 E+08$ & $1.0000000 E+08$ & $1.0000000 E+08$ & $1.0000000 E+08$ & $\mathbf{P a}$ & WP040514 & $\mathbf{Y}$ & NA \\
\hline 2432 & 3,4 & CUMULATME & $2.5200000 E+\infty 0$ & $9.4000000=-01$ & $1.1000000 E-01$ & $8.1000000 \mathrm{E}+00$ & NONE & WP030540 & $\mathbf{Y}$ & NA \\
\hline
\end{tabular}


CCA Parameter-Listing

\begin{tabular}{|c|c|c|c|c|c|c|c|c|c|c|}
\hline $\begin{array}{c}\text { Permintarn } \\
\text { Detabeen } \\
\text { (YAN) }\end{array}$ & 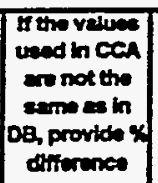 & DD & Maturial to & Mitarda Neme & $\begin{array}{l}\text { Permotar } \\
-10 .\end{array}$ & Parmater Mans & $\begin{array}{l}\text { PRPD } \\
\text { MPOA }\end{array}$ & $\begin{array}{c}\text { Dete Entry } \\
\text { Deto }\end{array}$ & 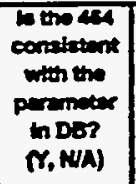 & 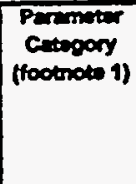 \\
\hline $\mathbf{Y}$ & vares & 2432 & CL_M_T4 & $\begin{array}{l}\text { Uppor Sabaco chy.50 } \\
\text { to } 100 \text { years }\end{array}$ & PORE_DIS & $\begin{array}{l}\text { Brocks-Corroy porio distribution } \\
\text { peremeter }\end{array}$ & 32198 & $20-50-06$ & $\mathbf{Y}$ & 1 \\
\hline$Y$ & varies & 2432 & CL_M_T4 & $\begin{array}{l}\text { Uppor Salodo clay.50 } \\
\text { to } 100 \text { years }\end{array}$ & PORE_DIS & $\begin{array}{l}\text { Erooks-coroy pore distrobution } \\
\text { peremetor }\end{array}$ & 32198 & $20-5=b-96$ & $\mathbf{Y}$ & 1 \\
\hline $\mathbf{Y}$ & varies & 2432 & CL_M_T4 & $\begin{array}{l}\text { Uppor Saledo ctay:50 } \\
\text { to } 100 \text { years }\end{array}$ & PORE_DIS & $\begin{array}{l}\text { Brooke-Corey pore disstioution } \\
\text { peremeter }\end{array}$ & 32198 & $20-50-96$ & $\mathbf{Y}$ & 1 \\
\hline $\mathbf{Y}$ & varios & 2432 & CL_M_T4 & $\begin{array}{l}\text { Uppor Salado cty:50 } \\
\text { to } 100 \text { years }\end{array}$ & PORE_DIS & $\begin{array}{l}\text { Brooks-Coroy pore distrbition } \\
\text { peranteter }\end{array}$ & 32198 & $20-F c 0-96$ & $Y$ & 1 \\
\hline $\mathbf{Y}$ & varies & 2432 & CL_M_T4 & $\begin{array}{l}\text { Upper Saleodo ctay.50 } \\
\text { to } 100 \text { years }\end{array}$ & PORE_DIS & $\begin{array}{l}\text { Brooks-Coroy poro distoution } \\
\text { parremeter }\end{array}$ & 32198 & $20+00.96$ & $\mathbf{Y}$ & 1 \\
\hline $\mathbf{Y}$ & & 2433 & CL_M_T4 & $\begin{array}{l}\text { Uppor Salado ctay.50 } \\
\text { to } 100 \text { years }\end{array}$ & POROSTTY & Effective porosity & 32199 & $13+$ eb-96 & $Y$ & 1 \\
\hline $\mathbf{Y}$ & & 2433 & CL_M_T4 & $\begin{array}{l}\text { Upper Salaco edyy.50 } \\
\text { to } 100 \text { years }\end{array}$ & POROSTr . & Effective porosity & 32199 & $13-F=b-96$ & $Y$ & 1 \\
\hline$Y$ & & 2433 & CL_M_T4 & $\begin{array}{l}\text { Uppor Salado ctry.50 } \\
\text { to } 100 \text { years }\end{array}$ & POROSTYY & Efrective porosity & 32199 & $13-F c b-96$ & $\mathbf{Y}$ & 1 \\
\hline $\mathbf{Y}$ & & 2434 & CL_M_T4 & $\begin{array}{l}\text { Upper Salaso day.50 } \\
\text { to } 100 \text { years }\end{array}$ & PO_MIN & $\begin{array}{l}\text { Mnimum brme pressure for } \\
\text { capilley model KPC }=3\end{array}$ & 32200 & 21 feb-s6 & $\boldsymbol{Y}$ & $A B$ \\
\hline $\mathbf{Y}$ & & 2434 & CL_M_T4 & $\begin{array}{l}\text { Uppor Salado ctay.50 } \\
\text { to } 100 \text { years }\end{array}$ & PO_MIN & $\begin{array}{l}\text { Mnimum brine prassure for } \\
\text { capillery model } \mathrm{KPC}=3\end{array}$ & 32200 & $21+5 a b-96$ & $\mathbf{Y}$ & $A B$ \\
\hline $\mathbf{Y}$ & & 2434 & CL_M_T4 & $\begin{array}{l}\text { Uppor Salado ctay.50 } \\
\text { to } 100 \text { years }\end{array}$ & PO_MIN & $\begin{array}{l}\text { Mrnmum brne prossure for } \\
\text { capillary modol } 1 P C=3\end{array}$ & 32200 & 21Fob-s6 & $\mathbf{Y}$ & $A B$ \\
\hline $\mathbf{Y}$ & varies & 2436 & CL_M_T4 & $\begin{array}{l}\text { Uppor Salado clay.50 } \\
\text { to } 100 \text { years }\end{array}$ & PRMX_LOG & $\begin{array}{l}\text { Log of intrnstc permesbility, } X \text { - } \\
\text { drection }\end{array}$ & 32202 & $19-50 b-96$ & $Y$ & 1 \\
\hline $\mathbf{Y}$ & varies & 2436 & $C L_{-} M_{-} T 4$ & $\begin{array}{l}\text { Uppor Salado clay.50 } \\
\text { to } 100 \text { years }\end{array}$ & PRMX_LOG & $\begin{array}{l}\text { Log of intrinsic permeabilty, } X \text { - } \\
\text { direction }\end{array}$ & 32200 & $19 F c b-96$ & $\mathbf{Y}$ & 1 \\
\hline $\mathbf{Y}$ & varies & 2436 & CL_M_T4 & $\begin{array}{l}\text { Upper Salado clay.50 } \\
\text { to } 100 \text { years }\end{array}$ & PRMX_LOG & $\begin{array}{l}\text { Log of mitrisic permeabilty, X- } \\
\text { drection }\end{array}$ & 32202 & $19 F a b-96$ & $\mathbf{Y}$ & 1 \\
\hline$Y$ & varios & 2436 & CL_M_T4 & $\begin{array}{l}\text { Upper Salado clay.50 } \\
\text { to } 100 \text { years }\end{array}$ & PRNX_LOG & 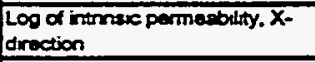 & 32202 & 19 reb-86 & $Y$ & 1 \\
\hline $\mathbf{Y}$ & vanes & 2436 & $C L \_M \_T 4$ & $\begin{array}{l}\text { Upper Salado clay.50 } \\
\text { to } 100 \text { yoars }\end{array}$ & PRMX_LOG & $\begin{array}{l}\text { Log of intrnsic permeability, } X- \\
\text { drection }\end{array}$ & 32202 & 19Feb-96 & $\mathbf{Y}$ & 1 \\
\hline $\mathbf{Y}$ & & 2437 & CL_M_T4 & $\begin{array}{l}\text { Uppoer Salaco clay.50 } \\
\text { to } 100 \text { years }\end{array}$ & PRMY_LOG & $\begin{array}{l}\text { Log of ntrmax permosallty, Y- } \\
\text { diroction }\end{array}$ & 32203 & $13-F=b-96$ & $Y$ & 1 \\
\hline$Y$ & & 2437 & $C L \_M_{-} T_{4}$ & $\begin{array}{l}\text { Uppor Salaso clay:50 } \\
\text { to } 100 \text { years }\end{array}$ & PRMY_LOG & 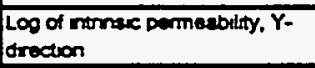 & 32203 & 13-Fab-96 & $\mathbf{Y}$ & 1 \\
\hline$Y$ & & 2437 & CL_M_T4 & $\begin{array}{l}\text { Upper Solsolo diny.50 } \\
\text { to } 100 \text { years }\end{array}$ & PRMY_LOG & $\begin{array}{l}\log \alpha \text { mirrac permeability, } Y- \\
\text { orwection }\end{array}$ & 32203 & $13+5 b-96$ & $\mathbf{Y}$ & 1 \\
\hline$Y$ & & 2437 & CL_M_T4 & $\begin{array}{l}\text { Upper Salado clay.50 } \\
\text { to } 100 \text { years }\end{array}$ & PRMY_LOG & $\begin{array}{l}\text { Log } \alpha \text { morrac pormesbility, } Y- \\
\text { arocton }\end{array}$ & 32203 & $13+50 b-96$ & $Y$ & 1 \\
\hline$Y$ & & 2437 & CL_M_T4 & $\begin{array}{l}\text { Uppor Salsos cay } 50 \\
\text { to } 100 \text { yeans }\end{array}$ & PRMY_LOG & $\begin{array}{l}\text { Lo d momec pormesbilty, } Y \text { - } \\
\text { arection }\end{array}$ & 32203 & 13feb-96 & $\mathbf{Y}$ & 1 \\
\hline$Y$ & & 2438 & CL_M_T4 & $\begin{array}{l}\text { Uppor Salaco clay } 50 \\
\text { to } 100 \text { years }\end{array}$ & PRAR_LOG & 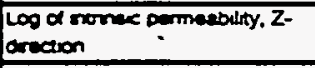 & 32204 & $13-506-96$ & $\mathbf{Y}$ & 1 \\
\hline $\mathbf{Y}$ & & 2438 & CL_M_T4 & $\begin{array}{l}\text { Uppor Salocos aly } 50 \\
\text { to } 100 \text { years }\end{array}$ & Pranz_LOG & $\begin{array}{l}\text { Log of norrece pormesoulty, Z- } \\
\text { orection }\end{array}$ & 32204 & 13-Fab-96 & $\mathbf{Y}$ & 1 \\
\hline$Y$ & & 2438 & CL_H_T4 & $\begin{array}{l}\text { Upper Salesco ary } 50 \\
\text { to } 100 \text { yean }\end{array}$ & Prakz_LOG & 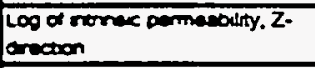 & 32204 & 13 feb-96 & $Y$ & 1 \\
\hline$Y$ & & 2438 & CL_H_T4 & $\begin{array}{l}\text { Uppor Selecos any } 50 \\
\text { to } 100 \text { years }\end{array}$ & Prove_100 & 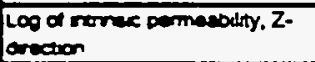 & 32204 & 13fab-96 &.$- Y$ & 1 \\
\hline$Y$ & & 2438 & CL_M_T4 & $\begin{array}{l}\text { Upper Salpos any } 50 \\
\text { to } 100 \text { yems }\end{array}$ & Prouz_106 & 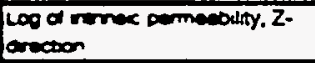 & 32204 & 13-Fob-96 & $\mathbf{Y}$ & 1 \\
\hline$Y$ & & 2442 & CL_M_T4 & $\begin{array}{l}\text { Upper Salsos any } 50 \\
\text { to } 100 \text { yeen }\end{array}$ & PELL_. & 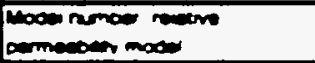 & $32209 A$ & $26-0 c t-85$ & $Y$ & 4B \\
\hline $\mathbf{Y}$ & & 2442 & CL_M_T4 & $\begin{array}{l}\text { Upper Saiscos an } 50 \\
\text { to } 100 \text { veme }\end{array}$ & REL_. & 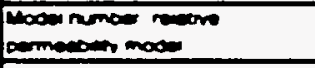 & 322094 & $26-0 c t-85$ & $\mathbf{Y}$ & 48 \\
\hline$Y$ & & 2442 & $C L_{2} M_{-} T_{4}$ & 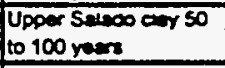 & 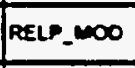 & nocou number revers & $32209 A$ & $26-0 c t-95$ & $\mathbf{Y}$ & $4 B$ \\
\hline $\mathbf{Y}$ & & 2442 & CL_M_TA & $\begin{array}{l}\text { Uppor Selecos an } 50 \\
\text { to } 100 \text { ram }\end{array}$ & RELP_. $=0$ & 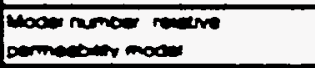 & 322094 & $26-0$ at.95 & $\mathbf{Y}$ & 48 \\
\hline$Y$ & & 2442 & $C L_{-} M_{-} T_{4}$ & 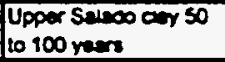 & 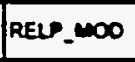 & 促 & 322094 & 26-0ct-95 & $\mathbf{Y}$ & $A B$ \\
\hline$Y$ & & 2442 & CL_M_TA & $\begin{array}{l}\text { Uppor Saloco cary } 50 \\
\text { to } 100 \text { vears }\end{array}$ & RELP_. & 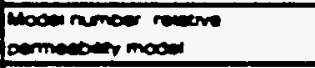 & 322094 & 26-0ct-95 & $\mathbf{Y}$ & AB \\
\hline $\mathbf{Y}$ & 100 & 244 & CL_M_T4 & $\begin{array}{l}\text { Upper Saleos aney } 50 \\
\text { to } 100 \text { veers }\end{array}$ & SAT_Menem & 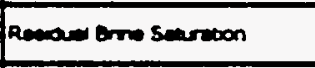 & 32225 & $19-F=0-96$ & $\mathbf{Y}$ & 1 \\
\hline$Y$ & 100 & 244 & CL_M_T4 & $\begin{array}{l}\text { Upper seiscos any } 50 \\
\text { to } 100 \text { yems }\end{array}$ & SAT_Rew & 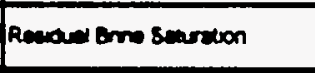 & 32225 & $19-F a b-96$ & $\mathbf{Y}$ & 1 \\
\hline Y & 100 & 244 & CL_M_T4 & $\begin{array}{l}\text { Wpper Selecos ary } 50 \\
\text { to } 100 \text { vars }\end{array}$ & Sat_mend & Readu ens Smartion & 32225 & $18 f a b-56$ & $\mathbf{Y}$ & 1 \\
\hline $\mathbf{Y}$ & 100 & 244 & $C_{2} M_{-} T_{4}$ & $\begin{array}{l}\text { Upopor Selecos any } 50 \\
\text { to } 100 \text { yeme }\end{array}$ & SAT_Agen & 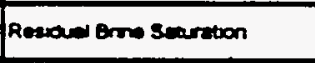 & 322025 & $19 F=0-96$ & $\mathbf{Y}$ & 1 \\
\hline
\end{tabular}


CCA-Parameter Listing-.

\begin{tabular}{|c|c|c|c|c|c|c|c|c|c|c|}
\hline 10 & 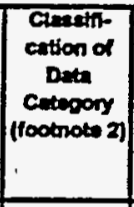 & $\begin{array}{l}\text { Distribertion } \\
\text { Type }\end{array}$ & $\operatorname{man}$ & modinn & Whamen & Axomim & Lrite & Rifimence id & $\begin{array}{l}\text { Wes the dit } \\
\text { demioped } \\
\text { underen } \\
\text { Hoh-1 } \\
\text { Proprem? }\end{array}$ & 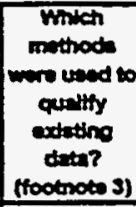 \\
\hline 2432 & 3,4 & CUMULATIVE & $2.5200000 E+\infty 0$ & $9.4000000 E-01$ & $1.1000000 E-01$ & $8.1000000 E+\infty$ & NONE & WP030094 & $\mathbf{Y}$ & NA \\
\hline 2432 & 3,4 & CUMULATIVE & $252000000+\infty 0$ & $9.4000000 E-01$ & $1.1000000 E-01$ & $8.1000000 E+\infty 0$ & NONE & WP052287 & $Y$ & NA \\
\hline 2432 & 3.4 & CUMULATIVE & $2.5200000 E+\infty 0$ & $9.4000000 E-01$ & $1.1000000 E-01$ & $8.1000000 E+\infty$ & MONE & WP035268 & $\gamma$ & NA \\
\hline 2432 & 3.4 & CUMULATIVE & $25200000 E+\infty 0$ & 9.4000000E.01 & $1.1000000 E-01$ & $8.1000000 E+\infty 0$ & NONE & WP035597 & $\mathbf{Y}$ & NA \\
\hline 2432 & 3,4 & CUMULATINE & $2.52000000+\infty 0$ & $9.4000000 E-01$ & $1.1000000 E-01$ & $8.1000000 E+\infty 0$ & NONE & WP041558 & $\mathbf{Y}$ & NA \\
\hline 2433 & $1,3,4$ & CONSTANT & $2.4000000 E-01$ & 2.4000000E-01 & $24000000 E-01$ & $24000000 E-01$ & $m^{2} 3 m^{n} 3$ & WPO30610 & $\mathbf{Y}$ & NA \\
\hline 2433 & $1,3,4$ & CONSTANT & 2.4000000E-01 & 24000000E-01 & 2.4000000E-01 & 2.4000000E-01 & $m^{\wedge} 3 m^{n} 3$ & WPososen & $\mathbf{Y}$ & NA \\
\hline 2433 & $1,3,4$ & CONSTANT & $2.4000000 E-01$ & $2.4000000=-01$ & 24000000E-01 & 2.4000000E-01 & $m \times 3 m=3$ & WP035597 & $\mathbf{Y}$ & NA \\
\hline 2434 & 1,3 & CONSTANT & $1.0132500 E+05$ & $1.0132500 E+0.5$ & $1.0132500 E+05$ & $1.0132500 E+05$ & Pa & WP035597 & $\mathbf{Y}$ & $N / A$ \\
\hline 2434 & 1,3 & CONSTANT & $1.0132500 \mathrm{E}+0.5$ & 1.0132500E+0.5 & $1.0132500 E+05$ & $1.0132500 E+05$ & $\mathrm{~Pa}$ & MPO36051 & $\mathbf{Y}$ & NA \\
\hline 2434 & 1,3 & CONSTANT & $1.0132500 E+05$ & $1.0132500 E+05$ & $1.0132500 E+05$ & $1.0132500 E+05$ & Pe & WPO40514 & $\mathbf{Y}$ & NA \\
\hline 2436 & $1,3,4$ & TRLANGULAR & $-1.8867000 E+01$ & $-1.8301000 E+01$ & $-21000000 E+01$ & $-1.7301000 E+01$ & $\log \left(m^{\wedge} 2\right)$ & WṔOS0640 & Y\&N & 1 \\
\hline 2436 & $1,3,4$ & TRLANGULAR & $-1.8867000 E+01$ & $-1.8301000 E+01$ & $-2.1000000 E+01$ & $-1.7301000 E+01$ & $\log \left(m^{n} 2\right)$ & upos0954 & Y\&N & 1 \\
\hline 2436 & $1.3,4$ & TRLANGULAR & $-1.8867000 E+01$ & $-1.8301000 E+01$ & $-2.1000000 E+01$ & $-1.7301000 E+01$ & $\log \left(m^{\wedge} 2\right)$ & WP035268 & Y\&N & 1 \\
\hline 2436 & $1,3,4$ & TRLANGULAR & $-1.8807000 E+01$ & $-1.8301000 E+01$ & $-2.1000000 E+01$ & $-1.7301000 E+01$ & $\log \left(m^{\wedge} 2\right)$ & WP035597 & YEN & 1 \\
\hline 2436 & $1,3,4$ & TRLANGULAR & $-1,8867000 E+01$ & $-1.8301000 E+01$ & $-21000000 E+01$ & $-1.7301000 E+01$ & $\log \left(m^{\wedge} 2\right)$ & WPOS1558 & Y8N & 1 \\
\hline 2437 & $1,3,4$ & TRLANGULAR & $-1.8867000 E+01$ & $-1.8301000 E+01$ & $-2.1000000 E+01$ & $-1.7301000 E+01$ & $\log \left(m^{\wedge} 2\right)$ & WPO30640 & Y\&N & 1 \\
\hline 2437 & $1,3,4$ & TRLANGULAR & $-1.8867000 E+01$ & $-1.8301000 E+01$ & $-21000000 E+01$ & $-1.7301000 E+01$ & $\log \left(m^{\wedge} 2\right)$ & WP030994 & YZN & 1 \\
\hline 2437 & $1,3,4$ & TRLANGULAR & $-1.8867000 E+01$ & $-1.8301000 E+01$ & $-2.1000000 E+01$ & $-1.7301000 E+01$ & $\log \left(m^{\wedge} 2\right)$ & WP035268 & YEN & 1 \\
\hline 2437 & $1,3,4$ & TRLANGULAR & $-1.8067000 E+01$ & $-1.8301000 E+01$ & $-2.1000000 E+01$ & $-1.7301000 E+01$ & $\log \left(m^{\wedge} 2\right)$ & WP035597 & YaN & 1 \\
\hline 2437 & $1,3,4$ & TRLANGULAR & $-1.8867000 E+01$ & $-1.8301000 E+01$ & $-2.1000000 E+01$ & $-1.7301000 E+01$ & $\log \left(m^{\wedge} 2\right)$ & WPOA1558 & YaN & 1 \\
\hline 2438 & $1,3,4$ & TRLANGULAR & $-1.8867000 E+01$ & $-1.8301000 E+01$ & $-2.1000000 E+01$ & $-1.7301000 \mathrm{E}+01$ & $\log \left(m^{\wedge} 2\right)$ & WP030640 & Yan & 1 \\
\hline 2438 & $1,3,4$ & TRIANGULAR & $-1,8067000 E+01$ & $-1.8301000 \mathrm{E}+01$ & $-2.1000000 E+01$ & $-1.7301000 E+01$ & $\log \left(m^{\wedge} 2\right)$ & up030994 & YaN & 1 \\
\hline 2438 & $1,3,4$ & TRIANGULAR & $-1.8867000 E+01$ & $-1.8301000 E+01$ & $-2.1000000 E+01$ & $-1.7301000 E+01$ & $\log \left(m^{\wedge} 2\right)$ & WP035268 & $\mathbf{r} \mathbf{N N}$ & 1 \\
\hline 2438 & $1,3,4$ & TRIANGULAR & $-1.8867000 E+01$ & $-1.0301000 E+01$ & $-2.1000000 \mathrm{E}+01$ & $-1.7301000 E+01$ & $\log \left(m^{\wedge} 2\right)$ & WP035597 & ZEN & 1 \\
\hline 2438 & $1,3,4$ & TRLANGULAR & $-1.8867000 E+01$ & $-1.8301000 E+01$ & $-2.1000000 E+01$ & $-1.7301000 E+01$ & $\log \left(m^{\wedge} 2\right)$ & WPOA1558 & YAN & 1 \\
\hline 2442 & $1,3,4$ & CONSTANT & $4.00000000 E+\infty$ & $4.0000000=+\infty 0$ & $4.0000000 E+00$ & $4.0000000 E+00$ & NONE & WP030540 & $\mathbf{Y}$ & NA \\
\hline 2442 & $1,3,4$ & CONSTANT & $4.00000000+00$ & $4.0000000=+00$ & $4.0000000 E+00$ & $4.0000000 E+\infty 0$ & NONE & WP030994 & $Y$ & N/A \\
\hline 2442 & $1,3,4$ & CONSTANT & $4.0000000 E+\infty 0$ & $4.00000000 E+00$ & $4.0000000 E+\infty$ & $4.0000000 E+\infty 0$ & NONE & WP035597 & $\mathbf{Y}$ & NA \\
\hline 2442 & $1,3,4$ & CONSTANT & $4.0000000 E+00$ & $4.0000000 E+\infty 0$ & $4.0000000 E+\infty 0$ & $4.0000000 E+\infty 0$ & NONE & WP037522 & $\mathbf{Y}$ & NA \\
\hline 2442 & $9,3,4$ & CONSTANT & $4.0000000 E+00$ & $4.0000000 E+\infty$ & $4.0000000 E+\infty$ & $4.0000000 E+\infty 0$ & NONE & WPO40406 & $\mathbf{Y}$ & NA \\
\hline 2442 & $1,3,4$ & CONSTANT & $4.0000000 E+\infty 0$ & $4.0000000 E+\infty$ & $4.0000000 E+\infty 0$ & $1.0000000 E+00$ & NONE & WPO40514 & $\mathbf{Y}$ & NA \\
\hline 2444 & 3,4 & CUMULATIVE & $25000000 E-01$ & 20000000E-01 & $0.0000000 E+\infty$ & $6.0000000 E-01$ & NONE & WPO30640 & $\mathbf{Y}$ & NA \\
\hline 2444 & 3,4 & CUMULATIVE & $2.5000000 E-01$ & $20000000 E-01$ & $0.0000000 E+\infty 0$ & $6.0000000 E-01$ & NONE & WP030994 & $\mathbf{Y}$ & NA \\
\hline 2444 & 3,4 & CURULATIVE & $25000000 E-01$ & $20000000 E-01$ & $0.00000000+\infty 0$ & $6.0000000 E-01$ & NONE & WP035268 & $\mathbf{Y}$ & NA \\
\hline 2444 & 3,4 & CUMULATIVE & $2.5000000 E-01$ & $2.0000000 E-01$ & $0.00000000+\infty 0$ & $6.0000000 E-01$ & NONE & WP035597 & $\mathbf{r}$ & $\mathrm{N} / \mathrm{A}$ \\
\hline
\end{tabular}


CCA Parameter Listing

\begin{tabular}{|c|c|c|c|c|c|c|c|c|c|c|}
\hline 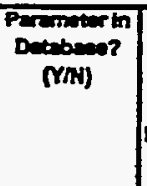 & 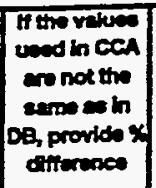 & to & Mexarial to & Anteriv Namo & $\begin{array}{c}\text { Permax } \\
\text { Dor }\end{array}$ & Perenterthe & $\begin{array}{l}\operatorname{PRP} \text { is } \\
\min =1\end{array}$ & $\begin{array}{c}\text { Decenty } \\
\text { Dent }\end{array}$ & 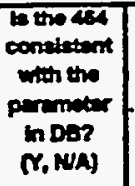 & 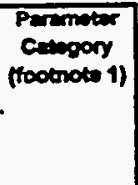 \\
\hline $\mathbf{Y}$ & 100 & 244 & $C L \_M I T A$ & $\begin{array}{l}\text { Upper Salaco clay.50 } \\
\text { to } 100 \text { years }\end{array}$ & SAT_RBRN & Residual Brime Seturation & 32025 & $19+a b-96$ & $\mathbf{Y}$ & 4 \\
\hline $\mathbf{Y}$ & 100 & 244 & CL_M_T4 & $\begin{array}{l}\text { Uppor Salaco ctry:50 } \\
\text { to } 100 \text { years }\end{array}$ & SAT_RBRN & Residual Brino Seturation & 32025 & $19 f a b-56$ & $\mathbf{Y}$ & 1 \\
\hline $\mathbf{Y}$ & 100 & 2445 & CL_M_T4 & $\begin{array}{l}\text { Uppor Stivido clay. } 50 \\
\text { to } 100 \text { yeers }\end{array}$ & SAT_RGAS & Reaidual Ges Sturation & 32226 & $13-F=b-96$ & $\mathbf{Y}$ & 1 \\
\hline $\mathbf{Y}$ & 100 & 2445 & CL_M_T4 & $\begin{array}{l}\text { Upper Salido ctry:50 } \\
\text { to } 100 \text { years }\end{array}$ & SAT_RGAS & Resicusal Ges Seturation & 32226 & $13-F a b-96$ & $\mathbf{Y}$ & 1 \\
\hline $\mathbf{Y}$ & 100 & 2445 & CL_M_T4 & $\begin{array}{l}\text { Upper Saledo day: } 50 \\
\text { to } 100 \text { years }\end{array}$ & SAT_RGAS & Reaidual Ges Seturation & 32226 & $13+a b-96$ & $\mathbf{Y}$ & 1 \\
\hline $\mathbf{Y}$ & 100 & 2445 & CL_M_T4 & $\begin{array}{l}\text { Upper Salado clay. } 50 \\
\text { to } 100 \text { years }\end{array}$ & SAT_RGAS & Residual Gas Seturation & 32226 & 13ffeb-96 & $\mathbf{Y}$ & 1 \\
\hline $\mathbf{Y}$ & 100 & 2445 & CL_M_T4 & $\begin{array}{l}\text { Upper Saledo clay:50 } \\
\text { to } 100 \text { years }\end{array}$ & SAT_RGAS & Residual Ges Suration & 32226 & 13Feb-9s & $\mathbf{Y}$ & 1 \\
\hline $\mathbf{Y}$ & 50 & 2446 & CL_M_T5 & $\begin{array}{l}\text { Upper Salado ctry:100 } \\
\text { to tOK yoars }\end{array}$ & CAP_MOD & $\begin{array}{l}\text { Model rumber, cepilloy preseire } \\
\text { model }\end{array}$ & $32228 A$ & C2+Nov-95 & $\mathbf{Y}$ & AB \\
\hline $\mathbf{Y}$ & 50 & 2446 & CL_M_T5 & $\begin{array}{l}\text { Upper Selado clay:100 } \\
\text { to 10K yeers }\end{array}$ & CAP_MOD & $\begin{array}{l}\text { Model number, cepithery preseure } \\
\text { model }\end{array}$ & 322281 & Oentov-95 & $\mathbf{Y}$ & 48 \\
\hline $\mathbf{Y}$ & 50 & 2446 & CL_H_T5 & $\begin{array}{l}\text { Uppor Salado cliny:100 } \\
\text { to 10K years }\end{array}$ & CAP_MOD & $\begin{array}{l}\text { Model number, expiliey preseurs } \\
\text { modil }\end{array}$ & 320284 & centroves & $\mathbf{Y}$ & 48 \\
\hline $\mathbf{Y}$ & 50 & 2446 & CL_M_T5 & $\begin{array}{l}\text { Upper Salado clay:100 } \\
\text { to 10K years }\end{array}$ & CAP_MOD & $\begin{array}{l}\text { Model namber, capiliay preserure } \\
\text { model }\end{array}$ & 322024 & C2Noviss & $\mathbf{Y}$ & 48 \\
\hline $\mathbf{Y}$ & 50 & 2446 & CL_M_T5 & \begin{tabular}{|l|} 
Upper Selodo clay:100 \\
to 40K years
\end{tabular} & CAP_MOD & $\begin{array}{l}\text { Model rumber, capillary pressurn } \\
\text { model }\end{array}$ & 322284 & C2Ator-35 & $\mathbf{Y}$ & AB \\
\hline $\mathbf{Y}$ & & 2447 & CL_M_T5 & $\begin{array}{l}\text { Upper Sulado clay:100 } \\
\text { to 10K yeers }\end{array}$ & COMP_RCK & Buk Compressibitity & 32230 & $13 f a b-96$ & $\mathbf{Y}$ & 1 \\
\hline$Y$ & & 2447 & CL_M_T5 & $\begin{array}{l}\text { Upper Salado clay. } 100 \\
\text { to toK years }\end{array}$ & COMP_RCK & Bulk Compressibitity & 32230 & 13 feb-96 & $\mathbf{Y}$ & 1 \\
\hline $\mathbf{Y}$ & & 2447 & CL_M_T5 & $\begin{array}{l}\text { Uppor Saledo ctay:100 } \\
\text { to 10K years }\end{array}$ & COMP_RCK & Buk Comprassibitity & 32230 & 13 Feb-s6 & $\mathbf{Y}$ & 1 \\
\hline $\mathbf{Y}$ & & 2448 & CL_M_TS & $\begin{array}{l}\text { Upper Salado ctay: } 100 \\
\text { to } 10 \mathrm{~K} \text { years }\end{array}$ & PC_MAX & $\begin{array}{l}\text { Maximum allowable capillery } \\
\text { presure }\end{array}$ & 322344 & $13-F e b-96$ & $\boldsymbol{Y}$ & $A B$ \\
\hline $\mathbf{Y}$ & & 2448 & CL_M_T5 & $\begin{array}{l}\text { Upper Saledo clay:100 } \\
\text { to } 10 K \text { years }\end{array}$ & PC_MAX & $\begin{array}{l}\text { Mexmum allowablo capillary } \\
\text { presure }\end{array}$ & 322344 & 13-Fab-96 & $\mathbf{Y}$ & 48 \\
\hline $\mathbf{Y}$ & & 2448 & CL_M_T5 & $\begin{array}{l}\text { Upper Salado clay: } 100 \\
\text { to 10K years }\end{array}$ & PC_MAX & $\begin{array}{l}\text { Maxinum allow blo cepillery } \\
\text { praseure }\end{array}$ & 322344 & 13-Fab-96 & $\mathbf{Y}$ & AB \\
\hline $\mathbf{Y}$ & & 2448 & CL_M_TS & $\begin{array}{l}\text { Upper Salacolo clay:100 } \\
\text { to 10K yeers }\end{array}$ & PC_MAX & $\begin{array}{l}\text { Meximum allowrable capillary } \\
\text { preasure }\end{array}$ & 322344 & 13Feb-96 & $\mathbf{Y}$ & 48 \\
\hline $\mathbf{Y}$ & & 2448 & CL_M_T5 & $\begin{array}{l}\text { Upper Salado elay.100 } \\
\text { to 10K years }\end{array}$ & PC_MAX & $\begin{array}{l}\text { Maxmum allowable copiliony } \\
\text { preasure }\end{array}$ & 320344 & 13-feb-96 & $\mathbf{Y}$ & 48 \\
\hline $\mathbf{Y}$ & varies & 2449 & CL_M_TS & $\begin{array}{l}\text { Upper Salado clay:100 } \\
\text { to 10K years }\end{array}$ & PORE_DIS & $\begin{array}{l}\text { Brocks-Corey pore distritution } \\
\text { parumoter }\end{array}$ & 32235 & 20-Feb-96 & $\mathbf{Y}$ & 1 \\
\hline $\mathbf{Y}$ & varies & 2449 & CL_M_T5 & $\begin{array}{l}\text { Uppor Salado day:100 } \\
\text { to } 10 \text { K years }\end{array}$ & PORE_DIS & $\begin{array}{l}\text { Brocks-Corey pore distribution } \\
\text { perameter }\end{array}$ & 32235 & 20-Fab-96 & $\mathbf{Y}$ & 1 \\
\hline $\mathbf{Y}$ & varies & 2449 & CL_M_T5 & $\begin{array}{l}\text { Upper Salado clay: } 100 \\
\text { to 10K years }\end{array}$ & PORE_DIS & $\begin{array}{l}\text { Brooks-Corny pore distribution } \\
\text { paremeter }\end{array}$ & 32235 & $20-F=b-96$ & $\mathbf{Y}$ & 1 \\
\hline $\mathbf{Y}$ & varios & 2449 & CL_M_T5 & $\begin{array}{l}\text { Upper Salado ctay.100 } \\
\text { to } 10 \mathrm{~K} \text { yours }\end{array}$ & PORE_DIS & $\begin{array}{l}\text { Brooks-Coray pore distribution } \\
\text { peremeter }\end{array}$ & 32235 & 20-Fob-96 & $\mathbf{Y}$ & 1 \\
\hline $\mathbf{Y}$ & varies & 2449 & CL_M_T5 & $\begin{array}{l}\text { Upper Saledo clay:100 } \\
\text { to 10K yeers }\end{array}$ & PORE_DIS & $\begin{array}{l}\text { Brooke-Corty pore distrocition } \\
\text { peremeter }\end{array}$ & 32235 & 20-Fab-96 & $=Y$ & 1 \\
\hline $\mathbf{Y}$ & varies & 2449 & CL_M_T5 & $\begin{array}{l}\text { Uppor Saledo ctay.100 } \\
\text { to } 10 \mathrm{~K} \text { years }\end{array}$ & PORE_DIS & $\begin{array}{l}\text { Erooks-Corny pore distribution } \\
\text { peremeter }\end{array}$ & 32235 & 20-Feb-96 & $\mathbf{Y}$ & 1 \\
\hline $\mathbf{Y}$ & & 2450 & CL_M_TS & $\begin{array}{l}\text { Upper Seledo clay. } 100 \\
\text { to 10K yeers }\end{array}$ & POROSITY & Effective porosity & 32236 & $93-$ feb-96 & $\mathbf{Y}$ & 1 \\
\hline $\mathbf{Y}$ & & 2450 & CL_M_T5 & $\begin{array}{l}\text { Upper Saledo clay:100 } \\
\text { to } 10 \mathrm{~K} \text { yours }\end{array}$ & POROSITY & Efinctive porosity & 32236 & $13-F e b-96$ & $\mathbf{Y}$ & 1 \\
\hline $\mathbf{Y}$ & & 2450 & $C L_{-} M_{-} T 5$ & $\begin{array}{l}\text { Upper Salado ctoy: } 100 \\
\text { to 10K yeers }\end{array}$ & POROsm & Efrective porosity & 32225 & $13-F=b-96$ & $\mathbf{Y}$ & 1 \\
\hline $\mathbf{Y}$ & & 2451 & CL_M_TS & $\begin{array}{l}\text { Upper Selado dity: } 100 \\
\text { to } 10 \mathrm{~K} \text { yers }\end{array}$ & PO_MIN & 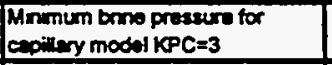 & 32237 & 21-Fab-96 & $\mathbf{Y}$ & AB \\
\hline $\mathbf{Y}$ & & 2451 & CL_M_T5 & $\begin{array}{l}\text { Upper Suledo day. } 100 \\
\text { to } 10 \mathrm{~K} \text { years }\end{array}$ & PO_MN & $\begin{array}{l}\text { Mnmum bone preseure for } \\
\text { capitieny model KPC=3 }\end{array}$ & 32237 & 21 feb-96 & $\mathbf{Y}$ & $4 B$ \\
\hline $\mathbf{Y}$ & & 2451 & CL_M_TS & $\begin{array}{l}\text { Upper Seledo clay: } 100 \\
\text { to } 10 K \text { yeers }\end{array}$ & PO_MIN & $\begin{array}{l}\text { Mnmum brome pressure for } \\
\text { capitery model KPC=3 }\end{array}$ & 32237 & 21 feb-96 & $\mathbf{Y}$ & 48 \\
\hline $\mathbf{Y}$ & varies & 2453 & CL_M_T5 & $\begin{array}{l}\text { Upper Seledo clay:100 } \\
\text { to } 10 K \text { yeers }\end{array}$ & PRAX_LOG & $\begin{array}{l}\text { Log of intrnac permesbilty, } X \text { - } \\
\text { direction }\end{array}$ & 32239 & 13-Fab-96 & $\mathbf{Y}$ & 1 \\
\hline $\mathbf{Y}$ & varios & 2453 & CL_M_TS & $\begin{array}{l}\text { Upper Selado cloy.100 } \\
\text { to 10K yaurs }\end{array}$ & PRMX_LOG & $\begin{array}{l}\text { Los of intrinax permesbilty, } X \text { - } \\
\text { diruction }\end{array}$ & 32039 & 13-feb-96 & $\mathbf{Y}$ & 1 \\
\hline $\mathbf{Y}$ & varios & 2453 & CL_M_T5 & $\begin{array}{l}\text { Upper Seledo ciry:100 } \\
\text { to } 10 k \text { yeer }\end{array}$ & PRIX_LOG & $\begin{array}{l}\text { Leg of intrinax parmesbility, } x \text { - } \\
\text { drection }\end{array}$ & 32239 & $13-f c b-96$ & $\mathbf{Y}$ & 1 \\
\hline $\mathbf{Y}$ & varies & 2453 & CL_M_TS & $\begin{array}{l}\text { Upper Seledo clay:100 } \\
\text { to } 10 \mathrm{~K} \text { year }\end{array}$ & PRMX_LOG & $\begin{array}{l}\text { Log of intrnac permesbitty, } X- \\
\text { direction }\end{array}$ & 32238 & $13-f c b-96$ & $\mathbf{Y}$ & 1 \\
\hline
\end{tabular}


CCA Parameter Listing

\begin{tabular}{|c|c|c|c|c|c|c|c|c|c|c|}
\hline 10 & $\begin{array}{c}\text { Clazelin- } \\
\text { cettion of } \\
\text { Deth } \\
\text { Crtagory } \\
\text { (footnote 2) }\end{array}$ & $\begin{array}{l}\text { Dhatribution } \\
\text { Type }\end{array}$ & $\operatorname{man}$ & Medim & Minitroum & Mextroum & Untas & Roforence id & $\begin{array}{l}\text { Wes the dint } \\
\text { omaloped } \\
\text { underen } \\
\text { NOA-1 } \\
\text { Progrem? }\end{array}$ & 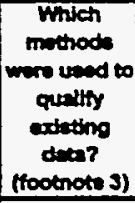 \\
\hline 2444 & 3,4 & CUMULATIVE & $25000000 \mathrm{E}-01$ & $2.0000000 E-01$ & $0.0000000=+\infty$ & $6.0000000 E-01$ & NONE & WPO41558 & $\mathbf{Y}$ & NA \\
\hline 244 & 3,4 & CUMULATIVE & $2.5000000 E-01$ & $2.0000000 E-01$ & $0.0000000 E+00$ & 6.0000000E-01 & NONE & WPO42751 & $\mathbf{Y}$ & NA \\
\hline 2445 & 3.4 & UNIFORM & $20000000 E-01$ & $20000000 E-01$ & $0.00000000+\infty$ & 4.0000000 E-01 & NONE & WP030640 & $Y$ & NA \\
\hline 2445 & 3,4 & UNIFORM & $2.0000000 E-01$ & $2.0000000 E-01$ & $0.00000000+\infty$ & $4.0000000 E-01$ & NONE & WPO30994 & $\mathbf{Y}$ & NA \\
\hline 2445 & 3,4 & UNIFORM & $2.0000000 E-01$ & $2.0000000 E-01$ & $0.0000000 E+\infty$ & 4.0000000E-01 & NONE & WP035268 & $Y$ & NA \\
\hline 2445 & 3,4 & UNIFORM & $20000000 E-01$ & 20000000E-01 & $0.0000000 E+\infty$ & 4.0000000E-01 & NONE & WP03559? & $\mathbf{Y}$ & NA \\
\hline 2445 & 3,4 & UNIFORM & $2.0000000 E-01$ & $2.0000000 E-01$ & $0.0000000 \mathrm{E}+\infty 0$ & 4.0000000E-01 & NONE & MPOA1558 & $Y$ & NA \\
\hline 2446 & $1,3,4$ & CONSTANT & $2.0000000 \mathrm{E}+\infty 0$ & $2.0000000 E+\infty$ & $20000000 E+\infty$ & $2.0000000 E+\infty 0$ & MONE & WP030610 & $\mathbf{Y}$ & NAA \\
\hline 2446 & $1,3,4$ & CONSTANT & $2.0000000 \mathrm{E}+00$ & $20000000 \mathrm{E}+\infty 0$ & $20000000 \mathrm{E}+\infty$ & $20000000 E+00$ & NONE & WP030994 & $\mathbf{Y}$ & NA \\
\hline 2446 & $1,3,4$ & CONSTANT & $20000000 E+00$ & $2.0000000 \mathrm{E}+\infty 0$ & $200000000+00$ & $2,0000000 E+\infty$ & NONE & WP035597 & $\mathbf{Y}$ & NA \\
\hline 2446 & $1,3,4$ & CONSTANT & $2.0000000 E+00$ & $20000000 E+\infty$ & $2.0000000 E+\infty 0$ & $2,0000000 E+\infty$ & NONE & WPO40486 & $Y$ & NA \\
\hline 2446 & $1,3,4$ & CONSTANT & $2.0000000 \mathrm{E}+00$ & $2.0000000 E+\infty 0$ & $2.0000000 \varepsilon+\infty$ & $200000000 E+00$ & NONE & WP040514 & $Y$ & NA \\
\hline 2447 & $1,3,4$ & CONSTANT & $1.8100000 E-09$ & $1.8100000 E-09$ & $1.8100000 E-09$ & $1.8100000 E-09$ & Pan-1 & WP030640 & $\mathbf{Y}$ & NA \\
\hline 2447 & $1,3,4$ & CONSTANT & $1.8100000 E-09$ & $1.8100000 E-09$ & $1.8100000 E-09$ & $1.8100000 E-09$ & $P x^{n-1}$ & WP030994 & $\mathbf{Y}$ & NA \\
\hline 2447 & $1,3,4$ & CONSTANT & $1.8100000 \mathrm{E}-09$ & $1.8100000 E-09$ & $1.8100000 E-09$ & $-1.8100000 E-09$ & Pan-1 & WP035597 & $\mathbf{Y}$ & NUA \\
\hline 2448 & 1,3 & CONSTANT & $1.0000000 \mathrm{E}+08$ & $1.0000000 \mathrm{E}+08$ & $1.0000000 E+08$ & $1.0000000 E+08$ & $\mathbf{P a}$ & WP030640 & $\mathbf{Y}$ & NAA \\
\hline 2448 & 1.3 & CONSTANT & $1.0000000 E+08$ & $1.0000000 E+08$ & $1.0000000 E+08$ & $1.0000000 E+08$ & $\mathbf{P a}$ & WPO30994 & $\mathbf{Y}$ & NA \\
\hline 2448 & 1,3 & CONSTANT & $1.0000000 \mathrm{E}+08$ & $1.0000000 E+08$ & $1.0000000 E+08$ & $1.0000000 E+08$ & Pa & WP035597 & $Y$ & NA \\
\hline 2448 & 1,3 & CONSTANT & $1.0000000 \mathrm{E}+08$ & $1.0000000 E+08$ & $1.0000000 E+08$ & $1.0000000 E+08$ & $\mathbf{P a}$ & WPO40486 & $Y$ & NA \\
\hline 2448 & 1,3 & CONSTANT & $1.0000000 E+08$ & $1.00000000+08$ & $1.0000000 E+08$ & $1.0000000 E+08$ & Pa & WP040514 & $Y$ & NA \\
\hline 2449 & 3,4 & CUMULATINE & $2.5200000 E+\infty$ & $9.4000000 \mathrm{E}-01$ & $1.1000000 E-01$ & $8.1000000 E+\infty$ & NONE & WP030640 & $\mathbf{Y}$ & NA \\
\hline 2449 & 3,4 & CUMULATINE & $2.5200000 E+\infty$ & 9.4000000E-01 & $1.1000000 E-01$ & $8.1000000 E+\infty 0$ & NONE & WP030994 & $\mathbf{Y}$ & NA \\
\hline 2449 & 3.4 & CUMULATINE & $2.5200000 E+00$ & $9.4000000 E-01$ & $1.1000000 E-01$ & $8.1000000 E+\infty$ & NONE & WP032287 & $\mathbf{Y}$ & NA \\
\hline 2449 & 3,4 & CUMULATINE & $2.5200000 E+\infty$ & $9.4000000 E-01$ & $1.1000000 E-01$ & $8.1000000 E+\infty 0$ & NONE & WP035268 & $Y$ & NA \\
\hline 2449 & 3,4 & CUMULATIVE & $2.5200000 E+\infty$ & $9,4000000 E-01$ & $1,1000000 E-01$ & 8. $1000000 E+\infty 0$ & NONE & WP035597 & $-Y Y$ & NA \\
\hline 2449 & 3,4 & CUMULATIVE & $2.52000000 E+\infty$ & 9.4000000E-01 & $1.1000000 E-01$ & B. $1000000 \mathrm{E}+\infty$ & NONE & WPO41558 & $\mathbf{Y}$ & NA \\
\hline 2450 & $1,3,4$ & CONSTANT & $2.4000000 E-01$ & $2.4000000 E-01$ & $2 \angle 000000 E-0.1$ & $24000000 E-01$ & $m^{\wedge} 3 m^{\wedge} \wedge 3$ & WPO30640 & $\mathbf{Y}$ & NA \\
\hline 2450 & $1,3,4$ & CONSTANT & 2.4000000E-01 & $24000000 E-01$ & $24000000 E-01$ & $2.4000000 E-01$ & $m^{\wedge} 3 / m^{\wedge} 3$ & WP030994 & $Y$ & NA \\
\hline 2450 & $1,3,4$ & CONSTANT & $2.4000000 E-01$ & $2.4000000 \varepsilon-01$ & $24000000 E-01$ & 2.4000000E-01 & $m^{\wedge} 3 / m^{\wedge} 3$ & WP035597 & $\mathbf{Y}$ & NA \\
\hline 2451 & 1,3 & CONSTANT & $1.0132500 E+05$ & $1.0132500 E+05$ & $1.0132500 E+05$ & $1.0132500 E+05$ & Pa & WP035597 & $\mathbf{Y}$ & NA \\
\hline 2451 & 1,3 & CONSTANT & $1.0132500 \mathrm{E}+05$ & $1.0132500 E+05$ & $1.0132500 E+05$ & $1.0132500 E+0.5$ & Pa & WP036051 & $\mathbf{Y}$ & NA \\
\hline 2451 & 1,3 & CONSTANT & $1.0132500 E+05$ & $1.0132500 E+05$ & $1.0132500 E+05$ & $1.0132500 E+05$ & Pa & WPO40514 & $\mathbf{Y}$ & NA \\
\hline 2453 & $1,3,4$ & TRLANGULAR & $-1.8857000 E+01$ & $-1.8301000 E+01$ & $-2.1000000 E+01$ & $-1.7301000 E+01$ & $\log \left(m^{\wedge} 2\right)$ & WP030640 & Y8N & 1 \\
\hline 2453 & $1,3,4$ & TRLANGULAR & $-1.8867000 \mathrm{E}+01$ & $-1.8301000 E+01$ & $-2.1000000 E+01$ & $-1.7301000 E+01$ & $\log \left(m^{\wedge} 2\right)$ & WP030994 & Yan & 1 \\
\hline 2453 & $1,3,4$ & TRIANGULAR & $-1.6867000 E+01$ & $-1.8301000 E+01$ & $-2.1000000 E+01$ & $-1.7301000 E+01$ & $\log \left(m^{\wedge} 2\right)$ & WP035268 & YSN & 1 \\
\hline 2453 & $1,3,4$ & TRLANGULAR & $-1.8867000 E+01$ & $-1.8301000 E+01$ & $-2.1000000 E+01$ & $-1.7301000 E+01$ & $\log \left(m^{\wedge} 2\right)$ & WP035597 & YEN & 1 \\
\hline
\end{tabular}


CCA Parameter Listing

\begin{tabular}{|c|c|c|c|c|c|c|c|c|c|c|}
\hline $\begin{array}{c}\text { Parmoter in } \\
\text { Datheae? } \\
\text { (Mai) }\end{array}$ & 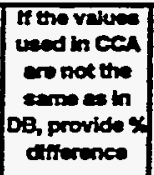 & 10 & Matardoto & Mrtede Nemo & $\begin{array}{c}\text { Permatiox } \\
0\end{array}$ & 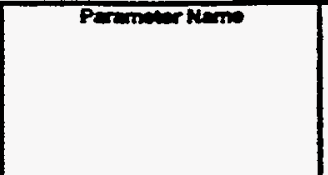 & $\begin{array}{l}\text { PRPD } \\
\text { MPOS }\end{array}$ & $\begin{array}{c}\text { Den Entry } \\
\text { Das }\end{array}$ & 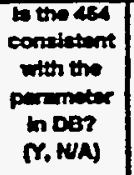 & 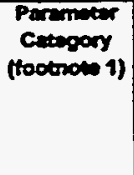 \\
\hline $\mathbf{Y}$ & vanes & 2453 & CL_M_T5 & $\begin{array}{l}\text { Upper Saledo clay. } 100 \\
\text { to toK years }\end{array}$ & PRMX_LOG & $\begin{array}{l}\text { Los of monsic permentilfy, } X \text { - } \\
\text { drection }\end{array}$ & 32239 & $13-F=b-96$ & $\mathbf{Y}$ & 1 \\
\hline $\mathbf{Y}$ & & 2454 & CL_M_TS & $\begin{array}{l}\text { Upper Satado clay.100 } \\
\text { to 10K years }\end{array}$ & PRAY_LOG & $\begin{array}{l}\text { Los of momsic permeability. Y- } \\
\text { direction }\end{array}$ & 32240 & $13-F=b-86$ & $\mathbf{Y}$ & 1 \\
\hline $\mathbf{Y}$ & & 2454 & CL_M_TS & $\begin{array}{l}\text { Upper Salodo day.100 } \\
\text { to 10K yaurs }\end{array}$ & PRAY_LOG & $\begin{array}{l}\text { Los of intrmsic pormadbilty. } Y \text { - } \\
\text { diroction }\end{array}$ & 32240 & $13 f a b-96$ & $\mathbf{Y}$ & 1 \\
\hline $\mathbf{Y}$ & & 2454 & CL_M_T5 & $\begin{array}{l}\text { Upper Salodo ctay.100 } \\
\text { to 10K years }\end{array}$ & PRAY_LOG & $\begin{array}{l}\text { Log of intmic permesbility, Y. } \\
\text { direction }\end{array}$ & 32240 & 13-Feb-96 & $\mathbf{Y}$ & 1 \\
\hline $\mathbf{Y}$ & & 2454 & CL_M_T5 & $\begin{array}{l}\text { Upper Salado clay:100 } \\
\text { to } 10 \mathrm{~K} \text { years }\end{array}$ & PRMY_LOG & $\begin{array}{l}\text { Log of intrinsxe permeability. Y- } \\
\text { direction }\end{array}$ & 32240 & $13+f e b-86$ & $Y$ & 1 \\
\hline $\mathbf{Y}$ & & 2454 & CL_M_T5 & $\begin{array}{l}\text { Upper Salado clay.100 } \\
\text { to } 10 \mathrm{~K} \text { years }\end{array}$ & PRWY_LOG & $\begin{array}{l}\text { Log of intrnase permesbility, Y. } \\
\text { direction }\end{array}$ & 32240 & 13feb-96 & $\mathbf{Y}$ & 1 \\
\hline $\mathbf{Y}$ & & 2455 & CL_M_T5 & $\begin{array}{l}\text { Upper Salado clay:100 } \\
\text { to 10K years }\end{array}$ & PRMZ_LOG & $\begin{array}{l}\text { Los of ntonste permesibilty, Z- } \\
\text { direction }\end{array}$ & 32241 & $13-F a b-56$ & $\mathbf{Y}$ & 1 \\
\hline $\mathbf{Y}$ & & 2455 & CL_M_T5 & $\begin{array}{l}\text { Upper Salado cisy:100 } \\
\text { to 10K yeers }\end{array}$ & PRMZ_LOG & $\begin{array}{l}\text { Log of intensic permanbildy, Z- } \\
\text { direction }\end{array}$ & 32241 & $13+a b-96$ & $Y$ & 1 \\
\hline$Y$ & & 2455 & CL_M_T5 & $\begin{array}{l}\text { Upper Salado clay: } 100 \\
\text { to } 10 \mathrm{~K} \text { yoars }\end{array}$ & PRAZ_LOG & $\begin{array}{l}\text { Log of ntrutse permesbility, Z- } \\
\text { direction }\end{array}$ & 32241 & $13-F a b-96$ & $\mathbf{Y}$ & 1 \\
\hline$Y$ & & 2455 & CL_M_T5 & $\begin{array}{l}\text { Upper Salaco clay: } 100 \\
\text { to 10K yuars }\end{array}$ & PRMZ_LOG & $\begin{array}{l}\text { Log of intrinsic permesbilty, Z- } \\
\text { direction }\end{array}$ & 32241 & 13-Feb-96 & $\mathbf{Y}$ & 1 \\
\hline $\mathbf{Y}$ & & 2455 & CL_M_T5 & $\begin{array}{l}\text { Upper Salsdo clay:100 } \\
\text { to 10K yoars }\end{array}$ & PRMZ_LOG & $\begin{array}{l}\text { Log of intrnssc permanbility, Z- } \\
\text { direction }\end{array}$ & 32241 & $13-F a b-96$ & $\mathbf{Y}$ & 1 \\
\hline$Y$ & & 2459 & CL_M_T5 & $\begin{array}{l}\text { Upper Salado cley: } 100 \\
\text { to tok yess }\end{array}$ & RELP_MOD & $\begin{array}{l}\text { Modal number, relative } \\
\text { pormeabifity model }\end{array}$ & $32248 A$ & 26-0ct-95 & $\mathbf{Y}$ & AB \\
\hline $\mathbf{Y}$ & & 2459 & CL_M_TS & $\begin{array}{l}\text { Upper Saledo clay.100 } \\
\text { to 10K yoars }\end{array}$ & RELP_MOD & $\begin{array}{l}\text { Hodel rumber, relative } \\
\text { pormasbility model }\end{array}$ & $32248 A$ & 26-0ct-95 & $\mathbf{Y}$ & 48 \\
\hline$Y$ & & 2459 & CL_M_T5 & $\begin{array}{l}\text { Uppor Salado ctay: } 100 \\
\text { to 10K years }\end{array}$ & RELP_MOD & $\begin{array}{l}\text { Model number, relativa } \\
\text { permeability model }\end{array}$ & 322484 & $26-0$ ct-95 & $\mathbf{Y}$ & 48 \\
\hline $\mathbf{Y}$ & & 2459 & CL_M_TS & $\begin{array}{l}\text { Upper Salado clay. } 100 \\
\text { to } 10 K \text { years }\end{array}$ & RELP_MOD & $\begin{array}{l}\text { Model number, rolatrive } \\
\text { permeability model }\end{array}$ & 32048A & $26-0 c t-95$ & $\mathbf{Y}$ & $4 B$ \\
\hline $\mathbf{Y}$ & & 2459 & CL_M_T5 & $\begin{array}{l}\text { Upper Salado ctay.100 } \\
\text { to 10K years }\end{array}$ & RELP_MOD & $\begin{array}{l}\text { Model number, relative } \\
\text { permesbility model }\end{array}$ & $32248 A$ & $26-0 c t-95$ & $\mathbf{Y}$ & AB \\
\hline $\mathbf{Y}$ & 100 & 2461 & CL_M_TS & $\begin{array}{l}\text { Uppor Salado clay:100 } \\
\text { to 10K years }\end{array}$ & SAT_RBRN & Residual Brine Saturation & 32254 & $13-5 a b-96$ & $\mathbf{Y}$ & 1 \\
\hline $\mathbf{Y}$ & 100 & 2461 & CL_M_T5 & $\begin{array}{l}\text { Upper Saledo clay.100 } \\
\text { to 10K years }\end{array}$ & SAT_RBRN & Residual Brine Seturation & 32254 & 13 Fob-96 & $\mathbf{Y}$ & 1 \\
\hline $\mathbf{Y}$ & 100 & 2461 & CL_M_T5 & $\begin{array}{l}\text { Upper Saledo cley,100 } \\
\text { to 10K years }\end{array}$ & SAT_RBRN & Residual Brine Seturation & 32254 & 13-Feb-96 & $\mathbf{Y}$ & 1 \\
\hline $\mathbf{Y}$ & 100 & 2461 & CL_M_T5 & $\begin{array}{l}\text { Upper Salado clay:100 } \\
\text { to 10K years }\end{array}$ & SAT_RBRN & Residual Brino Suturation & 32254 & 13-Feb-96 & $\mathbf{Y}$ & 1 \\
\hline $\mathbf{Y}$ & 100 & 2461 & CL_M_T5 & $\begin{array}{l}\text { Upper Salado clay.100 } \\
\text { to 10K years }\end{array}$ & SAT_RBRN & Residual Erine Seturetion & 32254 & 13 Feb-96 & $\mathbf{Y}$ & 1 \\
\hline $\mathbf{Y}$ & 100 & 2461 & $C L_{2} M_{-} T 5$ & $\begin{array}{l}\text { Upper Selado clay: } 100 \\
\text { to } 10 K \text { years }\end{array}$ & SAT_RBRN & Residual Brino Seturation & 32254 & 13 Feb-96 & $\mathbf{Y}$ & 1 \\
\hline $\mathbf{Y}$ & 100 & 2452 & CL_M_T5 & $\begin{array}{l}\text { Upper Salado clay.100 } \\
\text { to 10K years }\end{array}$ & SAT_RGAS & Residual Gas Saturation & 32255 & 13-Fab-96 & $\mathbf{Y}$ & 1 \\
\hline $\mathbf{Y}$ & 100 & 2462 & CL_M_T5 & $\begin{array}{l}\text { Upper Salado clay.100 } \\
\text { to } 10 \text { K yoars }\end{array}$ & SAT_RGAS & Resichal Ges Sxturation & 32255 & 13-Fab-96 & $\mathbf{Y}$ & 1 \\
\hline$Y$ & 100 & 2462 & $\mathrm{CL}_{-} \mathrm{M}_{-} \mathrm{T5}$ & $\begin{array}{l}\text { Upper Salado clay:100 } \\
\text { to 10K years }\end{array}$ & SAT_RGAS & Residual Ges Saturation & 32255 & 13-Fab-96 & $--Y$ & 1 \\
\hline $\mathbf{Y}$ & 100 & 2462 & CL_M_T5 & $\begin{array}{l}\text { Upper Salado clay: } 100 \\
\text { to 10K years }\end{array}$ & SAT_RGAS & Residual Gas Saturation & 32255 & 13 feb-96 & $\mathbf{Y}$ & 1 \\
\hline $\mathbf{Y}$ & 100 & 2462 & CL_M_TS & $\begin{array}{l}\text { Upper Saledo clay: } 100 \\
\text { to 10K years }\end{array}$ & SAT_RGAS & Residual Ges Seturation & 32255 & 13Fab-96 & $Y$ & 1 \\
\hline $\mathbf{Y}$ & 50 & 2453 & CONC_TI & $\begin{array}{l}\text { Concrute cotumn:0 to } \\
400 \text { years }\end{array}$ & CAP_MOD & $\begin{array}{l}\text { Model rumber, capillory prassume } \\
\text { model }\end{array}$ & 32549A & 14-Feb-96 & $\mathbf{Y}$ & 48 \\
\hline $\mathbf{Y}$ & 50 & 2463 & CONC_T1 & $\begin{array}{l}\text { Concreto cohmn:0 to } \\
400 \text { years }\end{array}$ & CAP_MOD & $\begin{array}{l}\text { Model mumber, capillery preasure } \\
\text { model }\end{array}$ & $32549 A$ & $14 f e b-95$ & $\mathbf{Y}$ & 48 \\
\hline $\mathbf{Y}$ & 50 & 2463 & CONC_T1 & $\begin{array}{l}\text { Concrite columnio to } \\
400 \text { yerers }\end{array}$ & CAP_MOD & $\begin{array}{l}\text { Model number, capilley pressure } \\
\text { model }\end{array}$ & 32549A & 14 Fob-96 & $\mathbf{Y}$ & $4 B$ \\
\hline $\mathbf{Y}$ & 50 & 2463 & CONC_TI & $\begin{array}{l}\text { Concrete cotumn:0 to } \\
400 \text { yesers }\end{array}$ & CAP_MOD & $\begin{array}{l}\text { Model number, capilisy pressuro } \\
\text { model }\end{array}$ & $32549 A$ & 14Fab-96 & $\mathbf{Y}$ & $4 B$ \\
\hline $\mathbf{Y}$ & 50 & 2453 & CONC_T1 & $\begin{array}{l}\text { Concrete column:0 to } \\
400 \text { yesers }\end{array}$ & CAP_MOD & $\begin{array}{l}\text { Modol number, capillary prossure } \\
\text { model }\end{array}$ & $32549 A$ & 14 feb-96 & $\mathbf{Y}$ & $4 \mathrm{~B}$ \\
\hline $\mathbf{Y}$ & & 2454 & CONC_T1 & $\begin{array}{l}\text { Concrute cokmn:0 to } \\
400 \text { years }\end{array}$ & COMP_RCK & Buk Compressibitity & 32556 & $02+4 a y-96$ & $\mathbf{Y}$ & $A B$ \\
\hline $\mathbf{Y}$ & & 2454 & CONC_T1 & $\begin{array}{l}\text { Concrote cokmn: } 0 \text { to } \\
400 \text { years }\end{array}$ & COMP_RCK & Buk Compressibility & 32556 & $02+$ May-96 & $\mathbf{Y}$ & $4 B$ \\
\hline $\mathbf{Y}$ & & 2464 & CONC_T1 & $\begin{array}{l}\text { Concrote colemnio to } \\
400 \text { years }\end{array}$ & COMP_RCK & Buk Compreasibitity & 32556 & $02+1 \times y+96$ & $\mathbf{Y}$ & 48 \\
\hline $\mathbf{Y}$ & & 2465 & CONC_TI & $\begin{array}{l}\text { Concrite column:0 to } \\
400 \text { vers }\end{array}$ & PC_MAX & $\begin{array}{l}\text { Mocontum allowloble capillary } \\
\text { pressure }\end{array}$ & $32567 \mathrm{~A}$ & O3-Hov-95 & $\mathbf{Y}$ & $4 B$ \\
\hline
\end{tabular}


CCA Parameter Listing

\begin{tabular}{|c|c|c|c|c|c|c|c|c|c|c|}
\hline 10 & 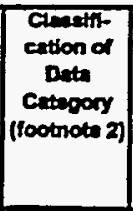 & $\begin{array}{l}\text { Destribertion } \\
\text { Type }\end{array}$ & nem & Medim & Minitriesn & Madmurn & Undts & Reformance to & $\begin{array}{l}\text { Wes the dert } \\
\text { denloped } \\
\text { onderen } \\
\text { Hol-1 } \\
\text { Progrem? }\end{array}$ & 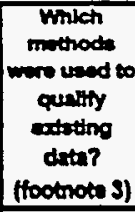 \\
\hline 2453 & $1,3,4$ & TRLANGULAR & $-1.8867000 E+01$ & $-1.8301000 E+01$ & $-21000000 E+01$ & $-1.7301000 E+01$ & $\log \left(m^{\wedge} 2\right)$ & UPO41558 & Y\&N & 1 \\
\hline 2454 & 1.3 .4 & TRLANGULAR & $-1.8967000 E+01$ & $-1.8301000 E+01$ & $-2.1000000 E+01$ & $-1.7301000 E+01$ & $\log \left(m^{2} 2\right)$ & WP030540 & YEN & 1 \\
\hline 2454 & $1,3,4$ & TRLANGULAR & $-1.8857000 E+01$ & $-1.8301000 E+01$ & $-2,1000000 E+01$ & $-1.7301000 E+01$ & $\cos \left(m^{2} 2\right)$ & WP030994 & YaN & 1 \\
\hline 2454 & $1,3,4$ & TRLANGULAR & $-1.8867000 E+01$ & $-1.8301000 E+01$ & $-21000000 E+01$ & $-1.7301000 E+01$ & $\log \left(m^{\wedge} 2\right)$ & WP035268 & YaN & 1 \\
\hline 2454 & $1,3,4$ & TRLANGULAR & $-1.8667000 E+08$ & $-1.8301000 E+01$ & $-2,1000000 E+01$ & $-1.7301000 E+01$ & $\log (m \wedge 2)$ & WP035597 & YZN & 1 \\
\hline 2454 & $1,3,4$ & TRLANGULAR & $-1.8867000 E+01$ & $-1.8301000 E+01$ & $-2.1000000 E+01$ & $-1.7301000 E+01$ & $\log \left(m^{\wedge} 2\right)$ & WPOA1558 & YAN & 1 \\
\hline 2455 & $1,3,4$ & TRLANGULAR & $-1.8867000 E+01$ & $-1.8301000 E+01$ & $-21000000 E+01$ & $-1.7301000 E+01$ & $\log \left(m^{2} 2\right)$ & WP030640 & Y\&N & 1 \\
\hline 2455 & $1,3,4$ & TRLANGULAR & $-1.8867000 E+01$ & $-1.8301000 E+01$ & $-2.1000000 E+01$ & $-1.7301000 E+01$ & $\log \left(m^{\wedge} 2\right)$ & WP030994 & YaN & 1 \\
\hline 2455 & $1,3,4$ & TRIANGULAR & $-1.8867000 E+01$ & $-1.8301000 E+01$ & $-2,1000000 E+01$ & $-1.7301000 E+01$ & $\log \left(m^{\wedge} 2\right)$ & WP035268 & YEN & 1 \\
\hline 2455 & $1,3,4$ & TRIANGULAR & $-1.8867000 E+01$ & $-1.8301000 E+01$ & $-2.1000000 E+01$ & $-1.7301000 E+01$ & $\log \left(m^{2} 2\right)$ & WP035597 & YEN & 1 \\
\hline 2455 & $1,3,4$ & TRLANGULAR & $-1.8867000 E+01$ & $-1.8301000 E+01$ & $-2.1000000 E+01$ & $-1.7301000 E+01$ & $\log \left(m^{\wedge} 2\right)$ & WPO41558 & YaN & 1 \\
\hline 2459 & $1,3,4$ & CONSTANT & $4.0000000 \mathrm{E}+\infty 0$ & $4.0000000 \mathrm{E}+\infty 0$ & $4.0000000 E+\infty 0$ & $4.0000000 \mathrm{E}+\infty$ & NONE & WP030640 & $\mathbf{Y}$ & NA \\
\hline 2459 & $1,3,4$ & CONSTANT & $4.0000000 E+\infty 0$ & $4.0000000 E+\infty 0$ & $4.0000000 E+\infty$ & $4.0000000 E+00$ & NONE & WP030994 & $\mathbf{Y}$ & NA \\
\hline 2459 & $1,3,4$ & CONSTANT & $4.00000000 E+00$ & $4.0000000 E+00$ & $4.0000000 E+\infty 0$ & $4.0000000 E+\infty 0$ & NONE & WP035597 & $\mathbf{Y}$ & NA \\
\hline 2459 & $1,3,4$ & CONSTANT & $4.0000000 \mathrm{E}+00$ & $4.0000000 E+00$ & $4.0000000 E+\infty 0$ & $4.0000000 E+\infty 0$ & NONE & WP040486 & $\mathbf{Y}$ & NA \\
\hline 2459 & $1,3,4$ & CONSTANT & $4.0000000 E+00$ & $4.0000000 \mathrm{E}+\infty 0$ & $4.0000000 \mathrm{E}+\infty 0$ & $4.0000000 \mathrm{E}+\infty 0$ & NONE & WP040514 & $\mathbf{Y}$ & NA \\
\hline 2461 & 3.4 & CUMULATIVE & $2.5000000 E-01$ & $2.0000000 E-01$ & $0.0000000 E+\infty$ & $6.0000000 E-01$ & NONE & WP030640 & $\mathbf{Y}$ & NA \\
\hline 2461 & 3,4 & CUMULATIVE & $2.5000000 E-01$ & $2.0000000 E-01$ & $0.0000000 E+\infty$ & $6.0000000 \mathrm{E}-01$ & NONE & WP030994 & $\mathbf{Y}$ & NA \\
\hline 2451 & 3,4 & CUMULATTVE & 2.5000000 E- 01 & $20000000 E-01$ & $0.0000000 \mathrm{E}+\infty$ & $6.0000000 E-01$ & NONE & WP035268 & $\mathbf{Y}$ & NA \\
\hline 2461 & 3,4 & CUMULATIVE & $2.5000000 E-01$ & 2.0000000E-01 & $0.0000000 E+\infty 0$ & 6.0000000E-01 & NONE & WP035597 & $\mathbf{Y}$ & NA \\
\hline 2461 & 3,4 & CUMULATIVE & $25000000 E-01$ & $20000000 E-01$ & $0.0000000 E+\infty$ & $6.0000000 E-01$ & NONE & WP041558 & $\mathbf{Y}$ & NA \\
\hline 2461 & 3,4 & CUMULATIVE & $2.5000000 E-01$ & $2.0000000 E-01$ & $0.0000000 \mathrm{E}+00$ & $6.0000000 E-01$ & NONE & WPO42751 & $Y$ & NA \\
\hline 2462 & 3,4 & UNIFORM & $2.0000000 \mathrm{E}-09$ & $20000000 E-01$ & $0.00000000+00$ & $4.0000000 E-01$ & NONE & WP030640 & $Y$ & NA \\
\hline 2462 & 3,4 & UNIFORM & $2.0000000 E-01$ & $20000000 E-01$ & $0.0000000 \mathrm{E}+\infty 0$ & $4.00000000-01$ & NONE & WP030994 & $\mathbf{Y}$ & N/A \\
\hline 2462 & 3,4 & UNIFORM & $2,0000000 \mathrm{E}-01$ & $2,0000000 E-01$ & $0.0000000 E+00$ & $4.0000000 E-01$ & NONE & WP035268 & $-Y Y$ & NAA \\
\hline 2462 & 3,4 & UNIFORM & $2.0000000 E-01$ & $2.0000000 E-01$ & $0.0000000 E+\infty$ & 4.0000000E-01 & NONE & WP035597 & $\mathbf{Y}$ & NA \\
\hline 2462 & 3,4 & UNIFORM & $20000000 E-01$ & $2,0000000 E-01$ & $0.0000000 E+\infty 0$ & 4.0000000E-01 & NONE & WPO41558 & $\mathbf{Y}$ & NA \\
\hline 2463 & $1,3,4$ & CONSTANT & $2.0000000 \mathrm{E}+\infty 0$ & $2.0000000 E+\infty 0$ & $2.0000000 E+\infty 0$ & $2.0000000 \mathrm{E}+00$ & NONE & WP030540 & $\mathbf{Y}$ & NA \\
\hline 2463 & $1,3,4$ & CONSTANT & $2.0000000 E+\infty 0$ & $2,0000000 E+\infty$ & $20000000 E+\infty$ & $2.0000000 E+\infty 0$ & NONE & WP030994 & $\mathbf{Y}$ & NA \\
\hline 2463 & $1,3,4$ & CONSTANT & $20000000 \mathrm{E}+\infty 0$ & $20000000 \mathrm{E}+00$ & $2.0000000 \mathrm{E}+00$ & $20000000 E+\infty 0$ & NONE & WP035597 & $\mathbf{Y}$ & NA \\
\hline 2463 & $1,3,4$ & CONSTANT & $2,0000000 E+\infty 0$ & $20000000 E+\infty 0$ & $2.0000000 E+\infty$ & $2.0000000 E+\infty$ & NONE & WPO40486 & $\mathbf{Y}$ & NA \\
\hline 2463 & $1,3,4$ & CONSTANT & $2.0000000 E+\infty 0$ & $2.00000000+\infty 0$ & $2.0000000 E+\infty 0$ & $2.0000000 \mathrm{E}+00$ & NONE & WP040514 & $Y$ & NA \\
\hline 2464 & $1,3,4$ & CONSTANT & $1.2000000 E-09$ & $1.2000000 \mathrm{E}-09$ & $1.2000000 E-09$ & $1.2000000 E-09$ & $P z^{n-1}$ & WPO30640 & $Y$ & NA \\
\hline 2464 & $1,3,4$ & CONSTANT & $1.2000000 E-09$ & $1.2000000 E-09$ & $1.2000000 E-09$ & $1.2000000 E-09$ & $P_{e}-1$ & WP035597 & $\mathbf{Y}$ & NA \\
\hline 2484 & $1,3,4$ & CONSTANT & $1.2000000 E-09$ & $1.2000000 \mathrm{E}-09$ & $1.20000000-09$ & $1.20000000-09$ & $P x^{n-1}$ & WPO40514 & $Y$ & NA \\
\hline 2465 & 1,3 & CONSTANT & $1.0000000 E+00$ & $1.0000000 E+08$ & $1.0000000 \mathrm{E}+00$ & $1.0000000 E+08$ & Pa & WP030640 & $\mathbf{Y}$ & NA \\
\hline
\end{tabular}


CCA Parameter Listing

\begin{tabular}{|c|c|c|c|c|c|c|c|c|c|c|}
\hline 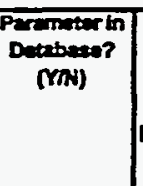 & 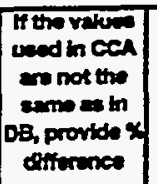 & 10 & Meteris io & Matadid Narto & $\begin{array}{c}\text { Paraneter } \\
\text { 10 }\end{array}$ & Panmeterimo & $\begin{array}{l}\text { PRPD } \\
\text { MPOA }\end{array}$ & $\begin{array}{c}\text { Deten Enty } \\
\text { Deste }\end{array}$ & 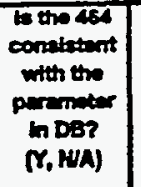 & $\begin{array}{l}\text { Paramecter } \\
\text { Cetegory } \\
\text { (tootnote 1) }\end{array}$ \\
\hline $\mathbf{Y}$ & & 2465 & CONC_TI & $\begin{array}{l}\text { Concrete cokimn:0 to } \\
400 \text { years }\end{array}$ & PC_MAX & $\begin{array}{l}\text { Maxumum allowrabue capillary } \\
\text { pressure }\end{array}$ & $32567 A$ & astiov-95 & $\mathbf{Y}$ & 48 \\
\hline $\mathbf{Y}$ & & 2465 & CONC_TI & $\begin{array}{l}\text { Concroto cokemn:D to } \\
400 \text { years }\end{array}$ & PC_maX & $\begin{array}{l}\text { Maxumum allowabto capiltary } \\
\text { pressuro }\end{array}$ & $32567 \mathrm{~A}$ & ortion-95 & $\mathbf{Y}$ & 48 \\
\hline $\mathbf{Y}$ & & 2465 & CONC_TI & $\begin{array}{l}\text { Concroto colemrio to } \\
400 \text { years }\end{array}$ & PC_max & $\begin{array}{l}\text { Maxmum sllowable capilery } \\
\text { pressure }\end{array}$ & $32567 \mathrm{~A}$ & O3+Nor-95 & $\mathbf{Y}$ & $A B$ \\
\hline $\mathbf{Y}$ & & 2465 & CONC_T1 & $\begin{array}{l}\text { Concrete column:0 to } \\
400 \text { years }\end{array}$ & PC_max & $\begin{array}{l}\text { Moxumum allowable capiltany } \\
\text { presseure }\end{array}$ & $32567 \mathrm{~A}$ & C3-Non-95 & $\mathbf{Y}$ & $4 B$ \\
\hline $\mathbf{Y}$ & varies & 2466 & CONC_T1 & $\begin{array}{l}\text { Concrete cokumn:0 to } \\
400 \text { yeers }\end{array}$ & PORE_DIS & $\begin{array}{l}\text { Brooks-Coray pore distribution } \\
\text { pareneter }\end{array}$ & 32570 & 20-Feb-86 & $\mathbf{Y}$ & 1 \\
\hline $\mathbf{Y}$ & veries & 2466 & CONC_t1 & $\begin{array}{l}\text { Concrote column:0 to } \\
400 \text { yoars }\end{array}$ & PORE_OIS & $\begin{array}{l}\text { Brooks-Corey pore distroution } \\
\text { parameter }\end{array}$ & 32570 & $20-7 c b-96$ & $\mathbf{Y}$ & 1 \\
\hline $\mathbf{Y}$ & varies & 2466 & CONC_T1 & $\begin{array}{l}\text { Concrete columnio to } \\
400 \text { yeurs }\end{array}$ & PORE_DIS & $\begin{array}{l}\text { Brooks-Corey pore distribution } \\
\text { parameter }\end{array}$ & 32570 & 20 fab-ss & $\mathbf{Y}$ & 1 \\
\hline $\mathbf{Y}$ & varies & 2466 & CONC_T1 & $\begin{array}{l}\text { Concrete column: } 0 \text { to } \\
400 \text { years }\end{array}$ & PORE_DIS & $\begin{array}{l}\text { Brooks-Corey pore distribution } \\
\text { parameter }\end{array}$ & 32570 & $20+50-96$ & $\mathbf{Y}$ & 1 \\
\hline $\mathbf{Y}$ & varies & 2466 & CONC_T1 & $\begin{array}{l}\text { Concrete colums:0 to } \\
400 \text { years }\end{array}$ & PORE_DIS & $\begin{array}{l}\text { Brooks-Corey pore distribution } \\
\text { parmeter }\end{array}$ & 32570 & $20-50 b-96$ & $\mathbf{Y}$ & 1 \\
\hline $\mathbf{Y}$ & varies & 2456 & CONC_T1 & $\begin{array}{l}\text { Concrote column:0 to } \\
400 \text { years }\end{array}$ & PORE_DIS & $\begin{array}{l}\text { Brooks-Corey pore distritution } \\
\text { paremeter }\end{array}$ & 32570 & 20fab-96 & $\mathbf{Y}$ & 1 \\
\hline $\mathbf{Y}$ & & 2467 & CONC_T1 & $\begin{array}{l}\text { Concroto columño to } \\
400 \text { years }\end{array}$ & POROSITY & Efrective porcsity & 32572 & 14Fob-96 & $\mathbf{Y}$ & 1 \\
\hline $\mathbf{Y}$ & & 2467 & CONC_TI & $\begin{array}{l}\text { Concreto columnio to } \\
400 \text { years }\end{array}$ & POROSTr & Enective porosity & 32572 & 14 Fab-96 & $\mathbf{Y}$ & 1 \\
\hline$Y$ & & 2467 & CONC_TI & $\begin{array}{l}\text { Concrote column:0 to } \\
400 \text { years }\end{array}$ & POROSTY & Efrective porosity & 32572 & $14-F a b-96$ & $\mathbf{Y}$ & 1 \\
\hline $\mathbf{Y}$ & & 2468 & CONC_T1 & $\begin{array}{l}\text { Concrete column:0 to } \\
400 \text { years }\end{array}$ & PO_MIN & $\begin{array}{l}\text { Minmum brne pressure for } \\
\text { capillary modol KPC=3 }\end{array}$ & .32576 & $21+f a b-96$ & $\mathbf{Y}$ & 48 \\
\hline $\mathbf{Y}$ & & 2468 & CONC_TI & $\begin{array}{l}\text { Concrate column:0 to } \\
400 \text { years }\end{array}$ & PO_MIN & $\begin{array}{l}\text { Mnmum bme pressere for } \\
\text { capillary model KPC }=3\end{array}$ & 32576 & 21 feb-96 & $\mathbf{Y}$ & $A B$ \\
\hline $\mathbf{Y}$ & & 2468 & CONC_T1 & $\begin{array}{l}\text { Concrate column: } 0 \text { to } \\
400 \text { yesss }\end{array}$ & PO_MIN & $\begin{array}{l}\text { Mrumum brie pressure for } \\
\text { capillery model KPC=3 }\end{array}$ & 32576 & 21facb-96 & $\mathbf{Y}$ & $4 B$ \\
\hline $\mathbf{Y}$ & & 2469 & CONC_T1 & $\begin{array}{l}\text { Conerate columnio to } \\
400 \text { years }\end{array}$ & PRESSURE & Brino far-fiold pore pressure & 32580 & 20 Feb-96 & $\mathbf{Y}$ & 1 \\
\hline$Y$ & & 2470 & CONC_T1 & $\begin{array}{l}\text { Concrote columnio to } \\
400 \text { yoars }\end{array}$ & PRMX_LOG & $\begin{array}{l}\text { Log of inturis } x \text { permesbiltty, X- } \\
\text { drrection }\end{array}$ & 32583 & 14-Fab-96 & $\mathbf{Y}$ & 1 \\
\hline $\mathbf{Y}$ & & 2470 & CONC_T1 & $\begin{array}{l}\text { Concrete column: } 0 \text { to } \\
400 \text { years }\end{array}$ & PRMX_LOG & $\begin{array}{l}\text { Log of intrnsxe permeability, } X \text { - } \\
\text { direction }\end{array}$ & 32583 & 14fab-96 & $\mathbf{Y}$ & 1 \\
\hline $\mathbf{Y}$ & & 2470 & CONC_T1 & $\begin{array}{l}\text { Concrete column: } 0 \text { to } \\
400 \text { years }\end{array}$ & PRMX_LOG & $\begin{array}{l}\text { Log of monnske permsability, } X- \\
\text { direction }\end{array}$ & 32583 & 14feb-96 & $\mathbf{Y}$ & 1 \\
\hline $\mathbf{Y}$ & & 2470 & CONC_TI & $\begin{array}{l}\text { Concrete column:0 to } \\
400 \text { years }\end{array}$ & PRMX_LOG & $\begin{array}{l}\text { Log of intonsic permeability, } X \text { - } \\
\text { direction }\end{array}$ & 32583 & $14 f \circ-96$ & $\mathbf{Y}$ & 1 \\
\hline $\mathbf{Y}$ & & 2470 & CONC_TI & $\begin{array}{l}\text { Concrete column:0 to } \\
400 \text { years }\end{array}$ & PRMX_LOG & $\begin{array}{l}\text { Log of antransx permeability, } X \text { - } \\
\text { drection }\end{array}$ & 32583 & $14-F 0 b-96$ & $\mathbf{Y}$ & 1 \\
\hline $\mathbf{Y}$ & & 2471 & CONC_T1 & $\begin{array}{l}\text { Concrete column: } 0 \text { to } \\
400 \text { years }\end{array}$ & PRMY_LOG & $\begin{array}{l}\text { Log of ntrmsic permsability, } Y \text { - } \\
\text { direction }\end{array}$ & 32585 & 14 fob-96 & $\mathbf{Y}$ & 1 \\
\hline $\mathbf{Y}$ & & 2471 & CONC_TI & $\begin{array}{l}\text { Conerete column:0 to } \\
400 \text { years }\end{array}$ & PRMY_LOG & $\begin{array}{l}\text { Log of intrinske permuabisty, Y- } \\
\text { drection }\end{array}$ & 32585 & $14 f a b-96$ & $Y$ & 1 \\
\hline $\mathbf{Y}$ & & 2471 & CONC_TI & $\begin{array}{l}\text { Concrote column: } 0 \text { to } \\
400 \text { years }\end{array}$ & PRMY_LOG & $\begin{array}{l}\text { Log of nitunske perm eability. } Y= \\
\text { direction }\end{array}$ & 32585 & $14-F-b-96$ & $--Y$ & 1 \\
\hline $\mathbf{Y}$ & & 2471 & CONC_TI & $\begin{array}{l}\text { Concrete columnio to } \\
400 \text { years }\end{array}$ & PRMY_LOG & $\begin{array}{l}\text { Log of mitrinsx permaabilty, } Y \text { - } \\
\text { direction }\end{array}$ & 32585 & 14 fob-96 & $\mathbf{Y}$ & 1 \\
\hline $\mathbf{Y}$ & & 2471 & CONC_T1 & $\begin{array}{l}\text { Concrete cokemn:0 to } \\
400 \text { years }\end{array}$ & PRMY_LOG & $\begin{array}{l}\text { Los of mimsic permeabilty, Y- } \\
\text { diroction }\end{array}$ & 32585 & 14Fob-96 & $\mathbf{Y}$ & 1 \\
\hline $\mathbf{Y}$ & & 2472 & CONC_TI & $\begin{array}{l}\text { Concrete column:0 to } \\
400 \text { years }\end{array}$ & PRMZ_LOG & $\begin{array}{l}\text { Log of intrinsx permesbulty, Z- } \\
\text { drection }\end{array}$ & 32587 & 14 Fob-96 & $\mathbf{Y}$ & 1 \\
\hline $\mathbf{Y}$ & & 2472 & CONC_T1 & $\begin{array}{l}\text { Concrule cokumn:0 to } \\
400 \text { years }\end{array}$ & PRMZ_LOG & $\begin{array}{l}\text { Log of intrnsic permesbulty. Z- } \\
\text { direction }\end{array}$ & 32587 & 14fab-96 & $\mathbf{Y}$ & 1 \\
\hline $\mathbf{Y}$ & & 2472 & CONC_TI & $\begin{array}{l}\text { Concrete column:0 to } \\
400 \text { years }\end{array}$ & PRMZ_LOG & $\begin{array}{l}\text { Log of mitrinsx permeabilty, } 2 \text { - } \\
\text { direction }\end{array}$ & 32587 & 14 feb-96 & $\mathbf{Y}$ & 1 \\
\hline $\mathbf{Y}$ & & 2472 & CONC_TI & $\begin{array}{l}\text { Concrite column:0 to } \\
400 \text { years }\end{array}$ & PRMZ_LOG & $\begin{array}{l}\text { Loog of intrinsx permesbility, Z- } \\
\text { drection }\end{array}$ & 32587 & 14Fab-96 & $\mathbf{Y}$ & 1 \\
\hline $\mathbf{Y}$ & & 2472 & CONC_TI & $\begin{array}{l}\text { Concrite colimn: } 0 \text { to } \\
400 \text { years }\end{array}$ & PRMZ_LOG & $\begin{array}{l}\text { Log of intrinsx permesbulty. Z- } \\
\text { direction }\end{array}$ & 32587 & 14 Feb-96 & $\mathbf{Y}$ & 1 \\
\hline $\mathbf{Y}$ & & 2476 & CONC_T1 & $\begin{array}{l}\text { Concrate column:0 to } \\
400 \text { years }\end{array}$ & RELP_MOD & $\begin{array}{l}\text { Model number, relative } \\
\text { permeability model }\end{array}$ & $32605 \mathrm{~A}$ & 14fob.96 & $\mathbf{Y}$ & 48 \\
\hline $\mathbf{Y}$ & & 2476 & CONC_T1 & $\begin{array}{l}\text { Concente columnio to } \\
400 \text { years }\end{array}$ & RELP_MOD & $\begin{array}{l}\text { Model number, relettre } \\
\text { permeability modal }\end{array}$ & $32605 A$ & 14-Fub-96 & $\mathbf{Y}$ & 48 \\
\hline $\mathbf{Y}$ & & 2476 & CONC_TI & $\begin{array}{l}\text { Concrete cokmnio to } \\
400 \text { years }\end{array}$ & RELP_MOD & $\begin{array}{l}\text { Model number, ralative } \\
\text { permeabilty moda! }\end{array}$ & $32605 A$ & 14febss & $\mathbf{Y}$ & $A B$ \\
\hline $\mathbf{Y}$ & & 2476 & CONC_TI & $\begin{array}{l}\text { Concrete colimn:0 to } \\
400 \text { years }\end{array}$ & RELP_MOD & $\begin{array}{l}\text { Model number, relative } \\
\text { permeability model }\end{array}$ & $32605 \mathrm{~A}$ & 14 fab-96 & $\mathbf{Y}$ & $4 B$ \\
\hline
\end{tabular}


CCA Parameter-Listing

\begin{tabular}{|c|c|c|c|c|c|c|c|c|c|c|}
\hline 10 & $\begin{array}{l}\text { Clactif- } \\
\text { cition of } \\
\text { Data } \\
\text { Catigory } \\
\text { (footnote 2) }\end{array}$ & $\begin{array}{c}\text { Datribution } \\
\text { Type }\end{array}$ & Menn & Wedien & Mintmum & Modinem & Units & Paterinco W & $\begin{array}{l}\text { Was the data } \\
\text { dombloped } \\
\text { undiren } \\
\text { non-1 } \\
\text { Progrm? }\end{array}$ & 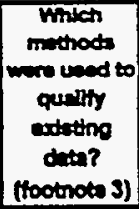 \\
\hline 2465 & 1,3 & CONSTANT & $1.0000000 E+08$ & $1.0000000 E+08$ & $1.0000000 E+08$ & $1.0000000 E+08$ & Pa & WP030994 & $\mathbf{Y}$ & NA \\
\hline 2465 & 1,3 & CONSTANT & $1.0000000 \varepsilon+08$ & $1.0000000 E+08$ & $1.0000000 E+08$ & $1.0000000 E+06$ & P* & WP035597 & $\mathbf{Y}$ & NA \\
\hline 2465 & 1,3 & CONSTANT & $1.0000000 E+08$ & $1.0000000 E+08$ & $1.0000000 E+08$ & $1.0000000 E+08$ & $\mathbf{P a}$ & WPO40486 & $\mathbf{Y}$ & NA \\
\hline 2465 & 1,3 & CONSTANT & $1.0000000 E+08$ & $1.0000000 E+08$ & $1.0000000 E+\infty 8$ & $1.0000000 E+08$ & $\mathrm{~Pa}$ & WPOL0514 & $\mathbf{Y}$ & NA \\
\hline 2466 & 3,4 & CUMULATINE & $25200000 E+\infty 0$ & 9.4000000E-01 & $1.1000000 E-01$ & $8.10000000+\infty 0$ & NONE & WP030640 & $\mathbf{Y}$ & NA \\
\hline 2466 & 3,4 & CUMULATIVE & $2.5200000 E+\infty$ & $9.4000000 E-01$ & $1.1000000 E-01$ & $8.1000000 E+\infty 0$ & NONE & WP030994; & $\mathbf{Y}$ & NAA \\
\hline 2466 & 3,4 & CUMULATIVE & $2.5200000 E+00$ & $9.4000000 E-01$ & $1.1000000 E-01$ & $8.1000000 E+\infty$ & NONE & WP032287 & $\mathbf{Y}$ & NA \\
\hline 2466 & 3,4 & CUMULATIVE & $2.5200000 E+00$ & $9.4000000 E-01$ & $1.1000000 E-01$ & $8.1000000 E+\infty$ & NONE & WP035268 & $\mathbf{Y}$ & NA \\
\hline 2466 & 3,4 & CUMULATIVE & $2.5200000 E+00$ & $9.4000000 \mathrm{E}-01$ & $1.1000000 E-01$ & $8.1000000 E+\infty 0$ & NONE & WP035597' & $\mathbf{Y}$ & N/A \\
\hline 2466 & 3,4 & CUMULATTVE & $2.5200000 E+00$ & $9.4000000 E-01$ & 1.1000000E-01 & $8.1000000 E+\infty 0$ & NONE & WPO41558. & $\mathbf{Y}$ & NA \\
\hline 2457 & 1,3 & CONSTANT & $5.0000000 E-02$ & $5.0000000 E-02$ & $5.0000000 E-02$ & $5.0000000 E-02$ & $m^{\wedge} 3 m^{\wedge} 3$ & WP030640 & Y\&N & 1 \\
\hline 2467 & 1,3 & CONSTANT & $5.0000000 E-02$ & $5.0000000 E-02$ & $5.0000000 \mathrm{E}-02$ & $5.0000000 E-02$ & $m^{\wedge} 3 m^{n} 3$ & WP030994 & Y $8 N$ & 1 \\
\hline 2457 & 1,3 & CONSTANT & $5.0000000 \mathrm{E}-02$ & $5.0000000 \mathrm{E}-02$ & $5.0000000 E-02$ & $5.0000000 E-02$ & $m^{\wedge} 3 m^{\wedge} 3$ & WP035597 & YaN & 1 \\
\hline 2468 & 3,5 & CONSTANT & $1.0132500 E+0.5$ & $1.0132500 E+05$ & $1.0132500 E+05$ & $1.0132500 E+05$ & $\mathbf{P a}$ & WP035597 & $\mathbf{Y}$ & NA \\
\hline 2468 & 3,5 & CONSTANI & $1.0132500 E+05$ & $1.0132500 E+05$ & $1.0132500 E+05$ & $1.0132500 E+05$ & $\mathbf{P a}$ & WP036051 & $\mathbf{Y}$ & NAA \\
\hline 2468 & 3,5 & CONSTANT & $1.0132500 E+05$ & $1.0132500 E+05$ & $1.0132500 E+05$ & $10132500 E+05$ & $\mathbf{P a}$ & WP040514 & $\mathbf{Y}$ & NA \\
\hline 2469 & 3,5 & CONSTANT & $1.0132500 E+05$ & $1.0132500 E+05$ & $1.0132500 E+05$ & $10132500 E+05$ & $\mathbf{P a}$ & WP030994 & $\mathbf{Y}$ & NA \\
\hline 2470 & 1,3 & TRIANGULAR & $-1.8816000 E+01$ & $-1.8749600 E+01$ & $-2.0699000 E+01$ & $-17000000 \mathrm{E}+01$ & $\cos \left(m^{\wedge} 2\right)$ & WPO30540 & Y\&N & 1 \\
\hline 2470 & 1,3 & TRIANGULAR & $-1.8816000 E+01$ & $-1.8749600 E+01$ & $-20699000 E-01$ & $-17000000 E+01$ & $\log \left(m^{\wedge} 2\right)$ & WP030994 & Y\&N & 1 \\
\hline 2470 & 1,3 & TRIANGULAR & $-1.8816000 E+01$ & $-1,8749600 E+01$ & $-20699000 E+01$ & $-17000000 E+01$ & $\log \left(m^{\wedge} 2\right)$ & WP035268 & YAN & 1 \\
\hline 2470 & 1,3 & TRIANGULAR & $-1.8816000 E+01$ & $-1,8749600 E+01$ & $-20699000 E+01$ & $-17000000 E+01$ & $\log \left(m^{\wedge} 2\right)$ & WP035597 & Y\&N & 1 \\
\hline 2470 & 1,3 & TRIANGULAR & $-1.8816000 \mathrm{E}+01$ & $-1.8749600 E+01$ & $.20690000 \times-01$ & $-17000000 E-0$, & $\log \left(m^{\wedge} 2\right)$ & WPO41558 & Y\&N & 1 \\
\hline 2471 & 1,3 & TRLANGULAR & $-1.8816000 E+01$ & $-1.8749600 E+01$ & $-20099000 \times 0.01$ & $-17000000 E \bullet 01$ & $\log \left(m^{\wedge} 2\right)$ & WP030540 & Y $8 N$ & 1 \\
\hline 2471 & 1,3 & TRIANGULAR & $-1.8816000 E+01$ & $-18749600 E+01$ & $-20695000<+01$ & 1 T000000E $\bullet 01$ & $\log \left(m^{\wedge} 2\right)$ & WP030994 & YaN & 1 \\
\hline 2471 & 1,3 & TRIANGULAR & $-1.8816000 E+01$ & $-18749600 E+01$ & $-20 \cos 000 \times-0.1$ & $.17000000<-01$ & $\log \left(m^{\wedge} 2\right)$ & WP035268 & YaN & 1 \\
\hline 2471 & 1,3 & TRLANGULAR & $-1.8816000 E+01$ & $-18749500 E-01$ & $-20000000 \times-01$ & -1 T0000000E -01 & $\log \left(m^{\wedge} 2\right)$ & WP035597 & ran & 1 \\
\hline 2471 & 1,3 & TRLANGULAR & $-1.8816000 E+01$ & $-18749600 E+01$ & $-20000000 \times-01$ & $17000000 \times+01$ & $\log (m \wedge 2)$ & WPO41558 & YaN & 1 \\
\hline 2472 & 1,3 & TRIANGULAR & $-1.8816000 E+01$ & $-18749600 E+01$ & $.20000000<-01$ & . 1 ro000000 -01 & $\log \left(m^{\wedge} 2\right)$ & WP030840 & Y\&N & 1 \\
\hline 2472 & 1,3 & TRLANGULAR & $-1.8816000 E+01$ & $-1.8749800 E<01$ & $-20 \cos 000 \times-01$ & $-17000000 E+01$ & $\log \left(m^{\wedge} 2\right)$ & WP030994 & Y\&N & 1 \\
\hline 2472 & 1,3 & TRIANGULAR & $-1.8816000 E+01$ & $-18749600 E+01$ & $-20 \cos c 00<-01$ & $.17000000 \times 01$ & $\log \left(m^{\wedge} 2\right)$ & WPO35268 & Y8N & 1 \\
\hline 2472 & 1,3 & TRIANGULAR & -1.8816000 E +01 & $-18749600 E+01$ & $-20 \operatorname{sen} 000 \times-01$ & $.170000006 \times 01$ & $\log \left(m^{\wedge} 2\right)$ & WP035597 & Y\&N & 1 \\
\hline 2472 & 1,3 & TRLANGULAR & $-1.8816000 E+01$ & $-18749600 E+01$ & $-2000000<-01$ & -1 70000000 +01 & $\log \left(m^{\wedge} 2\right)$ & WPOA1558 & Y8N & 1 \\
\hline 2476 & $1,3,4$ & CONSTANT & $4.0000000 E+\infty 0$ & $40000000 E \cdot 00$ & $4000000<=-\infty$ & $4000000 \times+\infty$ & NONE & WPO30640 & $\mathbf{Y}$ & NA \\
\hline 2476 & $1,3,4$ & CONSTANT & $4.0000000 E+\infty 0$ & $10000000 E+\infty$ & $4 \infty 00000 \times-\infty$ & $4000000 \times+\infty$ & NONE & WP030994 & $\mathbf{Y}$ & $\mathbf{N A}$ \\
\hline 2476 & $1,3,4$ & CONSTANT & $1.00000000+\infty 0$ & $10000000 \varepsilon+\infty$ & $400000000=00$ & $40000000 E+\infty$ & NONE & WP035597 & $\mathbf{Y}$ & NA \\
\hline 2476 & $1,3,4$ & CONSTANT & $4.0000000 E+\infty$ & $40000000-\infty$ & $4000000<+\infty$ & $40000000 E+\infty$ & NONE & WPO40486 & $\mathbf{Y}$ & $\mathbf{N A}$ \\
\hline
\end{tabular}


CCA Parameter Listing

\begin{tabular}{|c|c|c|c|c|c|c|c|c|c|c|}
\hline $\begin{array}{l}\text { Paranter th } \\
\text { Databean? } \\
\text { (YN) }\end{array}$ & 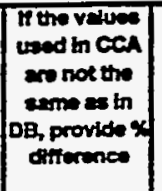 & ID & Intertad to & Matudereme & Parmation & Parmitar Nam & $\begin{array}{l}\text { PRPD } \\
\text { MPOA }\end{array}$ & Dententry & 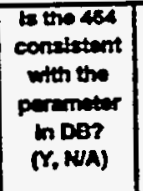 & $\begin{array}{l}\text { Parminar } \\
\text { Cationy } \\
\text { (footnote 1) }\end{array}$ \\
\hline $\mathbf{Y}$ & & 2476 & CONC_TI & $\begin{array}{l}\text { Concrete coliminio to } \\
400 \text { years }\end{array}$ & RELP_MOD & $\begin{array}{l}\text { Model number, relutive } \\
\text { permeabilty model }\end{array}$ & $32505 A$ & 14 fab-96 & $\mathbf{Y}$ & $4 B$ \\
\hline $\mathbf{Y}$ & & 2477 & CONC_TI & $\begin{array}{l}\text { Concrele columin o to } \\
400 \text { years }\end{array}$ & SAT_IBRN & Initial Brine Saturation & $32021 A$ & $28+a b-96$ & $\mathbf{Y}$ & 1 \\
\hline $\mathbf{Y}$ & & 247 & CONC_T4 & $\begin{array}{l}\text { Concrele columinio to } \\
400 \text { yours }\end{array}$ & SAT_IBRN & Initial Brin Seturation & $32521 A$ & 28-Feb-96 & $\mathbf{Y}$ & 1 \\
\hline $\mathbf{Y}$ & & 2477 & CONC_TI & $\begin{array}{l}\text { Concrite columio to } \\
400 \text { yours }\end{array}$ & SAT_IBRN & Iritial Brine Seturation & $32621 \mathrm{~A}$ & $28-F a b-96$ & $\mathbf{Y}$ & 1 \\
\hline $\mathbf{Y}$ & 100 & 2478 & CONC_T1 & $\begin{array}{l}\text { Concrete columi: } 0 \text { to } \\
400 \text { years }\end{array}$ & SAT_RBRN & Residual Brine Seturation & 32623 & $1450 b-66$ & $\mathbf{Y}$ & 1 \\
\hline $\mathbf{Y}$ & 100 & 2478 & CONC_T1 & $\begin{array}{l}\text { Concrete column:0 to } \\
400 \text { years }\end{array}$ & SAT_RBRN & Residual Brine Szturation & 32623 & 14Fob-96 & $\mathbf{Y}$ & 1 \\
\hline $\mathbf{Y}$ & 100 & 2478 & CONC_TI & $\begin{array}{l}\text { Concrote columnio to } \\
400 \text { years }\end{array}$ & SAT_RERN & Residual Brina Saturation & 32623 & $14 F=b-96$ & $\mathbf{Y}$ & 1 \\
\hline $\mathbf{Y}$ & 100 & 2478 & CONC_T1 & $\begin{array}{l}\text { Concreto cokennio to } \\
400 \text { years }\end{array}$ & SAT_RBRN & Residual Brinu Samration & 32623 & $14 F 6 b-96$ & $\mathbf{Y}$ & 1 \\
\hline $\mathbf{Y}$ & 100 & 2478 & CONC_TI & $\begin{array}{l}\text { Concrete column:0 to } \\
400 \text { years }\end{array}$ & SAT_RBRN & Resictual Brino Seturation & 32623 & $14-F a b-96$ & $\mathbf{Y}$ & 1 \\
\hline $\mathbf{Y}$ & 100 & 2478 & CONC_TI & $\begin{array}{l}\text { Concrete columrio to } \\
400 \text { years }\end{array}$ & SAT_RBRN & Recicusal Erine Seturation & 32023 & 14 Fob-96 & $\mathbf{Y}$ & 1 \\
\hline $\mathbf{Y}$ & 100 & 2479 & CONC_Tt & $\begin{array}{l}\text { Concrete columin: } 0 \text { to } \\
400 \text { years }\end{array}$ & SAT_RGAS & Residual Ges Seturation & 32625 & $14 f=b-96$ & $\boldsymbol{\gamma}$ & 1 \\
\hline $\mathbf{Y}$ & 100 & 2479 & CONC_TI & $\begin{array}{l}\text { Concrete column:0 to } \\
400 \text { years }\end{array}$ & SAT_RGAS & Residual Gas Suturation & 32625 & $14-F b-96$ & $\mathbf{Y}$ & 1 \\
\hline$Y$ & 100 & 2479 & CONC_TI & $\begin{array}{l}\text { Concrete colimn:0 to } \\
400 \text { yoars }\end{array}$ & SAT_RGAS & Residual Ges Seturation & 32625 & $14 F 0 b-96$ & $\mathbf{Y}$ & 1 \\
\hline $\mathbf{Y}$ & 100 & 2479 & CONC_T1 & $\begin{array}{l}\text { Concrote column:0 to } \\
400 \text { yoars }\end{array}$ & SAT_RGAS & Residual Ges Seturation & 32625 & $14 F \cdot b-96$ & $Y$ & 1 \\
\hline $\mathbf{Y}$ & 100 & 2479 & CONC_T4 & $\begin{array}{l}\text { Concrate colkmnio to } \\
400 \text { years }\end{array}$ & SAT_RGAS & Residuat Ges Selaration' . & 32625 & 14Fob-96 & $\mathbf{Y}$ & 1 \\
\hline$Y$ & 50 & 2480 & CONC_T2 & $\begin{array}{l}\text { Concrete column:400 } \\
\text { to } 10 \mathrm{~K} \text { years }\end{array}$ & CAP_MOD & $\begin{array}{l}\text { Model number, cepillery pressure } \\
\text { model }\end{array}$ & $32631 A$ & $14+50 b-96$ & $\mathbf{Y}$ & $4 B$ \\
\hline$Y$ & 50 & 2480 & CONC_T2 & $\begin{array}{l}\text { Concrete column:400 } \\
\text { to } 10 K \text { yesrs }\end{array}$ & CAP_MOD & $\begin{array}{l}\text { Model number, capillary pressure } \\
\text { model }\end{array}$ & $32631 A$ & $14 F$ ob-96 & $\mathbf{Y}$ & $4 B$ \\
\hline $\mathbf{Y}$ & 50 & 2480 & CONC_T2 & $\begin{array}{l}\text { Concreto column: } 400 \\
\text { to toK years }\end{array}$ & CAP_MOD & $\begin{array}{l}\text { Model number, cepillery pressure } \\
\text { model }\end{array}$ & $32631 A$ & $14 F=b-96$ & $\mathbf{Y}$ & AB \\
\hline $\mathbf{Y}$ & 50 & 2480 & CONC_T2 & $\begin{array}{l}\text { Concrete colimn:400 } \\
\text { to 10K years }\end{array}$ & CAP_MOD & $\begin{array}{l}\text { Model inumber, copillary prossure } \\
\text { model }\end{array}$ & $32631 A$ & 14-Fab-96 & $\mathbf{Y}$ & 48 \\
\hline $\mathbf{Y}$ & 50 & 2480 & CONC_T2 & $\begin{array}{l}\text { Concrete column:400 } \\
\text { to } 10 \mathrm{~K} \text { years }\end{array}$ & CAP_MOD & $\begin{array}{l}\text { Model number, cepillary pressure } \\
\text { model }\end{array}$ & $32631 A$ & 14 Feb-96 & $\mathbf{Y}$ & 48 \\
\hline$Y$ & & 2481 & CONC_T2 & $\begin{array}{l}\text { Concrete column:400 } \\
\text { to } 10 \text { K years }\end{array}$ & COMP_RCK & Buk Compresstbilty & 32638 & $02+19 y-96$ & $\mathbf{Y}$ & $4 B$ \\
\hline $\mathbf{Y}$ & & 2481 & CONC_T2 & $\begin{array}{l}\text { Concrete column: } 400 \\
\text { to } 10 \text { K yours }\end{array}$ & COMP_RCK & Bulk Compressibitity & 32638 & $02+$ any-96 & $" Y$ & $4 B$ \\
\hline $\mathbf{Y}$ & & 2481 & CONC_T2 & $\begin{array}{l}\text { Concrets column-400 } \\
\text { to } 10 \mathrm{~K} \text { years }\end{array}$ & COMP_RCK & Bulk Compressibdtify & 32638 & $02+118 y-96$ & $\mathbf{Y}$ & $4 B$ \\
\hline $\mathbf{Y}$ & & 2482 & CONC_T2 & $\begin{array}{l}\text { Concrite colkmn- } 400 \\
\text { to 10K years }\end{array}$ & PC_max & 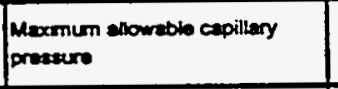 & $32657 \mathrm{~A}$ & 14Fab-96 & $Y$ & $4 B$ \\
\hline $\mathbf{Y}$ & & 2482 & CONC_T2 & $\begin{array}{l}\text { Concrete column:400 } \\
\text { to } 10 \mathrm{~K} \text { years }\end{array}$ & PC_MAX & $\begin{array}{l}\text { Maxinum atlown ble caillery } \\
\text { preasure }\end{array}$ & $32657 A$ & 14 feb-96 & $\mathbf{Y}$ & $4 B$ \\
\hline $\mathbf{Y}$ & & 2482 & CONC_T2 & $\begin{array}{l}\text { Concrete column:400 } \\
\text { to } 10 \mathrm{~K} \text { yesrs }\end{array}$ & PC_MAX & $\begin{array}{l}\text { Maxmum anowable capillery } \\
\text { preserre }\end{array}$ & $32657 \mathrm{~A}$ & 14 Feb-96 & $\mathbf{Y}$ & 48 \\
\hline $\mathbf{Y}$ & & 2482 & CONC_T2 & $\begin{array}{l}\text { Concrete column } 400 \\
\text { to } 10 \mathrm{~K} \text { years }\end{array}$ & PC_MAX & $\begin{array}{l}\text { Maxamim alloweble capiliary } \\
\text { pressure }\end{array}$ & $32657 A$ & 14Feb-96 & $\mathbf{Y}$ & $4 B$ \\
\hline $\mathbf{Y}$ & & 2482 & CONC_T2 & $\begin{array}{l}\text { Concrete column:400 } \\
\text { to } 10 \mathrm{~K} \text { years }\end{array}$ & PC_MAX & $\begin{array}{l}\text { Moximum allowble capilley } \\
\text { preseure }\end{array}$ & $32657 A$ & 14Feb-96 & $\mathbf{Y}$ & 48 \\
\hline $\mathbf{Y}$ & varies & 2483 & CONC_T2 & $\begin{array}{l}\text { Concrute columi:400 } \\
\text { to } 10 \mathrm{~K} \text { years }\end{array}$ & PORE_DIS & $\begin{array}{l}\text { Brooks-Corey pore dietribution } \\
\text { perameter }\end{array}$ & 32658 & $20-f=b-96$ & $\mathbf{Y}$ & 1 \\
\hline
\end{tabular}


CCA Parameter-Listing

\begin{tabular}{|c|c|c|c|c|c|c|c|c|c|c|}
\hline 10 & 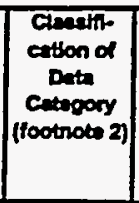 & $\begin{array}{l}\text { Deforibution } \\
\text { Type }\end{array}$ & Mem & Medien & Mlnirrem & Medimum & Whit & Retermea ID & $\begin{array}{l}\text { Wea the dete } \\
\text { dombloped } \\
\text { underen } \\
\text { Mon-1 } \\
\text { Progren? }\end{array}$ & 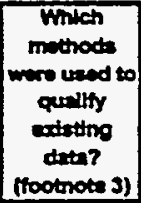 \\
\hline 2476 & $1,3,4$ & CONSTANT & $4.0000000 E+\infty 0$ & $4.00000000+\infty$ & $4.0000000 \mathrm{E}+\infty$ & $4.00000000=+\infty$ & NONE & WPO40514 & $\mathbf{Y}$ & NA \\
\hline 2477 & 1,3 & CONSTANT & 9.9999990E-01 & 9.9999990E-01 & 9.9999990E-01 & 9.9999990E-01 & NONE & WPO30610 & $\mathbf{Y}$ & NA \\
\hline 2477 & 1,3 & CONSTANT & 9.9999990E-01 & 9.9999990E-01 & 9.99PS9SOE-01| & 9.999999OE-01| & NONE & WP035597 & $Y$ & NA \\
\hline 2477 & 1,3 & CONSTANT & 9.9999990E-01 & 9.9999990E-01 & $9.9999990 E-01$ & 9.9999990E-01 & NONE & WP039169 & $\mathbf{Y}$ & NA \\
\hline 2478 & 3,4 & CUMULATINE & $2.5000000 E-01$ & $2.0000000 E-01$ & $0.0000000 E+00$ & $6.0000000 E-01$ & NONE & WP030540 & $\mathbf{Y}$ & NA \\
\hline 2478 & 3,4 & CUMULATIVE & $2.5000000 E-01$ & $20000000 E-01$ & $0.0000000 E+\infty$ & $6.0000000 E-01$ & NONE & WP030s9t & $\mathbf{Y}$ & NA \\
\hline 2478 & 3,4 & CUMULATIVE & $2.5000000 E-01$ & $-2,0000000 E-01$ & $0.0000000 E+\infty$ & $6.0000000=-01$ & NONE & WP035268 & $Y$ & NA \\
\hline 2478 & 3.4 & CUMULATIVE & $25000000 E-01$ & $2.0000000 E-01$ & $0.0000000 E+\infty$ & $6.0000000=-01$ & NONE - & WP035597 & $\mathbf{Y}$ & NA \\
\hline 2478 & 3,4 & CUMULATINE & 25000000E-01 & $2.0000000 E-01$ & $0.0000000 E+\infty 0$ & $6.0000000 E-01$ & NONE & WPO41558 & $\mathbf{Y}$ & NA \\
\hline 2478 & 3,4 & CUMULATIVE & $2.5000000 E-01$ & $2.0000000 E-01$ & $0.00000000 E+00$ & $6.0000000 E-01$ & NONE & WPO42751 & $\mathbf{Y}$ & NA \\
\hline 2479 & 3,4 & UNIFORM & $2.0000000 E-01$ & $20000000 E-01$ & $0.0000000 E+00$ & 4.0000000E-01 & NONE & WP030640 & $\mathbf{Y}$ & NA \\
\hline 2479 & 3,4 & UNIFORM & $2.0000000 E-01$ & $2.0000000 E-01$ & $0.0000000 \mathrm{E}+\infty 0$ & $4.0000000 E-01$ & NONE & WP030994 & $\mathbf{Y}$ & NA \\
\hline 2479 & 3,4 & UNIFORM & $2.0000000 E-01$ & $20000000 E-01$ & $0.00000000 \mathrm{E}+\infty 0$ & 4.0000000E-01 & NONE & WP035268 & $Y$ & NA \\
\hline 2479 & 3,4 & UNIFORM & $2.0000000 E-01$ & $20000000 E-01$ & $0.0000000 \mathrm{E}+00$ & 4.0000000E-01 & NONE & WP035597 & $\mathbf{Y}$ & NA \\
\hline 2479 & 3,4 & UNIFORM. & $2.0000000 E-01$ & $2.0000000 E-01$ & $0.0000000 E+\infty 0$ & $1.0000000 E-01$ & NONE & - WPO41558 & $\mathbf{Y}$. & NA \\
\hline 2480 & $1,3,4$ & CONSTANT & $2,0000000 E+\infty 0$ & $2.00000000+\infty 0$ & $2.0000000 E+\infty 0$ & $2.0000000 E+\infty 0$ & NONE & WP030640 & $\mathbf{Y}$ & NA \\
\hline 2480 & $1,3,4$ & CONSTANT & $20000000 E+\infty$ & $2.0000000 E+\infty$ & $20000000 E+\infty$ & $20000000 E+\infty$ & NONE & WP030994 & $Y$ & NA \\
\hline 2480 & $1,3,4$ & CONSTANT & $2,00000000+\infty$ & $2.0000000 \mathrm{E}+00$ & $2.0000000 \mathrm{E}+\infty$ & $20000000 E+\infty$ & NONE & WP035597 & $\mathbf{Y}$ & NA \\
\hline 2480 & $1,3,4$ & CONSTANT & $2.0000000 E+\infty$ & $2.0000000 E+\infty$ & $2.0000000 E+\infty 0$ & $2.0000000 E+\infty$ & NONE & WPO40486 & $\mathbf{Y}$ & NA \\
\hline 2480 & $1,3,4$ & CONSTANT & $2.0000000 E+\infty$ & $20000000 \mathrm{E}+\infty$ & $20000000 \mathrm{E}+\infty$ & $2.0000000 E+\infty$ & NONE & WPO40514 & $\mathbf{Y}$ & NA \\
\hline 2481 & $1,3,4$ & CONSTANT & $1.2000000 E-09$ & $1.2000000 E-09$ & $1.2000000 E-09$ & $1.2000000 E-09$ & $P z^{n}-1$ & WP030640 & $\mathbf{Y}$ & NA \\
\hline 2481 & $1,3,4$ & CONSTANT & $1.2000000 E-09$ & $1.2000000 E-09$ & $1.2000000 E-09$ & $1.2000000 E-09$ & Pan-1 & WP030994 & $\overline{\mathbf{Y}}$ & NA \\
\hline 2481 & $1,3,4$ & CONSTANT & $1.2000000 \mathrm{E}-09$ & $1.2000000 E-09$ & $1.2000000 E-09$ & $1.2000000 \mathrm{E}-09$ & $P a^{n}-1$ & WP040514 & $\mathbf{Y}$ & N/A \\
\hline 2482 & 1.3 & CONSTANT & $1.0000000 E+08$ & $1.00000000+08$ & $1.0000000 E+08$ & $1.0000000 E+08$ & $\mathrm{~Pa}$ & WP030640 & $\mathbf{Y}$ & NA \\
\hline 2482 & 1,3 & CONSTANT & $1.0000000 E+00$ & $1.00000000+08$ & $1.0000000 E+08$ & $1.0000000 E+08$ & Pa & WP030994 & $Y$ & NA \\
\hline 2482 & 1,3 & CONSTANT & $1.00000000 E+00$ & $1.00000000 E+08$ & $1.0000000 E+08$ & $1.0000000 \mathrm{E}+08$ & Pa & WP035597 & $Y$ & N/A \\
\hline 2482 & 1,3 & CONSTANT & $1.0000000 E+08$ & $1.0000000=+08$ & $1.0000000 E+08$ & $1.0000000 E+08$ & Pa & WPO40485 & $\mathbf{Y}$ & N/A \\
\hline 2182 & 1,3 & CONSTANT & $1.0000000 E+08$ & $1.0000000 \mathrm{E}+08$ & $1.0000000 E+08$ & $1.0000000 E+08$ & $\mathbf{P a}$ & WP040514 & $\mathbf{Y}$ & $N / A$ \\
\hline 2483 & 3,4 & CUMULATINE & $25200000 E+\infty$ & 9.4000000E-01 & $1.1000000 E-01$ & B.1000000E +00 & NONE & WP030640 & $\cdot \mathbf{Y}$ & NA \\
\hline
\end{tabular}


CCA Parameter Listing

\begin{tabular}{|c|c|c|c|c|c|c|c|c|c|c|}
\hline $\begin{array}{c}\text { Peremetion th } \\
\text { Detemese? } \\
\text { (YNN) }\end{array}$ & 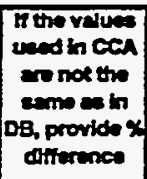 & 10 & Mrtartio & Metorla Nems & $\begin{array}{l}\text { Parmineter } \\
\text { to }\end{array}$ & Parmentar Hars & $\begin{array}{l}\text { PRPD DO } \\
\text { MPO }\end{array}$ & Denenty & 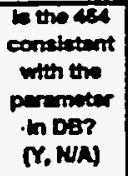 & 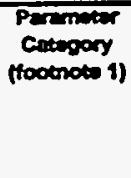 \\
\hline $\mathbf{Y}$ & verios & 2483 & CONC_T2 & $\begin{array}{l}\text { Concrote cokmnn:400 } \\
\text { to } 10 \mathrm{~K} \text { years }\end{array}$ & PORE_DIS & $\begin{array}{l}\text { Brooks-Coroy pore distrioution } \\
\text { parmeter }\end{array}$ & 32658 & $20-5 a b-96$ & $\mathbf{Y}$ & 1 \\
\hline $\mathbf{Y}$ & varies & 2483 & CONC_T2 & $\begin{array}{l}\text { Concrete column: } 400 \\
\text { to } 10 \text { K years }\end{array}$ & PORE_DIS & $\begin{array}{l}\text { Brooks-Coroy pore drstribution } \\
\text { permeter }\end{array}$ & 32658 & 20Fab-96 & $\mathbf{Y}$ & 1 \\
\hline$Y$ & varies & 2483 & CONC_T2 & $\begin{array}{l}\text { Concrete column:400 } \\
\text { to } 10 \mathrm{~K} \text { years }\end{array}$ & PORE_DIS & $\begin{array}{l}\text { Brocke-Coroy pore distribution } \\
\text { paraneter }\end{array}$ & 32658 & $20-F a b-96$ & $\mathbf{Y}$ & 1 \\
\hline $\mathbf{Y}$ & varies & 2483 & CONC_T2 & $\begin{array}{l}\text { Concrote column: } 400 \\
\text { to } 10 \mathrm{~K} \text { yours }\end{array}$ & PORE_DIS & $\begin{array}{l}\text { Brooks-Corey pore distribution } \\
\text { perameter }\end{array}$ & 32658 & $20-F a b-96$ & $\mathbf{Y}$ & 1 \\
\hline $\mathbf{Y}$ & varies & 2483 & CONC_T2 & $\begin{array}{l}\text { Concrote column: } 400 \\
\text { to } 10 K \text { years }\end{array}$ & PORE_DIS & $\begin{array}{l}\text { Brooks-Corey pore distribution } \\
\text { permeter }\end{array}$ & 32658 & $20-F 00-96$ & $\mathbf{Y}$ & 1 \\
\hline $\mathbf{Y}$ & & 2484 & CONC_T2 & $\begin{array}{l}\text { Concrote column: } 400 \\
\text { to } 10 \mathrm{~K} \text { years }\end{array}$ & POROSTYY & Efrective porosity & 32659 & $14 f a b-96$ & $\mathbf{Y}$ & 1 \\
\hline $\mathbf{Y}$ & & 2484 & CONC_T2 & $\begin{array}{l}\text { Concrete column:400 } \\
\text { to } 10 \% \text { yoars }\end{array}$ & POROSTY & Effective porosity & 32659 & $14+0 b-96$ & $\mathbf{Y}$ & 1 \\
\hline $\mathbf{Y}$ & & 2484 & CONC_T2 & $\begin{array}{l}\text { Concreto cokumn:400 } \\
\text { to } 10 \mathrm{~K} \text { yoars }\end{array}$ & POROSTY & Efrective porosity & 32659 & $14+50 b-96$ & $\mathbf{Y}$ & 1 \\
\hline $\mathbf{Y}$ & & 2485 & CONC_T2 & $\begin{array}{l}\text { Concrete column:400 } \\
\text { to } 10 \mathrm{~K} \text { years }\end{array}$ & PRESSURE & Brine far-fiold pore presure & 32661 & $20-5 e b-96$ & $\mathbf{Y}$ & $4 A$ \\
\hline$Y$ & & 2485 & CONC_T2 & $\begin{array}{l}\text { Concrete column:400 } \\
\text { to 10K years }\end{array}$ & PRESSURE & Brine far-fiald pore pressure & 32661 & $20-50 b-96$ & $\mathbf{Y}$ & $\mathbf{A}$ \\
\hline $\mathbf{Y}$ & & 2486 & CONC_T2 & $\begin{array}{l}\text { Concrote coltumnit } 400 \\
\text { to } 10 \mathrm{~K} \text { yoses }\end{array}$ & PRMX_LOG & $\begin{array}{l}\log \text { of intrinsic permesbility, } X \text { - } \\
\text { direction }\end{array}$ & 32662 & or-Nov-95 & $\mathbf{Y}$ & 1 \\
\hline $\mathbf{Y}$ & & 2486 & CONC_T2 & $\begin{array}{l}\text { Concrote cokmn:400 } \\
\text { to } 10 \text { K years }\end{array}$ & PRMX_LOG & $\begin{array}{l}\text { Log of intrinsic permesabilty, } x \text {. } \\
\text { direction }\end{array}$ & 32052 & O3-Atov-95 & $\mathbf{Y}$ & 1 \\
\hline $\mathbf{Y}$ & & 2486 & CONC_T2 & $\begin{array}{l}\text { Concrote column:400 } \\
\text { to } 10 \mathrm{~K} \text { years }\end{array}$ & PRMX_LOG & $\begin{array}{l}\text { Log of intrinsic permeabitity, } x \text {. } \\
\text { direction }\end{array}$ & 32662 & 03-Nov-95 & $\mathbf{Y}$ & 1 \\
\hline $\mathbf{Y}$ & & 2487 & CONC_T2 & $\begin{array}{l}\text { Concrete column:400 } \\
\text { to } 10 \mathrm{~K} \text { years }\end{array}$ & PRMY_LOG & $\begin{array}{l}\text { Los of intrinsic permesbility. } Y \text { - } \\
\text { direction }\end{array}$ & 32653 & C3-Nov-95 & $\mathbf{Y}$ & 1 \\
\hline $\mathbf{Y}$ & & 2487 & CONC_T2 & $\begin{array}{l}\text { Concrete column:400 } \\
\text { to } 10 \mathrm{~K} \text { years }\end{array}$ & PRMY_LOG & $\begin{array}{l}\text { Log of intrnsic permeability, Y- } \\
\text { direction }\end{array}$ & 32663 & O3-Nov-95 & $\mathbf{Y}$ & 1 \\
\hline $\mathbf{Y}$ & & 2487 & CONC_T2 & $\begin{array}{l}\text { Concrote column:400 } \\
\text { to } 10 \mathrm{~K} \text { yess }\end{array}$ & PRMY_LOG & $\begin{array}{l}\text { Log of intrnsic permeability. Y- } \\
\text { drection }\end{array}$ & 32653 & O3-Nov-95 & $\mathbf{Y}$ & 1 \\
\hline $\mathbf{Y}$ & & 2488 & CONC_T2 & $\begin{array}{l}\text { Concrele column: } 400 \\
\text { to } 10 \mathrm{~K} \text { years }\end{array}$ & PRMZ_LOG & $\begin{array}{l}\text { Log of ntrmsic permesbilty. Z- } \\
\text { drection }\end{array}$ & 32664 & 03-Nov-95 & $" Y$ & 1 \\
\hline $\mathbf{Y}$ & & 2488 & CONC_T2 & $\begin{array}{l}\text { Concrete column: } 400 \\
\text { to } 10 K \text { years }\end{array}$ & PRMZ_LOG & $\begin{array}{l}\text { Log of intronsx permeabilty, Z- } \\
\text { druction }\end{array}$ & 32664 & O3-Nov-95 & $\mathbf{Y}$ & 1 \\
\hline $\mathbf{Y}$ & & 2488 & CONC_T2 & $\begin{array}{l}\text { Concrete column:400 } \\
\text { to } 10 \mathrm{~K} \text { years }\end{array}$ & PRMZ_LOG & $\begin{array}{l}\text { Loo of intrnsic permesbility, Z- } \\
\text { drection }\end{array}$ & 32664 & 03-Nov-95 & $\mathbf{Y}$ & 1 \\
\hline $\mathbf{Y}$ & & 2492 & CONC_T2 & $\begin{array}{l}\text { Concrite column: } 400 \\
\text { to toK yaers }\end{array}$ & RELP_MOD & $\begin{array}{l}\text { Model number, relativo } \\
\text { permesbilty model }\end{array}$ & $32669 A$ & $14 f c b-86$ & $\mathbf{Y}$ & 48 \\
\hline $\mathbf{Y}$ & & 2492 & CONC_T2 & $\begin{array}{l}\text { Concrote column: } 100 \\
\text { to } 10 \mathrm{~K} \text { yerrs }\end{array}$ & RELP_MOD & $\begin{array}{l}\text { Model number, relative } \\
\text { permeability model }\end{array}$ & 326694 & 14Fob-96 & $\mathbf{Y}$ & $4 B$ \\
\hline $\mathbf{Y}$ & & 2492 & CONC_T2 & $\begin{array}{l}\text { Concerta column: } 400 \\
\text { to } 10 \mathrm{~K} \text { years }\end{array}$ & RELP_MOD & $\begin{array}{l}\text { Model number, relative } \\
\text { permesbility model }\end{array}$ & $32669 A$ & $14 f-b-96$ & $\mathbf{Y}$ & 48 \\
\hline $\mathbf{Y}$ & & 2492 & CONC_T2 & $\begin{array}{l}\text { Concrete cokumn:400 } \\
\text { to s0K yeers }\end{array}$ & RELP_MOD & $\begin{array}{l}\text { Modal number, relative } \\
\text { permeability modal }\end{array}$ & $32668 \mathrm{~A}$ & $14 F c b-96$ & $\mathbf{Y}$ & 48 \\
\hline$Y$ & & 2492 & CONC_T2 & $\begin{array}{l}\text { Concrete column:400 } \\
\text { to } 10 K \text { years }\end{array}$ & RELP_MOD & $\begin{array}{l}\text { Modvi mumber, relatrse } \\
\text { permeability model }\end{array}$ & 326594 & $14 f 0 b 06$ & $\mathbf{Y}$ & 48 \\
\hline
\end{tabular}


CCA Parameter-Listing

\begin{tabular}{|c|c|c|c|c|c|c|c|c|c|c|}
\hline tD & \begin{tabular}{|c|} 
Cleveltit \\
Cation of \\
Dant \\
Category \\
(footroto 2)
\end{tabular} & $\begin{array}{l}\text { Detaributton } \\
\text { Type }\end{array}$ & mean & medien & Wintremen & Medrimen & Onits & Refermine L & $\begin{array}{l}\text { Was the datal } \\
\text { dervoloped } \\
\text { under en } \\
\text { Nol-1 } \\
\text { Progrem? }\end{array}$ & 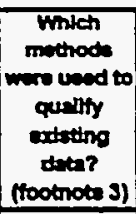 \\
\hline 2483 & 3,4 & CUMULATIVE & $2.5200000 E+\infty 0$ & $9.4000000 E-01$ & $1.1000000 E-01$ & $8.1000000 E+\infty$ & NONE & WP030994 & $\mathbf{Y}$ & N/A \\
\hline 2483 & 3,4 & CUMULATIVE & $2.5200000 \mathrm{E}+00$ & $9.4000000 E-01$ & $1.1000000 E-01$ & $8.1000000 E+\infty 0$ & NONE & WP032287 & $\mathbf{Y}$ & NA \\
\hline 2483 & 3,4 & CUMULATIVE & $2.52000000 E+00$ & $9.4000000 E-01$ & $1.1000000 E-01$ & $8.1000000 E+\infty$ & NONE & up035768 & $\mathbf{Y}$ & NA \\
\hline 2483 & 3,4 & CUMULATIVE & $2.5200000 E+\infty$ & $9.4000000 E-01$ & $1.1000000 E-01$ & $8.1000000 E+\infty 0$ & NONE & WP035597. & $\gamma$ & NA \\
\hline 2483 & 3,4 & CUMULATINE & $2.5200000 E+\infty 0$ & $9.4000000 E-01$ & $1.1000000 E-01$ & $8.1000000 E+\infty$ & NONE & UPOL1558 & $\mathbf{Y}$ & NA \\
\hline 2484 & 1.3 & CONSTANT & $5.0000000 E-02$ & $5.0000000 E-02$ & $5.0000000 E-\infty 2$ & $5.0000000 E-\infty 2$ & $m^{\wedge} 3 m^{\wedge} 3$ & WP030640 & Y8N & 1 \\
\hline 2484 & 1.3 & CONSTANT & $5.0000000 E-02$ & $5.0000000 E-02$ & $5.0000000 E-02$ & $5.0000000=-02$ & $m^{\wedge} 3 / m^{\wedge} 3$ & WPOs0094 & YAN & 1 \\
\hline 2484 & 1,3 & CONSTANT & $5.0000000 E-02$ & $5.0000000 E-02$ & $5.0000000 E-02$ & $5.0000000 E-02$ & $m^{\wedge} 3 m^{\wedge} \mathfrak{3}$ & WP035597 & YaN & 1 \\
\hline 2485 & 1.3 & CONSTANT & $1.0132500 E+05$ & $1.0132500 E+05$ & $1.0132500 E+05$ & $1.0132500 \mathrm{E}+05$ & Pa & WP035597 & $\mathbf{Y}$ & NA \\
\hline 2485 & 1,3 & CONSTAN! & $1.0132500 \mathrm{E}+05$ & $1.0132500 E+05$ & $1.0132500 E+05$ & $1.0132500 E+05$ & $\mathbf{P a}$ & WP036058 & $\mathbf{Y}$ & NA \\
\hline 2486 & 1,3 & CONSTANT & $-1.4000000 E+01$ & $-1.4000000 E+01$ & $-1,4000000 E+01$ & $-1.4000000 \mathrm{E}+01$ & $\log \left(m^{\wedge} 2\right)$ & WP030640 & $\mathbf{Y}$ & NA \\
\hline 2486 & 1,3 & CONSTANT & $-1.4000000 E+01$ & $-1.4000000 E+01$ & $-1.40000000+01$ & $-1,4000000 E+01$ & $\log \left(m^{\wedge} 2\right)$ & WP030994 & $\mathbf{Y}$ & N/A \\
\hline 2486 & 1,3 & CONSTANT & $-1.4000000 E+01$ & $-1.4000000 E+01$ & $-1.4000000 E+01$ & $-1.4000000 \mathrm{E}+01$ & $\log \left(m^{\wedge} 2\right)$ & WP035597 & $\mathbf{Y}$ & NA \\
\hline 2487 & 1,3 & CONSTANT & $-1.4000000 E+01$ & $-1.4000000 E+01$ & $-1.4000000 E+01$ & $-1.4000000 E+01$ & $\log \left(m^{\wedge} 2\right)$ & WP030640 & $\mathbf{Y}$ & N/A \\
\hline 2487 & 1,3 & CONSTANT & $-1.4000000 E+01$ & $-1.4000000 E+01$ & $-1.4000000 E+01$ & $-1.4000000 E+01$ & $\log \left(m^{\wedge} 2\right)$ & WP030994 & $\mathbf{Y}$ & NIA \\
\hline 2487 & 1,3 & CONSTANT & $-1.4000000 E+01$ & $-1.4000000 E+01$ & $-1.4000000 E+01$ & $-1.4000000 E+01$ & $\log \left(m^{\wedge} 2\right)$ & WP035597 & $\mathbf{Y}$ & NA \\
\hline 2488 & 1,3 & CONSTANT & $-1.4000000 E+01$ & $-1.4000000 E+01$ & $-1.4000000 E+01$ & $-1.4000000 E+01$ & $\log \left(m^{\wedge} 2\right)$ & UP030640 & $" Y$ & NA \\
\hline 2488 & 1,3 & CONSTANT & $-1.4000000 E+01$ & $-1.4000000 E+01$ & $-1.4000000 E+01$ & $-1.4000000 E+01$ & $\log \left(m^{\wedge} 2\right)$ & WP030994 & $\mathbf{Y}$ & NA \\
\hline 2488 & 1,3 & CONSTANT & $-1.4000000 E+01$ & $-1.4000000 E+01$ & $-1.4000000 E+01$ & $-1.4000000 E+01$ & $\log \left(m^{\wedge} 2\right)$ & WP035597 & $\mathbf{Y}$ & N/A \\
\hline 2492 & $1,3,4$ & CONSTANT & $4.0000000 E+\infty 0$ & $4.0000000 \mathrm{E}+00$ & $4.00000000 E+\infty 0$ & $4.0000000 E+\infty 0$ & NONE & WP030640 & $Y$ & NA \\
\hline 2492 & $1,3,4$ & CONSTANT & $4.0000000 E+\infty$ & $4.00000000 E+\infty 0$ & $4.00000000 E+00$ & $4.00000000 E+\infty 0$ & NONE & WP030994 & $\mathbf{Y}$ & N/A \\
\hline 2492 & $1,3,4$ & CONSTANT & $4.0000000 \varepsilon+\infty$ & $4.0000000 E+00$ & $4.0000000 E+00$ & $4.0000000 E+\infty$ & NONE & WP035597 & $Y$ & NA \\
\hline 2492 & $1,3,4$ & CONSTANT & $4.0000000 E+\infty 0$ & $4.0000000 E+\infty$ & $4.0000000 E+00$ & $4.00000000+00$ & NONE & WPO40485 & $\mathbf{Y}$ & NA \\
\hline 2492 & $1,3,4$ & CONSTANT & $4,00000000++\infty$ & $4.0000000 E+\infty 0$ & $1.0000000 E+\infty 0$ & $4.0000000 E+\infty 0$ & NONE & WPOLO514 & $\mathbf{Y}$ & NA \\
\hline
\end{tabular}


CCA-Parameter Listing

\begin{tabular}{|c|c|c|c|c|c|c|c|c|c|c|}
\hline $\begin{array}{c}\text { Parminter in } \\
\text { Datmbese? } \\
\text { (Mn) }\end{array}$ & 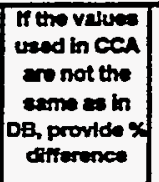 & $\mathbf{D}$ & Metarda iD & Mstard Name & Permetix & Perneter Nerse & $\begin{array}{c}\text { PRP D } \\
\text { (WPOS) }\end{array}$ & Deatity & $\begin{array}{l}\text { bo the aAd } \\
\text { conaletent } \\
\text { with the } \\
\text { prrnter } \\
\text { in DS? } \\
\text { N.NA) }\end{array}$ & $\begin{array}{c}\text { Parmits } \\
\text { Ceteocry } \\
\text { (foctincte } 1)\end{array}$ \\
\hline $\mathbf{Y}$ & & 2493 & CONC_T2 & $\begin{array}{l}\text { Concrete column: } 400 \\
\text { to } 10 K \text { yaars }\end{array}$ & SAT_IBRN & Initial Brino Seturation & 32675 & $29+50 b-96$ & $\mathbf{Y}$ & $4 A$ \\
\hline $\mathbf{Y}$ & & 2493 & CONC_T2 & $\begin{array}{l}\text { Concrete column:400 } \\
\text { to } 10 \mathrm{k} \text { years }\end{array}$ & SAT_IBRN & Initiel Brine Seturation & 32675 & 21feb-96 & $\mathbf{Y}$ & $4 A$ \\
\hline $\mathbf{Y}$ & 100 & 2494 & CONC_T2 & $\begin{array}{l}\text { Concrete colkmr: } 400 \\
\text { to } 10 K \text { years }\end{array}$ & SAT_RBRN & Residual Brina Seturation & 32676 & $14 F c b-96$ & $\mathbf{Y}$ & 1 \\
\hline $\mathbf{Y}$ & 100 & 2494 & CONC_T2 & $\begin{array}{l}\text { Concrete column:400 } \\
\text { to } 10 \% \text { years }\end{array}$ & SAT_RBRN & Residual Brim Saturation & 32676 & 14 Fob-96 & $\mathbf{Y}$ & 1 \\
\hline $\mathbf{Y}$ & 100 & 2494 & CONC_T2 & $\begin{array}{l}\text { Concrote column: } 400 \\
\text { to } 10 \mathrm{~K} \text { years }\end{array}$ & SAT_RBRN & Resicual Brin Saturation & 32676 & 14 Fab-96 & $\mathbf{Y}$ & 1 \\
\hline $\mathbf{Y}$ & 100 & 2494 & CONC_T2 & $\begin{array}{l}\text { Concrete columni } 400 \\
\text { to } 10 K \text { years }\end{array}$ & SAT_RBRN & Residtal Brino Saturation & 32676 & $14+5 e b-96$ & $\mathbf{Y}$ & 1 \\
\hline $\mathbf{Y}$ & 100 & 2494 & CONC_T2 & $\begin{array}{l}\text { Concrete cokumn: } 400 \\
\text { to } 10 \mathrm{~K} \text { yours }\end{array}$ & SAT_RBRN & Resicual Brime Saturation & 32576 & 14 Feb-96 & $\mathbf{Y}$ & 1 \\
\hline $\mathbf{Y}$ & 100 & 2494 & CONC_T2 & $\begin{array}{l}\text { Concrete column: } 400 \\
\text { to } 10 K \text { years }\end{array}$ & SAT_RBRN & Residual Brmo Saturation & 32676 & 14 Fob-s6 & $\mathbf{Y}$ & 1 \\
\hline $\mathbf{Y}$ & 100 & 2495 & CONC_T2 & $\begin{array}{l}\text { Concrete column:400 } \\
\text { to } 10 K \text { yoars }\end{array}$ & SAT_RGAS & Residual Gus Suturation & 32678 & 14 Feb-96 & $\mathbf{Y}$ & 1 \\
\hline $\mathbf{Y}$ & 100 & 2495 & CONC_T2 & $\begin{array}{l}\text { Concrete column: } 400 \\
\text { to } 10 \mathrm{~K} \text { years }\end{array}$ & SAT_RGAS & Residual Gas Saturation & 32678 & 14 feb-96 & $\mathbf{Y}$ & 1 \\
\hline $\mathbf{Y}$ & 100 & 2495 & CONC_T2 & $\begin{array}{l}\text { Concrete column: } 400 \\
\text { to } 10 \mathrm{~K} \text { years }\end{array}$ & SAT_RGAS & Residual Ges Suturation & 32678 & $14 F a b-96$ & $\mathbf{Y}$ & 1 \\
\hline $\mathbf{Y}$ & 100 & 2495 & CONC_T2 & $\begin{array}{l}\text { Concrete cohmn: } 400 \\
\text { to } 10 K \text { years }\end{array}$ & SAT_RGAS & Resicual Gen Seturation & 32678 & $14 F e b-96$ & $\mathbf{Y}$ & 1 \\
\hline $\mathbf{Y}$ & 100 & 2495 & CONC_T2 & $\begin{array}{l}\text { Concrote column: } 400 \\
\text { to } 10 \mathrm{~K} \text { years }\end{array}$ & SAT_RGAS & Resicual Ges Senration & 32678 & $14-50 b-96$ & $\mathbf{Y}$ & 1 \\
\hline $\mathbf{Y}$ & 50 & 2496 & EARTH & Earthen Fill & CAP_MOO & $\begin{array}{l}\text { Model number ceoltary pressure } \\
\text { model }\end{array}$ & $32916 A$ & 14 feb-96 & $\mathbf{Y}$ & $4 B$ \\
\hline $\mathbf{Y}$ & 50 & 2496 & EARTH & Eertimen Fix & CAP_MOO & $\begin{array}{l}\text { Mocol number capilary pressure } \\
\text { model }\end{array}$ & $32916 A$ & 14 Fob-96 & $\mathbf{Y}$ & $4 B$ \\
\hline $\mathbf{Y}$ & so & 2496 & EARTH & Earthen Fi: & CAP_MOO & $\begin{array}{l}\text { Model numoer ceplitery pressuro } \\
\text { model }\end{array}$ & 32916A & 14 feb-96 & $\mathbf{Y}$ & $4 B$ \\
\hline $\mathbf{Y}$ & so & 2496 & EARTH & Eartion Fi: & CAP_WOO & Model numoer ceollary pressure & 329164 & 14 Fob-96 & $\mathbf{Y}$ & $4 B$ \\
\hline $\mathbf{Y}$ & 50 & 2496 & EARTH & Eerthen Fit & CN_MO & $\begin{array}{l}\text { Hocei numoer ceollery pressure } \\
\text { mocer }\end{array}$ & 329164 & $14 f \circ b-96$ & $\mathbf{Y}$ & $4 B$ \\
\hline $\mathbf{Y}$ & & 2497 & EARTH & Earthen Fi: & com_nck & 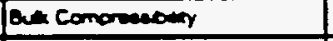 & 32918 & 14 Fob-96 & $Y$ & 1 \\
\hline $\mathbf{Y}$ & & 2497 & EARTH & Eartion Fil & con ancx & pue comormoniry & 32918 & 14-Fob-96 & $\mathbf{Y}$ & 1 \\
\hline$Y$ & & 2497 & EARTH & Earthen fill & com nack & Duen comoremonr & 32918 & 14 Feb-96 & $\mathbf{Y}$ & 1 \\
\hline $\mathbf{Y}$ & & 2498 & EARTH & Eartimen Fi: & $P C_{-} m$ & 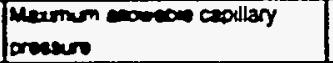 & $32922 \mathrm{~A}$ & OSHov.95 & $\mathbf{Y}$ & $4 B$ \\
\hline $\mathbf{Y}$ & & 2498 & EARTH & Eerthen Fa & PC_max & 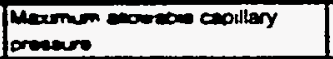 & $32920 A$ & O3-Nov-95 & $\mathbf{Y}$ & 48 \\
\hline $\mathbf{Y}$ & & 2498 & EARTH & Eerthen Fi & $P C_{-} m x$ & 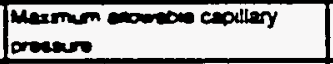 & $32922 A$ & O3-Nov-95 & $\mathbf{Y}$ & 48 \\
\hline $\mathbf{Y}$ & & 2498 & EARTH & Earthen Fa & $P C_{-} m x$ & 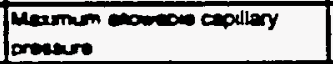 & $32920 A$ & O3NON-95 & $\mathbf{Y}$ & $4 B$ \\
\hline $\mathbf{Y}$ & & 2498 & EARTH & Eartim $\mathrm{Fi}$ & PC_max & 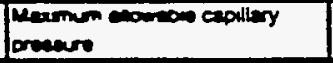 & $32922 A$ & O3-Nov-95 & $\mathbf{Y}$ & $4 B$ \\
\hline $\mathbf{Y}$ & vanes & 2499 & EARTH & Eartien Fu & POnE_DUS & 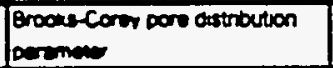 & 32923 & 20 feb-96 & $\mathbf{Y}$ & 1 \\
\hline $\mathbf{Y}$ & vanos & 2499 & EARTH & Eeromen Fa & Pone_DS & $\begin{array}{l}\text { Brocke-Corer Dore cistribution } \\
\text { Deremene }\end{array}$ & 32923 & $20+$ cb-96 & $\mathbf{Y}$ & 1 \\
\hline $\mathbf{Y}$ & vanes & 2499 & EARTH & Erthen Fa & POne_Des & 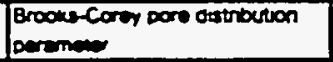 & 32923 & $20-f a b-86$ & $\mathbf{Y}$ & 1 \\
\hline $\mathbf{Y}$ & varies & 2499 & EARTH & Earthen Fa & PORE_DUS & 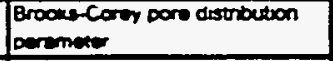 & 32923 & 20 Fab-96 & $\mathbf{Y}$ & 1 \\
\hline $\mathbf{Y}$ & veries & 2499 & ENRTH & Eartion Fa & POone_DS & $\begin{array}{l}\text { Brocke-Corer pore distribition } \\
\text { perammer }\end{array}$ & 32923 & 20 Feb-96 & $\mathbf{Y}$ & 1 \\
\hline
\end{tabular}


CCA Parameter Listing -

\begin{tabular}{|c|c|c|c|c|c|c|c|c|c|c|}
\hline ID & $\begin{array}{c}\text { Clesenti- } \\
\text { Catton of } \\
\text { Data } \\
\text { Cettegory } \\
\text { (footrote 2) }\end{array}$ & $\begin{array}{c}\text { Distribution } \\
\text { Type }\end{array}$ & $\operatorname{mean}$ & Medien & Mintmem & Madroun & Units & Reference id & $\begin{array}{l}\text { We the data } \\
\text { dwoloped } \\
\text { under an } \\
\text { NeA-1 } \\
\text { Progrem? }\end{array}$ & 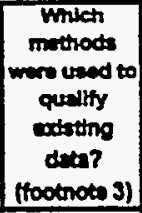 \\
\hline 2493 & Not Ured & CONSTANT & $1.0000000 E+\infty 0$ & $1.0000000 E+\infty 0$ & $1.0000000 E+\infty 0$ & $1.0000000 E+\infty$ & NONE & WP030994 & $\mathbf{Y}$ & N/A \\
\hline 2493 & Nol Used & CONSTANT & $1.0000000 E+\infty 0$ & $1.0000000 E+\infty$ & $1.0000000 E+\infty$ & $1.0000000 E+\infty$ & NONE & WP035597 & $\mathbf{Y}$ & $N / A$ \\
\hline 2494 & 3,4 & CUMULATIVE & 2.5000000E-01 & $2.0000000 E-01$ & $0.0000000 E+\infty 0$ & $6.0000000=-01$ & MONE & WP030640 & $\mathbf{Y}$ & NA \\
\hline 2494 & 3,4 & CUMULATIVE & $2.5000000 E-01$ & $2.0000000 E-01$ & $0.00000000+\infty$ & $6.0000000 E-01$ & NONE & WP030994 & $\mathbf{Y}$ & N/A \\
\hline 2494 & 3,4 & CUMULATIEE & $2.5000000 E-01$ & $2.0000000 E-01$ & $0.00000000 E+\infty 0$ & $6.0000000 E-01$ & NONE & WP035268 & $\mathbf{Y}$ & NA \\
\hline 2494 & 3,4 & CUMULATIVE & $2.5000000 E-01$ & $2.0000000 E-01$ & $0.0000000 E+\infty$ & $6.0000000 E-01$ & NONE & WP035597 & $\mathbf{Y}$ & NA \\
\hline 2494 & 3,4 & CUMULATINE & $2.5000000 E-01$ & $2.0000000 E-01$ & $0.0000000 E+\infty$ & $6.0000000 E-01$ & NONE & WPOA1558 & $\mathbf{Y}$ & NA \\
\hline 2494 & 3,4 & CUMULATIVE & $2.5000000 E-01$ & $2,0000000 E-01$ & $0.0000000 E+\infty$ & $6.0000000 E-01$ & NONE & WPO42751 & $\mathbf{Y}$ & NA \\
\hline 2495 & 3,4 & UNIFORM & 2.0000000E-01 & $2.0000000 E-01$ & $0.0000000 E+\infty$ & $4.0000000=-01$ & NONE & MP030640 & $\mathbf{Y}$ & NA \\
\hline 2495 & 3,4 & UNIFORM & $2.0000000 E-01$ & $2.0000000 E-01$ & $0.0000000 E+\infty 0$ & $4.0000000 E-01$ & NONE & WP030994 & $\mathbf{Y}$ & NA \\
\hline 2495 & 3,4 & UNIFORM & $2.0000000 E-01$ & $2.0000000 E-01$ & $0.0000000 E+\infty$ & $4.0000000 \mathrm{E}-01$ & NONE & WP035268 & $\mathbf{Y}$ & N/A \\
\hline 2495 & 3,4 & UNIFORM & $2.00000000-01$ & $2.0000000 E-01$ & $0.0000000 E+\infty$ & 4.0000000E-01 & NONE & WP035597 & $\mathbf{Y}$ & NA \\
\hline 2495 & 3,4 & UNIFORM & $2,0000000 E-01$ & $2,0000000 E-01$ & $0.0000000 E+\infty$ & $4.0000000 E-01$ & NONE & WPOA1558 & $\mathbf{Y}$ & NA \\
\hline 2496 & $1,3,4$ & CONSTANT & $2.0000000 E+\infty$ & $2.0000000 E+00$ & $2,0000000 E+\infty 0$ & $2.0000000 E+\infty 0$ & NONE & WP030540 & $\mathbf{Y}$ & NAA \\
\hline 2496 & $1,3,4$ & CONSTANT & $2.0000000 E+\infty 0$ & $200000000+\infty 0$ & $20000000 E+00$ & $200000000+\infty 0$ & NONE & WP030994 & $\mathbf{Y}$ & NA \\
\hline 2496 & $1,3,4$ & CONSTANT & $2.0000000 E+00$ & $2,0000000 E+\infty 0$ & $2.0000000 E+\infty 0$ & $20000000 E+\infty$ & NONE & WP035597 & $\mathbf{Y}$ & NAA \\
\hline 2496 & $1,3,4$ & CONSTANT & $2.0000000 E+00$ & $2.0000000 E+00$ & $2.0000000 E+\infty$ & $2.0000000 E+00$ & NONE. & WPO40486 & $\mathbf{Y}$ & N/A \\
\hline 2496 & $1,3,4$ & CONSTANT & $2.0000000 E+\infty)$ & $20000000 E+100$ & $20000000 E+\infty 0$ & $2.0000000 E+\infty$ & NONE & WPO40514 & $\mathbf{Y}$ & $N / A$ \\
\hline 2497 & 3,4 & CONSTANT & $3.10000000-08$ & $3.1000000 \mathrm{E}-08$ & $3.1000000 \mathrm{E}-00$ & $3.1000000 \mathrm{E}-08$ & $P e^{n-1}$ & WPO30540 & $\mathbf{Y}$ & N/A \\
\hline 2497 & 3,4 & CONSTANT & $3.1000000 \mathrm{E}-08$ & 3.1000000E-OS & $3.1000000 E-08$ & $3.1000000 E-08$ & $P a^{n}-1$ & WP030994 & $\mathbf{Y}$ & NA \\
\hline 2497 & 3,4 & CONSTANT & $3.1000000 E-08$ & $3.1000000 E-08$ & $3.1000000 E-08$ & 3.1000000E-08 & $P x^{n}-1$ & WP035597 & $Y$ & N/A \\
\hline 2498 & 3,5 & CONSTANT & $1.0000000 E+08$ & $1.0000000 E+08$ & $1.0000000 E+00$ & $1.0000000 E+08$ & $\mathbf{P a}$ & WPO30540 & $\mathbf{Y}$ & N/A \\
\hline 2498 & 3,5 & CONSTANT & $1.0000000 E+06$ & $1.0000000 E+08$ & $1.0000000 E+\infty 8$ & $1.0000000 E+08$ & $\mathbf{P a}$ & WP030994 & $\mathbf{Y}$ & NA \\
\hline 2498 & 3,5 & CONSTANT & $1.0000000 E+08$ & $1.0000000 E+08$ & $1.0000000 E+08$ & $1.0000000 E+08$ & Pa & WP035597 & $\mathbf{Y}$ & NA \\
\hline 2498 & 3,5 & CONSTANT & $1.0000000 E+08$ & $1.0000000 E+08$ & $1.0000000 E+06$ & $1.0000000 E+08$ & $P=$ & WPO40486 = & $\mathbf{Y} \cdot$ & . $\quad \mathbf{N} \mathbf{A}^{\prime}$ \\
\hline 2498 & 3,5 & CONSTANT & $1.0000000 E+08$ & $1.00000000 E+08$ & $1.0000000 \mathrm{E}+08$ & $1.0000000 E+08$ & Pa & WPO40514 & $\mathbf{Y}$ & $\mathbf{N A}$ \\
\hline 2499 & 3,4 & CUMULATIVE & $2.5200000 E+\infty$ & $9.4000000 E-01$ & 1.1000000 E-01 & $8.1000000 E+\infty$ & NONE & WPO30640 & $\mathbf{Y}$ & $\mathbf{N A}$ \\
\hline 2499 & 3,4 & CUMULATIVE & $2.5200000 E+\infty 0$ & $9.4000000 E-01$ & $1.1000000 E-01$ & 8.1000000E+00 & NONE & WP030994 & $\mathbf{Y}$ & NA \\
\hline 2499 & 3,4 & CUMULATINE & $25200000 E+\infty$ & 8.4000000E-01 & $1.1000000 E-01$ & $8.10000000+\infty$ & NONE & WP032287 & $Y$ & NA \\
\hline 2499 & 3,4 & CUMULATIVE & $2.5200000 E+\infty 0$ & $9.4000000 E-01$ & $1.1000000 E-01$ & $8.1000000=+\infty 0$ & NOME & WP035268 & $\mathbf{Y}$ & NA \\
\hline 2499 & 3.4 & CUMULATINE & $25200000 E+\infty 0$ & $9.4000000 E-01$ & $1.1000000 E-01$ & $8.1000000 E+\infty$ & NONE & WP035597 & $\mathbf{Y}$ & $\mathbf{N A}$ \\
\hline
\end{tabular}


CCA-Parameter Listing

\begin{tabular}{|c|c|c|c|c|c|c|c|c|c|c|}
\hline $\begin{array}{c}\text { Parmine in } \\
\text { Detabean? } \\
\text { (NN) }\end{array}$ & 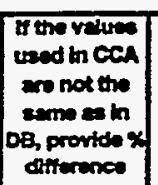 & 西 & Metertato & Metartar Name & $\begin{array}{l}\text { Persmets } \\
\mathbf{D}\end{array}$ & Perenower tivem & $\begin{array}{l}\text { PRP D } \\
\text { (MPOS) }\end{array}$ & $\begin{array}{l}\text { Deat Entry } \\
\text { Dato }\end{array}$ & 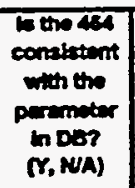 & 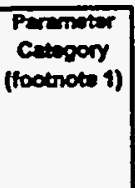 \\
\hline $\mathbf{Y}$ & varies & 2499 & EARTH & Earthen Fid & PORE_DIS & $\begin{array}{l}\text { Brooks-coroy pore distroution } \\
\text { peremeter }\end{array}$ & 32923 & $20-F a b-96$ & $\mathbf{Y}$ & 1 \\
\hline $\mathbf{Y}$ & & 2500 & EARTH & Earthen Fill & POROSTYY & Efrective porosity & 32924 & 14Fab-96 & $\mathbf{Y}$ & 1 \\
\hline $\bar{Y}$ & & 2500 & EARTH & Earthen Fill & POROSITY & Effectrve porosty & 32924 & 14Fob-96 & $y$ & 1 \\
\hline $\mathbf{Y}$ & & 2500 & EARTH & Earthen Ful & POROSIT & Effective porosity & 32924 & 14feb-96 & $Y$ & 1 \\
\hline $\mathbf{Y}$ & & 2501 & EARTH & Earthen Fit & PO_MIN & $\begin{array}{l}\text { Mnimum brine preassure for } \\
\text { capilley model KPC=3 }\end{array}$ & 32925 & $21-\mp \infty-96$ & $\mathbf{Y}$ & 48 \\
\hline $\mathbf{Y}$ & & 2501 & EARTH & Earthen Fill & PO_MIN & 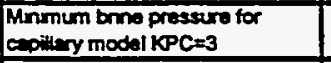 & 32925 & $21+f \infty b-96$ & $\mathbf{Y}$ & $4 B$ \\
\hline $\mathbf{Y}$ & & 2501 & EARTH & Earthen Fil & PO_MIN & $\begin{array}{l}\text { Minmum brne prossare for } \\
\text { capillany model KPC }=3\end{array}$ & 32925 & $21-F \infty 0-96$ & $\mathbf{\gamma}$ & AB \\
\hline$Y$ & & 2502 & EARTH & Earthen Fild & PRESSURE & Brine fer-field poro pressuro & 32926 & 20-Feb-96 & $Y$ & 1 \\
\hline $\bar{Y}$ & & 2502 & EARTH & Earthen Fill & PRESSURE & Brine fur-fiald pore presserme & 32926 & $20+c b-86$ & $\bar{Y}$ & 1 \\
\hline $\mathbf{Y}$ & & 25003 & EARTH & Earthon Fil & PRMX_LOG & $\begin{array}{l}\text { Log } \alpha \text { intrnsic permesbility, } X \text { - } \\
\text { direction }\end{array}$ & 32927 & 14 feb-96 & $\mathbf{\gamma}$ & 1 \\
\hline $\mathbf{Y}$ & & 2503 & EARTH & Exthen Fill & PRHX_LOG & $\begin{array}{l}\text { Log of intrinsic permaabilty, } X \text { - } \\
\text { diroction }\end{array}$ & 32927 & $14 f a b-96$ & $\mathbf{Y}$ & 1 \\
\hline $\mathbf{Y}$ & & 2503 & EARTH & Earthen Fill & PRMX_LOG & $\begin{array}{l}\text { Log of intrinsic permesbilty, } X- \\
\text { direction }\end{array}$ & 32927 & $14 F a b-96$ & $\mathbf{Y}$ & 1 \\
\hline $\mathbf{Y}$ & & 2503 & EARTH & Earthen Fill & PRMX_LOG & $\begin{array}{l}\text { Log of intomsic permarbilty, } X \text { - } \\
\text { direction }\end{array}$ & 32927 & $14 F a b-96$ & $\mathbf{Y}$ & 1 \\
\hline $\mathbf{Y}$ & & 2504 & EARTH & Eertien Fin & PRMY_LOG & $\begin{array}{l}\text { Log of intrinsic permeasbilaty, Y- } \\
\text { dinetion }\end{array}$ & 32928 & 14Fcb-96 & $\mathbf{Y}$ & 1 \\
\hline $\mathbf{Y}$ & & 2504 & EARTH & Eartien Fill & PRAY_LOG & $\begin{array}{l}\text { Log of intrinstic permestifity, Y- } \\
\text { direction }\end{array}$ & 32928 & $14 F=b-96$ & $\mathbf{Y}$ & 1 \\
\hline $\mathbf{Y}$ & & 2504 & EARTH & Earthen Fill & PRMY_LOG & $\begin{array}{l}\text { Log of intrnsic permeabilty, Y- } \\
\text { direction }\end{array}$ & 32928 & $14 f-b-96$ & $\mathbf{Y}$ & 1 \\
\hline $\mathbf{Y}$ & & 2504 & EARTH & Earthen Fid & PRAY_LOG & $\begin{array}{l}\text { Log of intrnste permesbilfy, Y- } \\
\text { direction }\end{array}$ & 32928 & 14Feb-96 & $\boldsymbol{Y}$ & 1 \\
\hline $\mathbf{Y}$ & & 2505 & EARTH & Earthen Fil & PRMZ_LOG & $\begin{array}{l}\text { Log of intrensic permesbilty, Z- } \\
\text { direction }\end{array}$ & 32944 & $14+a b-96$ & $\mathbf{Y}$ & 1 \\
\hline $\mathbf{Y}$ & & 2505 & EARTH & Earthen Fill & PRMZ_LOG & $\begin{array}{l}\text { Log of intomse permeability, Z- } \\
\text { direction }\end{array}$ & 32944 & 14-Fab-96 & $\mathbf{Y}$ & 1 \\
\hline $\mathbf{Y}$ & & 2505 & EARTH & Earthen Fill & PRMZ_LOG & $\begin{array}{l}\log \text { of intrinax permesbilty, } Z \text { - } \\
\text { direction }\end{array}$ & 32944 & 14Fob-96 & $\mathbf{Y}$ & 1 \\
\hline $\mathbf{Y}$ & & 2505 & EARTH & Earthen Fill & PRMZ_LOG & $\begin{array}{l}\text { Log of ntomsic permestilty, Z- } \\
\text { direction }\end{array}$ & 32944 & $14 f \cdot b-96$ & $\mathbf{Y}$ & 1 \\
\hline $\mathbf{Y}$ & & 2509 & EARTH & Earthen Fil & RELP_MOD & $\begin{array}{l}\text { Model nember, relative } \\
\text { permestility model }\end{array}$ & $32952 A$ & 14Feb-96 & $\mathbf{Y}$ & 48 \\
\hline $\mathbf{Y}$ & & 2509 & EARTH & Earthen Fit & RELP_MOD & $\begin{array}{l}\text { Model number, relatve } \\
\text { permesbility model }\end{array}$ & 32952A & 14 feb-96 & $\mathbf{Y}$ & 18 \\
\hline $\mathbf{Y}$ & & 2509 & EARTH & Earthen Ful & RELP_MOD & $\begin{array}{l}\text { Model number, relative } \\
\text { permeability modol }\end{array}$ & $32952 \mathrm{~A}$ & 14-Feb-96 & $\mathbf{Y}$ & 48 \\
\hline $\mathbf{Y}$ & & 2509 & EARTH & Earthen Fiti & RELP_MOD & $\begin{array}{l}\text { Model number, rolative } \\
\text { permeability modol }\end{array}$ & 32952A & 14f-b-9s & $\mathbf{Y}$ & $A B$ \\
\hline $\mathbf{Y}$ & & 2509 & EARTH & Earthen Fill & RELP_MOD & $\begin{array}{l}\text { Model number, relative } \\
\text { permesbility modal }\end{array}$ & $32952 A$ & 14-Fab-96 & $\mathbf{Y}$ & $A B$ \\
\hline$Y$ & -25 & 2510 & EARTH & Earthen Fill & SAT_IBRN & Iritial Brino Saturation & 32961 & O3-Nov-95 & $Y$ & 1 \\
\hline $\mathbf{Y}$ & -25 & 2510 & EARTH & Eerthen Fill & SAT_IBRN & Initial Brine Sturation & 32961 & O3-Nov-95 & $\mathbf{Y}$ & 1 \\
\hline $\mathbf{Y}$ & -25 & 2510 & EARTH & Earthen Fil & SAT_IBRN & Initial Brme Saturation & 32961 & O3-Nov.95 & $\mathbf{Y}$ & 1 \\
\hline $\bar{Y}$ & -25 & 2510 & EARTH & Exthen FrI & SAT_IBRN & Initial Bnno Saturation & 32961 & 03-Nov-95 & $\mathbf{Y}$ & 1 \\
\hline$Y$ & 100 & 2511 & EARTH & Emrtem Fat & SAT_RBRN & Residual Brine Saturation & 32963 & 14fob-86 & $Y$ & 1 \\
\hline$Y$ & 100 & 2511 & EARTH & Exthen Fal & SAT_RBRN & Residual Brmo Ssturation & 32963 & 14 Fob-96 & $\bar{Y}$ & 1 \\
\hline$Y$ & 100 & 2511 & EARTH & Earthen fin & SAT_RBRN & Resioval Brina Saturation & 32963 & 14Fab-95 & $Y$ & 1 \\
\hline$Y$ & 100 & 2511 & EARTH & Exrthen Fin & SAT_RBRN & Residual Brine Seturetion & 32963 & 14Fob-96 & $\mathbf{Y}$ & 1 \\
\hline$Y$ & 100 & 2511 & EARTH & Exthen Fit & SAT_RBRN & Residual Brine Seturation & 32963 & 14-Fob-96 & $Y$ & 1 \\
\hline $\bar{Y}$ & 100 & 2511 & EARTH & Enthen Fil & SAT_RBRN & Residual Brino Staration & 32963 & 14-Fab-96 & $\bar{Y}$ & 9 \\
\hline$Y$ & 100 & 2512 & EARTH & Exthen FiI & SAT_RGAS & Residual Ges Seturation & 32964 & 14-Fob-96 & $Y$ & 1 \\
\hline$Y$ & 100 & 2512 & EARTH & Emthen Fill & SAT_RGAS & Resictual Gas Saturation & 32964 & 14fob-96 & $\mathbf{Y}$ & 1 \\
\hline$Y$ & 100 & 2512 & EARTH & Exthen Fill & SAT_RGAS & Residual Ges Saturation & 32964 & $14+00-96$ & $r$ & 1 \\
\hline $\bar{Y}$ & 100 & 2512 & EARTH & Exthen Fin & SAT_RGAS & Residual Ges Saturation & 32964 & 14Fab-96 & $Y$ & 1 \\
\hline$Y$ & 100 & 2512 & EARTH & Earthen Fill & SAT_RGAS & Residual Ges Seturetion & 32964 & $14+06-86$ & $Y$ & 1 \\
\hline$Y$ & 50 & 2513 & SALT_TI & $\begin{array}{l}\text { Shan sat column } \\
\text { compactedtime } 0 \text { to } 10 \\
\text { yours }\end{array}$ & CAP_MOD & $\begin{array}{l}\text { Model number, capillery pressure } \\
\text { model }\end{array}$ & $33356 \mathrm{~A}$ & $27-0$ at.95 & $\mathbf{Y}$ & AB \\
\hline
\end{tabular}


CCA Parameter Listing

\begin{tabular}{|c|c|c|c|c|c|c|c|c|c|c|}
\hline ID & 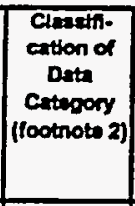 & $\begin{array}{l}\text { Dretribution } \\
\text { Type }\end{array}$ & $\operatorname{man}$ & $\begin{array}{c}\text { mexter } \\
.\end{array}$ & Mantmem & Maxdmun & Lits & Reformes id & 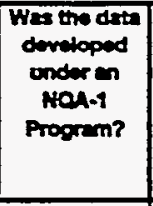 & 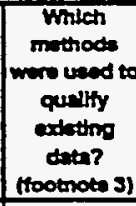 \\
\hline 2499 & 3,4 & CUMULATIVE & $25200000 E+00$ & $9.4000000 E-01$ & $1.1000000 \mathrm{E}-01$ & $8.1000000 E+\infty 0$ & NONE & WPO41558 & $\mathbf{Y}$ & NA \\
\hline 2500 & 3,4 & CONSTANT & $32000000 E-01$ & $3.2000000 E-01$ & $3.2000000 E-01$ & $3.2000000 E-01$ & $m^{\wedge} 3 / m^{\wedge} 3$ & WP030640 & $Y$ & NA \\
\hline 2500 & 3,4 & CONSTANT & $3.2000000 E-01$ & $3.2000000 \leq-01$ & $3.2000000 E-01$ & $3.2000000 E-01$ & $m^{\wedge} 3 m^{\wedge} 3$ & WP030994 & $Y$ & NA \\
\hline 2500 & 3,4 & CONSTANT & $3.2000000 E-01$ & $3.2000000 \mathrm{E}-01$ & $3.2000000 E-01$ & $3.2000000 E-01$ & $m^{\wedge} 3 m^{n} 3$ & WP035597 & $Y$ & NA \\
\hline 2501 & 3,5 & CONSTANT & $1.0132500 E+05$ & $1.0132500 E+05$ & $1.0132500 E+05$ & $1.0132500 E+05$ & Pa & WP035597 & $\gamma$ & NA \\
\hline 2501 & 3,5 & CONSTANT & $1.0132500 \mathrm{E}+05$ & $1.0132500 E+05$ & $1.0132500 E+05$ & $1.0132500 E+0.5$ & Po & WP036051 & $Y$ & N/A \\
\hline 2501 & 3,5 & CONSTANT & $1.0132500 E+05$ & $1.0132500 E+05$ & $1.0132500 \mathrm{E}+05$ & $1.0132500 E+05$ & Pa & WPO40514: & $\mathbf{Y}$ & NA \\
\hline 2502 & 3,5 & CONSTANT & $1.0132500 E+05$ & $1.0132500 E+05$ & $1.0132500 \mathrm{E}+05$ & $1.0132500 \mathrm{E}+05$ & $P_{2}$ & WP035597. & $Y$ & N/A \\
\hline 2502 & 3,5 & CONSTANT & $1.0132500 E+05$ & $1.0132500 E+05$ & $1.0132500 E+05$ & $1.0132500 \mathrm{E}+05$ & $\mathrm{~Pa}$ & WP036058 & $Y$ & NA \\
\hline 2503 & 3.4 & TALANGULAR & $-1,4333000 \mathrm{E}+01$ & $-1.4000000 E+01$ & $-1.7000000 E+01$ & $-1.2000000 E+01$ & $\log \left(m^{\wedge} 2\right)$ & WP030640 & $\mathbf{Y}$ & NA \\
\hline 2503 & 3,4 & TRLANGULAR & $-1.4333000 E+01$ & $-1.4000000 E+01$ & $-1.7000000 E+01$ & $-1.2000000 E+01$ & $\log \left(m^{\wedge} 2\right)$ & WP030994 & $\gamma$ & NAA \\
\hline 2503 & 3,4 & TRLANGULAR & $-1.4333000 E+01$ & $-1.4000000 E+01$ & $-1.7000000 E+01$ & $-1.2000000 E+01$ & $\log \left(m^{\wedge} 2\right)$ & WP035268 & $Y$ & N/A \\
\hline 2503 & 3,4 & TRIANGULAR & $-1.4333000 E+01$ & $-1.4000000 E+01$ & $-1.7000000 E+01$ & $-1.2000000 E+01$ & $\log \left(m^{\wedge} 2\right)$ & WP035597 & $Y$ & NA \\
\hline 2504 & 3,4 & TRLANGULAR & $-1.4333000 E+01$ & $-1.4000000 E+01$ & $-1.7000000 \mathrm{E}+01$ & $-1.2000000 E+01$ & $\log \left(m^{\wedge} 2\right)$ & WPO30640 & $\gamma$ & NA \\
\hline 2504 & 3,4 & TRIANGULAR & $-1.4333000 E+01$ & $-1.4000000 E+01$ & $-1.7000000 E+01$ & $-1.2000000 E+01$ & $\log \left(m^{\wedge} 2\right)$ & WP030994 & $\mathbf{Y}$ & NAA \\
\hline 2504 & 3.4 & TRLANGULAR & $-1.4333000 E+01$ & $-1.4000000 E+01$ & $-1.7000000 \mathrm{E}+01$ & $-1.2000000 \mathrm{E}+01$ & $\log \left(m^{\wedge} 2\right)$ & WP035268 & $\mathbf{Y}$ & NA \\
\hline 2504 & 3,4 & TRLANGULAR & $-1.4333000 E+01$ & $-1,4000000 E+01$ & $-1.7000000 \mathrm{E}+01$ & $-1.2000000 E+01$ & $\log \left(m^{\wedge} 2\right)$ & WP035597 & $\mathbf{Y}$ & NA \\
\hline 2505 & 3,4 & TRLANGULAR & $-1.4333000 E+01$ & $-1.4000000 E+01$ & $-1.7000000 E+01$ & $-1.2000000 E+01$ & $\log \left(m^{\wedge} 2\right)$ & WP030640 & $\mathbf{Y}$ & NA \\
\hline 2505 & 3.4 & TRLANGULAR & $-1.4333000 E+01$ & $-1.4000000 E+01$ & $-1.7000000 E+01$ & $-1.2000000 E+01$ & $\log \left(m^{\wedge} 2\right)$ & WP030994 & $\mathbf{Y}$ & NA \\
\hline 2505 & 3,4 & TRLANGULAR & $-1.4333000 E+01$ & $-1.4000000 E+01$ & $-1.7000000 E+01$ & $-1.2000000 E+01$ & $\log \left(m^{\wedge} 2\right)$ & WP035268 & $\gamma$ & NA \\
\hline 2505 & 3,4 & TRLANGULAR & $-1.4333000 E+01$ & $-1.4000000 E+01$ & $-1.7000000 E+01$ & $-1.2000000 E+01$ & $\log \left(m^{\wedge} 2\right)$ & WP035597 & $Y$ & NA \\
\hline 2509 & $1,3,4$ & CONSTANT & $4.00000000 E+00$ & 4.0000000 E +00 & $4.0000000 E+\infty$ & $4.0000000 \mathrm{E}+\infty 0$ & NONE & WPO30540 & $\mathbf{Y}$ & NA \\
\hline 2509 & $1,3,4$ & CONSTANT & $4.0000000 E+\infty 0$ & $4.0000000 E+\infty 0$ & $4.0000000 E+\infty$ & $1.0000000 E+\infty 0$ & NONE & WP030994 & $\mathbf{Y}$ & NA \\
\hline 2509 & $1,3,4$ & CONSTANT & $4.00000000+\infty 0$ & $4.00000000+00$ & $4.0000000 E+\infty$ & $4.0000000 E+\infty 0$ & NONE & WP035597 & $\mathbf{Y}$ & NAA \\
\hline 2509 & $1,3,4$ & CONSTANT & $4.0000000 \mathrm{E}+\infty 0$ & $4.0000000 E+00$ & $4.0000000 \mathrm{E}+00$ & $1.0000000 E+\infty$ & NONE & UPO40486 & $Y$ & NA \\
\hline 2509 & $1,3,4$ & CONSTANT & $4.0000000 E+\infty 0$ & $4.0000000 \mathrm{E}+\infty 0$ & $4.0000000 E+\infty 0$ & $4.0000000 E+\infty 0$ & NONE & WPO40514 & $\mathbf{Y}$ & NA \\
\hline 2510 & $1,3,4$ & CONSTANT & $8.0000000 \mathrm{E}-01$ & $8.0000000 E-01$ & $8.0000000 E-01$ & 80000000 E-01 & NONE & WPO30640 & $\bar{Y}$ & N/A \\
\hline 2510 & $1,3,4$ & CONSTANT & $8.0000000 E-01$ & $8.0000000 \mathrm{E}-01$ & $80000000 E-01$ & $80000000 E-01$ & NONE & WP030994 & $Y$ & NA \\
\hline 2510 & $1,3,4$ & CONSTANT & $8.0000000 E-01$ & $8.0000000 E-01$ & $80000000 E-01$ & $8.0000000 E-01$ & NONE & WP035597 & $Y$ & N/A \\
\hline 2510 & $1,3,4$ & CONSTANT & $8.0000000 E-01$ & $8.0000000 E-01$ & $80000000 E-01$ & $80000000 E-01$ & NONE & WPO40434 & $Y$ & NA \\
\hline 2511 & 3,4 & CUMULATINE & 2.5000000E-01 & 2.0000000E-01 & $00000000 E+\infty$ & $60000000 E-01$ & NONE & WP030640 & $Y$ & N/A \\
\hline 2511 & 3,4 & CUMULATIVE & $25000000 E-01$ & 2.0000000E-01 & $0.0000000 E+\infty 0$ & $60000000 E-01$ & NONE & WP030994 & $Y$ & NA \\
\hline 2511 & 3,4 & CUMULATIVE & $2.5000000 E-01$ & 20000000E-01 & $0.00000000+\infty 0$ & $6.00000005-01$ & NONE & WP035268 & $Y$ & NA \\
\hline 2511 & 3,4 & CUMULATIVE & $2.5000000 E-01$ & 2.0000000E-01 & $0.0000000 E+\infty$ & $6.0000000 E-01$ & NONE & WP035597 & $Y$ & NA \\
\hline 2511 & 3,4 & CUMULATIVE & $2.5000000 E-01$ & $20000000 \mathrm{E}-01$ & $0.0000000 E+\infty$ & $60000000 E-01$ & NONE & WPO41558 & $Y$ & NA \\
\hline 2511 & 3.4 & CUMULATIVE & 2.5000000E-01 & $2.0000000 \mathrm{E}-01$ & $0.0000000 \mathrm{E}+00$ & $6.0000000 E-01$ & NONE & WPO42751 & $Y$ & NA \\
\hline 2512 & 3,4 & UNIFORM & $2.0000000 \mathrm{E}-01$ & $20000000 E-01$ & $00000000 E+\infty$ & 4.0000000E-01 & NONE & WP030640 & $Y$ & NA \\
\hline 2512 & 3,4 & UNIFORM & 2.0000000E-01 & $20000000 E-01$ & $0.0000000 E+\infty$ & $4.0000000 E-01$ & NONE & WP030994 & $\bar{Y}$ & N/A \\
\hline 2512 & 3.4 & UNIFORM & $20000000 E-01$ & $2.0000000 \mathrm{E}-01$ & $0.0000000 E+\infty 0$ & $4.0000000 E-01$ & NONE & WP035268 & $Y$ & NAA \\
\hline 2512 & 3.4 & UNIFORM & 20000000E-01 & $2,0000000 \mathrm{E}-01$ & $0.0000000 E+00$ & 4.0000000E-01 & NONE & WP035597 & $Y$ & NA \\
\hline 2512 & 3,4 & UNIFORM & 20000000E-01 & $2.00000000-01$ & $0.0000000 E+\infty$ & 4.0000000E-01 & NONE & WPO41558 & $Y$ & NA \\
\hline 2513 & $1,3,4$ & CONSTANT & $2,0000000 E+\infty 0$ & $2.0000000 E+\infty 0$ & $2,0000000 E+\infty$ & $20000000 E+\infty 0$ & NONE & WP030640 & $Y$ & N/A \\
\hline
\end{tabular}


CCA Parameter Listing

\begin{tabular}{|c|c|c|c|c|c|c|c|c|c|c|}
\hline $\begin{array}{c}\text { Parmitic in } \\
\text { Databeat? } \\
\text { (YNI) }\end{array}$ & 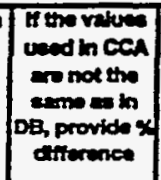 & 10 & Ifental io & Metarlal keme & Panminam & - Perendior Hama & $\begin{array}{l}\text { PRP D } \\
\text { (HTO }\end{array}$ & $\begin{array}{c}\text { Decantay } \\
\text { Dots }\end{array}$ & 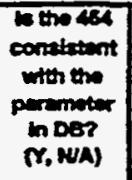 & $\begin{array}{l}\text { Paraneter } \\
\text { Cetegory } \\
\text { (footnote y) }\end{array}$ \\
\hline $\mathbf{Y}$ & 50 & 2513 & SALT_TI & $\begin{array}{l}\text { Shat salt column } \\
\text { compactect tine } 0 \text { to } 10 \\
\text { years }\end{array}$ & CAP_MOD & $\begin{array}{l}\text { Model number, capillery preseura } \\
\text { modal }\end{array}$ & $33356 \mathrm{~A}$ & $27-0 \mathrm{~cd}-95$ & $\mathbf{Y}$ & $4 B$ \\
\hline $\mathbf{Y}$ & 50 & 2513 & SALT_TY & $\begin{array}{l}\text { Shefl salt column } \\
\text { compectedtame } 0 \text { to } 10 \\
\text { yours }\end{array}$ & CAP_MOD & $\begin{array}{l}\text { Modal mumber, capilimy pressure } \\
\text { modal }\end{array}$ & $33358 \mathrm{~A}$ & 27-0ct-95 & $\mathbf{Y}$ & 48 \\
\hline $\mathbf{Y}$ & 50 & 2513 & SALT_TI & $\begin{array}{l}\text { Shan sat column } \\
\text { compecteditine } 0 \text { to } 10 \\
\text { yoars }\end{array}$ & CAP_MOD & $\begin{array}{l}\text { Model number, capillery preseuro } \\
\text { model }\end{array}$ & $33356 A$ & $27-00 t-35$ & $\mathbf{Y}$ & $4 B$ \\
\hline $\mathbf{Y}$ & 50 & 2513 & SALT_T1 & $\begin{array}{l}\text { Shat satt column } \\
\text { compactedimo } 0 \text { to } 10 \\
\text { yoars }\end{array}$ & CAP_MOO & $\begin{array}{l}\text { Model number, cepilary presure } \\
\text { model }\end{array}$ & $33356 A$ & $27-0 c t-95$ & $\mathbf{Y}$ & 48 \\
\hline $\mathbf{Y}$ & & 2514 & SALT_T1 & $\begin{array}{l}\text { Shuft salt cotumn } \\
\text { compactedtime } 0 \text { to } 19 \\
\text { years }\end{array}$ & COMP_RCK & Bulk Compressibitity & 33359 & $13-F=b-96$ & $\mathbf{Y}$ & 1 \\
\hline $\mathbf{Y}$ & & 2514 & SALT_TI & $\begin{array}{l}\text { Shaft salt coxumn } \\
\text { compactedtime } 0 \text { to id } \\
\text { yeurs }\end{array}$ & COMP_RCK & Buk Compressibitity & 33359 & 13 fab-96 & $\mathbf{Y}$ & 1 \\
\hline $\mathbf{Y}$ & & 2514 & SALT_T1 & $\begin{array}{l}\text { Shat salt column } \\
\text { compactedtime o to io } \\
\text { years }\end{array}$ & COMP_RCK & Buk Compressibitity & 33359 & 13 Fab-86 & $\mathbf{Y}$ & 1 \\
\hline $\mathbf{Y}$ & & 2515 & SALT_T1 & $\begin{array}{l}\text { Shaft salt column } \\
\text { compactedtime } 0 \text { to } 10 \\
\text { years }\end{array}$ & PC_MAX & $\begin{array}{l}\text { Mexinum allownto capittary } \\
\text { proseuro }\end{array}$ & 333734 & 27-0ct.95 & $\mathbf{Y}$ & $A B$ \\
\hline $\mathbf{Y}$ & & 2515 & SALT_T1 & $\begin{array}{l}\text { Shaft salt column } \\
\text { compactectime } 0 \text { to } 10 \text { f } \\
\text { years }\end{array}$ & PC_max & $\begin{array}{l}\text { Aeximum attowble capilery } \\
\text { pressure }\end{array}$ & $33373 A$ & 27-0ct-95 & $\boldsymbol{Y}$ & 48 \\
\hline $\mathbf{Y}$ & & 2515 & SALT_TI & $\begin{array}{l}\text { Shatt salt column } \\
\text { compactecttine } 0 \text { to } 10 \\
\text { yasers }\end{array}$ & PC_mAX & $\begin{array}{l}\text { Mexinum allowatio cepillary } \\
\text { pressure }\end{array}$ & $33373 A$ & 27-0ct.95 & $\mathbf{Y}$ & $4 B$ \\
\hline $\mathbf{Y}$ & & 2515 & SALT_TT & $\begin{array}{l}\text { Shat salt colvmn } \\
\text { compactedtime } 0 \text { to } 10 \text { f } \\
\text { yaars }\end{array}$ & PC_MAX & $\begin{array}{l}\text { Maximum allowable capitary } \\
\text { presare }\end{array}$ & $33373 A$ & $27-0<-95$ & $\mathbf{Y}$ & $4 B$ \\
\hline $\mathbf{Y}$ & & 2515 & SALT_TI & $\begin{array}{l}\text { Shaft salt column } \\
\text { compacted:time } 0 \text { to } 10 \\
\text { years }\end{array}$ & PC_MAX & $\begin{array}{l}\text { Maximum allownblo cepilliory } \\
\text { prestur }\end{array}$ & $33373 A$ & $27-0$ at.95 & $\mathbf{Y}$ & 48 \\
\hline $\mathbf{Y}$ & vanes & 2516 & SALT_TY & $\begin{array}{l}\text { Shaft salt column } \\
\text { compactedtime } 0 \text { to } 10 \\
\text { years }\end{array}$ & PORE_DIS & $\begin{array}{l}\text { Brooks-Corey pore distritution } \\
\text { paremeter }\end{array}$ & 33380 & 22 Fob-96 & $\mathbf{Y}$ & 1 \\
\hline $\mathbf{Y}$ & vanes & 2516 & SALT_T1 & $\begin{array}{l}\text { Shan sat column } \\
\text { compecteditume } 0 \text { to } 10 \\
\text { yaers }\end{array}$ & PORE_DIS & $\begin{array}{l}\text { Brooks-Coney pore distribution } \\
\text { perameter }\end{array}$ & 33380 & $22+50 b-96$ & $\mathbf{Y}$ & 1 \\
\hline $\mathbf{Y}$ & varies & 2516 & SALT_T1 & $\begin{array}{l}\text { Shint salt column } \\
\text { compectectime } 0 \text { to } 10 \\
\text { years }\end{array}$ & PORE_DIS & $\begin{array}{l}\text { Brocks-Coroy pore distribution } \\
\text { paraneter }\end{array}$ & 33390 & $22 F=b-96$ & $\mathbf{Y}$ & 1 \\
\hline$Y$ & varies & 2516 & SALT_TI & $\begin{array}{l}\text { Shan salt column } \\
\text { compacted:tuma } 0 \text { to } 10 \\
\text { years }\end{array}$ & PORE_DIS & $\begin{array}{l}\text { Brooks-Corey pore distribution } \\
\text { parameter }\end{array}$ & 33380 & 22F-b-96 & $Y$ & 4 \\
\hline $\mathbf{Y}$ & varies & 2516 & SALT_T1 & $\begin{array}{l}\text { Shaft satt column } \\
\text { compactect.time } 0 \text { to } 10 \\
\text { years }\end{array}$ & PORE_DIS & $\begin{array}{l}\text { Brocks-Corey pore distribution } \\
\text { permmeter }\end{array}$ & 33380 & 22-Fob-96 & $=\mathbf{Y}$ & 1 \\
\hline $\mathbf{Y}$ & varies & 2516 & SALT_TI & $\begin{array}{l}\text { Shath salt column } \\
\text { compactectime } 0 \text { to } 10 \\
\text { years }\end{array}$ & PORE_DIS & $\begin{array}{l}\text { Brooks-Corey pore distribution } \\
\text { paraneter }\end{array}$ & 33380 & $22 F c b-96$ & $\mathbf{Y}$ & 1 \\
\hline $\mathbf{Y}$ & varies & 2516 & SALT_TI & $\begin{array}{l}\text { Shan salt column } \\
\text { compectectume } 0 \text { to } 10 \\
\text { years }\end{array}$ & PORE_DIS & $\begin{array}{l}\text { Brooks-Corey pore distritution } \\
\text { parameter }\end{array}$ & 33380 & $22+00-96$ & $\mathbf{Y}$ & 1 \\
\hline $\mathbf{Y}$ & varias & 2516 & SALT_TI & $\begin{array}{l}\text { Shan ast column } \\
\text { compecteditine } 0 \text { to } 10 \\
\text { yours }\end{array}$ & PORE_DIS & $\begin{array}{l}\text { Brocks-Corey pore distribution } \\
\text { permeter }\end{array}$ & 33380 & 22fob-96 & $\mathbf{Y}$ & 1 \\
\hline $\mathbf{Y}$ & varies & 2516 & SALT_T1 & $\begin{array}{l}\text { Shat salt colmm } \\
\text { compectedtime } 0 \text { to } 10 \\
\text { yers }\end{array}$ & PORE_OIS & $\begin{array}{l}\text { Brooke-Corey pore distribution } \\
\text { peremeter }\end{array}$ & 33380 & 22fab-96 & $\mathbf{Y}$ & 1 \\
\hline$Y$ & & 2517 & SALT_TI & $\begin{array}{l}\text { Shan salt column } \\
\text { compectedtine } 0 \text { to } 10 \\
\text { yers }\end{array}$ & POROSTY & Efrective porosity & 33381 & $13-F a b-96$ & $\mathbf{Y}$ & 1 \\
\hline $\mathbf{Y}$ & & 2517 & SALT_TI & $\begin{array}{l}\text { Shat salt column } \\
\text { compactedtume } 0 \text { to } 10 \\
\text { years }\end{array}$ & Porosm & Eftective porosity & 33381 & $13+a b-96$ & $\mathbf{Y}$ & 1 \\
\hline $\mathbf{Y}$ & & 2517 & SALT_TI & $\begin{array}{l}\text { Shan ant colmonn } \\
\text { compectedtume } 0 \text { to } 10 \\
\text { yers }\end{array}$ & POROSIT & Effective porotity & 33381 & $13-f a b-98$ & $\mathbf{Y}$ & 1 \\
\hline
\end{tabular}




\begin{tabular}{|c|c|c|c|c|c|c|c|c|c|c|}
\hline 10 & $\begin{array}{l}\text { Cimestit- } \\
\text { Cation of } \\
\text { Deth } \\
\text { Cetteopory } \\
\text { (foctioto 2) }\end{array}$ & $\begin{array}{l}\text { Destribution } \\
\text { Type }\end{array}$ & maen & Medim & Mintrom & Mexkerum & Unt & Reforences id & 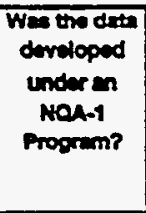 & 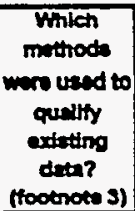 \\
\hline 2513 & $1,3,4$ & CONSTANT & $2.0000000 E+\infty$ & $20000000 E+\infty 0$ & $20000000 E+\infty$ & $2.0000000 E+\infty 0$ & NONE & WP030994 & $\mathbf{Y}$ & NA \\
\hline 2513 & $1,3,4$ & CONSTANT & $2.0000000 E+\infty 0$ & $2.0000000 E+\infty 0$ & $2.0000000 E+\infty 0$ & $2.0000000 E+\infty 0$ & NONE & WP035597 & $Y$ & NA \\
\hline 2513 & $1,3,4$ & CONSTANT & $2,0000000 E+\infty 0$ & $20000000 E+\infty$ & $2.0000000 E+\infty$ & $2.0000000 E+\infty$ & NONE & WPO40486 & $\mathbf{Y}$ & NA \\
\hline 2513 & $1,3,4$ & CONSTANT & $2.0000000 E+\infty 00$ & $2,0000000 E+\infty$ & $2.0000000 E+\infty 0$ & $20000000 E+\infty$ & NONE & WPO40514 & $\mathbf{Y}$ & NA \\
\hline 2514 & $1,3,4$ & CONSTANT & $1.6000000 \mathrm{E}-09$ & $1.6000000 E-09$ & $1.6000000 E-09$ & $1.6000000 E-09$ & $P a^{N}-1$ & WP030540 & Y\&N & 1.4 \\
\hline 2514 & $1,3,4$ & CONSTANT & $1.6000000 E-09$ & $1.6000000 \mathrm{E}-09$ & $1.6000000=-09$ & $1.6000000 \mathrm{E}-09$ & Pan-1 & WP030994 & YEN & 1.4 \\
\hline 2514 & $1,3,4$ & CONSTANT & $1.6000000 \mathrm{E}-09$ & $1.6000000 \mathrm{E}-09$ & $1.6000000 E-09$ & $1.6000000 E-09$ & $P x-1$ & WP035597 & YEN & 1.4 \\
\hline 2515 & 3,5 & CONSTANT & $1.0000000 E+08$ & $1.0000000 E+08$ & $1.00000000+08$ & $1.0000000 \mathrm{E}+08$ & Pa & WP030540 & $\mathbf{Y}$ & NA \\
\hline 2515 & 3,5 & CONSTANT & $1.0000000 E+08$ & $1.0000000 E+08$ & $1.0000000 E+08$ & $1.0000000 \mathrm{E}+08$ & $\mathbf{P a}$ & WP030994 & $Y$ & NA \\
\hline 2515 & 3.5 & CONSTANT & $1.0000000 \mathrm{E}+08$ & $1.0000000 E+08$ & $1.0000000 E+08$ & $1.0000000 E+08$ & Pa & WP035597 & $Y$ & NA \\
\hline 2515 & 3,5 & CONSTANT & $1.0000000 E+08$ & $1.0000000 E+08$ & $1.0000000 E+08$ & $1.0000000 E+08$ & Pa & WP040485 & $\gamma$ & NA \\
\hline 2515 & 3,5 & CONSTANT & $1.0000000 \mathrm{E}+08$ & $1.0000000 \mathrm{E}+08$ & $1.0000000 E+08$ & $1.0000000 \mathrm{E}+0 \mathrm{a}$ & $\mathbf{P a}$ & WPO40514 & $\mathbf{Y}$ & NA \\
\hline 2516 & 3.4 & CUMULATNE & $25200000 E+\infty$ & $9.4000000 E-01$ & $1.1000000 E-01$ & $8.1000000 E+00$ & NONE & WP030540 & $\mathbf{Y}$ & NA \\
\hline 2516 & 3,4 & CUMULATIVE & $2.5200000 \mathrm{E}+\infty$ & $9.4000000 E-01$ & $1.1000000 E-01$ & $8.1000000 E+\infty 0$ & NONE & WP030994 & $Y$ & NA \\
\hline 2516 & 3,4 & CUMULATIVE & $2.5200000 E+\infty 0$ & $9.4000000 E-01$ & $1.1000000 E-01$ & $8.1000000 E+\infty 0$ & NONE & WP031904 & $\mathbf{Y}$ & NA \\
\hline 2516 & 3,4 & CUMULATINE & $2.5200000 \mathrm{E}+\infty$ & $9.4000000 E-01$ & $1.1000000 E-01$ & $8.1000000 E+\infty 0$ & NONE & WP032287 & $\mathbf{Y}$ & NA \\
\hline 2516 & 3,4 & CUMULATIVE & $2.5200000 E+00$ & $9.4000000 E-01$ & $1.1000000 E-01$ & $8.1000000 E+\infty 0$ & NONE & WP035268 & $-Y$ & N/A \\
\hline 2516 & 3,4 & CUMULATIVE & $2.5200000 E+\infty 0$ & $9.4000000 E-01$ & $1.1000000 E-01$ & $8.1000000 E+\infty 0$ & NONE & WP035597 & $\boldsymbol{Y}$ & NA \\
\hline 2516 & 3,4 & CUMULATIVE & $2.5200000 E+\infty 0$ & $9.4000000 E-01$ & $1.1000000 E-01$ & $8.1000000 E+\infty 0$ & NONE & WP039058 & $\mathbf{Y}$ & NA \\
\hline 2516 & 3,4 & CUMULATINE & $2.5200000 E+\infty 0$ & $9.4000000 E-01$ & $1.1000000 E-01$ & $8.1000000 E+00$ & NONE & WPO41558 & $\mathbf{Y}$ & NA \\
\hline 2516 & 3,4 & CUMULATIVE & $2.5200000 E+\infty$ & $9.4000000 E-01$ & $1.1000000 E-01$ & $8.1000000 E+\infty 0$ & NONE & WPO42752 & $Y$ & N/A \\
\hline 2517 & $1,3,4$ & CONSTANT & $5.0000000 E-02$ & $5.0000000 E-02$ & $5.0000000 E-02$ & $5.0000000 E-02$ & $m^{\wedge} 3 m^{\wedge} 3$ & WP030610 & $\boldsymbol{Y}$ & NA \\
\hline 2517 & $1,3,4$ & CONSTANT & $5.0000000 \mathrm{E}-02$ & $5.0000000 E-02$ & $5.0000000 E-02$ & $5.00000000 E-02$ & $m^{\wedge} 3 m^{\wedge} \mathfrak{3}$ & WP030994 & $\mathbf{Y}$ & NA \\
\hline 2517 & 1.3.4 & CONSTANT & $5.0000000 E-02$ & $5.0000000 E-02$ & $5.0000000 E-02$ & $5.0000000 E-02$ & $m^{\wedge} 3 m^{\wedge} 3$ & WPO35597 & $Y$ & NA \\
\hline
\end{tabular}


CCA Parameter Listing

\begin{tabular}{|c|c|c|c|c|c|c|c|c|c|c|}
\hline $\begin{array}{c}\text { Parmition } \\
\text { Databees? } \\
\text { (YN) }\end{array}$ & 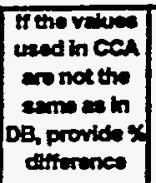 & D & Metertat to & Materal fome & $\begin{array}{l}\text { Permeter } \\
\text { iD }\end{array}$ & Perminar Ham & $\begin{array}{l}\text { PRP DD } \\
\text { PFO \& }\end{array}$ & $\begin{array}{c}\text { Dita Entry } \\
\text { Deto }\end{array}$ & 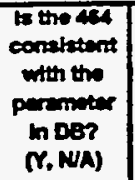 & $\begin{array}{l}\text { Parmintor } \\
\text { Cetegory } \\
\text { (footnoto 1) }\end{array}$ \\
\hline $\mathbf{Y}$ & & 2518 & SALT_T1 & $\begin{array}{l}\text { Shaft sat column } \\
\text { compactect time } 0 \text { to } 10 \\
\text { years }\end{array}$ & PO_MIN & $\begin{array}{l}\text { Mininum bnne pressure for } \\
\text { cepilley model KPC=3 }\end{array}$ & 33382 & $21-f \infty-96$ & $\mathbf{Y}$ & 48 \\
\hline $\mathbf{Y}$ & & 2518 & SALT_T1 & $\begin{array}{l}\text { Shan salt colemn } \\
\text { compecticttime } 0 \text { to } 10 \\
\text { yeers }\end{array}$ & PO_MIN & $\begin{array}{l}\text { Minimum brine presture for } \\
\text { capilary mosil KPC=3 }\end{array}$ & 33382 & $21+5 c b-96$ & $\mathbf{Y}$ & 48 \\
\hline $\mathbf{Y}$ & & 2518 & SALT_TI & $\begin{array}{l}\text { Shan satt column } \\
\text { compectedtime } 0 \text { to } 10 \\
\text { years }\end{array}$ & PO_MIN & $\begin{array}{l}\text { Minmum bros pressure for } \\
\text { capitary model KPC=3 }\end{array}$ & 33382 & $21+c 0-96$ & $\mathbf{Y}$ & 48 \\
\hline $\mathbf{Y}$ & & 2519 & SALT_TY & $\begin{array}{l}\text { Shaft salt column } \\
\text { compectect:time } 0 \text { to } 10 \\
\text { years }\end{array}$ & PRESSURE & Brine for-field pore preseure & 33386 & 20-Feb-s6 & $\mathbf{Y}$ & 1 \\
\hline $\mathbf{Y}$ & & 2519 & SALT_T1 & $\begin{array}{l}\text { Shaft salt column } \\
\text { compactect time } 0 \text { to } 10 \\
\text { years }\end{array}$ & PRESSURE & Brine far-field pore pressure & 33386 & $20-f e b-96$ & $\mathbf{Y}$ & 1 \\
\hline $\mathbf{Y}$ & & 2526 & SALT_T1 & $\begin{array}{l}\text { Shaft salt column } \\
\text { compsctect tine } 0 \text { to } 10 \\
\text { years }\end{array}$ & RELP_MOD & $\begin{array}{l}\text { Model mumber, rebativn } \\
\text { permeability model }\end{array}$ & $33405 A$ & $27-0 c-95$ & $\mathbf{Y}$ & $4 B$ \\
\hline $\mathbf{Y}$ & & 2526 & SALT_TI & $\begin{array}{l}\text { Shaf sat column } \\
\text { compactedine } 0 \text { to } 10 \\
\text { years }\end{array}$ & RELP_MOD & $\begin{array}{l}\text { Model number, neletive } \\
\text { permeability model }\end{array}$ & $33405 A$ & 27-Oct-95 & $\mathbf{Y}$ & AB \\
\hline $\mathbf{Y}$ & & 2526 & SALT_T1 & $\begin{array}{l}\text { Shaft salt colimn } \\
\text { compactedtime } 0 \text { to } 10 \\
\text { years }\end{array}$ & RELP_MOD & $\begin{array}{l}\text { Model number, niative } \\
\text { pormesbitity model }\end{array}$ & $33405 A$ & 27-Oct-95 & $\mathbf{Y}$ & $4 B$ \\
\hline $\mathbf{Y}$ & & 2526 & SALT_T1 & $\begin{array}{l}\text { Shaft salt column } \\
\text { compactedtime } 0 \text { to } 10 \\
\text { years }\end{array}$ & RELP_MOD & $\begin{array}{l}\text { Model number, relative } \\
\text { permeability model }\end{array}$ & $33405 A$ & 27-Oct-95 & $\mathbf{Y}$ & $\mathbf{A B}$ \\
\hline $\mathbf{Y}$ & & 2526 & SALT_TI & $\begin{array}{l}\text { Shaft salt column } \\
\text { compactedtimo } 0 \text { to } 10 \\
\text { years }\end{array}$ & RELP_MOD & $\begin{array}{l}\text { Model number, relative } \\
\text { permeability model }\end{array}$ & $33405 A$ & $27-0 c t-95$ & $\mathbf{Y}$ & $4 B$ \\
\hline $\mathbf{Y}$ & -212.5 & 2527 & SALT_T1 & $\begin{array}{l}\text { Shaft salt column } \\
\text { compactedtime } 0 \text { to } 10 \\
\text { years }\end{array}$ & SAT_IBRN & Initial Brina Seturation & 33416 & $13-F=b-96$ & $\mathbf{Y}$ & 1 \\
\hline $\mathbf{Y}$ & -2125 & 2527 & SALT_T1 & $\begin{array}{l}\text { Shaft salt column } \\
\text { compactedtime } 0 \text { to } 10 \\
\text { years }\end{array}$ & SAT_IBRN & Initial Brina Saturation & 33416 & 13 feb-96 & $\mathbf{Y}$ & 1 \\
\hline $\mathbf{Y}$ & -212.5 & 2527 & SALT_T1 & $\begin{array}{l}\text { Shaft salt colemn } \\
\text { compacteditime } 0 \text { to } 10 \\
\text { years }\end{array}$ & SAT_IBRN & Initial Brina Saturation & 33416 & 13 Fab-96 & $\mathbf{Y}$ & 1 \\
\hline$Y$ & -212.5 & 2527 & SALT_T1 & $\begin{array}{l}\text { Shoft salt column } \\
\text { compectectime } 0 \text { to } 10 \\
\text { years }\end{array}$ & SAT_IBRN & Initial Brino Seturation & 33416 & $13-F a b-96$ & $\mathbf{Y}$ & 1 \\
\hline$Y$ & 100 & 2528 & SALT_T1 & $\begin{array}{l}\text { Shaft salt column } \\
\text { compactoctine } 0 \text { to } 10 \\
\text { years }\end{array}$ & SAT_RBRN & Resichual Brine Saturation & 33418 & $13-F=b-96$ & $\mathbf{Y}$ & 1 \\
\hline $\mathbf{Y}$ & 100 & 2528 & SALT_T1 & $\begin{array}{l}\text { Shaf salt column } \\
\text { compactecttime } 0 \text { to } 10 \\
\text { years }\end{array}$ & SAT_RBRN & Residual Brine Saturation & 33418 & 13-Fob-96 & $\mathbf{Y}$ & 1 \\
\hline $\mathbf{Y}$ & 100 & 2528 & SALT_TI & $\begin{array}{l}\text { Shaft est column } \\
\text { compectectime } 0 \text { to } 10 \\
\text { years }\end{array}$ & SAT_RBRN & Resicusl Brine Suturation & 33418 & $13-f a b-96$ & $\bar{Y}$ & 1 \\
\hline $\mathbf{Y}$ & 100 & 2528 & SALT_TI & $\begin{array}{l}\text { Shaft sat column } \\
\text { compectedtime } 0 \text { to } 10 \\
\text { yerrs }\end{array}$ & SAT_RBRN & Residual Brine Saturation & 33418 & $13-f \circ b-96$ & $\mathbf{Y}$ & 1 \\
\hline $\mathbf{Y}$ & 100 & 2528 & SALT_TI & $\begin{array}{l}\text { Shat salt column } \\
\text { compectectime } 0 \text { to } 10 \\
\text { years }\end{array}$ & SAT_RBRN & Resictual Brine Seturation & 33418 & $13+f \circ b-96$ & $\mathbf{Y}$ & 1 \\
\hline $\mathbf{Y}$ & 100 & 2528 & SALT_TI & $\begin{array}{l}\text { Shet salt colvinn } \\
\text { compectactime } 0 \text { to } 10 \\
\text { years }\end{array}$ & SAT_RBRN & Residual Brina Saturation & 33418 & $13-F a b-96$ & $\mathbf{Y}$ & 1 \\
\hline $\mathbf{Y}$ & .100 & 2529 & SALT_TI & $\begin{array}{l}\text { Shon sak column } \\
\text { compectidtine } 0 \text { to } 10 \\
\text { years }\end{array}$ & SAT_RGAS & Residual Ges Seturation & 33420 & 13ffeb-9s & $\mathbf{Y}$ & 1 \\
\hline $\mathbf{Y}$ & 100 & 2529 & SALT_TI & $\begin{array}{l}\text { Shant sat column } \\
\text { compectedtume } 0 \text { to } 10 \\
\text { years }\end{array}$ & SAT_RGAS & Residual Gus Suturation & 33420 & 13Fob-96 & $\mathbf{Y}$ & 9 \\
\hline $\mathbf{Y}$ & 100 & 2529 & SNLT_TI & $\begin{array}{l}\text { Shat alif column } \\
\text { compectidtime } 0 \text { to } 10 \\
\text { yers }\end{array}$ & SAT_RGAS & Residual Ges Suturation & 33420 & $13-F c b-96$ & $\mathbf{Y}$ & 1 \\
\hline $\mathbf{Y}$ & 100 & 2529 & SALT_TI & $\begin{array}{l}\text { Shan sest colemn } \\
\text { compectedtume } 0 \text { to } 10 \\
\text { years }\end{array}$ & SAT_RGAS & Residual Ges Seturation & 33420 & 13-Fab-86 & $\mathbf{Y}$ & 1 \\
\hline
\end{tabular}


- CCA Parameter-Listing

\begin{tabular}{|c|c|c|c|c|c|c|c|c|c|c|}
\hline 10 & $\begin{array}{l}\text { Cleselfh- } \\
\text { costion of } \\
\text { Deth } \\
\text { Cettegory } \\
\text { (footrote 2) }\end{array}$ & $\begin{array}{l}\text { Dtetribertion } \\
\text { Type }\end{array}$ & maxn & Modien & Minkmum & Marinum & Untro & Reterences to & $\begin{array}{l}\text { Was the dith } \\
\text { doveloped } \\
\text { undoren } \\
\text { Hoh-1 } \\
\text { Progrem? }\end{array}$ & 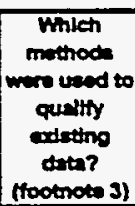 \\
\hline 2518 & 3,5 & CONSTANT & $1.0132500 E+0.5$ & $1.0132500 E+05$ & $1.0132500 E+05$ & 1.0132500E+0.5 & $\mathbf{P a}$ & WP035597 & $\mathbf{Y}$ & NA \\
\hline 2518 & 3.5 & CONSTANT & $1.0132500 E+05$ & $1.0132500 E+05$ & $1.0132500 E+05$ & $1.0132500 E+05$ & Pa & WP036051 & $\mathbf{Y}$ & NA \\
\hline 2518 & 3,5 & CONSTANT & $1.0132500 E+05$ & $1.0132500 E+05$ & $1.0132500 E+05$ & $1.0132500 E+05$ & Pa & WPO40514 & $\mathbf{Y}$ & NA \\
\hline 2519 & 3,5 & CONSTANT & $1.0132500 E+05$ & $1.0132500 E+05$ & $1.0132500 E+05$ & $1.0132500 E+05$ & $\mathbf{P a}$ & WP035597? & $\mathbf{Y}$ & NA \\
\hline 2519 & 3,5 & CONSTANT & $1.0132500 E+05$ & $1.0132500 E+05$ & $1.0132500 E+05$ & $1.0132500 E+05$ & $\mathbf{P a}$ & WP036058 & $\mathbf{Y}$ & N/A \\
\hline 2526 & $1,3,4$ & CONSTANT & $4.0000000 E+\infty 0$ & $4.0000000 \mathrm{E}+00$ & $4.0000000 E+\infty 0$ & $4.0000000 E+\infty 0$ & NONE & WP030640 & $\mathbf{Y}$ & NA \\
\hline 2526 & $1,3,4$ & CONSTANT & $4.0000000 E+\infty 0$ & $4.0000000 E+\infty$ & $4.0000000 E+\infty 0$ & $4.0000000 E+\infty 0$ & NONE & WPO3099 & $Y$ & NA \\
\hline 2526 & $1,3,4$ & CONSTANT & $4.0000000 E+\infty 0$ & $4.0000000 \mathrm{E}+\infty 0$ & $4.0000000 E+\infty 0$ & $4.0000000 E+00$ & NONE & WP035597 & $\mathbf{Y}$ & NA \\
\hline 2526 & $1,3,4$ & CONSTANT & $4.0000000 \bar{E}+\infty$ & $4.0000000 E+\infty 0$ & $4.0000000 E+\infty 0$ & $4.0000000 E+\infty$ & NONE & WPO40486 & $\mathbf{Y}$ & NA \\
\hline 2526 & $1,3,4$ & CONSTANT & $4.0000000 E+00$ & $4.0000000 E+00$ & $4.0000000 E+\infty$ & $4.0000000 E+\infty$ & NONE & WPO40514 & $\mathbf{Y}$ & NA \\
\hline 2527 & $1,3,4$ & CONSTANT & $3.2000000 E-01$ & $3.2000000 E-01$ & $3.2000000 E-01$ & $3.2000000 E-01$ & NONE & WP030640 & $\mathbf{Y}$ & NA \\
\hline 2527 & $1,3,4$ & CONSTANT & $3.2000000 E-01$ & 32000000 E-01 & $3.2000000 E-01$ & $3.2000000 E-01$ & NONE & WP030994 & $\mathbf{Y}$ & NA \\
\hline 2527 & $1,3,4$ & CONSTANT & $3.2000000 E-01$ & $3.2000000 E-01$ & $32000000 E-01$ & $32000000 E-01$ & NONE & WP035597 & $\mathbf{Y}$ & NA \\
\hline 2527 & $1,3,4$ & CONSTANT & $3.2000000 E-01$ & $3.2000000 E-01$ & $32000000 E-01$ & $32000000 E-01$ & NONE & WPO40434 & $\mathbf{Y}$ & NA \\
\hline 2528 & 3,4 & CUMULATIVE & $2.5000000 E-01$ & $20000000 E-01$ & $00000000 \mathrm{E}+\infty$ & $60000000-01$ & NONE & WP030640 & $\mathbf{Y}$ & NA \\
\hline 2528 & 3.4 & CUMULATIVE & $2.5000000 E-01$ & $20000000 E-01$ & $0,000000<-\infty$ & 60000000 E-01 & NONE & WP030994 & $\mathbf{Y}$ & NA \\
\hline 2528 & 3.4 & CUMULATINE & $2,5000000 E-01$ & $20000000 E-01$ & $0,0000000-\infty \infty$ & $60000000-01$ & NONE & WP035268 & $-\varphi$ & NA \\
\hline 2528 & 3,4 & CUMULATNEE & $2.5000000 E-01$ & $20000000 E-01$ & $00000000-\infty$ & 60000000 - 01 & NONE & WP035597 & $\mathbf{Y}$ & NA \\
\hline 2528 & 3,4 & CUMULATIVE & $2.5000000 E-01$ & $20000000 E-01$ & $0, \infty 00000 \times-\infty$ & - $00000000-01$ & NONE & WPO41558 & $\mathbf{Y}$ & NA \\
\hline 2528 & 3,4 & CUMULATIVE & $2.5000000 E-01$ & $200000006-01$ & $00000000<-\infty$ & 60000000.01 & NONE & WPO42751 & $\mathbf{Y}$ & NA \\
\hline 2529 & $\cdot 9,4$ & UNIFORM & $20000000 E-01$ & $20000000-01$ & $0,000000<-\infty$ & $40000000<-01$ & NONE & WP030640 & $\mathbf{Y}$ & NA \\
\hline 2529 & 3,4 & UNIFORM & $2.0000000 E-01$ & $20000000 E-01$ & $000000000 \times 00$ & 40000000 - 01 & NONE & WP030994 & $\mathbf{Y}$ & NA \\
\hline 2529 & 3,4 & UNIFORM & $20000000 E-01$ & $20000000 E-01$ & $00000000 \times-\infty$ & $40000000 E-01$ & NONE & WP035268 & $\mathbf{Y}$ & NA \\
\hline 2529 & 3,4 & UNIFORM & $20000000 E-01$ & $20000000 E-01$ & $0,000000<+\infty$ & 40000000 E-01 & NONE & WP035597 & $\mathbf{Y}$ & NAA \\
\hline
\end{tabular}


CCA Parameter Listing

\begin{tabular}{|c|c|c|c|c|c|c|c|c|c|c|}
\hline 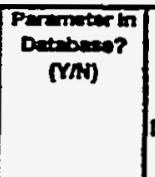 & 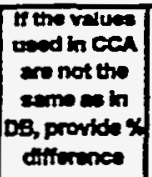 & का & Meterialo & menter Nem & $\int_{\text {to }}^{\text {Permer }}$ & 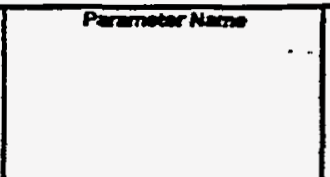 & $\begin{array}{l}\text { PRPDD } \\
\text { MPON }\end{array}$ & $\begin{array}{c}\text { Defentry } \\
\text { Date }\end{array}$ & 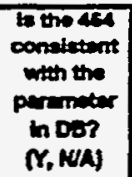 & 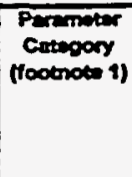 \\
\hline $\mathbf{Y}$ & 100 & 2529 & SALT_TI & $\begin{array}{l}\text { Shaft set column } \\
\text { compectectime } 0 \text { to } 10 \\
\text { years }\end{array}$ & SAT_RGAS & Residual Gas Seturation & 33420 & $13+50 b-96$ & $\mathbf{Y}$ & 1 \\
\hline $\mathbf{Y}$ & 50 & 2530 & SALT_T2 & $\begin{array}{l}\text { Shath ant cokmn } \\
\text { compactedime } 10 \text { to } \\
25 \text { years }\end{array}$ & CAP_MOD & $\begin{array}{l}\text { Model number, cepillery pressure } \\
\text { model }\end{array}$ & 334244 & $27-0 x-95$ & $\mathbf{Y}$ & $4 B$ \\
\hline $\mathbf{Y}$ & 50 & 2530 & SALT_T2 & $\begin{array}{l}\text { Shan sait colkmn } \\
\text { compactoctume } 10 \text { to } \\
25 \text { years }\end{array}$ & CAP_MOD & 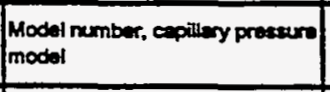 & 334249 & 27-0ct-55 & $\mathbf{Y}$ & 48 \\
\hline $\mathbf{Y}$ & 50 & 2530 & SALT_T2 & $\begin{array}{l}\text { Shaff salt column } \\
\text { compactoct tum } 10 \text { to } \\
25 \text { years }\end{array}$ & CAP_MOD & $\begin{array}{l}\text { Model nember, capiltary presururo } \\
\text { model }\end{array}$ & 334244 & 27-0ut-95 & $\mathbf{Y}$ & 4B \\
\hline $\mathbf{Y}$ & 50 & 2530 & SALT_T2 & $\begin{array}{l}\text { Shan set: colkmn } \\
\text { compectect time } 10 \text { to } \\
25 \text { years }\end{array}$ & CAP_MOD & $\begin{array}{l}\text { Modol number, capilary pressurno } \\
\text { modal }\end{array}$ & 334244 & 27-0ct-95 & $Y$ & AB \\
\hline$Y$ & 50 & 2530 & SALT_T2 & $\begin{array}{l}\text { Shant salt column } \\
\text { compsectectime } 10 \text { to } \\
25 \text { years }\end{array}$ & CAP_MOD & $\begin{array}{l}\text { Model number, capiliary pressuro } \\
\text { model }\end{array}$ & 334244 & $27-0 c t-95$ & $Y$ & AB \\
\hline $\mathbf{Y}$ & & 2531 & SALT_T2 & $\begin{array}{l}\text { Shath salt column } \\
\text { compactoct time } 10 \text { to } \\
25 \text { years }\end{array}$ & COMP_RCK & Buk Compressibilty & 33429 & $147 a b-96$ & $Y$ & 1 \\
\hline$\gamma$ & & 2531 & SALT_T2 & 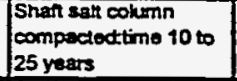 & COMP_RCK & Butk Compressibitity & 33429 & $14 F=0-96$ & $Y$ & 1 \\
\hline $\mathbf{Y}$ & & 2531 & SALT_T2 & $\begin{array}{l}\text { Shat sath column } \\
\text { compectodtime } 10 \text { to } \\
25 \text { years }\end{array}$ & COMP_RCK & Buk Compressibitity & 33429 & $14 f 0 b-96$ & $\mathbf{Y}$ & 1 \\
\hline$Y$ & & 2532 & SALT_T2 & $\begin{array}{l}\text { Shaft salt colkmn } \\
\text { compectodtime } 10 \text { to } \\
25 \text { yoars }\end{array}$ & PC_max & $\begin{array}{l}\text { Maximum abswablo capiliry } \\
\text { pressere }\end{array}$ & 334384 & 27-0ct-95 & $\boldsymbol{Y}$ & 48 \\
\hline$Y$ & & 2532 & SALT_T2 & $\begin{array}{l}\text { Shaft salt column } \\
\text { compoctodtime } 10 \text { to } \\
25 \text { yoars }\end{array}$ & PC_max & $\begin{array}{l}\text { Maximum allowable capiltary } \\
\text { prossere }\end{array}$ & 334384 & 27-0ct-95 & $\mathbf{Y}$ & 48 \\
\hline$Y$ & & 2532 & SALT_T2 & $\begin{array}{l}\text { Shaft sath column } \\
\text { compactodtime } 10 \text { to } \\
25 \text { years }\end{array}$ & PC_max & $\begin{array}{l}\text { Maximum alloweblo capilary } \\
\text { presure }\end{array}$ & $33438 A$ & 27-0ct.95 & $\mathbf{Y}$ & 48 \\
\hline $\mathbf{Y}$ & & 2532 & SALT_T2 & $\begin{array}{l}\text { Shant ealt column } \\
\text { compactodtime } 10 \text { to } \\
25 \text { years }\end{array}$ & PC_MAX & $\begin{array}{l}\text { Maximum allowablo capillary } \\
\text { pressure }\end{array}$ & 331384 & $27.0 \mathrm{dt}-95$ & $\mathbf{Y}$ & 18 \\
\hline $\mathbf{Y}$ & & 2532 & SALT_T2 & $\begin{array}{l}\text { Shat selt coturnn } \\
\text { compacted:tims } 10 \text { to } \\
25 \text { years }\end{array}$ & PC_max & $\begin{array}{l}\text { Meximum alloweblo capiliary } \\
\text { pressure }\end{array}$ & 334384 & 27-0ct-95 & $\mathbf{Y}$ & 48 \\
\hline$Y$ & varios & 2533 & SALT_T2 & $\begin{array}{l}\text { Shati ealt column } \\
\text { compactodtume } 10 \text { to } \\
25 \text { years }\end{array}$ & PORE_DIS & $\begin{array}{l}\text { Brooks-Corey pore distribution } \\
\text { paremeter }\end{array}$ & 33355 & $22+0 b-96$ & $\mathbf{Y}$ & 1 \\
\hline $\mathbf{Y}$ & varies & 2533 & SALT_T2 & $\begin{array}{l}\text { Shaff soth cohumn } \\
\text { compactoctume } 10 \text { to } \\
25 \text { years }\end{array}$ & PORE_DIS & $\begin{array}{l}\text { Brooks-Correy pore distribution } \\
\text { perametor }\end{array}$ & 33355 & $22-f e b-96$ & $\mathbf{Y}$ & 1 \\
\hline$Y$ & varios & 2533 & SALT_T2 & $\begin{array}{l}\text { Shat sall column } \\
\text { compectedtume } 10 \text { to } \\
25 \text { years }\end{array}$ & PORE_DIS & $\begin{array}{l}\text { Brooks-Corey pore destribution } \\
\text { parmeter }\end{array}$ & 33355 & 22 -Feb-96 & ${ }^{-Y} \mathrm{Y}$ & 1 \\
\hline $\mathbf{Y}$ & vanes & 2533 & SALT_T2 & $\begin{array}{l}\text { Shat sett column } \\
\text { compectodtums } 10 \text { to } \\
25 \text { yoars }\end{array}$ & PORE_DIS & $\begin{array}{l}\text { Brooke-Coray pore dustribution } \\
\text { pernmeter }\end{array}$ & 33355 & 22feb-96 & $Y$ & 1 \\
\hline $\mathbf{Y}$ & varies & 2533 & SALT_T2 & $\begin{array}{l}\text { Shat sath cotumn } \\
\text { compectodtumo } 10 \text { to } \\
25 \text { years }\end{array}$ & PORE_DIS & $\begin{array}{l}\text { Brooks-Corey pore deztribution } \\
\text { perameter }\end{array}$ & 33355 & $22-500-96$ & $\mathbf{Y}$ & 1 \\
\hline $\mathbf{Y}$ & varios & 2533 & SALT_T2 & $\begin{array}{l}\text { Shant ean colkmn } \\
\text { compectodtume } 10 \text { to } \\
25 \text { years }\end{array}$ & PORE_DIS & $\begin{array}{l}\text { Brooks-Corny porv deatribution } \\
\text { peremeter }\end{array}$ & 33355 & 22-fab-96 & $\mathbf{x}$ & 1 \\
\hline$Y$ & varies & 2533 & SNLT_T2 & $\begin{array}{l}\text { Shent alat column } \\
\text { compactod.tume } 10 \text { to } \\
25 \text { yeers }\end{array}$ & PORE_OIS & $\begin{array}{l}\text { Brooks-Corry pore dustribution } \\
\text { parameter }\end{array}$ & 33355 & 22-Feb-95 & $\mathbf{Y}$ & 1 \\
\hline$Y$ & varies & 2533 & SALT_T2 & $\begin{array}{l}\text { Shart eath column } \\
\text { compectodtume } 10 \text { to } \\
25 \text { years }\end{array}$ & FORE_DIS & $\begin{array}{l}\text { Brooks-Coroy pore distribution } \\
\text { peremeter }\end{array}$ & 33355 & 22 Feb-96 & $Y$ & 1 \\
\hline $\mathbf{Y}$ & varies & 2533 & SALT_T2 & 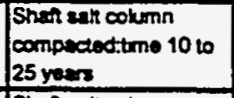 & PORE_OIS & $\begin{array}{l}\text { Brooks-Corry pore distribution } \\
\text { permenter }\end{array}$ & 33355 & $22+06-96$ & $Y$ & 1 \\
\hline $\mathbf{Y}$ & & 2534 & SALT_T & $\begin{array}{l}\text { Shatr ralt colkmn } \\
\text { compecectimn } 10 \text { to } \\
25 \text { years }\end{array}$ & POROSTr & Envective porosity & 33357 & $14+00-80$ & $r$ & 1 \\
\hline
\end{tabular}


CCA Parameter-Listing

\begin{tabular}{|c|c|c|c|c|c|c|c|c|c|c|}
\hline 10 & 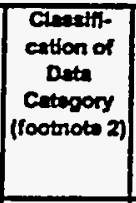 & $\begin{array}{l}\text { Detribution } \\
\text { Typo }\end{array}$ & $\operatorname{men}$ & Medien & Minkrium & Madmum & Walts & Rotermes ID & $\begin{array}{l}\text { Wes the dete } \\
\text { dematoped } \\
\text { undieren } \\
\text { Hou-1 } \\
\text { Progrem? }\end{array}$ & 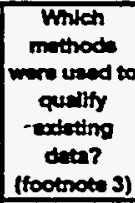 \\
\hline 2529 & 3,4 & UNIFORM & $2.0000000 E-01$ & $2.0000000 E-01$ & $0.0000000 E+00$ & $4.0000000 E-01$ & NONE & WPO11558 & $\mathbf{Y}$ & NA \\
\hline 2530. & $1,3,4$ & CONSTANT & $2.0000000 E+\infty 0$ & $20000000 E+\infty 0$ & $2,0000000 E+\infty 0$ & $20000000 E+\infty$ & NONE & WP030540 & $\mathbf{Y}$ & N/A \\
\hline 2530 & $1,3,4$ & CONSTANT & $2.0000000 E+\infty 0$ & $20000000 E+\infty 0$ & $2.0000000 E+00$ & $20000000 E+\infty 0$ & NONE & WP030994 & $\mathbf{Y}$ & NA \\
\hline 2530 & $1,3,4$ & CONSTANT & $2.0000000 \mathrm{E}+\infty 0$ & $20000000 E+\infty$ & $2.0000000 E+\infty$ & $20000000 \equiv+\infty 0$ & NONE & WP035598 & $\mathbf{Y}$ & NAA \\
\hline 2530 & $1,3,4$ & CONSTANT & $2.0000000 E+00$ & $20000000 E+\infty 0$ & $20000000 E+\infty 0$ & $2.0000000 E+00$ & NONE & UPO40485 & $\mathbf{Y}$ & NA \\
\hline 2530 & $1,3,4$ & CONSTANT & $20000000 E+00$ & $2.0000000 E+\infty 0$ & $2,0000000 E+\infty$ & $2.0000000 E+00$ & NONE & WPO40514 & $Y$ & NA \\
\hline 2531 & $1,3,4$ & CONSTANT & $1.6000000 E-09$ & $1.6000000 E-09$ & $1.6000000 E-09$ & $1.6000000 \mathrm{E}-09$ & Pan-1 & WP030540 & Y\&N & 1.4 \\
\hline 2531 & $1,3,4$ & CONSTANT & $1.6000000 E-09$ & $1.6000000 \mathrm{E}-09$ & $1.6000000 E-09$ & $1.6000000 \mathrm{E}-09$ & Par-1 & WP030994 & YEN & 1.4 \\
\hline 2531 & $1,3,4$ & CONSTANT & $1.6000000 E-09$ & $1.6000000 E-09$ & $1.6000000 E-09$ & $1.6000000 E-09$ & $P x-1$ & WP035597 & YEN & 1,4 \\
\hline 2532 & 1,3 & CONSTANT & $1.0000000 E+08$ & $1.0000000 \mathrm{E}+08$ & $1.0000000 E+08$ & $1.0000000 E+\infty 8$ & Pa & WP030640 & $\boldsymbol{\gamma}$ & NA \\
\hline 2532 & 1,3 & CONSTANT & $1.0000000 \mathrm{E}+00$ & $1.00000000 E+08$ & $1.0000000 E+08$ & $1.0000000 E+08$ & Pa & WP030994 & $Y$ & NA \\
\hline 2532 & 1,3 & CONSTANT & $1.0000000 E+08$ & $1.0000000 E+08$ & $1.0000000 E+08$ & $1.0000000 \mathrm{E}+08$ & Pa & WP035597 & $\mathbf{Y}$ & NA \\
\hline 2532 & 1,3 & CONSTANT & $1.0000000 E+\infty 6$ & $1.0000000 E+08$ & $1.0000000 E+08$ & $1.0000000 E+08$ & Pe & WPO40486 & $\mathbf{Y}$ & NA \\
\hline 2532 & 1,3 & CONSTANT & $1.0000000 E+08$ & $1.0000000 E+08$ & $1.0000000 E+08$ & $1.0000000 E+08$ & $\mathrm{~Pa}$ & WPO40514 & $Y$ & NA \\
\hline 2533 & 3,4 & CUMULATIVE & $2.5200000 E+\infty 0$ & $9.40000000-01$ & $1.1000000 \mathrm{E}-01$ & $8.1000000 E+\infty$ & NONE & WP030640 & $Y$ & NA \\
\hline 2533 & 3,4 & CUMULATIVE & $25200000 E+\infty 0$ & $9.4000000 E-01$ & $1.1000000 E-01$ & $8.1000000 E+\infty 0$ & NONE & WP030994 & $\boldsymbol{Y}$ & NA \\
\hline 2533 & 3,4 & CUMULATIVE & $2.5200000 E+\infty 0$ & 9.4000000E-01 & $1.1000000 E-01$ & $8.1000000 \mathrm{E}+\infty 0$ & NONE & WPO31904 & " & NA \\
\hline 2533 & 3,4 & CUMULATNE & $2.5200000 E+\infty 0$ & $9.4000000 E-01$ & $1.1000000 E-01$ & $8.1000000 E+\infty$ & NONE & WP032287 & $\mathbf{Y}$ & NA \\
\hline 2533 & 3,4 & CUMULATIVE & $2.5200000 \mathrm{E}+00$ & $9.4000000 E-01$ & $1.1000000 E-01$ & $8.1000000 E+00$ & NONE & WP035258 & $\mathbf{Y}$ & NA \\
\hline 2533 & 3,4 & CUMULATIVE & $25200000 E+\infty 0$ & $9.4000000 \mathrm{E}-01$ & $1.1000000 E-01$ & $8.1000000 E+\infty$ & NONE & WP035597 & $\mathbf{Y}$ & NA \\
\hline 2533 & 3,4 & CUMULATIVE & $2.5200000 E+\infty 0$ & $9.4000000 E-01$ & $1.1000000 E-01$ & $8.1000000 E+\infty 0$ & NONE & WP039058 & $\mathbf{Y}$ & NA \\
\hline 2533 & 3,4 & CUMULATNE & $2.5200000 E+\infty 0$ & $9.4000000 \mathrm{E}-01$ & $1.1000000 E-01$ & $8.1000000 E+\infty$ & NONE & WPOA1558 & $\mathbf{Y}$ & NA \\
\hline 2533 & 3,4 & CUMULATIVE & $2.5200000 E+\infty 0$ & $9.4000000 E-01$ & $1.1000000 E-01$ & $8.1000000 E+\infty$ & NONE & WPO12752 & $\mathbf{Y}$ & NA \\
\hline 2534 & $1,3,4$ & CONSTANT & $5.0000000 E-02$ & $5.00000000-02$ & $5.0000000 E-02$ & $5.0000000 E-02$ & $m^{\wedge} 3 m^{\wedge} 3$ & WP030640 & $\mathbf{Y}$ & NA \\
\hline
\end{tabular}


CCA Parameter Listing

\begin{tabular}{|c|c|c|c|c|c|c|c|c|c|c|}
\hline $\begin{array}{c}\text { Perametior th } \\
\text { Dotabae? } \\
\text { WNI }\end{array}$ & 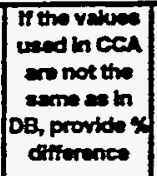 & ID & Metsaratio & Interid Name & Parmision & Parancer Nam & $\begin{array}{l}\text { PRP TD } \\
\text { (MPO })\end{array}$ & Dete Entory & 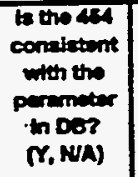 & $\begin{array}{l}\text { Parminis } \\
\text { Cotagory } \\
\text { (footnote } 1)\end{array}$ \\
\hline $\mathbf{Y}$ & & 2534 & SALT_T2 & $\begin{array}{l}\text { Shat sall column } \\
\text { compactedtime } 10 \text { to } \\
25 \text { years }\end{array}$ & POROSTYY & Efrective poresity & 33357 & 14Fab-96 & $\mathbf{Y}$ & 1 \\
\hline $\mathbf{Y}$ & & 2534 & SALT_T2 & $\begin{array}{l}\text { Shat salt colkmn } \\
\text { compectedtine } 10 \text { to } \\
25 \text { years }\end{array}$ & POROSITY & Effective porosity & 33357 & $14-500-96$ & $\mathbf{Y}$ & 1 \\
\hline $\mathbf{Y}$ & & 2535 & SALT_T2 & $\begin{array}{l}\text { Shent salt cokmn } \\
\text { compactedting } 10 \text { to } \\
25 \text { years }\end{array}$ & PO_MIN & $\begin{array}{l}\text { Minimum brime pressure for } \\
\text { expiltary model KPC=3 }\end{array}$ & 33352 & $21+$ eb-s6 & $\mathbf{Y}$ & 48 \\
\hline $\mathbf{Y}$ & & 2535 & SALT_T2 & $\begin{array}{l}\text { Shaft salt column } \\
\text { compecteditime } 10 \text { to } \\
25 \text { years }\end{array}$ & PO_MIN & $\begin{array}{l}\text { Mininum brine pressure for } \\
\text { expillery model KPC=3 }\end{array}$ & 33352 & 21 Fab-86 & $\mathbf{Y}$ & 48 \\
\hline $\mathbf{Y}$ & & 2535 & SALT_T2 & $\begin{array}{l}\text { Shaft calt column } \\
\text { compectedtine } 10 \text { to } \\
25 \text { years }\end{array}$ & PO_MIN & $\begin{array}{l}\text { Minimum brina preasere for } \\
\text { capillery model KPC=3 }\end{array}$ & 33352 & $29+e b-96$ & $\mathbf{Y}$ & 48 \\
\hline $\mathbf{Y}$ & & 2543 & SALT_T2 & $\begin{array}{l}\text { Shaf ealt column } \\
\text { compacteditime } 10 \text { to } \\
25 \text { years }\end{array}$ & RELP_MOD & $\begin{array}{l}\text { Model number, retative } \\
\text { permesbility model }\end{array}$ & $33379 A$ & 14 Fab-96 & $\mathbf{Y}$ & 48 \\
\hline $\mathbf{Y}$ & & 2543 & SALT_T2 & $\begin{array}{l}\text { Shaft salt column } \\
\text { compactectime } 10 \text { to } \\
25 \text { years }\end{array}$ & RELP_MOD & $\begin{array}{l}\text { Model number, ruktive } \\
\text { permesbitity model }\end{array}$ & 333794 & 14 Feb-96 & $\mathbf{Y}$ & 48 \\
\hline $\mathbf{Y}$ & & 2543 & SALT_T2 & $\begin{array}{l}\text { Shaft salt column } \\
\text { compactedtime } 10 \text { to } \\
25 \text { years }\end{array}$ & RELP_MOD & $\begin{array}{l}\text { Hodel number, relative } \\
\text { permoubility model }\end{array}$ & $33379 A$ & 14 Feb-96 & $\mathbf{Y}$ & 48 \\
\hline $\mathbf{Y}$ & & 2543 & SALT_T2 & $\begin{array}{l}\text { Shaft salt column } \\
\text { cornpactectime to to } \\
25 \text { years }\end{array}$ & RELP_MOD & $\begin{array}{l}\text { Model number, rulative } \\
\text { permesility model }\end{array}$ & $33379 A$ & $14-F 0 b-96$ & $\mathbf{Y}$ & $4 B$ \\
\hline $\mathbf{Y}$ & & 2543 & SALT_T2 & $\begin{array}{l}\text { Shaft salt column } \\
\text { compacted:time } 10 \text { to } \\
25 \text { years }\end{array}$ & RELP_MOD & $\begin{array}{l}\text { Model number, niative } \\
\text { permesbitiy modal }\end{array}$ & $33372 A$ & 14Fob-96 & $\mathbf{Y}$ & 48 \\
\hline$Y$ & 100 & 2545 & SALT_T2 & $\begin{array}{l}\text { Shaft salt cohimn } \\
\text { compactedtime } 10 \text { to } \\
25 \text { years }\end{array}$ & SAT_RBRN & Residual Brina Seturation & 33391 & $14+6 b-96$ & $\mathbf{Y}$ & 1 \\
\hline $\mathbf{Y}$ & 100 & 2545 & SALT_T2 & $\begin{array}{l}\text { Sheft salt column } \\
\text { compactedtime } 10 \text { to } \\
25 \text { years }\end{array}$ & SAT_RBRN & Residual Brine Seturation & 33391 & 14 Feb-96 & $\mathbf{Y}$ & 1 \\
\hline $\mathbf{Y}$ & 100 & 2545 & SALT_T2 & $\begin{array}{l}\text { Shat saht column } \\
\text { compactedtime } 10 \text { to } \\
25 \text { years }\end{array}$ & SAT_RBRN & Residual Brine Saturation & 33391 & 14Fab-96 & $\mathbf{Y}$ & 1 \\
\hline $\mathbf{Y}$ & 100 & 2545 & SALT_T2 & $\begin{array}{l}\text { Shaft salt column } \\
\text { compactodtime } 10 \text { to } \\
25 \text { years }\end{array}$ & SAT_RBRN & Residual Brine Seturation & 33391 & 14fob-9s & $\mathbf{Y}$ & 1 \\
\hline $\mathbf{Y}$ & 100 & 2545 & SALT_T2 & $\begin{array}{l}\text { Shaft salt column } \\
\text { compactedtime } 10 \text { to } \\
25 \text { years }\end{array}$ & SAT_RBRN & Residual Brine Saturation & 33391 & 14-Feb-96 & $\mathbf{Y}$ & 1 \\
\hline$Y$ & 100 & 2545 & SALT_T2 & $\begin{array}{l}\text { Shaft salt column } \\
\text { compactedtime } 10 \text { to } \\
25 \text { years }\end{array}$ & SAT_RBRN & Residua! Brino Saturation & 33391 & 14fab-96 & $\mathbf{Y}$ & 9 \\
\hline $\mathbf{Y}$ & 100 & 2546 & SALT_T2 & $\begin{array}{l}\text { Shat salt column } \\
\text { compactedtime } 10 \text { to } \\
25 \text { years }\end{array}$ & SAT_RGAS & Residual Gas Saturation & 33393 & 14Feb-9s & $\mathbf{Y}$ & 1 \\
\hline $\mathbf{Y}$ & 100 & 2546 & SALT_T2 & $\begin{array}{l}\text { Shen salt column } \\
\text { compactedtime } 10 \text { to } \\
25 \text { years }\end{array}$ & SAT_RGAS & Residual Ges Seturation & 33393 & 14Feb-96 & $\mathbf{Y}$ & 9 \\
\hline $\mathbf{Y}$ & 100 & 2546 & SALT_T2 & $\begin{array}{l}\text { Shaft selt column } \\
\text { compectedtime } 10 \text { to } \\
25 \text { years }\end{array}$ & SAT_RGAS & Resctual Ges Suturation & 33393 & 14 feb-96 & $\mathbf{Y}$ & 1 \\
\hline $\mathbf{Y}$ & 100 & 2546 & SALT_T2 & $\begin{array}{l}\text { Shent sen cokmn } \\
\text { compectuditime } 10 \text { to } \\
25 \text { years }\end{array}$ & SAT_RGAS & Resucual Ges Sesturation & 33393 & $14 F 0 b-96$ & $\mathbf{Y}$ & 1 \\
\hline $\mathbf{Y}$ & 100 & 2546 & SNT_T2 & $\begin{array}{l}\text { Shan sulf column } \\
\text { compectedtime } 10 \text { to } \\
25 \text { yers }\end{array}$ & SAT_RGAS & Residual Ges Suturation & 33393 & 14 Feb-96 & $\mathbf{Y}$ & 1 \\
\hline $\mathbf{Y}$ & 50 & 2547 & SALT_T3 & $\begin{array}{l}\text { Shan sell column } \\
\text { compectedtime } 25 \text { to } \\
50 \text { years }\end{array}$ & CAP_MOD & $\begin{array}{l}\text { Model number, ceplliery presure } \\
\text { model }\end{array}$ & $33397 \mathrm{~A}$ & 27-0ct-95 & $\mathbf{Y}$ & $4 \mathrm{~B}$ \\
\hline $\mathbf{Y}$ & 50 & 2547 & SALT_T3 & $\begin{array}{l}\text { Shan salt column } \\
\text { compectectime } 25 \text { to } \\
50 \text { years }\end{array}$ & CAP_MOD & $\begin{array}{l}\text { Model number, capillary pressure } \\
\text { model }\end{array}$ & $33397 A$ & $27-0$ ct-95 & $\mathbf{Y}$ & $4 B$ \\
\hline $\mathbf{Y}$ & 50 & 2547 & SALT_T3 & $\begin{array}{l}\text { Shat selt column } \\
\text { compectuctime } 25 \text { to } \\
\text { 50 yers }\end{array}$ & CAP_MOD & $\begin{array}{l}\text { Modal number, capillery presure } \\
\text { model }\end{array}$ & $33397 A$ & 27-0ct-95 & $\mathbf{Y}$ & 49 \\
\hline
\end{tabular}


CCA Parameter Listing

\begin{tabular}{|c|c|c|c|c|c|c|c|c|c|c|}
\hline 10 & 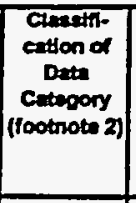 & $\begin{array}{l}\text { Distribution } \\
\text { Type }\end{array}$ & man & Modien & minkrim & Modmum & Unts & Refermen ID & $\begin{array}{l}\text { Wes the dent } \\
\text { domeloped } \\
\text { underen } \\
\text { Noh-1 } \\
\text { - Progrem? }\end{array}$ & 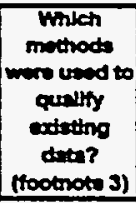 \\
\hline 2534 & $1,3,4$ & CONSTANT & $5.0000000 E-02$ & $5.0000000 E-02$ & $5.0000000 E-02$ & $5.0000000 E-02$ & $m^{\wedge} 3 m^{\wedge} 3$ & WP030994 & $Y$ & NA \\
\hline 2534 & $1,3,4$ & CONSTANT & $5.0000000 E-02$ & $5.0000000 E-02$ & $5.0000000 E-02$ & $5.0000000 E-02$ & $m^{\wedge} 3 m^{\wedge} 3$ & WP035597 & $\mathbf{Y}$ & NA \\
\hline 2535 & 1,3 & CONSTANT & $1.0132500 \mathrm{E}+0.5$ & $1.0132500 E+05$ & $1.0132500 E+05$ & $1.0132500 E+05$ & Pa & WP035597 & $\mathbf{Y}$ & NA \\
\hline 2535 & 1,3 & CONSTANT & $1.0132500 E+05$ & $1.0132500 E+05$ & $1.0132500 E+05$ & $1.0132500 E+05$ & Pa & WP03605? & Y & N/A \\
\hline 2535 & 1.3 & CONSTANT & $1.0132500 E+05$ & $1.0132500 E+05$ & $1.0132500 E+05$ & $1.0132500 \mathrm{E}+05$ & Pa & WPO40514 & $\mathbf{Y}$ & NA \\
\hline 2543 & 1,3 & CONSTANT & $4.0000000 E+\infty 0$ & $4.0000000 \mathrm{E}+\infty 0$ & $4.0000000 E+\infty 0$ & $4.0000000 \mathrm{E}+00$ & NONE & WP030540 & $\mathbf{Y}$ & N/A \\
\hline 2543 & 1,3 & CONSTANT & $4.0000000 E+\infty 0$ & $4.0000000 E+\infty 0$ & $4.0000000 E+\infty 0$ & $4.0000000 E+\infty 0$ & NONE & WP03099i & $Y$ & N/A \\
\hline 2543 & 1,3 & CONSTANT & $4.0000000 \mathrm{E}+\infty 0$ & $4.0000000 E+\infty 0$ & $4.00000000+00$ & $4.0000000 E+\infty 0$ & NONE & WP035597 & Y & NA \\
\hline 2543 & 1,3 & CONSTANT & 4.0000000E $+\infty 0$ & $4.00000000+\infty 0$ & $4.00000000 E+\infty 0$ & $4.0000000 E+\infty 0$ & NONE & WPO40486 & $Y$ & NA \\
\hline 2543 & 1.3 & CONSTANT & $4.00000000 E+00$ & $4,0000000 E+\infty 0$ & $4.00000000 E+\infty 0$ & $4.0000000 E+\infty 0$ & NONE & WPO40514 & $Y$ & NA \\
\hline 2545 & 3,4 & CUMUULATIVE & $2.5000000 E-01$ & $2.0000000 E-01$ & $0.0000000 E+\infty$ & 6.00000000E-01 & NONE & WPO30640 & $\mathbf{Y}$ & NA \\
\hline 2545 & 3.4 & CUMULLATIVE & $2.5000000 E-01$ & $20000000 E-01$ & $0.0000000 E+\infty$ & $6.0000000=-01$ & NONE & WP030994 & $\mathbf{r}$ & NA \\
\hline 2545 & 3,4 & CUMULATNE & $2.5000000 E-01$ & $2.0000000 E-01$ & $0.00000005+\infty$ & $6.0000000 E-01$ & NONE & WP035268 & $\gamma$ & NA \\
\hline 2545 & 3,4 & CUMULATNE & $2.5000000 \mathrm{E}-01$ & $2.0000000 \mathrm{E}-01$ & $0.0000000 E+\infty$ & $6.00000000-01$ & NONE & WP035597 & $\mathbf{Y}$ & NA \\
\hline 2545 & 3,4 & CUMULATIVE & $2.5000000 E-01$ & $2.0000000 E-01$ & $0.00000000+00$ & $6.0000000 E-01$ & NONE & WPO41558 & $\mathbf{Y}$ & NA \\
\hline 2545 & 3,4 & CUMULATIVE & $2.5000000 E-01$ & $2.0000000 E-01$ & $0.0000000 E+00$ & $6.0000000 E-01$ & NONE & WPO42751 & $\mathbf{Y}$ & NA \\
\hline 2546 & 3.4 & UNIFORM & $2.0000000 E-01$ & $2.0000000 E-01$ & $0.0000000 E+\infty$ & 4.0000000E-01 & NONE & WP030640 & $Y$ & NA \\
\hline 2546 & 3,4 & UNIFORM & $2.0000000 E-01$ & $2.0000000 E-01$ & $0.00000000+00$ & $4.0000000 E-01$ & NONE & WP030994 & $\mathbf{Y}$ & NA \\
\hline 2546 & 3,4 & UNIFORM & $2.0000000 E-01$ & $20000000 E-01$ & $0.0000000 E+00$ & $4.0000000 \mathrm{E}-01$ & NONE & WP035268 & $\mathbf{Y}$ & NA \\
\hline 2546 & 3,4 & UNIFORM & $2.0000000 E-01$ & $2.0000000=-01$ & $0.0000000 \mathrm{E}+\infty$ & 4.0000000E-01 & NONE & WP035597 & $\mathbf{Y}$ & NA \\
\hline 2546 & 3,4 & UNIFORM & $20000000=-01$ & $2.0000000 E-01$ & $0.0000000 E+\infty 0$ & $4.0000000 E-01$ & NONE & WPO41558 & $\mathbf{Y}$ & $N / A$ \\
\hline 2547 & $1,3,4$ & CONSTANT & $2.0000000 E+\infty$ & $2.0000000 E+\infty 0$ & $2.0000000 E+\infty 0$ & $2.0000000 \mathrm{E}+\infty 0$ & NONE & WP030640 & $\mathbf{Y}$ & NA \\
\hline 2547 & $1,3,4$ & CONSTANT & $2.0000000 E+\infty 0$ & $20000000 E+\infty$ & $2.0000000 E+\infty 0$ & $20000000 E+\infty 0$ & NONE & WP030994 & $\mathbf{Y}$ & NA \\
\hline 2547 & $1,3,4$ & CONSTANT & $20000000 E+\infty 0$ & $20000000 E+\infty$ & $200000000+\infty$ & $2.0000000 E+\infty 0$ & NONE & WP035597 & $\mathbf{Y}$ & NA \\
\hline
\end{tabular}


- CCA Parameter Listing -

\begin{tabular}{|c|c|c|c|c|c|c|c|c|c|c|}
\hline $\begin{array}{c}\text { Parmatin } \\
\text { Detabes? } \\
\text { (XN) }\end{array}$ & 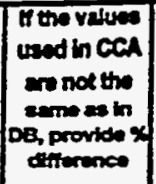 & D & Mandid & Matadal $\mathrm{k}$ mo & Purmetier & Parmithm & $\begin{array}{l}\text { PRP क } \\
\text { (HPO) }\end{array}$ & Den Entry & $\begin{array}{l}\text { to the dSA } \\
\text { corratetemt } \\
\text { with the } \\
\text { peremeter } \\
\text { in OB? } \\
X, N A)\end{array}$ & $\begin{array}{l}\text { Paramitar } \\
\text { Categony } \\
\text { (footnote 1) }\end{array}$ \\
\hline $\mathbf{Y}$ & 50 & 2547 & SALT_T3 & $\begin{array}{l}\text { Shat sat column } \\
\text { compactectime } 25 \text { to } \\
\text { s0 years }\end{array}$ & CAP_MOD & $\begin{array}{l}\text { Model number. cepillery pressuro } \\
\text { model }\end{array}$ & $33397 A$ & 27.0ct-95 & $\mathbf{Y}$ & 48 \\
\hline $\mathbf{Y}$ & 50 & 2547 & SALT_T3 & $\begin{array}{l}\text { Shat sall column } \\
\text { compactectime } 25 \text { to } \\
50 \text { years }\end{array}$ & CAP_MOD & $\begin{array}{l}\text { Model number, capittery prasuere } \\
\text { modil }\end{array}$ & $33397 A$ & $27-0<-95$ & $\mathbf{Y}$ & $4 B$ \\
\hline $\mathbf{Y}$ & & 2548 & SALT_T3 & $\begin{array}{l}\text { Shaft ealt colimn } \\
\text { compectedtimo } 25 \text { to } \\
50 \text { years }\end{array}$ & COMP_RCK & Bukk Compressibitity & 33400 & 14 Fab-96 & $\mathbf{Y}$ & 1 \\
\hline $\mathbf{Y}$ & & 2548 & SALT_T3 & $\begin{array}{l}\text { Shatr sah column } \\
\text { compactodtime } 25 \text { to } \\
50 \text { yoars }\end{array}$ & COMP_RCK & Buk Compressibitity & 33400 & 14fab-9s & $\mathbf{Y}$ & 1 \\
\hline $\boldsymbol{\gamma}$ & & 2548 & SALT_T3 & $\begin{array}{l}\text { Shat sah coiumn } \\
\text { compectedtime } 25 \text { to } \\
50 \text { years }\end{array}$ & COMP_RCK & Bulk Compressibitity & 33400 & $147 a b-96$ & $\mathbf{Y}$ & 1 \\
\hline $\mathbf{Y}$ & & 2549 & SALT_T3 & $\begin{array}{l}\text { Shat ralt column } \\
\text { compactodtimo } 25 \text { to } \\
50 \text { years }\end{array}$ & PC_MAX & $\begin{array}{l}\text { Macinum alfowable copillary } \\
\text { presturs }\end{array}$ & $33408 A$ & 27-Oct-95 & $\mathbf{Y}$ & $4 \mathrm{~B}$ \\
\hline$Y$ & & 2549 & SALT_T3 & $\begin{array}{l}\text { Shaft salt column } \\
\text { compactodtume } 25 \text { to } \\
50 \text { years }\end{array}$ & PC_MAX & $\begin{array}{l}\text { Maxinum allowable copillary } \\
\text { pressure }\end{array}$ & $33408 A$ & $27-0 x-95$ & $\mathbf{Y}$ & $A B$ \\
\hline $\mathbf{Y}$ & & 2549 & SALT_T3 & $\begin{array}{l}\text { Shat salt column } \\
\text { compacteditme } 25 \text { to } \\
50 \text { years }\end{array}$ & PC_MAX & $\begin{array}{l}\text { Maxinum allowabla captitery } \\
\text { pressure }\end{array}$ & $33402 n$ & $27-0 \mathrm{ct}-95$ & $\mathbf{Y}$ & $4 B$ \\
\hline $\mathbf{Y}$ & & 2549 & SALT_T3 & $\begin{array}{l}\text { Shaft anli column } \\
\text { compactedtime } 25 \text { to } \\
50 \text { yoars }\end{array}$ & PC_MAX & $\begin{array}{l}\text { Macimum atowable capillary } \\
\text { pressure }\end{array}$ & 33408 & $27-0 c t-95$ & $\mathbf{Y}$ & 48 \\
\hline $\mathbf{Y}$ & & 2549 & SALT_T3 & $\begin{array}{l}\text { Shaft salk column } \\
\text { compected:tme } 25 \text { to } \\
50 \text { years }\end{array}$ & PC_MAX & $\begin{array}{l}\text { Maximum allowabla capillery } \\
\text { pressure }\end{array}$ & $33408 A$ & 27-Oct-95 & $\mathbf{Y}$ & $4 B$ \\
\hline $\mathbf{Y}$ & varies & 2550 & SALT_T3 & $\begin{array}{l}\text { Shaft salt column } \\
\text { compacteditune } 25 \text { to } \\
50 \text { years }\end{array}$ & PORE_DIS & $\begin{array}{l}\text { Erocks-Corry pore distribution } \\
\text { paremeter }\end{array}$ & 33415 & $22+c a-96$ & $\mathbf{Y}$ & 1 \\
\hline $\mathbf{Y}$ & varies & 2550 & SALT_T3 & $\begin{array}{l}\text { Shath sall column } \\
\text { compactod:tme } 25 \text { to } \\
50 \text { years }\end{array}$ & PORE_DIS & $\begin{array}{l}\text { Brooks-Corey pore distribution } \\
\text { parameter }\end{array}$ & 33415 & 22 Fab-96 & $\mathbf{Y}$ & 1 \\
\hline $\mathbf{Y}$ & varies & 2550 & SALT_T3 & $\begin{array}{l}\text { Shat sal column } \\
\text { compactoditume } 25 \text { to } \\
50 \text { years }\end{array}$ & PORE_DIS & $\begin{array}{l}\text { Brooks-Corey pore distribution } \\
\text { perameter }\end{array}$ & 33415 & $22+5 e b-96$ & $\mathbf{Y}$ & 1 \\
\hline$Y$ & varies & 2550 & SALT_T3 & $\begin{array}{l}\text { Snath san colvmn } \\
\text { compacted tume } 25 \text { to } \\
50 \text { years }\end{array}$ & PORE_OAS & $\begin{array}{l}\text { Brooke-Corer Dore destribution } \\
\text { peremeter }\end{array}$ & 33415 & 22-Fob-96 & $\mathbf{Y}$ & 1 \\
\hline $\mathbf{Y}$ & varies & 2550 & SALT_T3 & $\begin{array}{l}\text { Shan rall column } \\
\text { compocted tum } 25 \text { to } \\
50 \text { years }\end{array}$ & PORE_DIS & $\begin{array}{l}\text { Brook-coney pore dutribution } \\
\text { peremen }\end{array}$ & 33415 & $22+50 b-96$ & $\mathbf{Y}$ & 1 \\
\hline $\mathbf{Y}$ & varies & 2550 & SALT_T3 & $\begin{array}{l}\text { Shat salt column } \\
\text { compected time } 25 \text { to } \\
50 \text { yours }\end{array}$ & PORE_DS & Drooke-coner pore dustibution & 33415 & 22f-6-96 & $\mathbf{Y}$ & 1 \\
\hline $\mathbf{Y}$ & varies & 2550 & SALT_T3 & $\begin{array}{l}\text { Shan sell column } \\
\text { compectec tmo } 25 \text { to } \\
50 \text { years }\end{array}$ & POAE_OS & 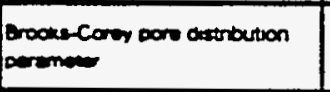 & 33415 & 22-Feb-96 & $\mathbf{Y}$ & 1 \\
\hline$Y$ & varies & 2550 & SALT_T3 & $\begin{array}{l}\text { Shein sely column } \\
\text { compected tmo is to } \\
50 \text { years }\end{array}$ & POAC_OS & 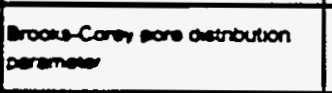 & 33415 & 22Feb-96 & $\mathbf{Y}$ & 1 \\
\hline $\mathbf{Y}$ & varies & 2550 & SALT_T3 & $\begin{array}{l}\text { Shent esx colem } \\
\text { compectect ome } 25 \text { io } \\
50 \text { yeers }\end{array}$ & Pone_Ds & brocke-cons dore ontroution & 33415 & $22+F=b-96$ & $\mathbf{Y}$ & 1 \\
\hline $\mathbf{Y}$ & & 2551 & SALT_T3 & $\begin{array}{l}\text { Shen ael colem } \\
\text { compected tome } 25 \text { to } \\
50 \text { yeers }\end{array}$ & POROSTr & Encow porom & 33417 & 14 feb-96 & $\mathbf{Y}$ & 1 \\
\hline $\mathbf{Y}$ & & 2551 & SALT_T3 & $\begin{array}{l}\text { Shan and colimn } \\
\text { compacted time } 25 \text { to } \\
50 \text { years }\end{array}$ & POROsir & 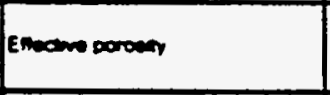 & 33417 & 14feb-96 & $\mathbf{Y}$ & 1 \\
\hline $\mathbf{Y}$ & & 2551 & SALT_T3 & $\begin{array}{l}\text { Shan atx colemn } \\
\text { compectectum } 25 \text { vo } \\
50 \text { years }\end{array}$ & Ponosir & Enscaw poroen & 33417 & 14 Fab-96 & $Y$ & 1 \\
\hline $\mathbf{Y}$ & & 2552 & SALT_T3 & $\begin{array}{l}\text { Shan sell colum } \\
\text { compectedtum } 25 \text { to } \\
50 \text { yers }\end{array}$ & PO_wa & 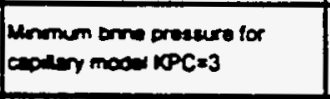 & 33419 & 21-feb-96 & $\mathbf{Y}$ & $A B$ \\
\hline $\mathbf{Y}$ & & 2552 & SALT_T3 & $\begin{array}{l}\text { Shan selk colum } \\
\text { compected tume } 25 \text { to } \\
50 \text { years }\end{array}$ & po_m & 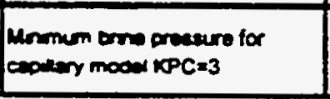 & 33419 & 21 feb-86 & $\mathbf{Y}$ & $\mathbf{A B}$ \\
\hline
\end{tabular}




\section{- CGAParameter-Listing}

\begin{tabular}{|c|c|c|c|c|c|c|c|c|c|c|}
\hline 10 & 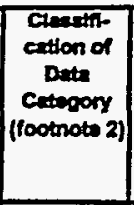 & $\begin{array}{l}\text { Detertibertion } \\
\text { Typo }\end{array}$ & man & modisn & Minimen & Medming & Thats & Reformance it & 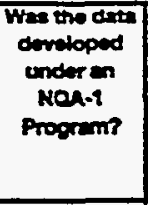 & 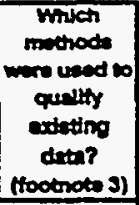 \\
\hline 2547 & $1,3,4$ & CONSTANT & $2.0000000 E+\infty 0$ & $20000000 E+\infty 0$ & $20000000 E+00$ & $2.0000000 E+\infty$ & NONE & WPO40485 & $Y$ & NA \\
\hline 2547 & $1,3,4$ & CONSTANT & $2.0000000 E+00$ & $2.0000000 E+\infty$ & $20000000 E+\infty 0$ & $20000000 E+\infty 0$ & NONE & WP040514 & $Y$ & NA \\
\hline 2548 & $1,3,4$ & CONSTANT & $1.6000000 E-09$ & $1.6000000 E-09$ & $1.6000000=-09$ & $1.6000000=-09$ & Pan-1 & WP030540 & YEN & 1.4 \\
\hline 2548 & $1,3,4$ & CONSTANT & $1.6000000 E-09$ & $1.6000000 E-09$ & $1.6000000 E-09$ & $1.6000000=.09$ & $P x^{n-1}$ & WP030994 & YEN & 1.4 \\
\hline 2548 & $1,3,4$ & CONSTANT & $1.6000000 E-09$ & $1.6000000 \mathrm{E}-09$ & $1.6000000 E-09$ & $1.6000000 E-09$ & Par-1 & WP035597 & YEN & 1.4 \\
\hline 2549 & 1,3 & CONSTANT & $1.0000000 E+08$ & $1.0000000 E+08$ & $1.0000000 \mathrm{E}+00$ & $1.0000000 E+08$ & $\mathrm{~Pa}$ & WP030510 & $\mathbf{Y}$ & NA \\
\hline 2549 & 1,3 & CONSTANT & $1.0000000 \mathrm{E}+08$ & $1.0000000 E+08$ & $1.0000000 E+08$ & $1.0000000 E+08$ & Pa & WP030994 & $\mathbf{Y}$ & NA \\
\hline 2549 & 1,3 & CONSTANT & $1.0000000 E+08$ & $1.0000000 E+00$ & $1.0000000 E+08$ & $1.0000000 E+08$ & $\mathbf{P a}$ & WP035597 & $\mathbf{Y}$ & NA \\
\hline 2549 & 1,3 & CONSTANT & $1.0000000 E+08$ & $1.0000000 E+08$ & $1.0000000 E+06$ & $1.0000000 E+08$ & Pa & WP040485 & $\mathbf{Y}$ & NA \\
\hline 2549 & 1,3 & CONSTANT & $1.0000000 E+08$ & $1.0000000 E+08$ & $1.0000000 E+08$ & $1.0000000 E+08$ & $\mathbf{P a}$ & WP040514 & $\mathbf{Y}$ & NA \\
\hline 2550 & 3,4 & CUMULATIVE & $2.5200000 E+\infty 0$ & 9.4000000E-01 & $1.1000000 E-01$ & $8.1000000 E+\infty 0$ & NONE & WP030640 & $Y$ & NA \\
\hline 2550 & 3,4 & CUMULATINE & $2.5200000 E+\infty 0$ & $9.4000000 E-01$ & $1.1000000 E-01$ & $8.1000000 E+\infty$ & NONE & WP030994 & $\boldsymbol{Y}$ & NA \\
\hline 2550 & 3,4 & CUMULATIVE & $2.5200000 E+\infty 0$ & $9.4000000 \equiv-01$ & $1.1000000=-01$ & $8.1000000 E+\infty 0$ & NONE & WP031904 & $\mathbf{Y}$ & NAA \\
\hline 2550 & 3,4 & CUMULATIVE & $2.5200000 E+\infty 0$ & $9.4000000 E-01$ & $1.1000000 E-01$ & $8.1000000 E+\infty$ & NONE & WP032287 & $Y$ & N/A \\
\hline 2550 & 3,4 & CUMULATINE & $2.5200000 E+\infty 0$ & 9.4000000E-01 & $1.1000000 E-01$ & $8.1000000 E+00$ & NONE & WP035268 & $\mathbf{Y}$ & NA \\
\hline 2550 & 3,4 & CUMULATIVE & $2.5200000 E+00$ & 9.4000000E-01 & $1.1000000 E-01$ & $8.1000000 E+\infty 0$ & NONE & WP035597 & $\mathbf{Y}$ & N/A \\
\hline 2550 & 3,4 & CUMULATIVE & $2.52000000+00$ & 9.4000000E-01 & $1.1000000=-01$ & $8.1000000 E+\infty$ & NONE & WP039058 & $Y$ & NA \\
\hline 2550 & 3,4 & CUMULATIVE & $2.5200000 E+C 0$ & $9.4000000 E-01$ & $1.1000000 E-01$ & $8.1000000 E+\infty 0$ & NONE & WPOS1558 & $Y$ & NA \\
\hline 2550 & 3,4 & CUMULATIVE & $2.5200000 E+\infty$ & $9.4000000 E-01$ & $1.1000000 E-01$ & $8.1000000 E+\infty 0$ & NONE & WPO42752 & $\mathbf{Y}$ & NA \\
\hline 2551 & $1,3,4$ & CONSTANT & $5.0000000 E-02$ & $5.0000000 E-02$ & $5.0000000 E-02$ & $5.0000000 E-02$ & $m^{\wedge} 3 / m^{\wedge} 3$ & WP030640 & $\mathbf{Y}$ & N/A \\
\hline 2551 & $1,3,4$ & CONSTANT & $5.0000000 E-02$ & $5.0000000 E-02$ & $5.0000000 E-02$ & $5.0000000 E-02$ & $m^{\wedge} 3 / m^{\wedge} 3$ & WP030994 & $\mathbf{Y}$ & N/A \\
\hline 2551 & $1,3,4$ & CONSTANT & $5.0000000 E-02$ & $5.00000000-02$ & $5.0000000 E-02$ & $5.0000000 E-02$ & $m^{\wedge} 3 / m^{\wedge} 3$ & WP035597 & $\mathbf{Y}$ & NAA \\
\hline 2552 & 1,3 & CONSTANT & $1.0132500 E+05$ & $1.0132500 E+05$ & $1.0132500 E+05$ & $1.0132500 \mathrm{E}+05$ & $\mathbf{P a}$ & WPO35597 & $\mathbf{Y}$ & N/A \\
\hline 2552 & 1,3 & CONSTANT & $1.0132500 E+05$ & $1.0132500 E+05$ & $1.0132500 E+05$ & $1.0132500 E+05$ & P\& & UP036051 & $Y$ & NA \\
\hline
\end{tabular}


CCA Parameter-Listing

\begin{tabular}{|c|c|c|c|c|c|c|c|c|c|c|}
\hline $\begin{array}{c}\text { Parmeter in } \\
\text { Detwoee? } \\
\text { (NN) }\end{array}$ & 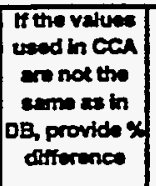 & ID & Metortalo & Matard Name & $\begin{array}{c}\text { Permetic } \\
\text { io }\end{array}$ & Peremone Natro & $\begin{array}{l}\text { PRP to } \\
\text { mpon }\end{array}$ & $\begin{array}{c}\text { Deta Entry } \\
\text { Dete }\end{array}$ & 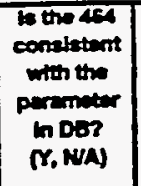 & $\begin{array}{l}\text { Parnetir } \\
\text { Cetegory } \\
\text { (footnote 1) }\end{array}$ \\
\hline $\mathbf{Y}$ & & 2552 & SALT_T3 & $\begin{array}{l}\text { Shath salt column } \\
\text { compectedtime } 25 \text { to } \\
50 \text { years }\end{array}$ & PO_MIN & $\begin{array}{l}\text { Minmum bone preseure for } \\
\text { capillery model KPC=3 }\end{array}$ & 33419 & $21+f a b-8 s$ & $\mathbf{Y}$ & 48 \\
\hline $\mathbf{Y}$ & & 2560 & SALT_T3 & $\begin{array}{l}\text { Snatt salt column } \\
\text { compactedtime } 25 \text { to } \\
50 \text { yoars }\end{array}$ & RELP_MOD & $\begin{array}{l}\text { Hodel number, relative } \\
\text { pormerbility model }\end{array}$ & 334364 & $27-0 \mathrm{ct}-95$ & $\mathbf{Y}$ & 48 \\
\hline $\mathbf{Y}$ & & 2560 & SALT_T3 & $\begin{array}{l}\text { Shaft salt colkmn } \\
\text { compactuctime } 25 \text { to } \\
50 \text { years }\end{array}$ & RELP_MOD & $\begin{array}{l}\text { Model number, relative } \\
\text { permosbitity model }\end{array}$ & $33436 \mathrm{~A}$ & 27-Oat-95 & $\mathbf{Y}$ & $4 \mathrm{~B}$ \\
\hline $\mathbf{Y}$ & & 2560 & SALT_T3 & $\begin{array}{l}\text { Shat salt column } \\
\text { compactedtime } 25 \text { to } \\
50 \text { years }\end{array}$ & RELP_MOD & $\begin{array}{l}\text { Model number, retative } \\
\text { pormasbilty model }\end{array}$ & $33436 A$ & 27-Oct-95 & $\mathbf{Y}$ & $4 B$ \\
\hline $\mathbf{Y}$ & & 2560 & SALT_T3 & $\begin{array}{l}\text { Shat sall column } \\
\text { compactodtimo } 25 \text { to } \\
50 \text { years }\end{array}$ & RELP_MOD & $\begin{array}{l}\text { Model number, reletrve } \\
\text { permesbility model }\end{array}$ & 334364 & 27-0et-95 & $\mathbf{Y}$ & 48 \\
\hline $\mathbf{Y}$ & & 2560 & SALT_TS & $\begin{array}{l}\text { Shaft sat column } \\
\text { compactedtine } 25 \text { to } \\
50 \text { years }\end{array}$ & RELP_MOD & $\begin{array}{l}\text { Model number, ratetive } \\
\text { permeability model }\end{array}$ & $33436 \mathrm{~A}$ & 27-0ct-95 & $\mathbf{Y}$ & $A B$ \\
\hline $\mathbf{Y}$ & 100 & 2562 & SALT_T3 & $\begin{array}{l}\text { Shatt salt column } \\
\text { compactedtime } 25 \text { to } \\
50 \text { years } \\
\end{array}$ & SAT_RBRN & Resideal Brino Sturation & 33446 & 14 Fab-96 & $\mathbf{Y}$ & 1 \\
\hline $\mathbf{Y}$ & 100 & 2562 & SALT_T3 & $\begin{array}{l}\text { Shaft salt column } \\
\text { compactedtime } 25 \text { to } \\
50 \text { yaers }\end{array}$ & SAT_RBRN & Residual Brine Seturation & 33446 & 14 fab-s6 & $\mathbf{Y}$ & 1 \\
\hline $\mathbf{Y}$ & 100 & 2562 & SALT_T3 & $\begin{array}{l}\text { Shat sal colimn } \\
\text { compactedtime } 25 \text { to } \\
50 \text { years }\end{array}$ & SAT_RBRN & Resictual Brin Seturation & 33446 & $14 f a b-96$ & $\mathbf{Y}$ & 1 \\
\hline $\mathbf{Y}$ & 100 & 2562 & SALT_T3 & $\begin{array}{l}\text { Shat salt column } \\
\text { compactectime } 25 \text { to } \\
50 \text { yoars }\end{array}$ & SAT_RBRN & Residual Brine Seturation & 33446 & 14Feb-96 & $\mathbf{Y}$ & 1 \\
\hline $\mathbf{Y}$ & 100 & 2562 & SALT_T3 & $\begin{array}{l}\text { Shaft salt cokmn } \\
\text { compactedtine } 25 \text { to } \\
50 \text { years } \\
\end{array}$ & SAT_RBRN & Residual Brine Seturation & 33446 & $14 f \infty b-96$ & $\mathbf{Y}$ & 1 \\
\hline $\mathbf{Y}$ & 100 & 2502 & SALT_T3 & $\begin{array}{l}\text { Shaft salt column } \\
\text { compectedtime } 25 \text { to } \\
50 \text { years }\end{array}$ & SAT_RBRN & Residual Brimo Seturation & 33446 & $14 F a b-96$ & $\mathbf{Y}$ & 1 \\
\hline $\mathbf{Y}$ & 100 & 2563 & SALT_T3 & $\begin{array}{l}\text { Snaft salt colkmn } \\
\text { compactedtime } 25 \text { to } \\
50 \text { years }\end{array}$ & SAT_RGAS & Resicual Gas Suturation & 33447 & 14 Feb-96 & $\mathbf{Y}$ & 1 \\
\hline $\mathbf{Y}$ & 100 & 2563 & SALT_T3 & $\begin{array}{l}\text { Shat salt column } \\
\text { compectedtime } 25 \text { to } \\
50 \text { years }\end{array}$ & SAT_RGAS & Residual Gas Seturation & 33447 & 14Feb-96 & $\mathbf{Y}$ & 1 \\
\hline $\mathbf{Y}$ & 100 & 2563 & SALT_T3 & $\begin{array}{l}\text { Shaft sell column } \\
\text { compactect tine } 25 \text { to } \\
50 \text { years }\end{array}$ & SAT_RGAS & Residual Ges Seturation & 33447 & 14 fab-96 & $\mathbf{Y}$ & 1 \\
\hline $\mathbf{Y}$ & 100 & 2563 & SALT_T3 & $\begin{array}{l}\text { Shaft salt column } \\
\text { compactectime } 25 \text { to } \\
50 \text { years }\end{array}$ & SAT_RGAS & Resicusal Ges Seturation & 33447 & $14-F \cdot b-96$ & $\mathbf{Y}$ & 1 \\
\hline $\mathbf{Y}$ & 100 & 2563 & SALT_T3 & $\begin{array}{l}\text { Shaft salt column } \\
\text { compactedtime } 25 \text { to } \\
50 \text { years }\end{array}$ & SAT_RGAS & Residual Ges Saturation & 33447 & $14-F e b-96$ & $\gamma$ & 1 \\
\hline $\mathbf{Y}$ & so & 2564 & SALT_T4 & $\begin{array}{l}\text { Shaft selt column } \\
\text { compectedtime } 50 \text { to } \\
100 \text { yours }\end{array}$ & CAP_HOD & $\begin{array}{l}\text { Model number, capillary pressure } \\
\text { model }\end{array}$ & $33449 A$ & $27-004-95$ & $\mathbf{Y}$ & $4 \mathrm{~B}$ \\
\hline $\mathbf{Y}$ & 50 & 2564 & SALT_T4 & $\begin{array}{l}\text { Shat sat column } \\
\text { compectectine } 50 \text { to } \\
100 \text { years }\end{array}$ & CAP_MOD & $\begin{array}{l}\text { Model number, capillary pressure } \\
\text { model }\end{array}$ & $33449 A$ & $27-0 c t-95$ & $\mathbf{Y}$ & $4 \mathrm{~B}$ \\
\hline $\mathbf{Y}$ & 50 & 2554 & SALT_TA & $\begin{array}{l}\text { Shaft salt column } \\
\text { compectedtime } 50 \text { to } \\
100 \text { years }\end{array}$ & CAP_MOD & $\begin{array}{l}\text { Modal number, copillery preasure } \\
\text { model }\end{array}$ & $33449 A$ & $27-0 c t-95$ & $\mathbf{Y}$ & 48 \\
\hline $\mathbf{Y}$ & 50 & 2554 & SALT_TA & $\begin{array}{l}\text { Shent sall colimn } \\
\text { compactedtime } 50 \text { to } \\
100 \text { years }\end{array}$ & CAP_MOD & $\begin{array}{l}\text { Model mumber, capiliery pressure } \\
\text { modil }\end{array}$ & 334494 & $27-0 c t-95$ & $\mathbf{Y}$ & 48 \\
\hline $\mathbf{Y}$ & 50 & 2564 & SALT_T4 & $\begin{array}{l}\text { Shat sell colimn } \\
\text { compectedtine } 50 \text { to } \\
100 \text { yeers }\end{array}$ & CAP_MOD & $\begin{array}{l}\text { Model mumber, cepillery preseure } \\
\text { model }\end{array}$ & 334494 & 27-0et-95 & $\mathbf{Y}$ & $A B$ \\
\hline $\mathbf{Y}$ & & 2565 & SALT_T4 & $\begin{array}{l}\text { Shar sen column } \\
\text { compectedtime } 50 \text { to } \\
100 \text { years }\end{array}$ & COMP_RCK & Buk Compressibilty & 33453 & $14 f a b-96$ & $\mathbf{Y}$ & 1 \\
\hline $\mathbf{Y}$ & & 2565 & SALT_T4 & $\begin{array}{l}\text { Shet set colimn } \\
\text { compectedtime } 50 \text { to } \\
100 \text { yeers }\end{array}$ & COMP_RCK & Bulk Compressibility & 33453 & $14 f \times b-96$ & $\mathbf{Y}$ & 1 \\
\hline
\end{tabular}


CCA Parameter-Listing

\begin{tabular}{|c|c|c|c|c|c|c|c|c|c|c|}
\hline 10 & $\begin{array}{l}\text { Ciesth- } \\
\text { Cation of } \\
\text { Date } \\
\text { Cetegory } \\
\text { (footnote 2) }\end{array}$ & $\begin{array}{c}\text { Didribution } \\
\text { Type }\end{array}$ & Men & Medien & Minimum & Modmens & Units & Reterince D & $\begin{array}{l}\text { Wes the deta } \\
\text { doveloped } \\
\text { undier an } \\
\text { NoA-1 } \\
\text { Program? }\end{array}$ & $\begin{array}{c}\text { minkh } \\
\text { mothode } \\
\text { more used to } \\
\text { quilly } \\
\text { codeting } \\
\text { deta? } \\
\text { (footnote 3) }\end{array}$ \\
\hline 2552 & 1,3 & CONSTANT & $1.0132500 E+05$ & $1.0132500 E+05$ & $1.0132500 E+05$ & $1.0132500 E+05$ & Pa & MPO40514 & $Y$ & NA \\
\hline 2560 & 1,3 & CONSTANT & $4.0000000 E+\infty 0$ & $4.0000000 E+\infty$ & $4.0000000 E+00$ & $1.0000000 E+\infty$ & NONE & ИPO30540 & $\mathbf{Y}$ & NA \\
\hline 2560 & 1,3 & CONSTANT & $4.0000000 E+\infty 0$ & $4.00000000 E+\infty$ & $1.0000000 E+\infty 00$ & $4.00000000+00$ & NONE & WP030994 & $\mathbf{Y}$ & NA \\
\hline 2560 & 1,3 & CONSTANT & $4.0000000 E+\infty 00$ & $4.0000000 E+\infty 0$ & $4.0000000 E+\infty 0$ & $1,0000000 E+\infty 00$ & NONE & $\begin{array}{r}\text { WPOS5597 } \\
1\end{array}$ & $\mathbf{Y}$ & WA \\
\hline 2560 & 1,3 & CONSTANT & $4.0000000 E+00$ & $1.0000000 \mathrm{E}+\infty$ & $4.00000000+00$ & $4.0000000 E+\infty$ & NONE & WPO40406 & $\mathbf{Y}$ & NA \\
\hline 2560 & 1,3 & CONSTANT & $4.0000000 \mathrm{E}+\infty 0$ & $4.0000000 E+\infty 0$ & $4.0000000 E+\infty 0$ & $4.00000000=+\infty$ & NONE & UPO40514 & $\mathbf{Y}$ & N/A \\
\hline 2562 & 3,4 & CUMULATIVE & $2.5000000 \mathrm{E}-01$ & $20000000 E-01$ & $0.0000000 E+00$ & $6.0000000 E-01$ & NONE & WP030640 & $\mathbf{Y}$ & NA \\
\hline 2562 & 3,4 & CUMULATIVE & $2.5000000 \mathrm{E}-01$ & 2,0000000E-01 & $0.0000000 \mathrm{E}+\infty$ & $6.0000000 \mathrm{E}-01$ & NONE & Wr030994 & $Y$ & $N A$ \\
\hline 2562 & 3,4 & CUMULATIVE & $2.5000000 E-01$ & $2.0000000 E-01$ & $0.0000000 E+00$ & $6.0000000 \leq-01$ & NONE & WPO35258 & $\mathbf{Y}$ & $N A$ \\
\hline 2562 & 3,4 & CUMULATIVE & $2.5000000 E-01$ & $2,0000000 E-01$ & $0.0000000 E+\infty 0$ & $6.0000000 E-01$ & NONE & WP035597 & $\mathbf{Y}$ & NA \\
\hline 2562 & 3,4 & CUMULATME & $25000000 E-01$ & $2,0000000 E-01$ & $0.00000000+\infty 0$ & $6.0000000 E-01$ & NONE & WP041558 & $\mathbf{Y}$ & NA \\
\hline 2562 & 3,4 & CUMULATIVE & $2.5000000 E-01$ & $2.0000000 E-01$ & $0.0000000 E+\infty$ & $6.0000000 E-01$ & NONE & WPO42751 & $\mathbf{Y}$ & NA \\
\hline 2563 & 3,4 & UNIFORM & $20000000 E-01$ & $2.0000000 E-01$ & $0.0000000 E+\infty$ & $4.0000000 E-01$ & NONE & WP030640 & $\mathbf{Y}$ & NA \\
\hline 2563 & 3,4 & UNIFORM & $2.0000000 E-01$ & $2.0000000 E-01$ & $0.0000000 E+\infty 0$ & $4.0000000 E-01$ & NONE & WPO30994 & $\mathbf{Y}$ & NA \\
\hline 2563 & 3,4 & UNIFORM & 2,0000000E-01 & $2,0000000 E-01$ & $0.00000000 E+\infty$ & $4.00000005-01$ & NONE & WP035268 & $\mathbf{Y}$ & NAA \\
\hline 2563 & 3,4 & UNIFORM & 2.0000000E-01 & $2.0000000 E-01$ & $0.0000000 E+\infty 0$ & $4.0000000=-01$ & NONE & WP035597 & $\mathbf{Y}$ & NA \\
\hline 2563 & 3,4 & UNIFORM & $20000000 E-01$ & $2.0000000 E-01$ & $0.0000000 E+\infty$ & $4,0000000 E-01$ & NONE & UPOAs5s8 & $-y$ & N/A \\
\hline 2564 & $1,3,4$ & CONSTANT & $2.0000000 E+\infty$ & $2.0000000 \mathrm{E}+\infty 0$ & $2.0000000 E+\infty 0$ & $2.0000000 E+00$ & NONE & WPO30640 & $\mathbf{Y}$ & NAA \\
\hline 2564 & $1,3,4$ & CONSTANT & $2.0000000 E+\infty 0$ & $2.0000000 E+\infty$ & $2.0000000 E+\infty$ & $2.0000000 E+\infty$ & NONE & WP030994 & $\mathbf{Y}$ & NA \\
\hline 2564 & $1,3,4$ & CONSTANT & $2.0000000 E+\infty 0$ & $2.0000000 E+\infty$ & $2.0000000 E+\infty$ & $2.0000000 \mathrm{E}+\infty$ & NONE & WP035597 & $\mathbf{Y}$ & N/A \\
\hline 2564 & $1,3,4$ & CONSTANT & $2.00000000 E+\infty 0$ & $2,0000000 E+\infty$ & $2.00000000+\infty 0$ & $2.0000000 E+\infty 0$ & NONE & WPO40485 & $\mathbf{Y}$ & NA \\
\hline 2564 & $1,3,4$ & CONSTANT & $2.0000000 E+\infty 0$ & $2.0000000 E+\infty$ & $2.0000000 E+\infty$ & $2.0000000 E+\infty$ & NONE & WPO40514 & $\mathbf{Y}$ & NA \\
\hline 2565 & $1,3,4$ & CONSTANT & $1.6000000 E-09$ & $1.6000000 E-09$ & $1.6000000 E-09$ & $1.60000000-09$ & $P e^{n}-1$ & WPO30640 & Y8N & 1,4 \\
\hline 2565 & $1,3,4$ & CONSTANT & $1.6000000=-09$ & $1.6000000 E-09$ & $1.6000000 E-09$ & $1.6000000 E-09$ & $P e^{n}-1$ & WP030994 & Y\&N & 1,4 \\
\hline
\end{tabular}


CCA Parameter tisting

\begin{tabular}{|c|c|c|c|c|c|c|c|c|c|c|}
\hline $\begin{array}{c}\text { Parmintar in } \\
\text { Detabea? } \\
\text { (YN) }\end{array}$ & 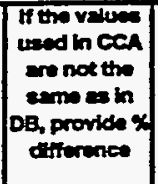 & D & Matariat Lo & Matarta kam & Permater & Parminater Nem & 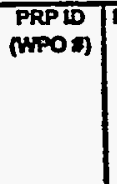 & $\begin{array}{c}\text { Deth Entry } \\
\text { Dex }\end{array}$ & 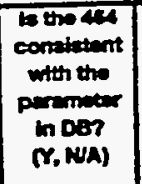 & 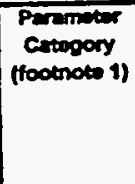 \\
\hline $\mathbf{Y}$ & & 2565 & SALT_T4 & $\begin{array}{l}\text { Shaft salt column } \\
\text { compactedtume } 50 \text { to } \\
100 \text { years }\end{array}$ & COMP_RCK & Bulk Compressibitity & 33453 & $14-F a b-96$ & $\mathbf{Y}$ & 1 \\
\hline $\mathbf{Y}$ & & 2566 & SALT_T4 & $\begin{array}{l}\text { Shat sall column } \\
\text { compectoctime } 50 \text { to } \\
100 \text { yeses }\end{array}$ & PC_MAX & $\begin{array}{l}\text { Maximum allowable copillery } \\
\text { pressure }\end{array}$ & $33459 A$ & $27-0 c t-05$ & $\mathbf{Y}$ & 48 \\
\hline $\mathbf{Y}$ & & 2566 & SALT_T4 & $\begin{array}{l}\text { Shaft salt column } \\
\text { compactectume } 50 \text { to } \\
100 \text { years }\end{array}$ & PC_MAX & $\begin{array}{l}\text { Mexinum allownble copillery } \\
\text { prevenure }\end{array}$ & $33459 A$ & 27-0ct-95 & $\mathbf{Y}$ & $A B$ \\
\hline $\mathbf{Y}$ & & 2565 & SALT_T4 & $\begin{array}{l}\text { Shaft salt cokmn } \\
\text { compactectime } 50 \text { to } \\
100 \text { years }\end{array}$ & PC_mAX & $\begin{array}{l}\text { Mexinum allowblo cepilary } \\
\text { presere }\end{array}$ & $33459 A$ & $27-0$ d- 95 & $\mathbf{Y}$ & 48 \\
\hline $\mathbf{Y}$ & & 2566 & SALT_T4 & $\begin{array}{l}\text { Shaft ralt column } \\
\text { compactedtime } 50 \text { to } \\
100 \text { years }\end{array}$ & PC_MAX & $\begin{array}{l}\text { Maximum allowable capillery } \\
\text { pressure }\end{array}$ & $33459 A$ & $27-0 c t-95$ & $\mathbf{Y}$ & 48 \\
\hline $\mathbf{Y}$ & & 2566 & SALT_T4 & $\begin{array}{l}\text { Shan salt column } \\
\text { compactedtime } 50 \text { to } \\
100 \text { yoars } \\
\end{array}$ & PC_MAX & $\begin{array}{l}\text { Maximum allownble capiliary } \\
\text { proseure }\end{array}$ & 334594 & $27-0$ ct.95 & $\mathbf{Y}$ & 48 \\
\hline $\mathbf{Y}$ & varies & 2567 & SALT_T4 & $\begin{array}{l}\text { Shaft salt cotumn } \\
\text { compacted:time } 50 \text { to } \\
100 \text { years } \\
\end{array}$ & PORE_DIS & $\begin{array}{l}\text { Brooks-Corey pore distribution } \\
\text { paraneter }\end{array}$ & 33465 & 22fab-s6 & $\mathbf{Y}$ & 1 \\
\hline $\mathbf{Y}$ & varies & 2567 & SALT_T4 & $\begin{array}{l}\text { Shaft sal column } \\
\text { compactoctine } 50 \text { to } \\
100 \text { years }\end{array}$ & PORE_DIS & $\begin{array}{l}\text { Brocka-Corey pore distribution } \\
\text { paremeter }\end{array}$ & 33465 & $22+F e b-96$ & $\mathbf{Y}$ & 1 \\
\hline $\mathbf{Y}$ & varies & 2567 & SALT_T4 & $\begin{array}{l}\text { Shat salt column } \\
\text { compactedtime } 50 \text { to } \\
100 \text { years }\end{array}$ & PORE_DIS & $\begin{array}{l}\text { Brooks-Corey pore distritution } \\
\text { parameter }\end{array}$ & 33465 & $22+F a b-96$ & $\mathbf{Y}$ & 1 \\
\hline $\mathbf{Y}$ & varies & 2567 & SALT_T4 & $\begin{array}{l}\text { Shaft salt column } \\
\text { compacted:time } 50 \text { to } \\
100 \text { years }\end{array}$ & PORE_DIS & $\begin{array}{l}\text { Brooks-Corey pore distribution } \\
\text { parameter }\end{array}$ & 33465 & 22+Fab-96 & $\mathbf{Y}$ & 1 \\
\hline $\mathbf{Y}$ & varies & 2567 & SALT_T4 & $\begin{array}{l}\text { Shaf salt column } \\
\text { compactecttme } 50 \text { to } \\
100 \text { years }\end{array}$ & PORE_DIS & $\begin{array}{l}\text { Brooks-Corey pore distribution } \\
\text { paraneter }\end{array}$ & 33465 & 22Feb-96 & $\mathbf{Y}$ & 1 \\
\hline $\mathbf{Y}$ & varies & 2567 & SALT_T4 & $\begin{array}{l}\text { Shaft salt column } \\
\text { compacted:time } 50 \text { to } \\
100 \text { years }\end{array}$ & PORE_DIS & $\begin{array}{l}\text { Brooks-Corey pore distribution } \\
\text { perameter }\end{array}$ & 33465 & $22 F c b-96$ & $\mathbf{Y}$ & 1 \\
\hline $\mathbf{Y}$ & varies & 2567 & SALT_T4 & $\begin{array}{l}\text { Shath sat column } \\
\text { compactedtime } 50 \text { to } \\
100 \text { years }\end{array}$ & PORE_DIS & $\begin{array}{l}\text { Brooks-Corey pore distribution } \\
\text { parameter }\end{array}$ & 33465 & 22Fab-96 & $\mathbf{Y}$ & 1 \\
\hline $\mathbf{Y}$ & varies & 2567 & SALT_T4 & $\begin{array}{l}\text { Shaft salt column } \\
\text { compactedtime } 50 \text { to } \\
100 \text { yoars }\end{array}$ & PORE_DIS & $\begin{array}{l}\text { Brocks-Corey pore distribution } \\
\text { parameter }\end{array}$ & 33465 & $22+50 b-96$ & $\mathbf{Y}$ & 1 \\
\hline $\mathbf{Y}$ & varies & 2567 & SALT_T4 & $\begin{array}{l}\text { Shaft salt cokmn } \\
\text { compactedtime } 50 \text { to } \\
100 \text { years }\end{array}$ & PORE_DIS & $\begin{array}{l}\text { Brooks-Corey pore distribution } \\
\text { perameter }\end{array}$ & 33465 & 22-Feb-96 & $\mathbf{Y}$ & 1 \\
\hline $\mathbf{Y}$ & & 2568 & SALT_T4 & $\begin{array}{l}\text { Shaf salh column } \\
\text { compactedtime } 50 \text { to } \\
100 \text { yoars }\end{array}$ & POROSTYY & Effective porosity & 33467 & $14 F a b-96$ & $\mathbf{Y}$ & 1 \\
\hline $\mathbf{Y}$ & & 2568 & SALT_T4 & $\begin{array}{l}\text { Shaft salt column } \\
\text { compectedtime } 50 \text { to } \\
100 \text { years }\end{array}$ & POROSTIY & Effective porosity & 33467 & 14 Feb-96 & ${ }^{-} Y$ & 1 \\
\hline $\mathbf{Y}$ & & 2568 & SALT_TA & $\begin{array}{l}\text { Shaf sath column } \\
\text { compectectime } 50 \text { to } \\
100 \text { years }\end{array}$ & POROSTY & Effectre porosity & 33467 & 14Feb-96 & $\mathbf{Y}$ & 1 \\
\hline $\mathbf{Y}$ & & 2569 & SALT_T4 & $\begin{array}{l}\text { Shan selt column } \\
\text { compectedtime } 50 \text { to } \\
100 \text { years }\end{array}$ & PO_MIN & $\begin{array}{l}\text { Mnamum brine pressure for } \\
\text { capeltery model KPC=3 }\end{array}$ & 33469 & 21-Feb-96 & $\mathbf{Y}$ & $4 B$ \\
\hline $\mathbf{Y}$ & & 2559 & SALT_TA & $\begin{array}{l}\text { Shat salk column } \\
\text { compactedtime } 50 \text { to } \\
100 \text { years }\end{array}$ & PO_MIN & $\begin{array}{l}\text { Mrimum brine pressure for } \\
\text { capillery model KPC=3 }\end{array}$ & 33469 & 21+fob-96 & $\mathbf{Y}$ & 48 \\
\hline $\mathbf{Y}$ & & 2569 & SALT_T4 & $\begin{array}{l}\text { Shant salt column } \\
\text { compectedtime } 50 \text { to } \\
100 \text { years }\end{array}$ & PO_MIN & $\begin{array}{l}\text { Minimum bono preseure for } \\
\text { capillery model } K P C=3\end{array}$ & 33469 & $21+F e b-96$ & $\mathbf{Y}$ & 48 \\
\hline $\mathbf{Y}$ & & 2577 & SALT_T4 & $\begin{array}{l}\text { Shat eal column } \\
\text { compactedtime } 50 \text { to } \\
100 \text { years }\end{array}$ & RELP_MOD & $\begin{array}{l}\text { Model number, reletive } \\
\text { permesbility model }\end{array}$ & $33553 A$ & $27-0 \mathrm{ct}-95$ & $\mathbf{Y}$ & $A B$ \\
\hline $\mathbf{Y}$ & & $25 \pi$ & SALT_T4 & $\begin{array}{l}\text { Shan salt column } \\
\text { compactectime } 50 \text { to } \\
100 \text { years }\end{array}$ & RELP_MOD & $\begin{array}{l}\text { Modal number, rolative } \\
\text { permesbilty model }\end{array}$ & $33553 \mathrm{~A}$ & 27-0ct-95 & $\mathbf{Y}$ & 48 \\
\hline $\mathbf{Y}$ & & 2577 & SALT_T4 & $\begin{array}{l}\text { Shein selt column } \\
\text { compectedtime } 50 \text { to } \\
100 \text { years }\end{array}$ & RELP_MOD & $\begin{array}{l}\text { Modili mumber, reiatrue } \\
\text { permeabitity model }\end{array}$ & $33553 \mathrm{~A}$ & $27-0 c t-95$ & $\mathbf{Y}$ & 48 \\
\hline
\end{tabular}


CCA Parameter Listing

\begin{tabular}{|c|c|c|c|c|c|c|c|c|c|c|}
\hline D & $\begin{array}{c}\text { Clesentit } \\
\text { cation of } \\
\text { Deth } \\
\text { Centegory } \\
\text { (footrot- 2) }\end{array}$ & $\begin{array}{c}\text { Distribution } \\
\text { Type }\end{array}$ & matn & madian & Alintromem & Mexinem & Untes & Reterence to & $\begin{array}{l}\text { Wes the deth } \\
\text { demoloped } \\
\text { underen } \\
\text { NOA-I } \\
\text { Progrem? }\end{array}$ & 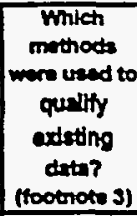 \\
\hline 2565 & $1,3,4$ & CONSTANT & $1.6000000 E-09$ & $1.6000000 E-09$ & $1.6000000 E-09$ & $1.6000000 E-09$ & Pan-1 & WP035597 & YON & 1,4 \\
\hline 2566 & 1,3 & CONSTANT & $1.0000000 \mathrm{E}+08$ & $1.0000000 E+08$ & $1.0000000 E+08$ & $1.0000000 E+08$ & $\mathbf{P a}$ & WP030640 & $\mathbf{Y}$ & NA \\
\hline 2566 & 1.3 & CONSTANT & $1.0000000 \mathrm{E}+08$ & $1.0000000 \mathrm{E}+\infty 0$ & $1.0000000 \mathrm{E}+\infty 8$ & $1.0000000 E+08$ & $\mathbf{P a}$ & WP030994 & $\mathbf{Y}$ & NA \\
\hline 2566 & 1,3 & CONSTANT & $1.0000000 E+08$ & $1.0000000 E+08$ & $1.0000000 E+\infty 0$ & $1.0000000 E+00$ & Pa & WP035597 & $Y$ & N/A \\
\hline 2566 & 1,3 & CONSTANT & $1.0000000 \mathrm{E}+08$ & $1.0000000 E+08$ & $1.0000000 E+08$ & $1.0000000 E+08$ & Pa & WPO40486 & $Y$ & NA \\
\hline 2566 & 1,3 & CONSTANT & $1.0000000 E+08$ & $1.0000000 E+08$ & $1.0000000 E+08$ & $1.0000000 E+\infty 8$ & Pa & WPO40514 & $\mathbf{Y}$ & N/A \\
\hline 2567 & 3,4 & CUMULATIVE & $2.5200000 E+\infty 0$ & $9.4000000 E-01$ & $1.1000000 E-01$ & $8.1000000 E+\infty 0$ & NONE & WP030640 & $Y$ & NA \\
\hline 2567 & 3,4 & CUMULATIVE & $2.5200000 E+\infty 0$ & $9.4000000 E-01$ & 1.1000000E-01 & $8.1000000 E+\infty$ & NONE & WP030994 & $\mathbf{Y}$ & NA \\
\hline 2567 & 3,4 & CUMULATIVE & $25200000 E+\infty 0$ & $9.4000000 E-01$ & $1.1000000=-01$ & $8.1000000 E+\infty$ & NONE & WP031904 & $\gamma$ & NA \\
\hline 2567 & 3,4 & CUMULATINE & $2.5200000 E+\infty 0$ & $9.4000000 E-01$ & $1.1000000 E-01$ & $8.1000000 \mathrm{E}+\infty$ & NONE & WP032287 & $Y$ & N/A \\
\hline 2567 & 3,4 & CUMULATIVE & $2.5200000 E+\infty 0$ & $9.4000000 E-01$ & $1.1000000 E-01$ & $8.1000000 E+\infty$ & NONE & WP035269 & $\mathbf{Y}$ & NA \\
\hline 2567 & 3,4 & CUMULATNE & $2.5200000 E+\infty$ & $9.4000000 E-01$ & $1.1000000 E-01$ & $8.10000000 \mathrm{E}+\infty$ & NONE & WP035597 & $\mathbf{Y}$ & NA \\
\hline 2567 & 3,4 & CUMULATNE & $2.5200000 E+00$ & $9.4000000 E-01$ & $1.1000000 E-01$ & $8.1000000 E+\infty$ & NONE & WPO39058 & $Y$ & NA \\
\hline 2567 & 3,4 & CUMULATNE & $2.5200000 E+\infty$ & $9.4000000 E-01$ & $1.1000000 E-01$ & $8.1000000 E+\infty 0$ & NONE & WPO41558 & $Y$ & NA \\
\hline 2567 & 3.4 & CUMULATINE & $2.5200000 E+\infty 0$ & 9.4000000E-01 & $1.1000000 E-01$ & $8.1000000 \mathrm{E}+00$ & NONE & WPO42752 & $Y$ & NA \\
\hline 2568 & $1,3,4$ & CONSTANT & $5.0000000 E-02$ & $5.0000000 E-02$ & $5.0000000 E-02$ & $5.0000000 E-02$ & $m^{\wedge} 3 m^{\wedge} 3$ & WPO30640 & $\mathbf{Y}$ & N/A \\
\hline 2568 & $1,3,4$ & CONSTANT & $5.0000000 E-02$ & $5.0000000 E-02$ & $5.0000000 E-02$ & $5.0000000 E-02$ & $m^{\wedge} 3 m^{\wedge} 3$ & WP030994 & $\gamma$ & NA \\
\hline 2568 & $1,3,4$ & CONSTANT & $5.0000000 E-02$ & $5.0000000 E-02$ & $5.0000000 E-02$ & $5.0000000 E-02$ & $m^{\wedge} 3 / m^{\wedge} 3$ & WP035597 & $Y$ & NA \\
\hline 2569 & 1,3 & CONSTANT & $1.0132500 E+05$ & $1.0132500 E+05$ & $1.0132500 E+05$ & $1.0132500 E+05$ & Pa & WP035597 & $Y$ & NA \\
\hline 2569 & 1,3 & CONSTANT & $1.0132500 E+05$ & $1.0132500 E+05$ & $1.0132500 E+05$ & $1.0132500 E+05$ & Pa & WP035051 & $Y$ & NA \\
\hline 2569 & 1,3 & CONSTANT & $1.0132500 E+05$ & $1.0132500 E+05$ & $1.0132500 E+05$ & $1.0132500 E+05$ & $P=$ & WPO40514 & $\mathbf{Y}$ & NA \\
\hline 2577 & 1,3 & CONSTANT & $4.0000000 E+00$ & $1.0000000 E+00$ & $4.0000000 E+\infty 0$ & $4.00000000+\infty$ & NONE & WPO30640 & $Y$ & NA \\
\hline 2577 & 1,3 & CONSTANT & $4.0000000 E+00$ & $4.0000000 E+00$ & $4.0000000 E+00$ & $4.0000000 E+\infty 0$ & NONE & WP030994 & $\mathbf{Y}$ & NA \\
\hline 2577 & 1,3 & CONSTANT & $4.0000000 E+00$ & $4.0000000 E+\infty$ & $4.0000000 E+\infty 0$ & $4.0000000 \mathrm{E}+00$ & NONE & WP035597 & $\mathbf{Y}$ & NA \\
\hline
\end{tabular}




\begin{tabular}{|c|c|c|c|c|c|c|c|c|c|c|}
\hline 8 & $\Lambda$ & $56-\infty 00-12$ & VELSEE & 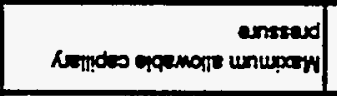 & $x+109$ & 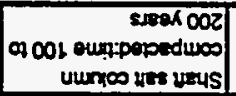 & SILITS & $\cos 2$ & & ᄉ \\
\hline 8 & $\boldsymbol{\Lambda}$ & S5:-200-L2 & VELSEE & 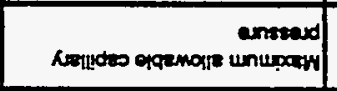 & $x+x^{-} 0 \mathrm{~d}$ & 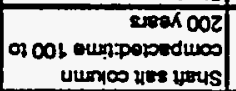 & SIITUS & cosz & & $\alpha$ \\
\hline 8 & $\lambda$ & S6-DO-L2 & V6LSEE & 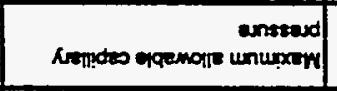 & xowiod & 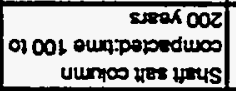 & S1-1TVS & $\cos 2$ & & 1 \\
\hline$b$ & $\lambda$ & $96-90 \pm t b$ & ZLSEE & 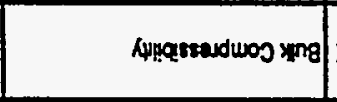 & ХУУ- dพOد & 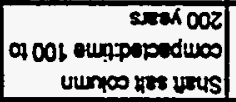 & S1'ITS & $\cos 2$ & & $\lambda$ \\
\hline 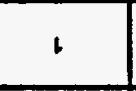 & 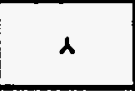 & $96-90 \pm+1$ & ZLSEE & Antqusendwor xng & X्रכУ- dพ०0 & 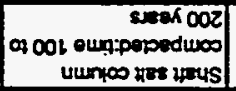 & S1-1TVS & $\cos 2$ & & $\lambda$ \\
\hline 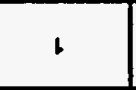 & $\Lambda$ & $96-90+t$ & ZLSEE & Anquseudwog $\times$ ma & xכy- dwo0 & 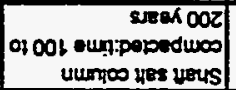 & SIITHS & $\cos 2$ & & $\boldsymbol{\lambda}$ \\
\hline $8 b$ & 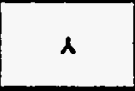 & $56-50-22$ & Vegser & 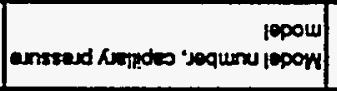 & gon-9R2 & 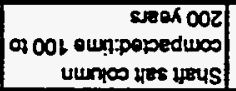 & SIITHS & I8SZ & os & $\lambda$ \\
\hline $8 b$ & $\lambda_{-}$ & S6-pO-LZ & VeOseE & 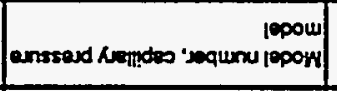 & Qow dY & 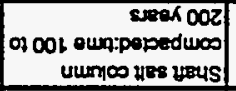 & SLITHS & เ8S2 & os & $\boldsymbol{A}$ \\
\hline ab & $\boldsymbol{1}$ & $56 \cdot p 0-\angle 2$ & Vosses & 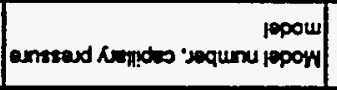 & gow dro & 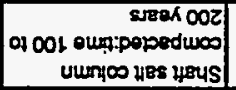 & SIITHS & เ852 & os & 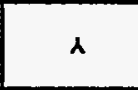 \\
\hline 8r & ᄉ & S6-PO-LZ & VegseE & 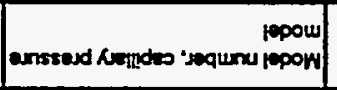 & gow'd dPo & 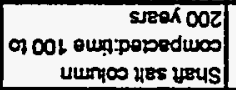 & SLITVS & LESZ & os & 人 \\
\hline Br & ᄉ & $56-100-\angle Z$ & VBSSEE & 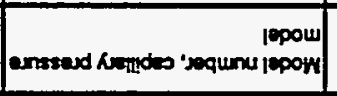 & oOW- aro & 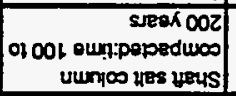 & SI-1THS & เ8S2 & os & 1 \\
\hline$t$ & 1 & $96-905+1$ & $\operatorname{ses} x$ & 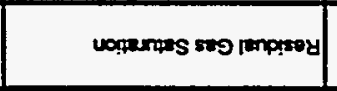 & SYפY-IVS & 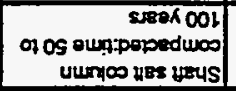 & $\nabla+1$ Trs & $\cos z$ & $\infty$ & 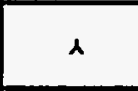 \\
\hline$\iota$ & $\lambda$ & $96-90+b 1$ & S9SEE & 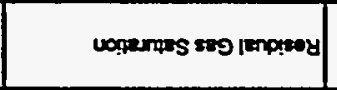 & sพפY'IVS & 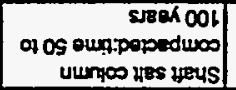 & เI-1TVS & 0892 & $\infty \mathbf{L}$ & ᄉ \\
\hline$\bullet$ & $\boldsymbol{\lambda}$ & $96-90 \pm+1$ & $\operatorname{sgses}$ & 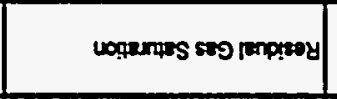 & SvפY'IYS & 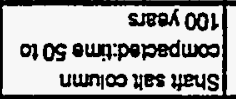 & PILTFS & $\cos 2$ & $\infty$ & 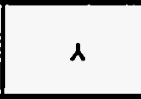 \\
\hline$\iota$ & $\boldsymbol{A}$ & $96-90+5 t$ & 59528 & 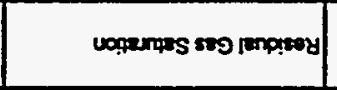 & 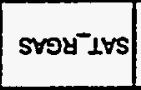 & 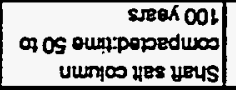 & TILTS & 0892 & $\infty$ & 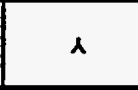 \\
\hline$b$ & $\boldsymbol{l}$ & $96-90 \pm+1$ & $\operatorname{ses} 28$ & 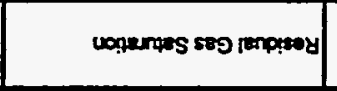 & SพפY-IVS & 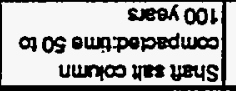 & HIITS & ossz & $\infty$ & $\lambda$ \\
\hline 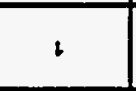 & 1 & $96-90+21$ & toser & 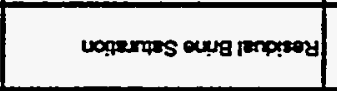 & Nygy $\perp \forall S$ & 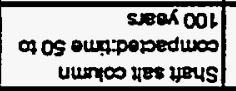 & VIITVS & $6 \angle s 2$ & $\infty$ & $\lambda$ \\
\hline 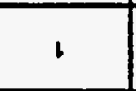 & ᄉ & $96-90+16$ & roser & 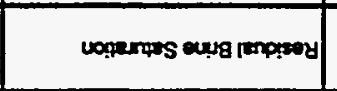 & N\&8Y-IVS & 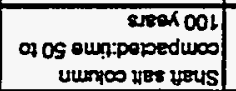 & DL'TWS & $6 \angle S Z$ & $\infty$ & $\lambda$ \\
\hline ! & $\lambda$ & $96-90 \pm+b$ & poses & 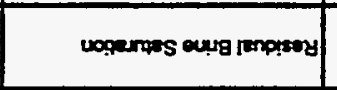 & 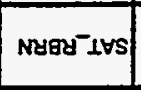 & 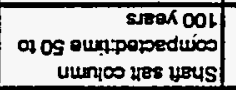 & $\nabla 1$ TTS & $6 \angle 5 Z$ & oor & 1 \\
\hline$b$ & h & $96-90+x \mid$ & POSE: & wopermes ouyg jexpssoy & 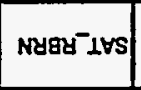 & 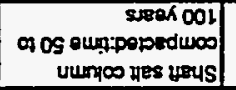 & TIITHS & $6 \angle 52$ & OOL & ᄉ \\
\hline 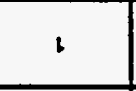 & $\lambda$ & $96-90+41$ & 1958E & 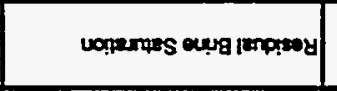 & NyeY IFS & 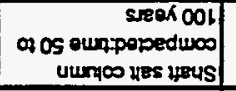 & $\nabla 1-17 \%$ & $6 \angle S Z$ & $\infty$ & $\boldsymbol{\lambda}$ \\
\hline 1 & $\boldsymbol{\Lambda}$ & $96-90 \pm+2$ & roses & 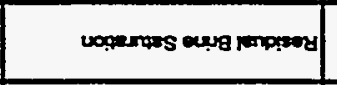 & Nugt $\perp$ VS & 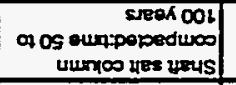 & WITHS & GLSZ & $\infty$ & 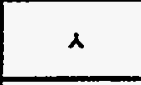 \\
\hline 8b & $\lambda$ & $56+\infty 0-22$ & Vassex & 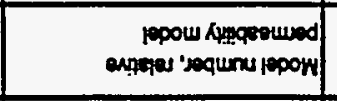 & cow- dTay & 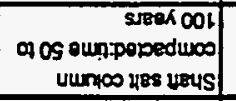 & TITHS & usz & & 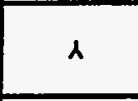 \\
\hline at & 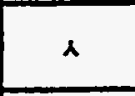 & S6-50-2Z & resses & 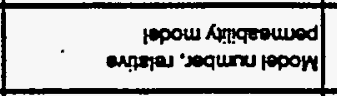 & cow'dtتy & 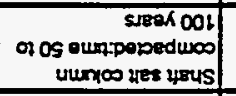 & $\$ 1 \perp T S$ & LSE & & $\lambda$ \\
\hline (6 16000001$)$ & 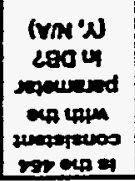 & Anบg & 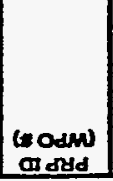 & 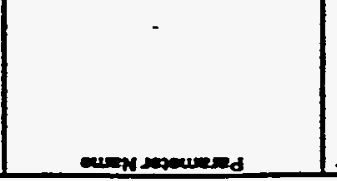 & d & 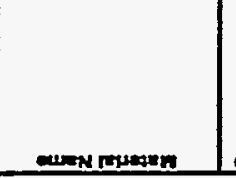 & at moseren & $a$ & 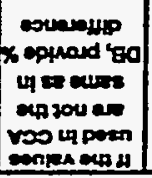 & (wiN) \\
\hline
\end{tabular}


CCA Parameter Listing

\begin{tabular}{|c|c|c|c|c|c|c|c|c|c|c|}
\hline TD & 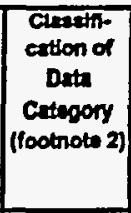 & $\begin{array}{l}\text { Detriburton } \\
\text { Typo }\end{array}$ & $\operatorname{man}$ & medien & Mintmern & Mextrum & Unts & Refermint Do & $\begin{array}{l}\text { Fas ofe dent } \\
\text { devaloped } \\
\text { ender en } \\
\text { Noh-1 } \\
\text { Progrum? }\end{array}$ & 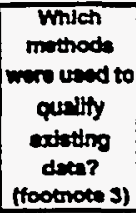 \\
\hline 2577 & 1,3 & CONSTANT & $4.0000000 E+\infty 0$ & $4.0000000 E+\infty 0$ & $4.0000000 E+\infty$ & $1.0000000 E+\infty 0$ & NONE & WPO40485 & $\mathbf{Y}$ & NA \\
\hline 2577 & 1,3 & CONSTANT & $4.0000000 \mathrm{E}+\infty 0$ & $4.0000000 E+\infty 0$ & $4.0000000=+\infty$ & $4.0000000 E+00$ & NONE & WPO40514 & $\mathbf{Y}$ & NA \\
\hline 2579 & 3,4 & CUMULATIVE & $2.5000000 E-01$ & $20000000 E-01$ & $0.0000000 E+\infty$ & $6.0000000 E-01$ & NONE & WP030640 & $\mathbf{r}$ & NA \\
\hline 2578 & 3,4 & CUMULATME & $25000000 E-01$ & $2.0000000 E-01$ & $0.0000000 E+\infty$ & $6.0000000 E-01$ & NONE & WP030s94 & $\mathbf{Y}$ & NA \\
\hline 2579 & 3,4 & CUMULATNE & $2.5000000 E-01$ & $2.0000000 \mathrm{E}-01$ & $0.0000000 E+\infty$ & $6.0000000 E-01$ & NONE & WP035268 & $\mathbf{Y}$ & NA \\
\hline 2579 & 3,4 & CUMULATIVE & $2.5000000 E-01$ & $2.0000000 E-01$ & $0.0000000 E+\infty$ & $6.0000000 E-01$ & NONE & WP035597 & $\mathbf{Y}$ & NA \\
\hline 2579 & 3,4 & CUMULATIVE & $25000000 E-01$ & $20000000 E-01$ & $0.00000000+\infty$ & $6.0000000 E-01$ & NONE & WPO41558 & $\mathbf{Y}$ & NA \\
\hline 2579 & 3,4 & CUMULATIVE & $2.5000000 E-01$ & $2.0000000 \mathrm{E}-01$ & $0.0000000 E+\infty$ & $6.00000000-01$ & NONE & WPOA2751 & $\mathbf{Y}$ & N/A \\
\hline 2580 & 3,4 & URIFORM & $20000000 E-01$ & $2.0000000 E-01$ & $0.0000000 \mathrm{E}+\infty$ & $4.0000000 E-01$ & NONE & WP030640 & $\mathbf{Y}$ & NA \\
\hline 2580 & 3,4 & UNIFORM. & $2.0000000 E-01$ & $2.0000000 E-01$ & $0.00000000 E+00$ & 4.0000000E-01 & NONE & WP030994 & $\mathbf{Y}$ & NA \\
\hline 2580 & 3,4 & UNIFORM & $2.0000000 E-01$ & $2.0000000 E-01$ & $00000000 E+\infty$ & $40000000 E-01$ & NONE & WP035268 & $\mathbf{Y}$ & NA \\
\hline 2580 & 3,4 & UNIFORM & $2.0000000 E-01$ & $2.0000000 \mathrm{E}-01$ & $00000000 E+\infty$ & $40000000 E-01$ & NONE & WP035597 & $\mathbf{Y}$ & NA \\
\hline 2580 & 3,4 & UNIFORM & $2.0000000 E-01$ & $2.0000000 E-01$ & $00000000 E+\infty$ & $40000000 E-01$ & NONE & WPO41558 & $\mathbf{Y}$ & NA \\
\hline 2581 & $1,3,4$ & CONSTANT & $2.0000000 E+\infty 0$ & $20000000 E+00$ & $20000000 E+\infty$ & $20000000 E+\infty 0$ & NONE & UP030640 & $\mathbf{Y}$ & NA \\
\hline 2581 & $1,3,4$ & CONSTANT & $2.0000000 E+\infty 0$ & $200000000+\infty$ & $20000000 \times \infty \infty$ & $20000000 E+\infty$ & NONE & WPO30994 & $\mathbf{\gamma}$ & NA \\
\hline 2581 & $1,3,4$ & CONSTANT & $2.0000000 E+\infty 0$ & $20000000 E+\infty$ & $20000000 \times+\infty$ & $200000006+\infty$ & NONE & UP035597 & $\mathbf{Y}$ & $N / A$ \\
\hline 2581 & $1,3,4$ & CONSTANT & $2.0000000 E+\infty 0$ & $20000000 E+\infty$ & $20000000<-\infty$ & $20000000<+\infty$ & NONE & WPO40486 & $-Y$ & NA \\
\hline 2581 & $1,3,4$ & CONSTANT & $2.0000000 \mathrm{E}+\infty$ & $20000000 E+\infty$ & $20000000<+\infty$ & $20000000 E \cdot \infty 0$ & NONE & WPO40514 & $\mathbf{Y}$ & N/A \\
\hline 2582 & $1,3,4$ & CONSTANT & $1.6000000 E-09$ & $16000000 \mathrm{E}-09$ & $10000000<-\infty$ & $10000000<-09$ & Pen-1 & WPO30640 & Y8N & 1.4 \\
\hline 2582 & $1,3,4$ & CONSTANT & $1.6000000 E-09$ & $16000000=-\infty 9$ & $10000000=-09$ & $180000006-09$ & $P e^{n-1}$ & WP030994 & Y8N & 1,4 \\
\hline 2582 & $1,3,4$ & CONSTANT & $1.6000000 E-09$ & $160000000-09$ & $10000000<-09$ & $10000000 \in-09$ & PEn-1 & WP035597 & YEN & 1.4 \\
\hline 2583 & 1,3 & CONSTANT & $1.0000000 \equiv+08$ & $10000000 E-00$ & $10000000 \mathrm{E}-\infty$ & $100000006+\infty 8$ & $\mathbf{P a}$ & WP030640 & $\mathbf{Y}$ & N/A \\
\hline 2583 & 1,3 & CONSTANT & $1.0000000 E+08$ & $100000000+00$ & $10000000<+\infty$ & $10000000 E+\infty$ & Pa & WP030994 & $\mathbf{Y}$ & N/A \\
\hline 2583 & 1,3 & CONSTANT & $1.0000000 E+08$ & $10000000 \mathrm{E}+\infty 0$ & $10000000<-08$ & $10000000 E+08$ & Pa & WP035597 & $\mathbf{Y}$ & NA \\
\hline
\end{tabular}


CCA Parameter Listing

\begin{tabular}{|c|c|c|c|c|c|c|c|c|c|c|}
\hline $\begin{array}{c}\text { Paramation in } \\
\text { Darabaea? } \\
\text { (Y/N) }\end{array}$ & 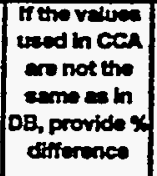 & $\mathbf{D}$ & Materald to & Metarial Nem & Paramix & Partunem Rim & $\begin{array}{l}\text { PFP DD } \\
\text { (MPOA) }\end{array}$ & $\underset{\text { Dete Entry }}{\text { Deto }}$ & 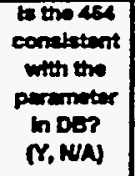 & $\begin{array}{l}\text { Peramitar } \\
\text { Cutiogory } \\
\text { (tootnote i) }\end{array}$ \\
\hline $\mathbf{Y}$ & & 2583 & SALT_TS & $\begin{array}{l}\text { Shaf salt column } \\
\text { compectedtime } 100 \text { to } \\
200 \text { years }\end{array}$ & PC_MAX & $\begin{array}{l}\text { Maximum allowable capillay } \\
\text { pressere }\end{array}$ & $33579 A$ & 27-0ct-95 & $\mathbf{Y}$ & 48 \\
\hline $\mathbf{Y}$ & & 2583 & SALT_T5 & $\begin{array}{l}\text { Shaft sak column } \\
\text { compectectime } 100 \text { to } \\
200 \text { years }\end{array}$ & PC_MAX & $\begin{array}{l}\text { Moxinum alloweble capillery } \\
\text { pressuro }\end{array}$ & $33579 A$ & 27-0ct-95 & $\mathbf{Y}$ & 48 \\
\hline $\mathbf{Y}$ & & 2585 & SALT_TS & $\begin{array}{l}\text { Shan sat column } \\
\text { compectedtine } 100 \text { to } \\
200 \text { years }\end{array}$ & POROSIT & Efrective porosity & 33590 & $14+F a b-96$ & $\mathbf{Y}$ & 1 \\
\hline $\mathbf{Y}$ & & 2585 & SALT_TS & $\begin{array}{l}\text { Shan salk column } \\
\text { compactedtime } 100 \text { to } \\
200 \text { yoars }\end{array}$ & POROSITY & Effoctive porosity & 33590 & $14 f a b-96$ & $\mathbf{Y}$ & 1 \\
\hline $\mathbf{Y}$ & & 2585 & SALT_T5 & $\begin{array}{l}\text { Shaft salt cokmn } \\
\text { compactectime } 100 \text { to } \\
200 \text { years }\end{array}$ & POROSITY & Effective porosity & 33590 & 14Fab-96 & $\mathbf{Y}$ & 1 \\
\hline $\mathbf{Y}$ & & 2594 & SALT_TS & $\begin{array}{l}\text { Sheft salt column } \\
\text { compectedtima } 100 \text { to } \\
200 \text { years }\end{array}$ & RELP_MOD & $\begin{array}{l}\text { Model number, relative } \\
\text { permeability modal }\end{array}$ & $33514 A$ & $27-0 \mathrm{ct}-95$ & $\mathbf{Y}$ & 48 \\
\hline $\mathbf{Y}$ & & 2594 & SALT_TS & $\begin{array}{l}\text { Sheft salt cokmn } \\
\text { compactectime } 100 \text { to } \\
200 \text { yoars }\end{array}$ & RELP_MOD & $\begin{array}{l}\text { Model number, relative } \\
\text { permesbility model }\end{array}$ & 336144 & $27-0 c t-95$ & $\mathbf{Y}$ & AB \\
\hline $\mathbf{Y}$ & & 2594 & SALT_T5 & $\begin{array}{l}\text { Shan salt colimn } \\
\text { compactiedtime } 100 \text { to } \\
200 \text { years }\end{array}$ & RELP_MOD & $\begin{array}{l}\text { Aodel number, relative } \\
\text { permeability model }\end{array}$ & $36614 A$ & 27-0ct-95 & $\mathbf{Y}$ & $A B$ \\
\hline $\mathbf{Y}$ & & 2594 & SALT_T5 & $\begin{array}{l}\text { Shaft sat column } \\
\text { compactedtime } 100 \text { to } \\
200 \text { years }\end{array}$ & RELP_MOD & $\begin{array}{l}\text { Model number, relative } \\
\text { permeability modol }\end{array}$ & $33614 A$ & $27-0 c t-95$ & $\mathbf{Y}$ & 48 \\
\hline $\mathbf{Y}$ & & 2594 & SALT_T5 & $\begin{array}{l}\text { Shat salt column } \\
\text { compacted time } 100 \text { to } \\
200 \text { years }\end{array}$ & RELP_MOD & $\begin{array}{l}\text { Model mumber, relative } \\
\text { pormenbitity model }\end{array}$ & $33614 A$ & $27-0 \mathrm{ct}-95$ & $\mathbf{Y}$ & 48 \\
\hline $\mathbf{Y}$ & 100 & 2596 & SALT_T5 & $\begin{array}{l}\text { Shaft salt column } \\
\text { compsctedtine } 100 \text { to } \\
200 \text { years }\end{array}$ & SAT_RBRN & Reaidual Brine Saturation & 33626 & $14 F=b-96$ & $\mathbf{Y}$ & 1 \\
\hline $\mathbf{Y}$ & 100 & 2590 & SALT_TS & $\begin{array}{l}\text { Shat salt cokenn } \\
\text { compectect time } 100 \text { to } \\
200 \text { yesrs }\end{array}$ & SAT_RBRN & Residual Bnns Saturation & 33626 & $14+a b-96$ & $\mathbf{Y}$ & 1 \\
\hline$Y$ & 100 & 2596 & SALT_TS & $\begin{array}{l}\text { Shaft sall colimn } \\
\text { compectectime } 100 \text { to } \\
200 \text { years }\end{array}$ & SAT_RBRN & Residual Brno Seturation & 33626 & $14 F a b-96$ & $\mathbf{Y}$ & 1 \\
\hline $\mathbf{Y}$ & 100 & 2596 & SALT_TS & $\begin{array}{l}\text { Sheft eall column } \\
\text { compacted:tine } 100 \text { to } \\
200 \text { yans }\end{array}$ & SAT_RBRN & Residual Bme Suturation & 33626 & $14 F a b-96$ & $\mathbf{Y}$ & 1 \\
\hline $\mathbf{Y}$ & 100 & 2596 & SALT_TS & $\begin{array}{l}\text { Shat salt cohmn } \\
\text { compectedtine } 100 \text { to } \\
200 \text { years }\end{array}$ & SAT_RBRH & Residual Bma Seturation & 33626 & 14 fob-96 & $\mathbf{Y}$ & 1 \\
\hline $\mathbf{Y}$ & 100 & 2596 & SALT_TS & $\begin{array}{l}\text { Shen salt cokmn } \\
\text { compectedtume } 100 \text { to } \\
200 \text { years }\end{array}$ & SAT_RBRN & Residual Bmo Saturation & 33626 & 14-Fob-96 & $\mathbf{Y}$ & 1 \\
\hline $\mathbf{Y}$ & 100 & 2597 & SALT_TS & $\begin{array}{l}\text { Shan sat colimn } \\
\text { compactedtume } 100 \text { to } \\
200 \text { years }\end{array}$ & SAT_RGAS & Reaucual Ges Saturation & 33628 & 14 Fab-96 & $\because Y$ & 1 \\
\hline $\mathbf{Y}$ & 100 & 2597 & SALT_T5 & $\begin{array}{l}\text { Shat sat cotumn } \\
\text { compacted:tume } 100 \text { to } \\
200 \text { years }\end{array}$ & SAT_RGAS & Residual Ges Senturation & 33628 & 14Fob-86 & $\mathbf{Y}$ & 1 \\
\hline $\mathbf{Y}$ & 100 & 2597 & SALT_TS & $\begin{array}{l}\text { Shat set colemn } \\
\text { compectectime } 100 \text { to } \\
200 \text { years }\end{array}$ & SAT_RGAS & Resictual Ges Seturration & 33628 & $14-F e b-96$ & $\mathbf{Y}$ & 1 \\
\hline $\mathbf{Y}$ & 100 & 2597 & SALT_T5 & $\begin{array}{l}\text { Shat sat column } \\
\text { compectedtime } 100 \text { to } \\
200 \text { yater }\end{array}$ & SAT_RGAS & Resucual Ges Seturation & 33628 & 14fob-96 & $\mathbf{Y}$ & 1 \\
\hline $\mathbf{Y}$ & 100 & 2597 & SALT_TS & $\begin{array}{l}\text { Shat sak column } \\
\text { compectedtume } 100 \text { to } \\
200 \text { yeers }\end{array}$ & SAT_RGAS & Rearcuil Ges Seturation & 33628 & $14 F a b-86$ & $\mathbf{Y}$ & 1 \\
\hline $\mathbf{Y}$ & & 2599 & ASPHALT & Asphat column & KPT & $\begin{array}{l}\text { Fieg for Permesedity Determined } \\
\text { Threshold }\end{array}$ & 31381 & $20-f=b-96$ & $\mathbf{Y}$ & $4 B$ \\
\hline $\mathbf{Y}$ & & 2599 & ASPHALT & Asphat cotumn & KPT & $\begin{array}{l}\text { Fleg for Permesbilty Determmed } \\
\text { Thro:hold }\end{array}$ & 31381 & $20-F a b-86$ & $\mathbf{Y}$ & $\mathbf{4 B}$ \\
\hline $\mathbf{Y}$ & & 2599 & ASPHALT & Asphat cokmn & KPT & $\begin{array}{l}\text { Fieg for Permeeblity Dotermined } \\
\text { Threahold }\end{array}$ & 31381 & $20-5 c b-96$ & $\mathbf{Y}$ & 48 \\
\hline $\mathbf{Y}$ & & 2599 & ASPHALT & Asphatt column & KPT & $\begin{array}{l}\text { Fieg for Permesidity Determined } \\
\text { Threshols }\end{array}$ & 31381 & $20-5 c b-96$ & $\mathbf{Y}$ & 48 \\
\hline $\mathbf{Y}$ & & 2599 & ASPHALT & Asphante column & KPT & $\begin{array}{l}\text { Fleg for Permesbidty Determined } \\
\text { Threshold }\end{array}$ & 31381 & 20 Fab-96 & $\mathbf{Y}$ & 48 \\
\hline
\end{tabular}




\begin{tabular}{|c|c|c|c|c|c|c|c|c|c|c|}
\hline DD & $\begin{array}{l}\text { Cleventin- } \\
\text { cation of } \\
\text { Deta } \\
\text { Cintegory } \\
\text { (footnote 2) }\end{array}$ & $\begin{array}{l}\text { Dtertibution } \\
\text { Type }\end{array}$ & man & Medien & Mntrem & Modrmang & Whats & Reforence DD & 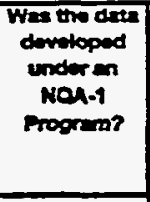 & 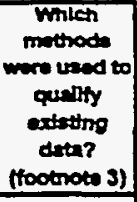 \\
\hline 2583 & 1,3 & CONSTANT & $1.0000000 E+08$ & $1.0000000 E+08$ & $1.0000000 E+08$ & $1.0000000 E+08$ & $\mathbf{P a}$ & WPO40486 & $\mathbf{Y}$ & NA \\
\hline 2583 & 1,3 & CONSTANT & $1.0000000 E+08$ & $1.0000000 E+08$ & $1.0000000 E+08$ & $1.0000000 E+08$ & $\mathbf{P a}$ & WPO40514 & $\mathbf{Y}$ & NA \\
\hline 2585 & $4,3,4$ & CONSTANT & $5.0000000 E-02$ & $5.0000000 E-02$ & $5.0000000 E-\infty 2$ & $5.0000000 E-02$ & $m^{\wedge} 3 m^{n} 3$ & WP030640 & $Y$ & NA \\
\hline 2585 & $1,3,4$ & CONSTANT & $5.0000000 E-02$ & $5.0000000 E-02$ & $5.0000000 E-\infty 2$ & $5.0000000 E-02$ & $m^{\wedge} 3 m^{\wedge} 3$ & $\begin{array}{r}\text { WPOs0s94 } \\
\text { i? }\end{array}$ & $\mathbf{Y}$ & NA \\
\hline 2585 & $1,3,4$ & CONSTANT & $5.0000000 E-02$ & $5.0000000 E-02$ & $5.0000000 E-02$ & $5.0000000 E-02$ & $m^{\wedge} 3 m^{\wedge} 3$ & WP035597 & $\mathbf{Y}$ & N/A \\
\hline 2594 & 1,3 & CONSTANT & $4.0000000 E+\infty 0$ & $4.00000000+00$ & $4.0000000 E+\infty 0$ & $4.0000000 E+00$ & NONE & WPO30S40 & $\mathbf{Y}$ & NA \\
\hline 2594 & 1,3 & CONSTANT & $4.0000000 E+00$ & $4.0000000 E+\infty 0$ & $4.0000000 E+00$ & $4.0000000 E+\infty$ & NONE & WP030394 & $Y$ & NA \\
\hline 2594 & 1,3 & CONSTANT & $4.0000000 \mathrm{E}+00$ & $4.0000000 \mathrm{E}+\infty$ & $4.0000000 E+00$ & $4.0000000 E+\infty 0$ & NONE & WP035597 & $\mathbf{Y}$ & NA \\
\hline 2594 & 1,3 & CONSTANT & $4.0000000 E+00$ & $4.0000000 E+\infty 0$ & $4.0000000 E+00$ & $4.00000000+00$ & NONE & WPO40486 & $\mathbf{Y}$ & NA \\
\hline 2594 & 1,3 & CONSTANT & $4.0000000 E+\infty 0$ & $4.0000000 E+00$ & $4.0000000 E+00$ & $4.00000000 \times+00$ & NONE & WP040514 & $\mathbf{Y}$ & $N A$ \\
\hline 2596 & 3,4 & CUMULATINE & $2.5000000 E-01$ & $20000000 E-01$ & $0.0000000 E+00$ & $6.0000000 E-01$ & NONE & WP030510 & $Y$ & NA \\
\hline 2596 & 3,4 & CUMULATIVE & $2.5000000 E-01$ & $20000000 E-01$ & $0.0000000 E+\infty$ & $6.0000000 E-01$ & NONE & WP030994 & $\mathbf{Y}$ & NA \\
\hline 2596 & 3,4 & CUMULATNE & 25000000E-01 & $20000000 E-01$ & $0.0000000 E+00$ & $6.0000000 E-01$ & NONE & WPO35268 & $\mathbf{Y}$ & N/A \\
\hline 2596 & 3,4 & CUMULATNE & $2.5000000 E-01$ & $2.0000000 E-01$ & $0.0000000 E+00$ & $6.0000000 E-01$ & NONE & WP035597 & $Y$ & NA \\
\hline 2596 & 3,4 & CUMULATIVE & $2.5000000 E-01$ & $2.0000000 E-01$ & $0.0000000 E+\infty 0$ & $6.0000000 E-01$ & NONE & WPOA1558 & $\mathbf{Y}$ & N/A \\
\hline 2596 & 3,4 & CUMULATINE & $2.5000000 E-01$ & $20000000 E-01$ & $0.0000000 E+\infty$ & $6.0000000 E-01$ & NONE & WPO42751 & $Y$ & NA \\
\hline 2597 & 3,4 & UNIFORM & $2.0000000 E-01$ & 20000000E-01 & $0.0000000 E+\infty 0$ & 4.0000000E-01 & NONE & WP030640 & $\bar{\gamma}$ & N/A \\
\hline 2597 & 3,4 & UNIFORM & $2.0000000 \mathrm{E}-01$ & $20000000 E-01$ & $0.0000000 E+\infty$ & $1.0000000 E-01$ & NONE & WP030994 & $\mathbf{Y}$ & NA \\
\hline 2597 & 3,4 & UNIFORM & $2.0000000 E-01$ & $20000000 E-01$ & $0.0000000 E+\infty 0$ & 4.0000000E-01 & NONE & WP035268 & $Y$ & NA \\
\hline 2597 & 3,4 & UNIFORM & $20000000 E-01$ & 20000000E-01 & $0.0000000 E+\infty$ & 4.0000000E-01 & NONE & WP035597 & $\gamma$ & N/A \\
\hline 2597 & 3,4 & UNIFORM & $2.0000000 E-01$ & $2.0000000 E-01$ & $0.0000000 E+\infty 0$ & $4.0000000 E-01$ & NONE & WPO41558 & $\mathbf{Y}$ & NA \\
\hline 2599 & 3,5 & CONSTANT & $0.0000000 E+\infty 0$ & $0.0000000 E+\infty$ & $0.0000000 E+100$ & $0.0000000 E+\infty 0$ & NONE & WPO30640 & $\mathbf{Y}$ & NA \\
\hline 2599 & 3,5 & CONSTANT & $0.0000000 E+\infty$ & $0.0000000 E+\infty 0$ & $0.0000000 E+\infty 0$ & $0.0000000 E+\infty 0$ & NONE & WP030994 & $\mathbf{Y}$ & N/A \\
\hline 2599 & 3,5 & CONSTANT & $0.0000000 E+00$ & $0.0000000 \mathrm{E}+\infty$ & $0.0000000 E+00$ & $0.0000000 E+00$ & NONE & WP035274 & $\mathbf{Y}$ & NA \\
\hline 2599 & 3,5 & CONSTANT & $0.0000000 E+00$ & $0.0000000 E+\infty$ & $0.0000000 E+\infty 0$ & $0.0000000 E+\infty 0$ & NONE & WP035333 & $\mathbf{Y}$ & NA \\
\hline 2599 & 3,5 & CONSTANT & $0.0000000 E+\infty$ & $0.0000000 E+\infty$ & $0.0000000 E+00$ & $0.0000000 E+\infty$ & NONE & WP035597 & $\mathbf{Y}$ & NAA \\
\hline
\end{tabular}


CCA Parameter Listing

\begin{tabular}{|c|c|c|c|c|c|c|c|c|c|c|}
\hline 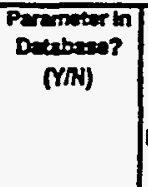 & 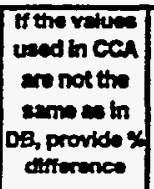 & DD & Actaristio & Meterlal Harme & $\begin{array}{c}\text { Perimier } \\
10\end{array}$ & Paremocer Mern & $\begin{array}{l}\text { PRP D } \\
\text { (WPO in) }\end{array}$ & Detanty & 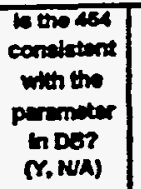 & $\begin{array}{l}\text { Peromits } \\
\text { Caxtegory } \\
\text { (footnots 1) }\end{array}$ \\
\hline $\mathbf{Y}$ & & 2599 & ASPHALT & Asphak cokumn & KPT & $\begin{array}{l}\text { Fleg for Permenbility Determinod } \\
\text { Threshold }\end{array}$ & 31381 & $20-500-96$ & $\mathbf{Y}$ & 18 \\
\hline $\mathbf{Y}$ & & 2600 & ASPHALT & Asphar column & PCT_A & $\begin{array}{l}\text { Trresinold Pressure Lneer } \\
\text { Parameter }\end{array}$ & 31382 & $16-f a b-96$ & $\mathbf{Y}$ & 1 \\
\hline $\mathbf{Y}$ & & 2600 & ASPHALT & Asphalt cokmn & PCT_A & $\begin{array}{l}\text { Threshold Pressurs Lunar } \\
\text { Permeter }\end{array}$ & 31382 & $16+f a-96$ & $\mathbf{Y}$ & 1 \\
\hline $\mathbf{Y}$ & & 2600 & ASPHALT & Asphalt cokumn & PCT_A & $\begin{array}{l}\text { Threshold Proserre Lneser } \\
\text { Permoter }\end{array}$ & 31382 & 16 foch-9s & $\mathbf{Y}$ & 1 \\
\hline $\mathbf{Y}$ & & 2601 & ASPHALT & Asphat cotumn & PCT_EXP & $\begin{array}{l}\text { Thresinold pressure exponental } \\
\text { paremeter }\end{array}$ & 31384 & $13-F a b-96$ & $\mathbf{Y}$ & 1 \\
\hline$Y$ & & 2601 & ASPHALT & Asphatt column & PCT_EXP & $\begin{array}{l}\text { Trrestrold pressure exponentad } \\
\text { peremeter }\end{array}$ & 31384 & $13-f a b-96$ & $Y$ & 1 \\
\hline$Y$ & & 2601 & ASPHALT & Asphats cokumn & PCT_EXP & $\begin{array}{l}\text { Threshold pressure exponential } \\
\text { peremeter }\end{array}$ & 31384 & $13-F a b-96$ & $\mathbf{Y}$ & 1 \\
\hline $\mathbf{Y}$ & & 2604 & OPS_AREA & Operations Region & KPT & $\begin{array}{l}\text { Flag for Permeabilay Dotentmined } \\
\text { Threshold }\end{array}$ & 32614 & 16-fab-96 & $\mathbf{Y}$ & $A B$ \\
\hline $\mathbf{Y}$ & & 2604 & OPS_AREA & Operations Region & KPT & $\begin{array}{l}\text { Fleg for Permecbilty Determined } \\
\text { Throshold }\end{array}$ & 32614 & 16 fab-96 & $\mathbf{Y}$ & 48 \\
\hline $\mathbf{Y}$ & & 2604 & OPS_AREA & Operations Region & KPT & $\begin{array}{l}\text { Fleg for Permesbilky Determined } \\
\text { Threstold }\end{array}$ & 32614 & $16-F a b-96$ & $\mathbf{Y}$ & 18 \\
\hline$Y$ & & 2604 & OPS_AREA & Operations Region & KPT & $\begin{array}{l}\text { Flag for Permeabifty Determnod } \\
\text { Threstrold }\end{array}$ & 32614 & $16-f a b-96$ & $\mathbf{Y}$ & 48 \\
\hline $\mathbf{Y}$ & & 2604 & OPS_AREA & Operations Region & KPT & $\begin{array}{l}\text { Flag for Permeabulty Determined } \\
\text { Throstiold }\end{array}$ & 32614 & 16 fab-96 & $\mathbf{Y}$ & 48 \\
\hline $\mathbf{Y}$ & & 2604 & OPS_AREA & Operations Region & KPT & $\begin{array}{l}\text { Flag for Permasiikty Detemned } \\
\text { Threshold }\end{array}$ & 32614 & $16-f a b-96$ & $Y$ & 48 \\
\hline $\mathbf{Y}$ & & 2605 & OPS_AREA & Oporations Rogion & PCT_A & $\begin{array}{l}\text { Throshold Prossure Luner } \\
\text { Parameter }\end{array}$ & 326164 & $16-F a b-96$ & $\mathbf{Y}$ & 48 \\
\hline $\mathbf{Y}$ & & 2605 & OPS_AREA & Operations Region & PCT_A & $\begin{array}{l}\text { Threshold Pressure Lineser } \\
\text { Pereneter }\end{array}$ & $32616 A$ & 16 fab-96 & - $Y$ & 48 \\
\hline $\mathbf{Y}$ & & 2605 & OPS_AREA & Operations Region & PCT_A & $\begin{array}{l}\text { Threshold Pressure Lnesr } \\
\text { Parmeter }\end{array}$ & 326164 & $16+5 c b-96$ & $\mathbf{Y}$ & $4 B$ \\
\hline $\mathbf{Y}$ & & 2605 & OPS_AREA & Operations Region & PCT_A & $\begin{array}{l}\text { Threshold Pressuro Linear } \\
\text { Paramoter }\end{array}$ & $32616 A$ & $16-f a b-96$ & $\mathbf{Y}$ & $d B$ \\
\hline $\mathbf{Y}$ & & 2606 & OPS_AREA & Operations Region & PCT_EXP & $\begin{array}{l}\text { Threshold pressure exponential } \\
\text { parameter }\end{array}$ & 32618 & $16-F b-96$ & $\mathbf{Y}$ & $4 B$ \\
\hline $\mathbf{Y}$ & & 2606 & OPS_AREA & Operations Rogion & PCT_EXP & $\begin{array}{l}\text { Threshold pressure exponential } \\
\text { parameter }\end{array}$ & 32618 & $16-f a b-96$ & $\mathbf{Y}$ & $4 B$ \\
\hline $\mathbf{Y}$ & & 2606 & OPS_AREA & Operations Region & PCT_EXP & $\begin{array}{l}\text { Threshold pressure exponential } \\
\text { parameter }\end{array}$ & 32618 & $16 f e b-96$ & $\mathbf{Y}$ & $4 B$ \\
\hline $\mathbf{Y}$ & & 2606 & OPS_AREA & Operations Region & PCT_EXP & $\begin{array}{l}\text { Threshold pressure exponential } \\
\text { paremeter }\end{array}$ & 32618 & $16-$ fob-96 & $Y$ & $A B$ \\
\hline $\mathbf{Y}$ & & 2608 & CASTILER & Custile Bine Reservoir & KPT & $\begin{array}{l}\text { Fieg for Permeability Determmed } \\
\text { Threshold }\end{array}$ & 31583 & $20-f c b-96$ & $\mathbf{Y}$ & 48 \\
\hline $\mathbf{Y}$ & & 2608 & CASTILER & Cestile Brine Reservoir & KPT & $\begin{array}{l}\text { Flag for Permeability Determined } \\
\text { Threstold }\end{array}$ & 31583 & $20+a b-96$ & $\mathbf{Y}$ & $4 B$ \\
\hline $\mathbf{Y}$ & & 2608 & CASTILER & Cestile Brino Reservoir & KPT & $\begin{array}{l}\text { Flag for Permeability Determmed } \\
\text { Threshold }\end{array}$ & 31583 & $20-f c b-96$ & $\mathbf{Y}$ & AB \\
\hline $\mathbf{Y}$ & & 2608 & CASTILER & Castile Brino Reservoin & KPT & $\begin{array}{l}\text { Flag for Pormosbility Determined } \\
\text { Throshold }\end{array}$ & 31583 & 20 Feb-86 & $-y$ & AB \\
\hline $\mathbf{Y}$ & & 2609 & CASTILER & Cestio Brine Ressorvoir & PCT_A & $\begin{array}{l}\text { Threshold Pressure Lnear } \\
\text { Peremotor }\end{array}$ & 31605 & 14-Nov-95 & $\mathbf{Y}$ & $A B$ \\
\hline $\mathbf{Y}$ & & 2609 & CASTILER | & Castile Brine Reservoir & PCT_A & $\begin{array}{l}\text { Threshold Pressure Lnear } \\
\text { Paranoter }\end{array}$ & 31605 & 14 Nor-95 & $\mathbf{Y}$ & 48 \\
\hline $\mathbf{Y}$ & & 2610 & CASTILER & Centile Brino Reservoir & PCT_EXP & $\begin{array}{l}\text { Threshold pressure exponental } \\
\text { paremeter }\end{array}$ & 31606 & 14-Nov-95 & $\mathbf{Y}$ & $4 \mathrm{~B}$ \\
\hline $\mathbf{Y}$ & & 2610 & CASTILER & Cestib Brine Reservoir & PCT_EXP & $\begin{array}{l}\text { Threshold pressure exponentzal } \\
\text { parameter }\end{array}$ & 31605 & 14 Nov-85 & $\mathbf{Y}$ & $4 B$ \\
\hline $\mathbf{Y}$ & & 2612 & CAVTTY_t & Cenity for Waste Aras & KPT & $\begin{array}{l}\text { Flag for Permeability Dotermmed } \\
\text { Threshold }\end{array}$ & 31650 & $17-F=b-96$ & $Y$ & $4 B$ \\
\hline $\mathbf{Y}$ & & 2612 & CAVIr_1 & Cevity for Wasto Arass & |RPT & $\begin{array}{l}\text { Fleg for Permesbility Determned } \\
\text { Thresthold }\end{array}$ & 31650 & $17+50 b-96$ & $\mathbf{Y}$ & $4 B$ \\
\hline $\mathbf{Y}$ & & 2612 & CAVIY_I & Cevity for Waste Aross & KPT & $\begin{array}{l}\text { Flag for Permeability Determined } \\
\text { Thre:thold }\end{array}$ & 31650 & $17+a b-96$ & $\mathbf{Y}$ & $4 B$ \\
\hline $\mathbf{Y}$ & & 2612 & CAVITY_t & Cenvity for Waste Aress & KOT & $\begin{array}{l}\text { Flog for Permeabidty Dotermined } \\
\text { Thre:hold }\end{array}$ & 31650 & $17+5 b-96$ & $\mathbf{Y}$ & 48 \\
\hline $\mathbf{Y}$ & & 2612 & CAVITY_1 & Cevity for Wasto Areas & KPT & $\begin{array}{l}\text { Fieg for Permeability Determned } \\
\text { Threshold }\end{array}$ & 31650 & $17 f \circ b-96$ & $\mathbf{Y}$ & $4 B$ \\
\hline $\mathbf{Y}$ & & 2613 & CAVIY_1 & Crvity for Whate Areas & PCT_A & $\begin{array}{l}\text { Threahold Pressure Lineser } \\
\text { Peranter }\end{array}$ & 31670 & $17+50-98$ & $\mathbf{Y}$ & 48 \\
\hline $\mathbf{Y}$ & & 2613 & CAVIT__1 & Corvity for Waste Areas & PCT_A & $\begin{array}{l}\text { Thrashold Pressure Linaer } \\
\text { Paremeter }\end{array}$ & 31670 & $17-f a b-96$ & $\mathbf{Y}$ & 48 \\
\hline
\end{tabular}


CCA Parameter Listing

\begin{tabular}{|c|c|c|c|c|c|c|c|c|c|c|}
\hline 10 & 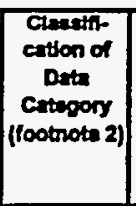 & $\begin{array}{l}\text { Distribution } \\
\text { Type }\end{array}$ & $\overline{m e n}$ & modien & Minkmum & Arodmim & Wintes & Rofermeato & 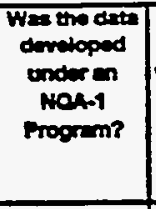 & 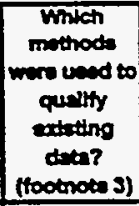 \\
\hline 2599 & 3,5 & CONSTANT & $0.0000000 \pm+\infty$ & $0.0000000 E+\infty$ & $0.0000000 E+\infty$ & $0.0000000 E+\infty$ & NONE & UPO40514 & $\mathbf{Y}$ & NA \\
\hline 2600 & 3,4 & CONSTANT & $0.0000000=+\infty$ & $0.0000000 E+\infty$ & $0.0000000 E+\infty 0$ & $0.0000000 E+\infty$ & Pe & WP030640 & $\mathbf{Y}$ & NA \\
\hline 2600 & 3,4 & CONSTANT & $0.0000000 \mathrm{E}+\infty$ & $0.0000000 \mathrm{E}+\infty$ & $0.0000000 E+\infty 0$ & $0.0000000 E+\infty 0$ & Pa & WP030994 & $\mathbf{Y}$ & NA \\
\hline 2600 & 3,4 & CONSTANT & $0.0000000 E+\infty$ & $0.0000000 \mathrm{E}+\infty 0$ & $0.0000000 \mathrm{E}+\infty$ & $0.0000000 E+\infty$ & Pa & WP035597 & $\mathbf{Y}$ & NA \\
\hline 2601 & 3,4 & CONSTANT & $0.0000000 \mathrm{E}+\infty 0$ & $0.0000000 \mathrm{E}+00$ & $0.0000000 E+\infty$ & $0.00000000 E+\infty$ & NONE & WP030640 & $Y$ & NA \\
\hline 2601 & 3.4 & CONSTANT & $0.0000000 E+\infty$ & $0.0000000 \mathrm{E}+00$ & $0.0000000 E+\infty$ & $0.0000000 E+\infty$ & NONE & WP03009s. & $Y$ & NA \\
\hline 2601 & 3,4 & CONSTANT & $0.0000000 E+\infty$ & $0.00000000 E+00$ & $0.0000000 E+\infty$ & $0.0000000 E+\infty 0$ & NONE & WP035597 & $Y$ & NA \\
\hline 2604 & 1,3 & CONSTANT & $0.0000000 E+\infty 0$ & $0.0000000 E+\infty 0$ & $0.0000000 E+\infty$ & $0.0000000 E+\infty$ & NONE & WP035271 & $\mathbf{Y}$ & NA \\
\hline 2604 & 1,3 & CONSTANT & $0.0000000 E+\infty$ & $0.0000000 E+\infty$ & $0.0000000 E+\infty$ & $0.0000000 E+\infty$ & NONE & WP035330 & $\mathbf{Y}$ & NA \\
\hline 2604 & 1.3 & CONSTANT & $0.0000000 E+\infty$ & $0.0000000 E+\infty 0$ & $0.0000000 E+00$ & $0.0000000 \mathrm{E}+\infty$ & NONE & WP035333 & $\mathbf{Y}$ & NA \\
\hline 2604 & 1,3 & CONSTANT & $0.0000000 \mathrm{E}+\infty$ & $0.0000000 E+00$ & $0.0000000 E+\infty$ & $0.0000000 \mathrm{E}+\infty 0$ & NONE & WP035597 & $\mathbf{Y}$ & NA \\
\hline 2604 & 1,3 & CONSTANT & $0.0000000 E+\infty 0$ & $0.0000000 E+\infty$ & $0.0000000 \mathrm{E}+\infty$ & $0.0000000 E+\infty$ & NONE & WP035320 & $\mathbf{Y}$ & NA \\
\hline 2604 & 1,3 & CONSTANT & $0.0000000 E+\infty 0$ & $0.0000000 E+\infty 0$ & $0.0000000 E+\infty 0$ & $0.0000000 E+\infty$ & NONE & WP040514 & $\mathbf{Y}$ & NA \\
\hline 2605 & 1,3 & CONSTANT & $0.0000000 E+00$ & $0.0000000 \mathrm{E}+\infty 0$ & $0.0000000 E+\infty$ & $0.0000000 E+\infty 0$ & $\mathbf{P a}$ & WPO35330 & $\mathbf{Y}$ & NA \\
\hline 2605 & 1,3 & CONSTANT & $0.0000000 E+\infty$ & $0.0000000 \mathrm{E}+\infty$ & $0.0000000 E+\infty$ & $0.00000000 E+\infty 0$ & $\mathbf{P a}$ & WP035597 & $\mathbf{Y}$ & NA \\
\hline 2605 & 1,3 & CONSTANT & $0.0000000 E+\infty$ & $0.0000000 E+\infty$ & $0.0000000 \mathrm{E}+\infty$ & $0.0000000 E+00$ & Pa & WPO36320 & $Y$ & NA \\
\hline 2605 & 1,3 & CONSTANT & $0.0000000 E+00$ & $0.0000000 \mathrm{E}+00$ & $0.0000000 \mathrm{E}+\infty$ & $0.0000000 E+\infty$ & Pa & WPO40514 & $\mathbf{Y}$ & NA \\
\hline 2606 & 1,3 & CONSTANT & $0.0000000 \mathrm{E}+\infty$ & $0.0000000 E+00$ & $0.0000000 E+\infty 0$ & $0.0000000 E+\infty$ & NONE & WP035330 & $\mathbf{Y}$ & NA \\
\hline 2606 & 1.3 & CONSTANT & $0.0000000 \mathrm{E}+00$ & $0.0000000 E+\infty$ & $0.0000000 E+\infty$ & $0.0000000 E+\infty 0$ & NONE & WP035597 & $\mathbf{Y}$ & N/A \\
\hline 2606 & 1,3 & CONSTANT & $0.0000000 \mathrm{E}+00$ & $0.0000000 \mathrm{E}+\infty$ & $0.0000000 \mathrm{E}+\infty 0$ & $0.0000000 E+\infty 0$ & NONE & WP035320 & $\mathbf{Y}$ & NA \\
\hline 2606 & 1,3 & CONSTANT & $0.0000000 \mathrm{E}+\infty 0$ & $0.0000000 E+\infty 0$ & $0.0000000 E+00$ & $0.0000000 E+00$ & NONE & WPO40514 & $\mathbf{Y}$ & NA \\
\hline 2608 & 3,5 & CONSTANT & $0.0000000 E+\infty 0$ & $0.00000000 E+\infty$ & $0.00000000 E+\infty 0$ & $0.0000000 E+\infty$ & NONE & WP035271 & $\mathbf{Y}$ & NA \\
\hline 2608 & 3,5 & CONSTANT & $0.0000000 E+\infty$ & $0.0000000 E+\infty$ & $0.0000000 E+\infty$ & $0.0000000 E+\infty 0$ & NONE & WPO35333 & $\mathbf{Y}$ & NA \\
\hline 2608 & 3,5 & CONSTANT & $0.0000000 E+\infty 0$ & $0.0000000 E+\infty$ & $0.0000000 E+\infty$ & $0.0000000 E+00$ & NONE & WP035597 & $Y$ & NA \\
\hline 2608 & 3,5 & CONSTANT & $0.0000000=+\infty$ & $0.0000000 E+\infty)$ & $0.0000000 E+\infty$ & $0.0000000 E+\infty$ & NONE & WP040514 & $-y$ & N/A \\
\hline 2609 & $1,3,4$ & CONSTANT & $5.6000000 E-01$ & $5.6000000 E-01$ & $5.6000000 E-01$ & $5.6000000 E-01$ & $\mathbf{P a}$ & WP038568 & $\mathbf{Y}$ & NA \\
\hline 2609 & $1,3,4$ & CONSTANT & $5.6000000 E-01$ & $5.6000000 E-01$ & $5.6000000 E-01$ & $5.6000000 \mathrm{E}-01$ & $\mathbf{P a}$ & WP040514 & $\mathbf{Y}$ & NA \\
\hline 2810 & $1,3,4$ & CONSTANT & $3.4600000 \mathrm{E}-01$ & $3.4600000 E-01$ & $-3.4600000 E-01$ & $-3.4500000 E-01$ & NONE & WP038568 & $\mathbf{Y}$ & NA \\
\hline 2610 & $1,3,4$ & CONSTANT & $3.4600000 \mathrm{E}-01$ & 3.4500000 E- 01 & $-3.4500000 E-01$ & $-3.4600000 E-01$ & NONE & WP040514 & $\mathbf{Y}$ & NA \\
\hline 2612 & 3,5 & CONSTANT & $0.00000000+\infty$ & $0.0000000 E+\infty 0$ & $0.0000000 E+\infty 0$ & $0.00000000 \pm+\infty$ & NONE & $\begin{array}{l}\text { MEMO:VAUGHN } \\
\text { TERNEY-9 }\end{array}$ & $\mathbf{Y}$ & NA \\
\hline 2612 & 3,5 & CONSTANT & $0.00000000+\infty$ & $0.00000000 E+00$ & $0.00000000+00$ & $0.00000000 E+00$ & NONE & WP035271 & $\mathbf{Y}$ & NA \\
\hline 2612 & 3.5 & CONSTANT & $0.0000000 E+\infty$ & $0.0000000 E+\infty$ & $0.0000000 E+\infty 0$ & $0.00000000+\infty$ & MONE & WP035333 & $\mathbf{Y}$ & NA \\
\hline 2612 & 3,5 & CONSTANT & $0.00000000 E+\infty 0$ & $0.0000000 E+\infty 0$ & $0.0000000 E+\infty$ & $0.00000000+00$ & NONE & WP035597 & $Y$ & NA \\
\hline 2812 & 3,5 & CONSTANT & $0.0000000=+\infty$ & $0.00000000+\infty$ & $0.0000000 E+00$ & $0.0000000 E+\infty$ & NONE & WPOS0514 & $\mathbf{Y}$ & NA \\
\hline 2613 & 3,5 & CONSTANT & $0.00000000 E+00$ & $0.00000000 E+\infty 0$ & $0.0000000 E+00$ & $0.0000000 E+\infty 0$ & Pa & $\begin{array}{c}\text { MEMO:VAUGHN } \\
\text { TIERNEY-9 }\end{array}$ & $\mathbf{Y}$ & NA \\
\hline 2613 & 3,5 & CONSTANT & $0.00000000+\infty$ & $0.00000000 E+\infty 0$ & $0.0000000 E+\infty 0$ & $0.0000000 \mathrm{E}+\infty$ & Pa & WP035597 & $Y$ & NA \\
\hline
\end{tabular}


CCA Parameter Listing-

\begin{tabular}{|c|c|c|c|c|c|c|c|c|c|c|}
\hline $\begin{array}{c}\text { Pamineter } \\
\text { Dutubes? } \\
\text { MaN) }\end{array}$ & 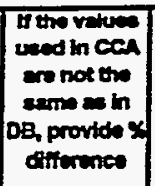 & $\bar{D}$ & Mecarda to & 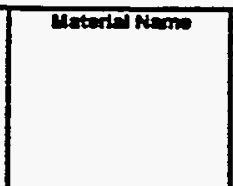 & Perrinotion & Permeter Name & $\begin{array}{l}\text { PRPD } \\
\text { MPO }\end{array}$ & $\begin{array}{l}\text { Deta Entry } \\
\text { Deto }\end{array}$ & 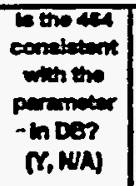 & 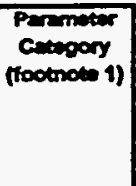 \\
\hline Y & & 2613 & CAVIr_I & Centy for Weste Aruss & PCT_A & $\begin{array}{l}\text { Throshold Prossuro Lnoerr } \\
\text { Paramotor }\end{array}$ & 31670 & 17 Feb-96 & $\mathbf{Y}$ & $4 B$ \\
\hline $\mathbf{Y}$ & & 2614 & CAVTTY_1 & Cavity for Waste Areas & PCT_EXP & $\begin{array}{l}\text { Throshold prossure exponertial } \\
\text { peremetor }\end{array}$ & 31672 & $17+06-06$ & $\mathbf{Y}$ & 48 \\
\hline $\mathbf{Y}$ & & 2614 & CAVITY_I & Cavity for Wasto Nraes & PCT_EXP & $\begin{array}{l}\text { Throshote prossure exponertial } \\
\text { peremeter }\end{array}$ & 31672 & $17 f=0-96$ & $\mathbf{Y}$ & 48 \\
\hline $\mathbf{Y}$ & & 2614 & CAVIT_1 & Cevity for Wasto Arous & PCT_EXP & $\begin{array}{l}\text { Threstrold prossum exponential } \\
\text { peremotor }\end{array}$ & 31672 & 17f-ab-96 & $\mathbf{Y}$ & 48 \\
\hline$Y$ & & 2616 & CAVITY_2 & $\begin{array}{l}\text { Covity for Non-wasto } \\
\text { Areas }\end{array}$ & KPT & $\begin{array}{l}\text { Fleg for Permesbithy Dotormned } \\
\text { imrestold }\end{array}$ & 31716 & 14Hoves & $\mathbf{Y}$ & 48 \\
\hline$Y$ & & 2616 & CAVTr_2 & $\begin{array}{l}\text { Cavity for Nor-weste } \\
\text { Areas }\end{array}$ & KPT & $\begin{array}{l}\text { Flog for Pormeablity Doterminod } \\
\text { inreshold }\end{array}$ & 39716 & 14Nov-95 & $\mathbf{Y}$ & $A B$ \\
\hline $\mathbf{Y}$ & & 2616 & CAVITY_2 & $\begin{array}{l}\text { Cavity for Non-waste } \\
\text { Areas }\end{array}$ & KPT & $\begin{array}{l}\text { Flag for Permesidty Dotermined } \\
\text { Throsthold }\end{array}$ & 31716 & 14Hov-95 & $\mathbf{Y}$ & 48 \\
\hline$Y$ & & 2616 & CAVITY_2 & $\begin{array}{l}\text { Cavity for Non-waste } \\
\text { Areas }\end{array}$ & KPT & $\begin{array}{l}\text { Fieg for Permeabity Determined } \\
\text { Threshold }\end{array}$ & $31716^{\circ}$ & 14-Nov-95 & $\mathbf{Y}$ & $A B$ \\
\hline $\mathbf{Y}$ & & 2616 & CAVIT_2 & $\begin{array}{l}\text { Cavity for Non-wasto } \\
\text { Areas }\end{array}$ & KPT & $\begin{array}{l}\text { Fteg for Permesbilfy Dotermined } \\
\text { Trreshotd }\end{array}$ & 31716 & 14Now-95 & $\mathbf{Y}$ & 48 \\
\hline $\mathbf{Y}$ & & 2617 & CAVITY_2 & $\begin{array}{l}\text { Cevity for Non-wasto } \\
\text { Avess }\end{array}$ & PCT_A & $\begin{array}{l}\text { Threashold Prossere Linosr } \\
\text { Peremetor }\end{array}$ & 31740 & 14Nov-95 & $\mathbf{Y}$ & $A B$ \\
\hline $\mathbf{Y}$ & & 2617 & CAVIT__2 & $\begin{array}{l}\text { Cervity for Nonwasto } \\
\text { Avens }\end{array}$ & PCT_A & $\begin{array}{l}\text { Tresthold Pressure Linear } \\
\text { Peremetor }\end{array}$ & 34740 & 14NHON-95 & $\mathbf{Y}$ & 48 \\
\hline $\mathbf{Y}$ & & 2617 & CAVTr_2 & $\begin{array}{l}\text { Cenvity for Non-wasto } \\
\text { Aross }\end{array}$ & PCT_A & $\begin{array}{l}\text { Throshold Prossura Linear } \\
\text { Paremetor }\end{array}$ & 31740 & 14Nor-95 & $\mathbf{Y}$ & AB \\
\hline $\mathbf{Y}$ & & 2618 & CAVITY_2 & $\begin{array}{l}\text { Covity for Non-wasto } \\
\text { Areas }\end{array}$ & PCT_EXP & $\begin{array}{l}\text { Threshold prossure exponential } \\
\text { parameter }\end{array}$ & 31741 & 14AKOV-95 & $\mathbf{Y}$ & 18 \\
\hline $\mathbf{Y}$ & & 2618 & CAVIT_2 & $\begin{array}{l}\text { Covity for Nornwasto } \\
\text { Areas }\end{array}$ & PCT_EXP & $\begin{array}{l}\text { Throshold presure exponential } \\
\text { parameter }\end{array}$ & 31741 & 14-Nov-95 & $\mathbf{Y}$ & 48 \\
\hline $\mathbf{Y}$ & & 2648 & CAVITY_2 & $\begin{array}{l}\text { Cavity for Non-wasto } \\
\text { Nreas }\end{array}$ & PCT_EXP & $\begin{array}{l}\text { Threshold pressure exponential } \\
\text { Daremetor }\end{array}$ & 31741 & 14Non-95: & $\mathbf{Y}$ & $4 B$ \\
\hline $\mathbf{Y}$ & & 2620 & CAVTr_3 & Cevity for Shan & KPT & $\begin{array}{l}\text { Flag for Pormesbilty Determined } \\
\text { Inreshotd }\end{array}$ & 31770 & $17-F a b-96$ & $\mathbf{Y}$ & 48 \\
\hline $\mathbf{Y}$ & & 2620 & CAVITY_3 & Cevity for Shath & KPT & $\begin{array}{l}\text { Flag for Pormesobity Dotermined } \\
\text { Threshold }\end{array}$ & 31770 & 17 fab-96 & $\mathbf{Y}$ & 48 \\
\hline $\mathbf{Y}$ & & 2620 & CAVTIY_3 & Covity for Shat & KPT & $\begin{array}{l}\text { Flag for Permecouty Delermned } \\
\text { Throshold }\end{array}$ & 31770 & 17 Feb-96 & $\mathbf{Y}$ & 18 \\
\hline$Y$ & & 2620 & CAVTIY3 & Cavity for Shat & KPT & $\begin{array}{l}\text { Flog for Permecolity Determined } \\
\text { Trroshold }\end{array}$ & 31770 & 17 Fab-56 & $\mathbf{Y}$ & $A B$ \\
\hline $\mathbf{Y}$ & & 2620 & CAVTr_3 & Cenvity for Shat & KPT & 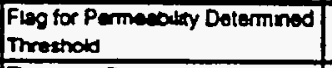 & 31770 & $17+$ fob-96 & $\mathbf{Y}$ & 48 \\
\hline Y & & 2621 & CAVIr__3 & Cavity for Sinat & PCT_A & $\begin{array}{l}\text { Treashow Procane Lnear } \\
\text { Peremere }\end{array}$ & 31773 & 17-feb-96 & $\mathbf{Y}$ & $A B$ \\
\hline $\mathbf{Y}$ & . & 2521 & CAVITY_3 & Covity tor Shen & PCT_A & 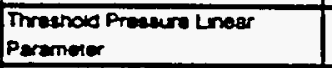 & 31773 & 17-Feb-96 & $\mathbf{Y}$ & $A B$ \\
\hline$\gamma$ & & 2621 & CAVTTY3 & Cenvity for Shen & $P C T_{-} A$ & 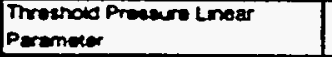 & 31773 & 17 -Fob-96 & $\mathbf{Y}$ & $4 B$ \\
\hline $\mathbf{Y}$ & & 2622 & CAVITY_3 & Convity tor Sinen & PCT_EXP & 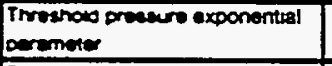 & 31774 & 17 Fab-96 & $\mathbf{Y}$ & $A B$ \\
\hline $\mathbf{Y}$ & & 2622 & CAVTTY_3 & Cavity tor Sinen & PCT_EXe & 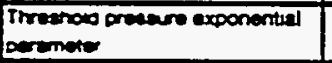 & 31774 & 17 fob-96 & $--Y$ & 48 \\
\hline $\mathbf{Y}$ & & 2622 & CAVIT_3 & Centry for simen & PCT_Ex & 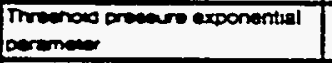 & 31774 & 17 -Fob-96 & $\gamma$ & 48 \\
\hline $\mathbf{Y}$ & & 2523 & CAVITY_3 & Centy for Sinen & PO_W & 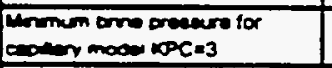 & 31779 & $17-F \cdot b-96$ & $\mathbf{Y}$ & 48 \\
\hline $\mathbf{Y}$ & & 2623 & CAVITY_3 & Cenvty for sinen & PO_nen & 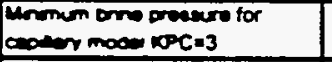 & 31779 & 17 fab-96 & $\mathbf{Y}$ & 48 \\
\hline $\mathbf{Y}$ & & 2623 & CAVTrY_3 & Centy tor Shan & PO_M & 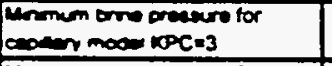 & 31779 & 17 Fob-96 & $\mathbf{Y}$ & 18 \\
\hline $\mathbf{Y}$ & & 2623 & CAVTrY_3 & Convay tox Shent & PO_Ln & 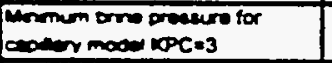 & 31779 & $17+0 b-96$ & $\mathbf{Y}$ & 48 \\
\hline $\mathbf{Y}$ & & 2625 & CAVITY_4 & Cevity tox 8orunow & |rot & 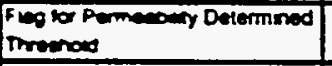 & 31796 & 17 fab-96 & $\mathbf{Y}$ & AB \\
\hline $\mathbf{Y}$ & & 2625 & CAVTY_4 & Conty for Bonmons & 100 & 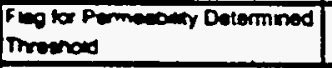 & 31786 & $17 \cdot F a b-96$ & $\mathbf{Y}$ & $4 B$ \\
\hline $\mathbf{Y}$ & & 2625 & CAVITY_4 & Conty for 80 runon & $|r|$ & 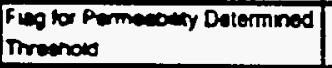 & 31756 & $17+f 0 b-96$ & $\mathbf{Y}$ & 48 \\
\hline $\mathbf{Y}$ & & 2625 & CAVITY_4 & Centy for Bormoles & KPT & 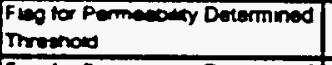 & 31796 & 17Fab-96 & $\mathbf{Y}$ & 48 \\
\hline $\mathbf{Y}$ & & 2625 & CAVITY_4 & Centy for Bcrunow & $\operatorname{lor}$ & 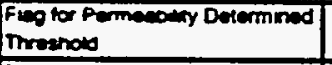 & 31796 & $17+f a b-96$ & $\mathbf{Y}$ & 4B \\
\hline$Y$ & & 2626 & CAVITY_A & Conty for Borunow & PCT_A & 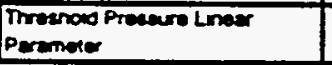 & 31787 & 17 feb-96 & $\mathbf{Y}$ & 18 \\
\hline
\end{tabular}


CCA Parameter Listing

\begin{tabular}{|c|c|c|c|c|c|c|c|c|c|c|}
\hline ID & 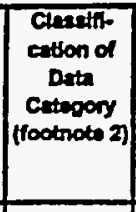 & $\begin{array}{l}\text { Distribution } \\
\text { Type }\end{array}$ & Man & Medien & Minkmum & Medints & Unts & Reformes id & $\begin{array}{l}\text { Was the dient } \\
\text { domeloped } \\
\text { underen } \\
\text { Nol-1 } \\
\text { Progrem? }\end{array}$ & 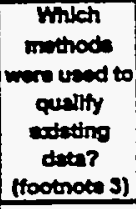 \\
\hline 2613 & 3,5 & CONSTANT & $0.0000000 E+\infty 0$ & $0.0000000 E+\infty 0$ & $0.0000000 E+\infty$ & $0.0000000 E+\infty$ & Pa & WP040514 & $\mathbf{Y}$ & NAA \\
\hline 2614 & 3,5 & CONSTANT & $0.0000000 E+00$ & $0.0000000 \mathrm{E}+00$ & $0.0000000 E+\infty$ & $0.0000000 E+\infty 0$ & NONE & $\begin{array}{c}\text { MEMO:VAUGHNN } \\
\text { TIERNEY-9 } \\
\end{array}$ & $\mathbf{Y}$ & NA \\
\hline 2614 & 3.5 & CONSTANT & $0.0000000 E+00$ & $0.0000000 E+\infty$ & $0.0000000 E+\infty 0$ & $0.0000000 E+\infty$ & NONE & WP035597 & $\mathbf{Y}$ & NA \\
\hline 2614 & 3,5 & CONSTANT & $0.00000000++\infty 0$ & $0.0000000 E+\infty$ & $0.0000000 E+\infty 0$ & $0.0000000 E+\infty 0$ & NONE & WPO40514 & $\mathbf{Y}$ & NA \\
\hline 2616 & 1,3 & CONSTANT & $0.0000000 \mathrm{E}+\infty 0$ & $0.0000000 E+\infty$ & $0.0000000 E+\infty 0$ & $0.0000000 E+\infty$ & NONE & $\begin{array}{c}\text { MEMO:VAUGHN } \\
\text { TIERNEY-9 } \\
\end{array}$ & $\mathbf{Y}$ & NA \\
\hline 2616 & 1,3 & CONSTANT & $\because 0.0000000 E+\infty$ & $0.0000000 E+\infty 0$ & $0.0000000 E+\infty$ & $0.0000000 E+\infty 0$ & NONE & WP03527\% & $\mathbf{Y}$ & NA \\
\hline 2616 & 1,3 & CONSTANT & $0.0000000 E+\infty 0$ & $0.0000000 E+\infty 0$ & $0.0000000 E+\infty 0$ & $0.0000000 E+\infty$ & NONE & WPO35333 & $\mathbf{Y}$ & NA \\
\hline 2616 & 1,3 & CONSTANT & $0.0000000 \in+\infty 0$ & $0.0000000 E+\infty$ & $0.0000000 E+\infty$ & $0.0000000 E+\infty$ & NONE & WP035597 & $\mathbf{Y}$ & NA \\
\hline 2616 & 1,3 & CONSTANT & $0.0000000 \mathrm{E}+\infty 0$ & $0.0000000 E+\infty$ & $0.0000000 E+\infty 0$ & $0.0000000 E+\infty$ & NONE & WPO40514 & $\mathbf{Y}$ & NA \\
\hline 2617 & 1,3 & CONSTANT & $0.0000000 E+00$ & $0.0000000 E+\infty 0$ & $0.0000000 E+\infty$ & $0.0000000 E+\infty 0$ & Pa & $\begin{array}{c}\text { MEMO:VAUGHN } \\
\text { TIERNEY-9 }\end{array}$ & $\mathbf{Y}$ & N/A \\
\hline 2617 & 1,3 & CONSTANT & $0.0000000 E+00$ & $0.0000000 E+\infty$ & $0.0000000 E+\infty$ & $0.0000000 E+00$ & $P=$ & WP035597 & $\mathbf{Y}$ & N/A \\
\hline 2617 & 1,3 & CONSTANT & $0.0000000 \mathrm{E}+00$ & $0.0000000 E+\infty 0$ & $0.0000000 E+\infty 0$ & $0.0000000 E+\infty$ & Pa & WPO40514 & $\mathbf{Y}$ & NA \\
\hline 2618 & 1,3 & CONSTANT & $0.0000000 \mathrm{E}+\infty 0$ & $0.0000000 \mathrm{E}+\infty 0$ & $0.0000000 E+\infty$ & $0.0000000 E+\infty 0$ & NONE & $\begin{array}{c}\text { MEMO:VAUGHN } \\
\text { TIERNEY-9 }\end{array}$ & $\mathbf{Y}$ & NA \\
\hline 2618 & 1,3 & CONSTANT & $0.0000000 E+\infty$ & $0.0000000 E+\infty$ & $0.0000000 E+\infty$ & $0.0000000 E+\infty 0$ & NONE & WP035597 & $\mathbf{Y}$ & NA \\
\hline 2618 & 1.3 & CONSTANT & $0.0000000 E+\infty$ & $0.0000000 E+\infty 0$ & $0.0000000 \mathrm{E}+\infty$ & $0.0000000 E+\infty$ & NONE & WPO40514 & $\mathbf{Y}$ & NA \\
\hline 2620 & 1,3 & CONSTANT & $0.0000000 E+\infty 0$ & $0.0000000 E+00$ & $0.00000000+\infty 0$ & $0.0000000 E+00$ & NONE & $\begin{array}{c}\text { MEMO:VAUGHN } \\
\text { TIERNEY-9 } \\
\end{array}$ & $\mathbf{Y}$ & NA \\
\hline 2620 & 1,3 & CONSTANT & $0.0000000 E+\infty$ & $0.0000000 \mathrm{E}+\infty$ & $0.0000000 E+\infty 0$ & $0.0000000 E+\infty 0$ & NONE & WP035271 & $\mathbf{Y}$ & NA \\
\hline 2620 & 1,3 & CONSTANT & $0.0000000 E+\infty 0$ & $0.0000000 E+\infty$ & $0.0000000 E+\infty$ & $0.0000000 E+00$ & NONE & WP035333 & $\mathbf{Y}$ & NA \\
\hline 2620 & 1,3 & CONSTANT & $0.0000000 E+\infty 0$ & $0.0000000 E+\infty$ & $0.0000000 E+\infty 0$ & $0.0000000 E+\infty$ & NONE & WP035597 & $\mathbf{Y}$ & NA \\
\hline 2620 & 1,3 & CONSTANT & $0.0000000 E+\infty$ & $0.0000000 \mathrm{E}+00$ & $0.0000000 E+\infty$ & $0.0000000 E+\infty)$ & NONE & WPO40514 & $\mathbf{Y}$ & NA \\
\hline 2621 & 1,3 & CONSTANT & $0.0000000 E+\infty$ & $0.0000000 E+\infty$ & $0.0000000 E+00$ & $0.0000000 E+\infty 0$ & Pa & $\begin{array}{c}\text { MEMO:VAUGHN } \\
\text { TIERNEY-9 } \\
\end{array}$ & $\mathbf{Y}$ & NA \\
\hline 2621 & 1,3 & CONSTANT & $0.0000000 E+\infty 0$ & $0.0000000 E+\infty 0$ & $0.0000000 E+00$ & $0.0000000 E+\infty 0$ & Pa & WP035597 & $\mathbf{Y}$ & NA \\
\hline 2621 & 1,3 & CONSTANT & $0.0000000 E+\infty$ & $0.0000000 E+\infty 0$ & $0.00000000+00$ & $0.0000000 E+\infty 0$ & Pa & WPO40514 & $\mathbf{Y}$ & $N A$ \\
\hline 2622 & 1,3 & CONSTANT & $0.0000000 E+\infty 0$ & $0.0000000 E+\infty 0$ & $0.0000000=+\infty$ & $0.0000000=+\infty$ & NONE & $\begin{array}{l}\text { MEMO:VAUGHN } \\
\text { TIERNEY-9 } \\
\end{array}$ & $\mathbf{Y}$ & NA \\
\hline 2622 & 1,3 & CONSTANT & $0.0000000 E+\infty$ & $0.0000000 E+00$ & $0.0000000 E+\infty$ & $0.0000000 E+\infty$ & NONE & WP035597 & $--Y$ & NA \\
\hline 2622 & 1,3 & CONSTANT & $0.0000000 E+\infty$ & $0.0000000 E+\infty$ & $0.0000000 E+\infty$ & $0.0000000 E+\infty$ & NONE & WPO40514 & $\mathbf{Y}$ & NA \\
\hline 2623 & 1,3 & CONSTANT & $1.0132500 E+05$ & $1.0132500 E+05$ & $1.0132500 E+05$ & $1.0132500 E+05$ & $\mathbf{P a}$ & $\begin{array}{c}\text { MEMO:VAUGHN } \\
\text { TIERNEY-9 }\end{array}$ & $\mathbf{Y}$ & N/A \\
\hline 2623 & 1,3 & CONSTANT & $1.0132500 E+05$ & $1.0132500 E+05$ & $1.0132500 \leqslant+05$ & $1.0132500 E+05$ & $P \approx$ & WP035597 & $\mathbf{Y}$ & NA \\
\hline 2623 & 1,3 & CONSTANT & $1.0132500 E+05$ & $1.0132500 E+05$ & $1.0132500 E+05$ & $1.0132500 E+05$ & Pa & WP035051 & $\mathbf{Y}$ & NA \\
\hline 2623 & 1,3 & CONSTANT & $1.0132500 E+05$ & $1.0132500 E+05$ & $1.0132500 E+05$ & $1.0132500 E+05$ & $P=$ & WPO40514 & $\mathbf{Y}$ & NA \\
\hline 2625 & 1,3 & CONSTANT & $0.0000000 E+\infty$ & $0.0000000 E+\infty$ & $0.0000000 E+00$ & $0.0000000 E+\infty$ & NONE & $\begin{array}{c}\text { MEMO:VAUGHN } \\
\text { TIERNEY.9 } \\
\end{array}$ & $\mathbf{Y}$ & NA \\
\hline 2625 & 1.3 & CONSTANT & $0.0000000 E+00$ & $0.0000000 E+\infty 0$ & $0.0000000 E+\infty$ & $0.0000000 E+\infty$ & NONE & WP035271 & $\mathbf{Y}$ & NA \\
\hline 2625 & 1,3 & CONSTANT & $0.0000000 E+00$ & $0.0000000 E+\infty 0$ & $0.0000000 E+\infty$ & $0.0000000 E+\infty 0$ & NONE & WP035333 & $\mathbf{Y}$ & NA \\
\hline 2625 & 1,3 & CONSTANT & $0.0000000 E+00$ & $0.0000000 E+\infty 0$ & $0.0000000 E+\infty 0$ & $0.0000000 E+\infty 0$ & NONE & WPO35597 & $\mathbf{Y}$ & NA \\
\hline 2625 & 1,3 & CONSTANT & $0.0000000 E+\infty$ & $0.0000000 E+\infty$ & $0.0000000 E+00$ & $0.00000005+00$ & NONE & WPO40514 & $Y$ & NA \\
\hline 2626 & 1,3 & CONSTANT & $0.0000000 E+\infty$ & $0.0000000 E+\infty$ & $0.0000000 E+00$ & $0.0000000 E+\infty$ & Pa & $\begin{array}{c}\text { MEMO:VAUGHNU } \\
\text { TIERNEY-9 } \\
\end{array}$ & $\mathbf{Y}$ & NAA \\
\hline
\end{tabular}


CCA Parameter Listing -

\begin{tabular}{|c|c|c|c|c|c|c|c|c|c|c|}
\hline $\begin{array}{c}\text { Paremeter in } \\
\text { Dertabeat? } \\
\text { (XNI) }\end{array}$ & 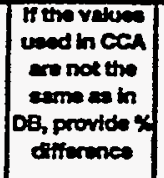 & 10 & meanted to & Meterel kems & Ponetion & Parminar Nimo & $\begin{array}{l}\text { PRPDD } \\
\text { (MPON) }\end{array}$ & $\begin{array}{c}\text { Deta Entry } \\
\text { Dets }\end{array}$ & $\begin{array}{l}\text { bo the dx } \\
\text { coneletent } \\
\text { with the } \\
\text { perameter } \\
\text { in DE? } \\
M, N A)\end{array}$ & $\begin{array}{l}\text { Peranieter } \\
\text { Centegory } \\
\text { (footnote 1) }\end{array}$ \\
\hline $\mathbf{Y}$ & & 2626 & CAVITY_4 & Cavity for Bonchale & PCT_A & $\begin{array}{l}\text { Threshold Pressuro Lnaser } \\
\text { Paremeter }\end{array}$ & 31797 & $17 f+b-96$ & $\mathbf{Y}$ & AB \\
\hline $\mathbf{Y}$ & & 2526 & CAVTY_4 & Centy tor Borchole & PCT_A & $\begin{array}{l}\text { Treashold Pressure Linest } \\
\text { Peremeter }\end{array}$ & 31797 & $17 f=b-96$ & $\mathbf{Y}$ & $4 B$ \\
\hline $\mathbf{Y}$ & & 2527 & CAVIT_4 & Cevity for Boraholo & PCT_EXP & $\begin{array}{l}\text { Threshold pressure exponential } \\
\text { pereneter }\end{array}$ & 31798 & 17 feb-96 & $\mathbf{Y}$ & 48 \\
\hline $\mathbf{Y}$ & & 2627 & CAVIT_4 & Cevity for Borehole & PCT_EXP & $\begin{array}{l}\text { Timoshold pressure exponented } \\
\text { permenter }\end{array}$ & 31798 & $17+f a b-86$ & $\mathbf{Y}$ & 48 \\
\hline $\mathbf{Y}$ & & 2027 & CAVTTY_4 & Cavity for Borchole & PCT_EXP & $\begin{array}{l}\text { Threshold presure exponential } \\
\text { paraneter }\end{array}$ & 31798 & $17+{ }^{2} b-96$ & $\mathbf{Y}$ & 48 \\
\hline $\mathbf{Y}$ & & 2628 & CAVITY_4 & Conity for Borehols & PO_MIN & $\begin{array}{l}\text { Minimum brne pressure for } \\
\text { capiltary modei KPC=3 }\end{array}$ & 31803 & 17 feb-96 & $\mathbf{Y}$ & $4 B$ \\
\hline $\mathbf{Y}$ & & 2528 & CAVIT_4 & Cenity for Boreholo & PO_MIN. & 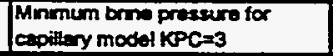 & 318003 & $17+f a b-96$ & $\mathbf{Y}$ & 48 \\
\hline $\mathbf{Y}$ & & 2628 & CAVITY_4 & Cavity for Borehols & PO_MIN & $\begin{array}{l}\text { Minimum brme pressure for } \\
\text { capiliary model KPC=3 }\end{array}$ & 31803 & $17-F a b-96$ & $\mathbf{Y}$ & AB \\
\hline $\mathbf{Y}$ & & 2628 & CAVTTY_4 & Cevity for Boretholo & PO_MIN & $\begin{array}{l}\text { Minimum brno prossure for } \\
\text { cepiliery model KPC=3 }\end{array}$ & 31803 & $17+f a b-96$ & $\mathbf{Y}$ & $4 B$ \\
\hline $\mathbf{Y}$ & & 2636 & CLAY_BOT & Shan Bottom Cliny & KPT & $\begin{array}{l}\text { Flag for Permeabilty Determined } \\
\text { Threshold }\end{array}$ & 31858 & $21+c b-96$ & $\mathbf{Y}$ & AB \\
\hline $\mathbf{Y}$ & & 2635 & CLAY_BOT & Shan Bottom Clay & KPT & $\begin{array}{l}\text { Fleg for Permeability Determned } \\
\text { Threshold }\end{array}$ & 31858 & $21+f c b-96$ & $\mathbf{Y}$ & AB \\
\hline $\mathbf{Y}$ & & 2636 & CLAY_BOT & Shat Bottom Clay & KPT & $\begin{array}{l}\text { Flog for Permeabilaty Dotermined } \\
\text { Throthold }\end{array}$ & 31858 & 21 Feb-96 & $\mathbf{Y}$ & $4 B$ \\
\hline $\mathbf{Y}$ & & 2636 & CLAY_BOT & Shant Bottom Clay & KPT & $\begin{array}{l}\text { Fleg for Permesbiliy Determined } \\
\text { Threstroid }\end{array}$ & 31858 & $21+c_{c}-96$ & $\mathbf{Y}$ & 48 \\
\hline $\mathbf{Y}$ & 100 & 2637 & CLAY_BOT & Shat Bottom Clay & PCT_A & $\begin{array}{l}\text { Throshold Pressurs Lneser } \\
\text { Parameter }\end{array}$ & 31859 & $21-F a b-96$ & $\mathbf{Y}$ & 48 \\
\hline $\mathbf{Y}$ & 100 & 2637 & CLAY_BOT & Shat Bottom Clay & PCT_A & $\begin{array}{l}\text { Threshold Pressure Lnear } \\
\text { Paremeter }\end{array}$ & 31859 & $21+c b-8$ & $\mathbf{Y}$ & 48 \\
\hline $\mathbf{Y}$ & 100 & 2637 & CLAY_BOT & Shat Bottom Cray & PCT_A & $\begin{array}{l}\text { Threstold Pressure Lnear } \\
\text { Parameter }\end{array}$ & 31859 & $21+5 a b-96$ & $\mathbf{Y}$ & $A B$ \\
\hline $\mathbf{Y}$ & 100 & 2637 & CLAY_BOT & Shan Bottom Clay & PCT_A & $\begin{array}{l}\text { Threshold Pressure Lnear } \\
\text { Paramoler }\end{array}$ & 31859 & 21-Fab-S6 & $\mathbf{Y}$ & AB \\
\hline $\mathbf{Y}$ & 100 & 2637 & CLAY_BOT & Shat Bottom Clay & PCT_A & $\begin{array}{l}\text { Threshold Pressure Linest } \\
\text { Parameter }\end{array}$ & 31859 & 21-Fab-96 & $\mathbf{Y}$ & 48 \\
\hline $\mathbf{Y}$ & 100 & 2637 & CLAY_BOT & Shan Bottom Cloy & PCT_A & $\begin{array}{l}\text { Trreshold Pressure Linear } \\
\text { Paramoter }\end{array}$ & 31859 & $21+F e b-96$ & $\mathbf{Y}$ & $\mathbf{A B}$ \\
\hline $\mathbf{Y}$ & 100 & 2638 & CLAY_BOT & Shar Bottom Clyy & PCT_EXP & $\begin{array}{l}\text { Throshold preseuro exponential } \\
\text { parameter }\end{array}$ & 31860 & 21-feb-96 & $\mathbf{Y}$ & 48 \\
\hline $\mathbf{Y}$ & 100 & 2638 & CLAY_BOT & Shen Bottom Clay & PCT_EXP & $\begin{array}{l}\text { Threshold pressure exponential } \\
\text { parameter }\end{array}$ & 31860 & 21-Fab-96 & $\mathbf{Y}$ & 48 \\
\hline $\mathbf{Y}$ & 100 & 2638 & CLAY_BOT & Shan Bottom Clyy & PCT_EXP & $\begin{array}{l}\text { Threshold pressure exponential } \\
\text { parameter }\end{array}$ & 31860 & $21+F e b-96$ & $\mathbf{Y}$ & $4 B$ \\
\hline $\mathbf{Y}$ & 100 & 2538 & CLAY_BOT & Shath Bottom Clay & PCT_EXP & $\begin{array}{l}\text { Thresthold pressure exponontial } \\
\text { pareneter }\end{array}$ & 31860 & $21+$ eb-96 & $\mathbf{Y}$ & 48 \\
\hline $\mathbf{Y}$ & 100 & 2538 & CLAY_BOT & Shatt Bottom Clay & PCT_EXP & $\begin{array}{l}\text { Threshold pressure exporientual } \\
\text { paremeter }\end{array}$ & 31860 & 21-Fab-96 & $\mathbf{Y}$ & $4 B$ \\
\hline $\mathbf{Y}$ & 100 & 2638 & CLAY_BOT & Shan Bottom Clay & PCT_EXP & $\begin{array}{l}\text { Threshold pressure exponentual } \\
\text { perameter }\end{array}$ & 31860 & $21+F u b-96$ & $--Y$ & 48 \\
\hline $\mathbf{Y}$ & & 2641 & CL_L_T1 & $\begin{array}{l}\text { Lower Salado clay.0 to } \\
10 \text { yoars }\end{array}$ & KPT & $\begin{array}{l}\text { Fleg for Permeability Dolermned } \\
\text { Threshotd }\end{array}$ & 31900 & 20 -Feb-96 & $\mathbf{Y}$ & 48 \\
\hline $\mathbf{Y}$ & & 2641 & $C L_{-} L_{-} T_{1}$ & $\begin{array}{l}\text { Lower Selado diry.0 to } \\
10 \text { yours }\end{array}$ & $K P T$ & $\begin{array}{l}\text { Fleg for Permesbilty Defermined } \\
\text { Threshold }\end{array}$ & 31900 & $20-F a b-96$ & $\mathbf{Y}$ & 48 \\
\hline$Y$ & & 2641 & CL_L_T1 & $\begin{array}{l}\text { Lower Salado ctay.0 to } \\
10 \text { years }\end{array}$ & KPT & $\begin{array}{l}\text { Fleg for Permesbility Determined } \\
\text { Threshold }\end{array}$ & 31900 & $20 F=b .96$ & $\mathbf{Y}$ & $4 B$ \\
\hline $\mathbf{Y}$ & & 2641 & $C L L L T H$ & $\begin{array}{l}\text { Lower Suledo cley.0 to } \\
10 \text { years }\end{array}$ & KPT & $\begin{array}{l}\text { Flag for Permesbility Determmed } \\
\text { Threshold }\end{array}$ & 31900 & $20+c b-96$ & $\mathbf{Y}$ & $4 B$ \\
\hline $\mathbf{Y}$ & & 2641 & $C L L L T 1$ & $\begin{array}{l}\text { Lower Salado day.0 to } \\
10 \text { years }\end{array}$ & KPT & $\begin{array}{l}\text { Flag for Permesbility Determned } \\
\text { Threshold }\end{array}$ & 31900 & $20-F a b-96$ & $\mathbf{Y}$ & $4 B$ \\
\hline $\mathbf{Y}$ & 100 & 2612 & CL_L_TI & $\begin{array}{l}\text { Lower Salado clay. } 0 \text { to } \\
10 \text { yoars }\end{array}$ & PCT_A & $\begin{array}{l}\text { Threshold Pressure Lnesr } \\
\text { Peremeter }\end{array}$ & 31901 & $13-F u b-96$ & $\mathbf{Y}$ & 1 \\
\hline $\mathbf{Y}$ & 100 & 2642 & $C L L L+T 1$ & $\begin{array}{l}\text { Lower Saledo clay.0 to } \\
10 \text { year }\end{array}$ & PCT_A & $\begin{array}{l}\text { Threshold Pressuro Lnear } \\
\text { Perenoter }\end{array}$ & 31901 & $13-F c b-96$ & $\mathbf{Y}$ & 1 \\
\hline $\mathbf{Y}$ & 100 & 2642 & $C L L_{-} T 1$ & $\begin{array}{l}\text { Lower Saledo ctry.0 to } \\
10 \text { years }\end{array}$ & PCT_A & $\begin{array}{l}\text { Threshold Pressure Lnear } \\
\text { Pareneter }\end{array}$ & 31901 & $13-F a b-96$ & $\mathbf{Y}$ & 1 \\
\hline$Y$ & 100 & 2642 & CL_L_T1 & $\begin{array}{l}\text { Lower Salado clay:0 to } \\
10 \text { yers }\end{array}$ & PCT_A & $\begin{array}{l}\text { Threshold Preasure Lnear } \\
\text { Pereneter }\end{array}$ & 31901 & 13ffebses & $\mathbf{Y}$ & 1 \\
\hline $\mathbf{Y}$ & 100 & 2613 & CL_L_T1 & $\begin{array}{l}\text { Lower Saledo ctiny. } 0 \text { to } \\
10 \text { years }\end{array}$ & PCT_EXP & $\begin{array}{l}\text { Threthotd preasure exponential } \\
\text { peremeter }\end{array}$ & 31902 & 13-Feb-96 & $\mathbf{Y}$ & 1 \\
\hline $\mathbf{Y}$ & 100 & 2643 & CL_L_T1 & $\begin{array}{l}\text { Lower Saledo clay.0 to } \\
10 \text { years }\end{array}$ & PCT_EXP & $\begin{array}{l}\text { Threstiold pressurv exponential } \\
\text { peremoter }\end{array}$ & 31902 & 13ffeb-86 & $\mathbf{Y}$ & 1 \\
\hline
\end{tabular}


CCA Parameter-Listing

\begin{tabular}{|c|c|c|c|c|c|c|c|c|c|c|}
\hline 10 & 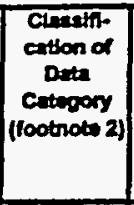 & $\begin{array}{l}\text { Defribution } \\
\text { Type }\end{array}$ & Men & Medien & Minimiman & Medroum & Unta & Refermenes 10 & $\begin{array}{l}\text { Was the data } \\
\text { derveloped } \\
\text { underen } \\
\text { Non-1 } \\
\text { Progrum? }\end{array}$ & 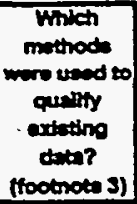 \\
\hline 2626 & 1,3 & CONSTANT & $0.0000000 E+\infty$ & $0.0000000 E+\infty$ & $0.0000000 E+\infty 0$ & $0.0000000 E+\infty$ & $\mathbf{P a}$ & WP035597 & $Y$ & NA \\
\hline 2626 & 1,3 & CONSTANT & $0.0000000 E+\infty$ & $0.0000000 E+\infty 0$ & $0.0000000 E+\infty$ & $0.0000000 E+\infty$ & Pa & WPOA0514 & $Y$ & N/A \\
\hline 2627 & 1,3 & CONSTANT & $0.0000000 E+\infty$ & $0.0000000 E+\infty 0$ & $0.0000000 E+\infty 0$ & $0.0000000 E+\infty$ & NONE & $\begin{array}{l}\text { MEMO:VAUGHN } \\
\text { TERNEYS9 }\end{array}$ & $Y$ & NA \\
\hline 2627 & 1,3 & CONSTANT & $0.0000000 E+\infty$ & $0.00000000+\infty 0$ & $0.0000000 E+\infty$ & $0.0000000=+\infty$ & NONE & WP035597 & $\mathbf{Y}$ & NA \\
\hline 2627 & 1,3 & CONSTANT & $0.0000000 E+\infty 0$ & $0.0000000 E+00$ & $0.0000000 E+\infty$ & $0.0000000 E+\infty$ & NONE & WP040514 & $\mathbf{Y}$ & NA \\
\hline 2628 & 1,3 & CONSTANT & $1.0132500 E+05$ & $1.0132500 E+05$ & $1.0132500 E+05$ & $1.0132500 E+05$ & Pa & $\begin{array}{l}\text { MENO:VAUGHN } \\
\text { TIERNEY-9, }\end{array}$ & $\mathbf{Y}$ & NA \\
\hline 2628 & 1,3 & CONSTANT & $1.0132500 E+05$ & $1.0132500 \mathrm{E}+05$ & $1.0132500 E+05$ & $1.0132500 E+05$ & Pa & UP035597 & $\mathbf{Y}$ & NA \\
\hline 2628 & 1,3 & CONSTANT & $1.0132500 E+05$ & $1.0132500 E+05$ & $1.0132500 E+05$ & $1.0132500 E+05$ & Pe & WP0s6051 & $\mathbf{Y}$ & N/A \\
\hline 2628 & 1,3 & CONSTANT & $1.0132500 E+05$ & $1.0132500 E+0.5$ & $1.0132500 E+05$ & $1.0132500 E+05$ & - $P_{a}$ & WPO40514 & $\mathbf{Y}$ & NA \\
\hline 2636 & 1,3 & CONSTANT & $0.0000000 E+\infty$ & $0.0000000 \mathrm{E}+\infty$ & $0.0000000 E+\infty$ & $0.0000000 E+\infty$ & NONE & WP035271 & $\mathbf{Y}$ & NA \\
\hline 2636 & 1,3 & CONSTANT & $0.0000000 E+00$ & $0.00000000+\infty$ & $0.0000000 E+00$ & $0.00000000+\infty 0$ & NONE & WP035333 & $\mathbf{Y}$ & NA \\
\hline 2636 & 1,3 & CONSTANT & $0.0000000 \mathrm{E}+\infty$ & $0.00000000+\infty$ & $0.0000000 \mathrm{E}+\infty$ & $0.0000000 E+\infty$ & NONE & WP035597 & $\mathbf{Y}$ & NA \\
\hline 2636 & 1,3 & CONSTANT & $0.0000000 E+\infty$ & $0.0000000 E+\infty$ & $0.0000000 E+\infty$ & $0.00000000 E+\infty$ & NONE & WPO40514 & $\mathbf{Y}$ & NA \\
\hline 2637 & $1,3,4$ & CONSTANT & $5.6000000 E-01$ & $5.6000000 E-01$ & $5.6000000 E-01$ & $5.6000000 E-01$ & Pa & WP030994 & $\mathbf{Y}$ & NA \\
\hline 2637 & $1,3,4$ & CONSTANT & $5.6000000 E-01$ & $5.6000000 E-01$ & $5.6000000 E-01$ & 5.6000000E-01 & Pa & WP035267 & $\mathbf{Y}$ & NA \\
\hline 2637 & $1,3,4$ & CONSTANT & $5.6000000 E-01$ & 5.6000000E-01 & $5.6000000 E-01$ & $5.6000000 E-01$ & $P=$ & WP035597 & $\mathbf{Y}$ & NA \\
\hline 2637 & $1,3,4$ & CONSTANT & $5.6000000 E-01$ & $5.6000000 E-01$ & $5.6000000 E-01$ & $5.6000000 E-01$ & $\mathrm{~Pa}$ & WP035383 & $\mathbf{Y}$ & NA \\
\hline 2637 & $1,3,4$ & CONSTANT & $5.6000000 E-01$ & $5.6000000 E-01$ & $5.6000000 E-01$ & S.6000000E-01 & $P_{2}$ & WPO40434 & $\mathbf{Y}$ & NA \\
\hline 2637 & $1,3,4$ & CONSTANT & $5.6000000 E-01$ & $5.6000000 E-01$ & 5.6000000E-01 & $5.6000000 \mathrm{E}-01$ & Pa & WPO40514 & $\mathbf{Y}$ & NA \\
\hline 2638 & $1,3,4$ & CONSTANT & $-3.4600000 \mathrm{E}-01$ & $3.4600000 E-01$ & $3.4600000 E-01$ & $3.4600000 E-01$ & NONE & WP030994 & $\mathbf{Y}$ & NA \\
\hline 2638 & $1,3,4$ & CONSTANT & $-3.4600000=-01$ & $-3.4600000 E-01$ & $-3.4600000 \mathrm{E}-01$ & $-3.4600000 \mathrm{E}-01$ & NONE & WP035267 & $\mathbf{Y}$ & N/A \\
\hline 2638 & $1,3,4$ & CONSTANT & $-3.4600000 E-01$ & $-3.4600000 E-01$ & $-34600000 E-01$ & $3.4600000 E-01$ & NONE & WP035597 & $\mathbf{Y}$ & NA \\
\hline 2638 & $1,3,4$ & CONSTANT & $3.46000005-01$ & $3.4600000 E-01$ & $-3.4600000 E-01$ & $3.4600000 \mathrm{E}-01$ & NONE & WP036383 & $\mathbf{Y}$ & N/A \\
\hline 2638 & $1,3,4$ & CONSTANT & $3,4600000 \mathrm{E}-01$ & $3.4600000 E-01$ & $3.4500000 E-01$ & $3.4600000 E-01$ & NONE & WPO40434 & $\mathbf{Y}$ & NA \\
\hline 2638 & $1,3,4$ & CONSTANT & $-3.4600000 E-01$ & 3.4600000 E-01 & $34600000 E-01$ & $3.4600000 E-01$ & NONE & WPO40514 & $-Y$ & NA \\
\hline 2841 & 3,5 & CONSTANT & $0.0000000 E+\infty 0$ & $0.0000000 E+\infty$ & $0,0000000 E+\infty$ & $0.0000000 E+\infty$ & NONE & WP030640 & $\mathbf{Y}$ & NA \\
\hline 2641 & 3,5 & CONSTANT & $0.0000000 \mathrm{E}+\infty$ & $0.0000000 E+\infty$ & $0.0000000 E+\infty$ & $0.00000000+\infty$ & NONE & WP030994 & $\mathbf{Y}$ & NA \\
\hline 2641 & 3,5 & CONSTANT & $0.00000000+\infty 0$ & $0.0000000 E+\infty$ & $0.0000000 E+\infty$ & $0,0000000 E+\infty$ & NONE & WP035333 & $\mathbf{Y}$ & N/A \\
\hline 2641 & 3,5 & CONSTANT & $0.0000000 \mathrm{E}+\infty$ & $0.0000000 E+\infty$ & $0.0000000 E+\infty 0$ & $0.0000000 E+\infty$ & NONE & WP035597 & $\mathbf{Y}$ & NA \\
\hline 2649 & 3,5 & CONSTANT & $0.00000000+\infty$ & $0.0000000=+\infty$ & $0.0000000 E+\infty$ & $0.0000000 \mathrm{E}+\infty$ & NONE & WP040514 & $\mathbf{Y}$ & $N / A$ \\
\hline 2642 & 3,4 & CONSTANT & $5.6000000 E-01$ & $5.60000006-01$ & $5.6000000 E-01$ & $5.6000000 E-01$ & Pa & WP030640 & $Y$ & NA \\
\hline 2642 & 3,4 & CONSTANT & $5.60000000-01$ & $5.6000000 E-01$ & $5.6000000 E-01$ & $5.6000000 E-01$ & $\mathbf{P a}$ & UP030994 & $\mathbf{Y}$ & NA \\
\hline 2642 & 3.4 & CONSTANT & $5.6000000 E-01$ & $5.6000000 \leqslant-01$ & $5.6000000 E-01$ & $56000000 E-01$ & Pa & WP035597 & $Y$ & NA \\
\hline 2642 & 3,4 & CONSTANT & $5.6000000 E-01$ & $5.6000000 E-01$ & $56000000 E-01$ & $5.6000000 E-01$ & Pa & WPO40434 & $\mathbf{Y}$ & NA \\
\hline 2643 & 3,4 & CONSTANT & $3.46000000-01$ & $3.4600000 \varepsilon-01$ & $34600000 E-01$ & $3.4600000 \varepsilon-01$ & NONE & WP030640 & $\mathbf{Y}$ & NA \\
\hline 2643 & 3,4 & CONSTANT & $3.4600000 E-01$ & $-3.4600000 \varepsilon-01$ & $-3.46000000-01$ & $3.4600000 E-01$ & NONE & WPOSO994 & $\mathbf{Y}$ & NA \\
\hline
\end{tabular}


CCA-Parameter Listing

\begin{tabular}{|c|c|c|c|c|c|c|c|c|c|c|}
\hline 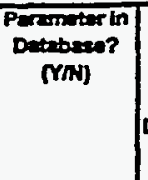 & 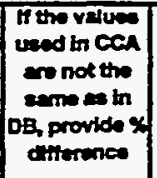 & 10 & Metariv to & Metarta NGm & Permotion & Parmeter Neme & $\begin{array}{l}\text { PRPD } \\
\text { INPO An }\end{array}$ & Den Entry & 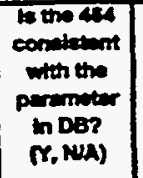 & $\begin{array}{l}\text { Parmintar } \\
\text { Category } \\
\text { (tootnoti 1) }\end{array}$ \\
\hline $\mathbf{Y}$ & 100 & 2643 & CL_L_T1 & $\begin{array}{l}\text { Lower Salado clay.0 to } \\
10 \text { years }\end{array}$ & PCT_EXP & $\begin{array}{l}\text { Threshold pressure exponental } \\
\text { pereneter }\end{array}$ & 31902 & 13 Feb-96 & $\mathbf{Y}$ & 1 \\
\hline $\mathbf{Y}$ & 100 & 2643 & CL_L_T1 & $\begin{array}{l}\text { Lower Salado clay.0 to } \\
10 \text { yoar }\end{array}$ & PCT_EXP & $\begin{array}{l}\text { Threshold pressure exporential } \\
\text { peremeter }\end{array}$ & 31902 & $13-f a b-96$ & $\mathbf{Y}$ & 1 \\
\hline $\mathbf{Y}$ & & 2646 & CL_L_T2 & $\begin{array}{l}\text { Lower Stado ciny:10 } \\
\text { to } 25 \text { years }\end{array}$ & KPT & $\begin{array}{l}\text { Flog for Permeability Determined } \\
\text { Threshold }\end{array}$ & 31927 & 20-Fab-96 & $\mathbf{Y}$ & 48 \\
\hline $\mathbf{Y}$ & & 2646 & CL_L_T2 & $\begin{array}{l}\text { Lowor Salado cty: } 10 \\
\text { to } 25 \text { years }\end{array}$ & KPT & $\begin{array}{l}\text { Flag for Permenobilty Determmed } \\
\text { Imreshold }\end{array}$ & 31927 & 20-Feb-96 & $\mathbf{Y}$ & 48 \\
\hline $\mathbf{Y}$ & & 2646 & CL_L_T2 & $\begin{array}{l}\text { Lower Salado cloy.10 } \\
\text { to } 25 \text { years }\end{array}$ & KPT & $\begin{array}{l}\text { Fleg for Peamiabilay Determined } \\
\text { Trreshold }\end{array}$ & 31927 & $20-f 0 b-96$ & $\mathbf{Y}$ & 48 \\
\hline $\mathbf{Y}$ & & 2645 & CL_L_T2 & $\begin{array}{l}\text { Lower Saloco clay. } 10 \\
\text { 10 } 25 \text { years }\end{array}$ & KPT & $\begin{array}{l}\text { Fleg for Permability Determined } \\
\text { Threshold }\end{array}$ & 31927 & $20-f<b-96$ & $\mathbf{Y}$ & 48 \\
\hline $\mathbf{Y}$ & & 2646 & CL_L_T2 & $\begin{array}{l}\text { Lower Salado day: } 10 \\
\text { to } 25 \text { years }\end{array}$ & KPT & $\begin{array}{l}\text { Flog for Permesbilky Determned } \\
\text { Throuhold }\end{array}$ & 31927 & $20-F=b-96$ & $\cdot \mathbf{Y}$ & $4 B$ \\
\hline $\mathbf{Y}$ & 100 & 2647 & $C L \_L \_T 2$ & $\begin{array}{l}\text { Lower Salado ciay: } 10 \\
\text { to } 25 \text { years }\end{array}$ & PCT_A & $\begin{array}{l}\text { Trreshold Pressure Linear } \\
\text { Parenoter }\end{array}$ & 31928 & 14Feb-96 & $\mathbf{Y}$ & 1 \\
\hline $\mathbf{Y}$ & 100 & 2647 & CL_L_T2 & $\begin{array}{l}\text { Lower Salado clay: } 10 \\
\text { to } 25 \text { years }\end{array}$ & PCT_A & $\begin{array}{l}\text { Threstrold Pressure Linaer } \\
\text { Parenoter }\end{array}$ & 31928 & $14 F c b-96$ & $\mathbf{Y}$ & 1 \\
\hline $\mathbf{Y}$ & 100 & 2647 & $C L L L+T 2$ & $\begin{array}{l}\text { Lower Salado clay. } 10 \\
\text { to } 25 \text { years }\end{array}$ & PCT_A & $\begin{array}{l}\text { Threshold Pressur Lnear } \\
\text { Paraneter }\end{array}$ & 31928 & $14 F c b-96$ & $\mathbf{Y}$ & 1 \\
\hline $\mathbf{Y}$ & 100 & 2647 & CL_L_T2 & $\begin{array}{l}\text { Lower Salado clay: } 10 \\
\text { to } 25 \text { years }\end{array}$ & PCT_A & $\begin{array}{l}\text { Threshold Preseura Linear } \\
\text { Parennoter }\end{array}$ & 31928 & 14 feb-96 & $\mathbf{Y}$ & 1 \\
\hline $\mathbf{Y}$ & 100 & 2648 & CL_L_T2 & $\begin{array}{l}\text { Lower Saloco clay:10 } \\
\text { to } 25 \text { yoars }\end{array}$ & PCT_EXP & $\begin{array}{l}\text { Threshold proserere exponential } \\
\text { paremeter }\end{array}$ & 31929 & $14 f a b-96$ & $\mathbf{Y}$ & 1 \\
\hline $\mathbf{Y}$ & 100 & 2648 & CL_L_T2 & $\begin{array}{l}\text { Lower Salado diny: } 10 \\
\text { to } 25 \text { years }\end{array}$ & PCT_EXP & $\begin{array}{l}\text { Threshoid pressure exponential } \\
\text { parameter }\end{array}$ & 31929 & $14 F a b-96$ & $\mathbf{Y}$ & 1 \\
\hline $\mathbf{Y}$ & 100 & 2648 & CL_L_T2 & $\begin{array}{l}\text { Lower Salado day: } 10 \\
\text { to } 25 \text { years }\end{array}$ & PCT_EXP & $\begin{array}{l}\text { Threshold pressure exponential } \\
\text { peraneter }\end{array}$ & 31929 & 14Fab-96 & $\mathbf{Y}$ & 1 \\
\hline $\mathbf{Y}$ & 100 & 2648 & CL_L_T2 & $\begin{array}{l}\text { Lower Salado cty. } 10 \\
\text { to } 25 \text { years }\end{array}$ & PCT_EXP & $\begin{array}{l}\text { Thrashold pressure exponential } \\
\text { perameter }\end{array}$ & 31929 & $14 f 0 b-95$ & $Y$ & 1 \\
\hline$Y$ & & 2651 & CL_L_T3 & $\begin{array}{l}\text { Lower Salado clay:25 } \\
\text { to } 50 \text { years }\end{array}$ & |KPT & $\begin{array}{l}\text { Flag for Pormeability Dotermined } \\
\text { Threshold }\end{array}$ & 31978 & 20 feb-96 & $\mathbf{Y}$ & $4 B$ \\
\hline $\mathbf{Y}$ & & 2651 & CL_L_T3 & $\begin{array}{l}\text { Lower Salado clay:25 } \\
\text { to } 50 \text { years }\end{array}$ & KPT & $\begin{array}{l}\text { Fleg for Permesbility Dotermined } \\
\text { Threshold }\end{array}$ & 31978 & $20-500-96$ & $\mathbf{Y}$ & 48 \\
\hline $\mathbf{Y}$ & & 2651 & CL_L_T3 & $\begin{array}{l}\text { Lower Salado clay:25 } \\
\text { to } 50 \text { years }\end{array}$ & KPT & $\begin{array}{l}\text { Fleg for Permesbildy Determined } \\
\text { Throshold }\end{array}$ & 31978 & 20-Feb-96 & $\mathbf{Y}$ & $4 B$ \\
\hline $\mathbf{Y}$ & & 2651 & $C L \_L / T 3$ & $\begin{array}{l}\text { Lower Salado diry.25 } \\
\text { to } 50 \text { years }\end{array}$ & KPT & $\begin{array}{l}\text { Fleg for Permeability Dotermned } \\
\text { Threshoid }\end{array}$ & 31978 & $20-F=b-96$ & $\mathbf{Y}$ & $A B$ \\
\hline $\mathbf{Y}$ & & 2651 & CL_L_T3 & $\begin{array}{l}\text { Lower Salado clay. } 25 \\
\text { to } 50 \text { years }\end{array}$ & KPT & $\begin{array}{l}\text { Fleg for Permeability Dotermned } \\
\text { Threshold }\end{array}$ & 31978 & 20-feb-96 & $\mathbf{Y}$ & $4 B$ \\
\hline$Y$ & & 2651 & CL_L_T3 & $\begin{array}{l}\text { Lower Salado ctay:25 } \\
\text { to } 50 \text { years }\end{array}$ & KPT & $\begin{array}{l}\text { Flag for Permeability Dotermmed } \\
\text { Throshold }\end{array}$ & 31978 & 20 fob-96 & $Y$ & $4 B$ \\
\hline $\mathbf{Y}$ & 100 & 2652 & CL_L_T3 & $\begin{array}{l}\text { Lowar Salado day.25 } \\
\text { to } 50 \text { years }\end{array}$ & PCT_A & $\begin{array}{l}\text { Threshold Pressure Lnear } \\
\text { Paraneter }\end{array}$ & 31979 & $14 f 0 b-96$ & $\mathbf{Y}$ & 1 \\
\hline $\mathbf{Y}$ & 100 & 2652 & CL_L_T3 & $\begin{array}{l}\text { Lower Salado clay.25 } \\
\text { to } 50 \text { years }\end{array}$ & PCT_A & $\begin{array}{l}\text { Threshold Pressure Linesr } \\
\text { Paremeter }\end{array}$ & 31979 & 14 Fob-96 & $\mathbf{Y}$ & 1 \\
\hline$Y$ & 100 & 2652 & $C L L L T 3$ & $\begin{array}{l}\text { Lower Salado clay } 25 \\
\text { to } 50 \text { years }\end{array}$ & PCT_A & $\begin{array}{l}\text { Threshoid Pressure Linear } \\
\text { Perameter }\end{array}$ & 31979 & 14Fob-96 & $\mathbf{Y}$ & 1 \\
\hline $\mathbf{Y}$ & 100 & 2652 & CL_L_T3 & $\begin{array}{l}\text { Lower Salado day: } 25 \\
\text { to } 50 \text { years }\end{array}$ & PCT_A & $\begin{array}{l}\text { Threchold Pressure Linear } \\
\text { Paremeter } \\
\end{array}$ & 31979 & 14Feb-96 & $-Y Y$ & 1 \\
\hline $\mathbf{Y}$ & 100 & 2653 & CL_L_T3 & $\begin{array}{l}\text { Lower Salado ctay:25 } \\
\text { to } 50 \text { years. }\end{array}$ & PCT_EXP & $\begin{array}{l}\text { Threshold pressure expornentual } \\
\text { parameter }\end{array}$ & 31980 & $14 F 0 b-96$ & $\mathbf{Y}$ & 1 \\
\hline $\mathbf{Y}$ & 100 & 2653 & CL_L_T3 & $\begin{array}{l}\text { Lower Salado clay } 25 \\
\text { to } 50 \text { years }\end{array}$ & PCT_EXP & $\begin{array}{l}\text { Throshodd pressure exponential } \\
\text { perameter }\end{array}$ & 31980 & $14 F \times b-96$ & $\mathbf{Y}$ & 1 \\
\hline $\mathbf{Y}$ & 100 & 2653 & $C L_{-} L_{-}{ }_{3}$ & $\begin{array}{l}\text { Lown Salado clay:25 } \\
\text { to } 50 \text { years }\end{array}$ & PCT_EXP & $\begin{array}{l}\text { Threshold preseure exponential } \\
\text { parameter }\end{array}$ & 31980 & $14 f=0-56$ & $\mathbf{Y}$ & 1 \\
\hline $\mathbf{Y}$ & 100 & 2653 & CL_L_T3 & $\begin{array}{l}\text { Lower Stledo cloy } 25 \\
\text { to } 50 \text { years }\end{array}$ & PCT_EXP & $\begin{array}{l}\text { Threahold prescure exponential } \\
\text { peramoter }\end{array}$ & 31980 & 14Fob-96 & $Y$ & 1 \\
\hline $\mathbf{Y}$ & & 2656 & CL_M_T1 & $\begin{array}{l}\text { Upper Selado clay.0 to } \\
10 \text { years }\end{array}$ & KPT & $\begin{array}{l}\text { Fieg for Permesbitty Daterminad } \\
\text { Thresthold }\end{array}$ & 32040 & $20-F a b-96$ & $\mathbf{Y}$ & AB \\
\hline $\mathbf{Y}$ & & 2656 & CL_M_T1 & $\begin{array}{l}\text { Upper Seledo doy.0 to } \\
10 \text { years }\end{array}$ & KPT & $\begin{array}{l}\text { Fleo for Permesbilty Detemnined } \\
\text { Threathold }\end{array}$ & 32040 & $20-F a b-56$ & $\mathbf{Y}$ & 48 \\
\hline $\mathbf{Y}$ & & 2656 & $C_{2} M_{2} T_{1}$ & $\begin{array}{l}\text { Upper Saledo diry.0 to } \\
10 \text { yeers }\end{array}$ & KPT & $\begin{array}{l}\text { Fleg for Permeabity Determned } \\
\text { Threahold }\end{array}$ & 32040 & 20-Fab-96 & $\mathbf{Y}$ & 48 \\
\hline$Y$ & & 2656 & CL_M_T1 & $\begin{array}{l}\text { Upper Salado ciay.0 to } \\
10 \text { years }\end{array}$ & KPT & $\begin{array}{l}\text { Flag for Permeabity Determned } \\
\text { Threshold }\end{array}$ & 32040 & $20-F e b-96$ & $\mathbf{Y}$ & $A B$ \\
\hline $\mathbf{Y}$ & & 2656 & CL_M_TI & $\begin{array}{l}\text { Upper Salado clay.0 to } \\
\text { to yours }\end{array}$ & KPT & $\begin{array}{l}\text { Fleg for Permeability Dotermined } \\
\text { Threshold }\end{array}$ & 32040 & $20-F a b-96$ & $\mathbf{Y}$ & $4 B$ \\
\hline $\mathbf{Y}$ & & 2656 & CL_H_T1 & $\begin{array}{l}\text { Uppor Saledo ctey.0 to } \\
\text { 10 yuers }\end{array}$ & KPT & $\begin{array}{l}\text { Fing for Permesbulty Determmed } \\
\text { Threahold }\end{array}$ & 32040 & $20-F c b-98$ & $\mathbf{Y}$ & $4 B$ \\
\hline $\mathbf{Y}$ & 100 & 2657 & CL_M_T1 & $\begin{array}{l}\text { Upper Saledo Clay.0 to } \\
\text { 10 years }\end{array}$ & PCT_A & $\begin{array}{l}\text { TTrashold Pressurs Liner } \\
\text { Peremeter }\end{array}$ & 32041 & $15-F=6-96$ & $\mathbf{Y}$ & 1 \\
\hline
\end{tabular}


-CCA Parameter Listing

\begin{tabular}{|c|c|c|c|c|c|c|c|c|c|c|}
\hline ID & $\begin{array}{c}\text { Cinietin- } \\
\text { catton of } \\
\text { Data } \\
\text { Cettegory } \\
\text { (footnota 2) }\end{array}$ & $\begin{array}{l}\text { Dietribution } \\
\text { Typ }\end{array}$ & Mexn & Medth & Dintment & Medinum & Lats: & Reference it & $\begin{array}{l}\text { Was tre deta } \\
\text { dereloped } \\
\text { underen } \\
\text { Noll-1 } \\
\text { Progrem? }\end{array}$ & $\begin{array}{l}\text { minich } \\
\text { mothods } \\
\text { wre used to } \\
\text { qualty } \\
\text { aditing } \\
\text { deta? } \\
\text { (tootnots 3) }\end{array}$ \\
\hline 2643 & 3,4 & CONSTANT & $-3.48000000-01$ & 3.4500000 E-01 & $-3.4500000 \mathrm{E}-01$ & $3.4600000 \mathrm{E}-01$ & NONE & WP035597 & $\mathbf{Y}$ & NA \\
\hline 2643 & 3,4 & CONSTANT & $34500000 E-01$ & -3.4600000 E- -01 & $-3.4600000 \mathrm{E}-01$ & $-3.4600000 \mathrm{E}=-01$ & NONE & WPO40434 & $\mathbf{Y}$ & NA \\
\hline 2646 & 1,3 & CONSTANT & $0.0000000 E+\infty$ & $0.0000000 E+\infty$ & $0.0000000 E+\infty$ & $0.0000000 E+\infty$ & NONE & WP030640 & $\mathbf{Y}$ & NA \\
\hline 2646 & 1,3 & CONSTANT & $0.0000000 \leq+\infty$ & $0.0000000 E+\infty$ & $0.00000000+\infty$ & $0.0000000=+\infty$ & NONE & WP030994 & $\mathbf{Y}$ & NA \\
\hline 2646 & 1,3 & CONSTANT & $0.00000000+\infty$ & $0.0000000 \mathrm{E}+\infty$ & $0.0000000 \mathrm{E}+\infty$ & $0.0000000 E+\infty 0$ & NONE & WPO35333 & $\mathbf{Y}$ & N/A \\
\hline 2646 & 1,3 & CONSTANT & $\because 0.0000000 E+\infty$ & $0.0000000 E+\infty 0$ & $0.0000000=+00$ & $0.0000000 E+\infty$ & NONE & WPOS559? & $\mathbf{Y}$ & NA \\
\hline 2646 & 1,3 & CONSTANT & $0.0000000 E+\infty$ & $0.0000000 E+\infty$ & $0.0000000 E+00$ & $0.00000005+\infty$ & NONE & WP040514 & $\mathbf{Y}$ & NA \\
\hline 2647 & 3,4 & CONSTANT & $5.60000000-01$ & $5.6000000=-01$ & $5.6000000 E-01$ & $5.6000000=-01$ & Pa & WP030540 & $\mathbf{Y}$ & NA \\
\hline 2647 & 3,4 & CONSTANT & $5.6000000 E-01$ & $5.6000000 E-01$ & $5.60000005-01$ & $5.6000000=-01$ & Pa & WP030994 & $\mathbf{Y}$ & NA \\
\hline 2647 & 3,4 & CONSTANT & 5.6000000E-01 & 5.6000000E-01 & $5.6000000 E-01$ & $5.6000000 E-01$ & Pa & WP035597 & $\mathbf{Y}$ & N/A \\
\hline 2647 & 3,4 & CONSTANT & $5.6000000 E-01$ & $5.6000000 \mathrm{E}-01$ & $5.6000000 E-01$ & $5.6000000 \mathrm{E}-01$ & Pa & WPO40434 & $\mathbf{Y}$ & NA \\
\hline 2648 & 3,4 & CONSTANT & $3.4600000 \mathrm{E}-01$ & $3.4600000 \mathrm{E}-01$ & $3.4600000 E-01$ & $3.4600000 \mathrm{E}-01$ & NONE & WP030640 & $\mathbf{Y}$ & NA \\
\hline 2648 & 3,4 & CONSTANT & $3.4600000 E-01$ & $3.4600000 \mathrm{E}-01$ & $-3.4600000 E-01$ & $3.4600000 E-01$ & NONE & WP030994 & $\mathbf{Y}$ & NA \\
\hline 2648 & 3,4 & CONSTANT & $3.4600000 \mathrm{E}-01$ & $3.46000000-01$ & $3.4600000 E-01$ & $3.4600000 E-01$ & NONE & WP035597 & $\mathbf{Y}$ & NA \\
\hline 2648 & 3,4 & CONSTANT & $-3.4600000 \mathrm{E}-01$ & $-3.4600000 E-01$ & $-3.4600000 E-01$ & $-3.4600000 E-01$ & NONE & WPO40434 : & $\mathbf{Y}$ & N/A \\
\hline 2651 & 1,3 & CONSTANT & $0.0000000 E+\infty$ & $0.00000000+\infty$ & $0.0000000 E+\infty$ & $0.00000000+\infty$ & NONE & WP030640 & $\mathbf{Y}$ & NA \\
\hline 2651 & 1,3 & CONSTANT & $0.0000000 E+\infty$ & $0.0000000 E+\infty$ & $0.0000000 E+00$ & $0.0000000 E+\infty$ & NONE & WP030994 & $\mathbf{Y}$ & N/A \\
\hline 2651 & 1,3 & CONSTANT & $0.0000000 E+00$ & $0.0000000 E+\infty$ & $0.0000000 E+\infty 0$ & $0.0000000 E+\infty$ & NONE & WP035271 & $\mathbf{Y}$ & NA \\
\hline 2651 & 1,3 & CONSTANT & $0.0000000 E+\infty 0$ & $0.0000000 E+\infty$ & $0.0000000 E+\infty 0$ & $0.0000000 E+\infty$ & NONE & WPO35333 & $\boldsymbol{Y}$ & NA \\
\hline 2651 & 1,3 & CONSTANT & $0.0000000 E+00$ & $0.0000000 E+\infty$ & $0.00000000+00$ & $0.0000000 E+\infty 0$ & NONE & WP035597 & $\mathbf{Y}$ & NA \\
\hline 2651 & 1,3 & CONSTANT & $0.0000000 E+\infty 0$ & $0.0000000 E+00$ & $0.0000000 E+\infty$ & $0.0000000 E+00$ & NONE & WP040514 & $\mathbf{Y}$ & NA \\
\hline 2652 & 3,4 & CONSTANT & $5.6000000 \mathrm{E}-01$ & $5.6000000 \mathrm{E}-01$ & $5.6000000 \mathrm{E}-01$ & 5.6000000E-01 & $\mathbf{P a}$ & WP030640 & $\mathbf{Y}$ & $N / A$ \\
\hline 2652 & 3,4 & CONSTANT & $5.6000000 E-01$ & $5.6000000 E-01$ & $5.6000000 E-01$ & $5.6000000 E-01$ & $\mathbf{P a}$ & WP030994 & $\mathbf{Y}$ & $N / A$ \\
\hline 2652 & 3,4 & CONSTANT & $5.6000000 \mathrm{E}-0.01$ & $5.6000000 \mathrm{E}-01$ & $5.6000000 E-01$ & $5.6000000 \mathrm{E}-01$ & Pa & WP035597 & $\mathbf{Y}$ & NAA \\
\hline 2652 & 3,4 & CONSTANT & $5.6000000 E-01$ & $5.60000000-01$ & $5.6000000 E-01$ & $5.6000000 E-01$ & Pa & WPO40434 & $-Y$ & NA \\
\hline 2653 & 3,4 & CONSTANT & $3.4600000 \mathrm{E}-01$ & $-3.4600000 \mathrm{E}-01$ & $3.4600000 E-01$ & $34600000 \mathrm{E}-01$ & NONE & WP030640 & $\mathbf{Y}$ & N/A \\
\hline 2653 & 3,4 & CONSTANT & $2.46000000-01$ & $-3.4600000 E-01$ & $-3.4600000 \mathrm{E}-01$ & $3.4600000 E-01$ & NONE & WP030994 & $\mathbf{Y}$ & NA \\
\hline 2653 & 3,4 & CONSTANT & $-3.4600000 E-01$ & $-3.4600000 E-01$ & $3.4600000 E-01$ & $3.4600000 \mathrm{E}-01$ & NONE & WP035597 & $Y$ & $N_{A}$ \\
\hline 2653 & 3,4 & CONSTANT & $-3.4600000=-01$ & $-3.4600000 E-01$ & $3.4500000 E-01$ & $3.4600000 \mathrm{E}-01$ & NONE & WPO40434 & $\mathbf{Y}$ & $N / A$ \\
\hline 2656 & 1,3 & CONSTANT & $0.0000000 E+\infty$ & $0.00000000+\infty$ & $0.0000000 E+00$ & $0.00000000 E+\infty$ & NONE & WP030640 & $\mathbf{Y}$ & $N A$ \\
\hline 2656 & 1,3 & CONSTANT & $0.00000000+\infty$ & $0.0000000 E+\infty$ & $0.0000000 E+\infty$ & $0.0000000 E+\infty$ & NONE & WP030994 & $\mathbf{Y}$ & $N A$ \\
\hline 2656 & 1,3 & CONSTANT & $0.0000000 E+\infty$ & $0.0000000 E+\infty$ & $0.0000000 E+\infty$ & $0.0000000 \mathrm{E}+00$ & NONE & WP035271 & $\mathbf{Y}$ & NA \\
\hline 2656 & 1,3 & CONSTANT & $0.00000000 E+\infty$ & $0.00000000+\infty$ & $0.0000000 E+\infty$ & $0.0000000 E+\infty$ & NONE & WPO35333 & $Y$ & N/A \\
\hline 2656 & 1,3 & CONSTANT & $0.00000000+00$ & $0.00000000 E+00$ & $0.0000000 E+\infty$ & $0.0000000 E+\infty$ & NONE & WP035597 & $Y$ & NA \\
\hline 2656 & 1,3 & CONSTANT & $0.00000000 E+\infty$ & $0.0000000 E+\infty 0$ & $0.0000000 E+\infty$ & $0.0000000 E+\infty$ & NONE & WPO40514 & $Y$ & NA \\
\hline 2657 & 3,4 & CONSTANT & $5.6000000=-01$ & $5.6000000 E-01$ & $5.6000000 E-01$ & $5.6000000 E-01$ & Pa & WP030640 & $\mathbf{Y}$ & N/A \\
\hline
\end{tabular}


CCA Parameter Listing

\begin{tabular}{|c|c|c|c|c|c|c|c|c|c|c|}
\hline 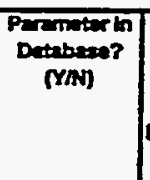 & 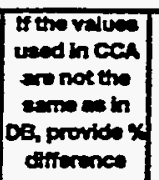 & ID & Meterdid & Mrtardal Name & $\begin{array}{l}\text { Pormmetar } \\
\text { of }\end{array}$ & Paramber Karen & $\begin{array}{l}\text { PRPID } \\
\text { IMPOA }\end{array}$ & $\begin{array}{c}\text { Dinte Enty } \\
\text { Dece }\end{array}$ & 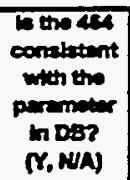 & 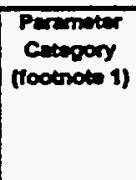 \\
\hline $\mathbf{Y}$ & 100 & 2657 & $C_{2} M_{-} T_{1}$ & $\begin{array}{l}\text { Upper Salado clay. } 0 \text { to } \\
10 \text { years }\end{array}$ & PCT_A & $\begin{array}{l}\text { Threshold Prossuro Lnear } \\
\text { Peremetor }\end{array}$ & 32041 & $15-F a b-96$ & $\mathbf{Y}$ & 1 \\
\hline$\gamma$ & 100 & 265 & $C L_{-} M_{-} T_{1}$ & $\begin{array}{l}\text { Uppor Salado ctay:0 to } \\
10 \text { years }\end{array}$ & PCT_A & $\begin{array}{l}\text { Throshold Pressure Lnear } \\
\text { Peremerer }\end{array}$ & 32041 & $15+\infty 0-96$ & $\mathbf{Y}$ & 9 \\
\hline $\mathbf{Y}$ & 100 & 2657 & CL_M_T1 & $\begin{array}{l}\text { Uppoer Sensodo ctoy.0 to } \\
\text { 10 yours }\end{array}$ & PCT_A & $\begin{array}{l}\text { Threshold Prossure L Lnoar } \\
\text { Peremeter }\end{array}$ & 32041 & $15-\infty b-96$ & $\mathbf{Y}$ & 1 \\
\hline$Y$ & 100 & 2658 & $C L_{-} M_{-} T_{1}$ & $\begin{array}{l}\text { Uppor Salado clay.0 to } \\
10 \text { years }\end{array}$ & PCT_EXP & $\begin{array}{l}\text { Thresthold pressurio esponential } \\
\text { perameter }\end{array}$ & 32012 & 15 feb-96 & $\mathbf{Y}$ & 1 \\
\hline $\mathbf{Y}$ & 100 & 2658 & CL_M_T1 & $\begin{array}{l}\text { Upper Selado clay.0 to } \\
10 \text { years }\end{array}$ & PCT_EXP & $\begin{array}{l}\text { Threathold pressure exponential } \\
\text { pereneter }\end{array}$ & 32042 & $15+\infty 6-96$ & $Y$ & 1 \\
\hline $\mathbf{Y}$ & 100 & 2658 & CL_M_TI & $\begin{array}{l}\text { Uppor Salado clay.0 to } \\
10 \text { years }\end{array}$ & PCT_EXP & $\begin{array}{l}\text { Trreshold pressure exponential } \\
\text { peramoter }\end{array}$ & 32042 & 15 fab-96 & $\mathbf{Y}$ & 1 \\
\hline $\mathbf{Y}$ & 100 & 2658 & $C L \_M \_T 1$ & $\begin{array}{l}\text { Upper Seledo clay:0 to } \\
10 \text { yeers }\end{array}$ & PCT_EXP & $\begin{array}{l}\text { Throstrotd prossice exponential } \\
\text { aremeter }\end{array}$ & 32012 & $15+\infty 0-96$ & $\mathbf{Y}$ & 1 \\
\hline $\mathbf{Y}$ & & 2661 & CL_M_T2 & $\begin{array}{l}\text { Upper Saledo ctay:10 } \\
\text { to } 25 \text { yours }\end{array}$ & KPT & $\begin{array}{l}\text { Flag for Permesbilty Dotermnod } \\
\text { Threshold }\end{array}$ & 32057 & $20+06-96$ & $\mathbf{Y}$ & 48 \\
\hline $\mathbf{Y}$ & & 2661 & CL_M_T2 & $\begin{array}{l}\text { Uppor Salado ctay. } 10 \\
\text { to } 25 \text { years }\end{array}$ & KPT & $\begin{array}{l}\text { Flisg for Permesbilty Dotermined } \\
\text { Throshold }\end{array}$ & 32057 & $20-00-96$ & $Y$ & 48 \\
\hline$\dot{\mathbf{Y}}$ & & 2661 & CL_M_T2 & $\begin{array}{l}\text { Upper Salado clay. } 10 \\
\text { to } 25 \text { years }\end{array}$ & KPT & $\begin{array}{l}\text { Fleg for Permeabity Determinod } \\
\text { Tresthold }\end{array}$ & 32067 & $20+a b-96$ & $\mathbf{Y}$ & 48 \\
\hline $\mathbf{Y}$ & & 2661 & CL_M_T2 & $\begin{array}{l}\text { Upper Salado clay: } 10 \\
\text { to } 25 \text { years }\end{array}$ & KPT & $\begin{array}{l}\text { Fleog for Permesbility Dotermined } \\
\text { Thresthold }\end{array}$ & 32007 & $20-F a b-96$ & $\mathbf{Y}$ & 48 \\
\hline$Y$ & & 2661 & $C L_{-} M_{-} T 2$ & $\begin{array}{l}\text { Upper Salado ctay: } 10 \\
\text { to } 25 \text { years }\end{array}$ & |KPT & 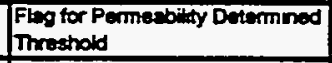 & 32067 & $20+\infty b-96$ & $\mathbf{Y}$ & 48 \\
\hline$Y$ & & 2651 & $C L_{-} M_{-} T_{2}$ & $\begin{array}{l}\text { Uppor Selado day.10 } \\
\text { to } 25 \text { years }\end{array}$ & KPT & $\begin{array}{l}\text { Fleog for Permesbity Dotermined } \\
\text { Threshold }\end{array}$ & 32067 & $20-700-96$ & $\mathbf{Y}$ & 48 \\
\hline $\mathbf{Y}$ & 100 & 2652 & CL_M_T2 & $\begin{array}{l}\text { Upper Salaco clay: } 10 \\
\text { to } 25 \text { years }\end{array}$ & PCT_A & $\begin{array}{l}\text { Timeshold Pressuro Linear } \\
\text { Paremeter }\end{array}$ & 32116 & $15-a b-96$ & $\mathbf{Y}$ & 1 \\
\hline $\mathbf{Y}$ & 100 & 2662 & CL_M_T2 & $\begin{array}{l}\text { Upper Salaco ctay.10 } \\
\text { to } 25 \text { yoars }\end{array}$ & PCT_A & $\begin{array}{l}\text { Throshtold Pressire Linaer } \\
\text { Perrenoter }\end{array}$ & 32116 & $15 F=b-96$ & $\gamma$ & 1 \\
\hline $\mathbf{Y}$ & 100 & 2662 & CL_M_T2 & $\begin{array}{l}\text { Uppor Salado clay: } 10 \\
\text { lo } 25 \text { years }\end{array}$ & PCT_A & $\begin{array}{l}\text { Threshold Pressure Linear } \\
\text { Perameter }\end{array}$ & 32116 & 15 fab- 96 & $Y$ & 1 \\
\hline $\mathbf{Y}$ & 100 & 2662 & CL_M_T2 & $\begin{array}{l}\text { Upper Salado clay. } 10 \\
\text { to } 25 \text { years }\end{array}$ & PCT_A & $\begin{array}{l}\text { Threshold Pressurs Lnear } \\
\text { Parmeter }\end{array}$ & 32116 & $15+06-96$ & $\mathbf{Y}$ & 1 \\
\hline$Y$ & 100 & 2653 & CL_M_T2 & $\begin{array}{l}\text { Upper Salado clay.10 } \\
\text { to } 25 \text { years }\end{array}$ & |PCT_EXP & $\begin{array}{l}\text { Trrashold prossuro exponential } \\
\text { perameter }\end{array}$ & 31117 & $15+a b-96$ & $Y$ & 1 \\
\hline$Y$ & 100 & 2663 & CL_M_T2 & $\begin{array}{l}\text { Upper Satado clay. } 10 \\
\text { to } 25 \text { years }\end{array}$ & PCT_EXP & $\begin{array}{l}\text { Thresthold pressure exponential } \\
\text { peremeter }\end{array}$ & 31117 & 15 -Fob- -86 & $Y$ & 1 \\
\hline$Y$ & 100 & 2663 & CL_M_T2 & $\begin{array}{l}\text { Upper Salado dry.10 } \\
\text { to } 25 \text { years }\end{array}$ & PCT_EXP & $\begin{array}{l}\text { Throshodd pressure exponontival } \\
\text { percmeter }\end{array}$ & 31117 & 15-Fab-96 & $Y$ & 1 \\
\hline $\mathbf{Y}$ & 100 & 2663 & $C L M_{-} T_{2}$ & $\begin{array}{l}\text { Uppor Salado ctay.10 } \\
\text { to } 25 \text { years }\end{array}$ & PCT_EXP & $\begin{array}{l}\text { Treashold pressure exponental } \\
\text { peraneter }\end{array}$ & 31117 & 15-Feb-96 & $\mathbf{Y}$ & 1 \\
\hline$Y$ & & 2686 & $C L H_{-}{ }_{-} T 3$ & $\begin{array}{l}\text { Uppor Salado cloy:25 } \\
\text { to } 50 \text { years }\end{array}$ & KPT & $\begin{array}{l}\text { Flos for Permesablity Dotormined } \\
\text { Threshold }\end{array}$ & 32142 & 20 fab- 86 & $\mathbf{Y}$ & 48 \\
\hline $\mathbf{Y}$ & & 2666 & $C L, M \_T 3$ & $\begin{array}{l}\text { Uppor Salado clay:25 } \\
\text { to } 50 \text { years }\end{array}$ & KPT & $\begin{array}{l}\text { Fleg for Perm estilty Determined } \\
\text { Throsthatd }\end{array}$ & 32142 & $20-706-96$ & $\mathbf{Y}$ & 48 \\
\hline $\mathbf{Y}$ & & 2656 & CL_M_T3 & $\begin{array}{l}\text { Upper Salado ciry:25 } \\
\text { to } 50 \text { years }\end{array}$ & KPT & $\begin{array}{l}\text { Fleog for Permeabilty Determned } \\
\text { Throshold }\end{array}$ & 32142 & 20-Feb- 56 & $Y$ & 48 \\
\hline $\mathbf{Y}$ & & 2666 & $C L \_M_{-} T 3$ & \begin{tabular}{|l} 
Upper Salado ctay.25 \\
to 50 yours
\end{tabular} & KPT & $\begin{array}{l}\text { Fiteg for Permeasility Determined } \\
\text { Threshold }\end{array}$ & 32142 & 20-Fob-96 & $-\gamma$ & 48 \\
\hline $\mathbf{Y}$ & & 2685 & CL_M_T3 & $\begin{array}{l}\text { Uppoer Salado ctay.25 } \\
\text { to } 50 \text { years }\end{array}$ & KPT & $\begin{array}{l}\text { Fleg for Pormoability Dotermined } \\
\text { Thresthok }\end{array}$ & 32142 & 20-Feb-96 & $\mathbf{Y}$ & 48 \\
\hline $\mathbf{Y}$ & & 2686 & CL_M_T3 & $\begin{array}{l}\text { Upper Saloso ctry.25 } \\
\text { to } 50 \text { yeare }\end{array}$ & KPr & $\begin{array}{l}\text { Fiog for Permeabilty Determined } \\
\text { Threshold }\end{array}$ & 32142 & 20-Fab-96 & $\mathbf{Y}$ & 48 \\
\hline $\mathbf{Y}$ & 100 & 2667 & CL_H_T3 & $\begin{array}{l}\text { Upper } 5 \text { alado ciay: } 25 \\
\text { to } 50 \text { years }\end{array}$ & PCT_A & \begin{tabular}{|l|} 
Throshold Prossure Lnear \\
Paremeter
\end{tabular} & 32143 & 15-Feb-86 & $\mathbf{Y}$ & 1 \\
\hline $\mathbf{Y}$ & 100 & 2667 & CL_M_T3 & \begin{tabular}{|l|} 
Upper Selecolo dey.25 \\
to 50 years
\end{tabular} & PCT_A & $\begin{array}{l}\text { Throshold Pressen Lumes } \\
\text { Peremeter }\end{array}$ & 32143 & $15-F a b-96$ & $\mathbf{Y}$ & 1 \\
\hline $\mathbf{Y}$ & 100 & 2667 & $C L \_M \_T 3$ & $\begin{array}{l}\text { Upper Salaco clay.25 } \\
\text { to } 50 \text { yeen }\end{array}$ & PCT_A & $\begin{array}{l}\text { Irreshold Pressure L Ineas } \\
\text { Paremetor }\end{array}$ & 32143 & 15 -Feb-96 & $\mathbf{Y}$ & 1 \\
\hline $\mathbf{Y}$ & 100 & 2657 & CL_M_T3 & $\begin{array}{l}\text { Uppoer Salado day.25 } \\
\text { to } 50 \text { years }\end{array}$ & PCT_A & 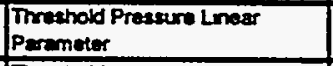 & 32143 & 15 fob-96 & $\mathbf{r}$ & 1 \\
\hline $\mathbf{Y}$ & 100 & 2668 & CL_M_T3 & $\begin{array}{l}\text { Uppor Salado clay.25 } \\
\text { to } 50 \text { yeers }\end{array}$ & PCT_EXP & $\begin{array}{l}\text { Trreshold pressure exponentsal } \\
\text { peremeter }\end{array}$ & 32144 & 15Feb-86 & $Y$ & 1 \\
\hline $\boldsymbol{Y}$ & 100 & 2668 & CL_M_T3 & $\begin{array}{l}\text { Upper Salodo clay } 25 \\
1050 \text { yeirs }\end{array}$ & PCT_EXP & $\begin{array}{l}\text { Threshold pressure exponential } \\
\text { parameler }\end{array}$ & 32144 & $15-F e b-86$ & $\mathbf{Y}$ & 1 \\
\hline $\mathbf{Y}$ & 100 & 2668 & CL_M_T3 & $\begin{array}{l}\text { Upper Salado ctay:25 } \\
\text { to } 50 \text { years }\end{array}$ & PCT_EXP & $\begin{array}{l}\text { Threshold prescuro exponential } \\
\text { perremeter }\end{array}$ & 32144 & $15-F<b-96$ & $\mathbf{Y}$ & 1 \\
\hline $\mathbf{Y}$ & 100 & 2668 & CL_M_T3 & $\begin{array}{l}\text { Upper Salaso day:25 } \\
\text { to } 50 \text { yeurs }\end{array}$ & PCT_EXP & $\begin{array}{l}\text { Throuthole prosturo exponented } \\
\text { perameter }\end{array}$ & 32144 & 15 feb-96 & $Y$ & 1 \\
\hline$\gamma$ & & 2671 & CL_M_T4 & $\begin{array}{l}\text { Upper Saledo ctoy.50 } \\
\text { to } 100 \text { years } \\
\end{array}$ & KPT & $\begin{array}{l}\text { Filog for Pormescity Determmed } \\
\text { Throzhold }\end{array}$ & 32194 & 20-Feb-86 & $Y$ & 48 \\
\hline
\end{tabular}


CCA-Parameter Listing

\begin{tabular}{|c|c|c|c|c|c|c|c|c|c|c|}
\hline 10 & $\begin{array}{c}\text { Crestit } \\
\text { cation ox } \\
\text { Datta } \\
\text { Citegory } \\
\text { (footnote 2) }\end{array}$ & $\begin{array}{l}\text { Ditublbution } \\
\text { Typ }\end{array}$ & Mean & Medien & Minimem & Modinimen & Units & Retermento & 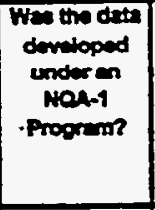 & $\begin{array}{c}\text { Which } \\
\text { methods } \\
\text { were used to } \\
\text { qually } \\
\text { axdsting } \\
\text { dece? } \\
\text { footnote 3) }\end{array}$ \\
\hline 2657 & 3,4 & CONSTANT & $5.6000000 E-01$ & $5.6000000 \varepsilon-01$ & $5.6000000=-01$ & $5.6000000 E-01$ & Pa & WP030994 & $\mathbf{Y}$ & NA \\
\hline 2657 & 3,4 & CONSTANT & $5.6000000 E-01$ & $5.6000000 E-01$ & $5.6000000 E-01$ & $5.6000000 E-01$ & Pa & WP035597 & $\mathbf{Y}$ & NA \\
\hline 2657 & 3,4 & CONSTANT & $5.6000000 \mathrm{E}-01$ & $5.6000000 E-01$ & 5.6000000E-01 & $5.6000000 E-01$ & Pa & WPO40434 & $\mathbf{Y}$ & NA \\
\hline 2658 & 3,4 & CONSTANT & $3.4600000 E-01$ & $3.4600000 E-01$ & $3.4600000 E-01$ & $-3.4600000 E-01$ & NONE & WP030540 & $\mathbf{Y}$ & NA \\
\hline 2658 & 3,4 & CONSTANT & $3.4600000 E-01$ & $3.4600000 E-01$ & $3.4600000 E-01$ & $3.4600000 E-01$ & NONE & WP030994 & $\mathbf{Y}$ & NA \\
\hline 2658 & 3,4 & CONSTANT & $3.4600000 E-01$ & $3.46000000-01$ & $3.4600000 E-01$ & $3.4500000 E-01$ & NONE & WP03559? & $\mathbf{Y}$ & NA \\
\hline 2658 & 3,4 & CONSTANT & $3.4600000 \mathrm{E}-01$ & $3.4600000 E-01$ & $3.4600000 E-01$ & $3.4600000 E-01$ & NOHE & MPO4O434 & $\mathbf{Y}$ & N/A \\
\hline 2661 & 1,3 & CONSTANT & $0.0000000 E+\infty 0$ & $0.0000000 E+\infty 0$ & $0.0000000 E+\infty 0$ & $0.0000000 E+\infty$ & NONE & WP030640 & $\mathbf{Y}$ & NA \\
\hline 2661 & 1,3 & CONSTANT & $0.0000000 E+\infty$ & $0.0000000 E+\infty 0$ & $0.0000000 E+\infty$ & $0.0000000 E+\infty 0$ & NONE & WP030994 & $\mathbf{Y}$ & NA \\
\hline 2681 & 1,3 & CONSTANT & $0.0000000 E+\infty$ & $0.0000000 E+\infty 0$ & $0.0000000 E+\infty$ & $0.0000000 E+\infty$ & NONE & WP035271 & $\mathbf{r}$ & NA \\
\hline 2661 & 1,3 & CONSTANT & $0.0000000 E+\infty 0$ & $0.0000000 E+\infty$ & $0.0000000 \varepsilon+\infty$ & $00000000 E+\infty$ & NONE & WP035333 & $\mathbf{Y}$ & NA \\
\hline 2661 & 1,3 & CONSTANT & $0.0000000 \mathrm{E}+\infty$ & $0.0000000 E+\infty$ & $0.0000000 E+\infty$ & $0.0000000 E+\infty$ & NONE & Wr035597 & $\mathbf{Y}$ & NA \\
\hline 2661 & 1,3 & CONSTANT & $0.0000000 \mathrm{E}+\infty$ & $0.0000000 E+\infty$ & $0.0000000 E+\infty$ & $0.0000000 E+\infty$ & NONE & WPO40514 & $\mathbf{Y}$ & NA \\
\hline 2662 & 3,4 & CONSTANT & $5.6000000 E-01$ & $5.6000000 E-01$ & $5.6000000 E-01$ & $5.6000000 E-01$ & Pa & WP030640 & $Y$ & NAA \\
\hline 2662 & 3,4 & CONSTANT & $5.6000000 E-01$ & $5.6000000 E-01$ & $5.6000000 E-01$ & $5.6000000 E-01$ & 'Pe & WP030994 & $\mathbf{Y}$ & NA \\
\hline 2662 & 3,4 & CONSTANT & $5.6000000 \mathrm{E}-01$ & $5.6000000 E-01$ & $56000000 E-01$ & $56000000 E-01$ & $\mathbf{P a}$ & WP035597 & $\boldsymbol{Y}$ & NA \\
\hline 2652 & 3,4 & CONSTANT & $5.6000000 E-01$ & $5.6000000 E-01$ & $56000000 E-01$ & $56000000 E-01$ & P: & WP040434 & $\mathbf{Y}$ & NA \\
\hline 2663 & 3,4 & CONSTANT & $-3.46000000 E-01$ & $34600000 E-01$ & $34600000 E-01$ & $34600000 E-01$ & NONE & WP030640 & $\mathbf{Y}$ & NA \\
\hline 2663 & 3,4 & CONSTANT & $3.4600000 E-01$ & $346000000-01$ & $34600000 E-01$ & $34600000 \mathrm{E}-01$ & NONE & WP030994 & $\mathbf{Y}$ & N/A \\
\hline 2663 & 3,4 & CONSTANT & $3.4600000 E-01$ & $34600000 E-01$ & $34 \infty 00000-01$ & $34600000 E-01$ & NONE & WP035597 & $Y$ & NA \\
\hline 2663 & 3,4 & CONSTANT & $3.4600000 \mathrm{E}-01$ & $34600000 \mathrm{E}-01$ & $34600000 E-01$ & $34000000-01$ & NONE & WPO40434 & $\mathbf{Y}$ & NA \\
\hline 2666 & 1,3 & CONSTANT & $0.0000000 E+00$ & $00000000 E+\infty$ & $00000000=-\infty$ & $00000000 E+\infty$ & NONE & WP030640 & $\mathbf{Y}$ & NA \\
\hline 2666 & 1,3 & CONSTANT & $0.0000000 E+\infty 0$ & $00000000 E+00$ & $00000000 E * \infty$ & $00000000 \times+\infty$ & NONE & Wp030994 & $\mathbf{Y}$ & NAA \\
\hline 2666 & 1,3 & CONSTANT & $0.0000000 E+00$ & $00000000 E+\infty$ & $0,000000<=+\infty$ & $00000000+\infty$ & NONE & WP035271 & $\mathbf{Y}$ & NA \\
\hline 2666 & 1,3 & CONSTANT & $0.0000000 E+\infty$ & $00000000 E+\infty$ & $00000000=\times \infty$ & $0<\infty \times 000 \times+\infty$ & NONE & WP035333 & $-Y Y$ & NA \\
\hline 2666 & 1,3 & CONSTANT & $0.0000000 E+\infty 0$ & $00000000 E+\infty$ & $00000000<-\infty$ & $00000000=-\infty 0$ & NONE & WP035597 & $\mathbf{Y}$ & NA \\
\hline 2666 & 1,3 & CONSTANT & $0.0000000 E+\infty 0$ & $00000000 E+\infty$ & $00000000<+\infty$ & $0000000<+\infty$ & NONE & WPO40514 & $\mathbf{Y}$ & NA \\
\hline 2657 & 3,4 & CONSTANT & $5.6000000 E-01$ & $56000000 E-01$ & $56000000<-01$ & $50000000<-01$ & Pa & WPO30540 & $\mathbf{Y}$ & NA \\
\hline 2657 & 3,4 & CONSTANT & $5.6000000 E-01$ & $56000000 E-01$ & s $0000000 E-0 t$ & $s+000000 E-01$ & Pa & WP030994 & $\mathbf{Y}$ & NA \\
\hline 2657 & 3,4 & CONSTANT & $5.6000000 E-01$ & $56000000=-01$ & $56000000<01$ & S 60000000 e or & Pa & WP035597 & $\mathbf{Y}$ & NA \\
\hline 2667 & 3,4 & CONSTANT & $5.6000000 E-01$ & $50000000-01$ & $S 0000000<0.01$ & $s$ se00000E-01 & $\mathbf{P a}$ & WPO40434 & $\mathbf{Y}$ & NA \\
\hline 2668 & 3,4 & CONSTANT & $-3.4600000 E-01$ & $34000000 E-01$ & $د 4000000$ e 01 & $3400000<-01$ & NONE & MP030540 & $\mathbf{Y}$ & NAA \\
\hline 2668 & 3,4 & CONSTANT & $2.4600000 E-01$ & $34600000 E-01$ & $3 \approx 00000$ E -01 & $3400000 \mathrm{E}-01$ & NONE & WP030994 & $\mathbf{Y}$ & NA \\
\hline 2668 & 3,4 & CONSTANT & $-3.4500000 E-01$ & $34600000 E-01$ & $3400000<-01$ & $3 \leftarrow 00000 E-01$ & NONE & WP035597 & $\mathbf{Y}$ & NA \\
\hline 2668 & 3,4 & CONSTANT & $-3.4600000 E-01$ & 3 400000E-O1 & 3400000 E- 01 & $3400000 E-01$ & NONE & WPO40434 & $\mathbf{Y}$ & NA \\
\hline 2671 & 1,3 & CONSTANT & $0.0000000 E+\infty$ & $00000000 E+\infty$ & $00000000 E+\infty$ & $00000000 E+\infty 0$ & NONE & WP030640 & $\mathbf{Y}$ & NA \\
\hline
\end{tabular}




\begin{tabular}{|c|c|c|c|c|c|c|c|c|c|c|}
\hline 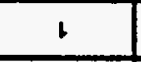 & $\lambda$ & $96-9+76$ & loseE & 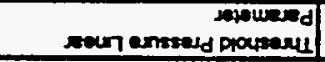 & $r-10 d$ & 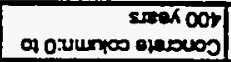 & แัวกOS & 2892 & $\infty$ & A \\
\hline$\vdash$ & $\alpha$ & $96-90+16$ & IOSze & 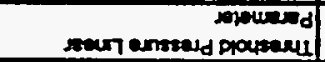 & $\nabla 10 d$ & 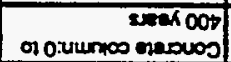 & แโวNOJ & $28 s z$ & oor & $d$ \\
\hline$\vdash$ & $\lambda$ & $96-90+16$ & ISSCE & 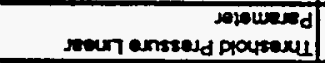 & $\forall-10 \mathrm{~d}$ & 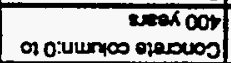 & แ־ONOS & 2092 & $\infty 01$ & $\alpha$ \\
\hline 8 & $\lambda$ & $96-q 9+0 z$ & 65sce & 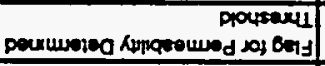 & $10 x$ & 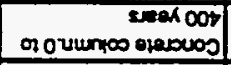 & แโอกOS & 1892 & & $\lambda$ \\
\hline ab & $\boldsymbol{\lambda}$ & $96-40+0 z$ & 65sce & 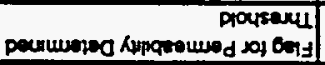 & $100 \times$ & 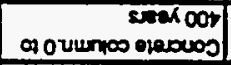 & แโวNOS & 1892 & & $\lambda$ \\
\hline$a b$ & $\alpha$ & $96-90 \pm 0 z$ & GSSCE & 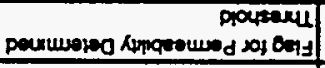 & $10 x$ & 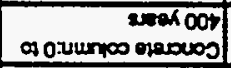 & II'כNOD & 1892 & & $\lambda$ \\
\hline 8 & 1 & $96-99 \pm 0 z$ & 6SSEE & 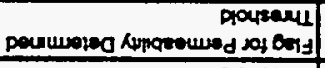 & $10 x$ & 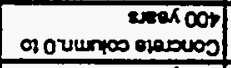 & \$־ JNOS & t89z & & 1 \\
\hline $8 r$ & 1 & $96-90+02$ & 6SSCE & 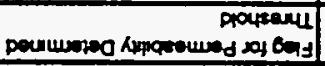 & $100 x$ & 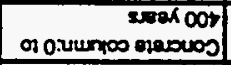 & แ־วNOכ & 1892 & & $\alpha$ \\
\hline gr & A & $96-99+02$ & 6SSER & 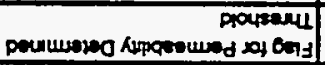 & $100 x$ & 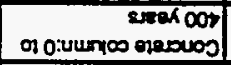 & แ־ัวOS & 1892 & & 1 \\
\hline$b$ & 1 & $96-90 \pm \varepsilon b$ & $\csc 2$ & 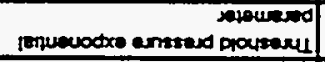 & $d \times a^{-} 10 d$ & $\begin{array}{r}\text { seak sol of } \\
\text { col:kep operes sodon }\end{array}$ & SL'W'T & $8 \angle 9 Z$ & $\infty$ & $\lambda$ \\
\hline$b$ & $\lambda$ & $96-90 \pm-\varepsilon b$ & $\operatorname{cescc}$ & 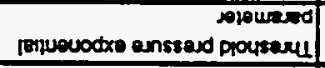 & $\alpha x^{-1} 12 d$ & $\begin{array}{r}\text { seak } 101 \text { of } \\
\text { ooliksp operes rodd? }\end{array}$ & ร1'พัาง & $8<9 z$ & Dol & $\lambda$ \\
\hline$b$ & A- & $96-90+\varepsilon b$ & esces & 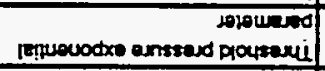 & $d \times 3^{-10} 10 d$ & 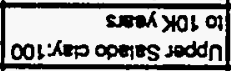 & S1'H־ & $8 L 9 Z$ & $\infty$ & $\lambda$ \\
\hline$b$ & 1 & $96-90 \pm \varepsilon b$ & $\operatorname{cecc} \varepsilon$ & 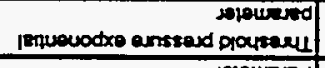 & $9 x e^{-10}+d$ & 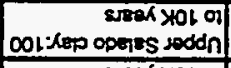 & Sเพั70 & $8 \angle 9 z$ & $\infty \mathrm{l}$ & 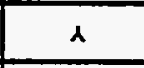 \\
\hline$\vdash$ & $\lambda$ & $96-90 \pm \varepsilon b$ & $28 \cos$ & 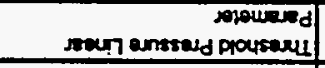 & $\forall 10 \mathrm{~d}$ & 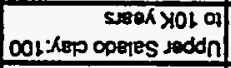 & 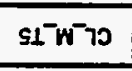 & $\angle 492$ & $\infty 01$ & $\Lambda$ \\
\hline$b$ & $\lambda$ & $96-90 \pm E t$ & $250 c 5$ & 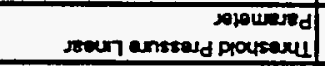 & $\forall-100$ & $\begin{array}{r}\text { suear xol of } \\
\text { ool:Rep operes soddn } \\
\end{array}$ & Sเพ-พา & $\angle 9 z$ & Dor & A \\
\hline$b$ & $\lambda$ & $96-90+8 b$ & टеटटе & $\begin{array}{l}\text { soputesed } \\
\text { soun anssay poustaul }\end{array}$ & $\forall^{-10 d}$ & 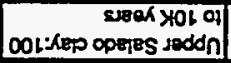 & S1' & $\angle Q r$ & $\infty$ & $\alpha$ \\
\hline$b$ & 1 & $96-40+\varepsilon l$ & zezces & 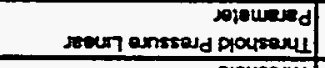 & $\forall 10 \mathrm{~d}$ & \begin{tabular}{|r|} 
seak \%Ol of \\
Dol:Kep operes soddn \\
\end{tabular} & S1'พั70 & $\angle 9 z$ & $\infty 01$ & 1 \\
\hline at & A & $96-90+02$ & เยž̨ & 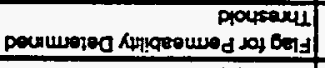 & $10 \mathrm{~d}$ & $\begin{array}{r}\text { seak Xol of } \\
\text { ool:kep operes soddn } \\
\end{array}$ & s1'w70 & $9 \angle S Z$ & & $\lambda$ \\
\hline $8 r$ & $\lambda$ & $96-q 9+0 z$ & เยzes & 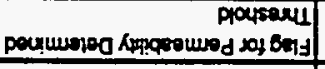 & $1 \mathrm{dD}$ & $\begin{array}{r}\text { sJeak \%ol ol } \\
\text { OOL:Kep operes roddn }\end{array}$ & s.' ${ }^{\top}{ }^{-} 70$ & $9 \angle 9 z$ & & $\lambda$ \\
\hline at & $\lambda$ & $96-\infty 0+02$ & teecs & 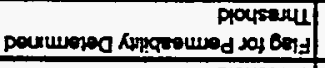 & 1001 & $\begin{array}{r}\text { suek Yol of } \\
\text { oOb:Kep opejes soddn }\end{array}$ & ร1พา & $9 \angle 9 Z$ & & $\lambda$ \\
\hline 8r & 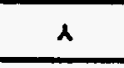 & $96-90 \pm 02$ & 1eces & 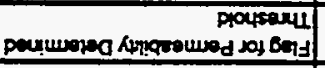 & $1 \mathrm{~d} \times \mathrm{A}$ & 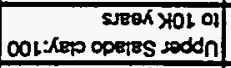 & 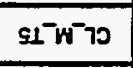 & $9 \angle 9 Z$ & & $\lambda$ \\
\hline 8r & $\lambda$. & $96-q 9+02$ & เฉटcट & 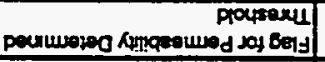 & 100 & 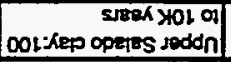 & 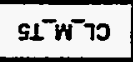 & $9 \angle 9 z$ & & $\boldsymbol{\lambda}$ \\
\hline 87 & 1 & $96-q+02$ & เEcce & 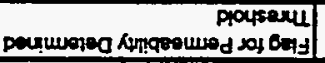 & $10 \times$ & $\begin{array}{r}\text { seer } \times 0 \text { ot of } \\
\text { Dor kep operes soddn }\end{array}$ & Sเพัพ-7อ & $9 \angle 9 z$ & & $\alpha$ \\
\hline 1 & $\lambda$ & $96-9 \pm 86$ & S6IEE & 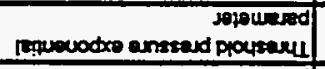 & $d \times g^{-} \perp 0 d$ & $\begin{array}{r}\text { seak ool of } \\
\text { os kep operis soddn }\end{array}$ & $\nabla \mathcal{L}^{-} \mathrm{H}^{-70}$ & $\varepsilon \angle 9 z$ & $\infty 06$ & 1 \\
\hline$\downarrow$ & $\lambda$ & $96-99+\varepsilon b$ & $9612 x$ & 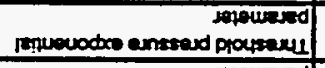 & $d \times g^{-} \perp 0 d$ & $\begin{array}{r}\text { sreak col of } \\
\text { os:rep operes soddn } \\
\end{array}$ & כา כ" & $\varepsilon<9 z$ & $\infty 0 \mathrm{~b}$ & 1 \\
\hline$b$ & $\lambda$ & $96-90+\varepsilon b$ & StLE & 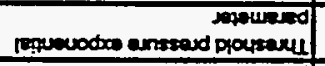 & $d x \theta^{-10 d}$ & $\begin{array}{r}\text { Sreak ool of } \\
\text { os kep operes sodd n } \\
\end{array}$ & $\$ 1$ พพ-70 & $\varepsilon<2$ & $\infty \mathrm{ot}$ & $\lambda$ \\
\hline I & 1 & $96-q 9 \pm \varepsilon l$ & S6IEe & 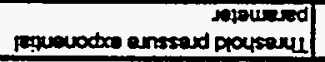 & $d x g^{-10 d}$ & 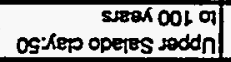 & 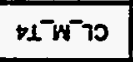 & $\varepsilon<9 z$ & $\infty$ & $\lambda$ \\
\hline$b$ & 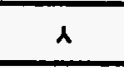 & $96-99+\varepsilon l$ & SGICE & 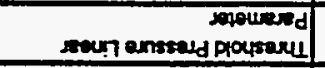 & $\forall-10 d$ & $\begin{array}{r}\text { seak oOL of } \\
\text { os kepp opejes sodd }\end{array}$ & 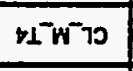 & $z\lfloor s z$ & $\infty$ & $\lambda$ \\
\hline$b$ & 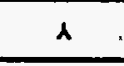 & $96-90 \pm \varepsilon 1$ & S612E & 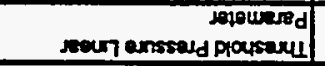 & $\forall \perp 0 d$ & $\begin{array}{r}\text { Sreok ool of } \\
\text { OS:kep opoies soddn }\end{array}$ & $+1 N^{-70}$ & $z<92$ & oob & $\lambda$ \\
\hline 1 & $\lambda$ & $S 6-q D \pm E L$ & sobize & 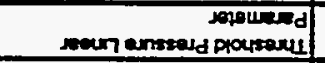 & $\forall-10 d$ & $\begin{array}{r}\text { seak oot of } \\
\text { og:kep operes soddh }\end{array}$ & כר־ & $z \not 9 z$ & $\infty$ & $\lambda$ \\
\hline$b$ & $\lambda$ & $96-90+\varepsilon l$ & stites & 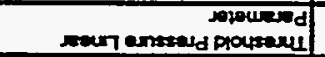 & $\forall-10 d$ & 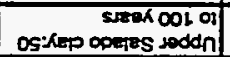 & 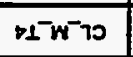 & $z \angle 9 z$ & $\infty 0 \mathrm{t}$ & $\lambda$ \\
\hline $8 b$ & $\Lambda$ & $96-9 \pm 0 z$ & 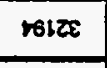 & 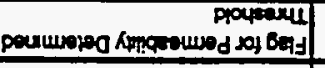 & $10 x$ & $\begin{array}{r}\text { seer oot ol } \\
\text { OS:Rep operes sodd? }\end{array}$ & 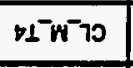 & $b \angle 92$ & & $\lambda$ \\
\hline 8 & $\lambda$ & $96-99 \pm 0 z$ & reter & 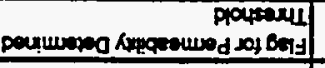 & $10 \mathrm{OPl}$ & 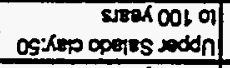 & เพพัพอ & $\$ \angle \mathscr{Z}$ & & 1 \\
\hline ab & A & $96-90 \pm 0 z$ & †เשE & 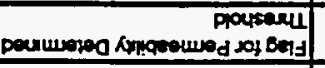 & Lexl & $\begin{array}{r}\text { seax col of } \\
\text { osikepopeins poddn }\end{array}$ & 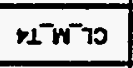 & $1 \angle S C$ & & $\alpha$ \\
\hline $8 b$ & $\lambda$ & $96-90+0 z$ & 6128 & 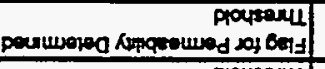 & $10 x$ & $\begin{array}{r}\text { seak col of } \\
\text { os:kep opeies soddn }\end{array}$ & ט1' & $1<9 z$ & & A \\
\hline ab & $\Lambda$ & $96-q \pm 0 z$ & notze & 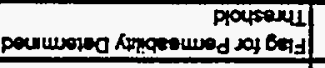 & LDP & $\begin{array}{r}\text { sear Dol of } \\
\text { OS:Kep operes sodd? } \\
\end{array}$ & เพพ-พาอ & $1<9 z$ & & ᄉ \\
\hline 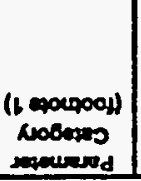 & 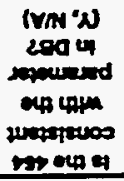 & 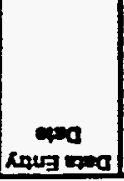 & $\begin{array}{l}\text { Goun } \\
\text { Goded }\end{array}$ & 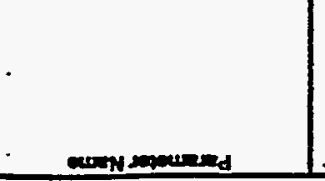 & d & (2) & a mpen & $\boldsymbol{a}$ & 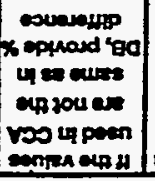 & 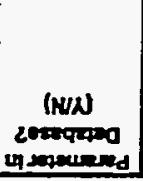 \\
\hline
\end{tabular}


CCA-Parameter Listing

\begin{tabular}{|c|c|c|c|c|c|c|c|c|c|c|}
\hline 10 & 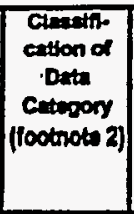 & $\begin{array}{l}\text { Dietribution } \\
\text { Type }\end{array}$ & $\operatorname{Tin}$ & Medien & Mininum & Modrmum & Units & Refernes id & $\begin{array}{l}\text { Wo the det } \\
\text { doweloped } \\
\text { unduren } \\
\text { Noh-t } \\
\text { Progrem - } \\
\end{array}$ & $\begin{array}{l}\text { Which } \\
\text { mothod } \\
\text { more ueed to } \\
\text { quelity } \\
\text { codoting } \\
\text { detan? } \\
\text { (footnote 3) }\end{array}$ \\
\hline 2671 & 1.3 & CONSTANT & $0.0000000 \mathrm{E}+\infty$ & $0.0000000 E+\infty 0$ & $0.0000000 E+\infty$ & $0.0000000 E+\infty 0$ & NONE & WPO30994 & $\mathbf{Y}$ & NA \\
\hline 2671 & 1,3 & CONSTANT & $0.0000000 E+\infty$ & $0.0000000 E+\infty 0$ & $0.0000000 E+\infty$ & $0.00000000+\infty 0$ & NONE & WP035271 & $\mathbf{Y}$ & NA \\
\hline 2671 & 1,3 & CONSTANT & $0.0000000 E+00$ & $0.0000000 E+\infty 0$ & $0.0000000 E+\infty 0$ & $0.0000000 E+\infty$ & NONE & WP035333 & $\mathbf{Y}$ & $N A$ \\
\hline 2671 & 1,3 & CONSTANT & $0.0000000 E+00$ & $0.0000000 E+\infty 0$ & $0.0000000 E+00$ & $0.0000000 E+\infty$ & NONE & WP035597 & $\mathbf{Y}$ & $N / A$ \\
\hline 2671 & 1,3 & CONSTANT & $0.0000000 E+\infty 0$ & $0.0000000 E+\infty 0$ & $0.0000000=+\infty$ & $0.0000000 E+\infty)$ & NONE & WPO40514 & $\mathbf{Y}$ & NA \\
\hline 2672 & 3,4 & CONSTANT & $5.6000000 E-01$ & 5.6000000E-01 & $5.6000000 E-01$ & $5.6000000 E-01$ & $P=$ & WP03064? & $\mathbf{Y}$ & NA \\
\hline 2672 & 3,4 & CONSTANT & 5.6000000E-01 & $5.6000000 E-01$ & $5.6000000 E-01$ & 5.6000000E-01 & Pa & WPOs0994 & $\mathbf{Y}$ & NA \\
\hline 2672 & 3,4 & CONSTANT & 5.6000000E-01 & $5,6000000 E-01$ & $5.6000000 E-01$ & $5.6000000 E-01$ & $\mathbf{P a}$ & WP035597 & $\mathbf{Y}$ & NA \\
\hline 2672 & 3,4 & CONSTANT & $5.6000000 E-01$ & 5.6000000E-01 & $5.6000000 E-01$ & 5.6000000E-01 & $\mathrm{Pa}$ & WPO40134 & $\mathbf{Y}$ & NA \\
\hline 2673 & 3,4 & CONSTANT & 3.4600000 E-01 & $3.4600000 E-01$ & $3.4600000 E-01$ & $3.4600000=-01$ & NONE & WPO30540 & $\mathbf{Y}$ & NA \\
\hline 2673 & 3,4 & CONSTANT & $-3.4600000 E-01$ & $-3.46000000 E-01$ & $-3.4600000 E-01$ & $-3.46000000 \mathrm{E}-01$ & NONE & WP030994 & $\mathbf{Y}$ & N/A \\
\hline 2673 & 3,4 & CONSTANT & $3.4600000 \mathrm{E}-01$ & $3.4600000 E-01$ & $3.4600000 E-01$ & $3.4600000 \mathrm{E}-01$ & NONE & WP035597 & $\mathbf{Y}$ & NA \\
\hline 2673 & 3,4 & CONSTANT & $-3.4600000 E-01$ & $-3.4600000 \mathrm{E}-01$ & $3.4600000 E-01$ & $3.4600000 E-01$ & NONE & WPOLO434 & $\mathbf{Y}$ & NA \\
\hline 2676 & 1,3 & CONSTANT & $0.0000000 E+\infty 0$ & $0.0000000 E+\infty$ & $0.0000000 E+\infty$ & $0.0000000 E+\infty$ & NONE & WP030540 & $\mathbf{Y}$ & NA \\
\hline 2676 & 1,3 & CONSTANT & $0.0000000 E+\infty$ & $0.0000000 E+\infty 0$ & $0.0000000 E+\infty$ & $0.0000000 E+\infty$ & NONE" & WP030994 & $\mathbf{Y}$ & NA \\
\hline 2676 & 1,3 & CONSTANT & $0.0000000 E+\infty 0$ & $0.0000000 E+\infty$ & $0.0000000 E+\infty$ & $0.0000000 E+\infty 0$ & NONE & WP035271 & $\mathbf{Y}$ & $\mathbf{N A}$ \\
\hline 2676 & 1,3 & CONSTANT & $0.0000000 E+\infty$ & $0.00000000+\infty 0$ & $0.0000000 \mathrm{E}+\infty$ & $0.0000000 E+00$ & NONE & WP035333 & $Y$ & NA \\
\hline 2676 & 1,3 & CONSTANT & $0.0000000 E+\infty$ & $0.0000000 E+\infty$ & $0.0000000 E+\infty$ & $0.0000000 E+\infty$ & NONE & WP035597 & $\mathbf{Y}$ & NA \\
\hline 2676 & 1,3 & CONSTANT & $0.0000000 E+\infty 0$ & $0.0000000 E+\infty 0$ & $0.0000000 E+\infty$ & $0.0000000 E+\infty$ & NONE & WPO40514 & $\mathbf{Y}$ & N/A \\
\hline 2677 & 3,4 & . CONSTANT & $5.6000000 \mathrm{E}-01$ & $5.6000000 E-01$ & $5.6000000 \mathrm{E}-01$ & $5.6000000 E-01$ & $\mathbf{P a}$ & WPOS0540 & $\mathbf{Y}$ & NA \\
\hline 2677 & 3,4 & CONSTANT & $5.6000000 E-01$ & $5.6000000 E-01$ & $5.6000000 E-01$ & $5.6000000 E-01$ & $\mathrm{~Pa}$ & WP030994 & $\mathbf{Y}$ & $N / A$ \\
\hline 2677 & 3,4 & CONSTANT & $5.6000000 E-01$ & $5.6000000 \mathrm{E}-01$ & $5.6000000 E-01$ & $5.6000000 E-01$ & $\mathbf{P a}$ & WP035597 & $\mathbf{Y}$ & NA \\
\hline 2677 & 3,4 & CONSTANT & $5.6000000 E-01$ & $5.6000000 E-01$ & 5.6000000E-01 & $5.6000000 \varepsilon-01$ & $\mathbf{P a}$ & WPO40434 & $\mathbf{Y}$ & NA \\
\hline 2678 & 3,4 & CONSTANT & $3.4600000 E-01$ & $-3.4600000 E-01$ & $3.4600000 E-01$ & $-3.4600000 E-01$ & NONE & MP030640 & $\mathbf{Y}$ & NA \\
\hline 2678 & 3,4 & CONSTANT & $-3.4600000 E-01$ & $3.4600000 E-01$ & $3.4600000 E-01$ & $3.4600000 E-01$ & NONE & wposos94 & $-Y$ & NA \\
\hline 2678 & 3,4 & CONSTANT & $-3.4600000 E-01$ & $3.4600000 E-01$ & $3.4600000 \mathrm{E}-01$ & $-3.4600000 E-01$ & NONE & WP035597 & $\mathbf{Y}$ & N/A \\
\hline 2678 & 3,4 & CONSTANT & $3.4600000 E-01$ & $3.4600000 E-01$ & $-3.4600000 E-01$ & $3.4600000 E-01$ & NONE & WPO40434 & $\mathbf{Y}$ & NA \\
\hline 2681 & 1,3 & CONSTANT & $0.0000000 E+\infty 0$ & $0.0000000 E+00$ & $0.0000000 E+\infty 0$ & $0.0000000 E+\infty$ & NONE & MP030640 & $\mathbf{Y}$ & $\mathbf{N} / \mathbf{A}$ \\
\hline 2681 & 1,3 & CONSTANT & $0.0000000 \varepsilon+\infty$ & $0.0000000 E+00$ & $0.0000000 E+00$ & $0.0000000 E+00$ & NONE & WPO30994 & $\mathbf{Y}$ & NA \\
\hline 2681 & 1,3 & CONSTANT & $0.0000000 E+\infty$ & $0.0000000 E+\infty$ & $0.0000000 E+\infty$ & $0.0000000 E+\infty$ & NONE & WP035271 & $\mathbf{Y}$ & NA \\
\hline 2681 & 1,3 & CONSTANT & $0.0000000 E+\infty$ & $0.00000000+00$ & $0.0000000 E+\infty$ & $0.0000000 E+\infty$ & NONE & WPO35333 & $\mathbf{Y}$ & $\mathbf{N A}$ \\
\hline 2681 & 1,3 & CONSTANT & $0.0000000 E+\infty 0$ & $0.00000000+\infty$ & $0.0000000 E+\infty 0$ & $0.0000000 E+00$ & NONE & WP035597 & $\mathbf{Y}$ & NA \\
\hline 2681 & 1,3 & CONSTANT & $0.0000000 E+\infty$ & $0.0000000 E+\infty$ & $0.00000000 \times+\infty$ & $0.0000000 E+\infty$ & NONE & WPO40514 & $\mathbf{Y}$ & NA \\
\hline 2682 & 3,4 & CONSTANT & $5.6000000 E-01$ & $5.6000000 E-01$ & $5.6000000 E-01$ & $5.6000000 E-01$ & $\mathbf{P a}$ & WPO30640 & $\mathbf{Y}$ & NA \\
\hline 2082 & 3,4 & CONSTANT & $5.6000000 E-01$ & $5.6000000=-01$ & $5.6000000 E-01$ & 5.6000000E-01 & $\mathrm{Pa}$ & WP030s94 & $\mathbf{Y}$ & NA \\
\hline 2682 & 3,4 & CONSTANT & $5.6000000 E-01$ & $5.6000000 E-01$ & $5.6000000 E-01$ & $5.6000000 E-01$ & Pa & WP035597 & $\mathbf{Y}$ & NA \\
\hline
\end{tabular}


CCA Parameter-Listing

\begin{tabular}{|c|c|c|c|c|c|c|c|c|c|c|}
\hline 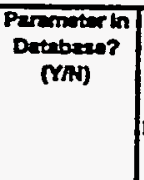 & 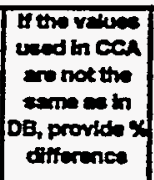 & DD & Hetation & Metorial Name & Parmation & Peremocer Name & $\begin{array}{l}\text { PFo } \\
\text { MPO }\end{array}$ & $\begin{array}{c}\text { Deterestry } \\
\text { Den }\end{array}$ & 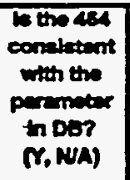 & 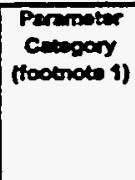 \\
\hline$Y$ & 100 & 2682 & CONC_T1 & $\begin{array}{l}\text { Concrole column:0 to } \\
400 \text { years }\end{array}$ & PCT_A & $\begin{array}{l}\text { Threshold Pressure Lnoar } \\
\text { Peramoter }\end{array}$ & 32561 & $14 F a b-56$ & $Y$ & 1 \\
\hline $\mathbf{Y}$ & 100 & 2683 & CONC_TI & $\begin{array}{l}\text { Concrote column:0 to } \\
400 \text { years }\end{array}$ & PCT_EXP & $\begin{array}{l}\text { Threshold pressure exponential } \\
\text { paremeter }\end{array}$ & 32563 & $14+0 b-86$ & $\mathbf{Y}$ & 1 \\
\hline$Y$ & 100 & 2683 & CONC_T1 & $\begin{array}{l}\text { Concoits colemnio to } \\
400 \text { years }\end{array}$ & PCT_EXP & $\begin{array}{l}\text { Trreshold pressuro exponential } \\
\text { parremoter }\end{array}$ & 325503 & 14.Fobros & $\mathbf{Y}$ & 1 \\
\hline $\mathbf{Y}$ & 100 & 2683 & CONC_T1 & $\begin{array}{l}\text { Concrote colemn:0 to } \\
400 \text { years }\end{array}$ & PCT_EXP & $\begin{array}{l}\text { Throshold pressure exponential } \\
\text { peremeter }\end{array}$ & 32563 & $|14 f c b-86|$ & $Y$ & 1 \\
\hline $\mathbf{Y}$ & 100 & 2683 & CONC_T1 & $\begin{array}{l}\text { Concrote column:0 to } \\
400 \text { years }\end{array}$ & PCT_EXP & $\begin{array}{l}\text { Throshole presture exponential } \\
\text { perenter }\end{array}$ & 32500 & $14 F=b-86$ & $\mathbf{Y}$ & 1 \\
\hline $\mathbf{Y}$ & & 2685 & CONC_T2 & $\begin{array}{l}\text { Concrote column:400 } \\
\text { to } 10 \mathrm{~K} \text { years }\end{array}$ & KPT & $\begin{array}{l}\text { Flag for Permesbility Determined } \\
\text { Threshold }\end{array}$ & 32640 & $20-F a b 56$ & $Y$ & 48 \\
\hline $\mathbf{Y}$ & & 2686 & CONC_T2 & $\begin{array}{l}\text { Concrets columni:400 } \\
\text { to } 10 \mathrm{~K} \text { yeurs }\end{array}$ & KPT & $\begin{array}{l}\text { Fles for Permesbitity Determined } \\
\text { Thresthold }\end{array}$ & 32640 & $20-F e b-96$ & $\mathbf{Y}$ & $A B$ \\
\hline $\mathbf{Y}$ & & 2686 & CONC_T2 & $\begin{array}{l}\text { Concrete cotumn:400 } \\
\text { to } 10 \mathrm{~K} \text { yeers }\end{array}$ & KRT & $\begin{array}{l}\text { Flag for Pormasbilty Dotermined } \\
\text { inroathold }\end{array}$ & 32640 & $20-f a b-96$ & $Y$ & 48 \\
\hline $\mathbf{Y}$ & & 2686 & CONC_T2 & $\begin{array}{l}\text { Concreto cotumn:400 } \\
\text { to } 10 \mathrm{~K} \text { yesers }\end{array}$ & KPT & $\begin{array}{l}\text { Fles for Permesoility Determined } \\
\text { Threstold }\end{array}$ & 32640 & 20 feb-96 & $\mathbf{Y}$ & $A B$ \\
\hline $\mathbf{Y}$ & & 2686 & CONC_T2 & $\begin{array}{l}\text { Concrots colemn:400 } \\
\text { to 10K years }\end{array}$ & KPT & $\begin{array}{l}\text { Fing for Permeability Determined } \\
\text { Throshold }\end{array}$ & 32540 & $20-F a b-96$ & $\mathbf{Y}$ & $4 B$ \\
\hline $\mathbf{Y}$ & & 2686 & CONC_T2 & $\begin{array}{l}\text { Concrote cokemn: } 400 \\
\text { to } 10 \mathrm{~K} \text { years }\end{array}$ & KPT & $\begin{array}{l}\text { Fileg for Permesbility Dotermined } \\
\text { Threshold }\end{array}$ & 32640 & $20-00-96$ & $Y$ & 4B \\
\hline $\mathbf{Y}$ & 100 & 2687 & CONC_T2 & $\begin{array}{l}\text { Concrete column:400 } \\
\text { to } 10 \mathrm{~K} \text { years }\end{array}$ & PCT_A & $\begin{array}{l}\text { Trreshold Pressure Linear } \\
\text { Pursernoter }\end{array}$ & 32652 & 14feb-96 & $\mathbf{Y}$ & 1 \\
\hline $\mathbf{Y}$ & 100 & 2687 & CONC_T2 & $\begin{array}{l}\text { Concrete column:400 } \\
\text { to 10K yoars }\end{array}$ & PCT_A & $\begin{array}{l}\text { Thresthold Prossure Linear } \\
\text { Paranetor }\end{array}$ & 32652 & 14 Feb-96 & $Y$ & 1 \\
\hline$Y$ & 100 & 2687 & CONC_T2 & $\begin{array}{l}\text { Concrote colkmn:400 } \\
\text { to } 10 \text { K years }\end{array}$ & PCT_A & $\begin{array}{l}\text { Throshold Pressure Linar } \\
\text { Peremetor }\end{array}$ & 32652 & $14 f-b-96$ & $\mathbf{Y}$ & 1 \\
\hline $\mathbf{Y}$ & 100 & 2687 & CONC_T2 & $\begin{array}{l}\text { Concrete column:400 } \\
\text { to } 10 \% \text { yeurs }\end{array}$ & PCT_A & $\begin{array}{l}\text { Throshold Pressure Linear } \\
\text { Persmeter }\end{array}$ & 32652 & 14Fob-96 & $\mathbf{Y}$ & 1 \\
\hline$Y$ & 100 & 2688 & CONC_T2 & $\begin{array}{l}\text { Concrete cokmn:400 } \\
\text { to 10K years }\end{array}$ & PCT_EXP & $\begin{array}{l}\text { Throeshold pressure exponential } \\
\text { parameter }\end{array}$ & 32656 & 14 Fab-96 & $\mathbf{Y}$ & 1 \\
\hline $\mathbf{Y}$ & 400 & 2688 & CONC_T2 & $\begin{array}{l}\text { Concrete conkenn: } 400 \\
1010 K \text { years }\end{array}$ & PCT_EXP & $\begin{array}{l}\text { Threshold pressure exponential } \\
\text { parameter }\end{array}$ & 32656 & $14 f \circ b-96$ & $\mathbf{Y}$ & 1 \\
\hline $\mathbf{Y}$ & 100 & 2688 & CONC_T2 & $\begin{array}{l}\text { Concrote cotumn:400 } \\
\text { to } 10 \text { K years }\end{array}$ & PCT_EXP & $\begin{array}{l}\text { Threshold pressure exponential } \\
\text { parameter }\end{array}$ & 32656 & 14Fob-96 & $\mathbf{Y}$ & 1 \\
\hline $\mathbf{Y}$ & 100 & 2688 & CONC_T2 & $\begin{array}{l}\text { Concrets column: } 400 \\
1010 \mathrm{~K} \text { yeurs }\end{array}$ & PCT_EXP & $\begin{array}{l}\text { Thrashold pressure exponential } \\
\text { persunter }\end{array}$ & 32656 & $14 F 0 b-96$ & $\mathbf{Y}$ & 1 \\
\hline $\mathbf{Y}$ & & 2691 & CULEBRA & $\begin{array}{l}\text { Culebra member of the } \\
\text { Rustior formstion }\end{array}$ & KPT & $\begin{array}{l}\text { Fleg tor Permeability Detormined } \\
\text { Tireshold }\end{array}$ & 32555 & $20-F a b-96$ & $Y$ & $4 B$ \\
\hline $\mathbf{Y}$ & & 2691 & CULEBRA & $\begin{array}{l}\text { Culabre member of the } \\
\text { Rustier formation }\end{array}$ & КPT & $\begin{array}{l}\text { Fleg tor Permesbility Determined } \\
\text { threashold }\end{array}$ & 32555 & $20-F 06-96$ & $\mathbf{Y}$ & 48 \\
\hline$Y$ & & 2691 & CULEBRA & $\begin{array}{l}\text { Culebre member of the } \\
\text { Rustier formution }\end{array}$ & KPT & $\begin{array}{l}\text { Flag for Permesability Determmed } \\
\text { Threshole }\end{array}$ & 32555 & $20-F b-96$ & $\mathbf{Y}$ & $4 B$ \\
\hline$Y$ & & 2691 & CULEBRA & $\begin{array}{l}\text { Culcora member of the } \\
\text { Rustier formation }\end{array}$ & KPT & $\begin{array}{l}\text { Fleg for Pormesbility Determined } \\
\text { Threshold }\end{array}$ & 32555 & 20 fob-96 & $Y$ & 40 \\
\hline$Y$ & & 2692 & CULEBRA & $\begin{array}{l}\text { Culebra member of the } \\
\text { Rustive formation }\end{array}$ & PCT_A & $\begin{array}{l}\text { Throshold Preasure Linear } \\
\text { Peramotor }\end{array}$ & 32752 & 20feb-96 & $\mathbf{Y}$ & 48 \\
\hline $\mathbf{Y}$ & & 2692 & CULEBRA & $\begin{array}{l}\text { Culbora manber } \alpha \text { tho } \\
\text { Rustier formation }\end{array}$ & PCT_A & $\begin{array}{l}\text { Threahold Preasure Linear } \\
\text { Perameter }\end{array}$ & 32752 & 20 Fab-96 & $Y$ & $A B$ \\
\hline $\mathbf{Y}$ & & 2692 & CULEBRA & $\begin{array}{l}\text { Cuibre member of the } \\
\text { Ruster formation }\end{array}$ & PCT_A & $\begin{array}{l}\text { Threshold Pressure Linaer } \\
\text { Paremeter }\end{array}$ & 32752 & 20-Fcb-96 & $\mathbf{Y}$ & $A B$ \\
\hline
\end{tabular}


CCA Parameter Listing

\begin{tabular}{|c|c|c|c|c|c|c|c|c|c|c|}
\hline 10 & 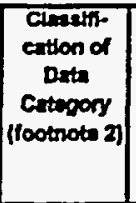 & $\begin{array}{c}\text { Dirtulbution } \\
\text { Type }\end{array}$ & $\operatorname{man}$ & Meden & Dintroum & Meximum & Uates & References in & $\begin{array}{l}\text { Was the deta } \\
\text { dowloped } \\
\text { onder an } \\
\text { Non-1 } \\
\text { Progren? }\end{array}$ & 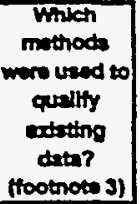 \\
\hline 2682 & 3,4 & CONSTANT & $5.60000000-01$ & $5.6000000 E-01$ & $5.6000000=-01$ & $5.6000000 E-01$ & $\mathbf{P a}$ & WPO40434 & $\mathbf{Y}$ & NA \\
\hline 2683 & 3,4 & CONSTANT & $3.4600000 E-01$ & $3.4600000 \mathrm{E}-01$ & $3.4600000 E-01$ & $-3.4600000 \mathrm{E}-01$ & NONE & WP030540 & $\mathbf{Y}$ & NA \\
\hline 2683 & 3,4 & CONSTANT & $3.4600000 E-01$ & $3.4600000 E-01$ & $3.4600000 \mathrm{E}-01$ & $3.4600000 E-01$ & NONE & WP030994 & $\mathbf{Y}$ & $N A$ \\
\hline 2683 & 3,4 & CONSTANT & $3.4600000 E-01$ & $3.4600000 E-01$ & $-3.4600000=-01$ & $-3.4500000=-01$ & NONE & WP035597 & $\mathbf{Y}$ & NA \\
\hline 2683 & 3,4 & CONSTANT & $-3.4600000 E-01$ & $3.4500000 \mathrm{E}-01$ & $-3.4600000 E-01$ & $-3.4600000 E-01$ & NONE & WPO4O434 & $\mathbf{Y}$ & NA \\
\hline 2686 & 1,3 & CONSTANT & $0.0000000 E+\infty$ & $0.0000000 E+\infty 0$ & $0.0000000 E+\infty$ & $0.0000000 E+\infty$ & NONE & WP030640 & $\mathbf{Y}$ & NA \\
\hline 2686 & 1,3 & CONSTANT & $0.0000000 E+\infty 0$ & $0.0000000 E+\infty$ & $0.0000000 E+\infty$ & $0.0000000 E+\infty$ & NONE & WP030994 & $\mathbf{Y}$ & NA \\
\hline 2686 & 1,3 & CONSTANT & $0.0000000 E+\infty$ & $0.0000000 E+\infty$ & $0.0000000 E+00$ & $0.0000000 E+\infty 0$ & NONE & WP035271 & $\mathbf{Y}$ & NAA \\
\hline 2686 & 1,3 & CONSTANT & $0.0000000 E+\infty 0$ & $0.0000000 \leq+\infty 0$ & $0.0000000 E+\infty 0$ & $0.0000000 E+00$ & NONE & WP035333 & $Y$ & NA \\
\hline 2686 & 1,3 & CONSTANT & $0.0000000 E+\infty 0$ & $0.0000000 E+00$ & $0.0000000 E+\infty$ & $0.0000000 E+\infty$ & NONE & WP035597 & $\mathbf{Y}$ & N/A \\
\hline 2686 & 1,3 & CONSTANT & $0.0000000 E+00$ & $0.0000000 E+\infty$ & $0.0000000 E+\infty$ & $0.0000000 E+\infty$ & NONE & WP040514 & $\mathbf{Y}$ & $N A$ \\
\hline 2687 & 3,4 & CONSTANİ & $5.6000000 \mathrm{E}-01$ & $5.6000000 \mathrm{E}-01$ & $5.6000000 \mathrm{E}-01$ & $5.6000000 E-01$ & $\mathbf{P a}$ & WP030540 & $\mathbf{Y}_{n}$ & NA \\
\hline 2687 & 3,4 & CONSTANT & $5.6000000 E-01$ & $5.6000000 E-01$ & $5.6000000 E-01$ & $5.6000000 E-01$ & Pa & WP030994 & $\mathbf{Y}$ & NA \\
\hline 2687 & 3,4 & CONSTANT & $5.6000000 E-01$ & $5.6000000 E-01$ & $5.6000000 E-01$ & $5.6000000 E-01$ & $\mathrm{~Pa}$ & WP035597 & $\mathbf{Y}$ & NA \\
\hline 2687 & 3,4 & CONSTANT & $5.6000000 E-01$ & $5.6000000 \mathrm{E}-01$ & $5.6000000 E-01$ & $5.6000000 \mathrm{E}-01$ & $\mathrm{~Pa}$ & WPO40434 & $\mathbf{Y}$ & NA \\
\hline 2688 & 3,4 & CONSTANT & $-3.4600000 E-01$ & $-3.4600000 \mathrm{E}-01$ & $3.4600000 E-01$ & $3.4600000 E-01$ & NONE & WP030640 & $\mathbf{Y}$ & NA \\
\hline 2688 & 3,4 & CONSTANT & $-3.4600000 \mathrm{E}-01$ & $3.4600000 E-01$ & $3.4600000 \mathrm{E}-01$ & $3.4600000 \mathrm{E}-01$ & NONE & WP030994 & $\mathbf{Y}$ & NA \\
\hline 2688 & 3,4 & CONSTANT & $-3.4600000 \mathrm{E}-01$ & $-3.4600000 \mathrm{E}-01$ & $-3.4600000 E-01$ & $3.4600000 \mathrm{E}-01$ & NONE & WP035597 & $\mathbf{Y}$ & NA \\
\hline 2688 & 3,4 & CONSTANT & $-3,4600000 \mathrm{E}-01$ & $3.4600000 E-01$ & $3.4600000 E-01$ & $3.4600000 E-01$ & NONE & WPO40434 & $\mathbf{Y}$ & NA \\
\hline 2691 & 3,5 & CONSTANT & $0.0000000 E+\infty 0$ & $0.0000000 E+00$ & $0.0000000 E+\infty 0$ & $0.0000000 E+00$ & NONE & WP035271 & $\mathbf{Y}$ & NA \\
\hline 2691 & 3,5 & CONSTANT & $0.0000000 E+\infty$ & $0.0000000 E+\infty$ & $0.0000000 E+\infty 0$ & $0.0000000 E+00$ & NONE & WP035333 & $\mathbf{Y}$ & N/A \\
\hline 2691 & 3,5 & CONSTANT & $0.0000000 E+\infty 0$ & $0.0000000 E+\infty$ & $0.0000000 E+\infty$ & $0.0000000 E+\infty$ & NONE & WP035597 & $\mathbf{Y}$ & N/A \\
\hline 2691 & 3,5 & CONSTANT & $0.0000000 E+\infty$ & $0.0000000 E+00$ & $0.0000000 E+\infty$ & $0.0000000 E+\infty$ & NONE & WP040514 & $\mathbf{Y}$ & N/A \\
\hline 2692 & $1,3,4$ & CONSTANT & $2.6000000 E-01$ & $2.6000000 E-01$ & $2.6000000 E-01$ & 2.6000000E-01 & $\mathbf{P a}$ & WP031041 & $\mathbf{Y}$ & NA \\
\hline 2692 & $1,3,4$ & CONSTANT & $2.6000000 E-01$ & $2.6000000 E-01$ & $2.6000000 E-01$ & $26000000 E-01$ & Pa & WP035597 & $\mathbf{Y}$ & NA \\
\hline 2692 & $1,3,4$ & CONSTANT & $2.60000000-01$ & $2.6000000=-01$ & $2.6000000 E-01$ & $2.6000000 E-01$ & Pa & WPO40514 & $Y$ & NA \\
\hline
\end{tabular}


CCA Parameter-Listing

\begin{tabular}{|c|c|c|c|c|c|c|c|c|c|c|}
\hline 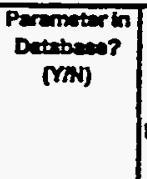 & 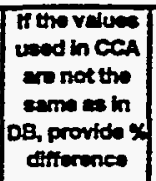 & D & Matada to & Mrtarid Mam & $\begin{array}{l}\text { Parmoter } \\
\text { to }\end{array}$ & Parmoter Ning & $\begin{array}{l}\text { PRPto } \\
\text { (MPOA) }\end{array}$ & Detentry & 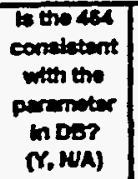 & $\begin{array}{c}\text { Permint } \\
\text { Cangory } \\
\text { (footnote 1) }\end{array}$ \\
\hline $\mathbf{Y}$ & & 2693 & CULEBRA & $\begin{array}{l}\text { Culabra member of the } \\
\text { Rustler formation }\end{array}$ & PCT_EXP & $\begin{array}{l}\text { Threshold presare axponential } \\
\text { paremeter }\end{array}$ & 32753 & $20+c b-96$ & $\mathbf{Y}$ & 48 \\
\hline $\mathbf{Y}$ & & 2693 & CULEBRA & $\begin{array}{l}\text { Culebra member of tho } \\
\text { Rustler formation }\end{array}$ & PCT_EXP & $\begin{array}{l}\text { Throshold pressure exponential } \\
\text { perameter }\end{array}$ & 32753 & 20 Feb-96 & $\mathbf{Y}$ & 48 \\
\hline $\mathbf{Y}$ & & 2693 & CULEBRA & $\begin{array}{l}\text { Culsbra member of tha } \\
\text { Rustier formation }\end{array}$ & PCT_EXP & $\begin{array}{l}\text { Threshotd pressure exponential } \\
\text { permeter }\end{array}$ & 32753 & $20-5 a-96$ & $\mathbf{Y}$ & 48 \\
\hline $\mathbf{Y}$ & & 2696 & DEWMLAE & Denwey Lake Red Beds & IFT & $\begin{array}{l}\text { Fiag for Permesbilty Determned } \\
\text { Imreshold }\end{array}$ & 32703 & $20-f a b-96$ & $\mathbf{Y}$ & 48 \\
\hline $\mathbf{Y}$ & & 2696 & DEWMAKE & Dowroy Lake Red Bods & KPT & $\begin{array}{l}\text { Fteg for Permesbility Determined } \\
\text { Threshold }\end{array}$ & 32703 & $20-F=b-56$ & $\mathbf{Y}$ & $A B$ \\
\hline $\mathbf{Y}$ & & 2696 & DEWMLAE & Denrey Lake Red Beds & KPT & $\begin{array}{l}\text { Flog for Permeability Determined } \\
\text { Threshold }\end{array}$ & 32703 & 20-feb-96 & $\mathbf{Y}$ & 48 \\
\hline $\mathbf{Y}$ & & 2696 & DEWMLAKE & Dewroy Lake Red Beds & KPT & $\begin{array}{l}\text { Flag for Permeabilty Determined } \\
\text { Thresthold }\end{array}$ & 32703 & 20 -Feb-96 & $\mathbf{Y}$ & $4 B$ \\
\hline $\mathbf{Y}$ & 100 & 2697 & DEWMLAKE & Dowoy Lake Red Beds & PCT_A & $\begin{array}{l}\text { Trmeshodd Presesure Lnoar } \\
\text { Parameter }\end{array}$ & 32725 & $20-F<b-96$ & $\mathbf{Y}$ & 48 \\
\hline $\mathbf{Y}$ & 100 & 2697 & DEWMLAKE & Dewey Loke Red Beds & PCT_A & $\begin{array}{l}\text { Throshold Pressure Linear } \\
\text { Paremeter }\end{array}$ & 32725 & $20-F=0-96$ & $\mathbf{Y}$ & $4 B$ \\
\hline $\mathbf{Y}$ & 100 & 2697 & DEWMAKE & Dewoy Lake Red Beds & PCT_A & $\begin{array}{l}\text { Threshold Pressure Lnear } \\
\text { Paramoter }\end{array}$ & 32725 & $20-F a b-96$ & $\mathbf{Y}$ & 48 \\
\hline $\mathbf{Y}$ & 100 & 2697 & DEWMAKE & Dowey Lake Rod Bads & PCT_A & $\begin{array}{l}\text { Threshold Pressure Linesr } \\
\text { Parameter }\end{array}$ & 32725 & $20-F c b-96$ & $\mathbf{Y}$ & 48 \\
\hline $\mathbf{Y}$ & 100 & 2698 & DEWMLAKE & Dewey Lako Red Beds & PCT_EXP & $\begin{array}{l}\text { Threshold pressure exponential } \\
\text { paremetor }\end{array}$ & 32727 & $20+50-6-86$ & $\mathbf{Y}$ & 48 \\
\hline $\mathbf{Y}$ & 100 & 2698 & DEWMAKE & Dowey Lake Rod Bods & PCT_EXP & $\begin{array}{l}\text { Threshold pressure exponential } \\
\text { peremoter }\end{array}$ & 32727 & $20-$ ab- 96 & $\mathbf{Y}$ & $4 B$ \\
\hline $\mathbf{Y}$ & 100 & 2698 & DEWMAKE & Dewey Lake Rod Bods & PCT_EXP & $\begin{array}{l}\text { Threshold pressure exponental } \\
\text { parameter }\end{array}$ & 32727 & 20-Feb-96 & $\mathbf{Y}$ & $4 B$ \\
\hline $\mathbf{Y}$ & 100 & 2698 & DEWMAKE & Dewry Lako Red Beds & PCT_EXP & $\begin{array}{l}\text { Threshold presuro exponential } \\
\text { perametor }\end{array}$ & 32727 & 20fob-96 & $\mathbf{Y}$ & $A B$ \\
\hline $\mathbf{Y}$ & & 2701 & $D R Z_{-} 0$ & $\begin{array}{l}\text { Disturbed rock zone; } \\
\text { time penod }-5 \text { to } 0 \\
\text { years }\end{array}$ & KPT & $\begin{array}{l}\text { Fieg for Permeculity Determined } \\
\text { Thresiold }\end{array}$ & 32801 & 20-Fob-96 & $\mathbf{Y}$ & 48 \\
\hline $\mathbf{Y}$ & & 2701 & DRZ_O & $\begin{array}{l}\text { Disturbed rock zone. } \\
\text { time period }-5 \text { to } 0 \\
\text { years }\end{array}$ & KPT & $\begin{array}{l}\text { Fieg for Permenatity Determined } \\
\text { Tmresthole }\end{array}$ & 32801 & $20-F=b-96$ & $\mathbf{Y}$ & $4 B$ \\
\hline $\mathbf{Y}$ & & 2701 & DRZ_O & $\begin{array}{l}\text { Disturbed rock zone; } \\
\text { time penod }-5 \text { to } 0 \\
\text { years }\end{array}$ & KPT & $\begin{array}{l}\text { Fleg for Permenainy Determined } \\
\text { Inreshold }\end{array}$ & 32801 & $20-F=b-96$ & $\mathbf{Y}$ & $A B$ \\
\hline $\mathbf{Y}$ & & 2701 & DRZ_o & $\begin{array}{l}\text { Disturbed rock zone, } \\
\text { time penod }-5 \text { to } 0 \\
\text { years }\end{array}$ & KాT & 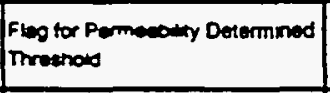 & 32801 & 20-Feb-96 & $\mathbf{Y}$ & $4 B$ \\
\hline$Y$ & & 2702 & $D R Z$ O & $\begin{array}{l}\text { Disturbed rock zono. } \\
\text { imo penod } 5 \text { to } 0 \\
\text { years }\end{array}$ & PCT_A & 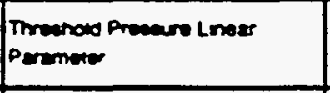 & 32832 & 14-Nov-95 & $\mathbf{Y}$ & 48 \\
\hline $\mathbf{Y}$ & & 2702 & $D R Z_{-} 0$ & $\begin{array}{l}\text { Disturted rock zone } \\
\text { ime pernod } 5 \text { to } 0 \\
\text { years }\end{array}$ & PCT_A & $\begin{array}{l}\text { Prounos Precers Lnear } \\
\text { Peramene }\end{array}$ & 32832 & 14-Nov-95 & ry & $4 B$ \\
\hline$Y$ & & 2703 & DRZ_o & $\begin{array}{l}\text { Disturbed rock zom } \\
\text { tume penod } 5 \text { vol } 0 \\
\text { years }\end{array}$ & PCT_EX & 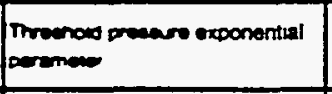 & 32833 & 14Nov-95 & $\mathbf{Y}$ & $4 B$ \\
\hline $\mathbf{Y}$ & & 2703 & $D R Z_{-} 0$ & $\begin{array}{l}\text { Desturbed roce } 2000 \\
\text { tume penco \& is } 0 \\
\text { years }\end{array}$ & PCT_EXP & 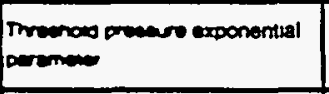 & 32833 & 14-Now-95 & $\mathbf{Y}$ & $4 B$ \\
\hline $\mathbf{Y}$ & & 2706 & EARTH & Eartien Fi & KాT & 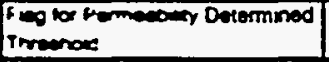 & 32919 & 20 -feb-9s & $\mathbf{Y}$ & $4 B$ \\
\hline $\mathbf{Y}$ & & 2706 & EARTH & Eertien Fi & nPt & 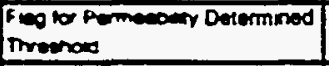 & 32919 & 20 -ab-96 & $Y$ & $A B$ \\
\hline $\boldsymbol{Y}$ & & 2706 & EARTH & Eartian $F=$ & KOT & 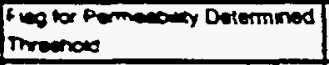 & 32919 & $20-50 b-96$ & $\mathbf{Y}$ & 48 \\
\hline $\mathbf{Y}$ & & 2706 & EARTH & Evarthen Fa & $|P|$ & 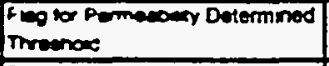 & 32918 & 20-Feb-96 & $\mathbf{Y}$ & 48 \\
\hline $\mathbf{Y}$ & & 2706 & EARTH & Eerthen Fi & RPT & 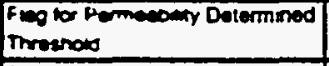 & 32919 & $20-5 a b-96$ & $Y$ & 48 \\
\hline $\mathbf{Y}$ & & 2706 & EARTH & Earthen Fi & का & 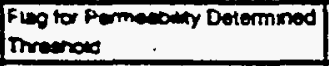 & 32919 & $20-F c b-96$ & $Y$ & 48 \\
\hline $\mathbf{Y}$ & 100 & 2707 & EARTH & Earthen Fa & PCT_A & $\begin{array}{l}\text { Throenodo Pressure Lnear } \\
\text { Peremete }\end{array}$ & 32920 & $14 F=b-96$ & $\mathbf{Y}$ & 1 \\
\hline $\mathbf{Y}$ & 100 & 2707 & EARTH & Eartion Fi & PCT_a & $\begin{array}{l}\text { Trrectiold Preature Lnes } \\
\text { Peremeter }\end{array}$ & 32920 & $14 f=b-86$ & $\mathbf{Y}$ & 1 \\
\hline
\end{tabular}




\section{- CCA Parameter-Listing}

\begin{tabular}{|c|c|c|c|c|c|c|c|c|c|c|}
\hline ID & $\begin{array}{l}\text { Chatif } \\
\text { cetbon of } \\
\text { Deta } \\
\text { Cetegory } \\
\text { (footnote 2) }\end{array}$ & $\begin{array}{l}\text { Dituiberton } \\
\text { Type }\end{array}$ & Men & Medien & Mlnimem & Meximuan & Units & Referunce to & $\begin{array}{l}\text { Wes the dete } \\
\text { demaloped } \\
\text { underen } \\
\text { NoA-1 } \\
\text { Progringt }\end{array}$ & 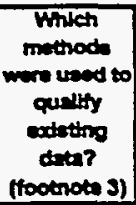 \\
\hline 2693 & $1,3,4$ & CONSTANT & $3.4800000 E-01$ & $3.4800000 E-01$ & $-3.4800000 E-01$ & $3.4800000 E-01$ & NONE & WP031041 & $\mathbf{Y}$ & N/A \\
\hline 2693 & $1,3,4$ & CONSTANT & $3.4800000 \mathrm{E}-01$ & $3.48000000 E-01$ & $3.4800000 E-01$ & 3.4800000 E-01 & NONE & WP035597 & $\mathbf{Y}$ & NA \\
\hline 2693 & $1,3,4$ & CONSTANT & $-3.4800000 \mathrm{E}-01$ & $-3.4800000 E-01$ & $-3.4800000 E-01$ & $3.4800000 \mathrm{E}-01$ & NONE & WP040514 & $\mathbf{Y}$ & NA \\
\hline 2696 & 3,5 & CONSTANT & $0.0000000 E+\infty$ & $0.0000000 \mathrm{E}+\infty 0$ & $0.00000000+\infty 0$ & $0.0000000 E+\infty$ & NONE & WP035271 & $\mathbf{Y}$ & NA \\
\hline 2696 & 3,5 & CONSTANT & $0.0000000 E+\infty$ & $0.0000000 E+\infty$ & $0.0000000 E+00$ & $0.0000000 E+\infty$ & NONE & WPO35333 & $\mathbf{Y}$ & NA \\
\hline 2696 & 3,5 & CONSTANT & $0.0000000 E+\infty$ & $0.0000000 E+\infty$ & $0.0000000 E+\infty 0$ & $0.0000000 E+\infty$ & NONE & WP035597 & $\mathbf{Y}$ & NA \\
\hline 2696 & 3,5 & CONSTANT & $0.0000000 \mathrm{E}+00$ & $0.0000000 E+\infty 0$ & $0.0000000 E+\infty$ & $0.0000000 E+\infty$ & NONE & WPO40514 & $\mathbf{Y}$ & NAA \\
\hline 2697 & $1,3,4$ & CONSTANT & $2.6000000 E-01$ & $2.6000000 E-01$ & $26000000 E-01$ & $26000000=-01$ & Pa & WP031041 & $\mathbf{Y}$ & NA \\
\hline 2697 & $1,3,4$ & CONSTANT & $2.6000000 E-01$ & $2.6000000 \mathrm{E}-01$ & $2.6000000 \mathrm{E}-01$ & $260000000-01$ & Pa & WP035597. & $\mathbf{Y}$ & $N / A$ \\
\hline 2697 & $1,3,4$ & CONSTANT & $2.6000000 E-01$ & $2.6000000=-01$ & $26000000=-01$ & $2.6000000 E-01$ & Pa & WPO40434 & $\mathbf{Y}$ & NA \\
\hline 2697 & $1,3,4$ & CONSTANT & $2.6000000 E-01$ & $2,6000000=-01$ & $2.6000000 E-01$ & $2.6000000 E-01$ & Pa & WPO40514 & $\mathbf{Y}$ & $N / A$ \\
\hline 2698 & $1,3,4$ & CONSTANT & $-3.4800000=-01$ & $-3.4800000=-01$ & $-3.4800000 E-01$ & $-3.4800000 \mathrm{E}-01$ & NONE & WP031041 & $\mathbf{Y}$ & NAA \\
\hline 2698 & $1,3,4$ & CONSTANT & $-3.4800000 \mathrm{E}-01$ & $-3.4800000 E-01$ & $3.4800000 E-01$ & $3.4800000 E-01$ & NONE & WP035597 & $\mathbf{Y}$ & NA \\
\hline 2698 & $1,3,4$ & CONSTANT & $3,4800000 E-01$ & $3.4800000 E-01$ & $3.4800000 \varepsilon-01$ & $-3.4800000 E-01$ & NONE & WPO40434 & $\cdot Y$ & NA \\
\hline 2698 & $1,3,4$ & CONSTANT & $3.48000000-01$ & $-3.4800000 \mathrm{E}-01$ & $3.4800000 E-01$ & $-3.4800000 E-01$ & NONE & WP040514 & $\mathbf{Y}$ & NA \\
\hline 2701 & 3,5 & CONSTANT & $0.0000000 E+\infty$ & $0.0000000 E+\infty 0$ & $0.00000000 E+\infty$ & $0.0000000 E+\infty$ & NONE & WP035271 & $\mathbf{Y}$ & NA \\
\hline 2701 & 3,5 & CONSTANT & $0.0000000 E+\infty 0$ & $0.0000000 E+\infty 0$ & $0.0000000 E+\infty 0$ & $0.0000000 E+\infty 0$ & NONE & WPO35333 & $Y$ & NA \\
\hline 2701 & 3,5 & CONSTANT & $0.0000000 \mathrm{E}+\infty 0$ & $0.00000000+00$ & $0.0000000 E+\infty$ & $0.0000000 E+\infty$ & NONE & WP035597 & $\mathbf{Y}$ & NA \\
\hline 2701 & 3,5 & CONSTANT & $0.0000000 E+\infty$ & $0.0000000 E+\infty$ & $0.0000000 \mathrm{E}+\infty$ & $0.0000000 E+\infty 0$ & NONE & WP040514 & $\mathbf{Y}$ & N/A \\
\hline 2702 & 3,5 & CONSTANT & $0.0000000 E+\infty$ & $0.0000000 E+\infty$ & $0.0000000 E+\infty 0$ & $0.0000000 E+\infty 0$ & Pa & WP038568 & $\mathbf{Y}$ & $N A$ \\
\hline 2702 & 3,5 & CONSTANT & $0.0000000 E+\infty$ & $0.0000000 E+\infty$ & $0.0000000 E+\infty 0$ & $0.0000000 E+\infty$ & $\mathbf{P a}$ & WPO40514 & $Y$ & NA \\
\hline 2703 & 3,5 & CONSTANT & $0.0000000 E+\infty$ & $0.0000000 E+00$ & $0.0000000 E+\infty 0$ & $0.0000000 E+\infty$ & NONE & WP038568 & $\mathbf{Y}$ & NA \\
\hline 2703 & 3,5 & CONSTANT & $0.0000000 E+\infty$ & $0.0000000 E+\infty$ & $0.0000000 E+\infty$ & $0.0000000 E+\infty$ & NONE & WPO40514 & $\mathbf{Y}$ & NAA \\
\hline 2706 & 3,5 & CONSTANT & $0.0000000 E+\infty$ & $0.0000000 E+\infty$ & $0.0000000 E+\infty 0$ & $0.0000000 E+\infty 0$ & NONE & WP030640 & $\mathbf{Y}$ & $N / A$ \\
\hline 2706 & 3,5 & CONSTANT & $0.0000000 E+\infty$ & $0.0000000 E+\infty 0$ & $0.0000000 E+\infty$ & $0.0000000 E+\infty$ & NONE & WP030994. & $\mathbf{Y}$ & N/A \\
\hline 2706 & 3,5 & CONSTANT & $0.0000000 E+\infty 0$ & $0.0000000 E+00$ & $0.0000000 E+\infty 0$ & $0.0000000 E+\infty 0$ & NONE & WP035271 & $\mathbf{Y}$ & $N A$ \\
\hline 2706 & 3,5 & CONSTANT & $0.00000000 E+\infty$ & $0.0000000 E+\infty$ & $0.0000000 E+\infty 0$ & $0.0000000 E+\infty$ & NONE & WP035333 & $\mathbf{Y}$ & $N A$ \\
\hline 2706 & 3.5 & CONSTANT & $0.0000000 E+\infty$ & $0.0000000 E+\infty 0$ & $0.00000000 E+\infty$ & $0.0000000 E+\infty$ & NONE & WP035597 & $\mathbf{Y}$ & NA \\
\hline 2706 & 3,5 & CONSTANT & $0.0000000 E+\infty$ & $0.0000000 E+\infty 0$ & $0.0000000 E+00$ & $0.0000000 E+\infty$ & NONE & WPO40514 & $\mathbf{Y}$ & NA \\
\hline 2707 & 3,4 & CONSTANT & $5.6000000 E-01$ & $5.6000000 E-01$ & $5.6000000 \mathrm{E}-01$ & $5.6000000 E-01$ & Pa & WPO30640 & $\mathbf{Y}$ & NA \\
\hline 2707 & 3,4 & CONSTANT & $5.6000000 \mathrm{E}-01$ & $5.6000000 E-01$ & $5.6000000 E-01$ & $5.6000000 E-01$ & $\mathbf{P e}$ & WP030994 & $\mathbf{Y}$ & NA \\
\hline
\end{tabular}


CCA Parameter-Listing

\begin{tabular}{|c|c|c|c|c|c|c|c|c|c|c|}
\hline $\begin{array}{c}\text { Peramutar in } \\
\text { Detabaes? } \\
\text { (Y/NI) }\end{array}$ & 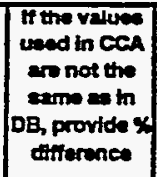 & ID & Axtactal bo & Matarid hams & $\begin{array}{c}\text { Parnater } \\
\text { D }\end{array}$ & Parencter Kerse & $\begin{array}{l}\text { PRP ID } \\
\text { (MPOE E) }\end{array}$ & $\begin{array}{c}\text { Dete Entry } \\
\text { Dets }\end{array}$ & 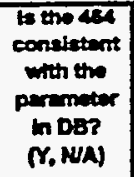 & $\begin{array}{l}\text { Parminiter } \\
\text { Catiogory } \\
\text { (footnote } 1 \text { ) }\end{array}$ \\
\hline $\mathbf{Y}$ & 100 & 2707 & EARTH & Earthen Fid & PCT_A & $\begin{array}{l}\text { Trreshold Pressura Lnear } \\
\text { Perameter }\end{array}$ & 32920 & $14 F 0 b-96$ & $\mathbf{Y}$ & 1 \\
\hline $\mathbf{Y}$ & 100 & 2707 & EARTH & Exthen Fill & PCT_A & $\begin{array}{l}\text { Throshoid Prossuro Lnaer } \\
\text { Paraneter }\end{array}$ & 32920 & 14 Fob-96 & $\mathbf{Y}$ & 1 \\
\hline $\mathbf{Y}$ & 100 & 2708 & EARTH & Exrthen Frl & PCT_EXP & $\begin{array}{l}\text { Trreshold preserte exponential } \\
\text { peraneter }\end{array}$ & 32921 & 22fFcb-96 & $\mathbf{Y}$ & 1 \\
\hline $\mathbf{Y}$ & 100 & 2708 & EARTH & Eerthen Fill & PCT_EXP & $\begin{array}{l}\text { Thresthold pressure exponential } \\
\text { paremeter }\end{array}$ & 32921 & $22+50-96$ & $\mathbf{Y}$ & 1 \\
\hline $\mathbf{Y}$ & 100 & 2708 & EARTH & Earthen Fill & PCT_EXP & $\begin{array}{l}\text { Threshold pressure exponentid } \\
\text { permeter }\end{array}$ & 32921 & 22Fab-96 & $\mathbf{Y}$ & 1 \\
\hline$Y$ & 100 & 2708 & EARTH & Earthen Frl & PCT_EXP & $\begin{array}{l}\text { Throshold pressure exponental } \\
\text { perameter }\end{array}$ & 32921 & 22Fob-96 & $\mathbf{Y}$ & 1 \\
\hline $\mathbf{Y}$ & & 2711 & EXP_AREA & Expenmental Area & KPT & $\begin{array}{l}\text { Fleg for Permenbilty Determned } \\
\text { Threshold }\end{array}$ & 33009 & 16 Feb-S6 & $\mathbf{Y}$ & $A B$ \\
\hline $\mathbf{Y}$ & & 2711 & EXP_AREA & Exporimental Area & KPT & $\begin{array}{l}\text { Flag for Permesbility Detemined } \\
\text { Threshold }\end{array}$ & 33009 & 16 feb-96 & $\mathbf{Y}$ & $4 B$ \\
\hline $\mathbf{Y}$ & & 2711 & EXP_AREA & Experinental Area & KPT & $\begin{array}{l}\text { Fleg for Permeability Determined } \\
\text { Trmeshold }\end{array}$ & 33009 & 16-Feb-96 & $\mathbf{Y}$ & 48 \\
\hline $\mathbf{Y}$ & & 2711 & EXP_AREA & Experimental Area & KPT & $\begin{array}{l}\text { Fleg for Permesbilty Datemined } \\
\text { Trreshold }\end{array}$ & 33009 & 16 feb-96 & $\mathbf{Y}$ & 48 \\
\hline $\mathbf{Y}$ & & 2712 & EXP_AREA & Expermental Area & PCT_A & $\begin{array}{l}\text { Threshold Pressure Lnes } \\
\text { Perameter }\end{array}$ & 33037 & 14Hov-95 & $\mathbf{Y}$ & $4 B$ \\
\hline $\mathbf{Y}$ & & 2712 & EXP_AREA & Experimental Area & PCT_A & $\begin{array}{l}\text { Threshold Pressure Lnear } \\
\text { Parameter }\end{array}$ & 33037 & $14+10 y-95$ & $\mathbf{Y}$ & 48 \\
\hline $\mathbf{Y}$ & & 2713 & EXP_AREA & Experinental Area & PCT_EXP & $\begin{array}{l}\text { Threshold prossure exponential } \\
\text { parameter }\end{array}$ & 33039 & 14tion-95 & $\mathbf{Y}$ & 48 \\
\hline $\mathbf{Y}$ & & 2713 & EXP_AREA & Experimental Area & PCT_EXP & $\begin{array}{l}\text { Threshold pressure exponential } \\
\text { peremoter }\end{array}$ & 33039 & 14-Atov-95 & $\mathbf{Y}$ & 48 \\
\hline $\mathbf{Y}$ & & $27-15$ & FORTMNIN & Forty Niner Member & KPT & $\begin{array}{l}\text { Flag for Pormeabilty Determined } \\
\text { Threshold }\end{array}$ & 32982 & 20-Fob-96 & $\mathbf{Y}$ & 48 \\
\hline $\mathbf{Y}$ & & 2715 & FORTYNIN & Forty Niner Member & KPT & $\begin{array}{l}\text { Flag for Permeability Determined } \\
\text { Threshold }\end{array}$ & 32962 & 20 Feb-96 & $\mathbf{Y}$ & 48 \\
\hline $\mathbf{Y}$ & & 2715 & FORTYNIN & Forty Niner Member & KPT & $\begin{array}{l}\text { Fleg for Permasbilty Determinad } \\
\text { Threstold }\end{array}$ & 32982 & 20-Fob-96 & $\mathbf{Y}$ & 48 \\
\hline $\mathbf{Y}$ & & 2715 & FORTYNIN & Forty Niner Member & KPT & $\begin{array}{l}\text { Flag for Permeability Determned } \\
\text { Threshold }\end{array}$ & 32982 & $20-F a b-96$ & $\mathbf{Y}$ & $4 B$ \\
\hline $\mathbf{Y}$ & & 2716 & FORTYNIN & Forty Niner Member & PCT_A & $\begin{array}{l}\text { Threstiold Pressuro Lnesr } \\
\text { Perameter }\end{array}$ & 32984 & 14-Nov-95 & $\mathbf{Y}$ & $4 B$ \\
\hline $\mathbf{Y}$ & & 2716 & FORTYNIN & Forty Niner Momber & PCT_A & $\begin{array}{l}\text { Throstold Pressure Lineser } \\
\text { Parameter }\end{array}$ & 32984 & 14-Nov-95 & $\mathbf{Y}$ & 48 \\
\hline $\mathbf{Y}$ & & 2717 & FORTYNIN & Forty Niner Member & PCT_EXP & $\begin{array}{l}\text { Threshold pressure exponential } \\
\text { paramoler }\end{array}$ & 32986 & 14Hov-95 & $\mathbf{Y}$ & 48 \\
\hline $\mathbf{Y}$ & & 2717 & FORTYNIN & Forty Niner Mernber & PCT_EXP & $\begin{array}{l}\text { Threshold pressure exponental } \\
\text { paremeter }\end{array}$ & 32986 & 14-Nov-95 & $\mathbf{Y}$ & $4 B$ \\
\hline $\mathbf{Y}$ & & 2718 & FORTMNIN & Forty Niner Member & PO_MIN & $\begin{array}{l}\text { Minimum bro pressure for } \\
\text { Capillary model KPC=3 }\end{array}$ & 32997 & 21Fab-96 & $\mathbf{Y}$ & 48 \\
\hline $\mathbf{Y}$ & & 2718 & FORTMNIN & Forty Niner Member & PO_MIN & $\begin{array}{l}\text { Mininum bnne proseure for } \\
\text { capillary model KPC }=3\end{array}$ & 32997 & 21-Feb-96 & $\mathbf{Y}$ & $4 B$ \\
\hline $\mathbf{Y}$ & & 2718 & FORTYNIN & Forty Niner Member & PO_MIN & $\begin{array}{l}\text { Mnimum brne pressure for } \\
\text { capillary model } \mathrm{KPC}=3\end{array}$ & 32997 & 21-Feb-96 &.$- Y$ & AB \\
\hline $\mathbf{Y}$ & & 2720 & IMPERM_Z & Impermesble Zones & KPT & $\begin{array}{l}\text { Fleg for Permeability Determined } \\
\text { Throshold }\end{array}$ & 33119 & 20 feb-96 & $\mathbf{Y}$ & $4 B$ \\
\hline $\mathbf{Y}$ & & 2720 & IMPERM_Z & Itinpermasble Zones & KPT & $\begin{array}{l}\text { Flag for Permeability Determned } \\
\text { Threshold }\end{array}$ & 33119 & 20Fab-96 & $\mathbf{Y}$ & AB \\
\hline$Y$ & & 2720 & MPERH_Z & Impermeable Zones & KPT & $\begin{array}{l}\text { Flag for Permeability Determined } \\
\text { Threshold }\end{array}$ & 33119 & $20-F=b-96$ & $\mathbf{Y}$ & AB \\
\hline $\mathbf{Y}$ & & 2720 & MPERM_Z & Inpermeable Zones & KPT & $\begin{array}{l}\text { Fleg for Permatbilty Determined } \\
\text { Threshold }\end{array}$ & 33119 & 20-Fob-96 & $\mathbf{Y}$ & $4 B$ \\
\hline $\mathbf{Y}$ & & 2721 & MPERM_Z & Impermesble Zones & PCT_A & $\begin{array}{l}\text { Threshold Pressure Lnas } \\
\text { Perameter }\end{array}$ & 33172 & 14-Nov-95 & $\mathbf{Y}$ & $4 B$ \\
\hline $\mathbf{Y}$ & & 2721 & AMERM_Z & Impermasble Zones & PCT_A & $\begin{array}{l}\text { Trreshold Pressuro Lnear } \\
\text { Peremeter }\end{array}$ & 33172 & 14-Nov-95 & $\mathbf{Y}$ & $4 B$ \\
\hline $\mathbf{Y}$ & & 2722 & MPERM_Z & Impermasbis Zones & PCT_EXP & $\begin{array}{l}\text { Throshold pressure exponentual } \\
\text { paremeter }\end{array}$ & 33174 & 14-Nov-95 & $\mathbf{Y}$ & 48 \\
\hline $\mathbf{Y}$ & & 2722 & MPERM_Z & limpermasble Zones & PCT_EXP & $\begin{array}{l}\text { Thrashold pressure exponemial } \\
\text { parnemeler }\end{array}$ & 33174 & 14Nov-95 & $\mathbf{Y}$ & 48 \\
\hline $\mathbf{Y}$ & & 2725 & MAGENTA & Meganta Momber & IPT & $\begin{array}{l}\text { Fleg for Permeabildy Determined } \\
\text { Throghold }\end{array}$ & 33254 & 20-Fab-96 & $\mathbf{Y}$ & $4 B$ \\
\hline $\mathbf{Y}$ & & 2725 & MAGENTA & Megenta Momber & KPT & $\begin{array}{l}\text { Fieg for Permeabilty Determined } \\
\text { Threahold }\end{array}$ & 33254 & 20fab-ss & $\mathbf{Y}$ & $4 B$ \\
\hline $\mathbf{Y}$ & & 2725 & MAGENTA & Maganta Mernber & KPT & $\begin{array}{l}\text { Fisg for Permesbility Detemned } \\
\text { Threshold }\end{array}$ & 33254 & 20 Fab-96 & $\mathbf{Y}$ & $4 B$ \\
\hline
\end{tabular}


CCA Parameter-Listing

\begin{tabular}{|c|c|c|c|c|c|c|c|c|c|c|}
\hline ID & 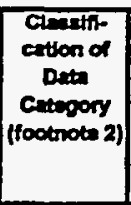 & $\begin{array}{l}\text { Dotribution } \\
\text { Type }\end{array}$ & maxn & Modim & Mintrum & maxinum & Lantes & Refermes D & 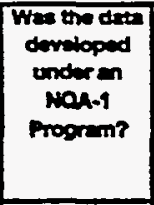 & $\begin{array}{c}\text { Wrich } \\
\text { mothods } \\
\text { more uned to } \\
\text { quinty } \\
\text { deting } \\
\text { (footnote 3) }\end{array}$ \\
\hline 2707 & 3,4 & CONSTANT & $5.6000000 E-01$ & $5.6000000 E-01$ & $5.6000000 \mathrm{E}-01$ & $5.6000000 E-01$ & $\mathbf{P a}$ & WP035597 & $Y$ & NA \\
\hline 2707 & 3,4 & CONSTANT & $5.6000000 E-01$ & $5.6000000 E-01$ & $5.6000000 E-01$ & $5.6000000 E-01$ & $\mathrm{~Pa}$ & WPO40434 & Y & NA \\
\hline 2708 & 3,4 & CONSTANT & 3.4600000 E-01 & $3.4600000 E-01$ & $3.4600000 E-01$ & $-3.46000005-01$ & NONE & WP030540 & $Y$ & NA \\
\hline 2708 & 3,4 & CONSTANT & $3.46000000-01$ & $3.4600000 E-01$ & $-3.4600000 \mathrm{E}-01$ & $3.4500000 E-01$ & NONE & WP030994 & $\mathbf{Y}$ & NA \\
\hline 2708 & 3,4 & CONSTANT & $3.4600000 E-01$ & $-3.4600000 E-01$ & $-3.4600000 E-01$ & $-3.4500000 E-01$ & NONE & WP035597 & $\mathbf{Y}$ & NA \\
\hline 2708 & 3,4 & CONSTANT & $3.4600000 \mathrm{E}-01$ & $-3.4600000 E-01$ & $3.4600000 E-01$ & $3.4600000=-01$ & NONE & WPO40434 & $Y$ & NA \\
\hline 2711 & 3,5 & CONSTANT & $0.00000000 E+00$ & $0.0000000 E+\infty 0$ & $0.0000000 E+\infty$ & $-0.0000000 E+\infty 0$ & NONE & WP035271 & $\mathbf{Y}$ & NA \\
\hline 2711 & 3,5 & CONSTANT & $0.0000000 E+\infty 0$ & $0.0000000 E+\infty 0$ & $0.0000000 E+\infty 0$ & $0.0000000=+\infty 0$ & NONE & WP035333 & $\mathbf{Y}$ & NA \\
\hline 2711 & 3,5 & CONSTANT & $0.0000000 E+\infty 0$ & $0.0000000 E+\infty 0$ & $0.0000000 E+\infty 0$ & $0.0000000 E+\infty$ & NONE & WP035597 & $Y$ & NA \\
\hline 2711 & 3,5 & CONSTANT & $0.0000000 E+00$ & $0.0000000 \mathrm{E}+00$ & $0.0000000 \approx+\infty 0$ & $0.0000000 E+\infty$ & NONE & WP040514 & $\mathbf{Y}$ & NA \\
\hline 2712 & 3,5 & CONSTANT & $0.0000000 \mathrm{E}+00$ & $0.0000000 E+\infty 0$ & $0.0000000 \approx+\infty 0$ & $0.0000000 E+\infty 0$ & $\mathrm{~Pa}$ & WP038568 & $Y$ & NA \\
\hline 2712 & 3,5 & CONSTANT & $0.0000000 E+00$ & $0.0000000 E+\infty$ & $0.0000000 \varepsilon+\infty$ & $0.0000000 E+\infty 0$ & Pa & WP040514 & $\mathbf{Y}$ & NA \\
\hline 2713 & 3,5 & CONSTANT & $0.0000000 \mathrm{E}+00$ & $0.0000000 E+\infty 0$ & $0.0000000 E+\infty 0$ & $0.0000000 E+\infty$ & NONE & WP038568 & $\mathbf{Y}$ & NA \\
\hline 2713 & 3,5 & CONSTANT & $0.0000000 E+\infty$ & $0.0000000 E+00$ & $0.0000000 \varepsilon+\infty 0$ & $0.0000000 E+\infty$ & NONE & WPO40514 & $\mathbf{Y}$ & NA \\
\hline 2715 & 1,3 & CONSTANT & $0.0000000 E+\infty$ & $0.0000000 E+\infty 0$ & $0.0000000 E+\infty$ & $0.0000000 E+\infty$ & MONE & WP035271 & $\mathbf{Y}$ & NA \\
\hline 2715 & 1,3 & CONSTANT & $0.0000000 E+\infty 0$ & $0.0000000 E+\infty 0$ & $0.0000000 E+\infty$ & $0.0000000 E+\infty$ & NONE & WPO35333 & $\mathbf{Y}$ & NA \\
\hline 2715 & 1,3 & CONSTANT & $0.0000000 \mathrm{E}+00$ & $0.0000000 E+\infty$ & $0.0000000 \varepsilon+\infty$ & $0.0000000 E+00$ & NONE & WP035597 & $\mathbf{Y}$ & NA \\
\hline 2715 & 1,3 & CONSTANT & $0.0000000 E+\infty 0$ & $0.0000000 E+\infty$ & $0.0000000 E+\infty$ & $0.0000000 \mathrm{E}+\infty 0$ & NOME & WPO40514 & $\mathbf{Y}$ & NA \\
\hline 2716 & 1,3 & CONSTANT & $0.0000000 E+00$ & $0.0000000 E+\infty$ & $0.0000000 E+\infty 0$ & $0.0000000 E+\infty 0$ & $\mathrm{~Pa}$ & WP038568 & $Y$ & NA \\
\hline 2716 & 1,3 & CONSTANT & $0.0000000 \mathrm{E}+\infty 0$ & $0.0000000 \mathrm{E}+\infty 0$ & $0.0000000 \mathrm{E}+\infty 0$ & $0.0000000 E+\infty 0$ & $\mathrm{~Pa}$ & WPO40514 & $\mathbf{Y}$ & NA \\
\hline 2717 & 1,3 & CONSTANT & $0.0000000 E+\infty 0$ & $0.0000000 E+\infty 0$ & $0.0000000 E+\infty 0$ & $0.0000000 E+\infty 0$ & NONE & WP038568 & $\mathbf{Y}$ & NA \\
\hline 2717 & 1,3 & CONSTANT & $0.0000000 E+00$ & $0.0000000 E+\infty 0$ & $0.0000000 \mathrm{E}+\infty 0$ & $0.0000000 E+\infty$ & NONE & WP040514 & $\mathbf{Y}$ & N/A \\
\hline 2718 & 1,3 & CONSTANT & $1.0132500 E+05$ & $1.0132500 E+0.5$ & $1.0132500 E+05$ & $1.0132500 E+0.5$ & $\mathrm{~Pa}$ & WP035597 & $Y$ & NA \\
\hline 2718 & 1,3 & CONSTANT & $1.0132500 E+05$ & $1.0132500 E+05$ & $1.0132500 \varepsilon+05$ & $1.0132500 E+05$ & $\mathrm{~Pa}$ & WP036051 & $\mathbf{Y}$ & NA \\
\hline 2718 & 1,3 & CONSTANT & $1.0132500 E+05$ & $1.0132500 E+05$ & $1.0132500 E+05$ & $1.0132500 E+05$ & $\mathrm{~Pa}_{2}$ & WP040514 &.$- Y$ & N/A \\
\hline 2720 & 3,5 & CONSTANT & $0.0000000 E+\infty$ & $0.0000000 E+\infty 0$ & $00000000 E+\infty$ & $00000000 E+\infty$ & NONE & WP035271 & $Y$ & NA \\
\hline 2720 & 3,5 & CONSTANT & $0.0000000 E+\infty$ & $0.0000000 E+\infty 0$ & $0.0000000 \varepsilon+\infty$ & $00000000 E+\infty 0$ & NONE & WP035333 & $\mathbf{Y}$ & N/A \\
\hline 2720 & 3.5 & CONSTANT & $0.0000000 E+00$ & $0.0000000 \mathrm{E}+00$ & $0.0000000 E+\infty$ & $00000000 E+00$ & NONE & WP035597 & $Y$ & NA \\
\hline 2720 & 3,5 & CONSTANT & $0.0000000 E+00$ & $0.0000000 E+00$ & $0.0000000 E+\infty$ & $00000000 E+\infty$ & NONE & WPO40514 & $\mathbf{Y}$ & NA \\
\hline 2721 & 3,5 & CONSTANIT & $0.0000000 E+\infty 0$ & $0.00000000=+\infty$ & $0.0000000 E+\infty 0$ & $0,0000000+\infty$ & $\mathrm{Pa}$ & WP038568 & $\mathbf{Y}$ & NA \\
\hline 2721 & 3,5 & CONSTANT & $0.0000000 E+\infty 0$ & $0.0000000 E+\infty 0$ & $0.0000000 E+\infty$ & $0.0000000 E+\infty 0$ & $\mathrm{~Pa}$ & WP040514 & $\mathbf{Y}$ & NA \\
\hline 2722 & 3,5 & CONSTANT & $0.0000000 \mathrm{E}+\infty 0$ & $0.0000000 E+\infty 0$ & $00000000 E+\infty 0$ & $0.0000000 \mathrm{E}+\infty$ & NONE & WP038568 & $\mathbf{Y}$ & NA \\
\hline 2722 & 3,5 & CONSTANT & $0.0000000 \leq+\infty$ & $0.0000000 E+\infty 0$ & $0.0000000 E+\infty$ & $0.0000000 E+\infty$ & NONE & WPO40514 & $\mathbf{Y}$ & NA \\
\hline 2725 & 1,3 & CONSTANT & $0.0000000 E+\infty 0$ & $0.0000000 E+\infty 0$ & $0.0000000 E+\infty$ & $0.0000000+\infty 0$ & NONE & WP035271 & $\mathbf{Y}$ & NA \\
\hline 2725 & 1,3 & CONSTANT & $0.0000000 E+\infty$ & $0.0000000 E+\infty$ & $0.0000000 E+\infty 0$ & $0.0000000 E+00$ & NONE & WP035333 & $\mathbf{Y}$ & NA \\
\hline 2725 & 1,3 & CONSTANT & $0.00000000+\infty 0$ & $0.0000000 E+\infty 0$ & $0.0000000 E+00$ & $0.0000000 E+00$ & NONE & WP035597 & $\mathbf{Y}$ & NA \\
\hline
\end{tabular}


CCA Parameter Listing -

\begin{tabular}{|c|c|c|c|c|c|c|c|c|c|c|}
\hline 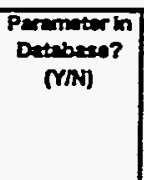 & 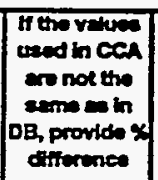 & .10 & Meridio & Mrtarial Neme & $\begin{array}{c}\text { Peramotar } \\
\text { wo }\end{array}$ & Parromion Name & $\begin{array}{l}\text { FRP ID } \\
\text { MPODI }\end{array}$ & $\begin{array}{c}\text { Denenty } \\
\text { Den }\end{array}$ & 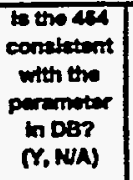 & $\begin{array}{l}\text { Parmmetar } \\
\text { Cutegary } \\
\text { (footnote 1) }\end{array}$ \\
\hline $\mathbf{Y}$ & & 2725 & MAGENTA & Magenta Member & KPT & $\begin{array}{l}\text { Flag for Permeability Delermined } \\
\text { Threshold }\end{array}$ & 33254 & $20-500-96$ & $\mathbf{Y}$ & 48 \\
\hline $\mathbf{Y}$ & & 2726 & MAGENTA & Megenta Member & PCT_A & $\begin{array}{l}\text { Throshold Pressuro Lrest } \\
\text { Paremeter }\end{array}$ & 33257 & $20-f e b-96$ & $Y$ & AB \\
\hline $\mathbf{Y}$ & & 2726 & MAGENTA & Mlagenti Mernber & PCT_A & $\begin{array}{l}\text { Throstiold Pressure Linear } \\
\text { Parmeter }\end{array}$ & 33257 & 20-Fob-s6 & $\mathbf{Y}$ & 48 \\
\hline $\mathbf{Y}$ & & 2726 & MAGENTA & Megents Member & PCT_A & $\begin{array}{l}\text { Throstiond Prossuro Lineer } \\
\text { Parenoter }\end{array}$ & 33257 & $20-00-96$ & $\mathbf{Y}$ & $4 B$ \\
\hline $\mathbf{Y}$ & & 2727 & MAGENTA & Magenta Member & PCT_EXP & $\begin{array}{l}\text { Throshold prosseurs exponential } \\
\text { peraneter }\end{array}$ & 33251 & $20+00-96$ & $Y$ & $4 B$ \\
\hline Y & & 2727 & MAGENTA & Magenta Member & PCT_EXP & $\begin{array}{l}\text { Threshold pressure exponental } \\
\text { peremotor }\end{array}$ & 33251 & $20-76 b-96$ & $\mathbf{Y}$ & $A B$ \\
\hline $\mathbf{Y}$ & & 2727 & MAGENTA & Magent Member & PCT_EXP & $\begin{array}{l}\text { Throshold pressure exponentiel } \\
\text { peranotior }\end{array}$ & 33281 & $20+00-90$ & $\mathbf{Y}$ & AB \\
\hline $\mathbf{Y}$ & & 2728 & MAGENTA & Magenta Momber & PO_MIN & $\begin{array}{l}\text { Mininium brno pressure for } \\
\text { ceppiltery model KPC=3 }\end{array}$ & 32536 & $21-f 0 b-96$ & $\mathbf{Y}$ & AB \\
\hline $\mathbf{Y}$ & & 2728 & MAGENTA & Megenta Member & PO_MIN & $\begin{array}{l}\text { Mnisium brino pressure for } \\
\text { capillary model KPC=3 }\end{array}$ & 32536 & $21+6 b-96$ & $\mathbf{Y}$ & 4B \\
\hline $\mathbf{Y}$ & & 2728 & MAGENTA & Magenta Member & PO_MIN & \begin{tabular}{|l|} 
Minimum brine pressure for \\
capillary model KPC=3
\end{tabular} & 32536 & 21 fab-96 & $\mathbf{Y}$ & 48 \\
\hline $\mathbf{Y}$ & & 2731 & PAN_SEAL & Penet Seal & KPT & $\begin{array}{l}\text { Fileg for Pormeability Doterm ned } \\
\text { Threstrold }\end{array}$ & 33009 & $20-F o b-96$ & $\mathbf{Y}$ & 4B \\
\hline $\mathbf{Y}$ & & 2731 & PAN_SEAL & Panel Seal & KPT & $\begin{array}{l}\text { Fing for Permeability Doterm ned } \\
\text { Threstoitd }\end{array}$ & 33089 & $20+\infty 6-96$ & $\mathbf{Y}$ & 4B \\
\hline $\mathbf{Y}$ & & 2731 & PAN_SEAL & Panot Sead & |KPT & $\begin{array}{l}\text { Flag for Permeability Dotermined } \\
\text { Threshold }\end{array}$ & 33089 & $20+60.96$ & $\boldsymbol{Y}$ & AB \\
\hline$Y$ & & 2731 & PAN_SEAL & Panel Seal & KPT & $\begin{array}{l}\text { Flog for Permeability Dotermined } \\
\text { Throshotd }\end{array}$ & 33009 & 20 feb-96 & $\mathbf{Y}$ & 4B \\
\hline $\mathbf{Y}$ & $100.00 \mathrm{hw}$ & 2732 & PAN_SEAL & Panel Seal & PCT_A & $\begin{array}{l}\text { Throshold Pressure Linear } \\
\text { Perameter }\end{array}$ & 33107A & $20-F a b-86$ & $-Y$ & $A B$ \\
\hline$Y$ & $100.00 \mathrm{mw}$ & 2732 & PAN_SEAL & Panol Seal & PCT_A & $\begin{array}{l}\text { Throshold Pressuro Linear } \\
\text { Parametor }\end{array}$ & $33107 A$ & $20-F 0 b-96$ & $Y$ & 48 \\
\hline $\mathbf{Y}$ & $100.00 \mathrm{hw}$ & 2732 & PAN_SEAL & Panel Sesl & PCT_A & $\begin{array}{l}\text { Threshold Pressuro Lunear } \\
\text { Peramoter }\end{array}$ & $33107 \mathrm{~A}$ & $20-f e b-96$ & $\mathbf{Y}$ & $A B$ \\
\hline $\mathbf{Y}$ & $100.00 \mathrm{hw}$ & 2733 & PAN_SEAL & Pand Seal & PCT_EXP & $\begin{array}{l}\text { Threshotd pressuro exponontal } \\
\text { parametor }\end{array}$ & 33108 & $20-F b-96$ & $\mathbf{Y}$ & 48 \\
\hline $\mathbf{Y}$ & $100.00 \mathrm{mw}$ & 2733 & PAN_SEAL & Penol Soal & PCT_EXP & $\begin{array}{l}\text { Threshold pressure exponential } \\
\text { parameter }\end{array}$ & 33108 & $20-F a b-96$ & $Y$ & 48 \\
\hline $\mathbf{Y}$ & $100.00 \mathrm{hw}$ & 2733 & PAN_SEAL & Panol Soal & PCT_EXP & $\begin{array}{l}\text { Throshold pressure exponential } \\
\text { paremeter }\end{array}$ & 33108 & 20 fob-96 & $\mathbf{Y}$ & 48 \\
\hline Y & & 2734 & PAN_SEAL & Panel Seal & SAT_IBRN & Initial Brine Saturation & $33134 A$ & 28-Fab-96 & $\mathbf{Y}$ & 48 \\
\hline$y$ & & 2734 & PAN_SEAL & Panel Seal & SAT_IBRN & Initial Brine Saturation & $33134 A$ & 28-Fob-96 & $\mathbf{Y}$ & 18 \\
\hline Y & & 2736 & REPOST & $\begin{array}{l}\text { Repository rogions } \\
\text { outside of Panel region }\end{array}$ & KPT & $\begin{array}{l}\text { Flag for Permeability Determinod } \\
\text { Threshold }\end{array}$ & 33238 & 20-Fob-96 & $\mathbf{Y}$ & 18 \\
\hline $\mathbf{Y}$ & & 2736 & REPOST & $\begin{array}{l}\text { Repository regions } \\
\text { outside of Panei rogion }\end{array}$ & KPT & $\begin{array}{l}\text { Flag for Permeability Determined } \\
\text { Throshold }\end{array}$ & 33238 & $20-70 b-96$ & $\begin{array}{r}Y \\
\ddots \\
\end{array}$ & $4 B$ \\
\hline Y & & 2735 & REPOSI & $\begin{array}{l}\text { Repository regions } \\
\text { outside of Panel region }\end{array}$ & KPT & $\begin{array}{l}\text { Fleg for Pormeabilty Delermined } \\
\text { Throstrold }\end{array}$ & 33238 & 20fob-96 & $\mathbf{Y}$ & AB \\
\hline $\mathbf{Y}$ & & 2736 & REPOST & $\begin{array}{l}\text { Repository regions } \\
\text { outside of Penel region }\end{array}$ & KPT & $\begin{array}{l}\text { Fleg for Permeability Delormined } \\
\text { Threshold }\end{array}$ & 33238 & $20-F=b-96$ & $\mathbf{Y}$ & AB \\
\hline$Y$ & & 2737 & REPOST & $\begin{array}{l}\text { Repositary regions } \\
\text { pectide of Panel region }\end{array}$ & PCT_A & $\begin{array}{l}\text { Tinreshold Pressure Linear } \\
\text { Percmeter }\end{array}$ & 33240 & ab-Feb-96 & $\mathbf{Y}$ & 48 \\
\hline$Y$ & & 2737 & REPOSIT & $\begin{array}{l}\text { Repository regions } \\
\text { actione of Parnol region }\end{array}$ & PCT_A & $\begin{array}{l}\text { Thresthotd Pressura Linear } \\
\text { Paramoter }\end{array}$ & 33240 & obfob-96 & $\cdot \gamma$ & 18 \\
\hline Y & & 2737 & REPOST & $\begin{array}{l}\text { Repocitory regions } \\
\text { outside of Panet region }\end{array}$ & PCT_A & $\begin{array}{l}\text { Threshold Pressure Linesr } \\
\text { Perwotot }\end{array}$ & 33240 & OB-fob-86 & $\mathbf{Y}$ & AB \\
\hline $\mathbf{Y}$ & & 2737 & REPOST & $\begin{array}{l}\text { Repository regions } \\
\text { octuide of Penel region }\end{array}$ & PCT_A & $\begin{array}{l}\text { Throshold Prossuro Linex } \\
\text { Parameter }\end{array}$ & 33240 & D8-Fcb-96 & $\mathbf{Y}$ & 48 \\
\hline $\mathbf{Y}$ & & 2738 & REPOSIT & $\begin{array}{l}\text { Repository regions } \\
\text { outside of Panet region }\end{array}$ & PCT_EXP & $\begin{array}{l}\text { Threshold preseure exporantial } \\
\text { perremeter }\end{array}$ & 33242 & $08+50 b-86$ & $\mathbf{Y}$ & 48 \\
\hline $\mathbf{Y}$ & & 2738 & REPOSIT & $\begin{array}{l}\text { Repocitory regions } \\
\text { outside of Penet region }\end{array}$ & PCT_EXP & $\begin{array}{l}\text { Throshold pressure exponontial } \\
\text { parameter }\end{array}$ & 33242 & $08-=b-96$ & 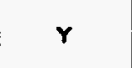 & 18 \\
\hline
\end{tabular}




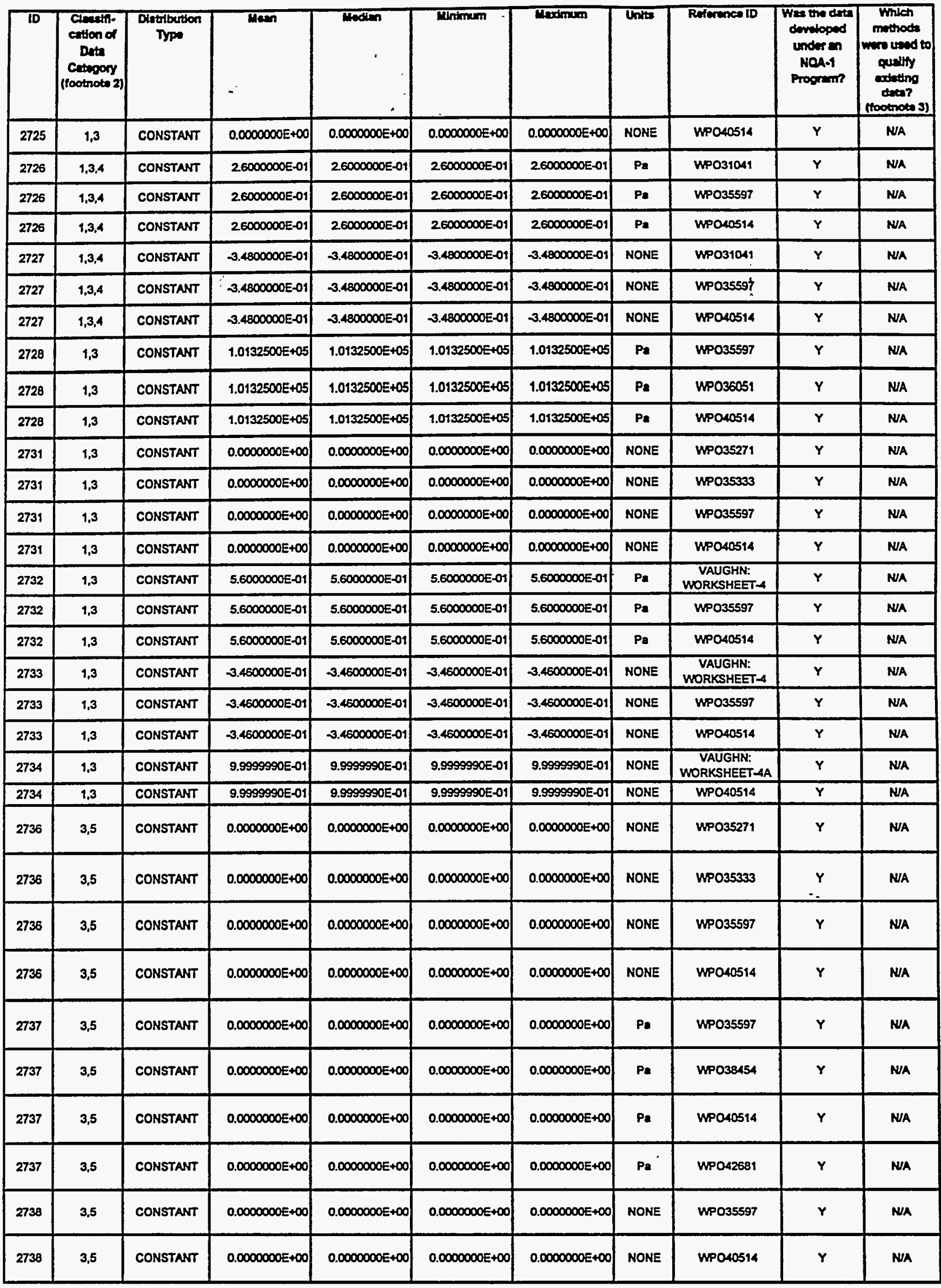


CCA Parameter Listing. -

\begin{tabular}{|c|c|c|c|c|c|c|c|c|c|c|}
\hline $\begin{array}{l}\text { Parmition in } \\
\text { Detablea? } \\
\text { (YN) }\end{array}$ & 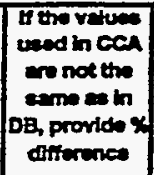 & 10 & Metaralo & Materd Kams & Perometer & 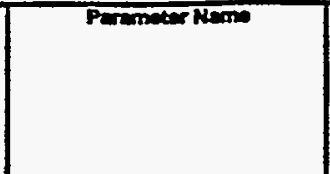 & $\begin{array}{c}\text { PRP DD } \\
\text { (WFO } 17 \\
\text { - }\end{array}$ & Detin Entry & 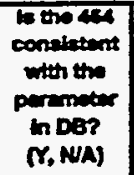 & $\begin{array}{l}\text { Parameter } \\
\text { Cetegory } \\
\text { (footnotio } 1)\end{array}$ \\
\hline $\mathbf{Y}$ & & 2738 & REPOST & $\begin{array}{l}\text { Repository regions } \\
\text { outtide of Pancl region }\end{array}$ & PCT_EXP & $\begin{array}{l}\text { Threschold pressire exponential } \\
\text { permeter }\end{array}$ & 33242 & $08-F=0-96$ & $\mathbf{Y}$ & 48 \\
\hline $\mathbf{Y}$ & & 2739 & REPOST & $\begin{array}{l}\text { Repository regons } \\
\text { outside of Pentl region }\end{array}$ & PO_MIN & $\begin{array}{l}\text { Minimum brine pressure for } \\
\text { capillery model KP }=3\end{array}$ & 33252 & 21.Feb-96 & $\mathbf{Y}$ & 48 \\
\hline $\mathbf{Y}$ & & 2739 & REPOSIT & $\begin{array}{l}\text { Repository regions } \\
\text { outaide of Panal region }\end{array}$ & PO_MIN & $\begin{array}{l}\text { Minimum brine preasere for } \\
\text { Capilary model KPC=3 }\end{array}$ & 33252 & $21+\ldots \infty-s 6$ & $\mathbf{Y}$ & 48 \\
\hline $\mathbf{Y}$ & & 2739 & REPOST & $\begin{array}{l}\text { Repositary regions } \\
\text { outside of Panol region }\end{array}$ & PO_MIN & $\begin{array}{l}\text { Minimum brine pressure for } \\
\text { copiltery model KPC=3 }\end{array}$ & 33252 & $21+F a b-96$ & $\mathbf{Y}$ & 48 \\
\hline $\mathbf{Y}$ & & 2740 & REPOSIT & $\begin{array}{l}\text { Repositary regions } \\
\text { cutside of Panol region }\end{array}$ & SAT_IBRN & Initial Brims Seturation & 33277 & $11+F a b-96$ & $\mathbf{Y}$ & $4 B$ \\
\hline $\mathbf{Y}$ & & 2740 & REPOST & $\begin{array}{l}\text { Repository regions } \\
\text { outside of Parvel region }\end{array}$ & SAT_IBRN & Initial Brino Seturation & $332 \pi 7$ & 11 Fob-s6 & $\mathbf{Y}$ & 48 \\
\hline $\mathbf{Y}$ & & 2740 & REPOSIT & $\begin{array}{l}\text { Repository regions } \\
\text { outside of Panol region }\end{array}$ & SAT_IBRN & Initial Brine Seturation & 33277 & 11Fob-96 & $\mathbf{Y}$ & $4 B$ \\
\hline $\mathbf{Y}$ & & 2740 & REPOSIT & $\begin{array}{l}\text { Repasitony regions } \\
\text { outside of Panel region }\end{array}$ & SAT_IBRN & Initial Brine Seturation & 33277 & 11 fab-s6 & $\mathbf{Y}$ & $4 B$ \\
\hline $\mathbf{Y}$ & & 2741 & REPOST & $\begin{array}{l}\text { Repositary regions } \\
\text { outside of Panel region }\end{array}$ & SAT_RBRN & Residual Brine Saturation & 33283 & 20-Fob-96 & $\mathbf{Y}$ & 48 \\
\hline $\mathbf{Y}$ & & $\begin{array}{c}2741 \\
-\end{array}$ & REPOST & $\begin{array}{l}\text { Repository regions } \\
\text { outside of Panel region }\end{array}$ & SAT_RBRN & Residual Brine Saturation & 33283 & 20-Fob-96 & $\mathbf{Y}$ & 48 \\
\hline $\mathbf{Y}$ & & 2741 & REPOST & $\begin{array}{l}\text { Repository regions } \\
\text { Qutside of Panel region }\end{array}$ & SAT_RBRN & Residual Brino Saturation & 33283 & 20 feb-9s & $\mathbf{Y}$ & AB \\
\hline $\mathbf{Y}$ & & 2741 & REPOST & $\begin{array}{l}\text { Repository regions } \\
\text { outside of Pand region }\end{array}$ & SAT_RBRN & Residual Brine Seturation & 33283 & 20 Feb-9s & $\mathbf{Y}$ & 48 \\
\hline $\mathbf{Y}$ & & 2744 & SALT_T1 & $\begin{array}{l}\text { Shan sat column } \\
\text { compectectime } 0 \text { to } 10 \\
\text { years }\end{array}$ & KPT & $\begin{array}{l}\text { Flag for Permeability Dotermined } \\
\text { Threshold }\end{array}$ & 33364 & $20-F \infty b-86$ & $\mathbf{Y}$ & $4 B$ \\
\hline $\mathbf{Y}$ & & 2744 & SALT_TI & $\begin{array}{l}\text { Shat salt column } \\
\text { compactectime } 0 \text { to to } \\
\text { years }\end{array}$ & KPT & $\begin{array}{l}\text { Flag for Permesbitity Determined } \\
\text { Thrusthold }\end{array}$ & 33364 & $20-F e b-96$ & $\mathbf{Y}$ & $4 B$ \\
\hline $\mathbf{Y}$ & & 2744 & SALT_T1 & $\begin{array}{l}\text { Shatt salt column } \\
\text { compectoditime } 0 \text { to } 10 \\
\text { years }\end{array}$ & IOT & $\begin{array}{l}\text { Fleg for Pormeability Detemined } \\
\text { Threshold }\end{array}$ & 33364 & $20-F=b-96$ & $\mathbf{Y}$ & 48 \\
\hline $\mathbf{Y}$ & & 2744 & SALT_T1 & $\begin{array}{l}\text { Shan satt colunn } \\
\text { compectedtime } 0 \text { to } 10 \\
\text { years }\end{array}$ & $\mathrm{KPT}$ & $\begin{array}{l}\text { Fisg for Pemeability Determined } \\
\text { Threshold }\end{array}$ & 33384 & 20 Fab-96 & $\mathbf{Y}$ & $4 B$ \\
\hline $\mathbf{Y}$ & & 2744 & SALT_T1 & $\begin{array}{l}\text { Shent elt column } \\
\text { compectedtime } 0 \text { to } 10 \\
\text { years }\end{array}$ & KPT & $\begin{array}{l}\text { Flag for Permeabilty Determined } \\
\text { Threshold }\end{array}$ & 33364 & $20-f-6-96$ & $-\mathbf{Y}$ & 48 \\
\hline $\mathbf{Y}$ & & 2744 & SALT_T1 & $\begin{array}{l}\text { Shaft salt colemn } \\
\text { compectedime } 0 \text { to id } \\
\text { yoars }\end{array}$ & KPT & $\begin{array}{l}\text { Fise for Permanbility Determined } \\
\text { Threathold }\end{array}$ & 33364 & $20-F a b-96$ & $\mathbf{Y}$ & $4 B$ \\
\hline $\mathbf{Y}$ & 100 & 2745 & SALT_T1 & $\begin{array}{l}\text { Sheft salt colkmn } \\
\text { compectect time } 0 \text { to } 10 \\
\text { years }\end{array}$ & PCT_A & $\begin{array}{l}\text { Thrashold Preseure Lnear } \\
\text { Perameter }\end{array}$ & 33369 & 13-Fab-96 & $\mathbf{Y}$ & 1 \\
\hline $\mathbf{Y}$ & 100 & 2745 & SALT_T1 & $\begin{array}{l}\text { Shat selt column } \\
\text { compactedtume } 0 \text { to } 10 \\
\text { yares }\end{array}$ & PCT_A & $\begin{array}{l}\text { Throshold Pressure Linas } \\
\text { Parnoter }\end{array}$ & 33369 & 13 Feb-S6 & $\mathbf{Y}$ & 1 \\
\hline $\mathbf{Y}$ & 100 & 2745 & SALT_T1 & $\begin{array}{l}\text { Shen sali column } \\
\text { compectudtime o to } 10 \\
\text { yoers }\end{array}$ & PCT_A & $\begin{array}{l}\text { Thro:hold Pressurn Lneser } \\
\text { Perenneter }\end{array}$ & 33369 & $13-f e b-96$ & $\mathbf{Y}$ & 4 \\
\hline $\mathbf{Y}$ & 100 & 2745 & SALT_T1 & \begin{tabular}{l|} 
Shat salt column \\
compectedtime 0 to id \\
yaurs
\end{tabular} & PCT_A & $\begin{array}{l}\text { Threshodd Pressure Lines } \\
\text { Pareneter }\end{array}$ & 33369 & $13+$ cb-g6 & $\mathbf{Y}$ & 1 \\
\hline $\mathbf{Y}$ & 100 & 2746 & SALT_TI & $\begin{array}{l}\text { Shat salt column } \\
\text { compectedtime } 0 \text { to } 10 \\
\text { yegr }\end{array}$ & PCT_EXP & $\begin{array}{l}\text { Threahold pressure exponential } \\
\text { peremeter }\end{array}$ & 33370 & $13-F=b-96$ & $\mathbf{Y}$ & 1 \\
\hline $\mathbf{Y}$ & 100 & 2746 & SALT_TI & $\begin{array}{l}\text { Shan salt colemn } \\
\text { compectedtime } 0 \text { to } 10 \\
\text { yeres }\end{array}$ & PCT_EXP & $\begin{array}{l}\text { Threshold presure exponemial } \\
\text { paremeter }\end{array}$ & 33370 & $13+F b-86$ & $\mathbf{Y}$ & 1 \\
\hline
\end{tabular}


CCA-Parameter Listing

\begin{tabular}{|c|c|c|c|c|c|c|c|c|c|c|}
\hline 10 & $\begin{array}{c}\text { Cinatifi- } \\
\text { cetton of } \\
\text { Dett } \\
\text { Cuttegory } \\
\text { (Iootnota 2) }\end{array}$ & $\begin{array}{l}\text { Dtatribution } \\
\text { Type }\end{array}$ & man & Medien & mintmom & Wnothem & Units & Refiorimea ID & $\begin{array}{l}\text { Was the dete } \\
\text { dowloped } \\
\text { under en } \\
\text { Hoh.1 } \\
\text { Progrem? }\end{array}$ & 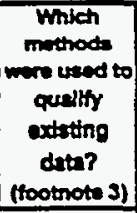 \\
\hline 2738 & 3,5 & CONSTANT & $0.0000000 E+\infty 0$ & $0.0000000 E+\infty$ & $0.0000000 E+\infty 0$ & $0.0000000 E+\infty$ & NONE & WPO42651 & $\mathbf{Y}$ & NA \\
\hline 2739 & 3.5 & CONSTANT & $1.0132500 \mathrm{E}+05$ & $1.0132500 \mathrm{E}+05$ & $1.0132500 E+05$ & $1.0132500 E+05$ & $P_{\mathbf{E}}$ & WPO35597 & $\gamma$ & N/A \\
\hline 2739 & 3,5 & CONSTANT & $1.0132500 E+05$ & $1.0132500 \mathrm{E}+05$ & $1.0132500 E+05$ & $1.0132500 E+05$ & Pa & WP036051 & $\mathbf{Y}$ & NA \\
\hline 2739 & 3,5 & CONSTANT & $1.0132500 E+05$ & $1.0432500 E+05$ & $1.0132500 E+05$ & $1.0132500 E+05$ & Pa & MP04051? & $\mathbf{r}$ & $N A$ \\
\hline 2740 & $1,3,4$ & CONSTANT & $1.5000000 E-02$ & $1.5000000 E-02$ & $1.5000000 E-02$ & $1.5000000 E-02$ & NONE & WP030S25 & $\mathbf{Y}$ & NA \\
\hline 2740 & $1,3,4$ & CONSTANT & $1.5000000 E-02$ & $1.5000000 E-02$ & $1.5000000 E-02$ & $1.5000000 E-02$ & NONE & WP035183 & $\mathbf{Y}$ & NA \\
\hline 2740 & $1,3,4$ & CONSTANT & $1.5000000 E-02$ & $1.5000000 E-02$ & $1.5000000 \mathrm{E}-02$ & $1.5000000 \mathrm{E}-02$ & NONE & WP035597 & $\mathbf{Y}$ & NA \\
\hline 2740 & $1,3,4$ & CONSTANT & $1.5000000 \mathrm{E}-02$ & $1.5000000 \mathrm{E}-02$ & $1.5000000 \mathrm{E}-02$ & $1.5000000 \mathrm{E}-02$ & NONE & WPO40514 & $\mathbf{Y}$ & N/A \\
\hline 2741 & $1,3,4$ & UNIFORM & $27600000 E-01$ & $2.7600000 E-01$ & $0.0000000 E+\infty$ & $5.5200000 E-01$ & NONE & $\begin{array}{l}\text { MEMO:VAUGHN } \\
\text { TIERNEY-11 }\end{array}$ & $\mathbf{Y}$ & N/A \\
\hline 2749 & $1,3,4$ & UNIFORH. & $27600000 E-01$ & $2.7600000 E-01$ & $00000000 E+\infty$ & $5.5200000 E-01$ & NONE & WP035268 & $\mathbf{Y}$ & NA \\
\hline 2741 & $1,3,4$ & UNIFORM & $27600000 E-01$ & $2.7600000 E-01$ & $0.0000000 E+\infty$ & $5.5200000 E-01$ & NONE & WP035597 & $\mathbf{Y}$ & NA \\
\hline 2741 & $1,3,4$ & UNIFORM & $2.7600000 \mathrm{E}-01$ & $2.7600000 E-01$ & $00000000 E+\infty 0$ & $55200000 E-01$ & NONE & WPO40514 & $\mathbf{Y}$ & N/A \\
\hline 2744 & 3,5 & CONSTANT & $0.0000000 E+\infty$ & $00000000 E+00$ & $00000000 E+\infty$ & $000000000+00$ & NONE & WP030640 & $\mathbf{Y}$ & NA \\
\hline 2744 & 3,5 & CONSTANT & $0.0000000 E+00$ & $00000000 E+\infty 0$ & $00000000 E+00$ & $0,0000000 E+\infty$ & NONE & WP030994 & $\mathbf{Y}$ & NA \\
\hline 2744 & 3,5 & CONSTANT & $0.0000000 \mathrm{E}+00$ & $000000000+\infty 00$ & $00000000 \times+\infty$ & $00000000 E+\infty$ & NONE & WP035271 & $\mathbf{Y}$ & N/A \\
\hline 2744 & 3,5 & CONSTANT & $0.0000000 E+00$ & $00000000 E+\infty 0$ & $0,0000000+\infty$ & $0,0000000 \leq+\infty$ & NONE & WP035333 & $\mathbf{Y}$ & NA \\
\hline 2744 & 3,5 & CONSTANT & $0.0000000 E+00$ & $00000000 \in+\infty$ & $0 \quad 200000<-\infty$ & $00000000+\infty$ & NONE & WP035597 & $\dot{\gamma}$ & NA \\
\hline 2744 & 3,5 & CONSTANT & $0.0000000 E+\infty$ & $00000000 E+\infty$ & $0,0000000 \times+\infty$ & $0<000000 \times+\infty$ & NONE & WPO40514 & Y & NA \\
\hline 2745 & 3,4 & CONSTANT & $5.6000000 \mathrm{E}-01$ & $560000006-01$ & 500000000.01 & S0000000E-01 & Pa & WP030640 & $\mathbf{Y}$ & NA \\
\hline 2745 & 3,4 & CONSTANT & $5.6000000 E-01$ & 56000000 - 01 & 58000000 e 01 & s 0000000 - 0 , & $P_{\mathbf{a}}$ & WP030s94 & $\mathbf{Y}$ & NA \\
\hline 2745 & 3,4 & CONSTANT & $5.6000000 E-01$ & $56000000 E-01$ & $50000000<-01$ & $50000000-0$. & Pa & WP035597 & $\mathbf{Y}$ & NA \\
\hline 2745 & 3,4 & CONSTANT & 5.6000000E-01 & $56000000 E-01$ & 560000000.01 & $S 60000000-01$ & $\mathrm{~Pa}$ & WPO40434 & $\mathbf{Y}$ & NA \\
\hline 2746 & 3.4 & CONSTANT & -3.4600000 E-01 & $34600000 E-01$ & $3=000006-01$ & $3 \backsim 00000 E-01$ & NONE & WP030640 & $\mathbf{Y}$ & NA \\
\hline 2746 & 3,4 & CONSTANT & $2.4600000 E-01$ & $34500000 E-01$ & $34500000<-08$ & $3<00000 E-01$ & NONE & WP030994 & $\mathbf{Y}$ & N/A \\
\hline
\end{tabular}


- CCA-Parameter-Listing

\begin{tabular}{|c|c|c|c|c|c|c|c|c|c|c|}
\hline $\begin{array}{c}\text { Parninder in } \\
\text { Dethese? } \\
\text { (XNI) }\end{array}$ & 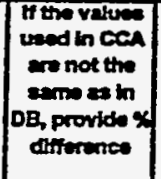 & ID & Meterial to & Metard Kmo & $\begin{array}{c}\text { Paramotar } \\
\text { id }\end{array}$ & Parmencer Nense & $\begin{array}{l}\text { PFP Do } \\
\text { MPOO }\end{array}$ & $\begin{array}{c}\text { Deta Entry } \\
\text { Dats }\end{array}$ & 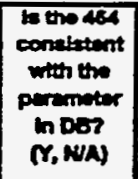 & $\begin{array}{c}\text { Parminter } \\
\text { Crtagory } \\
\text { (tootinote 1) }\end{array}$ \\
\hline$Y$ & 100 & 2746 & SALT_TI & $\begin{array}{l}\text { Shat salt column } \\
\text { compectectimie } 0 \text { to } 10 \\
\text { years }\end{array}$ & PCT_EXP & $\begin{array}{l}\text { Threstold presesure exponential } \\
\text { peremetor }\end{array}$ & 33370 & $13-F a b-96$ & $\mathbf{Y}$ & 1 \\
\hline $\mathbf{Y}$ & 100 & 2746 & SALT_TI & $\begin{array}{l}\text { Shat salt column } \\
\text { compactedtine } 0 \text { to } 10 \\
\text { yoars }\end{array}$ & PCT_EXP & $\begin{array}{l}\text { Thrushold pressure exponential } \\
\text { perconoter }\end{array}$ & 33370 & 13 Fob-96 & $\mathbf{Y}$ & 1 \\
\hline $\mathbf{Y}$ & & 2749 & SALT_T2 & $\begin{array}{l}\text { Shaft sah column } \\
\text { compactect:ono } 10 \text { to } \\
25 \text { years }\end{array}$ & KPT & $\begin{array}{l}\text { Fleg for Permeablity Determined } \\
\text { Threshold }\end{array}$ & 33431 & $20-F e b-96$ & $\mathbf{Y}$ & 48 \\
\hline $\mathbf{Y}$ & & 2749 & SALT_T2 & $\begin{array}{l}\text { Shaft salt column } \\
\text { compactectime } 10 \text { to } \\
25 \text { years }\end{array}$ & KPT & $\begin{array}{l}\text { Flag for Permesbilty Determined } \\
\text { Threshold }\end{array}$ & 33431 & $20+c b-9 s$ & $\mathbf{Y}$ & 48 \\
\hline $\mathbf{Y}$ & & 2749 & SALT_T2 & $\begin{array}{l}\text { Shat eall column } \\
\text { compectedtime } 10 \text { to } \\
25 \text { years }\end{array}$ & KPT & $\begin{array}{l}\text { Fileg for Permesbinty Determined } \\
\text { Threshold }\end{array}$ & 33431 & $20-F e b-95$ & $\mathbf{Y}$ & 48 \\
\hline $\mathbf{Y}$ & & 2749 & SALT_T2 & $\begin{array}{l}\text { Shaft salf column } \\
\text { compactedtume } 10 \text { to } \\
25 \text { yoars }\end{array}$ & KPT & $\begin{array}{l}\text { Fiteg for Permesidity Determined } \\
\text { Threahoid }\end{array}$ & 33431 & $20-F a b-96$ & $\mathbf{Y}$ & $4 B$ \\
\hline$Y$ & & 2749 & SALT_T2 & $\begin{array}{l}\text { Shaf salt column } \\
\text { compactediting } 10 \text { to } \\
25 \text { years }\end{array}$ & KPT & $\begin{array}{l}\text { Flag for Permescility Dotermined } \\
\text { Threshold }\end{array}$ & $3343 i$ & $20-$ feb-96 & $\mathbf{Y}$ & $4 B$ \\
\hline $\mathbf{Y}$ & & 2749 & SALT_T2 & $\begin{array}{l}\text { Shaft salt column } \\
\text { compectedtine } 10 \text { to } \\
25 \text { years }\end{array}$ & KPT & $\begin{array}{l}\text { Flag for Permesbility Determined } \\
\text { Threstoid }\end{array}$ & 33431 & $20 F a b-96$ & $\mathbf{Y}$ & $A B$ \\
\hline $\mathbf{Y}$ & 100 & 2750 & SALT_T2 & $\begin{array}{l}\text { Shaft salt column } \\
\text { compected:tine } 10 \text { to } \\
25 \text { years }\end{array}$ & PCT_A & $\begin{array}{l}\text { Throshold Pressure Linear } \\
\text { Paremeter }\end{array}$ & 33433 & $14 f e b-96$ & $\mathbf{Y}$ & 1 \\
\hline $\mathbf{Y}$ & 100 & 2750 & SALT_T2 & $\begin{array}{l}\text { Shat salt column } \\
\text { compactedtime } 10 \text { to } \\
25 \text { yoars }\end{array}$ & PCT_A & $\begin{array}{l}\text { Threshoid Pressure Linest } \\
\text { Peremeter }\end{array}$ & 33433 & 14 fob-96 & $\mathbf{Y}$ & 1 \\
\hline $\mathbf{Y}$ & 100 & 2750 & SALT_T2 & $\begin{array}{l}\text { Shat salt column } \\
\text { compactodtime } 10 \text { to } \\
25 \text { years }\end{array}$ & PCT_A & $\begin{array}{l}\text { Threshold Prossure Linear } \\
\text { Permeter }\end{array}$ & 33433 & 14 Fob-96 & $\mathbf{Y}$ & 1 \\
\hline $\mathbf{Y}$ & 100 & 2750 & SALT_T2 & $\begin{array}{l}\text { Shan salt column } \\
\text { compactodtimn } 10 \text { to } \\
25 \text { yours }\end{array}$ & PCT_A & $\begin{array}{l}\text { Threshold Pressure Linear } \\
\text { Peremoter }\end{array}$ & 33433 & $14 F a b-96$ & $\mathbf{Y}$ & 1 \\
\hline $\mathbf{Y}$ & 100 & 2751 & SALT_T2 & $\begin{array}{l}\text { Shan salt colemin } \\
\text { compactectime } 10 \text { to } \\
25 \text { years }\end{array}$ & PCT_EXP & $\begin{array}{l}\text { Threshold pressure exporiential } \\
\text { parameter }\end{array}$ & 33435 & 14 fob-96 & $\mathbf{Y}$ & 1 \\
\hline $\mathbf{Y}$ & 100 & 2751 & SALT_T2 & $\begin{array}{l}\text { Shaft salt cokmn } \\
\text { compacted.time } 10 \text { to } \\
25 \text { years }\end{array}$ & PCT_EXP & $\begin{array}{l}\text { Threshold pressure exporiential } \\
\text { pereneter }\end{array}$ & 33435 & 14Feb-96 & $\mathbf{Y}$ & 1 \\
\hline $\mathbf{Y}$ & 100 & 2751 & SALT_T2 & $\begin{array}{l}\text { Shaft salt colemn } \\
\text { compactedtime } 10 \text { to } \\
25 \text { years }\end{array}$ & PCT_EXP & $\begin{array}{l}\text { Threatold presure exponential } \\
\text { peremoter }\end{array}$ & 33435 & 14 feb-96 & $\mathbf{Y}$ & 1 \\
\hline $\mathbf{Y}$ & 100 & 2751 & SALT_T2 & $\begin{array}{l}\text { Shan sah column } \\
\text { compactodtime } 10 \text { to } \\
25 \text { years }\end{array}$ & PCT_EXP & $\begin{array}{l}\text { Threstiold pressure exponential } \\
\text { perameter }\end{array}$ & 33435 & $14 f$ eb-9s & $\mathbf{Y}$ & 1 \\
\hline$Y$ & & 2754 & SALT_T3 & $\begin{array}{l}\text { Shath sall column } \\
\text { compectectime } 25 \text { to } \\
50 \text { yoars }\end{array}$ & KPT & $\begin{array}{l}\text { Fieg for Permeability Determined } \\
\text { Thre:thold }\end{array}$ & 33402 & 20 fab-96 & $" Y$ & $4 B$ \\
\hline $\mathbf{Y}$ & & 2754 & SALT_T3 & $\begin{array}{l}\text { Shat salt cotumn } \\
\text { compactedtime } 25 \text { to } \\
50 \text { years }\end{array}$ & KPT & $\begin{array}{l}\text { Fieg for Permestulfy Determined } \\
\text { Throshold }\end{array}$ & 33402 & 20-Feb-96 & $\mathbf{Y}$ & $4 B$ \\
\hline $\mathbf{Y}$ & & 2754 & SALT_T3 & $\begin{array}{l}\text { Shaft sen column } \\
\text { compacteditine } 25 \text { to } \\
50 \text { years }\end{array}$ & KPT & $\begin{array}{l}\text { Fleo for Permesodity Dotermined } \\
\text { Thro:hold }\end{array}$ & 33402 & 20 feb-96 & $\mathbf{Y}$ & 48 \\
\hline$Y$ & & 2754 & SALT_T3 & $\begin{array}{l}\text { Shan salt column } \\
\text { compactedtime } 25 \text { to } \\
50 \text { yeers }\end{array}$ & KPT & $\begin{array}{l}\text { Flac tor Permastadity Determined } \\
\text { Trmenchold }\end{array}$ & 33402 & $20+50 b-96$ & $\mathbf{Y}$ & 48 \\
\hline $\mathbf{Y}$ & & 2754 & SALT_T3 & $\begin{array}{l}\text { Shen sall column } \\
\text { compectectume } 25 \text { to } \\
50 \text { years }\end{array}$ & KPT & $\begin{array}{l}\text { Fleg for Permeabilty Determined } \\
\text { Thre:hold }\end{array}$ & 33402 & $20-a b-86$ & $\mathbf{Y}$ & $4 B$ \\
\hline $\mathbf{Y}$ & & 2754 & SALT_T3 & $\begin{array}{l}\text { Shaf self column } \\
\text { compectedtune } 25 \text { to } \\
50 \text { years }\end{array}$ & KPT & $\begin{array}{l}\text { Flag for Permesculaty Determined } \\
\text { Threshold }\end{array}$ & 33402 & 20-Fob-96 & $\mathbf{Y}$ & 48 \\
\hline $\mathbf{Y}$ & 100 & 2755 & SALT_T3 & $\begin{array}{l}\text { Shaft salt column } \\
\text { compectect tme } 25 \text { to } \\
50 \text { years }\end{array}$ & PCT_A & $\begin{array}{l}\text { Throshold Proseure Lnear } \\
\text { Peremeter }\end{array}$ & 33404 & 14 feb-96 & $Y$ & 1 \\
\hline$Y$ & 100 & 2755 & SALT_T3 & $\begin{array}{l}\text { Shrit salt column } \\
\text { compectectime } 25 \text { to } \\
50 \text { yens }\end{array}$ & PCT_A & $\begin{array}{l}\text { Throshold Pressure Lunear } \\
\text { Parmenter }\end{array}$ & 33404 & 14feb-ses & $\mathbf{Y}$ & 1 \\
\hline
\end{tabular}


CCA-Parameter Listing

\begin{tabular}{|c|c|c|c|c|c|c|c|c|c|c|}
\hline ID & $\begin{array}{c}\text { Clesuth } \\
\text { cetion of } \\
\text { Dats } \\
\text { Cotegory } \\
\text { (lootrolo 2) }\end{array}$ & $\begin{array}{l}\text { Distribution } \\
\text { Type }\end{array}$ & Lem & Bedien & Mntrom & Medinum & Units & Reterines id & $\begin{array}{l}\text { Was the data } \\
\text { demeloped } \\
\text { underen } \\
\text { MoA-1 } \\
\text { Program? }\end{array}$ & $\begin{array}{l}\text { Wrich } \\
\text { methods } \\
\text { more used to } \\
\text { quilly } \\
\text { adeding } \\
\text { deta? } \\
\text { (tootnote 3) }\end{array}$ \\
\hline 2746 & 3.4 & CONSTANT & $-3,4600000 \mathrm{E}-01$ & $-3.4600000 E-01$ & $3.4600000 E-01$ & $3.4600000 E-01$ & NONE & WP035597 & $\mathbf{Y}$ & NA \\
\hline 2746 & 3,4 & CONSTANT & $3.4600000 E-01$ & 2.4600000 E- 01 & $3.4600000 \mathrm{E}-01$ & $3.4600000 E-01$ & NONE & MPO40434 & $\gamma$ & NA \\
\hline 2749 & 1,3 & CONSTANT & $0.0000000 E+\infty$ & $0.0000000 E+\infty$ & $0.0000000 E+\infty 0$ & $0.0000000 E+\infty 0$ & NONE & WP030640 & $\mathbf{Y}$ & $N A$ \\
\hline 2749 & 1,3 & CONSTANT & $0.0000000 E+\infty$ & $0.0000000 E+\infty$ & $0.0000000 E+\infty 0$ & $0.0000000 E+\infty 0$ & NONE & WP030994 & $\mathbf{Y}$ & N/A \\
\hline 2749 & 1.3 & CONSTANT & $0.0000000 E+\infty 0$ & $0.0000000 E+\infty 0$ & $0.0000000 E+\infty$ & $0.00000000+\infty$ & NONE & WP035271 & $\mathbf{Y}$ & N/A \\
\hline 2749 & 1,3 & CONSTANT & $0.0000000 \mathrm{E}+\infty$ & $0.0000000 E+\infty$ & $0.0000000 E+\infty 0$ & $0.0000000 E+\infty$ & NONE & WP035333 & $\mathbf{Y}$ & N/A \\
\hline 2749 & 1,3 & CONSTANT & $0.00000000+\infty 0$ & $0.0000000 E+\infty 0$ & $0.0000000 E+\infty 0$ & $0.0000000 E+\infty 0$ & NONE & WP035597: & $\mathbf{Y}$ & $N A$ \\
\hline 2749 & 1,3 & CONSTANT & $0.00000000+\infty$ & $0.0000000 E+00$ & $0.0000000 E+\infty 0$ & $0.00000000 E+00$ & NONE & WPO40514 & $Y$ & N/A \\
\hline 2750 & 3,4 & CONSTANT & $5.6000000=-01$ & $5.6000000 E-01$ & $5.6000000 E-01$ & $5.60000000-01$ & Pa & WP030640 & $\mathbf{Y}$ & NAA \\
\hline 2750 & 3,4 & CONSTANT & $5.6000000 \mathrm{E}-01$ & $5.6000000 \mathrm{E}-01$ & $5.6000000 E-01$ & $5.6000000 E-01$ & Pa & WP030994 & $Y$ & 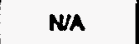 \\
\hline 2750 & 3,4 & CONSTANT & $5.6000000 E-01$ & $5.6000000 E-01$ & $5.6000000 E-01$ & 5.6000000E-01 & Pa & WP035597 & $\mathbf{Y}$ & NA \\
\hline 2750 & 3,4 & CONSTANT & $5.6000000 E-01$ & $5.6000000 \mathrm{E}-01$ & $5.6000000 E-01$ & $5.6000000 E-01$ & Pa & WPO40434 & $Y$ & NA \\
\hline 2751 & 3,4 & CONSTANT & $-3.4600000 \mathrm{E}-01$ & $3.4600000 E-01$ & $-3.4600000 \mathrm{E}-01$ & -3.4600000 E- 01 & NONE & WP030540 & $\mathbf{Y}$ & NA \\
\hline 2751 & 3,4 & CONSTANT & $-3.4600000 E-01$ & $3.4600000 E-01$ & $-3.4600000 E-01$ & $3.4600000 E-01$ & NONE & WP030994 & $\mathbf{Y}$ & NA \\
\hline 2751 & 3,4 & CONSTANT & $3.4600000 \mathrm{E}-01$ & $3.4600000 E-01$ & $-3.4600000 E-01$ & $3.4600000 E-01$ & NONE & WP035597 & $\mathbf{Y}$ & N/A \\
\hline 2751 & 3,4 & CONSTANT & $3.4600000 \mathrm{E}-01$ & $3.4600000 E-01$ & $-3.4600000 \mathrm{E}-01$ & $3.4600000=-01$ & NONE & WPO40434 & $\mathbf{Y}$ & NA \\
\hline 2754 & 1,3 & CONSTANT & $0.0000000 E+00$ & $0.0000000 E+\infty$ & $0.0000000 E+\infty$ & $0.0000000 E+\infty$ & NONE & WP030640 & $-\boldsymbol{Y}$ & N/A \\
\hline 2754 & 1,3 & CONSTANT & $0.00000000+00$ & $0.0000000 E+\infty$ & $0.0000000 \mathrm{E}+\infty 0$ & $0.00000000+\infty$ & NONE & WP030994 & $\mathbf{Y}$ & NA \\
\hline 2754 & 1,3 & CONSTANT & $0.0000000 E+\infty 0$ & $0.0000000 E+\infty$ & $0.0000000 E+\infty$ & $0.0000000 E+\infty 0$ & NONE & WP035271 & $\mathbf{Y}$ & N/A \\
\hline 2754 & 1,3 & CONSTANT & $0.0000000 E+\infty$ & $0.00000000+\infty$ & $0.0000000 \mathrm{E}+00$ & $0.0000000 E+\infty$ & NONE & WPO35333 & $\mathbf{Y}$ & NA \\
\hline 2754 & 1,3 & CONSTANT & $0.0000000 E+00$ & $0.0000000 E+\infty 00$ & $0.0000000 E+\infty$ & $0.0000000 E+\infty$ & NONE & WP035597 & $\mathbf{Y}$ & NA \\
\hline 2754 & 1,3 & CONSTANT & $0.0000000 E+\infty 0$ & $0.0000000 E+\infty 0$ & $0.0000000 E+\infty$ & $0.0000000 E+\infty 0$ & NONE & WPO40514 & $\mathbf{Y}$ & NA \\
\hline 2755 & 3,4 & CONSTANT & $5.6000000 E-01$ & $5.6000000 E-01$ & $5.6000000 E-01$ & $5.6000000 E-01$ & $\mathbf{P a}$ & WP030640 & $\mathbf{Y}$ & NA \\
\hline 2755 & 3,4 & CONSTANT & $5.6000000 E-01$ & $5.6000000 E-01$ & $5.6000000 E-01$ & $5.6000000 E-01$ & Pa & WP030934 & $\mathbf{Y}$ & $\mathbf{N A}$ \\
\hline
\end{tabular}


CCA Parameter Listing

\begin{tabular}{|c|c|c|c|c|c|c|c|c|c|c|}
\hline $\begin{array}{c}\text { Parametar hn } \\
\text { Datubese? } \\
\text { Man) }\end{array}$ & 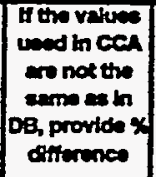 & DO & Metardalo io & Matarle Name & Permate & Parancter Nem & $\begin{array}{l}\text { PRP D } \\
\text { (NFO :) }\end{array}$ & $\begin{array}{c}\text { Dera Entry } \\
\text { Dute }\end{array}$ & 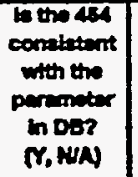 & $\begin{array}{l}\text { Parmiter } \\
\text { Cotepory } \\
\text { (tootnote I) } \\
\ldots\end{array}$ \\
\hline $\mathbf{Y}$ & 100 & 2755 & SALT_T3 & $\begin{array}{l}\text { Sheft salt column } \\
\text { compected:tme } 25 \text { to } \\
\text { so years }\end{array}$ & PCT_A & $\begin{array}{l}\text { Threshold Pressure Linaer } \\
\text { Parameter }\end{array}$ & 33404 & $14 F a b-96$ & $\mathbf{Y}$ & 1 \\
\hline $\mathbf{Y}$ & 100 & 2755 & SALT_T3 & $\begin{array}{l}\text { Shaft san colvenn } \\
\text { compactod:tume } 25 \text { to } \\
50 \text { years }\end{array}$ & PCT_A & $\begin{array}{l}\text { Threahold Pressern Linear } \\
\text { Pereneter }\end{array}$ & 33404 & 14 Feb-96 & $\mathbf{Y}$ & 1 \\
\hline$\cdot$ & 100 & 2756 & SALT_T3 & $\begin{array}{l}\text { Shaft sah column } \\
\text { compactedtime } 25 \text { to } \\
50 \text { years }\end{array}$ & PCT_EXP & $\begin{array}{l}\text { Threshold presedre exponential } \\
\text { perenoter }\end{array}$ & 33406 & $14 F a b-96$ & $\mathbf{Y}$ & 1 \\
\hline $\mathbf{Y}$ & 100 & 2756 & SALT_T3 & $\begin{array}{l}\text { Shaft salt column } \\
\text { compactedtime } 25 \text { to } \\
50 \text { years }\end{array}$ & PCT_EXP & $\begin{array}{l}\text { Thresthold prescure exponential } \\
\text { perenneter }\end{array}$ & 33406 & $14 F=b-96$ & $\mathbf{Y}$ & 1 \\
\hline $\mathbf{Y}$ & 100 & 2756 & SALT_T3 & $\begin{array}{l}\text { Shaft salt column } \\
\text { compactedtmo } 25 \text { to } \\
50 \text { years }\end{array}$ & PCT_EXP & $\begin{array}{l}\text { Threshotd proseure exponential } \\
\text { parmeter }\end{array}$ & 33406 & 14 Feb-96 & $\mathbf{Y}$ & 1 \\
\hline $\mathbf{Y}$ & 100 & 2756 & SALT_T3 & $\begin{array}{l}\text { Shan salt column } \\
\text { compactedtime } 25 \text { to } \\
50 \text { years }\end{array}$ & PCT_EXP & $\begin{array}{l}\text { Thre:chold presure exponential } \\
\text { peremeter }\end{array}$ & 33406 & 14fab-96 & $\mathbf{Y}$ & 1 \\
\hline $\mathbf{Y}$ & & 2759 & SALT_T4 & $\begin{array}{l}\text { Shaft salt column } \\
\text { compactedtime } 50 \text { to } \\
100 \text { years }\end{array}$ & KPT & $\begin{array}{l}\text { Flag for Permesbitity Determined } \\
\text { Threstiold }\end{array}$ & 33454 & 20-Feb-96 & $\mathbf{Y}$ & 48 \\
\hline $\mathbf{Y}$ & & 2759 & SALT_TA & $\begin{array}{l}\text { Shat salt cokmn } \\
\text { compectedtume } 50 \text { to } \\
100 \text { years }\end{array}$ & KPT & $\begin{array}{l}\text { Flag for Pormeability Determined } \\
\text { Threshold }\end{array}$ & 33454 & $20-50 b-96$ & $\mathbf{Y}$ & $4 B$ \\
\hline $\mathbf{Y}$ & & 2759 & SALT_T4 & $\begin{array}{l}\text { Shaft salt column } \\
\text { compactodtine } 50 \text { to } \\
100 \text { years }\end{array}$ & KPT & $\begin{array}{l}\text { Flag for Permasbility Doterminad } \\
\text { Threstoid }\end{array}$ & 33454 & 20 fab-96 & $\mathbf{Y}$ & 48 \\
\hline $\mathbf{Y}$ & & 2759 & SALT_T4 & $\begin{array}{l}\text { Shaft salt column } \\
\text { compactodtime } 50 \text { to } \\
100 \text { years }\end{array}$ & KPT & $\begin{array}{l}\text { Fiag for Permesbility Determined } \\
\text { Threshoid }\end{array}$ & 33454 & 20-Fab-96 & $\because$ & 48 \\
\hline $\mathbf{Y}$ & & 2759 & SALT_T4 & $\begin{array}{l}\text { Shaft salt column } \\
\text { compectedtime } 50 \text { to } \\
100 \text { years }\end{array}$ & KPT & $\begin{array}{l}\text { Fleg for Permeability Determined } \\
\text { Threshold }\end{array}$ & 33454 & 20 feb-96 & $\mathbf{Y}$ & $4 B$ \\
\hline $\mathbf{Y}$ & & 2759 & SALT_T4 & $\begin{array}{l}\text { Shaft salt column } \\
\text { compactectime } 50 \text { to } \\
100 \text { years }\end{array}$ & KPT & $\begin{array}{l}\text { Flag for Permeability Determined } \\
\text { Threshold }\end{array}$ & 33454 & $20+c b-96$ & $Y$ & $4 B$ \\
\hline $\mathbf{Y}$ & 100 & 2760 & SALT_T4 & $\begin{array}{l}\text { Shaft sah column } \\
\text { compactedtume } 50 \text { to } \\
100 \text { years }\end{array}$ & PCT_A & $\begin{array}{l}\text { Threshold Preseura Linear } \\
\text { Paramoter }\end{array}$ & 33456 & $14-F c b-96$ & $\mathbf{Y}$ & 1 \\
\hline $\mathbf{Y}$ & 100 & 2760 & SALT_T4 & $\begin{array}{l}\text { Shaft salt column } \\
\text { compacteditime } 50 \text { to } \\
100 \text { years }\end{array}$ & PCT_A & $\begin{array}{l}\text { Threchoid Pressure Linear } \\
\text { Parmeter }\end{array}$ & 33456 & 14freb-96 & $Y$ & 1 \\
\hline $\mathbf{Y}$ & 100 & 2760 & SALT_T4 & $\begin{array}{l}\text { Shatt salt column } \\
\text { compacted:tume } 50 \text { to } \\
100 \text { years }\end{array}$ & PCT_A & $\begin{array}{l}\text { Threshoid Pressure Linear } \\
\text { Parmeter }\end{array}$ & 33456 & 14 Fob-96 & $\mathbf{Y}$ & 1 \\
\hline $\mathbf{Y}$ & 100 & 2760 & SALT_TA & $\begin{array}{l}\text { Shaft salt cokmn } \\
\text { compecteditime } 50 \text { to } \\
100 \text { years }\end{array}$ & PCT_A & $\begin{array}{l}\text { Threshold Pressure Lnear } \\
\text { Parenoter }\end{array}$ & 33456 & 14Fab-96 & $\mathbf{Y}$ & 1 \\
\hline $\mathbf{Y}$ & 100 & 2761 & SALT_TA & $\begin{array}{l}\text { Shaft salt column } \\
\text { compactectime } 50 \text { to } \\
100 \text { years }\end{array}$ & PCT_EXP & $\begin{array}{l}\text { Threshold pressure exponential } \\
\text { perameter }\end{array}$ & 33457 & $14-f a b-96$ & $\because \gamma$ & 1 \\
\hline $\mathbf{Y}$ & 100 & 2761 & SALT_T4 & $\begin{array}{l}\text { Shan sak column } \\
\text { compactedtume } 50 \text { to } \\
100 \text { years }\end{array}$ & PCT_EXP & $\begin{array}{l}\text { Thrachold prassure exponential } \\
\text { permenter }\end{array}$ & 33457 & $14+F a b-96$ & $\mathbf{Y}$ & 1 \\
\hline $\mathbf{Y}$ & 100 & 2761 & SALT_T4 & $\begin{array}{l}\text { Shaft salt column } \\
\text { compectedtime } 50 \text { to } \\
100 \text { years }\end{array}$ & PCT_EXP & $\begin{array}{l}\text { Threshold preasure exponential } \\
\text { paremoter }\end{array}$ & 33457 & 14 Fob-96 & $\mathbf{Y}$ & 1 \\
\hline$Y$ & 100 & 2761 & SALT_T4 & $\begin{array}{l}\text { Shan salt cokmn } \\
\text { compactecttime } 50 \text { to } \\
100 \text { years }\end{array}$ & PCT_EXP & $\begin{array}{l}\text { Threshold pressure exponential } \\
\text { parameter }\end{array}$ & 33457 & 14 fob-96 & $\mathbf{Y}$ & 1 \\
\hline $\mathbf{Y}$ & & 2764 & SALT_T5 & $\begin{array}{l}\text { Shan satt column } \\
\text { compactedtime } 100 \text { to } \\
200 \text { years }\end{array}$ & KPT & $\begin{array}{l}\text { Fleg for Permesbility Determined } \\
\text { Threshold }\end{array}$ & 33573 & $20-F b b-96$ & $\mathbf{Y}$ & $4 B$ \\
\hline $\mathbf{Y}$ & & 2764 & SALT_TS & $\begin{array}{l}\text { Shath salt column } \\
\text { compacteditime } 100 \text { to } \\
200 \text { years }\end{array}$ & KPT & $\begin{array}{l}\text { Fleg for Penmesbility Datemnned } \\
\text { Threetiold }\end{array}$ & 33573 & 20-Feb-95 & $\mathbf{Y}$ & $A B$ \\
\hline$Y$ & & 2764 & SALT_TS & $\begin{array}{l}\text { Shath salt column } \\
\text { compectedtume } 100 \text { to } \\
200 \text { years }\end{array}$ & KPT & $\begin{array}{l}\text { Fleg for Permeability Determined } \\
\text { Trrethoid }\end{array}$ & 33573 & 20-Fab-96 & $\mathbf{Y}$ & $4 B$ \\
\hline $\mathbf{Y}$ & & 2764 & SALT_T5 & $\begin{array}{l}\text { Shat salt column } \\
\text { compectect tine } 100 \text { to } \\
200 \text { years }\end{array}$ & KPT & $\begin{array}{l}\text { Flog for Permeability Determined } \\
\text { Threshold }\end{array}$ & 33573 & 20 Fab-96 & $\mathbf{Y}$ & 48 \\
\hline
\end{tabular}


- CCA Parameter-Listing

\begin{tabular}{|c|c|c|c|c|c|c|c|c|c|c|}
\hline LD & $\begin{array}{c}\text { Creatin- } \\
\text { catton of } \\
\text { Data } \\
\text { Category } \\
\text { (footnote 2) }\end{array}$ & $\begin{array}{c}\text { Dastributton } \\
\text { Type }\end{array}$ & $\operatorname{man}$ & Medien & Minimen & Hadmum & Units & Reformence to & $\begin{array}{l}\text { Fea the diter } \\
\text { doveloped } \\
\text { under an } \\
\text { Noll-1 } \\
\text { Progrem? }\end{array}$ & 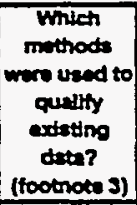 \\
\hline 2755 & 3,4 & CONSTANT & $5.6000000 E-01$ & $5.6000000 E-01$ & $5.6000000 E-01$ & $5.6000000 E-01$ & $P \approx$ & WP035597 & $\mathbf{Y}$ & NA \\
\hline 2755 & 3,4 & CONSTANT & $5.6000000 E-01$ & $5.6000000 \mathrm{E}-01$ & $5.6000000 E-01$ & $5.6000000 \mathrm{E}-01$ & Pa & WPO40134 & $Y$ & NA \\
\hline 2756 & 3,4 & CONSTANT & $3.4600000 E-01$ & $3.4600000 E-01$ & $3.4500000 E-01$ & $-3.4500000 E-01$ & NONE & WP030640 & $\mathbf{Y}$ & NA \\
\hline 2756 & 3,4 & CONSTANT & $3.4600000 \mathrm{E}-01$ & $3.4600000 \mathrm{E}-01$ & $3.4600000 E-01$ & $3.4600000 E-01$ & NONE & WPO30994 & $\mathbf{Y}$ & NA \\
\hline 2756 & 3,4 & CONSTANT & $-3.4600000 \mathrm{E}-01$ & $3.46000000-01$ & $3.46000000-01$ & $-3.45000000-01$ & NONE & WP035597 & $\mathbf{Y}$ & NA \\
\hline 2756 & 3,4 & CONSTANT & $-3.4600000 E-01$ & $-3.4600000 \mathrm{E}-01$ & $-3.4600000 \mathrm{E}-01$ & $-3.4500000 E-01$ & NONE & WPO4O434 & $\mathbf{Y}$ & N/A \\
\hline 2759 & 1,3 & CONSTANT & $0.0000000 E+\infty 0$ & $0.0000000 E+\infty 0$ & $0.0000000 E+\infty$ & $0.0000000 E+\infty 0$ & NONE & WP030640 & $\mathbf{Y}$ & NA \\
\hline 2759 & 1,3 & CONSTANT & $0.0000000 E+00$ & $0.0000000 E+\infty 0$ & $0.0000000 E+00$ & $0.0000000 E+\infty 0$ & NONE & WP030994 & $\mathbf{Y}$ & NA \\
\hline 2759 & 1,3 & CONSTANT & $0.0000000 E+\infty$ & $0.0000000 E+\infty 0$ & $0.0000000 E+00$ & $0.0000000 E+\infty$ & NONE & WP035271 & $\mathbf{\gamma}$ & NA \\
\hline 2759 & 1,3 & CONSTANT & $0.0000000 E+00$ & $0.0000000 \mathrm{E}+\infty 0$ & $0.0000000 \mathrm{E}+00$ & $0.0000000 E+\infty 0$ & NONE & WP035333 & Y. & NA \\
\hline 2759 & 1,3 & CONSTANT & $0.0000000 E+\infty$ & $0.0000000 E+00$ & $0.0000000 E+\infty 0$ & $0.0000000 E+00$ & NONE & WP035597 & $\mathbf{Y}$ & NA \\
\hline 2759 & 1,3 & CONSTANT & $0.0000000 E+00$ & $0.0000000 \mathrm{E}+\infty 0$ & $0.0000000 E+00$ & $0.0000000 E+\infty 0$ & NONE & WP040514 & $\mathbf{Y}$ & NA \\
\hline 2760 & 3,4 & CONSTANT & $5.6000000 E-01$ & $5.6000000 \mathrm{E}-01$ & $5.6000000 E-01$ & $5.6000000 E-01$ & $\mathbf{P a}$ & YP030640 & $\mathbf{Y}$ & NA \\
\hline 2760 & 3,4 & CONSTANT & $5.6000000 E-01$ & $5.6000000 \mathrm{E}-01$ & $5.6000000 E-01$ & $5.6000000 E-01$ & $\mathbf{P a}$ & WP030994 & $\mathbf{Y}$ & NA \\
\hline 2760 & 3.4 & CONSTANT & $5.6000000 E-01$ & $5.6000000 \mathrm{E}-01$ & $5.6000000 E-01$ & $5.6000000 E-01$ & $\mathbf{P a}$ & WP035597 & $\mathbf{Y}$ & NA \\
\hline 2760 & 3,4 & CONSTANT & $5.6000000 \mathrm{E}-01$ & $5.6000000 E-01$ & $5.6000000 E-01$ & $5.6000000 E-01$ & $\mathbf{P a}$ & WPO40434 & $Y$ & N/A \\
\hline 2761 & 3,4 & CONSTANT & $-3.4600000 \mathrm{E}-01$ & $3.4600000 E-01$ & $3.4600000 \mathrm{E}-01$ & $3.4600000 E-01$ & NONE & WPO30640 & ${ }^{-} \mathbf{Y}$ & NA \\
\hline 2761 & 3,4 & CONSTANT & $-3.4600000 \mathrm{E}-01$ & $3.4600000 E-01$ & $-3.4600000 \mathrm{E}-01$ & $-3.4600000 \mathrm{E}-01$ & NONE & WP030994 & $Y$ & N/A \\
\hline 2761 & 3,4 & CONSTANT & $-3.46000000-01$ & $-3.4600000 E-01$ & $-3.4600000 \mathrm{E}-01$ & $3.46000000-01$ & NONE & WP035597 & $\mathbf{Y}$ & NA \\
\hline 2769 & 3,4 & CONSTANT & $3,4600000 E-01$ & $3.4600000 \mathrm{E}-01$ & $3.4600000 E-01$ & $3.4600000 E-01$ & NONE & WPO40434 & $\mathbf{Y}$ & NA \\
\hline 2764 & 1,3 & CONSTANT & $0.0000000 E+\infty 0$ & $0.0000000 E+\infty 0$ & $0.0000000 E+00$ & $0.0000000 E+\infty 0$ & NONE & WP030640 & $\mathbf{Y}$ & $N / A$ \\
\hline 2764 & 1,3 & CONSTANT & $0.0000000 E+\infty 0$ & $0.00000000+\infty 0$ & $0.0000000 E+\infty$ & $0.0000000 \mathrm{E}+00$ & NONE & WP030994 & $\mathbf{Y}$ & N/A \\
\hline 2764 & 1,3 & CONSTANT & $0.0000000 E+\infty 0$ & $0.00000000+00$ & $0.0000000 E+\infty$ & $0.0000000 E+\infty$ & NONE & WP035271 & $\mathbf{Y}$ & N/A \\
\hline 2764 & 1.3 & CONSTANT & $0.0000000 E+\infty 0$ & $0.0000000 E+00$ & $0.0000000 E+\infty$ & $0.0000000 E+\infty 0$ & NONE & WP035333 & $Y$ & N/A \\
\hline
\end{tabular}


CCA Parameter Listing

\begin{tabular}{|c|c|c|c|c|c|c|c|c|c|c|}
\hline 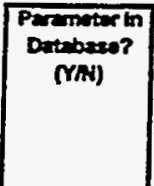 & 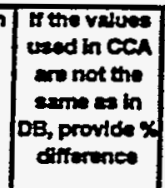 & Do & Material Do & Mxtarla Namo & Paremerter & Peremeter hems & $\begin{array}{l}\text { PRP DD } \\
\text { ATPO }\end{array}$ & $\begin{array}{c}\text { Detal Entry } \\
\text { Dats }\end{array}$ & 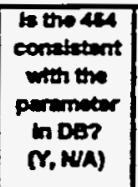 & $\begin{array}{l}\text { Parninitar } \\
\text { Cettegory } \\
\text { (foctiote i) }\end{array}$ \\
\hline $\mathbf{Y}$ & & 2764 & SALT_TS & $\begin{array}{l}\text { Shan cah column } \\
\text { compactedtume } 100 \text { to } \\
200 \text { years }\end{array}$ & KPT & $\begin{array}{l}\text { Fleg for Permasbitty Detemined } \\
\text { Threshold }\end{array}$ & 33573 & $20-F a b-96$ & $\mathbf{Y}$ & $4 B$ \\
\hline $\mathbf{Y}$ & & 2764 & SALT_T5 & $\begin{array}{l}\text { Shat san column } \\
\text { compactedtime } 100 \text { to } \\
200 \text { years }\end{array}$ & KPT & $\begin{array}{l}\text { Fleg for Permesbitty Determined } \\
\text { Throathoid }\end{array}$ & 33573 & 20-Feb-96 & $\mathbf{Y}$ & 48 \\
\hline $\mathbf{Y}$ & 100 & 2765 & SALT_TS & $\begin{array}{l}\text { Shar salt column } \\
\text { compectedtime } 100 \text { to } \\
200 \text { years }\end{array}$ & PCT_A & $\begin{array}{l}\text { Threshold Prossure Lnes } \\
\text { Parmeter }\end{array}$ & 33575 & $14+00-96$ & $\mathbf{Y}$ & 1 \\
\hline$Y$ & 100 & 2765 & SALT_T5 & $\begin{array}{l}\text { Shoft salt colemn } \\
\text { compactecttime } 100 \text { to } \\
200 \text { yasers }\end{array}$ & PCT_A & $\begin{array}{l}\text { Threshold Presturn Linas } \\
\text { Paremeter }\end{array}$ & 33575 & 14Feb-96 & $\mathbf{Y}$ & 1 \\
\hline $\mathbf{Y}$ & 100 & 2765 & SALT_T5 & $\begin{array}{l}\text { Shaf sak colemn } \\
\text { compected:tine } 100 \text { to } \\
200 \text { years }\end{array}$ & PCT_A & $\begin{array}{l}\text { Threstiold Presure Linar } \\
\text { Paraneter }\end{array}$ & 33575 & 14 Fob-96 & $\mathbf{Y}$ & 1 \\
\hline $\mathbf{Y}$ & 100 & 2765 & SALT_T5 & $\begin{array}{l}\text { Shaf salt column } \\
\text { compactedtume } 100 \text { to } \\
200 \text { years }\end{array}$ & PCT_A & $\begin{array}{l}\text { Threshold Pressure Linas } \\
\text { Peraneter }\end{array}$ & 33575 & 14 Fab-96 & $\mathbf{Y}$ & 1 \\
\hline $\mathbf{Y}$ & 100 & 2766 & SALT_T5 & $\begin{array}{l}\text { Shat ant cokmn } \\
\text { compactodt tme } 100 \text { to } \\
200 \text { years }\end{array}$ & PCT_EXP & $\begin{array}{l}\text { Threshold pressure exponential } \\
\text { parameter }\end{array}$ & 33577 & $14+0 b-96$ & $\mathbf{Y}$ & 1 \\
\hline $\mathbf{Y}$ & 100 & 2766 & SALT_T5 & $\begin{array}{l}\text { Shat salt column } \\
\text { compacted:time too to } \\
200 \text { years }\end{array}$ & PCT_EXP & $\begin{array}{l}\text { Throshold pressure exponential } \\
\text { paraneter }\end{array}$ & 3357 & 14 feb-96 & $\mathbf{Y}$ & 1 \\
\hline $\mathbf{Y}$ & 100 & 2766 & SALT_T5 & $\begin{array}{l}\text { Shat salt column } \\
\text { compscted:time } 100 \text { to } \\
200 \text { years }\end{array}$ & PCT_EXP & $\begin{array}{l}\text { Threshold proseure exponential } \\
\text { pareneter }\end{array}$ & 33577 & 14fab-96 & $\mathbf{Y}$ & 1 \\
\hline $\mathbf{Y}$ & 100 & 2766 & SALT_T5 & $\begin{array}{l}\text { Shaft sah column } \\
\text { compactedtme } 100 \text { to } \\
200 \text { years }\end{array}$ & PCT_EXP & $\begin{array}{l}\text { Threshold pressuro exponemial } \\
\text { peremeter }\end{array}$ & 33577 & 14 Fob-96 & $\mathbf{Y}$ & 1 \\
\hline$Y$ & & 2768 & SANTAROS & Santa Rosa Formation & KPT & $\begin{array}{l}\text { Flag for Permeability Determined } \\
\text { Threshold }\end{array}$ & 33510 & $20 F c b-96$ & $\mathbf{Y}$ & 48 \\
\hline$Y$ & & 2768 & SANTAROS & Senta Rase Formation & KPT & $\begin{array}{l}\text { Flag for Permeabitty Determined } \\
\text { Threshold }\end{array}$ & 33510 & $20-50 b-96$ & $Y$ & AB \\
\hline $\mathbf{Y}$ & & 2758 & SANTAROS & Santa Rosa Formation & KPT & $\begin{array}{l}\text { Flag for Permedodity Determmed } \\
\text { Throshold }\end{array}$ & 33510 & $20-F \circ b-96$ & $\mathbf{Y}$ & $A B$ \\
\hline $\mathbf{Y}$ & & 2768 & SANTAROS & Santa Rosa Formation & KPT & $\begin{array}{l}\text { Flag for Permenolty Determined } \\
\text { Threshold }\end{array}$ & 33510 & 20-Fob-96 & $\mathbf{Y}$ & 48 \\
\hline $\mathbf{Y}$ & & 2769 & SANTAROS & Santa Rosa Formatoon & PCT_A & $\begin{array}{l}\text { Threshold Proasure Lnesr } \\
\text { Peramoter }\end{array}$ & 33528 & 22-Fab-96 & $\mathbf{Y}$ & 48 \\
\hline $\mathbf{Y}$ & & 2769 & SANTAROS & Senta Rose Formation & PCT_A & $\begin{array}{l}\text { Trmeshold Preasure Lnear } \\
\text { Peramete }\end{array}$ & 33528 & 22frob-96 & $\mathbf{Y}$ & 48 \\
\hline $\mathbf{Y}$ & . & 2769 & SANTAROS & Santa Rose Formatoon & PCT_A & $\begin{array}{l}\text { Trreshow Proakre Linear } \\
\text { Parmete }\end{array}$ & 33528 & 22Fob-96 & $\mathbf{Y}$ & $4 \mathrm{~B}$ \\
\hline $\mathbf{Y}$ & & 2769 & SANTAROS & Sante Rose Fomation & PCT_A & $\begin{array}{l}\text { Trresnad Preacuro Linsar } \\
\text { Peremene }\end{array}$ & 33528 & $22+$ eb-9s & $\mathbf{Y}$ & AB \\
\hline $\mathbf{Y}$ & & 2770 & SANTAROS & Santa Rose Fommetion & PCT_EXP & 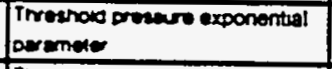 & 33529 & 22+Feb-96 & $\mathbf{Y}$ & $4 B$ \\
\hline $\mathbf{Y}$ & & 2770 & SANTAROS & Santa Rose Formecon & PCT_EXO & 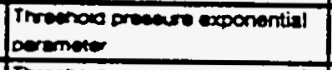 & 33529 & $22+50 b-96$ & $-\not \gamma$ & $4 B$ \\
\hline $\mathbf{Y}$ & & 2770 & SANTAROS & Santa Rose Formucon & PCT_EXP & $\begin{array}{l}\text { Trreshode preace exconentsal } \\
\text { peramete }\end{array}$ & 33529 & 22Fab-96 & $\mathbf{Y}$ & 48 \\
\hline $\mathbf{Y}$ & & 2770 & SANTAROS & Santa Roes Fomexon & PCT_EXP & $\begin{array}{l}\text { Irremos orewere exponentsal } \\
\text { oeramen }\end{array}$ & 33529 & 22-Fob-96 & $\mathbf{Y}$ & 48 \\
\hline $\mathbf{Y}$ & & 2773 & S_ANH_AB & 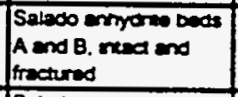 & rot & 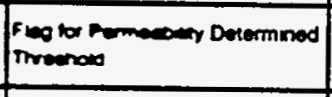 & 34165 & 20-Feb-96 & $\mathbf{Y}$ & $4 B$ \\
\hline $\mathbf{Y}$ & & 2773 & S_ANH_AB & 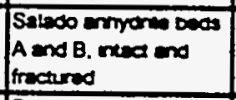 & IPT & 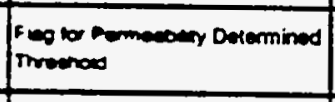 & 34165 & 20-Feb-9s & $\mathbf{Y}$ & $4 B$ \\
\hline $\mathbf{Y}$ & & 2773 & S_ANH_AB & $\begin{array}{l}\text { Saleco exmporte beds } \\
\text { A and B. rxict and } \\
\text { frectures }\end{array}$ & KPT & frop tor Pommony Doternined & 34165 & 20 feb-96 & $\mathbf{Y}$ & $A B$ \\
\hline $\mathbf{Y}$ & & 2773 & S_ANH_AB & $\begin{array}{l}\text { Saledo annorie onos } \\
\text { A and B. menct and } \\
\text { frectured }\end{array}$ & IOT & Fing for Pomeany Determined & 34165 & 20-fib-96 & $\mathbf{Y}$ & $A B$ \\
\hline $\mathbf{Y}$ & & 2774 & S_ANH_AB & $\begin{array}{l}\text { Saledo exmorie beds } \\
\text { A and B. ntind and } \\
\text { frecared }\end{array}$ & PCT_a & $\begin{array}{l}\text { Trreanoud Preacure Linear } \\
\text { Peremen }\end{array}$ & 34183 & ceffeb-s6 & $\mathbf{Y}$ & 44 \\
\hline$Y$ & & 2774 & S_ANH_AB & $\begin{array}{l}\text { Suleco enmores beces } \\
A \text { and } B \text {. ntect ind } \\
\text { frectured }\end{array}$ & $\mathrm{PCT}_{-4}$ & 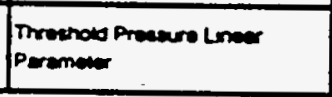 & 34183 & obfab-s & $\mathbf{Y}$ & $\mathbb{M}$ \\
\hline
\end{tabular}


CCA Parameter Listing

\begin{tabular}{|c|c|c|c|c|c|c|c|c|c|c|}
\hline D & \begin{tabular}{c|} 
Candin- \\
cation of \\
Deta \\
Cintegory \\
(footrota 2)
\end{tabular} & $\begin{array}{l}\text { Oistribution } \\
\text { Type }\end{array}$ & Main & Medten & Dintmum & Modimin & Whats & Rationsed LD & 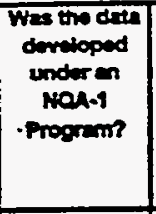 & 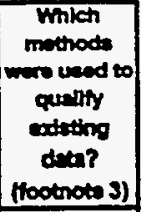 \\
\hline 2784 & 1,3 & CONSTANT & $0.0000000 \mathrm{E}+\infty 0$ & $0.0000000 E+\infty$ & $0.0000000 E+\infty 0$ & $0.00000000+\infty$ & NONE & WP035597 & $\mathbf{Y}$ & NA \\
\hline 2764 & 1,3 & CONSTANT & $0.00000000 E+00$ & $0.0000000 E+\infty$ & $0.0000000 E+\infty 0$ & $0.0000000 E+\infty 0$ & NONE & WPO40514 & $\mathbf{Y}$ & NA \\
\hline 2765 & 3,4 & CONSTANT & $5.6000000 E-01$ & $5.6000000 \varepsilon-01$ & $5.6000000 E-01$ & 5.6000000E-01 & Pa & WPO30640 & $\mathbf{Y}$ & NA \\
\hline 2765 & 3,4 & CONSTANT & 5.6000000E-01 & $5.6000000 E-01$ & $5.6000000 E-01$ & $5.6000000 E-01$ & $P_{2}$ & WP03099y & $\mathbf{Y}$ & NA \\
\hline 2765 & 3,4 & CONSTANT & 5.6000000E-01 & $5.6000000 E-01$ & $5.6000000=-01$ & $5.6000000 E-01$ & Pa & WP035597 & $\gamma$ & NAA \\
\hline 2765 & 3,4 & CONSTANT & $5.6000000 E-01$ & $5.6000000 E-01$ & $5.6000000 E-01$ & $5.6000000=-01$ & $\mathbf{P} \mathbf{a}$ & WPO40134; & $Y$ & N/A \\
\hline 2766 & 3,4 & CONSTANT & $-3.45000000-01$ & $-3.4600000 E-01$ & $-3.4600000 \mathrm{E}-01$ & $2.4600000=-01$ & NONE & WP030640 & $\mathbf{Y}$ & NA \\
\hline 2766 & 3,4 & CONSTANT & $-3.4600000 \mathrm{E}-01$ & $-3.4600000 E-01$ & $-3.4500000 E-01$ & $-3.4600000 E-01$ & NONE & WP030994 & $Y$ & NAA \\
\hline 2766 & 3,4 & CONSTANT & $-3.4500000 \mathrm{E}-01$ & $-3.4600000=-01$ & $-3.46000000-01$ & $-3.4600000=-01$ & NONE & WP035597 & $\mathbf{Y}$ & NA \\
\hline 2766 & 3,4 & CONSTANT & $-3.4600000 E-01$ & $-3.4600000 E-01$ & $-3.4500000 \mathrm{E}-01$ & $3.4600000 E-01$ & NONE & WPO40434 & $\mathbf{Y}$ & NA \\
\hline 2768 & 1,3 & CONSTANT & $0.0000000 E+00$ & $0.0000000 E+00$ & $0.0000000 E+\infty 0$ & $0.0000000 E+\infty 0$ & NONE & WP035271 & $\mathbf{Y}$ & NA \\
\hline 2768 & 1.3 & CONSTANT & $0.0000000 \mathrm{E}+\infty$ & $0.0000000 \mathrm{E}+00$ & $0.0000000 E+\infty 0$ & $0.0000000 E+00$ & NONE & WP035333 & $\mathbf{Y}$ & NA \\
\hline 2768 & 1,3 & CONSTANT & $0.0000000 \mathrm{E}+00$ & $0.0000000 \mathrm{E}+\infty$ & $0.0000000=+\infty$ & $0.0000000 E+\infty 0$ & NONE & WP035597 & $\mathbf{Y}$ & NA \\
\hline 2768 & 1,3 & CONSTANT & $0.0000000 E+00$ & $0.0000000 E+\infty 0$ & $0.0000000 E+\infty$ & $0.0000000 E+\infty$ & NONE & WP040514 & $\mathbf{Y}$ & NAA \\
\hline 2769 & 1,3 & CONSTANT & $0.0000000 E+\infty 0$ & $0.0000000 \mathrm{E}+00$ & $0.0000000 E+\infty 0$ & $0.0000000 \mathrm{E}+\infty 0$ & $\mathrm{~Pa}$ & WP031041 & $\mathbf{Y}$ & NA \\
\hline 2769 & 1,3 & CONSTANT & $0.0000000 E+\infty 0$ & $0.0000000 E+\infty 0$ & $0.0000000 E+00$ & $0.0000000 E+\infty$ & Pa & WP035597 & $\mathbf{Y}$ & NA \\
\hline 2769 & 1,3 & CONSTANT & $0.00000000+\infty 0$ & $0.0000000 E+\infty 0$ & $0.0000000 E+\infty$ & $0.0000000 E+00$ & Pa & WPO40514 & $\mathbf{Y}$ & NA \\
\hline 2769 & 1,3 & CONSTANT & $0.0000000 E+\infty$ & $0.0000000 E+\infty 0$ & $0.00000000+00$ & $0.0000000 E+\infty$ & $\mathbf{P a}$ & WS_PALMER & $\mathbf{Y}$ & NA \\
\hline 2770 & 1,3 & CONSTANT & $0.0000000 E+00$ & $0.00000000+00$ & $0.0000000 E+\infty 0$ & $0.0000000 E+\infty 0$ & NONE & WP031041 & $\mathbf{Y}$ & NA \\
\hline 2770 & 1,3 & CONSTANT & $0.0000000 \mathrm{E}+00$ & $0.0000000 E+00$ & $0.0000000 \mathrm{E}+00$ & $0.0000000 E+00$ & NONE & WP035597 & $--Y$ & NA \\
\hline 2770 & 1.3 & CONSTANT & $0.0000000 E+\infty 0$ & $0.0000000 E+\infty 0$ & $0.00000000+00$ & $0.0000000 \mathrm{E}+\infty$ & NONE & WP040514 & $Y$ & NA \\
\hline 2770 & 1,3 & CONSTANT & $0.0000000 E+00$ & $0.0000000 E+\infty$ & $0.0000000 \mathrm{E}+\infty 0$ & $0.0000000 E+00$ & NONE & WS_PALMER & $\mathbf{Y}$ & NA \\
\hline 2773 & 1,3 & CONSTANT & $0.00000000 E+\infty 0$ & $0.0000000 \mathrm{E}+00$ & $0.0000000 E+\infty 0$ & $0.0000000 E+\infty$ & NONE & WP035271 & $\mathbf{Y}$ & N/A \\
\hline 2773 & 1.3 & CONSTANT & $0.0000000 E+\infty 0$ & $0.0000000 E+\infty 0$ & $0.00000000 E+\infty$ & $0.0000000 E+\infty$ & NONE & WP035333 & $\mathbf{Y}$ & NAA \\
\hline 2773 & $\cdot 1,3$ & CONSTANT & $0.0000000 E+00$ & $0.0000000 E+\infty$ & $0.00000000=+\infty$ & $0.0000000 E+00$ & NONE & WP035597 & $\mathbf{Y}$ & NA \\
\hline 2773 & 1,3 & CONSTANT & $0.0000000 E+00$ & $0.0000000 E+\infty$ & $0.00000000 E+\infty 0$ & $0.0000000 E+\infty 0$ & NONE & WP040514 & $\mathbf{Y}$ & NA \\
\hline 2774 & 1.3 & CONSTANT & $2.6000000 E-01$ & $26000000 E-01$ & $2.6000000 E-01$ & $2.6000000 E-01$ & Pa & WP030643 & $\mathbf{Y}$ & NA \\
\hline 2774 & 1,3 & CONSTANT & $26000000 E-01$ & $26000000 E=-01$ & $2.6000000 E-01$ & $2,6000000 E-01$ & Pa & WPO35597 & $\mathbf{Y}$ & NA \\
\hline
\end{tabular}


CCA Parameter Listing

\begin{tabular}{|c|c|c|c|c|c|c|c|c|c|c|}
\hline $\begin{array}{l}\text { Peremitar in } \\
\text { Detabase? } \\
\text { (MaN) }\end{array}$ & 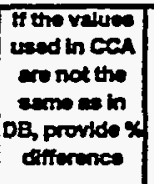 & DD & Metardia & Meterta Namo & $\begin{array}{l}\text { Peromeder } \\
\text { to }\end{array}$ & Permoter Karme & $\begin{array}{c}\text { PFP D } \\
\text { (MPO })\end{array}$ & $\begin{array}{c}\text { Dere Entry } \\
\text { Dut }\end{array}$ & 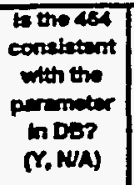 & $\begin{array}{l}\text { Parmoter } \\
\text { Cinegory } \\
\text { (footrote 1) }\end{array}$ \\
\hline$Y$ & & $2 \pi 74$ & S_ANH_AB & $\begin{array}{l}\text { Salado anhydrte beds } \\
\text { A and B, intad and } \\
\text { frectured }\end{array}$ & PCT_A & $\begin{array}{l}\text { Thrashold Pressura Lnoar } \\
\text { Parameter }\end{array}$ & 34183 & $08+50-96$ & $Y$ & 4 \\
\hline$Y$ & & 2775 & S_ANH_AB & 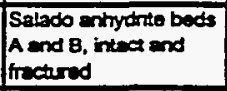 & PCT_EXP & $\begin{array}{l}\text { Threstold pressurs exponential } \\
\text { perenotor }\end{array}$ & 34284 & as-ab-96 & $\mathbf{Y}$ & $\mu$ \\
\hline $\mathbf{Y}$ & & 2775 & S_ANH_AB & $\begin{array}{l}\text { Saledo enhychite bedis } \\
\text { A and B. intact and } \\
\text { fractured }\end{array}$ & PCT_EXP & $\begin{array}{l}\text { Throstiold pressure exponential } \\
\text { perrenotor }\end{array}$ & 34184 & $08 F=0-6$ & $Y$ & $\mu$ \\
\hline$Y$ & & 2778 & S_HALTE & Salado halite, intact & KPT & $\begin{array}{l}\text { Fleg for Permesbitty Determined } \\
\text { Inreshold }\end{array}$ & 34233 & $20-F b-96$ & $\mathbf{Y}$ & AB \\
\hline $\mathbf{Y}$ & & 2778 & S_HALTE & Salado halito, intect & KPT & $\begin{array}{l}\text { Flag for Pormesbilty Determined } \\
\text { Inreathold }\end{array}$ & 34233 & 20 -fab-96 & $\mathbf{Y}$ & AB \\
\hline $\mathbf{Y}$ & & 2778 & S_HALTTE & Salado Ralito, intect & KPT & 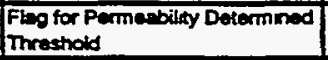 & 34233 & 20 -Feb-96 & $r$ & $1 B$ \\
\hline $\mathbf{Y}$ & & 2778 & S_HALTE & Salado halito, intact & |FT & $\begin{array}{l}\text { Flag for Pormeability Determined } \\
\text { Threshold }\end{array}$ & 34233 & $20-50-96$ & $\boldsymbol{\gamma}$ & $A B$ \\
\hline $\mathbf{Y}$ & $100.00 \mathrm{hw}$ & 2779 & S_MALTE & Selado halite, intact & PCT_A & $\begin{array}{l}\text { Threshold Prossure Lnear } \\
\text { Perameter }\end{array}$ & 34375 & $22+\infty-96$ & $\mathbf{Y}$ & AB \\
\hline $\mathbf{Y}$ & $100.00 \mathrm{hw}$ & 2779 & S_HALTE & Salado halite, intact & PCT_A & $\begin{array}{l}\text { Threashold Pressure Linaar } \\
\text { Perameter }\end{array}$ & 34375 & 22fab-96 & $\mathbf{Y}$ & AB \\
\hline $\mathbf{Y}$ & $100.00 \mathrm{hw}$ & 2779 & S_HALTTE & Salado halte, intect & PCT_A & $\begin{array}{l}\text { Threshold Pressure Lineser } \\
\text { Pareneter }\end{array}$ & 34375 & $22+80-96$ & $\mathbf{Y}$ & $4 B$ \\
\hline $\mathbf{Y}$ & $100.00 \mathrm{hw}$ & 2779 & S_HALTE & Satado halito, intact & PCT_A & $\begin{array}{l}\text { Thrashold Prestura Linadr } \\
\text { Paremotor }\end{array}$ & 34375 & $22 f-b-96$ & $\mathbf{Y}$ & $A B$ \\
\hline $\mathbf{Y}$ & $50.00 \mathrm{mw}$ & 2780 & S_HALTE & Salado halite, intact & PCT_EXP & $\begin{array}{l}\text { Thrashold pressure exponential } \\
\text { perameter }\end{array}$ & 34377 & 22-Feb-96 & $\mathbf{Y}$ & $A B$ \\
\hline $\mathbf{Y}$ & $50.00 \mathrm{hw}$ & 2780 & S_HALTTE & Saloco halite, intact & PCT_EXP & $\begin{array}{l}\text { Threshold pressuro exponential } \\
\text { parametor }\end{array}$ & 34377 & 22fob-96 & $\mathbf{Y}$ & $A B$ \\
\hline $\mathbf{Y}$ & $50.00 \mathrm{hw}$ & 2780 & S_HALTE & Salado halite, intect & PCT_EXP & $\begin{array}{l}\text { Throshold pressure axporrentsal } \\
\text { parameter }\end{array}$ & 34377 & 22 fab-96 & $\mathbf{Y}$ & 48 \\
\hline $\mathbf{Y}$ & $50.00 \mathrm{hw}$ & 2780 & S_HALTTE & Salaco malite, inted & PCT_EXP & $\begin{array}{l}\text { Threshold prossure exporiential } \\
\text { parameter }\end{array}$ & 34377 & 22fob-96 & $\mathbf{Y}$ & 18 \\
\hline$Y$ & $50.00 \mathrm{mw}$ & 2760 & S_HALITE & Salado halite, intact & PCT_EXP & $\begin{array}{l}\text { Throshold pressure exponential } \\
\text { parametor }\end{array}$ & 34377 & 22-Fob-96 & $\mathbf{Y}$ & $A B$ \\
\hline$Y$ & & 2783 & S_MB138 & $\begin{array}{l}\text { Salado marker bed } \\
138, \text { intact and } \\
\text { fractured }\end{array}$ & KPT & $\begin{array}{l}\text { Flag for Permesbility Determined } \\
\text { Throshold }\end{array}$ & 34479 & $20-F 0 b-96$ & $\mathbf{Y}$ & $A B$ \\
\hline$Y$ & & 2783 & S_MB138 & $\begin{array}{l}\text { Salado marker bed } \\
139 \text {, intact and } \\
\text { frectured }\end{array}$ & KPT & $\begin{array}{l}\text { Fleg tor Permeability Determined } \\
\text { Threshold }\end{array}$ & 34479 & 20-Feb-96 & $\mathbf{Y}$ & $A B$ \\
\hline $\mathbf{Y}$ & & 2783 & S_MB138 & $\begin{array}{l}\text { Salado marker bed } \\
\text { 133, intact and } \\
\text { frectured }\end{array}$ & KPT & $\begin{array}{l}\text { Fleg for Permeabilly Determined } \\
\text { Threshold }\end{array}$ & 34479 & 20-Feb-96 & $\mathbf{Y}$ & $A B$ \\
\hline $\mathbf{Y}$ & & 2783 & S_MB138 & $\begin{array}{l}\text { Salado marker bed } \\
\text { 138, intset and } \\
\text { fractured }\end{array}$ & KPT & $\begin{array}{l}\text { Flag for Permeability Determined } \\
\text { Threshold }\end{array}$ & 34479 & 20-fob-96 & $Y$ & AB \\
\hline $\mathbf{Y}$ & & 2784 & S_MB138 & $\begin{array}{l}\text { Salaob masker bed } \\
138 \text {, intact and } \\
\text { fractured }\end{array}$ & PCT_A & $\begin{array}{l}\text { Trreshold Pressuro Linear } \\
\text { Paremotor }\end{array}$ & 34505 & D8-Fob-96 &..$Y$ & $\mathbf{A A}$ \\
\hline $\mathbf{Y}$ & & 2784 & S_MB138 & $\begin{array}{l}\text { Salado marker bod } \\
138 \text {, intact and } \\
\text { fractured }\end{array}$ & PCT_A & $\begin{array}{l}\text { Threshold Pressure Linear } \\
\text { Perametor }\end{array}$ & 34505 & $08-50-86$ & $Y$ & $m$ \\
\hline $\mathbf{Y}$ & & 2784 & S_MB138 & $\begin{array}{l}\text { Saludo maker bed } \\
138 \text {, intiact and } \\
\text { fractured }\end{array}$ & PCT_A & $\begin{array}{l}\text { Threshold Pressure Lnear } \\
\text { Perameter }\end{array}$ & 34505 & $08-50 b-96$ & $\mathbf{Y}$ & $\mathbb{M}$ \\
\hline $\mathbf{Y}$ & & 2785 & S_MB138 & $\begin{array}{l}\text { Seledo matker bed } \\
\text { 138, intact and } \\
\text { frectured }\end{array}$ & PCT_EXP & $\begin{array}{l}\text { Threshold pressure exponential } \\
\text { peremmiter }\end{array}$ & 34507 & ab-feb-96 & $\mathbf{Y}$ & $\mathbb{A A}$ \\
\hline $\mathbf{Y}$ & & 2785 & S_MB138 & $\begin{array}{l}\text { Saledo manker bed } \\
138, \text { intect and } \\
\text { frectured }\end{array}$ & PCT_EXP & $\begin{array}{l}\text { Threshold presture exponential } \\
\text { parameter }\end{array}$ & 34507 & 08-0b-96 & $\mathbf{Y}$ & $\mu$ \\
\hline$Y$ & & 2785 & S_MB138 & $\begin{array}{l}\text { Seleco marker bad } \\
138 \text {, intact and } \\
\text { fractured }\end{array}$ & PCT_EXP & $\begin{array}{l}\text { Throsinold pressure exponential } \\
\text { parameter }\end{array}$ & 34507 & 08-feb-96 & $\mathbf{Y}$ & 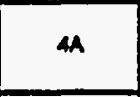 \\
\hline$Y$ & & 2788 & S_MB139 & $\begin{array}{l}\text { Saledo merker bed } \\
139 \text {, intiact and } \\
\text { inectured }\end{array}$ & KPT & $\begin{array}{l}\text { Fieg for Permeabilty Dotermined } \\
\text { Threstrold }\end{array}$ & 34825 & 20-Feb-86 & $\mathbf{Y}$ & $A B$ \\
\hline$Y$ & & 2788 & S_MB139 & $\begin{array}{l}\text { Saledo manker bed } \\
\text { 139, intuct and } \\
\text { iractured }\end{array}$ & KPT & $\begin{array}{l}\text { Fleg for Permeasbility Determinad } \\
\text { Threthold }\end{array}$ & 34825 & $20+50-96$ & $Y$ & 48 \\
\hline $\mathbf{Y}$ & & 2788 & S_MB139 & $\begin{array}{l}\text { Saledo maker bed } \\
139 \text {, intact and } \\
\text { fractured }\end{array}$ & KPT & $\begin{array}{l}\text { Fieg for Pormeability Determined } \\
\text { Threstots }\end{array}$ & 34825 & $20+\infty-96$ & $\mathbf{Y}$ & 18 \\
\hline
\end{tabular}


CCA Parameter-Listing

\begin{tabular}{|c|c|c|c|c|c|c|c|c|c|c|}
\hline DD & 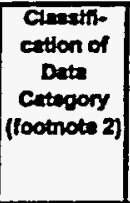 & $\begin{array}{c}\text { Distribution } \\
\text { Typ }\end{array}$ & Man & Wedien & Minimum & Modmun & Unite & $\begin{array}{c}\text { Reterwincs D } \\
.\end{array}$ & $\begin{array}{l}\text { Was the data } \\
\text { dewiloped } \\
\text { underen } \\
\text { Non-1 } \\
\text { Progren? }\end{array}$ & $\begin{array}{l}\text { Which } \\
\text { mothods } \\
\text { more used to } \\
\text { quilty } \\
\text { extetting } \\
\text { drta? } \\
\text { (footnots 3) }\end{array}$ \\
\hline 2774 & 1,3 & CONSTANT & $2.6000000 E-01$ & $26000000 E-01$ & $2.6000000 \mathrm{E}-01$ & $2.6000000 E-01$ & $\mathbf{P a}$ & WP038454 & $\mathbf{Y}$ & NA \\
\hline 2775 & 1,3 & CONSTANT & $3.4800000 E-01$ & $3.4800000 E-01$ & $-3.4800000 E-01$ & $3.4800000 E-01$ & NONE & WPO30643 & $\mathbf{Y}$ & $N / A$ \\
\hline 2775 & 1,3 & CONSTANT & $-3.4800000 \mathrm{E}-01$ & $3.4800000 E-01$ & -3.4800000 E- 01 & $3.4800000 E-01$ & NONE & WP035597 & $\mathbf{Y}$ & N/A \\
\hline 2778 & 3,5 & CONSTANT & $0.0000000 E+\infty 0$ & $0.0000000 E+\infty 0$ & $0.00000000 E+\infty$ & $0.00000000 E+\infty$ & NONE & WP035271 & $\mathbf{Y}$ & $\mathbf{N A}$ \\
\hline 2778 & 3,5 & CONSTANT & $0.0000000 E+00$ & $0.00000000 E+\infty 0$ & $0.0000000 E+\infty 0$ & $0.0000000 E+\infty$ & NONE & WPO35333 & $\mathbf{Y}$ & N/A \\
\hline 2778 & 3,5 & CONSTANT & $0.0000000 E+00$ & $0.0000000 E+\infty$ & $0.0000000 E+\infty 0$ & $0.0000000 E+\infty 0$ & NONE & WP035597 & $\mathbf{Y}$ & NA \\
\hline 2778 & $.3,5$ & CONSTANT & $0.0000000 E+\infty$ & $0.0000000 E+\infty$ & $0.0000000 E+\infty$ & $0.0000000 E+00$ & NONE & WP040514 & $\mathbf{Y}$ & N/A \\
\hline 2779 & $1,3,4$ & CONSTANT & $5.6000000 \mathrm{E}-01$ & 5.6000000E-01 & $5.6000000 E-01$ & $5.6000000 \mathrm{E}-01$ & Pa & SAND92-070033 & $\mathbf{Y}$ & NA \\
\hline 2779 & $1,3,4$ & CONSTANT & $5.6000000 E-01$ & $5.6000000 E-01$ & $5.6000000 E-01$ & $5.6000000 E-01$ & Pa & WP03559 & $\mathbf{Y}$ & N/A \\
\hline 2779 & $1.3,4$ & CONSTANT & $5.6000000 E-01$ & $5.6000000 E-01$ & $5.6000000 E-01$ & $5.6000000 E-01$ & $\mathbf{P a}$ & WP038454 & $\mathbf{Y}$ & NA \\
\hline 2779 & $1,3,4$ & CONSTANT & $5.6000000 E-01$ & 5.6000000E-01 & $5.6000000 E-01$ & $5.6000000 E-01$ & $\mathrm{~Pa}_{\mathbf{a}}$ & WP040514 & $\mathbf{Y}$ & NA \\
\hline 2780 & $1,3,4$ & CONSTANT & $-3.4600000 \mathrm{E}-01$ & $3.4600000 \mathrm{E}-01$ & $3.4600000 E-01$ & $3.4600000 \mathrm{E}-01$ & NONE & SAND92-0700/3 & $\mathbf{Y}$ & NA \\
\hline 2780 & $1,3,4$ & CONSTANT & $-3.4600000 \mathrm{E}-01$ & $-3.4600000 E-01$ & $-3.4600000 E-01$ & $-3.4600000 E-01$ & NOME & WP035271 & $\mathbf{Y}$ & NA \\
\hline 2780 & $1,3,4$ & CONSTANT & $-3.4600000 E-01$ & $-3.4600000 E-01$ & $-3.4600000 E-01$ & $-3.4600000 E-01$ & NONE & WP035333 & $\mathbf{Y}$ & NA \\
\hline 2780 & $1,3,4$ & CONSTANT & $-3.4600000 E-01$ & $3.4600000 \mathrm{E}-01$ & $3.4600000 E-01$ & $3.4600000 \mathrm{E}-01$ & NONE & WP035597 & $\mathbf{Y}$ & NAA \\
\hline 2780 & $1,3,4$ & CONSTANT & $-3.4600000 E-01$ & $3.4600000 \mathrm{E}-01$ & $3.4600000 E-01$ & $-3.4600000 E-01$ & NONE & WP040514 & $Y$ & N/A \\
\hline 2783 & 1,3 & CONSTANT & $0.0000000 \mathrm{E}+\infty$ & $0.00000000+00$ & $0.0000000 E+00$ & $0.0000000 E+00$ & NONE & WP035271 & $\mathbf{Y}$ & $\mathbf{N} / \mathbf{A}$ \\
\hline 2783 & 1,3 & CONSTANT & $0.0000000 E+\infty$ & $0.0000000 E+\infty 00$ & $0.0000000 E+00$ & $0.0000000 E+00$ & NONE & WP035333 & $\mathbf{Y}$ & NA \\
\hline 2783 & 1,3 & CONSTANT & $0.0000000 E+\infty 0$ & $0.0000000 E+\infty$ & $0.0000000 E+00$ & $0.0000000 E+\infty 0$ & NONE & WP035597 & $\mathbf{Y}$ & N/A \\
\hline 2783 & 1,3 & CONSTANT & $0.0000000 \mathrm{E}+\infty 0$ & $0.00000000+00$ & $0.0000000 E+\infty 0$ & $0.0000000 \mathrm{E}+\infty 0$ & NONE & WP040514 & $\mathbf{Y}$ & N/A \\
\hline 2784 & 1,5 & CONSTANT & 2.6000000E-01 & $2.6000000 E-01$ & $2.6000000 E-01$ & $26000000 E-01$ & $\mathbf{P a}$ & WP030643 & $\mathbf{Y} Y$ & NA \\
\hline 2784 & 1,5 & CONSTANT & $2.60000000-01$ & $2.6000000 E-01$ & $26000000 E-01$ & $260000000-01$ & $\mathrm{~Pa}$ & WP035597 & $\mathbf{Y}$ & N/A \\
\hline 2784 & 1,5 & CONSTANT & $2.6000000 E-01$ & $2.6000000 E-01$ & $2.6000000 E-01$ & $26000000 E-01$ & $\mathbf{P a}$ & WP042714 & $\mathbf{Y}$ & NA \\
\hline 2785 & 1,5 & CONSTANT & $3.4800000 E-01$ & $-3.48000000 E-01$ & $34800000 E-01$ & 34800000 E-01 & NONE & WP030543 & $\mathbf{Y}$ & NA \\
\hline 2785 & 1,5 & CONSTANT & $-3.48000000-01$ & $-3.4800000 \mathrm{E}-01$ & $3.4800000 E-01$ & $34800000 E-01$ & NONE & WP035597 & $\mathbf{Y}$ & NA \\
\hline 2785 & 1,5 & CONSTANT & $3.4800000=-01$ & $-3.4800000 E-01$ & $3.4800000 E-01$ & $34800000 E-01$ & NONE & WPO42714 & $\mathbf{Y}$ & NA \\
\hline 2788 & 3,5 & CONSTANT & $0.0000000 E+\infty 0$ & $0.0000000 E+\infty 0$ & $0.0000000 E+\infty$ & $0.0000000 E+00$ & NONE & WP035271 & $\mathbf{Y}$ & N/A \\
\hline 2788 & 3.5 & CONSTANT & $0.0000000 E+\infty$ & $0.0000000 E+\infty$ & $0.00000000+\infty 0$ & $0.0000000 E+\infty$ & NONE & WPO35333 & $\mathbf{Y}$ & N/A \\
\hline 2788 & 3,5 & CONSTANT & $0.00000000=+\infty$ & $0.0000000 E+\infty$ & $0.0000000 E+\infty$ & $0.0000000 E+\infty$ & NONE & WP035597 & $\mathbf{Y}$ & $\mathbf{N} / \mathbf{A}$ \\
\hline
\end{tabular}


CCA Parameter Listing

\begin{tabular}{|c|c|c|c|c|c|c|c|c|c|c|}
\hline $\begin{array}{c}\text { Paremetion in } \\
\text { Detabue? } \\
\text { (YNA) }\end{array}$ & 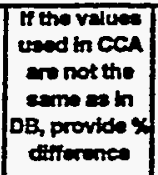 & D & Metartid & Deterd Mem & Parmin & Perneter Rams & $\begin{array}{l}\text { PRPD } \\
\text { (MPOD }\end{array}$ & Dete & 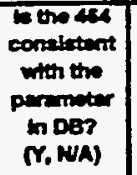 & $\begin{array}{c}\text { Parminter } \\
\text { Cateogry } \\
\text { (footnote 1) }\end{array}$ \\
\hline $\mathbf{Y}$ & & 2788 & S_MB139 & $\begin{array}{l}\text { Salado marker bed } \\
\text { 139. intact and } \\
\text { fractured }\end{array}$ & KPT & $\begin{array}{l}\text { Ftag for Permasbilty Determned } \\
\text { Threshold }\end{array}$ & 34825 & $20-F=b-56$ & $\mathbf{Y}$ & 48 \\
\hline $\mathbf{Y}$ & & 2789 & S_MB139 & $\begin{array}{l}\text { Salaco maker bed } \\
139 \text {, intact and } \\
\text { frectured }\end{array}$ & PCT_A & $\begin{array}{l}\text { Threshold Pressure Linear } \\
\text { Parameter }\end{array}$ & 34843 & $22+c b-96$ & $\mathbf{Y}$ & 1 \\
\hline $\mathbf{Y}$ & & 2789 & S_MB139 & $\begin{array}{l}\text { Salado marker bed } \\
139, \text { intact and } \\
\text { fractured }\end{array}$ & PCT_A & $\begin{array}{l}\text { Thrashold Pressurn Liner } \\
\text { Perameter }\end{array}$ & 34843 & 22fab-96 & $\mathbf{Y}$ & 1 \\
\hline $\mathbf{Y}$ & & 2790 & S_MB139 & $\begin{array}{l}\text { Salado marker bed } \\
139, \text { intact and } \\
\text { fractured }\end{array}$ & PCT_EXP & $\begin{array}{l}\text { Threstold presture exponential } \\
\text { peremeter }\end{array}$ & 34844 & as-fab-96 & $\mathbf{Y}$ & 1 \\
\hline $\mathbf{Y}$ & & 2790 & S_MB139 & $\begin{array}{l}\text { Salado marker bod } \\
\text { 139, intect and } \\
\text { fractured }\end{array}$ & PCT_EXP & $\begin{array}{l}\text { Throstold presture exponential } \\
\text { permenter }\end{array}$ & 34844 & $08-F a b-96$ & $\mathbf{Y}$ & 1 \\
\hline $\mathbf{Y}$ & & 2793 & TAMARISK & Ternarisk Member & $\mathrm{KPT}$ & $\begin{array}{l}\text { Fleg for Permesbility Dotermined } \\
\text { Threshold }\end{array}$ & 34558 & $20-50 b-96$ & $\mathbf{Y}$ & 48 \\
\hline $\mathbf{Y}$ & & 2793 & TAMARISK & Temariak Member & KPT & $\begin{array}{l}\text { Flag for Permeability Determined } \\
\text { Threshold }\end{array}$ & 34558 & 20-fab-96 & $\mathbf{Y}$ & $4 B$ \\
\hline $\mathbf{Y}$ & & 2793 & TAMARISK & Tamansk Member & KPT & $\begin{array}{l}\text { Flag for Permeability Dekermmed } \\
\text { Threstold }\end{array}$ & 34558 & 20-feb-96 & $\mathbf{Y}$ & 48 \\
\hline $\mathbf{Y}$ & & 2793 & TAMARISK & Tamarisk Momber & KPT & $\begin{array}{l}\text { Flag for Permeabilty Determined } \\
\text { Threshold }\end{array}$ & 34558 & $20-50 b-96$ & $\mathbf{Y}$ & $4 B$ \\
\hline $\mathbf{Y}$ & & 2794 & TAMARISK & Ternarisk Member & PCT_A & $\begin{array}{l}\text { Throstiold Pressure Lnes } \\
\text { Peremeter }\end{array}$ & 34560 & 14Nov-95 & $\mathbf{Y}$ & $4 B$ \\
\hline $\mathbf{Y}$ & & 2794 & TAMARISK & Temarisk Member & PCT_A & $\begin{array}{l}\text { Threshold Prescure Lniax } \\
\text { Paramoter }\end{array}$ & 34560 & 14-Nov-95 & $\mathbf{Y}$ & $4 B$ \\
\hline $\mathbf{Y}$ & & 2795 & TAMARISK & Temarisk Member & PCT_EXP & $\begin{array}{l}\text { Throshold pressure exponentral } \\
\text { parametor }\end{array}$ & 34562 & 14NON-95 & $\mathbf{Y}$ & 48 \\
\hline $\mathbf{Y}$ & & 2795 & TAMARISK & Tenerisk Member & PCT_EXP & $\begin{array}{l}\text { Threstrold pressure exponentual } \\
\text { parameter }\end{array}$ & 34562 & 14Hov-95 & $\mathbf{Y}$ & $4 B$ \\
\hline $\mathbf{Y}$ & & 2796 & TAMARISK & Temarisk Momber & PO_MIN & $\begin{array}{l}\text { Minimum brne pressure for } \\
\text { capillary model KPC=3 }\end{array}$ & 34571 & $21+F a b-96$ & $\mathbf{Y}$ & 48 \\
\hline $\mathbf{Y}$ & & 2796 & TAMARISK & Temansk Member & PO_MIN & $\begin{array}{l}\text { Minimum brne prossure for } \\
\text { capillary model KPC=3 }\end{array}$ & 34571 & 21 Feb-96 & $\mathbf{Y}$ & $4 \mathrm{~B}$ \\
\hline $\mathbf{Y}$ & & 2796 & TAMARISK & Tamarsk Member & PO_MIN & $\begin{array}{l}\text { Minmum brne pressure for } \\
\text { capilery model } \mathrm{KPC}=3\end{array}$ & 34571 & 21-Fob-96 & $\mathbf{Y}$ & $\mathbf{A B}$ \\
\hline $\mathbf{Y}$ & & 2799 & UNNAMED & $\begin{array}{l}\text { Unnamed Lower } \\
\text { Member of Rustior } \\
\text { Formation }\end{array}$ & KPT & $\begin{array}{l}\text { Flag for Permeability Determined } \\
\text { Threshold. }\end{array}$ & 34687 & 20-Feb-96 & $\mathbf{Y}$ & $4 B$ \\
\hline $\mathbf{Y}$ & & 2799 & UNNAMED & $\begin{array}{l}\text { Uminined Lower } \\
\text { Member of Rustler } \\
\text { Formation }\end{array}$ & KPT & $\begin{array}{l}\text { Flag for Permeability Determined } \\
\text { Threshold }\end{array}$ & 34687 & 20-Fob-96 & $\mathbf{Y}$ & $4 B$ \\
\hline $\mathbf{Y}$ & & 2799 & UNNAMED & $\begin{array}{l}\text { Ummamed Lower } \\
\text { Member of Rustlor } \\
\text { Formation }\end{array}$ & IFT & $\begin{array}{l}\text { Flag for Permesbility Determined } \\
\text { Threshold }\end{array}$ & 34687 & 20-Fob-96 & $Y$ & $4 B$ \\
\hline $\mathbf{Y}$ & & 2799 & UNNAMED & $\begin{array}{l}\text { Unnamed Lower } \\
\text { Member of Rustier } \\
\text { Formation }\end{array}$ & IRT & $\begin{array}{l}\text { Flag for Pormedbilty Determined } \\
\text { Threshold }\end{array}$ & 34687 & 20-feb-96 & $\mathbf{Y}$ & 48 \\
\hline $\mathbf{Y}$ & & 2800 & UNMAMED & $\begin{array}{l}\text { Umamed Lower } \\
\text { Member of Rustier } \\
\text { Formation }\end{array}$ & PCT_A & $\begin{array}{l}\text { Thrashold Pressure Linear } \\
\text { Peramotor }\end{array}$ & 34688 & 14-Nov-95 & $\mathbf{Y}$ & 48 \\
\hline $\mathbf{Y}$ & & 2800 & UNNAMED & $\begin{array}{l}\text { Umined Lower } \\
\text { Member of Rustler } \\
\text { Formation }\end{array}$ & PCT_A & $\begin{array}{l}\text { Threshold Pressura Linear } \\
\text { Perameter }\end{array}$ & 34688 & 14-Nov-95 & $\mathbf{Y}$ & 48 \\
\hline $\mathbf{Y}$ & & 2801 & UNNAMED & $\begin{array}{l}\text { Urmamed Lower } \\
\text { Hember of Rustler } \\
\text { Formation }\end{array}$ & PCT_EXP & $\begin{array}{l}\text { Threshold pressure exponential } \\
\text { parameter }\end{array}$ & 34689 & 14-Nov-95 & $\mathbf{Y}$ & $4 B$ \\
\hline $\mathbf{Y}$ & & 2801 & UNNAMED & $\begin{array}{l}\text { Unmemed Lower } \\
\text { Member of Rustler } \\
\text { Formation }\end{array}$ & PCT_EXP & $\begin{array}{l}\text { Threshold pressure exporiential } \\
\text { permenter }\end{array}$ & 34689 & 14Nov-95 & $Y$ & 48 \\
\hline$Y$ & & 2802 & UNNAMED & $\begin{array}{l}\text { Unmemed Lower } \\
\text { Member of Rustiox } \\
\text { Formation }\end{array}$ & PO_MIN & $\begin{array}{l}\text { Minimum brne preseure for } \\
\text { capiliey modol KPC=3 }\end{array}$ & 34693 & $21-f a b-96$ & $\mathbf{Y}$ & 48 \\
\hline $\mathbf{Y}$ & & 2802 & UNNAMED & $\begin{array}{l}\text { Unnimed Lawer } \\
\text { Member of Rustler } \\
\text { Formation }\end{array}$ & PO_MIN & $\begin{array}{l}\text { Mnmum brine pressure for } \\
\text { capillary model KPC=3 }\end{array}$ & 34693 & 21 Feb-96 & $\mathbf{Y}$ & 48 \\
\hline $\mathbf{Y}$ & & 2802 & UNNAMED & $\begin{array}{l}\text { Urnimed Lower } \\
\text { Member of Rustler } \\
\text { Formation }\end{array}$ & PO_MIN & $\begin{array}{l}\text { Minimum brine preseure for } \\
\text { cepillery model KePCx3 }\end{array}$ & 34693 & $21+50 b-96$ & $\mathbf{Y}$ & 48 \\
\hline $\mathbf{Y}$ & & 2804 & WAS_AREA & $\begin{array}{l}\text { Waste emplincernent } \\
\text { area and wasto }\end{array}$ & KPT & $\begin{array}{l}\text { Fleg for Permeabilty Determined } \\
\text { Threahold }\end{array}$ & 34942 & 20-F-b.96 & $\mathbf{Y}$ & $A B$ \\
\hline $\mathbf{Y}$ & & 2804 & WAS_AREA & $\begin{array}{l}\text { Wade emplacement } \\
\text { area and wasto }\end{array}$ & KPT & $\begin{array}{l}\text { Fteg for Permeability Detemned } \\
\text { Threahoid }\end{array}$ & 34942 & $20-f c b-96$ & $\mathbf{Y}$ & 48 \\
\hline
\end{tabular}


CCÁ.Parameter-Listing

\begin{tabular}{|c|c|c|c|c|c|c|c|c|c|c|}
\hline 10 & 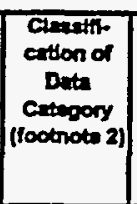 & $\begin{array}{l}\text { Detriburbon } \\
\text { Type }\end{array}$ & Mean & meden & Minfrimen & medrimen & What & $\begin{array}{l}\text { Reference to } \\
\text {. }\end{array}$ & $\begin{array}{l}\text { What the dint } \\
\text { doweloped } \\
\text { under an } \\
\text { Nol-1 } \\
\text { Progrem? }\end{array}$ & 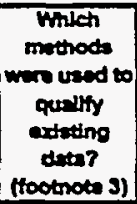 \\
\hline 2788 & 3,5 & CONSTANT & $0.0000000 E+\infty 0$ & $0.0000000 E+\infty$ & $0.00000000+\infty$ & $0.0000000 E+\infty$ & NONE & WPO40514 & $Y$ & NA \\
\hline 2789 & 1,5 & CONSTANT & $2.6000000 E-01$ & $26000000 E-01$ & $26000000 E-01$ & $26000000 E-01$ & $P=$ & WP030543 & $\boldsymbol{Y}$ & NA \\
\hline 2789 & 1.5 & CONSTANT & $2.6000000 E-01$ & $2.6000000 E-01$ & $26000000 E-01$ & $26000000 E-01$ & Pa & WP035597 & $\mathbf{Y}$ & NA \\
\hline 2790 & 1.5 & CONSTANT & $3.4800000 \mathrm{E}-01$ & $-3.4800000 E-01$ & $3.4800000=-01$ & $3.4800000 E-01$ & NONE & WP030643 & $\mathbf{Y}$ & NA \\
\hline 2790 & 1.5 & CONSTANT & 3.4800000 E-01 & $-3.4800000 E-01$ & $-3.4800000 E-01$ & $3.4800000 E-01$ & NONE & WP035597 & $\mathbf{Y}$ & NA \\
\hline 2793 & 1,3 & CONSTANT & $0.0000000 E+00$ & $0.0000000 E+00$ & $0.0000000 E+\infty$ & $0.0000000 E+00$ & NONE & WP035271 & $\mathbf{Y}$ & NA \\
\hline 2793 & 1,3 & CONSTANT & $0.0000000 E+\infty$ & $0.0000000 E+\infty$ & $0.0000000 E+\infty$ & $0.0000000 E+\infty$ & NONE & WP035333 & $\mathbf{Y}$ & NA \\
\hline 2793 & 1,3 & CONSTANT & $0.0000000 E+\infty$ & $0.0000000 E+00$ & $0.0000000 E+\infty$ & $0.0000000=+\infty$ & NONE & WP035597 & $Y$ & NA \\
\hline 2793 & 1,3 & CONSTANT & $0.0000000 E+00$ & $0.0000000 E+00$ & $0.0000000 E+\infty 0$ & $0.0000000 E+\infty 0$ & NONE & WP040514 & $\mathbf{Y}$ & NA \\
\hline 2794 & 1,3 & CONSTANT & $0.0000000 E+\infty$ & $0.0000000 E+\infty 0$ & $0.0000000 E+\infty$ & $0.0000000 E+\infty 0$ & Pa & WPO38568 & $\mathbf{Y}$ & NA \\
\hline 2794 & 1,3 & CONSTANT & $0.00000000 E+00$ & $0.0000000 E+\infty 0$ & $0.0000000 \mathrm{E}+\infty$ & $0.0000000 E+00$ & $\mathrm{~Pa}$ & WP040514 & $\mathbf{Y}$ & NA \\
\hline 2795 & 1,3 & CONSTANT & $0.0000000 \mathrm{E}+\infty 0$ & $0.0000000 E+\infty 0$ & $0.00000000+\infty$ & $0.0000000 E+\infty$ & NONE & WPO38568 & $Y$ & NA \\
\hline 2795 & 1.3 & CONSTANT & $0.0000000 \mathrm{E}+\infty 0$ & $0.0000000 E+\infty$ & $0.0000000 E+\infty$ & $0.00000000+\infty$ & NONE & WPO40514 & $Y$ & NA \\
\hline 2796 & 1,3 & CONSTANT & $1.0132500 E+05$ & $1.0132500 E+05$ & $1.0132500 E+05$ & $1.0132500 E+05$ & $\mathbf{P a}$ & WP035597 & $\mathbf{Y}$ & NA \\
\hline 2796 & 1,3 & CONSTANT & $1.0132500 E+05$ & $1.0132500 E+05$ & $1.0132500 E+05$ & $1.0132500 E+0.5$ & $\mathbf{P a}$ & WP036051 & $\mathbf{Y}$ & N/A \\
\hline 2796 & 1.3 & CONSTANT & $1.0132500 \mathrm{E}+05$ & $1.0132500 E+05$ & $1.0132500 E+05$ & $1.0132500 E+0.5$ & $\mathrm{~Pa}$ & WPO40514 & $\mathbf{Y}$ & NA \\
\hline 2799 & 1.3 & CONSTANT & $0.00000000 \mathrm{E}+\infty 0$ & $0.0000000 E+00$ & $0.00000000+\infty$ & $0.0000000 E+\infty$ & NONE & WP035271 & $\mathbf{Y}$ & NA \\
\hline 2799 & 1,3 & CONSTANT & $0.0000000 E+\infty 0$ & $0.0000000 E+\infty 0$ & $0.00000000+\infty$ & $0.0000000 E+00$ & NONE & WP035333 & $Y$ & NA \\
\hline 2799 & 1,3 & CONSTANT & $0.0000000 E+\infty 0$ & $0.0000000 E+\infty$ & $0.00000000 E+\infty$ & $0.0000000 E+\infty$ & NONE & WP035597 & $\mathbf{Y}$ & NA \\
\hline 2799 & 1,3 & CONSTANT & $0.0000000 E+00$ & $0.0000000 E+\infty 0$ & $0.0000000 E+\infty 0$ & $0.00000000+\infty$ & NONE & WP040514 & $\mathbf{Y}$ & N/A \\
\hline 2800 & 1,3 & CONSTANT & $0.0000000 E+00$ & $0.0000000 E+\infty$ & $0.0000000 E+\infty 0$ & $0.00000000+\infty 0$ & $\mathrm{~Pa}$ & UP038568 & $\mathbf{Y}$ & N/A \\
\hline 2800 & 1.3 & CONSTANT & $0.00000000+00$ & $0.0000000=+\infty$ & $0.0000000 E+\infty$ & $000000000+\infty$ & $\mathbf{P a}$ & WP040514 & $\mathbf{Y}$ & N/A \\
\hline 2801 & 1,3 & CONSTANT & $0.0000000 E+\infty$ & $0.0000000 E+\infty 0$ & $0.0000000 E+\infty$ & $0.0000000 E+\infty$ & NONE & WPO38568 & $\mathbf{Y}$ & NA \\
\hline 2801 & 1,3 & CONSTANT & $0.0000000 E+00$ & $0.0000000 E+\infty 0$ & $0.0000000 E+\infty 0$ & $0.0000000 E+\infty$ & NONE & WP040514 & $\mathbf{Y}$ & NA \\
\hline 2802 & 1,3 & CONSTANT & $1.0132500 E+05$ & $1.0132500 E+05$ & $1.0132500 E+05$ & $1.0132500 E+05$ & Pa & WP035597 & $\mathbf{Y}$ & N/A \\
\hline 2802 & 1,3 & CONSTANT & $1.0132500 E+05$ & $1.0132500 E+05$ & $1.0132500 E+05$ & $1.0132500 E+05$ & P. & WP035051 & $Y$ & NA \\
\hline 2802 & 1,3 & CONSTANT & $1.0132500 E+05$ & $1.0132500 E+05$ & $1.0132500 E+05$ & $1.0132500 E+05$ & Pa & WP040514 & $\mathbf{Y}$ & NA \\
\hline 2804 & 1,3 & CONSTANT & $0.00000000+\infty$ & $0.0000000 E+\infty$ & $0.00000000 E+\infty 0$ & $0.00000000 E+\infty 0$ & NONE & WP035279 & $\mathbf{Y}$ & N/A \\
\hline 2804 & 1,3 & CONSTANT & $0.00000000+\infty$ & $0.0000000 E+\infty 0$ & $0.00000000 E+\infty$ & $0.0000000 E+00$ & NONE & WP035333 & $Y$ & NA \\
\hline
\end{tabular}


CEA.Parameter-Listing

\begin{tabular}{|c|c|c|c|c|c|c|c|c|c|c|}
\hline $\begin{array}{l}\text { Permetarln } \\
\text { Detabese? } \\
\text { (YnN) }\end{array}$ & 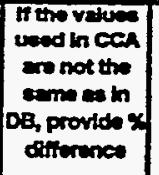 & D & Meterid to & Mrterlal Rtems & Permetar & Permeter Kams & $\begin{array}{l}\text { PRP DD } \\
\text { MPO कn }\end{array}$ & $\begin{array}{c}\text { Deda Enty } \\
\text { Deto }\end{array}$ & 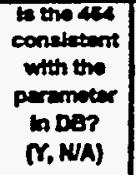 & $\begin{array}{l}\text { Parametir } \\
\text { Cetegory } \\
\text { flootnote } 1)\end{array}$ \\
\hline $\mathbf{Y}$ & & 2804 & WAS_AREA & $\begin{array}{l}\text { Wasto emplecement } \\
\text { erea end wasto }\end{array}$ & KPT & $\begin{array}{l}\text { Flag for Permesbilty Determined } \\
\text { Threshold }\end{array}$ & 34942 & $20-7 a b-96$ & $\mathbf{Y}$ & 48 \\
\hline $\mathbf{Y}$ & & 2804 & WAS_AREA & $\begin{array}{l}\text { Weste emplacement } \\
\text { erea end wasto }\end{array}$ & KPT & $\begin{array}{l}\text { Ftog for Pormesbilty Determmed } \\
\text { Throshold }\end{array}$ & 34942 & $20-F=b-96$ & $\mathbf{Y}$ & 48 \\
\hline $\mathbf{Y}$ & & 2805 & WAS_AREA & $\begin{array}{l}\text { Waste emplacement } \\
\text { area and weste }\end{array}$ & PCT_A & $\begin{array}{l}\text { Threstiold Prossure Lnear } \\
\text { Pyrameter }\end{array}$ & 34983 & $a 8-a b-96$ & $\mathbf{Y}$ & 48 \\
\hline $\mathbf{Y}$ & & 2005 & WAS_AREA & $\begin{array}{l}\text { Waste emplacement } \\
\text { ares end wasto }\end{array}$ & PCT_A & $\begin{array}{l}\text { Threstiold Pressuro Lnear } \\
\text { Peraneter }\end{array}$ & 34983 & obfrob-96 & $\mathbf{Y}$ & 48 \\
\hline$Y$ & & 2805 & WAS_AREA & $\begin{array}{l}\text { Weste emplacement } \\
\text { area and wasto }\end{array}$ & PCT_A & $\begin{array}{l}\text { Threshold Preseure Lnear } \\
\text { Perenoter }\end{array}$ & 34983 & $08-f a b-96$ & $\mathbf{Y}$ & 48 \\
\hline $\mathbf{Y}$ & & 2805 & WAS_AREA & $\begin{array}{l}\text { Waste emplacement } \\
\text { area and waste }\end{array}$ & PCT_A & $\begin{array}{l}\text { Thrashold Proserro Lnaer } \\
\text { Peremotor }\end{array}$ & 34983 & $a b+a b-96$ & $\mathbf{Y}$ & 48 \\
\hline $\mathbf{Y}$ & & 2806 & WAS_AREA & $\begin{array}{l}\text { Waste emplacement } \\
\text { area and wasto }\end{array}$ & PCT_EXP & $\begin{array}{l}\text { Thrashold pressure exporential } \\
\text { permeter }\end{array}$ & 34984 & $06-f a b-96$ & $\mathbf{Y}$ & 48 \\
\hline $\mathbf{Y}$ & & 2806 & WAS_AREA & $\begin{array}{l}\text { Wasto emplacenent } \\
\text { area and waste }\end{array}$ & PCT_EXP & $\begin{array}{l}\text { Threshold pressure exponential } \\
\text { paremeter }\end{array}$ & 34984 & DQfFb-96 & $\mathbf{Y}$ & $4 B$ \\
\hline $\mathbf{Y}$ & - & 2806 & WAS_AREA & $\begin{array}{l}\text { Weste emplacement } \\
\text { ares and wasto }\end{array}$ & PCT_EXP & $\begin{array}{l}\text { Threshold pressure exponential } \\
\text { parameter }\end{array}$ & 34984 & $08+f a-96$ & $\mathbf{Y}$ & $\mathbf{A B}$ \\
\hline $\mathbf{Y}$ & & 2808 & CONC_T2 & $\begin{array}{l}\text { Concrete column: } 400 \\
\text { to } 10 \mathrm{~K} \text { years }\end{array}$ & PO_MIN & $\begin{array}{l}\text { Minimem brine pressure for } \\
\text { capillery modil KPC=3 }\end{array}$ & 32660 & $21+a b-96$ & $\mathbf{Y}$ & 48 \\
\hline $\mathbf{Y}$ & & 2808 & CONC_T2 & $\begin{array}{l}\text { Concrete column: } 400 \\
\text { to } 10 \mathrm{~K} \text { years }\end{array}$ & PO_MIN & $\begin{array}{l}\text { Minimum brine pressure for } \\
\text { capillery model KPC=3 }\end{array}$ & 32660 & 21 Fob-96 & $\mathbf{Y}$ & 48 \\
\hline $\mathbf{Y}$ & & 2808 & CONC_T2 & $\begin{array}{l}\text { Concrete column:400 } \\
\text { to } 10 \mathrm{~K} \text { years }\end{array}$ & PO_MIN & $\begin{array}{l}\text { Mrimum brine pressure for } \\
\text { capilery model KPC=3 }\end{array}$ & 32660 & $21 F=6-96$ & $\mathbf{Y}$ & $4 B$ \\
\hline $\mathbf{Y}$ & varies & 2809 & SALT_TS & $\begin{array}{l}\text { Shan salt column } \\
\text { compectedtime } 100 \text { to } \\
200 \text { years }\end{array}$ & PORE_DIS & $\begin{array}{l}\text { Brooks-Corey pore distribution } \\
\text { parameter }\end{array}$ & 33588 & $26+f a b-96$ & $\mathbf{Y}$ & 1 \\
\hline $\mathbf{Y}$ & varies & 2809 & SALT_T5 & $\begin{array}{l}\text { Shat sak colimn } \\
\text { compactedtine } 100 \text { to } \\
200 \text { years }\end{array}$ & PORE_DIS & $\begin{array}{l}\text { Brooks-Coroy pore distribution } \\
\text { peremeter }\end{array}$ & 33588 & $26-F a b-96$ & $\mathbf{Y}$ & 1 \\
\hline$Y$ & varies & 2809 & SALT_T5 & $\begin{array}{l}\text { Shat salt colinn } \\
\text { compacted time } 100 \text { to } \\
200 \text { years }\end{array}$ & PORE_DIS & $\begin{array}{l}\text { Brooks-Corey pore ditribution } \\
\text { paremeter }\end{array}$ & 33588 & 26-Fob-96 & $\mathbf{Y}$ & 1 \\
\hline $\mathbf{Y}$ & varies & 2809 & SALT_TS & $\begin{array}{l}\text { Shat salt column } \\
\text { compsctedtime } 100 \text { to } \\
200 \text { years }\end{array}$ & PORE_DIS & $\begin{array}{l}\text { Brooks-Corey pore distribution } \\
\text { purameter }\end{array}$ & 33588 & $26-F a b-96$ & $\mathbf{Y}$ & 1 \\
\hline $\mathbf{Y}$ & vanes & 2809 & SALT_TS & $\begin{array}{l}\text { Shaft eal column } \\
\text { compacted:time } 100 \text { to } \\
200 \text { years }\end{array}$ & PORE_DIS & $\begin{array}{l}\text { Brooks-Corey pore distribution } \\
\text { parameter }\end{array}$ & 33588 & $26-F=b-96$ & $\mathbf{Y}$ & 1 \\
\hline$Y$ & varies & 2809 & SALT_TS & \begin{tabular}{|l|} 
Shan salt column \\
compacted:time 100 to \\
200 years
\end{tabular} & PORE_DIS & $\begin{array}{l}\text { Brocks-Corey poro distribution } \\
\text { pareneter }\end{array}$ & 33588 & 26 Fob-96 & $\mathbf{Y}$ & 1 \\
\hline $\mathbf{Y}$ & & 2810 & S_MB13B & $\begin{array}{l}\text { Salado marker bed } \\
138 \text {, intact and } \\
\text { fractured }\end{array}$ & IFRX & $\begin{array}{l}\text { Index for frecture perm. } \\
\text { enhancernent in X-direction }\end{array}$ & 34465 & $30-\operatorname{dan}-96$ & $\mathbf{Y}$ & $4 B$ \\
\hline $\mathbf{Y}$ & & 2810 & S_MB138 & $\begin{array}{l}\text { Salado marker bed } \\
\text { 138, intact and } \\
\text { frectured }\end{array}$ & IFRX & $\begin{array}{l}\text { Index for fracture perm. } \\
\text { eninencement in X-drection }\end{array}$ & 34465 & 30 Jen-96 & $Y$ & 48 \\
\hline $\mathbf{Y}$ & & 2810 & S_MB138 & $\begin{array}{l}\text { Salado maker bed } \\
\text { 138, intect end } \\
\text { trectured }\end{array}$ & IFRX & $\begin{array}{l}\text { Indax for trecture perm. } \\
\text { enhancoment in X-drection }\end{array}$ & 34465 & $30 \sqrt{a n-96}$ & $\mathbf{Y}$ & 48 \\
\hline $\mathbf{Y}$ & & 2811 & S_MB139 & $\begin{array}{l}\text { Saledo manker bed } \\
139 \text {, intect and } \\
\text { frectured }\end{array}$ & JFRX & $\begin{array}{l}\text { Index for frecture perm. } \\
\text { enhancernent in X-drection }\end{array}$ & 34818 & $30-\sqrt{a n}-96$ & $\mathbf{Y}$ & 48 \\
\hline $\mathbf{Y}$ & & 2811 & S_MB139 & $\begin{array}{l}\text { Salado maker bed } \\
139 \text {, intect and } \\
\text { frectured }\end{array}$ & IFRX & $\begin{array}{l}\text { Index for frecture perm. } \\
\text { enhercernent in X-direction }\end{array}$ & 34818 & 30 Jen-96 & $\mathbf{Y}$ & $A B$ \\
\hline $\mathbf{Y}$ & & 2811 & S_MB139 & $\begin{array}{l}\text { Saledo maker bed } \\
139 \text {, intect and } \\
\text { frectured }\end{array}$ & IFRX & $\begin{array}{l}\text { Index for frecturs perm. } \\
\text { enmencement in } X \text {-direction }\end{array}$ & 34818 & 30 Jen-56 & $\mathbf{Y}$ & $A B$ \\
\hline $\mathbf{Y}$ & & 2812 & S_ANH_AB & $\begin{array}{l}\text { Saludo antydito beds } \\
A \text { and } B \text {, intuct and } \\
\text { frectured }\end{array}$ & IFRX & $\begin{array}{l}\text { Index for tracture perm. } \\
\text { enhancement in X-direction }\end{array}$ & 34158 & 30 Jarses & $\mathbf{Y}$ & $4 B$ \\
\hline $\mathbf{Y}$ & & 2812 & S_ANH_AB & $\begin{array}{l}\text { Saledo antrydite beds } \\
\text { A end } B \text {, intect and } \\
\text { frectured }\end{array}$ & IFRX & $\begin{array}{l}\text { Index for trecture perm. } \\
\text { entrencoment in X-drection }\end{array}$ & 34158 & $30-\operatorname{den}-96$ & $\mathbf{Y}$ & AB \\
\hline$Y$ & & 2812 & S_ANH_AB & $\begin{array}{l}\text { Seledo entrydito beds } \\
\text { A and } B \text {, intact and } \\
\text { fractured }\end{array}$ & IFRX & $\begin{array}{l}\text { Index for frecture perm. } \\
\text { enhancenent in } X \text {-direction }\end{array}$ & 34158 & 30 Jen-96 & $\mathbf{Y}$ & $A B$ \\
\hline
\end{tabular}


CCA Parameter Listing-

\begin{tabular}{|c|c|c|c|c|c|c|c|c|c|c|}
\hline 10 & 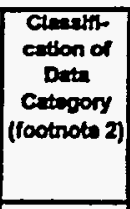 & $\begin{array}{l}\text { Dostribution } \\
\text { Type }\end{array}$ & Man & Heden & Minkmam & Mrodmention & Unts & Reformatio & 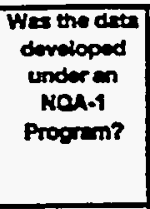 & 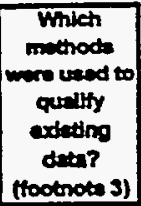 \\
\hline 2804 & 1.3 & CONSTANT & $0.0000000 E+\infty$ & $0.0000000 E+\infty$ & $0.0000000 E+\infty$ & $0.0000000 E+\infty$ & NONE & WP035597 & $Y$ & NA \\
\hline 2804 & 1,3 & CONSTANT & $0.0000000 E+\infty$ & $0.0000000 \mathrm{E}+\infty$ & $0.0000000 E+\infty 0$ & $0.0000000 E+\infty$ & NONE & WP040514 & Y & NA \\
\hline 2805 & 1,3 & CONSTANT & $0.0000000 E+\infty$ & $0.0000000 E+\infty$ & $0.00000000 \mathrm{E}+\infty 0$ & $0.0000000 E+\infty$ & Pa & WP035597 & $\mathbf{Y}$ & NA \\
\hline 2805 & 1,3 & CONSTANT & $0.0000000 \mathrm{E}+\infty$ & $0.0000000 E+\infty$ & $0.0000000 E+\infty$ & $0.0000000 E+\infty$ & Pa & WPO38454 & $\mathbf{Y}$ & NA \\
\hline 2805 & 1,3 & CONSTANT & $0.0000000 E+\infty 0$ & $0.0000000 E+\infty$ & $0.0000000 E+\infty$ & $0.00000000+\infty$ & Pa & WPO40514 & $\mathbf{Y}$ & NA \\
\hline 2805 & 1,3 & CONSTANT & . $0.0000000 \mathrm{E}+\infty$ & $0.0000000 E+\infty$ & $0.0000000 E+\infty$ & $0.0000000 E+\infty$ & Pa & WPO4268: & $Y$ & NAA \\
\hline 2806 & 1,3 & CONSTANT & $0.0000000 E+\infty$ & $0.0000000 E+\infty 0$ & $0.0000000 E+\infty$ & $0.0000000 E+\infty$ & NONE & WP035597 & $\gamma$ & N/A \\
\hline 2806 & 1,3 & CONSTANT & $0.0000000 E+\infty$ & $0.0000000 E+\infty$ & $0.0000000 E+\infty$ & $0.00000000+\infty$ & NONE & WP040514 & $\mathbf{Y}$ & NA \\
\hline 2806 & 1,3 & CONSTANT & $0.0000000 E+\infty 0$ & $0.0000000 E+\infty$ & $0.0000000 E+\infty$ & $0.0000000 E+\infty$ & NONE & WPO42681. & $Y$ & NA \\
\hline 2808 & 1,3 & CONSTANT & $1.0132500 E+05$ & $1.0132500 E+0.5$ & $1.0132500 \mathrm{E}+0.0$ & $1.0132500 \mathrm{E}+05$ & $\mathbf{P a}$ & WP035597" & $Y$ & NA \\
\hline 2808 & 1,3 & CONSTANT & $1.0132500 E+05$ & $1.0132500 E+05$ & $1.0132500 E+05$ & $1.0132500 E+0.5$ & Pa & WP036051 & $\mathbf{Y}$ & NA \\
\hline 2808 & 1,3 & CONSTANT & $1.0132500 E+05$ & $1.0132500 E+05$ & $1.0132500 E+0.5$ & $1.0132500 E+05$ & Pa & WP040514 & $Y$ & NA \\
\hline 2809 & 3,4 & CUMULATIVE & $2.5200000 E+\infty 0$ & 9.4000000E-01 & $1.1000000 E-01$ & $8.1000000 E+\infty$ & NONE & WP030640 & $\mathbf{Y}$ & NA \\
\hline 2809 & 3,4 & CUMULATINE & $25200000 E+\infty 0$ & $94000000 \mathrm{E}-01$ & $1.1000000 E-01$ & $8.1000000 E+00$ & NONE & WP030994 & $Y$ & NA \\
\hline 2809 & 3,4 & CUMULATINE & $2.5200000 \mathrm{E}+\infty 0$ & $94000000 E-01$ & $11000000 E-01$ & $81000000 E+\infty$ & NONE & WP035268 & $\mathbf{Y}$ & N/A \\
\hline 2809 & 3,4 & CUMULATINE & $2.5200000 E+\infty$ & $94000000 \mathrm{E}-01$ & 1 1000000E-01 & $81000000 E+\infty 0$ & NONE & WP035597 & $\mathbf{Y}$ & NA \\
\hline 2809 & 3,4 & CUMULATINE & $2.5200000 \mathrm{E}+\infty 0$ & $94000000 E-01$ & 1 1000000E-01 & $81000000 E+\infty$ & NONE & WPO41558 & $\mathbf{Y}$ & NA \\
\hline 2809 & 3,4 & CUMULATIVE & $2.5200000 \mathrm{E}+\infty$ & $94000000 E-01$ & $1,1000000 \mathrm{E}-01$ & 8 $1000000 \varepsilon+\infty$ & NONE & WPO42752 & $\mathbf{Y}$ & NA \\
\hline 2810 & 1,3 & CONSTANT & $1.00000000+\infty$ & $10000000 E+\infty$ & $10000000 \times+\infty$ & $10000000<+\infty$ & NONE & WP035597 & $\mathbf{Y}$ & N/A \\
\hline 2810 & 1,3 & CONSTANT & $1.0000000 \mathrm{E}+00$ & $10000000 E+\infty$ & $1000000<-\infty$ & $10000000+\infty$ & NONE & WPO3 5205 & •Y & NA \\
\hline 2810 & 1,3 & CONSTANT & $1.0000000 E+\infty$ & $10000000 E+\infty$ & $1000000<-\infty$ & $1 \infty 00000<+\infty$ & NONE & WP040514 & $\mathbf{Y}$ & NA \\
\hline 2811 & 3.5 & CONSTANT & $1.00000000+\infty$ & $10000000=-\infty$ & $10000000<-\infty$ & $1000000<+\infty$ & NONE & WP035597 & $Y$ & NA \\
\hline 2811 & 3,5 & CONSTANT & $1.0000000 E+\infty$ & $10000000 \in-\infty$ & $10000000<-\infty$ & $10000000=-\infty$ & NONE & WP035205 & $Y$ & NA \\
\hline 2811 & 3,5 & CONSTANT & $1.0000000 E+\infty$ & $10000000<+\infty$ & $1000000 \times+\infty$ & $10000000 E+\infty$ & NONE & WPO40514 & $Y$ & NA \\
\hline 2812 & 1,3 & CONSTANT & $1.0000000 E+\infty 0$ & $10000000=\infty$ & $10000000 \times \infty$ & $10000000<+\infty$ & NONE & WP035597 & $\mathbf{Y}$ & N/A \\
\hline 2812 & 1,3 & CONSTANT & $1.0000000 E+\infty$ & $10000000<+\infty 0$ & $10000000<-\infty$ & $1000000 \in+\infty$ & NONE & WP035205 & $\mathbf{Y}$ & NA \\
\hline 2812 & 1,3 & CONSTANT & $1,0000000 E+00$ & $10000000 \in+\infty$ & $10000000=00$ & $10000000+\infty$ & NONE & WPO40514 & $\mathbf{Y}$ & NA \\
\hline
\end{tabular}


CCA Parameter Listing-

\begin{tabular}{|c|c|c|c|c|c|c|c|c|c|c|}
\hline $\begin{array}{c}\text { Percinter in } \\
\text { Deribeas? } \\
\text { (MnI) }\end{array}$ & 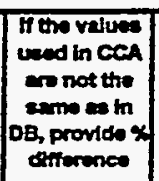 & 10 & Matertal ID & Whateris Narme & $\begin{array}{c}\text { Perameter } \\
\text { to }\end{array}$ & Parametar Kam & $\begin{array}{l}\text { PRPDD } \\
\text { (MPOA }\end{array}$ & $\begin{array}{c}\text { Deta Entry } \\
\text { Date }\end{array}$ & 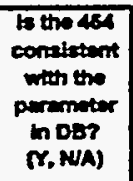 & 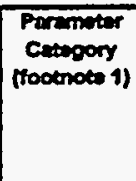 \\
\hline $\mathbf{Y}$ & & 2813 & S_MB138 & $\begin{array}{l}\text { Salado marker bed } \\
138 \text {, intuct and } \\
\text { fractured }\end{array}$ & IFRY & $\begin{array}{l}\text { Index for fracturo perm. } \\
\text { ontencoment in } Y \text {-direction }\end{array}$ & 34465 & 30-لven-96 & $\mathbf{Y}$ & 48 \\
\hline $\mathbf{Y}$ & & 2813 & S_MB138 & $\begin{array}{l}\text { Saloso merker bed } \\
138 \text {, intect and } \\
\text { frectured }\end{array}$ & |FRY & $\begin{array}{l}\text { Index for frecture perm. } \\
\text { enthencement in Y-direction }\end{array}$ & 3466 & $30 \operatorname{sen}-96$ & $\mathbf{Y}$ & 48 \\
\hline $\mathbf{Y}$ & & 2813 & S_MB138 & $\begin{array}{l}\text { Salsdo manker bod } \\
\text { 138, intect and } \\
\text { frectured }\end{array}$ & IFRY & 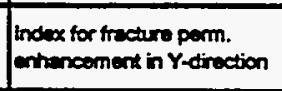 & 3406 & 30-van-96 & $\mathbf{Y}$ & 48 \\
\hline $\mathbf{Y}$ & & 2814 & S_MB139 & $\begin{array}{l}\text { Salado merker bed } \\
139 \text {, inted and } \\
\text { fractured }\end{array}$ & IFRY & $\begin{array}{l}\text { trodex for frecture perm. } \\
\text { onthencement in Y-direction }\end{array}$ & 34819 & 30 den-96 & $\mathbf{Y}$ & 48 \\
\hline $\mathbf{Y}$ & & 2814 & S_MB139 & $\begin{array}{l}\text { Seledob menker bed } \\
\text { 139, intad and } \\
\text { frectured }\end{array}$ & IFRY & $\begin{array}{l}\text { Index for fracture perm. } \\
\text { onthencoment in Y-direction }\end{array}$ & 34819 & $30 \sqrt{a n-96}$ & $\gamma$ & 48 \\
\hline $\mathbf{Y}$ & & 2814 & S_MB139 & $\begin{array}{l}\text { Saludo marker bod } \\
139, \text { intect and } \\
\text { fractured }\end{array}$ & IFRY & $\begin{array}{l}\text { Index for fracture perm. } \\
\text { ontancom ent in Y-orection }\end{array}$ & 34819 & 30-Jen-96 & $\mathbf{Y}$ & 48 \\
\hline $\mathbf{Y}$ & & 2815 & S_ANH_AB & $\begin{array}{l}\text { Salado antydrite beds } \\
\text { A and B, intact and } \\
\text { fractured }\end{array}$ & IFRY & $\begin{array}{l}\text { Index for frecture perm. } \\
\text { ontrancesnert in Y-iroction }\end{array}$ & 34159 & 30-Jen-96 & $\mathbf{Y}$ & $4 B$ \\
\hline $\mathbf{Y}$ & & 2815 & S_ANH_AB & $\begin{array}{l}\text { Salaco antydrte bods } \\
\text { A and B, intact and } \\
\text { tractured }\end{array}$ & IFRY & $\begin{array}{l}\text { Index for fractura perm. } \\
\text { onnancement in Yedrection }\end{array}$ & 34159 & 30-Jan-96 & $Y$ & 48 \\
\hline $\mathbf{Y}$ & & 2815 & S_ANH_AB & $\begin{array}{l}\text { Salado anhychto beds } \\
\text { A and B, intact and } \\
\text { fractured }\end{array}$ & IFRY & $\begin{array}{l}\text { Index for fracture perm. } \\
\text { enthancement in } Y \text {-direction }\end{array}$ & 34159 & $30-\operatorname{dan}-96$ & $\mathbf{Y}$ & 48 \\
\hline$Y$ & & 2816 & S_MB138 & $\begin{array}{l}\text { Salado marker bed } \\
\text { 138, intact und } \\
\text { fractured }\end{array}$ & IFRZ & $\begin{array}{l}\text { Index for fracture perm. } \\
\text { onhencoment in Z-direction }\end{array}$ & 3471 & 31 den-96 & $\mathbf{Y}$ & 18 \\
\hline $\mathbf{Y}$ & & 2816 & S_MB138 & $\begin{array}{l}\text { Salado manker bed } \\
138 \text {, intact and } \\
\text { fractured }\end{array}$ & IFRZ & $\begin{array}{l}\text { Indeax for frecture perm. } \\
\text { onthencement in Z-direction }\end{array}$ & 34471 & 31-Jan-96 & $\mathbf{Y}$ & AB \\
\hline $\mathbf{Y}$ & & 2816 & S_MB138 & $\begin{array}{l}\text { Salado marker bed } \\
\text { 138, intact and } \\
\text { frectured }\end{array}$ & IFRZ & $\begin{array}{l}\text { Index for fracture perm. } \\
\text { orthencoment in Z-drection }\end{array}$ & 3471 & $31 \sqrt{\operatorname{lan}-96}$ & $\mathbf{Y}$ & 48 \\
\hline $\mathbf{Y}$ & & 2817 & S_MB139 & $\begin{array}{l}\text { Salado marker bed } \\
139 \text {, intact and } \\
\text { fractured }\end{array}$ & IFRZ & $\begin{array}{l}\text { Index for frecture perm. } \\
\text { orhencentent in Z-drection }\end{array}$ & 34820 & 31-Jan-96 & $Y$ & 48 \\
\hline $\mathbf{Y}$ & & 2817 & S_MB139 & $\begin{array}{l}\text { Selado maker bed } \\
139 \text {, intuct and } \\
\text { fractured }\end{array}$ & IFRZ & $\begin{array}{l}\text { Index for fracture perm. } \\
\text { ornencoment } n z \text {-orroction }\end{array}$ & 34820 & 31 Jan-96 & $\mathbf{Y}$ & $4 B$ \\
\hline $\mathbf{Y}$ & & 2817 & S_MB139 & $\begin{array}{l}\text { Salado marker bod } \\
\text { 139, intact and } \\
\text { fractured }\end{array}$ & IFRZ & $\begin{array}{l}\text { Index for frecture perm. } \\
\text { montencoment in Z-droction }\end{array}$ & 348020 & 31-Jen-96 & $\mathbf{Y}$ & $4 B$ \\
\hline $\mathbf{Y}$ & & 2818 & S_ANH_AB & $\begin{array}{l}\text { Salado anhydinte beds } \\
\text { A and B, inted and } \\
\text { fractured }\end{array}$ & IFRZ & $\begin{array}{l}\text { index for trecturn perm. } \\
\text { ornencoment in Z-droction }\end{array}$ & 34160 & 31-Jen-95 & $Y$ & $A B$ \\
\hline $\mathbf{Y}$ & & 2818 & S_ANH_AB & $\begin{array}{l}\text { Saledo entrydinte beds } \\
\text { A and B, intact and } \\
\text { fractured }\end{array}$ & IFRZ & $\begin{array}{l}\text { Indeax for tracture perm. } \\
\text { orriencament in Z-drection }\end{array}$ & 34160 & 31Jen-9s & $\bar{Y}$ & AB \\
\hline$Y$ & & 2818 & S_ANH_AB & 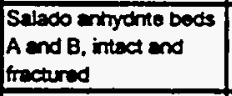 & IFRZ & $\begin{array}{l}\text { Index tor fracture perm. } \\
\text { imsencoment in Z-drection }\end{array}$ & 34160 & 31 Jan-96 & $\mathbf{Y}$ & AB \\
\hline $\mathbf{Y}$ & & 2819 & S_ANH_AB & 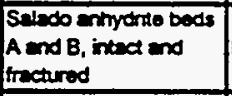 & BKINK & $\begin{array}{l}\text { Oankenberg B Corruction } \\
\text { Perumeters for HR gas }\end{array}$ & 34129A & 23-Apr-96 & $\mathbf{Y}$ & $4 A$ \\
\hline$Y$ & & 2819 & S_ANH_AB & 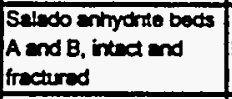 & BKLINK & $\begin{array}{l}\text { Kankenberg B Corruction } \\
\text { Purameters for H12 ges }\end{array}$ & 341294 & 23 -Apr-96 & $\mathbf{Y}$ & $4 A$ \\
\hline$Y$ & & 2819 & S_ANH_AB & $\begin{array}{l}\text { Selecos entryorte beds } \\
\text { A and B, intect and } \\
\text { frectured }\end{array}$ & BKLINK & $\begin{array}{l}\text { Karkentours B Correction } \\
\text { Peremotion for H2 gas }\end{array}$ & $34129 A$ & 23-Apr-96 & $\mathbf{Y}$ & 4 \\
\hline $\mathbf{Y}$ & & 2819 & S_ANH_AB & $\begin{array}{l}\text { Seliedo minyctinte beds } \\
A \text { and } B \text {, intect and } \\
\text { frectured }\end{array}$ & BKLINK & $\begin{array}{l}\text { Rankonberg B Cortaction } \\
\text { Perameters for } \mathrm{H} 2 \text { gas }\end{array}$ & 3A129A & 23-Apr-96 & $\mathbf{Y}$ & $M$ \\
\hline $\mathbf{Y}$ & & 2820 & S_ANH_AB & $\begin{array}{l}\text { Seleco entryorto beds } \\
\text { A end B, intact and } \\
\text { frectured }\end{array}$ & EXPIQINK & $\begin{array}{l}\text { Kinkenberg b correction } \\
\text { permeters for H12 oas }\end{array}$ & 381392 & 23-Apr-96 & $\mathbf{Y}$ & $\mu$ \\
\hline $\mathbf{Y}$ & & 2820 & S_ANH_AB & $\begin{array}{l}\text { Saleoso entryctite bede } \\
\text { A and B, intect and } \\
\text { frectured }\end{array}$ & EXPKLINK & $\begin{array}{l}\text { Ninkenbere b correction } \\
\text { peremeters for H. } 2 \text { gas }\end{array}$ & 341394 & 23-Apr.96 & $Y$ & 4 \\
\hline
\end{tabular}




\begin{tabular}{|c|c|c|c|c|c|c|c|c|c|c|}
\hline 10 & $\begin{array}{c}\text { Createn- } \\
\text { catton of } \\
\text { Deth } \\
\text { Cextopory } \\
\text { (footrota 2) }\end{array}$ & $\begin{array}{l}\text { Detertoutton } \\
\text { Type }\end{array}$ & $\operatorname{mans}$ & Medism & Mhrirmem & Madinum & Units & Referwea D & $\begin{array}{l}\text { Wes the dintal } \\
\text { imreloped } \\
\text { underen } \\
\text { Nol-1 } \\
\text { Progorem? }\end{array}$ & 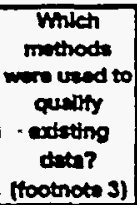 \\
\hline 2813 & 1,3 & CONSTANT & $1.0000000 E+\infty 0$ & $1.0000000 E+\infty 0$ & $1.0000000 E+\infty 0$ & $1.0000000 E+\infty$ & NONE & WP035597 & $Y$ & N/A \\
\hline 2813 & 1,3 & CONSTANT & $1.0000000 E+\infty 0$ & $1.0000000 E+\infty$ & $1.0000000 E+\infty 0$ & $1.0000000 E+\infty 0$ & NONE & WP036205 & $\mathbf{Y}$ & NA \\
\hline 2813 & 1,3 & CONSTANT & $1.0000000 E+\infty 0$ & $1.0000000 E+00$ & $1.0000000 E+\infty 0$ & $1.0000000 E+\infty 0$ & NONE & WPO40514 & $\mathbf{Y}$ & NA \\
\hline 2814 & 3,5 & CONSTANT & $1.0000000 E+\infty$ & $1.0000000 E+\infty 0$ & $1.0000000 E+\infty$ & $1.0000000 E+\infty$ & NONE & WPO35597 & $\mathbf{Y}$ & NA \\
\hline 2814 & 3,5 & CONSTANT & $1.0000000 \mathrm{E}+\infty 0$ & $1.0000000 E+\infty 0$ & $1.0000000 E+\infty$ & $1.0000000 E+\infty 0$ & NONE & WP036205 & $\mathbf{Y}$ & NA \\
\hline 2814 & 3,5 & CONSTANT & $1.0000000 E+\infty 0$ & $1.0000000 E+\infty 0$ & $1.0000000 E+\infty 0$ & $1.0000000 E+\infty 0$ & NONE & WPO40514 & $\mathbf{Y}$ & NA \\
\hline 2815 & 1,3 & CONSTANT & $1.0000000 E+00$ & $1.0000000 E+\infty 0$ & $1.0000000 E+\infty 0$ & $1.0000000 E+\infty 0$ & NONE & WP035597 & $\mathbf{r}$ & N/A \\
\hline 2815 & 1,3 & CONSTANT & $1.0000000 E+\infty 0$ & $.0000000 E+\infty 0$ & $1.0000000 E+\infty 0$ & $1.0000000 E+\infty$ & NONE & MP035205 & $\mathbf{Y}$ & NA \\
\hline 2815 & 1,3 & CONSTANT & $1.0000000 E+\infty 0$ & $1.0000000 E+\infty 0$ & $1.0000000 E+\infty 0$ & $1.0000000 E+00$ & NONE & WP040514 & $Y$ & NAA \\
\hline 2816 & 1,3 & CONSTANT & $0.0000000 E+\infty$ & $0.0000000 E+\infty 0$ & $0.0000000 E+\infty$ & $0.00000000 E+\infty$ & NONE & WP035597 & $\mathbf{Y}$ & NA \\
\hline 2816 & 1,3 & CONSTANT & $0.0000000 E+\infty$ & $0.0000000 E+00$ & $0.0000000 E+\infty 0$ & $0.0000000 E+\infty 0$ & NONE & WP035205 & $\mathbf{Y}$ & NA \\
\hline 2816 & 1.3 & CONSTANT & $0.0000000 E+\infty 0$ & $0.0000000 E+\infty 0$ & $0.0000000 E+00$ & $0.0000000 E+\infty$ & NONE & WPO40514 & $\mathbf{Y}$ & N/A \\
\hline 2817 & 3,5 & CONSTANT & $0.00000000+\infty$ & $0.0000000 E+\infty 0$ & $0.0000000 E+\infty 0$ & $0.0000000 E+\infty$ & NONE & WP035597 & $\mathbf{Y}$ & N/A \\
\hline 2817 & 3,5 & CONSTANT & $0.00000000 E+\infty$ & $0.0000000 E+\infty$ & $0.0000000 E+\infty 0$ & $0.0000000 E+\infty 0$ & NONE & WP036205 & $\mathbf{Y}$ & NA \\
\hline 2817 & 3.5 & CONSTANT & $0.00000000 E+00$ & $0.0000000 E+\infty 0$ & $0.0000000 E+\infty$ & $0.00000000 E+\infty$ & NONE & WP040514 & $\mathbf{Y}$ & N/A \\
\hline 2818 & 1,3 & CONSTANT & $0.0000000 E+\infty$ & $0.00000000 E+\infty$ & $0.00000000+\infty$ & $0.0000000 E+\infty$ & NONE & WP035597 & $Y$ & NA \\
\hline 2818 & 1,3 & CONSTANT & $0.0000000 E+\infty 0$ & $0.0000000 E+\infty 0$ & $0.0000000 E+\infty$ & $0.0000000 E+\infty 0$ & NONE & WP036205 & $" Y$ & NA \\
\hline 2818 & 1.3 & CONSTANT & $0.00000000 E+\infty 0$ & $0.00000000 E+\infty$ & $0.00000000+00$ & $0.0000000 E+\infty$ & NONE & WP040514 & $\mathbf{Y}$ & NA \\
\hline 2819 & 1,5 & CONSTANT & $2.7100000 E-01$ & $2.7100000 E-01$ & $2.7100000 E-01$ & $2.7100000 E-01$ & P: & WP030612 & $\mathbf{Y}$ & NA \\
\hline 2819 & 1,5 & CONSTANT & $27100000 E-01$ & $27100000 E-01$ & $27100000 \mathrm{E}-01$ & $2.7100000 E-01$ & P. & WP035597 & $Y$ & NA \\
\hline 2819 & 1,5 & CONSTANT & $2.7100000=-01$ & $27100000 E-01$ & $2.7100000 \mathrm{E}-01$ & $2.7100000 \mathrm{E}-01$ & Pa & WP038450 & $\mathbf{Y}$ & NA \\
\hline 2819 & 1,5 & CONSTANT & $2.7100000 E-01$ & $2.7100000 E-01$ & $2.7100000 E-01$ & $2.7100000 E-01$ & $\mathrm{~Pa}$ & WPO42714 & $\mathbf{Y}$ & NA \\
\hline 2820 & 1,5 & CONSTANT & 3.4100000 E- 01 & $2.4100000 E-01$ & $-3.4100000 E-01$ & $-3.4100000 E-01$ & NONE & WP030642 & $\mathbf{Y}$ & N/A \\
\hline 2820 & 1.5 & CONSTANT & $3.4100000 E-01$ & $-3.4100000 \mathrm{E}-01$ & $-3.4100000 E-01$ & $3.4100000 E-01$ & NONE & WP035597 & $\mathbf{Y}$ & NA \\
\hline
\end{tabular}


CCA Parameter Listing

\begin{tabular}{|c|c|c|c|c|c|c|c|c|c|c|}
\hline $\begin{array}{c}\text { Parroter In } \\
\text { Detibean? } \\
\text { (YMI) }\end{array}$ & 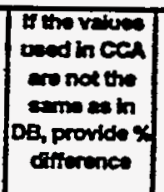 & $\overline{10}$ & Anterta 10 & पhended Nomo & $\begin{array}{c}\text { Perrovitar } \\
\text { D }\end{array}$ & Parmeter Mrm & $\begin{array}{l}\text { PRPTा } \\
\text { (MPON) }\end{array}$ & \begin{tabular}{|c|} 
Dowentry \\
Dast
\end{tabular} & 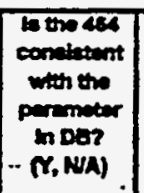 & 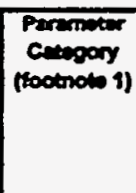 \\
\hline $\mathbf{Y}$ & & 2820 & S_ANH_AB & $\begin{array}{l}\text { Salado anthydrito bods } \\
\text { A and B, intact and } \\
\text { fractured }\end{array}$ & EXPKINK & $\begin{array}{l}\text { Klinkenberg b connction } \\
\text { perameters for H2 gas }\end{array}$ & 341394 & $23 x p r-96$ & $\mathbf{Y}$ & $\mu$ \\
\hline $\mathbf{Y}$ & & 2823 & WAS_AREA & $\begin{array}{l}\text { Wasto onplacoment } \\
\text { reas and wasto }\end{array}$ & PROBDEG & 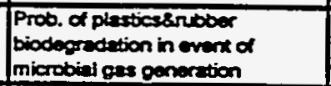 & $34 \$ 81$ & $23+00-96$ & $\mathbf{Y}$ & 48 \\
\hline $\mathbf{Y}$ & & 2823 & WAS_AREA & $\begin{array}{l}\text { Wasto emplacoment } \\
\text { ares and wasto }\end{array}$ & PROBDEG & 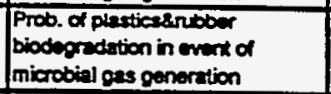 & 34881 & 23-Fcb-96 & $\mathbf{Y}$ & $4 B$ \\
\hline $\mathbf{Y}$ & & 2823 & WAS_AREA & $\begin{array}{l}\text { Wasto emplacement } \\
\text { area end wasto }\end{array}$ & PROBDEG & 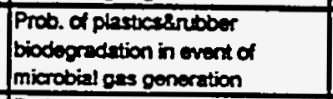 & 34881 & $23+0 b-96$ & $\mathbf{Y}$ & 48 \\
\hline $\mathbf{Y}$ & & 2823 & WAS_AREA & $\begin{array}{l}\text { Wasto emplacoment } \\
\text { area and wasto }\end{array}$ & PROBDEG & 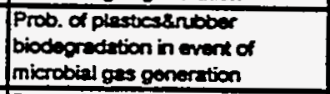 & 34881 & $23-F a b-96$ & $Y$ & 48 \\
\hline $\mathbf{Y}$ & & 2824 & REPOST & $\begin{array}{l}\text { Repostory regions } \\
\text { outsido of Pant region }\end{array}$ & PROBDEG & $\begin{array}{l}\text { Prob. of plastess sintober } \\
\text { biodegradetion in mont of } \\
\text { microbial gas peneration }\end{array}$ & 33264 & $23+c b-96$ & $\mathbf{Y}$ & 4B \\
\hline $\mathbf{Y}$ & & 2824 & REPOST & $\begin{array}{l}\text { Repository regions } \\
\text { outsido of Panel region }\end{array}$ & PROBOEG & $\begin{array}{l}\text { Prob. of plastices subbor } \\
\text { biodegredation in event of } \\
\text { mierobial gas generation }\end{array}$ & 33254 & $23-f e b-96$ & $\mathbf{Y}$ & 48 \\
\hline $\mathbf{Y}$ & & 2824 & REPOST & $\begin{array}{l}\text { Repository regions } \\
\text { outside of Panel region }\end{array}$ & PROBDEG & $\begin{array}{l}\text { Prob. of plasticas subber } \\
\text { biodegradation in mert of } \\
\text { microbiel gas generation }\end{array}$ & 33264 & $23 F=0-96$ & $Y$ & $4 B$ \\
\hline$Y$ & & 2824 & REPOSIT & $\begin{array}{l}\text { Repository repions } \\
\text { octside of Panel region }\end{array}$ & PROBDEG & $\begin{array}{l}\text { Prob. of plastcas anober } \\
\text { biodiegradation in evert of } \\
\text { microbial gas generation }\end{array}$ & 33264 & $23+50 b-96$ & $\mathbf{Y}$ & 48 \\
\hline $\mathbf{Y}$ & & 2825 & REFCON & Reference Constant & TC_H2 & $\begin{array}{l}\text { Critceal tomperatera:Hydrogen } \\
\text { (1H2) }\end{array}$ & 32429 & Osfob-96 & $\mathbf{r}$ & 3 \\
\hline r & & 2825 & REFCON & Reterencos Constant & TC_HR & $\begin{array}{l}\text { Crntical temporaturo:Hydrogen! } \\
\text { (H-2) }\end{array}$ & 32429 & $05+\infty b-96$ & $\mathbf{Y}$ & 3 \\
\hline $\mathbf{Y}$ & & 2826 & REFCON & Reference Constant & TC_CO2 & $\begin{array}{l}\text { Critical temperature:Cutbon } \\
\text { Dioxido (CO2) }\end{array}$ & 32428 & 05-Feb-96 & $\mathbf{Y}$ & 3 \\
\hline $\mathbf{Y}$ & & 2826 & REFCON & Roferences Constant & TC_CO2 & $\begin{array}{l}\text { Crnteal tomperature:Cartion } \\
\text { Dioxide (CO2) }\end{array}$ & 32428 & $a 5-F a b-96$ & $\mathbf{Y}$ & 3 \\
\hline $\mathbf{Y}$ & & 2827 & REFCON & Roferencos Constant & TC_CHA & $\begin{array}{l}\text { Critceal tomperature:Mothane } \\
\text { (CHA) }\end{array}$ & 32427 & 05-Feb-96 & $\boldsymbol{\gamma}$ & 3 \\
\hline $\mathbf{Y}$ & & 2827 & REFCON & Reforence Constent & $\mathrm{TC} \mathrm{CHA}_{2}$ & $\begin{array}{l}\text { Critcal tempercture:Methere } \\
\text { (CH4) }\end{array}$ & 32427 & $05-500-96$ & $\mathbf{Y}$ & 3 \\
\hline$Y$ & & 2828 & REFCON & Reforence Constant & TC_N & $\begin{array}{l}\text { Critical temperature:Nitrogen } \\
\text { (N2) }\end{array}$ & 32431 & $05-F=0-96$ & $\mathbf{Y}$ & 3 \\
\hline Y & & 2828 & REFCON & Roference Constent & TC_NO & $\begin{array}{l}\text { Critical tomperzure:Nitrogen } \\
\text { (N2) }\end{array}$ & 32431 & osfob-96 & $\mathbf{Y}$ & 3 \\
\hline $\mathbf{Y}$ & & 2829 & REFCON & Reference Constant & TC_HES & $\begin{array}{l}\text { Critical temperature: Hydrogen } \\
\text { Sulfice (H2S) }\end{array}$ & 32430 & 05-Fob-96 & $\boldsymbol{\gamma}$ & 3 \\
\hline Y & & 2829 & REFCON & Reforence Constant & TC_HES & $\begin{array}{l}\text { Cretical tomperature:Hydrogen } \\
\text { Sutifo (H2S) }\end{array}$ & 32430 & a5fob-96 & $\mathbf{Y}$ & 3 \\
\hline $\mathbf{Y}$ & & 2830 & REFCON & Reference Constant & TC_O2 & Critces tomperature:Oxpen (02) & 32432 & 05 -Feb- 96 & $\mathbf{Y}$ & 3 \\
\hline Y & & 2830 & REFCON & Reforence Constant & TC_02 & Crucal tomporature:Oxygen (02) & 32432 & $05-F a b-96$ & $Y$ & 3 \\
\hline $\mathbf{Y}$ & & 2831 & REFCON & Reference Constant & $\mathrm{ACF}_{2} \mathrm{HZ}$ & Acontine factors - H2 & 32351 & 12-Fob-96 & $\mathbf{Y}$ & 3 \\
\hline $\mathbf{Y}$ & & 2831 & REFCON & Reforence Constant & ACF_H2 & Acomatic Fectors - 12 & 32351 & 12-fob-96 & $\bar{Y}$ & 3 \\
\hline $\mathbf{Y}$ & & 2832 & REFCON & Reference Constent & ACF_CO2 & Acantric Fectors - $\mathrm{CO} 2$ & 32350 & $12-F e b-96$ & $\mathbf{Y}$ & 3 \\
\hline $\mathbf{Y}$ & & 2832 & REFCON & Reforence Constant & $\mathrm{ACF} \mathrm{CO}_{2}$ & Acentic Fectors - $\mathrm{CO} 2$ & 32350 & $12+F=b-96$ & $Y$ & 3 \\
\hline $\mathbf{Y}$ & & 2833 & REFCON & Reference constant & ACF_CH4 & Acentric Fectors - $\mathrm{CH}$ & 32349 & $12 f a b-96$ & $\mathbf{Y}$ & 3 \\
\hline $\mathbf{Y}$ & & 2833 & REFCON & Ruference Constant & ACF_CH4 & Acentric Factors - $\mathrm{CH} 4$ & 32349 & $12+6 b-96$ & $\mathbf{Y}$ & 3 \\
\hline $\mathbf{Y}$ & & 2834 & REFCON & Refermesce Constent & ACF_N2 & Acantric Factors - N2 & 32354 & 12 Fab- 96 & $\mathbf{Y}$ & 3 \\
\hline $\mathbf{Y}$ & & 2834 & REFCON & Reference Constent & ACF_ND & Acontic Factora - N2 & 32354 & $12 f c b-96$ & $Y$ & 3 \\
\hline Y & & 2835 & REFCON & Reference Constant & $A C F$ HISS & Acantric Fectors - H2S & 32352 & $12 f-b-96$ & $\mathbf{Y}$ & 3 \\
\hline $\mathbf{Y}$ & & 2835 & REFCON & Referenco Constant & ACF_H2S & Acentric Factors - $\mathrm{H} 2 \mathrm{~S}$ & 32352 & 12fob-96 & $\mathbf{Y}$ & 3 \\
\hline$Y$ & & 2836 & REFCON & Rutermes Conetent & ACF_O2 & Acantic Fectors - 02 & 32355 & 12 febses & $\mathbf{Y}$ & 3 \\
\hline $\mathbf{Y}$ & & 2836 & REFCON & Reference Constumt & ACF_02 & Acentric Fectors - 02 & 32355 & $12+F=b-96$ & $\bar{Y}$ & 3 \\
\hline Y & & 2837 & REFCON & Referencos Constent & PC_He & Critical Preseure of $\mathrm{H} 2$ & 32417 & |05-50-98 & $\mathbf{Y}$ & 3 \\
\hline
\end{tabular}


CCA Parameter-Listing

\begin{tabular}{|c|c|c|c|c|c|c|c|c|c|c|}
\hline ID & $\begin{array}{l}\text { Ciseatin- } \\
\text { cation of } \\
\text { Deta } \\
\text { Cetegory } \\
\text { (footnote 2) }\end{array}$ & $\begin{array}{l}\text { Dietribution } \\
\text { Type }\end{array}$ & Men & Medien & Minimum & Madion & Units & Reference id & $\begin{array}{l}\text { Was the data } \\
\text { demaloped } \\
\text { under an } \\
\text { Moh-1 } \\
\text { Program? }\end{array}$ & $\begin{array}{l}\text { Wmich } \\
\text { mithode } \\
\text { were uned to } \\
\text { quiltty } \\
\text { exdoting } \\
\text { dita? } \\
\text { (tootnots 3) }\end{array}$ \\
\hline 2820 & 1,5 & CONSTANT & $-3.4100000 E-01$ & $-3.4100000 E-01$ & $-3.4100000 E-01$ & $-3.4100000 E-01$ & NONE & WPO42714 & $\mathbf{Y}$ & N/A \\
\hline 2823 & 1,3 & DELTA & $2.0000000 E+\infty 0$ & $2.0000000 E+\infty$ & $0.00000000=+\infty 0$ & $2,0000000 E+\infty 0$ & NONE & $\begin{array}{c}\text { MEMO:TIERNEYI } \\
\text { FILE-1 }\end{array}$ & $\mathbf{Y}$ & NA \\
\hline 2823 & 1,3 & DELTA & $2.0000000 E+\infty 0$ & $2,0000000 E+\infty 0$ & $0.0000000 E+\infty)$ & $200000005+\infty$ & NONE & WP035268 & $\mathbf{Y}$ & NA \\
\hline 2823 & 1,3 & DELTA & $20000000 \mathrm{E}+00$ & $2,0000000 E+\infty$ & $0.0000000 E+\infty 0$ & $20000000 E+\infty 0$ & NONE & WP035597 & $\mathbf{Y}$ & NA \\
\hline 2823 & 1,3 & DELTA & $20000000 E+00$ & $2.0000000 E+\infty 0$ & $0.0000000 E+\infty 0$ & $2,0000000 E+\infty 0$ & NONE & WP040514 & $\mathbf{Y}$ & N/A \\
\hline 2824 & 3,5 & DELTA & $2.0000000 E+\infty$ & $2,0000000 E+\infty$ & $0.0000000 E+\infty 0$ & $2,0000000 E+\infty$ & NONE & $\begin{array}{c}\text { MEMO:TIERNEYI } \\
\text { FILE-1 }\end{array}$ & $\mathbf{Y}$ & N/A \\
\hline 2824 & 3,5 & DELTA & $2.0000000 E+\infty 0$ & $2,0000000 E+\infty 0$ & $0.0000000 E+\infty$ & $2.0000000 E+\infty 0$ & NONE & WP035268 & $\mathbf{Y}$ & NA \\
\hline 2824 & 3,5 & DELTA & $2.0000000 E+\infty 0$ & $2.0000000 E+\infty 0$ & $0.0000000 E+\infty$ & $2.0000000 E+00$ & NONE & WP035597 & $\mathbf{Y}$ & NA \\
\hline 2824 & 3,5 & DELTA & $2.0000000 E+\infty$ & $2.0000000 E+\infty$ & $0.0000000 E+\infty$ & $2.0000000 E+00$ & NONE & WPO40514 & $\mathbf{Y}$ & NA \\
\hline 2825 & 4 & CONSTANT & $4.3600000 E+01$ & $4.3600000 E+01$ & $4.3600000 E+01$ & $4.3600000 E+01$ & $\mathbf{K}$ & $\begin{array}{l}\text { VAPORLLOUID_ } \\
\text { EQUILIBRIA }\end{array}$ & $\mathbf{Y}$ & NAA \\
\hline 2825 & 4 & CONSTANT & $4.3600000 E+01$ & $4.3600000 E+01$ & $4.36000005+01$ & $4.3600000 E+01$ & $\mathbf{K}$ & WP035298 & $\mathbf{Y}$ & N/A \\
\hline 2826 & 4 & CONSTANT & $3.0415000 E+02$ & $3.0415000 E+02$ & $3.0415000 E+\infty 2$ & $3.0415000 E+\infty 2$ & $\mathbf{K}$ & $\begin{array}{l}\text { VAPOR-LIQUID_ } \\
\text { EQUILIBRLA }\end{array}$ & $\mathbf{Y}$ & NA \\
\hline 2826 & 4 & CONSTANT & $3.0415000 E+02$ & $3.0415000 E+02$ & $3.0415000 E+02$ & $3.0415000 E+02$ & $\mathbf{K}$ & WP035298 & $\mathbf{Y}$ & $N / A$ \\
\hline 2827 & 4 & CONSTANT & $1.9063000 \mathrm{E}+02$ & $1.9063000 \mathrm{E}+02$ & $1.9063000 E+02$ & $1.9053000 \mathrm{E}+02$ & $\mathbf{K}$ & $\begin{array}{l}\text { VAPOR-LIQUID_ } \\
\text { EQUILIBRIA }\end{array}$ & $\mathbf{Y}$ & N/A \\
\hline 2827 & 4 & CONSTANT & $1.9063000 E+\infty 2$ & $1.9063000 E+02$ & $1.9063000 E+02$ & $1.9063000 E+02$ & $\mathbf{K}$ & WP035298 & $\mathbf{Y}$ & N/A \\
\hline 2828 & 4 & CONSTANT & $1.2615000 E+02$ & $1.2615000 E+02$ & $1.2615000 E+02$ & $1.2615000 E+\infty 2$ & $\mathbf{K}$ & $\begin{array}{l}\text { VAPOR+LIQUID_ } \\
\text { EQUILIBRIA }\end{array}$ & $\mathbf{Y}$ & N/A \\
\hline 2828 & 4 & CONSTANT & $1.2615000 \leq+02$ & $1.2615000 E+02$ & $1.2615000 E+02$ & $1.2615000 E+02$ & $\mathbf{K}$ & WP036298 & $\mathbf{Y}$ & NA \\
\hline 2829 & 4 & CONSTANT & $3.7355000 E+02$ & $3.7355000 E+02$ & $3.7355000 \mathrm{E}+02$ & $3.7355000 E+\infty 2$ & $\mathbf{K}$ & $\begin{array}{l}\text { VAPOR-LIQUID_ } \\
\text { EQUILIBRIA }\end{array}$ & $\mathbf{Y}$ & NA \\
\hline 2829 & 4 & CONSTANT & $3.7355000 \mathrm{E}+02$ & $3.7355000 E+02$ & $3.7355000 E+\infty 2$ & $3.7355000 \mathrm{E}+02$ & $\mathbf{K}$ & WP035298 & $\mathbf{Y}$ & NA \\
\hline 2830 & 4 & CONSTANT & $1.5477000 \mathrm{E}+02$ & $1.5477000 E+02$ & $1.5477000 \mathrm{E}+02$ & $1.5477000 \mathrm{E}+02$ & $\mathbf{K}$ & $\begin{array}{l}\text { VAPOR-LIOUID_ } \\
\text { EQUILIBRIA }\end{array}$ & $\mathbf{Y}$ & NA \\
\hline 2830 & 4 & CONSTANT & $1.5477000 E+02$ & $1.5477000 E+02$ & $1.5477000 E+02$ & $1.5477000 \mathrm{E}+02$ & $\mathbf{K}$ & WP035298 & $\mathbf{Y}$ & NA \\
\hline 2831 & 4 & CONSTANT & $0.0000000 E+00$ & $0.0000000 E+00$ & $0.0000000 E+\infty 0$ & $0.0000000 E+00$ & NONE & $\begin{array}{l}\text { VAPOR-LIQUID_ } \\
\text { EQUILIBRIA }\end{array}$ & $\mathbf{Y}$ & NA \\
\hline 2839 & 4 & CONSTANT & $0.0000000 E+\infty 00$ & $0.0000000 E+00$ & $0.0000000 E+00$ & $0.0000000 E+\infty 0$ & MONE & WP036298 & $\mathbf{Y}$ & N/A \\
\hline 2832 & 4 & CONSTANT & $2.3100000 E-01$ & $23100000 E-01$ & $2.3100000 E-01$ & $2.3100000 E-01$ & NONE & $\begin{array}{l}\text { VAPOR-LIOUID_ } \\
\text { EOUILIBRIA }\end{array}$ & $\mathbf{Y}$ & NA \\
\hline 2832 & 4 & CONSTANT & 2.3100000E-01 & 2.3100000E-01 & $2.3100000 E-01$ & $2.3100000 E-01$ & NONE & WP036298 & $\mathbf{Y}$ & $N / A$ \\
\hline 2833 & 4 & CONSTANT & $1.0000000 E-02$ & $1.0000000 E-02$ & $1.0000000 \mathrm{E}-02$ & $1.0000000 \mathrm{E}-02$ & NONE & $\begin{array}{l}\text { VAPOR-LIQUID } \\
\text { EOUILIBRLA }\end{array}$ & $\mathbf{Y}$ & NA \\
\hline 2833 & 4 & CONSTANT & $1.0000000 E-02$ & $1.0000000 E-02$ & $1.0000000 \mathrm{E}-02$ & $1.0000000 \mathrm{E}-02$ & NONE & WP036298 & $\mathbf{Y}$ & N/A \\
\hline 2834 & 4 & CONSTANT & $4.5000000 E-02$ & $1.5000000 \mathrm{E}-02$ & $4.5000000 \mathrm{E}-02$ & $4.5000000 \mathrm{E}-02$ & NONE & $\begin{array}{l}\text { VAPORALIQUID_ } \\
\text { EQUILIBRLA }\end{array}$ & $\mathbf{Y}$ & NA \\
\hline 2834 & 4 & CONSTANT & $4.5000000 E-02$ & $4.5000000 E-02$ & $4.5000000 E-02$ & $4.5000000 E-02$ & NONE & WPO36298 & $\mathbf{Y}$ & N/A \\
\hline 2835 & 4 & CONSTANT & $1.0000000 E-01$ & $1.0000000 \in-01$ & $1.0000000 \mathrm{E}-01$ & $1.0000000 E-01$ & NONE & $\begin{array}{l}\text { VAPOR-LIQUID_ } \\
\text { EQUILIBRLA }\end{array}$ & $\mathbf{Y}$ & NA \\
\hline 2835 & 4 & CONSTANT & $1.0000000 E-01$ & $1.0000000 E-01$ & $1.0000000 E-01$ & $1.0000000 \mathrm{E}-01$ & NONE & WPO36298 & $\mathbf{Y}$ & N/A \\
\hline 2836 & 4 & CONSTANT & $1.9000000 E-00$ & $1.9000000 E-02$ & $1.5000000 E-02$ & $1.9000000 E-02$ & NONE & $\begin{array}{l}\text { VAPOR_LIQUID_ } \\
\text { EQUILIBRIA }\end{array}$ & $\mathbf{Y}$ & NA \\
\hline 2836 & 4 & CONSTANT & $1.9000000 E-02$ & $1.9000000 E-02$ & $1.9000000 \mathrm{E}-02$ & $1.9000000 \mathrm{E}-02$ & NONE & WP036298 & $\mathbf{Y}$ & NA \\
\hline 2837 & 4 & CONSTANT & $2.0470000 E+06$ & $2.0470000 E+06$ & 2.0470000E+06 & $2.0470000 E+06$ & Pa & $\begin{array}{l}\text { VAPOR-LIOUID_ } \\
\text { EQUILIBRU }\end{array}$ & $\mathbf{Y}$ & NA \\
\hline
\end{tabular}


CCA Parameter-Listing

\begin{tabular}{|c|c|c|c|c|c|c|c|c|c|c|}
\hline 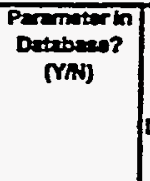 & 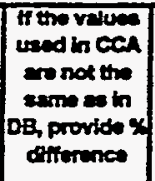 & to & Materia ID & Motord Name & $\begin{array}{c}\text { Paramoter } \\
\text { it }\end{array}$ & 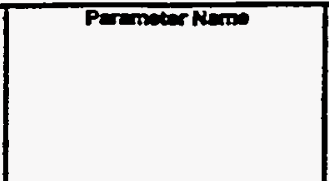 & $\begin{array}{c}\text { PRPDD } \\
\text { MPOON } \\
\end{array}$ & Derte Entry & 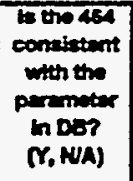 & $\begin{array}{l}\text { Parameter } \\
\text { Cetagory } \\
\text { (footnote 1) }\end{array}$ \\
\hline $\mathbf{Y}$ & & 2837 & REFCON & Referenco Constent & PC_H2 & Critical Pressure of $\mathrm{H}_{2}$ & 32417 & $05-5 e b-96$ & $Y$ & 3 \\
\hline $\mathbf{Y}$ & & 2838 & REFCON & Reference Constant & PC_CO2 & Critical Prescurs of $\mathrm{CO} 2$ & 32416 & asfab-96 & $\mathbf{Y}$ & 3 \\
\hline $\boldsymbol{\gamma}$ & & 2838 & REFCON & Referencos Constant & PC_CO2 & Cntical Prossuro of $\mathrm{CO} 2$ & 32416 & $05-F=0-96$ & $\mathbf{Y}$ & 3 \\
\hline $\mathbf{Y}$ & & 2839 & REFCON & Reference Constant & PC_CH4 & Critical Prossure of $\mathrm{CH}$ & 32415 & $05+500-96$ & $\mathbf{Y}$ & 3 \\
\hline $\mathbf{Y}$ & & 2839 & REFCON & Reference Constant & PC_CH4 & Critical Pressure of CH4 & 32415 & $05-\infty 6-96$ & $\mathbf{Y}$ & 3 \\
\hline $\mathbf{Y}$ & & 2840 & REFCON & Referenco Constent & PC_N2 & Critical Pressume of N2 & 32419 & $05+6 b-96$ & $\mathbf{Y}$ & 3 \\
\hline$Y$ & & 2840 & REFCON & Roferences Constent & PC_N2 & Critical Pressure of N2 & 32419 & 05-Feb-95 & $Y$ & 3 \\
\hline $\mathbf{Y}$ & & 2841 & REFCON & Reference Constant & PC_H2S & Critical Prosaure of H2S & 32418 & 05 fab-96 & $\mathbf{\gamma}$ & 3 \\
\hline $\mathbf{Y}$ & & 2841 & REFCON & Reference Constant & PC_H2S & Crntical Pressure of H2S & 32418 & 05-feb-96 & $Y$ & 3 \\
\hline $\mathbf{Y}$ & & 2842 & REFCON & Roferencos Constent & PC_O2 & Critical Pressure of $\mathrm{O} 2$ & 32420 & 05-feb-96 & $\mathbf{Y}$ & 3 \\
\hline$Y$ & & 2842 & REFCON & Reference Constant & PC_O2 & Critical Pressure o 02 & 32420 & 05feb-96 & $\mathbf{Y}$ & 3 \\
\hline$Y$ & & 2858 & REFCON & Reference Constant & MW_H2 & Motecular Whight - H12 & 32403 & $13+50-96$ & $Y$ & 3 \\
\hline $\mathbf{Y}$ & & 2858 & REFCON & Reference Constant & MW_H2 & Mobocutar Wheght - H2 & 32400 & $13+50-96$ & $Y$ & 3 \\
\hline $\mathbf{Y}$ & & 2864 & REFCON & Reference Constant & $\mathrm{MW}$ _H2O & Molocular Woight - $\mathrm{H}_{2} \mathrm{O}$ & 32405 & 13 feb-96 & $Y$ & 3 \\
\hline$Y$ & & 2804 & REFCON & Reference Constant & $M W_{-} \mathrm{H}_{2} \mathrm{O}$ & Molecular Weight - H2O & 32405 & $13-F \cdot b-96$ & $Y$ & 3 \\
\hline$Y$ & & 2865 & REFCON & Referenco Constant & MW_FE & Moloculer Whaght - FE & 32396 & 13 fob-96 & $Y$ & 3 \\
\hline$Y$ & & 2865 & REFCON & Referencos Constant & $M W_{-} F E$ & Molscular Weight - FE & 32396 & 13 feb-96 & $\mathbf{Y}$ & 3 \\
\hline$Y$ & & 2866 & REFCON & Reference Constant & $\mathrm{MW}$ - $\mathrm{CH} 20$ & Molocuter Wougth - CH2O & 32393 & 13-Fob-96 & $Y$ & 3 \\
\hline$Y$ & & 2866 & REFCON & Roference Constant & $\mathrm{MW}$ _CH2O & Molocular Weigox - CH2O & 32393 & $13-F e b-96$ & $\mathbf{Y}$ & 3 \\
\hline $\mathbf{Y}$ & & 2879 & REFCON & References Constant & BBLG & Converson trom barrel to gallion & 32360 & $05+50 b-96$ & $\mathbf{Y}$ & 3 \\
\hline $\mathbf{Y}$ & & 2879 & REFCON & Reference Constant & BBLG & Corversion from taerrol to gallon & 32360 & $05-F a b-96$ & $\mathbf{Y}$ & 3 \\
\hline $\mathbf{Y}$ & & 2880 & REFCON & References Constant & PASCP & Converson trom Pats to of & 32414 & 05 feb-96 & $\mathbf{Y}$ & 3 \\
\hline$Y$ & & 2880 & REFCON & Reference Constamt & PASCP & Conversion trom $\mathrm{Pa}^{*} \mathrm{~s}$ to of & 32414 & 05 -Fob-96 & $Y$ & 3 \\
\hline $\mathbf{Y}$ & & 2881 & REFCON & Reference Constant & FTM & Converson from foot to meter & 32385 & $06-F \cdot b-96$ & $\mathbf{Y}$ & 3 \\
\hline $\mathbf{Y}$ & & 2882 & REFCON & Referencos Constent & DARMQ & Converuon trom dercy to $m^{\wedge} 2$ & 32379 & $06+00-96$ & $\mathbf{Y}$ & 3 \\
\hline $\mathbf{Y}$ & & 2883 & REFCON & Referenco Constent & F3M3 & Conmereon trom $\mathrm{n}^{\wedge} 3$ to $\mathrm{m}^{\wedge} 3$ & 32384 & 06 -50b-s6 & $\mathbf{Y}$ & 3 \\
\hline$Y$ & & 2884 & REFCON & Reference Constent & GTB & Conmeruon trom pelton to in'3 & 32387 & 06 Feb-96 & $\mathbf{Y}$ & 3 \\
\hline $\mathbf{Y}$ & & 2885 & REFCON & Reference Conaters & LBKG & Converacon trom to wo ko & 32389 & $06 f=b-96$ & $\mathbf{Y}$ & 3 \\
\hline$Y$ & & 2806 & REFCON & Reforence Conatert & KGL & Commerson ram top to bo & 32388 & 06-Fab-96 & $\mathbf{Y}$ & 3 \\
\hline$Y$ & & 2886 & REFCON & Reforence Constem & KGLB & 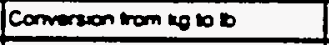 & 32388 & 06-Fob-96 & $Y$ & 3 \\
\hline $\mathbf{Y}$ & & 2887 & REFCON & Reforence Consense & parsec & Commension ars to seconds & 32383 & 06 Feb-96 & $\mathbf{Y}$ & 3 \\
\hline Y & & 2887 & REFCON & Reforence Consem & parsec & Comerson tom ors to roconds & 32383 & O6f-bob-86 & $\mathbf{Y}$ & 3 \\
\hline$Y$ & & 2888 & REFCON & Reference Coneierx & rasec & 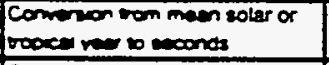 & 32434 & $06-f e b-96$ & $\mathbf{Y}$ & 3 \\
\hline $\mathbf{Y}$ & & 2888 & REFCON & Reforence constume & masec & 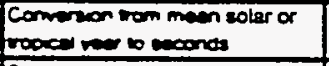 & 32434 & $06-f=b-96$ & $\mathbf{Y}$ & 3 \\
\hline$Y$ & 0.1510 .17 & 2889 & REFCON & Reference Coneters & Gruvace & 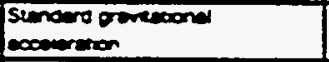 & 32386 & CS-Fob-96 & $\mathbf{Y}$ & 3 \\
\hline $\mathbf{Y}$ & $0.15 n .17$ & 2889 & REFCON & Reference Consterex & GRavace & 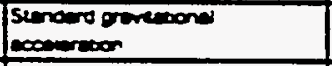 & 32385 & Doffob-96 & $\mathbf{Y}$ & 3 \\
\hline$Y$ & 0.1510 .17 & 2889 & REFCON & Roterence Conserent & gravace & Simpero orevational & 32386 & $06-F o b-96$ & $\mathbf{Y}$ & 3 \\
\hline $\mathbf{Y}$ & & 2890 & REFCON & Reverencia Conseurx & ATMEA & $\begin{array}{l}\text { Converson mon is etmosphore } \\
\text { o } P_{a}\end{array}$ & 32358 & o6-Fob-96 & $Y$ & 3 \\
\hline $\mathbf{Y}$ & & 2890 & REFCON & Retorenca Consterex & Arusa & $\begin{array}{l}\text { Comprean rom wo estmosphere } \\
\text { o } P_{0}\end{array}$ & 32358 & O6-Feb-86 & $r$ & 3 \\
\hline $\mathbf{Y}$ & & 2891 & REFCON & Reference constent & RTK & Commeruon trom Rerikne to $\mathrm{K}$ & 32425 & $06+50 b-96$ & $\mathbf{Y}$ & 3 \\
\hline $\mathbf{Y}$ & & 2891 & REFCON & Reference Conetert: & RTK & Convereon rom Rerkino to $\mathrm{K}$ & 32425 & $06-f a b-56$ & $\mathbf{Y}$ & 3 \\
\hline $\mathbf{Y}$ & & 2892 & REFCON & Reterenco Constent: & PSIPA & Conversen trom per to pascal & 32423 & 06fob-s6 & $\mathbf{Y}$ & 3 \\
\hline $\mathbf{Y}$ & & 2892 & REFCON & Reforence Constum & PSPPA & Cormereon trom peit to paseal & 32423 & 06-fob-96 & $Y$ & 3 \\
\hline
\end{tabular}


CCA Parameter-Listing

\begin{tabular}{|c|c|c|c|c|c|c|c|c|c|c|}
\hline DD & 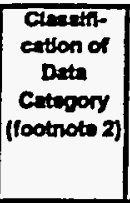 & $\begin{array}{l}\text { Detertibuttion } \\
\text { Typo }\end{array}$ & Mem & Mecten & Minkrown & Modmenn & Thits & Rotionence id & 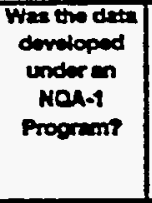 & 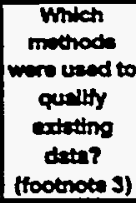 \\
\hline 2837 & 4 & CONSTANT & $20470000 E+06$ & $2.0470000 E+\infty 6$ & $20470000 \mathrm{E}+06$ & $2.0470000 E+06$ & $\mathbf{P a}$ & WP030298 & $Y$ & NA \\
\hline 2838 & 4 & CONSTANT & $7.3760000 E+06$ & $7.3760000 E++06$ & $7.3760000 E+06$ & $7.3760000 E+\infty 6$ & Pa & $\begin{array}{l}\text { VAPOR-UQUID_ } \\
\text { EQULIBRIA }\end{array}$ & $\mathbf{Y}$ & NA \\
\hline 2838 & 4 & CONSTANT & $7.3760000 E+\infty 6$ & $7.3760000 E+06$ & $7.3760000 E+06$ & $7.3760000 E+106$ & $\mathbf{P a}_{\mathbf{a}}$ & WP036290 & $\bar{Y}$ & N/A \\
\hline 2839 & 4 & CONSTANT & $4.6170000 E+06$ & $4.6170000 \approx+06$ & $4.6170000 E+06$ & $4.6170000 E+06$ & $\mathrm{~Pa}$ & $\begin{array}{l}\text { VAPOR-LOUID_ } \\
\text { EQUILIBRLA }\end{array}$ & $\boldsymbol{Y}$ & NA \\
\hline 2839 & 4 & CONSTANT & $4.6170000 E+06$ & $4.6170000 \mathrm{E}+06$ & $4.6170000 \mathrm{E}+06$ & $4.6170000 E+06$ & $\mathbf{P a}$ & WP036298 & $Y$ & NA \\
\hline 2840 & 4 & CONSTANT & $3.3940000 \mathrm{E}+06$ & $3.3940000 E+06$ & $3.3940000 E+05$ & $3.3940000 \mathrm{E}+06$ & Pa & $\begin{array}{l}\text { VAPOR-LIOUID } \\
\text { EQULIBRLA }\end{array}$ & $Y$ & NA \\
\hline 2840 & 4 & CONSTANT & $3.3940000 \mathrm{E}+06$ & $3.3940000 E+06$ & $3.3940000 E+06$ & $3.3940000 \mathrm{E}+\infty 6$ & $\mathbf{P a}$ & WP036298 & $\mathbf{Y}$ & NA \\
\hline 2841 & 4 & CONSTANT & $9.0070000 E+06$ & $9.0070000 E+06$ & $9.0070000 E+06$ & $9.0070000 E+06$ & $\mathrm{~Pa}$ & $\begin{array}{l}\text { VAPOR-LIOUID_ } \\
\text { EQUILIBRIA }\end{array}$ & $\mathbf{Y}$ & NA \\
\hline 2841 & 4 & CONSTANT & $9.0070000 E+06$ & $9.0070000 E+06$ & $9.0070000 \mathrm{E}+06$ & $9.0070000 E+06$ & $P_{\mathbf{a}}$ & WP036296 & $\mathbf{Y}$ & NA \\
\hline 2842 & 4 & CONSTANT & $5.0800000 E+05$ & $5.0800000 E+06$ & $5.0800000 \mathrm{E}+06$ & $5.06000000 E+06$ & $\mathrm{~Pa}$ & $\begin{array}{l}\text { VAPORLIQUID_ } \\
\text { EQUILIBRIA }\end{array}$ & $\mathbf{Y}$ & NA \\
\hline 2842 & 4 & CONSTANT & $5.0800000 \mathrm{E}+06$ & $5.0800000 \mathrm{E}+06$ & $5.0600000 \mathrm{E}+06$ & $5.0800000 \mathrm{E}+06$ & Pa & WP036298 & $Y$ & NA \\
\hline 2858 & 4 & CONSTANT & $20158800 E-03$ & $20158800 \mathrm{E}-03$ & $20158800 E-03$ & $20158000 E-\infty 3$ & ketmolo & CRC-72 & $Y$ & NA \\
\hline 2858 & 4 & CONSTANT & $2.0158800 \mathrm{E}-03$ & $2.0158800 E-03$ & 20158800E-03 & $20158800 E-03$ & ke/molo & WP036298 & $Y$ & NA \\
\hline 2864 & 4 & CONSTANT & $1.8015280 \mathrm{E}-02$ & $1.8015280 E-02$ & $1.8015280 E-02$ & $1.8015200 E-02$ & kotmole & CRC-72 & $Y$ & NA \\
\hline 2864 & 4 & CONSTANT & $1.8015280 \mathrm{E}-02$ & $1.8015280 E-02$ & $1.8015280 E-02$ & $1.8015200 E-\infty 2$ & kefinole & WP036298 & $\mathbf{Y}$ & N/A \\
\hline 2865 & 4 & CONSTANT & $5.5847000 E-02$ & $5.5847000 E-02$ & $5.5847000 \mathrm{E}-02$ & $5.5847000 E-02$ & kotmole & CRC-72 & $\mathbf{Y}$ & NA \\
\hline 2865 & 4 & CONSTANT & $5.5847000 E-02$ & $5.5847000 E-02$ & $5.5847000 \mathrm{E}-02$ & 5.5847000E-02 & ko/molo & WP035298 & $\bar{Y}$ & NA \\
\hline 2866 & 4 & CONSTANT & $3.0026280 E-02$ & $3.0025280 E-02$ & $3.0025280 E-02$ & $3.0026280 \mathrm{E}-02$ & ko/nolo & CRC.72 & $Y$ & NA \\
\hline 2866 & 4 & CONSTANT & $3.0026280 E-02$ & $3.0026280 E-02$ & $3.0026280 E-02$ & $3.0026280 E-02$ & $\mathrm{~kg} / \mathrm{mol}$ & WP035298 & $\mathbf{Y}$ & N/A \\
\hline 2879 & 4 & CONSTANT & $4.2000000 E+01$ & $4.2000000 E+01$ & $1.2000000 \mathrm{E}+01$ & $4.2000000 \mathrm{E}+01$ & ganbl & CRC-72 & $Y$ & NA \\
\hline 2879 & 4 & CONSTANT & $4.2000000 E+01$ & $4.2000000 \equiv+01$ & $4.2000000 E+01$ & $4.2000000 E+01$ & galsol & WP035298 & $\gamma$ & NA \\
\hline 2880 & 4 & CONSTANT & $1.0000000 E+03$ & $1.0000000 \mathrm{E}+\infty$ & $1.0000000 \mathrm{E}+03$ & $1.0000000 E+03$ & cPpays & CRC-72 & $\bar{Y}$ & NA \\
\hline 2880 & 4 & CONSTANT & $1.0000000 E+03$ & $1.0000000 E+03$ & $1.0000000 E+03$ & $1.0000000 \mathrm{E}+03$ & DPRes & WP036298 & $Y$ & NA \\
\hline 2881 & 4 & CONSTANT & $3.0480000 E-01$ & $3.0480000 E-01$ & $3.0480000 E-01$ & $3.0480000 E-01$ & $\mathrm{~m} / \mathrm{n}$ & $\begin{array}{l}\text { NUCLIDESI } \\
\text { ISOTOPES }\end{array}$ & $\mathbf{Y}$ & NA \\
\hline 2882 & 4 & CONSTANT & $9.8692330 E-13$ & $9.8692330 E-13$ & $9.8692330 E-13$ & $9.6692330 E-13$ & $m^{\wedge} 2$ darey & $\begin{array}{l}\text { NUCLIDESI } \\
\text { ISOTOPES }\end{array}$ & $Y$ & NA \\
\hline 2883 & 4 & CONSTANT & 2.8316850E-02 & $28316850 E-02$ & $28316850 E-02$ & $2.8316850 E-02$ & $m^{\wedge} 3 m^{\wedge} 3$ & $\begin{array}{l}\text { NUCLDES/ } \\
\text { ISOTOPES }\end{array}$ & $\mathbf{Y}$ & NA \\
\hline 2884 & 4 & CONSTANT & $2.3100010 E+02$ & $2.3100010 E+02$ & $23900010 E+02$ & $2.3100010 E+02$ & in+3/gal & $\begin{array}{l}\text { NUCLIDESI } \\
\text { ISOTTOPES }\end{array}$ & $\mathbf{Y}$ & NA \\
\hline 2885 & 4 & CONSTANT & $4.5359240 E-01$ & $4.5359240 E-01$ & 4.5359240E-01 & $4.5359240 E-01$ & $\mathrm{~kg} / \mathrm{b}$ & $\begin{array}{l}\text { NUCLIDES' } \\
\text { ISOTOPES }\end{array}$ & $\mathbf{Y}$ & NA \\
\hline 2886 & 4 & CONSTANT & $22045230 E+00$ & $2.2046230 E+\infty$ & $2.2040230 E+\infty 0$ & $2.2046230 E+\infty$ & bokp & $\begin{array}{l}\text { NUCLIDES } \\
\text { ISOTOPES }\end{array}$ & $\mathbf{Y}$ & NA \\
\hline 2886 & 4 & CONSTANT & $2.2040230 E+\infty 0$ & $2.2046230 E+00$ & $22046230 E+00$ & $2.2046230 E+\infty 0$ & Bnog & WP036298 & $Y$ & NA \\
\hline 2887 & 4 & CONSTANT & $0.6400000 E+04$ & $8.6400000 E+04$ & $8.6400000=+04$ & $8.6400000 E+04$ & I/dry & $\begin{array}{l}\text { NUCLIDESI } \\
\text { ISOTOPES }\end{array}$ & $\mathbf{Y}$ & NA \\
\hline 2887 & 4 & CONSTANT & 8.6400000E+0. & $8.6400000 E+04$ & $8.6400000 E+04$ & $8.6400000 E+04$ & eday & WP036298 & $\mathbf{Y}$ & NA \\
\hline 2888 & 4 & CONSTANT & $3.1556930 E+07$ & $3.1556930 E+07$ & $3.1556930 \mathrm{E}+07$ & $3.1556930 E+07$ & $2 y \pi$ & $\begin{array}{l}\text { NUCLIDESI } \\
\text { ISOTOPES }\end{array}$ & $\mathbf{Y}$ & NA \\
\hline $28 B 8$ & 4 & CONSTANT & $3.1556930 \mathrm{E}+07$ & $3.1556930 E+07$ & $3.1556930 E+07$ & $3.1556930 \mathrm{E}+07$ & $2 y r$ & WP035298 & $\mathbf{Y}$ & NA \\
\hline 2889 & 4 & CONSTANT & $9.8066500 E+00$ & $9.8066500 \mathrm{E}+\infty$ & $9.8066500 E+\infty$ & $9.8066500 \mathrm{E}+\infty 0$ & $m / s 2$ & $\begin{array}{l}\text { NUCLIDESI } \\
\text { ISOTOPES }\end{array}$ & $\mathbf{Y}$ & NA \\
\hline 2889 & 4 & CONSTANT & $9.8065500 E+\infty 0$ & $9.80665500 E+\infty 0$ & $9.8066500 \mathrm{E}+\infty 0$ & $9.0066500=+00$ & $\mathrm{~m} / \mathrm{s}^{\mathrm{n}} \mathrm{z}$ & WPO35298 & $\mathbf{Y}$ & NA \\
\hline 2889 & 4 & CONSTANT & $9.8065500 E+\infty$ & $9.8066500 E+\infty$ & $9.8056500 \leq+00$ & $9.8066500 E+\infty)$ & $m / s \times 2$ & WPO40434 & $Y$ & N/A \\
\hline 2890 & 4 & CONSTANT & $1.0132500 E+05$ & $1.0132500 E+05$ & $1.0132500 E+05$ & $1.0132500 \mathrm{E}+05$ & Paratm & $\begin{array}{l}\text { NUCLIDESI } \\
\text { ISOTOPES }\end{array}$ & $\mathbf{Y}$ & N/A \\
\hline 2890 & 4 & CONSTANT & $1.0132500 E+05$ & $1.0132500 E+05$ & $1.0132500 E+05$ & $1.0132500 E+05$ & Palatm & WP035298 & $\mathbf{Y}$ & N/A \\
\hline 2891 & 4 & CONSTANT & $5.5555560 E-01$ & $5.5555560 \mathrm{E}-01$ & $5.5555560 E-01$ & $5.5555560 E-01$ & Krrankine & $\begin{array}{l}\text { NUCLIDESI } \\
\text { ISOTOPES }\end{array}$ & $\mathbf{Y}$ & N/A \\
\hline 2891 & 4 & CONSTANT & $5.5555560 E-01$ & $5.5555560 \mathrm{E}-01$ & $5.5555560 \mathrm{E}-01$ & 5.5555560E-01 & Krankins & WP036298 & $Y$ & NA \\
\hline 2892 & 4 & CONSTANT & $6.8947570 E+03$ & $6.8947570 E+03$ & $6.8947570 E+\infty 3$ & $6.8917570 E+03$ & Patin'2/10 & $\begin{array}{l}\text { NUCLIDES } \\
\text { ISOTOPES }\end{array}$ & $\mathbf{Y}$ & NA \\
\hline 2892 & 4 & CONSTANT & $6.8947570 E+03$ & $6.8947570 E+03$ & $6.89 \times 757 \mathrm{OE}+03$ & $6.8947570 E+03$ & Parmento & WP036298 & $\mathbf{Y}$ & N/A \\
\hline
\end{tabular}


CCA Parameter-Listing

\begin{tabular}{|c|c|c|c|c|c|c|c|c|c|c|}
\hline 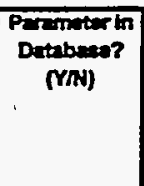 & 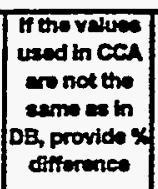 & D & Matedial Lo & Mrtared Neme & $\begin{array}{l}\text { Peremoter } \\
\text { D }\end{array}$ & Peremetar Rements & $\begin{array}{c}\text { PRPD } \\
\text { MPOA) } \\
-\end{array}$ & $\begin{array}{c}\text { Detenty } \\
\text { Dew }\end{array}$ & 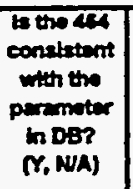 & 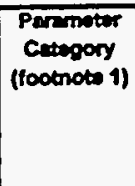 \\
\hline $\mathbf{Y}$ & & 2893 & REFCON & References Constant & $R$ & Gas constant $R$ & 32424 & $06+\infty 0-\infty$ & Y & 3 \\
\hline$Y$ & & 2893 & REFCON & Roference Constant & $R$ & Gas constant $R$ & 32424 & 06-Fob-96 & $Y$ & 3 \\
\hline $\mathbf{Y}$ & & 2894 & REFCON & Reforence Constant & OMEGAA & Constemts for RKS EOS & 32412 & 06 fab-96 & $\mathbf{Y}$ & 3 \\
\hline$\overline{\mathbf{Y}}$ & & 2894 & REFCON & Reference Constent & OMEGAA & Consterts for RKS EOS & 32412 & $06-500-96$ & $\mathbf{Y}$ & 3 \\
\hline Y & & 2895 & REFCON & Reference Constant & OAEGAB & Constants tor RDS EOS & 32413 & $\infty 6-\infty=b-96$ & $\mathbf{Y}$ & 3 \\
\hline $\mathbf{Y}$ & & 2895 & REFCON & Reforence Constant & OMEGAB & Constants for RDS EOS & 32413 & $06-f e b-96$ & $\mathbf{\gamma}$ & 3 \\
\hline$Y$ & cak & 2896 & REFCON & Reference Constant & PI & Mathematical constantPI & 32422 & 06 Fab-96 & $\mathbf{Y}$ & 3 \\
\hline$Y$ & cale & 2896 & REFCON & Reference Constant & $\mathrm{Pl}$ & Mathernatical constantPI & 32422 & $06-f \infty-96$ & $\mathbf{Y}$ & 3 \\
\hline $\mathbf{Y}$ & cak & 2896 & REFCON & Reference Constant & PI & Mathernatical constuntPI & 32422 & 06 Feb-96 & $\mathbf{Y}$ & 3 \\
\hline $\bar{Y}$ & & 2897 & REFCON & Reference Constant & AL2 & $\log 2$ & 32357 & $00-f<b-96$ & $\mathbf{Y}$ & 3 \\
\hline$Y$ & & 2897 & REFCON & Referenco Constant & Al2 2 & $\log 2$ & 32357 & 06-Fab-96 & $Y$ & 3 \\
\hline $\mathbf{Y}$ & & 2897 & REFCON & Reforence Constant & AL2 & $\log 2$ & 32357 & $06+20-96$ & $Y$ & 3 \\
\hline $\mathbf{Y}$ & & 2898 & STEEL & Generic steel in waste & STOIFX & Stoichiometric factor - $x$ & 34128 & $a 6$ fab-96 & $\mathbf{Y}$ & $\mathbb{A A}$ \\
\hline $\mathbf{Y}$ & & 2898 & STEEL & Generic steel in waste & STOIFX & Stoichiometric fector $-\mathrm{X}$ & 34128 & 06 -Fab-96 & $\mathbf{Y}$ & AA \\
\hline $\mathbf{Y}$ & & 2899 & FORTMNIN & Forty Niner Member & PRHX_LOG & 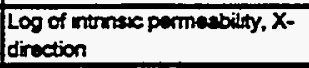 & 33002 & $\mid 11+\infty b-96$ & $\mathbf{Y}$ & AB \\
\hline$Y$ & & 2899 & FORTYNIN & Forty Nirer Member & PRMX_LOG & $\begin{array}{l}\text { Log of intrinsic pormeabildy, } X- \\
\text { diroction }\end{array}$ & 33002 & $\mid 11$ - eb-96 & $\mathbf{Y}$ & AB \\
\hline$Y$ & & 2899 & FORTYNIN & Forty Niner Hember & PRMX_LOG & $\begin{array}{l}\text { Log of intronsic permesubilty, } X- \\
\text { direction }\end{array}$ & 33002 & 11 fob-96 & $\mathbf{Y}$ & 4B \\
\hline$Y$ & & 2899 & FORTMNIN & Forty Niner Momber & PRMX_LOG & $\begin{array}{l}\text { Log of intrinsic permeability, } X \text { - } \\
\text { direction }\end{array}$ & 33002 & 11 fab-96 & $\mathbf{Y}$ & AB \\
\hline$Y$ & & 2900 & FORTYNIN & Forty Niner Member & PRMY_LG & $\begin{array}{l}\text { Log of ntrinste permenbidty, Y- } \\
\text { droction }\end{array}$ & 33000 & $11+00-96$ & $\mathbf{Y}$ & 4B \\
\hline$Y$ & & 2900 & FORTYNIN & Forty Niner Member & PRMY_LOG & $\begin{array}{l}\text { Log of intrinse permesbility, Y- } \\
\text { diroction }\end{array}$ & 33000 & 11 Feb-96 & $\mathbf{Y}$ & 4B \\
\hline $\mathbf{Y}$ & & 2900 & FORTYNIN & Forty Niner Momber & PRMY_LOG & $\begin{array}{l}\text { Loog of intrmsic permeabilly, Y. } \\
\text { direction }\end{array}$ & 33008 & 11 feb-s6 & $Y$ & 4B \\
\hline $\mathbf{Y}$ & & 2900 & FORTININ & Forty Niner Member & PRAY_LOG & $\begin{array}{l}\text { Log of intronsic pormeabilty, Y- } \\
\text { direction }\end{array}$ & 33008 & $\mid 11$ fob-96 & $\mathbf{Y}$ & 4B \\
\hline $\mathbf{Y}$ & & 2901 & FORTYNIN & Forty Niner Member & PRMZ_LOG & $\begin{array}{l}\text { Log of untunsic permosbilty, Z- } \\
\text { diroction }\end{array}$ & 33010 & 11.Fub-96 & $\mathbf{Y}$ & 48 \\
\hline $\mathbf{Y}$ & & 2901 & FORTMIN & Forty Niner Member & PRMZ_LOG & $\begin{array}{l}\text { Log of ntrinstx permeabilty, Z- } \\
\text { direction }\end{array}$ & 33010 & 11 fob-96 & $\mathbf{Y}$ & $4 B$ \\
\hline$Y$ & & 2901 & FORTYNIN & Forty Niner Member & PRMZ_LOG & $\begin{array}{l}\text { Log of intrnstic permeability, Z- } \\
\text { drection }\end{array}$ & 33010 & 11-Fob-96 & $\mathbf{Y}$ & 48 \\
\hline Y & & 2901 & FORTYNIN & Forty Niner Member & PRMZ_LOG & $\begin{array}{l}\text { Log of intmansic permesability, } 2 \text { - } \\
\text { direction }\end{array}$ & 33010 & 11 feb-96 & $\mathbf{Y}$ & 4B \\
\hline$Y$ & & 2902 & S_MB138 & $\begin{array}{l}\text { Seliedo marker bed } \\
138 \text {, intect and } \\
\text { frectured }\end{array}$ & EXPKLINK & $\begin{array}{l}\text { Kinkenberg b correction } \\
\text { paremeters for H12 gas }\end{array}$ & $34443 A$ & 23-Apr-96 & $\begin{aligned} & Y \\
- & \end{aligned}$ & AA \\
\hline $\mathbf{Y}$ & & 2902 & S_MB138 & $\begin{array}{l}\text { Salado marker bed } \\
\text { 138, intact and } \\
\text { fractured. }\end{array}$ & EXPKINK & $\begin{array}{l}\text { Klinkenterg b correction } \\
\text { paremeters for H12 ges }\end{array}$ & $34443 A$ & 23Apr-96 & $\mathbf{\gamma}$ & $\mathbf{A A}$ \\
\hline $\mathbf{Y}$ & & 2902 & S_MB138 & $\begin{array}{l}\text { Salado marker bed } \\
\text { 138. intact and } \\
\text { frectured }\end{array}$ & EXPKLINK & $\begin{array}{l}\text { Klinkenbere b comection } \\
\text { parameters for H2 gass }\end{array}$ & $34443 A$ & 23-Apr-96 & $\mathbf{r}$ & $\mathbb{A}$ \\
\hline $\mathbf{Y}$ & & 29003 & S_MB139 & $\begin{array}{l}\text { Seledo manker bed } \\
139, \text { intinct and } \\
\text { frectured }\end{array}$ & EXPKLINK & $\begin{array}{l}\text { Klinkenberg b correction } \\
\text { parameters for H2 ges }\end{array}$ & 34799A & 23-Apr.96 & $\mathbf{Y}$ & 1 \\
\hline $\mathbf{Y}$ & & 2900 & S_MB139 & $\begin{array}{l}\text { Seledo marker bed } \\
139 \text {, intact and } \\
\text { frectured }\end{array}$ & EXPKINK & $\begin{array}{l}\text { Kinkenberg b corruction } \\
\text { parameters for H2 gas }\end{array}$ & 347994 & 23-Apr-96 & $Y$ & 1 \\
\hline $\mathbf{Y}$ & & 2904 & S_MB138 & $\begin{array}{l}\text { Saledo marker bed } \\
138 \text {, intact and } \\
\text { frectured }\end{array}$ & BKLINK & $\begin{array}{l}\text { Minkenberg B Corruction } \\
\text { Paremeters for H2 gas }\end{array}$ & $34429 A$ & 23-Apr-96 & $\mathbf{Y}$ & 4A \\
\hline$Y$ & & 2904 & S_MB138 & $\begin{array}{l}\text { Salado marker bed } \\
138, \text { inted and } \\
\text { fractured }\end{array}$ & BKLINK & $\begin{array}{l}\text { Kinkenborg B Conrection } \\
\text { Parameters for H12 gas }\end{array}$ & 344294 & $23-A p r-96$ & $\mathbf{Y}$ & $\mathbb{4 A}$ \\
\hline $\mathbf{Y}$ & & 2904 & S_MB138 & $\begin{array}{l}\text { Saledo marker bed } \\
138 \text {, intect and } \\
\text { frectured }\end{array}$ & BLINK & $\begin{array}{l}\text { Kinkenberg B Convection } \\
\text { Peremeters for H2 gas }\end{array}$ & $34429 A$ & $23-x p r-56$ & $\mathbf{Y}$ & $\mathbb{A A}$ \\
\hline $\mathbf{Y}$ & & 2904 & S_MB138 & $\begin{array}{l}\text { Salado maker bad } \\
138 \text {, inted and } \\
\text { frectured }\end{array}$ & BKLINK & $\begin{array}{l}\text { Kinkmborg } 8 \text { Corroction } \\
\text { Parameters for H2 gas }\end{array}$ & $3428 A$ & 23 -Apr.96 & $\boldsymbol{Y}$ & $\mu$ \\
\hline
\end{tabular}


GCA Parameter Listing -

\begin{tabular}{|c|c|c|c|c|c|c|c|c|c|c|}
\hline 10 & 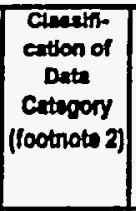 & $\begin{array}{l}\text { Dustribution } \\
\text { Type }\end{array}$ & mean & modien & Mintemem & Mexinum & Units & Raformen id & 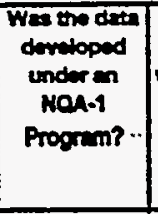 & 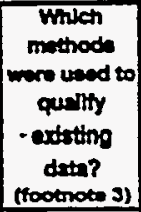 \\
\hline 2893 & 4 & CONSTANT & $8.3145100 E+\infty$ & $8.3145100 E+\infty$ & $8.3145100 E+\infty$ & $8.3145100 E+\infty$ & Jhork & $\begin{array}{l}\text { NUCLIDES } \\
\text { ISOTOPES }\end{array}$ & $\mathbf{Y}$ & N/A \\
\hline 2893 & 1 & CONSTANT & $8.3145100 E+\infty$ & $8.3145100 E+\infty$ & $8.3145100 E+\infty$ & $8.3145100 E+\infty$ & $I_{\text {morK }}$ & UP035298 & $Y$ & NA \\
\hline 2894 & 1 & CONSTANT & 4.2747000E-01 & $4.2747000 E-01$ & $4.2747000 E-01$ & $4.2747000 E-01$ & NONE & $\begin{array}{l}\text { PHASE_ } \\
\text { EQULIBRRA }\end{array}$ & $\mathbf{Y}$ & N/A \\
\hline 2894 & 4 & CONSTANT & $4.2747000 E-01$ & 4.2747000E-01 & 4.2747000E-01 & 1.2747000E-01 & NONE & MP036298 & $\mathbf{Y}$ & $N / A$ \\
\hline 2895 & 4 & CONSTANT & 8.6640000E-02 & $8.6640000 E-02$ & $8.6640000 E-02$ & $8.6640000 E-02$ & NONE & $\begin{array}{l}\text { PHASE } \\
\text { EQUILIBRIA }\end{array}$ & $\mathbf{Y}$ & NA \\
\hline 2895 & 4 & CONSTANT & $8.6640000 E-02$ & $8.6640000 \mathrm{E}-02$ & $8.6640000 E-02$ & $8.6640000 E-02$ & NONE & WP036298 & $\mathbf{Y}$ & NA \\
\hline 2896 & 4 & CONSTANT & $3.1415930 E+\infty 0$ & $3.1415930 E+\infty$ & $3.1415930 \mathrm{E}+\infty 0$ & $3.1415930 E+\infty$ & NONE & CRC-14 & $Y$ & NA \\
\hline 2896 & 4 & CONSTANT & $3.1415930 E+\infty$ & $3.1415930 E+\infty$ & $3.1415930 \mathrm{E}+\infty$ & $3.1415930 E+\infty$ & NONE & CRC-72 & $Y$ & NA \\
\hline 2896 & 4 & CONSTANT & $3.1415930 \mathrm{E}+\infty 0$ & $3.1415930 \mathrm{E}+00$ & $3.1415930 E+\infty)$ & $3.1415930 \mathrm{E}+\infty$ & NONE & WP036298 & $\bar{Y}$ & NA \\
\hline 2897 & 4 & CONSTANT & $6.9314720 \mathrm{E}-01$ & $6.9314720 E-01$ & $6.9314720 E-01$ & $6.9314720 E-01$ & NONE & CRC-14 & $Y$ & NA \\
\hline 2897 & 4 & CONSTANT & $6.9314720 E-09$ & 6.9314720E-01 & $6.9314720 \mathrm{E}-01$ & $6.9314720 E-01$ & NONE & CRG.72 & $\bar{Y}$ & NA \\
\hline 2897 & 4 & CONSTANT & $6.9394720 E-01$ & $6.9314720 E-01$ & 6.9314720E-01 & 6.9314720E-01 & NONE & WP036298 & $Y$ & $N A$ \\
\hline 2898 & 1,3 & CONSTANT & $1.0000000 \mathrm{E}+\infty 0$ & $1.0000000 E+\infty$ & $1.0000000 E+\infty$ & $1.0000000 E+\infty$ & NONE & WP030819 & $\mathbf{Y}$ & NA \\
\hline 2898 & 1,3 & CONSTANT & $1.00000000+\infty$ & $1.0000000 E+\infty$ & $1.0000000 E+\infty 0$ & $1.0000000 E+\infty 0$ & NONE & Wpos5597 & $Y$ & NA \\
\hline 2899 & 1,3 & CONSTANT & $3.5000000 E+01$ & $3.5000000 E+01$ & $-3.5000000 E+01$ & $-3.5000000 E+01$ & $\log \left(m^{\wedge} 2\right)$ & WP032291 & $\mathbf{Y}$ & N/A \\
\hline 2899 & 1,3 & CONSTANT & $-3.5000000 E+01$ & $3.5000000 E+01$ & $3.5000000 E+01$ & $-3.5000000 E+01$ & $\log (m \wedge 2)$ & WP035597 & $Y$ & NA \\
\hline 2899 & 1,3 & CONSTANT & $3.5000000 E+01$ & $3.5000000 E+01$ & $3.5000000 E+01$ & $3.5000000 E+01$ & $\log \left(m^{\wedge} 2\right)$ & WP038337 & $\mathbf{Y}$ & NA \\
\hline 2899 & 1,3 & CONSTANT & $3.5000000 E+01$ & $3.5000000 E+01$ & $-3.5000000 E+01$ & $3.5000000 E+01$ & $\log \left(m^{\wedge} 2\right)$ & WPO40514 & $\mathbf{Y}$ & NA \\
\hline 2900 & 1,3 & CONSTANT & $3.5000000 E+01$ & $3.5000000 E+01$ & $-3.5000000 E+01$ & $-3.5000000 E+01$ & $\log \left(m^{\wedge} 2\right)$ & WPO32291 & $Y$ & NA \\
\hline 2900 & 1,3 & CONSTANT & $3.5000000 \mathrm{E}+01$ & $3.5000000 E+01$ & $3.5000000 E+01$ & $-3.5000000 E+01$ & $\log \left(m^{\wedge} 2\right)$ & WP035597 & $\mathbf{Y}$ & NA \\
\hline 2900 & 1,3 & CONSTANT & $3.5000000 E+01$ & $3.5000000 E+01$ & $3.5000000 E+01$ & $3.5000000 \mathrm{E}+04$ & $\log \left(m^{\wedge} 2\right)$ & WP038337 & $\mathbf{Y}$ & NA \\
\hline 2900 & 1,3 & CONSTANT & $3.5000000 \mathrm{E}+01$ & $3.5000000 E+01$ & $3.5000000 E+01$ & $3.5000000 E+01$ & $\log \left(m^{\wedge} 2\right)$ & WP040514 & $\mathbf{Y}$ & NA \\
\hline 2901 & 1,3 & CONSTANT & $-3.5000000 \mathrm{E}+01$ & $-3.5000000 \mathrm{E}+01$ & $3.5000000 E+01$ & $-3.50000000+01$ & $\log \left(m^{\wedge} 2\right)$ & WP032291 & $\mathbf{Y}$ & NA \\
\hline 2901 & 1,3 & CONSTANT & $-3.5000000 E+01$ & $3.5000000 E+01$ & $3.5000000 E+01$ & $3.5000000 E+01$ & $\log \left(m^{\wedge} 2\right)$ & WP035597 & $\mathbf{Y}$ & NA \\
\hline 2901 & 1,3 & CONSTANT & $3.5000000 E+01$ & $3.5000000 E+01$ & $3.5000000 E+01$ & $3.5000000 E+01$ & $\log \left(m^{\wedge} 2\right)$ & WP038337 & $\mathbf{Y}$ & NA \\
\hline 2901 & 1,3 & CONSTANT & $3.5000000 E+01$ & $3.5000000 E+01$ & $3.5000000 E+01$ & $-3.5000000 E+01$ & $\log \left(m^{\wedge} 2\right)$ & WP040514 & $\mathbf{Y}$ & NA \\
\hline 2902 & 1,5 & CONSTANT & $3.41000000-01$ & $3.4100000 E-01$ & $34100000 E-01$ & $34100000 \mathrm{E}-01$ & NONE & WP030612 & $\mathbf{Y}$ & . NA \\
\hline 2902 & 1,5 & CONSTANT & $3.4100000 E-01$ & $-3.4100000 E-01$ & $-31100000 E-01$ & $34100000 E-01$ & NONE & WP035597 & $\mathbf{Y}$ & NA \\
\hline 2902 & 1,5 & CONSTANT & $3.4100000 E-01$ & $3.4100000 E-01$ & $34100000 E-01$ & $34100000 E-01$ & NONE & WPO42714 & $\mathbf{Y}$ & NA \\
\hline 2903 & 1,5 & CONSTANT & $-3.4100000 \mathrm{E}-01$ & $3.4100000 E-01$ & $31100000 E-01$ & $34100000 E-01$ & NONE & WP030642 & $\mathbf{Y}$ & NA \\
\hline 2903 & 1,5 & CONSTANT & $3.4100000 E-01$ & $3.4100000 E-01$ & $34100000 E-01$ & $34100000 E-01$ & NONE & WP035597 & $\mathbf{Y}$ & NA \\
\hline 2904 & 1.5 & CONSTANT & $2.7100000 E-01$ & $2.7100000 E-01$ & $2.7100000 E-01$ & $27100000 E-01$ & Pa & WP030642 & $\mathbf{Y}$ & NA \\
\hline 2804 & 1,5 & CONSTANT & $2.7100000 E-01$ & $2.7100000 E-01$ & $2.7100000 E-01$ & $2.7100000 E-01$ & $\mathrm{~Pa}$ & WP035597 & $\mathbf{Y}$ & NA \\
\hline 2904 & 1,5 & CONSTANT & $2.7100000 E-01$ & 27100000E-01 & $27100000=01$ & $2.7100000 E-01$ & Pa & WP038450 & $Y$ & N/A \\
\hline 2904 & 1,5 & CONSTANT & $2.7100000 E-01$ & $2.7100000 E-01$ & $2.7100000 \leqslant-01$ & $2.7100000 E-01$ & $P=$ & WPO42714 & $\mathbf{Y}$ & NA \\
\hline
\end{tabular}


CCA Parameter-Listing

\begin{tabular}{|c|c|c|c|c|c|c|c|c|c|c|}
\hline $\begin{array}{c}\text { Paramisen in } \\
\text { Detabaee? } \\
\text { (Yw) }\end{array}$ & 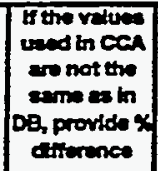 & ID & Meterial to & Metard Kam & Peremotar & Parminer Noms & $\begin{array}{l}\text { PFPDD } \\
\text { MPO }\end{array}$ & Denerty & 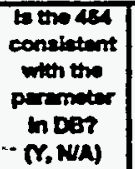 & $\begin{array}{c}\text { Parmiter } \\
\text { Catsogory } \\
\text { (footrote 1) } \\
\text {. }\end{array}$ \\
\hline $\mathbf{Y}$ & & 2905 & S_MB139 & $\begin{array}{l}\text { Salado marker bed } \\
\text { 139, intact and } \\
\text { fractured }\end{array}$ & BKINK & $\begin{array}{l}\text { Winkenberg B Correction } \\
\text { Parmeters for H2 gas }\end{array}$ & $34557 A$ & 23-Apr-96 & $\mathbf{Y}$ & 1 \\
\hline $\mathbf{Y}$ & & 2905 & S_MB139 & $\begin{array}{l}\text { Salado marker bed } \\
139, \text { intact and } \\
\text { fractured }\end{array}$ & BKLINK & $\begin{array}{l}\text { Kinkenberg } 8 \text { Correction } \\
\text { Parmeters for H2 oas }\end{array}$ & $34557 A$ & $23-A p \times-96$ & $\mathbf{Y}$ & 1 \\
\hline $\mathbf{Y}$ & & 2905 & S_MB139 & $\begin{array}{l}\text { Salodo manker bed } \\
139 \text {, intact and } \\
\text { fractured }\end{array}$ & BKLINK & $\begin{array}{l}\text { Kinkenberg } 8 \text { Correction } \\
\text { Paraneters for t } 12 \text { gas }\end{array}$ & 34557A & $23-4 p r-96$ & $\mathbf{Y}$ & 1 \\
\hline $\mathbf{Y}$ & & 2906 & NTTRATE & Nitrato & OINIT & Initial quentity of material in waste & 32335 & $22-F=b-96$ & $\mathbf{Y}$ & 2 \\
\hline $\mathbf{Y}$ & & 2906 & NTTRATE & Nitrate & aINT & Initial quantity of material in waste & 32335 & 22 Fabs 96 & $\mathbf{Y}$ & 2 \\
\hline $\mathbf{Y}$ & & 2906 & NITRATE & Nitrate & aINT & Initial quantity of material in waste. & 32335 & $22 f a b-96$ & $\mathbf{Y}$ & 2 \\
\hline $\mathbf{Y}$ & & 2907 & STEEL & Generic steol in wasto & CORRMCO2 & $\begin{array}{l}\text { Inundated corrosion rate for steel } \\
\text { without } \mathrm{CO} 2 \text { present }\end{array}$ & 34357 & $08+F a b-96$ & $\mathbf{Y}$ & 1 \\
\hline $\mathbf{Y}$ & & 2908 & STEEL & Generic steol in wasto & CORRMCO2 & $\begin{array}{l}\text { Inundsted corrosion rate for sted } \\
\text { with } \mathrm{CO} 2 \text { presert }\end{array}$ & 34358 & osfreb-ss & $\mathbf{Y}$ & 1 \\
\hline $\mathbf{Y}$ & & 2909 & SULFATE & Sulfato & QINT & Initial quantity of material in wacte & 32457 & 22 Fab-96 & $\mathbf{Y}$ & 2 \\
\hline $\mathbf{Y}$ & & 2909 & SULFATE & Sultate & QINIT & Initial quantity of material in wasted & 32457 & 22 Fab- 96 & $\mathbf{Y}$ & 2 \\
\hline $\mathbf{Y}$ & & 2909 & SULFATE & Sulfiate & OINIT & Initial quantity of material in waste & 32457 & 22Feb-96 & $\mathbf{Y}$ & 2 \\
\hline $\mathbf{Y}$ & & 2910 & STEEL & Generic steel in waste & HUMCORR & Humid corrosion rate for steel & 34127 & osfab-96 & $\mathbf{Y}$ & 1 \\
\hline $\mathbf{Y}$ & & 2910 & STEEL & Generic steo] in waste & HUMCORR & Humid corrosion rate for steol & 34127 & 08-Fab-96 & $\mathbf{Y}$ & 1 \\
\hline $\mathbf{Y}$ & & 2911 & UNNAMED & $\begin{array}{l}\text { Unnamed Lower } \\
\text { Member of Rustler } \\
\text { Formation }\end{array}$ & PRMX_LOG & $\begin{array}{l}\text { Log of intrintic permesbility, } x \text { - } \\
\text { direction }\end{array}$ & 34695 & $13-F a b-96$ & $\mathbf{Y}$ & $4 B$ \\
\hline $\mathbf{Y}$ & & 2911 & UNNAMED & $\begin{array}{l}\text { Urnamed Lower } \\
\text { Hember of Rustier } \\
\text { Fomation }\end{array}$ & PRMX_LOG & $\begin{array}{l}\text { Log of intrinsic permesbility, } X \text { - } \\
\text { drection }\end{array}$ & 34695 & $13 F a b-96$ & $\mathbf{Y}$ & AB \\
\hline $\mathbf{Y}$ & & 2911 & UNNAMED & $\begin{array}{l}\text { Unmamed Lower } \\
\text { Member of Rustler } \\
\text { Formation }\end{array}$ & PRMX_LOG & $\begin{array}{l}\text { Log of intrinsic permesbility, } X \text { - } \\
\text { drection }\end{array}$ & 34695 & 13-Fob-96 & $\mathbf{Y}$ & $4 B$ \\
\hline $\mathbf{Y}$ & & 2911 & UNNAMED & $\begin{array}{l}\text { Unnamed Lower } \\
\text { Member of Rustler } \\
\text { Formation }\end{array}$ & PRMX_LOG & $\begin{array}{l}\text { Loo of intrinsic permesbility, } X- \\
\text { drection }\end{array}$ & 34695 & 13-Fab-96 & $\mathbf{Y}$ & $4 B$ \\
\hline $\mathbf{Y}$ & & 2912 & UNNAMED & $\begin{array}{l}\text { Umamed Lower } \\
\text { Momber of Rustler } \\
\text { Formation }\end{array}$ & PRAY_LOG & $\begin{array}{l}\text { Lo of intrinsic permasbilty, } Y \text { - } \\
\text { direction }\end{array}$ & 34697 & $13-F e b-96$ & $\mathbf{Y}$ & 48 \\
\hline $\mathbf{Y}$ & & 2912 & UNMAMED & $\begin{array}{l}\text { Unnamed Lower } \\
\text { Member of Rustier } \\
\text { Formation }\end{array}$ & PRMY_LOG & $\begin{array}{l}\text { Log of intrinsic permeability, } Y \text {. } \\
\text { drection }\end{array}$ & 34697 & $13-F 0 b-96$ & $\mathbf{Y}$ & $4 B$ \\
\hline $\mathbf{Y}$ & & 2912. & UNNAMED & $\begin{array}{l}\text { Unnamed Lower } \\
\text { Momber of Rustler } \\
\text { Formation }\end{array}$ & PRMY_LOG & $\begin{array}{l}\text { Log of intrinsic permeability. Y- } \\
\text { drection }\end{array}$ & 34697 & 13-Fob-96 &.$-^{Y}$ & - 48 \\
\hline $\mathbf{Y}$ & & 2912 & UNNAMED & $\begin{array}{l}\text { Urmamod Lower } \\
\text { Member of Rustier } \\
\text { Formation }\end{array}$ & PRMY_LOG & $\begin{array}{l}\text { Log of intrinsic permesbility, Y- } \\
\text { drection }\end{array}$ & 34697 & 13-Fob-96 & $\mathbf{Y}$ & $4 B$ \\
\hline $\mathbf{Y}$ & & 2913 & UNRAMED & $\begin{array}{l}\text { Urnamed Lower } \\
\text { Member of Rustler } \\
\text { Fommetion }\end{array}$ & PRMZ_LOG & $\begin{array}{l}\text { Log of intrinsic pormasbility, } z \text { - } \\
\text { drection }\end{array}$ & 34699 & 13-Fab-96 & $\mathbf{Y}$ & AB \\
\hline$Y$ & & 2913 & UNNANED & $\begin{array}{l}\text { Unnemed Lower } \\
\text { Menber of Rustler } \\
\text { Formation }\end{array}$ & PRNR_LOG & $\begin{array}{l}\text { Loo of intrinsic permesbilty, } z \text { - } \\
\text { drection }\end{array}$ & 34699 & $13-F a b-96$ & $Y$ & $4 B$ \\
\hline $\mathbf{Y}$ & & 2913 & UNNANED & $\begin{array}{l}\text { Ummemed Lower } \\
\text { Member of Rustior } \\
\text { Formation }\end{array}$ & PRMZ_LOG & $\begin{array}{l}\text { Loo of intrnsic permeability, } z- \\
\text { drection }\end{array}$ & 34699 & 13fribss & $\mathbf{Y}$ & 48 \\
\hline $\mathbf{Y}$ & & 2913 & UNNAMED & $\begin{array}{l}\text { Unnamed Lowwer } \\
\text { Momber of Ruetter } \\
\text { Formation }\end{array}$ & PRMZ_LOG & $\begin{array}{l}\text { Loo of intrinaic permeabilty, } Z \text {. } \\
\text { drection }\end{array}$ & 34699 & 13-Fob-96 & $\mathbf{Y}$ & $4 B$ \\
\hline $\mathbf{Y}$ & & 2914 & TAMARISK & Temeriak Member & PRMX_LOG & $\begin{array}{l}\text { Log of untmnsic permeability, } X \text { - } \\
\text { droction }\end{array}$ & 34580 & 13Fib-96 & $\mathbf{Y}$ & $4 B$ \\
\hline $\mathbf{Y}$ & & 2914 & TAMARISK & Tamarisk Member & PRMX_LOG & $\begin{array}{l}\text { Log of intrinsic permesbilty, } X- \\
\text { droction }\end{array}$ & 34580 & $13 f-b-96$ & $\mathbf{Y}$ & 48 \\
\hline $\mathbf{Y}$ & & 2914 & TAMARISK & Tamerisk Hember & PRMX_LOG & $\begin{array}{l}\text { Log of intrmasic permasbilky, } X \text { - } \\
\text { direction }\end{array}$ & 34580 & 13Frab-96 & $\mathbf{Y}$ & AB \\
\hline $\mathbf{Y}$ & & 2915 & TAMARISK & Temariak Member & PRAY_LOG & $\begin{array}{l}\text { Los of intrnsic permesbility, } Y- \\
\text { direction }\end{array}$ & 34583 & $13-f c b-96$ & $\mathbf{Y}$ & $4 B$ \\
\hline
\end{tabular}


- CCA-Parameter-Listing

\begin{tabular}{|c|c|c|c|c|c|c|c|c|c|c|}
\hline 10 & $\begin{array}{c}\text { Clesefin- } \\
\text { catbon of } \\
\text { Date } \\
\text { Cutiegory } \\
\text { (footnots 2) }\end{array}$ & $\begin{array}{c}\text { Diatribution } \\
\text { Type }\end{array}$ & Man & Medien & Mintmum & Maximum & Units & Reference LD & 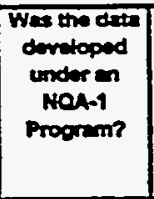 & $\begin{array}{c}\text { minich } \\
\text { mithods } \\
\text { were ueed to } \\
\text { qually } \\
\text { exteting } \\
\text { deta? } \\
\text { footnots } 3 \text { ) }\end{array}$ \\
\hline 2905 & 9,5 & CONSTANT & $2.7100000 \mathrm{E}-01$ & $2.7100000 \mathrm{E}-01$ & $27100000 E-01$ & $27100000 E-01$ & $\mathrm{~Pa}$ & WPO30642 & $\mathbf{Y}$ & NA \\
\hline 2905 & 1,5 & CONSTANT & $2.7100000 E-01$ & $27100000 E-01$ & $27100000 E-01$ & $2.7100000 E-01$ & $\mathrm{Pz}$ & WP035597 & $\boldsymbol{Y}$ & N/A \\
\hline 2905 & 1,5 & CONSTANT & $2.7100000 E-01$ & $2.7100000 E-01$ & $27100000 E-01$ & $2.7100000 \mathrm{E}-01$ & $\mathbf{P a}$ & WPO38450 & $\mathbf{Y}$ & NA \\
\hline 2906 & 2 & CONSTANT & $2.6100000 E+07$ & $2.6100000 E+07$ & $2.6100000 E+07$ & $2.6100000 E+07$ & moles & WPO30319 & $\mathbf{Y}$ & NA \\
\hline 2906 & 2 & CONSTANT & $2.6100000 E+07$ & $2.6100000 E+07$ & $2.6100000 E+07$ & $2.6100000 E+07$ & moles & WP031379 & $\mathbf{Y}$ & NA \\
\hline 2906 & 2 & CONSTANT & $2.6100000 E+07$ & $2.6100000 E+07$ & $2.6100000 E+07$ & $2.6100000 E+07$ & moles & WP035048 & $\mathbf{Y}$ & NA \\
\hline 2907 & 1 & UNIFORM & $7.9370000 \mathrm{E}-15$ & 7.9370000E-15 & $0.0000000 \mathrm{E}+\infty$ & $1.5870000 E-14$ & $\mathrm{~m} / \mathrm{s}$ & WPO30819 & $\mathbf{Y}$ & 4 \\
\hline 2908 & 1 & UNIFORM & $1.0318000 \mathrm{E}-13$ & $1.0318000 E-13$ & $0.0000000 E+\infty$ & $20535000 E-13$ & $\mathrm{~m} / \mathrm{s}$ & WP030819 & $\mathbf{Y}$ & 4 \\
\hline 2909 & 2 & CONSTANT & $6.5900000 E+06$ & $6.5900000 E+06$ & $6.5900000 \mathrm{E}+\infty 6$ & $6.5900000 E+06$ & moles & WP030819 & $\mathbf{Y}$ & $N A$ \\
\hline 2909 & 2 & CONSTANT & $6.5900000 E+06$ & $6.5900000 \mathrm{E}+06$ & $6.5900000 E+06$ & $6.5900000 \mathrm{E}+06$ & moles & WP031379 & $\mathbf{Y}$ & NA \\
\hline 2909 & 2 & CONSTANT & $6.5900000 \mathrm{E}+06$ & $6.5900000 E+06$ & $6.5900000 \mathrm{E}+06$ & $6.5900000 E+06$ & moles & WP036048 & $\mathbf{Y}$ & NA \\
\hline 2910 & 1 & CONSTANT & $0.0000000 E+00$ & $0.0000000 \mathrm{E}+\infty$ & $0.0000000 E+\infty$ & $0.0000000 E+\infty$ & $\mathrm{m} / \mathrm{s}$ & WP030819 & $\mathbf{Y}$ & 4 \\
\hline 2910 & 1 & CONSTANT & $0.0000000 E+\infty$ & $0.0000000 E+\infty$ & $0.0000000 E+00$ & $0.0000000 E+\infty$ & $\mathrm{m} / \mathrm{s}$ & WP035597 & $\mathbf{Y}$ & 4 \\
\hline 2911 & 1,3 & CONSTANT & $-3.5000000 E+01$ & $-3.5000000 E+01$ & $-3.5000000 E+01$ & $3.5000000 E+01$ & $\log \left(m^{\wedge} 2\right)$ & WP032291 & $\mathbf{Y}$ & NA \\
\hline 2919 & 1,3 & CONSTANT & $-3.5000000 E+01$ & $-3.5000000 \mathrm{E}+01$ & $3.5000000 E+01$ & $-3.5000000 E+01$ & $\log \left(m^{\wedge} 2\right)$ & WP035597 & $\mathbf{Y}$ & NA \\
\hline 2911 & 1,3 & CONSTANT & $-3.5000000 E+01$ & $-3.5000000 E+01$ & $3.5000000 E+01$ & $3.5000000 E+01$ & $\log \left(m^{\wedge} 2\right)$ & WP038337 & $\mathbf{Y}$ & NA \\
\hline 2911 & 1,3 & CONSTANT & $3.5000000 E+01$ & $-3.5000000 \mathrm{E}+01$ & $3.5000000 E+01$ & $3.5000000 E+01$ & $\log \left(m^{\wedge} 2\right)$ & WPO40514 & $\mathbf{Y}$ & NA \\
\hline 2912 & 1,3 & CONSTANT & $3.5000000 E+01$ & $-3.5000000 E+01$ & $3.5000000 \mathrm{E}+01$ & $3.5000000 E+01$ & $\log \left(m^{\wedge} 2\right)$ & WP032291 & $\mathbf{Y}$ & N/A \\
\hline 2912 & 1,3 & CONSTANT & $-3.5000000 E+01$ & $-3.5000000 E+01$ & $-3.5000000 E+01$ & $3.5000000 E+01$ & $\log \left(m^{\wedge} 2\right)$ & WP035597 & $\mathbf{Y}$ & N/A \\
\hline 2912 & 1,3 & CONSTANT & $-3.5000000 E+01$ & $-3.5000000 \mathrm{E}+01$ & $-3.5000000 E+01$ & $3.5000000 E+01$ & $\log \left(m^{\wedge} 2\right)$ & WPO38337 & $-Y$ & NA \\
\hline 2912 & 1,3 & CONSTANT & $-3.5000000 E+01$ & $-3.5000000 E+01$ & $3.5000000 E+01$ & $-3.5000000 \mathrm{E}+01$ & $\log \left(m^{\wedge} 2\right)$ & WP040514 & $\mathbf{Y}$ & NA \\
\hline 2913 & 1,3 & CONSTANT & $-3.5000000 E+01$ & $3.5000000 E+01$ & $3.5000000 E+01$ & $-3.5000000 E+01$ & $\log \left(m^{\wedge} 2\right)$ & WP032291 & $\mathbf{Y}$ & NA \\
\hline 2913 & 1,3 & CONSTANT & $-3.5000000 E+01$ & $3.5000000 E+01$ & $3.5000000 E+01$ & $3.5000000 E+01$ & $\log \left(m^{\wedge} 2\right)$ & WP035597 & $\mathbf{Y}$ & N/A \\
\hline 2913 & 1,3 & CONSTANT & $-3.5000000 E+01$ & $-3.5000000 E+01$ & $3.5000000 E+01$ & $-3.5000000 E+01$ & $\log \left(m^{\wedge} 2\right)$ & WP038337 & $\mathbf{Y}$ & NA \\
\hline 2913 & 1,3 & CONSTANT & $-3.5000000 E+01$ & $3.5000000 E+01$ & $3.5000000 E+01$ & $3.5000000 E+01$ & $\log \left(m^{\wedge} 2\right)$ & WPOS0514 & $\mathbf{Y}$ & NA \\
\hline 2914 & 1,3 & CONSTANT & $3.5000000 E+01$ & $3.5000000 E+01$ & $-3.5000000 E+01$ & $-3.5000000 E+01$ & $\log \left(m^{\wedge} 2\right)$ & WP032291 & $\mathbf{Y}$ & N/A \\
\hline 2914 & 1,3 & CONSTANT & $3.5000000 E+01$ & $3.5000000 E+01$ & $-3.5000000 E+01$ & $-3.5000000 E+01$ & $\log \left(m^{n} 2\right)$ & WP035597 & $\mathbf{Y}$ & NA \\
\hline 2914 & 1,3 & CONSTANT & $3.5000000 E+01$ & $-3.5000000 E+01$ & $3.5000000 E+01$ & $3.5000000 E+01$ & $\log \left(m^{\wedge} 2\right)$ & WPO40514 & $\mathbf{Y}$ & N/A \\
\hline 2915 & 1,3 & CONSTANT & $-3.5000000 E+01$ & $3.5000000 \mathrm{E}+01$ & $3.5000000 E+01$ & $3.5000000 E+01$ & $\log \left(m^{\wedge} 2\right)$ & WP032291 & $\mathbf{Y}$ & NA \\
\hline
\end{tabular}


CCA Parametertisting

\begin{tabular}{|c|c|c|c|c|c|c|c|c|c|c|}
\hline 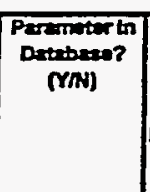 & 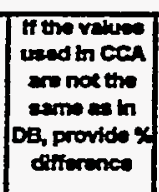 & $\bar{D}$ & Mxinto & Mender Name & $\begin{array}{l}\text { Parenter } \\
\text { to }\end{array}$ & Pernicier Mane & $\begin{array}{l}\text { PFPLD } \\
\text { (MrOA) }\end{array}$ & $\begin{array}{c}\text { Dete Enty } \\
\text { Dete }\end{array}$ & 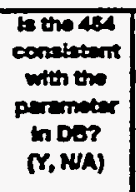 & 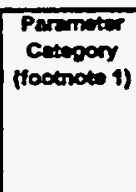 \\
\hline$Y$ & & 2915 & TAMARISK & Temarisk Monber & PRMYYLOG & $\begin{array}{l}\text { Log of mitrnsic permesabilty, Y- } \\
\text { direction }\end{array}$ & 34583 & $13-F e b-96$ & $\mathbf{Y}$ & 48 \\
\hline$Y$ & & 2915 & TAMARISK & Ternariak Member & PRMY_LOG & $\begin{array}{l}\text { Loo of nifmsic permeabulty, Y. } \\
\text { inection }\end{array}$ & 34583 & 13-Feb-96 & $\mathbf{Y}$ & 48 \\
\hline $\mathbf{Y}$ & & 2915 & TAYARISK & Tenarisk Member & PRMY_LOG & $\begin{array}{l}\text { Log of mirnsic permeability, Y- } \\
\text { orrection }\end{array}$ & 34583 & $13-5 e b-96$ & $\mathbf{Y}$ & 48 \\
\hline $\mathbf{Y}$ & & 2916 & TAMARISK & Temaristk Menber & PRNZ_LOG & $\begin{array}{l}\text { Log of intmensic pormeabitity, Z- } \\
\text { drection }\end{array}$ & 34586 & $16-F=b-96$ & $\mathbf{Y}$ & $4 B$ \\
\hline $\mathbf{Y}$ & & 2916 & TAMARISK & Tamarisk Hember & PRMZ_LOG & $\begin{array}{l}\text { Loo of intonsic permeability, Z- } \\
\text { direction }\end{array}$ & 34506 & 16 Feb-96 & $Y$ & $A B$ \\
\hline $\mathbf{Y}$ & & 2916 & TAMARISK & Temarisk Momber & PRMZ_LOG & $\begin{array}{l}\text { Log of intirnsic permeabilky, } 2- \\
\text { direction }\end{array}$ & 34586 & $16-f=b-96$ & $\mathbf{Y}$ & AB \\
\hline $\mathbf{Y}$ & & 2916 & TAMARISK & Tamerisk Mernber & PRNZ_LOG & $\begin{array}{l}\text { Loo of intrinsic permesabilty, Z- } \\
\text { direction }\end{array}$ & 34586 & 16 Fab-96 & $\mathbf{Y}$ & 48 \\
\hline $\mathbf{Y}$ & & 2918 & CASTILER & Castilo Brino Resonvir & VOLUME & Total Reservoir Vokme & $31625 A$ & 20-4m-96 & $\mathbf{Y}$ & 1 \\
\hline $\mathbf{Y}$ & & 2918 & CASTILER & Castile Brine Reservoir & VOLUME & Total Reservoir Volume & $31625 A$ & $20+1$ ar-96 & $\mathbf{Y}$ & 1 \\
\hline $\mathbf{Y}$ & & 2919 & CL_H_T4 & $\begin{array}{l}\text { Upper Salado clay:50 } \\
\text { to } 100 \text { years }\end{array}$ & RSH_AR & Air-supply shent redius $(3.09 \mathrm{~m})$ & $32212 A$ & $13-f a b-96$ & $\mathbf{Y}$ & 48 \\
\hline $\mathbf{Y}$ & & 2919 & CL_M_T4 & $\begin{array}{l}\text { Upper Salaco clay.50 } \\
\text { to } 100 \text { years }\end{array}$ & RSH_AIR & Air-supply atuent rediess $(3.09 \mathrm{~m})$ & $32212 A$ & 13Fab-96 & $\mathbf{Y}$ & 48 \\
\hline$Y$ & & 2919 & CL_M_T4 & $\begin{array}{l}\text { Uppor Sabado ctay.50 } \\
\text { to } 100 \text { years }\end{array}$ & RSH_ANR & Air-supply shent radies $(3.09 \mathrm{~m})$ & $32212 A$ & $13+5 e b-96$ & $\mathbf{Y}$ & 48 \\
\hline$Y$ & & 2919 & CL_M_T4 & $\begin{array}{l}\text { Upper Salado ctay.50 } \\
\text { to } 100 \text { years }\end{array}$ & RSH_AR & Ar-apply stuet radius (3.09 m) & 322124 & $13-\infty \omega-96$ & $\mathbf{Y}$ & 48 \\
\hline $\mathbf{Y}$ & & 2920 & CL_M_T4 & $\begin{array}{l}\text { Upper Selado clay. } 50 \\
\text { to } 100 \text { years }\end{array}$ & RSH_SAL & Salthanding shatt radius $(1.8 \mathrm{~m})$ | & $32214 a$ & $13-F a b-96$ & $\mathbf{Y}$ & 18 \\
\hline$Y$ & & 2920 & CL_M_T4 & $\begin{array}{l}\text { Uppor Salado clay.50 } \\
\text { to } 100 \text { years }\end{array}$ & RSH_SAL & Salthanding sthath radies $(1.8 \mathrm{~m}) \mid$ & 322144 & 13 fob-96 & $\mathbf{Y}$ & 48 \\
\hline$Y$ & & 2920 & CL_M_T4 & $\begin{array}{l}\text { Upper Salaco clay.50 } \\
\text { to } 100 \text { yoars }\end{array}$ & RSH_SAL & Salthending that radius $(1.8 \mathrm{~m})$ & 322144 & 13 feb-96 & $\mathbf{Y}$ & 48 \\
\hline $\mathbf{Y}$ & & 2920 & $C L \_M \_T 4$ & $\begin{array}{l}\text { Upper Salado clay.50 } \\
\text { to } 100 \text { years }\end{array}$ & RSH_SAL & Sathandling ahath radius $(1.8 \mathrm{~m})$ & 322144 & 13Feb-96 & $\mathbf{Y}$ & $4 B$ \\
\hline Y & & 2921 & CL_M_T4 & $\begin{array}{l}\text { Upper Salado ctay:50 } \\
\text { to } 100 \text { years }\end{array}$ & RSH_WAS & $\begin{array}{l}\text { Wasto-randing shan radus (3.5 } \\
\text { m) }\end{array}$ & $32215 A$ & $13+F a b-96$ & $\mathbf{Y}$ & 48 \\
\hline Y & & 2921 & CL_M_T4 & $\begin{array}{l}\text { Upper Salado y.50 } \\
\text { to } 100 \text { years }\end{array}$ & RSH_WAS & $\begin{array}{l}\text { Wastohanding shan radus (3.5 } \\
\text { (m) }\end{array}$ & $32215 A$ & $13-F a b-96$ & $\mathbf{Y}$ & 4B \\
\hline $\mathbf{Y}$ & & 2921 & CL_M_TA & $\begin{array}{l}\text { Uppor Satado clay.50 } \\
\text { to } 100 \text { years }\end{array}$ & RSH_WAS & $\begin{array}{l}\text { Wastohanding stan radius (3.5 } \\
\text { m) }\end{array}$ & $32215 A$ & 13-Feb-96 & $\mathbf{Y}$ & AB \\
\hline $\mathbf{Y}$ & & 2921 & $C L_{-} M_{-} T 4$ & $\begin{array}{l}\text { Upper Salado clay. } 50 \\
\text { to } 100 \text { years }\end{array}$ & RSH_WAS & $\begin{array}{l}\text { Wasto-handling shaft radies (3.5 } \\
\text { (m) }\end{array}$ & 32215A & 13Feb-96 & $\mathbf{Y}$ & 48 \\
\hline $\mathbf{Y}$ & & 2922 & $C L_{-} M_{-}{ }^{4}$ & $\begin{array}{l}\text { Uppor Salado clay.50 } \\
\text { to } 100 \text { years }\end{array}$ & RSH_EXH & Air-exhoust shat radius $(2.3 \mathrm{~m})$ & $32213 A$ & 13 feb-96 & $\mathbf{Y}$ & AB \\
\hline $\mathbf{Y}$ & & 2922 & CL_M_T4 & $\begin{array}{l}\text { Upper Salado clay:50 } \\
\text { to } 100 \text { years }\end{array}$ & RSH_EXH & Air-extheust that radives (2.3 m) & $32213 A$ & $13+F=b-96$ & $\mathbf{Y}$ & AB \\
\hline $\mathbf{Y}$ & & 2922 & CL_M_T4 & $\begin{array}{l}\text { Uppor Salado day.50 } \\
\text { to } 100 \text { years }\end{array}$ & RSH_EXH & Air-axhaust shat radius $(2.3 \mathrm{~m})$ & $32213 A$ & 13-Feb-96 & $\mathbf{Y}$ & 48 \\
\hline $\mathbf{Y}$ & & 2922 & CL_M_T4 & $\begin{array}{l}\text { Upper Selado ciry.50 } \\
\text { to } 100 \text { yeers }\end{array}$ & RSH_EXH & Air-axheust that radius $(2.3 \mathrm{~m})$ & 322134 & 13-Fob-96 & $-\boldsymbol{y}$ & 48 \\
\hline $\mathbf{Y}$ & & 2923 & $C L_{-} M_{-} T 4$ & $\begin{array}{l}\text { Upper Salacos clay:50 } \\
\text { to } 100 \text { yeers }\end{array}$ & RADN_DRZ & DRZ outor radus at each shan & 32207 & 13Fob-96 & $\mathbf{Y}$ & 1 \\
\hline $\mathbf{Y}$ & & 2923 & CL_M_TA & $\begin{array}{l}\text { Upper Saledo diay.50 } \\
\text { to } 100 \text { years }\end{array}$ & RADN_DRZ & DRZ outer radius at each shant & 32207 & $13-F 6 b-96$ & $\mathbf{Y}$ & 1 \\
\hline Y & & 2923 & $C L$ M_TA & $\begin{array}{l}\text { Uppor Salado clay.50 } \\
\text { to } 100 \text { years }\end{array}$ & RADN_DRZ & DRZ arter radius at each ahant & 32207 & 13Feb-96 & $\gamma$ & 1 \\
\hline $\mathbf{Y}$ & & 2924 & CL_M_T5 & $\begin{array}{l}\text { Upper Saledo clay. } 100 \\
\text { to 10K youss }\end{array}$ & RSH_AR & Air-aupply ehant redius $(3.09 \mathrm{~m})$ & 322494 & 13 Fob-96 & $Y$ & 4B \\
\hline$Y$ & & 2924 & CL_M_T5 & $\begin{array}{l}\text { Uppor Saledo ctay:100 } \\
\text { to 10K years }\end{array}$ & RSH_AIR & Ar-eupply entat radies $(3.09 \mathrm{~m})$ & 322494 & 13-feb-96 & $\mathbf{Y}$ & $A B$ \\
\hline $\mathbf{Y}$ & & 2924 & CL_M_T5 & $\begin{array}{l}\text { Upper Seledo day.100 } \\
\text { to 10K years }\end{array}$ & RSH_AR & Air-aupply ethath redius (3.09 m) & 322494 & 13 fob-96 & $Y$ & 4B \\
\hline $\mathbf{Y}$ & & 2924 & CL_M_T5 & $\begin{array}{l}\text { Upper Selecos day:100 } \\
\text { to 10K years }\end{array}$ & RSH_NR & Air-tupply ehan radicas $(3.09 \mathrm{~m})$ & 322494 & $13-F \mathrm{cb}-96$ & $Y$ & 48 \\
\hline$Y$ & & 2925 & CL_M_TS & $\begin{array}{l}\text { Upper Salado clay:100 } \\
\text { to 10K years }\end{array}$ & RSH_SAL & Salt thending sthat radius $(1.8 \mathrm{~m})$ & $32251 A$ & 13-Fob-86 & $\mathbf{Y}$ & $A B$ \\
\hline $\mathbf{Y}$ & & 2925 & $C L_{-} M_{-} T 5$ & $\begin{array}{l}\text { Uppor Saledo cay.100 } \\
\text { to 10K yours }\end{array}$ & RSH_SAL & Sallthending that radius $(1.8 \mathrm{~m})$ & $32251 A$ & $13-F .6-96$ & $\mathbf{Y}$ & 48 \\
\hline $\mathbf{Y}$ & & 2925 & $C L_{-} M_{2} T 5$ & $\begin{array}{l}\text { Upper Saindo diay.100 } \\
\text { to 10K yeirs }\end{array}$ & RSH_SAL & Sexthending etratt radius $(1.8 \mathrm{~m})$ & $32251 A$ & $13 f a b-96$ & $\mathbf{Y}$ & 48 \\
\hline $\mathbf{Y}$ & & 2925 & CL_M_TS & $\begin{array}{l}\text { Uppor Seledo ctay:100 } \\
\text { to 10K yeers }\end{array}$ & RSH_SAL & Selthendling shent redius $(1.8 \mathrm{~m})$ & $32251 \mathrm{~A}$ & $13-F-60$ & $\mathbf{Y}$ & 4B \\
\hline
\end{tabular}




\begin{tabular}{|c|c|c|c|c|c|c|c|c|c|c|}
\hline 10 & $\begin{array}{c}\text { Cimeth- } \\
\text { catton of } \\
\text { Deth } \\
\text { Category } \\
\text { (foctnota 2) }\end{array}$ & $\begin{array}{c}\text { Distriburtion } \\
\text { Type }\end{array}$ & $\operatorname{Man}$ & Medin & Mhinmem & Rexners & Units & Reference iD & 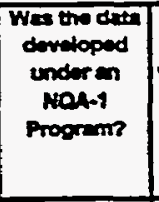 & 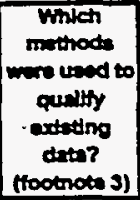 \\
\hline 2915 & 1,3 & CONSTANT & $3.5000000 \mathrm{E}+01$ & $3.5000000 \mathrm{E}+01$ & $3.5000000 E+01$ & $3.5000000 E+01$ & $\log \left(m^{\wedge} 2\right)$ & WP035597 & $\mathbf{Y}$ & NA \\
\hline 2915 & 1,3 & CONSTANT & $-3.5000000 E+01$ & $-3.5000000 \mathrm{E}+01$ & $-3.5000000 E+01$ & $3.5000000 E+01$ & $\log \left(m^{\wedge} 2\right)$ & WP038337 & $\mathbf{Y}$ & N/A \\
\hline 2915 & 1,3 & CONSTANT & $-3.5000000 E+01$ & $-3.5000000 E+01$ & $-3.5000000 E+01$ & $3.5000000 E+01$ & $\log \left(m^{\wedge} 2\right)$ & WP040514 & $\mathbf{Y}$ & NA \\
\hline 2916 & 1,3 & CONSTANT & $3.5000000 E+01$ & $3.5000000 E+01$ & $-3.5000000 E+01$ & $-3.5000000 E+01$ & $\log \left(m^{\wedge} 2\right)$ & WP032291 & $\mathbf{Y}$ & NA \\
\hline 2916 & 1.3 & CONSTANT & $-3.5000000 E+01$ & $3.5000000 \mathrm{E}+01$ & $-3.5000000 E+01$ & $-3.5000000 E+01$ & $\log \left(m^{\wedge} 2\right)$ & WP035597 & $\mathbf{Y}$ & NA \\
\hline 2916 & 1,3 & CONSTANT & $3.5000000 E+01$ & $-3.5000000 E+01$ & $-3.5000000 E+01$ & $-3.5000000 E+01$ & $\log \left(m^{\wedge} 2\right)$ & WP038337. & $\mathbf{Y}$ & $N A$ \\
\hline 2916 & 1,3 & CONSTANT & $3.5000000 E+01$ & $3.5000000 E+01$ & $-3.5000000=01$ & $-3.5000000 E+01$ & $\log \left(m^{n} 2\right)$ & WPO40514 & $\mathbf{Y}$ & NA \\
\hline 2918 & 3,5 & CONSTANT & $4.0000000 E+06$ & $4.0000000 E+06$ & $1.0000000 E+06$ & $4.0000000 E+05$ & $m^{\wedge} 3$ & WP031082 & $\mathbf{N}$ & 1 \\
\hline 2918 & 3,5 & CONSTANT & $4.0000000 E+06$ & $4.0000000 E+06$ & $4.0000000 E+06$ & $1.0000000 E+08$ & $m^{2} 3$ & WP035597 & $\mathbf{N}$ & 1 \\
\hline 2949 & 1,3 & CONSTANT & $3.0900000 E+\infty 0$ & $3.0900000 E+\infty$ & $3.0900000 E+\infty$ & $3.0900000 E+\infty$ & m & WPO30640 & $\mathbf{Y}$ & N/A \\
\hline 2919 & 1,3 & CONSTANT & $3.0900000 E+\infty$ & $3.0900000 E+\infty 0$ & $3.0900000 E+\infty$ & $3.0900000 E+\infty$ & m & WP030994 & $\mathbf{Y}$ & NA \\
\hline 2919 & 1,3 & CONSTANT & $3.0900000 E+00$ & $3.0900000 E+00$ & $3.0900000 E+\infty$ & $3.0900000 E+00$ & $m$ & WP035597 & $\mathbf{Y}$ & NA \\
\hline 2919 & 1,3 & CONSTANT & $3.0900000 E+00$ & $3.0900000 E+\infty 0$ & $3.0900000 E+\infty 0$ & $3.0900000 E+\infty 0$ & m & WPO40514 & $\mathbf{Y}$ & NA \\
\hline 2920 & 1,3 & CONSTANT & $1.8000000 E+\infty$ & $1.8000000 \mathrm{E}+\infty 0$ & $1.8000000 E+\infty$ & $1.8000000 E+\infty$ & $\mathbf{m}$ & WP030640 & $\mathbf{Y}$ & NA \\
\hline 2920 & 1,3 & CONSTANT & $1.8000000 E+\infty$ & $1.8000000 E+\infty$ & $1.8000000 \mathrm{E}+\infty$ & $1.8000000 E+\infty)$ & $\mathbf{m}$ & WP030994 & $\mathbf{Y}$ & NA \\
\hline 2920 & 1,3 & CONSTANT & $1.8000000 E+\infty 0$ & $1.8000000 E+\infty$ & $1.8000000 E+\infty 0$ & $1.8000000 E+\infty$ & $m$ & WP035597 & $\mathbf{Y}$ & N/A \\
\hline 2920 & 1,3 & CONSTANT & $1.8000000 \mathrm{E}+\infty 0$ & $1.8000000 E+\infty 0$ & $1.8000000 E+\infty 0$ & $18000000 E+\infty$ & $\mathbf{m}$ & WP040514 & $\mathbf{Y}$ & NA \\
\hline 2929 & 1,3 & CONSTANT & $3.5000000 E+00$ & $35000000 E+\infty$ & $3.5000000 E-\infty$ & $35000000 \leqslant+\infty$ & $\mathbf{m}$ & WP030540 & $\mathbf{Y}$ & N/A \\
\hline 2921 & 1,3 & CONSTANT & $3.5000000 E+\infty$ & $3.5000000 E+\infty 0$ & $3.5000000 E+\infty$ & $35000000 E+\infty$ & $m$ & WP030994 & $\mathbf{Y}$ & NA \\
\hline 2921 & 1,3 & CONSTANT & $3.5000000 E+\infty 0$ & $35000000 E+\infty$ & $35000000 E+\infty$ & $35000000 E+\infty$ & $m$ & WP035597 & $\mathbf{Y}$ & N/A \\
\hline 2921 & 1,3 & CONSTANT & $3.5000000 E+00$ & $35000000 E+\infty 0$ & $35000000<-\infty$ & $35000000 E+\infty$ & $\mathbf{m}$ & WPO40514 & $\mathbf{\gamma}$ & NA \\
\hline 2922 & 1,3 & CONSTANT & $2.3000000 E+\infty 0$ & $2.3000000 E+\infty 0$ & $23000000<+\infty$ & $23000000 E+\infty$ & m & WP030640 & $\mathbf{Y}$ & NA \\
\hline 2922 & 1,3 & CONSTANT & $2.3000000 E+\infty 0$ & $2.3000000 E+\infty$ & $23000000<+\infty$ & $23000000 \times+\infty$ & m & WP030994 & $\mathbf{Y}$ & $N A$ \\
\hline 2922 & 1,3 & CONSTANT & $2.3000000 E+\infty$ & $2.3000000 E+\infty$ & $23000000<-\infty$ & $23000000<+\infty$ & $m$ & WP035597 & $\mathbf{Y}$ & N/A \\
\hline 2922 & 1,3 & CONSTANT & $2.3000000 E+\infty 0$ & $23000000 E \cdot-\infty$ & $2300000<+\infty$ & $23000000 \times+\infty$ & $m$ & WPO40514 & $-Y$ & NA \\
\hline 2923 & $1,3,4$ & CONSTANT & $1.1070000 E+\infty$ & $11070000 E+\infty$ & $110+2000<-\infty$ & $1, c r+\infty 0<<-\infty$ & $\mathrm{m} / \mathrm{m}$ & WPO30640 & $\mathbf{Y}$ & NA \\
\hline 2923 & $1,3,4$ & CONSTANT & $1.1070000 E+\infty 0$ & $11070000 \mathrm{E}+\infty 0$ & $110: 0000<\infty \infty$ & $110,0000<-\infty$ & $\mathrm{m} / \mathrm{m}$ & WP030994 & $\mathbf{Y}$ & NA \\
\hline 2923 & $1,3,4$ & CONSTANT & $1.1070000 E+\infty 0$ & $11070000 E+\infty$ & 1 1070000e $\infty \infty$ & I 1c: $=000 \times+\infty$ & $\mathrm{m} / \mathrm{m}$ & WP035597 & $\mathbf{Y}$ & NA \\
\hline 2924 & 1,3 & CONSTANT & $3.0900000 E+\infty$ & $30900000<-\infty$ & $1000000 \times-\infty$ & $3000000<-\infty$ & m & WPO30540 & $\mathbf{Y}$ & NA \\
\hline 2924 & 1,3 & CONSTANT & $3.0900000 E+\infty 0$ & $30900000 E+\infty$ & $30 \infty 000<-\infty$ & $300000<<+\infty$ & m & WP030994 & $\mathbf{Y}$ & NA \\
\hline 2924 & 1,3 & CONSTANT & $3.0900000 E+\infty$ & $30 s 00000<-\infty$ & $30 \infty 000 \times-\infty$ & $32000000<-\infty$ & $m$ & WP035597 & $\mathbf{Y}$ & NA \\
\hline 2924 & 1,3 & CONSTANT & $3.0900000 E+\infty 0$ & $30900000 E-\infty$ & $30000000<-\infty$ & $30,00000<-\infty$ & $\mathbf{m}$ & WP040514 & $\mathbf{Y}$ & NAA \\
\hline 2925 & 1,3 & CONSTANT & $1.8000000 E+\infty$ & $18000000 \mathrm{E}+\infty$ & $1000000<-\infty$ & $10000000 E+\infty$ & $\mathrm{m}$ & WPO30640 & $\mathbf{Y}$ & $\mathbf{N A}$ \\
\hline 2925 & 1,3 & CONSTANT & $1.8000000 E+\infty 0$ & $18000000 E+\infty$ & $1, \infty \infty \infty \infty)<-\infty$ & $1+\infty 00000=+\infty$ & $m$ & WP030994 & $\mathbf{Y}$ & $N / A$ \\
\hline 2925 & 1,3 & CONSTANT & $1.8000000 E+00$ & $10000000 E+\infty$ & $10000000<+\infty$ & $1,000000 \in+\infty$ & m & WP035597 & $\mathbf{Y}$ & NA \\
\hline 2925 & 1,3 & CONSTANT & $1.8000000 E+\infty 0$ & $18000000 \mathrm{E}+\infty 0$ & $10000000<+\infty$ & $18000000 E+\infty$ & $m$ & WPO40514 & $\mathbf{Y}$ & NUA \\
\hline
\end{tabular}


CCA Parameter Listing

\begin{tabular}{|c|c|c|c|c|c|c|c|c|c|c|}
\hline $\begin{array}{c}\text { Parameter in } \\
\text { Datebees? } \\
\text { (YN) }\end{array}$ & 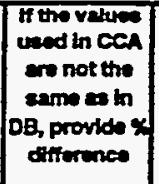 & W & Metortal to & Matorde Name & $\begin{array}{l}\text { Perantorar } \\
\text { DD }\end{array}$ & Perminter Kame & $\begin{array}{l}\text { PRPDD } \\
\text { MPO N) }\end{array}$ & Dete Entry & 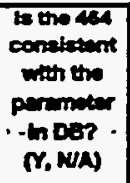 & $\begin{array}{l}\text { Paramiter } \\
\text { Catiogory } \\
\text { (footnote i) }\end{array}$ \\
\hline Y & & 2926 & CL_M_TS & $\begin{array}{l}\text { Uppor Salado clay: } 100 \\
\text { to 10K years }\end{array}$ & RSH_WAS & $\begin{array}{l}\text { Wasto-handling shan radus (3.5 } \\
\text { m) }\end{array}$ & 322524 & $13-f \infty-96$ & $\mathbf{Y}$ & AB \\
\hline Y & & 2926 & CL_M_TS & $\begin{array}{l}\text { Upper Saleso ctay. } 100 \\
\text { to 10K years }\end{array}$ & RSH_WAS & $\begin{array}{l}\text { Wastohtandthog shen roduss (3.5 } \\
\text { m) }\end{array}$ & $32252 A$ & $13-F a b-96$ & $\mathbf{Y}$ & AB \\
\hline $\mathbf{Y}$ & & 2926 & CL_M_T5 & $\begin{array}{l}\text { Uppor Saloco ciny:100 } \\
\text { to 10K years }\end{array}$ & RSH_WAS & $\begin{array}{l}\text { Wastothanding shen redes }(3.5 \\
\text { (m) }\end{array}$ & $32252 A$ & $13+a b-96$ & $\mathbf{Y}$ & AB \\
\hline $\mathbf{Y}$ & & 2926 & CL_M_T5 & $\begin{array}{l}\text { Uppor Seleob cisy.100 } \\
\text { to } 10 \mathrm{~K} \text { years }\end{array}$ & RSH_WAS & $\begin{array}{l}\text { Wastothanding shan rodus (3.5 } \\
\text { (m) }\end{array}$ & $32252 A$ & 13-Fob-96 & $\mathbf{Y}$ & 48 \\
\hline$Y$ & & 2927 & CL_M_T5 & $\begin{array}{l}\text { Upper Salado day.100 } \\
\text { to 10K years }\end{array}$ & RSH_EXH & Ar-echaust ahent radies $(2.3 \mathrm{~m})$ & $32250 \mathrm{~A}$ & 13 Fob-96 & $\mathbf{Y}$ & $4 B$ \\
\hline $\mathbf{Y}$ & & 2927 & CL_M_T5 & $\begin{array}{l}\text { Uppor Saledo clay:100 } \\
\text { to 10K years }\end{array}$ & RSH_EXH & Air-axhaust thent radius $(2.3 \mathrm{~m})$ & $32250 a$ & $13-5 \cos -96$ & $\mathbf{Y}$ & 48 \\
\hline $\mathbf{Y}$ & & 2927 & CL_H_T5 & $\begin{array}{l}\text { Uppor Salado ctory.100 } \\
\text { to } 10 \text { K years }\end{array}$ & RSH_EXH & Ait-extheust thent radius (2.3 m) & 322504 & $13+5 a b-96$ & $\mathbf{Y}$ & 48 \\
\hline $\mathbf{Y}$ & & 2927 & Cl_M_T5 & $\begin{array}{l}\text { Upper Salado clay:100 } \\
\text { to 10K years }\end{array}$ & RSH_EXH & Air-exthent ahent radies $(2.3 \mathrm{~m})$ & 322504 & 13 Feb-96 & $Y$ & 48 \\
\hline $\mathbf{Y}$ & & 2928 & CL_M_T5 & $\begin{array}{l}\text { Uppor Salaso clay: } 100 \\
\text { to } 10 \mathrm{~K} \text { years }\end{array}$ & RADN_DRZ & DRZ outer redius at each athent & 32245 & 13-Fab-96 & $\mathbf{Y}$ & 1 \\
\hline $\mathbf{Y}$ & & 2928 & CL_M_T5 & $\begin{array}{l}\text { Uppor Salado ciny:100 } \\
\text { to 10K yoars }\end{array}$ & RADN_DRZ & DRZ octer redies at each shent & 32245 & 13-Fab-96 & $\mathbf{Y}$ & 1 \\
\hline $\mathbf{Y}$ & & 2928 & CL_M_TS & $\begin{array}{l}\text { Upper Selado clay:100 } \\
\text { to 10K years }\end{array}$ & RADN_DRZ & DRZ outer radius at each shath & 32245 & 13 freb-96 & $\mathbf{Y}$ & 1 \\
\hline$Y$ & & 2929 & ASPHALT & Asphat column & RSH_AIR & Air-aupply stant radius (3.09 m) & 313999 & $13-500-96$ & $\mathbf{Y}$ & 48 \\
\hline$Y$ & & 2929 & ASPHALT & Asphath colemn & RSH_AIR & Air-suppty shant radius $(3.09 \mathrm{~m})$ & 31399 & $13-F e b-96$ & $\mathbf{Y}$ & AB \\
\hline $\mathbf{Y}$ & & 2929 & ASPHALT & Asphat cokmn & RSH_AR & Ar-eupply ehent redies $(3.09 \mathrm{~m})$ & 313992 & 13 feb-96 & $\mathbf{Y}$ & AB \\
\hline $\mathbf{Y}$ & & 2929 & ASPHALT & Asphell cokmn & RSH_ASR & Arrespply ahent rucius $(3.09 \mathrm{~m})$ & 313994 & 13Feb-96 & $\mathbf{Y}$ & 18 \\
\hline $\mathbf{Y}$ & & 2930 & ASPHALT & Asphall cokmn & RSH_SAL & Sellthending shath radius $(1.8 \mathrm{~m})$ & $31401 \mathrm{~A}$ & $13+$ feb-96 & $\mathbf{Y}$ & 48 \\
\hline $\mathbf{Y}$ & & 2930 & ASPHALT & Asphat column & RSH_SAL & Sathending then redius $(1.8 \mathrm{~m})$ & $31401 \mathrm{~A}$ & $13+f a b-96$ & $\mathbf{Y}$ & 48 \\
\hline $\mathbf{Y}$ & & 2930 & ASPHALT & Asphalt column & RSH_SAL & Salthanding shen radius $(1.8 \mathrm{~m})$ & $31401 A$ & $13-F a b-96$ & $\mathbf{Y}$ & AB \\
\hline$Y$ & & 2930 & ASPHALT & Asphats column & RSH_SAL & Salt thanding sthen radius $(1.8 \mathrm{~m})$ & $31401 A$ & $13-F a b-96$ & $\mathbf{Y}$ & $4 B$ \\
\hline$Y$ & & 2931 & ASPHALT & Asphat column & RSH_WAS & $\begin{array}{l}\text { Wasto-randing shat radus (3.5 } \\
\text { m) }\end{array}$ & $31402 A$ & 13-Feb-9s & $\mathbf{Y}$ & 48 \\
\hline Y & & 2931 & ASPHALT & Asphat column & RSH_WAS & $\begin{array}{l}\text { Wasto-hending shat radies (3.5 } \\
\text { m) }\end{array}$ & $31402 A$ & $13+a b-96$ & $\mathbf{Y}$ & 48 \\
\hline$Y$ & & 2931 & ASPHALT & Assphatt column & RSH_WAS & $\begin{array}{l}\text { Wasto hending enaft radus (3.5 } \\
\text { m) }\end{array}$ & $31402 A$ & 13-Feb-96 & $\mathbf{Y}$ & 48 \\
\hline $\mathbf{Y}$ & & 2931 & ASPHALT & Asphatt column & RSH_WAS & $\begin{array}{l}\text { Wasto-hending stunt radxus (3.5 } \\
\text { m) }\end{array}$ & $31402 A$ & 13-Fab-96 & $\mathbf{Y}$ & $4 B$ \\
\hline$Y$ & & 2932 & ASPHALT & Asphatit column & RSH_EXH & Ar-exthoust enthen radus $(2.3 \mathrm{~m})$ & $31400 A$ & 13-Feb-96 & $\mathbf{Y}$ & $4 B$ \\
\hline$Y$ & & 2932 & ASPHALT & Asphat cokmn & RSH_EXH & Ar-axtraurt shan redius $(2.3 \mathrm{~m})$ & 314004 & $13+F b-96$ & $-{ }_{-Y}$ & 48 \\
\hline $\mathbf{Y}$ & & 2932 & ASPHALT & Asphat column & RSH_EXH & Artaxheust entent radxes $(2.3 \mathrm{~m})$ & $31400 A$ & $13-F 0 b-96$ & $\mathbf{Y}$ & AB \\
\hline$Y$ & & 2932 & ASPHALT & Asphatt column & RSH_EXH & Ar-acheurst thant radus $(2.3 \mathrm{~m})$ & 314004 & 13-Feb-96 & $\mathbf{Y}$ & AB \\
\hline$Y$ & & 2933 & ASPHALT & Asphatr column & RADN_ORZ & DFiz ouker redies at esch shan & 31395 & 13 Fob-96 & $Y$ & 1 \\
\hline$Y$ & & 2933 & ASPHALT & Asphatt column & RAON_ORZ & Driz outer redius at esch shan & 31395 & 13-Fab-96 & $Y$ & 1 \\
\hline$Y$ & & 2933 & ASPHALT & Asphat column & RADN_DRZ & DRZ ater redives at eech shan & 31395 & $13-f a b-96$ & $\bar{Y}$ & 1 \\
\hline$Y$ & & 2934 & SALT_TI & 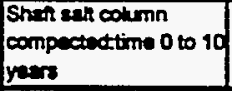 & RSH_ANR & Artapply then redus $(3.09 \mathrm{~m})$ & $33407 A$ & $13-c b-96$ & $\mathbf{Y}$ & 4B \\
\hline $\mathbf{Y}$ & & 2934 & SNT_TI & $\begin{array}{l}\text { Shat selt column } \\
\text { compectectime } 0 \text { to id } \\
\text { years }\end{array}$ & RSH_ANR & Ar-apoly athen radiess $(3.09 \mathrm{~m})$ & $33407 \mathrm{~A}$ & $13+506-96$ & $\mathbf{Y}$ & $A B$ \\
\hline $\mathbf{Y}$ & & 2934 & SALT_TI & $\begin{array}{l}\text { Shat sal colkmn } \\
\text { compectedtunt } 0 \text { to id } \\
\text { yean }\end{array}$ & RSH_NR & Artapply ahent radus $(3.09 \mathrm{~m})$ & $33407 \mathrm{~A}$ & 13-Feb-96 & $\mathbf{Y}$ & 48 \\
\hline$Y$ & & 2934 & SNLT_T1 & \begin{tabular}{|l|} 
Shan sal column \\
complatme 0 to id \\
years
\end{tabular} & RSH_NR & Artapply then radius (3.09 m) & $33407 A$ & $13-F=b-86$ & $\mathbf{Y}$ & AB \\
\hline $\mathbf{Y}$ & & 2935 & SALT_TI & 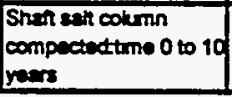 & RSH_SAL & Sethending thath radies $(9.8 \mathrm{~m})$ & $33411 A$ & $13-F a b-6$ & $\mathbf{Y}$ & $4 B$ \\
\hline
\end{tabular}


CCA Parameter-Listing

\begin{tabular}{|c|c|c|c|c|c|c|c|c|c|c|}
\hline 10 & 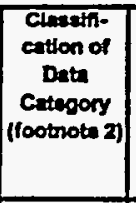 & $\begin{array}{l}\text { Distribution } \\
\text { Type }\end{array}$ & mean & uedien & mentinem & Mexionum & Units & Retwenca io & 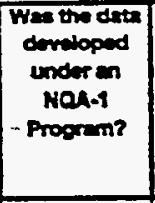 & 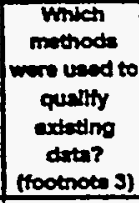 \\
\hline 2926 & 1,3 & CONSTANT & $3.5000000 \mathrm{E}+\infty 0$ & $3.5000000 \mathrm{E}+\infty 0$ & $3.5000000 \mathrm{E}+\infty 0$ & $3.5000000 E+\infty 0$ & $\mathrm{~m}$ & WP030540 & $\mathbf{Y}$ & NA \\
\hline 2926 & 1,3 & CONSTANT & $3.5000000 E+\infty 0$ & $3.5000000 \mathrm{E}+\infty 0$ & $3.5000000 E+\infty$ & $3.5000000 E+\infty 0$ & $\mathbf{m}$ & WP030994 & $\mathbf{Y}$ & NA \\
\hline 2926 & 1,3 & CONSTANT & $3.5000000 E+\infty 0$ & $3.5000000 E+\infty$ & $3.5000000 E+\infty$ & $3.5000000 \mathrm{E}+\infty$ & $m$ & WP035597 & $\mathbf{Y}$ & NA \\
\hline 2926 & 1,3 & CONSTANT & $3.5000000 E+\infty 0$ & $3.5000000 E+\infty$ & $3.5000000 E+00$ & $3.5000000 E+\infty$ & $m$ & WPO40514 & $\mathbf{Y}$ & NA \\
\hline 2927 & 1,3 & CONSTANT & $2.3000000 E+\infty 0$ & $2.3000000 E+\infty 0$ & $23000000 E+00$ & $2,3000000 E+00$ & $m$ & WP030540 & $\mathbf{Y}$ & NA \\
\hline 2927 & 1,3 & CONSTANT & $2.3000000 E+\infty 0$ & $2.3000000 \mathrm{E}+\infty 0$ & $2.3000000 E+\infty 0$ & $23000000 E+\infty 0$ & $m$ & WP030994 & $Y$ & NA \\
\hline 2927 & 1,3 & CONSTANT & $2.3000000 E+\infty 0$ & $23000000 E+\infty 0$ & $2.3000000 E+\infty 0$ & $230000000+\infty 0$ & $m$ & UP035597 & $\mathbf{Y}$ & NA \\
\hline 2927 & 1,3 & CONSTANT & $2.3000000 E+\infty 0$ & $2.3000000=+\infty 0$ & $2.3000000 E+\infty)$ & $23000000 E+\infty 0$ & $m$ & WPO40514 & $\mathbf{Y}$ & NA \\
\hline 2928 & $1,3,4$ & CONSTANT & $1.0000000 E+00$ & $1.0000000 \mathrm{E}+\infty$ & $1.0000000 E+\infty 0$ & $1.0000000 E+\infty 0$ & $\mathrm{~m} / \mathrm{m}$ & WP030540 & $Y$ & NA \\
\hline 2928 & $1,3,4$ & CONSTANT & $1.0000000 E+00$ & $1.00000000+\infty$ & $1.0000000 E+\infty 0$ & $1.0000000 E+\infty$ & $\mathrm{m} / \mathrm{m}$ & WP030994 & $\mathbf{Y}$ & NA \\
\hline 2928 & $1,3,4$ & CONSTANT & $1.0000000 E+\infty 0$ & $1.0000000 E+00$ & $1.0000000 E+00$ & $1.0000000 \mathrm{E}+00$ & $\mathrm{~m} / \mathrm{m}$ & WP035597 & $\mathbf{Y}$ & NA \\
\hline 2929 & 1,3 & CONSTANT & $3.0900000 E+\infty 0$ & $3.0900000 \mathrm{E}+\infty 0$ & $3.0900000 E+\infty 0$ & $3.0000000 E+\infty 0$ & $m$ & WP030640 & $\mathbf{Y}$ & NA \\
\hline 2929 & 1,3 & CONSTANT & $3.0900000 E+\infty$ & $3.0900000 \mathrm{E}+00$ & $3.0900000 E+\infty 0$ & $3.0900000 E+\infty 0$ & m & WP030994 & $Y$ & NA \\
\hline 2929 & 1,3 & CONSTANT & $3.0900000 E+00$ & $3.0900000 \mathrm{E}+\infty 0$ & $3.0900000 E+\infty$ & $3.0900000 \mathrm{E}+00$ & $m$ & WP035597 & $\mathbf{Y}$ & NA \\
\hline 2929 & 1,3 & CONSTANT & $3.0900000 \mathrm{E}+\infty 0$ & $3.0900000 \mathrm{E}+\infty 0$ & $3.0900000 \mathrm{E}+00$ & $3.0900000 \mathrm{E}+00$ & m & WPO40514 & $Y$ & NA \\
\hline 2930 & 1,3 & CONSTANT & $1.8000000 E+\infty 0$ & 1. $.8000000 \mathrm{E}+\infty$ & $1.8000000 E+\infty$ & $1.8000000 E+\infty 0$ & m & WPO30640 & $\mathbf{Y}$ & NA \\
\hline 2930 & 1,3 & CONSTANT & $1.8000000 E+\infty 0$ & $1.8000000 \mathrm{E}+\infty$ & $1.8000000 E+\infty$ & $1.8000000 \mathrm{E}+\infty$ & $\mathbf{m}$ & WP030994 & $\mathbf{Y}$ & NA \\
\hline 2930 & 1,3 & CONSTANT & $1.8000000 \mathrm{E}+\infty$ & $1.8000000 E+\infty 0$ & $1.8000000 E+\infty$ & $1.80000000 E+\infty$ & m & WP035597 & $\mathbf{Y}$ & NA \\
\hline 2930 & 1,3 & CONSTANT & $1.8000000 \mathrm{E}+00$ & $1.8000000 E+\infty$ & $1.8000000 E+\infty 0$ & $1.80000000 E+\infty 0$ & $\mathbf{m}$ & WP040514 & $\mathbf{Y}$ & NA \\
\hline 2931 & 1,3 & CONSTANT & $3.5000000 E+\infty 0$ & $3.5000000 \mathrm{E}+00$ & $3.5000000 E+\infty$ & $3.5000000 \mathrm{E}+\infty 0$ & $\mathbf{m}$ & WP030540 & $\mathbf{Y}$ & NIA \\
\hline 2931 & 1,3 & CONSTANT & $3.5000000 E+\infty$ & $3.5000000 E+00$ & $3.5000000 \mathrm{E}+\infty 0$ & $3.5000000 E+\infty$ & $m$ & WP030994 & $Y$ & NA \\
\hline 2931 & 1,3 & CONSTANT & $3.5000000 E+00$ & $3.5000000 E+\infty$ & $3.5000000 E+\infty$ & $3.5000000 E+\infty 0$ & $m$ & WP035597 & $\mathbf{Y}$ & NA \\
\hline 2931 & 1,3 & CONSTANT & $3.5000000 E+\infty$ & $3.5000000 E+\infty 0$ & $3.5000000 E+\infty$ & $3.5000000 E+\infty$ & $m$ & WPO40514 & $\mathbf{Y}$ & NA \\
\hline 2932 & 1,3 & CONSTANT & $2.3000000 E+\infty$ & $2.3000000 E+\infty 0$ & $2.3000000 \mathrm{E}+\infty 0$ & $2.3000000 E+\infty$ & m & WP030640 & $Y$ & NA \\
\hline 2932 & 1,3 & CONSTANT & $2.3000000 E+\infty 0$ & $2.3000000 \mathrm{E}+\infty$ & $2.3000000 E+00$ & $2,3000000 \varepsilon+\infty 0$ & $m$ & WP030994 &.$- Y$ & NA \\
\hline 2932 & 1,3 & CONSTANT & $2.3000000 E+\infty$ & $2.3000000 E+00$ & $2.3000000 E+\infty 0$ & $2.3000000 E+\infty 0$ & m & WP035597 & $\mathbf{Y}$ & NA \\
\hline 2932 & 1,3 & CONSTANT & $2.3000000 E+\infty$ & $2.3000000 E+\infty 0$ & $2.3000000 E+\infty 0$ & $2.3000000 \varepsilon+\infty 0$ & m & WPO40514 & $\mathbf{Y}$ & NA \\
\hline 2933 & $1,3,4$ & CONSTANT & $1.6290000 \mathrm{E}+\infty 0$ & $1.6290000 \mathrm{E}+\infty 0$ & $1.6290000 \mathrm{E}+\infty$ & $1.6290000 \mathrm{E}+00$ & $\mathrm{~m} / \mathrm{m}$ & WP030640 & $Y$ & NA \\
\hline 2933 & 1.3 .4 & CONSTANT & $1.6290000 \mathrm{E}+00$ & $1.6290000 \mathrm{E}+00$ & $1.6290000 \mathrm{E}+00$ & $1.6290000 E+00$ & $\mathrm{~m} / \mathrm{m}$ & WP030994 & $\mathbf{Y}$ & NA \\
\hline 2933 & $1,3,4$ & CONSTANT & $1.6290000 \mathrm{E}+00$ & $1.62900000+\infty 0$ & $1.6290000 \mathrm{E}+00$ & $1.62900000 \mathrm{E}+00$ & $\mathrm{~m} / \mathrm{m}$ & WP035597 & $Y$ & NA \\
\hline 2934 & 1,3 & CONSTANT & $3.09000000+\infty$ & $3.0900000 E+\infty$ & $3.0900000 E+\infty$ & $3.0900000 E+\infty 0$ & $m$ & WP030640 & $Y$ & N/A \\
\hline 2934 & 1.3 & CONSTANT & $3.0900000 E+\infty 0$ & $3.0900000 E+\infty$ & $3.0900000 E+\infty 0$ & $3.09000000+\infty$ & $\mathbf{m}$ & WP030994 & $\mathbf{Y}$ & N/A \\
\hline 2934 & 1,3 & CONSTANT & $3.0900000 E+\infty 0$ & $3.09000000+00$ & $3.0900000 E+00$ & $3.0900000 E+\infty$ & $m$ & WP035597 & $\mathbf{Y}$ & NA \\
\hline 2934 & 1,3 & CONSTANT & $3.09000000 E+\infty 0$ & $3.09000000+\infty$ & $3.09000000+\infty 0$ & $3.0900000 E+\infty 0$ & $m$ & WPO40514 & $\gamma$ & NA \\
\hline 2935 & 1,3 & CONSTANT & $1.80000000+\infty$ & $1.8000000 E+\infty 0$ & $1.8000000 \mathrm{E}+\infty 0$ & $1.80000000+00$ & $m$ & WPO30640 & $Y$ & NA \\
\hline
\end{tabular}


CCA Parameter Listing

\begin{tabular}{|c|c|c|c|c|c|c|c|c|c|c|}
\hline 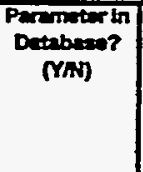 & 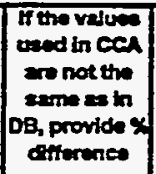 & TD & Matedal id & Matantal Nem & $\begin{array}{c}\text { Parematiox } \\
\text { D. }\end{array}$ & 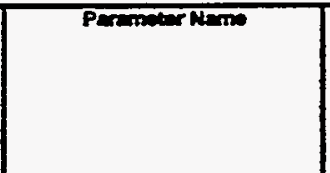 & $\begin{array}{c}\text { PRPD } \\
\text { (NPO) }\end{array}$ & Dents & 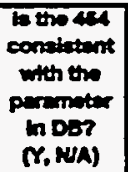 & $\begin{array}{l}\text { Parmiter } \\
\text { Cukgony } \\
\text { (footnote 1) }\end{array}$ \\
\hline $\mathbf{Y}$ & & 2935 & SALT_T1 & $\begin{array}{l}\text { Shaft salt colimn } \\
\text { compectedt tme } 0 \text { to } 10 \\
\text { years }\end{array}$ & RSH_SAL & Sathending then radxus $(1.8 \mathrm{~m})$ & $33491 A$ & $13-F a b-96$ & $\mathbf{Y}$ & 48 \\
\hline $\mathbf{Y}$ & & 2935 & SALT_T1 & $\begin{array}{l}\text { Shaft all column } \\
\text { compectedtine } 0 \text { to } 10 \\
\text { years }\end{array}$ & RSH_SNL & Saktharding shat radies $(1.8 \mathrm{~m})$ & 33411A & 13Fab-96 & $\mathbf{Y}$ & $A B$ \\
\hline $\mathbf{Y}$ & & 2935 & SALT_T1 & $\begin{array}{l}\text { Shan salt cotumn } \\
\text { compectedtime } 0 \text { to id } \\
\text { yoars }\end{array}$ & RSH_SAL. & Saxthanding ahaft radius $(1.8 \mathrm{~m})$ & $33411 \mathrm{~A}$ & 13 Feb-S6 & $\mathbf{Y}$ & 48 \\
\hline $\mathbf{Y}$ & & 2936 & SALT_TI & $\begin{array}{l}\text { Shaft sal colemn } \\
\text { compectedtime } 0 \text { to } 10 \\
\text { years }\end{array}$ & RSH_WAS & $\begin{array}{l}\text { Westo-hending that radius (3.5 } \\
\text { (m) }\end{array}$ & $33413 A$ & 13-feb-96 & $\mathbf{Y}$ & 48 \\
\hline $\mathbf{Y}$ & & 2936 & SALT_T1 & \begin{tabular}{|l|} 
Shath sak colimn \\
compectectine 0 to 10 \\
years
\end{tabular} & RSH_WAS & $\begin{array}{l}\text { Wasto-handing that redies (3.5 } \\
\text { m) }\end{array}$ & 334134 & $13-F a b-96$ & $\mathbf{Y}$ & 48 \\
\hline $\mathbf{Y}$ & & 2936 & SALT_TI & $\begin{array}{l}\text { Shat salt colemn } \\
\text { compactedtime } 0 \text { to } 10 \\
\text { years }\end{array}$ & RSH_WAS & $\begin{array}{l}\text { Wasto-handing shet redies (3.5 } \\
\text { m) }\end{array}$ & $33413 A$ & $13+50-96$ & $\mathbf{Y}$ & $4 B$ \\
\hline $\mathbf{Y}$ & & 2936 & SALT_T1 & $\begin{array}{l}\text { Shaft salt cohmn } \\
\text { compactectime } 0 \text { to id } \\
\text { years }\end{array}$ & RSH_WAS & $\begin{array}{l}\text { Wasto-handing that radius (3.5 } \\
\text { m) }\end{array}$ & $33413 A$ & 13-Feb-96 & $\mathbf{Y}$ & 48 \\
\hline $\mathbf{Y}$ & & 2937 & SALT_T1 & $\begin{array}{l}\text { Shaft salt column } \\
\text { compactedtime } 0 \text { to } 10 \\
\text { years }\end{array}$ & RSH_EXH & Air-exchaus shant radies $(2.3 \mathrm{~m})$ & 334104 & 13-Fob-96 & $\mathbf{Y}$ & $4 B$ \\
\hline $\mathbf{Y}$ & & 2937 & SALT_TI & $\begin{array}{l}\text { Shat salt column } \\
\text { compactedime } 0 \text { to } 10 \\
\text { years }\end{array}$ & RSH_EXH & Air-excheust shaft radius $(2.3 \mathrm{~m})$ & 334104 & $13-50 b-96$ & $\mathbf{Y}$ & 48 \\
\hline $\mathbf{Y}$ & & 2937 & SALT_T1 & $\begin{array}{l}\text { Shaft salt column } \\
\text { compactedtime } 0 \text { to } 10 \\
\text { years }\end{array}$ & RSH_EXH & Air-extheust atrant radius ( $2.3 \mathrm{~m}$ ) & 334104 & $13-F-b-96$ & $\mathbf{Y}$ & $4 B$ \\
\hline $\mathbf{Y}$ & & 2937 & SALT_T1 & $\begin{array}{l}\text { Shaft salt column } \\
\text { compactectims } 0 \text { to } 10 \\
\text { years }\end{array}$ & RSH_EXH & Air-excheust ghant rodius $(2.3 \mathrm{~m})$ & 33410A & $13-F a b-96$ & $\mathbf{Y}$ & $4 B$ \\
\hline $\mathbf{Y}$ & & 2938 & SALT_T1 & $\begin{array}{l}\text { Shaft salt column } \\
\text { compacted:time } 0 \text { to } 10 \\
\text { yoars }\end{array}$ & RADN_DRZ & DRZ outer radius at eacth shaft & 33401 & 13Fob-96 & $\mathbf{Y}$ & 1 \\
\hline $\mathbf{Y}$ & & 2938 & SALT_T1 & $\begin{array}{l}\text { Sheft selt column } \\
\text { compactedtime } 0 \text { to } 10 \\
\text { vears }\end{array}$ & RADN_DRZ & DRZ outer radius at each shath & 33401 & $13+F e b-96$ & $\mathbf{Y}$ & 1 \\
\hline $\mathbf{Y}$ & & 2938 & SALT_T1 & $\begin{array}{l}\text { Shaft salt cohumn } \\
\text { compactecttme } 0 \text { to } 10 \\
\text { years }\end{array}$ & RADN_DRZ & DRZ outer redus at each shant & 33401 & 13-Feb-96 & $\mathbf{Y}$ & 1 \\
\hline $\mathbf{Y}$ & & 2939 & SALT_T1 & $\begin{array}{l}\text { Shan salt column } \\
\text { compactedtime } 0 \text { to } 10 \\
\text { years }\end{array}$ & CUMPROB & Cumulative Probability & 33361 & $13-5 a b-96$ & $\mathbf{Y}$ & 48 \\
\hline $\mathbf{Y}$ & & 2939 & SALT_TI & $\begin{array}{l}\text { Shan salt column } \\
\text { compactedtume } 0 \text { to } 10 \\
\text { years }\end{array}$ & CUMPROB & Cumulative Probability & 33361 & 13-Fab-96 & $\mathbf{Y}$ & $4 B$ \\
\hline $\mathbf{Y}$ & & 2939 & SALT_T1 & $\begin{array}{l}\text { Shaft sal column } \\
\text { compactedtime } 0 \text { to } 10 \\
\text { years }\end{array}$ & CUMPROB & Cumulatrve Probability & 33361 & 13-Feb-96 & $=\mathbf{Y}$ & $A B$ \\
\hline $\mathbf{Y}$ & & 2939 & SALT_T1 & $\begin{array}{l}\text { Shati salt column } \\
\text { compactedtime } 0 \text { to } 10 \\
\text { years }\end{array}$ & CUMPROB & Cumulative Probebiltty & 33361 & 13-Fob-96 & $\mathbf{Y}$ & 48 \\
\hline $\mathbf{Y}$ & & 2939 & SALT_TI & $\begin{array}{l}\text { Shan salt column } \\
\text { compectectime } 0 \text { to } 10 \\
\text { yars }\end{array}$ & CUMPROB & Cumudativa Probability & 33361 & 13-Fob-96 & $\mathbf{Y}$ & $\mathbf{A B}$ \\
\hline $\mathbf{Y}$ & & 2939 & SALT_TI & $\begin{array}{l}\text { Shan salt column } \\
\text { compectedtime } 0 \text { to } 10 \\
\text { years }\end{array}$ & CUMPROB & Cumulativo Probebility & 33361 & $13-f e b-96$ & $\mathbf{Y}$ & 48 \\
\hline $\mathbf{Y}$ & varies & 2940 & SALT_TI & $\begin{array}{l}\text { Shan selt column } \\
\text { compectedtime } 0 \text { to } 10 \\
\text { yers }\end{array}$ & PMLT_MD & $\begin{array}{l}\text { Los trienguler distribution made } \\
\text { for permesbility }\end{array}$ & 33378 & 13Feb-96 & $\mathbf{Y}$ & 1 \\
\hline $\mathbf{Y}$ & varies & 2940 & SALT_T1 & $\begin{array}{l}\text { Shan salt column } \\
\text { compectectime } 0 \text { to } 10 \\
\text { years }\end{array}$ & PMLI_MO & $\begin{array}{l}\text { Log trimguler dietribution mode } \\
\text { for permestilty }\end{array}$ & 33378 & 13 Feb-96 & $\mathbf{Y}$ & 1 \\
\hline $\mathbf{Y}$ & varies & 2940 & SALT_T1 & $\begin{array}{l}\text { Shat salt colemn } \\
\text { compectedtume } 0 \text { to } 10 \\
\text { yers }\end{array}$ & PNLT_MO & $\begin{array}{l}\text { Log trianguler distribution mode } \\
\text { for permesbitity }\end{array}$ & 33378 & $13-F c b-96$ & $\mathbf{Y}$ & 1 \\
\hline $\mathbf{Y}$ & varies & 2941 & SALT_T1 & $\begin{array}{l}\text { Shm sill coimm } \\
\text { compectedtime } 0 \text { to } 10 \\
\text { years }\end{array}$ & PMLT_LO & $\begin{array}{l}\text { Log trianguter distribution baw } \\
\text { value for parmesbility }\end{array}$ & 33377 & $13-7 c b-96$ & $\mathbf{Y}$ & 1 \\
\hline
\end{tabular}




\begin{tabular}{|c|c|c|c|c|c|c|c|c|c|c|}
\hline 10 & $\begin{array}{l}\text { Clinstri- } \\
\text { cation of } \\
\text { Data } \\
\text { Cetegory } \\
\text { (footrota 2) }\end{array}$ & $\begin{array}{l}\text { Deteribertion } \\
\text { Type }\end{array}$ & $\operatorname{Men}$ & Medien & Minimem & Maximum & Lnits & Reference ID & 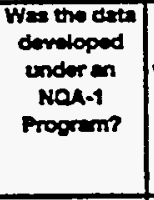 & $\begin{array}{l}\text { Which } \\
\text { mothod } \\
\text { wore used to } \\
\text { qually } \\
\text { exdeting } \\
\text { drtin? } \\
\text { (foctnote 3) }\end{array}$ \\
\hline 2935 & 1,3 & CONSTANT & $1.8000000 E+\infty 0$ & $1.8000000 \mathrm{E}+\infty$ & $1.8000000 E+\infty 0$ & $1.8000000 E+\infty 0$ & $\mathbf{m}$ & WP030994 & $\mathbf{Y}$ & NA \\
\hline 2935 & 1,3 & CONSTANT & $1.8000000 E+\infty 0$ & $1.8000000 \mathrm{E}+\infty$ & $1.8000000 E+\infty 0$ & $1.8000000 E+\infty 0$ & $m$ & WP035597 & $\mathbf{Y}$ & NA \\
\hline 2935 & 1,3 & CONSTANT & $1.8000000 E+\infty 0$ & $1.8000000 E+\infty 0$ & $1.8000000 E+\infty 0$ & $1.8000000 E+\infty 0$ & $m$ & WPO40514 & $\mathbf{Y}$ & NAA \\
\hline 2936 & 1,3 & CONSTANT & $3.5000000 E+\infty 0$ & $3.5000000 E+\infty 0$ & $3.5000000 E+\infty 0$ & $3.5000000 E+\infty)$ & $m$ & WP030640 & $\mathbf{Y}$ & $N / A$ \\
\hline 2936 & 1,3 & CONSTANT & $3.5000000 E+\infty 0$ & $3.5000000 E+\infty 0$ & $3.5000000 E+\infty 0$ & $3.5000000 E+\infty 0$ & m & WP030994 & $\mathbf{Y}$ & $N / A$ \\
\hline 2936 & 1,3 & CONSTANT & $3.5000000 E+00$ & $3.5000000 E+\infty 0$ & $3.5000000 E+\infty 0$ & $3.5000000 E+\infty$ & $\mathbf{m}$ & WP03559? & $\mathbf{Y}$ & NA \\
\hline 2936 & 1,3 & CONSTANT & $3.5000000 E+00$ & $3.5000000 E+\infty 0$ & $3.5000000 E+00$ & $3.5000000 E+\infty$ & $\mathbf{m}$ & WPO40514 & $\mathbf{Y}$ & NA \\
\hline 2937 & 1,3 & CONSTANT & $2.3000000 E+\infty 0$ & $2.3000000 E+\infty 0$ & $2.3000000 E+\infty 0$ & $2.3000000 E+\infty 0$ & $\mathbf{m}$ & WP030640 & $\mathbf{Y}$ & NAA \\
\hline 2937 & 1,3 & CONSTANT & $2.3000000 E+\infty 0$ & $2.3000000 E+\infty 0$ & $2.3000000 E+00$ & $2.3000000 E+00$ & $\mathbf{m}$ & WP030994 & $\mathbf{Y}$ & NA \\
\hline 2937 & 1,3 & CONSTANT & $2.3000000 E+\infty 0$ & $2.3000000 E+\infty 0$ & $23000000 E+\infty 0$ & $2.3000000 E+\infty 0$ & $\mathbf{m}$ & WP035597 & $\mathbf{Y}$ & NAA \\
\hline 2937 & 1,3 & CONSTANT & $2.3000000 E+\infty 0$ & $2.3000000 E+\infty 0$ & $2.3000000 E+\infty$ & $2.3000000 E+\infty 0$ & $m$ & WP040514 & $\mathbf{Y}$ & NA \\
\hline 2938 & $1,3,4$ & CONSTANT & $1.8140000 E+\infty 0$ & $1.8140000 E+\infty 0$ & $1.8140000 \varepsilon+\infty$ & $1.8140000 E+\infty 0$ & $\mathrm{~m} / \mathrm{m}$ & WP030640 & $\mathbf{Y}$ & NA \\
\hline 2938 & $1,3,4$ & CONSTANT & $1.8140000 E+\infty 0$ & $1.8140000 \mathrm{E}+\infty$ & $1.8140000 E+\infty 0$ & $1.8140000 E+00$ & $\mathrm{~m} / \mathrm{m}$ & WP030994 & $\mathbf{Y}$ & NA \\
\hline 2938 & $1,3,4$ & CONSTANT & $1.8140000 E+\infty 0$ & $1.8140000 \mathrm{E}+\infty 0$ & $1.8140000 E+00$ & $1.8140000 \mathrm{E}+\infty 0$ & $\mathrm{~m} / \mathrm{m}$ & WP035597 & $\mathbf{Y}$ & NA \\
\hline 2939 & 3,5 & UNIFORM & $5.0000000 E-01$ & $5.0000000 E-01$ & $0.0000000 E+\infty 0$ & $1.0000000 E+\infty$ & NONE & WP030540 & $\mathbf{Y}$ & N/A \\
\hline 2939 & 3,5 & UNIFORH & $5.0000000 E-01$ & $5.0000000 E-01$ & $0.0000000 E+00$ & $1.00000000+\infty$ & NONE & WP030994 & $\mathbf{Y}$ & N/A \\
\hline 2939 & 3,5 & UNIFORM & 5.0000000 E-01 & $5.0000000 E-01$ & $0.0000000 E+\infty 0$ & $1.0000000 E+\infty$ & NONE & WP035268 & $r$ & NA \\
\hline 2939 & 3,5 & UNIFORM & $5.0000000 \mathrm{E}-01$ & $5.0000000 E-01$ & $0.0000000 \varepsilon+\infty$ & $1.0000000 \mathrm{E}+\infty 0$ & NONE & WP035597 & $\mathbf{Y}$ & N/A \\
\hline 2939 & 3,5 & UNIFORM & $5.0000000=-01$ & $5.0000000 E-01$ & $0.0000000 E+00$ & $1.0000000 E+00$ & NONE & WP037542 & $\mathbf{Y}$ & NA \\
\hline 2939 & 3,5 & UNIFORM & $5.0000000 E-01$ & $5.0000000 E-01$ & $0.0000000 E+00$ & $1.0000000 E+\infty 0$ & NONE & WPO40514 & $\mathbf{Y}$ & NA \\
\hline 2940 & $9,3,4$ & CONSTANT & $-1.4782500 E+01$ & $-1.4782500 E+01$ & $-1.4782500 E+01$ & $-1.4782500 E+01$ & $\log \left(m^{\wedge} 2\right)$ & WPO30640 & YaN & 1.4 \\
\hline 2940 & $1,3,4$ & CONSTANT & $-1.4782500 E+01$ & $-1.4782500 E+01$ & $-1.4782500 E+01$ & $-1.4782500 E+01$ & $\log \left(m^{\wedge} 2\right)$ & WP030994 & YeN & 1,4 \\
\hline 2940 & $1,3,4$ & CONSTANT & $-1.4782500 E+01$ & $-1.4782500 \mathrm{E}+01$ & $-1.4782500 E+01$ & $-1.4782500 E+01$ & $\log \left(m^{\wedge} 2\right)$ & WP035597 & YaN & 1.4 \\
\hline 2941 & $1,3,4$ & CONSTANT & $-1.7301000 E+01$ & $-1.7301000 E+01$ & $-1.7301000 E+01$ & $-1.7301000=+01$ & $\log \left(m^{2} 2\right)$ & WPO30640 & Y8N & 1,4 \\
\hline
\end{tabular}


CCA Parameter-Listing - -

\begin{tabular}{|c|c|c|c|c|c|c|c|c|c|c|}
\hline 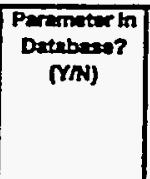 & 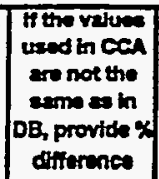 & W & Toteriat to & Mrterde $\mathrm{Nem}$ & $\begin{array}{l}\text { Parmwer } \\
\text { id }\end{array}$ & Permotar Nem & $\begin{array}{l}\text { PRPID } \\
\text { (KPO : }\end{array}$ & Data Entry & 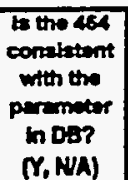 & $\begin{array}{l}\text { Parmetor } \\
\text { Catepory } \\
\text { (footiote 1) }\end{array}$ \\
\hline $\mathbf{Y}$ & vanes & 2941 & SALT_T1 & \begin{tabular}{|l|} 
Shaft salt column \\
compectedtume o to 10 \\
years
\end{tabular} & PMLT_LO & $\begin{array}{l}\text { Log trienoular distribution low } \\
\text { value for permesbility }\end{array}$ & 33377 & $13-F e b-96$ & $\mathbf{Y}$ & 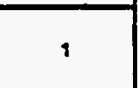 \\
\hline $\mathbf{Y}$ & venes & 2941 & SALT_T1 & \begin{tabular}{|l|} 
Shath sah column \\
compactect tme o to id \\
yoars
\end{tabular} & PALT_LO & 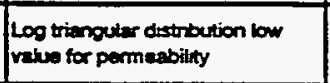 & 33377 & $13-F 0 b-96$ & $\mathbf{Y}$ & 1 \\
\hline $\mathbf{Y}$ & varies & 2942 & SALT_TI & $\begin{array}{l}\text { Shat salt column } \\
\text { compactect time } 0 \text { to } 10 \\
\text { yeers }\end{array}$ & PMLT_HI & $\begin{array}{l}\text { Log trianofier distribution high } \\
\text { value for permeability }\end{array}$ & 33375 & $13-F a b-96$ & $\mathbf{Y}$ & 1 \\
\hline $\mathbf{Y}$ & varies & 2942 & SALT_TI & $\begin{array}{l}\text { Shan satt column } \\
\text { compactedtime } 0 \text { to } 10 \\
\text { years }\end{array}$ & PMLT_HI & $\begin{array}{l}\text { Log trienguter distribution high } \\
\text { value for permeability }\end{array}$ & 33375 & $13+56-96$ & $\mathbf{Y}$ & 1 \\
\hline $\mathbf{Y}$ & veries & 2942 & SALT_T1 & $\begin{array}{l}\text { Shaft salt column } \\
\text { compactedtime } 0 \text { to id } \\
\text { years }\end{array}$ & PMLT_HI & $\begin{array}{l}\text { Log trienguler distribution high } \\
\text { value for pormeability }\end{array}$ & 33375 & $13-5.6-96$ & $\mathbf{Y}$ & 1 \\
\hline $\mathbf{Y}$ & & 2943 & SALT_T2 & $\begin{array}{l}\text { Shaft salt column } \\
\text { compactedtime } 10 \text { to } \\
25 \text { years }\end{array}$ & RSH_AR & Air-exply sheft radius $(3.09 \mathrm{~m})$ & 333834 & $13-F a b-96$ & $\mathbf{Y}$ & 48 \\
\hline $\mathbf{Y}$ & & 2943 & SALT_T2 & $\begin{array}{l}\text { Shaft sall column } \\
\text { compactodtime } 10 \text { to } \\
25 \text { years }\end{array}$ & RSH_ARR & Air-upply stant radius $(3.09 \mathrm{~m})$ & 333834 & $13-f a b-96$ & $\mathbf{Y}$ & 48 \\
\hline $\mathbf{Y}$ & & 2943 & SALT_T2 & $\begin{array}{l}\text { Shaft salt column } \\
\text { compactod:tume } 10 \text { to } \\
25 \text { years }\end{array}$ & RSH_AIR & Air-eupply shant radius $(3.09 \mathrm{~m})$ & $33383 \mathrm{~A}$ & $13-F e b-96$ & $\mathbf{Y}$ & $4 B$ \\
\hline $\mathbf{Y}$ & & 2943 & SALT_T2 & $\begin{array}{l}\text { Shat salt column } \\
\text { compacted:tme to to } \\
25 \text { years }\end{array}$ & RSH_AIR & Air-auppty enant radius (3.09 m) & $33383 A$ & 13 Fab-96 & $\mathbf{Y}$ & 48 \\
\hline $\mathbf{Y}$ & & 2944 & SALT_T2 & $\begin{array}{l}\text { Shath sah column } \\
\text { compactedtume } 10 \text { to } \\
25 \text { years }\end{array}$ & RSH_SAL & Salt-handing shan radius $(1.8 \mathrm{~m})$ ) & $33385 A$ & 13 Fob-96 & $\mathbf{Y}$ & $4 B$ \\
\hline $\mathbf{Y}$ & & 2944 & SALT_T2 & $\begin{array}{l}\text { Shaft salt column } \\
\text { compactedtume } 10 \text { to } \\
25 \text { years }\end{array}$ & RSH_SAL & Salthand $1 \mathrm{ng}$ shant radius $(1.8 \mathrm{~m})$ & $33385 A$ & $13+f \cdot b-96$ & $\mathbf{Y}$ & 48 \\
\hline $\mathbf{Y}$ & & 2944 & SALT_T2 & $\begin{array}{l}\text { Shaft sah column } \\
\text { compacted:tune } 10 \text { to } \\
25 \text { years }\end{array}$ & RSH_SN & Selthanding anen radius $(1.8 \mathrm{~m})$ & $33385 A$ & $13-F a b-96$ & $\mathbf{Y}$ & 48 \\
\hline $\mathbf{Y}$ & & 2944 & SALT_T2 & $\begin{array}{l}\text { Shath salt column } \\
\text { compacteditune } 10 \text { to } \\
25 \text { years }\end{array}$ & RSH_SN & Salt handing anan rades $(1.8 \mathrm{~m})$ & $33385 A$ & 13-Feb-96 & $\mathbf{Y}$ & $4 B$ \\
\hline $\mathbf{Y}$ & & 2945 & SALT_T2 & $\begin{array}{l}\text { Shaft salt column } \\
\text { compacted time to to } \\
25 \text { years }\end{array}$ & RSH_WAS & $\begin{array}{l}\text { Wasto-hending and radus (3.5 } \\
\text { m) }\end{array}$ & $33387 \mathrm{~A}$ & $13-F a b-96$ & $\mathbf{Y}$ & 48 \\
\hline $\mathbf{Y}$ & & 2945 & SALT_T2 & $\begin{array}{l}\text { Shaft salt conmn } \\
\text { compactod time } 10 \text { to } \\
25 \text { years }\end{array}$ & RSH_was & $\begin{array}{l}\text { Waste-nenano ent radius (3.5 } \\
\text { m) }\end{array}$ & $33387 \mathrm{~A}$ & 13-Feb-96 & $\mathbf{Y}$ & $4 B$ \\
\hline $\mathbf{Y}$ & & 2945 & SALT_T2 & $\begin{array}{l}\text { Shaft sati column } \\
\text { compactoo tme } 10 \text { to } \\
25 \text { years }\end{array}$ & RSH_was & 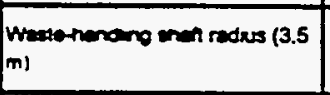 & $33387 \mathrm{~A}$ & 13-feb-96 & $\mathbf{Y}$ & 48 \\
\hline$Y$ & & 2945 & SALT_T2 & $\begin{array}{l}\text { Shath san column } \\
\text { compected trme } 10 \text { to } \\
25 \text { years }\end{array}$ & RSH_wns & $\begin{array}{l}\text { Wastenenon men radus (3.5 } \\
\text { m) }\end{array}$ & $33387 \mathrm{~A}$ & 13-Fob-96 & $\bar{y}$ & $4 B$ \\
\hline $\mathbf{Y}$ & & 2946 & SALT_T2 & $\begin{array}{l}\text { Shath sail column } \\
\text { compacted time } 10 \text { to } \\
25 \text { yoars }\end{array}$ & RSH_EXH & Ar moner and $(2.3 \mathrm{~m})$ & $33384 A$ & 13-Feb-96 & $\mathbf{Y}$ & 48 \\
\hline $\mathbf{Y}$ & & 2946 & SALT_T2 & $\begin{array}{l}\text { Shen san colum } \\
\text { compacted tome 10 to } \\
25 \text { years }\end{array}$ & RSH_EXY & 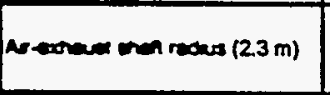 & $33384 A$ & 13-Fob-96 & $\mathbf{Y}$ & 48 \\
\hline $\boldsymbol{Y}$ & & 2946 & SALT_T2 & $\begin{array}{l}\text { Shath sal colimn } \\
\text { compected trme } 10 \text { to } \\
25 \text { years }\end{array}$ & RSH_EXM & 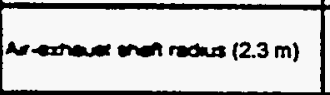 & $33384 A$ & 13-Feb-96 & $\mathbf{Y}$ & 48 \\
\hline $\mathbf{Y}$ & & 2946 & SALT_T2 & $\begin{array}{l}\text { Shath selt column } \\
\text { compected tme } 10 \text { to } \\
25 \text { years }\end{array}$ & RSH_ENH & Ar thant onen rectes $(2.3 \mathrm{~m})$ & 333844 & 13-Fob-96 & $\mathbf{Y}$ & $A B$ \\
\hline $\mathbf{Y}$ & & 2947 & SALT_T2 & $\begin{array}{l}\text { Shath sen column } \\
\text { compected tome } 10 \text { to } \\
25 \text { years }\end{array}$ & RAON_DRZ & DA2 our reoue a each shat & 33374 & 13-fob-96 & $\mathbf{Y}$ & 1 \\
\hline $\mathbf{Y}$ & & 2947 & SALT_T2 & $\begin{array}{l}\text { Shen sat colum } \\
\text { compecteo tim } 10 \text { wo } \\
25 \text { years }\end{array}$ & RAON_DRZ & DR2 aver rache at each shath & 33374 & $13-F \cdot b-96$ & $\mathbf{Y}$ & 1 \\
\hline $\mathbf{Y}$ & & 2947 & SALT_T2 & $\begin{array}{l}\text { Shath sal cokmn } \\
\text { compected time } 10 \text { to } \\
25 \text { years }\end{array}$ & RADN_ORZ & OR2 outer reace at esch shant & 33374 & $13-F a b-96$ & $\mathbf{Y}$ & 1 \\
\hline
\end{tabular}


CCA Parameter Listing

\begin{tabular}{|c|c|c|c|c|c|c|c|c|c|c|}
\hline ID & \begin{tabular}{c|} 
Cieselfi \\
cation of \\
Data \\
Cotegory \\
(footnote 2)
\end{tabular} & $\begin{array}{c}\text { Dietribution } \\
\text { Type }\end{array}$ & $\operatorname{Men}$ & Medien & Minimum & Maxdmun & Units & Reference L 10 & $\begin{array}{l}\text { Wes the drte } \\
\text { doveloped } \\
\text { under an } \\
\text { KOA-1 } \\
\text { Progrem? }\end{array}$ & $\begin{array}{l}\text { mich } \\
\text { mothods } \\
\text { wore used to } \\
\text { qually } \\
\text { exdeting } \\
\text { dete? } \\
\text { trootnoce 3) }\end{array}$ \\
\hline 2941 & $1,3,4$ & CONSTANT & $-1.7301000 E+01$ & $-1.7301000 E+01$ & $-1.7301000 E+01$ & $-1.7301000 E+01$ & $\log \left(m^{\wedge} 2\right)$ & WPO30994 & Y\&N & 1,4 \\
\hline 2941 & $1,3,4$ & CONSTANT & $-1.7301000 E+01$ & $-1.7301000 E+01$ & $-1.7301000 E+01$ & $-1.7301000 E+01$ & $\log \left(m^{n} 2\right)$ & WP035597 & YaN & 1.4 \\
\hline 2942 & $1,3,4$ & CONSTANT & $-1.2265200 E+01$ & $-1.2265200 E+01$ & $-1.2265200 E+01$ & $-1.2265200 E+01$ & $\log \left(m^{n} 2\right)$ & WP030640 & Y\&N & 1.4 \\
\hline 2942 & $1,3,4$ & CONSTANT & $-1.2265200 E+01$ & $-1.2265200 E+01$ & $-1.2265200 E+01$ & $-1.2265200 E+01$ & $\log \left(m^{\wedge} 2\right)$ & WP030994 & Y\&N & 1,4 \\
\hline 2942 & $1,3,4$ & CONSTANT & $-1.2265200 E+01$ & $-1.2265200 E+01$ & $-1.2265200 E+01$ & $-1.2265200 E+01$ & $\log \left(m^{\wedge} 2\right)$ & WP035597. & Y\&N & 1.4 \\
\hline 2943 & 1.3 & CONSTANT & $3.0900000 E+\infty 0$ & $3.0900000 E+00$ & $3.0900000 E+00$ & $3.0900000 E+00$ & $\mathbf{m}$ & WP030540 & $\mathbf{Y}$ & NA \\
\hline 2943 & 1,3 & CONSTANT & $3.0900000 E+\infty$ & $3.0900000 E+\infty 0$ & $3.0900000 E+00$ & $3.0900000 E+\infty 0$ & $\mathbf{m}$ & WP030994 & $Y$ & NA \\
\hline 2943 & 1,3 & CONSTANT & $3.0900000 E+\infty 0$ & $3.0900000 E+\infty 0$ & $3.0900000 E+\infty$ & $3.0900000 E+\infty$ & $\mathbf{m}$ & WP035597 & $\mathbf{Y}$ & NA \\
\hline 2943 & 1,3 & CONSTANT & $3.0900000 E+\infty 0$ & $3.0900000 E+00$ & $3.0900000 E+\infty 0$ & $3.0900000 E+\infty 0$ & $m$ & WP040514 & $\mathbf{Y}$ & NA \\
\hline 2944 & 1,3 & CONSTANI & $1.8000000 E+\infty$ & $1.8000000 E+\infty 0$ & $1.8000000 \mathrm{E}+\infty$ & $1.8000000 E+\infty 0$ & $\mathbf{m}$ & WP030640 & $\mathbf{Y}$ & NA \\
\hline 2944 & 1,3 & CONSTANT & $1.8000000 E+\infty 0$ & $1.8000000 E+00$ & $1.8000000 E+\infty$ & $1.8000000 E+\infty$ & $m$ & WP030994 & $\mathbf{Y}$ & N/A \\
\hline 2944 & 1,3 & CONSTANT & $1.8000000 E+\infty 0$ & $1.8000000 E+\infty$ & $1,8000000 E+\infty$ & $1.8000000 E+\infty 0$ & $m$ & WP035597 & $\mathbf{Y}$ & NA \\
\hline 2944 & 1,3 & CONSTANT & $1.8000000 E+00$ & $1.8000000 E+00$ & $18000000 E+\infty$ & $18000000 E+\infty$ & $\mathbf{m}$ & WP040514 & $\mathbf{Y}$ & NA \\
\hline 2945 & 1,3 & CONSTANT & $3.5000000 E+\infty 0$ & $3.5000000 E+\infty 0$ & $35000000 E+\infty 0$ & $35000000 E+\infty$ & $m$ & WP030540 & $\mathbf{Y}$ & N/A \\
\hline 2945 & 1,3 & CONSTANT & $3.5000000 E+00$ & $35000000 E+\infty 0$ & $35000000 E+\infty 0$ & $35000000=+\infty$ & $\mathbf{m}$ & WP030994 & $\mathbf{Y}$ & NA \\
\hline 2945 & 1,3 & CONSTANT & $3.5000000 E+\infty 0$ & $35000000 E+\infty$ & $35000000 E+\infty$ & $35000000 E+\infty$ & m & WP035597 & $\mathbf{Y}$ & $N A$ \\
\hline 2945 & 1,3 & CONSTANT & $3.5000000 E+\infty 0$ & $3.5000000 \varepsilon+00$ & $35000000<+\infty$ & $35000000 E+\infty$ & $m$ & WP040514 & $Y$ & N/A \\
\hline 2946 & 1,3 & CONSTANT & $23000000 E+\infty$ & $23000000 E+00$ & $23000000<-\infty 0$ & $23000000 \times+\infty$ & $m$ & WP030640 & $\mathbf{Y}$ & NAA \\
\hline 2946 & 1,3 & CONSTANT & $2.30000000 E+\infty 0$ & $2.3000000 \varepsilon+\infty$ & $230000006 \times 00$ & $23000000 \times+\infty$ & $\mathbf{m}$ & WP030994 & $\mathbf{Y}$ & NA \\
\hline 2946 & 1,3 & CONSTANT & $2.3000000 E+\infty 0$ & $2.3000000 E+\infty$ & $23000000<+\infty$ & $23000000 \varepsilon+\infty$ & $m$ & WP035597 & $\mathbf{Y}$ & NA \\
\hline 2946 & 1.3 & CONSTANT & $2,3000000 E+\infty 0$ & $2.3000000 E+\infty$ & $23000000 \times+00$ & $23000000 E+00$ & $m$ & WPO40514 & $\mathbf{Y}$ & N/A \\
\hline 2947 & $1,3,4$ & CONSTANT & $1.1100000 E+\infty 0$ & $11100000 E+\infty$ & $11100000<+\infty$ & $1100000 \mathrm{E}+\infty$ & $\mathrm{m} / \mathrm{m}$ & WP030640 & $\mathbf{Y}$ & N/A \\
\hline 2947 & $1,3,4$ & CONSTANT & $1.1100000 E+\infty$ & $11100000 E+\infty 0$ & 1 1 $1000000+\infty$ & 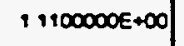 & $\mathrm{m} / \mathrm{m}$ & WP030994 & $\mathbf{Y}$ & $\mathbf{N} / \mathbf{A}$ \\
\hline 2947 & $1,3,4$ & CONSTANT & $1.1100000 E+\infty$ & $11100000 E+\infty$ & $11100000 E+\infty$ & $1100000 \mathrm{E}+\infty$ & $\mathrm{m} / \mathrm{m}$ & WP035597 & $\mathbf{Y}$ & $\mathbf{N A}$ \\
\hline
\end{tabular}


CCA Parameter Listing

\begin{tabular}{|c|c|c|c|c|c|c|c|c|c|c|}
\hline $\begin{array}{c}\text { Paremitor in } \\
\text { Drtabeas? } \\
\text { (YN) }\end{array}$ & 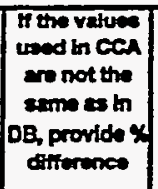 & $\overline{\mathbf{D}}$ & Antartal ID & Mrtarta Narme & $\begin{array}{c}\text { Peranoter } \\
\text { to }\end{array}$ & Peremeter Narese & $\begin{array}{l}\text { PFP DD } \\
\text { (MPOA) }\end{array}$ & $\begin{array}{c}\text { Dota Entry } \\
\text { Detta }\end{array}$ & $\begin{array}{l}\text { Is the } 454 \\
\text { consaletant } \\
\text { whth the } \\
\text { perameter } \\
\text { in DB? } \\
\text { (Y. NAY }\end{array}$ & 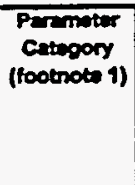 \\
\hline $\mathbf{Y}$ & varios & 2948 & SALT_T2 & $\begin{array}{l}\text { Shath salt column } \\
\text { compoctoctume } 10 \text { to } \\
25 \text { years }\end{array}$ & PMLT_MD & $\begin{array}{l}\text { Log trimouler distribution mode } \\
\text { for permedbility }\end{array}$ & 3345 & $13-F 0 b-96$ & $\mathbf{Y}$ & 1 \\
\hline $\mathbf{Y}$ & varies & 2948 & SALT_T2 & $\begin{array}{l}\text { Shat rat: column } \\
\text { compectedtime } 10 \text { to } \\
25 \text { years }\end{array}$ & PMLT_MD & $\begin{array}{l}\text { Log trianouter distribution mode } \\
\text { for permeability }\end{array}$ & 3345 & $13-F a b-96$ & $\mathbf{Y}$ & 1 \\
\hline$Y$ & varios & 2948 & SALT_T2 & $\begin{array}{l}\text { Shatt eatt colemn } \\
\text { compectodtime } 10 \text { to } \\
25 \text { years }\end{array}$ & PMLT_MD & $\begin{array}{l}\text { Log triengular distritution mods } \\
\text { for permeibitity }\end{array}$ & 3345 & 13 Feb-s6 & $\mathbf{Y}$ & 1 \\
\hline $\mathbf{Y}$ & varies & 2949 & SALT_T2 & $\begin{array}{l}\text { Shatt sat cokmn } \\
\text { compactedtime } 10 \text { to } \\
25 \text { years }\end{array}$ & PMLT_LO & $\begin{array}{l}\text { Log trienguter dietribution bow } \\
\text { valus for permenbilty }\end{array}$ & 33442 & $13-500-96$ & $\mathbf{Y}$ & 1 \\
\hline $\mathbf{Y}$ & varios & 2949 & SALT_T2 & $\begin{array}{l}\text { Shath salt colemn } \\
\text { compacteditime } 10 \text { to } \\
25 \text { years }\end{array}$ & PMLT_LO & $\begin{array}{l}\text { Log trienouter diestribution low } \\
\text { value for permescidty }\end{array}$ & 33442 & $13-50-96$ & $\mathbf{Y}$ & 1 \\
\hline $\mathbf{Y}$ & varies & 2949 & SALT_T2 & $\begin{array}{l}\text { Shat salt colemn } \\
\text { compactect time } 10 \text { to } \\
25 \text { years }\end{array}$ & PMLT_LO & $\begin{array}{l}\text { Log triangutar distribution low } \\
\text { valwo for permesbility }\end{array}$ & 33442 & $13-500-96$ & $\mathbf{Y}$ & 1 \\
\hline $\mathbf{Y}$ & varios & 2950 & SALT_T2 & $\begin{array}{l}\text { Shart salt column } \\
\text { compactoctime } 10 \text { to } \\
25 \text { yoars }\end{array}$ & PMLT_H! & $\begin{array}{l}\text { Log triangular distribution high } \\
\text { value for permesbility }\end{array}$ & 33440 & $13-$ feb- 96 & $Y$ & 1 \\
\hline$Y$ & varies & 2950 & SALT_T2 & $\begin{array}{l}\text { Shat salt column } \\
\text { compactodtime } 10 \text { to } \\
25 \text { years }\end{array}$ & PMLT_HI & $\begin{array}{l}\text { Log trimpular distribution high } \\
\text { velue for pertrestitiy }\end{array}$ & 3340 & $13-f e b-96$ & $\mathbf{\gamma}$ & 1 \\
\hline$\gamma$ & varies & 2950 & SALT_T2 & $\begin{array}{l}\text { Shatt salt column } \\
\text { compactodtime } 10 \text { to } \\
25 \text { years }\end{array}$ & PMLT_HI & $\begin{array}{l}\text { Log tienguler distribution high } \\
\text { value for permestility }\end{array}$ & 33440 & 13 Feb-96 & $\mathbf{Y}$ & 1 \\
\hline$Y$ & & 2951 & SALT_T3 & $\begin{array}{l}\text { Shatt sah colkmn } \\
\text { compactodtime } 25 \text { to } \\
50 \text { years }\end{array}$ & RSH_AR & Air-supply shat radies $(3.09 \mathrm{~m})$ & $33437 \mathrm{~A}$ & $13-F e b-96$ & $\mathbf{Y}$ & 4B \\
\hline $\mathbf{Y}$ & & 2951 & SALT_T3 & $\begin{array}{l}\text { Shat salt column } \\
\text { compactod:time } 25 \text { to } \\
50 \text { years }\end{array}$ & RSH_ARR & Air-supply shat rodiss (3.09 m) & $33437 A$ & 13Fab-96 & $\mathbf{Y}$ & AB \\
\hline $\mathbf{Y}$ & & 2951 & SALT_T3 & $\begin{array}{l}\text { Shaft sah column } \\
\text { compactodtitine } 25 \text { to } \\
50 \text { years }\end{array}$ & RSH_ANR & Air-eupply ahen recius $(3.09 \mathrm{~m})$ & $33437 \mathrm{~A}$ & 13-Feb-96 & $\mathbf{Y}$ & $4 B$ \\
\hline $\mathbf{Y}$ & & 2951 & SALT_T3 & $\begin{array}{l}\text { Shafh sall column } \\
\text { compactodtine } 25 \text { to } \\
50 \text { years }\end{array}$ & RSH_AR & Air-supply that radius (3.09 m) & $33437 A$ & $13+40 b-96$ & $Y$ & 48 \\
\hline $\mathbf{Y}$ & & 2952 & SALT_T3 & $\begin{array}{l}\text { Shant selt cokemn } \\
\text { compactootime } 25 \text { to } \\
\text { 50 years }\end{array}$ & RSH_SAL & Sattianding stant radius $(1.8 \mathrm{~m})$ & $33441 A$ & 13F-b-96 & $\mathbf{Y}$ & 4B \\
\hline $\mathbf{Y}$ & & 2952 & SALT_T3 & $\begin{array}{l}\text { Shath ealt coktumn } \\
\text { compactodtime } 25 \text { to } \\
50 \text { years }\end{array}$ & RSH_SAL & Sart thending shath rodius $(1.8 \mathrm{~m})$ & $33441 A$ & 13 -feb-96 & $\mathbf{Y}$ & 4B \\
\hline $\mathbf{Y}$ & & 2952 & SALT_T3 & $\begin{array}{l}\text { Shaft satt column } \\
\text { compactod:trne } 25 \text { to } \\
50 \text { years }\end{array}$ & RSH_SAL & Sathineding sthat radius $(1.8 \mathrm{~m})$ & $33411 A$ & 13-Fob-96 & $\mathbf{Y}$ & $A B$ \\
\hline$Y$ & & 2952 & SALT_T3 & $\begin{array}{l}\text { Shrifh selt colkmn } \\
\text { compactod:tme } 25 \text { to } \\
50 \text { year }\end{array}$ & RSH_SAL & Selthending ahan rodus $(1.8 \mathrm{~m})$ & $33441 A$ & 13-Feb-96 & 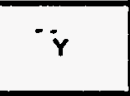 & 48 \\
\hline $\mathbf{Y}$ & & 2953 & SALT_T3 & $\begin{array}{l}\text { Shath eath column } \\
\text { compactoct.tme } 25 \text { to } \\
50 \text { years }\end{array}$ & RSH_WAS & $\begin{array}{l}\text { Whate-hencting ethatt radess (3.5 } \\
\text { m) }\end{array}$ & 33434 & 13-Fob-96 & $\mathbf{Y}$ & 48 \\
\hline$Y$ & & 2953 & SALT_T3 & $\begin{array}{l}\text { Shen ealt column } \\
\text { compuctodtime } 25 \text { to } \\
50 \text { years }\end{array}$ & RSH_WAS & $\begin{array}{l}\text { Wasto-handing shath radess (3.5 } \\
\text { m) }\end{array}$ & $33443 A$ & $13-F a b-96$ & $\mathbf{Y}$ & 48 \\
\hline$Y$ & & 2953 & SALT_T3 & $\begin{array}{l}\text { Shaft salt column } \\
\text { compectod:tme } 25 \text { to } \\
50 \text { yeers }\end{array}$ & RSH_WAS & $\begin{array}{l}\text { Whatto-hending enath radus (3.5 } \\
\text { m) }\end{array}$ & 334334 & $13-F e b-96$ & $\mathbf{Y}$ & AB \\
\hline $\mathbf{Y}$ & & 2953 & SALT_T3 & $\begin{array}{l}\text { Shat sall column } \\
\text { compactodtume } 25 \text { to } \\
50 \text { years }\end{array}$ & RSH_WAS & $\begin{array}{l}\text { Westo-hending anent redus (3.5 } \\
\text { m) }\end{array}$ & $33443 A$ & $13-F e b-96$ & $\mathbf{Y}$ & 48 \\
\hline $\mathbf{Y}$ & & 2954 & SALT_T3 & 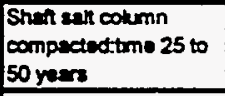 & RSH_EXH & Ar-atheust shant redius $(2.3 \mathrm{~m})$ & $33439 A$ & $13+e b-96$ & $\mathbf{Y}$ & 48 \\
\hline $\mathbf{Y}$ & & 2954 & SALT_T3 & $\begin{array}{l}\text { Shat salt column } \\
\text { compactoctime } 25 \text { to } \\
50 \text { years }\end{array}$ & RSH_EXH & Ar-extheunt then radus $(2.3 \mathrm{~m})$ & 334394 & $13+\infty 0-96$ & $\mathbf{Y}$ & 48 \\
\hline $\mathbf{Y}$ & & 2954 & SALT_T3 & $\begin{array}{l}\text { Shatt set cokemn } \\
\text { compectectume } 25 \text { to } \\
50 \text { years }\end{array}$ & RSH_EXH & Ar-extheust thant rediva $(23 \mathrm{~m})$ & $33439 A$ & $13-f e b-96$ & $\boldsymbol{Y}$ & 4B \\
\hline
\end{tabular}


CCA Parameter Listing

\begin{tabular}{|c|c|c|c|c|c|c|c|c|c|c|}
\hline 10 & 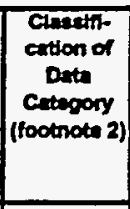 & $\begin{array}{l}\text { Distribution } \\
\text { Type }\end{array}$ & mann & Modim & Dintroum & Medrmum & Chits & Refertunce ID & $\begin{array}{l}\text { Wes the dita } \\
\text { developed } \\
\text { underen } \\
\text { Mon-1 } \\
\text { Progrum? }\end{array}$ & 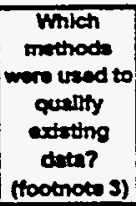 \\
\hline 2948 & $1,3,4$ & CONSTANT & $-1.4702500 \mathrm{E}+01$ & $-1,4782500 E+01$ & $-1.4782500 E+01$ & $-1.4782500 E+01$ & $\log \left(m^{\wedge} 2\right)$ & WP030640 & YEN & 1.4 \\
\hline 2948 & $1,3,4$ & CONSTANT & $-1.4782500 E+01$ & $-1.4782500 E+01$ & $-1.4782500 E+01$ & $-1.4782500 E+01$ & $\log \left(m^{\wedge} 2\right)$ & WP030994 & YSN & 1.4 \\
\hline 2948 & $1.3,4$ & CONSTANT & $-1.4782500 E+01$ & $-1.4782500 E+01$ & $-1.4782500 E+01$ & $-1.4782500 E+01$ & $\log \left(m^{\wedge} 2\right)$ & WP035597 & YaN & 1,4 \\
\hline 2949 & $1.3,4$ & CONSTANT & $-1.7301000 E+01$ & $-1.7301000 E+01$ & $-1.7301000 E+01$ & $-1.7301000 E+01$ & $\log \left(m^{\wedge} 2\right)$ & WP030640 & Y8N & 1.4 \\
\hline 2949 & $1,3,4$ & CONSTANT & $-1.7301000 E+01$ & $-1.7301000 E+01$ & $-1.7301000 E+01$ & $-1.7301000 E+01$ & $\log \left(m^{\wedge} 2\right)$ & WP030994 & YEN & 1.4 \\
\hline 2949 & $1,3,4$ & CONSTANT & $-1.7301000 E+01$ & $-1.7301000 E+01$ & $-1.7301000 E+01$ & $-1.7301000 E+01$ & $\log \left(m^{\wedge} 2\right)$ & WP035597 & Y\&N & 1,4 \\
\hline 2950 & $1,3,4$ & CONSTANT & $-1.2265200 E+01$ & $-1.2265200 E+01$ & $-1.2255200 E+01$ & $-1.2255200 E+01$ & $\log \left(m^{\wedge} 2\right)$ & WP030540 & YaN & 1,4 \\
\hline 2950 & $9,3,4$ & CONSTANT & $-1.2265200 E+01$ & $-1.2265200 E+01$ & $-1.2265200 E+01$ & $-1.2265200 E+01$ & $\log \left(m^{\wedge} 2\right)$ & WP030994 & YEN & 1.4 \\
\hline 2950 & $1,3,4$ & CONSTANT & $-1.2265200 E+01$ & $-1.2265200 E+01$ & $-1.2265200 E+01$ & $-1.2265200 \mathrm{E}+01$ & $\log \left(m^{\wedge} 2\right)$ & WP035597 & YEN & 1,4 \\
\hline 2951 & 1,3 & CONSTANT & $3.0900000 E+\infty 0$ & $3.0900000 \mathrm{E}+00$ & $3.0900000 E+\infty 0$ & $3.0900000 E+\infty 0$ & $\mathbf{m}$ & WP030640 & $\mathbf{Y}$ & NA \\
\hline 2951 & 1,3 & CONSTANT & $3.0900000 E+\infty 0$ & $3.0900000 \mathrm{E}+00$ & $3.0900000 E+\infty$ & $3.0500000 E+\infty 0$ & $\mathbf{m}$ & WP030994 & $\mathbf{Y}$ & NA \\
\hline 2951 & 1,3 & CONSTANT & $3.0900000 E+\infty 0$ & $3.0900000 E+\infty 0$ & $3.0900000 \mathrm{E}+00$ & $3.09000000+\infty$ & $m$ & WP035597 & $\mathbf{Y}$ & N/A \\
\hline 2951 & 1,3 & CONSTANT & $3.0900000 E+\infty 0$ & $3.0900000 E+\infty 0$ & $3.0900000 E+00$ & $3.0900000 \mathrm{E}+\infty 0$ & m & WPO40514 & $\mathbf{Y}$ & NA \\
\hline 2952 & 1,3 & CONSTANT & $1.8000000 E+00$ & $1.8000000 \mathrm{E}+\infty 0$ & $1.8000000 E+\infty 0$ & $1.8000000 E+\infty 0$ & $\mathbf{m}$ & WP030640 & $\mathbf{Y}$ & NA \\
\hline 2952 & 1,3 & CONSTANT & $1.8000000 \mathrm{E}+\infty 0$ & $1.8000000 E+\infty 0$ & $1.8000000 E+\infty 0$ & $1.8000000 E+\infty$ & m & WP030994 & $\mathbf{Y}$ & NA \\
\hline 2952 & 1,3 & CONSTANT & $1.8000000 E+\infty$ & $1.8000000 E+\infty$ & $1.8000000 E+\infty 0$ & $1.8000000 \mathrm{E}+\infty$ & $\mathrm{m}$ & WP035597 & $\mathbf{Y}$ & NA \\
\hline 2952 & 1,3 & CONSTANT & $1.8000000 E+\infty 0$ & $1.80000000+00$ & $1.8000000 E+\infty 0$ & $1.8000000 E+00$ & m & WPO40514 & $" Y$ & NA \\
\hline 2953 & 1,3 & CONSTANT & $3.5000000 E+\infty 0$ & $3.5000000 \mathrm{E}+\infty 0$ & $3.5000000 E+00$ & $3.5000000 E+\infty 0$ & $\mathbf{m}$ & WP030640 & $\mathbf{Y}$ & NA \\
\hline 2953 & 1,3 & CONSTANT & $3.5000000 \mathrm{E}+\infty 0$ & $3.5000000 E+\infty$ & $3.5000000 E+\infty 0$ & $3.5000000 E+\infty 0$ & $m$ & WP030994 & $\mathbf{Y}$ & NA \\
\hline 2953 & 1,3 & CONSTANT & $3.5000000 E+\infty 0$ & $3.5000000 E+\infty 0$ & $3.5000000 E+\infty 0$ & $3.5000000 E+\infty$ & $m$ & WP035597 & $\mathbf{Y}$ & N/A \\
\hline 2953 & 1,3 & CONSTANT & $3.5000000 E+\infty 0$ & $3.5000000 E+\infty 0$ & $3.5000000 E+\infty$ & $3.5000000 E+\infty 0$ & $\mathbf{m}$ & WPO40514 & $\mathbf{Y}$ & NA \\
\hline 2954 & 1,3 & CONSTANT & $2.3000000 E+\infty 0$ & $2.3000000 E+\infty$ & $2.3000000 E+\infty 0$ & $2.3000000 E+\infty$ & m & WP030640 & $\mathbf{Y}$ & NA \\
\hline 2954 & 1,3 & CONSTANT & $2.3000000 E+\infty 0$ & $2.3000000 E+\infty$ & $2.3000000 E+00$ & $2.3000000 E+\infty 0$ & $m$ & WP030994 & $Y$ & NA \\
\hline 2954 & 1.3 & CONSTANT & $2.3000000 E+\infty 0$ & $2.3000000 E+\infty$ & $2.3000000 E+\infty$ & $2.3000000 E+00$ & $m$ & WP035597 & $Y$ & NA \\
\hline
\end{tabular}


CCA Parameter Listing

\begin{tabular}{|c|c|c|c|c|c|c|c|c|c|c|}
\hline $\begin{array}{c}\text { Parameter in } \\
\text { Detabece? } \\
\text { (No) }\end{array}$ & 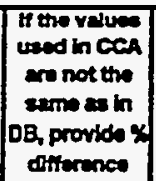 & ED & Metarta Lo & Mataria Nem & $\begin{array}{c}\text { Paramitar } \\
\text { it }\end{array}$ & Perment kero & $\begin{array}{c}\text { PRPD } \\
\text { (MPO \#) }\end{array}$ & Dete Entry & $\begin{array}{l}\text { to the } 464 \\
\text { consiatent } \\
\text { with the } \\
\text { prrmeter } \\
\text { in DB? } \\
(Y, N \text { N) }\end{array}$ & $\begin{array}{l}\text { Parenoter } \\
\text { Catiogony } \\
\text { (tootnote y) }\end{array}$ \\
\hline $\mathbf{Y}$ & & 2954 & SALT_T3 & $\begin{array}{l}\text { Shaft sett column } \\
\text { compectectume } 25 \text { to } \\
50 \text { years }\end{array}$ & RSH_EXH & Ar-echenst thent redies (2.3 m) & $33439 A$ & $13-F e b-96$ & $\mathbf{Y}$ & 48 \\
\hline $\mathbf{Y}$ & & 2955 & SALT_T3 & $\begin{array}{l}\text { Shan salt column } \\
\text { compectectime } 25 \text { to } \\
50 \text { yoars }\end{array}$ & RAON_DRZ & ORZ outer redus at esch ahen & 33432 & 13Fab-96 & $\mathbf{Y}$ & 1 \\
\hline $\mathbf{Y}$ & & 2955 & SALT_T3 & $\begin{array}{l}\text { Shaft alt colimn } \\
\text { compactedtume } 25 \text { to } \\
50 \text { years }\end{array}$ & RADN_DRZ & DRZ outer radess at each ghent & 33432 & 13Fab-96 & $\mathbf{Y}$ & 1 \\
\hline $\mathbf{Y}$ & & 2955 & SALT_T3 & $\begin{array}{l}\text { Shat salt column } \\
\text { compactectime } 25 \text { to } \\
50 \text { years }\end{array}$ & RADN_DRZ & DRZ outer radius at esch streft & 33432 & 13 Fob-96 & $\mathbf{Y}$ & 1 \\
\hline $\mathbf{Y}$ & varies & 2956 & SALT_T3. & $\begin{array}{l}\text { Shatt salt colturn } \\
\text { compsectedtime } 25 \text { to } \\
50 \text { years }\end{array}$ & PMLT_MD & $\begin{array}{l}\text { Loo triangular distribution modo } \\
\text { for permesabitity }\end{array}$ & 33414 & $13-F e b-96$ & $\mathbf{Y}$ & 1 \\
\hline $\mathbf{Y}$ & vanes & 2956 & SALT_T3 & $\begin{array}{l}\text { Shesf salt column } \\
\text { compactedtime } 25 \text { to } \\
50 \text { years }\end{array}$ & PMLT_MD & $\begin{array}{l}\text { Log trianqular distribution mode } \\
\text { for permability }\end{array}$ & 33414 & $13-F e b-96$ & $\mathbf{Y}$ & 1 \\
\hline $\mathbf{Y}$ & varies & 2956 & SALT_T3 & $\begin{array}{l}\text { Shaft salt column } \\
\text { compactectime } 25 \text { to } \\
50 \text { yoars }\end{array}$ & PMLT_MD & $\begin{array}{l}\text { Log trienouler diestribution mode } \\
\text { for penmesbilty }\end{array}$ & 33414 & 13Fob-96 & $\mathbf{Y}$ & 1 \\
\hline $\mathbf{Y}$ & varies & 2957 & SALT_T3 & $\begin{array}{l}\text { Shaft saft column } \\
\text { compactedtime } 25 \text { to } \\
50 \text { years }\end{array}$ & PMLT_LO & $\begin{array}{l}\text { Log triangutar distribution low } \\
\text { value for permesbility }\end{array}$ & 33412 & 13Feb-96 & $\mathbf{Y}$ & 1 \\
\hline $\mathbf{Y}$ & varies & 2957 & SALT_T3 & $\begin{array}{l}\text { Shaft salt column } \\
\text { compectedtime } 25 \text { to } \\
\text { 50 years }\end{array}$ & PMLT_LO & $\begin{array}{l}\text { Log trianguler distribution low } \\
\text { vakus for permesbility }\end{array}$ & 33412 & 13Fab-96 & $\mathbf{Y}$ & 1 \\
\hline $\mathbf{Y}$ & varies & 2957 & SALT_T3 & $\begin{array}{l}\text { Shan salt column } \\
\text { compectecttime } 25 \text { to } \\
50 \text { years }\end{array}$ & PMLT_LO & $\begin{array}{l}\text { Loo triangular distribution low } \\
\text { value for permeability }\end{array}$ & 33412 & 13-Fob-S6 & $\mathbf{Y}$ & 1 \\
\hline $\mathbf{Y}$ & varies & 2958 & SALT_T3 & $\begin{array}{l}\text { Shan salt column } \\
\text { compactedtime } 25 \text { to } \\
50 \text { years }\end{array}$ & PMLT_HI & $\begin{array}{l}\text { Log trianguler distribution high } \\
\text { value for permasbilty }\end{array}$ & 33409 & 13Fab-96 & $\mathbf{Y}$ & 1 \\
\hline $\mathbf{Y}$ & varies & 2958 & SALT_T3 & $\begin{array}{l}\text { Shath salt column } \\
\text { compactectime } 25 \text { to } \\
50 \text { yoars }\end{array}$ & PMLT_HI & $\begin{array}{l}\text { Log triargular distribution high } \\
\text { value for permesbility }\end{array}$ & 33409 & $13-F \circ b-96$ & $\mathbf{Y}$ & 1 \\
\hline $\mathbf{Y}$ & varies & 2958 & SALT_T3 & $\begin{array}{l}\text { Shen salt column } \\
\text { compacteditine } 25 \text { to } \\
50 \text { years }\end{array}$ & PMLT_HI & $\begin{array}{l}\text { Log triangular distribution high } \\
\text { value for permeability }\end{array}$ & 33409 & 13Fab-96 & $Y$ & 1 \\
\hline $\mathbf{Y}$ & & 2959 & SALT_T4 & $\begin{array}{l}\text { Shat salt column } \\
\text { compecteditus } 50 \text { to } \\
100 \text { years }\end{array}$ & RSH_AIR & Air-supply shat radius $(3.09 \mathrm{~m})$ & $33556 \mathrm{~A}$ & 13-Fob-95 & $\mathbf{Y}$ & 48 \\
\hline $\mathbf{Y}$ & & 2959 & SALT_T4 & $\begin{array}{l}\text { Shein salt column } \\
\text { compectedtime } 50 \text { to } \\
100 \text { years }\end{array}$ & RSH_AIR & Air-supply shan radius (3.09 m) & $33556 \mathrm{~A}$ & 13 Feb-96 & $\mathbf{Y}$ & 48 \\
\hline $\mathbf{Y}$ & & 2959 & SALT_T4 & $\begin{array}{l}\text { Shat salt column } \\
\text { compactectime } 50 \text { to } \\
100 \text { years }\end{array}$ & RSH_AR & Air-eupply shat radius (3.09 m) & $33556 \mathrm{~A}$ & 13-Feb-96 & $\mathbf{Y}$ & 48 \\
\hline $\mathbf{Y}$ & & 2959 & SALT_T4 & $\begin{array}{l}\text { Shat sak column } \\
\text { compactedtine } 50 \text { to } \\
100 \text { years }\end{array}$ & RSH_AIR & Air-supply shant radus (3.09 m) & $33556 \mathrm{~A}$ & 13Feb-96 & $\mathbf{Y}$ & $A B$ \\
\hline $\mathbf{Y}$ & & 2960 & SALT_T4 & $\begin{array}{l}\text { Sheft eat column } \\
\text { compectectime } 50 \text { to } \\
100 \text { years }\end{array}$ & RSH_SAL & Salthandling chath radus $(1.8 \mathrm{~m})$ & 335604 & 13-Fob-96 & $\mathbf{Y}$ & 48 \\
\hline $\mathbf{Y}$ & & 2960 & SALT_T4 & $\begin{array}{l}\text { Shat salt column } \\
\text { compacteditime } 50 \text { to } \\
100 \text { years }\end{array}$ & RSH_SAL & Salt-handing shat radius $(1.8 \mathrm{~m})$ & $33560 \mathrm{~A}$ & 13 Feb-96 & $\mathbf{Y}$ & 48 \\
\hline $\mathbf{Y}$ & & 2960 & SALT_T4 & $\begin{array}{l}\text { Shat ealt column } \\
\text { compectectime } 50 \text { to } \\
100 \text { yeers }\end{array}$ & RSH_SAL & Sekthanding shant radics $(1.8 \mathrm{~m})$ & 335604 & 13-Feb-96 & $\mathbf{Y}$ & $4 B$ \\
\hline $\mathbf{Y}$ & & 2960 & SALT_T4 & $\begin{array}{l}\text { Shat sak column } \\
\text { compectectimo } 50 \text { to } \\
100 \text { yeurs }\end{array}$ & RSH_SAL & Stathending thath radius $(1.8 \mathrm{~m})$ & 335604 & $13-F e b-96$ & $\mathbf{Y}$ & AB \\
\hline $\mathbf{Y}$ & & 2961 & SNLT_T4 & $\begin{array}{l}\text { Shan eax colemn } \\
\text { compuctedtime } 50 \text { to } \\
100 \text { years }\end{array}$ & RSH_WAS & $\begin{array}{l}\text { Wasto handing shaft radus (3.5 } \\
\text { m) }\end{array}$ & $33561 A$ & 13-Feb-96 & $\mathbf{Y}$ & $4 B$ \\
\hline $\mathbf{Y}$ & & 2961 & SALT_T4 & $\begin{array}{l}\text { Shat selt column } \\
\text { compactidtime } 50 \text { to } \\
100 \text { yers }\end{array}$ & RSH_WAS & $\begin{array}{l}\text { Wasto-hendling shaft redxes (3.5 } \\
\text { m) }\end{array}$ & $33561 A$ & $13+f a b-96$ & $\mathbf{Y}$ & 48 \\
\hline $\mathbf{Y}$ & & 2961 & SALT_TA & $\begin{array}{l}\text { Shat all column } \\
\text { compectedtime } 50 \text { to. } \\
100 \text { years }\end{array}$ & RSH_WAS & $\begin{array}{l}\text { Wastenthending that radius (3.5 } \\
\text { (m) }\end{array}$ & 335611 & 13-Fab-96 & $\mathbf{Y}$ & 48 \\
\hline
\end{tabular}


CCA Parameter Listing

\begin{tabular}{|c|c|c|c|c|c|c|c|c|c|c|}
\hline 10 & 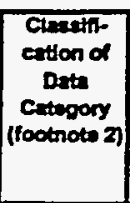 & $\begin{array}{c}\text { Destributton } \\
\text { Type }\end{array}$ & man & Medien & MLnistuman & Mnodmun & Chatte & Reference Lo & $\begin{array}{l}\text { Wes the dets } \\
\text { deviloped } \\
\text { under an } \\
\text { Noll-1 } \\
\text { Progrum? }\end{array}$ & 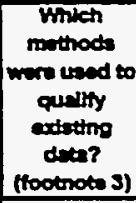 \\
\hline 2954 & 1,3 & CONSTANT & $2.3000000 E+00$ & $2.3000000 E+00$ & $2.3000000 E+\infty 0$ & $2.3000000 E+\infty 0$ & $m$ & WPO40514 & $\mathbf{Y}$ & NA \\
\hline 2955 & $1,3,4$ & CONSTANT & $1.0000000 \mathrm{E}+\infty$ & $1.0000000 E+00$ & $1.0000000 E+\infty 0$ & $1.0000000 E+\infty 0$ & $\mathbf{m} / \mathrm{m}$ & WP030540 & $Y$ & NA \\
\hline 2955 & $1,3,4$ & CONSTANT & $1.0000000 \mathrm{E}+00$ & $1.0000000 E+\infty 0$ & $1.0000000 E+\infty 0$ & $1.0000000 E+\infty 0$ & $\mathrm{~m} / \mathrm{m}$ & WPO30994 & $Y$ & NA \\
\hline 2955 & $1,3,4$ & CONSTANT & $1.0000000 E+00$ & $1.0000000 \mathrm{E}+\infty 0$ & $1.0000000 E+\infty 0$ & $1.0000000 E+\infty 0$ & $\mathrm{~m} / \mathrm{m}$ & WP035597? & $\mathbf{Y}$ & NA \\
\hline 2955 & $1,3,4$ & CONSTANT & $-1.4782500 E+01$ & $-1.4782500 E+01$ & $-1.4782500 E+01$ & $-1.4782500 E+01$ & $\log \left(m^{\wedge} 2\right)$ & WPO30540 & YaN & 1.4 \\
\hline 2956 & $1,3,4$ & CONSTANT & $-1.4782500 E+01$ & $-1.4782500 E+01$ & $-1.4782500 E+01$ & $-1.4782500 E+01$ & $\log \left(m^{\wedge} 2\right)$ & WPO30994 & YaN & 1.4 \\
\hline 2956 & $1,3,4$ & CONSTANT & $-1.4782500 E+01$ & $-1.4782500 E+01$ & $-1.4782500 E+01$ & $-1.4782500 E+01$ & $\log \left(m^{\wedge} 2\right)$ & WP035597 & YaN & 1.4 \\
\hline 2957 & $1,3,4$ & CONSTANT & $-1.7301000 E+01$ & $-1.7301000 E+01$ & $-1.7301000 E+01$ & $-1.7301000 E+01$ & $\log \left(m^{\wedge} 2\right)$ & UP030540 & Y\&N & 1.4 \\
\hline 2957 & $1,3,4$ & CONSTANT & $-1.7301000 E+01$ & $-1.7301000 \mathrm{E}+01$ & $-1.7301000 E+01$ & $-1.7301000 E+01$ & $\log \left(m^{\wedge} 2\right)$ & WP030994 & Y8N & 1.4 \\
\hline 2957 & $1,3,4$ & CONSTANT & $-1.7301000 E+01$ & $-1.7301000 E+01$ & $-1.7301000 E+01$ & $-1.7301000 E+01$ & $\log \left(m^{\wedge} 2\right)$ & WP035597 & Yan & 1.4 \\
\hline 2958 & $1,3,4$ & CONSTANT & $-1.2265200 E+01$ & $-1.2265200 E+01$ & $-1.2265200 E+01$ & $-1.2255200 \mathrm{E}+01$ & $\log \left(m^{\wedge} 2\right)$ & WP030540 & YEN & 1.4 \\
\hline 2958 & $1,3,4$ & CONSTANT & $-1.2265200 E+01$ & $-1.2265200 E+01$ & $-1.2265200 E+01$ & $-1.2265200 E+01$ & $\log \left(m^{\wedge} 2\right)$ & WP030994 & YRN & 1.4 \\
\hline 2958 & $1,3,4$ & CONSTANT & $-1.2265200 E+01$ & $-1.2265200 E+01$ & $-1.2285200 E+01$ & $-1.2265200 E+01$ & $\log \left(m^{\wedge} 2\right)$ & WP035597 & YEN & 1,4 \\
\hline 2959 & 1,3 & CONSTANT & $3.0900000 \mathrm{E}+\infty$ & $3.0900000 \mathrm{E}+\infty 0$ & $3.0900000 E+\infty 0$ & $3.0900000 E+00$ & m & WP030640 & $\mathbf{Y}$ & N/A \\
\hline 2959 & 1,3 & CONSTANT & $3.0900000 \mathrm{E}+\infty 0$ & $3.0900000 E+\infty 0$ & $3.0900000 \mathrm{E}+\infty 0$ & $3.0900000 E+\infty$ & m & WP030994 & $\mathbf{Y}$ & N/A \\
\hline 2959 & 1,3 & CONSTANT & $3.0900000 E+\infty 0$ & $3.0900000 \mathrm{E}+\infty 0$ & $3.0900000 E+00$ & $3.0900000 E+00$ & m & WP035597 & $Y$ & NA \\
\hline 2959 & 1,3 & CONSTANT & $3.0900000 E+\infty 0$ & $3.0900000 E+\infty$ & $3.09000000+\infty$ & $3.0900000 E+00$ & m & WPO40514 & $\dot{\gamma}$ & NA \\
\hline 2960 & 1,3 & CONSTANT & $1.8000000 \mathrm{E}+\infty 0$ & $1.80000000+\infty$ & $1.8000000 E+\infty$ & $1.8000000 E+\infty 0$ & m & WP030640 & $\mathbf{Y}$ & NA \\
\hline 2960 & 1,3 & CONSTANT & $1.8000000 E+\infty 0$ & $1.80000000+\infty 0$ & $1.8000000 E+\infty 0$ & $1.8000000 \mathrm{E}+\infty 0$ & m & WP030994 & $\mathbf{Y}$ & NA \\
\hline 2960 & 1,3 & CONSTANT & $1.8000000 E+00$ & $1.8000000 \mathrm{E}+\infty 0$ & $1.8000000 E+\infty 0$ & $1.8000000 E+\infty 0$ & $m$ & WP035597 & $\gamma$ & NA \\
\hline 2960 & 1,3 & CONSTANT & $1.8000000 E+00$ & $1.8000000 E+00$ & $1.8000000 E+00$ & $1.8000000 E+\infty$ & m & WPO40514 & $\mathbf{Y}$ & NA \\
\hline 2961 & 1,3 & CONSTANT & $3.5000000 E+\infty 0$ & $3.5000000 E+\infty 0$ & $3.5000000 E+\infty$ & $3.5000000 E+\infty 0$ & m & WPO30640 & $Y$ & N/A \\
\hline 2961 & 1,3 & CONSTANT & $3.5000000 E+00$ & $3.5000000 E+\infty$ & $3.5000000 E+\infty$ & $3.5000000 \mathrm{E}+\infty$ & $m$ & WP030994 & $Y$ & NA \\
\hline 2961 & 1.3 & CONSTANT & $3.5000000 \mathrm{E}+\infty 0$ & $3.5000000 \mathrm{E}+00$ & $3.5000000 E+\infty$ & $3.5000000 \varepsilon+\infty$ & $\mathbf{m}$ & WP035597 & $Y$ & NA \\
\hline
\end{tabular}


CCA Parameter Listing

\begin{tabular}{|c|c|c|c|c|c|c|c|c|c|c|}
\hline 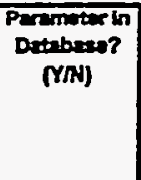 & 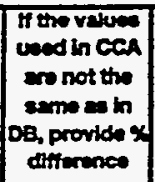 & ID & Metarlato & Matera Name & Parmotiax & Parenoter Merts & $\begin{array}{l}\text { PRP D } \\
\text { mPO }\end{array}$ & $\begin{array}{c}\text { Dete Enty } \\
\text { Dete }\end{array}$ & 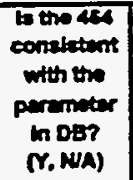 & $\begin{array}{l}\text { Parineter } \\
\text { Cetegory } \\
\text { (footnots i) }\end{array}$ \\
\hline $\mathbf{Y}$ & & 2961 & SALT_T4 & $\begin{array}{l}\text { Shaft salt colimn } \\
\text { compactedtime } 50 \text { to } \\
100 \text { years }\end{array}$ & RSH_WAS & $\begin{array}{l}\text { Wasto-handing shat radus (3.5 } \\
\text { m) }\end{array}$ & $33561 A$ & $13+500-96$ & $\mathbf{Y}$ & $4 B$ \\
\hline $\mathbf{Y}$ & & 2962 & SALT_T4 & $\begin{array}{l}\text { Shatt salt column } \\
\text { compectedtume } 50 \text { to } \\
100 \text { years }\end{array}$ & RSH_EXH & Air-exhaust sheth redius (2.3 m) & 335584 & $13-F a b-96$ & $\mathbf{Y}$ & $4 B$ \\
\hline $\mathbf{Y}$ & & 2962 & SALT_T4 & $\begin{array}{l}\text { Shat salt cokmn } \\
\text { compectedtune } 50 \text { to } \\
100 \text { years }\end{array}$ & RSH_EXH & Ar-exhent shen radis $(2.3 \mathrm{~m})$ & $33558 \mathrm{~A}$ & $13-F a b-96$ & $\mathbf{Y}$ & 48 \\
\hline $\mathbf{Y}$ & & 2962 & SALT_T4 & $\begin{array}{l}\text { Shaft walt column } \\
\text { compactectime } 50 \text { to } \\
100 \text { years }\end{array}$ & RSH_EXH & 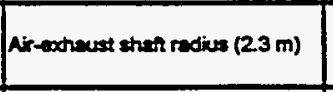 & $33558 A$ & 13 Frb-96 & $\mathbf{Y}$ & 48 \\
\hline $\mathbf{Y}$ & & 2952 & SALT_T4 & $\begin{array}{l}\text { Shath salt column } \\
\text { compectect time } 50 \text { to } \\
100 \text { years }\end{array}$ & RSH_EXH & Air -acheust that radius (2.3 m) & 335584 & 13-Fab-96 & $\mathbf{Y}$ & $4 \mathbf{B}$ \\
\hline $\mathbf{Y}$ & & 2963 & SALT_T4 & $\begin{array}{l}\text { Shaft salt column } \\
\text { compactectime } 50 \text { to } \\
100 \text { years }\end{array}$ & RADN_DRZ & DRZ outer redies at each ahent & 33540 & $13-F a b-96$ & $\mathbf{Y}$ & 1 \\
\hline $\mathbf{Y}$ & & 2963 & SALT_T4 & $\begin{array}{l}\text { Shaft salt column } \\
\text { compacteditime } 50 \text { to } \\
100 \text { years }\end{array}$ & RADN_DRZ & DRZ outer radies at each shaft & 33540 & $13-F \infty b-S 6$ & $\mathbf{Y}$ & 1 \\
\hline $\mathbf{Y}$ & & 2963 & SALT_TA & $\begin{array}{l}\text { Shaft salt cokmn } \\
\text { compectectime } 50 \text { to } \\
100 \text { yoers }\end{array}$ & RADN_DRZ & DRZ outer radius at each shaft & 33540 & 13 Feb-g6 & $\mathbf{Y}$ & 1 \\
\hline $\mathbf{Y}$ & varies & 2964 & SALT_TA & $\begin{array}{l}\text { Shath salt colemn } \\
\text { compactodtime } 50 \text { to } \\
100 \text { years }\end{array}$ & PMLT_MD & $\begin{array}{l}\text { Log trianoular distribution mode } \\
\text { for permeability }\end{array}$ & 33463 & 13-feb-96 & $\mathbf{Y}$ & 1 \\
\hline $\mathbf{Y}$ & varies & 2964 & SALT_T4 & $\begin{array}{l}\text { Shaft salt column } \\
\text { compactectime } 50 \text { to } \\
100 \text { years }\end{array}$ & PMLT_MD & $\begin{array}{l}\text { Log trienguler distribution mods } \\
\text { for permesbitity }\end{array}$ & 33463 & $13+50 b-96$ & $\mathbf{Y}$ & 1 \\
\hline $\mathbf{Y}$ & varies. & 2964 & SALT_T4 & $\begin{array}{l}\text { Shaft salt colemn } \\
\text { compactectime } 50 \text { to } \\
100 \text { years }\end{array}$ & PMLT_MD & $\begin{array}{l}\text { Log triengular distribution mode } \\
\text { for permeability }\end{array}$ & 33463 & $13-F=b-96$ & $\mathbf{Y}$ & 1 \\
\hline $\mathbf{Y}$ & varies & 2965 & SALT_T4 & $\begin{array}{l}\text { Shaft sal colimn } \\
\text { compactectime } 50 \text { to } \\
100 \text { years }\end{array}$ & PMLT_LO & $\begin{array}{l}\text { Log triangular distribution low } \\
\text { value for permeability }\end{array}$ & 33461 & 13 fob-96 & $\mathbf{Y}$ & 1 \\
\hline $\mathbf{Y}$ & varies & 2965 & SALT_T4 & $\begin{array}{l}\text { Shaft alt column } \\
\text { compactectime } 50 \text { to } \\
100 \text { years }\end{array}$ & PMLT_LO & $\begin{array}{l}\text { Log trianguler distrbution low } \\
\text { value for permasibity }\end{array}$ & 33461 & 13-Fob-96 & $\mathbf{Y}$ & 1 \\
\hline$Y$ & varies & 2965 & SALT_T4 & $\begin{array}{l}\text { Shet salt column } \\
\text { compected time } 50 \text { to } \\
100 \text { years }\end{array}$ & PMLT_LO & $\begin{array}{l}\text { Log trienguler distribution low } \\
\text { value for permescility }\end{array}$ & 33461 & $13-F a b-96$ & $\mathbf{Y}$ & 1 \\
\hline $\mathbf{Y}$ & varies & 2966 & SALT_T4 & $\begin{array}{l}\text { Shaft salt column } \\
\text { compectedtime } 50 \text { to } \\
100 \text { years }\end{array}$ & PMLT_HI & $\begin{array}{l}\text { Log triangular distribution high } \\
\text { value for permesbilty }\end{array}$ & 33460 & $13-F a b-96$ & $\mathbf{Y}$ & 1 \\
\hline $\mathbf{Y}$ & varies & 2966 & SALT_T4 & $\begin{array}{l}\text { Shaft salt column } \\
\text { compectedtime } 50 \text { to } \\
100 \text { years }\end{array}$ & PMLT_H! & $\begin{array}{l}\text { Log trangular distribution high } \\
\text { value for permeability }\end{array}$ & 33460 & 13-Fab-96 & $\mathbf{Y}$ & 1 \\
\hline $\mathbf{Y}$ & varies & 2966 & SALT_T4 & $\begin{array}{l}\text { Shaf eat column } \\
\text { compectedtime } 50 \text { to } \\
100 \text { years }\end{array}$ & PMLT_HI & $\begin{array}{l}\text { Log triengular distribution high } \\
\text { value for permeability }\end{array}$ & 33460 & 13 Fob-96 & $\because Y$ & 1 \\
\hline $\mathbf{Y}$ & & 2967 & SALT_T5 & $\begin{array}{l}\text { Shat salt column } \\
\text { compactedtime } 100 \text { to } \\
200 \text { years }\end{array}$ & RSH_ANR & Air-supply shat radies (3.09 m) & 33616A & 13 feb-gs & $\mathbf{Y}$ & 48 \\
\hline $\mathbf{Y}$ & & 2967 & SALT_T5 & $\begin{array}{l}\text { Shat salt column } \\
\text { compectedtime } 100 \text { to } \\
200 \text { years }\end{array}$ & RSH_AR & Air-upply chan radius $(3.09 \mathrm{~m})$ & $33616 A$ & 13-Feb-96 & $\mathbf{Y}$ & $4 B$ \\
\hline $\mathbf{Y}$ & & 2967 & SALT_T5 & $\begin{array}{l}\text { Shan sal column } \\
\text { compectect time } 100 \text { to } \\
200 \text { yeces }\end{array}$ & RSH_AR & Air-aupply thatt radius $(3.09 \mathrm{~m})$ & $33616 A$ & 13 feb-96 & $\mathbf{Y}$ & $4 B$ \\
\hline $\mathbf{Y}$ & & 2967 & SALT_TS & $\begin{array}{l}\text { Shan sat colemn } \\
\text { compectedtime } 100 \text { to } \\
200 \text { years }\end{array}$ & RSH_AR & Air-usply shan radius $(3.09 \mathrm{~m})$ & $33616 A$ & $13-F e b-96$ & $\mathbf{Y}$ & 48 \\
\hline $\mathbf{Y}$ & & 2968 & SALT_T5 & $\begin{array}{l}\text { Shin satt column } \\
\text { compected:time } 100 \text { to } \\
200 \text { years }\end{array}$ & RSH_SAL & Salt handing then radius $(9.8 \mathrm{~m})$ & $33620 \mathrm{~A}$ & 13Fob-96 & $\mathbf{Y}$ & 48 \\
\hline $\mathbf{Y}$ & & 2968 & SALT_T5 & \begin{tabular}{|l|} 
Shen salt column \\
compectedtime 100 to \\
200 yems
\end{tabular} & RSH_SAL & Salthending thest radius $(1.8 \mathrm{~m})$ & 336204 & $13-f a b-96$ & $Y$ & $4 B$ \\
\hline $\mathbf{Y}$ & & 2968 & SALT_TS & $\begin{array}{l}\text { Shan sat coumn } \\
\text { compectactime } 100 \text { to } \\
200 \text { years }\end{array}$ & RSH_SN & Salt hending shet redius $(1.8 \mathrm{~m})$ & 336204 & $13-F a b-96$ & $\mathbf{Y}$ & 48 \\
\hline
\end{tabular}


- CCA Parameter-Listing

\begin{tabular}{|c|c|c|c|c|c|c|c|c|c|c|}
\hline 10 & 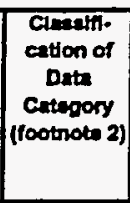 & $\begin{array}{l}\text { Distribution } \\
\text { Type }\end{array}$ & Mean & Modian & Mtalmem & Madrium & Untas & Reterence DD & $\begin{array}{l}\text { Was the dinta } \\
\text { doveloped } \\
\text { Under en } \\
\text { NoA-1 } \\
\text { Progrem? }\end{array}$ & 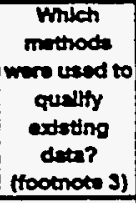 \\
\hline 2961 & 1.3 & CONSTANT & $3.5000000 \mathrm{E}+00$ & $3.5000000 E+\infty 0$ & $3.5000000 \varepsilon+\infty 0$ & $3.5000000 E+\infty 0$ & $m$ & wp040514 & $\mathbf{Y}$ & NA \\
\hline 2962 & 1,3 & CONSTANT & $2.3000000 \mathrm{E}+00$ & $2.3000000 E+\infty 0$ & $23000000 E+\infty 0$ & $2.3000000 \mathrm{E}+\infty 0$ & $m$ & WP030640 & $Y$ & NA \\
\hline 2962 & 1,3 & CONSTANT & $2.3000000 E+\infty 0$ & $2.3000000 E+\infty 0$ & $2.3000000 E+\infty 0$ & $2.3000000 E+\infty 0$ & $\mathbf{m}$ & WP030994 & $Y$ & NA \\
\hline 2962 & 1,3 & CONSTANT & $23000000 \mathrm{E}+\infty 0$ & $2.3000000 E+\infty 0$ & $2.3000000 E+\infty 0$ & $2.3000000=+\infty$ & $\mathbf{m}$ & WP03559? & $\mathbf{Y}$ & NA \\
\hline 2962 & 1,3 & CONSTANT & $2.3000000 E+\infty$ & $2.3000000 E+\infty 0$ & $23000000 E+\infty 0$ & $23000000 E+\infty 0$ & $\mathbf{m}$ & WPO40514 & $\mathbf{Y}$ & NA \\
\hline 2963 & $1,3,4$ & CONSTANT & $1,0000000 E+00$ & $1.0000000 E+00$ & $1.0000000 E+\infty 0$ & $1.0000000 E+\infty 0$ & $\mathrm{~m} / \mathrm{m}$ & WP030640 & Y & NA \\
\hline 2963 & $1,3,4$ & CONSTANT & $1.0000000 E+\infty 0$ & $1.0000000 E+\infty 0$ & $1.0000000 E+\infty 0$ & $1.0000000 E+\infty$ & $\mathrm{m} / \mathrm{m}$ & WP030994 & $\mathbf{Y}$ & NA \\
\hline 2963 & $1,3,4$ & CONSTANT & $1.0000000 E+00$ & $1.0000000 E+\infty 0$ & $1.0000000 E+\infty$ & $1.0000000 E+\infty 0$ & $\mathrm{~m} / \mathrm{m}$ & WP035597 & $\mathbf{Y}$ & NA \\
\hline 2964 & $1,3,4$ & CONSTANT & $-1.7165600 E+01$ & $-1.7165600 E+01$ & $-1.7165600 E+01$ & $-1.7165600 E+01$ & $\log \left(m^{\wedge} 2\right)$ & WP030640 & Y8N & 1.4 \\
\hline 2964 & $1,3,4$ & CONSTAN! & $-1.7165600 \mathrm{E}+01$ & $-1.7165600 E+01$ & $-1.7165600 \mathrm{E}+01$ & $-1.7165600 E+01$ & $\log \left(m^{\wedge} 2\right)$ & WP030994 & Y\&N & 1,4 \\
\hline 2964 & $1,3,4$ & CONSTANT & $-1.7165600 E+01$ & $-1.7165600 E+01$ & $-1.7165600 E+01$ & $-1.7165600 E+01$ & $\log \left(m^{n} 2\right)$ & wP035597 & Y\&N & 1.4 \\
\hline 2965 & $1,3,4$ & CONSTANT & $-22876100 E+01$ & $-2.2876100 E+01$ & $-2.2876100 E+01$ & $-22876100 E+01$ & $\log \left(m^{\wedge} 2\right)$ & WP030640 & YEN & 1.4 \\
\hline 2965 & $1,3,4$ & CONSTANT & $-2.2876100 E+01$ & $-2.2876100 E+01$ & $-2.2876100 E+01$ & $-2.2876100 E+01$ & $\log \left(m^{n} 2\right)$ & WP030994 & YEN & 1.4 \\
\hline 2965 & $1,3,4$ & CONSTANT & $-2.2876100 E+01$ & $-2.2876100 E+01$ & $-2.2876100 E+01$ & $-2.2876100 E+01$ & $\log \left(m^{\wedge} 2\right)$ & WP035597 & YaN & 1,4 \\
\hline 2966 & $1,3,4$ & CONSTANT & $-1.3950800 E+01$ & $-1.3950800 E+01$ & $-1.3950800 E+01$ & $-1.3950800 E+01$ & $\log \left(m^{\wedge} 2\right)$ & WP030540 & YEN & 1.4 \\
\hline 2966 & $1,3,4$ & CONSTANT & $-1.3950800 E+01$ & $-1,3950800 E+01$ & $-1.3950800 E+01$ & $-1.3950800 E+01$ & $\log \left(m^{\wedge} 2\right)$ & WP030994 & YBN & 1,4 \\
\hline 2966 & $1,3,4$ & CONSTANT & $-1.3950800 E+01$ & $-1.3950800 E+01$ & $-1.3950800 E+01$ & $-1.3950600 E+01$ & $\log \left(m^{\wedge} 2\right)$ & WP035597 & Y̌sN & 1.4 \\
\hline 2967 & 1,3 & CONSTANT & $3.0900000 E+00$ & $3.0900000 E+00$ & $3.0900000 E+00$ & $3.0900000 E+\infty 0$ & $\mathbf{m}$ & WP030640 & $\mathbf{Y}$ & NA \\
\hline 2967 & 1,3 & CONSTANT & $3.0900000 E+\infty 0$ & $3.0900000 E+\infty 0$ & $3.0900000 E+00$ & $3.0900000 E+\infty$ & m & WP030994 & $\mathbf{Y}$ & N/A \\
\hline 2967 & 1,3 & CONSTANT & $3.0900000 E+\infty 0$ & $3.0900000 E+\infty 0$ & $3.0900000 E+\infty$ & $3.0900000 E+00$ & $\mathbf{m}$ & WP035597 & $\mathbf{Y}$ & N/A \\
\hline 2967 & 1,3 & CONSTANT & $3.0900000 E+00$ & $3.0900000 E+\infty 0$ & $3.0900000 E+\infty 0$ & $3.0900000 \mathrm{E}+00$ & m & WP040514 & $\mathbf{Y}$ & NUA \\
\hline 2968 & 1.3 & CONSTANT & $1.6000000 E+00$ & $1.80000000+\infty 0$ & $1.8000000 E+\infty 0$ & $1.8000000 E+\infty$ & $\mathbf{m}$ & WP030640 & $\mathbf{Y}$ & N/A \\
\hline 2968 & 1,3 & CONSTANT & $1.8000000 E+00$ & $1.8000000 E+\infty 0$ & $1.80000000 E+00$ & $1.8000000 E+\infty$ & $\mathbf{m}$ & WP030994 & $Y$ & N/A \\
\hline 2968 & 1,3 & CONSTANT & $1.8000000 E+\infty 0$ & $1.8000000 E+\infty$ & $1.80000000+\infty 0$ & $1.8000000 E+\infty 0$ & $\mathbf{m}$ & WP035597 & $\mathbf{Y}$ & NA \\
\hline
\end{tabular}


CCA Parameter Listing

\begin{tabular}{|c|c|c|c|c|c|c|c|c|c|c|}
\hline $\begin{array}{c}\text { Parmotertin } \\
\text { Dumbete? } \\
\text { (Y/N) }\end{array}$ & 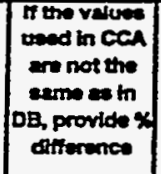 & D & Metertal iD & Mrterta Nom & $\begin{array}{c}\text { Parametar } \\
\text { Do } \\
-\end{array}$ & Parmier Nam & $\begin{array}{c}\text { PRP D } \\
\text { MPO \#) }\end{array}$ & Detan Entry & $\begin{array}{l}\text { b the ASA } \\
\text { conationtert } \\
\text { with the } \\
\text { permeter } \\
\text { in DeP? } \\
M, N A\end{array}$ & $\begin{array}{l}\text { Parminter } \\
\text { Cattegory } \\
\text { (footnote 1) }\end{array}$ \\
\hline $\mathbf{Y}$ & & 2968 & SALT_IS & $\begin{array}{l}\text { Shaft sat cotumn } \\
\text { compectedtume } 100 \text { to } \\
200 \text { years }\end{array}$ & RSH_SAL & Sakthanding sthef radius $(1.8 \mathrm{~m})$ & 335204 & $13-50 b-96$ & $\mathbf{Y}$ & 48 \\
\hline $\mathbf{Y}$ & & 2969 & SALT_T5 & $\begin{array}{l}\text { Shat salt colemn } \\
\text { compactectume } 100 \text { to } \\
200 \text { yoars }\end{array}$ & RSH_WAS & $\begin{array}{l}\text { Wasto-trancting ahet radis (3.5 } \\
\text { m) }\end{array}$ & $33622 A$ & $13-F a b-96$ & $\mathbf{Y}$ & 48 \\
\hline $\mathbf{Y}$ & & 2969 & SALT_TS & $\begin{array}{l}\text { Shen call column } \\
\text { compectedtime } 100 \text { to } \\
200 \text { years }\end{array}$ & RSH_WAS & $\begin{array}{l}\text { Wasto-handing athet radius (3.5 } \\
\text { m) }\end{array}$ & $33502 A$ & $13-F 0 b-96$ & $\mathbf{Y}$ & 48 \\
\hline $\mathbf{Y}$ & & 2969 & SALT_T5 & $\begin{array}{l}\text { Shaft salt column } \\
\text { compectedtime } 100 \text { to } \\
200 \text { years }\end{array}$ & RSH_WAS & $\begin{array}{l}\text { Wasto-handling ghat redies (3.5 } \\
\text { m) }\end{array}$ & $33622 A$ & 13Fob-96 & $\mathbf{Y}$ & $4 B$ \\
\hline $\mathbf{Y}$ & & 2969 & SALT_TS & $\begin{array}{l}\text { Shat sal column } \\
\text { compacteditime } 100 \text { to } \\
200 \text { years }\end{array}$ & RSH_WAS & $\begin{array}{l}\text { Wasto-handing atreft radius (3.5 } \\
\text { (m) }\end{array}$ & $35022 A$ & 13 Feb-96 & $\mathbf{Y}$ & 48 \\
\hline $\mathbf{Y}$ & & 2970 & SALT_T5 & $\begin{array}{l}\text { Shat salt colemn } \\
\text { compactedtine } 100 \text { to } \\
200 \text { years }\end{array}$ & RSH_EXH & Ar-exhaust shan radius $(2.3 \mathrm{~m})$ & 336184 & $13+F a b-S 6$ & $\mathbf{Y}$ & $4 B$ \\
\hline $\mathbf{Y}$ & & 2970 & SALT_T5 & $\begin{array}{l}\text { Shaft salt cotumn } \\
\text { compactedtime } 100 \text { to } \\
200 \text { years }\end{array}$ & RSH_EXH & Ar- acheust shent radies $(2.3 \mathrm{~m})$ & $33618 A$ & $13-F e b-96$ & $\mathbf{Y}$ & $4 B$ \\
\hline $\mathbf{Y}$ & & 2970 & SALT_T5 & $\begin{array}{l}\text { Shaft sak column } \\
\text { compactedtime } 100 \text { to } \\
200 \text { years }\end{array}$ & RSH_EXH & Air-extheust ahat radus $(2.3 \mathrm{~m})$ & $33618 A$ & 13 feb-gs & $\mathbf{Y}$ & $4 B$ \\
\hline $\mathbf{Y}$ & & 2970 & SALT_T5 & $\begin{array}{l}\text { Shaf salt column } \\
\text { compactecttime } 100 \text { to } \\
200 \text { years }\end{array}$ & RSH_EXH & Arr-exhaust shef radius (2.3 m) & $33618 A$ & 13-Fab-96 & $\mathbf{Y}$ & $4 B$ \\
\hline $\mathbf{Y}$ & & 2979 & SALT_TS & $\begin{array}{l}\text { Shaft sat column } \\
\text { compacted:tme } 100 \text { to } \\
200 \text { yoars }\end{array}$ & RADN_DRZ & DRZ outer radus at asch shat & 33609 & $13-F a b-96$ & $\mathbf{Y}$ & 1 \\
\hline $\mathbf{Y}$ & & 2971 & SALT_T5 & $\begin{array}{l}\text { Shafl salt cokmn } \\
\text { compectectume } 100 \text { to } \\
200 \text { yoars }\end{array}$ & RADN_DRZ & DRZ outer radus at each shat & 33609 & 13-Fob-96 & $\mathbf{Y}$ & 1 \\
\hline $\mathbf{Y}$ & & $297 i$ & SALT_T5 & $\begin{array}{l}\text { Sheit sat column } \\
\text { compectedtume } 100 \text { to } \\
200 \text { years }\end{array}$ & RADN_ORZ & DRZ outer recale et each shat & 33609 & $13+5 b-96$ & $\mathbf{Y}$ & 1 \\
\hline $\mathbf{Y}$ & varies & 2972 & SALT_T5 & $\begin{array}{l}\text { Shan sat cotumn } \\
\text { compectect.tme } 100 \text { to } \\
200 \text { years }\end{array}$ & PMLT_MO & 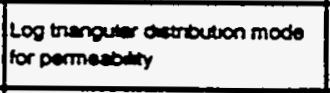 & 33585 & 13-Feb-ss & $\mathbf{Y}$ & 1 \\
\hline $\mathbf{Y}$ & varies & 2972 & SALT_T5 & $\begin{array}{l}\text { Shat sat conmn } \\
\text { compacted tume } 100 \text { to } \\
200 \text { years }\end{array}$ & PMLT_MD & 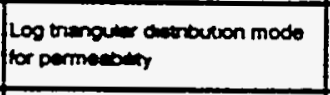 & 33585 & 13-Fob-96 & $\mathbf{Y}$ & 1 \\
\hline $\mathbf{Y}$ & varies & 2972 & SALT_T5 & $\begin{array}{l}\text { Shat san column } \\
\text { compacted time } 100 \text { to } \\
200 \text { years }\end{array}$ & PMLT_MO & 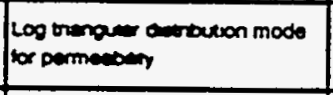 & 33585 & 13-Fob-96 & $Y$ & 1 \\
\hline $\mathbf{Y}$ & varies & 2973 & SALT_T5 & $\begin{array}{l}\text { Shaft set coumn } \\
\text { compacted trme } 100 \text { to } \\
200 \text { years }\end{array}$ & PMAT_LO & 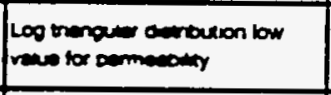 & 33583 & 13-Fab-96 & $\mathbf{Y}$ & 1 \\
\hline $\mathbf{Y}$ & varios & 2973 & SALT_T5 & $\begin{array}{l}\text { Shet sali colmm } \\
\text { compactect tme } 100 \text { to } \\
200 \text { vears }\end{array}$ & Puist_L & 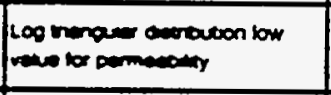 & 33583 & 13-Fob-96 & $-\bar{\gamma}$ & 1 \\
\hline $\mathbf{Y}$ & varies & 2973 & SALT_TS & $\begin{array}{l}\text { Shat sed coumn } \\
\text { compected tum ton wo } \\
200 \text { yeers }\end{array}$ & Pams_to & 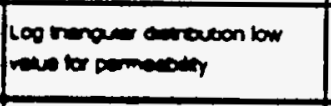 & 33583 & 13Fob-96 & $\mathbf{Y}$ & 1 \\
\hline $\mathbf{Y}$ & varies & 2974 & SALT_T5 & {$\left[\begin{array}{l}\text { Shith sall colmm } \\
\text { compacted tom ton to } \\
200 \text { years }\end{array}\right.$} & Punt_m & Log nenow & 33581 & 13-Fob-96 & $\mathbf{Y}$ & 1 \\
\hline $\mathbf{Y}$ & vanes & 2974 & SALT_T5 & $\begin{array}{l}\text { Shal sax coum } \\
\text { compected trm } 100 \text { to } \\
200 \text { yess }\end{array}$ & Pint_n & Lod & 33581 & 13-fob-96 & $\mathbf{Y}$ & 1 \\
\hline $\mathbf{Y}$ & varies & 2974 & SALT_TS & 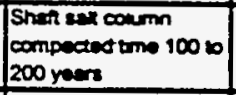 & Punt_a & 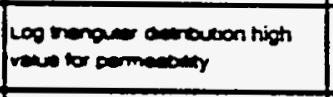 & 33581 & 13 Fab-96 & $\mathbf{Y}$ & 1 \\
\hline $\mathbf{Y}$ & & 2975 & SALT_T6 & $\begin{array}{l}\text { Shan sal coums } \\
\text { compecteot tome } 200 \text { to } \\
10 \text { K years }\end{array}$ & RSH_An & 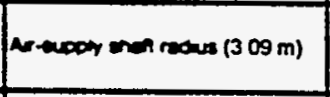 & $33478 A$ & 13-Feb-96 & $\mathbf{Y}$ & 48 \\
\hline $\mathbf{Y}$ & & 2975 & SALT_T6 & $\begin{array}{l}\text { Shat sax coumn } \\
\text { compectedtume } 200 \text { vo } \\
10 \text { K yees }\end{array}$ & $R_{\text {RS_An }}$ & Nrapour unen reoses $(3.09 \mathrm{~m})$ & $33479 A$ & $13+50-96$ & $\mathbf{Y}$ & 48 \\
\hline $\mathbf{Y}$ & & 2975 & SALT_T6 & $\begin{array}{l}\text { Shan sal colum } \\
\text { compectectume } 200 \text { to } \\
\text { 10K yeers }\end{array}$ & RSM_An & Ar-woon when nows $(3.09 \mathrm{~m})$ & 334784 & 13 -Feb-96 & $\mathbf{Y}$ & 48 \\
\hline
\end{tabular}




\begin{tabular}{|c|c|c|c|c|c|c|c|c|c|c|}
\hline - ID & $\begin{array}{c}\text { Cincint- } \\
\text { cation of } \\
\text { Dinta } \\
\text { Crategory } \\
\text { (foctnote 2) }\end{array}$ & $\begin{array}{l}\text { Distribution } \\
\text { Type }\end{array}$ & $\operatorname{man}$ & modien & Mintrinem & Drodimen & Unts & Referinee to & $\begin{array}{l}\text { Wha the detata } \\
\text { dorvioped } \\
\text { under an } \\
\text { Nol-1 } \\
\text { Proprem? }\end{array}$ & 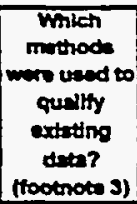 \\
\hline 2968 & 1,3 & CONSTANT & $1.8000000 E+\infty$ & $1.8000000 E+\infty$ & $1.8000000 E+\infty 0$ & $1.8000000 E+\infty$ & $m$ & WPO40514 & $\mathbf{Y}$ & NA \\
\hline 2969 & 1,3 & CONSTANT & $3.5000000 E+\infty 0$ & $3.5000000 E+\infty 0$ & $3.5000000 E+\infty$ & $3.5000000 E+\infty 0$ & $m$ & WPO30640 & $\mathbf{Y}$ & NA \\
\hline 2969 & 1,3 & CONSTANT & $3.5000000 E+\infty$ & $3.5000000 E+\infty$ & $3.5000000 E+\infty$ & $3.5000000 E+\infty$ & m & WP030994 & $\mathbf{Y}$ & NA \\
\hline 2969 & 1,3 & CONSTANT & $3.5000000 E+\infty$ & $3.5000000 E+\infty 0$ & $3.5000000 E+\infty 0$ & $3.50000000+\infty$ & $m$ & WP035597 & $Y$ & NA \\
\hline 2969 & 1,3 & CONSTANT & $3.5000000 E+\infty 0$ & $3.5000000 E+\infty 0$ & $3.5000000 E+\infty 0$ & $3.5000000 E+\infty 0$ & m & WP040514 & $\mathbf{Y}$ & NA \\
\hline 2970 & 1,3 & CONSTANT & $2.3000000 E+\infty$ & $2.3000000 E+\infty 0$ & $2.3000000 E+\infty 0$ & $2.3000000 E+\infty 0$ & $\mathbf{m}$ & WP030640 & $Y$ & NA \\
\hline 2970 & 1,3 & CONSTANT & $2.3000000 E+\infty 0$ & $23000000 E+\infty 0$ & $23000000 E+00$ & $2.3000000 E+\infty 0$ & $\mathbf{m}$ & WP030994 & $Y$ & N/A \\
\hline 2970 & 1,3 & CONSTANT & $2.3000000 E+\infty 0$ & $2.3000000 E+\infty 0$ & $2.3000000 E+00$ & $2.3000000 \equiv+\infty 0$ & m & WP035597 & $Y$ & NA \\
\hline 2970 & 1.3 & CONSTANT & $2.3000000 \mathrm{E}+00$ & $2.3000000 E+\infty 0$ & $2.3000000 E+\infty$ & $2.3000000 E+\infty 0$ & m & WPO40514 & $Y$ & NA \\
\hline 2971 & $1,3,4$ & CONSTANT & $1.0000000 E+\infty$ & $1.0000000 E+\infty 0$ & $1.00000000+\infty$ & $1.0000000 E+\infty$ & $\mathrm{m} / \mathrm{m}$ & WP030640 & $\mathbf{Y}$ & NUA \\
\hline 2971 & $1,3,4$ & CONSTANT & $1.00000000+00$ & $1.0000000 \mathrm{E}+\infty 0$ & $1.0000000 E+\infty 0$ & $1.0000000 E+\infty 0$ & $\mathrm{~m} / \mathrm{m}$ & WP030994 & $Y$ & NA \\
\hline 2971 & $1,3,4$ & CONSTANT & $1.00000000 \mathrm{E}+\infty 0$ & $1.0000000 E+00$ & $1.0000000 E+\infty$ & $1.0000000 \mathrm{E}+\infty 0$ & $\mathrm{~m} / \mathrm{m}$ & WP035597 & $\mathbf{Y}$ & NA \\
\hline 2972 & $1,3,4$ & CONSTANT & $-1.9278200 E+01$ & $-1.9278200 E+01$ & $-1.9278200 E+01$ & $-1.9278200 E+01$ & $\log \left(m^{\wedge} 2\right)$ & WPO30640 & Yas & 1.4 \\
\hline 2972 & $1,3,4$ & CONSTANT & $-1.9278200 E+01$ & $-1.9278200 E+01$ & $-1.9278300 E+01$ & $-1.9278200 E+01$ & $\log \left(m^{\wedge} 2\right)$ & WP030994 & YEN & 1,4 \\
\hline 2972 & $1,3,4$ & CONSTANT & $-1.9278200 E+01$ & $-1.9278200 E+01$ & $-1.9278200 E+01$ & $-1.9278200 E+01$ & $\log \left(m^{\wedge} 2\right)$ & WP035597 & YEN & 1.4 \\
\hline 2973 & $1,3,4$ & CONSTANT & $-2.2876100 E+01$ & $-2.2876900 E+01$ & $-2.2876100 E+01$ & $-2.2876100 E+01$ & $\log \left(m^{\wedge} 2\right)$ & WPO30S40 & YEN & 1,4 \\
\hline 2973 & $1,3,4$ & CONSTANT & $-2.2876100 E+01$ & $-2.2876100 E+01$ & $-2.2875100 E+01$ & $-2.2876100 E+01$ & $\log \left(m^{\wedge} 2\right)$ & WP030994 & $\dot{Y} 8 \mathrm{~N}$ & 1.4 \\
\hline 2973 & $1,3,4$ & CONSTANT & $-2.2876100 E+01$ & $-2.2876100 E+01$ & $-2.2878100 E+01$ & $-2.2876100 E+01$ & $\log \left(m^{\wedge} 2\right)$ & WP035597 & YEN & 1.4 \\
\hline 2974 & $1,3,4$ & CONSTANT & $-1.5426000 E+01$ & $-1.5426000 E+01$ & $-15426000 E+01$ & -1 5426000E+01 & $\log \left(m^{\wedge} 2\right)$ & WP030640 & YEN & 1.4 \\
\hline 2974 & $1,3,4$ & CONSTANT & $-1.5426000 E+01$ & $-1.5426000 E+01$ & $-1.5426000 E+01$ & $-1.5426000 E+01$ & $\log \left(m^{\wedge} 2\right)$ & WP030994 & YSN & 1,1 \\
\hline 2974 & $1,3,4$ & CONSTANT & $-1.5426000 E+01$ & $-1.5426000 E+01$ & $-1.5425000 E+01$ & $-1.5426000 E+01$ & $\log \left(m^{\wedge} 2\right)$ & WP035597 & YEN & 1.4 \\
\hline 2975 & 1.3 & CONSTANT & $3.0900000 E+\infty 0$ & $3.0900000 E+\infty$ & $3.0800000 E+\infty 0$ & $3.0900000=+00$ & m & WP030640 & $\mathbf{Y}$ & NA \\
\hline 2975 & 1,3 & CONSTANT & $3.0900000 E++00$ & $3.0900000 E+\infty 0$ & $3.0900000 \mathrm{E}+\infty$ & $3.0900000 E+\infty 00$ & $m$ & WP030994 & $\mathbf{Y}$ & NA \\
\hline 2975 & 1,3 & CONSTANT & $3.0900000 E+\infty$ & $3.0900000 \mathrm{E}+\infty 0$ & $3.0900000 \mathrm{E}+100$ & $3.0900000 E+\infty$ & m & WP035597 & $\mathbf{Y}$ & NA \\
\hline
\end{tabular}


CCA Parameter Listing

\begin{tabular}{|c|c|c|c|c|c|c|c|c|c|c|}
\hline $\begin{array}{c}\text { Parmetorin } \\
\text { Detabese? } \\
\text { (YhN) }\end{array}$ & 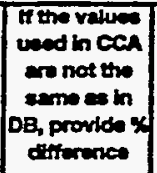 & $\mathbf{W}$ & Meterta io & Matard Nams & 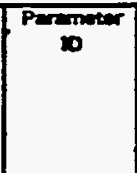 & Pardrater Nen & $\begin{array}{l}\text { PRP DO } \\
\text { MPOE }\end{array}$ & $\begin{array}{c}\text { Dationtry } \\
\text { Dets }\end{array}$ & 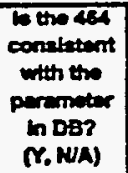 & $\begin{array}{c}\text { Perminter } \\
\text { Catrogory } \\
\text { (footnote 1) }\end{array}$ \\
\hline $\mathbf{Y}$ & & 2975 & SALT_T6 & $\begin{array}{l}\text { Sheft salt cokmn } \\
\text { compactect time } 200 \text { to } \\
10 \text { Y years }\end{array}$ & RSH_AR & Air-supply attant radex (3.09 m) & $33479 A$ & $13-F a b-96$ & $\mathbf{Y}$ & 48 \\
\hline $\mathbf{Y}$ & & 2976 & SALT_T6 & $\begin{array}{l}\text { Shaf salt colemn } \\
\text { compactectume } 200 \text { to } \\
\text { 10K years }\end{array}$ & RSH_SAL & Salt-randing thant radus $(1.8 \mathrm{~m})$ & $33481 A$ & 13 feo- 96 & $\mathbf{Y}$ & 48 \\
\hline $\mathbf{Y}$ & & 2976 & SALT_T6 & $\begin{array}{l}\text { Shat salt column } \\
\text { compacted time } 200 \text { to } \\
10 \text { K years }\end{array}$ & RSH_SN & Sall-wanding than radies $(1.8 \mathrm{~m})$ ] & $33481 A$ & $13-F a b-96$ & $\mathbf{Y}$ & $4 B$ \\
\hline $\mathbf{Y}$ & & 2976 & SALT_T6 & $\begin{array}{l}\text { Shaf saht column } \\
\text { compactedtime } 200 \text { to } \\
10 \text { Kears }\end{array}$ & RSH_SAL & Sall-handing then radus $(1.8 \mathrm{~m})$ & $33481 A$ & $13-F a b-96$ & $\mathbf{Y}$ & AB \\
\hline $\mathbf{Y}$ & & 2976 & SALT_T6 & $\begin{array}{l}\text { Shaft salt column } \\
\text { compactectime } 200 \text { to } \\
\text { jOK years }\end{array}$ & RSH_SAL & Salthending shat radius $(1.8 \mathrm{~m})$ & $33489 A$ & 13 Fob-96 & $\mathbf{Y}$ & $4 B$ \\
\hline $\mathbf{Y}$ & & 2977 & SALT_T6 & $\begin{array}{l}\text { Shaft salt cohmn } \\
\text { compacteditims } 200 \text { to } \\
10 \text { K years }\end{array}$ & RSH_WAS & $\begin{array}{l}\text { Wasto-hendting shat radius (3.5 } \\
\text { m) }\end{array}$ & 33482A & $13+F a b-96$ & $\mathbf{Y}$ & $4 B$ \\
\hline $\mathbf{Y}$ & & 2977 & SALT_T6 & $\begin{array}{l}\text { Shaft salt column } \\
\text { compactectime } 200 \text { to } \\
\text { 10K years }\end{array}$ & RSH_WAS & $\begin{array}{l}\text { Wasto-handing shan radius ( } 3.5 \\
\text { m) }\end{array}$ & $33482 A$ & $13-500-96$ & $\mathbf{Y}$ & 48 \\
\hline $\mathbf{Y}$ & & 2977 & SALT_T6 & $\begin{array}{l}\text { Shaft salt column } \\
\text { compsctectime } 200 \text { to } \\
\text { 10K years }\end{array}$ & RSH_WAS & $\begin{array}{l}\text { Waste-handing that rodius (3.5 } \\
\text { (ii) }\end{array}$ & $33482 A$ & $13-5 a b-96$ & $\mathbf{Y}$ & 48 \\
\hline $\mathbf{Y}$ & & 2977 & SALT_T6 & $\begin{array}{l}\text { Shaft salt column } \\
\text { compactect time } 200 \text { to } \\
\text { 10K years }\end{array}$ & RSH_WAS & $\begin{array}{l}\text { Wasto-handing that radus ( } 3.5 \\
\text { m) }\end{array}$ & $33482 A$ & $13-50 b-98$ & $\mathbf{Y}$ & $4 B$ \\
\hline $\mathbf{Y}$ & & 2978 & SALT_T6 & $\begin{array}{l}\text { Shaft salt column } \\
\text { compactect time } 200 \text { to } \\
\text { 10K yaars }\end{array}$ & RSH_EXH & Air-extheust thath radies $(2.3 \mathrm{~m})$ & 33480A & $13-F a b-96$ & $\mathbf{Y}$ & $\mathbf{4 8}$ \\
\hline $\mathbf{Y}$ & & 2978 & SALT_T6 & $\begin{array}{l}\text { Shaft salt column } \\
\text { compacteditinv } 200 \text { to } \\
10 \text { K years }\end{array}$ & RSH_EXH & Air-axhaust ahat radies $(2.3 \mathrm{~m})$ & 334804 & 13freb-9s & $\mathbf{Y}$ & $4 B$ \\
\hline $\mathbf{Y}$ & & 2978 & SALT_T6 & $\begin{array}{l}\text { Shaft salt column } \\
\text { compactectitime } 200 \text { to } \\
\text { iOK years }\end{array}$ & RSH_EXH & Air-exthaust that ractius $(23 \mathrm{~m})$ & 334804 & 13 Fob-s & $\mathbf{Y}$ & $4 B$ \\
\hline $\mathbf{Y}$ & & 2978 & SALT_T6 & $\begin{array}{l}\text { Shaft sal column } \\
\text { compactedtime } 200 \text { to } \\
10 \text { K years }\end{array}$ & RSH_EXH & Air-oxhaust shant radius $(2.3 \mathrm{~m})$ & 334804 & 13 Feb-96 & $\mathbf{Y}$ & 48 \\
\hline $\mathbf{Y}$ & & 2979 & SALT_T6 & $\begin{array}{l}\text { Shaft salt column } \\
\text { compactectime } 200 \text { to } \\
10 \text { K years }\end{array}$ & RAON_DRZ & DRZ outer radius at each shan & 33476 & 13-Fob-96 & $\mathbf{Y}$ & 1 \\
\hline $\mathbf{Y}$ & & 2979 & SALT_T6 & \begin{tabular}{|l|} 
Shaft salt column \\
compactectime 200 to \\
10 K years
\end{tabular} & RADN_ORZ & DRZ outer radius at each shaft & 33476 & $13-F e b-96$ & $\mathbf{Y}$ & 1 \\
\hline $\mathbf{Y}$ & & 2979 & SALT_T6 & $\begin{array}{l}\text { Shaft salt column } \\
\text { compactedtime } 200 \text { to } \\
\text { 10K years }\end{array}$ & RADN_DRZ & DRZ outer radius at wach shent & 33476 & 13-Feb-96 & $\mathbf{Y}$ & 1 \\
\hline $\mathbf{Y}$ & varias & 2980 & SALT_T6 & $\begin{array}{l}\text { Shat salt column } \\
\text { compactedtime } 200 \text { to } \\
10 \text { K years }\end{array}$ & PMLT_MD & $\begin{array}{l}\text { Log triangular distribution mode } \\
\text { for permesbility }\end{array}$ & 33651 & 13 feb-9s & $\cdot \mathbf{Y}$ & 1 \\
\hline $\mathbf{Y}$ & varies & 2980 & SALT_T6 & $\begin{array}{l}\text { Shaft salt column } \\
\text { compsetectime } 200 \text { to } \\
\text { 10K years }\end{array}$ & PMLT_MO & $\begin{array}{l}\text { Log triengular distribution mode } \\
\text { for permesbility }\end{array}$ & 33651 & 13-Fab-96 & $\mathbf{Y}$ & 1 \\
\hline $\mathbf{Y}$ & varies & 2980 & SNLT_T6 & $\begin{array}{l}\text { Shrft salt column } \\
\text { compactedtine } 200 \text { to } \\
\text { 10K years }\end{array}$ & PMLT_MD & $\begin{array}{l}\text { Log trangular distribution modo } \\
\text { for permesbilty }\end{array}$ & 33651 & 13-Fab-96 & $\mathbf{Y}$ & 1 \\
\hline$Y$ & varios & 2981 & SALT_T6 & $\begin{array}{l}\text { Sheft int column } \\
\text { compectedtine } 200 \text { to } \\
10 \text { K years }\end{array}$ & PMLT_LO & $\begin{array}{l}\text { Log trangular distribution low } \\
\text { vakue for permeability }\end{array}$ & 33650 & 13-Feb-96 & $\mathbf{Y}$ & 1 \\
\hline $\mathbf{Y}$ & varies & 2981 & SALT_T6 & $\begin{array}{l}\text { Shat alt column } \\
\text { compectedtime } 200 \text { to } \\
10 \text { K yoars }\end{array}$ & PMLT_LO & $\begin{array}{l}\text { Log trienguler distribution bow } \\
\text { value for permeability }\end{array}$ & 33650 & 13-Fab-96 & $\mathbf{Y}$ & 1 \\
\hline$Y$ & varins & 2981 & SALT_T6 & $\begin{array}{l}\text { Shat salt column } \\
\text { compactedtime } 200 \text { to } \\
\text { toK years }\end{array}$ & PMLT_LO & $\begin{array}{l}\text { Log trianguler distribution low } \\
\text { velwo for permeability }\end{array}$ & 33550 & 13-fob-96 & $\mathbf{Y}$ & 1 \\
\hline $\mathbf{Y}$ & varies & 2982 & SALT_T6 & $\begin{array}{l}\text { Shant salt column } \\
\text { compectedtime } 200 \text { to } \\
10 \text { Kyers }\end{array}$ & PMLT_HI & $\begin{array}{l}\text { Log tranguler distribution high } \\
\text { vake for permesbility }\end{array}$ & 33648 & $13+5 e b-96$ & $\mathbf{Y}$ & 1 \\
\hline $\mathbf{Y}$ & varies & 2982 & SALT_T6 & $\mid \begin{array}{l}\text { Shant satt column } \\
\text { compected:tmo } 200 \text { to } \\
10 \text { K years }\end{array}$ & PMLT_HI & $\begin{array}{l}\text { Log triengular distribution high } \\
\text { value for permeability }\end{array}$ & 33648 & $13+F a b-96$ & $\mathbf{Y}$ & 1 \\
\hline
\end{tabular}


CCA Parameter Listing

\begin{tabular}{|c|c|c|c|c|c|c|c|c|c|c|}
\hline ID & 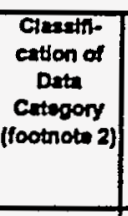 & $\begin{array}{l}\text { Distribution } \\
\text { Typo }\end{array}$ & $\operatorname{man}$ & Medim & Minitrum & Modinem & Thits & Reternonses ID & $\begin{array}{l}\text { Was the det? } \\
\text { demeloped } \\
\text { under an } \\
\text { Non-1 } \\
\text { Progrem? }\end{array}$ & 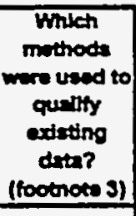 \\
\hline 2975 & 1,3 & CONSTANT & $3.0900000 E+\infty 0$ & $3.0900000 E+\infty 0$ & $3.0900000 \mathrm{E}+\infty$ & $3.0900000 E+\infty$ & m & WPO40514 & $\mathbf{Y}$ & N/A \\
\hline 2976 & 1,3 & CONSTANT & $1.8000000 E+00$ & $1.8000000 E+00$ & $1.8000000 E+\infty 0$ & $1.8000000 E+\infty 0$ & $\mathbf{m}$ & WP030540 & $\mathbf{Y}$ & N/A \\
\hline 2976 & 1,3 & CONSTANT & $1.8000000 \mathrm{E}+00$ & $1.8000000 E+\infty 0$ & $1.8000000 E+00$ & $1.8000000 E+\infty$ & m & WP030994 & $\mathbf{Y}$ & N/A \\
\hline 2976 & 1,3 & CONSTANT & $1.8000000 E+\infty 0$ & $1.8000000 E+\infty 0$ & $1.8000000 E+\infty 0$ & $1.8000000 \mathrm{E}+\infty 0$ & $\mathbf{m}$ & WP035597 & $\mathbf{Y}$ & NA \\
\hline 2976 & 1,3 & CONSTANT & $1.8000000 E+\infty 0$ & $1.8000000 E+\infty 0$ & $1.8000000 \mathrm{E}+00$ & $1.8000000 E+\infty 0$ & $m$ & WP040514 & $\mathbf{Y}$ & N/A \\
\hline 2977 & 1,3 & CONSTANT & $3.5000000 E+\infty 0$ & $3.5000000 \mathrm{E}+\infty 0$ & $3.5000000 E+\infty$ & $3.5000000 E+\infty 0$ & $\mathbf{m}$ & WP030640 & $\mathbf{Y}$ & N/A \\
\hline 2977 & 1,3 & CONSTANT & $3.5000000 E+\infty 0$ & $3.5000000 \mathrm{E}+\infty 0$ & $3.5000000 \mathrm{E}+00$ & $3.5000000 E+\infty 0$ & $m$ & WP030994 & $\mathbf{Y}$ & NA \\
\hline 2977 & 1,3 & CONSTANT & $3.5000000 E+\infty 0$ & $3.5000000 E+\infty 0$ & $3.5000000 E+00$ & $3.5000000 E+\infty 0$ & $m$ & WP035597 & $\mathbf{Y}$ & N/A \\
\hline 2977 & 1,3 & CONSTANT & $3.5000000 E+\infty 0$ & $3.5000000 E+00$ & $3.5000000 E+00$ & $3.5000000 \mathrm{E}+\infty 0$ & $m$ & WPO40514 & $\mathbf{Y}$ & N/A \\
\hline 2978 & 1,3 & CONSTANT & $2.3000000 \mathrm{E}+\infty 0$ & $2.3000000 E+\infty 0$ & $23000000 E+\infty 0$ & $23000000 E+\infty 0$ & $\mathbf{m}$ & WP030640 & $\mathbf{Y}$ & NA \\
\hline 2978 & 1.3 & CONSTANT & $2.3000000 E+00$ & $2.3000000 E+00$ & $2.3000000 E+00$ & $2.3000000 E+\infty$ & $\mathbf{m}$ & WP030994 & $\mathbf{Y}$ & NA \\
\hline 2978 & 1,3 & CONSTANT & $2.3000000 E+00$ & $2.3000000 E+\infty 0$ & $2.3000000 E+00$ & $2.3000000 E+\infty$ & $m$ & WP035597 & $\mathbf{Y}$ & NAA \\
\hline 2978 & 1,3 & CONSTANT & $2.3000000 E+00$ & $2.3000000 \mathrm{E}+00$ & $23000000 E+00$ & $2.3000000 E+\infty 0$ & $m$ & WP040514 & $\mathbf{Y}$ & NA \\
\hline 2979 & $1,3,4$ & CONSTANT & $1.0000000 E+00$ & $1.0000000 E+00$ & $1.0000000 E+\infty$ & $1.0000000 E+\infty$ & $\mathrm{m} / \mathrm{m}$ & WPO30640 & $\mathbf{Y}$ & N/A \\
\hline 2979 & $1,3,4$ & CONSTANT & $1.0000000 E+00$ & $1.0000000 E+00$ & $1.0000000 E+00$ & $1.0000000 E+\infty$ & $\mathrm{m} / \mathrm{m}$ & WP030994 & $Y$ & NA \\
\hline 2979 & $1,3,4$ & CONSTANT & $1.0000000 E+\infty 0$ & $1.0000000 \mathrm{E}+00$ & $1.0000000 \mathrm{E}+\infty 0$ & $1.0000000 E+00$ & $\mathrm{~m} / \mathrm{m}$ & WP035597 & $\mathbf{Y}$ & N/A \\
\hline 2980 & $1,3,4$ & CONSTANT & $-2.0271600 \mathrm{E}+01$ & $-2.0271600 E+01$ & $-2.0271600 E+01$ & $-2.0271600 \mathrm{E}+01$ & $\log \left(m^{\wedge} 2\right)$ & WP030640 & $\dot{Y} \mathbf{a} N$ & 1.4 \\
\hline 2980 & $1,3,4$ & CONSTANT & $-2.0271600 E+01$ & $-2.0271600 E+01$ & $-2.0271600 E+01$ & $-2.0271600 \mathrm{E}+01$ & $\log \left(m^{n} 2\right)$ & WP030994 & $\mathbf{Y a N}$ & 1,4 \\
\hline 2980 & $1,3,4$ & CONSTANT & $-2.0271600 E+01$ & $-2.0271600 E+01$ & $-2.0271600 E+01$ & $-2.0271600 E+01$ & $\log \left(m^{\wedge} 2\right)$ & WP035597 & YEN & 1,4 \\
\hline 2989 & $1,3,4$ & CONSTANT & $-2.2876100 E+01$ & $-2.2876100 E+01$ & $-2.2876100 E+01$ & $-2.2876100 E+01$ & $\log \left(m^{\wedge} 2\right)$ & WP030640 & YEN & 1,4 \\
\hline 2981 & $1,3,4$ & CONSTANT & $-2.2876100 E+01$ & $-2.2876100 E+01$ & $-2.2876100 E+01$ & $-2.2876100 E+01$ & $\log \left(m^{\wedge} 2\right)$ & WP030994 & YEN & 1.4 \\
\hline 2981 & $1,3,4$ & CONSTANT & $-2.2876100 E+01$ & $-2.2876100 E+01$ & $-2.2876100 E+01$ & $-2.2876100 E+01$ & $\log \left(m^{\wedge} 2\right)$ & WP035597 & Y8N & 1,4 \\
\hline 2982 & $1,3,4$ & CONSTANT & $-1.7667600 E+01$ & $-1.7667600 E+01$ & $-1.7667600 E+01$ & $-1.7667600 \mathrm{E}+01$ & $\log \left(m^{\wedge} 2\right)$ & UP030640 & YaN & 1.4 \\
\hline 2982 & $1,3,4$ & CONSTANT & $-1.7667600 E+01$ & $-1.7657600 E+09$ & $-1.7667600 \mathrm{E}+01$ & $-1.76676000+01$ & $\log \left(m^{*} 2\right)$ & WP030994 & YQN & 1.4 \\
\hline
\end{tabular}


CCA Parameter Listing

\begin{tabular}{|c|c|c|c|c|c|c|c|c|c|c|}
\hline $\begin{array}{c}\text { Permetion th } \\
\text { Databese? } \\
\text { (YNI) }\end{array}$ & 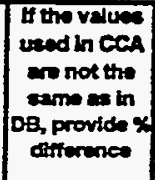 & D & Matarido & Material NEm & $\begin{array}{l}\text { Pernneter } \\
\text { ID }\end{array}$ & Permatiar Alarose & $\begin{array}{l}\text { PRP DD } \\
\text { MPOAI }\end{array}$ & $\begin{array}{c}\text { Denertry } \\
\text { Dets }\end{array}$ & 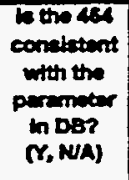 & $\begin{array}{l}\text { Parnmetar } \\
\text { Catiogory } \\
\text { (footonote } 4 \text { ) }\end{array}$ \\
\hline $\mathbf{Y}$ & varies & 2982 & SALT_T6 & $\begin{array}{l}\text { Shaft sall cotumn } \\
\text { compactect tme } 200 \text { to } \\
10 \text { K years }\end{array}$ & PMLT_HI & $\begin{array}{l}\text { Log trienguler distribution high } \\
\text { value for permesbitity }\end{array}$ & 33648 & $13-F a b-96$ & $\mathbf{Y}$ & 1 \\
\hline$Y$ & 50 & 2983 & SALT_T6 & $\begin{array}{l}\text { Sheft sall column } \\
\text { compactedtime } 200 \text { to } \\
\text { lok years }\end{array}$ & CAP_MOD & $\mid \begin{array}{l}\text { Model number, capillay prosture } \\
\text { model }\end{array}$ & 336334 & $13-500-96$ & $\mathbf{Y}$ & 48 \\
\hline$Y$ & 50 & 2983 & SALT_T6 & $\begin{array}{l}\text { Shean salt cotumn } \\
\text { compectodtime } 200 \text { to } \\
10 K \text { years }\end{array}$ & CAP_MOD & $\begin{array}{l}\text { Model number, capillary prosure } \\
\text { model }\end{array}$ & 336334 & $13-F b-96$ & $\mathbf{Y}$ & 48 \\
\hline$Y$ & 50 & 2983 & SALT_T6 & $\begin{array}{l}\text { Shath salt column } \\
\text { compacted:time } 200 \text { to } \\
\text { 10K years }\end{array}$ & CAP_MOD & $\begin{array}{l}\text { Model number, capillary proseuro } \\
\text { modal }\end{array}$ & $33633 \mathrm{~A}$ & 13-506-96 & $\mathbf{Y}$ & 48 \\
\hline $\mathbf{Y}$ & 50 & 2983 & SALT_TG & $\begin{array}{l}\text { Shaft salt column } \\
\text { compactedtime } 200 \text { to } \\
\text { 10K years }\end{array}$ & CAP_MOD & $\begin{array}{l}\text { Model number, cupitary presurn } \\
\text { model }\end{array}$ & 336334 & $13-F a b-96$ & $\mathbf{Y}$ & 48 \\
\hline $\mathbf{Y}$ & 50 & 2983 & SALT_T6 & $\begin{array}{l}\text { Shaft salt colkmn } \\
\text { compactect time } 200 \text { to } \\
\text { 10K yoars }\end{array}$ & CAP_MOD & $\begin{array}{l}\text { Model number, capiltary pressure } \\
\text { model }\end{array}$ & $33633 A$ & 13 -Feb-96 & $Y$ & 48 \\
\hline $\mathbf{Y}$ & & 2984 & SALT_T6 & $\begin{array}{l}\text { Shaft salt column } \\
\text { compsctedtime } 200 \text { to } \\
\text { 10K years }\end{array}$ & COMP_RCK & Butk Compressibitity & 33635 & 13 Feb-96 & $\mathbf{Y}$ & 1 \\
\hline$Y$ & & 2984 & SALT_T6 & \begin{tabular}{|l|} 
Shaft sah column \\
compactodt time 200 to \\
10K years
\end{tabular} & COMP_RCK & Buk Comprescibitity & 33635 & 13 Feb-96 & $\gamma$ & 1 \\
\hline $\mathbf{Y}$ & & 2984 & SALT_T6 & \begin{tabular}{|l|} 
Shaft sak column \\
compactoctime 200 to \\
10K years
\end{tabular} & COMP_RCK & Butk Compressibility & 33535 & 13-feb-96 & $Y$ & 1 \\
\hline$Y$ & & 2985 & SALT_T6 & $\begin{array}{l}\text { Shaft salt column } \\
\text { compactedtime } 200 \text { to } \\
\text { joK yesrs }\end{array}$ & KPT & $\begin{array}{l}\text { Fleg for Permesbility Determined } \\
\text { Throshold }\end{array}$ & 33640 & 20 Feb-96 & $Y$ & AB \\
\hline$Y$ & & 2985 & SALT_T6 & $\begin{array}{l}\text { Shat salt column } \\
\text { compactedtime } 200 \text { to } \\
\text { 10K years }\end{array}$ & KPT & $\begin{array}{l}\text { Fleg for Permeabilty Determined } \\
\text { Trreshold }\end{array}$ & 33640 & $20+06-96$ & $\mathbf{Y}$ & AB \\
\hline$Y$ & & 2985 & SALT_T6 & \begin{tabular}{|l|} 
Shat salt column \\
compacted:time 200 to \\
10K yoars
\end{tabular} & KPT & $\begin{array}{l}\text { Fleg for Permesbilly Dotermined } \\
\text { Threshold }\end{array}$ & 33640 & $20 F e b-96$ & $Y$ & AB \\
\hline$Y$ & & 2985 & SALT_T6 & $\begin{array}{l}\text { Shat salt colkmn } \\
\text { compactect time } 200 \text { to } \\
\text { 10K years }\end{array}$ & KPT & $\begin{array}{l}\text { Fieg for Permeabitity Dotermined } \\
\text { Threstrald }\end{array}$ & 33640 & $20-F 0 b-96$ & $\mathbf{Y}$ & AB \\
\hline$Y$ & & 2985 & SALT_T6 & \begin{tabular}{|l|} 
Shaft salt column \\
compacted:time 200 to \\
$10 \mathrm{~K}$ years
\end{tabular} & KPT & $\begin{array}{l}\text { Flag for Pormesbility Deternined } \\
\text { Threshold }\end{array}$ & 33640 & 20 Feb-96 & $Y$ & AB \\
\hline $\mathbf{Y}$ & & 2986 & SALT_T6 & \begin{tabular}{|l|} 
Shaft salt column \\
compactoct time 200 to \\
$10 \mathrm{~K}$ years
\end{tabular} & PC_max & $\begin{array}{l}\text { Maximum allowable capillary } \\
\text { pressure }\end{array}$ & $33647 \mathrm{~A}$ & $13+0 b-96$ & $\mathbf{Y}$ & $4 B$ \\
\hline$Y$ & & 2986 & SALT_T6 & $\begin{array}{l}\text { Shan salt column } \\
\text { compsctedt time } 200 \text { to } \\
\text { 10K years }\end{array}$ & PC_MAX & $\begin{array}{l}\text { Meximum allowable capillery } \\
\text { pressuro }\end{array}$ & $33647 \mathrm{~A}$ & $13-F a b-96$ & $\mathbf{Y}$ & AB \\
\hline$Y$ & & 2986 & SALT_T6 & $\begin{array}{l}\text { Shaff salt column } \\
\text { compactedtime } 200 \text { to } \\
\text { 10K years }\end{array}$ & PC_max & $\begin{array}{l}\text { Moximum allowablo capillary } \\
\text { preseuro }\end{array}$ & $33647 A$ & 13 Feb-96 & $" Y$ & 48 \\
\hline$Y$ & & 2986 & SALT_T6 & $\begin{array}{l}\text { Shan seal column } \\
\text { compactod:time } 200 \text { to } \\
10 \mathrm{~K} \text { years }\end{array}$ & PC_max & $\begin{array}{l}\text { Macumum allowable capillary } \\
\text { preseure }\end{array}$ & $33547 \mathrm{~A}$ & 13 feb-96 & $\mathbf{Y}$ & AB \\
\hline$Y$ & & 2986 & SALT_TE & $\begin{array}{l}\text { Sheft esth column } \\
\text { compected:tine } 200 \text { to } \\
\text { 10K yesers }\end{array}$ & $P C_{-}$max & $\begin{array}{l}\text { Maxmum alloweblo capillery } \\
\text { prescure }\end{array}$ & $33647 A$ & 13 feb-96 & $\mathbf{Y}$ & AB \\
\hline $\mathbf{Y}$ & 100 & 2997 & SNLT_T6 & \begin{tabular}{|l|} 
Shen selt column \\
compectodtime 200 to \\
IOK years
\end{tabular} & PCT_A & $\begin{array}{l}\text { Threshold Pressure Linear } \\
\text { Perameter }\end{array}$ & 33642 & 13-Fab-96 & $Y$ & 1 \\
\hline $\mathbf{Y}$ & 100 & 2987 & SALT_T6 & $\begin{array}{l}\text { Shith salt column } \\
\text { compectectime } 200 \text { to } \\
10 \mathrm{~K} \text { years }\end{array}$ & PCT_A & $\begin{array}{l}\text { Trreshold Pressure Linax } \\
\text { Peremetor }\end{array}$ & 33642 & 13-feb-96 & $Y$ & 1 \\
\hline$Y$ & 100 & 2987 & SALT_T6 & $\begin{array}{l}\text { Shent salf cotumn } \\
\text { compectectime } 200 \text { to } \\
\text { 10K years }\end{array}$ & PCT_A & $\begin{array}{l}\text { Threstold Pressure Linax } \\
\text { Peremeter }\end{array}$ & 33642 & 13Fob-96 & $Y$ & 1 \\
\hline$Y$ & 100 & 2987 & SALT_T6 & $\begin{array}{l}\text { Shan sall column } \\
\text { compactedtime } 200 \text { to } \\
10 \text { Kyears }\end{array}$ & PCT_A & $\begin{array}{l}\text { Threshold Pressure Linear } \\
\text { Peramoter }\end{array}$ & 33642 & 13Fab-96 & $\mathbf{Y}$ & 1 \\
\hline$Y$ & 100 & 2988 & SALT_T6 & $\begin{array}{l}\text { Shan salt colkmn } \\
\text { compactectime } 200 \text { to } \\
10 \text { K years }\end{array}$ & PCT_EXP & $\begin{array}{l}\text { Throshold preseure exponontial } \\
\text { perametior }\end{array}$ & 33645 & 13Fab-96 & $\mathbf{Y}$ & 1 \\
\hline
\end{tabular}


CCA Parameter-Listing

\begin{tabular}{|c|c|c|c|c|c|c|c|c|c|c|}
\hline DD & 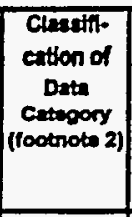 & $\begin{array}{l}\text { Ostribution } \\
\text { Typo }\end{array}$ & Mean . & Modien & Dintmum & Maximim & Units & Rofinnes ID & $\begin{array}{l}\text { Wes the dinta } \\
\text { demloped } \\
\text { under m } \\
\text { NoA-1 } \\
\text { Progrem? }\end{array}$ & 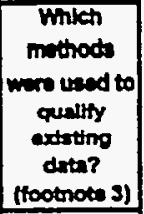 \\
\hline 2982 & $1,3,4$ & CONSTANT & $-1.7667600 \mathrm{E}+01$ & $-1.7667600 E+01$ & $-1.7667600 E+01$ & $-1.7667600 E+01$ & $\log \left(m^{n}{ }^{n}\right)$ & WP035597 & Y\&N & 1.4 \\
\hline 2983 & $1,3,4$ & CONSTANT & $2.0000000 E+\infty 0$ & $2.0000000 E+\infty 0$ & $20000000 E+\infty 0$ & $20000000 E+00$ & NONE & WPO30640 & $\mathbf{Y}$ & NA \\
\hline 2983 & $9,3,4$ & CONSTANT & $2.0000000 E+\infty$ & $2.0000000 E+\infty 0$ & $2,0000000 \leq+\infty$ & $20000000 E+\infty$ & NONE & WP030994 & $\mathbf{Y}$ & N/A \\
\hline 2883 & $1,3,4$ & CONSTANT & $2,0000000 E+\infty 0$ & $2.0000000 E+\infty 0$ & $2.0000000 E+\infty$ & $20000000 E+\infty 0$ & NONE & WP035597 & $\mathbf{Y}$ & NA \\
\hline 2983 & $1,3,4$ & CONSTANT & $2.0000000 E+\infty 0$ & $2.0000000 E+\infty 0$ & $2.0000000 E+\infty 0$ & $2.0000000 E+\infty 0$ & NONE & WPO40486 & $\mathbf{Y}$ & NA \\
\hline 2983 & $1,3,4$ & CONSTANT & $2.0000000 E+00$ & $2.0000000 E+00$ & $2.0000000 E+\infty$ & $20000000 E+\infty$ & NONE & WPO40514 & $Y$ & NA \\
\hline 2984 & $1,3,4$ & CONSTANT & $1.6000000 E-09$ & $1.6000000 E-09$ & $1.6000000 E-09$ & $1.6000000 E-09$ & Par & WP030640 & YEN & 1.4 \\
\hline 2984 & $1,3,4$ & CONSTANT & $1.6000000 E-09$ & $1.6000000 E-09$ & $1.6000000 E-09$ & $1.6000000 E-09$ & Part & WP030994 & YEN & 1.4 \\
\hline 2984 & $1,3,4$ & CONSTANT & $1.6000000 E-09$ & $1.6000000 E-09$ & $1.6000000 E-09$ & $1.6000000 E-09$ & Pel-1 & WP035597 & YEN & 1,4 \\
\hline 2985 & 1,3 & CONSTANT & $0.0000000 E+\infty 0$ & $0.00000000+\infty$ & $0.00000000 \hat{E}+\infty$ & $0.0000000 E+\infty 0$ & NONE & WPO30640 & $\mathbf{Y}$ & NA \\
\hline 2985 & 1,3 & CONSTANT & $0.0000000 E+\infty 0$ & $0.0000000 E+\infty$ & $0.0000000 E+\infty$ & $0.0000000 E+\infty$ & NONE & WP030994 & $\mathbf{Y}$ & N/A \\
\hline 2985 & 1,3 & CONSTANT & $0.0000000 E+\infty 0$ & $0.0000000 \mathrm{E}+\infty 0$ & $0.0000000 E+\infty$ & $0.00000000+\infty$ & NONE & WP035333 & $\mathbf{Y}$ & NA \\
\hline 2985 & 1,3 & CONSTANT & $0.0000000 E+\infty 0$ & $0.00000000+\infty$ & $0.0000000 E+\infty 0$ & $00000000 E+\infty$ & NONE & WP035597 & $\mathbf{Y}$ & NA \\
\hline 2985 & 1,3 & CONSTANT & $0.0000000 E+00$ & $0.0000000 E+00$ & $0,0000000 \leq+\infty$ & $00000000 E+\infty$ & NONE & WP040514 & $\mathbf{Y}$ & NA \\
\hline 2986 & 1,3 & CONSTANT & $1.0000000 E+08$ & $10000000 E+08$ & $10000000 E \cdot 08$ & $10000000<+08$ & Pa & WP030640 & $\mathbf{Y}$ & NA \\
\hline 2986 & 1,3 & CONSTANT & $1.0000000 E+08$ & $10000000 E+\infty 8$ & $10000000<-\infty 00$ & $10000000 E+08$ & Pa & WP030994 & $\mathbf{Y}$ & N/A \\
\hline 2986 & 1,3 & CONSTANT & $1.0000000 E+08$ & $1.0000000 E+08$ & $10000000 \mathrm{~N}+\infty$ & $10000000 \leq+\infty$ & $\mathrm{Pa}$ & WP035597 & $Y$ & NA \\
\hline 2986 & 1,3 & CONSTANT & $1.0000000 \mathrm{E}+08$ & $10000000 E+00$ & $10000000 \times+\infty$ & $10000000 \times \cdot 08$ & Pa & WPO40486 & $\mathbf{Y}$ & NAA \\
\hline 2986 & 1,3 & CONSTANT & $1.0000000 E+08$ & $1.00000000+00$ & $10000000<-\infty 0$ & $10000000 E+\infty 0$ & $\mathbf{P a}$ & WP040514 & $\mathbf{Y}$ & NA \\
\hline 2987 & 3,4 & CONSTANT & $5.6000000 E-01$ & $56000000 E-01$ & S60000000.0? & S6000000E-01 & $P=$ & WP030640 & $\mathbf{Y}$ & NAA \\
\hline 2987 & 3,4 & CONSTANT & $5.6000000 E-01$ & $56000000 E-01$ & S0000000E-01 & $30000000-01$ & Pa & WP030994 & $\mathbf{Y}$ & NA \\
\hline 2987 & 3,4 & CONSTANT & $5.6000000 E .01$ & $56000000 E-01$ & $56000000-01$ & $360000006-01$ & Pa & WP035597 & $\mathbf{Y}$ & NA \\
\hline 2987 & 3,4 & CONSTANT & $5.60000000-01$ & $56000000 E-09$ & S60000000-01] & $580000006-01$ & Pa & WP040434 & $\mathbf{Y}$ & NA \\
\hline 2988 & 3,4 & CONSTANT & $3.4600000 E-01$ & $-34600000 \mathrm{E}-01$ & $34500000 E-01$ & $34500000 E-01$ & NONE & WP030640 & $\mathbf{Y}$ & NUA \\
\hline
\end{tabular}


CCA Parameter-Listing

\begin{tabular}{|c|c|c|c|c|c|c|c|c|c|c|}
\hline 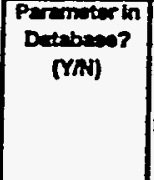 & 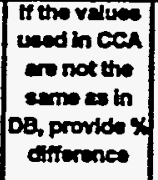 & ID & Meterial ID & Metarial Remente & $\begin{array}{c}\text { Parmotar } \\
\text { to }\end{array}$ & Parmiter Ramo & $\begin{array}{l}\text { PRP IO } \\
\text { MPON }\end{array}$ & $\begin{array}{c}\text { Deta Entry } \\
\text { Dex }\end{array}$ & 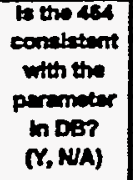 & 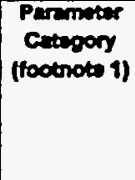 \\
\hline $\mathbf{Y}$ & 100 & 2988 & SALT_TS & $\begin{array}{l}\text { Shat rat column } \\
\text { oompacted time } 200 \text { to } \\
\text { 10K yoars }\end{array}$ & PCT_EXP & $\begin{array}{l}\text { Threshold pressure exponential } \\
\text { permeter }\end{array}$ & 33645 & $13-f a b-96$ & $\mathbf{Y}$ & 1 \\
\hline$Y$ & 100 & 2988 & SALT_T6 & $\begin{array}{l}\text { Shaf salt colemn } \\
\text { compactedtime } 200 \text { to } \\
10 \text { years }\end{array}$ & PCT_EXP & $\begin{array}{l}\text { Trrashold pressure exponential } \\
\text { peramotor }\end{array}$ & 33645 & 13-Fob-96 & $\mathbf{Y}$ & 1 \\
\hline$Y$ & 100 & 2988 & SALT_T6 & $\begin{array}{l}\text { Shan wak column } \\
\text { compactodtime } 200 \text { to } \\
\text { IOK years }\end{array}$ & PCT_EXP & $\begin{array}{l}\text { Throuthold presserv exponential } \\
\text { perameter }\end{array}$ & 33645 & 13 feb-96 & $\mathbf{Y}$ & 1 \\
\hline $\mathbf{Y}$ & varies & 2989 & SALT_TS & $\begin{array}{l}\text { Shaft rath cokumn } \\
\text { compected:time } 200 \text { to } \\
\text { 10K yesers }\end{array}$ & PORE_DIS & $\begin{array}{l}\text { Brooks-Corey pore distribution } \\
\text { permater }\end{array}$ & 33655 & $26+500-96$ & $\mathbf{Y}$ & 1 \\
\hline $\mathbf{Y}$ & varies & 2989 & SALT_TS & $\begin{array}{l}\text { Sheft ealt column } \\
\text { compectedtime } 200 \text { to } \\
10 \text { K years }\end{array}$ & PORE_DIS & $\begin{array}{l}\text { Brooks-Correy pors distribution } \\
\text { parmeter }\end{array}$ & 33655 & 26 Fob-96 & $\mathbf{Y}$ & 1 \\
\hline$Y$ & varias & 2989 & SALT_T6 & $\begin{array}{l}\text { Shaft salt colkmn } \\
\text { compectodtimo } 200 \text { to } \\
\text { 10K years }\end{array}$ & PORE_DIS & $\begin{array}{l}\text { Brocks-Coroy poro distribution } \\
\text { perameter }\end{array}$ & 33655 & 26-fob-96 & $\mathbf{Y}$ & 1 \\
\hline $\mathbf{Y}$ & varies & 2989 & SALT_T6 & \begin{tabular}{l|} 
Shaft sals colkmn \\
compectodtime 200 to \\
10 years
\end{tabular} & PORE_DIS & $\begin{array}{l}\text { Brooke-Corey pore distribution } \\
\text { puranter }\end{array}$ & 33655 & 26-Fab-96 & $\mathbf{Y}$ & 1 \\
\hline $\mathbf{Y}$ & varios & 2989 & SALT_T6 & $\begin{array}{l}\text { Shaft salt column } \\
\text { compactedtime } 200 \text { to } \\
\text { 10K years }\end{array}$ & PORE_DIS & $\begin{array}{l}\text { Brooks-Corny pore distribution } \\
\text { paremoter }\end{array}$ & 33655 & 26-Fob-96 & $Y$ & 1 \\
\hline$Y$ & veries & 2989 & SALT_TS & $\begin{array}{l}\text { Shant eaft colemn } \\
\text { compactoctime } 200 \text { to } \\
\text { I0K years }\end{array}$ & PORE_DIS & $\begin{array}{l}\text { Brooks-Corcy pore distribution } \\
\text { perameter }\end{array}$ & 33655 & 26-Fab-96 & $\mathbf{Y}$ & 1 \\
\hline $\mathbf{Y}$ & varies & 2999 & SALT_T6 & \begin{tabular}{|l|} 
Shaff selt coltumn \\
compsctodtime 200 to \\
toK years
\end{tabular} & PORE_DIS & $\begin{array}{l}\text { Brooks-Corvy pore distribution } \\
\text { perametor }\end{array}$ & 33655 & $26-7 c b-96$ & $Y$ & 1 \\
\hline$Y$ & & 2990 & SALT_T6 & \begin{tabular}{|l|} 
Shaft salt column \\
compactodtimo 200 to \\
10K years
\end{tabular} & POROSITY & Effectivo porosity & 33657 & $13-F-b-96$ & $\boldsymbol{Y}$ & 1 \\
\hline$Y$ & & 2990 & SALT_T6 & \begin{tabular}{l|} 
Shat salt colvmn \\
compactectime 200 to \\
10K years
\end{tabular} & POROSTYY & Effoctive porosity & 33657 & $13-F e b-96$ & $\mathbf{Y}$ & 1 \\
\hline $\mathbf{Y}$ & & 2990 & SALT_T6 & $\begin{array}{l}\text { Shaft selt colkmn } \\
\text { compectedtime } 200 \text { to } \\
\text { 10K years }\end{array}$ & POROSTY & Effective porosity & 33657 & 13 Feb-96 & $\mathbf{Y}$ & 1 \\
\hline $\mathbf{Y}$ & & 2991 & SALT_T6 & $\begin{array}{l}\text { Shat salt colkmn } \\
\text { compactedt time } 200 \text { to } \\
\text { IOK years }\end{array}$ & RELP_MOD & $\begin{array}{l}\text { Model number, relative } \\
\text { permeability model }\end{array}$ & $33478 A$ & 13-feb-96 & $\mathbf{Y}$ & 18 \\
\hline$Y$ & & 2991 & SALT_T6 & $\begin{array}{l}\text { Sheat saht column } \\
\text { compactodtume } 200 \text { to } \\
\text { 10K years }\end{array}$ & RELP_MOD & $\begin{array}{l}\text { Modal number, relative } \\
\text { permesbidty model }\end{array}$ & $33478 A$ & $13-f a b-96$ & $\mathbf{Y}$ & $4 B$ \\
\hline $\mathbf{Y}$ & & 2991 & SALT_T6 & $\begin{array}{l}\text { Shat salt cokmn } \\
\text { compactectime } 200 \text { to } \\
10 \text { 10K years }\end{array}$ & RELP_MOD & $\begin{array}{l}\text { Model rumber, reletive } \\
\text { permesbility model }\end{array}$ & 334784 & 13-Feb-96 & $Y$ & 48 \\
\hline $\mathbf{Y}$ & & 2991 & SALT_T6 & 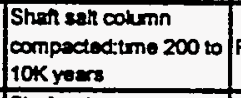 & RELP_MOD & $\begin{array}{l}\text { Model number, rotetve } \\
\text { permacbadty model }\end{array}$ & $33478 A$ & 13 Feb-96 & $e^{\gamma}$ & 48 \\
\hline $\mathbf{Y}$ & & 2991 & SALT_T6 & $\begin{array}{l}\text { Shen sal cotumn } \\
\text { compectodtume } 200 \text { to } \\
10 K \text { years }\end{array}$ & RELP_MOO & 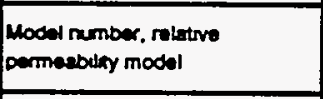 & $33478 A$ & 13-Feb-95 & $Y$ & 48 \\
\hline $\mathbf{Y}$ & 100 & 2992 & SALT_T6 & \begin{tabular}{|l|} 
Shant salt column \\
compected:tm 200 to \\
10K yoars
\end{tabular} & SAT_RBRA & Resudual Bme Seturation & 33483 & 13-Fab-96 & $\mathbf{Y}$ & 1 \\
\hline $\mathbf{Y}$ & 100 & 2992 & SALT_T6 & $\begin{array}{l}\text { Shath selt column } \\
\text { compactect tume } 200 \text { to } \\
10 \text { years }\end{array}$ & SAT_RBRY & Resudual Bmo Saturation & 33483 & 13-Fab-96 & $Y$ & 1 \\
\hline$Y$ & 100 & 2992 & SALT_T6 & 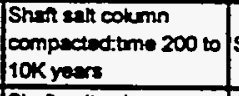 & SAT_RBRN & Residual Brrme Saturation & 33483 & 13Fab-96 & $Y$ & 1 \\
\hline $\mathbf{Y}$ & 100 & 2992 & SALT_T6 & $\begin{array}{l}\text { Shan eat column } \\
\text { compuctodtume } 200 \text { to } \\
10 \text { K years }\end{array}$ & SAT_RBRN & Ressoual Brme Seturation & 33483 & 13-Fob-96 & $\mathbf{Y}$ & 1 \\
\hline $\mathbf{Y}$ & 100 & 2992 & SALT_T6 & $\begin{array}{l}\text { Shert all colkmn } \\
\text { compectedt time } 200 \text { to } \\
10 \text { K year }\end{array}$ & SAT_RBRN & Residual Bmo Seturation & 33483 & 13-Fob-96 & $Y$ & 1 \\
\hline $\mathbf{Y}$ & 100 & 2992 & SNLT_T6 & $\begin{array}{l}\text { Shen sal column } \\
\text { compectedtume } 200 \text { to } \\
\text { 10K years }\end{array}$ & SAT_RBRN & Residual Brine Sturation & 33483 & $13-F a b-96$ & $\gamma$ & 1 \\
\hline
\end{tabular}


CCA Parameter-Listing

\begin{tabular}{|c|c|c|c|c|c|c|c|c|c|c|}
\hline 10 & 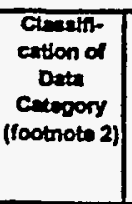 & $\begin{array}{l}\text { Oxetribertion } \\
\text { rypo }\end{array}$ & $\operatorname{man}$ & modien & Mintmem & Meximum & Untes & Retermos LD & $\begin{array}{l}\text { Wratho deta } \\
\text { demioped } \\
\text { underen } \\
\text { Noh-1 } \\
\text { Progrem? }\end{array}$ & 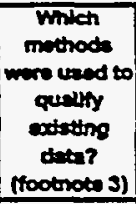 \\
\hline 2988 & 3.4 & CONSTANT & $3.4600000 E-01$ & $3.4600000 \mathrm{E}-01$ & $-3.4500000 \varepsilon-01$ & $-3.4600000 E-01$ & NONE & WP030994 & $\mathbf{Y}$ & NA \\
\hline 2988 & 3,4 & CONSTANT & $3.4600000 E-01$ & $3.4600000 E-01$ & $-3.4600000 E-01$ & $-3.4600000 E-01$ & NONE & WP035597 & $\mathbf{Y}$ & NA \\
\hline 2988 & 3,4 & CONSTANT & $3.4600000 E-01$ & $-3.4600000 E-01$ & $-3.46000000-01$ & $3.4600000 E-01$ & NONE் & WPO40434 & $\mathbf{Y}$ & NA \\
\hline 2989 & 3,4 & CUMULATIVE & $2.5200000 E+\infty 0$ & $9.4000000 E-01$ & 1.1000000E-01 & $8.1000000 E+\infty 0$ & NONE & WP030640 & $\mathbf{Y}$ & NA \\
\hline 2989 & 3,4 & CUMULATINE & $25200000 E+\infty 0$ & $9.4000000 E-01$ & $1.1000000 E-01$ & $8.1000000 E+\infty 0$ & NONE & UP030994 & $\mathbf{Y}$ & NA \\
\hline 2989 & 3,4 & CUMULATIVE & $2.5200000 E+\infty 0$ & $9.4000000 \mathrm{E}-01$ & $1.1000000 E-01$ & $8.1000000 E+\infty 0$ & NONE & WP032287 & $\mathbf{Y}$ & NA \\
\hline 2989 & 3,4 & CUMULATME & $2.5200000 E+\infty$ & $9.4000000 E-01$ & $1.1000000 E-01$ & $8.1000000 E+\infty$ & NONE & WP035268 & $\mathbf{Y}$ & NA \\
\hline 2989 & 3,4 & CUMULATIVE & $25200000 E+\infty 0$ & $9.4000000 \mathrm{C}-01$ & $1.1000000 E-01$ & $8.1000000 E+\infty 0$ & NONE & WP035597 & $\mathbf{Y}$ & NA \\
\hline 2989 & 3,4 & CUMULATME & $25200000 E+\infty$ & 9.4000000E-01 & $1.1000000 E-01$ & $8.1000000 E+00$ & NONE & WPO41558 & $\mathbf{Y}$ & NA \\
\hline 2989 & 3,4 & CUMULATIVE & $25200000 E+\infty$ & $9.4000000 E-01$ & $1.1000000=-01$ & $8.1000000 E+\infty$ & NONE & WPO42752 & $\mathbf{Y}$ & NA \\
\hline 2990 & $1,3,4$ & CONSTANT & $5.0000000 E-02$ & $5.0000000 \mathrm{E}-02$ & $5.0000000 E-02$ & $5.0000000 E-02$ & $m^{\wedge} 3 m^{\wedge} 3$ & WP030640 & $\mathbf{Y}$ & NA \\
\hline 2990 & $1,3,4$ & CONSTANT & $5.0000000 E-02$ & $5.0000000 E-02$ & $5.0000000 E-02$ & $5.0000000 \mathrm{E}-02$ & $m^{\wedge} 3 m^{\wedge} 3$ & WP030994 & $\mathbf{Y}$ & NA \\
\hline 2990 & $1,3,4$ & CONSTANT & $5.0000000 E-02$ & $5.0000000 E-02$ & $5.0000000=-02$ & $5.0000000 E-02$ & $m^{\wedge} 3 / \pi^{\wedge} 3$ & WP035597 & $\mathbf{Y}$ & N/A \\
\hline 2991 & 1,3 & CONSTANT & $4.0000000 E+\infty 0$ & $4.0000000 E+\infty 0$ & $4.0000000 \approx+\infty$ & $4.0000000=+\infty$ & NONE & WP030640 & $\mathbf{Y}$ & NA \\
\hline 2991 & 1,3 & CONSTANT & $4.0000000 E+\infty 0$ & $4.0000000 E+\infty 0$ & $4.00000000=+\infty$ & $4.0000000 E+\infty$ & NONE & WP030S94 & $\boldsymbol{Y}$ & NA \\
\hline 2991 & 1,3 & CONSTANT & $4.00000000 E+\infty 0$ & $4.00000000 E+\infty 0$ & $4.0000000 E+\infty 0$ & $4.0000000 E+00$ & NONE & WP035597 & $\mathbf{Y}$ & $N A$ \\
\hline 2991 & 1,3 & CONSTANT & $4.0000000 E+\infty$ & $4.0000000 E+\infty$ & $4.0000000 E+\infty 0$ & $4.0000000 E+\infty$ & NONE & upo4aces & $\gamma$ & NA \\
\hline 2999 & 1,3 & CONSTANT & $4.0000000 E+\infty 0$ & $4.0000000 E+\infty 0$ & $4.0000000 E+\infty$ & $4.0000000 E+\infty 0$ & NONE & WP040514 & $Y$ & NA \\
\hline 2992 & 3,4 & CUMULATINE & $2.5000000 E-01$ & $20000000 E-01$ & $0.0000000 E+\infty$ & $6.0000000 \mathrm{E}-01$ & NONE & WP030640 & $\mathbf{Y}$ & $N A$ \\
\hline 2992 & 3,4 & CUMULATIVE & $2.5000000 E-01$ & $20000000 E-01$ & $0.0000000 E+\infty$ & $6.0000000 E-01$ & NONE & WP030994 & $\mathbf{Y}$ & NA \\
\hline 2992 & 3,4 & CUMULATINE & $2.5000000 E-01$ & $20000000 E-01$ & $0.0000000 E+\infty 0$ & $6.0000000 E-01$ & NONE & WP035268 & $\mathbf{Y}$ & NA \\
\hline 2992 & 3,4 & CUMULATINE & $2.5000000 E-01$ & $2.0000000 E-01$ & $0.0000000 E+\infty 0$ & $6.0000000 E-01$ & NONE & WP035597 & $\mathbf{Y}$ & NA \\
\hline 2992 & 3,4 & CUMULATINE & $25000000 E-01$ & $2.0000000 E-01$ & $0.00000000 E+\infty 0$ & $6.0000000 E-01$ & NONE & WPO41558 & $\mathbf{Y}$ & NA \\
\hline 2992 & 3,4 & CUMULATIVE & $25000000 E-01$ & 20000000E-01 & $0.0000000 E+\infty$ & $6.0000000 E-01$ & NONE & WP042751 & $\mathbf{Y}$ & NA \\
\hline
\end{tabular}


CCA Parameter Listing

\begin{tabular}{|c|c|c|c|c|c|c|c|c|c|c|}
\hline 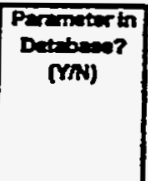 & 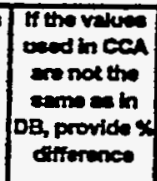 & D & Mecartato & Matterd Name & $\underset{\text { somex }}{P}$ & Pernew Herm & $\begin{array}{c}\text { PRPD } \\
\text { (MPON) }\end{array}$ & $\begin{array}{c}\text { Deta Entry } \\
\text { Date }\end{array}$ & 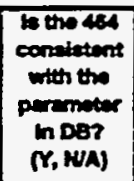 & 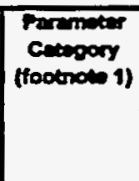 \\
\hline$Y$ & 100 & 2993 & SALT_T6 & $\begin{array}{l}\text { Shaf salt colvenn } \\
\text { compactedtune } 200 \text { to } \\
\text { 10K years }\end{array}$ & SAT_RGAS & Resiodal Gas Seturation & 33484 & $13+f a b-96$ & $\mathbf{Y}$ & 1 \\
\hline $\mathbf{Y}$ & 100 & 2993 & SALT_T6 & $\begin{array}{l}\text { Shaft walt column } \\
\text { compactect tume } 200 \text { to } \\
\text { 1OK yoars }\end{array}$ & SAT_RGAS & Resicusal Gas Sesturation & 33484 & 13-Feb-96 & $\mathbf{Y}$ & 1 \\
\hline $\mathbf{Y}$ & 100 & 2993 & SALT_T6 & $\begin{array}{l}\text { Shat san column } \\
\text { compactedtime } 200 \text { to } \\
\text { 10K yoars }\end{array}$ & SAT_RGAS & Resictal Ges Searation & 33484 & $13-F 00-96$ & $\mathbf{Y}$ & 1 \\
\hline $\mathbf{Y}$ & 100 & 2993 & SALT_T6 & $\begin{array}{l}\text { Sheft eah column } \\
\text { compractudtime } 200 \text { to } \\
10 \text { K years }\end{array}$ & SAT_RGAS & Residual Gess Seturation & 33484 & $13-F=b-96$ & $\mathbf{Y}$ & 1 \\
\hline $\mathbf{Y}$ & & 2994 & CELLULS & Colvilose & FBETA & $\begin{array}{l}\text { Factor bots for mierobual resction } \\
\text { rates }\end{array}$ & 31826 & 13 Feb-96 & $\mathbf{Y}$ & $M$ \\
\hline Y & & 2994 & CELLULS & Celluboso & FBETA & $\begin{array}{l}\text { Factor beta for microbual reaction } \\
\text { retes }\end{array}$ & 31826 & $13-F 0 b-96$ & $\mathbf{Y}$ & 44 \\
\hline $\mathbf{Y}$ & & 2994 & CELLULS & Colkutese & FBETA & $\begin{array}{l}\text { Factor beta for microbial nesction } \\
\text { rates }\end{array}$ & 31826 & 13-Fab-96 & $\mathbf{r}$ & 4 \\
\hline $\mathbf{Y}$ & & 2995 & REPOST & $\begin{array}{l}\text { Repository regions } \\
\text { outside of Panel region }\end{array}$ & DPLSCRHW & 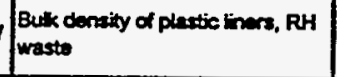 & 3240 & $13-F 0 b-96$ & $\mathbf{Y}$ & 2 \\
\hline $\mathbf{Y}$ & & 2995 & REPOST & $\begin{array}{l}\text { Repository regions } \\
\text { outside of Panal region }\end{array}$ & DPLSCRHW & $\begin{array}{l}\text { Buk density of plestic finers, RH } \\
\text { wasto }\end{array}$ & 32449 & 13-Feb-96 & $\mathbf{Y}$ & 2 \\
\hline $\mathbf{Y}$ & & 2996 & CLAY_RUS & $\begin{array}{l}\text { Rustlor Compactod } \\
\text { Clay Cokmn }\end{array}$ & RSH_AR & Arrsupply thent radius $(3.09 \mathrm{~m})$ & 318904 & 13 -Fob-s6 & $\mathbf{Y}$ & 48 \\
\hline$Y$ & & 2996 & CLAY_RUS & $\begin{array}{l}\text { Rustlor Compected } \\
\text { Clay Cohmn }\end{array}$ & RSH_AR & Ar-eupply shent redies $(3.09 \mathrm{~m})$ & 318904 & 13Feb-96 & $\mathbf{r}$ & 4B \\
\hline $\mathbf{Y}$ & & 2908 & CLAY_RUS & $\begin{array}{l}\text { Rustlor Compacted } \\
\text { Clay Column }\end{array}$ & RSH_AR & Air-eupply ahen radius (3.09 m) & $3189 a n$ & 13Føb-96 & $\mathbf{Y}$ & $4 B$ \\
\hline $\mathbf{Y}$ & & 2996 & CLAY_RUS & $\begin{array}{l}\text { Rustor Compacted } \\
\text { Ciay Column }\end{array}$ & RSH_AR & Air-eupply sheth redies $(3.09 \mathrm{~m})$ & $31890 A$ & 13-Feb-96 & $\mathbf{r}$ & 48 \\
\hline $\mathbf{Y}$ & & 2997 & CLAY_RUS & $\begin{array}{l}\text { Rustlor Compactod } \\
\text { Clay Cokmn }\end{array}$ & RSH_SAL & Salh-handing athan radus $(1.8 \mathrm{~m})$ & $31892 A$ & 13 feb-96 & $\mathbf{Y}$ & 48 \\
\hline $\mathbf{Y}$ & & 2997 & CLAY_RUS & $\begin{array}{l}\text { Rustior Compacted } \\
\text { Clay Column }\end{array}$ & RSH_SAL & Salthandting athent radiva $(1.8 \mathrm{~m})$ & $31892 A$ & 13 -Feb- 96 & $\mathbf{Y}$ & AB \\
\hline $\mathbf{Y}$ & & 2997 & CLAY_RUS & $\begin{array}{l}\text { Rustler Compactied } \\
\text { Clay Cokmn }\end{array}$ & RSH_SAL & Selthandling athat rectivs $(1.8 \mathrm{~m})$ & 31892A & 13 feb-96 & $\mathbf{Y}$ & 4B \\
\hline $\mathbf{Y}$ & & 2997 & CLAY_RUS & $\begin{array}{l}\text { Russtor Compactod } \\
\text { Clay Colkmn }\end{array}$ & RSH_SAL & Salt-hending shat radius $(1.8 \mathrm{~m})$ & 31892A & 13 -Feb-96 & $\mathbf{Y}$ & $A B$ \\
\hline$Y$ & & 2998 & CLAY_RUS & $\begin{array}{l}\text { Rustler Compected } \\
\text { Cloy Column }\end{array}$ & RSH_WAS & $\begin{array}{l}\text { Wastohinding shen rodves (3.5 } \\
\text { m) }\end{array}$ & $31893 A$ & 13-Feb-96 & $\mathbf{Y}$ & 48 \\
\hline Y & & 2998 & CLAY_RUS & $\begin{array}{l}\text { Rustler Compactod } \\
\text { Clay Column }\end{array}$ & RSH_WAS & $\begin{array}{l}\text { Whasto-handing shatt radxus (3.5 } \\
\text { m) }\end{array}$ & $31893 A$ & 13-Fob-96 & $Y$ & 48 \\
\hline$Y$ & & 2998 & CLAY_RUS & $\begin{array}{l}\text { Rustor Compactod } \\
\text { Clay Column }\end{array}$ & RSH_WAS & $\begin{array}{l}\text { Westohanding shen radues (3.5 } \\
\text { m) }\end{array}$ & $31893 A$ & 13 feb-9s & $\mathbf{Y}$ & 48 \\
\hline$Y$ & & 2998 & CLAY_RUS & $\begin{array}{l}\text { Rusiter Compacted } \\
\text { Clsy Column }\end{array}$ & RSH_WAS & $\begin{array}{l}\text { Wastothending enten radius (3.5 } \\
\text { m) }\end{array}$ & $31893 A$ & 13-Feb-96 & $\mathbf{Y}$ & 48 \\
\hline $\mathbf{Y}$ & & 2999 & CLAY_RUS & $\begin{array}{l}\text { Rustior Compected } \\
\text { Cley Column }\end{array}$ & RSH_EXH & Ar-axhaust ahat radius $(2.3 \mathrm{~m})$ & 31891A & 13 feb-96 &.$- Y$ & $4 B$ \\
\hline $\mathbf{Y}$ & & 2999 & CLAY_RUS & $\begin{array}{l}\text { Rurlter Compactod } \\
\text { Clay Column }\end{array}$ & RSH_EXH & Air-exheust shent redies $(2.3 \mathrm{~m})$ & $31891 A$ & 13-Fob-96 & $\mathbf{\gamma}$ & $A B$ \\
\hline $\mathbf{Y}$ & & 2999 & CLAY_RUS & $\begin{array}{l}\text { Rustier Compaciod } \\
\text { Clay Column }\end{array}$ & RSH_EXH & Air-exheust shent radus (2.3 m) & 318914 & 13 -Feb-95 & $\mathbf{Y}$ & AB \\
\hline $\mathbf{Y}$ & & 2999 & CLAY_RUS & $\begin{array}{l}\text { Rustior Compectod } \\
\text { Ciry Cotumn }\end{array}$ & RSH_EXH & Ar-extheust thent redius $(2.3 \mathrm{~m})$ & $31891 \mathrm{~A}$ & $13-F e b-96$ & $Y$ & AB \\
\hline $\mathbf{Y}$ & 50 & 3000 & CLAY_RUS & $\begin{array}{l}\text { Rustler Compacteos } \\
\text { Clay Column }\end{array}$ & CAP_MOD & $\begin{array}{l}\text { Model number, capillery pressure } \\
\text { model }\end{array}$ & $31877 \mathrm{~A}$ & $13+50 b-96$ & $\mathbf{Y}$ & 48 \\
\hline$\gamma$ & 50 & 3000 & CLAY_RUS & $\begin{array}{l}\text { Rustier Compected } \\
\text { Ciry Column } \\
\end{array}$ & CAP_MOD & $\begin{array}{l}\text { Modol number, copillary pressure } \\
\text { model }\end{array}$ & $31877 \mathrm{~A}$ & $13-F a b-96$ & $\mathbf{Y}$ & AB \\
\hline$Y$ & so & 3000 & CLAY_RUS & $\begin{array}{l}\text { Rustier Compactiod } \\
\text { Cley Column }\end{array}$ & CAP_MOD & $\begin{array}{l}\text { Model number, capiliery prossure } \\
\text { modo: }\end{array}$ & $31877 A$ & 13 Feb- 86 & $Y$ & $4 B$ \\
\hline $\mathbf{Y}$ & 50 & 3000 & CLAY_RUS & $\begin{array}{l}\text { Rester Compected } \\
\text { Cley Column }\end{array}$ & CAP_MOD & $\begin{array}{l}\text { Model number, cepillary pressume } \\
\text { model }\end{array}$ & $31877 A$ & 13Feb-86 & $\mathbf{Y}$ & 48 \\
\hline $\mathbf{Y}$ & 50 & 3000 & CLAY_RUS & \begin{tabular}{|l|} 
Rustier Compseted \\
Clay Column \\
\end{tabular} & CAP_MOD & $\begin{array}{l}\text { Mocel number, capillery prosecure } \\
\text { model }\end{array}$ & $31877 A$ & 13-Fob-96 & $\mathbf{Y}$ & 4B \\
\hline$Y$ & & 3001 & CLAY_RUS & $\begin{array}{l}\text { Rustier Compacted } \\
\text { Clay Cokmn }\end{array}$ & COMP_RCK & Buk Compraseribitity & 31878 & 13-Feb-96 & $\mathbf{Y}$ & 1 \\
\hline $\mathbf{Y}$ & & 3001 & CLAY_RUS & $\begin{array}{l}\text { Rustre Compucted } \\
\text { Clyy Cotumn }\end{array}$ & COMP_RCK & Buk Comprosecioiny & 31878 & $13+f a b-86$ & $\mathbf{Y}$ & 1 \\
\hline $\mathbf{Y}$ & & 3001 & CLAY_RUS & $\begin{array}{l}\text { Rustior Compuctod } \\
\text { Cliry Column }\end{array}$ & COMP_RCK & Buk Comprosecibitity & 31878 & 13-fab-96 & $Y$ & 1 \\
\hline
\end{tabular}


CCA Parameter Listing

\begin{tabular}{|c|c|c|c|c|c|c|c|c|c|c|}
\hline ID & $\begin{array}{c}\text { Creastit- } \\
\text { extion of } \\
\text { Data } \\
\text { Cextegory } \\
\text { (footnote 2) }\end{array}$ & $\begin{array}{l}\text { Dtertibution } \\
\text { Type }\end{array}$ & Man & Medien & Mintmem . & Meximum & Units & Rederence id & $\begin{array}{l}\text { Wos the dat } \\
\text { dovicoped } \\
\text { underen } \\
\text { Hen-1 } \\
\text { Progren? }\end{array}$ & 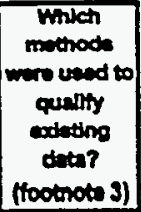 \\
\hline 2993 & 3,4 & UNIFORM & $20000000 E-01$ & $2.0000000 E-01$ & $0.0000000 E+\infty$ & $4.0000000 E-01$ & NONE & WP030540 & $\mathbf{Y}$ & NUA \\
\hline 2993 & 3,4 & UNIFORM & $2,0000000 E-01$ & $2.0000000 E-01$ & $0.0000000 E+\infty$ & $4.0000000 E-01$ & NONE & WP030994 & $\mathbf{Y}$ & NA \\
\hline 2993 & 3,4 & UNIFORM & $2.0000000 E-01$ & $2.0000000 E-01$ & $0.0000000 E+\infty$ & $4.0000000 E-01$ & NONE & WP035597 & $Y$ & N/A \\
\hline 2993 & 3,4 & UNIFORM & $2.0000000 E-01$ & $20000000 E-01$ & $0.0000000 E+\infty$ & $4.0000000 E-01$ & NONE & WPO41558 & $\mathbf{Y}$ & NA \\
\hline 2994 & 3,4 & UNIFORM & $5.0000000 E-01$ & $5.0000000 E-01$ & $0.0000000 E+\infty 0$ & $1.0000000=+\infty$ & MONE & WP030819 & $\mathbf{Y}$ & NA \\
\hline 2994 & 3,4 & UNIFORM & $5.0000000 E-01$ & $5.0000000 E-01$ & $0.0000000 E+\infty 0$ & $1.0000000 E+\infty 0$ & NONE & WP035268 & $\mathbf{Y}$ & NA \\
\hline 2994 & 3,4 & UNIFORM & $5.0000000 E-01$ & $5.0000000 E-01$ & $0.0000000 E+\infty$ & $1.0000000 E+\infty$ & NONE & WP035597 & $\mathbf{Y}$ & NA \\
\hline 2995 & 2 & CONSTANT & $3.1000000 E+\infty 0$ & $3.1000000 E+\infty$ & $3.1000000 E+\infty 0$ & $3.1000000 E+\infty 0$ & $k 0 / m^{\wedge} 3$ & WP032328 & $\mathbf{Y}$ & N/A \\
\hline 2995 & 2 & CONSTANT & $3.1000000 E+\infty 0$ & $3.1000000 E+\infty$ & $3.1000000 E+\infty 0$ & $3.1000000 E+\infty$ & $\mathrm{kg} m \times 3$ & WP035048 & $\mathbf{Y}$ & NA \\
\hline 2996 & 1,3 & CONSTANT & $3.0900000 E+\infty 0$ & $3.0900000 E+\infty 0$ & $3.0900000 E+00$ & $3.0900000 E+00$ & $m$ & WPO30640 & $\mathbf{Y}$ & NA \\
\hline 2996 & 1,3 & CONSTANT & $3.0900000 E+\infty$ & $3.0900000 E+\infty$ & $3.0900000 E+\infty 0$ & $3.0900000 E+\infty 0$ & $\mathbf{m}$ & WPOsos94 & $\mathbf{Y}$ & N/A \\
\hline 2996 & 1,3 & CONSTANT & $3.0900000 E+\infty 0$ & $3.0900000 \varepsilon+\infty$ & $3.0900000 E+\infty 0$ & $3.0900000 E+\infty 0$ & m & WP035597 & $\mathbf{Y}$ & N/A \\
\hline 2996 & 1,3 & CONSTANT & $3.0900000 E+\infty 0$ & $3.0900000 E+00$ & $3.0900000 E+\infty 0$ & $3.0900000 E+\infty 0$ & m & WP040514 & $\mathbf{Y}$ & NA \\
\hline 2997 & 1,3 & CONSTANT & $1.8000000 E+00$ & $1.8000000 E+\infty 0$ & $1.8000000 E+00$ & $1.8000000 E+\infty$ & $m$ & WP030640 & $\mathbf{Y}$ & NA \\
\hline 2997 & 1,3 & CONSTANT & $1.8000000 E+\infty 0$ & $1.8000000 E+00$ & $1.8000000 E+\infty 0$ & $1.8000000 E+\infty 0$ & $m$ & WP030994 & $\mathbf{Y}$ & NA \\
\hline 2997 & 1,3 & CONSTANT & $1.8000000 E+\infty 0$ & $1.8000000 E+\infty$ & $1.8000000 E+\infty$ & $1.8000000 E+\infty 0$ & $m$ & WP035597 & $\mathbf{Y}$ & $N A$ \\
\hline 2997 & 1,3 & CONSTANT & $1.8000000 E+00$ & $1.8000000 E+\infty$ & $1.8000000 E+\infty 0$ & $1.8000000 E+\infty 0$ & $m$ & WP040514 & $Y$ & NA \\
\hline 2998 & 1,3 & CONSTANT & $3.5000000 E+\infty 0$ & $3.5000000 E+\infty$ & $3.5000000 E+\infty$ & $3.5000000 E+\infty 0$ & $\mathbf{m}$ & WPO30640 & $Y$ & NA \\
\hline 2998 & 1,3 & CONSTANT & $3.5000000 E+\infty 0$ & $3.5000000 E+\infty 0$ & $3.5000000 E+\infty 0$ & $3.5000000 \mathrm{E}+\infty 0$ & $m$ & WP030994 & $\mathbf{Y}$ & NA \\
\hline 2998 & 1,3 & CONSTANT & $3.5000000 E+\infty$ & $3.5000000 E+\infty 0$ & $3.5000000 \mathrm{E}+00$ & $3.5000000 E+\infty$ & $\mathrm{m}$ & WP035597 & $\mathbf{Y}$ & N/A \\
\hline 2998 & 1,3 & CONSTANT & $3.5000000 E+\infty$ & $3.5000000 E+00$ & $3.5000000 E+\infty 0$ & $3.5000000 E+\infty 0$ & $\mathbf{m}$ & WPO40514 & $\mathbf{Y}$ & NA \\
\hline 2999 & 1,3 & CONSTANT & $2.3000000 E+\infty 0$ & $2.3000000 E+\infty 0$ & $2.3000000 E+\infty 0$ & $2.3000000 E+00$ & $m$ & WP030640 & $-Y$ & N/A \\
\hline 2999 & 1,3 & CONSTANT & $2.3000000 E+00$ & $2.3000000 E+\infty 0$ & $2.3000000 E+00$ & $2.3000000 E+\infty$ & $m$ & WP030994 & $\mathbf{Y}$ & N/A \\
\hline 2999 & 1,3 & CONSTANT & $2.3000000 E+00$ & $2.3000000 E+\infty 0$ & $23000000 E+\infty 0$ & $2.3000000 E+\infty 0$ & $m$ & WP035597 & $\mathbf{Y}$ & $\mathbf{N A}$ \\
\hline 2999 & 1,3 & CONSTANT & $2.3000000 E+\infty 0$ & $2.3000000 E+\infty 0$ & $2.3000000 E+\infty$ & $2.3000000 E+00$ & $\mathbf{m}$ & WP040514 & $\mathbf{Y}$ & NA \\
\hline 3000 & $1,3,4$ & CONSTANT & $2.0000000 E+\infty 0$ & $2.0000000 E+00$ & $2.0000000 E+\infty 0$ & $2,0000000 E+00$ & NONE & WPO30640 & $\mathbf{Y}$ & N/A \\
\hline 3000 & $1,3,4$ & CONSTANT & $20000000 E+\infty$ & $2,0000000 E+\infty$ & $2.0000000 E+\infty 0$ & $2,0000000 E+\infty 0$ & NONE & WP030994 & $\mathbf{Y}$ & NA \\
\hline 3000 & $1,3,4$ & CONSTANT & $2.0000000 E+\infty 0$ & $2.0000000 E+\infty$ & $2.0000000 E+\infty$ & $20000000 E+\infty$ & NONE & WP035597 & $\mathbf{Y}$ & NA \\
\hline 3000 & $1,3,4$ & CONSTANT & $2.0000000 E+\infty$ & $20000000 E+\infty 0$ & $2.0000000 \mathrm{E}+00$ & $2.0000000 E+00$ & NONE & WPO40486 & $\mathbf{Y}$ & NA \\
\hline 3000 & $1,3,4$ & CONSTANT & $2.0000000 E+\infty 0$ & $2.0000000 E+\infty 0$ & $2.0000000 E+\infty$ & $2.0000000 E+\infty 0$ & NONE & WPO405i4 & $\mathbf{Y}$ & NA \\
\hline 3001 & $1,3,4$ & CONSTANT & $1.9600000 E-09$ & $1.8600000 E-09$ & $1.96000000-09$ & $1.9600000 E-09$ & $P a^{n}-1$ & WPO30640 & $\mathbf{Y}$ & NA \\
\hline 3001 & $1.3,4$ & CONSTANT & $1.9600000 E-09$ & $1.9600000 E-09$ & $1.9600000 E-09$ & $1.9600000 E-09$ & $P x^{n}-1$ & WP030994 & $\mathbf{Y}$ & NA \\
\hline 3001 & $1,3,4$ & CONSTANT & $1.9600000 \mathrm{E}-09$ & $1.9600000 \mathrm{E}-09$ & $1.9600000 E-09$ & $1.9600000 E-09$ & $P a^{n}-1$ & WP035597 & $\mathbf{Y}$ & NA \\
\hline
\end{tabular}


CCA Parameter Listing

\begin{tabular}{|c|c|c|c|c|c|c|c|c|c|c|}
\hline $\begin{array}{c}\text { Parminter } \\
\text { Dacabae? } \\
\text { (Yin) }\end{array}$ & 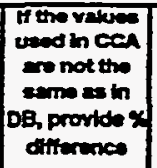 & $\mathbf{D D}$ & Menter io & Metertal nem & Pandin & Perenceor Nem & $\begin{array}{c}\text { Prop D } \\
\text { Mmo as } \\
-\end{array}$ & $\begin{array}{c}\text { Dan Entry } \\
\text { Date }\end{array}$ & 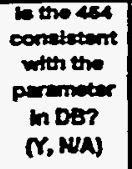 & $\begin{array}{l}\text { Parmineter } \\
\text { Caboony } \\
\text { (footinotes) }\end{array}$ \\
\hline $\mathbf{Y}$ & & 3002 & CLAY_RUS & $\begin{array}{l}\text { Rustler Compicted } \\
\text { Clay Cotumn }\end{array}$ & KPT & $\begin{array}{l}\text { Fleg for Permesbilty Determined } \\
\text { Thresthold }\end{array}$ & 31879 & $20-F a b-96$ & $\mathbf{Y}$ & $4 B$ \\
\hline $\mathbf{Y}$ & & 3002 & CLAY_RUS & $\begin{array}{l}\text { Rustier Compacted } \\
\text { Clay Column }\end{array}$ & KPT & $\begin{array}{l}\text { Fleg for Permeabidy Determined } \\
\text { Threshold }\end{array}$ & 31879 & 20 -Fab-96 & $Y$ & AB \\
\hline $\mathbf{Y}$ & & 3002 & CLAY_RUS & $\begin{array}{l}\text { Rustler Compected } \\
\text { Cloy Cohmn }\end{array}$ & KPT & $\begin{array}{l}\text { Fing for Permeability Detemmed } \\
\text { Threstrold }\end{array}$ & 31879 & $20+a b-96$ & $\mathbf{Y}$ & 48 \\
\hline $\mathbf{Y}$ & & 3002 & CLAY_RUS & $\begin{array}{l}\text { Rustior Compactod } \\
\text { Cley Cokumn }\end{array}$ & KPT & $\begin{array}{l}\text { Fleg for Permenbility Determined } \\
\text { Trroshold }\end{array}$ & 31879 & $20+\infty b-96$ & $\mathbf{Y}$ & 48 \\
\hline $\mathbf{Y}$ & & 3002 & CLAY_RUS & $\begin{array}{l}\text { Rutier Compected } \\
\text { Clay Cotumn }\end{array}$ & KPT & $\begin{array}{l}\text { Fleg for Permeabidy Determined } \\
\text { Thre:hoid }\end{array}$ & 31878 & $20-F<b-96$ & $\mathbf{Y}$ & 48 \\
\hline $\mathbf{Y}$ & & 3003 & CLAY_RUS & $\begin{array}{l}\text { Rustler Compacted } \\
\text { Clay Cokumn }\end{array}$ & PC_MAX & $\begin{array}{l}\text { Meximum allowablo cepiliory } \\
\text { pressure }\end{array}$ & 31880 A & $13+50-96$ & $\mathbf{Y}$ & 48 \\
\hline $\mathbf{Y}$ & & 3003 & CLAY_RUS & $\begin{array}{l}\text { Rustier Compectod } \\
\text { Clay Column }\end{array}$ & PC_MAX & $\begin{array}{l}\text { Moximum allownblo capillery } \\
\text { preasure }\end{array}$ & $31882 A$ & 13-Fabss & $\mathbf{Y}$ & $4 B$ \\
\hline $\mathbf{Y}$ & & 3003 & CLAY_RUS & $\begin{array}{l}\text { Rustior Compacted } \\
\text { Clay Cokmn }\end{array}$ & PC_MAX & $\begin{array}{l}\text { Meximum alloweble copiltery } \\
\text { prossure }\end{array}$ & 316024 & $13-F c b-96$ & $\mathbf{Y}$ & 48 \\
\hline $\mathbf{Y}$ & & 3003 & CLAY_RUS & $\begin{array}{l}\text { Rustier Compected } \\
\text { Clay Column }\end{array}$ & PC_max & $\begin{array}{l}\text { Mecimum allowlab copiliery } \\
\text { proaure }\end{array}$ & $31882 A$ & $13-f a b-96$ & $\mathbf{Y}$ & 48 \\
\hline $\mathbf{Y}$ & & 3003 & CLAY_RUS & $\begin{array}{l}\text { Rustier Compacted } \\
\text { Cley Cokumn }\end{array}$ & PC_max & $\begin{array}{l}\text { Mexmum allowable capittary } \\
\text { presaure }\end{array}$ & $31882 A$ & $13-F a b-96$ & $\mathbf{Y}$ & 48 \\
\hline$Y$ & 100 & 3004 & CLAY_RUS & $\begin{array}{l}\text { Rustler Compected } \\
\text { Cley Cokmn }\end{array}$ & PCT_A & $\begin{array}{l}\text { Threshold Presture Linear } \\
\text { Parmeter }\end{array}$ & 31880 & $13-50 b-96$ & $\mathbf{Y}$ & 1 \\
\hline $\mathbf{Y}$ & 100 & 3004 & CLAY_RUS & $\begin{array}{l}\text { Rustler Compacted } \\
\text { Clay Column } \\
\end{array}$ & PCT_A & $\begin{array}{l}\text { Threshold Pressurs Lineer } \\
\text { Pereneter }\end{array}$ & 31880 & $13+5 a-96$ & $\mathbf{Y}$ & 1 \\
\hline $\mathbf{Y}$ & 100 & 3004 & CLAY_RUS & $\begin{array}{l}\text { Rustier Compected } \\
\text { Clay Cohmn }\end{array}$ & PCT_A & $\begin{array}{l}\text { Throshold Pressure Linar } \\
\text { Peremeter }\end{array}$ & 31800 & 13 Feb-96 & $\mathbf{Y}$ & 1 \\
\hline $\mathbf{Y}$ & 100 & 3004 & CLAY_RUS & $\begin{array}{l}\text { Rustler Compected } \\
\text { Clay Column }\end{array}$ & PCT_A & $\begin{array}{l}\text { Threshold Prossure Linear } \\
\text { Peremeter }\end{array}$ & 31880 & 13-Fab-96 & $\mathbf{Y}$ & 1 \\
\hline $\mathbf{Y}$ & 100 & 3005 & CLAY_RUS & $\begin{array}{l}\text { Rustior Compacted } \\
\text { Clay Column }\end{array}$ & PCT_EXP & $\begin{array}{l}\text { Threshold presture exponential } \\
\text { perenoter }\end{array}$ & 31881 & $13-F a b-96$ & $\mathbf{Y}$ & 1 \\
\hline $\mathbf{Y}$ & 100 & 3005 & CLAY_RUS & $\begin{array}{l}\text { Ruxtler Compacted } \\
\text { Clay Cokumn }\end{array}$ & PCT_EXP & $\begin{array}{l}\text { Threathold pressure exponential } \\
\text { perameter }\end{array}$ & 31881 & $13-F a b-96$ & $\mathbf{Y}$ & 1 \\
\hline $\mathbf{Y}$ & 100 & 3005 & CLAY_RUS & $\begin{array}{l}\text { Rustier Compacted } \\
\text { Clay Column }\end{array}$ & PCT_EXP & $\begin{array}{l}\text { Threshold preseure exponential } \\
\text { permenoter }\end{array}$ & 31881 & $13-F 0 b-96$ & $\mathbf{Y}$ & 1 \\
\hline $\mathbf{Y}$ & 100 & 3005 & CLAY_RUS & $\begin{array}{l}\text { Rustler Compected } \\
\text { Clsy Column }\end{array}$ & PCT_EXP & $\begin{array}{l}\text { Threshold presere exponential } \\
\text { peremeter }\end{array}$ & 31881 & $13-F c b-56$ & $\mathbf{Y}$ & 1 \\
\hline $\mathbf{Y}$ & varies & 3006 & CLAY_RUS & $\begin{array}{l}\text { Rustier Compected } \\
\text { Clay Column }\end{array}$ & PORE_DIS & $\begin{array}{l}\text { Brooks-Corvy pore distribution } \\
\text { pereneter }\end{array}$ & 31883 & $20-70 b-96$ & $\mathbf{Y}$ & 1 \\
\hline $\mathbf{Y}$ & varies & 3006 & CLAY_RUS & $\begin{array}{l}\text { Rustier Compocted } \\
\text { Ctry Column }\end{array}$ & PORE_DIS & $\begin{array}{l}\text { Brooks-Corey pore distribution } \\
\text { purameter }\end{array}$ & 31883 & 20-feb-96 & $\mathbf{Y}$ & 1 \\
\hline $\mathbf{Y}$ & varies & 3006 & CLAY_RUS & $\begin{array}{l}\text { Rustler Compected } \\
\text { Clay Column }\end{array}$ & PORE_DIS & $\begin{array}{l}\text { Brooke-Corny poro distroution } \\
\text { perameter }\end{array}$ & 31883 & 20-Feb-96 & $\mathbf{Y}$ & 1 \\
\hline $\mathbf{Y}$ & varies & 3006 & CLAY_RUS & $\begin{array}{l}\text { Ruster Compacted } \\
\text { Clay Column }\end{array}$ & PORE_DIS & $\begin{array}{l}\text { Erocks-Corny pore distribution } \\
\text { perameter }\end{array}$ & 31883 & $20-5 a b-96$ & $\mathbf{Y}$ & 1 \\
\hline $\mathbf{Y}$ & vanes & 3006 & CLAY_RUS & $\begin{array}{l}\text { Rustier Compacted } \\
\text { Clay Cokmn }\end{array}$ & PORE_DIS & $\begin{array}{l}\text { Brocke-Corey pora distribution } \\
\text { peroneter }\end{array}$ & 31883 & $20-f c b-96$ & $\mathbf{Y}$ & 1 \\
\hline$Y$ & varies & 3006 & CLAY_RUS & $\begin{array}{l}\text { Rutior Compected } \\
\text { Cloy Cokmn }\end{array}$ & PORE_DIS & $\begin{array}{l}\text { Brooke-Corey pore distribution } \\
\text { peremeter }\end{array}$ & 31883 & $20-5 c b-96$ & $\mathbf{Y}$ & 1 \\
\hline $\mathbf{Y}$ & & 3007 & CLAY_RUS & $\begin{array}{l}\text { Rustior Compected } \\
\text { Clay Cohmn }\end{array}$ & POROSTr & Effictive porosity & 31884 & 13Fab-96 & $-Y$ & 1 \\
\hline $\mathbf{Y}$ & & 3007 & CLAY_RUS & $\begin{array}{l}\text { Rustler Compected } \\
\text { Cloy Column }\end{array}$ & POROSITY & Effective porosity & 31884 & 13 feb-s6 & $\mathbf{Y}$ & 1 \\
\hline $\mathbf{Y}$ & & 3007 & CLAY_RUS & $\begin{array}{l}\text { Rustler Compected } \\
\text { Cley Cokmn }\end{array}$ & POROSITY & Effective porosity & 31884 & 13-feb-96 & $\mathbf{Y}$ & 1 \\
\hline $\mathbf{Y}$ & & 3008 & CLAY_RUS & $\begin{array}{l}\text { Rustier Compected } \\
\text { Clay Column }\end{array}$ & PRESSURE & Brine for-field pore pressurs & 31885 & $20-50 b-96$ & $\mathbf{Y}$ & 1 \\
\hline $\mathbf{Y}$ & & 3008 & CLAY_RUS & $\begin{array}{l}\text { Rustler Compected } \\
\text { Cliny Column }\end{array}$ & PRESSURE & Brine ferfintd pore presure & 31885 & $20-F a b-96$ & $\mathbf{Y}$ & 1 \\
\hline $\mathbf{Y}$ & venes & 3009 & CLAY_RUS & $\begin{array}{l}\text { Ruetier Compected } \\
\text { Chy Coturnn }\end{array}$ & PRMX_LOG & 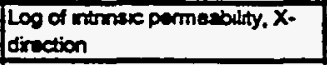 & 31886 & $13-f a b-86$ & $\mathbf{Y}$ & 1 \\
\hline $\mathbf{Y}$ & veries & 3009 & CLAY_RUS & $\begin{array}{l}\text { Rustor Compected } \\
\text { Cley Cotemn }\end{array}$ & PRMX_LOG & 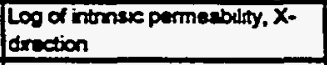 & $3 t 886$ & $13-f a b-96$ & $\mathbf{Y}$ & 1 \\
\hline $\mathbf{Y}$ & veries & 3009 & CLAY_RUS & $\begin{array}{l}\text { Rustier Compected } \\
\text { Cley Cokmn }\end{array}$ & PRMX_LOG & $\begin{array}{l}\text { Log of internaic permeability. } X \text { - } \\
\text { direction }\end{array}$ & 31886 & $13-F c b-96$ & $\mathbf{Y}$ & 1 \\
\hline $\mathbf{Y}$ & varies & 3009 & CLAY_RUS & $\begin{array}{l}\text { Rustier Compected } \\
\text { Clay Column }\end{array}$ & PRMX_LOG & $\begin{array}{l}\text { Log of intrinsic permasbilty, } x \text { - } \\
\text { direction }\end{array}$ & 31886 & $13-5=b-96$ & $\mathbf{Y}$ & 1 \\
\hline $\mathbf{Y}$ & varias & 3009 & CLAY_RUS & $\begin{array}{l}\text { Runter Compented } \\
\text { Clyy Cokmn }\end{array}$ & PRHX_LOG & $\begin{array}{l}\text { Log of intrinax permeability, } X \text { - } \\
\text { dinction }\end{array}$ & 31886 & $13-F e b-96$ & $\mathbf{Y}$ & 1 \\
\hline $\mathbf{Y}$ & & 3010 & CLAY_RUS & $\begin{array}{l}\text { Ruetier Compected } \\
\text { Clay Column }\end{array}$ & PRIYY_LOG & $\begin{array}{l}\text { Log of intrnax permesbilty, } Y \text {. } \\
\text { direction }\end{array}$ & 31887 & 13 febses & $\mathbf{Y}$ & 1 \\
\hline $\mathbf{Y}$ & & 3010 & CLAY_RUS & $\begin{array}{l}\text { Ruster Compected } \\
\text { Cloy Cokmn }\end{array}$ & PRAY_LOG & $\begin{array}{l}\text { Log of intrinsic permeabildy, } Y \text { - } \\
\text { direction }\end{array}$ & 31887 & 13Freb-96 & $\mathbf{Y}$ & 1 \\
\hline
\end{tabular}


CCA Parameter Listing

\begin{tabular}{|c|c|c|c|c|c|c|c|c|c|c|}
\hline To & $\begin{array}{c}\text { Clatint } \\
\text { cottion of } \\
\text { Deth } \\
\text { Cutteory } \\
\text { (foctnote 2) }\end{array}$ & $\begin{array}{l}\text { Dotetibution } \\
\text { Typo }\end{array}$ & Maen & medien & Mintmean & Heximen & Conts & Reference id & $\begin{array}{l}\text { Was the dint } \\
\text { domaloped } \\
\text { under an } \\
\text { KoA-1 } \\
\text { Progrem? }\end{array}$ & 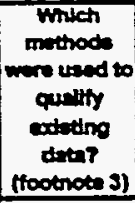 \\
\hline 3002 & 1.3 & CONSTANT & $0.00000000+\infty$ & $0.0000000 E+\infty$ & $0.00000000+\infty$ & $0.0000000 E+\infty$ & NONE & WP030ses & $Y$ & NA \\
\hline 3002 & 1,3 & CONSTANT & $0.00000000+\infty$ & $0.0000000 E+\infty 0$ & $0.0000000 \mathrm{E}+\infty$ & $0.00000000+\infty 00$ & NONE & WPO35271 & $Y$ & NA \\
\hline 3002 & 1,3 & CONSTANT & $0.0000000 E+\infty 00$ & $0.0000000 E+\infty$ & $0.0000000 E+\infty$ & $0.0000000 E+\infty$ & NONE & WP035333 & $\mathbf{Y}$ & NA \\
\hline 3002 & 1,3 & CONSTANT & $0.0000000 \mathrm{E}+\infty 0$ & $0.00000000+\infty 0$ & $0.00000000 E+00$ & $0.0000000 E+\infty$ & NONE & WP035597 & $\mathbf{Y}$ & NA \\
\hline 3002 & 1,3 & CONSTANT & $0.0000000 E+\infty$ & $0.00000000+\infty$ & $0.0000000 E+\infty 0$ & $0.00000000+\infty 00$ & NONE & WPO40514 & $\mathbf{Y}$ & NA \\
\hline 3003 & 1,3 & CONSTANT & - $1.0000000 E+08$ & $1.00000000+08$ & $1.0000000 E+00$ & $1.0000000 E+08$ & $\mathbf{P a}$ & WP030640 & $\mathbf{Y}$ & N/A \\
\hline 3003 & 1,3 & CONSTANT & $1.0000000 E+08$ & $1.0000000 E+08$ & $1.0000000 E+08$ & $1.0000000 E+08$ & Pa & WP030994 & $\mathbf{Y}$ & NA \\
\hline 3003 & 1,3 & CONSTANT & $1.0000000 E+08$ & $1.0000000 E+08$ & $1.00000000+08$ & $1.0000000 \mathrm{E}+00$ & Pa & WP035597 & $Y$ & NA \\
\hline 3003 & 1,3 & CONSTANT & $1.0000000 \mathrm{E}+08$ & $1.0000000 E+08$ & $1.0000000 E+08$ & $1.0000000 E+08$ & Pa & WPO40486 & $\mathbf{Y}$ & NA \\
\hline 3003 & 1,3 & CONSTANT & $1.0000000 E+08$ & $1.0000000 E+08$ & $1.0000000 E+08$ & $1.0000000 E+08$ & $\mathbf{P a}$ & WPO40514 & $\mathbf{Y}$ & NA \\
\hline 3004 & 3,4 & CONSTANT & $5.6000000 E-01$ & $5.6000000 E-01$ & $5.6000000 E-01$ & $5.6000000 E-01$ & $\mathbf{P a}$ & WP030640 & $\mathbf{Y}$ & NA \\
\hline 3004 & 3.4 & CONSTANT & $5.6000000 \mathrm{E}-01$ & $5.6000000 E-01$ & $5.6000000 E-01$ & 5.6000000E-01 & $\mathbf{P a}$ & WP030994 & $\mathbf{Y}$ & NA \\
\hline 3004 & 3.4 & CONSTANT & $5.6000000 E-01$ & $5.6000000 E-01$ & $5.6000000 E-01$ & $5.8000000 E-01$ & P: & WP035597 & $\mathbf{Y}$ & NMA \\
\hline 3004 & 3,4 & CONSTANT & $5.6000000 \mathrm{E}-01$ & 5.6000000E-01 & $5.6000000 E-01$ & $5.6000000 E-01$ & $\mathbf{P a}$ & WPO4O434 & $\mathbf{Y}$ & NA \\
\hline 3005 & 3,4 & CONSTANT & $3.4600000 \mathrm{E}-01$ & $3.4600000 E-01$ & $3.4600000 E-01$ & $3.4600000 \mathrm{E}-01$ & NONE & WP030640 & $\mathbf{Y}$ & NA \\
\hline 3005 & 3,4 & CONSTANT & $3.4600000 E-01$ & $3.4600000 E-01$ & $3.4600000 E-01$ & $3.4600000 \mathrm{E}-01$ & NONE & WP030994 & $\mathbf{Y}$ & NA \\
\hline 3005 & 3,4 & CONSTANT & $3.4600000 \mathrm{E}-01$ & $3.4600000 E-01$ & $3.4600000 \mathrm{E}-01$ & $3.4600000 \mathrm{E}-01$ & NONE & WP035597 & $\mathbf{Y}$ & NA \\
\hline 3005 & 3,4 & CONSTANT & $-3.4600000 E-01$ & $3.4600000 \mathrm{E}-01$ & $-3.46000000 E-01$ & $.3 .4600000 \mathrm{E}-01$ & NONE & WPO40434 & $\mathbf{Y}$ & NA \\
\hline 3006 & 3,4 & CUMULATIVE & $2.5200000 E+\infty$ & 9.4000000E-01 & $1.1000000 E-01$ & $8.1000000 E+\infty 0$ & NONE & WP030640 & $\mathbf{Y}$ & NA \\
\hline 3006 & 3,4 & CUMULATME & $2.5200000 E+\infty 0$ & 9.4000000E-01 & $1.1000000 E-01$ & $8.1000000 E+\infty$ & NONE & WP030994 & $Y$ & NA \\
\hline 3006 & 3,4 & CUMULATIVE & $2.5200000 E+\infty 0$ & 9.4000000E-01 & $1.1000000 \mathrm{E}-01$ & $8.10000000+\infty$ & NONE & WP032287 & $\mathbf{Y}$ & NA \\
\hline 3006 & 3,4 & CUMULATIVE & $2.5200000 E+\infty 0$ & $9.4000000 E-01$ & $1.1000000 E-01$ & $8.1000000 E+\infty$ & NONE & WP035268 & $\mathbf{Y}$ & NA \\
\hline 3006 & 3,4 & CUMULATNE & $2.52000000+\infty 0$ & $9.4000000 E-01$ & $1.1000000 E-01$ & $8.1000000 E+\infty 0$ & NONE & WP035597 & $\mathbf{Y}$ & N/A \\
\hline 3006 & 3,4 & CUMULATIVE & $25200000 E+\infty 0$ & $9.4000000 E-01$ & $1.1000000 E-01$ & $8.1000000 E+\infty 0$ & NONE & WPO41558 & $\mathbf{Y}$ & NA \\
\hline 3007 & $1,3,4$ & CONSTANT & 2.4000000E-01 & 2. $4000000 E-01$ & $2.4000000 E-01$ & 2.4000000E-01 & $m^{\wedge} 3 / m^{\wedge} 3$ & WP030640 &.$- y$ & NA \\
\hline 3007 & $1,3,4$ & CONSTANT & 2.4000000E-01 & $2.4000000 E-01$ & $2.4000000 E-01$ & $2.4000000 E-01$ & $m^{\wedge} 3 m^{\wedge} 3$ & WP030994 & $\mathbf{Y}$ & NA \\
\hline 3007 & $1,3,4$ & CONSTANT & 2.4000000E-01 & 2.4000000E-01 & $2.4000000 E-01$ & $2.4000000 E-01$ & $m^{\wedge} 3 m^{\wedge} 3$ & WP035597 & $\mathbf{Y}$ & NA \\
\hline 3008 & 3,5 & CONSTANT & $1.0132500 E+05$ & $1.0132500 E+05$ & $1.0132500 E+05$ & $1.0132500 E+05$ & P: & WP035597 & $\mathbf{Y}$ & NA \\
\hline 3000 & 3.5 & CONSTANT & $1.0132500 E+05$ & $1.0132500 E+05$ & $1.0132500 E+05$ & $1.0132500 E+05$ & Pa & WP036058 & $Y$ & NA \\
\hline 3009 & $1,3,4$ & TRLANGULAR & $-1.8857000 E+01$ & $-1.8301000 E+01$ & $-2.1000000 E+01$ & $-1.7301000 E+01$ & $\log \left(m^{\wedge} 2\right)$ & WP030640 & YEN & 1 \\
\hline 3009 & $1,3,4$ & TRLANGULAR & $-1.8867000 E+01$ & $-1.8301000 E+01$ & $-2.1000000 E+01$ & $-1,7301000 E+01$ & $\log \left(m^{\wedge} 2\right)$ & WP030994 & YoN & 1 \\
\hline 3009 & $1,3,4$ & TRLANGULAR & $-1.8857000 E+01$ & $-1.8301000 E+01$ & $-2.1000000 E+01$ & $-1.7301000 E+01$ & $\log \left(m^{\wedge} 2\right)$ & WP035268 & Yan & 1 \\
\hline 3009 & $1,3,4$ & TRLANGULAR & $-1.8867000 E+01$ & $-1.8301000 E+01$ & $-2.1000000 E+01$ & $-1.7301000 E+01$ & $\log \left(m^{\wedge} 2\right)$ & WP035597 & YaN & 1 \\
\hline 3009 & $1,3,4$ & TRLANGULAR & $-1.8867000 E+01$ & $-1.8301000 E+01$ & $-2.1000000 E+01$ & $-1.7301000 E+01$ & $\log \left(m^{\wedge} 2\right)$ & WPOA1558 & YEN & 1 \\
\hline 3010 & $1,3,4$ & TRLANGULAR & $-1.8867000 E+01$ & $-1.8301000 E+01$ & $-2.1000000 E+01$ & $-1.7301000 E+01$ & $\log \left(m^{\wedge} 2\right)$ & WP030540 & YEN & 1 \\
\hline 3010 & $1,3,4$ & TRLANGULAR & $-1.8657000 E+01$ & $-1.8301000 E+01$ & $-2.1000000 E+01$ & $-1.7301000 E+01$ & $\log \left(\mathrm{m}^{\wedge} 2\right)$ & WP030994 & Y\&N & 1 \\
\hline
\end{tabular}


CCA Parameter Listing

\begin{tabular}{|c|c|c|c|c|c|c|c|c|c|c|}
\hline $\begin{array}{l}\text { Parmetertin } \\
\text { Detabeso? } \\
\text { (YN) }\end{array}$ & 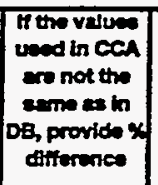 & D & mantalis & Mrtarde Name & 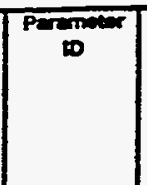 & Parmociar Name & $\begin{array}{l}\text { PRP DO } \\
\text { (MPOA) }\end{array}$ & $\begin{array}{c}\text { Deti Entry } \\
\text { Deto }\end{array}$ & 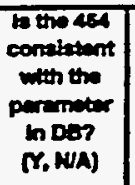 & $\begin{array}{l}\text { Paremoter } \\
\text { Cattegory } \\
\text { (footenote 1) }\end{array}$ \\
\hline $\mathbf{Y}$ & & 3010 & CLAY_RUS & $\begin{array}{l}\text { Rustler Compeced } \\
\text { Cley Cotumn }\end{array}$ & PRMY_LOG & $\begin{array}{l}\text { Log of minax permesolity, Y- } \\
\text { direction }\end{array}$ & 31887 & 13 fab-96 & $\mathbf{Y}$ & 1 \\
\hline $\mathbf{Y}$ & & 3010 & CLAY_RUS & $\begin{array}{l}\text { Rustior Compacted } \\
\text { Clay Column }\end{array}$ & PRAY_LOG & $\begin{array}{l}\text { Log of mantmax permesbility, Y- } \\
\text { drection }\end{array}$ & 31887 & $13 f=b-96$ & $\mathbf{Y}$ & 1 \\
\hline $\mathbf{Y}$ & & 3010 & CLAY_RUS & $\begin{array}{l}\text { Rustior Compacted } \\
\text { Clay Column }\end{array}$ & PRMY_LOG & $\begin{array}{l}\text { Log od mannax permesbilty, Y- } \\
\text { direction }\end{array}$ & 31887 & $13+=00-56$ & $\mathbf{Y}$ & 1 \\
\hline $\mathbf{Y}$ & & 3011 & CLAY_RUS & $\begin{array}{l}\text { Rustier Compectod } \\
\text { Cley Cokmn }\end{array}$ & PRMZ_LOG & $\begin{array}{l}\text { Log o intmasc pormesobitty, Z- } \\
\text { direction }\end{array}$ & 31888 & $13-F \infty-96$ & $\mathbf{Y}$ & 1 \\
\hline $\mathbf{Y}$ & & 3011 & CLAY_RUS & \begin{tabular}{|l} 
Rustiber Compected \\
Clay Column
\end{tabular} & PRMZ_LOG & $\begin{array}{l}\text { Log of intrense permesbiltry, Z- } \\
\text { diruction }\end{array}$ & 31888 & $13-700-96$ & $Y$ & 1 \\
\hline$Y$ & & 3011 & CLAY_RUS & $\begin{array}{l}\text { Rustor Compucted } \\
\text { Clay Column }\end{array}$ & PRUR_LOG & $\begin{array}{l}\text { Log of intraxs permeabilty, Z- } \\
\text { direction }\end{array}$ & 31888 & 13-Feb-96 & $\mathbf{Y}$ & 1 \\
\hline $\mathbf{Y}$ & & 3011 & CLAY_RUS & $\begin{array}{l}\text { Rustler Compscted } \\
\text { Clay Cotumn }\end{array}$ & PRMZ_LOG & $\begin{array}{l}\text { Log of nitimax permeability. Z. } \\
\text { direction }\end{array}$ & 31888 & $13-F a b-96$ & Y & 1 \\
\hline $\mathbf{Y}$ & . & 3011 & CLAY_RUS & $\begin{array}{l}\text { Rustler Compacted } \\
\text { Clay Cotumn }\end{array}$ & PRMZ_LOG & $\begin{array}{l}\text { Log of intronsex permestildy, Z- } \\
\text { drection }\end{array}$ & 31888 & 13-fab-96 & $\mathbf{Y}$ & 1 \\
\hline $\mathbf{Y}$ & & 3012 & CLAY_RUS & $\begin{array}{l}\text { Rustber Compacted } \\
\text { Clay Column }\end{array}$ & RELP_MOD & $\begin{array}{l}\text { Mocel number, relative } \\
\text { permeability model }\end{array}$ & 318894 & $13+5 e b-96$ & $\mathbf{Y}$ & AB \\
\hline $\mathbf{Y}$ & & 3012 & CLAY_RUS & $\begin{array}{l}\text { Rerstior Compacted } \\
\text { Cley Cotumn }\end{array}$ & RELP_MOD & $\begin{array}{l}\text { Model nember, rolative } \\
\text { permeability modol }\end{array}$ & 318894 & 13Fab-96 & $\mathbf{Y}$ & $4 B$ \\
\hline $\mathbf{Y}$ & & 3012 & CLAY_RUS & $\begin{array}{l}\text { Rustior Compreted } \\
\text { Clay Column }\end{array}$ & RELP_MOD & $\begin{array}{l}\text { Model number, rolititvo } \\
\text { permeabihty model }\end{array}$ & 318284 & $13-F a b-96$ & $\boldsymbol{\gamma}$ & $\triangle B$ \\
\hline $\mathbf{Y}$ & & 3012 & CLAY_RUS & $\begin{array}{l}\text { Rustler Compacted } \\
\text { Clay Column }\end{array}$ & RELP_MOD & $\begin{array}{l}\text { Model inmber, rolative } \\
\text { permeability model }\end{array}$ & $31889 A$ & 13 Fab-96 & $\mathbf{Y}$ & 4B \\
\hline $\mathbf{Y}$ & & 3012 & CLAY_RUS & $\begin{array}{l}\text { Rustler Compactod } \\
\text { Cloy Column }\end{array}$ & RELP_MOD & $\begin{array}{l}\text { Model number, rolative } \\
\text { permesbility model }\end{array}$ & 318894 & 13 Feb-96 & $Y$ & AB \\
\hline $\mathbf{Y}$ & -26.58 & 3013 & CLAY_RUS & $\begin{array}{l}\text { Rustlor Compacted } \\
\text { Cley Column }\end{array}$ & SAT_IBRN & Initial Bme Ssturation & 31894 & $13+f a b-96$ & $\mathbf{Y}$ & 1 \\
\hline $\mathbf{Y}$ & -26.58 & 3013 & CLAY_RUS & $\begin{array}{l}\text { Rustior Compactod } \\
\text { Clay Column }\end{array}$ & SAT_IBRN & |ritial Brno Seturation & 31894 & $13-F+6-96$ & $\mathbf{Y}$ & 1 \\
\hline $\mathbf{Y}$ & -26.58 & 3013 & CLAY_RUS & $\begin{array}{l}\text { Rustier Compected } \\
\text { Clay Cotumn } \\
\end{array}$ & SAT_IBRN & Initial Bmo Seturetion & 31894 & $13-00-96$ & $\mathbf{Y}$ & 1 \\
\hline Y & -26.58 & 3013 & CLAY_RUS & \begin{tabular}{|l} 
Rustior Compectod \\
Clay Column
\end{tabular} & SAT_IBRN & Nitral Brme Seturation & 31894 & 13-Fub-96 & $\mathbf{Y}$ & 1 \\
\hline $\mathbf{Y}$ & 100 & 3014 & CLAY_RUS & $\begin{array}{l}\text { Rustier Compectod } \\
\text { Clay Cotumn } \\
\end{array}$ & SAT_RBRN & Reschual Brme Securation & 31895 & 13fab-96 & $\mathbf{Y}$ & 1 \\
\hline $\mathbf{Y}$ & 100 & 3014 & CLAY_RUS & $\begin{array}{l}\text { Rustler Compactod } \\
\text { Ciny Cotumn }\end{array}$ & SAT_RBRK & Resictual $8 \mathrm{~mm}$ Smaration & 31095 & $13+00-96$ & $\mathbf{Y}$ & 1 \\
\hline $\mathbf{Y}$ & 100 & 3014 & CLAY_RUS & $\begin{array}{l}\text { Rustler Compaciod } \\
\text { Clay Column }\end{array}$ & SAT_RBRK F & Retudual eme Sauration & 31895 & 13-Feb-96 & $\mathbf{Y}$ & 1 \\
\hline Y & 100 & 3014 & CLAY_RUS & $\begin{array}{l}\text { Rustlor Compectod } \\
\text { Clay Cotumn }\end{array}$ & SAT_RBRH & Rescund Brme Searmon & 31895 & $13-F a b-96$ & $\mathbf{Y}$ & 1 \\
\hline$Y$ & 100 & 3014 & CLAY_RUS & $\begin{array}{l}\text { Rustiver Compecter } \\
\text { Cloy Column }\end{array}$ & SAT_RERA & Rotioner ens Seuration & 31895 & 13-Fab-96 & $\mathbf{Y}$ & 1 \\
\hline $\mathbf{Y}$ & 100 & 3014 & CLAY_RUS & $\begin{array}{l}\text { Rustlor Compeaded } \\
\text { Clizy Column }\end{array}$ & SAT_REAN & Rezous Brme Searation & 31895 & $13-50 b-96$ & $\mathbf{Y}$ & 1 \\
\hline $\mathbf{Y}$ & 100 & 3015 & CLAY_RUS & $\begin{array}{l}\text { Rustior Compeas } \\
\text { Cley Column }\end{array}$ & SAT_RGAS & 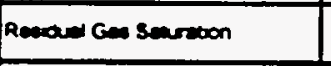 & 31896 & $13-f \circ b-86$ & $\mathbf{Y}$ & 1 \\
\hline $\mathbf{Y}$ & 100 & 3015 & CLAY_RUS & $\begin{array}{l}\text { Rustler Compected } \\
\text { Clay Column }\end{array}$ & SAT_mcas & 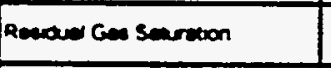 & 31896 & $13-f e b-96$ & $-\boldsymbol{Y}$ & 1 \\
\hline Y & 100 & 3015 & CLAY_RUS & $\begin{array}{l}\text { Rustior Compectod } \\
\text { Clay Column }\end{array}$ & SAT_RGas & Renaw Gen semrexon & 31896 & 13-Fob-96 & $\mathbf{Y}$ & 1 \\
\hline$Y$ & 100 & 3015 & CLAY_RUS & $\begin{array}{l}\text { Rustier Compecios } \\
\text { Clay Cotumn }\end{array}$ & sat_ncas & Remat Gen sempon & 31896 & $13-$ Fob-96 & $Y$ & 1 \\
\hline $\mathbf{Y}$ & 100 & 3015 & CLAY_RUS & $\begin{array}{l}\text { Rustiver Compeases } \\
\text { Ctry Colum }\end{array}$ & sat_ncas & 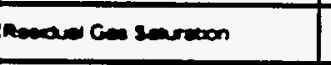 & 31896 & 13 feb-96 & $\mathbf{Y}$ & 1 \\
\hline $\mathbf{Y}$ & & 3016 & MAGENTA & Hagenta Mernoer & cons,ncx & 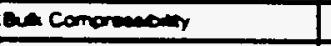 & 33249 & 13-fob-96 & $Y$ & 1 \\
\hline $\mathbf{Y}$ & & 3016 & MAGENTA & Magenes Mononor & cons_nex & 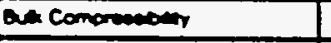 & 33249 & $13-506-96$ & $Y$ & 1 \\
\hline $\mathbf{Y}$ & & 3016 & MAGENTA & Magenen momoer & cols.ncx & at comonomoting & 33249 & 13 fab- 96 & $\mathbf{Y}$ & 1 \\
\hline $\mathbf{Y}$ & & 3017 & CL_L_TI & $\begin{array}{l}\text { Lower Saleco capro of } \\
10 \text { yean }\end{array}$ & RSH_an & 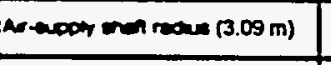 & $31916 A$ & $15+F a b-96$ & $\mathbf{Y}$ & AB \\
\hline $\mathbf{Y}$ & & 3017 & CL_L_T1 & $\begin{array}{l}\text { Lower Sateco ary } 0 \text { to } \\
10 \text { yers }\end{array}$ & RSH_An & 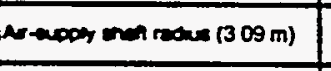 & $31916 \AA$ & $15-f a b-86$ & $\mathbf{Y}$ & 48 \\
\hline $\mathbf{Y}$ & & 3017 & CL_L_TI & $\begin{array}{l}\text { Lower Salwo con } 0 \text { of } \\
\text { 10 years }\end{array}$ & RSH_An & 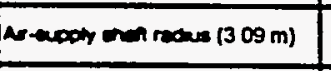 & 31916A & $15+a b-66$ & $\mathbf{Y}$ & $4 B$ \\
\hline $\mathbf{Y}$ & & 3017 & $C L_{-} L_{-} T I$ & $\begin{array}{l}\text { Lower Salcos asy } 0 \text { to } \\
10 \text { yeers }\end{array}$ & RSH_Ar & Nor-acosy and rectus $(3.09 \mathrm{~m})$ & 31916A & $15-f-b-96$ & $\mathbf{Y}$ & 48 \\
\hline $\mathbf{Y}$ & & 3018 & CL_L_TI & $\begin{array}{l}\text { Lower Saleco cery O D } \\
10 \text { yers }\end{array}$ & RSH_SN & sentranders men radks $(1.8 \mathrm{~m})$ & 31912A & $20-F a b-96$ & $\mathbf{Y}$ & $4 B$ \\
\hline $\mathbf{Y}$ & & 3018 & CL_L_TI & $\begin{array}{l}\text { Lower Selede car } \mathrm{O} \text { of } \\
10 \text { years }\end{array}$ & RSH_zul & 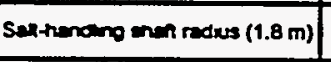 & 319184 & $20+c b-96$ & $\mathbf{Y}$ & 48 \\
\hline
\end{tabular}


CCA Parameter Listing

\begin{tabular}{|c|c|c|c|c|c|c|c|c|c|c|}
\hline DD & \begin{tabular}{|c|} 
Canestit \\
Cation of \\
Deta \\
Cetugory \\
(foctnots 2)
\end{tabular} & $\begin{array}{c}\text { Detriburbon } \\
\text { Type }\end{array}$ & מem & Medien & Mininem & Mediming & Chits & Retiones id & 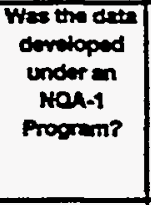 & 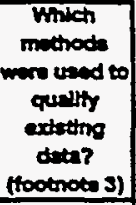 \\
\hline 3010 & $1.3,4$ & TRLANGULAR & $-1.8867000 E+01$ & $-1.8301000 E+01$ & $-2.1000000 E+01$ & $-1.7301000 E+01$ & $\log \left(m^{\prime} \alpha 2\right)$ & WP035268 & YEN & 1 \\
\hline 3010 & $1,3,4$ & TRLANGULAR & $-1,8867000 E+01$ & $-1,8301000 E+01$ & $-21000000 E+01$ & $-1.7301000 E+01$ & $\log \left(m^{2} 2\right)$ & WP035557 & YEN & 1 \\
\hline 3010 & $1,3,4$ & TRIANGULAR & $-1.8867000 E+01$ & $-1,8301000 E+01$ & $-2.1000000 E+01$ & $-1.7301000 E+01$ & $\log \left(m^{\wedge} 2\right)$ & WPO41558 & YAN & 1 \\
\hline 3011 & $1,3,4$ & TRLANGULAR & $-1.8867000 E+01$ & $-1.8301000 E+01$ & $-21000000 E+01$ & $-1.7301000 E+01$ & $\log \left(m^{2} 2\right)$ & WP030540 & YaN & 1 \\
\hline 3011 & $1,3,4$ & TRIANGULAR & $-1.8867000 E+01$ & $-1.8301000 E+01$ & $-2.1000000 E+01$ & $-1.7301000 E+01$ & $\log \left(m^{n} 2\right)$ & WP030S94 & YEN & 1 \\
\hline 3011 & $1,3,4$ & TRIANGULAR & $\therefore 1.8867000 E+01$ & $-1.8301000 E+01$ & $-2,1000000 E+01$ & $-1.7301000 E+01$ & $\log (m+2)$ & WP035268 & Y\&N & 1 \\
\hline 3011 & $1,3,4$ & TRLANGULAR & $-1.8867000 E+01$ & $-1.8301000 E+01$ & $-21000000 E+01$ & $-1.7301000 E+01$ & $\log \left(m^{\wedge} 2\right)$ & MP035597 & Y\&N & 1 \\
\hline 3011 & $1,3,4$ & TRIANGULAR & $-1.8867000 E+01$ & $-1.8301000 E+01$ & $-2.1000000 E+01$ & $-1.7301000 E+01$ & $\log \left(m^{\wedge} 2\right)$ & WPO41558 & Y8N & 1 \\
\hline 3012 & $1,3,4$ & CONSTANT & $4.0000000 \mathrm{E}+\infty 0$ & $4.0000000 E+\infty$ & $4.0000000 E+\infty$ & $1.0000000 E+\infty 0$ & NONE & WP030640 & $\mathbf{Y}$ & NA \\
\hline 3012 & $1,3,4$ & CONSTANT & $4.0000000 E+00$ & $4.00000000+\infty 0$ & $4.0000000 E+\infty 0$ & $4.0000000 E+\infty 0$ & NONE & WPO30994 & $\mathbf{Y}$ & NA \\
\hline 3012 & $1,3,4$ & CONSTANT & $4.0000000 E+\infty 0$ & $4.0000000 \mathrm{E}+\infty 0$ & $4.0000000 E+\infty 0$ & $4.00000000 E+\infty 0$ & NONE & WP035597 & $\mathbf{Y}$ & NA \\
\hline 3012 & $1,3,4$ & CONSTANT & $4.0000000 E+\infty 0$ & $4.0000000 E+\infty 0$ & $4.00000005+\infty 0$ & $4.0000000 E+\infty 0$ & NONE & WPO40486 & $\mathbf{Y}$ & NA \\
\hline 3012 & $1,3,4$ & CONSTANT & $4.0000000 E+\infty 0$ & $4.0000000 E+\infty 0$ & $4.0000000 E+\infty 0$ & $4.0000000 E+\infty 0$ & NONE & WPO40514 & $\mathbf{Y}$ & NA \\
\hline 3013 & $1,3,4$ & CONSTANT & $7.9000000 E-01$ & 7.9000000E-01 & $7.9000000 E-01$ & $7.9000000 E-01$ & NONE & WP030540 & $\mathbf{Y}$ & NA \\
\hline 3013 & $1,3,4$ & CONSTANI & $7.9000000 E-01$ & $7.9000000 E-01$ & 7.9000000E-01 & $7.9000000 E-01$ & NONE & WP030994 & $\mathbf{Y}$ & NA \\
\hline 3013 & $1,3,4$ & CONSTANT & $7.9000000 E-01$ & $7.9000000=-01$ & $7.9000000 E-01$ & $7.9000000 E-01$ & NONE & WP035597 & $\mathbf{Y}$ & N/A \\
\hline 3013 & $1,3,4$ & CONSTANT & 7.9000000E-01 & 7.9000000E-01 & $7.9000000 E-01$ & $7.9000000 E-01$ & NONE & WPO40434 & $\mathbf{Y}$ & NA \\
\hline 3014 & 3,4 & CUMULATIVE & $2.5000000 E-01$ & $2.0000000 E-01$ & $0.0000000 E+\infty$ & $6.0000000 E-01$ & NONE & WP030640 & $\mathbf{Y}$ & NA \\
\hline 3014 & 3,4 & CUMULATIVE & $25000000 E-01$ & $20000000 E-01$ & $0.0000000 E+\infty$ & $6.0000000 E-01$ & NONE & WPO30S94 & $Y$ & $N A$ \\
\hline 3014 & 3,4 & CUMULATIVE & $2.5000000 E-01$ & $20000000 E-01$ & $0.0000000 E+\infty 0$ & $6.0000000 E-01$ & NONE & WP035268 & $\mathbf{Y}$ & NA \\
\hline 3014 & 3,4 & CUMULATIVE & $2.5000000 E-01$ & $20000000 E-01$ & $0.0000000 E+\infty$ & $6.0000000 E-01$ & NONE & WP035597 & $\mathbf{Y}$ & $N A$ \\
\hline 3014 & 3,4 & CUMULATME & $2.5000000 E-01$ & $2.0000000 E-01$ & $0.0000000 E+\infty$ & $6.0000000 E-01$ & NONE & WPO41558 & $\mathbf{Y}$ & NA \\
\hline 3014 & 3,4 & CUMULATINE & 2.5000000E-01 & $20000000 E-01$ & $0.0000000 E+\infty$ & $6.0000000 E-01$ & NONE & WP042751 & $\mathbf{Y}$ & NA \\
\hline 3015 & 3,4 & UNIFORM & $20000000 E-01$ & $20000000 E-01$ & $0.00000000+\infty$ & $4.0000000 E-01$ & NONE & WP030540 & $\mathbf{Y}$ & NA \\
\hline 3015 & 3,4 & UNIFORM & $2.0000000 E-01$ & 20000000E-01 & $0.0000000 E+\infty$ & $4.0000000 E-01$ & NONE & WP030994 & $-\mathbf{Y}$ & NA \\
\hline 3015 & 3,4 & UNIFORM & $2.0000000 E-01$ & $2.0000000 E-01$ & $0.0000000 E+\infty$ & $4.0000000 E-01$ & NONE & WP035268 & $\mathbf{Y}$ & NA \\
\hline 3015 & 3,4 & UNIFORM & $20000000 E-01$ & $2.0000000 E-01$ & $0.0000000 E+\infty$ & $40000000 E-01$ & NONE & WP035597 & $\mathbf{Y}$ & NA \\
\hline 3015 & 3,4 & UNIFORM & $20000000 E-01$ & $20000000 E-01$ & $0.00000000+\infty$ & $40000000 E-01$ & NONE & WPO41558 & $\mathbf{Y}$ & NA \\
\hline 3016 & 1 & STUDENT & 2640000 E-10 & $2.640000 E-10$ & $11620000 E-10$ & $4.5530000 \mathrm{E}-10$ & $P x^{\wedge}-1$ & WP030511 & $Y$ & NA \\
\hline 3016 & 1 & STUDENT & $2.6440000 E-10$ & $2640000 E-10$ & $1.1620000 E-90$ & $45530000 E-10$ & PAn-1 & WP035597 & $Y$ & N/A \\
\hline 3016 & 1 & STUDENT & $26400006-10$ & $2.6440000 E-10$ & $1.1620000 \mathrm{E}-10$ & $4.5530000 \mathrm{E}-10$ & Px-1 & WPO35380 & $Y$ & NA \\
\hline 3017 & 1,3 & CONSTANT & $3.0900000 E+\infty$ & $3.0900000 E+\infty$ & $3.0900000 E+\infty$ & $3.0900000 E+\infty 0$ & $\mathbf{m}$ & WP030540 & $\mathbf{Y}$ & NA \\
\hline 3017 & 1,3 & CONSTANT & $3.0900000 \mathrm{E}+\infty$ & $3.0900000 E+\infty$ & $3.0900000 E+\infty$ & $3.0900000 \mathrm{E}+\infty 0$ & $\mathbf{m}$ & WP030994 & $Y$ & NA \\
\hline 3017 & 1,3 & CONSTANT & $3.09000000 E+00$ & $3.0900000 E+\infty 0$ & $3.09000000+\infty$ & $3.0900000 E+\infty$ & $m$ & WP035597 & $\mathbf{Y}$ & N/A \\
\hline 3017 & 1,3 & CONSTANT & $3.0900000 \mathrm{E}+00$ & $3.0900000 E+\infty 0$ & $3.0900000 E+\infty 0$ & $3.0900000 E+\infty 0$ & m & WPO40514 & $\mathbf{Y}$ & N/A \\
\hline 3018 & 1,3 & CONSTANT & $1.80000000+\infty 0$ & $1.8000000 E+\infty$ & $1.6000000 E+\infty 0$ & $1.8000000 \mathrm{E}+\infty 0$ & $\mathbf{m}$ & WP030640 & $\mathbf{Y}$ & WA \\
\hline 3018 & 1,3 & CONSTANT & $1.8000000 E+\infty 0$ & $1.8000000 E+\infty$ & $1.8000000 E+\infty 0$ & $1.8000000 E+\infty$ & $m$ & WP030994 & $\mathbf{Y}$ & $\mathrm{NAA}$ \\
\hline
\end{tabular}


CCA Parameter Listing

\begin{tabular}{|c|c|c|c|c|c|c|c|c|c|c|}
\hline 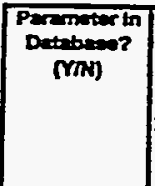 & 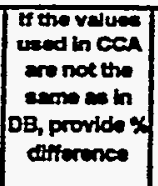 & $\mathbf{D}$ & Antardie to & Metwien Nem & $\begin{array}{c}\text { Paramenor } \\
\text { Do }\end{array}$ & 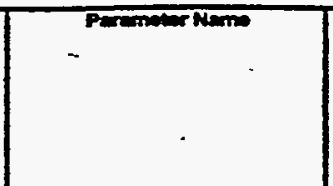 & 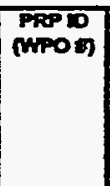 & Detenty & 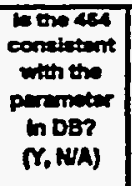 & $\begin{array}{l}\text { Parnmeter } \\
\text { Cetwoory } \\
\text { (foothotel 1) }\end{array}$ \\
\hline$\gamma$ & & 3018 & CL_L_T1 & $\begin{array}{l}\text { Lower Salado clay.0 to } \\
\text { to years }\end{array}$ & RSH_SAL & Salt-thending strat radus $(1.8 \mathrm{~m})$ & 319184 & $20-700-56$ & $Y$ & 48 \\
\hline$Y$ & & 3018 & CL_L_T1 & $\begin{array}{l}\text { Lower Sulado ciny.0 to } \\
10 \text { years }\end{array}$ & RSH_SAL & Sath thanding thin redies $(1.8 \mathrm{~m})$ & 319184 & $20+00-96$ & $\mathbf{Y}$ & $4 B$ \\
\hline$Y$ & & 3019 & CL_L_TI & $\begin{array}{l}\text { Lower Suledo ctry.0 to } \\
10 \text { yours }\end{array}$ & RSH_was & $\begin{array}{l}\text { Whato-handing athet redus (3.5 } \\
\text { m) }\end{array}$ & 31919A & $20-F 0 b-56$ & $\boldsymbol{\gamma}$ & $4 B$ \\
\hline $\mathbf{Y}$ & & 3019 & CL_L_TI & $\begin{array}{l}\text { Lower Selaco dey.0 to } \\
10 \text { years }\end{array}$ & RSH_WAS & $\begin{array}{l}\text { Weatohanding shaft raders (3.5 } \\
\text { m) }\end{array}$ & $31918 A$ & $20+0 b-96$ & $\mathbf{Y}$ & 48 \\
\hline$Y$ & & 3019 & CL_L_TI & $\begin{array}{l}\text { Lowor Salioso dity. } 0 \text { to } \\
10 \text { years }\end{array}$ & RSH_WAS & $\begin{array}{l}\text { Whastohendling thet rades (3.5 } \\
\text { (m) }\end{array}$ & 319184 & $20-7=6-96$ & $\mathbf{Y}$ & 48 \\
\hline$Y$ & & 3019 & CL_L_TI & $\begin{array}{l}\text { Lower Saludo day.0 to } \\
10 \text { years }\end{array}$ & RSH_WAS & $\begin{array}{l}\text { Whato-hending shet rades (3.5 } \\
\text { (m) }\end{array}$ & 31919A & $20-50 b-96$ & $\mathbf{Y}$ & 4B \\
\hline $\mathbf{Y}$ & & 3020 & CL_L_TI & $\begin{array}{l}\text { Lower Salodo doy.0 to } \\
10 \text { years }\end{array}$ & RSH_EXH & Air-exheust shant rectius $(2.3 \mathrm{~m})$ & 31917A & $15+\infty 0-96$ & $\mathbf{Y}$ & $4 B$ \\
\hline $\mathbf{Y}$ & & 3020 & CL_L_T1 & $\begin{array}{l}\text { Lower Salaco ctry.0 to } \\
10 \text { years }\end{array}$ & RSH_EXH & Air-whens stim radius $(2.3 \mathrm{~m})$ & 31917A & 15Feb-96 & $\mathbf{Y}$ & 4B \\
\hline $\mathbf{Y}$ & & 3020 & CL_L_TI & $\begin{array}{l}\text { Lower Salado doy.0 to } \\
10 \text { years }\end{array}$ & RSH_EXH & Air-exhauset ghent radivs $(2.3 \mathrm{~m})$ & 31917A & $15+e b-96$ & $\mathbf{Y}$ & $A B$ \\
\hline $\mathbf{Y}$ & & 3020 & CL_L_T1 & $\begin{array}{l}\text { Lower Salado ctay.0 to } \\
10 \text { years }\end{array}$ & RSH_EXH & 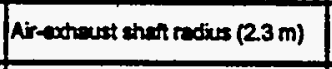 & 31917A & $15+a b-96$ & $\mathbf{Y}$ & 48 \\
\hline $\mathbf{Y}$ & & 3021 & CL_L_TI & $\begin{array}{l}\text { Lower Selado ctoy:0 to } \\
10 \text { years }\end{array}$ & RADN_ORZ & DRZ outer radius at each shath & 31913 & $15+f e b-96$ & $\mathbf{Y}$ & 1 \\
\hline $\mathbf{Y}$ & & 3021 & CL_L_TI & $\begin{array}{l}\text { Lowor Salado ciry.0 to } \\
10 \text { years }\end{array}$ & RADN_DRZ & DRZ outer radies at anch shath & 31913 & $15+\infty 6-96$ & $\mathbf{Y}$ & 1 \\
\hline $\mathbf{Y}$ & & 3021 & CL_L_TI & $\begin{array}{l}\text { Lower Salado clay. } 0 \text { to } \\
10 \text { years }\end{array}$ & RADN_DRZ & JRZ outer ractiss at eech shent & 31913 & $15-f e b-96$ & $\mathbf{Y}$ & 1 \\
\hline $\mathbf{Y}$ & & 3022 & CL_L_T2 & $\begin{array}{l}\text { Lower Salaco ctay. } 10 \\
\text { to } 25 \text { yours }\end{array}$ & RSH_AIR & Air-eupply thent radies $(3.09 \mathrm{~m})$ & $31964 A$ & $15-F \infty b-96$ & $\mathbf{Y}$ & 48 \\
\hline $\mathbf{Y}$ & & 3022 & CL_L_T2 & $\begin{array}{l}\text { Lower Salado clay: } 10 \\
\text { to } 25 \text { years }\end{array}$ & RSH_ANR & Air-eupply streft redives $(3.09 \mathrm{~m})$ & 319644 & $15 f c b-96$ & $\mathbf{Y}$ & 48 \\
\hline $\mathbf{Y}$ & & 3022 & $C L_{-} L_{-} T 2$ & $\begin{array}{l}\text { Lower Saloco diay. } 10 \\
\text { to } 25 \text { years }\end{array}$ & RSH_AIR & Air-supply shat redius (3.09 m) & $31964 A$ & 15 Feb-96 & $\mathbf{Y}$ & 18 \\
\hline $\mathbf{Y}$ & & 3022 & CL_L_T2 & $\begin{array}{l}\text { Lower Saledo clay: } 10 \\
\text { to } 25 \text { years }\end{array}$ & RSH_AIR & Air-supply strent radius $(3.09 \mathrm{~m})$ & $31964 A$ & $15-F a b-96$ & $\mathbf{Y}$ & $4 B$ \\
\hline$Y$ & & 3023 & CL_L_T2 & $\begin{array}{l}\text { Lower Salado clay.10 } \\
\text { to } 25 \text { years }\end{array}$ & RSH_SAL & Sakthandling ehath radies $(1.8 \mathrm{~m})$ & 319664 & $15+50 b-96$ & $\mathbf{Y}$ & 48 \\
\hline$Y$ & & 3023 & CL_L_T2 & $\begin{array}{l}\text { Lower Saledo clay: } 10 \\
\text { to } 25 \text { years }\end{array}$ & RSH_SAL & Salthandling stant radies $(1.8 \mathrm{~m})$ & $31968 A$ & 15Feb-96 & $Y$ & AB \\
\hline$Y$ & & 3023 & CL_L_T2 & $\begin{array}{l}\text { Lower Saledo clay.10 } \\
\text { to } 25 \text { years }\end{array}$ & RSH_SAL & Salthanding athan radies $(1.8 \mathrm{~m}) \mid$ & 319668 & 15feb-96 & $\mathbf{Y}$ & 48 \\
\hline$Y$ & & 3023 & CL_L_T2 & $\begin{array}{l}\text { Lower Salsodo ciay. } 10 \\
\text { to } 25 \text { yeers }\end{array}$ & RSH_SAL & Salthanding shath radas $(1.8 \mathrm{~m})$ & $31968 \mathrm{~A}$ & 15-Feb-96 & $\mathbf{Y}$ & 48 \\
\hline Y & & 3024 & $C L_{-} L_{-} T 2$ & $\begin{array}{l}\text { Lower Saludo day.10 } \\
\text { to } 25 \text { years }\end{array}$ & RSH_WAS & $\begin{array}{l}\text { Wasto-handing start redus (3.5 } \\
\text { (m) }\end{array}$ & $31967 A$ & 15 fab-96 & $\mathbf{Y}$ & 48 \\
\hline$Y$ & & 3024 & CL_L_T2 & $\begin{array}{l}\text { Lower Salado clay:10 } \\
\text { to } 25 \text { years }\end{array}$ & RSH_WAS & $\begin{array}{l}\text { Wrasto-handling shent radius (3.5 } \\
\text { (m) }\end{array}$ & $31967 A$ & 15 fab-96 & $\mathbf{Y}$ & 48 \\
\hline $\mathbf{Y}$ & & 3024 & CL_L_T2 & $\begin{array}{l}\text { Lower Saludo dry. } 10 \\
\text { to } 25 \text { years }\end{array}$ & RSH_WAS & $\begin{array}{l}\text { Wastohandling ahat redies (3.5 } \\
\text { m) }\end{array}$ & 31967A & 15 Fob-96 & $\mathbf{Y}$ & AB \\
\hline $\mathbf{Y}$ & & 3024 & CL_L_T2 & $\begin{array}{l}\text { Lower Selado diay: } 10 \\
\text { to } 25 \text { years }\end{array}$ & RSH_WAS & $\begin{array}{l}\text { Wasto-hending shaif reders (3.5 } \\
\text { m) }\end{array}$ & 31967A & $15-F$ bb-96 & $-\boldsymbol{Y}$ & AB \\
\hline $\mathbf{Y}$ & & 3025 & CL_L_T2 & $\begin{array}{l}\text { Lower Salado clay. } 10 \\
\text { to } 25 \text { years }\end{array}$ & RSH_EXH & Air-extreust thatt radius $(2.3 \mathrm{~m})$ & $31965 \mathrm{~A}$ & 15 Fob-96 & $\mathbf{Y}$ & 48 \\
\hline $\mathbf{Y}$ & & 3025 & CL_L_T2 & $\begin{array}{l}\text { Lower Seliado diny.10 } \\
\text { to } 25 \text { years }\end{array}$ & RSH_EXH & Ar-exthuset shent radius (23 m) & $31965 A$ & $15-F 0 b-96$ & $\mathbf{\gamma}$ & $4 B$ \\
\hline $\mathbf{Y}$ & & 3025 & CL_L_I2 & 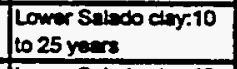 & RSH_EXH & Ar-ecthoust ghate radies (2.3 m) & $31965 A$ & $15-F a b-96$ & $\mathbf{Y}$ & AB \\
\hline $\mathbf{Y}$ & & 3025 & $C L_{-} L_{-} T 2$ & $\begin{array}{l}\text { Lower Saludo dity: } 10 \\
\text { io } 25 \text { yeare }\end{array}$ & RSH_EXH & Air-axtheunt ghent radive (2.3 m) & $31965 A$ & $15-F e b-86$ & $\mathbf{Y}$ & 48 \\
\hline$Y$ & & 3026 & CL_L_T2 & $\begin{array}{l}\text { Lower Salodo day:10 } \\
\text { to } 25 \text { vains }\end{array}$ & RADN_DRZ & DRZ outer ractius at exch chath & 31959 & $15+50 b-96$ & $\mathbf{Y}$ & 1 \\
\hline $\mathbf{Y}$ & & 3026 & CL_L_T2 & $\begin{array}{l}\text { Lower Salados clay: } 10 \\
\text { to } 25 \text { years }\end{array}$ & RADN_DRZ & DRZ outer recius at each that & 31959 & $15+50 b-96$ & $\mathbf{Y}$ & 1 \\
\hline$Y$ & & 3026 & CL_L_T2 & $\begin{array}{l}\text { Lowor Saledo cloy:10 } \\
\text { to } 25 \text { yeurs }\end{array}$ & RADN_DRZ & DRZ outer redies at each then & 31959 & 15-Fob-96 & $\mathbf{Y}$ & 1 \\
\hline $\mathbf{Y}$ & & 3027 & CL_L_T3 & $\begin{array}{l}\text { Lower Salado ctor:25 } \\
\text { to } 50 \text { years }\end{array}$ & RSH_AR & Ar-apply shat radies $(3.09 \mathrm{~m})$ & 319994 & $15 F a b-96$ & $\mathbf{Y}$ & 48 \\
\hline$Y$ & & 3027 & CL_L_T3 & $\begin{array}{l}\text { Lower Salado ciay } 25 \\
\text { to } 50 \text { years }\end{array}$ & RSH_NR & Air tupply shat radius $(3.09 \mathrm{~m})$ & 319994 & $15-F a b-96$ & $\mathbf{Y}$ & 48 \\
\hline $\mathbf{Y}$ & & 3027 & CL_L_T3 & $\begin{array}{l}\text { Lomor Saledo ctary-25 } \\
\text { to } 50 \text { years }\end{array}$ & RSH_AR & Ar-apoly athen radius $(3.09 \mathrm{~m})$ & 319924 & $15-F=b-96$ & $\mathbf{Y}$ & 48 \\
\hline $\mathbf{Y}$ & & 3027 & CL_L_T3 & \begin{tabular}{|l} 
Lomer Salodo diny.25 \\
to 50 years
\end{tabular} & RSH_NR & Ar-aupply stuat redius $(3.09 \mathrm{~m})$ & 318994 & 15 Fob-96 & $\mathbf{Y}$ & 48 \\
\hline
\end{tabular}


CCA Parameter Listing

\begin{tabular}{|c|c|c|c|c|c|c|c|c|c|c|}
\hline W & \begin{tabular}{c|} 
Creasint \\
coution ol \\
Deta \\
Centugory \\
(footnote 2)
\end{tabular} & $\begin{array}{l}\text { Dtatribution } \\
\text { Type }\end{array}$ & $\operatorname{men}$ & moden & Minkrom & Meximen & Units & Reformanca if & $\begin{array}{l}\text { Was the dete } \\
\text { dovetoped } \\
\text { under } \\
\text { Non-1 } \\
\text { Prosom? }\end{array}$ & 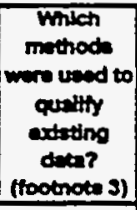 \\
\hline 3018 & 1,3 & CONSTANT & $1.8000000 \mathrm{E}+\infty 0$ & $1.80000000 E+00$ & $1.8000000 \mathrm{E}+\infty 0$ & $1.8000000 E+\infty$ & $\mathbf{m}$ & WP035597 & $Y$ & NA \\
\hline 3018 & 1,3 & CONSTANT & $1.8000000 E+\infty 0$ & $1,8000000 E+\infty$ & $1.8000000 E+\infty$ & $1.8000000=+\infty$ & $m$ & UPO40514 & $Y$ & NA \\
\hline 3019 & 1,3 & CONSTANT & $3.5000000 \mathrm{E}+\infty 0$ & $3.5000000 E+\infty$ & $3.5000000 E+\infty$ & $3.5000000 E+\infty$ & $m$ & WP030640 & $Y$ & NA \\
\hline 3019 & 1,3 & CONSTANT & $3.5000000 E+\infty 0$ & $3.50000000+00$ & $3.5000000 E+\infty 0$ & $3.5000000 E+\infty)$ & $m$ & WP030994 & $\mathbf{Y}$ & NA \\
\hline 3019 & 1,3 & CONSTANT & $3.5000000 E+\infty 0$ & $3.5000000 E+\infty$ & $3.5000000 E+\infty$ & $3.5000000 E+\infty$ & $m$ & WP035597 & $\mathbf{Y}$ & NA \\
\hline 3019 & 1,3 & CONSTANT & , $3.5000000 E+00$ & $3.5000000 \leq+\infty$ & $3.5000000 E+\infty 0$ & $3.5000000 E+\infty$ & $m$ & WPO40514 & $Y$ & NA \\
\hline 3020 & 1,3 & CONSTANT & $2.3000000 E+\infty 0$ & $2.3000000 E+\infty 0$ & $2.3000000 E+\infty$ & $23000000 E+\infty 0$ & $m$ & WP030640 & $\mathbf{Y}$ & NA \\
\hline 3020 & 1.3 & CONSTANT & $2.3000000 E+\infty 0$ & $23000000 E+\infty 0$ & $23000000 E+\infty$ & $23000000 E+\infty 0$ & $m$ & WPO30SS4 & $Y$ & NA \\
\hline 3020 & 1,3 & CONSTANT & $2.3000000 E+00$ & $2.3000000 E+\infty 0$ & $2.3000000 E+\infty 0$ & $2.3000000 E+\infty$ & m & WP035597 & $\mathbf{Y}$ & NA \\
\hline 3020 & 1,3 & CONSTANT & $2.3000000 E+\infty 0$ & $2.3000000 E+\infty 0$ & $2.3000000 \mathrm{E}+\infty$ & $2.3000000 \mathrm{E}+00$ & $\mathbf{m}$ & WPO40514 & $\mathbf{Y}$ & NA \\
\hline 3021 & $1,3,4$ & CONSTANT & $1.8580000=+\infty$ & $1.8580000 E+00$ & $1,8580000 E+\infty$ & $1.8580000 E+\infty$ & $\mathrm{mm}$ & WP030640 & $\gamma$ & NA \\
\hline 3021 & $1,3,4$ & CONSTANT & $1.85800000=00$ & $1.8580000 E+\infty$ & $1.8580000 E+\infty$ & $1.8580000 E+\infty 0$ & $\mathrm{~m} / \mathrm{m}$ & WP030994 & $\mathbf{Y}$ & NA \\
\hline 3021 & $1,3,4$ & CONSTANT & $1.8580000 E+\infty 0$ & $1.8580000 E+\infty$ & $1.8580000 E+\infty$ & $1.8580000 E+\infty 0$ & $\mathrm{~m} / \mathrm{m}$ & WP035597 & $Y$ & $N / A$ \\
\hline 3022 & 1,3 & CONSTANT & $3.0900000 E+00$ & $3.0900000 E+\infty 0$ & $3.0900000 E+\infty 0$ & $3.0900000 E+\infty 0$ & $m$ & WP030540 & $\mathbf{Y}$ & NA \\
\hline 3022 & 1,3 & CONSTANT & $3.0900000 E+\infty$ & $3.0900000 E+\infty$ & $3.0500000 \mathrm{E}+\infty$ & $3.09000000 E+\infty$ & $m$ & WP030994 & $\mathbf{Y}$ & NA \\
\hline 3022 & 1,3 & CONSTANT & $3.0900000 E+\infty$ & $3.09000000 E+00$ & $3.0900000 E+\infty$ & $3.0900000 \mathrm{E}+\infty 0$ & $\mathbf{m}$ & WP035597 & $\mathbf{Y}$ & N/A \\
\hline 3022 & 1,3 & CONSTANT & $3.0900000 \mathrm{E}+\infty 0$ & $3.0900000 E+\infty$ & $3.0900000 E+\infty 0$ & $3.0900000 \mathrm{E}+00$ & $\mathbf{m}$ & WPO40514 & $\mathbf{Y}$ & NA \\
\hline 3023 & 1,3 & CONSTANT & $1.8000000 \mathrm{E}+\infty 0$ & $1.8000000 \mathrm{E}+00$ & $1.80000000+\infty 0$ & $1.8000000 E+\infty$ & $m$ & WP030540 & $\mathbf{Y}$ & NA \\
\hline 3023 & 1,3 & CONSTANT & $1.80000000 E+\infty 0$ & $1.80000000 E+\infty 0$ & $1.8000000 \mathrm{E}+\infty 0$ & $1.80000000+\infty$ & $\mathbf{m}$ & WP030994 & $\mathbf{Y}$ & NA \\
\hline 3023 & 1,3 & CONSTANT & $1.8000000 E+\infty$ & $1.8000000 E+\infty$ & $1.80000000 E+\infty$ & $1.8000000 \mathrm{E}+00$ & $m$ & WF035597 & $Y$ & NA \\
\hline 3023 & 1,3 & CONSTANT & $1.8000000 E+\infty 0$ & $1.8000000 E+\infty 0$ & $1.8000000 E+\infty$ & $1.8000000 E+\infty$ & $m$ & WPO40514 & $\mathbf{Y}$ & NA \\
\hline 3024 & 1,3 & CONSTANT & $3.5000000 E+\infty$ & $3.5000000 E+\infty$ & $3.5000000 E+00$ & $3.5000000 E+\infty 0$ & $\mathbf{m}$ & WP030640 & $\mathbf{Y}$ & NA \\
\hline 3024 & 1,3 & CONSTANT & $3.5000000 E+\infty$ & $3.5000000 E+\infty$ & $3.5000000 E+\infty$ & $3.5000000 E+\infty$ & $\mathbf{m}$ & WP030994 & $Y$ & NA \\
\hline 3024 & 1,3 & CONSTANT & $3.5000000 \mathrm{E}+\infty 0$ & $3.50000000 E+00$ & $3.5000000 \mathrm{E}+\infty$ & $3.50000000+\infty$ & $m$ & WP035597 & $\mathbf{Y}$ & NA \\
\hline 3024 & 1,3 & CONSTANT & $3.5000000 E+00$ & $3.5000000 \mathrm{E}+\infty$ & $3.5000000 E+\infty 0$ & $3.5000000 E+\infty$ & $m$ & WPO40514 & $-y$ & NA \\
\hline 3025 & 1,3 & CONSTANT & $2.3000000 E+00$ & $2.3000000 E+\infty 0$ & $2.3000000 E+00$ & $2.30000000+\infty$ & $\mathrm{m}$ & WP030640 & $Y$ & NA \\
\hline 3025 & 1,3 & CONSTANT & $2.3000000 E+\infty 0$ & $2.3000000 E+\infty$ & $2.3000000 E+\infty 0$ & $23000000 E+\infty 0$ & $m$ & WP030994 & $Y$ & NA \\
\hline 3025 & 1,3 & CONSTANT & $2.3000000 E+\infty 0$ & $2.3000000 E+00$ & $2.3000000 E+\infty$ & $2.3000000 E+\infty$ & $m$ & WP035597 & $\mathbf{Y}$ & NA \\
\hline 3025 & 1,3 & CONSTANT & $23000000 E+\infty 0$ & $2,3000000 E+\infty$ & $2.3000000 E+\infty$ & $2.3000000 E+00$ & m & WPO40514 & $\mathbf{Y}$ & NA \\
\hline 3026 & $1,3,4$ & CONSTANT & $1.1620000 \mathrm{E}+\infty$ & $1.16200000 E+\infty 0$ & $1.1620000 \mathrm{E}+\infty 0$ & $1.16200000+\infty$ & $\mathrm{mm}$ & WP030640 & $\mathbf{Y}$ & NA \\
\hline 3026 & $1,3,4$ & CONSTANT & $1.1620000 \mathrm{E}+\infty 0$ & $1.1620000 E+\infty$ & $1.1620000 E+\infty$ & $1.1620000 E+00$ & $\mathrm{~m} / \mathrm{m}$ & WP030994 & $\mathbf{Y}$ & N/A \\
\hline 3026 & $1,3,4$ & CONSTANT & $1.1620000 \mathrm{E}+\infty$ & $1.1620000 \mathrm{E}+\infty$ & $1.1620000 \mathrm{E}+\infty 0$ & $9.1620000 \mathrm{E}+\infty 0$ & $\mathrm{~m} / \mathrm{m}$ & WP035597 & 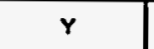 & NA \\
\hline 3027 & 4,3 & CONSTANT & $3.0900000 \varepsilon+\infty$ & $3.0900000 E+\infty$ & $3.0900000 E+00$ & $3.09000000+\infty 0$ & $\mathbf{m}$ & WPO30540 & $\mathbf{Y}$ & NA \\
\hline 3027 & 1,3 & CONSTANT & $3.0900000 E+\infty$ & $3.0900000 \mathrm{E}+\infty$ & $3.0900000 E+\infty$ & $3.09000000 E+\infty$ & $m$ & WP030994 & $Y$ & NA \\
\hline 3027 & 1,3 & CONSTANT & $3.09000000 E+\infty 0$ & $3.0900000=+\infty$ & $3.09000000=+\infty 0$ & $3.0900000 E+\infty$ & $m$ & WP035597 & $Y$ & NA \\
\hline 3027 & 1,3 & CONSTANT & $3.0900000 E+\infty$ & $3.0900000 \mathrm{E}+\infty 0$ & $3.0900000 \mathrm{E}+\infty 0$ & $3.0900000 E+\infty$ & $m$ & WPO40514 & $Y$ & MA \\
\hline
\end{tabular}


CCA Parameter Listing

\begin{tabular}{|c|c|c|c|c|c|c|c|c|c|c|}
\hline 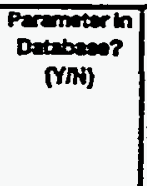 & 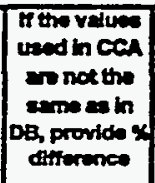 & D & Artartal io & Haterd Mams & Permatar & Parminar Rem & - & $\begin{array}{c}\text { Detentry } \\
\text { Deto }\end{array}$ & 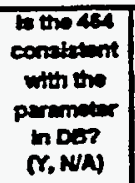 & $\begin{array}{l}\text { Parminder } \\
\text { Cutegory } \\
\text { (footrote } 1 \text { ) }\end{array}$ \\
\hline $\mathbf{Y}$ & & 3028 & CL_L_T3. & $\begin{array}{l}\text { Lower Salado clay.25 } \\
\text { to } 50 \text { years }\end{array}$ & RSH_SAL & Sexthanding ahat radius $(1.8 \mathrm{~m})$ & $32001 \mathrm{~A}$ & $15+a b-56$ & $\mathbf{Y}$ & $4 B$ \\
\hline $\mathbf{Y}$ & & 3028 & CL_L_T3 & $\begin{array}{l}\text { Lower Salaco ctay.25 } \\
\text { to } 50 \text { years }\end{array}$ & RSH_SAL & Sal handing than redies $(1.8 \mathrm{~m})$ & $32001 A$ & $15+F a b-96$ & $\mathbf{Y}$ & 48 \\
\hline $\mathbf{Y}$ & & 3028 & CL_L_T3 & $\begin{array}{l}\text { Lower Saludo ctay.25 } \\
\text { to } 50 \text { yoars }\end{array}$ & RSH_SAL & Sexthending than radies $(1.8 \mathrm{~m})$ & $32001 A$ & 15 Fab-86 & $\mathbf{Y}$ & 48 \\
\hline $\mathbf{Y}$ & & 3028 & $C L L_{-} T 3$ & $\begin{array}{l}\text { Lower Salado day.25 } \\
\text { to } 50 \text { years }\end{array}$ & RSH_SNL & Salthending that rodis $(1.8 \mathrm{~m})$ & $32001 A$ & 15 fab-96 & $\mathbf{Y}$ & $4 B$ \\
\hline $\mathbf{Y}$ & & 3029 & CL_L_T3 & $\begin{array}{l}\text { Lower Salado clay:25 } \\
\text { to } 50 \text { years }\end{array}$ & RSH_WAS & $\begin{array}{l}\text { Westo-handing shet radus (3.5) } \\
\text { (m) }\end{array}$ & 320001 & $15-F \infty b 06$ & $\mathbf{Y}$ & $4 B$ \\
\hline $\mathbf{Y}$ & & 3029 & CL_L_T3 & $\begin{array}{l}\text { Lower Saledo day:25 } \\
\text { to } 50 \text { years }\end{array}$ & RSH_WAS & 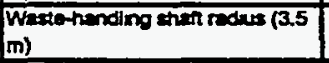 & 320001 & 15 Fab-96 & $\mathbf{Y}$ & 48 \\
\hline $\mathbf{Y}$ & & 3029 & $C L L L / T 3$ & $\begin{array}{l}\text { Lower Selaco clay:25. } \\
\text { to } 50 \text { years }\end{array}$ & RSH_WAS & $\begin{array}{l}\text { Wato-handing shet rodius (3.5 } \\
\text { (i) }\end{array}$ & 3200021 & 15 fab-96 & $\mathbf{Y}$ & 48 \\
\hline $\mathbf{Y}$ & & 3029 & CL_L_T3 & $\begin{array}{l}\text { Lower Saledo day. } 25 \\
\text { to } 50 \text { yeurs }\end{array}$ & RSH_WAS & $\begin{array}{l}\text { Westo-handing atiet radies (3.5 } \\
\text { m) }\end{array}$ & 320002 & 15 Fab-96 & $\mathbf{Y}$ & 48 \\
\hline $\mathbf{Y}$ & & 3030 & $C L_{-} L_{-} T 3$ & $\begin{array}{l}\text { Lower Salado ctay.25 } \\
\text { to } 50 \text { years }\end{array}$ & RSH_EXH & Air-extraust thent radius $(2.3 \mathrm{~m})$ & 320000 & 15 fab-s6 & $\mathbf{Y}$ & 48 \\
\hline $\mathbf{Y}$ & & 3030 & CL_L_T3 & $\begin{array}{l}\text { Lower Saledo ctay.25 } \\
\text { to } 50 \text { years }\end{array}$ & RSH_EXH & Air-extreust ethen radius (23 m) & $32000 \mathrm{~A}$ & 15 fab-96 & $\mathbf{Y}$ & 48 \\
\hline $\mathbf{Y}$ & & 3000 & $C L L L=T 3$ & $\begin{array}{l}\text { Lower Sulado day.25 } \\
\text { to } 50 \text { years }\end{array}$ & RSH_EXH & Air-excheust thant radius $(2.3 \mathrm{~m})$ & 320001 & $15-F a b-96$ & $\mathbf{Y}$ & $4 B$ \\
\hline $\mathbf{Y}$ & & 3030 & CL_L_T3 & $\begin{array}{l}\text { Lower Saledo doy } 25 \\
\text { to } 50 \text { years. }\end{array}$ & RSH_EXH & 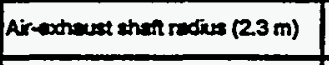 & 3200004 & 15-Fob-96 & $\mathbf{Y}$ & $4 B$ \\
\hline $\mathbf{Y}$ & & 3031 & CL_L_T3 & $\begin{array}{l}\text { Lower Salado ctay } 25 \\
\text { to } 50 \text { years }\end{array}$ & RADN_DRZ & DR2 outer radies at esch that & 31896 & 15 Feb-96 & $\mathbf{Y}$ & 1 \\
\hline $\mathbf{Y}$ & & 30031 & CL_L_T3 & $\begin{array}{l}\text { Lower Salado ctay.25 } \\
\text { to } 50 \text { years }\end{array}$ & RADN_DRZ & DRZ outer redius at eech then & 31996 & $15-F c b-98$ & $\mathbf{Y}$ & 1 \\
\hline $\mathbf{Y}$ & & 3031 & CL_L_T3 & $\begin{array}{l}\text { Lower Saledo dey: } 25 \\
\text { to } 50 \text { years }\end{array}$ & RAON_DRZ & DRZ outer radius at each shant & 31995 & 15-Fab-96 & $\mathbf{Y}$ & 1 \\
\hline $\mathbf{Y}$ & & 3032 & EARTH & Earthen Fill & RSH_AIR & Air-apply sthatt redius (3.09 m) & 32954 & $15+5 b-96$ & $\mathbf{Y}$ & $4 B$ \\
\hline $\mathbf{Y}$ & & 3032 & EARTH & Exthen Fid & RSH_AR & Air-aupply thant radius $(3.09 \mathrm{~m})$ & 329544 & 15 Fob-96 & $\mathbf{Y}$ & 48 \\
\hline $\mathbf{Y}$ & & 3032 & EARTH & Earthen Fin & RSH_AR & Airteupply shatt radies $(3.09 \mathrm{~m})$ & $32954 A$ & 15 Feb-96 & $\mathbf{Y}$ & 48 \\
\hline $\mathbf{Y}$ & & 3032 & EARTH & Earthen Fil & RSH_NR & Air-eupply thant redius $(3.09 \mathrm{~m})$ & $32954 A$ & 15-Feb-96 & $\mathbf{Y}$ & 48 \\
\hline $\mathbf{Y}$ & & 3033 & EARTH & Earthen Fill & RSH_SN & Salthanding than radius $(1.8 \mathrm{~m})$ & $32957 \mathrm{~A}$ & $15-F a b-96$ & $\mathbf{Y}$ & $4 \mathrm{~B}$ \\
\hline $\mathbf{Y}$ & & 3033 & EARTH & Earthen Fill & RSH_SAL & Salthendling sheft redius $(1.8 \mathrm{~m})$ & $32957 \mathrm{~A}$ & $15-F a b-96$ & $\mathbf{Y}$ & $4 B$ \\
\hline $\mathbf{Y}$ & & 3033 & EARTH & Eerthen Fit & RSH_SAL & Salthandling sthaft radies $(9.8 \mathrm{~m})$ & $32957 A$ & $15+c a-96$ & $\mathbf{Y}$ & 48 \\
\hline $\mathbf{Y}$ & & 3033 & EARTH & Earthen Fill & RSH_SAL & Salt handling shaft radices $(1.8 \mathrm{~m})$ & $32957 \mathrm{~A}$ & 15 Feb-96 & $\mathbf{Y}$ & 48 \\
\hline $\mathbf{Y}$ & & 3034 & EARTH & Earthen FiH & RSH_WAS & $\begin{array}{l}\text { Wasto-handling that redus (3.5 } \\
\text { m) }\end{array}$ & $32959 A$ & 15-Feb-96 & $\mathbf{Y}$ & AB \\
\hline $\mathbf{Y}$ & & 3034 & EARTH & Earthen Fill & RSH_WAS & $\begin{array}{l}\text { Waste-handling shan radus (3.5 } \\
\text { (i)) }\end{array}$ & 329594 & 15-Feb-S6 & $-\boldsymbol{\gamma}$ & $\mathbf{A B}$ \\
\hline $\mathbf{Y}$ & & 3034 & EARTH & Earthen Fill & RSH_WAS & $\begin{array}{l}\text { Wexte-handing shen radus ( } 3.5 \\
\text { m) }\end{array}$ & 329594 & 15 Fab-96 & $\mathbf{Y}$ & 48 \\
\hline $\mathbf{Y}$ & & 3034 & EARTH & Eerthen Fia & RSH_WAS & $\begin{array}{l}\text { Westo-handing shat radess (3.5 } \\
\text { (m) }\end{array}$ & $32959 A$ & $15+F a b-96$ & $\mathbf{Y}$ & $4 B$ \\
\hline $\mathbf{Y}$ & & 3035 & EARTH & Extren FT: & RSH_EXH & Ar-exthaust shent radius $(2.3 \mathrm{~m})$ & $32956 A$ & $15-F a b-96$ & $\mathbf{Y}$ & 48 \\
\hline $\mathbf{Y}$ & & 3035 & EARTH & Exthen Fid & RSH_EXH & Ar-exheust shat redius $(2.3 \mathrm{~m})$ & $32956 \mathrm{~A}$ & 15 Fab-96 & $\mathbf{Y}$ & 48 \\
\hline $\mathbf{Y}$ & & 3035 & EARTH & Earthon Fi: & RSH_EXH & Ar-exthast than radius $(23 \mathrm{~m})$ & 329564 & $15+c b-96$ & $\mathbf{Y}$ & 48 \\
\hline $\mathbf{Y}$ & & 3005 & EARTH & Eartien Fid & RSH_EXH & Ar-exheust shat radius $(2.3 \mathrm{~m})$ & 329564 & 15 Fob-96 & $\mathbf{Y}$ & 48 \\
\hline $\mathbf{Y}$ & & 30035 & EARTH & Extren Fid & RSH_EXH & Nir-exheust ahent radius $(2.3 \mathrm{~m})$ & $32956 \mathrm{~A}$ & 15 Fab-96 & $\mathbf{Y}$ & 48 \\
\hline $\mathbf{Y}$ & & 3036 & CONC_T1 & $\begin{array}{l}\text { Concrete cokinnio to } \\
400 \text { years }\end{array}$ & RSH_AR & Air-eupply shath radus (3.09 m) & $32607 A$ & 15 feb-s6 & $\mathbf{Y}$ & 48 \\
\hline $\mathbf{Y}$ & & 3036 & CONC_T1 & $\begin{array}{l}\text { Concento cokmini:0 to } \\
400 \text { yeurs }\end{array}$ & RSH_AR & Air-upply shat radius (3.09 m) & $32607 A$ & 15-Feb-96 & $\mathbf{Y}$ & $4 B$ \\
\hline $\mathbf{Y}$ & & 30036 & CONC_T1 & $\begin{array}{l}\text { Concrite column:0 to } \\
400 \text { yeurs }\end{array}$ & RSH_AYR & Air expply then radies $(3.09 \mathrm{~m})$ & $32607 A$ & 15Fab-83 & $\mathbf{Y}$ & 48 \\
\hline $\mathbf{Y}$ & & 3036 & CONC_T1 & $\begin{array}{l}\text { Concrate column:0 to } \\
400 \text { years }\end{array}$ & RSH_AR & Ar-upply chat radis (3.09 m) & $32607 A$ & 15-Fab-96 & $\mathbf{Y}$ & 48 \\
\hline
\end{tabular}


CCA Parameter Listing

\begin{tabular}{|c|c|c|c|c|c|c|c|c|c|c|}
\hline 10 & $\begin{array}{c}\text { Clatein- } \\
\text { cation of } \\
\text { Date } \\
\text { Category } \\
\text { (footrote 2) }\end{array}$ & $\begin{array}{l}\text { Diatribution } \\
\text { Type }\end{array}$ & $\operatorname{Man}$ & Median & Minkmeim & Medinum & Units & Reference id & $\begin{array}{l}\text { We the deta } \\
\text { dowbloped } \\
\text { underen } \\
\text { Nelet } \\
\text { Progrem? }\end{array}$ & 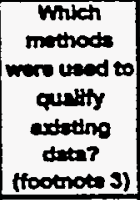 \\
\hline 3028 & 1,3 & CONSTANT & $1.8000000 E+\infty 0$ & $1.8000000 E+\infty$ & $1.8000000 E+\infty$ & $1.8000000 E+\infty 0$ & $\mathbf{m}^{\prime}$ & MP030640 & $\mathbf{Y}$ & NA \\
\hline 3028 & 1,3 & CONSTANT & $1.8000000 E+\infty 0$ & $1.8000000 E+\infty$ & $1.8000000 E+\infty$ & $1.8000000 E+\infty 0$ & $m$ & WPO30S94 & $\mathbf{Y}$ & N/A \\
\hline 3028 & 1,3 & CONSTANT & $1.8000000 E+00$ & $1.6000000 E+\infty 0$ & $1.8000000 E+\infty$ & $1.8000000 E+\infty$ & $\mathbf{m}$ & WP035597 & $Y$ & NAA \\
\hline 3028 & 1,3 & CONSTANT & $1.8000000 E+\infty 0$ & $1,8000000 E+00$ & $1.8000000 E+\infty$ & $1.8000000 E+\infty$ & $\mathbf{m}$ & WPO40514 & $Y$ & NAA \\
\hline 3029 & 1,3 & CONSTANT & $3.5000000 E+00$ & $3.5000000 E+\infty 0$ & $3.5000000 E+\infty 0$ & $3.5000000 E+\infty 0$ & m & WP030640 & $\mathbf{Y}$ & $\mathbf{N A}$ \\
\hline 3029 & 1,3 & CONSTANT & $3.5000000 E+\infty 0$ & $3.5000000 E+\infty 0$ & $3.5000000 E+\infty 0$ & $3.5000000 E+\infty$ & $m$ & WP030s9: & $\mathbf{Y}$ & NAA \\
\hline 3029 & 1,3 & CONSTANT & $3.5000000 \mathrm{E}+\infty 0$ & $3.5000000 E+00$ & $3.5000000=+\infty 0$ & $3.5000000 E+\infty$ & m & WP035597 & $\mathbf{Y}$ & NA \\
\hline 3029 & 1,3 & CONSTANT & $3.5000000 E+\infty$ & $3.5000000 E+\infty$ & $3.5000000 \leq+\infty$ & $3.5000000 E+\infty$ & $\mathbf{m}$ & WPO40514 & $\mathbf{Y}$ & NA \\
\hline 3030 & 1,3 & CONSTANT & $23000000 E+\infty 0$ & $2.3000000 E+\infty 0$ & $2.3000000 E+\infty$ & $23000000 E+\infty$ & $\mathbf{m}$ & WP030s40 & $\mathbf{Y}$ & NAA \\
\hline 3030 & 1,3 & CONSTANT & $2.3000000 E+\infty$ & $23000000 E+00$ & $2.3000000 E+00$ & $2.30000000+00$ & $m$ & WP030s94 & $\mathbf{Y}$ & NA \\
\hline 3030 & 1,3 & CONSTANT & $2.3000000 E+\infty 0$ & $2.3000000 E+\infty 0$ & $2.3000000=+\infty$ & $23000000 E+\infty$ & $m$ & WP035597 & $\mathbf{Y}$ & NA \\
\hline 3030 & 1,3 & CONSTANT & $2.3000000 E+\infty 0$ & $2.3000000 E+\infty$ & $2.3000000 E+\infty$ & $2.3000000=+\infty$ & $\mathbf{m}$ & WP040514 & $\mathbf{Y}$ & NA \\
\hline 3031 & $1,3,4$ & CONSTANT & $1.0020000 E+\infty 0$ & $1.0020000 E+\infty 0$ & $1.0020000 E+\infty$ & $1.0020000 E+00$ & $\mathrm{~m} / \mathrm{m}$ & WPO30640 & $\mathbf{Y}$ & NA \\
\hline 3031 & $1,3,4$ & CONSTANT & $1.0020000 E+00$ & $1.0020000 E+\infty$ & $1.0020000 E+00$ & $1.0020000 E+\infty 0$ & $\mathrm{~m} / \mathrm{m}$ & WPOS0994 & $\mathbf{Y}$ & NA \\
\hline 3031 & $1,3,4$ & CONSTANT & $1.0020000 E+00$ & $1.0020000 E+\infty$ & $1.0020000 E+\infty$ & $1.0020000=+\infty$ & $\mathrm{m} / \mathrm{m}$ & WP035597 & $\mathbf{Y}$ & NA \\
\hline 3032 & 1,3 & CONSTANT & $3.0900000 E+00$ & $3.0900000 E+\infty 0$ & $3.0900000 E+\infty 0$ & $3.0900000 E+\infty 0$ & m & WP030540 & $\mathbf{Y}$ & NA \\
\hline 3032 & 1,3 & CONSTANT & $3.0900000 E+00$ & $3.0900000 E+\infty$ & $3.0900000 E+\infty$ & $30900000 E+\infty$ & $m$ & WP030994 & $\mathbf{Y}$ & $N A$ \\
\hline 3032 & 1,3 & CONSTANT & $3.0900000 E+00$ & $3.0900000 E+\infty$ & $3.0900000 E+00$ & $30900000 E+\infty$ & $\mathbf{m}$ & WP035597 & $\mathbf{Y}$ & NAA \\
\hline 3032 & 1,3 & CONSTANT & $3.0900000 E+\infty 0$ & $3.0900000 E+\infty 0$ & $3.0000000 \in+\infty)$ & $3.0500000 E+\infty$ & $m$ & MPO40514 & $\mathbf{Y}$ & NAA \\
\hline 3033 & 1,3 & CONSTANT & $1.8000000 E+\infty 0$ & $1.8000000 E+\infty 0$ & $18000000+\infty$ & $18000000 E+\infty$ & $m$ & WPO30540 & $\mathbf{Y}$ & N/A \\
\hline 3033 & 1,3 & CONSTANT & $1.8000000 E+00$ & $1.8000000 E+\infty 0$ & $18000000 E+\infty$ & $18000000 E+\infty$ & $m$ & WP030994 & $\mathbf{Y}$ & N/A \\
\hline 3033 & 1,3 & CONSTANT & $1.8000000 E+\infty 0$ & $1.8000000 E+00$ & $18000000=+\infty$ & $10000000 E+\infty$ & $\mathrm{m}$ & WP035597 & $\mathbf{Y}$ & NA \\
\hline 3033 & 1,3 & CONSTANT & $1.8000000 E+\infty 0$ & $18000000 E+00$ & $18000000 \times+\infty$ & $18000000 E+\infty$ & $\mathrm{m}$ & WP040514 & $Y$ & NAA \\
\hline 3034 & 1,3 & CONSTANT & $3.5000000 E+\infty 0$ & $35000000 E+\infty$ & $35000000<-\infty$ & $35000000 E+\infty$ & $\mathbf{m}$ & WP030640 & $Y$ & NA \\
\hline 3034 & 1,3 & CONSTANT & $3.5000000 E+\infty 0$ & $35000000 E+\infty$ & $35000000 \times \infty \infty$ & $35000000 \times+00$ & m & WP030994 & $-Y$ & NA \\
\hline 3034 & 1,3 & CONSTANT & $3.5000000 E+\infty$ & $3.5000000 E+\infty$ & $35000000<-\infty 0$ & $35000000 \mathrm{E}+\infty$ & $m$ & WP035597 & $\mathbf{Y}$ & NA \\
\hline 3034 & 1,3 & CONSTANT & $3.5000000 E+00$ & $35000000 E+\infty$ & I s000000e $+\infty$ & $35000000=+\infty$ & $\mathbf{m}$ & WP040514 & $\mathbf{Y}$ & N/A \\
\hline 3035 & 1,3 & CONSTANT & $2.3000000 E+00$ & $23000000 \leq-\infty 0$ & $23000000 \leq-\infty$ & $23000000 E+00$ & m & WP030640 & $\mathbf{Y}$ & N/A \\
\hline 3035 & 1,3 & CONSTANT & $2.3000000 E+\infty$ & $2.3000000 E+\infty$ & $230000000-\infty$ & $23000000 \times+\infty$ & $\mathbf{m}$ & WPO30994 & $\mathbf{Y}$ & $N A$ \\
\hline 3035 & 1,3 & CONSTANT & $2.3000000 E+\infty 0$ & $2.3000000 E+\infty$ & $23000000 \times-\infty$ & $23000000 \times+\infty$ & $m$ & WP035333 & $\mathbf{Y}$ & $N A$ \\
\hline 3035 & 1,3 & CONSTANT & $2.30000005+\infty$ & $2.3000000 \leqslant+\infty$ & $230000 \times<\infty$ & $23000000<+\infty$ & $m$ & WP035597 & $\mathbf{Y}$ & $N A$ \\
\hline 3035 & 1,3 & CONSTANT & $2.3000000 E+00$ & $2,3000000 E+\infty 0$ & $23000000<\cdot 00$ & $23000000=+\infty$ & $m$ & WPO40514 & $\mathbf{Y}$ & NA \\
\hline 3036 & 1,3 & CONSTANT & $3.0900000 E+\infty 0$ & $30900000 E+\infty 0$ & $30000000 \times+\infty$ & $30,00000 \times+\infty$ & $m$ & WP030640 & $\mathbf{Y}$ & N/A \\
\hline 3036 & 1,3 & CONSTANT & $3.0900000 E+\infty 0$ & $3.0500000 E+00$ & $30000000 \times-\infty$ & $30000000 \leq+\infty$ & $m$ & WP030994 & $\mathbf{Y}$ & NA \\
\hline 3036 & 1,3 & CONSTANT & $3.0900000 E+\infty 0$ & $30900000<+\infty 0$ & $30,00000<-00$ & $3000000<+\infty$ & m & WP035597 & $\mathbf{Y}$ & NA \\
\hline 3036 & 1,3 & CONSTANT & $3.0900000 E+\infty 0$ & $3.09000000+00$ & $30000000 E-\infty 0$ & $30000000 E+\infty$ & m & WPO40514 & $\mathbf{Y}$ & NA \\
\hline
\end{tabular}


CCA Parameter Listing

\begin{tabular}{|c|c|c|c|c|c|c|c|c|c|c|}
\hline $\begin{array}{c}\text { Pomoter in } \\
\text { Detsbeco? } \\
\text { (InN) }\end{array}$ & 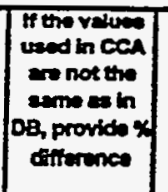 & प10 & Mrtarlatio & Mrtarla Name & Peromotion & Permoter Mrm & $\begin{array}{l}\text { PRPDD } \\
\text { MPOM }\end{array}$ & $\begin{array}{c}\text { Dete Entry } \\
\text { Dato }\end{array}$ & 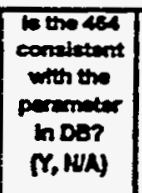 & 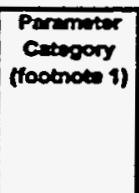 \\
\hline $\mathbf{Y}$ & & 3007 & CONC_TI & $\begin{array}{l}\text { Concrete colkmnto to } \\
400 \text { years }\end{array}$ & RSH_SNL & Sethimeling shat radies $(1.8 \mathrm{~m})$ & $32609 n$ & $15+06-96$ & $\mathbf{Y}$ & 48 \\
\hline $\mathbf{Y}$ & & 3037 & CONC_Ti & $\begin{array}{l}\text { Conceste colemn: } 0 \text { to } \\
400 \text { vers }\end{array}$ & RSH_SNL & Salk hawnding athat radius $(1.8 \mathrm{~m}) \mid$ & 326094 & 15 feb-96 & $Y$ & $4 B$ \\
\hline$Y$ & & 3037 & CONC_TI & $\begin{array}{l}\text { Concrote columito to } \\
400 \text { years }\end{array}$ & RSH_SAL & Sell handting ghast radive $(1.8 \mathrm{~m})$ & 326009 & $15-F a b-96$ & $\mathbf{Y}$ & 48 \\
\hline $\mathbf{Y}$ & & 3007 & CONC_Ts & $\begin{array}{l}\text { Concrote colemn:0 to } \\
400 \text { years }\end{array}$ & RSH_SNL & Salthending shent recius $(1.8 \mathrm{~m})$ & 320009 & $15+c b-96$ & $\mathbf{Y}$ & 48 \\
\hline $\mathbf{Y}$ & & 3038 & CONC_TI & $\begin{array}{l}\text { Concrete columnio to } \\
400 \text { yours }\end{array}$ & RSH_WAS & $\begin{array}{l}\text { Wastohanding ather reden (3.5 } \\
\text { (m) }\end{array}$ & $32617 A$ & $15+\infty 0-96$ & Y & 48 \\
\hline $\mathbf{Y}$ & & 3038 & CONC_TI & $\begin{array}{l}\text { Concreto colkmn:0 to } \\
400 \text { years }\end{array}$ & RSH_WAS & $\begin{array}{l}\text { Wartohending ghen raders (3.5 } \\
\text { m) }\end{array}$ & $32617 \mathrm{~A}$ & 15 fab-96 & $\mathbf{Y}$ & 48 \\
\hline $\mathbf{Y}$ & & 3038 & CONC_T1 & $\begin{array}{l}\text { Concrete colkmn:0 to } \\
400 \text { years }\end{array}$ & RSH_WAS & $\begin{array}{l}\text { Wastohanding anstit radus (3.5 } \\
\text { m) }\end{array}$ & $32617 A$ & $15-F a b-96$ & $\mathbf{Y}$ & $4 B$ \\
\hline $\mathbf{Y}$ & & 3038 & CONC_T1 & $\begin{array}{l}\text { Concrote colemnio to } \\
400 \text { yeers }\end{array}$ & RSH_WAS & $\begin{array}{l}\text { Westo-hending shat rodius (3.5 } \\
\text { m) }\end{array}$ & $32817 \mathrm{~A}$ & $15-F a b-96$ & $\mathbf{Y}$ & $4 B$ \\
\hline$Y$ & & 3039 & CONC_T1 & $\begin{array}{l}\text { Concrete colemn:0 to } \\
400 \text { years }\end{array}$ & RSH_EXH & Air-extrecest strat redies $(2.3 \mathrm{~m})$ & 326004 & $15+00-96$ & $\mathbf{Y}$ & 48 \\
\hline $\mathbf{Y}$ & & 3039 & CONC_T1 & $\begin{array}{l}\text { Concrete column:0 to } \\
400 \text { years }\end{array}$ & RSH_EXH & Ar-wheust shet raciess $(23 \mathrm{~m})$ & 326004 & 15 fab-s6 & $\mathbf{Y}$ & 48 \\
\hline $\mathbf{Y}$ & & 3039 & CONC_T1 & $\begin{array}{l}\text { Concrote column:0 to } \\
400 \text { years }\end{array}$ & RSH_EXH & Air-echeust athen radies $(2.3 \mathrm{~m})$ & $325000 \mathrm{~A}$ & $15-F a b-96$ & $Y$ & $4 B$ \\
\hline $\mathbf{Y}$ & & 3039 & CONC_T1 & $\begin{array}{l}\text { Concrote column:0 to } \\
400 \text { years }\end{array}$ & RSH_EXH & Ar-echoust ahath redies $(2.3 \mathrm{~m})$ & $32608 A$ & 15 Fob-96 & - $\mathbf{Y}$ & 48 \\
\hline $\mathbf{Y}$ & & 3039 & CONC_T1 & $\begin{array}{l}\text { Concrete column:0 to } \\
400 \text { years }\end{array}$ & RSH_EXH & Air-extaust athen ractius (23 m) & $32608 \mathrm{~A}$ & 15 feb-96 & $\mathbf{Y}$ & 48 \\
\hline$Y$ & & 3039 & CONC_T1 & $\begin{array}{l}\text { Concrete coirmn:0 to } \\
400 \text { yeums }\end{array}$ & RSH_EXH & Air-exhaust shat ractivs $(2.3 \mathrm{~m})$ & $32608 \mathrm{~A}$ & $15+5 b-96$ & $\mathbf{Y}$ & 48 \\
\hline $\mathbf{r}$ & & 3040 & CONC_T1 & $\begin{array}{l}\text { Concrote column to to } \\
400 \text { years }\end{array}$ & RADN_DRZ & DRZ outer redies at wexh strent & 32500 & 15-fob-96 & $\mathbf{\gamma}$ & 1 \\
\hline $\mathbf{Y}$ & & 3040 & CONC_TI & $\begin{array}{l}\text { Concrote column:0 to } \\
400 \text { yeers }\end{array}$ & RADN_DRZ & DRZ outer redius at asch strim & 32600 & $15+0 b-96$ & $\mathbf{Y}$ & 9 \\
\hline $\mathbf{Y}$ & & 3040 & CONC_TI & $\begin{array}{l}\text { Concrete columnt: to } \\
400 \text { years }\end{array}$ & RADN_ORZ & DRZ outer radies at eech shan & 32600 & $15-F e b-96$ & $\mathbf{Y}$ & 1 \\
\hline $\mathbf{Y}$ & & 3041 & CONC_T2 & $\begin{array}{l}\text { Concrete cokmn:400 } \\
\text { to } 10 \mathrm{~K} \text { years }\end{array}$ & RSH_AR & Air-eupply shath redius $(3.09 \mathrm{~m})$ & 326704 & 15-Fob-96 & $\mathbf{Y}$ & AB \\
\hline $\mathbf{Y}$ & & 3041 & CONC_T2 & $\begin{array}{l}\text { Concrote column:400 } \\
\text { to } 10 \text { K years }\end{array}$ & RSH_AR & Air-eupply sheff radius $(3.09 \mathrm{~m})$ & 326704 & 15 feb-96 & $\mathbf{Y}$ & $A B$ \\
\hline $\mathbf{Y}$ & & 3041 & CONC_T2 & $\begin{array}{l}\text { Concrete column:400 } \\
\text { to } 10 \mathrm{~K} \text { yours }\end{array}$ & RSH_AR & Air-supply shot redius $(3.09 \mathrm{~m})$ & 326704 & $15-F 0 b-96$ & $\mathbf{Y}$ & 48 \\
\hline $\mathbf{Y}$ & & 3041 & CONC_T2 & $\begin{array}{l}\text { Concrete column: } 400 \\
\text { to } 10 \mathrm{~K} \text { yoars }\end{array}$ & RSH_AR & Ar-eupply athen redies $(3.09 \mathrm{~m})$ & 326704 & 15-Feb-96 & $\mathbf{Y}$ & 48 \\
\hline $\mathbf{Y}$ & & 3042 & CONC_T2 & $\begin{array}{l}\text { Concrets colkmn: } 400 \\
\text { to } 10 \mathrm{~K} \text { years }\end{array}$ & RSH_SN & Senthending shen radies $(1.8 \mathrm{~m})$ & 326724 & $15-F-b-96$ & $\mathbf{Y}$ & 48 \\
\hline$Y$ & & 3042 & CONC_T2 & $\begin{array}{l}\text { Concrots column } 400 \\
\text { to } 10 \mathrm{~K} \text { years }\end{array}$ & RSH_SN & Sarthending shath radius $(1.8 \mathrm{~m})$ & $32672 A$ & $15+60 b-96$ & $\mathbf{Y}$ & 18 \\
\hline$Y$ & & 3012 & CONC_T2 & $\begin{array}{l}\text { Concrete column'400 } \\
\text { to } 10 \mathrm{~K} \text { yeims }\end{array}$ & RSH_SN & Sex-hending enat radus $(1.8 \mathrm{~m})$ & $32572 A$ & 15 Fab-96 & $\mathbf{Y}$ & 48 \\
\hline $\mathbf{Y}$ & & 3042 & CONC_T2 & $\begin{array}{l}\text { Concrote column:400 } \\
\text { to } 10 \mathrm{~K} \text { years }\end{array}$ & RSH_SN & Sakt hamoling shat radivs $(1.8 \mathrm{~m})$ & $32672 A$ & 15 Fob-96 & $\mathbf{Y}$ & $4 B$ \\
\hline $\mathbf{Y}$ & & 3012 & CONC_T2 & $\begin{array}{l}\text { Concrote column:400 } \\
\text { to } 10 \mathrm{~K} \mathrm{yedr}\end{array}$ & RSH_SNL & Sehthending shat redius $(1.8 \mathrm{~m})$ & $32572 A$ & $15-50 b-96$ & $\mathbf{Y}$ & $4 B$ \\
\hline $\mathbf{Y}$ & & 3043 & CONC_T2 & $\begin{array}{l}\text { Concrute column: } 400 \\
\text { to } 10 \mathrm{~K} \text { yeers }\end{array}$ & RSH_WAS & $\begin{array}{l}\text { Whatohanding ghan redivs (3.5 } \\
\text { m) }\end{array}$ & $32673 A$ & $15+0 b-96$ & $\mathbf{Y}$ & 48 \\
\hline $\mathbf{Y}$ & & 3043 & CONC_T2 & $\begin{array}{l}\text { Concrete column:400 } \\
\text { to 10K years }\end{array}$ & RSH_WAS & $\begin{array}{l}\text { Wasto-hending enam radies (3.5 } \\
\text { m) }\end{array}$ & $32673 A$ & $15+506-96$ & $\mathbf{Y}$ & $4 B$ \\
\hline $\mathbf{Y}$ & & 3043 & CONC_ 72 & $\begin{array}{l}\text { Concrule colemn } 400 \\
\text { to } 10 \% \text { years }\end{array}$ & RSH_WAS & $\begin{array}{l}\text { Wasto-handing strent radius (3.5 } \\
\text { m) }\end{array}$ & $32673 A$ & 15 fab-96 & $\mathbf{Y}$ & 48 \\
\hline $\mathbf{Y}$ & & 3043 & CONC_T2 & $\begin{array}{l}\text { Concrote column: } 400 \\
\text { to 10K yours }\end{array}$ & RSH_WAS & $\begin{array}{l}\text { Wasto-handing athan redies } 13.5 \\
\text { (n) }\end{array}$ & $32673 A$ & $15-F=b-96$ & $\mathbf{Y}$ & AB \\
\hline
\end{tabular}


CCA Parameter Listing

\begin{tabular}{|c|c|c|c|c|c|c|c|c|c|c|}
\hline 10 & 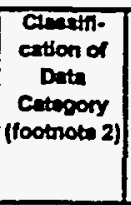 & $\begin{array}{l}\text { Diatulbertion } \\
\text { Type }\end{array}$ & Men & Medien & Mtritrum & Maximem & Conts & Porfermen iD & $\begin{array}{l}\text { Wea the dinta } \\
\text { doveloped } \\
\text { underen } \\
\text { Noh-1 } \\
\text { Program? }\end{array}$ & 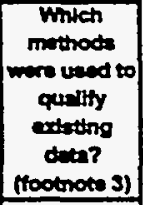 \\
\hline 3037 & 1,3 & CONSTANT & $1.8000000 \mathrm{E}+\infty 0$ & $1.80000000+\infty$ & $1.8000000 E+\infty$ & $1.8000000 \mathrm{E}+\infty 0$ & $m$ & WPO30540 & $Y$ & NA \\
\hline 3037 & 1,3 & CONSTANT & $1.8000000 E+\infty 0$ & $1.80000000+\infty$ & $1.8000000 E+\infty 0$ & $1.8000000 E+\infty 0$ & m & WP030094 & $\mathbf{Y}$ & NAA \\
\hline 3037 & 1,3 & CONSTANT & $1.8000000 E+\infty 0$ & $1.8000000 E+\infty$ & $1.8000000 E+\infty$ & $1.8000000 \mathrm{E}+\infty 0$ & $m$ & WP035597 & $Y$ & NA \\
\hline 3037 & 1,3 & CONSTANT & $1.8000000 \mathrm{E}+\infty 0$ & $1.80000000 \mathrm{E}+\infty$ & $1.8000000 E+\infty$ & $1.8000000 E+\infty$ & $\mathbf{m}$ & WPO40514 & $\mathbf{Y}$ & NA \\
\hline 3038 & 1.3 & CONSTANT & $3.5000000 E+\infty 0$ & $3.5000000 E+\infty$ & $3.5000000 E+\infty$ & $3.5000000 E+\infty 0$ & m & WPO30640 & $Y$ & NA \\
\hline 3038 & 1,3 & CONSTANT & $3.5000000 E+\infty 0$ & $3.5000000 E+\infty$ & $3.5000000 E+00$ & $3.5000000 E+\infty$ & $\mathbf{m}$ & WP030994 & $\mathbf{Y}$ & NA \\
\hline 3038 & 1,3 & CONSTANT & $3.5000000 E+00$ & $3.5000000 E+00$ & $3.5000000 E+00$ & $3.5000000 \leq+\infty 0$ & $m$ & WP035597 & Y & NA \\
\hline 3038 & 1,3 & CONSTANT & $3.5000000 E+\infty 0$ & $3.5000000 E+00$ & $3.5000000 E+00$ & $3.5000000 E+\infty$ & $\mathbf{m}$ & WPO40514 & $\mathbf{Y}$ & NA \\
\hline 3039 & 1,3 & CONSTANT & $23000000 E+\infty 0$ & $23000000 E+\infty 0$ & $2.3000000 \mathrm{E}+\infty 0$ & $2.3000000 E+\infty 0$ & $m$ & WP030540 & Y & NA \\
\hline 3039 & 1,3 & CONSTANT & $2.3000000 E+00$ & $2.3000000 E+\infty 0$ & $23000000 E+\infty$ & $2.3000000 E+\infty 0$ & $m$ & WP030994 & $Y$ & NA \\
\hline 3039 & 1,3 & CONSTANT & $2.3000000 E+00$ & $23000000 E+\infty$ & $2,3000000 E+\infty$ & $2.3000000 E+\infty 0$ & m & WP032287 & $\dot{Y}$ & NA \\
\hline 3039 & 1,3 & CONSTANT & $2.3000000 E+00$ & $2.3000000 E+\infty 0$ & $2.3000000 E+\infty$ & $2.3000000 \mathrm{E}+\infty 0$ & m & WP035268 & $\mathbf{Y}$ & NA \\
\hline 3039 & 1,3 & CONSTANT & $2.3000000 E+00$ & $2.3000000 E+\infty 0$ & $2.3000000 E+\infty 0$ & $2.3000000 E+\infty$ & $\mathbf{m}$ & WP035597 & $\mathbf{Y}$ & NA \\
\hline 3039 & 1.3 & CONSTANT & $23000000 E+00$ & $2.3000000 E+00$ & $2.3000000 E+00$ & $2.3000000 E+\infty 0$ & $\mathbf{m}$ & WP040514 & $\mathbf{Y}$ & NA \\
\hline 3040 & $1,3,4$ & CONSTANT & $1.0000000 E+\infty 0$ & $1.0000000 E+\infty 0$ & $1.0000000 E+\infty 0$ & $1.0000000 E+\infty$ & $\mathrm{m} / \mathrm{m}$ & WP030640 & $\mathbf{Y}$ & NA \\
\hline 3040 & $1,3,4$ & CONSTANT & $1.0000000 E+00$ & $1.0000000 E+\infty 0$ & $1.0000000 E+\infty 0$ & $1.0000000 E+00$ & $\mathrm{~m} / \mathrm{m}$ & WP030994 & $Y$ & NA \\
\hline 3040 & $1,3,4$ & CONSTANT & $1.0000000 E+\infty 0$ & $1.0000000 E+\infty 0$ & $1.0000000 \mathrm{E}+\infty 0$ & $1.0000000 E+\infty 0$ & $\mathrm{~m} / \mathrm{m}$ & WP035597 & $\mathbf{Y}$ & NA \\
\hline 3049 & 1,3 & CONSTANT & $3.0900000 E+\infty$ & $3.0900000 E+\infty 0$ & $3.0900000 E+\infty 0$ & $3.0900000 E+00$ & $\mathbf{m}$ & WP030640 & $Y$ & NA \\
\hline 3041 & 1,3 & CONSTANT & $3.0900000 \mathrm{E}+\infty$ & $3.0900000 \mathrm{E}+\infty 0$ & $3.0500000 \mathrm{E}+00$ & $3.0900000 E+\infty 0$ & $\mathbf{m}$ & WP030994 & $\mathbf{Y}$ & NA \\
\hline 3041 & 1,3 & CONSTANT & $3.0900000 E+\infty 0$ & $3.0900000 \mathrm{E}+00$ & $3.0900000 \mathrm{E}+00$ & $3.0900000 E+\infty$ & $\mathbf{m}$ & WP035597 & $\mathbf{Y}$ & NA \\
\hline 3041 & 1,3 & CONSTANT & $3.0900000 E+\infty 0$ & $3.0900000 E+\infty 0$ & $3.0800000 E+\infty 0$ & $3.0900000 \mathrm{E}+\infty 0$ & $m$ & WP040514 & $\mathbf{Y}$ & NA \\
\hline 3042 & 1.3 & CONSTANT & $1.8000000 E+\infty$ & $1.8000000 E+\infty$ & $1.8000000 E+00$ & $1.8000000 E+00$ & $\mathbf{m}$ & WP030540 & $\mathbf{Y}$ & N/A \\
\hline 3042 & 1,3 & CONSTANT & 1. $80000000 E+\infty 0$ & $1.8000000 E+\infty 0$ & $1.8000000 \mathrm{E}+\infty 0$ & $1.8000000 \mathrm{E}+00$ & $m$ & WP030994 & $\mathbf{Y}$ & NA \\
\hline 3042 & 1,3 & CONSTANT & $1.8000000 E+\infty$ & $1.8000000 E+\infty$ & $1.8000000 E+\infty$ & $1.8000000 E+\infty$ & $\mathbf{m}$ & WP035268 & $\mathbf{Y}$ & N/A \\
\hline 3042 & 1,3 & CONSTANT & $1.8000000 E+00$ & $1.8000000 E+\infty 0$ & $1.8000000 E+\infty 0$ & $1.8000000 E+\infty$ & m & WP035597 & $\mathbf{Y}$ & NA \\
\hline 3042 & 1,3 & CONSTANT & $1.6000000 E+\infty 0$ & $1.80000000 E+00$ & $1.8000000 E+\infty 0$ & $1.8000000 \mathrm{E}+\infty$ & $m$ & WP040514 & $\mathbf{Y}$ & N/A \\
\hline 3043 & 1.3 & CONSTANT & $3.5000000 E+\infty 0$ & $3.5000000 E+\infty$ & $3.5000000 \mathrm{E}+\infty 0$ & $3.50000000 E+\infty$ & $m$ & WP030640 & $\mathbf{Y}$ & NA \\
\hline 3043 & 1,3 & CONSTANT & $3.5000000 E+00$ & $3.5000000 E+\infty$ & $3.5000000 E+\infty$ & $3.5000000 E+\infty$ & $m$ & WP030994 & $\mathbf{Y}$ & NA \\
\hline 3043 & 1,3 & CONSTANT & $3.5000000 E+\infty$ & $3.5000000 \mathrm{E}+\infty 0$ & $3.50000000 \mathrm{E}+\infty 0$ & $3.50000000 E+\infty$ & m & WP035597 & $\mathbf{Y}$ & N/A \\
\hline 3043 & 1,3 & CONSTANT & $3.5000000 E+\infty$ & $3.5000000 E+\infty$ & $3.5000000 E+00$ & $3.5000000 E+\infty$ & m & UP040514 & $\mathbf{Y}$ & N/A \\
\hline
\end{tabular}


CCA Parameter Listing

\begin{tabular}{|c|c|c|c|c|c|c|c|c|c|c|}
\hline $\begin{array}{c}\text { Permitir in } \\
\text { Detabeise? } \\
\text { (YN) }\end{array}$ & 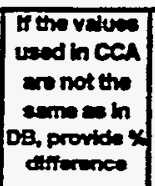 & DD & Matuda & 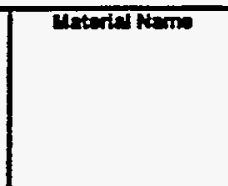 & Peremeter & Parenotion Nom & $\begin{array}{l}\text { PRPD DD } \\
\text { MIPO }\end{array}$ & $\begin{array}{c}\text { Defontry } \\
\text { Dente }\end{array}$ & 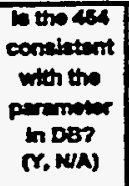 & 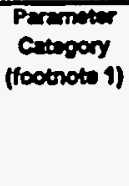 \\
\hline $\mathbf{Y}$ & & 3044 & CONC_T2 & $\begin{array}{l}\text { Concrote column: } 400 \\
\text { to } 10 \mathrm{~K} \text { yours }\end{array}$ & RSH_EXH & Ar-axtrevest shat radies $(2.3 \mathrm{~m})$ & $32571 \mathrm{~A}$ & $15-=b-96$ & $\mathbf{Y}$ & 48 \\
\hline $\mathbf{Y}$ & & 3044 & CONC_T2 & $\begin{array}{l}\text { Concrete column:400 } \\
\text { to } 10 \mathrm{~K} \text { years }\end{array}$ & RSH_EXH & Air-ectroust athant redess $(2.3 \mathrm{~m})$ & $32671 \mathrm{~A}$ & $15-a b-96$ & $Y$ & 48 \\
\hline $\mathbf{Y}$ & & 3044 & CONC_T2 & $\begin{array}{l}\text { Concrete column:400 } \\
\text { to 10K years }\end{array}$ & RSH_EXH & 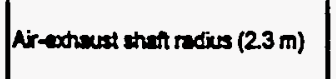 & $32671 A$ & $15+f e b-96$ & $\mathbf{Y}$ & 48 \\
\hline Y & & 3044 & CONC_T2 & $\begin{array}{l}\text { Concrote column: } 400 \\
\text { to } 10 \mathrm{~K} \text { years }\end{array}$ & RSH_EXH & Ar-axheust than redius $(23 \mathrm{~m})$ & $32671 \mathrm{~A}$ & $15-F 0 b-96$ & $\mathbf{Y}$ & 48 \\
\hline$Y$ & & 3044 & CONC_T2 & $\begin{array}{l}\text { Concroto column: } 400 \\
\text { to } 10 \mathrm{~K} \text { years }\end{array}$ & RSH_EXH & Ar-whened shant radius $(2.3 \mathrm{~m})$ & $32671 A$ & 15 Fab-86 & Y & $4 B$ \\
\hline $\mathbf{Y}$ & & 3045 & CONC_T2 & $\begin{array}{l}\text { Concrole column: } 400 \\
\text { to } 10 \mathrm{~K} \text { yoars }\end{array}$ & RADN_ORZ & DRZ outer redies at each that & 32657 & $15-F 0 b-96$ & $\mathbf{Y}$ & 1 \\
\hline $\mathbf{Y}$ & & 3045 & CONC_T2 & $\begin{array}{l}\text { Concroto cokmn: } 400 \\
\text { to } 10 \mathrm{~K} \text { years }\end{array}$ & RADN_DRZ & DRZ octer recius at each shent & 32657 & $15-0 b-96$ & $\mathbf{Y}$ & 1 \\
\hline $\mathbf{Y}$ & & 3045 & CONC_T2 & $\begin{array}{l}\text { Concrete column:400 } \\
\text { to } 10 \mathrm{~K} \text { years }\end{array}$ & RADN_DRZ & DRZ outer radus at exch shat & 32667 & 15 Fob- 96 & $\mathbf{Y}$ & 1 \\
\hline $\mathbf{Y}$ & & 3045 & CONC_T2 & $\begin{array}{l}\text { Concreto columint } 400 \\
\text { to 10K years }\end{array}$ & RADN_DRZ & DRZ outer radius at each ethath & 32667 & 15 fab-96 & $\mathbf{Y}$ & 1 \\
\hline $\mathbf{Y}$ & & 3046 & CONC_MON & Concrete Monolith & RSH_AIR & Air-eupply ahath radius $(3.09 \mathrm{~m})$ & 325324 & $15-F a b-96$ & $\mathbf{Y}$ & 48 \\
\hline $\mathbf{Y}$ & & 3045 & CONC_MON & Concreto Monolith & RSH_AR & Air-supply that radies (3.09 m) & $32532 A$ & $15+a b-96$ & $\mathbf{Y}$ & $4 B$ \\
\hline $\mathbf{Y}$ & & 3046 & CONC_MON & Concreto Monolith & RSH_AIR & Air-eupply then recius $(3.09 \mathrm{~m})$ & $32532 A$ & $15+00-96$ & $\mathbf{Y}$ & $A B$ \\
\hline$Y$ & & 3046 & CONC_MON & Concreto Monolith & RSH_AR & Ar-apply sthat radius (3.09 m) & $32532 A$ & $15-f c b-96$ & $\mathbf{Y}$ & $4 B$ \\
\hline $\mathbf{Y}$ & & 3047 & CONC_MON & Concrete Monolith & RSH_SAL & Salthanding shan radius $(1.8 \mathrm{~m})$ & $32537 \mathrm{~A}$ & 15 feb-96 & $\mathbf{Y}$ & 48 \\
\hline $\mathbf{Y}$ & & 3047 & CONC_MON & Concrete Monolith & RSH_SAL & Salthanding athent radius $(1.8 \mathrm{~m})$ & $32537 \mathrm{~A}$ & $15-F \infty b-96$ & $\mathbf{Y}$ & $A B$ \\
\hline Y & & 3047 & CONC_MON & Concrete Monolith & RSH_SAL & Salt thending shen radius $(1.8 \mathrm{~m})$ & $32537 \mathrm{~A}$ & $15+6 b-96$ & Y & AB \\
\hline $\mathbf{Y}$ & & 3047 & CONC_MON & Concrote Monolith & RSH_SAL & Salt thanding shat radius $(1.8 \mathrm{~m})$ & 32537A & 15-feb-96 & $Y$ & $A B$ \\
\hline $\mathbf{Y}$ & & 3048 & CONC_MON & Concrete Monolith & RSH_WAS & $\begin{array}{l}\text { Wast thendling ahan radius (3.5 } \\
\text { (i)) }\end{array}$ & $32538 A$ & $15-$ Feb-96 & $Y$ & 48 \\
\hline $\mathbf{Y}$ & & 3048 & CONC_MON & Concrete Honolith & RSH_WAS & $\begin{array}{l}\text { Wasto-handing shat radrus (3.5 } \\
\text { (m) }\end{array}$ & $32538 A$ & $15-f a b-96$ & $\mathbf{Y}$ & $A B$ \\
\hline $\mathbf{Y}$ & & 3048 & CONC_MON & Concrete Monolith & RSH_WAS & $\begin{array}{l}\text { Wastohandling shaft radus (3.5 } \\
\text { m) }\end{array}$ & $32538 A$ & 15 -Feb-96 & $Y$ & AB \\
\hline $\mathbf{Y}$ & & 3048 & CONC_MON & Concrete Monolith & RSH_WAS & \begin{tabular}{|l|}
$\begin{array}{l}\text { Wasto-handing shaft radus (3.5 } \\
\text { m) }\end{array}$ \\
\end{tabular} & 325384 & 15 Feb-96 & $\mathbf{Y}$ & AB \\
\hline $\mathbf{Y}$ & & 3049 & CONC_MON & Concrete Monolith & RSH_EXH & Ar-exheust entan radius $(23 \mathrm{~m})$ & 325344 & $15-F$ eb-96 & $\mathbf{Y}$ & $A B$ \\
\hline $\mathbf{Y}$ & & 3049 & CONC_MON & Concrete Monolith & RSH_EXH & Ar-extheurs thent radius $(2.3 \mathrm{~m})$ & $32534 A$ & $15-F a b-96$ & $\mathbf{Y}$ & 48 \\
\hline $\mathbf{Y}$ & & 3049 & CONC_MON & Concrete Monolith & RSH_EXH & Ar-wheust ethat radius $(2.3 \mathrm{~m})$ & 325344 & 15 fob-96 & $\mathbf{Y}$ & AB \\
\hline $\mathbf{Y}$ & & 3049 & CONC_MON & Conerete Morolith & RSH_EXH & Arrectheust etheft radius $(2.3 \mathrm{~m})$ & 325344 & 15 -Feb-96 & $\mathbf{Y}$ & $4 B$ \\
\hline $\mathbf{Y}$ & & 3050 & CONC_MON & Concrete Monolith & RADN_DRZ & DRZ ovier rodius at each shat & 32528 & 15-fob-96 & $Y$ & 1 \\
\hline $\bar{Y}$ & & 3050 & CONC_MON & Concrete Monolith & RADN_DRZ & DRZ outer redxes at each shath & 32528 & $15-F \circ b-96$ & $\mathbf{Y}$ & 1 \\
\hline $\mathbf{Y}$ & & 3050 & CONC_MON & Concrute Monolith & RADN_DRZ & DRZ outor radius at each thent & 32528 & $15-\mathrm{feb}-96$ & $\bar{Y}$ & 1 \\
\hline $\mathbf{Y}$ & 50 & 3051 & CONC_MON & Concrate Monolith & CAP_MOD & $\begin{array}{l}\text { Model number, capilley pressure } \\
\text { model }\end{array}$ & $32503 \mathrm{~A}$ & $15-5 a b-96$ & $\mathbf{Y}$ & AB \\
\hline $\mathbf{Y}$ & 50 & 3051 & CONC_MON & Concrete Monolith & CAP_MOD & $\begin{array}{l}\text { Model number, capiltwy prosedure } \\
\text { model }\end{array}$ & 325034 & 15-Fab- 86 & $\mathbf{Y}$ & 48 \\
\hline $\mathbf{Y}$ & 50 & 3051 & CONC_MON & Concrute Monorith & CAP_MOD & $\begin{array}{l}\text { Model number, ceppilley presesure } \\
\text { model }\end{array}$ & 325034 & $15-a b-86$ & $\mathbf{Y}$ & 48 \\
\hline $\mathbf{Y}$ & 50 & 3051 & CONC_MON & Concrete Monolith & CAP_MOD & $\begin{array}{l}\text { Model number, cepplisy pressure } \\
\text { model }\end{array}$ & $32503 \mathrm{~A}$ & $15+c b-96$ & $\mathbf{Y}$ & 48 \\
\hline $\mathbf{Y}$ & 50 & $305 i$ & CONC_MON & Conerete Monolith & CAP_MOD & $\begin{array}{l}\text { Model number, cappillsy pressure } \\
\text { mochl }\end{array}$ & $32503 \mathrm{~A}$ & 15 Fab- 96 & $Y$ & 48 \\
\hline
\end{tabular}


CCA Parameter Listing

\begin{tabular}{|c|c|c|c|c|c|c|c|c|c|c|}
\hline 10 & 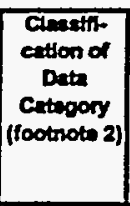 & $\begin{array}{l}\text { Destribution } \\
\text { Typu }\end{array}$ & Maen & medien & Mintromem & Mrodinum & Untes & Reformance id & 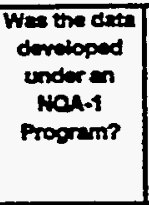 & 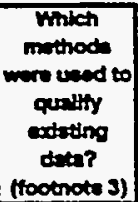 \\
\hline 3044 & 1,3 & CONSTANT & $2.3000000 E+\infty$ & $2.3000000 E+\infty 0$ & $2.3000000 \mathrm{E}+\infty 0$ & $2.3000000 E+\infty$ & $m$ & WP030S40 & $\mathbf{Y}$ & NA \\
\hline 3044 & 1,3 & CONSTANT & $2.3000000 E+00$ & $2.3000000 E+\infty 0$ & $2,3000000 E+00$ & $2.3000000 E+\infty 0$ & m & WP030819 & $\mathbf{Y}$ & NA \\
\hline 3044 & 1,3 & CONSTANT & $2.3000000 E+\infty$ & $2.3000000 E+00$ & $2.3000000 E+\infty$ & $2.3000000 E+\infty$ & $m$ & WP030994 & $\mathbf{Y}$ & N/A \\
\hline 3044 & 1,3 & CONSTANT & $2.3000000 E+00$ & $2.3000000 E+\infty 0$ & $2.3000000 E+\infty 0$ & $23000000 E+\infty 0$ & m & WP035597 & $\mathbf{Y}$ & NA \\
\hline 3044 & 1.3 & CONSTANT & $2.3000000 E+00$ & $2.3000000 E+\infty$ & $2.3000000 E+\infty 0$ & $2.3000000 E+\infty 0$ & $m$ & WP040514, & $\checkmark$ & N/A \\
\hline 3045 & $1,3,4$ & CONSTANT & $1.0000000 \mathrm{E}+\infty 0$ & $1.0000000 E+00$ & $1.0000000 E+\infty 0$ & $1.0000000 E+\infty 0$ & $\mathrm{~m} / \mathrm{m}$ & WP030640 & $\mathbf{Y}$ & NA \\
\hline 3045 & $1,3,4$ & CONSTANT & $1.0000000 E+00$ & $1.0000000 E+\infty 0$ & $1.0000000 E+\infty 0$ & $1.0000000 E+\infty$ & $\mathrm{m} / \mathrm{m}$ & upo30994 & $\mathbf{Y}$ & NA \\
\hline 3045 & $1,3,4$ & CONSTANT & $1.0000000 \mathrm{E}+\infty$ & $1.0000000 \mathrm{E}+\infty$ & $1.0000000 E+\infty 0$ & $1.0000000 E+\infty 0$ & $\mathrm{~m} / \mathrm{m}$ & WP035597 & $\mathbf{Y}$ & NA \\
\hline 3045 & $1,3,4$ & CONSTANT & $1.0000000 \mathrm{E}+00$ & $1.0000000 \mathrm{E}+\infty 0$ & $1.0000000 E+\infty 0$ & $1.0000000 E+\infty 0$ & $\mathbf{m} / \mathbf{m}$ & WP036048 & $\mathbf{Y}$ & NA \\
\hline 3046 & 1,3 & CONSTANT & $3.0900000 E+00$ & $3.0900000 E+\infty$ & $3.0900000 E+\infty 0$ & $3.0900000 E+00$ & $\mathbf{m}$ & WP030640 & $\mathbf{Y}$ & N/A \\
\hline 3046 & 1,3 & CONSTANT & $3.0900000 E+\infty 0$ & $3.0900000 E+\infty$ & $3.0900000 \mathrm{E}+\infty 0$ & $3.0900000 E+00$ & $\mathbf{m}$ & WP030991 & $\mathbf{Y}$ & NA \\
\hline 3046 & 1,3 & CONSTANT & $3.0900000 E+\infty$ & $3.0900000 E+\infty$ & $3.0900000 E+\infty$ & $3.0900000 \mathrm{E}+\infty 0$ & m & WPO35597 & $\mathbf{Y}$ & NA \\
\hline 3046 & 1,3 & CONSTANT & $3.0900000 E+\infty 0$ & $3.0900000 E+\infty 00$ & $3.0900000 \mathrm{E}+\infty$ & $3.0900000 E+\infty$ & $\mathbf{m}$ & WPO40514 & $\mathbf{Y}$ & NA \\
\hline 3047 & 1,3 & CONSTANT & $1.8000000 E+\infty$ & $1.8000000 E+\infty$ & $1.8000000 E+\infty$ & $1.8000000 \mathrm{E}+\infty$ & $m$ & WP030640 & $\mathbf{Y}$ & NA \\
\hline 3047 & 1,3 & CONSTANT & $1.8000000 E+\infty 0$ & $1.8000000 E+\infty$ & $1.8000000 E+\infty$ & $1.8000000 E+\infty 0$ & $\mathbf{m}$ & WP030994 & $\mathbf{Y}$ & NA \\
\hline 3047 & 1,3 & CONSTANT & $1,8000000 \mathrm{E}+\infty$ & $1.8000000 E+\infty$ & $1.8000000 E+\infty 0$ & $1.8000000 E+00$ & $\mathbf{m}$ & WP035597 & $\mathbf{Y}$ & NA \\
\hline 3047 & 1,3 & CONSTANT & $1.8000000 E+\infty 0$ & $1.8000000 \mathrm{E}+\infty$ & $1.8000000 E+\infty$ & $1.8000000 \mathrm{E}+\infty 0$ & $\mathbf{m}$ & WP040514 & $\mathbf{Y}$ & NA \\
\hline 3048 & 1.3 & CONSTANT & $3.5000000 E+\infty 0$ & $3.5000000 E+\infty$ & $3.5000000 E+\infty$ & $3.5000000 E+00$ & m & WP030640 & $\mathbf{Y}$ & N/A \\
\hline 3048 & 1,3 & CONSTANT & $3.5000000 E+\infty 0$ & $3.5000000 \mathrm{E}+\infty$ & $3.5000000 E+\infty$ & $3.5000000 E+\infty 0$ & $\mathbf{m}$ & WP030994 & $\mathbf{Y}$ & N/A \\
\hline 3048 & 1,3 & CONSTANT & $3.5000000 E+00$ & $3.5000000 E+\infty 0$ & $3.5000000 E+\infty 0$ & $3.5000000 E+\infty$ & m & WP035597 & $\mathbf{Y}$ & NA \\
\hline 3048 & 1,3 & CONSTANT & $3.5000000 \mathrm{E}+00$ & $3.5000000 \mathrm{E}+\infty 0$ & $3.5000000 \mathrm{E}+00$ & $3.5000000 E+00$ & $m$ & WPO40514 & $\mathbf{Y}$ & NA \\
\hline 3049 & 1,3 & CONSTANT & $2.3000000 E+\infty$ & $2.3000000 E+\infty 0$ & $2.3000000 E+00$ & $2.3000000 E+\infty$ & $\mathbf{m}$ & WP030640 & $\mathbf{Y}$ & NA \\
\hline 3049 & 1,3 & CONSTANT & $2.3000000 E+\infty 0$ & $2.3000000 E+\infty$ & $2.3000000 E+\infty$ & $2.3000000 E+\infty$ & $\mathbf{m}$ & WP030994 & $\mathbf{Y}$ & NA \\
\hline 3019 & 1,3 & CONSTANT & $2.3000000 E+\infty 0$ & $2.3000000 E+\infty$ & $23000000 E+\infty 0$ & $2.3000000 E+\infty 0$ & m & WP035597 & $\mathbf{Y}$ & NA \\
\hline 3049 & 1,3 & CONSTANT & $23000000 E+\infty 0$ & $2.3000000 E+\infty$ & $2.3000000 E+00$ & $2.3000000 E+\infty 0$ & $\mathbf{m}$ & WPO40514 & $\boldsymbol{\gamma}$ & N/A \\
\hline 3050 & 1,3 & CONSTANT & $1.0000000 E+\infty$ & $1.0000000 E+\infty$ & $1.0000000 E+00$ & $1.0000000 E+00$ & $\mathrm{~m} / \mathrm{m}$ & WP030540 & $\mathbf{Y}$ & NA \\
\hline 3050 & 1,3 & CONSTANT & $1.0000000 E+\infty$ & $1.0000000 E+\infty$ & $1.0000000 E+00$ & $1.0000000 E+00$ & $\mathrm{~m} / \mathrm{m}$ & WPO30994 & $\mathbf{Y}$ & N/A \\
\hline 3050 & 1,3 & CONSTANT & $1,0000000 E+\infty$ & $1.0000000 \mathrm{E}+\infty$ & $1.0000000 E+00$ & $1.0000000 E+\infty 0$ & $m / m$ & WP035597 & $\mathbf{Y}$ & NA \\
\hline 3051 & $1,3,4$ & CONSTANT & $2.0000000 E+\infty 0$ & $20000000 E+00$ & $2.0000000 E+\infty 0$ & $2.0000000 E+\infty 0$ & NONE & WPO30640 & $\boldsymbol{\gamma}$ & NA \\
\hline 3051 & $1,3,4$ & CONSTANT & $2.0000000 E+\infty 0$ & $2.0000000 E+\infty 0$ & $2,0000000 E+\infty 0$ & $2.0000000 E+\infty 0$ & NONE & WP030994 & $Y$ & NA \\
\hline 3051 & $1,3,4$ & CONSTANT & $20000000=+\infty$ & $2.0000000 E+\infty 0$ & $20000000 E+\infty$ & $20000000 E+\infty 0$ & NONE & WP035597 & $Y$ & NA \\
\hline 3051 & $1,3,4$ & CONSTANT & $2,0000000 E+\infty$ & $2.0000000 E+\infty$ & $2,0000000 E+\infty$ & $20000000 E+\infty$ & NONE & WPO40406 & $\mathbf{Y}$ & N/A \\
\hline 3051 & $1,3,4$ & CONSTANT & $20000000 E+\infty 0$ & $2.0000000 E+\infty 0$ & $2.0000000 E+\infty$ & $2.0000000 E+00$ & NONE & WPO40S14 & $\mathbf{Y}$ & NA \\
\hline
\end{tabular}


CCA Parameter Listing

\begin{tabular}{|c|c|c|c|c|c|c|c|c|c|c|}
\hline $\begin{array}{c}\text { Parminter In } \\
\text { Detabeen? } \\
\text { (Ya) }\end{array}$ & 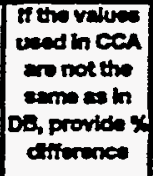 & D & Mintartato & Meterda kam & Permatar & Purninger hass & $\begin{array}{l}\text { PFP D } \\
\text { (NmOA) }\end{array}$ & $\begin{array}{l}\text { Denentyy } \\
\text { Dete }\end{array}$ & 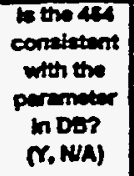 & 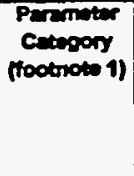 \\
\hline $\mathbf{Y}$ & & 3052 & CONC_MON & Concrete Monolith & COMP_RCK & Buk Compressibilty & 32504 & $02+$ ayy-96 & $\mathbf{Y}$ & $4 B$ \\
\hline $\mathbf{Y}$ & & 3052 & CONC_MON & Concrete Monolith & COMP_RCK & Bulk Compressibitay & 32504 & $02+4=y-96$ & $Y$ & 48 \\
\hline $\mathbf{Y}$ & & 3052 & CONC_MON & Concrete Monolith & COMP_RCK & Butk Compressibility & 32504 & $02+4 y-96$ & $Y$ & 48 \\
\hline $\mathbf{Y}$ & & 3053 & CONC_MON & Concrete Monolith & KPT & $\begin{array}{l}\text { Flag for Penmeabity Determned } \\
\text { Threahold }\end{array}$ & 32507 & $20-50 b-96$ & $\mathbf{Y}$ & $4 B$ \\
\hline $\mathbf{Y}$ & & 3053 & CONC_MON & Concroto Monolith & KPT & $\begin{array}{l}\text { Fleg for Permesbility Determined } \\
\text { Throutold }\end{array}$ & 32507 & $20 F=0-96$ & $\mathbf{Y}$ & 48 \\
\hline$Y$ & & 3053 & CONC_MON & Concrete Monolith & KPT & $\begin{array}{l}\text { Fleo for Pormesbity Dotermined } \\
\text { Throeshold }\end{array}$ & 32507 & 20F-b-96 & $\mathbf{Y}$ & $A B$ \\
\hline $\mathbf{Y}$ & & 3053 & CONC_MON & Concrote Mornolith & KPT & $\begin{array}{l}\text { Fleg for Pormesbilty Datermined } \\
\text { Throshold }\end{array}$ & 32507 & 20-5ab-s6 & $\mathbf{Y}$ & 48 \\
\hline $\mathbf{Y}$ & & 3054 & CONC_MON & Concrote Monolith & PC_MAX & $\begin{array}{l}\text { Maximum alloweble capillary } \\
\text { prossuro }\end{array}$ & 325124 & $15-7=b-96$ & $\mathbf{Y}$ & $4 B$ \\
\hline $\mathbf{Y}$ & & 3054 & CONC_HON & Concrete Monorith & PC_mAX & $\begin{array}{l}\text { Macinum allowable capillary } \\
\text { pressure }\end{array}$ & $32512 A$ & 15-Fob-96 & $\mathbf{Y}$ & $4 B$ \\
\hline $\mathbf{Y}$ & & 3054 & CONC_MON & Concreto Monolith & PC_mAX & $\begin{array}{l}\text { Meximim alloweble cepillary } \\
\text { pressure }\end{array}$ & 32512A & $15 F$ bb-s6 & $\mathbf{Y}$ & 48 \\
\hline $\mathbf{Y}$ & & 3054 & CONC_MON & Concrete Monolith & PC_MAX & $\begin{array}{l}\text { Moximum sllowabio cepiltey } \\
\text { prosenre }\end{array}$ & $32512 A$ & $15 F=b-96$ & $\mathbf{Y}$ & $4 B$ \\
\hline $\mathbf{Y}$ & & 3054 & CONC_MON & Concrete Monolith & PC_MAX & $\begin{array}{l}\text { Maximum allowable capiliny } \\
\text { pressure }\end{array}$ & $32512 A$ & 15Fab-96 & $\mathbf{Y}$ & $4 B$ \\
\hline $\mathbf{Y}$ & 100 & 3055 & CONC_MON & Concrote Monolith & PCT_A & $\begin{array}{l}\text { Threstrold Presture Linear } \\
\text { Pareneter }\end{array}$ & 32508 & 15 feb-96 & $\mathbf{Y}$ & 1 \\
\hline $\mathbf{Y}$ & 100 & 3055 & CONC_MON & Concrate Monolith & PCT_A & $\begin{array}{l}\text { Threshold Preseturo Linear } \\
\text { Peremeter }\end{array}$ & 32508 & 15 Feb-s6 & $\mathbf{Y}$ & 1 \\
\hline $\mathbf{Y}$ & 100 & 3055 & CONC_MON & Concrute Monolith & PCT_A & $\begin{array}{l}\text { Threshold Pressure Linear } \\
\text { Peremoter }\end{array}$ & 32508 & 15 fab-96 & $\mathbf{Y}$ & 1 \\
\hline $\mathbf{Y}$ & 100 & 3055 & CONC_MON & Concrate Monolith & PCT_A & $\begin{array}{l}\text { Trrestodd Proseure Liner } \\
\text { Pereneter }\end{array}$ & 32508 & 15 Feb-96 & $\mathbf{Y}$ & 1 \\
\hline $\mathbf{Y}$ & 100 & 3056 & CONC_MON & Concrete Monolith & PCT_EXP & $\begin{array}{l}\text { Threshoid pressure exponential } \\
\text { paremeter }\end{array}$ & 32510 & 15Fab-96 & $\mathbf{Y}$ & 1 \\
\hline $\mathbf{Y}$ & 100 & 3056 & CONC_MON & Concrete Monolith & PCT_EXP & $\begin{array}{l}\text { Threshold preasure exponential } \\
\text { peremeter }\end{array}$ & 32510 & 15Fab-96 & $\mathbf{Y}$ & 1 \\
\hline $\mathbf{Y}$ & 100 & 3056 & CONC_MON & Concrete Monolith & PCT_EXP & $\begin{array}{l}\text { Trreshold presure exponential } \\
\text { peremeter }\end{array}$ & 32510 & 15Feb-96 & $\mathbf{Y}$ & 1 \\
\hline $\mathbf{Y}$ & 100 & 3056 & CONC_MON & Concrete Monolith & PCT_EXP & $\begin{array}{l}\text { Threshoid pressure exponontinl } \\
\text { parameter }\end{array}$ & 32510 & $15 f a b-96$ & $\mathbf{Y}$ & 1 \\
\hline $\mathbf{Y}$ & varies & 3057 & CONC_MON & Concrete Monolith & PORE_OIS & $\begin{array}{l}\text { Brooks-Coroy pore distribution } \\
\text { perameter }\end{array}$ & 32514 & 20-Fab-96 & $Y$ & 1 \\
\hline $\mathbf{Y}$ & varias & 3057 & CONC_MON & Concrete Monolith & PORE_DIS & $\begin{array}{l}\text { Brocks-Corey pore distribution } \\
\text { parcmeter }\end{array}$ & 32514 & $20-F a b-96$ & $\mathbf{Y}$ & 1 \\
\hline $\mathbf{Y}$ & varies & 3057 & CONC_MON & Concrete Monolith & PORE_OIS & $\begin{array}{l}\text { Brooke-Corty pore distribution } \\
\text { paremeter }\end{array}$ & 32514 & 20 - ob-96 & $\mathbf{Y}$ & 1 \\
\hline $\mathbf{Y}$ & varies & 3057 & CONC_MON & Concrote Monolith & PORE_DIS & $\begin{array}{l}\text { Brooks-Corvy pore distribution } \\
\text { parameter }\end{array}$ & 32514 & 20-fob-96 & $\mathbf{Y}$ & 1 \\
\hline $\mathbf{Y}$ & varies & 3057 & CONC_MON & Concrete Monolith & PORE_DIS & $\begin{array}{l}\text { Brooks-Corey pore distribution } \\
\text { paremeter }\end{array}$ & 32514 & 20fab-96 & $\mathbf{Y}$ & 1 \\
\hline $\mathbf{Y}$ & varies & 3057 & CONC_MON & Concreto Monolith & PORE_DIS & $\begin{array}{l}\text { Brooks-Corey pore distribution } \\
\text { pareneter }\end{array}$ & 32514 & 20-Feb-ss & 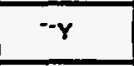 & 1 \\
\hline $\mathbf{Y}$ & varies & 3057 & CONC_MON & Concrets Monolith & PORE_DIS & $\begin{array}{l}\text { Brooks-Corey pore distribution } \\
\text { parameter }\end{array}$ & 32514 & $20-F 0 b-96$ & $\mathbf{Y}$ & 1 \\
\hline$Y$ & & 3058 & CONC_MON & Concrete Monolith & POROSITY & Eftuctive porosity & 32516 & 15 fob-96 & $Y$ & 1 \\
\hline $\mathbf{Y}$ & & 3058 & CONC_MON & Concreto Monolith & POROSITY & Effective porosiny & 32516 & $15-f a b-86$ & $\mathbf{Y}$ & 1 \\
\hline $\mathbf{Y}$ & & 3058 & CONC_MON & Concrets Monolth & POROSITY & Effective porosity & 32516 & 15 Fob-96 & $\mathbf{Y}$ & 1 \\
\hline $\mathbf{Y}$ & & 3059 & CONC_MON & Concrete Monolith & PRMX_LOG & $\begin{array}{l}\text { Loo of ntrmsic permesbilty, } X- \\
\text { drection }\end{array}$ & 32520 & 15 fob-96 & $\mathbf{Y}$ & 1 \\
\hline $\mathbf{Y}$ & & 3059 & CONC_MON & Concrete Manotith & PRMX_LOG & $\begin{array}{l}\text { Log of intrnac permesbilty, } X- \\
\text { direction }\end{array}$ & 32520 & 15 -Fob-96 & $\mathbf{Y}$ & 1 \\
\hline $\mathbf{Y}$ & & 3059 & CONC_MON & Concrite Honolith & PRMX LOG & $\begin{array}{l}\text { Log of intrasse permasulity, } X- \\
\text { dirnection }\end{array}$ & 32520 & $15+f a b-96$ & $\mathbf{Y}$ & 1 \\
\hline $\mathbf{Y}$ & & 3060 & CONC_MON & Concrete Monolith & PRMY_LOG & $\begin{array}{l}\text { Log of intersac permeability, } Y- \\
\text { dinection }\end{array}$ & 32522 & 15-Feb-95 & $\mathbf{Y}$ & 1 \\
\hline $\mathbf{Y}$ & & 3050 & CONC_MON & Conerete Monolth & PRIYY_LOG & $\begin{array}{l}\text { Loo of intrinalc permesbildy, } Y- \\
\text { direction }\end{array}$ & 32522 & 15 feb-9s & $\mathbf{Y}$ & 1 \\
\hline $\mathbf{Y}$ & & 3060 & CONC_MON & Concrete Monolith & PRAY_LOG & $\begin{array}{l}\text { Log of ntrosic permesbility, } Y \text { - } \\
\text { direction }\end{array}$ & 32522 & $15-F e b-96$ & $\mathbf{Y}$ & 1 \\
\hline $\mathbf{Y}$ & & 3061 & CONC_MON & Concrete Monolith & PRMZ_LOG & $\begin{array}{l}\text { Los of intrrac permeability, } z \text { - } \\
\text { orrection }\end{array}$ & 32527 & $15+F b-96$ & $\mathbf{Y}$ & 1 \\
\hline $\mathbf{Y}$ & & 3061 & CONC_MON & Concrete Monolith & PRMZ_LOG & $\begin{array}{l}\text { Loo of intrnaic permeability, Z- } \\
\text { drection }\end{array}$ & 32527 & $15+F b-96$ & $\mathbf{Y}$ & 9 \\
\hline
\end{tabular}


CCA Parameter Listing

\begin{tabular}{|c|c|c|c|c|c|c|c|c|c|c|}
\hline ID & $\begin{array}{l}\text { Cicestin- } \\
\text { carton of } \\
\text { Deth } \\
\text { Cuteoory } \\
\text { (footrote 2) }\end{array}$ & $\begin{array}{l}\text { Dentribution } \\
\text { Type }\end{array}$ & $\operatorname{man}$ & Hodien & Mintroum & modrines & Thits & Retermicos D & 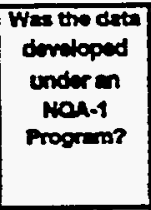 & 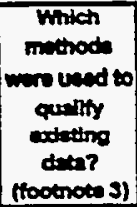 \\
\hline 3052 & $1,3,4$ & CONSTANT & $1.2000000 \mathrm{E}-09$ & $1.2000000 \mathrm{E}-09$ & $1.2000000 E-09$ & $1.2000000 \mathrm{E}-09$ & Prn-1 & WP030640 & $Y$ & NA \\
\hline 3052 & $1,3,4$ & CONSTANT & $1.2000000 \mathrm{E}-09$ & $1.2000000 \mathrm{E}-09$ & $1.2000000 E-09$ & $1.2000000 E-09$ & Par-1 & WP035597 & $Y$ & NA \\
\hline 3052 & $1,3,4$ & CONSTANT & $1.2000000 \mathrm{E}-09$ & $1.2000000 \mathrm{E}-09$ & $1.2000000 \mathrm{E}-09$ & $1.2000000 E-09$ & $P a^{n}-1$ & WPOS0514 & $\mathbf{Y}$ & NA \\
\hline 3053 & 3,5 & CONSTANT & $0.00000000+\infty$ & $0.0000000 E+\infty$ & $0.0000000 E+\infty$ & $0.0000000 E+\infty$ & NONE & WP035271 & $\mathbf{Y}$ & NA \\
\hline 3053 & 3,5 & CONSTANT & $0.0000000 E+\infty$ & $0.00000000 E+00$ & $0.0000000 E+\infty$ & $0.0000000=+\infty$ & NONE & WP035333 & $\mathbf{Y}$ & NA \\
\hline 3053 & 3,5 & CONSTANT & $0.0000000 E+\infty 0$ & $0.0000000 E+\infty$ & $0.00000000 E+\infty 0$ & $0.00000000+\infty$ & NONE & WP035597 & $\mathbf{Y}$ & NA \\
\hline 3053 & 3,5 & CONSTANT & $0.0000000 E+\infty$ & $0.0000000 E+\infty$ & $0.0000000 E+\infty 0$ & $0.0000000=+\infty$ & NONE & WPO40514 & $\mathbf{Y}$ & NA \\
\hline 3054 & 3,5 & CONSTANT & $1.0000000 E+08$ & $1.0000000 E+08$ & $1.0000000 E+08$ & $1.0000000 E+08$ & Pa & WP030610 & $\mathbf{Y}$ & NA \\
\hline 3054 & 3,5 & CONSTANT & $1.0000000 \mathrm{E}+08$ & $1.0000000 E+08$ & $1.0000000 E+08$ & $1.0000000 E+\infty 8$ & $\mathbf{P a}$ & WPOSOS94 & $\mathbf{Y}$ & NA \\
\hline 3054 & 3,5 & CONSTANT & $1.0000000 E+08$ & $1.0000000 E+08$ & $1.0000000 E+\infty 8$ & $1.0000000 E+\infty 8$ & $\mathbf{P a}$ & WP035597 & $\mathbf{Y}$ & NA \\
\hline 3054 & 3,5 & CONSTANT & $1.0000000 E+08$ & $1.0000000 E+08$ & $1.0000000 E+\infty 8$ & 1.0000000 E+08 & Pa & WPO4048s & $\boldsymbol{Y}$ & NA \\
\hline 3054 & 3,5 & CONSTANT & $1.0000000 E+08$ & $1.0000000 E+08$ & $1.00000000+00$ & $1.0000000 E+\infty 8$ & $\mathbf{P a}$ & WPO40514 & $\mathbf{Y}$ & NA \\
\hline 3055 & 3,4 & CONSTANT & $5.6000000 E-01$ & 5.6000000E-01 & $5.6000000 E-01$ & $5.6000000 E-01$ & Pa & WP030640 & $\mathbf{Y}$ & NA \\
\hline 3055 & 3.4 & CONSTANT & $5.6000000 E-01$ & $5.6000000 E-01$ & $5.6000000 E-01$ & $5.6000000 E-01$ & Pa & WP030994 & $\mathbf{Y}$ & NA \\
\hline 3055 & 3,4 & CONSTANT & $5.6000000 E-01$ & $5.6000000 E-01$ & $5.6000000 E-01$ & $5.6000000 E-01$ & $\mathbf{P a}$ & WP035597 & $Y$ & NA \\
\hline 3055 & 3.4 & CONSTANT & $5.6000000 E-01$ & $5.6000000 E-01$ & $5.6000000 E-01$ & $5.60000000-01$ & $\mathbf{P a}$ & WPO40434 & $Y$ & $N / A$ \\
\hline 3056 & 3,4 & CONSTANT & $3.4600000 \mathrm{E}-01$ & $3.4600000 E-01$ & $3.4600000 \mathrm{E}-01$ & $3.4600000 E-01$ & NONE & WPO30640 & $\mathbf{Y}$ & NA \\
\hline 3056 & 3,4 & CONSTANT & $3.4600000 E-01$ & $3.4600000 E-01$ & $3.4600000 \mathrm{E}-01$ & $3.4600000 E-01$ & NONE & WP030994 & $\mathbf{Y}$ & NA \\
\hline 3056 & 3,4 & CONSTANT & $-3.4600000 E-01$ & $-3.4600000 E-01$ & $3.4600000 E-01$ & $3.4600000 E-01$ & NONE & WP035597 & $\mathbf{Y}$ & NA \\
\hline 3056 & 3,4 & CONSTANT & $-3.4600000 \mathrm{E}-01$ & $-3.4600000 \mathrm{E}-01$ & 3.4600000 E-01 & $-3.46000000-01$ & NONE & WP040134 & $\mathbf{Y}$ & NA \\
\hline 3057 & 3.4 & CUMULATIVE & $2.5200000 E+\infty$ & 9.4000000E-01 & $1.1000000 E-01$ & $8.1000000 E+00$ & NONE & WP030640 & $\mathbf{Y}$ & NA \\
\hline 3057 & 3,4 & CUMULATIVE & $2.5200000 E+\infty 0$ & $9.4000000 E-01$ & $1.1000000 E-01$ & $8.1000000=+\infty 0$ & NONE & WP030994 & $\mathbf{Y}$ & N/A \\
\hline 3057 & 3,4 & CUMULATINE & $2.5200000 E+\infty 0$ & $9.4000000 \mathrm{E}-01$ & $1.1000000 E-01$ & $8.1000000 E+\infty 0$ & NONE & WP032287 & $\mathbf{Y}$ & NA \\
\hline 3057 & 3.4 & CUMULATINE & $2.5200000 E+\infty 0$ & $9.4000000 E-01$ & $1.1000000 E-01$ & $8.1000000 E+\infty$ & NONE & WP035268 & $\mathbf{Y}$ & NA \\
\hline 3057 & 3,4 & CUMULATINE & $2.5200000 E+00$ & $9.4000000 E-01$ & 9.1000000E-01 & $8.1000000 E+\infty$ & NONE & WP035597 & $\mathbf{Y}$ & NA \\
\hline 3057 & 3,4 & CUMULATIVE & $25200000 E+\infty 0$ & $9.4000000 E-01$ & $1.1000000 E-01$ & $8.1000000 E+\infty 0$ & NONE & WPO41558 & $-Y$ & NA \\
\hline 3057 & 3,4 & CUMULATIVE & $2.5200000 E+\infty 0$ & $9.4000000 E-01$ & $1.1000000 E-01$ & $8.1000000 E+\infty 0$ & NONE & WPO42752 & $\mathbf{Y}$ & NA \\
\hline 3058 & 3,4 & CONSTANT & $5.0000000 E-02$ & $5.0000000 E-02$ & $5.0000000 E-02$ & $5.0000000 \mathrm{E}-02$ & $m^{\wedge} 3 m^{\wedge} 3$ & WPO30540 & $N$ & 1 \\
\hline 3058 & 3,4 & CONSTANT & $5.0000000 E-02$ & $5.0000000 E-02$ & $5.0000000 \mathrm{E}-02$ & $5.0000000 E-02$ & $m^{\wedge} 3 / m^{\wedge} 3$ & WPO30994 & $N$ & 1 \\
\hline 3058 & 3,4 & CONSTANT & $5.0000000 E-02$ & $5.0000000 E-02$ & $5.0000000 E-02$ & $5.0000000 \mathrm{E}-02$ & $m^{\wedge} 3 / m^{\wedge} 3$ & WP035597 & $\mathbf{N}$ & 1 \\
\hline 3059 & 3,4 & CONSTANT & $-1.40000000 E+01$ & $-1.4000000 E+01$ & $-1.4000000 E+01$ & $-1.4000000 E+01$ & $\log \left(m^{\wedge} 2\right)$ & WP030640 & N & 1 \\
\hline 3059 & 3.4 & CONSTANT & $-1.4000000 E+01$ & $-1.4000000 E+01$ & $-1.4000000 E+01$ & $-1.4000000 E+01$ & $\log \left(m^{\wedge} 2\right)$ & WP030994 & $\mathbf{N}$ & 4 \\
\hline 3059 & 3,4 & CONSTANT & $-1.4000000 E+01$ & $-1.4000000 E+01$ & $-1.4000000 E+01$ & $-1.4000000 E+01$ & $\log \left(m^{\wedge} 2\right)$ & WP035597 & $\mathbf{N}$ & 1 \\
\hline 3060 & 3,4 & CONSTANT & $-1.4000000 E+01$ & $-1.4000000 E+01$ & $-1.4000000 E+01$ & $-1.4000000 E+01$ & $\log \left(m^{\wedge} 2\right)$ & WP030640 & N & 1 \\
\hline 3060 & 3,4 & CONSTANT & $-1.4000000 E+01$ & $-1.4000000 E+01$ & $-1.4000000 E+01$ & $-1.4000000 E+01$ & $\log \left(m^{\wedge} 2\right)$ & WP030994 & N & 1 \\
\hline 3060 & 3,4 & CONSTANT & $-1.4000000 E+01$ & $-1.4000000 E+01$ & $-1.4000000 E+01$ & $-1,4000000 \mathrm{E}+01$ & $\log \left(m^{\wedge} 2\right)$ & WP035597 & H & 1 \\
\hline 3061 & 3.4 & CONSTANT & $-1.4000000 E+01$ & $-1.4000000 E+01$ & $-1.4000000 E+01$ & $-1,4000000 E+01$ & $\log \left(m^{n} 2\right)$ & WP030640 & N & 1 \\
\hline 3061 & 3,4 & CONSTANT & $-1.4000000 E+01$ & $-1.4000000 E+01$ & $-1.4000000 E+01$ & $-1,4000000 E+01$ & $\log \left(\mathrm{m}^{\wedge} 2\right)$ & WP030994 & $\mathbf{N}$ & 1 \\
\hline
\end{tabular}


CCA Parameter-tisting

\begin{tabular}{|c|c|c|c|c|c|c|c|c|c|c|}
\hline $\begin{array}{c}\text { Pormotion } \\
\text { Dotembea? } \\
\text { (YN) }\end{array}$ & 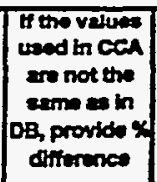 & ID & Metata to & Meterla Nerne & $\begin{array}{c}\text { Peranotior } \\
\text { to }\end{array}$ & $\begin{array}{l}\text { Parmitar Name } \\
. \quad . \quad .\end{array}$ & $\begin{array}{l}\text { PRPDO } \\
\text { (MPOA) }\end{array}$ & $\begin{array}{c}\text { Detan Entry } \\
\text { Dato }\end{array}$ & 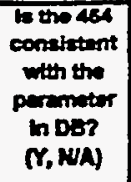 & $\begin{array}{l}\text { Perameter } \\
\text { Cetregory } \\
\text { (footinotie 1) }\end{array}$ \\
\hline $\mathbf{Y}$ & & 3061 & CONC_MON & Concrete Monolith & PRMZ_LOG & $\begin{array}{l}\text { Log of mornsic permesbidty, Z- } \\
\text { diroction }\end{array}$ & 32527 & 15-Fab-98 & $\mathbf{Y}$ & 1 \\
\hline $\mathbf{Y}$ & & 3052 & CONC_MON & Concriete Monolith & RELP_MOD & $\begin{array}{l}\text { Model number, roletive } \\
\text { permeabifity model }\end{array}$ & 325300 & 15 fab-86 & $\mathbf{Y}$ & 48 \\
\hline$Y$ & & 3052 & CONC_MON & Concrote Monolith & RELP_MOD & $\begin{array}{l}\text { Model nember, nolative } \\
\text { permenbifty model }\end{array}$ & 325304 & $15+F a b-96$ & $\mathbf{Y}$ & 48 \\
\hline $\mathbf{Y}$ & & 3052 & CONC_MON & Concreto monolith & RELP_MOD & $\begin{array}{l}\text { Model number, relative } \\
\text { permesbitity model }\end{array}$ & 325300 & $15+50-96$ & $\mathbf{Y}$ & 48 \\
\hline $\mathbf{Y}$ & & 3052 & CONC_MON & Concrete Monolith & RELP_MOD & $\begin{array}{l}\text { Modol number, nolativs } \\
\text { permeshatity model }\end{array}$ & $32530 n$ & 15-Fab-86 & $Y$ & 48 \\
\hline $\mathbf{Y}$ & & 3062 & CONC_MON & Concrets Monotith & RELP_MOD & $\begin{array}{l}\text { Model number, relativo } \\
\text { permoability model }\end{array}$ & 325304 & $15 F \infty b-86$ & $Y$ & 48 \\
\hline$Y$ & 100 & 3063 & CONC_MON & Concrote Monolith & SAT_RBRN & Resicual Brino Saturation & 32542 & 15 feo- 96 & $\mathbf{Y}$ & 1 \\
\hline$Y$ & 100 & 3053 & CONC_MON & Concruto Monolith & SAT_RBRN & Residual Brino Staration & 32542 & $15+c b-96$ & $Y$ & 1 \\
\hline$Y$ & 100 & 3053 & CONC_MON & Concrete Monolith & SAT_RBRN & Residual Brine Suturation & 32542 & 15-Feb-96 & $Y$ & 1 \\
\hline$Y$ & 100 & 3053 & CONC_MON & Concrete Monolith & SAT_RBRN & Resioual Brine Saturation & 32542 & $15-F a b-96$ & $Y$ & 1 \\
\hline$Y$ & 100 & 3063 & CONC_MON & Concrete Monolith & SAT_RBRN & Residual Brime Suturation & 32542 & 15 fab-96 & $Y$ & 1 \\
\hline$Y$ & 100 & 3053 & CONC_MON & Concroto Monolith & SAT_RBRN & Ressidual Brine Seturation & 32542 & $15+60-96$ & $Y$ & 1 \\
\hline $\mathbf{Y}$ & 100 & 3064 & CONC_MON & Concrete Monolith & SAT_RGAS & Resicual Ges Sraration & 32546 & $15-F \infty b-96$ & $Y$ & 1 \\
\hline $\mathbf{Y}$ & 100 & 3064 & CONC_MON & Concrete Monolith & SAT_RGAS & Resicual Gas Seturation & 32546 & $15+\infty 0-96$ & $Y$ & 1 \\
\hline$Y$ & 100 & 3064 & CONC_MON & Concrets Monolith & SAT_RGAS & Residual Gas Saturetion & 32546 & 15F-b-96 & $Y$ & 1 \\
\hline$Y$ & 100 & 3064 & CONC_MON & Concrete Monolith & SAT_RGAS & Residual Ges Saturation & 32546 & 15-Fob-96 & $Y$ & 1 \\
\hline $\bar{Y}$ & 100 & 3064 & CONC_MON & Concrete Monolith & SAT_RGAS & Residual Gas Saturation & 32546 & 15 -feb-96 & $Y$ & 1 \\
\hline $\mathbf{Y}$ & & 3065 & CL_L_TA & $\begin{array}{l}\text { Lower Selado clay.50 } \\
\text { to } 10 \mathrm{~K} \text { yeers }\end{array}$ & RSH_NR & Air-aupply athen redies (3.09 m) & $32021 \mathrm{~A}$ & 15-Fab-96 & $\mathbf{Y}$ & 48 \\
\hline $\mathbf{Y}$ & & 3065 & $C L_{-} L_{-} T 4$ & $\begin{array}{l}\text { Lower Selsoo clay.50 } \\
\text { to 10K years }\end{array}$ & RSH_NR & Ar-supply thent radius $(3.09 \mathrm{~m})$ & $32021 A$ & $15+06-96$ & $\boldsymbol{Y}$ & 48 \\
\hline $\mathbf{Y}$ & & 3065 & CL_L_T4 & $\begin{array}{l}\text { Lower Salaco clay:50 } \\
\text { to } 10 \mathrm{~K} \text { years }\end{array}$ & RSH_NR & Ar-upply ethen redies $(3.09 \mathrm{~m})$ & $32021 \mathrm{~A}$ & $15+c b-96$ & $\mathbf{Y}$ & $4 B$ \\
\hline $\mathbf{Y}$ & & 3065 & $C L \_L+T A$ & $\begin{array}{l}\text { Lower Salodo clay:50 } \\
\text { to } 10 \mathrm{~K} \text { years }\end{array}$ & RSH_ANR & Ar-eupply athen radus $(3.09 \mathrm{~m})$ & $32021 A$ & 15 fab-96 & $\mathbf{Y}$ & $4 B$ \\
\hline $\mathbf{Y}$ & & 3066 & $C L_{-} L$ T4 & $\begin{array}{l}\text { Lower Salaso clay:50 } \\
\text { to 10K years }\end{array}$ & RSH_SN & Selthending met radus $(1.8 \mathrm{~m})$ & 320234 & 15 fecb-85 & Y & 48 \\
\hline Y & & 3066 & CL_L_T4 & $\begin{array}{l}\text { Laver Saledo clay.50 } \\
\text { to } 10 \mathrm{~K} \text { years }\end{array}$ & RSH_SN & Sahtrancing aren radius $(1.8 \mathrm{~m})$ & $32023 A$ & $15-F a b-96$ & $\mathbf{Y}$ & 48 \\
\hline$Y$ & & 3056 & CL_L_T4 & $\begin{array}{l}\text { Lower Salado clay.50 } \\
\text { to 10K years }\end{array}$ & RSH_SN & Senhanang then redien $(1.8 \mathrm{~m})$ & $32023 A$ & $15 F-b-96$ & $\mathbf{Y}$ & 18 \\
\hline $\mathbf{Y}$ & & 3056 & CL_L_T4 & $\begin{array}{l}\text { Lower Salaco ctoy.50 } \\
\text { to } 10 k \text { yours }\end{array}$ & RSH_SN & Senthenaros ment rodus $(1.8 \mathrm{~m})$ & 320034 & 15 fab-96 & $\mathbf{Y}$ & 48 \\
\hline $\mathbf{Y}$ & & 3067 & $C L_{-} L_{-} T 4$ & $\begin{array}{l}\text { Lower Salecos ctory } 50 \\
\text { to 10K yeers }\end{array}$ & RSH_waS & 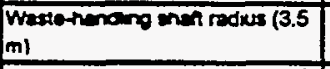 & 320244 & 15 Fab-96 & $\mathbf{Y}$ & AB \\
\hline $\mathbf{Y}$ & & 3057 & CL_L_T4 & $\begin{array}{l}\text { Lower Seleos dey } 50 \\
\text { to 10K yean }\end{array}$ & RSH_was & 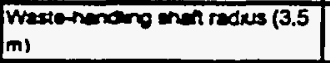 & 320244 & 15-Fab-96 & $Y$ & $A B$ \\
\hline $\mathbf{Y}$ & & 3067 & CL_L_T4 & $\begin{array}{l}\text { Lower Selecos car } 50 \\
\text { to } 10 \mathrm{~K} \text { yeen }\end{array}$ & RSH_mus & 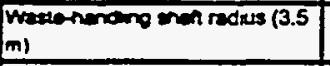 & $32024 \mathrm{~A}$ & 15Feb-96 & $\mathbf{Y}$ & 18 \\
\hline $\mathbf{Y}$ & & 3067 & CL_L_T4 & 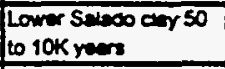 & RSH_mes & $\begin{array}{l}\text { mesorenoro men radius (3.5 } \\
m \text { ) }\end{array}$ & 320244 & 15 fob-96 & $\mathbf{Y}$ & 48 \\
\hline $\mathbf{Y}$ & & 3068 & CL_L_T4 & $\begin{array}{l}\text { Lower Salsos con } 50 \\
\text { to } 10 \mathrm{~K} \text { vaens }\end{array}$ & RSH_EN & 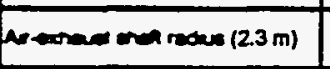 & $32022 \mathrm{~A}$ & $15+c b-96$ & $Y$ & 18 \\
\hline $\mathbf{Y}$ & & 3068 & CL_L_T4 & 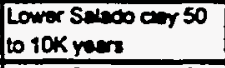 & RSA_EOH & 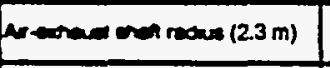 & $32022 A$ & $15-F c b-86$ & $\mathbf{Y}$ & 48 \\
\hline $\mathbf{Y}$ & & 3068 & CL_L_T4 & $\begin{array}{l}\text { Low selecos any } 50 \\
\text { to } 10 \mathrm{~K} \text { yeen }\end{array}$ & RSH_EOH & Ar & $32022 A$ & 15Fab-96 & $\mathbf{Y}$ & 18 \\
\hline $\mathbf{Y}$ & & 3068 & CL_L_T4 & $\begin{array}{l}\text { Lower Solwos any } 50 \\
\text { to } 10 \mathrm{~K} \text { yeers }\end{array}$ & RSH_EXM & 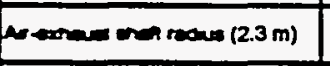 & $32002 A$ & $15+a b-96$ & $\mathbf{Y}$ & 48 \\
\hline $\mathbf{Y}$ & & 3069 & $C L_{-} L_{-} T 4$ & 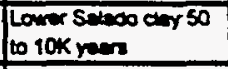 & RAON_Den & 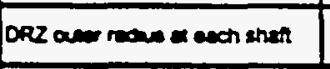 & 32019 & 15 feb-96 & $\mathbf{Y}$ & 1 \\
\hline$Y$ & & 3000 & CL_L_T4 & $\begin{array}{l}\text { Lower Selecos an } 50 \\
\text { to } 10 \mathrm{~K} \text { years }\end{array}$ & RAON_Der & 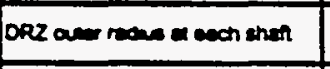 & 32019 & $15+a b-96$ & $\mathbf{Y}$ & 1 \\
\hline $\mathbf{Y}$ & & 3069 & CL_L_TA & $\begin{array}{l}\text { Lower Salecos cioy } 50 \\
\text { to } 10 \mathrm{~K} \text { vems }\end{array}$ & RuON_Donz & 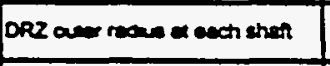 & 32019 & $15-F a b-96$ & $\mathbf{Y}$ & 1 \\
\hline $\mathbf{Y}$ & 50 & 3070 & CL_L_T4 & $\begin{array}{l}\text { Lowar selisos any } 50 \\
\text { to } 10 \mathrm{~K} \text { veers }\end{array}$ & CN_mon & 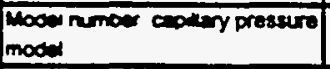 & $32007 A$ & 15 feb-96 & $\mathbf{Y}$ & 48 \\
\hline $\mathbf{Y}$ & 50 & 3070 & CL_L_T4 & $\begin{array}{l}\text { Lower Saledo cioy } 50 \\
\text { to lok yeers }\end{array}$ & CN__MOO & 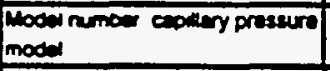 & $32007 A$ & $15-a b-96$ & $\mathbf{Y}$ & $4 B$ \\
\hline $\boldsymbol{Y}$ & 50 & 3070 & CL_L_T4 & $\begin{array}{l}\text { Low Selocos any } 50 \\
\text { to } 10 \mathrm{k} \text { yeers }\end{array}$ & CAP_MOO & 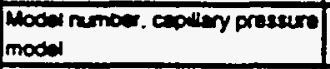 & $32007 A$ & $15+a b s e$ & $Y$ & $4 B$ \\
\hline $\mathbf{Y}$ & 50 & 3070 & CL_L_T4 & $\begin{array}{l}\text { Lower S sisoos ary } 50 \\
\text { to } 10 \mathrm{~K} \text { yeurs }\end{array}$ & CNP_MOO & $\begin{array}{l}\text { Moded number ceodiey preseurn } \\
\text { model }\end{array}$ & $32007 \mathrm{~A}$ & $15+a b-96$ & $\mathbf{Y}$ & 48 \\
\hline
\end{tabular}


CCA Parameter Listing

\begin{tabular}{|c|c|c|c|c|c|c|c|c|c|c|}
\hline ID & 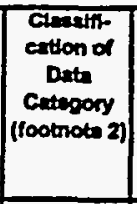 & $\begin{array}{l}\text { Disertioution } \\
\text { Typo }\end{array}$ & $\operatorname{man}$ & Medien & Mintmam & Anedrim & Untas & Retimne ID & $\begin{array}{l}\text { Wai the dete } \\
\text { dovaloped } \\
\text { underen } \\
\text { NOA-1 } \\
\text { Progrem? }\end{array}$ & 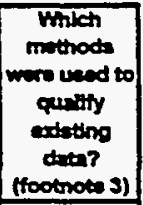 \\
\hline 3061 & 3,4 & CONSTANT & $-1.4000000 E+01$ & $-1.4000000 E+01$ & $-1,4000000 E+01$ & $-1.40000000 E+01$ & $\log \left(m^{n} 2\right)$ & WP035597 & $\mathbf{N}$ & 1 \\
\hline 3062 & $1,3,4$ & CONSTANT & $4.0000000 E+\infty$ & $4.00000000+\infty$ & $4.00000000 E+00$ & $4.00000000 E+00$ & NONE & WP030640 & $\mathbf{Y}$ & NA \\
\hline 3062 & $1,3,4$ & CONSTANT & $4.0000000 E+\infty$ & $4.0000000 E+\infty$ & $4.0000000 E+\infty 0$ & $4.00000000+\infty 00$ & NONE & WP030994 & $Y$ & NA \\
\hline 3062 & $1,3,4$ & CONSTANT & $4.0000000 E+\infty 0$ & $4.0000000 E+\infty$ & $4.0000000 E+\infty 0$ & $4.00000000+\infty 0$ & NONE & WP035597 & $Y$ & NA \\
\hline 3062 & $1,3,4$ & CONSTANT & $4.0000000 E+\infty 0$ & $4.0000000 E+\infty 0$ & $4.0000000 E+\infty$ & $4.0000000 E+\infty$ & NONE & WPO40486 & $\mathbf{Y}$ & N/A \\
\hline 3062 & $1,3,4$ & CONSTANT & $4.0000000 E+\infty$ & $4.00000000 E+\infty 0$ & $4.0000000 E+\infty$ & $4,00000000+\infty$ & NONE & WPO40514 & $Y$ & NA \\
\hline 3063 & 3,4 & CUMULATIVE & $2.5000000 E-01$ & $2.0000000 E-01$ & $0.0000000 E+\infty$ & 6.0000000E-01 & NONE & WP030640 & $Y$ & NA \\
\hline 3063 & 3,4 & CUMULATIVE & $2.5000000 E-01$ & $2.0000000 E-01$ & $0.0000000 E+\infty$ & $6.0000000 E-01$ & NONE & WP030994 & $\bar{Y}$ & N/A \\
\hline 3063 & 3,4 & CUMULATIVE & $2.5000000 E-01$ & $2.0000000 E-01$ & $0.0000000 E+\infty 0$ & $6.0000000 \mathrm{E}-01$ & NONE & WP035268 & $\bar{Y}$ & NA \\
\hline 3063 & 3,4 & CUMUULATIVE & $2.5000000 E-01$ & $2.0000000 E-01$ & $0.0000000 E+00$ & $6.0000000 E-01$ & NONE & WP035597 & $Y$ & NA \\
\hline 3063 & 3.4 & CUMULATIVE & $25000000 E-01$ & $2.0000000 E-01$ & $0.0000000 E+\infty 0$ & $6.0000000 E-01$ & NONE & WPO41558 & $\bar{Y}$ & NA \\
\hline 3063 & 3,4 & CUMULLATIVE & 2.5000000E-01 & $20000000 \mathrm{E}-01$ & $0.0000000 \mathrm{E}+\infty 0$ & $6.0000000 E-01$ & NONE & WPO42751 & $Y$ & NA \\
\hline 3064 & 3,4 & UNIFORM & $2.0000000 E-01$ & $2.0000000 \mathrm{E}-01$ & $0.0000000 E+\infty 0$ & $4.0000000 E-01$ & NONE & WP030640 & $Y$ & NA \\
\hline 3064 & 3,4 & UNIFORM & 2.0000000E-01 & $20000000 E-01$ & $0.0000000 E+\infty 0$ & 4.0000000E-01 & NONE & WP030994 & $Y$ & NA \\
\hline 3064 & 3,4 & UNIFORM & $2.0000000 E-01$ & $20000000 E-01$ & $0.0000000 E+\infty 0$ & 4.0000000E-01 & NONE & WP035268 & $\bar{Y}$ & N/A \\
\hline 3064 & $\overrightarrow{3,4}$ & UNIFORM & $20000000 E-01$ & $2.0000000 E-01$ & $0.0000000 E+\infty 0$ & 4.0000000E-01 & NONE & WP035597 & $\bar{Y}$ & NA \\
\hline 3064 & 3,4 & UNIFORM & $2.0000000 E-01$ & $2.0000000 E-01$ & $0.0000000 E+\infty 0$ & $4.0000000 E-01$ & NONE & WPOA1558 & $Y$ & NA \\
\hline 3065 & 1,3 & CONSTANT & $3.0900000 \mathrm{E}+00$ & $3.0900000 \mathrm{E}+\infty$ & $3.0800000 E+00$ & $3.0900000 E+\infty 0$ & $m$ & WP030640 & $Y$ & NA \\
\hline 3065 & 1,3 & CONSTANT & $3.0900000 E+\infty$ & $3.0900000 E+\infty$ & $3.0900000 E+\infty 0$ & $3.0900000 E+\infty 0$ & m & WP030994 & $Y$ & NA \\
\hline 3065 & 1,3 & CONSTANT & $3.0900000 E+\infty$ & $3.0900000 \equiv+\infty 0$ & $3.09000000+\infty$ & $3.0900000 E+\infty$ & $\mathbf{m}$ & WP035597 & $\mathbf{Y}$ & NA \\
\hline 3065 & 1,3 & CONSTANT & $3.0900000 E+\infty 0$ & $3.09000000+\infty$ & $3.0900000 E+\infty 0$ & $3.0900000 E+\infty$ & $\mathbf{m}$ & WP040514 & $Y$ & NA \\
\hline 3066 & 1,3 & CONSTANT & $1.8000000 \mathrm{E}+\infty 0$ & $1.8000000 \mathrm{E}+00$ & $1.8000000 E+\infty$ & $1.8000000 \mathrm{E}+\infty 0$ & $\mathbf{m}$ & WP030640 & $\mathbf{Y}$ & NA \\
\hline 3066 & 1,3 & CONSTANT & $1.8000000 E+00$ & $1.8000000 E+\infty 0$ & $1.8000000 E+\infty 0$ & $1.8000000 E+\infty 0$ & $\mathbf{m}$ & WP030994 & $\mathbf{Y}$ & NA \\
\hline 3066 & 1,3 & CONSTANT & $1.8000000 \mathrm{E}+\infty$ & $1.80000000+\infty$ & $1.80000000+\infty$ & $1.80000000+\infty$ & $\mathbf{m}$ & WP035597 & $\mathbf{Y}$ & NA \\
\hline 3066 & 1,3 & CONSTANT & $1.8000000 E+\infty$ & $1.8000000 E+\infty 0$ & $1.8000000 E+\infty 0$ & $1.8000000 E+\infty$ & $\mathbf{m}$ & WPO40514 & $\mathbf{Y}$ & NA \\
\hline 3067 & 1,3 & CONSTANT & $3.5000000 E+\infty 0$ & $3.5000000 \mathrm{E}+00$ & $3.5000000 E+\infty$ & $3.5000000 \mathrm{E}+\infty 0$ & $\mathbf{m}$ & WP030640 & $\mathbf{Y}$ & NA \\
\hline 3067 & 1,3 & CONSTANT & $3.5000000 E+\infty$ & $3.5000000 E+\infty$ & $3,5000000 \varepsilon+\infty$ & $3.5000000 E+00$ & $\mathbf{m}$ & WP030994 & $Y$ & NA \\
\hline 3067 & 1,3 & CONSTANT & $3.5000000 E+\infty 0$ & $3.5000000 E+\infty 0$ & $35000000 E+\infty$ & $35000000 E+\infty 0$ & $\mathrm{~m}$ & WPO35597 & $\mathbf{Y}$ & NA \\
\hline 3067 & 1,3 & CONSTANT & $3.5000000 E+00$ & $3.5000000 \equiv+\infty$ & $3.5000000 \in+\infty 0$ & $3.5000000 E+\infty$ & $\mathbf{m}$ & WPO40514 & $\mathbf{Y}$ & N/A \\
\hline 3068 & 1,3 & CONSTANT & $2.3000000 E+\infty 0$ & $2.3000000 E+\infty$ & $2.30000000+\infty$ & $2.3000000 E+\infty$ & $\mathbf{m}$ & WP030640 & $\mathbf{Y}$ & NA \\
\hline 3068 & 1,3 & CONSTANT & $2.3000000 \mathrm{E}+\infty$ & $2.3000000 E+\infty$ & $2.3000000 E+\infty 0$ & $23000000 E+\infty$ & $\mathbf{m}$ & WP030994 & $\mathbf{Y}$ & NA \\
\hline 3068 & 1,3 & CONSTANT & $2.3000000 E+\infty$ & $2.3000000 E+\infty$ & $2.3000000 E+\infty 0$ & $2,3000000 E+\infty$ & $m$ & WP035597 & $\mathbf{Y}$ & NA \\
\hline 3068 & 1,3 & CONSTANT & $23000000 E+\infty 0$ & $2.3000000 E+00$ & $2.3000000 E+\infty)$ & $2.3000000 E+\infty$ & $m$ & WPO40514 & $\mathbf{Y}$ & NA \\
\hline 3069 & $1,3,4$ & CONSTANT & $1.0000000 \mathrm{E}+\infty 0$ & $1.0000000=+\infty$ & $1.0000000 E+\infty 0$ & $1.0000000 E+\infty$ & $\mathrm{m} / \mathrm{m}$ & WP030640 & $Y$ & NA \\
\hline 3069 & $1,3,4$ & CONSTANT & $1.0000000 E+\infty 0$ & $1.0000000 E+\infty$ & $1.0000000 E+\infty$ & $1.0000000 E+\infty$ & $\mathrm{m} / \mathrm{m}$ & WP030994 & $\mathbf{Y}$ & NA \\
\hline 3069 & $1,3,4$ & CONSTANT & $1.0000000 E+\infty$ & $1.0000000 E+\infty$ & $1.0000000 E+\infty$ & $1.0000000 E+\infty$ & $\mathrm{m} / \mathrm{m}$ & WP035597 & $\mathbf{Y}$ & NA \\
\hline 3070 & $1,3,4$ & CONSTANT & $200000000+\infty$ & $20000000 E+\infty 0$ & $2.0000000 E+\infty$ & $20000000 E+\infty$ & NONE & WPO30540 & $Y$ & NA \\
\hline 3070 & $1,3,4$ & CONSTANT & $20000000 E+00$ & $2,0000000 E+\infty 0$ & $2.0000000 E+\infty$ & $2,0000000 E+\infty 0$ & NONE & WP030994 & $Y$ & NA \\
\hline 3070 & $1,3,4$ & CONSTANT & $2.00000000+\infty$ & $2.0000000 E+00$ & $2.0000000 E+00$ & $2.0000000 E+\infty 0$ & NONE & WP035597 & $Y$ & N/A \\
\hline 3070 & $1,3,4$ & CONSTANT & $2.0000000 E+00$ & $2.0000000 E+\infty$ & $2.0000000 E+\infty 0$ & $2.0000000 E+\infty 0$ & NONE & WPO40485 & $\mathbf{Y}$ & NA \\
\hline
\end{tabular}


CCA Parameter Listing

\begin{tabular}{|c|c|c|c|c|c|c|c|c|c|c|}
\hline 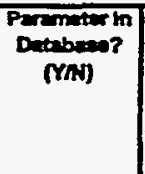 & 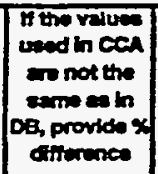 & to & Matariatio & Mrtard Name & Parenter & 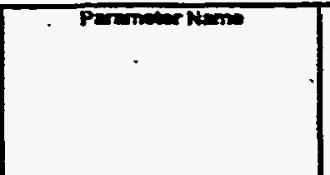 & $\begin{array}{l}\text { PRPT } \\
\text { MPOA }\end{array}$ & Denentry & 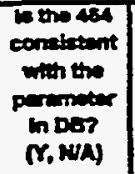 & $\begin{array}{l}\text { Parmits } \\
\text { Cumgory } \\
\text { (footenote 1) }\end{array}$ \\
\hline $\mathbf{Y}$ & 50 & 3070 & $C L_{-} L_{-} T_{4}$ & $\begin{array}{l}\text { Lower Salado ctay:50 } \\
\text { to } 10 \mathrm{~K} \text { years }\end{array}$ & CAP_MOD & $\begin{array}{l}\text { Model number, ceppliay preserts } \\
\text { model }\end{array}$ & $32007 A$ & $15-5 c b-56$ & $\mathbf{Y}$ & 48 \\
\hline $\mathbf{Y}$ & & 3071 & CL_L_TA & $\begin{array}{l}\text { Lower Salodo cty.50 } \\
\text { to } 10 \mathrm{~K} \text { yours }\end{array}$ & COMP_RCK & Bulk Compreasibitity & 32008 & $15-F a b-96$ & $\mathbf{Y}$ & 1 \\
\hline $\mathbf{Y}$ & & 3071 & CL_L_TA & $\begin{array}{l}\text { Lower Saludo cley:50 } \\
\text { to } 10 K \text { yours }\end{array}$ & COMP_RCK & Bulk Compressibitity & 32008 & $15-f c b-96$ & $\mathbf{Y}$ & 1 \\
\hline$Y$ & & 3071 & $C L_{-} L_{-} T 4$ & $\begin{array}{l}\text { Lower Saludo day.50 } \\
\text { to } 10 \mathrm{~K} \text { years }\end{array}$ & COMP_RCK & Buk Compressibity & 32,008 & $15-\mathrm{cb}-96$ & $\mathbf{Y}$ & 1 \\
\hline $\mathbf{Y}$ & & 3072 & CL_L_T4 & $\begin{array}{l}\text { Lower Salado ctay:50 } \\
\text { to } 10 \mathrm{~K} \text { yoers }\end{array}$ & KPT & $\begin{array}{l}\text { Fleg for Permenbithy Determned } \\
\text { Threshoid }\end{array}$ & 32009 & $20-f a b-86$ & $\mathbf{Y}$ & 48 \\
\hline $\mathbf{Y}$ & & 3072 & $C L \_L / T A$ & $\begin{array}{l}\text { Lower Salado clay.50 } \\
\text { to } 10 \mathrm{~K} \text { years }\end{array}$ & KPT & $\begin{array}{l}\text { Fleg for Penmesbility Delerminnd } \\
\text { Threatold }\end{array}$ & 32009 & 20 Feb-96 & $\mathbf{Y}$ & 48 \\
\hline $\mathbf{Y}$ & & 3072 & CL_L_T4 & $\begin{array}{l}\text { Lown Salado ctoy.50 } \\
\text { to } 10 \mathrm{~K} \text { years }\end{array}$ & KPT & $\begin{array}{l}\text { Flog tor Permesbility Determned } \\
\text { Threthold }\end{array}$ & 32009 & $20-F a b-96$ & $\mathbf{Y}$ & 48 \\
\hline $\mathbf{Y}$ & & 3072 & CL_L_T4 & $\begin{array}{l}\text { Lower Salado clay.50 } \\
\text { to 10K yeans }\end{array}$ & KPT & $\begin{array}{l}\text { Fleg for Permeabilty Determned } \\
\text { Threatrold }\end{array}$ & 32009 & $20 F a b-96$ & $\mathbf{Y}$ & 48 \\
\hline $\mathbf{Y}$ & & 3073 & $C L_{-} L_{-} T 4$ & $\begin{array}{l}\text { Lower Salado clay.50 } \\
\text { to } 10 \mathrm{~K} \text { yoess }\end{array}$ & $P C \_M A X$ & $\begin{array}{l}\text { Muxinum allow bla capiley } \\
\text { prespure }\end{array}$ & $32012 A$ & 15 Fab-96 & $\mathbf{Y}$ & 48 \\
\hline $\mathbf{Y}$ & & 3073 & $C L=L$ T4 & $\begin{array}{l}\text { Lower Saledo clay. } 50 \\
\text { to } 10 \mathrm{~K} \text { years }\end{array}$ & PC_MAX & $\begin{array}{l}\text { Maxinum allowbil capilary } \\
\text { presserv }\end{array}$ & 320124 & 15 Fab-96 & $\mathbf{Y}$ & 48 \\
\hline $\mathbf{Y}$ & & 3073 & CL_L_T4 & $\begin{array}{l}\text { Lower Sulado clay. } 50 \\
\text { to 10K yoers }\end{array}$ & PC_max & $\begin{array}{l}\text { Meximum allowable cepillory } \\
\text { pressure }\end{array}$ & $32012 A$ & $15+c b-86$ & $\mathbf{Y}$ & AB \\
\hline $\mathbf{Y}$ & & 3073 & CL_L_T4 & $\begin{array}{l}\text { Lower Salado day:50 } \\
\text { to tok years }\end{array}$ & PC_MAX & $\begin{array}{l}\text { Maximum allownble captitary } \\
\text { prossure }\end{array}$ & $32012 A$ & $15-F c b-96$ & $\mathbf{Y}$ & $4 B$ \\
\hline $\mathbf{Y}$ & & 3073 & CL_L_T4 & $\begin{array}{l}\text { Lower Selado ctay:50 } \\
\text { to 10K years }\end{array}$ & PC_MAX & $\begin{array}{l}\text { Moximum allowable cepillary } \\
\text { prossure }\end{array}$ & 320121 & $15-F c b-96$ & $\mathbf{Y}$ & $4 B$ \\
\hline $\mathbf{Y}$ & 100 & 3074 & $C L L L T 4$ & $\begin{array}{l}\text { Lower Salado day:50 } \\
\text { to tok years }\end{array}$ & PCT_A & $\begin{array}{l}\text { Threstiold Prossure Linear } \\
\text { Perameter }\end{array}$ & 32010 & $15-F=b-96$ & $\mathbf{Y}$ & 1 \\
\hline $\mathbf{Y}$ & 100 & 3074 & CL_L_T4 & $\begin{array}{l}\text { Lowor Salido clay.50 } \\
\text { to } 10 \mathrm{~K} \text { yars }\end{array}$ & PCT_A & $\begin{array}{l}\text { Threshold Pressure Lneer } \\
\text { Percneter }\end{array}$ & 32010 & 15 Fob- 96 & $\mathbf{Y}$ & 1 \\
\hline $\mathbf{Y}$ & 100 & 3074 & CL_L_T4 & $\begin{array}{l}\text { Lower Saledo clay.50 } \\
\text { to } 10 \text { K years }\end{array}$ & PCT_A & $\begin{array}{l}\text { Threshold Pressure Lnas } \\
\text { Paraneter }\end{array}$ & 32010 & 15 fab-86 & $Y$ & 1 \\
\hline $\mathbf{Y}$ & 100 & 3074 & CL_L_T4 & $\begin{array}{l}\text { Lower Salaco clay:50 } \\
\text { to toK years }\end{array}$ & PCT_A & $\begin{array}{l}\text { Thresinold Pressire Lneser } \\
\text { Parameter }\end{array}$ & 32010 & $15+56 b-96$ & $\mathbf{Y}$ & 1 \\
\hline $\mathbf{Y}$ & 100 & 3075 & $C L L_{-} T_{4}$ & $\begin{array}{l}\text { Lower Saledo ctry.50 } \\
\text { to } 10 K \text { yoars }\end{array}$ & PCT_EXP & $\begin{array}{l}\text { Threshotd pressure exponential } \\
\text { parameler }\end{array}$ & 32011 & $15+a b-96$ & $\mathbf{Y}$ & 1 \\
\hline $\mathbf{Y}$ & 100 & 3075 & $C L L_{-}{ }^{4}$ & $\begin{array}{l}\text { Lower Salodo clay.50 } \\
\text { to } 10 k \text { years }\end{array}$ & PCT_EXP & $\begin{array}{l}\text { Threshold pressure exponential } \\
\text { pareneter }\end{array}$ & 32011 & 15 Feb-96 & $\mathbf{Y}$ & 1 \\
\hline $\mathbf{Y}$ & 100 & 3075 & CL_L_T4 & $\begin{array}{l}\text { Lower Saledo ctay.50 } \\
\text { to } 10 \text { years }\end{array}$ & PCT_EXP & $\begin{array}{l}\text { Threshold pressure exponential } \\
\text { permemeter }\end{array}$ & 32011 & 15 Fab-96 & $\mathbf{Y}$ & 1 \\
\hline $\mathbf{Y}$ & 100 & 3075 & CL_L_T4 & $\begin{array}{l}\text { Lower Salado clay.50 } \\
\text { to 10K years }\end{array}$ & PCT_EXP & $\begin{array}{l}\text { Threstrold pressure exponential } \\
\text { peremeter }\end{array}$ & 32011 & $15-5 a b-96$ & $\mathbf{Y}$ & 1 \\
\hline $\mathbf{Y}$ & varies & 3076 & CL_L_T4 & $\begin{array}{l}\text { Lowar Salado day. } 50 \\
\text { to 10K years }\end{array}$ & PORE_DIS & $\begin{array}{l}\text { Brooks-Corey pors distribution } \\
\text { paremeter }\end{array}$ & 32013 & $20-F a b-96$ & $\mathbf{Y}$ & 1 \\
\hline $\mathbf{Y}$ & varies & 3076 & $C L L_{2} T 4$ & $\begin{array}{l}\text { Lower Saledo clay.50 } \\
\text { to toK years }\end{array}$ & PORE_DIS & $\begin{array}{l}\text { Brooks-Corey pore destribution } \\
\text { peremeter }\end{array}$ & 32013 & $20+0 b-96$ & $\mathbf{Y}$ & 1 \\
\hline $\mathbf{Y}$ & varies & 3076 & CL_L_T4 & $\begin{array}{l}\text { Lower Salado clay. } 50 \\
\text { to 10K yeers }\end{array}$ & PORE_DIS & $\begin{array}{l}\text { Brooks-Corey pore distribution } \\
\text { parameter }\end{array}$ & 32013 & 20 feb-96 & $\mathbf{Y}$ & 1 \\
\hline $\mathbf{Y}$ & varies & 3076 & CL_L_T4 & $\begin{array}{l}\text { Lower Seledo day.50 } \\
\text { to 10K years }\end{array}$ & PORE_DIS & $\begin{array}{l}\text { Brooks-Corey pore distribution } \\
\text { parameter }\end{array}$ & 32013 & 20 - & $--Y Y$ & 1 \\
\hline $\mathbf{Y}$ & varies & 3076 & $C L \_L / T 4$ & $\begin{array}{l}\text { Lowor Saledo ciny.50 } \\
\text { to } 10 \text { K years }\end{array}$ & PORE_DIS & $\begin{array}{l}\text { Brooks-Corey pore distribution } \\
\text { perameter }\end{array}$ & 32013 & 20 Fab-96 & $\mathbf{Y}$ & 1 \\
\hline $\mathbf{Y}$ & varies & 3076 & CL_L_T4 & $\begin{array}{l}\text { Lower Seledo ctoy.50 } \\
\text { to } 10 \text { k years }\end{array}$ & PORE_DIS & $\begin{array}{l}\text { Brooks-Corey poro distritution } \\
\text { parameter }\end{array}$ & 32013 & 20 Feb-96 & $\mathbf{Y}$ & 1 \\
\hline $\mathbf{Y}$ & varies & 3076 & CL_L_T4 & $\begin{array}{l}\text { Lower Saledo ctay.50 } \\
\text { to } 10 \text { K yeers }\end{array}$ & PORE_DIS & $\begin{array}{l}\text { Brooks-Corey pore destribution } \\
\text { peremeter }\end{array}$ & 32013 & $20-f c 0-96$ & $\mathbf{Y}$ & 1 \\
\hline $\mathbf{Y}$ & & 3077 & CL_L_T4 & $\begin{array}{l}\text { Lower Salado ctoy.50 } \\
\text { to } 10 \text { K yours }\end{array}$ & POROSIT & Effoctive porosity & 32014 & $15-5 b-96$ & $\mathbf{Y}$ & 1 \\
\hline $\mathbf{Y}$ & & 3077 & CL_L_T4 & $\begin{array}{l}\text { Lower Saledo diny:50 } \\
\text { to 10k yoes }\end{array}$ & POROSTY & Effective porosity & 32014 & 15 feb-96 & $\mathbf{Y}$ & 1 \\
\hline $\mathbf{Y}$ & & 3077 & $C L_{2} L_{-} T_{4}$ & $\begin{array}{l}\text { Lower Salado cty.50 } \\
\text { to 10k yeer }\end{array}$ & POROSTYY & Effective porouity & 32014 & $15-F a b-86$ & $\mathbf{Y}$ & 1 \\
\hline $\mathbf{Y}$ & varies & 3078 & CL_L_T4 & $\begin{array}{l}\text { Lower Salodo ctay.50 } \\
\text { to 10k years }\end{array}$ & PRUX_LOG & $\begin{array}{l}\text { Los of intrnac permesbilty, } X \text { - } \\
\text { drection }\end{array}$ & 32016 & $15 F a b-96$ & $\mathbf{Y}$ & 1 \\
\hline $\mathbf{Y}$ & veries & 3078 & CL_L_T4 & $\begin{array}{l}\text { Lower Saledo chy.50 } \\
\text { to } 10 \mathrm{~K} \text { yeass }\end{array}$ & PRMX_LOG & $\begin{array}{l}\text { Los of inturase permestidty, } X \text { - } \\
\text { orvetion }\end{array}$ & 32016 & 15-Fob-96 & $\mathbf{Y}$ & 1 \\
\hline $\mathbf{Y}$ & varias & 3078 & CL_L_T4 & $\begin{array}{l}\text { Lower Saledo ctay.50 } \\
\text { to 10K years }\end{array}$ & PRMX_LOG & $\begin{array}{l}\text { Log of intunate permestbilty, } X \text { - } \\
\text { diruction }\end{array}$ & 32016 & 15 fab-86 & $\mathbf{Y}$ & 1 \\
\hline $\mathbf{Y}$ & veries & 3078 & CL_L_T4 & $\begin{array}{l}\text { Lower Satado ctay.50 } \\
\text { to 10K youss }\end{array}$ & PRMK_LOG & $\begin{array}{l}\text { Log of nitrnax permasbilty, } X \text { - } \\
\text { dinction }\end{array}$ & 32016 & 15 Feb-es & $\mathbf{Y}$ & 1 \\
\hline $\mathbf{Y}$ & varies & 3078 & CL_L_T4 & $\begin{array}{l}\text { Lower Salado diny.50 } \\
\text { to 10K years }\end{array}$ & PRIX_LOG & $\begin{array}{l}\text { Los of mennsic permetbilay, } X- \\
\text { drection }\end{array}$ & 32016 & $15+4 c t-86$ & $\mathbf{Y}$ & 1 \\
\hline
\end{tabular}


CCA Parameter Listing

\begin{tabular}{|c|c|c|c|c|c|c|c|c|c|c|}
\hline 10 & $\begin{array}{c}\text { Cimeditit } \\
\text { cation of } \\
\text { Deth } \\
\text { Category } \\
\text { (footsoto 2) }\end{array}$ & $\begin{array}{c}\text { Otstrburson } \\
\text { Type }\end{array}$ & $\operatorname{man}$ & Moden & Minkmem & Hodrum & thats & Rifiermes b 10 & 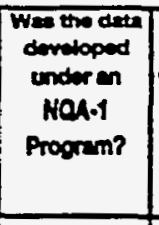 & 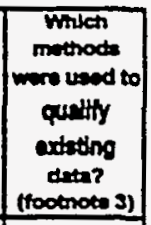 \\
\hline 3070 & $1,3,4$ & CONSTANT & $2.0000000 E+\infty 0$ & $2.0000000 E+\infty 0$ & $2.0000000 E+\infty$ & $20000000 E+\infty 0$ & NONE & WP040514 & $\mathbf{Y}$ & $N / A$ \\
\hline 3071 & $1,3,4$ & CONSTANT & $1.5900000 \mathrm{E}-09$ & $1.5900000=-09$ & $1.5900000 E-09$ & 1.5900000E-09 & $P a-1$ & UPO030640 & $\mathbf{Y}$ & NA \\
\hline 3071 & 1.3 .4 & CONSTANT & $1.5900000 \mathrm{E}-09$ & $1.5900000 \mathrm{E}-09$ & $1.5900000=-09$ & $1.5900000=-\infty 9$ & Pan-1 & WP030994 & $Y$ & NA \\
\hline 3071 & $1,3,4$ & CONSTANT & $1.59000000-09$ & $1.5900000=-09$ & $1.5900000 \leq-09$ & $1.5900000 E-09$ & Pan-1 & WP035597 & $\mathbf{Y}$ & NA \\
\hline 3072 & 1,3 & CONSTANT & $0.0000000 E+\infty 0$ & $0.0000000 E+\infty 0$ & $0.0000000 E+\infty 0$ & $0.0000000 E+\infty 0$ & NONE & WP035271 & $\mathbf{Y}$ & NA \\
\hline 3072 & 1,3 & CONSTANT & $0.0000000 E+\infty 0$ & $0.0000000 E+\infty$ & $0.0000000 E+\infty 0$ & $0.0000000 \mathrm{E}+\infty$ & NONE & WPO35335 & $\mathbf{Y}$ & NA \\
\hline 3072 & 1,3 & CONSTANT & $0.0000000 E+\infty 0$ & $0.0000000 E+\infty$ & $0.0000000 \mathrm{E}+\infty 0$ & $0.0000000 E+\infty$ & NONE & WP035597 & $\mathbf{Y}$ & NA \\
\hline 3072 & 1,3 & CONSTANT & $0.0000000 E+\infty 0$ & $0.0000000 E+\infty$ & $0.0000000 E+\infty$ & $0.0000000 E+\infty 0$ & NONE & WP040514 & $Y$ & NA \\
\hline 3073 & 1,3 & CONSTANT & $1.0000000 E+08$ & $1.0000000 E+08$ & $1.0000000 E+08$ & $1.0000000 E+08$ & $\mathbf{P a}$ & MP030640 & $Y$ & NA \\
\hline 3073 & 1,3 & CONSTANT & $1.0000000 E+08$ & $1.0000000 E+\infty 0$ & $1.0000000 E+08$ & $1.0000000 E+08$ & $P_{\mathbf{a}}$ & WP030994 & $\mathbf{Y}$ & NA \\
\hline 3073 & 1.3 & CONSTANT & $1.0000000 E+08$ & $1.0000000 E+08$ & $1.0000000 \varepsilon+08$ & $1.0000000 E+08$ & $P_{a}$ & WPO35597 & $\mathbf{Y}$ & NA \\
\hline 3073 & 1,3 & CONSTANT & $1.0000000 E+08$ & $1.0000000 E+08$ & $1.0000000 E+08$ & $1.0000000 \mathrm{E}+08$ & $\mathbf{P a}$ & WP040485 & $Y$ & NA \\
\hline 3073 & 1,3 & CONSTANT & $1.0000000 E+08$ & $1.0000000 E+00$ & $1.0000000 E+00$ & $1.0000000 E+08$ & $\mathrm{~Pa}$ & WPO40514 & $\mathbf{Y}$ & NA \\
\hline 3074 & 3,4 & CONSTANT & $5.6000000 E-01$ & $5.6000000 E-01$ & $5.6000000 E-01$ & $5.6000000 E-01$ & Pa & WP030640 & $\mathbf{Y}$ & NA \\
\hline 3074 & 3,4 & CONSTANT & $5.6000000 E-01$ & 5.6000000E-01 & $5.6000000 E-01$ & $5.6000000 E-01$ & $P=$ & WPO30994 & $\mathbf{Y}$ & $N / A$ \\
\hline 3074 & 3.4 & CONSTANT & $5.6000000 E-01$ & $5.6000000 E-01$ & 5.6000000E-01 & $5.6000000 E-01$ & $\mathbf{P a}$ & WP035597 & $\mathbf{Y}$ & NA \\
\hline 3074 & 3,4 & CONSTANT & $5.6000000 E-01$ & $5.6000000 E-01$ & $5.6000000 E-01$ & 5.6000000E-01 & $\mathrm{Pa}$ & WPO40434 & $\mathbf{Y}$ & NA \\
\hline 3075 & 3,4 & CONSTANT & $-3.4600000 E-01$ & $3.4600000 E-01$ & $-3.4600000=-01$ & $-3.4600000 E-01$ & NONE & WP030640 & $\mathbf{Y}$ & NUA \\
\hline 3075 & 3.4 & CONSTANT & $3,4600000 E-01$ & $3.4600000 E-01$ & $-3.4600000 E-01$ & $-3.4600000 \mathrm{E}-01$ & NONE & WP030994 & $Y$ & NA \\
\hline 3075 & 3.4 & CONSTANT & $3.4600000 \mathrm{E}-01$ & $-3.4600000 E-01$ & $-3.4600000 \leq-01$ & $-3.4600000 E-01$ & NONE & WP035597 & $Y$ & N/A \\
\hline 3075 & 3,4 & CONSTANT & $-3.4600000 \mathrm{E}-01$ & $3.4600000 E-01$ & $-3.46000005-01$ & $-3.4600000 E-01$ & NONE & WPO40434 & $\mathbf{Y}$ & $N / A$ \\
\hline 3076 & 3,4 & CUMULATIVE & $2.5200000 E+\infty$ & $9.4000000 \mathrm{E}-01$ & $1.1000000 \mathrm{E}-01$ & $8.1000000 E+\infty$ & NONE & WP030640 & Y & NA \\
\hline 3076 & 3,4 & CUMULATINE & $2.5200000 E+00$ & $9.4000000 E-01$ & $1.1000000 E-01$ & $8.1000000 E+\infty$ & NONE & Wp030994 & $Y$ & NA \\
\hline 3076 & 3.4 & CUMULATNE & $2.5200000 E+\infty$ & $9.4000000 E-01$ & $1.1000000 E-01$ & $8.1000000 E+\infty$ & NONE & WP032287 & $\mathbf{Y}$ & NA \\
\hline 3076 & 3.4 & CUMULATINE & $2.5200000 E+00$ & $9.4000000 E-01$ & $1.1000000 E-01$ & 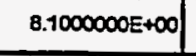 & NONE & WP035268 & $-Y$ & NA \\
\hline 3076 & 3,4 & CUMULATIVE & $2.5200000 E+\infty 0$ & $9.4000000 E-01$ & $1.1000000 E-01$ & $8.1000000 E+\infty 0$ & NONE & WP035597 & $\mathbf{Y}$ & N/A \\
\hline 3076 & 3,4 & CUMULATIVE & $2.52500000++\infty$ & $9.4000000 E-01$ & $1.1000000 E-01$ & $8.1000000 E+00$ & NONE & WPOA1558 & $\mathbf{Y}$ & N/A \\
\hline 3076 & 3,4 & CUMULATME & $2.5200000 \mathrm{E}+\infty 0$ & $9.4000000=-01$ & $1.1000000 E-01$ & $8.1000000 E+\infty$ & NONE & WPOA2752 & $\mathbf{Y}$ & $N A$ \\
\hline 3077 & $1,3,4$ & CONSTANT & 2.4000000E-01 & $21000000 E-01$ & $2.4000000 E-01$ & $24000000 E-01$ & $m^{\wedge} 3 m^{\wedge} 3$ & WP030640 & $\mathbf{Y}$ & NA \\
\hline $30 \pi 7$ & $1,3,4$ & CONSTANT & 2.4000000E-01 & $2.4000000 E-01$ & $24000000 E-01$ & $2.4000000 E-01$ & $m^{\wedge} 3 / m^{\wedge} 3$ & UP030994 & $Y$ & NA \\
\hline 307 & $1,3,4$ & CONSTANT & 2.4000000E-01 & 24000000E-01 & 24000000E-01 & $2.4000000 E-01$ & $m^{\wedge} 3 m^{\wedge} 3$ & WP035597 & $\mathbf{Y}$ & NA \\
\hline 3078 & $1,3,4$ & TRLANGULAR & $-1.8867000 E+01$ & $-1.8301000 E+01$ & $-2.1000000 E+01$ & $-1.7301000 E+01$ & $\log \left(m^{\wedge} 2\right)$ & WPO30640 & YEN & 1 \\
\hline 3078 & $1,3,4$ & TRIANGULAR & $-1.8857000 \mathrm{E}+01$ & $-1.8301000 E+01$ & $-2.1000000 E+01$ & $-1.7301000 E+01$ & $\log \left(m^{\wedge} 2\right)$ & WP030994 & YSN & 1 \\
\hline 3078 & $1,3,4$ & TRLANGULAR & $-1.8867000 \mathrm{E}+01$ & $-1.8301000 E+01$ & $-2.1000000 E+01$ & $-1.7301000 E+01$ & $\log \left(m^{\wedge} 2\right)$ & WP035258 & YEN & 1 \\
\hline 3078 & $1,3,4$ & TRLANGULAR & $-1.8867000 E+01$ & $-1.8301000 E+01$ & $-21000000 E+01$ & $-1.7301000 E+01$ & $\log \left(m^{n} 2\right)$ & WP035597 & Y\&N & 1 \\
\hline 3078 & $1,3,4$ & IRLANGULAR & $-1.6867000 E+01$ & $-1.830+000 E+01$ & $-2.1000000 E+01$ & $-1.7301000 E+01$ & $\log \left(m^{\wedge} 2\right)$ & WP0 41558 & Y8N & 1 \\
\hline
\end{tabular}


CCA Parameter Listing

\begin{tabular}{|c|c|c|c|c|c|c|c|c|c|c|}
\hline $\begin{array}{l}\text { Permeter in } \\
\text { Datubese? } \\
\text { (NN) }\end{array}$ & 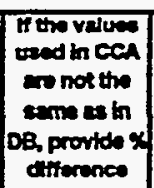 & का & Mataria id & Metartan & Parminar & $\begin{array}{l}\text { Permotar Narm } \\
- \\
-\end{array}$ & $\begin{array}{l}\text { PRPD } \\
\text { Mino on }\end{array}$ & $\begin{array}{c}\text { Dertantry } \\
\text { Dets }\end{array}$ & 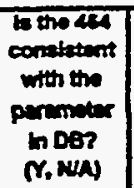 & $\begin{array}{l}\text { Parnester } \\
\text { Cotegory } \\
\text { (foounote i) }\end{array}$ \\
\hline $\mathbf{Y}$ & & 3079 & CL_L_T4 & $\begin{array}{l}\text { Lower Salado clay.50 } \\
\text { to } 10 \mathrm{~K} \text { years }\end{array}$ & PRMY_LOG & $\begin{array}{l}\text { Log of intensuc permesbilty, Y- } \\
\text { direction }\end{array}$ & 32017 & $15-F a b-96$ & $\mathbf{Y}$ & 1 \\
\hline $\mathbf{Y}$ & & 3079 & $C L_{2} L_{2} T_{4}$ & $\begin{array}{l}\text { Lower Salado clay.50 } \\
\text { to } 10 \text { K years }\end{array}$ & PRAY_LOG & $\begin{array}{l}\text { Los of inaresse permesbility, Y- } \\
\text { direction }\end{array}$ & 32017 & $15 F a b-96$ & $\mathbf{Y}$ & 1 \\
\hline $\mathbf{Y}$ & & 3079 & CL_L_T4 & $\begin{array}{l}\text { Lower Salado clay. } 50 \\
\text { to } 10 \mathrm{~K} \text { yoars }\end{array}$ & PRMY_LOG & $\begin{array}{l}\text { Loo of introste perineability, } Y \text { - } \\
\text { ofrection }\end{array}$ & 32017 & 15-fab-96 & $\mathbf{Y}$ & 1 \\
\hline $\mathbf{Y}$ & & 3079 & CL_L_TA & $\begin{array}{l}\text { Lower Saledo clay.50 } \\
\text { to } 10 \mathrm{~K} \text { years }\end{array}$ & PRUY_LOG & $\begin{array}{l}\text { Los of intricsic permentilaty, Y- } \\
\text { direction }\end{array}$ & 32017 & 15feb-96 & $\mathbf{Y}$ & 1 \\
\hline $\mathbf{Y}$ & & 3079 & CL_L_T4 & $\begin{array}{l}\text { Lower Solado clay:50 } \\
\text { to } 10 \mathrm{~K} \text { years }\end{array}$ & PRMY_LOG & $\begin{array}{l}\text { Log of intrinsic permestilty, } Y- \\
\text { direction }\end{array}$ & 32017 & $15+c b-96$ & $\mathbf{Y}$ & 1 \\
\hline $\mathbf{Y}$ & & 3080 & CL_L_T4 & $\begin{array}{l}\text { Lower Salado clay:50 } \\
\text { to 10K years }\end{array}$ & PRMZ_LOG & $\begin{array}{l}\text { Log of intmissic permeabildy, Z- } \\
\text { orrection }\end{array}$ & 32018 & $15+a b-96$ & $\mathbf{Y}$ & 1 \\
\hline $\mathbf{Y}$ & & 3000 & CL_L_TA & $\begin{array}{l}\text { Lower Salaco clay: } 50 \\
\text { to 10K years }\end{array}$ & PRMZ_LOG & $\begin{array}{l}\text { Log of intornax permeabilty, Z- } \\
\text { direction }\end{array}$ & 32018 & $15-F \Longleftrightarrow-96$ & $\mathbf{Y}$ & 1 \\
\hline $\mathbf{Y}$ & & 3080 & CL_L_T4 & $\begin{array}{l}\text { Lower Salado day.50 } \\
\text { to } 10 \mathrm{~K} \text { years }\end{array}$ & PRNZ_LOG & $\begin{array}{l}\text { Log of intrinsic permentidty, z- } \\
\text { orrection }\end{array}$ & 32018 & 15Fab-ss & $\mathbf{Y}$ & 1 \\
\hline $\mathbf{Y}$ & & 3080 & CL_L_T4 & $\begin{array}{l}\text { Lower Salado clay:50 } \\
\text { to } 10 \mathrm{~K} \text { years }\end{array}$ & PRMZ_LOG & $\begin{array}{l}\text { Log of intrinsic permenbilty, Z- } \\
\text { diruction }\end{array}$ & 32018 & 15-Feb-96 & $\mathbf{Y}$ & 1 \\
\hline $\mathbf{Y}$ & & 3081 & CL_L_T4 & $\begin{array}{l}\text { Lower Satado day.50 } \\
\text { to 10K years }\end{array}$ & RELP_MOD & $\begin{array}{l}\text { Model number, relative } \\
\text { permeability model }\end{array}$ & 320204 & 15-Feb-96 & $\mathbf{Y}$ & 48 \\
\hline $\mathbf{Y}$ & & 3081 & CL_L_T4 & $\begin{array}{l}\text { Lower Salado clay.50 } \\
\text { to 10K years }\end{array}$ & RELP_MOD & $\begin{array}{l}\text { Model number, relative } \\
\text { permenbitity model }\end{array}$ & 320204 & 15-fab-96 & $\mathbf{Y}$ & 48 \\
\hline $\mathbf{Y}$ & & 3081 & CL_L_T4 & $\begin{array}{l}\text { Lower Salado clay.50 } \\
\text { to 10K yoars }\end{array}$ & RELP_MOD & $\begin{array}{l}\text { Model number, relative } \\
\text { permesbitity model }\end{array}$ & 320204 & 15-fab-96 & $\mathbf{Y}$ & 48 \\
\hline $\mathbf{Y}$ & & 3081 & CL_L_T4 & $\begin{array}{l}\text { Lower Salado clay.50 } \\
\text { to } 10 \mathrm{~K} \text { years }\end{array}$ & RELP_MOD & $\begin{array}{l}\text { Model number, rotative } \\
\text { permeability modol }\end{array}$ & $32020 \mathrm{~A}$ & 15-Fob-96 & $\mathbf{Y}$ & 48 \\
\hline $\mathbf{Y}$ & & 3081 & CL_L_T4 & $\begin{array}{l}\text { Lower Salado ctay.50 } \\
\text { to 10K years }\end{array}$ & REIP_MOD & $\begin{array}{l}\text { Model number, relative } \\
\text { permesbility model }\end{array}$ & 320204 & $15+5 e b-96$ & $\mathbf{Y}$ & $4 B$ \\
\hline $\mathbf{Y}$ & 100 & 3082 & CL_L_T4 & $\begin{array}{l}\text { Lower Saledo clay.50 } \\
\text { to } 10 K \text { years }\end{array}$ & SAT_RBRN & Recideal Brino Saturation & 32025 & $15 f a b-96$ & $\mathbf{Y}$ & 1 \\
\hline $\mathbf{Y}$ & 100 & 3082 & CL_L_T4 & $\begin{array}{l}\text { Lower Salado clay.50 } \\
\text { to } 10 \mathrm{~K} \text { years }\end{array}$ & SAT_RBRN & Residual Brine Seturation & 32025 & $15+c b-96$ & $\mathbf{Y}$ & 1 \\
\hline $\mathbf{Y}$ & 100 & 3082 & CL_L_T4 & $\begin{array}{l}\text { Lower Salado chay.50 } \\
\text { to toK years }\end{array}$ & SAT_RBRN & Residual Brine Seturation & 32025 & $15+a b-96$ & $\mathbf{Y}$ & 1 \\
\hline $\mathbf{Y}$ & 100 & 3082 & CL_L_T4 & $\begin{array}{l}\text { Lower Salado clay.50 } \\
\text { to 10K years }\end{array}$ & SAT_RBRN & Residual Brino Seturation & 32025 & 15-fob-96 & $\mathbf{Y}$ & 1 \\
\hline $\mathbf{Y}$ & 100 & 3082 & CL_L_TA & $\begin{array}{l}\text { Lower Salado clay. } 50 \\
\text { to } 10 \text { K years }\end{array}$ & SAT_RBRN & Resicusal Brmo Suturation & 32025 & 15-Fob-96 & $\mathbf{Y}$ & 1 \\
\hline $\mathbf{Y}$ & 100 & 3082 & $C L_{-} L_{2} T 4$ & $\begin{array}{l}\text { Lower Salaco clay:50 } \\
\text { to } 10 \mathrm{~K} \text { years }\end{array}$ & SAT_RBRN & Residenal Brina Seturation & 32025 & $15+F b-96$ & $\mathbf{Y}$ & 1 \\
\hline $\mathbf{Y}$ & 100 & 3003 & CL_L_T4 & $\begin{array}{l}\text { Lower Saledo diny. } 50 \\
\text { to 10K years }\end{array}$ & SAT_RGAS & Residual Ges Saturation & 32027 & $15-F \cdot b-96$ & $\mathbf{Y}$ & 1 \\
\hline $\mathbf{Y}$ & 100 & 3083 & CL_L_T4 & $\begin{array}{l}\text { Lower Salodo ctoy.50 } \\
\text { to } 10 \text { K years }\end{array}$ & SAT_RGAS & Resicual Ges Seturation & 32027 & 15 feb-96 & $\mathbf{Y}$ & 1 \\
\hline $\mathbf{Y}$ & 100 & 3083 & $C L_{-} L_{-} T_{4}$ & $\begin{array}{l}\text { Lower Salado diy.50 } \\
\text { to 10K years }\end{array}$ & SAT_RGAS & Resicual Ges Suturation & 32027 & 15-Fob-96 & $\mathbf{Y}$ & 1 \\
\hline $\mathbf{Y}$ & 100 & 3083 & CL_L_T4 & $\begin{array}{l}\text { Lower Salado clay.50 } \\
\text { to 10K yeers }\end{array}$ & SAT_RGAS & Residual Ges Seturation & 32027 & $15+a b-96$ & $\mathbf{Y}$ & 1 \\
\hline $\mathbf{Y}$ & 100 & 3083 & CL_L_T4 & $\begin{array}{l}\text { Lower Saledo ctry.50 } \\
\text { to 10K yours }\end{array}$ & SAT_RGAS & Resicual Ges Seturation & 32027 & $15-F e b-96$ & $--\mathbf{Y}$ & 1 \\
\hline $\mathbf{Y}$ & & 3084 & CL_M_T1 & $\begin{array}{l}\text { Upper Salado clay:0 to } \\
10 \text { years }\end{array}$ & RSH_AR & Ar-eupply anant radies $(3.09 \mathrm{~m})$ & 320554 & 15-Feb-56 & $\mathbf{Y}$ & $A B$ \\
\hline $\mathbf{Y}$ & & 3084 & CL_M_T1 & $\begin{array}{l}\text { Upper Salado day.0 to } \\
10 \text { years }\end{array}$ & RSH_AR & Air-apply ahen radies $(3.09 \mathrm{~m})$ & $32056 \mathrm{~A}$ & 15-Fob-s6 & $\mathbf{Y}$ & $A B$ \\
\hline $\mathbf{Y}$ & & 3084 & CL_M_TI & $\begin{array}{l}\text { Upper Saledo dry:0 to } \\
10 \text { years }\end{array}$ & RSH_AR & Ar-upply shat redius $(3.09 \mathrm{~m})$ & 320564 & 15 fob-96 & $\mathbf{Y}$ & 48 \\
\hline $\mathbf{Y}$ & & 3084 & CL_M_TI & $\begin{array}{l}\text { Upper Saledo doy:0 to } \\
10 \text { years }\end{array}$ & RSH_ARR & Ar-apply shat redius $(3.09 \mathrm{~m})$ & 320564 & 15 Fab-96 & $\mathbf{Y}$ & 48 \\
\hline $\mathbf{Y}$ & & 3085 & CL_M_T1 & $\begin{array}{l}\text { Upper Salado clay.0 to } \\
10 \text { yaars }\end{array}$ & RSH_SAL & Sull handing that radies $(1.8 \mathrm{~m})$ & $32058 A$ & $15-F a b-86$ & $\mathbf{Y}$ & $\mathbf{A B}$ \\
\hline $\mathbf{Y}$ & & 3025 & CL_M_T1 & $\begin{array}{l}\text { Upper Saledo dey.0 to } \\
10 \text { yoars }\end{array}$ & RSH_SAL & Sall handting ghat radies $(1.8 \mathrm{~m})$ & 320584 & 15Fab-96 & $\mathbf{Y}$ & 48 \\
\hline $\mathbf{Y}$ & & 3085 & CL_M_Tt & $\begin{array}{l}\text { Upper Saledo diny.0 to } \\
10 \text { yeurs }\end{array}$ & RSH_SNL & Selthending that redius $(1.8 \mathrm{~m})$ & $32058 A$ & 15-feb-96 & $\mathbf{Y}$ & $4 B$ \\
\hline $\mathbf{Y}$ & & 3085 & CL_M_T1 & $\begin{array}{l}\text { Upper Saledo diny.0 to } \\
10 \text { yeers }\end{array}$ & RSH_SAL & Salthanding chen redus $(1.8 \mathrm{~m})$ & 320584 & $15-F a b-96$ & $\mathbf{Y}$ & 48 \\
\hline $\mathbf{Y}$ & & 3085 & CL_M_T1 & $\begin{array}{l}\text { Upper Salodo ctry.0 to } \\
10 \text { years }\end{array}$ & RSH_WAS & $\begin{array}{l}\text { Waste-handing shat redius (3.5 } \\
\text { m) }\end{array}$ & $32059 A$ & 15Fab-se & $\mathbf{Y}$ & 48 \\
\hline $\mathbf{Y}$ & & 3006 & CL_M_TI & $\begin{array}{l}\text { Uppor Selado clay. } 0 \text { to } \\
10 \text { years }\end{array}$ & RSH_WAS & $\begin{array}{l}\text { Wastohanding shan rades (3.5 } \\
\text { m) }\end{array}$ & $32059 A$ & $15-F a b-96$ & $\mathbf{Y}$ & 48 \\
\hline $\mathbf{Y}$ & & 3006 & CL_M_TI & $\begin{array}{l}\text { Uppor Salodo clary.0 to } \\
10 \text { years }\end{array}$ & RSH_WAS & $\begin{array}{l}\text { Wate-hendion shat redus (3.5 } \\
\text { (m) }\end{array}$ & 320594 & $15+50 b-96$ & $\mathbf{Y}$ & $4 B$ \\
\hline
\end{tabular}


CCA Parameter Listing

\begin{tabular}{|c|c|c|c|c|c|c|c|c|c|c|}
\hline DD & 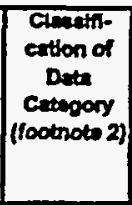 & $\begin{array}{c}\text { Detetbution } \\
\text { Type }\end{array}$ & Meen & Medin & MIntrmun & Medring & Units & Reterences to & $\begin{array}{l}\text { Was the data } \\
\text { dovioped } \\
\text { undiren } \\
\text { Noh-1 } \\
\text { Progrmm? }\end{array}$ & $\begin{array}{c}\text { Which } \\
\text { mothods } \\
\text { wore uned to } \\
\text { qually } \\
\text { extiting } \\
\text { dite? } \\
\text { (footnote 3) }\end{array}$ \\
\hline 3079 & $1,3,4$ & TRIANGULAR & $-1.8867000 E+01$ & $-1.8301000 E+01$ & $-2.1000000 E+01$ & $-1.7301000 E+01$ & $\log \left(m^{\wedge} 2\right)$ & WPO30640 & Y\&N & 1 \\
\hline 3079 & $1,3,4$ & TRLANGULAR & $-1.8867000 E+01$ & $-1.8301000 E+01$ & $-2.1000000 E+01$ & $-1.7301000 E+01$ & $\log \left(m^{n} 2\right)$ & WP030994 & YaN & 1 \\
\hline 3079 & $1,3,4$ & TRLANGULAR & $-1.8867000 E+01$ & $-1.8301000 E+01$ & $-2.1000000 E+01$ & $-1.7301000 E+01$ & $\log \left(m^{n} 2\right)$ & WP035268 & YEN & 1 \\
\hline 3079 & $1,3,4$ & TRLNGULAR & $-1.8867000 E+01$ & $-1.8301000 E+01$ & $-21000000 E+01$ & $-1.7301000 E+01$ & $\log \left(m^{\wedge} 2\right)$ & WPO35597 & Y\&N & 1 \\
\hline 3079 & $1,3,4$ & TRLANGULAR & $-1.8867000 E+01$ & $-1.8301000 E+01$ & $-2.1000000 E+01$ & $-1.7301000 E+01$ & $\log \left(m^{\wedge} 2\right)$ & WPOA1558 & YEN & 1 \\
\hline 3080 & $1,3,4$ & TRLANGULAR & $-1.8857000 E+01$ & $-1.8301000 E+01$ & $-21000000 E+01$ & $-1.7301000 E+01$ & $\log \left(m^{\wedge} 2\right)$ & WP030540 & YaN & 1 \\
\hline 3080 & $1,3,4$ & TRLANGULAR & $-1.8867000 E+01$ & $-1.8301000 E+01$ & $-2.1000000 E+01$ & $-1.7301000 E+01$ & $\log \left(m^{\wedge} 2\right)$ & WP030994 & YEN & 1 \\
\hline 3080 & $1,3,4$ & TRLANGULAR & $-1.8867000 E+01$ & $-1.8301000 E+01$ & $-2.1000000 E+01$ & $-1.7301000 E+01$ & $\log (m+2)$ & WP035597 & YaN & 1 \\
\hline 3080 & $1,3,4$ & TRLANGULAR & $-1.8867000 E+01$ & $-1.8301000 E+01$ & $-2.1000000 E+01$ & $-1.7301000 E+01$ & $\log \left(m^{\wedge} 2\right)$ & WPO41558 & Y\&N & 1 \\
\hline 3089 & $9,3,4$ & CONSTANT & $4.0000000 E+\infty 0$ & $4.0000000 E+\infty)$ & $4.0000000 E+\infty 0$ & $4.0000000 E+00$ & NORE & WP030640 & $\mathbf{Y}$ & NA \\
\hline 3081 & $1,3,4$ & CONSTANT & $4,0000000 E+\infty$ & $4.0000000 E+\infty 0$ & $4.0000000 E+\infty$ & $4.0000000 E+\infty)$ & NONE & WP030994 & $\mathbf{Y}$ & NA \\
\hline 3081 & $1,3,4$ & CONSTANT & $4,0000000 E+00$ & $4.0000000 E+\infty 0$ & $4.0000000 E+\infty$ & $4.0000000 E+00$ & NONE & WP035597 & $\mathbf{Y}$ & NA \\
\hline 3081 & $1,3,4$ & CONSTANT & $4.0000000 E+00$ & $4.00000000 E+\infty 0$ & $4.0000000 E+\infty$ & $4.00000000+\infty$ & NONE & WPO40405 & $\mathbf{Y}$ & NA \\
\hline 3081 & $1,3,4$ & CONSTANT & $4.0000000 E+\infty 0$ & $4.0000000 E+\infty 0$ & $4.0000000 E+\infty$ & $4.0000000 E+\infty$ & NONE & WPO40514 & $\mathbf{Y}$ & N/A \\
\hline 3082 & 3,4 & CUMULATINE & 25000000E-01 & $2,0000000 E-01$ & $0.0000000 E+\infty$ & $6.0000000 E-01$ & NONE & WP030540 & $Y$ & N/A \\
\hline 3082 & 3,4 & CUMULATIVE & $2.5000000 E-01$ & $2.0000000 E-01$ & $0.0000000 E+\infty$ & $6.0000000 E-01$ & NONE & WP030994 & $\mathbf{Y}$ & NA \\
\hline 3082 & 3,4 & CUMULATIVE & 2.5000000E-01 & $2.0000000 E-01$ & $0.00000000+\infty$ & $6.0000000 E-01$ & NONE & WP035268 & $\mathbf{Y}$ & N/A \\
\hline 3082 & 3,4 & CUMULATIVE & $2.5000000 E-01$ & $2,0000000 E-01$ & $0.0000000 E+\infty$ & $60000000 E-01$ & NONE & WP035597 & $\mathbf{Y}$ & NA \\
\hline 3082 & 3,4 & CUMULATIVE & $2.5000000 E-01$ & $2.0000000 E-01$ & $0,0000000 E+\infty$ & $60000000 E-01$ & NONE & WPO41558 & $\mathbf{Y}$ & N/A \\
\hline 3082 & 3,4 & CUMULATIVE & $2.5000000 E-01$ & $2.0000000 E-01$ & $00000000 E+\infty$ & $60000000 E-01$ & NONE & WPO42751 & $\mathbf{Y}$ & NA \\
\hline 3083 & 3,4 & UNIFORM & $20000000 \mathrm{E}-01$ & $2.0000000 \mathrm{E}-01$ & $0,0000000<+\infty$ & $40000000 E-01$ & NONE & WPO30540 & $\mathbf{Y}$ & NA \\
\hline 3083 & 3,4 & UNIFORM & $2.0000000 E-01$ & $2.0000000 E-01$ & $0,000000<-\infty$ & $4000000 \leq-01$ & NONE & WP030994 & $\mathbf{Y}$ & NA \\
\hline 3083 & 3,4 & UNIFORM & $2.0000000 E-01$ & $2.0000000 E-01$ & $0 \infty \infty \infty 000<-\infty$ & $4000000 E-01$ & NONE & WP035268 & $\mathbf{Y}$ & N/A \\
\hline 3083 & 3,4 & UNIFORM & $2.0000000 E-01$ & $2.0000000 E-01$ & $0,000000<=\infty$ & $\triangle 0000000 E-01$ & NONE & WP035597 & $\mathbf{Y}$ & $N / A$ \\
\hline 3083 & 3,4 & UNIFORM & $2,0000000 E-01$ & $20000000 \mathrm{E}-01$ & $0000000<+\infty$ & $10000000-01$ & NONE & WP041558 &.$- Y$ & NA \\
\hline 3084 & 1,3 & CONSTANT & $3.0900000 E+\infty$ & $3.0900000 E+\infty$ & $30000000<-\infty$ & $30,00000 \in+\infty$ & m & WPO30640 & $\mathbf{Y}$ & NA \\
\hline 3084 & 1,3 & CONSTANT & $3.0900000 E+\infty 0$ & $30900000 E+\infty$ & $30,00000<-\infty$ & $30,00000<+\infty$ & $m$ & WP030994 & $\mathbf{Y}$ & $N / A$ \\
\hline 3084 & 1,3 & CONSTANT & $3.0900000 E+\infty$ & $30 s 00000=+\infty$ & $3000000 \times-\infty$ & $3000000<+\infty$ & m & WP035597 & $\mathbf{Y}$ & NA \\
\hline 3084 & 1,3 & CONSTANT & $3.0900000 E+\infty$ & $3.0500000 E+\infty$ & $3000000<-\infty$ & $30, \infty \infty 00<+\infty$ & $m$ & WP040514 & $\mathbf{Y}$ & NAA \\
\hline 3085 & 1,3 & CONSTANT & $1.6000000 E+00$ & $1.0000000 E+\infty$ & $10000000 \times+\infty$ & $18000000 E+\infty$ & $m$ & WP030640 & $Y$ & NA \\
\hline 3085 & 1,3 & CONSTANT & $1.8000000 E+\infty 0$ & $1.8000000 E+\infty$ & $1+000000 \in-\infty$ & $1000000 E+\infty$ & $m$ & WP030994 & $\mathbf{Y}$ & NA \\
\hline 3085 & 1,3 & CONSTANT & $1.8000000 E+\infty$ & $18000000 E+\infty$ & $12000000 \times+\infty$ & $10000000=+\infty$ & m & WP035597 & $\mathbf{Y}$ & NA \\
\hline 3085 & 1,3 & CONSTANT & $1.8000000 E+\infty 0$ & $180000000+\infty$ & $10000000-\infty$ & $10000000=+\infty$ & $\mathbf{m}$ & WPO40514 & $\mathbf{Y}$ & NA \\
\hline 3086 & 1,3 & CONSTANT & $3.5000000 E+00$ & $3.5000000 E+\infty$ & $3500000=+\infty$ & $35000000 E+\infty$ & $m$ & WP030640 & $\mathbf{Y}$ & NA \\
\hline 3086 & 1,3 & CONSTANT & $3.5000000 E+\infty 0$ & $35000000 E \div 00$ & $35000000 E-\infty$ & $35000000 E+\infty$ & m & WP030994 & $\mathbf{Y}$ & NA \\
\hline 3085 & 1,3 & CONSTANT & $3.5000000 E+00$ & $35000000 E+\infty$ & $35000000 E+\infty$ & $35000000 E+\infty$ & $\mathbf{m}$ & WP035597 & $\mathbf{Y}$ & NA \\
\hline
\end{tabular}


CCA Parameter Listing

\begin{tabular}{|c|c|c|c|c|c|c|c|c|c|c|}
\hline $\begin{array}{c}\text { Permetar in } \\
\text { Dotabeee? } \\
\text { (Mn) }\end{array}$ & 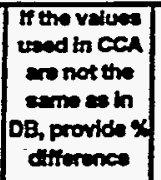 & ID & Matarialo & Metwara Name & $\begin{array}{l}\text { Parmoter } \\
\text { tD }\end{array}$ & $\begin{array}{l}\text { Parminer Nem } \\
\text { - }\end{array}$ & $\begin{array}{l}\text { PRPD } \\
\text { APPON }\end{array}$ & Den Entry & 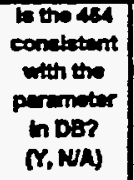 & $\begin{array}{l}\text { Paramitar } \\
\text { Cutegory } \\
\text { (tootnotio 1) }\end{array}$ \\
\hline $\mathbf{Y}$ & & 3086 & CL_M_TI & $\begin{array}{l}\text { Upper Salado clay.0 to } \\
10 \text { years }\end{array}$ & RSH_WAS & $\begin{array}{l}\text { Waste-rianding shan radues (3.5 } \\
\text { m) }\end{array}$ & 320594 & $15+\infty-\infty 6$ & $\mathbf{Y}$ & 48 \\
\hline $\mathbf{Y}$ & & 3087 & $C L_{-} M_{-} T 1$ & $\begin{array}{l}\text { Upper Salado elay.0 to } \\
10 \text { years }\end{array}$ & RSH_EXH & Ar-extheust thent rediess $(2.3 \mathrm{~m})$ & $32057 \mathrm{~A}$ & $15-F e b-96$ & $\mathbf{Y}$ & 48 \\
\hline $\mathbf{Y}$ & & 3087 & CL_M_TI & $\begin{array}{l}\text { Upper Salodo clay.0 to } \\
10 \text { years }\end{array}$ & RSH_EXH & Ar-whenas ghat radius $(2.3 \mathrm{~m})$ & $32057 A$ & $15-F a b-96$ & $\mathbf{Y}$ & 48 \\
\hline $\mathbf{Y}$ & & 3087 & $C L \_M \_T 1$ & $\begin{array}{l}\text { Upper Stado ctay.0 to } \\
10 \text { years }\end{array}$ & RSH_EXH & Ar-exheurt shath radies (23 m) & $32057 \mathrm{~A}$ & $15+c b-96$ & $\mathbf{Y}$ & 48 \\
\hline $\mathbf{Y}$ & & 3087 & CL_M_Tt & $\begin{array}{l}\text { Upper Salado clay.0 to } \\
10 \text { years }\end{array}$ & RSH_EXH & Ar-exheust ahat radies $(2.3 \mathrm{~m})$ & $32057 \mathrm{~A}$ & 15-Fab-96 & $\mathbf{Y}$ & 48 \\
\hline $\mathbf{Y}$ & & 3088 & CL_M_T1 & $\begin{array}{l}\text { Upper Salado ctay.0 to } \\
10 \text { years }\end{array}$ & RADN_DRZ & DRZ outer radius at esch sthent & 32053 & $15+$ ab-s6 & $\mathbf{Y}$ & $\mathfrak{1}$ \\
\hline $\mathbf{Y}$ & & 3088 & CL_M_T1 & $\begin{array}{l}\text { Upper Salodo clay.0 to } \\
10 \text { years }\end{array}$ & RADN_DRZ & DRZ outer radies at each athen & 32053 & $15+c b-96$ & $\mathbf{Y}$ & 1 \\
\hline $\mathbf{Y}$ & & 3088 & CL_M_TI & $\begin{array}{l}\text { Upper Saledo clay.0 to } \\
10 \text { years }\end{array}$ & RADN_DRZ & DRZ outer radius at each shant & 32053 & 15 -Fab-96 & $\mathbf{Y}$ & 1 \\
\hline $\mathbf{Y}$ & & 3089 & CL_M_T2 & $\begin{array}{l}\text { Upper Saledo clay:10 } \\
\text { to } 25 \text { years }\end{array}$ & RSH_AR & Air-exply that radius $(3.09 \mathrm{~m})$ & $32134 A$ & $05-500-96$ & $\mathbf{Y}$ & $4 \mathrm{~B}$ \\
\hline $\mathbf{Y}$ & & 3089 & CL_M_T2 & $\begin{array}{l}\text { Uppor Salaso cisy:10 } \\
\text { to } 25 \text { years }\end{array}$ & RSH_ARR & Air-upply shan radius $(3.09 \mathrm{~m})$ & $32131 A$ & $05+0 b-96$ & $\mathbf{Y}$ & $4 B$ \\
\hline $\mathbf{Y}$ & & 3089 & CL_M_T2 & $\begin{array}{l}\text { Upper Salado clay: } 10 \\
\text { to } 25 \text { years }\end{array}$ & RSH_ARR & Air-eupply athan radius $(3.09 \mathrm{~m})$ & $32139 A$ & 05 Fab-96 & $\mathbf{Y}$ & 48 \\
\hline $\mathbf{Y}$ & & 3009 & CL_M_M & $\begin{array}{l}\text { Upper Salado clay: } 10 \\
\text { to } 25 \text { years }\end{array}$ & RSH_AR & Air-eupply thent redies $(3.09 \mathrm{~m})$ & 321314 & $05+f a b-96$ & $\mathbf{Y}$ & $4 B$ \\
\hline $\mathbf{Y}$ & & 3090 & CL_M_T2 & $\begin{array}{l}\text { Upper Salado clay: } 10 \\
\text { to } 25 \text { years }\end{array}$ & RSH_SAL & Salthending ahet radius $(1.8 \mathrm{~m})$ & 321334 & $15+5 b-96$ & $\mathbf{Y}$ & 48 \\
\hline $\mathbf{Y}$ & & 3090 & CL_M_T2 & $\begin{array}{l}\text { Upper Salado ctay: } 10 \\
\text { to } 25 \text { years }\end{array}$ & RSH_SAL & Salthending shant radies $(1.8 \mathrm{~m})$ & $32133 A$ & 15-Fab-96 & $\mathbf{Y}$ & $4 B$ \\
\hline $\mathbf{Y}$ & & 3090 & CL_M_T2 & $\begin{array}{l}\text { Uppor Saledo cloy.10 } \\
\text { to } 25 \text { years }\end{array}$ & RSH_SAL & Salthanding shan radies $(1.8 \mathrm{~m})$ & $32133 A$ & 15-fab-96 & $\mathbf{Y}$ & 48 \\
\hline $\mathbf{r}$ & & 3090 & $C L \_M / T 2$ & $\begin{array}{l}\text { Upper Salado ciny:10 } \\
\text { to } 25 \text { years }\end{array}$ & RSH_SAL & Salt handling that radus $(1.8 \mathrm{~m})$ & 321334 & 15f-b-96 & $\mathbf{Y}$ & $4 B$ \\
\hline $\mathbf{Y}$ & & 3091 & CL_M_T2 & $\begin{array}{l}\text { Upper Salado clay: } 10 \\
\text { to } 25 \text { years }\end{array}$ & RSH_WAS & $\begin{array}{l}\text { Waste-hending thet radus (3.5 } \\
\text { m) }\end{array}$ & 321349 & 15 fob-96 & $\mathbf{Y}$ & 48 \\
\hline $\mathbf{Y}$ & & 3091 & CL_M_T2 & $\begin{array}{l}\text { Upper Salaco clay: } 10 \\
\text { to } 25 \text { years }\end{array}$ & RSH_WAS & $\begin{array}{l}\text { Wasto handing that radus (3.5 } \\
\text { (m) }\end{array}$ & 321344 & $15-50 b-96$ & $\mathbf{Y}$ & 48 \\
\hline $\mathbf{Y}$ & & 3091 & CL_M_T2 & $\begin{array}{l}\text { Upper Salado clay.10 } \\
\text { to } 25 \text { years }\end{array}$ & RSH_WAS & $\begin{array}{l}\text { Westo-handing thaft redus (3.5 } \\
\text { (m) }\end{array}$ & 321341 & 15 Feb-S6 & $\mathbf{Y}$ & 48 \\
\hline $\mathbf{Y}$ & & 3091 & CL_M_T2 & $\begin{array}{l}\text { Upper Salado clay:10 } \\
\text { to } 25 \text { yoars }\end{array}$ & RSH_WAS & $\begin{array}{l}\text { Wasto-handing chaft radus (3.5 } \\
\text { (n) }\end{array}$ & 321344 & 15-Fob-96 & $\mathbf{Y}$ & $4 B$ \\
\hline $\boldsymbol{Y}$ & & 3092 & CL_M_T2 & $\begin{array}{l}\text { Upper Salado clay: } 10 \\
\text { to } 25 \text { years }\end{array}$ & RSH_EXH & Ar-axhaust shatt radus $(2.3 \mathrm{~m})$ & 321324 & $15+a b-96$ & $\mathbf{Y}$ & $4 B$ \\
\hline $\mathbf{Y}$ & & 3092 & CL_H_T2 & $\begin{array}{l}\text { Upper Salado cloy.10 } \\
\text { to } 25 \text { years }\end{array}$ & RSH_EXH & Ar-exhoust shen radus $(23 \mathrm{~m})$ & 321324 & 15-Feb-96 & $\mathbf{Y}$ & $A B$ \\
\hline $\mathbf{Y}$ & & 3092 & CL_M_T2 & $\begin{array}{l}\text { Upper Saledo cisy: } 10 \\
\text { to } 25 \text { years }\end{array}$ & RSH_EXH & Ar-exheus thatt radius $(2.3 \mathrm{~m})$ & $32132 A$ & $15-F 0 b-96$ & $\mathbf{Y}$ & 48 \\
\hline $\mathbf{Y}$ & & 3092 & CL_M_T2 & $\begin{array}{l}\text { Upper Salado ctoy. } 10 \\
\text { to } 25 \text { years }\end{array}$ & RSH_EXH & Ar-achaust rodus $(2.3 \mathrm{~m})$ & 321324 & $15-F=b-96$ & $\mathbf{Y}$ & $4 B$ \\
\hline $\mathbf{Y}$ & & 3093 & CL_M_T2 & $\begin{array}{l}\text { Upper Salado ciay. } 10 \\
\text { to } 25 \text { years }\end{array}$ & RADN_DRZ & DRZ outer radus at esch shaft & 32128 & 15 fab-96 & $\because-Y$ & 1 \\
\hline $\mathbf{Y}$ & & 3093 & CL_H_t2 & $\begin{array}{l}\text { Upper Salado ciry. } 10 \\
\text { to } 25 \text { years }\end{array}$ & RADN_ORZ & Dr2 outer radus at each shan & 32128 & 15-Fab-96 & $\mathbf{Y}$ & 1 \\
\hline $\mathbf{Y}$ & & 3093 & $C_{2} M_{-} T 2$ & $\begin{array}{l}\text { Upper Saleco cior } 10 \\
\text { to } 25 \text { years }\end{array}$ & RADN_DRZ & ORZ outer redus at each ahent & 32128 & 15-Fab-96 & $\mathbf{Y}$ & 1 \\
\hline $\mathbf{Y}$ & & 3094 & CL_M_T3 & $\begin{array}{l}\text { Upper Seledo diry.25 } \\
\text { to } 50 \text { yeers }\end{array}$ & RSH_AR & Ar-auply andin ractess $(3.09 \mathrm{~m})$ & 321634 & 15-Fab-96 & $\mathbf{Y}$ & 48 \\
\hline $\mathbf{Y}$ & & 3094 & CL_M_T3 & $\begin{array}{l}\text { Upper Salodo ciny.25 } \\
\text { to } 50 \text { yoars }\end{array}$ & RSH_AR & Ar-eupoty anath radus $(3.09 \mathrm{~m})$ & $32163 \mathrm{~A}$ & 15-Feb-96 & $\mathbf{Y}$ & 48 \\
\hline $\mathbf{Y}$ & & 3094 & CL_M_T3 & $\begin{array}{l}\text { Upper Saledo cier.25 } \\
\text { to } 50 \text { years }\end{array}$ & RSH_AR & Ar-tepoly anden radus (3.09 m) & 321634 & 15 feb-96 & $\mathbf{Y}$ & 48 \\
\hline $\mathbf{Y}$ & & 3094 & 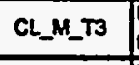 & $\begin{array}{l}\text { Upper Saledo dity.25 } \\
\text { to } 50 \text { years }\end{array}$ & RSH_NR & Ax-aupply ahan radius $(3.09 \mathrm{~m})$ & $32163 A$ & $15-F a b-96$ & $\mathbf{Y}$ & 48 \\
\hline $\mathbf{Y}$ & & 3095 & 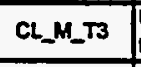 & $\begin{array}{l}\text { Upper Salado ctay } 25 \\
\text { to } 50 \text { yours }\end{array}$ & RSH_SN & Selthanding then radas $(1.8 \mathrm{~m})$ & $32165 A$ & 15-Feb-96 & $\mathbf{Y}$ & $4 B$ \\
\hline $\mathbf{Y}$ & & 3095 & CL_M_T3 & $\begin{array}{l}\text { Upper Sulado doy.25 } \\
\text { to } 50 \text { years }\end{array}$ & RSH_SNL & Salt-handing than radius $(1.8 \mathrm{~m})$ & 321654 & $15+c b-96$ & $\mathbf{Y}$ & $4 B$ \\
\hline $\mathbf{Y}$ & & 3095 & CL_M_T3 & $\begin{array}{l}\text { Upper Salado ciry.25 } \\
\text { to } 50 \text { years }\end{array}$ & RSH_SN & Sath handing then radius $(1.8 \mathrm{~m})$ & $32165 A$ & $15-f 0 b-96$ & $\mathbf{Y}$ & $4 B$ \\
\hline $\mathbf{Y}$ & & 3095 & CL_M_T3 & $\begin{array}{l}\text { Upper Salwoo ctor. } 25 \\
\text { to } 50 \text { years }\end{array}$ & RSH_SN & Selt thanding then rades $(9.8 \mathrm{~m})$ & $32165 A$ & $15+c b-96$ & $\mathbf{Y}$ & 48 \\
\hline $\mathbf{Y}$ & & 3096 & $C L_{-} M_{-} T_{3}$ & $\begin{array}{l}\text { Upper Saledo cty } 25 \\
\text { to } 50 \text { years }\end{array}$ & RSH_WAS & $\begin{array}{l}\text { Weste-handing ehant radius (3.5 } \\
\text { m) }\end{array}$ & 321664 & $15-f a b-86$ & $\mathbf{Y}$ & 48 \\
\hline
\end{tabular}


CCA Parameter Listing

\begin{tabular}{|c|c|c|c|c|c|c|c|c|c|c|}
\hline 10 & 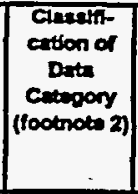 & $\begin{array}{c}\text { Dlatribetion } \\
\text { Type }\end{array}$ & mem & Median & Mintionim & Medrinep & Units & Retienance to & 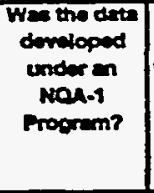 & 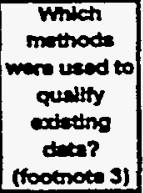 \\
\hline 3086 & 1,3 & CONSTANT & $3.5000000 E+\infty$ & $3.5000000 E+\infty$ & $3.5000000 E+00$ & $3.5000000 E+00$ & $m$ & WPO40514 & $\mathbf{Y}$ & NA \\
\hline 3067 & 1.3 & CONSTANT & $2.3000000 \varepsilon+\infty$ & $23000000 E+\infty 0$ & $2.3000000 E+00$ & $2.3000000 E+\infty 0$ & $\mathbf{m}$ & WP030640 & $\mathbf{Y}$ & NA \\
\hline 3087 & 1,3 & CONSTANT & $2.3000000 E+\infty$ & $23000000 E+\infty$ & $23000000 E+\infty 0$ & $23000000 E+\infty$ & $\mathbf{m}$ & WP030994 & $\mathbf{Y}$ & NA \\
\hline 3087 & 1,3 & CONSTANT & $2.3000000 E+00$ & $2.3000000 \varepsilon+\infty 0$ & $2.3000000 E+\infty 0$ & $23000000 E+00$ & $m$ & WP035597 & $Y$ & NA \\
\hline 3087 & 1,3 & CONSTANT & $2.3000000 \mathrm{E}+\infty$ & $2.3000000 E+\infty$ & $2,3000000 E+\infty$ & $2.3000000 E+\infty$ & m & WPO40514 & $Y$ & NA \\
\hline 3088 & $1.3,4$ & CONSTANT & $1.7090000 E+\infty 0$ & $1.7090000 E+\infty$ & $1.7090000 E+\infty 0$ & $1.7000000 E+\infty 0$ & $\mathrm{~m} / \mathrm{m}$ & WP030540 & $\mathbf{Y}$ & NA \\
\hline 3088 & $1,3,4$ & CONSTANT & $1.7090000 E+\infty 0$ & $1.7090000 E+\infty$ & $1.7090000 E+\infty$ & $1.7090000 E+00$ & $\mathrm{~m} / \mathrm{m}$ & WP030994 & $\mathbf{Y}$ & NA \\
\hline 3088 & $1,3,4$ & CONSTANT & $1.7090000 E+\infty 0$ & $1.7090000 E+\infty 0$ & $1.7090000 \mathrm{E}+00$ & $1.7090000 E+\infty 0$ & $\mathrm{~m} / \mathrm{m}$ & WP035597 & $\mathbf{Y}$ & NA \\
\hline 3089 & 1,3 & CONSTANT & $3.0900000 E+\infty 0$ & $3.0900000 E+00$ & $3.0900000 \mathrm{E}+00$ & $3.0900000 \mathrm{E}+\infty 0$ & $m$ & WP030640 & $\mathbf{Y}$ & NA \\
\hline 3089 & 1,3 & CONSTANT & $3.0900000 E+00$ & $3.0900000 E+00$ & $3.0900000 E+\infty 0$ & $3.0900000 \mathrm{E}+00$ & m & WP030994 & $\mathbf{Y}$ & NA \\
\hline 3089 & 1,3 & CONSTANT & $3.0900000 E+\infty 0$ & $3.0900000 E+\infty 0$ & $3.0900000 E+\infty 0$ & $3.0900000 E+\infty$ & $\mathbf{m}$ & WP035597 & $\mathbf{Y}$ & NA \\
\hline 3089 & 1,3 & CONSTANT & $3.0900000 E+\infty 0$ & $3.0900000 \mathrm{E}+\infty 0$ & $3.0900000 E+\infty$ & $3.0900000 E+\infty 0$ & $m$ & WP040514 & $\mathbf{Y}$ & NA \\
\hline 3090 & 1,3 & CONSTANT & $1.8000000 E+\infty 0$ & $1.8000000 E+\infty 0$ & $1.8000000 E+\infty 0$ & $1.8000000 E+\infty$ & $m$ & WP030640 & $\mathbf{Y}$ & NA \\
\hline 3090 & 1,3 & CONSTANT & $1.8000000 E+00$ & $1.8000000 E+00$ & $1.8000000 E+\infty 0$ & $1.8000000 E+\infty 0$ & $\mathbf{m}$ & WP030994 & $\mathbf{Y}$ & NA \\
\hline 3090 & 1,3 & CONSTANF & $1.8000000 \mathrm{E}+\infty 0$ & $1.8000000 E+\infty 0$ & $1.8000000 E+\infty$ & $1.8000000 E+\infty$ & $m$ & WP035597 & $\mathbf{Y}$ & NA \\
\hline 3090 & 1,3 & CONSTANT & $1.8000000 E+\infty 0$ & $1.8000000 E+\infty 0$ & $1.8000000 E+\infty$ & $1.8000000 E+\infty 0$ & $m$ & WPO40514 & $Y$ & NA \\
\hline 3091 & 1,3 & CONSTANT & $3.5000000 E+00$ & $3.5000000 E+\infty 0$ & $3.5000000 E+\infty$ & $3.5000000 E+\infty 0$ & $m$ & WP030640 & $\mathbf{Y}$ & N/A \\
\hline 3091 & 1,3 & CONSTANT & $3.5000000 E+00$ & $3.5000000 E+\infty 0$ & $3.5000000 E+\infty 0$ & $3.5000000 E+\infty$ & $\mathbf{m}$ & WP030994 & $\mathbf{Y}$ & NA \\
\hline 3091 & 1,3 & CONSTANT & $3.5000000 E+\infty 0$ & $3.5000000 E+\infty 0$ & $3.5000000 E+\infty 0$ & $3.5000000 E+\infty$ & $m$ & WP035597 & $\mathbf{Y}$ & NA \\
\hline 3091 & 1,3 & CONSTANT & $3.5000000 E+\infty 0$ & $3.5000000 E+00$ & $3.5000000 E+\infty$ & $3.5000000 E+\infty$ & $m$ & WP040514 & $\mathbf{Y}$ & $N / A$ \\
\hline 3092 & 1,3 & CONSTANT & $2.3000000 E+\infty 0$ & $2.3000000 E+00$ & $2.3000000 E+00$ & $2.3000000 E+\infty$ & $\mathbf{m}$ & WP030640 & $\mathbf{Y}$ & NA \\
\hline 3092 & 1,3 & CONSTANT & $2.3000000 E+\infty 0$ & $2.3000000 E+\infty 0$ & $2.3000000 E+\infty$ & $2.3000000 E+\infty$ & m & WP030994 & $\mathbf{Y}$ & WA \\
\hline 3092 & 1,3 & CONSTANT & $2.3000000 E+00$ & $2.3000000 E+00$ & $2.3000000 E+00$ & $2.3000000 E+\infty 0$ & $m$ & WP035597 & $\mathbf{Y}$ & $N / A$ \\
\hline 3092 & 1,3 & CONSTANT & $2.3000000 E+\infty 0$ & $2,3000000 E+\infty 0$ & $23000000 E+\infty$ & $23000000 E+00$ & $\mathbf{m}$ & WP040514 & $\mathbf{Y}$ & N/A \\
\hline 3093 & $1,3,4$ & CONSTANT & $1.4690000 E+\infty$ & $1.4690000 E+\infty 0$ & $1.4690000 E+\infty 0$ & $1: 4690000 E+\infty 0$ & $m / m$ & WP030540 & $--Y$ & NA \\
\hline 3093 & $1,3,4$ & CONSTANT & $1.4690000 E+\infty$ & $1.4690000 E+00$ & $1.4690000 E+\infty 0$ & $1.4690000 E+\infty$ & $\mathrm{m} / \mathrm{m}$ & WP030994 & $\mathbf{Y}$ & $N / A$ \\
\hline 3093 & $1,3,4$ & CONSTANT & $1.4690000 E+\infty$ & $1.4690000 E+\infty 0$ & $1.4690000 \mathrm{E}+00$ & $1.4690000 E+\infty$ & $\mathrm{m} / \mathrm{m}$ & WP035597 & $\mathbf{Y}$ & N/A \\
\hline 3094 & 1,3 & CONSTANT & $3.0900000 E+\infty$ & $3.0900000 \mathrm{E}+00$ & $3.0900000 \mathrm{E}+00$ & $3.0900000 E+00$ & $\mathbf{m}$ & WP030640 & $\mathbf{Y}$ & $N / A$ \\
\hline 3094 & 1,3 & CONSTANT & $3.0900000 E+\infty$ & $3.0900000 E+\infty$ & $3.0900000 E+\infty$ & $3.0900000 E+\infty$ & m & WP030994 & $\mathbf{Y}$ & NA \\
\hline 3094 & 1,3 & CONSTANT & $3.0900000 E+\infty 0$ & $3.0900000 E+00$ & $3.0500000 \mathrm{E}+00$ & $3.0900000 E+00$ & $m$ & WP035597 & $\mathbf{Y}$ & NA \\
\hline 3094 & 1,3 & CONSTANT & $3.0900000 E+\infty$ & $3.0900000 \mathrm{E}+00$ & $3.0900000 E+\infty 0$ & $3.0900000 \mathrm{E}+\infty$ & m & WPO40514 & $\mathbf{Y}$ & NA \\
\hline 3095 & 1,3 & CONSTANT & $1.8000000 E+00$ & $1.8000000 E+00$ & $1.8000000 E+\infty$ & $1.8000000 E+\infty 0$ & m & WPO30640 & $\mathbf{Y}$ & NA \\
\hline 3095 & 1,3 & CONSTANT & $1.8000000 \mathrm{E}+00$ & $1.8000000 E+\infty$ & $1.8000000 \mathrm{E}+\infty$ & $1.8000000 \mathrm{E}+\infty$ & $m$ & WP030994 & $\mathbf{Y}$ & N/A \\
\hline 3095 & 1,3 & CONSTANT & $1.8000000 E+\infty$ & $1.8000000 E+00$ & $1.8000000 E+\infty$ & $1.8000000 E+\infty 0$ & $m$ & WP035597 & $\mathbf{Y}$ & $\mathbf{N} / \mathbf{A}$ \\
\hline 3095 & 1,3 & CONSTANT & $1.8000000 E+\infty 00$ & $1.8000000 E+00$ & $1.0000000 E+00$ & $1.8000000 E+\infty$ & $m$ & WPO40514 & $Y$ & $\mathbf{N} / \mathbf{A}$ \\
\hline 3096 & 1,3 & CONSTANT & $3.5000000 E+\infty 0$ & $3.5000000 E+\infty 0$ & $3.5000000 E+00$ & $3.5000000 E+\infty 0$ & m & WP030640 & $\mathbf{Y}$ & $\mathbf{N A}$ \\
\hline
\end{tabular}


CCA Parameter Listing

\begin{tabular}{|c|c|c|c|c|c|c|c|c|c|c|}
\hline 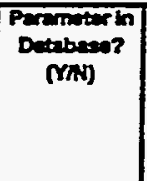 & 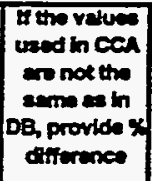 & ID & Mrataid & Mater Nem & $\begin{array}{c}\text { Permanar } \\
D\end{array}$ & $\begin{array}{c}\text { Permationtes } \\
.\end{array}$ & $\begin{array}{l}\text { Pro } 10 \\
\text { (MPOU) }\end{array}$ & $\begin{array}{c}\text { Denentry } \\
\text { Dew }\end{array}$ & 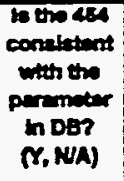 & $\begin{array}{l}\text { Paramour } \\
\text { Cotepory } \\
\text { (footencte 1) }\end{array}$ \\
\hline $\mathbf{Y}$ & & 3096 & CL_M_T3 & $\begin{array}{l}\text { Uppor Salaso clay:25 } \\
\text { to } 50 \text { years }\end{array}$ & RSH_WAS & $\begin{array}{l}\text { Wasto-handing shatt redues (3.5 } \\
\text { m) }\end{array}$ & $32166 A$ & $15-F 0 b-96$ & $\mathbf{Y}$ & $4 B$ \\
\hline $\mathbf{Y}$ & & 3096 & CL_M_T3 & $\begin{array}{l}\text { Uppor Saloso ciny } 25 \\
\text { to } 50 \text { years }\end{array}$ & RSH_WAS & $\begin{array}{l}\text { Wastohranding shent redes (3.5 } \\
\text { m) }\end{array}$ & $32165 A$ & $15+\infty 0-06$ & $Y$ & 48 \\
\hline $\mathbf{Y}$ & & 3006 & CL_M_T3 & $\begin{array}{l}\text { Uppor Saludo clay.25 } \\
\text { to } 50 \text { years }\end{array}$ & RSH_WAS & $\begin{array}{l}\text { Wasto-handing angath radus (3.5 } \\
\text { m) }\end{array}$ & $32165 A$ & 15 fab-96 & $\mathbf{Y}$ & 48 \\
\hline$Y$ & & 3097 & CL_M_T3 & $\begin{array}{l}\text { Uppor Salado clay:25 } \\
\text { to } 50 \text { years }\end{array}$ & RSH_EXH & Ar-exheust ahat redies $(23 \mathrm{~m})$ & 321641 & $15-a b-96$ & $\mathbf{Y}$ & $A B$ \\
\hline$Y$ & & 3097 & $C L_{-} M_{-} T 3$ & $\begin{array}{l}\text { Upper Salado clay:25 } \\
\text { to } 50 \text { years }\end{array}$ & RSH_EXH & Air-ectheust thath radius $(2.3 \mathrm{~m})$ & 321644 & $15-700-96$ & $\mathbf{Y}$ & $4 B$ \\
\hline$Y$ & & 3097 & CL_M_T3 & $\begin{array}{l}\text { Upper Saluso day:25 } \\
\text { to } 50 \text { yeers }\end{array}$ & RSH_EXH & Air-extheust shan radivs (23 m) & 321644 & $15-\infty 0-96$ & $\mathbf{Y}$ & 48 \\
\hline $\mathbf{Y}$ & & 3097 & CL_M_T3 & $\begin{array}{l}\text { Upper Selado clay-25 } \\
\text { to } 50 \text { years }\end{array}$ & RSH_EXH & Air-exthoust athath radive $(2.3 \mathrm{~m})$ & 321644 & 15fab-96 & $\mathbf{Y}$ & 48 \\
\hline$Y$ & & 3098 & CL_M_T3 & $\begin{array}{l}\text { Upper Salado ciny.25 } \\
\text { to } 50 \text { years }\end{array}$ & RADN_DRZ & DRZ outor radiess at asch shant & 32160 & 15freb-96 & $\mathbf{Y}$ & 1 \\
\hline $\mathbf{Y}$ & & 3098 & CL_M_T3 & $\begin{array}{l}\text { Upper Salado clay.25 } \\
\text { to } 50 \text { years }\end{array}$ & RADN_DRZ & DRZ outer radius at axch shin? & 32160 & 15-Feb-96 & $\mathbf{Y}$ & 1 \\
\hline $\mathbf{Y}$ & & 3098 & CL_M_T3 & $\begin{array}{l}\text { Upper Salado clay.25 } \\
\text { to } 50 \text { yaers }\end{array}$ & RADN_DRZ & DRZ acter radies at exch shent & 32160 & 15-Fob-96 & $\mathbf{Y}$ & 1 \\
\hline $\mathbf{Y}$ & & 3099 & CAVTTY1 & Cavity for Wasto Areas & SAT_IBRN & Initial Brino Suturation & 31686 & 17 Fob-96 & $\mathbf{Y}$ & 48 \\
\hline $\mathbf{Y}$ & & 3099 & CAVITY_1 & Crvity for Wasto Araes & SAT_IBRN & Initial Brino Saturation & 31686 & 17 Fab-96 & $Y$ & $4 B$ \\
\hline $\mathbf{Y}$ & & 3099 & CAVITY_I & Cervity for Westo Nress & SAT_IBRN & Initial Brina Srturation & 31686 & 17 fab-96 & $\mathbf{Y}$ & 48 \\
\hline$Y$ & & 3100 & CAVIr_2 & $\begin{array}{l}\text { Covity for Nor-waste } \\
\text { Areas }\end{array}$ & SAT_IBRN & Initivl Brine Saturation & 31760 & $17+a_{b-96}$ & $\mathbf{Y}$ & 48 \\
\hline $\mathbf{Y}$ & & 3100 & CAVIT__2 & $\begin{array}{l}\text { Comity for Non-waste } \\
\text { Araes }\end{array}$ & SAT_IBRN & Initial Brino Seturation & 31760 & 17 Fab-96 & $Y$ & $4 B$ \\
\hline $\mathbf{Y}$ & & 3100 & CAVITr_2 & $\begin{array}{l}\text { Covity for Non-wasto } \\
\text { Areas }\end{array}$ & SAT_IBRN & |initial Brine Seturation & 31760 & $17+\mp e b-96$ & $\mathbf{Y}$ & AB \\
\hline$Y$ & & 3101 & CAVITY_3 & Cavity for Shat & PRESSURE & Brine far-field pore pressure & 31780 & $17+$ ob-96 & $Y$ & 48 \\
\hline$Y$ & & 3101 & CAVITY_3 & Covity for Shan & PRESSURE & Brine ter-fietd pore pressure & 31780 & 17 Fob-96 & $Y$ & 48 \\
\hline$Y$ & & 3101 & CAVITY_3 & Covity for Shat & PRESSURE & Brine far field pore pressure & 31780 & 17 Fob-96 & $Y$ & $A B$ \\
\hline $\mathbf{Y}$ & & 3102 & CAVITY_3 & Cevity for Shat & SAT_IBRN & Initial Brine Seturation & 31786 & $17+50 b-96$ & $Y$ & $4 B$ \\
\hline$Y$ & & 3102 & CAVITY_3 & Cavity for Shat & SAT_IBRN & Intial Bme Seturation & 31786 & $17+06-96$ & $Y$ & $4 B$ \\
\hline$Y$ & & 3102 & CAVITY_3 & Cavity for Shan & SAT_IBRN & Initial Brino Saturation & 31786 & 17 fob- 96 & $Y$ & AB \\
\hline$Y$ & & 3103 & CAVITY_4 & Cevity for Borehole & PRESSURE & Brino far-field pore preseuro & 31815 & 17 fab-96 & $\mathbf{Y}$ & $4 B$ \\
\hline$Y$ & & 3103 & CAVITY_4 & Covity for Borenoto & PRESSURE & Brino far-field pore pressure & 31815 & 17 fab-96 & $Y$ & 48 \\
\hline$Y$ & & 3103 & CAVIT__4 & Crvity for Boreholo & PRESSURE & Brino far-field pore pressure & 31815 & $17+06-96$ & $Y$ & 48 \\
\hline $\mathbf{Y}$ & & 3104 & CAVITY_4 & Covity for Borehote & SAT_IBRN & Initial Erine Saturation & 31822 & $17+66-96$ & $\mathbf{Y}$ & AB \\
\hline$Y$ & & 3104 & CAVITY_4 & Cavity for Borehote & SAT_IBRN & Initial Brine Saturation & 31822 & 17 fob-96 & $Y$ & $4 B$ \\
\hline$Y$ & & 3104 & CAVTIY_4 & Cenvity for Boreholo & SAT_IBRN & Intial Bnne Saturation & 31822 & 17 fob-96 & $y$ & 48 \\
\hline$Y$ & & 3105 & REFCON & Reference Constent & VROOM & Volume of one room in repository & 33280 & 17 fab-s6 & $\mathbf{Y}$ & 48 \\
\hline$Y$ & & 3105 & REFCON & Reference Constant & VROOM & Volume of ore room in repository & 33280 & 17 F*b-96 & $\mathbf{Y}$ & $4 \mathrm{~B}$ \\
\hline $\mathbf{Y}$ & & 3105 & REFCON & Reforenco Constant & VROOM & Volume of one room in repository & 33280 & 17 fab-96 & $\mathbf{Y}$ & $4 B$ \\
\hline $\mathbf{Y}$ & & 3106 & REFCON & Reference Constimt & ASDRUM & $\begin{array}{l}\text { Surface areal of corrodeblo metal } \\
\text { per doum }\end{array}$ & 36370 & $17+f e b-96$ & $\mathbf{Y}$ & 48 \\
\hline$Y$ & & 3106 & REFCON & Reference Constemt & ASDRUM & $\begin{array}{l}\text { Surfoces mole of corrodeblo motal } \\
\text { per orm }\end{array}$ & 35370 & $17-F-b-86$ & $\mathbf{Y}$ & $4 B$ \\
\hline $\mathbf{Y}$ & & 3106 & REFCON & Reforence Constant & ASDRUM & $\begin{array}{l}\text { Surfaces wree of corrodeble metal } \\
\text { per chum }\end{array}$ & 36370 & 17 feb-96 & $\mathbf{Y}$ & AB \\
\hline $\mathbf{Y}$ & & 3107 & REFCON & Reference Constant & VPANLEX & Excrvated volume of ono pens! & 33273 & 17 Fob- 96 & $\mathbf{Y}$ & 48 \\
\hline$Y$ & & 3107 & REFCON & Ruference Constent & VPANLEX & Excavatod volume of one pene! & 33273 & 17 feb-96 & $Y$ & 48 \\
\hline$Y$ & & 3107 & REFCON & Rofierence Constent & VPANLEX & Excervated rolume of one penel & 33273 & $17+0 b-96$ & $\mathbf{Y}$ & AB \\
\hline $\mathbf{Y}$ & & 3108 & REFCON & Refierence Constemt & VREPOS & $\begin{array}{l}\text { Excervated storepe volume of } \\
\text { repositiony }\end{array}$ & 33276 & $17+F a b-96$ & $\mathbf{Y}$ & 48 \\
\hline $\mathbf{Y}$ & & 3108 & REFCON & Ruferenco Constant & VREPOS & $\begin{array}{l}\text { Execrited roorap volume of } \\
\text { reposition }\end{array}$ & 33276 & $17+F a b-96$ & $\mathbf{Y}$ & AB \\
\hline $\mathbf{Y}$ & & 3108 & REFCON & Ruference Constent & VREPOS & $\begin{array}{l}\text { Excaveted stores volum of } \\
\text { repocitory }\end{array}$ & 33276 & $17-F=b-96$ & $\mathbf{Y}$ & AB \\
\hline $\mathbf{Y}$ & & 3109 & REFCON & Reference Constent & AVOGAORO & Avogeadro's number & 32359 & $17+$ cbs 56 & $Y$ & 3 \\
\hline
\end{tabular}


CCA Parameter Listing

\begin{tabular}{|c|c|c|c|c|c|c|c|c|c|c|}
\hline 10 & 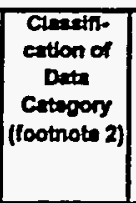 & $\begin{array}{l}\text { Diatribution } \\
\text { Type }\end{array}$ & $\operatorname{man}$ & Anedin & Minimem & Inotmiss & Whats & Reforence ID & $\begin{array}{l}\text { Was the difta } \\
\text { dervoloped } \\
\text { under an } \\
\text { Nol-1 } \\
\text { Progrum? }\end{array}$ & 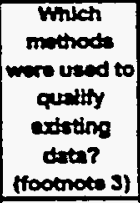 \\
\hline 3096 & 1,3 & CONSTANT & $3.5000000 E+00$ & $3.5000000 E+\infty$ & $3.5000000 E^{t}+\infty$ & $3.5000000 E+\infty 0$ & $m$ & WP030994 & $\mathbf{Y}$ & NA \\
\hline 3096 & 1,3 & CONSTANT & $3.5000000 E+\infty$ & $3.5000000 E+00$ & $3.5000000 \mathrm{E}+\infty$ & $3.5000000 E+\infty 0$ & $\mathbf{m}$ & WP035597 & $\mathbf{Y}$ & NA \\
\hline 3096 & 1,3 & CONSTANT & $3.5000000 E+\infty 0$ & $3.5000000 \mathrm{E}+\infty 0$ & $3.5000000 E+\infty$ & $3.5000000 E+\infty 0$ & m & WPO40514 & $\mathbf{Y}$ & NA \\
\hline 3097 & 1,3 & CONSTANT & $2.3000000 E+\infty 0$ & $2.3000000 E+\infty$ & $23000000 \mathrm{E}+00$ & $2.3000000 E+\infty$ & $\mathbf{m}$ & WP030610 & $\mathbf{Y}$ & NUA \\
\hline 3097 & 1,3 & CONSTANT & $2.3000000 E+\infty 0$ & $2.3000000 E+\infty 0$ & $23000000 \mathrm{E}+\infty 0$ & $2,3000000 E+\infty$ & $m$ & WP030s94 & $Y$ & NA \\
\hline 3097 & 1,3 & CONSTANT & $23000000 E+\infty 0$ & $23000000 E+00$ & $23000000 E+00$ & $2.3000000 E+\infty$ & $\mathbf{m}$ & WP035597 & $Y$ & N/A \\
\hline 3097 & 1,3 & CONSTANT & $2.3000000 E+\infty 0$ & $2.3000000 E+\infty 00$ & $2.3000000 E+\infty 0$ & $2.30000005+00$ & $m$ & WPO40514 & $\mathbf{Y}$ & NA \\
\hline 3098 & $1,3,4$ & CONSTANT & $1.2830000 E+\infty 0$ & $1.2830000 \mathrm{E}+00$ & $1.2830000 E+\infty 0$ & $1.2830000 E+\infty 0$ & $\mathrm{~m} / \mathrm{m}$ & WP030S40 & $\mathbf{Y}$ & NA \\
\hline 3098 & $1,3,4$ & CONSTANT & $; 1.2830000 E+\infty 0$ & $1.2830000 E+\infty 0$ & $1.2830000 E+\infty 0$ & $1.2830000 E+\infty 0$ & $\mathrm{~m} / \mathrm{m}$ & WP030994 & $\mathbf{Y}$ & NA \\
\hline 3098 & $1,3,4$ & CONSTANT & $1.2830000 E+\infty 0$ & $1.2830000 E+00$ & $1.2830000 E+\infty 0$ & $1.2830000 \mathrm{E}+\infty 0$ & $\mathrm{~m} / \mathrm{m}$ & WP035597 & $\mathbf{Y}$ & NA \\
\hline 3099 & 3,5 & CONSTANT & $0.0000000 E+\infty 0$ & $0.0000000 E+\infty 0$ & $0.0000000 E+\infty 0$ & $0.0000000 \mathrm{E}+\infty$ & NONE & $\begin{array}{l}\text { MEMO:VAUGHN } \\
\text { TIERNEY-9 }\end{array}$ & $\mathbf{Y}$ & N/A \\
\hline 3099 & 3,5 & CONSTANT & $0.0000000 E+\infty 0$ & $0.0000000 E+\infty$ & $0.0000000 E+\infty 0$ & $0.0000000 E+\infty 0$ & NONE & WP035597 & $\mathbf{Y}$ & NA \\
\hline 3099 & 3,5 & CONSTANT & $0.0000000 E+00$ & $0.0000000 E+\infty 0$ & $0.0000000 E+\infty 0$ & $0.0000000 E+\infty$ & NONE & WPO10514 & $\mathbf{Y}$ & NA \\
\hline 3100 & 1,3 & CONSTANT & $0.0000000 E+\infty 0$ & $0.0000000 E+\infty 0$ & $0.0000000 E+\infty 0$ & $0.0000000 E+\infty 0$ & NONE & $\begin{array}{c}\text { MEMO:VAUGHN } \\
\text { TIERNEY-9 }\end{array}$ & $Y$ & NA \\
\hline 3100 & 1,3 & CONSTANT & $0.0000000 E+\infty$ & $0.0000000 \mathrm{E}+\infty 0$ & $0.0000000 E+\infty$ & $0.0000000 E+\infty$ & NONE & WP035597 & $\mathbf{Y}$ & NA \\
\hline 3100 & 1,3 & CONSTANT & $0.0000000 E+\infty 0$ & $0.0000000=+\infty 0$ & $0.0000000 E+00$ & $0.0000000 E+00$ & NONE & WPO40514 & $\mathbf{Y}$ & N/A \\
\hline 3101 & 1.3 & CONSTANT & $1.0132500 E+05$ & $1.0132500 E+05$ & $1.0132500 E+05$ & $1.0132500 \mathrm{E}+05$ & $P_{a}$ & NONE & $Y$ & NA \\
\hline 3101 & 1,3 & CONSTANT & $1.0132500 E+05$ & $1.0132500 E+05$ & $1.0132500 E+05$ & $1.0132500 E+05$ & $\mathrm{~Pa}$ & WP035597 & $\mathbf{Y}$ & NA \\
\hline 3101 & 1,3 & CONSTANT & $1.0132500 E+05$ & $1.0432500 E+05$ & $1.0132500 E+05$ & $1.0132500 E+05$ & $\mathbf{P a}$ & WPO40514 & $\mathbf{Y}$ & NA \\
\hline 3102 & 1,3 & CONSTANT & $0.0000000 E+\infty 0$ & $0.00000000+\infty 0$ & $0.0000000 E+\infty$ & $0.0000000 E+\infty 0$ & NONE & $\begin{array}{c}\text { MEMO:VAUGHN } \\
\text { TIERNEY-9 } \\
\end{array}$ & $\mathbf{Y}$ & N/A \\
\hline 3102 & 1,3 & CONSTANT & $0.0000000 \mathrm{E}+\infty$ & $0.0000000 \mathrm{E}+00$ & $0.0000000 E+\infty 0$ & $0.0000000 E+\infty 0$ & NONE & WP035597 & $Y$ & N/A \\
\hline 3102 & 1.3 & CONSTANT & $0.0000000 E+\infty$ & $0.0000000 \mathrm{E}+00$ & $0.0000000 \mathrm{E}+\infty$ & $0.0000000 E+\infty$ & NONE & WPO40514 & $Y$ & NA \\
\hline 3103 & 1,3 & CONSTANT & $1.0132500 E+05$ & $1.0132500 E+05$ & $1.0132500 E+05$ & $1.0132500 E+05$ & $\mathbf{P a}$ & $\begin{array}{c}\text { MEMO:VAUGHN } \\
\text { TIERNEY-9 } \\
\end{array}$ & $\mathbf{Y}$ & NA \\
\hline 3103 & 1.3 & CONSTANT & $1.0132500 E+05$ & $1.0132500 \mathrm{E}+05$ & $1.0132500 E+05$ & $1.0132500 \mathrm{E}+\mathrm{CD}$ & $P_{\mathbf{a}}$ & WP035597 & $Y$ & NA \\
\hline 3103 & 1.3 & CONSTANT & $1.0132500 \mathrm{E}+05$ & $1.0132500 E+05$ & $1.0132500 \mathrm{E}+05$ & $1.0132500 E+05$ & $\mathbf{P a}$ & WPO40514 & $\bar{Y}$ & N/A \\
\hline 3104 & 1,3 & CONSTANT & $0.0000000 E+00$ & $0.0000000 E+\infty 0$ & $0.0000000 E+\infty 0$ & $0.0000000 E+\infty$ & NONE & $\begin{array}{c}\text { MEMO:VAUGHN } \\
\text { TIERNEY-9 } \\
\end{array}$ & $\mathbf{Y}$ & NA \\
\hline 3104 & 1.3 & CONSTANT & $0.0000000 E+\infty 0$ & $0.0000000 E+00$ & $0.0000000 E+00$ & $0.0000000 E+00$ & NONE & WP035597 & $\mathbf{Y}$ & NA \\
\hline 3104 & 1,3 & CONSTANT & $0.0000000 E+00$ & $0.0000000 E+\infty 0$ & $0.0000000 E+00$ & $0.0000000 E+00$ & NONE & WPO40514 & $\dot{Y}$ & NA \\
\hline 3105 & $1,3,4$ & CONSTANT & $3.6443780 E+\infty 03$ & $3.6443780 E+03$ & $3.6443780 E+03$ & $3.6443780 E+03$ & $m^{\wedge} 3$ & WP035597 & $Y$ & N/A \\
\hline 3105 & $1,3,4$ & CONSTANT & $3.6443780 E+03$ & $3.6443780 E+03$ & $3.6443780 E+03$ & $3.6443780 E+03$ & $m^{\wedge} 3$ & WP037713 & $\mathbf{Y}$ & NA \\
\hline 3105 & $1,3,4$ & CONSTANT & $3.6443780 E+\infty 03$ & $3.6443780 E+03$ & $3.6443780 E+03$ & $3.6443780 E+03$ & $m^{\wedge} 3$ & WP040514 & $\mathbf{Y}$ & N/A \\
\hline 3106 & 3,4 & CONSTANT & $6.0000000 E+00$ & $6.0000000 E+\infty 0$ & $6.0000000 E+00$ & $6.0000000 E+\infty$ & $m^{\wedge} 2$ & $\begin{array}{l}\text { SAND91-0893/3- } \\
\text { MEMOBRUSH }\end{array}$ & $\mathbf{Y}$ & N/A \\
\hline 3106 & 3,4 & CONSTANT & $6.0000000 E+00$ & $6.0000000 E+\infty$ & $6.0000000 E+\infty$ & $6.0000000 E+\infty$ & $m^{\wedge} 2$ & WP035597 & Y & N/A \\
\hline 3106 & 3,4 & CONSTANT & $6.0000000 E+00$ & $6.0000000 E+\infty 0$ & $6.0000000 E+\infty$ & $6.0000000 E+\infty 0$ & $m^{\wedge} 2$ & WPOC0514 & $Y$ & N/A \\
\hline 3107 & $1.3,4$ & CONSTANT & $4.6097650 E+04$ & 4.6097650E+04 & 4.6097650E+04 & 4.6097650z+04 & $m^{\wedge} 3$ & WP035597 & $\mathbf{Y}$ & N/A \\
\hline 3107 & $1,3,4$ & CONSTANT & 4.6097650E+04 & $4.6097650 \mathrm{E}+0 \mathrm{a}$ & 4.6097650E+04 & 4.6097650E+04 & $\mathrm{m}^{\wedge} 3$ & WP037713 & $\mathbf{Y}$ & NA \\
\hline 3107 & $1,3,4$ & CONSTANT & $4.6097650 E+04$ & $4.6097650 E+04$ & $4.6097650 E+04$ & 4.6097650E+04 & $m^{\wedge} 3$ & WP040514 & $\mathbf{Y}$ & N/A \\
\hline 3108 & $1,3,4$ & CONSTANT & $4.3602320 E+05$ & $4.3602320 E+05$ & $4.3602320 E+05$ & $4.3602320 E+05$ & $m^{\wedge} 3$ & WP035597 & $\mathbf{Y}$ & NA \\
\hline 3100 & $1,3,4$ & CONSTANT & $4.3602320 \mathrm{E}+05$ & $4.3602320 E+05$ & $4.36023205+05$ & $4.3602320 E+05$ & $m^{23}$ & WP037713 & $\mathbf{Y}$ & NA \\
\hline 3108 & $1,3,4$ & CONSTANT & $4.3602320 E+05$ & $4.3602320 E+05$ & $4.3602320 E+05$ & $4.3602320 E+05$ & $m^{\wedge} 3$ & WPO40514 & $\mathbf{Y}$ & NA \\
\hline 3109 & 4 & CONSTANT & $6.0021370 E+23$ & $6.0221370 E+23$ & $6.0221370 E+23$ & $6.0221370 E+23$ & $1 / \mathrm{mol}$ & CRC-72 & $\mathbf{Y}$ & NA \\
\hline
\end{tabular}


CCA Parameter Listing

\begin{tabular}{|c|c|c|c|c|c|c|c|c|c|c|}
\hline 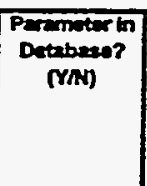 & 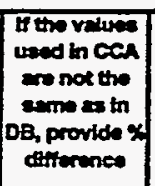 & ID & Mntortald & Meteria Mamo & $\begin{array}{l}\text { Permocior } \\
\text { Do }\end{array}$ & Permetion & $\begin{array}{l}\text { PrRDE } \\
\text { MPOAn }\end{array}$ & $\begin{array}{c}\text { Deta Entry } \\
\text { Doto }\end{array}$ & 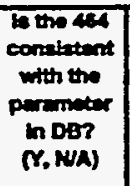 & $\begin{array}{l}\text { Perameter } \\
\text { Categony } \\
\text { (rootinote 1) }\end{array}$ \\
\hline$Y$ & & 3109 & REFCON & Reference Constant & AVOGADRO & Avogedro's number & 32359 & $17 f=0-96$ & $\mathbf{Y}$ & 3 \\
\hline$Y$ & & 3110 & REFCON & Reference Constant & ZCINK & Zero Celcuus in Ketvin & 32435 & $17+40-96$ & $\mathbf{Y}$ & 3 \\
\hline$Y$ & & 3110 & REFCON & Reforence Constant & ZCINK & Zero Colcies in Ketvin & 32435 & 17 fob-96 & $\mathbf{Y}$ & 3 \\
\hline $\bar{Y}$ & & 3111 & REFCON & Reference Constant & стово & Curie to Becquerd Combersion & $323 \pi$ & $17+F a b-96$ & $\bar{Y}$ & 3 \\
\hline$Y$ & & 3111 & REFCON & References Constant & сптово & Cirie to Becaunal Comversion & 32377 & $17+90-96$ & $\mathbf{Y}$ & 3 \\
\hline $\bar{Y}$ & 0.0004 & 3112 & REFCON & Reference Constant & SECYR & Seconds to years Corversion & 32426 & $17-F a b-96$ & $\bar{Y}$ & 3 \\
\hline $\bar{Y}$ & 0.0004 & 3112 & REFCON & Reference Constemt & SECYR & Seconds to yeers Corversion & 32426 & 17-Feb-96 & $\bar{Y}$ & 3 \\
\hline$Y$ & 0.0004 & 3112 & REFCON & Reference Constent & SECYR & Seconds to yeers Conversion & 32426 & $17+500-96$ & $\bar{Y}$ & 3 \\
\hline $\bar{Y}$ & & 3114 & CONC_MON & Concrets Monolith & PRESSURE & Brino far fietd pore presesere & 32518 & $21+f=b-96$ & $Y$ & 44 \\
\hline $\bar{Y}$ & & 3114 & CONC_MON & Concrote Monolith & PRESSURE & Brine ter field pore pressure & 32518 & $21 F=b-96$ & $\bar{Y}$ & $4 A$ \\
\hline Y & & 3115 & CONC_MON & Concreto Monolith & SAT_IBRN & Initial Brive Suturation & 325404 & $28-F a b-96$ & $\mathbf{Y}$ & 1 \\
\hline $\bar{Y}$ & & 3115 & CONC_MON & Concrete Monolith & SAT_IBRN & Initial Brine Seturation & 325404 & $28-50 b-96$ & $\mathbf{Y}$ & 7 \\
\hline $\bar{Y}$ & & 3115 & CONC_MON & Concrete Monolith & SAT_IBRN & | Initial Brino Seturation & 325404 & 28-Feb-96 & $Y$ & 1 \\
\hline $\mathbf{Y}$ & & 3116 & DRZ_1 & $\begin{array}{l}\text { Disturbed rock 20ne; } \\
\text { ime period } 0 \text { to } 1000 \\
\text { years }\end{array}$ & KPT & $\begin{array}{l}\text { Fiog for Permeabitity Determined } \\
\text { Trreshold }\end{array}$ & 32870 & $20+a b-96$ & $\mathbf{Y}$ & AB \\
\hline$Y$ & & 3116 & DRZ_1 & $\begin{array}{l}\text { Disturbed rock zorn: } \\
\text { time period o to } 1000 \\
\text { years }\end{array}$ & KPT & $\begin{array}{l}\text { Fleg for Permesbitify Determined } \\
\text { Threstiold }\end{array}$ & 32870 & $20-500-96$ & $\mathbf{Y}$ & 4B \\
\hline$Y$ & & 3116 & DRZ_1 & $\begin{array}{l}\text { Disturbed rock zone; } \\
\text { ime period } 0 \text { to } 1000 \\
\text { years }\end{array}$ & KPT & $\begin{array}{l}\text { Fiso for Permesbitty Dotermined } \\
\text { Throshold }\end{array}$ & 32870 & $2 a-c b-96$ & $\mathbf{Y}$ & $A B$ \\
\hline$Y$ & & 3116 & DRZ_1 & $\begin{array}{l}\text { Disturbed rock zone; } \\
\text { time pertiod } 0 \text { to } 1000 \\
\text { years }\end{array}$ & KPT & $\begin{array}{l}\text { Flag for Permesbitity Determined } \\
\text { Thresthold }\end{array}$ & 32870 & $20-506-96$ & $\mathbf{Y}$ & 48 \\
\hline$Y$ & & 3120 & BOREHOLE & Boroholo and Fill & PCT_A & $\begin{array}{l}\text { Throshold Presscure L inear } \\
\text { Prameter }\end{array}$ & $36362 A$ & 21fob-96 & $\mathbf{Y}$ & 48 \\
\hline$Y$ & & 3120 & BOREHOLE & Borehole and Fill & PCT_A & $\begin{array}{l}\text { Threshold Pressuro Lines } \\
\text { Paremuter }\end{array}$ & $36352 A$ & 21feb-96 & $\mathbf{Y}$ & 48 \\
\hline $\mathbf{Y}$ & & 3120 & BOREHOLE & Borethole and Fill & PCT_A & $\begin{array}{l}\text { Throshold Prossuro Lnear } \\
\text { Parameter }\end{array}$ & 363624 & $21-F=b-96$ & $Y$ & $4 B$ \\
\hline$Y$ & & 3121 & BOREHOLE & Boreholo and Fid & PCT_EXP & $\begin{array}{l}\text { Trroshold pressuro exponential } \\
\text { parameter }\end{array}$ & 36363 & 21Feb-96 & $\mathbf{Y}$ & $4 B$ \\
\hline $\mathbf{Y}$ & & 3121 & BOREHOLE & Borehole and Fill & PCT_EXP & $\begin{array}{l}\text { Throshold presure exponential } \\
\text { parameter }\end{array}$ & 35363 & 21-Feb-96 & $\mathbf{Y}$ & AB \\
\hline $\mathbf{Y}$ & & 3121 & BOREHOLE & Borehole and Fal & PCT_EXP & $\begin{array}{l}\text { Throshold pressure exporential } \\
\text { paremoter }\end{array}$ & 36363 & 21 feb-96 & $\mathbf{Y}$ & $A B$ \\
\hline$Y$ & & 3122 & BOREHOLE & Borehole and Fill & KPT & $\begin{array}{l}\text { Fles for Pormesbilty Dotermined } \\
\text { Inreshold }\end{array}$ & 36361 & 21-Feb-96 & $\mathbf{Y}$ & $4 B$ \\
\hline$Y$ & & 3122 & BOREHOLE & Boreholo and Fil & KPT & $\begin{array}{l}\text { Fles for Permesbility Detorminod } \\
\text { Trreshold }\end{array}$ & 36361 & 21-Feb-96 & $\boldsymbol{\gamma}$ & $4 B$ \\
\hline $\mathbf{Y}$ & & 3122 & BOREHOLE & Borehole and Fill & KPT & $\begin{array}{l}\text { Flag for Permeability Determined } \\
\text { Thresthold }\end{array}$ & 36361 & 21-Fob-9s & $\boldsymbol{Y}$ & $4 B$ \\
\hline $\mathbf{Y}$ & & 3122 & BOREHOLE & Borehole and Fill & KPT & $\begin{array}{l}\text { Fieg for Permeability Detormined } \\
\text { Throshootd }\end{array}$ & 36361 & 21Feb-96 & $\mathbf{Y}$ & 48 \\
\hline$Y$ & & 3123 & CL_L_T4 & $\begin{array}{l}\text { Lower Saledo clay.50 } \\
\text { to } 10 \mathrm{~K} \text { years }\end{array}$ & PO_MIN & $\begin{array}{l}\text { Minmum brne pressure for } \\
\text { capillary model KPC=3 }\end{array}$ & 36366 & 21-feb-96 & $\mathbf{Y}$ & 48 \\
\hline $\mathbf{Y}$ & & 3123 & CL_L_T4 & $\begin{array}{l}\text { Lower Salado clay.50 } \\
\text { to } 10 \mathrm{~K} \text { yeare }\end{array}$ & PO_MIN & $\begin{array}{l}\text { Minmum brre pressure for } \\
\text { capolitery model KPC }=3\end{array}$ & 36366 & 21-Fob-96 & $\mathbf{Y}$ & 48 \\
\hline$Y$ & & 3123 & CL_L_TA & $\begin{array}{l}\text { Lowor Saludo diny:50 } \\
\text { to 10K years }\end{array}$ & PO_MIN & $\begin{array}{l}\text { Mrnistum brone prossure for } \\
\text { capilluy model KPC=3 }\end{array}$ & 36366 & 21-Feb-96 & $\mathbf{Y}$ & $4 B$ \\
\hline $\mathbf{Y}$ & & 3124 & CONC_MON & Concrete Monoth & PO_MIN & $\begin{array}{l}\text { Minmum brime pressure for } \\
\text { capillery model KPC=3 }\end{array}$ & 36367 & 21-Feb-96 & $\mathbf{Y}$ & $A B$ \\
\hline$Y$ & & 3124 & CONC_MON & Concrite Monolith & PO_MIN & $\begin{array}{l}\text { Mnimum brmo pressure for } \\
\text { cappilery modal KPC=3 }\end{array}$ & 36367 & $21+\infty<-96$ & $\mathbf{Y}$ & 48 \\
\hline$Y$ & & 3124 & CONC_MON & Concrete Monolith & PO_MIN & 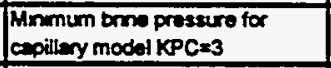 & 36357 & 21-Feb-96 & $\mathbf{Y}$ & $4 B$ \\
\hline$Y$ & & 3125 & SALT_T5 & $\begin{array}{l}\text { Shan sall column } \\
\text { compectedtimn } 100 \text { to } \\
200 \text { years }\end{array}$ & PO_MIN & $\begin{array}{l}\text { Mininum brne pressure for } \\
\text { capilimy modet KPC=3 }\end{array}$ & 36368 & 21Fob-96 & $Y$ & $4 B$ \\
\hline $\mathbf{Y}$ & & 3125 & SALT_TS & $\begin{array}{l}\text { Shen salt column } \\
\text { compactodtime } 100 \text { to } \\
200 \text { years }\end{array}$ & PO_MIN & $\begin{array}{l}\text { Minimum brino pressern tor } \\
\text { capiltery model } \mathrm{KPC}=3\end{array}$ & 36368 & 21 feb-96 & $\mathbf{Y}$ & 48 \\
\hline $\mathbf{Y}$ & & 3125 & SALT_TS & $\begin{array}{l}\text { Shan sal column } \\
\text { compactedtime } 100 \text { to } \\
200 \text { years }\end{array}$ & PO_MIN & $\begin{array}{l}\text { Minimum brme preasure for } \\
\text { capilliny modet KPC=3 }\end{array}$ & 36368 & $21+\infty e b-96$ & $\mathbf{Y}$ & AB \\
\hline
\end{tabular}


CCA Parameter Listing

\begin{tabular}{|c|c|c|c|c|c|c|c|c|c|c|}
\hline TD & \begin{tabular}{|c|} 
Cleveth- \\
cation of \\
Deth \\
Centegory \\
(footnote 2)
\end{tabular} & $\begin{array}{l}\text { Distibutton } \\
\text { Type }\end{array}$ & man & Medine & Mininum & Meximura & Thits & Retermen id & $\begin{array}{l}\text { We the dinta } \\
\text { devaloped } \\
\text { underen } \\
\text { Hoh-1 } \\
\text { Progrem? }\end{array}$ & 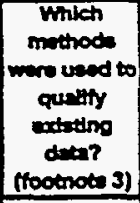 \\
\hline 3109 & 4 & CONSTANT & $6.0221370 \mathrm{E}+23$ & $6.0221370 E+23$ & $6.0221370 E+23$ & $6.0221370 E+23$ & $1 \mathrm{~mol}$ & WP030298 & $Y$ & NA \\
\hline 3110 & 4 & CONSTANT & $27315000 E+02$ & $27315000 E+02$ & $27315000 E+102$ & $27315000 E+02$ & $K$ & CRC-72 & $Y$ & NA \\
\hline 3110 & 4 & CONSTANT & $27315000 \mathrm{E}+02$ & $27315000 E+02$ & $2.7315000 E+02$ & $2.7315000 E+02$ & $\mathrm{k}$ & WP035298 & $Y$ & N/A \\
\hline 3111 & 4 & CONSTANT & $3.7000000 E+10$ & $3.7000000 E+10$ & $3.7000000 E+10$ & $3.7000000 E+10$ & $\mathrm{BaCl}$ & CRC. 72 & $Y$ & N/A \\
\hline 3111 & 4 & CONSTANT & $3.7000000 \mathrm{E}+10$ & $3.7000000 E+10$ & $3.7000000 \mathrm{E}+10$ & $3.7000000 E+10$ & $\mathrm{BQCC_{1 }}$ & WPO35298 & $Y$ & NA \\
\hline 3112 & 4 & CONSTANT & $3.1688770 E-08$ & $3.1689770 E-00$ & $3.1688770 E-08$ & $3.1688770 E-08$ & $\mathrm{yrrs}$ & CRG-72 & $Y$ & N/A \\
\hline 3112 & 4 & CONSTANT & $3.1688770 E-08$ & $3.1688770 E-08$ & $3.1688770 E-08$ & $3.1689770 E-08$ & yrts & WPOs5298 & $\bar{Y}$ & $N / A$ \\
\hline 3112 & 4 & CONSTANT & $3.1688770 E-08$ & $3.1688770 E-08$ & $3.1688770 E-00$ & $3.1689770 E-08$ & $y+/ 8$ & WPO38456 & $Y$ & NA \\
\hline 3114 & 3,5 & CONSTANT & $1.0132500 E+0.5$ & $1.0132500 E+05$ & 1.0132500E+05 & $1.0132500 E+0.5$ & $\mathrm{~Pa}_{\mathbf{2}}$ & WP035597 & $Y$ & NA \\
\hline 3114 & 3,5 & CONSTANT & $1.0132500 \mathrm{E}+05$ & $1.0132500 \mathrm{E}+05$ & $1.0132500 E+0.5$ & $1.0132500 \mathrm{E}+05$ & Pa & WP036058 & $\mathbf{Y}$ & NA \\
\hline 3115 & 3,4 & CONSTANT & 9.9999990E-01 & $9.9999990 E-01$ & 9.9999990E-01 & 9.9999990E-01 & NONE & $\begin{array}{c}\text { MEMO:VAUGHNW } \\
\text { TERNEYS } \\
\end{array}$ & $Y$ & NA \\
\hline 3115 & 3,4 & CONSTANT & 9.9999990E-01 & 9.99999SOE-01 & $9.9999990=-01$ & 9.9999990E-01 & NONE & WPO35597 & $Y$ & NA \\
\hline 3115 & 3,4 & CONSTANT & 9.9999990E-01 & 9.99999SOE-01 & 9.8999990E-01 & 9.9999990E-01 & NONE & WP039169 & $Y$ & NA \\
\hline 3116 & 3,5 & CONSTANT & $0.0000000 E+\infty 0$ & $0.0000000 E+00$ & $0.0000000 E+\infty$ & $0.0000000 E+\infty$ & NONE & WP035271 & $\mathbf{Y}$ & NA \\
\hline 3116 & 3,5 & CONSTANT & $0.00000000 E+00$ & $0.0000000 E+00$ & $0.0000000 E+\infty 0$ & $0.0000000=+\infty 0$ & NONE & WP035333 & $\mathbf{Y}$ & NA \\
\hline 3116 & 3,5 & CONSTANT & $0.0000000 \mathrm{E}+00$ & $0.0000000 \mathrm{E}+\infty 0$ & $0.0000000 E+\infty$ & $0.0000000 E+\infty 0$ & NONE & WP035597 & $\mathbf{Y}$ & NA \\
\hline 3116 & 3,5 & CONSTANT & $0.0000000 E+00$ & $0.0000000 E+\infty 0$ & $0.0000000 E+\infty 0$ & $0.0000000 E+00$ & NONE & WPO40514 & $Y$ & NA \\
\hline 3120 & Not Used & CONSTANT & $5.6000000 E-01$ & $5.6000000 E-01$ & $5.6000000 E-01$ & $5.6000000 E-01$ & $\mathbf{P a}$ & $\begin{array}{c}\text { MEMO:VAUGHNW } \\
\text { TIERNEY-7 }\end{array}$ & $\mathbf{Y}$ & N/A \\
\hline 3120 & Not Used & CONSTANT & $5.6000000 E-01$ & $5.6000000 E-01$ & $5.6000000 E-01$ & $5.6000000 E-01$ & $P=$ & WP035597 & $\mathbf{Y}$ & NA \\
\hline 3120 & Not Used & CONSTANT & $5.6000000 E-01$ & $5.6000000 E-01$ & $5.6000000 E-01$ & $5.6000000 E-01$ & Pa & WPO40514 & $\mathbf{Y}$ & NAA \\
\hline 3121 & Not Usod & CONSTANT & $3.4600000 E-01$ & $3.46000000-01$ & $3.4600000 \mathrm{E}-01$ & $3.4600000 E-01$ & NONE & $\begin{array}{c}\text { MEMO:VAUGHN } \\
\text { TERNEY-7 }\end{array}$ & $\mathbf{Y}$ & NA \\
\hline 3121 & Not Used & CONSTANT & $-3.4600000 E-01$ & $3.4600000 E-01$ & $3.46000000-01$ & $3.4600000 \mathrm{E}-01$ & NONE & WP035597 & $\mathbf{Y}$ & NA \\
\hline 3121 & Not Used & CONSTANT & $-3.46000000 E-01$ & $-3.46000000-01$ & $3.4600000 E-01$ & $-3.4600000 E-01$ & NONE & WPO40514 & $\mathbf{Y}$ & NA \\
\hline 3922 & Not Usod & CONSTANT & $0.0000000 E+\infty 0$ & $0.00000000 E+\infty 0$ & $0.0000000 E+00$ & $0.0000000 E+\infty 0$ & NONE & $\begin{array}{c}\text { MEMO:VAUGHNN } \\
\text { TIERNEY-7 }\end{array}$ & $\mathbf{Y}$ & N/A \\
\hline 3122 & Not Usod & CONSTANT & $0.0000000 \mathrm{E}+\infty 0$ & $0.0000000 E \pm 00$ & $0.0000000 E+\infty$ & $0.0000000 E+\infty$ & NONE & WPO35333 & $\gamma$ & NA \\
\hline 3122 & Not Usod & CONSTANT & $0.0000000 E+\infty 0$ & $0.0000000 E+\infty$ & $0.0000000 E+\infty 0$ & $0.0000000 E+\infty$ & NONE & WP035597 & $\mathbf{Y}$ & NA \\
\hline 3122 & Not Usod & CONSTANT & $0.00000000 E+00$ & $0.00000000 E+\infty$ & $0.0000000 E+\infty$ & $0.0000000 E+\infty 0$ & NONE & WPO40514 & $\bar{Y}$ & N/A \\
\hline 3123 & 1,3 & CONSTANT & $1.0132500 E+05$ & $1.0132500 E+05$ & $1.0132500 E+05$ & $1.0132500 \mathrm{E}+05$ & $P \approx$ & WP035597 & $\mathbf{Y}$ & NA \\
\hline 3123 & 1.3 & CONSTANT & $1.0132500 E+05$ & $1.0132500 E+05$ & $1.0132500 E+05$ & $1.0132500 E+05$ & $\mathbf{P z}$ & WP036051 & $\mathbf{Y}$ & NA \\
\hline 3123 & 1,3 & CONSTANT & $1.0132500 E+05$ & $1.0132500 E+05$ & $1.0132500 E+05$ & $1.0132500 E+05$ & $P a$ & UPO40514 & $\mathbf{Y}$ & NA \\
\hline 3124 & 3.5 & CONSTANT & $1.0132500 E+05$ & $1.0132500 E+05$ & $1.0132500 E+05$ & $1.0132500 E+05$ & P. & WP035597 & $\gamma$ & NA \\
\hline 3124 & 3,5 & CONSTANT & $1.0132500 E+05$ & $1.0132500 E+05$ & $1.0132500 E+05$ & $1.0132500 E+05$ & Pa & WP035051 & $\mathbf{Y}$ & NA \\
\hline 3124 & 3.5 & CONSTANT & $1.0132500 E+05$ & $1.0132500 E+05$ & $1.0132500 E+05$ & $1.0132500 E+05$ & Pa & WPO40514 & $\mathbf{Y}$ & NA \\
\hline 3125 & 1,3 & CONSTANT & $1.0132500 E+05$ & $1.0132500 E+05$ & $1.0132500 E+05$ & $1.0132500 E+05$ & $\mathrm{Pe}$ & WP035597 & $\mathbf{Y}$ & NA \\
\hline 3125 & 9,3 & CONSTANT & $1.0132500 E+05$ & $1.0132500 E+05$ & $9.0132500 E+05$ & $1.0132500 E+05$ & Pe & WP036051 & $\mathbf{Y}$ & $\mathrm{NA}$ \\
\hline 3125 & 1,3 & CONSTANT & $1.0132500 E+05$ & $1.0132500 E+05$ & $1.0132500 E+05$ & $1.0132500 E+05$ & $\mathbf{P a}$ & WPO40514 & $Y$ & NA \\
\hline
\end{tabular}


CCA Parameter Listing

\begin{tabular}{|c|c|c|c|c|c|c|c|c|c|c|}
\hline $\begin{array}{l}\text { Paramiter in } \\
\text { Dutabeen } \\
\text { (YN) }\end{array}$ & 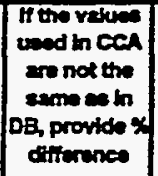 & 10 & Matara to & Metarid Nater & $\begin{array}{l}\text { Parmater } \\
\text { it }\end{array}$ & Permeter Name & $\begin{array}{l}\text { PRPD } \\
\text { MPON }\end{array}$ & $\begin{array}{c}\text { Dete Entry } \\
\text { Dete }\end{array}$ & $\begin{array}{l}\text { Ex the SA } \\
\text { consiatent } \\
\text { with the } \\
\text { parmeter } \\
\text { in DB? } \\
(N, N A)\end{array}$ & $\begin{array}{l}\text { Parmintar } \\
\text { Category } \\
\text { (footnote 1) }\end{array}$ \\
\hline $\mathbf{Y}$ & & 3126 & SALT_T6 & $\begin{array}{l}\text { Shaft salt column } \\
\text { compectedtume } 200 \text { to } \\
10 \text { K years }\end{array}$ & PO_MIN & $\begin{array}{l}\text { Minmum tone presture for } \\
\text { capillary modal } \mathrm{KPC}=3\end{array}$ & 36369 & $21-F a b-96$ & $\mathbf{Y}$ & 48 \\
\hline $\mathbf{Y}$ & & 3126 & SALT_T6 & $\begin{array}{l}\text { Shaft ent colemn } \\
\text { compectoctime } 200 \text { to } \\
10 \text { K years }\end{array}$ & PO_MIN & $\begin{array}{l}\text { Minimum brno preseure tor } \\
\text { Capilany model KPCCX3 }\end{array}$ & 36369 & $21+500-96$ & $\mathbf{Y}$ & $4 B$ \\
\hline $\mathbf{Y}$ & & 3126 & SALT_T6 & $\begin{array}{l}\text { Shaf selt colemn } \\
\text { compectedtine } 200 \text { to } \\
10 \text { K years }\end{array}$ & PO_MIN & $\begin{array}{l}\text { Animum brine presenre for } \\
\text { caplley model KPC=3 }\end{array}$ & 36369 & $21+c c b-96$ & $\mathbf{Y}$ & 48 \\
\hline $\mathbf{Y}$ & $0.0 \mathrm{mw}$ & 3128 & DRZ_1 & $\begin{array}{l}\text { Disturbed rock zone; } \\
\text { time period } 0 \text { to } 1000 \\
\text { years }\end{array}$ & PCT_A & $\begin{array}{l}\text { Throshold Pressure Linear } \\
\text { Parmoter }\end{array}$ & 36559 & 21 feb-96 & $\mathbf{Y}$ & 48 \\
\hline $\mathbf{Y}$ & $0.0 \mathrm{mw}$ & 3128 & DRZ_1 & $\begin{array}{l}\text { Disturbed rock zore: } \\
\text { tine period } 0 \text { to } 1000 \\
\text { years }\end{array}$ & PCT_A & $\begin{array}{l}\text { Threthold Pressure Linar } \\
\text { Peremoter }\end{array}$ & 36559 & $21-F a b-96$ & $\mathbf{Y}$ & 48 \\
\hline $\mathbf{Y}$ & $0.0 \mathrm{hw}$ & 3128 & DRZ_1 & $\begin{array}{l}\text { Disturbed rodx zone: } \\
\text { time period } 0 \text { to } 1000 \\
\text { years }\end{array}$ & PCT_A & $\begin{array}{l}\text { Threshoid Pressurs Lineer } \\
\text { Perameter }\end{array}$ & 36559 & $21+F e b-96$ & $\mathbf{Y}$ & 48 \\
\hline $\mathbf{Y}$ & $0.0 \mathrm{hw}$ & 3129 & $D R Z \_1$ & $\begin{array}{l}\text { Disturbed rock zone; } \\
\text { time period } 0 \text { to } 1000 \\
\text { years }\end{array}$ & PCT_EXP & $\begin{array}{l}\text { Threthold prossure exponential } \\
\text { permeter }\end{array}$ & 36560 & 21feb-gs & $\mathbf{Y}$ & 48 \\
\hline $\mathbf{Y}$ & $0.0 \mathrm{hw}$ & 3129 & DRZ_1 & $\begin{array}{l}\text { Disturbed rock zone: } \\
\text { time period } 0 \text { to } 1000 \\
\text { years }\end{array}$ & PCT_EXP & $\begin{array}{l}\text { Threachold pressure exponential } \\
\text { perenter }\end{array}$ & 35560 & $21+\ldots<-96$ & $\mathbf{Y}$ & 48 \\
\hline $\mathbf{Y}$ & $0.0 \mathrm{hw}$ & 3129 & DRZ_1 & $\begin{array}{l}\text { Disturbed rock zone; } \\
\text { time period } 0 \text { to } 1000 \\
\text { years }\end{array}$ & PCT_EXP & $\begin{array}{l}\text { Threshold pressure exponential } \\
\text { parameter }\end{array}$ & 36560 & $21+f e b-96$ & $\mathbf{Y}$ & $4 B$ \\
\hline $\mathbf{Y}$ & & 3131 & CLAY_RUS & $\begin{array}{l}\text { Rustler Compactod } \\
\text { Clay Cokumn }\end{array}$ & PO_MIN & $\begin{array}{l}\text { Mnimim brne prosstre for } \\
\text { cepiltery modol KPC=3 }\end{array}$ & 36561 & 26-Feb-96 & $\mathbf{Y}$ & 48 \\
\hline $\mathbf{Y}$ & & 3131 & CLAY_RUS & $\begin{array}{l}\text { Rustler Compacted } \\
\text { Clay Cohumn }\end{array}$ & PO_MIN & $\begin{array}{l}\text { Mrnimum brno preasure for } \\
\text { Capillary model KPC=3 }\end{array}$ & 36561 & 26 Feb-96 & $\mathbf{Y}$ & 48 \\
\hline $\mathbf{Y}$ & & 3131 & CLAY_RUS & $\begin{array}{l}\text { Rustler Compacted } \\
\text { Clsy Cohmn }\end{array}$ & PO_MIN & $\begin{array}{l}\text { Minimum brne pressure for } \\
\text { Capiltery model KPC=3 }\end{array}$ & 36561 & 26Feb-96 & $\mathbf{Y}$ & 48 \\
\hline $\mathbf{Y}$ & & 3132 & REFCON & Reference Constant & DRROOM & $\begin{array}{l}\text { Number of onms. per room, in } \\
\text { roval pectuno }\end{array}$ & 32372 & 26 Feb-96 & $\mathbf{Y}$ & 3 \\
\hline $\mathbf{Y}$ & & 3132 & REFCON & Reference Constant & DRROOM & $\begin{array}{l}\text { Number ot conms. per room, in } \\
\text { dosel pectino }\end{array}$ & 32372 & 26-Fob-96 & $\mathbf{Y}$ & 3 \\
\hline $\mathbf{Y}$ & & 3133 & SHFT_DRZ & $\begin{array}{l}\text { Desturbed Rock Around } \\
\text { Shan }\end{array}$ & PRMX_LOG & $\begin{array}{l}\text { Los of intrnec permesbilty, X- } \\
\text { orection }\end{array}$ & 36563 & $01+$ ther-96 & $\mathbf{Y}$ & 1 \\
\hline $\mathbf{Y}$ & & 3133 & SHFT_DRZ & $\begin{array}{l}\text { Dishurbed Rock Around } \\
\text { Shan }\end{array}$ & PRMK_LOG & 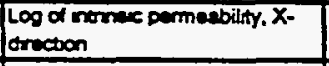 & 36563 & $01+$ Mar-96 & $\mathbf{Y}$ & 1 \\
\hline $\mathbf{Y}$ & & 3133 & SHFT_DRZ & $\begin{array}{l}\text { Oisturbed Rock Around } \\
\text { Shat }\end{array}$ & PRMX_LOG & 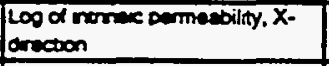 & 36563 & $01-$ Mar-96 & $\mathbf{Y}$ & 1 \\
\hline $\mathbf{Y}$ & & 3133 & SHFT_DRZ & $\begin{array}{l}\text { Disturbed Rock Around } \\
\text { Shan }\end{array}$ & PRUX_LOC & 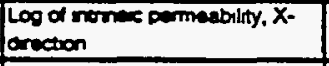 & 36563 & 01 thar-96 & $\mathbf{Y}$ & 1 \\
\hline $\mathbf{Y}$ & & 3133 & SHFT_DRZ & $\begin{array}{l}\text { Disturbod Rock Around } \\
\text { Shan }\end{array}$ & PRuX_lOG & $\begin{array}{l}\text { Log of restrac permasbility, } X \text { - } \\
\text { orection }\end{array}$ & 36563 & $01-$ Har-96 & $\mathbf{Y}$ & 1 \\
\hline $\mathbf{Y}$ & & 3134 & BH_OPEN & Borehole Unretionded & PRax_LOC & $\begin{array}{l}\text { Log of monic pormasblity, } X \text { - } \\
\text { arection }\end{array}$ & 36564 & 14Mar-96 & $Y$ & 48 \\
\hline $\mathbf{Y}$ & & 3134 & BH_OPEN & Borenow Utrostrated & Prace_100 & 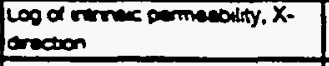 & 36564 & 14+Mar-96 & $\mathbf{Y}$ & 48 \\
\hline $\mathbf{Y}$ & & 3135 & BH_OPEN & Borehol Unrenand & POROsir & Ematom porom & 36565 & 14 Mer-96 & $\mathbf{Y}$ & 48 \\
\hline $\mathbf{Y}$ & & 3135 & BH_OPEN & Borahow Unrwenand & Ponosin & Ellocin porom & 36565 & 14 Mar-96 & $\mathbf{Y}$ & 48 \\
\hline $\mathbf{Y}$ & & 3136 & BH_OPEN & Borenol Urraturiad & com_nox & en comonmonr & 36566 & 14 Mar-96 & $\mathbf{Y}$ & 18 \\
\hline $\mathbf{Y}$ & & 3136 & BH_OPEN & Borahol Unrevioned & com_ncx & bu comprimeny & 36566 & $14410 r-96$ & $\mathbf{Y}$ & 48 \\
\hline $\mathbf{Y}$ & & 3137 & BH_OPEN & Borahol Urreatrand & REL_. & 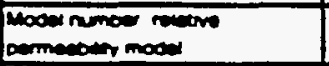 & 36567 & 14Mar-86 & $\mathbf{Y}$ & 48 \\
\hline $\mathbf{Y}$ & & 3137 & BH_OPEN & Boranow Unremand & REL_- $=\infty$ & 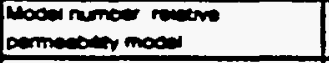 & 36567 & 14 Mer.e6 & $\mathbf{Y}$ & $A B$ \\
\hline $\mathbf{Y}$ & & 3138 & BH_OPEN & Borahole Urrosurad & CNP_MO & 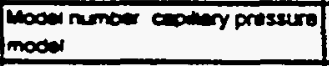 & 36568 & 14+kar-96 & $\mathbf{Y}$ & 48 \\
\hline $\mathbf{Y}$ & & 3138 & BH_OPEN & Borenole Unresorad & CNP_NOO & $\begin{array}{l}\text { mooes number copitery pressure } \\
\text { moow }\end{array}$ & 36568 & 14 Mar-96 & $\mathbf{Y}$ & 48 \\
\hline $\mathbf{Y}$ & & 3139 & BH_OPEN & Borahol Unrasorand & $P C_{-}$max & 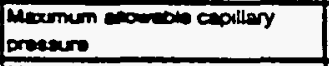 & 36569 & $14+M a r-96$ & $\mathbf{Y}$ & 48 \\
\hline $\mathbf{Y}$ & & 3139 & BH_OPEN & Borenow urrecticted & $P C_{-} m x$ & 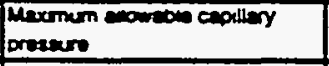 & 36569 & $1441=86$ & $\mathbf{Y}$ & 48 \\
\hline $\mathbf{Y}$ & & 3140 & BH_OPEN & Borenol Urrevitated & SAT_RBRN & Rearoun Brm Suaration & 36570 & 14 Her-96 & $\mathbf{Y}$ & $4 B$ \\
\hline
\end{tabular}


CCA Parameter Listing

\begin{tabular}{|c|c|c|c|c|c|c|c|c|c|c|}
\hline $\mathbf{1 0}$ & 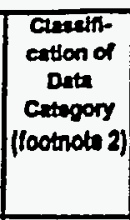 & $\begin{array}{l}\text { Dextribution } \\
\text { Type }\end{array}$ & maxn & Mndien & Mintrom & Mexdmen & Unite & Reforman to & $\begin{array}{l}\text { Wes the deth } \\
\text { demeloped } \\
\text { underem } \\
\text { Non-? } \\
\text { Proprem? }\end{array}$ & 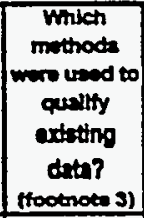 \\
\hline 3126 & 1,3 & CONSTANT & $1.0132500 E+05$ & $1.0132500 E+05$ & $1.0132500 E+05$ & $1.0132500 E+05$ & Pa & WP035597 & $\mathbf{Y}$ & NA \\
\hline 3926 & 1,3 & CONSTANT & $1.0132500 E+05$ & $1.0132500 E+05$ & $1.0132500 E+05$ & $1.01325005+05$ & $\mathbf{P a}$ & WP036051 & $Y$ & NA \\
\hline 3126 & 1,3 & CONSTANT & $1.0132500 E+05$ & $1.0132500 E+05$ & $1.0132500 E+05$ & $1.0132500 E+05$ & Pa & WPO40514 & $\mathbf{Y}$ & NA \\
\hline 3128 & 3,5 & CONSTANT & $0.0000000 E+\infty 0$ & $0.0000000 E+\infty 0$ & $0.0000000 E+\infty 0$ & $0.0000000 E+\infty$ & $\mathbf{P a}$ & $\begin{array}{c}\text { MEMO:LORD/ } \\
\text { TIERNEY-1 }\end{array}$ & $\mathbf{Y}$ & NA \\
\hline 3128 & 3,5 & CONSTANT & $0.0000000 E+\infty$ & $0.0000000 E+\infty 0$ & $0.0000000 E+\infty$ & $0.0000000 E+\infty 0$ & Pa & WP035597 & $\mathbf{Y}$ & N/A \\
\hline 3128 & 3,5 & CONSTANT & $0.0000000 \mathrm{E}+\infty$ & $0.0000000 E+00$ & $0.0000000 E+\infty 0$ & $0.0000000 E+\infty)$ & $\mathbf{P a}$ & WPOLO514 & $\mathbf{\gamma}$ & NA \\
\hline 3129 & 3,5 & CONSTANT & $0.0000000 E+\infty$ & $0.0000000 E+\infty$ & $0.0000000 E+\infty$ & $0.0000000 E+\infty 0$ & NONE & WP032288 & $\mathbf{Y}$ & NA \\
\hline 3129 & 3,5 & CONSTANT & $0.0000000 E+\infty 0$ & $0.0000000 E+00$ & $0.0000000 E+\infty 0$ & $0.00000000+\infty 0$ & NONE & WP035597 & $\mathbf{Y}$ & NA \\
\hline 3129 & 3,5 & CONSTANT & $0.0000000 E+\infty$ & $0.0000000 E+\infty 0$ & $0.00000000+\infty$ & $0.0000000 E+00$ & NONE & WP040514 & $\mathbf{Y}$ & NA \\
\hline 3131 & 1.3 & CONSTANT & $1.0132500 E+05$ & $1.0132500 E+05$ & $1.0132500 E+05$ & $1.0132500 E+05$ & Pa & WP035597 & $Y$ & N/A \\
\hline 3131 & 1,3 & CONSTANT & $1.0132500 E+05$ & $1.0132500 E+05$ & $1.0132500 E+05$ & $1.0132500 E+05$ & Pa & WP036051 & $Y$ & NA \\
\hline 3131 & 1,3 & CONSTANT & $1.0132500 E+05$ & $1.0132500 E+05$ & $1.0132500 \mathrm{E}+0.5$ & $1.0132500 E+05$ & $\mathrm{~Pa}$ & WP040514 & $Y$ & NA \\
\hline 3132 & 4 & CONSTANT & $6.8040000 \mathrm{E}+03$ & $6.8040000 E+03$ & $6.8040000 \mathrm{E}+03$ & $6.8040000 E+03$ & NONE & SAND92-0700/3 & $\mathbf{Y}$ & NA \\
\hline 3132 & 4 & CONSTANT & $6.80400000+03$ & $6.8040000 E+00$ & $6.8040000 E+03$ & $6.8040000 E+03$ & NONE & WP035298 & $\mathbf{Y}$ & NA \\
\hline 3133 & 1,3 & TRIANGULAR & $-1.5333330 \mathrm{E}+01$ & $-1.5000000 E+01$ & $-1.7000000 E+01$ & $-1.4000000 E+01$ & $\log \left(m^{\wedge} 2\right)$ & WP030640 & $\mathbf{Y}$ & NA \\
\hline 3133 & 1,3 & TRLANGULAR & $-1.5333330 E+01$ & $-1.5000000 E+01$ & $-1.7000000 E+01$ & $-14000000 E+01$ & $\log \left(m^{\wedge} 2\right)$ & WP030994 & $\mathbf{Y}$ & NA \\
\hline 3133 & 1,3 & TRLANGULAR & $-1.5333330 E+01$ & $-1.5000000 E+01$ & $-1.7000000 E+01$ & $-1.4000000 E+01$ & $\log \left(m^{\wedge} 2\right)$ & WP035268 & $Y$ & NA \\
\hline 3133 & 1,3 & TRLANGULAR & $-1.5333330 E+01$ & $-1.5000000 E+01$ & $-1.7000000 E+01$ & $-14000000 E+01$ & $\log \left(m^{\wedge} 2\right)$ & WP035597 & $Y$ & NA \\
\hline 3133 & 1,3 & TRLANGULAR & $-1.5333330 E+01$ & $-1.5000000 E+01$ & $-17000000 E+01$ & $-1<000000 E+01$ & $\log \left(m^{\wedge} 2\right)$ & WPO41558 & $\mathbf{Y}$ & NA \\
\hline 3134 & 3,5 & CONSTANT & $-9.0000000 E+\infty 0$ & $-9.0000000 E+\infty 0$ & $-90000000 E+\infty$ & $-90000000 E+\infty 0$ & $\log \left(m^{\wedge} 2\right)$ & WP035134 & $\mathbf{Y}$ & NA \\
\hline 3134 & 3,5 & CONSTANT & $-9.0000000 E+\infty 00$ & $-9.0000000 E+\infty$ & $-90000000 E+\infty 0$ & $-80000000 E+\infty$ & $\log \left(m^{\wedge} 2\right)$ & WPO40514 & $\mathbf{Y}$ & NA \\
\hline 3135 & 1,3 & CONSTANT & $3.2000000 E-01$ & $3.2000000 E-01$ & $32000000 E-01$ & $32000000 E-01$ & NONE & WP035134 & $\mathbf{Y}$ & NA \\
\hline 3135 & 1,3 & CONSTANT & $3.2000000 E-01$ & $3.2000000 E-01$ & $32000000 E-01$ & $32000000 E-01$ & NONE & WPO40514 & $Y$ & NA \\
\hline 3136 & 1,3 & CONSTANT & $0.0000000 E+00$ & $0.0000000 E+\infty 0$ & $00000000<-\infty$ & $00000000 E+\infty$ & Pen-1 & WP035134 & $Y$ & NA \\
\hline 3136 & 1.3 & CONSTANT & $0.00000000+\infty$ & $0.0000000 E+\infty$ & $00000000 E+\infty$ & $00000000 E+\infty 0$ & $P e^{n-1}$ & WP040514 & $\mathbf{Y}$ & NA \\
\hline 3137 & 3.5 & CONSTANT & $5.0000000 E+\infty$ & $5.0000000 E+\infty$ & $5.0000000 E+\infty$ & $50000000 E+\infty$ & NONE & WP035134 & $\mathbf{Y}$ & N/A \\
\hline 3137 & 3,5 & CONSTANT & $5.0000000 E+\infty 0$ & $5.0000000 E+\infty 0$ & $5.0000000 E+\infty 0$ & $5.0000000 E+\infty 0$ & NONE & WPO40514 & $\boldsymbol{Y}$ & NA \\
\hline 3938 & 1,3 & CONSTANT & $1.00000000+\infty 0$ & $1.0000000 E+\infty$ & $1.0000000 E+\infty$ & $1.0000000 E+\infty 0$ & NONE & WPO35134 & $\mathbf{Y}$ & NA \\
\hline 3138 & 1,3 & CONSTANT & $1.0000000 E+00$ & $1.0000000 E+\infty$ & $1.0000000 E+\infty$ & $1.0000000=+\infty 0$ & NONE & WPO40514 & $\mathbf{Y}$ & NA \\
\hline 3139 & 1,3 & CONSTANT & $1.0000000 E+08$ & $1.0000000 E+08$ & $1.0000000 E+08$ & $1.0000000 E+\infty 8$ & Pa & WP035134 & $\mathbf{Y}$ & NA \\
\hline 3139 & 1,3 & CONSTANT & $1.0000000 E+08$ & $1.0000000 E+08$ & $1.0000000 E+08$ & $1.0000000 E+\infty 8$ & Pa & WPO40514 & $\mathbf{Y}$ & NA \\
\hline 3140 & 1,3 & CONSTANT & $0.0000000 E+00$ & $0.0000000 E+\infty$ & $0.0000000 E+00$ & $0.0000000 E+\infty$ & NONE & WP035134 & $Y$ & NA \\
\hline
\end{tabular}


CCA Parameter Listing

\begin{tabular}{|c|c|c|c|c|c|c|c|c|c|c|}
\hline $\begin{array}{l}\text { Parmetar in } \\
\text { Databee? } \\
\text { (YiN) }\end{array}$ & 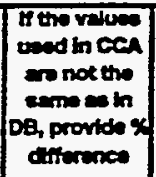 & D & Metenta io & Metard Nams & Pand & $\begin{array}{l}\text { Parmine Rers } \\
\cdots,\end{array}$ & $\begin{array}{l}\text { PRPD } \\
\text { MFO }\end{array}$ & Dentry & 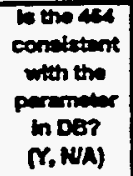 & $\begin{array}{l}\text { Panmiver } \\
\text { Cotegory } \\
\text { (foothote 1) }\end{array}$ \\
\hline $\mathbf{Y}$ & & 3140 & BH_OPEN & Borehoil Unrestricted & SAT_RBRN & Residual Brina Saturation & 36570 & 14Mer-96 & $\mathbf{Y}$ & 48 \\
\hline $\mathbf{Y}$ & & 3141 & BH_OPEN & Borehole Urrestricted & SAT_RGAS & Recidual Ges Suturation & 36571 & 14 Aer-96 & $\mathbf{Y}$ & 48 \\
\hline $\mathbf{Y}$ & & 3141 & BH_OPEN & Borahois Unrastricted & SAT_RGAS & Reaidual Ges Seturation & 36571 & 14 Mar-96 & $\mathbf{Y}$ & 48 \\
\hline $\mathbf{Y}$ & & 3142 & BH_OPEN & Borehola Urrestricted & PORE_DIS & $\begin{array}{l}\text { Brooks-Corey pore dietribution } \\
\text { peremeter }\end{array}$ & 36572 & 14Har-96 & $\mathbf{Y}$ & 48 \\
\hline $\mathbf{Y}$ & & 3142 & BH_OPEN & Boreholo Unrestricted & PORE_DIS & $\begin{array}{l}\text { Brooks-corey pore distribution } \\
\text { peremeter }\end{array}$ & 36572 & 14 Mer-96 & $\mathbf{Y}$ & 48 \\
\hline $\mathbf{Y}$ & & 3143 & BH_OPEN & Borehole Unrestricted & PO_MIN & $\begin{array}{l}\text { Mrinum brue presesure for } \\
\text { capillary model KPC=3 }\end{array}$ & 36584 & 14 Mar-96 & $\mathbf{Y}$ & $4 B$ \\
\hline $\mathbf{Y}$ & & 3143 & BH_OPEN & Borehois Urrestricted & PO_MIN & $\begin{array}{l}\text { Mininum brme preasure for } \\
\text { capilley model KPC=3 }\end{array}$ & 36584 & 14 Mer-56 & $\mathbf{Y}$ & 48 \\
\hline $\mathbf{Y}$ & & 3144 & BH_OPEN & Borohole Urrestricted & KPT & $\begin{array}{l}\text { Fleg for Permeability Determined } \\
\text { Threathold }\end{array}$ & 36585 & 14 Mr.96 & $\mathbf{Y}$ & $4 B$ \\
\hline $\mathbf{Y}$ & & 3144 & BH_OPEN & Borehole Unrestricted & KPT & $\begin{array}{l}\text { Fleg for Permeabildy Dotermined } \\
\text { Threshold }\end{array}$ & 36585 & 14Mer-96 & $\mathbf{Y}$ & $4 B$ \\
\hline $\mathbf{Y}$ & & 3145 & BH_OPEN & Borehoto Utrrestricted & PCT_A & $\begin{array}{l}\text { Thrashoid Pressure Linear } \\
\text { Parameter }\end{array}$ & 36585 & 14Mar-96 & $\mathbf{Y}$ & 48 \\
\hline $\mathbf{Y}$ & & 3145 & BH_OPEN & Boroholo Urrestricted & PCT_A & $\begin{array}{l}\text { Threabold Presure Linas } \\
\text { Paremeter }\end{array}$ & 36586 & 14Mir-96 & $\mathbf{Y}$ & $4 B$ \\
\hline $\mathbf{Y}$ & & 3146 & BH_OPEN & Borehole Urrestricted & PCT_EXP & $\begin{array}{l}\text { Threshold preseure exponential } \\
\text { peremeter }\end{array}$ & 36588 & $14+4 x-96$ & $\mathbf{Y}$ & $A B$ \\
\hline $\mathbf{Y}$ & & 3146 & BH_OPEN & Boraholo Unrestricted & PCT_EXP & $\begin{array}{l}\text { Threshold preseure exponential } \\
\text { peremoter }\end{array}$ & 36588 & 14 Har-96 & $\mathbf{Y}$ & 48 \\
\hline $\mathbf{Y}$ & & 3147 & CONC_PLG & $\begin{array}{l}\text { Concrote Plug, surface } \\
\text { and Rustior }\end{array}$ & POROSITY & Efrective porosity & 36589 & 14 Her-96 & $\mathbf{Y}$ & $4 B$ \\
\hline $\mathbf{Y}$ & & 3147 & CONC_PLG & $\begin{array}{l}\text { Concrete Plug. Eurfaco } \\
\text { and Rustior }\end{array}$ & POROSITY & Effective porosity & 36509 & 14 Har-96 & $\mathbf{Y}$ & $4 B$ \\
\hline $\mathbf{Y}$ & & 3148 & CONC_PLG & $\begin{array}{l}\text { Concrete Plug, surtece } \\
\text { and Rustior }\end{array}$ & COMP_RCK & Bulk Compressibility & 35591 & $02+4 x y-96$ & $\mathbf{Y}$ & 48 \\
\hline $\mathbf{Y}$ & & 3148 & CONC_PLG & $\begin{array}{l}\text { Concroto Phy, surtace } \\
\text { end Rustior }\end{array}$ & COMP_RCK & Bulk Compressibitity & 36591 & $02+4 x y-96$ & $\mathbf{Y}$ & 48 \\
\hline $\mathbf{Y}$ & & 3149 & CONC_PLG & $\begin{array}{l}\text { Concrete Plug, surtace } \\
\text { and Ruster }\end{array}$ & RELP_MOD & $\begin{array}{l}\text { Modal number, relatrve } \\
\text { Dermenbilty model }\end{array}$ & 36592 & 14tMar-96 & $\mathbf{Y}$ & 48 \\
\hline $\mathbf{Y}$ & & 3149 & CONC_PLG & $\begin{array}{l}\text { Concrete Plug, surtice } \\
\text { and Rustier }\end{array}$ & RELP_MOD & $\begin{array}{l}\text { Model mumber, relative } \\
\text { permenbility model }\end{array}$ & 36592 & 14Har-96 & $\mathbf{Y}$ & AB \\
\hline $\mathbf{Y}$ & & 3150 & CONC_PLG & $\begin{array}{l}\text { Concrete Plug. surtace } \\
\text { and Rustier }\end{array}$ & CAP_MOD & $\begin{array}{l}\text { Model number, cepillary pressuro } \\
\text { model }\end{array}$ & 36593 & 14 Mar-96 & $\mathbf{Y}$ & AB \\
\hline$Y$ & & 3150 & CONC_PLG & $\begin{array}{l}\text { Concrete Phy, surfaco } \\
\text { and Runtior }\end{array}$ & CAP_MOD & $\begin{array}{l}\text { Model number, ceppillary pressure } \\
\text { model }\end{array}$ & 36593 & 14Mar-96 & $\mathbf{Y}$ & AB \\
\hline $\mathbf{Y}$ & & 3151 & CONC_PLG & $\begin{array}{l}\text { Concrito Plug, surface } \\
\text { end Ruester }\end{array}$ & PC_MAX & $\begin{array}{l}\text { Maximum allowable capillary } \\
\text { pressure }\end{array}$ & 36595 & $14+4 a r-96$ & $\mathbf{Y}$ & $4 B$ \\
\hline $\mathbf{Y}$ & & 3151 & CONC_PLG & $\begin{array}{l}\text { Concrete Pho, surtace } \\
\text { and Rustler }\end{array}$ & PC_ MAX & $\begin{array}{l}\text { Meximum allowable cepillary } \\
\text { pressure }\end{array}$ & 36595 & 14Har-96 & $\mathbf{Y}$ & $4 B$ \\
\hline $\mathbf{Y}$ & & 3152 & CONC_PLG & $\begin{array}{l}\text { Concrate Phy, surface } \\
\text { and Rustler }\end{array}$ & SAT_RBRN & Residual Brine Seturation & 36596 & 14 Mar-96 & $\mathbf{Y}$ & $4 B$ \\
\hline $\mathbf{Y}$ & & 3152 & CONC_PLG & $\begin{array}{l}\text { Concrete Phy, surfeco } \\
\text { and Rustier }\end{array}$ & SAT_RBRN & Residual Brino Sturation & 36596 & 14 Her-96 & $--Y$ & 48 \\
\hline $\mathbf{Y}$ & & 3153 & CONC_PLG & $\begin{array}{l}\text { Concrete Pho, eurfece } \\
\text { and Rustier }\end{array}$ & SAT_RGAS & Residual Ges Seturation & 36597 & 14 Mar-96 & $\mathbf{Y}$ & 48 \\
\hline $\mathbf{Y}$ & & 3153 & CONC_PLG & $\begin{array}{l}\text { Concrete Phy, surfece } \\
\text { and Rustler }\end{array}$ & SAT_RGAS & Residual Ges Seturation & 36597 & 14 Mar-96 & $\mathbf{Y}$ & $4 \mathrm{~B}$ \\
\hline $\mathbf{Y}$ & & 3154 & CONC_PLG & $\begin{array}{l}\text { Concrete Pho, eurtece } \\
\text { end Rustier }\end{array}$ & PORE_DIS & $\begin{array}{l}\text { Brooks-Corey pore distribution } \\
\text { parameter }\end{array}$ & 36598 & 14ther-96 & $\mathbf{Y}$ & 48 \\
\hline $\mathbf{Y}$ & & 3154 & CONC_PLG & $\begin{array}{l}\text { Concrate Phy, surtaces } \\
\text { end Ruetior }\end{array}$ & PORE_DIS & $\begin{array}{l}\text { Brooke-Corty pore distribution } \\
\text { parameter }\end{array}$ & 36598 & 144Mer-96 & $\mathbf{Y}$ & 48 \\
\hline $\mathbf{Y}$ & & 3155 & CONC_PLG & $\begin{array}{l}\text { Concroto Phg, surtace } \\
\text { and Ruater }\end{array}$ & PO_MIN & $\begin{array}{l}\text { Mnimum brmo pressurs for } \\
\text { capillery modal KPC=3 }\end{array}$ & 36599 & 14Mar-96 & $\mathbf{Y}$ & 48 \\
\hline $\mathbf{Y}$ & & 3155 & CONC_PLG & $\begin{array}{l}\text { Concrato Plug, urtace } \\
\text { and Runter }\end{array}$ & PO_MIN & $\begin{array}{l}\text { Mnimum brine praseure for } \\
\text { capillery model KPC=3 }\end{array}$ & 36599 & 14-Mar-96 & $\mathbf{Y}$ & 48 \\
\hline $\mathbf{Y}$ & & 3156 & CONC_PLG & $\begin{array}{l}\text { Concrote Pug, eurtace } \\
\text { end Rustler }\end{array}$ & KPT & $\begin{array}{l}\text { Fleg for Permeabilty Dotermined } \\
\text { Threshold }\end{array}$ & 36500 & 14Har-96 & $\mathbf{Y}$ & 48 \\
\hline $\mathbf{Y}$ & & 3156 & CONC_PLG & $\begin{array}{l}\text { Concreis Phy, surfece } \\
\text { and Ruster }\end{array}$ & KPT & $\begin{array}{l}\text { Fleg for Permeability Determined } \\
\text { Threahold }\end{array}$ & 36600 & 14 Mar-96 & $\mathbf{Y}$ & AB \\
\hline $\mathbf{Y}$ & & 3157 & CONC_PLG & $\begin{array}{l}\text { Concrote Plug, surfeco } \\
\text { end Ruatier }\end{array}$ & PCT_A & $\begin{array}{l}\text { Threahold Pressure Lnaer } \\
\text { Peremeter }\end{array}$ & 36601 & 14Mar-96 & $\mathbf{Y}$ & 48 \\
\hline $\mathbf{Y}$ & & 3157 & CONC_PLG & $\begin{array}{l}\text { Correride Pleg, surtace } \\
\text { and Runtier }\end{array}$ & PCT_A & $\begin{array}{l}\text { Threshoid Presure Lnear } \\
\text { Perameter }\end{array}$ & 36501 & 14 Mer-96 & $\mathbf{Y}$ & $4 B$ \\
\hline $\mathbf{Y}$ & & 3158 & CONC_PLG & $\begin{array}{l}\text { Concreto Phy, surface } \\
\text { and Puxtior }\end{array}$ & PCT_EXP & $\begin{array}{l}\text { Trreshold preseure exponential } \\
\text { perameter }\end{array}$ & 36603 & 144Ax-96 & $\mathbf{Y}$ & $4 B$ \\
\hline
\end{tabular}


CCA Parameter Listing

\begin{tabular}{|c|c|c|c|c|c|c|c|c|c|c|}
\hline 10 & 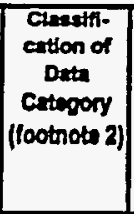 & $\begin{array}{c}\text { Distribution } \\
\text { Type }\end{array}$ & Man & Median & Mintmum & Mrodinum & Units & Reference tD & $\begin{array}{l}\text { Wes the det } \\
\text { dervloped } \\
\text { Ender an } \\
\text { Noh-1 } \\
\text { Progrem? }\end{array}$ & $\begin{array}{l}\text { Wrich } \\
\text { mothods } \\
\text { more uend to } \\
\text { qually } \\
\text { exdeting } \\
\text { date? } \\
\text { (footnots 3) }\end{array}$ \\
\hline 3140 & 1,3 & CONSTANT & $0.0000000 E+\infty$ & $0.0000000 E+\infty$ & $0.0000000^{\prime \prime} E+\infty$ & $0.0000000=+\infty$ & NONE & WP040514 & $\mathbf{Y}$ & NNA \\
\hline 3141 & 1,3 & CONSTANT & $0.0000000 E+\infty 0$ & $0.0000000 E+\infty$ & $0.0000000 E+\infty$ & $0.0000000 E+\infty$ & NONE & WP035134 & $\mathbf{Y}$ & NA \\
\hline 3141 & 1,3 & CONSTANT & $0.0000000 E+\infty 0$ & $0.00000000+\infty$ & $0.0000000 E+\infty$ & $0.0000000 E+\infty$ & NONE & WPO40514 & $\mathbf{Y}$ & N/A \\
\hline 3142 & 1,3 & CONSTANT & $7.0000000 E-01$ & $7.0000000 E-01$ & $7.0000000 E-01$ & $7.0000000 E-01$ & NONE & WP035134 & $\mathbf{Y}$ & N/A \\
\hline 3142 & 1,3 & CONSTANT & $7.0000000 E-01$ & $7.0000000 \mathrm{E}-01$ & $7.0000000 E-01$ & $7.0000000 E-01$ & NONE & WPO40514 & $\mathbf{Y}$ & N/A \\
\hline 3143 & 1,3 & CONSTANT & $1.0132500 \mathrm{E}+05$ & $1.0132500 E+05$ & $1.0132500 E+05$ & $1.0132500 E+05$ & Pa & WP035134 & $\mathbf{Y}$ & NA \\
\hline 3143 & 1,3 & CONSTANT & $1.0132500 E+05$ & $1.0132500 E+05$ & $1.0132500 E+05$ & $1.0132500 E+05$ & Pa & WP040514 & $\mathbf{Y}$ & NA \\
\hline 3144 & 1,3 & CONSTANT & $0.0000000 E+\infty$ & $0.0000000 E+00$ & $0.0000000 E+\infty$ & $0.0000000 E+\infty 0$ & NONE & WP035134 & $\mathbf{Y}$ & NA \\
\hline 3144 & 1,3 & CONSTANT & $0.0000000 E+\infty$ & $0.0000000 E+\infty$ & $0.0000000 E+\infty 0$ & $0.0000000 E+\infty$ & NONE & WP040514 & $\mathbf{Y}$ & NA \\
\hline 3145 & 1,3 & CONSTANT & $0.0000000 E+\infty 0$ & $0.0000000 E+\infty$ & $0.0000000 E+\infty$ & $0.0000000 E+\infty$ & $\mathrm{Pa}$ & WPO35134 & $\mathbf{Y}$ & NA \\
\hline 3145 & 1,3 & CONSTANT & $0.0000000 E+\infty$ & $0.0000000 E+\infty$ & $0.0000000 E+\infty)$ & $0.0000000=+\infty$ & Pa & WPO40514 & $\mathbf{Y}$ & NA \\
\hline 3146 & 1,3 & CONSTANT & $0.0000000 E+\infty 0$ & $0.0000000 E+\infty 0$ & $0.0000000=+\infty 0$ & $0.0000000 E+\infty$ & NONE & WP035134 & $\mathbf{Y}$ & $\mathbf{N A}$ \\
\hline 3146 & 1.3 & CONSTANT & $0.0000000 E+\infty$ & $0.0000000 E+\infty$ & $0.0000000 E+\infty 0$ & $0.0000000 E+\infty$ & NONE & WP040514 & $\mathbf{Y}$ & NA \\
\hline 3147 & 3,5 & CONSTANT & $3.2000000 \mathrm{E}-01$ & $3.2000000 E-01$ & $3.2000000 E-01$ & $3.2000000 E-01$ & NONE & WP035134 & $\mathbf{Y}$ & NA \\
\hline 3147 & 3,5 & CONSTANT & $3.2000000 E-01$ & $3.2000000 E-01$ & $3.2000000 \mathrm{E}-01$ & $3.2000000 E-01$ & NONE & WPO40514 & $\mathbf{Y}$ & NAA \\
\hline 3148 & $1,3,4$ & CONSTANT & $1.2000000 \mathrm{E}-09$ & $1.2000000 \mathrm{E}-09$ & $1.2000000 \mathrm{E}-\infty 9$ & $1.2000000 E-09$ & $P a^{a}-1$ & WP035134 & $\mathbf{Y}$ & NA \\
\hline 3148 & $1,3,4$ & CONSTANT & $1.2000000 \mathrm{E}-09$ & $1.2000000 \mathrm{E}-09$ & $1.2000000 E-09$ & $1.2000000 \mathrm{E}-09$ & $\operatorname{Pan}-1$ & WP040514 & $\mathbf{Y}$ & NA \\
\hline 3149 & $1,3,4$ & CONSTANT & $4.0000000 E+\infty 0$ & $4.0000000 E+\infty 0$ & $4.0000000 E+\infty 0$ & $4.0000000 \mathrm{E}+00$ & NONE & WP035134 & $\mathbf{Y}$ & NA \\
\hline 3149 & $1,3,4$ & CONSTANT & $4.0000000 E+00$ & $4.00000000+00$ & $4.0000000 E+\infty 0$ & $4.0000000 E+\infty$ & NONE & WP040514 & $\mathbf{Y}$ & NA \\
\hline 3150 & $1.3,4$ & CONSTANT & $1.0000000 E+\infty$ & $1.0000000 E+00$ & $1.0000000 E+00$ & $1.0000000 \mathrm{E}+\infty 0$ & NONE & WP035134 & $\mathbf{Y}$ & NA \\
\hline 3150 & $1,3,4$ & CONSTANT & $1.0000000 E+\infty 0$ & $1.0000000 E+\infty$ & $1.0000000 E+\infty$ & $1.0000000 E+\infty$ & NONE & WPO40514 & $\mathbf{Y}$ & N/A \\
\hline 3151 & 1,3 & CONSTANT & $1.0000000 E+08$ & $1.0000000 E+08$ & $1.0000000 E+08$ & $1.0000000 E+08$ & $\mathrm{~Pa}$ & WP035134 & $\mathbf{Y}$ & NA \\
\hline 3151 & 1,3 & CONSTANT & $1.0000000 \mathrm{E}+08$ & $1.0000000 E+08$ & $1.0000000 E+08$ & $1.0000000 E+08$ & $\mathrm{~Pa}$ & WP040514 & $\mathbf{Y}$ & $N / A$ \\
\hline 3152 & 3,5 & CONSTANT & $0.0000000 E+\infty$ & $0.0000000 E+\infty$ & $0.0000000 E+\infty$ & $0.0000000 E+\infty$ & NONE & WPO35134 & $\mathbf{Y}$ & N/A \\
\hline 3152 & 3,5 & CONSTANT & $0.0000000 \mathrm{E}+\infty 0$ & $0.0000000 E+\infty$ & $0.0000000 \mathrm{E}+\infty$ & $0.0000000 E+\infty$ & NONE & WPO40514 & $-Y$ & $N / A$ \\
\hline 3153 & 3,5 & CONSTANT & $0.0000000 E+\infty$ & $0.0000000 E+\infty$ & $0.0000000 E+\infty$ & $0.0000000 E+00$ & NONE & WPO35134 & $\mathbf{Y}$ & NA \\
\hline 3153 & 3,5 & CONSTANT & $0.0000000 E+\infty$ & $0.0000000 E+\infty$ & $0.0000000 E+\infty 0$ & $0.0000000 E+\infty$ & NONE & WP040514 & $\mathbf{Y}$ & NAA \\
\hline 3154 & 1,3 & CONSTANT & $9.4000000 E-01$ & $9.4000000 E-01$ & $9.4000000 E-01$ & $9.4000000 \mathrm{E}-01$ & NONE & WP035134 & $\mathbf{Y}$ & NA \\
\hline 3154 & 1,3 & CONSTANT & $9.4000000 E-01$ & $9.4000000 E-01$ & $9.4000000 E-01$ & $9.4000000 E-01$ & NONE & WP040514 & $\mathbf{Y}$ & NAA \\
\hline 3155 & 1,3 & CONSTANT & $1.0132500 E+05$ & $1.0132500 E+0 S$ & $1.0132500 E+05$ & $1.0132500 E+05$ & Pa & WP035134 & $Y$ & NA \\
\hline 3155 & 1,3 & CONSTANT & $1.0132500 E+05$ & $1.0132500 E+05$ & $1.0132500 E+05$ & $1.0132500 E+05$ & $\mathbf{P a}$ & WP040514 & $\mathbf{Y}$ & N/A \\
\hline 3156 & 1,3 & CONSTANT & $0.0000000 E+\infty 0$ & $0.0000000 E+\infty 0$ & $0.0000000 E+\infty$ & $0.0000000 E+\infty$ & NONE & WP035134 & $\mathbf{Y}$ & NA \\
\hline 3156 & 1,3 & CONSTANT & $0.0000000 E+\infty 0$ & $0.0000000 E+\infty 0$ & $0.0000000 E+\infty 0$ & $0.0000000 E+\infty$ & NONE & WPO40514 & $\mathbf{Y}$ & NA \\
\hline 3157 & 3,5 & CONSTANT & $0.0000000 E+\infty$ & $0.0000000 E+00$ & $0.0000000 E+\infty$ & $0.0000000 E+\infty$ & $\mathbf{P a}$ & WP035134 & $\mathbf{Y}$ & N/A \\
\hline 3157 & 3,5 & CONSTANT & $0.0000000=+\infty$ & $0.0000000 E+\infty 0$ & $0.0000000 E+\infty$ & $0.0000000 \varepsilon+\infty$ & $\mathbf{P a}$ & WPO40514 & $\mathbf{Y}$ & NA \\
\hline 3158 & 3,5 & CONSTANT & $0.0000000 E+\infty$ & $0.0000000 E+\infty$ & $0.0000000 E+\infty 0$ & $0.0000000 E+00$ & NONE & WPO35134 & $Y$ & NA \\
\hline
\end{tabular}


CCA Parameter Listing

\begin{tabular}{|c|c|c|c|c|c|c|c|c|c|c|}
\hline $\begin{array}{c}\text { Parmintar in } \\
\text { Databeae? } \\
\text { (YN) }\end{array}$ & 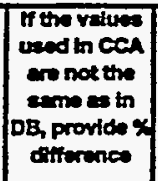 & ID & Metertalio & Mrtenda Mams & $\begin{array}{c}\text { Parmetar } \\
\text { to }\end{array}$ & Permontariars & $\begin{array}{l}\text { PRP D } \\
\text { nito }\end{array}$ & $\begin{array}{c}\text { Dotenty } \\
\text { Dew }\end{array}$ & 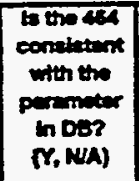 & $\begin{array}{l}\text { Parmine } \\
\text { Cateocy } \\
\text { footnow } 1 \text { ) }\end{array}$ \\
\hline $\mathbf{Y}$ & & 3158 & CONC_PLG & $\begin{array}{l}\text { Concreto Plug. surface } \\
\text { and Rustlor }\end{array}$ & PCT_EXP & $\begin{array}{l}\text { Threstiold pressure exponontal } \\
\text { parameter }\end{array}$ & 36600 & 14 Mur-96 & $\mathbf{Y}$ & $4 B$ \\
\hline $\mathbf{Y}$ & & 3159 & BH_SAND & $\begin{array}{l}\text { Borehole filled with sity } \\
\text { sand }\end{array}$ & POROSITY & Effective porosity & 30605 & 14 Mer-96 & $\mathbf{Y}$ & $4 B$ \\
\hline $\mathbf{Y}$ & & 3159 & BH_SAND & $\begin{array}{l}\text { Boroholo filled with stity } \\
\text { eard }\end{array}$ & POROSITY & Effective porosity & 36505 & 14 Mer-96 & $\mathbf{Y}$ & 48 \\
\hline $\mathbf{Y}$ & & 3160 & BH_SAND & $\begin{array}{l}\text { Borcholo filled with sity } \\
\text { sand }\end{array}$ & COMP_RCK & Bulk Comprestibility & 36510 & 14Mer-96 & $\mathbf{Y}$ & 48 \\
\hline $\mathbf{Y}$ & & 3160 & BH_SAND & $\begin{array}{l}\text { Borchole filled with sitty } \\
\text { sand }\end{array}$ & COMP_RCK & Butk Compressibitity & 36610 & 14 Mar-56 & $\mathbf{Y}$ & 48 \\
\hline $\mathbf{Y}$ & & 3161 & BH_SAND & $\begin{array}{l}\text { Borchole filled with sitty } \\
\text { sand }\end{array}$ & RELP_MOD & $\begin{array}{l}\text { Model number, relutw } \\
\text { pormeability model }\end{array}$ & 36611 & $144 \mathrm{mar} 96$ & $\mathbf{Y}$ & 48 \\
\hline $\mathbf{Y}$ & & 3161 & BH_SAND & $\begin{array}{l}\text { Borehole filled with sitty } \\
\text { sand }\end{array}$ & RELP_MOD & $\begin{array}{l}\text { Modal number, relative } \\
\text { permeability model }\end{array}$ & 36611 & 14 Ale 96 & $\mathbf{Y}$ & 48 \\
\hline $\mathbf{Y}$ & & 3162 & BH_SAND & $\begin{array}{l}\text { Borehole filled with sity } \\
\text { sand }\end{array}$ & CAP_MOD & $\begin{array}{l}\text { Model number, cepiltary pressure } \\
\text { model }\end{array}$ & 36612 & 14 Mer-86 & $\mathbf{Y}$ & 48 \\
\hline $\mathbf{Y}$ & & 3162 & BH_SAND & $\begin{array}{l}\text { Borehole filled with sitty } \\
\text { and }\end{array}$ & CAP_MOD & $\begin{array}{l}\text { Model rumber, capillery pressure } \\
\text { model }\end{array}$ & 35612 & 14 Mer-96 & $\mathbf{Y}$ & 48 \\
\hline $\mathbf{Y}$ & & 3163 & BH_SAND & $\begin{array}{l}\text { Borohole filled with efty } \\
\text { sand }\end{array}$ & PC_MAX & $\begin{array}{l}\text { Maxinum allowable capillery } \\
\text { pressure }\end{array}$ & 35613 & $1441=96$ & $\mathbf{Y}$ & 48 \\
\hline $\mathbf{Y}$ & & 3163 & BH_SAND & $\begin{array}{l}\text { Borcholo fillod with sitty } \\
\text { sand }\end{array}$ & PC_MAX & $\begin{array}{l}\text { Maximum allowable capillary } \\
\text { pressure }\end{array}$ & 36613 & $144=-86$ & $\mathbf{Y}$ & $4 B$ \\
\hline$Y$ & & 3164 & BH_SAND & $\begin{array}{l}\text { Borahole filled with sitty } \\
\text { sand }\end{array}$ & SAT_RBRN & Residual Brino Searetion & 36514 & $14-M a r-86$ & $\mathbf{Y}$ & $4 B$ \\
\hline $\mathbf{Y}$ & & 3164 & BH_SAND & $\begin{array}{l}\text { Boreholo filled with silty } \\
\text { send }\end{array}$ & SAT_RBRN & Resicual Brin Sturation & 36614 & 14 Mar-96 & $\mathbf{Y}$ & $4 B$ \\
\hline $\mathbf{Y}$ & & 3165 & BH_SAND & $\begin{array}{l}\text { Borehole filled whith sity } \\
\text { sand }\end{array}$ & SAT_RGAS & Residual Ges Suturation & 35615 & $14+14 r-96$ & $\mathbf{Y}$ & 48 \\
\hline $\mathbf{Y}$ & & 3165 & BH_SAND & $\begin{array}{l}\text { Borehole filled with sitty } \\
\text { send }\end{array}$ & SAT_RGAS & Residual Gas Seturation & 35815 & 14 Mar-96 & $\mathbf{Y}$ & 48 \\
\hline $\mathbf{Y}$ & & 3166 & BH_SAND & $\begin{array}{l}\text { Borohole filled with sitty } \\
\text { and }\end{array}$ & PORE_DIS & $\begin{array}{l}\text { Brooks-Coray pore detrbution } \\
\text { parameter }\end{array}$ & 35616 & $14 M=-96$ & $\mathbf{Y}$ & $4 B$ \\
\hline $\mathbf{Y}$ & & 3165 & BH_SAND & $\begin{array}{l}\text { Borohole filled with sitty } \\
\text { sand" }\end{array}$ & PORE_DIS & $\begin{array}{l}\text { Brooks-Corey pore distribution } \\
\text { paraneter }\end{array}$ & 36816 & $1444=-96$ & $\mathbf{Y}$ & $4 B$ \\
\hline $\mathbf{Y}$ & & 3167 & BH_SAND & $\begin{array}{l}\text { Borehole filled with sity } \\
\text { sand }\end{array}$ & PO_MIN & $\begin{array}{l}\text { Mnimum brine pressure tor } \\
\text { Capillary model KPC } x 3\end{array}$ & 36617 & 14 Mar-96 & $\mathbf{Y}$ & 48 \\
\hline $\mathbf{Y}$ & & 3167 & BH_SAND & $\begin{array}{l}\text { Borehole filled with sity } \\
\text { send }\end{array}$ & PO_MIN & $\begin{array}{l}\text { Minimum brme presture for } \\
\text { capilien model KPC=3 }\end{array}$ & 36617 & 144 er-96 & $\mathbf{Y}$ & $4 B$ \\
\hline $\mathbf{Y}$ & & 3168 & BH_SAND & $\begin{array}{l}\text { Borahole filled with sitty } \\
\text { end }\end{array}$ & KPT & $\begin{array}{l}\text { Fieg for Permasbility Determined } \\
\text { Threshold }\end{array}$ & 36619 & 14-Mar-96 & $\mathbf{Y}$ & 48 \\
\hline $\mathbf{Y}$ & & 3168 & BH_SAND & $\begin{array}{l}\text { Borwhole filled with sitty } \\
\text { send }\end{array}$ & KPT & $\begin{array}{l}\text { Fleg for Permeability Determined } \\
\text { Threshold }\end{array}$ & 36519 & $14+M=r-86$ & $\mathbf{Y}$ & 48 \\
\hline $\mathbf{Y}$ & & 3169 & BH_SAND & $\begin{array}{l}\text { Bornhole filled with sitty } \\
\text { sand }\end{array}$ & PCT_A & $\begin{array}{l}\text { Threshold Pressure Lrear } \\
\text { Perameter }\end{array}$ & 36520 & 14 Mar-96 & $\mathbf{Y}$ & 48 \\
\hline $\mathbf{Y}$ & & 3169 & BH_SAND & $\begin{array}{l}\text { Borshole filled with sitty } \\
\text { sand }\end{array}$ & PCT_A & $\begin{array}{l}\text { Threshold Pressure Lrear } \\
\text { Parameter }\end{array}$ & 36520 & 14Mer-96 & $\mathbf{Y}$ & 48 \\
\hline $\mathbf{Y}$ & & 3170 & BH_SAND & $\begin{array}{l}\text { Borehole filled with sitty } \\
\text { sand }\end{array}$ & PCT_EXP & $\begin{array}{l}\text { Threshold pressuro exponential } \\
\text { peremoter }\end{array}$ & 36621 & $14+$ Mar-96 & $\mathbf{Y}$ & 48 \\
\hline $\mathbf{Y}$ & & 3170 & BH_SAND & $\begin{array}{l}\text { Borehole filled with sity } \\
\text { sand }\end{array}$ & PCT_EXP & $\begin{array}{l}\text { Threshotd pressurs exponential } \\
\text { parenoter }\end{array}$ & 36521 & 14 Her-96 & $--Y$ & 48 \\
\hline $\mathbf{Y}$ & & 3171 & BH_CREEP & Creep Borehols Fill & POROSITY & Efrectrve porosity & 36524 & 14+Mar-96 & $Y$ & 48 \\
\hline$Y$ & & 3171 & BH_CREEP & Crewp Borchois Fill & POROSITY & Efrective porosity & 36524 & 14+1ker-96 & $\mathbf{Y}$ & 48 \\
\hline $\mathbf{Y}$ & & 3172 & BH_CREEP & Creop Borehole Fix & COMP_RCK & Bukk Compressibility & 36525 & 14Amer-96 & $Y$ & 48 \\
\hline $\mathbf{Y}$ & & 3172 & BH_CREEP & Creep Borehove Fill & COMP_RCK & Bulk Compressibility & 36525 & 14 Mar-96 & $\mathbf{Y}$ & 48 \\
\hline $\mathbf{Y}$ & & 3173 & BH_CREEP & Creep Borshole Fill & RELP_MOD & $\begin{array}{l}\text { Model number, retative } \\
\text { permeabilty model }\end{array}$ & 36526 & 14+Mar.96 & $\mathbf{Y}$ & 48 \\
\hline $\mathbf{Y}$ & & 3173 & BH_CREEP & Creep Borchole Fid & RELP_MOD & $\begin{array}{l}\text { Model mumber, nelativs } \\
\text { permesbilty model }\end{array}$ & 36626 & $14+4=4-96$ & $\mathbf{Y}$ & $4 B$ \\
\hline $\mathbf{Y}$ & & 3174 & BH_CREEP & Creep Borehole FX & CAP_MOD & $\begin{array}{l}\text { Model number, espillary preaser } \\
\text { model }\end{array}$ & 36527 & 14 mar-96 & $\mathbf{Y}$ & AB \\
\hline $\mathbf{Y}$ & & 3174 & BH_CREEP & Crenp Borahole FRI & CAP_MOD & $\begin{array}{l}\text { Model number, cepilieny preseure } \\
\text { model }\end{array}$ & 36627 & 14 Mar-96 & $\mathbf{Y}$ & $4 B$ \\
\hline $\mathbf{Y}$ & & 3175 & BH_CREEP & Creep Borahole Fid & PC_max & $\begin{array}{l}\text { Mexumum allowable capillary } \\
\text { presure }\end{array}$ & 36628 & 14 Mer-96 & $\mathbf{Y}$ & 48 \\
\hline $\mathbf{Y}$ & & 3175 & BH_CREEP & Creep Borehola Fil & Pc_max & $\begin{array}{l}\text { Meximum allowable capillery } \\
\text { presture }\end{array}$ & 36528 & $14+4 a r-96$ & $\mathbf{Y}$ & 48 \\
\hline $\mathbf{Y}$ & & 3176 & BH_CREEP & Creep Borahole Fit & SAT_RBRN & Resicual Brine Saturation & 36630 & 14turar-9s & $\mathbf{Y}$ & $4 B$ \\
\hline $\mathbf{Y}$ & & 3176 & BH_CREEP & Cresp Borchole Fill & SAT_RBRN & Residual Brino Saturation & 36630 & $14+$ Mer-96 & $\mathbf{Y}$ & 48 \\
\hline$Y$ & & 317 & BH_CREEP & Creep Borthola Fil & SAT_RGAS & Residual Ges Seturation & 36631 & $14+$ Mer-96 & $\mathbf{Y}$ & 48 \\
\hline$Y$ & & 317 & BH_CREEP & Creep Borchole Fil & SAT_RGAS & Residual Ges Seturation & 36531 & $14+$ Mar-96 & $\mathbf{Y}$ & 48 \\
\hline
\end{tabular}


CCA Parameter-Listing

\begin{tabular}{|c|c|c|c|c|c|c|c|c|c|c|}
\hline 10 & 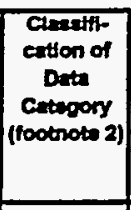 & $\begin{array}{l}\text { Distribution } \\
\text { Type }\end{array}$ & maen & modien & Menktom & 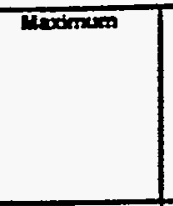 & Whats & Reforices to & 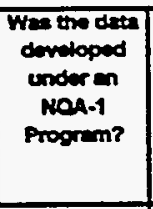 & 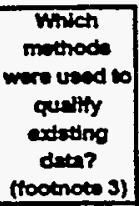 \\
\hline 3158 & 3,5 & CONSTANT & $0.0000000 \mathrm{E}+\infty 0$ & $0.0000000 E+\infty$ & $0.0000000 E+\infty$ & $0.0000000 E+\infty$ & NONE & WPO40514 & $\mathbf{Y}$ & NA \\
\hline 3159 & 1.3 & CONSTANT & $3.2000000 E-01$ & $3.2000000 E-01$ & $3.2000000 E-01$ & $3.2000000 E-01$ & NONE & WP035134 & $\mathbf{Y}$ & NA \\
\hline 3159 & 1,3 & CONSTANT & $3.2000000 E-01$ & $3.2000000 E-01$ & $3.2000000 E-01$ & $3.2000000 E-01$ & NONE & WPOLOS14 & $\mathbf{Y}$ & NA \\
\hline 3160 & 3,5 & CONSTANT & $0.0000000 E+\infty$ & $0.0000000 E+\infty$ & $0.0000000 E+\infty 0$ & $0.0000000 E+\infty$ & Pan-1 & WP035134 & $\mathbf{Y}$ & NA \\
\hline 3160 & 3,5 & CONSTANT & $0.0000000 E+00$ & $0.0000000 \varepsilon+00$ & $0.0000000 E+\infty$ & $0.0000000 E+00$ & $P M-1$ & WPO40514 & $Y$ & NA \\
\hline 3161 & 1,3 & CONSTANT & $4.0000000 E+00$ & $1.00000005+\infty$ & $1.0000000 \equiv+\infty$ & $4.0000000 E+\infty$ & NONE & WP035134: & $\mathbf{Y}$ & NA \\
\hline 3161 & 1,3 & CONSTANT & $4.0000000 E+\infty 0$ & $4.0000000 E+\infty)$ & $4.0000000 E+\infty$ & $4.0000000 E+\infty$ & NONE & WPO40514 & $\mathbf{Y}$ & NA \\
\hline 3162 & 3,5 & CONSTANT & $1.0000000 E+\infty$ & $1.0000000 E+\infty 0$ & $1.0000000 E+\infty 0$ & $1.0000000 E+\infty$ & NONE & WP035134 & $Y$ & NA \\
\hline 3162 & 3,5 & CONSTANT & $1.0000000 \mathrm{E}+\infty$ & $1.0000000 E+\infty 0$ & $1.0000000 E+\infty 0$ & $1.0000000 E+\infty$ & NONE & WP040514 & $\mathbf{Y}$ & N/A \\
\hline 3163 & 1,3 & CONSTANT & $1.0000000 \mathrm{E}+08$ & $1.0000000 E+08$ & $1.0000000 E+08$ & $1.0000000=+08$ & Pa & WP035134 & $\mathbf{Y}$ & NA \\
\hline 3163 & 1,3 & CONSTANT & $1.0000000 E+08$ & $1.0000000 E+08$ & $1.0000000 E+08$ & $1.0000000 E+08$ & $\mathbf{P a}$ & WP040514 & $\mathbf{Y}$ & NA \\
\hline 3964 & 3,5 & CONSTANT & $0.0000000 E+\infty$ & $0.0000000=+\infty 0$ & $0.0000000 E+\infty$ & $0.0000000 \leq+\infty$ & NONE & WP035134 & $\mathbf{Y}$ & NA \\
\hline 3164 & 3,5 & CONSTANT & $0.0000000 E+\infty$ & $0.0000000 E+\infty 0$ & $0.0000000 E+\infty$ & $0.0000000 E+\infty$ & NONE & WP040514 & $\mathbf{Y}$ & NA \\
\hline 3965 & 3,5 & CONSTANT & $0.0000000 \mathrm{E}+00$ & $0.0000000 \mathrm{E}+\infty 0$ & $0.0000000 E+\infty 0$ & $0.0000000 \varepsilon+\infty$ & NONE & WP035134 & $\mathbf{Y}$ & NA \\
\hline 3165 & 3,5 & CONSTANT & $0.0000000 E+\infty$ & $0.0000000 E+\infty$ & $0.0000000 E+\infty$ & $0.00000000+\infty 0$ & NONE & WPO40514 & $\mathbf{Y}$ & NA \\
\hline 3166 & 1,3 & CONSTANT & $9.4000000 E-01$ & $9.4000000 E-01$ & $94000000 E-01$ & 9.4000000E-01 & NONE & WP035134 & $\mathbf{Y}$ & NA \\
\hline 3166 & 1,3 & CONSTANT & $9.4000000 E-01$ & $9.4000000 E-01$ & $9.4000000 E-01$ & $94000000 E-01$ & NONE & WPO40514 & $\mathbf{Y}$ & NA \\
\hline 3167 & 1.3 & CONSTANT & $1.0132500 \mathrm{E}+05$ & $1.0132500 \equiv+05$ & $1.0132500 E+05$ & $1.0132500 E+05$ & $\mathbf{P a}$ & WP035134 & $\mathbf{Y}$ & NA \\
\hline 3967 & 1,3 & CONSTANT & $1.0132500 E+05$ & $1.0132500 E+05$ & $1.0132500 E+05$ & $1.0132500 E+05$ & $\mathbf{P a}$ & WPO40514 & $Y$ & NA \\
\hline 3168 & 1,3 & CONSTANT & $0.0000000 E+\infty$ & $0.0000000 \mathrm{E}+\infty 0$ & $00000000 E+\infty 0$ & $00000000 E+00$ & NONE & WP035134 & $Y$ & NA \\
\hline 3968 & 1.3 & CONSTANT & $0.0000000 E+\infty 0$ & $0.0000000 E+\infty$ & $00000000 E+\infty 0$ & $00000000 E+\infty 0$ & NONE & WP040514 & $Y$ & NA \\
\hline 3169 & 3,5 & CONSTANT & $0.0000000 \mathrm{E}+\infty$ & $00000000 E+\infty 0$ & $00000000 E+\infty$ & $0,000000 E+\infty$ & $P=$ & WP035134 & $\mathbf{Y}$ & N/A \\
\hline 3169 & 3,5 & CONSTANT & $0.0000000 E+\infty$ & $0.0000000 E+00$ & $0,0000000 \times-\infty$ & $0,000000 \times+\infty$ & $\mathbf{P a}$ & WPO40514 & $\mathbf{Y}$ & NA \\
\hline 3170 & 3,5 & CONSTANT & $0.0000000 \leqslant+\infty$ & $0.0000000 E+\infty$ & $00000000 \times+\infty$ & $0,000000<+00$ & NONE & WP035134 & $Y$ & NA \\
\hline 3170 & 3,5 & CONSTANT & $0.0000000 E+\infty$ & $0,0000000 E+\infty$ & $000000000 \times-\infty$ & $0000000<+\infty$ & NONE & WP040514 &.$- Y$ & NA \\
\hline 3171 & 1.3 & CONSTANT & $3.2000000 E-01$ & $32000000 \mathrm{E}-01$ & $32000000 \mathrm{E}-01$ & 32000000 E- I I & NONE & WPO35134 & $\mathbf{Y}$ & N/A \\
\hline 3171 & 1,3 & CONSTANT & $3.2000000 \mathrm{E}-01$ & $32000000 E-01$ & $320000006-01$ & $32000000 \mathrm{E}-01$ & NONE & WPO40514 & $Y$ & N/A \\
\hline 3172 & 3,5 & CONSTANT & $0.0000000 \mathrm{E}+\infty 0$ & $0,0000000 E+\infty 0$ & $00000000+\infty$ & 0 00000000 $+\infty$ & Pथn-1 & WP035134 & $\mathbf{Y}$ & NA \\
\hline 3172 & 3,5 & CONSTANT & $0.0000000 E+00$ & $00000000 E+00$ & $00000000 \times-\infty$ & $00000000 \times+\infty$ & $P e^{n-1}$ & WP040514 & $\mathbf{Y}$ & N/A \\
\hline 3173 & 1,3 & CONSTANT & $4.0000000 E+\infty 0$ & $40000000=+\infty$ & $40000000 \times+\infty$ & $4000000 \mathrm{x}+\infty$ & NOWE & WP035134 & $\mathbf{Y}$ & $N / A$ \\
\hline 3173 & 1,3 & CONSTANT & $4.0000000 E+\infty$ & $40000000 E+\infty 0$ & $<\infty<0000<-\infty$ & $40000000=-\infty$ & NONE & WP040514 & $\mathbf{Y}$ & $N / A$ \\
\hline 3174 & 3,5 & CONSTANT & $1.0000000 E+00$ & $1.0000000 E+\infty$ & $10000000-\infty$ & $10000000 E+\infty$ & NONE & WPO35134 & $\mathbf{Y}$ & NA \\
\hline 3174 & 3,5 & CONSTANT & $1.0000000 E+\infty 0$ & $1.0000000 E+\infty$ & $10000000=\infty 0$ & $10000000 \times+\infty$ & NONE & WPO40514 & $\mathbf{Y}$ & N/A \\
\hline 3175 & 1,3 & CONSTANT & $1.0000000 \mathrm{E}+\infty$ & $1.0000000 E+00$ & $10000000<-\infty$ & $10000000<+\infty$ & P. & WP035134 & $\mathbf{Y}$ & N/A \\
\hline 3175 & 1,3 & CONSTANT & $1.0000000 E+08$ & $10000000 E+\infty$ & $10000000 E+\infty \theta$ & $10000000 E+08$ & Pa & WPOS0514 & $Y$ & NA \\
\hline 3176 & 3,5 & CONSTANT & $0.0000000 E+00$ & $000000000+\infty 0$ & $00000000 E+\infty$ & $00000000 E+\infty 0$ & NONE & WP035134 & $\mathbf{Y}$ & NA \\
\hline 3176 & 3,5 & CONSTANT & $0.0000000 E+\infty$ & $00000000 E+00$ & $00000000 E+\infty$ & $00000000 E+00$ & NONE & WPO40514 & $\bar{Y}$ & NA \\
\hline 3177 & 3.5 & CONSTANT & $0.0000000 E+00$ & $0,0000000+\infty$ & $00000000 E+\infty$ & $00000000+\infty$ & NONE & WP035134 & $\mathbf{Y}$ & N/A \\
\hline 3177 & 3,5 & CONSTANT & $0.0000000 \mathrm{E}+\infty 0$ & $00000000 E+00$ & $00000000 E+\infty$ & $00000000 E+\infty$ & NOME & WP040514 & $\mathbf{Y}$ & N/A \\
\hline
\end{tabular}


CCA Parameter Listing

\begin{tabular}{|c|c|c|c|c|c|c|c|c|c|c|}
\hline 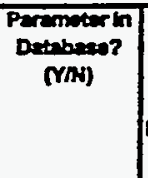 & 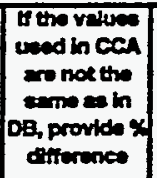 & $\mathbf{W}$ & Materla to & Wtandel Nmm & Parminar & Parmater Kanes & $\begin{array}{l}\text { PRPD } \\
\text { (WPO) }\end{array}$ & Dentery & 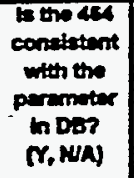 & $\begin{array}{l}\text { Parameter } \\
\text { Category } \\
\text { (footnota 1) }\end{array}$ \\
\hline $\mathbf{Y}$ & & 3178 & BH_CREEP & Creep Borehole Fill & PORE_DIS & $\begin{array}{l}\text { Brooks-Corey pore destribution } \\
\text { paremeter }\end{array}$ & 36533 & $1441=96$ & $\mathbf{Y}$ & $4 B$ \\
\hline $\mathbf{Y}$ & & 3178 & BH_CREEP & Cresp Borehole Fil & PORE_DIS & $\begin{array}{l}\text { Brooks-Cory pore destribution } \\
\text { peraneter }\end{array}$ & 36533 & 14 thar-56 & $\mathbf{Y}$ & $4 B$ \\
\hline $\mathbf{Y}$ & & 3179 & BH_CREEP & Creep Borehole Fil & PO_MIN & $\begin{array}{l}\text { Minimum brno pressure for } \\
\text { cepilitery model KPC=3 }\end{array}$ & 36534 & $14+4 x-96$ & $\mathbf{Y}$ & 48 \\
\hline $\mathbf{Y}$. & & 3179 & BH_CREEP & Creep Borchole Fill & PO_MIN & 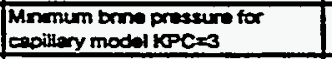 & 36534 & 14Mer-96 & $\mathbf{Y}$ & 48 \\
\hline $\mathbf{Y}$ & & 3180 & BH_CREEP & Creop Borehole Fid & KPT & $\begin{array}{l}\text { Fleg for Permesobiky Determined } \\
\text { Threghold }\end{array}$ & 30536 & $14 \mathrm{Mar}-96$ & $\mathbf{Y}$ & 48 \\
\hline $\mathbf{Y}$ & & 3180 & BH_CREEP & Creep Borehole Fill & KPT & $\begin{array}{l}\text { Fleg for Pemesbility Determned } \\
\text { Threathold }\end{array}$ & 36536 & 144 ar-96 & $\mathbf{Y}$ & 48 \\
\hline $\mathbf{Y}$ & & 3181 & BH_CREEP & Creep Borehole Fi & PCT_A & $\begin{array}{l}\text { Threahold Prassuro Lnaer } \\
\text { Peremoter }\end{array}$ & 36537 & 14 Mar-96 & $\mathbf{Y}$ & $\mathbf{4 B}$ \\
\hline $\mathbf{Y}$ & & 3181 & BH_CREEP & Creep Borehole Fill & PCT_A & $\begin{array}{l}\text { Throshold Proseuro Liness } \\
\text { Parameter }\end{array}$ & 36537 & $14+M r-56$ & $\mathbf{Y}$ & AB \\
\hline$Y$ & & 3182 & BH_CREEP & Creep Borehole Fill & PCT_EXP & $\begin{array}{l}\text { Threshold pressuro exponential } \\
\text { paremeter }\end{array}$ & 35539 & $14+4 a r-96$ & $\mathbf{Y}$ & 48 \\
\hline $\mathbf{Y}$ & & 3182 & BH_CREEP & Creep Borehole Fill & PCT_EXP & $\begin{array}{l}\text { Threshold pressure exponential } \\
\text { parmenter }\end{array}$ & 36539 & 14 Mar-96 & $\mathbf{Y}$ & 48 \\
\hline $\mathbf{Y}$ & varies & 3183 & BH_CREEP & Creep Borehole Fil & PRMX_LOG & $\begin{array}{l}\text { Log of intrinax permeability, } X- \\
\text { diroction }\end{array}$ & 36540 & 14Mar-96 & $\mathbf{Y}$ & 1 \\
\hline $\mathbf{Y}$ & veries & 3183 & BH_CREEP & Creop Borehole Fill & PRMX_LOG & $\begin{array}{l}\text { Los of internsic permentilty. X- } \\
\text { direction }\end{array}$ & 36840 & 14 Mar-96 & $\mathbf{Y}$ & 1 \\
\hline $\mathbf{Y}$ & varies & 3183 & BH_CREEP & Creep Borehole Fill & PRMX_LOG & $\begin{array}{l}\text { Los of intrinsic permesbilty, } X \text { - } \\
\text { direction }\end{array}$ & 36640 & $14+$ ther-96 & $\mathbf{Y}$ & 1 \\
\hline $\mathbf{Y}$ & & 3184 & BH_SAND & $\begin{array}{l}\text { Borehole filled with sity } \\
\text { sand }\end{array}$ & PRMX_LOG & $\begin{array}{l}\text { Log of intrinsic permeability, } X \text { - } \\
\text { direction }\end{array}$ & 36641 & 144tar-96 & $\mathbf{Y}$ & 1 \\
\hline $\mathbf{Y}$ & & 3184 & BH_SAND & $\begin{array}{l}\text { Borehole filled with sitty } \\
\text { sand }\end{array}$ & PRAX_LOG & $\begin{array}{l}\text { Log of intrinsic permesbilty, } x \text { - } \\
\text { direction }\end{array}$ & 36641 & 14 Mer-96 & $\mathbf{Y}$ & 1 \\
\hline $\mathbf{Y}$ & & 3185 & CONC_PLG & $\begin{array}{l}\text { Concrato Phy. unface } \\
\text { and Rustior }\end{array}$ & PRMX_LOG & $\begin{array}{l}\text { Log of intmssx permesbility, } X \text { - } \\
\text { drection }\end{array}$ & 36642 & 14 ther-96 & $\mathbf{Y}$ & 1 \\
\hline $\mathbf{Y}$ & & 3185 & CONC_PLG & $\begin{array}{l}\text { Concrete Phy, surface } \\
\text { and Rustier }\end{array}$ & PRMX_LOG & $\begin{array}{l}\text { Log of intrinsic permesbility, } X \text { - } \\
\text { direction }\end{array}$ & 36642 & 14 Mar-96 & $\mathbf{Y}$ & 1 \\
\hline $\mathbf{Y}$ & & 3185 & CONC_PLG & $\begin{array}{l}\text { Concrote Pkg. eurface } \\
\text { and Rustier }\end{array}$ & PRMX_LOG & $\begin{array}{l}\text { Log of intrnac permesbilty, X- } \\
\text { direction }\end{array}$ & 36642 & 14Aler-96 & $\mathbf{Y}$ & 1 \\
\hline $\mathbf{Y}$ & & 3186 & BH_OPEN & Boreholo Unrestricted & PRMY_LOG & $\begin{array}{l}\text { Los of intunse pormesbilty, Y- } \\
\text { direction }\end{array}$ & 36549 & $14-M a r-96$ & $\mathbf{Y}$ & 4B \\
\hline $\mathbf{Y}$ & & 3186 & BH_OPEN & Borehole Unrestricted & PRMY_LOG & $\begin{array}{l}\text { Log of intrinac permeability, Y. } \\
\text { droction }\end{array}$ & 36649 & 14+Mar-96 & $\mathbf{Y}$ & $A B$ \\
\hline $\mathbf{Y}$ & & 3187 & BH_OPEN & Borehol Unrestncted & PRMZ_LOG & $\begin{array}{l}\text { Log of intrase permesbility, Z- } \\
\text { drection }\end{array}$ & 36650 & 14 Har-96 & $\mathbf{Y}$ & $4 B$ \\
\hline$Y$ & & 3187 & BH_OPEN & Borehole Unrestricted & PRMZ_LOG & $\begin{array}{l}\text { Log of intrnsx permesbility, } Z \text { - } \\
\text { drection }\end{array}$ & 36650 & 14Awar-96 & $\mathbf{Y}$ & 48 \\
\hline $\mathbf{Y}$ & & 3188 & BH_CREEP & Creep Borahole Fin & PRAY_LOG & $\begin{array}{l}\text { Log of ntrinsic permasbitity, Y- } \\
\text { drection }\end{array}$ & 36652 & 14 Mar-96 & $\mathbf{Y}$ & 1 \\
\hline $\mathbf{Y}$ & & 3188 & BH_CREEP & Creep Borehole Fill & PRMY_LOG & $\begin{array}{l}\text { Log of nitrax permesbilty, } Y \text { - } \\
\text { drection }\end{array}$ & 36652 & 14Mlar.96 & $\mathbf{Y}$ & 1 \\
\hline $\mathbf{Y}$ & & 3189 & BH_CREEP & Creep Borenole Fill & PRMZ_LOG & $\begin{array}{l}\text { Log of intrixax pexmesbulty, } z \text {. } \\
\text { orvetion }\end{array}$ & 36653 & $14+$ tar-96 & $--Y$ & 1 \\
\hline$Y$ & & 3189 & BH_CREEP & Creep Boretrole Fill & PRMZ_LOG & $\begin{array}{l}\text { Los of nitmix permesbility, Z- } \\
\text { orrection }\end{array}$ & 36653 & 14Mar-96 & $\mathbf{Y}$ & 1 \\
\hline $\mathbf{Y}$ & & 3190 & BH_SAND & $\begin{array}{l}\text { Borahola filled with aling } \\
\text { sand }\end{array}$ & PRMY_LOG & $\begin{array}{l}\text { Los of nemax permeability, Y- } \\
\text { drection }\end{array}$ & 36654 & $14+4 a r-96$ & $\mathbf{Y}$ & 1 \\
\hline $\mathbf{Y}$ & & 3190 & BH_SAND & $\begin{array}{l}\text { Borahole filled when sulty } \\
\text { eand }\end{array}$ & PRMY_LOG & $\begin{array}{l}\text { Log of minsex permesbility, Y. } \\
\text { drection }\end{array}$ & 36654 & 14 Mar-96 & $\mathbf{Y}$ & 1 \\
\hline $\mathbf{Y}$ & & 3191 & BH_SAND & $\begin{array}{l}\text { Boruhols filled wath sity } \\
\text { send }\end{array}$ & PRMZ_LOG & $\begin{array}{l}\text { Lo } \alpha \text { momac permesbilty, } z \\
\text { orection }\end{array}$ & 36655 & $14+M=r .96$ & $\mathbf{Y}$ & 1 \\
\hline$Y$ & & 3191 & BH_SAND & $\begin{array}{l}\text { Borohole filled with suty } \\
\text { send }\end{array}$ & PRMZ_LOG & $\begin{array}{l}\text { Log of norrax permesbility, Z- } \\
\text { oriction }\end{array}$ & 36555 & $14+4 a r-96$ & $\mathbf{Y}$ & 1 \\
\hline $\mathbf{Y}$ & & 3192 & CONC_PLG & $\begin{array}{l}\text { Concrete Pug. surtece } \\
\text { end Ruetier }\end{array}$ & PRMY_LOG & $\begin{array}{l}\text { Los of normax permesbilty, } Y \text { - } \\
\text { orrection }\end{array}$ & 36656 & 14 Har-96 & $\mathbf{Y}$ & 1 \\
\hline $\mathbf{Y}$ & & 3192 & CONC_PLG & $\begin{array}{l}\text { Concrets Pho. surtece } \\
\text { end Rustior }\end{array}$ & PRMY_LOG & $\begin{array}{l}\text { Log of nimax permesbility, Y- } \\
\text { droction }\end{array}$ & 36656 & $14+$ Mar-96 & $\mathbf{Y}$ & 1 \\
\hline $\mathbf{Y}$ & & 3192 & CONC_PLG & $\begin{array}{l}\text { Concrete Pho. urfices } \\
\text { end Rustier }\end{array}$ & PRMY_LOG & $\begin{array}{l}\text { Log of mirrac pormesbality, Y- } \\
\text { droction }\end{array}$ & 36656 & 14-Har-96 & $\mathbf{Y}$ & 1 \\
\hline $\mathbf{Y}$ & & 3193 & CONC_PLG & $\begin{array}{l}\text { Concrute Plug. surfaco } \\
\text { end Rustior }\end{array}$ & PRMZ_LOG & $\begin{array}{l}\text { Log of intrnsic permesbildy, Z- } \\
\text { drection }\end{array}$ & 36657 & 14Amer.96, & $\mathbf{Y}$ & 4 \\
\hline $\mathbf{Y}$ & & 3193 & CONC_PLG & $\begin{array}{l}\text { Concrote Plug. surfece } \\
\text { and Rustior }\end{array}$ & PRMZ_LOG & $\begin{array}{l}\text { Log of ntomsac permenbitty, } 2 \text { - } \\
\text { direction }\end{array}$ & 36657 & 144mer-96 & $\mathbf{Y}$ & 1 \\
\hline $\mathbf{Y}$ & & 3193 & CONC_PLG & $\begin{array}{l}\text { Concrete Plug. sutece } \\
\text { end Rustior }\end{array}$ & PRMR_LOG & $\begin{array}{l}\text { Log of intensex permeability. Z- } \\
\text { drection }\end{array}$ & 36657 & 14 Mr-96 & $\mathbf{Y}$ & 1 \\
\hline
\end{tabular}


CCA Parameter Listing

\begin{tabular}{|c|c|c|c|c|c|c|c|c|c|c|}
\hline ID & 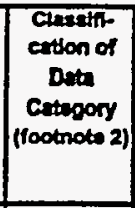 & $\begin{array}{l}\text { Detribution } \\
\text { Type }\end{array}$ & $\operatorname{Man}$ & Median & Minimim & Macimem & Unta & Roformes tD & $\begin{array}{l}\text { Was the dint } \\
\text { deraloped } \\
\text { undier an } \\
\text { Noh-1 } \\
\text { Program? }\end{array}$ & 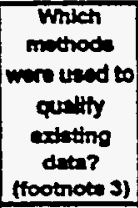 \\
\hline 3178 & 1,3 & CONSTANT & $9.4000000 E-01$ & $9.4000000 E-01$ & $9.4000000 E-01$ & $9.4000000 E-01$ & NONE & WP035134 & $\mathbf{Y}$ & N/A \\
\hline 3178 & 1,3 & CONSTANT & $9.4000000 E-01$ & 9.4000000E-01 & 9.4000000E-01 & $9.4000000 E-01$ & NONE & WP040514 & $\mathbf{Y}$ & NA \\
\hline 3179 & 1,3 & CONSTANT & $1.0132500 E+05$ & $1.0132500 \mathrm{E}+05$ & $1.0132500 E+C 5$ & $1.0132500 E+05$ & Pa & WPO35134 & $\mathbf{Y}$ & NA \\
\hline 3179 & 1,3 & CONSTANT & $1.0132500 \mathrm{E}+05$ & $1.0132500 E+05$ & $1.0132500 E+05$ & $1.0132500 E+05$ & $\mathbf{P a}$ & WP040514 & $Y$ & NA \\
\hline 3180 & 1,3 & CONSTANT & $0.0000000 \mathrm{E}+\infty 0$ & $0.0000000 E+00$ & $0.0000000 E+00$ & $0.0000000 E+\infty 0$ & NONE & WP035134 & Y & NA \\
\hline 3180 & 1,3 & CONSTANT & $0.0000000 E+\infty 0$ & $0.00000000 E+\infty 0$ & $0.0000000 E+\infty 0$ & $0.0000000 E+\infty$ & NONE & WPO40514 & $\mathbf{Y}$ & NA \\
\hline 3181 & 3,5 & CONSTANT & $0.0000000 E+00$ & $0.0000000 E+\infty$ & $0.0000000 E+\infty$ & $0.00000000+\infty$ & Pa & WP035134 & $\mathbf{Y}$ & NA \\
\hline 3181 & 3,5 & CONSTANT & $0.0000000 E+00$ & $0.0000000 E+00$ & $0.0000000 \mathrm{E}+00$ & $0.0000000 E+00$ & Pa & WPO40514 & $\mathbf{Y}$ & NA \\
\hline 3182 & 3,5 & CONSTANT & $0.0000000 E+\infty 0$ & $0.0000000 E+\infty$ & $0.0000000 E+\infty$ & $0.00000000+\infty 0$ & NONE & WP035134 & $Y$ & NA \\
\hline 3182 & 3,5 & CONSTANT & $0.0000000 E+\infty 0$ & $0.0000000 E+\infty 0$ & $0.0000000 E+\infty 0$ & $0.0000000 E+\infty$ & NONE & WP040514 & $\mathbf{Y}$ & NA \\
\hline 3183 & 3,5 & UNIFORM & $-1.3500000 E+01$ & $-1.3500000 E+01$ & $-1.5000000 E+01$ & $-1.2000000 E+01$ & $\log \left(m^{\wedge} 2\right)$ & WP035134 & $\mathbf{Y}$ & NA \\
\hline 3183 & 3,5 & UNIFORM & $-1.3500000 E+01$ & $-1.3500000 E+01$ & $-1.5000000 E+01$ & $-1.2000000 E+01$ & $\log \left(m^{\wedge} 2\right)$ & WP035268 & $\mathbf{Y}$ & NA \\
\hline 3183 & 3.5 & UNIFORM & $-1.3500000 E+01$ & $-1.3500000 E+01$ & $-1.5000000 \mathrm{E}+01$ & $-1.2000000 E+01$ & $\log \left(m^{\wedge} 2\right)$ & WPO4O134 & $\mathbf{Y}$ & NA \\
\hline 3184 & 3,5 & UNIFORM & $-1.2500000 \mathrm{E}+01$ & $-1.2500000 E+01$ & $-1.4000000 E+01$ & $-1.1000000 \mathrm{E}+01$ & $\log \left(m^{n} 2\right)$ & WP035134 & $\mathbf{Y}$ & NA \\
\hline 3184 & 3,5 & UNIFORM & $-1.2500000 E+01$ & $-1.2500000 E+01$ & $-1.4000000 E+01$ & $-1.1000000 E+01$ & $\log \left(m^{n} 2\right)$ & WP035268 & $\mathbf{Y}$ & NA \\
\hline 3185 & 3,5 & CONSTANT & $-1.6301000 E+01$ & $-1.6301000 E+01$ & $-1.6301000 E+01$ & $-1.6301000 E+01$ & $\log \left(m^{\wedge} 2\right)$ & WP035134 & $\mathbf{Y}$ & NA \\
\hline 3185 & 3,5 & CONSTANT & $-1.6301000 E+01$ & $-1.6301000 E+01$ & $-1.6301000 E+01$ & $-1.6301000 E+01$ & $\log \left(m^{\wedge} 2\right)$ & WP035041 & $\mathbf{Y}$ & NA \\
\hline 3185 & 3,5 & CONSTANT & $-1.6301000 E+01$ & $-1.6301000 E+01$ & $-1.6301000 E+01$ & $-1.6301000 E+01$ & $\log \left(m^{\wedge} 2\right)$ & WPO41131 & $Y$ & NA \\
\hline 3186 & 3,5 & CONSTANT & $-9.0000000 \mathrm{E}+\infty 0$ & $-9.0000000 E+00$ & $-9.0000000 E+\infty 0$ & $-9.0000000 E+\infty 0$ & $\log \left(m^{\wedge} 2\right)$ & WP035134 & $\mathbf{Y}$ & NA \\
\hline 3186 & 3,5 & CONSTANT & $-9.0000000 E+\infty 0$ & $-9.0000000 E+00$ & $-9.0000000 E+\infty 0$ & $-9.0000000 E+\infty 0$ & $\log \left(m^{\wedge} 2\right)$ & WP040514 & $\mathbf{Y}$ & N/A \\
\hline 3187 & 3,5 & CONSTANT & $-9.0000000 E+\infty 0$ & $-9.0000000 E+\infty 0$ & $-9.0000000 E+\infty 0$ & $-9.0000000 E+\infty 0$ & $\log \left(m^{\wedge} 2\right)$ & WP035134 & $\mathbf{Y}$ & NA \\
\hline 3187 & 3,5 & CONSTANT & $-9.0000000 E+\infty 0$ & $-9.0000000 \mathrm{E}+\infty 0$ & $-9.0000000 E+\infty 0$ & $-9.0000000 E+\infty 0$ & $\log \left(m^{\wedge} 2\right)$ & WPO40514 & $\mathbf{Y}$ & NA \\
\hline 3188 & 3,5 & UNIFORM & $-\{.3500000 E+01$ & $-1.3500000 E+01$ & $-1.5000000 E+01$ & $-1.2000000 E+01$ & $\log \left(m^{\wedge} 2\right)$ & WP035134 & $\mathbf{Y}$ & N/A \\
\hline 3188 & 3,5 & UNIFORM & $-1.3500000 E+01$ & $-1.3500000 E+01$ & $-1.5000000 E+01$ & $-1.2000000 E+01$ & $\log \left(m^{\wedge} \wedge 2\right)$ & WP035268 & $\mathbf{Y}$ & NA \\
\hline 3189 & 3,5 & UNIFORM & $-1.3500000 E+01$ & $-1.3500000 E+01$ & $-1.5000000 E+01$ & $-1.2000000 E+01$ & $\log \left(m^{2} 2\right)$ & WP035134 &.$- Y$ & NA \\
\hline 3189 & 3,5 & UNIFORM & $-1.3500000 E+01$ & $-1.3500000 E+01$ & $-1.5000000 E+01$ & $-1.2000000 E+01$ & $\log \left(m^{\wedge} 2\right)$ & WP035268 & $\mathbf{Y}$ & NA \\
\hline 3190 & 3,5 & UNIFORM & $-1,2500000 E+01$ & $-1.2500000 E+01$ & $-1.4000000 E+01$ & $-1.1000000 E+01$ & $\log \left(m^{\wedge} 2\right)$ & WPO35134 & $\mathbf{Y}$ & N/A \\
\hline 3190 & 3,5 & UNIFORM & $-1.2500000 E+01$ & $-1.2500000 E+01$ & $-1.4000000 E+01$ & $-1.1000000 E+01$ & $\log \left(m^{\wedge} 2\right)$ & WP035268 & $\mathbf{Y}$ & NA \\
\hline 3191 & 3,5 & UNIFORM & $-1.2500000 E+01$ & $-1.2500000 \mathrm{E}+01$ & $-1.4000000 E+01$ & $-1.1000000 E+01$ & $\log \left(m^{\wedge} 2\right)$ & WP035134 & $\mathbf{Y}$ & NA \\
\hline 3191 & 3,5 & UNIFORM & $-1.2500000 E+01$ & $-1.2500000 E+01$ & $-1.4000000 E+01$ & $-1.1000000 E+01$ & $\log \left(m^{\wedge} 2\right)$ & WP035268 & $\mathbf{Y}$ & NA \\
\hline 3192 & 3.5 & CONSTANT & $-1.6301000 E+01$ & $-1.6301000 E+01$ & $-1.6301000 E+01$ & $-1.6301000 E+01$ & $\log \left(m^{\wedge} 2\right)$ & WP035134 & $\mathbf{Y}$ & NA \\
\hline 3192 & 3,5 & CONSTANT & $-1.6301000 E+01$ & $-1.6301000 E+01$ & $-1.6301000 E+01$ & $-1.6301000 E+01$ & $\log \left(m^{\wedge} 2\right)$ & WPO36041 & $\mathbf{Y}$ & NA \\
\hline 3192 & 3,5 & CONSTANT & $-1.6301000 E+01$ & $-1.6301000 E+01$ & $-1.6301000 E+01$ & $-1.6301000 E+01$ & $\log \left(m^{\wedge} 2\right)$ & WPOS1131 & $\mathbf{Y}$ & N/A \\
\hline 3193 & 3,5 & CONSTANT & $-1,6301000 E+01$ & $-1.6301000 E+01$ & $-1.6301000 E+01$ & $-1.6301000 E+01$ & $\log \left(m^{\wedge} 2\right)$ & WP035134 & $\mathbf{Y}$ & NA \\
\hline 3193 & 3,5 & CONSTANT & $-1.6301000 E+01$ & $-1.6301000 \Sigma+01$ & $-1.6301000 E+01$ & $-1.6301000 E+01$ & $\log \left(m^{\wedge} 2\right)$ & WP036041 & $\mathbf{Y}$ & NA \\
\hline 3193 & 3,5 & CONSTANT & $-1.6301000 E+01$ & $-1.6301000 E+01$ & $-1.6301000 E+01$ & $-1.6301000 E+01$ & $\log \left(m^{\wedge} 2\right)$ & WPOA1131 & $\mathbf{Y}$ & NA \\
\hline
\end{tabular}


CCA Parameter Listing

\begin{tabular}{|c|c|c|c|c|c|c|c|c|c|c|}
\hline $\begin{array}{c}\text { Permeter in } \\
\text { Detebese? } \\
\text { (YhI) }\end{array}$ & 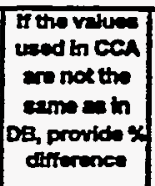 & $\bar{\omega}$ & Meterda 10 & 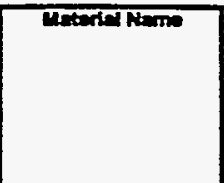 & Parnetior & Parnomer Mtanto & $\begin{array}{l}\text { PFP D } \\
\text { (MPON) }\end{array}$ & $\begin{array}{c}\text { Denentr| } \\
\text { Dens }\end{array}$ & 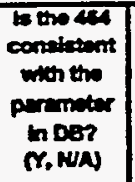 & $\begin{array}{l}\text { Parmedir } \\
\text { Celtegory } \\
\text { (tootnote 1) }\end{array}$ \\
\hline $\mathbf{Y}$ & & 3194 & CASTILER & Cestul Brine Reservoir & GRIDFLO & $\begin{array}{l}\text { lindex for Selecting a Brmo } \\
\text { Pocket }\end{array}$ & 366584 & 21 ther-96 & $\mathbf{Y}$ & 48 \\
\hline$Y$ & & 3184 & CASTILER & Custitio Brne Reservor & GRIDFLO & $\begin{array}{l}\text { Indox for Solocting \& Brro } \\
\text { Pocket }\end{array}$ & $36658 A$ & 21 ther-86 & $\mathbf{Y}$ & 4B \\
\hline$Y$ & & 3194 & CASTILER & Castio Brine Reservoir & GRIDFLO & $\begin{array}{l}\text { index for Solocting \& Bme } \\
\text { Pockel }\end{array}$ & $36658 \mathrm{~A}$ & $21+k=-86$ & $\mathbf{Y}$ & 48 \\
\hline $\mathbf{Y}$ & & 3194 & CASTILER & Certito Brmo Rosenvor & GRIDFLO & $\begin{array}{l}\text { index for Solecting a Brme } \\
\text { Pocket }\end{array}$ & $36658 \mathrm{~A}$ & 21 ther-96 & $\mathbf{Y}$ & 48 \\
\hline $\mathbf{Y}$ & & 3196 & $\pi 207$ & Thalliem 207 & ATWEIGHT & Atomic Weight in kotmols & 36659 & 10-4pr-96 & $\mathbf{Y}$ & 3 \\
\hline$Y$ & & 3196 & $\pi 207$ & Thallim 207 & ATWEIGHT & Atomic Whight in kemolo & 36659 & 10 apros & $\mathbf{Y}$ & 3 \\
\hline$Y$ & & 3197 & PA234M & $\begin{array}{l}\text { Protactinum } 234 \\
\text { Motastabto }\end{array}$ & ATWEIGHT & |Atonic Woight in ka/molo & 36660 & $10-4 p r-56$ & $\mathbf{Y}$ & 3 \\
\hline $\mathbf{Y}$ & & 3197 & PA234M & $\begin{array}{l}\begin{array}{l}\text { Protuctiniem } 234 \\
\text { Motastablo }\end{array} \\
\end{array}$ & ATWEIGHT & Atomic Woight in komole & 36560 & 10 Apr-96 & $\mathbf{Y}$ & 3 \\
\hline$Y$ & & 3198 & Y90 & Yutrien 90 & ATWEIGHT & Atomic Woight in kg/mote & 36703 & 10 Apr-96 & $Y$ & 3 \\
\hline$y$ & & 3158 & Yso & Vtrium 90 & ATWEIGHT & Atomic Woight in kolmolo & 35703 & 10-Apr-96 & $Y$ & 3 \\
\hline $\bar{Y}$ & & 3199 & $2 \mathrm{R} 90$ & Zrooniem 90 & ATWEIGHT & Atomic Weights in kemole & 35704 & $10-4 \times-96$ & $Y$ & 3 \\
\hline$Y$ & & 3199 & ZRSOO & Zirconium 90 & ATWEIGHT & Atomic Whight in kghole & 36704 & $10-4 p x+96$ & $Y$ & 3 \\
\hline $\bar{Y}$ & & 3200 & PB211 & Lead 211 & ATWEIGHT & Atomic Whight in $\mathrm{kg} / \mathrm{mol}$ & 36705 & $10-4 p x-96$ & $Y$ & 3 \\
\hline$Y$ & & 3200 & PB211 & Lead 211 & ATWEIGHT & Atomic Woight in kg/noto & 36705 & 10 -Apr-s6 & $Y$ & 3 \\
\hline $\bar{Y}$ & & 3201 & PB212 & Lead 212 & ATWEIGHT & Atomic Weight in kg/mole & 36706 & 10-Apr-96 & $\bar{Y}$ & 3 \\
\hline$Y$ & & 3201 & PB212 & Lead 212 & ATWEIGHT & Atomic Whight in ko/mols & 36706 & $10 \times$ pr -96 & $Y$ & 3 \\
\hline$Y$ & & 3202 & PB214 & Lead 214 & ATWEIGHT & Atomic Whight in kgholo & 36707 & 10-Apr-96 & $\bar{Y}$ & 3 \\
\hline $\bar{Y}$ & & 3202 & PB214 & Lead 214 & ATWEIGHT & Atomic Wexpht in kg/molo & 36707 & 10-Apr-96 & $\mathbf{Y}$ & 3 \\
\hline$Y$ & & 3203 & $\mathrm{PO} 212$ & Polonium 212 & ATWEIGHT & Atomic Woight in komolo & 36708 & 10-Apr-96 & $Y$ & 3 \\
\hline$Y$ & & 3203 & P0212 & Polonium 212 & ATWEIGHT & Atomic Woight in kolmolo & 36708 & $10-4 p x-96$ & $Y$ & 3 \\
\hline$Y$ & & 3204 & PO213 & Polonium 213 & ATWEIGHT & Atomic Woight in ko/molo & 36709 & 10-Apr-96 & $Y$ & 3 \\
\hline $\bar{Y}$ & & 3204 & $\mathrm{PO213}$ & Polonium 213 & ATWEIGHT & Alomic Weight in kg/molo & 36709 & $10-4 p r-96$ & $Y$ & 3 \\
\hline$Y$ & & 3205 & PO214 & Potonium 214 & ATWEIGHT & Asomic Woight in kolmolo & 36710 & 10-Apr-96 & $Y$ & 3 \\
\hline$Y$ & & 3205 & P0214 & Poionum 214 & ATWEIGHT & Alonic Woight in ko/molo & 36710 & 10-Apr-96 & $Y$ & 3 \\
\hline $\mathbf{Y}$ & & 3206 & PO215 & Polonium 215 & ATWEIGHT & Atonic Woight in ko/molo & 36711 & $10-4 p r-96$ & $\mathbf{Y}$ & 3 \\
\hline $\mathbf{Y}$ & & 3206 & P0215 & Potonium 215 & ATWEIGHT & Alomic Weight in ke/mols & 36711 & 10-Apr-96 & $\mathbf{Y}$ & 3 \\
\hline$Y$ & & 3207 & PO216 & Polonium 216 & ATWEIGHT & Alomic Woight in kg/mole & 36712 & 10-Apr-86 & $\mathbf{Y}$ & 3 \\
\hline$Y$ & & 3207 & P0216 & Poionium 216 & ATWEIGHT & Alomic Woight in kg/moto & 36712 & $10-40 x-96$ & $Y$ & 3 \\
\hline $\mathbf{Y}$ & & 3208 & PO218 & Polonium 218 & ATWEIGHT & Atomic Woight in $\mathrm{kg} /$ molo & 36713 & 10-Apr-96 & $\mathbf{Y}$ & 3 \\
\hline $\mathbf{Y}$ & & 3208 & PO218 & Potonium 218 & ATWEIGHT & Atornic Woight in kg/molo & 36713 & 10-Apr-96 & $\mathbf{Y}$ & 3 \\
\hline$Y$ & & 3209 & RA223 & Radium 223 & ATWEIGHT & Atomic Woight in kg/mole & 36714 & 10-Apr-96 & $\mathbf{Y}$ & 3 \\
\hline$Y$ & & 3209 & RA223 & Radium 223 & ATWEIGHT & Alomic Woight in kg/mole & 36714 & 10-Apr-96 & $Y$ & 3 \\
\hline$Y$ & & 3209 & RA223 & Rodum 223 & ATWEIGHT & Atomic Woight in kg/moto & 36714 & 10-Apr-96 & $\mathbf{Y}$ & 3 \\
\hline $\bar{Y}$ & & 3210 & RA224 & Radium 224 & ATWEIGHT & Atomic Weight in kg/mote & 36715 & 10-Apr-86 & $\bar{Y}$ & 3 \\
\hline $\mathbf{Y}$ & & 3210 & RA224 & Radiem 224 & ATWEIGHT & Alomic Woight in kg/molo & 36715 & 10-Apr-86 & $Y$ & 3 \\
\hline$Y$ & & 3210 & RA224 & Radiem 224 & ATWEIGHT & Alome Weigth in kghoto & 36715 & 10-Apr-96 & $\mathbf{Y}$ & 3 \\
\hline $\bar{Y}$ & & 3211 & RA225 & Rediem 225 & ATWEIGHT & Alomic Woight in kg/molo & 36716 & 10-Apr.96 & $\mathbf{Y}$ & 3 \\
\hline$Y$ & & 3211 & RA225 & Redum 225 & ATWEIGHT & Atomic Whight in kemols & 36716 & 10-Apr-96 & $\mathbf{Y}$ & 3 \\
\hline$Y$ & & 3211 & RA225 & Rradium 225 & ATWEIGHT & Atomic Woight in kghole & 36716 & 10-Apr-96 & $\mathbf{Y}$ & 3 \\
\hline$Y$ & & 3212 & RN219 & Redon 219 & ATWEIGHT & Nomx Woight in kemote & 35719 & 10-Apr-96 & $\bar{Y}$ & 3 \\
\hline$Y$ & & 3212 & RN219 & Redon 219 & ATWEIGHT & Atomx Whight in kg'moto & 36719 & $10-A p r-96$ & $\mathbf{Y}$ & 3 \\
\hline $\mathbf{Y}$ & & 3212 & RN219 & Radon 219 & ATWEIGHT & Atomx Weight in kgtmoto & 36719 & 10-Apr.96 & $\mathbf{Y}$ & 3 \\
\hline$Y$ & & 3213 & RNE220 & Raden 220 & ATWEIGHT & Atomic Whight in ko/mole & 35720 & 10-Apr-96 & $\mathbf{Y}$ & 3 \\
\hline$Y$ & & 3213 & RN220 & Risdon 220 & ATWEIGHT & Atomic Weight in kg/molo & 36720 & $10-4 p \times-96$ & $\mathbf{Y}$ & 3 \\
\hline$y$ & & 3213 & RN220 & Radion 220 & ATWEIGHT & Atomic Weight in kg/mole & 36720 & 10 Apr-96 & $y$ & 3 \\
\hline$Y$ & & 3214 & RNE222 & Redon 222 & ATWEIGHT & Atomic Weight in kotmole & 36732 & $10-A p r-96$ & $Y$ & 3 \\
\hline$Y$ & & 3214 & RN2222 & Redon 222 & ATWEIGHT & Alomic Whight in kg/mols & 36732 & 10-Apr-96 & $Y$ & 3 \\
\hline $\mathbf{Y}$ & & 3214 & RND22 & Redon 222 & ATWEIGHT & Atomic Whight in kghmole & 36732 & $10-1 p x-86$ & $Y$ & 3 \\
\hline$Y$ & 0.1 & 3215 & SR9O & Stronterm 90 & ATWEIGHT & Nomic Weight in kgmols & 36733 & 10-Apr-s6 & $Y$ & 3 \\
\hline$Y$ & 0.1 & 3215 & SRPO & Strontium 90 & ATWEIGHT & Alomic Weight in kg/molo & 36733 & $10-A p x-96$ & $Y$ & 3 \\
\hline $\mathbf{Y}$ & 0.1 & 3215 & SR9O & Strontum 90 & ATWEIGHT & Atornic Whight in ketmole & 36733 & 10-Apr-96 & $Y$ & 3 \\
\hline
\end{tabular}


CCA Parameter Listing

\begin{tabular}{|c|c|c|c|c|c|c|c|c|c|c|}
\hline D & $\begin{array}{c}\text { Cleastit- } \\
\text { cation of } \\
\text { Deta } \\
\text { Centegory } \\
\text { (footrote 2) }\end{array}$ & $\begin{array}{l}\text { Distribution } \\
\text { Typo }\end{array}$ & mean & modien & Mintroutr & Coxtrimen & Whits & Reference to & 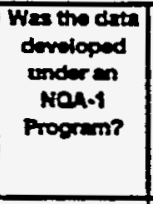 & 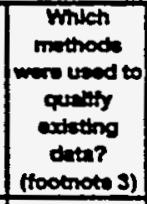 \\
\hline 3194 & 3,5 & DELTA & $1.6000000 E+01$ & $1.6000000 E+01$ & $1.0000000=+00$ & $3.2000000 \mathrm{E}+01$ & NONE & WP035268 & $\mathbf{Y}$ & NA \\
\hline 3194 & 3,5 & DELTA & $1.6000000 E+01$ & $1.6000000 E+01$ & $1.0000000 E+\infty 0$ & $3.2000000 \mathrm{E}+01$ & NONE & WP037147 & $\mathbf{Y}$ & NA \\
\hline 3194 & 3,5 & DELTA & $1.6000000 E+01$ & $1.6000000 E+01$ & $1.0000000 E+\infty 0$ & $3.2000000 E+01$ & NOHE & WP040514 & $\mathbf{Y}$ & NA \\
\hline 3194 & 3,5 & DELTA & $1.6000000 E+01$ & $1.6000000 E+01$ & $1.0000000 E+\infty$ & $3.2000000 \mathrm{E}+01$ & NOAE & WPOA16E2 & $\mathbf{Y}$ & N/A \\
\hline 3196 & 4 & CONSTANT & 20697700E-01 & 20697700E-01 & 2.0697700E-01 & 20697700E-01 & kolmowe & WPO36301 & $\mathbf{Y}$ & N/A \\
\hline 3196 & 4 & CONSTANT & $2.0697700 E-01$ & $2.0697700 E-01$ & 20697700E-01 & 2.0697700E-01 & kolmow & WP037404 & $Y$ & NA \\
\hline 3197 & 4 & CONSTANT & 2.3404300E-01 & $2.3404300 E-0 t$ & $234043005-01$ & $23404300 E-01$ & kgtmols & WPOs630! & $\mathbf{Y}$ & NA \\
\hline 3197 & 4 & CONSTANT & 2.3404300E-01 & 2.3404300E-01 & 23404300E-01 & $23404300 E-01$ & komoin & WP037404 & $\mathbf{Y}$ & NA \\
\hline 3198 & 4 & CONSTANT & 8.9907000E-02 & $8.9907000 E-02$ & $8.9907000 E-02$ & $8.9907000 \mathrm{E}-02$ & ko/molo & WP036301 & $\mathbf{Y}$ & NA \\
\hline 3198 & 4 & CONSTANT & $8.9907000 \mathrm{E}-02$ & $8.9907000 E-02$ & 8.9907000E-02 & $8.9907000 E-02$ & komole & WP037404 & $Y$ & NA \\
\hline 3199 & 4 & CONSTANT & $8.9905000 \mathrm{E}-02$ & 8.9905000E-02 & $8.9905000 \mathrm{E}-02$ & $8.9905000 \mathrm{E}-02$ & kolmols & WP035301 & $\mathbf{Y}$ & NA \\
\hline 3199 & 4 & CONSTANT & $8.9905000 \mathrm{E}-02$ & $8.9905000 \mathrm{E}-02$ & $8.9905000 E-02$ & $8.9905000 \mathrm{E}-02$ & komoles & WP037404 & $Y$ & $N / A$ \\
\hline 3200 & 4 & CONSTANT & $2.1098900 E-01$ & 2.1096900E-01 & 2.1090900E-01 & 2.1098900E-01 & kglmole & WP036301 & $\mathbf{Y}$ & NA \\
\hline 3200 & 4 & CONSTANT & 2.1058900E-01 & 21098900E-01 & 2.1098900E-01 & 2.1098900E-01 & komole & WP037404 & $Y$ & NA \\
\hline 3201 & 4 & CONSTANT & 2.1199200E-01 & 21199200E-01 & 2.1199200E-01 & 2.1199200E-01 & komolo & WP036301 & $Y$ & NA \\
\hline 3201 & 4 & CONSTANT & $2.1199200 E-01]$ & 2.1199200E-01 & 21199200E-01] & 2.1199200E-01 & kgmole & WF037404 & $\mathbf{Y}$ & NA \\
\hline 3202 & 4 & CONSTANT & $2.1400000 E-01$ & 21400000E-01 & $2.1400000 E-01$ & $2.1400000 E-01$ & kglmole & MP035301 & $Y$ & NA \\
\hline 3202 & 4 & CONSTANT & 2.1400000E-01 & 2.1400000E-01 & 2.1400000E-01 & 21400000E-01 & $\mathrm{kg} / \mathrm{mole}$ & WP037404 & 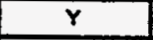 & NA \\
\hline 3203 & 4 & CONSTANT & 2.1198900E-01 & 21198900E-01 & 21198900E-01 & $2.1198900 E-01$ & kghole & WP035301 & $Y$ & N/A \\
\hline 3203 & 4 & CONSTANT & 2.1198900E-01 & $2.1199900 E-01$ & 2.1198900E-01 & 2.1198900E-01 & kemolo & WP037404 & $Y$ & NUA \\
\hline 3204 & 4 & CONSTANT & $2.1299300 E-01$ & 2.1299300E-01 & 2.1299300E-01 & 2.1299300E-01 & koinols & WP036301 & $Y$ & NA \\
\hline 3204 & 4 & CONSTANT & $2.1299300 E-01$ & 21299300E-01 & 2.1299300E-01 & $2.1299300 \mathrm{E}-01$ & $\mathrm{~kg}$ mole & WPO37404 & $Y$ & NA \\
\hline 3205 & 4 & CONSTANT & 2.1399500E-01 & 2.1399500E-01 & 21399500E-01 & 2.1399500E-01 & katmote & WP036301 & $Y$ & N/A \\
\hline 3205 & 4 & CONSTANT & 2.1399500E-01 & 2.1399500E-01 & 2.1399500E-01 & $2.1399500 E-01$ & kemolo & WP037404 & $Y$ & NAA \\
\hline 3206 & 4 & CONSTANT & 2.1499900E-01 & 2.1499900E-01 & $2.1499900 E-01$ & 2.1499900E-01 & komols & WP035301 & $Y$ & NA \\
\hline 3206 & 4 & CONSTANT & 2.1499900E-01 & 2.1499900E-01 & 21499900E-01 & 2.1499900E-01 & $\mathrm{kg} / \mathrm{mole}$ & WP037404 & $Y$ & N/A \\
\hline 3207 & 4 & CONSTANT & 2.1600200E-01 & $2.1600200 E-01$ & $2.1600200 \mathrm{E}-01$ & $2.1600200 E-01$ & kolmole & WP035301 & $\bar{Y}$ & NA \\
\hline 3207 & 4 & CONSTANT & 2.1600200E-01 & $2.1600200 E-01$ & $2.1600200 E-01$ & $2.1600200 \leq-01$ & kglnole & WP037404 & $\mathbf{Y}$ & NA \\
\hline 3208 & 4 & CONSTANT & 2.1800900E-01 & 2.1800900E-01 & $2.1800900 \mathrm{E}-01$ & $2.1800900 E-01$ & kofmole & WP035301 & $\mathbf{Y}$ & NA \\
\hline 3208 & 4 & CONSTANT & 2.1800900E-01 & $2.1800900 \mathrm{E}-01$ & $2.1800900 E-01$ & $2.1800900 \mathrm{E}-01$ & kefmote & WPO37404 & $Y$ & N/A \\
\hline 3209 & 4 & CONSTANT & 2.2301900E-01 & 2.2301900E-01 & 2.2301900E-01 & $22301900 E-01$ & kgimote & WPO35298 & $Y$ & N/A \\
\hline 3209 & 4 & CONSTANT & $2.2301900 E-01$ & 2.2301900E-01 & $2.2301900 E-01$ & $2.2301900 E-01$ & kolmow & WP036301 & $\mathbf{Y}$ & NA \\
\hline 3209 & 4 & CONSTANT & 2.2301900E-01 & 2.2301900E-01 & 2.2301900E-01 & $2.2301500 E-01$ & $\mathrm{~kg} / \mathrm{mole}$ & WPO37404 & $Y$ & NA \\
\hline 3210 & 4 & CONSTANT & 2.2402000E-01 & $2.24020000-01$ & $2.2402000 \mathrm{E}-01$ & $2.2402000 \mathrm{E}-01$ & kemole & WP036298 & $Y$ & NA \\
\hline 3210 & 4 & CONSTANT & 2.2402000E-01 & $2.2402000 \mathrm{E}-01$ & $2.2402000 E-01$ & $2.2402000 \mathrm{E}-01$ & kgimole & WP035301 & $Y$ & NA \\
\hline 3210 & 4 & CONSTANT & $2.2402000 E-01$ & $2.2402000 \mathrm{E}-01$ & $2.2402000 E-01$ & $2.2402000 \mathrm{E}-01$ & kgimole & WPO37404 & $\mathbf{Y}$ & N/A \\
\hline 3211 & 4 & CONSTANT & 2.2502400E-01 & $2.2502400 E-01$ & 2.2502400E-01 & $2.2502400 E-01$ & kgimale & WF036298 & $Y$ & N/A \\
\hline 3211 & 4 & CONSTANT & $2.2502400 E-01$ & $2.2502400 E .01$ & 2.2502400E-01 & 2.2502400E-01 & kgholo & WP036301 & $Y$ & NA \\
\hline 3219 & 4 & CONSTANT & $2.2502400 E-01$ & 2.2502400E-01 & $2.2502400 E-01$ & $2.2502400 E-01$ & kgholo & WP037404 & $Y$ & N/A \\
\hline 3212 & 4 & CONSTANT & 2.1900900E-01 & 2.1900900E-01 & 2.1900S00E-01 & 21900900E-01 & kg/molo & WP036298 & $Y$ & NA \\
\hline 3212 & 4 & CONSTANT & 2.1900900E-01 & 2.1900900E-01 & 21900900E-01 & $2.1900900 E-01$ & kgholo & WP036301 & $\bar{Y}$ & NA \\
\hline 3212 & 4 & CONSTANT & 2.1800900E-01 & 2.1900900E-01 & 2.1900900E-01 & $2.19009000-01$ & kolnows & WP037404 & $Y$ & N/A \\
\hline 3213 & 4 & CONSTANT & 2.2001100E-01 & 2.2001100E-01 & 22001100E-01 & $2.2001100 E-01$ & kghols & WP036298 & $Y$ & N/A \\
\hline 3213 & 4 & CONSTANT & $2.2001100 E-01$ & 22001100E-01 & 2.2001100E-01 & 2.2001100E-01 & kg/mote & WPO36301 & $Y$ & $N A$ \\
\hline 3213 & 4 & CONSTANT & $2.2001100 E-01$ & $2.2001100 E-01$ & 2.2001100E-01 & $22001100 E-01$ & $\mathrm{~kg} / \mathrm{mole}$ & WP037404 & $Y$ & NA \\
\hline 3214 & 4 & CONSTANT & $22201800 E-01$ & $2.2201800 E-01$ & 2.2201800E-01 & $22201800 E-01$ & kolmole & WP036298 & $\mathbf{Y}$ & N/A \\
\hline 3214 & 4 & CONSTANT & $2.2201800 \mathrm{E}-01$ & $22201800 E-01$ & $2.2201800 E-01$ & 22201800E-01 & $\mathrm{kg}$ mol & WP036301 & $Y$ & NA \\
\hline 3214 & 4 & CONSTANT & $2.2201800 E-01$ & $22201800 E-01$ & $22201800 \mathrm{E}-01$ & $22201800 E-01$ & komole & WP037404 & $\mathbf{Y}$ & NA \\
\hline 3215 & 4 & CONSTANT & $8.9908000 E-02$ & 8.9908000E-02 & $8.9908000 \mathrm{E}-02$ & $8.9908000 \mathrm{E}-02$ & ketmolo & WP036298 & $\mathbf{Y}$ & NA \\
\hline 3215 & 4 & CONSTANT & $8.9908000 E-02$ & $8.9906000 \mathrm{E}-02$ & $8.9908000 E-02$ & $8.9903000 E-02$ & kglinole & MP036301 & $\mathbf{Y}$ & NA \\
\hline 3215 & 4 & CONSTANT & B.9906000E-02 & $8.9908000 E-02$ & $8.9900000 \mathrm{E}-02$ & 8.9908000E-02 & kgholes & WP037404 & $\mathbf{Y}$ & NA \\
\hline
\end{tabular}


CCA Parameter-Listing

\begin{tabular}{|c|c|c|c|c|c|c|c|c|c|c|}
\hline $\begin{array}{c}\text { Paramecer in } \\
\text { Dutabese? } \\
\text { (NN) }\end{array}$ & 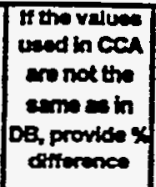 & $\overline{D D}$ & Metarie id & Mrtarial kemente & Permotar & Permotior Neme & $\begin{array}{c}\text { PRP DO } \\
\text { (WPOA) } \\
\cdot \\
\cdot\end{array}$ & Date & 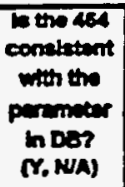 & $\begin{array}{l}\text { Perarnetar } \\
\text { Cextegony } \\
\text { (footrotes 1) }\end{array}$ \\
\hline $\boldsymbol{Y}$ & & 3216 & TH1227 & Thorem 227 & ATWEIGHT & Atomic Waght in kg/mole & 36734 & 10-Apr-96 & $\mathbf{Y}$ & 3 \\
\hline$Y$ & & 3216 & $\pi+227$ & Thorium 227 & ATWEIGHT & Atornic Woight in kg/mole & 36734 & 10 Apr-96 & $Y$ & 3 \\
\hline$Y$ & & 3217 & TH1228 & Thorium 228 & ATWEIGHT & Atome Woight in kglmole & 36735 & 10 Apr.96 & $\mathbf{Y}$ & 3 \\
\hline$Y$ & & 3217 & THL28 & Thorim 228 & ATWEIGHT & Atomic Whoght in kg/mols & 36735 & 10 -Apr-96 & $\mathbf{Y}$ & 3 \\
\hline $\mathbf{Y}$ & & 3218 & $\pi+231$ & Thorium 231 & ATWEIGHT & Atomic Whight in kormols & 36736 & 10-Apr-9s & $y$ & 3 \\
\hline $\mathbf{Y}$ & & 3218 & TH231 & Thoram 231 & ATWEIGHT & Asomic Whight in katmols & 36736 & $10-10 x-96$ & $\mathbf{Y}$ & 3 \\
\hline$Y$ & & 3219 & $\pi+234$ & Thorum 234 & ATWEIGHT & Atomic Woight in kg/molo & 36737 & 10-4pr-95 & $Y$ & 3 \\
\hline $\mathbf{Y}$ & & 3219 & TH234 & Thorium 234 & ATWEIGHT & Atomic Woight in komole & 36737 & 10-Apr-ss & $\mathbf{Y}$ & 3 \\
\hline $\mathbf{Y}$ & & 3220 & $A C 225$ & Aetinim 225 & ATWEIGHT & Atomic Woight in $\mathrm{kg} / \mathrm{mol}$ & 36738 & $10-4 p r-96$ & $Y$ & 3 \\
\hline $\mathbf{Y}$ & & 3220 & AC225 & Actiniem 225 & ATWEIGHT & Atomic Woight in ke/nols & 36738 & $10-4 p r \cdot 96$ & $\mathbf{Y}$ & 3 \\
\hline $\mathbf{Y}$ & & 3221 & AC227 & Aetiniem 227 & ATWEIGHT & Atomic Woight in $\mathrm{kg} / \mathrm{mol}$ & 36739 & $10-1 p r-96$ & $\mathbf{Y}$ & 3 \\
\hline$Y$ & & 3221 & $A C 227$ & Actinium 227 & ATWEIGHT & Atomic Whight in kg/mole & 36739 & $10-4 p x-96$ & $Y$ & 3 \\
\hline $\mathbf{r}$ & & 302 & AC228 & Actinitum 228 & ATWEIGHT & Atomic whions in ke/nolo & 36740 & $10-A p r-96$ & $Y$ & 3 \\
\hline $\mathbf{Y}$ & & 3222 & AC228 & Actinium 228 & ATWEIGHT & Atomic Whipht in ko/molo & 36740 & 10 -Apr-96 & $\mathbf{Y}$ & 3 \\
\hline $\mathbf{Y}$ & 0.03 & 3223 & All243 & Americium 243 & ATWEIGHT & Atomic Whight in kefinolo & 36741 & 10 -Apr-96 & $\mathbf{Y}$ & 3 \\
\hline$Y$ & 0.03 & 3223 & AM1243 & Americium 243 & ATWEIGHT & Atomic Woight in kghole & 36741 & $10-4 p r-96$ & $Y$ & 3 \\
\hline$Y$ & & 3224 & AT217 & Astatine 217 & ATWEIGKT & Atomic Weight in kg/nole & 36742 & 10-Apr-96 & $Y$ & 3 \\
\hline$Y$ & & 3224 & AT217 & Astatine 217 & ATWEIGHT & Atomic Weight in ketnols & 36742 & $10-10 x+6$ & $Y$ & 3 \\
\hline$Y$ & & 3225 & BA137 & Bariem 137 & ATWEIGHT & Atomic Whaght in kg/mole & 36743 & $10-4 p r-86$ & $\mathbf{Y}$ & 3 \\
\hline$Y$ & & 3225 & BA137 & Barium 137 & ATWEIGHT & Atomic Whight in kgimole & 36743 & 10 apres & $Y$ & 3 \\
\hline $\mathbf{Y}$ & & 3226 & BA137M & Barium 137 Melastablo & ATWEIGHT & Atomic Whight in kg/nols & 3674 & 10 -Apr-96 & $\mathbf{Y}$ & 3 \\
\hline $\mathbf{Y}$ & & 3226 & BA137M & Benum 137 Metastable & ATWEIGHT & Atomic Whight in komolo & 36744 & $10-4 p r \cdot 96$ & $\mathbf{Y}$ & 3 \\
\hline$Y$ & & 3227 & 81211 & Bismuth 219 & ATWEIGHT & Atomic Woight in kghrolo & 36746 & 10 -Apr-96 & $Y$ & 3 \\
\hline $\mathbf{Y}$ & & 3227 & 81211 & Bismuth 211 & ATWEIGHT & Atomic Woight in kg/mole & 36746 & $10-40 \mathrm{x}-96$ & $Y$ & 3 \\
\hline$Y$ & & 3228 & $B 1212$ & Bismuth 212 & ATWEIGHT & Atomic Whigtt in kg/mols & 36748 & 10-Apr-96 & $Y$ & 3 \\
\hline $\mathbf{Y}$ & & 3228 & 81212 & Bismuth 212 & ATWEIGHT & Atonic Woight in $\mathrm{kg} / \mathrm{mole}$ & 36748 & $10-1 p r-86$ & $y$ & 3 \\
\hline$Y$ & & 3229 & 81213 & Bismuth 213 & ATWEIGHT & Atomic Woight in $\mathrm{kg} / \mathrm{mol}$ & 36749 & 10-Apr-96 & $Y$ & 3 \\
\hline$\gamma$ & & 3229 & 81213 & Bismuth 213 & ATWEIGHT & Atomic Whight in kg/molo & 36749 & 10 apr-96 & $Y$ & 3 \\
\hline$Y$ & & 3230 & 81214 & Bismuth 214 & ATMEIGHT & Atomic Woight in $\mathrm{kg} / \mathrm{mol}$ & 36750 & $10-4 p x-96$ & $Y$ & 3 \\
\hline$Y$ & & 3230 & B1214 & Bismuth 214 & ATWEIGHT & Atomic Whight in $\mathrm{kg}$ mole & 36750 & 10 -Apr-96 & $Y$ & 3 \\
\hline$Y$ & 0.03 & 3231 & $\mathrm{CM} 243$ & Curiem 243 & ATWEIGHT & Atomic Whight in kg/nolo & 36751 & 10-Apr-96 & $Y$ & 3 \\
\hline Y & 0.03 & 3231 & CN1243 & Curium 243 & ATWEIGHT & Alomic Woight in kg/mole & 36751 & $10-A p x-96$ & $Y$ & 3 \\
\hline$Y$ & 0.03 & 3232 & CM245 & Curium 245 & ATWEIGHT & Atornic Woight in kg/mole & 36752 & 10-Apr.96 & $Y$ & 3 \\
\hline$Y$ & 0.03 & 3232 & CM1245 & Curium 245 & ATWEIGHT & Atomic Weight in $\mathrm{kg}$ /mole & 36752 & 10 -Apr-96 & $Y$ & 3 \\
\hline$Y$ & 0.03 & 3233 & $\mathrm{CH} 248$ & Curium 248 & ATWEIGHT & Atomic Woight in kg/mols & 36754 & $10-4 p r-96$ & $Y$ & 3 \\
\hline$Y$ & 0.03 & 3233 & CM1248 & Curium 248 & ATWEIGHT & Alomic Weight in kginole & 36754 & $10-\operatorname{Apr}-96$ & $Y$ & 3 \\
\hline$Y$ & & 3234 & FR221 & Francium 221 & ATWEIGHT & Atomic Woight in kg/mote & 36755 & $10-4$ pr-96 & $Y$ & 3 \\
\hline$Y$ & & 3234 & FR221 & Francium 221 & ATWEIGHT & Alomic Whight in kg/mole & 36755 & $10-A p r-96$ & $Y$ & 3 \\
\hline$Y$ & & 3235 & ND143 & Neodymixm 143 & ATWEIGHT & Atomic Weipht in ko/mole & 36757 & 10-Apr-86 & $Y$ & 3 \\
\hline $\mathbf{Y}$ & & 3235 & ND143 & Noodymium 143 & ATWEIGHT & Atomic Whight in ke/mole & 36757 & $10-A p x-96$ & $Y$ & 3 \\
\hline$Y$ & & 3236 & NP239 & Neptunium 239 & ATWEIGHT & Atomic Whight in ketmols & 36758 & $10-A p r-96$ & $Y$ & 3 \\
\hline$Y$ & & 3236 & NP239 & Neptunimm 239 & ATWEIGHT & Nlomse Wright in kg/nolo & 36758 & $10-1 p x-96$ & $Y$ & 3 \\
\hline $\mathbf{Y}$ & & 3237 & PA233 & Protuctiniem 233 & ATWEIGHT & Atomic Woight in kg/mote & 36759 & 10-Apr-96 & $Y$ & 3 \\
\hline $\mathbf{Y}$ & & 3237 & PA233 & Prodactinium 233 & ATWEIGHT & Atornic Whight in $\mathrm{kg}$ /nole & 36759 & $10-4 p r-86$ & $Y$ & 3 \\
\hline $\mathbf{Y}$ & & 3238 & ASPHALT & A Apphat cokemn & DNSGRAN & Material Grain Densaty & 36760 & $12 \times p x-86$ & $\gamma$ & 1 \\
\hline $\mathbf{Y}$ & & 3238 & ASPHALT & Aephate column & DNSGRAIN & Mererial Grein Densaty & 36760 & 12Apr-96 & $Y$ & 1 \\
\hline $\mathbf{Y}$ & & 3239 & BOREHOLE & Borchole and Fill & INN_AR & $\begin{array}{l}\text { The men of the repostory in the } \\
\text { cuTrinas moose }\end{array}$ & 37081 & 25-Apr-96 & $\mathbf{Y}$ & 4B \\
\hline $\mathbf{Y}$ & & 3239 & BOREHOLE & Boreholo and Fil & INV_AR & $\begin{array}{l}\text { The meas of the rupository in the } \\
\text { CUTTINGS model }\end{array}$ & 37001 & 25 Apr.96 & $\mathbf{Y}$ & 48 \\
\hline $\mathbf{Y}$ & & 3239 & BOREHOLE & Borenole and Fil & INN_AR & $\begin{array}{l}\text { The erat of the rapolitory n the } \\
\text { CUTINGS model }\end{array}$ & 37081 & 25 Apr-96 & $Y$ & 48 \\
\hline $\mathbf{Y}$ & & 3240 & BOREHOLE & Boretrolo and Ful & ROUGHP & \begin{tabular}{|l|} 
Frittion fictor for vary rough papos \\
in CUTTINGS model
\end{tabular} & 37082 & 25-Apr-86 & $\mathbf{Y}$ & 3 \\
\hline $\mathbf{Y}$ & & 3240 & BOREHOLE & Borthole and Fy & ROUGHP & $\begin{array}{l}\text { Fretion factor for very rough pipo } \\
\text { in CUTTINGS model }\end{array}$ & 37002 & 25 Apr-96 & $\mathbf{Y}$ & 3 \\
\hline
\end{tabular}


CCA Parameter Listing

\begin{tabular}{|c|c|c|c|c|c|c|c|c|c|c|}
\hline ID & 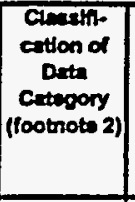 & $\begin{array}{l}\text { Distribution } \\
\text { Typo }\end{array}$ & $\operatorname{man}$ & Modien & Minhmem & Medinarn & Units & Rotermeses id & 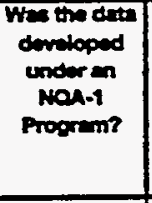 & 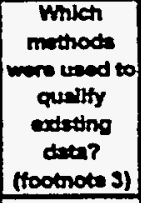 \\
\hline 3216 & 4 & CONSTANT & 2.2702800E-01 & 22702800E-01 & 22702800E-01 & $22702800 \mathrm{E}-01$ & kgmolo & WP036301 & $\mathbf{Y}$ & NA \\
\hline 3216 & 4 & CONSTANT & $2.2702800 E-01$ & 222702800E-01 & $22702800 E-01$ & $2.2702800 E-01$ & kgtmolo & WP037404 & $Y$ & NA \\
\hline 3217 & 4 & CONSTANT & 2.2802900E-01 & 22802900E-01 & 22802900E-01 & $2.2802900 E-01$ & kglmole & WP036501 & $Y$ & NA \\
\hline 3217 & 4 & CONSTANT & 2.2802900E-01 & $2.2802900 E-01$ & 2.2802900E-01 & $228002900 E-01$ & kemolo & WP037404 & $Y$ & NA \\
\hline 3218 & 4 & CONSTANT & $2.3103600 E-01$ & $23103600 E-01$ & $2.3103600 E-01$ & $23103600 E-01$ & komoia & WP036301 & $Y$ & NA \\
\hline 3218 & 4 & CONSTANT & $2.3103600 \mathrm{E}-01$ & 2.3103600E-01 & $23103600 E-01$ & 2.3103600E-01 & kotmolo & WP037404 & $Y$ & NA \\
\hline 3219 & 4 & CONSTANT & $23404400 E-01$ & 2.3404400E-01 & 2.3404400E-01 & $2.3404400 E-01$ & kolmoin & WP036301 & $Y$ & NA \\
\hline 3219 & 4 & CONSTANT & $23404400 E-01$ & $23404400=-01$ & 23404400E-01 & $23404400 E-01$ & kolmole & WP03740 & $Y$ & NA \\
\hline 3220 & 4 & CONSTANT & $2.2502300 E-01$ & 2.2502300E-01 & $22502300 E-01$ & 2.2502300E-01 & katmolo & WP036301: & $Y$ & NA \\
\hline 3220 & 4 & CONSTANT & 2.2502300E-01 & 2.2502300E-01 & $22502300 E-01$ & $22502300=-01$ & $\mathrm{~kg} / \mathrm{mol}$ & WP037404 & $Y$ & NA \\
\hline 3221 & 4 & CONSTANT & $2.2702800 E-01$ & 2.2702800E-01 & 22702800E-01 & 227002000E-01 & katmols & WP036301 & $Y$ & NA \\
\hline 3221 & 4 & CONSTANT & $2.2702800 \mathrm{E}-01$ & 2.2702800E-01 & 22702800E-01 & $2.2702800 E-01$ & $\mathrm{~kg} / \mathrm{mols}$ & WP037404 & $Y$ & NA \\
\hline 3220 & 4 & CONSTANT & 2.2803100E-01 & 2.2803100E-01 & 2.2803100E-01 & $22803100 E-01$ & kghols & WP036301 & $Y$ & NA \\
\hline 3222 & 4 & CONSTANT & $2.2803100 E-01$ & 22803100E-01 & $22803100 E=01$ & $2.2803100 E-01$ & kg/molo & WP037404 & $Y$ & N/A \\
\hline 3223 & 4 & CONSTANT & $2.4306100 E-01$ & 2.4306100E-01 & 24306100E-01 & 24306100E-01 & katmole & WPO36301 & $\mathbf{Y}$ & N/A \\
\hline 3223 & 4 & CONSTANT & 2.4306100E-01 & 2.4306100E-01 & 2.4306100E-01 & 2.4306100E-01 & kgtmolo & WP037404 & $\mathbf{Y}$ & NA \\
\hline 3224 & 4 & CONSTANT & 2.1700500E-01 & $21700500 E-01$ & $2.1700500 E-01$ & 2.1700500E-01 & kgmolo & WP036301 & $Y$ & NA \\
\hline 3224 & 4 & CONSTANT & $2.1700500 E-01$ & $2.1700500 E-01$ & 2.1700500E-01 & $2.1700500 \leq-01$ & kginols & WP037404 & $\mathbf{Y}$ & N/A \\
\hline 3225 & 4 & CONSTANT & $1.3690600 E-01$ & 1.3690600E-01 & 1.3690600E-01 & $1.3690600 E-01$ & ketmols & WP036301 & $Y$ & NA \\
\hline 3225 & 4 & CONSTANT & $1.3690600 \mathrm{E}-01$ & $1.3690600 \mathrm{E}-01$ & $1.3690600 \mathrm{E}-09$ & $1.3690600 E-01$ & katmolo & WP037404 & $Y$ & NAA \\
\hline 3226 & 4 & CONSTANT & 1.3690700E-01 & $1.3690700 E-01$ & $1.3690700 E-01$ & 1.3690700E-01 & kgtmolo & WP036296 & $Y$ & N/A \\
\hline 3226 & 4 & CONSTANT & 1.3690700E-01 & $1.3690700 E-01$ & $1.3690700 E-01$ & $1.3690700 E-01$ & komolo & WP037404 & $\mathbf{Y}$ & NA \\
\hline 3227 & 4 & CONSTANT & 2.1098700E-01 & 2.1098700E-01 & 2.1098700E-01 & $2.1098700 E-01$ & kg/moto & WP036301 & $Y$ & NA \\
\hline 3227 & 4 & CONSTANT & 2.1098700E-01 & $2.1098700 E-01$ & 2.1098700E-01 & 2.1098700E-01 & kemolo & WP037404 & $\mathbf{Y}$ & N/A \\
\hline 3228 & 4 & CONSTANT & 2.1199100E-01 & 2.1199100E-01 & 2.1199100E-01 & 2.1199100E.09 & kg/mols & WP036301 & $\mathbf{Y}$ & NA \\
\hline 3228 & 4 & CONSTANT & 2.1199100E-01 & 2.1199100E-01 & 2.1199100E-01 & $21199100 E-01$ & $\mathrm{~kg}$ mole & WP037404 & $\mathbf{Y}$ & N/A \\
\hline 3229 & 4 & CONSTANT & 2.1299400E-01 & $2.1299400 E-01$ & $2.1299400 E-01$ & $21299400 E-01$ & kglmols & WP036301 & $Y$ & N/A \\
\hline 3229 & 4 & CONSTANT & 2.1299400E-01 & $2.1299400 E-01$ & 2.1299400E-01 & 2.1299400E-01 & kgtindo & WP037404 & $Y$ & NA \\
\hline 3230 & 4 & CONSTANT & 2.1399900E-01 & $2.1399900 E-01$ & 2.1399900E-01 & $2.1399900=-01$ & komole & WP036301 & $Y$ & NA \\
\hline 3230 & 4 & CONSTANT & 2.1399900E-01 & 2.1399900E-01 & 2.1399900E-01 & $2.1399900 E-01$ & kgindo & WP037404 & $Y$ & NA \\
\hline 3231 & 4 & CONSTANT & $2.4306100 E-01$ & 2.4305100E-01 & $2.4305100 E-01$ & 2.4306100E-01 & kg/molo & WP0350301 & $Y$ & NA \\
\hline 3231 & 4 & CONSTANT & $2.4306100 \mathrm{E}-01$ & 2.4305100E-01 & 2.4306100E-01 & $2.4306100 E-01$ & $\mathrm{~kg} / \mathrm{molo}$ & WP037404 & $Y$ & NA \\
\hline 3232 & 4 & CONSTANT & 2.4506500E-01 & $2.4506500 E-01$ & 2.4506500E-01 & 2.4506500E-01 & kg/mole & WP036301 & $Y$ & NA \\
\hline 3232 & 4 & CONSTANT & 2.4506500E-01 & 2.4506500E-01 & 2.1506500E-01 & 2.4506500E-01 & kgholo & WP037404 & $Y$ & N/A \\
\hline 3233 & 4 & CONSTANT & 2.4807200E-01 & 2.4807200E-01 & 2.4807200E-01 & 2.4807200E-01 & $\mathrm{kg} / \mathrm{mole}$ & WP036301 & $\mathbf{Y}$ & N/A \\
\hline 3233 & 4 & CONSTANT & 24807200E-01 & $2.4807200 E-01$ & 2.4807200E-01 & $2.4807200 E-01$ & kglinols & WPO37404 & $Y$ & NA \\
\hline 3234 & 4 & CONSTANT & $22101400 E-01$ & 2.2101400E-01 & 2.2101400E-01 & $2.2101400 E-01$ & kgmols & WPO35301 & $Y$ & N/A \\
\hline 3234 & 4 & CONSTANT & $2.2101400 E-01$ & 22101400E-01 & 2.2101400E-01 & 2.2101400E-01 & $\mathrm{kg}$ molo & WP037404 & $Y$ & N/A \\
\hline 3235 & 4 & CONSTANT & 1.4291000E-01 & 1.4291000E-01 & 1.4291000E-01 & 1.4291000E-01 & $\mathrm{kg} / \mathrm{mol}$ & WPO36301 & $Y$ & NA \\
\hline 3235 & 4 & CONSTANT & $1.4291000 \mathrm{E}-01$ & 1.4291000E-01 & $1.4291000 \mathrm{E}-01$ & 1.4291000E-01 & kelmolo & WPO37404 & $Y$ & N/A \\
\hline 3236 & 4 & CONSTANT & $2.3905300 \mathrm{E}-01$ & $2.3905300 E-01$ & 2.3905300E-01 & $2.3905300 E-01$ & kemolo & WP036301 & $\mathbf{Y}$ & N/A \\
\hline 3236 & 4 & CONSTANT & 23905300E-01 & $23905300 E-01$ & $2.3905300 E-01$ & $2.3905300 E-01$ & kginolo & WP037404 & $Y$ & N/A \\
\hline 3237 & 4 & CONSTANT & 23304000E-01 & 23304000E-01 & $23304000 E-01$ & $2.3304000 E-01$ & kgimols & WP036301 & $Y$ & NA \\
\hline 3237 & 4 & CONSTANT & $2.3304000 E-01$ & $23304000 E-01$ & 2.3304000E-01 & 2.3304000E-01 & $\mathrm{kg} / \mathrm{mole}$ & WP037404 & $\mathbf{Y}$ & NA \\
\hline 3238 & 1,3 & CONSTANT & $2,0222000 \mathrm{E}+03$ & $2,0222000 E+00$ & $2.0222000 \mathrm{E}+03$ & $2.02220005+03$ & $\mathrm{~kg} / \mathrm{m}^{\wedge} 3$ & WP030640 & $\mathbf{Y}$ & N/A \\
\hline 3238 & 1,3 & CONSTANT & $2.0222000 \mathrm{E}+03$ & $2.0222000 E+03$ & $2.0222000 E+03$ & $2.0222000 \mathrm{E}+03$ & $\mathrm{~kg} / \mathrm{m}^{\wedge} 3$ & WP035854 & $Y$ & NA \\
\hline 3239 & $1,3,4$ & CONSTANT & $1.1152000 \mathrm{E}+05$ & $1.1152000 E+05$ & $1.1152000 E+05$ & $1.1152000 E+05$ & $m^{\wedge 2}$ & SAND91-0693/3 & $\mathbf{Y}$ & NA \\
\hline 3239 & $1,3,4$ & CONSTANT & $1.1152000 E+05$ & 1.1152000E+0.5 & $1.1152000 E+05$ & $1.1152000 E+05$ & $\mathbf{m}^{\wedge} \mathbf{2}$ & WP036766 & $\mathbf{Y}$ & NA \\
\hline 3239 & $1,3,4$ & CONSTANT & $1.1152000 E+05$ & 1.1152000E+05 & $1.1152000 E+05$ & $1.1152000 E+00$ & $m^{\wedge} 2$ & UPO40521 & $\mathbf{Y}$ & N/A \\
\hline 3240 & 4 & CONSTANT & $8.0000000 E-02$ & $8.0000000 E-02$ & $8.0000000 E-02$ & $8.0000000 E-02$ & NONE & STREETER-2 & $\mathbf{Y}$ & N/A \\
\hline 3240 & 4 & CONSTANT & $8.0000000 E-02$ & $8.0000000 E-02$ & $8.0000000 E-02$ & $8.0000000 E-02$ & NONE & WP036301 & $Y$ & NA \\
\hline
\end{tabular}


CCA Parameter Listing

\begin{tabular}{|c|c|c|c|c|c|c|c|c|c|c|}
\hline $\begin{array}{c}\text { Parmetion in } \\
\text { Detubece? } \\
\text { (MN) }\end{array}$ & 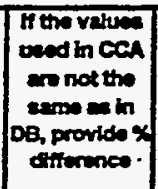 & ID & Meterlat & Mothen & $\begin{array}{c}\text { Peranotiar } \\
\text { Do }\end{array}$ & 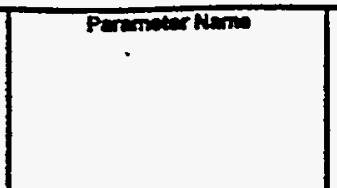 & $\begin{array}{l}\text { PRPLD } \\
\text { (MPOA) }\end{array}$ & $\begin{array}{c}\text { Dententy } \\
\text { Dete }\end{array}$ & 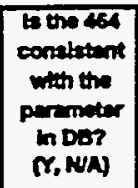 & 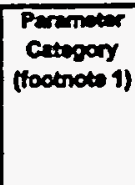 \\
\hline$Y$ & & 3240 & BOREHOLE & Borchalo and Fill & ROUGHP & $\begin{array}{l}\text { Frection fector for very rough ppo } \\
\text { in CUITINGS model }\end{array}$ & 37002 & 25-Apr-96 & $Y$ & 3 \\
\hline $\mathbf{Y}$ & & 3240 & BOREHOLE & Borahole and Fill & ROUGHP & $\begin{array}{l}\text { Friction tactor for viry rough pipe } \\
\text { in CUTIINGS model }\end{array}$ & 37002 & 25-Apr-96 & $\mathbf{Y}$ & 3 \\
\hline $\mathbf{Y}$ & & 3241 & BOREHOLE & Boreholo and Fit & PIPED & $\begin{array}{l}\text { Donl ppe demexer n CUTrinGS } \\
\text { model }\end{array}$ & 37003 & 25 -npr-96 & $\mathbf{Y}$ & 48 \\
\hline $\mathbf{Y}$ & & 3241 & BOREHOLE & Borehole and Fill & PIPED & $\begin{array}{l}\text { Dril pipe dimoter in CUTTINGS } \\
\text { model }\end{array}$ & 37003 & 25 Apr-96 & $\mathbf{Y}$ & 48 \\
\hline$Y$ & & 3241 & BOREHOLE & Borehole and Fx & PIPED & $\begin{array}{l}\text { Drill ppe dimeter n CUTTINGS } \\
\text { model }\end{array}$ & 37063 & 25-Apr-96 & $\mathbf{Y}$ & 48 \\
\hline$Y$ & & 3242 & BOREHOLE & Borehole and Fid & COLDLA & $\begin{array}{l}\text { Drill coller demeter in CUTrinGs } \\
\text { model }\end{array}$ & 37004 & 25 apr-96 & $\mathbf{Y}$ & 48 \\
\hline$Y$ & & 3242 & BOREHOLE & Borehole and Ful & COLOLA & $\begin{array}{l}\text { Drill coller diemeter h CUTINGS } \\
\text { model }\end{array}$ & 37064 & 25 Apr-96 & $\mathbf{Y}$ & 48 \\
\hline $\mathbf{Y}$ & & 3242 & BOREHOLE & Borehole and Fta & COLOLA & $\begin{array}{l}\text { Drily coltar dremeter in CUTINGS } \\
\text { model }\end{array}$ & 37084 & 25-Apr.96 & $\mathbf{Y}$ & $4 B$ \\
\hline $\mathbf{Y}$ & & 3243 & BOREHOLE & Borahole and Frd & 12 & $\begin{array}{l}\text { Dril pipe longth when repository } \\
\text { penpetrated, CUTTINGS model }\end{array}$ & 37085 & 25 Apr.96 & $\mathbf{Y}$ & $A B$ \\
\hline $\mathbf{Y}$ & & 3243 & BOREHOLE & Boreholo and Fill & 12 & $\begin{array}{l}\text { Dril pipo ingith when repository } \\
\text { penetrated, CUTTINGS model }\end{array}$ & 37005 & 25 Apr-96 & $\mathbf{Y}$ & AB \\
\hline $\mathbf{Y}$ & & 3243 & BOREHOLE & Borcholo and Fil & 12 & $\begin{array}{l}\text { Drill pipe ing ith when repository } \\
\text { penetrated, CUTIINGS modil }\end{array}$ & 37085 & $25-4 p r-86$ & $Y$ & $A B$ \\
\hline $\mathbf{Y}$ & & 3244 & BOREHOLE & Borotiolo and Fit & L1 & $\begin{array}{l}\text { Drill coller longth in CUTingGS } \\
\text { model }\end{array}$ & 37006 & 25-Apr.96 & $\mathbf{Y}$ & $4 B$ \\
\hline $\mathbf{Y}$ & & 3244 & BOREHOLE & Borehole and Fill & L1 & $\begin{array}{l}\text { Driil collar longet n CUTIINGS } \\
\text { modol }\end{array}$ & 37066 & 25-Apr-96 & $\mathbf{Y}$ & $4 B$ \\
\hline $\mathbf{Y}$ & & 3244 & BOREHOLE & Boreholo and Fill & L1 & $\begin{array}{l}\text { Donll coller longen } n \mathrm{CU} T \mathrm{TINGS} \\
\text { model }\end{array}$ & 37006 & 25 -4pr-96 & $\mathbf{Y}$ & 48 \\
\hline $\mathbf{Y}$ & & 3245 & BLOWOUT & $\begin{array}{l}\text { BRAGFLOW Drect } \\
\text { Brine Releasos }\end{array}$ & CEMENT & Waste Comentation Strength & 37007 & 25 Apr-96 & $\mathbf{Y}$ & 48 \\
\hline $\mathbf{Y}$ & & 3245 & BLOWOUT & $\begin{array}{l}\text { BRAGFLOW Dired } \\
\text { Brine Roleases }\end{array}$ & CEMENT & Wasto Cementation Strength & 37007 & 25-Apr-96 & $\mathbf{Y}$ & $4 B$ \\
\hline$Y$ & & 3245 & BLOWOUT & $\begin{array}{l}\text { BRAGFLOWDrod } \\
\text { Brine Releases }\end{array}$ & CEMENT & Waste Cornentevoon Strength & 37087 & 25-Apr-96 & $\mathbf{Y}$ & AB \\
\hline $\mathbf{Y}$ & & 3246 & BLOWOUT & $\begin{array}{l}\text { BRAGFLOWDired } \\
\text { Brine Releases }\end{array}$ & PARTDU & 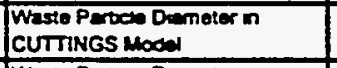 & 37068 & |03+May-96 & $\mathbf{Y}$ & 1 \\
\hline $\mathbf{Y}$ & & 3246 & BLOWOUT & $\begin{array}{l}\text { BRAGFLOWDred } \\
\text { Brine Rolossos }\end{array}$ & PARTDU & $\begin{array}{l}\text { Warte Pertoci Dameter n } \\
\text { CUTTINGS Ulodel }\end{array}$ & 37088 & |03-May-96| & $\mathbf{Y}$ & 1 \\
\hline $\mathbf{Y}$ & & 3246 & BLOWOUT & $\begin{array}{l}\text { BRAGFLOWDract } \\
\text { Brino Reloases }\end{array}$ & PARTOU & 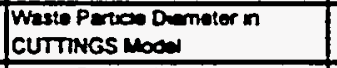 & 37088 & 03-14ay-96 & $\gamma$ & 1 \\
\hline $\mathbf{Y}$ & & 3247 & BLOWOUT & $\begin{array}{l}\text { BRAGFLOW Druc: } \\
\text { Brine Robases }\end{array}$ & RHOS & 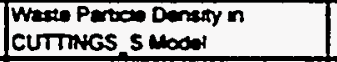 & 37009 & 25 Apr-96 & $Y$ & AB \\
\hline $\mathbf{Y}$ & & 3247 & BLOWOUT & $\begin{array}{l}\text { BRAGFLOW Druad } \\
\text { Brine Relazes }\end{array}$ & RHOS & 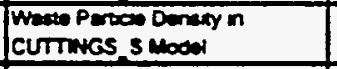 & 37009 & 25-Apr-96 & $\mathbf{Y}$ & $4 B$ \\
\hline $\mathbf{Y}$ & & 3247 & BLOWOUT & $\begin{array}{l}\text { BRAGFLOW Drea } \\
\text { Brins Rolases: }\end{array}$ & RHOS & 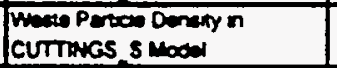 & 37009 & $25-A p r-96$ & $\mathbf{Y}$ & 48 \\
\hline $\mathbf{Y}$ & & 3247 & BLOWOUT & $\begin{array}{l}\text { BRAGFLOW Drea } \\
\text { Brine Retoenes }\end{array}$ & RHOS & 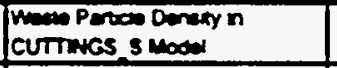 & 37009 & 25-Apr-96 & $\mathbf{Y}$ & 48 \\
\hline $\mathbf{Y}$ & & 3248 & BLOWOUT & $\begin{array}{l}\text { BRAGFLOW Dred } \\
\text { Brme Rovanes: }\end{array}$ & Room & 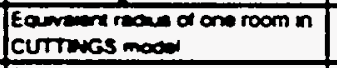 & 37090 & 25-Apr-96 & $\mathbf{Y}$ & 48 \\
\hline $\mathbf{Y}$ & & 3248 & BLOWOUT & $\begin{array}{l}\text { BRAGFLOW Drea } \\
\text { Brme Rolanem }\end{array}$ & ROON & 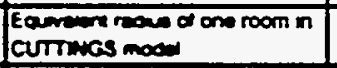 & 37090 & 25Apr-96 & $Y$ & 48 \\
\hline $\mathbf{Y}$ & & 3248 & BLOWOUT & $\begin{array}{l}\text { BRAGFLOW Drsa } \\
\text { Brino Rovenen }\end{array}$ & RoON & 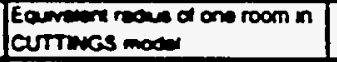 & 37090 & 25-Apr-96 & $\boldsymbol{Y}$ & AB \\
\hline $\mathbf{Y}$ & & 3249 & BLOWOUT & $\begin{array}{l}\text { BRAGFLOW Drea } \\
\text { Brine Revaeses }\end{array}$ & RPPANEL & 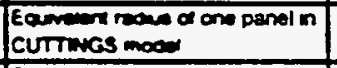 & 37091 & 25 -Apr-86 & $Y$ & 48 \\
\hline$Y$ & & 3249 & BLOWOUT & $\begin{array}{l}\text { BRAGFLOWDruca } \\
\text { Brino Relaseas }\end{array}$ & RPANCL & 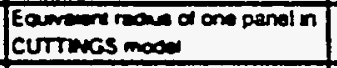 & 37091 & 25-Apx-96 & $\mathbf{Y}$ & AB \\
\hline $\mathbf{Y}$ & & 3249 & BLOWOUT & $\begin{array}{l}\text { BRAGFLOW Drea } \\
\text { Brine Relazes: }\end{array}$ & RPANEL & 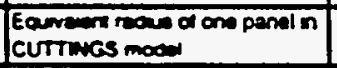 & 37091 & 25 -Apr-96 & $Y$ & 48 \\
\hline$Y$ & & 3250 & BLOWOUT & $\begin{array}{l}\text { BRAGFLOW Draca } \\
\text { Brino Reveses }\end{array}$ & HREPO & 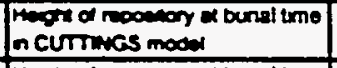 & 37092 & $25-\wedge p r-96$ & $Y$ & 48 \\
\hline$Y$ & & 3250 & BLOWOUT & $\begin{array}{l}\text { BRAGFLOW Drua } \\
\text { Brine Rebases }\end{array}$ & HRESO & 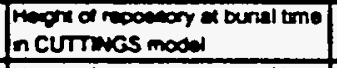 & 37092 & 25 -Apr-86 & $Y$ & $A B$ \\
\hline$Y$ & & 3250 & BLOWOUT & $\begin{array}{l}\text { BRAGFLOW Drea } \\
\text { Brine Relesene }\end{array}$ & HRESO & 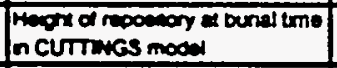 & 37092 & $25-\wedge p r-96$ & $\mathbf{Y}$ & 48 \\
\hline$Y$ & & 3251 & BLOWOUT & $\begin{array}{l}\text { BRAGFLOW Drea } \\
\text { Brime Rewaven }\end{array}$ & psue & 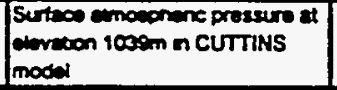 & 37093 & 25 -Apr-96 & $\mathbf{Y}$ & $\mu$ \\
\hline
\end{tabular}


CCA Parameter Listing

\begin{tabular}{|c|c|c|c|c|c|c|c|c|c|c|}
\hline 10 & \begin{tabular}{c|} 
Cleasifi- \\
catton of \\
Duth \\
Catiogony \\
(footnote 2)
\end{tabular} & $\begin{array}{l}\text { Disuribution } \\
\text { Type }\end{array}$ & Mean & medien & Mintmum & Maxtmun & Unt & Reformes to & 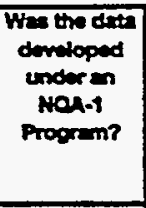 & 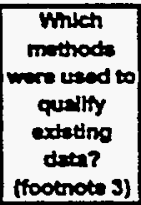 \\
\hline 3240 & 4 & CONSTANT & $8.0000000 E-02$ & $8.0000000 E-02$ & $8.0000000 E-02$ & $8.0000000 E-02$ & NONE & WP036766 & $\mathbf{Y}$ & NAA \\
\hline 3240 & 4 & CONSTANT & $8.0000000 E-02$ & $8.0000000 E-02$ & $8.0000000 E-02$ & $8.0000000 E-02$ & NONE & WP033455 & $\mathbf{Y}$ & NA \\
\hline 3241 & $1,3,4$ & CONSTANT & $1.1430020 E-01$ & $1.1430020 E-01$ & $1.1430020 E-01$ & $1.1430020 E-01$ & $\mathbf{m}$ & UP026051 & $Y$ & NA \\
\hline 3241 & $1,3,4$ & CONSTANT & $1.1430020 \mathrm{E}-01$ & $1.1430020 E-01$ & $1.1430020 E-01$ & $1.1430020 E-01$ & $\mathrm{~m}$ & WP035766 & $Y$ & NA \\
\hline 3241 & $1,3,4$ & CONSTANT & $1.1430020 E .01$ & $1.1430020 E-01$ & $1.1430020 E-01$ & $1.1130020 E-01$ & $m$ & WPOLO521 & $Y$ & NA \\
\hline 3242 & $1,3.4$ & CONSTANT & $2.0320040 E-01$ & $20320040 E-01$ & $20320040 E-01$ & $2.0320040 E-01$ & $\mathbf{m}$ & WP02505i : & $Y$ & N/A \\
\hline 3242 & $1,3,4$ & CONSTANT & 2.0320040E-01 & $2.0320040 E-01$ & $2.0320040 E-01$ & $2.0320040 E-01$ & $\mathbf{m}$ & WP035765 & $Y$ & NA \\
\hline 3242 & $1,3,4$ & CONSTANT & $2.0320040 E-01$ & $2.0320040 E-01$ & $20320040 E-01$ & $2.0320040 E-01$ & $m$ & WPO40521 & $Y$ & NA \\
\hline 3243 & $1,3,4$ & CONSTANT & $4.7212000 E+02$ & $4.7212000 E+02$ & $4.7212000 E+02$ & $4.7212000 E+02$ & $\mathbf{m}$ & SAND91-00sa3 & $\mathbf{Y}$ & NA \\
\hline 3243 & $1,3,4$ & CONSTANT & $4.7212000 E+02$ & 4.7212000E+02 & $4.7212000 E+02$ & $4.7212000 E+02$ & $m$ & WP036766 & $Y$ & NA \\
\hline 3243 & $1,3,4$ & CONSTANT & 4.7212000E+02 & $4.7212000 \mathrm{E}+02$ & $4.72120000+002$ & $4.7212000 \mathrm{E}+02$ & m & WPO40521 & $\gamma$ & NA \\
\hline 3244 & $1,3,4$ & CONSTANT & $1.82880000 E+02$ & $1.8283000 \mathrm{E}+\infty 2$ & $1.82880000 E+02$ & $1.8288000= \pm+02$ & $m$ & WP026051 & $Y$ & NA \\
\hline 3244 & $1,3,4$ & CONSTANT & $1.8288000 E+\infty 2$ & $1.8288000 \mathrm{E}+02$ & $1.8288000 E+02$ & $1.8288000 \mathrm{E}+02$ & $m$ & WP036765 & $\mathbf{Y}$ & NA \\
\hline 3244 & $1,3,4$ & CONSTANT & $1.8288000 \mathrm{E}+02$ & $1.8288000 \mathrm{E}+02$ & $1.8283000 \mathrm{E}+02$ & $1.8288000 E+02$ & $m$ & WP040521 & $\mathbf{Y}$ & NA \\
\hline 3245 & 3,4 & CONSTANT & $6.8950000 E+03$ & $6.8950000 E+03$ & $6.8950000 E+03$ & $6.8950000 \varepsilon+03$ & Pa & WP035695 & $Y$ & NA \\
\hline 3245 & 3,4 & CONSTANT & $6.8950000 \mathrm{E}+03$ & $6.8950000 \mathrm{E}+03$ & $6.8950000 E+03$ & $6.8950000 E+03$ & Pa & WP036723 & $Y$ & NA \\
\hline 3245 & 3,4 & CONSTANT & $6.8950000 \approx+\infty 03$ & $6.8950000 E+03$ & $6.8950000 E+03$ & $6.8950000 E+03$ & Pe & WPO40521 & $\mathbf{Y}$ & NA \\
\hline '3246 & 1 & LOGUNIFORM & $2.3500000 \mathrm{E}-02$ & $2.8000000 E-03$ & $4.0000000 E-05$ & $2.0000000 E-01$ & $m$ & WP035268 & N & 1 \\
\hline 3246 & 1 & LOGUNIFORM & $2.3500000 E-02$ & $2.8000000 E-03$ & $4.0000000 E-05$ & $2.0000000 E-01$ & m & WP035695 & $N$ & 1 \\
\hline 3245 & 1 & LOGUNIFORM & $2.3500000 E-02$ & $20000000 E-03$ & $4.0000000 E-05$ & $2.0000000 E-01$ & m & WP036766 & $N$ & 1 \\
\hline 3247 & 3,4 & CONSTANT & $2.6500000 \mathrm{E}+03$ & $2.6500000 E+03$ & $2.6500000 E+03$ & $2.6500000 E+03$ & $\mathrm{~kg} / \mathrm{m} \times 3$ & WP035695 & $Y$ & NA \\
\hline 3247 & 3,4 & CONSTANT & $2.6500000 E+03$ & $2.6500000 E+03$ & $2.6500000 E+03$ & $2.6500000 E+03$ & $\mathrm{~kg} / \mathrm{m}^{\mathrm{A}} 3$ & WP035766 & $Y$ & N/A \\
\hline 3247 & 3,4 & CONSTANT & $2.6500000 E+03$ & $2.6500000 E+03$ & $2.6500000 E+03$ & $2.6500000 E+03$ & $\mathrm{~kg} / \mathrm{m}^{\wedge} 3$ & WPO40521 & $Y$ & NA \\
\hline 3247 & 3,4 & CONSTANT & $2.6500000 E+03$ & $2.65000000+003$ & $26500000 E+00$ & $.26500000 \mathrm{E}+03$ & $\mathrm{~kg} / \mathrm{m}^{\wedge} 3$ & WPO42263 & $\mathbf{Y}$ & NA \\
\hline 3248 & $1,3,4$ & CONSTANT & $1.7100000 E+01$ & $1.7100000 E+01$ & $17100000 E+01$ & $17100000 E+01$ & $\mathbf{m}$ & SAND91-0893/3 & $\mathbf{Y}$ & NA \\
\hline 3248 & $1,3,4$ & CONSTANT & $1.7100000 E+01$ & $1.7100000 E+01$ & $171000006-01$ & $17100000 E+01$ & $\mathbf{m}$ & WP035766 & $\mathbf{Y}$ & NA \\
\hline 3248 & $1,3,4$ & CONSTANT & $1.7100000 E+01$ & $1.7100000 E+01$ & $1.7100000 E+01$ & $17100000 E+01$ & $m$ & WPO40521 & $\mathbf{Y}$ & NA \\
\hline 3249 & $1,3,4$ & CONSTANT & $6.0870000 E+01$ & $6.0870000 E+01$ & $60870000 E+01$ & $60870000 E+01$ & $m$ & SAND91-06933 & $\mathbf{Y}$ & NA \\
\hline 3249 & $1,3,4$ & CONSTANT & $6.0870000 E+01$ & $6.0670000 E+01$ & $6.0670000 E+01$ & $60870000 E+01$ & $m$ & WP036766 & $\mathbf{Y}$ & NA \\
\hline 3249 & $1,3,4$ & CONSTANT & $6.0870000 E+01$ & $6.0070000 E+01$ & $6.0670000<+01$ & $6.0870000 E+01$ & $m$ & WPO40521 & $\mathbf{Y}$ & NA \\
\hline 3250 & $1,3,4$ & CONSTANT & $3.9600000 \mathrm{E}+00$ & $3.9600000 E+\infty 0$ & $3.9600000 \times+\infty$ & $3.9600000 E+\infty$ & $\mathbf{m}$ & SAND91-08933 & $\mathbf{Y}$ & NA \\
\hline 3250 & $1,3,4$ & CONSTANT & $3.96000000+\infty$ & $3.9600000 E+\infty$ & $3.9600000 E+00$ & $3.9600000 E+\infty$ & $m$ & WP036766 & $\mathbf{Y}$ & NA \\
\hline 3250 & $1,3,4$ & CONSTANT & $3.9600000 E+\infty 0$ & $3.9600000 \in+\infty$ & $39600000 E+\infty$ & $3.9600000 E+\infty$ & $m$ & WPO40521 & $\mathbf{Y}$ & NA \\
\hline 3251 & $3,4,5$ & CONSTANT & $8.9465000 E+04$ & $8.9465000 E+01$ & $8.9465000 E+04$ & $8.9465000 E+04$ & Pa & FOX-1 & $\mathbf{Y}$ & N/A \\
\hline
\end{tabular}


CCA Parameter Listing

\begin{tabular}{|c|c|c|c|c|c|c|c|c|c|c|}
\hline $\begin{array}{c}\text { Parriner in } \\
\text { Detabees? } \\
\text { N/NI) }\end{array}$ & $\begin{array}{l}\text { fitw value } \\
\text { used in cen } \\
\text { ere not the } \\
\text { exmes in } \\
\text { og, provide } x \\
\text { difierence }\end{array}$ & DI & Mecartab & Materin Nam & Pormeter & Paranter Nans & 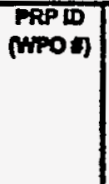 & Deta Entry & 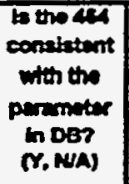 & $\begin{array}{l}\text { Permiter } \\
\text { Cetagony } \\
\text { (footiots 1) }\end{array}$ \\
\hline $\mathbf{Y}$ & & 3251 & BLOWOUT & $\begin{array}{l}\text { BRAGFLOW Direct } \\
\text { Brina Relesses }\end{array}$ & PSUF & $\begin{array}{l}\text { Surface utmosphenc pressure at } \\
\text { slevation 1039m in CUTTINS } \\
\text { model }\end{array}$ & 37003 & $25-A p r-96$ & $\mathbf{Y}$ & $\mu$ \\
\hline $\mathbf{Y}$ & & 3251 & BLOWOUT & $\begin{array}{l}\text { BRAGFLOW Direa } \\
\text { Brine Rolateses }\end{array}$ & PSUF & 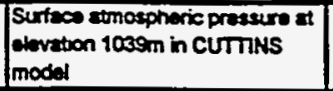 & 37093 & 25 Apr-96 & $\mathbf{Y}$ & 4 \\
\hline $\mathbf{Y}$ & & 3252 & BLOWOUT & $\begin{array}{l}\text { BRAGFLOW Dreed } \\
\text { Brme Roteases }\end{array}$ & unsc & $\begin{array}{l}\text { Hydrogen Vecosity in CUTTINGS } \\
\text { Model }\end{array}$ & 37094 & 25 Apr-96 & $\mathbf{Y}$ & 3 \\
\hline $\mathbf{Y}$ & & 3252 & BLOWOUT & $\begin{array}{l}\text { BRAGFLOWDred } \\
\text { Brine Retaseses }\end{array}$ & visc & $\begin{array}{l}\text { Hyctoopen Viscosty in CUTTINGS } \\
\text { Model }\end{array}$ & 37094 & 25-Apr-95 & $\mathbf{Y}$ & 3 \\
\hline $\mathbf{Y}$ & & 3252 & BLOWOUT & $\begin{array}{l}\text { BRAGFLOWDired } \\
\text { Brine Reloases }\end{array}$ & visc & $\begin{array}{l}\text { Hydropen Viscosity in CUTTINGS } \\
\text { Model }\end{array}$ & 37094 & $25-\wedge p r-96$ & $\mathbf{Y}$ & 3 \\
\hline $\mathbf{Y}$ & & 3253 & BLOWOUT & $\begin{array}{l}\text { BRAGFLOW Dred } \\
\text { Brine Releases }\end{array}$ & RGAS & Ges Coratunt for Hytrogen & 37005 & 25 -Apr-95 & $\mathbf{Y}$ & 3 \\
\hline $\mathbf{Y}$ & & 3253 & BLOWOUT & $\begin{array}{l}\text { BRAGFLOW Drect } \\
\text { Brine Releases }\end{array}$ & RGAS & Ges Constent for Hydrogen & 37005 & $25-A p x-96$ & $\mathbf{Y}$ & 3 \\
\hline $\mathbf{Y}$ & & 3254 & BLOWOUT & $\begin{array}{l}\text { BRAGFLOW Droct } \\
\text { Brino Roleases }\end{array}$ & KGAS & $\begin{array}{l}\text { Ratio of eppecific heats for } \\
\text { Hydrogen in CUTTINGS model }\end{array}$ & 37096 & $25-4 p r-96$ & $\mathbf{Y}$ & 3 \\
\hline $\mathbf{Y}$ & & 3254 & ELOWOUT & $\begin{array}{l}\text { BRAGFLOW Dred } \\
\text { Brine Releases }\end{array}$ & KGAS & $\begin{array}{l}\text { Ratio of epecific hexts for } \\
\text { Hydrogen in CUTTINGS model }\end{array}$ & 37056 & 25-Apr-96 & $\mathbf{Y}$ & 3 \\
\hline $\mathbf{Y}$ & & 3254 & BLOWOUT & $\begin{array}{l}\text { BRAGFLOW Diruct } \\
\text { Brino Releases }\end{array}$ & KGAS & $\begin{array}{l}\text { Ratio of specific hants for } \\
\text { itydrogen in CUTTINGS model }\end{array}$ & 37056 & $25-A p r-96$ & $\mathbf{Y}$ & 3 \\
\hline $\mathbf{Y}$ & & 3255 & BLOWOUT & $\begin{array}{l}\text { BRAGFLOWDrect } \\
\text { Brine Reloseses }\end{array}$ & FSE & $\begin{array}{l}\text { Stress offectivenss factor in } \\
\text { CUTMNGS modes }\end{array}$ & 37097 & $25-A p x-96$ & $\mathbf{Y}$ & $A B$ \\
\hline $\mathbf{Y}$ & & 3255 & BLOWoUT & $\begin{array}{l}\text { BRAGFLOW Direct } \\
\text { Brino Roleases }\end{array}$ & FSE & $\begin{array}{l}\text { Struss effectivents factor in } \\
\text { CUTTINGS model }\end{array}$ & 37097 & 25-Apr-96 & $\mathbf{Y}$ & 48 \\
\hline $\mathbf{Y}$ & & 3255 & BLOWOUT & $\begin{array}{l}\text { BRAGFLOW Droct } \\
\text { Brine Roloases }\end{array}$ & FSE & $\begin{array}{l}\text { Stress effectrmas factor in } \\
\text { cUTMNGS model }\end{array}$ & 37097 & $25-A p r-96$ & $\mathbf{Y}$ & 48 \\
\hline $\mathbf{Y}$ & & 3255 & BLOWOUT & $\begin{array}{l}\text { BRAGFLOW Drect } \\
\text { Brina Reloases }\end{array}$ & FSE & $\begin{array}{l}\text { Stross efioctivenss fector in } \\
\text { CUTTINGS model }\end{array}$ & 37097 & 25-Apr-96 & $\mathbf{Y}$ & 48 \\
\hline$Y$ & & 3256 & BLOWOUT & $\begin{array}{l}\text { BRAGFLOW Direct } \\
\text { Brino Relaases }\end{array}$ & FGE & $\begin{array}{l}\text { Gravily offectreness factor in } \\
\text { CUTINGS model }\end{array}$ & 37098 & $25-A p r-96$ & $\mathbf{Y}$ & 48 \\
\hline$Y$ & & 3256 & BLOWOUT & $\begin{array}{l}\text { BRAGFLOWDirect } \\
\text { Brine Relases }\end{array}$ & FGE & $\begin{array}{l}\text { Gravity offectiveness factor in } \\
\text { CUTTINGS model }\end{array}$ & 37098 & $25-A p \times .96$ & $\mathbf{Y}$ & 48 \\
\hline $\mathbf{Y}$ & & 3256 & BLOWOUT & $\begin{array}{l}\text { BRAGFLOW Drect } \\
\text { Brine Reloases }\end{array}$ & FGE & $\begin{array}{l}\text { Gravity effoctweness factor in } \\
\text { CUTTINGS modol }\end{array}$ & 37098 & 25-Apr-96 & $Y$ & AB \\
\hline$Y$ & & 3256 & BLOWOUT & $\begin{array}{l}\text { BRAGFLOW Direct } \\
\text { Brine Relenses }\end{array}$ & FGE & $\begin{array}{l}\text { Grovity effectiveness fector in } \\
\text { CUTTINGS model }\end{array}$ & 37098 & 25-Apr-96 & $\mathbf{Y}$ & 48 \\
\hline $\mathbf{Y}$ & & 3256 & BLOWOUT & $\begin{array}{l}\text { BRAGFLOWDirect } \\
\text { Brino Relaeses }\end{array}$ & FGE & $\begin{array}{l}\text { Gravity effectiveness factor in } \\
\text { CUTTINGS model }\end{array}$ & 37098 & 25 -Apr-96 & $\mathbf{Y}$ & 48 \\
\hline $\mathbf{Y}$ & & 3257 & BLOWDUT & $\begin{array}{l}\text { BRAGFLOW Direct } \\
\text { Brine Releases }\end{array}$ & SUFTEN & $\begin{array}{l}\text { Surfuce tension of brne in } \\
\text { cUTTINGS model }\end{array}$ & 37100 & $25-A p r-96$ & $\mathbf{Y}$ & 3 \\
\hline$Y$ & & 3257 & BLOWOUT & $\begin{array}{l}\text { BRAGFLOW Direct } \\
\text { Brine Releseses }\end{array}$ & SUFTEN & $\begin{array}{l}\text { Sutece tension of brino in } \\
\text { CUTITINGS model }\end{array}$ & 37100 & 25-Apr-96 & $\mathbf{Y}$ & 3 \\
\hline$Y$ & & 3257 & BLOWOUT & $\begin{array}{l}\text { BRAGFLOW Direct } \\
\text { Brine Rolasses }\end{array}$ & SUFTEN & $\begin{array}{l}\text { Surfece tension of brme in } \\
\text { CUTTINGS model }\end{array}$ & 37100 & 25-Apr-96 & $\mathbf{Y}$ & 3 \\
\hline $\mathbf{Y}$ & & 3257 & BLOWOUT & $\begin{array}{l}\text { BRAGFLOW Drect } \\
\text { Brine Relauses }\end{array}$ & SUFTEN & $\begin{array}{l}\text { Surfece tension of brna in } \\
\text { CUTMINGS model }\end{array}$ & 37100 & $25-A p r-96$ & $\mathbf{Y}$ & 3 \\
\hline $\mathbf{Y}$ & & 3258 & BLOWOUT & $\begin{array}{l}\text { BRAGFLOW Direct } \\
\text { Brine Reloases }\end{array}$ & TREPO & $\begin{array}{l}\text { Temporature of repository m } \\
\text { CUTTINGS modal }\end{array}$ & 37101 & 25-Apr-96 &.$- Y$ & $4 A$ \\
\hline $\mathbf{Y}$ & & 3259 & BLOWOUT & $\begin{array}{l}\text { BRAGFLOW Direct } \\
\text { Brine Relanses }\end{array}$ & APORO & $\begin{array}{l}\text { Waste permesbility in CUTTINGS } \\
\text { model }\end{array}$ & 37102 & 25-Apr-96 & $\mathbf{Y}$ & 1 \\
\hline $\mathbf{Y}$ & & 3260 & BLOWOUT & $\begin{array}{l}\text { BRAGFLOW Direct } \\
\text { Brine Releseses }\end{array}$ & INPORO & $\begin{array}{l}\text { Defaut value for initial repository } \\
\text { porosity in CUTTINGS model }\end{array}$ & 37103 & 25-Apr-96 & $\mathbf{Y}$ & 48 \\
\hline $\mathbf{Y}$ & & 3260 & BLOWOUT & $\begin{array}{l}\text { BRAGFLOW Dired } \\
\text { Brim Relasses }\end{array}$ & INPORO & $\begin{array}{l}\text { Defoutt value for initial repository } \\
\text { porosity in CUTINGS modol }\end{array}$ & 37103 & 25 -Apr-96 & $\mathbf{Y}$ & 48 \\
\hline $\mathbf{Y}$ & & 3281 & BOREHOLE & Borahoin and Fid & RHW_AR & $\begin{array}{l}\text { The tolli erea of the remote- } \\
\text { handled waste in the CUTTINGS } \\
\text { model }\end{array}$ & 37104 & $25-A p x \cdot 96$ & $\mathbf{Y}$ & $4 B$ \\
\hline $\mathbf{Y}$ & & 3261 & BOREHOLE & Boruhois and fill & RHW_AR & $\begin{array}{l}\text { The total ares of the renote- } \\
\text { hendled waste in the CUTTINGS } \\
\text { model }\end{array}$ & 37104 & $25-\operatorname{Apr}-95$ & $\mathbf{Y}$ & $A B$ \\
\hline $\mathbf{Y}$ & & 3262 & SOLAM3 & $\begin{array}{l}\text { Solubility Mutiplier for } \\
\text { An+3 }\end{array}$ & SOLSIM & $\begin{array}{l}\text { Solubility Muth in Salado Brine, } \\
\text { Inorganic Chm Controlied by } \\
M g(\mathrm{OH}) 2+\mathrm{MgO} 3\end{array}$ & $37105 A$ & $24+12 y-95$ & $\mathbf{Y}$ & 1 \\
\hline$Y$ & & 3202 & SOLAMB & $\begin{array}{l}\text { Soltitity Muliplier tor } \\
\text { Am+3 }\end{array}$ & SOLSAM & $\begin{array}{l}\text { Soldbitity Muth in Salado Brine. } \\
\text { inorganic Chem Controtied by } \\
\text { Mo(OH)2 HgCO3 }\end{array}$ & 371054 & 24+Mey-95 & $Y$ & 1 \\
\hline
\end{tabular}


CCA Parameter Listing

\begin{tabular}{|c|c|c|c|c|c|c|c|c|c|c|}
\hline ID & $\begin{array}{c}\text { Climenti } \\
\text { catton of } \\
\text { Dita } \\
\text { Certegory } \\
\text { (lootnote 2) }\end{array}$ & $\begin{array}{c}\text { Distilbution } \\
\text { Type }\end{array}$ & Mean & Medien & Mtnimum & Moximin & Units & Raference D & $\begin{array}{l}\text { Wes the dith } \\
\text { deniloped } \\
\text { under en } \\
\text { Non-? } \\
\text { Program? }\end{array}$ & 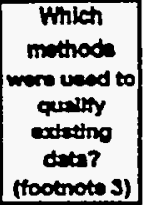 \\
\hline 3251 & $3,4,5$ & CONSTANT & B.9465000E+04 & $8.9465000 E+04$ & $8.9465000 E+04$ & $8.9465000 E+04$ & $\mathbf{P a}$ & SAND91-009333 & $\mathbf{Y}$ & NAA \\
\hline 3251 & 3.4 .5 & CONSTANT & $8.9465000 E+04$ & $8.9465000 E+04$ & $8.9465000 E+04$ & $8.9455000 E+04$ & $\mathrm{~Pa}$ & WP036766 & $\mathbf{Y}$ & NA \\
\hline 3252 & 4 & CONSTANI & $9.2000000 E-06$ & $9.2000000 E-06$ & $92000000 E-06$ & $9.2000000=-06$ & Pa's & SAND92-07003 & $\mathbf{Y}$ & NA \\
\hline 3252 & 4 & CONSTANT & $9.2000000 E-06$ & $9.2000000 E-06$ & $9.2000000 E-06$ & $9.2000000 E-06$ & Pacs & WPO36301 & $\mathbf{Y}$ & NA \\
\hline 3252 & 4 & CONSTANT & $9.2000000 E-06$ & $9.2000000 E-06$ & $9.2000000 E-06$ & $9.2000000 E-06$ & Pars & WP036765, & $\mathbf{Y}$ & NA \\
\hline 3253 & 4 & CONSTANT & $4.1160000 E+\infty 3$ & $4.1160000 E+\infty 03$ & $4.1160000 E+03$ & $4.1160000 \mathrm{E}+03$ & mikologot & OBERT-1. & $\mathbf{Y}$ & NA \\
\hline 3253 & 4 & CONSTANT & $4.1160000 E+03$ & $4.1160000 \mathrm{E}+03$ & $4.1160000 E+03$ & $4.1160000 E+03$ & mikg/degl & WP035766 & $\mathbf{Y}$ & NA \\
\hline 3254 & 4 & CONSTANT & $1.4100000 E+\infty$ & $1.4100000=+\infty$ & $1.4100000 E+\infty 0$ & $1.4100000 E+\infty$ & NONE & OBERT-1 - & $Y$ & NA \\
\hline 3254 & 4 & CONSTANT & $1.4100000 E+\infty$ & $1.4100000 E+\infty$ & $1.1100000 E+\infty 0$ & $1.4100000 E+\infty$ & NONE & WP036301. & $Y$ & NA \\
\hline 3254 & 4 & CONSTANT & $1.4100000 E+\infty$ & $1.4100000 E+\infty 0$ & $1.4100000 E+\infty$ & $1.4100000=+\infty$ & NONE & WP036766 & $\mathbf{Y}$ & NA \\
\hline 3255 & 1,3 & CONSTANT & $0.0000000 E+\infty 0$ & $0.0000000 E+\infty 0$ & $0.0000000 E+\infty$ & $0.0000000 E+\infty$ & NONE & WP035595 & $\mathbf{Y}$ & NA \\
\hline 3255 & 1,3 & CONSTANT & $0.0000000 E+00$ & $0.0000000 E+\infty$ & $0.0000000 E+\infty$ & $0.0000000 E+00$ & NONE & WP036766 & $\mathbf{Y}$ & NA \\
\hline 3255 & 1,3 & CONSTANT & $0.0000000 E+00$ & $0.0000000 E+\infty 0$ & $0.0000000 E+\infty 0$ & $0.0000000 E+\infty$ & NONE & WPO40521 & $\mathbf{Y}$ & NA \\
\hline 3255 & 1,3 & CONSTANT & $0.0000000 E+\infty$ & $0.0000000 E+\infty$ & $0.0000000 E+\infty$ & $0.0000000 E+\infty$ & NONE & WP042263 & $\mathbf{Y}$ & NA \\
\hline 3256 & 1,3 & CONSTANT & $1.8100000 \mathrm{E}+01$ & $1.8100000 E+01$ & $1.8100000 E+01$ & $1.8100000 E+01$ & NONE & WP035695 & $\mathbf{Y}$ & NA \\
\hline 3256 & 1,3 & CONSTANT & $1.8100000 E+01$ & $1.8100000 E+01$ & $1.8100000 E+01$ & $1.8100000 \varepsilon+01$ & NONE & WPO35723 & $\mathbf{Y}$ & NA \\
\hline 3256 & 1,3 & CONSTANT & $1.8100000 E+01$ & $1.8100000 E+01$ & $1,8100000 E+01$ & $1.8100000 E+01$ & NONE & WP036766 & $\mathbf{Y}$ & NA \\
\hline 3256 & 1,3 & CONSTANT & $1.8100000 E+01$ & $1.8100000 E+01$ & $1.8100000 E+01$ & $1.8100000 \mathrm{E}+01$ & NONE & WP040521 & $Y$ & N/A \\
\hline 3256 & 1,3 & CONSTANT & $1.8100000 E+01$ & $1.8100000 E+01$ & $1.8100000 E+01$ & $1.8100000 E+01$ & NONE & WPO42263 & $\mathbf{Y}$ & NA \\
\hline 3257 & 4 & CONSTANT & B.0000000E-02 & $8.0000000 E-02$ & $8.0000000 E-02$ & $8.0000000 E-02$ & $\mathrm{~N} / \mathrm{m}$ & CRC-49 & $\mathbf{Y}$ & NA \\
\hline 3257 & 4 & CONSTANT & $8.0000000 E-02$ & $8.0000000 E-02$ & $8.0000000 E-02$ & $8.0000000 E-02$ & $\mathrm{~N} / \mathrm{m}$ & WP036301 & $\mathbf{Y}$ & NA \\
\hline 3257 & 4 & CONSTANT & $8.0000000 E-02$ & $8.0000000 \mathrm{E}-02$ & $8.0000000 \mathrm{E}-02$ & $8.0000000 E-02$ & $\mathrm{~N} / \mathrm{m}$ & WP036766 & $\mathbf{Y}$ & N/A \\
\hline 3257 & 4 & CONSTANT & $8.0000000 E-02$ & $8.0000000 E-02$ & $8.0000000 E-02$ & $8.0000000 E-02$ & $\mathrm{~N} / \mathrm{m}$ & WPO38455 & $\mathbf{Y}$ & NA \\
\hline 3258 & $1,3,5$ & CONSTANT & $3.0000000 \mathrm{E}+02$ & $3.0000000 E+02$ & $3.0000000 \mathrm{E}+\infty 2$ & $3.0000000 \mathrm{E}+02$ & $\mathbf{K}$ & WP036766 & $-Y$ & NA \\
\hline 3259 & 1 & CONSTANT & $1.7000000 E-13$ & $1.7000000 E-13$ & $1.7000000 E-13$ & $1.7000000 \mathrm{E}-13$ & $m^{\wedge} 2$ & WP030921 & $\mathbf{N}$ & 1 \\
\hline 3260 & 1,3 & CONSTANT & $8.4900000 \mathrm{E}-01$ & $8.4900000 E-01$ & $8.4900000 E-01$ & $8,4900000 E-01$ & NONE & WP036766 & $\mathbf{Y}$ & N/A \\
\hline 3260 & 1,3 & CONSTANT & $8.4900000 E-01$ & 8.4900000E-01 & $8.4900000 E-01$ & 8.4900000E-01 & NONE & WP040521 & $\mathbf{Y}$ & N/A \\
\hline 3261 & $1,3,4$ & CONSTANT & $1.5760000 E+04$ & $1.5760000 E+04$ & $1.5760000 E+04$ & $1.5760000 \mathrm{E}+04$ & $m^{\wedge} 2$ & WP034618 & $\mathbf{Y}$ & NA \\
\hline 3261 & $1,3,4$ & CONSTANT & $1.5760000 E+04$ & $1.5760000 \mathrm{E}+04$ & $1.5760000 E+04$ & $1.5760000 E+04$ & $m^{\wedge} 2$ & WPO40521 & $\mathbf{Y}$ & NA \\
\hline 3262 & 1,3 & CUMULATIVE & $1.8000000 E-01$ & $-9.0000000 E-02$ & $-2.0000000 E+00$ & $1.4000000 E+\infty 0$ & NONE & WP035268 & $\mathbf{Y}$ & 1 \\
\hline 3262 & 1,3 & CUMULATIVE & 1.8000000E-01 & $-9.0000000=-02$ & $-2,0000000 E+\infty 0$ & $1.4000000 E+\infty$ & NONE & WPO35835 & $Y$ & 1 \\
\hline
\end{tabular}


CCA-Parameter Listing

\begin{tabular}{|c|c|c|c|c|c|c|c|c|c|c|}
\hline $\begin{array}{l}\text { Permeturla } \\
\text { Detabea? } \\
\text { (YN) }\end{array}$ & 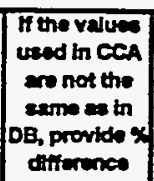 & 10 & Meterid ID & Materid Nam & Parmitar & 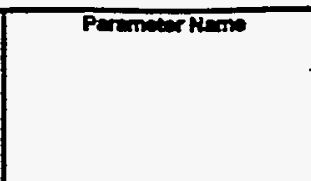 & $\begin{array}{l}\text { PRo D } \\
\text { (Mro })\end{array}$ & $\begin{array}{c}\text { Deta Entry } \\
\text { Dets }\end{array}$ & 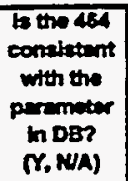 & $\begin{array}{l}\text { Parumiter } \\
\text { Citegory } \\
\text { (footinote 1) }\end{array}$ \\
\hline $\mathbf{Y}$ & & 3262 & SOLAMB & $\begin{array}{l}\text { Solubility Mutiplier for } \\
\text { An+3 }\end{array}$ & SOLSM & $\begin{array}{l}\text { Solubilaty Mull in Salodo Brme. } \\
\text { inoromic Chem Controllod by } \\
\text { Mo(OH)2+HoCO3 }\end{array}$ & $37105 A$ & $24-\mu m y-96$ & $\mathbf{Y}$ & 1 \\
\hline $\mathbf{Y}$ & & 3262 & SOLAMB & $\begin{array}{l}\text { Solubility Muttiplier for } \\
\text { Am+3 }\end{array}$ & SOLSM & 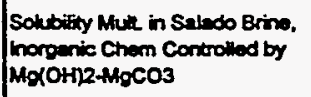 & $37105 A$ & $24+16-96$ & $\mathbf{Y}$ & 1 \\
\hline $\mathbf{Y}$ & & 3262 & SOLANB & $\begin{array}{l}\text { Solubility Mutiplier for } \\
\mathrm{Am}+3\end{array}$ & SOLSaM & $\begin{array}{l}\text { Solubilty Mutt in Salado Brine, } \\
\text { inoromic Chem Controlled by } \\
\mathrm{Mg}(\mathrm{OH}) 2+\mathrm{MgCO}\end{array}$ & $37105 A$ & $24+$ Myy-96 & $\mathbf{Y}$ & 1 \\
\hline $\mathbf{Y}$ & & 3263 & SOLAMB & $\begin{array}{l}\text { Solubility Multiplier for } \\
\text { Am+3 }\end{array}$ & SOLCAN & $\begin{array}{l}\text { Solubity Muth in Castilo Brime, } \\
\text { Inorganic Chem Cortrolted by } \\
\mathrm{Mg}(\mathrm{OH}) 2+\mathrm{HOCO}\end{array}$ & 371064 & $24+40 y-96$ & $\mathbf{Y}$ & 1 \\
\hline $\mathbf{Y}$ & & 3263 & SOLANB & $\begin{array}{l}\text { Solubility Muttiplier for } \\
\text { Am+3 }\end{array}$ & SOLCM & 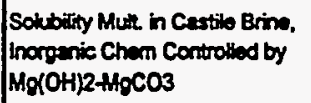 & 371064 & $24+14 y-96$ & $\mathbf{Y}$ & 1 \\
\hline $\mathbf{Y}$ & & 3263 & SOLAMB & $\begin{array}{l}\text { Solubility Multiplier for } \\
\text { Am+3 }\end{array}$ & SOLCM & 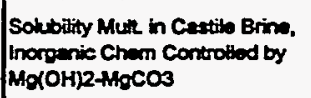 & $37106 \mathrm{~A}$ & $24+4 m y-96$ & $\mathbf{Y}$ & 1 \\
\hline $\mathbf{Y}$ & & 3263 & SOLAM3 & $\begin{array}{l}\text { Sohubility Muttiplier for } \\
\mathrm{Am}+3\end{array}$ & SOLCAM & 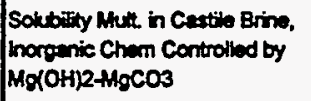 & $37106 A$ & $24 M x y-96$ & $\mathbf{Y}$ & 1 \\
\hline $\mathbf{Y}$ & & $\stackrel{\cdot}{3263}$ & SOLAM3 & $\begin{array}{l}\text { Solubility Murtiplior for } \\
\text { Am+3 }\end{array}$ & SOLCM & $\begin{array}{l}\text { Solubifty Muth in Castio Brine. } \\
\text { Inorganic Chem Controlled by } \\
\text { Mg(OH)2 } 1 \mathrm{HCO} 3\end{array}$ & $37106 A$ & $24-M+y-96$ & $\mathbf{Y}$ & 1 \\
\hline $\mathbf{Y}$ & & 3264 & SOLPU3 & $\begin{array}{l}\text { Solubility Multiplier for } \\
\text { Put3 }\end{array}$ & SOLCIM & $\begin{array}{l}\text { Solubility Mult in Cestile Brine. } \\
\text { Inorganc Chem Controlled by } \\
\text { Mg(OH)2HgCO3 }\end{array}$ & $37108 A$ & $24-\operatorname{may}-96$ & $\mathbf{Y}$ & 1 \\
\hline $\mathbf{Y}$ & & 3264 & SOLPUS & $\begin{array}{l}\text { Solubility Muttiplier for } \\
\text { Pu+3 }\end{array}$ & SOLCIM & 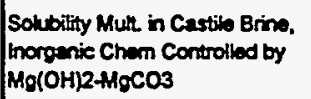 & $37108 A$ & $24+4 a y-96$ & $\mathbf{Y}$ & 1 \\
\hline $\mathbf{Y}$ & & 3264 & SOLPU3 & $\begin{array}{l}\text { Solubitity Muttiplier for } \\
\text { Put3 }\end{array}$ & SOLCIM & $\begin{array}{l}\text { Solubility Mult in Castile Brine, } \\
\text { Inorganic Chem Controlled by } \\
\mathrm{Mg}(\mathrm{OH}) 2 \mathrm{HgCO}_{2}\end{array}$ & $37108 A$ & $24+1=y-96$ & $\mathbf{Y}$ & 1 \\
\hline $\mathbf{Y}$ & & 3264 & SOLPU3 & $\begin{array}{l}\text { Solubility Multipler for } \\
\text { Pu+3 }\end{array}$ & SOLCIM & $\begin{array}{l}\text { Solubility Mult in Castile Brivo, } \\
\text { thorganic Chem Controlled by } \\
\text { Mg(OH)2-MgCO3 }\end{array}$ & $37108 A$ & $24+18 y-96$ & $\mathbf{Y}$ & 1 \\
\hline $\mathbf{Y}$ & & 3264 & SOLPU3 & $\begin{array}{l}\text { Solubility Multiplier for } \\
\text { Put3 }\end{array}$ & SOLCM & $\begin{array}{l}\text { Solubility Mult in Cestile Brine, } \\
\text { Inorganic Chem Controlled by } \\
\text { Mg(OH)2MgCO3 }\end{array}$ & $37108 \mathrm{~A}$ & 24 Mry-96 & Y & 1 \\
\hline $\mathbf{Y}$ & & 3265 & SOLPU3 & $\begin{array}{l}\text { Solubitity Mutipher for } \\
\text { Pu+3 }\end{array}$ & SOLSIM & $\begin{array}{l}\text { Solubilty Mult in Saludo Brine, } \\
\text { Inorganic Chem Controlied by } \\
\mathrm{Mg}(\mathrm{OH}) 2+\mathrm{MgCO} 3\end{array}$ & $37109 \mathrm{~A}$ & 24 May-96 & $\mathbf{Y}$ & 1 \\
\hline$Y$ & & 3265 & SOLPU3 & $\begin{array}{l}\text { Solubility Mutiplier for } \\
\text { Pu+3 }\end{array}$ & SOLSIM & $\begin{array}{l}\text { Solubility Muth in Salado Brine. } \\
\text { inorganic Chem Controlled by } \\
\text { Mg(OH)2 } 2 \mathrm{ggCO} 3\end{array}$ & 37109A & $24+4 m y-96$ & $\mathbf{Y}$ & 1 \\
\hline $\mathbf{Y}$ & & 3265 & SOLPU3 & $\begin{array}{l}\text { Solubity Mutipiper for } \\
\text { Put3 }\end{array}$ & SOLSM & $\begin{array}{l}\text { Solubilty Mul in Salado Brina. } \\
\text { inorganic Cham Controlled by } \\
\mathrm{Mg}(\mathrm{OH}) 2+\mathrm{HgO} 3\end{array}$ & $37109 A$ & 24 Aау-86 & $\mathbf{Y}$ & 1 \\
\hline $\mathbf{Y}$ & & 3265 & SOLPUS & $\begin{array}{l}\text { Solubitity Muttipliar for } \\
\text { Put3 }\end{array}$ & SOLSM & $\begin{array}{l}\text { Sotubility Muk in Saledo Brin, } \\
\text { lnorganic Chem Controllod by } \\
\text { Mg(OH)2HgCO3 }\end{array}$ & $37109 \mathrm{~A}$ & $24+4 x y-96$ & $\mathbf{Y}$ & 1 \\
\hline $\mathbf{Y}$ & & 3266 & SOLPU4 & $\begin{array}{l}\text { Solubitty Multiplier for } \\
\text { Pund }\end{array}$ & SOLSM & $\begin{array}{l}\text { Solubilty Muth in Selsdo Brim, } \\
\text { thorganic Chem Controlled by } \\
\text { Mg(OH)2MgCO3 }\end{array}$ & 371104 & 24+4ay-96 & $\mathbf{Y}$ & 1 \\
\hline
\end{tabular}


CCA Parameter Listing

\begin{tabular}{|c|c|c|c|c|c|c|c|c|c|c|}
\hline 10 & 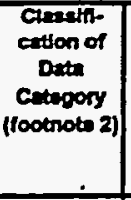 & $\begin{array}{l}\text { Deteribution } \\
\text { Typo }\end{array}$ & $\operatorname{man}$ & Medien & Minkrom & $\begin{array}{l}\text { Hodinum } \\
\text { - }\end{array}$ & Units & Roternece tD & 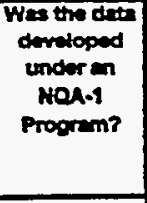 & $\begin{array}{c}\text { Which } \\
\text { mothode } \\
\text { wore used to } \\
\text { qually } \\
\text { extoting } \\
\text { drtin? } \\
\text { (footnote 3) }\end{array}$ \\
\hline 3262 & 1.3 & CUMULATINE & $1.8000000 E-01$ & $-9.0000000 E-02$ & $-20000000 E+\infty \mid$ & $1.4000000 E+\infty 0$ & NONE & WP037536 & $\mathbf{Y}$ & 1 \\
\hline 3262 & 1,3 & CUMULATTEE & $1.8000000 E-01$ & $-9.0000000=-02$ & $-2.0000000 E+\infty$ & $1.4000000 E+\infty$ & NONE & WP037545 & $Y$ & 1 \\
\hline 3262 & 1,3 & CUMULATINE & $1.8000000 E-01$ & $-9.0000000 E-02$ & $-20000000 E+\infty 0$ & $1.4000000 E+\infty 0$ & NONE & WP040512 & Y & 1 \\
\hline 3263 & 1,3 & CUMULATINE & $1.8000000 E-01$ & $-9.0000000 E-02$ & $-20000000 E+\infty 0$ & $1.4000000 E+\infty 0$ & NONE & WP035268 & $\mathbf{Y}$ & 1 \\
\hline 3263 & 1,3 & CUMULATIVE & $1.8000000 E-01$ & $-9.0000000 E-02$ & $-20000000 E+\infty 0$ & $1.4000000 E+\infty$ & NONE & WP035835 & $\mathbf{Y}$ & 1 \\
\hline 3263 & 1,3 & CUMULATIVE & $1.8000000 E-01$ & $-9.0000000 E-02$ & $-2.0000000 E+\infty 0$ & $1.4000000 E+\infty 0$ & NONE & WP037536 & $\mathbf{Y}$ & 1 \\
\hline 3263 & 1,3 & CUMULATIVE & $1.8000000 E-01$ & $-9.0000000 E-02$ & $-2.0000000 E+\infty 0$ & $1.4000000 E+00$ & NONE & WP037545 & $\mathbf{Y}$ & 1 \\
\hline 3263 & 1,3 & CUMULATIVE & $1.8000000 E-01$ & $-9.0000000 E-02$ & $-2.0000000 E+\infty 0$ & $1.4000000 E+00$ & NONE & WP040512 & $\mathbf{Y}$ & 1 \\
\hline 3264 & 1.3 & CUMULATINE & $1.8000000 E-01$ & $-9.0000000 E-02$ & $-2.0000000 E+\infty 0$ & $14000000 E+\infty$ & NONE & WP035268 & $\mathbf{Y}$ & 1 \\
\hline 3264 & 1,3 & CUMULATIVE & $1.6000000 E-01$ & $-9.0000000 E-02$ & $-2.0000000 E+\infty 0$ & $14000000 E+00$ & NONE & WP035835 & $\mathbf{Y}$ & 1 \\
\hline 3264 & 1,3 & CUMULATNE & $1.8000000 E-01$ & $-9.0000000 E-02$ & $-20000000 E+\infty 0$ & $14000000=+\infty$ & NONE & WP037536 & $\mathbf{Y}$ & 1 \\
\hline 3264 & 1.3 & CUMULATIVE & 1.8000000E- -01 & $-90000000 E-02$ & $-20000000 E+\infty 0$ & $14000000+\infty 0$ & NONE & WP037545 & $\mathbf{Y}$ & 1 \\
\hline 3264 & 1,3 & CUMULATNE & $1.8000000 E-01$ & $-8.0000000 E-02$ & $-20000000 \in+\infty$ & $10000000=+\infty$ & NONE & WP040512 & $\gamma$ & 1 \\
\hline 3265 & 1,3 & CUMULATIVE & $1.8000000 E-01$ & $-9.0000000 E-02$ & $-200000000 \times-\infty 0$ & $10000000+\infty$ & NONE & WP035268 & $\mathbf{Y}$ & 1 \\
\hline 3265 & 1,3 & CUMULATME & $1.8000000 E-01$ & $-90000000 E-02$ & $-20000000 \times \infty 00$ & $1+000000 E+\infty$ & NONE & WP035835 & $\mathbf{Y}$ & 1 \\
\hline 3265 & 1,3 & CUMULATIVE & $1.8000000 E-01$ & $-900000000-02$ & $20000000=-\infty$ & $1+000000 E+\infty$ & NONE & WP037536 & $\mathbf{Y}$ & 1 \\
\hline 3265 & 1,3 & CUMULATNE & $1.8000000 E-01$ & $-90000000 E-02$ & $-20000000 E+\infty$ & $14000000 E+\infty 0$ & NONE & WP040512 & $\mathbf{Y}$ & 1 \\
\hline 3266 & 1,3 & CUMULATIVE & $1.8000000 E-01$ & $-90000000 E-02$ & $-200000006+\infty$ & $14000000 E+\infty$ & NONE & WP035268 & $\mathbf{Y}$ & 1 \\
\hline
\end{tabular}


CCA Parameter-Listing

\begin{tabular}{|c|c|c|c|c|c|c|c|c|c|c|}
\hline $\begin{array}{c}\text { Parrinetion in } \\
\text { Detibew? } \\
\text { (MNIN) }\end{array}$ & 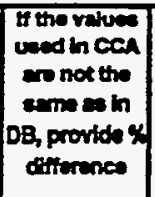 & 10 & Materdat iD & 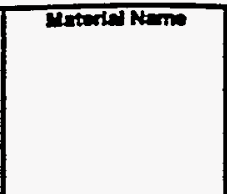 & $\begin{array}{c}\text { Permeter } \\
\text { D }\end{array}$ & Parnment Mersos & $\begin{array}{l}\text { PRPD } \\
\text { (MPOA) }\end{array}$ & $\begin{array}{c}\text { Don Entry } \\
\text { Den }\end{array}$ & 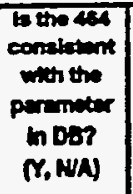 & $\begin{array}{l}\text { Pernintar } \\
\text { Catiogony } \\
\text { (footnotio 1) }\end{array}$ \\
\hline $\mathbf{Y}$ & & 3266 & SOLPUA & $\begin{array}{l}\text { Solubitay Mutiplier for } \\
\text { Putd }\end{array}$ & SOLSDM & 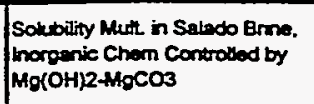 & $37110 \mathrm{~A}$ & 24 uny-s6 & $Y$ & 1 \\
\hline$Y$ & & 3266 & SOLPUA & $\begin{array}{l}\text { Solubility Mutiolier for } \\
\text { Put4 }\end{array}$ & SOLSM & 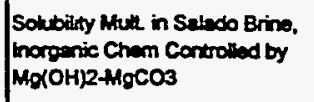 & 371104 & $2444 x y-96$ & $Y$ & 1 \\
\hline $\mathbf{Y}$ & & 3266 & SOLPU4 & $\begin{array}{l}\text { Salubilty Mutiplior for } \\
\text { Puth }\end{array}$ & SOLSIM & 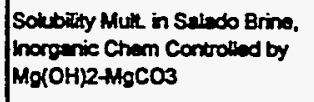 & 371104 & $24+k+y-96$ & $\mathbf{Y}$ & 1 \\
\hline $\mathbf{Y}$ & & 3266 & SOLPUA & $\begin{array}{l}\text { Solubitity Mutipliar for } \\
\text { Put4 }\end{array}$ & SOLSM & $\begin{array}{l}\text { Solubitity Muth in Salado Brine, } \\
\text { inorganic Chem Controlled by } \\
\text { Mo(OH)2 MgCO3 }\end{array}$ & 371104 & 24 Mary-96 & $\mathbf{Y}$ & 1 \\
\hline$Y$ & & 3267 & AC225 & Actinium 225 & HALFLIFE & Hallifo & 35795 & 16-Apr-96 & $\mathbf{Y}$ & 3 \\
\hline$Y$ & & 3267 & AC2225 & Actinium 225 & HALFLIFE & Halnifo & 36795 & 16-Apr-96 & $Y$ & 3 \\
\hline $\mathbf{Y}$ & & 3268 & $A C 227$ & Actiniem 227 & HALFUFE & Halnife & 36796 & 16-Apr-96 & $Y$ & 3 \\
\hline $\bar{Y}$ & & 3268 & $A C 227$ & Actinium 227 & HALFLIFE & Hanite & 36796 & 16-Apr-96 & $\bar{Y}$ & 3 \\
\hline$Y$ & & 3269 & AC228 & Actinium 228 & HALFUFE & Hanife & 36797 & 16-Apr-96 & $Y$ & 3 \\
\hline$Y$ & & 3269 & AC228 & Actinium 228 & HALFUFE & Halnito & 35797 & 16 Apr-86 & $Y$ & 3 \\
\hline$Y$ & & 3270 & AT217 & Astatine 217 & HALFLIFE & Hanite & 36799 & 16-Apr-96 & $Y$ & 3 \\
\hline$Y$ & & 3270 & AT217 & Astatne 217 & HALFLIFE & Hantifo & 36799 & 16 -Apr-96 & $\mathbf{Y}$ & 3 \\
\hline$Y$ & & 3271 & BA137 & Beriem 137 & HALFUFE & Halfito & 36800 & 16-1pr-96 & $Y$ & 3 \\
\hline$Y$ & & 3271 & BA137 & Barim 137 & HALFLIFE & Halnifo & 36800 & $16-4 x-96$ & $Y$ & 3 \\
\hline $\mathbf{Y}$ & & 3272 & BA137M & Barium 137 Motastablo & HALFLIFE & Hanifo & 36801 & 16-Apr-96 & $\mathbf{Y}$ & 3 \\
\hline Y & & 3272 & BA137M & Barium 137 Motastable & HALFLIFE & Halnife & 36801 & 16 -Apr-96 & $\mathbf{Y}$ & 3 \\
\hline$Y$ & & 3273 & 81211 & Bismuth 211 & HALFUFE & Hamifo & 36803 & 16-Apr-96 & $Y$ & 3 \\
\hline$Y$ & & 3273 & B1211 & Bismuth 211 & HALFLIFE & Hamfo & 36803 & $16-4 p r-96$ & $\bar{Y}$ & 3 \\
\hline$Y$ & & 3274 & $B 1212$ & Bismutin 212 & HALFUFE & Halnifo & 36804 & 16-Apr-96 & $Y$ & 3 \\
\hline $\bar{Y}$ & & 3274 & 81212 & Bismuth 212 & HALFLIFE & Haindo & 36804 & 16-Apr-96 & $\bar{Y}$ & 3 \\
\hline$Y$ & & 3275 & $B 1213$ & Bismuth 213 & HALFUFE & Halifite & 36806 & 16-Apr-96 & $Y$ & 3 \\
\hline $\bar{Y}$ & & 3275 & B1213 & Bismuth 213 & HALFLIFE & Hahtide & 30806 & 16 Apr-96 & $\bar{Y}$ & 3 \\
\hline$Y$ & & 3276 & B1214 & Bisenuth 214 & HALFLIFE & Halfitio & 36807 & 16 Apr.96 & $Y$ & 3 \\
\hline$Y$ & & 3276 & $B 1214$ & Biamith 214 & HALFUFE & Halitite & 36807 & 16-Apr-96 & $Y$ & 3 \\
\hline$Y$ & .100 & 3277 & CM1243 & Curium 243 & HALFLIFE & Hantite & 36809 & 16-Apr-96 & $Y$ & 3 \\
\hline$Y$ & 100 & 3277 & CH243 & Curium 243 & HALFLIFE & Hainto & 36809 & 16 Apr.96 & $Y$ & 3 \\
\hline$Y$ & 100 & 3278 & CM245 & Curium 245 & HALFLIFE & Manto & 36811 & 16-Apr-96 & $Y$ & 3 \\
\hline$Y$ & 100 & 3278 & CM1245 & Curium 245 & HALFLIFE & Hemfo & 36811 & 16-Apr-96 & $Y$ & 3 \\
\hline $\mathbf{Y}$ & & 3279 & FR221 & Francaum 221 & HALFLIFE & Heinfo & 36812 & $16-A p r-96$ & $Y$ & 3 \\
\hline$Y$ & & 3280 & ND143 & Noodymium 143 & HULFLIFE & Hanto & 36813 & 16-Apr-96 & $Y$ & 3 \\
\hline$Y$ & & 3281 & NP239 & Neptuniem 239 & HALFLIFE & Hainto & 36815 & 16 -Apr-96 & $Y$ & 3 \\
\hline$Y$ & & 3282 & PA233 & Protuctiniem 233 & HALFLIFE & Mente & 36828 & 16-Apr-96 & $Y$ & 3 \\
\hline $\mathbf{Y}$ & & 3283 & PA234M & $\begin{array}{l}\text { Protictinum } 234 \\
\text { Motastablo }\end{array}$ & HALFLIFE & Hanto & 36829 & $16-A p r-86$ & $\mathbf{Y}$ & 3 \\
\hline$Y$ & & 3284 & PB209 & Leed 209 & HULLLIFE & Hanco & 36830 & $16-4 p r+86$ & $\mathbf{Y}$ & 3 \\
\hline$Y$ & & 3285 & PB211 & Leed 211 & HALFUFE & Halnife & 36831 & 16-Apr-96 & $Y$ & 3 \\
\hline$Y$ & & 3286 & PB212 & Lead 212 & HULFLIFE & Hente & 36832 & 16 Apr.96 & $Y$ & 3 \\
\hline $\mathbf{Y}$ & & 3287 & PB214 & Loed 214 & HULFLIFE & Kante & 36833 & 16-Apr-96 & $Y$ & 3 \\
\hline$Y$ & & 3288 & PO212 & Polonium 212 & HALFLIFE & Hante & 36834 & $16-\wedge p r-96$ & $y$ & 3 \\
\hline$Y$ & & 3289 & $P 0213$ & Potonium 213 & HALFLIFE & Henifo & 36835 & 16-Apr-98 & $Y$ & 3 \\
\hline$Y$ & & 3290 & PO214 & Potonium 214 & HALFLIFE & Hamte & 36836 & 16-Apr-s6 & $y$ & 3 \\
\hline$Y$ & & 3291 & PO215 & Potonium 215 & HALFLIFE & Hente & 36837 & $16-A p r-96$ & $Y$ & 3 \\
\hline$y$ & & 3292 & PO216 & Polonum 216 & HMLFUFE & Hanife & 36038 & $16-1 p x-96$ & $y$ & 3 \\
\hline$Y$ & & 3293 & PO218 & Potonium 218 & HALFUFE & Hanide & 36839 & $16-4 p r-86$ & $Y$ & 3 \\
\hline$Y$ & & 3294 & RA223 & Radium 223 & HWLFLIFE & Haluifo & 36840 & 16-Apr-96 & $Y$ & 3 \\
\hline$Y$ & & 3295 & RA224 & Radium 224 & HWLFLIFE & Halinte & 36841 & 16-Apr-86 & $Y$ & 3 \\
\hline
\end{tabular}


CCA Parameter Listing

\begin{tabular}{|c|c|c|c|c|c|c|c|c|c|c|}
\hline WD & 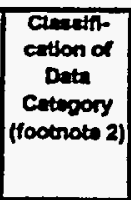 & $\begin{array}{l}\text { Dofortubution } \\
\text { Type }\end{array}$ & $\operatorname{man}$ & Modien & Mintrient & mexirnum & Whits & Refinence if & $\begin{array}{l}\text { Wes ore deta } \\
\text { dombloped } \\
\text { underen } \\
\text { NoA-1 } \\
\text { Progrem? }\end{array}$ & 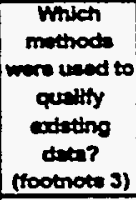 \\
\hline 3266 & 1,3 & CUMULATIVE & $1.8000000 E-01$ & $-9.0000000 E-02$ & $-20000000 E+\infty 0$ & $1.4000000 E+\infty 0$ & NONE & WP035835 & $\mathbf{Y}$ & $i$ \\
\hline 3266 & 1,3 & CUMULATIVE & $1.8000000 E-01$ & $-9.0000000 E-02$ & $-2,0000000 E+\infty 0$ & $1.4000000 E+\infty 0$ & HONE & WP037536 & $\mathbf{Y}$ & 1 \\
\hline 3266 & 1,3 & CUMULATIVE & $1.8000000 E-0\}$ & $-9.0000000 E-02$ & $-20000000 E+\infty 0$ & $1.40000000+\infty 0$ & NONE & WP037545 & $\mathbf{Y}$ & 1 \\
\hline 3266 & 1,3 & CUMULATNE & $1.8000000 E-01$ & $-9.0000000 E-02$ & $-2,0000000 E+\infty 0$ & $1.4000000 E+\infty$ & NONE & WP040512 & $\mathbf{Y}$ & 1 \\
\hline 3267 & 4 & CONSTANT & $8.6400000 \mathrm{E}+0.5$ & $8.6400000 E+05$ & $8.6400000 E+05$ & $8.6400000 E+05$ & 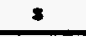 & WP036301 & $\gamma$ & NA \\
\hline 3257 & 4 & CONSTANT & $8.6400000 \mathrm{E}+05$ & $8.6400000 E+05$ & $8.6400000 E+05$ & $8.6400000 E+05$ & $s$ & WP037404 & $\mathbf{Y}$ & NA \\
\hline 3268 & 4 & CONSTANT & $6.8710000 \mathrm{E}+08$ & $6.8710000 E+08$ & $6.8710000 E+\infty 8$ & $6.8710000 E+\infty$ & 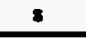 & WP036301 & $Y$ & NA \\
\hline 3268 & 4 & CONSTANT & $6.8710000 E+08$ & $6.8710000 \mathrm{E}+08$ & $6.8710000 E+08$ & $6.8710000 E+08$ & 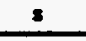 & WP037404 & $Y$ & NA \\
\hline 3269 & 4 & CONSTANT & $2.2070000 E+04$ & $2.2070000 \mathrm{E}+04$ & $2.2070000 E+04$ & $22070000 E+04$ & s & WP035298 & $Y$ & NA \\
\hline 3269 & 4 & CONSTANT & $2.2070000 \mathrm{E}+0.4$ & $2.2070000 \mathrm{E}+04$ & $22070000 E+04$ & $2.20700000+04$ & $s$ & WP037404 & $Y$ & NA \\
\hline 3270 & 4 & CONSTANT & $3.2300000 \mathrm{E}-02$ & $3.2300000 \mathrm{E}-02$ & $3.2300000 E-02$ & $3.2300000 E-02$ & $s$ & WP035298 & $Y$ & NA \\
\hline 3270 & 4 & CONSTANT & $3.2300000 E-02$ & $3.2300000 E-02$ & $3.2300000 \mathrm{E}-02$ & $3.2300000 \mathrm{E}-02$ & 8 & WPO37404 & $Y$ & NA \\
\hline 3271 & 4 & CONSTANT & $1.0000000 E+38$ & $1.0000000 E+38$ & $1.0000000 E+38$ & $1.0000000 E+38$ & 8 & WP036298 & $Y$ & NA \\
\hline 3271 & 4 & CONSTANT & $1.0000000 \mathrm{E}+38$ & $1.0000000 \mathrm{E}+38$ & $1.0000000 E+38$ & $1.0000000 E+3 \theta$ & 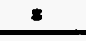 & WP037404 & $Y$ & NA \\
\hline 3272 & 4 & CONSTANT & $1.5310000 E+02$ & $1.5310000 E+02$ & $1.5310000 E+02$ & $1.5310000 E+02$ & $=$ & WP036298 & Y & N/A \\
\hline 3272 & 4 & CONSTANT & $1.5310000 \mathrm{E}+02$ & $1.5310000 E+02$ & $1.5310000 E+02$ & $1.5310000 E+02$ & 5 & WP037404 & $Y$ & NA \\
\hline 3273 & 4 & CONSTANT & $1.2780000 E+02$ & $1.2780000 E+02$ & $1.2780000 E+02$ & $1.2780000 E+02$ & $=$ & WP035298 & $\mathbf{Y}$ & NA \\
\hline 3273 & 4 & CONSTANT & $1.2780000 E+02$ & $1.2780000 E+02$ & $1.2780000 E+02$ & $1.2780000 E+02$ & $=$ & WP037404 & $\mathbf{Y}$ & NA \\
\hline 3274 & 4 & CONSTANT & $3.6330000 \mathrm{E}+03$ & $3.6330000 \mathrm{E}+03$ & $3.6330000 \mathrm{E}+03$ & $3.6330000 E+03$ & $s$ & WP035298 & $Y$ & NA \\
\hline 3274 & 4 & CONSTANT & $3.6330000 E+03$ & $3.63300000+03$ & $3.6330000 E+03$ & $3.6330000 \mathrm{E}+03$ & - & WP037404 & $Y$ & NA \\
\hline 3275 & 4 & CONSTANT & $2.7390000 \mathrm{E}+03$ & $2.7390000 \mathrm{E}+03$ & $2.7390000 E+03$ & $2.7390000 \mathrm{E}+03$ & 3 & WP036298 & $Y$ & NA \\
\hline 3275 & 4 & CONSTANT & $27390000 \mathrm{E}+03$ & $2.7390000 E+03$ & $2.7390000 E+03$ & $2.7390000 E+03$ & $s$ & WP037404 & $Y$ & N/A \\
\hline 3276 & 4 & CONSTANT & $1.1940000 \mathrm{E}+03$ & $1.1940000 \mathrm{E}+03$ & $1.1940000 E+03$ & $1.1940000 \mathrm{E}+03$ & 3 & WP036301 & $Y$ & NA \\
\hline 3276 & 4 & CONSTANT & $1.1940000 \mathrm{E}+03$ & $1.1940000 \mathrm{E}+03$ & $1.1940000 E+03$ & $1.1940000 \mathrm{E}+03$ & 3 & WP037404 & $Y$ & NA \\
\hline 3277 & 4 & CONSTANT & $8.9940000 E+08$ & $8.9940000 E+06$ & $8.9940000 E+08$ & $8.9940000 \mathrm{E}+00$ & 3 & WP037404 & $\mathbf{Y}$ & N/A \\
\hline $32 \pi 7$ & 4 & CONSTANT & $8.9940000 E+08$ & $8.9940000 \mathrm{E}+08$ & $8.9940000 E+08$ & $8.9940000 E+08$ & 8 & WPO40434 & $Y$ & NAA \\
\hline 3278 & 4 & CONSTANT & $2.6820000 E+11$ & $2.6820000 \mathrm{E}+11$ & $2.6820000 E+11$ & $2.6820000 \mathrm{E}+11$ & 3 & WP037404 & $Y$ & NA \\
\hline 3278 & 4 & CONSTANT & $2.6820000 E+11$ & $2.6820000 \mathrm{E}+11$ & $2.6820000 E+11$ & $26820000 E+11$ & 3 & WPO40434 & $Y$ & NA \\
\hline 3279 & 4 & CONSTANT & $2.8800000 E+02$ & $2.8800000 \mathrm{E}+02$ & $2.8800000 E+02$ & $2.8800000 \mathrm{E}+02$ & $s$ & WP037404 & $\mathbf{Y}$ & NA \\
\hline $32 B O$ & 4 & CONSTANT & $1.0000000 \mathrm{E}+38$ & $1.0000000 \mathrm{E}+38$ & $1.0000000 E+38$ & $1.0000000 E+38$ & 3 & WP037404 & $Y$ & NA \\
\hline 3281 & 4 & CONSTANT & $2.0350000 E+05$ & $2.0350000 E+05$ & $2.0350000 E+05$ & $2.0350000 \mathrm{E}+0.5$ & $s$ & WP037404 & $\mathbf{Y}$ & NA \\
\hline 3282 & 4 & CONSTANT & $2.3330000 E+06$ & $23330000 E+06$ & $2.3330000 E+06$ & $23330000 E+06$ & $=$ & WP03740 & $\mathbf{Y}$ & NA \\
\hline 3283 & 4 & CONSTANT & $7.0200000 E+01$ & $7.02000000+01$ & $7.0200000 E+01$ & $7.00200000 E+01$ & s & WP037404 & $\mathbf{Y}$ & N/A \\
\hline 3284 & 4 & CONSTANT & $1.1880000 \mathrm{E}+0 \mathrm{~A}$ & $1.1880000 E+04$ & $1.1880000 E+04$ & $1.1880000 E+04$ & 3 & WP037404 & $\mathbf{Y}$ & N/A \\
\hline 3285 & 4 & CONSTANT & $2.16600005+03$ & $2.1660000 E+03$ & $2.1660000 \mathrm{E}+03$ & $2.1660000 E+03$ & $=$ & WP037404 & $\mathbf{Y}$ & $\mathrm{NuA}$ \\
\hline 3286 & 4 & CONSTANT & $3.8300000 \mathrm{E}+0 \mathrm{~A}$ & $3.8300000 \mathrm{E}+0 \mathrm{~s}$ & $3.8300000 \mathrm{E}+0 \mathrm{~A}$ & $3.8300000 E+04$ & s & WP037404 & $\mathbf{Y}$ & NA \\
\hline 3287 & 4 & CONSTANT & $1.6000000 E+00$ & $1.6000000 \mathrm{E}+03$ & $1.6080000 E+03$ & $1.6030000 \mathrm{E}+03$ & $s$ & WP037404 & $\mathbf{Y}$ & NAA \\
\hline 3288 & 4 & CONSTANT & $3.0000000 E-07$ & $3.0000000 \mathrm{E}-07$ & $3.0000000 E-07$ & 3.0000000 E- 07 & 8 & WP037404 & $\mathbf{Y}$ & NA \\
\hline 3289 & 4 & CONSTANT & 4.2000000E-06 & 4.2000000E- -06 & 4.2000000E-06 & $4.2000000 E-06$ & $s$ & WP037404 & $Y$ & NA \\
\hline 3290 & 4 & CONSTANT & $1.6430000 E-04$ & $1.6430000 E-04$ & 1.6430000E-04 & $1.6430000 E-01$ & $s$ & WP037404 & $Y$ & NA \\
\hline 3291 & 4 & CONSTANT & $1.7800000 \mathrm{E}-03$ & $1.7800000 E-03$ & $1.78000000-\infty 3$ & $1.7800000 E-03$ & $=$ & WP037404 & $\gamma$ & NAA \\
\hline 3292 & 4 & CONSTANT & $1.5000000 E-01$ & $1.5000000 E-01$ & $1.5000000 E-01$ & $1.5000000 E-01$ & 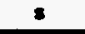 & WP037404 & $\mathbf{Y}$ & NA \\
\hline 3293 & 4 & CONSTANT & $1.8300000 \mathrm{E}+02$ & $1.8300000 E+02$ & $1.8300000 E+02$ & $1.8300000 E+02$ & $s$ & WP037404 & $Y$ & NA \\
\hline 3294 & 4 & CONSTANT & $9.8790000 E+05$ & $9.8790000 E+0.5$ & $9.8790000 E+05$ & $9.8790000 E+05$ & 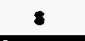 & WP037404 & $\mathbf{Y}$ & NA \\
\hline 3295 & 4 & CONSTANT & $3.1620000 \mathrm{E}+0.5$ & $3.1620000 \mathrm{E}+05$ & $3.1620000 \mathrm{E}+05$ & $3.1620000 \mathrm{E}+05$ & $\mathrm{~s}$ & WP037404 & $\mathbf{Y}$ & NA \\
\hline
\end{tabular}


CCA Parameter-tisting-

\begin{tabular}{|c|c|c|c|c|c|c|c|c|c|c|}
\hline $\begin{array}{c}\text { Permenth } \\
\text { Detabese? } \\
\text { (YN) }\end{array}$ & 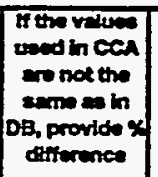 & tD & Metardio & Mrtorian $N=10$ & $\begin{array}{c}\text { Paremetar } \\
\text { to }\end{array}$ & Parmecer itars & $\begin{array}{l}\text { PRPDO } \\
\text { MPOA }\end{array}$ & $\begin{array}{c}\text { Dete Entry } \\
\text { Dot }\end{array}$ & 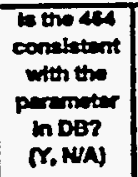 & $\begin{array}{l}\text { Permeter } \\
\text { Cetegory } \\
\text { (footinote 1) }\end{array}$ \\
\hline $\bar{Y}$ & & 3296 & RA225 & Radiem 225 & HALFLIFE & Haltifo & 36842 & 16 -apr-96 & $Y$ & 3 \\
\hline$Y$ & & 3297 & RN219 & Radon 219 & HALFLIFE & Hainfe & 36843 & 16-Apr-96 & $Y$ & 3 \\
\hline $\bar{Y}$ & & 3298 & RND20 & Radon 220 & HALFLIFE & Hamite & 36844 & 16-Apr-96 & $\bar{Y}$ & 3 \\
\hline $\bar{Y}$ & & 3299 & RN2222 & Redon 222 & HALFLIFE & Halinito & 36845 & $16-A p x-96$ & $Y$ & 3 \\
\hline$Y$ & & 3300 & TH1227 & Thorium 227 & HALFLIFE & Hanite & 36846 & $16-4 x-96$ & $Y$ & 3 \\
\hline $\mathbf{Y}$ & & 3301 & 71228 & Thorium 228 & HALFLIFE & Hainto & 36847 & 16-Apr.96 & $Y$ & 3 \\
\hline$Y$ & & 3302 & THE231 & Thorium 231 & HALFLIFE & Heifito & 36848 & 16-Apr-96 & $\bar{Y}$ & 3 \\
\hline $\bar{Y}$ & & 3303 & THE234 & Thorum 234 & HALFUFE & Mantife & 36849 & 16-Apr-96 & $\bar{Y}$ & 3 \\
\hline$Y$ & & 3304 & $\pi 207$ & Thalliem 207 & HALFUFE & Heinifo & 36850 & 16-Apr-56 & $Y$ & 3 \\
\hline$Y$ & & 3305 & Y90 & Yutrixm 90 & HALFLIFE & Heafitite & 36851 & 16-4pr-96 & $\bar{Y}$ & 3 \\
\hline$Y$ & & 3306 & ZR9O & Zreoniem 90 & HALFUFE & Halitite & 36852 & $16-1 p x-96$ & $Y$ & 3 \\
\hline $\mathbf{Y}$ & & 3307 & $u$ & Urenium & CONCINT & $\begin{array}{l}\text { Actinide Concentration with } \\
\text { Mobito Actinide intrinstic Colloids }\end{array}$ & 36854 & $16-A x-96$ & $\mathbf{Y}$ & 1 \\
\hline$Y$ & & 3307 & $u$ & Uranium & CONCINT & $\begin{array}{l}\text { Actinide Concantration with } \\
\text { Mobil Actinide Intrinatic Colbids }\end{array}$ & 36854 & 16-Apr-96 & $\mathbf{Y}$ & 1 \\
\hline$Y$ & & 3308 & u & Uraniem & CAPMIC & $\begin{array}{l}\text { Maximum Concontration of } \\
\text { Actinide on Mierobe Cossoids }\end{array}$ & 36855 & 16 -Apr-96 & $\mathbf{Y}$ & 1 \\
\hline $\mathbf{Y}$ & & 3300 & u & Urenium & CAPMIC & $\begin{array}{l}\text { Maximum Concentration of } \\
\text { Actinide on Mierobe Colloids }\end{array}$ & 36855 & $16-4 x-96$ & $\mathbf{Y}$ & 1 \\
\hline$Y$ & & 3309 & u & Uranium & PROPMIC & $\begin{array}{l}\text { Molos of Actinide Mobilized on } \\
\text { Microbe Colbids per Moles } \\
\text { Dissolved }\end{array}$ & 36856 & 24-May-96 & $\mathbf{Y}$ & 1 \\
\hline $\mathbf{Y}$ & & 3309 & u & Uranium & PROPMIC & $\begin{array}{l}\text { Moles of Actinido Mobilized on } \\
\text { Mierobe Colloids per Molos } \\
\text { Dissolved }\end{array}$ & 36856 & $24+4 a y-96$ & $\mathbf{Y}$ & 1 \\
\hline $\mathbf{Y}$ & & 3309 & u & Ureniem & PROPMIC & $\begin{array}{l}\text { Moles of Actinide Mobilzed on } \\
\text { Microbe Colloids per Moles } \\
\text { Diesolved }\end{array}$ & 36856 & 24 May-96 & $Y$ & 1 \\
\hline $\mathbf{Y}$ & & 3310 & AM & Americium & CONCINT & $\begin{array}{l}\text { Actunide Concentration with } \\
\text { Mobite Actinido thtrinsic Colloids }\end{array}$ & 36857 & 16 -Apr-96 & $Y$ & 1 \\
\hline$Y$ & & 3310 & $A M$ & Americium & CONCINT & $\begin{array}{l}\text { Actinide Corcantration with } \\
\text { Mobilo Actinido Intrinsic Colloids }\end{array}$ & 36857 & $16-4 p r-96$ & $\mathbf{Y}$ & 1 \\
\hline $\mathbf{Y}$ & & 3311 & AM & Americium & PROPMIC & $\begin{array}{l}\text { Moles of Act nide Mobilzed on } \\
\text { Microbe Colbids per Moles } \\
\text { Dissolved }\end{array}$ & 36858 & 16 Apr-96 & $\mathbf{Y}$ & 1 \\
\hline$\gamma$ & & 3311 & AM & Annericium & PROPMIC & $\begin{array}{l}\text { Moles of Actinide Mobilized on } \\
\text { Mrorobe Colloids per Moles } \\
\text { Dissolved }\end{array}$ & 36858 & 16-Apr-96 & $\mathbf{Y}$ & 1 \\
\hline$Y$ & & 3312 & NP & Neptunium & CONCINT & $\begin{array}{l}\text { Actride Concentration with } \\
\text { Mobib Actinide thitrinsic Colloids }\end{array}$ & 36859 & 16 Apr-96 & $\mathbf{Y}$ & 1 \\
\hline$Y$ & & 3312 & NP & Neptunium & CONCINT & $\begin{array}{l}\text { Actinide Concentration with } \\
\text { Mobile Actinide Intrinsic Colbids }\end{array}$ & 36859 & 16 Apr-98 & $\mathbf{Y}$ & 1 \\
\hline $\mathbf{Y}$ & & 3313 & NP & Noptunium & CAPMIC & $\begin{array}{l}\text { Mexmum Concentration of } \\
\text { Actinide on Microse Colloids }\end{array}$ & 36060 & 16 -Apr-96 & $\mathbf{Y}$ & 1 \\
\hline$Y$ & & 3313 & NP & Neptunium & CAPMic & $\begin{array}{l}\text { Mexmum Concentration } \alpha \\
\text { Aetinido on Microbe Colloids }\end{array}$ & 36860 & 16 Apr-96 & $Y$ & 1 \\
\hline$Y$ & & 3314 & NP & Neptunium & PROPMIC & $\begin{array}{l}\text { Moles of Actinide Hobilesed on } \\
\text { Microbe Colbits per Moles } \\
\text { Disselved }\end{array}$ & 36861 & $16-A p x-96$ & $\mathbf{Y}$ & 1 \\
\hline$Y$ & & 3314 & NP & Neptunium & PROPMIC & $\begin{array}{l}\text { Moles of Actnido Mobilezed on } \\
\text { Merobe Colbids per Moles } \\
\text { Dassotred }\end{array}$ & 36861 & 16-Apr-96 & $Y$ & 1 \\
\hline$Y$ & & 3315 & PU & Ptetonim & Capmic & $\begin{array}{l}\text { Maximum Concentration of } \\
\text { Actiniob on Microbo Colloids }\end{array}$ & 36862 & 16 Apr-96 & $\mathbf{Y}$ & 1 \\
\hline$Y$ & & 3315 & PU & Putonium & CAPMIC & $\begin{array}{l}\text { Meximum Concentration of } \\
\text { Actinide on Microbe Colloids }\end{array}$ & 36862 & 96 -Apr.96 & $Y$ & 1 \\
\hline $\mathbf{Y}$ & & 3316 & PU & Plutoniem & CONCINT & 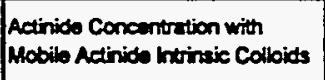 & 36063 & 16-Apr-96 & $Y$ & 1 \\
\hline $\mathbf{Y}$ & - & 3316 & PU & Plutonim & CONCINT & $\begin{array}{l}\text { Aetinido Concentration with } \\
\text { Mobilo Aetinido hromaic Colloids }\end{array}$ & 36853 & $16-A p r-96$ & $\mathbf{Y}$ & 1 \\
\hline
\end{tabular}


CCA Parameter Listing

\begin{tabular}{|c|c|c|c|c|c|c|c|c|c|c|}
\hline W & 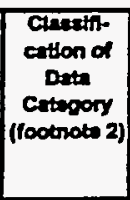 & $\begin{array}{l}\text { Destribution } \\
\text { Typo }\end{array}$ & $\operatorname{man}$ & $\operatorname{modin}$ & Whanom & Modrium & Whits & Roterence is & $\begin{array}{l}\text { Was the dinte } \\
\text { doreloped } \\
\text { underen } \\
\text { Non-1 } \\
\text { Progrem? }\end{array}$ & 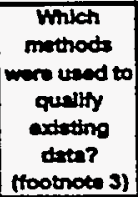 \\
\hline 3296 & 4 & CONSTANT & $1.2790000 \mathrm{E}+06$ & $1.2790000 E+06$ & $1.2790000 E+\infty 6$ & $1.2790000 E+06$ & $s$ & WP037404 & $\mathbf{Y}$ & N/A \\
\hline 3297 & 4 & CONSTANT & $3.9600000 \mathrm{E}+00$ & $3.9600000 E+00$ & $3.9600000 \mathrm{E}+\infty 0$ & $3.9600000 \mathrm{E}+\infty$ & 3 & WP037404 & $Y$ & NA \\
\hline 3298 & 4 & CONSTANT & $5.5600000 \mathrm{E}+01$ & $5.5600000 \mathrm{E}+01$ & $5.5600000 E+01$ & $5.5600000 E+01$ & $s$ & WP-037404 & $Y$ & NA \\
\hline 3299 & 4 & CONSTANT & $3.3040000 E+05$ & $3.3040000 E+05$ & $3.3040000 E+05$ & $3.3040000 \mathrm{E}+05$ & $s$ & WP037404 & $Y$ & N/A \\
\hline 3300 & 4 & CONSTANT & $1.6170000 E+06$ & $1.6170000 E+06$ & $1.6170000 E+\infty 6$ & $1.6170000 E+06$ & $=$ & WP037404 & $Y$ & NA \\
\hline 3301 & 4 & CONSTANT & $6.0370000 \mathrm{E}+07$ & $6.0370000 \mathrm{E}+07$ & $6.03700005+07$ & $6.0370000 \mathrm{E}+07$ & $s$ & WP037404 & $Y$ & NA \\
\hline 3302 & 4 & CONSTANT & $9.1870000 E+04$ & $9.1870000 E+04$ & $9.1870000 \mathrm{E}+04$ & $9.1870000 E+04$ & 8 & WP037404 & $\mathbf{Y}$ & NA \\
\hline 3303 & 4 & CONSTANT & $2.0820000 E+06$ & $2.0820000 \mathrm{E}+\infty 6$ & $20080000 E+06$ & $20820000 E+06$ & - & WP037404 & $\mathbf{Y}$ & NA \\
\hline 3304 & 4 & CONSTANT & $2.86200000 \mathrm{E}+02$ & $2.8620000 \mathrm{E}+02$ & $2.80520000 E+02$ & $2.8620000 \mathrm{E}+02$ & 3 & WP037404 & $Y$ & NA \\
\hline 3305 & 4 & CONSTANT & $2,3040000 E+05$ & $2.3040000 E+05$ & $2.3040000 E+05$ & $23040000 E+05$ & $s$ & WP037404 & $\mathbf{Y}$ & NA \\
\hline 3306 & 4 & CONSTANT & $1.0000000 E+38$ & $1.0000000 \mathrm{E}+38$ & $1.0000000 E+38$ & $1.0000000 \mathrm{E}+38$ & 8 & WP037404 & $\mathbf{Y}$ & NA \\
\hline 3307 & 4 & CONSTANT & $0.0000000 E+\infty 0$ & $0.0000000 E+00$ & $0.0000000 E+\infty 0$ & $0.0000000 E+\infty 0$ & moteshiter & WP035852 & $\mathbf{Y}$ & NA \\
\hline 3307 & 4 & CONSTANT & $0.0000000 E+\infty 0$ & $0.0000000 E+\infty$ & $0.0000000 E+\infty 0$ & $0.0000000 E+\infty$ & moleskiter & WP035771 & $\mathbf{Y}$ & NA \\
\hline 3308 & 1 & CONSTANT & $2.1000000 E-03$ & $2.1000000 E-03$ & $2,1000000 E-03$ & $21000000 E-03$ & molestitor & WP035855 & $\mathbf{Y}$ & NA \\
\hline 3308 & 1 & CONSTANT & $2.1000000 E-03$ & $2.1000000 E-03$ & $2.1000000 E-03$ & $2.1000000 E-03$ & molesfiter & WP035771 & $\mathbf{Y}$ & NA \\
\hline 3309 & 1 & CONSTANT & $2.1000000 E-03$ & $2.1000000 E-03$ & $21000000 E-03$ & $21000000 E-03$ & NONE & WP035855 & $\mathbf{Y}$ & NA \\
\hline 3309 & 1 & CONSTANI & $2.1000000 E-03$ & $2.1000000 E-03$ & $2.1000000 E-03$ & $2.1000000 E-03$ & NONE & WP035771 & $\mathbf{Y}$ & NA \\
\hline 3309 & 1 & CONSTANT & $2.1000000 E-03$ & $2.1000000 E-03$ & $2.1000000 E-03$ & $2.1000000 E-03$ & NONE & WP037804 & $\mathbf{Y}$ & NA \\
\hline 3310 & 4 & CONSTANT & $0.0000000 \mathrm{E}+\infty$ & $0.0000000 E+\infty 0$ & $0.0000000 E+\infty$ & $0.0000000 E+\infty$ & molesfiter & WP035852 & $\mathbf{Y}$ & N/A \\
\hline 3310 & 4 & CONSTANT & $0.0000000 E+00$ & $0.0000000 E+\infty$ & $0.0000000 E+00$ & $0.00000000++\infty 0$ & moteshiter & WP036771 & $\mathbf{Y}$ & NA \\
\hline 3311 & 1 & CONSTANT & $3.6000000 E+00$ & $3.6000000 \mathrm{E}+\infty$ & $3.6000000 E+\infty 0$ & $3.60000000+\infty 0$ & NONE & WP035856 & $Y$ & NA \\
\hline 3311 & 1 & CONSTANT & $3.6000000 E+\infty 0$ & $3.6000000 E+\infty 0$ & $3.6000000 E+\infty 0$ & $3.6000000 E+\infty$ & NONE & WP036771 & $\mathbf{Y}$ & N/A \\
\hline 3312 & 4 & CONSTANT & $0.0000000 E+\infty 0$ & $0.00000000+\infty$ & $0.00000000+\infty$ & $0.0000000 E+\infty$ & moleshiter & WP035852 & $\mathbf{Y}$ & NA \\
\hline 3312 & 4 & CONSTANT & $0.0000000 E+00$ & $0.00000000 \mathrm{E}+00$ & $0.0000000 E+00$ & $0.0000000 E+\infty$ & molesniter & WP036771 & $\mathbf{Y}$ & NA \\
\hline 3313 & 1 & CONSTANT & $2.7000000 E-03$ & $2.7000000 E-03$ & $2.7000000 E-03$ & $27000000 \mathrm{E}-03$ & moleshiter & WP035856 & $\mathbf{Y}$ & N/A \\
\hline 3313 & $1^{\circ}$ & CONSTANT & $2.7000000 E-03$ & $2.7000000 E-03$ & $2.7000000 E-03$ & $2.7000000 E-03$ & moleshiter & WP036771 & $\mathbf{Y}$ & NA \\
\hline 3314 & 1 & CONSTANT & $1.2000000 E+01$ & $1.2000000 E+01$ & $1.20000000+01$ & $1.2000000 E+01$ & NONE & WP035856 & $\mathbf{Y}$ & NA \\
\hline 3314 & 1 & CONSTANT & $1.2000000 E+01$ & $1.2000000 \mathrm{E}+01$ & $1.2000000 \mathrm{E}+01$ & $1.2000000 E+01$ & NONE & WP036771 & $\mathbf{Y}$ & NA \\
\hline 3315 & 1 & CONSTANT & $6.8000000 E-0.5$ & $6.8000000 E-05$ & $6.8000000 E-05$ & $6.8000000 E-05$ & moleshiter & WP035856 & $\mathbf{Y}$ & N/A \\
\hline 3315 & 1 & CONSTANT & $6.8000000 E .05$ & $6.8000000 E-05$ & $6.8000000 E-05$ & $6.8000000 E-05$ & molosititer & WP036771 & $\mathbf{Y}$ & NA \\
\hline 3316 & 1 & CONSTANT & $1.0000000 E-09$ & $1.0000000 E-09$ & $1.0000000 E-09$ & $1.0000000 E-09$ & molestiter & WP035852 & $\mathbf{Y}$ & N/A \\
\hline 3316 & 1 & CONSTANT & $1.0000000 \mathrm{E}-09$ & $1.0000000 E-09$ & $1.0000000 E-09$ & $1.0000000 E-09$ & moloshiter & WP036771 & $\mathbf{Y}$ & NA \\
\hline
\end{tabular}


CCA Parameter Listing

\begin{tabular}{|c|c|c|c|c|c|c|c|c|c|c|}
\hline 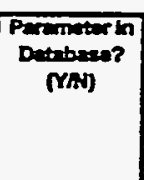 & 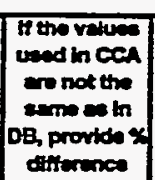 & ID & Mexerdio & Metara Name & Peremotar & 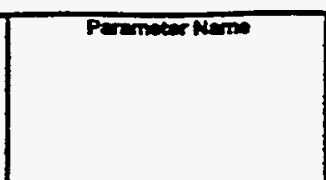 & $\begin{array}{l}\text { PRPD } \\
\text { MPO }\end{array}$ & $\underset{\text { Detery }}{\text { Deto }}$ & 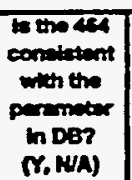 & $\begin{array}{l}\text { Paremitar } \\
\text { Compory } \\
\text { (footion 1) }\end{array}$ \\
\hline $\mathbf{Y}$ & & 3317 & PU & Pistonium & PROPMIC & $\begin{array}{l}\text { Moles of Actinde Mobileed on } \\
\text { Microbe Colloids per Moles } \\
\text { Dissetred }\end{array}$ & 36864 & $16-40 x-96$ & $\mathbf{Y}$ & 1 \\
\hline $\mathbf{Y}$ & & 3317 & Pu & Plutonim & PROPMIC & $\begin{array}{l}\text { Moles of Actincol Mobitzed on } \\
\text { Mierobe Calloids per Moles } \\
\text { Disseotred }\end{array}$ & 36064 & $16-\operatorname{apr}-\infty 6$ & $\mathbf{Y}$ & 1 \\
\hline $\mathbf{Y}$ & & 3318 & TH & Thorium & CAPMIC & $\begin{array}{l}\text { Maxemum Concantration of } \\
\text { Actinido on Microbe Collsids }\end{array}$ & 36865 & $16-A p r .96$ & $\mathbf{Y}$ & 1 \\
\hline $\mathbf{Y}$ & & 3318 & TH & Thorium & CAPMIC & $\begin{array}{l}\text { Meximum Concantration of } \\
\text { Actinide on Mierobe Colloids }\end{array}$ & 36865 & $16-A p r-96$ & $\mathbf{Y}$ & 1 \\
\hline $\mathbf{Y}$ & & 3319 & TH & Thoriun & CONCINT & $\begin{array}{l}\text { Aetinide Concartsation with } \\
\text { Mobile Actinide Intrinsic Colloids }\end{array}$ & 36066 & 16 Apr-96 & $\mathbf{Y}$ & 1 \\
\hline $\boldsymbol{\gamma}$ & & 3319 & TH & Thoriem & CONCINT & $\begin{array}{l}\text { Actinide Concentration with } \\
\text { Mobile Actinide Intmsic Coltoidts }\end{array}$ & 36066 & 16-Apr-96 & $\mathbf{Y}$ & 1 \\
\hline $\mathbf{Y}$ & & 3320 & $\mathrm{TH}$ & Thorium & PROPMIC & $\begin{array}{l}\text { Moles of Actinido Mobileced on } \\
\text { Microbe Colbids per Moles } \\
\text { Dissolved }\end{array}$ & 36067 & 16-Apr-96 & $\mathbf{Y}$ & 1 \\
\hline $\mathbf{Y}$ & & 3320 & $\pi H$ & Thorium & PROPMIC & $\begin{array}{l}\text { Moles of Actinich Mobilized on } \\
\text { Microbe Colloids per Moles } \\
\text { Dissolved }\end{array}$ & 36067 & 16-Apr-06 & $\mathbf{Y}$ & 1 \\
\hline$Y$ & & 3321 & $A \operatorname{cos25}$ & Actinium 225 & EPAREL & EPA Relessoso Limit & 36068 & 16-Apr-DS & $\mathbf{Y}$ & 3 \\
\hline$Y$ & & 3321 & AC225 & Actinium 225 & EPAREL & EPA Rolesse Limit & 36868 & 16-Apr-96 & $Y$ & 3 \\
\hline$Y$ & & 3322 & AC228 & Actinium 228 & EPAREL & EPA Relesse Limit & 36869 & $16-A p r-96$ & $\mathbf{Y}$ & 3 \\
\hline $\bar{Y}$ & & 3322 & AC228 & Actinium 228 & EPAREL & EPA Releseso Limit & 36869 & 16-Apr-96 & $\bar{Y}$ & 3 \\
\hline$Y$ & & 3323 & AT217 & Astatine 217 & EPAREL & EPA Relases Limit & 36870 & 16-Apr-96 & $\bar{Y}$ & 3 \\
\hline $\mathbf{Y}$ & & 3323 & AT217 & Astatine 217 & EPAREL & EPA Reloses Limit & 36870 & $16-A p r-96$ & $Y$ & 3 \\
\hline$Y$ & & 3324 & BA137 & Barium 137 & EPAREL & EPA Reteases Limit & 36871 & 16 -Apr-96 & $\mathbf{Y}$ & 3 \\
\hline $\bar{Y}$ & & 3324 & BA137 & Barium 137 & EPAREL & EPA Roloaso Limit & 36871 & 16-Apr-96 & $Y$ & 3 \\
\hline$Y$ & & 3325 & BA137M & Barium 137 Metastablo & EPAREL & EPA Relesso Limit & 36872 & $16-A p r-96$ & $Y$ & 3 \\
\hline $\mathbf{Y}$ & & 3325 & BA137M & Berium 137 Metastabio & EPAREL & EPA Releaso Limit & 36872 & 16 -Apr-96 & $\mathbf{Y}$ & 3 \\
\hline $\mathbf{Y}$ & & 3325 & BA137M & Barium 137 Metastablo & EPAREL & EPA Rolosso Limit & 36872 & 16-Apr-96 & $\mathbf{Y}$ & 3 \\
\hline $\mathbf{Y}$ & & 3326 & B1211 & Bismuth 219 & EPAREL & EPA Rolesso Limit & 36873 & 16-Apr-96 & $\mathbf{Y}$ & 3 \\
\hline$Y$ & & 3326 & B1211 & Bismuth 211 & EPAREL & EPA Relesse Limit & 36873 & 16 Apr-96 & $Y$ & 3 \\
\hline$Y$ & & 3327 & $B 1212$ & Biemuth 212 & EPAREI & EPA Retesso Limit & 36874 & $16-A p r-96$ & $Y$ & 3 \\
\hline$Y$ & & 3327 & 81212 & Bismuth 212 & EPAREL & EPA Reiosse Limit & 36874 & 16-Apr-96 & $Y$ & 3 \\
\hline $\mathbf{Y}$ & & 3328 & 81213 & Bismuth 213 & EPAREL & EPA Rejesso Limit & 36875 & 16-Apr-96 & $\mathbf{Y}$ & 3 \\
\hline$Y$ & & 3328 & $B 1213$ & Bismuth 213 & EPAREL & EPA Release Limit & 36875 & 16-Apr-96 & $Y$ & 3 \\
\hline$Y$ & & 3329 & 81214 & Bisonuth 214 & EPAREL & EPA Roloassa Limit & 36876 & 16-Apr-96 & $\mathbf{Y}$ & 3 \\
\hline$Y$ & & 3329 & 81214 & Bismuth 214 & EPAREL & EPA Rotesso Limit & 36876 & 16-Apr-96 & $-Y$ & 3 \\
\hline$Y$ & & 3330 & CF252 & Catforinum 252 & EPAREL & EPA Relosse Limit & 36877 & 16-Apr-96 & $Y$ & 3 \\
\hline$Y$ & & 3330 & CF252 & Celffornium 252 & EPAREL & EPA Rolesso Limit & 36877 & 16-Apr-96. & $Y$ & 3 \\
\hline$Y$ & & 3331 & CM1244 & Curium 244 & EPAREL & EPA Rolestse Limit & 36878 & 16-Apr-96 & $Y$ & 3 \\
\hline$Y$ & & 3331 & CM244 & Curium 24 & EPAREL & EPA Relazes Limit & 36878 & $16-A p r-96$ & $\mathbf{Y}$ & 3 \\
\hline$Y$ & & 3332 & FR221 & Frencium 221 & EPAREL & EPA Relesse Limit & 36879 & $16-1 p x-56$ & $Y$ & 3 \\
\hline $\mathbf{Y}$ & & 3332 & FR221 & Frmaium 221 & EPAREL & EPA Release Limit & 36879 & 16-Apr-96 & $\mathbf{Y}$ & 3 \\
\hline $\bar{Y}$ & & 3333 & ND143 & Neodymium 143 & EPAREL & EPA Relaseo Limit & 36880 & 16-Apr-96 & $Y$ & 3 \\
\hline $\mathbf{Y}$ & & 3333 & ND143 & Neodymium 143 & EPAREI & EPA Roleaseo Linit & 36880 & 16-Apr-86 & $\mathbf{Y}$ & 3 \\
\hline$Y$ & & 3334 & NP239 & Neptunim 239 & EPAREL & EPA Roloses Limit & 36881 & 16-Apr-96 & $Y$ & 3 \\
\hline$Y$ & & 3334 & NP239 & Neptunimm 239 & EPAREL & EPARoiases Limil & 36881 & 16 -Apr-86 & $Y$ & 3 \\
\hline $\mathbf{Y}$ & & 3335 & PA233 & Protactinium 233 & EPAREL & EPA Reloses Limit & 36882 & $16-A p r-96$ & $Y$ & 3 \\
\hline$Y$ & & 3335 & PA233 & Protsctinium 233 & EPAREL & EPA Relesese Limt & 36882 & 16-Apr-96 & $\bar{Y}$ & 3 \\
\hline $\mathbf{Y}$ & & 3336 & PAZZ4M & \begin{tabular}{|l} 
Protictinum 234 \\
Motastable
\end{tabular} & EPAREL & EPA Rolasen Limt & 36883 & 16-Apr.96 & $\mathbf{Y}$ & 3 \\
\hline $\mathbf{Y}$ & & 3336 & PA234M & $\begin{array}{l}\text { Protictniem } 234 \\
\text { Mretartable }\end{array}$ & EPAREL & EPA Releseso Limit & 36883 & 16 Apr-98 & $\mathbf{Y}$ & 3 \\
\hline$Y$ & & 3337 & PB209 & Lend 209 & EPAREL & EPA Rolasese Limit & 36884 & 16-Apr-96 & $Y$ & 3 \\
\hline $\mathbf{Y}$ & & 3337 & PB209 & Lead 209 & EPAREI & EPA Rolas:so Limit & 36884 & $16-4 p \times-96$ & $\mathbf{Y}$ & 3 \\
\hline
\end{tabular}


CCA Parameter Listing

\begin{tabular}{|c|c|c|c|c|c|c|c|c|c|c|}
\hline ID & 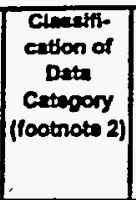 & $\begin{array}{l}\text { Detroution } \\
\text { Typo }\end{array}$ & mean & medien & Munhroum & Anodmem & Units & 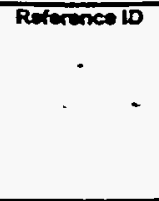 & $\begin{array}{l}\text { We the deta } \\
\text { developed } \\
\text { under an } \\
\text { Nol-1 } \\
\text { Program? }\end{array}$ & 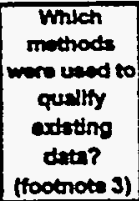 \\
\hline 3317 & 1 & CONSTANT & $3.0000000 E-01$ & 3.0000000E-01 & $3.0000000 E-01$ & $3.0000000 E-01$ & NONE & WP035855 & $\mathbf{Y}$ & NA \\
\hline 3317 & 1 & CONSTANT & $3,0000000 E-01$ & $3.0000000 E-01$ & $3.0000000 \mathrm{E}-01$ & $3.0000000 E-01$ & NONE & WP036771 & $Y$ & NA \\
\hline 3318 & 1 & CONSTANT & $1.9000000 E-03$ & $1.9000000 E-03$ & $1.9000000 E-03$ & $1.9000000 E-03$ & molestiter & WP035856 & $\mathbf{Y}$ & N/A \\
\hline 3318 & 1 & CONSTANT & $1.9000000 E-03$ & $1.9000000 E-03$ & $1.9000000 E-03$ & $1.9000000 \leq-03$ & molessitar & WP036771 & $Y$ & NA \\
\hline 3319 & 4 & CONSTANT & $0.0000000 E+\infty 0$ & $0.0000000 E+\infty$ & $0.00000000+\infty$ & $0.0000000 E+00$ & molentiter & WP035852 & $\mathbf{Y}$ & NA \\
\hline 3319 & 4 & CONSTANT & $0.0000000 E+\infty$ & $0.0000000 E+\infty 0$ & $0.0000000 E+\infty 0$ & $0.0000000 E+\infty$ & molostiter & WP036771 & $\mathbf{Y}$ & NA \\
\hline 3320 & 1 & CONSTANT & $3.1000000 E+\infty 0$ & $3.10000000+00$ & $3.1000000 \mathrm{E}+\infty$ & $3.1000000 E+\infty 0$ & NONE & WP035856 & $Y$ & NA \\
\hline 3320 & 1 & CONSTANT & $3.1000000 E+\infty$ & $3.1000000 E+\infty$ & $3.1000000 E+\infty$ & $3.1000000 E+\infty$ & NONE & WP036771 & $\mathbf{Y}$ & NAA \\
\hline 3321 & 4 & CONSTANT & $0.0000000 E+\infty$ & $0.0000000 E+\infty$ & $0.0000000 E+\infty$ & $0.0000000 E+\infty$ & Cinut & 40CFR191 & $Y$ & NA \\
\hline 3321 & 4 & CONSTANT & $0.0000000 \mathrm{E}+\infty$ & $0.0000000 \mathrm{E}+\infty 0$ & $0.0000000 \mathrm{E}+00$ & $0.0000000 E+\infty)$ & Cinut & WP035301 & $Y$ & NAA \\
\hline 3322 & 4 & CONSTANT & $0.0000000 E+\infty 0$ & $0.0000000 E+\infty$ & $0.0000000 E+\infty$ & $0.0000000 E+\infty$ & Cimut & 40CFR191 & $Y$ & NA \\
\hline 3322 & 4 & CONSTANT & $0.0000000 \mathrm{E}+00$ & $0.0000000 E+00$ & $0.0000000 E+\infty$ & $0.0000000 E+\infty$ & Cimut & WP036301 & $\mathbf{Y}$ & NA \\
\hline 3323 & 4 & CONSTANT & $0.0000000 \mathrm{E}+00$ & $0.0000000 E+\infty 0$ & $0.0000000 E+\infty$ & $0.0000000 \mathrm{E}+00$ & Cinct & 40CFR191 & $Y$ & NA \\
\hline 3323 & 4 & CONSTANT & $0.0000000 E+\infty$ & $0.0000000 E+\infty$ & $0.0000000 \mathrm{E}+00$ & $0.0000000 E+\infty 0$ & Cinut & WP036301 & $Y$ & N/A \\
\hline 3324 & 4 & CONSTANT & $0.0000000 \mathrm{E}+00$ & $0.0000000 \mathrm{E}+\infty$ & $0.0000000 \mathrm{E}+00$ & $0.0000000 E+00$ & Cimst & 40CFR191 & $Y$ & NA \\
\hline 3324 & 4 & CONSTANT & $0.0000000 E+\infty$ & $0.0000000 E+00$ & $0.0000000 E+\infty$ & $0.0000000 E+00$ & Cirmut & WP035301 & $Y$ & NA \\
\hline 3325 & 4 & CONSTANT & $0.0000000 E+00$ & $0.0000000 E+\infty 0$ & $0.0000000 E+\infty$ & $0.0000000 E+\infty 0$ & Cirunt & LOCFR191 & $\mathbf{Y}$ & N/A \\
\hline 3325 & 4 & CONSTANT & $0.0000000 E+00$ & $0.0000000=+\infty 0$ & $0.0000000 E+\infty 0$ & $0.0000000 E+\infty$ & Cimus & WP036298 & $\mathbf{Y}$ & NA \\
\hline 3325 & 4 & CONSTANT & $0.0000000 \mathrm{E}+\infty 0$ & $0.0000000 E+\infty$ & $0.0000000 E+\infty 0$ & $0.00000000 E+\infty 0$ & Cimus & WP035301 & $Y$ & NA \\
\hline 3326 & 4 & CONSTANT & $0.0000000 \mathrm{E}+00$ & $0.0000000 E+\infty 0$ & $0.0000000 E+\infty$ & $0.0000000 E+00$ & Ciwof & 40CFR191 & $Y$ & NA \\
\hline 3326 & 4 & CONSTANT & $0.0000000 E+\infty$ & $0.0000000 \mathrm{E}+\infty 0$ & $0.0000000 E+\infty$ & $0.0000000 E+00$ & Cimut & WPO36301 & $Y$ & NA \\
\hline 3327 & 4 & CONSTANT & $0.0000000 E+\infty$ & $0.0000000 \mathrm{E}+00$ & $0.0000000 E+00$ & $0.0000000 \mathrm{E}+\infty 0$ & Cinus & 40CFR191 & $Y$ & NA \\
\hline 3327 & 4 & CONSTANT & $0.00000000 E+\infty$ & $0.00000000+\infty 0$ & $0.00000000+\infty 00$ & $0.0000000 \mathrm{E}+\infty$ & Cinur & WP036301 & $\bar{Y}$ & NA \\
\hline 3328 & 4 & CONSTANT & $0.0000000 E+\infty$ & $0.00000000+00$ & $0.0000000 \mathrm{E}+00$ & $0.0000000 E+\infty$ & Cihusf & 40CFR191 & $\mathbf{Y}$ & NA \\
\hline 3328 & 4 & CONSTANT & $0.0000000 E+\infty 0$ & $0.0000000 E+\infty$ & $0.0000000 E+\infty$ & $0.0000000 E+\infty 0$ & Cimut & WPO36301 & $Y$ & NA \\
\hline 3329 & 4 & CONSTANT & $0.0000000 E+\infty$ & $0.0000000 \mathrm{E}+00$ & $0.0000000 E+\infty$ & $0.0000000 E+00$ & Cimot & 40CFR191 & $\bar{Y}$ & N/A \\
\hline 3329 & 4 & CONSTANT & $0.0000000 \mathrm{E}+00$ & $0.0000000 E+\infty 0$ & $0.0000000 \mathrm{E}+\infty$ & $0.0000000 E+00$ & Cilunf & WP036301 & $-Y$ & N/A \\
\hline 3330 & 4 & CONSTANT & $0.0000000 E+\infty 0$ & $0.0000000 E+\infty$ & $0.0000000 E+00$ & $0.0000000 E+00$ & Ciwof & 40CFR191 & $Y$ & N/A \\
\hline 3330 & 4 & CONSTANT & $0.0000000 E+00$ & $0.0000000 E+00$ & $0.0000000 E+\infty$ & $0.0000000 E+00$ & Cimuff & WPO36301 & $\mathbf{Y}$ & NA \\
\hline 3331 & 4 & CONSTANT & $0.0000000 E+\infty 0$ & $0.0000000 E+\infty 0$ & $0.0000000 E+00$ & $0.0000000 E+00$ & Cimut & 40CFRI91 & $Y$ & N/A \\
\hline 3331 & 4 & CONSTANT & $0.0000000 E+\infty 0$ & $0.0000000 E+\infty$ & $0.0000000 E+\infty$ & $0.0000000 E+00$ & Cimus & WP035301 & $Y$ & N/A \\
\hline 3332 & 4 & CONSTANT & $0.0000000 E+\infty$ & $0.0000000 E+\infty$ & $0.0000000 E+\infty$ & $0.00000000+00$ & Cimut & 40CFR191 & $Y$ & N/A \\
\hline 3332 & 4 & CONSTANT & $0.00000000 E+\infty$ & $0.0000000 E+\infty 0$ & $0.0000000 E+\infty 0$ & $0.0000000 E+00$ & Cimut & WPO36301 & $\mathbf{Y}$ & N/A \\
\hline 3333 & 4 & CONSTANT & $0.00000000+00$ & $0.0000000 \mathrm{E}+\infty 0$ & $0.0000000 \mathrm{E}+00$ & $0.0000000 E+\infty$ & Cihus & 40CFR191 & $Y$ & NA \\
\hline 3333 & 4 & CONSTANT & $0.0000000 E+\infty$ & $0.0000000 \mathrm{E}+00$ & $0.0000000 \mathrm{E}+00$ & $0.0000000 E+\infty$ & Cimut & WPO36301 & $\mathbf{Y}$ & NA \\
\hline 3334 & 4 & CONSTANT & $0.0000000 E+00$ & $0.0000000 E+00$ & $0.0000000 E+\infty$ & $0.0000000 \mathrm{E}+00$ & Cimus & 40CFR191 & 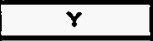 & NA \\
\hline 3334 & 4 & CONSTANT & $0.0000000 \mathrm{E}+00$ & $0.0000000 E+\infty 0$ & $0.0000000 E+\infty 0$ & $0.0000000 E+\infty$ & Cinunt & WP036301 & $Y$ & N/A \\
\hline 3335 & 4 & CONSTANT & $0.0000000 E+\infty 0$ & $0.0000000 \mathrm{E}+\infty 0$ & $0.0000000 \mathrm{E}+00$ & $0.0000000 \mathrm{E}+00$ & Cimst & 40CFR191 & $Y$ & NA \\
\hline 3335 & 4 & CONSTANT & $0.00000000+\infty$ & $0.00000000 \mathrm{E}+00$ & $0.0000000 E+00$ & $0.0000000 E+\infty$ & Citwut & WP036301 & $\mathbf{Y}$ & $N A$ \\
\hline 3336 & 4 & CONSTANT & $0.00000000 E+\infty$ & $0.0000000 E+\infty 0$ & $0.0000000 \mathrm{E}+\infty 0$ & $0.0000000 \mathrm{E}+\infty 0$ & Cimut & 40CFR191 & Y & NA \\
\hline 3336 & 4 & CONSTANT & $0.00000000+\infty 0$ & $0.0000000 \mathrm{E}+00$ & $0.0000000 E+00$ & $0.0000000 E+\infty$ & Cunst & WP036301 & $\mathbf{Y}$ & NA \\
\hline 3337 & 4 & CONSTANT & $0.0000000 E+00$ & $0.0000000 E+00$ & $0.0000000 E+00$ & $0.0000000 E+\infty$ & Cimest & 40CFR191 & $Y$ & N/A \\
\hline 3337 & 4 & CONSTANT & $0.0000000 \mathrm{E}+\infty$ & $0.0000000 E+\infty 0$ & $0.0000000 E+\infty$ & $0.0000000 E+\infty$ & Cims & WP036301 & $\mathbf{Y}$ & N/A \\
\hline
\end{tabular}


CCA Parameter Listing

\begin{tabular}{|c|c|c|c|c|c|c|c|c|c|c|}
\hline 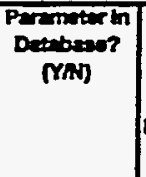 & 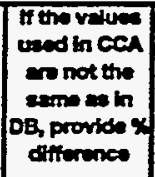 & DD & Meterta to & Wateria $\mathrm{Nam}$ & $\begin{array}{c}\text { Peramotin } \\
\text { D }\end{array}$ & Parmetir Reme & $\begin{array}{l}\text { PRP DD } \\
\text { MPO In }\end{array}$ & $\begin{array}{c}\text { Deta Entry } \\
\text { Deta }\end{array}$ & 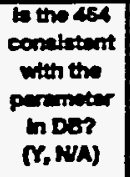 & $\begin{array}{l}\text { Parmetar } \\
\text { Cettegory } \\
\text { (footrota 1) }\end{array}$ \\
\hline$Y$ & & 3338 & PB211 & Lead 211 & EPAREL & EPA Rolesse Limit & 36885 & $16-4 p \times-96$ & $Y$ & 3 \\
\hline$Y$ & & 3338 & PB211 & Lead 211 & EPAREL & EPA Retense Limit & 36885 & 16-Apr-96 & $Y$ & 3 \\
\hline $\mathbf{Y}$ & & 3339 & PB212 & Load 212 & IEPAREI & EPA Reteese Limil & 36886 & 16-Apr-96 & $\mathbf{Y}$ & 3 \\
\hline$Y$ & & 3339 & PB212 & Loed 212 & EPARE & EPA Rotosts Linit & 36886 & 16 -Apr-96 & $Y$ & 3 \\
\hline $\mathbf{Y}$ & & 3340 & PB214 & Lead 214 & EPAREL & EPA Ralasea Limi & 36888 & 16-Apr-96 & $\mathbf{Y}$ & 3 \\
\hline$Y$ & & 3340 & PB21a & Lead 214 & EPAREL & EPA Relasse Limit & 36883 & 16-Apr-96 & $Y$ & 3 \\
\hline$Y$ & & 3341 & PM147 & Promethiem 147 & EPAREL & EPA Relosse Limil & 36889 & 16-Apr-96 & $Y$ & 3 \\
\hline$Y$ & & 3341 & PM147 & Promethiem 147 & EPAREL & EPA Reloseso Limit & 36889 & $16-4 p r-96$ & $Y$ & 3 \\
\hline$y$ & & 3342 & PO212 & Potonium 212 & EPAREL & EPA Relesse Limit & 36891 & $16-4 p x-96$ & $Y$ & 3 \\
\hline$Y$ & & 3342 & PO212 & Potonium 212 & EPAREL & EPA Relosse Limi & 36891 & 16-Apr-86 & $\mathbf{Y}$ & 3 \\
\hline$Y$ & & 3343 & $\mathrm{PO} 213$ & Poionium 213 & EPAREL & EPA Relosese Limit & 36892 & 16-Apr-96 & $Y$ & 3 \\
\hline$Y$ & & 3343 & PO213 & Polonium 213 & EPAREL & EPA Retoseso Limil & 36892 & 16 Apr-96 & $Y$ & 3 \\
\hline$y$ & & 3344 & PO214 & Polonium 214 & EPAREL & EPA Rolateso Limi & 36893 & $16-A p r-96$ & $Y$ & 3 \\
\hline$Y$ & & 3344 & P0214 & Polonium 214 & EPAREL & EPA Roleazes Limit & 36893 & 16-Apr-96 & $Y$ & 3 \\
\hline$Y$ & & 3345 & P0215 & Polonium 215 & EPAREL & EPA Reterse Limit & 36994 & 16-Apr-96 & $Y$ & 3 \\
\hline$Y$ & & 3345 & P0215 & Polonum 215 & EPAREL & EPA Retesse Lmit & 36894 & $16-4 p r-96$ & $\mathbf{Y}$ & 3 \\
\hline$Y$ & & 3346 & P0216 & Polonum 216 & EPAREL & EPA Rolease Limit & 36895 & 16-Apr-96 & $\mathbf{Y}$ & 3 \\
\hline$Y$ & & 3346 & P0216 & Polonium 216 & EPAREL & EPA Rolease Limil & 36395 & 16-Apr-96 & $Y$ & 3 \\
\hline$Y$ & & 3347 & P0218 & Polonium 218 & EPAREL & EPA Rolease Lmit & 36896 & 16 -Apr-96 & $\mathbf{Y}$ & 3 \\
\hline$Y$ & & 3347 & P0218 & Polonium 218 & EPAREL & EPA Relosese Lmi & 36896 & 16-Apr-96 & $Y$ & 3 \\
\hline$Y$ & & 3348 & PU241 & Phutonium 241 & EPAREL & EPA Reinasen Lmit & 36898 & 16-Apr-96 & $\mathbf{Y}$ & 3 \\
\hline $\mathbf{Y}$ & & 3348 & PU241 & Phetonium 241 & EPAREL & EPA Roteme Lmt & 36898 & 16-Apr-96 & $\mathbf{Y}$ & 3 \\
\hline$Y$ & & 3349 & RA223 & Redium 223 & EPAREL & EPA Roloase Lmt & 36899 & 16-Apr-96 & $\mathbf{Y}$ & 3 \\
\hline $\mathbf{Y}$ & & 3349 & RA223 & Radium 223 & EPAREL & EPA Retoses Lmt & 36899 & 16-Apr-96 & $\mathbf{Y}$ & 3 \\
\hline$Y$ & & 3350 & RA224 & Radxm 224 & EPAREL & EPA Rotanso Lmt & 36901 & 16-Apr-96 & $y$ & 3 \\
\hline$Y$ & & 3350 & RA224 & Radium 224 & EPAREL & EPA Relosise Lmx & 36901 & 16-Apr-96 & $y$ & 3 \\
\hline$Y$ & & 3351 & RA225 & Radium 225 & EPAREL & EPA Reloses LmR & 36902 & $16-A p r .96$ & $\mathbf{Y}$ & 3 \\
\hline$Y$ & & 3351 & RA225 & Radium 225 & EPAREL & EPA Rolouse LimR & 36902 & 16-Apr-96 & $\mathbf{Y}$ & 3 \\
\hline$Y$ & & 3352 & RA228 & Rodum 228 & EPAREL & EPA Rotasen $1 \mathrm{mr}$ & 36903 & 16-Apr-96 & $Y$ & 3 \\
\hline$Y$ & & 3352 & RA228 & Radium 228 & EPAREL & EPA Roloesen Lms & 36903 & 16-Apr-96 & $Y$ & 3 \\
\hline $\mathbf{Y}$ & & 3353 & RN219 & Radon 219 & EPAREL & EPA Roleaso Lms & 36904 & 16-Apr-96 & $\mathbf{Y}$ & 3 \\
\hline$Y$ & & 3353 & RN1219 & Radon 219 & EPAREL & EPA Rolenen Lme & 36904 & 16-Apr-96 & $Y$ & 3 \\
\hline$Y$ & & 3354 & RN220 & Radon 220 & EPAREL & EPA Rowam Lome & 36906 & 16-Apr-96 & $Y$ & 3 \\
\hline$Y$ & & 3354 & RN220 & Rodon 220 & EPAREL & EPA Rowacen LmR & 36906 & 16-Apr-96 & $Y$ & 3 \\
\hline$Y$ & & 3355 & RNE22 & Radon 222 & EPAREL & EPA Rancen Lme & 36907 & 16-Apr-96 & $Y$ & 3 \\
\hline$Y$ & & 3355 & RN222 & Redon 222 & EPAREL & EPA Romene Lma & 36907 & 16-Apr-96 & $Y$ & 3 \\
\hline$Y$ & & 3356 & TH1227 & Thonum 227 & EPAREL & EPA Romeno Lma & 36909 & 16-Apr-96 & $Y$ & 3 \\
\hline $\mathbf{Y}$ & & 3356 & THL227 & Thorum 227 & EPAREL & EPA Romome Lma & 36909 & 16 Apr-96 & $Y$ & 3 \\
\hline$Y$ & & 3357 & TH1228 & Thorum 228 & EPAREL & EPA REm L L & 36910 & 16 Apr-96 & $Y$ & 3 \\
\hline$Y$ & & 3357 & TH1228 & Thorum 228 & EPANEL & EPA R & 36910 & 16-Apr-96 & $Y$ & 3 \\
\hline$Y$ & & 3358 & THE231 & Thorum 231 & EPANEL & EPA Romenos Lme & 36911 & 16-Apr.96 & $y$ & 3 \\
\hline$Y$ & & 3358 & THE231 & Thorum 231 & EPAREL & 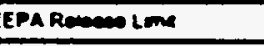 & 36911 & 16-Apr-96 & $Y$ & 3 \\
\hline$Y$ & & 3359 & TH1234 & Thorum 234 & EPAPEL & EPA Romenos lima & 36921 & 16-Apr.96 & $Y$ & 3 \\
\hline$Y$ & & 3359 & TH1234 & Thorum 234 & EPAREL & EPA Roween ime & 36921 & $16-40 \times-96$ & $Y$ & 3 \\
\hline$Y$ & & 3360 & $\pi 207$ & Thaliem 207 & EPAPEL & EPA Rowan Lme & 36922 & 16 Apr-96 & $Y$ & 3 \\
\hline$Y$ & & 3360 & $\pi 20$ & Thallem 207 & EPARES & EPA Remen Lome & 36922 & 16-Apr-96 & $Y$ & 3 \\
\hline$Y$ & & 3361 & Y9o & Yrtrum 90 & EPAREL & EPA Romear Lme & 36923 & 16-Apr-96 & $Y$ & 3 \\
\hline$Y$ & & 3361 & Y90 & Yutum 90 & EPAPEL & EPA Rancon Lone & 36923 & 16-Apr-96 & $Y$ & 3 \\
\hline$y$ & & 3352 & ZR90 & Erconem so & EPAREL & EPA Ranem Loms & 36924 & 16-npr-96 & $Y$ & 3 \\
\hline$Y$ & & 3352 & ZR90 & Zrconam 90 & EPAREL & EPARoman Lome & 36924 & 16-4pr-96 & $Y$ & 3 \\
\hline$Y$ & & 3363 & AN241 & Amercam 241 & EPAPEL: & EPA Rowem Lome & 36925 & 16-Apr-96 & $Y$ & 3 \\
\hline$Y$ & & 3353 & AN241 & Amencum 241 & EPAREL & EPA Rowane LmR & 36925 & 16-Apr-86 & $Y$ & 3 \\
\hline$Y$ & & 3354 & AC227 & Actinem 227 & EPAREL & EPA Ronesen Limx & 36926 & 16 Apr-96 & $Y$ & 3 \\
\hline $\mathbf{Y}$ & & 3354 & AC227 & Actinum 227 & EPAREL & EPA Rovecen LmR & 36926 & 16-Apr-86 & $\mathbf{Y}$ & 3 \\
\hline
\end{tabular}


CCA Parameter-Listing

\begin{tabular}{|c|c|c|c|c|c|c|c|c|c|c|}
\hline 10 & 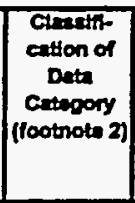 & $\begin{array}{l}\text { Dentribution } \\
\text { Type }\end{array}$ & man & Medien & Mintroum & Mexdmen & Units & Rationimes id & $\begin{array}{l}\text { Whe the dith } \\
\text { dowaloped } \\
\text { underen } \\
\text { Nol-1 } \\
\text { Progrem? }\end{array}$ & 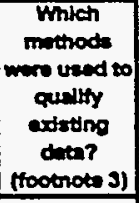 \\
\hline 3338 & 4 & CONSTANT & $0.0000000 E+00$ & $0.0000000 E+\infty$ & $0.0000000 E+\infty 0$ & $0.0000000 E+\infty$ & Cimest & 40CFR191 & $\mathbf{Y}$ & NA \\
\hline 3338 & 4 & CONSTANT & $0.0000000 \mathrm{E}+\infty$ & $0.0000000 E+00$ & $0.0000000 E+\infty 0$ & $0.0000000 E+\infty$ & Cimus & WP035301 & $Y$ & NA \\
\hline 3339 & 4 & CONSTANT & $0.0000000 \mathrm{E}+00$ & $0.0000000 E+\infty$ & $0.0000000 E+\infty 0$ & $0.0000000 E+\infty 0$ & Cibuns & 40CFR191 & $Y$ & NA \\
\hline 3339 & 4 & CONSTANT & $0.0000000 E+\infty 0$ & $0.0000000 \mathrm{E}+\infty 0$ & $0.0000000 E+00$ & $0.0000000 E+\infty$ & Cimus & WPO35301 & $Y$ & NA \\
\hline 3340 & 4 & CONSTANT & $0.0000000 E+\infty$ & $0.0000000 \mathrm{E}+\infty 0$ & $0.0000000 \mathrm{E}+\infty 0$ & $0.0000000 E+\infty 0$ & Cimonf & 40CFR191 & $Y$ & $N A$ \\
\hline 3340 & 4 & CONSTANT & $0.0000000 E+\infty 0$ & $0.0000000 E+\infty$ & $0.0000000 E+\infty$ & $0.0000000 E+\infty$ & Cimut & WPO35301 & $\mathbf{Y}$ & NA \\
\hline 3341 & 4 & CONSTANT & $0.0000000 \mathrm{E}+00$ & $0.0000000 E+\infty$ & $0.0000000 \mathrm{E}+\infty 0$ & $0.0000000 \mathrm{E}+\infty 0$ & Cirunf & 40CFR191 & $\mathbf{Y}$ & NA \\
\hline 3341 & 4 & CONSTANT & $0.0000000 E+00$ & $0.0000000 \mathrm{E}+\infty 0$ & $0.0000000 E+\infty$ & $0.0000000 \mathrm{E}+\infty 0$ & Cimer & WPO35301 & $\mathbf{Y}$ & NA \\
\hline 3342 & 4 & CONSTANT & $0.0000000 \mathrm{E}+00$ & $0.0000000 \mathrm{E}+\infty$ & $0.0000000 \mathrm{E}+\infty 0$ & $0.0000000 \mathrm{E}+\infty 0$ & Cims & 40CFR191. & $\bar{Y}$ & N/A \\
\hline 3342 & 4 & CONSTANT & $0.0000000 E+00$ & $0.0000000 \mathrm{E}+00$ & $0.0000000 \mathrm{E}+00$ & $0.0000000 E+\infty 0$ & Cirunf & WP036301 & $Y$ & NA \\
\hline 3343 & 4 & CONSTANT & $0.0000000 E+00$ & $0.0000000 \mathrm{E}+\infty$ & $0.0000000 \mathrm{E}+\infty 0$ & $0.0000000 \mathrm{E}+\infty$ & cimes & 40CFR191 & $\mathbf{Y}$ & NA \\
\hline 3343 & 4 & CONSTANT & $0.0000000 \mathrm{E}+\infty$ & $0.0000000 E+\infty$ & $0.0000000 E+00$ & $0.0000000 E+\infty$ & Cinent & WP036301 & $Y$ & NAA \\
\hline 3344 & 4 & CONSTANT & $0.0000000 \mathrm{E}+\infty 0$ & $0.0000000 E+\infty 0$ & $0.0000000 \mathrm{E}+00$ & $0.0000000 E+\infty 0$ & Cimer & 40CFR191 & $Y$ & NA \\
\hline 3344 & 4 & CONSTANT & $0.0000000 E+00$ & $0.0000000 E+\infty$ & $0.0000000 \mathrm{E}+\infty 0$ & $0.0000000 E+\infty$ & Cinent & WP036301 & $Y$ & NA \\
\hline 3345 & 4 & CONSTANT & $0.0000000 \mathrm{E}+\infty 0$ & $0.0000000 E+\infty 0$ & $0.0000000 \mathrm{E}+\infty 0$ & $0.0000000 E+\infty 0$ & Cimut & 40CFR191 & $Y$ & $N A$ \\
\hline 3345 & 4 & CONSTANT & $0.0000000 \mathrm{E}+\infty 0$ & $0.0000000 E+\infty 0$ & $0.0000000 E+00$ & $0.0000000 \mathrm{E}+00$ & Cimut & WP036301 & $Y$ & $N / A$ \\
\hline 3346 & 4 & CONSTANT & $0.0000000 \mathrm{E}+00$ & $0.0000000 E+\infty 0$ & $0.0000000 E+\infty 0$ & $0.0000000 E+\infty$ & Cimut & 40CFR191 & $Y$ & NA \\
\hline 3346 & 4 & CONSTANT & $0.0000000 \mathrm{E}+00$ & $0.00000005+\infty$ & $0.0000000 E+\infty 0$ & $0.0000000 E+\infty$ & Cinnt & WP036301 & $y$ & NA \\
\hline 3347 & 4 & CONSTANT & $0.0000000 \mathrm{E}+00$ & $0.0000000 \mathrm{E}+00$ & $0.0000000 E+\infty 0$ & $0.0000000 E+\infty 0$ & Cimut & 40CFR191 & $\bar{Y}$ & NA \\
\hline 3347 & 4 & CONSTANT & $0.0000000 \mathrm{E}+00$ & $0.0000000 \mathrm{E}+\infty 0$ & $0.0000000 E+00$ & $0.0000000 E+\infty$ & Cirunt & WP036301 & $Y$ & NA \\
\hline 3348 & 4 & CONSTANT & $0.0000000 \mathrm{E}+\infty 0$ & $0.0000000 E+\infty$ & $0.0000000 E+\infty 0$ & $0.0000000 E+00$ & Cimnt & 40CFR191 & $Y$ & N/A \\
\hline 3348 & 4 & CONSTANT & $0.0000000 E+00$ & $0.0000000 \mathrm{E}+00$ & $0.0000000 \mathrm{E}+00$ & $0.0000000 \mathrm{E}+00$ & Cinent & WP036301 & $Y$ & N/A \\
\hline 3349 & 4 & CONSTANT & $0.0000000 \mathrm{E}+00$ & $0.0000000 \mathrm{E}+\infty 0$ & $0.0000000 \mathrm{E}+00$ & $0.0000000 \mathrm{E}+00$ & Cimut & 40CFR191 & $Y$ & N/A \\
\hline 3349 & 4 & CONSTANT & $0.0000000 E+00$ & $0.0000000 E+00$ & $0.0000000 E+\infty$ & $0.0000000 \mathrm{E}+00$ & Cimut & WP036301 & $Y$ & N/A \\
\hline 3350 & 4 & CONSTANT & $0.0000000 \mathrm{E}+00$ & $0.0000000 \mathrm{E}+00$ & $0.0000000 E+00$ & $0.0000000 E+\infty 0$ & Cinut & 40CFR191 & $Y$ & N/A \\
\hline 3350 & 4 & CONSTANT & $0.0000000 \mathrm{E}+00$ & $0.0000000 \mathrm{E}+\infty 0$ & $0.0000000 \mathrm{E}+00$ & $0.0000000 \mathrm{E}+\infty 0$ & Cinurf & WP036301 & $Y$ & N/A \\
\hline 3351 & 4 & CONSTANT & $0.0000000 \mathrm{E}+00$ & $0.0000000 \mathrm{E}+00$ & $0.0000000 \mathrm{E}+00$ & $0.0000000 E+00$ & Cinus & 40CFR191 & $Y$ & N/A \\
\hline 3351 & 4 & CONSTANT & $0.0000000 \mathrm{E}+\infty 0$ & $0.0000000 E+\infty$ & $0.0000000 E+\infty$ & $0.0000000 E+\infty$ & Cimut & WP036301 & $Y$ & $N A$ \\
\hline 3352 & 4 & CONSTANT & $0.0000000 E+00$ & $0.0000000 E+\infty 0$ & $0.0000000 E+00$ & $0.0000000 \mathrm{E}+00$ & Cinut & 40CFR191 & $\bar{Y}$ & NA \\
\hline 3352 & 4 & CONSTANT & $0.0000000 E+00$ & $0.0000000 \mathrm{E}+00$ & $0.0000000 \mathrm{E}+00$ & $0.0000000 \mathrm{E}+\infty 0$ & Cinus & WP036301 & $Y$ & NA \\
\hline 3353 & 4 & CONSTANT & $0.0000000 E+\infty 0$ & $0.0000000 E+00$ & $0.0000000 \mathrm{E}+00$ & $0.0000000 \mathrm{E}+00$ & Cirunt & 10CFR191 & $Y$ & NAA \\
\hline 3353 & 4 & CONSTANT & $0.0000000 E+00$ & $0.0000000 \mathrm{E}+\infty$ & $0.0000000 E+\infty$ & $0.0000000 \mathrm{E}+00$ & Cimut & WP036301 & $Y$ & NA \\
\hline 3354 & 4 & CONSTANT & $0.0000000 \mathrm{E}+00$ & $0.0000000 E+\infty$ & $0.0000000 \mathrm{E}+00$ & $0.0000000 \mathrm{E}+00$ & Cimut & 40CFR191 & $\bar{Y}$ & NA \\
\hline 3354 & 4 & CONSTANT & $0.0000000 E+\infty 0$ & $0.0000000 E+\infty 0$ & $0.0000000 \mathrm{E}+\infty 0$ & $0.0000000 \mathrm{E}+\infty 0$ & Cilund & WPO36301 & $Y$ & NA \\
\hline 3355 & 4 & CONSTANT & $0.00000000+00$ & $0.0000000 E+\infty 0$ & $0.0000000 \mathrm{E}+00$ & $0.0000000 \mathrm{E}+00$ & Cilwed & 40CFR191 & $\mathbf{Y}$ & NA \\
\hline 3355 & 4 & CONSTANT & $0.00000000 E+00$ & $0.00000000+\infty 00$ & $0.0000000 E+\infty$ & $0.0000000 E+\infty$ & Cimut & WPO36301 & $Y$ & NA \\
\hline 3356 & 4 & CONSTANT & $0.0000000 E+\infty 0$ & $0.0000000 E+\infty 0$ & $00000000 E+\infty$ & $0,0000000+\infty 0$ & Cimus & 40CFR191 & $Y$ & NA \\
\hline 3356 & 4 & CONSTANT & $0.0000000 E+\infty 0$ & $0.0000000 E+00$ & $0,0000000 E+00$ & $0,0000000 \mathrm{E}+\infty$ & Cimu & WPO36301 & $Y$ & $N / A$ \\
\hline 3357 & 4 & CONSTANT & $0.00000000 E+\infty$ & $0.0000000 E+\infty$ & $00000000 E+\infty$ & $00000000 E+00$ & Cirmes & 40CFR191 & $Y$ & NA \\
\hline 3357 & 4 & CONSTANT & $0.0000000 \mathrm{E}+00$ & $0.0000000 \mathrm{E}+00$ & $00000000 E+\infty 0$ & $00000000 \mathrm{E}+00$ & Cimus & WP036301 & $Y$ & NA \\
\hline 3358 & 4 & CONSTANT & $0.0000000 E+00$ & $0.0000000 E+00$ & $0.0000000 \mathrm{E}+00$ & $00000000 \mathrm{E}+\infty$ & Cisurf & 40CFR191 & $Y$ & N/A \\
\hline 3358 & 4 & CONSTANT & $0.0000000 E+\infty 0$ & $0.0000000 E+\infty 0$ & $00000000 E+00$ & $00000000 E+\infty$ & Cims & WP035301 & $\mathbf{Y}$ & $N / A$ \\
\hline 3359 & 4 & CONSTANT & $0.0000000 E+00$ & $0.00000000+00$ & $00000000 E+\infty$ & $00000000 E+\infty$ & Cimut & 40CFR191 & $Y$ & NA \\
\hline 3359 & 4 & CONSTANT & $0.0000000 \mathrm{E}+00$ & $0.0000000 \mathrm{E}+00$ & $0,0000000 E+\infty$ & $0.0000000 \mathrm{E}+\infty$ & Ciment & WP036301 & $\mathbf{Y}$ & N/A \\
\hline 3360 & 4 & CONSTANT & $0.0000000 \mathrm{E}+\infty 0$ & $0.0000000 \mathrm{E}+00$ & $0.0000000 \mathrm{E}+00$ & $00000000 E+\infty 0$ & Cimnt & 10CFR191 & $Y$ & NA \\
\hline 3360 & 4 & CONSTANT & $0.00000005+00$ & $0.0000000 E+\infty 0$ & $0.0000000 E+\infty$ & $0.0000000 E+\infty$ & Ciment & WPO36301 & $Y$ & N/A \\
\hline 3361 & 4 & CONSTANT & $0.000000 \mathrm{CE}+00$ & $0.0000000 E+\infty$ & $00000000 E+\infty$ & $0.0000000 E+\infty 0$ & Cimens & 40CFR191 & $Y$ & NA \\
\hline 3381 & 4 & CONSTANT & $0.0000000 \mathrm{E}+\infty 0$ & $0.0000000 E+\infty 0$ & $00000000 E+00$ & $0.0000000 \mathrm{E}+00$ & Cinus & WPO36301 & $Y$ & NA \\
\hline 3362 & 4 & CONSTANT & $0.0000000 E+00$ & $0.0000000 E+00$ & $00000000 E+\infty$ & $00000000 E+00$ & Cimut & 40CFR191 & $Y$ & NA \\
\hline 3362 & 4 & CONSTANT & $0.0000000 \mathrm{E}+\infty$ & $0.0000000 E+\infty$ & $0.0000000 \mathrm{E}+\infty$ & $0.0000000 \mathrm{E}+\infty$ & Cimut & WP036301 & $Y$ & N/A \\
\hline 3363 & 4 & CONSTANT & $1.0000000 \mathrm{E}+02$ & $1.0000000 \mathrm{E}+02$ & $10000000 \mathrm{E}+02$ & $1.0000000 \mathrm{E}+02$ & Cimnt & 40CFR191 & $Y$ & $N A$ \\
\hline 3363 & 4 & CONSTANT & $1.0000000 \mathrm{E}+02$ & $1.0000000 \mathrm{E}+02$ & $1.0000000 \mathrm{E}+02$ & $1.0000000 \mathrm{E}+02$ & cimst & WP036301 & $\mathbf{Y}$ & $\mathrm{N} / \mathrm{A}$ \\
\hline 3364 & 4 & CONSTANT & $1.00000000 \mathrm{E}+02$ & $1.0000000 \mathrm{E}+02$ & $1.0000000 \mathrm{E}+02$ & $1.0000000 \mathrm{E}+02$ & Cimut & 40CFR191 & $\bar{Y}$ & NA \\
\hline 3364 & 4 & CONSTANT & $1.0000000 \mathrm{E}+02$ & $1.0000000 E+02$ & $10000000 \mathrm{E}+02$ & $1.0000000 \mathrm{E}+02$ & Cimut & WP036301 & $\mathbf{Y}$ & N/A \\
\hline
\end{tabular}


CCA Parameter Listing

\begin{tabular}{|c|c|c|c|c|c|c|c|c|c|c|}
\hline $\begin{array}{c}\text { Permoter in } \\
\text { Detabeae? } \\
\text { (NoN) }\end{array}$ & 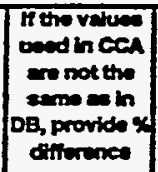 & $\mathbf{1 0}$ & Matarato & Metored Neme & $\begin{array}{c}\text { Perinetix } \\
\text { DD }\end{array}$ & Parmention & $\begin{array}{l}\text { PRP DO } \\
\text { MPOA }\end{array}$ & $\begin{array}{c}\text { Deta Enty } \\
\text { onte }\end{array}$ & 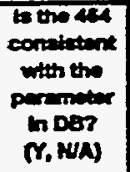 & 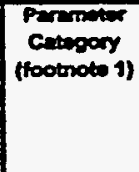 \\
\hline$Y$ & & 3365 & AN243 & Americium 243 & EPAREL & EPA Release Limit & 36927 & $16-1 \times x-96$ & $Y$ & 3 \\
\hline$Y$ & & 3365 & AN243 & Americiem 243 & EPAREL & EPA Rotasese Limik & 36927 & 16-Apr-96 & $Y$ & 3 \\
\hline$Y$ & & 3366 & CM243 & Curium 243 & EPAREL & EPA Rclaeses Limit & 36928 & $16-4 \times x-96$ & $Y$ & 3 \\
\hline$Y$ & & 3366 & CH243 & Curium 243 & EPAREL & EPA Relosise Limit & 35928 & 16-Apr-96 & $Y$ & 3 \\
\hline$Y$ & & 3367 & CN1245 & Curiem 245 & EPAREL & EPA Retasse Limit & 36929 & 16-Apr-96 & $Y$ & 3 \\
\hline$Y$ & & 3367 & CM245 & Curium 245 & EPAREL & EPA Rowane Limi & 36929 & 16 ppr-06 & $Y$ & 3 \\
\hline $\bar{Y}$ & & 3368 & CN1248 & Curium 248 & EPAREL & EPA Releasso Limit & 36330 & 16-Apr-96 & $\mathbf{Y}$ & 3 \\
\hline$Y$ & & 3368 & CH248 & Curium 248 & EPAREL & EPA Releases Limik & 36930 & $16-4 p r-96$ & $Y$ & 3 \\
\hline$Y$ & & 3369 & CS137 & Cesiem 137 & EPAREL & EPA Relasse Limit & 36931 & 16 Apr-96 & $Y$ & 3 \\
\hline $\bar{Y}$ & & 3369 & CS137 & Cesium 137 & EPAREL & EPA Releaso Limik & 36931 & 16-Apr-96 & $Y$ & 3 \\
\hline$Y$ & & 3370 & NP237 & Noptunium 237 & EPAREL & EPA Roloese Limit & 36932 & 16-Apr-96 & $Y$ & 3 \\
\hline$Y$ & & 3370 & NP237 & Nephanium 237 & EPAREL & EPA Retause Limit & 36932 & 16 Apr-96 & $Y$ & 3 \\
\hline$Y$ & & 3371 & PA231 & Protactinium 231 & EPAREL & EPA Rolos:o Limik & 36933 & 16-Apr-96 & $Y$ & 3 \\
\hline $\bar{Y}$ & & 3371 & PA231 & Protactinium 231 & EPAREL & EPA Relenese Limik & 36933 & 16 Apr-s6 & $Y$ & 3 \\
\hline$Y$ & & 3372 & PB210 & Lead 210 & EPAREI & EPA Rotosse Limit & 36934 & 16-Apr-96 & $Y$ & 3 \\
\hline$Y$ & & 3372 & P8210 & Lead 210 & EPAREL & EPA Roloases Limit & 36934 & 16-Apr.86 & $Y$ & 3 \\
\hline$Y$ & & 3373 & PU238 & Putonium 238 & EPAREL & EPA Roleasse Limit & 36935 & 16 -Apr-96 & $Y$ & 3 \\
\hline$Y$ & & 3373 & PU238 & Phtonium 238 & EPAREL & EPA Retense Limit & 36935 & 16 -Apr-96 & $Y$ & 3 \\
\hline $\mathbf{Y}$ & & 3374 & PU239 & Putonium 239 & EPAREL & EPA Reloseso Limik & 36936 & 16-Apr.96 & $\mathbf{Y}$ & 3 \\
\hline$Y$ & & 3374 & PU239 & Purtonium 239 & EPAREL & EPA Retanse Limit & 36936 & 16-Apr-86 & $Y$ & 3 \\
\hline$y$ & & 3375 & PU240 & Putonium 240 & EPAREL & EPA Releases Limit & 36937 & 16-Apr-96 & $Y$ & 3 \\
\hline$Y$ & & 3375 & PU240 & Putoniem 240 & EPAREL & EPA Roiness Limi & 36937 & 16 Apr-96 & $Y$ & 3 \\
\hline$Y$ & & 3376 & PU242 & Plutonium 242 & EPAREL & EPA Roiness Limit & 36938 & 16Apr-96 & $Y$ & 3 \\
\hline $\bar{Y}$ & & 3376 & PU242 & Pletonium 242 & EPAREL & EPA Revesso Limit & 36938 & 16-Apr-96 & $\bar{Y}$ & 3 \\
\hline$Y$ & & 3377 & PU244 & Phtorium 244 & EPAREL & EPA Roiases Limit & 36939 & 16-Apr-96 & $Y$ & 3 \\
\hline$Y$ & & 3377 & PU244 & Pletonium 244 & EPAREL & EPA Retanse Limit & 36939 & 16-Apr-96 & $y$ & 3 \\
\hline$\gamma$ & & 3378 & RA226 & Radium 226 & EPAREL & EPA Rolosaso Limit & 36940 & 16-Apr-96 & $Y$ & 3 \\
\hline$Y$ & & 3378 & RA226 & Rediem 226 & EPAREL & EPA Roterse Limit & 36960 & 16 Apr.96 & $Y$ & 3 \\
\hline$Y$ & & 3379 & SM147 & Samerium 147 & EPAREL & EPA Retaenso Linit & 36941 & 16-Apr-96 & $Y$ & 3 \\
\hline$Y$ & & 3379 & SM147 & Samarium 147 & EPAREL & EPA Relasso Limir & 36941 & $16-A p r-96$ & $Y$ & 3 \\
\hline$Y$ & & 3380 & SR9O & Strontiem 90 & EPAREL & EPA Relasso Limit & 36942 & 16-Apr-96 & $Y$ & 3 \\
\hline$Y$ & & 3380 & SR9O & Strontium 90 & EPAREL & EPA Rotesso Limit & 36942 & 16 -Apr-96 & $Y$ & 3 \\
\hline $\bar{Y}$ & & 3381 & $\pi+1229$ & Thorium 229 & EPAREL & EPA Release Limit & 36943 & 16-Apr-96 & $Y$ & 3 \\
\hline$y$ & & 3381 & $T+1229$ & Thorium 229 & EPAREL & EPA Release Limit & 36943 & 16 Apr.96 & $Y$ & 3 \\
\hline$Y$ & & 3382 & TH1230 & Thorium 230 & EPAREL & EPA Releaso Limit & 36944 & 16-Apr.96 & $y$ & 3 \\
\hline$Y$ & & 3382 & TH230 & Thorium 230 & EPAREL & EPA Rolease Limit & 36944 & 16-Apr.96 & $Y$ & 3 \\
\hline$Y$ & & 3383 & TH1232 & Thorium 232 & EPAREL & EPA Retease Limit & 36945 & 16-Apr-96 & $Y$ & 3 \\
\hline$Y$ & & 3383 & $\pi+1232$ & Thorium 232 & EPAREL & EPA Releass Limit & 36945 & 16 -Apr.96 & $\bar{Y}$ & 3 \\
\hline$Y$ & & 3384 & U233 & Urmium 233 & EPAREL & EPA Rolanses Limit & 36946 & 16 Apr-96 & $Y$ & 3 \\
\hline$Y$ & & 3384 & 4233 & Uranium 233 & EPAREL & EPA Rotanso Limit & 36946 & 16-Apr-96 & $Y$ & 3 \\
\hline$Y$ & & 3385 & $\mathbf{U 2 3 4}$ & Uranim 234 & EPAREL & EPA Rolosso Limit & 36947 & 16-Apr-96 & $Y$ & 3 \\
\hline$Y$ & & 3385 & U234 & Ureniem 234 & EPAREL & EPA Roleane Linit & 36947 & 16-Apr.96 & $Y$ & 3 \\
\hline$Y$ & & 3386 & U235 & Urmiem 235 & EPAREL & EPA Reiasas Limit & 36948 & 16-Apr-96 & $Y$ & 3 \\
\hline$y$ & & 3386 & $\mathbf{U 2 3 5}$ & Uranium 235 & EPAREL & EPA Relanse Limit & 36948 & 16-Apr-96 & $Y$ & 3 \\
\hline$Y$ & & 3387 & $\mathbf{u 2 3 6}$ & Urmiem 236 & EPAREL & EPA Retease Limit & 36949 & 16-Apr-96 & $Y$ & 3 \\
\hline$Y$ & & 3387 & 0236 & Urenium 236 & EPAREL & EPA Releaso Limit & 36949 & 16 -npr-96 & $Y$ & 3 \\
\hline$Y$ & & 3383 & U238 & Uranium 238 & EPAREL & EPA Rolanas Linit & 36950 & 16-Apr-96 & $Y$ & 3 \\
\hline$Y$ & & 3388 & 4238 & Urenium 238 & EPAREL & EPA Rolessos Limit & 36950 & 16-Apr-96 & $Y$ & 3 \\
\hline $\mathbf{Y}$ & & 3389 & SOLPLS & $\begin{array}{l}\text { Solubillty Multiptier for } \\
\text { Pund }\end{array}$ & SOLCA & 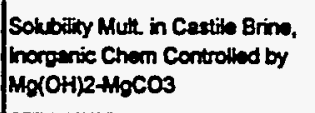 & $37111 A$ & $24+4 a y-96$ & $\mathbf{Y}$ & 9 \\
\hline $\mathbf{Y}$ & & 3389 & SOLPUA & $\begin{array}{l}\text { Solubitity Muthiplier for } \\
\text { Puth }\end{array}$ & SOLCIM & 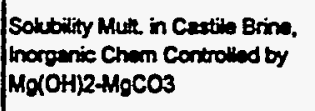 & $37191 A$ & 24+14y-s6 & $\mathbf{Y}$ & 1 \\
\hline
\end{tabular}


CCA Parameter-Listing

\begin{tabular}{|c|c|c|c|c|c|c|c|c|c|c|}
\hline 10 & 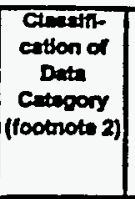 & $\begin{array}{l}\text { Distibertion } \\
\text { Type }\end{array}$ & $\operatorname{man}$ & moden & Mintrinkm & Hodrimem & Wats & 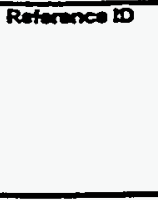 & $\begin{array}{l}\text { Fentwe deta } \\
\text { developed } \\
\text { underen } \\
\text { Nole-1 } \\
\text { Progrem? }\end{array}$ & 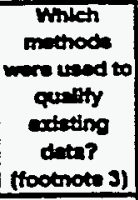 \\
\hline 3365 & 4 & CONSTANT & $1.0000000 E+02$ & $1.0000000 E+02$ & $1.0000000 \mathrm{E}+\infty 2$ & $1.0000000 E+02$ & Cihure & LOCFR191 & $\mathbf{Y}$ & NA \\
\hline 3365 & 4 & CONSTANT & $1.0000000 \mathrm{E}+02$ & $1.0000000 \mathrm{E}+02$ & $1.0000000 E+02$ & $1.0000000 \mathrm{E}+02$ & Cinut & MP036301 & $Y$ & NA \\
\hline 3366 & 4 & CONSTANT & $1.0000000 \mathrm{E}+02$ & $1.0000000 E+02$ & $1.0000000 \mathrm{E}+02$ & $1.0000000 E+02$ & Cinver & LOCFR191 & $\mathbf{Y}$ & NA \\
\hline 3366 & 4 & CONSTANT & $1.0000000 \mathrm{E}+02$ & $1.0000000 \mathrm{E}+02$ & $1.0000000 \mathrm{E}+02$ & $1.0000000=+02$ & Cimst & WP036301 & $\mathbf{Y}$ & NA \\
\hline 3367 & 4 & CONSTANT & $1.0000000 \mathrm{E}+02$ & $1.0000000 \mathrm{E}+002$ & $1.0000000 E+02$ & $1.0000000 \mathrm{E}+02$ & Cimand & 40CFR191 & $\mathbf{Y}$ & $N / A$ \\
\hline 3367 & 4 & CONSTANT & $1.0000000 \mathrm{E}+02$ & $1.0000000 E+02$ & $1.0000000 \mathrm{E}+02$ & $1.0000000 \mathrm{E}+02$ & Cimnt & WP035301 & $\mathbf{Y}$ & NA \\
\hline 3368 & 4 & CONSTANT & $1.0000000 E+02$ & $1.0000000 \mathrm{E}+02$ & $1.0000000 \mathrm{E}+02$ & $1.0000000 E+02$ & Cimut & 40CFR191 & $Y$ & NA \\
\hline 3368 & 4 & CONSTANT & $1.0000000 E+02$ & $1.0000000 E+02$ & $1.0000000 \mathrm{E}+02$ & $1.0000000 \mathrm{E}+02$ & Cimut & WP035301 & $Y$ & NA \\
\hline 3369 & 4 & CONSTANT & $1.0000000 E+03$ & $1.0000000 E+03$ & $1.0000000 E+03$ & $1.0000000 E+03$ & Cimf & 4OCFR191. & $Y$ & NA \\
\hline 3369 & 4 & CONSTANT & $1.0000000 E+03$ & $1.0000000 E+03$ & $1.0000000 \mathrm{E}+03$ & $1.0000000 \mathrm{E}+03$ & Cimnt & WP036301. & $\mathbf{Y}$ & NA \\
\hline 3370 & 4 & CONSTANT & $1.0000000 E+02$ & $1.0000000 \mathrm{E}+02$ & $1.0000000 E+02$ & $1.0000000 E+02$ & Cimut & 40CFR191 & $\mathbf{Y}$ & NA \\
\hline 3370 & 4 & CONSTANT & $1.0000000 \mathrm{E}+02$ & $1.0000000 \mathrm{E}+02$ & $1.0000000 \mathrm{E}+02$ & $1.0000000 E+02$ & Cimut & WP035301 & $\mathbf{Y}$ & NA \\
\hline 3371 & 4 & CONSTANT & $1.0000000 E+02$ & $1.0000000 \mathrm{E}+02$ & $1.0000000 \mathrm{E}+02$ & $1.0000000 \mathrm{E}+02$ & Cimut & 40CFR191 & $\mathbf{Y}$ & NA \\
\hline 3371 & 4 & CONSTANT & $1.0000000 E+02$ & $1.0000000 \mathrm{E}+02$ & $1.0000000 \mathrm{E}+02$ & $1.0000000 \mathrm{E}+02$ & Cimus: & WP036301 & $\mathbf{Y}$ & NA \\
\hline 3372 & 4 & CONSTANT & $1.0000000 \mathrm{E}+02$ & $1.0000000 E+02$ & $1.0000000 \mathrm{E}+02$ & $1.0000000 \mathrm{E}+02$ & Cimus & 40CFR191 & $Y$ & NA \\
\hline 3372 & 4 & CONSTANT & $1.0000000 \mathrm{E}+02$ & $1.0000000 E+02$ & $1.0000000 E+02$ & $1.0000000 \mathrm{E}+102$ & Cinut & WP036301 & $Y$ & NA \\
\hline 3373 & 4 & CONSTANT & $1.0000000 \mathrm{E}+02$ & $1.0000000 \mathrm{E}+02$ & $1.0000000 E+02$ & $1.0000000 E+02$ & Cinns: & 40CFR191 & $\mathbf{Y}$ & N/A \\
\hline 3373 & 4 & CONSTANT & $1.0000000 E+02$ & $1.00000000+02$ & $1.0000000 E+02$ & $1.0000000 \mathrm{E}+02$ & Cimns & WP036301 & $Y$ & NA \\
\hline 3374 & 4 & CONSTANT & $1.0000000 \mathrm{E}+02$ & $1.0000000 \mathrm{E}+02$ & $1.0000000 E+02$ & $1.0000000 E+02$ & Cimont & LOCFR191 & $\mathbf{Y}$ & NA \\
\hline 3374 & 4 & CONSTANT & $1.0000000 \mathrm{E}+02$ & $1.0000000 E+02$ & $1.0000000 \mathrm{E}+02$ & $1.0000000 \mathrm{E}+02$ & Cinut & WP036301 & $\mathbf{Y}$ & NA \\
\hline 3375 & 4 & CONSTANT & $1.0000000 \mathrm{E}+02$ & $1.0000000 \mathrm{E}+02$ & $1.0000000 \mathrm{E}+02$ & $1.0000000 E+02$ & Cinuf & 4OCFR191 & $\mathbf{Y}$ & N/A \\
\hline 3375 & 4 & CONSTANT & $1.0000000 \mathrm{E}+02$ & $1.0000000 \mathrm{E}+02$ & $1.0000000 \mathrm{E}+02$ & $1.0000000 E+02$ & Cinns & WP036301 & $Y$ & NA \\
\hline 3376 & 4 & CONSTANT & $1.0000000 \mathrm{E}+02$ & $1.0000000 \mathrm{E}+02$ & $1.0000000 \mathrm{E}+02$ & $1.0000000 E+02$ & Cimut & LOCFR191 & $\mathbf{Y}$ & NA \\
\hline 3376 & 4 & CONSTANT & $1.0000000 E+02$ & $1.0000000 E+02$ & 1.0000000E+02 & $1.0000000 E+02$ & Cimnt & WP036301 & $Y$ & $N / A$ \\
\hline 3377 & 4 & CONSTANT & $1.0000000 \mathrm{E}+02$ & $1.0000000 \mathrm{E}+02$ & $1.0000000 \mathrm{E}+02$ & $1.0000000 \mathrm{E}+02$ & Cimur & 40CFR191 & $Y$ & NA \\
\hline 3377 & 4 & CONSTANT & $1.0000000 \mathrm{E}+02$ & $1.0000000 \mathrm{E}+02$ & $1.0000000 E+02$ & $1.0000000 \mathrm{E}+02$ & cinut & WP036301 & $Y$ & NA \\
\hline 3378 & 4 & CONSTANT & $1.0000000 \mathrm{E}+02$ & $1.0000000 \mathrm{E}+02$ & $1.0000000 \mathrm{E}+02$ & $1.0000000 \mathrm{E}+02$ & Cimut & 40CFR191 & $\mathbf{Y}$ & N/A \\
\hline 3378 & 4 & CONSTANT & $1.0000000 E+02$ & $1.0000000 \mathrm{E}+02$ & $1.0000000 \mathrm{E}+02$ & $1.0000000 \mathrm{E}+02$ & Cinut & WP036301 & $\mathbf{Y}$ & NA \\
\hline 3379 & 4 & CONSTANT & $1.0000000 \mathrm{E}+02$ & $1.0000000 \mathrm{E}+02$ & $1.0000000 E+02$ & $1.0000000 E+02$ & Cimus & 40CFR191 & $Y$ & NA \\
\hline 3379 & 4 & CONSTANT & $1.00000000 E+02$ & $1.0000000 E+02$ & $1.0000000 E+02$ & $1.0000000 \mathrm{E}+02$ & Cimut & WP036301 & $\mathbf{Y}$ & N/A \\
\hline 3380 & 4 & CONSTANT & $1.0000000 E+03$ & $1.0000000 E+03$ & $1.0000000 \mathrm{E}+03$ & $1.0000000 E+03$ & Cimut & 40CFR191 & $\mathbf{Y}$ & N/A \\
\hline 3380 & 4 & CONSTANT & $1.0000000 \mathrm{E}+03$ & $1.0000000 E+03$ & $1.0000000 \mathrm{E}+03$ & $1.0000000 \mathrm{E}+03$ & Cinus & WP036301 & $Y$ & NA \\
\hline 3381 & 4 & CONSTANT & $1.0000000 \mathrm{E}+02$ & $1.0000000 \mathrm{E}+02$ & $1.0000000 E+02$ & $1.0000000 \mathrm{E}+02$ & Cinut & 40CFR191 & $Y$ & NA \\
\hline 3381 & 4 & CONSTANT & $1.0000000 \mathrm{E}+02$ & $1.0000000 E+02$ & $1.0000000 \mathrm{E}+02$ & $1.0000000 \mathrm{E}+02$ & Cinurf & WP036301 & $Y$ & NA \\
\hline 3382 & 4 & CONSTANT & $1.0000000 \mathrm{E}+01$ & $1.0000000 \mathrm{E}+01$ & $1.0000000 E+01$ & $1.0000000 \mathrm{E}+01$ & Cimut & 40CFR191 & $Y$ & NA \\
\hline 3382 & 4 & CONSTANT & $1.0000000 \mathrm{E}+01$ & $1.0000000 E+01$ & $1.0000000 E+01$ & $1.0000000 \mathrm{E}+01$ & Cinut & WP036301 & $\mathbf{Y}$ & NA \\
\hline 3393 & 4 & CONSTANT & $1.0000000 E+01$ & $1.0000000 E+01$ & $1.0000000 E+01$ & $1.0000000 E+01$ & Cimut & 40CFR191 & $\mathbf{Y}$ & NA \\
\hline 3383 & 4 & CONSTANT & $1.0000000 E+01$ & $1.0000000 E+01$ & $1.0000000 E+01$ & $1.0000000 E+01$ & Cinut & WP036301 & $Y$ & NA \\
\hline 3304 & 4 & CONSTANT & $1.0000000 \mathrm{E}+02$ & $1.0000000 \mathrm{E}+02$ & $1.0000000 E+02$ & $1.0000000 E+02$ & Ciswout & 40CFR191 & $Y$ & N/A \\
\hline 3384 & 4 & CONSTANT & $1.0000000 \mathrm{E}+02$ & $1.0000000 E+02$ & $1.0000000 E+02$ & $1.0000000 \mathrm{E}+02$ & Cimut & WP036301 & $\mathbf{Y}$ & NA \\
\hline 3385 & 4 & CONSTANT & $1.0000000 \mathrm{E}+02$ & $1.0000000 \mathrm{E}+02$ & $1.0000000 E+02$ & $1.0000000 \mathrm{E}+02$ & Cisurd & 40CFR191 & $Y$ & N/A \\
\hline 3385 & 4 & CONSTANT & $1.0000000 \mathrm{E}+02$ & $1.0000000 E+02$ & $1.0000000 E+02$ & $1.0000000 \mathrm{E}+02$ & Cinns & WP036301 & $Y$ & NA \\
\hline 3386 & 4 & CONSTANT & $1.0000000 \mathrm{E}+02$ & $1.0000000 \mathrm{E}+02$ & $1.0000000 E+02$ & $1.0000000 \mathrm{E}+02$ & Cinnet & 40CFR191 & $\mathbf{Y}$ & NA \\
\hline 3386 & 4 & CONSTANT & $1.0000000 E+02$ & $1.0000000 E+02$ & $1.0000000 E+02$ & $1.0000000 \mathrm{E}+02$ & Cirwut & WP035301 & $Y$ & NA \\
\hline 3387 & 4 & CONSTANT & $1.0000000 \mathrm{E}+02$ & $1.0000000 E+02$ & $1.0000000 E+02$ & $1.0000000 E+02$ & Cimst & 40CFR191 & $\boldsymbol{Y}$ & NA \\
\hline 3387 & 4 & CONSTANT & $1.0000000 \mathrm{E}+02$ & $1.0000000 \mathrm{E}+02$ & $1.0000000 E+02$ & $1.0000000 E+02$ & Cimunf & WP036301 & $Y$ & NA \\
\hline 3388 & 4 & CONSTANT & $1.0000000 \mathrm{E}+02$ & $1.0000000 E+02$ & $1.0000000 \mathrm{E}+02$ & $1.0000000 E+02$ & Cimnt & 40CFR191 & $Y$ & N/A \\
\hline 3388 & 4 & CONSTANT & $1.0000000 \mathrm{E}+02$ & $1.0000000 \mathrm{E}+02$ & $1.0000000 E+02$ & $1.0000000 E+02$ & Cinurf & WPO36301 & $Y$ & NA \\
\hline 3389 & 1,3 & CUMULATTVE & $1.8000000 \mathrm{E}-01$ & $-9.0000000 \mathrm{E}-02$ & $-2.0000000 E+\infty 0$ & $1.4000000 E+\infty 0$ & NONE & WP035268 & $\mathbf{Y}$ & 1 \\
\hline 3389 & 1,3 & CUMULATIVE & $1.8000000 E-01$ & $-9.00000000-02$ & $-2.0000000 E+\infty 0$ & $1.4000000 E+\infty 0$ & NONE & WP035835 & $\mathbf{Y}$ & 1 \\
\hline
\end{tabular}


CCA Parameter Listing

\begin{tabular}{|c|c|c|c|c|c|c|c|c|c|c|}
\hline $\begin{array}{c}\text { Permotar in } \\
\text { Datebe:e? } \\
\text { (YN) }\end{array}$ & 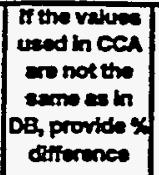 & $\mathbf{D}$ & Meteratio & Metarial Neme & Parmiar & Parmax Kers & $\begin{array}{c}\text { PRP D } \\
\text { fWP }\end{array}$ & $\begin{array}{c}\text { Deta Entry } \\
\text { Dets }\end{array}$ & 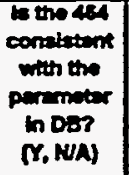 & $\begin{array}{c}\text { Parmiter } \\
\text { Cathoory } \\
\text { (footinote 1) }\end{array}$ \\
\hline $\mathbf{Y}$ & & 3389 & SOLPU4 & $\begin{array}{l}\text { Solubidty Mutiplier for } \\
\text { Puth }\end{array}$ & SOLCM & 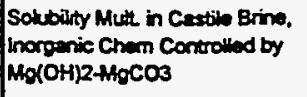 & $37111 A$ & $24+M=y-96$ & $\mathbf{Y}$ & 1 \\
\hline $\mathbf{Y}$ & & 3389 & SOLPU & $\begin{array}{l}\text { Solubility Mulliplies for } \\
\text { Putd }\end{array}$ & SOLCA & 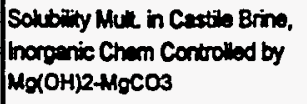 & $37111 A$ & $24+4 a y-96$ & $\mathbf{Y}$ & 1 \\
\hline $\mathbf{Y}$ & & 3390 & SOLU4 & $\begin{array}{l}\text { Solubility Multiplicer for } \\
\text { Uta }\end{array}$ & SOLSAM & $\begin{array}{l}\text { Solubitity whin in Seledo Bring. } \\
\text { norganic Chem Controlled by } \\
\text { Mg(O+1)2+MgCO3 }\end{array}$ & $37112 A$ & $24+4 a y-96$ & $\mathbf{Y}$ & 1 \\
\hline $\mathbf{Y}$ & & 3390 & SOLUA & $\begin{array}{l}\text { Solubility Muttiplior for } \\
\text { UtA }\end{array}$ & SOLSA & $\begin{array}{l}\text { Solubity Hul in Sulado Brino. } \\
\text { horganic Chem Controlled by } \\
\mathrm{Mg}(\mathrm{OH}) 2+\mathrm{HCO}\end{array}$ & $37112 A$ & 24May-96 & $\mathbf{Y}$ & 1 \\
\hline $\mathbf{Y}$ & & 3390 & SOLUA & $\begin{array}{l}\text { Solubitity Multiplier for } \\
\text { U+4 }\end{array}$ & SOLSM & 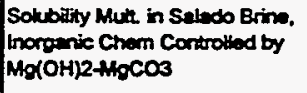 & $37112 A$ & 24 May-96| & $\mathbf{Y}$ & 1 \\
\hline $\mathbf{Y}$ & & 3390 & SOLU4 & $\begin{array}{l}\text { Solubility Multiplier for } \\
\text { U }+ \text { a }\end{array}$ & SOLSM & 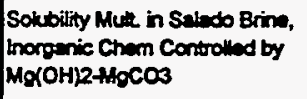 & $37112 A$ & $24+4 a y-96$ & $\mathbf{Y}$ & 1 \\
\hline $\mathbf{Y}$ & & 3390 & SOLU4 & $\begin{array}{l}\text { Solubility Multiplier for } \\
U+4\end{array}$ & SOLSM & $\begin{array}{l}\text { Solubitity mule in Saludo Brino, } \\
\text { morganic Chem Controlied by } \\
\text { Mg(OH)2 MgCO3 }\end{array}$ & $37112 A$ & 244ay-96 & $\mathbf{Y}$ & 1 \\
\hline $\mathbf{Y}$ & & 3391 & SOLU6 & $\begin{array}{l}\text { Solubility Multiplior for } \\
\text { U*6 }\end{array}$ & SOLSIM & $\begin{array}{l}\text { Solubitity Mult in Sulado Brine, } \\
\text { Inorgmic Chem Controtied by } \\
\mathrm{Mg}(\mathrm{OH}) 2 \mathrm{HgCO} 3\end{array}$ & $37113 A$ & 24May-96 & $\mathbf{Y}$ & 1 \\
\hline $\mathbf{Y}$ & & 3391 & SOLUG & $\begin{array}{l}\text { Solubility Muttiplior for } \\
\text { U+6 }\end{array}$ & SOLSIM & $\begin{array}{l}\text { Solubility Mute in Seledo Brime. } \\
\text { Inoroanic Chom Controlied by } \\
\text { Mg(OH)2+HgCO3 }\end{array}$ & $37113 A$ & 24May-96 & $\mathbf{Y}$ & 1 \\
\hline $\mathbf{Y}$ & & 3391 & SOLU6 & $\begin{array}{l}\text { Solkbility Muttiplier for } \\
U \neq 6\end{array}$ & SOLSM & 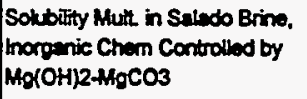 & $37113 A$ & 24-May-96 & $\mathbf{Y}$ & 1 \\
\hline $\mathbf{Y}$ & & 3391 & SOLUS & $\begin{array}{l}\text { Solubirity Muliplier for } \\
\text { U+6 }\end{array}$ & SOLSIM & $\begin{array}{l}\text { Solubitty Mutc in Salado Brine, } \\
\text { Inorganic Chem Controlled by } \\
\mathrm{Mg}(\mathrm{OH}) 2+\mathrm{HgCO} 3\end{array}$ & $37113 A$ & $24+4 a y-96$ & $\mathbf{Y}$ & 1 \\
\hline $\mathbf{Y}$ & & 3391 & SOLU6 & $\begin{array}{l}\text { Solubility Muttiplior for } \\
\text { U+6 }\end{array}$ & SOLSIM & $\begin{array}{l}\text { Solubility Mult in Salado Brine, } \\
\text { Inorgenic Chem Contolled by } \\
\mathrm{MgOH)2+MgCO3}\end{array}$ & $37113 A$ & 24-May-96 & $\mathbf{Y}$ & 1 \\
\hline $\mathbf{Y}$ & & 3392 & SOLUG & $\begin{array}{l}\text { Solubility Multiplier for } \\
\text { U+6 }\end{array}$ & SOLCM & $\begin{array}{l}\text { Solubility Muth in Cestila Brine, } \\
\text { Inorgenic Chem Controlied by } \\
\mathrm{Mg}(\mathrm{OH}) 2 \mathrm{HgCO} 3\end{array}$ & $37114 A$ & 24-May-96 & $\boldsymbol{Y}$ & 9 \\
\hline $\mathbf{Y}$ & & 3392 & SOLUS & $\begin{array}{l}\text { Solubility Muliplier for } \\
\text { U+6 }\end{array}$ & SOLCIM & $\begin{array}{l}\text { Solubility Mult in Cestile Brine, } \\
\text { tinorganic Chem Controlled by } \\
\mathrm{Mg}(\mathrm{OH}) 2+\mathrm{HgCO} 3\end{array}$ & $37114 \mathrm{~A}$ & $24+M=y-56$ & $\mathbf{Y}$ & 1 \\
\hline $\mathbf{Y}$ & & 3392 & SOLUS & $\begin{array}{l}\text { Solubitity Mutuiptior for } \\
\text { U+6 }\end{array}$ & SOLCIM & $\begin{array}{l}\text { Solubity Mul in Ceatil Brine, } \\
\text { inorganic Cham Controllad by } \\
\mathrm{M}(\mathrm{OH}) 2 \mathrm{Hg} \mathrm{CO} 3\end{array}$ & $37114 A$ & 24+May-S6 & $\mathbf{Y}$ & 1 \\
\hline $\mathbf{Y}$ & & 3392 & SOLUS & $\begin{array}{l}\text { Solubility Mutiplior for } \\
\text { U+6 }\end{array}$ & SOLCA & $\begin{array}{l}\text { Sohbility Mult in Castiva Brin, } \\
\text { Inorganic Chem Controlled by } \\
\left.\mathrm{Mg}_{\mathrm{O}} \mathrm{OH}\right) 2+\mathrm{HOCO} 3\end{array}$ & $37114 A$ & $24+4 a y-96$ & $\mathbf{Y}$ & 1 \\
\hline$Y$ & & 3392 & SOLUS & $\begin{array}{l}\text { Solubitity Mutiplier for } \\
\text { U+6 }\end{array}$ & SOLCM & $\begin{array}{l}\text { Solubility Muth in Castile Brino, } \\
\text { thorganic Chem Controlled by } \\
\mathrm{Mg}_{(\mathrm{OH}) 2 \mathrm{HgCO} 3}\end{array}$ & 371144 & 24 May-96 & $\mathbf{Y}$ & 1 \\
\hline $\mathbf{Y}$ & & 3393 & SOLTHA & $\begin{array}{l}\text { Solubitity Mutiptior for } \\
\text { Then }\end{array}$ & SOLSEM & 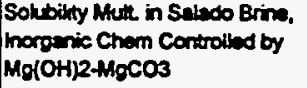 & $37115 A$ & 24+May-96 & $Y$ & 1 \\
\hline
\end{tabular}


CCA Parameter Listing

\begin{tabular}{|c|c|c|c|c|c|c|c|c|c|c|}
\hline 10 & $\begin{array}{c}\text { Criatin- } \\
\text { cattion of } \\
\text { Data } \\
\text { Category } \\
\text { (footnote 2) }\end{array}$ & $\begin{array}{l}\text { Dherfbution } \\
\text { Type }\end{array}$ & Man & Medien & Mlntmum & Merdroun & Units & Reterwesce id & $\begin{array}{l}\text { Wes tho dett } \\
\text { doveloped } \\
\text { under } \\
\text { Noi-1 } \\
\text { Progrem? }\end{array}$ & 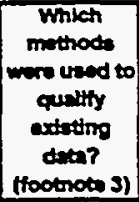 \\
\hline 3389 & 1,3 & CUMULATNE & $1.8000000 E-01$ & $-9.0000000 E-02$ & $-2.0000000 E+\infty 0$ & $1.4000000 E+\infty 0$ & NONE & WPO37545 & $\mathbf{Y}$ & 1 \\
\hline 3389 & 1,3 & CUMULATINE & $1.8000000 E-01$ & $-9.0000000 E-02$ & $-2.0000000 E+\infty$ & $1.4000000 E+\infty 0$ & NONE & WPO40512 & $\mathbf{Y}$ & 1 \\
\hline 3390 & 1,3 & CUMULATIVE & $1.8000000 E-01$ & $-9.0000000 E-02$ & $-2.0000000 E+\infty 0$ & $1.4000000 E+\infty 0$ & NONE & WP035268 & $\mathbf{Y}$ & 1 \\
\hline 3390 & 1,3 & CUMULATIVE & $1.8000000 E-01$ & $-9.0000000 E-02$ & $-2,0000000 E+00$ & $1.4000000 E+00$ & NONE & WP035835 & $\mathbf{Y}$ & 1 \\
\hline 3390 & 1,3 & CUMULATIVE & $1.8000000 E-01$ & $-9.0000000 E-02$ & $-20000000 E+\infty 0$ & $1.4000000 E+\infty 0$ & NONE & WP037536 & $\mathbf{Y}$ & 1 \\
\hline 3390 & 1,3 & CUMULATIVE & $1.8000000 E-01$ & $-9.0000000 \mathrm{E}-02$ & $-2,0000000 E+\infty 0$ & $1.4000000 \mathrm{E}+00$ & NONE & WP037515 & $\mathbf{Y}$ & 9 \\
\hline 3390 & 1,3 & CUMULATIVE & $1.8000000 E-01$ & $-9.0000000 \mathrm{E}-02$ & $-2,0000000 E+\infty 0$ & $1.4000000 E+\infty 0$ & NONE & WPO40512 & $\mathbf{Y}$ & 1 \\
\hline 3391 & 1,3 & CUMULATINE & $1.8000000 E-01$ & $-9.0000000 E-02$ & $-2.0000000 E+\infty$ & $1.4000000 E+\infty 0$ & NONE & WP035268 & $\mathbf{Y}$ & 1 \\
\hline 3391 & 1,3 & CUMULATIVE & $1.8000000 E-01$ & $-9.0000000 E-02$ & $-2.0000000 E+\infty$ & $14000000 E+\infty)$ & NONE & WP035835 & $\mathbf{Y}$ & 1 \\
\hline 3391 & 1,3 & CUMULATINE & $1.8000000 E-01$ & $-9.0000000 \mathrm{E}-02$ & $-20000000 E+\infty$ & $14000000 E+00$ & NONE & WP037536 & $\mathbf{Y}$ & 1 \\
\hline 3391 & 1,3 & CUMULATNE & $1.8000000 E-01$ & $-90000000 E-02$ & $-20000000 E+\infty 0$ & $1+000000+\infty$ & NONE & WP037545 & $\mathbf{Y}$ & 1 \\
\hline 3391 & 1,3 & CUMULATNE & $1.8000000 E-01$ & $-90000000 E-02$ & $-20000000 E+\infty 0$ & $14000000 E+\infty$ & NONE & WPO40512 & $\mathbf{Y}$ & 1 \\
\hline 3392 & 1,3 & CUMULATIVE & $1.8000000 E-01$ & $-90000000 E-02$ & $-20000000 \in+\infty$ & $1+000000 E+\infty$ & NONE & WP035268 & 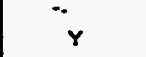 & 1 \\
\hline 3392 & 1,3 & CUMULATIVE & $1.8000000 \mathrm{E}-01$ & $-90000000 E-02$ & $.20000000 \in+00$ & $1,0000000 E+\infty 0$ & NONE & WP035835 & $\gamma$ & 1 \\
\hline 3392 & 1,3 & CUMULATTVE & $1.8000000 E-01$ & $-90000000 E-02$ & $-20000000 E+\infty 0$ & $1+000000 \mathrm{E}+\infty 0$ & NONE & WP037536 & $\mathbf{Y}$ & 1 \\
\hline 3392 & 1,3 & CUMULATINE & $1.8000000 E-01$ & $-9.00000000-02$ & $-20000000<-\infty$ & $14000000 E+\infty$ & NONE & WP037545 & $\mathbf{Y}$ & 1 \\
\hline 3392 & 1,3 & CUMULATNE & $1.8000000 E-01$ & $-90000000 \mathrm{E}-02$ & $-20000000 E+00$ & $1+0000000+\infty$ & NONE & WPO40512 & $\mathbf{Y}$ & 1 \\
\hline 3393 & 1.3 & CUMULATIVE & $1.8000000 E-01$ & $-9.0000000 E-02$ & $-20000000 E+\infty$ & $14000000 E+\infty$ & NONE & WP035268 & $\mathbf{Y}$ & 1 \\
\hline
\end{tabular}


CCA Parameter Listing

\begin{tabular}{|c|c|c|c|c|c|c|c|c|c|c|}
\hline $\begin{array}{c}\text { Parmination in } \\
\text { Dotabeas? } \\
\text { (r/N) }\end{array}$ & \begin{tabular}{|c|} 
If the velues \\
uned in cach \\
ore not the \\
seme an in \\
OB, provide $x$ \\
difference
\end{tabular} & ID & Materale iD & Mntard Nem & $\begin{array}{c}\text { Paremitar } \\
\text { D }\end{array}$ & Perminer Ken & $\begin{array}{l}\text { PRP to } \\
\text { NhPo a }\end{array}$ & Detentry & 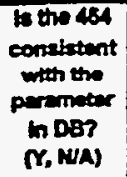 & $\begin{array}{l}\text { Parnmeter } \\
\text { Cortepory } \\
\text { (footinote 1) }\end{array}$ \\
\hline $\mathbf{Y}$ & & 3393 & SOLTHA & $\begin{array}{l}\text { Solubilty Mulliplier for } \\
\text { Th+4 }\end{array}$ & SOLSIM & $\begin{array}{l}\text { Solubildy Muth in Salado Brino, } \\
\text { inorganic Chem Controlied by } \\
\text { Hg(OH)2HoCO3 }\end{array}$ & $37115 A$ & 244ky-96 & $\mathbf{Y}$ & 1 \\
\hline $\mathbf{Y}$ & & 3393 & SOLTH4 & $\begin{array}{l}\text { Solubitity Multiptiar for } \\
\text { Th+4 }\end{array}$ & SOLSAM & $\begin{array}{l}\text { Solubility Muth in Saledo Brine, } \\
\text { inoroanic Chem Controlled by } \\
\text { Mo(OH)2-MoCO3 }\end{array}$ & $37115 A$ & 24 May-96 & $\mathbf{Y}$ & 1 \\
\hline $\mathbf{Y}$ & & 3393 & SOLTHA & $\begin{array}{l}\text { Sokbillty Multiplier for } \\
\text { Th+4 }\end{array}$ & SOLSIM & $\begin{array}{l}\text { Solubitity Mut in Saludo Bring. } \\
\text { Inorganic Chem Controlind by } \\
\text { Mo(OH)2+16CO3 }\end{array}$ & $37115 A$ & 24-4hay-96 & $\mathbf{Y}$ & 1 \\
\hline $\mathbf{Y}$ & & 3393 & SOLTHA & $\begin{array}{l}\text { Solubility Muttiplier for } \\
\text { Th+4 }\end{array}$ & SOLSEM & $\begin{array}{l}\text { Solubitity Mult in Salado Brino, } \\
\text { Irorganic Chem Controlled by } \\
\text { Mo(OH)2+19CO3}\end{array}$ & $37115 A$ & $24+\operatorname{Mry}-96$ & $\mathbf{Y}$ & 1 \\
\hline $\mathbf{Y}$ & & 3402 & SOLMOD3 & $\begin{array}{l}\text { Oxidation state III } \\
\text { model }\end{array}$ & SOLCM & $\begin{array}{l}\text { Solubility Mut in Cestib Brino. } \\
\text { Inorganic Chan Controlied by } \\
\text { MglOH)2MgCO3 }\end{array}$ & $37125 A$ & 24May-96 & $\mathbf{Y}$ & 1 \\
\hline $\mathbf{Y}$ & & 3402 & SOLMOD3 & $\begin{array}{l}\text { Oxidation state III } \\
\text { modal }\end{array}$ & SOLCIM & 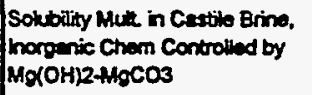 & $37125 A$ & $24+4=y-96$ & $\mathbf{Y}$ & 1 \\
\hline $\mathbf{Y}$ & & 3402 & SOLMOD3 & $\begin{array}{l}\text { Oxidation state [i] } \\
\text { modei }\end{array}$ & SOLCM & $\begin{array}{l}\text { Soltbitity Mult in Cestio Brme, } \\
\text { thorganic Cham Controlied by } \\
\text { Mg(OH)2HgCO3 }\end{array}$ & $37125 A$ & 24 May-96 & $\mathbf{Y}$ & 1 \\
\hline $\mathbf{Y}$ & & 3402 & SOLMOD3 & $\begin{array}{l}\text { Oxidetion date III } \\
\text { model }\end{array}$ & SOLCIM & $\begin{array}{l}\text { Solubility Mull in Cestib Brino, } \\
\text { Inorganic Chem Controlled by } \\
\mathrm{Mg}(\mathrm{OH}) 2+\mathrm{NoCO} 3\end{array}$ & $37125 A$ & $24+4 a y-96$ & $\mathbf{Y}$ & 1 \\
\hline$-\mathbf{Y}$ & & 3402 & SOLMOD3 & $\begin{array}{l}\text { Oxidation state III } \\
\text { model }\end{array}$ & SOLCIM & $\begin{array}{l}\text { Solubility Mull in Cestib Brine. } \\
\text { Inorganic Chem Controlted by } \\
\mathrm{Mg}(\mathrm{OH}) 2+\mathrm{HCO}\end{array}$ & $37125 A$ & $24+4=y-96$ & $\mathbf{Y}$ & 1 \\
\hline $\mathbf{Y}$ & & 3402 & SOLMOD3 & $\begin{array}{l}\text { Oxidation state III } \\
\text { model }\end{array}$ & SOLCIM & $\begin{array}{l}\text { Solubility Mulh in Cestib Brine, } \\
\text { Inorganic Chem Controlled by } \\
\mathrm{Mg}(\mathrm{OH}) 2+\mathrm{HCO} 3\end{array}$ & $37125 A$ & $24+M n y-96$ & $\mathbf{Y}$ & 1 \\
\hline $\mathbf{Y}$ & & 3402 & SOLMOD3 & $\begin{array}{l}\text { Oxidation state III } \\
\text { model }\end{array}$ & SOLCIM & $\begin{array}{l}\text { Solubility Mull in Castile Brine. } \\
\text { Inoromic Chem Controlled by } \\
\text { Mo(OH)2 MgCO3 }\end{array}$ & $37125 A$ & 24+May-86 & $\mathbf{Y}$ & 1 \\
\hline $\mathbf{Y}$ & & 3402 & SOLMOD3 & $\begin{array}{l}\text { Oxidation state III } \\
\text { mode! }\end{array}$ & SOLCM & $\begin{array}{l}\text { Solubility Muth n Castie Brine, } \\
\text { Inoranic Chem Controlled by } \\
\text { Mg(OH)2+MgCO3 }\end{array}$ & $37125 A$ & $24-M a y-96$ & $\mathbf{Y}$ & 1 \\
\hline $\mathbf{Y}$ & & 3403 & SOLMOD4 & $\begin{array}{l}\text { Oxidation state } N \\
\text { modal }\end{array}$ & SOLCM & $\begin{array}{l}\text { Soludity Mull in Cestile Brine, } \\
\text { norganc Chem Controlled by } \\
\text { Mg(OH)2 } 2 \mathrm{MgCO} 3\end{array}$ & 37126 & 24-Apr-96 & $\begin{array}{r}-\cdot \\
Y\end{array}$ & 1 \\
\hline$Y$ & & 3403 & SOLMODA & $\begin{array}{l}\text { Oxidation state } N \\
\text { model }\end{array}$ & SOLCM & 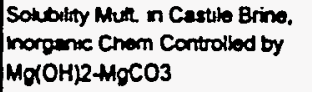 & 37126 & 24 Apr-SS & $\mathbf{Y}$ & 1 \\
\hline $\mathbf{Y}$ & & 3403 & SOLMODA & $\begin{array}{l}\text { Oxidation state } N \\
\text { model }\end{array}$ & SOLCM & $\begin{array}{l}\text { Soludithy Muth in Castule Brine. } \\
\text { Imorganc Chem Controlied by } \\
\text { MoOH)2 MoCO3 }\end{array}$ & 37125 & 24Apr-96 & $\mathbf{Y}$ & 1 \\
\hline $\mathbf{Y}$ & & 3403 & SOLMODA & $\begin{array}{l}\text { Oxidation atate } N \\
\text { model }\end{array}$ & SOLCAM & 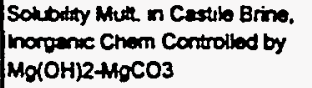 & 37126 & 24-Apr-96 & $\mathbf{Y}$ & 1 \\
\hline $\mathbf{Y}$ & & 3404 & SOLMODS & $\begin{array}{l}\text { Oxidetion state V } \\
\text { model }\end{array}$ & SOLCIM & $\begin{array}{l}\text { Solubility Muk in Cestive Brino, } \\
\text { Ithorganc Chem Controlled by } \\
\text { Mg(OH)2 } \mathrm{MgCO} 3\end{array}$ & 37127 & 24-Apr-86 & $\mathbf{Y}$ & 1 \\
\hline $\mathbf{Y}$ & & 3404 & SOLMOD5 & $\begin{array}{l}\text { Oxidation state } V \\
\text { modal }\end{array}$ & SOLCM & 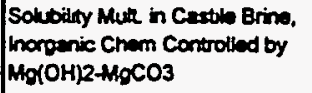 & 37127 & $24-1 p r-96$ & $\mathbf{Y}$ & 1 \\
\hline
\end{tabular}


CCA Parameter Listing

\begin{tabular}{|c|c|c|c|c|c|c|c|c|c|c|}
\hline 10 & $\begin{array}{l}\text { Cluseffi- } \\
\text { cestion of } \\
\text { Duta } \\
\text { Cintegory } \\
\text { (footnote 2) }\end{array}$ & $\begin{array}{l}\text { Detribution } \\
\text { Type }\end{array}$ & Man & Medien & Mintroum & Meximum & Units & Reforence ID & $\begin{array}{l}\text { Was the dath } \\
\text { dereloped } \\
\text { underen } \\
\text { NoA-1 } \\
\text { Progrem? }\end{array}$ & 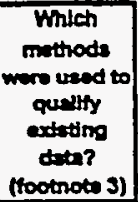 \\
\hline 3393 & 1,3 & CUMULATIVE & $1,8000000 E-01$ & $-9.0000000 \mathrm{E}-02$ & $-20000000 E+\infty 0$ & $1.4000000 E+\infty 0$ & NONE & WP035835 & $\mathbf{Y}$ & 1 \\
\hline 3393 & 1,3 & CUMULATNE & $1.8000000 E-01$ & $-9.0000000 E-02$ & $-2,0000000 E+00$ & $1.4000000=+\infty$ & NONE & WP037536 & $\mathbf{Y}$ & 1 \\
\hline 3393 & 1,3 & CUMULATINE & 1.8000000E-01 & $-9.0000000 E-02$ & $-2.0000000 E+\infty 0$ & $1.4000000=+\infty$ & NONE & WP037545 & $\mathbf{Y}$ & 1 \\
\hline 3393 & 1,3 & CUMULATIVE & $1.8000000 E-01$ & $-9.0000000 \mathrm{E}-02$ & $-2.0000000 E+\infty 0$ & $1.4000000 E+\infty 0$ & NONE & WP040512 & $\mathbf{Y}$ & 1 \\
\hline 3402 & 1 & CUMULATIVE & $1.9700000 E-07$ & $6.5200000 E-08$ & $6.5200000=-09$ & $6.5200000 E-07$ & moleshiter & WP035268 & $\boldsymbol{\gamma}$ & 1 \\
\hline 3402 & 1 & CUMULATIVE & $1.9700000 E-07$ & $6.5200000 E-08$ & $6.5200000 E-09$ & $6.5200000 E-07$ & molesfitor & WP035835 & $\mathbf{Y}$ & 1 \\
\hline 3402 & 1 & CUMULATINE & $1.9700000 \mathrm{E}-07$ & $6.5200000 E-08$ & $6.5200000 E-09$ & $6.5200000 E-07$ & molesiter & WPO36053 & $\mathbf{Y}$ & 1 \\
\hline 3402 & 1 & CUMULATIVE & $1.9700000 E-07$ & $6.5200000 E-08$ & $6.5200000 E-09$ & $6.5200000 E-07$ & moloshter & WP037536 & $\mathbf{Y}$ & 1 \\
\hline 3402 & 1 & CUMULATINE & $1.9700000 E-07$ & $6.5200000 E-08$ & $6.5200000 E-09$ & $6.5200000 \mathrm{E}-07$ & moleshiter & WP037545 & $\mathbf{Y}$ & 1 \\
\hline 3402 & 1 & CUMULATIVE & $1.9700000 E-07$ & 6.5200000 E- -08 & $6.5200000 E-09$ & $6.5200000 \mathrm{E}-07$ & moleshiter & WP037822 & $\boldsymbol{Y}$ & 1 \\
\hline 3402 & 1 & CUMULATINE & $1.9700000 E-07$ & $6.5200000 E-08$ & $6.5200000 E-09$ & $6.5200000 \mathrm{E}-07$ & molesiter & WP040512 & $\mathbf{Y}$ & 1 \\
\hline 3402 & 1 & CUMULATIVE & $1.9700000 E-07$ & $6.5200000 E-08$ & $6.5200000 E-09$ & $6.5200000 \mathrm{E}-07$ & motastitor & WP041556 & $\mathbf{Y}$ & 1 \\
\hline 3403 & 1 & CUMULATIVE & $1.8000000 E-08$ & $6.0000000 E-09$ & $6.0000000 E-10$ & $6.0000000 E-08$ & moteshiter & WPO35268 & $\bar{Y}$ & 1 \\
\hline 3403 & 1 & CUMULATME & $1.8000000 E-08$ & $6.0000000 E-09$ & $6.0000000 E-10$ & $6.0000000 E-08$ & molesniter & WPO35835 & $\mathbf{Y}$ & 1 \\
\hline 3403 & 1 & CUMULATIVE & $1.8000000 E-08$ & $6.0000000 \mathrm{E}-09$ & $6.0000000 E-10$ & $6.0000000 \mathrm{E}-08$ & moleshitor & WP036053 & $\mathbf{Y}$ & 1 \\
\hline 3403 & 1 & CUMULATIVE & $1.8000000 E-00$ & $6.0000000=-09$ & $6.0000000 E-10$ & $6.0000000 \mathrm{E}-08$ & moleshiter & WPOA1556 & $\mathbf{Y}$ & 1 \\
\hline 3404 & 1 & CUMULATIVE & $6.7000000 E-06$ & $2.2000000 E-06$ & 2.2000000E-07 & $2.2000000 E-05$ & molositer & WP035268 & $\mathbf{Y}$ & 1 \\
\hline 3404 & 1 & CUMULATIVE & $6.7000000=-06$ & $2.2000000 E-06$ & 2.2000000E-07 & $2.2000000 E-05$ & moleahiter & WP035835 & $\mathbf{\gamma}$ & 1 \\
\hline
\end{tabular}


CCA Parameter Listing

\begin{tabular}{|c|c|c|c|c|c|c|c|c|c|c|}
\hline $\begin{array}{c}\text { Paremoter tn } \\
\text { Databeen? } \\
\text { (YhI) }\end{array}$ & 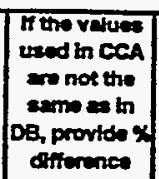 & DD & Matura to & Material kans & $\begin{array}{c}\text { Purmiter } \\
10\end{array}$ & Parmoter Nerno & $\begin{array}{l}\text { PRP To } \\
\text { MPOA }\end{array}$ & $\begin{array}{c}\text { Deinentry } \\
\text { Dute }\end{array}$ & $\begin{array}{l}\text { is the } 464 \\
\text { contabtant } \\
\text { whth the } \\
\text { pormmeter } \\
\text { in De? } \\
(X, N A)\end{array}$ & $\begin{array}{l}\text { Permetar } \\
\text { Cotegory } \\
\text { (foconote 1) }\end{array}$ \\
\hline $\mathbf{Y}$ & & 3404 & SOLMOD5 & $\begin{array}{l}\text { Oxidation state } V \\
\text { model }\end{array}$ & SOLCM & 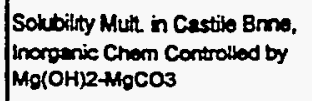 & 37127 & $24-4 p r+96$ & $\mathbf{Y}$ & 1 \\
\hline $\mathbf{Y}$ & & 3404 & SOLMODS & $\begin{array}{l}\text { Oxidation stute } V \\
\text { model }\end{array}$ & SOLCAM & $\begin{array}{l}\text { Solubilty Mute in Castile Brme, } \\
\text { inorganic Chem Controliad by } \\
\text { Mg(OH)2+HgCO3 }\end{array}$ & 37127 & 24 pr-86 & $\mathbf{Y}$ & 1 \\
\hline $\mathbf{Y}$ & & 3404 & SOLMOD5 & $\begin{array}{l}\text { Oxidation state V } \\
\text { model }\end{array}$ & SOLCIM & $\begin{array}{l}\text { Solubility Muth in Castile Brine. } \\
\text { inorganic Chem Contsolled by } \\
M O(\mathrm{OH}) 2+\mathrm{HCO}_{3}\end{array}$ & 37127 & 24-Apr-96 & $\mathbf{Y}$ & 1 \\
\hline $\mathbf{Y}$ & & 3404 & SOLMODS & $\begin{array}{l}\text { Oxidation state V } \\
\text { model }\end{array}$ & SOLCM & $\begin{array}{l}\text { Solubihty Huth in Cestive Brine, } \\
\text { Irorganic Chem Controlled by } \\
\text { Mg(OH)2 } \mathrm{HgCO} 3\end{array}$ & 37127 & 24Apr-96 & $\mathbf{Y}$ & 1 \\
\hline $\mathbf{Y}$ & & 3404 & SOLMODS & $\begin{array}{l}\text { Oxidation state V } \\
\text { model }\end{array}$ & SOLCM & $\begin{array}{l}\text { Solubilty Mult in Cestib Brins, } \\
\text { Inorganic Chem Controlied by } \\
\text { Mo(OH)2HoCO3 }\end{array}$ & 37127 & $24 A p r-56$ & $\mathbf{Y}$ & 1 \\
\hline $\mathbf{Y}$ & & 3405 & SOLMODS & $\begin{array}{l}\text { Oxidation state } \mathrm{V} \\
\text { model }\end{array}$ & SOLCAM & $\begin{array}{l}\text { Solubility Mutt in Cestila Brine, } \\
\text { Inorgenic Chem Controlled by } \\
\mathrm{Mo}(\mathrm{OH}) 2+\mathrm{MgO} 3\end{array}$ & 37128 & 24-Apr-96 & $\mathbf{Y}$ & 1 \\
\hline $\mathbf{Y}$ & & 3405 & SOLMODS & $\begin{array}{l}\text { Oxidation state V } \\
\text { model }\end{array}$ & SOLCIM & $\begin{array}{l}\text { Solubility Muth in Castive Brine, } \\
\text { Inorganic Chem Controliad by } \\
\text { Mg(OH)2 } \mathrm{MgCO} 3\end{array}$ & 37128 & $24-A p r-96$ & $\mathbf{Y}$ & 1 \\
\hline $\mathbf{Y}$ & & 3405 & SOLMODS & $\begin{array}{l}\text { Oxidation state VI } \\
\text { model }\end{array}$ & SOLCIM & $\begin{array}{l}\text { Solubilty Muth in Cestiv Brine, } \\
\text { Inorganic Chem Controlled by } \\
\mathrm{Mg}(\mathrm{OH}) 2+\mathrm{gCO} 3\end{array}$ & 37128 & $24-A p r-96$ & $\mathbf{Y}$ & 1 \\
\hline $\mathbf{Y}$ & & 3405 & SOLMOD6 & $\begin{array}{l}\text { Oxidation state V } \\
\text { modal }\end{array}$ & SOLCa & $\begin{array}{l}\text { Solubitity Muth in Cestile Brino, } \\
\text { inorganic Chem Controlled by } \\
\text { MgOH) } 2+\mathrm{HgCO} 3\end{array}$ & 37128 & 24Apr-96 & $\mathbf{Y}$ & 1 \\
\hline $\mathbf{Y}$ & & 3405 & SOLMODS & $\begin{array}{l}\text { Oxidation stato VI } \\
\text { modol }\end{array}$ & SOLCM & $\begin{array}{l}\text { Sohubilty Mult in Castilo Brine, } \\
\text { Inorganic Chom Controlled by } \\
\mathrm{Mg}(\mathrm{OH}) 2+\mathrm{HgCO} 3\end{array}$ & 37128 & 24-Apr-96 & $\mathbf{Y}$ & 1 \\
\hline$Y$ & & 3405 & SOLMODG & $\begin{array}{l}\text { Oxidation state v } \\
\text { model }\end{array}$ & SOLCIM & $\begin{array}{l}\text { Solubilty Mult, in Castile Brino, } \\
\text { Inorganic Chem Controlled by } \\
\mathrm{Mg}(\mathrm{OH}) 2+\mathrm{HgCO}\end{array}$ & 37128 & $24-4 p x-96$ & $\mathbf{Y}$ & 1 \\
\hline $\mathbf{Y}$ & & 3405 & SOLMODS & $\begin{array}{l}\text { Oxidation state V } \\
\text { model }\end{array}$ & SOLCIM & $\begin{array}{l}\text { Solubility Mult. in Castile Bmmo. } \\
\text { Inorganic Chem Controlled by } \\
\mathrm{Mg}(\mathrm{OH}) 2 \mathrm{MgCO} 3\end{array}$ & 37128 & 24-Apr-96 & $\mathbf{Y}$ & 1 \\
\hline $\mathbf{Y}$ & & 3406 & SOLMOD3 & $\begin{array}{l}\text { Oxidation state III } \\
\text { model }\end{array}$ & SOLSIM & $\begin{array}{l}\text { Solubilty Mult. in Salado Brine, } \\
\text { inorganic Chem Controlled by } \\
\mathrm{Mg}(\mathrm{OH}) 2+\mathrm{HgCO} 3\end{array}$ & $37129 A$ & 24+Aay-96 & $\mathbf{Y}$ & 1 \\
\hline $\mathbf{Y}$ & & 3406 & SOLMOD3 & $\begin{array}{l}\text { Oxidation state III } \\
\text { model }\end{array}$ & SOLSIM & $\begin{array}{l}\text { Solubilty Mutt in Saledo Brine, } \\
\text { Inorganx Chem Controllod by } \\
M g(O H) 2+\mathrm{HCO}\end{array}$ & 371294 & $24+1$ ay-96 & $\mathbf{Y}$ & 1 \\
\hline $\mathbf{Y}$ & & 3406 & SOLMOD3 & $\begin{array}{l}\text { Oxidation atate III } \\
\text { model }\end{array}$ & SOLSM & $\begin{array}{l}\text { Solubilty Mult in Salado Bmo, } \\
\text { horgenic Chem Controlled by } \\
\text { Mg(OH)2 } 2 \text { gCO3 }\end{array}$ & $37129 A$ & $24+1=y-96$ & $\mathbf{Y}$ & 1 \\
\hline $\mathbf{Y}$ & & 3406 & SOLMOD3 & $\begin{array}{l}\text { Oxidetion stete III } \\
\text { model }\end{array}$ & SOLSM & $\begin{array}{l}\text { Solubifity Muth in Salado Brine. } \\
\text { Inorganic Chem Controlled by } \\
\mathrm{Mg}(\mathrm{OH}) 2 \mathrm{MgCO}\end{array}$ & $37129 A$ & 24+May-96 & $\mathbf{Y}$ & 1 \\
\hline $\mathbf{Y}$ & & 3406 & SOLMOD3 & $\begin{array}{l}\text { Oxidetion state III } \\
\text { model }\end{array}$ & SOLSIM & $\begin{array}{l}\text { Solubilty Mult in Salado Bmro. } \\
\text { Inorganic Chem Controliod by } \\
\mathrm{Mg}(\mathrm{OH}) 2+\mathrm{gCO} 3\end{array}$ & 371284 & $24+40 y-96$ & $\mathbf{Y}$ & 1 \\
\hline $\mathbf{Y}$ & & 3406 & SOLMOD3 & $\begin{array}{l}\text { Oxidation atate III } \\
\text { model }\end{array}$ & SOLSM & 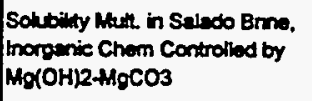 & $37129 A$ & $24+115 y-96$ & $\mathbf{Y}$ & 1 \\
\hline
\end{tabular}


CCA Parameter Listing

\begin{tabular}{|c|c|c|c|c|c|c|c|c|c|c|}
\hline ID & 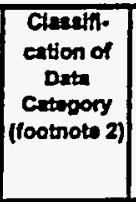 & $\begin{array}{l}\text { Dtatribution } \\
\text { Typo }\end{array}$ & Man & Medien & Mintmum & Medimum & Untes & Reference iD & $\begin{array}{l}\text { Wes the detal } \\
\text { developed } \\
\text { underen } \\
\text { Noh-1 } \\
\text { Progrem? }\end{array}$ & 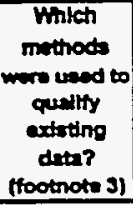 \\
\hline 3404 & 1 & CUMULATIVE & $6.7000000 E-06$ & $22000000 E-06$ & $22000000 E-07$ & $22000000 E-05$ & nolesinter| & WP036053 & $\mathbf{Y}$ & 1 \\
\hline 3404 & 1 & CUMULATIVE & $6.7000000 E-06$ & $2.2000000 E-06$ & $22000000 E-07$ & $2.2000000 \mathrm{E}-05$ & nolestiter & WP037536 & $\mathbf{Y}$ & 1 \\
\hline 3404 & 1 & CUMULATIVE & $6.7000000 \mathrm{E}-06$ & $2.2000000 E-06$ & $22000000 E-07$ & $22000000=-05$ & motesintor & WP037545 & $\mathbf{Y}$ & 1 \\
\hline 3404 & 1 & CUMULATIVE & $6.7000000 E-06$ & $22000000 E-06$ & 2.2000000E-07| & $22000000 E-05$ & moleshiter| & WP040512 & $\mathbf{Y}$ & 1 \\
\hline 3404 & 1 & CUMULATINE & $6.7000000 \mathrm{E}-06$ & $2.2000000 E-06$ & $2.2000000 E-07$ & $22000000 E-05$ & moveshiter & WP041556 & $\mathbf{Y}$ & 1 \\
\hline 3405 & 1 & CUMULATIVE & $27000000 E-05$ & $8.8000000 E-06$ & $8.8000000 E-07$ & $8.8000000 E-05$ & molosititer & WP035268 & $\mathbf{Y}$ & 1 \\
\hline 3405 & 1 & CUMULATIVE & $27000000 E-0.5$ & $8.8000000 E-06$ & $8.8000000 E-07$ & 8.8000000E-0.5 & moloshiter & WP035835 & $\mathbf{Y}$ & 1 \\
\hline 3405 & 1 & CUMULATIVE & $27000000 E-05$ & B.8000000E-06 & $8.8000000 E-07$ & 8.8000000E-05 ? & moleshiter & WP036053 & $\mathbf{Y}$ & 1 \\
\hline 3405 & 1 & CUMULATIVE & $2.7000000 \mathrm{E}-05$ & $8.8000000 E-06$ & $8.8000000 E-07$ & $8.8000000 E-05$ & moleshiter & WP037536 & $\mathbf{Y}$ & 1 \\
\hline 3405 & 1 & CUMULATIVE & $2.7000000 E-0.5$ & $8.8000000 E-06$ & $8.8000000 E-07$ & 8.8000000E-05 & moleshiter & WP037545 & Y & 1 \\
\hline 3405 & 1 & CUMULATIVE & $2.7000000 E-05$ & $8.8000000 E-06$ & $8.8000000 E-07$ & $8.8000000 E-05$ & molesiter & WP040512 & $\mathbf{Y}$ & 1 \\
\hline 3405 & 1 & CUMULATNE & $27000000 E-05$ & $8.8000000 E-06$ & $8.8000000 E-07$ & $8.8000000 E-05$ & molesniter & WPO41556 & $\mathbf{Y}$ & 1 \\
\hline 3406 & 1 & CUMULATNE & $1.7600000 E-06$ & $5.8200000 E-07$ & $5.8200000 \mathrm{E}-08$ & $5.0200000 E-06$ & moles/itior & WP035268 & ${ }^{\prime} \mathrm{r}$ & 1 \\
\hline 3406 & 1 & CUMULATIVE & $1.7600000 E-06$ & $5.8200000 \mathrm{E}-07$ & $5.8200000 E-08$ & $5.8200000 \mathrm{E}-06$ & molosititor & WP035835 & $\mathbf{Y}$ & 1 \\
\hline 3406 & 1 & CUMULATNE & $1.7600000 E-06$ & $5.8200000 E-07$ & $5.8200000 \mathrm{E}-08$ & $5.8200000 E-06$ & molasiter & WP036053 & $\mathbf{Y}$ & 1 \\
\hline 3406 & 1 & CUMULATINE & $1.7600000 E-06$ & $5.8200000 E-07$ & $5.6200000 \mathrm{E}-08$ & $5.8200000 E-06$ & molesinter & WP037536 & $\mathbf{Y}$ & 1 \\
\hline 3406 & 1 & CUMULATNE & $1.7600000 E-06$ & $5.8200000 E-07$ & $5.8200000 E-08$ & $5.8200000 E-06$ & motesniter & WPO37545 & $\mathbf{Y}$ & 1 \\
\hline 3406 & 1 & CUMULATIVE & $1.7600000 E-06$ & $5.8200000 E-07$ & $5.8200000 E-08$ & $5.8200000 E-06$ & moleshiter & WP037822 & $\mathbf{Y}$ & 1 \\
\hline
\end{tabular}


CCA Parameter Listing

\begin{tabular}{|c|c|c|c|c|c|c|c|c|c|c|}
\hline $\begin{array}{l}\text { Permet in } \\
\text { Decabsea? } \\
\text { (YNI) }\end{array}$ & 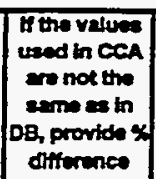 & DD & Materted ID & Mataral Nam & $\begin{array}{c}\text { Paremeter } \\
\text { to }\end{array}$ & Porvetar kerse & $\begin{array}{l}\text { PRP DO } \\
\text { MPO in }\end{array}$ & $\begin{array}{l}\text { Dete Entry } \\
\text { Deto }\end{array}$ & 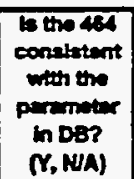 & $\begin{array}{l}\text { Parmintar } \\
\text { Cattegory } \\
\text { (footinote 1) }\end{array}$ \\
\hline $\mathbf{Y}$ & & 3406 & SOLMOD3 & $\begin{array}{l}\text { Oxudation state IIt } \\
\text { model }\end{array}$ & SOLSEM & $\begin{array}{l}\text { Solubility Muth in Salado Brim. } \\
\text { Inorganic Chem Controtlod by } \\
\text { Mg(OH)2 MoCO3 }\end{array}$ & 371294 & $24 \mathrm{Mry}-96$ & $\mathbf{Y}$ & 1 \\
\hline $\mathbf{Y}$ & & 3406 & SOLMOD3 & $\begin{array}{l}\text { Oxidetion state III } \\
\text { model }\end{array}$ & SOLSM & $\begin{array}{l}\text { Solubility Meth in Selado Brine, } \\
\text { inorganic Chem Controled by } \\
\mathrm{Mg}(\mathrm{OH}) 2 \mathrm{HgCO} 3\end{array}$ & $37129 A$ & 24-May-S6 & $\mathbf{Y}$ & 1 \\
\hline $\mathbf{Y}$ & & 3407 & SOLMODA & $\begin{array}{l}\text { Oxidabon state } N \\
\text { model }\end{array}$ & SOLSM & $\begin{array}{l}\text { Solubility Mult in Salado Brire, } \\
\text { inorganic Chem Controlied by } \\
\text { Mo(OH)2 } \mathrm{MgCO}^{2}\end{array}$ & $\mathbf{3 7 1 3 0}$ & $24 A p \times-96$ & $\mathbf{Y}$ & 1 \\
\hline $\mathbf{Y}$ & & 3407 & SOLMOD4 & $\begin{array}{l}\text { Oxidation stato } N \\
\text { Imodel }\end{array}$ & SOLSIM & $\begin{array}{l}\text { Solubitity Mult in Salado Brine, } \\
\text { Inorganic Chem Controlled by } \\
\text { Mg(OH)2 HgCO3 }\end{array}$ & 37130 & 24Apr-96 & $\mathbf{Y}$ & 1 \\
\hline$Y$ & & 3407 & SOLMOD4 & $\begin{array}{l}\text { Oxidation state } N \\
\text { model }\end{array}$ & SOLSIM & $\begin{array}{l}\text { Solubity Mutt in Salado Brine, } \\
\text { Inorganic Chem Contothed by } \\
\text { MoXOH)2HoCO3 }\end{array}$ & 37130 & 24 Apr-96 & $\mathbf{Y}$ & 1 \\
\hline $\mathbf{Y}$ & & 3407 & SOLMOD4 & $\begin{array}{l}\text { Oxidation state } N \\
\text { model }\end{array}$ & SOLSM & $\begin{array}{l}\text { Solubitity Muth in Salado Bring, } \\
\text { Inorganic Chem Controlied by } \\
\text { Mg(OH)2MgCO3 }\end{array}$ & 37130 & 24Apr-96 & $\mathbf{Y}$ & 1 \\
\hline $\mathbf{Y}$ & & 3407 & SOLMOD4 & $\begin{array}{l}\text { Oxidation state N } \\
\text { model }\end{array}$ & SOLSIM & $\begin{array}{l}\text { Solubility Muth in Salado Brine, } \\
\text { Inorganic Chem Controlled by } \\
\mathrm{Hg}(\mathrm{OH}) 2+\mathrm{HOCO} 3\end{array}$ & 37130 & $24 A p r-96$ & $\mathbf{Y}$ & 1 \\
\hline $\mathbf{Y}$ & & 3407 & SOLMOD4 & $\begin{array}{l}\text { Oxidation state N } \\
\text { model }\end{array}$ & SOLSIM & $\begin{array}{l}\text { Solubitity Murt in Salado Brine, } \\
\text { inorganic Chem Controlled by } \\
\text { Mg(OH)2 } 2 \text { gCO3 }\end{array}$ & 37130 & 24-Apr-96 & $\cdot \mathbf{Y}$ & 1 \\
\hline $\mathbf{Y}$ & & 3407 & SOLMOD4 & $\begin{array}{l}\text { Oxidation state } N \\
\text { model }\end{array}$ & SOLSM & $\begin{array}{l}\text { Solubilty Muth in Salado Brine, } \\
\text { Inorganic Chem Controllod by } \\
\text { Mg(OH)2 } M g C O 3\end{array}$ & 37130 & 24Apr-96 & $\mathbf{Y}$ & 1 \\
\hline $\mathbf{Y}$ & & 3408 & SOLMOD5 & $\begin{array}{l}\text { Oxidation state V } \\
\text { model }\end{array}$ & SOLSIM & $\begin{array}{l}\text { Solubility Mult, in Salado Brime, } \\
\text { inorganic Chem Controlled by } \\
\text { Mg(OH)2MgCO3 }\end{array}$ & 37131 & $24-A p r-96$ & $\mathbf{Y}$ & 1 \\
\hline $\mathbf{Y}$ & & 3408 & SOLMOD5 & $\begin{array}{l}\text { Oxidation state V } \\
\text { model }\end{array}$ & SOLSIM & $\begin{array}{l}\text { Solubilty Muth in Salado Brme, } \\
\text { Inorganic Chem Controlled by } \\
\mathrm{Mg}(\mathrm{OH}) 2 \mathrm{HgCO} 3\end{array}$ & 37131 & 24 Apr-96 & $\mathbf{Y}$ & 1 \\
\hline $\mathbf{Y}$ & & 3408 & SOLMODS & $\begin{array}{l}\text { Oxidation state V } \\
\text { model }\end{array}$ & SOLSM & $\begin{array}{l}\text { Solubility Muth in Salado Eme, } \\
\text { Inorganic Chem Controlied by } \\
\text { Mg(OH)2+HgCO3 }\end{array}$ & 37131 & 24-Apr-96 & $\mathbf{Y}$ & 1 \\
\hline $\mathbf{Y}$ & & 3408 & SOLMODS & $\begin{array}{l}\text { Oxidation state V } \\
\text { model }\end{array}$ & SOLSLM & $\begin{array}{l}\text { Soktuility Muth in Salado Brine, } \\
\text { inorganx Chem Controlled by } \\
\text { Mo(OH)2+4gCO3 }\end{array}$ & 37131 & 24-Apr-96 & $\ddot{Y}$ & 1 \\
\hline $\mathbf{Y}$ & & 3408 & SOLMODS & $\begin{array}{l}\text { Oxidation state V } \\
\text { model }\end{array}$ & SOLSIM & $\begin{array}{l}\text { Solubilty Mult in Salado Brino, } \\
\text { Inorganic Chem Controlled by } \\
\text { Mg(OH)2+HgCO3 }\end{array}$ & 37131 & 24 Apr-96 & $\mathbf{Y}$ & 1 \\
\hline $\mathbf{Y}$ & & 3408 & SOLMOD5 & $\begin{array}{l}\text { Oxidation state V } \\
\text { model }\end{array}$ & SOLSIM & $\begin{array}{l}\text { Solubitity Mulk in Salado Brine, } \\
\text { Inorpanic Chem Controlied by } \\
\text { Mo(OH)2+HgCO3 }\end{array}$ & 37131 & 24 Apr-96 & $\mathbf{Y}$ & 1 \\
\hline $\mathbf{Y}$ & & 3408 & SOLMODS & $\begin{array}{l}\text { Oxidation atute } V \\
\text { model }\end{array}$ & solstM & $\begin{array}{l}\text { Solubility Mult in Salado Brine, } \\
\text { Inoromic Chem Comsolled by } \\
\text { Mg(OH)2+MgCO3 }\end{array}$ & 37131 & $24 n p x-96$ & $\mathbf{Y}$ & 1 \\
\hline $\mathbf{Y}$ & & 3409 & SOLMODS & $\begin{array}{l}\text { Oxidation state V } \\
\text { model }\end{array}$ & SOLSIM & $\begin{array}{l}\text { Solubilty Mult, in Saledo Brine. } \\
\text { Inorganic Chem Controllod by } \\
\text { Mg(OH)2HgCO3 }\end{array}$ & 37132 & 24-Apr-96 & $Y$ & 1 \\
\hline $\mathbf{Y}$ & & 3409 & SOLMODS & $\begin{array}{l}\text { Oxidetion state V } \\
\text { model }\end{array}$ & SOLSM & $\begin{array}{l}\text { Sokbitity Muth in Salodo Brms. } \\
\text { Inorganic Chom Controlled by } \\
\mathrm{Mg}(\mathrm{OH}) 2 \mathrm{HgCO} 3\end{array}$ & 37132 & $24-1 p r-96$ & $\mathbf{Y}$ & 1 \\
\hline
\end{tabular}


CCA Parameter Listing

\begin{tabular}{|c|c|c|c|c|c|c|c|c|c|c|}
\hline ID & 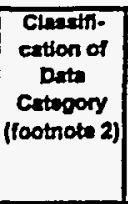 & $\begin{array}{l}\text { Otatibution } \\
\text { Type }\end{array}$ & $\operatorname{mean}$ & medisn & minhmum & Madrinum & Units & Reference ID & $\begin{array}{l}\text { Was the deta } \\
\text { demuloped } \\
\text { undaren } \\
\text { Nol-1 } \\
\text { Progrum? }\end{array}$ & 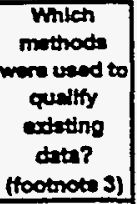 \\
\hline 3406 & 1 & CUMULATNE & $1.7600000 E-06$ & $5.8200000 E-07$ & $5.8200000 E-09$ & $5.8200000 \mathrm{E}-06$ & molesniter & WP0.6512 & $Y$ & 1 \\
\hline 3406 & 1 & CUMULATIVE & $1.7600000 E-06$ & $5.8200000=-07$ & $5.8200000 E-08$ & $5.8200000 E-06$ & molvestiter & WP041556 & $\mathbf{Y}$ & 1 \\
\hline 3407 & 1 & CUMULATINE & $1.3000000 E-05$ & $4.4000000 E-06$ & 4.4000000E-07 & 4.4000000 E- -05 & molventiter & WP035268 is & $\mathbf{Y}$ & 1 \\
\hline 3407 & 1 & CUMULATIVE & $1.3000000 E-05$ & $4.4000000 E-06$ & $4.4000000 E-07$ & 1.40000000E-05 & moliashititer & WP035835 & $\mathbf{Y}$ & 1 \\
\hline 3407 & 1 & CUMULATIVE & $1.3000000 E-05$ & 4.4000000E-06 & $4.4000000 \varepsilon-07$ & $4.4000000 E-05$ & molessiter & WP036053 & $Y$ & 1 \\
\hline 3407 & 1 & CUMULATIVE & $1.3000000 E-05$ & $4.4000000 E-06$ & $4.4000000 E-07$ & 4.4000000E-05 & moleshiter & WP037536 & $\mathbf{Y}$ & 1 \\
\hline 3407 & 1 & CUMULATIVE & $1.3000000 E-05$ & $4.4000000 E-06$ & 4.4000000E-07 & $4.4000000 E-05$ & moleshitior & WP037545 & $\mathbf{Y}$ & 1 \\
\hline 3407 & 1 & CUMULATIVE & $1.3000000 E-05$ & $4.4000000 E=-06$ & 4.4000000E-07 & 4.4000000E-05 & molesinter & WP040512 & $\mathbf{Y}$ & 1 \\
\hline 3407 & 1 & CUMULATIVE & $1.3000000 E-05$ & $4.4000000 E-06$ & $4.4000000 E-07$ & $4.4000000 E-05$ & molosinter & MP041556 & $Y$ & 1 \\
\hline 3408 & 1 & CUMULATNE & $7.0000000 E-06$ & $23000000 E-06$ & $2.3000000 E-07$ & $23000000 E-05$ & moleshiter & WP035268 & $\mathbf{Y}$ & 1 \\
\hline 3408 & 1 & CUMULATNE & $7.0000000 E-06$ & $2.3000000 E-06$ & $2.3000000 E-07$ & $2.3000000 E-05$ & molesitter & WP035835 & $\mathbf{Y}$ & 1 \\
\hline 3408 & 1 & CUMULATIVE & $7.0000000 E-06$ & $23000000 E-06$ & $2.3000000 E-07$ & $2.3000000 E-05$ & molesititer & WP036053 & $\mathbf{Y}$ & 1 \\
\hline 3408 & 1 & CUMULATIVE & $7.0000000 E-06$ & $23000000 E-06$ & $2.3000000 E-07$ & $2.3000000 E-05$ & molashiter & WP037536 & $\bar{\gamma}$ & 1 \\
\hline 3408 & 1 & CUMULATME & $7.0000000 E-06$ & $23000000 E-06$ & $23000000 E-07$ & $2.3000000 E-05$ & motesnitior & WPO37545 & $\mathbf{Y}$ & 1 \\
\hline 3408 & 1 & CUMULATNE & $7.0000000 E-06$ & $23000000 E-06$ & $2.3000000 E-07$ & $2.3000000 E-05$ & molesfiter & WPO40512 & $\gamma$ & 1 \\
\hline 3408 & 1 & CUMULATIVE & $7.0000000 E-06$ & $2.3000000 E-06$ & $2.3000000 E-07$ & $2.3000000 E-05$ & molesititor & WPO41556 & $\mathbf{Y}$ & 1 \\
\hline 3409 & 3,4 & CUMULATTVE & $2.6000000 \mathrm{E}-05$ & B.7000000E-06 & $8.7000000 E-07$ & $8.7000000 E-05$ & molesniter & WP035268 & $\mathbf{Y}$ & 1 \\
\hline 3409 & 3.4 & CUMULATIVE & $26000000 E-0.5$ & $8.7000000 E-06$ & $8.7000000 E-07$ & $8.7000000 E-05$ & moveliter & WP035835 & $\mathbf{Y}$ & 1 \\
\hline
\end{tabular}


CCA Parameter Listing

\begin{tabular}{|c|c|c|c|c|c|c|c|c|c|c|}
\hline $\begin{array}{c}\text { Paramitar in } \\
\text { Datubes? } \\
\text { (YN) }\end{array}$ & 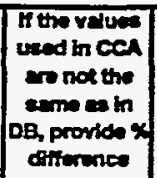 & ID & Mxarta to & Matard Kim & Permitar & Paramiar Kam & $\begin{array}{l}\text { PRPD } \\
\text { MPON) }\end{array}$ & Detery & 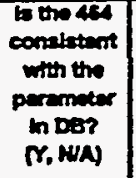 & $\begin{array}{l}\text { Parminter } \\
\text { Ceteocy } \\
\text { footiote } 1)\end{array}$ \\
\hline $\mathbf{Y}$ & & 3409 & SOLMODG & $\begin{array}{l}\text { Oxidation stato V } \\
\text { model }\end{array}$ & SOLSIM & $\begin{array}{l}\text { Solubifty Mult in Solado Bmo, } \\
\text { inorgenic Chem Controllod by } \\
\mathrm{MO(OH)2+4OCO3}\end{array}$ & 37132 & $24-A p r-96$ & $\mathbf{Y}$ & 1 \\
\hline $\mathbf{Y}$ & & 3409 & SOLMOD6 & $\begin{array}{l}\text { Oxidation stato VI } \\
\text { model }\end{array}$ & SOLSM & 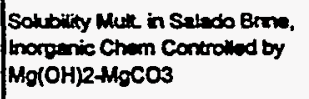 & 37132 & 24 Apr-96 & $\mathbf{Y}$ & 1 \\
\hline $\mathbf{Y}$ & & 3409 & SOLMODS & $\begin{array}{l}\text { Oxidation state V } \\
\text { model }\end{array}$ & SOLSiM & $\begin{array}{l}\text { Solubility Muth in Salado Brino, } \\
\text { trorganic Chem Controllod by } \\
\mathrm{MO}(\mathrm{OH}) 2 \mathrm{HgCO} 3\end{array}$ & 37132 & 24 Apr-96 & $\mathbf{Y}$ & 1 \\
\hline $\mathbf{Y}$ & & 3409 & SOLMODS & $\begin{array}{l}\text { Oxidation state V } \\
\text { model }\end{array}$ & SOLSIM & $\begin{array}{l}\text { Solubility Muth in Salado Brine, } \\
\text { Inorganic Chem Controliad by } \\
\text { Mg(OH)2+HgCO3 }\end{array}$ & 37132 & 24 Apr-96 & $\mathbf{Y}$ & 1 \\
\hline $\mathbf{Y}$ & & 3409 & SOLMODS & $\begin{array}{l}\text { Oxidation state V } \\
\text { model }\end{array}$ & SOLSAY & $\begin{array}{l}\text { Solubitity Mut. in Salado Brine, } \\
\text { inorganic Chem Controlled by } \\
\mathrm{Mg}(\mathrm{OH}) 2+\mathrm{HOCO} 3\end{array}$ & 37132 & 24-Apr-96 & $\mathbf{Y}$ & 1 \\
\hline $\mathbf{Y}$ & & 3410 & CM243 & Curium 243 & INVCHD & $\begin{array}{l}\text { Inventory of Contect Handied } \\
\text { Design }\end{array}$ & $37133 A$ & 290 act 96 & $\mathbf{Y}$ & 2 \\
\hline $\mathbf{Y}$ & & 3410 & CM243 & Curium 243 & INVCHD & $\begin{array}{l}\text { Inventory of Cortect Handled } \\
\text { Design }\end{array}$ & 371334 & 29-0ct-96 & $\mathbf{Y}$ & 2 \\
\hline $\mathbf{Y}$ & & 3410 & CN1243 & Curium 243 & INNCHO & $\begin{array}{l}\text { Inventory of Contect Hendled } \\
\text { Desion }\end{array}$ & $37133 A$ & 29-0ct-96 & $\mathbf{Y}$ & 2 \\
\hline $\mathbf{Y}$ & & 3410 & $\mathrm{CH} 243$ & Curium 243 & INVCHD & $\begin{array}{l}\text { Inventory of Contact Handled } \\
\text { Design }\end{array}$ & 371334 & 29-0ct-96 & $\mathbf{Y}$ & 2 \\
\hline $\mathbf{Y}$ & & 3411 & CM243 & Cunum 243 & INVRHD & $\begin{array}{l}\text { Iimentory of Remote Hendred } \\
\text { Design }\end{array}$ & 371344 & $29-0 c t .96$ & $\mathbf{Y}$ & 2 \\
\hline $\mathbf{Y}$ & & 3411 & CM243 & Curium 243 & INVRHD & $\begin{array}{l}\text { Imventory of Rernote Hendied } \\
\text { Design }\end{array}$ & $37134 A$ & 290 ct.96 & $\mathbf{Y}$ & 2 \\
\hline$Y$ & & 3411 & CM1243 & Curium 243 & INVRHD & $\begin{array}{l}\text { Imventory of Remote Handled } \\
\text { Design }\end{array}$ & $37134 A$ & $29-0$ at-96 & $\mathbf{Y}$ & 2 \\
\hline $\mathbf{Y}$ & & 3411 & CM1243 & Curium 243 & INNRHD & $\begin{array}{l}\text { inventiony of Remote Handled } \\
\text { Desuon }\end{array}$ & 371344 & $2900 t-96$ & $\mathbf{Y}$ & 2 \\
\hline $\mathbf{Y}$ & & 3412 & CM245 & cunum 245 & INVCHD & $\begin{array}{l}\text { Inventory of Conted Handied } \\
\text { Design }\end{array}$ & $37135 A$ & $29-0$ c.95 & $\mathbf{Y}$ & 2 \\
\hline $\mathbf{Y}$ & & 3412 & CM1245 & Curum 245 & INVCHD & $\begin{array}{l}\text { timuntory of Coneact Handled } \\
\text { Desegn }\end{array}$ & $37135 A$ & $29-0$ ct.96 & $\mathbf{Y}$ & 2 \\
\hline $\mathbf{Y}$ & & 3412 & CM1245 & Curium 245 & INVCHO & $\begin{array}{l}\text { Invertory of Coneact Handled } \\
\text { Desion }\end{array}$ & $37135 A$ & $29-0$ t-96 & $\mathbf{Y}$ & 2 \\
\hline $\mathbf{Y}$ & & 3412 & CM1245 & Cumen 245 & IINCKD & $\begin{array}{l}\text { Imventory of Corteca Handled } \\
\text { Desion }\end{array}$ & $37135 A$ & $29-0$ ct-96 & $\mathbf{Y}$ & 2 \\
\hline $\mathbf{Y}$ & & 3413 & CH245 & Currum 245 & IANRAO & $\begin{array}{l}\text { Imrencory of Remote Handled } \\
\text { Doepen }\end{array}$ & $37136 \mathrm{~A}$ & $29-0$ ct.96 & $\mathbf{Y}$ & 2 \\
\hline $\mathbf{Y}$ & & 3413 & CM:45 & Curium 245 & INAROD & $\begin{array}{l}\text { mumeon of Remote Handlod } \\
\text { Dexpn }\end{array}$ & $37136 A$ & $29-0$ ct-96 & $\mathbf{Y}$ & 2 \\
\hline $\mathbf{Y}$ & & 3413 & CH1245 & Cumm 245 & banaono & 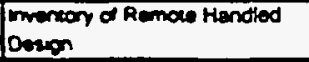 & $37136 \mathrm{~A}$ & $29-0$ ct.96 & $\cdot \cdot \cdot Y$ & 2 \\
\hline $\mathbf{Y}$ & & 3413 & CH245 & Cunum 245 & AnRoco & 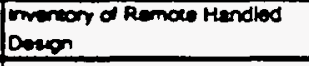 & $37136 A$ & 29-0ct-96 & $Y$ & 2 \\
\hline$Y$ & & 3414 & BOREHOLE & Borehote and Fal & me & Unt of num & 37137 & O4-Dec-96 & $\mathbf{Y}$ & 2 \\
\hline $\mathbf{Y}$ & & 3414 & BOREHOLE & Borthote and Fit & mer & une of numes & 37137 & $04-000-96$ & $Y$ & 2 \\
\hline $\mathbf{Y}$ & & 3414 & BOREHOLE & Borento end FA & mer & ine of num & 37137 & O4-Dec-96 & $\mathbf{Y}$ & 2 \\
\hline $\mathbf{Y}$ & & 3414 & BOREHOLE & Borehole and Fa & mor & une of numer & 37137 & 04-Dec-86 & $Y$ & 2 \\
\hline $\mathbf{Y}$ & & 3415 & A4243 & Ammearn 243 & incero & 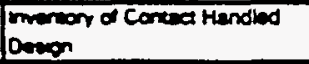 & 371384 & $29-0$ ct-96 & $\mathbf{Y}$ & 2 \\
\hline $\mathbf{Y}$ & & 3415 & AN243 & Amencam 243 & mancro & 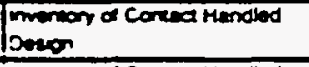 & $37138 A$ & 29-0ct-96 & $\mathbf{Y}$ & 2 \\
\hline $\mathbf{Y}$ & & 3415 & AMR43 & Amencaun 243 & mancos & $\begin{array}{l}\text { Inverion of Cortect Hendled } \\
\text { Dever }\end{array}$ & $37138 A$ & $29-0 \mathrm{ct}-96$ & $\mathbf{Y}$ & 2 \\
\hline $\mathbf{Y}$ & & 3415 & AH2 & Amencium 243 & invero & $\begin{array}{l}\text { mentary of Conect Hendied } \\
\text { Dopen }\end{array}$ & $37138 A$ & $29-0 c t-96$ & $\mathbf{Y}$ & 2 \\
\hline $\mathbf{Y}$ & & 3416 & $A N 1243$ & Anencam 243 & WNato & $\begin{array}{l}\text { Imerion of Remote Handied } \\
\text { Deson }\end{array}$ & $37139 A$ & $29-0$ ct-96 & $\mathbf{Y}$ & 2 \\
\hline $\mathbf{Y}$ & & 3416 & AMr243 & Anenceum 243 & INARE & $\begin{array}{l}\text { imenory of Remoto Hendled } \\
\text { Dewan }\end{array}$ & $37139 A$ & $29-0 c t-96$ & $\mathbf{Y}$ & 2 \\
\hline $\mathbf{Y}$ & & 3416 & AMR13 & Amencaum 243 & IANRTOD & $\begin{array}{l}\text { menteory of Remoto Handied } \\
\text { Deengen }\end{array}$ & 371384 & $22-0$ ct-96 & $\mathbf{Y}$ & 2 \\
\hline $\mathbf{Y}$ & & 3416 & AH243 & Amencoum 243 & INVRO & $\begin{array}{l}\text { Imention of Remoto Handled } \\
\text { Desion }\end{array}$ & $37139 A$ & $29-0 c t-93$ & $\mathbf{Y}$ & 2 \\
\hline
\end{tabular}


CCA Parameter tisting

\begin{tabular}{|c|c|c|c|c|c|c|c|c|c|c|}
\hline ID & $\begin{array}{l}\text { Clesedin- } \\
\text { Cation of } \\
\text { Dete } \\
\text { Cetagory } \\
\text { (footnote 2) }\end{array}$ & $\begin{array}{l}\text { Dietribution } \\
\text { Type }\end{array}$ & Men & Medien & $\begin{array}{l}\text { Mintmim } \\
\therefore \therefore\end{array}$ & Modinim & Latis & Refermen ID & $\begin{array}{l}\text { Was the deta } \\
\text { demaloped } \\
\text { underen } \\
\text { NOA-1 } \\
\text { Progren? }\end{array}$ & $\begin{array}{c}\text { Which } \\
\text { methods } \\
\text { were usend to } \\
\text { quility } \\
\text { existing } \\
\text { dete? } \\
\text { (tootrote 3) }\end{array}$ \\
\hline 3409 & 3,4 & CUMULATIVE & $26000000 E-05$ & $8.7000000 E-06$ & $8.7000000 E-07$ & $8.7000000 E-05 / n$ & molosititer & WP036053 & $\mathbf{Y}$ & 1 \\
\hline 3409 & 3,4 & CUMULATIVE & $2.6000000 E-05$ & $8.7000000 E-06$ & 8.7000000E-07 & $8.7000000 E-05$ & moleshiter & UP037536 & $\mathbf{Y}$ & 1 \\
\hline 3409 & 3,4 & CUMULATIVE & 26000000E-0.5 & $8.7000000 E-05$ & $8.7000000 E-07$ & $8.7000000 E-05$ & moles/liter & WP037545 & $\mathbf{Y}$ & 1 \\
\hline 3409 & 3,4 & CUMULATIVE & $2.6000000 \mathrm{E}-05$ & $8.7000000 E-06$ & 8.7000000E-07 & $8.7000000 E-05$ & molessiter & MP040512 & $Y$ & 1 \\
\hline 3409 & 3,4 & CUMULATME & $26000000 E-05$ & $8.7000000 E-05$ & 8.7000000E-07 & $8.7000000 E-0.5$ & moleshiter & WPOs1556 & $\mathbf{Y}$ & 1 \\
\hline 3410 & 2 & CONSTANT & $1.0800000 E+\infty 0$ & $1.0800000 E+\infty 0$ & $1.0800000 E+\infty 0$ & $1.0800000 \mathrm{E}+\infty 0$ & Ci & WP037061 & $\mathbf{Y}$ & N/A \\
\hline 3410 & 2 & CONSTANT & $1.0800000 E+00$ & $1.0800000 \mathrm{E}+00$ & $1.0800000 E+00$ & $1.0800000 E+\infty 0$ & $\mathbf{C i}$ & WPO39260 & $\mathbf{Y}$ & NAA \\
\hline 3410 & 2 & CONSTANT & $1.0800000 E+00$ & $1.0800000 E+\infty$ & $1.0800000 E+00$ & $1.0000000 E+00$ & $\mathrm{Ci}$ & WPOA1559 & $\mathbf{Y}$ & NA \\
\hline 3410 & 2 & CONSTANT & $1.0800000 E+\infty 0$ & $1.0800000 E+\infty$ & $1.0800000 E+\infty$ & $1.0800000 E+\infty 0$ & $\mathbf{C i}$ & WPO41550 & $\mathbf{Y}$ & NA \\
\hline 3411 & 2 & CONSTANT & $1.9700000 E+01$ & $1.9700000 \mathrm{E}+01$ & $1.9700000 E+01$ & $1.9700000 E+01$ & $\mathbf{C i}$ & WP037061 & $\mathbf{Y}$ & NA \\
\hline 3411 & 2 & CONSTANT & $1.9700000 E+01$ & $1.9700000 E+01$ & $1.9700000 E+01$ & $1.9700000 E+01$ & $\mathbf{C i}$ & WP039260 & $\mathbf{Y}$ & NA \\
\hline 3411 & 2 & CONSTANT & $1.9700000 E+01$ & $1.9700000 E+01$ & $1.9700000 E+01$ & $1.9700000 \mathrm{E}+01$ & $\mathbf{C i}$ & WPO41559 & $\mathbf{Y}$ & NA \\
\hline 3411 & 2 & CONSTANT & $1.9700000 E+01$ & $1.9700000 E+01$ & $1.9700000 E+01$ & $1.9700000 E+01$ & $\mathbf{C i}$ & WPO41560 & $\mathbf{Y}$ & NA \\
\hline 3412 & 2 & CONSTANT & $1.1500000 E+02$ & $1.1500000 E+02$ & $1.1500000 \varepsilon+\infty 2$ & $1.1500000 E+\infty 2$ & $\mathbf{C i}$ & WPO37051 & $\mathbf{Y}$ & N/A \\
\hline 3412 & 2 & CONSTANT & $1.1500000 \mathrm{E}+02$ & $1.1500000 E+02$ & $1.1500000 E+\infty 2$ & $1.1500000 E+\infty 02$ & $\mathrm{Ci}_{\mathrm{i}}$ & WP039260 & $\mathbf{Y}$ & $N / A$ \\
\hline 3412 & 2 & CONSTANT & $1.1500000 E+02$ & $1.1500000 E+02$ & $1.1500000 E+02$ & $1.1500000 E+02$ & $\mathrm{Ci}$ & WP041559 & $\mathbf{Y}$ & NA \\
\hline 3412 & 2 & CONSTANT & $1.1500000 \mathrm{E}+02$ & $1.1500000 E+02$ & $1.1500000 E+02$ & $1.1500000 E+02$ & $\mathbf{C i}$ & WPO41560 & $\mathbf{Y}$ & $N A$ \\
\hline 3413 & 2 & CONSTANT & $1.4800000 E-05$ & $1.4800000 E-05$ & $14800000 E-05$ & $1.4800000 \mathrm{E}-05$ & $\mathbf{C i}$ & WP037061 & $\mathbf{Y}$ & NA \\
\hline 3413 & 2 & CONSTANT & $1.4800000 \mathrm{E}-05$ & $1.4800000=-0.5$ & $14800000 E-05$ & $1.4800000 E-05$ & $\mathrm{Ci}$ & WPO39260 & $\mathbf{Y}$ & N/A \\
\hline 3413 & 2 & CONSTANT & $1.4800000=-05$ & $1.4800000=-05$ & $14800000 E-05$ & $14800000 E-05$ & ci & WP041559 & $-\because Y$ & NA \\
\hline 3413 & 2 & CONSTANT & $1.4800000 E-05$ & $1.4800000 E-05$ & $14000000 E-05$ & $14800000 E-05$ & $\mathbf{C i}$ & WPO41560 & $\mathbf{Y}$ & N/A \\
\hline 3414 & 2 & CONSTANT & $3.4400000 E+\infty 0$ & $3.4400000 E+\infty 0$ & $3400000 E+\infty$ & $3 \backsim 00000 E+00$ & Curies & WP037051 & $\mathbf{Y}$ & NA \\
\hline 3414 & 2 & CONSTANT & $3.4400000 E+\infty 0$ & $3.4400000 E+\infty 0$ & $3400000 E+\infty)$ & $34000000+00$ & Curies & WP039260 & $\mathbf{Y}$ & N/A \\
\hline 3414 & 2 & CONSTANT & $3.4400000 E+00$ & $3.4400000 E+\infty 0$ & $3400000+\infty$ & $3400000 E+\infty$ & Cunes & WPO41559 & $Y$ & NA \\
\hline 3414 & 2 & CONSTANT & $3.4400000 E+\infty$ & $3.4400000 E+\infty 0$ & $34400000 E+00$ & $34400000 E+00$ & Cunes & WPOA1560 & $\mathbf{Y}$ & N/A \\
\hline 3415 & 2 & CONSTANT & $3.2500000 E+01$ & $3.2500000 E+01$ & $3.2500000 E+01$ & $32500000 E+01$ & $\mathbf{C i}$ & WP037061 & $\mathbf{Y}$ & NA \\
\hline 3415 & 2 & CONSTANT & $3.2500000 E+01$ & $3.2500000 E+01$ & $3.25000000+01$ & $3.2500000 E+01$ & $\mathrm{Ci}$ & WP039260 & $\mathbf{Y}$ & N/A \\
\hline 3415 & 2 & CONSTANT & $3.2500000 E+01$ & $3.2500000 E+01$ & $3.2500000 \mathrm{E}+01$ & $3.2500000 E+01$ & $\mathbf{C i}$ & WPO41559 & $\mathbf{Y}$ & $N / A$ \\
\hline 3415 & 2 & CONSTANT & $3.2500000 E+01$ & $3.2500000 E+01$ & $3.2500000 E+01$ & $3.2500000 E+01$ & Ci & WPO41560 & $\mathbf{Y}$ & NA \\
\hline 3416 & 2 & CONSTANT & $2.2700000 \mathrm{E}-04$ & $2.2700000 E-04$ & $2.2700000 \mathrm{E}-04$ & $2.2700000 \mathrm{E}-04$ & $\mathbf{C i}$ & WP03706t & $\mathbf{Y}$ & NA \\
\hline 3416 & 2 & CONSTANT & $2.2700000 E-04$ & 2.2700000E.04 & 2.2700000E-04 & $2.2700000=-04$ & $C_{i}$ & WP039260 & $\mathbf{Y}$ & NA \\
\hline 3416 & 2 & CONSTANT & $2.2700000 E-04$ & $2.2700000 E-04$ & $2.2700000=-04$ & $2.2700000 \leq-04$ & Ci & WPOA1559 & $\mathbf{Y}$ & NIA \\
\hline 3416 & 2 & CONSTANT & $2.2700000 \mathrm{E}-04$ & $2.2700000 E-04$ & $22700000 \mathrm{E}-04$ & $2.2700000 \mathrm{E}-0.4$ & $\mathbf{C i}$ & WPO41560 & $\mathbf{Y}$ & NA \\
\hline
\end{tabular}


CCA Parameter Listing

\begin{tabular}{|c|c|c|c|c|c|c|c|c|c|c|}
\hline $\begin{array}{c}\text { Parmoter In } \\
\text { Databea? } \\
\text { (XM) }\end{array}$ & 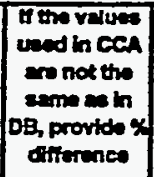 & D & Materatio & Metrardi NAm & $\begin{array}{l}\text { Peramoter } \\
\text { to }\end{array}$ & Parmocor Neme & $\begin{array}{l}\text { PRP DO } \\
\text { (NPO \#) }\end{array}$ & $\begin{array}{c}\text { Dent Entry } \\
\text { Dete }\end{array}$ & 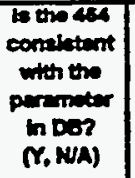 & $\begin{array}{l}\text { Peranetar } \\
\text { Cotegory } \\
\text { (foctinote 1) }\end{array}$ \\
\hline $\mathbf{Y}$ & & 3417 & GLOBAL & $\begin{array}{l}\text { Information that apples } \\
\text { globally }\end{array}$ & DXXTAT & Index for the Oxidrtion State & 37663 & $01+10 y-96$ & $\mathbf{Y}$ & 1 \\
\hline $\mathbf{Y}$ & & 3417 & GLOBAL & $\begin{array}{l}\text { Infiormation that applios } \\
\text { grobally }\end{array}$ & OXSTAT & Index for the Oxidation State & 37663 & $01+$ Mary -96 & $\mathbf{Y}$ & 1 \\
\hline $\mathbf{Y}$ & & 3417 & GLOBAL & $\begin{array}{l}\text { Information that epplies } \\
\text { globalty }\end{array}$ & DXXSTAT & Inolex for the Oxadetion State & 376003 & $01+\operatorname{moy}-96$ & $\mathbf{\gamma}$ & 1 \\
\hline $\mathbf{Y}$ & & 3417 & GLOBAL & $\begin{array}{l}\text { Intormation that apphes } \\
\text { globally }\end{array}$ & OXSTAT & | Irocex for the Oxidation State & 37603 & $\mid 01$ May-96 & $\mathbf{Y}$ & 1 \\
\hline $\mathbf{Y}$ & & 3418 & CULEBRA & $\begin{array}{l}\text { Culabra member of tho } \\
\text { Rustior formation }\end{array}$ & MEA_STOR & Measund Stordivity & 37664 & $\mid 01+$ may $-\infty \mid$ & $Y$ & 1 \\
\hline $\mathbf{Y}$ & & 3419 & CULEBRA & $\begin{array}{l}\text { Culiebra member of the } \\
\text { Rustier formation }\end{array}$ & MINP_FAC & Mining Transmissivity Mukiplior & 37666 & $\mid 01+$ may-96| & $\mathbf{Y}$ & 1 \\
\hline $\mathbf{Y}$ & & 3419 & CULEBRA & $\begin{array}{l}\text { Culabra member of tho } \\
\text { Rustler formation }\end{array}$ & MINP_FAC & Mining Transmissivity Multiplier & 37666 & $\mid 01+$ May-96| & $\mathbf{Y}$ & 1 \\
\hline $\mathbf{Y}$ & & 3419 & CULEBRA & $\begin{array}{l}\text { Culobra member of the } \\
\text { Rustler formation }\end{array}$ & MINP_FAC & Mining Trensmissivity Mutiplier & 37666 & $\mid 01$ they-96 & $\mathbf{Y}$ & 1 \\
\hline $\mathbf{Y}$ & & 3420 & BLOWOUT & $\begin{array}{l}\text { BRAGFLOW Dirod } \\
\text { Brino Relesses }\end{array}$ & FCE & Comentation Scating Fuctor & 37668 & $|02+M a y-96|$ & $\mathbf{Y}$ & AB \\
\hline $\mathbf{Y}$ & & 3420 & BLOWOUT & \begin{tabular}{|l} 
BRAGFLOW Direct \\
Brino Roleases
\end{tabular} & FCE & Cermentation Scaling Fuctor & 37668 & Dany-86 & $\mathbf{Y}$ & $4 B$ \\
\hline Y & & 3421 & P8209 & Lead 200 & ATWEIGHT & Atomic Weight in kemolo & 37670 & CotMay-96 & $\mathbf{Y}$ & 3 \\
\hline $\mathbf{Y}$ & & 3421 & PB209 & Lead 209 & ATWEIGHT & Atomic Woight in kgmolo & 37670 & 02+May-96 & $\mathbf{Y}$ & 3 \\
\hline$Y$ & & 3429 & PHUMOX3 & \begin{tabular}{|l|}
$\begin{array}{l}\text { Proportionality } \\
\text { Constant }+3 \text { State. } \\
\text { Humic Colloids }\end{array}$ \\
\end{tabular} & PHUMCM & $\begin{array}{l}\text { Proportionality Const, Humic } \\
\text { Colfoids, Cestill Brine, MgO } \\
\text { controlts pH }\end{array}$ & 37683 & $08+\operatorname{may}-96$ & $\mathbf{Y}$ & 1 \\
\hline$Y$ & & 3429 & PHUMOX3 & $\begin{array}{l}\text { Proportionalidy } \\
\text { Constont }+3 \text { State, } \\
\text { Humic Colloids }\end{array}$ & PHUMCQM & $\begin{array}{l}\text { Proportionality Const., Humic } \\
\text { Colloids, Castilo Brino, MgO } \\
\text { controlls pH }\end{array}$ & 37683 & Do-Mey-96 & $\mathbf{Y}$ & 1 \\
\hline $\mathbf{Y}$ & & 3429 & PHUMOX3 & $\begin{array}{l}\text { Proportionality } \\
\text { Constant, }+3 \text { State. } \\
\text { Humic Colloids } \\
\end{array}$ & PHUMCM & $\begin{array}{l}\text { Proportionality Const, Humic } \\
\text { Cotbids, Cestito Brine, MgO } \\
\text { controlls pH }\end{array}$ & 37683 & Dothay-96 & $\mathbf{Y}$ & 1 \\
\hline $\mathbf{Y}$ & & 3430 & PHUMOX4 & $\begin{array}{l}\text { Proportionality } \\
\text { onstant wath humic } \\
\text { onloids for actinide in } \\
\text { oxidation stato N }\end{array}$ & PHUMCIM & $\begin{array}{l}\text { Proportionality Const, Humic } \\
\text { Cotbids. Cestile Brino, MgO } \\
\text { controlis pH }\end{array}$ & 37685 & Dotmay-96 & $Y$ & 1 \\
\hline $\mathbf{Y}$ & & 3430 & PHUMOX4 & $\begin{array}{l}\text { Proportionality } \\
\text { constant with hemic } \\
\text { colloids for actinice in } \\
\text { axidation state N }\end{array}$ & PHUMCLM & $\begin{array}{l}\text { Proportionalty Const. Humic } \\
\text { Colloids, Castilo Brino, MgO } \\
\text { controlls pH }\end{array}$ & 37685 & C8+May-96 & $\mathbf{Y}$ & 1 \\
\hline$Y$ & & 3431 & PHUMOX5 & $\begin{array}{l}\text { Proportionality } \\
\text { constant with humic } \\
\text { colbids for actinide in } \\
\text { exidation state V }\end{array}$ & PHUMCIM & $\begin{array}{l}\text { Proportionality Const. Humic } \\
\text { Colbids, Castilo Brme. MgO } \\
\text { controlls pH }\end{array}$ & 37687 & Domay-96 & $Y$ & 1 \\
\hline $\mathbf{Y}$ & & 3431 & PHUMOX5 & $\begin{array}{l}\text { Proportionality } \\
\text { constisnt with humic } \\
\text { colbids for actinice in } \\
\text { oxidation state V }\end{array}$ & PHUMCIM & $\begin{array}{l}\text { Proportionalty Const., Humic } \\
\text { Colloids, Cestule Brino, MgO } \\
\text { controlls pH }\end{array}$ & 37687 & DBMay-96 & $\bar{Y}$ & 1 \\
\hline $\mathbf{Y}$ & & 3432 & PHUMOX6 & $\begin{array}{l}\text { Proportorality } \\
\text { constant with humic } \\
\text { colloids for actinide in } \\
\text { oxidation state VI }\end{array}$ & PHUMCLM & $\begin{array}{l}\text { Proportionality Const.,Humic } \\
\text { Colbids, Castilo Brino, MgO } \\
\text { controlls pH }\end{array}$ & 37688 & O8-May-96 & $\mathbf{Y}$ & 1 \\
\hline $\mathbf{Y}$ & & 3432 & PHUMOX6 & 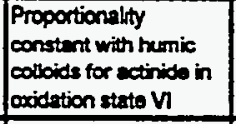 & РНUMCIM & $\begin{array}{l}\text { Proportionality Const, Hurnic } \\
\text { cosoids, Castito Brino. MgO } \\
\text { controlls pH }\end{array}$ & 37688 & $08+$ may-96 & $Y$ & 1 \\
\hline$Y$ & & 3433 & PHUMOX3 & $\begin{array}{l}\text { Proportionalty } \\
\text { Constent, +3 State. } \\
\text { Hemic Colsoids }\end{array}$ & PHUMSA & $\begin{array}{l}\text { Proportionality Const of Actinides } \\
\text { in Saleso Brime w/Humic Colloids. } \\
\text { inoromic }\end{array}$ & 37690 & O8-May-96| & $\mathbf{Y}$ & 1 \\
\hline $\mathbf{Y}$ & & 3433 & PHUMOX3 & \begin{tabular}{|l} 
Proportionality \\
Constint, +3 Sututo. \\
Humic Colioids \\
\end{tabular} & PHUMSIM & 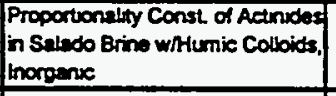 & 37690 & 08-14ay-96| & $\mathbf{Y}$ & 1 \\
\hline $\mathbf{Y}$ & & 3434 & PHUMOXA & 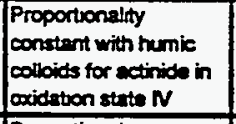 & PHUMSEM & $\begin{array}{l}\text { Proportionalty Const. of Actinides } \\
\text { in Subudo Brine w/turnic Colloids. } \\
\text { inorgenic }\end{array}$ & 37691 & $08+1$ ay 96 & $\mathbf{Y}$ & 1 \\
\hline $\mathbf{Y}$ & & 3434 & PHUMOXA & $\begin{array}{l}\text { Proportionality } \\
\text { constemt with humic } \\
\text { oothoids for actinich in } \\
\text { oxidation strate } N\end{array}$ & PHUMSA & 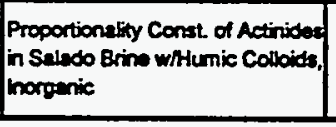 & 37691 & $03-16 y-66$ & $\mathbf{Y}$ & 1 \\
\hline
\end{tabular}


CCA Parameter Listing

\begin{tabular}{|c|c|c|c|c|c|c|c|c|c|c|}
\hline TD & $\begin{array}{c}\text { Clansin- } \\
\text { cation of } \\
\text { Datt } \\
\text { Centegory } \\
\text { (footnots 2) }\end{array}$ & $\begin{array}{c}\text { Distribution } \\
\text { Typo }\end{array}$ & $\operatorname{man}$ & woden & Minisinem & Merdinem & Thros & Referraxed id & $\begin{array}{l}\text { Fes the dintl } \\
\text { demeloped } \\
\text { under m } \\
\text { Nol-1 } \\
\text { Proprem? }\end{array}$ & 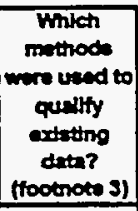 \\
\hline 3417 & 1,4 & UNIFORM & $5.0000000 E-01$ & $5.0000000 \mathrm{E}-01$ & $0.00000000+\infty$ & $1.0000000 \leq+\infty$ & NONE & WP035194 & $\mathbf{Y}$ & NA \\
\hline 3417 & 1,4 & UNIFORM & $5.0000000 E-01$ & $5.0000000 E-01$ & $0.0000000 E+\infty$ & $1.0000000 E+\infty$ & NONE & WP035268 & $\mathbf{Y}$ & NA \\
\hline 3417 & 1,4 & UNIFORM & $5.0000000 E-01$ & $5.0000000 E-01$ & $0.0000000 E+\infty$ & $1.0000000 \approx+\infty 0$ & NONE & WPO35835 & $\mathbf{Y}$ & NA \\
\hline 3417 & 1,4 & UNIFORM & $5.0000000 E-01$ & $5.0000000 E-01$ & $0.0000000 E+\infty 0$ & $1.0000000 E+\infty 0$ & NONE & WP037536 & $\mathbf{Y}$ & NA \\
\hline 3418 & 1 & CONSTANT & $1.0000000 E-05$ & $1.0000000 E-05$ & $1.0000000 E-05$ & $1.0000000 E-05$ & NONE & WP036456 & $\mathbf{Y}$ & 1 \\
\hline 3419 & 4 & UNIFORM & $5.0050000 \mathrm{E}+02$ & $5.0050000 E+\infty 2$ & $1.0000000 E+00$ & $1.0000000 E+03$ & NONE & WP035268, & $\mathbf{Y}$ & 1 \\
\hline 3419 & 4 & UNIFORM & $5.0050000 E+02$ & $5.0050000 E+02$ & $1.0000000 E+\infty 0$ & $1.0000000 E+03$ & NONE & WP036489 & $\mathbf{Y}$ & 1 \\
\hline 3419 & 4 & UNIFORM & $5.0050000 E+02$ & $5,0050000 \mathrm{E}+102$ & $1.0000000 E+\infty 0$ & $1.0000000 E+03$ & NONE & WP039355 & $\mathbf{Y}$ & 1 \\
\hline 3420 & 3,5 & CONSTANT & $1.0000000 E+\infty$ & $1.0000000 E+\infty 0$ & $1.0000000 E+00$ & $1.0000000 E+\infty$ & NONE & WP036723 & $\mathbf{Y}$ & N/A \\
\hline 3420 & 3.5 & CONSTANT & $1.0000000 E+00$ & $1.0000000 E+\infty 0$ & $1.0000000 E+\infty 0$ & $1.0000000 E+\infty 0$ & NONE & WP040521 & $\mathbf{Y}$ & NA \\
\hline 3421 & 4 & CONSTANT & 2.0898100E-01 & $2.0898100 E-01$ & 20898100E-01 & 2008s8100E-01 & kolmole & WP036301 & $\mathbf{Y}$ & NA \\
\hline 3421 & 4 & CONSTANT & 2.0898900E-01 & $2.0858100 E-01$ & 2.0898100E-01 & $2.0898100 E-01$ & $\mathrm{~kg}$ molo & WP037404 & $\mathbf{Y}$ & NA \\
\hline 3429 & 1.4 & CUMULATIVE & $1.1000000 E+\infty 0$ & $1.3700000 E+\infty 0$ & $6.5000000 E-02$ & $1.6000000 E+\infty$ & NONE & WP035268 & $\mathbf{Y}$ & N/A \\
\hline 3429 & 1.4 & CUMULATIVE & $1.1000000 E+\infty 0$ & $1.3700000 E+\infty$ & $6.5000000 E-02$ & $1.6000000 E+\infty$ & NONE & WP035855 & $\mathbf{Y}$ & NA \\
\hline 3429 & 1,4 & CUMULATIVE & $1.1000000 E+\infty$ & $1.3700000 E+\infty 0$ & $6.5000000 E-02$ & $1.6000000 E+\infty$ & NONE & WP037522 & $\mathbf{Y}$ & NA \\
\hline 3430 & 1.4 & CONSTANT & $6.3000000 \mathrm{E}+00$ & $6.3000000 E+\infty 0$ & $6.3000000 E+\infty$ & $6.3000000 E+\infty 0$ & NONE & WP035855 & $\mathbf{Y}$ & NA \\
\hline 3430 & 1.4 & CONSTANT & $6.3000000 E+\infty 0$ & $6.3000000 E+\infty 0$ & $6.3000000 E+\infty 0$ & $6.3000000 E+\infty$ & NONE & WPO37522 & $\mathbf{Y}$ & NA \\
\hline 3431 & 1,4 & CONSTANT & $7.4000000 E-03$ & $7.4000000 E-03$ & $7.4000000 E-03$ & $7.4000000 E-03$ & NONE & WPO35855 & $\mathbf{Y}$ & NA \\
\hline 3431 & 1,4 & CONSTANT & $7.4000000 E-03$ & $7.4000000 E-03$ & $7.4000000 E-03$ & $7.4000000 E-03$ & NONE & Wp037522 & $\bar{Y}$ & N/A \\
\hline 3432 & 1,4 & CONSTANT & $5.1000000 E-01$ & $5.1000000 E-01$ & $5.1000000 E-01$ & $5.1000000 E-01$ & NONE & WPO35855 & $\mathbf{Y}$ & N/A \\
\hline 3432 & 1,4 & CONSTANT & $5.1000000 E-01$ & $5.1000000 E-01$ & $5.1000000 E-01$ & $5.1000000 E-01$ & NONE & WP037522 & $\mathbf{Y}$ & NA \\
\hline 3433 & 1.4 & CONSTANT & $1.9000000 E-01$ & $1.9000000 E-01$ & $1.9000000 E-01$ & $1.9000000 E-01$ & NONE & WPO35855 & $\mathbf{Y}$ & N/A \\
\hline 3433 & 1,4 & CONSTANT & $1.9000000 E-01$ & $1.9000000 E-01$ & $1.9000000 \mathrm{E}-01$ & $1.9000000 \mathrm{E}-01$ & NONE & UP037522 & $\mathbf{Y}$ & N/A \\
\hline 3434 & 1,4 & CONSTANT & $6.3000000 E+00$ & $6.3000000 E+\infty$ & $6.3000000 E+\infty$ & $6.3000000 E+\infty$ & NONE & WPO35855 & $\mathbf{Y}$ & N/A \\
\hline 3434 & 1,4 & CONSTANT & $6.3000000 E+\infty 00$ & $6.3000000 E+\infty$ & $6.3000000 E+\infty 0$ & $6.3000000 E+\infty 0$ & NONE & WP037522 & $\mathbf{Y}$ & NA \\
\hline
\end{tabular}


CCA Parameter Listing

\begin{tabular}{|c|c|c|c|c|c|c|c|c|c|c|}
\hline 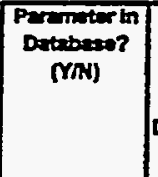 & 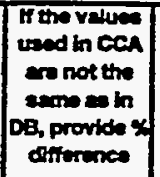 & 10 & Mrearta bo & Matade Nem & Parmeter & Perniter Rem & $\begin{array}{l}\text { PRPDD } \\
\text { MPOA }\end{array}$ & $\begin{array}{c}\text { Deten Entry } \\
\text { Decte }\end{array}$ & 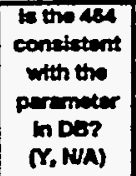 & $\begin{array}{l}\text { Parmineter } \\
\text { Categary } \\
\text { (tootnote 1) }\end{array}$ \\
\hline $\mathbf{Y}$ & & 3435 & PHUAOX5 & $\begin{array}{l}\text { Proportionsality } \\
\text { constent with rumic } \\
\text { colloids for actinich in } \\
\text { oxidation state V }\end{array}$ & PHUMSm & $\begin{array}{l}\text { Proportionalty Const of Actinided } \\
\text { in Salado Brim whimic Colloids, } \\
\text { inorganic }\end{array}$ & 37694 & $08+4 x y-96$ & $\mathbf{Y}$ & 1 \\
\hline $\mathbf{Y}$ & ? & 3435 & PHUMOXS & $\begin{array}{l}\text { Proportionality } \\
\text { constant with humic } \\
\text { colloids for actinide in } \\
\text { oxidation state V }\end{array}$ & PHUMSM & $\begin{array}{l}\text { Proportionslity Const. of Actinides } \\
\text { in Suledo Brine w/Humic Colloids, } \\
\text { Inorgenic }\end{array}$ & 37694 & 08 Moy-96 & $\mathbf{Y}$ & 1 \\
\hline $\mathbf{Y}$ & & 3436 & PHUMOXE & $\begin{array}{l}\text { Proportionality } \\
\text { constent with humic } \\
\text { colbids for actinide in } \\
\text { exidation state VV }\end{array}$ & PHUMSIM & $\begin{array}{l}\text { Proportionality Const of Actinided } \\
\text { in Saledo Brino w/Humic Colloids, } \\
\text { inorganic }\end{array}$ & 37695 & 08-14ay-96 & $\mathbf{Y}$ & 1 \\
\hline $\mathbf{Y}$ & & 3436 & PHUMOX6 & $\begin{array}{l}\text { Proportionality } \\
\text { constent with humic } \\
\text { colbids for metinido in } \\
\text { oxidation state VI }\end{array}$ & PHUMSIM & $\begin{array}{l}\text { Proportionality Const of Actinides } \\
\text { in Saludo Brin w/Hemic Colloids. } \\
\text { lnorganic }\end{array}$ & 37685 & $08+4 x y-96$ & $\mathbf{Y}$ & 1 \\
\hline $\mathbf{Y}$ & & 3437 & $\mathbf{T H}$ & Thorium & CONCAIN & $\begin{array}{l}\text { Actinide Concenteation with } \\
\text { Mobide Minerd Fregment Colloids }\end{array}$ & 37697 & 08-May-96 & $\mathbf{Y}$ & 1 \\
\hline $\mathbf{Y}$ & & 3437 & $\mathbf{T H}$ & thorium & CONCMIN & $\begin{array}{l}\text { Actinide Concentration with } \\
\text { Mobilo Mineral Frasenent Colloids }\end{array}$ & 37697 & $08+14 y-96$ & $\mathbf{Y}$ & 1 \\
\hline $\mathbf{Y}$ & & 3438 & $\mathbf{U}$ & Uranium & CONCMIN & $\begin{array}{l}\text { Actinide Concentration with } \\
\text { Mobilo Mineral Fregnent Colloids }\end{array}$ & 37698 & ob+11ay-96 & $\mathbf{Y}$ & 1 \\
\hline $\mathbf{Y}$ & & 3438 & $\mathbf{u}$ & Uranium & CONCMIN & $\begin{array}{l}\text { Actinide Concentration with } \\
\text { Mobile Mineral Fregment Colloids }\end{array}$ & 37698 & 08-May-96 & $\mathbf{Y}$ & 1 \\
\hline $\mathbf{Y}$ & & 3439 & NP & Neptunium & CONCMIN & $\begin{array}{l}\text { Actinide Concertration with } \\
\text { Mobilo Mineral Fregment Colroids }\end{array}$ & 37700 & O8-May-96 & $\mathbf{Y}$ & 1 \\
\hline $\mathbf{Y}$ & & 3439 & NP & Neptunium & CONCMIN & $\begin{array}{l}\text { Actinide Concentration with } \\
\text { Mobile Mineral Fragment Colloids }\end{array}$ & 37700 & 08-May-96 & $\mathbf{Y}$ & 1 \\
\hline $\mathbf{Y}$ & & 3440 & PU & Plutonium & CONCMIN & $\begin{array}{l}\text { Actinide Concentration with } \\
\text { Mobilo Mineral Fregment Colloids }\end{array}$ & 37703 & 08-May-96 & $\mathbf{Y}$ & 1 \\
\hline $\mathbf{Y}$ & & 3440 & PU & Plutonium & CONCMIN & $\begin{array}{l}\text { Actinide Concentration with } \\
\text { Mobile Mineral Fregment Colloids }\end{array}$ & 37703 & $08+1 a y-96$ & $\mathbf{Y}$ & 1 \\
\hline $\mathbf{Y}$ & & 3441 & AM & Americium & CONCMIN & $\begin{array}{l}\text { Actinide Concentration with } \\
\text { Mobile Mineral Fregment Colbids }\end{array}$ & 37704 & 08-May-96 & $\mathbf{Y}$ & 1 \\
\hline $\mathbf{Y}$ & & 3441 & AM & Americium & CONCMIN & $\begin{array}{l}\text { Actinide Concentration with } \\
\text { Mobise Mineral Fragment Colloids }\end{array}$ & 37704 & 08-May-96 & $\mathbf{Y}$ & 1 \\
\hline $\mathbf{Y}$ & & 3442 & PU+3 & Phutonium III & $M D O$ & Molecular dittusion in pure fluid & 37705 & 17 -May-96 & $\mathbf{Y}$ & 1 \\
\hline $\mathbf{Y}$ & & 3443 & PU+4 & Putonium N & MDO & Molocular ditrusion in pure fiuid & 37708 & $17+$ thay-96 &.$- Y$ & 1 \\
\hline$Y$ & & 3444 & $A M+3$ & Americium III & MDO & Molocular datusion in pure fuvid & 37709 & 17 May-96 & $\mathbf{Y}$ & 1 \\
\hline $\mathbf{Y}$ & & 3446 & $u+4$ & Ureniem N & MDO & Molocular dittusion in pure fluid & 37711 & 17 thay-96 & $\mathbf{Y}$ & 9 \\
\hline $\mathbf{Y}$ & & 3447 & AM & Americim & CAPMIC & $\begin{array}{l}\text { Mexinum Concentration of } \\
\text { Actinide on Microbe Cossoids }\end{array}$ & 37712 & D8-May-86 & $\mathbf{Y}$ & 1 \\
\hline $\mathbf{Y}$ & & 3447 & AM & Americiun & CAPMIC & $\begin{array}{l}\text { Meximum Concentration of } \\
\text { Actinide on Microbe Colbids }\end{array}$ & 37712 & $08+4 a y-96$ & $\mathbf{Y}$ & 1 \\
\hline $\mathbf{Y}$ & & 348 & $U+6$ & Urarium V & MDO & Moleculer ditusion in pure furid & 37714 & 17 May-96 & $\mathbf{Y}$ & 1 \\
\hline $\mathbf{Y}$ & & 349 & $\mathrm{TH}+\mathrm{A}$ & Thorium N & $M D O$ & Molecular ditrusion in pure fluid & 37715 & 17 may-96 & $\mathbf{Y}$ & 1 \\
\hline $\mathbf{Y}$ & & 3454 & WAS_AREA & $\begin{array}{l}\text { Watte emptecement } \\
\text { erea end wasto }\end{array}$ & CLOSHOD & Closure Surface Model & 38200 & 04Jun-96 & $\mathbf{Y}$ & $4 \mathrm{~B}$ \\
\hline $\mathbf{Y}$ & & 3454 & WAS_AREA & $\begin{array}{l}\text { Wasti emplacement } \\
\text { area end wasto }\end{array}$ & CLOSMOD & Closure Sutace Model & 38200 & 04-Jun-96 & $\mathbf{Y}$ & 48 \\
\hline $\mathbf{Y}$ & & 3454 & WAS_AREA & $\begin{array}{l}\text { Wasto emplacement } \\
\text { erea end westo }\end{array}$ & CLOSMOD & Closure Surtace Model & 38200 & O4Jun-96 & $\mathbf{Y}$ & 48 \\
\hline $\mathbf{Y}$ & & 3455 & REPOST & $\begin{array}{l}\text { Repository regons } \\
\text { outside of Panti region }\end{array}$ & CLOSMOD & Clowre Surface Model & 38207 & Ohten-96 & $\mathbf{Y}$ & 48 \\
\hline $\mathbf{Y}$ & & 3455 & REPOST & $\begin{array}{l}\text { Repostory regions } \\
\text { cutside of Pentil rogion }\end{array}$ & CLOSHOD & Closure Surfece Hodel & 38207 & Ohlun-96 & $\mathbf{Y}$ & 48 \\
\hline
\end{tabular}


CCA Parameter Listing

\begin{tabular}{|c|c|c|c|c|c|c|c|c|c|c|}
\hline ID & 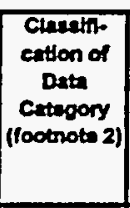 & $\begin{array}{l}\text { Duturbution } \\
\text { Type }\end{array}$ & $\operatorname{man}$ & Medien & Mintrixem & Moximum & Units & Reference ID & $\begin{array}{l}\text { Wes the dath } \\
\text { doviloped } \\
\text { under m } \\
\text { Nol-1 } \\
\text { Progran? }\end{array}$ & 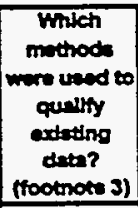 \\
\hline 3435 & 1,4 & CONSTANT & $9.1000000 E-04$ & $9.1000000 E-04$ & $9.1000000 E-04$ & $9.1000000 E-04$ & NONE & WP035855 & $\mathbf{Y}$ & NA \\
\hline 3435 & 1,4 & CONSTANT & $9.1000000 E-01$ & $9.1000000 E-04$ & $9.1000000 E-04$ & $9.1000000 E-04$ & NONE & WP037522 & Y & NA \\
\hline 3436 & 1,4 & CONSTANT & $1.2000000 E-01$ & $1.2000000 E-01$ & $1.2000000 E-01$ & $1.2000000 E-01$ & NONE & WP035855 & $\mathbf{Y}$ & N/A \\
\hline 3436 & 1,4 & CONSTANT & $1.2000000 E-01$ & $1.2000000 E-01$ & $1.2000000 E-01$ & $1.2000000 E-01$ & NONE & WPO37522 & $\mathbf{Y}$ & NA \\
\hline 3437 & 1,4 & CONSTANT & $26000000 E-08$ & $26000000 E-08$ & 26000000E-08 & $2.6000000 E-08$ & moleshitex & WP035850 & $\mathbf{Y}$ & NA \\
\hline 3437 & 1,4 & CONSTANT & $2.6000000 E-08$ & $26000000 E-08$ & 26000000E-08 & $2.6000000 E-08$ & molesnter & WP037522 & $\mathbf{Y}$ & NA \\
\hline 3438 & 1,4 & CONSTANT & $2.6000000 E-08$ & $2.6000000 E-08$ & $26000000 E-08$ & $2.6000000 \mathrm{E}-08$ & moleshiter & WP035850 & $Y$ & NA \\
\hline 3438 & 1,4 & CONSTANT & $2.6000000 E-08$ & $2.6000000 E-08$ & $26000000 E-08$ & $2.6000000 E-00$ & molesniter & WP037522 & $\mathbf{Y}$ & N/A \\
\hline 3439 & 1,4 & CONSTANI & $2.6000000 E-08$ & $2.6000000 E-08$ & $2.6000000 E .08$ & $2.6000000 E-08$ & moles/iter & WP035850 & $\mathbf{Y}$ & NA \\
\hline 3439 & 1,4 & CONSTANT & $2.6000000=-08$ & $2.6000000 E-08$ & $2.6000000 E-08$ & $26000000 E-08$ & moleshititor & WP037522 & $\mathbf{Y}$ & N/A \\
\hline 3440 & 1,4 & CONSTANT & $2.6000000 E-08$ & 2.6000000E-08 & $2.6000000 E-08$ & $26000000 \mathrm{E}-08$ & moleshiter & WP035850 & $\mathbf{Y}$ & NA \\
\hline 3440 & 1.4 & CONSTANT & $2.6000000 E-08$ & $2.6000000 E-08$ & $2.6000000 E-08$ & $26000000 E-08$ & moleshiter & WP037522 & $\mathbf{Y}$ & NA \\
\hline 3441 & 1,4 & CONSTANT & $2.6000000 E-08$ & $26000000 E-08$ & $26000000 E-08$ & $26000000 \mathrm{E}-08$ & molesniter & WP035850 & $Y$ & NA \\
\hline 3441 & 1,4 & CONSTANT & $2.6000000 E-08$ & $26000000 E-08$ & $26000000 E-08$ & $20000000 E-0 \theta$ & moleshiter & WP037522 & $\mathbf{Y}$ & N/A \\
\hline 3442 & 4 & CONSTANT & $3.0000000 E-10$ & $30000000 E-10$ & 30000000 - 10 & $30000000 E-10$ & $m^{\wedge} 2 / s$ & WP037533 & $\mathbf{Y}$ & NA \\
\hline 3443 & 4 & CONSTANT & $1.5300000 \mathrm{E}-10$ & $15300000 E \cdot 10$ & $13300000=-10$ & $15300000 E \cdot 10$ & $m^{\wedge} 2 / s$ & WP037533 &.$- Y$ & N/A \\
\hline 3444 & 4 & CONSTANT & $3.0000000 E-10$ & $30000000 E \cdot 10$ & J0000000E.10 & J0000000E-10 & $m^{\wedge} 2 / s$ & WP037533 & $\mathbf{Y}$ & NA \\
\hline 3446 & 4 & CONSTANT & $1.5300000 E-10$ & $15300000 E-10$ & $130000006 \cdot 10$ & I SN00000E-10 & $m^{\wedge} 2 / s$ & WP037533 & $\mathbf{Y}$ & N/A \\
\hline 3447 & 1 & CONSTANT & $1,0000000 \mathrm{E}+\infty 0$ & $10000000 \in+\infty$ & $10000000 \times-\infty$ & $10000000=-\infty$ & molesnter & WP035856 & $\mathbf{Y}$ & N/A \\
\hline 3447 & 1 & CONSTANT & $1.0000000 E+\infty$ & $10000000 E+\infty 0$ & $100000000+\infty$ & $100000006+\infty$ & molesulter & WP036771 & $\mathbf{Y}$ & NA \\
\hline 3448 & 4 & CONSTANT & 4.2600000E-10 & $42600000 E-10$ & $42000000<-10$ & A ZEO0000E-10 & $m^{\wedge} 2 / s$ & WP037533 & $\mathbf{Y}$ & $\mathrm{NA}$ \\
\hline 3449 & 4 & CONSTANT & $1.5300000 E-10$ & $15300000 E-10$ & $15300000-10$ & I SU000000E-10 & $m^{\wedge} 2 / s$ & WP037533 & $\mathbf{Y}$ & NA \\
\hline 3454 & 1,3 & CONSTANT & $4.00000000 E+\infty 0$ & $40000000 E+\infty 0$ & $40000000<+\infty$ & $40000000 E+\infty$ & NONE & WP030703 & $Y$ & NA \\
\hline 3454 & 1,3 & CONSTANT & $4.0000000 E+\infty 0$ & $10000000 E+\infty 0$ & $40000000-\infty$ & $40000000 E+\infty$ & NONE & WP031368 & $\mathbf{Y}$ & NA \\
\hline 3454 & 1,3 & CONSTANT & $4.0000000 E+\infty 0$ & $40000000 E+\infty 0$ & $4000000<-\infty$ & $40000006+\infty$ & NONE & WP040514 & $\mathbf{Y}$ & NA \\
\hline 3455 & 1,3 & CONSTANT & $4.0000000 E+00$ & $4000000 E+\infty$ & $4 \infty 000000 E-\infty$ & $40000000 E+\infty 0$ & NONE & WP030703 & $\mathbf{Y}$ & NA \\
\hline 3455 & 1,3 & CONSTANT & $4.0000000 E+\infty 0$ & $400000000+00$ & $40000000 E+00$ & $40000000 E+\infty$ & NONE & WP031368 & $\mathbf{Y}$ & NA \\
\hline
\end{tabular}


CCA Parameter Listing

\begin{tabular}{|c|c|c|c|c|c|c|c|c|c|c|}
\hline $\begin{array}{c}\text { Parameter hn } \\
\text { Datubee? } \\
\text { (rN) }\end{array}$ & 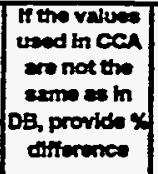 & 10 & Metara to & Metarianem & Parmetor & Peremeter Marno & $\begin{array}{l}\text { PRPD } \\
\text { ANPOE }\end{array}$ & Deca Entry & 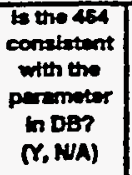 & $\begin{array}{l}\text { Parminter } \\
\text { Criegory } \\
\text { (foodiote 1) }\end{array}$ \\
\hline $\mathbf{Y}$ & & 3455 & REPOST & $\begin{array}{l}\text { Repositary reglons } \\
\text { outsida of Penol rogion }\end{array}$ & CLOSMOD & Clowere Surface Moder & 38207 & a4vin-s6 & $\mathbf{Y}$ & 48 \\
\hline $\mathbf{Y}$ & & 3456 & BLOWOUT & $\begin{array}{l}\text { BRAGFLOW Drect } \\
\text { Brino Releases }\end{array}$ & RE_CAST & $\begin{array}{l}\text { External dramage redess for the } \\
\text { Cestile formation }\end{array}$ & 38200 & Osven-96 & $\mathbf{Y}$ & 48 \\
\hline $\mathbf{Y}$ & & 3456 & BLOWOUT & $\begin{array}{l}\text { BRAGFLOW Drect } \\
\text { Brine Rolesses }\end{array}$ & RE_CAST & $\begin{array}{l}\text { External drainepo redius for the } \\
\text { Castile formation. }\end{array}$ & 38206 & oster-96 & $\mathbf{Y}$ & 48 \\
\hline $\mathbf{Y}$ & & 3457 & AM & Americium & CAPHUM & $\begin{array}{l}\text { Maxamum Concentraton of } \\
\text { Actinide with Mobile Humic } \\
\text { Colloids }\end{array}$ & 37721 & OB-May-96 & $\mathbf{Y}$ & 1 \\
\hline $\mathbf{Y}$ & & 3457 & AM & Americium & CAPHUM & $\begin{array}{l}\text { Maxmum Concentration of } \\
\text { Actinide with Mobito Humic } \\
\text { Colloids }\end{array}$ & 37721 & $08-M n y-96$ & $\mathbf{Y}$ & 1 \\
\hline $\mathbf{Y}$ & & 3458 & NP & Nopturium & CAPHUM & $\begin{array}{l}\text { Maximum Concentration of } \\
\text { Actinido with Mobila Humic } \\
\text { Colbids }\end{array}$ & 37723 & $08+45 y-96$ & $\mathbf{Y}$ & 1 \\
\hline $\mathbf{Y}$ & & 3458 & NP & Neptuniem & CAPHUM & $\begin{array}{l}\text { Moxinum Concentration of } \\
\text { Actinide with Mobils Hemic } \\
\text { Colloids }\end{array}$ & 37723 & Domby-96 & $\mathbf{Y}$ & 1 \\
\hline $\mathbf{Y}$ & & 3459 & PU & Putonium & CAPHUM & $\begin{array}{l}\text { Maximum Concentration of } \\
\text { Actinide with Mobila Humic } \\
\text { Colloids }\end{array}$ & 37724 & De-4ay-96 & $\mathbf{Y}$ & 1 \\
\hline $\mathbf{Y}$ & & 3459 & PU & Phitonium & CAPHUM & $\begin{array}{l}\text { Maximum Concentration of } \\
\text { Actinide with Mobile Humic } \\
\text { Colloids }\end{array}$ & 37724 & Cos4ay-96 & $\mathbf{Y}$ & 1 \\
\hline $\mathbf{Y}$ & & 3460 & $\mathbf{U}$ & Uranium & CAPHUM & $\begin{array}{l}\text { Maxinum Concentration of } \\
\text { Actinide with Mobila Humic } \\
\text { Colloids }\end{array}$ & 37725 & 08-May-96 & $\mathbf{Y}$ & 1 \\
\hline $\mathbf{Y}$ & & 3460 & $\mathbf{u}$ & Uranium & CAPHUM & $\begin{array}{l}\text { Maximum Concentraton of } \\
\text { Actinide with Mobile Humic } \\
\text { Colbids }\end{array}$ & 37725 & O8-May-96 & $\mathbf{Y}$ & 1 \\
\hline $\mathbf{Y}$ & & 3461 & $T H$ & Thorium & CAPHUM & $\begin{array}{l}\text { Maximum Concentraton of } \\
\text { Actinide with Mobile Hurnic } \\
\text { Colloids }\end{array}$ & 37726 & 08-Mry-96 & $\mathbf{Y}$ & 1 \\
\hline $\mathbf{Y}$ & & 3461 & $\pi H$ & Thorim & CAPHUH & $\begin{array}{l}\text { Maxmum Concentretion of } \\
\text { Actinide with Mobils Hunic } \\
\text { Colloids }\end{array}$ & 37726 & 084tay-96 & $\mathbf{Y}$ & 1 \\
\hline$Y$ & & 3452 & CULEBRA & $\begin{array}{l}\text { Culebra member of the } \\
\text { Rustlar formation }\end{array}$ & ETHICK & Elfective Thickness & 37727 & 07 May-96 & $\mathbf{Y}$ & 1 \\
\hline $\mathbf{Y}$ & & 3462 & CULEBRA & $\begin{array}{l}\text { Culabra member of tho } \\
\text { Rustler formation }\end{array}$ & ETHICK & Effective Thickness & 37727 & 07 May-96 & $\mathbf{Y}$ & 1 \\
\hline $\mathbf{Y}$ & & 3469 & CULEBRA & $\begin{array}{l}\text { Culwbre member of the } \\
\text { Rustier fomation }\end{array}$ & SKIN_RES & Skn Resustenco & 37735 & DSMay-96 & $\mathbf{Y}$ & 48 \\
\hline $\mathbf{Y}$ & & 3469 & CULEBRA & $\begin{array}{l}\text { Culebra member of the } \\
\text { Rustler formetion }\end{array}$ & SKIN_RES & Sten Resistenco & 37735 & OS-May-95 & $\mathbf{Y}$ & 48 \\
\hline $\mathbf{Y}$ & & 3469 & CULEBRA & $\begin{array}{l}\text { Culbore member of the } \\
\text { Rustlex formation }\end{array}$ & SKIN_RES & Sten Resistancos & 37735 & O9May-96 & $\mathbf{Y}$ & $4 B$ \\
\hline $\mathbf{Y}$ & & 3470 & BLOWOUT & $\begin{array}{l}\text { BRAGFLOW Dred } \\
\text { Brine Releseses }\end{array}$ & GAS_MIN & Ges rme an-of & 38209 & 06-Jun-96 & $Y$ & 48 \\
\hline $\mathbf{Y}$ & & 3470 & BLOWOUT & $\begin{array}{l}\text { BRAGFLOW Drect } \\
\text { Brmo Roleaces }\end{array}$ & GAS_MIN & Ges rute axtof & 38209 & 06-Jun-96 & $\mathbf{Y}$ & $4 B$ \\
\hline $\mathbf{Y}$ & & 3471 & BLOWOUT & $\begin{array}{l}\text { BRAGFLOW Drect } \\
\text { Brine Releases }\end{array}$ & MAXFLOW & Mexmum blowout fiow & 38210 & 06-Jun-96 & $\mathbf{Y}$ & 48 \\
\hline $\mathbf{Y}$ & & 3471 & BLOWOUT & $\begin{array}{l}\text { BRAGFLOW Drect } \\
\text { Brime Roleseses }\end{array}$ & MAXFLOW & Mexmum blowrout fiow & 39210 & CosJun-96 & $\mathbf{Y}$ & 4B \\
\hline $\mathbf{Y}$ & & 3472 & BLOWOUT & $\begin{array}{l}\text { BRAGFLOW Dreat } \\
\text { Brine Releses }\end{array}$ & MINFLOW & Mnmum blowout flow & 38211 & 05 Jun-96 & $\mathbf{Y}$ & 48 \\
\hline $\mathbf{Y}$ & & 3472 & BLOWOUT & $\begin{array}{l}\text { BRAGFLOW Drect } \\
\text { Bino Revases }\end{array}$ & MINFLOW & Mnmum blowout fiow & 38211 & 06-Jun-96 & $\mathbf{Y}$ & 48 \\
\hline $\mathbf{Y}$ & & 3473 & BLOWOUT & $\begin{array}{l}\text { BRAGFLOW Dreat } \\
\text { Brime Relases: }\end{array}$ & THCK_CAS & $\begin{array}{l}\text { Thedoness of the Castile } \\
\text { formation }\end{array}$ & 38213 & 06-Jun-96 & $\mathbf{Y}$ & $A B$ \\
\hline $\mathbf{Y}$ & & 3473 & BLOWOUT & $\begin{array}{l}\text { BRAGFLOWDroct } \\
\text { Bnno Rolesses }\end{array}$ & THCK_CAS & $\begin{array}{l}\text { Thudenass of the Cestule } \\
\text { formation }\end{array}$ & 38213 & Co-vin-96 & $\mathbf{Y}$ & $4 B$ \\
\hline $\mathbf{Y}$ & & 3474 & CULEBRA & $\begin{array}{l}\text { Cuinbre member of the } \\
\text { Rustler formation }\end{array}$ & DTORT & Ditusive Tortucity & 38345 & $90-J u n-96$ & $\mathbf{Y}$ & 1 \\
\hline $\mathbf{Y}$ & & 3474 & CULEBRA & $\begin{array}{l}\text { Cukbre member of the } \\
\text { Rustier formetion }\end{array}$ & DTORT & Difirive Tortuosity & 38345 & 10.an-96 & $\mathbf{Y}$ & 1 \\
\hline
\end{tabular}


CCA Parameter Listing

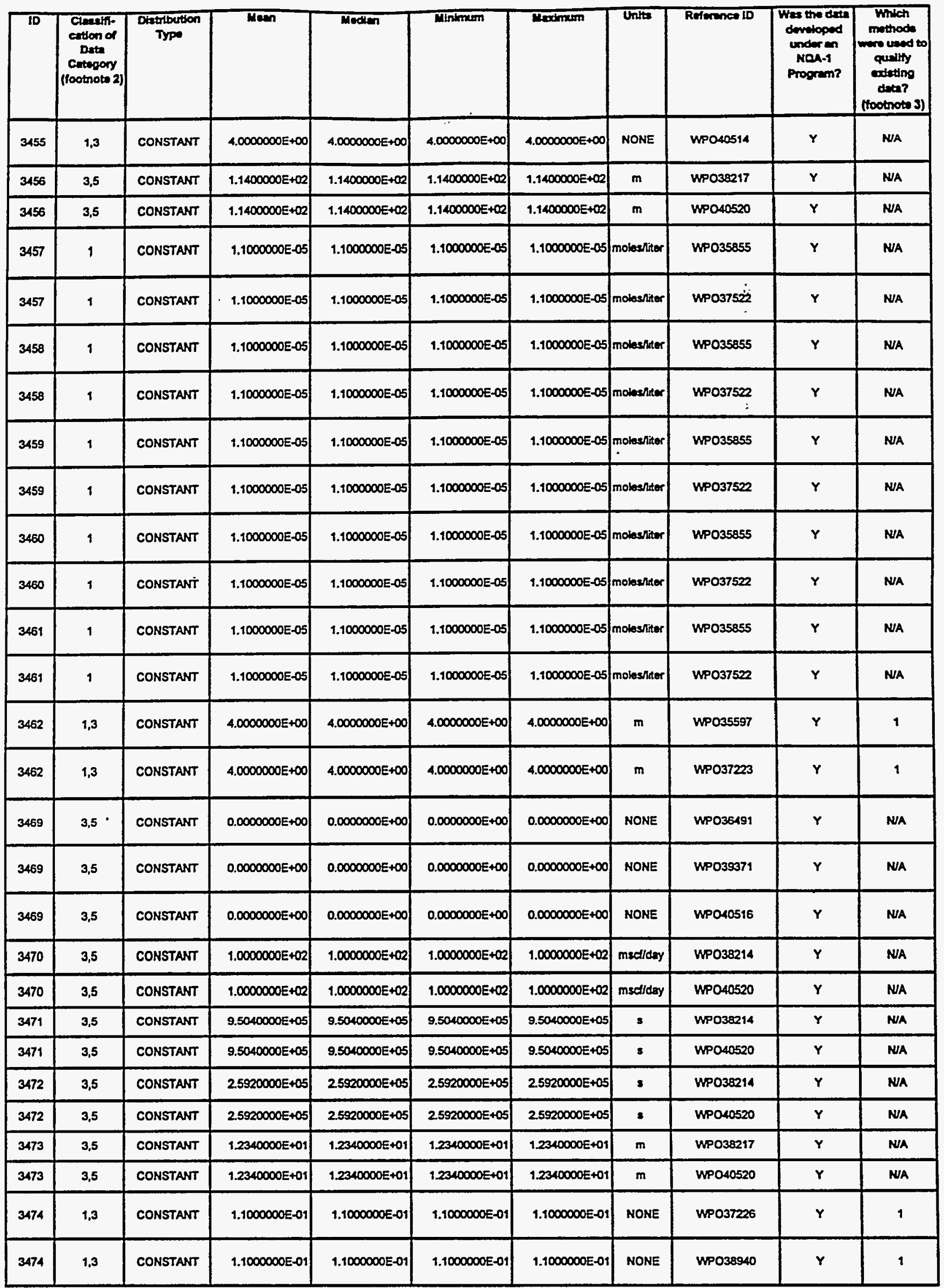


CCA Parameter tisting

\begin{tabular}{|c|c|c|c|c|c|c|c|c|c|c|}
\hline $\begin{array}{c}\text { Perwotar in } \\
\text { Dothoues? } \\
\text { (YN) }\end{array}$ & 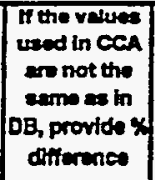 & ID & Mataralo & Materia Namo & Permeter & Permear kams & $\begin{array}{l}\text { PRP ID } \\
\text { (NPO D) }\end{array}$ & Dente Entry & $\begin{array}{l}\text { is the } 464 \\
\text { conciationt } \\
\text { wth the } \\
\text { permeter } \\
\text { in DB? } \\
(N, N A)\end{array}$ & $\begin{array}{l}\text { Parametar } \\
\text { Category } \\
\text { (footenote 1) }\end{array}$ \\
\hline $\mathbf{Y}$ & & 3475 & $U+6$ & Urasum V & MKD_U & $\begin{array}{l}\text { Matrox Partion Coofficuent for } \\
\text { Uranium }\end{array}$ & 38346 & $12 \sqrt{2}+96$ & $\mathbf{Y}$ & 1 \\
\hline $\mathbf{Y}$ & & 3475 & $U+6$ & Uranium V & MKD_U & $\begin{array}{l}\text { Matix Partition Coofficient for } \\
\text { Uranimn }\end{array}$ & 38346 & 12-kn-96 & $\mathbf{Y}$ & 1 \\
\hline $\mathbf{Y}$ & & 3475 & $U+6$ & Uranium V & MKD_U & $\begin{array}{l}\text { Matsu Pertion Coeficicent for } \\
\text { Uranium }\end{array}$ & 38346 & 12- $\sqrt{2}-96$ & $\mathbf{Y}$ & 1 \\
\hline $\mathbf{Y}$ & & 3475 & $U+6$ & Uranium VI & MKD_U & $\begin{array}{l}\text { Matrax Partiton Coefficient for } \\
\text { Urenium }\end{array}$ & 38346 & 12-Jun-96 & $\mathbf{Y}$ & 1 \\
\hline $\mathbf{Y}$ & & 3475 & $u+6$ & Uranium V & MKD_U & $\begin{array}{l}\text { Metsix Partion Cooficient for } \\
\text { Uranium }\end{array}$ & 38346 & 12Jun-96 & $\mathbf{Y}$ & 1 \\
\hline $\mathbf{Y}$ & & 3476 & $N P+5$ & Neptunium V & MKD_NP & $\begin{array}{l}\text { Matrix Partion Coefficient for } \\
\text { Neptenium }\end{array}$ & 38347 & 12Jun-96 & $\mathbf{Y}$ & 1 \\
\hline $\mathbf{Y}$ & & 3476 & $N P+5$ & Neptunium V & MKD_NP & $\begin{array}{l}\text { Matrx Partiton Cooficiond for } \\
\text { Nopturiem }\end{array}$ & 38347 & $12-$ tun-s & $\mathbf{Y}$ & 1 \\
\hline $\mathbf{Y}$ & & 3476 & NP+5 & Neptunium V & MKD_NP & $\begin{array}{l}\text { Matrix Partition Coefficiont for } \\
\text { Noptunium }\end{array}$ & 38347 & 12 Jun-96 & $\mathbf{Y}$ & 1 \\
\hline $\mathbf{Y}$ & & 3476 & $N P+5$ & Neptenium V & $M K D \_N P$ & $\begin{array}{l}\text { Matro Partition Cosficient for } \\
\text { Neptunium }\end{array}$ & 38347 & 12-Jun-96 & $\mathbf{Y}$ & 1 \\
\hline $\mathbf{Y}$ & & 3476 & $N P+5$ & Neptunium V & MKD_NP & $\begin{array}{l}\text { Matrix Pertition Coefficient for } \\
\text { Neptunium }\end{array}$ & 38347 & 12Vun-96 & $\mathbf{Y}$ & 1 \\
\hline $\mathbf{Y}$ & & 3477 & $N P+4$ & Noptunium N & MKD_NP & $\begin{array}{l}\text { Matrix Partition Coofficient for } \\
\text { Neptunisan }\end{array}$ & 38348 & 12-Jun-96 & $\mathbf{Y}$ & 1 \\
\hline $\mathbf{Y}$ & & 3477 & $N P+4$ & Neptenium N & MKD_NP & $\begin{array}{l}\text { Matrix Partition Coofficent for } \\
\text { Neptunium }\end{array}$ & 38348 & 12-Jun-96 & $\mathbf{Y}$ & 1 \\
\hline $\mathbf{Y}$ & & 3477 & $N P+4$ & Neptunium N & MKD_NP & $\begin{array}{l}\text { Matrix Partiton Coefficient for } \\
\text { Neptunium }\end{array}$ & 38348 & 12-Jun-96 & $\mathbf{Y}$ & 1 \\
\hline $\mathbf{Y}$ & & 3477 & NP+4 & Noptunium N & MKD_NP & $\begin{array}{l}\text { Matrix Partition Coofficient for } \\
\text { Neptunium }\end{array}$ & 38348 & 12Jun-96 & $\mathbf{Y}$ & 1 \\
\hline$Y$ & & 3477 & $N P+4$ & Neptunum IV & MKD_NP & $\begin{array}{l}\text { Matrix Partion Coefficient for } \\
\text { Neptunium }\end{array}$ & 38348 & 12Jur-96 & $\mathbf{Y}$ & 1 \\
\hline $\mathbf{Y}$ & & 3478 & $T H+4$ & Thorium N & MKD_TH & $\begin{array}{l}\text { Matrix Partition Coofficient for } \\
\text { Thorium }\end{array}$ & 38349 & 12-Jur-96 & $\mathbf{Y}$ & 1 \\
\hline $\mathbf{Y}$ & & 3478 & $\mathrm{TH}+4$ & Thorium N & MKD_TH & $\begin{array}{l}\text { Matrx Partition Cosficient for } \\
\text { Thorium }\end{array}$ & 38349 & 12 Jur-96 & $\mathbf{Y}$ & 1 \\
\hline $\mathbf{Y}$ & & 3478 & TH+4 & Thorium N & MKD_TH & $\begin{array}{l}\text { Matrix Pertition Coefficient for } \\
\text { Thonum }\end{array}$ & 38349 & 12-Jun-96 & $\mathbf{Y}$ & 1 \\
\hline $\mathbf{Y}$ & & 3478 & $T H+4$ & Thorium $\mathbf{N}$ & MKD_TH & $\begin{array}{l}\text { Matrox Partition Coefficient for } \\
\text { Tharium }\end{array}$ & 38349 & 12 Jun-96 & $\mathbf{Y}$ & 1 \\
\hline $\mathbf{Y}$ & & 3478 & $T H+4$ & Thorium N & MKO_TH & $\begin{array}{l}\text { Matrix Partition Coefficient for } \\
\text { Thorium }\end{array}$ & 38349 & $12 \sqrt{u n-96}$ & $\mathbf{Y}$ & 1 \\
\hline $\boldsymbol{Y}$ & & 3479 & $U+4$ & Uranium $\mathbf{N}$ & MKD_U & $\begin{array}{l}\text { Matrox Partition Coefficenent for } \\
\text { Uranium }\end{array}$ & 38350 & 12-Jun-96 & $Y$ & 1 \\
\hline $\mathbf{Y}$ & & 3479 & $u+4$ & Uranium $\mathbf{N}$ & MKD_U & $\begin{array}{l}\text { Matrox Partition Coofficient for } \\
\text { Uranium }\end{array}$ & 38350 & 12-Jun-96 & $\mathbf{Y}$ & 1 \\
\hline $\mathbf{Y}$ & & 3479 & $U+4$ & Uraniem N & MKD_U & $\begin{array}{l}\text { Matrox Partion Coefficient for } \\
\text { Uranium }\end{array}$ & 38350 & 12ปJun-96 & $\mathbf{Y}$ & 1 \\
\hline $\mathbf{Y}$ & & 3479 & $u+4$ & Uranium $\mathbf{N}$ & MKD_U & $\begin{array}{l}\text { Matrox Partion Cosficent for } \\
\text { Uranium }\end{array}$ & 38350 & 12-Jun-96 & $\mathbf{Y}$ & 1 \\
\hline $\mathbf{Y}$ & & 3479 & $U+4$ & Uranium N & MKD_U & $\begin{array}{l}\text { Matrox Partion Coefficient fox } \\
\text { Uranium }\end{array}$ & 38350 & 12-Jun-96 & $--Y$ & 1 \\
\hline $\mathbf{Y}$ & & 3480 & PU+3 & Phetonium III & MKD_PU & $\begin{array}{l}\text { Matsx Partion Cosficient for } \\
\text { Phitonum }\end{array}$ & 38351 & 12-Jun-96 & $\mathbf{Y}$ & 1 \\
\hline $\mathbf{Y}$ & & 3480 & $\mathrm{PU}+3$ & Plutoritum III & MKD_PU & $\begin{array}{l}\text { Matrox Partition Coefficient for } \\
\text { Phitonium }\end{array}$ & 38351 & 12-Jun-96 & $\mathbf{Y}$ & 1 \\
\hline $\mathbf{Y}$ & & 3480 & $\mathrm{PU}+3$ & Phtonium III & MKD_PU & $\begin{array}{l}\text { Matrax Partion Coefficient for } \\
\text { Phitonium }\end{array}$ & 38351 & 12-Jun-96 & $\mathbf{Y}$ & 1 \\
\hline $\mathbf{Y}$ & & 3480 & PU+3 & Pktonium III & $M K D_{2} P U$ & $\begin{array}{l}\text { Metra Partion Coefficient for } \\
\text { Phtonium }\end{array}$ & 38351 & 12-Jun-96 & $\mathbf{Y}$ & 1 \\
\hline $\mathbf{Y}$ & & 3480 & PU+3 & Plutorium IfI & MKD_PU & $\begin{array}{l}\text { Matrox Partion Coefficaent for } \\
\text { Phitonium }\end{array}$ & 38351 & 12-Jun-96 & $\mathbf{Y}$ & 1 \\
\hline $\mathbf{Y}$ & & 3481 & Put4 & Pktorium N & MKD_PU & $\begin{array}{l}\text { Metrx Partition Coefficent for } \\
\text { Phtonium }\end{array}$ & 38352 & 12-Jun-96 & $\mathbf{Y}$ & 1 \\
\hline $\mathbf{Y}$ & & 3481 & PU+4 & Pltonium N & MKD_PU & $\begin{array}{l}\text { Matrx Partion Coefficient for } \\
\text { Plutonium }\end{array}$ & 38352 & 12 Jun-96 & $\mathbf{Y}$ & 1 \\
\hline $\mathbf{Y}$ & & 3481 & PU+4 & Pletorium N & MKD_PU & $\begin{array}{l}\text { Matrox Pertion Confficent for } \\
\text { Phtonium }\end{array}$ & 38352 & 12-Jun-96 & $\mathbf{Y}$ & 1 \\
\hline $\mathbf{Y}$ & & 3481 & PU+4 & Phetonicm N & $M K D \_P U$ & $\begin{array}{l}\text { Matrox Pertion Coefficient for } \\
\text { Phitoniam }\end{array}$ & 38352 & 12.Jun-96 & $\mathbf{Y}$ & 1 \\
\hline $\mathbf{Y}$ & & 3481 & PUt4 & Pheorium N & MKD_PU & $\begin{array}{l}\text { Metrox Partion Comficent for } \\
\text { Phtoriem }\end{array}$ & 38352 & 12Jun-96 & $\mathbf{Y}$ & 1 \\
\hline $\mathbf{Y}$ & & 3482 & $A H+3$ & Americium III & MKO_AM & $\begin{array}{l}\text { Metrox Pertion Coefilenent for } \\
\text { Anericium }\end{array}$ & 38353 & 12-Jun-96 & $Y$ & 1 \\
\hline
\end{tabular}


CCA Parameter Listing

\begin{tabular}{|c|c|c|c|c|c|c|c|c|c|c|}
\hline ID & \begin{tabular}{c|} 
Cisetin- \\
cartion of \\
Onta \\
Cuttegory \\
(footrote 2)
\end{tabular} & $\begin{array}{l}\text { Distribertion } \\
\text { Type }\end{array}$ & mean & Medien & Hinimum & Modinim & Units & Reterence ID & $\begin{array}{l}\text { Westio dets } \\
\text { developed } \\
\text { underen } \\
\text { Nol-9 } \\
\text { Progrem? }\end{array}$ & 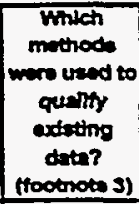 \\
\hline 3475 & 1 & UNIFORM & $1.5000000 E-02$ & $1.5000000=-02$ & $3.0000000 E-05$ & $3.0000000 E-02$ & $m^{\wedge} 3 \times 0$ & WP035268 & $\mathbf{Y}$ & NA \\
\hline 3475 & 1 & UNIFORM & $1.5000000=-02$ & $1.5000000 E-02$ & $3.0000000 E-05$ & $3.0000000 E-02$ & $m^{\wedge} 3 \times 0$ & WP035269 & $\mathbf{Y}$ & N/A \\
\hline 3475 & 1 & UNIFORM & $1.5000000 E-02$ & $1.5000000 E-02$ & $3.0000000 E-05$ & $3.0000000=-02$ & $m^{\wedge} 3 \mathrm{~kg}$ & WP038231 & $\mathbf{Y}$ & NA \\
\hline 3475 & 1 & UNIFORM & $1.5000000 E-02$ & $1.5000000 E-02$ & $3.0000000 E-05$ & $3.0000000 E-02$ & $m^{\wedge} 3 / x 0$ & WP038so1 & $\mathbf{Y}$ & NA \\
\hline 3475 & 1 & UNIFORM & $1.5000000 E-02$ & $1.5000000 E-02$ & $3.0000000 E-0.5$ & $3.0000000 E-02$ & $m^{\wedge} 3 \times x$ & WP04155? & $\mathbf{Y}$ & N/A \\
\hline 3476 & 1 & UNIFORM & $1.0000000 E-01$ & $1.0000000 E-01$ & $1.0000000=-03$ & $20000000=-01$ & $m^{\wedge} 3 / k 0$ & WPO35268' & $\gamma$ & NA \\
\hline 3476 & 1 & UNIFORM & $1.0000000 E-01$ & $1.0000000 E-01$ & $1.0000000 E-03$ & $2.0000000 E-01$ & $m^{n} 3 k_{0}$ & WP035269 & $Y$ & NA \\
\hline 3476 & 1 & UNIFORM & $1.0000000 E-01$ & $1.0000000 E-01$ & $1.0000000 E-03$ & $20000000=-01$ & $m^{\wedge} 3 / k_{0}$ & WP038231 & $\mathbf{Y}$ & NA \\
\hline 3476 & 1 & UNIFORM & $1.0000000 E-01$ & $1.0000000 E-01$ & $1.0000000 \mathrm{E}-03$ & $20000000 E-01$ & $m^{n} 3 \times x_{0}$ & WP038801 & $\mathbf{Y}$ & NA \\
\hline 3476 & 1 & UNIFORM & $1.0000000 E-01$ & $1.0000000 \mathrm{E}-01$ & $1.0000000 E-03$ & $20000000=-01$ & $m^{n} 3 / k_{0}$ & WP041557 & $\mathbf{Y}$ & NA \\
\hline 3477 & 1 & UNIFORM & $1.0000000 E+01$ & $1.0000000 E+01$ & 9.0000000E-0t & $20000000 E+01$ & $m \wedge 3 m 0$ & WP035268 & $\mathbf{Y}$ & NA \\
\hline 3477 & 1 & UNIFORM & $1.0000000 E+01$ & $1.0000000 E+01$ & $9.0000000 E-01$ & $2,0000000 E+01$ & $m^{\wedge} 3 / k g$ & WP035269 & $\mathbf{Y}$ & NA \\
\hline 3477 & 1 & UNIFORM & $1.00000000 E+01$ & $1.0000000 E+01$ & $9.0000000 E-01$ & $200000000 \mathrm{E}+01$ & $m^{\wedge} 3 / k 0$ & WPO38231 & $\mathbf{Y}$ & NA \\
\hline 3477 & 1 & UNIFORM & $1.0000000 \mathrm{E}+01$ & $1.0000000 E+01$ & $9.0000000 E-01$ & $2,0000000 E+01$ & $m^{\wedge} 3 / k_{0}$ & WP038s01 & $\mathbf{Y}$ & NA \\
\hline 3477 & 1 & UNIFORA & $1.0000000 E+01$ & $1.0000000 E+01$ & $9.0000000 E-01$ & $2.0000000 E+01$ & $m^{\wedge} 3 \mathbf{k g}$ & WPO11557 & $\mathbf{Y}$ & NA \\
\hline 3478 & 1 & UNIFORM & $1.0000000 E+01$ & $1.0000000 \mathrm{E}+01$ & $9.0000000 E-01$ & $2.0000000 E+01$ & $m^{n} 3 / k g$ & WP035268 & $\mathbf{Y}$ & N/A \\
\hline 3478 & 1 & UNIFORM & $1.0000000 E+01$ & $1.0000000 E+01$ & $9.0000000 E-01$ & $20000000 E+01$ & $m^{\wedge} 3 / k g$ & WP035269 & $\mathbf{Y}$ & N/A \\
\hline 3478 & 1 & UNIFORM & $1.0000000 E+01$ & $1.0000000 \mathrm{E}+01$ & 9.0000000E-01 & $2.0000000 E+01$ & $m^{\wedge} 3 k_{00}$ & WP038231 & $\mathbf{Y}$ & N/A \\
\hline 3478 & 1 & UNIFORM & $1.0000000 \mathrm{E}+01$ & $1.0000000 E+01$ & $9.0000000 E-01$ & $20000000 E+01$ & $m^{\wedge} 3 \times n$ & WP038801 & $\mathbf{Y}$ & NA \\
\hline 3478 & 1 & UNIFORM & $1.0000000 E+01$ & $1.0000000 E+01$ & $9.0000000 E-01$ & $2,0000000 E+01$ & $\mathrm{~m}^{\wedge} 3 \mathrm{~kg}$ & WPO41557 & Y & NA \\
\hline 3479 & 1 & UNIFORM & $1.0000000 E+01$ & $1.0000000 \mathrm{E}+01$ & $9.0000000 E-01$ & $2.0000000 E+01$ & $m^{\wedge} 3 / k g$ & WP035268 & $Y$ & NA \\
\hline 3479 & 1 & UNIFORM & $1.0000000 E+01$ & $1.0000000 E+01$ & $9.0000000 E-01$ & $2.0000000 E+01$ & $m^{\wedge} 3 k_{0}$ & WP035269 & $\mathbf{Y}$ & NA \\
\hline 3479 & 1 & UNIFORM & $1.0000000 E+01$ & $1.00000000 E+01$ & $9.0000000 E-01$ & $2.0000000 \mathrm{E}+01$ & $m^{\wedge} 3 \mathrm{~kg}$ & WP038231 & $\mathbf{Y}$ & NA \\
\hline 3479 & 1 & UNIFORM & $1.0000000 E+01$ & $1.0000000=+01$ & $9.0000000 E-01$ & $2.0000000 E+01$ & $m^{\wedge} 3 / k g$ & WP038801 & $Y$ & NA \\
\hline 3479 & 1 & UNIFORM & $1.0000000 E+01$ & $1.0000000 E+01$ & $9.0000000 E-01$ & $2.0000000 E+01$ & $m^{\wedge} 3 k g$ & WP041557 & $\because \cdot Y$ & N/A \\
\hline 3480 & 1 & UNIFORM & 2.6000000E-01 & $2,6000000 E-01$ & $20000000 E-02$ & $5.0000000 E-01$ & $m^{\wedge} 3 \times g$ & WP035268 & $Y$ & N/A \\
\hline 3480 & 1 & UNIFORM & 2.6000000E-01 & $2,6000000 E-01$ & $2.0000000 E-02$ & $5.0000000 E-01$ & $m^{\wedge} 3 \times x$ & WP035269 & $Y$ & N/A \\
\hline 3480 & 1 & UNIFORM & $2.6000000 E-01$ & $2.6000000 E-01$ & $2.0000000 E-02$ & $5.0000000 E-01$ & $m^{\wedge} 3 \times g$ & WP038231 & $\mathbf{Y}$ & N/A \\
\hline 3480 & 1 & UNIFORM & $2.6000000 E-01$ & $26000000 E-01$ & $20000000 E-02$ & $5.0000000 E-01$ & $m^{\wedge} 3 / x g$ & WPO38801 & $\mathbf{Y}$ & N/A \\
\hline 3480 & 1 & UNIFORM & 26000000E-01 & $2.6000000 E-01$ & $20000000 E-02$ & $5.0000000 E-01$ & $m^{\wedge} 3 / k g$ & WPO41557 & $\mathbf{Y}$ & NA \\
\hline 3481 & 1 & UNIFORM & $1.0000000 E+01$ & $1.0000000 \mathrm{E}+01$ & $9.0000000 E-01$ & $2.0000000 E+01$ & $m^{\wedge} 3 k_{0}$ & WP035268 & $\mathbf{Y}$ & NA \\
\hline 3481 & 1 & UNIFORM & $1.0000000 E+01$ & $1.0000000 E+01$ & $9.0000000 E-01$ & $20000000 E+01$ & $\mathbf{m}^{\wedge} 3 / \mathbf{k g}$ & WP035269 & $Y$ & NA \\
\hline 3481 & 1 & UNIFORM & $1.00000000+01$ & $1.0000000 E+01$ & $9.0000000 E-01$ & $200000000+01$ & $m^{\wedge} 3 / k 0$ & WPO38231 & $\mathbf{Y}$ & NA \\
\hline 3481 & 1 & UNIFORM & $1.0000000 E+01$ & $1.0000000 E+01$ & $9.0000000 E-01$ & $200000000 E+01$ & $m^{\wedge} 3 / k 0$ & WP038801 & $\mathbf{Y}$ & NAA \\
\hline 3481 & 1 & UNIFORN & $1.0000000 E+01$ & $1.0000000 E+01$ & $9.0000000 E-01$ & $20000000 E+01$ & $\mathrm{~m}^{\wedge} 3 / \mathrm{kg}$ & WPO41557 & $\mathbf{Y}$ & NA \\
\hline 3482 & 1 & UNIFORM & $26000000 E-01$ & $2.6000000 E-01$ & $2.0000000 E-02$ & $5.0000000 E-01$ & $m^{\wedge} 3 / k_{0}$ & WP035268 & $\mathbf{Y}$ & $N A$ \\
\hline
\end{tabular}


CCA Parameter Listing

\begin{tabular}{|c|c|c|c|c|c|c|c|c|c|c|}
\hline $\begin{array}{c}\text { Parwiter in } \\
\text { Detabaee? } \\
\text { (YM) }\end{array}$ & 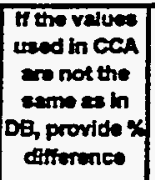 & ID & Metartalo & Materde Kam & $\begin{array}{c}\text { Parametar } \\
\text { to }\end{array}$ & Peramitar Harme & $\begin{array}{l}\text { PRPLD } \\
\text { (MPO *) }\end{array}$ & $\begin{array}{l}\text { Data Entor } \\
\text { Dete }\end{array}$ & 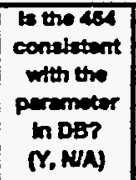 & $\begin{array}{l}\text { Parameter } \\
\text { Category } \\
\text { (footiote 1) }\end{array}$ \\
\hline$Y$ & & 3482 & $A M+3$ & Americum III & MKD_AM & $\begin{array}{l}\text { Matrix Partition Coefficiont for } \\
\text { Amoricien }\end{array}$ & 38253 & 12.tun-96 & $\mathbf{Y}$ & 1 \\
\hline $\mathbf{Y}$ & & 3482 & $A M+3$ & Americium III & MKD_AH & $\begin{array}{l}\text { Matro Partion Coefficient for } \\
\text { Americium }\end{array}$ & 38353 & $12 \sqrt{\mathrm{l} u n-96}$ & $\mathbf{Y}$ & 1 \\
\hline $\mathbf{Y}$ & & 3482 & $A M+3$ & Americium III & MKD_AM & $\begin{array}{l}\text { Matrix Partion Coefficient for } \\
\text { Americium }\end{array}$ & 38353 & 12-Jun-S6 & $\mathbf{Y}$ & 1 \\
\hline $\mathbf{Y}$ & & 3482 & $A M+3$ & Americiem III & MKD_AM & $\begin{array}{l}\text { Mestrix Partution Coefficiont for } \\
\text { Americium }\end{array}$ & 38353 & 12 dun-96 & $\mathbf{Y}$ & 1 \\
\hline $\mathbf{Y}$ & 100 & 3483 & CULEBRA & $\begin{array}{l}\text { Culebra momber of thoo } \\
\text { Rustier formation }\end{array}$ & DISP_L & Longitudinal dispersivity & 38354 & 12 Jun- 96 & $\mathbf{Y}$ & 1 \\
\hline $\mathbf{Y}$ & 100 & 3483 & CULEBRA & $\begin{array}{l}\text { Culebra member of the } \\
\text { Rustler formation }\end{array}$ & DISP_L & Longitudinal dispercivity & 38254 & 12Jun-96 & $\mathbf{Y}$ & 1 \\
\hline $\mathbf{Y}$ & 100 & 3483 & CULEBRA & \begin{tabular}{|l|} 
Culabra momber of tho \\
Rustior formation
\end{tabular} & DISP_L & Longitudinal dispersivity & 38354 & 12-hen-96 & $Y$ & 1 \\
\hline $\mathbf{Y}$ & 100 & 3483 & CULEBRA & $\begin{array}{l}\text { Culebra member of tho } \\
\text { Rustier formation }\end{array}$ & DISP_L & Longitudiral dispersivity & 38354 & 12-Jun-96 & $\mathbf{Y}$ & 1 \\
\hline $\mathbf{Y}$ & 100 & 3483 & CULEBRA & $\begin{array}{l}\text { Culebra momber of the } \\
\text { Rustier formation }\end{array}$ & DISP_L & Longitudinal dispersivity & 38354 & 12-Jun-96 & $\mathbf{Y}$ & 1 \\
\hline $\mathbf{Y}$ & 100 & 3484 & CULEBRA & $\begin{array}{l}\text { Cullebra menber of tho } \\
\text { Rustier formation }\end{array}$ & DISPT_L & Transverse dispersivity & 38355 & 12-tun-96 & $\mathbf{Y}$ & 1 \\
\hline $\mathbf{Y}$ & 100 & 3484 & CULEBRA & $\begin{array}{l}\text { Culebra member of the } \\
\text { Rustior formation }\end{array}$ & DISPT_L & Trensverse dispersivity & 38355 & 12.Jun-96 & $\mathbf{Y}$ & 1 \\
\hline $\mathbf{Y}$ & 100 & 3484 & CULEBRA & $\begin{array}{l}\text { Culebra member of the } \\
\text { Rustler formation }\end{array}$ & DISPT_L & Trunsverse dispersivity & 38355 & 12Jur-S6 & $\mathbf{Y}$ & 1 \\
\hline$Y$ & 100 & 3484 & CULEBRA & $\begin{array}{l}\text { Culebra member of tho } \\
\text { Rustler formation }\end{array}$ & DISPT_L & Trunsverze dispersivity & 38355 & 12-Jun-96 & $\mathbf{Y}$ & 1 \\
\hline$Y$ & 100 & 3484 & CULEBRA & $\begin{array}{l}\text { Culebra menber of the } \\
\text { Rustler formation }\end{array}$ & DISPT_L & Transverse dispersivity & 38355 & 12-Jun-86 & $\mathbf{Y}$ & 1 \\
\hline $\mathbf{Y}$ & & 3485 & CULEBRA & $\begin{array}{l}\text { Culabra momber of tho } \\
\text { Rustler formation }\end{array}$ & HMBLKLT & Culobra Half Matrix-Block Length & 38356 & 13-Jun-96 & $\mathbf{Y}$ & 1 \\
\hline $\mathbf{Y}$ & & 3485 & CULEBRA & $\begin{array}{l}\text { Culebra member of the } \\
\text { Rustlor formation }\end{array}$ & HMBLKLT & Culebra Hef Matrix-Block Longth & 38356 & 13-Jun-96 & $\mathbf{Y}$ & 1 \\
\hline$Y$ & & 3485 & CULEBRA & $\begin{array}{l}\text { Culebra momber of the } \\
\text { Rustier formaton }\end{array}$ & HMBLKLT & Culebra Hall Matrix-Block Longth & 38356 & $13-\sqrt{\operatorname{lon}} 96$ & $\mathbf{Y}$ & 1 \\
\hline $\mathbf{Y}$ & & 3485 & CULEBRA & $\begin{array}{l}\text { Cuibora member of tho } \\
\text { Rustifer formation }\end{array}$ & HMBLLLT & Culebra Half Matrix-Block Longth & 38356 & 13-Jun-96 & $Y$ & 1 \\
\hline $\mathbf{Y}$ & & 3486 & CULEBRA & $\begin{array}{l}\text { Culebra member of tho } \\
\text { Rustier formation }\end{array}$ & DPOROS & $\begin{array}{l}\text { Dimisave Porosity for Culebra } \\
\text { Dolomite }\end{array}$ & 38357 & 13-Jun-96 & $\mathbf{Y}$ & 1 \\
\hline $\mathbf{Y}$ & & 3486 & CULEBRA & $\begin{array}{l}\text { Culebra member of the } \\
\text { Rustler formation }\end{array}$ & DPOROS & $\begin{array}{l}\text { Diflusivi Porosty for Culetera } \\
\text { Dolomite }\end{array}$ & 38357 & 13-Jun-96 & $\mathbf{Y}$ & 1 \\
\hline $\mathbf{Y}$ & & 3486 & CULEBRA & $\begin{array}{l}\text { Culdora member of tho } \\
\text { Rustler formation }\end{array}$ & DPOROS & $\begin{array}{l}\text { Diflusive Porosity for Culetra } \\
\text { Dolomte }\end{array}$ & 38357 & 13-Jun-96 & $Y$ & 1 \\
\hline $\mathbf{Y}$ & & 3486 & CULEBRA & $\begin{array}{l}\text { Culebra mernber of the } \\
\text { Rustier formation }\end{array}$ & DPOROS & $\begin{array}{l}\text { Diffusive Porosity for Culebra } \\
\text { Dolomite }\end{array}$ & 38357 & 13Jun-96 & $\mathbf{Y}$ & 1 \\
\hline $\mathbf{Y}$ & & 3487 & CULEBRA & $\begin{array}{l}\text { Culebra member of the } \\
\text { Ruster formation }\end{array}$ & APOROS & Culobra Advedivo Porosity & 38358 & 14tur-96 & Y & 1 \\
\hline $\mathbf{Y}$ & & 3487 & CULEBRA & $\begin{array}{l}\text { Culebre member of the } \\
\text { Rustior formation }\end{array}$ & APOROS & Culobra Aovectivo Porosity & 38358 & 14ann-96 & $\mathbf{Y}$ & 1 \\
\hline $\mathbf{Y}$ & & 3487 & CULEBRA & $\begin{array}{l}\text { Culeora member of the } \\
\text { Rustier formation }\end{array}$ & APOROS & Culbera Advedive Porosity & 38358 & 14 un- 86 & $\mathbf{Y}$ & 1 \\
\hline$Y$ & & 3487 & CULEBRA & $\begin{array}{l}\text { Culebra mamber of the } \\
\text { Rustler formation }\end{array}$ & APOROS & Culebre Advective Porosity & 38358 & 14Jun-86 & $Y$ & 1 \\
\hline
\end{tabular}


CCA Parameter Listing

\begin{tabular}{|c|c|c|c|c|c|c|c|c|c|c|}
\hline DD & $\begin{array}{c}\text { Clasedit- } \\
\text { cation of } \\
\text { Deta } \\
\text { Cettegory } \\
\text { (footinote 2) }\end{array}$ & $\begin{array}{c}\text { Distibution } \\
\text { Typ }\end{array}$ & Maen & Medien & Minimum & Madmuen & Units & Reference to & $\begin{array}{l}\text { Wes the dital } \\
\text { doreloped } \\
\text { under an } \\
\text { Nel-1 } \\
\text { Progran? }\end{array}$ & $\begin{array}{c}\text { miles } \\
\text { mothods } \\
\text { wre used to } \\
\text { quallfy } \\
\text { exteting } \\
\text { data? } \\
\text { (footnote 3) }\end{array}$ \\
\hline 3482 & 1 & UNIFORM & $2.6000000 \mathrm{E}-01$ & $26000000=-01$ & $20000000 E-02$ & $5.0000000 E-01$ & $m^{\wedge} 3 \times 100$ & WP035269 & $\mathbf{Y}$ & NAA \\
\hline 3482 & 1 & UNIFORM & $2.6000000 \mathrm{E}-01$ & $2.6000000 \mathrm{E}-01$ & $2,0000000 E-02$ & $5.0000000 E-01$ & $m^{n} 3 / k 0$ & WP038231 & $Y$ & NA \\
\hline 3482 & 1 & UNIFORM & $2.6000000 E-01$ & $26000000 E-01$ & $2,0000000 E-02$ & 5.0000000E-01 & $m^{\wedge} \mathbf{3} / \mathrm{kp}_{0}$ & WP038801 & $\mathbf{Y}$ & $N / A$ \\
\hline 3482 & 1 & UNIFORM & $2.6000000 E-01$ & $2.6000000 E-01$ & $20000000 E-02$ & $5.0000000 E-01$ & $m^{\wedge} 3 \times 0$ & WPOA1557 & $\mathbf{Y}$ & N/A \\
\hline 3483 & $3,4,5$ & CONSTANT & $0.0000000 \mathrm{E}+\infty 0$ & $0.0000000 E+\infty$ & $0.0000000 E+\infty$ & $0.0000000 E+\infty$ & $\mathbf{m}$ & WP035270 & $\mathbf{Y}$ & NA \\
\hline 3483 & $3,4,5$ & CONSTANT & $0.0000000 E+\infty 0$ & $0.0000000 E+\infty 0$ & $0.0000000 E+00$ & $0.0000000 E+\infty$ & $m$ & WP037230 & $\mathbf{Y}$ & NAA \\
\hline 3483 & $3,4,5$ & CONSTANT & $0.0000000 E+00$ & $0.0000000 E+\infty 0$ & $0.0000000 E+\infty$ & $0.0000000 E+\infty$ & $\mathbf{m}$ & WP037231 & $\mathbf{Y}$ & NA \\
\hline 3483 & $3,4,5$ & CONSTANT & $0.0000000 E+\infty 0$ & $0.0000000 E+\infty 0$ & $0.0000000 E+\infty 0$ & $0.0000000 E+\infty 0$ & $m$ & WP038941 & $\mathbf{Y}$ & NA \\
\hline 3483 & $3,4,5$ & CONSTANT & $0.0000000 E+\infty 0$ & $0.00000000+\infty 0$ & $0.0000000 E+\infty 0$ & $0.0000000 E+\infty 0$ & m & WPO40434 & $\mathbf{Y}$ & NA \\
\hline 3484 & $3,4,5$ & CONSTANT & $0,0000000 E+\infty 0$ & $0.0000000 E+00$ & $0.0000000 E+\infty 0$ & $0.0000000 E+00$ & $\mathbf{m}$ & WP035270 & $\mathbf{Y}$ & N/A \\
\hline 3484 & $3,4,5$ & CONSTANT & $0.0000000 E+\infty$ & $0.0000000 E+\infty$ & $0.0000000 E+\infty 0$ & $0.0000000 E+\infty 0$ & $\mathbf{m}$ & WP037230 & $Y$ & NA \\
\hline 3484 & $3,4,5$ & CONSTANT & $0.0000000 E+\infty$ & $0.0000000 E+\infty 0$ & $0.0000000 E+\infty 0$ & $0.0000000 E+\infty$ & $\mathbf{m}$ & WP037231 & $\mathbf{Y}$ & NA \\
\hline 3484 & $3,4,5$ & CONSTANT & $0.0000000 \varepsilon+\infty$ & $0.0000000 E+\infty 0$ & $0.0000000 E+00$ & $0.0000000 E+\infty$ & $m$ & WP038941 & $\mathbf{Y}$ & NA \\
\hline 3484 & $3,4,5$ & CONSTANT & $0.0000000 \mathrm{E}+00$ & $0.0000000 E+\infty$ & $0.0000000 E+00$ & $0.0000000 E+\infty 0$ & m & WPO40434 & $Y$ & N/A \\
\hline 3485 & 1,3 & UNIFORM & $27500000 \mathrm{E}-01$ & $2.7500000 E-01$ & $5.0000000 \mathrm{E}-02$ & $5.0000000 E-01$ & $m$ & WP035268 & $\mathbf{Y}$ & 1 \\
\hline 3485 & 1,3 & UNIFORM & $2.7500000 \mathrm{E}-01$ & $2.7500000 \mathrm{E}-01$ & $5.0000000 \mathrm{E}-02$ & $5.0000000 E-01$ & $m$ & WP035597 & $Y$ & 1 \\
\hline 3485 & 1,3 & UNIFORM & $27500000 E-01$ & $2.7500000 \mathrm{E}-01$ & $5.0000000 \mathrm{E}-02$ & $5.0000000 \mathrm{E}-01$ & m & WP037225 & $Y$ & 1 \\
\hline 3485 & 1,3 & UNIFORM & $27500000 E-01$ & $2.7500000 E-01$ & $5.0000000 \mathrm{E}-02$ & $5.0000000 E-01$ & m & WP038928 & $\underline{Y}$ & 1 \\
\hline 3486 & 1,3 & CUMULATME & $1.6000000 \mathrm{E}-01$ & $1.6000000 \mathrm{E}-01$ & $1.00000000-01$ & $2.5000000 E-01$ & NONE & WP035268 & $\mathbf{Y}$ & 1 \\
\hline 3486 & 1,3 & CUMULATIVE & $1.6000000 E-01$ & $1.6000000 E-01$ & $1.0000000 E=01$ & $2.5000000 E-01$ & NONE & WP035597 & $\mathbf{Y}$ & 1 \\
\hline 3486 & 1,3 & CUMULATIVE & $1.6000000 \mathrm{E}-01$ & $1.6000000 E-01$ & $1.0000000 E-01$ & $2.5000000 \mathrm{E}-01$ & NONE & WP037228 & $\mathbf{Y}$ & 1 \\
\hline 3486 & 1,3 & CUMULATINE & $1.6000000 \mathrm{E}-01$ & $1.6000000 E-01$ & $1.0000000 E-01$ & $2.5000000 E-01$ & NONE & WP038773 & $\mathbf{Y}$ & 1 \\
\hline 3487 & 1,3 & LOGUNIFORM & $2.10000000-03$ & $1.0000000 \mathrm{E}-03$ & $1.0000000 E-04$ & $1.0000000 E-02$ & NONE & WP035268 & $\mathbf{Y}$ & 1 \\
\hline 3487 & 1,3 & LOGUNIFORM & $2.1000000 E-03$ & $1.0000000 E-\infty 3$ & $1.0000000 E-04$ & $1.0000000 E-02$ & NONE & WPO35597 & $\mathbf{Y}$ & 1 \\
\hline 3487 & 1,3 & LOGUNIFORM & $2.1000000 E-03$ & $1.0000000 \mathrm{E}-03$ & $1.00000000=-04$ & $1.0000000 \mathrm{E}-02$ & NONE & WP037227 & $\mathbf{Y}$ & 1 \\
\hline 3487 & 1,3 & LOGUNIFORM & $2.1000000 \mathrm{E}-03$ & $1.0000000 E-03$ & $1.0000000 E-04$ & $1.0000000 E-02$ & NONE & WP039928 & $\mathbf{Y}$ & 1 \\
\hline
\end{tabular}


CCA Parameter Listing

\begin{tabular}{|c|c|c|c|c|c|c|c|c|c|c|}
\hline 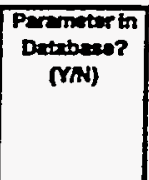 & 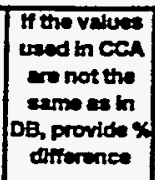 & 10 & Materia D & Mxtordiname & $\begin{array}{c}\text { Permintiar } \\
\text { to }\end{array}$ & Ponmoter Kamo & $\begin{array}{l}\text { PRP DD } \\
\text { (MPO \&) }\end{array}$ & $\begin{array}{c}\text { Dite Entry } \\
\text { Deten }\end{array}$ & 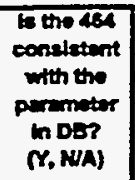 & $\begin{array}{l}\text { Parmeter } \\
\text { Cettepory } \\
\text { (footurote 1) }\end{array}$ \\
\hline $\mathbf{Y}$ & & 3488 & REFCON & Reference Constant & AREA_ZRO & $\begin{array}{l}\text { Aras in Waste Panols Not Used } \\
\text { For Dispocell (CCDFGF Model) }\end{array}$ & 38727 & 26 hen-96 & $\mathbf{Y}$ & $4 B$ \\
\hline $\mathbf{Y}$ & & 3488 & REFCON & References Constent & AREA_ZRO & $\begin{array}{l}\text { Avat in Waste Panats NoX Ured } \\
\text { For Dispocel (CCDFGF Modal) }\end{array}$ & 38727 & 26-Jun-96 & $Y$ & $A B$ \\
\hline $\mathbf{Y}$ & & 3489 & REFCON & Referencos Constent & AREA_CH & $\begin{array}{l}\text { Aran For CH Waste Desposed in } \\
\text { CCDFGF Model }\end{array}$ & 39728 & 26-Jun-96 & $Y$ & $A B$ \\
\hline$Y$ & & 3489 & REFCON & Reforenco Constent & AREA_CH & $\begin{array}{l}\text { Arae For CH Wasto Disposal in } \\
\text { CCDFGF Model }\end{array}$ & 38728 & $26 \sqrt{u n-96}$ & $\mathbf{Y}$ & AB \\
\hline$Y$ & & 3490 & REFCON & Reference Constant & VOLWP & $\begin{array}{l}\text { Uncompected Volume of Waste } \\
\text { Pemels in CCDFGF Model }\end{array}$ & 38729 & $26-\sqrt{4}-96$ & $\gamma$ & AB \\
\hline $\mathbf{Y}$ & & 3490 & REFCON & Referencos Constant & VOLWP & $\begin{array}{l}\text { Uncompactod Volumo of Whasto } \\
\text { Panols in CCDFGF Model }\end{array}$ & 38729 & 26rtur-96 & $Y$ & 48 \\
\hline $\mathbf{Y}$ & & 3491 & GLOBAL & $\begin{array}{l}\text { Information that epplios } \\
\text { olobally }\end{array}$ & TA & $\begin{array}{l}\text { Time Active hostitutional Controls } \\
\text { ot WiPP Sito Ave Efiective }\end{array}$ & 38730 & 26-Jun-96 & $\mathbf{Y}$ & $4 B$ \\
\hline$Y$ & & 3491 & GLOBAL & $\begin{array}{l}\text { Information that applied } \\
\text { olobally }\end{array}$ & TA & $\begin{array}{l}\text { Trme Active Institutional Controts } \\
\text { at WIPP Sito Are Effective }\end{array}$ & 39730 & $26 \sqrt{2}-96$ & $Y$ & 4B \\
\hline $\mathbf{Y}$ & & 3492 & REFCON & Reference Constant & Fuw & $\begin{array}{l}\text { Fraction of Repostory Votume } \\
\text { Ocaspied By Waste in CCDFGF } \\
\text { Moda! }\end{array}$ & 38731 & 28-hn-96 & $\mathbf{Y}$ & $4 B$ \\
\hline$Y$ & & 3492 & REFCON & Reforence Constant & Fuw & $\begin{array}{l}\text { Fraction of Repository Volume } \\
\text { Ocoupiod By Wasto in CCDFGF } \\
\text { Model }\end{array}$ & 38731 & 28-Jun-96 & $\mathbf{Y}$ & $4 B$ \\
\hline$Y$ & & 3492 & REFCON & Reforence Constant & FUw & $\begin{array}{l}\text { Frection of Repository Volume } \\
\text { Ocapiod By Wasto in CCDFGF } \\
\text { Model }\end{array}$ & 38731 & $28-\sqrt{1}-96$ & $\mathbf{Y}$ & $4 B$ \\
\hline $\mathbf{Y}$ & & 3493 & GLOBAL & $\begin{array}{l}\text { Information that epplies } \\
\text { coboutly }\end{array}$ & PBRINE & $\begin{array}{l}\text { Prob. that Donling intrusion in } \\
\text { Excavated Arae Encournteres } \\
\text { Pressureed Brne }\end{array}$ & 38732 & 26 Jun-96 & $\mathbf{Y}$ & 1 \\
\hline$Y$ & & 3493 & GLOBAL & $\begin{array}{l}\text { Information that applies } \\
\text { globally }\end{array}$ & PBRINE & 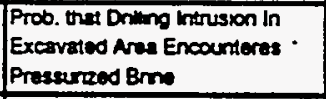 & 38732 & $26 \sqrt{k}-96$ & $\mathbf{Y}$ & 1 \\
\hline$Y$ & & 3493 & GLOBAL & $\begin{array}{l}\text { Information that applies } \\
\text { globally }\end{array}$ & PBRINE & 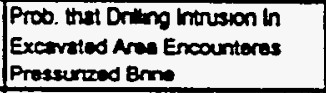 & 38732 & 26-hun-96 & $\mathbf{Y}$ & 1 \\
\hline$Y$ & & 3493 & GLOBAL & $\begin{array}{l}\text { Information that appleses } \\
\text { globalty }\end{array}$ & PBRINE & 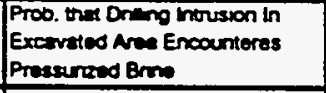 & 38732 & 26-Jun-96 & $\mathbf{Y}$ & 1 \\
\hline$Y$ & & 3494 & GLOBAL & $\begin{array}{l}\text { Information unat moplios } \\
\text { globally }\end{array}$ & unsono & Driting Reso Per Una Aroa & 38733 & 26-Jun-96 & $\mathbf{Y}$ & 1 \\
\hline $\mathbf{Y}$ & & 3495 & GLOBAL & $\begin{array}{l}\text { Information that explios } \\
\text { globally }\end{array}$ & PLGPAT & 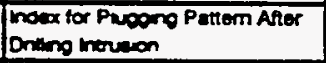 & 38734 & 26-VIun-96 & $\mathbf{Y}$ & 1 \\
\hline $\mathbf{Y}$ & & 3496 & REFCON & Reterences Constem & AREA_Nor & 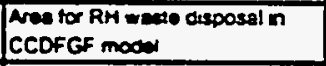 & 38735 & 27 Jun-96 & $\mathbf{Y}$ & 48 \\
\hline $\mathbf{Y}$ & & 3496 & REFCON & Refermase Convetere & AREA_RH & 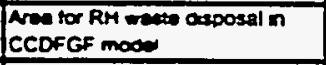 & 38735 & 27 Jun-96 & $\mathbf{Y}$ & 48 \\
\hline $\mathbf{Y}$ & & 3497 & GLOBAL & $\begin{array}{l}\text { Intormation the mootien } \\
\text { glotally }\end{array}$ & MUEEAT & 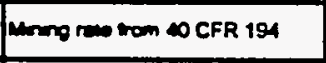 & 38736 & 27-Jun-96 & $\mathbf{Y}$ & 1 \\
\hline $\mathbf{Y}$ & & 3498 & GLOBAL & 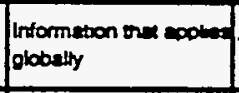 & Trxan & 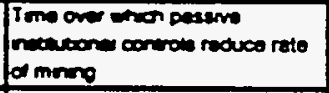 & 38737 & 27-Jun-96 & $Y$ & 48 \\
\hline $\mathbf{Y}$ & & 3498 & GLOBAL & $\begin{array}{l}\text { Intormation them copine: } \\
\text { globelly }\end{array}$ & TPICN & 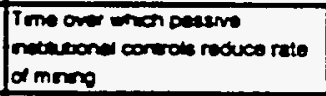 & 38737 & 27-Jun-96 & $\mathbf{Y}$ & 18 \\
\hline$Y$ & & 3499 & GLOBAL & 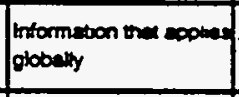 & TPXO & 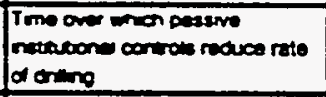 & 38738 & 27 Jun-96 & $Y$ & 1 \\
\hline$Y$ & & 3499 & GLOBAL & 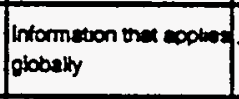 & TPKCo & 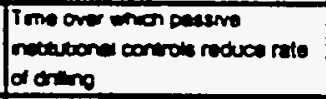 & 38738 & 27 Jun-96 & $Y$ & 1 \\
\hline$Y$ & & 3500 & GLOBAL & \begin{tabular}{|l|} 
Intormation the epoliat \\
globally
\end{tabular} & FPXCA & $\begin{array}{l}\text { PIC mutiolicions fector for } \\
\text { humen reneon of mnng }\end{array}$ & 38742 & 27-Jun-96 & $\mathbf{Y}$ & 48 \\
\hline$Y$ & & 3500 & GLOBAL & 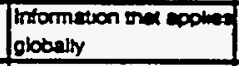 & FPICA & 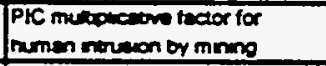 & 38742 & 27-Jun-96 & $\mathbf{Y}$ & 48 \\
\hline $\mathbf{Y}$ & & 3500 & GLOBAL & 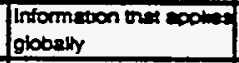 & FPICN & 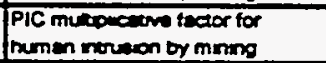 & 38742 & 27-Jun-96 & $Y$ & 48 \\
\hline$Y$ & & 3501 & GLOBAL & 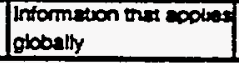 & FPICO & 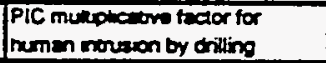 & 38743 & 27-Jun-96 & $\mathbf{Y}$ & 48 \\
\hline
\end{tabular}


CCA Parameter Listing

\begin{tabular}{|c|c|c|c|c|c|c|c|c|c|c|}
\hline 10 & 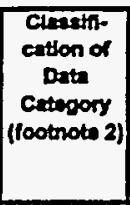 & $\begin{array}{l}\text { Destrbution } \\
\text { Type }\end{array}$ & Mam & Modien & Mintmotm & Mexinum & Untas & Reference ID & $\begin{array}{l}\text { We tho darte } \\
\text { dorveloped } \\
\text { underen } \\
\text { NoA-1 } \\
\text { Program? }\end{array}$ & 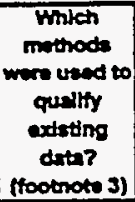 \\
\hline 3488 & 1,3 & CONSTANT & $4.1330000 \mathrm{E}+03$ & $4.1330000 E+\infty 03$ & $4.1330000 E+03$ & $4.1330000 \mathrm{E}+00$ & $m^{\wedge} 2$ & SAND92-070013 & $Y$ & NA \\
\hline 3488 & 1,3 & CONSTANT & $4.1330000 \mathrm{E}+\infty 03$ & $4.1330000 E+\infty 0$ & $4.1330000 \mathrm{E}+03$ & $4.1330000 \mathrm{E}+03$ & $m^{\wedge} 2$ & WP040524 & $\mathbf{Y}$ & NA \\
\hline 3489 & 1,3 & CONSTANT & $1.1150000 E+05$ & $1.1150000 E+05$ & $1.1150000 \mathrm{E}+05$ & $1.1150000 \mathrm{E}+05$ & $m^{\wedge} \mathbf{2}$ & SAND92-070013 & Y & NA \\
\hline 3489 & 1,3 & CONSTANT & $1.1150000 E+05$ & $1.1150000 \mathrm{E}+05$ & $1.1150000 \mathrm{E}+0.5$ & $1.1150000 E+05$ & $m^{\wedge} \mathbf{2}$ & WP040524 & $\mathbf{Y}$ & NA \\
\hline 3490 & $1,3,4$ & CONSTANT & $4.3600000 \mathrm{E}+05$ & 4.3600000E+05 & $4.3600000 E+05$ & $4.3600000 \mathrm{E}+05$ & $m^{\wedge} 3$ & SAND92-070013 & $\mathbf{Y}$ & NA \\
\hline 3490 & $1,3,4$ & CONSTANT & $4.3600000 \mathrm{E}+05$ & $4.3600000 E+05$ & $4.3600000 E+05$ & $4.3500000 E+05$ & $m^{\wedge} 3$ & WP040524 & $\mathbf{Y}$ & NA \\
\hline 3491 & 3,4 & CONSTANT & $1.0000000 E+02$ & $1.0000000 \mathrm{E}+02$ & $1.0000000 E+02$ & $1.0000000 E+02$ & $y$ & $\begin{array}{l}\text { FEDERAL_: } \\
\text { REGISTER }\end{array}$ & $\mathbf{Y}$ & NA \\
\hline 3491 & 3,4 & CONSTANT & $1.0000000 \mathrm{E}+02$ & $1.0000000 E+02$ & $1.0000000 E+\infty 2$ & $1.0000000 E+02$ & $y$ & WPO40524 & Y & NAA \\
\hline 3492 & 1,3 & CONSTANT & $3.6600000 \mathrm{E}-01$ & $3.8600000 E-01$ & $3.86000000-01$ & $3.8600000 E-01$ & NONE & SAND92-07003 & $\mathbf{Y}$ & NA \\
\hline 3492 & 1,3 & CONSTANT & $3.8600000 E-01$ & $3.6600000 E-01$ & $3.8600000 E-01$ & $3.8600000 E-01$ & NONE & WP032328 & $\mathbf{Y}$ & NA \\
\hline 3492 & 1,3 & CONSTANT & $3.8600000 E-01$ & $3.8600000=-01$ & $3.85000000 E-01$ & $3.0600000 E-01$ & NONE & WPO40524 & $\mathbf{Y}$ & NA \\
\hline 3493 & 1,3 & CONSTANT & $8.0000000 E-02$ & $8.0000000 E-02$ & $8.0000000 E-02$ & $8.0000000 E-02$ & NONE & $\begin{array}{c}\text { MEMO:LEONARDI } \\
\text { MARIETTA-2 }\end{array}$ & $\mathbf{Y}$ & NAA \\
\hline 3493 & 1,3 & CONSTANT & $8.0000000 E-02$ & $8.0000000 E-02$ & $8.0000000 E-02$ & $8.0000000 E-02$ & NONE & $\begin{array}{c}\text { MEMO:LEONAROI } \\
\text { MARIETTA-3 }\end{array}$ & Y & NA \\
\hline 3493 & 1,3 & CONSTANT & $8.0000000 E-02$ & $8.0000000 E-02$ & $8.0000000 E-02$ & $8.0000000 E-02$ & NONE & WP031235 & $\mathbf{Y}$ & NA \\
\hline 3493 & 1,3 & CONSTANT & $8.0000000 E-02$ & $8.0000000 E-02$ & $8.0000000 E-02$ & $8.0000000 E-02$ & NONE & WPO40199 & $\mathbf{Y}$ & N/A \\
\hline 3494 & 1,3 & CONSTANT & $4.6800000 \mathrm{E}-03$ & $4.6800000 \mathrm{E}-03$ & $4.68000000 \mathrm{E}-03$ & $46800000 E-03$ & $\left.k m^{n}-2\right) y m^{n}-$ & $\begin{array}{l}\text { MENO:LEONARDI } \\
\text { MARIETTA }\end{array}$ & $\mathbf{Y}$ & NA \\
\hline 3495 & 1,3 & DELTA & $0.0000000 E+\infty$ & $0.0000000 E+\infty 0$ & $10000000 E+\infty$ & $3.0000000 E+\infty$ & NONE & $\begin{array}{l}\text { MEMO:HOWARDI } \\
\text { MARIETTA-C }\end{array}$ & $\mathbf{Y}$ & NA \\
\hline 3496 & 1,3 & CONSTANT & $1.5760000 E+04$ & $1.5760000 \mathrm{E}+04$ & $15760000 \mathrm{E}+0.4$ & $1.5760000 £+04$ & $m^{\wedge} 2$ & WP034618 & $Y$ & NA \\
\hline 3496 & 1,3 & CONSTANT & $1.5760000 \mathrm{E}+04$ & $1.5760000 \mathrm{E}+04$ & $157600000+04$ & $15760000 E+04$ & $m^{\wedge} \mathbf{2}$ & WPO40524 & $\mathbf{Y}$ & NA \\
\hline 3497 & 4 & CONSTANT & $1.0000000 E-04$ & $1.0000000 E-04$ & 10000000 E. OS & $10000000 E-04$ & $y r^{n-1}$ & $\begin{array}{l}\text { FEDERAL- } \\
\text { REGISTER }\end{array}$ & $\mathbf{Y}$ & N/A \\
\hline 3498 & $1,3,4$ & CONSTANT & $6.0000000 \mathrm{E}+02$ & $6.0000000 E+02$ & $60000000 E+02$ & $60000000 E+02$ & $y r$ & WP038819 & $\mathbf{Y}$ & NA \\
\hline 3498 & $1,3,4$ & CONSTANT & $6.0000000 \mathrm{E}+02$ & $6.0000000 E+02$ & $60000000 E+02$ & $6.0000000 \mathrm{E}+02$ & $r$ & WPO40524 & $\mathbf{Y}$ & NA \\
\hline 3499 & 3,4 & CONSTANT & $6.0000000 \mathrm{E}+02$ & $6.0000000 E+02$ & $60000000 E+02$ & $6.0000000 \mathrm{E}+02$ & $y$ & WP038819 & $\mathbf{Y}$ & 1 \\
\hline 3499 & 3,4 & CONSTANT & $6.0000000 E+02$ & $6.0000000 E+\infty 2$ & $60000000 E+00$ & $6.0000000 E+00$ & $y$ & WPO42701 & $Y$ & 1 \\
\hline 3500 & 3,4 & CONSTANT & $1.0000000 \mathrm{E}-02$ & $1.0000000 E-02$ & $10000000 E-02$ & $1.00000000-02$ & NONE & WP038819 & $\mathbf{Y}$ & N/A \\
\hline 3500 & 3,4 & CONSTANT & $1.0000000 E-02$ & $1.0000000 E-02$ & $10000000 E-02$ & $1.0000000 E-02$ & NONE & WPO40524 & $Y$ & N/A \\
\hline 3500 & 3,4 & CONSTANT & $1.0000000 \mathrm{E}-02$ & $1.0000000 E-02$ & $1.0000000=-02$ & $1.0000000 E-02$ & NONE & WPO42701 & $\mathbf{Y}$ & NA \\
\hline 3501 & 3.4 & CONSTANT & $1.0000000 E-02$ & $1.0000000 \mathrm{E}-02$ & $10000000 E-02$ & $1.0000000 \mathrm{E}-02$ & NONE & WP038819 & $\mathbf{Y}$ & NA \\
\hline
\end{tabular}


CCA Parameter Listing

\begin{tabular}{|c|c|c|c|c|c|c|c|c|c|c|}
\hline $\begin{array}{c}\text { Paramotertin } \\
\text { Databeve? } \\
\text { (X/N) }\end{array}$ & 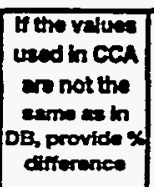 & DD & Mrtardato & Whated Neme & $\begin{array}{c}\text { Paremoter } \\
\text { DD }\end{array}$ & Parconoter Ranse & $\begin{array}{l}\text { PRP D } \\
\text { (MPO D) }\end{array}$ & Datentry & 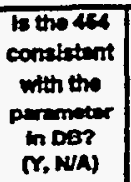 & 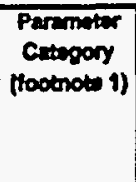 \\
\hline $\mathbf{Y}$ & & 3501 & GLOBAL & $\begin{array}{l}\text { Information that applies } \\
\text { globally }\end{array}$ & FPICD & $\begin{array}{l}\text { PIC mutepleatre factor for } \\
\text { tumen intrusion by dilling }\end{array}$ & 38743 & $27 \sin -96$ & $\mathbf{Y}$ & 48 \\
\hline $\mathbf{Y}$ & & 3501 & GLOBAL & $\begin{array}{l}\text { Information that applies } \\
\text { globally }\end{array}$ & FPICD & 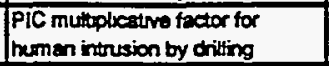 & 38743 & $27 \sqrt{t} n-96$ & $\mathbf{Y}$ & $4 B$ \\
\hline $\mathbf{Y}$ & & 3502 & REFCON & Referenco Constemt & $\mathrm{HRH}$ & $\begin{array}{l}\text { Emplaced Height of Remole } \\
\text { Hindind Wasto in CCDFGF } \\
\text { Model }\end{array}$ & 38744 & 27 Jum-96 & $\mathbf{Y}$ & 48 \\
\hline$Y$ & & 3502 & REFCON & Referencos Constent & HRH & $\begin{array}{l}\text { Emplecos Hexpht of Remoce } \\
\text { Hendiod Weste in CCDFGF } \\
\text { Model }\end{array}$ & 3874 & 27 Jun-96 & $\mathbf{Y}$ & 18 \\
\hline $\mathbf{Y}$ & & 3503 & REFCON & Referenco Constant & ABERM & $\begin{array}{l}\text { Araa of Berm Placed Over Wasto } \\
\text { Penel }\end{array}$ & 38745 & 27-2hn-96 & $Y$ & AB \\
\hline $\mathbf{Y}$ & & 3503 & REFCON & Reference Constant & ABERM & $\begin{array}{l}\text { Araes of Berm Placed Over Wasto } \\
\text { Pane! }\end{array}$ & 38745 & $27 \sqrt{t} n-\infty 6$ & $\mathbf{Y}$ & 4B \\
\hline $\mathbf{Y}$ & & 3503 & REFCON & Reference Constant & ABERM & $\begin{array}{l}\text { Aras of Berm Placed Over Waste } \\
\text { Panel }\end{array}$ & 38745 & 27Jun-96 & $\mathbf{Y}$ & $4 B$ \\
\hline $\mathbf{N}$ & & 9000 & WAS_AREA & $\begin{array}{l}\text { Wasto Emplocoment } \\
\text { Area and Westo }\end{array}$ & HEIGHT & Crusted Panol Height (meters) & NA & 18-Feb-97 & & 48 \\
\hline $\mathbf{N}$ & & 9001 & WAS_AREA & $\begin{array}{l}\text { Wasto Emplacoment } \\
\text { Area and Wasto }\end{array}$ & PRESPANI & \begin{tabular}{|l|} 
Brme Prossure at intusion \\
Trme:Region 1 (Updip)
\end{tabular} & NA & $18-\mp a b-97$ & & 4B \\
\hline N & & 9002 & WAS_AREA & $\begin{array}{l}\text { Wasto Emplecement } \\
\text { Area and Wasto }\end{array}$ & PRESPANR & $\begin{array}{l}\text { Brine Pressure et Intusion Time: } \\
\text { Region2 (Up-Middle) }\end{array}$ & NA & $18-\mp a b-97$ & & 48 \\
\hline $\mathbf{N}$ & & 9003 & WAS_AREA & $\begin{array}{l}\text { Warte Emplacement } \\
\text { Area and Wasto }\end{array}$ & PRESPANB & $\begin{array}{l}\text { Brine Prossure at hrousion Time: } \\
\text { Rogion3 (Lower-Midolo) }\end{array}$ & N/A & $18-\mp 0 b-97$ & & 48 \\
\hline N & & 9004 & WAS_AREA & $\begin{array}{l}\text { Waste Emplacemert } \\
\text { Area and Wasto }\end{array}$ & PRESPAN4 & $\begin{array}{l}\text { Brino Prossure on hrouson Trme: } \\
\text { Regiont (Lower) }\end{array}$ & NA & 18-Fab-97 & & 48 \\
\hline N & & 9005 & WAS_AREA & $\begin{array}{l}\text { Wasto Emplacomont } \\
\text { Area and Waste }\end{array}$ & GPRSPAN1 & $\begin{array}{l}\text { Ges Pressure at Intrusion Time: } \\
\text { Region } 1 \text { (Updip) }\end{array}$ & NA & 18-Fob-97 & & 48 \\
\hline N & & 9006 & WAS_AREA & $\begin{array}{l}\text { Waste Emplacernem } \\
\text { Area and Weste }\end{array}$ & GPRSPAN2 & $\begin{array}{l}\text { Gas Pressure at thrusiosion Tmo: } \\
\text { Region 2(Up-Middlo) }\end{array}$ & NA & 18-Fob-97 & & $4 B$ \\
\hline $\mathbf{N}$ & & 9007 & WAS_AREA & $\begin{array}{l}\text { Wasto Emplacement } \\
\text { Ares and Wasto }\end{array}$ & GPRSPAN3 & $\begin{array}{l}\text { Gas Pressure at lintusion Tmo: } \\
\text { Rogion 3(Lower Hiddio) }\end{array}$ & NA & $18-F=b-97$ & & AB \\
\hline N & & 9008 & WAS_AREA & $\begin{array}{l}\text { Waste Emplacement } \\
\text { Aroa and Waste }\end{array}$ & GPRSPANA & $\begin{array}{l}\text { Ges Pressure at Intrusion Trmo: } \\
\text { Region 4(Lower) }\end{array}$ & NA & $18-F=b-97$ & & $4 B$ \\
\hline $\mathbf{N}$ & & 9009 & WAS_AREA & $\begin{array}{l}\text { Waste Emplacoment } \\
\text { Area and Wasto }\end{array}$ & BSATPAN1 & $\begin{array}{l}\text { Bmo Seturation at intrustion } \\
\text { Ime: Region } 1 \text { (Updip) }\end{array}$ & NA & $18-F a b-97$ & & 48 \\
\hline N & & 9010 & WAS_AREA & $\begin{array}{l}\text { Waste Emplacernent } \\
\text { Avoa and Waste }\end{array}$ & BSATPANQ & $\begin{array}{l}\text { Brme Soturation at Intrusion } \\
\text { Trme: Rogion } 2 \text { (Up-Midetio) }\end{array}$ & NA & 18-feb-97 & & 48 \\
\hline N & & 9011 & WAS_AREA & $\begin{array}{l}\text { Wasto Emplacoment } \\
\text { Area and Weste }\end{array}$ & BSATPAN3 & $\begin{array}{l}\text { 8rino Seturation at thtrusion } \\
\text { Trme: Region } 3 \text { (Lower-Middle) }\end{array}$ & N/A & 18-feb-97 & & 48 \\
\hline N & & 9012 & WAS_AREA & $\begin{array}{l}\text { Wasto Emplecoment } \\
\text { Area end Wasto }\end{array}$ & BSATPANA & \begin{tabular}{|l|} 
Brmo Seturation at intusion \\
Trme: Region 4 (Lower)
\end{tabular} & NA & $18-F 0 b-97$ & & 48 \\
\hline N & & 9013 & WAS_AREA & $\begin{array}{l}\text { Wasto Emplecement } \\
\text { Area and Wasto }\end{array}$ & GSATPAN1 & $\begin{array}{l}\text { Gess Saturation at intrusion Tirno: } \\
\text { Region1 (Updip) }\end{array}$ & N/A & 18-fob-97 & & 48 \\
\hline $\mathbf{N}$ & & 9014 & WAS_AREA & $\begin{array}{l}\text { Wasto Emplacoment } \\
\text { Area and Waste }\end{array}$ & GSATPAN2 & $\begin{array}{l}\text { Gas Saturation at Intrusion Tme: } \\
\text { Region2 (Up-Hiddto) }\end{array}$ & NA & $18-f e b-97$ & & 48 \\
\hline $\mathbf{N}$ & & 5015 & WAS_AREA & $\begin{array}{l}\text { Wasto Emplecoment } \\
\text { Area and Wasto }\end{array}$ & GSATPAN3 & $\begin{array}{l}\text { Gas Saturation at Intrusion Time: } \\
\text { Rogion3 (Lower Middle) }\end{array}$ & N/A & 18-feb-97 & $\because$ & 48 \\
\hline $\mathbf{N}$ & & 9016 & WAS_AREA & $\begin{array}{l}\text { Waste Emplecement } \\
\text { Area and Wasto }\end{array}$ & GSATPAN4 & $\begin{array}{l}\text { Gas Seturation at Intrusion Tmo: } \\
\text { Rogions (Lower) }\end{array}$ & N/A & 18Fab-97 & & 48 \\
\hline $\mathbf{N}$ & & 9017 & DRZ_I & $\begin{array}{l}\text { Disturbed Rock Zone: } \\
\text { True Period } 0 \text { to } 1000 \\
\text { Years }\end{array}$ & HEIGHT & Crusthed Penel Heighte (meters) & N/A & $18-f e b-97$ & & 4B \\
\hline $\mathbf{N}$ & & 9018 & S_HALTIE & Salado Halito, intuct & HEIGHT & Crushed Panel Height (moters) & N/A & 18-Feb-97 & & 48 \\
\hline $\mathbf{N}$ & & 5019 & PAN_SEAL & Penol Sal & HEIGHT & Crusched Panol theight (meters) & NA & $18+00-97$ & & $4 B$ \\
\hline $\mathbf{N}$ & & 9020 & WELLBORE & None Given & INTR_TME & Intruston Time & NA & $18+c b-97$ & & 48 \\
\hline $\mathbf{N}$ & & 8021 & WEULORE & None Given & BITSIZE & Drill Bit Diameter & N/A & 18-feb-97 & & 48 \\
\hline $\mathbf{N}$ & & 9022 & WELLBORE & None Given & SKIN & $\begin{array}{l}\text { Skin fector for will delvwrability } \\
\text { equation }\end{array}$ & NA & 18-feb-97 & & AB \\
\hline $\mathbf{N}$ & & 9023 & WELLBORE & None Given & WELLPI & Well Productivity index & NA & 18-F0b-97 & & 48 \\
\hline N & & 9024 & WELUORE & Nome Given & DRANRAD & Equivalent Grid Drainage Redess & NA & $18-F e b-97$ & & $4 B$ \\
\hline N & & 9025 & WELLBORE & None Given & PRM_OPEN & $\begin{array}{l}\text { Abendoned E1 Woil Open } \\
\text { Boretholo Perm: } 0 \text { to } 200 \mathrm{ym}\end{array}$ & NA & $18-f e b-97$ & & $4 B$ \\
\hline $\mathbf{N}$ & & 9026 & WELLBORE & Nono Given & PRM_SAND & $\begin{array}{l}\text { Abandoned EI Wall Send } \\
\text { Borahole Perm:20010 } 1200 \text { yrs }\end{array}$ & N/A & $18-f=b-97$ & & $4 B$ \\
\hline N & & 9027 & WELLBORE & None Given & PRM_CREP & $\begin{array}{l}\text { Abondoned El Whall Croep } \\
\text { Borehote Perm: } 1200 \text { mis on }\end{array}$ & NA & 10-fab-97 & & AB \\
\hline
\end{tabular}


CCA Parameter Listing

\begin{tabular}{|c|c|c|c|c|c|c|c|c|c|c|}
\hline $\mathbf{W D}$ & \begin{tabular}{c|} 
Clesefitit \\
crition of \\
Dith \\
Cetwoory \\
(footnote 2)
\end{tabular} & $\begin{array}{l}\text { Detertoutson } \\
\text { Type }\end{array}$ & meen & Medim & Mintrem & Mradrnens & Whits & Roforence id & 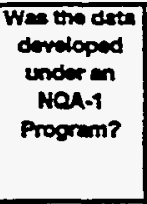 & 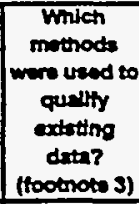 \\
\hline 3501 & 3,4 & CONSTANT & $1.0000000 \mathrm{E}-02$ & $1.0000000 E-02$ & $1.0000000 E-\infty 2$ & $1.0000000 \mathrm{E}-02$ & NONE & WPO40524 & $Y$ & NA \\
\hline 3501 & 3,4 & CONSTANT & $1.0000000 E-02$ & $1.0000000 E-02$ & $1.0000000 E-02$ & $1.0000000 E-02$ & NONE & WPO42701 & $\mathbf{Y}$ & NA \\
\hline 3502 & 1,3 & CONSTANT & $5.0800000 E-01$ & $5.0900000 \mathrm{E}-01$ & $5.0900000 E-01$ & 5.0900000E-01 & $\mathbf{m}$ & WD & $Y$ & N/A \\
\hline 3502 & 1,3 & CONSTANT & $5.0900000 E-01$ & 5.0900000E-01 & $5.0900000 E-01$ & $5.0500000 E-01$ & $m$ & WPO40524 & $\mathbf{Y}$ & NA \\
\hline 3503 & 1,3 & CONSTANT & $-6.2850000 \mathrm{E}+0.5$ & $6.2850000 \mathrm{E}+05$ & $6.2850000 E+0.5$ & $6.2850000 E+05$ & $m^{\wedge} 2$ & WPO40133. & $\mathbf{Y}$ & N/A \\
\hline 3503 & 1,3 & CONSTANT & $6.2850000 E+05$ & $6.2850000 E+05$ & $6.2850000 \mathrm{E}+0.05$ & 6.2850000E+05 & $m^{2} 2$ & WPO40524 & $\mathbf{Y}$ & NA \\
\hline 3503 & 1,3 & CONSTANT & $6.2850000 E+05$ & $6.2850000 \equiv+05$ & $6.2850000 E+0.5$ & $6.2850000 E+05$ & $m^{\wedge} 2$ & WPO43751 & $\mathbf{Y}$ & NA \\
\hline 9000 & 3 & CONSTANT & $1.5000000 \mathrm{E}+00$ & $1.5000000 E+\infty 0$ & $1.5000000=+\infty 0$ & $1.5000000 E+\infty 0$ & $m$ & wPO40520 & & NA \\
\hline 9001 & 3 & CONSTANT & $0.0000000 E+\infty 0$ & $0.0000000 E+\infty 0$ & $0.0000000 E+\infty 0$ & $0.0000000 E+\infty 0$ & Pa & WPO40520 & & N/A \\
\hline 9002 & 3 & CONSTANT & $0.0000000 E+\infty 0$ & $0.0000000 E+\infty 0$ & $0.0000000 E+\infty 0$ & $0.0000000 E+\infty 0$ & Pa & WPO40520 & & NA \\
\hline 8003 & 3 & CONSTANT & $0.0000000 E+\infty$ & $0.0000000 E+00$ & $0.0000000 E+\infty$ & $0.0000000 E+\infty$ & Pa & WPO40520 & & N/A \\
\hline 9004 & 3 & CONSTANT & $0.00000000 E+\infty 0$ & $0.0000000 E+00$ & $0.0000000 \mathrm{E}+\infty$ & $0.0000000 E+00$ & $\mathrm{~Pa}$ & WP040520 & & NA \\
\hline 9005 & 3 & CONSTANT & $0.0000000 E+\infty$ & $0.0000000 E+00$ & $0.0000000 E+\infty$ & $0.0000000 E+\infty 0$ & Pa & WPO40520 & & N/A \\
\hline 9006 & 3 & CONSTANT & $0.0000000 E+\infty 0$ & $0.0000000 E+00$ & $0.00000000+\infty$ & $0.0000000 \mathrm{E}+\infty 0$ & Pa & WPO40520 & & NA \\
\hline 9007 & 3 & CONSTANT & $0.00000000+00$ & $0.0000000 E+\infty 0$ & $0.0000000 E+\infty$ & $0.00000000+00$ & Pa & WPO40520 & & NA \\
\hline 9008 & 3 & CONSTANT & $0.0000000 E+00$ & $0.0000000 E+\infty 0$ & $0.0000000 E+\infty$ & $0.0000000 E+\infty 0$ & Pe & WP040520 & & NA \\
\hline 9009 & 3 & CONSTANT & $0.0000000 \mathrm{E}+\infty$ & $0.0000000 E+\infty 0$ & $0.0000000 E+\infty$ & $0.0000000 E+\infty$ & NONE & WPO40520 & & NA \\
\hline 9010 & 3 & CONSTANT & $0.0000000 E+\infty 0$ & $0.0000000 E+\infty 0$ & $0.00000000+\infty$ & $0.0000000 E+\infty 0$ & NONE & WPO40520 & & NA \\
\hline 9011 & 3 & CONSTANT & $0.0000000 E+\infty$ & $0.0000000 E+\infty 0$ & $0.0000000 E+\infty 0$ & $0.0000000 E+\infty$ & NONE & WPO40520 & & N/A \\
\hline 9012 & 3 & CONSTANT & $0.0000000 E+\infty$ & $0.0000000 E+\infty 0$ & $0.00000000+00$ & $0.0000000 E+\infty 0$ & NONE & WPO40520 & & NA \\
\hline 9013 & 3 & CONSTANT & $0.0000000 E+\infty 0$ & $0.0000000 E+\infty 0$ & $0.0000000 E+\infty$ & $0.0000000 E+\infty$ & NONE & WPO40520 & & NA \\
\hline 9014 & 3 & CONSTANT & $0.0000000 \mathrm{E}+\infty$ & $0.0000000 E+\infty 0$ & $0.0000000 E+\infty 0$ & $0.0000000 E+\infty 00$ & NONE & WP040520 & & NA \\
\hline 9015 & 3 & CONSTANT & $0.0000000 E+\infty$ & $0.0000000 E+\infty 0$ & $0.00000000+\infty 0$ & $0.0000000 E+\infty$ & NONE & WPO40520 & - & N/A \\
\hline 9016 & 3 & CONSTANT & $0.0000000 E+\infty$ & $0.0000000 E+\infty 0$ & $0.00000000+\infty 00$ & $0.0000000 E+\infty$ & NONE & WPO40520 & & NA \\
\hline 9017 & 3 & CONSTANT & $8.8800000 E+\infty$ & $8.9800000 E+\infty 0$ & 8. $9800000 E+00$ & $8.9800000 E+\infty 0$ & $\mathbf{m}$ & WPO40520 & & N/A \\
\hline 9018 & 3 & CONSTANT & $8.9800000 E+\infty$ & $8.9800000 E+\infty 0$ & $8.9800000 E+\infty 0$ & $8.9800000 \mathrm{E}+00$ & $m$ & wPO40520 & & NA \\
\hline 9019 & 3 & CONSTANT & $3.9600000 E+\infty$ & $3.9600000 \mathrm{E}+\infty 0$ & $3.9600000 E+\infty$ & $3.9600000 E+\infty$ & $m$ & WP040520 & & NA \\
\hline 9020 & 3 & CONSTANT & $0.0000000 E+\infty$ & $0.0000000 E+\infty$ & $0.0000000 E+00$ & $0.0000000 E+\infty 0$ & 8 & WPO40520 & & NA \\
\hline 9021 & 3 & CONSTANT & $0.0000000 E+00$ & $0.0000000 E+\infty 0$ & $0.0000000 E+00$ & $0.0000000 \mathrm{E}+00$ & $m$ & WPO40520 & & N/A \\
\hline 9022 & 3 & CONSTANT & $0.0000000 E+\infty 0$ & $0.0000000 E+\infty$ & $0.0000000 E+00$ & $0.0000000 E+\infty 0$ & NONE & WPO40520 & & NA \\
\hline 9023 & 3 & CONSTANT & $0.0000000 \mathrm{E}+\infty$ & $0.0000000 E+\infty 0$ & $0.00000000+00$ & $0.00000000+00$ & $m^{n} 3 / s+P a$ & WPO40520 & & N/A \\
\hline 9024 & 3 & CONSTANT & $0.0000000 E+\infty$ & $0.0000000 E+\infty 0$ & $0.00000000+\infty 0$ & $0.0000000 E+\infty$ & m & WPO40520 & & N/A \\
\hline 9025 & 3 & CONSTANT & $0.00000000+\infty 0$ & $0.0000000 E+\infty 0$ & $0.0000000 E+\infty$ & $0.0000000 \mathrm{E}+\infty 0$ & $m^{\wedge} \mathbf{2}$ & WPO40520 & & NA \\
\hline 9026 & 3 & CONSTANT & $0.0000000 E+\infty$ & $0.0000000 E+\infty$ & $0.00000000+\infty$ & $0.0000000 E+\infty 0$ & $m^{\wedge 2}$ & WPO 40520 & & NA \\
\hline 9027 & 3 & CONSTANT & $0.00000000+\infty$ & $0.0000000 E+\infty$ & $0.00000000 E+\infty$ & $0.00000000+\infty$ & $m^{\wedge} 2$ & WPO40520 & & N/A \\
\hline
\end{tabular}


CCA Parameter-Listing

\begin{tabular}{|c|c|c|c|c|c|c|c|c|c|c|}
\hline $\begin{array}{c}\text { Parameter in } \\
\text { Detabese? } \\
\text { (YNT) }\end{array}$ & 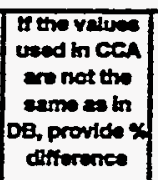 & 10 & Materialo & Prtarde $\mathrm{Nem}$ & $\begin{array}{c}\text { Parementer } \\
\text { to }\end{array}$ & 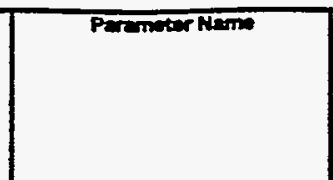 & $\begin{array}{l}\text { PRPD } \\
\text { MPO * }\end{array}$ & $\begin{array}{c}\text { Detentoy } \\
\text { Dete }\end{array}$ & $\begin{array}{l}\text { is thic } 484 \\
\text { consulatent } \\
\text { with the } \\
\text { purameter } \\
\text { in DB? } \\
(Y, N A)\end{array}$ & $\begin{array}{l}\text { Parmintar } \\
\text { Cetwoory } \\
\text { (footinote i) }\end{array}$ \\
\hline $\mathbf{N}$ & & 9028 & WELLORE & None Given & AREA_TOT & $\begin{array}{l}\text { Tolal Area Soldds remored by } \\
\text { CuttingshCervings/Spallings }\end{array}$ & NA & $18-f e b-\theta 7$ & & AB \\
\hline $\mathbf{N}$ & & 9029 & WELLBORE & None Given & VOLU_TOT & 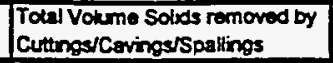 & N/A & 18-feb-97 & & 48 \\
\hline N & & 5030 & WELLBORE & None Given & CAST_RE & $\begin{array}{l}\text { Pressure on Cestulio Bmo Res at } \\
\text { Boundary: } 10,000 \text { yr model }\end{array}$ & NA & $18-F \infty b-97$ & & 48 \\
\hline $\mathbf{N}$ & & 9031 & WELLBORE & None Given & CAST_WB & $\begin{array}{l}\text { Presecuro in Castilia Brino Res at } \\
\text { Woubore: } 10,000 \text { y model }\end{array}$ & NA & $18-f=b-97$ & & 48 \\
\hline $\mathbf{N}$ & & 9032 & WELLBORE & None Given & PRM_CAST & $\begin{array}{l}\text { Permeabilly in Cestile } \\
\text { Reservoir.10.000 yr model }\end{array}$ & NA & $18-F=6-97$ & & 4B \\
\hline $\mathbf{N}$ & & 9033 & WELLBORE & None Given & WELL_PAN & $\begin{array}{l}\text { Prescure at Wellocore in Penal: } \\
10.000 \text { yr model }\end{array}$ & NA & $18+c b-97$ & & 48 \\
\hline N & & 9034 & REFCON & Reforences Constart & DIP_DEG & $\begin{array}{l}\text { Formation dip through excevetiod } \\
\text { region }\end{array}$ & N/A & 18f-cb-97 & & AB \\
\hline N & & 9035 & & None Given & TIME & $\begin{array}{l}\text { Stert tmo for all Drect Brme } \\
\text { Retease Modols }\end{array}$ & N/A & & & AB \\
\hline N & & 9036 & & None Given & SATBREL & $\begin{array}{l}\text { Initial Bmo Saturation, Boundary } \\
\text { Cond Well Elom }\end{array}$ & NA & & & AB \\
\hline N & & 9037 & WELLORE & None Given & EO1_A & $\begin{array}{l}\text { Contunt for Equaston } 1 \text { FBHP } \\
\text { lookup function }\end{array}$ & NA & 18-Feb-97 & & AB \\
\hline $\mathbf{N}$ & & 9038 & WELLBORE & None Given & EO1_B & $\begin{array}{l}\text { Contant for Equation } 1 \text { FBMP } \\
\text { bolken function }\end{array}$ & NA & $18-F 00-97$ & & 48 \\
\hline $\mathbf{N}$ & & 9039 & WEUBORE & None Given & EQ1_C & $\begin{array}{l}\text { Cortume for Equation } 1 \text { FBHP } \\
\text { lookep tunction }\end{array}$ & NA & $18-F \infty-97$ & & $A B$ \\
\hline $\mathbf{N}$ & & 9040 & WEULORE & None Given & EQ1_D & $\begin{array}{l}\text { Content for Equation } 1 \text { FBHP } \\
\text { lookus function }\end{array}$ & NA & $18-f 0 b-97$ & & 48 \\
\hline $\mathbf{N}$ & & S041 & WELLBORE & Nond Given & EQ1_E & $\begin{array}{l}\text { Contunt for Equation 1 FBHP } \\
\text { lookup tunction }\end{array}$ & NA & 18-feb-97 & & 48 \\
\hline N & & 9042 & WELLBORE & None Given & EQ2_A & $\begin{array}{l}\text { Contunt for Equation } 2 \text { FBHP } \\
\text { bokep function }\end{array}$ & NA & 18-feb-97 & & 48 \\
\hline N & & 9043 & WEULORE & None Given & E02_B & $\begin{array}{l}\text { Contant for Equation } 2 \text { F8HP } \\
\text { lookep function }\end{array}$ & N/A & 18-Fab-97 & & $A B$ \\
\hline N & & 9044 & WEULORE & None Given & EQ2_C & $\begin{array}{l}\text { Content for Equation } 2 \text { FBHP } \\
\text { lookep function }\end{array}$ & N/A & 18-Feb-87 & & AB \\
\hline N & & 9045 & WELLBORE & None Given & $E 02$ _D & $\begin{array}{l}\text { Content for Equation } 2 \text { FBHP } \\
\text { lookep tunction }\end{array}$ & N/A & 18-fab-97 & & $A B$ \\
\hline N & & 9046 & WELLBORE & Nonś Given & E02_E & $\begin{array}{l}\text { Content for Equartion } 2 \text { FBHP } \\
\text { bolkop tunction }\end{array}$ & NA & 18-Fob-97 & & $A B$ \\
\hline N & & 9047 & WELLBORE & None Given & EQ2_F & $\begin{array}{l}\text { Contant for Equation } 2 \text { FBHP } \\
\text { bokup tunction }\end{array}$ & N/A & $18-f a b-97$ & & 48 \\
\hline N & & 9048 & WELLBORE & None Given & $E 02 \_G$ & $\begin{array}{l}\text { Contant for Equation } 2 \text { FBHP } \\
\text { lookup function }\end{array}$ & NA & 18-Feb-97 & & 48 \\
\hline $\mathbf{N}$ & & 9049 & WELLBORE & None Given & EO2_H & $\begin{array}{l}\text { Contant for Equation } 2 \text { FBHP } \\
\text { lookup function }\end{array}$ & N/A & 18-Fab-97 & & $4 B$ \\
\hline N & & 9050 & WELLBORE & None Given & E03_A & $\begin{array}{l}\text { Contant for Equation } 3 \text { FBHP } \\
\text { lookup function }\end{array}$ & NA & 18-Fob-97 & & 18 \\
\hline $\mathbf{N}$ & & 9051 & WELLOORE & None Given & EQ3_B & $\begin{array}{l}\text { Contant for Equation } 3 \text { FBHP } \\
\text { lookup function }\end{array}$ & NA & 18fab-97 & & 48 \\
\hline $\mathbf{N}$ & & 9052 & WELLBORE & None Given & EQ3_C & $\begin{array}{l}\text { Content for Equation } 3 \text { FBHP } \\
\text { lookup tunction }\end{array}$ & NA & 18-Fab-97 & $\because$ & AB \\
\hline N & & 9053 & WELLBORE & None Given & EQ3_D & $\begin{array}{l}\text { Content for Equation } 3 \text { FBHP } \\
\text { lookup function }\end{array}$ & NA & 18-fab-97 & & $A B$ \\
\hline N & & 9054 & WELLOORE & None Given & EQ3_E & $\begin{array}{l}\text { Contunt for Equation } 3 \mathrm{FBHP} \\
\text { lookup function }\end{array}$ & N/A & 18Fob-97 & & $A B$ \\
\hline $\mathbf{N}$ & & 9055 & All & None Gimen & ZORIGIN & $\begin{array}{l}\text { Sea-tevel Elevation of Repository } \\
\text { at ahan }\end{array}$ & NA & $18-F \cup b-97$ & & 48 \\
\hline N & & $\cos 6$ & NL & None Given & YORIGIN & $\begin{array}{l}\text { Horcontal distanco from Direct } \\
\text { Rel meahnode }(1,1) \text { to shatt-m }\end{array}$ & NA & $18-F \in b-97$ & & $4 B$ \\
\hline $\mathbf{N}$ & & 9057 & WEUBORE & None Given & LEN_BC & $\begin{array}{l}\text { Longth of abendonod BH from } \\
\text { Cesstive torepository }\end{array}$ & N/A & 18-Fob-97 & & 48 \\
\hline $\mathbf{N}$ & & 9058 & WEUBORE & Nono Given & PREV_TME & Provious E1 intrusion tume & NA & 18-Fob-97 & & 48 \\
\hline N & & 9059 & WELLBORE & None Given & WELPI_BC & $\begin{array}{l}\text { Boundery condition well } \\
\text { productivityindex }\end{array}$ & N/A & $18-f e b-97$ & & $4 B$ \\
\hline $\mathbf{N}$ & & 9060 & WEULORE & None Given & BHP_ABAN & $\begin{array}{l}\text { Actual flowing pres for aban. E2 } \\
\text { well used in BRAGFLO }\end{array}$ & NA & 18-Fab-97 & & 48 \\
\hline $\mathbf{N}$ & & 9061 & & Nono Given & PCUT & $\begin{array}{l}\text { Boundery for press. above which } \\
\text { spall effects possiblo }\end{array}$ & NA & $18+a b-97$ & & $A B$ \\
\hline $\mathbf{N}$ & & 9002 & & Nono Given & PR_max & $\begin{array}{l}\text { Greatest repos. pore press. } \\
\text { besed on lithostatic pressure }\end{array}$ & N/A & $18+f a b-97$ & & 48 \\
\hline $\mathbf{N}$ & & 9053 & & None Given & PE_max & $\begin{array}{l}\text { Gremest wasto pormeabilty } \\
\text { considered }\end{array}$ & NA & $18-F 00-97$ & & $4 B$ \\
\hline
\end{tabular}


CCA Parameter Listing

\begin{tabular}{|c|c|c|c|c|c|c|c|c|c|c|}
\hline D & 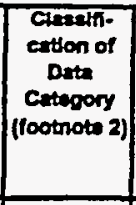 & $\begin{array}{l}\text { Oistribution } \\
\text { Type }\end{array}$ & Men & Medven & Mintmem & Maximum & Unts & Reforence 10 & $\begin{array}{l}\text { Wes tho dete } \\
\text { developed } \\
\text { under an } \\
\text { Nol-4 } \\
\text { Progrum? }\end{array}$ & $\begin{array}{c}\text { Wrich } \\
\text { mothode } \\
\text { wore used to } \\
\text { quilly } \\
\text { oxteting } \\
\text { dete? } \\
\text { (tootinote J) }\end{array}$ \\
\hline 9028 & 3 & CONSTANT & $0.0000000 E+\infty 0$ & $0.0000000 E+\infty$ & $0.0000000 E+\infty$ & $0.0000000 E+\infty$ & $m^{\wedge} 2$ & WPO40520 & & NA \\
\hline 9029 & 3 & CONSTANT & $0.0000000 E+\infty 0$ & $0.0000000 \mathrm{E}+\infty 0$ & $0.0000000 E+\infty$ & $0.0000000 E+\infty 0$ & $m^{\wedge 2}$ & WPO40520 & & NA \\
\hline 9030 & 3 & CONSTANT & $1.7000000 E+07$ & $1.7000000 E+07$ & $1.7000000 E+07$ & $1.7000000 E+07$ & $\mathbf{P a}_{2}$ & WPO40520 & & NA \\
\hline 9031 & 3 & CONSTANT & $1.7000000 E+07$ & $1.7000000 E+07$ & $1.70000005+07$ & $1.7000000 E+07$ & Pa & WPO40520 & & NA \\
\hline 9032 & 3 & CONSTANT & $1.0000000 E-12$ & $1.0000000 E-12$ & $1.0000000 E-12$ & $1.0000000 E-12$ & $m^{\wedge} 2$ & WPO40520 & & NA \\
\hline 9003 & 3 & CONSTANT & . $1.4000000 \mathrm{E}+07$ & $1.4000000 E+07$ & $1.4000000 \mathrm{E}+07$ & $1.4000000 E+07$ & Pa & WPO40520 & & NA \\
\hline 9034 & 3 & CONSTANT & $1.0000000 E+00$ & $1.0000000 E+\infty$ & $1.0000000 E+\infty 0$ & $1.0000000 E+\infty$ & degrees & WPO40520 & & NA \\
\hline 9035 & 3 & & $0.0000000 E+\infty$ & $0.0000000 E+\infty$ & $0.00000000+\infty$ & $0.0000000 E+\infty 0$ & & WPO40520 & & NA \\
\hline 9036 & 3 & & $1.0000000 E+\infty 0$ & $0.0000000 E+\infty$ & $0.00000000+\infty$ & $0.00000000=+\infty$ & & WP040520 & & NA \\
\hline 9037 & 3 & CONSTANT & $8.0025800 E+06$ & $8.0025800 E+06$ & $8.0025800 E+06$ & $8.0025800 E+06$ & NONE & WPO40520 & & NA \\
\hline 9038 & 3 & CONSTANT & $8.2138000 E+66$ & $8.2138000 E+66$ & $8.2138000 E+66$ & $82138000 E+66$ & NONE & WPO40520 & & N/A \\
\hline 9039 & 3 & CONSTANT & 2.4916100E-02 & $2.4916100 E-02$ & $2.4916100 E-02$ & $2.4916100 E-02$ & NONE & WPO40520 & & NA \\
\hline 9040 & 3 & CONSTANT & $1.0264800 E-01$ & $1.0264800 E-01$ & $1.0264800 E-01$ & $1.0264800 E-01$ & NONE & WPO40520 & & NA \\
\hline 9041 & 3 & CONSTANT & $3.1235800 E-09$ & $0.0000000 E+\infty 0$ & $0.00000000 E+\infty$ & $0.0000000 E+00$ & NONE & WPO40520 & & NA \\
\hline 9042 & 3 & CONSTANT & $8.4708300 E+05$ & $0.0000000 E+\infty$ & $0.00000000 E+\infty$ & $0.0000000 E+\infty 0$ & NONE & WPO40520 & & NA \\
\hline 9043 & 3 & CONSTANT & $2.7881500 E+06$ & $0.0000000 E+\infty$ & $0.0000000 E+\infty$ & $0.0000000 E+\infty 0$ & NONE & WP040520 & & NA \\
\hline 9044 & 3 & CONSTANT & $3.4510600 E+06$ & $0.0000000 E+\infty$ & $0.00000000+\infty 0$ & $00000000 \mathrm{E}+\infty$ & NONE & WPO40520 & & NA \\
\hline 9045 & 3 & CONSTANT & $-5.4884400 E+04$ & $0.0000000 E+\infty$ & $0.0000000 E+\infty$ & $00000000 E+\infty$ & NONE & WPO40520 & & NA \\
\hline 9046 & 3 & CONSTANT & $-1.7079500 E-02$ & $0.0000000 E+\infty$ & $00000000 E+00$ & $00000000 E+\infty$ & NONE & WPO40520 & & NA \\
\hline 9047 & 3 & CONSTANT & $8.9536000 E-01$ & $0.00000000+\infty$ & $00000000 E+\infty$ & $0,0000000 E+\infty$ & NONE & WP040520 & & NAA \\
\hline 9048 & 3 & CONSTANT & $5.4041500 E-01$ & $0.0000000 E+00$ & $00000000 E+\infty)$ & $0,0000000 E+\infty$ & NONE & WP040520 & & NA \\
\hline 8049 & 3 & CONSTANT & $-4.9369100 E-09$ & $0,0000000 E+00$ & $000000000+\infty$ & $00000000 E+\infty$ & NONE & WP040520 & & NA \\
\hline 9050 & 3 & CONSTANT & $8.9214600 E+\infty 0$ & $0,0000000 E+\infty$ & $0,0000000 \times \cdot 00$ & $00000000 E+\infty$ & NONE & WPO40520 & & N/A \\
\hline 9051 & 3 & CONSTANT & $-22742800 E-01$ & $0.00000000+\infty$ & $00000000 \times+\infty$ & $00000000 E+\infty$ & NONE & WPO40520 & & NAA \\
\hline 9052 & 3 & CONSTANT & $1.3680600 E+\infty 0$ & $0.0000000 E+\infty$ & $00000000<-\infty$ & $00000000 \times+\infty$ & NONE & WPO40520 & $\because$ & N/A \\
\hline 9053 & 3 & CONSTANT & $1.8350100 E+\infty 0$ & $00000000 E+\infty$ & $0,0000000=\infty$ & $0,0000000+\infty$ & NONE & WP040520 & & NA \\
\hline 9054 & 3 & CONSTANT & $4.5726200 E-01$ & $00000000<=+\infty$ & $00000000<-\infty$ & $0,000000 \times-\infty$ & NONE & WPO40520 & & NA \\
\hline 9055 & 3 & CONSTANT & $3.8267100 E+02$ & $80025000 E+06$ & $-0 \cos s 000-00$ & $-10058000+06$ & $\mathbf{m}$ & WPO40520 & & NA \\
\hline 9056 & 3 & CONSTANT & $1.00000000 E+03$ & $0.0000000 \mathrm{E}+\infty$ & $00000000<-\infty$ & $00000000 \times-\infty$ & $m$ & WPO40520 & & $N / A$ \\
\hline 9057 & 3 & CONSTANT & $24700000 E+\infty 2$ & $00000000 E+\infty$ & $00000000 \times-\infty$ & $00000000=-\infty$ & $m$ & WP040520 & & NA \\
\hline 9058 & 3 & CONSTANT & $0.0000000 E+\infty 0$ & $0.0000000<+\infty$ & $000000000 \times \infty$ & $000000006+00$ & yrs & WPO40520 & & N/A \\
\hline 9059 & 3,5 & CONSTANT & $0.00000000+\infty 0$ & $0.0000000 \mathrm{E}+\infty 0$ & $0, \infty \infty 0000 \times-\infty$ & $0,0000000 E+\infty$ & $m^{\wedge} 3$ & WP040530 & & NA \\
\hline 9060 & 3 & CONSTANT & $0.00000000+\infty$ & $00000000<+\infty$ & $0000000<-\infty$ & $0,0000000 \times+\infty$ & $\mathbf{P a}$ & WPO40520 & & NA \\
\hline 9061 & 3 & CONSTANT & $8.0000000 E+00$ & $00000000<\cdot 00$ & $00000000-\infty$ & $00000000<+\infty$ & MPa & WPO40521 & & NA \\
\hline 9062 & 3 & CONSTANT & $1.5000000 E+01$ & $00000000 \varepsilon+\infty$ & $0, \infty 00000 E+\infty$ & $0,0000000+\infty$ & MPa & WP040521 & & NA \\
\hline 9063 & 3 & CONSTANT & $1.0000000 E-12$ & $0,0000000 \leqslant+\infty$ & $0,0000000 E+\infty$ & $0,0000000 E+\infty$ & $m^{\wedge} \mathbf{2}$ & WPO40521 & & N/A \\
\hline
\end{tabular}


-CCA-Parameter-Listing

\begin{tabular}{|c|c|c|c|c|c|c|c|c|c|c|}
\hline $\begin{array}{l}\text { Paramite in } \\
\text { Databaan? } \\
\text { (X) }\end{array}$ & 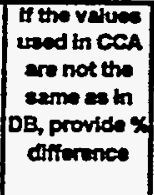 & ID & Metarelo & Matertal Nemb & $\begin{array}{l}\text { Parmeter } \\
\text { iD }\end{array}$ & Parincen Nam & $\begin{array}{c}\text { PRP DD } \\
\text { (WFO t) }\end{array}$ & $\begin{array}{c}\text { Dete Entry } \\
\text { Deto }\end{array}$ & $\begin{array}{l}\text { be the } 464 \\
\text { consistemt } \\
\text { with the } \\
\text { permenter } \\
\text { in DBP } \\
(\mathrm{N}, N A)\end{array}$ & $\begin{array}{l}\text { Parminitar } \\
\text { Cattegory } \\
\text { (foctnote 1) }\end{array}$ \\
\hline $\mathbf{N}$ & & 9064 & & None Given & KCRT & $\begin{array}{l}\text { Wasto permeabitity that } \\
\text { ceperetes blowout spell responsed } \\
\text { from gas erosion end it }\end{array}$ & NA & 18-Fab-97 & & 48 \\
\hline $\mathbf{N}$ & & 8065 & & None Given & PGAS & $\begin{array}{l}\text { Repository pore presenre thist } \\
\text { seperates gas erosion and etuck } \\
\text { pipe responso in }\end{array}$ & NA & $18-f a b-97$ & & $A B$ \\
\hline $\mathbf{N}$ & & 9066 & & None Given & SPECKEN & Activity cormersion factor & $N / A$ & 18feb-97 & & 3 \\
\hline
\end{tabular}

Footnote 1: Parameter Category

1. Based on site specific information used as initial input to a MPP PA numerical modal that specifies the physical, chemical, or hydrologic properties of the rock formations, sals, badfils, and wasto form, or eny other netural or engneared feature.

2. Parameters representing the inventory of the wasto to be emplaced in the WPP as defined in the WPP Basaline Inventory Report

3. Parameters ropresonting precisoly known, tabulated physical constants.

4A. Parameters that are assignod based on a similarity of proporties between sinilar miterials or features.

4B. Perameters that are model configuration parmeters not based on epecific MPP properties or features but are necoed to make PA models nn.

5. Parameters not used in curtent compliance calculations. 
CCA Parameter Listing

\begin{tabular}{|c|c|c|c|c|c|c|c|c|c|c|}
\hline $\mathbf{D O}$ & $\begin{array}{l}\text { Cinastin- } \\
\text { cation of } \\
\text { Deth } \\
\text { Centegory } \\
\text { (footnote 2) }\end{array}$ & $\begin{array}{l}\text { Distribution } \\
\text { Typo }\end{array}$ & $\operatorname{man}$ & modien & Ifthimum & moxtmum & Untas & Reference id & $\begin{array}{l}\text { Wes the deta } \\
\text { dereloped } \\
\text { underen } \\
\text { Nou-1 } \\
\text { Progrem? }\end{array}$ & 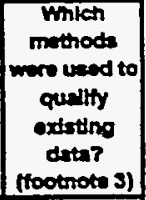 \\
\hline 9064 & 3 & CONSTANT & $1.0000000 E-16$ & $0.0000000 E+\infty$ & $0.0000000 E+\infty 0$ & $0.0000000 E+\infty 0$ & $\mathrm{~m}^{\wedge} \mathbf{2}$ & WP040521 & & NA \\
\hline 8065 & 3 & CONSTANT & $1.0000000 E+01$ & $0.00000000 E+\infty 0$ & $0.0000000 E+\infty 0$ & $0.0000000 E+\infty 0$ & $\mathrm{MPa}$ & WP040521 & & NA \\
\hline 9066 & 1 & CONSTANT & $1.1281700 E+13$ & $0.0000000 E+\infty 0$ & $0.00000000 \mathrm{E}+\infty$ & $0.0000000 E+\infty$ & Curso'stom & WP040521 & & NA \\
\hline
\end{tabular}

Footnote 2: Classification of Data Category

Footnote 3: Methods Used to Qualify Existing Data

1. Site or Waste Specific

1. Peer Review

2. BIR

2. Corroborating Data

3. Protessional Judoment

4. General Literature

3. Confimatory Testing

4. Demonstration that the data were colbeted under a quality assurance program equivalent to NQA-1/3.

5. If none of the above methods were used, then the data remain unqualfied.

6. Non-WPP published iterature (includes joumal articies, conference papers, textbooks, handbooks, etc).

7. QED 
-

Page intentionally blank

M1-300 


\section{Appendix M2}

Table from EPA Parameter Database: Additional Sources 
Additional Sources

\begin{tabular}{|c|c|c|c|c|}
\hline ID & Material ID & Param ID & \multicolumn{2}{|c|}{ Technical Traceablitty Documentation } \\
\hline 3310 & AM & CONCINT & $\begin{array}{l}\text { WPO\#36771 Memo:Hans Papenguth to } \\
\text { Christine Stockman "Colloidal Actinide Source } \\
\text { Term parameters" 3/28/96 }\end{array}$ & $\begin{array}{l}\text { PRP WPO\#35852 Table } 4 \text { "Mobile Colloidal } \\
\text { Actinide Source Term } 2 \text { Actinide Intrinsic } \\
\text { Colloids Hans Papenguth. }\end{array}$ \\
\hline 3312 & NP & CONCINT & $\begin{array}{l}\text { WPO\#36771 Memo:Hans Papenguth to } \\
\text { Christine Stockman "Colloidal Actinide Source } \\
\text { Term parameters" 3/28/96 }\end{array}$ & $\begin{array}{l}\text { PRP WPO\#35852 Table } 4 \text { "Mobile Colloidal } \\
\text { Actinide Source Term } 2 \text { Actinide Intrinsic } \\
\text { Colloids Hans Papenguth. }\end{array}$ \\
\hline 3319 & TH & CONCINT & $\begin{array}{l}\text { WPO\#36771 Memo:Hans Papenguth to } \\
\text { Christine Stockman "Colloidal Actinide Source } \\
\text { Term parameters" 3/28/96 }\end{array}$ & $\begin{array}{l}\text { PRP WPO\#35852 Table } 4 \text { "Mobile Colloidal } \\
\text { Actinide Source Term } 2 \text { Actinide Intrinsic } \\
\text { Colloids Hans Papenguth. }\end{array}$ \\
\hline 3307 & $\mathrm{U}$ & CONCINT & $\begin{array}{l}\text { WPO\#36771 Memo:Hans Papenguth to } \\
\text { Christine Stockman "Colloidal Actinide Source } \\
\text { Term parameters" 3/28/96 }\end{array}$ & $\begin{array}{l}\text { PRP WPO\#35852 Table } 4 \text { "Mobile Colloidal } \\
\text { Actinide Source Term } 2 \text { Actinide Intrinsic } \\
\text { Colloids Hans Papenguth. }\end{array}$ \\
\hline 3311 & AM & PROPMIC & $\begin{array}{l}\text { WPO\#36771 Memo:Hans Papenguth to } \\
\text { Christine Stockman "Colloidal Actinide Source } \\
\text { Term parameters" 3/28/96 }\end{array}$ & $\begin{array}{l}\text { PRP WPO\#35856 Table } 6 \text { "Mobile Colloidal } \\
\text { Actinide Source Term } 4 \text { Microbes. Hans } \\
\text { Papenguth. }\end{array}$ \\
\hline 3482 & $A M+3$ & MKD_AM & $\begin{array}{l}\text { WPO\#38801 memo L.H. Brush to M.S. } \\
\text { Tiemey June 10, 1996. Additional information } \\
\text { is PRP WPO\#38231. }\end{array}$ & $\begin{array}{l}\text { WPO\#40846 lists basis for recommendations } \\
\text { of values }\end{array}$ \\
\hline 2283 & ASPHALT & PRMX_LOG & $\begin{array}{l}\text { WPO\#30640: WIPP Shaft Seal System } \\
\text { BRAGFLO Parameters, SWCF- } \\
\text { A:1.1.03.2.1:QA:PDD Sec II Vol } 2 \text { 2.2.1; Sec I } \\
\text { Vol 4 4.5 }\end{array}$ & $\begin{array}{l}\text { Literature: Barth (1962) p. 229: Brown (1960) } \\
\text { pg 27; Engelmann et al. (1989) p. 159; Zakar } \\
\text { (1971) p. } 15\end{array}$ \\
\hline 2284 & ASPHALT & PRMY_LOG & $\begin{array}{l}\text { WPO\#30640: WIPP Shaft Seal System } \\
\text { BRAGFLO Parameters, SWCF- } \\
\text { A:1.1.03.2.1:QA:PDD Sec II Vol } 2 \text { 2.2.1; Sec I } \\
\text { Vol 4 4.5 }\end{array}$ & $\begin{array}{l}\text { Literature: Barth (1962) p. 229: Brown (1960) } \\
\text { p. 27; Engelmann et al. (1989) p. 159; Zakar } \\
\text { (1971) p. } 15\end{array}$ \\
\hline 2285 & ASPHALT & PRMZ_LOG & $\begin{array}{l}\text { WPO\#30640: WIPP Shaft Seal System } \\
\text { BRAGFLO Parameters, SWCF- } \\
\text { A:1.1.03.2.1:QA:PDD Sec II Vol. 2 2.2.1; Sec I } \\
\text { Vol. 4 4.5 }\end{array}$ & $\begin{array}{l}\text { Literature: Barth (1962) p. 229: Brown (1960) } \\
\text { p. 27; Engelmann et al. (1989) p. 159; Zakar } \\
\text { (1971) p. } 15\end{array}$ \\
\hline 3173 & BH_CREEP & RELP_MOD & $\begin{array}{l}\text { WPO\#40514 Sec. } 4.2 .4 \text { p. } 4-166 \text { (the } \\
\text { BRAGFLO analysis write-Up). RELP_MOD is } \\
\text { the relative permeability "model number" for } \\
\text { the mixed Brooks and Corey model. Sec. } \\
3.4 .2 \text { p. } 3-23 \text { gives discussion for } \\
\text { implementation of model. }\end{array}$ & $\begin{array}{l}\text { WPO\#30703 WIPP PA User's Manual for } \\
\text { BRAGFLO, Version } 4.00 \text { January } 31,1996 \text {, } \\
\text { pg- } 115-116 \text { lists } 8 \text { models for relative } \\
\text { permeability. }\end{array}$ \\
\hline 3134 & BH_OPEN & PRMX_LOG & $\begin{array}{l}\text { WPO\#35134 Memo:Vaughn to Tiemey } \\
\text { "Borehole Properties" } 3 / 13 / 96^{\prime} \text { (handwritten } 4 \\
\text { page note with parameter values - contains an } \\
\text { email cover page). }\end{array}$ & $\begin{array}{l}\text { Additional Info in WPO\#36564 (this is the } 464 \\
\text { form). }\end{array}$ \\
\hline 3186 & BH_OPEN & PRMY_LOG & $\begin{array}{l}\text { WPO\#35134 Memo:Vaughn to Tiemey } \\
\text { "Borehole Properties" } 3 / 13 / 96 \text { " (handwritten } 4 \\
\text { page note with parameter values - contains an } \\
\text { email cover page). }\end{array}$ & $\begin{array}{l}\text { Additional Info in WPO\#36649 (this is the } 464 \\
\text { form). }\end{array}$ \\
\hline 3187 & BH_OPEN & PRMZ_LOG & $\begin{array}{l}\text { WPO\#35134 Memo:Vaughn to Tierney } \\
\text { "Borehole Properties" } 3 / 13 / 96 \text { " (handwritten } 4 \\
\text { page note with parameter values - contains an } \\
\text { email cover page). }\end{array}$ & $\begin{array}{l}\text { Additional Info in WPO\#36650 (this is the } 464 \\
\text { form). }\end{array}$ \\
\hline 3423 & No EPA data & & & \\
\hline 3422 & No EPA data & & & \\
\hline 3159 & BH_SAND & POROSITY & $\begin{array}{l}\text { WPO\#35134 Memo:Vaughn to Tiemey } \\
\text { "Borehole Properties" } 3 / 13 / 96 \text { " (handwritten } 4 \\
\text { page note with parameter values - contains an } \\
\text { email cover page). }\end{array}$ & \\
\hline
\end{tabular}




\begin{tabular}{|c|c|c|}
\hline ID & \multicolumn{2}{|c|}{ Technical Traceability Documentation } \\
\hline 3310 & & \\
\hline 3312 & & v \\
\hline 3319 & & \\
\hline 3307 & & \\
\hline 3311 & & \\
\hline 3482 & & \\
\hline 2283 & $\begin{array}{l}\text { WPO\#30994 - Contains tables of BRAGFLO parameters used } \\
\text { to model WIPP shaft seal system. Contains listings of the } \\
\text { extent of DRZ and figures showing proposed seal design for } \\
\text { WIPP Air Intake Shaft. }\end{array}$ & \\
\hline 2284 & $\begin{array}{l}\text { WPO\#30994 - Contains tables of BRAGFLO parameters used } \\
\text { to model WIPP shaft seal system. Contains listings of the } \\
\text { extent of DRZ and figures showing proposed seal design for } \\
\text { WIPP Air intake Shaft. }\end{array}$ & \\
\hline 2285 & $\begin{array}{l}\text { WPO\#30994 - Contains tables of BRAGFLO parameters used } \\
\text { to model WIPP shaft seal system. Contains listings of the } \\
\text { extent of DRZ and figures showing proposed seal design for } \\
\text { WIPP Air Intake Shaft. }\end{array}$ & \\
\hline 3173 & $\begin{array}{l}\text { WPO\#38367 S.W. Webb to P. Vaughn, August } 29,1995 \text {, } \\
\text { "Mixed Brooks and Corey Two-Phase Characteristic Curves } \\
\text { "Describes the correct model to use which is consistent with } \\
\text { the relative permeability Model \#4 described in WPO\#30703. }\end{array}$ & $\begin{array}{l}\text { SAND91-2378 SAND92-0700/3 (early discussions found } \\
\text { here). WPO\#35134 Memo:Vaughn to Tiemey "Borehole } \\
\text { Properties" 3/13/96" (handwritten } 4 \text { page note with parameter } \\
\text { values - contains an email cover page). }\end{array}$ \\
\hline 3134 & $\begin{array}{l}\text { WPO\#40514 3-52,53, and 4-159 "Analysis Package for the } \\
\text { Salado Flow Calculations (Task1) of the PA Supporting } \\
\text { Compliance Certification Application". }\end{array}$ & \\
\hline 3186 & $\begin{array}{l}\text { WPO\#40514 3-52,53, and 4-159 "Analysis Package for the } \\
\text { Salado Flow Calculations (Task1) of the PA Supporting } \\
\text { Compliance Certification Application" }\end{array}$ & \\
\hline 3187 & $\begin{array}{l}\text { WPOH0514 3-52,53, and 4-159 "Analysis Package for the } \\
\text { Salado Flow Calculations (Task1) of the PA Supporting } \\
\text { Compliance Certification Application" }\end{array}$ & $\begin{array}{l}\text { WPO\#38568 states this is a 'legacy parameter' whose value } \\
\text { appeared in the baseline WIPP Parameter database of March } \\
2,1996 .\end{array}$ \\
\hline 3423 & & Not Used in CCA \\
\hline 3422 & & Not Used in CCA \\
\hline 3159 & $\cdot$ & \\
\hline
\end{tabular}




\section{Additional Sources}

\begin{tabular}{|c|c|c|c|c|}
\hline ID & Material ID & Param ID & \multicolumn{2}{|c|}{ Technical Trafeability Documentation } \\
\hline 3171 & BH_CREEP & POROSITY & $\begin{array}{l}\text { WPO\#35134 Memo:Vaughn to Tiemey } \\
\text { "Borehole Properties" } 3 / 13 / 96^{\prime \prime} \text { (handwritten } 4 \\
\text { page note with parameter values - contains an } \\
\text { email cover page). }\end{array}$ & \\
\hline 3135 & BH_OPEN & POROSITY & $\begin{array}{l}\text { WPO\#35134 Memo:Vaughn to Tiemey } \\
\text { "Borehole Properties" 3/13/96" (handwritten } 4 \\
\text { page note with parameter values - contains an } \\
\text { email cover page). }\end{array}$ & \\
\hline 3184 & BH_SAND & PRMX_LOG & $\begin{array}{l}\text { Report WPO\#39626 Records and } \\
\text { documentation package for Permeability of } \\
\text { Borehole filled with Sitty Sand. Section } 3.0 \\
\text { provides discussion on conceptual models of } \\
\text { plugged boreholes. Section } 6.0 \text { provides a } \\
\text { summary on the permeabilities. }\end{array}$ & $\begin{array}{l}\text { WPO } 41118 \text { Freeze and Cherry, } 1979, \text { p29, } \\
\text { Table } 2.2 \text { (sitty sand). The borehole sitty sand } \\
\text { permeability derives from the 92PA pg } 4-2 \\
\text { range }-14.0 \text { to }-11.0 \text {. }\end{array}$ \\
\hline 3190 & BH_SAND & PRMY_LOG & $\begin{array}{l}\text { Report WPO\#39626 Records and } \\
\text { documentation package for Permeability of } \\
\text { Borehole filled with Silty Sand. Section } 3.0 \\
\text { provides discussion on conceptual models of } \\
\text { plugged boreholes. Section } 6.0 \text { provides a } \\
\text { summary on the permeabilities. }\end{array}$ & $\begin{array}{l}\text { WPO } 41118 \text { Freeze and Cherry. } 1979, \mathrm{p} 29 \text {, } \\
\text { Table } 2.2 \text { (sitty sand). The borehole silty sand } \\
\text { permeability derives from the 92PA pg } 4-2 \\
\text { range }-14.0 \text { to }-11.0 \text {. }\end{array}$ \\
\hline 3191 & BH_SAND & PRMZ_LOG & $\begin{array}{l}\text { Report WPO\#39626 Records and } \\
\text { documentation package for Permeability of } \\
\text { Borehole filled with Silty Sand. Section } 3.0 \\
\text { provides discussion on conceptual models of } \\
\text { plugged boreholes. Section } 6.0 \text { provides a } \\
\text { summary on the permeabilities. }\end{array}$ & $\begin{array}{l}\text { WPO\#41118 Freeze and Cherry, } 1979, \text { p29, } \\
\text { Table } 2.2 \text { (silty sand). The borehole silty sand } \\
\text { permeability derives from the 92PA pg } 4-2 \\
\text { range }-14.0 \text { to }-11.0 \text {. }\end{array}$ \\
\hline 3259 & BLOWOUT & APORO & $\begin{array}{l}\text { WPO\#30921 Memo: B.M. Butcher to } \\
\text { M.S.Tierney 1/29/96 }\end{array}$ & \\
\hline 3470 & BLOWOUT & GAS_MIN & $\begin{array}{l}\text { WPO\#38214 Memo: Dan Stoelzel to Mel } \\
\text { Marietta "Response to Westinghouse memo } \\
\text { on Brine blowout duration" May } 31,1996 \\
\text { (contains Dan Stoelzel's reasoning for } \\
\text { selecting value). }\end{array}$ & $\begin{array}{l}\text { Parameter value is based on Dan Stoelzel's } \\
\text { discussion. He defines his position by citing } \\
\text { other statistical data taken from drilling reports } \\
\text { from within the Delaware Basin near the WIPP } \\
\text { site. }\end{array}$ \\
\hline 3472 & BLOWOUT & MINFLOW & $\begin{array}{l}\text { WPO\#38214 Memo: Dan Stoelzel to Mel } \\
\text { Marietta "Response to Westinghouse memo } \\
\text { on Brine blowout duration" May } 31,1996 \\
\text { (contains Dan Stoelzel's reasoning for } \\
\text { selecting value). }\end{array}$ & $\begin{array}{l}\text { Parameter value is based on Dan Stoelzel's } \\
\text { discussion. He defines his position by citing } \\
\text { other statistical data taken from drilling reports } \\
\text { from within the Delaware Basin near the WIPP } \\
\text { site. }\end{array}$ \\
\hline 3456 & BLOWOUT & RE_CAST & $\begin{array}{l}\text { WPO\#38217 Memo: Dan Stoelzel to Martin } \\
\text { Tiemey "Castile thickness and extemal } \\
\text { drainage radius values for BRAGFLO-DBR" } \\
\text { June 6, } 1996 \text { (number chosen because it } \\
\text { represents physical dimensions of castile brine } \\
\text { pocket reservoir }(12.34 \mathrm{~m}) .\end{array}$ & $\begin{array}{l}\text { Memo: Larson to SWCF, "Rationale for } \\
\text { Selection of Parameter } 3456 \text { value blowout } \\
\text { re_cast } 3 / 19 / 97 \text { located in } 464 \text { defines how } \\
\text { value was calculated. }\end{array}$ \\
\hline 3473 & BLOWOUT & THCK_CAS & $\begin{array}{l}\text { WPO\#38217 Memo: Dan Stoelzel to Martin } \\
\text { Tiemey "Castile thickness and external } \\
\text { drainage radius values for BRAGFLO-DBR" } \\
\text { June 6, } 1996 \text { (number chosen because it } \\
\text { represents physical dimensions of castile brine } \\
\text { pocket reservoir }(12.34 \mathrm{~m}) .\end{array}$ & $\begin{array}{l}\text { Memo: K Larson to SWCF "Rationale for value } \\
\text { chosen for CCA Parameter } 3473 \text { blout } \\
\text { thick_cast" located in } 464 \text { package. Value is } \\
\text { based on data from and interpretation of WIPP } \\
12 \text { reservoir testing. }\end{array}$ \\
\hline 3245 & BLOWOUT & CEMENT & $\begin{array}{l}\text { Analysis Package for the Cuttings and Spalling } \\
\text { Calculations WPO\#40521 - Sec } 7 \text { "Input } \\
\text { Parameters and Sources pg } 39 .\end{array}$ & $\begin{array}{l}\text { WPO\#35695 by Barry M. Butcher, PI } \\
\text { Parameter Package, Record 1.B.VII "Waste } \\
\text { Cementation Strength is discussed in a Memo } \\
\text { from Butcher to Memorandum of Record } \\
5 / 30 / 96 \text {. Justification of value is discussed. }\end{array}$ \\
\hline
\end{tabular}




\begin{tabular}{|c|c|c|c|}
\hline ID & \multicolumn{3}{|c|}{ Technical Traceability Documentation } \\
\hline 3171 & & & \\
\hline 3135 & & & \\
\hline 3184 & $\begin{array}{l}\text { SAND92-700/3 p. } 4-6 \text { which provides additional reference to } \\
\text { Harar 1987, Table 1.8.1. WPO\#41131 Inadvertent Intrusion } \\
\text { Borehole Permeability (report). }\end{array}$ & & \\
\hline 3190 & $\begin{array}{l}\text { SAND92-700/3 p. } 4-6 \text { which provides additional reference to } \\
\text { Harar 1987, Table 1.8.1. WPO\#41131 Inadvertent Intrusion } \\
\text { Borehole Permeability (report). }\end{array}$ & & \\
\hline 3191 & $\begin{array}{l}\text { SAND92-700/3 p. } 4-6 \text { which provides additional reference to } \\
\text { Harar } 1987, \text { Table 1.8.1. WPO\#41131 Inadvertent Intrusion } \\
\text { Borehole Permeability (report). }\end{array}$ & & \\
\hline 3259 & & & \\
\hline 3470 & & & \\
\hline 3472 & & & \\
\hline 3456 & $\begin{array}{l}\text { Memo: Larson to Chu "Error in derivation of value for } \\
\text { parameter } 3456 \text { blowout re_cast and impact assessment " } \\
\text { 3/19/97, defines a mistake in Larson to SWCF memo and } \\
\text { redefines the value. }\end{array}$ & & - \\
\hline 3473 & $\begin{array}{l}\text { Additional information WPO\#38213 (this is the } 464 \text { form). } \\
\text { WPO\#42263 SAND96-2800 "Blowout Experiments using Fine- } \\
\text { Grained Sands in an Axisymmetric Geometry" L. Lenke, J. } \\
\text { Berglund, R. Cole 1997/7/32250 NMERI UNM Mar96 p.27. }\end{array}$ & & \\
\hline 3245 & $\begin{array}{l}\text { WPO\#36723 Memo from J.W. Berglund to M.S.Tierney } \\
4 / 17 / 96 \text { suggests a value for this parameter. This memo } \\
\text { supplements WPO\#36766 (Berglund to Tiemey } 4 / 1 / 96 \\
\text { Parameters Required for the CUTTING-S Code) which did not } \\
\text { include this parameter. }\end{array}$ & & \\
\hline
\end{tabular}


Additionar-Sources

\begin{tabular}{|c|c|c|c|c|}
\hline ID & Material ID & Param ID & \multicolumn{2}{|c|}{ Technical Traceability Documentation } \\
\hline 3256 & BLOWOUT & FGE & $\begin{array}{l}\text { WPO\#36766 Memo:J.W. Berglund to M.S. } \\
\text { Tierney "Parameters required for the } \\
\text { CUTTING_S code for use in WIPP PA" } 4 / 1 / 96 \\
\text { included in } 464 \text { package (states values were } \\
\text { taken from a draft report SAND96-2800 prior } \\
\text { to publication, pg 27). }\end{array}$ & $\begin{array}{l}\text { WPO\#36723 Corrections on memo from J.W. } \\
\text { Berglund to M.S.Tiemey } 4 / 17 / 96 \text { suggests a } \\
\text { value for this parameter. WPO\#42600 } \\
\text { Memo: Martell to Lattier } 11 / 21 / 96 \text { lists } \\
\text { WPO\#42263 and WPO\#35695 that refer to } \\
\text { this parameter (located in } 464 \text { form) }\end{array}$ \\
\hline 3471 & BLOWOUT & MAXFLOW & $\begin{array}{l}\text { WPO\#38214 Memo: Dan Stoelzel to Mel } \\
\text { Marietta "Response to Westinghouse memo } \\
\text { on brine blowout duration" May } 31,1996 \\
\text { (contains Dan Stoelzel's reasoning for } \\
\text { selecting value). }\end{array}$ & $\begin{array}{l}\text { Parameter value is based on Dan Stoelzel's } \\
\text { discussion. He defines his position by citing } \\
\text { other statistical data taken from drilling reports } \\
\text { from within the Delaware Basin near the WIPP } \\
\text { site. }\end{array}$ \\
\hline 3246 & BLOWOUT & PARTDIA & $\begin{array}{l}\text { Analysis Package for the Cuttings and Spalling } \\
\text { Calculations WPO\#40521 - Sec } 7 \text { "Input } \\
\text { Parameters and Sources pg } 39 .\end{array}$ & $\begin{array}{l}\text { BLOWOUT: PARTDIA } d=4.0 E-5 \text { to } 0.2(\mathrm{~m}) \\
\text { uniform distribution }\end{array}$ \\
\hline 3247 & BLOWOUT & RHOS & $\begin{array}{l}\text { WPO\#36766 Memo:J.W. Berglund to M.S. } \\
\text { Tiemey "Parameters required for the } \\
\text { CUTTING_S code for use on WIPP PA" } \\
\text { 4/1/96. Also see WPO\#42600 contained in } \\
\text { 464 Form. }\end{array}$ & $\begin{array}{l}\text { WPO\#42600 Memo: Martell to Lattier } 11 / 21 / 96 \\
\text { which lists WPO numbers WPO\#42263 and } \\
\text { WPO\#35695 that refer to this parameter } \\
\text { (located in } 464 \text { form) }\end{array}$ \\
\hline 27 & BOREHOLE & DOMEGA & $\begin{array}{l}\text { Analysis Package for the Cuttings and Spalling } \\
\text { Calculations WPO\#40521- Sec } 7 \text { "Input } \\
\text { Parameters and Sources" pg } 39 \text {. WPO\#38568 } \\
\text { states this is a placeholder parameter. }\end{array}$ & $\begin{array}{l}\text { BOREHOLE:DOMEGA DW=7.8 drilling string } \\
\text { angular velocity (rad/s). Value cited in SAND92 } \\
\text { o700/3 pg 4-2 as a constructed distribution } \\
\text { with a range of } 4.2-23.0 \text { rad/s and median of } \\
7.7 \mathrm{rad} / \mathrm{s} \text {. Tech review has value of } 7.8 \mathrm{rad} / \mathrm{s} \\
\text { in accordance with QAP 9-5. }\end{array}$ \\
\hline 34 & BOREHOLE & PRMX_LOG & $\begin{array}{l}\text { WPO\#40514 Sec. } 4.2 .3 \text { pg. 4-142 (the } \\
\text { BRAGFLO analysis write-up). }\end{array}$ & $\begin{array}{l}\text { WPO\#38568 states this is a 'placeholder } \\
\text { parameter' is a parameter whose value is not } \\
\text { used by the BRAGFLO code, but is left in the } \\
\text { database to facilitate the correct reading of all } \\
\text { parameters for BRAGFLO from the SCMS } \\
\text { files. }\end{array}$ \\
\hline 35 & BOREHOLE & PRMY_LOG & $\begin{array}{l}\text { WPO\#40514 Sec. } 4.2 .3 \text { pg. } 4-142 \text { (the } \\
\text { BRAGFLO analysis write-up). }\end{array}$ & $\begin{array}{l}\text { WPO\#38568 states this is a 'placeholder } \\
\text { parameter' is a parameter whose value is not } \\
\text { used by the BRAGFLO code, but is left in the } \\
\text { database to facilitate the correct reading of all } \\
\text { parameters for BRAGFLO from the SCMS } \\
\text { files. }\end{array}$ \\
\hline 36 & BOREHOLE & PRMZ_LOG & $\begin{array}{l}\text { WPO\#40514 Sec. } 4.2 .3 \text { pg. } 4-142 \text { (the } \\
\text { BRAGFLO anaiysis write-up). }\end{array}$ & $\begin{array}{l}\text { WPO\#38568 states this is a 'placeholder } \\
\text { parameter' is a parameter whose value is not } \\
\text { used by the BRAGFLO code, but is left in the } \\
\text { database to facilitate the correct reading of all } \\
\text { parameters for BRAGFLO from the SCMS } \\
\text { files. }\end{array}$ \\
\hline 2254 & BOREHOLE & TAUFAIL & $\begin{array}{l}\text { WPO\#36766 Memo:J.W.Berglund to M.S. } \\
\text { Tiemey 4/1/96. Additional Info in } \\
\text { WPO\#36766. }\end{array}$ & $\begin{array}{l}\text { WPO\#36766 Memo:J.W. Berglund to M.S. } \\
\text { Tiemey "Parameters required for the } \\
\text { CUTTING_S code for use in WIPP PAn 4/1/96 } \\
\text { included in } 464 \text { package (states values were } \\
\text { taken from a draft report SAND96-2800 prior } \\
\text { to publication, pg 27). }\end{array}$ \\
\hline 3414 & BOREHOLE & WUF & $\begin{array}{l}\text { WPO\#37061 Memo: L.C. Sanchez to M. } \\
\text { Martell "Radionuclides for use in WIPP PA } \\
\text { Calculations" 4/23/96 }\end{array}$ & \\
\hline 43 & BRINECST & COMPRES & Not used in 1996 CCA calculation. & \\
\hline 44 & BRINECST & DNSFLUID & Not used in 1996 CCA calculation. & \\
\hline
\end{tabular}




\begin{tabular}{|c|c|c|}
\hline ID & \multicolumn{2}{|c|}{ Technical Traceability Documentation } \\
\hline 3256 & $\begin{array}{l}\text { WPO\#35695 "Cuttings Release of Solids Caused by Blowout", } \\
\text { WPO\#42263 SAND96-2800 "Blowout Experiments using Fine- } \\
\text { Grained Sands in an Axisymmetric Geometry" L. Lenke, J. } \\
\text { Berglund, R. Cole 1997/7/32250 NMERI UNM Mar96 p.28. } \\
\text { (Nalue was taken from this report) }\end{array}$ & $\begin{array}{l}\text { WPO\#36723 Memo from J.W. Berglund to M.S.Tiemey } \\
4 / 17 / 96 \text { suggests a value for this parameter. This memo } \\
\text { supplements WPO\# } 36766 \text { (Berglund to Tiemey } 4 / 1 / 96 \\
\text { Parameters Required for the CUTTING-S Code) which did not } \\
\text { include this parameter. }\end{array}$ \\
\hline 3471 & & \\
\hline 3246 & $\begin{array}{l}\text { Based on peer reviewed waste permeability- Peer Review } \\
\text { Report Engineered Systems }\end{array}$ & \\
\hline 3247 & $\begin{array}{l}\text { WPO\#35695 "Cuttings Release of Solids Caused by Blowout", } \\
\text { WPO\#42263 SAND96-2800 "Blowout Experiments using Fine- } \\
\text { Grained Sands in an Axisymmetric Geometry" L. Lenke, J. } \\
\text { Berglund, R. Cole 1997/7/32250 NMERI UNM Mar96 p.28. } \\
\text { (Value was taken from this report) }\end{array}$ & \\
\hline 27 & $\begin{array}{l}\text { Domega is directly traceable to SAND89-2408 Pg A-168 } \\
\text { showing a graph of drilling speed percentages in RPM units. } \\
\text { Values are converted to units of RAD/s. Data is from Barrid } \\
\text { Drilling Fluids Co. field data. See EPA CCA Review Personnel } \\
\text { Request Form 4/16/97. }\end{array}$ & $\begin{array}{l}\text { Two references in SAND92-700/3 92 PA are to Pace, } 1990 \\
\text { (Sand89-2408 pg A-165 to A-170), a memo from a person } \\
\text { working in field, and to Austin, 1983 Drilling Engineering } \\
\text { Handbook, Boston, MA, International Human Resources Dev. } \\
\text { Corp. }\end{array}$ \\
\hline 34 & WPO\#41118 Freeze and Cherry, 1979, p29, Table 2.2. & \\
\hline 35 & WPO\#41118 Freeze and Cherry, 1979, p29, Table 2.2. & \\
\hline 36 & WPO\#41118 Freeze and Cherry, 1979, p29, Table 2.2. & \\
\hline 2254 & $\begin{array}{l}\text { 35695, Cuttings Release of Solids Caused by Blowout". } \\
\text { Value Taken From Parthenaicles, E. R. E Paaswell, } 1970 \\
\text { "Erodability of Channels w/Cohesive Boundary "J. of } \\
\text { Hydraulics Div. Proceedings of the ASCE Vol } 96 \text { No. HY3, } 755 \\
771 .\end{array}$ & \\
\hline 3414 & & \\
\hline 43 & Not Used in CCA & \\
\hline 44 & Not Used in CCA & \\
\hline
\end{tabular}


Additional Sources

\begin{tabular}{|c|c|c|c|c|}
\hline ID & Material ID & Param ID & \multicolumn{2}{|c|}{ Technical Traceability Documentation } \\
\hline 48 & BRINESAL & COMPRES & $\begin{array}{l}\text { WPO\#41247 pg 4-54 "WIPP Natural Barriers } \\
\text { Data Qualification Peer Review Report" Aug } \\
96 \text { states value was generated from lab } \\
\text { samples. }\end{array}$ & \\
\hline 49 & BRINESAL & DNSFLUID & $\begin{array}{l}\text { WPO\#41247 pg } 4-51 \text { "WIPP Natural Barriers } \\
\text { Data Qualification Peer Review Report" Aug } \\
96 \text { states value is the mean of } 10 \text { brine density } \\
\text { sample values. Report lists DOEMIPP87-010 } \\
\text { Table 3-1, p27 as geochemical analysis } \\
\text { results. }\end{array}$ & See parameter package \\
\hline 58 & CASTILER & AREA_FRC & Not used in 1996 CCA calculation. & $\begin{array}{l}\text { Changes in modeling made this parameter } \\
\text { obsolete. }\end{array}$ \\
\hline 61 & CASTILER & COMP_RCK & $\begin{array}{l}\text { WPO\#31084 SWCF- } \\
\text { A:W:351.2.07.1:PDD:QA:Nonsalado Pkg } \\
\text { 19:Castile Brine Reservoir Rock } \\
\text { Compressibility. Additional info found in } \\
\text { WPO\#31561 located in Form } 464 .\end{array}$ & $\begin{array}{l}\text { WPO\#41247 pg 5-1 "WIPP Natural Barriers } \\
\text { Data Qualification Peer Review Report" Aug } \\
96 \text { states value was derived from laboratory } \\
\text { strain tests and acoustic logs in borehole } \\
\text { WIPP-12. High end of value was taken from } \\
\text { Freeze and Cherry, Groundwater } 1979 .\end{array}$ \\
\hline 3194 & CASTILER & GRIDFLO & $\begin{array}{l}\text { WPO\#41662,Memo from Jon Helton to SWCF- } \\
\text { A Records Center, "Clarification of CASTILER } \\
\text { GRIDFLO (ID\#3194) Parameter definition and } \\
\text { Usage", Oct 30, 1996 (defines the } 5 \text { different } \\
\text { brine pocket volumes) WPO\#44401 has } \\
\text { additional clarification (located in 464). }\end{array}$ & $\begin{array}{l}\text { WPO\#44401 has additional clarification } \\
\text { (graphic roadmap located in } 464 \text { ). } \\
\text { WPO\#37147 Volume Probabilities Derivation } \\
\text { (1/32 probability of occurring), WPO\#37971 } \\
\text { Supporting info and rationale, WPO\#31070 Cr } \\
\text { data values, Popielak et al, 1983, TME-3153 } \\
\text { (N \&P). }\end{array}$ \\
\hline 2608 & CASTILER & KPT & $\begin{array}{l}\text { WPO\#40514, SWCF- } \\
\text { A:1.2.07.4.1:PA:QA:CCA:Analysis Package for } \\
\text { Salado Flow (Task 1) pg 4-149. }\end{array}$ & $\begin{array}{l}\text { KPT is a flag that allows threshold capillary } \\
\text { pressure to change with permeability. Since } \\
\text { this feature is not used in the } 1996 \text { CCA } \\
\text { calculation, the parameter value is specified as } \\
\text { "O". }\end{array}$ \\
\hline 2599 & ASPHALT & KPT & $\begin{array}{l}\text { WPO\#40514, SWCF- } \\
\text { A:1.2.07.4.1:PA:QA:CCA:Analysis Package for } \\
\text { Salado Flow (Task 1) pg 4-149. }\end{array}$ & $\begin{array}{l}\text { KPT is a flag that allows threshold capillary } \\
\text { pressure to change with permeability. Since } \\
\text { this feature is not used in the } 1996 \text { CCA } \\
\text { calculation, the parameter value is specified as } \\
\text { "O". }\end{array}$ \\
\hline 2604 & OPS_AREA & KPT & $\begin{array}{l}\text { WPO\#40514, SWCF- } \\
\text { A:1.2.07.4.1:PA:QA:CCA:Analysis Package for } \\
\text { Salado Flow (Task 1) pg 4-150 through 4-159. }\end{array}$ & $\begin{array}{l}\text { KPT is a flag that allows threshold capillary } \\
\text { pressure to change with permeability. Since } \\
\text { this feature is not used in the } 1996 \text { CCA } \\
\text { calculation, the parameter vajue is specified as } \\
\text { "O". }\end{array}$ \\
\hline 2612 & CAVITY_1 & KPT & $\begin{array}{l}\text { WPO } 40514, \text { SWCF- } \\
\text { A:1.2.07.4.1:PA:QA:CCA:Analysis Package for } \\
\text { Salado Flow (Task 1) pg 4-149 }\end{array}$ & $\begin{array}{l}\text { KPT is a flag that allows threshold capillary } \\
\text { pressure to change with permeability. Since } \\
\text { this feature is not used in the } 1996 \text { CCA } \\
\text { calculation, the parameter value is specified as } \\
\text { "O". }\end{array}$ \\
\hline 2616 & CAVITY_2 & KPT & $\begin{array}{l}\text { WPO\#40514, SWCF- } \\
\text { A:1.2.07.4.1:PA:QA:CCA:Analysis Package for } \\
\text { Salado Flow (Task 1) pg 4-150 through 4-159. }\end{array}$ & $\begin{array}{l}\text { KPT is a flag that allows threshold capillary } \\
\text { pressure to change with permeability. Since } \\
\text { this feature is not used in the } 1996 \text { CCA } \\
\text { calculation, the parameter value is specified as } \\
\text { "O". }\end{array}$ \\
\hline 2620 & CAVITY_3 & KPT & $\begin{array}{l}\text { WPO\#40514, SWCF- } \\
\text { A:1.2.07.4.1:PA:QA:CCA:Analysis Package for } \\
\text { Salado Flow (Task 1) pg 4-150 through 4-159. }\end{array}$ & $\begin{array}{l}\text { KPT is a flag that allows threshold capillary } \\
\text { pressure to change with permeability. Since } \\
\text { this feature is not used in the } 1996 \text { CCA } \\
\text { calculation, the parameter value is specified as } \\
\text { "O". }\end{array}$ \\
\hline
\end{tabular}


Additional Sources

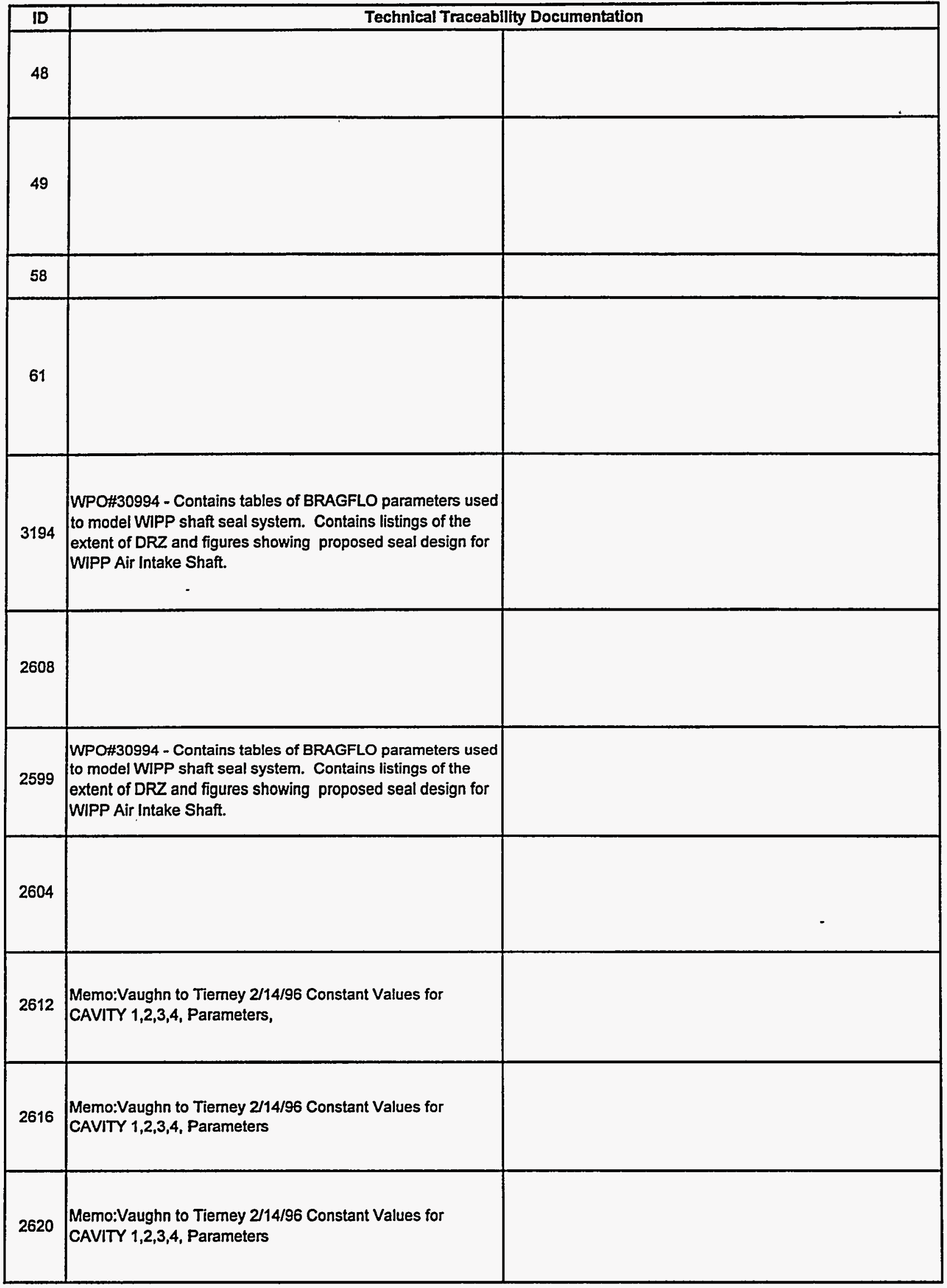


Additional Sources

\begin{tabular}{|c|c|c|c|c|}
\hline ID & Material ID & Param ID & \multicolumn{2}{|c|}{ Technical Traceability Documentation } \\
\hline 2625 & CAVITY_4 & KPT & $\begin{array}{l}\text { WPO40514, SWCF- } \\
\text { A:1.2.07.4.1:PA:QA:CCA:Analysis Package for } \\
\text { Salado Flow (Task 1) pg 4-150 through 4-159. }\end{array}$ & $\begin{array}{l}\text { KPT is a flag that allows threshold capillary } \\
\text { pressure to change with permeability. Since } \\
\text { this feature is not used in the } 1996 \mathrm{CCA} \\
\text { calculation, the parameter value is specified as } \\
\text { "0". }\end{array}$ \\
\hline 2636 & CLAY_BOT & KPT & $\begin{array}{l}\text { WPO 40514, SWCF- } \\
\text { A:1.2.07.4.1:PA:QA:CCA:Analysis Package for } \\
\text { Salado Flow (Task 1) pg 4-150 through 4-159. }\end{array}$ & $\begin{array}{l}\text { KPT is a fiag that allows threshold capillary } \\
\text { pressure to change with permeability. Since } \\
\text { this feature is not used in the } 1996 \text { CCA } \\
\text { calculation, the parameter value is specified as } \\
\text { "0". }\end{array}$ \\
\hline 2641 & CL_L_T1 & KPT & $\begin{array}{l}\text { WPO\#40514, SWCF- } \\
\text { A:1.2.07.4.1:PA:QA:CCA:Analysis Package for } \\
\text { Salado Flow (Task 1) pg 4-149. }\end{array}$ & $\begin{array}{l}\text { KPT is a flag that allows threshold capillary } \\
\text { pressure to change with permeability. Since } \\
\text { this feature is not used in the } 1996 \mathrm{CCA} \\
\text { calculation, the parameter value is specified as } \\
\text { "0". }\end{array}$ \\
\hline 2646 & CL_L_T2 & KPT & $\begin{array}{l}\text { WPO\#40514, SWCF- } \\
\text { A:1.2.07.4.1:PA:QA:CCA:Analysis Package for } \\
\text { Salado Flow (Task 1) pg 4-150 through 4-159. }\end{array}$ & $\begin{array}{l}\text { KPT is a flag that allows threshold capillary } \\
\text { pressure to change with permeability. Since } \\
\text { this feature is not used in the } 1996 \text { CCA } \\
\text { calculation, the parameter value is specified as } \\
\text { "O". }\end{array}$ \\
\hline 2651 & CL_L_T3 & KPT & $\begin{array}{l}\text { WPO\#40514, SWCF- } \\
\text { A:1.2.07.4.1:PA:QA:CCA:Analysis Package for } \\
\text { Salado Flow (Task 1) pg 4-150 through 4-159. }\end{array}$ & $\begin{array}{l}\text { KPT is a flag that allows threshold capillary } \\
\text { pressure to change with permeability. Since } \\
\text { this feature is not used in the } 1996 \text { CCA } \\
\text { calculation, the parameter value is specified as } \\
\text { "O". }\end{array}$ \\
\hline 2656 & CL_M_T1 & KPT & $\begin{array}{l}\text { WPO\#40514, SWCF- } \\
\text { A:1.2.07.4.1:PA:QA:CCA:Analysis Package for } \\
\text { Salado Flow (Task 1) pg 4-150 through 4-159. }\end{array}$ & $\begin{array}{l}\text { KPT is a flag that allows threshold capillary } \\
\text { pressure to change with permeability. Since } \\
\text { this feature is not used in the } 1996 \text { CCA } \\
\text { calculation, the parameter value is specified as } \\
\text { "0". }\end{array}$ \\
\hline 2661 & CL_M_T2 & KPT & $\begin{array}{l}\text { WPO\#40514, SWCF- } \\
\text { A:1.2.07.4.1:PA:QA:CCA:Analysis Package for } \\
\text { Salado Flow (Task 1) pg 4-150 through 4-159. }\end{array}$ & $\begin{array}{l}\text { KPT is a flag that allows threshold capillary } \\
\text { pressure to change with permeability. Since } \\
\text { this feature is not used in the } 1996 \text { CCA } \\
\text { calculation, the parameter value is specified as } \\
\text { " } 0 \text { ". }\end{array}$ \\
\hline 2666 & CL_M_T3 & KPT & $\begin{array}{l}\text { WPO\#40514, SWCF- } \\
\text { A:1.2.07.4.1:PA:QA:CCA:Analysis Package for } \\
\text { Salado Flow (Task 1) pg 4-150 through 4-159. }\end{array}$ & $\begin{array}{l}\text { KPT is a flag that allows threshold capillary } \\
\text { pressure to change with permeability. Since } \\
\text { this feature is not used in the } 1996 \mathrm{CCA} \\
\text { calculation, the parameter value is specified as } \\
\text { " } 0 \text { ". }\end{array}$ \\
\hline 2671 & CL_M_T4 & KPT & $\begin{array}{l}\text { WPO\#40514, SWCF- } \\
\text { A:1.2.07.4.1:PA:QA:CCA:Analysis Package for } \\
\text { Salado Flow (Task 1) pg 4-150 through 4-159. }\end{array}$ & $\begin{array}{l}\text { KPT is a flag that allows threshold capillary } \\
\text { pressure to change with permeability. Since } \\
\text { this feature is not used in the } 1996 \mathrm{CCA} \\
\text { calculation, the parameter value is specified as } \\
\text { "O". }\end{array}$ \\
\hline 2676 & CL_M_T5 & KPT & $\begin{array}{l}\text { WPO\#40514, SWCF. } \\
\text { A:1.2.07.4.1:PA:QA:CCA:Analysis Package for } \\
\text { Salado Flow (Task 1) pg 4-150 through 4-159. }\end{array}$ & $\begin{array}{l}\text { KPT is a flag that allows threshold capillary } \\
\text { pressure to change with permeability. Since } \\
\text { this feature is not used in the } 1996 \mathrm{CCA} \\
\text { calculation, the parameter value is specified as } \\
\text { "O". }\end{array}$ \\
\hline 2681 & CONC_T1 & KPT & $\begin{array}{l}\text { WPO\#40514, SWCF- } \\
\text { A:1.2.07.4.1:PA:QA:CCA:Analysis Package for } \\
\text { Salado Flow (Task 1) pg 4-150 through 4-159. }\end{array}$ & $\begin{array}{l}\text { KPT is a flag that allows threshold capillary } \\
\text { pressure to change with permeability. Since } \\
\text { this feature is not used in the } 1996 \text { CCA } \\
\text { calculation, the parameter value is specified as } \\
\text { "0". }\end{array}$ \\
\hline
\end{tabular}




\section{Additional Sources}

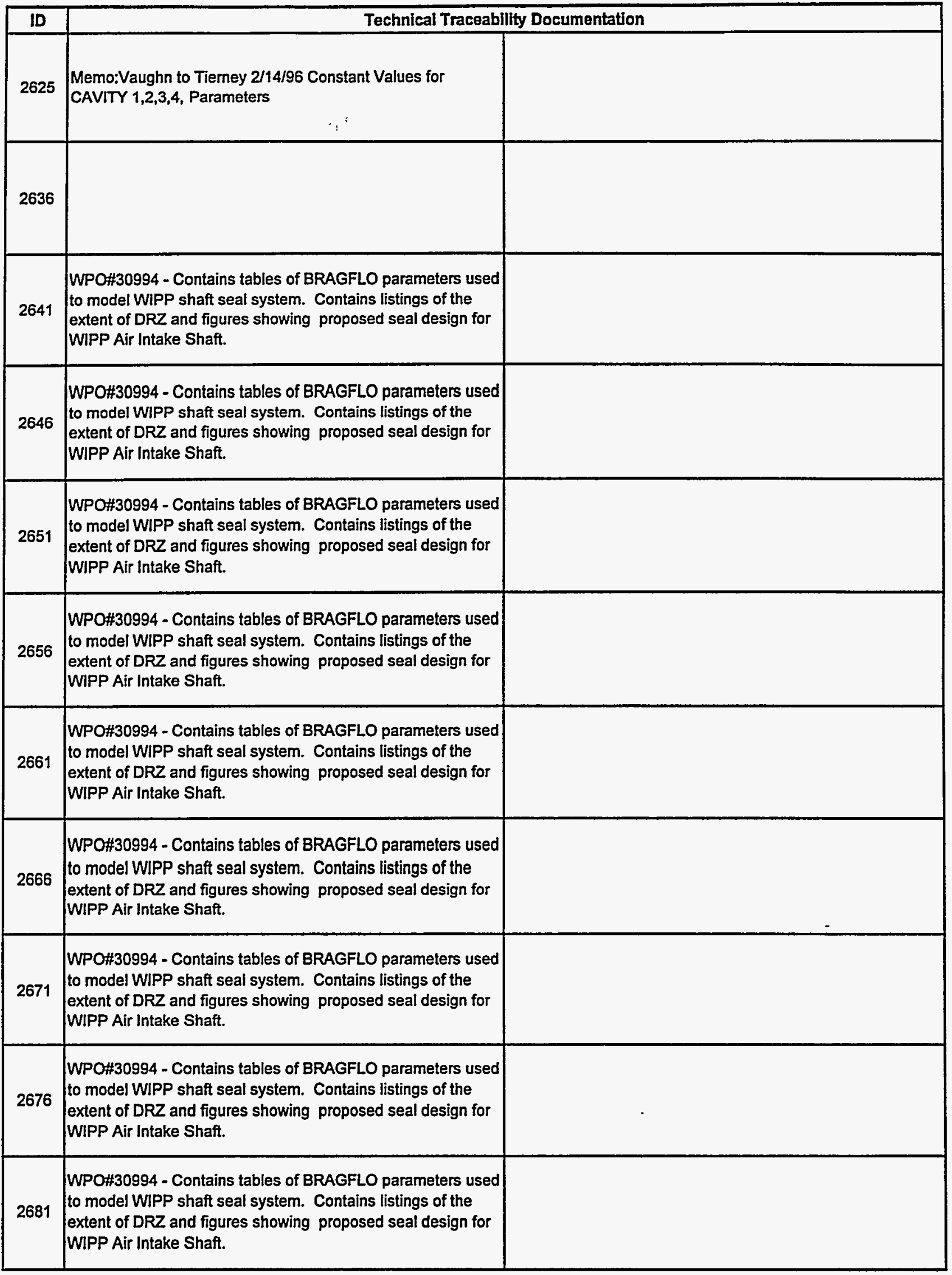




\begin{tabular}{|c|c|c|c|c|}
\hline ID & Material ID & Param ID & \multicolumn{2}{|c|}{ Technical Traceability Documentation } \\
\hline 2686 & CONC_T2 & KPT & $\begin{array}{l}\text { WPO\#40514, SWCF. } \\
\text { A:1.2.07.4.1:PA:QA:CCA:Analysis Package for } \\
\text { Salado Flow (Task 1) pg 4-150 through 4-159. }\end{array}$ & $\begin{array}{l}\text { KPT is a flag that allows threshold capillary } \\
\text { pressure to change with permeability. Since } \\
\text { this feature is not used in the } 1996 \text { CCA } \\
\text { calculation, the parameter value is specified as } \\
\text { "0". }\end{array}$ \\
\hline 2691 & CULEBRA & KPT & $\begin{array}{l}\text { WPO\#40514, SWCF- } \\
\text { A:1.2.07.4.1:PA:QA:CCA:Analysis Package for } \\
\text { Salado Flow (Task 1) pg 4-149. }\end{array}$ & $\begin{array}{l}\text { KPT is a flag that allows threshold capillary } \\
\text { pressure to change with permeability. Since } \\
\text { this feature is not used in the } 1996 \text { CCA } \\
\text { calculation, the parameter value is specified as } \\
\text { "O". }\end{array}$ \\
\hline 2696 & DEWYLAKE & KPT & $\begin{array}{l}\text { WPO\#40514, SWCF- } \\
\text { A:1.2.07.4.1:PA:QA:CCA:Analysis Package for } \\
\text { Salado Flow (Task 1) pg 4-149. }\end{array}$ & $\begin{array}{l}\text { KPT is a flag that allows threshold capillary } \\
\text { pressure to change with permeability. Since } \\
\text { this feature is not used in the } 1996 \text { CCA } \\
\text { calculation, the parameter value is specified as } \\
\text { "0". }\end{array}$ \\
\hline 2701 & $D R Z$ O & KPT & $\begin{array}{l}\text { WPO\#40514, SWCF- } \\
\text { A:1.2.07.4.1:PA:QA:CCA:Analysis Package for } \\
\text { Salado Flow (Task 1) pg 4-149. }\end{array}$ & $\begin{array}{l}\text { KPT is a flag that allows threshold capillary } \\
\text { pressure to change with permeability. Since } \\
\text { this feature is not used in the } 1996 \text { CCA } \\
\text { calculation, the parameter value is specified as } \\
\text { "O". }\end{array}$ \\
\hline 2706 & EARTH & KPT & $\begin{array}{l}\text { WPO40514, SWCF- } \\
\text { A:1.2.07.4.1:PA:QA:CCA:Analysis Package for } \\
\text { Salado Flow (Task 1) pg 4-149. }\end{array}$ & $\begin{array}{l}\text { KPT is a flag that allows threshold capillary } \\
\text { pressure to change with permeability. Since } \\
\text { this feature is not used in the } 1996 \text { CCA } \\
\text { calculation, the parameter value is specified as } \\
\text { "0". }\end{array}$ \\
\hline 2711 & EXP_AREA & KPT & $\begin{array}{l}\text { WPO\#40514, SWCF- } \\
\text { A:1.2.07.4.1:PA:QA:CCA:Analysis Package for } \\
\text { Salado Flow (Task 1) pg 4-150. }\end{array}$ & $\begin{array}{l}\text { KPT is a flag that allows threshold capillary } \\
\text { pressure to change with permeability. Since } \\
\text { this feature is not used in the } 1996 \mathrm{CCA} \\
\text { calculation, the parameter value is specified as } \\
\text { "O". }\end{array}$ \\
\hline 2715 & FORTYNIN & KPT & $\begin{array}{l}\text { WPO\#40514, SWCF- } \\
\text { A:1.2.07.4.1:PA:QA:CCA:Analysis Package for } \\
\text { Salado Flow (Task 1) pg 4-150 through 4-159. }\end{array}$ & $\begin{array}{l}\text { KPT is a flag that allows threshold capillary } \\
\text { pressure to change with permeability. Since } \\
\text { this feature is not used in the } 1996 \mathrm{CCA} \\
\text { calculation, the parameter value is specified as } \\
\text { "O". }\end{array}$ \\
\hline 2720 & IMPERM_Z & KPT & $\begin{array}{l}\text { WPO\#40514, SWCF- } \\
\text { A:1.2.07.4.1:PA:QA:CCA:Analysis Package for } \\
\text { Salado Flow (Task 1) pg 4-150. }\end{array}$ & $\begin{array}{l}\text { KPT is a flag that allows threshold capillary } \\
\text { pressure to change with permeability. Since } \\
\text { this feature is not used in the } 1996 \text { CCA } \\
\text { calculation, the parameter value is specified as } \\
\text { "O". }\end{array}$ \\
\hline 2725 & MAGENTA & KPT & $\begin{array}{l}\text { WPO\#40514, SWCF- } \\
\text { A:1.2.07.4.1:PA:QA:CCA:Analysis Package for } \\
\text { Salado Flow (Task 1) pg 4-150 through 4-159. }\end{array}$ & $\begin{array}{l}\text { KPT is a flag that allows threshold capillary } \\
\text { pressure to change with permeability. Since } \\
\text { this feature is not used in the } 1996 \text { CCA } \\
\text { calculation, the parameter value is specified as } \\
\text { "0". }\end{array}$ \\
\hline 2731 & PAN_SEAL & KPT & $\begin{array}{l}\text { WPO\#40514, SWCF- } \\
\text { A:1.2.07.4.1:PA:QA:CCA:Analysis Package for } \\
\text { Salado Flow (Task 1) pg 4-150 through 4-159. }\end{array}$ & $\begin{array}{l}\text { KPT is a flag that allows threshold capillary } \\
\text { pressure to change with permeability. Since } \\
\text { this feature is not used in the } 1996 \text { CCA } \\
\text { calculation, the parameter value is specified as } \\
\text { "O". }\end{array}$ \\
\hline 2736 & REPOSIT & KPT & $\begin{array}{l}\text { WPO\#40514, SWCF- } \\
\text { A:1.2.07.4.1:PA:QA:CCA:Analysis Package for } \\
\text { Salado Flow (Task 1) pg 4-150. }\end{array}$ & $\begin{array}{l}\text { KPT is a flag that allows threshold capillary } \\
\text { pressure to change with permeability. Since } \\
\text { this feature is not used in the } 1996 \text { CCA } \\
\text { calculation, the parameter value is specified as } \\
\text { "0". }\end{array}$ \\
\hline
\end{tabular}




\section{Additional Sources}

\begin{tabular}{|c|c|c|}
\hline ID & \multicolumn{2}{|c|}{ Technical Traceability Documentation } \\
\hline 2686 & $\begin{array}{l}\text { WPO\#30994 - Contains tables of BRAGFLO parameters used } \\
\text { to model WIPP shaft seal system. Contains listings of the } \\
\text { extent of DRZ and figures showing proposed seal design for } \\
\text { WIPP Air Intake Shaft. }\end{array}$ & \\
\hline 2691 & $\begin{array}{l}\text { WPO\#30994 - Contains tables of BRAGFLO parameters used } \\
\text { to model WIPP shaft seal system. Contains listings of the } \\
\text { extent of DRZ and figures showing proposed seal design for } \\
\text { WIPP Air Intake Shaft. }\end{array}$ & \\
\hline 2696 & & \\
\hline 2701 & & \\
\hline 2706 & $\begin{array}{l}\text { WPO\#30994 - Contains tables of BRAGFLO parameters used } \\
\text { to model WIPP shaft seal system. Contains listings of the } \\
\text { extent of DRZ and figures showing proposed seal design for } \\
\text { WIPP Air Intake Shaft. }\end{array}$ & \\
\hline 2711 & & \\
\hline 2715 & & \\
\hline 2720 & & \\
\hline 2725 & & \\
\hline 2731 & & \\
\hline 2736 & & \\
\hline
\end{tabular}




\begin{tabular}{|c|c|c|c|c|}
\hline & & & -Additional-Sources-'- & - \\
\hline ID & Material ID & Param ID & \multicolumn{2}{|c|}{ Technical Traceability Documentation } \\
\hline 2744 & SALT_T1 & KPT & $\begin{array}{l}\text { WPO\#40514, SWCF- } \\
\text { A:1.2.07.4.1:PA:QA:CCA:Analysis Package for } \\
\text { Salado Flow (Task 1) pg 4-150. }\end{array}$ & $\begin{array}{l}\text { KPT is a flag that allows threshold capillary } \\
\text { pressure to change with pemeability. Since } \\
\text { this feature is not used in the } 1996 \text { CCA } \\
\text { calculation, the parameter value is specified as } \\
\text { " } 0^{n} \text {. }\end{array}$ \\
\hline 2749 & SALT_T2 & KPT & $\begin{array}{l}\text { WPO\#40514, SWCF- } \\
\text { A:1.2.07.4.1:PA:QA:CCA:Analysis Package for } \\
\text { Salado Flow (Task 1) pg 4-150 through 4-159. }\end{array}$ & $\begin{array}{l}\text { KPT is a flag that allows threshold capillary } \\
\text { pressure to change with permeability. Since } \\
\text { this feature is not used in the } 1996 \text { CCA } \\
\text { calculation, the parameter value is specified as } \\
\text { "0". }\end{array}$ \\
\hline 2754 & SALT_T3 & KPT & $\begin{array}{l}\text { WPO\#40514, SWCF- } \\
\text { A:1.2.07.4.1:PA:QA:CCA:Analysis Package for } \\
\text { Salado Flow (Task 1) pg 4-150 through 4-159. }\end{array}$ & $\begin{array}{l}\text { KPT is a flag that allows threshold capillary } \\
\text { pressure to change with permeability. Since } \\
\text { this feature is not used in the } 1996 \mathrm{CCA} \\
\text { calculation, the parameter value is specified as } \\
\text { "O". }\end{array}$ \\
\hline 2759 & SALT_T4 & KPT & $\begin{array}{l}\text { WPO\#40514, SWCF- } \\
\text { A:1.2.07.4.1:PA:QA:CCA:Analysis Package for } \\
\text { Salado Flow (Task 1) pg 4-150 through 4-159. }\end{array}$ & $\begin{array}{l}\text { KPT is a flag that allows threshold capillary } \\
\text { pressure to change with permeability. Since } \\
\text { this feature is not used in the } 1996 \text { CCA } \\
\text { calculation, the parameter value is specified as } \\
\text { "0". }\end{array}$ \\
\hline 2764 & SALT_T5 & KPT & $\begin{array}{l}\text { WPO } 40514, \text { SWCF- } \\
\text { A:1.2.07.4.1:PA:QA:CCA:Analysis Package for } \\
\text { Salado Flow (Task 1) pg 4-150 through 4-159. }\end{array}$ & $\begin{array}{l}\text { KPT is a flag that allows threshold capillary } \\
\text { pressure to change with permeability. Since } \\
\text { this feature is not used in the } 1996 \text { CCA } \\
\text { calculation, the parameter value is specified as } \\
\text { "O". }\end{array}$ \\
\hline 2768 & SANTAROS & KPT & $\begin{array}{l}\text { WPO\#40514, SWCF- } \\
\text { A:1.2.07.4.1:PA:QA:CCA:Analysis Package for } \\
\text { Salado Flow (Task 1) pg 4-150 through 4-159. }\end{array}$ & $\begin{array}{l}\text { KPT is a flag that allows threshold capillary } \\
\text { pressure to change with permeability. Since } \\
\text { this feature is not used in the } 1996 \text { CCA } \\
\text { calculation, the parameter value is specified as } \\
\text { "O". }\end{array}$ \\
\hline 2773 & S_ANH_AB & KPT & $\begin{array}{l}\text { WPO\#40514, SWCF- } \\
\text { A:1.2.07.4.1:PA:QA:CCA:Analysis Package for } \\
\text { Salado Flow (Task 1) pg 4-150 through 4-159. }\end{array}$ & $\begin{array}{l}\text { KPT is a flag that allows threshold capillary } \\
\text { pressure to change with permeability. Since } \\
\text { this feature is not used in the } 1996 \text { CCA } \\
\text { calculation, the parameter value is specified as } \\
\text { "O". }\end{array}$ \\
\hline 2778 & S_HALITE & KPT & $\begin{array}{l}\text { WPO\#40514, SWCF- } \\
\text { A:1.2.07.4.1:PA:QA:CCA:Analysis Package for } \\
\text { Salado Flow (Task 1) pg 4-150. }\end{array}$ & $\begin{array}{l}\text { KPT is a flag that allows threshold capillary } \\
\text { pressure to change with permeability. Since } \\
\text { this feature is not used in the } 1996 \text { CCA } \\
\text { calculation, the parameter value is specified as } \\
\text { "O". }\end{array}$ \\
\hline 2783 & S_MB138 & KPT & $\begin{array}{l}\text { WPO\#40514, SWCF- } \\
\text { A:1.2.07.4.1:PA:QA:CCA:Analysis Package for } \\
\text { Salado Flow (Task 1) pg 4-150 through 4-159. }\end{array}$ & $\begin{array}{l}\text { KPT is a flag that allows threshold capillary } \\
\text { pressure to change with permeability. Since } \\
\text { this feature is not used in the } 1996 \text { CCA } \\
\text { calculation, the parameter value is specified as } \\
\text { " } 0 \text { ". }\end{array}$ \\
\hline 2788 & S_MB139 & KPT & $\begin{array}{l}\text { WPO\#40514, SWCF- } \\
\text { A:1.2.07.4.1:PA:QA:CCA:Analysis Package for } \\
\text { Salado Flow (Task 1) pg 4-150. }\end{array}$ & $\begin{array}{l}\text { KPT is a flag that allows threshold capillary } \\
\text { pressure to change with permeability. Since } \\
\text { this feature is not used in the } 1996 \text { CCA } \\
\text { calculation, the parameter value is specified as } \\
\text { "0". }\end{array}$ \\
\hline 2793 & TAMARISK & KPT & $\begin{array}{l}\text { WPO\#40514, SWCF- } \\
\text { A:1.2.07.4.1:PA:QA:CCA:Analysis Package for } \\
\text { Salado Flow (Task 1) pg 4-150 through 4-159. }\end{array}$ & $\begin{array}{l}\text { KPT is a flag that allows threshold capillary } \\
\text { pressure to change with permeability. Since } \\
\text { this feature is not used in the } 1996 \mathrm{CCA} \\
\text { calculation, the parameter value is specified as } \\
\text { "O". }\end{array}$ \\
\hline
\end{tabular}




\section{Additional Sources}

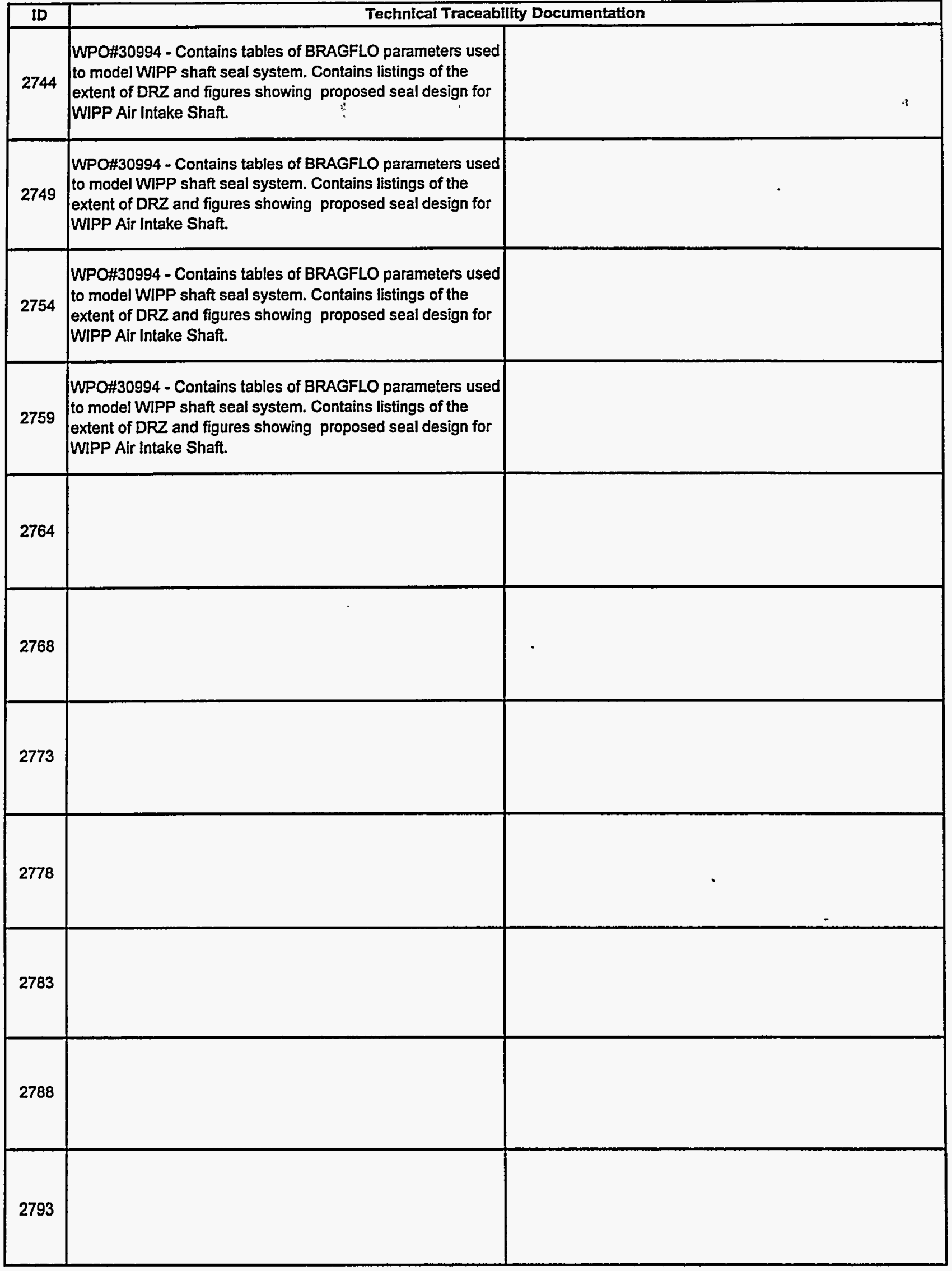


Additional-Sources

\begin{tabular}{|c|c|c|c|c|}
\hline ID & Material ID & Param ID & \multicolumn{2}{|c|}{ Technical Traceabllity Documentation } \\
\hline 2799 & UNNAMED & KPT & $\begin{array}{l}\text { WPO\#40514, SWCF- } \\
\text { A:1.2.07.4.1:PA:QA:CCA:Analysis Package fort } \\
\text { Salado Flow (Task 1) pg 4-150 through 4-159. }\end{array}$ & $\begin{array}{l}\text { KPT is a flag that allows threshold capillary } \\
\text { pressure to change with permeability. Since } \\
\text { this feature is not used in the } 1996 \text { CCA } \\
\text { calculation, the parameter value is specified as } \\
\text { "O". }\end{array}$ \\
\hline 2804 & WAS_AREA & KPT & $\begin{array}{l}\text { WPO\#0514, SWCF- } \\
\text { A:1.2.07.4.1:PA:QA:CCA:Analysis Package for } \\
\text { Salado Flow (Task 1) pg 4-150 through 4-159. }\end{array}$ & $\begin{array}{l}\text { KPT is a flag that allows threshold capillary } \\
\text { pressure to change with permeability. Since } \\
\text { this feature is not used in the } 1996 \text { CCA } \\
\text { calculation, the parameter value is specified as } \\
\text { "O". }\end{array}$ \\
\hline 2985 & SALT_T6 & KPT & $\begin{array}{l}\text { WPO\#40514, SWCF- } \\
\text { A:1.2.07.4.1:PA:QA:CCA:Analysis Package for } \\
\text { Salado Flow (Task 1) pg 4-150 through 4-159. }\end{array}$ & $\begin{array}{l}\text { KPT is a flag that allows threshold capillary } \\
\text { pressure to change with permeability. Since } \\
\text { this feature is not used in the } 1996 \text { CCA } \\
\text { calculation, the parameter value is specified as } \\
\text { "O". }\end{array}$ \\
\hline 3002 & CLAY_RUS & KPT & $\begin{array}{l}\text { WPO\#40514, SWCF- } \\
\text { A:1.2.07.4.1:PA:QA:CCA:Analysis Package for } \\
\text { Salado Flow (Task 1) pg 4-150 through 4-159. }\end{array}$ & $\begin{array}{l}\text { KPT is a flag that allows threshold capillary } \\
\text { pressure to change with permeability. Since } \\
\text { this feature is not used in the } 1996 \text { CCA } \\
\text { calculation, the parameter value is specified as } \\
\text { "O". }\end{array}$ \\
\hline 3053 & CONC_MON & KPT & $\begin{array}{l}\text { WPO\#40514, SWCF- } \\
\text { A:1.2.07.4.1:PA:QA:CCA:Analysis Package for } \\
\text { Salado Flow (Task 1) pg 4-149. }\end{array}$ & $\begin{array}{l}\text { KPT is a flag that allows threshold capillary } \\
\text { pressure to change with permeability. Since } \\
\text { this feature is not used in the } 1996 \text { CCA } \\
\text { calculation, the parameter value is specified as } \\
\text { "O". }\end{array}$ \\
\hline 3072 & CL_L_T4 & KPT & $\begin{array}{l}\text { WPO\#40514, SWCF- } \\
\text { A:1.2.07.4.1:PA:QA:CCA:Analysis Package for } \\
\text { Salado Flow (Task 1) pg 4-150 through 4-159. }\end{array}$ & $\begin{array}{l}\text { KPT is a flag that allows threshold capillary } \\
\text { pressure to change with permeability. Since } \\
\text { this feature is not used in the } 1996 \mathrm{CCA} \\
\text { calculation, the parameter value is specified as } \\
\text { "O". }\end{array}$ \\
\hline 3116 & DRZ_1 & KPT & $\begin{array}{l}\text { WPO\#40514, SWCF- } \\
\text { A:1.2.07.4.1:PA:QA:CCA:Analysis Package for } \\
\text { Salado Flow (Task 1) pg 4-149. }\end{array}$ & $\begin{array}{l}\text { KPT is a flag that allows threshold capillary } \\
\text { pressure to change with permeability. Since } \\
\text { this feature is not used in the } 1996 \text { CCA } \\
\text { calculation, the parameter value is specified as } \\
\text { " } 0^{n} \text {. }\end{array}$ \\
\hline 3122 & BOREHOLE & KPT & & $\begin{array}{l}\text { KPT is a flag that allows threshold capillary } \\
\text { pressure to change with permeability. Since } \\
\text { this feature is not used in the } 1996 \text { CCA } \\
\text { calculation, the parameter value is specified as } \\
\text { " }{ }^{\text {". }}\end{array}$ \\
\hline 3144 & BH_OPEN & KPT & $\begin{array}{l}\text { WPO\#40514, SWCF- } \\
\text { A:1.2.07.4.1:PA:QA:CCA:Analysis Package for } \\
\text { Salado Flow (Task 1) pg 4-150 through 4-159. }\end{array}$ & $\begin{array}{l}\text { KPT is a flag that allows threshold capillary } \\
\text { pressure to change with permeability. Since } \\
\text { this feature is not used in the } 1996 \text { CCA } \\
\text { calculation, the parameter value is specified as } \\
\text { "0". }\end{array}$ \\
\hline 3156 & CONC_PLG & KPT & $\begin{array}{l}\text { WPO\#40514, SWCF- } \\
\text { A:1.2.07.4.1:PA:QA:CCA:Analysis Package for } \\
\text { Salado Flow (Task 1) pg 4-150 through 4-159. }\end{array}$ & $\begin{array}{l}\text { KPT is a flag that allows threshold capillary } \\
\text { pressure to change with permeability. Since } \\
\text { this feature is not used in the } 1996 \text { CCA } \\
\text { calculation, the parameter value is specified as } \\
\text { "0". }\end{array}$ \\
\hline 3168 & BH_SAND & KPT & $\begin{array}{l}\text { WPO\#40514, SWCF- } \\
\text { A:1.2.07.4.1:PA:QA:CCA:Analysis Package for } \\
\text { Salado Flow (Task 1) pg 4-150 through 4-159. }\end{array}$ & $\begin{array}{l}\text { KPT is a flag that allows threshold capillary } \\
\text { pressure to change with permeability. Since } \\
\text { this feature is not used in the } 1996 \text { CCA } \\
\text { calculation, the parameter value is specified as } \\
\text { "0". }\end{array}$ \\
\hline
\end{tabular}




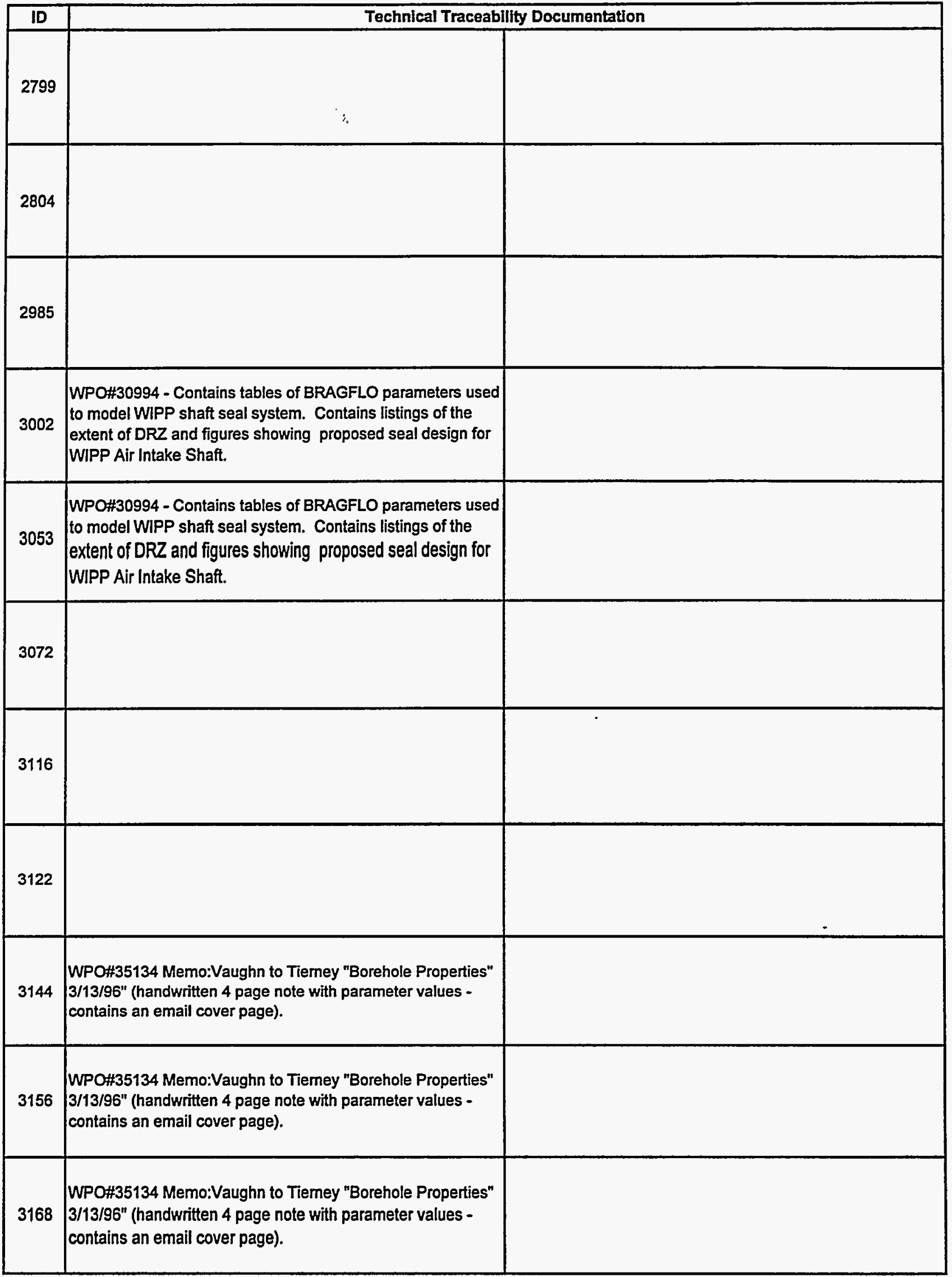


AdditionatSources

\begin{tabular}{|c|c|c|c|c|}
\hline ID & Material ID & Param ID & \multicolumn{2}{|c|}{ Technical Traceability Documentation } \\
\hline 3180 & BH_CREEP & KPT & $\begin{array}{l}\text { WPO\#40514, SWCF- } \\
\text { A:1.2.07.4.1:PA:QA:CCA:Analysis Package for } \\
\text { Salado Flow (Task 1) pg 4-150 through 4-159. }\end{array}$ & $\begin{array}{l}\text { KPT is a flag that allows threshold capillary } \\
\text { pressure to change with permeability. Since } \\
\text { this feature is not used in the } 1996 \text { CCA } \\
\text { calculation, the parameter value is specified as } \\
\text { "O". }\end{array}$ \\
\hline 62 & CASTILER & PC_MAX & $\begin{array}{l}\text { WPO\#30703 WIPP PA User's Manual (N1.01) } \\
\text { for BRAGFLO, Version } 4.00 \text { January } 31,1996 \\
\text { pg } 115-116 . \text { Pg } 115 \text { gives a discussion for } \\
\text { PC_MAX with the recommended value (1.0 E8 } \\
\text { Pa). Pg. } 116 \text { gives a discussion on the } \\
\text { CAP_MOD models for capillary pressure; }\end{array}$ & $\begin{array}{l}\text { WPO\#40514 pg 3-25 states ..."Capillary } \\
\text { pressure is an unbounded function as brine } \\
\text { saturation approaches residual brine } \\
\text { saturation. (PC_Max =1.0E8 Pa) and is a large } \\
\text { number chosen to represent infinity, in a } \\
\text { modeling sense based on analyst's } \\
\text { experience. }\end{array}$ \\
\hline 64 & CASTILER & POROSITY & $\begin{array}{l}\text { WPO\#41247 pg 3-1 (table), pg 5-6. States } \\
\text { there were } 3 \text { measured values/student T } \\
\text { distribution - used mean value for porosity. T- } \\
\text { distribution was run on } 3 \text { anhydrite samples } \\
\text { located north of WIPP. A conservative } \\
\text { porosity value was established. }\end{array}$ & $\begin{array}{l}\text { WPO\#31083 SWCF- } \\
\text { A:1.2.07.1:PDD:QA:Nonsalado:Pkg 19. Castile } \\
\text { Brine Reservoir Porosity }\end{array}$ \\
\hline 66 & CASTILER & PRESSURE & $\begin{array}{l}\text { WPO\#31072 Memo: Freeze, Larson to } \\
\text { Tiemey. Additional info WPO\#31612. See } \\
\text { attachment } 2 \text { in } 464 \text { Form (original } \\
\text { interpretation by M. Tiemey 1/12/96). See } \\
\text { attachment } 3 \text { in } 464 \text { Form (suggested changes } \\
\text { by Geoff Freeze } 1 / 24 / 96 \text { ). }\end{array}$ & $\begin{array}{l}\text { WPO\#41247, pg 5-9 "WIPP Natural Barriers } \\
\text { Data Qualification Peer Review Report" states } \\
\text { pressures were measured at various wells, } \\
\text { WIPP-12 well was most representative. } 85 \% \\
\text { estimation of this wells pressure was used for } \\
\text { value. }\end{array}$ \\
\hline 67 & CASTILER & PRMX_LOG & $\begin{array}{l}\text { WPO\#31070 SWCF- } \\
\text { A:1.2.07.1:PDD:Nonsalado:Pkg 19: Castile } \\
\text { Brine reservoir Permeability. Additional info in } \\
\text { WPO\#31613, WPO\#31614, WPO\#31615. }\end{array}$ & PI PRP WPO\#31083 \\
\hline 68 & CASTILER & PRMY_LOG & $\begin{array}{l}\text { WPO\#31070 SWCF- } \\
\text { A:1.2.07.1:PDD:Nonsalado:Pkg 19: Castile } \\
\text { Brine resenoir Permeability. Additional info in } \\
\text { WPO\#31613, WPO\#31614, WPO\#31615. }\end{array}$ & PI PRP WPO\#31083 \\
\hline 69 & CASTILER & PRMZ_LOG & $\begin{array}{l}\text { WPO\#31070 SWCF- } \\
\text { A:1.2.07.1:PDD:Nonsalado:Pkg 19: Castile } \\
\text { Brine reservoir Permeability. Additional info in } \\
\text { WPO\#31613, WPO\#31614, WPO\#31615. }\end{array}$ & PI PRP WPO\#31083 \\
\hline 2918 & CASTILER & VOLUME & $\begin{array}{l}\text { WPO\#31082 } \\
\text { SWCFA:1.2.07.1:PDD:Nonsalado:Pkg 19: } \\
\text { Castile Brine Reservoir Volume. }\end{array}$ & $\begin{array}{l}\text { WPO\#37149 Memo: Larson to Tierney } 3 / 20 / 96 \\
\text { discusses meeting held on continguous } \\
\text { reservoirs and a new volume value to use. }\end{array}$ \\
\hline 2994 & CELLULS & FBETA & $\begin{array}{l}\text { WPO\#35162, Memo from Yifeng Wang and } \\
\text { Larry Brush to Martin S.Tierney, Jan 26,1996; } \\
\text { "Estimates of Gas-Generation Parameters for } \\
\text { the Long-Term WIPP Performance } \\
\text { Assessment" P.9, Table } 1 \text { (with SI conversion } \\
\text { described on } 464 \text { Form). }\end{array}$ & $\begin{array}{l}\text { Raw data found in WPO\#42357 and } \\
\text { WPO\#38474 }\end{array}$ \\
\hline 2334 & CL_L_T1 & PRMX_LOG & $\begin{array}{l}\text { WPO\#41809 Sec 4.6 p.4-36. WPO\#30994 - } \\
\text { Contains tables of BRAGFLO parameters used } \\
\text { to model WIPP shaft seal system. Contains } \\
\text { listings of the extent of DRZ and figures } \\
\text { showing proposed seal design for WIPP Air } \\
\text { Intake Shaft. }\end{array}$ & $\begin{array}{l}\text { WPO\#30640 WIPP Shaft Seal System } \\
\text { BRAGFLO Parameters, SWCF- } \\
\text { A:1.1.03.2.1:QA:PDD Sec II Vol 2, 2.1.1; Sec I } \\
\text { Vol 4 4.2. }\end{array}$ \\
\hline
\end{tabular}




\begin{tabular}{|c|c|c|}
\hline ID & \multicolumn{2}{|c|}{ Technical Traceability Documentation } \\
\hline 3180 & $\begin{array}{l}\text { WPO\#35134 Memo:Vaughn to Tiemey "Borehole Properties" } \\
\text { 3/13/96" (handwritten } 4 \text { page note with parameter values - } \\
\text { contains an email cover page). }\end{array}$ & \\
\hline 62 & $\begin{array}{l}\text { WPO\#40514 Sec. } 4.2 .4 \text { pg 4-150 (the BRAGFLO analysis } \\
\text { write-up) CASTILER:PC_MAX - } 1.0 \text { E8. CASTILER:PC_MAX } \\
=1.0 \text { E (pg 3-25) Maximum capillary pressure is controlled by } \\
\text { CAP-MOD and is bounded by PC_MAX. }\end{array}$ & $\begin{array}{l}\text { WPO } 38568 \text { states this is a 'legacy parameter' whose value } \\
\text { appeared in the baseline WIPP Parameter database of March } \\
2,1996 .\end{array}$ \\
\hline 64 & $\begin{array}{l}\text { WPO\#31175 PI PRP - has table in back that lists many } \\
\text { references used for various WPO numbers. }\end{array}$ & 40434 \\
\hline 66 & & \\
\hline 67 & $\begin{array}{l}\text { WPO\#41247, pg 5-14 "WIPP Natural Barriers Data } \\
\text { Qualification Peer Review Report" states permeability was } \\
\text { based on testing of Castile wells near WIPP. GTFM code was } \\
\text { used. }\end{array}$ & \\
\hline 68 & $\begin{array}{l}\text { WPO\#41247, pg 5-14 "WIPP Natural Barriers Data } \\
\text { Qualification Peer Review Report" states permeability was } \\
\text { based on testing of Castile wells near WIPP. GTFM code was } \\
\text { used. }\end{array}$ & \\
\hline 69 & $\begin{array}{l}\text { WPO\#41247, pg 5-14 "WIPP Natural Barriers Data } \\
\text { Qualification Peer Review Report" states permeability was } \\
\text { based on testing of Castile wells near WIPP. GTFM code was } \\
\text { used. }\end{array}$ & 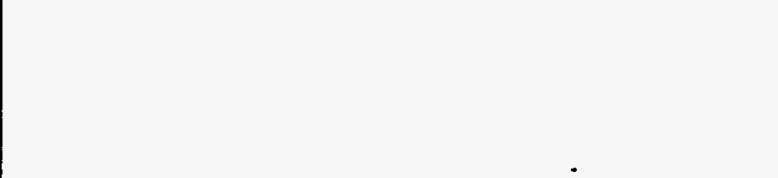 \\
\hline 2918 & $\begin{array}{l}\text { WPO\#41247, pg 5-18 "WIPP Natural Barriers Data } \\
\text { Qualification Peer Review Report" states volumes were based } \\
\text { on brine collected during testing and estimates of brine } \\
\text { release during open borehole periods. }\end{array}$ & \\
\hline 2994 & $\begin{array}{l}\text { WPO\#30994 - Contains tables of BRAGFLO parameters used } \\
\text { to model WIPP shaft seal system. Contains listings of the } \\
\text { extent of DRZ and figures showing proposed seal design for } \\
\text { WIPP Air Intake Shaft. }\end{array}$ & \\
\hline 2334 & $\begin{array}{l}\text { Literature: Cheung et al. (1987) pg 406, knowles and Howard } \\
\text { (1996) pg 5, Pusch et al. (1987) pg 61, Ran and Daemen } \\
\text { (1965) pg 1; Data packages Knowles (1995) WPO\#28745 }\end{array}$ & \\
\hline
\end{tabular}


Additional-Sources

\begin{tabular}{|c|c|c|c|c|}
\hline ID & Material ID & Param ID & \multicolumn{2}{|c|}{ Technical Traceability Documentation } \\
\hline 2335 & CL_L_T1 & PRMY_LOG & $\begin{array}{l}\text { WPO\#41809 Sec } 4.6 \text { p.4-36. WPO\#30994 - } \\
\text { Contains tables of BRAGFLO parameters used } \\
\text { to model WIPP shaft seal system. Contains } \\
\text { listings of the extent of DRZ and figures } \\
\text { showing proposed seal design for WIPP Air } \\
\text { Intake Shaft. }\end{array}$ & $\begin{array}{l}\text { WPO\#30640: WIPP Shaft Seal System } \\
\text { BRAGFLO Parameters, SWCF- } \\
\text { A:1.1.03.2.1:QA:PDD Sec II Vol 2, 2.1.1; Sec I } \\
\text { Vol 4 4.2. }\end{array}$ \\
\hline 2336 & CL_L_T1 & PRMZ_LOG & $\begin{array}{l}\text { WPO\#41809 Sec 4.6 p.4-36. WPO\#30994 - } \\
\text { Contains tables of BRAGFLO parameters used } \\
\text { to model WIPP shaft seal system. Contains } \\
\text { listings of the extent of DRZ and figures } \\
\text { showing proposed seal design for WIPP Air } \\
\text { Intake Shaft. }\end{array}$ & $\begin{array}{l}\text { WPO\#30640: WIPP Shaft Seal System } \\
\text { BRAGFLO Parameters, SWCF- } \\
\text { A:1.1.03.2.1:QA:PDD Sec II Vol 2, 2.1.1; Sec I } \\
\text { Vol 4 4.2. }\end{array}$ \\
\hline 3009 & CLAY_RUS & PRMX_LOG & $\begin{array}{l}\text { WPO\#41809 Sec } 4.6 \text { p.4-36. WPO\#30994 - } \\
\text { Contains tables of BRAGFLO parameters used } \\
\text { to model WIPP shaft seal system. Contains } \\
\text { listings of the extent of DRZ and figures } \\
\text { showing proposed seal design for WIPP Air } \\
\text { Intake Shaft. }\end{array}$ & $\begin{array}{l}\text { WPO\#30640: WIPP Shaft Seal System } \\
\text { BRAGFLO Parameters, SWCF- } \\
\text { A:1.1.03.2.1:QA:PDD Sec II Vol 2, 2.1.1; Sec I } \\
\text { Vol 4 4.2. }\end{array}$ \\
\hline 3010 & CLAY_RUS & PRMY_LOG & $\begin{array}{l}\text { WPO\#41809 Sec } 4.6 \text { p.4-36. WPO\#30994 - } \\
\text { Contains tables of BRAGFLO parameters used } \\
\text { to model WIPP shaft seal system. Contains } \\
\text { listings of the extent of DRZ and figures } \\
\text { showing proposed seal design for WPP Air } \\
\text { intake Shaft. }\end{array}$ & $\begin{array}{l}\text { WPO\#30640: WIPP Shaft Seal System } \\
\text { BRAGFLO Parameters, SWCF- } \\
\text { A:1.1.03.2.1:QA:PDD Sec II Vol 2, 2.1.1; Sec I } \\
\text { Vol 4 4.2. }\end{array}$ \\
\hline 3011 & CLAY_RUS & PRMZ_LOG & $\begin{array}{l}\text { WPO\#1809 Sec } 4.6 \text { p.4-36. WPO\#30994 - } \\
\text { Contains tables of BRAGFLO parameters used } \\
\text { to model WIPP shaft seal system. Contains } \\
\text { listings of the extent of DRZ and figures } \\
\text { showing proposed seal design for WIPP Air } \\
\text { Intake Shaft. }\end{array}$ & $\begin{array}{l}\text { WPO\#30640: WIPP Shaft Seal System } \\
\text { BRAGFLO Parameters, SWCF- } \\
\text { A:1.1.03.2.1:QA:PDD Sec II Vol 2, 2.1.1; Sec I } \\
\text { Vol 4 4.2. }\end{array}$ \\
\hline 2351 & CL_L_T2 & PRMX_LOG & $\begin{array}{l}\text { WPO\#41809 Sec 4.6 p.4-36. WPO\#30994 - } \\
\text { Contains tables of BRAGFLO parameters used } \\
\text { to model WIPP shaft seal system. Contains } \\
\text { listings of the extent of DRZ and figures } \\
\text { showing proposed seal design for WIPP Air } \\
\text { Intake Shaft. }\end{array}$ & $\begin{array}{l}\text { WPO\#30640: WIPP Shaft Seal System } \\
\text { BRAGFLO Parameters, SWCF- } \\
\text { A:1.1.03.2.1:QA:PDD Sec II Vol 2, 2.1.1; Sec I } \\
\text { Vol 4 4.2. }\end{array}$ \\
\hline 2352 & CL_L_T2 & PRMY_LOG & $\begin{array}{l}\text { WPO\#41809 Sec } 4.6 \text { p.4-36. WPO\#30994 - } \\
\text { Contains tables of BRAGFLO parameters used } \\
\text { to model WIPP shaft seal system. Contains } \\
\text { listings of the extent of DRZ and figures } \\
\text { showing proposed seal design for WIPP Air } \\
\text { Intake Shaft. }\end{array}$ & $\begin{array}{l}\text { WPO\#30640: WIPP Shaft Seal System } \\
\text { BRAGFLO Parameters, SWCF- } \\
\text { A:1.1.03.2.1:QA:PDD Sec II Vol 2, 2.1.1; Sec I } \\
\text { Vol 4 4.2. }\end{array}$ \\
\hline 2353 & CL_L_T2 & PRMZ_LOG & $\begin{array}{l}\text { WPO\#41809 Sec } 4.6 \text { p.4-36. WPO\#30994 - } \\
\text { Contains tables of BRAGFLO parameters used } \\
\text { to model WIPP shaft seal system. Contains } \\
\text { listings of the extent of DRZ and figures } \\
\text { showing proposed seal design for WIPP Air } \\
\text { Intake Shaft. }\end{array}$ & $\begin{array}{l}\text { WPO\#30640: WIPP Shaft Seal System } \\
\text { BRAGFLO Parameters, SWCF- } \\
\text { A:1.1.03.2.1:QA:PDD Sec II Vol 2, 2.1.1; Sec I } \\
\text { Vol 4 4.2. }\end{array}$ \\
\hline 2368 & CL_L_T3 & PRMX_LOG & $\begin{array}{l}\text { WPO\#41809 Sec } 4.6 \text { p.4-36. WPO\#30994 - } \\
\text { Contains tables of BRAGFLO parameters used } \\
\text { to model WIPP shaft seal system. Contains } \\
\text { listings of the extent of DRZ and figures } \\
\text { showing proposed seal design for WIPP Air } \\
\text { Intake Shaft. }\end{array}$ & $\begin{array}{l}\text { WPO\#30640: WIPP Shaft Seal System } \\
\text { BRAGFLO Parameters, SWCF- } \\
\text { A:1.1.03.2.1:QA:PDD Sec II Vol 2, 2.1.1; Sec I } \\
\text { Vol 4 4.2. }\end{array}$ \\
\hline
\end{tabular}




\section{Additional Sources}

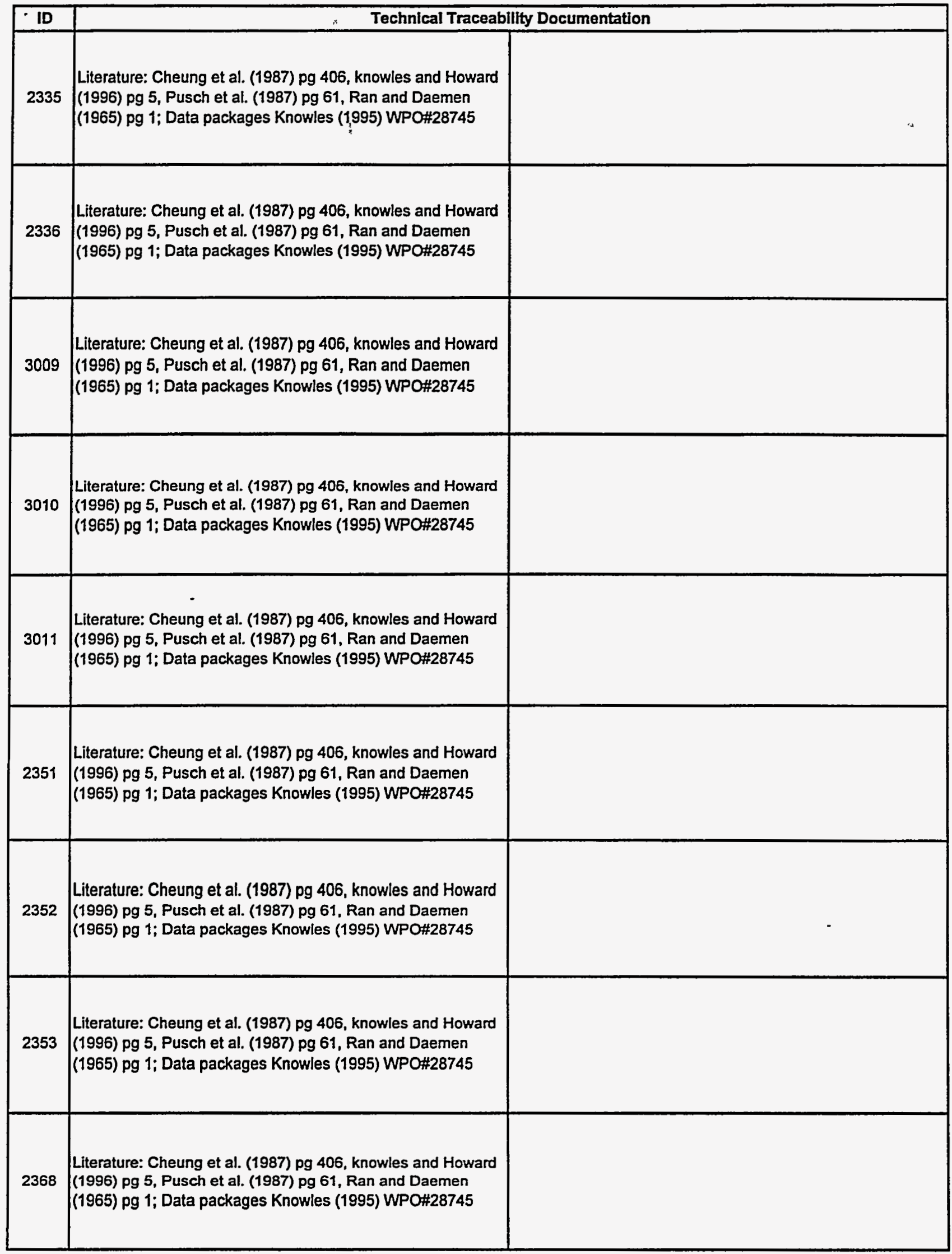




\section{Additional Sources}

\begin{tabular}{|c|c|c|c|c|}
\hline ID & Material ID & Param ID & \multicolumn{2}{|c|}{ Technical Traceability Documentation } \\
\hline 2370 & CL_L_T3 & PRMZ_LOG & $\begin{array}{l}\text { WPO\#41809 See 4.6 p.4-36. WPO\#30994 - } \\
\text { Contains tables of BRAGFLO parameters used } \\
\text { to model WIPP shaft seal system. Contains } \\
\text { listings of the extent of DRZ and figures } \\
\text { showing proposed seal design for WIPP Air } \\
\text { Intake Shaft. }\end{array}$ & $\begin{array}{l}\text { WPO\#30640: WIPP Shaft Seal System } \\
\text { BRAGFLO Parameters, SWCF- } \\
\text { A:1.1.03.2.1:QA:PDD Sec II Vol 2, 2.1.1; Sec I } \\
\text { Vol 4 4.2. }\end{array}$ \\
\hline 3078 & CL_L_T4 & PRMX_LOG & $\begin{array}{l}\text { WPO\#41809 Sec 4.6 p.4-36. WPO\#30994 - } \\
\text { Contains tables of BRAGFLO parameters used } \\
\text { to model WIPP shaft seal system. Contains } \\
\text { listings of the extent of DRZ and figures } \\
\text { showing proposed seal design for WIPP Air } \\
\text { Intake Shaft. }\end{array}$ & $\begin{array}{l}\text { WPO\#30640: WIPP Shaft Seal System } \\
\text { BRAGFLLO Parameters, SWCF- } \\
\text { A:1.1.03.2.1:QA:PDD Sec II Vol 2, 2.1.1; SeC I } \\
\text { Vol 4 4.2. }\end{array}$ \\
\hline 3079 & CL_L_T4 & PRMY_LOG & $\begin{array}{l}\text { WPO\#41809 Sec 4.6 p.4-36. WPO\#30994 - } \\
\text { Contains tables of BRAGFLO parameters used } \\
\text { to model WIPP shaft seal system. Contains } \\
\text { listings of the extent of DRZ and figures } \\
\text { showing proposed seal design for WPP Air } \\
\text { Intake Shaft. }\end{array}$ & $\begin{array}{l}\text { WPO\#30640: WIPP Shaft Seal System } \\
\text { BRAGFLO Parameters, SWCF- } \\
\text { A:1.1.03.2.1:QA:PDD Sec II Vol 2, 2.1.1; Sec I } \\
\text { Vol 44.2. }\end{array}$ \\
\hline 3080 & CL_L_T4 & PRMZ_LOG & $\begin{array}{l}\text { WPO\#41809 Sec 4.6 p.4-36. WPO\#30994 - } \\
\text { Contains tables of BRAGFLO parameters used } \\
\text { to model WIPP shaft seal system. Contains } \\
\text { listings of the extent of DRZ and figures } \\
\text { showing proposed seal design for WIPP Air } \\
\text { Intake Shaft. }\end{array}$ & $\begin{array}{l}\text { WPO\#30640: WIPP Shaft Seal System } \\
\text { BRAGFLO Parameters, SWCF- } \\
\text { A:1.1.03.2.1:QA:PDD Sec II Vol 2, 2.1.1; Sec I } \\
\text { Vol 4 4.2. }\end{array}$ \\
\hline 2385 & CL_M_T1 & PRMX_LOG & $\begin{array}{l}\text { WPO\#41809 Sec 4.6 p.4-36. WPO\#30994 - } \\
\text { Contains tables of BRAGFLO parameters used } \\
\text { to model WIPP shaft seal system. Contains } \\
\text { listings of the extent of DRZ and figures } \\
\text { showing proposed seal design for WIPP Air } \\
\text { intake Shaft. }\end{array}$ & $\begin{array}{l}\text { WPO\#30640: WIPP Shaft Seal System } \\
\text { BRAGFLO Parameters, SWCF- } \\
\text { A:1.1.03.2.1:QA:PDD Sec II Vol 2, 2.1.1; Sec I } \\
\text { Vol 4 4.2. }\end{array}$ \\
\hline 2386 & CL_M_T1 & PRMY_LOG & $\begin{array}{l}\text { WPO\#41809 Sec 4.6 p.4-36. WPO\#30994 - } \\
\text { Contains tables of BRAGFLO parameters used } \\
\text { to model WIPP shaft seal system. Contains } \\
\text { listings of the extent of DRZ and figures } \\
\text { showing proposed seal design for WIPP Air } \\
\text { Intake Shaft. }\end{array}$ & $\begin{array}{l}\text { WPO\#30640: WIPP Shaft Seal System } \\
\text { BRAGFLO Parameters, SWCF- } \\
\text { A:1.1.03.2.1:QA:PDD Sec II Vol 2, 2.1.1; SeC I } \\
\text { Vol 4 4.2. }\end{array}$ \\
\hline 2387 & CL_M_T1 & PRMZ_LOG & $\begin{array}{l}\text { WPO\#41809 Sec } 4.6 \text { p.4-36. WPO\#30994 - } \\
\text { Contains tables of BRAGFLO parameters used } \\
\text { to model WIPP shaft seal system. Contains } \\
\text { listings of the extent of DRZ and figures } \\
\text { showing proposed seal design for WIPP Air } \\
\text { Intake Shaft. }\end{array}$ & $\begin{array}{l}\text { WPO\#30640: WIPP Shaft Seal System } \\
\text { BRAGFLO Parameters, SWCF- } \\
\text { A:1.1.03.2.1:QA:PDD Sec II Yol 2, 2.1.1; Sec I } \\
\text { Vol 4 4.2. }\end{array}$ \\
\hline 2402 & CL_M_T2 & PRMX_LOG & $\begin{array}{l}\text { WPO\#41809 Sec 4.6 p.4-36. WPO\#30994 - } \\
\text { Contains tables of BRAGFLO parameters used } \\
\text { to model WIPP shaft seal system. Contains } \\
\text { listings of the extent of DRZ and figures } \\
\text { showing proposed seal design for WIPP Air } \\
\text { Intake Shaft. }\end{array}$ & $\begin{array}{l}\text { WPO\#30640: WIPP Shaft Seal System } \\
\text { BRAGFLO Parameters, SWCF- } \\
\text { A:1.1.03.2.1:QA:PDD Sec II Vol 2, 2.1.1; Sec I } \\
\text { Vol 4 4.2. }\end{array}$ \\
\hline 2403 & CL_M_T2 & PRMY_LOG & $\begin{array}{l}\text { WPO\#41809 Sec 4.6 p.4-36. WPO\#30994- } \\
\text { Contains tables of BRAGFLO parameters used } \\
\text { to model WIPP shaft seal system. Contains } \\
\text { listings of the extent of DRZ and figures } \\
\text { showing proposed seal design for WIPP Air } \\
\text { intake Shaft. }\end{array}$ & $\begin{array}{l}\text { WPO\#30640: WIPP Shaft Seal System } \\
\text { BRAGFLO Parameters, SWCF- } \\
\text { A:1.1.03.2.1:QA:PDD Sec II Vol 2, 2.1.1; SeC I } \\
\text { Vol 4 4.2. }\end{array}$ \\
\hline
\end{tabular}




\section{Additional Sources}

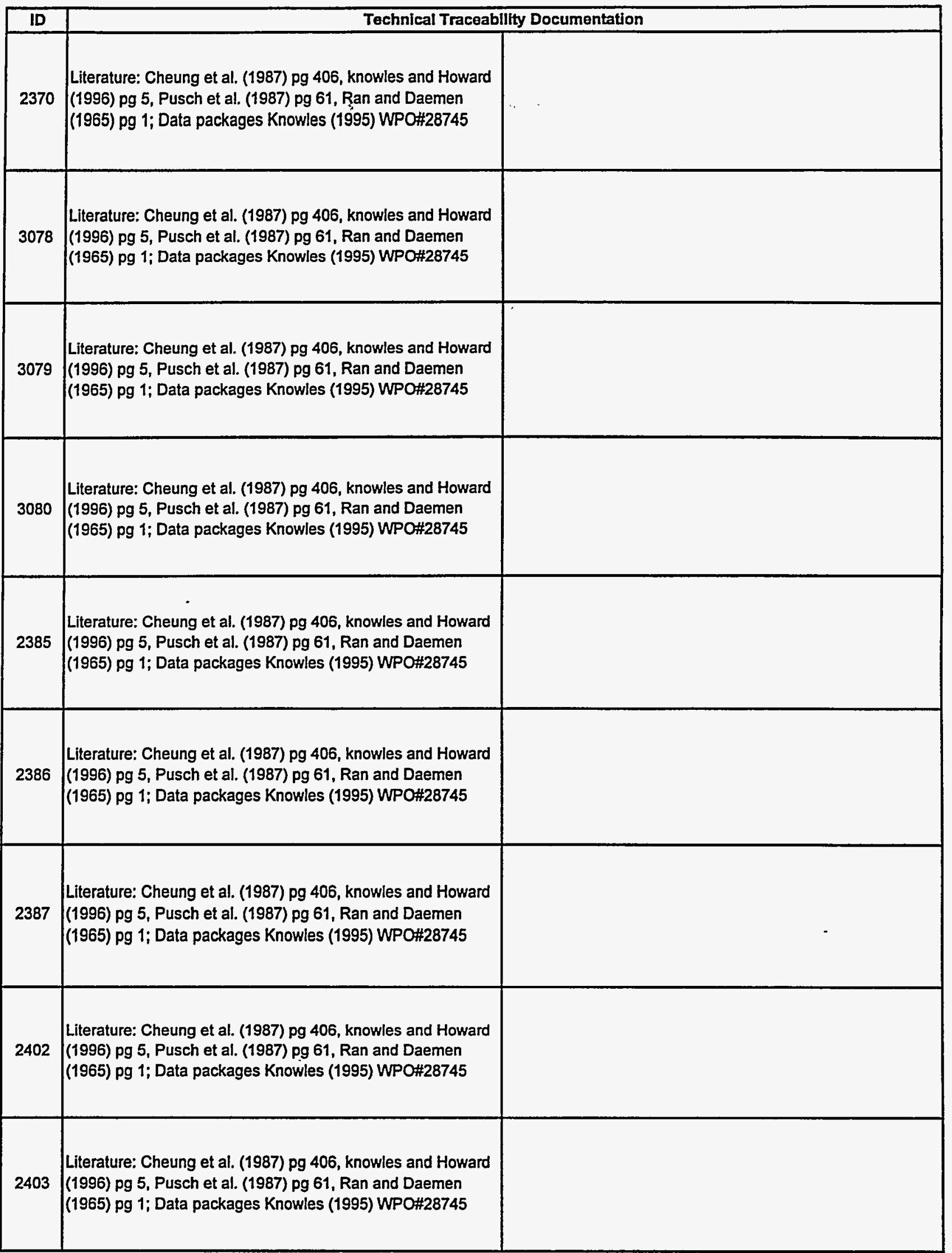


Additional Sources

\begin{tabular}{|c|c|c|c|c|}
\hline ID & Material ID & Param ID & \multicolumn{2}{|c|}{ Technical Traceabllity Documentation } \\
\hline 2404 & CL_M_T2 & PRMZ_LOG & $\begin{array}{l}\text { WPO\#41809 Sec } 4.6 \text { p.4-36. WPO\#30994 - } \\
\text { Contains tables of BRAGFLO parameters useo } \\
\text { to model WIPP shaft seal system. Contains } \\
\text { listings of the extent of DRZ and figures } \\
\text { showing proposed seal design for WIPP Air } \\
\text { Intake Shaft. }\end{array}$ & $\begin{array}{l}\text { WPO\#30640: WIPP Shaft Seal System } \\
\text { BRAGFLO Parameters, SWCF- } \\
\text { A:1.1.03.2.1:QA:PDD Sec II Vol 2, 2.1.1; Sec I } \\
\text { Vol 4 4.2. }\end{array}$ \\
\hline 2419 & CL_M_T3 & PRMX_LOG & $\begin{array}{l}\text { WPO\#41809 Sec } 4.6 \text { p.4-36. WPO\#30994 - } \\
\text { Contains tables of BRAGFLO parameters used } \\
\text { to model WIPP shaft seal system. Contains } \\
\text { listings of the extent of DRZ and figures } \\
\text { showing proposed seal design for WIPP Air } \\
\text { Intake Shaft. }\end{array}$ & $\begin{array}{l}\text { WPO\#30640: WIPP Shaft Seal System } \\
\text { BRAGFLO Parameters, SWCF- } \\
\text { A:1.1.03.2.1:QA:PDD Sec II Vol 2, 2.1.1; Sec I } \\
\text { Vol 4 4.2. }\end{array}$ \\
\hline 2420 & CL_M_T3 & PRMY_LOG & $\begin{array}{l}\text { WPO\#41809 Sec } 4.6 \text { p.4-36. WPO\#30994- } \\
\text { Contains tables of BRAGFLO parameters used } \\
\text { to model WIPP shaft seal system. Contains } \\
\text { listings of the extent of DRZ and figures } \\
\text { showing proposed seal design for WIPP Air } \\
\text { Intake Shaft. }\end{array}$ & $\begin{array}{l}\text { WPO\#30640: WIPP Shaft Seal System } \\
\text { BRAGFLO Parameters, SWCF- } \\
\text { A:1.1.03.2.1:QA:PDD Sec II Vol 2, 2.1.1; Sec I } \\
\text { Vol 4 4.2. }\end{array}$ \\
\hline 2421 & CL_M_T3 & PRMZ_LOG & $\begin{array}{l}\text { WPO\#41809 Sec } 4.6 \text { p.4-36. WPO\#30994- } \\
\text { Contains tables of BRAGFLO parameters useo } \\
\text { to model WIPP shaft seal system. Contains } \\
\text { listings of the extent of DRZ and figures } \\
\text { showing proposed seal design for WIPP Air } \\
\text { Intake Shaft. }\end{array}$ & $\begin{array}{l}\text { WPO\#30640: WIPP Shaft Seal System } \\
\text { BRAGFLO Parameters, SWCF- } \\
\text { A:1.1.03.2.1:QA:PDD Sec II Vol 2, 2.1.1; Sec I } \\
\text { Vol 4 4.2. }\end{array}$ \\
\hline 2436 & CL_M_T4 & PRMX_LOG & $\begin{array}{l}\text { WPO\#41809 Sec } 4.6 \text { p.4-36. WPO\#30994 - } \\
\text { Contains tables of BRAGFLO parameters used } \\
\text { to model WIPP shaft seal system. Contains } \\
\text { listings of the extent of DRZ and figures } \\
\text { showing proposed seal design for WIPP Air } \\
\text { Intake Shaft. }\end{array}$ & $\begin{array}{l}\text { WPO\#30640: WIPP Shaft Seal System } \\
\text { BRAGFLO Parameters, SWCF- } \\
\text { A:1.1.03.2.1:QA:PDD Sec II Vol 2, 2.1.1; Sec I } \\
\text { Vol 4 4.2. }\end{array}$ \\
\hline 2437 & CL_M_T4 & PRMY_LOG & $\begin{array}{l}\text { WPO\#41809 Sec } 4.6 \text { p.4-36. WPO\#30994 - } \\
\text { Contains tables of BRAGFLO parameters used } \\
\text { to model WIPP shaft seal system. Contains } \\
\text { listings of the extent of DRZ and figures } \\
\text { showing proposed seal design for WIPP Air } \\
\text { Intake Shaft. }\end{array}$ & $\begin{array}{l}\text { WPO\#30640: WIPP Shaft Seal System } \\
\text { BRAGFLO Parameters, SWCF- } \\
\text { A:1.1.03.2.1:QA:PDD Sec II Vol 2, 2.1.1; Sec I } \\
\text { Vol 4 4.2. }\end{array}$ \\
\hline 2438 & $C L_{-} M_{-} T_{4}$ & PRMZ_LOG & $\begin{array}{l}\text { WPO\#41809 Sec } 4.6 \text { p.4-36. WPO\#30994- } \\
\text { Contains tables of BRAGFLO parameters useo } \\
\text { to model WIPP shaft seal system. Contains } \\
\text { listings of the extent of DRZ and figures } \\
\text { showing proposed seal design for WIPP Air } \\
\text { Intake Shaft. }\end{array}$ & $\begin{array}{l}\text { WPO\#30640: WIPP Shaft Seal System } \\
\text { BRAGFLO Parameters, SWCF- } \\
\text { A:1.1.03.2.1:QA:PDD Sec II Yol 2, 2.1.1; Sec I } \\
\text { Vol 4 4.2. }\end{array}$ \\
\hline 2317 & CLAY_BOT & PRMX_LOG & $\begin{array}{l}\text { WPO\#41809 Sec } 4.6 \text { p.4-36. WPO\#30994 - } \\
\text { Contains tables of BRAGFLO parameters used } \\
\text { to model WIPP shaft seal system. Contains } \\
\text { listings of the extent of DRZ and figures } \\
\text { showing proposed seal design for WIPP Air } \\
\text { Intake Shaft. }\end{array}$ & $\begin{array}{l}\text { WPO\#30640: WIPP Shaft Seal System } \\
\text { BRAGFLO Parameters, SWCF- } \\
\text { A:1.1.03.2.1:QA:PDD Sec II Vol 2, 2.1.1; Sec I } \\
\text { Vol 4 4.2. }\end{array}$ \\
\hline 2318 & CLAY_BOT & PRMY_LOG & $\begin{array}{l}\text { WPO\#41809 Sec } 4.6 \text { p.4-36. WPO\#30994- } \\
\text { Contains tables of BRAGFLO parameters used } \\
\text { to model WIPP shaft seal system. Contains } \\
\text { listings of the extent of DRZ and figures } \\
\text { showing proposed seal design for WIPP Air } \\
\text { Intake Shaft. }\end{array}$ & $\begin{array}{l}\text { WPO\#30640: WIPP Shaft Seal System } \\
\text { BRAGFLO Parameters, SWCF- } \\
\text { A:1.1.03.2.1:QA:PDD Sec II Vol 2, 2.1.1; Sec I } \\
\text { Vol 4 4.2. }\end{array}$ \\
\hline
\end{tabular}




\section{Additional Sources}

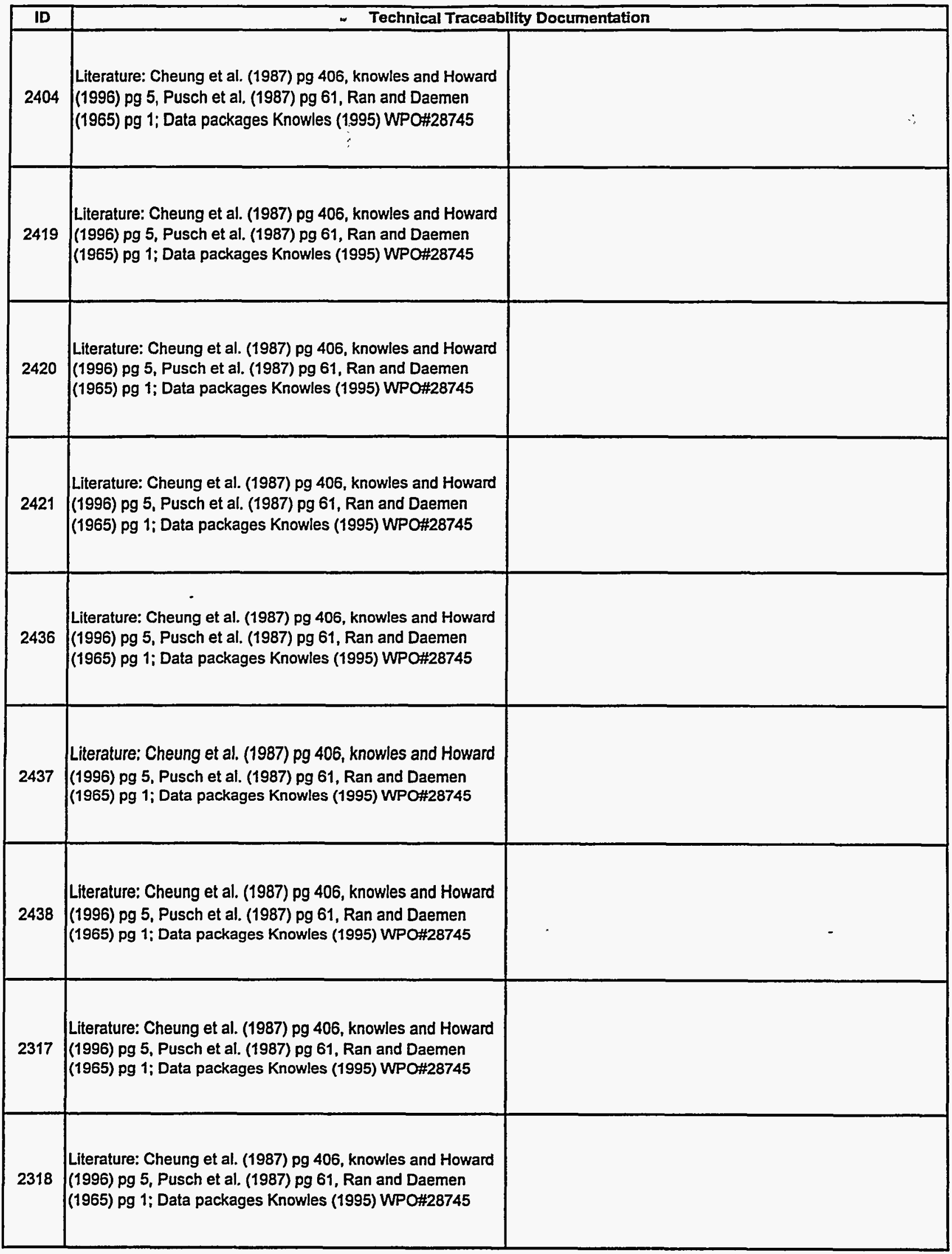




\section{Additional Sources}

\begin{tabular}{|c|c|c|c|c|}
\hline ID & Material ID & Param ID & \multicolumn{2}{|c|}{ Technical Traceability Documentation } \\
\hline 2319 & CLAY_BOT & PRMZ_LOG & $\begin{array}{l}\text { WPO\#41809 Sec 4.6 p.4-36. WPO\#30994 - } \\
\text { Contains tables of BRAGFLO parameters used } \\
\text { to model WIPP shaft seal system. Contains } \\
\text { listings of the extent of DRZ and figures } \\
\text { showing proposed seal design for WIPP Air } \\
\text { Intake Shaft. }\end{array}$ & $\begin{array}{l}\text { WPO\#30640: WIPP Shaft Seal System } \\
\text { BRAGFLO Parameters, SWCF. } \\
\text { A:1.1.03.2.1:QA:PDD Sec II Vol 2, 2.1.1; Sec I } \\
\text { Vol 4 4.2. }\end{array}$ \\
\hline 3147 & CONC_PLG & POROSITY & $\begin{array}{l}\text { WPO\#35134 Memo:Vaughn to Tierney } \\
\text { "Borehole Properties" } 3 / 13 / 96 " \text { (handwritten } 4 \\
\text { page note with parameter values - contains an } \\
\text { email cover page). }\end{array}$ & \\
\hline 3185 & CONC_PLG & PRMX_LOG & $\begin{array}{l}\text { WPO\#35134 Memo:Vaughn to Tiemey } \\
\text { "Borehole Properties" 3/13/96" (handwritten } 4 \\
\text { page note with parameter values - contains an } \\
\text { email cover page). }\end{array}$ & $\begin{array}{l}\text { Report WPO\#39626 Records and } \\
\text { documentation package for Permeability of } \\
\text { Borehole filled with Silty Sand. Section } 3.0 \\
\text { provides discussion on conceptual models of } \\
\text { plugged boreholes. Section } 6.0 \text { provides a } \\
\text { summary on the permeabilities. }\end{array}$ \\
\hline 3192 & CONC_PLG & PRMY_LOG & $\begin{array}{l}\text { WPO\#35134 Memo:Vaughn to Tiemey } \\
\text { "Borehole Properties" } 3 / 13 / 96^{n} \text { (handwritten } 4 \\
\text { page note with parameter values - contains an } \\
\text { email cover page). }\end{array}$ & $\begin{array}{l}\text { Report WPO\#39626 Records and } \\
\text { documentation package for Permeability of } \\
\text { Borehole filled with Silty Sand. Section } 3.0 \\
\text { provides discussion on conceptual models of } \\
\text { plugged boreholes. Section } 6.0 \text { provides a } \\
\text { summary on the permeabilities. }\end{array}$ \\
\hline 3193 & CONC_PLG & PRMZ_LOG & $\begin{array}{l}\text { WPO\#35134 Memo:Vaughn to Tierney } \\
\text { "Borehole Properties" } 3 / 13 / 96 " \text { (handwritten } 4 \\
\text { page note with parameter values - contains an } \\
\text { email cover page). }\end{array}$ & $\begin{array}{l}\text { Report WPO\#39626 Records and } \\
\text { documentation package for Permeability of } \\
\text { Borehole filled with Silty Sand. Section } 3.0 \\
\text { provides discussion on conceptual models of } \\
\text { plugged boreholes. Section } 6.0 \text { provides a } \\
\text { summary on the permeabilities. }\end{array}$ \\
\hline 2464 & CONC_T1 & COMP_RCK & $\begin{array}{l}\text { WPO\#30640: WIPP Shaft Seal System } \\
\text { BRAGFLO Parameters, SWCF- } \\
\text { A:1.1.03.2.1:QA:PDD Sec III Vol } 2 \text { 2.2.3; Sec } \\
\text { II Vol } 2 \text { 2.4.1; Sec II Vol } 2 \text { 2.3.3; Sec I Vol } 4 \\
\begin{array}{ll}\text { 4.4. } & \end{array}\end{array}$ & $\begin{array}{l}\text { Literature - RE/SPEC (1994) pg 3, Van } \\
\text { Sambeek (1987) pg } 9\end{array}$ \\
\hline 3148 & CONC_PLG & COMP_RCK & $\begin{array}{l}\text { WPO\#30640: WIPP Shaft Seal System } \\
\text { BRAGFLO Parameters, SWCF- } \\
\text { A:1.1.03.2.1:QA:PDD Sec III Vol } 2 \text { 2.2.3; Sec } \\
\text { II Vol } 2 \text { 2.4.1; Sec II Vol } 2 \text { 2.3.3; Sec I Vol } 4 \\
\text { 4.4. }\end{array}$ & $\begin{array}{l}\text { Literature - RE/SPEC (1994) pg 3, Van } \\
\text { Sambeek (1987) pg } 9\end{array}$ \\
\hline 2467 & CONC_T1 & POROSITY & 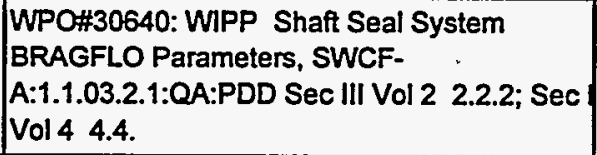 & $\begin{array}{l}\text { Literature: Dennis (1992) pg-2; Data Package } \\
\text { WPO\#28380 Wakely et al. (1995) }\end{array}$ \\
\hline 2470 & CONC_T1 & PRMX_LOG & $\begin{array}{l}\text { WPO\#30640: WIPP Shaft Seal System } \\
\text { BRAGFLO Parameters, SWCF. } \\
\text { A:1.1.03.2.1:QA:PDD Sec III Vol } 2 \text { 2.2.1; Sec I } \\
\text { Vol 4 4.4. }\end{array}$ & $\begin{array}{l}\text { Knowles et al. (1995), WPO \#39631 and } \\
\text { WPO\#39632; WPO\#41392 RE/SPEC (1996), } \\
\text { pg } 20\end{array}$ \\
\hline 2471 & CONC_T1 & PRMY_LOG & $\begin{array}{l}\text { WPO\#30640: WIPP Shaft Seal System } \\
\text { BRAGFLO Parameters, SWCF- } \\
\text { A:1.1.03.2.1:QA:PDD Sec III Vol } 2 \text { 2.2.1; Sec I } \\
\text { Vol 4 4.4. }\end{array}$ & $\begin{array}{l}\text { Knowles et al. (1995), WPO \#39631 and } \\
\text { WPO\#39632; WPO\#41392 RE/SPEC (1996), } \\
\text { pg } 20\end{array}$ \\
\hline 2472 & CONC_T1 & PRMZ_LOG & $\begin{array}{l}\text { WPO\#30640: WIPP Shaft Seal System } \\
\text { BRAGFLO Parameters, SWCF- } \\
\text { A:1.1.03.2.1:QA:PDD Sec III Vol } 2 \text { 2.2.1; Sec I } \\
\text { Vol 4 4.4. }\end{array}$ & $\begin{array}{l}\text { Knowles et al. (1995), WPO \#39631 and } \\
\text { WPO\#39632; WPO\#41392 REJSPEC (1996), } \\
\text { pg } 20\end{array}$ \\
\hline 120 & CULEBRA & COMP_RCK & $\begin{array}{l}\text { WPO\#45770 Freeze R.A. and J.A.Cherry } \\
\text { 1979, Groundwater. Englewood Cliffs, NJ } \\
\text { Prentice Hall, Inc. Table } 2.5 \text { p55. }\end{array}$ & \\
\hline
\end{tabular}


Additional Sources

\begin{tabular}{|c|c|c|}
\hline ID & \multicolumn{2}{|c|}{ Technical Traceability Documentation } \\
\hline 2319 & $\begin{array}{l}\text { Literature: Cheung et al. (1987) pg 406, knowles and Howard } \\
\text { (1996) pg 5, Pusch et al. (1987) pg 61, Ran and Daemen } \\
\text { (1965) pg 1; Data packages Knowles (1995) WPO\#28745 }\end{array}$ & \\
\hline 3147 & & \\
\hline 3185 & $\cdot$ & \\
\hline 3192 & & \\
\hline 3193 & $\cdot$ & \\
\hline 2464 & $\begin{array}{l}\text { WPO\#37738 Memo: Diane Hurtado to Martin Tiemey } \\
\text { "Modification of Concrete Compressibility Parameter" 4/26/96 } \\
\text { (located in WPO\#30640) }\end{array}$ & \\
\hline 3148 & $\begin{array}{l}\text { WPO\#37738 Memo: Diane Hurtado to Martin Tierney } \\
\text { "Modification of Concrete Compressibility Parameter" 4/26/96 } \\
\text { (located in WPO\#30640) }\end{array}$ & $\begin{array}{l}\text { WPO\#35134 Memo:Vaughn to Tiemey "Borehole Properties" } \\
\text { 3/13/96" (handwritten } 4 \text { page note with parameter values - } \\
\text { contains an email cover page) }\end{array}$ \\
\hline 2467 & Additional info WPO\#32572 & - \\
\hline 2470 & Additional info in WPO\#41809 Sec 4.6 & \\
\hline 2471 & Additional info in WPO\#41809 Sec 4.6 & \\
\hline 2472 & Additional info in WPO\#41809 Sec 4.6 & \\
\hline 120 & & \\
\hline
\end{tabular}


Additional Sources

\begin{tabular}{|c|c|c|c|c|}
\hline ID & Material ID & Param ID & \multicolumn{2}{|c|}{ Technical Traceability Documentation } \\
\hline 861 & CULEBRA & FTORT & $\begin{array}{l}\text { WPO\#40516(Analysis write-up for Culebra } \\
\text { flow and Transport) Appendix D Table D2. }\end{array}$ & $\begin{array}{l}\text { Memo: M. Wallace to M. Tiemey, } 5 / 2 / 96 \text {, } \\
\text { Distribution for Non-Salado Parameter for } \\
\text { SECOTP2D:Fracture Tortuosity (Distribution } \\
\text { for Non-Salado Parameter for } \\
\text { SECOTP2D:Fracture Tortuosity }\end{array}$ \\
\hline 139 & CULEBRA & PORE_DIS & $\begin{array}{l}\text { WPO\#31041 Memo:McCord to Tiemey } \\
1 / 12 / 96\end{array}$ & \\
\hline 140 & CULEBRA & POROSITY & $\begin{array}{l}\text { WPO\#30608 SWCF- } \\
\text { A:WBS1.2.07.01:PDD:QA:Nonsalado:Pkg } 22 \\
\text { NonSalado Effective Porosity }\end{array}$ & $\begin{array}{l}\text { WPO\#41247, pg 6-1 "WIPP Natural Barriers } \\
\text { Data Qualification Peer Review Report" states } \\
\text { porosity was determined from core analyses } \\
\text { and borehole data. }\end{array}$ \\
\hline 142 & CULEBRA & PRESSURE & $\begin{array}{l}\text { WPO\#30713 SWCF- } \\
\text { A:WBS1.2.07.01:PDD:QA:Nonsalado:Pkg } 24 \\
\text { NonSalado Pressure. WPO\#32290 Memo:M. } \\
\text { Tierney to L. Dotson "Initial Pressure } \\
\text { Parameter Values for NonSalado" 2/1/96. }\end{array}$ & $\begin{array}{l}\text { WPO\#41247, pg 6-8 "WIPP Natural Barriers } \\
\text { Data Qualification Peer Review Report" states } \\
\text { pore pressure tests were conducted on several } \\
\text { isolated well units to determine value. }\end{array}$ \\
\hline 143 & CULEBRA & PRMX_LOG & $\begin{array}{l}\text { PC_MAX (1.0E8) is a large number chosen to } \\
\text { represent infinity in a modeling sense. }\end{array}$ & $\begin{array}{l}\text { WPO\#31167, Documentation attached to } \\
\text { WPO\#'s } 32775,32776 \text {, and } 32777 \text { respectively }\end{array}$ \\
\hline 144 & CULEBRA & PRMY_LOG & $\begin{array}{l}\text { PC_MAX (1.0E } 8) \text { is a large number chosen to } \\
\text { represent infinity in a modeling sense. }\end{array}$ & $\begin{array}{l}\text { WPO\#31167, Documentation attached to } \\
\text { WPO\#'s } 32775,32776 \text {, and } 32777 \text { respectively }\end{array}$ \\
\hline 145 & CULEBRA & PRMZ_LOG & $\begin{array}{l}\text { PC_MAX (1.OE8) is a large number chosen to } \\
\text { represent infinity in a modeling sense. }\end{array}$ & $\begin{array}{l}\text { WPO\#31167, Documentation attached to } \\
\text { WPO\#'s } 32775,32776 \text {, and } 32777 \text { respectively }\end{array}$ \\
\hline 3487 & CULEBRA & APOROS & $\begin{array}{l}\text { WPO\#41247, pg 6-61 "WIPP Natural Barriers } \\
\text { Data Qualification Peer Review Report" states } \\
\text { this value was derived from } 3 \text { steps, 1) trace } \\
\text { test in field, 2) numerical simulations, 3) } \\
\text { integration of simulation results to generate } \\
\text { value for SECOTP. }\end{array}$ & PI PRP 37227 \\
\hline 3484 & CULEBRA & DISPT_L & PRP 37231 & PRP WPO\#37230, WPO\#42672 \\
\hline 3483 & CULEBRA & DISP_L & PRP 37231 & PRP WPO\#37230, WPO\#42672 \\
\hline 3486 & CULEBRA & DPOROS & $\begin{array}{l}\text { WPO\#38773 Memo:Lucy Meigs to Jim } \\
\text { Ramsey June 6, } 1996 \text { See also WPO\#37228 } \\
\text { and WPO\#38357. }\end{array}$ & $\begin{array}{l}\text { WPO } 41247 \text {, pg 6-76 "WIPP Natural Barriers } \\
\text { Data Qualification Peer Review Report" states } \\
\text { this value was derived based on laboratory } \\
\text { measurements of } 100 \text { core samples from } 21 \\
\text { boreholes. }\end{array}$ \\
\hline 3474 & CULEBRA & DTORT & $\begin{array}{l}\text { WPO\#37226 SWCF- } \\
\text { A:WBS1.2.07.1:PDD:QA:NonSalado:Culebra } \\
\text { Diffusive Tortuosity ....WPO\#38940 } \\
\text { Memo:Lucy Meigs to Jim Ramsey "Nonsalado } \\
\text { diffusive tortuosity for the Culebra Dolomite" } \\
\text { May 16, 1996. }\end{array}$ & $\begin{array}{l}\text { WPO\#41247, pg 6-80 "WIPP Natural Barriers } \\
\text { Data Qualification Peer Review Report" states } \\
\text { this value was derived based on } 36 \text { core } \\
\text { analyses reported in SAND90-7011 and from } \\
\text { H19b4 cores. Value is the median of the } 13 \\
\text { borehole average tortuosity values. }\end{array}$ \\
\hline
\end{tabular}




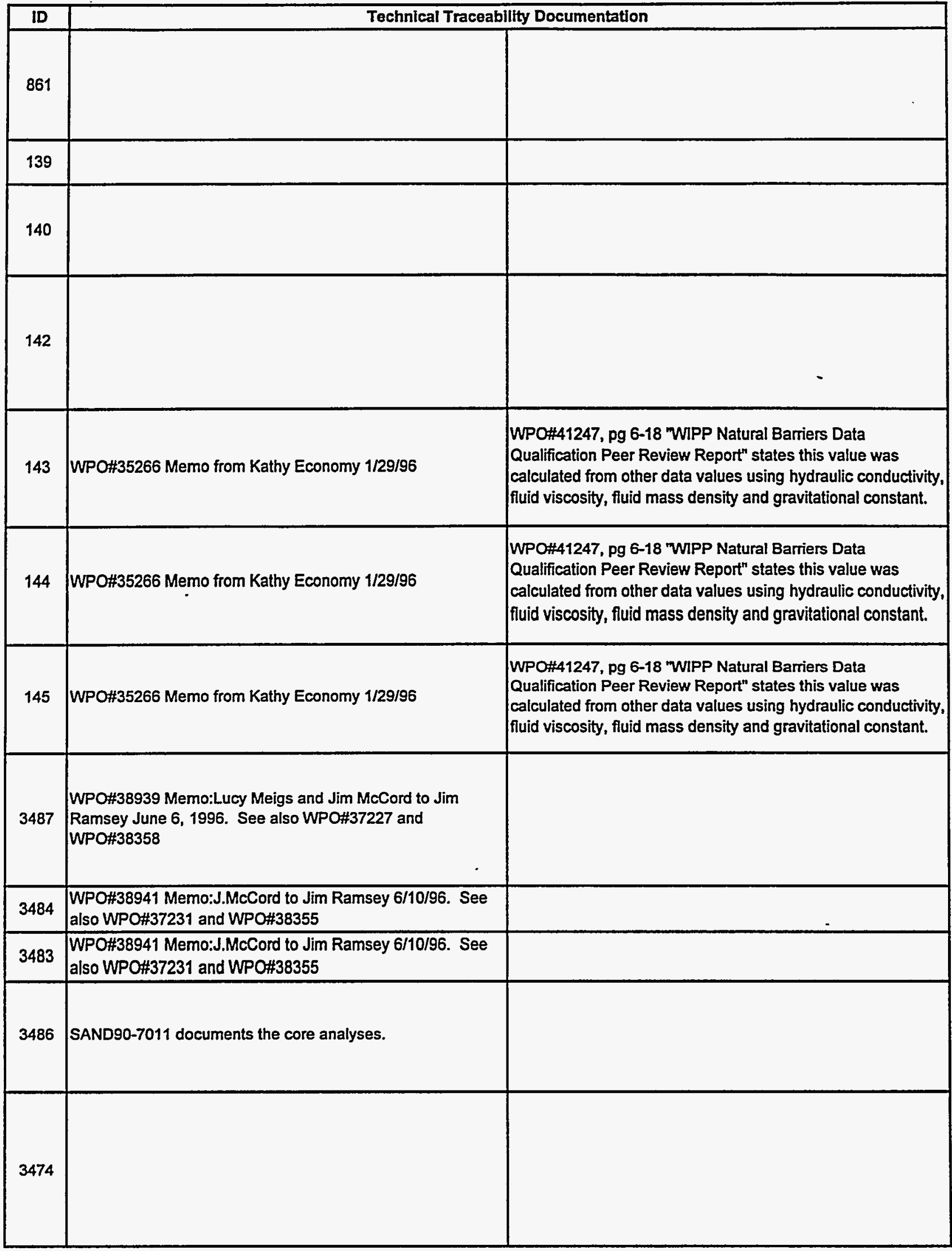


Additional Sources

\begin{tabular}{|c|c|c|c|c|}
\hline ID & Material ID & Param ID & \multicolumn{2}{|c|}{ Technical Traceability Documentation } \\
\hline 3485 & CULEBRA & HMBLKLT & $\begin{array}{l}\text { WPO\#38928 Memo:Lucy Meigs and Jim } \\
\text { McCord to Jim Ramsey June 7, } 1996 \text { See also } \\
\text { WPO\#37225, and WPO\#38356 }\end{array}$ & $\begin{array}{l}\text { WPO\#41247, pg 6-69 "WIPP Natural Barriers } \\
\text { Data Qualification Peer Review Report" states } \\
\text { this value was derived from } 3 \text { steps, 1) trace } \\
\text { test in field, 2) numerical simulations, 3) } \\
\text { integration of simulation results to generate } \\
\text { value for SECOTP. }\end{array}$ \\
\hline 3419 & CULEBRA & MINP_FAC & $\begin{array}{l}\text { WPO\#36489 memo:M. Wallace to M. Tiemey } \\
\text { "Distribution for NonSalado parameter for } \\
\text { SECOFL2D: Mining Transmissivity Multiplier" } \\
\text { 4/18/96 See also WPO\#39355. }\end{array}$ & \\
\hline 158 & DEWYLAKE & POROSITY & $\begin{array}{l}\text { Terra Tek AA-2896 Data Package } \\
\text { WPO\#38234 }\end{array}$ & $\begin{array}{l}\text { WPO\#41247, pg 6-1 WIPP Natural Barriers } \\
\text { Data Qualification Peer Review Report" states } \\
\text { porosity was determined from core analyses } \\
\text { and borehole data. }\end{array}$ \\
\hline 161 & DEWYLAKE & PRMX_LOG & $\begin{array}{l}\text { WPO\#30607, SWCF- } \\
\text { A:1.2.07.1:PDD:QA:PKG.\#2:Non-Salado } \\
\text { Permeability, p.4 (Calculations on } 464 \text { Forms } \\
\text { WPO\#'s 32734, 32735, } 32736 \text {, Raw data } \\
\text { contained in WPO\#38234 respectively). }\end{array}$ & $\begin{array}{l}\text { WPO\#43088 Memo:Vaughn to Tiemey } \\
\text { 2/22/96 "Dewey lake logs of intrinsic }\end{array}$ \\
\hline 162 & DEWYLAKE & PRMY_LOG & $\begin{array}{l}\text { WPO\#30607, SWCF- } \\
\text { A:1.2.07.1:PDD:QA:PKG.\#2:Non-Salado } \\
\text { Permeability, p.4 (Calculations on } 464 \text { Forms } \\
\text { WPO\#'s 32734, 32735, 32736, Raw data } \\
\text { contained in WPO\#38234 respectively). }\end{array}$ & $\begin{array}{l}\text { WPO\#43088 Memo:Vaughn to Tierney } \\
\text { 2/22/96 "Dewey lake logs of intrinsic". }\end{array}$ \\
\hline 163 & DEWYLAKE & PRMZ_LOG & $\begin{array}{l}\text { WPO\#30607, SWCF- } \\
\text { A:1.2.07.1:PDD:QA:PKG.\#2:Non-Salado } \\
\text { Permeability, p.4 (Calculations on } 464 \text { Forms } \\
\text { WPO\#'s 32734, 32735, 32736, Raw data } \\
\text { contained in WPO\#38234 respectively). }\end{array}$ & $\begin{array}{l}\text { WPO } 43088 \text { Memo:Vaughn to Tierney } \\
\text { 2/22/96 "Dewey lake logs of intrinsic ". }\end{array}$ \\
\hline 167 & DEWYLAKE & SAL_USAT & $\begin{array}{l}\text { WPO\#40514 (the BRAGFLO analysis write-up) } \\
\text { Sec. 4.2.4 pg 4-170, and discussion on Dewey } \\
\text { Lake Sec 3.2.2.4 pg 3-13. }\end{array}$ & \\
\hline 171 & DRILLMUD & DNSFLUID & $\begin{array}{l}\text { Analysis Package for the Cuttings and Spalling } \\
\text { Calculations WPO\#40521- Section "Input } \\
\text { Parameters and Sources" pg } 39 .\end{array}$ & $\begin{array}{l}\text { DRILLMUD DNSFLUED } 5=1210 \mathrm{~kg} / \mathrm{m} 3 \text {. The } \\
\text { 1992 PA (SAND92-0700/3) range pg } 4-1 \text { is } \\
1.139 E 3 \text { to } 1.378 E 3 \mathrm{~kg} / \mathrm{m} 3 \text {, with a median of } \\
\text { 1.211E3. Reference for } 92 \text { PA is SAND89- } \\
2408 \text { Pace, 1990. INGRES DB shows median } \\
\text { as 1.21E3 (28 Jun 91) in agreement with value } \\
\text { used. }\end{array}$ \\
\hline 172 & DRILLMUD & VISCO & $\begin{array}{l}\text { Analysis Package for the Cuttings and Spalling } \\
\text { Calculations WPO\#40521- Section "Input } \\
\text { Parameters and Sources" pg } 39 .\end{array}$ & $\begin{array}{l}\text { DRILLMUD VISCO hO=0.00917 (Pa). The } \\
1992 \text { PA range is (pg 4-1) 5E-3 to 3E-2 Pa, } \\
\text { with a median of 9.17E-3. The } 92 \mathrm{PA} \\
\text { reference is SAND89-2408 pg A-159-A170, } \\
\text { Pace, 1990. See also EPA CCA Review } \\
\text { Personnel Request Form Joel Miller 4/16/97. }\end{array}$ \\
\hline 173 & DRILLMUD & YLDSTRSS & $\begin{array}{l}\text { Analysis Package for the Cuttings and Spalling } \\
\text { Calculations WPO\#40521- Section "Input } \\
\text { Parameters and Sources" pg } 39 .\end{array}$ & $\begin{array}{l}\text { DRILLMUD YLDSTRSS } y=4.4(\mathrm{~Pa}) \text {. The } \\
1992 \mathrm{PA} \text { range (pg 4-1) is } 2.4 \text { to } 19.2 \mathrm{~Pa} \text {, with } \\
\text { a median of } 4 \text {. The } 92 \mathrm{PA} \text { reference is } \\
\text { SAND89-2408 Pace, } 1990 \text {. INGRES DB } \\
\text { shows median as } 4.4 \text { ( } 28 \text { Jun } 91 \text { ) in } \\
\text { accordance with value used. }\end{array}$ \\
\hline
\end{tabular}


Additional Sources

\begin{tabular}{|c|c|c|}
\hline ID & \multicolumn{2}{|c|}{ Technical Traceability Documentation } \\
\hline 3485 & 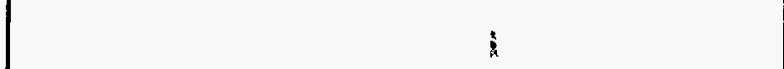 & \\
\hline 3419 & & \\
\hline 158 & & \\
\hline 161 & $\begin{array}{l}\text { WPO\#41247, pg 6-14 "WIPP Natural Barriers Data } \\
\text { Qualification Peer Review Report" states transmissivity was } \\
\text { derived from one of transmissivity test results obtained from } \\
\text { USGS } 83-4016 \text { report. }\end{array}$ & \\
\hline 162 & $\begin{array}{l}\text { WPO\#41247, pg 6-14 "WIPP Natural Barriers Data } \\
\text { Qualification Peer Review Report" states transmissivity was } \\
\text { derived from one of transmissivity test results obtained from } \\
\text { USGS } 83-4016 \text { report. }\end{array}$ & \\
\hline 163 & $\begin{array}{l}\text { WPO\#41247, pg 6-14 "WIPP Natural Barriers Data } \\
\text { Qualification Peer Review Report" states transmissivity was } \\
\text { derived from one of transmissivity test results obtained from } \\
\text { USGS } 83-4016 \text { report. }\end{array}$ & \\
\hline 167 & & \\
\hline 171 & $\begin{array}{l}\text { WPO\#38568 states this is a 'legacy parameter' whose value } \\
\text { appeared in the baseline WIPP Parameter database of March } \\
2,1996 .\end{array}$ & - \\
\hline 172 & $\begin{array}{l}\text { Drillmud viscosity is traced back to } 92 \text { PA page A-169 or } \\
\text { SAND89-2408. See EPA CCA Review Personnel Request } \\
\text { Form 4/18/97 by Joel Miller }\end{array}$ & $\begin{array}{l}\text { SAND92-0700/3.WPO\#38568 states this is a "legacy } \\
\text { parameter' whose value appeared in the baseline WIPP } \\
\text { Parameter database of March } 2,1996 \text {. }\end{array}$ \\
\hline 173 & $\begin{array}{l}\text { Drillmud yldstress is traced back to } 92 \text { PA page A-169 or } \\
\text { SAND89-2408. See also EPA CCA Review Personnel } \\
\text { Request Form 4/18/97 by Joel Miller }\end{array}$ & $\begin{array}{l}\text { SAND92-0700/3.WPO\#38568 states this is a 'legacy } \\
\text { parameter' whose value appeared in the baseline WIPP } \\
\text { Parameter database of March } 2.1996 \text {. }\end{array}$ \\
\hline
\end{tabular}


Additional Sources

\begin{tabular}{|c|c|c|c|c|}
\hline ID & Material ID & Param ID & \multicolumn{2}{|c|}{ Technical Traceability Documentation } \\
\hline 178 & DRZ_ó & POROSITY & $\begin{array}{l}\text { WPO\#32288 Memo: P. Vaughn to M. Chu } \\
\text { "Revision to DRZ and TZ properties to be used } \\
\text { in BRAGFLO CCA calculation" } 1 / 24 / 96 \text {. }\end{array}$ & \\
\hline 195 & DRZ_1 & POROSITY & $\begin{array}{l}\text { WPO\#32288 Memo: P. Vaughn to M. Chu } \\
\text { "Revision to DRZ and TZ properties to be used } \\
\text { in BRAGFLO CCA calculation" } 1 / 24 / 96 \text {. }\end{array}$ & \\
\hline 181 & $D R Z$ D & PRMX_LOG & $\begin{array}{l}\text { WPO\#32288 Memo: P. Vaughn to M. Chu } \\
\text { "Revision to DRZ and TZ properties to be used } \\
\text { in BRAGFLO CCA calculation" } 1 / 24 / 96 \text {. }\end{array}$ & $\begin{array}{l}\text { WPO\#38568 Memo: M. Tiemey and P. } \\
\text { Vaughn to file "Designation of Legacy } \\
\text { Parameters and Placeholders in WIPP } \\
\text { Parameter Database" 6/17/96 states this is a } \\
\text { "legacy parameter' whose value appeared in } \\
\text { the baseline WIPP Parameter database of } \\
\text { March 2,1996. }\end{array}$ \\
\hline 182 & DRZ_0 & PRMY_LOG & $\begin{array}{l}\text { WPO\#32288 Memo: P. Vaughn to M. Chu } \\
\text { "Revision to DRZ and TZ properties to be used } \\
\text { in BRAGFLO CCA calculation" } 1 / 24 / 96 \text {. }\end{array}$ & $\begin{array}{l}\text { WPO\#38568 Memo: M. Tiemey and P. } \\
\text { Vaughn to file "Designation of Legacy } \\
\text { Parameters and Placeholders in WIPP } \\
\text { Parameter Database" } 6 / 17 / 96 \text { states this is a } \\
\text { 'legacy parameter' whose value appeared in } \\
\text { the baseline WIPP Parameter database of } \\
\text { March } 2,1996 \text {. }\end{array}$ \\
\hline 183 & DRZ_0 & 'PRMZ_LOG & $\begin{array}{l}\text { WPO\#32288 Memo: P. Vaughn to M. Chu } \\
\text { "Revision to DRZ and TZ properties to be used } \\
\text { in BRAGFLO CCA calculation" } 1 / 24 / 96 \text {. }\end{array}$ & $\begin{array}{l}\text { WPO\#38568 Memo: M. Tiemey and P. } \\
\text { Vaughn to file "Designation of Legacy } \\
\text { Parameters and Placeholders in WIPP } \\
\text { Parameter Database" 6/17/96 states this is a } \\
\text { 'legacy parameter' whose value appeared in } \\
\text { the baseline WIPP Parameter database of } \\
\text { March 2,1996. }\end{array}$ \\
\hline 198 & DRZ_1 & PRMX_LOG & $\begin{array}{l}\text { WPO\#32288 Memo: P. Vaughn to M. Chu } \\
\text { "Revision to DRZ and TZ properties to be used } \\
\text { in BRAGFLO CCA calculation" } 1 / 24 / 96 \text {. }\end{array}$ & $\begin{array}{l}\text { WPO\#38568 Memo: M. Tiemey and P. } \\
\text { Vaughn to file "Designation of Legacy } \\
\text { Parameters and Placeholders in WIPP } \\
\text { Parameter Database" 6/17/96 states this is a } \\
\text { "legacy parameter' whose value appeared in } \\
\text { the baseline WIPP Parameter database of } \\
\text { March } 2,1996 .\end{array}$ \\
\hline 199 & DRZ_1 & PRMY_LOG & $\begin{array}{l}\text { WPO\#32288 Memo: P. Vaughn to M. Chu } \\
\text { "Revision to DRZ and TZ properties to be used } \\
\text { in BRAGFLO CCA calculation" 1/24/96. }\end{array}$ & $\begin{array}{l}\text { WPO\#38568 Memo: M. Tiemey and P. } \\
\text { Vaughn to file "Designation of Legacy } \\
\text { Parameters and Placeholders in WIPP } \\
\text { Parameter Database" 6/17/96 states this is a } \\
\text { "legacy parameter' whose value appeared in } \\
\text { the baseline WIPP Parameter database of } \\
\text { March 2,1996. }\end{array}$ \\
\hline 200 & DRZ_1 & PRMZ_LOG & $\begin{array}{l}\text { WPO\#32288 Memo: P. Vaughn to M. Chu } \\
\text { "Revision to DRZ and TZ properties to be used } \\
\text { in BRAGFLO CCA calculation" 1/24/96. }\end{array}$ & $\begin{array}{l}\text { WPO\#38568 Memo: M. Tierney and P. } \\
\text { Vaughn to file "Designation of Legacy } \\
\text { Parameters and Placeholders in WIPP } \\
\text { Parameter Database" } 6 / 17 / 96 \text { states this is a } \\
\text { 'legacy parameter' whose value appeared in } \\
\text { the baseline WIPP Parameter database of } \\
\text { March } 2,1996 \text {. }\end{array}$ \\
\hline 223 & GLOBAL & CLIMTIDX & $\begin{array}{l}\text { WPO\#33031 Memo from T. Corbet and P. } \\
\text { Swift to M. Tiemey, } 3 / 12 / 96 \text { (attached to } 464 \\
\text { form). Supporting documentation is in } \\
\text { WPO\#36425. }\end{array}$ & $\begin{array}{l}\text { WPO\#41247, pg 6-22 "WIPP Natural Barriers } \\
\text { Data Qualification Peer Review Report" states } \\
\text { this value was derived from numerous } \\
\text { computer runs using different values of } \\
\text { infiltration and hydraulic conductivities. }\end{array}$ \\
\hline
\end{tabular}


Additional-Sources

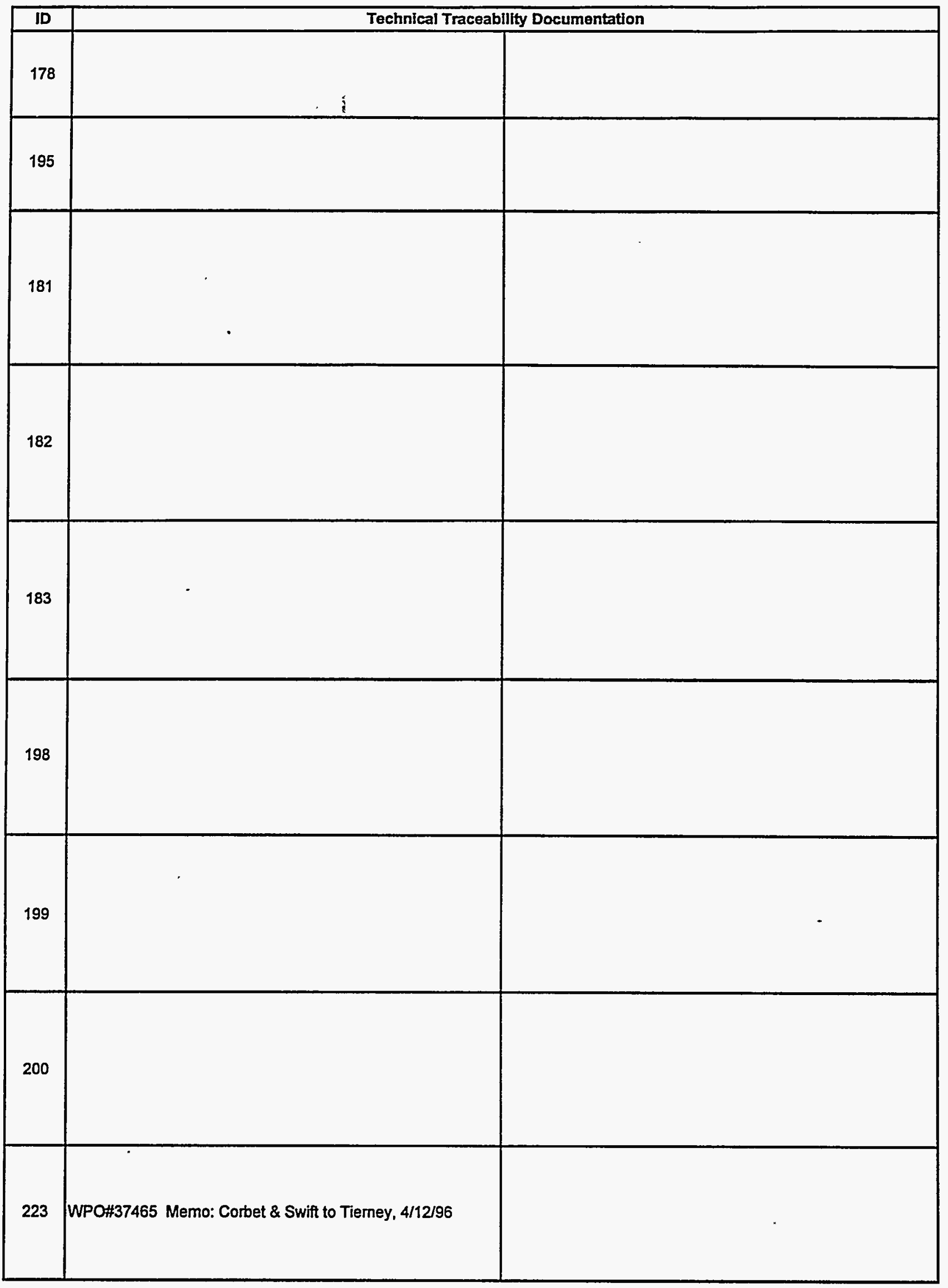


Additional Sources

\begin{tabular}{|c|c|c|c|c|}
\hline ID & Material ID & Param ID & \multicolumn{2}{|c|}{ Technical Traceability Documentation } \\
\hline 225 & GLOBAL & TRANSIDX & $\begin{array}{l}\text { WPO\#35193, Memo from Greg Ruskauff to } \\
\text { Martin Tiemey, 3/13/96, "Culebra } \\
\text { Transmissivity Field Index, GLOBAL, } \\
\text { TRANSIDX. Legacy parameter value same as } \\
\text { in WPO\#38568 SAND92-0700/3 p. 2-86- } \\
\text { IDXTRANS parameter. }\end{array}$ & PI PRP WPO\#36425 \\
\hline 3501 & GLOBAL & FPICD & $\begin{array}{l}\text { WPO\#42852 WIPP/CAO-96-3168, Chapter 6, } \\
\text { 4/19/96, Effectiveness of Passive Institutional } \\
\text { Controls in Reducing Inadvertent Human } \\
\text { Intrusion into the WIPP for Use in Performance } \\
\text { Assessments. }\end{array}$ & \\
\hline 3500 & GLOBAL & FPICM & $\begin{array}{l}\text { WIPP/CAO-96-3168, Chapter 6, 4/19/96, } \\
\text { Effectiveness of Passive Institutional Controls } \\
\text { in Reducing Inadvertent Human Intrusion into } \\
\text { the WIPP for Use in Performance } \\
\text { Assessments. }\end{array}$ & \\
\hline 3497 & GLOBAL & MINERT & $\begin{array}{l}\text { Federal Register Vol } 61 \text { No. } 28 \text { 40CFR194 } \\
\text { Section } 194.32 \text { parag (b). }\end{array}$ & \\
\hline 3417 & GLOBAL & OXSTAT & $\begin{array}{l}\text { WPO\#37536 memo:C. Stockman to M. } \\
\text { Tiemey "Reducing the number of sampled } \\
\text { parameters" } 4 / 16 / 96 \text {. }\end{array}$ & \\
\hline 3493 & GLOBAL & PBRINE & $\begin{array}{l}\text { WPO\#40199 contains WPO\#40849 } \\
\text { memo:R.J.Leonard to Mel Marietta 6/13/96 } \\
\text { and WPO\#40850 M.S.Y.Chu to SWCF_A } \\
\text { Record Center (both memos are located in } \\
464 \text { Form). }\end{array}$ & \\
\hline 233 & IMPERM_Z & POROSITY & $\begin{array}{l}\text { WPO\#38568 Memo: M. Tiemey and P. } \\
\text { Vaughn to file "Designation of Legacy } \\
\text { Parameters and Placeholders in WIPP } \\
\text { Parameter Database" 6/17/96 }\end{array}$ & $\begin{array}{l}\text { WPO\#38568 states this is a 'placeholder } \\
\text { parameter' whose value is not used by the } \\
\text { BRAGFLO code, but is left in the database to } \\
\text { facilitate the correct reading of all parameters } \\
\text { for BRAGFLO from the SCMS files. }\end{array}$ \\
\hline 2100 & MAGENTA & POROSITY & $\begin{array}{l}\text { WPO\#30608 SWCF-A:WBS } \\
\text { 1.2.07.1:PDD:QA:NonSalado:Pkg } 22 \\
\text { NonSalado Effective Porosity. }\end{array}$ & $\begin{array}{l}\text { WPO\#41247, pg 6-1 "WIPP Natural Barriers } \\
\text { Data Qualification Peer Review Report" states } \\
\text { porosity was determined from core analyses } \\
\text { and borehole data. }\end{array}$ \\
\hline 2102 & MAGENTA & PRMX_LOG & $\begin{array}{l}\text { WPO\#30607, SWCF- } \\
\text { A:1.2.07.1:PDD:QA:PKG\#2:Non-Salado } \\
\text { Permeability p.4. Raw data contained in } \\
\text { WPO\#38234. }\end{array}$ & $\begin{array}{l}\text { WPO\#41247, pg 6-14 "WIPP Natural Barriers } \\
\text { Data Qualification Peer Review Report" states } \\
\text { transmissivity was derived form one of } \\
\text { transmissivity test results obtained from USGS } \\
83-4016 \text { report. }\end{array}$ \\
\hline 2103 & MAGENTA & PRMY_LOG & $\begin{array}{l}\text { WPO\#30607, SWCF- } \\
\text { A:1.2.07.1:PDD:QA:PKG\#2:Non-Salado } \\
\text { Permeability p.4. Raw data contained in } \\
\text { WPO\#38234. }\end{array}$ & $\begin{array}{l}\text { WPO } 41247, \text { pg 6-14 "WIPP Natural Barriers } \\
\text { Data Qualification Peer Review Report" states } \\
\text { transmissivity was derived form one of } \\
\text { transmissivity test results obtained from USGS } \\
83-4016 \text { report. }\end{array}$ \\
\hline 2104 & MAGENTA & PRMZ_LOG & $\begin{array}{l}\text { WPO\#30607, SWCF- } \\
\text { A:1.2.07.1:PDD:QA:PKG\#2:Non-Salado } \\
\text { Permeability p.4. Raw data contained in } \\
\text { WPO\#38234. }\end{array}$ & $\begin{array}{l}\text { WPO\#41247, pg 6-14 "WIPP Natural Barriers } \\
\text { Data Qualification Peer Review Report" states } \\
\text { transmissivity was derived form one of } \\
\text { transmissivity test results obtained from USGS } \\
83-4016 \text { report. }\end{array}$ \\
\hline 253 & PAN_SEAL & COMP_RCK & $\begin{array}{l}\text { WPO\#40514 pg 4-146 (the BRAGFLO } \\
\text { analysis write-up) }\end{array}$ & $\begin{array}{l}\text { Reason this is different from values used for } \\
\text { concrete shaft seal members is not clear. } \\
\text { They use } 1.2 E-9 \text { and the engineered Systems } \\
\text { peer panel recommended } 0.9 e-9 \text { (although } \\
\text { value } 1.2 e-9 \text { was acceptable with no impact) }\end{array}$ \\
\hline
\end{tabular}




\begin{tabular}{|c|c|c|}
\hline ID & \multicolumn{2}{|c|}{ Technlcal Traceabllity Documentation } \\
\hline 225 & $\begin{array}{l}\text { WPO\#38568 states this is a 'legacy parameter' whose value } \\
\text { appeared in the baseline WIPP Parameter database of March } \\
2,1996 \text {. }\end{array}$ & \\
\hline 3501 & & \\
\hline 3500 & & \\
\hline 3497 & & \\
\hline 3417 & & \\
\hline 3493 & & \\
\hline 233 & & \\
\hline 2100 & & \\
\hline 2102 & & \\
\hline 2103 & & \\
\hline 2104 & & \\
\hline 253 & $\begin{array}{l}\text { Value used for PAN_SEAL COMP_RCK (2.64E-9) was based } \\
\text { on early memorandum provided by PI. A revised value (1.2E- } \\
\text { 9) was provided by PI after completion of calculation. }\end{array}$ & Vaughn worksheet, no date ,Panel Seal \\
\hline
\end{tabular}




\section{Additional Sources}

\begin{tabular}{|c|c|c|c|c|}
\hline ID & Material ID & Param ID & \multicolumn{2}{|c|}{ Technical Traceability Documentation } \\
\hline 256 & PAN_SEAL & POROSITY & $\begin{array}{l}\text { Value for PAN_SEAL is from WPO\#40514 pg } \\
\text { 4-158 (the BRAGFLO analysis write-up). } \\
\text { Reason it is different from values used for } \\
\text { concrete shaft seal members (0.05) is not } \\
\text { clear. WPO\#32278, a concurrence memo from } \\
\text { Knowles and Tillerson to Vaughn } 2 / 8 / 96 \\
\text { "Panel.." }\end{array}$ & $\begin{array}{l}\text { A concurrence memo (WPO\#32278) from } \\
\text { Knowles and Tillerson to Vaughn 2/8/96 } \\
\text { "Panel Seal Porosity" provides some } \\
\text { discussion. }\end{array}$ \\
\hline 259 & PAN_SEAL & PRMX_LOG & $\begin{array}{l}\text { WPO\#40514 pg 4-160 (the BRAGFLO } \\
\text { analysis write-up) }\end{array}$ & $\begin{array}{l}\text { PAN_SEAL: PRMX_LOG=15 log (m2) Also } \\
\text { Sec 3.2.6 pg 3-17 discusses panel closure. }\end{array}$ \\
\hline 260 & PAN_SEAL & PRMY_LOG & $\begin{array}{l}\text { WPO } 40514 \text { pg 4-160 (the BRAGFLO } \\
\text { analysis write-up) }\end{array}$ & $\begin{array}{l}\text { PAN_SEAL: PRMX_LOG=-15 log (m2) Also } \\
\text { Sec 3.2.6 pg 3-17 discusses panel closure. }\end{array}$ \\
\hline 261 & PAN_SEAL & PRMZ_LOG & $\begin{array}{l}\text { WPO\#40514 pg 4-160 (the BRAGFLO } \\
\text { analysis write-up) }\end{array}$ & $\begin{array}{l}\text { PAN_SEAL: PRMX_LOG=-15 log (m2) Also } \\
\text { Sec 3.2.6 pg 3-17 discusses panel closure. }\end{array}$ \\
\hline 265 & PAN_SEAL & SAT_RBRN & $\begin{array}{l}\text { WPO\#40514 pg 4-172 (the BRAGFLO } \\
\text { analysis write-up) }\end{array}$ & $\begin{array}{l}\text { PAN_SEAL:SAT_RBRN }=0.20 \text {. This is linked } \\
\text { to value of SAT_RBRN for CONC_T1 which is } \\
\text { traceable through WPO } 30640 \text { Shaft Seals } \\
\text { Parameter Package. }\end{array}$ \\
\hline 3429 & PHUMOX3 & PHUMCIM & $\begin{array}{l}\text { WPO\#42261 Memo: Hans Papenguth to } \\
\text { Christine Stockman "Colloidal Actinide Source } \\
\text { Term Parameters Rev. 2" } 4 / 22 / 96 \text { (located in } \\
\text { Form 464). }\end{array}$ & $\begin{array}{l}\text { Package WPO\#35852 contains WPO\#37522 } \\
\text { memo from Papenguth to Stockman "Colloidal } \\
\text { Actinide Source Term Parameters Rev. 2" } \\
4 / 22 / 96\end{array}$ \\
\hline 3433 & PHUMOX3 & PHUMSIM & $\begin{array}{l}\text { WPO\#42261 Memo: Hans Papenguth to } \\
\text { Christine Stockman "Colloidal Actinide Source } \\
\text { Term Parameters Rev. 2" 4/22/96 (located in } \\
\text { Form 464). }\end{array}$ & $\begin{array}{l}\text { Package WPO\#35852 contains WPO\#37522 } \\
\text { memo from Papenguth to Stockman "Colloidal } \\
\text { Actinide Source Term Parameters Rev. } 2^{\text {" }} \\
4 / 22 / 96\end{array}$ \\
\hline 3459 & PU & CAPHUM & $\begin{array}{l}\text { WPO\#42261 Memo: Hans Papenguth to } \\
\text { Christine Stockman "Colloidal Actinide Source } \\
\text { Term Parameters Rev. 2" 4/22/96 (located in } \\
\text { Form 464). }\end{array}$ & $\begin{array}{l}\text { Package WPO\#35852 contains WPO\#37522 } \\
\text { memo from Papenguth to Stockman "Colloidal } \\
\text { Actinide Source Term Parameters Rev. 2" } \\
4 / 22 / 96\end{array}$ \\
\hline 3457 & $A M$ & CAPHUM & $\begin{array}{l}\text { WPO\#42261 Memo: Hans Papenguth to } \\
\text { Christine Stockman "Colloidal Actinide Source } \\
\text { Term Parameters Rev. 2" } 4 / 22 / 96 \text { (located in } \\
\text { Form 464). }\end{array}$ & $\begin{array}{l}\text { Package WPO\#35852 contains WPO\#37522 } \\
\text { memo from Papenguth to Stockman "Colloidal } \\
\text { Actinide Source Term Parameters Rev. } \text { 2" }^{\text {" }} \\
4 / 22 / 96\end{array}$ \\
\hline 3458 & NP & CAPHUM & $\begin{array}{l}\text { WPO\#42261 Memo: Hans Papenguth to } \\
\text { Christine Stockman "Colloidal Actinide Source } \\
\text { Term Parameters Rev. 2" } 4 / 22 / 96 \text { (located in } \\
\text { Form 464). }\end{array}$ & $\begin{array}{l}\text { Package WPO\#35852 contains WPO\#37522 } \\
\text { memo from Papenguth to Stockman "Colloidal } \\
\text { Actinide Source Term Parameters Rev. 2" } \\
4 / 22 / 96\end{array}$ \\
\hline 3460 & $\mathrm{U}$ & CAPHUM & $\begin{array}{l}\text { WPO\#42261 Memo: Hans Papenguth to } \\
\text { Christine Stockman "Colloidal Actinide Source } \\
\text { Term Parameters Rev. } 2^{n} 4 / 22 / 96 \text { (located in } \\
\text { Form 464). }\end{array}$ & $\begin{array}{l}\text { Package WPO\#35852 contains WPO\#37522 } \\
\text { memo from Papenguth to Stockman "Colloidal } \\
\text { Actinide Source Term Parameters Rev. 2" } \\
4 / 22 / 96\end{array}$ \\
\hline 3461 & TH & CAPHUM & $\begin{array}{l}\text { WPO\#42261 Memo: Hans Papenguth to } \\
\text { Christine Stockman "Colloidal Actinide Source } \\
\text { Term Parameters Rev. 2" 4/22/96 (located in } \\
\text { Form 464). }\end{array}$ & $\begin{array}{l}\text { Package WPO\#35852 contains WPO\#37522 } \\
\text { memo from Papenguth to Stockman "Colloidal } \\
\text { Actinide Source Term Parameters Rev. 2" } \\
4 / 22 / 96\end{array}$ \\
\hline 3315 & PU & CAPMIC & $\begin{array}{l}\text { WPO\#36771 Memo: Hans Papenguth to } \\
\text { Christine Stockman "Colloidal Actinide Source } \\
\text { Term Parameters " } 3 / 29 / 96 \text {. }\end{array}$ & \\
\hline 3316 & PU & CONCINT & $\begin{array}{l}\text { WPO\#36771 Memo: Hans Papenguth to } \\
\text { Christine Stockman "Colloidal Actinide Source } \\
\text { Term Parameters " 3/29/96. }\end{array}$ & \\
\hline 3440 & PU & CONCMIN & PRP WPO\#35850 & $T=0$ memo for SNL activities (in prep) \\
\hline
\end{tabular}




\section{Additional Sources}

\begin{tabular}{|c|c|c|}
\hline ID & \multicolumn{2}{|c|}{ Technical Traceabllity Documentation } \\
\hline 256 & $\begin{array}{l}\text { Vaughn worksheet, 2-5-96 CCA Panel seal Parameter Values } \\
\text { for BRAGFLO }\end{array}$ & $\begin{array}{l}\text { Vaughn worksheet, 2-5-96 CCA Panel seal Parameter Values } \\
\text { for BRAGFLO }\end{array}$ \\
\hline 259 & $\begin{array}{l}\text { Vaughn worksheet, no date, Panel Seal parameter values are } \\
\text { listed. }\end{array}$ & \\
\hline 260 & $\begin{array}{l}\text { Vaughn worksheet, no date, Panel Seal parameter values are } \\
\text { listed. }\end{array}$ & \\
\hline 261 & $\begin{array}{l}\text { Vaughn worksheet, no date, Panel Seal parameter values are } \\
\text { listed. }\end{array}$ & \\
\hline 265 & $\begin{array}{l}\text { Vaughn worksheet, 2-5-96 CCA Panel Seal Parameter Values } \\
\text { for BRAGFLO. }\end{array}$ & \\
\hline 3429 & & \\
\hline 3433 & - & \\
\hline 3459 & & \\
\hline 3457 & & \\
\hline 3458 & & \\
\hline 3460 & & \\
\hline 3461 & & \\
\hline 3315 & & \\
\hline 3316 & & \\
\hline 3440 & $\begin{array}{l}\text { WPO\#36771 Memo: Hans Papenguth to Christine Stockman } \\
\text { "Colloidal Actinide Source Term Parameters " 3/29/96. }\end{array}$ & \\
\hline
\end{tabular}


Additional-Sources

\begin{tabular}{|c|c|c|c|c|}
\hline ID & Material ID & Param ID & \multicolumn{2}{|c|}{ Technical Traceabllity Documentation } \\
\hline 3437 & $T H$ & CONCMIN & $\begin{array}{l}\text { PRP WPO\#35850, T=0 memo for SNL } \\
\text { activities (in prep) }\end{array}$ & $\begin{array}{l}\text { WPO\#36771 Memo: Hans Papenguth to } \\
\text { Christine Stockman "Colloidal Actinide Source } \\
\text { Term Parameters " 3/29/96. }\end{array}$ \\
\hline 3438 & U & CONCMIN & $\begin{array}{l}\text { PRP WPO\#35850, T=0 memo for SNL } \\
\text { activities (in prep) }\end{array}$ & $\begin{array}{l}\text { WPO \#6771 Memo: Hans Papenguth to } \\
\text { Christine Stockman "Colloidal Actinide Source } \\
\text { Term Parameters " 3/29/96. }\end{array}$ \\
\hline 3439 & NP & CONCMIN & $\begin{array}{l}\text { PRP WPO\#35850, T=0 memo for SNL } \\
\text { activities (in prep) }\end{array}$ & $\begin{array}{l}\text { WPO\#36771 Memo: Hans Papenguth to } \\
\text { Christine Stockman "Colloidal Actinide Source } \\
\text { Term Parameters " 3/29/96. }\end{array}$ \\
\hline 3441 & AM & CONCMIN & $\begin{array}{l}\text { PRP WPO\#35850, T=0 memo for SNL } \\
\text { activities }\end{array}$ & $\begin{array}{l}\text { WPO\#36771 Memo: Hans Papenguth to } \\
\text { Christine Stockman "Coltoidal Actinide Source } \\
\text { Term Parameters " 3/29/96. }\end{array}$ \\
\hline 3317 & PU & PROPMIC & $\begin{array}{l}\text { WPO\#36771 Memo:Hans Papenguth to } \\
\text { Christine Stockman "Colloidal Actinide Source } \\
\text { Term parameters" 3/28/96. }\end{array}$ & $\begin{array}{l}\text { PRP WPO\#35856 Table } 6 \text { "Mobile Colloidal } \\
\text { Actinide Source Term } 4 \text { Microbes. Hans } \\
\text { Papenguth. }\end{array}$ \\
\hline 3442 & $\mathrm{PU}+3$ & MDO & $\begin{array}{l}\text { WPO\#37533 Memo: L.H.Brush to M. Tiemey } \\
\text { 5/11/96. }\end{array}$ & \\
\hline 3480 & $P U+3$ & MKD_PU & $\begin{array}{l}\text { WPO\#37533 Memo: L.H.Brush to M. Tiemey } \\
\text { 5/11/96. }\end{array}$ & \\
\hline 3481 & PU+4 & MKD_PU & PRP WPO\#38231 & LANL AN-1789 T=0 memo for SNL activities \\
\hline 3478 & $\mathrm{TH}+4$ & MKD_TH & PRP WPO\#38231 & LANL AN-1789 T=0 memo for SNL activities \\
\hline 297 & PU239 & INVCHD & $\begin{array}{l}\text { WPO\#41560 Memo:L.C.Sanchez to M. Martell } \\
\text { "Updated (2033yr) Radionuclides for use in } \\
\text { WIPP PA Calculations" 10/28/96. }\end{array}$ & \\
\hline 298 & PU239 & INVRHD & $\begin{array}{l}\text { WPO\#41560 Memo:L.C.Sanchez to M. Martell } \\
\text { "Updated (2033yr) Radionuclides for use in } \\
\text { WIPP PA Calculations" 10/28/96. }\end{array}$ & \\
\hline 2832 & REFCON & ACF_CO2 & $\begin{array}{l}\text { Knapp, et al. Vapor-Liquid Equilibria for } \\
\text { Mixtures of Low Boiling Substances, Chemical } \\
\text { Data Ser. Vol Vl (1982) DECHEMA p. 795- } \\
\text { 797. (no WPO number - info is contained } \\
\text { within } 464 \text { Form). }\end{array}$ & \\
\hline 2831 & REFCON & ACF_H2 & $\begin{array}{l}\text { Knapp, et al. Vapor-Liquid Equilibria for } \\
\text { Mixtures of Low Boiling Substances, Chemical } \\
\text { Data Ser. Vol VI (1982) DECHEMA p. 795- } \\
\text { 797. (no WPO number - info is contained } \\
\text { within } 464 \text { Form). }\end{array}$ & \\
\hline 2883 & REFCON & F3M3 & G.E.Nuclides and Isotopes 14th Edition p.52. & $\cdot$ \\
\hline 2859 & $\begin{array}{l}\text { No EPA data } \\
\text { gn }\end{array}$ & & Was not used in CCA & \\
\hline 2865 & REFCON & MW_FE & $\begin{array}{l}\text { WPO\#44691 CRC Handbook of Chemistry and } \\
\text { Physies 72nd edition p. 17-19. }\end{array}$ & \\
\hline 2864 & REFCON & $\mathrm{MW} \mathrm{H} 2 \mathrm{O}$ & DRZ Perm/Porosity & \\
\hline 2863 & $\begin{array}{l}\text { No EPA data } \\
\text { gn }\end{array}$ & & Was not used in CCA & \\
\hline 2826 & REFCON & TC_CO2 & $\begin{array}{l}\text { Knapp, et al. Vapor-Liquid Equilibria for } \\
\text { Mixtures of Low Boiling Substances, Chemical } \\
\text { Data Ser. Vol Vl (1982) DECHEMA p. 795- } \\
\text { 797. (no WPO number - info is contained } \\
\text { within } 464 \text { Form) }\end{array}$ & \\
\hline
\end{tabular}




\section{Additional Sources}

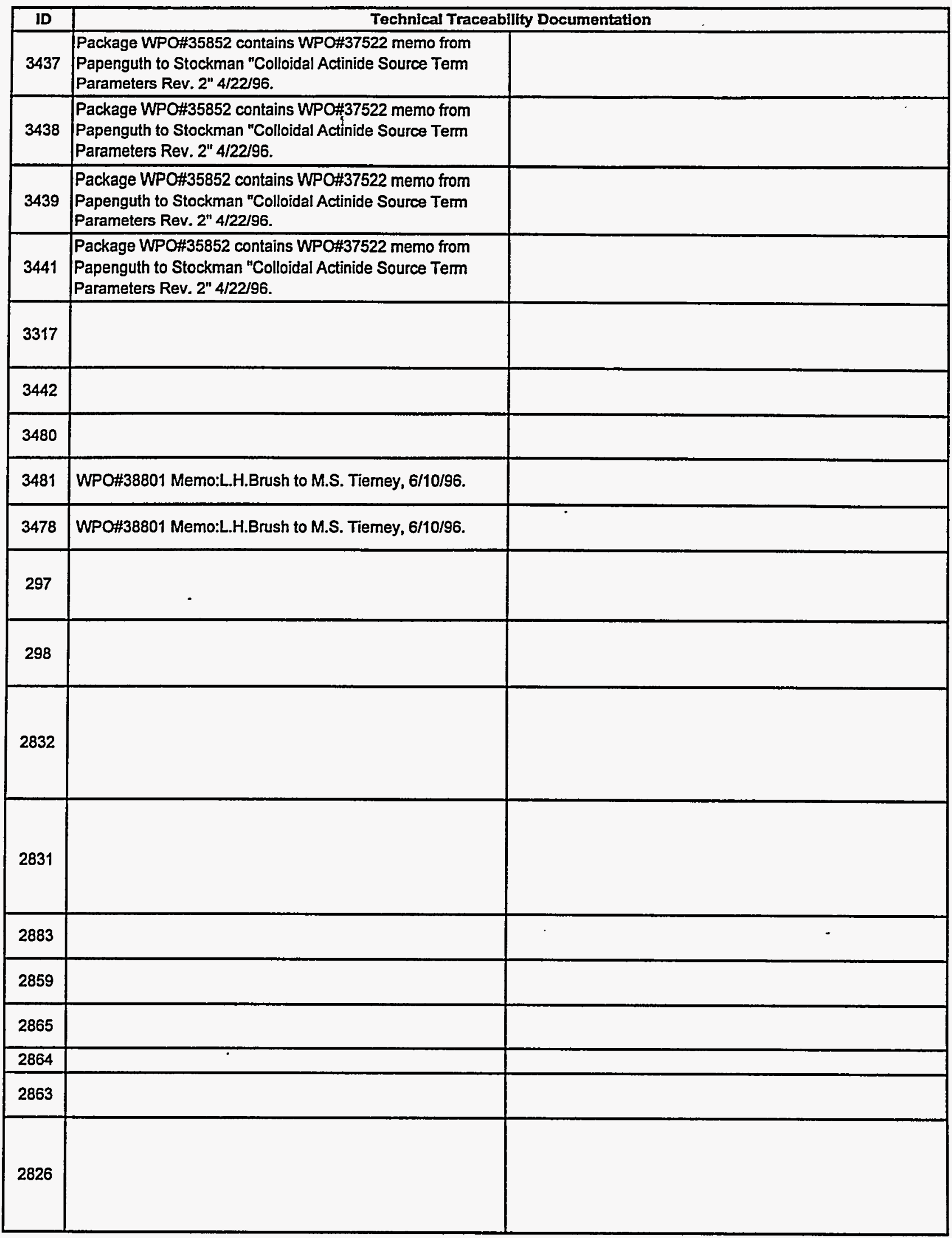


Additional Sources

\begin{tabular}{|c|c|c|c|c|}
\hline ID & Material ID & Param ID & \multicolumn{2}{|c|}{ Technical Traceabllity Documentation } \\
\hline 2825 & REFCON & TC_H2 & $\begin{array}{l}\text { Knapp, et al. Vapor-Liquid Equilibria for } \\
\text { Mixtures of Low Boiling Substances, Chemical } \\
\text { Data Ser. Vol VI (1982) DECHEMA p. 795- } \\
\text { 797. (no WPO number - info is contained } \\
\text { within } 464 \text { Form). }\end{array}$ & \\
\hline 2830 & REFCON & TC_O2 & $\begin{array}{l}\text { Knapp, et al. Vapor-Liquid Equilibria for } \\
\text { Mixtures of Low Boiling Substances, Chemical } \\
\text { Data Ser. Vol VI (1982) DECHEMA p. 795- } \\
\text { 797. (no WPO number - info is contained } \\
\text { within } 464 \text { Form). }\end{array}$ & \\
\hline 3503 & REFCON & ABERM & $\begin{array}{l}\text { WPO\#40133 Memo: K. Trauth to Jon Helton } \\
\text { 06/29/96. }\end{array}$ & \\
\hline 3489 & REFCON & AREA_CH & $\begin{array}{l}\text { WPO\#23529 Numbers taken from Table 3.1-1 } \\
\text { p.3-4 SAND92-0700/3. }\end{array}$ & \\
\hline 3496 & REFCON & AREA_RH & $\begin{array}{l}\text { WPO\#34618 memo:Michael McFadden to Rip } \\
\text { Anderson 3/01/96 (located in Form 464). }\end{array}$ & \\
\hline 3132 & REFCON & DRROOM & WPO\#23529 SAND92-0700/3 p.3-12 (92 PA). & \\
\hline 3492 & REFCON & $\mathbf{F W}$ & $\begin{array}{l}\text { WPO\#23529 SAND92-0700/3 Sec 3.1-1 } \\
\text { Table 3.1-1....WPO\#32328 DOE/CAO-95-1121 } \\
\text { (BIR-Rev2) MST 6/27/96 }\end{array}$ & \\
\hline 3105 & REFCON & VROOM & $\begin{array}{l}\text { See analyst's calculation in } 464 \text { Form. } \\
\text { WPO } \# 33280\end{array}$ & \\
\hline 2130 & REPOSIT & POROSITY & $\begin{array}{l}\text { WPO\#40514 pg 4-159 (the BRAGFLO } \\
\text { analysis write-up) }\end{array}$ & $\begin{array}{l}\text { REPOSIT: POROSITY }=0.848 \text {. Also, see } \\
\text { WPO\#35697 Principal Investigator } \\
\text { Documentation Package for: Final Porosity } \\
\text { Surface Data. }\end{array}$ \\
\hline 2131 & REPOSIT & PRMX_LOG & $\begin{array}{l}\text { See WPO\#40514 Sec. } 4.2 .4 \mathrm{pg} .4-174 \text { (the } \\
\text { BRAGFLO analysis write-up) and WPO\#38152 } \\
\text { the composite Phase I FEP Records Package. }\end{array}$ & \\
\hline 2132 & REPOSIT & PRMY_LOG & $\begin{array}{l}\text { See WPO\#40514 Sec. } 4.2 .4 \mathrm{pg} .4-174 \text { (the } \\
\text { BRAGFLO analysis write-up) and WPO\#38152 } \\
\text { the composite Phase I FEP Records Package. }\end{array}$ & \\
\hline 2133 & REPOSIT & PRMZ_LOG & $\begin{array}{l}\text { See WPO\#40514 Sec. } 4.2 .4 \text { pg. 4-174 (the } \\
\text { BRAGFLO analysis write-up) and WPO\#38152 } \\
\text { the composite Phase I FEP Records Package. }\end{array}$ & \\
\hline 520 & S_ANH_AB & CAP_MOD & $\begin{array}{l}\text { WPO\#30643 SWCF- } \\
\text { A:WBS.1.2.07.1:PDD:QA:Salado:Pkg 10: } \\
\text { Anh2 Phase Parameters. }\end{array}$ & \\
\hline 559 & S_MB138 & CAP_MOD & $\begin{array}{l}\text { WPO\#30643 SWCF- } \\
\text { A:WBS.1.2.07.1:PDD:QA:Salado:Pkg 10: } \\
\text { Anh2 Phase Parameters. }\end{array}$ & \\
\hline 579 & S_MB139 & CAP_MOD & $\begin{array}{l}\text { WPO\#30643 SWCF- } \\
\text { A:WBS.1.2.07.1:PDD:QA:Salado:Pkg 10: } \\
\text { Anh2 Phase Parameters. }\end{array}$ & $\begin{array}{l}\text { WPO\#43487 Memo: Archuleta to SWCF } \\
1 / 20 / 97 \text { defining } T=0 \text {, initial date when data } \\
\text { collection by Intera was performed under } \\
\text { conditions equal to that of a qualified QA } \\
\text { program. }\end{array}$ \\
\hline 2158 & S_ANH_AB & DPHIMAX & $\begin{array}{l}\text { WPO\#43487 Memo: Archuleta to SWCF } \\
1 / 20 / 97 \text { defining } T=0 \text {, initial date when data } \\
\text { collection by Intera was performed under } \\
\text { conditions equal to that of a qualified QA } \\
\text { program. }\end{array}$ & $\begin{array}{l}\text { WPO\#44704 Memo: Larson, Beauheim, Weart } \\
\text { to Shephard "Experimental Data and } \\
\text { BRAGFLO Fracture Model Parameter Values } \\
3 / 3 / 1 / 97 \text { " discusses the derivation of the } \\
\text { fracture model input parameters and how they } \\
\text { are consistent with experimental data. (in } 464 \\
\text { form) }\end{array}$ \\
\hline
\end{tabular}


Additional Sources

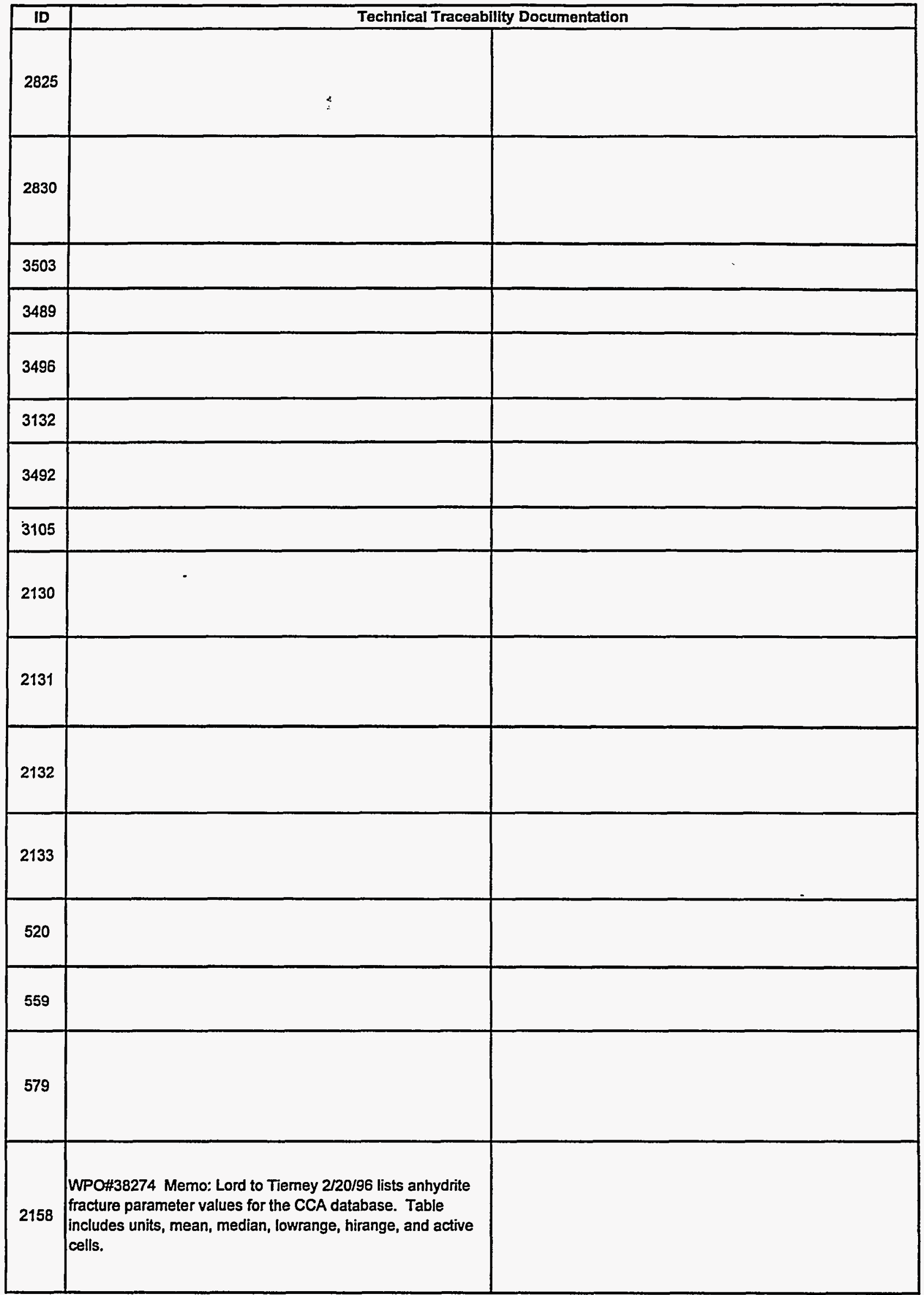


Additional Sources

\begin{tabular}{|c|c|c|c|c|}
\hline ID & Material ID & Param ID & \multicolumn{2}{|c|}{ Technical Traceability Documentation } \\
\hline 522 & S_ANH_AB & PC_MAX & $\begin{array}{l}\text { WPO\#30643 SWCF- } \\
\text { A:WBS.1.2.07.1:PDD:QA:Salado:Pkg 10: } \\
\text { Anh2 Phase Parameters. }\end{array}$ & \\
\hline 561 & S_MB138 & PC_MAX & $\begin{array}{l}\text { WPO\#30643 SWCF- } \\
\text { A:WBS.1.2.07.1:PDD:QA:Salado:Pkg 10: } \\
\text { Anh2 Phase Parameters. }\end{array}$ & \\
\hline 582 & S_MB139 & PC_MAX & $\begin{array}{l}\text { WPO\#30643 SWCF- } \\
\text { A:WBS.1.2.07.1:PDD:QA:Salado:Pkg 10: } \\
\text { Anh2 Phase Parameters. }\end{array}$ & \\
\hline 541 & S_HALITE & COMP_RCK & $\begin{array}{l}\text { WPO\#30598 SWCF-A:WBS } \\
\text { 1.2.07.1:PDD:QA:Salado:Pkg 5:Salado halite } \\
\text { Rock Compressibility. See also WPO\#31220 }\end{array}$ & PI PRP WPO\#31220 \\
\hline 544 & S_HALITE & POROSITY & $\begin{array}{l}\text { WPO\#30601 SWCF-A:WBS } \\
\text { 1.2.07.1:PDD:QA:Salado:Pkg 8:effective } \\
\text { porosity/Hal }\end{array}$ & \\
\hline 546 & S_HALITE & PRESSURE & $\begin{array}{l}\text { WPO\#31221 SWCF-A:WBS } \\
\text { 1.2.07.1:PDD:QA:Salado:Pkg 4:halite } \\
\text { pressure }\end{array}$ & $\begin{array}{l}\text { WPO\#41247 p } 4-7 \text { "WIPP Natural Barriers } \\
\text { Data Qualification Peer Review Repott" Aug } \\
96 \text { states undisturbed halite pore pressure is } \\
\text { based on interpretation of GTFM } 6.0 \\
\text { simulation on two boreholes adjacent to Room } \\
\text { Q. }\end{array}$ \\
\hline 547 & S_HALITE & PRMX_LOG & $\begin{array}{l}\text { WPO\#36772 Memo from Peter Davies to } \\
\text { Martin Tiemey } 3 / 7 / 96 \text {. }\end{array}$ & $\begin{array}{l}\text { WPO\#31218 SWCF-A:WBS } \\
\text { 1.2.07.1:PDD:QA:Salado:Pkg 7:Salado Halite } \\
\text { Permeability }\end{array}$ \\
\hline 548 & S_HALITE & PRMY_LOG & $\begin{array}{l}\text { WPO\#36772 Memo from Peter Davies to } \\
\text { Martin Tiemey } 3 / 7 / 96 .\end{array}$ & $\begin{array}{l}\text { WPO\#31218 SWCF-A:WBS } \\
\text { 1.2.07.1:PDD:QA:Salado:Pkg 7:Salado Halite } \\
\text { Permeability }\end{array}$ \\
\hline 549 & S_HALITE & PRMZ_LOG & $\begin{array}{l}\text { WPO\#36772 Memo from Peter Davies to } \\
\text { Martin Tiemey } 3 / 7 / 96 \text {. }\end{array}$ & $\begin{array}{l}\text { WPO\#31218 } \text { SWCF-A:WBS } \\
\text { 1.2.07.1:PDD:QA:Salado:Pkg 7:Salado Halite } \\
\text { Permeability }\end{array}$ \\
\hline 580 & S_MB139 & COMP_RCK & $\begin{array}{l}\text { WPO\#41247 pg 4-38 'WIPP Natural Barriers } \\
\text { Data Qualification Peer Review Report" Aug } \\
96 \text { states value was derived from } 2 \text { hydraulic } \\
\text { tests in boreholes using GTFM } 6.0 \text { code to } \\
\text { estimate permeability. }\end{array}$ & $\begin{array}{l}\text { WPO } 31186 \text { SWCF- } \\
\text { A:WBS1.2.07.1:PDD:QA:Salado:Pkg19:Rock } \\
\text { Compressibility }\end{array}$ \\
\hline 560 & S_MB138 & COMP_RCK & $\begin{array}{l}\text { WPO\#41247 pg 4-38 "WIPP Natural Barriers } \\
\text { Data Qualification Peer Review Report" Aug } \\
96 \text { states value was derived from } 2 \text { hyoraulic } \\
\text { tests in boreholes using GTFM } 6.0 \text { code to } \\
\text { estimate permeability. }\end{array}$ & $\begin{array}{l}\text { WPO } 31186 \text { SWCF- } \\
\text { A:WBS1.2.07.1:PDD:QA:Salado:Pkg19:Rock } \\
\text { Compressibility }\end{array}$ \\
\hline 521 & S_ANH_AB & COMP_RCK & $\begin{array}{l}\text { WPO } 41247 \text { pg 4-38 "WIPP Natural Barriers } \\
\text { Data Qualification Peer Review Report" Aug } \\
96 \text { states value was derived from } 2 \text { hydraulic } \\
\text { tests in boreholes using GTFM } 6.0 \text { code to } \\
\text { estimate permeability. }\end{array}$ & $\begin{array}{l}\text { WPO } 31186 \text { SWCF- } \\
\text { A:WBS1.2.07.1:PDD:QA:Salado:Pkg19:Rock } \\
\text { Compressibility }\end{array}$ \\
\hline 2177 & S_MB139 & DPHIMAX & $\begin{array}{l}\text { WPO\#43487 Memo: Archuleta to SWCF } \\
1 / 20 / 97 \text { defining T=0, initial date when data } \\
\text { collection by Intera was performed under } \\
\text { conditions equal to that of a qualified QA } \\
\text { program. }\end{array}$ & $\begin{array}{l}\text { WPO\#38274 Memo: Lord to Tierney } 2 / 20 / 96 \\
\text { lists anhydrite fracture parameter values for } \\
\text { the CCA database. Table includes units, } \\
\text { mean, median, lowrange, hirange, and active } \\
\text { cells. }\end{array}$ \\
\hline
\end{tabular}




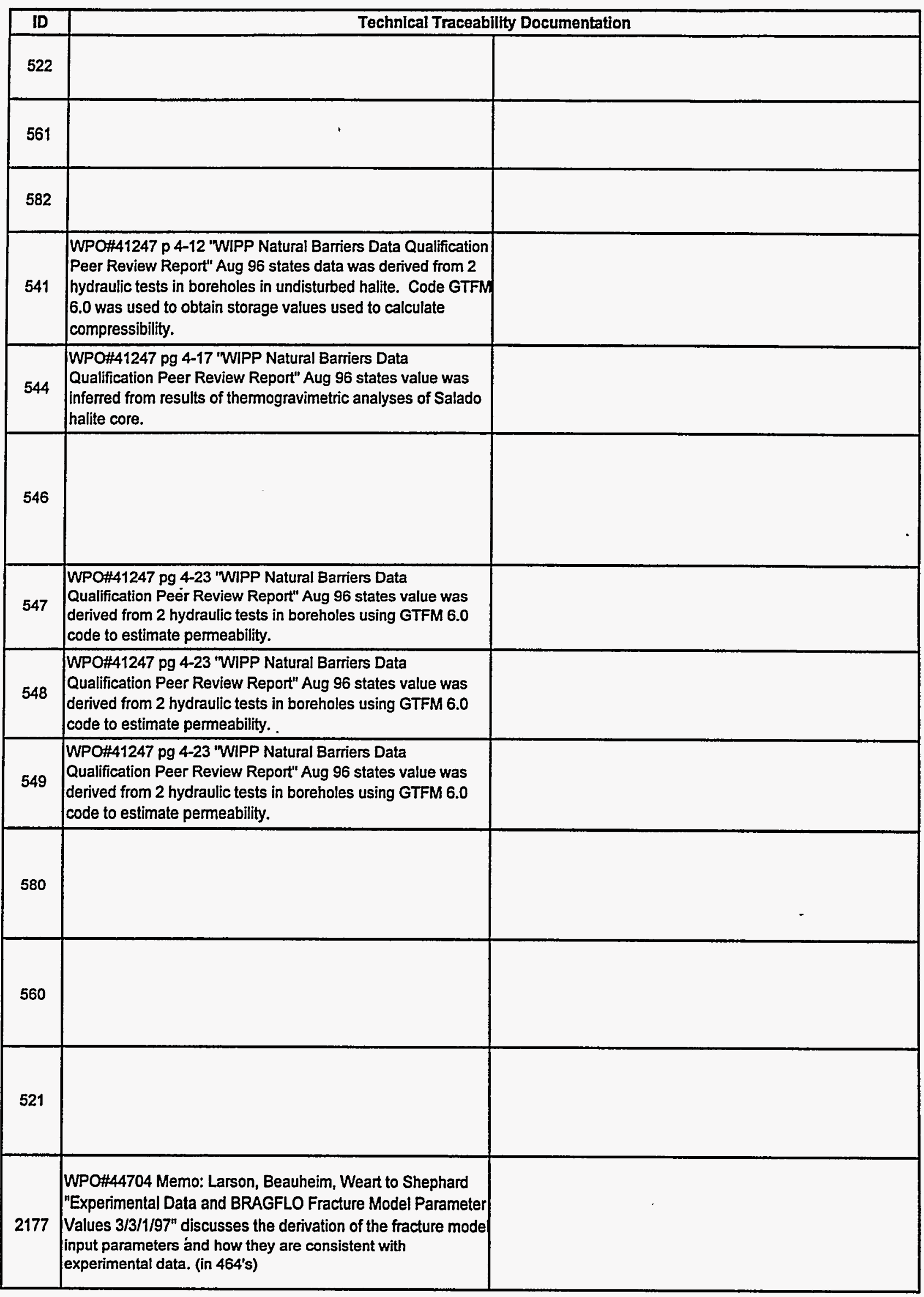




\section{Additional Sources}

\begin{tabular}{|c|c|c|c|c|}
\hline ID & Material ID & Param ID & \multicolumn{2}{|c|}{ Technical Traceability Documentation } \\
\hline 2169 & S_MB138 & DPHIMAX & $\begin{array}{l}\text { WPO\#43487 Memo: Archuleta to SWCF } \\
1 / 20 / 97 \text { defining T=0, initial date when data } \\
\text { collection by Intera was performed under } \\
\text { conditions equal to that of a qualified QA } \\
\text { program. }\end{array}$ & $\begin{array}{l}\text { WPO } 38274 \text { Memo: Lord to Tierney } 2 / 20 / 96 \\
\text { lists anhydrite fracture parameter values for } \\
\text { the CCA database. Table includes units, } \\
\text { mean, median, lowrange, hirange, and active } \\
\text { cells. }\end{array}$ \\
\hline 2811 & S_MB139 & IFRX & $\begin{array}{l}\text { WPO\#43487 Memo: Archuleta to SWCF } \\
1 / 20 / 97 \text { defining } T=0 \text {, initial date when data } \\
\text { collection by Intera was performed under } \\
\text { conditions equal to that of a qualified } Q A \\
\text { program. }\end{array}$ & $\begin{array}{l}\text { WPO\#36205 Memo: P. Vaughn to M. Chu } \\
\text { 1/22/96 }\end{array}$ \\
\hline 2814 & S_MB139 & IFRY & $\begin{array}{l}\text { WPO\#43487 Memo: Archuleta to SWCF } \\
1 / 20 / 97 \text { defining T=0, initial date when data } \\
\text { collection by Intera was performed under } \\
\text { conditions equal to that of a qualified QA } \\
\text { program. }\end{array}$ & $\begin{array}{l}\text { WPO\#36205 Memo: P. Vaughn to M. Chu } \\
\text { 1/22/96 }\end{array}$ \\
\hline 2817 & S_MB139 & IFRZ & $\begin{array}{l}\text { WPO\#43487 Memo: Archuleta to SWCF } \\
\text { 1/20/97 defining T=0, initial date when data } \\
\text { collection by Intera was performed under } \\
\text { conditions equal to that of a qualified QA } \\
\text { program. }\end{array}$ & $\begin{array}{l}\text { WPO\#36205 Memo: P. Vaughn to M. Chu } \\
\text { 1/22/96 }\end{array}$ \\
\hline 2178 & S_MB139 & KMAXLOG & $\begin{array}{l}\text { WPO\#43487 Memo: Archuleta to SWCF } \\
1 / 20 / 97 \text { defining } T=0 \text {, initial date when data } \\
\text { collection by Intera was performed under } \\
\text { conditions equal to that of a qualified QA } \\
\text { program. }\end{array}$ & $\begin{array}{l}\text { WPO\#44704 Memo: Larson, Beauheim, Weart } \\
\text { to Shephard "Experimental Data and } \\
\text { BRAGFLO Fracture Model Parameter Values } \\
3 / 3 / 1 / 97 \text { " discusses the derivation of the } \\
\text { fracture model input parameters and how they } \\
\text { are consistent with experimental data. (in } \\
464 \text { 's) }\end{array}$ \\
\hline 2159 & S_ANH_AB & KMAXLOG & $\begin{array}{l}\text { WPO\#43487 Memo: Archuleta to SWCF } \\
1 / 20 / 97 \text { defining T=0, initial date when data } \\
\text { collection by Intera was performed under } \\
\text { conditions equal to that of a qualified QA } \\
\text { program. }\end{array}$ & $\begin{array}{l}\text { WPO\#44704 Memo: Larson, Beauheim, Weart } \\
\text { to Shephard "Experimental Data and } \\
\text { BRAGFLO Fracture Model Parameter Values } \\
3 / 3 / 1 / 97 \text { " discusses the derivation of the } \\
\text { fracture model input parameters and how they } \\
\text { are consistent with experimental data. }\end{array}$ \\
\hline 2170 & S_MB138 & KMAXLOG & $\begin{array}{l}\text { WPO\#43487 Memo: Archuleta to SWCF } \\
1 / 20 / 97 \text { defining T=0, initial date when data } \\
\text { collection by Intera was performed under } \\
\text { conditions equal to that of a qualified QA } \\
\text { program. }\end{array}$ & $\begin{array}{l}\text { WPO\#4704 Memo: Larson, Beauheim, Weart } \\
\text { to Shephard "Experimental Data and } \\
\text { BRAGFLO Fracture Model Parameter Values } \\
\text { 3/3/1/97" discusses the derivation of the } \\
\text { fracture model input parameters and how they } \\
\text { are consistent with experimental data. }\end{array}$ \\
\hline 2789 & S_MB139 & PCT_A & $\begin{array}{l}\text { WPO\#30643 SWCF- } \\
\text { A:1.2.07.1:PDD:QA:Salado:Pkg10:Anh2 - } \\
\text { Phase Parameters }\end{array}$ & \\
\hline 2784 & S_MB138 & PCT_A & $\begin{array}{l}\text { WPO\#30643 SWCF- } \\
\text { A:1.2.07.1:PDD:QA:Salado:Pkg10:Anh2 - } \\
\text { Phase Parameters }\end{array}$ & \\
\hline 2774 & S_ANH_AB & PCT_A & $\begin{array}{l}\text { WPO\#30643 SWCF- } \\
\text { A:1.2.07.1:PDD:QA:Salado:Pkg10:Anh2 - } \\
\text { Phase Parameters }\end{array}$ & \\
\hline 2790 & S_MB139 & PCT_EXP & $\begin{array}{l}\text { WPO\#30643 SWCF- } \\
\text { A:1.2.07.1:PDD:QA:Salado:Pkg10:Anh2 - } \\
\text { Phase Parameters }\end{array}$ & \\
\hline 2785 & S_MB138 & PCT_EXP & $\begin{array}{l}\text { WPO\#30643 SWCF- } \\
\text { A:1.2.07.1:PDD:QA:Salado:Pkg10:Anh2 - } \\
\text { Phase Parameters }\end{array}$ & \\
\hline
\end{tabular}


Additional Sources

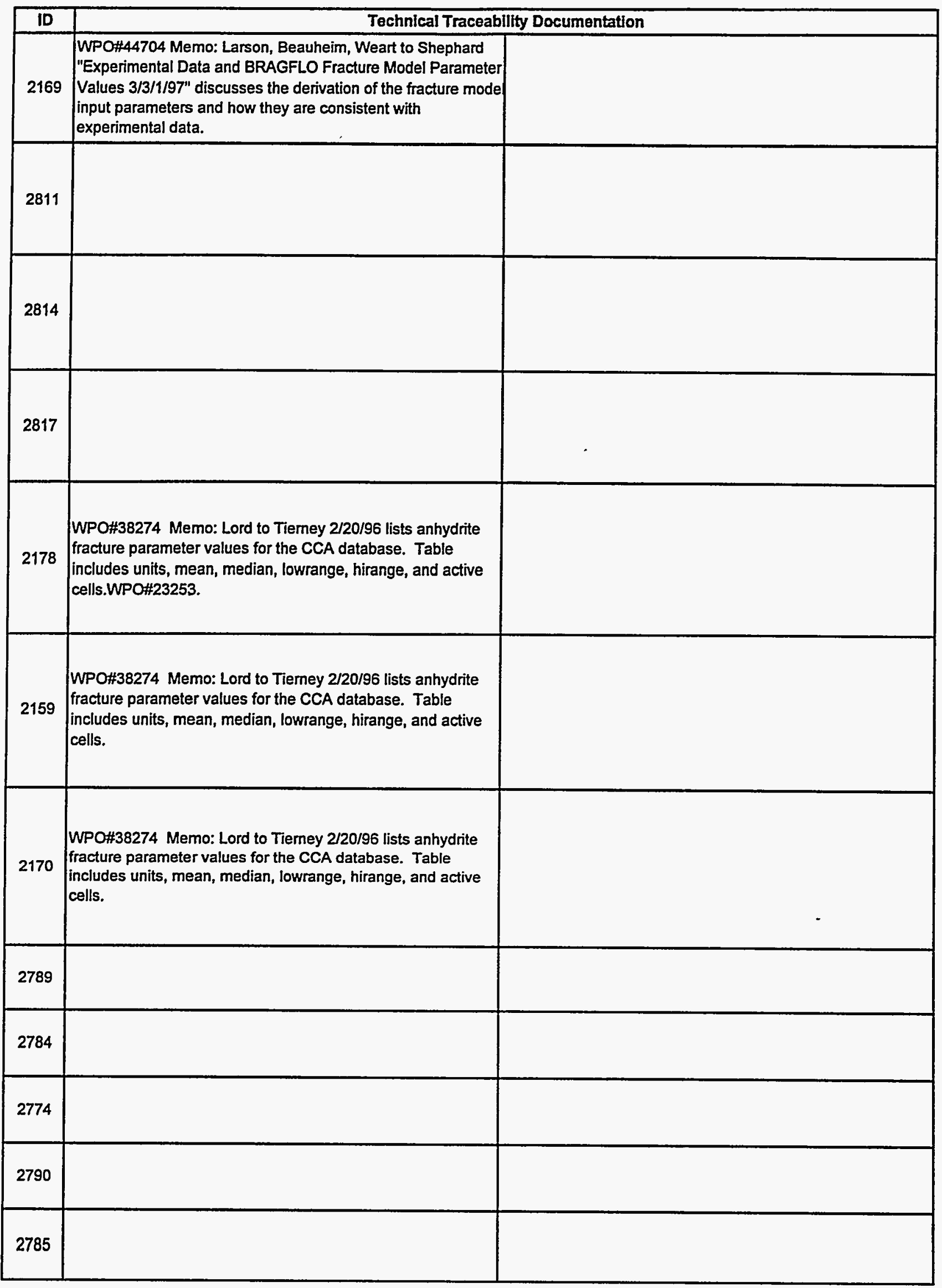


Additional Sources

\begin{tabular}{|c|c|c|c|c|}
\hline ID & Material ID & Param ID & \multicolumn{2}{|c|}{ Technical Traceability Documentation } \\
\hline 2775 & S_ANH_AB & PCT_EXP & $\begin{array}{l}\text { WPO\#30643 SWCF- } \\
\text { A:1.2.07.1:PDD:QA:Salado:Pkg10:Anh2 - } \\
\text { Phase Parameters }\end{array}$ & \\
\hline 2180 & S_MB139 & PF_DELTA & $\begin{array}{l}\text { WPO\#43487 Memo: Archuleta to SWCF } \\
1 / 20 / 97 \text { defining T=0, initial date when data } \\
\text { collection by Intera was performed under } \\
\text { conditions equal to that of a qualified QA } \\
\text { program. }\end{array}$ & $\begin{array}{l}\text { WPO\#44704 memo: Larson, Beauheim, } \\
\text { Weart to Shepard "Experimental Data and } \\
\text { BRAGFLO Fracture Model Parameter Values" } \\
\text { 3/31/97 (located in 464) }\end{array}$ \\
\hline 524 & S_ANH_AB & PF_DELTA & $\begin{array}{l}\text { WPO\#43487 Memo: Archuleta to SWCF } \\
1 / 20197 \text { defining T=0, initial date when data } \\
\text { collection by intera was performed under } \\
\text { conditions equal to that of a qualified QA } \\
\text { program. }\end{array}$ & $\begin{array}{l}\text { WPO\#44704 Memo: Larson, Beauheim, Weart } \\
\text { to Shephard "Experimental Data and } \\
\text { BRAGFLO Fracture Model Parameter Values } \\
\text { 3/3/1/97" discusses the derivation of the } \\
\text { fracture model input parameters and how they } \\
\text { are consistent with experimental data. }\end{array}$ \\
\hline 563 & S_MB138 & PF_DELTA & $\begin{array}{l}\text { WPO\#43487 Memo: Archuleta to SWCF } \\
1 / 20 / 97 \text { defining T=0, initial date when data } \\
\text { collection by Intera was performed under } \\
\text { conditions equal to that of a qualified QA } \\
\text { program. }\end{array}$ & $\begin{array}{l}\text { WPO\#44704 Memo: Larson, Beauheim, Weart } \\
\text { to Shephard "Experimental Data and } \\
\text { BRAGFLO Fracture Model Parameter Values } \\
\text { 3/3/1/97" discusses the derivation of the } \\
\text { fracture model input parameters and how they } \\
\text { are consistent with experimental data. }\end{array}$ \\
\hline 586 & S_MB139 & PI_DELTA & $\begin{array}{l}\text { WPO\#44704 Memo: Larson, Beauheim. Weart } \\
\text { to Shephard "Experimental Data and } \\
\text { BRAGFLO Fracture Model Parameter Values } \\
3 / 3 / 1 / 97 \text { " discusses the derivation of the } \\
\text { fracture model input parameters and how they } \\
\text { are consistent with experimental data. }\end{array}$ & $\begin{array}{l}\text { WPO\#43487 Memo: Archuleta to SWCF } \\
1 / 20 / 97 \text { defining T=0, initial date when data } \\
\text { collection by intera was performed under } \\
\text { conditions equal to that of a qualified QA } \\
\text { program. }\end{array}$ \\
\hline 526 & S_ANH_AB & PI_DELTA & $\begin{array}{l}\text { WPO\#44704 Memo: Larson, Beauheim. Weart } \\
\text { to Shephard "Experimental Data and } \\
\text { BRAGFLO Fracture Model Parameter Values } \\
\text { 3/3/1/97" discusses the derivation of the } \\
\text { fracture model input parameters and how they } \\
\text { are consistent with experimental data. }\end{array}$ & $\begin{array}{l}\text { WPO\#43487 Memo: Archuleta to SWCF } \\
1 / 20 / 97 \text { defining } T=0 \text {, initial date when data } \\
\text { collection by Intera was performed under } \\
\text { conditions equal to that of a qualified QA } \\
\text { program. }\end{array}$ \\
\hline 565 & S_MB138 & PI_DELTA & $\begin{array}{l}\text { WPO\#44704 Memo: Larson, Beauheim, Weart } \\
\text { to Shephard "Experimental Data and } \\
\text { BRAGFLO Fracture Model Parameter Values } \\
\text { 3/3/1/97" discusses the derivation of the } \\
\text { fracture model input parameters and how they } \\
\text { are consistent with experimental data. }\end{array}$ & $\begin{array}{l}\text { WPO\#43487 Memo: Archuleta to SWCF } \\
1 / 20 / 97 \text { defining } T=0 \text {, initial date when data } \\
\text { collection by Intera was performed under } \\
\text { conditions equal to that of a qualified } Q A \\
\text { program. }\end{array}$ \\
\hline 589 & S_MB139 & PO_MIN & $\begin{array}{l}\text { WPO\#40514 Sec. } 4.2 .4 \text { (BRAGFLO Analysis } \\
\text { Write-Up) pg. 4-155 through 4-156. }\end{array}$ & $\begin{array}{l}\text { PO_MIN is the minimum brine pressure. It is } \\
\text { only used when capillary pressure model } \\
\text { (CAP_MOD) } 3 \text { is specified; Since models } \\
\text { (CAP_MOD) } 1 \& 2 \text { were used in } 1996 \text { CCA } \\
\text { calculation, PO_MIN value was a placeholder } \\
\text { and had no effect on the simulations. (Line } \\
\text { 7.34) }\end{array}$ \\
\hline 12 & OPS_AREA & PO_MIN & $\begin{array}{l}\text { WPO\#40514 Sec. } 4.2 .4 \text { (BRAGFLO Analysis } \\
\text { Write-Up) pg. 4-155 through 4-156. }\end{array}$ & $\begin{array}{l}\text { PO_MIN is the minimum brine pressure. It is } \\
\text { only used when capillary pressure model } \\
\text { (CAP_MOD) } 3 \text { is specified; Since models } \\
\text { (CAP_MOD) } 1 \& 2 \text { were used in } 1996 \text { CCA } \\
\text { calculation; PO_MIN value was a placeholder } \\
\text { and had no effect on the simulations. (Line } \\
\text { 7.34) }\end{array}$ \\
\hline
\end{tabular}


Additional Sources

\begin{tabular}{|c|c|c|}
\hline ID & \multicolumn{2}{|c|}{ Technical Traceability Documentation } \\
\hline 2775 & & \\
\hline 2180 & WPO\#23253. & $\begin{array}{l}\text { WPO\#38274 Memo: Lord to Tiemey } 2 / 20 / 96 \text { lists anhydrite } \\
\text { fracture parameter values for the CCA database. Table } \\
\text { includes units, mean, median, lowrange, hirange, and active } \\
\text { cells. }\end{array}$ \\
\hline 524 & $\begin{array}{l}\text { WPO\#38274 Memo: Lord to Tiemey } 2 / 20 / 96 \text { lists anhydrite } \\
\text { fracture parameter values for the CCA database. Table } \\
\text { includes units, mean, median, lowrange, hirange, and active } \\
\text { cells. }\end{array}$ & \\
\hline 563 & $\begin{array}{l}\text { WPO\#38274 Memo: Lord to Tiemey } 2 / 20 / 96 \text { lists anhydrite } \\
\text { fracture parameter values for the CCA database. Table } \\
\text { includes units, mean, median, lowrange, hirange, and active } \\
\text { cells. }\end{array}$ & \\
\hline 586 & $\begin{array}{l}\text { WPO\#38274 Memo: Lord to Tierney } 2 / 20 / 96 \text { lists anhydrite } \\
\text { fracture parameter values for the CCA database. Table } \\
\text { includes units, mean, median, lowrange, hirange, and active } \\
\text { cells. }\end{array}$ & WPO $40514 \mathrm{pg}$ 4-155 (the BRAGFLO analysis write-up) \\
\hline 526 & $\begin{array}{l}\text { WPO\#38274 Memo: Lord to Tiemey } 2 / 20 / 96 \text { lists anhydrite } \\
\text { fracture parameter values for the CCA database. Table } \\
\text { includes units, mean, median, lowrange, hirange, and active } \\
\text { cells. }\end{array}$ & WPO\#40514 pg 4-155 (the BRAGFLO analysis write-up) \\
\hline 565 & $\begin{array}{l}\text { WPO\#38274 Memo: Lord to Tierney } 2 / 20 / 96 \text { lists anhydrite } \\
\text { fracture parameter values for the CCA database. Table } \\
\text { includes units, mean, median, lowrange, hirange, and active } \\
\text { cells. }\end{array}$ & WPO\#40514 pg 4-155 (the BRAGFLO analysis write-up) \\
\hline 589 & WPO\#36051 Memo: P. Vaughn to M. Tierney $2 / 20 / 96$ & \\
\hline 12 & WPO\#36051 Memo: P. Vaughn to M. Tierney 2/20/96 & \\
\hline
\end{tabular}


Additional Sources

\begin{tabular}{|c|c|c|c|c|}
\hline ID & Material ID & Param ID & \multicolumn{2}{|c|}{ Technical Traceability Documentation } \\
\hline 32 & BOREHOLE & PO_MIN & $\begin{array}{l}\text { WPO\#40514 Sec. } 4.2 .4 \text { (BRAGFLO Analysis } \\
\text { Write-Up) pg. 4-155 through 4-156. }\end{array}$ & $\begin{array}{l}\text { PO_MIN is the minimum brine pressure. It is } \\
\text { only used when capillary pressure model } \\
\text { (CAP_MOD) } 3 \text { is specified; Since models } \\
\text { (CAP_MOD) } 1 \& 2 \text { were used in } 1996 \text { CCA } \\
\text { calculation, PO_MIN value was a placeholder } \\
\text { and had no effect on the simulations. (Line } \\
7.34 \text { ) }\end{array}$ \\
\hline 65 & CASTILER & PO_MIN & $\begin{array}{l}\text { WPO\#40514 Sec. } 4.2 .4 \text { (BRAGFLO Analysis } \\
\text { Write-Up) pg. 4-155 through 4-156. }\end{array}$ & $\begin{array}{l}\text { PO_MIN is the minimum brine pressure. It is } \\
\text { only used when capillary pressure model } \\
\text { (CAP_MOD) } 3 \text { is specified; Since models } \\
\text { (CAP_MOD) } 1 \& 2 \text { were used in } 1996 \text { CCA } \\
\text { calculation, PO_MIN value was a placeholder } \\
\text { and had no effect on the simulations. (Line } \\
\text { 7.34) }\end{array}$ \\
\hline 81 & CAVITY_1 & PO_MIN & $\begin{array}{l}\text { WPO\#40514 Sec. } 4.2 .4 \text { (BRAGFLO Analysis } \\
\text { Write-Up) pg. 4-155 through 4-156. }\end{array}$ & $\begin{array}{l}\text { PO_MIN is the minimum brine pressure. It is } \\
\text { only used when capillary pressure model } \\
\text { (CAP_MOD) } 3 \text { is specified; Since models } \\
\text { (CAP_MOD) } 1 \& 2 \text { were used in } 1996 \text { CCA } \\
\text { calculation, PO_MIN value was a placeholder } \\
\text { and had no effect on the simulations. (Line } \\
\text { 7.34) }\end{array}$ \\
\hline 96 & CAVITY_2 & PO_MIN & $\begin{array}{l}\text { WPO\#40514 Sec. } 4.2 .4 \text { (BRAGFLO Analysis } \\
\text { Write-Up) pg. 4-155 through 4-156. }\end{array}$ & $\begin{array}{l}\text { PO_MIN is the minimum brine pressure. It is } \\
\text { only used when capillary pressure model } \\
\text { (CAP_MOD) } 3 \text { is specified; Since models } \\
\text { (CAP_MOD) } 1 \text { \& } 2 \text { were used in } 1996 \text { CCA } \\
\text { calculation, PO_MIN value was a placeholder } \\
\text { and had no effect on the simulations. (Line } \\
\text { 7.34) }\end{array}$ \\
\hline 141 & CULEBRA & PO_MIN & $\begin{array}{l}\text { WPO\#40514 Sec. } 4.2 .4 \text { (BRAGFLO Analysis } \\
\text { Write-Up) pg. 4-155 through 4-156. }\end{array}$ & $\begin{array}{l}\text { PO_MIN is the minimum brine pressure. It is } \\
\text { only used when capillary pressure model } \\
\text { (CAP_MOD) } 3 \text { is specified; Since models } \\
\text { (CAP_MOD) } 1 \& 2 \text { were used in } 1996 \text { CCA } \\
\text { calculation, PO_MIN value was a placeholder } \\
\text { and had no effect on the simulations. (Line } \\
7.34 \text { ) }\end{array}$ \\
\hline 159 & DEWYLAKE & PO_MIN & $\begin{array}{l}\text { WPO\#40514 Sec. } 4.2 .4 \text { (BRAGFLO Analysis } \\
\text { Write-Up) pg. 4-155 through 4-156. }\end{array}$ & $\begin{array}{l}\text { PO_MIN is the minimum brine pressure. It is } \\
\text { only used when capillary pressure model } \\
\text { (CAP_MOD) } 3 \text { is specified; Since models } \\
\text { (CAP_MOD) } 1 \text { \& } 2 \text { were used in } 1996 \text { CCA } \\
\text { calculation, PO_MIN value was a placeholder } \\
\text { and had no effect on the simulations. (Line } \\
7.34 \text { ) }\end{array}$ \\
\hline 179 & $D R Z \_0$ & PO_MIN & $\begin{array}{l}\text { WPO\#40514 Sec. } 4.2 .4 \text { (BRAGFLO Analysis } \\
\text { Write-Up) pg. 4-155 through 4-156. }\end{array}$ & $\begin{array}{l}\text { PO_MIN is the minimum brine pressure. It is } \\
\text { only used when capillary pressure model } \\
\text { (CAP_MOD) } 3 \text { is specified; Since models } \\
\text { (CAP_MOD) } 1 \& 2 \text { were used in } 1996 \text { CCA } \\
\text { calculation, PO_MIN value was a placeholder } \\
\text { and had no effect on the simulations. (Line } \\
7.34 \text { ) }\end{array}$ \\
\hline 196 & DRZ_1 & PO_MIN & $\begin{array}{l}\text { WPO\#40514 Sec. } 4.2 .4 \text { (BRAGFLO Analysis } \\
\text { Write-Up) pg. 4-155 through 4-156. }\end{array}$ & $\begin{array}{l}\text { PO_MIN is the minimum brine pressure. It is } \\
\text { only used when capillary pressure model } \\
\text { (CAP_MOD) } 3 \text { is specified; Since models } \\
\text { (CAP_MOD) } 1 \& 2 \text { were used in } 1996 \text { CCA } \\
\text { calculation, PO_MIN value was a placeholder } \\
\text { and had no effect on the simulations. (Line } \\
7.34 \text { ) }\end{array}$ \\
\hline
\end{tabular}


Additional Sources

\begin{tabular}{|c|c|c|}
\hline ID & Technical T & litity Documentation \\
\hline 32 & WPO\#36051 Memo: P. Vaughn to M. Țierney 2/20/96 & \\
\hline 65 & WPO\#36051 Memo: P. Vaughn to M. Tierney 2/20/96 & \\
\hline 81 & WPO\#36051 Memo: P. Vaughn to M. Tiemey 2/20/96 & 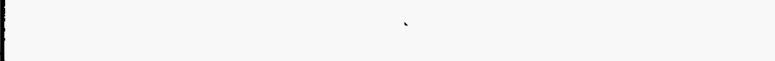 \\
\hline 96 & WPO\#36051 Memo: P. Vaughn to M. Tierney 2/20/96 & $\begin{array}{l}\text { Memo: Vaughn to Tierney 2/14/96 Constant Values for Cavity } \\
1,2,3,4 \text { Parameters }\end{array}$ \\
\hline 141 & WPO\#36051 Memo: P. Vaughn to M. Tierney 2/20/96 & \\
\hline 159 & WPO\#36051 Memo: P. Vaughn to M. Tierney 2/20/96 & \\
\hline 179 & WPO\#36051 Memo: P. Vaughn to M. Tierney 2/20/96 & \\
\hline 196 & WPO\#36051 Memo: P. Vaughn to M. Tierney 2/20/96 & \\
\hline
\end{tabular}


Additional-Sources

\begin{tabular}{|c|c|c|c|c|}
\hline ID & Material ID & Param ID & \multicolumn{2}{|c|}{ Technical Traceabllity Documentation } \\
\hline 212 & EXP_AREA & PO_MIN & $\begin{array}{l}\text { WPO\#40514 Sec. } 4.2 .4 \text { (BRAGFLO Analysis } \\
\text { Write-Up) pg. 4-155 through 4-156. }\end{array}$ & $\begin{array}{l}\text { PO_MIN is the minimum brine pressure. It is } \\
\text { only used when capillary pressure model } \\
\text { (CAP_MOD) } 3 \text { is specified; Since models } \\
\text { (CAP_MOD) } 1 \text { \& } 2 \text { were used in } 1996 \text { CCA } \\
\text { calculation, PO_MIN value was a placeholder } \\
\text { and had no effect on the simulations. (Line } \\
7.34 \text { ) }\end{array}$ \\
\hline 234 & IMPERM_Z & PO_MIN & $\begin{array}{l}\text { WPO\#40514 Sec. } 4.2 .4 \text { (BRAGFLO Analysis } \\
\text { Write-Up) pg. 4-155 through 4-156. }\end{array}$ & $\begin{array}{l}\text { PO_MIN is the minimum brine pressure. It is } \\
\text { only used when capillary pressure model } \\
\text { (CAP_MOD) } 3 \text { is specified; Since models } \\
\text { (CAP_MOD) } 1 \& 2 \text { were used in } 1996 \text { CCA } \\
\text { calculation, PO_MIN value was a placeholder } \\
\text { and had no effect on the simulations. (Line } \\
7.34 \text { ) }\end{array}$ \\
\hline 257 & PAN_SEAL & PO_MIN & $\begin{array}{l}\text { WPO\#40514 Sec. } 4.2 .4 \text { (BRAGFLO Analysis } \\
\text { Write-Up) pg. 4-155 through 4-156. }\end{array}$ & $\begin{array}{l}\text { PO_MIN is the minimum brine pressure. It is } \\
\text { only used when capillary pressure model } \\
\text { (CAP_MOD) } 3 \text { is specified; Since models } \\
\text { (CAP_MOD) } 1 \& 2 \text { were used in } 1996 \text { CCA } \\
\text { calculation, PO_MIN value was a placeholder } \\
\text { and had no effect on the simulations. (Line } \\
7.34 \text { ) }\end{array}$ \\
\hline 342 & SANTAROS & PO_MIN & $\begin{array}{l}\text { WPO\#40514 Sec. } 4.2 .4 \text { (BRAGFLO Analysis } \\
\text { Write-Up) pg. 4-155 through 4-156. }\end{array}$ & $\begin{array}{l}\text { PO_MIN is the minimum brine pressure. It is } \\
\text { only used when capillary pressure model } \\
\text { (CAP_MOD) } 3 \text { is specified; Since models } \\
\text { (CAP_MOD) } 1 \& 2 \text { were used in } 1996 \text { CCA } \\
\text { calculation, PO_MIN value was a placeholder } \\
\text { and had no effect on the simulations. (Line } \\
7.34 \text { ) }\end{array}$ \\
\hline 529 & S_ANH_AB & PO_MIN & $\begin{array}{l}\text { WPO\#40514 Sec. } 4.2 .4 \text { (BRAGFLO Analysis } \\
\text { Write-Up) pg. 4-155 through 4-156. }\end{array}$ & $\begin{array}{l}\text { PO_MIN is the minimum brine pressure. It is } \\
\text { only used when capillary pressure model } \\
\text { (CAP_MOD) } 3 \text { is specified; Since models } \\
\text { (CAP_MOD) } 1 \& 2 \text { were used in } 1996 \text { CCA } \\
\text { calculation, PO_MIN value was a placeholder } \\
\text { and had no effect on the simulations. (Line } \\
7.34 \text { ) }\end{array}$ \\
\hline 545 & S_HALITE & PO_MIN & $\begin{array}{l}\text { WPO\#40514 Sec. } 4.2 .4 \text { (BRAGFLO Analysis } \\
\text { Write-Up) pg. 4-155 through 4-156. }\end{array}$ & $\begin{array}{l}\text { PO_MIN is the minimum brine pressure. It is } \\
\text { only used when capillary pressure model } \\
\text { (CAP_MOD) } 3 \text { is specified; Since models } \\
\text { (CAP_MOD) } 1 \& 2 \text { were used in } 1996 \text { CCA } \\
\text { calculation, PO_MIN value was a placeholder } \\
\text { and had no effect on the simulations. (Line } \\
7.34 \text { ) }\end{array}$ \\
\hline 568 & S_MB138 & PO_MIN & $\begin{array}{l}\text { WPO\#40514 Sec. } 4.2 .4 \text { (BRAGFLO Analysis } \\
\text { Write-Up) pg. 4-155 through 4-156. }\end{array}$ & $\begin{array}{l}\text { PO_MIN is the minimum brine pressure. It is } \\
\text { only used when capillary pressure model } \\
\text { (CAP_MOD) } 3 \text { is specified; Since models } \\
\text { (CAP_MOD) } 1 \& 2 \text { were used in } 1996 \text { CCA } \\
\text { calculation, PO_MIN value was a placeholder } \\
\text { and had no effect on the simulations. (Line } \\
7.34 \text { ) }\end{array}$ \\
\hline 661 & WAS_AREA & PO_MIN & $\begin{array}{l}\text { WPO\#40514 Sec. } 4.2 .4 \text { (BRAGFLO Analysis } \\
\text { Write-Up) pg. 4-155 through 4-156. }\end{array}$ & $\begin{array}{l}\text { PO_MIN is the minimum brine pressure. It is } \\
\text { only used when capillary pressure model } \\
\text { (CAP_MOD) } 3 \text { is specified; Since models } \\
\text { (CAP_MOD) } 1 \& 2 \text { were used in } 1996 \text { CCA } \\
\text { calculation, PO_MIN value was a placeholder } \\
\text { and had no effect on the simulations. (Line } \\
7.34 \text { ) }\end{array}$ \\
\hline
\end{tabular}




\begin{tabular}{|c|c|c|}
\hline ID & Technical Ti & Illity Documentation \\
\hline 212 & WPO\#36051 Memo: P. Vaughn to M. Tierney 2/20/96 & \\
\hline 234 & WPO\#36051 Memo: P. Vaughn to M. Tierney 2/20/96 & \\
\hline 257 & WPO\#36051 Memo: P. Vaughn to M. Tiemey 2/20/96 & \\
\hline 342 & WPO\#36051 Memo: P. Vaughn to M. Tiemey 2/20/96 & \\
\hline 529 & WPO\#36051 Memo: P. Vaughn to M. Tierney 2/20/96 & \\
\hline 545 & WPO\#36051 Memo: P. Vaughn to M. Tierney 2/20/96 & \\
\hline 568 & WPO\#36051 Memo: P. Vaughn to M. Tierney 2/20/96 & \\
\hline 661 & WPO\#36051 Memo: P. Vaughn to M. Tierney 2/20/96 & \\
\hline
\end{tabular}


Additional-Sources

\begin{tabular}{|c|c|c|c|c|}
\hline ID & Material ID & Param ID & \multicolumn{2}{|c|}{ Technical Traceability Documentation } \\
\hline 2281 & ASPHALT & PO_MIN & $\begin{array}{l}\text { WPO\#40514 Sec. } 4.2 .4 \text { (BRAGFLO Analysis } \\
\text { Write-Up) pg. 4-155 through 4-156. }\end{array}$ & $\begin{array}{l}\text { PO_MIN is the minimum brine pressure. It is } \\
\text { only used when capillary pressure model } \\
\text { (CAP_MOD) } 3 \text { is specified; only Models } 1 \& 2 \\
\text { were used in } 1996 \text { CCA calculation; PO_MIN } \\
\text { value is a placeholder. }\end{array}$ \\
\hline 2315 & CLAY_BOT & PO_MIN & $\begin{array}{l}\text { WPO\#40514 Sec. } 4.2 .4 \text { (BRAGFLO Analysis } \\
\text { Write-Up) pg. 4-155 through 4-156. }\end{array}$ & $\begin{array}{l}\text { PO_MIN is the minimum brine pressure. It is } \\
\text { only used when capillary pressure model } \\
\text { (CAP_MOD) } 3 \text { is specified; only Models } 1 \& 2 \\
\text { were used in } 1996 \text { CCA calculation; PO_MIN } \\
\text { value is a placeholder. }\end{array}$ \\
\hline 2332 & CL_L_T1 & PO_MIN & $\begin{array}{l}\text { WPO\#40514 Sec. } 4.2 .4 \text { (BRAGFLO Analysis } \\
\text { Write-Up) pg. 4-155 through 4-156. }\end{array}$ & $\begin{array}{l}\text { PO_MIN is the minimum brine pressure. It is } \\
\text { only used when capillary pressure model } \\
\text { (CAP_MOD) } 3 \text { is specified; only Models } 1 \& 2 \\
\text { were used in } 1996 \text { CCA calculation; PO_MIN } \\
\text { value is a placeholder. }\end{array}$ \\
\hline 2349 & CL_L_T2 & PO_MIN & $\begin{array}{l}\text { WPO\#40514 Sec. } 4.2 .4 \text { (BRAGFLO Analysis } \\
\text { Write-Up) pg. 4-155 through 4-156. }\end{array}$ & $\begin{array}{l}\text { PO_MIN is the minimum brine pressure. It is } \\
\text { only used when capillary pressure model } \\
\text { (CAP_MOD) } 3 \text { is specified; only Models } 1 \& 2 \\
\text { were used in } 1996 \text { CCA calculation; PO_MIN } \\
\text { value is a placeholder. }\end{array}$ \\
\hline 2366 & CL_L_T3 & PO_MIN & $\begin{array}{l}\text { WPO\#40514 Sec. } 4.2 .4 \text { (BRAGFLO Analysis } \\
\text { Write-Up) pg. 4-155 through 4-156. }\end{array}$ & $\begin{array}{l}\text { PO_MIN is the minimum brine pressure. It is } \\
\text { only used when capillary pressure model } \\
\text { (CAP_MOD) } 3 \text { is specified; only Models } 1 \& 2 \\
\text { were used in } 1996 \text { CCA calculation; PO_MIN } \\
\text { value is a placeholder. }\end{array}$ \\
\hline 2383 & CL_M_T1 & PO_MIN & $\begin{array}{l}\text { WPO\#40514 Sec. } 4.2 .4 \text { (BRAGFLO Analysis } \\
\text { Write-Up) pg. 4-155 through 4-156. }\end{array}$ & $\begin{array}{l}\text { PO_MIN is the minimum brine pressure. It is } \\
\text { only used when capillary pressure model } \\
\text { (CAP_MOD) } 3 \text { is specified; only Models } 1 \& 2 \\
\text { were used in } 1996 \text { CCA calculation; PO_MIN } \\
\text { value is a placeholder. }\end{array}$ \\
\hline 2400 & CL_M_T2 & PO_MIN & $\begin{array}{l}\text { WPO\#40514 Sec. } 4.2 .4 \text { (BRAGFLO Analysis } \\
\text { Write-Up) pg. 4-155 through 4-156. }\end{array}$ & $\begin{array}{l}\text { PO_MIN is the minimum brine pressure. It is } \\
\text { only used when capillary pressure model } \\
\text { (CAP_MOD) } 3 \text { is specified; only Models } 1 \text { \& } 2 \\
\text { were used in } 1996 \text { CCA calculation; PO_MIN } \\
\text { value is a placeholder. }\end{array}$ \\
\hline 2417 & $C L_{-} M_{-} T 3$ & PO_MIN & $\begin{array}{l}\text { WPO\#40514 Sec. } 4.2 .4 \text { (BRAGFLO Analysis } \\
\text { Write-Up) pg. 4-155 through 4-156. }\end{array}$ & $\begin{array}{l}\text { PO_MIN is the minimum brine pressure. It is } \\
\text { only used when capillary pressure model } \\
\text { (CAP_MOD) } 3 \text { is specified; only Models } 1 \& 2 \\
\text { were used in } 1996 \text { CCA calculation; PO_MIN } \\
\text { value is a placeholder. }\end{array}$ \\
\hline 2434 & $C_{2} M_{-} T_{4}$ & PO_MIN & $\begin{array}{l}\text { WPO\#40514 Sec. } 4.2 .4 \text { (BRAGFLO Analysis } \\
\text { Write-Up) pg. 4-155 through 4-156. }\end{array}$ & $\begin{array}{l}\text { PO_MIN is the minimum brine pressure. It is } \\
\text { only used when capillary pressure model } \\
\text { (CAP_MOD) } 3 \text { is specified; only Models } 1 \& 2 \\
\text { were used in } 1996 \text { CCA calculation; PO_MIN } \\
\text { value is a placeholder. }\end{array}$ \\
\hline 2451 & CL_M_T5 & PO_MIN & $\begin{array}{l}\text { WPO\#40514 Sec. } 4.2 .4 \text { (BRAGFLO Analysis } \\
\text { Write-Up) pg. 4-155 through 4-156. }\end{array}$ & $\begin{array}{l}\text { PO_MIN is the minimum brine pressure. It is } \\
\text { only used when capillary pressure model } \\
\text { (CAP_MOD) } 3 \text { is specified; only Models } 182 \\
\text { were used in } 1996 \text { CCA calculation; PO_MIN } \\
\text { value is a placeholder. }\end{array}$ \\
\hline 2501 & EARTH & PO_MIN & $\begin{array}{l}\text { WPO\#40514 Sec. } 4.2 .4 \text { (BRAGFLO Analysis } \\
\text { Write-Up) pg. 4-155 through 4-156. }\end{array}$ & $\begin{array}{l}\text { PO_MIN is the minimum brine pressure. It is } \\
\text { only used when capillary pressure model } \\
\text { (CAP_MOD) } 3 \text { is specified; only Models } 1 \& 2 \\
\text { were used in } 1996 \text { CCA calculation; PO_MIN } \\
\text { value is a placeholder. }\end{array}$ \\
\hline
\end{tabular}


Additional-Sources

\begin{tabular}{|c|c|c|}
\hline ID & Technical Ti & lity Documentation \\
\hline 2281 & WPO\#36051 Memo: P. Vaughn to M. Tiemey 2/20/96 & \\
\hline 2315 & WPO\#36051 Memo: P. Vaughn to M. Tiemey 2/20/96 & \\
\hline 2332 & WPO\#36051 Memo: P. Vaughn to M. Tiemey 2/20/96 & \\
\hline 2349 & WPO\#36051 Memo: P. Vaughn to M. Tierney 2/20/96 & \\
\hline 2366 & WPO\#36051 Memo: P. Vaughn to M. Tierney 2/20/96 & \\
\hline 2383 & WPO\#36051 Memo: P. Vaughn to M. Tierney 2/20/96 & \\
\hline 2400 & WPO\#36051 Memo: P. Vaughn to M. Tierney 2/20/96 & \\
\hline 2417 & WPO\#36051 Memo: P. Vaughn to M. Tierney 2/20/96 & \\
\hline 2434 & WPO\#36051 Memo: P. Vaughn to M. Tierney 2/20/96 & \\
\hline 2451 & WPO\#36051 Memo: P. Vaughn to M. Tierney 2/20/96 & \\
\hline 2501 & WPO\#36051 Memo: P. Vaughn to M. Tierney 2/20/96 & \\
\hline
\end{tabular}


Additional Sources-

\begin{tabular}{|c|c|c|c|c|}
\hline ID & Material ID & Param ID & \multicolumn{2}{|c|}{ Technical Traceabllity Documentation } \\
\hline 2518 & SALT_T1 & PO_MIN & $\begin{array}{l}\text { WPO\#40514 Sec. } 4.2 .4 \text { (BRAGFLO Analysis } \\
\text { Write-Up) pg. 4-155 through 4-156. }\end{array}$ & $\begin{array}{l}\text { PO_MIN is the minimum brine pressure. It is } \\
\text { only used when capillary pressure model } \\
\text { (CAP_MOD) } 3 \text { is specified; only Models } 1 \text { \& } 2 \\
\text { were used in } 1996 \text { CCA calculation; PO_MIN } \\
\text { value is a placeholder. }\end{array}$ \\
\hline 2535 & SALT_T2 & PO_MIN & $\begin{array}{l}\text { WPO\#40514 Sec. } 4.2 .4 \text { (BRAGFLO Analysis } \\
\text { Write-Up) pg. 4-155 through 4-156. }\end{array}$ & $\begin{array}{l}\text { PO_MIN is the minimum brine pressure. It is } \\
\text { only used when capillary pressure model } \\
\text { (CAP_MOD) } 3 \text { is specified; only Models } 1 \text { \& } 2 \\
\text { were used in } 1996 \text { CCA calculation; PO_MIN } \\
\text { value is a placeholder. }\end{array}$ \\
\hline 2552 & SALT_T3 & PO_MIN & $\begin{array}{l}\text { WPO\#40514 Sec. } 4.2 .4 \text { (BRAGFLO Analysis } \\
\text { Write-Up) pg. 4-155 through 4-156. }\end{array}$ & $\begin{array}{l}\text { PO_MIN is the minimum brine pressure. It is } \\
\text { only used when capillary pressure model } \\
\text { (CAP_MOD) } 3 \text { is specified; only Models } 1 \text { \& } 2 \\
\text { were used in } 1996 \text { CCA calculation; PO_MIN } \\
\text { value is a placeholder. }\end{array}$ \\
\hline 2569 & SALT_T4 & PO_MIN & $\begin{array}{l}\text { WPO\#40514 Sec. } 4.2 .4 \text { (BRAGFLO Analysis } \\
\text { Write-Up) pg. 4-155 through 4-156. }\end{array}$ & $\begin{array}{l}\text { PO_MIN is the minimum brine pressure. It is } \\
\text { only used when capillary pressure model } \\
\text { (CAP_MOD) } 3 \text { is specified; only Models } 1 \text { \& } 2 \\
\text { were used in } 1996 \text { CCA calculation; PO_MIN } \\
\text { value is a placeholder. }\end{array}$ \\
\hline 2623 & CAVITY_3 & PO_MIN & $\begin{array}{l}\text { WPO\#40514 Sec. } 4.2 .4 \text { (BRAGFLO Analysis } \\
\text { Write-Up) pg. 4-155 through 4-156. }\end{array}$ & $\begin{array}{l}\text { PO_MIN is the minimum brine pressure. It is } \\
\text { only used when capillary pressure model } \\
\text { (CAP_MOD) } 3 \text { is specified; only Models } 1 \text { \& } 2 \\
\text { were used in } 1996 \text { CCA calculation; PO_MIN } \\
\text { value is a placeholder. }\end{array}$ \\
\hline 2628 & CAVITY_4 & PO_MIN & $\begin{array}{l}\text { WPO\#40514 Sec. } 4.2 .4 \text { (BRAGFLO Analysis } \\
\text { Write-Up) pg. 4-155 through 4-156. }\end{array}$ & $\begin{array}{l}\text { PO_MIN is the minimum brine pressure. It is } \\
\text { only used when capillary pressure model } \\
\text { (CAP_MOD) } 3 \text { is specified; only Models } 1 \text { \& } 2 \\
\text { were used in } 1996 \text { CCA calculation; PO_MIN } \\
\text { value is a placeholder. }\end{array}$ \\
\hline 2718 & FORTYNIN & PO_MIN & $\begin{array}{l}\text { WPO } 40514 \text { Sec. } 4.2 .4 \text { (BRAGFLO Analysis } \\
\text { Write-Up) pg. 4-155 through 4-156. }\end{array}$ & $\begin{array}{l}\text { PO_MIN is the minimum brine pressure. It is } \\
\text { only used when capillary pressure model } \\
\text { (CAP_MOD) } 3 \text { is specified; only Models } 1 \& 2 \\
\text { were used in } 1996 \text { CCA calculation; PO_MIN } \\
\text { value is a placeholder. }\end{array}$ \\
\hline 2728 & MAGENTA & PO_MIN & $\begin{array}{l}\text { WPO\#40514 Sec. } 4.2 .4 \text { (BRAGFLO Analysis } \\
\text { Write-Up) pg. 4-155 through 4-156. }\end{array}$ & $\begin{array}{l}\text { PO_MIN is the minimum brine pressure. It is } \\
\text { only used when capillary pressure model } \\
\text { (CAP_MOD) } 3 \text { is specified; only Models } 1 \text { \& } 2 \\
\text { were used in } 1996 \text { CCA calculation; PO_MIN } \\
\text { value is a placeholder. }\end{array}$ \\
\hline 2739 & REPOSIT & PO_MIN & $\begin{array}{l}\text { WPO\#40514 Sec. } 4.2 .4 \text { (BRAGFLO Analysis } \\
\text { Write-Up) pg. 4-155 through 4-156. }\end{array}$ & $\begin{array}{l}\text { PO_MIN is the minimum brine pressure. It is } \\
\text { only used when capillary pressure model } \\
\text { (CAP_MOD) } 3 \text { is specified; only Models } 1 \text { \& } 2 \\
\text { were used in } 1996 \text { CCA calculation; PO_MIN } \\
\text { value is a placeholder. }\end{array}$ \\
\hline 2796 & TAMARISK & PO_MIN & $\begin{array}{l}\text { WPO\#40514 Sec. } 4.2 .4 \text { (BRAGFLO Analysis } \\
\text { Write-Up) pg. 4-155 through 4-156. }\end{array}$ & $\begin{array}{l}\text { PO_MIN is the minimum brine pressure. It is } \\
\text { only used when capillary pressure model } \\
\text { (CAP_MOD) } 3 \text { is specified; only Models } 1 \text { \& } 2 \\
\text { were used in } 1996 \text { CCA calculation; PO_MIN } \\
\text { value is a placeholder. }\end{array}$ \\
\hline 2802 & UNNAMED & PO_MIN & $\begin{array}{l}\text { WPO\#40514 Sec. } 4.2 .4 \text { (BRAGFLO Analysis } \\
\text { Write-Up) pg. 4-155 through 4-156. }\end{array}$ & $\begin{array}{l}\text { PO_MIN is the minimum brine pressure. It is } \\
\text { only used when capillary pressure model } \\
\text { (CAP_MOD) } 3 \text { is specified; only Models } 1 \text { \& } 2 \\
\text { were used in } 1996 \text { CCA calculation; PO_MIN } \\
\text { value is a placeholder. }\end{array}$ \\
\hline
\end{tabular}




\begin{tabular}{|c|c|c|}
\hline ID & \multicolumn{2}{|c|}{ Technical Traceabillty Documentation } \\
\hline 2518 & WPO\#36051 Memo: P. Vaughn to M. Tiemey 2/20/96 & \\
\hline 2535 & WPO\#36051 Memo: P. Vaughn to M. Tiemey 2/20/96 & \\
\hline 2552 & WPO\#36051 Memo: P. Vaughn to M. Tierney 2/20/96 & \\
\hline 2569 & WPO\#36051 Memo: P. Vaughn to M. Tierney 2/20/96 & \\
\hline 2623 & WPO\#36051 Memo: P. Vaughn to M. Tierney 2/20/96 & $\begin{array}{l}\text { Memo:Vaughn to Tierney } 2 / 14 / 96 \text { Constant Values for } \\
\text { CAVITY 1,2,3,4, Parameters }\end{array}$ \\
\hline 2628 & WPO\#36051 Memo: P. Vaughn to M. Tierney 2/20/96 & $\begin{array}{l}\text { Memo:Vaughn to Tierney } 2 / 14 / 96 \text { Constant Values for } \\
\text { CAVITY } 1,2,3,4 \text {, Parameters }\end{array}$ \\
\hline 2718 & WPO\#36051 Memo: P. Vaughn to M. Tierney 2/20/96 & \\
\hline 2728 & WPO\#36051 Memo: P. Vaughn to M. Tierney 2/20/96 & \\
\hline 2739 & WPO\#36051 Memo: P. Vaughn to M. Tiemey 2/20/96 & \\
\hline 2796 & WPO\#36051 Memo: P. Vaughn to M. Tiemey 2/20/96 & \\
\hline 2802 & WPO\#36051 Memo: P. Vaughn to M. Tiemey 2/20/96 & \\
\hline
\end{tabular}


Additional Sources

\begin{tabular}{|c|c|c|c|c|}
\hline ID & Matorial ID & Param ID & \multicolumn{2}{|c|}{ Technical Traceabllity Documentation } \\
\hline 2808 & CONC_T2 & PO_MIN & $\begin{array}{l}\text { WPO\#40514 Sec. } 4.2 .4 \text { (BRAGFLO Analysis } \\
\text { Write-Up) pg. 4-155 through 4-156. }\end{array}$ & $\begin{array}{l}\text { PO_MIN is the minimum brine pressure. It is } \\
\text { only used when capillary pressure model } \\
\text { (CAP_MOD) } 3 \text { is specified; only Models } 1 \& 2 \\
\text { were used in } 1996 \text { CCA calculation; PO_MIN } \\
\text { value is a placeholder. }\end{array}$ \\
\hline 3123 & $C L_{-} L_{-} T 4$ & PO_MIN & $\begin{array}{l}\text { WPO\#40514 Sec. } 4.2 .4 \text { (BRAGFLO Analysis } \\
\text { Write-Up) pg. 4-155 through 4-156. }\end{array}$ & $\begin{array}{l}\text { PO_MIN is the minimum brine pressure. It is } \\
\text { only used when capillary pressure model } \\
\text { (CAP_MOD) } 3 \text { is specified; only Models } 1 \& 2 \\
\text { were used in } 1996 \text { CCA calculation; PO_MIN } \\
\text { value is a placeholder. }\end{array}$ \\
\hline 3124 & CONC_MON & PO_MIN & $\begin{array}{l}\text { WPO\#40514 Sec. } 4.2 .4 \text { (BRAGFLO Analysis } \\
\text { Write-Up) pg. 4-155 through 4-156. }\end{array}$ & $\begin{array}{l}\text { PO_MIN is the minimum brine pressure. It is } \\
\text { only used when capillary pressure model } \\
\text { (CAP_MOD) } 3 \text { is specified; only Models } 1 \& 2 \\
\text { were used in } 1996 \text { CCA calculation; PO_MIN } \\
\text { value is a placeholder. }\end{array}$ \\
\hline 3125 & SALT_T5 & PO_MIN & $\begin{array}{l}\text { WPO\#40514 Sec. } 4.2 .4 \text { (BRAGFLO Analysis } \\
\text { Write-Up) pg. 4-155 through 4-156. }\end{array}$ & $\begin{array}{l}\text { PO_MIN is the minimum brine pressure. It is } \\
\text { only used when capillary pressure model } \\
\text { (CAP_MOD) } 3 \text { is specified; only Models } 182 \\
\text { were used in } 1996 \text { CCA calculation; PO_MIN } \\
\text { value is a placeholder. }\end{array}$ \\
\hline 3126 & SALT_T6 & PO_MIN & $\begin{array}{l}\text { WPO\#40514 Sec. } 4.2 .4 \text { (BRAGFLO Analysis } \\
\text { Write-Up) pg. 4-155 through 4-156. }\end{array}$ & $\begin{array}{l}\text { PO_MIN is the minimum brine pressure. It is } \\
\text { only used when capillary pressure model } \\
\text { (CAP_MOD) } 3 \text { is specified; only Models } 1 \text { \& } 2 \\
\text { were used in } 1996 \text { CCA calculation; PO_MIN } \\
\text { value is a placeholder. }\end{array}$ \\
\hline 3131 & CLAY_RUS & PO_MIN & $\begin{array}{l}\text { WPO } 40514 \text { Sec. } 4.2 .4 \text { (BRAGFLO Analysis } \\
\text { Write-Up) pg. 4-155 through 4-156. }\end{array}$ & $\begin{array}{l}\text { PO_MIN is the minimum brine pressure. It is } \\
\text { only used when capillary pressure model } \\
\text { (CAP_MOD) } 3 \text { is specified; only Models } 1 \& 2 \\
\text { were used in } 1996 \text { CCA calculation; PO_MIN } \\
\text { value is a placeholder. }\end{array}$ \\
\hline 3143 & BH_OPEN & PO_MIN & $\begin{array}{l}\text { WPO\#40514 Sec. } 4.2 .4 \text { (BRAGFLO Analysis } \\
\text { Write-Up) pg. 4-155 through 4-156. }\end{array}$ & $\begin{array}{l}\text { PO_MIN is the minimum brine pressure. It is } \\
\text { only used when capillary pressure model } \\
\text { (CAP_MOD) } 3 \text { is specified; only Models } 1 \text { \& } 2 \\
\text { were used in } 1996 \text { CCA calculation; PO_MIN } \\
\text { value is a placeholder. }\end{array}$ \\
\hline 3155 & CONC_PLG & PO_MIN & $\begin{array}{l}\text { WPO\#40514 Sec. } 4.2 .4 \text { (BRAGFLO Analysis } \\
\text { Write-Up) pg. 4-155 through 4-156. }\end{array}$ & $\begin{array}{l}\text { PO_MIN is the minimum brine pressure. It is } \\
\text { only used when capillary pressure model } \\
\text { (CAP_MOD) } 3 \text { is specified; only Models } 182 \\
\text { were used in } 1996 \text { CCA calculation; PO_MIN } \\
\text { value is a placeholder. }\end{array}$ \\
\hline 3167 & BH_SAND & PO_MIN & $\begin{array}{l}\text { WPO\#40514 Sec. } 4.2 .4 \text { (BRAGFLO Analysis } \\
\text { Write-Up) pg. 4-155 through 4-156. }\end{array}$ & $\begin{array}{l}\text { PO_MIN is the minimum brine pressure. It is } \\
\text { only used when capillary pressure model } \\
\text { (CAP_MOD) } 3 \text { is specified; only Models } 1 \text { \& } 2 \\
\text { were used in } 1996 \text { CCA calculation; PO_MIN } \\
\text { value is a placeholder. }\end{array}$ \\
\hline 3179 & BH_CREEP & PO_MIN & $\begin{array}{l}\text { WPO\#40514 Sec. } 4.2 .4 \text { (BRAGFLO Analysis } \\
\text { Write-Up) pg. 4-155 through 4-156. }\end{array}$ & $\begin{array}{l}\text { PO_MIN is the minimum brine pressure. It is } \\
\text { only used when capillary pressure model } \\
\text { (CAP_MOD) } 3 \text { is specified; only Models } 1 \& 2 \\
\text { were used in } 1996 \text { CCA calculation; PO_MIN } \\
\text { value is a placeholder. }\end{array}$ \\
\hline 587 & S_MB139 & PORE_DIS & PRP WPO\#30643 & Core Labs AF-3945 T=0 memo WPO 42351 \\
\hline 566 & S_MB138 & PORE_DIS & PRP WPO\#30643 & Core Labs AF-3945 T=0 memo WPO 42351 \\
\hline
\end{tabular}




\begin{tabular}{|c|c|c|}
\hline ID & \multicolumn{2}{|c|}{ Technical Traceabillty Documentation } \\
\hline 2808 & WPO\#36051 Memo: P. Vaughn to M. Tierney $2 / 20 / 96$ & - \\
\hline 3123 & WPO\#36051 Memo: P. Vaughn to M. Tierney $2 / 20 / 96$ & \\
\hline 3124 & WPO\#36051 Memo: P. Vaughn to M. Tierney 2/20/96 & \\
\hline 3125 & WPO\#36051 Memo: P. Vaughn to M. Tiemey 2/20/96 & \\
\hline 3126 & WPO\#36051 Memo: P. Vaughn to M. Tierney 2/20/96 & \\
\hline 3131 & WPO\#36051 Memo: P. Vaughn to M. Tierney 2/20/96 & \\
\hline 3143 & WPO\#36051 Memo: P. Vaughn to M. Tierney 2/20/96 & $\begin{array}{l}\text { WPO\#35134 Memo:Vaughn to Tiemey "Borehole Properties" } \\
\text { 3/13/96" (handwritten } 4 \text { page note with parameter values - } \\
\text { contains an email cover page). }\end{array}$ \\
\hline 3155 & WPO\#36051 Memo: P. Vaughn to M. Tierney 2/20/96 & $\begin{array}{l}\text { WPO\#35134 Memo:Vaughn to Tiemey "Borehole Properties" } \\
\text { 3/13/96" (handwritten } 4 \text { page note with parameter values - } \\
\text { contains an email cover page). }\end{array}$ \\
\hline 3167 & WPO\#36051 Memo: P. Vaughn to M. Tierney $2 / 20 / 96$ & $\begin{array}{l}\text { WPO\#35134 Memo:Vaughn to Tiemey "Borehole Properties" } \\
\text { 3/13/96" (handwritten } 4 \text { page note with parameter values - } \\
\text { contains an email cover page). }\end{array}$ \\
\hline 3179 & WPO\#36051 Memo: P. Vaughn to M. Tiemey 2/20/96 & $\begin{array}{l}\text { WPO\#35134 Memo:Vaughn to Tiemey "Borehole Properties" } \\
\text { 3/13/96" (handwritten } 4 \text { page note with parameter values -. } \\
\text { contains an email cover page). }\end{array}$ \\
\hline 587 & $\begin{array}{l}\text { WPO\#30643 SWCF- } \\
\text { A:WBS1.2.07.1:PDD:QA:Salado:PKG10:Anh2 Phase } \\
\text { Parameters }\end{array}$ & \\
\hline 566 & $\begin{array}{l}\text { WPO\#30643 SWCF- } \\
\text { A:WBS1.2.07.1:PDD:QA:Salado:PKG10:Anh2 Phase } \\
\text { Parameters }\end{array}$ & \\
\hline
\end{tabular}


Additional Sources

\begin{tabular}{|c|c|c|c|c|}
\hline ID & Material ID & Param ID & \multicolumn{2}{|c|}{ Technical Traceablilty Documentation } \\
\hline 527 & S_ANH_AB & PORE_DIS & PRP WPO\#30643 & Core Labs AF-3945 T=0 memo WPO 42351 \\
\hline 588 & S_MB139 & POROSITY & & \\
\hline 567 & S_MB138 & POROSITY & & \\
\hline 528 & S_ANH_AB & POROSITY & & \\
\hline 590 & S_MB139 & $\begin{array}{c}\text { PRESSURE } \\
.\end{array}$ & $\begin{array}{l}\text { Not used per BRAGFLO analysis p. 4-2 - see } \\
\text { also BRAGFLO anaiysis p. 4-124 and Section } \\
\text { 3.2.3 }\end{array}$ & $\begin{array}{l}\text { WPO\#31185 SWCF- } \\
\text { A:WBS1.2.07.1:PDD:QA:Salado:PKG12:Anyh } \\
\text { drite Pressure }\end{array}$ \\
\hline 569 & S_MB138 & PRESSURE & $\begin{array}{l}\text { Not used per BRAGFLO analysis p. } 4-2-\text { see } \\
\text { also BRAGFLO analysis p. } 4-124 \text { and Section } \\
\text { 3.2.3 }\end{array}$ & $\begin{array}{l}\text { WPO\#31185 SWCF- } \\
\text { A:WBS1.2.07.1:PDD:QA:Salado:PKG12:Anyh } \\
\text { drite Pressure }\end{array}$ \\
\hline 591 & S_MB139 & PRMX_LOG & $\begin{array}{l}\text { WPO\#30603, WPO\#30605, WPO\#30606, } \\
\text { WPO\#31217 SWCF- } \\
\text { A:WBS1.2.07.1:PDD:QA:Salado:Pkg13:Perme } \\
\text { ability }\end{array}$ & $\begin{array}{l}\text { WPO\#41677 Memo: Tiemey to Dotson } \\
\text { "Justification memo for choice of anhydrite } \\
\text { permeabilities (MB138, MB139, Anh } \\
\text { A\&B",10/30/96. Discusses defining the values } \\
\text { for anhydrite permeabilities (a somewhat } \\
\text { condensed version of WPO\#39065). }\end{array}$ \\
\hline 592 & S_MB139 & PRMY_LOG & $\begin{array}{l}\text { WPO\#30603, WPO\#30605, WPO\#30606, } \\
\text { WPO\#31217 SWCF. } \\
\text { A:WBS1.2.07.1:PDD:QA:Salado:Pkg13:Perme } \\
\text { ability }\end{array}$ & $\begin{array}{l}\text { WPO\#41677 Memo: Tierney to Dotson } \\
\text { "Justification memo for choice of anhydrite } \\
\text { permeabilities (MB138, MB139, Anh } \\
\text { A\&B",10/30/96. Discusses defining the values } \\
\text { for anhydrite permeabilities (a somewhat } \\
\text { condensed version of WPO\#39065). }\end{array}$ \\
\hline 593 & S_MB139 & PRMZ_LOG & $\begin{array}{l}\text { WPO\#30603, WPO\#30605, WPO\#30606, } \\
\text { WPO\#31217 SWCF- } \\
\text { A:WBS1.2.07.1:PDD:QA:Salado:Pkg13:Perme } \\
\text { ability }\end{array}$ & $\begin{array}{l}\text { WPO\#41677 Memo: Tierney to Dotson } \\
\text { "Justification memo for choice of anhydrite } \\
\text { permeabilities (MB138, MB139, Anh } \\
\text { A\&B",10/30/96. Discusses defining the values } \\
\text { for anhydrite permeabilities (a somewhat } \\
\text { condensed version of WPO\#39065). }\end{array}$ \\
\hline 570 & S_MB138 & PRMX_LOG & $\begin{array}{l}\text { WPO\#30603, WPO\#30605. WPO\#30606, } \\
\text { WPO\#31217 SWCF- } \\
\text { A:WBS1.2.07.1:PDD:QA:Salado:Pkg13:Perme } \\
\text { ability }\end{array}$ & $\begin{array}{l}\text { WPO\#41677 Memo: Tiemey to Dotson } \\
\text { "Justification memo for choice of anhydrite } \\
\text { permeabilities (MB138, MB139, Anh } \\
\text { A\&B",10/30/96. Discusses defining the values } \\
\text { for anhydrite permeabilities (a somewhat } \\
\text { condensed version of WPO\#39065). }\end{array}$ \\
\hline 571 & S_MB138 & PRMY_LOG & $\begin{array}{l}\text { WPO\#30603, WPO\#30605, WPO\#30606, } \\
\text { WPO\#31217 SWCF- } \\
\text { A:WBS1.2.07.1:PDD:QA:Salado:Pkg13:Perme } \\
\text { ability }\end{array}$ & $\begin{array}{l}\text { WPO\#41677 Memo: Tiemey to Dotson } \\
\text { "Justification memo for choice of anhydrite } \\
\text { permeabilities (MB138, MB139, Anh } \\
\text { A\&B",10/30/96. Discusses defining the values } \\
\text { for anhydrite permeabilities (a somewhat } \\
\text { condensed version of WPO\#39065). }\end{array}$ \\
\hline
\end{tabular}




\section{Additional Sources}

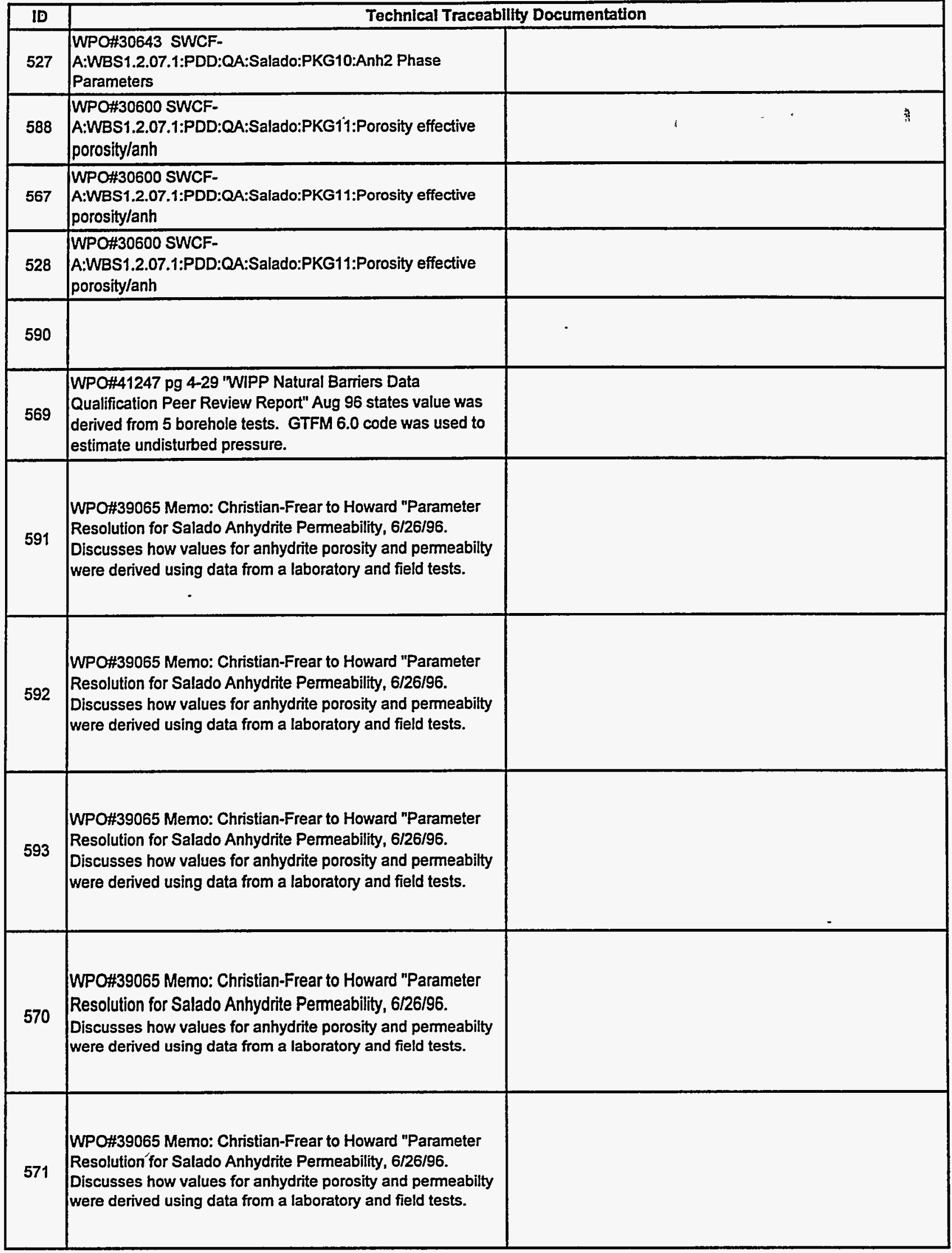


Additional Sources

\begin{tabular}{|c|c|c|c|c|}
\hline ID & Material ID & Param ID & \multicolumn{2}{|c|}{ Technical Traceability Documentation } \\
\hline 572 & S_MB138 & PRMZ_LOG & $\begin{array}{l}\text { WPO\#30603, WPO\#30605, WPO\#30606, } \\
\text { WPO\#31217 SWCF- } \\
\text { A:WBS1.2.07.1:PDD:QA:Salado:Pkg13:Perme } \\
\text { ability }\end{array}$ & $\begin{array}{l}\text { WPO\#41677 Memo: Tiemey to Dotson } \\
\text { "Justification memo for choice of anhydrite } \\
\text { permeabilities (MB138, MB139, Anh } \\
\text { A\&B",10/30/96. Discusses defining the values } \\
\text { for anhydrite permeabilities (a somewhat } \\
\text { condensed version of WPO } 39065 \text { ). }\end{array}$ \\
\hline 531 & S_ANH_AB & PRMX_LOG & $\begin{array}{l}\text { WPO\#30603, WPO\#30605, WPO\#30606, } \\
\text { WPO\#31217 SWCF- } \\
\text { A:WBS1.2.07.1:PDD:QA:Salado:Pkg13:Perme } \\
\text { ability }\end{array}$ & $\begin{array}{l}\text { WPO\#41677 Memo: Tiemey to Dotson } \\
\text { "Justification memo for choice of anhydrite } \\
\text { permeabilities (MB138, MB139, Anh } \\
\text { A\&B",10/30/96. Discusses defining the values } \\
\text { for anhydrite permeabilities (a somewhat } \\
\text { condensed version of WPO\#39065). }\end{array}$ \\
\hline 532 & S_ANH_AB & PRMY_LOG & $\begin{array}{l}\text { WPO\#30603, WPO\#30605, WPO\#30606, } \\
\text { WPO\#31217 SWCF- } \\
\text { A:WBS1.2.07.1:PDD:QA:Salado:Pkg13:Peme } \\
\text { ability }\end{array}$ & $\begin{array}{l}\text { WPO\#41677 Memo: Tiemey to Dotson } \\
\text { "Justification memo for choice of anhydrite } \\
\text { permeabilities (MB138, MB139, Anh } \\
\text { A\&B",10/30/96. Discusses defining the values } \\
\text { for anhydrite permeabilities (a somewhat } \\
\text { condensed version of WPO\#39065). }\end{array}$ \\
\hline 533 & S_ANH_AB & PRMZ_LOG & $\begin{array}{l}\text { WPO\#30603, WPO\#30605, WPO\#30606, } \\
\text { WPO\#31217 SWCF- } \\
\text { A:WBS1.2.07.1:PDD:QA:Salado:Pkg13:Perme } \\
\text { ability }\end{array}$ & $\begin{array}{l}\text { WPO\#41677 Memo: Tierney to Dotson } \\
\text { "Justification memo for choice of anhydrite } \\
\text { permeabilities (MB138, MB139, Anh } \\
\text { A\&B",10/30/96. Discusses defining the values } \\
\text { for anhydrite permeabilities (a somewhat } \\
\text { condensed version of WPO\#39065). }\end{array}$ \\
\hline 596 & S_MB139 & RELP_MOD & $\begin{array}{l}\text { WPO\#30643 SWCF- } \\
\text { A:WBS.1.2.07.1:PDD:QA:Salado:PKG10:Anh } \\
\text { 2 Phase Parameters. }\end{array}$ & \\
\hline 575 & S_MB138 & RELP_MOD & $\begin{array}{l}\text { WPO\#30643 SWCF- } \\
\text { A:WBS.1.2.07.1:PDD:QA:Salado:PKG10:Anh } \\
\text { 2 Phase Parameters. }\end{array}$ & \\
\hline 536 & S_ANH_AB & RELP_MOD & $\begin{array}{l}\text { WPO\#30643 SWCF- } \\
\text { A:WBS.1.2.07.1:PDD:QA:Salado:PKG10:Anh } \\
\text { 2 Phase Parameters. }\end{array}$ & \\
\hline 597 & S_MB139 & SAT_IBRN & $\begin{array}{l}\text { WPO\#30643 SWCF- } \\
\text { A:WBS.1.2.07.1:PDD:QA:Salado:PKG10:Anh } \\
\text { 2 Phase Parameters. }\end{array}$ & \\
\hline 576 & S_MB138 & SAT_IBRN & $\begin{array}{l}\text { WPO\#30643 SWCF- } \\
\text { A:WBS.1.2.07.1:PDD:QA:Salado:PKG10:Anh } \\
\text { 2 Phase Parameters. }\end{array}$ & \\
\hline 537 & S_ANH_AB & SAT_IBRN & $\begin{array}{l}\text { WPO\#30643 SWCF- } \\
\text { A:WBS.1.2.07.1:PDD:QA:Salado:PKG10:Anh } \\
\text { 2 Phase Parameters. }\end{array}$ & \\
\hline 598 & S_MB139 & SAT_RBRN & $\begin{array}{l}\text { WPO\#30643 SWCF- } \\
\text { A:WBS.1.2.07.1:PDD:QA:Salado:PKG10:Anh } \\
\text { 2 Phase Parameters. }\end{array}$ & \\
\hline 577 & S_MB138 & SAT_RBRN & $\begin{array}{l}\text { WPO\#30643 SWCF- } \\
\text { A:WBS.1.2.07.1:PDD:QA:Salado:PKG10:Anh } \\
\text { 2 Phase Parameters. }\end{array}$ & \\
\hline 538 & S_ANH_AB & SAT_RBRN & $\begin{array}{l}\text { WPO\#30643 SWCF- } \\
\text { A:WBS.1.2.07.1:PDD:QA:Salado:PKG10:Anh } \\
\text { 2 Phase Parameters. }\end{array}$ & \\
\hline 599 & S_MB139 & SAT_RGAS & $\begin{array}{l}\text { WPO\#30643 SWCF- } \\
\text { A:WBS.1.2.07.1:PDD:QA:Salado:PKG10:Anh } \\
\text { 2 Phase Parameters. }\end{array}$ & \\
\hline
\end{tabular}




\section{Additional Sources}

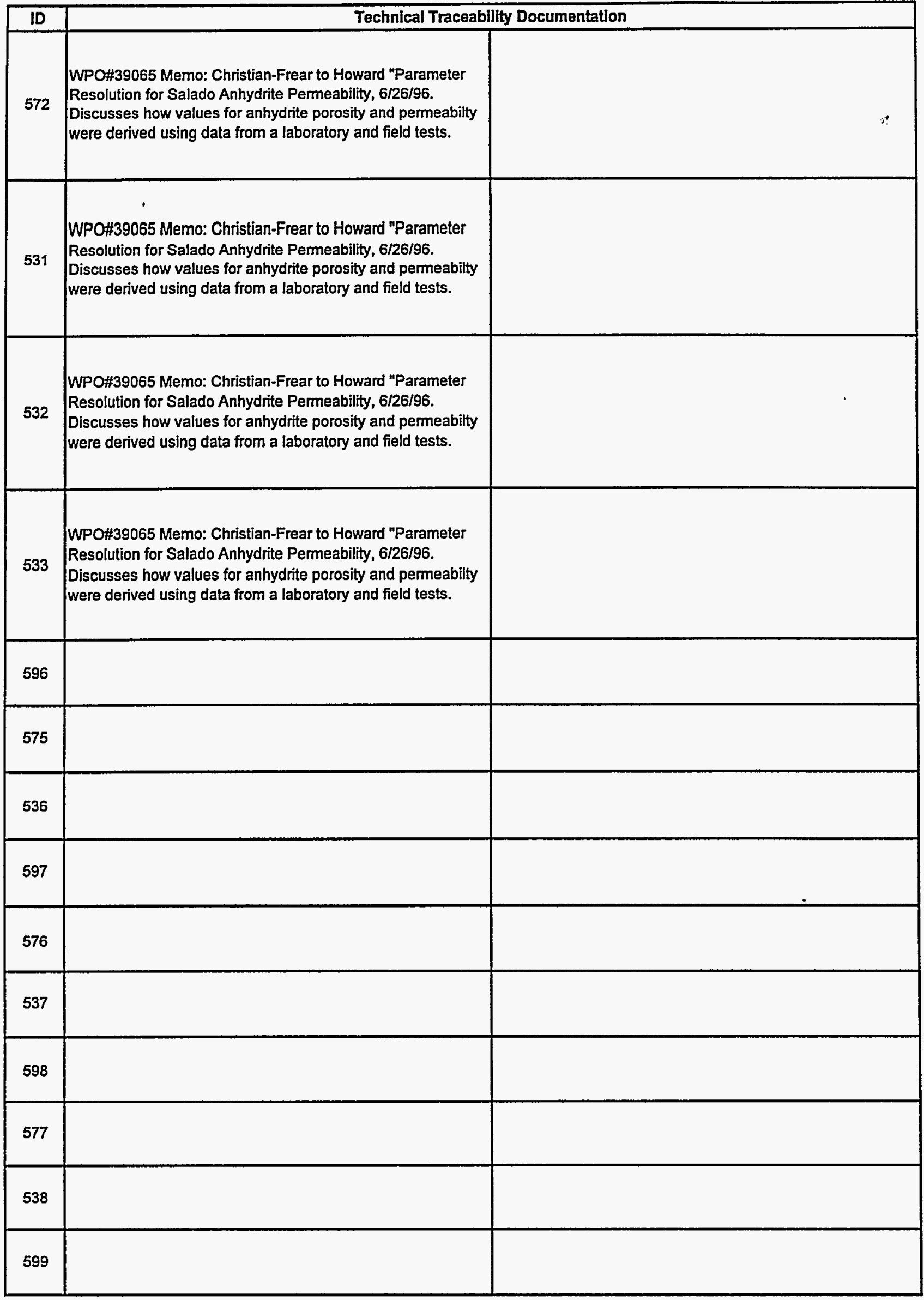


Additional Sources

\begin{tabular}{|c|c|c|c|c|}
\hline ID & Material ID & Param ID & \multicolumn{2}{|c|}{ Technical Traceability Documentation } \\
\hline 578 & S_MB138 & SAT_RGAS & $\begin{array}{l}\text { WPO\#30643 SWCF- } \\
\text { A:WBS.1.2.07.1:PDD:QA:Salado:PKG10:Anh } \\
\text { 2 Phase Parameters. }\end{array}$ & \\
\hline 539 & S_ANH_AB & SAT_RGAS & $\begin{array}{l}\text { WPO\#30643 SWCF- } \\
\text { A:WBS.1.2.07.1:PDD:QA:Salado:PKG10:Anh } \\
\text { 2 Phase Parameters. }\end{array}$ & \\
\hline 2939 & SALT_T1 & CUMPROB & $\begin{array}{l}\text { WPO\#37542 Memo: P.Vaugn and D. McArthur } \\
\text { to M. Tierney "CUMPROB Parameter } \\
\text { Definition and Usage" 5/20/96 }\end{array}$ & \\
\hline 2516 & SALT_T1 & PORE_DIS & $\begin{array}{l}\text { WPO } 30640: \text { WIPP Shaft Seal System } \\
\text { BRAGFLO Parameters, SWCF- } \\
\text { A:1.1.03.2.1:QA:PDD Sec II Vol 2, 2.5.7; Sec I } \\
\text { Vol 4 4.3 }\end{array}$ & $\begin{array}{l}\text { WPO\#32287 Memo: Hurtado to Tiemey } \\
2 / 12 / 90 \text {. WPO\#39058 memo: McCord to } M \text {. } \\
\text { Tierney "Statement of impact of pore } \\
\text { distribution range error on BRAGFLO CCA } \\
\text { calculation 7/3/96. }\end{array}$ \\
\hline 2533 & SALT_T2 & PORE_DIS & $\begin{array}{l}\text { WPO\#30640: WIPP Shaft Seal System } \\
\text { BRAGFLO Parameters, SWCF- } \\
\text { A:1.1.03.2.1:QA:PDD Sec II Vol 2, 2.5.7; Sec I } \\
\text { Vol 4 4.3 }\end{array}$ & $\begin{array}{l}\text { WPO\#32287 Memo: Hurtado to Tiemey } \\
2 / 12 / 90 \text {. WPO\#39058 memo: McCord to M. } \\
\text { Tierney "Statement of impact of pore } \\
\text { distribution range error on BRAGFLO CCA } \\
\text { calculation 7/3/96. }\end{array}$ \\
\hline 2550 & SALT_T3 & PORE_DIS & $\begin{array}{l}\text { WPO\#30640: WIPP Shaft Seal System } \\
\text { BRAGFLO Parameters, SWCF- } \\
\text { A:1.1.03.2.1:QA:PDD Sec II Vol 2, 2.5.7; Sec I } \\
\text { Vol 4 4.3 }\end{array}$ & $\begin{array}{l}\text { WPO\#32287 Memo: Hurtado to Tiemey } \\
2 / 12 / 90 \text {. WPO\#39058 memo: MeCord to M. } \\
\text { Tierney "Statement of impact of pore } \\
\text { distribution range error on BRAGFLO CCA } \\
\text { calculation 7/3/96. }\end{array}$ \\
\hline 2567 & SALT_T4 & PORE_DIS & $\begin{array}{l}\text { WPO\#30640: WIPP Shaft Seal System } \\
\text { BRAGFLO Parameters, SWCF- } \\
\text { A:1.1.03.2.1:QA:PDD Sec II Vol 2, 2.5.7; Sec I } \\
\text { Vol 4 4.3 }\end{array}$ & $\begin{array}{l}\text { WPO\#32287 Memo: Hurtado to Tierney } \\
2 / 12 / 90 \text {. WPO\#39058 memo: McCord to M. } \\
\text { Tiemey "Statement of impact of pore } \\
\text { distribution range error on BRAGFLO CCA } \\
\text { calculation } 7 / 3 / 96 \text {. }\end{array}$ \\
\hline 2809 & SALT_T5 & PORE_DIS & $\begin{array}{l}\text { WPO\#30640: WIPP Shaft Seal System } \\
\text { BRAGFLO Parameters, SWCF. } \\
\text { A:1.1.03.2.1:QA:PDD Sec II Vol 2, 2.5.7; Sec I } \\
\text { Vol 4 4.3 }\end{array}$ & $\begin{array}{l}\text { WPO\#32287 Memo: Hurtado to Tiemey } \\
2 / 12 / 90 \text {. WPO\#39058 memo: McCord to M. } \\
\text { Tiemey "Statement of impact of pore } \\
\text { distribution range error on BRAGFLO CCA } \\
\text { calculation } 7 / 3 / 96 \text {. }\end{array}$ \\
\hline 2989 & SALT_T6 & PORE_DIS & $\begin{array}{l}\text { WPO\#30640: WIPP Shaft Seal System } \\
\text { BRAGFLO Parameters, SWCF- } \\
\text { A:1.1.03.2.1:QA:PDD Sec II Vol 2, 2.5.7; Sec I } \\
\text { Vol 44.3 }\end{array}$ & $\begin{array}{l}\text { WPO\#32287 Memo: L. D. Hurtado to M. } \\
\text { Tierney "Correction of Lamda Distribution" } \\
2 / 12 / 96\end{array}$ \\
\hline 2499 & EARTH & PORE_DIS & $\begin{array}{l}\text { WPO\#30640: WIPP Shaft Seal System } \\
\text { BRAGFLO Parameters, SWCF- } \\
\text { A:1.1.03.2.1:QA:PDD Sec II Vol 2, 2.1.9 }\end{array}$ & $\begin{array}{l}\text { WPO\#32287 Memo:L. D. Hurtado to M. } \\
\text { Tierney "Correction of Lamda Distribution" } \\
\text { 2/12/96. }\end{array}$ \\
\hline 3006 & CLAY_RUS & PORE_DIS & $\begin{array}{l}\text { WPO\#30640: WIPP Shaft Seal System } \\
\text { BRAGFLO Parameters, SWCF- } \\
\text { A:1.1.03.2.1:QA:PDD Sec II Vol 2, 2.1.9; Sec I } \\
\text { Vol 4 4.2 }\end{array}$ & $\begin{array}{l}\text { WPO\#32287 Memo:L. D. Hurtado to M. } \\
\text { Tiemey "Correction of Lamda Distribution" } \\
\text { 2/12/96. }\end{array}$ \\
\hline 2330 & CL_L_T1 & PORE_DIS & $\begin{array}{l}\text { WPO\#30640: WIPP Shaft Seal System } \\
\text { BRAGFLO Parameters, SWCF- } \\
\text { A:1.1.03.2.1:QA:PDD Sec II Vol 2, 2.1.9; Sec I } \\
\text { Vol 4 4.2 }\end{array}$ & $\begin{array}{l}\text { WPO\#32287 Memo:L. D. Hurtado to M. } \\
\text { Tiemey "Correction of Lamda Distribution" } \\
2 / 12 / 96 .\end{array}$ \\
\hline 2347 & CL_L_T2 & PORE_DIS & $\begin{array}{l}\text { WPO\#30640: WIPP Shaft Seal System } \\
\text { BRAGFLO Parameters, SWCF- } \\
\text { A:1.1.03.2.1:QA:PDD Sec II Vol 2, 2.1.9; Sec I } \\
\text { Vol 4 4.2 }\end{array}$ & $\begin{array}{l}\text { WPO\#32287 Memo:L. D. Hurtado to M. } \\
\text { Tierney "Correction of Lamda Distribution" } \\
2 / 12 / 96 .\end{array}$ \\
\hline 2364 & CL_L_T3 & PORE_DIS & $\begin{array}{l}\text { WPO\#30640: WIPP Shaft Seal System } \\
\text { BRAGFLO Parameters, SWCF- } \\
\text { A:1.1.03.2.1:QA:PDD Sec II Vol 2, 2.1.9; Sec I } \\
\text { Vol 4 4.2 }\end{array}$ & $\begin{array}{l}\text { WPO\#32287 Memo:L. D. Hurtado to M. } \\
\text { Tierney "Correction of Lamda Distribution" } \\
2 / 12 / 96 .\end{array}$ \\
\hline
\end{tabular}


Additional Sources

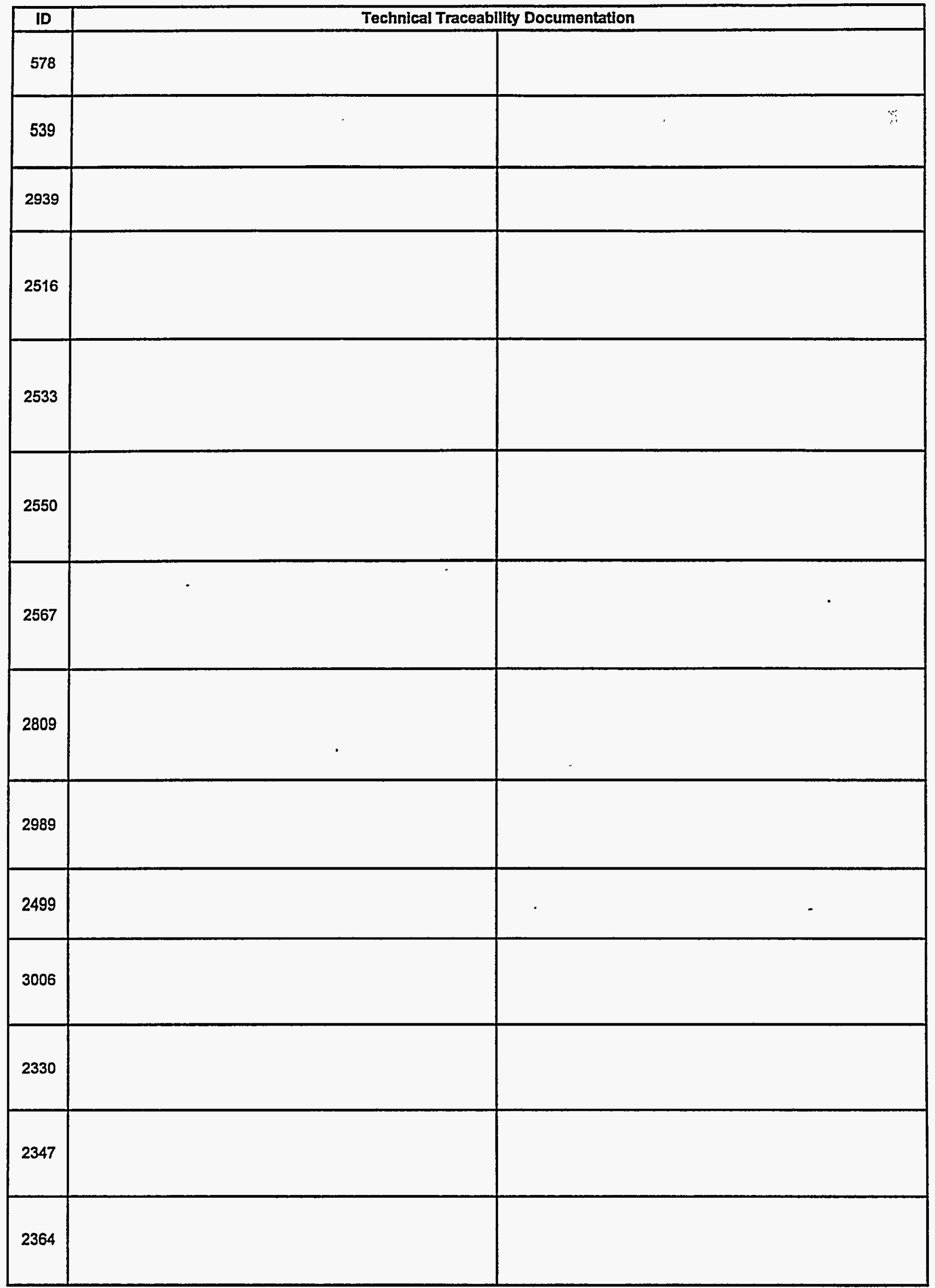


Additional Sources

\begin{tabular}{|c|c|c|c|c|}
\hline ID & Material ID & Param ID & \multicolumn{2}{|c|}{ Technical Traceability Documentation } \\
\hline 3076 & CL_L_T4 & PORE_DIS & $\begin{array}{l}\text { WPO\#30640: WIPP Shaft Seal System } \\
\text { BRAGFLO Parameters, SWCF- } \\
\text { A:1.1.03.2.1:QA:PDD Sec II Vol 2, 2.1.9; Sec I } \\
\text { Vol 4 4.2 }\end{array}$ & $\begin{array}{l}\text { WPO\#32287 Memo:L. D. Hurtado to M. } \\
\text { Tierney "Correction of Lamda Distribution" } \\
2 / 12 / 96 .\end{array}$ \\
\hline 2381 & CL_M_T1 & PORE_DIS & $\begin{array}{l}\text { WPO\#30640: WIPP Shaft Seal System } \\
\text { BRAGFLO Parameters, SWCF- } \\
\text { A:1.1.03.2.1:QA:PDD Sec II Vol 2, 2.1.9; Sec I } \\
\text { Vol 4 4.2 }\end{array}$ & $\begin{array}{l}\text { WPO\#32287 Memo:L. D. Hurtado to M. } \\
\text { Tiemey "Correction of Lamda Distribution" } \\
2 / 12 / 96 .\end{array}$ \\
\hline 2398 & CL_M_T2 & PORE_DIS & $\begin{array}{l}\text { WPO\#30640: WIPP Shaft Seal System } \\
\text { BRAGFLO Parameters, SWCF- } \\
\text { A:1.1.03.2.1:QA:PDD Sec II Vol 2, 2.1.9; Sec I } \\
\text { Vol 4 4.2 }\end{array}$ & $\begin{array}{l}\text { WPO\#32287 Memo:L. D. Hurtado to M. } \\
\text { Tiemey "Correction of Lamda Distribution" } \\
2 / 12 / 96 .\end{array}$ \\
\hline 2415 & CL_M_T3 & PORE_DIS & $\begin{array}{l}\text { WPO\#30640: WIPP Shaft Seal System } \\
\text { BRAGFLO Parameters, SWCF- } \\
\text { A:1.1.03.2.1:QA:PDD Sec II Vol 2, 2.1.9; Sec I } \\
\text { Vol 4 4.2 }\end{array}$ & $\begin{array}{l}\text { WPO\#32287 Memo:L. D. Hurtado to M. } \\
\text { Tiemey "Correction of Lamda Distribution" } \\
2 / 12 / 96 .\end{array}$ \\
\hline 2432 & CL_M_T4 & PORE_DIS & $\begin{array}{l}\text { WPO\#30640: WIPP Shaft Seal System } \\
\text { BRAGFLO Parameters, SWCF- } \\
\text { A:1.1.03.2.1:QA:PDD Sec II Vol 2, 2.1.9; Sec I } \\
\text { Vol 4 4.2 }\end{array}$ & $\begin{array}{l}\text { WPO\#32287 Memo:L. D. Hurtado to M. } \\
\text { Tiemey "Correction of Lamda Distribution" } \\
2 / 12 / 96 .\end{array}$ \\
\hline 2449 & CL_M_T5 & PORE_DIS & $\begin{array}{l}\text { WPO\#30640: WIPP Shaft Seal System } \\
\text { BRAGFLO Parameters, SWCF- } \\
\text { A:1.1.03.2.1:QA:PDD Sec II Vol 2, 2.1.9; Sec I } \\
\text { Vol 4 4.2 }\end{array}$ & $\begin{array}{l}\text { WPO\#32287 Memo:L. D. Hurtado to M. } \\
\text { Tiemey "Correction of Lamda Distribution" } \\
2 / 12 / 96 .\end{array}$ \\
\hline 2313 & CLAY_BOT & PORE_DIS & $\begin{array}{l}\text { WPO\#30640: WIPP Shaft Seal System } \\
\text { BRAGFLO Parameters, SWCF- } \\
\text { A:1.1.03.2.1:QA:PDD Sec II Vol 2, 2.1.9; Sec I } \\
\text { Vol 4 4.2 }\end{array}$ & $\begin{array}{l}\text { WPO\#32287 Memo:L. D. Hurtado to M. } \\
\text { Tierney "Correction of Lamda Distribution" } \\
2 / 12 / 96 \text {. }\end{array}$ \\
\hline 2466 & CONC_T1 & PORE_DIS & $\begin{array}{l}\text { WPO\#30640: WIPP Shaft Seal System } \\
\text { BRAGFLO Parameters, SWCF- } \\
\text { A:1.1.03.2.1:QA:PDD Sec II Vol 2, 2.1.9 }\end{array}$ & $\begin{array}{l}\text { WPO\#32287 Memo:L. D. Hurtado to M. } \\
\text { Tiemey "Correction of Lamda Distribution" } \\
2 / 12 / 96 .\end{array}$ \\
\hline 2483 & CONC_T2 & PORE_DIS & $\begin{array}{l}\text { WPO\#30640: WIPP Shaft Seal System } \\
\text { BRAGFLO Parameters, SWCF. } \\
\text { A:1.1.03.2.1:QA:PDD Sec II Vol 2, 2.1.9 }\end{array}$ & $\begin{array}{l}\text { WPO\#32287 Memo:L. D. Hurtado to M. } \\
\text { Tiemey "Correction of Lamda Distribution" } \\
2 / 12 / 96 \text {. }\end{array}$ \\
\hline 3057 & CONC_MON & PORE_DIS & $\begin{array}{l}\text { WPO\#30640: WIPP Shaft Seal System } \\
\text { BRAGFLO Parameters, SWCF- } \\
\text { A:1.1.03.2.1:QA:PDD Sec II Vol 2, 2.4.7; Sec I } \\
\text { Vol } 4 \text { 4.4 }\end{array}$ & $\begin{array}{l}\text { WPO\#32287 Memo:L. D. Hurtado to M. } \\
\text { Tiemey "Correction of Lamda Distribution" } \\
2 / 12 / 96 .\end{array}$ \\
\hline 2279 & ASPHALT & PORE_DIS & $\begin{array}{l}\text { WPO\#30640: WIPP Shaft Seal System } \\
\text { BRAGFLO Parameters, SWCF- } \\
\text { A:1.1.03.2.1:QA:PDD Sec II Vol 2, 2.1.9; Sec I } \\
\text { Vol 4 4.5 }\end{array}$ & $\begin{array}{l}\text { WPO\#32287 Memo:L. D. Hurtado to M. } \\
\text { Tierney "Correction of Lamda Distribution" } \\
2 / 12 / 96 \text {. }\end{array}$ \\
\hline 2528 & SALT_T1 & SAT_RBRN & $\begin{array}{l}\text { WPO\#30640: WPP Shaft Seal System } \\
\text { BRAGFLO Parameters, SWCF- } \\
\text { A:1.1.03.2.1:QA:PDD Sec II Vol 2, 2.5.5; Sec } 1 \\
\text { Vol 4 4.3 }\end{array}$ & \\
\hline 2545 & SALT_T2 & SAT_RBRN & $\begin{array}{l}\text { WPO\#30640: WIPP Shaft Seal System } \\
\text { BRAGFLO Parameters, SWCF- } \\
\text { A:1.1.03.2.1:QA:PDD Sec II Vol 2, 2.5.5; Sec } 1 \\
\text { Vol 4 4.3 }\end{array}$ & \\
\hline 2562 & SALT_T3 & SAT_RBRN & $\begin{array}{l}\text { WPO\#30640: WIPP Shaft Seal System } \\
\text { BRAGFLO Parameters, SWCF- } \\
\text { A:1.1.03.2.1:QA:PDD Sec II Vol 2, 2.5.5; Sec } 1 \\
\text { Vol 4 4.3 }\end{array}$ & \\
\hline 2579 & SALT_T4 & SAT_RBRN & $\begin{array}{l}\text { WPO\#30640: WIPP Shaft Seal System } \\
\text { BRAGFLO Parameters, SWCF- } \\
\text { A:1.1.03.2.1:QA:PDD Sec II Vol 2, 2.5.5; Sec } 1 \\
\text { Vol 4 4.3 }\end{array}$ & \\
\hline
\end{tabular}


Additional Sources

\begin{tabular}{|c|l|l|}
\hline ID & \multicolumn{2}{|c|}{ Technical Traceability Documentation } \\
\hline 3076 & & \\
\hline 2381 & & \\
\hline 2398 & & \\
\hline 2415 & & \\
\hline 2432 & & \\
\hline 2449 & & \\
\hline 2313 & & \\
\hline 2466 & & \\
\hline 2483 & & \\
\hline 3057 & & \\
\hline 2579 & & \\
\hline & & \\
\hline
\end{tabular}

M2-65 
Additional Sources

\begin{tabular}{|c|c|c|c|c|}
\hline ID & Material ID & Param ID & \multicolumn{2}{|c|}{ Technical Traceabillty Documentation } \\
\hline 2596 & SALT_T5 & SAT_RBRN & $\begin{array}{l}\text { WPO\#30640: WIPP Shaft Seal System } \\
\text { BRAGFLO Parameters, SWCF- } \\
\text { A:1.1.03.2.1:QA:PDD Sec II Vol 2, 2.5.5; Sec } 1 \\
\text { Vol 4 4.3 }\end{array}$ & \\
\hline 2992 & SALT_T6 & SAT_RBRN & $\begin{array}{l}\text { WPO\#30640: WIPP Shaft Seal System } \\
\text { BRAGFLO Parameters, SWCF- } \\
\text { A:1.1.03.2.1:QA:PDD Sec II Vol 2, 2.5.5; Sec } 1 \\
\text { Vol 4 4.3 }\end{array}$ & \\
\hline 2511 & EARTH & SAT_RBRN & $\begin{array}{l}\text { WPO\#30640: WIPP Shaft Seal System } \\
\text { BRAGFLO Parameters, SWCF- } \\
\text { A:1.1.03.2.1:QA:PDD Sec II Vol 2, 2.2.7 }\end{array}$ & \\
\hline 3014 & CLAY_RUS & SAT_RBRN & $\begin{array}{l}\text { WPO\#30640: WIPP Shaft Seal System } \\
\text { BRAGFLO Parameters, SWCF- } \\
\text { A:1.1.03.2.1:QA:PDD Sec II Vol 2, 2.2.7; Sec I } \\
\text { Vol } 4 \text { 4.2 }\end{array}$ & \\
\hline 2342 & CL_L_T1 & SAT_RBRN & $\begin{array}{l}\text { WPO\#30640: WiPP Shaft Seal System } \\
\text { BRAGFLO Parameters, SWCF- } \\
\text { A:1.1.03.2.1:QA:PDD Sec II Vol 2, 2.2.7; Sec I } \\
\text { Vol 4 4.2 }\end{array}$ & \\
\hline 2359 & CL_L_T2 & SAT_RBRN & $\begin{array}{l}\text { WPO\#30640: WIPP Shaft Seal System } \\
\text { BRAGFLO Parameters, SWCF- } \\
\text { A:1.1.03.2.1:QA:PDD Sec II Vol 2, 2.2.7; Sec I } \\
\text { Vol 4 4.2 }\end{array}$ & \\
\hline 2376 & CL_L_T3 & .SAT_RBRN & \begin{tabular}{|l|} 
WPO\#30640: WIPP Shaft Seal System \\
BRAGFLO Parameters, SWCF- \\
A:1.1.03.2.1:QA:PDD Sec II Vol 2, 2.2.7; Sec I \\
Vol 4 4.2
\end{tabular} & \\
\hline 3082 & CL_L_T4 & SAT_RBRN & $\begin{array}{l}\text { WPO\#30640: WIPP Shaft Seal System } \\
\text { BRAGFLO Parameters, SWCF- } \\
\text { A:1.1.03.2.1:QA:PDD Sec II Vol 2, 2.2.7; Sec I } \\
\text { Vol 4 4.2 }\end{array}$ & \\
\hline 2393 & CL_M_T1 & SAT_RBRN & $\begin{array}{l}\text { WPO\#30640: WIPP Shaft Seal System } \\
\text { BRAGFLO Parameters, SWCF- } \\
\text { A:1.1.03.2.1:QA:PDD Sec II Vol 2, 2.2.7; Sec I } \\
\text { Vol 4 4.2 }\end{array}$ & \\
\hline 2410 & CL_M_T2 & SAT_RBRN & $\begin{array}{l}\text { WPO\#30640: WIPP Shaft Seal System } \\
\text { BRAGFLO Parameters, SWCF. } \\
\text { A:1.1.03.2.1:QA:PDD Sec II Vol 2, 2.2.7; Sec I } \\
\text { Vol 4 4.2 }\end{array}$ & \\
\hline 2427 & CL_M_T3 & SAT_RBRN & \begin{tabular}{|l|} 
WPO\#30640: WIPP Shaft Seal System \\
BRAGFLO Parameters, SWCF- \\
A:1.1.03.2.1:QA:PDD Sec II Vol 2, 2.2.7; Sec I \\
Vol 4 4.2
\end{tabular} & \\
\hline 2444 & CL_M_T4 & SAT_RBRN & $\begin{array}{l}\text { WPO\#30640: WIPP Shaft Seal System } \\
\text { BRAGFLO Parameters, SWCF- } \\
\text { A:1.1.03.2.1:QA:PDD Sec II Vol 2, 2.2.7; Sec I } \\
\text { Vol 4 4.2 }\end{array}$ & \\
\hline 2461 & CL_M_T5 & SAT_RBRN & $\begin{array}{l}\text { WPO\#30640: WIPP Shaft Seal System } \\
\text { BRAGFLO Parameters, SWCF. } \\
\text { A:1.1.03.2.1:QA:PDD Sec II Vol 2, 2.2.7; Sec I } \\
\text { Vol 4 4.2 }\end{array}$ & \\
\hline 2325 & CLAY_BOT & SAT_RBRN & $\begin{array}{l}\text { WPO\#30640: WIPP Shaft Seal System } \\
\text { BRAGFLO Parameters, SWCF- } \\
\text { A:1.1.03.2.1:QA:PDD Sec II Vol 2, 2.2.7; Sec I } \\
\text { Vol 4 4.2 }\end{array}$ & \\
\hline
\end{tabular}


Additional Sources

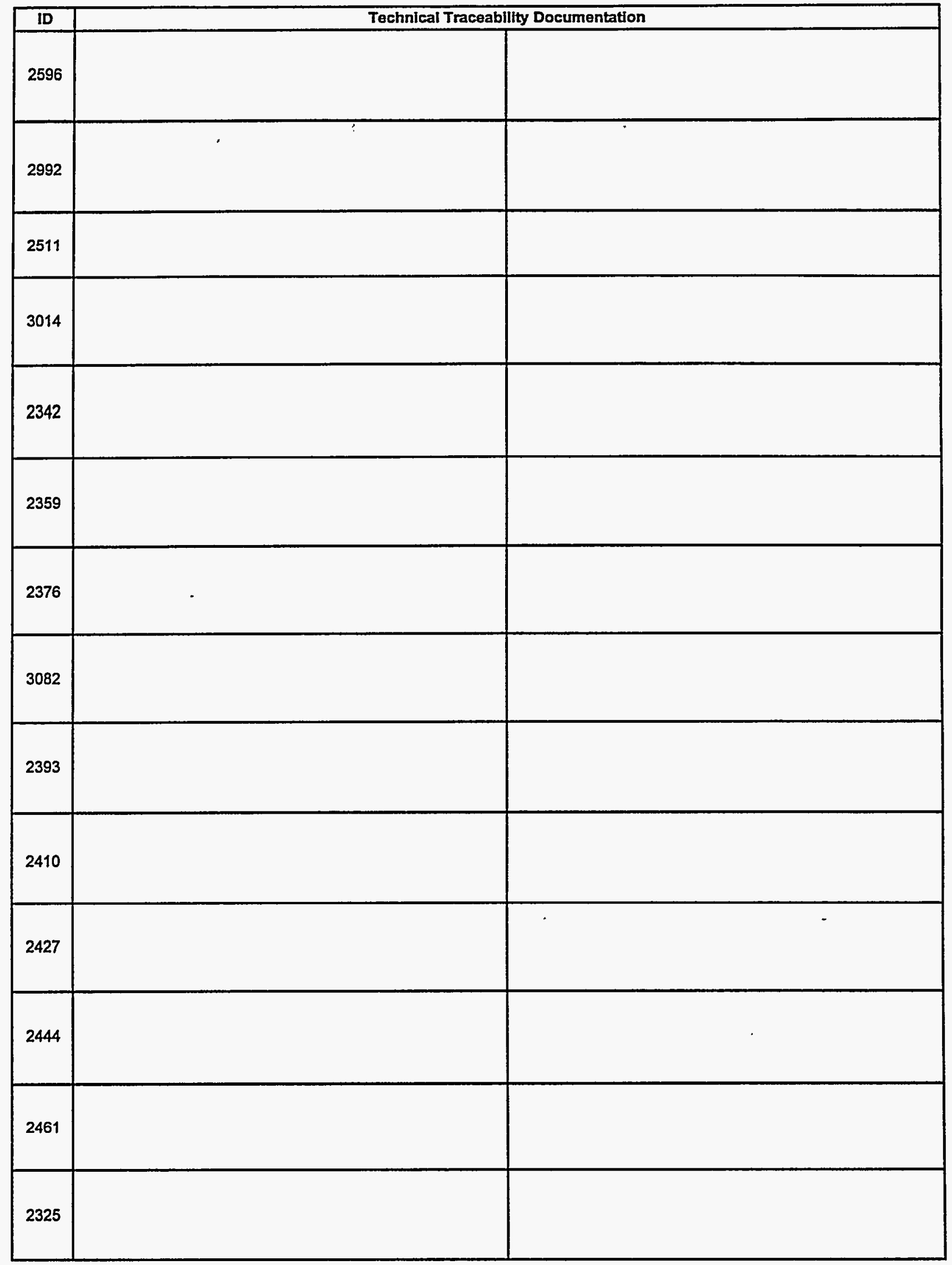


Additional Sources

\begin{tabular}{|c|c|c|c|c|}
\hline ID & Material ID & Param ID & \multicolumn{2}{|c|}{ Technical Traceability Documentation } \\
\hline 2478 & CONC_T1 & SAT_RBRN & $\begin{array}{l}\text { WPO\#30640: WIPP Shaft Seal System } \\
\text { BRAGFLO Parameters, SWCF- } \\
\text { A:1.1.03.2.1:QA:PDD Sec II Vol 2, 2.4.5; Sec I } \\
\text { Vol } 4 \text { 4.4 }\end{array}$ & \\
\hline 2494 & CONC_T2 & SAT_RBRN & $\begin{array}{l}\text { WPO\#30640: WIPP Shaft Seal System } \\
\text { BRAGFLO Parameters, SWCF. } \\
\text { A:1.1.03.2.1:QA:PDD Sec II Vol 2, 2.4.5; Sec I } \\
\text { Vol } 4 \text { 4.4 }\end{array}$ & 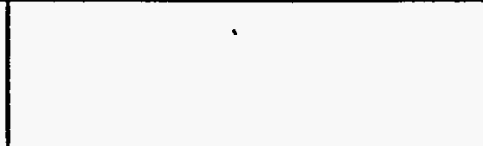 \\
\hline 3063 & CONC_MON & SAT_RBRN & $\begin{array}{l}\text { WPO\#30640: WIPP Shaft Seal System } \\
\text { BRAGFLO Parameters, SWCF- } \\
\text { A:1.1.03.2.1:QA:PDD Sec II Vol 2, 2.4.5; Sec I } \\
\text { Vol } 4 \text { 4.4 }\end{array}$ & \\
\hline 2291 & ASPHALT & SAT_RBRN & $\begin{array}{l}\text { WPO\#30s40: WIPP Shaft Seal System } \\
\text { BRAGFLO Parameters, SWCF- } \\
\text { A:1.1.03.2.1:QA:PDD Sec II Vol 2, 2.2.7; Sec I } \\
\text { Vol 4, 4.5 }\end{array}$ & \\
\hline 2529 & SALT_T1 & SAT_RGAS & $\begin{array}{l}\text { WPO\#30640: WIPP Shaft Seal System } \\
\text { BRAGFLO Parameters, SWCF- } \\
\text { A:1.1.03.2.1:QA:PDD Sec II Vol 2, 2.5.6; Sec I } \\
\text { Vol 4 4.3 }\end{array}$ & Literature: Mayer et al. (1992) pg 173 \\
\hline 2546 & SALT_T2 & SAT_RGAS & $\begin{array}{l}\text { WPO\#30640: WIPP Shaft Seal System } \\
\text { BRAGFLO Parameters, SWCF- } \\
\text { A:1.1.03.2.1:QA:PDD Sec II Vol 2, 2.5.6; Sec I } \\
\text { Vol 4 4.3 }\end{array}$ & Literature: Mayer et al. (1992) pg 173 \\
\hline 2563 & SALT_T3 & SAT_RGAS & $\begin{array}{l}\text { WPO\#30640: WIPP Shaft Seal System } \\
\text { BRAGFLO Parameters, SWCF- } \\
\text { A:1.1.03.2.1:QA:PDD Sec II Vol 2, 2.5.6; Sec I } \\
\text { Vol 4 4.3 }\end{array}$ & Literature: Mayer et al. (1992) pg 173 \\
\hline 2580 & SALT_T4 & SAT_RGAS & $\begin{array}{l}\text { WPO\#30640: WIPP Shaft Seal System } \\
\text { BRAGFLO Parameters, SWCF- } \\
\text { A:1.1.03.2.1:QA:PDD Sec II Vol 2, 2.5.6; Sec I } \\
\text { Vol 4 4.3 }\end{array}$ & Literature: Mayer et al. (1992) pg 173 \\
\hline 2597 & SALT_T5 & SAT_RGAS & $\begin{array}{l}\text { WPO\#30640: WIPP Shaft Seal System } \\
\text { BRAGFLO Parameters, SWCF- } \\
\text { A:1.1.03.2.1:QA:PDD Sec II Vol 2, 2.5.6; Sec I } \\
\text { Vol 4 4.3 }\end{array}$ & Literature: Mayer et al. (1992) pg 173 \\
\hline 2993 & SALT_T6 & SAT_RGAS & $\begin{array}{l}\text { WPO 30640: WIPP Shaft Seal System } \\
\text { BRAGFLO Parameters, SWCF. } \\
\text { A:1.1.03.2.1:QA:PDD Sec II Vol 2, 2.5.6; Sec I } \\
\text { Vol 4 4.3 }\end{array}$ & Literature: Mayer et al. (1992) pg 173 \\
\hline 2512 & EARTH & SAT_RGAS & $\begin{array}{l}\text { WPO\#30640: WIPP Shaft Seal System } \\
\text { BRAGFLO Parameters, SWCF- } \\
\text { A:1.1.03.2.1:QA:PDD Sec II Vol 2, 2.1.8 }\end{array}$ & \\
\hline 3015 & CLAY_RUS & SAT_RGAS & $\begin{array}{l}\text { WPO\#30640: WIPP Shaft Seal System } \\
\text { BRAGFLO Parameters, SWCF- } \\
\text { A:1.1.03.2.1:QA:PDD Sec II Vol 2, 2.1.8; Sec I } \\
\text { Vol 4 4.2 }\end{array}$ & Literature: Mayer et al. (1992) pg 173 \\
\hline 2343 & CL_L_T1 & SAT_RGAS & $\begin{array}{l}\text { WPO\#30640: WIPP Shaft Seal System } \\
\text { BRAGFLO Parameters, SWCF- } \\
\text { A:1.1.03.2.1:QA:PDD Sec II Vol 2, 2.1.8; Sec } 1 \\
\text { Vol 4 4.2 }\end{array}$ & Literature: Mayer et al. (1992) pg 173 \\
\hline 2360 & CL_L_T2 & SAT_RGAS & $\begin{array}{l}\text { WPO\#30640: WIPP Shaft Seal System } \\
\text { BRAGFLO Parameters, SWCF- } \\
\text { A:1.1.03.2.1:QA:PDD Sec II Vol 2, 2.1.8; Sec I } \\
\text { Vol 4 4.2 }\end{array}$ & Literature: Mayer et al. (1992) pg 173 \\
\hline
\end{tabular}


Additional Sources

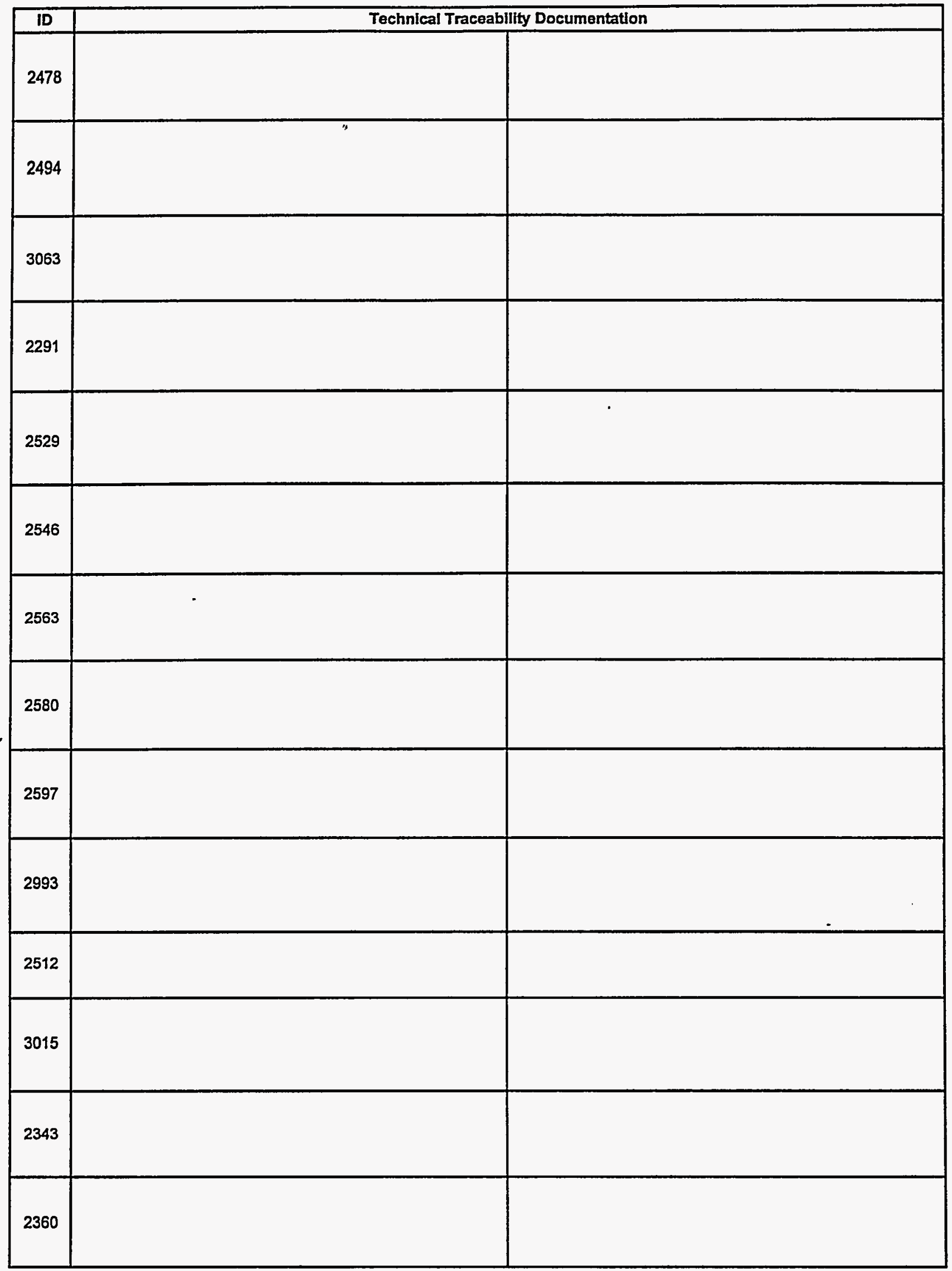


Additional Sources

\begin{tabular}{|c|c|c|c|c|}
\hline ID & Material ID & Param ID & \multicolumn{2}{|c|}{ Technical Traceability Documentation } \\
\hline 2377 & CL_L_T3 & SAT_RGAS & $\begin{array}{l}\text { WPO\#30640: WIPP Shaft Seal System } \\
\text { BRAGFLO Parameters, SWCF- } \\
\text { A:1.1.03.2.1:QA:PDD Sec II Vol 2, 2.1.8; Sec I } \\
\text { Vol 4 4.2 }\end{array}$ & Literature: Mayer et al. (1992) pg 173 \\
\hline 3083 & CL_L_T4 & SAT_RGAS & $\begin{array}{l}\text { WPO\#30640: WIPP Shaft Seal System } \\
\text { BRAGFLO Parameters, SWCF- } \\
\text { A:1.1.03.2.1:QA:PDD Sec II Vol 2, 2.1.8; Sec I } \\
\text { Vol 4 4.2 }\end{array}$ & Literature: Mayer et al. (1992) pg 173 \\
\hline 2394 & CL_M_T1 & SAT_RGAS & $\begin{array}{l}\text { WPO\#30640: WIPP Shaft Seal System } \\
\text { BRAGFLO Parameters, SWCF- } \\
\text { A:1.1.03.2.1:QA:PDD Sec II Vol 2, 2.1.8; Sec I } \\
\text { Vol 4 4.2 }\end{array}$ & Literature: Mayer et al. (1992) pg 173 \\
\hline 2411 & CL_M_T2 & SAT_RGAS & $\begin{array}{l}\text { WPO\#30640: WIPP Shaft Seal System } \\
\text { BRAGFLO Parameters, SWCF- } \\
\text { A:1.1.03.2.1:QA:PDD Sec II Vol 2, 2.1.8; Sec I } \\
\text { Vol 4 4.2 }\end{array}$ & Literature: Mayer et al. (1992) pg 173 \\
\hline 2428 & CL_M_T3 & SAT_RGAS & $\begin{array}{l}\text { WPO\#30640: WIPP Shaft Seal System } \\
\text { BRAGFLO Parameters, SWCF- } \\
\text { A:1.1.03.2.1:QA:PDD Sec II Vol 2, 2.1.8; Sec I } \\
\text { Vol 4 4.2 }\end{array}$ & Literature: Mayer et al. (1992) pg 173 \\
\hline 2445 & CL_M_T4 & SAT_RGAS & $\begin{array}{l}\text { WPO\#30640: WIPP Shaft Seal System } \\
\text { BRAGFLO Parameters, SWCF- } \\
\text { A:1.1.03.2.1:QA:PDD Sec II Vol 2, 2.1.8; Sec I } \\
\text { Vol 4 4.2 }\end{array}$ & Literature: Mayer et al. (1992) pg 173 \\
\hline 2462 & CL_M_T5 & SAT_RGAS & $\begin{array}{l}\text { WPO\#30640: WIPP Shaft Seal System } \\
\text { BRAGFLO Parameters, SWCF- } \\
\text { A:1.1.03.2.1:QA:PDD Sec II Vol 2, 2.1.8; Sec I } \\
\text { Vol 4 4.2 }\end{array}$ & Literature: Mayer et al. (1992) pg 173 \\
\hline 2326 & CLAY_BOT & SAT_RGAS & $\begin{array}{l}\text { WPO\#30640: WIPP Shaft Seal System } \\
\text { BRAGFLO Parameters, SWCF- } \\
\text { A:1.1.03.2.1:QA:PDD Sec II Vol 2, 2.1.8; Sec I } \\
\text { Vol 4 4.2 }\end{array}$ & Literature: Mayer et al. (1992) pg 173 \\
\hline 2479 & CONC_T1 & SAT_RGAS & $\begin{array}{l}\text { WPO\#30640: WIPP Shaft Seal System } \\
\text { BRAGFLO Parameters, SWCF- } \\
\text { A:1.1.03.2.1:QA:PDD-Sec II Vol 2, 2.4.6; Sec I } \\
\text { Vol } 4 \text { 4.4 }\end{array}$ & Mayer et al. (1992) pg 173 \\
\hline 2495 & CONC_T2 & SAT_RGAS & $\begin{array}{l}\text { WPO\#30640: WiPP Shaft Seal System } \\
\text { BRAGFLO Parameters, SWCF- } \\
\text { A:1.1.03.2.1:QA:PDD Sec II Vol 2, 2.4.6; Sec I } \\
\text { Vol } 4 \text { 4.4 }\end{array}$ & Mayer et al. (1992) pg 173 \\
\hline 3064 & CONC_MON & SAT_RGAS & $\begin{array}{l}\text { WPO\#30640: WIPP Shaft Seal System } \\
\text { BRAGFLO Parameters, SWCF- } \\
\text { A:1.1.03.2.1:QA:PDD Sec II Vol 2, 2.4.6; Sec I } \\
\text { Vol } 4 \text { 4.4 }\end{array}$ & Mayer et al. (1992) pg 173 \\
\hline 2292 & ASPHALT & SAT_RGAS & $\begin{array}{l}\text { WPO\#30640: WIPP Shaft Seal System } \\
\text { BRAGFLO Parameters, SWCF- } \\
\text { A:1.1.03.2.1:QA:PDD Sec II Vol 2, 2.1.8; Sec I } \\
\text { Vol 4 4.5 }\end{array}$ & \\
\hline 344 & SANTAROS & PRMX_LOG & $\begin{array}{l}\text { WPO\#41118 Groundwater. Freeze and } \\
\text { Cherry, } 1979 \text { p.29 Table } 2-2 \text { gives a best } \\
\text { estimate range for sandstone permeability's. } \\
\text { See form } 464 \text { for citation. }\end{array}$ & $\begin{array}{l}\text { SAND 78-1596 the full name of the formation } \\
\text { is the Santa Rosa Sandstone. There is no } \\
\text { empirical data for this formation, therefore a } \\
\text { literature value was used. }\end{array}$ \\
\hline 345 & SANTAROS & PRMY_LOG & $\begin{array}{l}\text { WPO\# } 4118 \text { Groundwater, Freeze and } \\
\text { Cherry, } 1979 \text { p. } 29 \text { Table } 2-2 \text { gives a best } \\
\text { estimate range for sandstone permeability's. } \\
\text { See form } 464 \text { for citation. }\end{array}$ & $\begin{array}{l}\text { SAND 78-1596 the full name of the formation } \\
\text { is the Santa Rosa Sandstone. There is no } \\
\text { empirical data for this formation, therefore a } \\
\text { literature value was used. }\end{array}$ \\
\hline
\end{tabular}


Additional Sources

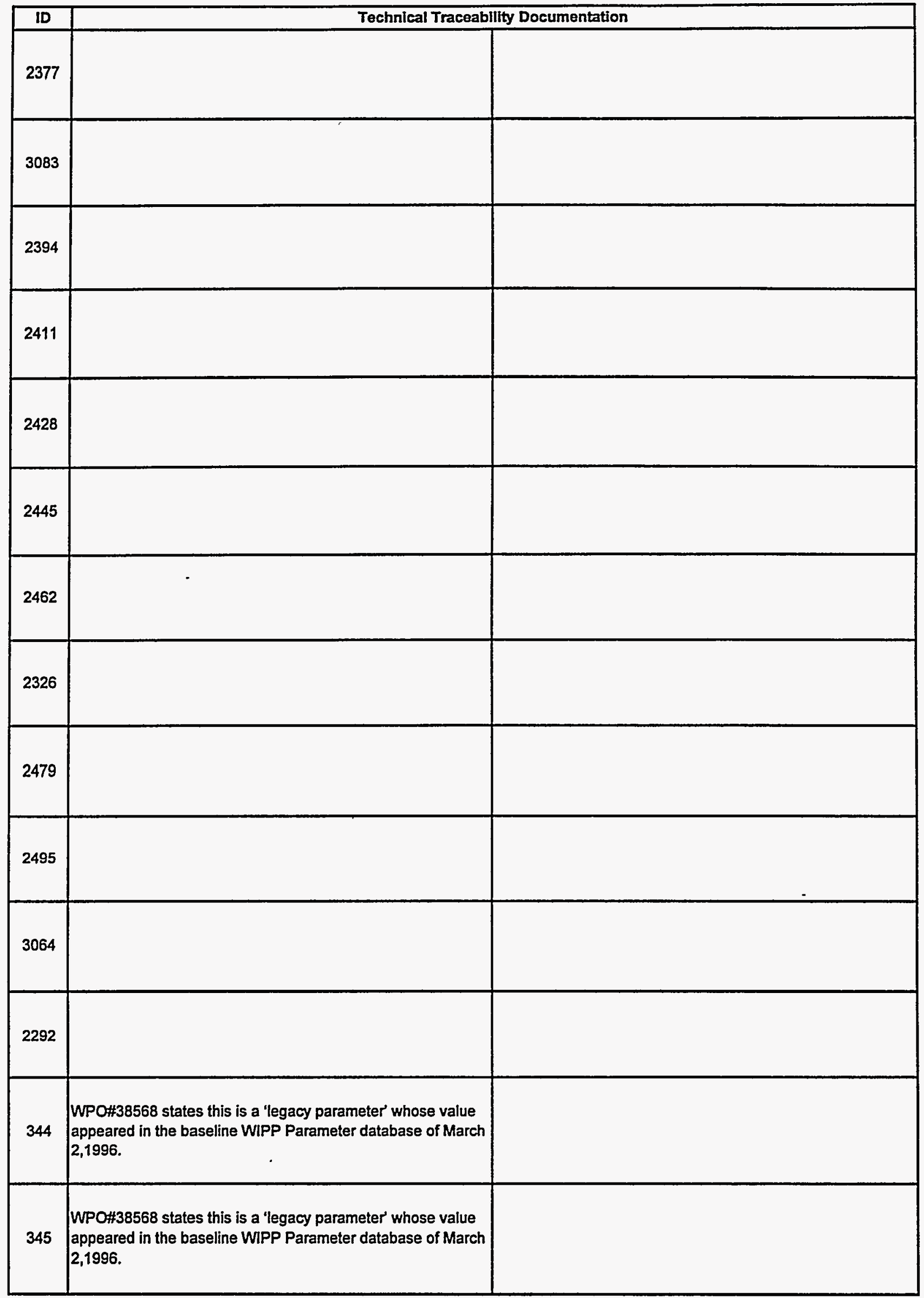


Additional Sources

\begin{tabular}{|c|c|c|c|c|}
\hline ID & Material ID & Param ID & \multicolumn{2}{|c|}{ Technical Traceability Documentation } \\
\hline 346 & SANTAROS & PRMZ_LOG & $\begin{array}{l}\text { WPO\#41118 Groundwater, Freeze and } \\
\text { Cherry, } 1979 \text { p.29 Table } 2-2 \text { gives a best } \\
\text { estimate range for sandstone permeability's. } \\
\text { See form } 464 \text { for citation. }\end{array}$ & $\begin{array}{l}\text { SAND 78-1596 the full name of the formation } \\
\text { is the Santa Rosa Sandstone. There is no } \\
\text { empirical data for this formation, therefore a } \\
\text { literature value was used. }\end{array}$ \\
\hline 3133 & SHFT_DRZ & PRMX_LOG & PRP WPO\#30640 & $\begin{array}{l}T=0 \text { for SNL activities (AIS and Rm D DRZ } \\
\text { testing) }\end{array}$ \\
\hline 3262 & SOLAM3 & SOLSIM & WPO\#41808 pg 4-2, 4-14, PI PRP\#35835 & $\begin{array}{l}\text { WPO } \$ 37791 \text { Memo: R.V.Byrum to M.S. } \\
\text { Tiemey and C. Stockman,"Revised Update of } \\
\text { Uncertainty Range and Distribution for Actinide } \\
\text { Solubility Used in CCA Nuts Calculation" } \\
5 / 23 / 96 \text {. }\end{array}$ \\
\hline 3263 & SOLAM3 & SOLCIM & WPO\#41808 pg 4-2, 4-14, PI PRP\#35835 & $\begin{array}{l}\text { WPO\#37791 Memo: R.V.Byrum to M.S. } \\
\text { Tiemey and C. Stockman,"Revised Update of } \\
\text { Uncertainty Range and Distribution for Actinide } \\
\text { Solubility Used in CCA Nuts Calculation" } \\
\text { 5/23/96. }\end{array}$ \\
\hline 3265 & SOLPU3 & SOLCIM & WPO\#41808 pg 4-2, 4-14, PI PRP\#35835 & $\begin{array}{l}\text { WPO\#37791 Memo: R.V.Byrum to M.S. } \\
\text { Tiemey and C. Stockman,"Revised Update of } \\
\text { Uncertainty Range and Distribution for Actinide } \\
\text { Solubility Used in CCA Nuts Calculation" } \\
\text { 5/23/96. }\end{array}$ \\
\hline 3264 & SOLPU3 & SOLCIM & WPO\#41808 pg 4-2, 4-14, PI PRP\#35835 & $\begin{array}{l}\text { WPO\#37791 Memo: R.V.Byrum to M.S. } \\
\text { Tiemey and C. Stockman,"Revised Update of } \\
\text { Uncertainty Range and Distribution for Actinide } \\
\text { Solubility Used in CCA Nuts Calculation" } \\
5 / 23 / 96 \text {. }\end{array}$ \\
\hline 3266 & SOLPU4 & SOLSIM & WPO\#41808 pg 4-2, 4-14, PI PRP\#35835 & $\begin{array}{l}\text { WPO\#37791 Memo: R.V.Byrum to M.S. } \\
\text { Tiemey and C. Stockman,"Revised Update of } \\
\text { Uncertainty Range and Distribution for Actinide } \\
\text { Solubility Used in CCA Nuts Calculation" } \\
5 / 23 / 96 \text {. }\end{array}$ \\
\hline 3389 & SOLPU4 & SOLCIM & WPO\#41808 pg 4-2, 4-14, PI PRP\#35835 & $\begin{array}{l}\text { WPO\#37791 Memo: R.V.Byrum to M.S. } \\
\text { Tierney and C. Stockman,"Revised Update of } \\
\text { Uncertainty Range and Distribution for Actinide } \\
\text { Solubility Used in CCA Nuts Calculation" } \\
5 / 23 / 96 \text {. }\end{array}$ \\
\hline 3390 & SOLU4 & SOLSIM & WPO\#41808 pg 4-2, 4-14, PI PRP\#35835 & $\begin{array}{l}\text { WPO\#37791 Memo: R.V.Byrum to M.S. } \\
\text { Tiemey and C. Stockman,"Revised Update of } \\
\text { Uncertainty Range and Distribution for Actinide } \\
\text { Solubility Used in CCA Nuts Calculation" } \\
5 / 23 / 96 \text {. }\end{array}$ \\
\hline 3391 & SOLU6 & SOLSIM & WPO\#41808 pg 4-2, 4-14, PI PRP\#35835 & $\begin{array}{l}\text { WPO\#37791 Memo: R.V.Byrum to M.S. } \\
\text { Tiemey and C. Stockman,"Revised Update of } \\
\text { Uncertainty Range and Distribution for Actinide } \\
\text { Solubility Used in CCA Nuts Calculation" } \\
\text { 5/23/96. }\end{array}$ \\
\hline 3392 & SOLU6 & SOLCIM & WPO\#41808 pg 4-2, 4-14, PI PRP\#35835 & $\begin{array}{l}\text { WPO\#37791 Memo: R.V.Byrum to M.S. } \\
\text { Tierney and C. Stockman, "Revised Update of } \\
\text { Uncertainty Range and Distribution for Actinide } \\
\text { Solubility Used in CCA Nuts Calculation" } \\
\text { 5/23/96. }\end{array}$ \\
\hline 3393 & SOLTH4 & SOLSIM & WPO\#41808 pg 4-2, 4-14, PI PRP\#35835 & $\begin{array}{l}\text { WPO\#37791 Memo: R.V.Byrum to M.S. } \\
\text { Tiemey and C. Stockman,"Revised Update of } \\
\text { Uncertainty Range and Distribution for Actinide } \\
\text { Solubility Used in CCA Nuts Calculation" } \\
5 / 23 / 96 .\end{array}$ \\
\hline
\end{tabular}




\section{Additional Sources}

\begin{tabular}{|c|c|c|}
\hline ID & \multicolumn{2}{|c|}{ Technical Traceability Documentation } \\
\hline 346 & $\begin{array}{l}\text { WPO\#38568 states this is a "legacy parameter" whose value } \\
\text { appeared in the baseline WIPP Parameter database of March } \\
2,1996 \text {. }\end{array}$ & \\
\hline 3133 & $\begin{array}{l}\text { WPO\#30640: WIPP Shaft Seal System BRAGFLO } \\
\text { Parameters, SWCF-A:1.1.03.2.1:QA:PDD, Sec III, Vol 3, } \\
\text { 2.5.1; Sec I Vol } 4 \text { 4.6 }\end{array}$ & $\begin{array}{l}\text { Literature - Dale and Hurtado (1996); 'Data Packages - } \\
\text { WPO\#39631 Knowles et al. (1995), WPO\#28103 Hurtado } \\
\text { (1995) }\end{array}$ \\
\hline 3262 & & \\
\hline 3263 & & \\
\hline 3265 & & \\
\hline 3264 & $\cdot$ & \\
\hline 3266 & & \\
\hline 3389 & & \\
\hline 3390 & . & - \\
\hline 3391 & & \\
\hline 3392 & & \\
\hline 3393 & & \\
\hline
\end{tabular}


Additional Sources

\begin{tabular}{|c|c|c|c|c|}
\hline ID & Material ID & Param ID & \multicolumn{2}{|c|}{ Technical Traceabllity Documentation } \\
\hline 2907 & STEEL & CORRMCO2 & $\begin{array}{l}\text { WPO\#35162, Memo from Yifeng Wang and } \\
\text { Larry Brush to Martin S.Tierney, Jan 26,1996; } \\
\text { "Estimates of Gas-Generation Parameters for } \\
\text { the Long-Term WIPP Performance } \\
\text { Assessment" P.9, Table } 1 \text { (with SI conversion } \\
\text { described on 464 form). }\end{array}$ & $\begin{array}{l}\text { Raw data found in WPO\#42357 and } \\
\text { WPO\#38474 }\end{array}$ \\
\hline 2898 & STEEL & STOIFX & $\begin{array}{l}\text { WPO\#35162, Memo from Yifeng Wang and } \\
\text { Larry Brush to Martin S.Tierney, Jan 26,1996; } \\
\text { "Estimates of Gas-Generation Parameters for } \\
\text { the Long-Term WIPP Performance } \\
\text { Assessment" P.9, Table } 1 \text { (no SI conversion } \\
\text { required) }\end{array}$ & $\begin{array}{l}\text { Raw data found in WPO } 42357 \text { and } \\
\text { WPO\#38474 }\end{array}$ \\
\hline 2909 & SULFATE & QINIT & $\begin{array}{l}\text { WPO\#31379 Memo: Paul Drez to File } 1 / 26 / 96 \text {, } \\
\text { WPO\#30819 Memo:Y.Wang and L. Brush to } \\
\text { M. Tiemey 1/26/96. }\end{array}$ & \\
\hline 3479 & $U+4$ & MKD_U & $\begin{array}{l}\text { WPO\#338801 Memo:L.H. Brush to M. Tiemey } \\
6 / 10 / 96\end{array}$ & \\
\hline 3475 & $\mathrm{U}+6$ & MKD_U & $\begin{array}{l}\text { WPO\#338801 Memo:L.H. Brush to M. Tierney } \\
6 / 10 / 96\end{array}$ & \\
\hline 651 & WAS_AREA & ABSROUGH & $\begin{array}{l}\text { Analysis Package for the Cuttings and Spalling } \\
\text { Calculations WPO\#40521- Sec. } 7 \text { "Input } \\
\text { Parameters and Sources" pg } 39 .\end{array}$ & $\begin{array}{l}\text { WAS_AREA:ABSROUGH epsilon }=0.025 \\
\text { Absolute roughness (m). Equation } 9 \mathrm{pg} 21 \text { of } \\
\text { WPO } \$ 40521 \text { uses absolute roughness. Value } \\
0.025 \text { can be traced to WPO } 40521 \text { SAND92- } \\
0700 / 3 \mathrm{pg} 3-57 \text { a drilling erosion parameter } \\
\text { with a uniform distribution, range of } 001 \text { to } \\
0.04 \text {. }\end{array}$ \\
\hline 653 & WAS_AREA & COMP_RCK & $\begin{array}{l}\text { See WPO\#40514 Sec. } 4.2 .4 \text { pg. 4-146 (the } \\
\text { BRAGFLO analysis write-up). }\end{array}$ & $\begin{array}{l}\text { In BRAGFLO, the dependence of porosity on } \\
\text { pressure in regions WAS_AREA and } \\
\text { REPOSIT is described through "porosity } \\
\text { surface" or closure surface, rather than pore } \\
\text { volume compressibility }\end{array}$ \\
\hline 2041 & WAS_AREA & DCELLCHW & $\begin{array}{l}\text { WPO\#32328 Transuranic Waste Baseline } \\
\text { Inventory Report Revision } 2 \text { Vol.1 DOE/CAD- } \\
\text { 95-1121-Dec 95. }\end{array}$ & \\
\hline 2274 & WAS_AREA & DCELLRHW & $\begin{array}{l}\text { WPO\#32328 Transuranic Waste Baseline } \\
\text { Inventory Report Revision } 2 \text { Vol.1 DOE/CAD- } \\
\text { 95-1121-Dec } 95 .\end{array}$ & \\
\hline 1992 & WAS_AREA & DIRNCCHW & $\begin{array}{l}\text { WPO\#32328 Transuranic Waste Baseline } \\
\text { Inventory Report Revision } 2 \text { Vol.1 DOE/CAD- } \\
\text { 95-1121-Dec } 95 .\end{array}$ & - \\
\hline 1993 & WAS_AREA & DIRNCRHW & $\begin{array}{l}\text { WPO\#32328 Transuranic Waste Baseline } \\
\text { Inventory Report Revision } 2 \text { Vol.1 DOE/CAD- } \\
\text { 95-1121-Dec } 95 .\end{array}$ & \\
\hline 2040 & WAS_AREA & DIRONCHW & $\begin{array}{l}\text { WPO\#32328 Transuranic Waste Baseline } \\
\text { Inventory Report Revision } 2 \text { Vol.1 DOE/CAD- } \\
\text { 95-1121-Dec } 95 .\end{array}$ & \\
\hline 2044 & WAS_AREA & DIRONRHW & $\begin{array}{l}\text { WPO\#32328 Transuranic Waste Baseline } \\
\text { Inventory Report Revision } 2 \text { Vol.1 DOE/CAD- } \\
\text { 95-1121-Dec 95. }\end{array}$ & \\
\hline 1994 & $\begin{array}{l}\text { No EPA data } \\
\text { gn }\end{array}$ & & Not used in 96 CCA calculation. & \\
\hline 2043 & WAS_AREA & DPLASCHW & $\begin{array}{l}\text { WPO\#32328 Transuranic Waste Baseline } \\
\text { Inventory Report Revision } 2 \text { Vol.1 DOE/CAD. } \\
\text { 95-1121-Dec } 95 .\end{array}$ & \\
\hline 2275 & WAS_AREA & DPLASRHW & $\begin{array}{l}\text { WPO\#32328 Transuranic Waste Baseline } \\
\text { Inventory Report Revision } 2 \text { Vol.1 DOE/CAD- } \\
\text { 95-1121-Dec } 95 .\end{array}$ & \\
\hline
\end{tabular}




\begin{tabular}{|c|c|c|}
\hline ID & \multicolumn{2}{|c|}{ Technical Traceability Documentation } \\
\hline 2907 & $\begin{array}{l}\text { Data package WPO\#45549 QED package for PNL } \\
\text { WPO\#23213 PNL AJ-1365 T=0 memo WPO\#44760 }\end{array}$ & \\
\hline 2898 & & \\
\hline 2909 & & \\
\hline 3479 & & \\
\hline 3475 & & \\
\hline 651 & $\begin{array}{l}\text { and median of } 0.025 \mathrm{~m} \text {. Ref given in WPO\#23529 Sand92- } \\
\text { 0700/3 (92 PA) is Streeter and Wylie, 1975, Fluid Mechanics } \\
\text { 6th ed. NYNY:McGraw-Hill Boo Co fig } 5.32 \text { pg } 297 \text {. See also } \\
\text { EPA CCA Review Personnel Request Form by Joel Miller } \\
\text { dated 4/17/97. }\end{array}$ & $\begin{array}{l}\text { Moody Diagram Pg } 297 \text { Fig } 5.32 \text { of Fluid Mechanics, Streeter } \\
\& \text { Wylie. Graph contains table of values. Value of } 0.025 \mathrm{~m} \text { was } \\
\text { chosen by Jerry Berglund to be conservative and at upper end } \\
\text { of range shown on table of Streeter \& Wylie. }\end{array}$ \\
\hline 653 & $\begin{array}{l}\text { See also WPO\#30703 (BRAGFLO User's Manual) p. } 130 \text {, } \\
\text { p.55. WPO\#31368 has further info on closure surface. }\end{array}$ & WPO\#35188 Worksheet by Palmer Vaughn 1/29/96. \\
\hline 2041 & & \\
\hline 2274 & & \\
\hline 1992 & & - \\
\hline 1993 & & \\
\hline 2040 & & \\
\hline 2044 & & \\
\hline 1994 & & \\
\hline 2043 & & \\
\hline 2275 & & \\
\hline
\end{tabular}


Additional Sources

\begin{tabular}{|c|c|c|c|c|}
\hline ID & Material ID & Param ID & \multicolumn{2}{|c|}{ Technical Traceabllity Documentation } \\
\hline 1995 & WAS_AREA & DPLSCCHW & $\begin{array}{l}\text { WPO\#32328 Transuranic Waste Baseline } \\
\text { Inventory Report Revision } 2 \text { Vol.1 DOE/CAD- } \\
\text { 95-1121-Dec } 95 .\end{array}$ & \\
\hline 2228 & WAS_AREA & DPLSCRHW & $\begin{array}{l}\text { WPO\#32328 Transuranic Waste Baseline } \\
\text { Inventory Report Revision } 2 \text { Vol.1 DOE/CAD- } \\
\text { 95-1121-Dec 95. }\end{array}$ & \\
\hline 2042 & WAS_AREA & DRUBBCHW & $\begin{array}{l}\text { WPO\#32328 Transuranic Waste Baseline } \\
\text { Inventory Report Revision } 2 \text { Vol.1 DOE/CAD- } \\
\text { 95-1121-Dec 95. }\end{array}$ & \\
\hline 2046 & WAS_AREA & DRUBBRHW & $\begin{array}{l}\text { WPO\#32328 Transuranic Waste Baseline } \\
\text { Inventory Report Revision } 2 \text { Vol.1 DOEJCAD- } \\
\text { 95-1121-Dec } 95 \text {. }\end{array}$ & \\
\hline 656 & WAS_AREA & GRATMICH & $\begin{array}{l}\text { WPO\#35162, Memo from Yifeng Wang and } \\
\text { Larry Brush to Martin S.Tierney, Jan 26,1996; } \\
\text { "Estimates of Gas-Generation Parameters for } \\
\text { the Long-Term WIPP Performance } \\
\text { Assessment" P.9, Table } 1 \text { (with SI conversion } \\
\text { described on } 464 \text { form); }\end{array}$ & $\begin{array}{l}\text { Raw data found in WPO\#42357 and } \\
\text { WPO\#38474 }\end{array}$ \\
\hline 2127 & REPOSIT & GRATMICH & $\begin{array}{l}\text { WPO\#35162, Memo from Yifeng Wang and } \\
\text { Larry Brush to Martin S.Tierney, Jan 26,1996; } \\
\text { "Estimates of Gas-Generation Parameters for } \\
\text { the Long-Term WPP Performance } \\
\text { Assessment" P.9, Table } 1 \text { (with SI conversion } \\
\text { described on } 464 \text { form); }\end{array}$ & $\begin{array}{l}\text { Raw data found in WPO } 42357 \text { and } \\
\text { WPO\#38474 }\end{array}$ \\
\hline 657 & WAS_AREA & GRATMICI & $\begin{array}{l}\text { WPO\#35162, Memo from Yifeng Wang and } \\
\text { Larry Brush to Martin S.Tiemey, Jan 26,1996; } \\
\text { "Estimates of Gas-Generation Parameters for } \\
\text { the Long-Term WIPP Performance } \\
\text { Assessment" P.9, Table } 1 \text { (with SI conversion } \\
\text { described on } 464 \text { form); }\end{array}$ & $\begin{array}{l}\text { Raw data found in WPO\#42357 and } \\
\text { WPO\#38474 }\end{array}$ \\
\hline 2128 & REPOSIT & GRATMICI & $\begin{array}{l}\text { WPO\#35162, Memo from Yifeng Wang and } \\
\text { Larry Brush to Martin S.Tiemey, Jan 26,1996; } \\
\text { "Estimates of Gas-Generation Parameters for } \\
\text { the Long-Term WIPP Performance } \\
\text { Assessment" P.9, Table } 1 \text { (with SI conversion } \\
\text { described on } 464 \text { form); }\end{array}$ & $\begin{array}{l}\text { Raw data found in WPO\#42357 and } \\
\text { WPO\#38474 }\end{array}$ \\
\hline 663 & WAS_AREA & PRMX_LOG & $\begin{array}{l}\text { WPO\#30921 Memo:B.Butcher to M. Tierney } \\
\text { "QAP 9-2 Documentation for the Overall } \\
\text { Waste Permeability and Flow Property Values } \\
\text { for the CCA" 1/29/96. Also WPO\#35188 } \\
\text { Worksheet by Palmer Vaughn 1/29/96 }\end{array}$ & $\begin{array}{l}\text { WPO\#30925 Memo B. Butcher to M. Tierney } \\
\text { "Documentation of Initial Water Content for } \\
\text { CCA" 1/29/96. }\end{array}$ \\
\hline 664 & WAS_AREA & PRMY_LOG & $\begin{array}{l}\text { WPO\#30921 Memo:B.Butcher to M. Tiemey } \\
\text { "QAP 9-2 Documentation for the Overall } \\
\text { Waste Permeability and Flow Property Values } \\
\text { for the CCA" 1/29/96. Also WPO\#35188 } \\
\text { Worksheet by Paimer Vaughn } 1 / 29 / 96 \text {. }\end{array}$ & $\begin{array}{l}\text { WPO\#30925 Memo B. Butcher to M. Tiemey } \\
\text { "Documentation of Initial Water Content for } \\
\text { CCA" 1/29/96. }\end{array}$ \\
\hline 665 & WAS_AREA & PRMZ_LOG & $\begin{array}{l}\text { WPO\#30921 Memo:B.Butcher to M. Tiemey } \\
\text { "QAP 9-2 Documentation for the Overall } \\
\text { Waste Permeability and Flow Property Values } \\
\text { for the CCA" } 1 / 29 / 96 \text {. Also WPO\#35188 } \\
\text { Worksheet by Palmer Vaughn } 1 / 29 / 96 \text {. }\end{array}$ & $\begin{array}{l}\text { WPO\#30925 Memo B. Butcher to M. Tiemey } \\
\text { "Documentation of Initial Water Content for } \\
\text { CCA" 1/29/96. }\end{array}$ \\
\hline
\end{tabular}


Additional Sources

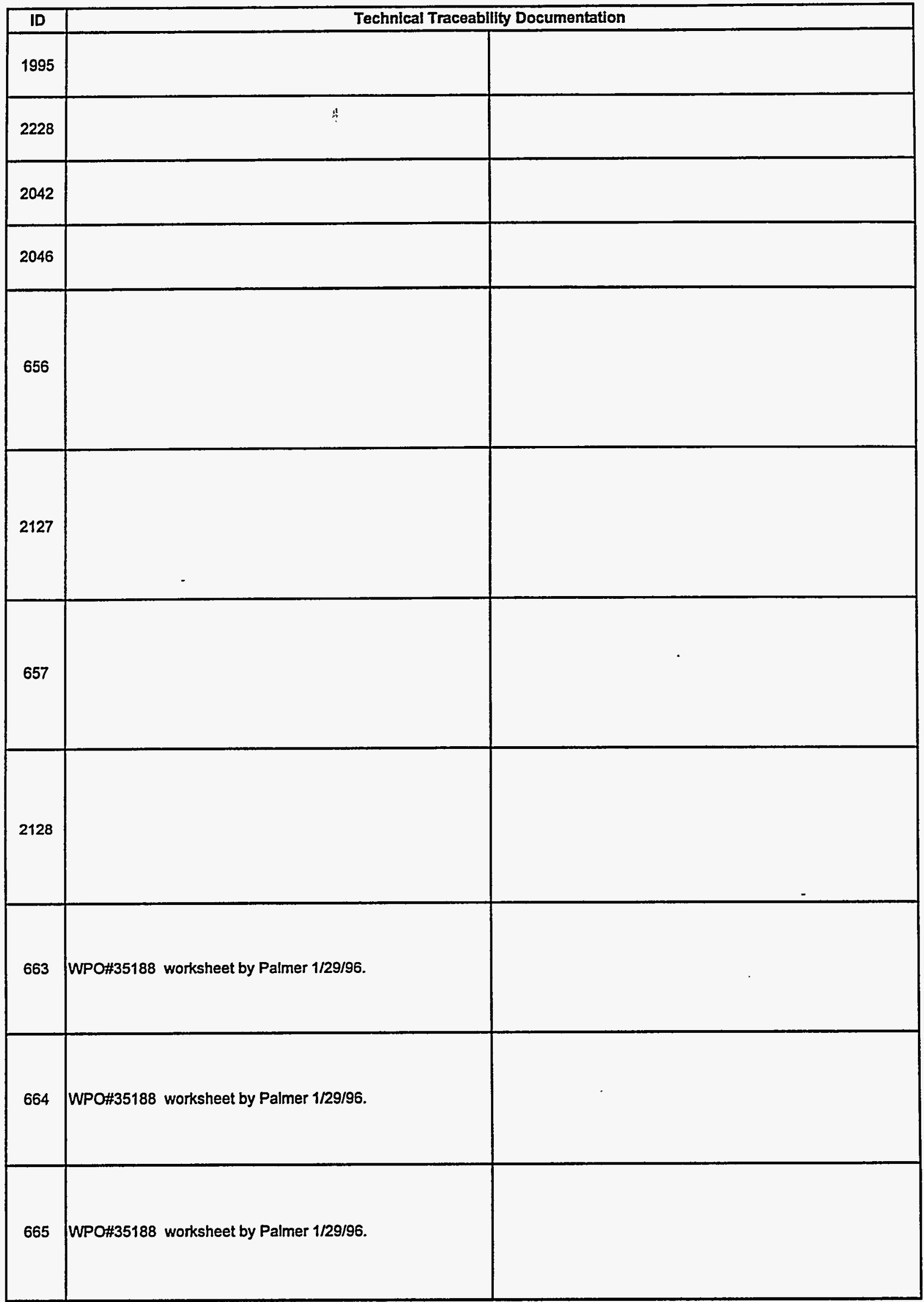




\section{Additional Sources}

\begin{tabular}{|c|c|c|c|c|}
\hline ID & Material ID & Param ID & \multicolumn{2}{|c|}{ Technical Traceability Documentation } \\
\hline 2823 & WAS_AREA & PROBDEG & $\begin{array}{l}\text { Memo: Tiemey to File } 3 / 29 / 96 \text { (no WPO } \\
\text { Number - memo is located in } 464 \text { Form). }\end{array}$ & \\
\hline 2824 & REPOSIT & PROBDEG & $\begin{array}{l}\text { Memo: Tiemey to File } 3 / 29 / 96 \text { (no WPO } \\
\text { Number - memo is located in } 464 \text { Form). }\end{array}$ & \\
\hline 670 & WAS_AREA & SAT_RBRN & $\begin{array}{l}\text { See WPO\#40514 Sec. } 4.2 .4 \text { pg. 4-172 (the } \\
\text { BRAGFLO analysis write-up). }\end{array}$ & $\begin{array}{l}\text { Supported by SAND91-0893/3 pg. 3-121, } \\
\text { which is in turn supported by SAND89-2408, } \\
\text { which is supported by Brooks and Corey, } 1964 \\
\text { (included in refs in WPO\#30640 Shaft Seals } \\
\text { Parameter Package). }\end{array}$ \\
\hline 2741 & REPOSIT & SAT_RBRN & $\begin{array}{l}\text { See WPO\#40514 Sec. } 4.2 .4 \text { pg. } 4-172 \text { (the } \\
\text { BRAGFLO analysis write-up). }\end{array}$ & $\begin{array}{l}\text { Supported by SAND91-0893/3 pg. 3-121, } \\
\text { which is in tum supported by SAND89-2408, } \\
\text { which is supported by Brooks and Corey, } 1964 \\
\text { (included in refs in WPO\#30640 Shaft Seals } \\
\text { Parameter Package). }\end{array}$ \\
\hline 671 & WAS_AREA & SAT_RGAS & $\begin{array}{l}\text { See WPO } 40514 \text { Sec. } 4.2 .4 \text { pg. } 4-174 \text { (the } \\
\text { BRAGFLO analysis write-up). }\end{array}$ & $\begin{array}{l}\text { WPO\#35188 Worksheet by Palmer Vaughn } \\
\text { 1/29/96. }\end{array}$ \\
\hline 2137 & REPOSIT & SAT_RGAS & $\begin{array}{l}\text { See WPO \#40514 Sec. } 4.2 .4 \text { pg. } 4-174 \text { (the } \\
\text { BRAGFLO analysis write-up). }\end{array}$ & $\begin{array}{l}\text { WPO\#35188 Worksheet by Palmer Vaughn } \\
1 / 29 / 96 \text {. }\end{array}$ \\
\hline 2231 & WAS_AREA & SAT_WICK & $\begin{array}{l}\text { See WPO } 40514 \text { Sec. } 4.2 .4 \text { pg. 4-174 (the } \\
\text { BRAGFLO analysis write-up) and WPO\#38152 } \\
\text { the composite Phase I FEP Records Package. }\end{array}$ & Appendix D \\
\hline 2138 & No EPA data & & Not used in CCA Calculation & \\
\hline 214 & EXP_AREA & PRMX_LOG & $\begin{array}{l}\text { WPO\#36320 Memo:Vaughn to Tiemey } \\
\text { "Northend Regions (Backfill and Experimental) } \\
\text { Porosity" 1/30/96 }\end{array}$ & \\
\hline
\end{tabular}


Additional Sources

\begin{tabular}{|c|c|c|}
\hline ID & \multicolumn{2}{|c|}{ Technical Traceability Documentation } \\
\hline 2823 & & \\
\hline 2824 & & \\
\hline 670 & WPO\#35188 Worksheet by Palmer Vaughn 1/29/96. & $\begin{array}{l}\text { Memo: Vaughn to Tiemey } 2 / 13 / 96 \text { WAS_AREA and } \\
\text { REPOSIT/SAT RBRN distribution. }\end{array}$ \\
\hline 2741 & WPO\#35188 Worksheet by Palmer Vaughn 1/29/96. & \\
\hline 671 & & \\
\hline 2137 & WPO\#35188 Worksheet by Palmer Vaughn 1/29/96. & \\
\hline 2231 & $\begin{array}{l}\text { WPO\#38568 Memo: M. Tiemey and P. Vaughn to file } \\
\text { "Designation of Legacy Parameters and Placeholders in WIPP } \\
\text { Parameter Database" 6/17/96. }\end{array}$ & $\begin{array}{l}\text { WPO\#38568 states this is a 'legacy parameter' whose value } \\
\text { appeared in the baseline WIPP Parameter database of March } \\
2,1996 \text {. }\end{array}$ \\
\hline 2138 & & \\
\hline 214 & & \\
\hline
\end{tabular}


Page intentionally blank 


\section{Appendix M3}

Table from EPA Parameter Database:

Enhanced Sources 
Enhanced Sources

\begin{tabular}{|c|c|c|c|}
\hline ID & \multicolumn{3}{|c|}{ Enhanced Source or Other Additional Information } \\
\hline 60 & $\begin{array}{l}\text { See WPO \#40514, page 4-145. Used anhydrite } \\
\text { capillary pressure properties as analog. }\end{array}$ & & \\
\hline 62 & $\begin{array}{l}\text { See WPO \#40514, page 4-150. The capillary } \\
\text { pressure model selected permits capillary } \\
\text { pressure to vary with the degree of saturation. In } \\
\text { this model, capillary pressure approaches } \\
\text { infinity at a brine saturation equal to residual } \\
\text { brine saturation. }\end{array}$ & $\begin{array}{l}\text { The numerical implementation of } \\
\text { this model requires an } \\
\text { approximation to infinity. A value } \\
\text { of } 1.0 \mathrm{E}+08 \mathrm{~Pa} \text { is sufficiently large } \\
\text { to capture infinity and does not } \\
\text { alter the shape of the capillary } \\
\text { pressure curve. Furthermore, for } \\
\text { halite, }\end{array}$ & $\begin{array}{l}\text { gas is able to enter the brine- } \\
\text { saturated pore space of the halite, } \\
\text { so the halite remains fully brine } \\
\text { saturated for all time. The value } \\
\text { of PC_MAX is irrelevant as long } \\
\text { as it is larger than the value for } \\
\text { the capillary threshold pressure } \\
\text { value. }\end{array}$ \\
\hline 64 & $\begin{array}{l}\text { There is data to support this parameter, See } \\
\text { also WPO \#31610 }\end{array}$ & & \\
\hline 65 & No effect. See WPO \#40514, page 4-155 & & \\
\hline 76 & Rationale in WPO \#40514, page 4-144 & & \\
\hline 77 & Rationale in WPO \#40514, page 4-146 & & \\
\hline 78 & No effect. See WPO \#40514, page 4-150 & & \\
\hline 79 & Not important. See WPO \#40514, page 4-157 & & \\
\hline 80 & Rationale in WPO \#40514, page 4-158 & & \\
\hline 81 & No effect. See WPO \#40514, page 4-155 & & \\
\hline 83 & Rationale in WPO \#40514, page 4-159 & & \\
\hline 84 & Rationale in WPO \#40514, page 4-161 & & \\
\hline 85 & Rationale in WPO \#40514, page 4-162 & & \\
\hline 88 & $\begin{array}{l}\text { See WPO \#40514, page 4-166. Used } \\
\text { baseline/default relative permeability model } \\
\text { (Brooks-Corey Model). Two phase flow } \\
\text { characteristics of the cavity regions do not effect } \\
\text { the flow behavior of the cavity regions because } \\
\text { of } 2 \text { things: }\end{array}$ & $\begin{array}{l}\text { 1) the intrinsic permeability of the } \\
\text { DRZ is much lower (by } 7 \text { orders of } \\
\text { magnitude) than that of the cavity, } \\
\text { therfore the DRZ controls brine } \\
\text { inflow to the cavity, and }\end{array}$ & $\begin{array}{l}\text { 2) the cavity is in place for only } 5 \\
\text { years and the increased brine } \\
\text { volume or saturationin the cavity } \\
\text { resulting from drainage of the } \\
D R Z \text { during this time is not } \\
\text { sufficent to alter the rate of brine } \\
\text { inflow from the DRZ. }\end{array}$ \\
\hline 89 & Rationale in WPO \#40514, page 4-171 & & \\
\hline 90 & Rationale in WPO \#40514, page 4-173 & & \\
\hline 137 & $\begin{array}{l}\text { Rationale in WPO \#40514, pages 4-151 and 8- } \\
15\end{array}$ & & \\
\hline 141 & No effect. See WPO \#40514, page 4-156 & & \\
\hline 156 & $\begin{array}{l}\text { Rationale in WPO \#40514, pages 4-151 and 8- } \\
15\end{array}$ & & \\
\hline 159 & No effect. See WPO \#40514, page 4-156 & & \\
\hline 174 & Rationale in WPO \#40514, page 4-144 & & \\
\hline 176 & $\begin{array}{l}\text { See WPO \#40514, page 4-151. PC_MAX is } \\
\text { irrelevant and not used for the capillary pressure } \\
\text { model characteristic of these regions. }\end{array}$ & & \\
\hline 179 & No effect. See WPO \#40514, page 4-156 & & \\
\hline 188 & Rationale in WPO \#40514, page 4-171 & & \\
\hline 189 & Rationale in WPO \#40514, page 4-173 & & \\
\hline 190 & Rationale in WPO \#40514, page 4-144 & & \\
\hline 193 & $\begin{array}{l}\text { Rationale in WPO \#40514, page 4-151. } \\
\text { PC_MAX is irrelevant and not used for the } \\
\text { capillary pressure model characteristic of these } \\
\text { regions. }\end{array}$ & & \\
\hline 194 & $\begin{array}{l}\text { See WPO \#40514, page 4-157. Used halite and } \\
\text { DRZ_0 as analog. }\end{array}$ & & \\
\hline 196 & No effect. See WPO \#40514, page 4-156 & & \\
\hline
\end{tabular}




\section{Enhanced Sources}

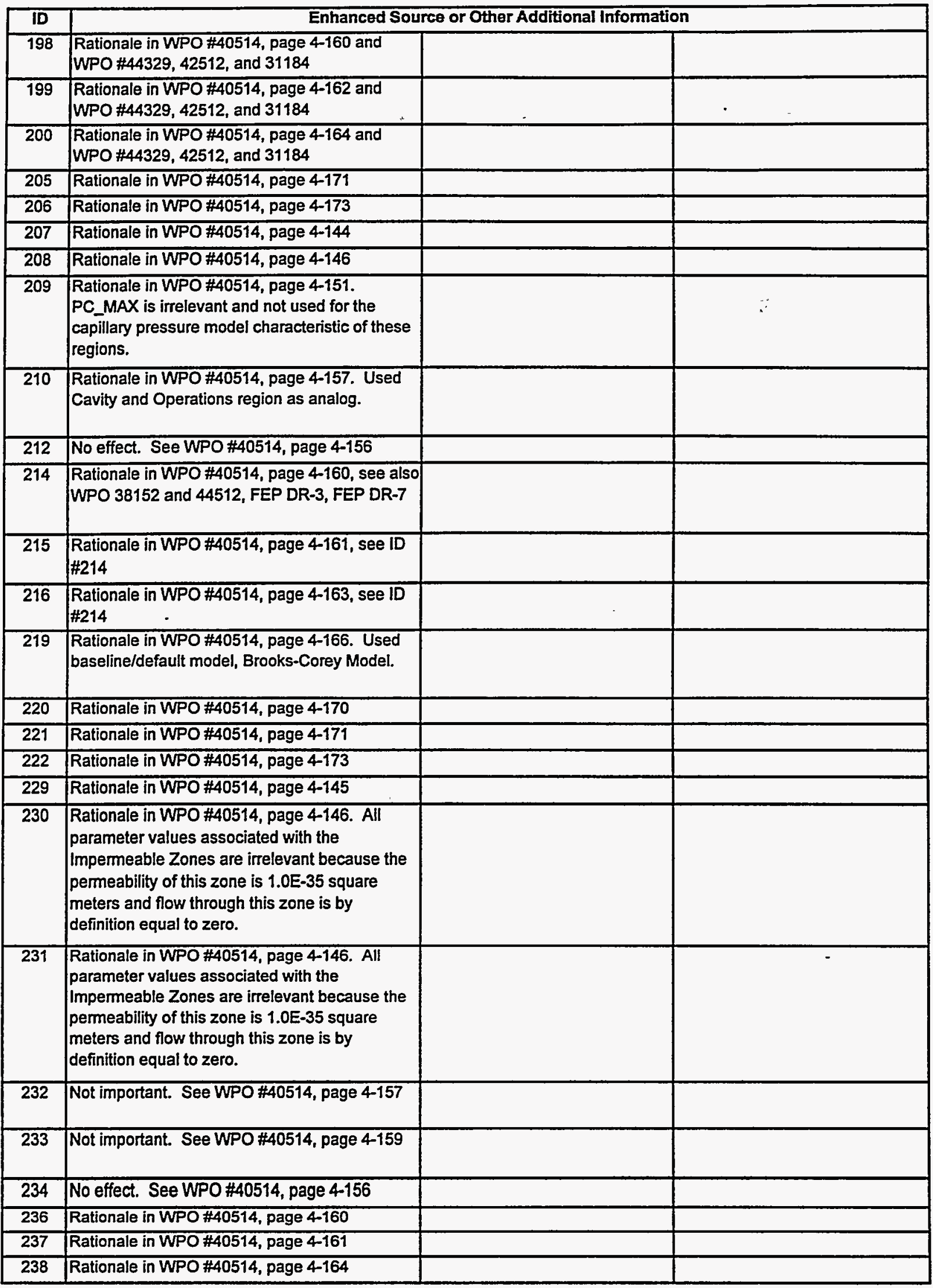


Enhanced Sources

\begin{tabular}{|c|c|c|c|}
\hline ID & \multicolumn{3}{|c|}{ Enhanced Source or Other Additional Information } \\
\hline 241 & $\begin{array}{l}\text { Rationale in WPO \#40514, page 4-166. All } \\
\text { parameter values associated with the } \\
\text { Impermeable Zones are irrelevant because the } \\
\text { permeability of this zone is } 1.0 \mathrm{E}-35 \text { square } \\
\text { meters and flow through this zone is by } \\
\text { definition equal to zero. }\end{array}$ & & \\
\hline 243 & Rationale in WPO \#40514, page 4-171 & & \\
\hline 244 & Rationale in WPO \#40514, page 4-173 & & \\
\hline 256 & $\begin{array}{l}\text { Not important, insensitive. See WPO \#40514, } \\
\text { page 4-158 }\end{array}$ & & \\
\hline 542 & $\begin{array}{l}\text { Rationale in WPO \#40514, page 4-151. The } \\
\text { capillary pressure model selected permits } \\
\text { capillary pressure to vary with the degree of } \\
\text { saturation. In this model, capillary pressure } \\
\text { approaches infinity at a brine saturation equal to } \\
\text { residual brine saturation. }\end{array}$ & $\begin{array}{l}\text { The numerical implementation of } \\
\text { this model requires an } \\
\text { approximation to infinity. A value } \\
\text { of } 1.0 \mathrm{E}+08 \mathrm{~Pa} \text { is sufficiently large } \\
\text { to capture infinity and does not } \\
\text { alter the shape of the capillary } \\
\text { pressure curve. Furthermore, for } \\
\text { halite, }\end{array}$ & $\begin{array}{l}\text { gas is able to enter the brine- } \\
\text { saturated pore space of the halite } \\
\text { so the halite remains fully brine } \\
\text { saturated for all time. The value } \\
\text { of PC_MAX is irrelevant as long } \\
\text { as it is larger than the value for } \\
\text { the capillary threshold pressure } \\
\text { value. }\end{array}$ \\
\hline 545 & No effect. See WPO \#40514, page 4-156 & & \\
\hline 586 & $\begin{array}{l}\text { Rationale in WPO } \# 40514 \text {, page 4-155 and } \\
\text { WPO } \# 44405\end{array}$ & & \\
\hline 589 & Rationale in WPO \#40514, page 4-156 & & \\
\hline 2110 & Rationale in WPO \#40514, page 4-145 & & \\
\hline 2112 & Rationale in WPO \#40514, page 4-146 & & \\
\hline 2177 & 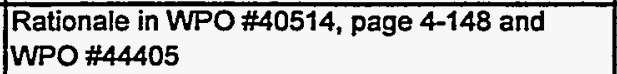 & & \\
\hline 2178 & $\begin{array}{l}\text { Rationale in WPO \#40514, page 4-149 and } \\
\text { WPO \#44405 }\end{array}$ & & \\
\hline 2180 & $\begin{array}{l}\text { Rationale in WPO \#40514, page 4-154 and } \\
\text { WPO \#44405}\end{array}$ & & \\
\hline 2242 & $\begin{array}{l}\text { Rationale in WPO \#40514, page 4-151. } \\
\text { PC_MAX is irrelevant and not used for the } \\
\text { capillary pressure model characteristic of these } \\
\text { regions. }\end{array}$ & & \\
\hline 2254 & $\begin{array}{l}\text { Literature, was also part of EPA's PA } \\
\text { Verification Runs }\end{array}$ & & \\
\hline 2276 & Rationale in WPO \#40514, page 4-144 and 8-17 & & \\
\hline 2278 & $\begin{array}{l}\text { Rationale in WPO \#40514, page 4-150, 8-17, } \\
\text { and 8-18 }\end{array}$ & & - \\
\hline 2281 & No effect. See WPO \#40514, page 4-155 & & \\
\hline 2289 & $\begin{array}{l}\text { Rationale in WPO \#40514, page 4-166, 8-17, } \\
\text { and 8-18 }\end{array}$ & & \\
\hline 2329 & $\begin{array}{l}\text { Rationale in WPO \#40514, page 4-150, 8-17, } \\
\text { and 8-18 }\end{array}$ & & \\
\hline 2332 & No effect. See WPO \#40514, page 4-155 & & \\
\hline 2468 & No effect. See WPO \#40514, page 4-156 & & \\
\hline 2498 & $\begin{array}{l}\text { Rationale in WPO \#40514, page 4-151, 8-17, } \\
\text { and 8-18 }\end{array}$ & & \\
\hline 2501 & No effect. See WPO \#40514, page 4-156 & & \\
\hline 2515 & $\begin{array}{l}\text { Rationale in WPO \#40514, page 4-151, 8-17, } \\
\text { and 8-18 }\end{array}$ & & \\
\hline 2518 & No effect. See WPO \#40514, page 4-156 & & \\
\hline 2599 & $\begin{array}{l}\text { Rationale in WPO \#40514, page 4-149 Model } \\
\text { Configuration Parameter, toggle }\end{array}$ & & \\
\hline
\end{tabular}




\section{Enhanced-Sources-}

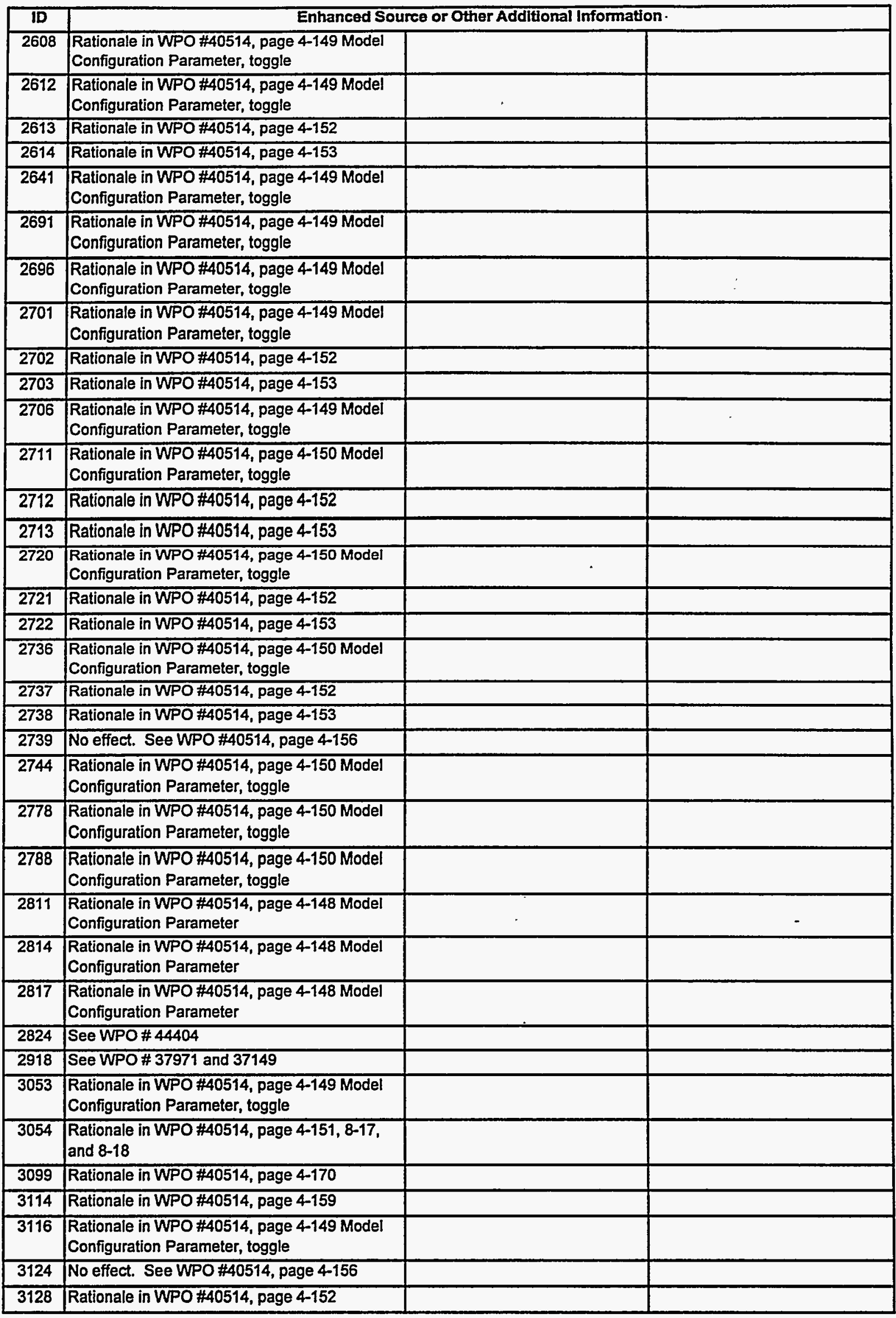




\section{Enhanced Sources}

\begin{tabular}{|c|c|c|c|}
\hline ID & Enhanced Sou & ree or Other Additional Informatio & \\
\hline 3129 & Rationale in WPO \#40514, page 4-153 & & \\
\hline 3134 & Rationale in WPO \#40514, page 4-159 & & \\
\hline$\overline{3137}$ & Rationale in WPO \#40514, page 4-167 & & \\
\hline 3147 & $\begin{array}{l}\text { No impact. Rationale in WPO \#40514, page } 4- \\
158\end{array}$ & & \\
\hline 3152 & Rationale in WPO \#40514, page 4-171 & & \\
\hline 3153 & Rationale in WPO \#40514, page 4-173 & & \\
\hline 3157 & Rationale in WPO \#40514, page 4-152 & & \\
\hline 3158 & Rationale in WPO \#40514, page 4-153 & & \\
\hline 3160 & Rationale in WPO \#40514, page 4-146 & & \\
\hline 3162 & $\begin{array}{l}\text { Rationale in WPO \#40514, page 4-144. These } \\
\text { regions consist of high permeability earthy } \\
\text { material. Shaft earth fill material is used as an } \\
\text { analog for capillary pressure treatment in these } \\
\text { regions. }\end{array}$ & & \\
\hline 3164 & Rationale in WPO \#40514, page 4-171 & & \\
\hline 3165 & Rationale in WPO \#40514, page 4-173 & & \\
\hline 3169 & Rationale in WPO \#40514, page 4-152 & & \\
\hline$\overline{3170}$ & Rationale in WPO \#40514, page 4-153 & & \\
\hline$\overline{3172}$ & Rationale in WPO \#40514, page 4-146 & & \\
\hline 3174 & $\begin{array}{l}\text { Rationale in WPO \#40514, page 4144. These } \\
\text { regions consist of high permeability earthy } \\
\text { material. Shaft earth fill material is used as an } \\
\text { analog for capillary pressure treatment in these } \\
\text { regions. }\end{array}$ & & - \\
\hline 3176 & Rationale in WPO \#40514, page 4-171 & & \\
\hline$\overline{3177}$ & Rationale in WPO \#40514, page 4-173 & & \\
\hline 3181 & Rationale in WPO \#40514, page 4-152 & & - \\
\hline$\overline{3182}$ & Rationale in WPO \#40514, page 4-153 & & \\
\hline$\overline{3186}$ & Rationale in WPO \#40514, page 4-161 & & \\
\hline 3187 & Rationale in WPO \#40514, page 4-162 & & \\
\hline 3194 & $\begin{array}{l}\text { Rationale in WPO \#40514, page 4-148, see also } \\
\text { WPO \#44401 }\end{array}$ & & \\
\hline 3258 & See WPO \#36766, 40521, and 21200 & & \\
\hline 3420 & $\begin{array}{l}\text { Scaling factor was not used, therefore set to 1.0. } \\
\text { See WPO \#36723 }\end{array}$ & & \\
\hline 3456 & See WPO \#44520 and WPO \#42749 & & \\
\hline 3469 & See WPO \#37735 and WPO \#39371 & & \\
\hline$\overline{3470}$ & See WPO \#38214 & & \\
\hline$\overline{3471}$ & See WPO \#38214 & & \\
\hline$\overline{3472}$ & See WPO \#38214 & & \\
\hline$\overline{3473}$ & See WPO \#44519 & & \\
\hline
\end{tabular}


Appendix M4

Table from EPA Parameter Database: Source Cross Reference 


\section{Source Cross-Reference}

\begin{tabular}{|c|c|c|}
\hline Ref ID & Title & Joumal \\
\hline 40CFR191 & $\begin{array}{l}40 \text { CFR Part } 191 \text { - Environmental Radiation Protection } \\
\text { Standards for Management and Disposal of Spent Nuclear } \\
\text { Fuel, High-Level and Transuranic Radioactive Wastes; } \\
\text { Appendix A }\end{array}$ & \\
\hline CRC-14 & CRC Handbook of Chemistry and Physics, 14th Edition & \\
\hline CRC-49 & Handbook of Chemistry and Physics, 49th edition & \\
\hline CRC-72 & $\begin{array}{l}\text { CRC Handbook of Chemistry and Physics, 72nd Edition, } \\
\text { "Table of Isotopes" }\end{array}$ & \\
\hline FEDERAL_REGISTER & $\begin{array}{l}\text { Federal Register, Volume 61, No. 28, Rules and Regulations } \\
\text { (dtd 2/9/96) }\end{array}$ & Federal Register \\
\hline FINLEY-1 & Seal and Backfill Information (memo) & $\begin{array}{l}\text { Preliminary Performance Assessment } \\
\text { for the Waste Isolation Pilot Plant, Dec } \\
\text { 1992. Volume 5: Uncertainty and } \\
\text { Sensitivity Analyses for Gas and Brine } \\
\text { Migration for Undisturbed Performance }\end{array}$ \\
\hline FOX-1 & Introduction to Fluid Mechanics & \\
\hline FREEZE-1 & Groundwater & \\
\hline HYDRAULICSIASCE-3/70 & Erodibility of Channels with Cohesive Boundary & $\begin{array}{l}\text { Joumal of the Hydraulics Division, } \\
\text { Proceedings of the American Society of } \\
\text { Civil Engineers }\end{array}$ \\
\hline $\begin{array}{l}\text { MEMO:HOWARDI } \\
\text { MARIETTA-C }\end{array}$ & Memo: B. Howard to M. Marietta, 6/26/96 & \\
\hline $\begin{array}{l}\text { MEMO:LEONARD/ } \\
\text { MARIETTA }\end{array}$ & $\begin{array}{l}\text { Memo: R. Leonard to M. Marietta, 7/30/96, Geographical } \\
\text { Definition of the Delaware Basin and its Drilling Rates } \\
\text { (w/Attachments) }\end{array}$ & \\
\hline $\begin{array}{l}\text { MEMO:LEONARDI } \\
\text { MARIETTA-2 }\end{array}$ & $\begin{array}{l}\text { Memo: R. Leonard to M. Marietta, 7/31/96, Commonality of } \\
\text { Pressurized Brine Encounters within the Delaware Basin } \\
\text { (w/attachments) }\end{array}$ & . \\
\hline $\begin{array}{l}\text { MEMO:LEONARD/ } \\
\text { MARIETTA-3 }\end{array}$ & $\begin{array}{l}\text { Memo: R. Leonard to M. Marietta, } 6 / 13 / 96, \text { Geostatistical } \\
\text { Analysis of Brine Reservoir Occurrences in the Area of WIPP } \\
\text { for WIPP Performance Assessment (PA) }\end{array}$ & \\
\hline MEMO:LORD/TIERNEY-1 & $\begin{array}{l}\text { Memo: } M \text {. Lord to } M \text {. Tiemey, dtd } 2 / 21 / 96 \text {, subj:Additional } \\
\text { DRZ Parameters to be used in the BRAGFL Calculation }\end{array}$ & \\
\hline MEMO:TIERNEYIFILE-1 & $\begin{array}{l}\text { Memo: M. Tiemey to File, } 3 / 29 / 96 \text {, Reasons for Choice of the } \\
\text { PROBDEG parameter (id nos. } 2824 \text { and } 2823 \text { ) on February } \\
\text { 22, } 1996\end{array}$ & - \\
\hline $\begin{array}{l}\text { MEMO:VAUGHN/TIERNEY } \\
10\end{array}$ & $\begin{array}{l}\text { Memo: P. Vaughn to M. Tiemey, 2/22/96, DEWYLAKE Logs of } \\
\text { Intrinsic Permeability }\end{array}$ & \\
\hline $\begin{array}{l}\text { MEMO:VAUGHN/TIERNEY } \\
11\end{array}$ & $\begin{array}{l}\text { Memo: P. Vaughn to } M \text {. Tiemey, 2/13/96, WAS_AREA and } \\
\text { REPOSIT/SAT_RBRN Distribution }\end{array}$ & \\
\hline $\begin{array}{l}\text { MEMO:VAUGHN/TIERNEY } \\
5\end{array}$ & Memo: P. Vaughn to M. Tierney, 2/19/96, Concrete Monolith & \\
\hline $\begin{array}{l}\text { MEMO:VAUGHN/TIERNEY } \\
7\end{array}$ & Memo: P. Vaughn to M. Tierney, 2/20/96, Borehole Properties & \\
\hline $\begin{array}{l}\text { MEMO:VAUGHN/TIERNEY } \\
9\end{array}$ & $\begin{array}{l}\text { Memo: P. Vaughn to M. Tiemey, 2/14/96, Constant Values for } \\
\text { CAVITY_1,_2,_3, } 4 \text { Parameters }\end{array}$ & \\
\hline NONE & NO DOCUMENTATION AVAILABLE - LEGACY PARAMETER & \\
\hline NOVAKMEMO-1 & $\begin{array}{l}\text { Parameter Recommendations for Porosity and Thickness of } \\
\text { Clay Fracture Linings for the } 1992 \text { WIPP Performance } \\
\text { Assessment Calculations }\end{array}$ & $\begin{array}{l}\text { Preliminary Performance Assessment } \\
\text { for the Wastelsolation Pilot Plant, } \\
\text { December 1992. Volume 3: Modle } \\
\text { Paramenters. A-125 through A-131. }\end{array}$ \\
\hline
\end{tabular}


Source Cross-Reference

\begin{tabular}{|c|c|c|c|c|c|}
\hline Ref ID & Author & Editor & Date & Publisher & Location \\
\hline 40CFR191 & U.S. EPA & & 1985 & & \\
\hline \multicolumn{6}{|l|}{ CRC-14 } \\
\hline CRC-49 & & R. Weast & & $\begin{array}{l}\text { The Chemical Rubber } \\
\text { Co. }\end{array}$ & Cleveland, $\mathrm{OH}$ \\
\hline CRC-72 & N.E. Holden & $\begin{array}{l}\text { D.R. Lide, Editor- } \\
\text { in-Chief }\end{array}$ & $1991-1992$ & CRC Press, Inc. & Boca Raton, FL \\
\hline FEDERAL_REGISTER & & & 09-Feb-96 & & \\
\hline FINLEY-1 & $\begin{array}{l}\text { R.E. Finley and } \\
\text { Palmer Vaughn }\end{array}$ & & 17-Feb-93 & $\begin{array}{l}\text { Sandia National } \\
\text { Laboratories }\end{array}$ & Albuquerque, NM \\
\hline FOX-1 & $\begin{array}{l}\text { R. Fox and A. } \\
\text { McDonald }\end{array}$ & & 1934 & John Wiley \& Sons, Inc. & New York, NY \\
\hline FREEZE-1 & $\begin{array}{l}\text { R.A. Freeze, J.A. } \\
\text { Cherry }\end{array}$ & & 1979 & Prentice-Hall, Inc. & Englewood Cliffs, NJ \\
\hline HYDRAULICS/ASCE-3/70 & $\begin{array}{l}\text { E. Partheniades } \\
\text { and R. Paaswell }\end{array}$ & & Mar-70 & & \\
\hline $\begin{array}{l}\text { MEMO:HOWARD/ } \\
\text { MARIETTA-C }\end{array}$ & B. Howard & & 26-Jun-96 & & \\
\hline $\begin{array}{l}\text { MEMO:LEONARD/ } \\
\text { MARIETTA }\end{array}$ & R. Leonard & & 30-Jul-96 & $\cdot$ & Carisbad, NM \\
\hline $\begin{array}{l}\text { MEMO:LEONARD/ } \\
\text { MARIETTA-2 }\end{array}$ & R. Leonard & & 31-Jul-96 & & Carisbad, NM \\
\hline $\begin{array}{l}\text { MEMO:LEONARD/ } \\
\text { MARIETTA-3 }\end{array}$ & R. Leonard & & 13-Jun-96 & & Carisbad, NM \\
\hline MEMO:LORD/TIERNEY-1 & Michael Lord & & 21-Feb-96 & & Albuquerque, NM \\
\hline MEMO:TIERNEY/FILE-1 & M. Tiemey & & 29-Mar-96 & & Albuquerque, NM \\
\hline $\begin{array}{l}\text { MEMO:VAUGHN/TIERNEY- } \\
10\end{array}$ & P. Vaughn & & 22-Feb-96 & $\begin{array}{l}\text { Sandia National } \\
\text { Laboratories }\end{array}$ & Albuquerque, NM \\
\hline $\begin{array}{l}\text { MEMO:VAUGHN/TIERNEY } \\
11\end{array}$ & P. Vaughn & & 13-Feb-96 & $\begin{array}{l}\text { Sandia National } \\
\text { Laboratories }\end{array}$ & Albuquerque, NM \\
\hline $\begin{array}{l}\text { MEMO:VAUGHNTIERNEY } \\
5\end{array}$ & Palmer Vaughn & & 19-Feb-96 & . & $\begin{array}{l}\text { Sandia National } \\
\text { Laboratories }\end{array}$ \\
\hline $\begin{array}{l}\text { MEMO:VAUGHN/TIERNEY- } \\
7\end{array}$ & Palmer Vaughn & & 20-Feb-96 & & Albuquerque, NM \\
\hline $\begin{array}{l}\text { MEMO:VAUGHN/TIERNEY } \\
9\end{array}$ & P. Vaughn & & 14-Feb-96 & \begin{tabular}{|l|} 
Sandia National \\
Laboratories \\
\end{tabular} & Albuquerque, NM \\
\hline \multicolumn{6}{|l|}{ NONE } \\
\hline NOVAKMEMO-1 & $\begin{array}{l}\text { C.F. Novak, F. } \\
\text { Gelbard, H.W. } \\
\text { Paperguth, } 6119\end{array}$ & & 20-Jul-92 & $\begin{array}{l}\text { Sandia National } \\
\text { Laboratories }\end{array}$ & Albuquerque, NM \\
\hline
\end{tabular}


Source Cross-Reference

\begin{tabular}{|c|c|c|}
\hline Ref ID & Tite & Joumal \\
\hline NUCLIDES/ISOTOPES & G.E. Nuclides and Isotopes, 14th Edition & \\
\hline OBERT-1 & Thermodynamics & \\
\hline PHASE_EQUILIBRIA & Phase Equilibria in Chemical Engineering, pp. 52-54 & \\
\hline SAND86-7167 & $\begin{array}{l}\text { Modeling of Ground-Water Flossing the Culebra Dolomite at } \\
\text { the Waste Isolation Pilot Plant (WIPP)Site: Interim Report }\end{array}$ & SAND86-7167 \\
\hline SAND89-0462 & $\begin{array}{l}\text { Systems Analysis, Long-Term Radionuclide Transport and } \\
\text { Dose Assessments, Waste Isolation Pilot Plant(WIPP), } \\
\text { Southeastern New Mexico; March } 1989\end{array}$ & SAND89-0462 \\
\hline SAND89-2408 & $\begin{array}{l}\text { Data Used in Preliminary Performance Assessment ofthe } \\
\text { Waste Isolation Pilot Plant (1990) }\end{array}$ & SAND89-2408 \\
\hline SAND89-7068/1 & $\begin{array}{l}\text { Ground-Water Flow Modeling of the Culebra Dolomite Volume } \\
\text { I: Model Calibration }\end{array}$ & SAND89-7068/1 \\
\hline SAND91-0893/3 & $\begin{array}{l}\text { Preliminary Comparison with 40CFR Part 191, Subpart B for } \\
\text { the Waste Isolation Pilot Plant, Volume 3:Reference Data }\end{array}$ & \\
\hline $\begin{array}{l}\text { SAND91-0893/3- } \\
\text { MEMOBEAUHEIM }\end{array}$ & $\begin{array}{l}\text { Review of Salado Parameter Values to be Used in } 1991 \\
\text { Performance Assessment Calculations }\end{array}$ & $\begin{array}{l}\text { Preliminary Comparison with } 40 \text { CFR } \\
\text { Part 191, Subpart B for the Waste } \\
\text { Isolation Pilot Plant, December } 1991 . \\
\text { Volume 3: Reference Dta. WoO } \\
\text { Performance Assessment Division. A-19 } \\
\text { through A-23. }\end{array}$ \\
\hline $\begin{array}{l}\text { SAND91-0893/3- } \\
\text { MEMOBEAUHEIM2 }\end{array}$ & $\begin{array}{l}\text { Recommendations for the } 1991 \text { Perfomance Assessment } \\
\text { Calculations on Parameter Uncertainty and Model } \\
\text { Implementation for Culebra Transport Under Undisturbed and } \\
\text { Brine-Reservoir-Breach Conditions }\end{array}$ & $\begin{array}{l}\text { Preliminary Comparison with } 40 \text { CFR } \\
\text { Part 191, Subpart B for the Waste } \\
\text { Isolation Pilot Plant, December1991. } \\
\text { Volume 3: Reference Data. WIPP } \\
\text { PerformanceAssessment Division. A-7 } \\
\text { through A-18. }\end{array}$ \\
\hline $\begin{array}{l}\text { SAND91-0893/3 } \\
\text { MEMOBRUSH }\end{array}$ & $\begin{array}{l}\text { Current Estimates of Gas Production Rates, Gas Production } \\
\text { Potentials, and Expected Chemical Conditions Relevant to } \\
\text { Radionuclide Chemistry for the Long-Term WIPP Performance } \\
\text { Assessment }\end{array}$ & $\begin{array}{l}\text { Preliminary Comparison with } 40 \text { CFR } \\
\text { Part 191, Subpart B for the Waste } \\
\text { Isolation Pilot Plant, December } 1991 . \\
\text { Volume 3: Reference Data WIPP } \\
\text { Performance Assessment Division. A-25 } \\
\text { through A-36. }\end{array}$ \\
\hline SAND92-0479 & $\begin{array}{l}\text { The Use of Expert Judgement to Quantify Uncertainty in } \\
\text { Solubility and Sorption Parameters for the Waste Isolation } \\
\text { Pilot Plant Performance Assessment }\end{array}$ & SAND92-0479 \\
\hline SAND92-0700/3 & $\begin{array}{l}\text { Preliminary Performance Assessment for the Waste Isolation } \\
\text { Pilot Plant, December 1992. Vol. } 3 \text { Model Parameters }\end{array}$ & SAND92-0700/3 \\
\hline $\begin{array}{l}\text { SAND92-0700/3- } \\
\text { MEMOPETERSON }\end{array}$ & $\begin{array}{l}\text { Preliminary Contact Handled (CH) Radionuclide and } \\
\text { Nonradionuclide Inventories and Remote Handled (RH) } \\
\text { Radionuclide Inventory for Use in } 1992 \text { Performance } \\
\text { Assessment }\end{array}$ & $\begin{array}{l}\text { Preliminary Performance Assessment } \\
\text { for the Waste Isolation Pilot Plant, } \\
\text { December 1992. Volume } 3 \text { Model } \\
\text { Parameters. Sandia WIPP Project. A- } \\
133 \text { throughA-140. }\end{array}$ \\
\hline STREETER-1 & Fluid Mechanics, 6th edition & \\
\hline STREETER-2 & Fluid Mechanics, 2nd edition & \\
\hline
\end{tabular}


Source Cross-Reference

\begin{tabular}{|c|c|c|c|c|c|}
\hline Ref ID & Author & Editor & Date & Publisher & Location \\
\hline NUCLIDES/ISOTOPES & $\begin{array}{c}\text { F.W. Walker, J.R. } \\
\text { Parrington, F. } \\
\text { Feiner }\end{array}$ & & 1989 & $\begin{array}{l}\text { General Electric } \\
\text { Company }\end{array}$ & San Jose, CA \\
\hline OBERT-1 & E. Obert & & 1948 & $\begin{array}{l}\text { McGraw-Hill Book } \\
\text { Company, Inc. }\end{array}$ & New York, NY \\
\hline PHASE_EQUILIBRIA & S. M. Walas & & 1985 & Butterworth Publishers & Boston, MA \\
\hline SAND86-7167 & $\begin{array}{l}\text { A. Haug, V.A. } \\
\text { Kelley, A.M. } \\
\text { LaVenue, J.F. } \\
\text { Pickens }\end{array}$ & & 1987 & $\begin{array}{l}\text { Sandia National } \\
\text { Laboratories }\end{array}$ & Albuquerque, NM \\
\hline SAND89-0462 & & $\begin{array}{l}\text { A.R. Lappin, R.L. } \\
\text { Hunter, D.P. } \\
\text { Garber, P.B. } \\
\text { Davies }\end{array}$ & Mar-89 & $\begin{array}{l}\text { Sandia National } \\
\text { Laboratories }\end{array}$ & Albuquerque, NM \\
\hline SAND89-2408 & $\begin{array}{l}\text { R.P. Rechard, H. } \\
\text { luzzolino, J.S. } \\
\text { Sandha }\end{array}$ & & 1990 & $\begin{array}{l}\text { Sandia National } \\
\text { Laboratories }\end{array}$ & Albuquerque, NM \\
\hline SAND89-7068/1 & $\begin{array}{l}\text { A.M. LaVenue, } \\
\text { T.L. Cauffman, } \\
\text { J.F. Pickens }\end{array}$ & . & 1990 & $\begin{array}{l}\text { Sandia National } \\
\text { Laboratories }\end{array}$ & Albuquerque, NM \\
\hline SAND91-0893/3 & & & Dec-91 & $\begin{array}{l}\text { Sandia National } \\
\text { Laboratories }\end{array}$ & Albuquerque, NM \\
\hline $\begin{array}{l}\text { SAND91-0893/3- } \\
\text { MEMOBEAUHEIM }\end{array}$ & R.L. Beauheim & & 1991 & $\begin{array}{l}\text { Sandia National } \\
\text { Laboratories }\end{array}$ & Albụquerque, NM \\
\hline $\begin{array}{l}\text { SAND91-0893/3- } \\
\text { MEMOBEAUHEIM2 }\end{array}$ & $\begin{array}{l}\text { R.L. Beauheim, } \\
\text { T.F. Corbet., P.B. } \\
\text { Davies, and J.F. } \\
\text { Pickens }\end{array}$ & & 1991 & $\begin{array}{l}\text { Sandia National } \\
\text { Laboratories }\end{array}$ & Albuquerque, NM \\
\hline $\begin{array}{l}\text { SAND91-0893/3- } \\
\text { MEMOBRUSH }\end{array}$ & L.H. Brush & & 1991 & $\begin{array}{l}\text { Sandia National } \\
\text { Laboratories }\end{array}$ & Albuquerque, NM \\
\hline SAND92-0479 & $\begin{array}{l}\text { K.M Trauth., S.C. } \\
\text { Hora, R.P. } \\
\text { Rechard, and D. } \\
\text { R. Anderson }\end{array}$ & & 1992 & $\begin{array}{l}\text { Sandia National } \\
\text { Laboratories }\end{array}$ & Albuquerque, NM \\
\hline SAND92-0700/3 & $\begin{array}{l}\text { Sandia WIPP } \\
\text { Project }\end{array}$ & & Dec-92 & $\begin{array}{l}\text { Sandia National } \\
\text { Laboratories }\end{array}$ & Albuquerque, NM \\
\hline $\begin{array}{l}\text { SAND92-0700/3- } \\
\text { MEMOPETERSON }\end{array}$ & A. Peterson 6342 & & 28-Oct-92 & $\begin{array}{l}\text { Sandia National } \\
\text { Laboratories }\end{array}$ & Albuquerque, NM \\
\hline STREETER-1 & $\begin{array}{c}\text { V.L. Streeter, E.B. } \\
\text { Wylie }\end{array}$ & & 1975 & $\begin{array}{l}\text { McGraw-Hill Book } \\
\text { Company, Inc. }\end{array}$ & New York, NY \\
\hline STREETER-2 & V.L. Streeter & & 1958 & $\begin{array}{l}\text { McGraw-Hill Book } \\
\text { Company, Inc. }\end{array}$ & New York, NY \\
\hline
\end{tabular}




\section{Source Cross-Reference}

\begin{tabular}{|c|c|c|}
\hline Ref ID & Title & Joumal \\
\hline $\begin{array}{l}\text { VAPOR- } \\
\text { LIQUID_EQUILIBRIA }\end{array}$ & $\begin{array}{l}\text { Vapor-Liquid Equilibria for Mixtures of Low-Boiling Substances } \\
\text { (Vol 6) }\end{array}$ & Chem Data Series, Vol. VI \\
\hline VAUGHN:WORKSHEET- 1 & $\begin{array}{l}\text { Worksheet by P. Vaughn, dtd 2/5/96, CCA Panel Seal } \\
\text { Parámeter Values for BRAGFLO }\end{array}$ & \\
\hline VAUGHN:WORKSHEET-4 & Worksheet by P. Vaughn, no date, Panel Seal & \\
\hline VAUGHN:WORKSHEET-4A & Worksheet by P. Vaughn, 2/20/96, Panel Seal & \\
\hline WID & Westinghouse WID Operations Drawing Number H-2-91273-1 & \\
\hline WPO22443 & FEP S-6, "Dynamic Alteration of the DRZTRANSITION Zone" & \\
\hline WPO26051 & $\begin{array}{l}\text { Memo: J.P. Marquis to J. Berglund, 9/22/95, SWCF- } \\
\text { A:1.1.1.2.7:PA:QA:Summary of Gas/Oil Well Drill Collar Size } \\
\text { Survey }\end{array}$ & \\
\hline WPO30598 & $\begin{array}{l}\text { SWCF-A:1.2.07.1:PDD:QA:SALADO:PKG\#5:Salado Halite } \\
\text { Rock Compressibility:COMP_RCK }\end{array}$ & \\
\hline WPO30599 & $\begin{array}{l}\text { SWCF-A:1.2.07.1:PDD:QA:SALADO:PKG\#10:DNSGRAIN } \\
\text { Material Grain Density/Anh }\end{array}$ & \\
\hline WPO30600 & $\begin{array}{l}\text { SWCF-A:1.2.07.1:PDD:QA:NON- } \\
\text { SALADO:PKG\#11:POROSITY:Effective Porosity/Anh }\end{array}$ & \\
\hline WP030601 & $\begin{array}{l}\text { SWCF-A:1.2.07.1:PDD:QA:SALADO:PKG\#8:Porosity Effective } \\
\text { Porsity/hal }\end{array}$ & \\
\hline WPO30603 & $\begin{array}{l}\text { SWCF- } \\
\text { A:1.2.07.1:PDD:QA:SALADO:PKG\#13:PRMX_LOG:Log of } \\
\text { Permeability in X-Direction/Anh }\end{array}$ & \\
\hline WPO30605 & $\begin{array}{l}\text { SWCF- } \\
\text { A:1.2.07.1:PDD:QA:SALADO:PKG\#13:PRMY_LOG:Log of } \\
\text { Permeability in Y-Direction/Anh }\end{array}$ & \\
\hline WP030606 & $\begin{array}{l}\text { SWCF- } \\
\text { A:1.2.07.1:PDD:QA:SALADO:PKG\#13:PRMZ_LOG:Log of } \\
\text { Permeability in Z-Direction/Anh }\end{array}$ & \\
\hline WP030607 & $\begin{array}{l}\text { SWCF-A:1.2.07.1:PDD:QA:NON-SALADO:PKG\#21:Non- } \\
\text { Salado Permeability }\end{array}$ & \\
\hline WPO30608 & $\begin{array}{l}\text { SWCF-A:1.2.07.1:PDD:QA:NON-SALADO:PKG\#22:Non- } \\
\text { Salado Effective Porosity }\end{array}$ & \\
\hline WPO30611 & $\begin{array}{l}\text { SWCF-A:1.2.07.1:PDD:QA:NON-SALADO:PKG\#23:NON- } \\
\text { SALADO Bulk Compressibility }\end{array}$ & \\
\hline WPO30640 & $\begin{array}{l}\text { Memo: D. Hurtado to M. Tiemey, 4/24/96, Modification of } \\
\text { Concrete Compressibility Parameter }\end{array}$ & $\cdot$ \\
\hline WPO30641 & $\begin{array}{l}\text { SWCF-A:1.2.07.1:PDD:QA:SALADO:PKG\#2:H2 } \\
\text { Viscosity:VISCO }\end{array}$ & \\
\hline WPO30642 & $\begin{array}{l}\text { SWCF-A:1.2.07.1:PDD:QA:SALADO:PKG\#2:Anh H2 Gas } \\
\text { Parameters:BKLINK:EXPKLINK }\end{array}$ & \\
\hline WPO30643 & $\begin{array}{l}\text { SWCF-A:1.2.07.1:PDD:QA:SALADO:PKG\#10:Anh2 - Phase } \\
\text { Parameters }\end{array}$ & \\
\hline WPO30703 & BRAGFLO User's Manual & \\
\hline WPO30713 & $\begin{array}{l}\text { SWCF-A:1.2.07.1:PDD:QA:NON-SALADO:PKG\#24:Non- } \\
\text { Salado Pressure }\end{array}$ & \\
\hline WPO30721 & $\begin{array}{l}\text { SWCF-A:1.2.07.1:PDD:QA:SALADO:PKG\#7:Salado Halite } \\
\text { Permeability:PERMX_LOG, PERMY_LOG, PRMZ_LOG }\end{array}$ & \\
\hline WPO30819 & $\begin{array}{l}\text { Memo: Wang and Brush to Tiemey, 1/26/96, Estimates of Gas } \\
\text { Generation Parameters for Long-Term WIPP PA }\end{array}$ & \\
\hline WPO30921 & $\begin{array}{l}\text { Memo: B. Butcher to M. Tierney, 1/29/96, QAP 9-2 } \\
\text { Documentation of the Overall Waste Permeability and Flow } \\
\text { Property Values for the CCA }\end{array}$ & \\
\hline
\end{tabular}


Source Cross-Reference -

\begin{tabular}{|c|c|c|c|c|c|}
\hline Ref ID & Author & Editor & Date & Publisher & Location \\
\hline $\begin{array}{l}\text { VAPOR- } \\
\text { LIQUID_EQUILIBRIA }\end{array}$ & H. Kapp, et al. & $\begin{array}{l}\text { D. Behrens, R. } \\
\text { Eckermann }\end{array}$ & 1982 & DECHEMA & \\
\hline VAUGHN:WORKSHEET-1 & P. Vaughn & & 05-Feb-96 & & Albuquerque, NM \\
\hline VAUGHN:WORKSHEET-4 & P. Vaughn & & & & \\
\hline VAUGHN:WORKSHEET-4A & P. Vaughn & & 20-Feb-96 & & Albuquerque, NM \\
\hline WID & $\begin{array}{c}\text { Rockwell Hanford } \\
\text { Operations }\end{array}$ & & 02-Nov-83 & US DOE & Richland, Washington \\
\hline WPO22443 & $\begin{array}{l}\text { P. Vaughn, M. } \\
\text { Lord, B. } \\
\text { MacKinnon }\end{array}$ & & & & \\
\hline WPO26051 & J.P. Marquis & & Sep-95 & & \\
\hline WPO30598 & & & & & \\
\hline WPO30599 & & & & & \\
\hline WPO30600 & & & & & \\
\hline WP030601 & & & & & \\
\hline WPO30603 & & & & & \\
\hline WPO30605 & & & & & \\
\hline WP030606 & & & & & \\
\hline WPO30607 & & & & & \\
\hline WPO30608 & & & & & \\
\hline WPO30611 & & & & & \\
\hline WPO30640 & D. Hurtado & & 24-Apr-96 & $\begin{array}{l}\text { Sandia National } \\
\text { Laboratories }\end{array}$ & Albuquerque, NM \\
\hline WPO30641 & & & & & \\
\hline WPO30642 & & & & & . \\
\hline WPO30643 & & & & & \\
\hline WPO30703 & & & & & \\
\hline WPO30713 & & & & & \\
\hline WPO30721 & & & & & \\
\hline WPO30819 & Wang, Brush & & 02-Jan-96 & & Albuquerque, NM \\
\hline WPO30921 & B. Butcher & & 29-Jan-96 & & Albuquerque, NM \\
\hline
\end{tabular}




\section{Source Cross-Reference}

\begin{tabular}{|c|c|c|}
\hline Ref ID & Titie & Joumal \\
\hline WPO30925 & $\begin{array}{l}\text { Memo: B. Butcher to M. Tiemey, 1/29/96, QAP 9-2 } \\
\text { Documentation of the Initial Waste Water Content for the CCA }\end{array}$ & \\
\hline WPO30994 & $\begin{array}{l}\text { Memo: M. Knowles to M. Tierney, 1/17/96, Transmittal of Seal } \\
\text { System BRAGFLO Parameters (Intera Memo attached) }\end{array}$ & \\
\hline WP031041 & $\begin{array}{l}\text { Memo: J. McCord to M. Tierney, 1/12/96, Non-Salado } \\
\text { Multiphase Flow Parameters Used in BRAGFLO Simulations }\end{array}$ & \\
\hline WPO31070 & $\begin{array}{l}\text { SWCF-A:1.2.07.1:PDD:QA:NON-SALADO:PKG\#19:Castile } \\
\text { Brine Reservoir Permeability }\end{array}$ & \\
\hline WPO31072 & $\begin{array}{l}\text { SWCF-A:1.2.07.1:PDD:QA:NON-SALADO:PKG\#19:Castile } \\
\text { Brine Reservoir Pressure }\end{array}$ & \\
\hline WP031082 & $\begin{array}{l}\text { SWCF-A:1.2.07.1:PDD:QA:NON-SALADO:PKG\#19:Castile } \\
\text { Brine Reservoir Volume }\end{array}$ & . \\
\hline WP031083 & $\begin{array}{l}\text { SWCF-A:1.2.07.1:PDD:QA:NON-SALADO:PKG\#19:Castile } \\
\text { Brine Reservoir Porosity }\end{array}$ & \\
\hline WPO31084 & $\begin{array}{l}\text { SWCF-A:1.2.07.1:PDD:QA:NON-SALADO:PKG\#19:Castile } \\
\text { Brine Reservoir Rock Compressibility }\end{array}$ & \\
\hline WP031168 & SWCF-A:1.2.07.1:PDD:QA:SALADO:PKG\#4:Brine Viscosity & \\
\hline WPO31171 & SWCF-A:1.2.07.1:PDD:QA:SALADO:PKG\#1:WTF & \\
\hline WPO31174 & $\begin{array}{l}\text { SWCF-A:1.2.07.1:PDD:QA:SALADO:PKG\#1:Brine } \\
\text { Compressibility }\end{array}$ & \\
\hline WPO31175 & SWCF-A:1.2.07.1:PDD:QA:SALADO:PKG\#1:Brine Density & \\
\hline WPO31185 & $\begin{array}{l}\text { SWCF-A:1.2.07.1:PDD:QA:SALADO:PKG\#12:Anydrite } \\
\text { Pressure }\end{array}$ & \\
\hline WPO31186 & $\begin{array}{l}\text { SWCF-A:1.2.07.1:PDD:QA:SALADO:PKG\#19:Rock } \\
\text { Compressibility }\end{array}$ & \\
\hline WPO31217 & $\begin{array}{l}\text { SWCF-A:1.2.07.1:PDD:QA:SALADO:PKG\#13:Anhydrite } \\
\text { Permeability }(x, y, z)\end{array}$ & \\
\hline WPO31218 & $\begin{array}{l}\text { SWCF-A:1.2.07.1:PDD:QA:SALADO:PKG\#7:Halite } \\
\text { Permeability }(x, y, z)\end{array}$ & \\
\hline WPO31220 & $\begin{array}{l}\text { SWCF-A:1.2.07.1:PDD:QA:SALADO:PKG\#5:Halite Rock } \\
\text { Compressibility }\end{array}$ & \\
\hline WPO31221 & SWCF-A:1.2.07.1:PDD:QA:SALADO:PKG\#4:Halite Pressure & \\
\hline WPO31235 & Design Document for CCDFGF and GRIDFLO - (page 14) & \\
\hline WPO31368 & $\begin{array}{l}\text { Memo: C. Stone to B. Butcher, } 12 / 19 / 95 \text {. Transfer of Porosity } \\
\text { and Gas Curves from SANTOS for the Final Porosity Surface }\end{array}$ & - \\
\hline WPO31379 & $\begin{array}{l}\text { Memo: P. Drez to File, 1/26/96, Preliminary Estimate for } \\
\text { SNLNM PA Calculations of Nitrate. Sulphate, and Phosphate } \\
\text { Content in Transuranic Solidified Wastes Destine for Disposal } \\
\text { in WIPP }\end{array}$ & \\
\hline WPO31904 & Form 464 for id=2330, idmtrl=CL_L_T1, idpram=PORE_DIS & \\
\hline WPO32038 & $\begin{array}{l}\text { SWCF-A:1.2.07.1:PDD:QA:SALADO:PKG\#16:DRZ } \\
\text { Permeability }(x, y, z)\end{array}$ & \\
\hline WPO32281 & $\begin{array}{l}\text { Memo: B. Butcher to M. Tiemey, 2/6/96, Porosity of the WIPP } \\
\text { North End Excavations }\end{array}$ & \\
\hline WPO32287 & $\begin{array}{l}\text { Memo: D. Hurtado to M. Tierney, 2/12/96, Correction of } \\
\text { Lambda Distribution }\end{array}$ & \\
\hline WPO32288 & $\begin{array}{l}\text { Memo: P. Vaughn to M. Chu, } 1 / 24 / 96, \text { Revision to DRZ \& TZ } \\
\text { Properties to be Used in BRAGFLO CCA Calculations }\end{array}$ & \\
\hline WPO32290 & $\begin{array}{l}\text { Memo: L. Dotson to M. Tiemey, 2/1/96, Initial Pressure } \\
\text { Parameter Values for Non-Salado }\end{array}$ & \\
\hline
\end{tabular}


Source Cross-Reference

\begin{tabular}{|c|c|c|c|c|c|}
\hline Ref ID & Author & Editor & Date & Publisher & Location \\
\hline WP030925 & B. Butcher & & 29-Jan-96 & & Albuquerque, NM \\
\hline WP030994 & M. K. Knowles & & 17-Jan-96 & $\begin{array}{l}\text { Sandia National } \\
\text { Laboratories }\end{array}$ & Albuquerque, NM \\
\hline WPO31041 & J. McCord & & 12-Jan-96 & & \\
\hline WPO31070 & & & & & \\
\hline WPO31072 & & & & & \\
\hline WPO31082 & & & & & \\
\hline WP031083 & & & & & \\
\hline WPO31084 & Geoff Freeze & & 18-Jan-96 & & $\begin{array}{l}\text { Sandia National } \\
\text { Laboratories }\end{array}$ \\
\hline WPO31168 & & & & & \\
\hline WPO31171 & & & & & \\
\hline WPO31174 & & & & & \\
\hline WPO31175 & & & & & \\
\hline WPO31185 & & & & & \\
\hline WPO31186 & & & & & \\
\hline WP031217 & & & & & \\
\hline WPO31218 & & & & & \\
\hline WPO31220 & & & & & \\
\hline WP031221 & & & & & \\
\hline WPO31235 & J. Johnson & & 15-Feb-96 & & \\
\hline WP031368 & C.M. Stone & & 19-Dec-95 & & - \\
\hline WPO31379 & P. Drez & & 26-Jan-96 & & Albuquerque, NM \\
\hline WPO31904 & & & 27-Feb-96 & & \\
\hline WPO32038 & & & & & \\
\hline WPO32281 & B. Butcher & & 06-Feb-96 & & Albuquerque, NM \\
\hline WPO32287 & D. Hurtado & & 12-Feb-96 & & Albuquerque, NM \\
\hline WPO32288 & P. Vaughn & & 24-Jan-96 & & Albuquerque, NM \\
\hline WPO32290 & L. Dotson & & 01-Feb-96 & & \\
\hline
\end{tabular}


Source Cross-Reference

\begin{tabular}{|c|c|c|}
\hline Ref ID & Title & Journal \\
\hline WPO32291 & $\begin{array}{l}\text { Memo: L. Dotson to M. Tiemey, 2/1/96, Parameter Values for } \\
\text { Forty-Niner, Tamarisk, and Unnamed Lower Members of the } \\
\text { Rustler Formation (Addundum to Records \# 21, 22, 23, and } \\
\text { 24) }\end{array}$ & \\
\hline WPO32328 & $\begin{array}{l}\text { Transuranic Waste Baseline Inventory Report, Revision 2, Deo } \\
1995 \text { (Nol 1) }\end{array}$ & DOE/CAD-95-1121 \\
\hline WPO34618 & $\begin{array}{l}\text { Memo: M. McFadden(DOE) to R. Anderson, dtd 3/1/96, } \\
\text { subj:Emplacement Strategy for Remote-Handled Transuranic } \\
\text { Wast Canisters }\end{array}$ & \\
\hline WPO35134 & E-Mail: P. Vaughn, 3/13/96, Borehole Properties & \\
\hline WPO35148 & $\begin{array}{l}\text { Memo: A. Shinta to M. Tiemey, 2/8/96, NUTS Salado } \\
\text { Parameters List }\end{array}$ & \\
\hline WPO35183 & $\begin{array}{l}\text { E-Mail: P. Vaughn, 2/5/96, Initial Brine Saturation in } \\
\text { Repository }\end{array}$ & \\
\hline WPO35193 & $\begin{array}{l}\text { Memo: G. Ruskauff to M. Tiemey, dtd 3/13/96, Culebra } \\
\text { Transmissivity Field Index, GLOBAL, TRANSIDX (\#225) }\end{array}$ & \\
\hline WPO35194 & Oxidation State Distribution & \\
\hline WPO35266 & $\begin{array}{l}\text { Memo: K. Economy to M. Tiemey, 1/29/96, Culebra Hydraulic } \\
\text { Conductivity Value to be Used by BRAGFLO }\end{array}$ & \\
\hline WPO35267 & $\begin{array}{l}\text { Memo: D. MCArthur to M. Tierney, 3/20/96, CLAY_BOT is } \\
\text { Required by BRAGFLO Modeling of the Shaft }\end{array}$ & \\
\hline WPO35268 & Memo: M. Tierney to Distribution, 3/21/96, Distributions & \\
\hline WPO35269 & $\begin{array}{l}\text { Memo: J. Ramsey to J. Nowak, 03/18/96, subj: Culebra } \\
\text { Dissolved Actinide Parameter Request }\end{array}$ & \\
\hline WPO35270 & $\begin{array}{l}\text { Memo: J. Ramsey to A. Lappin, 3/18/96, Culebra } \\
\text { Transmissivity and Physical Transport Parameter Request }\end{array}$ & \\
\hline WPO35271 & $\begin{array}{l}\text { Memo: P. Vaughn to M. Tiemey, 2/19/96, KPT Modelling } \\
\text { Feature }\end{array}$ & \\
\hline WPO35329 & $\begin{array}{l}\text { Memo: P. Vaughn to M. Tiemey, 2/23/96, Salado Borehole } \\
\text { Diameter }\end{array}$ & \\
\hline WPO35330 & $\begin{array}{l}\text { Memo: P. Vaughn to } M \text {. Tiemey, 2/26/96, Change BACKFILL } \\
\text { Material Name to OPS_AREA }\end{array}$ & \\
\hline WPO35333 & $\begin{array}{l}\text { Memo: P. Vaughn to M. Tiemey, 2/20/96, Change KTP } \\
\text { Material Name to KPT }\end{array}$ & \\
\hline WPO35597 & $\begin{array}{l}\text { Memo: M. Martell to File, } 3 / 25 / 96 \text {, Support Documentation } \\
\text { (Salado, Non-Salado, and Shaft Seals) to Request the Need } \\
\text { or Intended Use of a WIPP Parameter }\end{array}$ & \\
\hline WPO35695 & $\begin{array}{l}\text { Memo: J. Berglund to B. Butcher, 10/28/96, subj: Effective } \\
\text { Shear Resistance to Erosion TAUFAlL (in "Principal } \\
\text { investigator Documentation Package for: Cuttings Release of } \\
\text { Solids Caused by Blowout") }\end{array}$ & - \\
\hline WPO35835 & $\begin{array}{l}\text { Memo: R. Bynum to M. Tiemey and C. Stockman, 5/23/96, } \\
\text { Revised Update of Uncertainty Range and Distribution for } \\
\text { Actinide Solubility to be Used in CCA Nuts Calculations }\end{array}$ & \\
\hline WPO35850 & $\begin{array}{l}\text { PRP: Mobile Colloidal Actinide Source Term. 1. Mineral } \\
\text { Fragment Colloids }\end{array}$ & \\
\hline WPO35852 & $\begin{array}{l}\text { PRP: Mobile Colloidal Actinide Source Term. 2. Actinide } \\
\text { Intrinsic Colloids }\end{array}$ & \\
\hline WPO35854 & $\begin{array}{l}\text { Memo: K. Knowles to M. Tiemey, 3/14/96, Grain Density for } \\
\text { Asphalt Seal Components }\end{array}$ & \\
\hline WPO35855 & $\begin{array}{l}\text { PRP: Mobile Colloidal Actinide Source Term. 3. Humic } \\
\text { Substances }\end{array}$ & \\
\hline WPO35856 & PRP: Mobile Colloidal Actinide Source Term. 4. Microbes & \\
\hline WPO36041 & Analysis of Bell Canyon Test Results & SAND80-7044C \\
\hline
\end{tabular}




\section{- Source Cross-Reference}

\begin{tabular}{|c|c|c|c|c|c|}
\hline Ref ID & Author & Editor & Date & Publisher & Location \\
\hline WPO32291 & Lori Dotson & & 01-Feb-96 & $\begin{array}{l}\text { Sandia National } \\
\text { Laboratories }\end{array}$ & \\
\hline WPO32328 & & & Dec-95 & & \\
\hline WPO34618 & M. McFadden & & 01-Mar-96 & & \\
\hline WP035134 & P. Vaughn & & 13-Mar-96 & & Albuquerque, NM \\
\hline WPO35148 & A. Shinta & & 08-Feb-96 & & Albuquerque, NM \\
\hline WPO35183 & P. Vaughn & & 05-Feb-96 & & \\
\hline WPO35193 & Greg Ruskauff & & 13-Mar-96 & & \\
\hline WPO35194 & Ruth Weiner & & $19-03-96$ & & \\
\hline WPO35266 & K. Economy & & 29-Jan-96 & & \\
\hline WPO35267 & D. McArthur & & 20-Mar-96 & \begin{tabular}{|l|} 
Sandia National \\
Laboratories
\end{tabular} & Albuquerque, NM \\
\hline WPO35268 & M. Tierney & & 04-Mar-96 & & \begin{tabular}{|l|} 
Sandia National \\
Laboratories \\
\end{tabular} \\
\hline WPO35269 & J. Ramsey & & 18-Mar-96 & SNL & Albuquerque, NM \\
\hline WPO35270 & Jim Ramsey & & 18-Mar-96 & & \\
\hline \multicolumn{6}{|l|}{ WPO35271 } \\
\hline WPO35329 & P. Vaughn & & 23-Feb-96 & $\begin{array}{l}\text { Sandia National } \\
\text { Laboratories }\end{array}$ & Albuquerque, NM \\
\hline WPO35330 & P. Vaughn & & 26-Feb-96 & & Albuquerque, NM \\
\hline WPO35333 & P. Vaughn & & 20-Feb-96 & \begin{tabular}{|l} 
Sandia National \\
Laboratories
\end{tabular} & Albuquerque, NM \\
\hline WPO35597 & M. Martell & & 26-Mar-96 & $\begin{array}{l}\text { Sandia National } \\
\text { Laboratories }\end{array}$ & Albuquerque, NM \\
\hline WPO35695 & $\begin{array}{l}\text { J.W. Berglund } \\
\text { (memo) \& B. } \\
\text { Butcher (PI } \\
\text { Package) }\end{array}$ & & 28-Oct-96 & & - \\
\hline WPO35835 & R. V. Bynum & & 23-May-96 & & Albuquerque, NM \\
\hline WPO35850 & Hans Papenguth & & & & \\
\hline WPO35852 & Hans Papenguth & & & & \\
\hline WPO35854 & Karen Knowles & & 14-Mar-96 & & $\begin{array}{l}\text { Sandia National } \\
\text { Laboratories }\end{array}$ \\
\hline WPO35855 & Hans Papenguth & & & & \\
\hline WP035856 & Hans Papenguth & & & & \\
\hline WP036041 & $\begin{array}{l}\text { E.W. Peterson and } \\
\text { C.L. Christensen }\end{array}$ & & 1980 & SNL & Albuquerque, NM \\
\hline
\end{tabular}


Source Cross-Reference

\begin{tabular}{|c|c|c|}
\hline Ref ID & Titie & Joumal \\
\hline WPO36048 & $\begin{array}{l}\text { Memo: M. Tierney to File, 3/29/96, Reasons for Change of } \\
\text { Category } 2 \text { (Baseline Inventory Report) Data }\end{array}$ & \\
\hline WPO36050 & $\begin{array}{l}\text { Memo: P. Vaughn to M. Tiemey, 2/22/96, CLAY_BOT } \\
\text { Analogue of Lower Salado Clay }\end{array}$ & \\
\hline WPO36051 & Memo: P. Vaughn to $M$. Tiemey, 2/20/96, idpram PO_MIN & \\
\hline WP036053 & $\begin{array}{l}\text { Memo: C. Stockman to M. Siegel, 3/28/96, subj: Request for } \\
\text { Solubility Parameters }\end{array}$ & \\
\hline WPO36058 & $\begin{array}{l}\text { Memo: P. Vaughn to M. Tierney, 2/22/96, Brine Far-Field Pore } \\
\text { Pressure }\end{array}$ & \\
\hline WPO36203 & $\begin{array}{l}\text { Memo: M. Tiemey to File, } 3 / 29 / 96, \text { Assignment of Brooks- } \\
\text { Corey Pore Distribution Parameters to Castille Formation }\end{array}$ & \\
\hline WPO36205 & $\begin{array}{l}\text { Memo: P Vaughn to M. Chu, } 1 / 22 / 96, \text { Strawman for Altered } \\
\text { Anhydrite Model in Support of the CCA }\end{array}$ & \\
\hline WPO36298 & $\begin{array}{l}\text { Memo: M. Martell to File, } 4 / 2 / 96, \text { Reason for Category } 3 \\
\text { Parameters }\end{array}$ & \\
\hline WPO36301 & $\begin{array}{l}\text { Memo: D. Stoelzel to M. Tiemey, 4/3/96, Request for } \\
\text { Parameters for Use in CUTTINGS_S }\end{array}$ & \\
\hline WPO36320 & $\begin{array}{l}\text { Memo: P. Vaughn to M. Tiemey, } 1 / 30 / 96 \text {, Northend Regions } \\
\text { ('Backfill' and Experimental) Porosity }\end{array}$ & \\
\hline WPO36380 & $\begin{array}{l}\text { Memo: M. Martell to Distribution, 4/4/96, Student-T } \\
\text { Distribution (w/attachment) }\end{array}$ & \\
\hline WPO36383 & $\begin{array}{l}\text { Memo: P. Vaughn to M. Tierney, 3/29/96, CLAY_BOT } \\
\text { Analogue of Lower Salado Clay }\end{array}$ & \\
\hline WPO36455 & $\begin{array}{l}\text { SWCF-A:1.2.07.1:PDD:QA:NON-SALADO:Culebra Thickness } \\
\text { Data }\end{array}$ & \\
\hline WPO36456 & $\begin{array}{l}\text { SWCF-A:1.2.07.1:PDD:QA:NON-STORATIVITY:Culebra } \\
\text { Storativity Data }\end{array}$ & • \\
\hline WPO36489 & Mining Transmissivity Multiplier [Non-Salado] & \\
\hline WPO36491 & SWCF-A:PDD, 1.2.07.1, Skin Resistence & . \\
\hline WPO36723 & $\begin{array}{l}\text { Memo: J.W. Berglund to M.S. Tiemey, 4/17/96, Parameters } \\
\text { Required for the CUTTINGS_S Code for Use in WIPP } \\
\text { Performance Assessment (Additional Parameters) }\end{array}$ & \\
\hline WPO36724 & $\begin{array}{l}\text { Memo: E. Nowak to C. Stockman, 4/11/96, Reply to Request } \\
\text { for Solubility Parameters for } \mathrm{Ra}, \mathrm{Cs}, \mathrm{Sr}\end{array}$ & \\
\hline WP036766 & $\begin{array}{l}\text { Memo: J.W. Berglund to M.S. Tiemey, 4/1/96, Parameters } \\
\text { Required for the CUTTINGS_S Code for use in WIPP } \\
\text { Performance Assessment }\end{array}$ & \\
\hline WPO36771 & $\begin{array}{l}\text { Memo: H. Papenguth to C. Stockman, 3/29/96, Colloidal } \\
\text { Actinide Source Term Parameters }\end{array}$ & - \\
\hline WPO36772 & $\begin{array}{l}\text { Memo: P. Davies and R. Beauheim to M. Tierney, } 3 / 7 / 96 \text {, } \\
\text { Necessary Changes to the Parameter Records Package and } \\
\text { Form } 464 \text { for Far-Field Permeability of Salado (ID\#s: } 547,548 \text {, } \\
549 ; \text { idmtrl: S_HALITE; idpr }\end{array}$ & \\
\hline WP037061 & $\begin{array}{l}\text { Memo: L. Sanchez to M. Martell, 4/23/96, Radionuclides for } \\
\text { Use in WIPP PA Calculations }\end{array}$ & \\
\hline WPO37147 & $\begin{array}{l}\text { Memo: J. Helton to M. Tiemey, 3/21/96, Addition of Discrete } \\
\text { Parameter }\end{array}$ & \\
\hline WPO37148 & $\begin{array}{l}\text { Memo: G. Freeze/K. Larson to M. Tiemey, 3/20/96, Initial } \\
\text { Pressure in the Castile Brine Reservoir }\end{array}$ & \\
\hline WPO37223 & $\begin{array}{l}\text { SWCF-A:1.2.07.1:PDD:QA:Non-Salado:Culebra Effective } \\
\text { Thickness (Culebra Transport Parameter) }\end{array}$ & \\
\hline WPO37225 & $\begin{array}{l}\text { SWCF-A:1.2.07.1:PDD:QA:Non-Salado:Culebra Half Matrix } \\
\text { Block Length (Culebra Transport Parameter) }\end{array}$ & \\
\hline WP037226 & $\begin{array}{l}\text { SWCF-A:1.2.07.1:PDD:QA:Non-Salado:Culebra Diffusive } \\
\text { Tortuosity (Culebra Transport Parameter) }\end{array}$ & \\
\hline
\end{tabular}


Source Cross-Reference

\begin{tabular}{|c|c|c|c|c|c|}
\hline Ref ID & Author & Editor & Date & \begin{tabular}{|l|} 
Publisher \\
\end{tabular} & Location \\
\hline WPO36048 & M. Tierney & & 29-Mar-96 & $\begin{array}{l}\text { Sandia National } \\
\text { Laboratories }\end{array}$ & Albuquerque, NM \\
\hline WPO36050 & P. Vaughn & - & 22-Feb-96 & $\begin{array}{l}\text { Sandia National } \\
\text { Laboratories }\end{array}$ & Albuquerque, NM \\
\hline WP036051 & Paimer Vaughn & & 20-Feb-96 & & $\begin{array}{l}\text { Sandia National } \\
\text { Laboratories }\end{array}$ \\
\hline WPO36053 & C.T. Stockman & & 28-Mar-96 & SNL & Albuquerque, NM \\
\hline WPO36058 & P. Vaughn & & 22-Feb-96 & & Albuquerque, NM \\
\hline WPO36203 & M.S. Tierney & & 29-Mar-96 & \begin{tabular}{|l} 
Sandia National \\
Laboratories
\end{tabular} & Albuquerque, NM \\
\hline WPO36205 & P. Vaughn & & 22-Jan-96 & & Albuquerque, NM \\
\hline WPO36298 & M. Martell & & 04-Apr-96 & \begin{tabular}{|l} 
Sandia National \\
Laboratories
\end{tabular} & Albuquerque, NM \\
\hline \multicolumn{6}{|l|}{ WP036301 } \\
\hline WPO36320 & P. Vaughn & & 30-Jan-96 & & Albuquerque, NM \\
\hline WPO36380 & M. Martell & & 04-Apr-96 & & Albuquerque, NM \\
\hline WPO36383 & P. Vaughn & & 29-Mar-96 & \begin{tabular}{|l|} 
Sandia National \\
Laboratories
\end{tabular} & Albuquerque, NM \\
\hline WPO36455 & R. Beauheim & & . & & \\
\hline WPO36456 & R. Beauheim & & & & Albuquerque, NM \\
\hline WPO36489 & Wallace, M.G. & & 1996 & & SWCF \\
\hline WPO36491 & M. Wallace & & & & \\
\hline WPO36723 & J.W. Berglund & & 17-Apr-96 & & Albuquerque, NM \\
\hline WPO36724 & E.J. Nowak & & 11-Apr-96 & $\begin{array}{l}\text { Sandia National } \\
\text { Laboratories }\end{array}$ & Albuquerque, NM \\
\hline WP036766 & J.W. Berglund & & 01-Apr-96 & & Albuquerque, NM \\
\hline WPO36771 & $\begin{array}{l}\text { Hans W. } \\
\text { Papenguth }\end{array}$ & & 29-Mar-96 & & $\begin{array}{l}\text { Sandia National } \\
\text { Laboratories }\end{array}$ \\
\hline WP036772 & $\begin{array}{l}\text { P. Davies and R. } \\
\text { Beauheim }\end{array}$ & & 07-Mar-96 & & Albuquerque, NM \\
\hline WPO37061 & L. C. Sanchez & & 23-Apr-96 & & Albuquerque, NM \\
\hline WPO37147 & Jon Helton & & 21-Mar-96 & & Albuquerque, NM \\
\hline WPO37148 & $\begin{array}{c}\text { G. Freeze and } \mathrm{K} \text {. } \\
\text { Larson }\end{array}$ & & 20-Mar-96 & & Albuquerque, NM \\
\hline \multicolumn{6}{|l|}{ WPO37223 } \\
\hline \multicolumn{6}{|l|}{ WPO37225 } \\
\hline WP037226 & & & & $\begin{array}{l}\text { Sandia National } \\
\text { Laboratories }\end{array}$ & Albuquerque, NM \\
\hline
\end{tabular}


Source Cross-Reference

\begin{tabular}{|c|c|c|}
\hline Ref ID & \begin{tabular}{|c|} 
Title \\
\end{tabular} & Joumal \\
\hline WPO37227 & $\begin{array}{l}\text { SWCF-A:1.2.07.1:PDD:QA:Non-Salado:Culebra Advective } \\
\text { Porosity (Culebra Transport Parameter) }\end{array}$ & \\
\hline WPO37228 & $\begin{array}{l}\text { SWCF-A:1.2.07.1:PDD:QA:Non-Salado:Culebra Diffusive } \\
\text { Porosity (Culebra Transport Parameter) }\end{array}$ & \\
\hline WPO37230 & SWCF-A:1.2.07.1:PDD:QA:Culebra Longitudinal Dispersivity & \\
\hline WPO37231 & $\begin{array}{l}\text { SWCF-A:1.2.07.1:PDD:QA:Ratio of Longitudinal to Transverse } \\
\text { Dispersivity for the Culebra }\end{array}$ & \\
\hline WPO37232 & \begin{tabular}{|l|} 
SWCF-A:1.2.07.1:PDD:QA:Non-Salado:Grain Density \\
(Culebra Transport Parameter)
\end{tabular} & \\
\hline WPO37314 & $\begin{array}{l}\text { Memo: M. Siegel to M. Tiemey, 3/29/96, Solubility Parameters } \\
\text { for Use in the CCA NUTS and GRIDFLOW Calculations }\end{array}$ & \\
\hline WPO37400 & $\begin{array}{l}\text { Memo: L. Brush to M. Tiemey, 5/2/96, Free-Soloution Tracer } \\
\text { Diffusion Coefficients (DSOLS) for Dissolved Pu, Am, U, Th, } \\
\text { Np, Cm, and Ra in Boreholes and the Culebra for Use in the } \\
\text { PA Calculations to Su }\end{array}$ & \\
\hline WPO37401 & $\begin{array}{l}\text { Memo: M. Martell to C. Stockman, 5/10/96, Solubility Update, } \\
\text { Request } \mathrm{Pb} \text { to Database, Further Justifcation for } \mathrm{Cs}, \mathrm{Ra} \text {, and } \\
\mathrm{Sr}\end{array}$ & \\
\hline WPO37402 & $\begin{array}{l}\text { Memo: P. Vaughn to M. Tierney, 5/1/96, Additional Borehole } \\
\text { Parameters }\end{array}$ & \\
\hline WPO37404 & $\begin{array}{l}\text { Memo: L. Sanchez to M. Martell, 3/28/96, Radionuclide Half- } \\
\text { lives and Specific Activities Obtained From ORIGEN2 Data }\end{array}$ & \\
\hline WPO37465 & $\begin{array}{l}\text { Memo: T.Corbet to M. Tierney, 4/12/96, Distribution for Non- } \\
\text { Salado Parameters for SECOFL2D:Climate Index }\end{array}$ & \\
\hline WPO37522 & $\begin{array}{l}\text { Memo: H. Papenguth to C. Stockman, 4/22/96, Colloidal } \\
\text { Actinide Source Term Parameters, Revision } 2\end{array}$ & \\
\hline WPO37533 & $\begin{array}{l}\text { Memo: L. Brush to M. Tiemey, 5/11/96, Revised Free-Solution } \\
\text { Tracer Diffusion Coefficients (DsolS) for Dissolved Pu, Am, U, } \\
\text { Th, Np, CM, \& Ra in Boreholes and the Culebra for Use in the } \\
\text { PA Calculations }\end{array}$ & \\
\hline WPO37536 & $\begin{array}{l}\text { Memo: C. Stockman to M. Tiemey, 4/16/96, Implementation of } \\
\text { Chemistry Parameters in PA }\end{array}$ & \\
\hline WPO37542 & $\begin{array}{l}\text { Memo: P. Vaughn to M. Tierney, 5/20/96, CUMPROB } \\
\text { Parameter Definition and Usage }\end{array}$ & \\
\hline WPO37545 & $\begin{array}{l}\text { Memo: C. Stockman to M. Martell, 5/10/96, Proposed Method } \\
\text { of implementation of Updated Solubility Distributions }\end{array}$ & \\
\hline WPO37713 & $\begin{array}{l}\text { Analyst Calculation: Schreiber, 7/26/91, Updated Waste } \\
\text { Storage Volumes (12 pgs) }\end{array}$ & . \\
\hline WPO37799 & $\begin{array}{l}\text { Memo: R. Bynum to M. Tiemey, } 5 / 17 / 96, \text { Revision of +III } \\
\text { Solubility Parameters for Use in CCA NUTS Calculations }\end{array}$ & \\
\hline WPO37804 & $\begin{array}{l}\text { Memo: C. Lattier to SNL WIPP Managers, 5/22/96, Error } \\
\text { Corrections to the CCA Database, Calculation Name: CCA5 }\end{array}$ & \\
\hline WP037822 & $\begin{array}{l}\text { Memo: M. Siegel to M. Tiemey and C. Stockman, 5/9/96, } \\
\text { Revised + III Solubility Parameters for Use in the CCA NUTS } \\
\text { and GRIDFLOW Calculations }\end{array}$ & \\
\hline WPO37841 & $\begin{array}{l}\text { Memo: S. Altman, L. Meigs, L. Brush to J. Ramsey, 5/15/96, } \\
\text { Parameter Records Package Non-Salado: Culebra Dolomite } \\
\text { Grain Density (WPO\#37232) }\end{array}$ & \\
\hline WPO37970 & $\begin{array}{l}\text { Memo: M. Tierney to Memo of Record, 5/30/96, List of Legacy } \\
\text { Parameters that are Category } 1\end{array}$ & \\
\hline WPO38214 & $\begin{array}{l}\text { Memo: D. Stoelzel to M. Marietta, 5/31/96, Response to } \\
\text { Westinghouse Memo on Brine Blowout Duration }\end{array}$ & \\
\hline
\end{tabular}


Source Cross-Reference

\begin{tabular}{|c|c|c|c|c|c|}
\hline Ref ID & Author & Editor & Date & Publisher & Location \\
\hline WPO37227 & & & & & \\
\hline WPO37228 & & & & & \\
\hline WPO37230 & J. McCord & & & & \\
\hline WPO37231 & J. McCord & & & & \\
\hline WPO37232 & & & & $\begin{array}{l}\text { Sandia National } \\
\text { Laboratories }\end{array}$ & Albuquerque, NM \\
\hline WPO37314 & M. Siegel & & 29-Mar-96 & $\begin{array}{l}\text { Sandia National } \\
\text { Laboratories }\end{array}$ & Albuquerque, NM \\
\hline WP037400 & L. H. Brush & & 02-May-96 & & Albuquerque, NM \\
\hline WP037401 & C. Stockman & & 10-May-96 & & Albuquerque, NM \\
\hline WPO37402 & P. Vaughn & & 01-May-96 & & Albuquerque, NM \\
\hline WPO37404 & Larry Sanchez & & 28-Mar-96 & & $\begin{array}{l}\text { Sandia National } \\
\text { Laboratories }\end{array}$ \\
\hline WPO37465 & T. Corbet & & 12-Apr-96 & $\begin{array}{l}\text { Sandia National } \\
\text { Laboratories }\end{array}$ & Albuquerque, NM \\
\hline WPO37522 & H. Papenguth & & 22-Apr-96 & $\begin{array}{l}\text { Sandia National } \\
\text { Laboratories }\end{array}$ & Albuquerque, NM \\
\hline WPO37533 & L. Brush & & 11-May-96 & & Albuquerque, NM \\
\hline WPO37536 & C. Stockman & & 16-Apr-96 & $\begin{array}{l}\text { Sandia National } \\
\text { Laboratories }\end{array}$ & Albuquerque, NM \\
\hline WPO37542 & P. Vaughn & & 20-May-96 & & Albuquerque, NM \\
\hline WPO37545 & C.T. Stockman & & 10-May-96 & $\begin{array}{l}\text { Sandia National } \\
\text { Laboratories }\end{array}$ & Albuquerque, NM \\
\hline WPO37713 & J. Schreiber & & 26-Jul-91 & & Albuquerque, NM \\
\hline WPO37799 & R. V. Bynum & & 17-May-96 & & Albuquerque, NM \\
\hline WP037804 & C. Lattier & & 22-May-96 & & Albuquerque, NM \\
\hline WPO37822 & M. Siegel & & $09-$ May-96 & & Albuquerque, NM \\
\hline WPO37841 & $\begin{array}{l}\text { S. Altman, L. } \\
\text { Meigs, L. Brush }\end{array}$ & & 15-May-96 & $\begin{array}{l}\text { Sandia National } \\
\text { Laboratories }\end{array}$ & Albuquerque, NM \\
\hline WPO37970 & M. Tiemey & & 30-May-96 & & Albuquerque, NM \\
\hline WP038214 & D. Stoelzel & & 31-May-96 & & Albuquerque, NM \\
\hline
\end{tabular}




\section{Source Cross-Reference}

\begin{tabular}{|c|c|c|}
\hline Ref ID & Title & Joumal \\
\hline WP038217 & $\begin{array}{l}\text { Memo: D. Stoelzel to M. Tiemey, 6/6/96, Castile Thickness } \\
\text { and Extemal Drainage Radius Values fo BRAGFLO_DBR }\end{array}$ & \\
\hline WPO38231 & Culebra Dissolve Actinide Distribution Coefficients (Kd's) & \\
\hline WPO38274 & $\begin{array}{l}\text { Memo: M. Lord to M. Tiemey, 2/20/96, Anhydrite Fracture } \\
\text { Parameters for the CCA Data Base }\end{array}$ & \\
\hline WPO38333 & $\begin{array}{l}\text { Memo: P. Vaughn to M. Tierney, 2/15/96, Impermeable Zone } \\
\text { Parameters }\end{array}$ & \\
\hline WPO38337 & $\begin{array}{l}\text { Memo: P. Vaughn to M. Tiemey, 1/31/96, Some Possible Non- } \\
\text { Salado Disconnects for CCA }\end{array}$ & \\
\hline WPO38343 & \begin{tabular}{|l|} 
Memo: S. Howarth to SWCF, 6/14/96, Technical Data \\
Package for Borehole Diameter (w/attachments)
\end{tabular} & \\
\hline WPO38344 & $\begin{array}{l}\text { Memo: S. Howarth to SWCF, 6/14/96, Parameter Record } \\
\text { Package for Nominal Wellbore Diameter }\end{array}$ & \\
\hline WPO38449 & $\begin{array}{l}\text { Memo: L. Sanchez to Memo of Record, 6/12/96, Qualification } \\
\text { Justified for ID Nos. } 1993 \text { and } 2116 \text { (Category 2) }\end{array}$ & \\
\hline WPO38450 & $\begin{array}{l}\text { Memo: M. Martell to Memo of Record, } 6 / 11 / 96, \text { Unit Changes } \\
\text { to ID Nos. } 2819,2904,2905 \text { (Category 1) }\end{array}$ & \\
\hline WPO38454 & $\begin{array}{l}\text { Memo: M. Martell to Memo of Record, 6/11/96, Unit Changes } \\
\text { to ID Nos. } 2737,2779,2805,2774 \text { (Category 4B) }\end{array}$ & \\
\hline WPO38455 & $\begin{array}{l}\text { Memo: M. Tiemey to Memo of Record, 6/11/96, Qualification } \\
\text { Justified for ID Nos. } 3240 \text { and } 3257\end{array}$ & \\
\hline WPO38456 & $\begin{array}{l}\text { Memo: M. Martell to Memo of Record, 5/31/96, Qualification } \\
\text { Justified for ID No. } 3112\end{array}$ & \\
\hline WPO38568 & $\begin{array}{l}\text { Memo: M. Tiemey and P. Vaughn to File, 6/17/96, } \\
\text { Designation of "Legacy Parameters" and "Placeholders" in the } \\
\text { WIPP Parameter Database }\end{array}$ & \\
\hline WPO38569 & $\begin{array}{l}\text { Memo: M. Martell to Memo of Record, 6/19/96, Error } \\
\text { Correction for Source Document }\end{array}$ & \\
\hline WPO38769 & $\begin{array}{l}\text { Memo: D. O'Brien, 11/15/95, Critical Gas Saturation } \\
\text { Recommendations for WIPP }\end{array}$ & \\
\hline WPO38773 & $\begin{array}{l}\text { Memo: L. Meigs to J. Ramsey, dtd 6/6/96, subj:Non- } \\
\text { Salado:Diffusive Porosity for the Culebra Dolomite (WPO\# } \\
\text { 37228) }\end{array}$ & \\
\hline WPO38801 & $\begin{array}{l}\text { Memo: L. Brush to M. Tierney, 6/10/96, Ranges and } \\
\text { Probability Distributions of Kds for Dissolved Pu, Am, U, Th, } \\
\text { and Np in the Culebra for the PA Calculations to Support the } \\
\text { WIPP CCA }\end{array}$ & $=$ \\
\hline WPO38819 & $\begin{array}{l}\text { WIPP/CAO-96-3168, Chapter 6, 4/19/96, Effectiveness of } \\
\text { Passive Institutional Controls in Reducing Inadvertent Human } \\
\text { intrusion into the WIPP for Use in Performance Assessments }\end{array}$ & WIPP/CAO-96-3168 \\
\hline WP038928 & $\begin{array}{l}\text { Memo: L. Meigs and J. McCord to J. Ramsey, 6/6/96, Non- } \\
\text { Salado: Culebra Half Matrix Block Length (WPO\# 37225) }\end{array}$ & \\
\hline WPO38928 & $\begin{array}{l}\text { Memo: L. Meigs and J. McCord to J. Ramsey, 6/6/96, } \\
\text { subj:Non-Salado: Advective Porosity for the Culebra Dolomite } \\
\text { (see also WPO37227) }\end{array}$ & \\
\hline WPO38940 & $\begin{array}{l}\text { Memo: L. Meigs to J. Ramsey, 5/16/96, Non-Salado:Diffusive } \\
\text { Tortuosity for the Culebra Dolomite (WPO37226) }\end{array}$ & \\
\hline WP038941 & $\begin{array}{l}\text { Memo: J. McCord to J. Ramsey, 6/10/96, Non-Salado: } \\
\text { Longitudinal Dispersivity and Longitudinal to Transverse } \\
\text { Dispersivity Ratio for the Culebra Dolomite (WPO\#s } 37230 \\
\text { and 37231) }\end{array}$ & \\
\hline
\end{tabular}


Source Cross-Reference

\begin{tabular}{|c|c|c|c|c|c|}
\hline & Author & Editor & Date & Publisher & Location \\
\hline WPO38217 & D. Stoelzel & & 06-Jun-96 & & Albuquerque, NM \\
\hline WPO38231 & Brush, L.H. & & & & \\
\hline WPO38274 & M. Lord & & 20-Feb-96 & & Albuquerque, NM \\
\hline WPO38333 & P. Vaughn & & 15-Feb-96 & & Albuquerque, NM \\
\hline WPO38337 & P. Vaughn & & 31-Jan-96 & & \\
\hline WPO38343 & S. Howarth & & 14-Jun-96 & . & Albuquerque, NM \\
\hline WPO38344 & S. Howarth & & 14-Jun-96 & & Albuquerque, NM \\
\hline WPO38449 & L. Sanchez & & 12-Jun-96 & & Albuquerque, NM \\
\hline WPO38450 & M. Martell & & 11-Jun-96 & & Albuquerque, NM \\
\hline WPO38454 & M. Martell & & 11-Jun-96 & & Albuquerque, NM \\
\hline WPO38455 & M. Tiemey & & 11-Jun-96 & & Albuquerque, NM \\
\hline WPO38456 & M. Martell & & 31-May-96 & & Albuquerque, NM \\
\hline WPO38568 & $\begin{array}{l}\text { M. Tiemey, P. } \\
\text { Vaughn }\end{array}$ & & 17-Jun-96 & & Albuquerque, NM \\
\hline WPO38569 & M. Martell & & 19-Jun-96 & & Albuquerque, NM \\
\hline WPO38769 & $\begin{array}{l}\text { D. O'Brien, } \\
\text { Solutions } \\
\text { Engineering }\end{array}$ & & 15-Nov-95 & & \\
\hline WPO38773 & L. Meigs & & 06-Jun-96 & $\begin{array}{l}\text { Sandia National } \\
\text { Laboratories }\end{array}$ & Albuquerque, NM \\
\hline WP038801 & L.H. Brush & & 10-Jun-96 & & Albuquerque, NM \\
\hline WP038819 & USDOEICAO & & 19-Apr-96 & USDOE & CAO \\
\hline WPO38928 & $\begin{array}{l}\text { L. Meigs and J. } \\
\text { McCord }\end{array}$ & & 06-Jun-96 & $\begin{array}{l}\text { Sandia National } \\
\text { Laboratories }\end{array}$ & Albuquerque, NM \\
\hline WPO38928 & $\begin{array}{l}\text { L. Meigs and J. } \\
\text { McCord }\end{array}$ & & 06-Jun-96 & $\begin{array}{l}\text { Sandia National } \\
\text { Laboratories }\end{array}$ & Albuquerque, NM \\
\hline WP038940 & L. Meigs & & 16-May-96 & $\begin{array}{l}\text { Sandia National } \\
\text { Laboratories }\end{array}$ & Albuquerque, NM \\
\hline WPO38941 & J. McCord & & 10-Jun-96 & & Albuquerque, NM \\
\hline
\end{tabular}


Source Cross-Reference

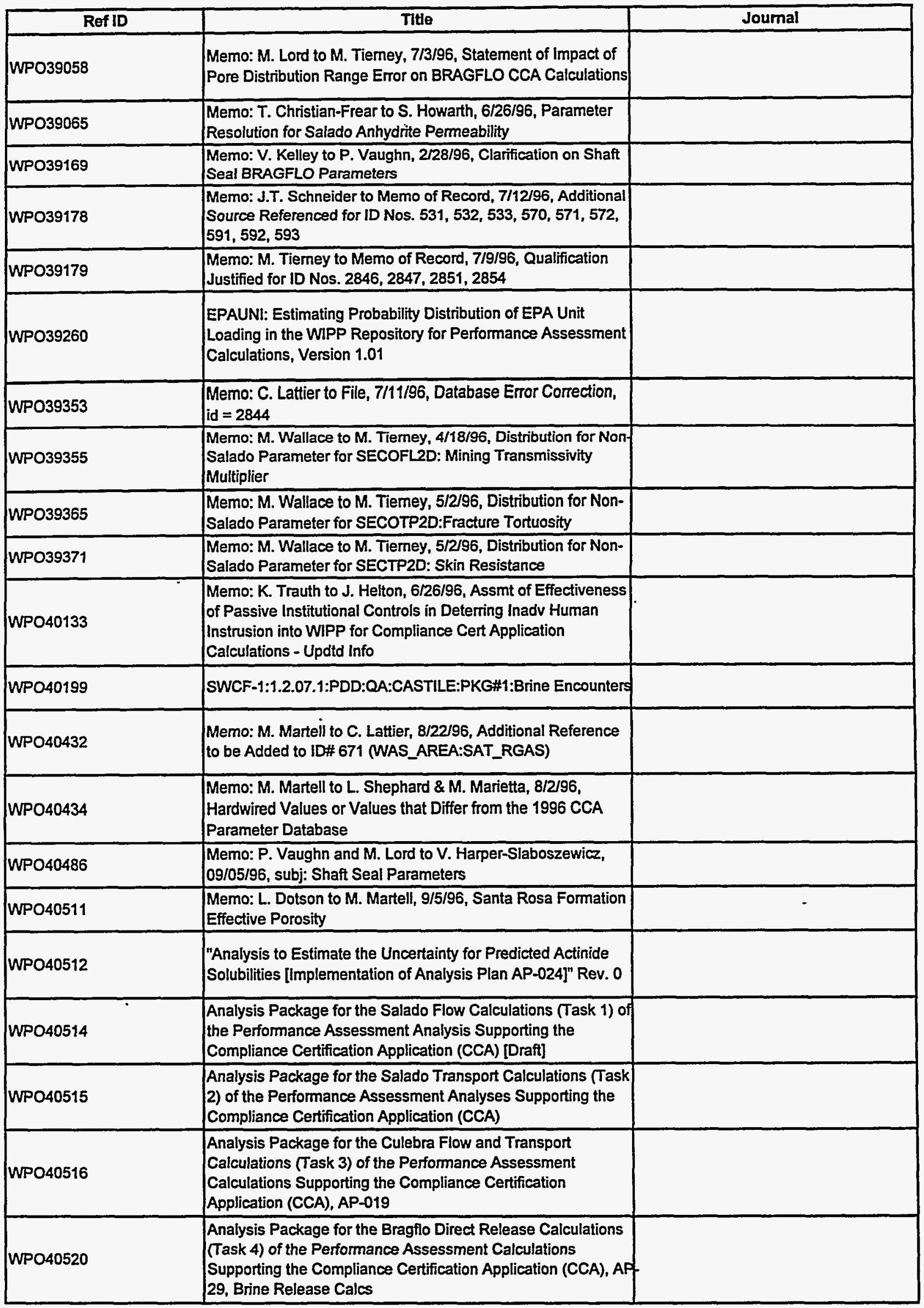


Source Cross-Reference

\begin{tabular}{|c|c|c|c|c|c|}
\hline Ref ID & Author & Editor & Dato & Publlsher & Location \\
\hline WPO39058 & M. Lord & & 03-Jul-96 & & Albuquerque, NM \\
\hline WP039065 & T. Christian-Frear & & 26-Jun-96 & & Albuquerque, NM \\
\hline WPO39169 & $\begin{array}{l}\text { Van Kelley, } \\
\text { INTERA Inc. }\end{array}$ & & 28-Feb-96 & & \\
\hline WPO39178 & J.T. Schneider & & 12-Jul-96 & & Albuquerque, NM \\
\hline WPO39179 & M. Tierney & & 09-Jul-96 & & Albuquerque, NM \\
\hline WPO39260 & $\begin{array}{l}\text { L.C. Sanchez, J.L. } \\
\text { Powell, J.S. Rath, } \\
\text { H.R. Trellue }\end{array}$ & & & & $\cdot$ \\
\hline WPO39353 & C. Lattier & & $11-J u l-96$ & & Albuquerque, NM \\
\hline WP039355 & M. Wallace & & 18-Apr-96 & & Albuquerque, NM \\
\hline WPO39365 & m. Wallace & & 02-May-96 & & \\
\hline WPO39371 & M. Wallace & & 02-May-96 & & Albuquerque, NM \\
\hline WPO40133 & K.Trauth & & 26-Jun-96 & & Albuquerque, NM \\
\hline WP040199 & M. Chu & & & & \\
\hline WPO40432 & M. Martell & & 22-Aug-96 & & Albuquerque, NM \\
\hline WPO40434 & M. Martell & & 20-Aug-96 & & Albuquerque, NM \\
\hline WPO40486 & $\begin{array}{l}\text { Palmer Vaughn } \\
\text { and Mike Lord }\end{array}$ & & 05-Sep-96 & & \\
\hline WPO40511 & L. Dotson & & 05-Sep-96 & & Albuquerque, NM \\
\hline WPO40512 & Byrum, R.V. & & 1996 & & \\
\hline WPO40514 & Vaughn, P.M. & & 1996 & $\begin{array}{l}\text { Sandia National } \\
\text { Laboratories }\end{array}$ & SWCF \\
\hline WPO40515 & Shinta, A.A. & & 1996 & $\begin{array}{l}\text { Sandia National } \\
\text { Laboratories }\end{array}$ & SWCF \\
\hline WPO40516 & Ramsey, J.L. & & 1996 & $\begin{array}{l}\text { Sandia National } \\
\text { Laboratories }\end{array}$ & SWCF \\
\hline WPO40520 & Stoelzel, D.M. & & 1996 & $\begin{array}{l}\text { Sandia National } \\
\text { Laboratories }\end{array}$ & SWCF \\
\hline
\end{tabular}




\section{Source Cross-Reference}

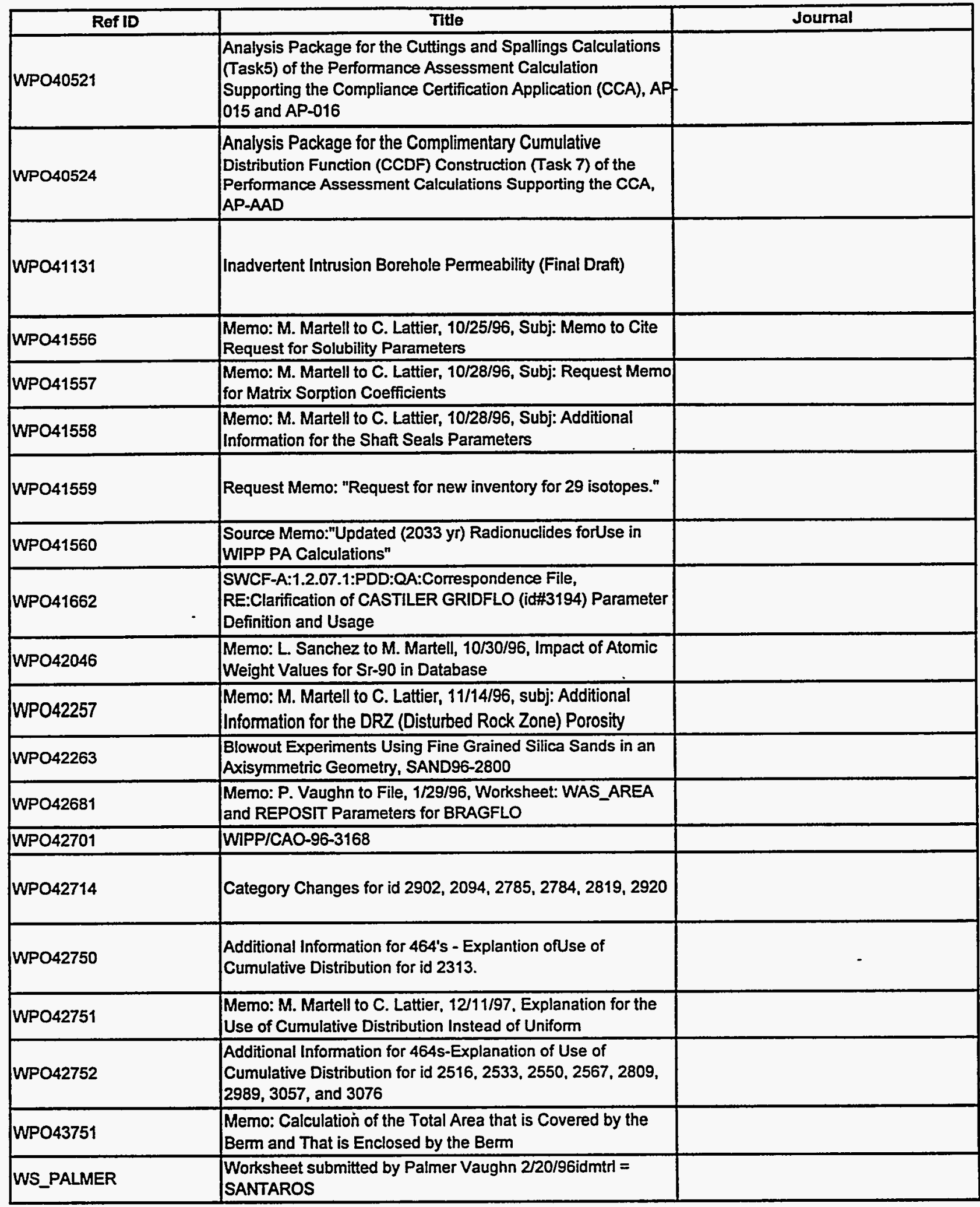


Source Cross-Reference

\begin{tabular}{|c|c|c|c|c|c|}
\hline Ref ID & Author & Editor & Date & Publisher & Location \\
\hline WP040521 & Berglund, J.W. & & 1996 & $\begin{array}{l}\text { Sandia National } \\
\text { Laboratories }\end{array}$ & SWCF \\
\hline WPO40524 & Helton, J.C. & & 1996 & $\begin{array}{l}\text { Sandia National } \\
\text { Laboratories }\end{array}$ & SWCF \\
\hline WPO41131 & $\begin{array}{l}\text { T.W. Thompson, } \\
\text { W.E. Coons, J.L. } \\
\text { Krumhans!, and } \\
\text { F.D. Hansen }\end{array}$ & & 20-May-96 & $\begin{array}{l}\text { Sandia National } \\
\text { Laboratories }\end{array}$ & Albuquerque, NM \\
\hline WP041556 & Mary-Alena Martell & & 25-Oct-96 & & \\
\hline WPO41557 & Mary-Alena Martell & & 28-0ct-96 & & \\
\hline WPO41558 & Mary-Alena Martell & & 28-Oct-96 & & \\
\hline WPO41559 & \begin{tabular}{|c|} 
Christine \\
Stockman to Larry \\
Sanchez
\end{tabular} & & $28-10-96$ & & \\
\hline WPO41560 & $\begin{array}{l}\text { L.C. Sanchez to } \\
\text { M. Martell }\end{array}$ & & $28-10-96$ & & \\
\hline WPO41662 & Jon Helton & & & & \\
\hline WPO42046 & Larry Sanchez & & $30-$ Oct-96 & & $\begin{array}{l}\text { Sandia National } \\
\text { Laboratories }\end{array}$ \\
\hline WPO42257 & M. Martell & & 14-Nov-96 & & \\
\hline WPO42263 & & & & & \\
\hline WPO42681 & P. Vaughn & & 25-Nov-96 & & Albuquerque, NM \\
\hline WPO42701 & & & 04-Jun-96 & & \\
\hline WPO42714 & $\begin{array}{l}\text { Mary-Alena Martell } \\
\text { to Charlene Lattier }\end{array}$ & & 10-Dec-96 & & \\
\hline WPO42750 & $\begin{array}{l}\text { Mary-Alena Martell } \\
\text { to Charlene Lattier }\end{array}$ & & 11-Dec-96 & & - \\
\hline WPO42751 & Mary-Alena Martell & & 11-Dec-96 & & $\begin{array}{l}\text { Albuquerque, New } \\
\text { Mexico }\end{array}$ \\
\hline WPO42752 & $\begin{array}{l}\text { Mary-Alena Martell } \\
\text { to Charlene Lattier }\end{array}$ & & 12-Dec-96 & & \\
\hline WPO43751 & K. Trauth & & 12-Jan-97 & & \\
\hline WS_PALMER & P. Vaughn & & 20-Feb-96 & & \\
\hline
\end{tabular}


Page intentionally blank

M4-22 


\section{Appendix M5}

Table from EPA Parameter Database: Model Cross Reference 
Model-Cross-Reference

\begin{tabular}{|c|c|}
\hline ID & Codename \\
\hline 2 & CUTTINGS S \\
\hline 2 & GRIDFLO \\
\hline 2 & NUTS \\
\hline 2 & SECOFL \\
\hline 2 & SECOTP \\
\hline 3 & CUTTINGS_S \\
\hline 3 & NUTS \\
\hline 4 & CUTTINGS_S \\
\hline 4 & NUTS \\
\hline 4 & PANEL \\
\hline 5 & CUTTINGS_S \\
\hline 5 & NUTS \\
\hline 5 & PANEL \\
\hline 6 & CUTTINGS_S \\
\hline 6 & NUTS \\
\hline 7 & BRAGFLO \\
\hline 8 & BRAGFLO \\
\hline 9 & BRAGFLO \\
\hline 10 & BRAGFLO \\
\hline 11 & BRAGFLO \\
\hline 12 & BRAGFLO \\
\hline$\overline{13}$ & BRAGFLO \\
\hline$\overline{14}$ & BRAGFLO \\
\hline 15 & BRAGFLO \\
\hline 16 & BRAGFLO \\
\hline 19 & BRAGFLO \\
\hline 20 & BRAGFLO \\
\hline 21 & BRAGFLO \\
\hline 22 & BRAGFLO \\
\hline 23 & BRAGFLO \\
\hline 25 & BRAGFLO \\
\hline 26 & BRAGFLO \\
\hline 27 & CUTTINGS_S \\
\hline 29 & BRAGFLO \\
\hline 30 & BRAGFLO \\
\hline 31 & BRAGFLO \\
\hline 32 & BRAGFLO \\
\hline 34 & BRAGFLO \\
\hline 35 & BRAGFLO \\
\hline 36 & BRAGFLO \\
\hline 40 & BRAGFLO \\
\hline 41 & BRAGFLO \\
\hline 42 & BRAGFLO \\
\hline 48 & BRAGFLO \\
\hline 49 & BRAGFLO \\
\hline 50 & BRAGFLO \\
\hline 51 & BRAGFLO \\
\hline 55 & BRAGFLO \\
\hline 57 & BRAGFLO \\
\hline 60 & BRAGFLO \\
\hline 61 & BRAGFLO \\
\hline
\end{tabular}

\begin{tabular}{|c|c|}
\hline ID & Codename \\
\hline 62 & BRAGFLO \\
\hline 63 & BRAGFLO \\
\hline 64 & BRAGFLO \\
\hline 65 & BRAGFLO \\
\hline 66 & BRAGFLO \\
\hline 67 & BRAGFLO \\
\hline 68 & BRAGFLO \\
\hline 69 & BRAGFLO \\
\hline 72 & BRAGFLO \\
\hline 73 & BRAGFLO \\
\hline 74 & BRAGFLO \\
\hline 75 & BRAGFLO \\
\hline 76 & BRAGFLO \\
\hline 77 & BRAGFLO \\
\hline 78 & BRAGFLO \\
\hline 79 & BRAGFLO \\
\hline 80 & BRAGFLO \\
\hline 81 & BRAGFLO \\
\hline 82 & BRAGFLO \\
\hline 83 & BRAGFLO \\
\hline 84 & BRAGFLO \\
\hline 85 & BRAGFLO \\
\hline 88 & BRAGFLO \\
\hline 89 & BRAGFLO \\
\hline 90 & BRAGFLO \\
\hline 91 & BRAGFLO \\
\hline 92 & BRAGFLO \\
\hline 93 & BRAGFLO \\
\hline 94 & BRAGFLO \\
\hline 95 & BRAGFLO \\
\hline 96 & BRAGFLO \\
\hline 97 & BRAGFLO \\
\hline 98 & BRAGFLO \\
\hline 99 & BRAGFLO \\
\hline 100 & BRAGFLO \\
\hline 103 & BRAGFLO \\
\hline 104 & BRAGFLO \\
\hline 105 & BRAGFLO \\
\hline 106 & CUTTINGS_S \\
\hline 106 & GRIDFLO \\
\hline 106 & NUTS \\
\hline 106 & SECOFL \\
\hline 106 & SECOTP \\
\hline 107 & CUTTINGS_S \\
\hline 107 & NUTS \\
\hline$\overline{108}$ & CUTTINGS_S \\
\hline 108 & NUTS \\
\hline 108 & PANEL \\
\hline 109 & CUTTINGS_S \\
\hline 109 & NUTS \\
\hline 109 & PANEL \\
\hline
\end{tabular}

\begin{tabular}{|c|c|}
\hline ID & Codename \\
\hline 110 & CUTTINGS_S \\
\hline 110 & GRIDFLO \\
\hline 110 & NUTS \\
\hline 110 & SECOFL \\
\hline 110 & SECOTP \\
\hline 111 & CUTTINGS_S \\
\hline 111 & NUTS \\
\hline 112 & CUTTINGS_S \\
\hline 112 & NUTS \\
\hline 112 & PANEL \\
\hline 113 & CUTTINGS_S \\
\hline 113 & NUTS \\
\hline 113 & PANEL \\
\hline 115 & CUTTINGS_S \\
\hline 115 & NUTS \\
\hline 116 & CUTTINGS_S \\
\hline 116 & GRIDFLO \\
\hline 116 & NUTS \\
\hline 116 & SECOFL \\
\hline 116 & SECOTP \\
\hline 117 & CUTTINGS_S \\
\hline 117 & NUTS \\
\hline 118 & CUTTINGS_S \\
\hline 118 & NUTS \\
\hline 118 & PANEL \\
\hline 119 & BRAGFLO \\
\hline 120 & BRAGFLO \\
\hline 137 & BRAGFLO \\
\hline 139 & BRAGFLO \\
\hline 140 & BRAGFLO \\
\hline 141 & BRAGFLO \\
\hline 142 & BRAGFLO \\
\hline 143 & BRAGFLO \\
\hline 144 & BRAGFLO \\
\hline 145 & BRAGFLO \\
\hline 148 & BRAGFLO \\
\hline 149 & BRAGFLO \\
\hline 150 & BRAGFLO \\
\hline 151 & BRAGFLO \\
\hline 153 & BRAGFLO \\
\hline 154 & BRAGFLO \\
\hline 156 & BRAGFLO \\
\hline 157 & BRAGFLO \\
\hline 158 & BRAGFLO \\
\hline 159 & BRAGFLO \\
\hline 160 & BRAGFLO \\
\hline 161 & BRAGFLO \\
\hline 162 & BRAGFLO \\
\hline 163 & BRAGFLO \\
\hline 166 & BRAGFLO \\
\hline 167 & BRAGFLO \\
\hline
\end{tabular}


Model Cross-Reference

\begin{tabular}{|c|c|}
\hline ID & Codename \\
\hline 168 & BRAGFLO \\
\hline 169 & BRAGFLO \\
\hline 170 & BRAGFLO \\
\hline 171 & CUTTINGS_S \\
\hline 172 & CUTTINGS_S \\
\hline 173 & CUTTINGS_S \\
\hline 174 & BRAGFLO \\
\hline 175 & BRAGFLO \\
\hline 176 & BRAGFLO \\
\hline 177 & BRAGFLO \\
\hline 178 & BRAGFLO \\
\hline 179 & BRAGFLO \\
\hline 181 & BRAGFLO \\
\hline 182 & BRAGFLO \\
\hline 183 & BRAGFLO \\
\hline 186 & BRAGFLO \\
\hline 187 & BRAGFLO \\
\hline 188 & BRAGFLO \\
\hline 189 & BRAGFLO \\
\hline 190 & BRAGFLO \\
\hline 191 & BRAGFLO \\
\hline 193 & BRAGFLO \\
\hline 194 & BRAGFLO \\
\hline 195 & BRAGFLO \\
\hline 196 & BRAGFLO \\
\hline 198 & BRAGFLO \\
\hline 199 & BRAGFLO \\
\hline 200 & BRAGFLO \\
\hline 203 & BRAGFLO \\
\hline 205 & BRAGFLO \\
\hline 206 & BRAGFLO \\
\hline 207 & BRAGFLO \\
\hline 208 & BRAGFLO \\
\hline 209 & BRAGFLO \\
\hline 210 & BRAGFLO \\
\hline 211 & BRAGFLO \\
\hline 212 & BRAGFLO \\
\hline 213 & BRAGFLO \\
\hline 214 & BRAGFLO \\
\hline 215 & BRAGFLO \\
\hline 216 & BRAGFLO \\
\hline 219 & BRAGFLO \\
\hline 220 & BRAGFLO \\
\hline 221 & BRAGFLO \\
\hline 222 & BRAGFLO \\
\hline 223 & SECOFL \\
\hline 225 & SECOTP \\
\hline 228 & BRAGFLO \\
\hline 229 & BRAGFLO \\
\hline 230 & BRAGFLO \\
\hline 231 & BRAGFLO \\
\hline
\end{tabular}

\begin{tabular}{|c|c|}
\hline ID & Codename \\
\hline 232 & BRAGFLO \\
\hline 233 & BRAGFLO \\
\hline 234 & BRAGFLO \\
\hline 236 & BRAGFLO \\
\hline 237 & BRAGFLO \\
\hline 238 & BRAGFLO \\
\hline 241 & BRAGFLO \\
\hline 243 & BRAGFLO \\
\hline 244 & BRAGFLO \\
\hline 246 & CUTTINGS_S \\
\hline 246 & GRIDFLO \\
\hline 246 & NUTS \\
\hline 246 & SECOFL \\
\hline 246 & SECOTP \\
\hline 247 & CUTTINGS_S \\
\hline 247 & NUTS \\
\hline 248 & CUTTINGS_S \\
\hline 248 & NUTS \\
\hline 248 & PANEL \\
\hline 249 & CUTTINGS_S \\
\hline 249 & NUTS \\
\hline 249 & PANEL \\
\hline 250 & CUTTINGS_S \\
\hline 250 & GRIDFLO \\
\hline 250 & NUTS \\
\hline 250 & SECOFL \\
\hline 250 & SECOTP \\
\hline 251 & CUTTINGS_S \\
\hline 251 & \begin{tabular}{|l|} 
NUTS \\
\end{tabular} \\
\hline 252 & BRAGFLO \\
\hline 253 & BRAGFLO \\
\hline 254 & BRAGFLO \\
\hline 255 & BRAGFLO \\
\hline 256 & BRAGFLO \\
\hline 257 & BRAGFLO \\
\hline 258 & BRAGFLO \\
\hline 259 & BRAGFLO \\
\hline 260 & BRAGFLO \\
\hline 261 & BRAGFLO \\
\hline 264 & BRAGFLO \\
\hline 265 & BRAGFLO \\
\hline 266 & BRAGFLO \\
\hline 282 & PANEL \\
\hline 283 & CUTTINGS_S \\
\hline 283 & GRIDFLO \\
\hline 283 & NUTS \\
\hline 283 & SECOFL \\
\hline 283 & SECOTP \\
\hline 284 & CUTTINGS_S \\
\hline 284 & NUTS \\
\hline 285 & CUTTINGS_S \\
\hline
\end{tabular}

\begin{tabular}{|c|c|}
\hline ID & Codename \\
\hline 285 & NUTS \\
\hline 285 & PANEL \\
\hline 286 & CUTTINGS_S \\
\hline 286 & NUTS \\
\hline 286 & PANEL \\
\hline 287 & CUTTINGS_S \\
\hline 287 & GRIDFLO \\
\hline 287 & NUTS \\
\hline 287 & SECOFL \\
\hline 287 & SECOTP \\
\hline 288 & CUTTINGS_S \\
\hline 288 & NUTS \\
\hline 289 & CUTTINGS_S \\
\hline 289 & NUTS \\
\hline 289 & PANEL \\
\hline 291 & CUTTINGS_S \\
\hline 291 & GRIDFLO \\
\hline 291 & NUTS \\
\hline 291 & SECOFL \\
\hline 291 & SECOTP \\
\hline 292 & CUTTINGS_S \\
\hline 292 & INUTS \\
\hline 293 & CUTTINGS_S \\
\hline 293 & NUTS \\
\hline 293 & PANEL \\
\hline 294 & CUTTINGS_S \\
\hline 294 & NUTS \\
\hline 294 & PANEL \\
\hline 295 & CUTTINGS_S \\
\hline 295 & GRIDFLO \\
\hline 295 & NUTS \\
\hline 295 & SECOFL \\
\hline 295 & SECOTP \\
\hline 296 & CUTTINGS_S \\
\hline 296 & NUTS \\
\hline 297 & CUTTINGS_S \\
\hline 297 & NUTS \\
\hline 297 & PANEL \\
\hline 298 & CUTTINGS_S \\
\hline 298 & NUTS \\
\hline 298 & PANEL \\
\hline 299 & CUTTINGS_S \\
\hline 299 & GRIDFLO \\
\hline 299 & NUTS \\
\hline 299 & SECOFL \\
\hline 299 & SECOTP \\
\hline 300 & CUTTINGS_S \\
\hline 300 & NUTS \\
\hline 301 & CUTTINGS_S \\
\hline 301 & NUTS \\
\hline 301 & PANEL \\
\hline
\end{tabular}


Model'Cross=Reference

\begin{tabular}{|c|c|}
\hline ID & Codename \\
\hline 302 & CUTTINGS_S \\
\hline 302 & NUTS \\
\hline 302 & PANEL \\
\hline 303 & CUTTINGS_S \\
\hline 303 & GRIDFLO \\
\hline 303 & NUTS \\
\hline 303 & SECOFL \\
\hline 303 & SECOTP \\
\hline 304 & CUTTINGS_S \\
\hline 304 & NUTS \\
\hline 305 & CUTTINGS_S \\
\hline 305 & NUTS \\
\hline 305 & PANEL \\
\hline 306 & CUTTINGS_S \\
\hline 306 & NUTS \\
\hline 306 & PANEL \\
\hline 307 & CUTTINGS_S \\
\hline 307 & GRIDFLO \\
\hline 307 & NUTS \\
\hline 307 & SECOFL \\
\hline 307 & SECOTP \\
\hline 308 & CUTTINGS_S \\
\hline 308 & NUTS \\
\hline 309 & CUTTINGS_S \\
\hline 309 & NUTS \\
\hline 309 & PANEL \\
\hline 310 & CUTTINGS_S \\
\hline 310 & NUTS \\
\hline 310 & PANEL \\
\hline 311 & CUTTINGS_S \\
\hline 311 & GRIDFLO \\
\hline 311 & \begin{tabular}{|l} 
NUTS \\
\end{tabular} \\
\hline 311 & SECOFL \\
\hline 311 & SECOTP \\
\hline 312 & CUTTINGS_S \\
\hline 312 & NUTS \\
\hline 313 & NUTS \\
\hline 314 & CUTTINGS_S \\
\hline 314 & GRIDFLO \\
\hline 314 & NUTS \\
\hline 314 & SECOFL \\
\hline 314 & SECOTP \\
\hline 315 & CUTTINGS_S \\
\hline 315 & NUTS \\
\hline 316 & CUTTINGS_S \\
\hline 316 & NUTS \\
\hline 316 & PANEL \\
\hline 317 & CUTTINGS_S \\
\hline 317 & NUTS \\
\hline 317 & PANEL \\
\hline 318 & CUTTINGS_S \\
\hline
\end{tabular}

\begin{tabular}{|c|c|}
\hline ID & Codename \\
\hline 318 & GRIDFLO \\
\hline 318 & NUTS \\
\hline 318 & SECOFL \\
\hline 318 & SECOTP \\
\hline 319 & CUTTINGS_S \\
\hline 319 & NUTS \\
\hline 336 & BRAGFLO \\
\hline 337 & BRAGFLO \\
\hline 339 & BRAGFLO \\
\hline 340 & BRAGFLO \\
\hline 341 & BRAGFLO \\
\hline 342 & BRAGFLO \\
\hline 343 & BRAGFLO \\
\hline 344 & BRAGFLO \\
\hline 345 & BRAGFLO \\
\hline 346 & BRAGFLO \\
\hline 349 & BRAGFLO \\
\hline 350 & BRAGFLO \\
\hline 351 & BRAGFLO \\
\hline 352 & BRAGFLO \\
\hline 514 & CUTTINGS_S \\
\hline 514 & GRIDFLO \\
\hline 514 & NUTS \\
\hline 514 & SECOFL \\
\hline 514 & SECOTP \\
\hline 515 & CUTTINGS_S \\
\hline 515 & GRIDFLO \\
\hline 515 & NUTS \\
\hline 515 & SECOFL \\
\hline 515 & SECOTP \\
\hline 517 & CUTTINGS_S \\
\hline 517 & NUTS \\
\hline 518 & CUTTINGS_S \\
\hline 518 & NUTS \\
\hline 518 & PANEL \\
\hline 520 & BRAGFLO \\
\hline 521 & BRAGFLO \\
\hline 522 & BRAGFLO \\
\hline 524 & BRAGFLO \\
\hline 526 & BRAGFLO \\
\hline 527 & BRAGFLO \\
\hline 528 & BRAGFLO \\
\hline 529 & BRAGFLO \\
\hline 531 & BRAGFLO \\
\hline 532 & BRAGFLO \\
\hline 533 & BRAGFLO \\
\hline 536 & BRAGFLO \\
\hline 537 & BRAGFLO \\
\hline 538 & BRAGFLO \\
\hline 539 & BRAGFLO \\
\hline 540 & BRAGFLO \\
\hline
\end{tabular}

\begin{tabular}{|c|c|}
\hline ID & Codename \\
\hline 541 & BRAGFLO \\
\hline 542 & BRAGFLO \\
\hline 543 & BRAGFLO \\
\hline 544 & BRAGFLO \\
\hline 545 & BRAGFLO \\
\hline 546 & BRAGFLO \\
\hline 547 & BRAGFLO \\
\hline 548 & BRAGFLO \\
\hline 549 & BRAGFLO \\
\hline 553 & BRAGFLO \\
\hline 554 & BRAGFLO \\
\hline 555 & BRAGFLO \\
\hline 556 & BRAGFLO \\
\hline 559 & BRAGFLO \\
\hline 560 & BRAGFLO \\
\hline 561 & BRAGFLO \\
\hline 563 & BRAGFLO \\
\hline 565 & BRAGFLO \\
\hline 566 & BRAGFLO \\
\hline 567 & BRAGFLO \\
\hline 568 & BRAGFLO \\
\hline 569 & BRAGFLO \\
\hline 570 & BRAGFLO \\
\hline 571 & BRAGFLO \\
\hline 572 & BRAGFLO \\
\hline 575 & BRAGFLO \\
\hline 576 & BRAGFLO \\
\hline 577 & BRAGFLO \\
\hline 578 & BRAGFLO \\
\hline 579 & BRAGFLO \\
\hline 580 & BRAGFLO \\
\hline 582 & BRAGFLO \\
\hline 586 & BRAGFLO \\
\hline 587 & BRAGFLO \\
\hline 588 & BRAGFLO \\
\hline 589 & BRAGFLO \\
\hline 590 & BRAGFLO \\
\hline 591 & BRAGFLO \\
\hline 592 & BRAGFLO \\
\hline 593 & BRAGFLO \\
\hline 596 & BRAGFLO \\
\hline 597 & BRAGFLO \\
\hline 598 & BRAGFLO \\
\hline 599 & BRAGFLO \\
\hline 603 & CUTTINGS_S \\
\hline 603 & GRIDFLO \\
\hline 603 & NUTS \\
\hline 603 & SECOFL \\
\hline 603 & SECOTP \\
\hline 604 & CUTTINGS_S \\
\hline 604 & NUTS \\
\hline
\end{tabular}


Model Cross-Reference

\begin{tabular}{|c|c|}
\hline ID & Codename \\
\hline 605 & CUTTINGS_S \\
\hline 605 & NUTS \\
\hline 605 & PANEL \\
\hline 606 & CUTTINGS_S \\
\hline 606 & NUTS \\
\hline 606 & PANEL \\
\hline 607 & CUTTINGS_S \\
\hline 607 & GRIDFLO \\
\hline 607 & NUTS \\
\hline 607 & SECOFL \\
\hline 607 & SECOTP \\
\hline 608 & CUTTINGS_S \\
\hline 608 & NUTS \\
\hline 609 & CUTTINGS_S \\
\hline 609 & NUTS \\
\hline 609 & PANEL \\
\hline 610 & CUTTINGS_S \\
\hline 610 & NUTS \\
\hline 610 & PANEL \\
\hline 611 & CUTTINGS_S \\
\hline 611 & GRIDFLO \\
\hline 611 & NUTS \\
\hline 611 & SECOFL \\
\hline 611 & SECOTP \\
\hline 612 & CUTTINGS_S \\
\hline 612 & NUTS \\
\hline 613 & CUTTINGS_S \\
\hline 613 & NUTS \\
\hline 613 & PANEL \\
\hline 614 & CUTTINGS_S \\
\hline 614 & \begin{tabular}{|l|} 
NUTS \\
\end{tabular} \\
\hline 614 & PANEL \\
\hline 632 & CUTTINGS_S \\
\hline 632 & GRIDFLO \\
\hline 632 & NUTS \\
\hline 632 & SECOFL \\
\hline 632 & SECOTP \\
\hline 633 & CUTTINGS_S \\
\hline 633 & NUTS \\
\hline 634 & CUTTINGS_S \\
\hline 634 & NUTS \\
\hline 634 & PANEL. \\
\hline 635 & CUTTINGS_S \\
\hline 635 & NUTS \\
\hline 635 & PANEL \\
\hline 636 & CUTTINGS_S \\
\hline 636 & GRIDFLO \\
\hline 636 & NUTS \\
\hline 636 & SECOFL \\
\hline 636 & SECOTP \\
\hline 637 & CUTTINGS_S \\
\hline
\end{tabular}

\begin{tabular}{|c|c|}
\hline ID & Codename \\
\hline 637 & NUTS \\
\hline 638 & CUTTINGS_S \\
\hline 638 & NUTS \\
\hline 638 & PANEL \\
\hline 639 & CUTTINGS_S \\
\hline 639 & NUTS \\
\hline 639 & PANEL \\
\hline 640 & CUTTINGS_S \\
\hline 640 & GRIDFLO \\
\hline 640 & NUTS \\
\hline 640 & SECOFL \\
\hline 640 & SECOTP \\
\hline 641 & CUTTINGS_S \\
\hline 641 & NUTS \\
\hline 642 & CUTTINGS_S \\
\hline 642 & \begin{tabular}{|l} 
NUTS \\
\end{tabular} \\
\hline 642 & PANEL \\
\hline 643 & CUTTINGS_S \\
\hline 643 & NUTS \\
\hline 643 & PANEL \\
\hline 644 & CUTTINGS_S \\
\hline 644 & GRIDFLO \\
\hline 644 & NUTS \\
\hline 644 & SECOFL \\
\hline 644 & SECOTP \\
\hline 645 & CUTTINGS_S \\
\hline 645 & NUTS \\
\hline 646 & CUTTINGS_S \\
\hline 646 & NUTS \\
\hline 646 & PANEL \\
\hline 647 & CUTTINGS_S \\
\hline 647 & GRIDFLO \\
\hline 647 & NUTS \\
\hline 647 & SECOFL \\
\hline 647 & SECOTP \\
\hline 648 & CUTTINGS_S \\
\hline 648 & NUTS \\
\hline 649 & CUTTINGS_S \\
\hline 649 & NUTS \\
\hline 649 & PANEL \\
\hline 650 & CUTTINGS_S \\
\hline 650 & NUTS \\
\hline 650 & PANEL \\
\hline 651 & CUTTINGS_S \\
\hline 652 & BRAGFLO \\
\hline 653 & BRAGFLO \\
\hline 656 & BRAGFLO \\
\hline 657 & BRAGFLO \\
\hline 658 & BRAGFLO \\
\hline 659 & BRAGFLO \\
\hline 660 & BRAGFLO \\
\hline
\end{tabular}

\begin{tabular}{|c|l|}
\hline ID & Codename \\
\hline 661 & BRAGFLO \\
\hline 662 & BRAGFLO \\
\hline 663 & BRAGFLO \\
\hline 664 & BRAGFLO \\
\hline 665 & BRAGFLO \\
\hline 668 & BRAGFLO \\
\hline 669 & BRAGFLO \\
\hline 670 & BRAGFLO \\
\hline 671 & BRAGFLO \\
\hline 838 & NUTS \\
\hline 841 & NUTS \\
\hline 843 & NUTS \\
\hline 843 & SECOTP \\
\hline 861 & SECOTP \\
\hline 1659 & NUTS \\
\hline 1661 & BRAGFLO \\
\hline 1661 & NUTS \\
\hline 1743 & BRAGFLO \\
\hline 1743 & NUTS \\
\hline 1784 & BRAGFLO \\
\hline 1784 & NUTS \\
\hline 1992 & BRAGFLO \\
\hline 1993 & BRAGFLO \\
\hline 1995 & BRAGFLO \\
\hline 2037 & CUTTINGSSS \\
\hline 2037 & NUTS \\
\hline 2037 & PANEL \\
\hline 2038 & CUTTINGS_S \\
\hline 2038 & NUTS \\
\hline 2038 & PANEL \\
\hline 2039 & CUTTINGSSS \\
\hline 2039 & NUTS \\
\hline 2039 & PANEL \\
\hline 2040 & BRAGFLO \\
\hline 2041 & BRAGFLO \\
\hline 2042 & BRAGFLO \\
\hline 2043 & BRAGFLO \\
\hline 2044 & BRAGFLO \\
\hline 2046 & BRAGFLO \\
\hline 2049 & BRAGFLO \\
\hline 2051 & BRAGFLO \\
\hline 2052 & BRAGFLO \\
\hline 2053 & BRAGFLO \\
\hline 2054 & BRAGFLO \\
\hline 2055 & BRAGFLO \\
\hline 2056 & BRAGFLO \\
\hline 2059 & BRAGFLO \\
\hline BRAGFLO \\
\hline BRAGFLO \\
\hline BRAGFLO \\
\hline BRAGFLO \\
\hline
\end{tabular}


Model Gross-Reference

\begin{tabular}{|c|l|}
\hline ID & Codename \\
\hline 2064 & BRAGFLO \\
\hline 2065 & BRAGFLO \\
\hline 2066 & BRAGFLO \\
\hline 2067 & BRAGFLO \\
\hline 2069 & BRAGFLO \\
\hline 2070 & BRAGFLO \\
\hline 2071 & BRAGFLO \\
\hline 2071 & SECOFL \\
\hline 2085 & BRAGFLO \\
\hline 2087 & BRAGFLO \\
\hline 2088 & BRAGFLO \\
\hline 2093 & BRAGFLO \\
\hline 2094 & BRAGFLO \\
\hline 2097 & BRAGFLO \\
\hline 2098 & BRAGFLO \\
\hline 2099 & BRAGFLO \\
\hline 2100 & BRAGFLO \\
\hline 2101 & BRAGFLO \\
\hline 2102 & BRAGFLO \\
\hline 2103 & BRAGFLO \\
\hline 2104 & BRAGFLO \\
\hline 2106 & BRAGFLO \\
\hline 2107 & BRAGFLO \\
\hline 2110 & BRAGFLO \\
\hline 2112 & BRAGFLO \\
\hline 2113 & BRAGFLO \\
\hline 2114 & BRAGFLO \\
\hline 2115 & BRAGFLO \\
\hline 2116 & BRAGFLO \\
\hline 2117 & BRAGFLO \\
\hline 2118 & BRAGFLO \\
\hline 2119 & BRAGFLO \\
\hline 2120 & BRAGFLO \\
\hline 2121 & BRAGFLO \\
\hline 2122 & BRAGFLO \\
\hline 2123 & BRAGFLO \\
\hline 2127 & BRAGFLO \\
\hline 2128 & BRAGFLO \\
\hline 2129 & BRAGFLO \\
\hline 2130 & BRAGFLO \\
\hline 2131 & BRAGFLO \\
\hline 2132 & BRAGFLO \\
\hline 2133 & BRAGFLO \\
\hline 2135 & BRAGFLO \\
\hline 2137 & BRAGFLO \\
\hline 2141 & BRAGFLO \\
\hline BRA & BRAGFLO \\
\hline BRAGFLO \\
\hline BRAGFLO \\
\hline 2170 & BRAGFLO \\
\hline
\end{tabular}

\begin{tabular}{|c|c|}
\hline ID & Codename \\
\hline 2177 & BRAGFLO \\
\hline 2178 & BRAGFLO \\
\hline 2180 & BRAGFLO \\
\hline 2183 & BRAGFLO \\
\hline 2185 & BRAGFLO \\
\hline 2186 & BRAGFLO \\
\hline 2191 & BRAGFLO \\
\hline 2192 & BRAGFLO \\
\hline 2216 & CUTTINGS_S \\
\hline 2216 & NUTS \\
\hline 2216 & PANEL \\
\hline 2217 & BRAGFLO \\
\hline 2218 & BRAGFLO \\
\hline 2219 & BRAGFLO \\
\hline 2220 & BRAGFLO \\
\hline 2225 & BRAGFLO \\
\hline 2226 & BRAGFLO \\
\hline 2228 & BRAGFLO \\
\hline 2231 & BRAGFLO \\
\hline 2232 & BRAGFLO \\
\hline 2233 & BRAGFLO \\
\hline 2234 & BRAGFLO \\
\hline 2235 & BRAGFLO \\
\hline 2236 & BRAGFLO \\
\hline 2237 & BRAGFLO \\
\hline 2238 & BRAGFLO \\
\hline 2239 & BRAGFLO \\
\hline 2240 & BRAGFLO \\
\hline 2241 & BRAGFLO \\
\hline 2242 & BRAGFLO \\
\hline 2243 & BRAGFLO \\
\hline 2244 & BRAGFLO \\
\hline 2245 & BRAGFLO \\
\hline 2247 & BRAGFLO \\
\hline 2248 & BRAGFLO \\
\hline 2254 & CUTTINGS_S \\
\hline 2265 & CUTTINGS_S \\
\hline 2265 & NUTS \\
\hline 2265 & PANEL \\
\hline 2266 & CUTTINGS_S \\
\hline 2266 & NUTS \\
\hline 2266 & PANEL \\
\hline 2267 & CUTTINGS_S \\
\hline 2267 & NUTS \\
\hline 2267 & PANEL \\
\hline 2268 & CUTTINGS_S \\
\hline 2268 & NUTS \\
\hline 2268 & PANEL \\
\hline 2269 & CUTTINGS_S \\
\hline 2269 & NUTS \\
\hline 2269 & PANEL \\
\hline
\end{tabular}

\begin{tabular}{|c|c|}
\hline ID & Codename \\
\hline 2270 & CUTTINGS_S \\
\hline 2270 & NUTS \\
\hline 2270 & PANEL \\
\hline 2271 & CUTTINGS_S \\
\hline 2271 & NUTS \\
\hline 2271 & PANEL \\
\hline 2272 & CUTTINGS_S \\
\hline 2272 & NUTS \\
\hline 2272 & PANEL \\
\hline 2274 & BRAGFLO \\
\hline 2275 & BRAGFLO \\
\hline 2276 & BRAGFLO \\
\hline 2277 & BRAGFLO \\
\hline 2278 & BRAGFLO \\
\hline 2279 & BRAGFLO \\
\hline 2280 & BRAGFLO \\
\hline 2281 & BRAGFLO \\
\hline 2282 & BRAGFLO \\
\hline 2283 & BRAGFLO \\
\hline 2284 & BRAGFLO \\
\hline 2285 & BRAGFLO \\
\hline 2289 & BRAGFLO \\
\hline 2290 & BRAGFLO \\
\hline 2291 & BRAGFLO \\
\hline 2292 & BRAGFLO \\
\hline 2310 & BRAGFLO \\
\hline 2311 & BRAGFLO \\
\hline 2312 & BRAGFLO \\
\hline 2313 & BRAGFLO \\
\hline 2314 & BRAGFLO \\
\hline 2315 & BRAGFLO \\
\hline 2316 & BRAGFLO \\
\hline 2317 & BRAGFLO \\
\hline 2318 & BRAGFLO \\
\hline 2319 & BRAGFLO \\
\hline 2323 & BRAGFLO \\
\hline 2324 & BRAGFLO \\
\hline 2325 & BRAGFLO \\
\hline 2326 & BRAGFLO \\
\hline 2327 & BRAGFLO \\
\hline 2328 & BRAGFLO \\
\hline 2329 & BRAGFLO \\
\hline 2330 & BRAGFLO \\
\hline 2331 & BRAGFLO \\
\hline 2332 & BRAGFLO \\
\hline 2333 & BRAGFLO \\
\hline 2334 & BRAGFLO \\
\hline 2335 & BRAGFLO \\
\hline 2336 & BRAGFLO \\
\hline 2340 & BRAGFLO \\
\hline 2341 & BRAGFLO \\
\hline
\end{tabular}


Model Cross-Reference

\begin{tabular}{|c|c|}
\hline ID & Codename \\
\hline 2342 & BRAGFLO \\
\hline 2343 & BRAGFLO \\
\hline 2344 & BRAGFLO \\
\hline 2345 & BRAGFLO \\
\hline 2346 & BRAGFLO \\
\hline 2347 & BRAGFLO \\
\hline 2348 & BRAGFLO \\
\hline 2349 & BRAGFLO \\
\hline 2351 & BRAGFLO \\
\hline 2352 & BRAGFLO \\
\hline 2353 & BRAGFLO \\
\hline 2357 & BRAGFLO \\
\hline 2359 & BRAGFLO \\
\hline 2360 & BRAGFLO \\
\hline 2361 & BRAGFLO \\
\hline 2362 & BRAGFLO \\
\hline 2363 & BRAGFLO \\
\hline 2364 & BRAGFLO \\
\hline 2365 & BRAGFLO \\
\hline 2366 & BRAGFLO \\
\hline 2368 & BRAGFLO \\
\hline 2369 & BRAGFLO \\
\hline 2370 & BRAGFLO \\
\hline 2374 & BRAGFLO \\
\hline 2376 & BRAGFLO \\
\hline 2377 & BRAGFLO \\
\hline 2378 & BRAGFLO \\
\hline 2379 & BRAGFLO \\
\hline 2380 & BRAGFLO \\
\hline 2381 & BRAGFLO \\
\hline 2382 & BRAGFLO \\
\hline 2383 & BRAGFLO \\
\hline 2384 & BRAGFLO \\
\hline 2385 & BRAGFLO \\
\hline 2386 & BRAGFLO \\
\hline 2387 & BRAGFLO \\
\hline 2391 & BRAGFLO \\
\hline 2392 & BRAGFLO \\
\hline 2393 & BRAGFLO \\
\hline 2394 & BRAGFLO \\
\hline 2395 & BRAGFLO \\
\hline 2396 & BRAGFLO \\
\hline 2397 & BRAGFLO \\
\hline 2398 & BRAGFLO \\
\hline 2399 & BRAGFLO \\
\hline 2400 & BRAGFLO \\
\hline 2402 & BRAGFLO \\
\hline 2403 & BRAGFLO \\
\hline 2404 & BRAGFLO \\
\hline 2408 & BRAGFLO \\
\hline 2410 & BRAGFLO \\
\hline
\end{tabular}

\begin{tabular}{|c|c|}
\hline ID & Codename \\
\hline 2411 & BRAGFLO \\
\hline 2412 & BRAGFLO \\
\hline 2413 & BRAGFLO \\
\hline 2414 & BRAGFLO \\
\hline 2415 & BRAGFLO \\
\hline 2416 & BRAGFLO \\
\hline 2417 & |BRAGFLO \\
\hline 2419 & BRAGFLO \\
\hline 2420 & BRAGFLO \\
\hline 2421 & BRAGFLO \\
\hline 2425 & BRAGFLO \\
\hline 2427 & BRAGFLO \\
\hline 2428 & BRAGFLO \\
\hline 2429 & BRAGFLO \\
\hline 2430 & BRAGFLO \\
\hline 2431 & BRAGFLO \\
\hline 2432 & BRAGFLO \\
\hline 2433 & BRAGFLO \\
\hline 2434 & BRAGFLO \\
\hline 2436 & BRAGFLO \\
\hline 2437 & BRAGFLO \\
\hline 2438 & BRAGFLO \\
\hline 2442 & BRAGFLO \\
\hline 2444 & BRAGFLO \\
\hline 2445 & BRAGFLO \\
\hline 2446 & BRAGFLO \\
\hline 2447 & BRAGFLO \\
\hline 2448 & BRAGFLO \\
\hline 2449 & BRAGFLO \\
\hline 2450 & BRAGFLO \\
\hline 2451 & BRAGFLO \\
\hline 2453 & BRAGFLO \\
\hline 2454 & BRAGFLO \\
\hline 2455 & BRAGFLO \\
\hline 2459 & BRAGFLO \\
\hline 2461 & BRAGFLO \\
\hline 2462 & BRAGFLO \\
\hline 2463 & BRAGFLO \\
\hline 2464 & BRAGFLO \\
\hline 2465 & BRAGFLO \\
\hline 2466 & BRAGFLO \\
\hline 2467 & BRAGFLO \\
\hline 2468 & BRAGFLO \\
\hline 2469 & BRAGFLO \\
\hline 2470 & BRAGFLO \\
\hline 2471 & BRAGFLO \\
\hline 2472 & BRAGFLO \\
\hline 2476 & BRAGFLO \\
\hline 2477 & BRAGFLO \\
\hline 2478 & BRAGFLO \\
\hline 2479 & BRAGFLO \\
\hline
\end{tabular}

\begin{tabular}{|c|c|}
\hline ID & Codename \\
\hline 2480 & BRAGFLO \\
\hline 2481 & BRAGFLO \\
\hline 2482 & BRAGFLO \\
\hline 2483 & BRAGFLO \\
\hline 2484 & BRAGFLO \\
\hline 2485 & BRAGFLO \\
\hline 2486 & BRAGFLO \\
\hline 2487 & BRAGFLO \\
\hline 2488 & BRAGFLO \\
\hline 2492 & BRAGFLO \\
\hline 2493 & BRAGFLO \\
\hline 2494 & BRAGFLO \\
\hline 2495 & BRAGFLO \\
\hline 2496 & BRAGFLO \\
\hline 2497 & BRAGFLO \\
\hline 2498 & BRAGFLO \\
\hline 2499 & BRAGFLO \\
\hline 2500 & BRAGFLO \\
\hline 2501 & BRAGFLO \\
\hline 2502 & BRAGFLO \\
\hline 2503 & BRAGFLO \\
\hline 2504 & BRAGFLO \\
\hline 2505 & BRAGFLO \\
\hline 2509 & BRAGFLO \\
\hline 2510 & BRAGFLO \\
\hline 2511 & BRAGFLO \\
\hline 2512 & BRAGFLO \\
\hline 2513 & BRAGFLO \\
\hline 2514 & BRAGFLO \\
\hline 2515 & BRAGFLO \\
\hline 2516 & BRAGFLO \\
\hline 2517 & BRAGFLO \\
\hline 2518 & BRAGFLO \\
\hline 2519 & BRAGFLO \\
\hline 2526 & BRAGFLO \\
\hline 2527 & BRAGFLO \\
\hline 2528 & BRAGFLO \\
\hline 2529 & BRAGFLO \\
\hline 2530 & BRAGFLO \\
\hline 2531 & BRAGFLO \\
\hline 2532 & BRAGFLO \\
\hline 2533 & BRAGFLO \\
\hline 2534 & BRAGFLO \\
\hline 2535 & BRAGFLO \\
\hline 2543 & BRAGFLO \\
\hline 2545 & BRAGFLO \\
\hline 2546 & BRAGFLO \\
\hline 2547 & BRAGFLO \\
\hline 2548 & BRAGFLO \\
\hline 2549 & BRAGFLO \\
\hline 2550 & BRAGFLO \\
\hline
\end{tabular}


Model Cross-Reference

\begin{tabular}{|c|c|}
\hline ID & Codename \\
\hline 2551 & BRAGFLO \\
\hline 2552 & BRAGFLO \\
\hline 2560 & BRAGFLO \\
\hline 2562 & BRAGFLO \\
\hline 2563 & BRAGFLO \\
\hline 2564 & BRAGFLO \\
\hline 2565 & BRAGFLO \\
\hline 2566 & BRAGFLO \\
\hline 2567 & BRAGFLO \\
\hline 2568 & BRAGFLO \\
\hline 2569 & BRAGFLO \\
\hline 2577 & BRAGFLO \\
\hline 2579 & BRAGFLO \\
\hline 2580 & BRAGFLO \\
\hline 2581 & BRAGFLO \\
\hline 2582 & BRAGFLO \\
\hline 2583 & BRAGFLO \\
\hline 2585 & BRAGFLO \\
\hline 2594 & BRAGFLO \\
\hline 2596 & BRAGFLO \\
\hline 2597 & BRAGFLO \\
\hline 2599 & BRAGFLO \\
\hline 2600 & BRAGFLO \\
\hline 2601 & BRAGFLO \\
\hline 2604 & BRAGFLO \\
\hline 2605 & BRAGFLO \\
\hline 2606 & BRAGFLO \\
\hline 2608 & BRAGFLO \\
\hline 2609 & BRAGFLO \\
\hline 2610 & BRAGFLO \\
\hline 2612 & BRAGFLO \\
\hline 2613 & BRAGFLO \\
\hline 2614 & BRAGFLO \\
\hline 2616 & BRAGFLO \\
\hline 2617 & BRAGFLO \\
\hline 2618 & BRAGFLO \\
\hline 2620 & BRAGFLO \\
\hline 2621 & BRAGFLO \\
\hline 2622 & BRAGFLO \\
\hline 2623 & BRAGFLO \\
\hline 2625 & BRAGFLO \\
\hline 2626 & BRAGFLO \\
\hline 2627 & BRAGFLO \\
\hline 2628 & BRAGFLO \\
\hline 2636 & BRAGFLO \\
\hline 2637 & BRAGFLO \\
\hline 2638 & BRAGFLO \\
\hline 2641 & BRAGFLO \\
\hline 2642 & BRAGFLO \\
\hline 2643 & BRAGFLO \\
\hline 2646 & BRAGFLO \\
\hline
\end{tabular}

\begin{tabular}{|c|c|}
\hline ID & Codename \\
\hline 2647 & BRAGFLO \\
\hline 2648 & BRAGFLO \\
\hline 2651 & BRAGFLO \\
\hline 2652 & BRAGFLO \\
\hline 2653 & BRAGFLO \\
\hline 2656 & BRAGFLO \\
\hline 2657 & BRAGFLO \\
\hline 2658 & BRAGFLO \\
\hline 2661 & BRAGFLO \\
\hline 2662 & BRAGFLO \\
\hline 2663 & BRAGFLO \\
\hline 2666 & BRAGFLO \\
\hline 2667 & BRAGFLO \\
\hline 2668 & BRAGFLO \\
\hline 2671 & BRAGFLO \\
\hline 2672 & BRAGFLO \\
\hline 2673 & BRAGFLO \\
\hline 2676 & BRAGFLO \\
\hline 2677 & BRAGFLO \\
\hline 2678 & BRAGFLO \\
\hline 2681 & BRAGFLO \\
\hline 2682 & BRAGFLO \\
\hline 2683 & BRAGFLO \\
\hline 2686 & BRAGFLO \\
\hline 2687 & BRAGFLO \\
\hline 2688 & BRAGFLO \\
\hline 2691 & BRAGFLO \\
\hline 2692 & BRAGFLO \\
\hline 2693 & BRAGFLO \\
\hline 2696 & BRAGFLO \\
\hline 2697 & BRAGFLO \\
\hline 2698 & BRAGFLO \\
\hline 2701 & BRAGFLO \\
\hline 2702 & BRAGFLO \\
\hline 2703 & BRAGFLO \\
\hline 2706 & BRAGFLO \\
\hline 2707 & BRAGFLO \\
\hline 2708 & BRAGFLO \\
\hline 2711 & BRAGFLO \\
\hline 2712 & BRAGFLO \\
\hline 2713 & BRAGFLO \\
\hline 2715 & BRAGFLO \\
\hline 2716 & BRAGFLO \\
\hline 2717 & BRAGFLO \\
\hline 2718 & BRAGFLO \\
\hline 2720 & BRAGFLO \\
\hline 2721 & BRAGFLO \\
\hline 2722 & BRAGFLO \\
\hline 2725 & BRAGFLO \\
\hline 2726 & BRAGFLO \\
\hline 2727 & BRAGFLO \\
\hline
\end{tabular}

\begin{tabular}{|c|c|}
\hline ID & Codename \\
\hline 2728 & BRAGFLO \\
\hline 2731 & BRAGFLO \\
\hline 2732 & BRAGFLO \\
\hline 2733 & BRAGFLO \\
\hline 2734 & BRAGFLO \\
\hline 2736 & BRAGFLO \\
\hline 2737 & BRAGFLO \\
\hline 2738 & BRAGFLO \\
\hline 2739 & BRAGFLO \\
\hline 2740 & BRAGFLO \\
\hline 2741 & BRAGFLO \\
\hline 2744 & BRAGFLO \\
\hline 2745 & BRAGFLO \\
\hline 2746 & BRAGFLO \\
\hline 2749 & BRAGFLO \\
\hline 2750 & BRAGFLO \\
\hline 2751 & BRAGFLO \\
\hline 2754 & BRAGFLO \\
\hline 2755 & BRAGFLO \\
\hline 2756 & BRAGFLO \\
\hline 2759 & BRAGFLO \\
\hline 2760 & BRAGFLO \\
\hline 2761 & BRAGFLO \\
\hline 2764 & BRAGFLO \\
\hline 2765 & BRAGFLO \\
\hline 2766 & BRAGFLO \\
\hline 2768 & BRAGFLO \\
\hline 2769 & BRAGFLO \\
\hline 2770 & BRAGFLO \\
\hline 2773 & BRAGFLO \\
\hline 2774 & BRAGFLO \\
\hline 2775 & BRAGFLO \\
\hline 2778 & BRAGFLO \\
\hline 2779 & BRAGFLO \\
\hline 2780 & BRAGFLO \\
\hline 2783 & BRAGFLO \\
\hline 2784 & BRAGFLO \\
\hline 2785 & BRAGFLO \\
\hline 2788 & BRAGFLO \\
\hline 2789 & BRAGFLO \\
\hline 2790 & BRAGFLO \\
\hline 2793 & BRAGFLO \\
\hline 2794 & BRAGFLO \\
\hline 2795 & BRAGFLO \\
\hline 2796 & BRAGFLO \\
\hline 2799 & BRAGFLO \\
\hline 2800 & BRAGFLO \\
\hline 2801 & BRAGFLO \\
\hline 2802 & BRAGFLO \\
\hline 2804 & |BRAGFLO \\
\hline 2805 & BRAGFLO \\
\hline
\end{tabular}


Model Cross-Reference ..

\begin{tabular}{|c|c|}
\hline ID & \begin{tabular}{|c|} 
Codename \\
\end{tabular} \\
\hline 2806 & BRAGFLO \\
\hline 2808 & BRAGFLO \\
\hline 2809 & BRAGFLO \\
\hline 2810 & BRAGFLO \\
\hline 2811 & BRAGFLO \\
\hline 2812 & BRAGFLO \\
\hline 2813 & BRAGFLO \\
\hline 2814 & BRAGFLO \\
\hline 2815 & BRAGFLO \\
\hline 2816 & BRAGFLO \\
\hline 2817 & BRAGFLO : \\
\hline 2818 & BRAGFLO \\
\hline 2819 & BRAGFLO \\
\hline 2820 & BRAGFLO \\
\hline 2823 & BRAGFLO \\
\hline 2824 & BRAGFLO \\
\hline 2825 & BRAGFLO \\
\hline 2826 & BRAGFLO \\
\hline 2827 & BRAGFLO \\
\hline 2828 & BRAGFLO \\
\hline 2829 & BRAGFLO \\
\hline 2830 & BRAGFLO \\
\hline 2831 & BRAGFLO \\
\hline 2831 & CUTTINGS_S \\
\hline 2831 & GRIDFLO \\
\hline 2831 & NUTS \\
\hline 2831 & SECOFL \\
\hline 2831 & SECOTP \\
\hline 2832 & BRAGFLO \\
\hline 2832 & CUTTINGS_S \\
\hline 2832 & GRIDFLO \\
\hline 2832 & NUTS \\
\hline 2832 & SECOFL \\
\hline 2832 & SECOTP \\
\hline 2833 & BRAGFLO \\
\hline 2833 & CUTTINGS_S \\
\hline 2833 & GRIDFLO \\
\hline 2833 & NUTS \\
\hline 2833 & SECOFL \\
\hline 2833 & SECOTP \\
\hline 2834 & BRAGFLO \\
\hline 2834 & CUTTINGS_S \\
\hline 2834 & GRIDFLO \\
\hline 2834 & \begin{tabular}{|l} 
NUTS \\
\end{tabular} \\
\hline 2834 & SECOFL \\
\hline 2834 & SECOTP \\
\hline 2835 & BRAGFLO \\
\hline 2835 & CUTTINGSSS \\
\hline 2835 & GRIDFLO \\
\hline 2835 & NUTS \\
\hline 2835 & SECOFL \\
\hline
\end{tabular}

\begin{tabular}{|c|c|}
\hline ID & Codename \\
\hline 2835 & SECOTP \\
\hline 2836 & BRAGFLO \\
\hline 2836 & CUTTINGS_S \\
\hline 2836 & GRIDFLO \\
\hline 2836 & NUTS \\
\hline 2836 & SECOFL \\
\hline 2836 & SECOTP \\
\hline 2837 & BRAGFLO \\
\hline 2838 & BRAGFLO \\
\hline 2839 & BRAGFLO \\
\hline 2840 & BRAGFLO \\
\hline 2841 & BRAGFLO \\
\hline 2842 & BRAGFLO \\
\hline 2858 & BRAGFLO \\
\hline 2858 & CUTTINGS_S \\
\hline 2858 & GRIDFLO \\
\hline 2858 & NUTS \\
\hline 2858 & SECOFL \\
\hline 2858 & SECOTP \\
\hline 2864 & BRAGFLO \\
\hline 2864 & CUTTINGS_S \\
\hline 2864 & GRIDFLO \\
\hline 2864 & NUTS \\
\hline 2864 & SECOFL \\
\hline 2864 & SECOTP \\
\hline 2865 & BRAGFLO \\
\hline 2865 & CUTTINGS_S \\
\hline 2865 & GRIDFLO \\
\hline 2865 & NUTS \\
\hline 2865 & SECOFL \\
\hline 2865 & SECOTP \\
\hline 2866 & BRAGFLO \\
\hline 2866 & CUTTINGS_S \\
\hline 2866 & GRIDFLO \\
\hline 2866 & NUTS \\
\hline 2866 & SECOFL \\
\hline 2866 & SECOTP \\
\hline 2879 & BRAGFLO \\
\hline 2880 & BRAGFLO \\
\hline 2881 & BRAGFLO \\
\hline 2881 & CUTTINGS_S \\
\hline 2881 & GRIDFLO \\
\hline 2881 & NUTS \\
\hline 2881 & SECOFL \\
\hline 2881 & SECOTP \\
\hline 2882 & BRAGFLO \\
\hline 2882 & CUTTINGS_S \\
\hline 2882 & GRIDFLO \\
\hline 2882 & NUTS \\
\hline 2882 & SECOFL \\
\hline 2882 & SECOTP \\
\hline
\end{tabular}

\begin{tabular}{|c|c|}
\hline ID & Codename \\
\hline 2883 & BRAGFLO \\
\hline 2883 & CUTTINGS_S \\
\hline 2883 & GRIDFLO \\
\hline 2883 & NUTS \\
\hline 2883 & SECOFL \\
\hline 2883 & SECOTP \\
\hline 2884 & BRAGFLO \\
\hline 2884 & CUTTINGS_S \\
\hline 2884 & GRIDFLO \\
\hline 2884 & NUTS \\
\hline 2884 & SECOFL \\
\hline 2884 & SECOTP \\
\hline 2885 & BRAGFLO \\
\hline 2885 & CUTTINGS_S \\
\hline 2885 & GRIDFLO \\
\hline 2885 & NUTS \\
\hline 2885 & SECOFL \\
\hline 2885 & SECOTP \\
\hline 2886 & BRAGFLO \\
\hline 2886 & CUTTINGS_S \\
\hline 2886 & GRIDFLO \\
\hline 2886 & NUTS \\
\hline 2886 & SECOFL \\
\hline 2886 & SECOTP \\
\hline 2887 & BRAGFLO \\
\hline 2887 & CUTTINGS_S \\
\hline 2887 & GRIDFLO \\
\hline 2887 & NUTS \\
\hline 2887 & SECOFL \\
\hline 2887 & SECOTP \\
\hline 2888 & BRAGFLO \\
\hline 2888 & CUTTINGS_S \\
\hline 2888 & GRIDFLO \\
\hline 2888 & NUTS \\
\hline 2888 & SECOFL \\
\hline 2888 & SECOTP \\
\hline 2889 & BRAGFLO \\
\hline 2889 & CUTTINGS_S \\
\hline 2889 & GRIDFLO \\
\hline 2889 & NUTS \\
\hline 2889 & SECOFL \\
\hline 2889 & SECOTP \\
\hline 2890 & BRAGFLO \\
\hline 2890 & CUTTINGS_S \\
\hline 2890 & GRIDFLO \\
\hline 2890 & NUTS \\
\hline 2890 & SECOFL \\
\hline 2890 & SECOTP \\
\hline 2891 & BRAGFLO \\
\hline 2891 & CUTTINGS_S \\
\hline 2891 & GRIDFLO \\
\hline
\end{tabular}




\section{Model Cross-Reference}

\begin{tabular}{|c|c|}
\hline 10 & \begin{tabular}{|c|} 
Codename \\
\end{tabular} \\
\hline 2891 & NUTS \\
\hline 2891 & SECOFL \\
\hline 2891 & SECOTP \\
\hline 2892 & BRAGFLO \\
\hline 2892 & CUTTINGS_S \\
\hline 2892 & GRIDFLO \\
\hline 2892 & NUTS \\
\hline 2892 & SECOFL \\
\hline 2892 & SECOTP \\
\hline 2893 & BRAGFLO \\
\hline 2893 & CUTTINGS_S \\
\hline 2893 & GRIDFLO \\
\hline 2893 & NUTS \\
\hline 2893 & SECOFL \\
\hline 2893 & SECOTP \\
\hline 2894 & BRAGFLO \\
\hline 2895 & BRAGFLO \\
\hline 2896 & BRAGFLO \\
\hline 2897 & BRAGFLO \\
\hline 2898 & BRAGFLO \\
\hline 2899 & BRAGFLO \\
\hline 2900 & BRAGFLO \\
\hline 2901 & BRAGFLO \\
\hline 2902 & BRAGFLO \\
\hline 2903 & BRAGFLO \\
\hline 2904 & BRAGFLO \\
\hline 2905 & BRAGFLO \\
\hline 2906 & BRAGFLO \\
\hline 2907 & BRAGFLO \\
\hline 2908 & BRAGFLO \\
\hline 2909 & BRAGFLO \\
\hline 2910 & BRAGFLO \\
\hline 2911 & BRAGFLO \\
\hline 2912 & BRAGFLO \\
\hline 2913 & BRAGFLO \\
\hline 2914 & BRAGFLO \\
\hline 2915 & BRAGFLO \\
\hline 2916 & BRAGFLO \\
\hline 2918 & BRAGFLO \\
\hline 2919 & BRAGFLO \\
\hline 2920 & BRAGFLO \\
\hline 2921 & BRAGFLO \\
\hline 2922 & BRAGFLO \\
\hline 2923 & BRAGFLO \\
\hline 2924 & BRAGFLO \\
\hline 2925 & BRAGFLO \\
\hline 2926 & BRAGFLO \\
\hline 2927 & BRAGFLO \\
\hline 2928 & BRAGFLO \\
\hline 2929 & BRAGFLO \\
\hline 2930 & BRAGFLO \\
\hline
\end{tabular}

\begin{tabular}{|c|c|}
\hline ID & Codename \\
\hline 2931 & BRAGFLO \\
\hline 2932 & BRAGFLO \\
\hline 2933 & BRAGFLO \\
\hline 2934 & BRAGFLO \\
\hline 2935 & BRAGFLO \\
\hline 2936 & BRAGFLO \\
\hline 2937 & BRAGFLO \\
\hline 2938 & BRAGFLO \\
\hline 2939 & BRAGFLO \\
\hline 2940 & BRAGFLO \\
\hline 2941 & BRAGFLO \\
\hline 2942 & BRAGFLO \\
\hline 2943 & BRAGFLO \\
\hline 2944 & BRAGFLO \\
\hline 2945 & BRAGFLO \\
\hline 2946 & BRAGFLO \\
\hline 2947 & BRAGFLO \\
\hline 2948 & BRAGFLO \\
\hline 2949 & BRAGFLO \\
\hline 2950 & BRAGFLO \\
\hline 2951 & BRAGFLO \\
\hline 2952 & BRAGFLO \\
\hline 2953 & BRAGFLO \\
\hline 2954 & BRAGFLO \\
\hline 2955 & BRAGFLO \\
\hline 2956 & BRAGFLO \\
\hline 2957 & BRAGFLO \\
\hline 2958 & BRAGFLO \\
\hline 2959 & BRAGFLO \\
\hline 2960 & BRAGFLO \\
\hline 2961 & BRAGFLO \\
\hline 2962 & BRAGFLO \\
\hline 2963 & BRAGFLO \\
\hline 2964 & BRAGFLO \\
\hline 2965 & BRAGFLO \\
\hline 2966 & BRAGFLO \\
\hline 2967 & BRAGFLO \\
\hline 2968 & BRAGFLO \\
\hline 2969 & BRAGFLO \\
\hline 2970 & BRAGFLO \\
\hline 2971 & BRAGFLO \\
\hline 2972 & BRAGFLO \\
\hline 2973 & BRAGFLO \\
\hline 2974 & BRAGFLO \\
\hline 2975 & BRAGFLO \\
\hline 2976 & BRAGFLO \\
\hline 2977 & BRAGFLO \\
\hline 2978 & BRAGFLO \\
\hline 2979 & BRAGFLO \\
\hline 2980 & BRAGFLO \\
\hline 2981 & BRAGFLO \\
\hline
\end{tabular}

\begin{tabular}{|c|c|}
\hline ID & Codename \\
\hline 2982 & BRAGFLO \\
\hline 2983 & BRAGFLO \\
\hline 2984 & BRAGFLO \\
\hline 2985 & BRAGFLO \\
\hline 2986 & BRAGFLO \\
\hline 2987 & BRAGFLO \\
\hline 2988 & BRAGFLO \\
\hline 2989 & BRAGFLO \\
\hline 2990 & BRAGFLO \\
\hline 2991 & BRAGFLO \\
\hline 2992 & BRAGFLO \\
\hline 2993 & BRAGFLO \\
\hline 2994 & BRAGFLO \\
\hline 2995 & BRAGFLO \\
\hline 2996 & BRAGFLO \\
\hline 2997 & BRAGFLO \\
\hline 2998 & BRAGFLO \\
\hline 2999 & BRAGFLO \\
\hline 3000 & BRAGFLO \\
\hline 3001 & BRAGFLO \\
\hline 3002 & BRAGFLO \\
\hline 3003 & BRAGFLO \\
\hline 3004 & BRAGFLO \\
\hline 3005 & BRAGFLO \\
\hline 3006 & BRAGFLO \\
\hline 3007 & BRAGFLO \\
\hline 3008 & BRAGFLO \\
\hline 3009 & BRAGFLO \\
\hline 3010 & BRAGFLO \\
\hline 3011 & BRAGFLO \\
\hline 3012 & BRAGFLO \\
\hline 3013 & BRAGFLO \\
\hline 3014 & BRAGFLO \\
\hline 3015 & BRAGFLO \\
\hline 3016 & BRAGFLO \\
\hline 3017 & BRAGFLO \\
\hline 3018 & BRAGFLO \\
\hline 3019 & BRAGFLO \\
\hline 3020 & BRAGFLO \\
\hline 3021 & BRAGFLO \\
\hline 3022 & BRAGFLO \\
\hline 3023 & BRAGFLO \\
\hline 3024 & BRAGFLO \\
\hline 3025 & BRAGFLO \\
\hline 3026 & BRAGFLO \\
\hline 3027 & BRAGFLO \\
\hline 3028 & BRAGFLO \\
\hline 3029 & BRAGFLO \\
\hline 3030 & BRAGFLO \\
\hline 3031 & BRAGFLO \\
\hline 3032 & BRAGFLO \\
\hline
\end{tabular}




\section{II-SW}

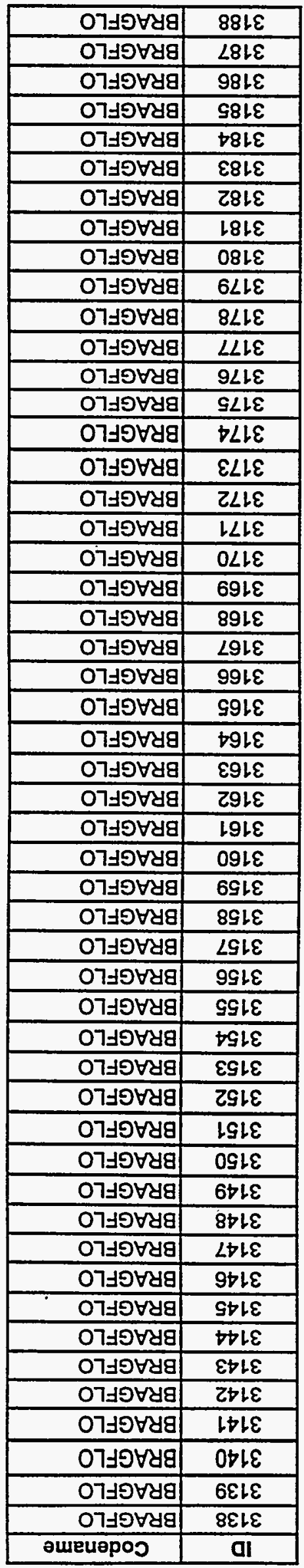

\begin{tabular}{|c|c|}
\hline 0า 5 $\forall y 9$ & $\angle E L E$ \\
\hline 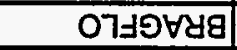 & $9 \varepsilon\llcorner\varepsilon$ \\
\hline 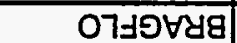 & SELE \\
\hline 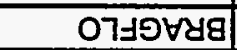 & 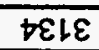 \\
\hline 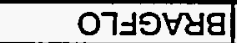 & $\varepsilon \varepsilon\llcorner\varepsilon$ \\
\hline 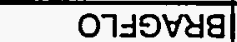 & 乙EเE \\
\hline 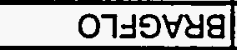 & LELE \\
\hline 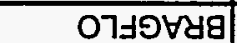 & 6ZLE \\
\hline Oางจ४บ8 & $8 Z L E$ \\
\hline 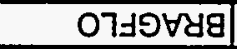 & 9ZLE \\
\hline 0างจษะด & SZLE \\
\hline 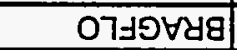 & tZLE \\
\hline 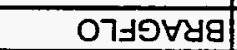 & $\varepsilon Z\llcorner\varepsilon$ \\
\hline 0างอซช8 & ZZLE \\
\hline 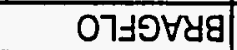 & LZLE \\
\hline 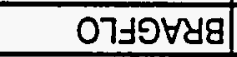 & $0 Z 1 \varepsilon$ \\
\hline oา & 9เLE \\
\hline 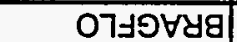 & SLLE \\
\hline 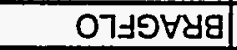 & DLLE \\
\hline $07 \pm 9 \forall y a$ & ZLLE \\
\hline 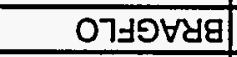 & เレE \\
\hline SInN & LLE \\
\hline SLnN & OLLE \\
\hline 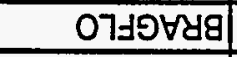 & OLLE \\
\hline $\operatorname{S\perp \cap N}$ & $601 \varepsilon$ \\
\hline 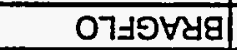 & $601 \varepsilon$ \\
\hline 0ามอหชด & $801 \varepsilon$ \\
\hline 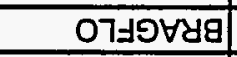 & $\angle O L \varepsilon$ \\
\hline 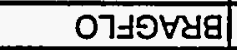 & $901 \varepsilon$ \\
\hline 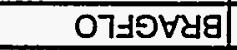 & SOLE \\
\hline 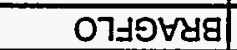 & $\$ 01 \varepsilon$ \\
\hline 0719648 & $\varepsilon 01 \varepsilon$ \\
\hline 0างอषช9 & ZOLE \\
\hline 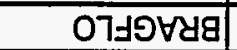 & LOLE \\
\hline 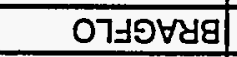 & $001 \varepsilon$ \\
\hline 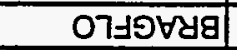 & $660 \varepsilon$ \\
\hline 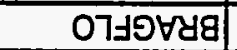 & $860 \varepsilon$ \\
\hline 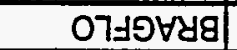 & $\angle 60 \varepsilon$ \\
\hline 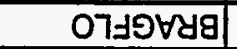 & $960 \varepsilon$ \\
\hline 07งอษบ8 & S60ع \\
\hline 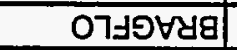 & $t 60 \varepsilon$ \\
\hline 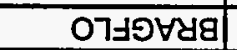 & $\varepsilon 60 \varepsilon$ \\
\hline 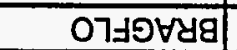 & $260 \varepsilon$ \\
\hline 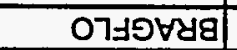 & L60ع \\
\hline 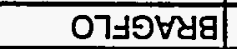 & $060 \varepsilon$ \\
\hline 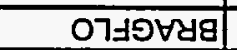 & $680 \varepsilon$ \\
\hline $07 \pm 9 \forall y$ & $880 \varepsilon$ \\
\hline 0ามจ৮บg & $\angle 80 \varepsilon$ \\
\hline 07」จ৮yg & $980 \varepsilon$ \\
\hline 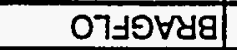 & \$80ع \\
\hline 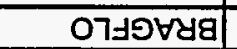 & $\not 80 \varepsilon$ \\
\hline өuruapos & al \\
\hline
\end{tabular}

\begin{tabular}{|c|c|}
\hline 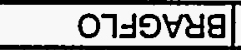 & $\varepsilon 80 \varepsilon$ \\
\hline 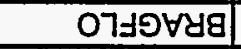 & $280 \varepsilon$ \\
\hline 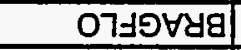 & $180 \varepsilon$ \\
\hline 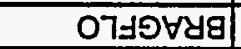 & $080 \varepsilon$ \\
\hline 0าปפษชด & $6 \angle 0 \varepsilon$ \\
\hline 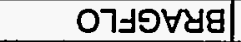 & $8 \angle 0 \varepsilon$ \\
\hline 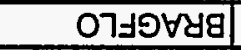 & $\angle L O E$ \\
\hline 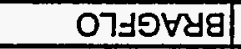 & $9 \angle 0 \varepsilon$ \\
\hline 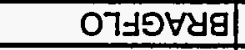 & SLOE \\
\hline 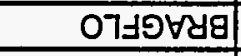 & $b \angle 0 \varepsilon$ \\
\hline 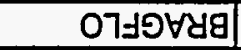 & $\varepsilon \angle 0 \varepsilon$ \\
\hline 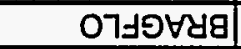 & $Z \angle 0 \varepsilon$ \\
\hline 0ามอชช8 & $\not \angle O \varepsilon$ \\
\hline 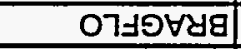 & $0 \angle 0 \varepsilon$ \\
\hline 07d5ชบg & $690 \varepsilon$ \\
\hline 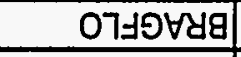 & $890 \varepsilon$ \\
\hline 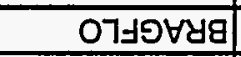 & $\angle 90 \varepsilon$ \\
\hline 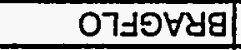 & $990 \varepsilon$ \\
\hline 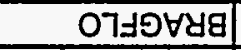 & G90ع \\
\hline oางอซyg & $790 \varepsilon$ \\
\hline 07ปอชบ9 & $\varepsilon 90 \varepsilon$ \\
\hline 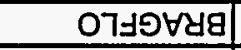 & $290 \varepsilon$ \\
\hline 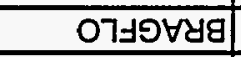 & L90E \\
\hline 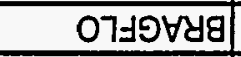 & $090 \varepsilon$ \\
\hline 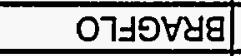 & $690 \varepsilon$ \\
\hline 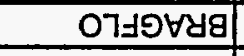 & $890 \varepsilon$ \\
\hline 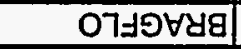 & $\angle G 0 \varepsilon$ \\
\hline 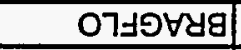 & $990 \varepsilon$ \\
\hline 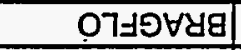 & GSOE \\
\hline 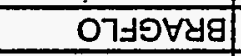 & $\forall G 0 \varepsilon$ \\
\hline O7jO৮ya & $\varepsilon 90 \varepsilon$ \\
\hline 07„5ชช9 & Z90ह \\
\hline 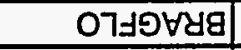 & LSOE \\
\hline $07 \pm 9 \forall 48$ & $0 S 0 \varepsilon$ \\
\hline 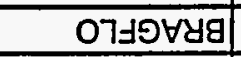 & 6 6t0ع \\
\hline 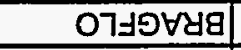 & $860 \varepsilon$ \\
\hline 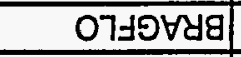 & $\angle t 0 \varepsilon$ \\
\hline 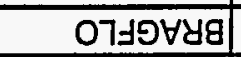 & $960 \varepsilon$ \\
\hline 0าฺอषบg & St0\& \\
\hline 0างอหมด & 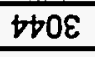 \\
\hline $07 \pm 9 \forall 49$ & $\varepsilon+0 \varepsilon$ \\
\hline 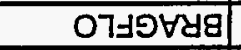 & Z†0द \\
\hline 0าปอชบด & ltOE \\
\hline 07ঊอ $\forall y 8$ & $0 \nmid 0 \varepsilon$ \\
\hline 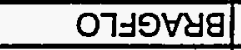 & $6 \varepsilon 0 \varepsilon$ \\
\hline 0าะอหมด & $8 \varepsilon 0 \varepsilon$ \\
\hline 0าjอ৮ya & $\angle \varepsilon O \varepsilon$ \\
\hline 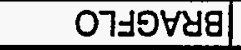 & $9 \varepsilon 0 \varepsilon$ \\
\hline 07」อ৮yg & $\mathcal{S \varepsilon 0 \varepsilon}$ \\
\hline 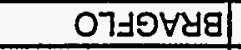 & 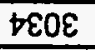 \\
\hline 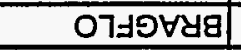 & $\varepsilon \varepsilon 0 \varepsilon$ \\
\hline әureuөpos & al \\
\hline
\end{tabular}

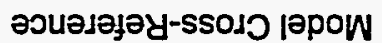


- -Model-Cross-Reference

\begin{tabular}{|c|c|}
\hline ID & Codename \\
\hline 3189 & BRAGFLO \\
\hline 3190 & BRAGFLO \\
\hline 3191 & BRAGFLO \\
\hline 3192 & BRAGFLO \\
\hline 3193 & BRAGFLO \\
\hline 3194 & BRAGFLO \\
\hline 3194 & CCDFGF \\
\hline 3194 & GRIDFLO \\
\hline 3196 & CUTTINGS_S \\
\hline 3196 & NUTS \\
\hline 3197 & CUTTINGS_S \\
\hline 3197 & NUTS \\
\hline 3198 & CUTTINGS_S \\
\hline 3198 & NUTS \\
\hline 3199 & CUTTINGS_S \\
\hline 3199 & NUTS \\
\hline 3200 & CUTTINGS_S \\
\hline 3200 & NUTS \\
\hline 3201 & CUTTINGS_S \\
\hline 3201 & NUTS \\
\hline 3202 & CUTTINGS_S \\
\hline 3202 & NUTS \\
\hline 3203 & CUTTINGS_S \\
\hline 3203 & NUTS \\
\hline 3204 & CUTTINGS_S \\
\hline 3204 & NUTS \\
\hline 3205 & CUTTINGS_S \\
\hline 3205 & NUTS \\
\hline 3206 & CUTTINGS_S \\
\hline 3206 & NUTS \\
\hline 3207 & CUTTINGS_S \\
\hline 3207 & NUTS \\
\hline 3208 & CUTTINGS_S \\
\hline 3208 & NUTS \\
\hline 3209 & CUTTINGS_S \\
\hline 3209 & NUTS \\
\hline 3210 & CUTTINGS_S \\
\hline 3210 & NUTS \\
\hline 3211 & CUTTINGS_S \\
\hline 3211 & NUTS \\
\hline 3212 & CUTTINGS_S \\
\hline 3212 & NUTS \\
\hline 3213 & CUTTINGS_S \\
\hline 3213 & NUTS \\
\hline 3214 & CUTTINGS_S \\
\hline 3214 & NUTS \\
\hline 3215 & CUTTINGS_S \\
\hline 3215 & NUTS \\
\hline 3216 & CUTTINGS_S \\
\hline 3216 & NUTS \\
\hline 3217 & CUTTINGS_S \\
\hline
\end{tabular}

\begin{tabular}{|c|c|}
\hline ID & Codename \\
\hline 3217 & NUTS \\
\hline 3218 & CUTTINGS_S \\
\hline 3218 & NUTS \\
\hline 3219 & CUTTINGS_S \\
\hline 3219 & NUTS \\
\hline 3220 & CUTTINGS_S \\
\hline 3220 & NUTS \\
\hline 3221 & CUTTINGS_S \\
\hline 3221 & NUTS \\
\hline 3222 & |CUTTINGS_S \\
\hline 3222 & NUTS \\
\hline 3223 & CUTTINGS_S \\
\hline 3223 & NUTS \\
\hline 3224 & CUTTINGS_S \\
\hline 3224 & NUTS \\
\hline 3225 & CUTTINGS_S \\
\hline 3225 & NUTS \\
\hline 3226 & CUTTINGS_S \\
\hline 3226 & NUTS \\
\hline 3227 & CUTTINGS_S \\
\hline 3227 & NUTS \\
\hline 3228 & CUTTINGS_S \\
\hline 3228 & NUTS \\
\hline 3229 & CUTTINGS_S \\
\hline 3229 & NUTS \\
\hline 3230 & CUTTINGS_S \\
\hline 3230 & NUTS \\
\hline 3231 & CUTTINGS_S \\
\hline 3231 & NUTS \\
\hline 3232 & CUTTINGS_S \\
\hline 3232 & NUTS \\
\hline 3233 & CUTTINGS_S \\
\hline 3233 & NUTS \\
\hline 3234 & CUTTINGS_S \\
\hline 3234 & NUTS \\
\hline 3235 & CUTTINGS_S \\
\hline 3235 & NUTS \\
\hline 3236 & CUTTINGS_S \\
\hline 3236 & NUTS \\
\hline 3237 & CUTTINGS_S \\
\hline 3237 & NUTS \\
\hline 3238 & BRAGFLO \\
\hline 3238 & NUTS \\
\hline 3239 & CUTTINGS_S \\
\hline 3240 & CUTTINGS_S \\
\hline 3241 & CUTTINGS_S \\
\hline 3242 & CUTTINGS_S \\
\hline 3243 & CUTTINGS_S \\
\hline 3244 & CUTTINGS_S \\
\hline 3245 & CUTTINGS_S \\
\hline 3246 & CUTTINGS_S \\
\hline
\end{tabular}

\begin{tabular}{|c|c|}
\hline ID & Codename \\
\hline 3247 & CUTTINGS_S \\
\hline 3248 & CUTTINGS_S \\
\hline 3249 & CUTTINGS_S \\
\hline 3250 & CUTTINGS_S \\
\hline 3251 & CUTTINGS_S \\
\hline 3252 & CUTTINGS_S \\
\hline 3253 & CUTTINGS_S \\
\hline 3254 & CUTTINGS_S \\
\hline 3255 & CUTTINGSS \\
\hline 3256 & CUTTINGS_S \\
\hline 3257 & CUTTINGS_S \\
\hline 3258 & CUTTINGS S \\
\hline 3259 & CUTTINGS_S \\
\hline 3260 & CUTTINGS_S \\
\hline 3261 & CUTTINGS_S \\
\hline 3262 & GRIDFLO \\
\hline 3262 & NUTS \\
\hline 3262 & PANEL \\
\hline 3263 & GRIDFLO \\
\hline 3263 & NUTS \\
\hline 3263 & PANEL \\
\hline 3264 & GRIDFLO \\
\hline 3264 & NUTS \\
\hline 3264 & PANEL \\
\hline 3265 & GRIDFLO \\
\hline 3265 & NUTS \\
\hline 3265 & PANEL \\
\hline 3266 & GRIDFLO \\
\hline 3266 & NUTS \\
\hline 3266 & PANEL \\
\hline 3267 & CUTTINGS_S \\
\hline 3267 & NUTS \\
\hline 3268 & CUTTINGS_S \\
\hline 3268 & NUTS \\
\hline 3269 & CUTTINGS_S \\
\hline 3269 & NUTS \\
\hline 3270 & CUTTINGS_S \\
\hline 3270 & NUTS \\
\hline 3271 & CUTTINGS_S \\
\hline 3271 & NUTS \\
\hline 3272 & CUTTINGS_S \\
\hline 3272 & NUTS \\
\hline 3273 & |CUTTINGS_S \\
\hline 3273 & INUTS \\
\hline 3274 & CUTTINGSS \\
\hline 3274 & |NUTS \\
\hline 3275 & |CUTTINGS_S \\
\hline 3275 & NUTS \\
\hline 3276 & |CUTTINGS_S \\
\hline 3276 & NUTS \\
\hline 3277 & CUTTINGS_S \\
\hline
\end{tabular}


Model-Cross-Reference

\begin{tabular}{|c|c|}
\hline ID & Codename \\
\hline 3277 & NUTS \\
\hline 3278 & CUTTINGS_S \\
\hline 3278 & NUTS \\
\hline 3279 & CUTTINGS_S \\
\hline 3279 & NUTS \\
\hline 3280 & CUTTINGS_S \\
\hline 3280 & NUTS \\
\hline 3281 & CUTTINGS_S \\
\hline 3281 & NUTS \\
\hline 3282 & CUTTINGS_S \\
\hline 3282 & NUTS \\
\hline 3283 & CUTTINGS_S \\
\hline 3283 & NUTS \\
\hline 3284 & CUTTINGS_S \\
\hline 3284 & NUTS \\
\hline 3285 & CUTTINGS_S \\
\hline 3285 & NUTS \\
\hline 3286 & CUTTINGS_S \\
\hline 3286 & NUTS \\
\hline 3287 & CUTTINGS_S \\
\hline 3287 & NUTS \\
\hline 3288 & CUTTINGS_S \\
\hline 3288 & NUTS \\
\hline 3289 & CUTTINGS_S \\
\hline 3289 & NUTS \\
\hline 3290 & CUTTINGS_S \\
\hline 3290 & NUTS \\
\hline 3291 & CUTTINGS_S \\
\hline 3291 & NUTS \\
\hline 3292 & CUTTINGS_S \\
\hline 3292 & NUTS \\
\hline 3293 & CUTTINGS_S \\
\hline 3293 & NUTS \\
\hline 3294 & CUTTINGS_S \\
\hline 3294 & NUTS \\
\hline 3295 & CUTTINGS_S \\
\hline 3295 & NUTS \\
\hline 3296 & CUTTINGS_S \\
\hline 3296 & NUTS \\
\hline 3297 & CUTTINGS_S \\
\hline 3297 & NUTS \\
\hline 3298 & CUTTINGS_S \\
\hline 3298 & NUTS \\
\hline 3299 & CUTTINGS_S \\
\hline 3299 & NUTS \\
\hline 3300 & CUTTINGS_S \\
\hline 3300 & NUTS \\
\hline 3301 & CUTTINGS_S \\
\hline 3301 & NUTS \\
\hline 3302 & CUTTINGS_S \\
\hline 3302 & NUTS \\
\hline
\end{tabular}

\begin{tabular}{|c|c|}
\hline ID & Codename \\
\hline 3303 & CUTTINGS_S \\
\hline 3303 & NUTS \\
\hline 3304 & CUTTINGS_S \\
\hline 3304 & NUTS \\
\hline 3305 & CUTTINGS_S \\
\hline 3305 & NUTS \\
\hline 3306 & CUTTINGS_S \\
\hline 3306 & NUTS \\
\hline 3307 & GRIDFLO \\
\hline 3307 & NUTS \\
\hline 3308 & GRIDFLO \\
\hline 3308 & NUTS \\
\hline 3309 & GRIDFLO \\
\hline 3309 & NUTS \\
\hline 3310 & GRIDFLO \\
\hline 3310 & NUTS \\
\hline 3311 & GRIDFLO \\
\hline 3311 & \begin{tabular}{|l} 
NUTS \\
\end{tabular} \\
\hline 3312 & GRIDFLO \\
\hline 3312 & NUTS \\
\hline 3313 & GRIDFLO \\
\hline 3313 & NUTS \\
\hline 3314 & GRIDFLO \\
\hline 3314 & NUTS \\
\hline 3315 & GRIDFLO \\
\hline 3315 & NUTS \\
\hline 3316 & GRIDFLO \\
\hline 3316 & NUTS \\
\hline 3317 & GRIDFLO \\
\hline 3317 & NUTS \\
\hline 3318 & GRIDFLO \\
\hline 3318 & NUTS \\
\hline 3319 & GRIDFLO \\
\hline 3319 & NUTS \\
\hline 3320 & GRIDFLO \\
\hline 3320 & NUTS \\
\hline 3321 & CUTTINGS_S \\
\hline 3322 & CUTTINGS_S \\
\hline 3323 & CUTTINGS_S \\
\hline 3324 & CUTTINGS_S \\
\hline 3325 & CUTTINGS_S \\
\hline 3326 & CUTTINGS_S \\
\hline 3327 & CUTTINGS_S \\
\hline 3328 & CUTTINGS_S \\
\hline 3329 & CUTTINGS_S \\
\hline 3330 & CUTTINGS_S \\
\hline 3331 & CUTTINGS_S \\
\hline 3332 & CUTTINGS_S \\
\hline 3333 & CUTTINGS_S \\
\hline 3334 & CUTTINGS_S \\
\hline 3335 & |CUTTINGS_S \\
\hline
\end{tabular}

\begin{tabular}{|c|l|}
\hline ID & Codename \\
\hline 3336 & CUTTINGS_S \\
\hline 3337 & CUTTINGS_S \\
\hline 3338 & CUTTINGS_S \\
\hline 3339 & CUTTINGS_S \\
\hline 3340 & CUTTINGS_S \\
\hline 3341 & CUTTINGS_S \\
\hline 3342 & CUTTINGS_S \\
\hline 3343 & CUTTINGS_S \\
\hline 3344 & CUTTINGS_S \\
\hline 3345 & CUTTINGS_S \\
\hline 3346 & CUTTINGS_S \\
\hline 3347 & CUTTINGS_S \\
\hline 3348 & CUTTINGS_S \\
\hline 3349 & CUTTINGS_S \\
\hline 3350 & CUTTINGS_S \\
\hline 3351 & CUTTINGS_S \\
\hline 3352 & CUTTINGS_S \\
\hline 3353 & CUTTINGS_S \\
\hline 3354 & CUTTINGS_S \\
\hline 3355 & CUTTINGS_S \\
\hline 3356 & CUTTINGS_S \\
\hline 3357 & CUTTINGS_S \\
\hline 3358 & CUTTINGS_S \\
\hline 3359 & CUTTINGS_S \\
\hline 3360 & CUTTINGS_S \\
\hline 3361 & CUTTINGS_S \\
\hline 3362 & CUTTINGS_S \\
\hline 3363 & CUTTINGS_S \\
\hline 3364 & CUTTINGS_S \\
\hline 3365 & CUTTINGS_S \\
\hline 3366 & CUTTINGS_S \\
\hline 3367 & CUTTINGS_S \\
\hline 3368 & CUTTINGS_s \\
\hline 3369 & CUTTINGS_S \\
\hline 3370 & CUTTINGS_S \\
\hline 3371 & CUTTINGS_S \\
\hline 3372 & CUTTINGS_S \\
\hline 3373 & CUTTINGS_S \\
\hline 3374 & CUTTINGS_S \\
\hline 3375 & CUTTINGS_S \\
\hline 3376 & CUTTINGS_S \\
\hline 3377 & CUTTINGS_S \\
\hline 3378 & CUTTINGS_S \\
\hline 3379 & CUTTINGS_S \\
\hline 3380 & CUTTINGS_S \\
\hline 3381 & CUTTINGS_S \\
\hline 3383 & CUTTINGS_S \\
\hline 3384 & CUTTINGS_S \\
\hline CUTTINGS_S \\
\hline CUTTINGS_S \\
\hline CUTTINGS_S \\
\hline 336
\end{tabular}


Model Cross-Reference

\begin{tabular}{|c|l|}
\hline ID & \multicolumn{1}{|c|}{ Codename } \\
\hline 3387 & CUTTINGS_S \\
\hline 3388 & CUTTINGS_S \\
\hline 3389 & GRIDFLO \\
\hline 3389 & NUTS \\
\hline 3389 & PANEL \\
\hline 3390 & GRIDFLO \\
\hline 3390 & NUTS \\
\hline 3390 & PANEL \\
\hline 3391 & GRIDFLO \\
\hline 3391 & NUTS \\
\hline 3391 & PANEL \\
\hline 3392 & GRIDFLO \\
\hline 3392 & NUTS \\
\hline 3392 & PANEL \\
\hline 3393 & GRIDFLO \\
\hline 3393 & NUTS \\
\hline 3393 & PANEL \\
\hline 3402 & GRIDFLO \\
\hline 3402 & NUTS \\
\hline 3402 & PANEL \\
\hline 3403 & GRIDFLO \\
\hline 3403 & NUTS \\
\hline 3403 & PANEL \\
\hline 3404 & GRIDFLO \\
\hline 3404 & NUTS \\
\hline 3404 & PANEL \\
\hline 3405 & GRIDFLO \\
\hline 3405 & NUTS \\
\hline 3405 & PANEL \\
\hline 3406 & GRIDFLO \\
\hline 3406 & NUTS \\
\hline 3406 & PANEL \\
\hline 3407 & GRIDFLO \\
\hline 3407 & NUTS \\
\hline 3407 & PANEL \\
\hline 3408 & GRIDFLO \\
\hline 3408 & NUTS \\
\hline 3408 & PANEL \\
\hline 3409 & GRIDFLO \\
\hline 3409 & NUTS \\
\hline 3409 & PANEL \\
\hline 3410 & CUTTINGS_S \\
\hline 3410 & NUTS \\
\hline 3410 & PANEL \\
\hline 3411 & CUTTINGS_S \\
\hline 3411 & NUTS \\
\hline 3412 & PANEL \\
\hline 3412 & NUTTINGS_S \\
\hline PANEL \\
\hline CUTTINGS_S \\
\hline 343 & \\
\hline 340 \\
\hline 340
\end{tabular}

\begin{tabular}{|c|c|}
\hline ID & Codename \\
\hline 3413 & NUTS \\
\hline 3413 & PANEL \\
\hline 3414 & CUTTINGS_S \\
\hline 3415 & CUTTINGS_S \\
\hline 3415 & NUTS \\
\hline 3415 & PANEL \\
\hline 3416 & CUTTINGS_S \\
\hline 3416 & NUTS \\
\hline 3416 & PANEL \\
\hline 3417 & NUTS \\
\hline 3418 & GRASP_INV \\
\hline 3419 & SECOFL \\
\hline 3420 & CUTTINGS_S \\
\hline 3421 & CUTTINGS_S \\
\hline 3421 & NUTS \\
\hline 3429 & GRIDFLO \\
\hline 3429 & NUTS \\
\hline 3430 & GRIDFLO \\
\hline 3430 & NUTS \\
\hline 3431 & GRIDFLO \\
\hline 3431 & NUTS \\
\hline 3432 & GRIDFLO \\
\hline 3432 & NUTS \\
\hline 3433 & GRIDFLO \\
\hline 3433 & NUTS \\
\hline 3434 & GRIDFLO \\
\hline 3434 & NUTS \\
\hline 3435 & GRIDFLO \\
\hline 3435 & NUTS \\
\hline 3436 & GRIDFLO \\
\hline 3436 & NUTS \\
\hline 3437 & GRIDFLO \\
\hline 3437 & NUTS \\
\hline 3438 & GRIDFLO \\
\hline 3438 & NUTS \\
\hline 3439 & GRIDFLO \\
\hline 3439 & NUTS \\
\hline 3440 & GRIDFLO \\
\hline 3440 & NUTS \\
\hline 3441 & GRIDFLO \\
\hline 3441 & NUTS \\
\hline 3442 & NUTS \\
\hline 3442 & SECOTP \\
\hline 3443 & NUTS \\
\hline 3443 & SECOTP \\
\hline 3444 & NUTS \\
\hline 3444 & SECOTP \\
\hline 3446 & NUTS \\
\hline 3446 & SECOTP \\
\hline 3447 & GRIDFLO \\
\hline 3447 & NUTS \\
\hline
\end{tabular}

\begin{tabular}{|c|c|}
\hline ID & Codename \\
\hline 3448 & NUTS \\
\hline 3448 & SECOTP \\
\hline 3449 & NUTS \\
\hline 3449 & SECOTP \\
\hline 3454 & BRAGFLO \\
\hline 3455 & BRAGFLO \\
\hline 3456 & BRAGFLO_DBR \\
\hline 3457 & GRIDFLO \\
\hline 3457 & NUTS \\
\hline 3458 & GRIDFLO \\
\hline 3458 & NUTS \\
\hline 3459 & GRIDFLO \\
\hline 3459 & NUTS \\
\hline 3460 & GRIDFLO \\
\hline 3460 & NUTS \\
\hline 3461 & GRIDFLO \\
\hline 3461 & NUTS \\
\hline 3462 & SECOFL \\
\hline 3462 & SECOTP \\
\hline 3469 & SECOTP \\
\hline 3470 & BRAGFLO_DBR \\
\hline 3471 & BRAGFLO_DBR \\
\hline 3472 & BRAGFLO_DBR \\
\hline 3473 & BRAGFLO_DBR \\
\hline 3474 & SECOTP \\
\hline 3475 & NUTS \\
\hline 3475 & SECOTP \\
\hline 3476 & NUTS \\
\hline 3476 & SECOTP \\
\hline 3477 & NUTS \\
\hline 3477 & SECOTP \\
\hline 3478 & NUTS \\
\hline 3478 & SECOTP \\
\hline 3479 & NUTS \\
\hline 3479 & SECOTP \\
\hline 3480 & NUTS \\
\hline 3480 & SECOTP \\
\hline 3481 & NUTS \\
\hline 3481 & SECOTP \\
\hline 3482 & NUTS \\
\hline 3482 & SECOTP \\
\hline 3483 & SECOTP \\
\hline 3484 & SECOTP \\
\hline 3485 & SECOTP \\
\hline 3486 & SECOTP \\
\hline 3487 & SECOTP \\
\hline 3488 & CCDFGF \\
\hline 3488 & GRIDFLO \\
\hline 3489 & CCDFGF \\
\hline 3489 & GRIDFLO \\
\hline 3490 & CCDFGF \\
\hline
\end{tabular}


Model-Cross-Reference

\begin{tabular}{|c|l|}
\hline ID & Codename \\
\hline 3490 & GRIDFLO \\
\hline 3491 & CCDFGF \\
\hline 3491 & GRIDFLO \\
\hline 3492 & CCDFGF \\
\hline 3492 & GRIDFLO \\
\hline 3493 & CCDFGF \\
\hline 3493 & GRIDFLO \\
\hline 3494 & CCDFGF \\
\hline 3494 & GRIDFLO \\
\hline 3495 & CCDFGF \\
\hline 3495 & GRIDFLO \\
\hline 3496 & CCDFGF \\
\hline 3496 & GRIDFLO \\
\hline 3497 & CCDFGF \\
\hline 3497 & GRIDFLO \\
\hline 3498 & CCDFGF \\
\hline 3498 & GRIDFLO \\
\hline 3499 & CCDFGF \\
\hline 3499 & GRIDFLO \\
\hline 3500 & CCDFGF \\
\hline 3500 & GRIDFLO \\
\hline 3501 & CCDFGF \\
\hline 3501 & GRIDFLO \\
\hline 3502 & CCDFGF \\
\hline 3502 & GRIDFLO \\
\hline 3503 & CCDFGF \\
\hline 3503 & GRIDFLO \\
\hline 9000 & BRAGFLO \\
\hline 9001 & BRAGFLO \\
\hline 9002 & BRAGFLO \\
\hline 9003 & BRAGFLO \\
\hline 9004 & BRAGFLO \\
\hline & \\
\hline
\end{tabular}

\begin{tabular}{|c|l|}
\hline ID & Codename \\
\hline 9036 & BRAGFLO \\
\hline 9037 & BRAGFLO \\
\hline 9038 & BRAGFLO \\
\hline 9039 & BRAGFLO \\
\hline 9040 & BRAGFLO \\
\hline 9041 & BRAGFLO \\
\hline 9042 & BRAGFLO \\
\hline 9043 & BRAGFLO \\
\hline 9044 & BRAGFLO \\
\hline 9045 & BRAGFLO \\
\hline 9046 & BRAGFLO \\
\hline 9047 & BRAGFLO \\
\hline 9048 & BRAGFLO \\
\hline 9049 & BRAGFLO \\
\hline 9050 & BRAGFLO \\
\hline 9051 & BRAGFLO \\
\hline 9052 & BRAGFLO \\
\hline 9053 & BRAGFLO \\
\hline 9054 & BRAGFLO \\
\hline 9055 & BRAGFLO \\
\hline 9056 & BRAGFLO \\
\hline 9057 & BRAGFLO \\
\hline 9058 & BRAGFLO \\
\hline 9059 & BRAGFLO \\
\hline 9060 & BRAGFLO \\
\hline 9061 & CUTTINGS_S \\
\hline 9062 & CUTTINGS_S \\
\hline 9063 & CUTTINGS_S \\
\hline 9064 & CUTTINGS_S \\
\hline 9065 & CUTTINGS_S \\
\hline 9066 & CUTTINGS_S \\
\hline & \\
\hline
\end{tabular}


Page intentionally blank

M5-16 
Appendix M6

\section{Memoranda: Description of EPA Parameter \\ Database Contents}


date: July 31, 1997

Albuquerque. New IVicxico $87185-1341$

to: Sandia WIPP Central Files, SWCF-A:1.2.07.1:PDD:QA:EPA

Database

from: Lori J. Dotson, MS 1341 (6848)

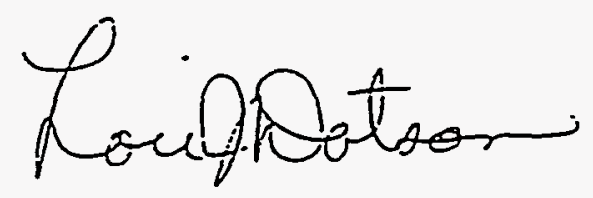

subject: Submittal of Intermediate Tables from "EPA Database"

(wipp_reference) to Sandia WIPP Central Files

The WIPP EPA Database contains information which supports the parameter values used in the 1996 WIPP CCA calculations. The database is maintained in INGRES and is named wipp_reference. Several views from this database have been downloaded into an EXCEL workbook titled epa_072297.xls. This workbook contains six sheets (described below) which are currently available on the following ftp site:

ftp.nwer.sandia.gov/clatii/epa_072297.xls. A hardcopy of these six sheets are contained as attachments to this memorandum and should be filed in the Sandia WIPP Central Files.

\section{ATTACHMENT 1: Footnotes for Attachment 2 (Worksheet titled "footnotes")}

This sheet defines footnotes 1 (Parameter category), 2 (Classification of data category) and 3 (Methods used to qualify existing data) which are used in the following sheet of the workbook "epa_072297" (See also Attachment 2).

\section{ATTACHMENT 2: Main EPA Database -7/22/97}

(Worksheet titled "epa_072297")

This sheet contains a comprehensive listing of all parameters contained in the WIPP

PA Database. There are several columns which are defined below:

\section{Column "艹1: "Parameter in Database? (YN)"}

Identifies whether or not values for the parameter are stored in the WIPP PA Database.

Column $\# 2:$ "If the values used in CCA are not the same as in DB. provide \% difference" 
Provides the percent difference between a parameter value used in the $1996 \mathrm{CCA}$ calculation versus the value stored in the WIPP PA Database for the rare case where a parameter value from the database was not used.

\section{Column H3: "ID"}

Unique parameter identification number assigned by the Database Administrator.

\section{Column ${ }^{\prime 4}:$ "Material ID"}

Abbreviated name of the material region represented by the parameter value.

\section{Column "H5: "Material Name"}

Full name of the material region.

\section{Column "̈6: "Parameter ID"}

Abbreviated name of the properry.

\section{Column "1: "Parameter Name"}

Full name of the property.

\section{Column \#8: "PRP ID (NPO:ँ)"}

The WIPP Project Office (WPO) number assigned to the Parameter Records Package (PRP). The PRP consists of the 464 Form plus any attached information.

\section{Column "U9: "Data Entrv Date"}

Date the parameter value was entered into the WIPP PA Database.

\section{Column $"$ 10: "Is the 464 consistent with the parameter in the DB? N. N/A)"}

Indicates whether the parameter value contained in the WIPP PA Database is the same as the value contained in the 464 Form $(Y=$ Yes, N/A = Not Applicable).

\section{Column "11: "Parameter Categorv (footnote 1)"}

(See also Attachment 1)

Parameter category descriptions as contained in Q.AP 9-2, Rev 2.

1) Parameters based on site-specific information used as initial input to a WIPP PA numerical model that specifies the physical, chemical, or hydrological properties of the 
rock formations, seals, backfills, and waste form, or any other natural or engineered feature of the WIPP

2) Parameters representing the inventory of the waste to be emplaced in the WIPP as defined in the Waste Isolation Pilot Plant Transuranic Waste Baseline Inventory Report (BIR)

3) Parameters representing precisely known, tabulated physical constants (e.g., the half-life of a radionuclide, gravitational constant).

4a) Parameters that are assigned based on similarity of properties between similar material or features

4b) Parameters that are model configuration parameters not based on specific WIPP properties or features but are need to make PA models nun (e.g. time-step limits).

5) Parameters not used in current compliance calculations.

\section{Column "12: "Classification of Data Categorv (footnote 2)"} (See also Attachment 1)

General classification for data and other information used in the development of parameters. (Note that a parameter may have more than one data category assigned to it). 1) Site-specific or waste-specific data. This category includes information obtained from in-situ experiments and research conducted at off-site laboratories (for example, permeability data, microbial gas generation). This category also includes simulated waste experiments and may indicate correlations made with other material regions based on professional judgement.

2) Waste-specific observational data. This category includes data obtained through observation or empirical analysis, such as semi-quantitative and qualitative visual characterization or acceptable knowledge of transuranic (IRU) waste (for example, waste components).

3) Professional Judgement. This category of information may involve the use of experimental or observational data from other non-WIPP contexts; interpreting information obtained from the general literature; or may be based on general engineering knowledge (see below). In some cases, professional judgement can be used in assigning values to category 1 parameters.

4) General Literature Data. This category of information includes that obtained from reports, joumal articles, or handbooks relevant to systems or processes being modeled in the performance assessment.

5) General Engineering Knowledge. This category of information identifies parameter values obtained from knowledge of standard engineering principles.

\section{Column}

Parameter probability distribution type:

CONSTANT $=$ Single value (no distribution)

TRIANGULAR = Triangular probability distíbution

UNIFORM = Uniform probability distribution 
CUMULATIVE = Cumulative probability distribution

NORMAL $=$ Normal probability distribution

STUDENT = Student's $t$ (or simply " $t$ ") probability distribution

DELTA = Delta probability distribution

LOGUNIFORM = Loguniform probability distribution

LOGCUMULATIVE = Logcumulative probability distribution

LOGNORMAL = Lognormal probability distribution

This list is inclusive for the CCA WIPP Database.

\section{Column H14: "Mean"}

The mean of the distribution.

\section{Column \#15: "Median"}

The median of the distribution.

Column 눈: "Minimum"

The minimum value in the distribution.

\section{Column "17: "Maximum"}

The maximum value in the distribution.

\section{Column "18: "Units"}

The SI units of the parameter value.

\section{Column H19: "Reference ID"}

(See also Attachment 5)

The reference(s) which support the parameter value.

\section{Column \#20: "Was the data developed under an NOA-1 Program?"}

Identifies whether or not the parameter values were developed under a NQA-1 Program (i.e., the American Society of Mechanical Engineers (ASME) "Quality Assurance Program Requirements for Nuclear Facilities").

Column ":21: "Which methods were used to qualifv existing data? (footnote 3)" (See also Attachment 1)

Methods used to qualify existing data: 
1) Peer review

2) Corroborating data

3) Confirmatory testing

4) Demonstration that the data was collected under a quality assurance program equivalent to NQA-1/3

5) If none of the above methods were used, then the data remains unqualified

6) Non-WIPP published literature (includes journal articles, conference papers, textbooks, handbooks, etc.)

7) QED (same as "1" aböve)

$N / A=$ Not Applicable

\section{ATTACHMENT 3: Parameters based on Empirical Data (Worksheet titled "sorted - empirical data")}

This sheet lists those parameters which are based, in whole or part, on empirical data. Attachment 3 does not contain the entire spreadsheet as presented on the FTP site; only key columns are presented in Attachment 3 since this sheet is a subset of "epa_072297" which is printed out in its entirety in Attachment 2 of this memorandum. The column headings for this sheet are the same as defined above for Attachment 2.

\section{ATTACHMENT 4: Codes}

(Worksheet titled "model cross-reference")

This sheet lists the code(s) in which each parameter is used. The sheet identifies parameters by id number only; parameter names are identified in the sheet "epa_072297" (See also Attachment 2).

ATTACHMENT 5: References for Attachment 2

(Worksheet titled "source cross-reference")

This sheet provides details for the references cited in Column "19" of the sheet "epa_072297" (See also Attachment 2).

ATTACHMENT 6: Additional Sources (Worksheet titled "additional sources")

This sheet provides detailed technical documentation for a select group of parameters. 
Copy (w/ attachments) to:

MS-1341, Lori J. Dotson, 6848

MS-1341, Susan Howarth, 6801

MS-1328, Gary Froehlich, 6801

Copv (w/o attachments) to:

MS-1395, Les Shephard, 6800

MS-1335, Margaret Chu; 6801

MS-1328, Charlene Lattièr, 6848

MS-1328, Gretchen Newman, 6848

MS-1335, Mel Marietta, 6821

MS-1328, Mary-Alena Martell, 6849

DOE/CAO Marc Italiano

DOE/CAO George Basabilvazo 


\section{Sandia National Laboratories}

Albuquerque, New Mexico 87185-1341

date: August 7, 1997

to: Sandia WIPP Central Files, SWCF-A:1.2.07.1:PDD:QA:EPA

Database

from: Lori J. Dotson, MS 1341 (6848)

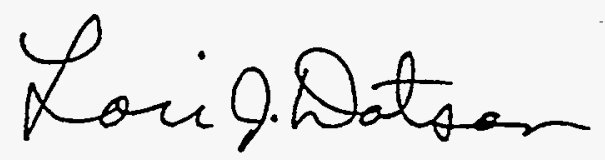

subject: Replacement of Attachment 6 and Addition of Attachment 7 to WIPP "EPA Database" (WPO\# 46761)

Please replace Attachment 6 "Additional Sources" and add Attachment 7 "Enhanced Sources" to the WIPP "EPA Database" memorandum which was submitted to the Sandia WIPP Central Files on July 31, 1997 (WPO\# 46761). The complete updated workbook now contains seven sheets which are currently available on the following ftp site: ftp.nwer.sandia.gov/clatti/epa_080797.xls. Attachment 6 was described in my memorandum dated July 31,1997 . Attachment 7 contains enhanced source information for the 149 parameters previously classified as being based on professional judgement. $\mathrm{A}$ hardcopy of the new sheets are contained as attachments to this memorandum.

Copy (w/ attachments) to:

MS-1341, Lori J. Dotson, 6848

MS-1341, Susan Howarth, 6801

MS-1328, Gary Froehlich, 6801

Copy (w/o attachments) to:

MS-1395, Les Shephard, 6800

MS-1335, Margaret Chu, 6801

MS-1328, Charlene Lattier, 6848

MS-1328, Gretchen Newman, 6848

MS-1335, Mel Marietta, 6821

MS-1328, Mary-Alena Martell, 6849

DOE/CAO Marc Italiano

DOE/CAO George Basabilvazo 
Page intentionally blank

M6-10 


\section{Appendix $\mathbf{N}$}

\section{Listing of Parameters Used in CCA PA Codes}

which Differ from Database Values 
Listing of Parameters Used in BRAGFLO which Differ from the WIPP 1996 Compliance Certification Application Parameter Database

\begin{tabular}{|c|c|c|c|c|c|c|c|}
\hline $\begin{array}{l}\text { Dutabase " } \\
\text { material } \\
\end{array}$ & $\begin{array}{l}\text { Database } \\
\text { parameter: }\end{array}$ & . Modeling valüe used & Distribution type & Database value & 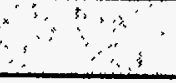 & Units & Wimo: \\
\hline REFCON & GRAVACC & 9.79 & CONSTANT & 9.8067 & & $\mathrm{~m} / \mathrm{s}^{\wedge} 2$ & 32386 \\
\hline DRZ_0 & POROSITY & $0.0029+$ (realization value S_HALITE) & CONSTANT & $3.90 \mathrm{E}-03$ & $3.29 \mathrm{E}-02$ & $\log \left(m^{\wedge} 2\right)$ & 32839 \\
\hline DRZ_1 & POROSITY & $0.0029+$ (realization value $S \_$HALITE) & CUMULATIVE & $1.00 \mathrm{E}+00$ & $1.03 \mathrm{E}+00$ & $\log \left(m^{\wedge} 2\right.$ & 32902 \\
\hline DEWYLAKE & PCT_A & 0.00 & CONSTANT & 2.60E-01 & & $\mathrm{Pa}$ & 32725 \\
\hline DEWYLAKE & PCT_EXP & 0.00 & CONSTANT & $-3.48 \mathrm{E}-01$ & & none & 32727 \\
\hline BH_CREEP & PRMX_LOG & $0.1^{*}+$ (realization value BH_SAND) & UNIFORM & $-1.50000 \mathrm{E}+01$ & $-1.20000 \mathrm{E}+01$ & $\log \left(m^{\wedge} 2\right)$ & 36640 \\
\hline CL_L_TI & PCT_A & 0.00 & CONSTANT & 0.56 & & $\mathrm{~Pa}$ & 31901 \\
\hline CL_L_T2 & PCT_A & 0.00 & CONSTANT & 0.56 & & $\mathrm{~Pa}$ & 31928 \\
\hline CL_L_T3 & PCT_A & 0.00 & CONSTANT & 0.56 & & $\mathrm{~Pa}$ & 31979 \\
\hline CL_L_T4 & PCT_A & 0.00 & CONSTANT & 0.56 & & $\mathrm{~Pa}$ & 32010 \\
\hline CL_M_Tl & PCT_A & 0.00 & CONSTANT & 0.56 & & $\mathrm{~Pa}$ & 32041 \\
\hline CL_M_T2 & PCT_A & 0.00 & CONSTANT & 0.56 & & $\mathrm{~Pa}$ & 32116 \\
\hline CL_M_T3 & PCT_A & 0.00 & CONSTANT & 0.56 & & $\mathrm{~Pa}$ & 32143 \\
\hline CL_M_T4 & PCT_A & 0.00 & CONSTANT & 0.56 & & $\mathrm{~Pa}$ & 32195 \\
\hline CL_M_T5 & PCT_A & 0.00 & CONSTANT & 0.56 & & $\mathrm{~Pa}$ & 32232 \\
\hline CLAY_BOT & PCT_A & 0.00 & CONSTANT & 0,56 & & $\mathrm{~Pa}$ & 31859 \\
\hline CLAY_RUS & PCT_A & 0.00 & CONSTANT & 0.56 & & $\mathrm{~Pa}$ & 31880 \\
\hline CONC_MON & PCT_A & 0.00 & CONSTANT & 0.56 & & $\mathrm{~Pa}$ & 32508 \\
\hline CONC_Tl & PCT_A & 0.00 & CONSTANT & 0.56 & & $\mathrm{~Pa}$ & 32561 \\
\hline CONC_T2 & PCT_A & 0.00 & CONSTANT & 0.56 & & $\mathrm{~Pa}$ & 32652 \\
\hline EARTH & PCT_A & 0.00 & CONSTANT & 0.56 & & $\mathrm{~Pa}$ & 32920 \\
\hline SALT_T1 & PCT_A & 0.00 & CONSTANT & 0.56 & & $\mathrm{~Pa}$ & 33369 \\
\hline SALT_T2 & $\mathrm{PCT} \_\mathrm{A}$ & 0.00 & CONSTANT & 0.56 & & $\mathrm{~Pa}$ & 33433 \\
\hline SALT_T3 & PCT_A & 0.00 & CONSTANT & 0.56 & & $\mathrm{~Pa}$ & 33404 \\
\hline SALT_T4 & PCT_A & 0.00 & CONSTANT & 0.56 & & $\mathrm{~Pa}$ & 33456 \\
\hline SALT_T5 & PCT_A & 0.00 & CONSTANT & 0.56 & & $\mathrm{~Pa}$ & 33575 \\
\hline SALT_T6 & PCT_A & 0.00 & CONSTANT & 0.56 & & $\mathrm{~Pa}$ & 33642 \\
\hline CONC_TI & PCT_EXP & 0.00 & CONSTANT & $-3.46000 \mathrm{E}-01$ & & none & 32563 \\
\hline CONC_T & PCT_EXP & 0.00 & CONSTANT & $-3.46000 \mathrm{E}-01$ & & none & 32656 \\
\hline CLAY_RUS & PCT_EXP & 0.00 & CONSTANT & $-3.46000 \mathrm{E}-01$ & & none & 31881 \\
\hline CLAY_BOT & PCT_EXP & 0.00 & CONSTANT & $-3.46000 \mathrm{E}-01$ & & none & 31860 \\
\hline CL_M_T1 & PCT_EXP & 0.00 & CONSTANT & $-3.46000 \mathrm{E}-01$ & & none & 32042 \\
\hline
\end{tabular}

NOTES:

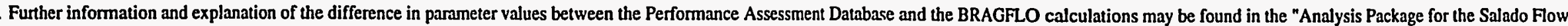

Calculations (TASK 1) of the Performance Assessment Analysis Supporting the Compliance Certification Application," SNL, 1996.

2. Pressure is specified in the Salado Halite at the horizon of MB139 and other pressures in the Salado are calculated, assuming a hydrostatic pressure gradient. 
Listing of Parameters Used in PANEL which Differ from the WIPP 1996 Compliance Certification Application Parameter Database

\begin{tabular}{|c|c|c|c|c|c|c|c|c|c|c|c|c|}
\hline 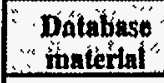 & $\begin{array}{l}\text { Dintabase } \\
\text { pairameter }\end{array}$ & $\begin{array}{l}\text { PANEL halnilte } \\
\text { (years) }\end{array}$ & $\begin{array}{l}\text { Databuse hainite } \\
(\mathrm{sec})\end{array}$ & $\begin{array}{l}\text { Datáabase } \\
\text { (séctyr) }\end{array}$ & $\begin{array}{l}\text { PANEL } \\
\text { yr to sec }\end{array}$ & $\begin{array}{l}\text { Mginifó } \\
\text { o difr }\end{array}$ & Impact & $\begin{array}{l}\text { Datubise } \\
\text { páranieter }\end{array}$ & $\begin{array}{l}\text { PAN } \\
\text { atomic wétght }\end{array}$ & $\begin{array}{l}\text { Daliduses } \\
\text { âtomic wrejglat }\end{array}$ & $\begin{array}{l}\text { Atomite } \\
\text { weighí wodín }\end{array}$ & Impact \\
\hline SR-90 & HALFLIFE & $2.91200 \mathrm{E}+01$ & $9.19000 \mathrm{E}+08$ & $3.155693 \mathrm{E}+07$ & $9.18938 \mathrm{E}+08$ & $0.01 \%$ & b & ATWEIGHT & 90 & 8.99080E-02 & $0.10 \%$ & a \\
\hline CS-137 & HALFLIFE & $3.00000 \mathrm{E}+01$ & $9.46700 \mathrm{E}+08$ & & $9.46708 \mathrm{E}+08$ & $0.00 \%$ & $\mathbf{b}$ & ATWEIGHT & 137 & $1.36907 \mathrm{E}-01$ & $0.07 \%$ & a \\
\hline PB-210 & HALFLIFE & $2.23000 \mathrm{E}+01$ & $7.03700 \mathrm{E}+08$ & & $7.03720 \mathrm{E}+08$ & $0.00 \%$ & $\mathbf{b}$ & ATWEIGHT & 210 & $2.09984 \mathrm{E}-01$ & $0.01 \%$ & $\mathbf{a}$ \\
\hline RA-226 & HALFLIFE & $1.60000 \mathrm{E}+03$ & $5.04900 \mathrm{E}+10$ & - & $5.04911 \mathrm{E}+10$ & $0.00 \%$ & b & ATWEIGHT & 226 & 2.26025E-01 & $0.01 \%$ & $\mathbf{a}$ \\
\hline RA-228 & HALFLIFE & $5.75000 \mathrm{E}+00$ & $2.11430 \mathrm{E}+08$ & & $1.81452 E+08$ & $14.18 \%$ & $\mathbf{b}$ & ATWEIGHT & 228 & 2.28031E-01 & $0.01 \%$ & $\mathbf{a}$ \\
\hline TH-229 & HALFLIFE & $7.34000 \mathrm{E}+03$ & $2.31600 \mathrm{E}+11$ & & $2.31628 \mathrm{E}+11$ & $0.01 \%$ & b & ATWEIGHT & 229 & 2.29032E-01 & $0.01 \%$ & a \\
\hline TH-230 & HALFLIFE & $7.70000 \mathrm{E}+04$ & $2.43000 \mathrm{E}+12$ & & $2.42988 \mathrm{E}+12$ & $0.00 \%$ & $\mathbf{b}$ & ATWEIGHT & 230 & $2.30033 \mathrm{E}-01$ & $0.01 \%$ & $\mathbf{a}$ \\
\hline TH-232 & HALFLIFE & $1.40500 \mathrm{E}+10$ & 4.43400E+17 & & $4.43375 \mathrm{E}+17$ & $0.01 \%$ & $\mathbf{b}$ & ATWEIGHT & 232 & 2.32038E-01 & $0.02 \%$ & a \\
\hline PA-231 & HALFLIFE & $3.27600 \mathrm{E}+04$ & $1.03400 \mathrm{E}+12$ & & $1.03381 \mathrm{E}+12$ & $0.02 \%$ & b & ATWEIGHT & 231 & $2.31036 \mathrm{E}-01$ & $0.02 \%$ & $\mathbf{a}$ \\
\hline$U-233$ & HALFLIFE & $1.58500 \mathrm{E}+05$ & $5.00200 \mathrm{E}+12$ & & $5.00177 \mathrm{E}+12$ & $0.00 \%$ & b & ATWEIGHT & 233 & 2.33040E-01 & $0.02 \%$ & $\mathbf{a}$ \\
\hline$U-234$ & HALFLIFE & $2.44500 \mathrm{E}+05$ & $7.71600 \mathrm{E}+12$ & & $7.71567 \mathrm{E}+12$ & $0.00 \%$ & b & ATWEIGHT & 234 & 2.34041E-01 & $0.02 \%$ & a \\
\hline$U-235$ & HALFLIFE & $7.03800 \mathrm{E}+08$ & $2.22100 \mathrm{E}+16$ & & $2.22098 \mathrm{E}+16$ & $0.00 \%$ & b & ATWEIGHT & 235 & 2.35044E-01 & $0.02 \%$ & a \\
\hline$U-236$ & HALFLIFE & $2.34200 \mathrm{E}+07$ & $7.38900 E+14$ & & 7.39063E+14 & $0.02 \%$ & b & ATWEIGHT & 236 & $2.36046 \mathrm{E}-01$ & $0.02 \%$ & a \\
\hline$U-238$ & HALFLIFE & $4.46800 \mathrm{E}+09$ & $1.41000 \mathrm{E}+17$ & & $1.40996 \mathrm{E}+17$ & $.0 .00 \%$ & b & ATWEIGHT & 238 & 2.38051E-01 & $0.02 \%$ & a \\
\hline NP-237 & HALFLIFE & $2.14000 \mathrm{E}+06$ & $6.75300 \mathrm{E}+13$ & & $6.75318 \mathrm{E}+13$ & $0.00 \%$ & b & ATWEIGHT & 237 & $2.37048 \mathrm{E}-01$ & $0.02 \%$ & $\mathbf{a}$ \\
\hline PU-238 & HALFLIFE & $8.77400 \mathrm{E}+01$ & $2.76900 \mathrm{E}+09$ & & $2.76881 E+09$ & $0.01 \%$ & b & ATWEIGHT & 238 & $2.38050 \mathrm{E}-01$ & $0.02 \%$ & a \\
\hline PU-239 & HALFLIFE & $2.40700 \mathrm{E}+04$ & $7.59400 \mathrm{E}+11$ & & $7.59575 \mathrm{E}+11$ & $0.02 \%$ & b & ATWEIGHT & 239 & 2.39052E-01 & $0.02 \%$ & $\mathbf{a}$ \\
\hline PU-240 & HALFLIFE & $6.53700 \mathrm{E}+03$ & $2.06300 \mathrm{E}+11$ & & $2.06288 \mathrm{E}+11$ & $0.01 \%$ & b & ATWEIGHT & 240 & $2.40054 \mathrm{E}-01$ & $0.02 \%$ & $\mathbf{a}$ \\
\hline PU-241 & HALFLIFE & $1.44000 \mathrm{E}+01$ & $4.54400 \mathrm{E}+08$ & & $4.54420 \mathrm{E}+08$ & $0.00 \%$ & b & ATWEIGHT & 241 & 2.41057E-01 & $0.02 \%$ & a \\
\hline PU-242 & HALFLIFE & $3.76300 \mathrm{E}+05$ & $1.22100 \mathrm{E}+13$ & & $1.18749 \mathrm{E}+13$ & $2.74 \%$ & $\mathbf{b}$ & ATWEIGHT & 242 & $2.42059 \mathrm{E}-01$ & $0.02 \%$ & a \\
\hline PU-244 & HALFLIFE & $8.26000 \mathrm{E}+07$ & $2.60700 \mathrm{E}+15$ & & $2.60660 \mathrm{E}+15$ & $0.02 \%$ & b & ATWEIGHT & 244 & 2.44064E-01 & $0.03 \%$ & a \\
\hline AM-241 & HALFLIFE & $4.32200 \mathrm{E}+02$ & $1.36400 \mathrm{E}+10$ & & $1.36389 \mathrm{E}+10$ & $0.01 \%$ & b & ATWEIGHT & 241 & 2.41057E-01 & $0.02 \%$ & a \\
\hline CM-244 & HALFLIFE & $1.81100 \mathrm{E}+01$ & $5.71500 \mathrm{E}+08$ & & $5.71496 \mathrm{E}+08$ & $0.00 \%$ & b & ATWEIGHT & 244 & 2.44063E-01 & $0.03 \%$ & $\mathbf{a}$ \\
\hline CM-248 & HALFLIFE & $3.39000 \mathrm{E}+05$ & $1.07000 \mathrm{E}+13$ & & $1.06978 \mathrm{E}+13$ & $0.02 \%$ & b & ATWEIGHT & 248 & 2.48072E-01 & $0.03 \%$ & $\mathbf{a}$ \\
\hline CF-252 & HALFLIFE & $2.63800 \mathrm{E}+00$ & $8.32500 E+07$ & & $8.32472 E+07$ & $0.00 \%$ & b & ATWEIGHT & 252 & 2.52082E-01 & $0.03 \%$ & a \\
\hline PM-147 & HALFLIFE & $2.62340 \mathrm{E}+00$ & $8.27900 E+07$ & & $8.27865 \mathrm{E}+07$ & $0.00 \%$ & b & ATWEIGHT & 147 & 1.46915E-01 & $0.06 \%$ & $\mathbf{a}$ \\
\hline SM-147 & HALFLIFE & $1.06000 \mathrm{E}+11$ & $3.37700 E+18$ & & $3.34503 \mathrm{E}+18$ & $0.95 \%$ & b & ATWEIGHT & 147 & 1.46915E-01 & $0.06 \%$ & a \\
\hline AM-243 & HALFLIFE & $7.37000 \mathrm{E}+03$ & $2.32900 \mathrm{E}+11$ & & $2.32575 \mathrm{E}+11$ & $0.14 \%$ & b & ATWEIGHT & 243 & 2.43061E-01 & $0.03 \%$ & $\mathbf{a}$ \\
\hline CM-243 & HALFLIFE & $2.91000 \mathrm{E}+01$ & $8.99400 \mathrm{E}+08$ & & $9.18307 \mathrm{E}+08$ & $2.10 \%$ & $\mathbf{b}$ & ATWEIGHT & 243 & $2.43061 \mathrm{E}-01$ & $0.03 \%$ & $\mathbf{a}$ \\
\hline CM-245 & HALFLIFE & $8.53000 \mathrm{E}+03$ & $2.68200 \mathrm{E}+11$ & & $2.69181 \mathrm{E}+11$ & $0.37 \%$ & b & ATWEIGHT & 245 & 2.45065E-01 & $0.03 \%$ & $\mathbf{a}$ \\
\hline
\end{tabular}

NOTES:

1. The variables for PANEL can be found in block dala GE_CHART, taken from u1:[jwgarne.panel]cpanel.for;99 dated 10-may-1996 10:56

2. PANEL uses halfife in years. The value is converted to seconds using the sec/yr stored in the database.

3. The difference columns were added to show the variation of the values for halfife and atomic weight.

4. Further information and explanation of the difference in parameter values between the Performance Assessment Database and the PANEL calculations may be found in the "Analysis Package for the

Salado Transport (TASK 2) of the Performance Assessment Analysis Supporting the Compliance Certification Application, ${ }^{, ~ S N L, ~} 1996$. 
Impact/Justification:

a. The atomic weight values used by the PANEL code were extracted from the isotope names. The database values were obtained from the memo: L.C. Sanchez to M. Martell, March 281996. "Radionuclide Half-lives and Specific Activities Obtained From ORIGEN2 Data" WPO\#37404. The percent enor between the two methods was calculated by the following equation

relative error $=[(($ database value - PANEL value $) / d a t a b a s e ~ v a l u e) * 100$
The difference column for atomic weights shows that values are less than $1 \%$ for all the radionuclides, therefore, there is no impact.

b. The halflives used by the PANEL code were from the ICRP and GE Chart of the Nuclides. The database values were obtained from the memo: L.C. Sanchez to M. Martell, March 28 1996.

"Radionuclide Half-lives and Specific Activities Obtained From ORIGEN2 Data" WPO\#37404. The percent difference was calculated using the above equation. The difference column for half-lives

shows that except for Ra228, Pu242, and Cm243 the percent error is less than 1\%. Because Ra228 is not one of the isotopes for which PANEL calculates release, it's properties did not effect the CCDF.

The Pu242 and CM243 are both less than 3\% error. Cm243 has a short halflife (29.1 yrs) and is a minor contributor to the total EPA units at $6.03 \mathrm{e}-2$ (see WIPP PA Analysis Repont for EPAUNI (Sanchez et al., (1996), WPO\#39259). Pu242 has a maximum of 3 EPA units, Pu239 has a maximum of 2310 EPA units. 3/2310 = 1.29E-3. Pu242 overall addition to the EPA units is less than 0.01\% contribution for the total Plutonium EPA units. A 2.74\% difference in the halfife for Pu242 would have no impact in the final results.

References:

ICRP:

Radionuclide Transformations Energy and Intensity of Emissions, 1983, Publication 38, Publisher Pergamon Press New York, NY.

GE Chart of the Nuclides:

Nuclides and Isotopes, Fourteenth Edition. General Electric Company (Nuclear Energy Operations), San Jose, CA. 1989.

Listing of Parameters Used in SWIFT II for GRASP_INVERSE which Differ from the WIPP 1996 Compliance Certification Application Parameter Database

\begin{tabular}{|c|c|c|c|c|c|c|}
\hline $\begin{array}{c}\text { swrirt u } \\
\text { parameter } \\
\text { name } \\
\end{array}$ & Modeling value & $\because \begin{array}{c}\text { Databise } \\
\text { material }\end{array}$ & $\begin{array}{c}\text { Database" } \\
\text { parameter }\end{array}$ & Datubase value & $\begin{array}{ccc}3 \\
3\end{array}$ & 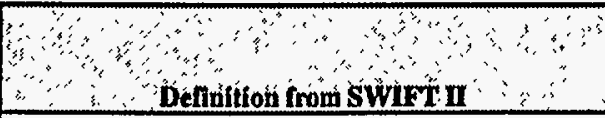 \\
\hline GRAV & 9.792 & REFCON & GRAVACC & 9.8067 & $\mathrm{~m} / \mathrm{s}^{\wedge} \mathbf{2}$ & Gravitational constant \\
\hline $\mathbf{C R}$ & $7.57 \mathrm{E}-10$ & CULEBRA & COMP_RCK & $1.00 \mathrm{E}-10$ & $P a^{\wedge}-1$ & compressibility of pore structure \\
\hline ALPHL & 50 & CULEBRA & DISP_L & 0.00 & $\mathbf{m}$ & Longitudinal dispersivity factor \\
\hline ALPHT & 2.5 & CULEBRA & DISPT_L & 0.00 & $\mathbf{m}$ & Transverse dispersivity factor \\
\hline BROCK & $2.50 \mathrm{E}+03$ & CULEBRA & DNSGRAIN & $2.82 E+03$ & $\mathrm{~kg} / \mathrm{m}^{\wedge} 3$ & Rock density \\
\hline
\end{tabular}

1. Further information and explanation of the parameter values may be found in the "Analysis of the Generation of Transmissivity Fields for the Culebra Dolomite," SNL, 1996.

2. SWIFT II for GRASP_INVERSE parameters and values are not stored or read from the database. The database equivalent column was used to show comparable parameters and values stored. 
Page intentionally blank

N-6 


\section{WIPP \\ UC721 - DISTRIBUTION LIST \\ SAND98-0180}

\section{Federal Agencies}

US Department of Energy (4)

Office of Civilian Radioactive Waste Mgmt.

Attn: Deputy Director, RW-2

Acting Director, RW-10

Office of Human Resources \& Admin.

Director, RW-30

Office of Program Mgmt. \& Integ.

Director, RW-40

Forrestal Building

Office of Waste Accept., Stor., \& Tran.

Washington, DC 20585

Yucea Mountain Site Characterization Office Director, RW-3

Office of Quality Assurance

Attn: Project Director

P. O. Box 30307 .

Las Vegas, NV 89036-0307

Yucca Mountain Site Characterization Office

Attn: L. E. Shephard

P. O. Box 30307

MS 523

Las Vegas, NV 89036-0307

US Department of Energy

Research \& Waste Management Division

Attn: Director

P.O. Box E

Oak Ridge, TN 37831

US Department of Energy (4)

Carlsbad Area Office

Attn: D. Galbraith

M. McFadden

R. Lark

J. A. Mewhinney

P.O. Box 3090

Carlsbad, NM 88221-3090

US Department of Energy

Office of Environmental Restoration and

Waste Management

Attn: M. Frei, EM-30

Forrestal Building

Washington, DC 20585-0002
US Department of Energy (3)

Office of Environmental Restoration and

Waste Management

Attn: J. Juri, EM-34, Trevion II

Washington, DC 20585-0002

US Department of Energy

Office of Environmental Restoration and

Waste Management

Attn: S. Schneider, EM-342, Trevion II

Washington, DC 20585-0002

US Department of Energy (2)

Office of Environment, Safety \& Health

Attn: C. Borgstrom, EH-25

R. Pelletier, EH-231

Washington, DC 20585

US Department of Energy (2)

Idaho Operations Office

Fuel Processing \& Waste Mgmt. Division 785 DOE Place

Idaho Falls, ID 83402

US Environmental Protection Agency (2)

Radiation Protection Programs

Attn: M. Oge

ANR-460

Washington, DC 20460

\section{Boards}

Defense Nuclear Facilities Safety Board

Attn: D. Winters

625 Indiana Ave. NW, Suite 700

Washington, DC 20004

Nuclear Waste Technical Review Board (2)

Attn: Chairman

J.L. Cohon

1100 Wilson Blvd., Suite 910

Arlington, VA 22209-2297 
State Agencies

Attorney General of New Mexico

P.O. Drawer 1508

Santa Fe, NM 87504-1508

Environmental Evaluation Group (3)

Attn: Library

7007 Wyoming NE

Suite F-2

Albuquerque, NM 87109

NM Environment Department (3)

Secretary of the Environment

Attn: Mark Weidler

1190 St. Francis Drive

Santa Fe, NM 87503-0968

NM Bureau of Mines \& Mineral Resources Socorro, NM 87801

\section{Laboratories/Corporations}

Battelle Pacific Northwest Laboratories Battelle Blvd.

Richland, WA 99352

Los Alamos National Laboratory

Attn: B. Erdal, INC-12

P.O. Box 1663

Los Alamos, NM 87544

Tech Reps, Inc. (3)

Attn: J. Chapman (1)

Loretta Robledo (2)

5000 Marble NE, Suite 222

Albuquerque, NM 87110

Westinghouse Electric Corporation (5)

Attn: Library

J. Epstein

J. Lee

B. A. Howard

R. Kehrman

P.O. Box 2078

Carlsbad, NM 88221

S. Cohen \& Associates

Attn: Bill Thurber

1355 Beverly Road

Mclean, VA 22101
National Academy of Sciences, WIPP Panel

Tom Kiess (15)

Staff Study Director

GF456

2101 Constitution Ave.

Washington, DC 20418

\section{Universities}

University of New Mexico

Geology Department

Attn: Library

141 Northrop Hall

Albuquerque, NM 87131

University of Washington

College of Ocean \& Fishery Sciences

Attn: G. R. Heath

583 Henderson Hall, HN-15

Seattle, WA 98195

\section{Libraries}

Thomas Brannigan Library

Attn: D. Dresp

106 W. Hadley St.

Las Cruces, NM 88001

Goverument Publications Department

Zimmerman Library

University of New Mexico

Albuquerque, NM 87131

New Mexico Junior College

Pannell Library

Attn: R. Hill

Lovington Highway

Hobbs, NM 88240

New Mexico State Library

Attn: N. McCallan

325 Don Gaspar

Santa Fe, NM 87503

New Mexico Tech

Martin Speere Memorial Library

Campus Street

Socorro, NM 87810 
WIPP Public Reading Room

Carisbad Public Library

$101 \mathrm{~S}$. Halagueno St.

Carisbad, NM 88220

\section{Foreign Addresses}

Atomic Energy of Canada, Ltd.

Whiteshell Laboratories

Attn: B. Goodwin

Pinawa, Manitoba, CANADA ROE ILO

Francois Chenevier (2)

ANDRA

Parc de la Croix Blanche

1-7 rue Jean Monnet

92298 Chatenay-Malabry, Cedex

FRANCE

Claude Sombret

Centre d'Etudes Nucleaires de la Vallee Rhone CEN/VALRHO

S.D.H.A. B.P. 171

30205 Bagnols-Sur-Ceze

FRANCE

Commissariat a L'Energie Atomique

Attn: D. Alexandre

Centre d'Etudes de Cadarache

13108 Saint Paul Lez Durance Cedex

FRANCE

Bundesanstalt fur Geowissenschaften und

Rohstoffe

Attn: M. Langer

Postfach 510153

D-30631 Hannover

GERMANY

Bundesministerium fur Forschung und

Technologie

Postfach 200706

5300 Bonn 2

GERMANY

Institut fur Tieflagerung

Attn: K. Kuhn

Theodor-Heuss-Strasse 4

D-3300 Braunschweig

GERMANY
Gesellschaft fur Anlagen und Reaktorsicherheit (GRS)

Attn: B. Baltes

Schwertnergasse 1

D-50667 Cologne

GERMANY

Shingo Tashiro

Japan Atomic Energy Research Institute

Tokai-Mura, Ibaraki-Ken, 319-11

JAPAN

Netherlands Energy Research Foundation ECN

Attn: J. Prij

3 Westerduinweg

P.O. Box 1

1755 ZG Petten

THE NETHERLANDS

Svensk Karnbransleforsorjning $A B$

Attn: F. Karisson

Project KBS (Karnbranslesakerhet)

Box 5864

S-102 48 Stockholm

SWEDEN

Nationale Genossenschaft fur die Lagerung

Radioaktiver Abfalle (2)

Attn: S. Vomvoris

P. Zuidema

Hardstrasse 73

CH-5430 Wettingen

SWITZERLAND

AEA Technology

Attn: J. H. Rees

D5W/29 Culham Laboratory

Abington, Oxfordshire OX14 3DB -.

UNITED KINGDOM

AEA Technology

Attn: W. R. Rodwell

044/A31 Winfrith Technical Centre

Dorchester, Dorset DT2 8DH

UNITED KINGDOM

AEA Technology

Attn: J. E. Tinson

B4244 Harwell Laboratory

Didcot, Oxfordshire OX11 ORA

UNITED KINGDOM 
Internal

$\begin{array}{lll}\text { MS } & \text { Org. } & \\ \mathbf{0 7 3 5} & \mathbf{6 1 1 5} & \text { P. B. Davies } \\ 0737 & 6831 & \text { E. J. Nowak } \\ 0737 & 6833 & \text { J. R. Tillerson } \\ 0779 & \mathbf{6 8 4 9} & \text { D. R. Anderson } \\ 0779 & 6848 & \text { H. N. Jow } \\ 0771 & \mathbf{6 8 0 0} & \text { M. Chu } \\ 1395 & 6821 & \text { M. Marietta } \\ 0773 & 6821 & \text { S. M. Howarth (10) } \\ 0718 & 6341 & \text { R. F. Weiner (3) } \\ 1395 & 6821 & \text { M.A. Martell } \\ 0731 & 6811 & \text { K. Hart (2) } \\ 0731 & 4415 & \text { NWM Library (20) } \\ 9018 & 8940-2 & \text { Central Technical Files } \\ 0899 & 4916 & \text { Technical Library (2) } \\ 0619 & 12690 & \text { Review and Approval Desk, } \\ & & \text { For DOE/OSTI (2) }\end{array}$

